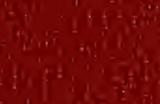

[6 15.

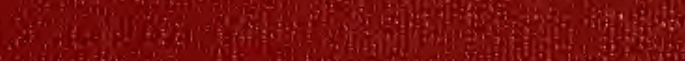

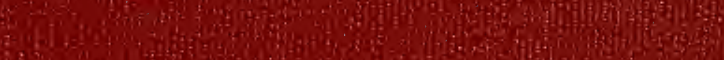

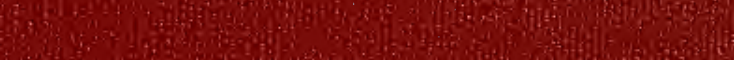

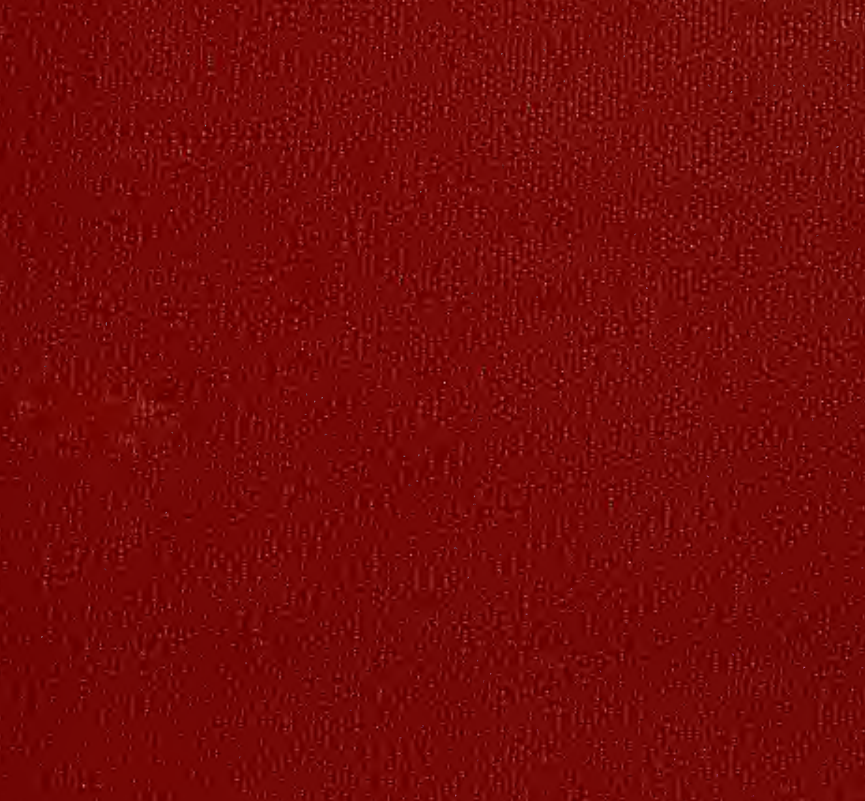

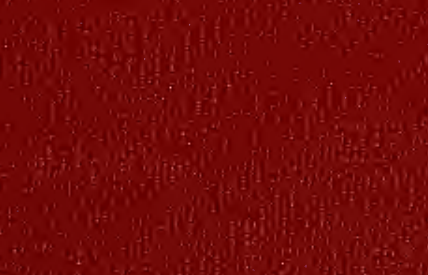
H.

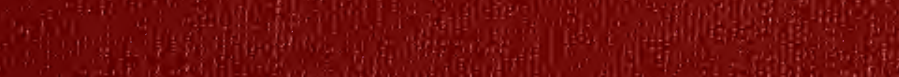

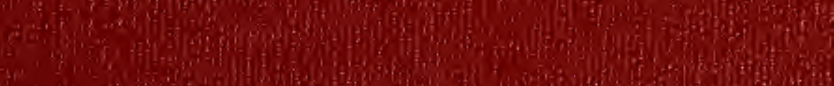

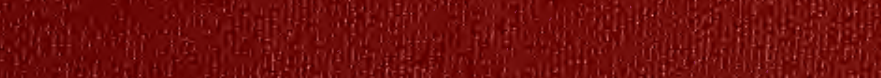

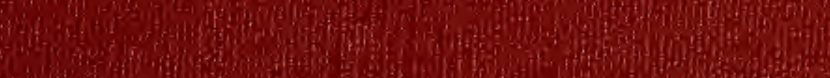
cas 



\section{Digitized by the Internet Archive}

in 2010 with funding from Boston Library Consortium Member Libraries 

PHILOSOPHICAL AND MATHEMATICAL

D I C T I O N A R Y.

V O L. I. 


PRIN'TED BY S. HAMILTON,
Weylridge, Surrey.




\title{
PHILOSOPHICAL AND MATHEMATICAL
}

\section{I C T I O N A R Y:}

conransise

AN EXPLANATION OF THE TERMS, AND AN ACCOUNT OF THE SEVERAL SUBJECTS, COMPRISED UNDER THE HEADS

\section{MATHEMATICS, ASTRONOMY, AND PHILOSOPHY}

\author{
BOTH NATURAL AND EXPERIMENTAL;
}

WITH AN

HISTORICAL ACCOUNT OF THE RISE, PROGRESS, AND PRESENT STATE OF THESE SCIENCES;

ALSO

BOSTON DOLAEGE LIIRART

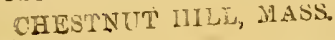

MEMOIRS OF THE LIVES AND WRITINGS OF THE MOST EMINENT AUTHORS, BOTH ANCIENT AND MODERN,

WHO BY TMEIR DISCOVERIES OR IMPROVEMENTS HAVE CONTRIBUTED TO TIE ADVANCEMENT OF THEM.

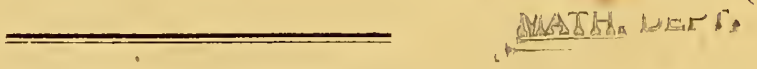

BY CHARLES HUTTON, LL.D.

FELLOW OF THE ROYAL SOCIETIES OF LONDON AND EDINBURGII, AND OF THE PHILOSOPHICAL SOCIETIES OF IIAARLEM AND AMERICA; AND EMERITUS PROFESSOR OF MATHEMATICS IN TIIE ROYAL MILITARY AĆCADEMY, WOOLWICII.

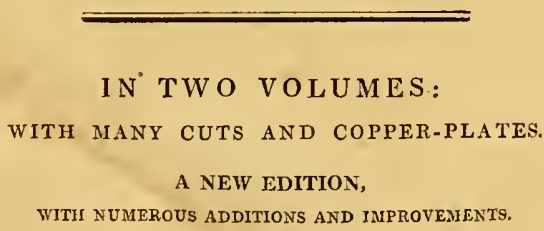

V O L. I.

L O N D O N :

PRINTED FOR THE AUTHOR;

F. C. AND J. RIVINGTON; J. CUTHELL; LAW AND WHITTAKER; LONGMAN, HURST, REES, ORME, AND BROWN; CADELL AND DAVIES; J. MAWMAN; BLACK, PARRY, AND CO; BALDWIN, CRADOCK AND JOY; J. HOOKER; G. AND 5. ROBINSON; T. HAMLLTON; WALIEER AND EDWARDS; JOHN ROBINSON; B. REYNOLDS; AND SIMPKIN AND MARSHALL, 
Q

121

. 149

DEC 2 3 mas

BOSTOK GOLLEAE UBRARY

CHESTNET IML, ALA ORHG

160154 


\title{
$\begin{array}{lllllll}P & R & E & F & A & C & E\end{array}$
}

\begin{abstract}
Arong the Dictionaries of Arts and Sciences which have been published, of late years, in various parts of Europe, it is matter of surprise that Philosophy and Mathematics should have been so far overlooked as not to be thought worthy of a separate Treatise, in this form. These sciences constitute a large portion of the present stock of human knowledge, and have been usually considered as possessing a degrce of importance to which few others are entitled; and yet we have hitherto had no distinct Lexicon, in which their constituent parts and techuical terms have been explained, with that amplitude and precision, which the great improvements of the Moderns, as well as the rising dignity of the Subject, seem to demand.
\end{abstract}

THE only works of this kind in the English language, deserving of notice, are Harris's Lexicon Technicum, and Stone's Mathematical Dictionary; the former of which, though a valuable performance at the time it was written, is now become too dry and obsolete to be referred to with pleasure or satisfaction: and the latter, consisting only of one volume in 8ro, must be regarded merely as an unfinished sketch, or brief compendium, extremely limited in its plan, and necessarily deficient in useful information.

IT became, therefore, the only resource of the Reader, in many cases where explanation was wanted, to have recourse to Chambers's Dictionary, in four large volumes folio, or to the Encyclopædia Britannica, now in eighteen large volumes 4 to, or the still more stupendous performance of the French Encyclopedists; and even here his expectations might be frequently disappointed. These great and useful works, aiming at a general comprehension of the whole circle of the Sciences, are sometimes very deficient in their descriptions of particular branches; it being almost impossible, in such extensive undertakings, to appreciate, with exactness, the due value of every article. They are, besides, so voluminous and hetero- 
geneous in their nature, as to render a frequent reference to them extremely inconvenient ; and even if this were not the case, their high price puts thein out of the reach of the generality of readers.

W1TH a view to obviate these defects, the Public are here presented with a Dictionary of a moderate size and price, which is devoted solely to Philosophical and Mathematical subjects. It is a work for which materials have been collecting through a course of many years; and is the result of great labour and reading. Not only most of the Encyclopedias already extant, and the various publications of the Learned Societies throughout Europe, have been carefully consulted, but also all the original works, of any reputation, which have hitherto appeared on these subjects, from the earliest writers down to the present times.

From the latter of these sources, in particular, a considerable portion of information has been obtained, which the curious reader will find, in many cases, to be highly interesting and important. The History of Algebra, for instance, which is detailed at considerable length in the first volume, under the head of that article, will afford sufficient evidence to show in what a superficial and partial way the inquiry has been hitherto investigated, cven by professed writers on the subject; tlie principal of whom are M. Montucla, our countryman the celebrated Dr. Wallis, and the Abbé De Gua, a late French author, who has pretended to correct the Doctor's errors and misrepresentations.

RegUlak historical details are in like manner given of the origin and progress of each of these Sciences, as well as of the inventions and injprovements by which they have been gradually brought from their first rude beginnings to their present advanced state.

IT is also to be observed, that besides the articles common to the generality of Dictionaries of this kind, an interesting Biographical Account is here introduced of the most celebrated Philosophers and Mathematicians, both ancient and modern; among which will be found the Lives of eminent characters, who have hitherto been either wholly overlooked, or very imperfectly recorded. Complete lists of their works are also subjoined to eacl article, when they could be procured; which cannot but prove highly acceptable to that class of readers, who are desirous of obtaining the most salisfactory information on the subjects of their particular inquiries and pursuits. On the head of Biography, however, the Author has still to lament the want of many other respcctable names which lie was desirous to have 
added to his list of authors, not having been able to procure any circumstantial accounts of their lives. He could have wished to have comprised in his list, the lives of all such public literary characters as the University Professors of Astronomy, Philosophy, and Mathematics, as well as those of the other more respectable class of authors on those sciences. IIe will therefore thankfully receive the communication of any such memoirs from the hands of gentlemen possessed of them; as well as hints and information on such useful improvements in the sciences as may have been overlooked in this Dictionary, or any articles that may here have been imperfectly or incorrectly treated; that he may at some future time, by adding them to this work, render it still more complete and deserving the public notice.

As this work is an attempt to separate the words in the sciences of Astronomy, Mathematics, and Philosophy, from those of other arts or sciences, in several of which there are already separate Dictionaries ; as in Chemistry, Geography, Music, Marine and Naval affairs, \&c; words sometimes occurred which it was rather doubtful whether they could be considered as properly belonging to the present work or not; in which case many of such words have been here inserted. But such as appeared clearly and peculiarly to belong to any of those other subjects, have been either wholly omitted, or else have had a very short account only given of them. The readers of this work therefore, recollecting that it is not a General Dictionary of all the Arts and Sciences, will not expect to find all sorts of words and subjects here treated of; but such only as peculiarly appertain to the proper matter of the work. And therefore, although some few words may inadvertently have been oinitted; yet when the Reader does not immediately find cvery word which he wishes to consult, he will not always consider them as omissions of the Author, but for the most part as relating to some other science foreign to this Dictionary.

In all cases where it could bc conveniently done, the nccessary figures and diagrams are inserted in the samc page with the subjects which they are designed to elucidate; a method which will be found much morc commodious than that of putting them in separate plates at the end of cach volumc, but, which has added very considerably to the expence of the undertaking. Where the subjects are of such a nature that they could not be otherwise well represented, thicy are cugraved on copperplates.

$\Lambda$ s the whole of this work was written before it was put to the press, the Reader will find it of an equal and uniform uature and construction throughout; in which respect many 
publications of this kind are very defective, from the subjects being diffusely treated under the first letters of the alphabet, while articles of equal inportance in the latter part are so much abridged as to be rendered almost useless, in order that the whole might be comprised in a limited number of sheets, according to proposals made before the works were composed. The present Dictionary having been completed without any of these unfavourable circumstances, will be found in most cases equally instructive and useful, and may be consulted with no less advantage by the Man of Science than the Student.

IN addition to the foregoing original account of this work, we have to observe, that the whole has been several times carefully perused, in order to correct, enlarge, and amend the several articles; that all the new discoveries and improvements in the sciences, since the first composing of the work, have been attentively collected and abridged, for introducing in this new edition; that many new articles have been inserted in their places through the volumes, and even several of the chief articles of some of the other sciences \&c, have now been included,-as Electricity, Galvanism, Voltaic pile, Acid, Alkali, Freezing, New Planets and Comets, with numerous other similar articles; that a large collection of new and interesting lives of eminent authors have been inserted, as far as materials could be obtained, and the merits of the authors were deemed worthy of notice in this work; that several new plates and figures have been added; that the pages have been considerably enlarged, and the printing so arranged and contrived as to include more matter in the sheets. In short, the alterations, additions, and other improvements, are so numerous, and so large, as entitle the present edition to be considered alnost as an entire new work. 


\title{
PHILOSOPHICAL AND MATHEMATICAL
}

\author{
D I C T I O A R Y.
}

A.

ABACIST, an Arithmetician. In this sense we find the word used by William of Malmesbury, in his IIistory de Gestis Anglorum, written about the year 1150; where he shows that one Gerbert, a learned monk of France, who afterwards became pope of Rome in the year 998 or 999 , by the name of Silvester the $2 \mathrm{~d}$, was the first who got from the Saracens the abacus, and that taught such rules concerning it, as the Abacists themselves could hardly understand.

ABACUS, in Arithmetic, an ancient instrument used by most nations for casting up accounts, or perfurming aritbmetical calculations. It is by some derived from the Greek $\alpha E \alpha \xi$, which signifies a cupboard or beaufet, perhaps from the similarity of the form of this instrument: and by others it is derived from the Phœuician abak, which signifies dust or powder, because it was said that this instrument was sometimes a square board or tablet, which was powdered over with fine sand or dust, in which were traced the figures or characters used in making calculations, which could thence be casily defaced, and the abacus refitted for usc. But Lucas Paciolus, in the first part of his second distinction, thinks it is a corruption of Arabicus, by which he meant their Algorithm, or the method of numeral computation received from them.

We find this instrument in use for computation, under st)me variations, with most nations; as the Greeks, Roinans, Germans, French, Chinese, \&c.

The Grecian $A$ bacus was an oblong frame, over which were stretched several brass wires, strung with little ivory balls, like the beads of a necklace; by the various arrangements of which, all kinds of computations were easily made. Mahudel, in Ilist. Acad. R. Inscr. tom. 3. p. 390 .

The lioman Abacus was a little varied from the Gre: cian, liaving pins sliding in grooves, instead of strings or wires and heads. Philos. Trans. No. 180.

The Chinese Abacus, or Shwan-pan, like the Grccian, consists of several series of beads strung on brass wires, stretched from the top to the bottom of the instrument, and divided in the mirdlle by a cross piece from side to side. In the upper space every string has two beads, which are: each counted for 5 ; and in the lower space "very string has five beads, of different values, the first being counted as 1 , the second as 10 , the thirt as 100 , and so on, as with us. See SHWAN-PAN.

VoL. I.
The Abacus chiefly used in Enropean countries, is nearly on the same principles, though the use of it is here more limited, because of the arbitrary and unequal divisions of moncy, weights, and measures, which, in China, are all divided in a tenfold proportion, like our scale of common numbers. This is made by drawing any number of parallel lines, like paper ruled for music, at such a distance as may be at least equal to twice the diameter of the calculus, or counter. Then the value of these lines, and of the spaces between them, increases, from the lowest to the highest, in a tcnfold proportion. Thus, counters placed upon the first line, signify so many units or ones; on the second line 10 's, on the third line 100 's, on the fourth line 1000's, and so on. In like manner a counter placed in the first space, betwcen the first and second line, denotes 5 , in the second space 50 , in the third space 500 , in the fourth 5000 , and so on. So that there are never more than four counters placed on any line, nor more than one placed in any space, this being of the same value as five cuunters on the next line below. Thus, the counters on the Abacus, in the following figure, express the number or sum 47382 .

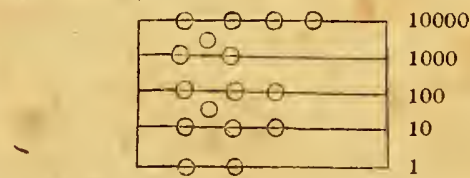

Besides the above instrunents of cuntputation, several others have been invented by different persons: as, $\mathrm{Na}$ pier's Rods or Bones, described in his Rhabdulogia, which see, under the word NAPIER; atso the Abacus Rhabdologicus, a variation of Napier's, which is described in the first vol. of Machines et Inventions approuvées par l'Academie Royale des Scionces. An ingenious and general one was also invented by $\mathrm{Mr}$. Gamalici smethurst, and is described in the Philosophical Transactions, vol. 46; where the inventor remarks that computations by it are much quicker and easier perturned than by the pen, are also less burthensome to the menory, and can be performed by blind persons, or in the dark as welt as in the light. A very comprehensive instument of this kind was also contrived by the late learned Dr. Nicholas Saunderson, the blind professor of mathenatics, by which he performed 
very intricate calculations : an account of it is prefised to the first volume of his Algebra, where it is called by the editor Palpable Arithmetic: which see.

In the Edinb. Rev. vol. 18, p. 205, is the following neat account of this instrument:- It is curious to observe, that the term Calculation itself claims no higher descent than from calculus, a pebble. A table strewed with fine sand, the pulvis eruditus of the poets, served both for tracing geometrical diagrams, and teaching the elements of writing; a very primitive contrivance, but universally used throughout the East, from which it has lately been imported into this country. The board on which arithmetical operations were performed, at first by means of pebbles, was named Abacus, from the Greek $\alpha$ bi $\alpha$; probably because originally it was only a writing-table of a compendious form, and designed for instructing children in their alphabet.

Nec qui Aliaco numeros, et secto in pulvere metas
Scit disisse vafer.
Pers. Sat. i. 138.

The Abacus was divided, from the right to the left hand, by vertical columns, on which the pebbles were placed, to denote, units, tens, hundreds, thousands, \&c, The labour of counting and arranging those pebbles was afterwards sensibly abridged, by drawing across the board a horizontal line, above which, each single pebble had the power of 5. In the progress of luxury, tali, or dies made of ivory, were used instead of pebbles, and small silver coins came to supply the place of counters. But the operations with the abacus were rendered still more commodious, by substituting for such tali or counters, small beacts strung on parallel threads, and sometimes pegs stuck along grooves.-Accountants by profession, ainong the Romans, were hence styled calculatores, or rationarii. Various expedients seem to have been employed for shortening the arithmetical operations. The different positions of the fingers were, for that purpose, used to a certain extent. Boethius treated largely of the subject; and even the vencrable Bede has given very diffuse rules for what was called digital arithmetic.

But the application of the abacus itsclf was not entirely forgotten at a much later period. Witness the small volume of arithmetic, quaintly composed in the form of dialogue by Robert Recorde, teacher of mathematics and practitioner in physic at Cambridge, during the reign of Edward vI ; in which round dots, placed on perpendiculal. lines, and employed to express the succession of units, tens, hundreds, \&c, are made to perform some of the simpler numerical operations.-A small instrument, entirely resembling the abacus, has likewise at different times been recommended, for teaching the elements of ciphering, under the name of palpable arithmetic.

A sacus, P'ythagorean, so denominated from its inventor, Pythagoras, is a table of numbers, contrived for readily learning the principles of arithmetic; being probably what is now called the multiplication-table.

ABacus, or Abaciscus, in Architecture, the upper part or member of the capital of a column; serving as in crowning both to the capital and to the whole column. Vitruvius informs us that the Abacus was originally intended to represent a square flat tile laid over an urn, or a basket; and the invention is ascriber to Calimachus, an magenious statuary of Athens, who, it is said, adopted it on ubserving a small basket, covered with a tile, over the loot ul an $A$ canthus plant, which grew on the grave of a young lady; the plant shooting up, cucompassed the basket, till meeting with the tile, it curled back in a kind of scroll: Calimachus passing by, took the hint, and immediately executed a capital on this plan; representing the tile by the Abacus, the leaves of the acanthus by the volutes, and the basket by the vase or body of the capital. Sec ACanthus.

A EACUS is also used by Scamozzi for a concave moulding in the capital of the Tuscan pedestal. And the word is used by Palladio for other nembers, which he describes. In ancient architecture, the same term is used to denote cerain compartments in the incrustation or lining of the walls of state-rooms, mosaic-pavements, anc the like. There were Alaci of marble, porphyry, jasper, alabaster, and even glass; variously shaped, as square, triangular, and such-like.

A вacus Logisticus is a right-angled triangle, whose sides, about the right-angle, contain all the numbers from 1 to 60 ; and its area, the products of each two of the opposite numbers. This is also called a canon of sexagesimals, and is no other than a multiplication-table carried to 60 both ways.

A в Acus et Palmula, in the Ancient Music, denote the machinery by which the strings of the polyplectra, or instruments of many strings, were struck, with a plectrum made of quills.

A васUs Harmonicus is used by Kircher for the structure and disposition of the keys of a musical instrument, either to be'touched with the hands or fect.

Ав ACUs, in Geometry, a table or slate upon which schemes or diagrams are drawn.

ABATIS, or ABATTIS, from the French abattre, to throw down, or beat down, in the Military Art, denotes a kind of retrenchment made by a quantity of whole trees cut down, and laid lengthways beside each other, the closer the better, having all their branches pointed towards the enemy, which prevents his approach, at the same time that the trunks serve as a breast-work before the men. The Abattis is a very useful work on most occasions, especially on sudden emergencies, when trees are near at hand; and has always been practised with considerable success, by the ablest commanders in all ages and nations.

ABAUZIT, Firmin, a learned and ingenious mathematician and philosopher, born at Usez, in Languedoc, on the 1 Ith of November 1679; his parents being protestants, he was obliged to leave France by the revocation of the edict of Nantes, and after suffering many hardships, at length found an asylum in Geneva, where he prosccuted his studies with the greatest success; and though his chicf attention was directed to the mathematics, he neverthcless made considerable progress in every department of literature; he was acquainted with most of the learned men of his time, and among others with our illustrious countryman Sir Isáac Newton, who in an epistolary correspondence does him the honour to say, " you are a very fit person to judge between Leibnitz and me." He seems ind eel to have perfectly understood the Principia, laving undertaken the defence of it against father Castell, wherein he displayed great talents and ability: he discovered an error in that work when it first appeared, which was corrected in the next edition. After having signalized himself as much for his virtue as his learning, he died on the 20 h of March 1767 , at the adraneed age of 87 years, lumented and regretted by all whom he honoured with his fricndship. 
ABRREVIATE; to abbreviate fractions, in Arithmetic and Algebra, is to lessen proportionally their terms, or the numerator and denominator; which is performed by dividing those terms by any number or quantity, which will tivide them without leaving a remainder. And when the terms cannot be any further so divided, the fraction is said to be in its least terms.

$$
\text { So } \frac{16}{24}=\frac{8}{12}=\frac{4}{6}=\frac{2}{3} \text {, }
$$

by dividing the terms continually by 2 .

$$
\text { And } \frac{294}{504}=\frac{147}{252}=\frac{49}{84}=\frac{7}{12}
$$

by dividing by 2,3 , and 7 .

$$
\text { Also } \frac{3 \times 8 \times 5}{6}=\frac{8 \times 5}{2}=4 \times 5=20 \text {. }
$$

by dividing by 3 and by 2 .

$$
\begin{aligned}
& \text { And } \frac{12 n b x^{3}}{4 a c x}=\frac{3 l x}{c} \text {, by dividing by } 4 a x \text {. } \\
& \text { And } \frac{a l^{2}+b^{2} x}{a x+x^{3}}=\frac{l^{2}}{x} \text {, by dividing by } a+x
\end{aligned}
$$

ABBREVIATION, of fractions, in Aritbmetic and Algebra, is the reducing them to lower terms, as in the examples above.

ABERRATION, in Astronomy, an apparent motion of the celestial bodies, occasioned by the progressive motion of light, and the earth's annual motion in its orbit.

This effect may be explained and familiarized by the motion of a line parallel to itself, much after the manner of the composition and resolution of forces. For this purpose, let the ratio of the velocity of light to that of the earth in its orbit, be as the line $\mathbf{B} \mathbf{c}$ to $\mathrm{AC}$; then, by the composition of these two motions, the particle of light will seem to describe the line BA or DC, instead of its real course B C; and will appear in the direction $A B$ or $C D$, instead of its true direction с в. So that, if $\mathrm{AlB}$ represent a tube, carried with a parallel motion by an observer along the line $A c$, in the time that a particle of light would move over the space $\mathrm{B}$ c, the different places of

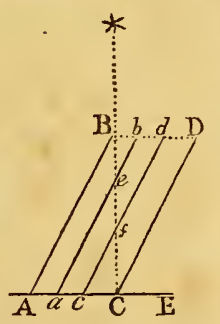
the tube being A $\mathrm{B}, a b, c d, \mathrm{CD}$; and when the eye, or end of the tube, is at $A$, let a particle of light enter the other end at $\mathrm{B}$; then, when the tube is at $a b$, the particle of light will be at $e$, exactly in the axis of the tube; and when the tube is at $c d$, the particle of light will arrive at $f$, still in the axis of the tube; and lastly, when the tube arrives at $\mathrm{CD}$, the particle of light will arrive at the eye or point $\mathrm{c}$, and consequently will appear to come in the direction $D C$ of the tube, instead of the true direction $\mathrm{B} C$; and so on; one particle succceding another, and forming a continued stream or ray of light in the apparent direction DC. So that the apparent angle made by the ray of light with the line $\mathrm{AE}$, is the angle $\mathrm{DCE}$, instead of the true angle $\mathrm{BCE}$; and the difference, $\mathrm{BCD}$ or $\mathrm{ABC}$, is the quantity of the aberration.

M. de Maupertuis, in his Elements of Geography, gives also a familiar and ingenious idea of the aberration, in this manner: "It is thus," says he, " concerning the direction in which a gun must be pointed to strike a bird in its flight; instead of pointing it straight to the bird, the fuwler will point a little before it, in the path of its fight, and that so much the more as the flight of the bird is more rapid, with respect to the flight of the shot." In this way of considering the matter, the flight of the bird represents the motion of the earth, or the line AC, in our scheme above, and the flight of the shot represents the motion of the ray of light, or the line в $\mathrm{c}$.

M. Clairaut too, in the Memoirs of the Academy of Sciences for the year 1746 , illustrates this effect in a familiar way, by supposing drops of rain to fall rapidly and quickly after each other from a cloud, under which a person moves with a very narrow tube; in which case it is evident that the tube must have a certain inclination, in order that a drop which enters at the top, may fall freely through the axis of the tube, without touching its sides; which inclination must be more or less according to the velocity of the drops, in respect to that of the tube: then the angle made by the direction of the tube and of the falling drops, is the aberration arising from the combination of those two motions.

Another ingenious and familiar illustration of this effect is given in the Encyclopédie Methodique; which is nearly as follows.

Let C F A represent a boát, or other vessel, moving parallel to itself from $\mathrm{c}$ to $\mathrm{D}$, andsuppose a person at $\mathrm{c}$ to throw a stoue towards $A$, with such a velocity that it exactly arrives there when the point $A$ arrives at $B$ by the motion of the boat: now a person situated at $\mathrm{A}$ will na-

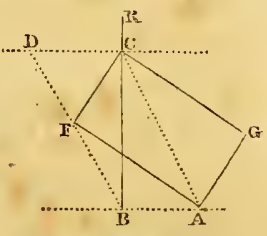
turally refer the direction of the stone to the diagonal $\mathrm{c}_{\mathrm{A}}$ or $\mathrm{DB}$ of the boat, over which it has actually passed, notwithstanding its real direction is that of св; for the same appearances would have taken place, had the stone been cast from $\mathrm{C}$ to $\mathrm{B}$, provided its velocity had been such as to cause it to arrive at $\mathrm{B}$, in the same time as before.

This therefore explains the nature of the aberration of the celestial bodies; for if we now consider the stone above mentioned as a particle of light issuing from a body at $\mathrm{C}$, in the direction $\mathrm{CB}$, and that it arrives there in the same time that a person moves from $\mathrm{A}$ to $\mathrm{B}$; then it is evident, from what is said above, that he will refer its direction to the line $\mathrm{D} \mathrm{B}$; but all bodies appear in the direction of the visual rays emitted by them, and conscquently the point $D$ will be the apparent place of the body c. And thus the effect of aberration is always to make bodies appear more advanced in the direction we are moving, than they really are.

This discovery; which is one of the brightest that have been made in the last age, we owe to the accuracy and ingenuity of the late Dr. Bradley, afterwards Astronomer Royal; to which lie was occasionally led by the result of some accurate observations which he had made with another view, namely, to determine the annual parallax of the fixed stars, or that which arises from the motion of the earth in its annual orbit about the sun.

This motion of the earth, about the sun, had been often doubted, and warmly contested. The defenders of that doctrine, among other proofs of its reality, conceived the idea of adducing an incontestable one from the annual parallax of the fixed stars, if they should be within such a distance, or if instruments and observations could be made with such accuracy, as to render that B 2 


\section{A B E}

[

parallax sensible; and with this view various attempts have been made. Before the observations of M. Picard, made in 1672 , it was the general opinion, that the stars did not change their position during the course of a year. Tycho Brahe and Riccioli fancied that they had assured themselves of it from their observations; from which they concluded that the earth did not move round the sun, and that there was no annual parallax in the fixed stars. M. Picard, in the account of his Voyage d'Uranibourg, made in 1672 , says that the pole star, at different times of the year, has certain variations which he bad observed for about 10 years, and which amounted to near $40^{\prime \prime}$ a year: whence sume, who favoured the annual motion of the earth, were led to conclude that these variations were the effect of the parallax of the earth's orbit. But it was impossible to explain it by that parallax; because this motion was in a manner contrary to what ought to follow only from the motion of the earth in its orbit.

In 1674 Dr. Hook published an account of observations, made by him in 1669 , by which he had found that the star $\gamma$ Draconis was $23^{\prime \prime}$ more northerly in July than in October: observations which, at that time, seemed to favour the upinion of the earth's motion, though it be now known that there could not be any accuracy in them.

Flamsteed having observed the pole star with his mural quadrant, in 1689 and the following years, found that its declination was $40^{\prime \prime}$ less in July than in December; which observations, though very just, were yet, however, improper for proving the annual parallax: and he recommended the making of an instrument of 15 or 20 feet radius, to be firmly fixed on a strong foundation, for deciding a doubt which was otherwise not soon likely to be brought to a conclusion.

In this state of uncertainty and doubt, Dr. Bradley, in conjunction with Mr. Samuel Molineux, in the year 1725, formed the project of verifying, by a series of new observations, those which Dr. Hook had communicated to the public almost 50 years before. And as it was his attempt that chiefly gave rise to this undertaking, so it was his method in making the observations, in some measure, that they followed; for they made choice of the same star, and their instrument was constructed on nearly the same principles: but bad it not greatly exceeded the former in exactness, they might still have continued in uncertainty and doubt, as to the parallax of the fixed stars. And this was chichy owing to the accuracy of the ingenious Mr. George Graham, to whom the lovers of astronomy are also indebted for several other exact and convenicnt instruments.

The success then of the intended experiment, evidently depending very much on the accuracy of the instrument, that leading object was first to be well secured. Mr. Molineux's apparatus having been completed, and fitted fur observing, about the end of November 1725 , on the third day of December following, the bright star in the head of Draco, marked $\gamma$ by Bayer, was for the first time wherved, as jt passed near the zenith, and its situation carcfully taken with the instrument. The like observations were inade frequeptly, on many days, both in that and the succeding montbs, when they found a gradual variation in the star's position towards the south. They took care to examine very nicely, at the time of each obsurvation, how much the variation was; till about the bergining of March 1726, when the star was found to be
A B E

$20^{\prime \prime}$ more southerly than at the time of the first observation: : it now indeed seemed to have arrived at its utmost limit southward, as in several trials, made about this time, no sensible difference was observed in its situation. By the middle of April, it appeared to be returning back again towards the north; and about the beginning of June, it passed at the same distance from the zonith, as it had done in December, when it was first observed.

From the quick alteration in the declination of the star about this tine, increasing about one stcond in 3 days, it was conjectured that it would now proceed northward of its present situation, as it laad before gone soutlward; which happened accordingly; for the star continued to move northward till September following, when it became stationary again; being then near $20^{\prime \prime}$ more northeFly than in June, and upwards of $39^{\prime \prime}$ more northerly than it had been in March. From Septemlir the star again returned towards the south, till, in Decomber, it arrived at the same situation in which it bad bren obsorved twelve months before, allowing for the difference of declination on account of the precession of the equinox.

This was a sufficient proof that the instrument had not been the cause of this apparent motion of the star; and yet it seemed difficult to devise one that should be adequate to such an unusual effect. A nutation of the earth's axis was one of the first things that offered itself on this occasion; but it was soon found to be insufficient.

The great regularity of the observations left no room to doubt, but that there was some uniform cause by which this unexpected motion was produced, and which did not depend on the uncertainty or variety of the seasons of the year. But not being able to frame any hypothesis, sufficient to account for all the phenomena, and being very desirous to search a little further into this matter, Dr. Bradley began to think of erecting an instrument for himself at Wanstead; that, having it always at hand, he might with the more ease and certainty inquire into the laws of this new motion. The consideration likewise of being able, by another instrument, to confirm the truth of the observations hitherto made with that of Mr. Molineux's, was no small inducement to the undertaking; but the principal one was, the opportunity he should thereby have of trying in what maner other stars should be affected by the same cause, whatever it might be. For Mr. Nolineux's instrument being originally designed for observing $y$ Draconis, to try whether it had any' sensible parallax, it was so contrived, as to be capable of but little alteration in its direction; not above 7 or 8 minutes of a degree: and there being but few stars, within half that distance from the zenith of $\mathrm{Kcw}$, bright enough to be well observed, he could not, with his instrument, thoroughly cxamine how this cause affected stars that were difierently situated, with respect to the cquinoctial and solsticial points of the celiptic.

These considerations determined lim; and by the contrivance and dircetion of the same ingenious person, Mr. Grabain, his instrument was fixed upon the 19)th of $\Lambda$ ugust 1727. $\Lambda$ s he had no convenient place where he couli make use of so long a telescope as Mr. Molineux's, he contented himself with one of but little more than hali the length, namely, of 12 feet and a half, the other being 24. feet and a hall long, jutging, from the expericuce he had already had, that this radius would be long enough to 
adjust the instrument to a sufficient degree of exactuess : and he had no reason afterwards to change his opinion; for by all his trials he was very well satisfied, that when it was carelully rectified, its situation might be securely depended on to half a second.

His instrument being fixed, he immediately began to obscrve such stars as be judged most proper to give him any light into the cause of the motion already mentioned. There was a sufficient variety of small ones, and not less than twelve that he could observe through all seasons of the year, as they were bright enough to be seen in the daytime, when nearest the sun.

When the year was completed, be began to examine and compare his observations; and having pretty well satisfied himself as to the general laws of the plienomena, he then endeavoured to find ont the cause of them. He was already convinced that the apparent motion of the stars was not owing to a nutation of the earth's axis. The next idea/that occurred to him, was an alteration in the direction of the plumb-line, by which the instrument was constantly adjusted; but this, upon trial, proved insufficient. He then considered what refraction might do; but here also he 'met with no satisfaction. At length, through an amazing sagacity, he conjectured that all the phenomena hitherto inentioned, proceeded from the progressive motion of light, and the earth's annual motion in its orbit : for he conceived, that if light were propagated in time, the apparent place of a fixed object would not be the same when the eye is at rest, as when it is moving in any uther direction except that of the line passing through the object and the eye ; and that when the eye is moving in different directions, the apparent place of the object would be different.

He considered this matter in the following manner. He imagined $\mathrm{CA}$ to be a ray of light, falling perpendicularly on the line BD: then, if the ey'e be at rest at $A$, the object must appear in the direction $A \mathrm{c}$, whether light be propagated in time, or instantaneously. But if the eye be moving from $B$ towards $A$, and light be propagated in time, with a velocity that is to the velocity of the eye, as $\mathrm{A} C$ to $A B$; then, light moving from $\mathrm{C}$ to $A$, while the eye moves from $B$ to $A$, that particle of it by which the object will be discerned, when the eye in its motion comes to $\mathrm{A}$, is at $c$ when the eye is at $\mathbf{B}$. Jolning the points $B, C$, he supposed the line $B \mathrm{C}$ to be a tilbe, inclined to the line $B D$ in the angle

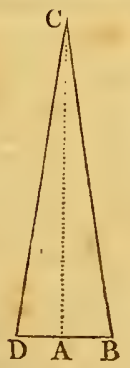

$C B D$, and of such a diameter as to adnit of but one particle of light: then it was easy to conceive, that the particle of light at $c$, by which the object must be seen when the eye arrives at $\Lambda$, would pass through the tube $\mathrm{BC}$, so inclined to the line $\mathrm{B} \mathrm{D}$, and accompanying the eye in its motion from $\mathrm{B}$ to $\mathrm{A}$; and that it would not come to the eye, placed behind such a tube, if it had any nther inclination to the line B D. But it, instead of suppusing B $\mathrm{C}$ so small a tube, we conceive it to be the axis of a larger; then, for the same reason, the particle of light at $\mathbf{c}$ cannot pass through that axis, unless it be inclined (1) Is $b$ in the same angle $C$ \& $D$.

lit like manner, if the eye move the contrary way, from 1) terwards $\Lambda$, with the sane velocity; then the tube must be incliner in the angle B DC. Nithough therefore the true or real. place of an object, be perpendicular to. the line in which the eye is moving, yet the visible place will not be sa ; since that must doubtless be in the direction of the tube. But the difference between the true and apparent place, will be, cæteris paribus, greater or less, according to the different proportions between the velucity of light and that of the eye: so that, if we could suppose light to be propagated in an instant, then there would be no difference between the real and visible place of an object, though the eye were in motion; for in that case, $\mathrm{AC}$ being infinite with respect to $\mathrm{A} \mathrm{B}$, the angle $A C B$, which is the difference between the true and visible place, valuishes, But if light be propagated in time, which was then allowed by most philosophers, then it is evident, from the foregoing considerations, that there will always be a difference between the true and visible place of an object, except when the eye is moving either directly towards, or from the object. And in all cases, the sine of the difference between the true and visible place of the object, will be to the sine of the visible inclination of the object to the line in which the eye is moving, as the velocity of the eye, is to the velocity of light.

If light moved only 1000 times faster than the eye, and an object, strpposed to be at an infinite distance, were really placed perpendicularly over the plane in which the eye is moving; it follows, from what has becn said, that the apparent place of such object will always be inclined to that plane, in an angle of $89^{\circ} 56^{\prime} \frac{1}{2}$; so that it will constantly appear $3 \frac{x}{2}$ from its true place, and will seem so much less inclined to the plane, that way towards which the eye tends. That is, if $\mathrm{A} C$ be to $\mathrm{A} B$ or $\mathrm{A} D$, as 1000 to 1 , the angle $\mathrm{A} \mathrm{B} \mathrm{C}$ will be $89^{\circ} 56^{\prime} \frac{\mathrm{x}}{2}$, and the angle $\triangle \mathrm{CB} 3^{\prime} \frac{\pi}{2}$, and B CD or $2 \mathrm{ACB}$ will be $7^{\prime}$, if the direction of the motion of the eye be contrary at one time to what it is at another.

If the earth revolve about the sun annually, and the velocity of light were to the velocity of the earth's motion in its orbit, as 1000 is to 1; then it is easy to conceive, that a star really placed in the pole of the ccliptic, would to an eye carried along with the earth, seem to change its place continually; and, neglecting the small difference on account of the earth's diurnal revolution on its axis, it would seem to describe a circle about that pole, every where distant from it by $3 \frac{x}{2}$. So that its longitude would be varied through all the points of the ecliptic every year, but its latitude would always remain the same. Its right ascension would also change, and its declination, according to the different situations of the sun in respect of the equinoctial points; and its apparent distance from the worth pole of the equator, would be $7^{\prime}$ less at the autumnal, than at the vernal equinox.

The greatest alteration of the place of a star, in the pole of the ecliptic, or, which in effect amounts to the same thing, the proportion between the velocity of light and the earth's motion in its orbit, being known, it will not be difficult to find what would be the difference, on this account, between the true and apparent place of any other star at any time: and, on the contrary, the difference between the true and apparent place being given, the proportion between the velocity of light, and the earth's motion in its orbit, may be found.

After the history of this curious discovery, related by the author (in the Philos. 'Jrans. of that year), be gives the results of a multitude of accurate obscrvations, made oin a great number of stars, at all seasons of the year. From all which abservations, and the loregoing theory, he 
found that every star, in consequence of the earth's inotion in its orbit and the progressive motion of light, appears to describe a small ellipse in the heavens, the transverse axis of which is cqual to the same quantity for every star, namely $40^{\prime \prime}$ nearly; and that the conjugate axis of the ellipse, for differcnt stars, varies as the sine of the star's latitude; that is, radius is to the sine of the star's latitude, as the transverse axis to the conjugate axis : and consequently a star in the pole of the ecliptic, its latitude being there $90^{\circ}$, whose sine is equal to the radius, will appear to describe a small circle about that pole as a centre, whose radius is equal to $20^{\prime \prime}$. He also gives the following law of the rariation of the star's declination : if $A$ denote the angle of position, or the angle at the star made by two great circles drawn from it through the poles of the ecliptic and equator, and $\mathrm{B}$ another angle, whose tangent is to the tangent of $A$, as radius is to the sine of the star's latitude; then $B$ will be equal to the difference of longitude between the sun and the star, when the true and apparent declination of the star are the same. And if the sun's longitude in the ecliptic be reckoned from that point in which it is when this bappers; then the difference between the true and apparent declination of the star, will be always as the sine of the sun's longiturle from that point. It will also be found that the greatest difference of declination that can be between the true and apparent place of the star, will be to $20^{\prime \prime}$, the semitransverse axis of the ellipse, as the sine of $A$ to the sine of $B$.

The author then shows, by the comparison of a number of observations made on different stars, that they exactly agree with the theory deluced from the progressive motion of light, and that consequently it is highly probable that such motion is the cause of those variations in the situation of the stars. From which he infers, that the parallax of the fixed stars is much smaller than hitherto supposed, by those who pretended to deduce it from observations. He thinks he may venture to say that, in the stars he had observed, the parallax does not amount to $2^{\prime \prime}$; nay, that if it had amounted to $\mathbf{1}^{\prime \prime}$, he should certainly have perceived $i t$, in the great number of observations that he made, especially of $\gamma$ Draconis : which agreeing with the hypothesis, without allowing any thing for parallax, nearly as well when the sun was in conjunction with, as in opposition to, this star, it seems very probable that its parallax is not so much as one second; and consequently that it is above 400000 times farther from us than the sun.

From the greatest variation in the place of the stars, namely $40^{\prime \prime}, \mathrm{Dr}$. Bradley deduces the ratio of the velocity of light, in comparison with that of the earth in its orbit. In the preceding figure, $\mathrm{AC}$ is to $\mathrm{AB}$, as the velucity of light to that of the earth in its orbit, the angle $\triangle \mathrm{CB}$ heing cqual to $20^{\prime \prime}$; so that the ratio of those velocities is that of radius to the tangent of $20^{\prime \prime}$, or of radius to the arc of $20^{\prime \prime}$, since in very small angles the tangent has no sensible difference from the arc itsell": but the radius of a circle is equal to the arc of $57^{\circ} x^{3}$ nearly, or equal to $206260^{\prime \prime}$; therefore the velocity of light is to the velocity of the earth, as 206260 to 20 , or as 10313 to 1 .

And hence also the time, in which light passes over the space from the sun to the earth, is easily deduced; for this time will be to one year, as $A$ is or $20^{\prime \prime}$ to $360^{\circ}$ or the whole circle; that is, $360^{\circ}: 20^{\prime \prime}:: 365 \frac{x}{4}$ days : $8^{\mathrm{m}} 7^{\mathrm{s}}$, that is, light will pass from the sun to the earth in 8 minutes, 7 seconds; and this will be the same, whatever may be the distance of the sun. See Abridg. Philos. Trans. vol. $\tau$, p. 308 , and vol. 9 , p. 4.17 .

Dr. Bradley having annexed to his theory the rules or formula for computing the aberration of the fixed stars in declination and right ascension; these rules have bcen rariously demonstrated, and reduced to other practical forms, by Clairaut, in the Memoirs of the Academy of Sciences for 1737 ; by Simpson, in bis Essays in 1740; by Fontaine, in 1744; and by several other persolis. The results of these rules are as follow: Every star appears 10 describe in the course of a year, by means of the aberrition, a small cllipse, whose greater axis is $40^{\prime \prime}$, and the less axis, perpendicular to the ecliptic, is equal to $40^{\prime \prime}$ multiplicd by the sine of the star's latitude, the radius being 1 . The eastern extremity of the longer axis, mark : the apparent place of the star, on the day of the opnosition; and the extremity of the less axe, which is farthest from the ecliptic, marks its situation three months after.

The greatest aberration in longitude, -is equal to $20^{\prime \prime} \mathrm{di}$ vided by the cusine of its latitude, or multiplied by its secant. $\Lambda$ nd the aberration for any time, is equal to $20^{\prime \prime}$ multiplied by the cosine of the elongation of the star found for the same time, and divided by the cosine of its latitude, or multiplicd by its secant. This aberration is subtractive in the first and last quadrants of the argument, or of the difference between the longitudes of the sun and star; and additive in the second and third quadrants. The greatest aberration in latitude, is equal to $20^{\prime \prime}$ multiplied by the sine of the star's latitude. And the aberration in latitude for any time, is equal to $20^{\prime \prime}$ multiplied by the sine of the star's latitude, and also by the sine of the elongation. The aberration is subtractive before the opposition, and additive after it.

The greatest aberration in declination, is equal to $20^{\prime \prime}$ multiplied by the sine of the angle of position $A$, and divided by the sine of $\mathrm{B}$ the difference of longitude between the sun and star when the aberration in declination is nothing. And the aberration in declination at any other time, will be equal to the greatest aberration multiplied by the sine of the difference between the sun's place at the given time and his place when the aberration is nothing. Also the sine of the latitude of thestar, is to radius, as the tangent of $A$ the angle of position at the star, is to the tangent of $\mathrm{B}$, the difference of longitude between the sun and star when the aberration in declination is nothing. The greatest aberration in right-ascension, is equal to $20^{\prime \prime}$ multiplied by the cosine of $A$ the angle of position, and divided by the sine of $\mathrm{c}$ the difierence in longitude, between the sun and star when the aberration in right-ascension is nothing. And the aberration in right-ascension at any other time, is equal to the greatest aberration multiplied by the sine of the difference between the surs's place at the given time, and his place when the aberration is nothing. Also the sine of the latitude of the star is to radius, as the cotangent of $A$ the angle of position, to the tangent of $c$.

In the first article of Mr. Simpson's Essays (an. 1740), is given a geometrical demonstration of a star's apparent patll, resulting from the motion of light combined with the earth's motion in its annual orbit; accompanied with rules thence delluced for practical calculations. A nother curious demonstration of the same was given by $\mathrm{Dr}$. Brook 'Taylor in the ycar 1729, as soon as 1)r. Bratley's discovery was known. It is preserved in Robins's Tracts, vol. 2, 1.276, and is as follows.-Let $\mathrm{ABC}$ be the orbit of 


\section{A B E}

the earth, in which it moves from $\mathrm{B}$ to $\mathrm{c}$ and $A$, jound the sun in $D$. Then, the earth being in $c$, if the, tangent $\mathrm{CE}$ be drawn, and on the transverse axis $A$ B as a diameter the circle $\mathrm{A}$ E $\mathrm{B} F$ be described, $\mathrm{I} \mathrm{D}$ being drawn and continued to $\mathrm{F}, \mathrm{D} E$ is perpendicular to the tangent $\mathrm{EC}$; and therefore the velocity of the earth in $B$, is to the velocity in c, reciprocally as D B to D E; that is, directly as $A D$ to $D G$, since the rectangles under A D B and E D G are equal. Now $\mathrm{C} 11$ being drawn from the earth in $\mathrm{C}$, toward the true place of any star, take $\mathrm{c}_{1}$ to $\mathbf{A D}$ as the velocity of light to the velocity of the earth in $B$. Through $I$ draw $K I I$ in a situation perpendicular to $A B$, and making $\mathrm{K} 1$ equal to $\mathrm{AD}$, and $\mathrm{I} \mathrm{L}$ equal to $\mathrm{DB}$, on the diameter $\mathrm{k} \mathrm{L}$ describe the circle $\mathrm{K}$ M L N parallel to the plane A C B F. Lastly, make the angle under K 10 equal to that under A D G, which is nearly the arithmetical mean between the mean and true distance of the earth from the aphelion, and

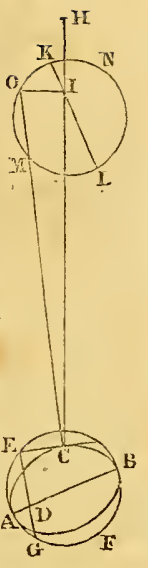
draw $\mathrm{C} 0$.- Now $\mathrm{K} \mathbf{L}$ being in a situation perpendicular to $\mathrm{A} \mathrm{B}$, and the angles under $\mathrm{K} \mathrm{IO}, \mathrm{A} \mathrm{DG}$ equal, or will be equal to $D G$, and be situated perpendicular to $G E$, and therefore is parallel to $\mathrm{c} E$, or to the direction of the earth's motion in $\mathrm{C}$; and is to $\mathrm{k} \mathrm{r}$, as the velocity of the earth.in $\mathrm{c}$, to its volocity in $\mathrm{B}$. Consequently, since $\mathbf{k} \mathbf{1}$ or $A$ D is to $\mathrm{C} 1$ as the velocity of the earth in $B$ is to the velocity of light, by equality or is to I $\mathrm{C}$, as the velocity of the earth's motion in $\mathrm{c}$ to the velocity of light. Therefore the star will be seen from the earth at $\mathrm{c}$ in the direction co. Hence it follows, that every fixed star is viewed from the earth as moving in a circle parallel to the plane of the ecliptic, the diameter of that circle which is parallel (1) the lesser axis of the earth's orbit being divided by the true place of the star, in the same proportion, as the greater axis of the earth's orbit is divided by the sun.

A BER R A TrON of the Planets, is equal to their geocentric motion, or the space they appear to move through as seen from the earth, during the time that light employs in passing from the planet to the earth. Thus, in the sun, the aberration in longitude is constantly $20^{\prime \prime}$, that being the space moved by the sun, or, which is the same thing, by the earth, in $8^{m} 7^{\mathrm{s}}$, that being the time in which light passes fiom the sun to the earth, as we have secn in the foregoing article. In like manner, knowing the distance of any planet from the carth, by proportion it will be, as the distance of the sun is to the distance of the planet, so is $8^{m} 7^{3}$ to the time of light passing from the planct to the earth : then computing the planet's geocentric motion in this time, that will be the aberration of the planet, whether it be in longitude, latitude, right-ascension, or declination.

It is evident that the aberration will be greatest in the longitude, and very small in latitude, because the planets deviate very little from the plane of the ecliptic or path of the earth; so that the aberration in the latitudes of the planets, is commonly neglected, as insensible; the greatest in Mercury being only $4^{\prime \prime} \frac{1}{3}$, and much less in the other planets. $\Lambda$ s to the aberrations in declination and rightascension, they must depend on the situation of the planet in the zoliac. The aberration in longitude, bring equal to the gencentric motion, will be more or less according as that motion is; it will therefore be least, or nothing at all,

when the planet is stationary; and greatest in the superior planets, Mars, Jupiter, Saturn, \&c, when they are in opposition to the sun; but in the inferior planets, V'enus and Mercury, the aberation is greatest at the time of their superior conjunction. These maxima of aberration for the several planets, when their distance from the sun is least, are as annexed, viz, for

Saturn - $27^{\prime \prime} \cdot 0$
Jupiter - $29 \cdot 8$
Mars - $37 \cdot 8$
Venus - $43 \cdot 2$
Mercury $59 \cdot 0$
The Moon
$\frac{2}{3}$

And between these numbers and nothing, the aberrations of the planets, in longitude, vary according to their situations. But that of the sun varies not, being constantly $20^{\prime \prime}$, as has been before observed. And this may alter bis declination by a quantity, which varies from 0 to near $8^{\prime \prime}$; being greatest or $8^{\prime \prime}$ about the equinoxes, and vanishing in the solstices.

The methods of computing these, and the formulas for all cases, are given by M. Clairaut, in the Memoirs of the Academy of Sciences for the year 1746 , and by M. Euler in the Berlin Memoirs, vol. 2, for 1746 .

AвERRATION, in Optics, is the deviation or dispersion of the rays of light, when reflected by a speculum, or refracted by a lens, by which they are prevented from ineeting or uniting in the same point, called the geometrical focus, but are spread over a small space, and produce a confusion of images. This aberration is either lateral or longitudinal: the lateral aberration is measured by a perpendicular to the axis of the speculum or lens, drawn from the focus to meet the refracted or reflected ray: the longitudinal aberration is the distance, on the axis, between the focus and the point where the ray meets the axis. The aberrations are very amply treated in Sinitb's Complete System of Optics, in 2 volumes 4 to.

There are two species of aberration, distinguished according to their different causes: the one arises from the figure of the speculum or lens, producing a geometrical dispersion of the rays, when these are perfectly equal in all respects : the other arises from the unequal refrangibility of the rays of light thenselves; a discovery that was made by Sir Isaac Newton, and for this reason it is often called the Newtonian aberration. As to the former species of aberration, or that arising from the figure, it is well known that if rays issue from a point at a given distance, then they will be reflected into the other focus of an ellipse having the given luminous point lor one focus, or directly from the other focus of an hyperbola; and will be variously dispersed by all other figures. But if the luminous point be infinitely distant, or, which is the same, the incident rays be parallel, then they will be reflected by a parabola into its focus, and variously dispersed by all other figures. But those figures are very difficult to make, and therefore curved specula are commonly marle spherical, the figure of which is generated by the revolution of a circular arc, which produces an aberration of all rays, whether they are parallel or not, and therefore it has no accurate geometrical focus which is common to all the rays. Let $B \vee F$ represent a concave spherical speculum, whose centre is $\mathrm{c}$; and let $\Lambda \mathrm{B}, \mathrm{E} F$ be incirlent rays parallel to the axis $\mathrm{cv}$, Because the angle ol incidence

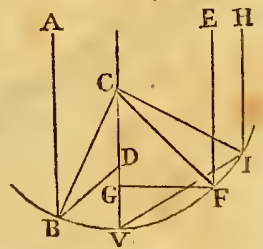


is equal to the angle of reflection in all cases, therefore if the radii с в, C $\mathrm{F}$ be drawn to the points of incidence, and thence $B$ D making the angle $C$ B D equal to the angle $\mathrm{CBA}$, and $\mathrm{F} \&$ making the angle $\mathrm{CFG}$ equal to the angle CFE; tben BD, FG will be the reflected rays, and D, G, the points where they meet the axis. Hence it appears that the point of coincidence with the axis, is equally. distant from the point of incidence and the centre: for because the angle $C B$ D is equal to the angle $\mathrm{C} B \mathrm{~A}$, which is cqual to the alternate angle в $\mathrm{CD}$, therefore their opposite sides $\mathrm{C} D, \mathrm{DB}$ are equal: and in like manner, in any other, $\mathrm{G} F$ is equal to $\mathrm{G} c$. And bence it is evident that when $\mathrm{B}$ is indefinitely near the vertex $\mathrm{v}$, then $\mathrm{D}$ is in the middle of the radius $\mathrm{cv}$; and the nearer the incident ray is to the axis $\mathbf{c v}$, the nearer will the reflected ray come to the middle point $\mathrm{D}$; and the contrary. So that the aberration $\mathrm{DG}$ of any ray $\mathbf{L F G}$, is always more and more, as the incident ray is farther from the axis, or the incident point $\mathrm{F}$ from the vertex $\mathrm{v}$; till when the distance $\mathrm{r} x$ is 60 degrees, then the reflected ray falls in the vertex $\mathrm{v}$, making the aberration equal to the whole length $\mathrm{D} v$. And this shows the reason why specula are made of a very small segment of a sphere, namcly, that all their reflected rays may arrive very near the middle point or focus $D$, to produce an image the most distinct, by the least aberration of the rays. And in like manner for rays refracted through lenses.

In spherical lenses, M. Huygens has demonstrated that the aberration from the figure, in different lenses, is as follows.

1. In all plano-convex lenses, having their plane surface exposed to parallel rays, the longitudinal aberration of the extreme ray, or that remotest from the axis, is equal to $\frac{9}{2}$ of the thickness of the lens.

2 . In all plano-convex lenses, having their convex surface exposed to parallel rays, the longitudinal aberration of the extreme ray, is equal to $\frac{7}{6}$ of the thickness of the lens. So that in this position of the same plano-convex lens, the aberration is but about onc-fourth of that in the former; being to it only as 7 to 27 .

3. In all double convex lenses of equal spheres, the aberration of the extreme ray is equal to $\frac{5}{3}$ of the thickness of the lens.

4. In a double convex lens, the radii of whose spheres are as 1 to 6 , if the more convex surface be exposed to parallel rays, the aberration from the figure is less than in any other spherical lens; being no more than $\frac{15}{14}$ of its thickness.

But the foregoing species of aberration, arising from the figure, is very small, and casily remedied, in comparison with the other, arising from the unequal refrangibility of the rays of light; insomuch that Sir lsaac Newton shows in his Optics, p. 84 of the 8vo edition, that if the object-glass of a telescope be plano-convex, the plane side being turned towards the object, and the diamster of the sphere, to which the convex side is ground, be 100 feet, the diameter of the aperture bring 4 inches, and the ratio of the sine of incidence out of glass into air, be to thit of refraction, as 20 to 31 ; then the diameter of the circle of aberrations will in this case be only $\frac{7061}{200000}$ parts of an inch: while the diamcter of the little circle, through which the same rays are scattered by unequal refrangibility, will be about the 55 th part of the aperture of the object-glass, which here is 4 inches. And therefore the error arising from the spherical figure of the glass, is to the error arising from the different refrangibility of the rays, as $\frac{961}{7200000}$ to $\frac{4}{55}$, that is as 1 to 544.4 .

So that it may seem strange that objects appear through telescopes so distinct as they do, considering that the error arising from the different refrangibility, is almost incomparably larger than that of the figure. Newton however solves the difficulty by observing that the rays, uniler their various aberrations, are not scattered uniformly over all the circular space, but collected infinitely more dense in the centre than in any other part of the circle; and that, in the way from the centre to the circumference, they grow more and more rare, so as at the circumference to become infinitely rare; and, by reason of their rarity, they are not strong enough to be visible, unless in the centre, and very near it.

In consequence of the discovery of the unequal refrangibility of light, and the apprehension that equal refractions must produce equal divergencies, in every kind of medium, it was supposed that all spherical objectglasses of telescopes would be equally affected by the dilferent refrangibility of light, in proportion to their aperture, of whatever materials they might be constructed: and therefore, that the only improvement that could be made in refracting telescopes, was that of increasing their length. So that Sir Isaac Newton, and other persons after him, despairing of success in the use and fabric of lenses, directed their chief attention to the construction of reflecting telescopes.

However, about the year 1747, M. Euler applied himself to the subject of refraction; and pursued a hint suggested by Newton, for the design of making objectglasses with two lenses of glass inclosing water betwren them ; hoping that, by constructing them of difiercut, materials, the refractions would balance one another, and so the usual aberration be prevented. Mr. John Dollond, an ingenious optician in London, minutely examined this scheme, and found that M. Euler's principles were not satisfactory. M. Clairaut likewise, whose attention lad been excited to the same subject, concurred in opinion that Euler's speculations were more ingenious than useful. This controv rsy, which seemed to be of great importance in the science of optics, engaged also the attention of Ml. Klingenstierna of Sweden, who was led to make a careful examination of the sthexperiment in the second part of Newton's Optics, with the conclusions there drawn from it. The consequence was, that he found that the rays of light, in the circumstances there mentioned, did not lose their colour, as Sir lsaac had imagined. This hint of the Swelish philosopher led Mr. Dollond to rcexamine the same experiment: and after several trials it appeared, that different substances caused the light to diverge very differently, in proportion to their general refractive powers. In the year 1757 therefore he procured wedges of diflerent kinds of glass, and applied them together so, that the refractions might be mate in contrary directions, that he might discover whether the retraction and divergency of colour would vanish together. 'I'he result of his tirst trials encouraged him to persevere; for be discovered at difierence far beyous his hopes in the qualities of different kinds of glass, with respect to their divergency of colours. 'The Venice glass and linglish crown ghass were found to be nearly allied in this respect: the common linglish plate glass made the rays diverge more; and the linglish tlint glass most of all. But, without inçuirng into the cause of this diflerence, he pro- 


\section{A B E}

ceeded to adapt wedges of crown glass, and of white flint glass, ground to different angles, to each other, so as to refract in different directions; till the refincted light was entirely free from colours. Having measured the refractions of each wedge, he found that the refraction of the white glass, was to that of the crown glass, nearly as 2 to 3 : and he hence concluded in general, that any two wedges made in this proportion, and applied together so as to refract in contrary directions, would refract the light without any aberration of the rays.

Mr. Dollond's next object was to make similar trials with spherical glasses of different materials, with the view of applying his discovery to the improvement of telescopes : and here he perceived that, to obtain a refraction of light in contrary directions, the one glass must be concave, and the other convex; and the latter, which was to refract the most, that the rays might converge to a real focus, he made of crown glass, the other of white flint glass. And as the refractions of spherical glasses are inversely as their focal distances, it was necessary that the focal distances of the two glasses, should be inversely as the ratios of the refractions of the wedges; because that, being thus proportioned, every ray of light that passes through this compound glass, at any distance from its axis, will constantly be refracted, by the difference between two contrary refractions, in the proportion required; and therefore the different refrangibility of the light will be entirely removed.

But in the applications of this ingenions discovery to practice, Mr. Dollond met with many and great difficulties. At length however, after many repeated trials, by a resolute perseverance, lie succeeded so far as to construct refracting telescopes much superior to any that had hitherto been made; representing objects with great distinctness, and in their true colours.

M. Clairaut, who harl interested himself from the begiuning in this discovery, now endeavoured to ascertain the principles of Mr. Dollond's theory, and to lay down rules to facilitate the construction of these new telescopes. With this view he made several experiments, to determine the refractive power of different kinds of glass, and the proportions in which they scparated the rays of light: and from these experiments he deduced several theorems of general use. M. d'Alembert made likewise a great variety of calculations to the same purpose; and lie showed how to correct the errors to which these telescopes are subject, sometimes by placing the object-glasses at a small distance from each other, and sometimes by using eye-glasses of different refractive powers. But though foreigners were hereby supplied with the most accurate calculations, they wcre very defectice in practice; and the English telescopes, made, as they imagined, withnut any precise rule, were greatly superior to the best of their construction.

M. Euler, whose speculations had first given occasion to this important and useful inquiry, was very reluctant in admitting Mr. Dullond's improvements, because they militated against a pre-conceived theory of lis own. At last however, after several altercations, being convinced of their reality and importance by $\mathrm{M}$. Clairaut, he assent'd; and he soon after received further satisfaction from the experiments of MI. Zeiher, of Petcrshurg.

M. Zeiher showed by experiments, that it is the leat, in the composition of glass, which eives it this renarkable VOL. I.
A B S

property, namely, that while the refraction of the mean rays is nearly the same, that of the extreme rays considerably differs. And, by increasing the lead, he produced a kind of glass, which occasioned a much greater separation of the extreme rays than that of the flint glass used by Mr. Dollond, and at the same time considerably increased the mean refraction. M. Zeiher, in the course of his experiments, made glass of minium and lead, with a mixture also of alkaline salts; and he found that this mixture greatly diminished the mean refraction, while it made hardly any change in the dispersion: and he at length obtained a kind of glass greatly superior to the flint glass of Mr. Dollond, for the construction of telescopes; as it occasioned three times as great a dispersion of the rays as the common glass, while the mean refraction was only as 1.61 to 1 .

Other improvements were also made on the new or achromatic telescopes by the inventor Mr. John Dollond, and by his son Peter Dollond; which may be scen under the proper words. For various dissertations also on the subject of the aberration of light, colours, and the figure of the glass, see Philos. Trans. vols. $35,48,50,51,52$, 55,60; Memuirs of the Academy of Sciences of Patis, for the years $1737,1746,1752,1755,1756,1757,1762$, $1764,1765,1767,1770$; the Berlin Acad. 1746,1769 , 1766; Swed. Mlem. vol. 16; Com. Nov. Petripol. 1762; M. Euler's Dioptrics; M. d'Alembert's Opuscules Math.; M. de Rochon Opuscules; \&zc, \&:c.

ABRIDGING, in Algebra, is the reducing a componud equation, or quantity, to a more simple form of expression. This is done either to save room, or the trouble of writing a number of symbols ; or to simplify the expression, and ease the memory, or to render the formula more easy and general:

So the equation $x^{3}-a x^{2}+a b x-a b c=0$, by putting $p=a, q=a b$, and $r=a b c$, becomes $x^{3}-p x^{2}+q x-r=0$.

And the equation $x^{2}+(a+b) x-\frac{a b}{c}=0$, by putting $p=a+b$, and $q=\frac{a h}{c}$, bccomes $x^{2}+p x-q=0$.

ABSCISS, Anscisse, or ABscissa, is a part or segment cut off a line, terminated at some certain point, by an ordinate to a curve; as $A$ P or B P.
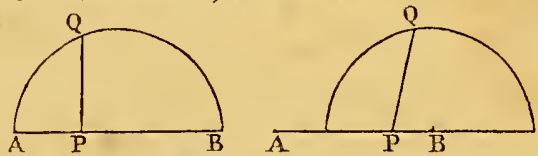

So that the absciss may either commence at the vertex of the curve, or at any other fixed point. And it may be taken either upon the axis or diameter of the curve, or upon any other line given in position.

Hence there are an infinite number of variable abscisses, terminated at the same fixed point at one end, the other end being at any point of the given line or diameter.

When an absciss and its corresponding ordinate are considered together, they are called the co-ordinates of the curve.

In the common parabola, each ordinate $\mathrm{P} Q$ has but one absciss, $\triangle \mathrm{P}$; in the cllipse or circle, the ordinate has two abscisses, A P, B P, lying on the opposite sides of it; and in the hyperbola, the ordinate $P Q$ has also two abscissee, but they lie both on the same side of it. That is, in ge- 

A B S

neral, a line of the second kind, or a curve of the first kind, may have two abscisses to each ordinate. But at

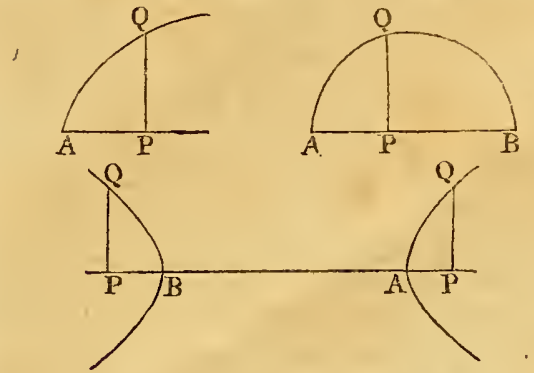

line of the third order may have three abscisses to each ordinate; a line of the fourth order may have four; and so on.

The use of the abscisses is, in conjunction with the ordinates, to express the nature of the curves, either by some proportion or equation including the absciss and its ordinate, with some other fixed invariable line or lines. Every different curve has its own peculiar equation, or property, by which it is expressed, and different from all others : and that equation or expression is the same for every ordinate and its abscisses, whatever point of the curve be taken. So, in the circle, the square of any orclinate is equal to the rectangle of its two abscisses, or A P . PB $=\mathrm{PQ}^{2}$; in the parabola, the square of the ordisate is equal to the rectangle of the absciss and the parameter ; in the ellipse and hyperbola, the square of the ordinate is always in a certain constant proportion to the rectangle of the two abscisses, namely, as the square of the conjugate to the square of the transverse, or as the parameter to the transverse asis; and so other properties in other curves.

When the natures or properties of curves are expressed by algebraic equations, any genera! absciss, as $A P$, is commonly denoted by the letter $x$, and the ordinate $\mathrm{P}$ Q by the letter $y$; the other or constant lines being represented by other letters. Then the equations expressing the nature of these curres are as follow; namely, for the

circle - $d x-x^{2}=y^{2}$, where $d$ is the diameter $A \mathrm{~B}$; parabola - $p x=y^{2}$, where $p$ is the parameter; ellipse $-t^{2}: c^{2}:: t x-x^{2}: y^{2}$, $\{$ where $t$ is the transverse, lyyperbola $t^{2}: c^{2}:: t x+x^{2}: y^{2},\{$ and $c$ the conjugate axis.

But if the abscisses are taken from the ceritre; then in the $\left.\begin{array}{l}\text { circle - } b^{2}-x^{2}=y^{2} \text {, } \\ \text { rllipse - } b^{2}: c^{2}:: b^{2}-x^{2}: y^{2},\end{array}\right\}$ where $b$ is the semiaxis,

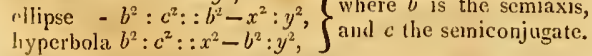

Or we may likewise employ the parameter $p$ in the cquation to the ellipse and hyperbola; then in the cllipse - $\left.t: p:: t x-x^{2}: y^{2},\right\}$ when the abscisses are taken hyperbola $\left.t: p:: x^{2} \pm t x: y^{2},\right\}$ from the vertex, ellipse $-t: p^{\prime}:: \frac{1}{4} t^{2}-x^{2}: y^{2}$, when the abscisses are taken hyperbolat $\left.t: p:: x^{2}-\frac{1}{4} t^{2}: y^{2},\right\}$ from the centre, where $t$ is the tratusverse axis, and the parameter.

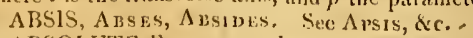

ABSOLUTE EoUation, in $\Lambda$ stmonomy, is the sum of the optic and excentric equations. The apparent incepuality of' a planct's motion, arising from its not being equally distant from the earth at all times, is called its optic equation; and this would subsist even if the planet's real motion were uniform. The excentric inequality is causer by the [lanet's motion being not uniform. To illustrate this, conceive the sun to move, or to appear to move, in the curcuinference of a circle, in whose centre the earth is placed. It is manifest, that if the sun move uniformly in this circle, he mast appear to move uniformly to a spectator at the earth; and in this case there will be no optic nor excentric equation. But suppose the earth to be placed out of the centre of the circle: then, though the sun's motion should be really uniform, it would not appear to be so, being seen from the earth; and in this case there would be all optic equation, without an excentric one. But if we imagine the sun's orbit not to be circular, but elliptical, and the earth in its focus; it will be fuil as evident that the sun cannot appear to have a uniform motion in such ellipse; so that his motion will then be subject to two equations; that is, the optic equation, and the cxcentric equation. See EQUATrox, and Optic Inequality.

Absolute Number, in Algebra, is that term or member of an equation that is completely known, and whicl is equal to all the other, or unkwown terms, taken together; and is the same as what Vieta calls the homogeneum comparationis. So, of the equation $x^{2}+16 x-$ $36=0$, or $x^{2}+16 x=36$, the absolute number, or known term, is 36 .

Assolute Gravity, Motion, Space, Time, \&c. Sce the respective substantives.

ABSTRACT Maturmatics, otherwise called pure mathematics, is that which treats of the properties of magnitude, figure, or quantity, absclutely and gencrally considered, without restriction to any species in particular: such as Arithmetic and Geometry. In this sense, abstract, or pure mathematics, is opposed to mixed mathematics, in which simple and abstract properties, and the relations of quantities, primitively considered in pure mathematics, are applied to sensible objcet"; as in astronomy, hydrostatics, optics, \&c.

A в sтr A ст Number, is a number, or collection of units, considered in itself, without being applied to denote a collection of any particular and determinate things. So, for example, 3 is an abstract number, so far as it is not applied to something: but when we say 3 feet, or 3 persons, the 3 is no longer an alstract, but a concrete number.

ABSURD, or $A$ BSURDUn, a term commonly used in demoustrating converse propositions; a mode of demonstration, in which the proposition intended is not proved in a direct manner, by principles before latid down; but it proves that the contrary is absurd or impossible; and so indirectly, as it were, proves the proposition itsclf. The 4th proposition in the first book of Euclid, is the first in which he makes use of this mode of proof; where he shows that if the extremities of two lines coincide, those lines will coincide in all their parts, otherwise they would inclose a space, which is absurd or contrary to the loth axjom. Most converse propositions are proved in this way, which mode of prout is called reductio ad absturlum.

ABUNDANT NUaner, in Arithuetic, is a number whose aliquot parts, added all together, make is sum which is greater than the number itsclf. Thus 12 is ant abundant uumber, because its aliquot parts, mumely 1, 2, 


\section{A C A}

[

$3,4,6$, when added together, make 16 , which is greater than the number 12 itself.

An abundant number is opposed to a deficient one, which is tess than the sum of its aliquot parts taken together, as the number 14, whose aliquot parts, $1,2,7$, make no more than 10; and to a perfect number, which is exactly equal to the sum of all its aliquot parts, as the number 6 , which is equal to the sum of $1,2,3$, its aliquot parts.

ACADENICIAN, a member of a society called an academy, instituted for the promotion of arts, sciences, or natural knowledge in general.

ACADEMICS, an ancient sect of philosophers, who followed the doctrine of Socrates and Plato, as to the uncertainty of knowlerge, and the incomprehensibility of truth.

Academic, in this sense, amounts to much the same with Platonist; the difference between them being only in point of time. Those who embraced the system of Plato, among the ancients, were called Academici, academician or academic; whereas those who did the same since the restoration of learning, have assumed the denomination of Platonists.

There are usually reckoned three sects of academics; though some make five. The aucient academy was that of which Plato was the chief. Arcessilas, one of Plato's successars, introducing some alterations into the philosophy of this sect, founded what is called the second academy. The establishment of the third, called also the new academy, is attributed to Lacydes, or rather to Carneades. Some authors add a fourth; founded by Philo; and a fifth, by Antiochus, called the Antiochan, which tempered the ancient academy with Stoicism.

The ancient academy doubted of every thing; and carried this principle so far as to make it a doubt, whether or no they ought to doubt. It was a kind of a principle with them, never to be certain or satisfied of any thing; never to affirm or to deny any thing, either for true or false.

The new academy was somewhat more reasonable; they acknowledged several things for truths, but without attaching themselves to any with entire assurance. These philosophers bad found that the ordinary commerce of life and society was inconsistent with the absolute and universal doubtfulness of the ancient academy: and yet it is evident that they looked upon things rather as probable, than as true and certain: by this amendment thinking to secure thenselves from those absurdities into which the ancient academy bad fallen.

ACADEMIST, the same as Academician.

ACADEMY, ACA DEM 1A, in Antiquity, a fine villa or pleasure-house, in one of the suburbs of Athens, about a nile from the city; where Plato, and the wise men who followed him, held assemblies for disputes and philosophical conference; which gave the name to the sect of Açitlemies. The house took its name, Academy, from one Academus, or Ecademus, a citizen of Athens, to whom it originally belonged: he lived in the time of Thescus; and here he used to have gymnustic sports or texercises.

The academy was further improved by Cimon, and adorned with fountains, trees, shady walks, \&c, for the ronvenience of the philosophers and men of learning, who hete inct to sobfer and dispute for their mutual improvement. lt ves surrounded with a wall by Hipparchus,
A C A

the son of Pisistratus; and it was also used as the brirying-place for illustrious persons, who had deserved well of the republic.

It was here that Plato taught his philosophy; and hence it was that all public places, destined for the assemblies of the learned and ingenious, have been since called Academies.-Sylla sacrificed the delicious walks and groves of the academy, which had been planted by Cimon, to the ravages of war; and employed those very trees in constructing machines to batter the walls of the city which they had adorned.-Cicero too had a villa, or country retirement, near Puzzuoli, which he called by the same name, Academia. Here he used to entertain his philosophical friends; and here it was that he composed his Academical Questions, and his books De Naturâ Deorum.

ACADEMY, among the moderns, denotes a regular society or company of learned persons, instituted under the protection of some prince, or other public authority, for the cultivation and improvement of arts or sciences.

Some authors confound Academy with University; but though much the same in Latin, they are very different things in English. An university is properly a body composed of graduates in the several faculties; of professors, who teach in the public schools; of regents or tutors, and students who learn under them, and aspire likewise to degrees. Whereas, an academy is not intended to teach, or profess any art or science, but to improve it : it is not for novices to be instructed in, but for persons of learning to confer in, and communicate their lights and discoveries to each other, for their mutual benefit and improvement.

The first modern academy we read of, was established by Charlemagne, by the advice of Alcuin, an English monk: it was composed of the most intelligent men of the court, the emperor himself being a member. In their academical conferences, every person was to give some account of the ancient authors he had read; and cach one assumed the name of some ancient author, that pleased him most, ог some celebrated person of antiquity. Alcuin, from whose letters we learn these particulars, took that of Flaccus, the surname of Horace; a young lord, named Augilbert, took that of Homer; Adelard, bishop of Corbie, was called Augustin; Recluse, bishop of Mentz, was Dametas; and the king himself, David.

Since the revival of learning in Europe, academies have multiplied greatly, most nations being furnished with several, and from their communications the chief improvements have been made in the arts and sciences, and in cultivating natural knowledge. There are now academies for almost every art, or species of knowledge; but we shall give a short account only of those institutions of this kind, which regard the cultivation of mathematical or philosophical subjects, which are the proper and peculiar objects of our undertaking.

Italy abounds more in academics than any other country; there being enumerated by Jarckius not less than five hundred and fifty; and eren to the amount of twentyfive in Milan itself. These are, however, mostly of a private and inferior nature.

The first academy of a philosophical kind was established at Naples, in the house of Baptista Porta, about the year 1560 , under the name of $\Lambda$ cademia Secretorum Naturx; being formed for the improvement of natural and mathematical hnowledge. This was succeded by the C 2 


\section{A C \&}

ACADEMY of Lyncei, fuunded at Roine by prince Frederick Cesi, towards the end of the same century. It was rendered famous by the notable discoveries made by several of its members; among whom was the cclebrated $\mathrm{Ga}$ lileo Galilei.

Several other academies contributed also to the advancement of the sciences; but it was by speculations, rather than by repeated experiments on the phenomena of nature: such were the academy of Bessarian at Rome, and that of Laurence de Medicis at Florence, in the 15th century; and in the $16 \mathrm{th}$ those of Infiammati at Padua, of Vegna Juoli at Rome, of Ortolani at Placentia, and of Umidi at Florence, were constituted. The first of these studied fire and pyrotechnia; the second, wine and vineyards; the third, por-herbs and gardens; the fourth, water and hydraulics. To these may be added that of Venice, called La Veneta, and founded by Frederick Badoara, a noble Venctian; another in the same city, of which Campegio, bishop of Feltro, appears to have been the chief; also that of Cosenza, or La Consentina, of which Bernadin Telesio, Sertorio Quatromanni, Paulus Aquinas, Julio Cavalcanti, and Fabio Cical:, celebrated philusophers, were the chief members. The cumpositions of all these academies, of the 16ith century, were good in their kind; but none of them comparable to those of the Lyncei.

ACADEMY del Cimento, that is, of Expcriments, arose at Florence, some years after the death of Torricelli, namely in the year 1657 , under the protection of prince Leopold of Tuscany, afterwards cardinal de Medicis, and brother to the Grand Duke Fcrdinand the Second. Galileo, Toriccll, Aggiunti, and Viviani had prepared the way for it: and one of its chief nembers was Paul del Buono, who in 1657 invented the instrument for trying the incompressibility of water, which was a thick globular shell of gold, having its cavity filled with water; the globe being compressed by a strong screw, the water came through the pores of the gold, rather than yicld to the strong compression: there were also, Alphonsus Borelli, wcll known for his ingenions treatise De Motu Animalium, and other works; Candicle del Buono, brother of Paul; Alexander Marsilli, Vincent Viviani, Francis Rhedi, and the Count Laurence Magalotti, secretary of the academy, who published a volume of their curious experiments in 1667, under the title of Saggi di Naturali Esperienze; a copy of which being prescnted to the Royal Society, it was translated into English by Mr. Waller, and published at London, in 4to, 1684: being $\mathrm{A}$ curious collection of tracts, containing ingenious experiments on the pressure of the air, on the compressing of water, on cold, heat, ice, magnets, electricity, odours, the mution of sound, projectiles, light, \&c, \&c. But we have heard little or nothing more of the academy since that time. It may not be improper to observe here, that the Grand Duke Ferdinand, above mentioned, was himself no mean philosopher and chemist, and that he invented thermoncters, of which the construction and use may be seen in the collection of the academy del Cimento.

ACADEM X degl' Inquicti, at Bologna, incorporated afterwards into that della traccia in the same city, followed the example of that del Cimento. 'The members met at the house of the abbot Antonio Sampieri; and here Geminiano Montanari, one of the chicf members, male excellent discourses on mathematical and philosophical subjucts, some parts of which were published in 1667, under
A C A

the title of Pensieri Fisico-Mathematici. This acadeny afterwards met in an apartment of Eustachio Manfredj; and then in that of Jacob Sandri; but it arrived at its chief lustre while its assemblies were held in the palace Marsilli.

ACADEMY of Rossano, in the kingdom of Naples, called La Societu Scientifica Rossanese degl' Incuriosi, was founded about the year 1540 , under the name of Naviganti; and was renewed under that of Spensicrati, by Camillo Tuscano, about the year 1600 . It was then an academy of belles-lettres; but was afterwards transformed into an acaderny of sciences, on the solicitation of the learned abbot Don Giacinto Gimma; who, bring marle president umler the title of promoter-general, in 1695 , gave it a new set of regulations. IJe divided the acadeinicians into several classes, namely, grammarians, rhetoricians, poets, historians, philosophers, physiciaris, mathematicians, lawycrs, and divines; with a separate class for carclinals and persons of quality. To be admitted a member, it was necessary that the candiclate should have degrees in some faculty. Members, in the title of their books, are not allowed to use the name Academist without a written permission from the president, which is not granted till the work has been examined by the censors of the academy. This permission is the highest honour the academy can confer; since they hereby, as it were, adopt the work, and engage to answer for it against any criticisms that may be made upon it. The president himself is not exempt from this law : nor is any academician permitted to publish any tbing against the writings of another, without leave obtained from the society.

There have been several other academies of sciences in Italy, but they have not long subsisted, for want of the support of the reigning princes. Sucb were at Naplcs that of the Investiganti, founded about the year 1679 , by the marquis d'Arena, Don Andrea Concubletto; and that which, about the year 1698 , met in the palace of Don Lewis della Cerda, the duke de Mledina, and viceroy of Naples: at Rome, that of Fisico-Matematici, which in 1686 met in the house of Signior Ciampini : at Verona, that of Alitafili, founded the same year Ly Signior Joseph Gazola, and which met in the house of the count Screnghi della Cucca: at Brescia, that of Filesotici, founded the same year for the cultivation of philosophy and mathematics, and terminated the year following: that of $\mathrm{F}$. Francisco Lana, a jesuit of great skill in these sciences: and lastly, that of Fisico-Critici at Sienna, founded in 1691, by Signior Peter Maria Gabrielli.

Some other academies, still or lately subsisting in I taly, repair with advantage the loss of the former. One of the principal is the acadenny of Filarmonici at Verona, supported by the marquis Scipio Maffei, one of the most learned men in Italy; the members of which academy, though they cultivate the belles-lettres, do not neglect the sciences. The academy of Ricorrati at Padua still subsists with reputation; in which, from time to tine, learnod discourses are held on philosophical subjects. The like may be said of the academy of the Muti di Reggio, ut Modena. At Bologna is an ucademy of sciences, in a flourishing condition, known by the name of The Institute of Bologna; which was founded in 1712 by count Marsigli, for cultivating physics, mathematics, medicine, chemistry, and natural history. 'The history of it is written by $\mathrm{Ml}$. de Liniers, from inemoirs furmislicd by the founder himself. Among the new academies, that which ranks 


\section{A C A}

first, after the Institute of Bolagna, is given to that of the Countess Donna Clelio Grillo Buromeo, one of the most learned ladies of the age, to whom Signior Gimma dedicates his literary history of Italy. She had lately established an academy of experimental philosophy in her palace at Milan; of which Signior Vallisnieri was noninated president, and had already drawn up the regulations for it, though we do not find it has ever taken place. In the number of these academies may also be ranked the assembly of the learned, who of late year's met at $\mathrm{Ve}-$ nice in the house of Signior Cristino Martinelli, a noble Venetian, and a great patron of learning.

ACADEMIa Cosmografica, or that of the Argonauts, was instituted at Venice, at the instance of $\mathrm{F}$. Coronelli, for the improvement of geography; the design being to procure exact maps, geographical, topographical, hydrographical, and ichnographical, of the celestial as well as terrestrial globe, and their several regions or parts, together with geographical, historical, and astronomical descriptions, accommodated to them: to promote which purposes, the several members oblige themselves, by their subscription, to take one copy or more of each piece published under the direction of the acadeny; and to advance the money, or part of it, to defray the charge of publication. 'To this end thrce societies were settled, namely at Venice, Paris, and Rome; the first under F. Moro, provincial of the Minorites of Hungary; the second under the abbot Laurence au Rue Payenne au Marais; the third under $F$. Ant. Baldigiani, jesuit, professor of mathematics in the Roman college; to whom those addressed themselves who were willing to engage in this design. The Argonauts numbered near 200 meinbers, in the different countries of Europe; and their device is the terraqueous globe, with the motto Plus ultra. All the globes, maps, and geographical writings of $\mathbf{F}$. Coronelli have been published at the expense of this academy.

The Academy of Apatists, or Impartial Academy, deserves to be mentioned on account of the extent of its plan, including universally all arts and sciences. It holds from time to time public meetings at Florence, where any person, whether academician or not, may read his works, in whatever form, language, or subject; the academy receiving all with the great'st impartiality.

In France there are many acarlemies for the improvement of arts and sciences. F. Mersenne, it is said, gave the first idea of a philosophical academy in France, about the beginning of the seventecnth century, by the conferences of inathematicians and naturalists, held occasionally at his lorlgings; at which Des Cartes, Gassendus, Holsbes, Roberval, Pascal, Blondel, and others, assisted. $F$. Mersenne proposed to each of them certain problems to examine, or certain experiments to be made. These private assemblies were succeeded by more public ones, tormed by M. Monmort, and M. 'Thevenot, the celebrated traveller. The Frencli example animated several Englishmen of rank and learning to erect a kind of phiJosophical academy at Oxford, towards the close of Cromwell's administration; which after the restoration was rrected, by public authority, into a Royal Society: an account of which sce under the word. The English example, in its turn, aninated the French. In 1666 Louis xiv, assisted by the counsels of M. Colbert, founded an academy of sciences at Paris, called The

Aca Deme Royale des Sciences, or Royal Academy of Siciences, for the improvement of philosophy, mathema- tics, chemistry, medicine, belles-lettres, \&c. Among the principal meinbers, at the comnencement in 1666, were the respectable nanies of Carcavi, Huygens, Roberval, Frenicle, Auzout, Picard, Buot, Du Hamel the Secretary, and Mariotte. There was a perfect equality among all the inembers, and many of them received salaries from the king. By the rules of the academy, every class was to meel twice a week ; the philosophers and geometricians were to ineet, separately, every Wednesday, and then both together on the Saturday, in a room of the king's library, where the philosophical and mathematical hooks were kept : the history class was to meet on the Monday and Thursday, in the room of the historical books: and the class of belles-lettres on the Tuesday and Friday: and on the first Thursday of every month all the classes met together, and by their secretaries made a mutual report of what had been transacted by each, during the preceding month.

In 1099, on the application of the president, the abbé Bignon, the academy received, under royal authority and protection, a new form and constitution; by the articles of which, the acadenyy was to consist of four sorts of members, namely honorary, pensionary, associates, and clèves. The honorary class to consist of ten persons, and the other three classes of twenty persons eacl. The president to be chosen annually out of the lonorary class, and the secretary and treasurcr to be perpetual, and of the pensionary class. The meetings to be twice a week, on the Wednesday and Saturday; besides two public meetings in the year.

Of the pensionaries, or those who receive salarjes, three to be geometricians, three astronomers, three mechanists, three anatomists, three botanists, and three chemists, the other two being the secretary and treasurer. Of the twenty associates, of which twelve to be French, and eight might be foreigners, two were to cultivate geometry, two astronomy, two mechanics, two anatomy, two botany, and two chemistry. Of the twenty elèves, one to be attached to each pensionary, and to cultivate lis peculiar branch of science. The pensionaries and their elèves to reside at Paris. No regulars nor religious to be admitted, except into the honorary class : nor any person to be admitted a pensioner who was not known by some considerable work, or some remarkable discovery.

In 1716 the Duke of Orleans, then regent of France, by the king's authority made some alteration in their constitution. The class of elères was suppressed; and instead of them were instituted twelve adjuncts, two to cacls of the six classes of pensioners. The honorary mentbers were increased to twelve: and atclass of six free associates was made, who were not under the obligation of cultivating any particular branch of science, and in this class only could the regulars or religious be adnitted. A president and vice-president to be appointed annually from the lionorary class, and a director and sub-director aunually from that of the pensioners. And no person to be allowed to make use of his quality of academician, in the title of any of his books that he published, unless such book were first approved by the academy. The academy had for a device or motto, Invenit et perficit. And the meetings, which were formerly held in the king's library, since the year 1699 were beld in a fine hall of the old Louvre.

Finally, in the year 1785, the king confirmed, by letters patent, dated April 23, the establishinent of the aca- 
demy of sciences, making the following alterations, and adding classes of agriculture, natural history, mineralogy, and physics; incorporating the associates and adjuscts, and limiting the number of members to six each class, namely three pensioners and three associates; by which the former received an increase of salary, and the latter approached nearer to becoming pensioners.

By the articles of this instrument it was ordained, that - the academy shall consist of eight classes, 1st geometry, $2 \mathrm{~d}$ astronomy, $3 \mathrm{~d}$ mechanics, 4th general plysics, 5th anatomy, 6 th chemistry and metallurgy, 7 th botany aud auriculture, and 8 th natural history and mineralogy. That each class shall remain irrevocably fixed at six members; namely, three pensioners and three associates, independent however of a perpetual secretary and treasurer; of twelve free-associates and of eight associate strangers or foreigners, the same as before, except that the adjunct-geographer for the future to be called the associate-geographer.

The classes at first to be filled by the following persons, namely, that of geometry by Messieurs de Borda, Jeaurat, Vandermonde, as pensioners; and Messieurs Cousin, Meusnier, and Charles, as associates: that of astronomy by Messieurs lc Monnicr, de la Lande, and le Gentil, as pensioners; and Messieurs Messicr, de Cassini, and Dagelat, as associates: that of mechanics by Messieurs l'abbé Bossut, l'abbé Rochon, and de la Place, as peusioners; and Messieurs Coulomb, le Gendre, and Perrier, as associates: that of general physics by Messieurs Leroy, Brisson, and Bailly, as pensioners; and Messieurs Monge, Mechain, and Quatremere, as associates: that of anatomy by Messieurs Daubluton, Tenon, and Portal, as pensioners; and Messieurs Sabatier, Vicq-d'azir, and Broussonet, as associates: that of chemistry and metallurgy by Messicurs Cadet, Lavoisier, and Beaume, as pensioners; and Messieurs Cornette, Berthollet, and Fourcroy, as. associates: that of botany and agriculture by Messieurs Guettard, Fougeroux, and Adanson, as pensioners; and Messicurs de Jussieu, de la Marck, and Desfontaines, as associates : and that of natural history and mineralogy by Messieurs Desmaretz, Saye, and l'abbé de Gua, as pensinners; and Messieurs Darcet, l'abbé Hauy, and l'abbe Tessier, as associates. All natmes respectable in the commonwealth of letters; and from whom the world might expect still further improvements in the several branches of science.

The late M. Roville de Mcslay, counsellor of the parliament of Paris, founded two prizcs, the one of 2500 livres, the other of 2000 livres, which the academy distributed altermately every year: the subjects of the former prize respecting physical astronomy; and of the latter, navigation and commerce.

The world is highly indebted to this academy for the many valuable works they have exceuted, or published, both individually and as a body collectively, especially by their memoirs, making upwards of a hundred volumes in 4.to, with the machines, indexes, \&c. in which may be found most excellent compositions in every branch of science. They published a volume of these memoirs every year, with the history of the academy, and eloges of remarkable men lately deceased: also a gencral index to the volumes every ten years. An alteration was introduced into the volume for $17 \mathrm{~s} 3$, which it seems was to be continued in future, by omitting, in the listory, the minutes or extracts from the reglsters, containing some preliminary account of the subjects of the memorr: but still however retaining the eloges of distinguished men, lately deceased.

Mi. l'abbé Rozier also published, in four guarto volumes, an excellent index of the contents of all the volumes, and the writings of all the members, from the beginning of their publications to the year 1770; with convenient blank spaces for continuing the articies in writing.

Their history also, to the year 1697, was written by M. Du Hamel; and after that time continued from yeat to year by M. Fontenelle, under the following titles, J) u Ilamel Historia Regiæ Academiæ Scientiarum, Paris, tio. Histoire de l'Academie Royale des Sciences, arcc les Mi. moires de Mathematique et de Physique, tirez c'es Registres de l'Academie, Paris, 4to. Histoire de l'Acadcinie Royale des Sciences depuis son etablissement en 1666 , jusqu'en 1699, en 13 tomes, 4to. A new history. frol! the institution of the academy, to the period from whence M. de Fontenelle commences, has been formed; with a series of the works published under the name of this academy, luring the first interval.

The French revolution, Lowever, gave occasion to the new-modeling of the Academy in 1793, as well as an alteration in its nalne. (Sce Institute.) The memoirs of the academy are comprebended in 139 volumes, 4to; viz. 11 vols. from its foundation in 1666 , to its renewal in $1699 ; 92$ vols. from 1699 to $1790 ; 11$ vols. of memoirs presented to the acadeny, 9 vols. of prizes, 9 of tables, and 7 of drawings of machines.

Besides the academies in the capital, there were a great many in other parts of France. The

ACADEMIE Royale, at Caen, was established by letters patent in the year 1705; but it had its rise fifty years earlier, in private conferences, held first in the house of M. de Brieux. M. de Segrais retiring to this city, to spend the rest of his days, restored and gave new lustre to their meetings. In $1707 \mathrm{M}$. Foucault, intendant of the generality of Caen, procured the king's letters patent for erecting them into a perpetual academy, of which M. Foucault was to be protector for the time, and the choice afterwards left to the members, the number of whom was fixed to thirty, chosen for this time by M. Foucault. But besides the thirty original members, leave was given to add six supernumerary members, from the ecclesiastical communities in that city.

At Toulouse was the Academic des jeux foraux, conposed of forty persons, the oldest of the kingdom: besides an academy of sciences and belles-lettres, founded in 1750 .

At Montpelier, the royal society of sciences; which, after 1708 , was united with the royal academy of sciences at Paris.

Other academies were also established: at Bourcleanx, founded in 1703; at Soissons in 1674, at Mlarseilles in 1726 , at Lyons in 1700, at Pau in Bearn in 1721, it Montauban in 1744, at Angers in 1685 , at Amictis in 1750 , at Villefranche in 1679 , at Dijon in 1740 , at Nîmes in 1682, at Besançon in 1752, at Chalons in 1775, ut Rochelle in 1734, at Beziers in 1723, at houen in $17+4$, nt Metz in 1760, at Arras in 1773 , \&.c. The number of these academies is continually augmenting; and even in such towns as have no ucademies, the literati form themselves into literary socicties, having nearly the same objects aud pursuits. 
In Germany and other parts of Europe there are varions açademies of sciences, \&c. The

AcaDemie Royule des Sciences et des Belles-Lettres of Prussia, was founded at Berlin, in the year 1700 , by Frederic I. king of Prussia, of which the celebrated M. Leibnitz was the first presilent, and its great promoter. The academy received a new form and a new set of statutes in 1710 ; by which it was ordained, that the president shall be one of the counsellors of state; and that the members be divided into four clisses; the first to cultivate physics, medicine, and chemistry; the second, mathematics, astronomy, and mechanics; the third, the German language, and the history of the country; and the fourth, oriental learning, particularly as it may concern the propagation of the gospel among infidels. That each class elect a director for themselves, who shall hold his post for life. That they meet in the castle called the New Marshal, the classes to meet in their turns, one each week. And that the members of any of the classes have free access into the asscmblies of the rest. Several volumes of their transactions have been published in Latin, from time to time, under the title of Miscellanea Berolinensia.

In 1743 Frederic 11 . king of Prussia made grcat alterations and improvements in the academy. Instead of a great lord or minister of state, who bad usually presided over the academy, he wisely judged that office would be better filled by a man of letters; and he honoured the French academy of sciences by fixing upon one of its members for a president, namely M. Maupertuis, a distinguished character in the litcrary world, and whose conduct in improving the academy was a proof of the sound judgment of the king, who at the same time made new rcgulations for the academy, and took the title of its Protictor. From that time the transactions of the academy have been published annually, under the title of Histoire de l'Academie Royale des Sciences et Belles Lettres à Berlin, much in the manner of those of the French academy of sciences, and in the French language; particularly enriched with the compositions of the celebrated Euler, and other lcarned men. Bcsides the ordinary private meetings of the academy, it has two public ones in the year, in January and May; at the latter of which is given a prize gold medal, of the value of 50 ducats, or about 20 guineas. The subject of the prize is successively physics, mathematics, metaphysics, and general literature. For the academy has this peculiar circumstance, that it embraces also metaphysics, logic, and morality; having one class particularly appropriated to these objects, called the class of Speculative Philosophy.

Imperiul ACA DEMY of Petersburg. This acndemy was projected by the czar Peter I, commonly called Peter the Great, who in so many other instances also was instrumental in raising Russia from the state of barbarity in which it had been immerged for so many ages. Having visited France in 1717 , and among other things informed limself of the advantages of an academy of arts and sciences, he resolved to establish one in his new capital, whither he had drawn by noble encouragements several learned strangers, and made other preparations, when his death prevented him from fully accomplishing that object, in the beginning of the year 1725 . Those preparations and intentions however were carried into execotion the same year, loy the cstablishment of the academy, by bis consoit the ezarina Catharine, who succeded hin.
And soon after the acadomy composer the first volume of their works, published in $\mathbf{1 7 2 8}$, under the title of Commentarii Academice Scientiarum Imperialis Petropolitanæ; which they continued almost annually till $17+6$, the whole amounting to 14 volumes, which were published in Latin, and the subjects divicled and classed under the following heads, namely mathematics, pliysics, history, and astronomy. Their device a tree bearing fruit not ripe, with the modest motto Paullatim.

Most part of the strangers who composed this academy being dead, or having retired, it was rather in a languishing state at the beginning of the reign of the empress Elizabeth, when the count Rasomowski was happily aypointed president, who was instrumental in tecovering its vigour and labours. This empress renewed and alteres] its constitution, by letters patent dated July 24, 17.47 , giving it a new form and regulations. It consists of two chief parts, an academy, and a university, having regular professors in the several faculties, who read lectures as in our colleges. The ordinary assemblies are held twice a week, and public or solemu ones thrice in the year; in which an account is given of what has been done in the private ones. The academy has a noble building for their meetings, \&ce, with a good apparatus of instruments, a fine library, observatory, \&c. Their first volume, after this renovation, was published for the years 1747 and 17.4 , and they have been since continued from year to year, now to a great number of volumes, under the title of Novi Commentarii Academiz Scicntiarum Imperialis Petropolitanæ. They are printed in the Latin language, and contain many excellent comprositions in all the sciences, espccially the mathematical papers of the late excellent 11. L. Enler, which always made a considerable portion of evcry volume. The subjects are classed under heads in the following order, mathematics, physicomathcmatics, physics, which include botany, anatomy, \&c, and astronomy; the whole prefaced by historical extracts, or minutes, rclating to each paper or memoir, after the manner of the volumes of the French academy; but wanting however the eloges of deceased cminent men. Their device is a heap of ripe fruits piled on a table, with the motto En addit Fructus atate recentes. After the death of Euler, in 1783 , who lad teen many years the president, that important literary office was, by the empress Catharine, conferred on the princess Daschiof.

Imperiul and Royal ACA DEM Lettres, at Brussels. This acadeiny was founded in the year 1773 ; and several volumes of their memoirs bave bcen published.

Royal ACADEMY of Sciences, at Stockholm, was instituted in 1739 , and since that time it has published a great many volumes of transactions, quarterly, in Svo, in the Swedish language.

For an account of the Royal Society of London, and several other similar institutions, see the words JournaL, Societr, \&c.

Americun Aca Demy of Arts and Sciences, was cstablished in 7780 by the council and louse of representatives in the province of Massachusct's Bay, for promoting the knowledge of the antiquities of Americn, and the natural history of the comntry; for determining the uses to which its various natural productions may be applied; for encouraging medicinal discoveries, mathematical disquisitions, philosophical incquiries and experiments, astronomical, metcorological, and geographical observations, 
and improvements in agriculture, manufactures, and commerce; and, in short, for cultivating crery art and sci. ence, which may tend to advancc the interest, honour, dignity, and happiness, of a free, independent, and virtuous people. The members of this academy are never to be less than forty, nor more than two hundred.

ACADEMY is also used amung us for a kind of collegiate school, or seminary; where youth are instructed in the liberal arts and sciences in a private way: now indecd it is used for all kinds of schools.

Frederic I, king of Prussia, established an academy at Berlin in 1703, for educating the young nobility of the court, suitable to their extraction. The expence of the students was very moderate, the king having undertaken to pay the extraordinarics. This illustrious school, which was then called the academy of princes, has now lost much of its first splendour.

The Romans bad a kind of military academies established in all the citics of Italy, under the name of Campi Martis. Here the youth were admitted to be trained for war at the public expense. And the Grecks, besides academies of this kind, had military professors, called Tactici, who taught all the higher offices of war, \&c.

We have three royal academies of this kind in England, the expenses of which are defrayed by the government; the one at Woolwich, for the artillery and military engineers; another at Portsmouth, for the navy; and the third at Sandhurst near Bagshot, called the Royal Military College, for educating officers for the army in general. (See COLLEGE.) The first was established by his majesty king George 11, by warrants dated A pril the 30 th and November the 18th, 1741, for instructing persons belonging to the military part of the ordnance, in the several branches of mathematics, fortification,' \&c, proper to qualify them for the service of artillery and the office of engineers. This institution is under the direction of the master-general and board of ordnance for the time being: and at first the lectures of the masters in the academy were attended by the practitioner-engineers, with the officers, serjeants, corporals, and private men of the artillery, besides the cadets. At present, however, none are cducated there but the gentlemen cadets, to the number of 1 so. The master-general of the ordnance is always captain of the cadets' company, and governor of the acadeny; under him are a lieutenant-governor, and an inspector of of studies. The masters have been gradually increased, from two or three at first, now (in is14) to almost twenty; namcly, a professor of mithematics; and six or seven other mathematical masters, a professor of fortification, and two assistants, two drawing-masters, two French tnasters, with masters for fencing, dancing, and chemistry. This institution is of the greatest consequence to the state, and it is bardly credible that so important an object should be accomplished at so trifing an expense.

The Royal Naval Acarlemy at Portsmouth was founded by George 1, in 1722, for instructing young gentlemen in the sciences uscful for navigation, to educate them for the royal navy. The establishment is under the direction of the boird of admiralty, who give salaries to the different inasters, by one of whom the students are bonrded and lodged, the expense of which is defrayed by their own friends, nothing being supplied by the govermment but their education. - This academy has been lately new-modeled, and its nane changed to the Royal Naval College.
see Col Leice.
ACANTHUS, in Architecture, the lraves of a plant which forms the ornament of the capital of the Corinthian order. See ABAcus.

ACCELERATED Motion, is that which receives fresh accessions of velocity; and the acceleration may be cither equable or variable: if the accessions of velocity be always equal in equal times, the motion is said to be equably or uniformly accelerated: but if the accessions, in equal times, cither incrcase or decrease, then the motion is unequably or variably accelerated.

Acccleration is directly opposite to retardation, which denotes a diminution of velocity.

Acceleration' comes more particularly under consideration in physics, in respect to the descent of heary bodies, falling freely towards the centre of the carth.

That bodies arc accelerated in their descent is evident from various circumstances, which immediately strike the cye of an attentive observer: thus, the grcater the hcight from whence a body falls, the greater inpression it makes on the object upon which it impinges; and this increase of action upon the fixed body, must necessarily arisc from an increase of velocity in the moving one, or from its acceleration. Before the law of gravitation was known, which is now generally acknowledged to be the immediate cause of the accelcration of bodies, various fantastical hypotheses were advanced to account for this phænomenon; some ascribing it to the action of the atmosphere; others to a very subtile fluid, which they introduced for that particular purpose; others again, attributed it to a kind of instinct in bodies, by means of which they accelerated their motion as they approacbed towards the centre of the earth, that being considered as the place appropriated to their element: thesc and various other hypotheses of a similar nature are now cutirely rejected, and would be wholly unworthy of being mentioned, did they not scrie to show how mucl the knowledge of man is increased, and the solid foundation upon which the modern philosophy is establislied, when compared with the vague and hypothetical theoril's of the ancients.

Leaving thereforc all suci visionary causes of acceleration, and only admitting the existence of such a force as grävity, so evidently inherent in all bodics, without regard to what may be the cause of it, the whole mystery of acceleration will be cleared up. Consider gravity then, with Galileo, only as a cause or force which acts continually on heavy bodics; and it will be easy to conccive that the force of gravitation, which determines bodies to descend, must by a necessary consequence accelerate them in falling.

A body then having once begun to descerid, by the impulse of gravity will continue to descend by Ncwton's first law of nature, even though the first cause of its descent should cease. But besides this determination to move, impressed upon it by the first cause of motion, and which would be sufficient to continue to infinity the degree of motion already begun, new impulses are continually superadded by the same cause; which continues to act upon the body already in motion, in the same manner as if it had remained at rest. There being now then two cause's of motion, acting both in the same direction; it necessurily follows, that the motion which they unitedly produce, must be more considerable than that which either could produce separately. And as long as the motion is thus continued, the same cause still operating to increase it, the desceut must necessarily be continually accrlerated. 


\section{A C C}

厂 17

Supposing then that gravity, from whatever principle it arises, acts uniformly upon all bodies at the same distamce from the centre of the earth; and conceive the time which a heavy body takes in falling to the earth, to be divided into indefinitely small equal parts; gravity will impel the body toward the centre, in the first indefinitely short instant of the descent; and if after this we suppose the action of gravity to cease, the body will continue perpetually to advance uniformly toward the earth's centre, with an indefinitely small velocity, equal to that which resulted from the first impulse.

But if we suppose that the action of gravity still continues the same after the first impulse; in the second instant, or small part of time, the body will receive a new impulse toward the earth, equal to that which it rcceived in the first instant; and consequently its velocity will be doubled; in the third instant, it will be tripled; in the fourth, quadrupled; in the fifth, quintupled; and so on continually: for the impulse made in any preceding instant, is no ways altered by that which is made in the following one; but they are, on the contrary, always accumulated on each other.

So that the instants of time being supposed indefinitely small, and all equal, the velocity acquired by the falling body, will be, at every instant, proportional to the times from the beginning of the descent; and consequently the velocity will be proportional to the time in which it is produced. So that if a body, by this constant force, acquire a velocity of $32 \frac{\pi}{6}$ feet in one second of time; it will acquire a velocity of $64 \frac{\pi}{3}$ feet in 2 seconds, $96 \frac{\pi}{2}$ feet in 3 seconds, $128_{\frac{2}{3}}$ in 4 seconds, and so on. Further, all bodies, great or small, acquire, by the force of gravity, the same velocity in the same time. For every equal particle of matter being endued with an equal impelling force, namely its gravity or weight, the sum of all the forces, in any compound mass of matter, will be proportional to the sum of all the weights, or quantities of matter to be moved; consequently, the forces and masses moved, bcing thus constantly increased in the same proportion, the velocities generated will be the same in all bodies, great or small. That is, a double force moves a double mass of matter, with the same velocity that the single force moves the single mass; and so on. Or otherwise, the whole compound mass falls all together with the same velocity, and in the same manner, as if its particles werc not united, but as if each fell by itself, separated all from one another. And thus all being let go at once, they would fall together, just as if they were united into one mass.

The foregoing law of the descent of falling bodics, namely, that the velocities are always proportional to the times of descent, as wcll as the following laws concerning the spaces passed over, \&c, were first discovered and tanght by the great Galileo, and that nearly in the following inanner.

Bccause the constant velocity with which any body moves, or the space it passes ovcr in a given time, as suppose one second, being multiplied by the time, or number of scconds it is in motion, expresses the space passed over in that time; and the area or space of a rectangular figure bring denoted by the length multiplied by the breadth; therefore the space so run over, may be considered as a rectangle compounded of the time and velocity, that is a rectangle of which the time denotes the lingth, and the velocity the breadth. Suppose then a to Vol. I. to be the beavy body which descends, and $\boldsymbol{A}$ B to denote the whole time of its descent; which let be divided into a certain number of equal parts, denoting intervals or portions of the A C C

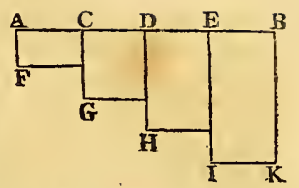
given time, as A C, C D, D E, \&c ; and imagine the body to descend, during the time expressed by the first of the divisions $\mathrm{A}$ c, with a certain uniform velocity arising from the force of gravity acting on it, which let be denoted by $A F$, the breadth of the rectangle $\mathbf{c} F$; then the space run through during the time denoted by $\mathrm{A} c$, with the velocity denoted by $\mathrm{A} F$, will be expressed by the rectangular space $\mathbf{C} \mathbf{F}$.

Now the action of gravity having produced, in the first moment, the velocity A F, in the body, before at rest; in the first tivo moments it will produce the velocity $\mathrm{cG}$, the double of the former; in the third moment, to the velocity $\mathrm{C} G$ will be added onc degree more, by which means will be produced the velocity D H, triple of the first; and so of the rest; so that, during the whole time A B, the body will have acquired the velocity B $\mathrm{K}$. Hence, taking the divisions of the line $\mathbf{A} \mathbf{B}$ at pleasure; for example, the divisions A C, C D, \& c, for the times; the spaces run through during those times, will be as the areas or rectangles $\mathrm{CP}$, D G, \&C; and so the space described by the moving body during the whole time $\mathrm{A} \mathrm{B}$, will be equal to all the rectangles, that is, equal to the whole indented space A B K I HGF. And thus it would happen if the increments of velocity were produced at the end of certain portions of time; for instance at $\mathrm{C}, \mathrm{D}, \& \mathrm{c}$; so that the degree of motion remains the same to the instant that a new acceleration takes place.

By conceiving the divisions of time to be shorter, for example but half as long as the former, the indentures of the figure will be proportionably more contracted, and it will approach nearer to a triangle; and if these divisions be supposed infinitely small, that is, if increments of the velocity be supposed to be acquired continually, and at each indivisible particle of time, which is rally the case, the rectangles so successively produced, will form a triangle, as A BC; the whole time A B consisting of minute portions $A 1,12,23, \& \mathrm{C}$; and the arca of the triangle

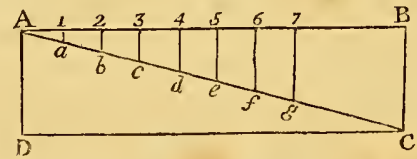

A B C, of all the minute surfaces, or trapeziums, which answer to the divisions of the times; the area of the whole triangle A B c, will denote the space run through during the whole time $A \mathrm{~B}$; and the area of any smaller triangle A $7 \mathrm{~g}$, denoting the space run through during the corresponding time $A 7$. But the triangles $A 1$ a, a $7 \mathrm{~g}, 8 \mathrm{c}$, being similar, have thcir areas to ench other as the squares of their like sides A $1, \mathbf{A} 7, \& \mathrm{c}$; and consequently the spaces gone through, in any times counted from the beginning, are to each other as the squares of the times.

Hence, in any right-angled triangle, as $\mathrm{A}$ в $\mathrm{C}$, the onc side A B represents the time, the other side BC the velocity acquired in that time, and the area of the triangle the space described by the falling body. 


\section{A C C}

From the preceding demonstration is also drawn this uther guneral theorem, in inotions that are uniformly accelerated; namely, that a body descending with a uniformly accelerated motion, describs in the whole time of its deseent, a space, which is exactly the half of that which it would deseribe uniformly in the same time, with the velocity t has aequired at the end of its aecelerated fall. For it has veen shown that the whole space which the falling body has run through in the time $\mathbf{A} \mathbf{B}$, is represented by the triangle $A$ B C; the last velocity being Ic; and the space which the body would run through uniformly in the same time $\mathbf{A} \mathbf{B}$, constantly with the said greatest velocity $B C$, is represented by the rectangle $A B C D$ : but it is well known that the rectangle $A B C D$ is dou ole the triangle $A B C$; and therefore the latter space run through, is double the former; that is, the space run through by the accelerated motion, is just half of that which the body would describe in the same time, moving uniformly with its last-acquired velocity.

Hence then, from the foregoing considerations are deduced the following general laws of uniformly accelerated motions, namely,

1st. That the velocities acquired, are coustantly proportional to the times; in a double time a double velocity, \&c.

2d. That the spaces deseribed in the whole times, each counted from the commencement of the motion, are proportional to the squares of the times, or to the squares of the velucities; that is, in twice the time, the body will describe 4 times the space; in thrice the rime, it will describe 9 times the space; in quadruple the time, 16 times the space; and so on. In short, if the times are proportional to the numbers - $1,2,3,4,5, \& c$, the spaces will be as $1,4,9,16,25, \& c$, which are the squares of the former. So that, if a body, by the natural force of gravity, fall through the space of $16 \frac{1}{12}$ feet in the first second of time; then in the first 2 seconds of time it will fall.through 4 times as much, or $64 \frac{1}{3}$ feet; in the first 3 seconds it will fall 9 times as much, or $144_{4}^{3}$ feet; and so on. And as the spaces fallen through are as the squares of the tines, or of the velocities; therefore the times, or the velocities, are proportional to the square roots of the spaces.

$3 \mathrm{~d}$. The spaces described by falling bodies, in a series of equal instants or intervals of time, will be as the odd numbers - - 1,3,5, 7,9 , \&c, which are the differences of $\} 1,4,9,16,25,8 \mathrm{c}$, that is, the body which has run through $16_{\frac{1}{12}}$ feet in the tirst second, will in the next seconil ruin through $48 \frac{1}{3}$ feet, in the thirel second $80_{\gamma^{5}}^{5}$, and so on.

4th. A body having lallen through any space in a certain time, it will have acquired a velncity equal to double that space; that is, in an equal time, with the last veloeity acquired, if 'miformly continued, it would pass over just double the space. So, if a body fiall through $16_{\frac{1}{x}}$ feet in tle first second of time, it nequires a velocity of $32 \frac{1}{6}$ leet in a second; that is, if the b.'sy move uniformly tior one second, with the velocity acquired, it will pass over $32 \frac{1}{6}$ feet in this one second: and if in any time the body fall through 100) feet; then in anuther equal time, if it move uniformly with the last acquired velocity, it will pass over 200 leet. And so on.

But, as the methorl of demonstration used by Galileo, by incins of infinitely small parts forming a regular tri- angle, though very ingenious, is not so elegant as miglit be wished, the areas of triangles being rather unnatural representations of the spaces descended by talting is dies; the same may be otherwise demonstrated thus: Let the whole time of a body's free descent be divided into any number of parts, calling tach of these parts $t$; and let $a$ denote the velocity acquired at the end of the first part of time; then will $2 a, 3 u, 4 a, \& c$, represent the velocities at the end of the $2 \mathrm{~d}, 3 \mathrm{~d}, 4 \mathrm{th}, \& \mathrm{c}$, part of time, bccause the velocities are us the times; and for the same reason $\frac{1}{2} a, \frac{3}{2} a, \frac{5}{2} a, \& c$, will be the relocities at the niddle point of the first, second, third, \&c, part of time. But now as the velocities increase uniformly, the space described in any one of these parts of time, may be eonsidered as unitormly described with its middle velocity, or the velocity in the middle of that part of time; and therefore, multiplying those mean veleciti's each by their common time $l$, we have the same urations $\frac{\pi}{2} a, \frac{3}{2} a, \frac{5}{2} a, \& \mathrm{c}$, for the spaces passed orer in the surcissive parts of the time; that is, the space $\frac{1}{2} a$ in the first time, $\frac{3}{2} a$ in the second, $\frac{5}{2}$ in the third, and so on: then add these spaces successively to one another, and we obtain $\frac{\pi}{2} a, \frac{4}{2} a, \frac{9}{2} a$, $\frac{16}{2} a$, \&e, for the whole spaces described from the beginning of the motion, to the end of the first, second, third, \&c, portion of time; namely $\frac{1}{2} \Omega$ space in one time, $\frac{4}{2} a$ in 2 times, $\frac{9}{2} a$ in 3 times, and so on: and it is evident that these spaces are as the numbers $1,4,9,16, \& c$, which are as the squares of the times.

And from this mode of demonstration, all the properties above-mentioned evidently following; $i$. $e$. the whole spaces - - - - $\quad \frac{\pi}{2} a, \frac{4}{2} a, \frac{9}{2} a, \& c$, are as the squares of the times - $1,2,3, \& c$, that the separate spaces - $\frac{1}{2} a, \frac{3}{2} a, \frac{5}{2} a, \& c$, described in the successive times, $\} 1,3,5, \& c$, and that the velocity $a$ acquired in any time 1 , is double the space - - $\frac{1}{2} a$ described in the same time.

As the laws of acceleration are very important, I shall here insert the two following propositions, sent me by my learned friend Mr. Abram Robertson, of Christ Church College, Oxford, in which those laws are demonstrated in a manner somewhat different.

"Proposi'r 1ov I.-If from the point $P$ in the straight line $\mathrm{A} B$, the points $\mathrm{M}, \mathrm{N}$ begin to move at the same tima, namely, $M$ towards $A$ with a motion uniformly retarded, and $\mathrm{N}$ from rest towards $\mathbf{B}$ with a motion uniformly accelerated; and if the velocity of $\mathrm{N}$ decreases as much as the velocity of $\mathrm{N}$ increases in the same time; then the space $\mathrm{M} N$ is generated by a uniform motion, equal to the velocity with which $\mathrm{M}$ begins to move.

$$
\begin{array}{ccc}
M & P & N \\
\hline & \text { I } & \text { B }
\end{array}
$$

For, by hypothesis, whatever is lost in the velocity of $M$ by retardation, is added to the velocity of $\mathrm{N}$ by acceleration: the joint velocities therefore of $\mathrm{N}$ and $\mathrm{N}$, must always be equal. But it is by the joint velocities of $M$ and $\mathrm{N}$ that the space $\mathrm{M} \mathrm{N}$ is generated. Consequently $\mathrm{MN}$ is generated by a uniform motion, which is evidently equal to the velocity with which $M$ begins to move.

"Proposition II.- If a point begins to move in the direction of a straight line, and continues to move in the same direction with a velocity uniformly accelerated; the space passcd over in any given time, will be equal to half 
the spase passed over in the same time with the velocity with which the acceleration ends.

Let the point $\mathrm{D}$ begin to move from $\mathrm{A}$ towards $\mathrm{B}$, along the straight line $\mathrm{A}$, , with a motion uniformly accelerated; the space A D passed over, is equal to half the space which the point would pass over, in the same time with the acquired velocity at $D$.

$$
\text { A— D }
$$

Let the points $M, N$ begin to move in the straight line f $\mathbf{H}$, at the same time, with equal velocities uniformly acceleratel; $\mathrm{M}$ beginning to move from $\mathrm{G}$, and $\mathrm{N}$ from $\mathrm{P}$; and at the same time that $M$ comes to the point $\mathbf{P}$, let $\mathbf{N}$ come to $H$. Then as $M$ and $N$ move with equal veloci-

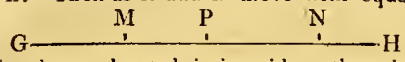

ties, uniformly accelerated, it is evident that the spaces, which they pass over in the same time, are equal to one another ; consequently the space $\mathbf{G} P$ is equal to the space PH. Now as al begins to move from $\mathrm{G}$ with a relocity uniformly accelerated, it will arrive at $P$ with an acquired velocity. Hence it is evident, if it be supposed to begin to move from $\mathbf{P}$ with this acquired velocity, and proceed toward $G$ with a velocity uniformly retarded in the same degree that it was accelerated when it began to move from $G$, that it will pass over the same space $G P$ in the same time. Therefore, supposing the two points $\mathrm{M}, \mathrm{N}$ to begin to move from $P$ at the same time, namely the point $M$ beginning to move with the acquired velocity mentioned above, and proceeding towards $\mathrm{G}$ with the velocity uniformly retarded, described above; and the point $\mathrm{N}$ as before with the velocity uniformly accelerated: then, as the acceleration and retardation are uniform, they will be equal in equal spaces of time. Again; as $M$ is retarded in the same degree that it was accelerated when it began to move from $G$, that is, in the same degree that $N$ is accelerated, by the former prop. $M \mathrm{~N}$ is generated by an uniform velocity. But when the point $M$ arrives at $G$, its velocity becomes equal to 0 or nothing; and at the time that $\mathrm{M}$ arrives at $\mathrm{G}, \mathrm{N}$ arrives at $\mathrm{H}$ with the acquired velocity. Therefore, as the velocities of $M$ and $N$ taken jointly are equal, and consequently uniform, the space $\mathrm{GH}_{\mathrm{H}}$ is passed over with the velocity of $\mathrm{N}$ at $\mathbf{r}$, in the same time that $\mathrm{PH}$ is passed over by $\mathrm{N}$ beginning to move from $\mathbf{P}$ with a velocity uniformly accelerated to $\mathbf{m}$. But PH is half of G II. "Hence the prop. is manifest."

And hence the other laws of the spaces, before-mentioned, easily follow.

Since the spaces descended are as the squares of the times, and the abscisses of a parabola are as the squares of the ordinates, therefore the relation of the times and spaces descended may be very well represented by the ordinates and abscisses of tbat figure. Thus, if $A$ B be the axis of the parabola $A b d f h$, and $A C$ a tangent at the vettex perpendicular to the axis, be divided into any number of equal parts $\mathrm{A} a, a c, c e, \& \mathrm{c}$, to represent the times; and if there be drawn $a b, c d, e f, \& c$, parallol to the axis: then, if $a b$ be the space descended in the time $A a, c d$ will be the space descended in the time $A c$, and $f f$ the space descended in the time $A e$, and so on continually; because, by this property of the parabola, $a b, c d, e f, \& c$, are to each otler respectively as $A a^{2}, A c^{2}, A c^{2}, \& c$.

From the properties above demonstrated, are derived the following practical formulas or theorems. Namely, if $g$ denote the space passed over in the first second ot time, by a body urged by any constant force, denoted by 1 , and $t$ denote the time or number of seconds in which the body passes over any other space $s$, and $v$ the velocity acquired at the end of that time; then, from the foregoing laws, we have $v=2 g t$, and $s=g t^{2}$; and from these two equations result the following general formulas:

$$
\begin{array}{ll}
s=\frac{x}{2} t v=g t^{2}=\frac{v^{2}}{4} . & v=2 g t=\frac{2 s}{t}=2 \sqrt{g s} . \\
t=\frac{v}{2 g}=\frac{2 s}{v}=\sqrt{ } \frac{s}{g} . & g=\frac{v}{2} t=\frac{s}{t^{2}}=\frac{v^{2}}{4 s} .
\end{array}
$$

And when the constant foree 1 , is the natural force of gravity, then the distance $g$ descended in the first second, in the latitude of London, is $16_{\frac{x}{12}}$ feet: but if it be any other constant force, the value of $g$ will be different, in proportion as the force is more or less.

'The motion of an ascending body, or of one that is im=pelled upwards, is diminisbed or retarded by the same principle of gravity, acting in a contrary direction, after the same manner that a falling body is acceleratcd.

A body projected upwards, ascends until it has lost all its motion; which it does in the same time as, in falling, would have been necessary for it to have acquired that velocity. And consequently the heights to which bedies ascend. when projected upwards with different velocities, are to each other as the squares of those velocities.

It should be observed that in what has been said respecting the ascent and descent of bodies, the resistance of the atmosphere has not been considered, and therefore, in practice, the results will not accurately agree with the above theory. And further, that we have considered the force of gravity as being constant at all heights, which is not strictly true, as it varies inverscly as the square of the distance from the earth's centre, (see Atrraction). But as the greatest height, to which we can ascend, is very small when compared with the radius of the earth, this assumption inay not commonly produce any sensible error.

Acceler a ted Motion of Bodies on Inclined Planes. The same general laws obtain here, as in bodies falling freely, or perpendicularly; namely, that the velocities are as the times, and the spaces descended down the planes as the squares of the times, or of the velocitics. But those velocities are less, according to the sine of the plane's inclination; and the spaces less, according to the square of the sine. See Incuined Plane.

Acceler ated Motion of Pendulums. See Pen ntrevm. Accelne ated Motion of Projectiles. See Prujectile. Accelerated Motion of Compressed Bodics, in expanding or restoring themselves. See Dilatation, ComPRESSion, and Elasticity.

Accelerating Force, in Physics, is the force that accclerates the motion or velocity of bodies; and it is equal to, or expressed by, the quotient arising from the motive or absolute force, divided by the mass or weight of the body that is moved. In treating of physical subjects respecting forces, where velocities, times, and spaces are considered, the first inquiry is the accelerating or accclerative force. This force is greater or less in pro1) 2 
portion to the velocity it generates in the same time, and by this velocity it is measured. All accelerating furces that have the motive forces directly proportional to the quantities of matter are equal, and generate equal velocities; so a double motive furce will move a double quantity of matter, with the same velocity, a triple motive force a triple quantity, a quadruple force a quadruple quantity, \&c. And this is the reason why all bodies fall equally swift by the force of gravity; for the motive force is exactly proportional to their weight or mass. In general the accelerating force is in the direct ratio of the motive force, and inverse ratio of the quantity of matter. When a body is left freely to descend by the force of its natural gravity, it has been found by experiment that in the latitude of London, it falls through $16_{\frac{1}{x^{2}}}$ fect in the first second of time, and acquires a velocity of $32 \frac{x}{6}$ feet in that time: but if the quantity of matter be doubled, and the motive force remain the same as before, which may be done by connecting the falling body to another of equal weight by ineans of a thread, the latter body being placed on a sinooth horizontal plane, along which it may be drawn without friction by the former body, which hangs freely over the side of the plane; then the accelerating force will be only half of what it was before, and the space fallen through in one second will be only $\delta_{\frac{1}{24}}$ feet, and the velocity acquired $16 \frac{x}{1^{2}}$ : also, if the quantity of matter be tripled, or the body drawn along the plane doubled; then the accclerating force will be only one-third of what it was at first, and the space desccnded in one second, and the velocity acquired, will be each one-third of the first :- and so on.

But accelerating forces are sometimes variable, and sometimes constant; and the variation may be cither increasing or decressing. The nature of constant and variable accelerating forces, may be illustrated in the following manner. Let two weights $\mathrm{w}, w$, be conrected by a
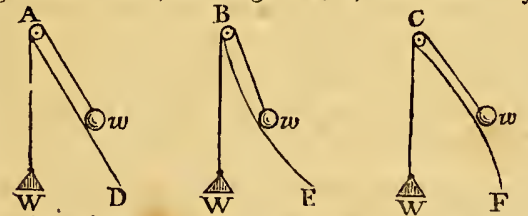

thread passing over a pully, at $\Lambda, \mathrm{B}$, or $\mathrm{C}$; and let the weight $\mathrm{w}$ descend perpendicularly down, while it draws the smaller weight $w$ up the line $\lambda \mathrm{D}$, or $\mathrm{B} \mathrm{E}$, or $\mathrm{C} \mathrm{F}$, the first being a straiglit inclined plane, and the other two curves, the one convex and the other concave to the perpendicular. 'Then the small weight $w$ will alivays make some certain resistance to the free descent of the large weight $\mathrm{w}$, and that resistance will be constantly the same in every part of the plane A D, the difficulty to draw it up being the same in every point of $\mathrm{it}$, because every part of it has the same inclination to the horizon, or to the perpendicular; and consequently the acressions to the velocity of the descending weight $w$, will be always equal in equal time's; that is, in this case w descends by a uniformly accelerating force. But in the two curves B E, C $v$, the resistance or opposition of the small weight $w$ will be constantly varying, as it is dirawn up the curves, becausc crury part of them lias a different inclination to the horizon, or to the perpendienlatr. In the former curve, the direction becomes more and more upright, or nearer perpendicular, as the small weight $w$ ascends, and the opposition it makes to the descent of w necessarily becomes greater; and consequently the accessions to the velocity of $w$ will be always less and less in equal times; that is, $w$ descends by a decreasing accelerating force. But in the latter curve $\mathbf{C}$, as $w$ iscends, the direction of the curve becomes less and less upright, and the opposition it makes to the descent of $w$, becomes always less and less: consequently the accessions to the velocity of $w$ will be always more and more in equal times; that is, w descends by an increasing accelerating force. So that though the velocity continually increases in all these cases, yet while it increases in a constant ratio to the times of motion, in the plane $A \mathrm{D}$; the velocity increases in a less ratio than the time it ascended up $\mathrm{B} \mathrm{S}$, and in a greater ratio than the time increases in the other curve $\mathbf{c} \mathbf{F}$.

Now the relations between the times and velocities in all these cases, may be very well represented by the relations betwcen the abscisses and ordinates of certain lines. Thus let $A B$ and $A C$ be two straight lines, making any angle $\mathrm{BAC}$; and $\mathrm{AD}, \mathrm{A} \mathrm{E}$ two curves, the former concave, and the latter convex towards $A B$ : divide $A B$ into any parts $\mathrm{A} a, \mathrm{~A} b, \& \mathrm{c}$, representing the times of motion; and draw the perpendiculars acde, $b f g h$, \&c, representing the velocities. Then in the right line $\mathrm{A} C$, theordinates

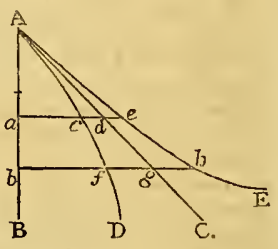
$a d, b g$, being as the abscisses $A a, A b$, this represents the case of uniformly accelerated motion, in which the velocities are always as the times: but in the curve $A \mathrm{D}$, the ordinates $a c, b f$ increase in a less ratio than the abscisses $\Delta a, \mathrm{~A} b$; and therefore this represents the case of decrcasing acceleration, in which the velocitics increase in a less ratio than the times: and in the other curve $\mathrm{A} \mathrm{E}$, the ordinates $a e, b h$ increase in a greater ratio than the abscisses; and therefore this represents the case of increasing acceleration, in which the velocities increase in a greater ratio than the times.

The several algebraic formulas or theorems, respecting the time, velocity, and space, for constint accolerating forces, have been delivered above, at the article Accelerated Motion, where the value of each is expressed in finite determinate quantities. But, in the cases of variably accelerated motions, the formulas will require the assistance of fluxions to express, not those general relations themselves, but the fluxions of them ; and consequently, tiking the fluents of those expressions, in particular cases, the reJations of time, space, velocity, \&c, are obtained.

Thus, if $t$ denote the time in motion, $v$ the velocity generated by uny force, $s$ the spacc passed over,

and $s g$ the variable force at any part of the motion, or the velucity the force would generate in one second of time, if it should continue invariable, like the force of gravity, during that time; and therefore the value of this velocity $2 \mathrm{~g}$, will be to $32 \frac{\mathrm{x}}{\mathrm{s}}$ fect, as that variable force, is to 1 the force of gravity. 'Then, because the force may be supposed constant during the indefinitely smalt time $i$, and that in uniform motions the spaces and velocitics are proportional to the times, we from thence ubtain these two general fundamental proportions, 


\section{$\triangle \mathrm{CC}$}

$$
\begin{aligned}
v: \dot{s}:: 1^{\prime \prime}: \dot{t}, \text { or } \dot{s} & =\dot{t} v ; \\
2 g: \dot{v}:: 1^{\prime \prime}: \dot{t}, \text { or } \dot{q} & =2 \mathrm{~g} \dot{t} .
\end{aligned}
$$

From which are derived the four following formulas, in which the value of each quantity is cxpressed in terms of the rest.

$$
\begin{array}{ll}
\dot{s}=v \dot{t}=\frac{v \dot{v}}{2 g} . & \dot{v}=2 g \dot{t}=\frac{2 g \dot{s}}{v} . \\
\dot{t}=\frac{\dot{s}}{v}=\frac{\dot{v}}{2 g} . & 2 g=\frac{\dot{v}}{\dot{t}}=\frac{v \dot{v}}{\dot{s}} .
\end{array}
$$

And these theorems equally hold good for the destruction of motion'and velocity, by means of retarding forces, as tor the generation of the same by means of accelerating forces.

ACceleration, in Mechanics, the increase of velocity in a moving borly.

Acceleration, Astron. The Diurnal Acceleration of the fixed stars, is the time which the stars, in one diurnal revolution, anticipate the mean diurnal revolution of the sun; which is $3^{\mathrm{m}} 55^{\mathrm{s}}$ 学 of mean time, or nearly $3^{\mathrm{m}} 56^{\mathrm{s}}$ : that is, a star rises, or sets, or passes the meridian, about $3^{\mathrm{m}} 56^{\mathrm{s}}$ sooner each day. This acceleration of the stars, which is only apparent in them, arises from real retardation of the sun, owing to his apparent motion in his orbit towards thc east, which is about $59^{\prime} \mathrm{s}^{\prime \prime}{ }^{2} \mathrm{O}$ of a degrce every day. So that the star which passed the meridian yesterday at the same moment with the sun, is to-day about $59^{\prime}$ $\delta^{\prime \prime}$ past the meridian to the west, when the sun arrives at it; which will take him up about $3^{\text {m }} 56^{\text {s }}$ of time to pass over; and therefore the star passes by $3^{\mathrm{m}} 56^{3}$ sooner than the sun each day, or anticipates his motion at that rate. The true quantity of this anticipation, or acceleration, is found by this proportion, $360^{\circ} 59^{\prime} \mathrm{s}^{\prime \prime} \frac{x}{5}: 59^{\prime} 8^{\prime \prime} \frac{1}{5}:: 24$ hours : $3^{\text {m }} 55^{\mathrm{s}} \frac{9}{90}$, the fourth term of wbich is the acceleration

The diurnal acceleratiou serves to regulate the lengths or vibration of pendulums in clocks. If I ubserve a fixed star set or pass behind a hill, steeple, or such like, when the cluck marks for instance $8^{\mathrm{h}} 10^{\mathrm{m}}$; and the next day, the eye being in the same place as before, the passige be at $8^{\mathrm{h}} 6^{\mathrm{m}} 4^{\mathrm{s}} ; 1$ thence conclude that the clock is well regulated, or truly measures mean time.

Acceleration of a Planet. A planet is said to be accelcrated in its motion, when its real diurnal motion exceeds its mean diurnal motion. . And, on the other hand, the planet is said to be retarded in its motion, when the mean excceds the real diurnal motion. This inequality arises from the change in the distance of the planet from the sun, which is continually varying; the planet moving always quicker in its orbit when nearer the sun, and slower when farther off.

Acceleration of the Moon, is a term used to express the increase of the moon's mean motion from the sull, compared with the diurnal motion of the earth; by which it appears that, from some uncertain cause, it is now a little quicker than it was formerly. Dr. Halley was led to the discovery, or suspicion, of this acceleration, by comparing the ancient eclipses observed at Babylon, \&c, and those observed by Albategnius in the ninth century, with some of his own time; as may be seen in $N^{\circ} 218$ of the Plilosujhical T'ransactions. He could not however ascertain the quantity of the acceleration, because the longitudics of liagdat, Alexandria, and Aleppo, where the observatious were made, had not been accurately determined. But since lis time the longitude of Alexandria lias been ascer-

\section{A C C}

tained by Chazelles; and Babylon, according to Ptolemy's account, lies $50^{\prime}$ east of Alexandria. From these data, Mr. Dunthorne, vol. 46 Philos. Transactions, compared the recorded times of several ancient and modern eclipses, with the calculations of them by his own tables, and thereby verified the suspicion that had been started by Dr. Halley; for he found that the same tables gave the moon's pläce more backward than her true place in ancient eclipses ; and more forward than her true place in later eclipses; and thence he justly inferred that her motion in ancient times was slôwer, and in later times quicker, than the tables give it.

Not content however with barely ascertaining the fact, he proceeded to determine, as well as the observations would allow, the quantity of the acceleration; and by means of an eclipse, of which the most anthentic account remains, observed at Babylon in the year 721 before Christ, he found that the beginning of this eclipse was about an hour and three quarters sooner than the beginning by the tables; and that therefore the moon's true place preceded her place by computation, by about $50^{\circ}$ of a degree at that time. Then, admitting the acceleration to be uniform, and the aggregate of is $a$ s the square of the time, it will ve at the raie of about $10^{\prime \prime}$ or $9^{\prime \prime}$ by later conıputations, in too years.

M. de la Place announced to the Acadeiny of Sciences the cause of this very interesting discovery in physical astronomy. Dr. llalley had remarked, as above-mentioned, that modern observations had given a more rapid motion to the moon than the ancient ones, and M. Lalande bad inserted some proof of it in the memoirs of the Academy in 1757. The cause of this acceleration had been for a long time the discnssion of geometers. The academy had proposed at three different times, in 1762,70 , and 72 , a preminin for the elucidation of this subject, but Euler himself could find nothing in the theory of attraction, which conld explain this acceleration; and the learned began almost to conclude, that the difference between the ancient and modern observations was to be regarded as donbtful, and it was very possible that the Arabs, who formed the most substantial proof, might be mistaken; but M. Delambre, having calcnlated in 1783 the observations made by M. le Paute d'Agelet at the Military Academy, bas found a new proof of this singular phenomenon. We were impatiently waiting for theory's giving us a satisfactory account of it, when M. Laplace, who so ingeniously explained in 1786 the secular equations of Jupiter and Saturn, succeedel so far, after several attempts, as to reduce that of the moon to the laws of universal weight. It is the diminution which exists for many centuries in the equation of the solar orbit, that is the cause of this inequality in the moon: but as the diminution, produced by the attraction of plancts, will become, in the revolution of ages, a real augmentation, so what appears now to us an acceleration in the moun's motion, will likewise become a retardment, and it is $\mathrm{I}$, thing more than a periodical inequality. M. Laplace finds it eleven seconds for the first century, and as this increases within a little as the square of the time, so for the year 720 before our æra, the nearest date for the most ancient observations that have reached us, it produces a difference that perfectly agrees with the observation. It is to M. Laplace, therefore, we owe a confirmation of the attraction of celestial bodies, which will prove as useful, as it is ingrenious and learned. 
'Thus then the moon's mean motion appears to accelerate continually; and the accelerations appear to be in the duplicate ratio of the times. The acceleration for the end of the 17 th century was about $9^{\prime \prime}$; so that the whole motion of the moon during the 17 th century must be increased $9^{\prime \prime}$, to obtuin its motion during the 18 th ; and as much must be takin from it, or aided to the computed longitude, to obtain its motion during the $16 \mathrm{th}$; and the double of this must be taken from the motion during the 16 th, to obtain its motion during the 15 th, \&c.-To cxplain the theory, it must be considered that the lunar period which we observe, is not that which would have obtained, had the moon been intluenced by the earth alone. We should not have known that her natural perivd was incrased, had the disturbing action of the sun remained unchanged; whereas this varies, and that in the inverse triplicate ratio of the earth's distance from the sun, and is therefore greater in our winter, when the earth is nearer to the sun. This is the source of the annual equation, by which the lunar period in January is made to exceed that in July nearly 24 minutes. The angular velocity of the moon in general is diminished by $\frac{1}{7} \frac{1}{9}$, and this numerical coefficient varies inversely as the cube of the earth's distance from the sun. If this ratio be expanded into a series arranged accurding to the simes and co-sines of the earth's mean motion, making the carth's mean distance 1, then the series will contain a term equal to $\frac{1}{7} \bar{g}$ of this velocicy multiplied by $\frac{3}{2}$ of the square of the earth's excentricity. Had this excentricity remained constant, this product would also be constant, and would still be confounded with the general diminution, making a constant part of it: but it is known that the excentricity of the earth's orbit diminishes, and its diminution is the result of the universality of the Newtonian law of the planetary deflections. Though this diminution is exceedingly small, its effect on the lunar motion becomes sensible by accumulation in the course of ages. The excentricity diminishing, the diminution of the moon's angular motion inust also diminish, that is, the angular motion must increase.

During the 18 th century, the square of the earth's excentricity has diminished 0.0000015325 , the mean distance from the sun being 1 . In that time this has increased the moon's angular motion 0.00000001285 . As this augmentation is gradual, we must multiply the angular motion during the century by the half of this quantity, in order to obtain its accumulated effect. This will be found to be $9^{\prime \prime}$ very nearly; which exceeds that deduced from a most careful comparison of the motion in the last two centuries, only by a fraction of a second! While the diminution of the square of the excentricity of earth's orbit can be supposed proportional to the time, this effect will be as the squares of the times. When this theory is compared with observations, the coincidence is wonderful indeed. The effect on the moon's motion is periodical, as the change of the solar excentricity is, and its period includes millions of years. Its effect on the moon's longitude will amount to several degrees before the secular acceleration change to a retardation. Eneyclopedia Brit.

By the solution of this problem, which had for a long time engaged the attention of mathematicians, M. Laplace has given the last degree of perfection to theoretical astronomy; ws there is now no anomaly, or irregularity, in the motion of the hearenly lodies, that is not satisfac- torily accounted for, on the simple Newtonian laws of gravitation.

ACCELERATIVE Force, $\& c$, the same as AcceleR A IING.

ACCESSIBLE, something that may be approached, or to which we can come. In Surveying, it is such a place as will admit of havitig a distance or length of ground measured from it; or such a height or depth as can be measured by actually applying a proper instrument to it. For the mcans of doing which, sce A LTrMeTRY, LONGIMETRY, Or HEIGHTS-AN D-DISTANCES.

ACCIDENS, Accident, Philos.

Per Accinens is a term often used among philosopliers, to denote what does not follow from the nature of a thing, but trom some accidental quality of it: in this sense it stands opposed to per se, which denotes the nature and essence of a thing. Thus, fire is said to burn per se, or considered as fire, and not per accidens; but a piece of iron, though red-hot, only burns per aceidens, by a quality accidental to it, and not considered as irom.

ACCIDENTAL, something that partakes of the nature of an accident; or that is indifferent, or not essential to its subject.-Thus, whiteness is accidental to marble, and sensible heat to jron.

Accidental Colours, so called by M. Buffon, are those which depend on the affections of the eye, in contradistinction to such as belong to light itself.

'The impressions made upon the eye, by looking stedfastly on objects of a particular colour, are various according to the single colour, or assemblage of colours, in the object; and they continue for some time after the eye is withdrawn, and give a false colouring to other objects that are viewed during their continuance. $M$. Buffon has endeavoured to trace the connection between these accidental colours, and those that are natural, in a variety of instances. M. d'Arcy contrived a machine for measuring the duration of those impressions on the eye; and from the result of several trials he inferred, that the effect of the action of light on the eye continued about $S$ thirds of a minute.

The subject has also been considered by M. de la Hire, and M. Aepinus, \&c. See Mem. Acad. Paris 1743, and 1765; Nov. Com. Petrop. vol. 10; also Dr. Priestley's Hist. of Discoveries relating to Vision, pa. 631 .

Accidental Point, in Perspective, is the point in which a right line drawn from the eye, parallel to anotber right line, cuts the picture or perspective plane.

Let $A$ B be the line given to be put in perspective, C F D the picture or perspective plane, and $\mathrm{E}$ the eye: draw E parallel to $A B$; so shall $\mathbf{F}$ be the accidental point of the line $A B$, and indeed of all lines parallel to it,

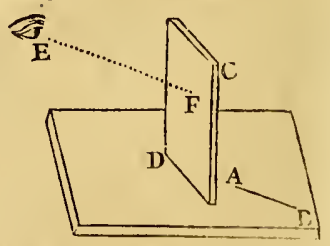
since only one parallel to them, namely $\mathbf{E}$, can be drawu from the same point $\mathrm{E}$ : and in the accidental point $r$, there concur or meet the representations of all the parallels to $A \mathrm{~B}$, when produced.

It is called the accidental point, to distinguish it from the principal point, or point of view, where a line drawn from the eye perpcudicular to the perspective plane, meets this plane, and which is the acci- 
dicital point to all lines that are perpendicular to the same plane.

ACCLIVITY, the slope or stecpness of a line or plane inclined to the horizon, taken upwards; in contradistinction to declivity, which is taken downwards. So, the ascent of a hill, is an acclivity; the descent of the same, a declivity. Some writers on fortification use acclivity for talus: though more commonly the word talus is used to denote the slope, whether in ascending or descending.

ACCOMMOdATION, in Physics, or Philosophy, the application of one thing to another by analogy.

ACCOMPANIMENT, in Music, denutes either the different parts of a piece of music for the different instruinents, or the instruments themselves which accompany a voice, to sustain it, as well as to make the music more full. The Accompaniment is used in recitative, as well as in song; on the stage, as well as in the choir, \&c.

The ancients had likewise their accompaniments on the theatre; and they had even different kinds of instruments to accompany the chorus, from those which accompanied the actors in the recitation. The accompaniment among the moderns, is often a different part, or melody, trom the song it accompanies. But it is disputed whether it was so among the ancients. Organists sometimes apply the word to several pipes, which they occasionally touch to accompany the treble; as the drone, the flute, Sc.

ACCOMPT. See Account.

ACCORD, according to the modern French music, is the union of two or more sounds heard at the same time, and forming together a regular harmony.

Accords are divided into perfect and imperfect; and again into consonances and dissonances. Accord is more commonly called ConcorD; which see.

AccorD is also spoken of the state of an instrument, when its fixed sounds have among themselves all the justness that they ought to have.

ACCOUNT, or Accom PT, in Arithmetic, \&c, a calcuIation or computation of the number or order of certain things; as the computation of time, \&c. There are various ways of accounting; as, by enumcration, or telling one by one; or by the rules of arithmetic, addition, subtraction, \&c.

Accoun $\mathrm{T}$, in Chronology, is nearly synonymous with style. Thus, we say the English, the foreign, the Julian, the Gregorian, the Old, or the New account, or style. We account time by years, months, \&c; the Greeks accounted it by olympiads; the Romans, by indictions, lustres, \&c.

ACCRETION, in Physics, the growth or jncrease of an organical body.

ACHLRNER, or AcianNer, in Astronomy, a star of the first magnitude in the southern extremity of the constellation Eridanus, marked $\alpha$ by Bilyer. Its longitude for 1761, ( $11^{\circ} 55^{\prime} 1^{\prime \prime}$; and latitude south $59^{\circ} 22^{\prime} 4^{\prime \prime}$.

ACHILLES, a name given by the schools to the principal argument alleged by each sect of philosophers in behalf of their system. In this sense we say, this is his Achilles; that is, his master-proof: alluding to the strength and importance of the hero Achilles among the Greeks.

Zeno's argument against motion is peculiarly termed Achilles. That philosopher made a comparison between the swiftness of Achilles and the slowness of a tortoise, pretending that a very swift animal could never overtake a slow oue that was before it, and that therefore there is no such thing as motion: for, said he, if the tortoise were one mile before $\Lambda$ chilles, and the motion of Achilles 100 times swifter than that of the tortuise, yet he would never overtake it ; and for this reason, namely, that while Achilles runs over the mnile, the tortoise will creep over one hundredth part of a mile, and will be so inuch the foremost ; again, while Achilles runs over this $\frac{3}{10 \delta}$ th part, the tortoise will creep over the 100th part of that $\frac{x}{100}$ th part, and will still be this last part the foremost; and so on continually, according to an infinite series of 100 th parts: from which he concluded that the swifter could never overtake the slower in any finite time, but that they must go on approaching to infinity. But this sophism lay in Zeno's considering as an infinite time, the sum of the infinite series of small times in which Achilles could run over the infinite series of spaces, $1+\frac{1}{\pi \delta 0}+\frac{1}{0}+\frac{x}{000}+$ Toס to- \&c, not knowing that the sum of this infinite series is equal to the finite small quantity $1 \frac{1}{95}$ of a mile, and that therefore Achilles will overtake the tortoise when the latter has crawled over $\frac{1}{9}$ th part of a mile.

ACHROMATIC, in Optics, without colvur; a term which, it seems, was first used by Dr. Bevis, to denote telescupes of a new invention, contrived to remedy abcrrations and colours. See Aberration and Telescope.

Aсn ro matrc Telescope, a singular species of refracting telescope, said to be invented by the late Mr. John Dollond, optician to the king, and since improved by his son Mr. Peter Dollond, and others.

Every ray of light passing obliquely from a rarer into a deuser medium, changes its direction towards the perpendicular; and every ray passing obliquely from a denser into a rarer medium, changes its direction from the perpendicular. This bending of the ray, caused by the change of its direction, is called its refraction; and the quality of light which subjects it to this refraction, is called its refrangibility. Every ray of light, before it is refracted, is white, though it consists of a number of component rays, each of which is of a different colour. As soon as it is refracted, it is separated into its constitucnt rays, before mentioned, which, from that time, proceed diverging from each other, like emanations from a centre: and this divergency is caused by the different refrangibility of the component rays, in such sort, that the more the original or component ray is refracted, the more will the compounding rays diverge when the light is refracted by one given medium only. Hence it has been cuncluded, that any two different mediums, that can be made to produce equal refractions, will necessarily produce equal divergencies: whence it should also follow, that equal and contrary refractions should not only destroy each other, but that the divergency of the colours caused by one refraction, should be corrected by the other; and that, to produce refraction which would not be affected by the different refrangibility of light, is impossible.

But Mr. Dollond proved, by many experiments, that these conclusions are not well founded: from his experiments it appeared, that a ray of light, after equal and contrary refractions, was still spread into eomponent rays differently coloured: in other words, that two different mediums may cause equal refraction, but different divergency; and equal divergency, with different refraction. It follows therefore, that refraction may be produced, which is not affected by the different refrangibility of light; in other words, that, if the mediums be different, different refractions may be produced, though at the same time the divergency caused by one refraction, shall be 
exactly counteracted by the divergency caused by the other; and so an object may be seen through mediums which, together, cause the rays to converge, without appearing of different colours.

This is the foundation of Mr. Dollond's improvement of refracting telescopes. By subsequent experiments he found, that different kinds of glass differed greatly in their refractive qualities, with respect to the divergency of colours. At the same time be found that crown glass causes the least divergency, and white flint the most, when they are wrought into forms that produce equal refractions. He therefore ground a piece of white flint glass into a wedge, the angle of which was a 25 degrees; and a piece of crown glass to another, whose angle was about 29 degrees; and these he found refracted nearly alike, but that their divergency of tolours was very different.

After this, be ground several other pieces of crown glass to wedges of different angles, till he got one that was equal, in the divergency it produced, to that of a wedge of flint glass of 25 degrees; so that when they were put together, in such a manner as to refract in contrary directions, the refracted light was perfectly free from colour; then measuring the reftactions of each wedge, he found that that of the white-flint glass, was to that of the crown glass, nearly as 2 to 3 . And hence any two wredges, made of these two substances, and in this proportion, would, when applied together so as to refract in contrary directions, refract the light without any effect arising from the different refrangibility of the component rays.

Therefore, to make two spherical glasses that refract the light in contrary directions, one must be concave, and the other convex; and as the rays, after passing through both, must meet in a focus, the excess of the refraction must be in the convex one: and as the convex is to refract most, it appears from the experiment that it must be made of crown glass; and as the concave is to refract least, it must be made of white flint.

And farther, as the refractions of spherical glasses are in an inverse ratio of their focal distances, it follows, that the focal distances of the two glasses should be in the ratio of the refractions of the wedges: for, being thus proportioned, every ray of light that passes through this combined glass, at whatever distance it may be from its axis, will constantly be refracted hy the difference between two contrary refractions, in the proportion required; and therefore the effect of the different refrangibility of light will be prevented.

The removal of this impediment, however, produced another: for the two glasses, which were thus combined, being segments of very deep spheres, the aberrations from the spherical surfaces became so considerable, as greatly to disturh the distinctness of the image. Yct, considering that the surfaces of spherical glasses admit of great variations, though the focal distance be limited; and that by these variations their aberration might be made more or less at pleasure; Mr. Dollond plainly saw that it was possible to make the aberrating of any two glasses equal; and that, as in this case the refractions of the two glasses were contrary to each other, and their aberrations being equal, these would destroy each other.

Thus he obtained a perfect theory of making objectglasses, to the apertures of which he could hardly perceive any limits: for if the practice could answer to the theory, they must admit of apertures of great extent, and consequently bear great magnifying powers.
The difficulties of the practice are, however, still rery considerable. For first, the focal distances, as well as the particular surfaces, must be proportioned with the utmo.t accuracy to the densities and refracting powers of the glasses, which vary even in the same kind of glass, when made at different times. Secondly, there are four surfaces to be wrought perfectly spherical. However, Mr. Dollond could construct retracting telescopes on these principles, with such apertures and magnitying powers, under limited lengths, as greatly exceed any that were produced before, in forming the images of objects bright, distinct, and uninfected with colours about the edges, through the whole extent of a very large field or compass of view; of which he has given abundant and undeniable testimony. See Telescope.

The defects which were yet unremedied in Mr. Dollond's telescopes, have called forth the exertions of other philosophers, and many contrivances have been invented. Father Boscovich, to whom every branch of optics is much indebted, has displayed much ingenuity in his attempts for this purpose. But the philosopher whose exertions have been attended with most success, and who appears to liave made the most important discovery, in this branch of science, since the time of Newton, is Dr. Robert Blair, regius professor of astronony in Edinburgh. By a judicious set of experiments, ably conducted, he has proved, that the quality of dispersing the rays in a greater degree than crown glass, is not confined to a few mediums, but is possessed by many fluids, and by some of them in a most extraordinary degree. He has shown, that though the greater refrangibility of the violet rays than of the red, when light passes from any medium into a vacuum, may be considered as a law of nature; yet, in the passage of light from one medium into another, it depends entirely on the qualities of the mediums as to which of these rays shall be the most refrangible, or whether there shall be any difierence in their refrangibility. In order to correct the observation arising from difference of refrangibility among the rays of light, he instituted a set of experiments, by which lie detected a very singular and important quality in the muriatic acid. In all the dispersive mediums hitherto exansined, the green rays, which are the mean refrangible in crown glass, were found among the less refrangible; but in the muriatic acid, he found that these same rays made a part of the more refrangible.

This discovery led to complete success in removing the great defect in optical instruments, viz, that dissipation or aberration of the rays which arises from their unequal refrangibility, and has bitherto rendered it impossible to converge all of them in one point, either by single or opposite refractions. A fluid, in which the particles of marime and metalline particles hold a due proportion, at the same time that it separates the extreme rays of the spectrum much more than crown glass, refracts all the orders of the rays in the sanc proportion that glass does: and hence rilys of all colours, made to diverge by the refraction of the glass, may cither be rendered parallel, by a subsequent rutraction made in the confine of the glass and this fluid; or, by weakening the refractive density of the fluid, the refraction which takes place in the confine of it and glass, may be rendered as regular as reflection, without any the least colour whatever. 'T'he doctor, it scems, has a telescope, not ubove 15 inches in length, with a compound object-glass of this kind, which equals in all 
respects, if it does not surpass, the best of Dollond's 42 inches long. Of this object-glass, a figure may be seen in the Sd volume of the Transactions of the Royal Society of Edinburgh; to which volume the reader must be referred for a full and clear account of the experiments which led to this discovery, as well as of the important purposes to which it may be applied. A judicious abridgment of this paper may also be seen in Nicholson's Journal, vol. i, 4to. p. 1.

In the Gentleman's Magazine (1790, p. 890) a paper was published on the refracting telescope, by a person who signs Veritas, in which the invention is ascribed to another persun, not heretofore mentioned, in these words: "As the invention has been claimed by M. Euler, M. Klingenstierna, and some other foreigners, we ought, for the honour of Eigland, to assert our right, and give the merit of the discovery to whom it is due; and therefore, without farther preface, I shall observe, that the inventor was Chester More Hall, Esq. of More-hall, in Essex, who, about 1729 , as appears by his papers, considering the different humours of the eye, imagined they were placed so as to correct the different refrangibility of light. He then conceived, that if he could find substances having such properties as he supposed these humours unight possess, he should be enabled to construct an object-glass that would show objects colourless. $\Lambda$ fter many experiments, he had the good fortune to find those properties in two different kinds of glass; and making them disperse the rays of light in different directions, he succeeded. About 1733 he completed several achromatic object-glasses, though he did not give them this name, that bore an aperture of more than $2 \frac{1}{2}$ inches, though the focal length did not exceed 20 inches; one of which is now in the possession of the Rev. Mr. Smith, of Cliarlotte Street, Rathbone Place. This glass has been examined by several gentlemen of eminence and scientific abilities, and found to possess the properties of the present achromatic glasses.

"Mr. Hall used to employ the working opticians to grind his lenses; at the same time he furnished them with the radii of the surfaces, not only to correct the different refrangibility of rays, but also the aberration arising from the spherical figure of the lenses. Old Mr. Bass, who at that time lived in Bridewell precinct, was' one of these working opticians, from whom Mr. Hall's invention seems to have been obtained.

"In the trial at Westminster-hall about the patent for making achromatic telescopes, $\mathrm{Mr}$. Hall was allowed to be the inventor; but Lord Mansfield observed, that 'It was not the person that locked up his invention in his scrutoire that ought to profit by a patest for such an invention, but he who brought it forth for the benefit of the public.' 'This, perhaps, might be said with some degree of justice, as Mr. Hall was a gentleman of property, and did not look to any pecuniary advantage from his discovery; and consequently it is very.probable that he might not have an intention to make it generally known at that tine.

"That Mr. Ayscough, optician on Ludgate Hill, was in possession of one of Mr. Hall's achromatic telescopes in 1754 , is a fact which at this time will not be disputed"

ACHRONICAL, or Achronycal. See ACron YCuAL.

ACIDS. All trliose substances' which impress the organs of taste with a sharp; sourg, and cooling sensation, VoL. 1. are commonly denominated acids. But as there are som. bodies destitute of this property, which are nevertheirss classed by all chemical writers as acids, this pupular characteristic must be abandoned as essential, for one that is more comprehensive.

The characteristic properties of acids may be enumerated as follows:

1. When taken into the mouth they occasion a sour taste.

2. They change certain vegetable blue colours to red; such as tincture of litnus, or syrup of violets; and restore the blue colours which have turned green, or the red colours which have been turned blue, by an' alkali.

3. They have a stronger affinity for alkalies, than they have for any other substance.

4. They unite with eartbs, with alkalies, and metallic oxyds, forming the numerous and very important classes of neutral, earthy, and metallic salts.

As this article is entirely chemical, it does not accord with our plan to extend it to any considerable length; we shall therefore content ourselves with giving the most approved arrangement of acids, referring the reader, for farther information on this important subject, to Thomson's Chemistry. See also Aikin's and Nicholson's Chemical Dictionaries, Encycloped. Method., Lavoisier's Elements of Chemistry, Priestley on Air, Fourcroy Systeme de Connois. Chimiq. \&c. \&c. MODERN ARRANGEMENT OF ACIDS.

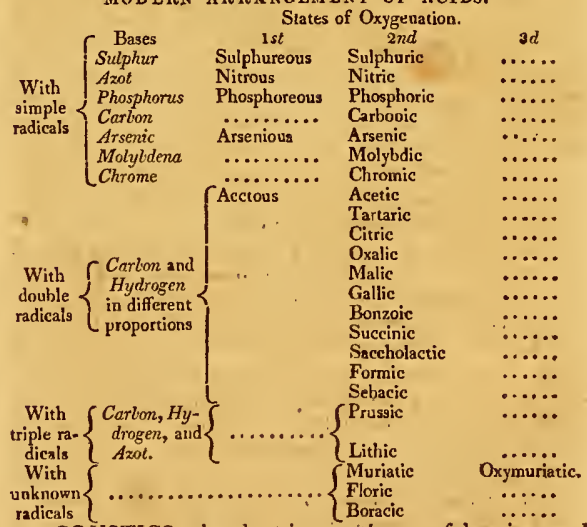

ACOUSTICS, the doctrine or theory of hearing, and of sound.

Sturmins, in his Elements of Universal Mechanics, treating of Acoustics, after examining into the nature of sounds, describes the several parts of the external and internal ear, and their several uses ind connexions with each other ; and from thence deduces the mechanism of hearing : and lastly, he trichts of the means of adding an intensity of force to the voice and other sounds; and explains the nature of echors, otacoustic tubes, and speaking trumpets.' Sce Sounn,' Ėri, Vorce, and Ecro.

Dr. Hook, in the preface to his Micrography, asserts that the lowest whisper, by certain means, may be heard at the distance of a furrlong; and that he knew a way by which it is easy to hear any one speak through a wall of three feet thick; 'also' that by meals of an extended wire, 


\section{$\mathrm{ACO}$}

[ 26

sound may be conveyed to a very great distance, almost in an instant.

The ancients seem to have cousidered sounds under no other point of view than that of music; that is to say, as affecting the ear in an agreeable manner. It is cven very doubtful whether they were acquainted with any thing more than melody, and whether they had any art similar to what we call composition. The modesis, by studying the philosophy of sounds, so much neglected by the ancients, have given birtl to a new science distinguished by the name of acoustics, which has for its object the nature of sounds, and treats also of the theory of hearing, and the best means of assisting that sense. This science is divided by some writers into diacoustics, which explain the properties of those sounds that come directly from the sonorous body to the ear; and catacoustics, which treats of reflected sounds; but such distinction does not appear to be of any real utility.

On many particulars connected with this science opinions are still muci divided; and a sufficient number of experiments have not been made to determine what is essential to the nature of a vehicle of sound; how it is transmitted, reflected, or destroyed, in many instances; or whether its velocity be uniform or variable..

Thus, with respect to these vehicles; as a bell or other sonorous body when struck in vacuo transmits no sound, it therefore necessarily follows that air is such a vehicle, or that some subtle fuid is contained in its composition which performs the office of transmitting sound: this last seems the more probable, because we know, that air is not the only vehicle, nor the best, for this transmission; and on this supposition it follows, that bodies of any density or texture will transmit sound more or less perfect, according to the quantity of this fluid contained in them.

The aqueducts constructing at Paris have lately given M. Biot an opportunity of making some interesting experiments on the propagation of sound through solid bodies, being on a larger scale than any hitherto attempted. The total length of the pipes was $3 \mathrm{t} 1 \mathrm{~s}$ feet. A blow with a hammer at one extremity was heard at the other, producing two distinct sounds, the interval of which, measured in more than 200 trials, was $2 \cdot 5^{\prime \prime}$. And according to the experiments of the academy, the time of propagation of sound to this distance through the air should be $2 \cdot 79^{\prime \prime}$, from which deducting $2 \cdot 5^{\prime \prime}$ the interval observed, we have $29^{\prime \prime}$ for the time that sound was in being propagated through the solid substance to the distance above stated. These experiments were afterwards varied; the result however generally agrced with that ahove stated.

Some have considered the electrical fluid as the agent by which this office is performed; it has however been objected to by others, and many ingenious arguments have been advanced on both sjeles, but without coming to a satisfactory conclusion. One thing however is certain, that whatever the fluid may be, the sound we hear is produced by the stroke that the sounding body makes against $\mathrm{it}$, and is thence conveyed to the organ of hearing. But the manner in which this conveyance is made is still disjuted: whether the sound be diffused into air in circle beyoud circle, like the waves of water when we disturb the smoothness of its surface by dropping in a stone, or whether it travels alorg, like rays diflused from a centre, somewhat in the swift manuer that electricity runs along a lod of iron, are questions which lave greatly divjded the opinions of the learned.
A $\mathrm{C} \mathrm{K}$

Newton was of the former opinion; but John Bernouilli confessed, that he did not understand this part of the Principia-and attempted to give a more perspicuous demonstration of his own, that might confirm and illustrate the Newtonian theory; but the subject seemed to reject elucidation; for his theory is obviously wrong, as $\mathrm{D}^{\prime} \mathrm{A}$ lembert has proved in his treatise on fluids.

The Newtonian theory has however found an able advocate in the ingenious Dr. Young, of Trinity College, Dublin, who has given a clear, explanatury, and able defence of the 47 th proposition of the $2 \mathrm{~d}$ book of the Principia, which has been considéred the most objectionable; and some late rexperiments have contributed in a great degree to remove the difficulties with which this subject is clouded, and which will be explained in their proper places. isee the articles CHoRd, Ecro, Elastic string, Ear, Sound, Transmission, Velocity, ViBRATION, \&c.

ACRE, is a measure of land, containing, by the nrdinance for measuring land, made in the $33 \mathrm{~d}$ and 34 th of Edward I, 160 perches or square poles of land; and as the statute length of a pole is $5 \frac{1}{2}$ yards, or $16 \frac{x}{2}$ feet, therefore the acre will contain 4840 square yards, or 43560 square feet. The chain with which land is commonly measured, and which was invented by Gunter, is 4 poles or 22 yards in length; and therefore the acre is just 10 square chains. Further, as a mile contains 1760 yards, or 80 chains in length, therefore the square mile contains 640 acres.

The acre, in surveying, is divided into 4 roods, and the the rood is 40 perches.

The French acre, arpent, is equal to $1 \frac{1}{4}$ English acre;

The Strasburg contains about $\frac{1}{2}$ an English acre;

The Welch acre contains about 2 English acres;

The Irish acre contains 1 ac. 2 r. $19 \frac{27}{T 21}$ p. English.

The Scotch acre is near $1 \frac{1}{4}$ English, being equal to 6150 square yards.

Sir Villiam Petty, in his Political Arithmetic, reckons that England contains 39 million acres : but Dr. Greve shows, in the Philos. Trans. No. 330, that England contains not less than 46 million acres. Whence he infers that England is above 46 times as large as the province of Holland, which it is said contains but about one million of acres.

By a statute of the 31 st of Elizabeth, it is ordained, that if any man-erect a cottage, he shall annex four acres of land to it. But this statute was repealed by the 15 th of Geo. 111. cap. 32.

ACROA'TICS, a name given to Aristotle's lectures in the more difficult and nice parts of philosophy; to which none but his disciples and intimate friends were admitted; whereas the exoteric were public or open to all.

ACRONYCHAL, or Acron Ycal, in Astronony, is said of a stur or planet, when it is opposite to the sun. It is from the Greek axporuxos, the point or extremity of night, because the star rose at sun-set, or the begiming of night, and set at sun-rise, or the end of night; and so it shone all the night.

'I'he acronychal is one of the three Greck poetic risings and settings of the stars; and stands distinguished from Cosmical and Heliacal. And by means of which, for want of accurate instruments, and other observations, they might regulate the length of thejr year.

AClROTlili $\Lambda$, or Acro'sens, in Architecture, small pedestals, usually without bases, placed on pediments, and serving to support statues. Those at the extremities 
ought to be half the height of the tympanum; and that in the middle, according to Vitruvius, one eighth part more.

Acroteria also are sometimes used to signify figures, whether of stone or metal, placed as ornaments or crownings, on the tops of temples, or other buildings.

It is also sometimes used to denote those sharp pinacles or spiry battlements, that stand in ranges about flat buildings, with rails and balustres.

ACTION, in Mechanics or Physics, a term used to denote, sometimes the effort which a body or power exerts against another body or power, and sometimes it denotes the effects resulting from such effort.

Action is either instantaneous or continued; that is, either by collision or percussion, or by pressure. These two sorts of action are beterogenenus quantitics, and are not comparable, the sinallest action by percussion exceeding the greatest action of pressure, as the smallest surface exceeds the longest line, or as the smallest solid exceeds the largest surface : thus, a man by a small blow with a hammer, will drive a wedge below the greatest ship on the stocks, or under any other weight; that is, the smallest percussion overcomes the pressure of the greatest weight. These actions then cannot be measured the one by the other, but each must have a measure of its own kind, like as solids must be measured by solids, and surfaces by surfaces: time being concerned in the one, but not in the other.

It is one of the laws of nature, that action and reaction are always equal, and contrary to each other in their directions. Therefore, if a body be urged at the same time by equal and contrary forces, it will remain at rest. But if one of these forces be greater than its opposite one, motion will ensue towards the part which is least urged.

The actions of bodies on each other, in a space that is carried uniformly forward, are the same as if the space were at rest; and any powers or forces that act upon bodies, so as to produce equal velocities in them in the same, or in parallel right lines, have no effect on their mutual actions, or relative motions. Thus the motion of bndies on board of a ship that is carried uniformly forward, are performed in the same manner as if the ship were at rest. And the motion of the earth about its axis has $n o$ effect on the actions of bodies and agents at its surface, except so far as it is not uniform and rectilincal. In general, the actions of bodies upon cach other, depend not on their absnlute, but relative motion.

Quantity of Action, in Mechanics, a name given by Maupertuis, in the Memoirs of the Academy of Sciences of Paris for 1744, and in those of Berlin for 1746, to the continual product of the mass of a body, by the space which it runs through, and by its celerity. He lays it down as a gencral law, that in the changes made in the state of $"$ i body, the quantity of action necessiry to produce such change is the least possible. This principle he applies to the investigation of the laws of refraction, and even to what he calls the laws of rest, that is, of the equilibrium or equipollency of pressures; and even to the modes of acting of the Supreme Being. III this way Maupertuis attempts to connect the metaphysics of fiual causes with the fundamental trutlıs of mechanics; to show the dependence of the collision of hoth elastic and hard bodies, upon one and the same law, which before had always been referred to separate laws; and to reduce the laws of motion, and those of eyuilibrium, to one and the same principle.
But this quantity of motion, or of action, of Maupertuis, which is defined to be the product of the mass, the space passed over, and the celerity, comes to the same thing as the mass multiplice by the square of the velocity, when the space passed over is equal to that by which the velocity is measured; and so the quantity of force will be proportional to the mass multiplied by the square of the velocity; since, the space is measured by the velocity continued for a certain time.

In the same year that Maupertuis communicated the idea of his principle, Euler, in the supplenent to his book, intitled Methodus inveniendi lineas curvas maximi zel minimi proprietate gaudentes, demonstrates, that in the trajectories which bodies describe by central forces, the velocity multiplied by what the foreign mathematicians call the element of the curve, always makes a minimum: which Maupertuis considered as an application of his principle to the motion of the planets.

It appears from Maupertuis's Memoir of 1744, that it was his reflections on the laws of refractions, that led him to the theorem above mentioned. The principle which Fermat, and after him Leibnitz, made use of, in accounting for the laws of refraction, is sufficiently known. Those mathematicians asserted, that a particle of light, in its passage from one point to another, through two mediums, in each of which it moves with a different velocity, must do it in the shortest time possible; and from this principle they have demonstrated geometrically, that the particle cannot go from the one point to the other in a right line; but being arrived at the surface that separates the two mediums, it must alter its direction in such a manner, that the sine of its incidence shall be to the sine of its refraction, as its velocity in the first medium is to its velocity in the secnnd: whence they deduced the well known law of the constant ratio of those sines.

This explanation, though very ingenious, is liable to this pressing difficulty, namely, that the particle must approach tuwards the perpendicular, in that medium where its velocity is the least, and which consequently resists it the most: which seems contrary to all the mechanical explanations of the refraction of bodies, that have hitherto been advanced, and of the refraction of light in particular.

Sir Isaac Newton's manner of accounting for $\mathrm{it}$, is the most satisfactory of any that has hitherto been offered, and gives a clear reason for the constant ratio of the sines, by ascribing the refraction to the attractive force of the mediums; from which it follows, that the densest mediums, whose attraction is the strongest, should cause the ray to approach the perpendicular; a fact confirmed by experiment. But the attraction of the medium could not cause the ray to approacl towards the perpendicular, without increasing its velocity; as may easily be demonstrated. Thus then, according to Newton, the refraction must be towards the perpendicular, when the velocity is increased: contrary to the law of Fermat and Leibnitz.

Maupertuis has attempted to rcconcile Newton's explanation with metaphysical principles. Instead of supposing, with Fermat and Leibnitz, that a particle of light proceeds from one point to another in the shortest time possible; he contends that a particle of light passes from one point to another in such a manner, that the quantity of action shall be the least possible. This quantity of action, says be, is a real expense, in which nature is always frugal. In virtue of this philosophical principle he 
discovers, that not only the sines are in a constant ratio, but also that they are in the inverse ratio of the velocities, according to Newton's explanation, and not in the direct ratio, as had been asserted by Fermat and Leibnitz.

Now according to this principle, the time, that is, the space divided by the velocity, should be a minimum; so that calling the space run through in the first medium $\mathrm{s}$, with the velocity $\mathrm{v}$, and the space run through in the second medium $s$, with the velocity $v$, we shall have $\frac{\mathrm{s}}{\mathrm{v}}+\frac{s}{v}=$ a minimum; hence $\frac{\dot{\mathrm{s}}}{\mathrm{v}}+\dot{s}=0$, or $\frac{\dot{\mathrm{s}}}{\mathrm{v}}=-\frac{\dot{s}}{v}$. Now it is easy to perceive, that the sines of incidence and refraction are to each other, as $\dot{s}$ to $-\dot{s}$; whence it follows, that those sines are in the direct ratio of the velocities $\mathrm{v}, v$; which is exactly what Fermat makes it. But in order to have those sines in the inverse ratio of the velocities, it is only supposing $\mathrm{v} \dot{\mathrm{s}}+v \dot{s}=0$; which gives $\mathrm{sv}+s v$ or $\mathrm{s} \times \mathrm{v}+s \times v$ a minimum: which is Maupertuis's principle.

In the Memoirs of the Academy of Berlin, above cited, may be seen all the other applications which Maupertuis has made of this principle. And however unfounder his metaphysical basis of it may be, as also the irica he has annexed to the quantity of action, it will still hold good, that the product of the space by the velocity is a minimum in some of the most general laws of nature.

ACTIVE, the quality of an agent for communicating motion or action to some body. In this sense the word stands opposed to passive: thus we say an active cause, active principle, \&c.-Sir Isaac Newton shows, that the quantity of motion in the world must be always decreasing, in consequence of the vis ilicrtix, \&c. So that there is a necessity for certain active principles to recruit it : such, he takes the cause of gravity to be, and the cause of fermentation; adding, that we see but little motion in the universe, except what is owing to these active principles.

ACTIVITY, the virtue or faculty of acting. As the activity of an acid, a poison, \&c : the activity of fire excecds all imagination.-According to Sir Isaac Newton, bodies derive their activity from the principle of attraction.

Sphere of Acrivity, is the space which surrounds a body, as far as its efficacy or virtue extends to produce any sensible effect. Thus we say, the sphere of activity of a luadstone, of an electric body, \&c.

ACUBENE, in Astronomy, the Arabic name of a star of the fourth magniturle, in the southern claw of Cancer, marke'd $\alpha$ bv Bayer. lis longitude for $1761, \Omega 10^{\circ} 18^{\prime} 9^{\prime \prime}$, south latitude $5^{\circ} 5^{\prime} 56^{\prime \prime}$.

ACUTE, or sharp; a term opposed to obtuse. Thus, Acute, Angle, in Geometry, is that which is less than a right angle; and is measured by less than $90^{\circ}$, or a quandrant of a circle. As the angle $A B C$.
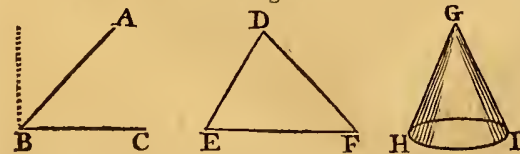

Acute angle Triangle, is that whose three angles are all acute; and is otherwise called an oxygenous triangle. As the triangle DEF.

Acutz-angled Cone, is that whose opposite sides make an acute angle at the vertex, or whose $a x i_{2}$, in a right cone, makes less than half a right angle with the side. As the core $\mathrm{GH}$ I.

Pappus, in his Mathematical Collections, says, this name was given to such a cone by Euclid and the ancients, before the time of Apollonius. And they called an

Acure-angled Section of a Cone, an Ellipsis, which was made by a plane cutting both sides of an acute-angled conc; it bcing not known that such a section conld be generated from any cone whatever, till it was shown by Apollonius.

Acute, in Music, is uuderstood of a tone, or sound. which is high, sharp, or shrill, with regard to some other: in which sense the word stands opposed to grave. And both these sounds are independent of loudness or force: so that the tone may be acute or high, without bcing loud; and loud without being high or acutc. For both the affections of acute and grave, depend entircly on the quickness or slowncss of the vibrations by which they are produced.- Sounds considercd as grave and acute, that is, in the relation: of gravity and acuteness, constitute what is called tune, the foundation of all harmony. ...

ADAGIO, in Music, one of the terms used by the Italians to express a degree or distinction of time:

Adagio denotes the slowest time except,grave.

Sometimes the word is, repeated, as adugio, adagio, to denote a still slower time than the former.

Adagio'also signifies a slow movement, when used substantively..

$A D A R$, in the Hebrew Chronology, is the 6 th month of their civil year, but the 12 th of their ecclesiastical year. It contains only 29 days; and it answers to our February; but sometimes entering into the month of March, according to the course of the moon.

ADDITION, the uniting or joining of two or more quantities together.

ADDITION, in Arithmetic, is the first of the four fundamental rules or operations of that science; and. consists in finding a number equal to several others taken together, or in finding the most simple expression of a number according to the, established mode of notation; and the quantity so found: is named their, sum.

The sign or character of addition is + , and is called plus. This character is set between the quantities to be added, to denote their sum: thus, $3+6=9$; that is, 3 plus 6 are equal to 9 .

Simple numbers are either added as above; or else by placing them under one another, as in the margin, and adding them togcther, one after another, beginning at the bottom : thus 2 and 4 make 6 , and 6 make 12 .

Numbers consisting of several figures are added, by first ranging the numbers in columns under each other, placing always the numbers of the same denomination under each other, that is, units under units, tens under tens, and so on; and theu adding up each column separately, beginning at the right hand, setting down the sum of cich column below it, unless it amount to ten or some number of tens, and in that case setting down only the overplus, and carrying one for each ten to the next co. lumn. Thus, to add 4.51 and 326 ,

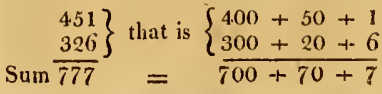




\section{A D D}

Also to add the numbers $329+1562+$ $20347+712048$; set them down as in the margin, and beginning at the lowest number on the right hand, say 8 and 7 make 15 , and 2 make 17 , and 9 make 26 ; set down 0 , and carry 2 to the next column, saying 2 and 4 make 6 , and 4 make 10 , and 6 make 16 , and 2 make 18 ; set down 8 , and carry 1 , saying 1 and 3 make 4 , and 5 make 9 , and 3 make 12 ; set down 2 , and carry 1 , saying 1 and 2 make 3 , and 1 make 4 , which set down; then 1 and 2 make 3 ; and 7 is 7 to set down : so the sum of all together is 734286 . Or it is the same as; the sums of the columns set, under one, another, as in the margin, and then these added up in the same:manner.

When a great number of separate sums or numbers are to be added, as in, long accounts, it is easier to break or separate them into two or more parcéls, which are added up severally; and then their sums added together for the total sum. And thus also the truth of the addition may be proved, by dividing the numbers into parcels different ways, as the totals must be the same in both cases when the operation is right.

Another method of proving addition, was given by $\mathrm{Dr}$. Wallis, in his Arithmetic, as well as by Lucas de Burgo, and probably by former authors, alsop, by casting out the nines, which method of proof extends also to the other rules of arithmetic. The method is this : add the figures of each line of numbers together severally, .casting. out always 9 from the sums, as they arise.in so, adding, carrying the overplus to the next figure, and setting at the end of each line what is over the nine or nines; then do the same by thesum-total, as also by the former excesses of 9 , so shall the last excesses be equal when the work is right. Thus, the former example will be proved as annexed:

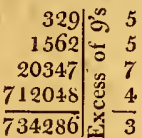

When the numbers are of, different denominations; as pounds, shillings, and pence; or yards, feet, and inches; place the numbers of the same kind under, each other, as pence under pence, shillings under shillings, \&c ; then add each colunis separately, and carry the overplus as before, from one column to another. As in the following examples :

\begin{tabular}{|c|c|c|c|c|c|c|}
\hline l. & s. & d. & & Yards & Feet & nches, \\
\hline 271 & 12 & 3 & & 271 & 10 & 3 \\
\hline 94 & 14 & 7 & & 36 & 2 & 7 \\
\hline 42 & 5 & 10 & & 14 & 2 & 5 \\
\hline 408 & 12 & 8 & sums & $\overline{323}$ & 0 & \\
\hline
\end{tabular}

ADDITson of Vulgar Fractions, is performed by bringing all the proposed fractions to the same denomination, when necessary, and all compound fractions and mixed numbers to simple fractions; then reduce all the fractions to a common denominator, and the sum of the numerators placed over the common denominator will be the answer sought.

Su $\frac{2}{7}+\frac{2}{7}=\frac{5}{7}$, And $\frac{1}{5}+\frac{2}{5}+\frac{3}{5}=\frac{6}{5}=1 \frac{1}{5}$.

And $\frac{7}{2}+\frac{2}{3}=\frac{3}{6}+\frac{4}{6}=\frac{7}{6}=1 \frac{1}{6}$.

And $\frac{5}{6}+\frac{2}{3}$ of $\frac{3}{4}=\frac{5}{6}+\frac{3}{6}=\frac{8}{6}=\frac{4}{3}=1 \frac{x}{3}$.

When the fractions are of different denominations, some farther operations are necessary: thus,

Add. $\frac{2}{5} \mathrm{G}+\frac{3}{7} \mathrm{~L}+\frac{5}{6} \mathrm{~s}$ together.

Here $\frac{2}{5} \mathrm{c}=\frac{2}{3} \times \frac{21}{\mathrm{~T}}=\frac{42}{5} \mathrm{~s}$

A D D

$$
\begin{aligned}
& \frac{3}{7} \mathrm{~L}=\frac{3}{7} \times \frac{20}{\mathrm{~T}}=\frac{60}{7} \mathrm{~s} \\
& \frac{5}{6} \mathrm{~S}=
\end{aligned}
$$

and $\frac{42}{5}+\frac{60}{7}+\frac{5}{6}=\frac{1764}{210}+\frac{1800}{210}+\frac{175}{210}=\frac{3739}{210} \mathrm{~s}=$ 17s. 9 d. $\frac{2}{3} \frac{3}{5}$ Ans.

ADDition of Decimals, is performed in the same manner as that of whole numbers, placing the numbers of the same denomination under each other, in which case the decimal separating points will range straight in one column; as in this example, to add together these numbers 371.0496 $+25 \cdot 213+1 \cdot 704+924 \cdot 61+\cdot 0962$

$371 \cdot 0496$ $25 \cdot 213$

$1 \cdot 704$ $924 \cdot 61$ 0962

$\overline{1322 \cdot 6728}$

Andition of Circulating. Decimals is perforined by changing each of them into its equivalent vulgar fraction, and finding the sum of such fractions.-For the method of changing, recurring or circulating decirnals to vulgar fractions, see Circulating Dẹcimals.

AD DITION in Algebra, is the uniting or incorporating of indeterminate quantities denoted by letters into one contracted expression. The operation is performed by connecting the quantities together with their proper signs, and collecting all similar quantities into one sum; namely, by adding their co-efficients together if their signs are the same, but subtracting them when different. Tlius the quantity $a$ added to the quantity $b$, makes $a_{3}+b^{\prime} ;$ and $a$ added to $-b$, gives $a-b$; 2lso the compound quantity $(5 a-b)$ added to $(4 a+3 b)$ produces the sum $(9 a+2 b)$. See the following examples:

$$
\begin{aligned}
& 5 x y \\
& 4 a x \\
& -x y \text {. } \\
& -4 a x \\
& 4 x y \quad \overline{6 x^{2} y^{2}-7 x^{2} y-2 a x y} \\
& 2 a x-150+2 x^{\frac{x}{2}} \\
& 3 x^{2}+2 a x+6 x^{2} \\
& 5 x y+50-3 x^{\frac{x}{2}} \\
& -5 x^{2}+100+x^{\frac{1}{2}} \\
& 4 a x^{3}+4 x^{2}+5 x y \\
& \begin{array}{l}
6 x^{2} y^{2} \\
-4 x^{2} y
\end{array} \\
& -2 a x y \\
& -2 a x y \\
& 7 a b \\
& 4 a^{2} b^{2} \\
& -3 a b \\
& -2 a^{2} b^{2}
\end{aligned}
$$

ADDITION of Algebraic Fractions; is performed by the same rule, as has been given for addition of vulgar fractions; as will be seen by the following exámples :

Ex. 1. $\frac{x-4}{3}+\frac{x+3}{7}=\frac{7 x-28}{21}+\frac{3 \ddot{x}+9}{21}=\frac{10 x-19}{21}$ Ans.

Ex. $2 \cdot y+\frac{3 y-2 a}{4}+7 y-\frac{4 y-3 a}{6}=8 y+\frac{y}{12}$ Ans.

ADDITIon of Surds. In this rule all the given quantities must be reduced to the same denomination, or to the same radical, if that can be done; then adding or uniting the rational parts, and subjoining the common surd. Otherwise comnecting them with their own signs.

So $\sqrt{ } 8+\sqrt{ } 18=\sqrt{ }(4 \times 2)+\sqrt{ }(9 \times 2)=2 \sqrt{ } 2$ $+3 \sqrt{ } 2=5 \sqrt{ } 2=\sqrt{ } 50$;

and $\sqrt{ } 12+\sqrt{ } 27=\sqrt{ }(4 \times 3)+\sqrt{ }(9 \times 3)=2 \sqrt{ } 3$ $+3 \sqrt{ } 3=5 \sqrt{ } 3=\sqrt{ } 75$;

and $\sqrt{ } 50+\sqrt{ } 75=\sqrt{ }(25 \times 2)+\sqrt{ }(25 \times 3)=5 \sqrt{ } 2$ $+5 \sqrt{ } 3=(\sqrt{ } 2+\sqrt{ } 3) 5$

but of $\sqrt{ } 5$ and $\sqrt{ } 6$ the sum is set down $\sqrt{ } 5+\sqrt{ } 6$, because the terms are incommensurable, and not reducible to a coinmon surd.

ADDITION of Logarithms. Sce Logarithms.

ADDITION of Ratios, the same as composition of ratios; which see.

ADDITIVE, denotes something to be added to another, in contradistinction to something to be taken away or 
subtracted. So astronomers speak of additive equations, and geometricians of additive ratios.

ADELARD, or ATHELARD, was a learned monk of Bath, in England, who flourished about the year 1130, as appears by some manuscripts of his in Corpus Christi, and Trinity Colleges, Oxford. Vossius says he was universally learned in all the sciences of his time ; and that, to acquire all sorts of knowledge, he travelled into France, Germany, Italy, Spain, Egypt, and Arabia. He wrote many books himself, and translated others from different languages: thus he translated, from Arabic into Latin, Euclid's Elements, at a time before any Greek copies had been discovered; also Erichiafarim, on the seven planets. He wrote a book on the seven liberal arts, another on the astrolabe, another on the causes of natural compositions, besides several on physics and on medicine.

Though Vossius refers to Oxford for some of these manuscripts, it would yet seem they were not to be found there in Wallis's time; for the Doctor, speaking of this author, and other English authors and travellers about the same age, says, "A particular account of these travels of Shelley and Morley was a while since to be seen in two prefaces or two manuscript books of theirs in the library of Corpus-Christi College in Oxford, but hath lately (by some unknown band) been cut out, and carried away; which prefaces (one or both of them) did also make mention of the travels of Athelardus Bathoniensis, and are, to that purpose, cited by Vossius out of that manuscript copy. Whoever hath them, would do a kindness (by some way or other) to restore them, or at least a copy of then." Wallis's Algebra, pa. 6.

ADELM, Aldhelmus, or Althelmus, a learned Englishman, who flourished about the year 680 . He was first abbot of Malmsbury, and afterward bishop of Shirburn. He died in the year 709 , in the monastery of Malmsbury.-Adelm was the son of Kenred or Kenten, who was the brother of Ina, king of the West Saxons in England. Besides certain books on theology, he composed several on the mathematical sciences, \&c ; as Arithmetic, and Astrology, and librum de philosophorum disciplinis. See Bede's History, lib. 5. cap. 19. He is also mentioned by Bale and William of Malmsbury.

ADERAIMIN, or ALDERAImis, the Arabic name of a star of the third magnitude, in the left shoulder of Cepheus marked $\alpha$ by Bayer. Its longitude for 1761, $\Upsilon$ $9^{\circ} 30^{\prime} 8^{\prime \prime}$, north latitude $68^{\circ} 56^{\prime} 20^{\prime \prime}$.

ADFECTED, see APFECTED.

ADHeSION, AвHerence, in Physics and Chemistry, a species of attraction which takes place betwcen the surfaces of bodies, that are either similar or dissimilar, and which in a certain degree connects them together; thus, water adheres to the fingers, mercury to gold, two pieces of lead or brass to each other, \& c. Hence arises an important distinction between two words, that in a louse and popular sense are often confounded, that is, Adhesion and Colesion.-The former denotes an union to a certain point between two similar or dissimilar substances; and the latter, that which retains together the component particles of the same mass.

The proximate cause of adhesion, has been variously stated by different philosophers. James Bernoulli attributed it to the pressure of the air; but this was afterwards found to be incorrect by some experiments made by Mr. Hauksbee; and it has since been proved, that the power of adhesion is proportional to the number of touching points, and this depends on the figure of the particles of which the body is formed; and in solid bodies on the degree in which their surfaces are polished and compressed. Dr. Brook Taylor first observed, in 1713 , the ascent of water between two planes of glass; this led bim to make several experiments on the adhesive power of surfaces; from which be cuncluded that the degree of this force might be measured by the weight required to separate them. This idea was taken up and pursued with considerable success by $\mathbf{M}$. de Morveau (now M. Guyton).

He procured cylinders, or rather plates, of different metals in their highest state of purity, which were made perfectly circular, highly polished, and furnished with a small ring fixed to their centre, each plate being exactly an inch in diameter. Those plates were in turn suspended to the arm of an assay balance, and being exactly counterpoised by weights placed in the scale attached to the opposite arm, were applied to mercury in a ressel about $\frac{x}{6}$ of an inch below them. After sliding them along the surface to prevent any air from lodging between them and the mercury, weights were successively added to the scale attached to the opposite arm, till the adhesion between the plate and the mercury was broken: and fresh mercury was used for each experiment. The results of which are exhibited in the following table.

Gold adheres to mercury with a force of 446 grains.

Silver ......................................... 429

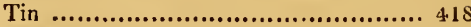

Lead .................................... 397

Bismuth .................................... 372

Zinc .......................................... 204

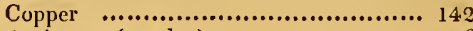

Antimony (regulus) $\ldots \ldots \ldots \ldots \ldots \ldots \ldots \ldots \ldots 126$

Iron.......................................... 115

Cobalt .................................... 8

These experiments clearly proved, as indeed bad bren before done by Hauksbee, that the atmosphere was not the agent or cause of adhesion; for had this been the case, it is evident that, the surfaces being the same in each experiment, the force of adhesion in each must have been equal also. The above method has been since pursued to greater lengths, and with more accuracy by M. Achard and others, who took into their account the temperature of the fluids. And from the result of these interesting experiments we may conclude-That there exists a tendency to adhesion between many, and probably between all substances in nature, absolutely independent of atmospherical or any other external pressure; that the force of this adhesion between solids is in the order of their chemical affinities; and between solids and fluids in an inverse ratio to the thermonnetrical temperature, and in a direct ratio to the squares of the surfaces; also that every solid adheres with a peculiar force to each fluid; that this force is truly expressed by the weight necessary to break the adhesion, in all cases where the solid comes out clear from the Ruid; but that when any particles of the fluid adhere to the solid, the weight of the counterpoise is then expressive of the mixed force of adliesion between the surface of the solid and the fluid, and of the cohesion bitween the component particles of the latter.

In order to keep a commected view of this subject in its progress townerds perfuction, such experiments only have been related as most materially tended to the accomplishment of that desirable end; and consequemely many cu- 
rious and entertaining experiments have not been mentioned. As, however, some of these may afford a degree of satisfaction and amusement to the reader, we have detailed a few of the most interesting ones, as they are related by Musschenbroek and Martin. The former, in his Philosophy, as translated by Colson, relates that two cylinders of glass, whose diameters were not quite two iuches, being beated to the degree of boiling water, and joined together by means of melted tallow lightly put between them, acllered with a force of 130 pounds: lead of the same diameter, and in similar circumstances, adhered with a force of 275 pounds, and soft iron with a force of 300 pounds. And Martin, in his Plilosophia Britannica, vol. 1, says, that with two leaden balls, not weighing above a pound each, nor touching on more than $\frac{1}{30}$ of a square inch of surface, he has lifted more than 130 pounds weight: the balls being first very finely planed with the edge of a sharp pen-knife, and then equally pressed together with a considerable force, and a gentle turn of the hand. The force of adhesion between two brass plates, of about $4 \frac{5}{3}$ inches diameter, and smeared witlı grease or fat, is so powerful, as to require more than the strength of two strong men to separate them. Those who wish to see more on this subject, may consult to advantage the Supplement to Encyclopedia Britannica, art. Chemistry, and Rees' Cyclopedia, art. Adhesion; as also the two works above mentioned.

ADHIL, in Astronomy, a star, of the 6th magnitude, on the garment of Andromeda, under the last star in her foot.

ADJACENT, whatever lies immediately by the side of a nother.

AdJAcEnt Angle, in Geometry, is said of an angle when it is immediately contiguous to another, so that they have both one common side. And the term is more particularly used when the two angles have not only one common side, but also when the other two sides form one continued right line.

ADJACEN T Bodies, in Physics, are understood of those that are near, or next to, some other body.

ADJUTAGL, or rather AJUTAGE; which see.

ADSCRIPTS, in Trigonometry, is used by some mathematicians, for the tangents of arcs. Vieta calls them also prosines.

AUVANCE-Fosse, in Fortification, a ditch thrown round the esplanade or glacis of a place, to prevent its being surprised by the besiegers.-The ditch sometimes made in that part of the lines or retrenchments nearest the enemy, to prevent him from attacking them, is also called the advince-fosse.-The advance-fosse should always be full of water, otherwise it will serve to cover the enenty from the fire of the place, if he should become mastir of it. Beyond the advance-fosse it is usual to construct lunctes, redouts, \&c.

ADVENT, Adventus, in the Calendar, the time immediately preceding Christmas; and was anciently employed in pious preparation. for the adventus, or coming on, of the feast of the Nativity.

Advent includes four Sundays, or weeks; commencing either with the Sunday which falls on St. Andrew's day, namely the 30 th day of November, or the nearest Sunday to that day, either before or after.

FOLII'LL, Eolipila, in Hydraulics, a hollow ball of metal, with a very sinall hole or opening; chicfly used to blow the convertibility of water into elastic steam. 'The best way of fitting up this instrument, is with a very slender neck or pipe, to screw on and off, for the convenience of introducing the water into the inside; for, by unscrewing the pipe, and immerging the ball in water, it readily fills, tbe hole being pretty large; and then the pipe is screwed on. But if the pipe do not unscrew, its orifice is too small to force its way in against the included air; and therefore to expel most of the air, the ball is heated red-hot, and suddenly plunged with its orifice into water, which will then rush in till the ball is about twothirds filled with it. The water having been introduced, the ball is set upon the fire, which gradually heats the contained water, and converts it into elastic steam, this rushes out by the pipe with great violence and noise; and thus continues till all the water is so discharged; though not with a constant and uniform blast, but by sudden unes: and the stronger the fire is, the more elastic will the steam be, and the greater the force of the blast. Care should be taken that the ball be not set upon a violent fire with very little water in it, and that the small pipe be not stopped with any thing; for in sucl case, the included elastic steam will suddenly burst the ball with a very dangerous explosion.

This instrument was known to the ancieuts, being mentioned by Vitruvius, lib. 1, cap. 6. It is also treated of, or mentioned, by several modern authors; as Descartes, $i_{11}$ his Meteor. cap. 4; and Father Mersenne, in prop. 29 Phædom. Pneumat. uses it to weigh the air, by first weighing the instrument when red-hot, and having no water in it; and afterwards weighing the same when it becomes cold. But the conclusion gained by this means, cannot be quite accurate, as there is supposed to be no air in the ball when it is red-hot; whereas it is shown by Varenius, in his Geography, cap. 19, sect. 6, prop. 10, that the air is rarefied but about 70 times; and cunsequently the weight obtained by the above process, will be about one-70th too small, or more or less according to the intensity of the heat.

Descartes, and others, have made use of this instrument, to account for the natural cause and production of the wind. And hence its name, Eolipila, q. d. pila Eoli, Æolus' ball.

In Italy it is said that the Eolipile is often used to cure smoaky chimneys: for, being hung over the fire, the blast arising from it carries up the loitering smoke along with it.

And some have imagined that the aolipile might be employed as bollows to blow up a fire, having the blast from the pipe directed into the fire: but experience would soon convince them of their mistake; for it would rather blow the fire out than up, as it is not air, but rarefied water, that is thus violently blown through the pipe.

EOLUS, in Mechanics, a small portable machine, invented by Mr. Tidd, for refreshing and changing the air in rooms which are made too close. The machine is adapted to supply the place of a square of glass in a sashwindow, where it works with little or no noise, on the principle of the sails of a mill, or a smoke jack; and thus admitting an agreeable quantity of air, at a convenient part of the room.

EoLus's Harp, or IEolian Harp, an instrument so named, from its producing an agreeable melody, merely by the action of the wind. Neither the inventor, nor the date of the invention, of this instrument is accurately kuown. Kircher, in his Magia Phenotactica and Phousur- 
gia, has given the first description of it ; and hence the invention of it has by some authors been attributed to him.

Its construction is extremely simple, as it consists of little more than a number of cat-gut or wire strings, distended in parallel lines over a box of thin deal, having a top of the same thin wood, with sounding-holes. Then the strings being tuned in unison, and the instrument placed in a proper situation for receiving a current of air, as an open window, \&c, it produces by the tremulous motion which the wind communicates to the strings, a soft murmuring, and pleasing combination of sounds.

The late Rev. W. Jones, constructed an ÆEolian harp on an improved plan; the strings of which, instead of being on the outside, are fixed to a sounding-board within the box, and the wind is admitted to them through an horizontal aperture. In this form the instrument is portable and may be used in the open air. Several attempts to explain the principles of the wild harmony of this instrument have been made by different authors: of these Dr. Young's is considered as the most satisfactory, which is here subjoined. The particles of a current of air, which strike against the middle point of a stretched elastic string, will move the whole string from its rectilinear position; and as no breeze continues of the same strength for any considerable time; although it be sufficient to remove the string from its rectilineal position, yet, unless it be very rapid and violent, it will not be able to keep it bent; and consequently a vibration will ensue, and pulses in the air will be excited, which will produce the tone of the entire.string. But if the current of air be too strong and rapid, when the string is bent from the rectilineal position it will not be able to recover that position, but will continue bent, like the cordage of a ship in a brisk gale. However, though the whole string cannot perform its vibrations, the subordinate aliquot parts may, which will be of different lengths in different cases according to the rapidity of the brecze. Thus, when the velocity of the current of air increases so as to prevent the vibration of the whole string, those particles, which strike against the middle points of the halves of the string agitate those halves; and as these vibrate in half the time : that the whole string does, notwithstanding the breeze may be too rapid to admit of the vibration of the whole string, yet it can have no more effect in preventing the motion of the halves, than it would have on the whole were its tension quadruple; ; for the-times of vibrations of strings of different lengths, agreeing in other circumstances, are directly as the lengths; and in strings of different tensions, and agreeing in other circumstances, inversely as the square roots of the tensions (see CнопD): and therefore, their vibiations may become strong enough to excite such pulses as will affect the drum of the ear: and the same may be said of the other aliquot. divisions of the string. Those particles which strike against such points of the string as are not in the middle or aliquot parts, will:interrupt and counteract each other's vibration, as is the case of sympathetic and secondary tones, and therefore. will not produce a sensible effect. When Eolian notes are heard, which are not próduced by any submultiples of the string, they are very transitory, and im nediately vary thrir pitch, till they coincide with the Hotes the next above or below them, which are produced by the exact aliquot parts of the whole string: and thus the harmony is never interrupted by long-continued discords.
ERA, in Chronology, is the same as epoch, or epocha, and means a fixed point of time, from which to begin a computation of the years ensuing. The word is sometimes also written era in ancient authors. Its origin is contested, though it is commonly supposed that it had its rise in Spain, others say from the Arabs. Some imagine that it is formed from $a$. er. $a$. the abbreviations of the words, unnus erat Augusti, or from a. e. $r$. $a$. the initials of the words annus erat regni Augusti, because the Spaniards began their computation from the time that their country came under the dominion of Augustus. Others derive it from ass, brass, the tribute money with which Augustus taxed the world. It is also said that ara originally signified a number staniped on money to determine its current value; and that the ancients used as or cra as an article, as we do the word item, to each particular of an account; and hence it came to stand for a sum or number itself.

$E_{R A}$ also means the way or mode of accounting time. Thus we say, such a year of the Christiar æra, \&c.

Spanish $\mathbb{E}_{\mathrm{R}}$, otherwise called the year of Cæsar, was introduced after the second division of the Ruman provinces, between Augustus, Anthony, and Lepidus, in the 714 th year of Rome, the 4676 th year of the Julian period, and the 38 th year before Christ. In the 447 th year of this æra, the Alani, the Vandals, Suevi, \&c, entered Spain. It is frequently montioned in the Spanish aftairs; their councils, and other public acts, being all dated according to it. Some say it was abolished under Peter ir, king of Arragon, in the year of Christ 1358, and the Christian ara introduced instead of it. But Mariana observes that it cersed in the year of Christ 1383, under John I, king of Castile. The like was afterwards done in Portugal.

Christian Æen A.. It is. generally allowed by Chronologers, that: the computation of time from the birth of Christ, was only introduced.in the sixth century in the reign of Justinian; and it is commonly ascribed to Dionysius. Exiguus. "This æra came then into use in deeds, \&c; before which time, either the olympiads, or the year of Rome, or that of the reign of the emperors, was used for such purposes.

See un account of the other principal æras under the word Epoch.

AERIAL Perspective; is that which represents bodies diminished and weakened, in proportion to their distance from the eye.

Aërial Perspective chiefly relates to the colours of objects, whose force and lustre it díminishes more or less, to make them appear as if more or less remote. It is founded upon this, that the longer the column of air an object is seen through, the more feebly do the visual rays emitted from it affect:the.eye.

AEROGRAPHY; a description of the air, or atmosphere, its limits, dimensions, properties, \&c.

AEROLI'THS, or AEROLLTES, i. e. Air-stones, or stones from the atmosphere, are those compound kind of masses whicl sometimes fall from the atmosphere, in all countries, and which are therefore often called meteoric stones.-The descent of any such bodies was for a long time doubted, the popular opinion that attested the reality being mostly regarded as a vulgar prejudice; but the fact hasibeen often proved of late, in such a manner as to leave no reasonable doubt of the existence of the phiznomenon. Scc an account of many of these descents, 


\section{A E R}

[ 33

described in my paper on this subject, in the Abridg. Philos. Trans. vol. 6 , pa. 100, as well as several other accounts there referred to.

The following table, drawn up by M. Izaron, a philosopher who has paid considerable attention to the history

Substances fallen.
Shower of sulphur .........
Shower of stones ............
Shower of stones ...........
Shower of iron .............
Shower of Mercury........
A very large stone...........
Three large stones ..........
Mass of iron, 14 quintals...
Large stone, 26 (nlbs .........

A bout 1200 stones; one? $120 \mathrm{lbs}$. another of 6olbs. $\}$ Another, of 591 bs.............

Sulphurious rain ............

The same......................

Shower of viscid matter....

Stone of $72 \mathrm{lbs}$

Shower of fire $\ldots \ldots \ldots \ldots \ldots .$.

Shower of sand for 15 hours

Shower of sulphur...........

A stony mass .................

Shower of stones.............

Two stones, wt. 20lbs .......

Two do. 200 and 3001 bs ...

A stone of $7 \frac{1}{2}$ lbs ...........

A stone.......................

A stone.......................

Shower of stones ............

Extensive do. .

About 12 stane ..............

Large stone of $56 \mathrm{lbs}$

A stone of $10 \mathrm{lbs}$

A stone of zolbs

A stone of 20 bs

Shower of stones

Mass of iron, 70 cubic $f$ t....

Somestones, from 10 to 17 lbs

\section{Places where they fell.} Sodom and Gomorrah....... At Rome......................

At Rome.....................

In Lucania..................... In Italy ........................ Near the river Negos, Thrace In Thrace................... Abakaick, Siberia ............ Ensisheim, Upper Rhine... Near Padua, in Italy ........ Mount Vaiser, Provence ... Copenhagen... Duchy of Mansfeld............ Iceland...................... Macedonia........................... Quesnoy ......................... Atlantic Ocean................ Brunswick .................... Niort, Normandy ............. Near Tabor, Bohemia ....... Liponas in Bresse............. Near Verona ................. At Luce, in Le Maine...... Aire, in Artois ............... In Le Cotentin.

Near Roquefort .............. Environs of Agen............ Sienna, Tuscany ............. Wold-Cottage, Yorks. ...... In Portugal ................... Sales, near Ville-Franche... Sale, dept. of the IRhone... Benares, East Indies ....... A mirica

L'Aigle, Normandy ..........

To this list might be added inany more instances, and anong them several of more recent date. Of these masses, the larger kinds have been seen as luminous bodies moving with very great velocities, descending in oblique directions, commonly with a loud hissing noise, resembliug that of a mortar shell, or cannon ball, or rather that of an irregular hard mass, violently projected through the air; surrounded by a blaze or flame, tapering off to a narrow stream in the hinder part; they are heard ti) explode or burst, and even to fyy in pieces, the larger jarts going foremost, and the smaller following in succession: they are thus seen to full on the earth, striking it with great violence; and, on examining the place of the fall, the prarts are found scattered about, being still considerably warin, and mostly entered several inches deep in the earth. But their most remarkable character is, that they bave a perfect resemblance to one another. They are always found to be different from the neighbouring bodies, and show in cvery case the same appearance of senimetallic matter, coated on the outside with a thin black incrustation, and bearing strong marks of $1 \mathrm{e}$ Vot. $)$ of these bodies, exhibits a collection of some of the best authenticated instances of the falling of stones, and otler substances, from the atmosphere, that have been observed, with the times when they fell, and the authorities or names of the persons on whose evidence the facts rest.

\begin{tabular}{|c|c|}
\hline Times of their fall. & Testimonies. \\
\hline Year before J. C. $1898 \ldots \ldots$. & Moses \\
\hline Under Tullius Hostilius.... & Livy \\
\hline $\begin{array}{c}\text { Consuls C. Marti } \\
\text { Torquatus... }\end{array}$ & J. Obsequens \\
\hline Year bef. defeat of Crassus & Pliny \\
\hline (................... & Dion \\
\hline $2 \mathrm{~d}$ year of 78 th Olympiad & Pliny \\
\hline Year before J. C. $452, \ldots \ldots$. & Ch. of Count Marcellin \\
\hline Very old ...................... & Pallas, Chladni, \&c \\
\hline Nov. 7 th, 1492 & Butenschoen \\
\hline In $1510 \ldots \ldots \ldots$ & Cardin, Varait \\
\hline Nov. 27,1627 & Gassendi \\
\hline In $1646 \ldots \ldots \ldots$ & Olaus Wormius \\
\hline In $1658 \ldots \ldots \ldots$ & Spaugenberg \\
\hline In $1695 \ldots \ldots \ldots$ & Muschenbroeck \\
\hline January 1706 .. & Paul Lucas \\
\hline Jan. 4th, 1717 . & Geoffroy le Cadet \\
\hline April $6,1719 \ldots$ & Pere le Feuillée \\
\hline October $1721 \ldots$ & Siegesber \\
\hline In $1750 \ldots \ldots \ldots$ & Lalande \\
\hline July $3 \mathrm{~d}, 1753 \ldots$ & B. de Born \\
\hline September 1753 & Lalande \\
\hline In $1762 \ldots \ldots \ldots$ & Acad. de Bourd \\
\hline Sept. 13,1768 . & Bachelay \\
\hline In $1768 \ldots \ldots$ & Gurson de Boyàval \\
\hline In $1768 \ldots \ldots$ & Morand \\
\hline July $1789 . \ldots \ldots \ldots$. & Darcet, jun. Lomet, \&c \\
\hline July $24,1790 \ldots \ldots$ & St. Amand, Baudin, \&c \\
\hline July $1794 \ldots \ldots$ & Earl of Bristol \\
\hline Dec. $13,1795 \ldots$ & Capt. Topham \\
\hline Feb. $19,1796 \ldots \ldots$ & Southey \\
\hline March $12,1798 \ldots$ & De Drée \\
\hline March $17,1798 \ldots$ & Lelievre \& De Drée \\
\hline Dec. $19,1798 \ldots .$. & J. Lloyd Williams \\
\hline April 5, $1800 \ldots \ldots \ldots \ldots \ldots$ & Philos. Magazine \\
\hline April 20, $1803 . . . \ldots \ldots . . .$. & Fourcroy \\
\hline
\end{tabular}

cent fusion. Besides, several of these singular substances have been very carefully examined and analysed by Fourcroy, Howard, Bournois, Lavoisier, Vauquelin, and other eminent cliemists and naturalists in Europe; and it has been found that all the substances examined agree very nearly in nature and composition, having the same component materials, and in nearly the same proportions: thus, the stones examined by Count de Bournon and $\mathrm{Mr}$. Howard, were found to consist of four distinct substances, a peculiar martial pyrites, a number of globular and elliptical bolies, also of a peculiar nature, and an earthy cement surrounding the other component parts. The nature of the metallic particles was the same in all, bcing in each an alloy of iron and nikel. In the pyrites, nikc.l as well as iron was detected; and the easy decomposition of the pyrites, by muriatic acid, afforded a distinguishing character of that substance. 'The globules containcd mingnesia, silica, with oxides of nikel and iron. The eartliy. coment consisted of the same substances nearly in the same proportions. With regard to the proportions of these constituents, the celebrated stone which fell at linI. 
sisheim, in Alsace, in 1492, and those which fell at l'Aigle, in France, in 1803, were found to yield, by the analyses of Fourcroy and Vauquelin, the proportions following, viz, at

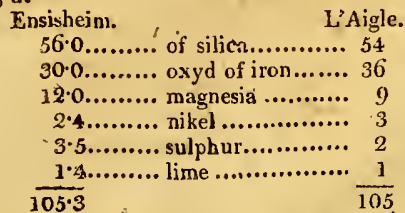

It likewise appears that the specific gravities of these bodies are nearly alike, and such as greatly to exceed all the known ordinary stones, and approach that of the metallic ores. Thus, the specific gravity of

the Ensisheim stone was 3233

Benares...................... 3352

Sienna...................... 3418

Gassendi's ................ 3456

Yorkshire ................. 3508

Bach

of such parts as that of water is 1000 .

These common and constant characters indicate, with strong evidence, a common origin. Yet, it is to be remarked, that iron is hardly ever found in the metallic state in terrestrial bodies : volcanic bodies only containing it in the state of oxyd. Nikel is also very rare, being never found on the surface of the earth. Whence it appears natural to conclude, that aerolites have an origin foreign to our globe, or at least that they are not produced by phænomena that have been commonly observed. Various hypotheses have therefore been devised to account for the origin of these stones. Some philosophers think that they are nothing else than small planets, that circulate in space after the manner of the other celestial bodies; and which, when found engaged in the earth's atmosphere, become there inflamed, by the resistance and friction they experience; that they lose little by little their velocity, and at length fall to the earth by their gravitation. Mr. King, Sir Wm. Hamilton, and others, consider these stones as concretions actually formed in the atmosphere: while others have inagined such phænomena as resulting from electricity. But, in my $\mathrm{Abridg}$. of the Philos. Trans. (vol 6, p. 100), 1 have shown not only that it is possible, but even bighly probable, that they may have been projected on the earth by lunar volcanoes. On subjecting this idea to calculation, it has been found that a projectile force only the triple of that sometimes given to cannon balls, would be sufficient to carry at butly out of the splice of the moon's influence, so that 11.. hirrestrial gravitation would draw it towards our planet. Nor is it at all improbable. that lunar volcanues might impress such a force on a body, since terrestrial voluanoes can communicate a much greater impulsion. This opinion acquire's a fresh degree of probability from the recent observations of $M$. Schroeter on the intun, with respect to thi great bright of some of the lunar volcanoes, and the frequent variations observed on the surfare of thit ghobe. 'Tlus, then, the probability of this origin of the aerolites appoinrs to be greatly in favour of this last opmion; though, in the present state of our knowledge of these substinces and of metrorology, t is not possible as yet to decide. For accutunts of the phanomena of many acrolites, the inquisitive reader may consult Mr. Howaru's valuable paper in the Plilos. Trans. for the year 1\$02, as well as my disscriation above referred to.

A EROLOGY, the doctrine or science of the air, and its phænomena, its properties, good and bad qualities, Sc. It is much the same with the foregoing word, Aerography.

ALROMETRY, Acrometria, the science of mensuring the air, its powers and properties; comprehending not only the quantity of the air itself, as a fluid body, but also its pressure or weight, its elasticity, rarefaction, condensation, \&c.

The term is not much used at present; this branch of natural philosophy being usually called pneunatics, which see. Wolfus, late professor of mathematics at Hail, having reduced several propertics of the air to geometrical demonstrations, first published at Leipsic his Elements of Aerometry, in the German language, and afterwards more enlarged in Latin, which have since been inserted in his Cursus Mathematicus, in five volumes in 4 to.

AERONAUTICA, the pretended art of sailing through the air, or atmosphere, in a vessel, sustained as a ship in the sea.

AEROSTATICA, is properly the doctrine of the weight, pressure, and balance of the air and atinosphere.

AEROSTATION, in its proper and primary sense, denotes the science of weights suspended in the air; but in the modern application of the tcrm, it signifies the art of navigating or floating in the air, both as to the practice and principles of it. Hence also the machines which are employed for this purpose, are called aerostats, or aerostatic machines; and which, on account of their form, are otherwise called air-balloons. Also aeronaut is the name given to the person who navigates or floats in the air by neans of such machines.

Principles of AEROSTATloN. The fundamental principles of this art have been long and generally known, as well as speculations on the theory of it; but the successful application of them to practice seems to be altogether a modern discovery. These principles chiefly respect the wright or pressure, and elasticity of the air, with its specific gravity, and that of the other bodies to be raised or floated in it: the particular detail of which principles may be seen under the respective words in this dictionary. Suffice it therefore in this place to observe, that any body which is specifically lighter than the atmosphere, will be buoyed up by it, and ascend, as wood, or a cork, or a blown bladder, ascends in water. And thus the body would continue to ascend to the top of the atmosphere, if it were every where of the same density as at the surface of the earth. But as the air is compressible and elastic, its density decreases continually in ascending, on account of the diminished pressure of the superincumbent air, at the higher clevations above the earth; and therefore the body will ascend only to that height where the air is of the saine spicific gravity with itself; there the body will float, and move along with the wind or current of air which it may meet with at that place. This body then is an acrostatic machine, of whatever form or nature it may be. And an air-balloon is a body of this kind, the whole inass of which, including its covering and contents, and the weights annexed to it, is of less weight than the same bulk of air in which it rises.

We know of no solid bodic's however that are light cnoughis thus to ascend and float in the atmosphere; and 
therefore recourse must be had to some fluid or aeriform substance.

Among these, that which is called hydrogen gas, or inflammable air, is the most proper of any that has hitherto been discovered. It is very elastic, and from 6 to 10 or 12 times lighter than common atinospheric air at the surface of the earth, according to the different methods of preparing it. If therefore a sufficient quantity of this gas be inclosed in a very thin bag or covering, the weight of the two together will be less than the weight of the same bulk of common air; and consequently this compound mass will rise in the atmosphere, and continue to ascend till it attain a height at which the atmosphere is of the same specific gravity as itself; where it will remain or float with the current of air, as long as the inflammable air does not escape through the pores of its covering. And this is an inflammable-ajr balloon.

Another way is to make use of common.air, rendered lighter by warming it, instead of the inflammable air. Heat, it is well known, rarefies and expands common air, and consequently lessens its specific gravity; and the diminution of its weight is proportional to the heat applicd. If therefore the air, inclosed in any kind of a bag or covering, be heated, and consequently dilated, to such a degree, that the excess of the wcight of an equal bulk of common air, above the weight of the hcated air, be greater than the weight of the covering and its appendages, the whole compound mass will ascend in the atmosphere, the same as in the former case; till, by the cooling and condensation of the included air, the balloon shall gradually contract and descend again, unless the heat is renewed or kept up. And such is a beated-air or fire balloon, otherwise called a Montgolfier, from its inventor.

Now it has been discovered, by various experiments, that one degree of heat, according to the scale of Fahrenheit's thermometer, expands the ait about one five-hundredth part; and therefore that it will require about 500 degrees, or nearer 484 degrees of heat, to expand the air to just double its bulk. Which is a degree of heat far above what it is practicable to give it on such occasions. And therefore, in this respect, common air heated, is much inferior to hydrogen gas.

But, before entering more particularly into this subject, it will be proper to give a short historical account of its rise and progress, with the various experiments and aerial voyages, that have been made of later years by divers aeronauts.

History of Aerostation. Various schemes for rising in the air, and passing through it, have been devised and attempted, both by the ancients and moderns, and that upon different principles, and with various success. Of these, some attempts have been upon mechanical principles, or by virtue of the powers of mechanism : and such are conceived to be the instunces related of the flying pi. geon made by Archytas, the Aying eagle and fly by Regiomontanus, and various others. Again, some projects have been forined, by attaching wings to some part of the human body, which were to be moved either by the hands or feet, by the help of mechanical powers; so that, striking the air with them, after the manner of the wings of a bird, the person might raise himself in the atmosphere, and transport himself through it, in imitation of that animal. But of these, and various other devices of the like naturr, a particular account will be given under the article artificial fying, as belonging rather to that species or principle of motion, than to our present subject of aerostation, which is properly the sailing or floating in the air by means of a machine rendered specitically lighter than that element, in imitation of aqueous navigation, or the sailing upon the water in a ship, or vessel, which is specifically lighter than the water.

The first rational account upon record, for this kind of sailing, is perhaps that of our countryman Roger Bacon, who died in the year 1292. He not only affirms that the art is practicable; but assures us that he himself knew how to make an engine, in which a man sitting might be able to carry himself through the air like a bird; and he farther affirms, that there was another person who had tried it with success. The secret it seems consisted in having a couple of large thin shells, or hollow globes, of copper, exhausted of air; so that the whole being thus rendered lighter than air, they would support a chair, in which a person might sit.

Bishop Wilkins too, who died in 1672, in several of his works, makes mention of similar jdeas being entertained by divers persons. "It is a pretty notion to this purpose, says he, (in his Discovery of a New IVorld, prop. 14), mentioned by Albertus de Saxonia, and out of him by Francis Mendoza, that the air is in some part of it navigable. And that upon this statick principle, any brass or iron vessel (suppose a kettle), whose substance is much heavier than that of the water; yet being filled with the lighter air, it will swim upon it, and not sink." And again, in his Dedalus, chap. 6, "Scaliger conceives the framing of such volant automata to be very easy. Volantis columbx maclinulam, cujus autorem Archytam tradunt, vel facillime profiteri audco. Those ancient motions were thought to be contrived by the force of some included air: So Gellius, Ita erat scilicet libramentis suspensum, et aura spiritus inclusa, atque occulta consitum, \&c. . As if there had been some lamp, or other fire within it, which might produce such a forcible rarefaction, as should give a motion to the whole frame." From which it would seem that Bishop Wilkins had some confused notion of sucl a thing as a heated-air balloon.

Again, F. Francisco Lana, in his Prodroma, printed in 1670 , proposes the stume method with that of Roger Bacon, as his own thought. He considered that a hollow vessel, exhausted of air, would weigh less than when filled with that fluid; he also reasoned that, as the capacity of spherical vessels increases much faster than their surficc, if there were two spherical vessels, of which the diameter of one is double the diameter of the other; then the capacity of the former will be equal to 8 times the capacity of the latter, but the surface of that only equal to 4 times the surface of this: and if the one sphere have its diameter equal to triple the diameter of the other, then the capacity of the greater will be equal to 27 times the capacity of the less, while its surface is only 9 times greater : and so on, the capacities increasing as the cubes of the diameters, while the surfaces increase only as the squares of the same. And from this mathematical principle, father Lana deduces, that it is possible to make a spherical vessel of any given matter, and thickness, and of such a size as, when emptied of air, it will be lighter than an equal bulk of that air, and consequently that it will ascend in that element, together with some additional weight attached to it. After stating these principles, father Lana computes that a round vessel of plate brass, 14 feet in diameter, weighing 3 ounces the square foot, will 
only weigh 1848 ounces; whereas a quantity of air of the sane bulk will weigb $2155 \frac{2}{3}$ ounces, allowing only one ounce to the cubic foot; so that the glube will not only ascend in the air, but will also carry up a weight of $307 \frac{2}{3}$ ounces: and by increasing the bulk of the globe, withont increasing the thickness of the metal, he adds, a vessel might be made to carry a much greater weight.

Such then were the ingenious speculations of learned men, and the gradual approaches towards the perfection of this art. But one thing more was yet wanted: though acquainted in some degree with the weight of any quantity of air, considered as a detached substance, it seems they were not aware of its great elasticity, and the universal pressure of the atmosphere; by which pressure, a globe of the dimensions above-described, and exbausted of its air, would immediately be crushed inwards, for want of the equivalent internal counter pressure, to be sought for in some element, much lighter than common air, and yet nearly of equal pressure or elasticity with it; a property or circumstance attending inflammable gas, and also common air when considerably heated.

It is evident then, that the schemes of ingenious men hitherto must have gone no farther than mere speculation; otherwise they could never have recorded fancies, which, on the first attempt to put in practice, must have manifested their own insufficiency, by an immediate failure of success. For, instead of exhausting the vessel, it must either be filled with common air heated, or with some other equally elastic and lighter fluid. So that upon the whole it appears, that the art of traversing the air, is an invention of our own time; and the whole history of it is comprehended within a very short period:

Mr. Cavendish was the first who discovered with any degree of accuracy the specific gravity of inflammable air; and his experiments and observations upon it, are published in the 56th volume of the Philosophical Transactions for the year 1766 .

Soon after this discovery of Mr. Cavendish, it occurred to the ingenious Dr. Black of Edinburgh, that if a bladder, or other vessel, sufficiently light and thin, were filled with this air, it would form altogether a mass lighter than the sime bulk of atmospheric air, and consequently that it would ascend in it. This idea he mentioned in his chemical lectures in the year 1767 or 1768 ; and he further proposed to exhibit the experiment, by filling the allantois of a calf with such air. The allantois, however, was not prepared just at the time when he was at that part of his lectures, and other avocations afterwards preveuted his design: so that, considering it only as an anusing experiment, and being fully satisfied of the trutb of so evident an cffect, he contented hinself with barely mentioning the experiment from time to time in his lectures.

Abont the year 1777 or 1778 it also occurred to Mr. Cavallo, that it might be possible to construct a vessel, which, when filled with inflammable air, would ascend in the atmosphere: and there is no doubt but that similar ideas occurred to many otluer persons, of so cvident a consequence of Mr. Cavendish's discovery. But it serms to have been Mr. Cavallo who first actually attempterl the experiment, in which however he succeded no lurther than in being able to raise soap bubbles of two or thres inches dianeter: a thing which had been done by children for their amisement from time immemorial. These experiments Ar. Cavallo made in the beginning of the year 1782, and an account of them was read at a public meeting of the Royal Socicty on the 20 th of June of that year. From which it apptars that he cricid blad. ders and paper of various kinds. But the bladders, how ever thin they were made by scraping, \& c, were stlll found too heavy to ascend in the atmospluere, when sully inflated with the inflammable air: and in using China paper, be found that this arr passed through its pores, like water throughe a sieve. So that, having falled of success by blowng the same air into a thick solution of gum, thich varniblirs, and oil paint, he was obliged to rest satisfied with soap-balls or bubble's, which, being filled with inflanmable air, by dipping the end of a small glass tube, connected with a bladder containing the air, into a thick soluition of soap, and gently comprissing the bladder, ascended rapiclly, and broke against the ceiling of the roon.

Here, however, it seems the matter might have restid, had it not bcen for experiments made in France soon after, by the two brothers Stephen and Joseph Montgolfier, on principles suggested, not by the levity of inflammable air, which probably they had never heard of, but by that of smoke ancl clouds ascending in the atmosphere. These two brothers it secms were natives of Amnonay, a town in the Vivarais, about 36 miles from Lyons; and that in their youth, Stephen, the elder, had assictuously studied the mathenatics, but the other had applicil himself more particularly to natural pbilosophy and chemistry. They were not intended for any particular way of business, but the death of a brother obliged them to put themselves at the head of a considerable paper mantifactory at Annonay. In the intervals of time allowed by their business they amused themselves in seicral'philosophical pursuits, and particularly with the experiments in aerostation, of which we are now to give some account. It would be perhaps impossible to know all the particular steps and ideas which finally produced this discorery: but it has been saicl that the real principle, on which the effect of the aerostatic machine depends, was unknown cren for a considerable time after its discovery: that Montgolfier attributed the effect of the machine, not to the rarefaction of the air, which is the true cause, but to a certain gas, specifically lighter than common air, which was supposed to be developed trom hurning subitances, and which was commonly called Montgolfier's gas. Be this however as it may, it is well known that the two brothers began to think of the experiment of the acrostatic macline about the midrle or the latter part of the jear 1782 . The natural ascension of the suoke and the clouds in the atunosphere, suggested the first idea; and to imitate those bodies, or to inclose a cloud in a bag, and let the latter be lifted up by the buoyancy of the former, was the first project of those gentlemen.

Accordingly, the first experiment was made at A vignon by Stephen, about the midale of November 1782. Hiving prepared a bag of fine silk, in the shape of a parallalopipedon, and of about 40 cubic fect in capucity, he applied burning paper to an aperture in the bottom, which rarefied the air, and thus lormed a kind of clotid in the bag ; and when it became sufficiently expanded, it ascended rapidly to the ceiling.

Soon afterwards the experiment was repeated with the same machine at Annonay, by the two brothers, in the open air; when the bag ascended to the height of about 70 feet. Encouraged by this success, they constructerl another machine, of abous 650 cubic feet capacity; which, when indated as belurí, broke the cords which 
confined it, and after ascending rapidly to the beight of about 600 feet, descended and fell on the adjoining ground. With another larger machine, of 37 feet diameter, they repeated the experiment on the 25th of April, which answered exceedingly well : the machine had such force of ascension, that, breaking abruptly from its confincment of 1 opes, it rose to the height of more than 1000 feet, and then, being carried by the wind, descended and fell at a place about three quarters of a mile from the place of its ascension. The capacity of this machine, was equal to above 23 thousand cubic feet, and, being nearly globular, when inflated, it meąsured 117 English feet in circunference. The covering was formed of linen. lined with paper; and its aperture at the bottom was fixed to a wooden frame, of about 4 feet square, or 16 feet in surfacc. When filled with vapour, which it was conjectured might be about half as heavy as common air, it was capable of lifting up about 490 pounds, besides its own weight, which, together wiil that of the wooden frame, was eynal to about 500 pounds. With this machine the next experiment was publicly performed at Annonay, on the 5th of "June 1783 , before a great multitude of spectators. The flaccid bag wis suspended on a pole 35 feet high; straw and chopped wool were burned under the opening at the bottom; the vaponr, or rather smoke, soon inflated the bag, so as to distend it in all its parts; and this enrormous mass ascended in the air with such velocity, that in less than ten minutes it reached the height of above 6 thousand fect; when a breeze carricd it in an horizontal ditection to the distance of near a mile and a half, where it descended gently to the ground.

As soun as the nows of this experiment reached Paris, the philusophers of that cit!, concriving that a new species of gas, of ahout half the weight of common air, had heen discovered by Messrs. Montgolfier; and knowing that the weight of inflammable air was but aocut the 8 or 10th part of the weight of common air, they justly concluiled that intlammable air would answer the purpose of this experiment better than the gas of Montgolfier, and accordingly they resolved to make trial of it.

A subscription was opened by M. Faujas de St. Fond, towards defraying the expense of the experiment. A sufficient sum of money baving soon been raiscd, Messrs. Roberts were appointed to construct the machine, and M. Charles, professor of experimental philosophy, to superintend the work. After a consicierable time spent, and surmounting many difficultics in obtaining a sufficient quantity of this gas, and searching out a substance light cnough for the coverin:, they at length constructed a globe of the silk called lutestring, which was rendered impervious to the inclosed air by a varnish of elastic gum or caeutchouc, dissolved in some kind of spirit or essential oil. The diameter of this globe was about $13 \mathrm{fret}$; and it had only one aperture, like a bladder, to which a stopcock was arlapted: the weight of this covering, when empty, togetlier with that of the stop cock, was 25 pounds.

On the 231 of August 1783, they began to fill the globe; but this, being their first attempt, was attended with many obstructions and disappointments, which took up two or three days to overcome. At length, however, it was prepared for exhibition, and on the 27 th it was carried to the Champs de Mars, a spacious open ground in the front of the Military School, where, after introducing some more infammable air, and disengaging it from the cords by which it was held down, it rose, in less than two minutes, to the height of 3123 lect : the weight of the balloon, when it went up, being 35 pounds less than that of an equal bulk of common air. Aiter floating in the air for about three quarters of an hour, it fell in a field at 15 miles from the place of its ascent ; where, as we may easily imagine, it occasioned great a mazemrent to the peasants who found it. Its fall was owing to a rent in the covering, probably occasioned by the superior elasticity of the inflammable air, over that of the rare part of the atmosphere to which it had ascended.

In consequence of this brilliant experiment, numberless snall balloons were made, mustly of gold-beater's skin, from 6 and 9 to 18 or 20 inche dianeter; their cheapness putting it in the power of almost every family to satisfy its curiosity relative to the new experiment; and in a f'w days balloons were seen flymg all about $\mathrm{Pa}$ ris, whence the'y were soon after sent abroad.

M. Joseph Montgulfier repeitled an experiment witl a machine of his construction before the commissaries of the Academy of Sciences, on the 11th and 12th of September. The machine was about 74 feet high, and 43 feet in diameter; it was made of canvass, covered with paper both within and without, and weighed 1000 pounds. It was filled with rarefied air in 9 minutes, and in one trial "the weight of $S$ men was not sufficient to keep it down. It was not suffered to go up, as it had been intended for exhibition before the Royal Family, a fow days after. By the violence of the rain, however, which fell about this time, it was so much spoiled, that he thought proper to construct another for that purpose, in which he uscd so great di-patch, that it was completed in the short space of four days. This machine was constructed of cloth made of linen and cotton thread, and painted with water colours both within and without. lis height was 60 feet, and diameter 43 feet. Having made the necessary preparation for inflating it, the operation was begun about one o'clock on the igth of the same month, before the king and queen, the court, and the inhabitants of the place, as well as all the Parisians who conld procure a conveyance to Versailles. The balloun was soon filled, and in 11 minutes after the commencement of the operation, the ropes being cut, it ascended, bearing up with it a wicker cage, containing a cock, a duck, and a shcep, the first animals that ever ascencled into the atmosphere with ari aernstatic machine. Its power of ascension, er the weight by which it was lighter than an equal bulk of common air, allowing for the animals and their cage, was 696 pounds. The ballonn rose to the height of $1+40$ feet; and being driven hy the wind for about 8 minutes, it gradually descrinded, in consequence of two large rents made in the covering by the wind, and fill in a wood at the distance of about two mile's from Versailles. The animals landed again as safe as when they went up, and the sheep was found feeding.

The success of this experiment induced M. Pilatre de Rozier, with a philosophical intrepidity which will be recorded with applause in the history of acrostation, to offer himself as the first adventurer in this aerial navigation. For this purpose, M. Montgolfier constructed a new machine, of an oval shape, in a garden of the fauxbourg St. Antoine; its diameter being about 48 feet, and height 74 feet. To the aperture in the lower part was annexed a wicker gallery about threc feet broad, with a ballustrade of three feet bigh. From the middle of the aperture an 
iron grute, or urazier, was suspended by chains, descendilys from the sides of the machine, in which a fire was lighted for inflating the machine; and towards the aperture port-holes were opened in the gallery, through which ary person, who might venture to ascend, might feed the fire on the grate with fucl, and regulate at pleasure the dilatation of the air inclosed in the machine: the weight of the whole being upwards of 1600 pounds. On the 15 th of October 1783, the fire being lighted, and the balloon inflated, M. P. de Rozier placed himself in the gallery, and, to the astouishment of a multitude of spectators, ascended as high as the lenth of the restraining cords would permit, which was about 84 feet from the ground, and there kept the machine afloat near 5 minutes, by repeatedly throwing straw and wool upon the fire: the machine then descended gradually and gently, through a medium of increasing density, to the ground; and the intrcpid adventurer assured the admiring spectators that he had not $\mathrm{ex}$ perienced the least inconvenience in this aerial excursion. This experiment was repcated on the 17 th with nearly the same success; and again several times on the 19 th, when M. Rozier, by a partial ascent and descent, several times repeated, evinced to the multitude of observers, that the machine may be made to ascend and descend at the pleasure of the aeronaut, by merely increasing or diminishing the fire in the grate. The balloon having been hauled down, by the ropes which always confined it, M. Gironde de Villette placed himself in the gallery opposite to $M$. Rozier, and the machine being suffered to ascend, it hovered for about 9 minutes over Paris, in the sight of all its inhabitants, at the height of 330 feet. And on their descending, the marquis of Arlandes ascended with M. Rozier much in the same manner.

In consequence of the report of these experiments, signed by the commissaries of the Academy of Sciences, it was ordered that the annual prize of 600 livres should be given to Messrs. Montgolfier for the year 1783 .

In the experiments above-recited, the machine was always secured by long ropes, to prevent its entire escape : but they were soon succeeded by unconfined aerial navigation. For this purpose, the same balloon, of 74 feet in height, was conveyed to $\mathrm{La}$ Muette, a royal palace in the Bois de Boulugne: and all things being ready, on the 21 st of November 1783, M. Rozier and the marquis d'Arlandes took their post in opposite sides of the gallery, and at $2 n$ 'clock the machine was abandoned to the element, when it ascended calmly and majestically in the atmosphere. On reaching the height of about 280 feet the intrepid atronauts waved their hats to the astonished multitude: but they soon after rose too high to be distinguished, their greatest height being estimated at 3000 feet. At first they were drivel, by a north-west wind, horizontally over the river Seine and part of Paris, taking care to clear the steeples and high buildings by increasing the fire; and in rising they met with a current of air which carried them southward. Having thus passed the Boulevard, and finally desisting from supplying the fire with fuel, they descended very gently in a field beyond the new Boulevard, a little nore than 5 miles from the palace de La Muette, having been between 20 and 25 minutes in the air. The wight of the whole apparatus, including that of the two travelters, was between 1600 and 1700 prounds.

Notwitlistanding the rapid progress of acrostation in France, it is remarkable that we have no authentic account of any experiments of this kind being attempted in other countries. Even in our own island, whare all arts and sciences find an indulgent nursery, and many their birth, no aerostatic machine was seen before the month of November 1783 . Various speculations have been made on the reasons of this strange neglect of so novel and brilliant an experiment. But none seemed to carry auy show of probability except that it was said to be discouraged by the leader of a philosophical society, expressly instituted for the improvement of natural knowledge, for the reason, as it was said, that it was the discovery of a neighbouring nation. Be this however as it may, it is a fact that the first aerostatic experiment was exhibited in Fingland by a foreigner unconnected and unsupported. This was a count Zambeccari, an ingenious Italian, who bappened to be in London about that time. He made a balloon of oiled-silk, 10 feet in diameter, weighing only 11 pounds : it was gilt, both for ornament, and to render it more impermeable to the inflammable air with which it was to be filled. The balloon, after having been publicly shown for several days in London, was carried to the Artillery Ground, and there being about three-quarters filled with inflammable air, and having a direction inclosed in a tin box for any person by whom it should afterwards be found, it was launched about one o'clock on the 25th of November 1783. At lialf paist 3 it was taken up near Petworth in Sussex, 48 miles distant from London; so that it travelled at the rate of near 20 miles an hour. Its descent was occasioned by a rent in the silk, the effect of the rarefaction of the inflammable air, when the balloon ascended to a rarer part of the atmosphere.

The French philosophers having executed the first aerial voyage with a balloon inflated by heated air, resolved to attempt a similar voyage with a balloon filled with inflammable air, which seemed to be preferable to dilated air in every respect, the expense of preparing it only excepted. A subscription was opened however to defray that expense, which was estimated at about ten thousand livres; and the balloon was constructed by Messrs. Roberts, of gores of silk, varnished with a solution of elastic gum. Its form was spherical, and it me'asured $27 \frac{1}{2}$ feet in diameter. The upper hemisphere was covered by a net, which was fastened to a hoop encircling its middle, and called its equator. To this equator was suspended by ropes a car or boat, covered with painted linen, and beautifully ornamented, which swung a few fect below the balloon. To prevent the bursting of the machine by the expansion of the inflammable air in a rarer medium, or to cause the balloon to descend, it was furnished with a valve, which might be opened by means of a string descending from it, for discharging a part of the internal air, without admitting the external to enter: And the car was ballasted with bags of sand, for the purpose of lightening it occasionally, and causing it to ascend: so that by letting some of the air, cscape through the valve, they might descend; and by discharging some of their sand ballast, ascend. Th this balloon was likewise annexed a long pipe by which it was filled. The apparatus for this purpose consisted of several casks placed round a large tub of water, each having a long tin iube, that terminated under a vessel or fumel, which was inverted into the water of the tub, and communicated with the long pipe anmexed to the lower part of the balloon. Iron filings and diluted vitriolic acid being put into the casks, the inflammable air which was produced from these materials, passed through the tin tubes, thence through the 
water of the tubs to the inverted funnel, and so through the pipe into the balloon. When inflated, the weight of the common air which was cqual in bulk to the balloon; uas $771 \frac{1}{2}$ pounds; also the power of ascension, or weight just necessary to keep it from ascending, was 20 pounds, and weight of the balloon, with its car, passengers, and all its appendages, was $604 \frac{1}{2}$ pounds, making in all $624 \frac{1}{2}$ pounds: and this talven from $771 \frac{\mathrm{T}}{2}$ pounds, the weight of common air displaced, leaves 147 pounds for the weight of the inflammable air contained in the balloon, and which is to $771 \frac{x}{2}$ pounds, the weight of the same bulk of common air, nearly as 1 to $5 \frac{x}{4}$; that is, the inflammable air used in this experiment was $5 \frac{x}{4}$ times lighter than commoń air.

The first of December was fixed on for the display of this grand experiment; and every preparation was made for conducting it with advantage. The garden of the Thuilleries at Paris was the scene of operation; which was soon crowded and encompassed with a prodigious multitude of observers. Signals were given, from time to time, by the firing of cannon, waving of flags, \& $\mathrm{c}$ : and a small montgolfier was launched, for showing the direction of the wind, and for the amusement of the people previous to the general display. At a quarter betore 1 o'clock, M. Charles and one of the Roberts, having seated themselves in the boat attached to the balloon, and beng furnished with proper instruments, cloathing, and provisions, left the ground, and ascended with a moderate velocity to the beight of about 600 yards; the surrounding multitude stauding silent with lear and amazement ; while the aerial navigators at this height made signals of their safety. When they.left the ground, the thermometer, according to Fahrenheit's scale stood at $59^{\circ}$; and the barometer, at 30.18 inches: at the urmost height to which they ascended, the barometer fell to 27 inches; from which they deduced their height as above, tō be 600 yards, or one third part of a mile. During the rest of the voyage the quicksilver in the barometer was generally between 27 and 27.65 inches, rising and falling, as part of the ballast was thr wn out, or some of the inflammable air escaped from the balloon. The thermometer generally stood between 53 and 57 degrees. Soon after their ascent, they remained stationary for some time: they then moved horizontally in a direction N.N.W.: and having crossed the Seine, and passed over several towns and villages, to the great amazement of the inhabitants, they descended in a field, about 27 miles frum Paris, at threequarters past 3 o'clock; so that they had travelled at the rate of near 15 miles an hour, without feeling the least inconvenience.

The balloon still containing a cousiderable quantity of inflammable air, M. Charle's re-ascended alone, and it was computed he went to the height of 3100 yards, or near 2 miles, the barometer being then at 20 English inches : having amused himsclf in the air about $33 \mathrm{mi}-$ nutes, he pulled the string of the valve, and descended at 3 miles distance from the place of his ascent. All the inconvenience he experienced in his great elevation, was a dry sharp cold, with a pain in one of his cars and a part of his face, which he ascribed to the dilatation of the internal air: a circumstance that usually happens to persons "ho suddenly change the density of their atmosphere, either by ascending into a 1 arer, or descending into a denser one The small ballown, laumcled at the beginning by M. Montgoltier, was found to have moved in a direction opposite to that of the aeronauts; from which it is uiffrred that there were two currents of air at different heights above the earth.

In the month of December this year, several experiments, were made at Philadelphia in America with airballoons, by Messrs. Rittenhouse and Hopkins. They constructed and filled a great many small balloons, and connected them together; by which a man went up several times, and was drawn down aguin; and finally, the ropes being cat, he ascended to the height of 100 feet, and floated to a considerable distance; but, being afraid, he cut open the balloons with a knife, and so descended.

About the close of this year small balloons were sent up in many places, and were become very common in soine parts of France and Fingiand. In the beginning of the year following, their number and magnitude increased considerably. Some of the more remarkable ones were as follow :-On the 19tls of January M. Joseph Montgolfier, accompanied by six other persons, ascended from Lyons with a rarefied-air balloon, to the height of 1000 yards. This was the largest machine that had been hitherto made, being 131 feet ligh, and 104 feet in diameter: it was formed of a double covering of linen, with three layers of paper between them; and it weighed, when it went up, 1600 pounds, including the gallery, passengers, \&c. It was at first intended for six passengers; but before it went up, it was not judged safe to freisht it with more than three: bowever, no authority nor solicitations could prevail upon any of the six to quit their place, nor even to cast lots which three should resign their pretensions: so that the spectators saw them all ascend with terror and anxiety; and to add to their distress, when the ropes were cut, and the machine had ascended a foot or two from the ground, a scventh person suddenly leaped into the gallery, and the fire being increased, the whole ascended together. To add to the terror of the scene, after being in the air about 15 minutes, a large rent of about 50 feet in length was made by the balloon taking fire, in consequence of which it descemded very rinpidly to the ground, thougl fortunately withont injury to any of the aeronauts.

On the 22d of February an inflammable-air balloon was launched from Sandwich in Kent. It was but a small one, being only 5 feet in diameter; but it was rendered remarkable by being the first machine that crossed the sea from England to France. It was found in a field at Warneton, about 9 miles from Lisle in French Flanders, two hours and a half after it left Sandwich, the distance being about 74 miles; so that it floated at the rate of about 30 miles an hour.

The chevalier Paul Andreani, of Milan, was the first aerial traveller in ltaly. The chevalier himself was at the sole expense of this machine, but was assisted in the construction by two brothers of the name of Gerli. They all three ascended together near Milan on the 25th of February, and remained in the atmosphere about $20 \mathrm{mi}-$ nutes, when they descended, all their fuel being exhausted. This machine was a montgolfier, of a spherical shape, and about $6 \mathrm{~s}$ feet in diameter. From calculations made on the power of this, and other machines of the same sort, it appeass that the included air is rarefied commonly but about one-third, or that the included warm air weighs about two thirds of the same bulk of the cxternal or common air.

The next aerial voyage was performed on the $2 \mathrm{~d}$ of 
March 1784 , by M. Jean Pierre Blanchard, a man who has since that time made more voyages than any other person, except M. Garnerin, and who has attained great celebrity by being the first who has floated in the air over the channel froin England to France. M. Blanchard it seens had for many years heen in pursuit of mechanical means for flying through the air; but on hearing of the Jate invented air-balloons, he dropped his former pursuits, and turned his attention to them. He accordingly constructed a balloon of 27 fect diameter, to which a boat was suspended, with two wings, and a rudder to steer it by, as also a large parachute spread horizontally between the boat and the balloon, designed to check the fall in case the balloon should burst. The machine being filled with inflammable air, he ascended, from the Charnps de Mars at Paris, to the height of near 2 niles; and after loating in the air for an hour and a quarter, he descended at Billancourt, near Seve, having experienced by turns heat, cold, hunger, and an excessive slrowsiness. It appears from his own account, and as might have been expected, that the wings and rudder of his boat had little or no power in turning the balloon from the direction of the wind.

In the course of this year, 1784, acrostatic experiments and aerial voyages became so frequent, that the limits of this article will not allow of any thing farther than mentioning those that were attended with any remarkable circumstances. On the 25th of April, Messrs. de Morseau and Bertrand ascended from Dijon, with an inflammable-air balloon, to the beight of near 2 miles and a half, where the thermometer marked $25^{\circ}$. They were in the air an hour and 25 minutes, in which time they floated 18 miles.

On the 20th of May, four ladies and a gentleman ascended from Paris, in a large montgolfier, above the highest buildings, and remained suspended there a considerable time, the balloon being confined by ropes from flying away.

On the 23rl of May, M. Blanchard, with the same balloon as before, ascended from Rouen, to such height that the mercury in the barometer stuod at 20.57 inches, which on the earth had been at $30 \cdot 16$. It was observed that in this voyage M. Blanchard's wings or oars could not turn him aside from the direction of the wind.

On the 4th of June M. Fleurant and Madame Thible, the first lady who made an aerial voyage, ascended at Lyons in a machine of 70 feet diameter. They went to the height of 8500 ) fect, and floated about 2 iniles in 45 minute's.

On the 14th of Juue, M. Constard de Massi and M. Nolichet ascended at Nantes to a great height, with a balloon of 32 feet cliamet.r, filled with inflimmable air extracted from zinc; and they floated to the distance of 27 miles in 58 uinutes.

On the asd of June, the first ascrial traveller \$1. Rozier, arcompanied with M. Prouts, atcended at Versulles, in tive presence of the royal fanily and the king of Sweden, with at large montgutlier, whose diameter was 79 fret, and its height 91 fret ind a halt. Thicy thonted to the distance of 36 miles in three-quarters of an hour, when they descenderd, which is at the rate of 48 miles an hour. In consiquarice of this experiment, the king granted to $\mathbf{M}$. de Regzicr a pension of 2000 livres.

On the 15 th of July the duke of Chartres, the two brothers Roberts, and a fourth persou, ascencied from the park of St. Cloud, with an inftammable-air machine, of an oblong form, its diameter being 34 feet, and its length, which went in a direction parallel to the horizon, was $55 \frac{7}{2}$ feet: they remained in the atmosphere 45 minntes, in the greatest fear and danger. The machine contained an interior small balloon, filled with conmon air, by means of which it was proposed to cause the machine to ascend or descend without the loss of any inflammable air or ballast : and the boat was furnished with a helm and oars, which were intended to guide it. Three minutes after ascending, the machine was lost in the clouds, and involved in a dense vapour. A violent agitation of the air, resembling a whirlwind, greatly alarmed the aeronauts, turned the machine three times very rapidly, and gave it such shocks as prevented them from using any of their instruments for managing the machine. After many struggles, with great difficulty they tore about 7 or 8 feet of the lower part of the covering, by which the inflammable air escaped, and they descended to the ground with great rapidity, though without any hurt. At the place of departure the barometer stood at 30.12 inches, and at their greatest elcvation it descended to $24 \cdot 36$; so that their ascent was nearly a mile.

On the 18 th of July, M. Blanchard, with a Mr. Boley, made his tbird voyage with the same balloon as he had before, and rose so high as to sink the barometer from $30^{\circ} 1$ to 25.34 inches, answering to a height of about 4600 feet. In 2 hours and a quarter they Hoated 45 miles, which is at the rate of 20 miles an hour. In this voyage M. Blanchard thought that he was able to turn the machine with his wings, and to make it ascend and descend at pleasure. After descending, it is said the balloon remained all the night at anchor full of air ; and that the next day several ladies amused themselves by ascending successively to the height of 80 feet, the lengtb of the ropes by which it was anchored.

In the cuurse of this summer fwo persons bad nearly lost their lives by ascending with machines of heated air. The one in Spain, on the 5 th of June, by the machine taking fire, was much burnt, and so hurt by the fall that his life was long despaired of. The latter liaving ascended a few feet, the machinery entangled under the eaves of a house, which broke the ropes, and the man fell about twenty feet: the machine presently took firc, and was consumed. 'Other montgolfiers were also burned about London, by taking fire, through the defects of their construction.

The first aerial voyage performed in England was by one Vincent Lunarli, a native of Italy, who ascended from the Artillery Ground, London, wish an inftumınable-air balloon on 15 th of September. His machine was made of oiled silk, painted in alternate stripes of bluc and red; and jts diameter was 33 feet. From n iset, which covered about two thirds of the balloon, 45 cords descended to at hoop hanging below it, to which the gallery was attached. The machine had no valve; and jts weck, which terminated in the form of a pear, was the aperture through which the inflammable air was introluced, and through which it might be let out. The balloon was filted with air produced from zinc by means of clituted vitriolic acid. And when the acronatut departed at 2 o'clock he took up with him a dog, a cat, and a pigeon. After throwing out some sand, to clear the houses, he ascenfled to a considerable beight ; and the direction of his motion at first was north-west by west; but as the balloon rose higlier, it came into another current of air, which curried 
it nearly north. In the course of his voyage the thermometer was as low as $29^{\circ}$, and the drops of water which had collected round the balloon were frozen. About half after 3 he descended very near the ground, and landed the cat, which was almost dead with cold: then rising, he prosecuted his voyage, till at 10 minutes past 4. o'clock he landed near Ware in Hertfordshire. He ascribes his descent to the action of his oars; but as he was under the necessity of throwing out ballast in order to reascend, his descent was more probably occasioned by the loss of inflammable air.

The longest and most interesting voyage performed about this time, was that of Messrs. Roberts and M. Colin Hullin, who ascended at Paris, at noon on the 19th of September, with a ballonn, filled with infammable air, which was $27 \frac{3}{4}$ feet in diameter, and $46 \frac{3}{4}$ feet long, the machine being made to float with its longest part parallel to the horizon, and having a boat of near 17 feet long attached to it. The boat was fitted up with several wings or oars, shaped like an umbrella; and they ascended at 12 o'clock with 450 pounds of sand ballast, and after'various manœurres finally descended, at 40 minutes past 6 o'clock, near Arras in Artois, 150 miles from Paris, having still 200 pounds of ballast renaining in the boat. In one part they found the current of air uniform from 600 to 4200 feet high, which it seems was their greatcst height, and the fall of the barometer had been near $5 \cdot 6 \mathrm{de}$ grees. They found that by means of their oars they could accelerate their course a little in the direction of the wind, when it moved slowly, whicb may be true; but there is great reason to doubt of the accuracy of their experiments by which they pretended to cause their path to deviate about 22 degrees from the wind, going with a considerable velocity.

The second aerial voyage in England, was performed by Mr. Blanchard, and MIr. Sheldon, professor of anatomy to the Royal Academy, being the first Englishman who ascended with an aerostatic nacline. They ascended at Chelsea the 16 th of October, at 9 minutes past 12 o'clock. Mr. Blanchard having landed Mr. Sheldon at about 14 miles from Chelsca, re-ascended alone, and finally landed near Rumsey in Hampshire, about 75 miles from London, having gone nearly at the rate of 20 miles an hour. The wings used on this occasion it seems produeed no deviation from the direction of the wind. MIr. Blanchard said that he ascended so high as to feel a great difficulty of breathing: and that a pigeon, which flew away from the boat, laboured for some time to sustain itself with its wings in the rarefeed air, but after wandering a good while, returned, and rested on the side of the boat.

On the 4th of October, Mr. Sadler, an ingenious tradesman at Oxford, ascended at that place with an inflammab]e-air balloon, of his own construction and filling. And again on the 12th of the same month he ascended at $\mathrm{Ox}$ ford, and floated to the distance of 14 miles in 17 minutes, which is at the ratc of near 50 miles an hour.

The 30th of November this year Mr. Blanchard's fifth aerial voyage, still with his old inachine, was performed in company with Dr. J. Jeffries, a native of America. Their voyage was about 21 miles; and it does not appeart hat the greatest action of their oars produced any effect in directing the course of the balloon.

On the 4th of January, 1785, a Mr. Harper ascended at Birmingham with an inflammable-iir balloon, and went to the distance of 50 miles in an hour and a quarter: he Vou. 1. suffered no other inconvenience than a temporary deafuess, and what might be expected from the changes of wet and cold. The thermometer descended from 40 to 28 degrees.

On the 7 th of January, Mr. Blanchard, accompanied by $D r$. Jefiries, performed his sixth aerial voyage, by actually crossing the British chanuel from Dover to Calais, with the same balloon which had five times before carried him successfully through the air. They ascended with only 30 pounds of sand ballast, besides their provisions, some books, instruments, and other necessaries. The machine parted with the gas very rapidly, and their ballast was soon all exhausted; after which, from time to time they threw out every thing else in the boat, to prevent themselves from dropping into the sea. In this way they disposed of all their provisions, their books and instruments, and finally with part of their cloches. This however bringing them near the French coast, they gradually ascended, cleared the cliffs and houses, and landed in the forest of Guiennes. In consequence of this voyage, the king of France presented M. Blanchard with a gift of 12000 livres, and granted hin a pension of 1200 livres a year.

On the 19 th of January, Mr. Crosbie ascended at Dublin in Ireland with au inflammable-air balloon to a great height. He rose so rapidly, that he was out of sight in 3 minutes and a half. By suddenly opening the valve, he descended just at the edge of the sea, as he was driving towards the channel, being unprovided for properly passing over to England.

On the 23d of March, Count Zambeccari and Admiral Sir Edward Vernon ascended at London, and sailed to Horsham in Sussex, at the distance of 35 miles in less than an hour. The voyage proved very dangerous, owing to some of the machinery about the valve being damaged, which obliged them to cut open some part of the balloon when they were about 2 miles perpendicular height above the earth, the barometer having fallen from $30^{\circ} 4$ to $20^{\circ} \cdot 8$ inches. In descending, they passed through a dense cloud, which felt very cold, and covered them with snow. The observations they made were, that the balloon kept perpetually turning round its vertical axis, sometimes so rapidly as to make each revolution in 4 or 5 seconds; that a peculiar noise, like rustling, was heard among the clouds, and that the balloon was greatly agitated in the descent.

On May the 5th, Mr. Sadler, and William Windham, esq. member of parliament for Norwich, ascended at Moulsey-hurst. The machine took a south-east course, and the current of air was so strong, that they were in great danger of being driven to sea. They had the good fortune however to descend near the conflux of the Thames and Medway, not a mile from the water's edge. By an accident they lost their balloon: for while the aeronauts were busied in securing their instruments, the country people, whom they had employed in holding down the machine, suddenly let go the cords, when the balloon instantly ascended, and was driven many miles out to sea, where it fell, and was taken up by a trading vessel. It was afterwards restored again, and another voyage made with it from Manchester to Pontefract, in which Mr. Sadler was still more unfortunate; for no person being near when he descended, and not being able to confine the balloon by his own strength, he was dragged by it over trees and hedges; and at last was forced to quit it at the utmost peril of his life; after which it rose again, 
and was out of sight in a few minutes. It was afterwards found near Gainsborough.

On the 12th of May, Mr. Crosbie ascended, at Dublin, as high as the tops of the houses; but soon descended again with a velocity that alarmed all the spectators for his safety. On his stepping out of the car, in an instant Mr. M'Guire, a college youth, sprang into it, on which the balloon ascended with him to the astonishment of the beholders, and presently he was carried with great velocity towards the channel in the direction of Holyhead. This being observed, a crowd of horsemen pursucd full speed the course he seemed to take, and could plainly perceive the balloon descending into the sea. Lord $\mathrm{H}$. Fitzgerald, who was among the foremost, instantly dispatched a swift-sailing vessel mounted with oars, and all the boats that could be got, to the relief of the gallant youth; whom they found almost spent with swimming, just time enough to save his life.

The fate of M. Pilatre de Rozier, the first aerial traveller, and his companion M. Romain, has been much lamented. They ascended at Boulogne the 15th of June, with intent to cross the channel to England : for the first 20 minutes they seemed to take the proper direction; when presently the whole apparatus was seen in flames, and the unfortunate adventurers fell to the ground from the height of more than a thousand yards, and were killed on the spot, their bones being broken, and their bodies crushed in a shocking manner. The machine in which they ascended, consisted of a spherical balloon, 37 feet in diameter, filled with inflammable air; and under this was suspended a small montgolfier, or fire balloon, of 10 feet diameter; the gallery whicb suspended the aeronauts, was attached to the net of the upper balloon by cords, which were fastened to a hoop rather larger than the montgolfier, and descended perpeudicularly to the gallery. The montgolfier was intended to promote and prolong the ascension, by rarefying the atmospheric air.

On the 19 th of July, at 20 minutes past 2 o'clock, Mr. Crosbie a third time ascended at Dublin, with intent to cross the channel to Holyhead in Eingland. The usual form of the boat had been changed, for a capacious wicker basket, of a circular form; and round its upper edges were fastened a great many bladders, which were intended to render his gallery buoyant, in case of a disaster at sea. About 300 pounds of ballast were put into the basket, but he discharged half a hundred on his first rise. At first a current of air carried him due west; but it soon changed his course to north-east, pointing towards Whitchaven. At upwards of 40 miles from the Irish shore, he found himself within sight of both lands, and he said it was impossible to give an adequate idea of the beauties which the scenery of the sea, bounded by both lands, presented. He rose at one time so high, that by the intense cold his ink was frozen, and the mercury sunk quite into the ball of the thermoneter. He was sick, and felt a strong prepulsion on the tympanum of the ears. $\Delta t$ his utmost height he thought himself stationary ; but on liberating some gas, he descended to a current of air blowing north, and extremely rough. He now entered a thick cloud, and encountered strong blasts of wind, with thunder and lightning, which brouglit him rapidly towards the surface of the water. Here the balloon made a circuit, but falling lower, the water entered his car, and he lost his notes of observation. $\Lambda l l$ his cndeavours to throw out ballast were of no avail; the force of the wind plunged him into the occan; and with much difficulty he put on his cork jacket. The propriety of his idea was now very manifest in the construction of his boat; as by the admission of the water into the lower part of it, and the suspension of his bladders, which were arranged at the top, the water, added to his owll weight, became proper ballast; and the balloon maintaining its poise, it became a powerful sail, by means of which, and a suatch-block to his car, he went before the wind as regularly as a sailing vessel. In this situation he found himself inclined to eat, and he took a little fowl. At the distance of a league he discovered some vessels crowding after him; but as his progress outstripped all thcir endeavours, he lengthened the space of the balloon from the car, which gave a check to the rapidity of his sailing, and he was at lengih overtaken and saved by the Dumleary barge, which took him on board, and steered to Dunleary, towing the balloon after them.

A similar accident happened to Major (now General) Moncy, who ascended at Norwich, on the 22d of July, at 20 minutes past 4 in the afternoon; when meeting with an improper current, and not being able to let himself down, on account of the smalluess of the valve, hẹ was driven out to sea, where, after blowing about for npar two hours, he dropped into the water. Here the struggles were astonishing which he made to keep the balloon up, which was torn, and hung only like an umbrella over his bead. A ship was once within a mile, but, whether from want of humanity, or by mistaking the balloon for a sea munster, they shecred off, and left him to his fate: but a boat chased him for two hours, till just dark, and then bore away. He now gave up all hopes, and began to wish that Providence had given him the fate of Rozicr, rather than such a lingcring death. Exerting himself howerer to preserve life as long as possible, by keeping the ballcon floating over his head, to keep himself out of the water, into which nevertheless he sunk gradually inch by inch, as it lost its power, till lye was at length breast dcep in water, when he was taken up by a rvenue cutter, at half past eleven at night, but so weak that he was obliged to be lifted out of the car into the ship.

About the latter end of August, the longest aerial voyage hitherto made, was performed by Mr. Blanchard, who ascended at Lisle, accompanied by the Chevalier de L'Epinard, and travelled 300 miles in their balloon before it descended. On this occasion, as on some former ones, Mr. Blanchard made trial of a parachute, like a large umbrella, invented to brok the fall in case of an accident happening the balloon: with this machine he dropped a dog from the car soon after his ascension, which descended gently and unhurt.

On the Sth of September, Thomas Balclwin, esq. ascended from the city of Chester, and ascended at RixtonMoss, at 25 miles' distance, after a voyage of 2 hours and a quarter. The greatest perpendicular altitude ascended was about a mile and a half, and the aeronnut computed that in some parts of the royage he moved at the rate of 30 miles an hout. Mr. Baldwin published a very circumstantial account of his voyage, with many ingenious philosophical remarks relating to acrostation, of which subject his book may be considered as one of the best treatises yet given to the public.

October the $5 \mathrm{th}, \mathrm{Mr}$. Lunardi made the first aerial voyage in Scotland, He ascended at Ediuburgh, and, after. various turnings, landed near Cupar in Fife, luving de- 
scribed a track of 40 miles over the sca, and 10 over the land, in an hour and a half. The mercury in the barometer sunk as low as $18^{\circ} 3$ inches at his greatest elevation.

November the 19th, the celebrated Blanchard ascended at Ghent to a great height, and after many dangers descended at Delft without his car, which he cut away to lighten the machine when he was descending too rapidly, and slung himself by the cords to the balloon, which served him then in the nature of a parachute. On his first ascent, when he was almost out of sight, he let down a dog, by means of a parachute, which came easily to the ground.

November the 25th, Mr. Lunardi ascended at Glasgow, and in two hours it is said he described a track of 125 miles. It is further remarkable that, being overcome with drowsiness, he slept for about 20 minutes in the bottom of the car, during this voyage.

In order to obviate the danger of a fall, arising from any accident happening to the balloon, some experiments were made with a parachute first by Mr. Blanchard, who performed several other excursions in different parts of Europe, and made some improvements in the parachute, wings, \&c. An account of the two following voyages performed by him will be best related in Mr. Blanchard's own words, taken from his letter, dated Leipsick, October the 9 th, 1787 , to the editors of the Paris Journal. "I did not mention," says he, "in your interesting paper, my ascension at Strasburg on the 26th of last August: the weather was so horrible, that I mounted only for the sake of contenting the astonishing crowd of strangers, assembled there from all parts of the country. The only remarkable thing that occurred at that time, was the following circumstance: At the height of about 2000 yards, or a mile and half a quarter, I let down a dog tied to the parachute, which, instead of descending gently, was forcibly carried by a whirlwind above the clouds. I met him soon after, bending his course directly downwards: when he perceived me, he began to bark a little, and $I$ was going to take hold of the parachute, when another whirlwind lifted him again to a great height. I lost him for about 6 minutes, and perceived him afterwards, with my telescope, as if sleeping in the cradle or basket belonging to the machine. Continually agitated, and impetuously tossed through every point of the eompass, by the violence of the different currents of air, I determined to end my vuyage on the other side of the Rhine, after having passed vertically over Zell. I descended at a small village, with an intention to be assisted a little, and about thirty men soon came within reach of the balloon very a-propos, and fixed me to the ground. The wind was so violent that anchors or ropes would have been of no service. I had however added to the large aerostatic globe a smaller one, of 60 pounds ascensional force, which would have contributed to fix me, when once I let it loose; but, notwithstanding this precaution, the men's assistance was very necessary to me. The parachute was yet wavering in the air, and did not come down till 12 minutes after."

"1 perfc:med my 27th ascension at Leipsick the 29th of September, in the midst of an ineredible number of spectators, forming one of the rnost brilliant assemblies I ever beheld. The sky was as clear and serene as possible, and the air so ealm that many of my friends, and multitudes of others, could follow me on horseback, and even on foot. I was sometimes so near them that they thought they could reach me, but I could soon find the means of rising; and once, when they had actually taken hold of the cords, to see me float with the strings in their hands, I suddenly cut them, and mounted agan in the air. All these amusing evolutions were in sight of the town and its environs. At length I yielded to the earnest solicitations of the company, and entered the town. The next day I emptied the inflammable air into another globe, with which I intended to try some experiments; and I let it off with a cradle, in which a dog was fixed. The balloon, having reached a considerable height, made an explosion in its under part, as I had imagined it would, having previously disposed it in a proper manner for that purpose ; by which means the little animal fell gently to the ground."

" The day before yesterday having repeated this experiment, I prepared the globe in such a manner as to cause an explosion in its upper part, and added a parachute with two small dogs fixed to it. They went so high that, notwithstanding the serenity of the sky, the balloon was lost in its immense expanse. Telescopes of the best sort became useless, and I began to be apprehensive for the death of the little animals, on account of the severity of the cold. They descended however about two hours after, quite safe and well, in the town of Delitzsch, 3 miles from Leipsick. I went yesterday to claim them, and found them again over the town in the air with the parachute. Such experiments had been already tried many times in the course of the day, and some officers had thrown them from the top of a steeple, in the sight of all the inhabitants, of Delitzsch, from which they descended safe."

We shall conclude this historical sketch with a shurt account of the aerial voyages performed by M. Garmerin, in London, in 1802. His first aseent in London was on June 29th, accompanied by Captain Sowden : they rose from Ranelagh, and alighted near Colchester, in less than three quarters of an hour, having in that short period travelled full 60 miles. During this voyage the aeronants did not experience any inconvenience from the rapidity of their motion, until they began to descend, when they were much affected by the hoisterousness of the wind: their descent was attended with danger, and occupied somc millutes.

On the 21 st of September, M. Garnerin undertook the singular and desperate experiment of ascending with an air balloon, and descending in a parachute. He ascended from St. George's Parade, North Audley-street, and descended safe into a feld near the small-pox hospital at Pancras. The balloon was as usual made of oiled silk, covered with a net, from which ropes proceeded that terminated in, or were fixed to, a single rope, a few feet below the balloon, and to this rope the parachute was fastened in the following manner.

The parachute was about 30 feet in cliameter; and several ropes of about 30 feet in length, which proceeded from its edge, terminated in a conmon joining, from which shorter ropes proceeded, and to the extremities of these a circular basket was attached, in which M. Garnerin placed himself. Now the single rope that has been said above to proceed from the balluon, passed through a hole in the centre of the parachute, and through tin tubes, which were placed one after another, and was lastly fastened to the basket; so that when the balloon was in the air, by cutting this rope, the parachute with the basket would be separated from it, and in falling downwards would naturally open by the resistance of the air. The G 2 


\section{A E R}

use of the tin tubes was to let the rope slip off with the greater certainty, and to prevent it from getting entangled with any part of the machinery belonging to the balloon.

About 6 o'clock, every thing being ready, M. Garnerin placed bimself in the basket, and ascended majestically amidst the acclamations of innumerable spectators. The weather was the clearest and pleasantest imaginable, the wind blew a gentle breeze from $\mathrm{w}$ by $\mathrm{s}$, and in about 8 minutes the balloon and parachute had ascended to an immense height, when M. Garnerin, in the basket, could scarcely be perceived. While every spectator was contemplating the grand sight before them, M. Garnerin cut the rope, and in an instant he was separated from the balloon, trusting his safety to the parachute. At first, viz, before the parachute opened, be fell with a great velocity; but as soon as it was expanded, which took place almost immediately after, the descent became very gentle and gradual.

In this descent a remarkable circumstance was observed, namely, that the parachute with the appendage of cords and basket, soon began to vibrate like the pendulum of a clock, and the vibrations were so great, that more than once the basket with M. Garnerin seemed to lie horizontally, which to the spectators appeared extremely dangerous: however, the extent of the vibrations diminished as he approached the ground. On coming to the earth the aeronaut experienced some pretty severe shocks, and when he came out of the basket he was muclı discomposed; but he soon recovered his spirits, and sustained no material injury from this desperate undertaking.

For several figures of balloons, see plate 1.

Practice of Aerostation. The first consideration in the practice of aerostation, is the form and the size of the machine. Various shapes have been tried and proposed, but the globular, or the egg-like figures, are the most proper and convenient, for all purposes; and this form also will require less cloth or silk than any other shape of the same capacity; so that it will both come cheaper, and have a greater power of ascension. The bag or cover of an inflammable-air balloon, is best made of the silk stuff called lustring, varnished over. But for a montgolfier, or heated-air balloon, on account of its great size, linen cloth has been used, lined within or without with paper, and varnished. Small balloons are made either of varnished paper, or simply of paper unvarnished, or of gold-beater's skin, or such-like light substances.

With respect to the form of a balloon, it will be necessary that the operator remember the common proportions betwcen the diameters, circumferences, surfaces, and solidities of spheres; for instunce, that of different spheres, the circumferences are as the diameters; that the surfaces are as the squares of the diamcters; and the solidities as the cubes of the same: that any diameter is to its circumference as 7 to 22 , or as 1 to $3 \frac{1}{7}$; so that if the diameter of a balloon be 35 feet, its circumference will be 110 feet. $\Lambda$ nd if the diameter be multiplied by the circumference, the product will be the surface of the sphere; thus 35 multiplicd by 110 gives 3850 , which is the surface of the same sphere in square feet: and if this surface be divided by the breadth of the stuff, in feet, which the balloon is to be made of, the quotiont will be the number of feet in length necessary to construct the ballorn; so if the stuff be 3 feet wide, then 3850 divided by 3 , gives $1283 \frac{2}{3}$ feet, or 428 yards nearly, the requisite quantity of yard-wide stuff, to form the balloon of 35 fcet diameter. Hence also, by knowing the weight of a given piece of the stutt, as of a square foot, or square yard, it is easy to find the weight of the whole bag, namely by multiplying the surface, in square feet or yards, by the weight of a square foot or yard: so if each square yard weigh 1 pound, then the whole bag will weigh 428 pounds. Again, the capacity, or solid contents, of the sphere, will be found by multiplying $\frac{1}{6}$ of the surface by the diameter; which gives 224.58 cubic feet for the capacity of the said balloon, that is, it will contain, or displace, $2245 \mathrm{~s}$ cubic feet of air. From the content and surface of the balloon, so found, is to be derived its power of ascension, thus : On an average, a cubic foot of common air weighs $1 \frac{1}{5}$ ounce, and therefore to the number 22458 , which is the content of our balloon, adding its $\frac{1}{5}$ part, we have 26950 ounces, or 1684 pounds, for the weight of the common air displaced or occupied by the balloon. From this weight must be deducted the weight of the bag, namely 428 pounds, and then there remains a power of 1256 pounds.

If inflammable air be used, as it is of different wcights, from $\frac{x}{4}$ to $\frac{1}{10}$ or $\frac{x}{12}$ the weight of common air, according to the modes of preparing it : let vs suppose for instance, that it is $\frac{1}{6}$ of the weight of common air; then $\frac{x}{6}$ of 1684 . is $26 \mathrm{I}$ pounds, which is the weight of the bag full of that air; which being taken from 1250 , leaves 495 pounds for the power of the balloon when so filled with that inflammable air, or the weight which it will carry up, consisting of the car, the ropes, the passengers, ballast, \&c. But if heated air be used; then as it is known from experiment that, by heat, the contained air is diminished in density about one-third only, therefore from 1684 , take $\frac{1}{3}$ of itself, and there remains 1123 for the weight of the contained warm air; and this being subtracted from 1256 , leaves only 133 pounds for the power of the balloon in this case; which being too small to carry up the car, passengers, \&cc, it shows that for those purposes a larger balloon is necessary, on Montgolfier's principles. But if now, from the preceding computation, it be required to find how much the size of the balloon must be increased, that its power of ascension may be equal to aluy given weight, as suppose 1000 pounds; then because the power's are nearly as the cubcs of the cliameters, therefore the diameters will be nearly as the cube roots of the powers; but 133 and 1000 are nearly as 1 to 8 , the cube roots of which are as 1 to 2 , and consequently $1: 2:: 35: 70$ feet, which is the diameter of a montgolfier, made of the same thickness of stuff as the former, capable of lifting 1000 pounds.

On the same principles we can easily find the size of a balloon that shall just float in air when made of stuff of a given thickness or weight, and filled with air of a given density; the rule for which is this: From the weight of a cubic foot of common air, subtract that of a cubic foot of the lighter or contained air; then divide 6 times the weight of a square foot of the stuff, by the remainder; and the quotient will be the diameter, in feet, of the balloon that will just float at the surface of the earth. Suppose, for instance, that the materials are as before, namely, the stuff 1 pound to the square yard, or $\frac{16}{9}$ ounces to the squate foot, which taken 6 times is $\frac{32}{3}$; then the cubic foot of common air weighing $1 \frac{\pi}{5}$ ounce, and of heated air $\frac{2}{3}$ of the same, whose difference is $\frac{2}{5}$; the refore $\frac{32}{3}$ divided by $\frac{2}{5}$, gives $26 \frac{2}{3}$ fect, which is the diameter of a montgolfier that will just float : but if inlammable air be 
used of $\frac{1}{6}$ the weight of common air, the difference between $1 \frac{x}{5}$ and $\frac{t}{6}$ of it, is 1 ; by which dividing $\frac{32}{3}$ or $10 \frac{2}{3}$, the quotient is the same $10 \frac{2}{3}$ feet, which therefore is the diameter of an inflammable-air balloon that will just float. And if the diameter be more than these dimensions, the balloons will ascend into the atmosphere.

An estinate of the height to which a given balloon will rise in the atmosphere, may be made as follows: having given only the diameter of the balloon, and the weight which just balances it, or that is just necessary to keep it from rising: compute the capacity or content of the globe in cubic feet, and divide its restraining weight in ounces by that content, then the quotient will be the difference between the density or specific gravity of the atmosphere at the earth's surface, and that at the height to which the balloon will rise; therefore subtract that difference or quotient from $1 \frac{\pi}{5}$ or $1 \cdot 2$, the density at the earth's surface, and the remainder will be the rlensity at that height: then the height answering to that density will be found sufficiently near in the annesed table. Thus, in the for'going examples, in which the diameter of the balloon is 35 feet, its catpacity 22458 , and the power of the first 995 pounds, or 15920 ounces, the quotient of the latter number divided by the former, is .709 , which is the density at the utmost

\begin{tabular}{|c|c|}
\hline $\begin{array}{c}\text { Height } \\
\text { in miles. }\end{array}$ & Density. \\
\hline 0 & $1 \cdot 200$ \\
$\frac{3}{4}$ & $1 \cdot 141$ \\
$\frac{1}{2}$ & $1 \cdot 085$ \\
$\frac{3}{4}$ & $1 \cdot 031$ \\
1 & $0 \cdot 980$ \\
$1 \frac{1}{4}$ & $0 \cdot 932$ \\
$1 \frac{1}{2}$ & $0 \cdot 886$ \\
$1 \frac{3}{4}$ & $0 \cdot 842$ \\
2 & $0 \cdot 800$ \\
$2 \frac{1}{4}$ & $0 \cdot 761$ \\
$2 \frac{1}{2}$ & 0723 \\
$2 \frac{3}{4}$ & $0 \cdot 687$ \\
3 & 0.653 \\
\hline
\end{tabular}
beight, and to which in the table answers a little more than $2 \frac{1}{2}$ miles, or $2 \frac{5}{8}$ miles rearly, which therefore is the height to which the balloon will ascend. And when the same balloon was filled with heated air, its power was found equal to only 133 pounds, or 2128 ounces, and dividing this by 22458 the capacity, the quotient $0 y 5$ taken from 1.200 , leave's $1 \cdot 105$ for the density; which in the table corresponds to half a mile, or nearer $\frac{3}{8}$ of a mile. And so high would these balloons ascend, if they keep the same figure, and lost none of the contained air; or rather, those are the heights they would settle at: for their acquired velocity would first carry them above that height, so far as till all their motion should be destroyed; then they would descend and pass below that height, but not so inuch as they had gone above; after which they would re-ascend, and pass that height again, but not so far as they had gone below it; and so on for many times, vibrating alternately above and below that point, but always less and less every time. The foreguing rule, for finding the height to which the ballon will ascend, is independent of the different states of the thermometer at that lighe'st point, and at the surface of the earth; but for greater accuracy, including the allowances depending on the different states of the thermometer, see under the word ATNOSPHERE, where the more accurate rules are given at large.

The best way to make up the whole coating of the balloon, is by different pieces or slips joined length ways from end to end, like the picces composing the surface of a geographical globe, and contained between one meridian and another, or like the slices into which a melon is usually cit, and supposed to be spread flat out. Now the edges of such pieces cannot be exactly described by a pair of compass's, not being circular, but flatter or less round than circular arches; but if the slips are sufficiently nar- row, or numerous, they will differ the less from circles and may be described as such. But more accurately, the breadths of the slip, at the several distances from the points to the mildle, where it is broadest, are directly as the sines of those distances, radius being the sine of the half length of the slip, or of the distance of either point from the middle of the slip: that is, If A C B D be one of the slips, $A$ d being half the circumference, or $A \mathrm{E}$ a quadrant conceived to be equal to $A C$ or $A D$; then will $C D$ be to $a b$, as radius or the sine of $A c$, to the sine of $A a$. So that if the quadrant $A \mathrm{E}$ or AC be divided into any number of equal parts, as here suppose 9 , then divide the quadrant or 90 degrees by the number of parts 9 , and the quotient 10 is the number of degrees

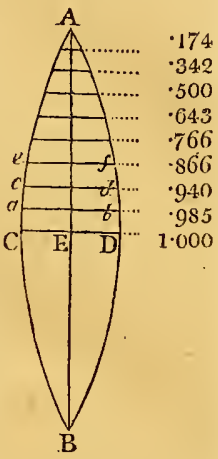
in each part; and hence the $\operatorname{arcs} \Lambda \mathrm{c}, A a, \mathrm{Ac}, \& \mathrm{c}$, will be respectively $90^{\circ}, 80^{\circ}, 70^{\circ}, \& \mathrm{c}$; and, $\mathrm{CD}$ being radius, the several breadiths $a b, c d, e f, \& c$, will be respectively the sines of $80^{\circ}, 70^{\circ}, 60^{\circ}$, \&ce, which are here placed opposite to them, the 1adius being 1 . Therefore, when it is proposed to cut out slips for a globe of a given diameter; compute the circumference, and make $A \mathrm{E}$ or $\mathrm{AC}$ a quarter of that circumference, and $C D$ of any breadth, as 3 feet, or 2 feet, or any other quantity; then multiply each of the decimal numbers, set opposite the figure, by that quantity, or breadth of $\mathrm{CD}$, so shall the products be the several breadths $a b, c d, e f, \& c$.

Various schemes have been devised for conducting balloons in any direction, whether vertical or sideways. As to the vertical directions, naincly upwards or downwards, the means are obvious, viz, in order to ascend, the aeronaut throws out some ballast; and that he may descend, he opens a valve in the top of his machine by means of a string, to let some of the gas escape; or if it be a montgolfier, he increases or diminishes the fire, as be would ascend or descend. But to direct the machine in a side or horizontal course, is a very difficult operation, and what has hitherto not been accomplished, except in a small degree, and when the current of air is very gentle indeed. The difficulty of moving the balloon sideways, arises from the want of wind blowing upon it ; for as it fluats along with the current of air, it is relatively in a $\mathrm{calm}$, and the aeronaut feels no more wind than if the macline were at rest in a perfect calm. For this reason, any thing in the nature of sails can be of no use; and all that can be hoped for, is to be attemptrd by means of oars; and how small the effect of these must be, may easily be conceived from the rarity of the medium against which they must act, and the great magnitucle of the machine to be forced through it. We can easily assign what force is necessary to move a given machine in the air with any proposed velocity. From very accurate experiments I lave determincd, that a globe of $6 \frac{3}{8}$ inches in diameter, and moving with a velocity of 20 fect per second of time, suffers a resistance from the air which is just equal to the weight or pressure of one ounce Averlupois : and further, that with different surfaces, and the same velocity, the resistances are dircetly proportional to the surface nearly, at 


\section{A E R}

double surface baving a double resistance, a triple surface a triple resistance, and so on; and also, that with different velocities, the resistances are proportional to the squares of the velocities nearly, so that a double velocity produced a quadruple resistance, and 3 times the velocity 9 times the resistance, and so on. And hence we carn assign the resistance necessary to move a given balloon, with any velocity. Thus, take the balloon as before of 35 feet diameter; then by comparison as above it is found that this globe, if moved with the velocity of 20 feet per second, or almost 14 miles per hour, will suffer a resistance equal to 271 pounds; to move it at the rate of 7 miles an hour, the resistance will be 68 pounds; and to move it $3 \frac{1}{2}$ miles an hour, the resistance will be 17 pounds; and so on: and with such force must the aeronauts act on the air in a contrary direction, to communicate such a motion to the machine. But, if the balloon move through a rarer part of the atmosphere, than that at the surface of the earth, as $\frac{x}{3}$, or $\frac{x}{4}$, \&c, rarer, and consequently the resistance be less in the same proportion; yet the force of the oars will be diminished as much; and therefore the same difficulty still remains. In general, the aeronaut must strike the air, by means of his oars, with a force just equal to the resistance of the air on the balloon, and therefore he must strike that air with a velocity which must be greater as the surface of the oar is less than the resisted surface of the globe, but not in the same proportion, because the force is as the square of the velocity.

Now, suppose the aeronaut act with an oar equal to 100 square feet of surface, to move the balloon above mentioned at the rate of 20 feet per second, or 14 miles an hour; then must be move this oar with the great velocity of 62 feet per second, or near 43 miles per hour: and so in proportion for other velocities of the balloon. Whence it is highly probable, that it will never be in the power of man to guide such machine with any tolerable degree of success, especially when any considerable wind blows, which is almost always the case.

The compositions for varnishing balloons have been variously modified; but upon the whole, the most approved appears to be the bird-lime varnish of M. Faujas St. Fond. Mr. Blan'chard used a varnish made of elastic rosin, known commonly by the name of India rubber, dissolved in five times its weight of rectified essential oil of turpentine; which is also held in great esteem.

The methods of obtaining inflammable air for filling balloons is generally, by applying acids to certain metals, by exposing animal, vegetable, and some mineral substances, in a close vessel to a strong fire, or by transmitting the vapour of certain fluids through red-hot tubes.

In the first of these methods, iron, zinc, and vitriolic acid, are the materials most generally used. The vitriolic acid must be diluted by five or six parts of water. Iron may be expected to yield in the common way 1700 times its own bulk of gas; or one foot of inflammable air to be produced by $4 \frac{1}{2}$ ounces of iron, the same weight of oil of vitriol, and $22 \frac{\mathrm{r}}{2}$ ounces of water. Six ounces of zinc, an equal wcight of oil of vitriol, and 30 ounces of water, are necessary for producing the same quantity of gas.

And here it should be obscrved, that the turnings or chippings of great pieces of iron, as of cannons, \&c, are preferable to the filings of that metal. The weight of the inflammable air tlus obtained, by means of acid of vitriol, is in the common way of procuring it, generally about one seventh part of the weight of coinmon

\section{A E R}

air; but with the necessary precautions, for philosophical experiments, less than one tenth of the weight of common air. Two other sorts of elastic fluids are sometimes generated with the inflammable air, which may be separated from $i t$, by passing the gas through water, in which quick lime has been dissolved. The water will absorb these fluids, cool the inflammable air, and prevent its over beatiug the balloon when introduced into it.

Inflammable air may be obtained at a much cheaper rate by the action of fire upon various substances; but the gas that they yield is not so light as that produced by the effervescence of acids and metals. The substances proper for this are, pit-coal, asphaltum, amber, rock-oil, and other minerals; wood and particularly oak, camphoroil, spirits of wine, ether, and animal substances; all of which yield air in different degrees, and of various specific gravities: but pit-coal is the preferable substance.

Another method of obtaining inflammable air was discovered by M. Lavoisier, and also by Dr. Priestley; which is, by making the steam of boiling water pass through the barrel of a gun, kept red-hot by burning coal. Dr. Priestley used, instead of the gun barrel, a red-hot tube of brass, upon which the steam of water bas no eflect; and which he filled with the pieces of iron separated by the boring of cannon; and by this means be obtained an inflammable air $\frac{1}{3}$ th part the weight of common air. But of these three methods, the former is the ouly one that can be safely depended on for obtairing proper gas for filling large balloons; and here it has been recommended to use zinc instead of iron turnings, because the white vitriol, the salt produced by the union of the vitriolic acid and zinc, is much more valuable than the green sort produced by the union of the same acid with irou. But though this is undoubtedly the case, it will certainly be found upon trial, that the superior price of the zinc, will be more than an equivalent for all the advantage that can be derived from the additional price of the white vitriol.

The method of filling balloons, being merely mechanical, is omitted in this place; but ample information on this, and many other particulars, may be met with in several books expressly written on the subject; as in $\mathrm{Ca}$ vallo's History and Practice of Acrostation, Svo, 1785; in Baldwin's Airopaidia, Svo, 1786; and the Description des Experiences, \&c, par M. Faujus St. Fond. See also an ingenious and learned treatise on the mathematical and physical principles of $\Lambda \mathrm{ir}$-balloons, by the late $\mathrm{Dr}$. Damen, professor of philosophy and mathematics in the University of Leyden, entitled, Physical and Alaihematical Contemplations on Aerostatic Balloons, \&c; in 8vo, at Utrecht, 1784.

It has often been discussed, says the former of these gentlemen, whether the preference should be given to machines raised by inflammable air, or to those raised by heated air. Each of them has its peculiar advantages and disadvantages; a just consideration of which seems to decide in favour of those made with inflammable air. The principal comparative advantages of the other surt are, that they do not require to be made of so cxpensive materials; that they are filled with little or no expense; and that the combustibles necessary to fill them are found almost every where; so that, when the stock of fuel is exhausted, the acronaut may descend and recruit it again, in order to proceed on his voyago. But then this kind of machines must be made larger than the other, to take up 
the same weight; and the presence of a fire is a contiwual trouble, as well as danger: in fact, among the many aerial voyages that have been made and attempted with such machines, very few have succeeded without an inconvenience, or an accident; and some indeed bave been attended with dangerous and even fatal consequences; from which the other kind is in a great measure exempt. But, on the other hand, the inflammable-air balloon must be made of a substance impermeable to the subtle gas: the gas itself cannot be produced without a considerable expense; and it is not easy to find the materials and apparatus necessary for the production of it in every place. However, it has been found that an infammable-air balloon, of 30 feet in diameter, may be made so elose as to sustain two persons, and a considerable quantity of ballast, in the air for more than 24. hours, when properly managed; and possibly one man might be supported by the same inachine for three days: and it is probable that the stuff for these balloons may be so far improved, as to be quite inspermeable to the gas, or very nearly so; in which ease, the machine, once filled, would continue to float for a long time. At Paris they have already attained to a great degree of perfection in this point; and small balloons have been kept floating in a room for many weeks, without losing any considerable quantity of their levity: but the difficulty lies in the large machines: for in these, the weight of the stuff itself, with the weight and stress of the ropes and boat, and the folding them up, may easily erack and rub off the varnish, and make them leaky.

With regard to the uses to which balloons can be applied, it must be observed, that the perfection of all arts, and the application of them to the useful purposes of rnan, have always been progressive, and not the inmediate result of a fortunate discovery; if therefore at present no great advantages have been derived from the art of aerostation, we ought not to despair, nor discourage any future attempts, which it is to be hoped may prove more successful in this respect. But even in the present iucomplete state of this art, some advantages have been derived from it. Thus, the French ascribe to the elevation of a balloon, and the infurmation obtained in conscquence of thus reconnoitring the army of the encmy, the signal victory gained in the battle of Fleurus in 1794. Balloons may likewise serve to explore and ascertain the nature of the air in the higher regions of the atmosphere. One of the finest experiments made on this point, is that of GayLusac, who being elevated in a balloon to the height of nearly eight miles, the greatest ever attained by any person, brought some atmospheric air from those regions, which, on being analysed, was found to furnish the principles of oxygen, azotc, hydrogen, and earbonic acid gas, in the same proportions as at the surface of the earth. In fact, the application of these machines to the advancement of our knowledge of the various phænomena in meteorology, stands prominent, as perhaps the only means of maturing our acquaintance with causes yet known only by their effects.

RESTIVAL, see EstivaL.

ESTUARY, or EsTUARY, in Geography, an arm of the sea, running up a good way into the land. Such as Bristol channel, many of the friths in Scotland, and suchlike.

ÆTTHER, see ETHER.

AFFECTED, or A DFECTED, Equation, in Algebra, is an equation in which the unknown quantity rises to two or more sereral powcrs or degrecs. Such, for example, is the equation $x^{3}-p x^{2}+q x=r$, in which there are three different powers of $x$, llamely, $x^{3}, x^{2}$, and $x$.

The term, affected, is also used somerimes in algebra, when speaking of quantities that have coefficients. Thus, in the quantity $2 a, a$ is said to be affected with the coefficient 2. It is also said, that an algebraic quantity is affected with the sign + or - , or with a radical sign; meaning no more than that it has the sign + or - , or that it includes a radical sign. The term adfected, or affected, I think, was introduced by Vieta.

AFFECTION, in Geometry, a term used by some ancient writers, signifying the same as property.

AFFECTION. Phys. The affections of a body are certain modifications oceasioned or induced by motion; in virtue of which the body is disposed after such or such a inaniser.

The affections of bodies, are sometimes divided into primary and secondary.

Primary Affections, are those which arise either out of the idea of matter, as magnitude, quantity, and figure; or out of the idea of form, as quality and power; or out of both, as motion, place, and time.

Secondary, or derivative Affections, are such as arise out of primary ones, as divisibility, continuity, contiguity, $\& c$, which arise out of quantity; regularity, irregularity, $\& c$, which anise out of figure, \&c.

AFFINITY, in natural philosophy, the tendency which the particles of matter have to be attracted, or united to each other.

AFFIRMative Quantity, or Positive Quantity, one which is to be added, or taken effectively; in contradistinction to one that is to be subtracted, or takels defectively. - The term affirmative was introduced by Vieta.

Affirmative Srgn, or Positive Sign, in Algebra, the sign of addition, thus marked + , and is called plus, or more, or added to. When set before any single quantity, it serves to denote that it is an affirmative or a positive quantity; when set between two or more quantities, it denotes their sum, showing that the latter are to be added to the former. So +6 , and $+a$, and $+A$ B, are affirmative quantitics; also $6+8+10$ denote the sum of 6,8 , and 10 , which is 24 , and are read thus, 6 plus 8 plus 10 . Also $a+b+c$ denote the sum of the quantities represented by $a, b$, and $c$, when added together. It scems now not easy to ascertain with eertainty, when, or by whom, this sign was first introduced; but it was probably by the Germans, as 1 find it first used by Stifelius in his Arithmetic, printed in 1544. When quantities are set down without a prefixed sign, as $a$, or $b$, or $x$, $\& e$, they are understood to be affrmative ones.

'The early writers on Algebra used the word plus in Latin, or piu in Italian, for addition, and afterwards the initial $p$ only, as a contraction; like as they used minus, or meno, or the initial $m$ only, for subtraction : and thus these operations were denuted in Italy by Lucas de Burgo, Tartalea, and Cardan, while the signs + and - were employed much about the same time in Germany by Stifelius, Scheubelius, and others, for the same operations.

AGE, in Chronology, is used for a century, being a system or period of a hundred years.

Chronologists also divide the time since the creation of the world into three ages: The first, from Adam to Moses, which they call the age of nature; the second from 
Moses to Jesus Christ, called the age of the law; and the third, or age of grace, from Jesus Christ till the end of the world.

AGE of the Moon, in Astronomy, is the number of days elapsed since the last new moon. 'To find the moon's age, for any time nearly, for ordinary uses; add together the epact, the day of the current month, and the number of months from March to the present month inclusive; the sum is the moon's age : but if the sum exceed 29, deduct this number from it in months that have 30 days, or 30 in those that have 31 ; and the remainder will be the age. - At the end of 19 years the moon's age returns upon the same day of the month, but falls a little short of the same hour of the day.

AGENT, Agens, in Physics, that by which a thing is done or effected; or any thing having a power by which it acts on another, called the object.

AGGREGATE, the sum or result of several things added together. See Sum.

AGITATION, in Physics, a brisk intestine motion, excited among the particles of a body. Thus, fire agitates the subtlest particles of bodies. Fermentations, and effervescences, are produced by a brisk agitation of the particles of the fermenting body.

AGNESI (Maria Gaetana), an Italian young lady, highly celebrated for her learning and talents. Signora Agnesi was born at Milan in 1718, and by her genius and industry soon became profoundly learned in several languages, as well as in the mathematical and philosophical sciences. While very young she composed an excellent treatise on the doctrine of fluxions, which was published at Milan 1748, under the title of Analytical Institutions, in 2 volumes 4 to; a very iseful work, which was honoured by a French translation, at Paris, by Cousin, in 1775 ; and by an English translation, made many years since by the late professor Colson, of Cambridge, and lately published at the expense of Mr. Baron Maseres, under the care of the Rev. John Hollins, in 2 vols. 4to, 1801. In just consideration of such eminent qualifications, S. Agnesi was appointed public professor of mathematics at Bologna in 1750, an office which she discharged with the greatest credit for some time.

It is with regret however we add, that neither her inclination for those honourable intellectual pursuits, nor a desire of preserving and increasing the celebrity she had obtained, nor the entreaties of her family and friends, could prevent her from devoting herself to a monastic life, among the sisters known by the name of the Blue Nuns. From the period of her retiring to the cloister, it may be concluded that her life was not chequered with any variety of incidents. Accordingly, we only further learn that this illustrious ornament of her sex dicd about the year 1770 .

AGUILON, or AQUiLon, (Francis), was a jesuit of Brussels, and professor of philosophy at Doway, and of theology at Antwerp. He was one of the first that introduced mathematical studies into Flanders. He wrote a large work on Optics, in 6 books, which was published in folio, at Antwerp, in 1613; and a treatise of Projections of the Spherc. He promised also to treat upon $\mathrm{Ca}$ toptrics and Dioptrics; but this was prevented by his death, which happened at Seville, in the year 1617 .

AlR, in Natural Philosophy and Chemistry, a general term used to denote such invisible rare fluids as possess a very high degree of elasticity, and are not condensable into a visible fluid by any degree of colls; in this respect air is distinguished from vapour, which may be thus condensed.

This term was originally applied to the air of which our atmosphere is composed, and considered by the ancients as a simple element; but the researches of modern chemists have shown it to be a compound, and that it may be resolved into its several component parts, (see $\mathrm{G}_{\mathrm{AS}}$ ); but at present we shall only consider it as relating to the atmosphere, without entering into its chemical properties.

Air, Atmospheric, is an invisible, insipid, inodorous, heavy, and elastic fluid, possessing great mobility, susceptible of rarefaction, and of condensation; it surrounds the terrestrial globe to a certain height, the entire mass constituting the atmosphere. The consideration of this fluid cannot but be a highly interesting subject, since it is the depository of the signs of our thoughts and our affections; at the same time, that it serves for the preservation of life in every animated being.

Mechanical Properties and Effects of AIr. Of these the most considerable are its fluidity, its weight, and its elasticity.

1. Its Fluidity.-The great fluidity of the air is manifest from the facility with which bodies traverse it; as in the propagation of, and easy conveyance it aftords to, sounds, odours and other effluvia and emanations that escape from bodies: for these effects prove that it is a body whose parts give way to any force, and in yielding are easily moved among themselves; which is the definition of a fluid. That the air is a fluid is also proved from this circumstance, that it is found to exert an equal pressure in all directions; an effect which could not take place otherwise than from its extreme fluidity. Neither has it been found that the air can be deprived of this property, whether it be kept for many years together confined in glass vessels, or be exposed to the greatest natural or artificial culd, or condensed by the most powerful pressure; for in none of these circumstances has it ever bcen reduced to a solid state.

2. Its Weight or Gravity.-The weight or gravity of the air, is a property belonging to it as a body; for gravity is a property essential to matter, or at least a property found in all bodies. But independent of this, we have many direct proofs of its gravity from sense and experiment: thus, the hand laid cluse upon the end of a vessel, out of which the air is drawn at the other end, soon feels the load of the incumbent atmosphere: thus also, two hollow segments of a sphere, of about 4 inches in diameter, cxactly fitting each other, being emptied of air, are, by the weight of the ambient air, pressed together with a force which requires the weight of ISS pounds to separate then; and that they are thus forcibly held together by the pressure of the air, is made evident by suspending them in un exhausted receiver, for then they quickly separate of themselves, and fall asunder. Again, if a tubc, close at one end, be filled with quicksilver, and the open end be immerged in a bason of the same fluid, and so held upright, the quicksilver in the tube will be kept raised up in it to the height of about 30 inclics above the surface of that in the bason, being supported and balanced by the pressure of the external air upon that surface: and that this is the cause of the suspension of the quicksilver in the tube, is made evident by placing the whole apparatus under the receiver of an air-pump; for then the 
fluid descends in the tube in proportion as the receiver is exhausted of its air; and on gradually letting in the air again, the quicksilver reascends to its lormer beight: and this is the common barometer, or what is called, from its inventor, the Torricellian experiment. Nay farther, air can actually be weighed like any other body: for a rigid vessel, full even of common air, by a nice balance is found to weigh more than when the air is exhausted from it; and the effect is proportionally more sensible, if the ressel be weighed full of condensed air, and more still if it be weighed in a receiver void of air.

But, though we have innumerable proofs of the gravitating property of the air, yet the full discovery of the laws and circumstances of it are certainly due to the moderns. It cannot indeed be denied, that several of the ancients had some confused notions about this property: thus Aristotle says, that all the elements have gravity, and even air itself; and as a proof of it, says that a bladder inflated with air, weighs more than the same when empty; and Plutarch and Stobæus quote him as teaching that the air in its weight is between that of fire and of earth; and farther, he himself, treating of respiration, reports it as the opinion of Empedocles, that he ascribes the cause of it to the weight of the air, which by its pressure forces itself into the lungs: and much in the same way are the sentiments of Asclepiades expressed by Plutarch, who represents him as saying, among other things, that the external air, by its weight, forcibly upened its way into the breast. But nevertheless it is certain, however unreasonable it may seem, that Aristotle's followers departed in this instance from their master, by asserting the contrary for many ages together. Indeed several of the phænomena arising from this property, "have been remarked from the highest antiquity. Many centuries since, it was kuown that by sucking the air from an open pipe, having its extremity inmersed in water, this fluid rises above its level, and occupies the place of the air. In consequence of such observations, sucking pumps were contrived, and various other hydraulic machines; as Heron's syphons, described in his Spiritalia or Pneumatics, and the watering-pots known in Aristotle's time under the name of clepsydra, which alternately stop or run as the finger closes or opens their upper orifice. Indeed the reason assigned, by philosophers many ages after, for this phænomenon, was a pretended horror that nature conceives for a vacuum, which, rather then endure it, makes a body ascend contrary to the powerful solicitation of its gravity. Even Galileo, with all his sagacity, could not for some time hit upon any thing more satisfactory; for he only assigned a limit to this dread of vacuity : having observed that sucking pumps would not raise water higher than 16 brasses, or 34 English feet, be limited this abhorring force of nature, to one that was equivalent to the weight of a column of water 34 feet high, on the same base as the void space. Consequently he pointed out a way of making a vacuuin, by means of a hollow cylinder, whose piston is charged with a weiglit sufficient to detach it from the close bottom turned upwards: this effort he called the measure of the force of vacuity, and made use of it for explaining the cohesion of the parts of bodies.

Galileo, however, was well apprised of the weight of the air as a body: in his Dialogues he shows two ways of temonstrating it, by weighing it in bottles: the transition was easy from one discovery to another: yet still Galilco's knowledge of the matter was imperfect, that is, as to Vor. I. the particular instance of the suspension of a flud above its level, by the pressure of the external air.

At length, Torricelli conceived the happy idea, that the counterpoise which keeps fluids above their level, when nothing presses upon their internal surface, is the mass of air resting upon the external one. He discovered it in the following manner: In the year 1643 this disciple of Galileo, on occasion of executing an experiment on the vacuum formed in pumps, above the column of water, when it exceeas 34 feet, thought of using some heavier fluid, such as quicksilver. He conceived, that whatever might be the cause by which a column of water of 34 feet high is sustained above its level, the same force would sustain a column of any other fluid, which weighed as much as that column of water, on the same base; whence he concluded that quicksilver, being about 14 times as heavy as water, would not be sustained higher than 29 or 30 inches. He therefore took a glass tube of several feet in length, sealed it hermetically at one end, and filled it with quicksilver; then inverting it, and holding it upright, by pressing his finger against the lower or open orifice, he immersed that end in a vessel of quicksilver; when removing his finger, and suffering the fluid to run out, the event verified his conjecture; the quicksilver, faithful to the laws of hydrostatics, descended till the column of it was about 30 inches high above the surface of that in the vessel below. And hence Torricelli concluded, that it was no other than the weight of the air incumbent on the surface of the external quicksilver, which counterbalanced the fluid contained in the tube.

By this experiment Torricelli not only proved, what Galileo had done before, that the air had weight, but also that it was its weight which kept water and quicksilver raised in pumps and tubes, and that the weight of the whole column of it was equal to that of a like column of quacksilver of 30 inches in height, or of water 34 or 35 feet high; but he did not ascertain the weight of any particular quantity of it, as a gallon, or a cubic foot of it, nor its specific gravity to water, which had been done by Galileo, though to be sure with no great accuracy, for he only proved that water was more than 400 times heavier than air.

Father Mersenne, who kept up a correspondence with most of the literati in Italy, was informed of Torricelli's experiment in 1644, and communicated it to those of France, who presently repeated the experiment: Messrs. Pascal and Petit made it first, and varied it several ways; which gave occasion to the ingenious treatise which Pascal published at 23 years of age, entitled Experiences Nourvelles touchant la Vuide. In this treatise indeed he makes use of the old principle of fuga vacui; but afterwards getting some notion of the weight of the air, he soon adopted Torricelli's idea, and devlsed several experiments to confirm it. One of these was to procure a vacuum above the reservoir of quicksilver; in which case, he found the column sink down to the common level : but this appearing to him not sufficiently powerful to dissipate the prejudices of the ancient philosoplyy, he prevailed on M. Perier, his brother-in-law, to execute the celebrated experiment of Puy-de-Donme, who found that the height of the quicksilver half-way up the mountain was less, by some inches, than at the foot of it, and still less at the top: so that it was now put out of doubt, that it was the weight of the atmosplere which counterpoised the quicksilver. 


\section{A I R}

[ 50

Des Cartes too had a right notion of this effect of the air, to sustain fluids above their level, as appears by some of his letters about this time, and some years before; and in one of those he lays claim to the idea of the Puy-deDomme experiment. After having desired M. de Carcavi to inform him of the success of the experiment, made by M. Pascal, he adds, "I had reason to expect this from him, rather than from you, because $I$ first proposed it to him two years since, assuring him at the same time, that though I had not tried it, yet I could not doubt of the consequence; but as he is a friend of M. Roberval, who professes himself no friend to me, I suppose he is guided by that gentleman's passions." See more of this history under BAROM ETER.

As to the actual weight of any given portion of common air, it seems that Galileo was the first who determined it experimentally; and he gives two different methods, in his Dialogues, for weighing it in bottles: he did not however perform the experiment very accurately, as he stated froin the result that the gravity of water was to - that of air rather above 400 to 1 , as before said.

A quantity of air was next weighed by Mersenne in a very ingenious manner. His idea was to weigh a vessel both when full of air, and when emptied of it: to make the vacuum for this purpose, he knew no better way than by expelling the air out of an eolipile by heating it red-hot: by weighing it both when cold and hut, he found a certain difference; which however was not the exact weight of that capacity of air, because the vacuum was not perfect. For, by plunging the colipile, when red-hot, into water, just so much water entered as was equal in bulk to the air that had been expelled; be then took it out and weighed it with the water, which gave the weight of the same bulk of water; and on comparing this with the former difference, or weight of air expelled, he found their proportion to be as 1300 to 1 ; which is as wide of the truth as Galileo's proportion, namely 400 to $\mathrm{J}$, but the contrary way. And it is remarkable that the mean between the two, namely 850 to 1 , happens to be very near the true proportion, as settled by other more accurate expcriments.

Mr. Boyle, by a more accurate experiment, found the proportion to be that of 938 to 1. And Mr. Hauksbee found it as 850 to 1 , proceeding on the same principles as Mersenne, with a three-gallon glass bottle, but extracting the air out of it with the air-pump, instead of expelling it by fire; the height of the barometer being at that time 29.7 inches. Also by other experiments made before the Royal Society by Mr. Hauksbee, Dr. Halley, $\mathrm{Mr}$. Cotes, and others, the proportion was always between 800 and 900 to 1 , but rather nearer the latter, namely, being first found as 840 to 1 , then as 852 to 1 , and a third time as 860 to 1 ; the barometer then standing at $29 \frac{3}{4}$ inches, and the weather warm. Mr. Cavendish deiermines the ratio 800 to 1 , the barometer being. 293, and the thermometer at $50^{\circ}$; and Sir George Shuckburgh, by a very accurate experiment; finds it 836 to 1 , the barometer being at that time $29^{\circ} 27$, and the thermometer at $51^{\circ}$. The medium of all these is about 832 or 833 to 1 , when reduced to the pressure of 30 inches of the barometer, and the mean temperature $55^{\circ}$ of the thermometer. Upon the whole thercfore, it may be safely concluded that, when the barometer is at 30 inches, and the thermoineter at the mean temperature $55^{\circ}$, the density or gravity of water, is to that of air, as $833 \frac{r}{3}$ to 1 , that is as
A I R

$\frac{2500}{3}$ to 1 , or as 2500 to 3 ; and that for any changes in the height of the barometer, the ratio varies proportionally; also that the density of the air is altered by the $4^{x} \delta^{\text {th }}$ part for every degree of the thermometer above or below temperate.

This number, which is a very good medium among then all, we have chosen with the fraction $\frac{I}{5}$, because it gives exactly $1 \frac{x}{5}$ ounce for the mean wejght of a cubic foot of air, the weight of the cubic foot of water being just $\mathbf{1 0 0 0}$ ounces averdupois, and that of quicksilver equal to 13600 ounces.

Air, then, having been shown to be a heary fluid substance, the laws of its gravitation and pressure must be the same as those of water and other fluids; and consequently its pressure must be proportional to its perpendicular altitude. Which is exactly conformable to experiment; for on removing the Torricellian tube to different heights, where the column of air is shorter, the columi of quicksilver which it sustains is shorter also, and that nearly at the rate of 100 feet for $\frac{r}{10}$ of an inch of quicksilver. And on these principles depend the structure and use of the barometer.

From the same principle it likewise follows that air, like other fluids, presses equally in all directions. And hence it happens, that soft bodies endure this pressure without change of figure, and hard or brittle bodies without breaking; being equally pressed on all parts : but if the pressure be taken off, or diminished, on one side, the effect of it is immediately perceived on the other. See Atmosprean, for the total quantity of effects and pressure, and the laws of different altitudes, \&c.

From the weight and fluidity of the air, jointly considered, many effects and uses of it may easily be deduced. By the combination of these two qualities, it closely invests the earth, with all the bodies upon it, constringing and binding them down with a great force, namely a pressure equal to about 15 pounds upon every square inch. Hence, for example, it prevents the arterial vessels of plants and animals from being too much distended by the impetus of the circulating juices, or by the elastic force of the air so copiously abounding in them. For hence it happens, that on a diminution of the pressure of the air, in the operation of cupping, we see the parts of the body grow tumid, which causes an alteration in the circulation of the fluids in the capillary vessels. And the same cause binders the fluids from transpiring through the pores of their containing vessels, which would otherwise cause the greatest debility, and often destroy the animal. To the same two qualities of the air, weight and fluidity, is owing the mixture of bodies contiguous to one another, especially fuids; for several liquids, as oils and salts, which readily mix of themselves in air, will not mix at all in vacuo. With many other natural phanomena.

3. Elasticity. Another quality of the air, from whence arise a multitude of effects, is its elasticity; a quality by which it yields to the pressure of any other bodies, by contracting its rolume; and dilates and expands itselt again on the removal or diminution of the pressure. "l'his quality is the chief distinctive property of air, the other two being common to all fluids.

Of this property we have innumerable instances. 'Thus, for example, a blown bladder being squeezed in the hand, we find a sensible resistance from the included air; and, on taking of the pressure, the compressed parts immediately restore themselves to their former round igure. 
And on this property of elasticity depend the structure and uses of the air-pump.

- Every particle of air makes a continual effort to dilate itself, thus acting forcibly against all the neighbouring particles, which also exert the like force in return; but if their resistance happen to cease, or be weakened, the particle immediately expands to an immense extent. Hence it is that thin glass bubbles, or bladders, filled with air, and placed under the receiver of an air-pump, will, on pumping out the air, burst asunder by the force of the air which they contain. So likewise a close flaccid bladder, containing only a small quantity of air, being put under the receiver, swells as the receiver is exhausted, and at length appears quite full. And the same thing happens by carrying the flaccid bladder to the top of a very high mountain.

The same experiment shows that this elastic property of the air is very different from the elasticity of solid bodies, and that their dilatation arises from a different cause. For when air ceases to be compressed, it not only dilates, but occupies a far greater space, and exists under a volume immensely larger than before; whereas solid elastic bodies only resume their former figure.

It is plain that the weight or pressure of the air does not at all depend on its elasticity, and that it is neither more nor less heavy than if it were not at all elastic. But from its being elastic, it follows, that it is susceptible of a pressure, which reduces it to such a space, that the force of its elasticity, which re-acts against the pressing weight, is exactly equal to that weight. Now the law of the elasticity is such, that it increases in proportion to the density of the air, and that its density increases in proportion to the forces or weights which compress it. Now, there is a necessary equality between action and re-action; that is, the gravity of the air, which effects its compression, and the elasticity of it, which gives it its tendency to expansion, are equal.

So that, the elasticity increasing or diminishing, in the same proportion as the density increases or diminishes, that is, as the distances among its particles decrease or increase; it is no matter whether the air be compressed, and retained in any space, by the weight of the atmosphere, or by any other cause; as in either case it must endcavour to expand with the same force. And therefore, if such air as is near the earth be inclosed in a vesscl, so as to have no comınunication with the external air, the pressure of such inclosed air will be exactly equal to that of the whole external atmosphere. And accordingly we find that quicksilver is sustained to the same height, by the elastic force of air inclosed in a glass vessel, as by the whole pressure of the atmosphere.-And on this principle of the condensation and elasticity of the air, depends the structure and use of the air-gun.

That the density of the air is al ways directly proportionol to the force or weight which compresses it, was proved by Boyle and Mariotte, at least as far as their experiments go on this head: and Mr. Mariotte has shown that the same rule takes place in condensed air. However, this is not to be admitted as scrupulously exact; for when air is very forcibly compressed, so as to be reduced to $\frac{\pi}{4}$ th of its ordinary bulk, the effect does not answer precisely to the rulc; for in this case the air begins to make a. greater rcsistance, and requires a stronger compression, than is given by the above proportion. And hence it appears, that the particles of air canuot, by means of any pussible weight or pressure, however great, be brought into perfect contact, or that it cannot thus be reduced to a solid mass; and consequently, that there must be a Iimit to which this condensation of the air can never arrive. The same remark is true with regard to the rarefaction of air, namely, that in very high degrees of rarefaction, the elasticity is decreased rather more than in proportion to the weight or density of the air: and hence there must also be a limit to the rarefaction and expansion of the air, by which it is prevented from expanding to infinity.

To what degree air is susceptible of condeusation, by compression, is not certainly known. Mr. Boyle condensed it 13 times more than in its natural state, by this means: others have compressed it into $\frac{T}{60}$ th part of its ordinary volume; Dr. Hales made it 38 times more dense, by means of a press; but by freezing water in a hollow cast-iron ball or shell, he reduced it to 1522 times less space than it naturally occupies; in which state it must have been of almost double the density or specific gravity of water: And as water is not compressible, except in a very small degree, it follows from this experiment, that the particles of air must be of a nature very different from those of water; since it would otherwise be impossible to reduce air to a volume above 800 times less than in its common state; there is however some reason to doubt of the accuracy of this experiment, as has been asserted by Dr. Halley, from some experiments performed in London, and others at Florence by the Academy del Cimento, that it may be safely concluded that no force whatever is capable to reduce air into a space 800 times less than that which it naturally occupies near the surface of the earth, at least by pressure. But others, and particularly M. Amontons, in the Memoirs of the French Academy, maintain, that there is no affixing any limits to its condensation; that greater and greater forces will reduce it into less and less compass; and that it is only elastic in virtue of the fire it contains; and that, as it is impossible ever absolutely to drive out all its fire, it is impossible cver to make the utmost condensation. We may further add, that the air contained in nitre, is probably condensed about 1600 times. See my Tracts, vol. 3, p. 303.

The elasticity of the air exerts its force equally in all directions; and when it is at liberty, and freed from the cause which compressed it, it expands equally on all sides, and in consequence always assumes a spherical figure in the interstices of the fluids in which it is lodged. This is evident in liquors placed in the receiver of an air-pump, by exhausting the air; at first there appears a multitude of exceeding small bubbles, like grains of fine sand, dispersed through the fluid mass, and rising upwards; and as more air is pumped out, they enlarge in size; but still they continue round. Also if a plate of metal be immerged in the liquor, on pumping, its surface will be seen covered over with small round bubbles, composed of the air which adhered to it, now expanding itself. And for the same reason it is that large glass globes are always blown up of a spherical shape, by blowing air through an iron tube into a piece of melted glass at the end of the pipe.

The expansion of the air, by virtue of its elastic property, when only the compressing force is taken off, or dininished, is found to be surprisingly great; and yet we are far from knowing the utmost dilatation of which it is capable. In several experiments made by Mr. Boyle, it 


\section{A I R}

[ 5

expanded first into 9 times its former space; then into 31 times; then into 60 , and then into 150 times. Afterwards, it was brought to dilate into 8060 times its first space; then into 10000 , and at last even into 13679 times its space; and all this solely by its own natural expansive foree, by only removing the pressure, but without the help of fire. And on this principle depends the construction and use of the MANOMETRR.

The elasticity of the air, under one and the same pressure, is still farther increased by heat, and dininished by cold, and that, by some late accurate experiments made by Sir George Shuckburgh, at the rate of the 440 th part of its volume nearly, for each degree of the variation of heat, from that of temperate, in Fahrenheit's thermometer.

Mr. Hauksbee observed, that a portion of air inclosed in a glass tube, when the temperature was at the freezing point, formed a volume which. was to that of the same quantity of air in the greatest heat of summer in England, as 6 to 7. And it has been found by several experiments, that air is expanded $\frac{x}{3}$ of its natural bulk by applying the heat of boiling water to it.

Dr. Hales found that the air in a retort, when the bottom of the vessel just became red-bot, was dilated into double its former space; and that in a white, or almost melting heat, it filled thrice its former space: but Mr. Robins found that air was expanded, by means of the white or fusing heat of iron, to 4 times its former bulk. See also several ingenious experiments on the elasticity of the air, in the Philos. Trans. for the year 1777, by Sir Genrge Shuckburgh and Colonel Roy.

This properly explains the common effect observed on bringing a close flaccid bladder near the fire to warm it; when it is presently found to swell as if more air were blown into it. And on this principle depends the structure and office of the thermometer.

M. Amontons first discovered that, with the same heat, air will expand in a degree proportioned to its density. And on this foundation the ingenious author has formed a discourse, to prove " that the spring and weight of the air, with a moderate degree of warmth, may enable it to produce even earthquakes, and others of the most vehement commotions of nature." He computes, that at the depth of the 74th part of the earth's radius bclow the surface, the natural pressure of the air would reduce to the density of gold; and thence infers that all matter below that depth, is probably heavier than the heavicst metal that we know of. And hence again, as it is proved that the more the air is compressed, the more does the same degree of fire increase the force of its clasticity; we may infer that a degree of heat, which in our orb can produce only a moderate effect, may have a very violent one in such lower orb; and that, as there are many degrees of heat in nature, beyond that of boiling water, it is probable there may, be some whose violence, thus assisted by the density of the air, inay be sufficiently powerful to tear asunder the solid globe. Mem. de l'Acead. 1703.

Many philosophers have supposed that the elastic property of the air depends on the figure of its corpuseles, which they take to be ramous: some maintain that they are so many minute flocculi, resembling flecces of wool: others rouccive them rolled up like hoops, and curled like wires, or shavings of wood, or coited like the springs of watches, and endeavouring to expand themselves by virtue of their texture.
A I R

But Sir Isaac Newton (Optics, Qu. 31, \&c,) explains the matter in a different way : such a contexture of parts he thinks by no means sufficient to accuunt for that amazing power of elasticity observed in air, which is capable of dilating itself into abore a million of times more space than it occupied before: but, he observes, as it is known that all bodjes have an attractive and a repelling power; and as both these are stronger in bodies, the denser, more compact, and solid they are; hence it follows that when, by heat, or any other powerful agent, the attractive force is overcome, and the particles of the body separated so far as to be out of the sphere of attraction; the repelling power, then commencing, makes them recede from each other with a strong foree, proportionable to that with which they before cohered; and thus they become permanent air.

And hence, he says, it is, that as the particles of air are grosser, and rise from denser bodies, than those of transient air, or vapour, true air is more ponderous than vapour, and a moist atmosphere lighter than a dry one.

And M. Amontons makes the elasticity of air to arise from the fire it contains; so that by augmenting the degree of heat, the rarefaction will be increased to a far greater degree than by a mere spontaneous dilatation.

The clastic power of the air becomes the second great source of the remarkable effects of this important fluid. By this property it insinuates itself into the pores of bodies, where, by means of this virtue of expancling, which is so easily excited, it must put the particles of those bodies into perpetual vibrations, and maintain a continual motion of dilatation and contraction in all budies, by the incessant changes in its gravity and deisity, and consequently its elasticity and expansion.

This reciprocation is observable in several instances, particularly in plants, in which the tracheæ or air-vessels perform the office of lungs; for, as the beat increases or diminishes, the air alternately dilatẹs and contracts, and so by turns compresses the vessels, and eases them again; thus pronoting a eirculation of their juices And hence it is found that wo regetation or germination is carried on in vacuo.

It is from the same cause too, that ice is burst by the continual action of the air contained in its bubbles. Thus too, glasses and other vessels are frcquently cracked, when their contained liquors are frozen; and thus also large blocks of stone, and entire columns of marble, sometimes split in the winter season, from some little bubble of included air acquiring an increased elasticity : and for the same reason it is that so few stones will bear to be heated by a fire, without eracking into many picces, by the incrtased expansive force of some air confined within their pores. From the same source arise also all putrefaction and fermentation; neither of which can be carried on in vacuo, even in the best-disposed subjects. And eren respiration, and animal life itsclf, are supprosed, by many authors, to be conducted, in a great measure, by the same principle of the air. And as we find such great quantities of air generated by the solution of aninul and vegetable substances, a gond deal must constantly be raised from the dissolution of these elements in the stomach and bowels. In fact, all natural corruption and alteration seem to depend on air; and even metals, particularly gold, only scein to bo durable and isicorruptible, in so far as thcy are impervious to air.

Composition, \&c, of the air. We have already ot- 
served, that for many ages the air we breathe was considered as a simple homogeneous fluid: various exhalations and particles of bodies arising from the eartl were indeed observed to mix with it, and on these its different degrees of salubrity were supposed to depend; but the aeriform fluid itself was never, till within a few years, known to be a compound substance. This point bowever has been clearly ascertained by the discoveries of modern chemists, particularly those of Priestley, Black, Cavendish, Lavoisier, Fourcroy, \&c. From the united testimonies of these discoveries we learn, that atmospheric air-is composed of at lcast two species of air, or elastic fluids, which arc called vital air, or oxygen gas, and azotic gas. The furmer is the great agent in respiration and combustion; and upon the proper proportion of both depends the purity of the atmosphere. The latter possesses contrary qualities, is noxious to animals, and incapable of maintaming combustion. The proportion of these in 100 parts of atmospheric air, is commonly about 26 or 27 parts of oxygen gas, and 74 or 73 parts of azotic gas, by wcight; or about 22 parts of the former, and 78 of the latter, in bulk.

'These two gases however, though they constitute the principal part of atmospheric air, are not the only ones that enter into its composition; for beside the numerous particle's of water and other substances, it contains about 1 part in 100 of carbonic acid gas, and a still smaller quantity of hydrogen gas; and from experiments made upon atir brought from the higher regions of the atmosphcre, by means of air-balloons, these' constitueust parts of it were found in nearly the same proportion as at the surface of the earth. For further particulars on this subject, see Azote, Gas, OXYGEN, \&c.

For the resistance of the air, sce Resistance.

Air-Balloon. See Afrostation.

AIR-Gun, in PNeUmatics, is a machine for propelling bullets with great violence, by the sole means of condensed air.

The first accourit we meet with of an air-gun, is in the Filemens d'Artillerie of David Rivaut, who was preceptor to Louis xulı, of France. IIe ascribes the invention to Marin, a burgher of Lisieux, who presented one to Henry iv.

To construct a machine of this kind, it is only necessary to take a strong vessel of any sort, into which the air is to be thrown or condensed by means of a syringe, or otherwise, the more the better; then a valve is suddenly opened, which lets the air escape by a small tube in which a bullet is placed, and which is thus violently forced out before the air.

It is evident then that the cffect is produced by virtue of the elastic property of the air; the force of which, as has been shown in the last article, is nearly proportional to its condensation; and therefore the greater quantity that can be forced into the engine, the greater will be the effect. Now this effect will be exactly similar to that of a gun charged with powder, and therefore we can easily form a comparison betwcen them : for inflamed gun-powder is nothing morc than very condensed elastic air; so that the two forces are exactly similar. It is shown by Mr. Robins, in his New Principles of Gunnery, that the fluid of inflamed gun-powder, has, at the first moment, a forec of elasticity equal to ubout 1000 times that of common air; and I have shown (vol. 3 of my Tracts) that the first force of the inflamed powder is often more than 2000 times that of the common air. 'Therefore it is necessary that air should be condensed a 1000 or 2000 times more than in its natural state, to produce the same effect as gun-powder. But then it is to be considered, that the velocities with which equal balls are impelled, are directly proportional to the square roots of the forces; so that if the air in an air-gun be condensed only 10 times, the velocity it will project a ball with, will be, by

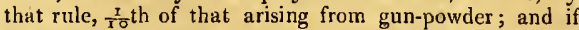
the air were condensed 20 times, it would communicate a velocity of $\frac{x}{7}$ th of that of gun-powder. But in reality the air-gun shoots its ball with a much greater proportion of velocity than as above, and for this reason, namely, that as the reservoir, or magazine of condensed air, is commonly very large in proportion to the tube which contains the ball, the density of the air is very little altered by expanding through that narrow tube, and consequently the ball is urged all the way by nearly the same uniform force as at the first instant ; whereas the elastic fluid arising from inflamed gun-powder is but very small in proportion to the tube or barrel of the gun, occupying at first indeed but a very small portion of it next the butend; and therefore, by dilating into a comparatively large space, as it urges the ball along the barrel, its elastic furce is proportionally more weakened, and it acts alivays less and less on the ball in the tube. From which cause it happens, that air condensed into a good large machine only 10 times, will sboot $j$ ts ball with a velocity but little inferior to that given by the gun-powder. And if the valve of communication be suddenly shut again by a spring, after opening it to let some air escape, then the same collection of $i t$ serves to impel many balls, one after another.

In all cases in which a considerable force is required, and consequently a great condensation of air, it will be requisite to have the condensing syringe of a small bore, perbaps not more than half an inch in diameter: otherwise the force to produce the compression will become so great, that the operator camnot work the machine: for, as the pressure against every square inch is about 15 pounds, and against every circularinch, or a circle of 1 inch diameter, about 12 pounds, if the syringe be one inch in diameter, when one injection is made, there will be a resistance of 12 pounds against the piston; when 2 , of 24 pounds; and when 10 are injected, there will be a force of 120 pounds to overcome; whereas 10 injections act against the half-inch piston, whose area is but $\frac{t}{4}$ of the former, with $\frac{x}{4}$ of the foree only, namely, 30 pounds; and 40 injections may be made with such a syringe, as well as 10 with the larger.

Air-guns are of various constructions: an easy and portable one is represented in Plate 2 , fig. 1 , which is a section lengthways through the axis, to show the inside. It is made of brass, and has two barrels : the inner barrel $D A$ of a small bore, from which the bullets are discharged; and a larger barrel ES C D R, on the outside of it. In the stock of the gun there is a. syringe $M N M S$, whose rod $\mathrm{M}$ draws out to take in air; and by peshing it in again, the piston $\mathrm{SN}$ drives the air before it, through the valve PE, into the cavity between the two barrels. The ball $\mathrm{K}$ is put down into its place in the small barrel, with the rammer, as in another: gun. There is another valve at $\mathrm{S \textrm {L }}$, which, being opened by the trigger 6 , permits the air to come behind the ball, so as to drive it ont with great force. If this valve be opened and shut sud- 
denly, one charge of condensed air may make several discharges of bullets; because only part of the injected air then goes out at a time, and another bullet may be put into the place $\mathrm{K}$ : but if the whole air be discharged on a single bullet, it will impel it more forcibly. This discharge is effected by means of a lock (fig. 2) when fixed to its place as usual in other guns; for the trigger being pulled, the cock will go down, and drive a lever which opens the valve.

Dr. Macbride (Exper. lss. p. 81) mentions an improvement of the air-gun, made by Dr. Ellis; in which the chamber for containing the condensed air is not in the stock, which renders the machine heavy and unwieldy, but has five or six hollow spheres belonging to it, of about 3 inches diameter, fitted to a screw on the lock of the gun. These spheres are contrived with valves, to confine the air which is forced into their cavities, so that a servant may carry them ready charged with condensed air : and thus the gun of this construction is rendered as light and portable as one of the smallest forvling-pieces.

Fig. 3 represents one made by the late Mr. B. Martin of London, and now by several of the mathematical instrument and gun-makers of the metropolis; which, for simplicity and perfection, perbaps exceeds any other that has been contrived. $\mathbf{A}$ is the gun-barrel, of the size and weight of a common fowling-piece, with the lock, stock, and ramrod. Under the lock, at $b$, is a round steel tube, having a small moveable pin in the inside, which is pushed out when the trigger $a$ is pulled, by the springwork within the lock; to this tube $b$ is screwed a bollow copper ball, perfectly air-tight. This copper ball is fully charged with condensed air by means of a syringe, before it is applied to the tube $b$. Hence, if a bullet be rammed down in the barrel, the copper ball screwed fast at $b$, and the trigger $a$ be pulled; the pin in $b$ then will forcibly push open a valve within the copper ball, and let out a portion of the condensed air; which will rush up through the aperture of the lock, and forcibly act against the bullet, driving it to the distance of 60 or 70 yards, or farther. If the air be strongly condensed at every discharge, only a portion of the air escapes from the ball ; therefore, by re-cocking the piece, another discharge may be made; and this repeated 15 or 16 times. An additional barret is sometimes made, and applied for the discharge of shot, instead of the ball above describerl.

Sometimes the syringe is applied to the end of the barrel c (fig. 4); the lock and trigger shut up in a brass case $d$; and the trigger pulled, or the discharge made, by pulling the chain $b$. In this contrivance there is a round chamber for the condensed air at the end of the spring at $e$, and it has a valve acting in a similar manner to that of the copper ball. When this instrument is not in use, the brass case $d$ is made to slide off, and the instrument then becomes a walking-stick: from which circumstance, and the barrel being made of cane, or brass, \&c, it has been called the Air-cane. The head of the cane unscrews and takes off at $a$, where the extremity of the piston-rod in the barrel is shown. An iron rod is placed in a ring at the end of this, and the air is condensed in the barrel in a manner similar to that of the gun as above; but its force and action is not near so strong as in the gun.

Magazine Air-Gun. This is an improvement of the common air-gun, made by an ingenious artist, L. Colbe. By his contrivance, ten bullets are so lodged in a cavity, near the place of discharge, that they may be succes- sively drawn into the barrel, and shot so quickly, as to be nearly of the same use as so many different guns: the only motion required, after the air has been injected, being that of shutting and opening the hammer, and cocking and pulling the trigger. Fig. 5 is a longitudinal section of this gun, as large in every part as the gun itself; and as much of its length is shown as is peculiar to this construction; the rest of it being like the ordinary airgun. $E E$ is part of the stock; $G$ is the end of the injecting syringe, with its valve Is, opening into the cavity FFFF between the barrels. $\mathrm{K} K$ is the small or shooting barrel, which receives the bullets, one at a time, from the magazine $\mathrm{D} E$, being a serpentine cavity, in which the bullets $b, b, b, \& c$, are lodged, and closed at the end D; whence, by one motion of the hammer, they are brought into the barrel at 1 , and thence are shot out by the opening of the valve $v$, which lets in the condensed air from the cavity F FF into the channel $\mathrm{V}_{\mathrm{K}} \mathrm{I}$, and so along the inner barrel $\mathrm{K} \mathrm{K} \mathrm{K}$, whence the bullet is discharged. $s \mathrm{I} s i$ a $k$ is the key of a cock, luaving a hole through it; which loole, in the present situation, makes part of the barrel $\mathrm{K} K$, being just of the same bore: so that the air, which is let in at every opening of the valve $\mathrm{v}$, comes behind this cock, and taking the ball out of it, carries it forward, and so out of the mouth of the piece.

To bring in another bullet to succeed $\mathrm{I}$, which is done in an instant, bring the cylindrical cavity of the key of the cock, which made part of the barrel $\mathrm{K} \mathrm{KK}$, into the situation $i k$, so that the part $\mathrm{x}$ may be at $\mathrm{K}$; then turning the gun upside-down, one bullet next the cock will fall into it out of the magazine, but will go no farther into this cylindrical cavity, than the two little pieces $s s$ will permit it; by which means only one bullet at a time will be taken in to the place 1 , to be discharged again as before.

A more particular description of the several parts may be seen in Desaguliers' Exper. Philos. rol. ii. pa. 399 et seq..

Arr-Pump, in Pneumatics, is a machine for exlausting the air out of a proper vessel, and thus making what is commonly called a vacuum; though in reality the air in the receiver is only rarefied to a certain deyree, so as to take off the ordinary effects of the atmosphere. By this machine we learn, in some measure, what our earth would be without air; and how much all vital, generative, nutritive, and alterative powers depend upon it.

The principle on which the air-pump is constructed, is the spring or elasticity of the air; as that on which llace common, or water pump is formed, is the effect of its gravity: the one gradually exhausting the air from a v'ssel by means of a piston, with a proper valve, working in a cylindrical barrel or tube; and the other exhausting water in a similar manner.

The invention of this instrument, to which the present age is indebted for so many fine discoveries, is ascribed to Otto Guericke, the celebrated magistrate of Magdeburg, who exhibited his first public experiments with it, betore the emperor and the states of Germany, at the breaking up of the imperial diet at Ratisbon, in the year $165 t$. But it was not till the year 1672 that Guericke published a description of the instrument, with an account of his experiments, in his Experimenta Nova Magdeburgica de Vacue Spatia: though an account of them had been published by Schottus in 1057, in his Mechanica Ilydrantico Pneumatica, by his consent. 
Dr. Hooke and M. Duhamel ascribe the invention of the air-pump to Mr. Boyle. But that great man frankly confesses that Guericke was boforehand with him in the execution. Some attempts, he assures us, he had indecd made on the same fousdation, before he knew any thing of what had been done abroad: but the information he afterwards received from the account given by Schottus, enabled him, with the assistance of Dr. Hooke, after two or three unsuccessful trials, to bring his design to maturity. The product of their labours was a new air-pump, much more easy, convenient and manageable, than the German one. And hence, or rather from the great variety of experiments to which this illustrious author applied the machine, it was afterwards called Machina Boyliana, and the vacuum produced by it, Vacuum Buylianum.

Structure of the Air-Pump. Most of the air-pumps that were first made consisted of only one barrel, or hollow cylinder, of brass, with a valve at the bottom, opening inwards ; and a moveable embolus or piston, having likewise a valve opening upivards, and so exactly fitted to the barrel, that when it is drawn up from the bottom, by means of an indented iron rod or rack, and a handle turning a small indented wheel, playing in the teeth of that rod, all the air is drawn up from the cavity of the barrel : there is also a small pipe opening into the bottom of the barrel, by means of which it communicates with any proper vessel to be exhausted of air, which is called a receiver, from its office in riceiving the subjects on which experiments are to be made in vacuo: the whole being fixed in a convenient frame of wood-work, where the end of the pipe turns up into a horizontal plate, on which the receiver is placed, just over that end of it.

The other parts of tbe machine, being only accidental circumstances, chiefly respecting conveniency, have been diversified and improved from time to time, according to the address and several views of the makers. That of Otto Guericke was very rude and inconvenient, requiring the labour of two strong men, for more than two hours, to extract the air from a glass, which was also placed unter water; and which allowed of no change of subjects for experiments.

Mr. Boyle, from time to time, removed several of these inconveniences, and lessened others: but still the working of his pump, which had but one barrel, was laborious, by reason of the pressure of the atmospliere, a great part of which was to be removed at every lift of the piston, when the exhaustion was nearly completed. Various improvernents were successively made in the machine, by the philosophers about that time, and soon after, who cultivated this new and important branch of pneumatics; as Papin, Mersenne, Mariotte, and others; but still they laboured under a difficulty of working them, from the circumstance of the single barrel, till Papin, in his further improvements, removed that inconvenience, by the use of a second barrel and piston, contrived to rise, as the other fell, and to fall as that rose; by which means, and the great improvements made by Mr. Hankshee, the pressure of the atmosphere on the descending piston, always ncarly balanced that of the ascending one; so that the winch, which worked them up and down, was easily moved by a very gentle force with one hand: and besides, the exhaustion was hercby made in less than half the time.

Some of the Germans, and others also, made improvements in the air-pump, and contrived it to perform the counter office of a condenser, in order to examine the properties of the air depending on its condensation.

Mr. Boyle contrived a mercurial gange or index to the air-pump, which is described in his first and second Physico-Mechanical Continuations, for measuring the degrees of the air's rarefaction in the receiver. This gauge is similar to the barometer, being a long glass tube, having its lower end immersed in an open bason of quicksilver, but its other end, which was open also, communicating with the receiver: which being exhausted, this tube is equally exhausted of air at the same time, and the external air presses the quicksilver up into the tube, to a height proportioned to the degree of exhaustion.

Mr. Vream, an ingenious pneumatic operator, made an improvement in Hauksbee's air-pump, by reducing the alternate up-and-down motion of the hand and winch, to a circular one. In his method, the winch is turned quite round, and yet the pistons are alternately raised and depressed: by which the trouble of shifting the hand backwards and forwards, as well as the loss of time, and the shaking of the pump, are prevented.

The air-pump, thus improved, is represented in plate iii, fig. 1; where $O 0$ is the receiver to be exhausted, ground truly level at the bottom, set over a hole in the plate, from which descends the bent pipe $h h$ to the cistern $d d$, with which the two barrels $a$ a communicate, where the pistons are worked by a toothed wheel, by turning the handle $b b$; thus the racks $c c$, with the pistons, are worked alternately up and down. $l l$ is the gauge tube, immersed in a bason of quicksilver $m$ at the bottom, and communicating with the receiver at top; from which however it may be occasionally disengaged, by turning a cock. And $n$ is another cock, by turning of which, the air is again let in to the exhausted receiver; into which it is heard to rush with a considerable hissing noise.

Notwithstanding the great excellency of Mr. Hauksbee's air-pump, it was still subject to inconveniences, from which it was in a great measure relieved by some contrivances of Mlr. Smeaton, which are described at large in the Philos. Trans. for the year 1752 . The principal improvements suggested by Mr. Smeaton, relate to the gauge, the valves of the piston, and the piston going closer down to the bottom of the barrel; for his pump has only one. By the last of these, the air was extracted more perfectly at each stroke. By the second, he remedied an inconvenience arising from the valve hole of the piston being too wide properly to support the bladder valve which covered it : instead of the usual circular orifice, Mr. Smeaton perforated the piston with seven small and equal hexagonal holes, one in the centre, and the other six around, forming tugether the appearance of a transverse section of a honey-comb; the bars.or divisions between which served to support the pressure of the air on the valve. His gauge consists of a bulb of glass, of a pear-like shape, and capable of bolding about half a pound of quicksilver: it is open at the lower end, the other terminating in a tube hermetically sealed: and it has annexed to it a scale, divided into parts of about $T^{\mathrm{T}} \mathrm{O}$ of an inch, and answering to the 1000th part of the whole capacity. During the exhaustion of the receiver, the gange is suspended in it by a wire; but when the pump has been worked as much as necessary, the gauge is pushed down, till the open end be immersed in a bason of quicksilver placed below. The air is then let into the receiver again, and the quicksilver is driven by it from the bason, up into the gauge, till the 
air remaining in it become of the same density as that without; and as the air always takes the highest place, the tube being uppermost, the expansion is determined by the number of divisions occupied by the air at the top. This air-pump is made to act also as a condensing engine, as some German maclines had done before, by the very simple operation of turning a cock.

By means of this gauge, Mr. Smeaton judged that his machine was incomparably better than any former one, as it seemed to rarefy the air in the receiver 1000 , or even 2000 times, while the best of these only rarefied it about 140 times: and as an implicit confidence was placed in Mr. Smeaton's accuracy, it was generally understood in this light, till the fallacy was accidentally detected in the manner related at large by $\mathrm{Mr}$. Nairne in the Philos. Trans, for the year 1777. This accurate and ingenious artist wanting to make trial of Mr. Smeaton's pear-gauge, executed an air-pump of his improved construction, in the best manner possible; which, in various experiments made with it, appeared, by the pear-gauge, to rarefy the air to an amazing degree indeed, being at times from 4000

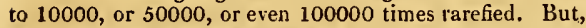
on measuring the same expansion by the usual long and short tube gauges, which both accurately agreed together, he found that these never slowed a rarefaction of more than 600 times: widely different from the same as measured by the pear or internal gauge, by experiments often repeated. ' Finding, says Mr. Nairne, 'still this disagreement between the pear-gauge and the other gauges, I tried a variety of experiments; but none of them appeared to me satisfactory, till one day in April 1776, when showing an experiment with one of these pumps to the honourable Henry Cavendish, Mr. Smeaton, and several other gentlemen of the Royal Society, the two gauges differed some thousand times from one another, Mr. Cavendish accounted for it in the following manner. "It appeared, he said, from some experiments of his father's, Lord Charles Cavendisb; that water, whenever the pressure of the atmosphere on it is diminished to a certain degree, is immediately turned into vapour, and is à immediately turned back again into water on restoring the pressure. This degree of pressure is different according to the heat of the water: when the heat is $72^{\circ}$ of Fahrenheit's scale, it turns jnto vapour as soon as the pressure is no greater than that of three quarters of an inch of quicksilver, or about 1-40th of the usual pressure of the atmospliere; but when the heat is only $41^{\circ}$, the pressure must be reduced to that of a quarter of an inch of quicksilver before the water turns into vapour. It is true, that water exposed to the open air, will evaporate at any heat, and with any pressure of the atmosphere; but that evaporation is entirely owing to the action of the air upon it; whereas the evaporation here spoken of is performed without any assistance from the air. Hence it follows, that when the receiver is exhausted to the above-mentioned degree, the moisture adhering to the different parts of the machine will turn into vapour, and supply the place of the air, which is continually drawn away by the working of the pump; so that the fluid in the pear-gauge, as well as that in the receiver, will consist in a great measurc of vapour. Now letting the air into the receiver, all the vapour within the pear-gauge will be reduced to water, and only the real air will remain uncondensed; consequently the pear-gauge shows only how much real air is left in the recciver, and not how much the pressure or spring of the included fuid is diminished; whereas the common gauges show how much the pressure of the inclucked fluid is diminished, and that equally, whether it consist of air or of vapour." Mr. Cavendish having explained so satisfactorily the cause of the disagreement between the two gauges, Mr. Nairne considered that, if he were to avoid moisture as much as pussible, the two gauges should nearly agree. Andin fact they were found to do so, each showing a rarefaction of about 600 , when all moisture was perfectly cleared away from the pump, and the plate and the edges of the receiver were secured by a cement, instead of setting it upon a soaked leather, as in the usual way. But, by future experiments, Mr. Nairne found that the same excellent machine would not exhaust more than 50 or 60 times, when the receiver was set upon leather soaked in water, the heat of the room being about $57^{\circ}$. And from hence he concludes, that the air-pump of Otto Guericke, and those contrived by Mr. Gratorix and Dr. Hooke, and the improved one by Mr. Papin, both used by Mr. Boyle, as also Hauksbee's, Gravesande's, Muschenbroech's, and those of all who have used water in the barrels of their pumps, could never have exliausted to more than between 40 and 50 , if the heat of the place was about $57^{\circ}$. And though $\mathrm{Mr}$. Smeaton, with his pump. where no water was in the barrel, but where leather soaked in a inixture of water and spirit of wine was used on the pump-plate, to set the receiver upon, may have exhausted all but a thousaudth, or even a ten-thousandth part of the common air, according to the testimony of his pear-gauge; yet so much vapour must have arisen from the wet leather, that the contents of the receiver could never be less tban a 70 th or 80 th part of the density of the atmosphere. But when nothing of moisture is used about this machine, it will, when in its greatest perfection, rarcfy its contents of air about 600 times.

It is evident that, by means of these two gauges, we can ascertain the several quantities of vapour and permanent air which make up the contents of the receiver, after the exhaustion is made as perfect as can be ; for the usual external gauge determincs the whole contents made up of the vapour and air, while the pear-gauge shows the quantity of real permanent air; consequently the difference is the quantity of vapour.

The principal cause which prevents this pump from exhausting beyond the limit above mentioned, is the weakened elasticity of the air within the receiver, which, decreasing in proportion as the quantity of the air within is diminished, becomes at last incapable of lifting up the valve of communication between the receiver and the barrel; and consequently no more air can then pass from the former to the latter.

Several ingenious persons have used their endeavours to remove this imperfection in the best air-pumps. Among these it seems that one Mr. Haas has succeeded tolerably well; having by means of a contrivance to open the cominunication valve in the bottom of the barrel, made his machine so correct, that when every thing is in the greatest perfection, it rarefies the contents of the recciver as far as 1000 times, cven when measured by the exterior gauge. The description of this machine, and an account of some experiments performed with it, are given by Mr. Cavallo in the Philos. Trans, for the year 1783.

But the imperfections it secms have inore recently been removed by an ingenious contrivance of Mr. Cuthberison, a mathomatical instrument maker at Amstcrdam, now of 
London, whose air-pump has neither cocks nor valves, and is so constructed, that what supplies their place has the advantages of both, without the inconveniences of cither. He has also made improvements in the gauges, by means of which he determines the height of the mercury in the tube, by which the degree of exhaustion is indicated, to the hundredth part of an inch. And to obviate the inconvenience of the elastic vapour arising from the wet leather, upon which the receiver is placed, for common experiments, he recommends the use of leather dressed with alum, and soaked in hog's lard, which he found to yield very little of this yapour; but when the utmost degree of exhaustion is required, his advice is, to dry the receiver well, and set it upon the plate without any leather, only smearing its outer edges with hog's lard, or with a mixture of three parts of hog's lard and one of oil. But indeed the use of the leather has long been laid aside by our English instrument-makers, a circumstance which probably bad not come to Mr. Cuthbertson's knowledge. An account of this instrument, and of some experiments performed with it, was published at Amsterdam in the year 1787 ; from which it appears that, by a coincidence of the several gauges, a rarefaction of 1200 times was shown; but when the atmosphere was very dry, the exhaustion has bcen so complete, that the gauges haveshown the air in the receiver to be rarefied above 2400 times.

There are made also by different persons, portable, or small air-pumps, of various constructions, to set upon a table, to perform experiments with. In these, the gauge is varied according to the fancy of the maker, but commonly it cousists of a bent glass tube, like a syphon, open only at one end. The gauge is placed under a small receiver communicating, by a pipe, with the principal one lcading from the general receiver to the barrels. 'The close end of the gange, of 3 or 4 inches long, before the exhaustion, has the quicksilver forced close up to the top by the pressure of the air on the open end; but when the exhaustion is considerably advanced, it begins to descend, and then the difference of the heiglits of the quicksilver in the two legs, compared with the height in the barometrical tubr, determines the degree of exhaustion: so, if the difference between the two be one inch, when the harometer stands at 30 , the air is rarcfied 30 times; but if the difference be only half an inch, the rarefaction is 60 times, and so on. See Plate rix. fig.r.

The Use of the Air-Pump. In whatever manner or form this machine is made, the use and operation of it are always the same. 'The handle, or winch, works the rackbar and piston up anil down in the barrel; by which mcans a barrel of the contained air is drawn out at every stroke of the piston, in the following manner: by pushing the piston down to the bottom of tlue barrel, where the air is prevented from cscaping downwards, by its elasticity it opens the valve of the piston, and escapes upwards; then raising the piston up, the extcrnal atmosphere shuts down its valve, and a vacuum would be made below it, but for the air in the receiver, pipe, \&c, which now raising the vilve in the bottom of the barrel, rushes in and fills it agnin, till the whole air in the recciver and barrel be of one uniform density, but less than it was beforc the stroke, in proportion as the sum of all the capacities of the receiver, pipe, and barrel together, is to the same sum winting the barrel. And thus the air in the receiver is diminished at each stroke of the piston, by the quantity or content of

Vi). I. the barrel or cylinder, and therefore always in the same proportion: 'so that by thus repeating the operation a number of times, the air is rarefied to any proposed degree, or till it has not elasticity enough to open the valve of the piston or of the barrel, after which the exhaustion cunnot be any farther carried on : the gauge, in comparison with the barometer, showing at any time what the degree of exhaustion is, according to the particular nature and construction of it.

But supposing no vapour from moisture, \&c, to rise in the receiver, the degree of exhaustion, after any number of strokes of the piston, may be determined by knowing the respective capacities of the barrel and the receiver, including the pipe, \&c. For as we have seen above that every stroke diminishes the density in a constant proportion, namely'as much as the whole content exceeds that of the cylinder or barrel; consequently the sum of as many diminutions as there are strokes of the piston, will show the whole diminution by all the strokes. So, if the capacity of the barrel be equal to that of the receiver, in which the communication pipe is always to be included; then, the barrel being half the sum of the whole contents, half the air will be drawn out at one stroke; and consequently the remaining halt, being dilated through the whole or first capacity, will be of only half the density of the first: in like manner, after the second stroke, the density of the remaining contents will be only half that of the former, that is only $\frac{x}{4}$ of the original density : continuing this operation, it follows that the density of the remaining air will be $\frac{1}{8}$, after 3 strokes of the piston, $\frac{1}{16}$, after 4 strokes, $\frac{1}{3 \frac{1}{2}}$, after 5 strokes, and so on, according to the powers of the ratio $\frac{\pi}{2}$; that is, such power of the ratio as is denoted by the number of the strokes. In like manner, if the barrel be $\frac{1}{3}$ of the whole contents, that is, the receiver double of the barrel, or $\frac{2}{3}$ of the whole contents; then the ratio of diminution of density being $\frac{2}{3}$, the density of the contents, after any number of strokes of the piston, will be denoted by such power of $\frac{2}{3}$ whose exponent is that number; namely, the density will be $\frac{2}{3}$ after one stroke, $\left(\frac{2}{3}\right)^{2}$ or $\frac{4}{9}$ after two strokes, $\left(\frac{2}{3}\right)^{3}$ or $\frac{8}{2}$ after 3 strokes, and in general it will be $\left(\frac{2}{3}\right)^{\mathbf{n}}$ after $n$ strokes : the original density of the air being 1. Hence then, universally, if $s$ denote the sum of the contents of the receiver and barrel, and $r$ that of the receiver only without the barrel, and $n$ any number of strokes of the piston; then, the original density of the air being 1 , the density after $n$ strokes will be $\left(\frac{r}{s}\right)^{n}$ or $\frac{r}{s^{n}}$, namely the $n$ power of the ratio $\frac{r}{s}$. So, for example, if the capacity of the receiver he equal to 4 times that of the barrel ; then their sum $s$ is 5 , and $r$ is 4 ; hence the density of the contents after 30 strokes, will be $\left(\frac{4}{5}\right)^{30}$, or the 30th power of $\frac{4}{5}$, which is $8 \frac{1}{8}$ nearly; so that the air in the receiver is rarefied Sos times.

See also the Memoires de l'Acad, Royale des Sciences for the ycars 1693 and 1705 .

From the same formula, namcly $\left(\frac{r}{s}\right)^{n}=d$ the density, we easily derive a rule for finding the number of strokes of the piston, necessary to rarcfy the air any number of times, or to reduce it to a given density $d$, that of the natural air being 1 . For since $\left(\frac{r}{s}\right)^{n}=d$, by taking the logarithm of this equation, it is $n \times \log$. of $\frac{r}{s}=\log$. of $d$; 
and hence $n=\frac{\log \cdot d}{\log \cdot \frac{r}{s}}=\frac{\log \cdot d .}{\log \cdot r-\log \cdot s}$; that is, divide the log. of the proposed density by the log. of the ratio of the receiver to the sum of the receiver and barrel together, and the quotient will show the number of strokes of the piston requisite to produce the degree of exhaustion required. So, for example, if the receiver be equal to 5 times the barrel, and it be proposed to find how many strokes of the piston will rarefy the air 100 times; then $r=5, s=6$, $d=\frac{I}{\text { roo }}$, whose $\log$, is -2 , and $\frac{r}{s}=\frac{5}{6}$, whose log. is $-\cdot 07918$; therefore $\frac{2}{.07918}=25 \frac{x}{4}$ nearly, wbich is the number of strokes required.

And, farther, the same formula reduced, will give us the proportion between the receiver and barrel, when the air is rarefied to any degree by an assigned number of strokes of the piston. For since $\left(\frac{r}{s}\right)^{\mathrm{n}}=d$ the density, therefore, extracting the $n$ root of both sides, it is $\frac{r}{s}=\sqrt[n]{ } d$ : that is, the $n$ root of the density is equal to the ratio of the receiver to the sum of the receiver and barrel. So, if the density $d$ be $\frac{1}{12} \overline{8}$, and the number of strokes $n=7$; then the 7 th root of $\frac{1}{12} 8$ is $\frac{1}{2}$; which shows that the receiver is cqual to half the receiver and barrel together, or that the capacity of the barrel is just equal to that of the receiver.

Some of the principal effects and phæmomena of the air-pump, are the following: That, in the exhausted receiver, heavy and light bodies fall equally swift: so, a guinea and feather fall from the top of a tall receiver to the bottom exactly together. That most animals die in a minute or two: but however, That vipers and frogs, though they swell much, live an hour or two; and after being seemingly quite dead, come to life again in the open air. That snails survive about 10 hours; efts, or slowworms, two or three days; and leeches five or six. That pysters live for 24 hours. That the heart of an eel taken out of the body, continues to beat for great part of an hour, and that more briskly than in the air. That warm blood, milk, gall, \&c, undergo a considerable intumescence and ebullition. That a mouse or other animal may be brought, by degrees, to survive longer in a rarefied air, than naturally it does. That air may retain its usual pressure, after it is become unfit for respiration. That the eggs of silk-worms hatch in vacuo. That vegetation stops. That fire extinguishes; the flame of a candle usually going out in one minute; and a charcoal in about five minutes. That red-hot iron, however, scems not to be affected; and yet sulphur or gun-powder are not lighted by it, but only fused. That a match, after lying scemingly cxtinct a long time, revives again on re-admitling the air. That a flint and stcel strike sparks of fire as copiously, and in all dircetions, as in air. 'That magnets, and magnetic needles, act the same as in air. 'That the smoke of an extinguished luminary gradually settles to the bottom in a darkish body, leaving the upper part of the receiver clear and transparent; and that on inclining the vessel sometimes to one side, and sometimes to another, the fume preserves its surface horizontal, after the nature of otlicr fluids. That heat may be produced by attrition. 'l'hat camphire will not take fire; and that gun-powder, though some of the grains of a licap of it be kindled by a burning glass, will not give fire to the contiguous grains. That glow-worms lose their light in proportion as the air is exhausted, and at length become totally obscure; but on re-admitting the air, they presently recover it all. That a bell, on being struck, is not heard to ring, or very faintly. That water freezes. But that a syphon will not run. That electricity appears like the aurora borealis. With multitudes of other curious and important particulars, to be met with in the numerous writings on this machine, namely, besides the Philos. Transactions of mosi academies and societies, in the writings of Torricelli, Pascal, Mersenne, Guericke, Schottus, Boyle, Hooke, Duhamel, Mariotte, Hauksbee, 'Hales, Muschenbroeck, Gravesande, Desaguliers, Frankliu, Cotes, Helsham, and a great many other authors.

AIR-VESSEL, in Hydraulics, is a vessel of air contained within some water engines, which being compressed, by forcing in a considerable quantity of water, will, by its uniform spring, force it out at the pipe in a constant uninterrupted stream, to a great height.

Air-vessel too, in the improved fire engines, is a metallic cylinder, placed between the two forcing pumps: by the action of the pistons the water is forced into this ressel, through two other pipes, with valves; then the air, previously contained in it, is compressed by the water, in proportion to the quantity admitted, and this, by its spring, forces the water through a pipe by a constant and equal stream; whereas in the common squirting engine, the stream is discontinued between the several strokes.

AJUTAGE, or ADJUTAGE, in Hydraulics, part of the apparatus of a jet d'eau, or artificial fountain: being a kind of tube fitted to the aperture or mouth of the cistern, or the pipe; through which the water is to be played in any direction, and in any shape or figure.

It is chiefly the diversity in the ajutage, that makes the different kinds of fountains. So that, by having several ajutages, to be applied occasionally, one fountain is made to have the effect of many.

Mariotte, Gravesande, Desaguliers, and Buat, have written pretty fully on the nature of ajutages, or spouts for jets d'eau, and especially the former. He affirms, from experiment, that an even polished round holc, made in the thin end of a pipe, gives a bigher jet than either a cylindrical or a conical ajutage; but that, of these two latter however, the conical is bctter than the cylindrical figure. See his Traité du Mouvement des Eanx, part 4.

The quantity of water discharged by ajutages of equal area, but of different figures, is the same. And for like figures, but of different sizes, the quantity discharged, is directly proportional to the area of the ajutage, or to the square of its diameter, or of any side or otlier linear dimension: so, an ajutage of a double diameter, or side, will discharge 4 times the quantity of water; of a triple diameter, 9 times the quantity; and so on; supposing them at an equal depth below the surface or head of water. But if the ajutage be at different depths below the head, then the celerity with which the water issues, and consequently the quantity of it run out in any given time, is directly proportional to the square root of the altitude of the head, or depth of the hole: so at 4 times the depth, the celerity and quantity is double; at 9 times the depth, triple ; and so on.

It has bren found that jets do not rise quite so high as the head of water; owing chicfly to the resistance of the air against it, and the pressure of the upper parts of the 
jet upon the lower: and for this reason it is, that if the direction of the ajutage be turned a very little from the perpendicular, it is found to spout rather higher, than when the jet is exactly upright.

It is found by experiment too, that the jet is higher or lower, according to the size of the ajutage; that a circular hole of about an inch and a quarter in diameter, jets highest; and that the nearer that size, the better. Expericnce also shows, that the pipe leading to the ajutage, should be much larger than it; and if the pipe be a long one, that it should be wider the farther it is from the ajutage.

For the other circumstances relating to jets and the issuing of water under various circumstances, see ExHAUstion, Flux, Fountain, Jet d'EaU, \&c, to which they more properly belong.

ALBATEGNI, an Arabic prince of Batan in Mesopotamia, who was a celebrated astronomer, about the year of Christ 880 , as appears by his observations. He is also called Mubammed ben Geber Albatani, Mahomet the son of Geber, and Mubamedes Aractensis. He made astronomical observations at Antioch, and at Racab or Aracta, a town of Chaldea, which some authors call a town of Syria or of Mesopotamia. He is highly spoken of by Dr. Halley, as a vir admirandi acuminis, ac in administrandis observationibus exercitatissimus.

Finding that the tables of Ptolemy were imperfect, he computed new ones, which were long used as the best among the Arabs: these were adapted to the meridian of Aracta or Racah. Albategni composed in $j$ Arabic a work under the title of The Science of the Stars, comprising all parts of astronomy, according to his own observations and those of Ptolemy. This work, translated into Latin by Plato of Tibur, was published at Nuremberg in 1537, with some additions and demonstrations of Regiomontanus; and the same was reprinted at Bologna in 1645 , with this author's notes. Dr. Halley detected many faults in these editions: Philos. Trans. for $1693, N^{\circ} 204$.

In this work, Albategni gives the motion of the sun's apogee since Ptolemy's time, as well as the motion of the stars, which he makes 1 degree in 70 years. He made the longitude of the first star of $\Lambda$ ries to be $18^{0} 2^{\prime}$; and the obliquity of the ecliptic $23^{\circ} 35^{\prime}$. And upon Albategni's observations were founded the Alphonsine tables of the moon's motions; as is observed by Nic. Muler, in the Tab. Frisicæ, pa. 248.

ALBERTUS MAGNUS, a very learned man in the 13 th century, who, among a multitude of books, wrote several on the various mathematical sciences, as Arithmetic, Geometry, Perspective or Optics, Music, Astrology and Astronomy, particularly under the titles, de sphæri, de astris, de astronomia, item speculum astronomicum.

Albertus Maguus was born at Lawingen on the Danube, in Suabia, in 1205, or according to some in 1193 ; and be died at a great age, at Cologn, November 1s, 1280. Vossius and other anthors speak of him as a great genius, and decply skilled in all the learning of the age. His writiugs were so numerous, that they make 21 volumes in folio, in the Lyons edition of 1615 . He has passed also for the author of some writings relating to nidwifery, \&c, under the title of De natura rerum, and De secretis mulierum, in which there are many phrascs and expressions unavoidable on such a subject, which gave great offence; and raised a clamour against him as the supposed author, and inconsistent with his character, being a Dominican friar, and sometime bishop of Ratisbon; which dignity however he soon resigned, through his love for solitude, to enter again into the monastic life. But the advocates of Albert assert, that he was not the author of either of these two works. It must be acknowledged however, that there are, in his Comment on the Master of Sentences, some questions concerning the practice of conjugal duty, in which he has used some words rather too gross for chaste and delicate ears: but they allege what he himself used to say in his own vindication, that he came to the knowledge of so many monstrous things at coniession, that it was impossible to avoid touching upon such questions. Albert was certainly a man of a most curious and inquisitive turn of mind, which gave rise to other accusations against him; such as, that he laboured to find out the pbilosopher's stone; that he was a magician; and that he made a macline in the shape of a man, which was an oracle to him, and explained all the difficulties he proposed: the conmon cant accusations of those times of ignorance and superstition. But having great knowledge in the mathematics and mechanics, by his skill in these sciences he probably formed a head, with springs capable of articulate sounds; like the machines of Boetius and others.

Jobn Matthæus de Luna, in his treatise De Rerum Inventoribus, has attributed the invention of fire-arms to Albert; but in this he is refuted by Naude, in his Apologie des grands hommes.

ALBUMAZAR, otherwise called ABUASSAR, and $\mathbf{J}_{A \mathbf{P H A R}}$, was a celebrated Arabian philosopher and aśtrologer, of the 9 th or 10 th century, or according to some authors much earlier. Blancanus, Vossius, \&c, speak of him as one of the most learned astronomers of his time, or astrologer, which was then the same thing. He wrote a work De Magnis Conjunctionibus Annorum Revolutionibus, ac eorum Perfectionibus, printed at Venice in 1515, at the expense of Melchior Sessa, a work chiefly astrological.

He wrote also Introductio in Astronomiam, printed in the year 1489. And it is reported that he observed a comet in his time, above the orb of Venus.

ALDEBARAN, the Arabian name of a fixed star, of the first magnitude, just in the eye of the sigu or constellation Taurus, or the bull, and hence it is popularly called the bull's eye. For the beginning of the year 1800 , its

Right Ascension is............ $66^{\circ} 6^{\prime} \quad 51^{\prime \prime} 10$

Annual variation in AR...... 0 . $0051: 31$

Declination .................. $16 \quad 5 \quad 52.00 \mathrm{~N}$.

And Annual variat. in Decl. $\begin{array}{cccc}0 & 0 & 8.30\end{array}$

ALDERAIMIN, a star of the third magnitude in the right shoulder of the constellation Cepheus.

ALDHAFERA, or Aldhaplira, in the Arabian Astronomy, denotes a fixed star of the third magnitude, in the mane of the sign or constellation Leo, the lion.

ALEMBERT (John le Rond d'). See Dalembert.

ALFECCA, or Alfeta, a name given to the star conmonly called Lucida Coronæ.

alfragan, Alvergani, or Fargani, a celebrated Arabic astronomer, who flourished about the year 800. He was so called from the place of his nativity, Fergan, in Sogdiana, now called Maracanda, or Samarcand, anciently a part of Bactria. He is also called Ahmed (or Muhammed) ben-Cothair, or Katir. Ile wrote 
the Elements of Astronomy, in 30 chapters or sections. In this work the author chiefly follows Ptolemy, using the same hypotheses, and the same terms, and frcquently citing him.

There are three Latin translations of Alfragan's work. The first was made in the 12th century, by Joannes Hispalensis; and was published at Ferrara in 1493, and at Nuremberg in 1537, with a preface by Melancthon. The second was by James Christman, from the Hebrew version of James Antoli, and appeared at Frankfort in 1590. Christman added to the first cbapter of the work an ample commeutary, in which he compares together the calendars of the Romans, the Egyptians, the Arabians, the Persians, the Syrians, and the Hebrews, and shows the correspondence of their years.

The third and best translation was made by Golius, professor of mathematics and Oriental languages at Leyden: this work, which came out in 1669 , after the death of Golius, is accompanied with the Arabic text, and many learned notes upon the first nine chapters; for this author was not spared to carry them farther.

ALGAROTI, commonly called Count Algaroti, a celebrated Italian of the last century, well skilled in Architecture and the Newtonian philosophy, \&c. Algaroti was born at Padua, but in what year has not been mentioned. Led by curiosity, as well as a desire of improvement, he travelled early into foreign countries; and was very young when be arrived in France in 1736. It was here that he composed his Newtonian Philosophy for the Ladies, as Fontenelle bad done his Cartesian Astronomy, in the work entitled The Plurality of Worlds. He was much noticed by the king of Prussia, who conferred on him many marks of his esteem. He died at Pisa the 23d of May, 1764, and gave orders for his own mausolcum, with this inscription upon it ; Hic jacet Algarotus, sed non omnis. He was esteemed to be well skilled in painting, sculpture, and architecture. His works, which are numerous, and on a variety of subjects, abound with vivacity, elegance, and wit: a collection of them has lately been made, and printed at Leghorn; but that for which he is chiefly cntitled to a place in this work is his Newtonian Philosophy for the Ladies, a sprightly, ingenious, and popular work.

ALGEBRA is usually understood to be a general method of resolving mathematical problems by means of equations. Or, it is a method of performing the calculations of all kinds of quantities by means of general signs or characters. At first, numbers and things were expressed ly their names at full length; but afterwards these were abridged, and the initials of the worcls used instead of them; and, as the art advanced farther, the letters of the alphabet came to be employed as general representations of all kinds of quantities. Other marks were also gradually introduced, to express all sorts of operations and combinations; so as to entitle it to different appellations-as, universal arithmetic, and literal arithmetic, and the arithmetic of signs.

The etymology of the name, Algebra, is given in various ways. It is, however, pretty generally considered, that the word is $\Lambda$ rabian, and that from those people we had the name, as well as the art itself, as is testified by Lucas de Burgo, the first European author on this art, whose treatise was printed, and who also refers to former authors and masters, from whose writings he had learned

\section{A L G}

it. The Arabic name be gives it, is $\Lambda$ lghebra e $A \operatorname{lmu}$ cabala, which is explained to signify the art of restitution and comparison, or opposition and restoration, or resolution and equation, all which agree wcll enough with the nature of this art. Some, however, derive it from various other Arabic words; as from Geber, a celebrated philosopher, chemist, and matbematician, to whom also they ascribe the invention of this science: and some derive it from the word Geber, which, with the particle al, makes Algeber, which is purély Arabic, and signifies the reduction of broken numbers or fractions to integers.

But Peter Ramus, in the beginning of his algebra, says, " the name algebra is Syriac, signifying the art and doctrine of an excellent man. For Geber, in Syriac, is a name applied to men, and is sometimes a term of honour, as master or doctor among us. That these was a certain learned mathematician, wbo sent his algebra, written in the Syriac language, to Alexander the Great, and he named it Almucabala, that is, the book of dark or mysterious things, which others would rather call the doctrine of algebra. And to this day the same book is in great estimation among the learned in the oriental nations, and by the Indians who cultivate this art it is called aljabra, and alboret; though the name of the author himself is not known." But Ramus gives no authority for this singular paragraph. It has, howcver, on various cocasions been distinguished by other names. Lucas Paciolus, or de Burgo, in. Italy, called it l'Arte Magiore: ditta dal vulgo la Regola de la Cosa over Alghebra e Alnucabala; calling it l'Arte Magiore, or the greater art, to distinguish it from common arithmetic, which is called l'Arte $\mathrm{Mi}$ nore, or the lesser art. It seems too that it had been long and commonly known in his country by the lame Regola de la Cosa, or Rule of the Thing; whence came our rule of coss, cosic numbers, and such-like terms. Some of his countrymen followed his denomination of the art: but other Italian and Latin writers called it Regula rei ct census, the rule of the thing and the product, or the root and the square, as the unknown quantity in their equations commonly ascended no higher than the square or second power. From this Italian word census, pronounced chensus, came the barbarous word zenzus, used by the Germans and others, for quadratics; with the several zenzic or square roots. And hence $2,3, \pi$, which are derived from the letters $r, z, c$, the initials of res, zenzus, cubus, or root, square, cube, came to be the signs or characters of these words: like as $\mathrm{R}$ and $\sqrt{ }$, derived from the letters $R, r$, became the signs of radicality.

Later authors, and other nations, used some the one of those names, and some another. It was also called specious arithmetic by Vieta, on account of the species, or letters of the alphabet, which he brought into general use; and by Newton it was called universal arithmetic, from the manner in which it performs all arithmetical operations by general symbols, or indeterminate quantities.

Some authors define algebra to be the art of resolving mathematical problems : but this is the idea of analysis, or the analytic art in general, rather thun of algebra, which is only one particular species of it. Indecel, algebra properly consists of two parts: first, the method of calculating magnitudes or quantities, as represented by letters or other characters; and secondly, the mamer 
of applying these calculations in the solution of problens.

In algebra, as applied to the resolution of problems, the first business is to translate the problem out of the common, into the algebraic language, by expressing all the conditions and quantities, both known and unknown, by their proper characters, arranged in an equation, or several equations if necessary, and treating the unknown quantity, whether it be number, or line, or any other thing, in the same way as if it were a known one: this forms the composition. Then the resolution, or analytic part, is the disentangling the unknown quantity from the several others with which it is connected, so as to retain it alone on one side of the equation, while all the other, or known quantities, are collected on the other side, and so giving the value of the unknown one. And as this disentangling of the quantity sought, is performed by the converse of the operations by which it is connected with the others, taking them always backwards in the contrary order, it hence becomes a species of the analytic art, and is called the 'modern analysis, in contradistinction to the ancient analysis, which chiefly respected geometry, and its applications.

There have arisen great controversies and sharp disputes among authors, concerning the history of the progress and improvements of algebra; arising partly from the partiality and prejuclices which are natural to all nations, and partly from the want of a closer examination of the works of the older authors on this subject. From these causes it has happened, that the improvements made by the writers of one nation, have been ascribed to those of another; and the discoveries of an earlier author, to some one of much later date. Add to this also, that the peculiar methods of many author's have been described so little in detail, that our information derived from such histories is but very imperfeet, and amounting only to some general and vague ideas of the true state of the arts. To remerly this inconvenience therefore, and to reform this article, I have taken the pains carefully to read over in succession all the older authors on the subject, which I have been able to mcet with, and to write down distinctly a particular account and description of their several compositions, as to their contents, notition, improvements, and peculiarities; from the comparison of all which, 1 have acquired an idea more precise and accurate than it was possible to obtain from other histories, and in a great many instances very different from them. 'I'he full detail of these descriptions would employ a volume of itself, and would be far tor extensive for this place: I must therefore limit this article to a very brief abriclgment of my notes', reniarking only the most material circumstances in cach author; from which a general idea of the chain of improvements may be perceived, from the first rude beginnings, down to the more perfect state; from which it will appear that the discoveries and improvements marle by any one single author, are scarcely ever either very great ur nunserous; but that, on the contrary, the improvements are almost always very slow and gradual, from former writers, successively made, not by great leaps, and after long intervals of time, but by gradations which, viewed in succession, become almost imperceptible.

Of Diopifantus's Algebia. As to the origin of the analytic art, of which algebra is a species, it is doubtless as old as any science in the world, bcing the natural. method by which the mind investigates truths, causcs, and theories, from their observed effects and properties. Accordingly, traces of it are observable in the works of the earliest philosophers and mathematicians, the subject of whose enquiries most of any require the aid of such an art. And this process constituted their analytics. Of that part of analytics, however, which is properly called algebra, the oldest treatise which has come down to us, is that of Diopliantus of Alexandria, who flourished abuut the year 150 after Christ, and who wrote, in the Grcek language, 13 books of algebra or arithmetic, as mentioned by himself at the end of his address to one Dionysius, though only 6 of them have hitherto been printed; and an imperfect book on multangular numbers, namely, in a Latin translation only, by Xilander, in the year 1575 , and afterwards in 1621 and 1670 in Greek and Latin by Gaspar Bachet. These books, however, do not contain a treatise on the elementary parts of algebra, but only collections of difficult questions relating to square and cube numbers, and other curious properties of numbers, with their solutions. And Diophantus only prefaces the books by an address to Dionysius, for whose use it was probably written, in which he just mentions certain precognita, as it were to prepare him for the problems themselves. In these remarks he shows the names and generation of the powers, the square, cubc, $4 \mathrm{th}, 5 \mathrm{th}, 6 \mathrm{th}, \mathrm{kc}$, which he calls dynamis, cubus, dynamodinamis, dynamocubus, cubocubus, according to the sum or ardition of the indices of the powers; and he marks these powers with the initials thus $\delta \vec{v}, x^{\bar{v}}, \delta \vec{v}, \delta x^{\vec{v}}, x x^{\bar{v}}, \& c$ : the un-

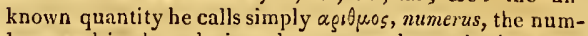
ber; and in the solutions he commonly marks it by the final thus $\bar{s}$; also he denotes the monades, or indefinite unit, by $\mu^{\overline{0}}$. Diophantus then remarks on the multiplication and division of simple species together, showing what powers or species they produce; declares that minus ( $\lambda \varepsilon i \psi(s)$ multiplied by minus produces plus ( $\left(\pi \alpha \xi_{\xi} \xi v\right)$; but that minus multiplied by plus, produces minus; and that the mark used for minus is $\uparrow$, namely the $\psi$ inverted and curtailed; but he uses no mark for plus, but a word: or conjunction copulative.

As to the operations, viz, of addition, subtraction, multiplication, and division of compound species, or those connected by plus and minus, Diophantus does uot teach, but supposes his reader to know them. He then remarks on the preparation or simplifying the equations that are derived from the questions, which we call reduction of cquations, by collecting like quantitics tugether, adding quantities that are minus, and subtracting such as are plus, called by the moderns transposition, so as to bring. the equation to simple terms, and then depressing it to a lower degree by equal division, when the powers of the unknown quantity are in every term : which preparation, or reduction of the complex equation, bcing now made, or reduced to what we call a final cquation, Diophantus goes no farther, but barely says what the root or res ignota is, without giving any rules for finding it, or for the resulution of equations; thereby intimating that such rules were to be found in some other work, douc either by himself or others.

Of the body of the work, lib. 1 contains $4: 3$ questions, concerning one, two, three, or four unkuown numbers, having certain relations to cacli other, viz, concerning 

A L G
$\left[\begin{array}{ll}62 & \end{array}\right]$
A L G

their sums, differences, ratios, products, squares, sums and differences of squares, \&c, \&c; but none of them concerning either square or cubic numbers. Lib. 2 contains 36 questions. The first 5 questions are concerning two numbers, though only one condition is given in each question; but he supplies another by assuming the numbers in a given ratio, viz, as 2 to 1 . The 6 th and 7 th contain each two conditions: then in the 8 th question he first comes to treat of square numbers, which is this, to divide a given square number into two other squares; and the 9 th is the same, but performed in a different way: the rest, to the end, are almost all about one, two, or three squares. Lib. 3 contains 24 questions concerning squares, chiefly including three or four numbers. Lib. 4 begins with cubes; the first of which is this, to divide a given number into two cubes whose sides shall have a given sum : bere he has occasion to cube the two binomials $5+n$ and $5-n$; the manner of doing which shows that he was acquainted with the composition of the cube of a binomial; and many other places manifest the same thing. Only part of the questions in this book are concerning cubes; the rest are relating to squares. Two or three questions in this book have general solutions, and the theorems deduced are general, and tor any numbers indefinitely; but all the other questions, in all the four books, are employed in finding only particular numbers. Lib. 5 is also concerning square and cube numbers, but of a more difficult kind, beginning with some that relate to numbers in geometrical progression. Lib. 6 contains 26 propositions, concerning right-angled triangles; such as to make their sides, areas, perimeters, \&c, \&c, squares or cubes, or rational numbers, \&c. In some parts of this book it appears, that Diophantus was acquainted with the composition of the 4 th power of the binomial root, as he sets down all the terms of it; and, from his great skill in such matters, it seems probable that he was acquainted with the composition of other higher powers, and with other parts of algebra, besides what are here treated of. At the end is part of a book, in 10 propositions, concerning arithmetical progressions, and multangular or polygonal numbers. Diophantus once mentions a compound quadratic equation; but the resolution of his questions is by simple equations, and by means of only one unknown letter or character, which he chooses or assumes so ingeniously, that all the other unknown quantities in the question are easily expressed by it, and the final equation reduced to the simplest form which it seems the question can admit of. Sonnetimes he substitutes for a number sought immediately, and then expresses the other numbers or comlitions by it: at other times he substitutes for the sum or difference, \&c, and thence derives the rest, so as always to obtain the expressions in the simplest form. Thus, if the sum of two numbers be given, he substitutes for their difference; and if the difference be gtven, he substitutes for their sum: in both cases he has the two numbers easily expressed by adding and subtracting the half sum and half difference; and so in other cases he uses other similar ingenious notations. In short, the chief excellence in this collection of questions, which seems to be only a set of exercises to some rules which had been given elsewhere, is the neat mode of substitution or notation; which being once made, the reduction to the final equation is easy and evident: and there he leaves the solution, only mentioning that the root or apsonos is so much. On the whole, this work is treated in a very able and masterly manner, manifesting the utmost address and knowledge in the solutions, and inducing a persuasion that the author was deeply skilled in the science of algebra, to some of the most abstruse parts of which these questions or exercises relate. However, as be contrives his assumptions and notations so as to reduce all his conditions to a simple equation, or at least a simple quadratic, it does not appear what his knowledge was in the resolution of compound or affected equations.

But though Diophantus was the first author on algebra that we now know of, it was not from him, but from the Muors or Arabians that we received the knowledge of algebra in Europe, as well as that of most other sciences. And it is matter of dispute who were the: first inventors of it ; some ascribing the invention to the Grecks, while others say that the Arabians had it from the Persians, and these from the Indians, as well as the arithmetical method of computing by ten characters, or digits; but the Arabians themselves say it was invented among them by one Mahomet ben Musa, or son of Moses, who it seems flourished about the 8 th or 9 th century. It is more probable, however, that Mahomet was not the inventor, but only a person well skilled in the art; and it is further probable, that the Arabians drew their first knowledge of it, either from the Indians, or from Diophantus and other Greek writers, as they did that of geometry and other sciences, which they improved and translated into their own language; and from them it was that Europe received these sciences, before the Greek authors were known to us, after the Moors settled in Spain, and after the Europeans began to hold communication with them, and began to travel among them to learn the sciences. And according to the testimony of Abulpharagius, in the year 969 , the arithmetic of Diophantus had been trans. lated into Arabic by Mahomet ben-yahya Buziani. But whoever were the inventors and first cultirators of algebra, it is certain that the Europeans first received the knowledge, as well as the name, from the Arabians or Moors, in consequence of the close intercourse which subsisted between them for several centuries. And it appears that the art was pretty generally known, and much cultivated, at least in Italy, if not in Spain, as well as other parts of Europe also, long before the invention of printing, as many writings on the art are still extant in the libraries of manuscripts; and the first authors, presently after the invention of printing, speak of many former writers on this subject, from whom they learncd the art.

Of the Jnvian Algenra. Some notices have lately been obtained of the science of algebra among the Indians; and it is very probable that, through the intercourse of learned Englishmen with that country, we shall receive still inore considerable information on that head.

There lias long existed cause to suspect that the principles of this art came to Europe through the Arabians and Moors, as well as the Indian numeration and arithmetic; and every cxtension of our concerns among them, serves further to increase the probability of that opinion. For more than a century past, evidence has been received in Europe, at various times, of the existence of very learned works on astronomy among the Indians. Such notices were first imported by certain learned Frenchmen, and communicated through the Memoirs of the Academy; whence a very ingenious and learned account of such works was given in the Astronomie Indienne of the unfortunate NI. Bailly. Since then, many other valuable cum- 
munications have been made by several of our own learned countrymen, belonging to the Bengal Society, and other persons curious in the sciences; as sir William Jones, Samuel Davis, esq., Edward Strachey, esq., and many others. Hence the strongest evidence has been obtained, that, at a period several thousand years (at least three or four) before the Christian era, the Indians must have possessed very correct astronomical observations and rules of calculation; rules that require a considerable knowledge of geometry and of trigonometry, both plane and spherical; and even accompanied with regular tables of sines and versed sines: at a time when all Europe was in a state of gross barbarity, if it was at all inhabited. See a valuable paper in the $2 \mathrm{~d}$ volume of the Asiatic Researches, by Samuel Davis, Esq. on the Astronomical Computations of the Hindus; also two learned dissertations on the Indian Astronomy and Trigonometry, by Professor Playfair, in the Edinburgh Philosophical Transactions, vols. $1 \mathrm{I}$. and IV.

What we have now, however, particularly to attend to, is the algebra of that country. It has long been thought that a people, possessing so much knowledge of many other branches of mathematical science, could not well be unacquainted with algebra; and we have now received incontestable proofs of their very critical skill in that branch. Several specimens of such works have been seen in that country, both in the native language and in Persian translations. Some of the latter are also now in the hands of S. Davis, esq. of Portman Square, one of the directors of the East-India Company, with a partial translation into English; and similar translations of some others have been sent to England by Mr. Edward Strachey, before mentioned. And as I have heen favoured with the perusal of these, $I$ am enabled to give some account of them.

The first of these communications, by Mr. Strachey, of the Bengal civil establishment, is a printed account of some observations on the originality, extent, and importance, of the mathematical science of the Hindoos; with extracts from Persian translations of the Lilawati or Leelawuttee, and the Beej Gunnit; or the Bija Ganita, as it is written by Mr. Davis. These two works, Mr. Strachey informs us, were both written by Bhasker Acharij, a famous Hindoo mathematician and astronomer, who lived about the beginning of the 13 th century of the Christian era; the latter of these two treatises being on algebra, ivith some of its applications; and the former on arithmetic, and algebra, and mensuration or practical geometry. The Bcja or Bija Ganita was translated into Persian in 1634, by Utta Ulla Rushcedee, at Agra or Dehli probably; and the Leelawuttee in 1587 , by the celebrated Fyzce.

It is well known, Mr. Strachey says, that the only Persian science is Arabian, and that the Arabs had much of their mathematical knowledge fiom the Greeks; $i t$ is certain, however, that they had their arithmetic from the Indians, and most likely their algebra was drawn from the same source; but the time, and other circumstances respecting the introduction of these sciences among the Arabs, is unknown. It appears, however, that the first account of any Indian mathernatical science among the Arabs, was of their astronomy, which was known in the reign of At Mamoon. In later times, many Mahoinmedais have lad access to the Hindoo books; accounts of several are in the $\Lambda$ ycen $\Lambda$ chbery, and in D'Herbelot.
Abul Fuzl gives a list of Sanscrit books, which were translated into Persian in Akbar's time; among which the Leelawuttee is the only mathematical work.

From a comparison of the algebra of the Arabians and Grecks, and that of the inodern Europeans, with the Persian translations of the Beja Ganita and Lelawati, it would probably appear, that the algebra of the Arabs is quite different from that of Diophantus, and not taken the one from the other: that if the Arabs did learn from the Indians, as is most probable, they did not borrow largely from them; that the Persian translations of the Beej Gunnit and Leelawuttee, contain principles, which are sufficient for the solution of any propositions in the Arabian, or in the Diophantine algebra; that these translations contain propositions, which are not to be solved on any principles that could be supplied by the Arabian or the Diophantine algebra; and that the Hindoos were farther advanced in some branches of this science than the modern Europeans, with all their improvements, till the middle of the eighteenth century.

These premises are exemplified by several extracts from the Lilawati and the Beja Ganita, too long to be repeated herc, but are to be seen in my Tracts, vol. 2, p. 154, \&c; where we trace many instances of the Hindoos' knowledge in geometry, and in mensuration, as well as in the various parts of algebra, as far as compound quadratic equations inclusively, and that after a more perfect manner, in some respects, than our own practice.

On Indeterminate Problems of the Second Degree, Dioplanttus, and the Bija Ganita.

'The 16 th question of the 6 th book of Diophantus, is as follows:-

"Having two numbers given, if one of these drawn into a ccrtain square, and the other subtracted from the product, make a square; it is required to find another square, greater than the former, which shall do the same thing. For instance, given the two numbers 3 and 11, and a certain square 25 , which drawn into 3 , and 11 taken from the product, leaves the square of $\delta$; to find another square greater than 25 having the same property. Put its side $1 \mathrm{~N}+5$, then its square is $1 \mathrm{Q}+10 \mathrm{~N}+25$; triple of this diminished by 11 leaves $3 Q+30 N+64$ equal a square; letits side be $2 \mathrm{~N}-8$, which gives $\mathrm{N}=62$; then $62+5=67$ is the side, and 4489 the square sought."

In the Bija Ganita this problem is solved very gencrally and scientifically, by the assistance of another, which was unknown in Europe till the middle of the 17 th century; and first applied to questions of this nature by Euler, in the middle of the 18 th century.- With the affirmative sign, the Bija Ganita rule for finding new values of $a x^{2}+b$ $=y^{2}$, is this: Suppose $a g^{2}+b=h^{2}$ a particular case: find $m$ and $n$ such that $a x^{2}+1=m^{2}$; then is $x=m g+n h$, and $y=m h+a n g$.

General methods, according to the Hindus, for the soIution of indeterminate problems of the first and second degrces, are found in the 4 th and 5 th chapters of the Bija Ganita, which differ much from Diophantus's work. It contains, a great deal of knowledge and skill, which the Greeks had not; such as, the use of an indefinite number of unknown quantitics, and the use of arbitrary marks to express them; a good arithmetic of surds; a perfect theory of indeterininate problems of the first degree; a very extensive and general knowledge of those of the second degrec; a perfect acquaintance with quadratic cquations, \&c. The arrangement and manner of the two works 
A L G

[ 6

are as different as their substance: the one constitutes a regular bouly of science; the other does not: the Bija Ganita is quite connected and well digested, and abounds in general rules, which suppose great learning; the rules are illustrated by examples, and the solutiuns are performed with skill. Diophantus, though not entirely without method, gives very few general propositions, being chiefly remarkable for the dexterity and ingenuity with which he makes assumptions for the simple solution of his questions. The former teaches algebra as a science, by treating it systematically; the latter sharpens the wit, by solving a variety of abstruse and complicated problems in an ingenious manner. The author of the Bija Ganita goes deeper into his subject, treats it more abstractedly, and more methodically; though not more acutely than Diophantus. The former has every characteristic of an assiduous and learned compiler; thc latter of a man of genius in the infancy of science.

Besides the foregoing remarks, derived chiefly from the printed notices of Mr. Strachey, I have lately been favoured with communications of several other curious particulars relating to the same two books, by $\mathrm{S}$. Davis, esq. The late Mr. Reuben Burrow collected, in Inclia, many oriental manuscripts on the mathematical sciences, both in the Sanscrit and the Persian languages, the lat ter being translations only of the former : most of these he bequcathed by will to one of his sons there, but with an injunction not to be delivered to him till he should have learned those languages and the sciences. But one or two of these Burrow left to his friend $\mathrm{Mr}$. Dalby, mathematical professor at the Royal Military College, Farnham. These are now in Mr. Dalby's possession, bcing the Persian translations of the Bija Ganita and Lilawati, with an attempt at an English translation of some parts of them by Mr. Burrow; but these attempts being mostly interlineations written with a black-lead pencil, are in danger of being obliterated. Mr. Strachey also, lately arrived from India, has an entire English translation of the Bija, of which he has favoured me with the perusal, and besides communicated by letter many descriptive remarks of those works, from all which sources I have collected the following curious particulars, till such time as he may be pleased to give the whole work to the public.

The first work is called the Beej, or the Beej Gunnit (as they are pronounced, but written Bija Ganita), and secms to have been translated into Persian about the year 1634. The Persian introduction calls it "Beej Gunnit, the author Blıasker Acharya, author of the Lilawati." It is there also said, that "this excellent method of computation was translated from the Hindu into Persian, and is called the Book of Composition and Resolution;" and " that it is not written in any book, Persian or Arabic." The two words here translated " composition and resolution," are elsewhere simply called "algebra." The two Persian words for "Beej Gunnit," are totally different from them; so that the Persians and Arabians have adopted the meaning, not the pronunciation, of the Sanscrit. Part of the MS. is a commentary on the original Hindu work, by the Persian translator.

The work consists of five parts. It commences with explaining affirmatives and negatives, which he characterises by two terus denotiug cxisting aud non-cxisting, also properiy and debt. 'Then follow the first rules, as with us. Next, surds are given at great length; and there seems here a general method of finding the number of surds in
A L G

any powers raised from inultinomial surd roots, something like our method of finding the number of combinations. Next follow questions about squares; such as (using our own notation), finding $67 x^{2}+1$, and $61 x^{2}+1$, and $13 x^{2}-1$, \&c, squares; also a general way or method to make expressions of this kind $a x^{2}+b$ squares.

Then questions are treated producing simple equations; with the application to some questions about triangles. Then more questions about squares; as, to find $x \pm y$ and $x y$ both squares; also $x^{3}+y^{3}$ and $x^{2}+y^{2}$ both squares.

Next, some questions producing quadratics. Something is also said of a cubic; but it seems to hint that it cannot be solved generally: a straight ruler is mentioned, which it is suspected may allude to some mechanical method of solution, for there is an omission in the translation. Then more about squares; such as, to find $x-y\}$ both $\left.7 x^{2}+8 y^{2}\right\}$ both $\left.x^{2}+y^{3}\right\}$ $\left.x^{2}+y^{2}\right\}$ squares; $\left.7 x^{2}-8 y^{2}+1\right\}$ squares; $\left.x+y\right\}$ squares; $\left.\begin{array}{l}x-y+2 \\ x+y+2\end{array}\right\}$ squares; $\left.\begin{array}{l}x^{2}-y^{2}+1 \\ x^{2}+y^{2}+1\end{array}\right\}$ squares; $\left.\begin{array}{l}x-y \text { a square } \\ x^{2}+y^{2} \text { a cube }\end{array}\right\}$ in whole numbers; $\frac{x^{3}-a}{b}$ a whole number, \&c, \&c.

Lastly, more indeterminate questions. In short, a grcat part is about indeterminate, and what we call questions of the Diophantine kind, yet without any one being the very same as in that author; which alone seems to show a ditferent origin; besides, they are mostly treated in a very different way; and several of them are not easy.

There are three or four questions about 1 ight-angled triangles, done algebraically; and here one might have expected to have found Eucl. 47 , I, quoted; but, instead of that, a reference is made to the "figurc of wedding chair." But from these, and many other parts of their writings, wc perceive tliat they were possessed of, not this property only, but most others the same as in our geometry; and here it was probably that Pythagoras acquired his mathematical knowledge, which he carried back with him, and taught to his countrymen. The results of the operations by the cipher 0 , are the same as we make them: the quotient $\frac{a}{0}$ Burrow at first translated " infinite," but afterwards crossed it with his pencil, and substituted " cannot be comprehended :" probably it means unbounded, or unassignable.

Respecting this notation in this at $\mathrm{S}$. it appears that unknown quantities are represented by letters or characıers, which they call "colours." Thus, when only onc unknown is used, it is denoted by a character callud "Majool ;" when two unknowns enter into the computation, the second is "Aswad" (black); a third is Ncelok" (blue); the fourth yellow, \&x. The unknown, or quantity sought, is called technically the thing, meaning emphatically the thing about which the question or enquiry is male. All their marks, denoting words expressing the operation, are Arabian. $\Lambda \mathrm{nd}$ it is remarkable that the first lialian writers on algebra, used a worel of the same import for the unknown quantity, viz, $\cos a$, the thing; whence, in Europe, the science came to be called the cossic art, and such quantities cossic numbers, \&c.

There is also a character set next an unknown, to denote its squarc; another for the cube, \&c: also a mark for the square root; and one for the cube root: but there does not appear to be any thing answering to a vinculum used in compound quantities. The higher powers are formed, and named, by repeating und combining these 


\section{A L G}

$[65$

marks; so, the 4 th power is called the square square; the 5 th power, the square eube; the 6 th power, the cube cube; and so on, meaning those powers multiplied together. For equality they use the expression "I equalize," or " equalizing," and, indeed, all their operations are expressed in words at length.

When unknowns are multiplied together, they are placed next one another, in our inanner; though there seems occasionally to be a mark for product. It is observable too that the affirmative, or the negative sign, and the coefficient, are always placed on contrary sides of the unknown, not $+2 x y$, as with us, but $2 x y+$, or $+x y 2$; this latter is in the Persian way, as they read from right to left; but the Hindus from left to right, like the Europeans. But, though the Persians write from right to left, they do not use, or translate the Sanscrit numerals backwards; a circumstance which renders the opinion very probable, that the Persians derived their numeration characters and calculation from the Indians, but probably at second hand through the Arabians.

Compounds are multiplied as we fill the multiplication table: for example, to multiply $-3 x+2 y-2$ by $+4 y-$ $x+1$, they proceed as in the

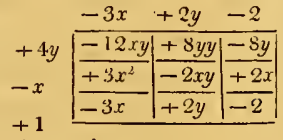
margin, and afterwards collect the products.

In the process of their sulutions, there is no distinct arrangement of the different steps, as in the modern algebra; but it is carried on without any breaks in the lines, something like de Burgo, or Bombelli, \&c. 'The language is written in the first person, as, -1 do so and so-I then equalize-next I multiply, \&c.

We have already noticed their method of resolving compound quadratic equations, the same as ours, by completing the square; and better than ours in such cases as $a x^{2}$ $+b x=c$, when the first term has a coefficient $a$, which does not divide the two $b$ and $c$, by which they avoid the operation in fractions. In imitation of this, also, I see they have a method of complcting the powers in some cases of cubics and biquadratics: Thus, baving the cubic equation $x^{3}+12 x=6 x^{2}+35$; first subtracting $6 x^{2}$, gives $x^{3}-6 x^{2}+12 x=35$; next subtracting 8 , gives $x^{3}-$ $6 x^{2}+12 x-8=27$, which completes the binomial cubic, and the roots are $x-2=3$, or $x=5$. Again, having given the imperfect biquadratic $x^{4}-400 x-2 x^{2}=9999$, a case which it is not very obvious how to bring it to a complete power, but which is managed with much address, in this manuer. First add $400 x+1$ to both sides, this gives $x^{4}-$ $2 x^{2}+1=10000+400 x$, where the first side is a complete sqinire, and the roots are $x^{2}-1=\sqrt{ }(10000+400 x)$; but as the latter side is not a complete square, the author goes back again, and tries another course; thus, to the original equation be adds $4 x^{2}+400 x+1$, which gives $x^{4}+2 x^{2}+1$ $=4 x^{2}+400 x+10000$, two complete squares, the roots of which are $x+1=2 x+100$; again, subtract $2 x$, and it becomes $x^{2}-2 x+1=100$, which are again two complete squares, the roots of which are $x-1=10$, and hence $x=$ 11. And this process has some resemblance to that which was afterwards plactised, if not imitated, by Lewis Ferrari. It appears, however, that the Indians had no general method for all equations of these two powers, but only depended on their own ingenuity for artfully managing some particular cases of them; for, at the couclusion of the ubove process, the author emphatically adds, "The soluVor. I.
A L G

tion of such questions as these depends on correct judgment, aided by the assistance of God."

At the conclusion of the first book occur some curious circumstances in the solution of certain problems in the application of algebra to geometry, particularly some relating to rightitangled triangles, by which it appears that they were well acquainted with the most remarkable properties in Euclid's Elements, some of which are cited under names peculiar to themselves, and sometimes, $\mathrm{Mr}$. Stracbey thinks, by numbers of the books and propositions in Euclid's collection, unless these last references have been added by the translator, or some transcriber, which is to be suspected.

ln the $3 \mathrm{~d}$ book the author comes to treat of questions and equations having several unknown quantities in them. These he directs to exterminate one after another, much after our modern way, till they are reduced to one unknown only. The number of independent equations are stated to be equal to the number of the unknown quantities; but when there are not so many equations, the defect is supplicd by assuming values for as many of the ulıknown quantities, \&c, as with us. The unknown quantities are represented and called by so many different characters and names, as is our own practicc also. We denote then usually by the letters $x, y, z, \& \mathrm{c}$; the Hindus by different. colours, or letters, or other marks also. "Thus," says our author, "suppose the first unknown, and the $2 \mathrm{~d}$ black, and the $3 \mathrm{~d}$ blue, and the 4 th yellow, and the 5 th red, and the 6 th green, and the 7 th parti-coloured, and so on, giving whatever names you please to the unkuown quantities which you wish to discover; and if, instead of these colours, other names are supposed, such as letters, and the like, it may be done. For what is required, is to find out the unknown quantities, and the object in giving names, is that you may distinguish the things required."

ln this $3 \mathrm{~d}$ book occur many examples of making ccrtain analytical expressions to be squares and cubes, \&z. These are mostly very curious, many of them difficult, and often involving several unknown quantities; they are generally solved in a masterly way, and that very tifferent from the manner of Diophantus. The two other, books are on similar indeterminate problems, but gradually more and more complex and difficult.

Besides the above, there are many curious things in the Bija Ganita, and it may be presumed, adds Mr. Strachey, that the Hindu books contain much interesting matter respecting this branch of algebra; it is probable that thcy have some system of continued-fractions; and perhaps methods of approximation, and theories of serics and equations. From the rules of the 5 th book, and their application, there is some ground for an opinion, that the Hindus may liave had a knowledge of curves, and much of the application of mathematics to natural philosuplay.

On the whole, it would seem that the origin of algebra, as well as of aritlumetic, was probably Judian. We have however as yct but little certain information on this subject. It has been said that the Arabs ascribe the inworion of algebra to the Grecks; but the algebra of Diophantus is widely different from that of the Arabs; and it is very doubtful whether the Grecks ever had any other than that of Diophantus. If there be any doubt of Diophantus's algebra being of Cireek origin, it may be worthy of remark, that at Alexandria he mightit liave had the means of access K 
to Indian literature. The Bija Ganita is indeed of comparatively modern date; but we must not forget that it is extracted from other books, as stated by Mr. Davis, in the Asiatic Researches, in a very learned article on the cycle of 60 years. And it is not unlikely that there are old Hindu treatises, from which not only the Bija Ganita, but even the algebra of Diophantus, and that of the Arabs, may have been derived.

The other work, the Lilawati, as before mentioned, is on arithmetic, mensuration, \&c. In the introduction, we find that "the collector of the book Lilawati was Bhasker Acharya, of Bidder city," on the northern confines of Hindustan. And "though the date of it is not known, yet in another book, made in the year 1105 of Salbahan, the reason of making the Lilawati is given." In the Ayeen Akbery (a Persian work on the manners, history, laws, $\& c$, of the Hindus, translated by Gladwin), we find " that Acharya among the followers of Jine, is one who explains any difficulties that may occur to noviciates;" and therefore we may conclude that Bhasker was an instructor in mathematics. Now, the Salbahan, according to the Hindu chronology, commenced Anno Dom. so; hence this book must have been written in 1185. But all that can be inferred from this is, that in 1185 they did not know when Bhasker lived.

The Lilawati begins with the first rules of arithmetic, and goes through fractions, the extraction of roots, \&c, with a good deal of what we should call alligation. Like us, they mark every $3 \mathrm{~d}$ figure from the place of units, in extracting the cube root. A part towards the end is upon "forms", somewhat similar it seems to our permutations. The numeral figures are nearly the same shape as those mentioned in p. 8 of Wallis's Algebra.

From which it appears that our present figures have been derived from them, hy gradual and successive alterations in the shape, by different transcribers. And hence it appears also, that these figures, as well as their use, and the arts of arithmetic, algebra, \&cc, have come to us from India, in their progress through Persia, Arabia, Africa, Spain, Italy, \&c; and that we ought rather to call them Indian figures, \&c, than Arabian.

\section{Translation of Fyzi's Preface to the Lilawati.}

"By order of the king Akber, Fyzi translates into Persian, from the Indian language, the book Lilawati, so famous for the rare and wonderful arts of calculation and mensuration. He (Fyzi) begs leave to mention, that the compiler of this book was Bhascara Acharya, whose birth place, and the abode of his ancestors, was the city of Biddur, in the country of the Deccan. Though the date of compiling this work is not mentioned, yet it may be nearly known from the circumstance, that the author made anwther book on the Construction of Almanacks, called Kurrun Kuttohul, in which the date of compiling it is mentioned to be 1105 years from the date of the Salibahn, an era famous in India. From that year, to this, which is the 32nd Jlahi year, corresponding with the Hejira year 995, there have passed 37.3 years." (answering nearly to 1585 of the Christian era).

"It is said that the composing the Lilawati was occasioned by the following circumstance. Lilawati was the name of the author's (Bhascara's) daughter, concerning whom it appeared, from the qualities of the Ascendant at her birth, that she was destined to pass her life unmarried, and to remain without children. The father ascertained a lucky hour for contracting licr in inarriage, that she might be firmly connected, and have children. It is said that when that hour approached, he brought his daugliter and his intended son near him. He left the bour cup on the vessel of water, and kept in attendance a time-knowing astrologer, in order that when the cup should subside in the water, those two precious jewels should be united. But, as the intended arrangement was not according to destiny, it happened that the girl, from a curiosity natural to children, looked into the cup, to observe the water coming in at the hole; when by chance a pearl separated from her bridal dress, fell into the cup, and, rolling down to the hole, stopped the influx of the water. So the astrologer waited in expectation of the promised hour. When the operation of the cup had thus been delayed beyond all moderate time, the father was in consternation, and examining, he found that a small pearl bad stopped the course of the water, and that the long-expected hour was passed. In short, the father, thus disappointed, said to his unfortunate daughter, I will write a book of your name, which shall remain to the latest times-for a good name is a sccond life, and the ground-work of eternal existence." Fyzi's preface then proceeds:

"The arrangement of this translation was made with the assistance of men learned in this science, particularly the astrologers of the Deccan. Some Indian words, for which corresponding expressions were not to be found in books of this science, have been retained, as thcy were in the Indian language. The work has been divided into an Introduction, some Rules, and a Conclusion."

Introduction. In this part are given explanations of some expressions in the science of calculation; with the meaning of certain terms employed in operations of the art of numbers; the divisions and terms of money, weights, measures, time, and mensurations; some of which are curious, as bearing some analogy to our own, of which they might possibly be the origin. The grain, or barleycorn, is given by the author, as the element of weights as well as measures. Thus, two barleycorns make 1 soorkh. The breadth of $S$ barleycorns is the quantity of 1 finger; 24 fingers make the Arm or cubit; and 10 cubits make a Bamboo or rod, nearly the same as our own. The element of time is given thus: The time in which a word of two letters, as $T_{a}$ or $K a$, can be uttered 10 times, "neither slowly nor quickly," is called Pran; then 6 prans make 1 Pul ; 60 puls 1 Ghurry; and 60 ghurrys one day and night. So that it hence appears that,

$$
\begin{aligned}
& 60 \text { ghurrys are }=\text { our } 24 \text { hours } \\
& 1 \text { gliurry }-=-24 \text { minutes } \\
& 1 \text { pul }-=-24 \text { seconds } \\
& 1 \text { pran }-=-4 \text { secomls } \\
& 1 \text { ka uttered in } \\
& \text { ON THE } \triangle \text { RABIAN ALGEBMA. }
\end{aligned}
$$

Mr. Strachey says, "the Greek algebra may be secu in Diophanus, who is the only Greek writer on the subject that we have hoard of.-The Indian algebra may be seen in Bija Ganita, and in the Lilawati, by the author of the Bija: and as the Persian translations of these works contain a degree of knowledge, which did not exist in any of the ordinary sources of science, extant in the time of the translators, they may be safely taken as Indian, and of ancient origin. To give some idea of the algebra of the Arabians, by which we may be enabled to judge whether, on the one hand, it could have been derived from Diophantus, or, on the other, that of the Hindus could have been 
taken from them, the work entitled Khulasat-ul-Hisab, may be taken as a specimen; especially because there is a part of this book which marks the limits of algebraical knowledge, in the time of the writer.

"We have seen that the first European Algebraists learnt of the Arabians; but no account bas been given of the nature, the extent, and the origin of A rabian algebra. No distinct abstract or translation of any Arabic book, on the subject, has appeared in print; nor has it been establisbed beyond controversy, who taught the Arabians. The Khulasat-ul-Hisab is of considerable repute in India: it is thought to be the best treatise on algebra, and it is almost the only book on the subject read there. I selected it, because I understood that, as well as the shortest, it was the best treatise that could be procured. Besides general report, I was guided by the authority of Maulavi Roshen Ali, an acknowledged good judge of such matters, who assured me that, among the learned Moslems, it was considered as a most complete work, and that he knew of no Arabian algebra beyond what it contained. In the Sulafat-ul-Asr, a book of biography, by Nizam-al-dinAhmed, there is this account of Baha-ul-din, the author of the Khulasat-ul-Hisab. "He was born at Balbec, in the month of D'hi lhaj, $953 \mathrm{Hijri}$ (anno Chr. 1575) and died at Isfahan in Shawal, 1031,' (anno Chr. 1653). Mention is made of many writings of Baha-ul-din, on religion, law, grammar, \&c, a treatise on astronomy, and one on the astrolabe. In this list of his works, no notice is taken of his great treatise on algebra, the Behr-ulHisab, which is alluded to in the Khulasat-ul-Hisab. Mawlavi Roshen Ali tells me the commentators say, it is not extant. There is no reason to believe that the Arabians ever knew more than appears in Baha-ul-din's book, for their learning was at its height long before his time.

"From what has been stated it will appear, that from the Khalasat-ul-Ilisab, an adequate conception may be formed of the nature and extent of the algebraical knowledge of the Arabians; and hence I am induced to bope, that a short analysis of its contents will not be unacceptable herc. I deem it necessary here to state that, possessing nothing more than the knowledge of a few words in Arabic, I made the translation, from which the following summary is abstracted, from the viva voce interpretation into Persian of Maulavi Roshen Ali, who perfectly understood the subject and both languages, and afterwards collated it with a Persian translation, which was made about 60 years after Baba-ul-din's death, (anno Chr, 1713), and which Roshen Ali allowed to be perfectly correct.

" The work, as stated by the anthor in his preface, consists of an introduction, 10 books, and a conclusion. The introduction contains definitions of arithmetic, of number, which is its object, and of various classes of numbers. The author distiuctly ascribes to the Indian sages the invention of the nine figures, to express the numbers from 1 to 9 . l3ook 1 comprises the arithmetic of integers. The rules enumerated under this head are, Addition, Duplation, Subtraction, Halving, Multiplication, Division, and the Extraction of the Square Root. The method of proving the operation, by casting out the 9's is described under each of these rules. The author gives the following remarkable definitions of multiplication and division : viz, 'Multiplication is finding a number such, that the ratio which one of the factors bears to it, shall be the same which unity bears to the other factor; and clivision is finding a number, which has the same ratio to unity, as the dividend has to the divisor.'

"For the multiplication of even tens, hundreds, \&c, into one another, the author delivers the following rule, which is remarkable in this respect, that it exhibits an application of something resembling the indices of logarithims." "Take the nombers as if they were units, and multiply them together, writing down the product, Then add the numbers of the rasks together, the place of units being 1 , that of tens $2, \& c$; subtract 1 from the sum, and call the remainder the number of the rank of the product.' This is similar to our own practice in such cases, when we say, Multiply the significant numbers together, and subjoin to their product all the ciphers that are in both factors."

There next follow several other ingenious contrivances, and compendiums, such as, To multiply figures between 5 and 10. To multiply units into numbers between units and 20. To multiply together numbers between 10 and 20. To multiply numbers between 10 and 20 into compuund numbers between 20 and 100 . To multiply numbers between 20 and 100 , where the digits in the place of tens are the same. To multiply numbers between 10 and 100 , when the digits in the place of tens are different. To multiply two unequal numbers, whose sum is even ; from the square of the half sum subtract the square of the halt difference. For multiplying numbers consisting each of several places of figures, the method described by this author, under the name of Sbabacah, or network, and illustrated by the following example, has some resemblance to the operation by Napier's bones; where, instead of our way, the method is, to set dlown the whole of each product in the alternate chequers, then add up the columns diagonal wise. On the other rules, nothing is delivered differing so much from those contained in our common books of arithmetic, as to require particula: mention.

Book 2d contains the arithmetic of fractions. Book $3 d$, the rule of thrce, or to find an unknown number by four proportionals. Book 4 th delivers the rule of position, both single and double, or to find an uuknown number by assuming a number once or twice, and comparing the errors. Book 5th gives the method of finding an unknown number by reversing all the steps of the process described in the question. This last rule has a near affinity to the reduction of equations in algebra, and might very naturally lead to the invention of algebra itself, being easily and generally applicable to the solution of questions usually given in the rules of position, and in the reduction of equations. For instance, taking the first example in Double Position, in vol. 1, of my Course of Mathematics: viz, What number is that, which being multiplied by 6 , the product increased by 18 , and the sum divided by 9 , the quotient slıall be 20 ?

Now, beginning with the last result 20 , and performing in a retrograde order all the reverse operations, they will be thus: because the last operation was ditiding by 9 , to give the result 20; and multiplication being the reverse of division, therefore multiply the result 20 by the 9 , and it gives 180 ; then because the next preceding operation was adding 18 , therefore subtracting 18 from the 180 , leaves 162 ; lastly, because the next preceding operation was multiplying by 6 , therefore diride the 162 by 6 , and it gives 27 , which must be the number sought, or that K 2 
first begun with. The proof is thus : $27 \times 6=162$, then $162+18=180$, and $180 \div 9=20$. Or thus, expressing all the operations by their respective marks or characters, it will give $\frac{27 \times 6+18}{9}$; now first multiplying by the divisor 9 , gives $27+6+18$; then subtracting the increase 18 , leaves $2 \bar{T}+6$ : and lastly, dividing by the divisor 6 , must give the first or number required.

And this natural process is exactly the same as the general rule laid stown at the beginning of Simple Equations in the same volume, as the most general and natural method for the reduction of all equations. Thus, assuming $x$ for the number sought, in the same example: then by the question, $\frac{6 x+18}{9}=20$;

multiply by 9 , and it becomes $-\quad 6 x+18=1 \mathrm{~s} 0$; subtract 1s, and it is - - $-6 x=162$ :

lastly, divide by 6 , and it gives - $\quad x=27$.

So that the mode of opcration is exactly the same in all these ways.

Book 6 th treats of Mensuration. The introduction contains geometrical definirions. Chap. 1 treats on the mensuration of rectilincar surfaces. Under this head, the two following articles are deserving of notice. 1st, To fud the point in the base of a triangle, where it will be cut by a perpendicular, let fall from the opposite angle. Call the greatest side the base; multiply the sum of the two less sides by their difference; divide the product by the base, and subtract the quotient from the base; half the remainder will show the point on the base, where the perpendicular falls towards the least side. This property will easily be recognized as similar to that which we usually employ in resolving that case in plane trigonometry when the three sides are given, probably borrowed from the orientals; it is also the same property as that in the 35 th theorem of my Gcometry.

2nd, To find the area of an equilateral triangle. Multiply the square of a quarter of the square of one of the sides by 3 ; then the square root of the product is the area. That is, the side being $a$, its square is $a^{2}$, the quarter of this is $\frac{1}{4} a^{2}$, the square of which is $\frac{x}{16} a^{4}$, multiplied by 3 , it is $\frac{3}{16} a^{4}$, its root $\sqrt{ } \frac{3}{16} a^{4}$ is the area. This is naturally derived from the property of right-angled triangles, and, by extracting the roots, soon reduces to $\frac{x}{4} a^{2} \sqrt{ } 3$, the same as our own rule for the same purpose.

Chap. 2nd treats on the mensuration of curvilinear surfaces. We here find the same rule for the area of the circle, as one of those employed by ourselves, for the same purpose, viz, multiply the square of the diameter by 11 , and divisle the product by 14 .

Chap. $3 \mathrm{~d}$, on the mensuration of solids, contains nothing sufficiently remarkable to incrit particular notice. This cliapter concludes with the following sentence: "The de. monstrations of all these rules are contained in my greater work entitled Bahr-ul-Hisab (the ocean of calculations), may God grant me grace to finish it.'

Book the 7 th treats on Practical Geometry. Of which, the first chapter is on levelling, for the purpose of making canals. In this are described the plummetlevel, and the water-level, on the same principle with our spirit-level.

'The 2 nd chapter is on the mensuration of heights, accessible and inaccessible. Under the former of these hearls are delivered the common methouts, by bringing the top of a pole (whose heightis is known) in a line betiven the eye and the top of the height required; or by viewing the image of the top in a horizontal inirror; also by taking the proportion between a perpendicular stick of known length, and its shadow; or by taking the length of the shadow of the height when the sun's altitucle is $45 \mathrm{dt}$ grees. The concluding method is this: "Place the index of the astrolabe at the mark of $45^{\circ}$, and stand at a place where the hejght of the object is seen through the sights;' then measure from the place where you stand, to the place where a stone would fall from the top; add your own height, and the sum is the quantity required.

For the mensuration of inaccessible heights, the following rule is delivered: "Observe the top of the object through the sights, and mark on what shadow-line (division) the lower end of the index falls. Then move the index a step forward or backward, and advance or recede till you see the top of the object again. Measure the distance between your stations, and multiply by 7 if the index is moved a Dliil-Kadam, and by 12 if it is moved a Dhil-Asba, according to the shadow lines on the astrolabe; this is the quantity reyuired."

The $3 \mathrm{~d}$ chapter is on measuring the breadth of rivers and depth of wells. Stand on the bank of the river, and through the two sights look at the opposite bank; then turn round and look at any thing on the land side, kecping the astrolabe even: the distance from the observer to the place is the same as the breadth of the river.

Book 8 th is on finding unknown quantities by algebra, In this book are two chapters. The first is introductory, chiefly relating to the formation and operation of powers of the unknown quantity, thus. Call the unknown quantity Shai (thing), its product into itself Mal (possession), the product of Mal into Shai, Cab (a die or cube), of Shai into $\mathrm{Cab}, \mathrm{Mal}-\mathrm{Mal}$; of Shai into Mal-i-Mal, MIal-Cab; Shai into Mal-i-Cab, Cab-i-Cab; and so on, without end. For one Cab write two Mals, and of these two Mals one becomes Cab; afterwards both Mals become Cab. Thus the 7 th power is Mal-i-Mal-i-Cab, and the Sth Mal-i-Cabi-Cab, in the 9th $\mathrm{Cab}-\mathrm{i}-\mathrm{Cab}-\mathrm{Cab}$, and so on. All these powers are in continued proportion, eitlier ascending or descending. Thus the ratio of Mal-i-Mal to Cab, is like (or similar or equal to) the ratio of Cab to Mal, of Mal to Shai, and of Shai to 1 , and of 1 to 1 divided by Shai, and of 1 divided by Shai to 1 divided by Mal, and of 1 divided by Mal to 1 divided by $\mathrm{Cab}$, and of $\mathrm{I}$ divided by $\mathrm{Cab}$ to 1 divided by Mal-i-Mal. All this means, that all the terms in the successive powers are in continued proportion, viz, in our notation,

$$
\frac{1}{x^{4}}, \quad \frac{1}{x^{3}}, \quad \frac{1}{x^{3}}, \frac{1}{x}, 1, x, x^{2}, x^{3}, x^{4}, \& \mathrm{c},
$$$$
\text { or } x^{-4}, x^{-3}, x^{-2}, x^{-1}, x^{\circ}, x^{1}, x^{2}, x^{3}, x^{4} \text {, scc. }
$$

To nultiply one of these powers by another. If they are both on the same side, (viz, of unity) add the exponents of their powers together; the prorluct will have the same denomination as this sum. For example, to multiply Mal-i-Cab by Mal-i-Mal-i-Cab, the first is the 5 th power, and the other is the 7 th; the result then is Cab-iCab-i-Cab-i-Cab, or four Cabs, which is the 12th power. If the fictors are on difierent sides, the product will be the excess on the sille of the greater.-So, the product of 1 divided by Mlal-i-Mlal into Mal-i-Cäb, is Slıai; and the product of 1 divided by Cab-i-Cab-Cab into Cab-i-Mal-iMal, is 1 divided by Mal. And if the factors are at the same distance (from 1 ), the product is 1. The author 
adds, "The particulars of the methods of division, with extraction of roots, and other rules, I have given in my greater book."

"The rules of algebra which have been discovered by leafned men are six, and they relate to number and Shai and Mal." 'That is, to $n$ and $x$ and $x^{2}$, including what we call simple and quadratic equations.

\section{Cri p pter II. On the Six Rules of Algebra.}

To find unknown quantities by algebra depends on acuteness and sagacity, with an attentive consideration of the terms of the question, and a successfui application of the invention to such things as may serve to bring out the quantity required. Call the unknown quantity Shai (the Thing), and proceed with it according to the terms of the questiun, till the operation ends with an equation. Let that side where there are negative quantities be made perfect, adding the negative quantity to the other side, which is called restoration, Jebr, (hence doubtless, with the article $A l$, comes the name Algebra.) Let those things which are of the same kind, and equal on both sides, be thrown away which is called opposition (Mrakabalah).

Equality is eitber of one species to another, which is of three kinds, called simple (Mufridat), or of one species to two species, which is of three kiuds, called compound (Multarinat).

CASE 1st. Mufridat. When number is equal to things $(a x=n)$; divide the number by the coefficient of the thing, and the unknown quantity will be found. Example. A person admitted that he owed to Zaid 1000 and one half of what leowed Amer; also that he owed Amer 1000 all but half of what he uwed to Zaid. Call Zaid's debt Shai $(x)$. Then Amer's debt is 1000 wanting half of Shai $\left(1000-\frac{1}{2} x\right)$; and Zaid's is 1500 wanting a fourth of Shai $\left(1500-\frac{x}{4} x\right)$. This is equal to Shai $\left(1500-\frac{1}{4} x=x\right)$. After Jebr, 1500 is equal to one Shai and a quarter of Shai $\left(1500=1 \frac{\pi}{4} x\right)$. So for Zaid is 1200 , and for Amer 400.

CAsE 2nd. Multiples of Shai equal to multiples of Mal $\left(a x=b x^{2}\right)$. Divide the coefficient of the thing by that of Mal; the quotient is the unknown quantity $\left(\frac{a}{b}=x\right)$. Ex. ample. Some sons plundered their father's inheritance, which consisted of Dinars. One took 1, another 2, the third 3 , and so on increasing by 1 . The ruling power took back what they had plundererl, and divided it among them in equal shares, by which each received 7 . How many sons were there, and how many Dinars? Suppose the number of Sons Shai $(x)$, and take the sum of the extremes, viz, 1 and Shai $(1+x)$. Multiply them by half of Shai $\left(\frac{1}{2} x\right)$; this is the number of dinars $\left(\frac{1}{2} x+\frac{1}{2} x^{2}\right)$; for the sum of any series of numbers in arithmetical progression, is equal to the product of the sum of the two extremes, into half the number of terms. Divide the nuraber of the Dinars by Shai $(x)$, which is the number of the sous, the quotient according to the terins of the question, will be $7\left(\frac{1}{2} x^{2}+\frac{r}{2} x\right) \div x=7$ ). Multiply 7 by Shai $(x)$, the divisor, 7 Shai is the product, which is equal to $\frac{1}{2}$ Mal and $\frac{r}{2}$ Shai $\left(7 x=\frac{1}{2} \mathrm{r}^{2}+\frac{1}{2} x\right)$. Alter Jebr and Mutucbalah, 1 Alal is equal to 13 Shai $\left(x^{2}=13 x\right)$; Shai then is $13(x=13)$; and this is the number of sons. Multiply this by 7 , and the number of Jinars will be found 91 .

It is added, that questions of this kind may be solved by position. "Thus, suppose the number of sons to be 5 , the first errur is 4 in defect; then suppose it to be 9 , the

\section{A L G}

second error is 2 in defect. The first Mafudh is 10 , and the second is 36 ; their difference is 26 , and the difference of the errors is 2." - "Another method, which is short, is this: Double the quotient $(\tau)$ is 14 ; subtract 1 , and the result (13) is the number of sons."

CAse 3d. Number equal to Mal $\left(n=a x^{2}\right)$. Divide the number by the coefficient of the Mal; the root of the quotient $\left(\sqrt{ } \frac{n}{a}=x\right.$ is the unknown quantity. Example. A person admitted that he owed Zaid the greater of two sums of money, the sum of which was 20 , and the product 96 . Suppose one of them to be 10 and Shai $(10+x)$, and the other 10 wanting Shai $(10-x)$. The product, which is 100 all but Mal $\left(100-x^{2}\right)$ is equal to 96 ; and after $J c b r$ and Mukabalah, 1 Mal is equal to $4\left(x^{2}=4\right)$, and Shai equal to $2(x=2)$. One of the sums then is 8 , and the other 12, which is the debt of Zaid. Here, by substituting for the half-sum and half-difference the equation, comes out a simple one.

- Finst Case of Maktarinat. Number equal to Mal and Shai $\left(a x^{2}+b x=n\right)$. Complete the Mal to unit if it is deficient, or reduce it to the same if it exceeds, and reduce the number and Shai in the same ratio, by dividing all by the coefficient of the Mal. Then square one half the coefficient of the Shai, and add this square to the number. From the ront of the sum subtract half the coefficient of the Shai, and the unknown will remain. An example follows as usual : and the method is evidently the same as ours at present.

CASE 2nd. Shai equal to number and Mal $\left(b x=x^{2}+n\right)$. After completing or rejecting, subtract the number from the square of half the coefficient of Shai; then add the ruot of the remainder to half the coefficient of the Shai, or subtract the former from the latter, and the result is the unknown quantity. This also is the same as the present method, and both the two roots are noticed in this case, by taking the root of the known quantity either positive or negative. An example is added as usual.

CASE 3d. Mal equal to number and Shai $\left(x^{2}=n+b x\right)$. After completion or rejection, add the square of balf the coefficient of the Shai to the number, and add the root of the sum to half the coefficient of the Shai ; this is the unknown quantity. Example. What number is that which being subtracted from its square, and the remainder added to its square, is 10 ? Subtract Shai from Mal and go on. with the operation, than $2 \mathrm{M}$ al all but Shai is equal to $10\left(2 x^{2}-x=10\right)$; and after $J e b r$ and Radd, Mal is equal to 5 and half of Shai $\left(x^{2}=5+\frac{1}{2} x\right)$. The square of half the coefficient of Shai and 5 , is 5 and half an eighth $\left(5 \frac{1}{16}\right)$, and its root is $2 \frac{\pi}{4}$; to this add $\frac{\pi}{4}$, the result $2 \frac{\pi}{2}$ is the number sought.

Book 9th, contains 12 rules respecting the properties of numbers. $\Delta s, 1$ st. To find the sum of the products multiplied into itself and into all numbers below it. Add 1 to the number; and multiply the sum by the square of the number; half the product is the number sought.

2nd. To add the odd numbers in their regular order. Add 1 to the last number, and take the square of half the sum.

3d. To add the even numbers from 2 upwards. Multiply half the last even number by a number greater by 1 than that half.

4th. To add the squares of the numbers in order. Add 1 to twice the last number, and multiply a $3 \mathrm{~d}$ of the sum by the sum of the numbers. 


\section{A L $\mathrm{G}$}

\section{[ 70}

5th. To find the sum of the cubes in succession. Take the square of the sum of the numbers.

6 th. To find the product of the roots of two numbers. Multiply one by the other, and the root of the product is the answer.

7 th. To divide the root of one number by that of another. Divide one by the other, the root of the quotient is the answer.

Sth. To find a perfect number; that is, a number which is equal to the sum of its aliquot parts, (Eucl. book 7, def. 22). The rule is that delivered by Euclid, book 9, prop. 36.

9 th. To find a square in a given ratio to its root. Divide the first number of the ratio by the second; the square of the quotient is the square required.

10th. If any number be multiplied and divided by another, the product multiplied by the quotient, is the square of the first number.

11 th. The difference of two squares is equal to the product of the sum and difference of the roots.

12th. If two numbers be divided by each other, and the quotients multiplied together, the result is always 1 .

Book 10th contains 9 practical examples, all of which are capable of solution by simple equations, or by position, ol by retracing the steps of the operation, and some of them by simple proportion; so that it is needless to specify them.

The conclusion, which marks the limits of algebraical knowledge in the age of the writer, is here given entire, in the autlior's words.

"Conclusion. There are many questions in this science which learned men have to this time in vain attempted to solve; and they have stated some of these questions in their writings, to prove that this science contains difficulties, to silence those who pretend they find nothing in it above their ability, to warn arithmeticians against undertaking to answer every question that may be proposed, and to excite men of genius to attempt their solution. Of these 1 have selected seven.-1st. To divide 10 into two parts, such, that when each part is added to its square root, and the sums are inultiplied together, the product is equal to a supposed number.-2d. What square number is that, which being increased or diminished by 10 , the sum and remainder are both square numbers ?-3d. $\Lambda$ person said he owed to Zaid 10 all but the square root of what he owed to Amer, and that he owed Amer 5 all but the square root of what he owed Zaid. -4 th. To divide a cube number into two cube numbers.-5th. To divide 10 into two parts such, that if each is divided by the other, and the two quoticnts are added together, the sum is equal to one of the parts. $-6 \mathrm{th}$. There are three square numbers in continued geometric proportion, such, that the sum of the three is a square number.-7th. There is a square, such, that when it is increased and diminished by its root and 2 , the sum and the difierence are squares.-Know, reader, that in this treatise $I$ have collected in a small space the most beautilul and best rules of this science, more than were ever collected before in one book. Do not underrate the value of this bride; hide her from the view of those who are unworthy of her, and let her go to the liouse of him only who aspires to wed her."

From the preceding account of this Arabian treatise on Algebra, the Khulasent-ul-Hisab, of Baha-ul-din, which is estecmed the best in that language, we pretty clearly per-
A L G

ceive what was the state of science in that nation; that it was much the same as the first treatises among the Italians, derived directly from the former; but that it was much inferior to the same.science among the Indians. It does not appear that the Arabians used algebraic notation or abbreviating symbols; that they had any knowledge of the Diophantine algebra, or of any but the easiest and elementary parts of the science. We have seen that Bahaul-din ascribes the invention of the numeral figures, in the decimal scale, to the Indians; as is done indeed by all the Arabic and Persian books of arithmetic.

The following is an extract from a Persiañ treatise of arithmetic in Mir. Strachey's possession.

"The Indian sages wishing to express numbers conveniently, invented these nine figures, $\mid \sim \sim \vee \diamond Y \vee \wedge q \ldots$ The first figure on the right hand they made stand for units, the second for tens, the thir' for handreds, the fourth for thousands. Thus, after the third rank, the next following is units of thousands, the second tens of thousands, the third hundreds of thousands, and so on. Every figure therefore in the first rank is the number of units it cxpresses; every figure in the second, the number of tens which the figure expresses; in the third, the number of hundreds; and so on. When in any rank a figure is wanting, write a cipher like a small circlc $\left({ }^{\circ}\right)$ to preserve the rank. Thus, ten is written 10, a hundred 100 , five thousand and twenty-five 5025."

In short, of the Indian algcbra, in its full extent, the Arabians seem to have been ignorant; but most likely they had their algebra from the same source as their arithmetic. The Arabian and Persian treatises on algebra, like the first and old European ones, begin with arithmetic, called in those treatises the arithmetic of the Indians, and have a second part on algebra; but no notice is taken of the origin of the latter. Must likely their algebra, being numeral, was considered by the autbors as a part of arithmetic.

Though part only of the Khulasat-al-Hisab, Mr. Strachey says, is concerning algebra, the rest, relating to arithmetic and mensuration, must be thought not wholly unconnected with the subject. It is to be hoped that ere long we shall have either translations from the Sanscrit of the Bija Ganita and Lilawati, or perfect accounts from the originals; and that other Ifindu books of algebra will be found, and made known to the world. But in the mean time the Persian translations will be found well deserving of attention; observing carefully to distinguish between what is interpolated, and what is original.

From the preceding account of this Arabic treatise, also, is clearly secn the origin of the name of Algebra, being an Arabic compound, viz, of the article $a l$, and jebr, which denotes one of the modes of relucing the equations, riz, by transposing or adding the negative terms, to make them all affirmative.

For a fuller account of the lndian and Arabian algebra, sce my Tracts, vol. 2, p. 151, \&c.

OF ALGEBRA IN ITALY AND OTHER PARTS OF EURope. We have seen that algebra had probably its rise in Hindustan, as well as our present numeration and arithmetic, all the rules of it having been lound in the ancient is ss. of that country, much the same, in matter and form, as they appear in the first Italian authors; and among these, the two rules of falsc position, which are ncarly al- 
lied to algebra, and the extension of which probably led to, and ultimately became, the art of algebra itself. Hence it appears that these arts passed successively into Persia, Arabia, Africa, and Europe. In this last quarter it seems doubtful, whether their introduction was first into Spain or into Italy: the probability would appear to be on the side of the former, as it would most likely be introduced by the Moors on their settlement in the peninsula in the sth, $9 \mathrm{th}$, and 10 th centuries; whence it might be communicater to Gaul and Germany and England, \&c. 'This route is rendered the more probable by the circumstance, that the early state of the algebraic art in these countries was very different, and even more perfect, in several respects, than that in Italy, in the contemporary stages. And yet, on the other hand, against this probability of the first introduction of the art into Spain by means of the Moorish conquest, it may reasonably be objected, that it does not appear that the Arabians themselves had cultivated it at a time so early as the date of that conquest; and besides that we have not heard of any early works on Algebra having ever been found in the Spanish peninsula. It appears, however, at any rate, that ltaly received the art by a different route, though probably at a later date, viz, immediately from the eastern Arabians themselves, without the knowledge of any other source or communication: and hence it was watural for the Italians to ascribe the invention of the art to that people.

It has usually been thought, that the first introduction of algebra into Italy, was about the 14th century. But we have lately, viz, in 1797 , been favoured with a new and very diffuse history of algebra, by Sig. Cossali, in two large volumes, 4 to, by which it appears, that the art was first imported into ltaly, from the east, by Leonard Bonacci, of Pisa, who composed his arithmetic in the year 1202, and again in 1228; adding the algebra at the end, as a part of $i$.

In this work of Cossali's, accounts are given of several other old authors, as well Italian as Arabian and Persian, \&c, among which some were much earlier than Leonard: as, a Mohamed ben Musa or Moses, called also Mohamed of Buziani, a place in Corasan, near the south-east point of the Caspian Sea. This Mohaned, it seems, in the year 959, travelled eastward to the confines of India, to learn the mathematical sciences, and afterwards to teach them; and who, according to Abulfaragio, in the year 969 , wrote a Commentary on Diophantus; he wrote also Demonstrations of the Propositions in the same; and another work on the Universal Logistic Art, in three books.

It seems, however, that it was Leonard Bonacci, of Pisa, who first introduced the art in Italy, as before mentioned. Leonard's work, it appears, was a very orderly and regular treatise on inithmetic and algebra, as far as it was then known; teaching and demonstrating all the rules, and illustrating them with many examples; being also much occupied with questions about square and cube numbers, like those of Diophantus, or rather like what we have seen and described anong the Indians and Arabians. - In algebra, Leonard distinguisherl three kinds of numbers, viz, the absolute known number in any question; then the unknown number, which he calls radice, the root; and its square, which he calls census in Latill, or censo in Italian; for his algebra extended only to the solution of (quations of the 1st and 21 degree, the same as that of the Indians and Arabians. The language in wbich the work wals written, was in barbarous Latin, or something between Latin and Italian, when the language of the country was changing from the one to the other.

In treating his subject, Leonard had no such notation as is used by modern authors; on the contrary, he expressed every thing, both the quantities and the several operations, by their names, or words at full length. Those equations he treated of in six different forms, which are as follow, when expressed in the modern notation. 1st, $x^{2}=u x$; $2 \mathrm{~d}, x^{2}=n ; 3 \mathrm{~d}, a x=n ; 4$ th, $x^{2}+a x=n ; \quad 5 \mathrm{th}, x^{2}=a x$ $+n$; $6 \mathrm{th}, x^{2}+n=a x$; where $x$ denutes the radice, or quantity sought, $x$ ? its censo or square, $a$ the number multiplying $x$, and $n$ the absolute known number; and where it is remarkable that he places the terms, more or less, on the ore side or the other, so as they may be all affirmative: all which cases and forms are exactly the same as before given by their masters the Arabians, \&c.

Now omitting the simple forms, as including but little reniarkable, we shall only consider the compound ones, or the solution of quadratic equations, by the method of completing the square, which Leonard founds on geometrical demonstration, as follows:- In the first case, if $x^{2}+a x=n$, then the rule is $x=\sqrt{ }\left(\frac{x}{4} a^{2}+n\right)-\frac{1}{2} a$; which he thus demonstrates.

Demonstr. If upon a right line $\mathrm{Bc}$, greater than $\frac{x}{2} a$, the square $A B C D$ be constructed, on the sides of which there are taken the parts $\mathrm{CG}, \mathrm{CH}, \mathrm{DF}$, $\mathrm{TE}$, each equal to $\frac{1}{2} a$, and drawing the right lines $\mathrm{EH}, \mathrm{FG}$, intersecting in $I$, they form the square IGCH $=\frac{1}{4} a^{2}$.

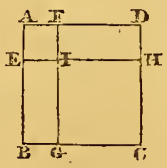

Supposing $\mathrm{EI}_{\mathrm{I}}=\mathrm{AF}=\mathrm{EA}=1 \mathrm{~F}=\mathrm{BG}=\mathrm{DH}$, to denote the required quantity $x$; then will the square $\mathrm{Al}$ or of $\mathrm{EI}=x^{2}$, the rectangle $\mathbf{B I}=\frac{1}{2} a x=$ also to the rectangle $\mathrm{ID}$. Therefore the whole square $\mathrm{A} \mathrm{BCD}=x^{2}+a x+\frac{\pi}{4} a^{2}:$ but $x^{2}+a x$ $=n$; therefore is the same square $A \mathrm{BCD}=n+\frac{\pi}{4} a^{2}$ : consequently the side $\mathrm{BC}=\sqrt{ }\left(n+\frac{\pi}{4} a^{2}\right)$; and the quantity sought $x=\mathrm{BC}-\mathrm{GC}=\mathrm{BC}-\frac{1}{2} a=\sqrt{ }\left(n+\frac{1}{4} a^{2}\right)-\frac{1}{2} a$.

2ndly. If $x^{2}=a x+n$; then will $x=\sqrt{ }\left(\frac{1}{4} a^{2}+n\right)+\frac{1}{2} a$.

Demonstr. Let the right line $\mathrm{BC}=x$, and the square upon it $A \mathrm{BCD}=x^{2}$. If $\mathrm{CE}$ be taken $=a$, and the perp. $\mathrm{EF}=x$. Then the rectangle $\mathrm{FD}=a x$. Therefore the remaining rectangle $A E=n$. But $\mathrm{AE}=\mathrm{AB} \times \mathrm{BE}=\mathrm{BC} \times \mathrm{BE}$; therefore $n$ $=\mathrm{BC} \times \mathrm{BE}=\mathrm{BE}^{2}+\mathrm{BE} \times \mathrm{EC}$. If $\mathrm{EC}$ be bisected in $\mathrm{G}$, then will $n+\mathrm{EG}^{2}=\mathrm{BE}^{2}+\mathrm{BE} \times \mathrm{EC}+\mathrm{EG}^{2}$. Hence $\sqrt{ }\left(n+\mathrm{EG}^{2}\right)=\mathrm{BE}+\mathrm{EG}$; and $\mathrm{GC}+\sqrt{ }\left(n+\mathrm{EG}^{2}\right)$ $=\mathrm{GC}+\mathrm{BG}$. So that, it being $\mathrm{GC}=\mathrm{EG}=\frac{\mathrm{r}}{2} a$, and $\mathrm{BC}$ $=x$, it will be $\frac{x}{2} a+\sqrt{ }\left(n+\frac{1}{4} a^{2}\right)=x$.

3 dly. Let $x^{2}+n=a x$. - Here he says, if $\frac{x}{4} a^{2}<n$, the equation is impossible. - If $\frac{1}{4} a^{2}=n$, then is $x=\frac{1}{2} a$. - If $\frac{\pi}{4} a^{2}>n$, then is $x=\frac{\pi}{2} a-\sqrt{ }\left(\frac{1}{4} a^{2}-n\right)$, or $=\frac{1}{2} a+\sqrt{ }\left(\frac{1}{4} a^{2}\right.$ $-n)$.

Demonstr. Draw $\Delta \mathrm{B}$ representing $a$, bisected in $G$, and unequally divided in $\mathrm{D}$; and upon one of the unequal parts, supposed $=x$, if there be made a square $=x^{2}$. Now, if, in the first place, it be formed on

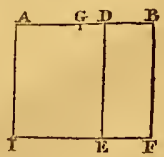
the less part $\mathrm{DB}$, supposed $=x$; let $\mathrm{FE}$ be produced to $\mathrm{I}$, till $\mathrm{FI}$ be $=\mathbf{A B}$, and let $\mathbf{A 1}$ be drawn. Then the whole rectangle $A Y=A B \times B F=a x$; hence, taking away the square $\mathrm{BE}=x^{2}$, there remains the rectangle $\mathrm{AE}=\mathrm{AD} \times$ $\mathrm{DE}=\mathrm{AD} \times \mathrm{DB}=n$; and adding to both the square $G \mathrm{D}^{2}$, gives $A D \times D B+C D^{2}=n+G D^{2}$. But, by Eucl. Ir, 5 , it 

A L $G$
[ $\begin{array}{ll}72 & ]\end{array}$
A L G

is $\mathrm{AD} \times \mathrm{DB}+\mathrm{GD}^{2}=\mathrm{BG}^{2}$; therefore $\mathbf{B O}^{2}=n+\mathrm{GD}^{2}$; hence $\mathrm{BG}^{2}-n=\mathrm{GD}^{2}$, and $\sqrt{ }\left(\mathrm{BG}^{2}-n\right)=\mathrm{GD}$; then $\mathrm{BG}-$ $\sqrt{ }\left(\mathrm{BG}^{2}-n\right)=\mathrm{BG}-\mathrm{GD}=\mathrm{DB}$, or $\frac{\mathrm{r}}{2} u-\sqrt{ }\left(\frac{1}{4} a^{2}-n\right)=x$. -If the square $x^{2}$ be constituted on the greater part $A D$, considered as $=x$; then by a like process it will be $\sqrt{ }\left(\mathrm{AG}^{2}-n\right)=\mathrm{GD}$; hence $\mathrm{AG}+\sqrt{ }\left(\mathrm{AG}^{2}-n\right)=\mathrm{AG}+$ $\mathrm{GD}=\mathrm{AD}$, that is $\frac{\mathrm{T}}{2} a+\sqrt{ }\left(\frac{1}{4} a^{2}-n\right)=x$.

Thus we see that Leonard derived the rules for quadratic equations from geonetrical considerations, and even the double values in the possible case of $x^{2}+n=a x$; as it is probable his predecessors had done, and as we find his successors Lucas de Burgo and others did also. And this method shows the reason why these writers never admitted those values of $x$, in which $n$ is greuter than $\frac{1}{4} a^{2}$. We farther find, that neither Leonard, nor the Arabians, nor Indians, made any use of the form $x^{2}+a x+n=0$, nor of the negative roots of the two forms $x^{2}+a x=n$, and $x^{2}=a x+n$ : it being Harriot who first departed from the usual custom (of equalizing the positive terms to the positive in an equation), by placing all the terins on one side, with their signs + or - , and made $=0$ on the other side, thus $x^{2} \pm a x \pm n=0$; and it was Cardan who had the honour of sbowing that, of the two forms $x^{2}+a x$ $=n$, and $x^{2}=a x+n$, the negative root of the one is the positive root of the other.

lt appears that Leonard Pisanus ivas well skilled in the various ways of reducing equations to their final simple state, by all the usual methods; as addition, subtraction, multiplication, division, powers and roots, to free them from radicals, \&c. He was also well acquainted with the modes of substitution, so as to bring out the equation in the lowest degree; such as, iu cases of two unknown quantities, instead of finding either of them separately, he would first scarch out their sum or difference. So, for instance, in this problem, "To divide the number 10 into two such parts, that from the greater part taking its own squareroot, and to the less part adding its square root, the two results should be equal." Now, in this case, if in the common way $x$ be made to denote one of the parts, as the greater suppose, then $10-x$ will denote the less part, and the equation will be $x-\sqrt{ } x=10-x+\sqrt{ }(10-x)$, the reduction of which leads to a complete equation of the 4 th degree. But, instead of this methorl, Leonard employs the way of the half-sum and half-difference, as used by Diophantus and the Arabians and Hindus, which has been accounted by some persons an artifice of the modern algebraists. Thus, if $x$ be put to denote the balf-difference of the two parts; then 5 being their half-sum, $5+x$ will be the greater, and $5-x$ the less, and the equation will be $5+x-\sqrt{ }(5+x)=5-x+\sqrt{ }(5-x)$, which reduces to a final quadratic, as is done by this author.

From Pisa, as from a centre, it seenis the art gradually spread through Tuscany and all Italy; in conscquence of which, many other authors in that country bad respectable names before the period of the art of priuting. So, we read that Raffuello Canacci, a Florentine mathematician, was author of a " Ragionamento di Algelıra," who praises another that preceded him, named Gugliclmo di Lunis; of whom Canacci writes, at the beginning of his Ragionamento, "La regola dell' algibra, la quale regola Ghughelmo di Lunis la translato d'৯rabico a nostra lingua;" whence some have thought, though without probability, that the honour of liaving made ltaly aequainted with algebra was due to Guglielmo di Lunis, rather than to Lconardo Bonucci di Pisa. Many other early authors are mentioned.
Bombelli, in the preface to his book, writes that a work of Mohamed ben Musa had been shown, but it was of little value. And it is said that Lucas de Burgo, the first author in print, was instructed in this science at Venice, by Dumanico Bragadini, successor in the public chair to the learned Paolo della Pergola, his preceptor, who died in 1366 .

Proceed we now to the consideration of the books of Lucas de Burgo, and other authors, whose works we are possessed of $\mathrm{in}$ print.

LUCAS PACIULUS, OR DE BURGO.

It was chiefiy among the ltalians that this art was first . cultivated in Europe. And, the tirst author whose works were in print, was Lucas Paciolus, or Lucas de Burgo, a cordelier, or minorite friar. He wrote several treatises of aritnmetic, algebra, and geometry, which were printed in the years $1470,1476,1481,1487$, and in 1494 his principal work, entitled Summa de Arithmetica, Geomctria, Proportioni, et Proportionalita, which is a very masterly and complete treatise on those sciences, as they then stood. In this work he mentions various former writers, as Luclid, St. Augustine, Sacrobosco or Halifax, Buetius, Prodocimo, Giordano, Biagio da Parma, and Leonardus Pisanus, from whom he learued those sciences.

The order of the work is, 1st arithinetic, $2 \mathrm{~d}$ algebra, and $3 \mathrm{~d}$ geometry. Of the arithmetic, the contents, and the order of them, are nearly as follow. First, of numbers figurate, odd and even, perfect, prime and composite, and many others. Then of common arithmetic in seven parts, namely, numeration or notation, addition, subtraction, multiplication, division, progression, and extraction of roots. Before him, he says, duplation and mediation, or doubling and halving, were accounted two rules in arithmetic; but that he omits them, as being included in inultiplication and division. He ascribes the present notation and method of arithmetic to the Arabs; and says that according to some the word $A$ baco is a corruption of Modo Arabico, but that according to others it was from a Greek word. This, however, must be a mistake; for though the Italians had those arts from the Arabs, these latter had them probably from the Indians. All those primary operations he both performs and demonstrates in various ways, many of which are not in use at present; proving them not only by what is called casting out the nines, but also.by casting out the sevens, and otherwise. In the extraction of roots he uses the initial $\mathrm{R}$ for a root; and when the roots can be cxtracted, he calls them discrete or rational; otherwise surd, or indiscrete, or irrational. The square root is extracted much the same way as at present, namely, dividing always the last remainder by double the root found; and so be continues the surd rows continually nearer and nearer in vulgar finctions. Thus, for the root of 6 , he first finds the nearest whole number 2, and the remainder 2 also; then $\frac{2}{4}$ or $\frac{1}{2}$ is the first correction, and $2 \frac{1}{2}$ the sccond root: its square is $6 \frac{x}{4}$, therefore $\frac{1}{4}$ divided by 5 , or $\frac{1}{2}$, is the next currection, and $2 \frac{\mathrm{r}}{2}$ minus $\frac{x}{20}$, or $2 \frac{9}{2}$ is the $3 \mathrm{~d}$ root: its square is $6 \frac{1}{400}$, therefore $\frac{1}{40}$ divider by $4 \frac{9}{20}$, or $\frac{1}{960}$, is the $3 \mathrm{~d}$ correction, which gives $2 \frac{98}{1960}$ for the $4 . t h$ root, whose square exceeds 6 by only $\frac{1}{384}$ ? this process he calls approximation. Ilo observes that fractions, which he sets down the same way as we do at present, are extracted, by taking the root of the denominator, and of the denominated, for so be calls the numerator: and when mixed numbers occur, he directs to re- 
duce the whole to a fraction, and then extract the roots of its two terms as above: as if it be $12 \frac{\pi}{4}$; this he reduces to $\frac{49}{4}$, and then the roots give $\frac{7}{2}$ or $3 \frac{1}{2}$ : in like manner he finds that $4 \frac{x}{2}$ is the root of $20 \frac{x}{4} ; 5 \frac{\pi}{2}$ the rnot of $30 \frac{1}{4} ; "$ and so on (he adds) in infinitum;" which shows that he knew how to form the series of squares by addition. He then extracts the cuhe root, by a rule much the same as that which is used at present; from which it appears that he was well acquainted with the co-efficients of the binomial cubed, namely, 1, 3, 3, 1; and he directs how the operation may be continued " in infinitum" in fractions, Jike as in the square root. After this, be describes geometrical methods for extracting the square and cube roots instrumentally: he then treats professedly of vulgar fractions, their reductions, addition, subtraction, and other operations, much the same as at present: then of the rule-ofthree, gain-and-loss, and other rules used by merehants.

Paciolus next enters on the algebraical part of this work, which he calls "L'Arte Magiore; ditta dal vulgo la Regola de la Cosa, over Alghebra e Almucabala:" which last name he explains by restauratio et oppositio, and assigns as a reason for the first name, that it treats of things above the common affairs in business, which make the arte minore. Here he mistakenly ascribes the invention of algebra to the Arabians; and he says that the Arabian algebra means in Italian position, or rather opposition. He denominates the series of powers, with their marks or abbreviations, as $n^{\circ}$. or numero, the absolute or known number; co. or cosa, the thing or 1st power of the unknown quantity; ce. or censo, the product or square; cu. or cubo, the cube, or $3 \mathrm{~d}$ power; ce. ce. or censo de censo, the square-squared, or 4 th power; $p^{\circ} . r^{\circ}$. or primo relato, or 5 th power; ce. cu. or censo de cubo, the square of the cube, or 6th power; and so on, compounding the names or indices according to the multiplication of the numbers 2,3 , \&c, and not according to their sum or addition, as used by Diophantus, with the Arabians and Indians. He describes also the other characters made use of in this part, which are for the most part no more than the initials or other abbreviations of the words themselves, after the manner of the Indians; as for Be radici, the root; B2. B2. radici de radici, the root of the root; R2. u. radici universale, or radici legata, or radici unita; $\mathrm{B} \mathbf{c u}$. radici cuba; and $\overline{q 3}^{2}$ quantita, quantity; $p$ for piu or plus, and $m$ for meno or minus; and he remarks that the necessity and use of these two last eharacters are for connecting, by addition or subtraction, different powers together; as 3 co.p. $4 c e$. m. 5 cu. p. $2 c e . c e$. m. $6 n^{\mathrm{i}}$. that is, 3 cosa, piu 4 censa, meno 5 cubo, piu 2 censa-censa, meno 6 numeri, or, as we now write the same thing, $3 x+$ $4 x^{2}-5 x^{3}+2 x^{4}-6$.

He first treats very fully of proportions and proportionalities, both arithmetical and geometrical, accompanied with a large collection of questions concerning numbers in continued proportion, resolved by a kind of algebra. He then treats of el Cataym, which he says, according to some, is an Arabic or Phenician word, and signifies the Double Rule of False Position : but he bere treats of both single and double position, as we do at present, dividing the el Cataym into single and double, as the Arabians and Indians did. He gives also a geometrical demonstration of both the cases of the errors in the double rule, namely, when the errors are both plus or both minus, and when the one error is plus and the other minus; and adds a large collection of questions, as usual. He then goes Vou. J. through the common operations of algebra, with all the variety of signs, as to plus and minus; proving that, in multiplication and division, like signs give plus, and unlike signs give minus. He next treats of different roots in infinitum, and the extraction of roots; giving also a copious treatise on radicals or surds, as to their addition, subtraction, multiplication, and division, and that both in square roots and cube roots, and in the two together, much the same as at present. He makes here a digression concerning the 15 lines in the 10th book of Euclid, treating them as surd numbers, and teaching the extraction of the roots of the same, or of compound surds or binomials, such as of $23 p$ B 448, or of $\mathrm{B} 1 \mathrm{1S} p$ B 10 ; and gives this rule, among several others, namely: Divide the first term of the binomial into two such parts, that their product may be $\frac{x}{4}$ of the number in the second term; then the roots of those two parts, connected by their proper sign $p$ or $m$, is the root of the binomial; as in this $23 p$ B 448 , the two parts of 23 are 7 and 16 , whose product, 112, is $\frac{1}{4}$ of 448 , therefore their roots give 4 . $p$ \& 7 for the root $\mathrm{BZ}$ u. 23 $p$ BX 448 .

He next treats of equations both simple and quadratic, or simple and compound, as he calls it; and this latter he performs by completing the square, and then extracting the root, just as usual. He also resolves equations of the simple 4 th power, and of the 4 th combined with the $2 \mathrm{~d}$ power, which he treats the same way as quadratics; expressing his rules in a kind of bad verse, and giving geometrical demonstrations of all the cases, the same as those of Leonard of Pisa, before deseribed. He uses both the roots or values of the unknown quantity, in that case of the quadratics which has two positive roots; but he takes no notice of the negative roots in the other two cases. As to any other compound equations, such as the cube and any other power, or the 4th and 1st, or 4th and $3 \mathrm{~d}$, \&ce, he gives them up as impossible, or at least he says that no general rule has yet been found for them, any nore, he adds, than fur the quadrature of the circle. - The remainder of this part is employed on rules in trade and merchandise, such as Fellowship, Barter, Exchange, Interest, Composition or Alligation, with various other cases in trade. And in the third part of the work, he treats of Geometry, both theoretical and practical.

From this account of Lucas de Burgo's book, we may perceive what was the state of algebra about the year 1500 , in Europe; and probably it was much the same in Africa and Arabia, whence the Europeans had it. It appears that their knowledge extended no farther than quadratic equations, of which they used only the positive roots; that they used only one unknown quantity; that they had no marks or signs for either quantities or operations, excepting only some few atbreviations of the works or names themselves; and that the art was only employed in resolving certain numeral problems. So that either the orientals had not carried algebra beyond quadratic equaiions, or else the Europeans bad not learned the whole of the art, as it was then known to the former. And indeed it is not impossible but this miglut be the case: for whether the art was brought to us by an European, who, travelling into the East, there learned it ; or whether it was brought to us by those people; in either case we might receive the art only in an imperfect state, and perhaps far sliort of the degree of perfection to which it had been carried by their best authors. And this suspicion is rendered rather probable by the circumstance of an $\Lambda$ rabic manuscript, said $\mathbf{L}$ 


\section{A L G}

[ 74

to be on cubic equations, deposited in the hibrary of the university of Leyden by the celebrated Warner, bearing a title, which in Latin signifies Omar Ben Ibrahim al'Ghajamai Algebra cubicarum aquationum, size de problematum solidorum resolutione. At any rate, however, we have found that the Hindus, if not the Arabians, treated of problems including several unknown quantities, with applications of algebra to geometrical and to indeterminate problems.

TERREUS AND CARDAN.

After the publication of the books of Lucas de Burgo, the science of algebra became nore generally known, and improved, especially by many persons in Italy ; and about this time, or soon after, namely, about the year 1505, the first rule was there found out by Scipio Ferreus, for resolving one case of a compound cubic equation. But this science, as well as other branches of mathematics, was most of all cultivated and improved there by Hieronymus Cardan of Bononia, a very learned man, whose arithmetical writings were the next that appeared in print, namely, in the year 1539, in nine books, in the Latin language, at Milan, where he practised physic, and read public lectures on mathematics; and in the year 154.5 came out a 10th book, containing the whole doctrine of cubic equations, which had been in part revealed to him by Tartalea, about the time of the publication of his first nine books. And as it is only this 10 th book which contains the new discoveries in algebra, I shall here confine myself to it alone, as it will also afford sufficient occasion to speak of his manner of treating algebra in general. This book is divided into 40 chapters, in which the whole science of cubic equations is most amply and ably treated. Chap. 1, treats of the nature, number, and properties, of the roots of equations, and particularly of single equations that have double roots. He begins with a few remarks on the invention and name of the art: calls it Ars Magna, or Cosa, or Rules of Algebra, after Lucas de Burgo and others: says it was invented by Mahomet, the son of one Moses, an Arabian, as is testified by Leonardus Pisanus; and that he left four rules or cases, which only included quadratic equations: that afterwards three derivatives were added by an unknown author, though some think by Lucas Paciolus; and after that again three other derivatives, for the cube and 6 th power, by another unknown author; all which were resolved like quadratics: that then Scipio Ferreus, professor of mathematics at Bononia, about 1505 , found out the rule for the case cubun et rerum numero aqualium, or, as we now write it, $x^{3}+b x=c$, which he speaks of as a thing admirable: that the same thing was next afterwards found out, in 1535 , by Tartalca, who revealed it to him, Cardan, after the most eamest entreaties: that finally, by himself and his quondam pupil Lewis Ficrrari, the cases were greatly augmented and extended, namely, by all that is not here expressly ascribed to others; and that all the demonstrations of the rules are his own, except only three adopterl from Mahomet for the quadratics, and two of Ferrari for cubics.

Cardan then delivers some remarks, showing that all square numbers have two roots, the one positive, and the other nigative, or, as he calls them, verce fictu, true and fictitious or false; so the astimatio rei, or root, of 9 , is (ither 3 or -3 ; of 16 it is + or -4 ; the 4 th root of 81 is 3 or -3 ; and so on for all ('ve'n denominations or powers. And the same is remarked on compound cases of even powers that are added together; as if $x^{4}+3 x^{2}=28$, then the estimatio $x$ is $=2$ or -2 ; but that the form $x^{4}+12$ $=7 x^{2}$ has four answers or roots, in real numbers, two plus and two minus, viz, 2 or -2 , and $\sqrt{ } 3$ or $-\sqrt{ } 3$; while the case $x^{4}+12=6 x^{2}$ bas no real roots; and the case $x^{4}=2 x^{2}+8$ has two, namely, 2 and -2 ; and in like manner for other even powers. So that he includes both the positive and negative roots; but rejects what we now call imaginary ones. I here express the cases in our modern notation, for brevity sake, as he commonly expresses the terms by words at full length, calling the absolute or known term the numero, the 1 st power the res, the $2 \mathrm{~d}$ the quadratum, the $3 \mathrm{~d}$ the cubum, and so on, using no mark for the unknown quantity, and only the initials $p$ and $m$ for plus and minus, and $B$ for radix or root. The res he sometimes calls positio (as derived from the like quantity in the rule of position), and quantitas ignota; and in stating or setting down his equations, he as well as Lucas de Burgo before him, sets down the terms on that side where they will be plus, and not minus.

On the other hand, he remarks that the odd denominations, or powers, have only one æstimatio, or root, and that true or positive, but none fictitious or negative, and for this reason, that no negative number raised to an odd power, will give a positive number; so of $2 x=16$, the root is S only; and if $2 x^{3}=16$, the root is 2 only: and if there be ever so many odd denominations, added together, cqual to a number, there will be only one astimatio or root; as if $x^{3}+6 x=20$, the only root is 2 . But that when the signs of some of the terms are different, as to 'plus and minus, they may have more roots; and he shows certain relations of the coefficients, when they have two or more roots : so the equation $x^{3}+16=12 x$ has two astimatios, the one true or 2 , and the other fictitious or -4 , which he observes is the same is the true astimatio of the case $x^{3}=12 x+16$, having only the sign of the absolute number changed from the former, the $3 \mathrm{~d}$ root 2 being the same as the first, which therefure be does not count. He next shows what are the relations of the coefficients when a cubic equation has three roots, of which two are true, and the $3 \mathrm{~d}$ fictitiuus, which is al ways cqual to the sum of the other two, and also equal to the true root of the same equation with the sign of the absulute number changed: thus, in the equation $x^{3}+9=12 x$, the two true roots are 3 and $\sqrt{ } 5 \frac{x}{4}-1 \frac{x}{2}$, and the fictitious one is $-\sqrt{ } 5 \frac{x}{4}-1 \frac{x}{2}$, which last is the same as the true root of $x^{3}=12 x+9$, viz, $\sqrt{ } 5 \frac{x}{4}+1 \frac{x}{2}$; and he here infers generally that the fictitious astimatio of the case $x^{3}+$ $c=b x$, always answers to the true root of $x^{2}=b x+c$. Cardan alsu shows what the relation of the coeflicients is, when the case has no true roots, but only one tictitious root, which is the same as the true root of the reciprocal case, formed by changing the sign of the absolute number. Thus, the case $x^{3}+21=2 x$ has no truc root, and only one false root, viz, -3 , which is the same as the true root of $x^{3}=2 x+21$ : and he shows in general, that changing the sign of the absolute number in such cases as want the 2d term, or chan ing the signs of the even terms when it is not wanting, changes the signs of all the three roots, which he also illustrates by many examples; thus, the roots of $x^{3}+11 x^{2}=72$, are $+\sqrt{ } 40-4$, and -3 , and $-\sqrt{ } 40-4 ;$ and the roots of $x^{3}+72=11 x^{2}$, are $\sqrt{ } 40+4$, and +3 , ind $+\sqrt{ } 40+4$.

And he further observes, that the sum of the three roots, or the difference between the true and fictitions. roots, is equal to 11 , the coeflicicnt of the $2 \mathrm{~d}$ term. 1le 
also shows how certain cubic cases have one, or two, or three roots, according to circumstances: that the case $x^{4}+d=b x^{2}$ has sometimes four roots, and sometimes none at all, that is, no real ones: that the case $x^{3}+b x=a x^{2}+c$ may have three true æstimatios, or positive roots, but no fictitious or negative ones; and for this reason, that the odd powers of minus being minus, and the even powers plus, the two terms $x^{3}+b x$ would be negative, and equal to a positive sum $a x^{2}+c$, which is absurd: and further, that the case $x^{3}+a x^{2}+b x=c$ has three roots, one plus and two minus, which are the same, with the signs changed, as the roots of the case $x^{3}+b x+c=a x^{2}$. He also shows the relation of the coefficients when the equation has only one real root, in a variety of cases: but that the case $x^{3}+c=a x^{2}+b x$ has always one negative root, and either two positive roots, or none at all; the uumber of roots failing by pairs, or the impossible roots, as we now call them, being always in pairs. Of all these circumstances Cardan gives a great many particular examples in numeral coefficients, and subjoins geometrical demonstrations of the properties here enumerated; such as, that the two corresponding or reciprocal cases have the same root or roots, but with different signs or affections; and how many true or positive roots each case has.

On the whole, it appears from this short chapter, that Cardan had discovered most of the principal properties of the roots of equations, and could point out the number and nature of the roots, partly from the signs of the terms, and partly from the magnitude and relations of the coefficients. He shows in effect, that when the case has all its roots, or when none are impossible, the number of $i$ ts positive roots is the same as the number of changes in the signs of the terms, when they are all brought to one side : that the co-efficient of the $2 \mathrm{~d}$ term is equal to the sum of all the roots positive and negative collected together, and consequently that when the $2 \mathrm{~d}$ term is wanting, the positive roots are equal to the negative ones: and that the signs of all the roots are changed, by changing only the signs of the even terms: with many other just remarks conceruing the nature of equations.

In chap. 2, Cardan enumerates all the cases of compound equations of the $2 \mathrm{~d}$ and $3 \mathrm{~d}$ order, namely 3 quadratics, and 19 cubics; with 44 derivatives of these two, that is, of the same kind, with higher denominations.

In chap. 3 are treated the roots of simple cases, or simple equations, or at least that will reduce to such, having only two terms, the one equal to the other. He dircets to depress the denominations equally, as much as they will, according to the height of the least; then divide by the number or coefficient of the greatest; and lastly extract the root on both sides. So if $20 x^{3}=180 x^{5}$, then $20=180 x^{2}$, and $\frac{x}{9}=x^{2}$, and $x=\frac{r}{3}$.

Chap. 4 treats of both general and particular roots, and contains various definitions and observations concerning them. It is here shown, that the several cases of quadratics and cubics have their roots of the following forms or kinds, namcly, that the case

$x^{2}=a x+b$ has its root of this kind $\sqrt{ } 19+3$,

$x^{2}+a x=b$ has its root of this kind $\sqrt{ } 19-3$,

$x^{2}+b=a x$ has two roots like $3+\sqrt{ } 2$ and $3-\sqrt{ } 2$,

$x^{3}=b x+c$ bas its root of this kind $\sqrt[3]{4}+\sqrt[3]{2}$,

$x^{3}+b x=c$ has its root of this kind $\sqrt[3]{4}-\sqrt[3]{2}$,

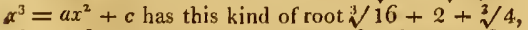

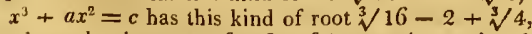

where the three parts $\sqrt[3]{16}, 2, \sqrt[3]{4}$, are in continual propurtion.
Chap. 5 treats of the æstimatio of the lowest degree of compound cases, that is, affected quadratic equations; giving the rule for each of the three cases, which consists in completing the square, \&c, as at present, and which was the method given by the Arabians and Hindus; and proving them by geometrical demonstrations from Eucl. i, 43 , and $i$, 4 and 5 ; in which be makes some improvement on the demonstrations of Mahomet. And hence it appears that the work of this Arabian author was in being, and well known in Cardan's time.

Chap. 6 , on the methods of finding new rules, contains some curious speculations concerning the squares and cubes of binomial and residual quantities, and the proportions of the terms of which they consist, shown from geometrical demonstrations, with many curious remarks and properties, forming a foundation of principles for investigating the rules for cubic equations.

Chap. 7 is on the transmutation of equations, showing how to change them from one form to another, by taking away certain terms out of them; as $x^{3}+a x^{2}=c$, to $x^{3}=b x+d, \& c$. The rules are demonstrated geometrically; and a table is added, of the forms into wbich any given cases will reduce; which transformations are extended to equations of the 4 th and 5 th order. And hence it appears that Cardan knew how to take away any term out of an equation.

Chap. 8 shows generally how to find the root of any such equation as this, $a x^{\mathrm{m}}=x^{\mathrm{n}}+b$, where $m$ and $n$ are any exponents whatever, but $n$ the greater; and the rule is, to separate or divide the coefficient $a$ into two such parts $z$ and $a-z$, as that the absolute number $b$ shall be equal to $(a-z) \cdot \frac{\mathrm{m}}{z^{\mathrm{n}}-\mathrm{m}}$, the product of the one part $a-z$, and the $\frac{m}{n-m}$ power of the other part: then the root $x$ is $=$ 1

$\overline{z^{n-m}}$. The rule is general for quadratics, cubics, and all the higher powers: and could not have been formed without the knowledge of the composition of the terms from the roots of the equation.

Chap. 9 and 10 contain the resolution of various questions producing equations not higher than quadratics.

Chap. 11 is on the case or form $x^{3}+3 b x=2 c$. Cardan now comes to the actual resolution of the first case of cubic equations. He begins with relating a short listory of the invention of it, observing that it was first found out, about thirty years before, by Scipio Ferreus, of Bononia, and by him taught to Antonio Maria Florido, of Venice, who having a contest afterwards with Nicolas Tartalea, of Brescia, it gave occasion to Tartaleg. to find it out himself, who after great entreaties taught it to Cardan, but suppressed the demonstration. By help of the rule alone, however, Cardan of himself discovered the source or geometrical investigation, which he gives here at large, from Euc. ii, 4. In this process he makes use of the Greek letters $\alpha, \varsigma, \gamma, \delta, \& c$, to denote certain indefinite numbers or quantities, to render the investigation general; which may be considered as the first instance of such literal notation in algebra. He then gives the rule in words at length, which comes to this, $x=\sqrt[3]{ }\left[\sqrt{ }\left(c^{2}+b^{3}\right)+c\right]$ $-\sqrt[3]{ }\left[\sqrt{ }\left(c^{2}+b^{3}\right)-c\right]$; illustrating it by a variety of examples; in the resolution of which, he extracts the cubic roots of such of the binomials as will admit of it, by some rule which he had for that purpose; snch as $x^{3}+6 x=20$, where $x=\sqrt[3]{ }(\sqrt{ } 108+10)-\sqrt[3]{ }(\sqrt{ } 108-10)=(\sqrt{ } 3$ $+1)-(\sqrt{ } 3-1)=2$. 
Chap. 12, on the case $x^{3}=3 b x+2 c$. This he treats exactly as the last, and finds the rule to be $x=\sqrt[3]{ }\left[c+\sqrt{ }\left(c^{2}\right.\right.$ $\left.\left.-b^{3}\right)\right]+\sqrt[3]{ }\left[c-\sqrt{ }\left(c^{2}-b^{3}\right)\right]$; which he illustrates by many examples, as usual. But when $b^{3}$ exceeds $c^{2}$, which has since been called the irreducible case, he refers to another following book, called Aliza, for other rules of solution, to overcome this difficulty, about which he took infinite pains.

Chap. 13 , on the case $x^{3}+2 c=3 b x$. This case, by a geometrical process, he reduces to the case in the last chapter : thus, find the xstimatio $y$ of the case $y^{3}=3 b y$ $+2 c$, having the same coefficients as the given case $x^{3}+2 c=3 b x$; then is $x=\frac{1}{2} y \pm \frac{1}{2} \sqrt{ }\left(12 b-3 y^{2}\right)$, giving two roots. He shows also how to find the second root, when the first is known, independent of the foregoing case. From this relation of these two cases he deduces several corollaries, one of which is, that the æstimatio or roct of the case $y^{3}=3 b y+2 c$, is equal to the sum of the roots of the case $x^{3}+2 c=3 b x$. As in the example $y^{3}=16 y+21$, whose æstimatio is $\sqrt{ } 9 \frac{x}{4}+1 \frac{x}{2}$, which is equal to the sum of 3 and $\sqrt{ } 9 \frac{x}{4}-1 \frac{r}{2}$, the two roots of the case $x^{3}+21=16 x$;

In chapters 14,15 , and 16 , he treats of the three cases, which contain the $2 \mathrm{~d}$ and $3 \mathrm{~d}$ powers, but wasting the $1 \mathrm{st}$ power, according to all the varieties of the signs; which he performs by exterminating the $2 \mathrm{~d}$ term, or that which contains the $2 \mathrm{~d}$ power of the unknown quantity $x$, by substituting $y \pm \frac{r}{3}$ the coefficient of that term for $x$, and so reducing these cases to one of the former. In these chapters Cardan sometimes also gives other rules; thus, for the case $x^{3}+3 a x^{2}=2 c$, find first the æstimatio $y$ of the case $y^{3}=3 a y+\sqrt{ } 2 c$, then is $x=y^{2}-3 a$ : also for the case $x^{3}+2 c=3 a x^{2}$, first find the two roots of $y^{3}+2 c$, $=3 a y \sqrt[3]{2 c}$, then is $x=\frac{\sqrt[3]{4 c^{2}}}{y}$ the two values of $x$ according to the two values of $y$. He here also gives another rule, by which a second æstimatio or root is found, when the first is known, namely, if $e$ be the first æstimatio, or value of $x$ in the case $x^{3}+2 c=3 a x^{2}$, then is the other value of $x=\frac{1}{2} \sqrt{ }[(3 a-e) \cdot(3 a+3 e)]+\frac{1}{2}(3 a-e)$.

In chapters $17,18,19,20,21,22,23$, Cardan treats of the cases in which all the four terms of the equation are present; and this he always effects by taking away the $2 \mathrm{~d}$ term out of the equation, and so reducing it to one of the foregoing cases which want that term, giving always geometrical investigations, and adding a great many examples of every case of the equations.

Chap. 24, is on the 44 derivative cases; which are only higher powers of the forms of quadratics, and cubics.

Chap. 25, on imperfect and special cases; containing many particular examples when the coefficients have certain relations among them, with casy rulcs for finding the roots; also 8 other rules for the irreducible case $x^{3}=b x$ $+c$.

Chap. 26, in like manner, contains easy rules for biquadratics, when the coefficients have certain special relations.

Then the following chapters, from chap. 27 to chap. 38 , contain a great number of questions and applications of various kinds, the titles of which are these: De transitu capituli specialis in capitulum speciale; De operrationibus ruticum pronicarum seu mixtarum et Allellarum; De regula modi; De regula Aurea; De regula Magna, or the method of funding out solutions to certain questions; De regula aqualis positionis, being a method of substituting for the half sum and half difference of two quantities instead of the quantities themselves; De regula incequaliter ponendi, seu proportionis; De regula medii; De regula aggregati; De regula libera pasitionis; De regula falsum ponendi, in which some quantities come out negative; Quomodo excidant partes et denominationes multiplicando. Among the foregoing collection of questions, which are chiefly about numbers, there are some geometrical ones, being the application of algebra to geometry; such as, In a rightangled triangle, given the sum of each leg and the adjacent segnient of the hypotenuse, made by a perpendicular from the right angle, to determine the area, \&c; with other such geometrical questions, resolved algebraically.

Chap. 39 , De regula qua pluribus positionibus invenimius ignotam quantitatem; which is employed on biquadratic equations. After some examples of his own, Cardan gives a rule of Lewis Ferrari's, for resolving all biquadratics, namely, by means of a cubic equation, which Ferrari investigated at his request, and which Cardan here demonstrates, and applies in all its cases. The method is very general, and consists in forming three squares, thus: first, complete one side of the equation up to a square, by adding or subtracting some multiples or parts of some of its own terms on both sides, which it is always easy to do: $2 \mathrm{~d}$, supposing now the three terms of this square to bebut one quantity, viz, the first term of another square to which this same side is to be completed, by annexing the square of a new and assumed indeterminate quantity, with double the product of the roots of both; which cvidently forms the square of a binomial, consisting of the assumed indeterminate quantity and the root of the first square: $3 \mathrm{~d}$, the other side of the equation is then made to become the square of a binomial also, by supposing the product of its $1 \mathrm{st}$ and $3 \mathrm{~d}$ terms to be equal to the square of half its $2 \mathrm{~d}$ term; for it consists of only three terms, or three different denominations of the original unknown quantity: then this equality will determine the value of the assumed indeterminate quantity, by mcans of a cubic equation, and from it, that of the original ignota, by the equal roots of the $2 \mathrm{~d}$ and $3 \mathrm{~d}$ squares. Here we have a notable example of the use of assuming a new indeterminate quantity to introduce into an equation, long before Des Cartes was born, who made use of a like assumption for a similar purpose. And this method is very general, and is here applied to all forms of biquadratics, either having all their terms, or wanting some of them. To illustrate this rule, I shall here set down the process in one of his examples, which is this, $x^{4}+4 x+8=10 x^{2}$. Now first subtract $2 x^{2}+4 x+7$ from both sides, then the first becomes a square, viz, $x^{4}-2 x^{2}+1$ or $\left(x^{2}-1\right)^{2}=8 x^{2}$ $-4 x-7$. Next assume the indeterminate $y$, and subtract $2 y\left(x^{2}-1\right)-y^{2}$ from both sides, making the first side again a square, viz, $(x-1)^{2}-2 y(x-1)+y^{2}$ or $\left(x^{2}-1-y\right)^{2}=(8-2 y) x^{2}-4 x+y^{2}+2 y-7$. Of this latter side, make the product of the 1 st and $3 \mathrm{~d}$ terms equal tn the square of half the $2 \mathrm{~d}$ term, that is, $(8-2 y)$. $\left(y^{2}+2 y-7\right)=2^{2}$, which reduces to $y^{3}+30=2 y^{2}+$ $15 y$; the positive roots of which are $y=2$ or $\sqrt{ } 15$ : and hence, using 2 for $y$, the equation of equal squares becomes $\left(x^{2}-1-y\right)^{2}$ or $\left(x^{2}-3\right)^{2}=4 x^{2}-4 x+1$, the roots of which give $x^{2}-3=2 x \sim 1$; and hence $x^{2}=2 x+2$, or $x^{2}+2 x=4$; the two positive roots of which are $\sqrt{ } 5+1$ and $\sqrt{ } 5-1$, which are two of the values of $x$ in the given equation $x^{4}+4 x+8=10 x^{2}$. The other roots he leaves to be tried by the reader.

The 40th, or last, chap. is cntitlerl, On modes of general supposition relating to this art; with some rules of an un- 
usual kind; and æstimatios or roots of a nature different from the foregoing ones. Some of these are as follow: If $x^{3}=a x^{2}+c$, and $x-a=y$, and $x: y:: c: d$; then is $y^{3}+a y^{2}=d$.

Secondly, if $x^{3}+a x^{2}=c$, and $y^{3}=a y^{2}+c$, then is $x+a: y-a:: y^{2}: x^{2}$.

Thirdly, when $x^{3}+c=a x^{2}$, the square will be taken away, by putting $x=y+\frac{1}{3} a$; and then the equation becomes $y^{3}+c-2\left(\frac{x}{3} a\right)^{3}=\frac{1}{3} a^{2} y$.

Cardan adds some other remarks concerning the solutions of certain cases and questions, all evincing the accuracy of his skill, and the extent of his practice; he then concludes the book with a remark concerning a certain transformation of equations, which quite astonishes us, to find that the same person who, through the whole work, has shown such a profound and critical skill in the nature of equations, and the solution of problems, should yet be ignorant of one of the most obvious transmutations attending them, namely, increasing or diminishing the roots in any proportion. Cardan having observed that the form $x^{3}=b x+c$ may be changed into another similar one, viz, $y^{3}=\frac{b}{c} y+\sqrt{ } \frac{1}{c}$, of which the coefficient of the term $y$ is the quotient arising from the coefficient of $x$ divided by the absolute number of the first equation; and that the absolute number of the $2 \mathrm{~d}$ equation, is the root of the quotient of 1 divided by the said absolute number of the first ; he then adds, that finding the æstimatio or root of the one equation from that of the other is very difficult, valde difficilis.

It is matter of wonder that Cardan, among so many transmutations, should never think of substituting, instead of $x$ in such equations, another positio or root, greater or less than the former in any indefinite proportion, that is, multiplied or divided by a given number; for this would have led him immediately to the same transformation as be makes above, and that by a way which would have shown the constant proportion between the two roots. Thus, instead of $x$ in the given form $x^{3}=b x+c$, substitute $d y$, and it becomes $d^{3} y^{3}=b d y+c$; and this divided by $d^{3}$ becomes $y^{3}=\frac{b}{d^{2}} y+\frac{c}{d^{3}}$; and here if $d$ be taken $=$ $\sqrt{ } c$, it becomes $y^{3}=\frac{b}{c} y+\sqrt{ } \frac{1}{c}$; the very transformation in question, and in which it is evident that $x$ is $=y \sqrt{ } c$, and $y=\frac{x}{\sqrt{c}}$. Instead of this, Cardan gives the following strange way of finding the one root $x$ from the other $y$, when this latter is by any means known; viz, Multiply the first given cquation by $y^{2} x+1$, then add $\frac{x^{2}}{4 y^{2}}$ to both sicles, and lastly extract the roots of both, which can always be done, as they will always be both of them squares; and the roots will give the value of $x$ by a quadratic equation.

Thus, $x^{3}=b x+c$ multiplied by $y^{2} x+1$ gives $y^{2} x^{4}+x^{3}=b y^{2} x^{2}+\left(c y^{2}+b\right) x+c ;$ add $\frac{x^{2}}{4 y^{2}}$ then $y^{2} x^{4}+x^{3}+\frac{x^{2}}{4 y^{2}}=b y^{2}+\frac{1}{4 y^{2}} \cdot x^{2}+\left(b+c y^{2}\right) x+c$; and the roots are $y x^{2}+\frac{x}{2 y}=\sqrt{ }\left[\left(b y^{2}+\frac{1}{4 y^{2}}\right) x^{2}+\left(b+c y^{2}\right)\right.$ $x+c]$; and this $2 \mathrm{~d}$ side of the equation, he says, will always have a root also. It is indeed true, that it will have all exact root; but the reason of it is not obvious, which is, because $y$ is the root of the cquation $y^{3}=\frac{b}{c} y+$ $\sqrt{ } \frac{1}{c}$. Cardan has not shown the reason why this happens; but $I$ apprehend he made it out in this manner, viz, similar to the way in which he forms the last square in the case of biquadratic equations, namely, by making the product of the 1 st and $3 \mathrm{~d}$ terms equal to the square of half the $2 \mathrm{~d}$ term: thus, in the present case, it is $4 c\left(b y^{2}+\frac{1}{4 y^{2}}\right)$ $=\left(b+c y^{2}\right)^{2}$, which reduces to $y^{3}=\frac{b}{c} y+\sqrt{ } \frac{1}{c}$ the equation in question. Therefore taking $y$ the root of the equation $y^{3}=\frac{b}{c} y+\sqrt{c}_{c}^{1}$, and substituting its value in the quantity $\left(b y^{2}+\frac{1}{4 y^{2}}\right) x^{2}+\left(b+c y^{2}\right) x+c$, this becomes a complete square.

Of CA RDAN's Libellus de Aliza Regula. Subjoined to the above treatise on cubic equations, is this Libellus de Aliza Regula, or the a]gebraic logistics, in which the author treats of some of the more abstruse parts of arithmetic and algebra, especially cubic equations, with many more attempts on the irreducible case $x^{3}=b x+c$. This book is divided into 60 chapters; but $I$ shall only set down the titles of some few of them, whose contents require more particular notice.

Chap. 4. De modo redigendi quantitates omnes, qua dicuntur latera prima, ex decino Euclidis in compendium. He treats here of all Euclid'sirrational lines, as surd numbers, and performs various operations with them.

Chap. 5. De consideratione binoniorum et recisorum, \& c ; $u b i$ de astimatione capitulorum. Contains various operations of multiplying compound numbers and surds.

Chap. 6. De operationibus p: et $\mathrm{m}$ : (i. e. + and -) secundum communem usum. Here it is shown that, in multiplication and division, plus always gives the same signs, and minus gives the contrary signs. So also in addition, every quantity retains its own sign; but in subtraction they change the signs. That the $\sqrt{+}$, or the square root of plus, is + ; but the $\sqrt{ }-$, or the square root of minus is nothing as to common use: (but of this below.) That $\sqrt[3]{ }-$ is - , as $\sqrt[3]{ }-8$ is -2 . That a residual, composed of + and - may have a ront also composed of + and - : so $\sqrt{ }(5-\sqrt{24})$ is $=\sqrt{ } 3-\sqrt{ } 2$. The rules for the signs in multiplication and division are illustrated by this example; to divide 8 by $2+\sqrt{ } 6$ or $\sqrt{ } 6$ +2 . Take the two corresponding residuals $2-\sqrt{ } 6$ and $\sqrt{ } 6-2$, and by these multiply both the divisor and dividend; then the products are + and - respectively, and the quotients still both alike. Thus,

$$
\begin{array}{cc|cc}
\text { Divid. } & \text { Divis. } & \text { Divid. } & \text { Divis. } \\
8 & \sqrt{ } 6+2 & 8 & 2+\sqrt{ } 6 \\
\sqrt{ } 6-2 & \frac{\sqrt{ } 6-2}{\sqrt{384-16}} \text { divide+2 } & \frac{2-\sqrt{ } 6}{16-\sqrt{ } 384} \text { div. } \frac{2-\sqrt{ } 6}{-2} \\
\text { Quot. } \sqrt{ } 96-8 . & \text { Quot. } \sqrt{ } 96-8 .
\end{array}
$$

And this method of performing division of compound surds, was fully tanglit before him, by Lucas de Burgo, namely, reducing the compound divisor to a simple quantity, by multiplying by the corresponding quantity, having the sign changed.

In chap. 11 and 18 , and elsewhere, Cardan makes a general notation of $a, b, c, d, e, f$, for any indefinite quantities, and treats of them in a general way.

Chap. 2. De contemplatione $\mathrm{p}:$ et $\mathrm{m}:$ (or + and - ) et guod $\mathrm{m}:$ in $\mathrm{m}:$ facit $\mathrm{p}$ : et de causis horum juxta veritatem. Cardan bere demonstrates gcometrically that, in multipli- 
A L G

cation and division, like signs give plus, and unlike signs give minus. And he illustrates this numerically, by squaring the quantity 8 , or $6+2$, or $10-2$, which must all produce the same thing, namely, 64 .

Among many of the chapters which treat of the irreducible case $x^{3}=b x+c$, there is a peculiar kind of way given in chap. 31, which is entitled Deastimatione generali $x^{3}=b x+c$ solida vocata, et operationibus ejus; in which he shows how to approximate to the root of that case, in a manner similar to approximating the square root and cube root of a number. The rule he uses for this purpose, is the $3 \mathrm{~d}$ in chap. 25 of the last book, and it is this: Divide $b$ into two parts, such that the sum of the products of each, multiplied by the square root of the other, may be equal to $\frac{\mathrm{r}}{2} \mathrm{c}$; then the sum of the roots of these parts is the æstimatio or value of $x$ required. So, of this equation $x^{3}=10 x$ +24 ; the two parts are 9 and 1 , and their +24 ; the two parts are 9 and 1 , and their $3+9$ or $12=\frac{x}{2} c$,
roots 3 and 1 , and their sum $4=x$, as $x=3+1=4$. in the margin. Again, take $x^{3}=6 x+1$. Here he invents a new notation to express the root or radix, which he calls solida, viz, $x=\sqrt{ }$ solida 6 in $\frac{\mathrm{T}}{2}$, that is, the roots of the two parts of 6 , so that each part multiplied by the root of the other, the two products may be $\frac{r}{2}$ or $\frac{\pi}{2} c$. Then to free this from fractions, and make the operation easier, multiply that root by some number, as suppose 4 , that is, the square part 6 by the square of 4 , and the solid part $\frac{\pi}{2}$

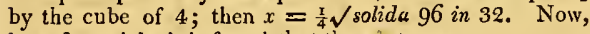
by a few trials, it is found that the parts are

nearly $95 \frac{8}{9}$ and $\frac{\pi}{9}$, which give too much,

or $95 \frac{9}{20}$ and $\frac{x}{x \sigma}$, which give too little,

and thereof $95 \frac{1}{19}$ and $\frac{2}{19}$ are still nearer. Divide both by $4^{2}$ or 16 , then $5 \frac{151}{S^{2}}$ and $\frac{T}{1 \frac{1}{5}}$ are the quotients. And the sum of their roots, or $x=\sqrt{ } 5 \frac{15}{15 \frac{1}{2}}+\sqrt{\frac{\pi}{5} \frac{2}{2}}$ is nearly the value of the root $x$.

Chap. 42. De duplici aquatione comparanda in capitulo cubi et numeri aqualium rebus. Treats of the two positive roots of that case, neglecting the negative one; and showing, not only that the case has two such roots, but that the same number may be the common root of inuumerable equations.

Chap. 57. De tractatione astimationis generaliscapituli $x^{3}$ $=b x+c$. Cardan here again resumes the consideration of the irreducible case, making ingenious observations upon it, but still without obtaining the root by a general rule: In this place also, as well as elsewhere, he shows how to form an equation in this case, that shall have a given binomial root, as suppose $\sqrt{ } m+n$, where the equation will be $x^{3}=\left(m+3 n^{2}\right) x+2 n\left(m-n^{2}\right)$, having $\sqrt{ } m+n$ for one root, namely the positive root. From which it appears that he was well acquainted with the composition of cubic equations from given roots.

Chap. 59. De ordine et exemplis in binonizis secundo et quinto. Contains a great many numeral forms of the same irreducible case $x^{3}=b x+c$, with their roots; from which are derived these following cases, with many curious remarks. As, when

$$
\begin{aligned}
& x^{3}=(c+1) x+c, \text { then } x=\sqrt{ }\left(c+\frac{1}{4}\right)+\frac{1}{2} \\
& x^{3}=\left(\frac{1}{2} c+4\right) x+c \text {, then } x=\sqrt{ }\left(\frac{1}{2} c+\frac{4}{4}\right)+\frac{2}{2} \\
& x^{3}=\left(\frac{1}{3} c+9\right) x+c \text {, then } x=\sqrt{ }\left(\frac{1}{3} c+\frac{9}{4}\right)+\frac{3}{2} \\
& x^{3}=\left(\frac{1}{4} c+16\right) x+c, \text { then } x=\sqrt{ }\left(\frac{1}{4} c+\frac{16}{4}\right)+\frac{4}{2} . \\
& x^{3}=\left(\frac{1}{n} c+n^{2}\right) x+c, \text { then } x=\sqrt{ }\left(\frac{1}{n} c+\frac{n^{2}}{4}\right)+\frac{n}{2} .
\end{aligned}
$$

Chap.60. Demonstratio generalis capituli cubi aqualis rebus et numero. This demonstration of the irreducible case
A L G

is geometrical, like all the rest. Some more ingenious remarks are again added, as if he reluctantly finished the book, without perfectly overcoming the difficulty of the irreducible case. Cardan here also uses the letters $a$ and $b$ for any two indefinite numbers, in order to show the form and manner of the arithmetical operations : thus $\frac{a}{b}$ is the fraction for theirquotient, also $\sqrt{ } \frac{a}{b}$ or $\frac{\sqrt{ } a}{\sqrt{b}}$ the square root of that quotient, and $\sqrt[3]{\frac{a}{b}}$ or $\frac{\sqrt[3]{a}}{\sqrt[3]{b}}$ the cube root of it, \&c.

Having considered the chief contents of Cardan's algebra, it will now be proper to sum them up, and set down a list of the improvements made by him, as collected from lis writings.

And 1st, Tartalea baving only communicated to him the rules for resolving these three cases of cubic equations, viz,

$\left.x^{3}+b x=c,\right\}$ he thence raised a very large and complete $\left.x^{3}=b x+c,\right\}$ work, laying down rules for all forms and $x^{3}+c=b x,\{$ varieties of cubic equations, having all their terins, or wanting any of them, and having all possible varieties of signs; demonstrating all these rules geometrically; and treating very fully of almost all kinds of transfornations of equations, in a manner before unkaown.

$2 \mathrm{~d}$, It appears that he was well acquainted with all the roots of equations that are real, both positive and negative; or, as he calls them, true and fictitious; and that he made use of them both occasionally. He also showed that the even roots of positive quantities, are either positive or negative; that the odd roots of negative quantities, are real and negative; but that the even roots of them are impossible, or nothing as to common use. He was also acquainted with the

$3 \mathrm{~d}$, Number and nature of the roots of an equation, and that partly from the signs of the terms, and partly from the magnitude and relation of the coefficients. He also knew,

4 th, That the number of positive roots is equal to the number of changes of the signs of the terms.

5 th, That the coefficient of the second term of the equation, is the difference bctween the positive and negative roots.

6 th, That when the second term is wanting, the sum of the negative roots is equal to the sum of the positive roots.

7 th, How to compose equations that shall lave given roots.

Sth, That, changing the signs of the even terms, changes the signs of all the roots.

9 th, That the number of roots failed in pairs ; or what we now call impossible roots were always in pairs.

10th, To change the equation from one forw to another, by taking away any term out of $i t$.

1 Ith, To increase or diminish the roots by a given quantity. It appears also,

12 th, That he had a rule for extracting the cube root of such binomials as admit of extraction. \&c.

13th, 'That he often used the literal notation $a, b, c, d$, cc.

14th, That he gave a rule for biquadratic equations, suiting all their cases; and that, in the investigation of that rulc, he made nse of an assumed incleterminate quantity, and alterwards found its value by the arbitrary assumption of a relation between the terms. 
15 th, That he applied algebra to the resolution of geometrical problems. And,

16 th, That be was well acquainted with the difficulty of what is called the irreducible case, viz, $x^{3}=b x+c$, upon which he spent a great deal of time, in attempting to overcome it. And though he did not fully succeed in this case, any more than other persons have done since, be nevertheless made many ingenious observations about $i t$, laying down rules for many particular forms of it, and showing how to approximate very nearly to the root in all cases whatever.

\section{Cardan died at Rome in the year 1575 .}

$$
\text { OF TARTALEA: }
$$

Nicholas Tartalea, or Tartaglia, of Brescia, was contemporary with Cardan, and was probably older than he was; but I do not know of any book of algebra published by him till the year 1546 , the year after the date of Cardan's work on Cubic Equations, when he printed his Quesiti et Inventioni diverse, at Venice, where he resided as a public lecturer on mathematics. This work is dedicated to our king Henry the Sth of England, and consists of nine books, containing answers to various questions whicl, had been proposed to him at different times, concerning mechanics, statics, hydrostatics, \&c ; but it is only the 9 th, or last book, that we shall have occasion to take notice of in this place, as it contains all those questions which relate to arithmetic and algebra. These are all set down in chronological order, forming a pretty collection of questions and solutions on those subjects, with a short account of the occasion of each of them. Among these, the correspondence between him and Cardan forms a remarkable part, as we have here the history of the invention of the rules for cubic equations, which he communicated to Cardan, under the promise, and indced oath, to keep them secret, on the 25th of March, 1539. But, notwithstanding his oath, finding that Cardan published them in 1545 , as above related, it scems Tartalea published the correspondence between them, in revenge for his breach of faith ; and it elsewhere appears, that many other sharp bickerings passed between them on the same account, which only ended with the the death of 'Tartalea, in the year 1557 .

It seems it was a common practice among the mathematicians, and others, of that time, as in most other times, to send to each other curious and difficult questions, as trials of skill; and to this circumstance it is that we owe the principal questions and discoveries in this collection, as well as many of the best discoveries of other authors. The collection now before us contains questions and solutions, with their dates, in a regular order, from the year 1521 , and ending in $15+1$, in 42 dialogues, the last of which is with an English gentleman, namely, Mr. Richard Wentworth, who it seems was no mean mathematician, and who learned some algebra, \&c, of Tartalea, while he residerl at Venice. The questions at first are mostly very easy on's in arithmetic, but gradually become more difficult, and exercising simple and quadratic equations, with complex calculations of radical quantitics: all showing that he was well skilled in the art of algebra as it then stood, and that he was very ingenious in applying it to the solution of yuestions. Tartalea made no alteration in the notation or forms of expression used by Lucas de Burgo, calling the first power of the unknown quantity, in his language, cosi, the second power censa, the third cubo, \&rc, and writing the names of all the operationsin words at length, without using any contractions, except the initial $\mathbb{B}_{\infty}$ for root or radicality. So that the only thing remarkable in this collection, is the discovery of the rules for cubic equations, witb the curions circumstances attending the same.

The first two of these were discovered by Tartalea in the year 1530 , namely, for the two cases $x^{3}+a x^{2}=c$, and $x^{3}=a x^{2}+c$, as appears by Quest. 14 and 25 of this collection, on occasion of a question then proposed to him by one Zuanni de Tonini da Coi or Colle, John Hill, who kept a school at Brescia. And from the 25th question we learn, that he discovered the rules for the other two cases $x^{3}+b x=c$, and $x^{3}=b x+c$, on the 12 th and 13 th of February, 1535, at.Venice, where he had come to reside the year before. And the occasion of it was this: There was then at Venice one Antonio Maria Fiore or Florido, who, by his own account, had received from his preceptor Scipio Farreo, about thirty years before, a general rule for resolving the case $x^{3}+b x=c$. Being a captious man, and presuming on this discovery, Florido used to brave his contemporaries, and by his insults provoked Tartalea to enter into a wager with him, that each should propose to the other 30 different questions; and that he who soonest resolved those of his adversary, should win from him as many treats for himself and friends. These questions were to be proposed on a certain day at some weeks distance; and Tartalea made such good use of his time, that eight days before the time appointed for delivering the propositions, he discovered the rules both for the case $x^{3}+b x$ $=c$, and the case $x^{3}=b x+c$. He therefore proposed several of his questions, so as to fall either on this latter case, or on the cases of the cube and square, expecting that his adversary would propose his in the former. And what he suspected fell out accordingly; the consequence of which was, that on the day of meeting 'Tartalea resolved all his adversary's questions in the space of two hours, without receiving one answer from Florido in return; to whom, however, Tartalea generously remitted the forfeit of the 30 treats won of him.

Question 31 first brings us acquainted with the correspondence between Tartalea and Cardan. This correspondence is very curious, and would well deserve to be given at full length in their own worls, if it were not too long for this placc. I may enlarge farther upon it under the article Cubic Equations; but must here be content with a brief abstract only. Cardan was then a respectable physician, and lecturer in mathematics, at Milan; who having nearly finished the printing of a large work on arithmetic, algebra, and gcometry, and having heard of Tartalea's discoveries in cubic equations, he was very desirous of drawing those rules from him, that he might add them to his book before it was finished. For this purpose, be first applied to Tártalea, by means of a third person, a bookseller, whom he sent to him, in the beginning of the year 1539 , with many flattering compliments, and offers of his services and friendship, \&c, accompanied with some critical questions for him to resolve, according to the custom of the times. Tartalea however refused to disclose his rules to any one; as the knowledge of them gained him great reputation among all people, and gave him great advantage over his competitors for fame, who were commonly afraid of him on account of those very rules. He only sent Cardan therefore, at his request, a copy of the 30 questions which had been proposed to hin in the contest with Florido.

Not to be rebuffed so casily, Cardan next applied, in 
the most urgent manner, by letter, to Tartalea; which however procured from him only the solution of some other questions proposed by Cardan, and of a few of the questions that had been proposed to Florido, but none of their solutions. Finding he could not thus prevail, with all his fair promises, Cardan then fell upon another scheme. There was at Milan a certain Marquis dal Vasto, a great patron of Cardan, and, it was said, of learned men in general. Cardan conceived the idea of making use of the influence of this nobleman to draw Tartalea to Milan, hoping that then, by personal entreaties, he should succeed in drawing the long-concealcd rules from him. Accordingly he wrote a second letter to Tartalea, much in the same strain with the former, strongly inviting bim to come and spend a few days in his house at Milan, and representing that, having often commended him in the bighest terms to the marquis, this nobleman desired much to see him; for which reason Cardan advised him, as a friend, to come to visit them at $\mathrm{Mi}$ lan, as it might be greatly to his interest, the marquis being very liberal and bountiful; and he besides gave Tartalea to understand, that it might be dangerous to offend such a man by refusing to come, who might, in that case, take offence, and do him some injury. This manœurre had the desired effect: Tartalea on this occasion laments to bimself in these words, "By this I am reduced to a great dilemma ; for if I go not to Milan, the marquis may take it amiss, and some evil nay befall me on that account; I shall therefore go, though very unwillingly." When he arrived at Milan, however, the marquis was gone to Vigeveno; and Tartalea was prevailed on to stay three days with Cardan, in expectation of the marquis returning; at the end of which time he set out from Milan, with a letter from Cardan, to go to Vigeveno to that nobleman. While Tartalea was at Milan the three days, Cardan plied him by all possible means, to draw from him the rules for the cubic equations; and at leigth, just as Tartalea was about to depart from Milan, on the 25 th of March, 1539, he was overcome by the most solemn protestations of secrecy that could be made. Cardan says, "I shall swear to you on the holy evangelists, and by the honour of a gentleman, not only never to publish your inventions, if you reveal them to me; but I also promise to you, and pledge my faith as a true Christian, to note them down in cyphers, so that after.my death no other person may be able to understand them." To this Tartalea replies, "If I refuse to give credit to these assurances, I should deservedly be accounted utterly roid of belief. But as I intend to ride to Vigeveno, to see his excrllency the marquis, as I have been here now these three days, and am weary of waiting so long; whenever I return therefore, I promise to show you the whole." Cardan answers, "Since you determine at any rate to go to Vigeveno to the inarquis, I shall give you a letter for his excellency, that he may know who you are. But now before you depart, I entreat you to show me the rule for the equations, as you have promised." "I am content," says Tartalca: " but yon must know, that to be able on all occasions to remember such operations, I have brought the rule into rhyme; for if I had not used that precaution, I should often have forgot it; and though my rhymes are not very good, 1 do not value that, as it is sufficiont that they serve to bring the rule to mind as often as I repeat them. I shall here write the rule with my own hand, that you may be sure I give you the discovery exactly."
'These rude verses contain, in rather dark and enigmatical language, the rule for these three cases, viz, $x^{3}+b x=c, 7$ which differ however only in the sign of $\left.x^{3}=b x+c,\right\}$ one quantity, and the rule amounts to $x^{3}+c=b x, \int$ this: Find two numbers, $z$ and $y$, such that their difference in the first case, and their sum in the $2 \mathrm{~d}$ and $3 \mathrm{~d}$, may be equal to $c$ the absolute number, and their product equal to the cube of $\frac{1}{3}$ of $b$ the coefficient of the less power; then the difference of their cube roots will be equal to $x$ in the first case, and the sum of their cube roots equal to $x$ in the $2 \mathrm{~d}$ and $3 \mathrm{~d}$ cases: that is, taking $z-y=c$ in the first case, or $z+y=c$ in the $2 d$ and $3 \mathrm{~d}$, and $z y=\left(\frac{1}{3} b\right)^{3}$; then $x=\sqrt[3]{z}-\sqrt[3]{y}$ in the first case, and $x=\sqrt[3]{2}+\sqrt[3]{y}$ in the other two. At parting, Tartalea, fails not again to remind Cardan of his obligation: "Now your excellency will remember not to break your promised faith, for if unhappily you should insert these rules either in the work you are now printing, or in any other, though you should even give them under my name, and as of my invention, I promise and swear that 1 shall immediately print another work that will not be very pleasing to you," "Doubt not," says Cardan, "but that I shall observe what I have promised: Go, and rest secure as to that point; and give this letter of mine to the marquis." It should seem, however, that Tartalea was much displeased at having suffered himself to be worried as it were out of his rules; for as soon as be quitted Milan, instead of going to wait upon the marquis, he turned his horse's head, and rode straight home to Venice, saying to himself, "By my faith I shall not go to Vigeveno, but shall return to Venice, come of it what will."

After 'Tartalea's departure, it seems Cardan applied himself immediately to resolving some examples in the cubic equations by the new rules, but not succeeding in them; for indeed he had mistaken the words, as it was very easy to do in such bad verses, having mistaken $\left(\frac{1}{3} b\right)^{3}$ for $\frac{x}{3} b^{3}$, or the cube of $\frac{x}{3}$ of the coefficient, for $\frac{x}{3}$ of the cube of the coefficient; accordingly we find him writing to 'lartalea in 14 days after the above, blaming him much for his abrupt departure without seeing the marquis, who was so liberal a prince, be said, and rcquesting Tartalea to resolve him the example $x^{3}+3 x=10$. This Tartalea did to his sutisfaction, rightly guessing at the nature of his mistake; and concludes his auswer with these emphatical words, "Remember your promise." On the 12th of May following, Cardan returns him a letter of thanks, togcther with a copy of his book, saying, "As to my work, just fuished, to remove your suspicion, I send you a copy, but unbound, as it is yet too fresh to be beaten. But as to the doubt you express lest I may print your inventions, my faith, which I gave you with an oath, should satisfy you; for as to the finishing of my book, that could be no security, as $I$ could always add to it whenever 1 please. But on account of the dignity of the thing, I excuse you for not relying on that which you ought to have done, namcly, on the faith of a gentleman, instead of the finishing of a book, which might at any time be cnlarged by the addition of new chapters; and there are besides a thousand other ways. But the sccurity consists in this, that there is no greater treachery than to break one's faith, and to agorieve those who have given us pleasure. And when you shall try me, you will find whether I be your friend or not, and whether I shall make an ungrateful return for your friendship, and the satisfaction you have given me." 
It was within less than two months after this, hovever, that Tartalea received the alarming news of Cardan's showing some symptoms of breaking the faith he had so lately pledged to him; this was in a letter from a quondam pupil of his, in which he writes, "A friend of mine at Milan has written to me, that Dr. Cardano is composing another algebraical work, coneerning some lately-diseovered rules; hence I imagine they may be those same rules wbich you told me you had taught him; so that I fear be will deceive you." "To which Tartalea replies, "I am heartily grieved at the news you inform me of, concerning Dr. Cardano of Milan; for if it be true, they can be no other rules but those I gave him; and therefore the proverb truly says, "That which you wish not to be known, tell to nobody.' Pray endeavour to learn more of this matter, and inform me of it."

Tartalea, after this, kept on the reserve with Cardan, not answering several letters he sent him, till one written on the 4th of August the same year, 1539, complaining greatly of Tartalea's neglect of him, and farther requesting his assistance to elear up the difficulty of the irreducible ease $x^{3}=b x+c$, which Cardan had thus early been embarrassed with: he says that when $\left(\frac{1}{3} b\right)^{3}$ exceeds $\left(\frac{\pi}{2} c\right)^{2}$, the rule cannot be applied to the equation in hand, beeause of the square root of the negative quantities. On this occasion Tartalea turns the tables on Cardan, and plays his own game baek upon him; for being aware of the above difficulty, and unable to overeome it himself, he wanted to try if Cardan could be encouraged to accomplish it, by pretending that the ease might be done, though in another way. He says thus to himself, "I have a good mind to give no answer to this letter, no more than to the other two. However I will answer it, if it be but to let him know what I have been told of him. And as I perceive that a suspicion has arisen concerning the difficulty or obstacle in the rule for the case $x^{3}=b x$ $+c$, I have a mind to try if he can alter the data in hand, so as to remove the said obstacle, and to change the rule into another forim, though I believe indeed that it eannot be done; however there is no harm in trying." - "M. Ilieronime, I have received your letter, in which you write that you understand the rule for the case $x^{3}=$ $b x+c$; but that when $\left(\frac{3}{3} b\right)^{3}$ exceeds $\left(\frac{1}{2} c\right)^{2}$, you cannot resolve the equation by following the rule, and therefore you request me to give you the solution of this equation $x^{3}=9 x+10$. To which I reply, that you have not used a good method in that ease, and that your whole process is entirely false. And as to resolving you the equation you have sent, I must say that I am very sorry that I have already given you so much as I have done; for I have been informed, by a credible person, that you are about to publish another algebraical work, and that you have been boasting through Milan of baving discovered some new rules in algebra. But, take notice, that if you break your faith with me, I shall certainly keep my word with you, nay, I cven assure you to do more than I promised." In Cardan's answer to this he says, "You have been misinformed as to my intention to publish more on algebra. But I suppose you have heard something about my work De Mysteriis Aiternitatis, which you take for some algebra $I$ intend to publish. As to your repenting of having given me your rules, I am not to be moved from the faith $\mathbf{j}$ promised you for any thing you say." T'o this, and many other things contained in the sarne letter, Tartalea returned no answer, being still

Vor. I. suspicious of Cardan's intentions, and declining any more correspondence with him.

This however did not disconrage $\mathrm{C}$. for we find hin writing again to $\mathrm{T}$. on the 5th of January, 1540, to clear up another difficulty which had occurred in this business, namely, to extract the cube root of the binomials, ot which the two parts of the rule always consisted, and for which purpose it seems $C$. had not yet found out a rule. On this oceasion he informs $T$. that his quondam competitor Zuanne Colle had come to Milan, where, in some contests between them, Colle gave Cardan to understand that he had found out the rules for the two cases $x^{3}+$ $b x=c$, and $x^{3}=b x+c$, and further that be bad discovered a general rule for extracting the cube roots of all such binomials as can be extracted; and that, in particular, the cube root of $\sqrt{ } 108+10$ is $\sqrt{ } 3+1$, and that of $\sqrt{ } 108-10$ is $\sqrt{ } 3-1$, and consequently that $\sqrt[3]{ }(\sqrt{ } 108$ $+10)-\sqrt[3]{ }(\sqrt{ } 108-10)$ is $=(\sqrt{ } 3+1)-(\sqrt{ } 3-1)$ $=2$. He then earnestly entreats $\mathrm{T}$. to try to find out the rule, and the solution of certain other questions which had been proposed to him by Colle. By this lettcr 'T. is still more eonfirmed in his resolutions of silence; so that, without returning any answer, he only sets down among his own memorandums some curious remarks on the contents of the letter, and then coneludes to himsclf, "Wherefore I do not choose to answer him again, as I have no more affection for him than for M. Zuanne, and therefore I shall leave the matter betiveen them." Among those remarks he sets down a rule for extracting the cube root of such binomials as ean be extracted, and that is done from either member of the binomial alone, thus : Take either term of the binomial, and divide it into two such parts that one of them may be a complete cube, and the other part exactly divisible by 3 ; then the cube root of the said eubic part will be one term of the required root, and the square root of the quotient arising from the division of $\frac{1}{3}$ of the $2 \mathrm{~d}$ part by the cube root of the first, will be the other member of the root sought. This rule will be better understood in charaeters thus : let $m$ be one member of the given binomial, whose cube root is sought; and let it be divided into the two parts $a^{3}$ and $3 b$, so that $a^{3}+3 b$ be $=m$; then is $a+\sqrt{ } \frac{b}{a}$ the cube root required, if it has one. Thus, in the quantity $\sqrt{ } 108+10$, taking the term 10 for $m$, then 10 divides into 1 and 9 , where $a^{3}=1$ or $a=1$, and $3 b=9$ or $b=3$ : therefore $a+$ $\sqrt{ } \frac{b}{a}$ becomes $1+\sqrt{ } 3$ for the cube root of $\sqrt{ } 108+10$. And taking the other member $\sqrt{ } 108$, this divides into the two equal parts $\sqrt{ } 27$ and $\sqrt{ } 27$, making $a^{3}=\sqrt{ } 27$, and $3 b=\sqrt{ } 27$; hence $a=\sqrt{ } 3$, and $b=\sqrt{ } 3$ also; consequently $a+\sqrt{ } \frac{b}{a}$ is $=\sqrt{ } 3+\sqrt{ } \frac{3}{3}$ or $\sqrt{ } 3+1$ for the cube root of the binomial sought, the same as before. "And thus," headds, "we may know whetherany proposed binomial or residual be a cube or a noneube; for if it be a cube, the same two terms for the root must arise from both the given terms separately; and if the two terms of the root cannot thus be brought to àgree both ways, stich binomial or residual will not be a cube." And thus ends the correspondence between them, at least for this time. But it seems they bad still more violent disputes when C. in violation of his faith, so often pledged to the contrary, publisbed his work on cubic equations 4 years afterwards, viz, in the jear $15 \pm 5$, of which we have before given an 
account, which disputes, it appears, continued till the death of Tartalea in the year 1557.

The last article in the volume contains a dialogue on some other forms of cubic equations, in the year 1541, between 'T. and Mr. Richard Wentworth, the English gentleman before-mentioned, who it seems had resided some time at Venice, on some public service from Eıgland, as $T$. in the dedication of the volume to Henry vil 1 , king of England, makes mention of him as " a gentleman of his sacred majesty." Mr. Wentworth had learned some mathematics of 1 ' and being about to depart for England, requests $T$. to show him his newly discovered rules for cubic equations, as a farewell lesson; and it is worth while to note a few particulars in this conference, as they show pretty well the limited knowledge of $\mathrm{T}$. at that time, as to the nature and roots of such equations. . T. had before, it seems, showed Mr. W. the rules for the cases of the $3 \mathrm{~d}$ and 1 st powers, and now the latter desires him to do the same as to the three cases in which the $3 \mathrm{~d}$ and $2 \mathrm{~d}$ powers only are concerned. On this T. professes great gratitude to Mr. W. for many obligations, but desires to be excused from giving him the rules for these, because he says he intends soon to compose a new work on Arithmetic, Geometry, and Algebra, which he intends to dedicate to him, and in which he means to insert all his new discoveries, On Mr. W. urging him further, however, $\mathrm{T}$. gives him the roots of some equations of that kind, as for instance:

If $x^{3}+6 x^{2}=100$, then

$x=\sqrt[3]{ }(42+\sqrt{17000})+\sqrt[3]{ }(42-\sqrt{17000})-2$,

If $x^{3}+9 x^{2}=100$, then $x=\sqrt{ } 24-2$.

If $x^{3}+3 x^{2}=2$, then $x=\sqrt{ } 3-1$.

If $x^{3}+4=5 x^{2}$, then $x=\sqrt{ } 8+2$.

If $x^{3}+6=7 x^{2}$, then $x=\sqrt{ } 15+3$.

But he does not mention the rules for finding them.

In the course of the conversation T. tells him that "all such equations admit of two different answers, and perhaps more; and hence it follows that they have, or admit of, two different rules, and perhaps more, the one more difficult than the other." And on Mr. W. expressing his wonder at this circumstance of a plurality of roots, $T$. replies, "It is however very true, though hardly to be believed, and indeed if experience had not confirmed it, I should scarcely have believed it myself." He then commits a strange blunder in an example which he takes to illustrate this by, namely the equation $x^{3}+3 x=14$, which, he says, it is evident has the number 2 for one of its roots; and yet, he adds, "Whoever shall resolve the same equation by my rule, will find the value of $x$ to be $\sqrt[3]{ }(7+\sqrt{ } 50)+\sqrt[3]{ }(7-\sqrt{ } 50)$, which is proved to be a true root by substituting it in the equation for $x$. And therefore," continues he, "it is manifest that the case $x^{3}+$ $b x=c$ admits of two rules, namely, one (as in the above example) which ought to give the value of $x$ rational, viz, 2, and the other is my rule, which gires the value of $x$ irrational, as appears above; and there is reason to think that there may be such a rulc as will give the valuc of $x=2$, though our alucestors may not have found it out." " And these two different answers will be found not only in every equation of this form $x^{3}+b x=c$, when the value of $x$ happens to be rational, as in the exumple $x^{3}+3 x=14$ above, but the same will also happen in all the other five forms of cubic equations: and therefore there is reason to think that they also admit of two different rules; and by certain circumstances attending some of them, I am almost certain that they admit of more than two rules, as, God willing, I shall soon demonstrate."

Now all this discourse shows a strange mixture of knowledge and ignorance. It is very probable that he had met with some equations'which admit of a plurality of roots; indeed it was hardly possible for bim to avoid it: but it seems he had no suspicion what the number of roots might be, nor that lis reasoning in this instance was founded on an error of his own, mistaking the root $x=$ $\sqrt[3]{ }(7+\sqrt{ } 50)+3 /(7-\sqrt{ } 50)$, of the equation $x^{3}+$ $3 x=14$, for a different root from the number or root 2 , when in reality it is the very same, as he might easily have found, if he had extracted the cube roots of the binomials by the rule which he himself had.just given above for that purpose: for by that rule he would have found $\sqrt[3]{ }(7+\sqrt{ } 50)=1+\sqrt{ } 2$, and $\sqrt[3]{ }(7-\sqrt{ } 50)=1-\sqrt{ } 2$, and therefore their sum is $2=x$, the samer root as the other, which $\mathrm{T}$. thought had been different. And besides this root 2 , the equation in question, $x^{3}+3 x=14$, admits of no other real roots. Nor indeed does any equation of the same form, $x^{3}+b x=c$, admit of more than one real root.

It seems also they had not yet discovered that all cases belong to the rules and forms for quadratic equations, which have only two powers in them, in which the exponent of the one is just double of the exponent of tie other, as $x^{2 n}+b x^{n}=c$; but some particular cases only of this sort they had as yet ventured to refer to quadratics, as the case $x^{4}+b x^{2}=c$. But, in the conclusion of this dialogue, T. informs W. of another case of this sort which he had accomplished, as a notable discovery, in these words : "I well remember," says he, "that in the year 1536 , on the night of St. Martin, which was on a Saturday, meditating in bed when I could not sleep, I discovered the general rule for the case $x^{6}+b x^{3}=c$, and also for the other two, its accompanying cases, in the same night." And then he directs that they are to be resolved like quadratics, by completing the square, \&c. And in these resolutions it is remarkable that he uses only the positive roots, without taking any notice of the negative ones.

Tartalea also published at $V^{\top}$ enice, in 1556 , \&c, a very large work, in folio, on Arithmetic, Gcometry, and Algebra. This is a very complete and curious work on the first two branches; but that of Algebra is carried no farther than quadratic equations, called book the first, with which the work terminates. It is evidently incomplete, owing to the death of the author, which happened before this latter part of the work was printed, as appears by the dates, and by the prefaces. It appears also, from several parts of this work, that the author had many severe conflicts with Cardan and his friend Lewis Ferrari: and particularly, there was a public trial of skill between them, in the year 1547 ; in which it would seem that Tartalea had greatly the advantage, his questions mostly remaining unanswered by his antagonists.

\section{OF MICHAEL STIFELIUS.}

After the foregoing analysis of the works of the first algebraic writers in Italy, it will now be proper to consider those of their contemporarics in Germany; where it is remarkable that, excepting for the discoveries in cubic equations, the art was in a more advanced state, and of a form approaching nearer to that of our modern algebra; the states and circumstances indeed being so 
different, that one would almost be led to suppose they had derived their knowledge of it from a different origin.

Here Stifelius and Scheubelius were writers of the same time with Cardan and Tartalea, and even before their discoveries, or publication, concerning the rules for cubic equations, Stifelius's Arithmetica Integra, being published at Norimberg in 1544, the year before Cardan's work on çubic equations, and is an excellent treatise, both on arithmetic and algebra. The work is divided into three books, and is prefaced with an Introduction by the famous Melanchthon. The first book contains a complete and ample Treatise on Arithmetic, the second an Exposition of the 10th book of Euclid's Elements, and the third a Treatise on Algebra, being therefore properly the part with which we are at present concerned. In the dedication of this part, he ascribes the invention of algebra to Geber, an Arabic astronomer; and mentions besides, the authors Campanus, Christ. Rudolph, and Adam Ris, Risen, or Gigas, whose rules and examples be has chiefiy given. In other parts of the book he speaks, and makes use also, of the works of Boetius, Campanus, Cardan (i. e. his Arithmetic published in 1539, before the work on cubic equations appeared), de Cusa, Euclid, Jordan, Milichius, Schonerus, and Stapulensis. So that he appears to have been very little acquainted indeed with any besides the German authors!

Chap. 1. On the Rule of Algelra, and its parts. Stifelius bere describes the notation and marks of powers or denominations as he calls them, which marks for the several powers are thus:

$$
\begin{aligned}
& \text { 1st, 2d, 3d, 4th, 5th, 6th, \&c, } \\
& 2, \quad 3, \quad s, 33, \quad s s, \quad 3 \pi, 8 \mathrm{c} \text {, }
\end{aligned}
$$

being formed from the initials of the barbarous way in which the Germans pronounced and wrote the Latin and Italic names of the powers, namely, res or cusa, zensus, cubo, zensi-zensus, sursolid, zensi-cubo, \&c. And the coss or first power 2 , he calls the radix or root, which is the first time that we meet with this word in the printed authors. He also here uses the signs or characters, + and -, for addition and subtraction, and the first of any that $I$ know of: for in Italy they used none of these characters for a long time after. He has no mark however for equality, but makes use of the word itself.

Chap. 2. On the Parts of the Rule of Geber or Algebra: teaching the various reductions by addition, subtraction, multiplication, division, involution, and evolution, \&c.

Cliap. 3. On the Algorithm of Cossic numbers; teaching the usual operations of addition, subtraction, multiplication, division, involution, and extraction of roots, much the same as they are at present. Single terms, or powers, he calls simple quantities; but such as $13+12 p$ a composite or compound, and $2 \vartheta_{p}-8$ a defective one. In multiplication and division, he proves that like signs give + , and unlike signs -. He shows that the powers $1,2,3, \quad, \quad z$, form a geometrical progression from unity; and that the natural series of numbers $0,1,2,3$, $\&$ e, from 0 , are the exponents of the cossic powers; and he, for the first time, expressly calls them exponents : thus, Exponents, 0, 1, 2, 3, 4, 5, 6, \&x.

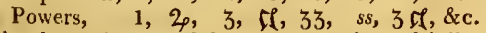
And lie shows the use of the exponents, in multiplication, division, powers, and roots, as we do at present; viz, adding the exponents in multiplication, and subtracting them in division, \&ec. And these operations he demonstrates from the nature of arithmetical and geometrical progressions. It is remarkable that these compound denominations of the powers are formed from the simple ones according to the products of the exponents; while those of Diophantus are formed according to the sums of them; thus the $6 t_{1}$ power here is $3 c$ or quadrate-cubi, but with Diophantus it is cubo-cubi ; and so of others. Which is presumptive evidence that the Furopeans had not taken their Algebra immediately from him, independent of other proofs.

Chap. 4. On the extraction of the roots of cossic numbers. He here treats of quadratic equations, which he resolves by completing the square, from Euclid ii, 4, \&c. Also quadratics of the higher orders, showing how to resolve them in all cases, whatever the height may be, provided the exponents be but in arithmetical progression, as $2,1,0)$

$4,2,0 \quad \& c$; where it is plain that he slways counts 0 $6,3,0\}$ for the exponent of the unknown quantity in $8,4,0\}$ the absolute term.

Chap. 5. Of irrational cossic numbers, and of surd or negative numbers. In this treatise of radicals or irrationals, he first uses the character $\sqrt{ }$ to denote a root, and sets after it the mark of the power whose root is intended; as $\sqrt{ } 320$ for the square root of 20 , and $\sqrt{ } \leqslant 20$ for the cube root of the same, and so on. He treats here also of negative numbers, or what he calls surd or fictitious, or numbers less than 0 . On which he takes occasion to observe, that when a geometrical progression is continued downwards below 1, then the exponents of the terms, or the arithmetical progression, will go below 0 into negative numbers, and will yet be the true exponents of the former; as in these,

$$
\begin{aligned}
& \text { Expon. -3, }-2,-1,0,1,2,3, \\
& \text { Pow. } \frac{x}{8}, \frac{x}{4}, \frac{\pi}{2}, 1,2,4,8,
\end{aligned}
$$

And he gives examples to show that these negative exponents perform their office the saine as the positive ones, in all the operations.

Chap. 6. On the perfection of the Rule of Algebra, and of Secondary Roots. In the reduction of equations, he uses a more general rule than those who had preceded him, who detailed the rule in a multitude of cases; instead of which, he directs to multiply or divide the two sides equally, to transpose the terms with + or - , and lastly to extract such root as may be denoted by the exponent of the highest power.

As to secondary roots, Cardan treated of a $2 \mathrm{~d}$ ignota or unknown, which he called quantitas, and denoted it by the initial $q$, to distinguish it from the first. But here Stifelius, for distinction sake, and to prevent one root from being mistaken for others, assigns literal marks to all of them, as A, B, C, D, \& c , and then performs all the usual operations with them, joining them together as we do now, except that he subjoins the initial of the power, instead of its numeral exponent: thus,

$3 \mathrm{~A}$ into $9 \mathrm{~B}$ makes $27 \mathrm{AB}$, 33 into $4 \mathrm{~B}$ makes $123 \mathrm{~B}$, $2 S$ into $4 A 3$ makes 8 SIA, $1 \mathrm{~A}$ squared makes $1 \mathrm{~A} 3$, 6 into $3 \mathrm{c}$ makes $18 \mathrm{c}$, $2 \mathrm{~A} 3$ into $5 \mathrm{~A}$ makes $10 \mathrm{Ass}, \& \mathrm{c}, \& \mathrm{c}$, $8,1 \wedge 3$ divided by 4 负 makes $2 \wedge \overline{3}, \& c$, The square root of $25 \mathrm{~A} 3$ is $5 \mathrm{~A}, \mathrm{Kc}$, Also $2 A$ added to 22 makes $2 \%+2 A$, and 2 A subtr. from $2 q_{\text {makes } 2} q_{p}-2_{A}$. 
And he shows how to use the same, in questions concerning several unknown numbers; where he puts a different character for each of them, as $\mathcal{\psi}, \Delta, \mathrm{B}, \mathrm{c}, \& \mathrm{c}$; he then makes out, from the conditions of the question, as many equations as there are characters; from these he finds the value of each letter, in termis of some one of the rest; and so, expelling them all but that one, reduces the whole to a final equation, as we do at present.

The remainder of the book is employed with the solutions of a great number of questions, to exercise all the rules and methods; some ot which are geometrical ones.

From this account of the state of Algebra in Stifelius, it appears that the improvements made by himself, or other Germans, beyond those of the Italians, as contained in Cardan's book of 1539, were as follow:

Ist. He introduced the characters,,$+- \sqrt{ }$, for plus, ininus, and root, or radix, as he calls it.

2d. The initials $2,3, \pi, \& c$, for the powers.

$3 \mathrm{~d}$. He treated all the higher orders of quadratics by the same general rule.

4 th. He introduced the numeral exponents of the powers, $-3,-2,-1,0,1,2,3, \& \mathrm{c}$, both positive and negative, so far as integral numbers, but not fractional ones; calling them by the name exponens, exponent: and he taught the general uses of the exponents, in the several operations of powers, as we now use them, or the logarithms.

5 th. And lastly, he used the general literal notation $A, B, C, D, \& C$, for so many different unknown or general quantities.

OF SCHEUBRLIUS.

John Scheubelius published several books on Arithmetic and Algebra. The one now before me, is entitled Algebræ Compendiosa Facilisque Descriptio, quâ depromuntur magna Arithmetices miracula. Authore Johanne Scheubelio Mathematicarum Professore in Academia Tubingensi. Parisiis 1552. But at the end of the book it is dated 1551. The work is most beautifully printed, and is a very clear though succinct treatise; and both in the form and matter much resembles a modern printed book. He says that the writers ascribe this art to Diophantus, which is the first time that $I$ find this Greek author mentioned by the modern algebraists: he further observes, that the Latins call it Regula Rei et Census, the rule of the thing and the square, or of the 1st and $2 d$ power; and the Arabs, Algebra. His characters and operations are much the same as those of Stifelius, using the signs and characters,,$+- \sqrt{ }$, and the powers $8,2,3$, , il, \&ce, where the character $S$ is used for 1 or unity, or a number, or the 0 power; prefixing also the numerical coefficients; thus $44 s 3+113+318-53 \pi$. He uses also the exponents $0,1,2,3$, \&c, of the powers, the same way as Stifelius, before him. Ife performs the algebraical calculations, first in integers, and then in fractions, much the same as we do at present. Then of equations, which he says may be of iufinite degrees, though he treats only of two, namely the first and second orders, or what we call simple and quadratic equations, in the usual way, taking however only the positive roots of these; and adverting to all the higher orders of quadratics, namely,

$x^{4}, a x^{2}, b$; and $x^{6}, a x^{3}, b$; and $x^{8}, a x^{4}, b ; s c$.

Next follow's a tract on surds, both simple and compound, quadratic, cubic, binomial, and residual. IIere lhe first marks the notation, observing that the root is ci- ther denoted by the initial of the word, or, after some authors, by the mark $\sqrt{ }:$, viz, the sq. root $\sqrt{ }:$, the cube root $\sim \vee \sqrt{ }$ :, and the 4 th root, or root of the root thus $\sqrt{ }$ :, which latter method he mostly uses. He then gives the Arithmetic of surds, in multiplication, division, addition, and subtraction. In these last two rules he squares the sum or difference of the surds, and then sets the root to the whole compound, which he calls radix collecti, what Cardan calls radix universalis. Thus $\sqrt{ } 12 \pm \sqrt{ } 20$ is ra. col. $32 \pm \sqrt{ } 960$. But when the terms will reduce to a common surd, he then unites then into one number; as $\sqrt{ } 27+\sqrt{ } 12$ is equal $\sqrt{ } 75$. Also of cubic surds, and 4 th roots. In. binomial and residual surds, he remarks the different kinds of them which answer to the several irrational lines in the 10th book of Euclid's elements; and then gives this general rule for extracting the root of any binomial or residual $a \pm b$, where one or both parts are surds, and $a$ the greater quantity, namely, that the square root of it is $\sqrt{ } \frac{a+\sqrt{ }\left(a^{2}-b^{2}\right)}{2} \pm \sqrt{ } \frac{a-\sqrt{ }\left(a^{2}-b^{2}\right)}{2}$; which he illustrates by many examples. This rule will only succeed however, so as to come out in simple terms, in certain cases, namely, either when $a^{2}-b^{2}$ is a square, or when $a$ and $\sqrt{ }\left(a^{2}-b^{2}\right)$ will reduce to a common surd, and unite : in all other cases the root is in two compound surds, instead of one. He gives also another rule, which comes however to the same thing as the former, though by the words of them they seem to be different.

Scheubelius wrote much about the time of Cardan and Stifelius. And as he takes no notice of cubic equations, it is probable he had neither seen nor leard any thing about them; which might very well happen, the one living in Italy, and the otber in Germany. And besides, I know not if this be the first edition of Scheubel's book: it is rather likely it is not, as it is printed at Paris, and he himself was professor of mathematics at Tubingen in Germany.

ROBERT RECORDE.
To this ingenious man we are indebted for the first treatise on algebra, then named the Cossic Art, in the English language; but his meritorious labours, like those of the greatest benefactors of mankind, appear to have been ill requited, since, after removing to the capital, he died under confinement for debt in the Fleet-prison. In his book on Arithmetic he is styled " teacher of mathematics and practitioner in physic at Cambridge." It was for many ages the custom to unite the title as well as the practice of medicine with those of chemistry, alchymy, mathematics, and astrology, by the Moors, and after them by the Europeans, and is still continued among the almanacmakers. And it is remarkable, that as the Moors were not less famed in Europe for their skill in medicine, than their dexterity in calculation, the term Physician and Algebraist appear at first to have been regarded as almost synonymous. Thus, it is curious to remark, that in the celebrated romance of Don Quixote, published about this time, the bachelor Samson Carrasco, who in his rencounter with the kuight was thrown from his horse, and had his ribs broken, sent in quest of an Algebrista to heal his bruises.

The first part of his Arithmetic was puhlished in 1552; and the second part in 1557, under the title of "The Whetstone of Witte, which is the seconde parte of Arithmetike: containing the lixtraction of Rootes: The Cossike Practise, with the Rule of Equation: and the Workes of 


\section{$A$ L G}

Surde Nombers." The work is in dialogue between the master and scholar; and is nearly after the manner of the Germans, Stifelius and Scheubelius, but especially the latter, whom he often quotes, and takes examples from. The chief parts of the work are, 1st, The properties of abstract and figurate numbers. 2d, The extraction of the square and cube roots, much the same as at present. Here, when the number is not in exact power, but having some remainder over, he either continues the root into decimals as far as he pleases, by adding to the remainders always periods of ciphers; or clse makes a vulgar fraction for the remaining part of the root, by taking the remainder for the numerator, and double the root for the denominator, in the square root; but in the cube root he takes, for the denominator, either the triple square of the root, which is Caroan's rule, or the triple square and triple root, with one more, which is Scheubel's rule. 3d, Of Algebra, or "Cossike Nombers." He uses the notations of powers with their exponents the same as Stifel, with all the operations in simple and compound quantities, or integers and fractions. And he gives also many examples of extracting the roots of compound algebraic quantities, even when the roots are from two to six terms, in imitation of the same process in numbers, just as, we do at present; which is the first instance of this kind that I have observed. As of this quantity:

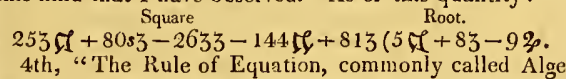
ber's Rule." He here, first of any, introduces the character $=$, for brevity sake. His words are, "And to avoid the tediouse repetition of these woordes : is equalle to: I will sette as I doe often in woorke use, a paire of paralleles, or gemowe lines of one lengthe, thus: $=$, bicause noe 2 thynges can be moare equalle." He gives the rules for simple and quadratic equations, with many examples. He gives also some examples in higher compound equations, with a root for each of them, but gives no rule how to find it. 5th, "Of Surde Nombers." This is a very ample treatise on surds, both simple and compound, and surds of various degrees, as square, cubic, and biquadratic, marking the roots in Scheubel's manner, thus: $\sqrt{ }, 4 \sqrt{ }, \sqrt{ }$. He here uses the names bimedial, binomial, and residual; but says they have been used by others before him, and indeed by Lucas de Burgo, as in cart. 120, edit. 1494.-Hence it appears that the things which chiefly are new in this a uthor, are these two, viz,

1. The extraction of the roots of compound algebraic quantities.

2. The use of the sign of equality, or $=$. OF PELETARIUS.

The first edition of this author's algebra was printed in 4 to at Paris, in 1558, under this title, Jocobi Peletarii Cenomiani, de occulta parte Numerorum, quam Algebram vocant. Lib. duo.

In the preface he speaks of the supposed anthors of Algebra, namely Geber, Mahomet the son of Moses, an Arabian, and Diophantus. But he thinks tlue art older, and mentions some of his contemporary writers, or a very little before him, as Cardan, Stifel, Scheubel, Chr. Januarius; and a little carlier again, Lucas Paciolus of Florcnce, and Stephen Villafrancus a Gaul.

Of the two books, into which the work is diviled, the first is on rational, and the second on irrational or surd quantities; each veing divided into many chapters. It will be sufficient to mention only the principal articles.

He calls the series of powers numeri creati, or derived numbers, or also radicals, because they are all raised from one root or radix. He names them thus, radix, quadratus, cubus, quadrato-quadratus, or biquadratus, supersolidus, quadrato-cubus, \&c; and marks them thus $\mathbb{B}, q, \& q, q q$, $s \delta, q \delta f ; b s s, \& c$. Of these he gives the following series in numbers, having the common ratio 2 , with their narks set over them, and the exponents set over these again, in an arithmetical series, beginning at 0 , thus:

$\begin{array}{ccccccccc}0 & 1 & 2 & 3 & 4 & 5 & 6 & 7 & 8 \\ 1 & \mathrm{R} & 9 & \pi & q q & s s & q 5 & b s s & q q q \\ 1 & 2 & 4 & 8 & 16 & 32 & 64 & 128 & 256 \quad \& \mathrm{c} .\end{array}$

And he shows the use of the exponents, the same as Stifel and Scheubel; like them also be prefixes coefficients to quantities of all kinds, as also the radical $\sqrt{ }$. But he does not follow them in the use of the sigus + and -, but employs the initials $p$ and $m$ for the same purpose. After the operations of addition, \&ce, he performs involution, and evolution also, much the same way as at present: thus, in powers, raise the coefficient to the power required, and multiply the exponent, or sign, as he calls it, by 2 , or 3 , or 4 , \& c, for the $2 \mathrm{~d}, 3 \mathrm{~d}, 4 \mathrm{th}, 8 \mathrm{c}$, power; and the reverse for extraction: and hence he observes, if the number or coefficient will not exactly extract, or the sign do not exactly divide, the quantity is a surd.

After the operations of compound quantities, and fractions, and reduction of equations, namely simple and quadratic equations, as usual, in chap. 16, De Inveniendis generatim Radicibus Denominatorum, he gives a method of finding the roots of equations among the divisors of the absolute number, when the root is rational, whether it be integral or fractional ; for then, he observes the root always lies hid in that number, and is some one of its divisors. This is exemplified in scveral instances, both of quadratic and cubic equations, and both for integral and fractional roots. And he here observes, that he knows not of any person who has yet given general rules for the solution of cubic equations; which shows that when be wrote this book, eithei Cardan's last book was not published, or else it had not yet come to his knowledge.

Chap. 17 contains, in a few words, directions for bringing questions to equations, and for reducing these. He here observes, that some authors call the unknown number res, and others the positio; but that he calls it radix, or root, and marks it thus $\mathbb{B}$ : bence the term, ront of an equation. But it was before called radix by Stifelius.

Chap. $21 \& \mathrm{seg}$. treat of secondary roots, or a plurality of roots, denoted by $A, \mathrm{~B}, \mathrm{c}$, \&c, after Stifelius.

The $2 \mathrm{~d}$ book contains the like operations in surds, or irrational numbers, and is a very complete work on this subject indeed. He treats first of simple or single surds, then of binomial surds, and lastly of trinomial surds. He gives here the same rule for extracting the root of a binomial and residual as Scheubelius, viz, $\sqrt{ }(a \pm b)=$

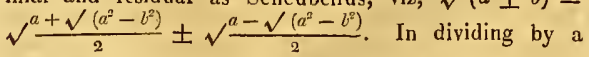
binomial or residual, he proceeds as all others before him had done, namely, reducing the divisor to a simple quantity, by multiplying it by the same two terms with the sign of one of them clanged, that is by the binomial if it be a residual, or by the same residual if it be a bino- 


\section{A L G}

[ 86

mial; and multiplying the dividend by the same thing: thus $\frac{3}{\sqrt{5-2}}=\frac{3}{\sqrt{5-2}} \times \frac{\sqrt{5}+2}{\sqrt{5+2}}=\frac{3 \sqrt{ } 5+6}{5-4}=3 \sqrt{ } 5+6$. And, in imitation of this method, in division by trinomial surds, he directs to reduce the trinomial divisor first to a binomial or residual, by multiplying it by the same trinomial with the sign of one term changed, and then to reduce this binomial or residual to a simple nominal as above; observing to multiply the dividend by the same quantities as the divisor. Thus, if the divisor be $4+\sqrt{ } 2$ $-\sqrt{ } 3$; nultiplying this by $4+\sqrt{ } 2+\sqrt{ } 3$, the product is $15+8 \sqrt{ } 2$; then tbis binomial multiplied by the residual $15-8 \sqrt{ } 2$, gives $225-128$ or 97 for the simple divisor: and the dividend, whatever it is, must also be multiplied by the two $4+\sqrt{ } 2+\sqrt{ } 3$ and $15-8 \sqrt{2}$. Or in general if the divisor be $a+\sqrt{ } b-\sqrt{ } c$;

multiply it by $a+\sqrt{ } b+\sqrt{ } c$,

which gives $(a+\sqrt{ } b)^{2}-c=a^{2}+b-c+2 a \sqrt{ } b$;

then multiply this by - $a^{2}+b-c-2 a \sqrt{ } b$,

and it gives - - - - $\left(a^{2}+b-c\right)^{2}-4 a^{2} b$, which will be rational, and will all collect into one single term. But Tartalea must have been in possession of some such rule as this, as one of the questions he proposed to Florido was of this nature, namely to find such a quantity as multiplied by a given trinomial surd, shall make it rational: and it appears, from what is done above, that, the given trinomial being $a+\sqrt{ } b-\sqrt{ } c$, the answer will be $(a+$ $\sqrt{ } b+\sqrt{ } c) \times\left(a^{2}+b-c+2 \sqrt{ } b\right)$.

Chap. 24 shows the composition of a cube of a binomial or residual, and thence remarks on the root of the case or equation 1 i $p 3 \mathrm{~B}$ equal to 10 , which he seems to know something about, though he had not Cardan's rules.

Chap. 30, which is the last, treats of certain precepts relating to square and cubic numbers, with a table of such squares and cubes from all numbers to 140 ; also showing how to compute them both, by adding always their differences.

He then concludes with remarking that there are many curious properties of these numbers, one of which is this, that the sum of ally number of the cubes, taken from the beginning, always makes a square number, the root of which is the sum of the roots of the cubes; so that the series of squares so formed, have for their roots $1,3,6,10$, 15,21 , \& $c$, whose diff. are the natural $n^{\circ s} 1,2,3,4,5$, 6 , \&c. Namely, $1^{3}=1^{2} ; 1^{3}+2^{3}=3^{3} ; 1^{3}+2^{3}+3^{3}=6^{2}$, \&c. Or in general, $1^{3}+2^{3}+3^{3}-\cdots n^{3}=(1+2+3 \cdots n)^{2}$ $=\left[\frac{1}{2} n \cdot(n+1)\right]^{2}=\frac{1}{4} n^{2}(n+1)^{2}$.

This work of Peletarius is a very ingenious and inasterly composition, treating in an able manner of the several parts of the subject then known, excepting the cubic equations. But his real discoveries, or improvements, may be reduced to these three, viz.

1st. That the root of an equation is one of the divisors of the absolute term.

$2 d$. He taught how to reduce trinomials to simple terms, by multiplying them by compound factors.

3d. He taught curious precepts and properties concerning square and cube numbers, and the method of constructing a series of each by addition only, mamely by adding successively their several orders of differences.

PETER RAMUS.

Peter Ramus wrote his arithmetic and algebra about the year 1560. His notation of the powers is thus, $l, q$, $c, b q$, being the initials of latus, quadratus, cubus, biqua-
A L G

dratus. He treats only of simple and quadratic equations. And the only thing remarkable in his work, is the first article, on the name and invention of Algebra, which we have noticed at the beginning of this history.

OF PEDRO NUGNEZ OR NUNEE, OR IN LATIN NONYUS.

Peter Nunez or Nonius, was a very ingenious and eminent physician and mathematician, for the time in which he lived. He was born in 1497, at Alcazar in Portugal, and died in 1577 , at 80 years of age. He was professor of mathematics in the university of Coimbra, where he published several ingenious and useful pieces on different branches of the mathematics, as may be seen by the account of his life given in the $2 \mathrm{~d}$ vol. of this Dictionary; but it is only with his Algebra that our business is at present.

This work he had composed. in Portuguese, but translated it into the Castilian tongue, when he resolved on making it public, which he thought would render his book more useful, as this language was more generally known than the former. The dedication, to his former pupil, prince Henry, was dated from Lisbon, Dec. 1, 1564; and the work contains 341 leaves, equal to 682 closely printed yages, in the Antwerp edition of 1567 , in Svo; the folios being numbered only on one side.

The work is very methodically and plainly treated; being divided into regular and distinct chapters or sections; leading the reader gradually through the several operations of computation, in integers and fractions, in powers and roots, in surds and in proportions, \&c. The rules for dignities or powers are given; and these he denominates trom the product of their indices; thus, for the powers of 2 , with their names, and denominations under them:

$$
\begin{array}{ccccc}
2 & 4 & 16 & 32 & 64 \\
\text { Co. Ce. Cu. Ce. Ce. Re. } p^{\circ} \text {. Ce. Ca. or }{ }_{1} & 2 & 3 & 4 & 5
\end{array}
$$

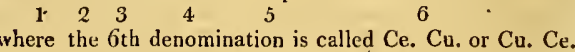
that is Censo-Cubo or Cubo-Censo, meaning the square cubed or the cube squared, the index 6 denoted by $2 \times 3$, the product of the indices of the powers; after the manner of the former European authors, Lucas de Burgo, Tartalea, and Cardan. And exactly after the manner of these also is his practice in every other part, with little or no variation, as far as he goes, which is to quadratic equations; without treating on cubics, further than giving some account of the dispute between Tartalea and Cardan concerning their invention; and that in such a manner as shows he did not very well understand them. Like those authors are his names and rules for Raizes, roots or radicals, which he sometimcs calls Sorda, surds. In their marks or signs also; as $R$ for root ; $\tilde{p}$ for + ; $\tilde{m}$ for minus; $R . u$. for root universal, instead of the vinculi used by the moderns; also $L$ for ligature or composition, such as $L$. R. 9 with $R 4$, composed of 3 which is $R 9$, and of 2 which is $R$ 4, making 5 . He places likewise the name of the root after the $R$; as $R . c u .25 . \tilde{m} . R . c u .15 . \tilde{p} . R . c u$. 9 ; that is, $\sqrt[3]{25}-\sqrt[3]{15}+\sqrt[3]{9}$

After all the usual preparatory rules, Nunez then treats of equations, simple and quadratic, in the common way, and giving geometrical denonstrations of the rules, as had been done before. He then applies these equations in the solution of a great number of examples, of questions or problems, first in numbers or aritlimetic, and then in geometrical problems or figures; in which he procceds orderly through the several kinds; as squarcs, rectangles, 
triangles, rhombs, rhomboids, trapeziums, pentagons, \&c: all which be calls by the same names as at present. The content of a figure too he calls its Area, as, De Burgo did before him; and after the same author also he gives the geometrical demonstration of the common rule for finding the area of a triangle from the three sides given. He treats on the inscription of circles and squares in triangles of various kinds; and the division of triangles into several parts in different ways.

In an address to his readers, at the end of the book, Nunez informs them what are the authors whose books, on his subject, are to be found in Spain, which consist only of De Burgo, Tartalea, and Cardan; stating his ideas on the merits of their works, with critical remarks on many parts of them, in which he more particularly approves of those of Tartalea. Upon the whole, the merit of $\mathrm{Nunez}$, in this art, consists chiefly, or wholly, in having given a very neat and orderly treatise on it, after the manner of those authors; but without having made any improvements or inventions of his own, in the art.

On this occasion it is very remarkable to observe, that Nunez appears not to have been at all acquainted with any of the Germanic authors, several of whom were contemporaries of Tartalea and Cardan, and who treated the subject in a better manner, in some respects, than these did. Another thing may be here noticed on this occasion, as remarkable; not only that we have never heard of any other early writers on this subject, in Spain or Portugal; but that we have never heard of writings on it by the Moors, who occupied great part of that peninsula during several centuries, by whom we have always been taught to believe that the arts of arithmetic and algebra were brought into that country.-Had that people left any such works in the country, would not some of them have been fousd in some of the great cities or the universities, or could they yet be in existence there?-Or could the people have carried all their books away with them when they were expelled from the country?

$$
\text { BOMBELLI. }
$$

Raphacl 13ombelli's Algebra was published at Bologna in the year 1572 , in the Italian language. In a short, but neat, introduction, he first adverts, in a few words, to the great excellence and usefulness of arithmetic and algebra. He then laments that it had hitherto been treated in so imperf'ct and irregular a way; and declares it his intention to remedy all defects, and to make the science and practice of it as easy and perfect as may be. And for this purpose he first resolved to procure and study all the former authors. Ile then mentions several of these, with a short history or character of them; as Mahomet the son of Moses, an Arabian; Leonard Pisano; Lucas de Burgn, the first printed author in Europe; Oroncius; Scribelius; Boglione Francesi; Stifelius in Germany; a certain Spaniard, perhaps meaning Nunez or Nouius; and lastly Cardan, Ferrari, and Tartalea; with some others since, whose names he omits. He then adds a curious paragraph concerning Diophantus: he says that some years since there had been found, in the Vatican library, a Greek work on this art, composed by a certain Diophantus, of Alexandria, a Greek author, who lived in the time of Intoninus Pius; which work having been shown to him by Mr. Antonio Maria Pazzi Reggiano, public lecturer on mathematics at Rome; and finding it to be a good work, these two formed the design of giving it to the world; and he says that they had already translated fise books, of the seven which were then extant, being as yet hindered by other avocations from completing the work. He then adds the following strange circumstance, viz, that they had found that in the said work the Indian authors are often cited; by which he learned that this science was known among the Indians before the Arabians liad it: a paragraph the more remarkable as I have never unlerstood that any other person could ever find, in Diophantus, any reference to Indian writers: and I have examined his work with some attention, for that purpose. Probably the copy which Bombelli saw, contained marginal remarks by Planudes, or some other scholiast, making mention of the Indian works on the science, or some such remarks; which might be mistaken for part of the text of Diophantus.

Bombelli's work is divided into three books. In the first, are laid down the definitions and operations of powers and roots, with various kinds of radicals, simple and compound, binomial, residual, \&c ; mostly after the rules and manner of former writers, excepting in some few instances, which I shall bere take notice of. And first of his rule for the cube root of binomials or residuals, which, for the sake of brevity, may be expressed in modern notation as follows: let $\sqrt{ } b+a$ be the binomial, the term $\sqrt{ } b$ being greater than $a$; then the rule for the cube root of $\sqrt{ } b+a$ comes to this, $\mathrm{p}-\mathrm{Q}+\sqrt{ }\left[(\mathrm{P}-\mathrm{Q})^{2}+\sqrt[3]{ }(b-\right.$ $\left.\left.a^{2}\right)\right]$; where $\mathrm{P}=\sqrt[3]{ }\left(\sqrt{ }\left(\frac{a^{2}}{64}+\frac{b-a^{2}}{64}+\frac{a}{8}\right)\right.$; and $Q=$ $\sqrt[3]{ }\left(\sqrt{ }\left(\frac{a^{2}}{64}+\frac{b-a^{2}}{64}\right)-\frac{a}{8}\right)$. Which is a rule that can be of little or no use. For in the first place, $(\mathrm{P}-Q)^{2}+3^{3}\left(b-a^{2}\right)$ is the same as $(\mathrm{P}+\mathrm{Q})^{2}$; and $\mathrm{P}$ or $\sqrt[3]{ }\left(\sqrt{ }\left(\frac{a^{2}}{64}+\frac{b-a^{2}}{64}\right)+\frac{a}{8}\right)$ is $=\sqrt[3]{ }\left(\sqrt{ } \frac{b}{64}+\frac{a}{8}\right)=\sqrt[3]{ } \frac{\sqrt{ } b+a}{8}=\frac{1}{2} \sqrt[3]{ }(\sqrt{ } b+a)$; therefore the whole $P-Q+\sqrt{ }(P-Q)^{2}+\sqrt[3]{ }(b-a)^{2}$ reduces to $\mathrm{P}-\mathrm{Q}+\mathrm{P}+\mathrm{Q}=2 \mathrm{P}=2 \times \frac{1}{2} \sqrt[3]{ }(\sqrt{ } b+a)=\sqrt[3]{ }(\sqrt{ } b$ $+a$ ), the original quantity first proposed.

The next thing remarkable in this 1 st book, is his method for the square roots of negative quantities, and his rule for the cube roots of such inaginary binomials as arise from the irreducible case in cubic equations. His words, translated, are these: "I have found another sort of cubic root, very different from the former, which arises from the case of the cube equal to the first power and a number, when the cube of the $\frac{1}{3} d$ part of the (coef. of the) 1st power, is greater than the square of lialf the absolute number, which kind of square root hath, in its algorism, names and operations different from the others; for in that case, the excess cannot be called either plus or minus; I therefore call it plus of minus when it is to be added, and minus of minus when it is to be subtracted." He then gives a set of rules for the signs when such roots are multiplied, and illustrates them by a great many examples. His rule for the cube roots of such binomials, viz, such as $a+\sqrt{ }-b$, is this : First find $\sqrt[3]{ }\left(a^{2}+b\right)$; then, by trials search out a number $c$, and a sq. root, $\sqrt{ } d$, such, that the sum of their squares $c^{2}+d$ may be $=\sqrt[3]{ }\left(a^{2}\right.$ $+b)$, and also $c^{3}-3 c d=a$; then sluall $c+\sqrt{ }-d$ be $=\mathfrak{V}(a+\sqrt{ }-b)$ sought. Thus, to extract the cube root of $2+\sqrt{ }-121$ : here $\sqrt[3]{ }\left(a^{2}+b\right)=\sqrt[3]{125}=5$; then taking $c=2$, and $d=1$, it is $c^{2}+d=5=\sqrt[3]{ }\left(a^{2}+b,\right)$ and $c^{3}-3 c d=8-6=2=a$, as it ought; and therefore $2+\sqrt{ }-1$ is $=$ the cube root of $2+\sqrt{ }-121$, as re quired. 


\section{A L G}

[. 88

The notation in this book, is the initial $R$ for root, with $q$ or $c, \& c$, after it, for quadrate or cubic, \&c root. Also $p$ for $p l u s$, and $m$ for minus.

In the $2 d$ book, Bombelli treats of the algorism with unknown quantities, and the resolution of equations. He first gives the definitions and characters of the unknown quantity and its powers; in which he deviates from the former authors, but professes to imitate Diophantus. He calls the unknown quantity tanto, and marks it

thus -

Its cube - - - cubo, 3 ,

and the higher names are compounded of these, and marked $4,5,6,7, \& c$; so that he denotes all the powers by their exponents set over the common character $\_$. And all these powers he calls by the general name dignita, dignity. He then performs all the algorism of these powers, by means of their exponents, as we do at present, viz, adding them in multiplication, subtracting in division, multiplying them by the index in involution, and dividing by the same in evolution.

In equations he goes regularly through all the cases, and varieties of the signs and terms; first all the simple or single powers, and then all the compound cases; demonstrating the rules geometrically, and illustrating them by many examples.

In compound quadratics, lie gives two rules: the first is by freeing the potenza or square from its coefficient by division, and then completing the square, \&c, in the usual way: and the $\hat{2} \mathrm{~d}$ rule, when the first term has its coeffcient, may be thus expressed; if $a x^{2}+b x=c$, then $x=$ $\frac{\sqrt{ }\left(a c+\frac{1}{4} b^{2}\right)-\frac{1}{2} b}{a}$, being a way that was practised by the Indians. He takes only the positive root or roots; and in the case $a x^{2}+c=b x$, which has two, he observes that the nature of the problem must show which of the two is the proper one.

- In the cubic equations, he gives the rules and transformations, \&c, after the manner of Cardan; remarking that some of the cases have only one root, but others two or three, of which some are true, and others false or negalive. And in one place he says that by means of the case $x^{3}=b x+c$, he trisects or divides an angle into three equal parts.

When he arrives at biquadratic equations, and particuIarly to this case $x^{4}+a x=b$, he says, "Since I have seen Diophantus's work, I have always been of opinion that his chief intention was to come to this equation, because 1 observe he labours at finding always square numbers, and such, that adding some number to them, may make squares; and I believe that the six books, which are lost, may treat of this cquation, \&c."-" But Lewis Ferrari," he adds, "of this city, also laboured in this way, and found out a rule for such cases, which was a very fine invention, and therefore 1 shall here treat of it the best 1 can." This he accordingly does, in all the cases of biquadratics, both with respect to the number of terms in the equation, and the signs of the terms, except 1 think this most general case only $r x-q x^{2}+p x^{3}-x^{4}=s$; fully applying Ferrari's method in all cases. Which concludes the $2 \mathrm{~d}$ book.

The $3 \mathrm{~d}$ book consists only of the resolution of near 300 practical questions, as exercises in all the rules and equations, many of which are taken from other authors, cspecially from the first five books of Diophantus.
A L G

Upon the whole it appears, that this is a plain, explicit, and very orderly treatise on algebra, in which are very well explained the rules and methods of former writers. But Bombelli does not produce much of improvement or invention of his own, except his notation, which varies from others, and is by means of one general cbaracter, with the numeral indices of Stifelius. He also first remarks that angles are trisected by a cubic equation. GULIELMI GOSSALINI, \&c.

De Arte Magna, seu de Occulta Parte Numeromm que et Algebra et Almucabala vulgo dicitur, libri quatuor. In quibus explicantur aquationes Diophanti, Regula 2uantitatis Simplicis, et Quantitutis Surda.-Paris, 1577, in small $8 v n, 86$ leaves, numbered only on one side.

In the dedication, this author notices the term Cossic Numbers; and mentions many of the former writers; as, Diophantus, Forcadel, Scheubel, Peletare, Stifel, Cardan, Lucas, Villafranc, Nunez, and Tartaglia, whose work he says be had translated and published in French. The present work seems meant as an elementary bork of instruction, being regularly divided into chapters, and treated methodically, though not deeply. The chapters are, on quantity, on the methods of jumeration, or the object of algebra, on the names of numbers with respect to their powcrs, which are thus denoted: I the latus or number, $Q$ the quadrant or square, $c$ the cube, $Q$ the squaresquared or 4th power, $R$ P the relatus-primus or 5 th power (called by Diophantus quadratocubus), $\mathrm{RS}$ the relatussecundus or 7 th power, QQ Q quadrato-quadrato-quadratus or 8 th power, $\mathrm{c} c$ the cubocubus or 9 th power, \&c.Chap. 7 , gives a long account of the composition of the cube of a binomial, and the cxtraction of the cube root, from the theorem $c^{3}=(a+b)^{3}=a^{3}+b^{3}+3 a b . a+3 a b$ .$b$, from Tartaglia.-Chap. 8 , \&c, treats on proportion, arithínetical, geometrical, and harmonical, with tlic arithmetic of ratios.-Chap. 15, 16, on the single and double rule of Hypothesis, or Position.-Chap. 17, on finding several mean proportionals, by the double rule of Hypothesis, with its application to the celebrated problem of doubling the cube; also to its tripling, and quadrupling, \&c.

Book 11. Chap. 1. On the values of the powers, (or what are now called their exponents), being the numbers of their situation in the scale of powers, $1,2,3,4$, \&c. Chap. 2, on the addlition of the powers, by means of their signs $p$ and $m$, that is, plus and minus.-Cliap. 3, 4, on the multiplication and division of powers, by adding and subtracting their values or indices.-Chap. 5, 6, addition and subtraction of complex quantities, witl mixed signs, $p$ and $m$, and various powers.-Chap. 7,8 , multiplication and division of mixed quantities. Here the author states that in these operations, like signs produce $p$ (plus), but mnlike signs $m$ (minus); and he says he has proved these in lis Arithmetic, which no one has done before him.-Chap. 9, $10, \& c$, on fincling the sides or roots of simple and compound quantities.

Book III. On Equations. Chap. 1, 2, 3, on simple cquations.-Chap. $4,5,6,7,8,9$, on quadratic equations, by the usual method of completing the square, \&c. In the case $x^{2}+12=a x$, the author assigns the two roots. In the other two cases, he finds one root only, without noticing the negative roots.-In chap. 10 , he speaks of the cubic equations trated of by Cardan and 'Tartaglia, but without stating the rules for their solution. In chap. 11, 12, 13, some Diuphantine problems are treated of. 
Book Iv, titled De 2uantitate Absoluta et Surda, contains two chapters. These include various problems or questions, in which several unknown quantities are required to be found, from as many proposed independent relations. In the solution of these questions, the author uses the capitals $A, B, C, D, \& C$, for the unknown quantities to be found, which he employs in the same way as we do now the letters $x, y, z, \& \mathrm{c}$, making out with them as many independent equations as are the unknown letters, A, B, c, \&c; by the reduction of which equations, in the usual way, the values of these letters are determined.-But Stifelius, before him, had used A, B, c, \&c, for the unknown quantities.

$$
\text { clavius. }
$$

Christopher Clavius wrote his Algebra about the year 1580 , though it was not published till 1608 , at Orlcans. He mostly follows Stifelius and Scheubelius in his notation and method, \&c, having scarcely any variations from them; nor does he treat of cubic equations. He mentions the names given to the art, and the opinions about its origin, in which be inclines to ascribe it to Diopbantus, from what Diophantus says in his preface to Dionysius.

$$
\text { STEVINUS. }
$$

The Arithmetic of Simon Stevin of Bruges, was published in 1585, and the same with his Algebra in 1605, in the Flemish dialect, and containing a free translation of the first 4 books of Diophantus. They were also printed in a French edition of his works at Leyden in 1634, with some notes and additions of Albert Girard, who it seems died the year before, this edition being published for the benefit of Girard's widow and childrên. This edition contains all the 6 books of Diophantus.

The Algebra is an ingenious and original work. He denotes the res, or unknown quantity, in a way of his own, namely by a small circle $O$, within which he places the numeral exponent of the power, as (a), (3, (2), (3), \&c, which are the $0,1,2,3, \& \mathrm{c}$, power of the quantity $O$; where @, or the 0 power, is the beginning of quantity, or arithmetical unit. He also extends this notation to roots or fractional exponents, and even to radical ones.

Thus (2), (과, $6, \mathrm{sc}$, are the square root, cube root, 4 th root, \&c;

also 3 is the cube root of the square;

and (-7) is the square root of the cube. And so of others.

The first three powers, (3), (2), (3), be also calls coste (side), quarre (square), cube (cube); and the first of them, (1), the prine quantity, which he obstrves is also metaphorically called the racine or root, (the mark of which is also $\sqrt{ }$,) because it represents the root or origin from which all other quantitics spring or arise, called the potences or powers of it. He condemns the terms sursolids, and numbers absurr, irrational, irregular, inexplicable, or surd, and shows that all numbers are denoted the same way, and are all equally proper expressions of some length or magnitude, or sume power of the same root. He also rejects all the compound expressions of square-squared, cube-squared, cube-cubed, \&cc; and observes that it is best to name them all by their exponents, as the 1st, $2 \mathrm{~d}, 3 \mathrm{~d}$, 4 th, 5 th, 6 th, \&cc, power or quantity in the series. And on his extension of the new notation he justly observes, that what was before obscure, laborions, and tiresome, will by these inarks be clear, easy, and pleasant. He also makes the notation of algebraic quautities more general in their coefficients, including in them not only integers, as 3 (1), but also fractions and radicals, as $\frac{3}{4}(2)$, and $\sqrt{2}$ (3), \&rc. He has yarious other peculiarities in his notations ; all showing an original and inventive mind. A quantity of several terms, he calls a multinomial, and also binomial, trinomial, \&c, according to the number of the terms. He uses the signs + and - , and sometimes : for equality: also $\times$ for division of fractions, or to multiply crosswise thus, $\frac{5}{7} \times \frac{2}{3}: \frac{15}{14}$.

He teaches the generation of powers by means of the annexed table of numbers, which are the cocfficients of all the terms, except the first and last. And he makes use of the same numbers also for extract- $\begin{array}{lllll}6 & 15 & 20 & 15 & 6\end{array}$ ing all roots whatever: both which things \&c. had first been done by Stifelius. In ex-

tracting the roots of non-quadrate or non-cubic numbers, he has the same approximations as at present, viz, either to continue the extraction indefinitely in decimals, by adding periods of ciphers, or by making a fraction of the remainder in this manner, viz, $\sqrt{ } \mathrm{N}=n+\frac{N-n^{2}}{2 n+1}$ nearly, and $\sqrt[3]{ } \mathrm{N}=n+\frac{\mathrm{N}-n^{3}}{3 n^{2}+3 n+1}$ nearly; where $n$ is the nearest exact root of $\mathrm{N}$; which is Peletarius's rule, and which differs from Tartalea's rule, as this wants the 1 in the denominator. And in like manner he goes on to the roots of higher powers.

He then treats of equations, and their inventors, which according to him are thus :

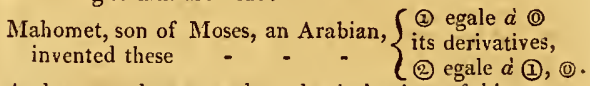
And some unknown author, the derivatives of this.

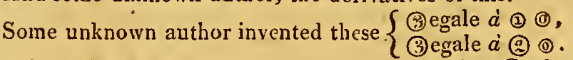

But afterwards he mentions Ferreus, Tartalea, Cardan, $\& c$, as being also concerned in the invention of them.

Lewis Ferrari invented - (4)egale $a$ (2) (2) (1) ().

He says also that Diophantus once resulves the case (2) egale $d$ (3) (.). In his reduction of equations, which is full and masterly, he always places the highest power on one side alone, equal to all the other terms, set in their order, on the other side, whetlier they be + or - . And he demonstrates all the rules both arithmetically and geometrically. In cubics, he gives up the irreducible case, as hopeless: but says that Bombelli resolves it by plus of minus, and minus of minus:

thus, if 1 (3) $=30$ (3) +36 , then 1 (1) $=\sqrt[3]{ }(18+$ of -26$)$

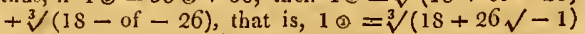
$+\sqrt[3]{ }(18-26 \sqrt{ }-1)$. He resolves biquadratics by means of cubics and quadratics. In quadratics, he takes both the two roots, but looks for no more than two in cllbics or biquadratics. He gives also a general methorl of approaching indefinitely near, in decimals, to the ront of any equation whatever; but it is very laborious, being little more than trying all numbers, one after another, finding thus the 1st figure, then the $2 \mathrm{~d}$, then the $3 \mathrm{~d}, \& \mathrm{c}$, among these ten characters $0,1,2,3,4,5,6,7,8,9$. And finally he applies the rules, in the resolution of a great many practical questions.

Though a general air of originality and improvement runs through the whole of Stevinus's work, yet his more remarkable or peculiar inventions, may be reduced to these few following: viz,

1st. He inventerl not only a new character for the unknown quantity, but greatly improved the notation of $\mathrm{N}$ 
powers, by numeral indices, first given by Stifelius as to integral exponents; which Stevinus extended to fractional and all other sorts of exponents, thereby denoting all kinds of roots the same way as powers, by numeral exponents. A circumstance hitherto thought to be of much later inrention.

2d. He improved and extended the use and notation of coefficients, including in them fractions and radicals, and all sorts of numbers in general.

3d. A quantity of several terms, he called generally a multinomial; and he denoted all nomials whatever by particular names expressing the number of their terms, binomial, trinomial, quadrinomial, \&rc.

4th. A numeral solution of all equations whatever, by one general method.

Besjdes which, he hints at some nnknown author as the first inventor of the rules for cubic cquations; by whom may perhaps be intended the author of the Arabic manuscript treatise on cubic equations, given to the library at Leyden by the celebrated Warner.

$$
\text { vIETA. }
$$

Most of Vieta's algebraical works were written about or before the year 1600 , but some of them were not published till after his death, which bappened in the year 1603 , in the $63 \mathrm{~d}$ year of his age. And his whole mathematical works were collected together by Francis Schooten, and elegantly printed in a folio volume in 1646. Of these, the algebraical parts are as follow :

- 1. Isagoge in Artem Analyticam.

2. Ad Logisticen Speciosam Notæ Priores.

3. Zeteticorum libri quinque.

4. De Æquationum Recognitione, et Emendatione.

5. De Numerosâ Potestatum ad Exegesin Resolutione.

Of all these I shall give a particular account, especially in such parts as contain any discoveries, as we here meet with more improvements and inventions on the nature of equations, than in almost any former author. And first of the Isagoge, or Introduction to the Analytic Art. In this short introduction Vieta lays down certain præcognita in this art; as definitions, axioms, notations, common precepts or operations of addition, subtraction, multiplication, and division, with rules for questions, \&c. From which we find, 1st, That the namcs of his powers are latus, quadratum, cubus, quadrato-quadratum, quadrato-cubus, cubo-cubus, \&c; in which he follows the method of Diophantus, and not that derived from the $\Lambda$ rabians. 2d, That he calls powers pure or allected, and first here uscs the terms coefficient, affirmative, negative, specious logistics or calculations, homogeneum comparationis, or the absolute known term of an equation, homogeneum, adfectionis, or the $2 d$ or other term which makes the equation alfected, \&c. 3d, That he uses the capital letters to denote the known as well as unknown qquantities, to render his rules and calculations general; namely the vowels $A, \mathbf{E}, \mathrm{I}, \mathrm{O}, \mathrm{U}, \mathrm{Y}$ for the unknown quantities, and the consonants, B, C, D, \& $\mathrm{C}$, for the known ones. 4th, That he uses the sign + between two terms for addition; - for subtraction, placing the greater before the less; and when it is not known which term is the greater, he places $=$ between them for the difference, as we now use $\sim$; thus $A=B$ is the same as $A \sim B$; that he expresses division by placing the terms like a fraction, as at present; though lie was not first in this. But that he uses no characters for multiplication or cquality, but writes the words themselves, as well as the names of all the powers, as he uscs no exponents, which causes much trouble and prolixity in the progress of his work; and the numeral coefficients set after the litcral quantities, have a disagrecable effect.

II. Ad Logisticen Speciosam Notce Priores. These consist of various theorems conccrning sums, differences, products, powers, proportionals, \&cc, with the genesis of powers from binomial and residual roots, and certain properties of rational riglit-angled triangles.

IlI. Zeteticorum libri quinque. The zetetics or questions, in these 5 books, are chiefly from Diophantus, but resolved more generally by literal arithmetic. And in these questions are also investigated rules for the resolution of quadratic and cubic equations. In these also Vieta first uses a line drawn over compound quantities, as a vinculum.

IV. De AEquationum Recognitione, et Emendatione. These two hooks, which contain Vieta's chief improvements in algebra, werc not published till the year 1615, by Alexander Anderson, a learned and ingenious Scotchman, with various corrections and additions. The 1st of these two books consists of 20 chapters. In the first six chapters, rules are drawn from the zetetics for the resolution of quadratic and cubic equations. These rules are by means of certain quantities in continued proportion, but in the solution they come to the same thing as Cardan's rules. In the cubics, Vieta sometimes changes the negative roots into affirmative, as Cardan had done, but he finds only the affirmative roots. And he here refers the irreducible case to angular sections for a solution, a method which had been mentiened by Bombelli.

Chap. 7 treats of the general method of transforming equations, which is done either by changing the root in various ways, namely by substituting another instead of it which is either increased or diminished, or multiplied or divided, by some known number, or raised or depressed in some known proportion ; or by retaining the same root, and equally multiplying all the terms. Which kinds of transformation, it is cvident, are intended to make the equation become simpler, or more convenient for solution. And all or most of these reductions and transformations were also practised by Cardan.

Chap. $S$ shows what purposes are answered by the foregoing transformations; such as taking away some of the terms out of an equation, and particularly the od term, which is done by incrcasing or diminishing the root by the coefficient of the $2 \mathrm{~d}$ term, divided by the index of the first: by which means also the afficted quadratic is reduced to a simple onc. And various other effects are produced.

Chap. 9. shows how to deduce compound quadratic equations from pure ones, which is done by increasing or diminishing the root by a given quantity, being one application of the foregoing reductions.

Chap. 10, the reduction of cubic cquations affected with the 1st power, to such as are affected with the $2 \mathrm{~d}$ power; by the same means.

In chap. 11, by the same means also the $2 \mathrm{~d}$ term is restored to such cubic equations as want it.

In chap. 12, quadratic and cubic equations are raised to higher degrees, by substituting for the root, the square or cube of another root divided by a given quantity.

In chap. 13, affected biquadratic equations are deduced from affected quadratics in this manner, when expressed in the modern notation. If $A^{2}+B A=7$, then shall $A^{4}+\overline{B^{3}+2 B z}, A=Z^{2}+B^{2} z$. 


\section{A L G}

For since $A^{2}+B A=z$, therefore $A^{2}=z-B A$, and its square is $A^{4}=Z^{2}-2 B A Z+B^{2} A^{2}$ : but $B^{2} A^{2}=B^{2} Z-B^{3} A$, therefore $A^{4}=z^{2}-2 B A Z+R^{2} Z-B^{3} A$, or $A^{4}+\overline{B^{3}+2 B z} \cdot A=z^{2}+B^{2} z$.

And in like manner for the biquadratic affected with its other terms. And in a similar nnanner also, in chap. 14, affected cubic equations are deduced from the affected quadratics.

In chap. 15 it is shown that the quadratic $\mathbf{B A}-\mathrm{A}^{2}=\mathrm{z}$ bas two values of the root $A$, or has ambiguous roots, as he calls them; and also that the cubics, biquadratics, \&c, which are raised or deduced from that quadratic, have also double roots.

Having in the foregoing chapters, shown how the coefficients of equations of the $3 \mathrm{~d}$ and 4 th degree are formed from those of the $2 \mathrm{~d}$ degree, of the same root; and that certain quadratics, and others raised from them, have double roots; then in the 16 th chap. Vieta shows what relation those two roots bear to the coefficients of the two lowest terms of an equation consisting of only three terms.

Chap. 17 contains several theorems concerning quantities in continued geometrical progression. Which are preparatory to what follows, concerning the double roots of equations, the nature of which he expounds by means of such properties of proportional quantities.

Chap. 18, Eqquationum ancipitum constitutiva ; trcating of the nature of the double roots of equations.

Next follows the $2 \mathrm{~d}$ of the pieces published by Alexander Anderson, namely,

De Emendatione Equationum, in 14 chapters.

Chap. 1. Of preparing equations for their resolution in numbers, by taking away the $2 \mathrm{~d}$ term; by which affected quadratics are reduced to pure ones, and cubic equations affected with the $2 \mathrm{~d}$ term are reduced to such as are affected with the $3 \mathrm{~d}$ only. Several examples of both kinds of equations are given. He here too remarks on the method of taking away any other term out of an equation, when the highest power is combined with that other term only; and this Vieta effects by means of the coefficients, or, as he calls them, the uncixe of the power of a binomial. All which was also performed by Cardan for the same purpose.

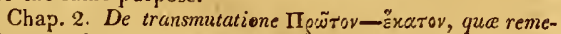
dium est adversus vitium negationis. Concerning the transformations by changing the given root $\mathrm{A}$ for another root $\mathrm{E}$, which is equal to the homogeneum comparationis divided by the first root $A$; by which means negative terms are changed to affirmative, and radicals are taken out of the equation when they are contained in the homogeneum comparationis.

Chap. 3. De Anastrophe, showing the relation between the roots of corrclate equations; whence, having given the root of the one equation, that of the other becomes known.

Chap. 4. De Isomaria, adversus vitium fractionis. To take away fractions out of an equation. Thus,

If $A^{3}+\frac{B}{D} A=z$. Put $A=\frac{E}{D}$; then $E^{3}+B D E=Z D^{3}$.

Chap. 5. De Symmetrica Climactismo, adversus vitium asymmetria. 'l'o take atway radicals or surds out of equavions, by squaring, \&c, the otber side of the equation.

Chap. 6. 'To reduce biquadratic equations by means of cubics and quadratics, by methods which are small varianons from those of Ferruri and Cardan.

Chap. 7 . The resolution of cubic equations by rules which are the same with Cardan's.

Chap. 8. De Canonica aquationum transmutatione, ut coefficientes subgraduales sint qua prascribuntur. To transmute the equation so that the coefficient of the lower term, or power, may be any given number, he changes the root in the given proportion, thus: Let $\mathrm{A}$ be the root of the equation given, $\mathbf{E}$ that of the transmuted equation, $B$ the given coefficient, and $x$ the required one; then take $A=\frac{B E}{x}$, which substitute in the given equation, and it is done.- $-\mathrm{He}$ commonly changes $\mathrm{jt}$ so, that $\mathrm{x}$ may be 1 ; which he does, that the numeral root of the equation may be the easier found; and this lie bere performs by trials, by taking the nearest root of the highest power alone: and if that does not turn out to be the root of the whole equation, he concludes that it has no rational root.

Chap. 9. To reduce certain peculiar forms of cubics to quadratics, or to simpler forms, inuch the same as Cardan had done.

Chap. 10. Similium reductionum continuatio. Being some more similar theorems, when the equation is affected with all the powers of the unknown quantity $A$.

Chap. 11, 12, 13 relate also to certain peculiar forms of equations, in which the root is one of the terms of a certain series of continued proportionals.

Chap. 14, which is the last in this tract, contains in four theorems, the general relation between the roots of an equation and the coefficients of its terms, when all its roots are positive.

And from these last four theorems it appears, that Vieta was acquainted with the composition of these equations, that is, when all their roots are positive, for he never adverts to negative roots; and from other parts of the work it appears, that he was not aware that the same properties will obtain in all sorts of roots whatever. But it is not certain in what mamer he obtained these theorems, as he has not given any account of the investigations, though that was usually his way on other occasions; but he here contents bimself with barely announcing the theorems, and for this strange reason, that he might at length bring his work to a conclusion.

To this piece is added, by Alexander Anderson, an Appendix, containing the construction of cubic equations by the trisection of an angle; and a demonstration of the property referred to by Vieta for this purpose.

De Numerosa Potestatum Purarum Resolutione. Vieta here gives some cxamples of extracting the roots of pure powers, in the way that had been long before practised, by pointing the number into periods of figures according to the index of the root to be extracted, and then proceeding from one period to another, in the usual way.

De Numerosa Potestatum adfectarum Resolutione. And here, in close initation of the above method for the roots of pure powers, Vieta extracts those of adfected ones; or finding the roots of affected equations, placing always the homogeneum comparationis, or absolute term, on one side, and all the terms affected with the unknown quantity, and their proper signs, on the other side, The method is very laborious, and is but little more than what was before done by Stevinus on this subject, depending not a little on trials. The examples he uses are such as have either one or two roots, and indeed such as are affected commonly with only two powers of the unknown quantity, and which therefore adnit only of these two 
varieties as to the number of roots, namely according as the higher of the two powers is affirmative or negative, the homogeneum comparationis, on the other side of the equation, being always affirmative; and he remarks this general rule, if the higher power be negative, the equation has two roots, otherwise only one; that is, affirmative roots; for as to negative and imaginary ones, Vieta knew nothing about them, or at least he takes no notice of them. By the foregoing extraction, Vieta finds both the greater and less root of the two that are contained in the equation, and either of them that he pleases; having first, for this purpose, laid down some observations concerning the limits within which the two roots are contained. Also, having found one of the roots, he shows how the other root may be found by means of another equation, which is a degree lower than the given one; though not by depressing the given equation, by dividing it as is now done; but from the nature of proportionals, and the theorems relating to equations, as given in the former tracts, he finds the terms of another equation, different from that last mentioned, from the root \&c of which, the $2 \mathrm{~d}$ root of the original equation may be obtained.

In the course of this work, Vieta makes also some observations on equations that are ambiguous, or have three roots; namely, that the equation $1 \mathrm{c}-6 \mathrm{Q}+11 \mathrm{~N}=6$, or as we write it $x^{3}-6 x^{2}+11 x=6$, is ambiguous, when the $2 \mathrm{~d}$ terin is negative, and the $3 \mathrm{~d}$ term affirmative, and when $\frac{x}{3}$ of the square of 6 , the coefficient of the $2 \mathrm{~d}$ term, exceeds 11, the coefficient of the $3 d$ term, and bas then three roots. Or in general, if $x^{3}-a x^{2}+b x=c$, and $\frac{1}{3} a^{2}>b$, the equation is ambiguous, and has three roots. He shows also, from the relation of the coefficients, how to find whetber the roots are in arithmetical progression or not, and how far the middle root differs from the extremes, by means of a cubic equation of this form $x^{3}-$ $b x=c$. In all or most of which remarks be was preceded by Cardan.- Vieta also remarks that the case $x^{3}-$ $9 x^{2}+24 x=20$, has three roots by the same rule, viz, $2,2,5$, but that two of them are equal. And further, that when $\frac{x}{3} a^{2}$ is $=b$, then all the three roots are equal, as in the case $x^{3}-6 x^{2}+12 x=8$, the three roots of which are $2,2,2$. But when $\frac{x}{3} a^{2}$ is less than $b$, the case is not ambiguous, having but one root. And when $a b=c$, then $a=x$ is one root itself.

Many curious notes are added at the cnd, with remarks on the method of furding the approximate ronts, when they are not-rational, which is done in two ways, in imitation of the same thing in the extraction of pure powers, viz, the one by forming a fraction of the temainder after all the figures of the homogeneum comparationis are exhausted; the other by increasing the root of the equation in a 10 fold, or 100 fold. \&c, proportion, and then dividing the root which results by 10 , or $100,8 \mathrm{c}$ : and this is a decimal approximation. And lieta ohserves, that the roots will be incriased 10 or 100 fold, \&e, by adding the corresponting number of ciphers to the corfiscient of the $2 \mathrm{~d}$ term, double that number to the $3 \mathrm{~d}$, triple the same number to the $4 \mathrm{th}$, and so on. So if the equation were $1 \mathrm{c}+4 \mathrm{Q}+6 \mathrm{~N}=8$, then $1 \mathrm{c}+40 \mathrm{Q}+600 \mathrm{~N}=$ 8000 will have its root 10 fold, and $1 \mathrm{c}+400 \mathrm{Q}+60000 \mathrm{~N}$ $=8000000$ will have it 100 fold.

Besides the foregoing algebraical works, Vieta gave various constructions of equations by means of circles and right lines, and angular sections, which may be considered as an algebraical tract, or a method of exhibiting the roots of certain equations having all their roots affir- mative, and by means of which he resolved the celebrated equation of 45 powers, proposed to all the world by Adrianus Romanus.

Having now delivered a particular analysis of Vieta's algebraical writings, it will be proper, as with other anthors, to collect into one view the particulars of his more remarkable peculiarities, inventions, and improvements.

And first it may be observed, that his writings show great originality of genius and invention, and that he made alterations and improvements in most parts of algebra; though in other parts and respects his method is inferior to some of his predecessors; as, for instance, where he neglects to avail bimself of the negative roots of Cardan; the numeral exponents of Stifelius, instead of which he uses the names of the powers themselves; or the fractional exponents of Stevinus; or the commodious way of prefixing the coefficient before the quantity or factor; and such like circumstances; the want of which gives bis Algebra the appearance of an age much earlier than its own. But his real inventions of things before not known, may be reduced to the following particulars.

1st. Vieta introduced the general use of the letters of the alphabet, to denote indefinite given quantitic's; which had only been done on some particular occasions before his time. But the general use of letters, for the unknown quanticies, was before pretty common with Stifelius and his successors. Vieta uses the vowels $A, E, X, 0, U, x$ for the unknown quantities, and the consonants, B, C, D, \& c, for known ones.

2d. He invented, and introduced, many expressions or terms, several of which are in use to this day: such as coefficient, affirmative and negative, pure and adfected or affected, uncix, homogencum adfectionis, homogeneum comparationis, the line or vinculum over compound quantities, thus $\overline{A+B}$. And his method of setting down his equations, is to place the lomogeneum comparationis, or absolute known term, on the right-hand sile alone, and on the other side all the terms which contain the unknown quantity, with their proper signs.

$3 \mathrm{~d}$. In most of the rules and reductions for cubic and other equations, he made some improvements, and variations in the modes.

4th. He showed how to change the root of an equation in a given proportion.

5. He derived or raised the cubic and biquadratic, \&c, equations, from quadratics; not by composition in Harriot's way, but by squaring and otherwise multiplying certain parts of the quadratic. And as some quadratic cquations have two roots, therefore the cubics and others raised from them, have also the same two roots, and no more. And hence he comes to know what relation these two roots brar to the coefticients of the two lowest terms of cubic and other equations, when they have only 3 terms, namely, by comparing them with similar equations so raised from quadratics. And, on the contrary, what the roots are, in terms of such coefficients.

6 . He madr some observations on the limits of the two roots of certain equations.

7. He stated the general relation between the roots of certain cquations and the cocflicicnts of its terms, when the terms are alternately plus and minus, and none of them are wanting, or the roots all positive.

8. He extracted the rosts of affected equations, by a method of approximation similar to that for pure powers.

9. He gave the construction of certain cquations, and 
exhibited their roots by means of angular sections; before adverted to by Boinbelli.

\section{OF ALIIRT GIRARD.}

Albert Girard was an ingenious Dutclu or Flemisl mathematician, who died about the year 1633 . He published an edition of Steviuus's Arithmetic in 1625, augmented with many notes; and the year after his death was published, by his widow, an edition of the whole works of Stevinus, in the same manner, which Girard had left ready for the press. But the work which entitles him to a particular notice in this history, is his "Invention Nouvelle en l'Algebre, tant pour la solution dés equations, que pour recoguoistre le nombre des solutions qu'elles reçoivent, avec plusieurs choses qui sont necessaires a $\mathrm{l}_{i 2}$ perfection de ceste divine science;" which was printed at Amsterdam 1629, in small quarto, in 63 pages, viz, 49 pages on arithmetic and algebra, and the rest on the measure of the superficies of spherical triangles and polygons, by him then lately discovered.

In this work, Girard first premises a short tract on Arithmetic; in the notation of which he has something peculiar, viz, dividing the numbers into the ranks of millions, billıuns, trillions, \&c.

He next delivers the common rules of algebra, not only in integers and fractions, but radicals also; with the notation of the quantities and signs. In this part he uses sometimes the letters $A, B, C, \& c$, after the manner of Vieta, but more commonly the characters of Stevinus, viz, (1), (1), (2), , \&c, for the powers of the unknown

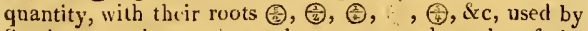
Stevinus; and somitimes the more usual marks of the roots, as $\sqrt{ }$ or $\sqrt[2]{ }, \sqrt[3]{ }, \sqrt[4]{ }, \& \mathrm{c}$; prefixing the coefficients, as 6 , or $3 \sqrt[5]{3}$, or 24 . In the signis he follows his predecessurs so far as to have + for plus, - or $\div$ for minus, $=$ for general or indefinite difference, $A+B$ for the sum, $\mathrm{A}-\mathrm{B}$ or $\mathrm{A}=\mathrm{B}$ for the difference, $\mathrm{AB}$ the product, and $\frac{A}{B}$ for the quotient of $A$ and $B$. He uses the parentheses ( ) for the vinculum or bond of compound quantities, as is now coinmonly practised; as $A(A B+B q)$, or $\sqrt[2]{ }(\mathrm{A}$ cub. $-3 \mathrm{AqB})$; and he introduces the new characters ff for greater than, and $\S$ for less than; but he uses no character for equality, only the word itsclf.

Girard gives a new rule for extracting the cube root of binomials, which however is in a good measure tentative, and which he explains thus: To extract the cube root of $72+\sqrt{ } 5120$.

$$
\text { The squares of the terms }\left\{\begin{array}{l}
5184 \\
5120
\end{array}\right.
$$

their difference 64 , and its cube root 4. Which shows that the difference between the squares of the terms required is 4 ; and the rational part 72 being the greater, the greater tern of the ront will be rational also; and further, that the greatcr terms of the power and root are commensurable, as also the two less terms. Then having made a table as in the margin, where the square of the rational term always cx-

$$
\begin{aligned}
& 2+\sqrt{ } 0 \\
& 3+\sqrt{ } 5 \\
& 4+\sqrt{ } 12 \\
& 5+\sqrt{ } 21
\end{aligned}
$$
ceeds that of the other, by the number 4 above mentioned, one of these binomials must be the cubic root sought, if the given quantity have such a root; and it must be one of these four forms; for it is known to be carried far enough by observing that the cube root of 72 is less than 5 , and the cube root of 5120 - less than 21 ; indeed, this being the casc, the last binomial is excluded, as cvidently too great; and the first is excluded because one of its terins is 0 ; therefore the root must be either $3+\sqrt{ } 5$ or $4+\sqrt{ } 12$. And to know whether of these two it must be, try which of them has its two terms exact divisors of the corresponding terms of the given quantity; then it is fonnd that 3 and 4 are both divisors of 72 , but that only 5 , and not 12 , is a divisor of 5120 ; therefore $3+\sqrt{ } 5$ is the root sought, which upon trial is fuund to answer. It is remarkable here, that Girard uses $4+\sqrt{ } 20$ instearl of $4+\sqrt{ } 12$, and $5+\sqrt{ } 29$ instead of $5+\sqrt{ } 20$, contrary to his own rule.

Girard then gives distinct and plain rules for bringing questions to equations, and for the reduction of those equations to their simplest form, for solution, by the usual modes, and also by the way called by Vieta Isomeria, multiplying the terms of the equation by the terms of a geometrical progression, by which means the roots are altered in the proportion of 1 to the ratio of the progression. He then treats of the methods of finding the roots of the several kinds of equations, quadratic, cubic, \&x ; and adds remarks on the proper number of conditions or equations for limiting questions. The quadratics are resolved by completing the square, and both the positive and negative routs are taken; and he observes that sometimes the equation is impossible, as (2) equ. 6 (1) -25 , whose roots, he adds, are $3+\sqrt{ }-16$ and $3-\sqrt{ }-16$.

The cubic equations he resulves by Cardan's rule, except the irreducible case, which he the first of any resolves by a table of sines; the other cases he also resolves by tables of sines and tangents; and adds geometrical constructions by means of the hyperbola or the trisection of angles. He next adds a particular mode of resolving all kinds of equations, that have rational roots, on the principle of the ruots being divisors of the last or absolute term, as before mentioned by Peletarins; and then gives the method of approximating to other roots that are not rational, much in the same way as Stevinus.

Having found one ront of an equation, by any of the former methods, by means of it he depresses the equation one degree lower, then finds another root, and so on till they are all found; for he shows that every algebraic equation, admits of as many solutions or ronts, as there are nnits in the index of the highest power, which roots may be either positive or negative, or imaginary, or, as he calls them, greater than nothing, or less than nothing, or involved; so the roots of the equation 1 (3) equ. 7 (1) -6 , are 2,1 , and -3 ; and the roots of the equation 1 (4) equ. 4 (1) -3 are, 1 , and 1 , and $-1+\sqrt{ }-2$, and $-1-$ $\sqrt{ }-2$

In depressing an cquation to lower degrees, he does not use the method of resolution of Harriot, but that which is derived from the general relation of the roots and coefficients of the terms, which be here fully and universally states, viz, that the coefficient of the $2 \mathrm{~d}$ term is equal to the sum of all the roots; that of the $3 \mathrm{~d}$ term equal to the sum of all the products of the roots, taken two by two; that of the 4th term, the sum of the products, taken three by three; and so on, to the last ar absolute term, which is the continual product of all the roots; a properry which was before stated by Victa, as to the equations that have all their roots positive; and here extended by Girard to all kinds of roots whatever: but how cither Vieta or he came by this property, no where appears that I know of. From this general property, among other deductions, Girard shows how to find the sums of the powers of the 


\section{A L, G}

roots of an equation; thus, let $A, P, C, D, \& C$, be the $1 \mathrm{st}, 2 \mathrm{~d}, 3 \mathrm{~d}, 4 \mathrm{th}, \& \mathrm{c}$, coefficient, after the first term, or the sums of the products taken one by one, two by two, tbree by three, \&c; then, in all kinds of equations,

$$
\left.\begin{array}{l}
A \mathrm{Aq}-2 \mathrm{~B} \\
\mathrm{~A} \text { cub. }-3 \mathrm{AB}+3 \mathrm{C} \\
\mathrm{Aqq}-4 \mathrm{~A} \mathrm{~B}+4 \mathrm{AC}+2 \mathrm{~Bq}-4 \mathrm{D}
\end{array}\right\} \begin{aligned}
& \text { roots, } \\
& \text { squares, } \\
& \text { cubcs, } \\
& \text { biquadrates. }
\end{aligned}
$$

Girard next cxplains the use of negative roots in geometry, showing that they represent lines only drawn in a direction contrary to those representing the positive roots; and he remarks that this is a thing bitherto unknown. He ther terminates the algebra by some questions having two or more unknown quantities. And be subjoins to the whole a tract on the mensuration of the surfaces of spherical triangles and polygons, by him lately discovered.

From the foregoing account it appenrs that,

1st, He was the first person who understood the general doctrine of the formation of the coefficients of the powers, from the sums of their roots, and their products, \&c.

$2 \mathrm{~d}$, He was the first who understood the use of negative roots in the solution of geometrical problems.

$3 \mathrm{~d}$, He was the first who spoke of the imaginary roots, and understood that every equation might have as many roots, real and inaginary, and no more, as there are units in the index of the highest power. And he was the first who gave the whimsical name of quantities less than nothing to the negative ones.

4th, He was also the first who discovered the rules for summing the powers of the roots of any cquation.

$$
\text { OF HARRIOT. }
$$

Thomas Harriot, an excellent astronomer, philosopher, and mathematician, was born at Oxford in 1560 . After taking the degree of bachelor of arts in 1579, be accompanied the famous Sir Walter Raleigh in an expedition to Virginia, where the first English establishment was made. Harriot here drew the map of the country, and in 1588 gave a relation of the voyage. Being returned to his country, it appears that he gave himself up entirely to the study of the mathematics, and especially to that of algebra. He remained not long unknown to the earl of Northumberland, a great encourager of the sciences, who maintained several learned men, such as Rob. Hues, Walter Warner, and Nathanicl Tarporley. This enlightened nobleman entertained Harriot in his house, with a salary of $£ 300$ sterling a-year, a great sum in those times; and in this situation it was that Harriot finished his days, in the year 1621, at 61 years of age. It appears by Kepler's letters, that he held a correspondence with this astronomer, chiefly on the theory of the rainbow. Harriot's manuscripts, lately discovered in the castle of the earl of Egremont, inform us of many of his astronomical observations, and particularly of those of the spots in the sun as early as the beginuing of December 1610 , while the first of those of Galilco were not made before the preceding month. So that Harriot must then either have made himself a telescope, or procured one from Holland. He made in the same year also, observations on Jupiter's satellites, and on the remarkable comets of the years 1607 and $161 \mathrm{~S}$.

His Algebra was left behind him unpublished, as well as those other papers, at his death, which happened in the year 1621 , as before mentioned, and but 6 years after the first publication of the principal parts of Victa's Algebra, by Alexander Andcrson; so that it is probable that
A L G

Harriot's Algebra was written long before this time, and indeed that he bad never scen these pieces.

Harriot's Algebra was published by his friend Walter Warmer, in the year 1631: and it would doubtless be highly grateful to the learned in these sciences, if his other curious algebraical and astronomical works were published, from his original papers in the possession of the earl of Egremont, to whom they hare descended from Henry Percy, the earl of Northumberland, that noble Mæcenas of his day. The book is in folio, and entitled Artis Analyticæ Praxis, ad Æquationes Algebraicas nora expedita, ct generali mcthodo, resolvendas; a work in all parts of it showiıg marks of great genius and originality', and is the first instance of the modern form of algebra, in which it has ever since appeared. It is prefaced by 18 definitions, which are these: 1st, Logistica Speciosa 2d, Equation; 3d, Synthesis; 4, Analysis; 5, Composition and Resolution; 6, Furming an Lquation; 7, Reduction of an Equation; 8, Verification; 9, Numerosa et Speciosa; 10, Excogitata ; 11, Resolution; 12, Roots; 13 and 14, The kinds and generation of equations by multiplication, from binomial roots or factors, called original equations:

$$
\begin{aligned}
& \text { as } a+b \mid=a a+l a \quad \text { or } a+l \mid=a a a+l a a+7 c a
\end{aligned}
$$

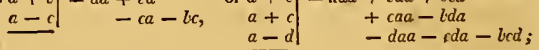

where be puts $a$ for the unknown quantity, and the small consonants, $b, c, d, \& c$, for its literal values or roots. 15 , The first form of canonical equations, which ale derived from the above originals, by transposing the hoinogeneum, or absolute term, thus $a a+b a$

$$
-c a=+b c, \& c \text {. }
$$

16 , The secondary canonicals, formed from the primary by expelling the $2 \mathrm{~d}$ term, thus $a a=+b b$,

$$
\text { or } \begin{aligned}
a a a & -b b a \\
& -b c a \\
& -c c a=+b b c \\
& +b c c .
\end{aligned}
$$

17 , That these are called canonicals, because they are adapted to canons or rules for finding the numeral roots, \&c. 18 , Reciprocal equations, in which the homogeneum is the product of the coefficients of the other terms, and the first term, or highest power of the root, is equal to the product of the powers in the other terms, as aa $c a a+b b a=+b b c$.

After these definitions, the work is divided into two principal parts; 1 st, of various generations, reductions, and preparations of equations for their resolution in the $2 \mathrm{~d}$ part. The former is divided into 6 secions as follows.

Sect. 1. Logistices Speciosa, exemplified in the 4 operations of addition, subtraction, multiplication, and division; as also the reduction of algebraic fractions, and the ordinary reduction of irregular equations to the form proper for the resolution of them, namely, so that all the unknown terms be on one side of the equation, and the known term on the other, the powers in the terms ranged in order, the greatest first, and the first or highest power made positive, and freed from its coefficient; as $a a+b a=c d$; or $a a a+b a a-c d a=-c^{2} d$.

In this part he explains some unusual characters which he introduces, namcly, $=$ for equality, as $a=b$. - for majority, as $a>b$, $<$ for minority, as $a<b$;

but the first had bcen before introduced by Robert Recorde. 
Sect. 2. The generation of original equations from binomial factors or roots, and the deducing of canonicals from the originals. He supposes that every equation has as many roots as dimensions in its highest power; then supposing the values of the unknown letter $a$ in any equation to be $b, c, d, f, \& \mathrm{c}$, that is $a=b$, and $a=c$, and $a=d, \& c$; by transposition, or equal subtraction, these become $a-b=0$, and $a-c=0$, and $a-d=0, \& \mathrm{c}$, or the same letters with contrary signs for negative values or roots; then two of these binomial factors, multiplied together, give a quadratic equation, three of them a cubic, four of them a biquadratic, and so on, with all the terms on one side of the equation, and 0 on the other side, since, every binomial factor being $=0$, the continual product of all of them must also be $=0$.

And these operations are carricd through all the cases of the $2 d, 3 d$, and 4 th powers, as to the varieties of the signs + and -, and the proportions of the roots as to equal and unequal, with the reciprocals, \&c. From which are made evident, at one glance of the cye, all the relations and properties between the roots of equations, and the coefficients of the terms.

Sect. 3. Eqquationum canonicarum secundariarum a primariis reductio per gradus alicujus parodici sublationem radice supposititia invariat a manente. Containing a great many examples of preparing equations, by taking away the $2 \mathrm{~d}$, $3 d$, or any other of the intermediate terms, which is done by making the positive coefficients in that term, equal to the negative ones, by which meaus the whole term vanishes, or becomes equal to nothing. They are extended as far as cquations of the 5 th degree; and at the end are collected, and placed in regular urder, all the secondary canonicals, so redured; so that by the uniform law which is visible through them all, the series may be continued to the higher degrees as far as we please.

Sect. 4. Equationum canonicarum tam primariarum, quam secunduriarum, radicum designatio. A great many literal equations are here set down, and their roots assigned from the furm of the equation, that is, all their positive routs; for their negative roots are not nuticed here; and it is every where proved that they cannot have any more positive roots than these, and consequently the rest are negative. 'I'hat those are roots, he proves by substituting them instead of the unknown letter $a$ in the equation, when they make all the terms on one side come to the same thing as the hornogeneum on the other side.

Sect. 5. In qua aquationum communium per canonicarum aquipollentiam, radicum numerus determinatur. On the number of the roots of common equations, that is the positive roots. This Harriot determines by comparing them with the like cases found among his canonical forms, which two equations, having the same number of terms with the same signs, and the relations of the coefficients and hoinngeneum correspondent, he calls equipollents. And whatever was the number of positive roots used in the composition of the canonical, the same, he infers, is the number in the proposed common equation. It is remarkable that, in all the examples here used, the number of positive roots is just equal to the number of the changes in the signs from + to - and from - to + , which is a circumstance, though not here expressly inentioned, that could not escape the observation, or the eye, of any one, much less of so clear and comprehensive a sight as that of Harriot.

In this section are contained many ingenious disquisi-
A L G

tions, concerning the limits aud magnitudes of quantities, with sevcral curious lemmas laid down to demonstrate the propositions by, which lemmas are themselves demonstrated in a pure mathematical way, from the magnitudes thenselves, independent of geometrical figures; such as, 1 , If a quantity be divided into any two unequal parts, the square of half the quantity will be greater than the product of the two unequal parts. 2 , In three continued proportionals, the sum of the extremes is greater than clouble the mean. 3, In four continued proportionals, the sum of the extremes is greater than the sum of the two mcans. 4, In any two quantities, one-fourth the square of the sum of the cubes, is greater than the cube of the product of the two quantities. 5, Of any two quantities $q$ and $r$, then $\frac{r}{27}(q q+q r+r r)^{3}>\frac{1}{4}\left(q q^{2}+\right.$ $q r r)^{2} .6$, If any quantity be divided into three unequal parts, the square of $\frac{1}{3}$ of the whole quantity is greater than $\frac{x}{3}$ of the sum of the three products made of the three unequal parts. 7 , Also the cube of the $\frac{1}{3}$ part of the whole, is greater than the solid or continual product of the three unequal parts.

Sect.6. AEquationumi communium reductio per gradus alicujus parodici cxclusionem et radicis supposititia mutationem. Here are a great many examples of reducing and transforming equations of the $2 \mathrm{~d}, 3 \mathrm{~d}$, and 4 th degrees; chiefly either by multiplying the roots of equations in any proportion, as was done-by Vieta, or increasing or diminishing the root by a given quantity, after the manner of Cardan. The former of these reductions is performed by multiplying the terms of the equation by the corresponding terms of a geometrical progression, the first term being 1 , and the $2 \mathrm{~d}$ term the quantity by which the root is to be multiplied. And the other reduction, or transfurming to another root, which may be greater or less than the given root by a given quantity, is performed commonly by substituting $e+$ or $-b$ for the given root $a$, by which the equation is reduced to a simpler form. Other modes of substitution are also used; one of which is this, viz, substituting $\frac{e e \pm l v}{e}$ or $c \pm \frac{l l}{e}$ for the root $a$ in the given equation $a a a \mp 3 . b b a=2 . c c c$, by which it reduces to this quadratic form $e^{6} \mp 2 c^{3} e^{3}=-b^{6}$, from which Cardan's lorms are immediately deduced; namely, $e=\sqrt{ } 3) c^{3}-\sqrt{ } c^{6} \pm l^{6}$, and thercfore $a$ or $e \pm \frac{l b}{e}=$ $\left.\sqrt{ } 3) c^{3}+\sqrt{ } c^{6} \pm b^{6} \pm \sqrt{ } 3\right) c^{3}-\sqrt{ } c^{6} \pm b^{6}$; where he denotes the cubic or $3 \mathrm{~d}$ root thus, $\sqrt{ } 3)$, but without any vinculum over the compound quantities.

In this section, Harriot makes various remarks as they occur: thus, he remarks, and demonstrates, that ece $3 . b b=-c c c-2 . b b b$ is an impossible equation, or has no affirmative root. He remarks also that the three cases of the equation aaa $-3.6 b a=+2 . c c c$ are similar to the three conic sections; nanely to the hyperbola when $c=b$, to the parabola when $c=b$, or to the ellipsis when $c<b$, and for which reason this case is not generally resoluble in species.

Having thus shown how to simplify equations, and prepare them for solution, Harriot enters next upon the second part of his work, bcing the Exegetice Numerosa,

or the numeral resolution of all kinds of equations by a general method, which is excmplified in a grcat number of equations, both simple and affected, as far as the 5 th power inclusive; and they are commonly prepared, by 
the foregoing parts, by freeing them from their $2 \mathrm{~d}$ term, \&c. These extractions are explained and performed in a way different from that of Vieta; and the examples are first in perfect or terminate roots, and afterwards for irrational or interminate ones, to which Harriot approximates, by adding always periods of ciphers to the given number or resolvend, as far as necessary in decimals, which are continued and set down as such, but with their proper denominator 10 , or 100 , or 1000 , \&c.

He then concludes the work with

Canones Directorii,

which form a collection of the cases or theorems for making the foregoing numeral extractions, ready arranged for use, under the various forms of equations, with the factors necessary to form the several resolvends and subtrahends.

And from a review of the whole work, it appears that Harriot's inventions, peculiarities, and improvements in algebra, may be comprised in the following particulars.

1st. He introduced the uniform use of the small letters, $a, b, c, d, \& \mathrm{c}$, viz, the vowels $a, e, \& c$ for unknown quantities, and the consonants $b, c, d, f, \& c$ for the known ones; which he joins together like the letters of a word, to represent the multiplication or product of any number of these literal quantities, and prefixing the numeral coefficieht as we do at present, except only separated by a point, thus 5.bbc. For a root, he set the index of the root after the mark $\sqrt{ }$; as $\sqrt{ } S$ ) for the cube root. He also introduced the characters $>$ and $<$, for greater and Iess; and in the reduction of equations, he arranged the operations in separate steps or lines, setting the explanations in the margin on the left hand, for each line. By which, and other means, he may he considered as the introdncer of the modern state of algebra, which quite changed its form under his hands.

$2 d$. He showed the universal generation of all the compound or affected equations, by the continual multiplication of as many simple ones, or binomial roots; thereby plainly exhibiting to the eye the whole circumstances of the nature, mystery and number of the roots of equations; with the composition and relations of the coefficients of the terms; and from which many of the most important properties have since been deduced.

$3 \mathrm{~d}$. He greatly improved the numeral exegesis, or extraction of the roots of all equations, by clear and explicit rules and methods, drawn from the foregoing generation or composition of affected equations of all degrces.

OF OUGHTRED's CLAVIS.

Oughtred was contemporary with Harriot, but lived a long time after him, as he died only in 1660 , at $\$ 7$ years of age. His Clavis was first published in 1631 , the same year in which Harriot's Algebra was published by his friend Warner. In this work, Oughtred chicfly follows Vieta, in the notation by the capitals $\mathrm{A}, \mathrm{B}, \mathrm{C}, \mathrm{D}, \& \mathrm{c}$, in the designation of products, powers, and roots, though with some few variations. His work may be comprehended under the following particulars.

1. Notation. 'This extends to both algebra and arithmetic, vulgar and decimal. The algebra chiefly after the manner of Vieta, as abovesaid. And he separates the decimals from the integers thus $21\lfloor 56$, which is the first timc I have observed such a separation, and the decimals sct down without their denominator.

2. The common rules or operations of arithmetic and
A L G

algebra. In algebraic multiplication, he either joins the letters together like a word, or connects them by the mark $x$, which is the first introduction of this character of multiplication: thus $\mathrm{A} \times \mathrm{A}$ or AA or A $q$. But omiting the vinculum over compound factors, used by Vieta. He introduces bere many neat and useful contractions in multiplication and division of decimals : as that common one of inverting the multiplier, to have fewer decimals, and abridge the work; that of omitting always one figure at a time, of the divisur, for the sanic purpose; dividing by the component factors of a number insteact of the number itself; as 4 and 6 for 24 ; and many other neat contractions. He states his proportions thus $7.9:: 28.36$, and denotes continued proportion thus $\because \frac{\pi}{}$; which is the first time I have observed these characters.

3. Invents and describes variuus symbolical marks or abbreviations, which are not now used.

4. The genesis and analysis of powers. Denotes powers like Vieta, and also roots, thus $\sqrt{ } q 6, \sqrt{ } c 20, \sqrt{ } q q 24$, \&c; and much in his manner too performs the numeral extraction of roots. He here gives a table of the powers of the binomial $\mathrm{A}+\mathrm{E}$ as far as the 10 th power, with all their terms and coefficients, or uncix as he calls them, after Vieta.

5. Equations. He here gives express and particular directions for the several kinds of reductions, according as the form of the equation may require. He uses the letter $u$ after $\sqrt{ }$, for universal, instead of the vinculum of Vieta. And he observes that the signs of all the terms of the powers of $\mathrm{A}+\mathrm{E}$ are positive, but those of $\mathrm{A}-\mathrm{E}$ are alternately positive and negative.

6 . Next follow many properties of triangles and other geometrical figures; and the first instance of applying algebra to geometry, so as.to investigate new geometrical properties; also, alter the algebraical resolution of each problem, he commonly deduces and gives a geometrical construction adapted to it. He gives also a good tract on angular scctions.

7. The work concludes with the numeral solution of affected equations, in which he follows the manner of Vieta, though he is more explicit.

\section{OF DEscartes.}

Descartes's Geometry was first published in 1637 , being six years after the publication of Harriot's Algebra. That work was rather an application of algebra to geometry, than the science either of algebra or geometry itself, purely and properly so called. And yet he made improvements in both. We must observe, however, that all the properties of equations, \&c, which he sets down, are not to be considered as cven meant by himself for new inventions or discoveries; but as statements and cnumerations of properties, before known and taught by other authors, wbich he is about to make some use or application of, and for which reason it is that he mentions those properties.

Descartes's Geometry consists of three books. The first of these is, De Problematibus, quæ construi possunt, adhibendo tautum rectas lineas ct circulos. He here accommodates or performs arithmetical operations by geometry, supposing some line to represent unity, and then, by meaus of proportionals, showing how to multiply, divide, and extract roots, by lines. He next describes the notation he uses, but not because it is a new one, for it is the samc as had been used by former autbors, viz, $a+b$ for the addition of $a$ and $b$, also $a-b$ for their subtrac- 
tion, $a b$ multiplication, $\frac{a}{b}$ division, $a a$ or $a^{2}$ the square of $a, a^{3}$ its cube, \&c: also $\sqrt{a^{2}+b^{2}}$ for the square root of $a^{2}+b^{2}$, and $\sqrt{\mathrm{c} \cdot a^{3}-b^{3}+a b b}$ for the cube root, \&c, with a bar over the quantities. He then observes, after Stifelius, that there must be as many equations as there are unknown lines or quantities; and that they must be reduced all to one final equation, by exterminating all the unkuown letters except one; when the final equation will appear like these,

$$
\begin{aligned}
& z=0, \text { or } \\
& z^{2} \infty 0-a z+b^{2}, \text { or } \\
& z^{3}>0+a z^{2}+b^{2} z-c^{3}, \text { or } \\
& z^{1} \infty 0+a z^{3}+b^{2} z^{2}-c^{3} z+d^{4}, \& \mathrm{c} .
\end{aligned}
$$

Where be uses $\supset$ for $=$ or equality, setting the highest term or power alone on one side of the equation, and all the other terms on the other side, with their proper signs.

Descartes next defines plane problems, namely, such as can be resolved by right lines and circles, described on a plane superficies; and then the final equation rises only to the $2 \mathrm{~d}$ power of the unknown letter. He then constructs "such equations, viz, quadratics, by the circle, thus finding geometrically the root or roots, that is, the positive ones. But when the lines, by which the roots are determined, neither cut nor touch, he observes that the equation has then no possible root, or that the problem is impossible. He then concludes this book with the algebraical solution of the celebrated problem, before treated of by the ancients, namely, to find a point, or the locus of all the points, from which a line being drawn, to meet any number of given lines in given angles, the product of the segments of some of them shall have a given ratio to that of the rest.

Book 2. De Natura Linearum Curvarum. This is a good algebraical treatise on curve lines in general, and the first of the kind that has been produced by the nooderns. Hcre the nature of the curve is expressed by an equation containing two unknown or variable lines, and others that are known or constant, as $y^{2} \infty c y-\frac{c x y}{b}+a y-a c$. But, not relating to pure algebra, the particulars will be most properly placed under the article of curve lines, and other terms relating to them. Only one discovery, among many ingenious applications of algebra to geometry, may here be particularly noticed, as it may be considered as the first step towards the arithmetic of infinites; and that is the method of tangents, here given, or, which comes to the same thing, of drawing a line perpendicular to a curve at any point; which is an ingenious application of the gencral form of an equation, generated in Harriot's way, that has two equal roots, to the equation of the curve. Of which a particular accosnt will be given at the article TANGENTS.

Buok 3. De Constructione Problematum Solidorum, et Solida excedentium. Descartes begins this book with remarks on the nature and roots of equations, observing, that they have as many roots as dimensions, which he slows, after Harriot, by multiplying a certain number of simple binomial equations together, as $x-2 \infty 0$, and $r-3>0$, and $x-4>0$, producing $x^{3}-9 x x+26 x$ $-24>0$. He here remarks that equations may sometimes have their roots false, or what we call negative, which he opposcs to those that are positive, or as he calls them truc, as Cardan had done before. $\Delta s$ a natural deduction from the gencration or composition of equations, VOL. 1. by multiplication, be infers their resolution, or depression, or decomposition, namely, dividing them by the binomial factors which were multiplied to produce the equation: and he observes, that, by this operation it is known that this divisor is one of the binomial roots, and that there can be no more roots than dimensions, or than those which form with the unknown letter $x$, binomials that will exactly divide the equation, as Harriot had shown before.

Descartes adverts to several other properties, mostly known before, which he has occasion to make use of in the progress of his work; such as, that equations may have as many true roots as the terms have changes of the signs + and - , and as many false ones as successions of the same signs; which number and nature of the roots had before been partly shown by Cardan and Vieta, from the relation of the coefficients, and their signs, and the same thing appears more fully by Harriot's 5th section. And hence Descartes infers the method of changing the true roots to false, and the false to true, namely, by changing the signs of the even terms only, as Cardan had taught before. Descartes then adverts to other reductions and transmutations which had been taught by Cardan, Vieta, and Harriot, such as, To increase or diminish the roots by any quantity; To take away the $2 \mathrm{~d}$ term; To alter the roots in any proportion, and thence to fre the equation from fractions and radicals.

Descartes next remarks that the roots of cquationg, whether true or false, may be either real or imaginary; as in the equation $x^{3}-6 x x+13 x-10>0$, which has only one real root, namely 2 . The imaginary roots were first noticed by Albert Girard, as before mentioned. He then treats of the depression of a cubic equation to a quadratic, or plane problem, that it may be coustructed by the circle, by dividing it by some one of the binomial factors, which, in Harriot's way, compose the equation. Peletarius having shown that the simple root is one of the divisors of the known term of the equation, and Harriot that that term is the continual product of all the roots, Descartes therefore tries all the simple divisors of that term, till he finds one of them which, connected with the unknown letter $x$, by + or - , will exactly divide the equation. And the process is the same for higher powers than the cube. But when a divisor cannot be thus found, for depressing a biquadratic equation to a cubic, he gives another rule, which is a new one, for dissolving it into two qualratics, by means of a cubic equation, in this manner:

Let the given biqu. be $+x^{4}{ }_{*} \cdot p x x \cdot q x, r$ is 0 ;

Which suppose?

$\left.\begin{array}{l}\text { equal to the } \\ \text { product of } \\ \text { these two }\end{array}\right\}+x x+y x+\frac{x}{2} y y \cdot \frac{1}{2} p \cdot \frac{q}{2 y} \infty 0$,

where the sign of $\frac{T}{2} p$ in the two quadratics must be the same as the sign of $p$ in the given equation, and in the 1st quadratic the sign of $\frac{q}{2 y}$ must be the same as the sign of $q$, but in the $2 \mathrm{~d}$ quadratic the contrary. Then if there be found the root $y y$ of this cubic equation $y^{6} \cdot 2 p y^{4}+$ ${ }_{4}^{p p} y y-q q>0$, where the sign $2 p$ is the same as of $p$ in the given biquadratic, but the sign of $4 r$ contrary to that of $r$ in the same; then the value of $y$, hence deduced, being substituted for it in the two quadratic equations, and their two pairs of roots taken, they will be the four roots of the proposed biquadratic. And thus slso, be 


\section{$A L G$}

t 98

hints, may equations of the 6 th power be reduced to those of the 5th, and those of the 8th power to those of the 7 th, and so on. Descartes does not give the investigation of this rule; but it has evidently been done, by assuming indeterminate quantities, after the manner of Ferrari and Cardan, as coefficients of the terms of the two quadratic equations, and after multiplying the two together, determining their values by comparing the resulting terms with those of the proposed biquadratic equation.

After these reductions, which are only mentioned for the sake of the geometrical constructions which follow, by simplifying and depressing the equations as much as they will admit, Descartes then gives the construction of solid and other higher problems, or of cubic and higher equations, by means of parabolas and circles; where be observes, that the false roots are denoted by the ordinates to the parabola lying on the contrary sille of the axis to the true roots, as before mentioned by Girard. Finally, these constructions are illustrated by various problems concerning the trisecting of an angle, and the finding of two or four mean proportionals; which concludes this ingenious work.

From the foregoing analysis may easily be collected the real inventions and improvements made in algebra by Descartes. His work, as has been observed before, is not algebra itself, but the application of algebra to geometry, and the algebraical doctrine of curve lines, expressing and explaining their nature by algebraical equations; and, on the contrary, constructing and explaining equations by means of the curve lines. What respects the geometrical parts of this tract we shall have occasion to advert to elsewhere; and therefore shall here only enumerate the circumstances which belong more peculiarly to the science of algebra, which I shall distinguish into the two heads, of improvements, and inventions. And

1st. Of his improvements.-That he might fit equations the better for their application in the construction of problems, Descartes mentions, as it were by-the-by, many things concerning the nature and reduction of equations, without troubling himself about the first inventors of them, stating them in his own terms and manner, which is commonly more clear and explicit, and often with improvements of his own. And under this head we find that he chiefly followed Cardan, Vieta, and Harriot, but especially the last, and explains some of their rules and discoveries more distinctly, and varies but a little in the notation, putting the first letters of the-alphabet for the known, and the latter letters for the unknown quantities; a]so $x^{3}$ for $a a a, \& c$; and $\infty$ for $=$. But Herigone used the numeral exponents in the same manner some years before, as well as several older authors. Descartes explained or improved most parts of the reductions of equations, in their various transmutations, the number and nature of their roots, true and false, real and what he denominates imaginary, called involved by Girard; and the depression of equations to lower degrees.

2al. As to his inventions and discoveries in algebra, they may be comprised in these particulars : namely, the alpplication of algebra to the geometry of curve lines, the constructing equations of the higher orders, and a rule for resolving biquadratic equations by means of a cubic and two quadratics.

Having now traced the science of algebra from its origin and rudes state, down to its modern and mnre polished form, in which it has ever since continued, with very
A L G

little variation; having analysed all or most of the principal authors, in a chronological order, and deduced the inventions and improvements made by each of them; from this time the authors both become too numerous, and their improvements too inconsiderable, to merit a detail in the same minute and circumstantial way: and besides, these will be better explained in a particular manner under the word or article to which each of them severally belongs. It may therefore now suffice to enumerate, or announce only in a cursory way, the chief improvements and authors on algebra down to the present time.

After the publication of the Geometry of Descartes, a great many other ingenious men followed the same course, applying themselves to algebra and the new geometry, to the mutual improvement of them both; which was done chiefly by reasoning on the nature and forms of equations, as generated and composed by Harriot. Before proceeding upon these, however, it is but proper to take notice here of Fermat, a learned and ingenious mathematician, who was contemporary and a competitor of Descartes, for his brightest discoveries, which le was in possession of before the geometry of Descartes appeared. Namely, the application of algebra to curve lines, which he expressed by an algebraical equation, and hy them constructed equations of the $3 \mathrm{~d}$ and 4 th orders; also a method of tangents, and a method de maximis et minimis, which approach very near to the method of fluxions or increments, which they strikingly resemble, both in the manner of treating the problenss, and in the alyebraic notation and process. The particulars of which, ste under the proper heads. Besides these, Furmat. was deeply learned in the Diophantine problems, and the best edition of Diophantus's Arithmetic, is that which contains the notes of Fermat on that ingenious work.

But to return to the successors of Descartes. His geometry having been published in Holland, several learned and ingenious mathematicians, of that country, presently applied themselves to cultivate and improve it; as Schootell, Hudde, Van-Heuraet, De Witte, Slusius, Huygens, \&c; besides M. de Beaune, and perhaps some other's in France.

Francis Schooten, profcssor of mathematics in the university of Leyden, was one of the first cultivators of the new geometry. He translaterl Descartes's geometry out of French into Latin, and published it in 1649, with his commentary on it, as also Brief Notes of M. de Benune; both of them containing many ingenious and useful things. And in 1659 he gave a new edition of the same, in two volumes, with the addition of several other ingenious piecrs: as, two posthumons tracts of de Bcaune, the one on the nature and constitution, the other on the limits of cquations, showing how to assign the limits between which are contained the greatest and lenst roots of equations, cxtended and completed by Erasmus Bartholine: two letters of M. Hudde on the reduction of equatious, and on the maxima and minima of quantities, containing inany ingenious rules; anong. which are sime concerning the drawing of tangents, and on the equitl roots of equatinns, which he determines by multiplying the terms of the equation by the terms of uny arithuetical progression, 0 being one of the terins, the equation is commonly depressed one degree lower ; abo a tract of Van Heuract on the rectification of curve lines; the elements of curves by $\mathrm{De}$ Witte; Bertholine's principles of universal mathe- 
matics, or introduction to Descartes's geometry, which had before been published by itself in 1651; and to the end of the work is added a posthumous piecc of Schooten's (for he died while the $2 \mathrm{~d}$ vol. was printing) entitled Tractatus de concinnandis demonstrationibus geometricis ex calculo algebraico. Schooten also published, in 1657 , Exercitationes Mathematice, in which are contained many curious algebraical and analytical pieces, among others of a geometrical nature.

An elaborate commentary on Descartes's geometry was also published by F. Rabuel, a Jesuit; and James Bernoulli, enriched with notes, an edition of the same, printed at Basil in 169--

The celebrated Huygens also, among his great discoveries, very much cultivated the algebraical analysis: and he is often cited by Schooten, who relates divers inventions of his, while he was his pupil.

Slusius, a canon of Liege, published in 1659 , Mesolabum, seu duæ mediæ propor. per circulum et ellips. vel hyperb. infinitis modis exhibita; by which, any solid problem may be constructed by infinite different ways: And in 1668, he gave a second edition of the same, with the addition of the analysis, and a miscellaneous collection of curious and important problems, relating to spirals, centres of gravity, maxima and minima, points of inflexion, and some Diophantine problems; all sbowing him deeply skilled in algebra and geometry.

'There have been a great number of other writers and improvers of algebra, of which it may suffice slightly to mention the chief part, as in the following catalogue.

In 1619 several pieces of Van Collen, or Ceulen, were translated out of Dutch into Latin, and published at Leyden by W. Snell; among which are contained a particular treatise on surds, and his proportion of the circumference of a circle to its diameter.

In 1621 Bachet published, in Greek and Latin, an edition of Diophantus, with many notes. And another edition of the same was published in 1670 , with additions by Fermat.

In 1624 Bachet's Problemes Plaisans et Delectables, being curious problems in mathematical recreations.

In 1634 Herigone published, at Paris, the first course of mathematics, in 5 vols. 8 vo; in the $2 \mathrm{~d}$ of which is contained a good treatise on algebra; in which he uses the notation by small letters, introduced by the algebra of Harriot, which was published three years before, though the rest of it does not resemble that work, and one would suspect that Herigone had not seen it. The whole of this piece bears evident marks of originality and ingenuity. Besides + for plus, he uses os for minus, and $2 / 2$ for equality, with several other useful abbreviations and marks of his own. In the notation of powers, he does not repeat the letters like Hariot, but subjoins the numeral exponents to the letter, as Descartes did two ycars afterwards. And Herigone uses the same numeral exponents for roots, as $\sqrt{ } 3$ for the cube root.

In 1635 Cavalerius published his Indivisibles; which proved a new xa in analytics, and gave rise to other new morles of computation in analytics.

A bout 1640, et seq. Roberval made several notable improvements in analytics, which are published in the carly volumes of the Memoirs of the Academy of Sciences; as, 1, A tract on the composition of motion, and a method of tangents. 2, De recognitione aquationum. 3, De geo- metrica planarum et cubicarum equationum resolutione. 4, A treatise on indivisibles, \&c.

In 1643 De Billy published Nova Geometriæ Clavis Algebra. And in 1670 Diophantus Redivivus. He was an.author particularly well skilled in Diophantine problems.

In 1644 Renaldine published, in 4to, Opus Algebraicum, both ancient and modern, with mathematical resolution and composition. And in 1665 , in folio, the same, greatly enlarged, or rather a new work, which is very heavy and tedious. In this work Renaldine uses the parentlieses $\left(a^{2}+b^{2}\right)$ as a vinculum, instead of the line over, as $\overline{a^{2}+b^{2}}$.

In 1655 was published Wallis's Arithmetica Infinilorum, being a new method of reasoning on quantities, or a great improvement on the indivisibles of Cavalerius, and which in a great measure led the way to infinite series, the binomial theorem, and the method of fluxions. Wallis hen treats ingeniously of quadratures and many other protblems, and gives the first expression for the quadrature of the circle by an infinite series. Another series is bere added for the same purpose, by the Lord Brouncker, "the first pres. of the R.S.

In 1659 was published, at Zurich in Switzerland, Algcbra Rhonii Germanice; which was, in 1668, translated into English by $\mathrm{Mr}$. Thomas Brancker, with additions and alterations by Dr. John Pell.

In 1661 was published in Dutch, a neat piece of $\mathrm{Al}$ gebra by Mr. Kinckhuysen; which Sir Isaac Newton, while he was professor of mathematics at Cambridge, marle use of and improved, and he meant to republish it, with the addition of his method of fluxions and infinite series; but was prevented by the accidental burning of some of his papers.

In 1665 or 1666 Sir Isaac Newton made several of his brightest discoveries, though they were not published till afterwards: such as the binomial theorem; the method of fluxions and infigite series; the quadrature, rectification, Src, of curves; to find the roots of all kinds of equations, both numeral and literal, in infinite converging series; the reversion of series, \&c. Of each of which a particular account may be seen in their proper articles.

In $1666 \mathrm{M}$. Frenicle gave several curious tracts concerning combinations, magic squares, triangular numbers, \&c; which were printed in the early volumes of the $\mathrm{Me}$ moirs of the Academy of Sciences.

In 1668 Thomas Brancker published a translation of Rhonius's Algebra, with many additions by Dr. John Pell, who used a peculiar method of registering the steps in any algebraical process, by means of marks and abbreviations in a small column drawı down the margin, by which each line, or step, is clearly explained, as was before done by Harriot in words at length. This book contains a valuable table of prime numbers.

In 1668 Mercator publisbed his Logarithmotechnia, or method of constructing logarithms; in which he gives the quadrature of the hyperbola, by means of an infinite series of algebraical terms, found by dividing a simple algebraic quantity by a compound one, and for the first time that this operation was given to the public, though Newton bad before that expanded all sorts of compound algebraical quantities into infuite series.

In the same year was published James Gregory's Excrcitationes Geometrice, containing, among other O 2 
tbings, a demonstration of Mercator's quadrature of the hyperbola, by the same series.

And in the same year was published, in the Philosophical Transactions, Lord Brouncker's quadrature of the hyperbola, by unother infinite series of simple rational terms, which he harl been in possession of since the year 1657 , when it was announced to the public by Dr. Wallis. Lord Brouncker's series for the quadrature of the circle had been published by IVallis in his Arithmetic of Infinites, as before mentioned.

In 1669 Dr. Isaac Barrow published his Optical and Geometrical Lectures, abounding with profound researches on the dimensions and properties of curve lines; but particularly to be noticed liere for his method of tangents, by a mode of calculation similar to that of fluxions, or increments, from which these differ but little, except in the notation. Of these lectures, the 13 th merits the most special notice, being entirely employed on equations, and delivered in a very curious way. He there treats of the nature and number of their roots, andi the limits of their magnitudes, from the description of lines accommodated to each, viz, treating the subject as a branch of the doctrine of maxima and minima, which in the opinion of some persons is the right way of considering thern, and as far preferable to the so much boasted invention of the generation of equations from eacl other, explained by Harriot and Descartes.

In 1673 was published, in 2 vols. folio, Elements of Algebra, by John Kersey; a very ample and complete work, in which Diophantus's problems are fully explained.

In 1675 were published Nouveaux Elemens des Mathematiques, par J. Prestet, prêtre: a prolix and tedious work, which he presumptuously dedicated to God Almighty.

About 16 7 Leibnitz discovered his Methodus Differentialis, or else made a variation in Newton's Fluxions, or an extension of Barrow's method, for it is not certain which. He gave the first instance of it in the Leipsic Acts for the year 1684 . He also improved infinite series, and gave a simple one for the quadrature of the circle, in the same acts for 1682 . But this serics bad before been found by Mr. James Gregory, viz, in 1671.

In 1682 Isnael Bulliaid published, in folio, his Opus Novum ad Arithmeticam Infinitorum, being a large am • plification of Wallis's Arithmetic of Infinites.

In 1683 Tschirnausen gave a memoir, in the Leipsic Acts, conceming the extraction of the roots of all equations in a general way; in which he promised too much, as the method did not succeed.

In 1684 came out, in English and Latin, 4to, Thomas Baker's Geometrical Key, or Gate of Equations Unlock'd ; being an improvement of Duscartes's construction of all equations under the 5 th degree, by means of a circle and willy one and the same parabolit for all equations, using any diameter instead of the axis of the parabola.

In 1685 was published, in folio, Wallis's Treatise of Algebra, both Historical and Practical, with the addition of several other pieces; exhibiting the origin, progress, and advancement of that science, from time to time. It cannot be denied that, in this work, Wallis has shown too much partiality to the Algebra of Harriot. Yct, on the other liand, it is as true, that M. de Gua, in his account of it, in the Nemoirs of the Acadeny of Sciences for 1741, has run at least as far into the like extreme on the contrary side, with respect to the discoveries of Vieta; and both these I believe from the same cause, namely, the want of examining the works of all former writers or algebra, and specifying their several discoveries; as has been done in the course of this article.

In 1687 Dr. Halley gave, in the Philos. Trans. the construction of cubic and biquadratic equations, by a parabola and circle; with improvements on whst had been done by Descartes, Baker, \&c. Also, in the same Transactions, a memoir on the number of the routs of equations, with their limits and signs.

In 1690 was published, in 4to, by M. Rolle, Traité d'Algébre; in 1699, Une Methode pour la Resolution des Problcmes indeterminés; and in 1704, Memoires sur l'inverse des tangents; and other pieces.

In 1690 Joseph Raphson published Analysis Æquationum Universalis; being a general method of approximating to the roots of cquations in numbers. And in 1715 he published the History of Fluxions, toth in English and Latin.

In 1690 was also published, in 4 vols 4to, Dechale's Cursus seu mundus mathematicus; in which is a piece of algebri, of a very old-fashioned sort, considering the time when it was written.

About 1692 , and at different times afterwards, De Lagny published many pieces on the resolution of equations in numbers, with many theorems and rules for that purpose.

In 1693 was published, in a neat little volume, Symopsis Algebraica, opus posthumum Johannis Alexandri.

In 1694 Dr. Halley gave, in the Philos. Trans. an ingenious tract on the numeral extraction of all roots, without any previous reduction. And this tract is also added to some editions of Newton's Universal Arithmetic.

In $1695 \mathrm{Mr}$. John Ward, of Chester, published, in 8vo, A Compendium of Algebra, containing plain, easy, and concise rules, with examples in an casy and clear way. And in 1706 he published the first edition of his Foung Mathematician's Guide, or a plain and easy introduction to the mathematics: a book which is still in great request, especially with beginness, and which has been ever since the ordinary introduction of the greatest part of the mathematicians of this countrv.

In 1696 the Marquis de l'Hôpital published his Analyse des infiniment petits; and gave several papers to the Leipsic Acts and the Memoirs of the Academy of Sciences. He left hehind him also an ingenious treatise, which was published in 1707, entitled Traité analytique des Sections Coniques, et de la construction des lieux geometriques.

In 1697, and several other years, M. Ab. Demoivre gave various papers in the Philos. Trans. containing improvements in algebra; viz, in 1697 , a method of raising an infinite multinomial to any power, or extracting any root of the same. In 1698, The extraction of the root of an infinite equation. In 1707, Analytical solution of certain equations of the $3 \mathrm{~d}, 5 \mathrm{th}, 7 \mathrm{th}$, \& $\mathrm{c}$, degre. In 1722, Of algebraic fractions and recurring series. In 1738, The reduction of rudicals into more simple forms. Also in 1730, he published Miscellanca analytica de serichus et quadraturis, containing great juprovements in series, \&c.

In 1699 Mr. Richard Sault published, in 4to, A New Treatise of Algebra, apply'd to numeral yluestions and 
geonetry. With a converging series for all manner of Adfected Equations. The series here alluded to, is $\mathrm{Mr}$. Raphison's nethod of approximation, which had been lately published.

In 1699 Hyac. Christopher published at Naples, in 4to, De constructione æequationum.

In 1702 was published Ozanam's Algebra; which is chiefly remarkable for the Diophantine analysis. He had published his mathematical dictionary in 1691, and in 1693 his course of mathematics, in 5 vols. 8 vo, contairing also a piece on algebra.

In 1704, Dr. John Harris published his Lexicon Technicum, the first dictionary of arts and sciences : a very plain and useful book, especially in the mathematical articles. And in 1705, a neat little picce on algebra and fluxions.

In $1705 \mathrm{M}$. Guisnée published, in 4to, his Application de l'algebre a la geometrie: a useful book.

In $1706 \mathrm{Mr}$. William Jones published his Synopsis Palmariurum Natheseos, or a new introduction to the mathematics: a very useful compendium in the mathematical sciences. And in 1711 he published, in 4to, a collection of sir Isaac Newton's papers, entitled Analysis per quantitatum series, fluxiones, ac differentias: cum enumeratione linearum tertii ordinis.

In 1707 was published, by Mr. Whiston, the first edition of sir lsaac Newton's Arithmetica Universalis: sive de compositjone et resolutione arithmetica liber: and many editions have been published since. This work, it seems, was the text-book used by our great author in his lectures, while he was professor of mathematics in the university of Cambridge. And though it was never in'tended for publication, it contains many and great improvements in analytics; particularly in the nature and transmutation of equations; the limits of the roots of equations; the number of impossible roots; the invention of divisors, both surd and rational; the resolution of problems, arithmetical and geonetrical; the linear construction of equations; approximating to the roots of all equations, \&c. To the later editions of the book is commonly subjoined Dr. Halley's method of finding the roots of equations. As the principal parts of this work are not adapted to the circumstance of beginuers, there have been published commentaries upon it by several persons, as s'Gravesande, Castillion, Wilder, \&rc.

In 1708 M. Reyneau published his Analysé Demontrée, in 2 vols, 4to. And in 1714, La Scieenc du Calcul, \&c.

In 1709 was published an English translation of Alexander's Algebra. With an ingenious appendix by Humphry Ditton.

In 1715 Dr. Brooke Taylor published his Methodus Incrementorum : an ingenious and learned work. And in the Philns. Trans. for 1718, An improvement of the method of approsimating to the ruots of equations in numbers.

In $1717 \mathrm{M}$. Nicole gave, in the memoirs of the academy of sciences, a tract on the calculation of finite differences. And in several following years, he gave various other tracts on the same subject, and on the resolution of equations of the $3 d$ degree, and particularly on the irreducible case in cubic equations: those tracts are grounded on a production of $\mathrm{M}$. Leibnitz in the year 1677 .

Also in 1717 was published a Treatise on Algebra by Philip Ronayne.

Also in $1717 \mathrm{Mr}$. James Sterling published Lineæ tertii Ordinis; an ingenious work, containing good improvements in analytics. And in 1730 , Methodus Differentialis : sive tractatus de summatione et interpolatione serierum infinitarum: with great improvements on infinite series.

In 1726 and 1729 Maclaurin gave, in the Philos. Trans. tracts on the imaginary roots of equations. And afterwards was published, from his posthumous papers, his Treatise on Algebra, with its application to curve lines.

In 1727 came out s'Gravesande's Algebra, with a specimen of a commentary on Newton's universal arithmetic.

In $1728 \mathrm{Mr}$. Campbell gave, in the Philos. Trans. an ingenious paper on the number of impossible "roots of equations.

In 1732 was published Wolfius's Algebra, in his course of mathematics, in 5 vols, 4 to.

In $1735 \mathrm{Mr}$. John Kirby published his arithmetic and algebra. And in 1748 his doctrine of ultimators.

In 7740 were published Mr. Thomas Simpson's Essays; in 1743 his Dissertations, and in 1757 his Tracts; in all which are contained several improvements in series and other parts of algebra. As also in his Algebra, first printed in 174.5, and in his Select Exercises, in 1752.

Also in 1740 was published professor Saunderson's Elèments of Algebra, in 2 vols, 4 to; being a very useful work for beginners.

In $1741 \mathrm{M}$ : de la Caille published Leçons de mathematiques; ou elemens d'algebre et de geometrie.

Also in 1741 , in the Memoirs of the academy of sciences, were given two articles by M. de Gua, on the number of positive, negative, and imaginary roots of equations. Vith an historical account of the improvements in algebra; in which he severely censures Wallis for his partiality: a circumstance in which he himself is not less faulty.

In 1746 M. Clairaut published his Elemens d'algebre, in which are contained several improvements, cspecially on the irreducible case in cubic equations; chiefly after the manner of Leibnitz and Nicole. He has also several good papers on different parts of analytics, in the Memoirs of the academy of sciences.

In $1747 \mathrm{M}$. Fontaine gave, in the Mcinoirs of the academy of sciences, a paper on the resolution of equations. Besides sume analytical papers in the memoirs of other years.

II 1761 M.Castillion published, in 2 vols, 4 to, Newton's universal arithmetic, with a large commentary.

In $1763 \mathrm{Mr}$. Emerson published his Increments. In 1764 his Algeb'ra, \&c.

In $1764 \mathrm{Mr}$. Landen published his Residual Analysis. In 1765 his Mathematical Lucubrations. And in 1780 his Mathematical Memoirs. All containing good improvements in infinite series, \& $c$.

In 1770 was published, in the German langnage, Elements of Algebra by M. Euler, which have been since translated into French and English. And in 1774 a French translation of the same. The memoirs of Berlin and Petersburg academies also abound with various improvements in series and other branches of analysis, by this cclebrated mathematician.

In 1775 was published at Bologna, in 2 vols, 4 to, Compendio d'Analisi di Girólamo Seladin.

In 1797 was published, by Signor Cossali, in the Italian language, in 2 vols, 4 to, a very claborate history of the origin of the first introduction of algebra into Italy, and 
of its progress and more early improvements among his countrymen. In this work, the author clearly sliows, what however was well known long before, that the science was imported into Italy from the Arabians; but he seems erroneously to ascribe the very invention of algebra to these latter people, from his ignorance of the discoveries lately made of the writings on this science among the Hindus.

IE GENDRE.

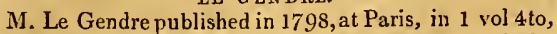
his Essai sur la Theorie des Nombres, in which all the most interesting properties of numbers are recorded, and faithfilly attributed to their respective authors, which is I believe the first book of any importance that was dedicated to this particular subject. The work is divided into four parts, each containing several theorems besides an Introduction at considerable length; and at the end of the book are arded several tables relating to the particular forms of divisors which belong exclusively to certain algebraical fornulæ, the use and application of which are explained in the body of the work.

The Introduction treats of the particular forms under which prime numbers are coritained, and the number of the latter is proved to be infinite. It is also demonstrated that no algebraical formula can express prime numbers only. Lastly, the number of prime numbers $b$, contained between unity and any given number $a$, is shown to be very nearly expressed by $b=\frac{a}{2 \log \cdot a}$.

Part 1, Contains an explanation of Continued Fractions, and their application to various cases in the indeterminate analysis; among others, a general solution is given in integer numbers to every possible indeterminate equation of the second degree, and a method of judging of the possibility, or impossibility of such equations; this solution, however, is due to $\mathrm{La}$ Grange.

The second part commences with the demonstration of several general properties of numbers, most of which are mentioned under that article in this Dictionary. The rest of this part is cmployed in discovering the forms of divisors which belong exclusively to certain algebraical formulæ, and the application of them for finding prime numbers, with an explanation of the law of reciprocity between the latter.

Part 3. Here numbers arc considered as decomposed into three squares, and many properties relating to them are demonstrated. Every number is shown to be thesum of one, two, or three triangular numbers, which is the first demonstration that was given to this part of the celebrated theorem of Fermat.

The fourth part contains the demonstration of some other properties, in which the possibility and impossibility of various formulæ are demonstrated with regard to their being particular powers, thus $x^{3} \pm y^{3}=z^{3}$ is impossible, as also $x^{4} \pm y^{4}=z^{4}$, and generally $x^{n} \pm y^{n}=2^{n}$ is impossible, when $n$ is greater than 2 , but this last has never yet been demonstrated.

In the course of this work some new symbols are occasionally introduced, which however our limits will not permit us to explain.

Several other works have been published by the same author, but as they do not properly fall under this arricle, they are omitted.

G A Uss.

Soon afur the above Essays appeared, M. Gauss pub- lished a similar work in Latin, afterwards translated into French in 1 rol, 4to, under the title of Recherches $A$ rithmetique. The subject of which is much the same as the former, these two able mathematicians having undertaken the same task at nearly the same time, without knowing each other's intentions; but here the subject is carried to a greater length than in the former. M. Gauss has by an extension of his analysis bcen able to express the side of a polygon of 17 sides inscribed in a circle by means of a quadratic equation, which was before thought to be impossible; and the same method may be applied to other polygons when the number of their sides is a prime number of a particular form. There are likewise some bints given for forming a table of logarithms, or numbers which may be employed in algebra to great advantage, as common logarithms are in arithmetical computations. In this work inany new symbols are introduced, such as $\equiv$ for congruity, that is $a \equiv b$ (to modulus $c$ ), when $a \pm b$ is divisible by $c$, and many others, which cannot be properly understood without an attentive perusal of the work itself.

We are informed by M. Gauss that he had another work in hand, which will carry this interesting subject much farther.

ARBOGAST.

M. Arbogast, professor of mathematics at Strasburgh, published in 1800 , in 1 vol, 4 to, a work entitled Du Calcul des Derivations, whieh is appropriated entirely to the doctrine of series; where their transformation, summation, interpolation, and reversion, as also various other particulars relating to this intricate branch of analysis, are treated of in a ininner, as new and ingenious, as it is profound and learned. By the method here introduced many difficulties are surmounted relating to the higher branches of astronomy, which the Calcul Differential was inadequatc to encounter with success. It seems, however, that some hints of this method was first given by Dr. Waring in his Meditationes Analytica, and which, perhaps, furnished Arbogast with the germ of his iden. In this work many new characters are introduced, not to represent quantitics, but the operations performed upon them. Thus, let $\mathrm{r}(a+x)$ be any function of the binomial $a+x$, we can expand this function in a series procecding according to the powers of $x$, in this form, $\mathrm{F}(a+x)=a+b x+\frac{c}{1,2} x^{2}+\frac{d}{1,2,3} x^{3}$ \&c. Now, wc know that $a=\mathrm{F} a$, that $b$ is derived from $a$, or from $\mathrm{F} a$; $c$ from $b, d$ from $c \& c$, by the same law; therefore, if we conceive the manner in which we derive $b$ from $a$, in the same manner we may derive $c$ from $b, d$ from $c$, \&ic. Thus representing by $D$, the operation that must be performed on $\mathrm{F} a$ in order to obtain $b$, we shall have $b=\mathrm{D} r a$, $c=\mathrm{D}^{2} \mathrm{~F} a, d=\mathrm{D}^{3} \mathrm{~F} a$ \& $\mathrm{C}$; so that the above series niay be expressed thus, $\mathrm{F}(a+x)=\mathrm{F} a+\frac{11 \mathrm{~F} a}{1} x+\frac{\mathrm{n}^{2} \mathrm{~F} u}{1 \cdot 2} x^{2}+$ $\frac{1)^{3} \mathrm{~F} a}{1.2 .3} x^{2}$ \& $\mathrm{c}$.

it is however impossible to give any thing like an adequate idea of the method, or notation, employed in this profound work, in the narrow limits to which we are under the necessity of confining our observations, which have already exceded the bounds we had at first prescribed to this articlc.

Besides the foregoing, there have been many other authors who have given treatises on algcbra, or who have 
made improvements on series and other parts of algebra ; as Schonerus, Coignet, Salignat, Laloubere, Hemischius, Degraave, Mescher, Henischius, Roberval, the Bernoullis, Malbranche, $\Lambda$ gnesi, Wells, Dodson, Manfredi, Begnault, Bezout, Bossut, Rowning, Maseres, Waring, Lorgna, de la Grange, de la Place, Bertrand, Kuhnius, Hales, and many other authors. Dr. Waring and the Rev. Mr. Vince, of Cambridge, have both given many improvements and discoveries in series, and in other branches of analysis. Those of Mr. Vince are chiefly contained in the latter volumes of the Philos. Trans.; where also are several of Dr. Waring's: but the bulk of this gentleman's improvements are contained in his separate publications, particularly the Meditationes Algebraica published in 1770; the Proprietates Algebraicarum Curvarum, in 1772; and the Meditationes Analyticæ, in 1776.

For a more enlarged history of algebra, see my Tracts, vol. 2 , tract 33 .

AlgEBRAICAL, something relating to algebra. Thus we say algebraical solutions, curves, characters or symbols, \&c.

ALgEBRa ICA C Curve, is a curve in which the general relation between the abscisses and ordinates can be expressed by a common algebraical equation. These are also called geometrical lines or curves, in contradistinction to mechanical or trinscendental ones.

ALGEBRAIST, a person skilled in algebra.

ALGENEB, or ALGENIB, a fixed star of the second magnitude, on the right side of Perseus.

ALGOL, or Medusi's Head, a fixed star of the third magnitude, in the constellation Perseus.

$\triangle L G O R A B$, a fixed star of the third magnitude, in the right wing of the constellation Corvus.

ALGORITHII, is similar to logistics, signifying the art of computing in any particular way, or about some particular.subject; or the common rules of computing in any art. As the algorithm of numbers, of algebru, of integers, of fractions, of surds, \&c; meaning the common rules for performing the operations of arithmetic, or algebra, or fractions, \&c.

ALHAZEN, Allacen, or Abprlazum, was a learned Arabian, who lived in Spain about the year 1100 , according to his editor Risner, and Weidler. He wrote upon astrology; and his work on optics was printed in Latin, at Basil, in 1572, under the title of Optica Thesaurus, by Risner. Alhazen was the first who showed the importance of refractions in astronomy, so little known to the ancients. He is also the first author who has treated on the twilight, upon which he wrote a viork, in which he likewise speaks of the height of the clouds.

ALIDADE, an Arabic nane for the label, index, or ruler, whicl is moveable about the centre of an astrolabe, quadrant, \&ce, and carrying the sights or telescope, and by which are shown the degree's cut off the limb or arch of the instrument.

ALIQUAN'l' part, is that part which will not exactly measure or divide the whole, without leaving some remainder. Or the aliquant part is such, as being taken or repeated any number of times, does not make up the whole exactly, but is either greater or less than it. Thus 4 is an aliquant part of 10 ; for 4 , twice taken makes 8 , which is less than 10 ; and three times taken makes 12, which is greater thas 10 .

ALIQUO' part, is such a part of a number as will exactly divide it without a remainder. Or the aliquot part is such, as being taken or repeated a certain number of times, exactly makes up, or is equal to the whole. So 1 is an aliquot part of 6 , or of any other whole number; 2 is also an aliquot part of 6 , being contained just 3 times in 6 ; and 3 is also an aliquot part of 6 , being contained just 2 times; so that all the aliquot parts of 6 are $1,2,3$.

All the aliquot parts of any number may be thus found: Divide the given number by its least divisor; then divide the quotient also by its least divisor; and so on, always dividing the last quotient by its least divisor, till the quotient 1 is obtạined; and all the divisors, thus taken, are the prime aliquot parts of the given number. Then multiply continually together these prime divisors, viz, every two of them, every three of them, every four of them, \&c; and the products will be the other or compound aliquot parts of the given number. So, if the aliquot parts of 60 be required; first divide it by 2 , and the quotient is 30 : then 30 divided by 2 also gives 15 , and 15 divided by 3 gives 5 , and 5 divided by 5 gives 1 : so that all the prime divisors or aliquot parts are $1,2,2,3,5$. Then the compound ones, by multiplying every two, are $4,6,10,15$; and every three 10,20, 30. So that all the aliquot parts of the given number 60 , are $1,2,3,4,5,6,10,12,15$, 20,30 .- In like manner it will be found that all the aliquot parts of 360 , are $1,2,3,4,5,6,8,9,10,12,15$, $18,20,24,30,36,40,45,60,72,180$.

ALLEN (THOMAS), a celebrated mathematician of the 16 th century. He was born at Uttoxeter in Staffordshire, in 1542 ; was admitted a scholar of Trinity college, Oxford, in 1561; where he took his degree of master of arts in 1567 . In 1570 he quitted his college and fellowship, and retired to Glocester hall, where he studied very closely, and became celebrated for his knowledge in antiquity, philosophy, and mathematics. He received an invitation from Henry earl of Northumberland, the great friend and patron of mathematicians, and he spent some time at the earl's house; where he became acquainted with those able mathematicians Thomas Harriot, John Dee, Walter Warner, and Nathaniel 'Turporley. Robert earl of Leicester, too, had a great esteen for Allen, and would have conferred a bishopric upon him; but his love for solitude and retirement made him decline the offer. His great skill in the mathematics gare occasion to the ignorant and vulgar to look upon him as a magician or conjurer. Allen was very curious and indefatigable in collecting scattered manuscripts relating to histury, antiquity, astronomy, philosuphy, and mathematics: which collections have beeti quoted by several learned authors, and mentioned as in the Bibliotheca Alleniana. He published in Latin the second and third books of Ptolemy, Concerning the judgement of the Stars, or, as it is usually called, of the quadripartite construction, with an exposition. He wrote also notes on many of Lilly's books, and some on John Bale's work, De scriptoribus Maj. Britanniæ. He died at Glocester hall in 1632 , at 90 years of age.

Mr. Burton, the author of his funeral oration, calls him the very soul and sun of all the mathematicians of his age. And Selden mentions him as a person of the most extensive learning and consummate judgment, the brightest ornanient of the university of Oxford. Camden also says he was skilled in most of the best arts and sciences. A. Wood has also transcribed part of his character from a manuscript in the library of Trinity college, in these words: "He studied polite literature with great application; he was strictly tenacious of academic discipline; 


\section{A L L}

always highly esteemed both by foreigners and those of the university, and by all of the highest stations in the church of England, and the university of Oxford. He was a sagacious observer, an agreeable companion, \&c." See Wood, vol. i, p. 492 .

ALLIGATION, one of the rules in arithmetic, by which are resolved questions which relate to the compounding or mixing together of divers simples or ingredients, being so called from alligare, to tie or connect together, probably from certain vincula, or crooked ligatures, commonly used to connect or bind the numbers together:

It is probable that this rule came to us from the Moorish or Arabic writers, as we find it, with all the other rules of arithmetic, in Lucas de Burgo, and other early authors in Europe.

Alligation is of two kinds, medial and alternate.

Alligation medial is the method of finding the rate or quality of the composition, from having given the rates and quantities of the simpless or ingredients.

The rule of operation is this : multiply each quantity by its rate, and add all the products together; then divide the sum of the products by the sum of the quantities, or whole compound, and the quotient will be the rate sought.

For example; Suppose it were required to mix together 6 gallons of wine, at $5 s$. a gallon; 8 gallous, at $6 s$. the gallon; and 4 gallons, at 8s. the gallon; and to find the worth or value, per gallon, of the whole mixture.

$$
\begin{aligned}
& \text { Here } \cdot \stackrel{\text { Gall. }}{6} \times 5=\text { s. }=30 \\
& 8 \times 6=48 \\
& 4 \times 8=32 \text {. } \\
& \text { whole comp. } \overline{18} \quad \overline{110} \text { sum of prod. } \\
& \text { Then } 18) 110\left(6 \frac{2}{18} \text { or } 6 \frac{x}{9} s\right. \text {, is the rate sought. } \\
& \frac{108}{2}
\end{aligned}
$$

ALLIGATION alternate is the method of finding the quantities of ingredients or simples, necessary to form a compound of a given rate.

The rule of operation is this: 1st, Place the given rates of the simples in a column, under each other. 2d, Connect or link with a continued line, each rate which is less than the proposed compound rate, with one or any number of those that are greater; and every greater rate with one or any number of the less. $3 \mathrm{~d}$, Write the difference between the given compound rate and that of cach simple opposite the rates with which they are linked. $4 \mathrm{th}$, Then if only one difference stand opposite any rate, it will be the quantity belonging to that rate; but when there are several, their sum will be the quantity.

For example, Suppose it were required to mix together gold of various degrees of fineness, viz, of 19 , of 21 , and of 23 carats fine, so that the mixture shall be of 20 carats fine. Here,

$$
\text { Comp. rate } 20\left\{\begin{array} { l } 
{ \text { Rates } \ldots } \\
{ 2 1 ) \cdots 1 } \\
{ 1 9 - \ldots 1 + 3 } \\
{ 2 3 ) \ldots 1 }
\end{array} \left\{\begin{array}{c}
1 \text { of } 21 \text { carats fine, } \\
4 \text { of } 19 \text { carats fine, } \\
1 \text { of } 23 \text { carats fine. }
\end{array}\right.\right.
$$

That is, there must be an equal quantity of 21 and 23 carats fine, and 4 times as much of 19 carats fine.

Various linitations, both of the compound and the ingredients, may be conccived; and in such cases, the differences are to be altered proportionally.

Questions of this sort are however cummonly best and casiest resulved by commun algebra, of which they form
A L M

a species of indeterminate problems, as they adnit of many, or an indefinite number of answers.

There is recorded a remarkable instance of a discovery made by Archimedes, both by alligation and specific gravity at the same time, namely, cuncerning the crowil of Hiero, king of Syracuse. The king had ordered a crown to be made of pure gold; but when brought to hin, a suspicion arose that it was nixed with alloy of either silver or copper, and the king recommended it to Archimedes to discover the cheat,' without defacing the crown. Archimedes, after long thinking on the matter, without lighting on the means of doing it, being one day in the bath, and observing how his body raised the water higher, conceived the idea that different metals of the same weight would occupy different spaces, and so raise or expel different quantities of water. Upon which he procured two other masses, each of the same weight with the crown, the one of pure gold, and the other of alloy; then immersing them all three, separately, in water, and observing the space each occupied, by the quantity it raised the water, he thence computed the quantities of gold and alloy contained in the crown.

ALLIOTH, a star in the tail of the great bear. The word in Arabic denotes a horse; and they gave this name to each of the three stars, in the tail of the great bear, as they are placerl like three horses, thus arranged for the purpose of drawing the waggon commonly called Charles's wain, represented by the four stars on the body of the same constellation.

ALMACANTAR, or ALMICANTAR, from the Arabic almocantharat, are circles parallel to the horizon, and conceived to pass through every degree of the meridian; serving to show the height of the sun, inoon, or stars, \&c ; and are the same as the parallels of altitude.

AlMaCantar's Staff, was an instrument formerly used at sea for observing the sun's amplitude at rising or setting, and thence to determine the variation of the compass, \&.c.

ALMIAGEST, the name of a celebrated book cumposed by Ptolemy; being a collection of a great number of the observations and problens of the ancients, relating to geometry and astronomy, but especially the latter. Being the first work of this kind which has come down to us, and containing a catalogue of the fixed stars, with their places, besides numerous recurds and observations of eclipses, the motions of the planets, \&c, this work will ever be held dear and valuable to the cultivators of astrononiy.

In the original Greek it is called $\sigma v \tau_{\alpha} \xi_{5}, \mu \varepsilon \gamma / \sigma \tau \%$, the great composition or collection. And to the word $\mu E \gamma i \sigma \tau \gamma$, megiste, the Arabians joined the particle $a l$, and thence called it Almaghesi $i$, or, as we call it, frons them, the Almagest.

Ptolemy was born about the year of Christ 69 , and died in 147: he wrote this work, consisting of 13 books, at Alexandria in ligypt, where the Arabians found it on the capture of that kingdom. It was by them translated out of Greek, into Arabic, by order of the caliph Almismon, about the year $\mathrm{S} 27$; and first into Latin about 1230 , by fivour of the emperor Frederic II. The Greck text howerer was not known in Europe till about the beginning of the 15 th century, when it was brought from Constantinople, then taken by the Turks, by George, a monk of 'Trabezond, who translated it into Litin, which trauslation has several times been published. 
Riecioli, an Italian jesuit, also published, in $\mathbf{1 6 5 1}$, a body of Astronomy, which, in initation of Ptolemy, he called Almagestum Novum, the New Almagest; being a large collection of ancient and modern observations and discoveries, in the seience of astronomy.

ALMAMON, caliph of Bagdat, a philosopher and astronomer, in the beginning of the 9th century, having aseended the throne in the year 814. He was son of Harun Al-Rashid, and grandson of Almansor. His name is otherwise written Mamon, Almaon, Almamun, Alamoun, or Al-Maimon. Having been educated witl great care, and possessing a love for the liberal seiences, he applied himself to eultivate and encourage them in his own country. For this purpose he requested the Greek emperors to supply him with sueh books on philosoply as they had among them; and he collected skilful interpreters to translate them into the Arabic language. He also eneouraged his subjects to study them; frequenting the meetings of the learned, assisting at their exereises and deliberations. He caused Ptolemy's Almagest to be translated in 827 , by Isaac Ben-honain, and Thabet Ben-korah, aecording to Herbelot, but aceording to others by Sergius, and Alhazen, the son of Joseph. In his reign, and doubtless by his encouragement, an astronomer of Bagdat, named Habash, composed three sets of astronomical tables.

Almamon himself made many astronomical observations, and determined the obliquity of the ecliptic to be then $23^{\circ} 35^{\prime}$ (or $23^{\circ} 33^{\prime}$ in some manuscripts), but Vossius says $23^{\circ} 31^{\prime}$ or $23^{\circ} 34^{\prime}$. He also caused skilful observers to procure proper instruments to be made, and to exercise themselves in astronomical observations; which they did accordingly at Shemasi in the province of Bagdat, and upon Mount Casius near Damas.

Under the auspiees of Mamon also a degree of the meridian was measured on the plains of Sinjar or Sindgiar (or according to some Fingar), on the borders of the Red Sea; by which the degree was found to contain $56 \frac{2}{3}$ miles, of 4000 coudces each, the condce being a foot and a half: but it is not known what foot is here meant, whether the Roman, the Alexandrian, or some other. Riccioli makes this measure of the degree amount to 81 ancient Roman miles, which value answers to 62046 French toises; a quantity more than the true value of the degree by almost one-third.

Finally, Mamon revived the seiences in the East to such a degree, that many learned men were found, not only in his own time, but after him, in a country where the study of the seienees had been long forgotten. This learned king died near Tarsus in Ciilicia, by having enten too freely of some dates, on his return from a military expedition, in the year 833 .

ALIINAC, a ealendar or table, in which are set down and marked the days and feasts of the year, the common ecclesiastical notes, the course and phases of the moon, \&c, for each month: and answers to the fasti of the ancicut Romans.

The rtymology of the word is much controverted among grammarians.-Some derive it from the Arabic, viz, from the particte al, and manah, to count. While Scaliger, and others, derive it from the same al, and the Greek pavainos, the eourse of the inonths. But Golius eontroverts these opinions, and aseribes the word to another origin, though he still malse's it of Arabic extract, which it more evidently is. IIe says that, in the liast, it is the custom for the people, at the beginning of the yeur, to

VOL. I. make presents to their prinee; and that, among the resk, the astrologers present them with their almanaes, or ephemerides, for the year ensuing; whence these came to be called almanha, that is, new-year's gifts. But this derivation seems rather strained and improbable; for, by the same rule, the gifts or productions of other artists, or classes of men, might also be called almanaes. There are other guesses at the etymology; and Verstegan writes the word almonac, and makes it of German original. Our ancestors, he observes, used to carve the courses of the moon, for the whole year, upon a square piece of wood, which they ealled al-monaght, which is as much as to say, in old English or Saxon, all-moon-heed.

An instrument of this kind, it scems, of a very ancient date, is to be seen in St. John's college at Cambridge; and that in the midland counties there are still several remains of them. An engraved figure of one of them, with a particular description, may be seen in Brady's Clavis Calendaria, vol. $1,1812$.

Almanacs are of various kinds and composition, some books, others shects, \&c, some annual, others perpetual. The essential part is the calendar of months, weeks, and days; the motions, changes, and phases of the moon; with the rising and setting of the sun and moon. 'To these are commonly added various matters, astronomical, asirologieal, chronological, meteorologieal, and even political, rural, medieal, \&e ; as also eelipses, solar ingresses, "aspects, and configurations of the heavenly bodies, lunations, helioeentric and geoeentric motions of the planets, prognostications of the weather, predictions of other events, the tides, twilight, equation of time, \&c.

Till about the 4 th eentury, almanaes bore the marks of heathenism only; from thence to the 7 th century, they were a mixture of heathenisn 2 and Christianity; and ever since they have been altogether Christian : but at all times astrological and other predictions have been considered as an 'ssential part, and are so to this day with several of them, notwithstanding that most people affect to disbelieve in such predictions.

Natuicul ALMANAC, and Astronomical Ephemeris, is a kind of national almanae, ehiefly for nautical purposs, which was begun in the year 1767 under the direction of the Board of Longitude, on the recommendation of the late worthy Astronomer Royal, Dr. Maskelyne, who had the inmediate conducting of it. It is still published by anticipation for several years before-band, for the convenence of ships going out upon long voyages, for which purpose it is highly useful, and was found eminently so in the course of the late voyages round the world for making discoveries. Besides most things essential to general usc, that are to be found in other almanacs, it contains many new and important particulars; more especially, the di. stanees of the moon from the sun and lixed stars, which are computed for the meridian of the Royal Observatory of Greenwieh, and set down to every three hours of time, expressly designed for eomputing the longitude at sea, by comparing these with the like distane's observed there.

For an explanation of the Hindu almanacs, sec Philos. Trans. Abridg. vol. 17, p. 250, \&c.

ALPHONSINE T'ables, are astronomical tables compilerl by order of Alphonsus, king of Castile, in the compiling of which, it is thought that prince himself assisted. See Astronomical Tubles.

ALPHONSUS the 10ih, king of Leon and Castile, who has been surnamed 'l'he Wise, on account of his attachment $\mathrm{P}$ 


\section{A L P}

[ 100

to literature, and is now more celebrated for having been an astronomer than a king. He was born in 1203; succeeded his father Ferdinand the $3 \mathrm{l}$, in 1252 ; and died in 1284 , at the age of 81 .

The affirs of the reign of Alphonsus were very extraordinary, and unfortunate for lim. But we shall here only.consider him in that part of bis character, on accoust of which he has a place in this work, namely, as an astronomer and man of letters. He understood astronony, philosophy, and history; and composed books upon the motions of the heavens, and on the history of Spain, which are highly commended. "What can be more surprising," says Mariana, "than that a prince, educated in a camp, and handling arms from his childhood, should have such a knowledge of the stars, of philosophy, and the transactious of the world, as men of leisure can scarcely acquire in their retirements? There are extant some bonks of Alphonsus on the motions of the stars, and the history of Spain, writien with great skill and incredible care." In his astronomical pursuits he discovered that the tables of Ptolemy were full of errors; and thence he conceived, the first of any, the resolution of correcting them. For this purpose, about the year 1240, and duriug the life of his father, he assembled at Toledo the most skilful astronomers of his time, Christians, Moors, or Jews, when a plan was formed for constructing new tables. This task was accomplished about 1252, the first year of his reign; the tables being drawn up chiefly by the skill and pains of Rabbi Isaac Hasan, a learned Jew, and the work called the Alphonsine Tubles, in honour of the prince, who was at vast expenses concerning them. He fixed the epoch of the tables to the 30th of May 1252, being the day of his accession to the throne. They were printed for the first time in 1483 , at Venice, by Radtolt, who excelled in printing at that time; which edition is extremely rare: there are others of $1492,1521,1545$, \&c. (Weiller, p. 280.)

We must not omit a memorable saying of Alphonsus, which has been recorded for its boldness and pretended impiety; namely, " that if he had been of God's privy council when he made the world, he could have advised him better." Mariana however says only in general, that Alphonsus was so buld as to blame the works of Providence, and the construction of our bodies; and he says that this story concerning him rested only on a vulgar tradition. The jesuit's worts are curious: "Emanucl, the uncle of Sanchez (the son of Alphonsus), in his own name, and in the name of other nobles, deprived Alphousus of his kingrlom by a public sentence: which that prince merited, for daring severely and boldly to censure the works of Divine Providence, and the construction of the human body, as tradition says he did. Heaven most justly punished the folly of his tongue." Though the silence of such an historian as Mariana, in regard to Ptolemy's system, ouglit to be of some weight, yet we cannot think it improbable, that if Alphonsus did pass so bold a censure on any part of the universe, it was on the celestial sphere, and merat to glance upon the contrivers and supporters of that system. lior, besides that he studied nothing more, it is certain that at that time astronomers explained the motions of the heavens by intricate and confused hypotheses, which did no honour to God, nor anywise answered the idea of an able operator. So that, from considering the multitule of splacrers composing the system of Ptolemy, and those numerous eccentric cycles and epi-
A L T

cycles with which it is embarrassed, if we suppose Alphonsus to have said, "That if God had asked his advice when lie made the world, he would have given him better council," the boldness and impiety of the censure will be greatly diminished, if not entirely removed, as probably meant for a satirical sncer at the intricate devices and contrivances of mortals.

ALSTED (JoHn-HENRY), a German protestant divine, and one of the nost indefatigable writers of the 17 th century. He was some time professor of philosophy and divinity at Herborn, in the county of Nassau: from thence he went into Transylvania, to be professor at Alba Julia; where he continued till his death, which happened in 1638 , at 50 years of age. He applied himself chiefly to compose methods, and to reduce the several branches of arts and sciences into systems. His Encyclopadia has been much esteemed, even by Roman catholics: it was printed at Lyons, and sold very well throughout all France. Vossius mentions the Encyclopædia in general, but speaks of his treatise of Aritbmetic more particularly, and allows the author to have been a man of great reading and universal erudition. His Thesaurus Chronologicus is by some esteemed one of his best works, and has gone throtigh several editions, though others speak of it with contempt. In his Triumphus Biblicus, Alsted endeavours to prove, that the materials and principles of all the arts and sciences may be found in the Scriptures; but he gained very few to his opinion. John Himmclius wrote a piece against his Theologia Polemica, which was one of Alsted's best performances. It seems he was a millenarian, having published, in 1672 , a treatise De Mille Annis, in which he asserts that the faithful shall reign with Jesus Christ upon earth a thousand years; after which will be the general resurrection, and the last judgment; and he pretended that this reign would commence in the year 1694.

ALTERNATE angles, in Geometry, are the internal angles, $A$ and $\mathrm{B}$, or $\boldsymbol{u}$ and $b$, made by a line cutting two parallels, and lying on opposite sides of the cutting line. It is the property of these angles to be always equal to each other, namely the angle $\Lambda=$ the angle $B$, and the angle

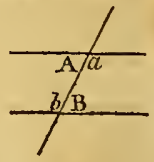
$a=$ the angle $b$. And the exterior alternate angles are also equal.

Aztenvate Ratio or Proportion, is the ratio of the one antecedent to the other, or of one consequent to the other, in any proportion, in which the quantitics are of the same kind.

So if $A: B:: C: D$, then ilternately, or by alternation, $\mathrm{A}: \mathrm{c}:: \mathbf{B}: \mathrm{D}$.

ALTIRNATION, or Permutation, of quantities or things, is the varying or changing the order or position of them.

$A$ s suppose two things $a$ and $b$; these may be placed either thus, $a b$ or $b a$, that is two ways, or $1 \times 2$. li there be three things, $a, b, c$ : then the $3 d$ thing $c$, may be placed threc diflerent ways with respect to cich of the two positions $a b$ and $b a$ of the other two things; it may stand either before them, or between them, or after them both, that is, it may stand either $1 \mathrm{st}, 2 \mathrm{~d}$, or $\mathrm{Sd}$; and therefore, with three things there will be thre times as many changes as with two, that is $1 \times 2 \times 3$ or 6 changes with three tlings. Again, if there be four things $a, b, c$, $d$; then the fourth thing $d$ may be placed in four difierent ways with respect to each of the six positions of the other three; for it may be set eitluer 1 st or $2 \mathrm{~d}$ or $3 \mathrm{~d}$ or 4 th in 
the order of each position; consequently, from four things there will be four times as many alternations as there are from three things; and therefore $1 \times 2 \times 3 \times 4=24$ is the number of changes with four things. And so on, always multiplying the last found number of alternations by the next number of things; or to find the number of changes for any number of things, as $n$, multiply the series of natural numbers $1,2,3,4,5$, \&c, to $n$, continually together, and the last product will be the number of alternations sought; as, $1 \times 2 \times 3 \times 4 \times 5 \cdots-n$ is the number of changes in $n$ things.

So if, for example, it were-required to find how many changes may be rung on 12 bells; it would be

$1 \times 2 \times 3 \times 4 \times 5 \times 6 \times 7 \times 8 \times 9 \times 10 \times 11 \times 12$ $=479001600$, the number of changes. Now supposing there might be rung 10 changes in one minute, that is $10 \times 12$ or 120 strokes in a minute, or 2 strokes in each second of time; then, according to this rate, it would take upwards of 91 years, without ever ceasing day or night, to ring over all these changes on the 12 bells only. Also, if but two more bells were added, making 14 bells; then, at the same rate of ringing, it would require about 16575 years to ring all the changes on the 14 bells but once over. And if the number of bells were 24, it would réquire more than 117000000000000000 years to ring all the different changes upon them!

ALTIMETRY, ALTIMETRIA, the art of taking or measuring altitudes or heights, whether accessible or inaccessible. Or Altimetria is that part of practical geometry which relates to the theory and practice of measuring both heights and depths, either in perpendicular or oblique lines.

AL'TING (JAMES), was born at Heidelberg in 1618. He travelled into England in 1640 , where he was ordained by the learned Dr. Prideaux, bishop of Worcester. He afterwards succeeded Gomarus in the professorship of Groninghen. He died in 1697; and recommended the edition of his works to Menso Alting (author of Notitia German. Infer. Antiqua); but they were published in 5 vols folio, witl his life, by Bekker of Amsterdam. They contain various analytical, exegetical, practical, problematical, and philosophical tracts, which show his great industry and knowledge.

ALTITUDE, in Geometry, is the height of an object, or its elevation above that plane to which its base is referred; so that the altitude of the same object is different in different places; thus, two persons taking the height of a mountain, being on contrary sides of it, will each consider its base as on a level with the place where he stands, and therefore in many cases they will give different results.

ALTITUDE of a figure, is the distance of its, vertex from the base, or the length of a perpendicular let fall from its vertex to the base. The altitudes of figures are necessary to be known in computing their areas or solidities.

AlTituDE, or Height of any point of a terrestrial object, is the perpendicular let fall from that point to the plane of the horizon.

To measure the ALTITUDE of bodies or oljects. If this cannot be done by stretching a line from top to bottom, which is the direct and nost accurate way, then some indirect way is used, by measuring some other line or distance which muy scrve as a basis, in conjunction with angles, or proportional lines, either to compute, or geometrically determine, the altitude of the object sought.

There are various ways of measuring altitudes, or depths, by means of different instruments, and by shadows or reflected images, on optical principles. There are also various ways of computing the altitude in numbers, from the measurements taken as above, either by geometrical construction, or trigonometrical calculation, or by simple numerical computation from the property of parallel lines, \&c.

The instruments mostly used in measuring altitudes, are the quadrant, theodolite, geometrical square, line of shadows, \&c; the descriptions of each of which may be seen under their respective names.

To measure an altitude geometrically, when we can have access to the base of the object. Suppose the height of the accessible tower А В be required. First, by means of two rods, or staves, of different lengths. Plant the longer staff perpendicularly at $\mathbf{C}$; then move the shorter backwards, till by trials you find such a place, $D$, that the eye placed at the top of it at E, may see the top of the other, $F$, and that of the object $\mathbf{B}$ in a straight line : next measure the distances DA Or EG and $\mathrm{DC}$ or $\mathbf{E H}$, also $\mathrm{HF}$ the difference between the heights of the rods: then, by similar triangles; as EH : EG : : HF : the 4 th proportional GB; to which add $A G$ or $D E$, and the sum will be the whole altitude A B sought. Or, as EH : IA : : F F : BA.

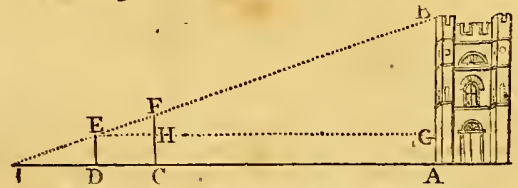

Or, with one rod CF only. Plant it at such a place $c$, that the cye at the ground, or near it, at $\mathbf{r}$, may see the tops $F$ and $\mathrm{E}$, in a right line: then, having measured $I C$, IA, CF, the 4th proportion to these will be the altitude AB sought.

Or thus, by means of shadows. Plant a rod $a b$ perpendicularly at $a$, and measure its shadow $a c$, as also the shadow $\triangle C$ of the object $A B$, at the same instant; then the 4 th proportional to $a c, a b, A \mathrm{C}$ will be the altitude $A B$ sought. For the angles at $B$ and $c$ being equal, the triangles are similar.

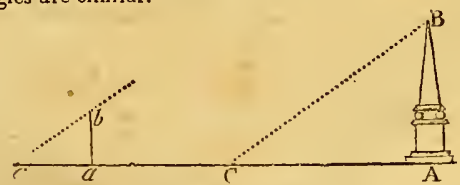

Or thus, by means of optical reflection. Place a vessel of water, or a mirror or other reflecting surface, horizontal at $\mathrm{C}$; and go to such a distance, $\boldsymbol{v}$, that the eye $\mathrm{E}$ may see the image of the top of the object in the mirror at $\mathrm{c}$ : then, by similar figures, $C D: D E:: C A: A B$ the altitucle sought. This depends on a property in optics, namely, that the angle of incidence is equal to the angle of reflection, that is, the angle $\mathrm{BCA}$ is equal to the angle $\mathrm{keD}$; and the augles at $A$ and $D$ bcing right angles, the two triangles are similar: hence the above proportion. Sice Optics. 
A L T

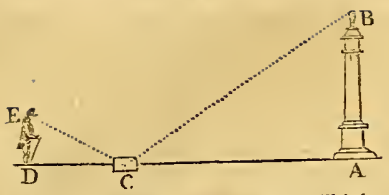

Or thus, by the geometrical square. Which consists of a plain piece of board, a foot square, one of the sides of which, as GF, is divided into a number of equal parts, and having a plumb line suspended at the angular point $\mathrm{E}$, with two sights or a telescope placed on the side DE; and then the whole fixed to a stand at $D$. At any place, as at $\mathrm{c}$, fix the stand, and turn the square about the centre of inotion, $D$, till the eye there sec the top of the object through the sights or telescope on the side $D E$ of the square, and note the number of divisions cut off the other side by the plumb line $\mathrm{EG}$ : then, by similar triangles, as $E F: F G:: D H: H B$; to which add $A H$ or $C D$, for the whole height $\mathrm{AB}$. If the side $\mathrm{GF}$ be divided into 100 equal parts, we have only to multiply the length of $\mathrm{DH}$ in feet by the number of parts cut off by the plumb line, and to cut off two figures from the product for decimals; and we shall have the height of $\mathrm{BH}$ in feet.

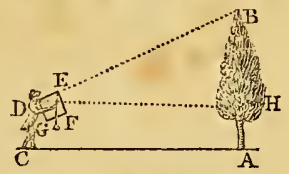

To measure the altitude of an accessible object trigonometrically. At any convenient station, c, with a quadrant, theodolite, or other graduated instrument, observe the angle of elevation $\mathrm{ACB}$ above the horizontal line $\mathrm{AC}$ : and measure the distance $A \mathrm{C}$. Then, $\mathrm{A}$ being a right angle, it will be, as radius is to the tangent of the angle $c$, so is AC to A B sought.

If $\mathrm{AC}$ be not horizontal, but an inclined plane; then the angle above it must be observed at two stations $\mathrm{C}$ and $\mathrm{D}$ in a right line, and the distances $A \mathrm{C}, \mathrm{CD}$ both measured. Then, from the angle $\mathrm{c}$ take the angle $\mathrm{D}$, and there remains the angle are given the angles and the side $\mathrm{DC}$, to find the side св; and then in the triangle $A B C$, are given the two sides $C_{A}$ and $C B$, with the included angle $C$, to find the third side $\mathrm{AB}$.
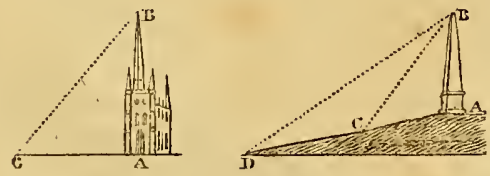

Or thus, measure only the distance $\Lambda \mathrm{c}$, and the angles $A$ and $\mathrm{C}$ : then, in the triangle $\triangle \mathrm{BC}$, are given all the angles and the side $\mathrm{AC}$, to find the side $\mathrm{AB}$.

To meusure the altitude of an inatcessible object, as a hill, cloud, or otber body. 'This is commonly done, by observing the angle of its altitude at two stations, and ineasuring the distance between them. 'Thus, for the leeight $A B$ of a lill, measure the distance $C D$ at the foot of $i t$, and observe the quantity of the two angles $\mathrm{C}$ and $\mathrm{D}$.
A L T

Then, from the angle $\mathrm{c}$ taking the angle $\mathrm{D}$, leaves the angle Св D; hence

As sine $\angle C B D:$ sine $\angle D:: C D: C B$; and

As rad. : sine $\angle A C B:$ : $C B: A B$, the altitude.

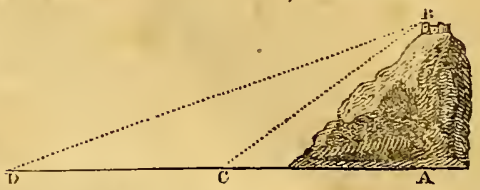

And for a balloon, or cloud, or other moveable object $\mathrm{c}$, let two observers at $\mathbf{A}$ and $\mathbf{B}$, in the same plane with $\mathbf{c}$, takc at the same time the angles $A$ and $B$, and ineasure the distance A $\mathrm{B}$ between them; then

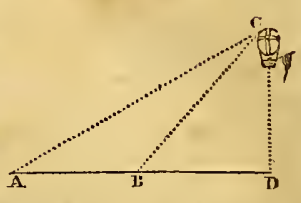
calculate the altitude $\mathrm{CD}$ exactly as in the last example.

It must however be by mere accident that $A, B$, and $c$, are in the same vertical plane; and consequently this method, though it be true in theory, is of no use in practice: we will therefore show how the altitude may be obtained when the observers are not in a plane with the object.

In this case, there must be three observers, who take the angle of elevation at the same instant, being at given distances from each other in the same right line, as at $\Lambda$, $\mathrm{B}$, and $\mathrm{C}$, in the annexed figure; now admitting those three angles to be known, it is required to find the altitude of the balloon.

Demit the perpendicular D P to the horizontal plane $A C P$, and draw the other lines as in the figure. Make $t, t^{\prime}, t^{\prime \prime}$, represent respectively the nat. cotan. of the angles observed at $\mathrm{A}, \mathrm{B}$, and $\mathrm{C}$; also put $\mathrm{AB}=a, \mathrm{BC}=b$, and $\mathrm{AC}=c$; and call $\mathrm{DP}=x$. Then $\mathrm{AP}$

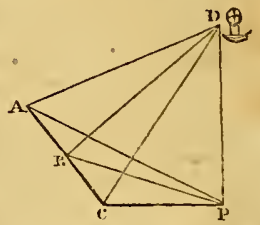
$=t x, \mathrm{BP}=t^{\prime} x$, and $\mathrm{CP}=t^{\prime \prime} x$ : hence from the common

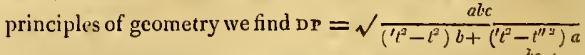
for the altitude sought. If $\angle A=\angle B$, then $D P=\sqrt{\frac{b c}{t^{3}-t^{\prime \prime 3}}}$; if $\angle \mathrm{B}=\angle \mathrm{C}$, then $\mathrm{DP}=\sqrt{\frac{a c}{t^{2}-t^{\prime \prime}}}$; and if $a=b$, then will $\mathrm{DP}=\sqrt{\frac{\frac{1}{2} c^{2}}{2 t^{\prime 2}-t^{3}-t^{\prime \prime 2}}}$.

To find the leight of an object, by knowing the utmost distance at which its top can be just seen in the horizon. As, suppose the top 11 of a tower Fu can be just scen from $E$ when the distance $\mathrm{E} F$ is 25 miles, supposing the circumference of the earth to be 25000 milcs, or the

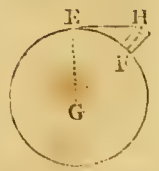
radius 3979 miles or 21009120 feet. First, as 25000 : $25:: 360^{\circ}: 21^{\prime} 36^{\prime \prime}$ equal to the angle $\mathrm{G}$; then as radius : scc. $\angle G::$ EG : (ill, which will be found to be 21009536 feet; from which take ra or $\mathrm{GR}$, ind there remains $416 \mathrm{fect}$, for $\mathrm{F}$ the licight of the tower sought.Or rather thus, as 10000000 madius : $198=$ scc. $\angle 0-$ radius : : $21009120=\mathrm{EC}: 416=\mathrm{r} 11$, as before. 
Or the same may be found casier thus: The horizon dips nearly 8 inches or $\frac{2}{3}$ of a foot at the distance of 1 mile, and according to the square of the distance for other distances; therefure as $1^{8}$ or $1: 25^{2}$ or $625:: \frac{2}{3}$ : $\frac{2}{3}$ of 625 or 416 feet, the same as bcfore.

Or conversely, to find the distance $\mathrm{s} F$, from the known leight $F 11$ : Here $\frac{2}{3}: 1$, or as $2: 3:: 416: 625$, the square root of which gives 25 miles, for the distance $\mathbf{E F}$. That is, in general, take the square root of $\frac{3}{2}$ of the altitude, in feet, for the distance in miles.

There is a very easy method of taking great terrestrial altitudes, such as mountains, or the heights to which balloons ascend, \&zc, by means of the difference between the heights of the mercury in the barometer observed at the bottom and top of the same. Which see under the article BAROMETER.

Altitude of the Eye, in Perspective, is a right line let fall from the eye, perpendicular to the geometrical plane.

AltiT UDE, in Astronomy, is the arch of a vertical circle, measuring the beight of the sun, moon, star, or other celestial object, above the horizon.

This altitude may be either true or apparent. The apparent altitude, is that which appears by sensible observations, made at any place on the surface of the earth. And the true altitude, is that which results by correcting the apparent, on account of refraction and parallax.

'The quantity of the refraction is different at different altitudes; and the quantity of the parallax is different according to the distance of the different luminaries: in the fixed stars, this is too small to be obserred; in the sun, it is but about $S \frac{3}{3}$ seconds; but in the moon it is about 52 minutes.

Altitudes are observed by a quadrant, or sextaut, or by the shadow of a gnomon or high pole, and by various other ways, as may be scen in most books of astronomv.

Mİeridian A LTITUDE, is an arch of the meridian, intercepted between any point in it and the horizon. So, if $\mathrm{HO}$ be the horizon, and HEzo the meridian; then the arch if $E$, or the angle HCE, is the meridian altitude of an object in the meridian at the

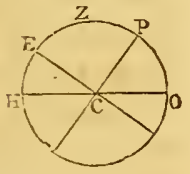
point $\mathrm{E}$.

Altitude, or elevation, of the Pole, is the angle $.0 \mathrm{CP}$, or arch of uf the incridian, intercepted between the borizon and pole $P$.

This is equal to the latitude of the place; and it may be found by observing the meridian altitude of the polar star, when it is both abrve and below the pole, and taking half the sum, when currected on account of refraction. Or the same may be found by the declination and meridian altitude of the sun.

ALTITUDE, or elecation, of the equator, is the angle HCE, or arch IIE of the meridian, between the horizon and the equator at $\mathrm{E}$; and it is cqual to $\mathrm{zP}$ the co-latitude of the place.

A LTIT UDE of the Tropies, the same as what is otherwise called the solstitial altitude of the sun, or his meridian altitude when in the solstitial points.

Altitune, or height, of the horizon, or of stars, \&c, seen in it, is the quantity by which it is raised by refraction.

Refraction of Altitude, is an arch of a vertical circle, by which the true altitude of the moon, or a star, or other object, is increased by means of the refraction; and is different at different altitudes, being nothing in the zenitb, and greatest at the horizon, where it is about $33^{\prime}$.

Parallax of Altit ude, is an arch of a vertical circle, by which the true altitude, observed at the centre of the earth, exceeds that which is observed on the surface; or the difference between the an-

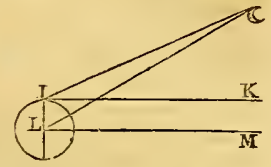
gles $D \mathrm{LM}$ and $D \mathrm{IK}$ of altitude there; and is equal to the angle IDL, formed at the moon or other body, and subtended by the radius $I L$ of the earth.

It is evident that this angle is less, as the luminary is fartber distant from the earth; and also less, for any one luminary, as it is higher abore the horizon; being greatest tbere, and nothing in the zenith.

Altitude of the Nonagesimal, is the altitude of the 90th degree of the ecliptic, counted upon it from where it cuts the horizon, or of the middle or highest point of it which is above the horizon, at any time; and is equal to the angle made by the ecliptic and horizon where they intersect at that time.

ALTITUDE of the cone of the earth's or moon's shadow, the beight of the shadow of the body, made by the sun, and measured from the centre of the body. To find it, say, As the tangent of the angle of the sun's apparent semjdiameter, is to radius, so is 1 to a th proportional, which will be the beight of the shadow, in semidiameiers of the body.

So, the greatest height of the earth's shadow, is 217.8 senidiameters of the earth, when the sun is at his greatest distance, or his semidiameter subtends an angle of about $15^{\prime} 47^{\prime \prime}$; and the height of the same is 210.7 semidiameters of the earth, when the sun is nearest the earth, or when his semidiameter is about $16^{\prime} 19^{\prime \prime}-$ And proportionally between these limits, for the intermediate distances or semidiameters of the sun.

The altitudes of the shadows of the earth and moon, are nearly as 11 to 3 , the proportion of their diameters.

ALTITUDE of notion, is a term used by Dr. Wallis, for the measure of any motion, estimated in the line of direction of the moving force.

ALTITUDE, in speaking of fluids, is more frequently expressed by the term depth. The pressure of fluids, in every direction, is in propurtion to their altitude or depth. And ou this principle is constructed the sea-gage, for finding the depth of the water of the occin. SFE GAGE.

AlTITUDE of the merciny, in the basaneter and thermometer, is marked by degıe's, or equal divisions, placed by the side of the tube of those instruments.

The altitude of the barometer, or of the mercury in its tube, at London, is usually comprised between the limits of 28 and 31 inches; and the mean height, observed for every day in several years, is found nearly $29^{\circ} 87$ inches.

Altitude of the pyramids. in Egypt, was measured so long since as the time of Thales, which he effected by means of their sliadow, and that of a pole set upright beside them, making the altitudes of the pole and pyramicl proportional to the lengths of their shadows. Plutarch has given an account of the manner of this operation, which is one of the first geometrical observations we bave an exact account of. 
ALTITU DE, circles of, parallels of, quadrant of, \&c. Sec the respective words.

Equal ALTITUDE. Instrument, is an instrument used to observe a celestial object, when it has the same or an equal altitude, on both sides of the meridian, or before and after it passes the meridian: an instrument very useful in adjusting clocks, \&c, and for comparing equal and apparent time.

AMALGAM, in Chemistry and the Arts, a mixture or alloy of mercury with any of the other metals.-For a description of the amalgam used by electricians, and the method of preparing it, see Exectricrtr.

AMBIENT, encompassing round about; as the bodies which are placed round any other body, are called ambient bodies, and sometimes circum-ambient bodies; and the whole mass of the air or atinosphere, because it encompasses all things on the face of the earth, is called the ambient air.

AMBIGENAL Hyperbola, a name given by Newton, in his Enumeratio linearum tertii ordinis, to one of the triple hyperbolas EGF of the second order, having one of its infinite legs, as EG, falling within the angle $A C D$, formed by the

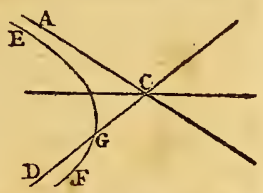
asymptotes $A C, C D$, and the other leg $G \mathrm{r}$ falling without that angle.

AMBIT, of a figure, in Geometry, is the perimeter, or line, or sum of the lines, by which the figure is bounded.

AMBLIGON, or A м ВLIG ONAL, in Geometry, signifies obtuse-angular, as a triangle which has one of its angles obtuse, or consisting of more than 90 degrees.

ANICABLE numbers, denote pairs of numbers, of which each of them is mutually equal to the sum of all the aliquot parts of the other. So the first or least pair of amicable numbers are 220 and 284 ; all the aliquot parts of which, with their sums, are as follow, viz, of 220 , they are $1,2,4,5,10,11,20,22,44,55$,

110 , their sum being - - - 284; of 284 , they are $1,2,4,71,142$, and their sum is 220 .

The $2 \mathrm{~d}$ pair of amicable numbers are 6232 and 6368 , which have the same properties.

The $3 \mathrm{~d}$ pair of amicable numbers are 17296 and 18416 , which have also the same property as above.

And the 4th pair of amicable numbers are 9363584 and 9437056 .

The 1st, $3 \mathrm{~d}$, and 4th pairs of amicable numbers were found out by F.Scbooten, sect. 9 of his Exercitationes Mathematicæ, who I believe first gave the name of amicable to such numbers, though their properties it seems had before been treated of by Rudolphus, Descartes, and others.

To find the first pair, Schooten puts $4 x$ and $4 y z$, or $a^{2} x$ and $a^{2} y z$ for the two numbers, where $a=2$; then making each of these equal to the sum of the aliquot parts of the other, gives two equations, from which are found the values of $x$ and $z$; and consequently, assuming a proper value for $y$, the two amicable numbers themscilves $4 x$ and $4 y z$.

In like manncr for the otler pairs of such numbers; in which he finds it necessary to assume $16 x$ and $16 y z$ or $a^{4} x$ and $a^{1} y z$ for the $3 \mathrm{~d}$ pair, and $128 x$ and $128 y z$ or $a^{7} x$ and $c^{7} y z$ for the 4 th pair.

Schooten then gives this practical rule, from Descartes, for finding amicable numbers, viz, Assume the number 2 , or some power of the number 2, such that if unity or 1 be subtracted from each of these three folloxing quantities, viz;

from three times the assumed number,

also from six times the assumed number,

anil from 18 times the square of the assumed number, the three remainders may be all primes; then the last prime number being multiplied by double the assumed number, the product will be one of the amicable numbers sought; and the sum of jts aliquot parts will be the other. That is, if $a$ be put $=$ the number 2 , and $n$ some integer number, such that $3 a^{\mathrm{n}}-1$, and $6 a^{\mathrm{n}}-1$, and $18 a^{2 \mathrm{n}}-1$ be all three prime numbers; then is $\left(18 a^{2 n}-1\right) \times 2 a^{\mathrm{n}}$ one of the anicable number; and the sum of its aliquot parts is the other.

Or this rule may be otherwise expressed thus. Suppose $A$ and $B$ are the pairs of a micable numbers sought; then find such a power of 2 , that is, $a=2^{2}$, such that $3 a-1=b$, $6 a-1=c, 1 \& a^{2}-1=d$, the numbers $b, c$ and $d$ may be primes; then will the numbers sought be $A=2^{n+1} \cdot d$, and $\mathrm{B}=2^{n+1} \cdot b c$.

The demonstration of which rule is as follows. Let $f_{A}$ represent the sum of the divisors of $A$, and $f_{B}$ the sum of the divisors of $B$, the whole values of $A$ and $B$ not being comprised, then since $1+2^{1}+2^{2}+2^{3} \ldots+2^{n+1}=$ $2^{n+2}-1$, we shall have $f_{A}=\left(2^{n+2}-1\right) \cdot(1+d)-$ $2^{n+1} \cdot d=(4 a-1) 18 a^{2}-2 a\left(18 a^{2}-1\right)=\mathrm{B}$, and $f_{B}=\left(2^{n+2}-1\right) \cdot(1+b) \cdot(1+c)-2^{n+1} \cdot b c=(4 a$ $-1) \cdot 18 a^{2}-2 a(3 a-1) \cdot(6 a-1)=A$, as is evident; because $\left(1+2^{1}+2^{2} \therefore+2^{n+1}\right) \cdot(d+1)$ will be the sum of all the divisors of $A$; and in the same man. ner $\left(1+2^{1}+2^{2} \ldots+2^{n+1}\right) \cdot(b+1) \cdot(c+1)$ is the sum of all the divisors of $\mathrm{B}$.

Mr. John Gough has lately investigated some properties of these numbers, especially of the Cartesian form, where $a x$ and $a y z$ are amicable numbers, consisting of a common measure $a$, multiplied by the primes $x, y, z$. See Leybourn's Math. Repos. $n^{\circ} .7, n, s$.

AMONTONS (WILL1AM), an ingenious French experimental philosopher, was born in Normandy the 31st of $A$ ugust 1663 . While at the grammar-school, he by sickness contracted a deainess that almost excluded him from the conversation of mankind. In this situation lie applied himself to the study of geonetry and mechanics; with which he was so delighted, that it is said he refused to try any remedy for his disorder, either because he deemed it incurable, or becausc it increased his attention to his studics. Among other objects of his study, were the arts of drawing, of land-surveying, and of building; and shortly after he acquired some knowledge of those more sublime laws by which the universe is regulated. He studiecl with grent care the nature of barometers and thermometcrs; and wrote his treatise of Observations and Experiments concerning a new Hour-glass, and concerning Barometers, Thermoneters, and Iygroscopres; as also some pieces in the' Journal des Savans. In 1657 , he presented a new hygroscope to the Acadeny of sciences, which was much approvect. He reconimended a methed of conveying intelligence to a great distance in a short space of tine: this was by making signals from one person to another, placed at as great distances from iach other as they coulcl sce the strnals by means of telescopes. When the Royal $\Lambda$ cadeng 
was new regulated in 1699, Amontons was chosen a member of it, as an elève under the third Astronomer; and he read there his New Theory of Friction, in which he happily cleared up an important object in mechanics. In fact he had a particular genius for making experiments: his notions were just and delicate: and he knew how to prevent the inconveniences of his new inventions, and bad a wonderful skill in executing them. He died of an inflammation in his bowels, the 11 th of October 1705 , being only 42 years of age.

The eloge of Amontons may be seen in the volume of the Memoirs of the Academy of Sciences for the year 1705 , Hist. pa. 150. And his pieces contained in the different volumes of that work, which are pretty numerous, and un various subjects, as the air, action of fire, barometers, thermom ters, hygrometers, friction, machines, heat, cold, rarefactions, puinp; \& $\&$, may be seen in the volumes for the years $1696,1699,1702,1703,1704$, and 1705 .

AMPHISCil, or AmPHiscians, are the pcople who inhabit the torrid zone; which are so called, bccause they have their shadow at noon turned sometimes one way and sometimes another, namely, at one time of the year towards the north, and at the other towards the south.

AMPLITUDE, in Gunnery, the range of a projectile, or the right line upon the ground subtending the curvilinear path in which it moves.

AMPLITUDE, in Astronomy, is an arch of the horizon, intercepted between the true east or west point, and the centre of the sun or a star at its rising or setting: so that the amplitude is of two kinds; ortive or eastern, and occiduous or western. Each of these anıplitudes is also either northern or soutbern, according as the point of rising or setting is in the northern or southern part of the horizon: and the complement of the amplitude, or the arch of distance of the point of rising or setting, from the north or south point of the horizon, is the azimutl.

The amplitude is of use in navigation, to find the variation of the compass or magnetic ncedle. And the rule to find it is this: As the cosine of the latitude is to radius, so is the sine of the sun's or star's declination, to the sine of the amplitude. So in the latitude of London, viz, $51^{\circ} 31^{\prime}$, when the sun's declination is $23^{\circ} 28^{\prime}$; then

cos. $51^{0} 31^{\prime}$ the lat. - $\quad-97939907$

sir. 2328 the decl. - + 96001181

sin. 3947 the ampl. - 9.8061274

That is, the sun then rises or sets $39^{\circ}+7^{\prime}$ from the east or west point, to the north or south according as the declination is north or south.

Magnetical AMPL1TUDE, is an arch of the horizon contained between the sun or star, at the rising or setting, and the magnetical east nr west point of the horizon, pointed out by the magnetical compass, or the amplitude or azimuth compass. And the difference between this magnetical amplitide, so observed, and the true amplitude, as computed in the last article, is the variation of the compass.

So if, for instance, the magnetical amplitude be observed, by the compass, to be $61^{0} 4 \tau^{\prime}$, at the time when it is computed to be - 3947 ,

then the difference - - 220 is the variation west.

ANABIBAZON, a name sometimes given to the dragon's tail, or northern node of the moon.

ANACAMPTICS, or the science of the reflections of sounds, frequently used in reference to echoes, which are said to be sounds produced anacamptically, or by refection: And in this sense it was used by the ancients for that part of optics which is othetwise called Catoptrics.

ANACHRONISM, in Chronology, an error in computation of time, by which an event is placed earlicr than it really happened. Such is that of Virgil, who makes Dido to reign at Carthage in the time of Fneas, though, to reality, she did not arrive in Africa till 300 years after the taking of Troy.

An error on the other side, by which a fact is placed later, or lower than it should be, is called a parachronisrn. But in common use, this distinction, though proper, is not attended to; and the word anachronism is used indifferently for the mistake on both sides.

ANACLASTICS, or ANACLATICS, an ancient name for that part of optics which considers refracted light; being the same as what is more usually called dioptrics. See the Compendium of Ambrosius Rhodius, lib. 3. Opticæ, pa. 384. \& seq.

Ana cLastic Curzes, a name given by M. de Mairan to certain apparent curves formed at the bottom of a vessel full of water, to an eye placed in the air; or the vault of the heavens, seen by refraction through the atmosphere. M. de Mairan determines these curves by a principle not admitted by all authors; but Dr. Barrow, at the end of his Optics, determines the same curves by other principles.

ANALEMMA, a planisphere, or projection of the sphere, orthographically made on the plane of the meridian, by perpendiculars from every point of that plane, the cye supposed to be at an infinite distance, and in the east or west point of the horizon. In this projection, the solstitial colure, and all its parallels, are projected into concentric circles, equal to the real circles in the sphere; and all circles whose planes pass through the eye, as the horizon and its parallels, are projected into right lines equal to their diameters; but all oblique circles are projected into ellipses, having the diameter of the circle for the transverse axis. This instrument, having the furniture drawn on a plate of wood or brass, with an horizon fitted to it, is used for resolving many astronomical problems; as the time of the sun's rising and setting, the length and hour of the day, \&c. It is also useful in dialling, for laying down the signs of the zodiac, with the lengths of days, and other matters of furniture, upou dials.

The oldest treatise we have on the analemma, was written by Ptolemy, which was printed at Rome 1562, with a commentary by F. Commandine. Pappus also treated of the same. Since that time, many other authors have treated very well of the analenma; as Aguilonius, Taquet, Dechales, Witty, \& $c$.

ANALOGY, the same as proportion, or equality, or similitude of ratios. Which sce.

ANALYSIS, is, gencrally, the resolution of any thing into its component parts, in order to discover it or its composition. And in mathematies it is properly the method of resolving problems, by reducing them to equations. Analysis may be distinguished into the anciont and the modern.

The ancient analysis, as described by Pappus, is the method of proceeding from the thing sought as taken for granted, through its conseyuences, to sumething that is really granted or known; in which sense it is the reverse of synthcsis or composition, in which we lay that duwn 
nirst which was the last step of the analysis, and tracing the steps of the analysis back, making that antecedent here which was consequent there, till we arrive at the thing sought, wbich "as taken or assumed as granted in the first step of the analysis. This chiefly respected geoinetrical inquiries.

The principal authors on the ancient analysis, as recounted by Pappus, in the 7 th book of his Mathematical Collections, are Euclid in his Data, Porismata, et de Locis ad Snperficiem; Apollonios de Sectione Rationis, de Sectione Spatii, de Tactionibus, de Inclinationibus, de Locis Plaris, et de Sectionibus Conicis; Aristæus, de Locis Solidis; and Eratosthenes, de Mediis Proportionalibus: from which Pappus gives many examples in the same bouk. To these authors we may add Pappus himself. The same sort of analysis has also been well cultivated by many of the moderns; as Fermat, Viviani, Ghetaldus, Snellius, Huygens, Simson, Stewart, Lawson, Playfair, Leslie, Sc, and more especially Hugo d'Omerique, in his Analysis Geometrica, in which he has endeavoured to restore the analysis of the ancients. And on this bead, Dr. Pemberton tells us, "that sir Isaac Newton. used to censure himself for not lollowing the ancients more closely than he did; and spoke with regret of his mistake, at the beginning of his mathematical studies, in applying himself to the works of Descartes, and other' algebraical writers, before he had considered the Elements of Euclid with that attention so excellent a writer deserves:, that he highly approved the laudable attempt of Hugo d'Omerique to restore the ancient analysis."

In the application of the ancient analysis in geometrical problems, every thing cannot be brought within strict rules; nor any invariable directions given, by which we may succeed in all cases; but some previous preparation is necessary, a kind of mental contrivance and construction, to form a connexion between the data and quxsita, which must be left to every one's judgment to find out; being various, according to the various nature of the problems proposed. Right lines must be drawn in particular dircctions, or of particular magnitudes; bisecting perhaps a given angle, or perpendicular to a given line; or perhaps taugents in ust be drawn to a given curve, from a given point; or circles described from a given centre, with a given radius, or touching given lines, or other circles; or such-like operations. Whoever is conversant with the works of Archinedes, Apollonius, or Pappos, well knows that they founded their analysis on some such previous operations; and the great skill of the analyst consists in discovering the most proper affections on which to found his analysis: for the same problem may often be effected in many different ways: of which it may be proper to gire bere nu example ur twu. Let there be taken, for instance, this problem, which is the $155 \mathrm{th}^{\mathrm{p}}$ prop. of the $7 \mathrm{th}$ book of Pappus.

From the extremities of the base $\mathrm{A}$ B, of a given segment of a circle, requiled to draw two lines ac, Bc, mecting at a point $\mathrm{c}$ in the circumlerence, so that they shatl] liave a given ratio to each other, suppose that of $F$ to $\mathbf{c}$.

The solution of this problem, as given by Pappus, is thes.

ANALYSIS.

Suppose the thing done, and that the poine $\mathrm{c}$ is found: and let $\mathrm{C} n$ be drawn a tangent to the circle at $\mathbf{c}$, and meeting the line $\mathbf{A} \mathbf{B}$ produced in the point $\mathrm{D}$. Now by the hypothesis $\mathrm{AC}: \mathrm{BC}:: \mathrm{F}: \mathrm{G}$, and also $\mathrm{AC}^{2}: \mathrm{BC}^{2}:: \mathrm{DA}$ : $D B$, as inay be thus proved.

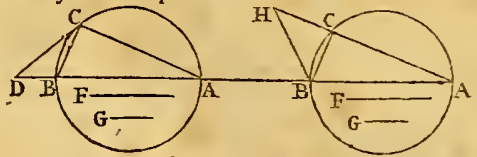

Since DC touches the circle, and BC cuts it, the angle BCD is equal to BAC by Euc. iii 32 ; also the angle $D$ is common to both the triangles $D C A, D C B$; these are therefore similar, and so, by ri $4, \mathrm{DA}: \mathrm{DC}:: \mathrm{DC}: \mathrm{DB}$, and hence $\mathrm{DA}^{2}: \mathrm{DC}^{2}:: \mathrm{DA}: \mathrm{DB}$ by cor. vi 20 . But also, by vi $4, \mathrm{DA}: \mathrm{AC}:: \mathrm{DC}: \mathrm{CB}$, and by permutation $\mathrm{DA}: \mathrm{DC}$ : : $\mathbf{A C}: \mathbf{B C}$, or $\mathbf{D A}^{2}: \mathbf{D C}^{2}:: \mathbf{A C}^{2}: \mathbf{B C}^{2}$; and hence, by equality, $-\mathrm{AC}^{2}: \mathrm{BC}^{2}:: \mathrm{DA}: \mathrm{DB}$.

But the ratio of $\mathrm{AC}^{2}$ to $\mathrm{BC}^{2}$ is given, because the ratin of $A C$ to $B C$ is given, and consequently that of $D A$ to $D B$ is given. Now since the ratio of $\mathrm{DA}$ to $\mathrm{DB}$ is given, therefore also, by Data vi, that of $D A$ to $A B$, and hence, by Data $\mathrm{ii}, \mathbf{D A}$ is given in magnitude.

And here the analysis properly ends. For it baving bcen shown that $\mathrm{DA}$ is given, or that a point $\mathrm{D}$ may be found in AB produced, such, that a tangent being drann from it to the circumference, the point of contact will be the point sought; we may now begin the composition, or synthetical demonstration; which must be done by finding the point $\mathrm{D}$, or laying down the line $\wedge \mathrm{D}$, which, it was affirmed, was given, in the last step of the analysis.

\section{SYNTHESIS.}

Construction. Make as $\mathrm{F}^{2}: \mathrm{G}^{2}:: \mathrm{AD}: \mathrm{DB}$, (which may be done, since $A B$ is given, by making it as $F^{2}-G^{2}: G^{2}:$ : $A B: D B$, and then by composition it will be as $F^{2}: G^{2}:$ : $A D: D E$ ); then from the point $D$, thus found, draw a tangent to the circle, and from the point of contact $\mathrm{c}$ drawing $\mathbf{C A}$ and $\mathrm{cB}$, the thing is done.

Demonstration. Since, by the constr. $\mathrm{F}^{2}: \mathrm{G}^{3}:: \mathrm{AD}$ : $\mathbf{D B}$, and also $\mathbf{A D}: \mathbf{D B}:: \mathbf{A C}^{2}: \mathbf{B C}^{2}$, which has been already demonstrated in the analysis, and might be here proved in the same manner. Therefore $\mathrm{F}^{2}: \mathrm{G}^{2}:: A \mathrm{C}^{2}$ : $\mathrm{BC}^{2}$, and consequently $\mathrm{F}: \mathrm{G} \cdot:$ : $\mathrm{AC}:$ в C. 2. $E$. $D$.

Here we sce an instance of the method of resulution and composition, as it was practised by the ancients, the solution here given being that of Pappus himself. But as the inethod of reterring and reducing every thing to the Data, and constantly quoting the same, may appear now to be tedious and troublesome: and indeed it is unnecessary to those who have already made themselves mastcrs of the substance of that valuable book of Eucticl, and have hy practice and experience acquired a tacility of reasoning in such matters: I shall therefore now sluw how we may abate sonething of the rigour and strict form of the ancient muthod of solution, without aliminishing any part of its admirable elegance and perspicuity. And this may be done by means of another solution, ot the many more which might be given, of the same problem, as fullows.

\section{Ax A Lysis.}

Let us agin suppose that the thing is done, siy, ac: вс : : $\mathbf{r}: \mathbf{6}$, and let there be driwn wn miking the angle A Hu cqual to the angle $\mathrm{AC}$, and mecting $\mathrm{AC}$ produced in' is. 'Then, the angle a being also common, the two triangles $A \mathrm{BC}$ and $\mathrm{A} B \mathrm{~B}$ al'e eguiangular, and therefore, by vi $4, \mathbf{A C}: \mathbf{B C}:: \mathbf{A} \mathbf{B}: \mathbf{B H}$, in al given ratio; and, $A x$ 

A N A
[ 113$]$
A N A

being given, therefore $\mathrm{BH}$ is given in position and magnitude.

\section{SYNTHESIS.}

Construction. Draw $\mathrm{RH}$ making the angle $\mathrm{ABH}$ equal to that which may be contained in the given segment, and take $A B$ to $B H$ in the given ratio of $F$ to G. Draw $A C H$, and $\mathrm{BC}$.

Demonstration. The triangles $\mathrm{ABC}, \mathrm{ABH}$ are equiangular, therefore, vi $4, A C: \mathrm{CB}:: \mathrm{AB}: \mathrm{BH}$, which is the given ratio by construction.

Modern ANALIsis, consists chiefly of algebra, arithmetic of infinites, infinite series, increments, fluxions, \& c; of each of which a particular account may be seen under their respective articles.

These form a kind of arithmetical and symbolical analysis, depending partly on modes of arithmetical computation, partly on rules peculiar to the symbols made use of, and partly on rules drawn from the nature and species of the quantities they represent, or from the modes of their existence or generation.

The modern analysis is a general instrument, by which the finest inventions, and the greatest improvements, have been made in mathematics and philosophy, for two centuries past. It furnishes tbe most perfect examples of the manner in which the art of reasoning should be employed; it gives to the mind a wonderful skill for discovering things unknown, by means of a small number that are given; and by employing short and easy symbols for expressing ideas, it presents to the understanding things which otherwise would seem to lie above its sphere: By this means, geometrical demonstrations may ke greatly abridged; a long train of arguments, in which the mind cannot, without the greatest effort of attention, discover the connexion of ideas, is converted into visible symbols; and the various operations which they require, are simply effected by the combination of those symbols. And, what is still more extraordinary, by this artifice, a great number of truths are often expressed iu one line only : instead of which, by following the ordinary way of cxplanation and demonstration, the same truths would occupy whole pages or volumes. And thus, by the bare contem. plation of one line of calculation, we may understand in a short time whole sciences, which otherwise could hardly be comprehended in several years.

It is true that Newton, who best knew all the advantages of analysis in geometry and other sciences, laments, in several parts of his works, that the study of the ancient genmetry is abandoned or neglected. And indeed the method employed by the ancients in their geometrical writings, is commonly regarded as more rigorous than that of the modern analysis: and though it be greatly inferior to the latter, in point of dispatch and facility of invention; it is nevertheless highly useful in strengthening the mind, improving the reasoning faculties, and in accustoming the young mathematician to a pure, clear, and accurate mode of investigation and demonstration, though by a long and laboured process, which he would with difficulty have submitted to if his taste lad before been vitiated, as it were, by the more piquant sweets of the modern analysis. And it is principally on this that the complaints of Newton are founded, who feared lest by the too early and frequent use of the modern analysis, the science of geometry should lose that rigour and purity which characterise its investigations, and the mind become debilitated by the facility of our analysis. This great man

VoL. 1 . was therefore well founded, in recommending, to a certain extent, the study of the ancient geometricians : for, their demonstrations being more difficult, give more exercise to the mind, accustom it to a closer application, give it a greater scope, and habituate it to patience and resolution, so necessary for making discoveries. But this is the only or principal advantage from it; for if we should look no farther than the method of the ancients, it is probable that, even with the best genius, we should have made but few or small discoveries, in comparison with those obtained by means of the medern analysis. And even with regard to the advantage given to investigations made in the manner of the ancients, namely of being more rigorous, it may perhaps be doubted whether this pretension be well founded. For, to instance in those of Newton himself, though his demonstrations be managed in the manner of the ancients; yet at the same time it is evident that be investigates his theorems by a method different from that employed in the demonstrations, which are commonly analytical calculations, disguised by substituting the name of lines for their algebraical value: and though it be true that his demonstrations are rigorous, it is no less so that they would be the same when translated and delivered in algebraic language; and what difference can it make in this respect, whether we call a line $A B$, or denote it by the algebraic character $a$ ? Indeed this last designation lias this peculiarity, that when all the lines are denoted by algebraic characters, many operations can be performed upon them, without thinking of the lines or the figure. And this circumstance proves of no small advantage: the mind is relieved, and spared as much as possible, that its whole force may be employed in overcoming the natural difficulty of the problem alone.

Upon the whole therefore, the state of the comparisan seems to be this; That the method of the ancients is fittest to begin our studies with, to form the mind and to establish proper habits; and that of the moderns to succeed, by extending our views beyond the present fimits, and enabling us to make new discoveries and improvements.

Analysis is divided, with respect to its object, into that of finites, and that of infinites.

Analysis of finite quantities, is what is otherwise called algebra, or specious arithmetic.

Analysis of infinites, called also the new analysis, is that which is concerned in calculating the relations of quantities which are considered as infinite, or infinitely small; one of its chief branches being the method of fluxions, or the differential calculus. And the great advantage of the modern mathematicians over the ancients, arises chiefly from the use of this modern analysis.

ANALYsis of porvers, is the same as resolving them into their roots, and is otherwise called Evolutions.

AN ALYSiss of curve lines, shows their constitution, nature and properties, their points of inflection, station, rotrogradation, variation, \&c.

ANALYST, a person who analyses something, or makes use of the analytical method. In mathematics it is a person skilled in algebra, or in the mathematical analysis in general.

ANALYST, the title of an ingenious, though sophistical book, written by the celebrated Dr. Berkeley, against the doctrine of fluxions.

ANALYTIC, or ANALYTICAL, something belonging to, or partaking of, the nature of analysis; or performed by the method of analysis. 
Thus we say analytical demonstration, analytical enquiry, analytical table or scheme, analytical method, \&c. The analytical stands opposed to the synthetical, or that which proceeds by the way of synthesis.

ANALYTICS, the science, or doctrine, and use of analysis."

ANAMORPHOSIS, in perspective and painting, a monstrous projection; or a representation of some image, either on a plane or curve surface, deformed or distorted; but which in a certain point of view shall appear regular, and drawn in just proportion.

To construct an Anamorphosis, or monstrous projection, on a plane.-Draw the square $\mathrm{ABCD}$ (fig. 1), of any size at pleasure, and divide it by crossing lines into a number of areolæ or smaller squares : and then in this square, or reticle, called also the craticular prototype, draw the regular image which is to be distorted.--Or, about any image, proposed to be distorted, draw a reticle of small squares.
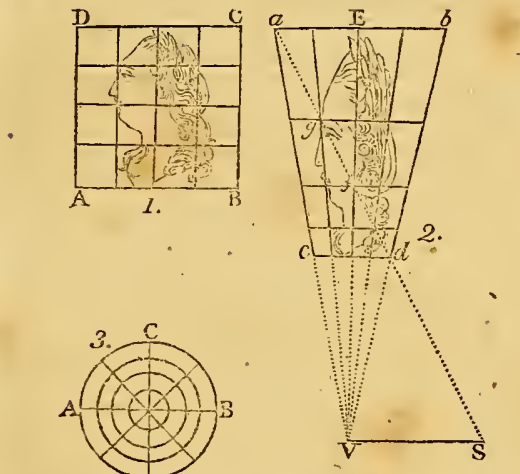

Then draw the line $a b$ (fig. 2) equal to $\mathrm{AB}$, dividing it into the same number of equal parts, as the side of the prototype $A \mathrm{~B}$; and on its middle point $\mathrm{E}$ erect the perpendicular $\mathrm{Ev}$, as also vs perpendicular to $\mathrm{kv}$, making rv so much the longer, and vs so much the shorter, as it is intended the image shall be more distorted. From each of the points of division draw right lines to the point $\mathbf{v}$, and draw the right line $a$ s. Lastly, through the points $c, e, f, g$, \&c, draw lines parallel to $a b$ : So shall abcd be the space upon which the monstrous projection is to be drawn; which is called the craticular ectype.

Then, in every areoli, or small trapezium, of the space abcd, draw what appcars contained in the corresponding areola of the original space $A B C D$ : so shall there be produced a deformed image in the space abd, which yet will appear in just proportion to an eye distant from it the letrgth of $\mathrm{Ev}$, and raised above it by a height equal to vs.

It will be amusing to contrive it so, that the deformed image may not represent a mere chaos, but some certain figure: thus, a river with soldiers, waggons, and other objects on the side of it, have been so drawn and distorted, that when viewed by an eye at $S$, it appeared like the face of a satyr.

An image may also be distorted inechanically, by perforating it through in several places with a fiue pin; then, placing it against a candle or lamp, obscrve where the rays, which pass through these small holes, fall on any plane or curve superficies; for they will give the correspondent point: of the image deformed, and by means of which the deformation may be completed.

To draw an Anamorphosis on the convex surface of a cone. It appears from the construction above, that we have only to make a craticular ectype on the surface of the cone, which may appear equal to the craticular prototype, to an eye placed at a proper height above the vertex of the cone. Hence,

Let the base, or circumference, $A \mathrm{SCD}$, of the cone (fig. 3) be divided by radii into any number of equal parts; and let some one radius be likewise divided into equal parts; then through each point of division draw concentric circles: so shall the craticular prototype be formed.

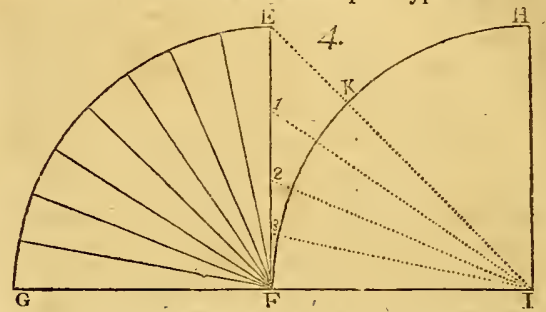

With double the diameter $\mathrm{AB}$, as a radius; describe the quadrant EFG (fig. 4) so as the arch IG be equal to the whole periphery; then this quadrant, being plied or bent round, will form the superficies of a cone, whose base is the circle.

Next divide the arch $\mathbf{E} \mathbf{G}$ into the same number of equal parts as the craticular prototype is divided into; and draw radii from all the points of division. Produce GE to I, so that $\mathrm{FI}$ be equal to $\mathrm{Fr}$; from the centre I, with the radius IF, describe the quadrant FKн; and draw the right line IE. Then divide the arch $\mathrm{KF}$ into the same number of equal parts as the radius of the craticular prototype is divided into; and from the ceutre I draw radii through all the points of division, meeting $\mathrm{E} F$ in $1,2,3$, \&c. Lastly, from the centre $\mathrm{w}$, with the radii $\mathrm{Fl}, \mathrm{F} 2$, F3, \&c, describe concentric circles. So will the craticular cctype be formed, whose areolas will appear cqual to each other.

Hence, what is delineated in every areola of the craticular prototype, being transferred into the arealas of the craticular ectype, the images will be distorted or deformed; and yet they will appear in just proportion to an eye elevated above the vertex at a height equal to the height of the cone itself.

If the chords of the quadrants be drawn in the craticular prototype, and chords of each of the th parts in the craticulitr ectype, every thing else remaining the same, there will be obtained the craticular ectype in a quadrangular pyramid.

And hence it will be casy to deform an image, in any other pyramid, whose base is any regulitr polygon.

Because the illusion is more perfect when the eye, by the contiguous objects, cabnot estimate the distance of the parts of the deformed image, it is therefore proper to view it through a small hole.

Anamorploses, or monstrous images, may also be made to appear in their natural shape and just proportions, by menels of mirrors of certain shapes, from which those 
Images are reflected again; and then they are said to be reformed. For farther particulars, see Wolfius's Catoptrics and Dioptrics, and some other optical authors.

ANASTROUS signs, in Astronony, a name given to the duodecatemoria, or the 12 portions of the ecliptic, which the signs posscssed anciently, but have since deserted by the precession of the equinox.

ANAXAGORAS, one of the most celebrated philosophers among the ancients. He was born at Clazomene in Ionia, about the 70th Olympiad. He was a disciple of Anaximenes; and he gave up bis patrimony, to be more at leisure for the study of philosophy, giving lectures in that science at Athens. Being persecuted in this place, and at last banished from it, he opened a school at Lampsacum, where he was greatly bonoured during his life, and still more after his death, statues having been erected to bis memory. It is said he made predictions relative to the phenomena of nature, as earthquakes, \&c, upon which he wrote some treatises. His principal tenets may be reduced to the following:-All things were in the beginning confusedly mixed together, without order and without motion. The principle of things is at the same time one and multiplex, which had the name of homcemeries, or similar particles, deprived of life. But there is beside this, from all eternity, another principle, an infinite and incorporeal spirit, who gave motion to these particles; in virtue of which, such as were homogeneal united, and such as were beterogeneal separated according to their different kinds. All things being thus put into motion by the spirit, and every thing being united to such as are similar, those that had a circular motion produced heavenly bodies, the lighter particles ascending, while those that were heavier descended. The rocks of the earth, being drawn up by the whirling force of the air, took fire, and became stars, beneath which the sun and moon took their stations. - It was said he also wrote upon the quadrature of the circle; the treatise on which, Plutarch says, he composed during his imprisonment at Athens.

ANAXIMANUER, a very celebrated Greek philosopher, was born at Miletus in the $42 \mathrm{~d}$ Olympiad ; for, according to Apollodorus, he was 64 ycars of age in the $2 \mathrm{~d}$ year of the 58th Olympiad. He was one of the first who publicly taught philosophy, and wrote upon philosophical subjects. He was the kinsman, companiou, and disciple of 'Thales. He wrote also upon the spliere and on geometry, \&c. Ant be carried his researches into nature very far, for the time in which he lived. It is said that he discovered the obliquity of the zodiac; that he first published a geographical table; that he invented the gnomon, and set up the first sun-dial in an open place at Lacedæmon. He taught that infinity of things was the principal and universal clement ; that this infinite always preserved its unity, but that its parts underwent changes; that all things came from it; and that all were about to return to it. By this obscuie and indeterminate principle lie probably meant the chaos of other philosophers. He held that the worlds are infinite; that the stars are composed of air and fire, which are carried about in their spheres, and that these spheres are gods; and that the earth is placed in the midst of the universe, as in a common centre. Further, that infinite worlds were the produce of infinity; and that corruption proceeded from separation.

ANAXJMENES, an eminent Greek philosopher, born at Miletus, the fritend, scholar, and successor of Anaxi- mander. He diffused some legree of light upon the obscurity of his master's system. He made the first principle of things to consist in the air, which he considered as infinite or immense, and to which he ascrlbed a perpetual motion; that this air was the same as spirit or God, since the livine power resided in it, and agitated it. The stars were as fiery nails in the heavens; the sun a flat plate of fire; the eartl an extended flat surface, \&c.

ANDERSON (ALEXANDER), one of the brightest ornaments of the mathematical world, who flourished about 200 years ago. It appears that he was born at $A$ berdeen in Scotland, towards the latter part of the 16 th century, and he was professor of mathematics at Paris in the early part of the $17 \mathrm{th}$, where he published several ingenious works in geometry and algebra, both of his own, and of his friend Vieta's. He published his

1. "Supplementum Apollonii Redivivi; sive analysis problematis hactenus desiderati ad Apollonii Pergæi doctrinam $\pi \varepsilon \rho s \nu \varepsilon v \sigma \varepsilon \omega v$, a Marino Glietaldo Patritio Ragusino bujusque, non ita.pridem restitutan. In qua exhibetur mechanice æqualitatum tertii gradus sive solidarum, in quibus magnitudo omnino data, æquatur homogeneæ sub altero tantum coefficiente ignoto. Huic subnexa est variorum problematum practice." Paris, 1612, in 4to.

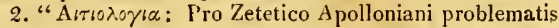
a se jam pridem edito in supplemento Apollonii Redivivi. Ad clarissimum et ornatissimum virum Marinum Ghetaldum Patritium Ragusinum. In qua ad ea quae obiter mihi perstrinxit Ghetaldus rcspondetur, et analytices clarius detegitur." Paris, 1615, in 4to.

3. "Francisci Vietæ Fontenacensis de Aequationum Recognitione et Emendatione Tractatus duo." Paris, 1615, in 4to; with a Dedication, Preface, and an Appendix, by Anderson.

4. "Ad Angularium Sectionum Analyticen Theo-

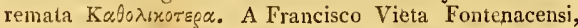
excogitata, at absque ulla demonstratione ad nos transmissa, jam taniem demonstrationibus confirmata. Opera et studio Alexandri Andersoni Scoti." Parisiis, 16t5, in 4 to.

5. "Vindiciæ Archimedis. Sive, Elenchus Cyclometriæ nova, a Philippo Lansbergio nuper edita. Per Alexandrum Andersonum Scotum." Parisiis, 1616, in 4.to.

6. "Alexandri Andersoni Scoti Exercitationum Mathematicarum Dicas Prina. Continens, Quastionum aliquot, quæ nobilissimorum tum hujus tum veteris æri, Nathematicorum ingenia exercuere, Enodationem." $\mathrm{Pa}$ risiis, 1619 , in 4 to.

All these pieces, of this excellent gcometrician, are replete with the finest specimens of pure geometrical exerciss's that have perhaps ever been prouluced by any authors, ancient or modern. So that it is to be lamented that the limits of this work will not admit of giving the full account of all the particulars contained in them, which I had prepared indeed expressly for that purpose. Besides these, I am not aware ot any more pablications of our author, though perhaps there may have been others. Incleed from the last piece, it fully appears that he had at least written, if not published, another, viz. A treatise on the mensuration of solids, perhaps with a reference to ganging; as, in several problems, where he critically examines the treatise on cask-gauging of Keplsr, our author olien refers to his own work on stercometry, which 1 have not learned had ever been published. 
A N D

[ 116

Alexander was cousin-german to a Mr. David Anderson, of Finshaugh, a gentleman who also possessed a singular turn for mathematical and mechanical knowledge. This mathematical genius appears to have been hereditary in the family of the Andersons, and from them transmitted to their descendants of the name of Gregory in the same country: the daughter of the said David Anderson having besn the mother of the celebrated mathematician James Gregory, and who herself first instructed her son James in the elements of the mathematics, on observing in him, while yet a child, a strong propensity to those sciences.

The time either of the birth or death of our author Alexander, has not come to my knowledge.

ANDERSON (RовеRT), an ingenious mathematician, who flourished in the latter half of the 17th century, and was much noticed and encouraged in those studies by the mathematical John Collins, among others, so often mentioned in the early labours of the Royal Society. Mr. Anderson was not in the profession of mathematics, or of those other branches which it appears he usefully cultivated and improved; but was a silk-weaver by trade, and must have been a person of some consideration and substance, as he was able to make, at his own charge, some thousands of experiments with canron, for improving the art of gunnery; which be did in a considerable degree, as appears by the tracts on that art which he published, as deduced from those experiments; viz, The Genuine Use and Effects of the Gun, in 1674; To Hit a Mark, in 1690; and, To Cut the Rigging, \&c. in 1691, all in 4to. He published also, Stereometrical Propositions, variously applicable, but particularly intended for Gauging: in small $8 \mathrm{vo}, 1668$. This little work is an elaborate treatise on the measurement of all kinds of solids that can be generated by the rotation of circles and the conic sections, or the ellipse, hyperbola, and parabola; viz, all spheres, spheroids, conoids, and spindles, \&c, with their segments, zones, ungulas, \&c.

ANDROMEDA, in Astronomy, a constellation of the northern hemisphere, representing the figure of a woman almost nakéd, her feet at a distance from each other, and her arms extended and chained; being one of the original 48 asterisms, or figures under which the ancients comprehended the stars, as derived to us from the Greeks, who probably had them from the Egyptians or Indians, and who, it is suspected, altered their names, and accompanied them with fabulous stories of their own. According to them, Cepheus, the father of Andromeda, was obliged to give her up to be devoured by a monster, to preserve his kingdom from the plagne; but that she was delivered by Perseus, who slew the monster, and espoused her. And the family were all translated by Minerva to heaven, the mother being the constellation Cassiopeia.

She is somctimcs called, in Latin, Persea, Mulier catenata, Virgo devota, \&c. The Arabians, whose religion did not permit them to draw the figure of the human body on any occasion whatever, have changed this constcllation into the figure of a sea-calf. Schickard has changed the name for that of the Scripture name Abigail. And Schiller has also changed the figure of the constellation, for that of a sepulchre, and calls it the Holy Sepulchre.

This constellation contains about 27 stars, that are visible to the naked eye; of which the principal are, $\alpha$ Andromeda's head; $\beta$ in the girdle, and called Mirach or Mizar; $\gamma$ on the south foot, and llamed Alamak, and sometimes Alhames.

\section{A N E}

The number of stars placed in this constellation, by the catalogue of Ptolemy is 23, by that of Tycho Brahe also 23, by that of Hevelius 47 , and by that of Flamsteed 66 .

ANEMOMETER, an instrument for measuring the force of the wind.

An instrument of this sort, it scems, was first invented by Wolfius; in the year 1708, and first published in his Areometry in 1709, also in the Acta Eruditorum, of the same year; afterwards in his Matbematical Dictionary, and in his Elem. Matheseos. He says he tried the goodness of it, and observes that the internal structure may be preserved, so as to measure the force of running water, or that of men or horses when they draw or pull. 'The machine consists of sails, A, B, c, like those of a wind-mill, against which the wind blows, and by turning them about raises an arm $K$ with a weight $L$ upon it, to different angles of elevation, shown by the index $M$, according to the force of the wind. (Plate iii. fig. 3.)

Mr. Leutmann improved on Wolfius's anemometer, by placing the sails horizontal, instead of vertical, which are easier then to move, and turn whatever way the wind blows.

Mr. Benjamin Martin also (Plate iii. fig. 5) improved on the same. He rnade the axis like the fusee of a watch, having a cord winding upon it, with two weights at the ends, which make always a balance to the force of the wind on the sails. See his Philos. Britan.

And M. D'Ons-en-Bray invented a new anemometer, which of itself expresses on paper, not only the several winds that have blown during the space of 24 hours, and at what hour each began and ended, but also their different velocity. Sce Mem. Acad. Scienc, an. 1734.

In the Philos.'Trans. another anemometer is described, in which the wind being supposed to blow directly against a flat side, or board, which moves along the graduated arch of a quadrant, the number of degrees it advances shows the comparative force of the wind.

In the same Transactions, for the year 1766, Mr. Alex. Brice describes a method, successfully practised by him, of measuring the velocity of the wind, by means of that of the shadow of clouds passing over a plane upon the earth.

Also in the same Transactions, for the year 1775 , Dr. Lind gives a description of a very ingenious portable Wind-Gauge, by which the force of the wind is casily measured; and which seems to have been snggcsted by a hint of Dr. Hales, in his Description of Ventilators, pa.12, and described in the Huetiana, long before Dr. Lind's time. A brief description of the principal parts of it here follows. This simple instrument consists of two glass tubes, $\triangle \mathrm{B}, \mathrm{CD}$ (Plate iii. fig. 4), which should not be less than 8 or 9 inches long, the bore of each being about $4^{4}$ of an inch diameter, and connected together by a small bent glass tube $a$, only of about ${ }_{\text {To }}^{2}$ of an inch dianeter, to check the undulations of the water caused by a sudden gust of wind. On the upper end of the $\operatorname{leg} A \mathrm{~B}$ is fixed at right angles to it a thin metal tube, and having its mouth open to receive the wind blowing horizontally into it. The two tubes, or rather the two branches of the tube, are connected to a steel spindle $\mathrm{K} \mathrm{L}$, by slips of brass near the top and bottom, on the sockets of which at $e$ and $f$, by the wind blowing in at the orifice at $\mathbf{F}$, the whole instrument turus casily about the spindle, which is fixed into a block by a screw in its bottom. When the instru- 
ment is used, a quantity of water is poured in, till the tubes are about balf full; then exposing the instrument to the wind, by blowing in at the orifice $F$, it forces the water down lower in the tube $A B$, and raises it so much higher in the other tube; then the distance between the surfaces of the water in the two tubes, estimated by a scale of incbes and parts $\mathrm{H} 1$, placed by the sides of the tubes, will be the beight of a column of water whose weight is equal to the force or momentum of the wind, blowing or striking against an equal base. And as a cubic foot of water weighs 1000 ounces, or $62 \frac{1}{2}$ pounds, the 12 th part of which is $5 \frac{5}{24}$ or $5 \frac{1}{5}$ pounds nearly, thercfore for every inch the surface of the water is raised, the force of the wind will be equal to so many times $5 \frac{\mathrm{t}}{5}$ pounds on a square foot. Thus, suppose the water stand 3 inches higher in the one tube, than in the other; then 3 times $5 \frac{\pi}{5}$, or $15 \frac{3}{5}$ pounds, is equal to the pressure or force of the wind on the surface of a square foot.

This instrument of Dr. Lind's, measures only the force or momentum of the wind, but not its velocity. However, the velocity of the wind may be deduced from its force so obtained, by help of some experiments performed by me at the Royal Military Academy, in the years 1786 , 1787 , and 1788 ; from which it appears that a plane surface of a square foot suffers a resistance of 12 ounces, or $\frac{3}{4}$ of a pound, from the wind, when blowing with a velocity of 20 feet per second ; and that the force is nearly as the square of the velocity. Hence then, taking the force of $15 \frac{3}{5}$ pounds, above found, for the force of the wind when it sustains 3 inches of water, and taking the square roots of the forces, it will be, as $\sqrt{\frac{3}{4}}: \sqrt{ } 15 \frac{3}{5}:$ : $20: 91 \frac{x}{5}$ the 4 th proportional, that is a velucity of $91 \frac{x}{5}$ feet per second, or 62 miles per bour, is the rate or velocity at which the wind blows, when it raises the water 3 inches higher in the one tube than the other. And farther, as the said height is as the force, and the force as the square of the velocity, we shall have the force and velocity, corresponding to several heights of the water in the one tube, above that in the other, as in the following table.

Table of the corresponding height of water, force of the wind on a square foot, and its velocity per hour.

\begin{tabular}{|c|c|c|}
\hline $\begin{array}{l}\text { Height of } \\
\text { water. }\end{array}$ & $\begin{array}{l}\text { Force of } \\
\text { wind }\end{array}$ & $\begin{array}{l}\text { Velocity of } \\
\text { wind per hour. }\end{array}$ \\
\hline$\overline{\text { Inches. }}$ & $\overline{\text { Pountls. }}$ & Miles. \\
\hline $0 \frac{\pi}{4}$ & $1 \cdot 3$ & $18^{\circ} 0$ \\
\hline $0 \frac{x}{2}$ & $2 \cdot 6$ & $25 \cdot 6$ \\
\hline 1 & $5 \cdot 2$ & 36.0 \\
\hline 2 & 104 & $50 \cdot 8$ \\
\hline 3 & 15.6 & $62 \cdot 0$ \\
\hline 4 & $20 \cdot 8$ & 76.0 \\
\hline 5 & $26^{\circ} 0$ & $80 \%$ \\
\hline 6 & $31 \cdot 25$ & 88.0 \\
\hline 7 & $36 \cdot 5$ & $95 \cdot 2$ \\
\hline 8 & $41 \cdot 7$ & $101 \cdot 6$ \\
\hline 9 & 46.9 & 1080 \\
\hline 10 & $52 \cdot 1$ & 113.6 \\
\hline 11 & $57 \cdot 3$ & $119 \cdot 2$ \\
\hline 12 & $62 \cdot 5$ & 124.0 \\
\hline
\end{tabular}

In one instance, Dr. Lind found, that the force of the wind was such as to be equal to 34 T $^{2}$ o pounds, on a square foot; and this by proportion, in the following table, will be found to answer to a velocity of 93 miles per hour.

Sec also Wind-Gauge.

ANENIOSCOPE, is sometimes used to denote a ma. chine invented to foretell the changes of the wind, or weather ; and sometımes for an instrument showing by an index what the present direction of the wind is. Of this latter kind, it seens, was that used by the ancients, as described by Vitruvius; and we have many of them at present in large or public buildings, where an index withinside a room or hall, points to the name of the quarter from which the wind blows without; which is simply effected by connecting an index to the lower end of the spindie of a weather-cock.

It has been observed that hygroscopes made of cat-gut, or such like, prove very good anemoscopes; seldom failing, by the turning of the index, to foretell the shifting of the wind. See accounts of two different anemoscopes; one by Mr. Pickering, vol. 43 Philos. Trans. the other by Mr. B. Martin, vol. 2 of his Philos. Britan.

Otto Guericke also gave the title anemoscope to a machine invented by him to foretell the change of the weather, as to rain ant fair. It consisted of the small wooden figure of a man, which' rose and fell in a glass tube, as the atmosphere was more or less heavy. Which was only an application of the common barometer, as shown by $\mathbf{M}$. Couriers in the Acta Eruditorum for 1684.

ANGLE, Angulus, in Geometry, the opening or mutual inclination of two lines, or two planes, meeting in a point, called the vertex or angular point. Such as the angle formed by, or between the two lines $A B$ and $A C$, at the vertex or angular point $A .-$ Also the two lines $\mathrm{AB}$ and $\mathrm{AC}$, are called the legs or the sides of the angle.
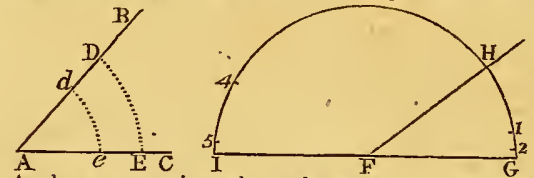

Angles are sometimes denoted, or named, by the single letter placed at the angular point, as the angle $\mathrm{A}$; and sometimes by threc letters, as RAc, placing always that of the vertex in the middle. The former nethod is used when only one angle has the same vertex; and the latter method it is necessary to use when several angles have the same vertex, to distinguish the en from one another.

The measure of all angle, by which its quantity or magniturle is expressed, is an arch, as $D E$, described from the centre $A$, with any radius at pleasure, and contained bctween its legs $\mathbf{A B}$ and $\mathbf{A C}$.- Hence angles are compared and distingnished by the ratio of the arcs which subtend them, to the whole circumfercnee of the circle; or by the number of degrees contained in the arc De by which they are measure $A$, to 360 , the number of degrees in the whole circumference of the circle. And thus an angle is said to be of so many degrees as are contained in the are DE, by which it is measured.

Hence it matters not with what radius the are is described, for the arcs $\mathrm{DE}$, de, being similar, they have the same ratio as their respretice radii or circumferences, and therefore they contain the same number of degrees.Hence it follows, that the quantity or magnitude of the angle remains still the same, though the legs be ever so much incrcased or diminished.-And thus, in sinilar figures, the like or corresponding angles are equal.

The tuking or measuring of angles, is an operation of great use and extent in surveying, navigation, geography, astronomy, \&c. And the instruments chiefly used for this purpose, are quadrants, sextants, octants, theodolites, circumferentors, \&c. Mr. Hadley invcnted an excellent instrument for taking the larger kind of angles, where. 

A N G
[ 118 ]
A N G

much accuracy is required, or where the motion of the object, or any circumstance causing an unsteadiness in the common instruments, renters the observations difficult, or uncertain. And Mr. Dollond contrived an instrument for measuring small angles See Hadley's 2uadront, MTicrometer, and the Philos. Trans. Numbers 420 , 425 , and vol. 48 .

To measure the Quantity of an Angle.

1. On paper. Apply the centre of a protractor to the vertex $A$ of the angle, so that the radius may coincide with one leg, as $A B$; then the degree on the arch that is cut by the other leg $A C$, will give the measure of the angle required.

Or thus, by a line of chords. Take off the chord of 60 with a pair of compasses; and with that radius, from the centre $A$, describe an arc, as DE. Then take this arc $\mathrm{DE}$ between the compasses, and apply the extent to the scale of chords, which will give the degrees in the angle as before.

M. De Lagny gave, in several memoirs of the Royal Academy of Sciences, a new method of measuring angles, which he called Goniometry. The method consists in measuring with a pair of compasses, the arc which subtends the proposed angle; not by applying its extent to a preconstructed scale of chords, but in the following manner: From the angular point as a centre, with a pretty large radius, describe a circle, producing one leg of the angle backwards to cut off a semicircle; then search out what part of the semicircle the arc is which measures the given angle in this manner; viz. take the extent of this arc with a very fine pair of compasses, and apply it several times to the arc of the semicircle, to find how often it is contained, with a small part remaining over; in the same manner take the extent of this small part, and apply it to the first arc, to find how often it is contained in it ; and what remains this $2 \mathrm{~d}$ time, apply in like manner to the first remainder; then the 3 il remainder apply to the $2 \mathrm{~d}$, and so on, always counting how often the last remainder is contained in the next foregoing, till nothing remain, or till the remainder is insensible, and too small to be measured. Then, begiuning at the last, and returning backwards, make a series of fractions, of which the numerators are always 1 , and the denominators are the number of times each remainder is contained in its next remainder, with the fractional part more, as derived from the following remainder; then the last fraction, thus obtained, will show what part the given angle is of $180^{\circ}$, or the semicircle; and being turned into degrees \&c, will be the measure of the angle, and nearer, it is asserted, than it can be obtaines by any other means; whether it be measuring, or calculating by trigonometrical tables.Thus, if it be reyuircl to measure the angle $G$ Fir: With a large radius describe the semicircle Gu, meeting the leg $F G$ produccd in 1 ; then take the extent of the arc $\mathrm{G} H$ in the compasses, and ápplying it from $G$ upon the semicircle, suppose it contains 4 times to the point 4 , and the part 4 i over; take 41 and apply it from 11 to 1 , so that kG contains 41 once, and 16 over; also apply this remainder to the former $4 \mathrm{i}$, and it is contained 5 times, from 4 to 5 , and 51 over; and lastly the remainder $5 \mathrm{I}$ is just twice contained in the former remainder $1 \mathrm{G}$ or 12 , withott any remainler. Here then, the series of quotients, or 'numbers of times contained, are 4, 1, 5, 2; therefore, beginning at the list, the first fraction is $\frac{1}{2}$, or the last remainder is half the preceding one; and the $2 \mathrm{~d}$ fraction is $\frac{1}{5 \frac{x}{2}}$ or $\frac{2}{x 1}$; the $3 \mathrm{~d}$ is $\frac{1}{1 \frac{2}{11}}$ or $\frac{1}{1 \frac{x}{3}}$; and the fourth is $\frac{1}{4 \frac{11}{13}}$ or $\frac{1}{6} \frac{3}{3}$; that is, the arc $\mathrm{GH}$ is $\frac{13}{6} \frac{3}{3}$ of a semicircle, or the angle GFH is $\frac{1}{6} \frac{3}{3}$ of two right angles, or of $180^{\circ}$, which is equivalent to $37 \frac{\mathrm{x}}{7}$ degrees, or $37^{\circ} \mathrm{8}^{\prime} 34^{\prime \prime} \frac{2}{7}$.

2. On the ground. Place a surveying instrument with its centre over the angular point to be measured, turning the instrument about till 0 , the beginning of its arch, fall in the line or direction of one leg of the angle; then turn the index about to the direction of the other leg, and it will cut off from the arch the degrees answering to the given angle.

To plot or lay down any given angle, either on paper or on the ground, may be performed in the same manner; and the method is farther explained under the articles Plotering and Protracting, also under the names of the several instruments.

To biscct a given angle, as suppose the angle $\mathrm{L} \mathrm{K} \mu$. From the centre $\mathrm{K}$, with any radius, describe the arc $L M$; then with the centres $\mathbf{L}$ and $\mathbf{M}$, describe two arcs intersecting in $\mathrm{N}$; and draw the line $\mathrm{KN}$, which will bisect the given angle $\mathbf{L K M}$, dividing it into the two equal angles $L K N$, $\mathrm{MKN}$.
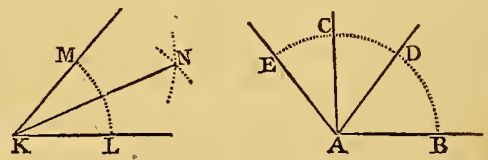

To trisect an angle, see TrISEction, and ANGULAR Sr.ctions.

- ANGLES are of various kinds and denominations. With regard to the form of thcir legs, they are divided into rectilinear, curvilinear, and mixed.

Rectilinear, or right-lined $\mathrm{ANGLE}$, is that whose legs are both right lines; as the foregoing angle is $\mathrm{K} \mathrm{L}$.

Curvilinear A NGLE, is that whose legs are both of them curves.

Mixt, or mixtilinear $\Lambda_{\mathrm{NGLE}}$, is that of which one leg is a right line, and the other a curve.

With regard to their magnitude, angles are again divided into right and oblique, acute and obtuse.

Right AngLe, is that which is formed by one line perpendicular to another; or that which is subtended by a quadrant of a circle. As the angle BAc.-Therefore the measure of a right angle is a quadrant of a circle, or $90^{\circ}$; and consequently all right angtes are equal to each other.

Oblique ANGLE, is a common name for any angle that is not a right one; and it is either acute or obtuse.

Acute ANGLE, is that which is less than a right angle, or less than 00 degrees; as the angle $B A D$.

Obtuse AxGLe, is grenter than a right angle, or whose measure exceeds 90 degrees; as the angle в $A$ F.

With regard to their situation in respect of eacb other, angles are distinguished into contiguous, adjacent, vertical, opposire, and altemate.

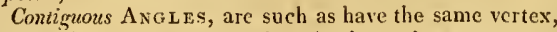
and one leg common to both. As the angles BAD, CAD, which have $A \mathrm{D}$ common.

Adjacent $A_{\mathrm{NGL}} \mathrm{s}$, are those of which a leg of the one produced forms a leg of the other: as the angles avn and Iv', which have the legs $1 F$ and $r g$ in a straight line.Hence, adjacent angles are supplements to each other, makiug together 180 degres. And therefore if one of 
these be given, the other will be known by subtracting the given one from 180 degrees. Which property is useful in surveying, to find the quantity of an inaccessible angle; viz, measure its adjacent accessible one, and subtract this from 180 degrees.
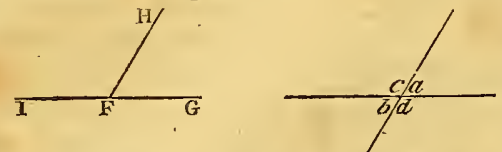

Vertical or opposite ANGLES, are such as have their legs mutually continuations of each other; as the two angles $a$ and $b$, or $c$ and $d$. -The property of these is, that the vertical of opposite angles are always equal to each other, viz, $\angle a=\angle b$, and $\angle c=\angle d$. And bence the quantity of an inaccessible angle of a field, \&c, may be found, by measuring its accessible opposite angle.

Altemate ANGLES, are those made on the opposite sides of a line cutting two parallel lines; so, the angles $e$ and $f$, or, $g$ and $h$, are alternates. And these are always equal to each other; viz, the $\angle e=\angle f$, or $\angle g=\angle h$.
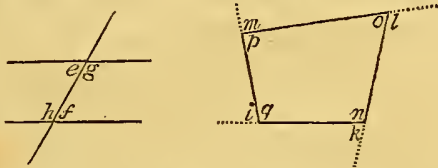

External ANGLES, are the angles of a figure made without it, by producing its sides; as the angles $i, k, l, m$. All the external angles of any right-lined figure, taken together, are equal to 4 right angles; and the external angle of a triangle is equal to both the internal opposite ones taken together; also any cxternal angle of a trapezium inscribed in a circle, is equal to the internal opposite angle.

Internal $\mathrm{AXGLES}$, are the angles within any figure, made by the sides of it; as the angles $n, o, p, q$. - In any rightlined figure, an internal angle as $n$, and its adjacent external angle $k$, together make two right angles, or 180 degrees; and all the internal angles $n, o, p, q$, taken together, inake twice as many right angles, wanting 4 , as the figure has sides; also any two opposite internal angles of a trapczium inscribed in a circle, taken together, make two right angles, or $\mathbf{1 8 0}$ degrees.

Homologous, or like ANGLES, are such angles in two figures, as ritain the same order in both.

ANGIE, at the centre, is that whose vertex is in the centre; as the angle $\mathrm{AFC}$, formed by two radii $\mathrm{AF}, \mathrm{FC}$, and it is measured by the arc $A D C .-\Lambda n$ angle at the centre, as $A F C$, is always double the angle $\triangle B C$ at the circumference, standing on the same arc $A 1) \mathrm{C}$; and all angles at the centre are "qual that stand upon the same or equal arcs: also all angles at the centre are proportional to the ares they stand upon; and so also are all angles at the circumference.
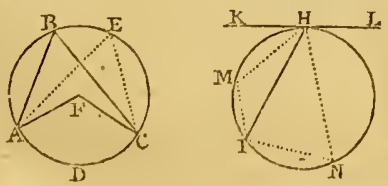

ANGLE at the circumference, is that whose vertex is somewhere in the circumference of a circle; as the angle $A B C$.

ANGLE in a segment, is that whose legs meet the extremities of the base of the segment, and its vertex is anywhere in its arch; as the angle $B$ is in the segment $A \mathbf{B C}$; and is coniprehencled between two chords $A B$ and $\mathrm{BC}$.An angle at the circumference is measured by half the $\operatorname{arc} A \mathrm{DC}$ on which it stands; and all the angles $A \mathbf{B C}, \mathbf{A E C}$, in the same segment, are equal to each other.

ANGLE in a semicircle is an angle at the circumference standing on a semicircle, or on a diameter.-An angle in a semicircle, is a right angle; in a greater segment, the angle is less, and in a less segment is greater, than a right angle.

ANGLE of a segment, is that made by a chord with a tangent, at the point of contact. So' 1 н $\mathrm{K}$ is the angle of the less segment Iми, and IнL, the angle of the greater segment INH.-And the measure of each of these angles, is half the alteruate or supplemental segment, or equal to the angle in it; viz, the $\angle \mathrm{IHK}=\angle \mathrm{INH}$, and the $\angle \mathrm{IHL}=\angle \mathrm{IMH}$.

ANGLE out of the centre, as $G$, is one whose vertex is not in the centre of the circle.-And its measure is half the sum $\frac{1}{2} a+\frac{x}{2} b$ of the arcs intercepted by its legs when it is within the circle, or half the difference $\frac{x}{2} a-\frac{1}{2} b$ when it is without.
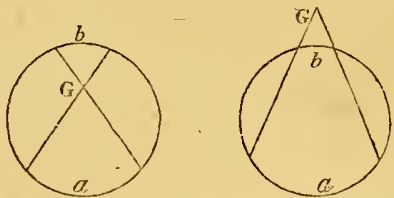

ANGLE of a semicircle, is the angle which the diameter of a circle makes with the circumference. Euclid demonstrates that this is less than a right angle, but greater than any acute angle.
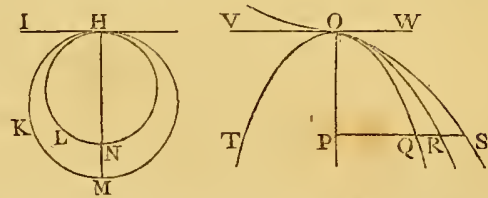

ANG LE of contact, is that made by a curve line and a taugent to $\mathrm{jt}$, at the point of contact; as the angle $\mathbf{I H K}$. It is proved by Euclid, that the angle of contact between a right line and a circle, is less than any right-lined angle whatever; though it does not therefore follow that it is of no magnitude or quantity. This has been the subject of great disputes among geometricians, in which Peletarius, Clavius, Taquet, IVallis, \&c, bore a considerable share; Peletarius and Wallis contending that it is no angle at all, against Clavius, who rightly asserts that it is not absolutely nothing in itself, but only of no magniturle in comparison with a right-lined angle, being a quantity of a different kind or nature; like as a line in respect to a surface, or a surface in respect to a solid, \&c. And sinee lis time, it has been proved by sir Isaac Newton, and others, that angles of contact can be compared to each other, though not to right-lined angles, and what 
the proportions are which they bear to each other. Thus, the circular angles of contact $1 \mathrm{HK}, \mathrm{IHL}$, are to each other in the reciprocal subduplicate ratio of the diameters II $\mathrm{M}, \mathrm{H}$. And hence the circular angle of contact may be divided, by describing intermediate circles, into-any number of parts, and in any proportion. And if, instead of circles, the curves be parabolas, and the point of contact 3 or o the common vertex of their axes; the angles of contact will then be reciprocally in the subduplicate ratio of their parameters. But in such elliptical and hyperbolical angles of contact, these will be reciprocally in the subduplicate of the ratio compounded of the ratios of the parameters, and the transverse axes. Moreover, if TOQ be a common parabola, to the axis $O P$, and tangent vow, and whose equation is $1 \times x=y^{2}$, or $x=y^{2}$, where $x$ is the absciss $a P$, and $y$ the ordinate $\mathrm{PQ}$, the parameter being 1 : and if or, os, \&c, be other parabolas to the same axis, tangent, and parameter, their ordinate $y$ being $\mathrm{PR}_{\mathbf{z}}$ or PS, \& C, and their equations $x=y^{3}, x=y^{4}, x=y^{5}$, \&c: then the series of angles of contact will be in succession infinitely greater than each other, viz, the angle of contact wOQ infinitely greater than wOR, and this infinitely greater than wos, and so in infinitum.

And farther, between the angles of contact of any two of this kind, may other angles of contact be found ad infinitum, which shall infinitely exceed each other, and yet the greatest of them be infinitely less than the smallest right-lined angle. So ālso $x^{2}=y^{3}, x^{3}=y^{4}, x^{4}=y^{5}$, \&c, denote a series of curves, of which every succeeding one makes an angle with its tangent, infinitely greater than the preceding one; and the least of these, viz, that whose equation is $x^{2}=y^{3}$, or the semicubical parabola, is infinitely greater than any circular angle of contact.

ANGLEs are again divided into plane, spherical, and solid.

Plane ANGLES, are all those above treated of; which are defined by the inclination of two lines in a plane, meeting in a point.

Spherical ANGLE, is an angle formed on the surface of a sphere by the intersection of two great circles; or, it is the inclination of the planes of those circles.

The measure of a spherical angle, is the arc of a great circle of the sphere, intercepted between the two planes which form the angle, and which cuts the said planes at right angles. For their properties, \&c, see SPHERE, SPnerical, and SPnerical Trigonometry.

Solid ANGLE, is the mutual inclination of more than two planes, or plane angles, meeting in a point, and not contained in the same plane; like the angles or corners of solid bodies. For their measure, properties, \&c, see Soun Angle.

Angles of other less usual kinds and denominations, are also $t o$ be found in some buoks of Geonetry. As,

Horned ANGLE, angulus cornutus, that which is made by a right line, whether a tangent or secunt, with the circumference of a circle.

Lunular $\Lambda_{\mathrm{N}} \mathrm{LE}$, angulus lunularis, is that which is formed by the intersection of two curve lines, the onc concave, and the other convex.

Cissoid ANGLE, angulus cissoides, the inner angle made by two spherical convex lines intersecting each other.

Sistroid $A N G \mathrm{LE}$, angulus sistroides, is that which is in the form of a sistrum.

Pelccoid ANGLE, angulus pelecoides, is that in the form of a hatchet.
Angle, in Trigonometry. See Triangle, TrigonoNetr $\mathrm{Y}$, Sine, TANGENT, \&ic.

ANG LE, in Mechanics.-Angle of Direction, is that which is comprehended between the lines of direction of two conspiring forces.

ANGLE of Elecation, is that which is comprehended between the line of direction and any plane on which the projection is made, whether horizontal or oblique.

ANGLE of Incidence, is that made by the line of direction of an impinging body, at the point of impact. As the angle $\mathrm{ABC}$.

ANGLE of Reflection, is that made by the line of direction of the refected body, at the point of impact. As the angle $\mathrm{DBE}$.

Instead of the angles of incidence and reflection being estimated from the plane on which the body impinges, sometimes the complements of these are understood, viz, as estimated from a perpendicular to the reflecting plane; as the two angles $A B F$ and $D B F$.
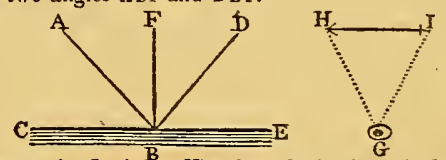

AxGLE, in Optics.-Visual or Optic Angle, is the angle included between the two rays drawn from the two extreme points of an object to the centre of the pupil of the eye : as the angle HGI. The apparent magnitude of objects, when their distance cannot be compared, is greater or less, according to the angle under which they appear. -Objects seen under the same or an equal angle, appear equal.-But there is an exception to this rule in the case of the heavenly bodies, which are really seen under a less angle in the horizon than when they have attained any sensible degree of elevation (see Rerraction); notwithstanding which, it must bave been observed by every one that they appear larger in the horizon, than in any other part of their course; this becomes still more sensible if we observe the distance of two stars at their rising, and again when they are near the zenith, their apparent distance in the former case being considerably greater than in the latter, yet if their angular distance be measured by a sextant, it will be found to increase as their distance from the zenith decreases. (See Apparent Magnitude.)

The least visible angle, or least angle under which a body can be seen, according to Dr. Hooke, is one minute; but Dr. Jurin shows, that at the time of his debate with Hevelius on this subject, the latter could probably discover a single star under so snall an angle as $20^{\prime \prime}$. But bodies are visible under smaller angles as they are more bright or luminous. Dr. Jurin states the grounds of this controversy, and discusses the question at large, in his Essay on distinct and indistinct Vision, published in Smith's Optics, pa, 148, $e l$ seq.

ANG $\mathrm{x}$ of the interval, of two places, is the angle subtended by two lines directed from the eye to those places.

ANGLE of incidence, or reffction, or refraction, Sc. See the respective words Incidence, Refliction, ReFRACTION, \&C.

ANgIe, in Astronomy. - Angle of Commutation. Sce Commuration.

Avgue of elongation, or Angle at the Earth. Sce Elongation. 
Parallactic ANGLE, or the parallax, is the angle made at the centre of a star, or the sun, \&c, by two lines drawn, the one to the centre of the earth, and the other to its surface. See Parallactic, and Parallax.

ANGLE of the position of the sun, of the sun's npparent semi-diameter, \&c. See the respective words.

A NGLE at the sun, is the angle under which the distance of a planet from the ecliptic is seen from the sun.

Angle of the East. See Nonagesimal.

ANGLE of obliquity, of the ecliptic, or the angle of inclination of the axis of the earth to the axis of the ecliptic, is now nearly $23^{\circ} 28^{\prime}$. See Obliquitr, and ECLiptic.

ANGLE of longitude, is the angle which the eircle of a star's longitude makes with the meridian, at the pole of the ecliptic.

A NGLE of right ascension, is the angle which the circle of a star's right ascension makes with the meridian at the pole of the equator.

ANGLE, in Navigation. ANGLE of the rhumb, or loxodromic angle: See RII M B and LoXodromic.

ANGLEs, in Fortification, are understood of those formed by the several lines used in fortifying, or making a place defensible.

These are of two sorts; real and inaginary.-Real angles are those which actually, exist and appear in the works. Such as the flanked angle, the angle of the epaule, angle of the fiank, and the re-entering angle of the counterscarp. Imaginary, or occult angles, are those which are only subservient to the eonstruction, and which exist no more after the fortification is drawn. Such as the angle of the centre, angle of the polygon, flanking angle, saliant angle of the countersearp, \&c.

ANGLE of, or at, the centre, is the angle formed at the centre of the polygon, by two radii drawn from the centre to two adjacent angles, and subtended by a side of it, as the angle $\mathrm{ACB}$. This is found by dividing 360 degrees by the number of sicles in the regular polygon.

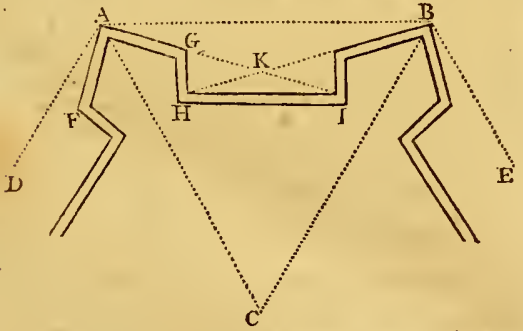

ANGLE of the Polygon, is the angle intercepted between two sides of the polygon; as DAB, or ABE. This is the supplement of the angle at the centre, and is therefore found by subtracting the angle $\mathrm{c}$ from 180 degrees.

ANGLE of the Triangle, is half the angle of the polygon; as CAB or CBA; and is therefore half the supplement of the angle $c$ at the centre.

ANGLE of the Brstion, is the angle FAG, made by the two faces of the bastion. And is otherwise called the flanked angle.

Diminished ANGLE, is the angle $\mathbf{B A G}$, madk: by the meeting of the exterior side of the polygun with the face $A$ (: of the bastion.

VOL. I.
ANGLE of the curtin, or of the flank, is the angle GII made between the curtin and the flank.

ANGLE of the epaule, or shoulder, is the angle AGH, made by the flank and the face of the bastion.

ANGLE of the tenaille, or exterior flanking angle, is the angle $A$ K $B$, made by the two rasant lines of defence, or the faces of two bastirns produced.

ANGLE of the counterscarp, is the angle made by the two sides of the counterscarp, meeting before the middle of the curtin.

ANGLE flunking inward, is the angle made by the flanking line with the curtin.

A NG LF, forming the flank, is that consisting of one fank and one demigorge.

ANGLE forming the face, is that composed of one flank and one face.

ANGLE of the moat, is that made before the curtin, where it is intersected.

Re-entering, or re-entrant ANGLE, is that whose vertex is turued invards, towards the place; as $\mathbf{H}$ or 1 .

Saliant, or sortant ANGLE, is that turned outwards, advancing its point towards the field; as $A$ or $G$.

Dead ANGLE, is a re-entering angle, which is not flanked or defended.

ANGLE of a wall, in Architecture, is the point or corner where the two sides or faces of a wall meet.

ANGUINEAL Hyperbola, a name given by Sir Isaac Newton to four of his curves of the second order, viz, species $33,3+, 35,36$, expressed by the equation $x y^{2}+e y$ $=-a x^{3}+b x^{2}+e x+d$; being bypertuolas of a selpentine figure. See Curves.

ANGULAR, something relating to, or that hath angles.

At a distance, angular bodies appear round; the angles and small inequalities disappearing at a much less distance. than the butk of the body.

ANGULAR Motion, is the motion of a body which moves circularly about a point; or the variation in the angle described by a line, or radius, connecting a body with the centre about which it moves.-Thus, a pendulum has an angular motion about its centre of motion; and the planets have an angular motion about the sun.Two moveable points $\mathrm{M}$ and $\mathrm{o}$, of which the one describes the arc $\mathrm{MN}$, and the other the arc op, in the same time, have an equal, or the same angular motion, though the real motion of the point $o$ be much greatcr than that of the point $M$, viz, as the arc $O P$ is greater than the arc MN. The angular motions of revolving bodies, as of the planets about the sun, are reciprocally proportional to their periodic times. And they are also, as their real or absolute motions directly, and as their radii of motion inversely.

ANGULAR motion is also a kind of compound motion composed of a circular and a rectilinear motion; like the wheel of a coach, or other vehicle.

Angular Sections, the dividing of angles into some number of equal parts; as, to bisect, or to trisect an angle, \&c.

Euclid taught how to bisect an angle, viz, in prop. 9, bonk 1 ; but his geometry goes not farther. The other ancients after him endeavoured to trisect an angle; in which attempt, as Luclid's geometry failed them, they had recourse to the conic sections, which had been ad. $\mathrm{R}$ 
mitted into geometry, by means of which, and a problem in Apollonius's Inclinations, they effected the trisection; which was equivalent to solving a cubic equation. Pappus, in the $34 \mathrm{th}$, and following props. of book 4 , treats of the trisection; and in the 36 th et seq. of any section in general. Many of the moderns have also treated of angular sections; as, Vieta, Alex. Anderson, Wallis, Briggs, I'Hopital, Guisne, Emerson, \&c. See Secrion and Trisectiox, \&c.

ANNUAL, in Astronomy, something that returns every year, or which terminates with the year.

ANNUAL motion of the earth. See EARTH.

ANNUAL argument of longitude. See ARGUMENT.

ANNUAL epacts. See EPact.

ANNUAL equation of the mean motion of the sun and moon, and of the moon's apogee and nodes. See EQUATION.

ANNUITIES, any periodical incomes, arising from money lent, or from houses, lands, salaries, pensions, \&c; payable from time to time; either annually, or at other intervals of time.

Annuities may be divided into such as are certain, and such as depend on some contingency, as the continuance of a life, \&ic. Sée LifE AN NuTizs.

Annuities are also divided into annuities in possession, and annuities in reversion; the former meaning such as have commenced; and the latter such as will not commence till some particular event has happened, or till soine given period of time has elapsed.

Annuities may be farther considered as payable either yearly, or half yearly, or quarterly, \&c.

The present value of an annuity, is that sum, which, being improved at interest, will be sufficient to pay the annuity.

The present value of annuity certain, payable yearly, is calculated in the following manner.-Let the annuity be $1 l$ : and let $r$ denote the amount of $1 l$. for a year, or $1 l$. increased by its interest for one year. Then, 1 being the present value of the sum $r$, and having to find the present value of the sum 1 , it will be, by proportion thus, $r: 1:$ : $1: \frac{1}{r}$ the present value of $1 l$. due a year hence. And for the same reason $\frac{a}{r}$ is the present value of $a$ pounds due a year hence; the present value being always in direct proportion to the yearly payment. So that, in what follows, we shall only consider the annuity as $1 l$, ; and whatever results are thus obtained, being multiplied by $a$, will correspond to an annuity of $a$ pounds.

Now since $\frac{1}{r}$ is the present value of $1 l$, due a year hence, so likewise $\frac{1}{y^{2}}$ will be the present value of $1 l$. due 2 years hence; for $r: 1:: \frac{1}{r}: \frac{1}{r^{2}}$. So also $\frac{1}{r^{3}}, \frac{1}{r^{4}}, \frac{1}{r^{5}}$, \&c, will be the present value of $1 l$. due at the end of $3,4,5$, \&c, years, respectively; and in general, $\frac{12}{r^{\mathrm{u}}}$ will be the value of $1 l$, to be received after the expiration of $n$ years. Consequently the sum of all these, or $\frac{1}{r}+\frac{1}{r^{3}}+\frac{1}{r^{3}}+\frac{1}{r^{2}}+$ \& c, continued to $n$ terms, will be the present value of all the $n$ years annuities. And the value of the perpetuity, is the sum of the series continued to infinity.

But this series, it is evident, is a geometrical progres- sion, the first term and common ratio being each $\frac{1}{r}$, and the number of its" terms $n$; therefore the sum $s$ of all the terms, or the present value of all the annual payments, will be $s=\frac{1}{r-1}-\frac{1}{r-1} \times \frac{1}{r^{n}}$.

When the annuity is a perpetuity, it is plain that the last quantity $\frac{1}{r^{\mathbf{n}}}$ vanishes, and therefore $\frac{1}{r-1} \times \frac{1}{r^{\mathbf{n}}}$ also vanishes; consequently the expression becomes barely $s=$ $\frac{1}{r-1}$; that is, $\frac{1}{r-1}$ is the present value of the perpetuity of an annuity of $1 l$. per ann. and consequently $\frac{a}{r-1}$ will be the present value of the perpetuity of $a$ pounds per ann.; hence any annuity divided by the interest of $1 l$. for one year, is the value of the perpetuity. So, if the rate of interest be 5 per cent. and yearly payments, then $\frac{x 00}{5} a=$ $20 a$ is the value of the perpetuity. Also $\frac{100}{4} a=25 a$ is the value of the perpetuity at 4 per cent. And $\frac{100}{3} a=$ $33 \frac{1}{3} a$ is the value of the perpetuity at 3 per cent. interest. And so on.

If the annuity is not to be entered on immediately, but after a certain time, as $m$ years; then the present value of the reversion is equal to the difference between two present values, the one of the first tern of $m$ years, and the other for the end of the last term $n$ : that is, equal to the difference between $\frac{1}{r-1}-\frac{1}{r-1} \times \frac{1}{r^{\mathrm{n}}}$ and $\frac{1}{r-1}-\frac{1}{r-1} \times$ $\frac{1}{r^{\mathrm{m}}}, \mathrm{or}=\frac{1}{r-1} \times\left(\frac{1}{r^{\mathrm{m}}}-\frac{1}{r^{\mathrm{n}}}\right)$.

Annuities certain differ in value, as they are made payable yearly, half-yearly, or guarterly. And by proceeding as above, using the interest or amount of a half year, or a quarter, as those for the whole year were used, the following set of theorems will arise; where $r$ denotes, as before, the amount of $1 l$. and its interest for a year, and $n$ the number of years, during which, any annuity is to be paid; also $\mathbf{P}$ denotes the perpetuity $\frac{1}{r-1}, \mathrm{Y}$ denotes $\frac{1}{r-1}$ $-\frac{1}{r-1} \times \frac{1}{r^{n}}$ the present value of the annuity supposed payable yearly, iI the present value of the same when it is payable lialf-yearly, and a the same when payable quarterly; or universally, a the present value when it is payable every $m$ part of a year.

$$
\begin{aligned}
& \text { Theor. 1. } \mathbf{Y}=\mathrm{P}-\mathrm{P} \times\left(\frac{1}{r}\right)^{\mathrm{n}} . \\
& \text { Theor. 2. } \mathrm{I}=\mathrm{P}-\mathrm{P} \times\left(\frac{2}{r+1}\right)^{2 \mathrm{n}} . \\
& \text { Theor. 3. } \mathrm{Q}=\mathrm{P}-\mathrm{P} \times\left(\frac{4}{r+3}\right)^{4 \mathrm{n}} . \\
& \text { Theor. 4. } \mathrm{M}=\mathrm{P}-\mathrm{P} \times\left(\frac{m}{r+m-1}\right)^{\mathrm{mn}} .
\end{aligned}
$$

Exam. 1.-Let the rate of interest be 4 per cent, and the term 5 years; and consequently $r=1 \cdot 04, n=5$, P $=25 a$; also let $m=12$, or the interest payable monthly in thorem 4 : then the present value of an ammity of $a$ pounds a year, for 5 years, according as it is supposed payable yearly, or every half year, or every quarter, or every month or $\frac{1}{2}$ th part of a year, will be as follows:

$$
\begin{aligned}
& \mathrm{x}=25-25 \times \cdot 821.928=4.451 \mathrm{~s} \\
& \mathrm{u}=25-25 \times \cdot 8203.18=4.413 \\
& \mathrm{Q}=25-25 \times \cdot 81.9543=.15114 \\
& \mathrm{u}=25-25 \times \cdot 818996=4.525 \mathrm{t}
\end{aligned}
$$

Exam. 2. Supposing the annuity to contimue 25 

A N N

years, the rate of interest and every thing clse being as before; then the values of the annuities for 25 years will be

$$
\begin{aligned}
& \mathrm{y}=25-25 \times 375118=15.6221 \\
& \mathrm{r}=25-25 \times 371527=15.711 \mathrm{~S} \\
& \mathrm{Q}=25-25 \times \cdot 369709=15.7573 \\
& \mathrm{M}=25-25 \times \cdot 368477=15.7881
\end{aligned}
$$

Exam. 3. And if the term be 50 years, the values will be

$$
\begin{aligned}
& \mathrm{Y}=25-25 \times \cdot 14.0713=21.4822 \\
& \mathrm{H}=25-25 \times \cdot 138032=21.5492 \\
& \mathrm{Q}=25-25 \times \cdot 136685=21.5829 \\
& \mathrm{M}=25-25 \times \cdot 135775=21.6056
\end{aligned}
$$

Exam. 4. Also if the term be 100 years, the values will be

$$
\begin{aligned}
& \mathrm{Y}=25-25 \times 019800=24 \cdot 5050 \\
& \mathrm{H}=25-25 \times \cdot 019053=24.5237 \\
& \mathrm{Q}=25-25 \times 018683=24.5329 \\
& \mathrm{M}=25-25 \times \cdot 018435=24.5391
\end{aligned}
$$

Hence the difference in the value by making periods of payments smaller, for any given term of years, is the more as the intervals are smaller, or the periods more frequent. Tlue same difference is also variable, both as the rate of inlerest varies, and also as the whole term of years $n$ varies; and, for any given rate of interest, it is evident that the difference, for any periods $m$ of payments, first increases from nolhing as the term $n$ increases, from 0 , to some certain finite term or value of $n$, when the difference $D$ is the greatest or a maximum; and that afterwards, as $n$ increases more, that difference will continually decrease to nothing again, and vanish when $n$ is infinite: also the term or value of $n$, for the maximum of the difference, will be different according to the periods of payment, or value of $m$.

Anmities may also be considered as in arrears, or as forborn, for any number of years; in which case each payment is to be considered as a som put out to interest for the remainder of the term atter the time it becomes due. And as 1l. due at the end of 1 year, amounts to $r$ at the end of another year, and to $r^{2}$ at the end of the $3 \mathrm{~d}$ year, and to $r^{3}$ at the end of the 4th year, and so on; therefore, by adding always the last year's annuity, or 1, to the amounts of all the former years, the sum of all the annuities and their intcrests, will be the sum of the fol-

\begin{tabular}{|c|c|c|c|c|c|c|}
\hline & The Am & int of $a$ & $\begin{array}{l}\text { T A B } \\
\text { Annuit }\end{array}$ & 1. & amp. $I$ & \\
\hline 5 & $\begin{array}{l}\text { at } 3 \text { per } \\
\text { cent. }\end{array}$ & $\begin{array}{l}3 \frac{1}{2} \text { yer } \\
\text { cent. }\end{array}$ & $\begin{array}{l}4 \text { per } \\
\text { ceut. }\end{array}$ & $\begin{array}{l}4 \frac{1}{2} \text { per } \\
\text { cent. }\end{array}$ & $\begin{array}{l}5 \text { per } \\
\text { ccnt. }\end{array}$ & $\begin{array}{l}6 \text { per } \\
\text { cent. }\end{array}$ \\
\hline$\sigma_{1}$ & 1.00000 & 1.00000 & 1.00000 & $1 \cdot 00000$ & $1 \cdot 00000$ & $1 \cdot 00000$ \\
\hline 2 & 2.03000 & $2 \cdot 03500$ & $2 \cdot 04000$ & 2.04500 & $2 \cdot 05000$ & $2 \cdot 06000$ \\
\hline 3 & 3.09090 & $3 \cdot 10623$ & $3 \cdot 12160$ & $3 \cdot 13703$ & 3.15250 & $3 \cdot 18360$ \\
\hline 4 & $4 \cdot 18363$ & 4.21494 & $4 \cdot 24646^{\circ}$ & $4 \cdot 27819$ & 4.31013 & $4 \cdot 37462$ \\
\hline 5 & $5 \cdot 30914\}$ & $5 \cdot 3624$ ㄱ & 5.41632 & 5.47071 & $5 \cdot 52563$ & $5 \cdot 63709$ \\
\hline
\end{tabular}
lowing geometrical series, $1+r+r^{2}+r^{3}+r^{4}-\ldots . .-$

\begin{tabular}{|c|c|c|c|c|c|c|}
\hline & The Amo & unt of an & $\begin{array}{l}\text { LE } 1 .-\mathrm{Co} \\
\text { Annity }\end{array}$ & $\begin{array}{l}\text { ontinucd } \\
\text { of } 1 l \text {. at }\end{array}$ & $a m p . I$ & rest. \\
\hline 4 & $\begin{array}{l}\text { at } 3 \text { per } \\
\text { cent. }\end{array}$ & $\begin{array}{l}3 \frac{1}{2} \text { per } \\
\text { cent. }\end{array}$ & $\begin{array}{l}4 \text { per } \\
\text { cent. }\end{array}$ & $\begin{array}{l}4 \frac{l}{2} \text { per } \\
\text { centit. }\end{array}$ & $\begin{array}{l}5 \text { per } \\
\text { cent. }\end{array}$ & $\begin{array}{l}6 \text { per } \\
\text { cent. }\end{array}$ \\
\hline-6 & 6.46841 & 6.55015 & 6.63298 & 6.71689 & 6.80191 & 6.97532 \\
\hline 7 & $7 \cdot 66246$ & $7 \cdot 77941$ & $7 \cdot 89829$ & $8 \cdot 01915$ & $8 \cdot 14201$ & $8 \cdot 39384$ \\
\hline 8 & 8.89234 & $9 \cdot 05169$ & $9 \cdot 21423$ & $9 \cdot 38001$ & $9 \cdot 54911$ & 0.89747 \\
\hline 9 & $10 \cdot 15911$ & $10 \cdot 36850$ & 10.58280 & $10 \cdot 80211$ & $11 \cdot 02656$ & 11.49132 \\
\hline 10 & $11 \cdot 46388$ & $11 \cdot 73139$ & 12.00611 & $12 \cdot 28821$ & 12.57789 & $13 \cdot 18079$ \\
\hline 11 & $12 \cdot 80780$ & $13 \cdot 14199$ & 13.48635 & $13 \cdot 84118$ & 14.20679 & 14.97164 \\
\hline 12 & $14 \cdot 19203$ & $14 \cdot 60196$ & $15 \cdot 02581$ & 15.46403 & 15.91713 & 9994 \\
\hline 13 & 15.61779 & $16 \cdot 11303$ & 16.62684 & $17 \cdot 15991$ & $17 \cdot 71298$ & $18 \cdot 88214$ \\
\hline 14 & $17 \cdot 08632$ & $17 \cdot 67699$ & $18 \cdot 29191$ & $18 \cdot 93211$ & 19.59863 & 21.01507 \\
\hline 15 & 18.59891 & $19 \cdot 29568$ & $20 \cdot 02359$ & $20 \cdot 78405$ & 21.57856 & $23 \cdot 27597$ \\
\hline 16 & $20 \cdot 15688$ & $20 \cdot 97103$ & 21.82453 & 22.71934 & $23 \cdot 65749$ & $25 \cdot 67253$ \\
\hline 17 & 21.76159 & $22 \cdot 70502$ & $23 \cdot 69751$ & $24 \cdot 74171$ & 34037 & 1288 \\
\hline 18 & 23.41444 & $24 \cdot 49969$ & $25 \cdot 6454 \mathrm{I}$ & 26.85508 & 3238 & 90565 \\
\hline 19 & $25 \cdot 11687$ & 26.35718 & $27 \cdot 67123$ & $29 \cdot 06356$ & 3900 & 5999 \\
\hline 20 & $26 \cdot 87037$ & 28.27968 & $29 \cdot 77808$ & $31 \cdot 37142$ & 33.06595 & 36.78559 \\
\hline 21 & $28 \cdot 67649$ & $30 \cdot 26947$ & 31.96920 & $33 \cdot 78314$ & $35 \cdot 71925$ & 39.99273 \\
\hline 22 & 3678 & $32 \cdot 32890$ & $34 \cdot 24797$ & $36 \cdot 30338$ & 521 & \\
\hline 23 & $32 \cdot 45288$ & 34.46041 & $36 \cdot 61789$ & 38.93703 & $41 \cdot 43048$ & 46.99583 \\
\hline 24 & $34 \cdot 42647$ & $36 \cdot 666 \vdots 3$ & $39 \cdot 08260$ & $41 \cdot 68920$ & $44 \cdot 50200$ & $50 \cdot 81558$ \\
\hline 25 & $36 \cdot 45926$ & 38.94986 & $41 \cdot 64591$ & $44 \cdot 56521$ & 2710 & 451 \\
\hline 26 & $38 \cdot 55304$ & 41.31310 & $44 \cdot 31174$ & $47 \cdot 57064$ & $51-11345$ & 5638 \\
\hline 27 & $40 \cdot 70963$ & $43 \cdot 75906$ & 47.08421 & $50 \cdot 7$ & 6913 & 0577 \\
\hline 28 & $42 \cdot 9$ & $46 \cdot 29063$ & $49 \cdot 96758$ & 53.99333 & 10258 & 52811 \\
\hline 29 & $45 \cdot 2$ & 48.91080 & 52.96629 & $57 \cdot 4$ & 2271 & 3980 \\
\hline 30 & $47 \cdot 57542$ & $51 \cdot 62268$ & $56 \cdot 08494$ & 61.00707 & $66 \cdot 43885$ & 79.05819 \\
\hline 31 & $50 \cdot 00268$ & $54 \cdot 42947$ & $59 \cdot 32834$ & 64.75239 & $70 \cdot 76079$ & $84 \cdot 80168$ \\
\hline 32 & $52 \cdot 5$ & $57 \cdot 33450$ & $62 \cdot 70147$ & $68 \cdot 6$ & 9883 & 978 \\
\hline 33 & $55 \cdot 07784$ & $60 \cdot 34121$ & $66 \cdot 20953$ & $72 \cdot 75623$ & 80.06377 & $97 \cdot 34316$ \\
\hline 34 & $57 \cdot 73018$ & 63.45315 & $69 \cdot 85791$ & $77 \cdot 03026$ & $85 \cdot 06696$ & $104-18375$ \\
\hline 35 & $60 \cdot 46208$ & $66 \cdot 67401$ & $73 \cdot 65222$ & $81 \cdot 49662$ & $90 \cdot 3$ & 3478 \\
\hline 36 & $63 \cdot 27594$ & 70.00760 & $77 \cdot 59831$ & $86 \cdot 16397$ & $95 \cdot 8$ & $119 \cdot 1$ \\
\hline 37 & $66 \cdot 17422$ & 73.45787 & $81 \cdot 70225$ & 1134 & & $81 \%$ \\
\hline 38 & $69 \cdot 15945$ & $77 \cdot 02889$ & $85 \cdot 97034$ & $96 \cdot 1$ & & 0421 \\
\hline 39 & $72 \cdot 2$ & $80 \cdot 72491$ & 90. & iol. & & 846 \\
\hline 40 & 75.40126 & & & & 120 & $154 \cdot 76197$ \\
\hline 41 & $78 \cdot 66330$ & $88 \cdot 50954$ & $99 \cdot 82654$ & 112 & 127 & \\
\hline 42 & 82.02320 & & & & & \\
\hline 43 & 85.48389 & $96 \cdot 84863$ & 110.01238 & $125 \cdot 2$ & 142 & 187 \\
\hline 44 & $89 \cdot 04841$ & & & & 151 & $199 \cdot 75803$ \\
\hline 45 & $92 \cdot 71986$ & & & $138 \cdot 84997$ & $159 \cdot 70016$ & $212 \cdot 74351$ \\
\hline 46 & 96.50146 & $110 \cdot 48403$ & $126 \cdot 87057$ & 146.09821 & $168 \cdot 68516$ & 226.50812 \\
\hline 47 & $100 \cdot 39650$ & $115 \cdot 350$ & 132.94539 & 153.67263 & $178 \cdot 11942$ & $241 \cdot 09861$ \\
\hline 48 & & & & & & \\
\hline 49 & & & & & & \\
\hline 50 & 112.796 & 13 & 70 & 303 & $209 \cdot 34$ & $290 \cdot 3$ \\
\hline $5 \mathrm{I}$ & $117 \cdot 18077$ & $136 \cdot 5828$ & 159 & & $220 \cdot 815$ & 308 \\
\hline & & & & & & \\
\hline 53 & & $148 \cdot 34595$ & 174.85131 & 206.8386 & & (1) \\
\hline & & & & & & \\
\hline
\end{tabular}
to $r^{n-1}$, continued till the last term be $r^{n-1}$, or till the number of terms be $n$, the number of years the annuity is fiorborn. But the sum of this geometrical progression is $\frac{r^{n}-1}{r-1}$, which therefore is the amount of $1 l$. annuity for born for $n$ yeaŕs. And this quantity being multiplied by $a$ instead of 1 , will produce the amount for the annuity $a$.

But the amounts of annuities, or their present values, are easiest found by the two following tables of numbers for the annuily of $\mathbf{1}$. ready computed irom the foregoing principles.

\section{TIIE Use of Table I.}

To find the Amount of an annuity forborn any number of years. Take out the amount from the 1 st table, for the proposed years and rate of interest; then multiply it by the annuity in question; and the product will be its amount, for the same number of years, and rate of interest.-And the converse, to find the rate or time.

Exem. 1. To find how much an annuity of $50 l$. will amount to in 20 years at $3 \frac{1}{2}$ per cent. compound interest. -On the line of 20 years, and in the column of $3 \frac{x}{2}$ per cent, stands $28 \cdot 27968$, which is the amount of an annuity of $1 l$. for the 20 years; and therefore $28 \cdot 27968$ multiplied by 50 , gives $1413.984 l$. or $14.13 l .19 s .8 d$. for the answer.

Exam. 2. In what time will an annuity of $20 l$. amount to $1000 l$. at 4 per cent. compound interest?-Here the amount of $1000 l$. divided by $20 l$, the annuity, gives 50 , the amount of $1 l$. annuity for lie same time and rate. R 2 
Then, the nearest tabular number in the column of 4 per cent. is 49.96758 , which standing on the line of 28 , shows that 28 years is the answer.

Exam.3. If it be required to find at what rate of interest an annuity of $20 \mathrm{l}$. will amount to $1000 \mathrm{l}$. forborn for 28 years. - llere 1000 divided by 20 gives 50 as before: Then looking along the line of 28 years, for the nearest to this number 50 , we find 49.96758 in the column of 4 per cent. which is therefore the rate of interest required.

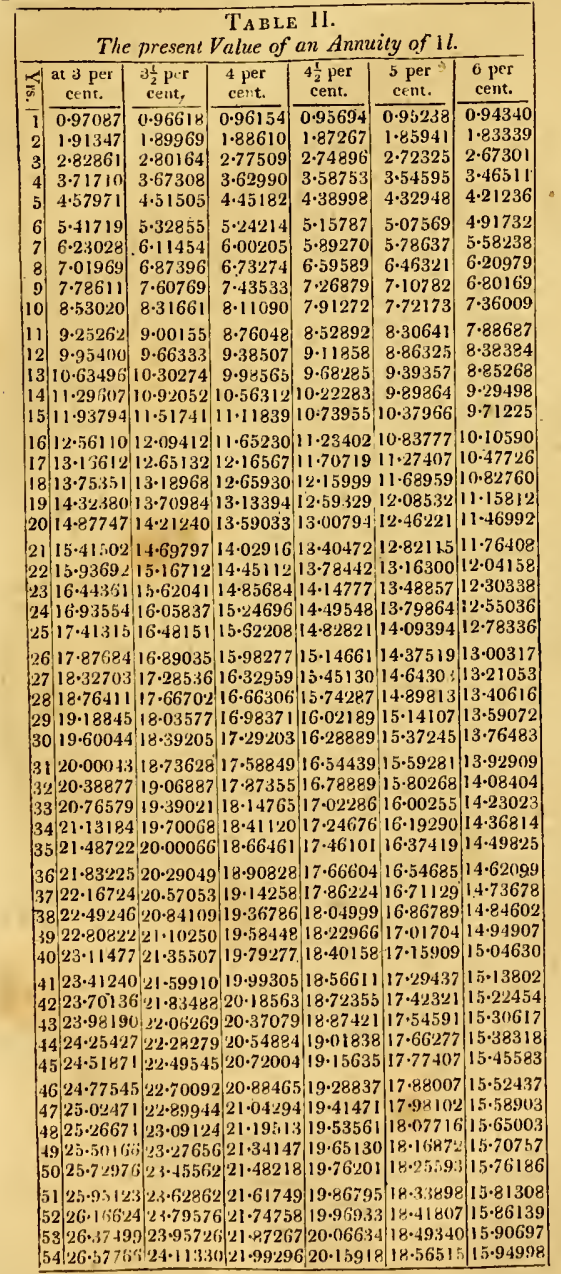

THE USE OF TABLE II.

Exam. 1. To find lie presint value of an annuity of $50 \%$. which is to continue 20 years, at $3 \frac{x}{2}$ per cent.-By the table, the present value of $1 l$. for the same rate and time, is 14.21240 ; therefore $14.2124 \times 50=710^{\circ} 62 l$. or $710 l$. 12s. $4 d$. is the present valuc sought.

Exam.2. To find the present value of an annuity of 20l. to commence 10 years bence, and then to continue for 40 years, or to terminate 50 years hence, at 4 per cent. interest.-In such cases as this, it is plain we bave to find the difference between the present values of two equal annuities, for the two given times; which therefore will be effected by subtracting the tabular value of the one term from that of the other, and multiplying by the annuity. Thus,

tabular value for 50 years $21 \cdot 48218$

tabular value for 10 years 8.11090

the difference $\overline{13.37128}$

mult. by - - 20

gives - $-\overline{267.4256}$

or - $\quad-26 \pi l .8 s .6 d$. the answer.

The foregoing observations, rules, and tables, contain all that is important in the doctrine of annuities certain. Ansl for further information, refertnce may be had to arithmetical writings, particularly Malcolın's A rithmetic, page 595; Simpson's Algebra, sect. 16; Dodson's Mathematical Repository, page 298, \&c ; Jones's Synopsis; cb. 10; Philos. Trans. vol. lsvi, page 109.

For what relates 10 the doctrine of annuities on lives, see Assurance, Cumplenent, Expectation, Life ANNuities, Reversions, \&ic

ANNULETS, in Architecture, are small square mombers, in the Doric capital, placed under the quarter round.

Annulet is also used for a narrow flat moulding, common to other parts of a column, as well as to the capital; and so called, because it cncompasses the column around. In which sense annulet is frequently used for baguette, or little astragal.

ANNULUS, a species of Voluta. Sce also Ring.

ANOMALISTICAL Year, in Astronomy, culled also periodical year, is the space of time in which the earth, or a planet, passes through its orbit. 'The anomalistical, or common year, is somewhat longer thati the tropical year; by reason of the prece'ssion of the cquinos.

And the apses of all the plants have a like progressive motion; by which it happens, that a longer time is nocessary to arrive at the aphelion, which has advanced a little, than to arrive at the same fixed star, For example, the tropical revolution of the sun, with respect to the equinox, is - - - $\quad-365^{\mathrm{da}} 5^{\mathrm{h}} 4 \mathrm{~s}^{\mathrm{m}} 45^{\mathrm{s}}$; but the sidereal, or return to the

same star, - $\quad-\quad-365 \quad 6 \quad 9 \quad 10 \frac{x}{2}$ and the anomalistic revolution is $365 \quad 6 \quad 1520$, because the sun's apogee advances each your $65^{\prime \prime \frac{1}{2}}$ with respect to the equimoses, and the sun camot arrive at the apogee till he las passed over the $65^{\prime \prime} \frac{1}{2}$ more that the revolution of the year answering to the equiness's.

To find the anomalistic revolution, sily, is the whole secular mution of a planet minus the molion of its aphelion, is to 100 years or 3155760000 sccontis, so is $360^{\circ}$, to the duration of the anomalistic revolution.

ANOMALOUS, is sometring irregular, or that deviates from the ordinary tule and method of other things of the same kind.

ANOMALY, in Astronomy, is an irregularity in the motion of a planet, by which it deviate's from the apholion or apogee; or it is the angular distance of the planet from 


\section{A N O}

the aphelion or apogee; that is, the angle formed by the line of the apses, and another line drawn through the planet.

Kepler distinguishes three kinds of anomaly; mean, eccentric, and true.

Mean or Simple AxomaIY, in the ancient astronomy, is the distance of a planet's mean place from the apogce. Which Ptolemy calls the angle of the mean motion.

But in the modern astronomy, in which a planet $P$ is considered as describing an ellipse $\mathrm{APB}$ about the sun s, placed in one focus, it is the time in which the planet moves froin its aphelion $\mathrm{A}$, to the incan place or point of its orbit.

Hence, as the elliptic area $\mathbf{A S P}$ is proportional to

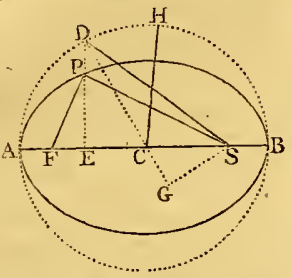
the time in which the-planet describes the arc $A P$, that area may represent the mean anomaly.-Or, if $P D$ be drawn perpendicular to the transverse axis $\Lambda \mathrm{B}$, and meet the circle in $D$ described on the same axis; then the mean anomaly may also be represented by the circular trilineal $\Lambda \mathrm{SD}$, which is always proportional to the elliptic one $\mathbf{A} \mathbf{S P}$, as is proved in my Mensuration, pr. 8 , paye 223, 4th erlition - Or, drawing sG perpendicular to the radius DC produced ; then the mean anomaly is also proportional to $S G+$ the circular arc $A D$, as is demonstrated by Keil in his Lect. Astron.-Hence, taking DH = Sr, the arc A $\mathrm{Al}$, or angle $\mathrm{ACH}$ will be the mean amomaly in practice, as expressed in degrees of a circle, the number of those degrees being to $360^{\circ}$, as the elliptic trilineal area ASP, is to the whole area if the ellipse; the degrecs of mean anomaly, besng those in the arc A 11 , or angle $\mathrm{A}$ Cı.

Eccentric AvomaLy, or of the centre, in the modern astronomy, is the arc $\Lambda \mathrm{D}$ of the circle $A D B$ intercepted between the aphelion $A$, and the point $D$, determined by the perpenslicular DPE to the line of the apses, drawn through the place $P$ of the planet. Or it is the angle $A C D$ at the centre of the circle-Hence the eccentric anomaly is to the mean anomaly, as $A D$ to $A D+S G$, or as $A D$ to $A 1$, or as the angle $A C D$ to the angle $A C r$.

True or Equated ANomALY, is the angle Asp at the sun, which the planet's distance AP from the aphelion, appears under; or the angle formed by the radius vector, or line $s \mathbf{P}$, drawn from the sun to the planet, with the line of the rpses.

The true anomaly being given, it is easy from it to find the mean anomaly. For the angle $\mathbf{A} \mathbf{S P}$, which is the true anomaly, being given, the point $p$ in the ellipse is given, and thence the proportion of the area $A$ sp to the ivhole ellipse, or of the mean anomaly to 360 degrees. And for this purpose, the following easy rules for practice are deduced from the properties of the ellipse, by M. de lis Caille, in his Elements of Astronomy, and M. de la Lande, art. 1240 \&c of his Astronomy: Ist, as the square root of $\mathrm{s} B$ the perihelion distance, is to the square root of s a the aphelion distance, so is the tangent of half the truc anomaly $\Lambda \$ P$, to the tangent of half the eccentric anomaly $A C D$. $2 d$, The difference DII or SG between the -ccentric and mean anomaly, is equal to the product of the eccentricity cs, by the sine of sca the eccentric anomaly just found. And in this case, it is proper to express the eccentricity in seconds of a degree, which will be found by this proportion, as the mean distance 1 : the eccentricity : : $206264 \cdot 8$ seconds, or $57^{\circ} 17^{\prime} 44^{\prime \prime} \cdot 8$, in the arch whose length is equal to the radius, to the seconds in the arc which is equal to the eccentricity cs; which being multiplied by the sine of the eccentric anomaly, to radius 1 , as above, gives the seconds in $\mathrm{SG}$, or in the arc $\mathrm{DH}$, being the difference between the mean and eccentric anomalies. $3 \mathrm{~d}$, To find the radius vector $\mathrm{s} P$, or distance of the planet from the sun, say either, as the sine of the true anomaly is to the sine of the eccentric anomaly, so is half the less axis of the orbit, to the radius vector $\mathrm{SP}$; or as the sine of half the true anomaly is to the sine of half the eccentric anomaly, so is the square root of the perihelion distance $\mathrm{SB}$, to the square root of the radius vector, or planet's distance $\mathbf{S} \mathbf{P}$.

But the mean anomaly being given, it is not so easy to find the true anomaly, at least by a direct process. Kepler, who first proposed this problem, could not find a direct way of resolving it, and therefore made use of an indirect one, by the rule of false position, as may be seen page 695 of Kepler's Epitom. Astron. Copernic. See also $\$ 628$ Wolfus Elem. Astron. Now the easiest method of performing this operation, would be to work first for the eccentric anomaly, viz, assume it nearly, and from it, so assumed, compute what would be its mean anomaly by the rule above given, and find the difference between this result and the mean anomaly given; then assume another eccentric anomaly, and proceed in the same way with it, finding another computed mean anomaly, and its difference from the given one; and treating these differences as in the rule of position for a nearer value of the eccentric anomaly: repeating the operation till the result comes out exact. Then, from the eccentric anomaly, thus found, compute the true anomaly by the 1st rule above laid down.

Of this problem, Dr. Wallis first gave the geometrical solution by means of the protracted cycloid; and Sir Isaac Newton did the same at prop. 31, lib. 1 Princip. But these methods being unfit for the purpose of the practical astronomer, various series for approximation have been given, viz, several by Sir Isaac Newton in his Fragmenta Epistolaruı, page 26, as also in the Schol. to the prop. above-mentioned, which is his best, being not only proper for the plancts, but alsu for the comets, whose orbits are very eccentric. Dr. Gregory, in his Astron. lib. 3 , has also given the solution by a series, as well as M. Reyneau, in his Analyse Demontrée, p. 713, \&c. And a better still for converging is given by Keil in his Prælect. Astron. p. 375: he says, if the arc An be the mean anomaly, calling its sine $e$, cosine $f$, the eccentricity $g$, also putting $z=g e$, and $a=1+f g$; then the eccentric anomaly $\triangle \mathrm{D}$ will be $=\frac{\tau x}{a} \times\left(1-\frac{x^{3}}{a^{2}} \& c\right)$, supposing $r=$ $57 \cdot 2957 \mathrm{~S}$ degrees; of which the frist term $\frac{r z}{a}$ is sufficient for all the planets, even for Mars itself, where the error will not exceed the 200th part of a degree; and ill the orbit of the earth, the error is less than the 10000 th part of a rlegree.

Dr. Seth Ward, in his Astronomia Geometrica, takes the angle $A F P$ at the other focus, where the sun is not, for the mean anomaly, and thence gives an elegant solution. But this method is not sufficiently accurate when the orbit is very eccentric, as in that of the planet Mars, as is shown by Bullialdus, in his defence of the Philolaic. Astron, against Dr. Ward. However, when Ncwion's cor- 
rection is made, as in the schol. above-mentioned, and the problem resolved according to Ward's hypothesis, Sir Isaac affirms that, even in the orbit of Mars, there will scarce ever be an error of more than one second.

ANS $Æ, A N s E s$, in Astronomy, those scemingly prominent parts of the ring of the planet Saturn, perceived in its opening, and appearing like handles to the body of the planet; from which appearance the name ansæ is taken.

ANSER, in Astronomy, a small star, of the 5 th or 6 th magnitude, in the inilky-way, between the eagle and swan, first brought into order by Hevelius.

ANTARCTIC pole, denotes the southern pole, or southern end of the earth's axis.-'The stars near the antarctic pole never appear above our horizon in these northern latitudes.

ANTARCTIC circle, is a small circle parallel to the equator, at the distance of $23^{\circ} 28^{\prime}$ from the antarctic or south pole.-At one time of the year the sun never rises above the horizon of any part within this circle; and at other times he never sets.

ANTARES, in Astronomy, the scorpion's heart; a fixed star of the first magnitule, in the constellation Scorpio.

ANTECANIS, is used by some astronomers, to denote the constellation otherwise called Canis Minor, or the star Procyon. It is so called, as preceding, or being the forerunner of, the Canis Major, and rising a little bcfore it.

ANTECEDENT, of a ratio, denotes the first of the two terms of the ratio, or that term which is compared with the other. Thus, if the ratio be 2 to 3 , or $a$ to $b$; then 2 or $a$ is the antecedent.

ANTECEDENTAL Method, is a branch of general geometrical proportion, or universal comparison, and is derised from an examination of the Antecedents of ratios, having given consequents, and a given standard of comparison, in the various degrees of augmentation and diminution, which they undergo by composition and decomposition. This is a method invented by Mr. James Glenie, and published by him in 1793; a method which he says he always used instead of the fluxional and differential methods, and which is totally unconnected with the ideas of motion and time. See the author's treatise abovementioned, and also his Doctrine of Universal Comparison, or General Proportion, 1789, upon which it is founded.

ANTECEDENTIA, a term used by astronomers when a planet \& $c$ moves westward, or contrary to the order of the signs aries, taurus, \&c. When it moves eastward, or according to the order of the signs arics, taurus, \&c, it is then said to move in consequentia.

ANTECIANS, or ANTOECr, in Geography, the inhabitants of the earth which occupy the same semicircle of the same meridian, but cqually distant from the equator, the one north and the other south; as Peloponnesus and the Cape of Good Ilope.

These have their noon, or midnight, or any other hour at the same time; but their sensons are contrary, being spring to the one, when it is antumn with the other; and summer with the one, when it is winter with the other; also the length of the day to the one, is equal to the length of night to the other.

$\Lambda N^{\prime} T E S$, in Architecture, are small pilastres placed at the corners of buildings.

ANTICS, in Architecture, figures of men, beasts, \&c, placed as ormaments to buildings.
ANTICUM, in Architecture, a porch before a door also that part of a temple, which is called the outer temple, and lies between the body of the temple and the portico.

ANTILOGARITHM, the complement of the logarithm of a sine, tangent, secant, \&c, to that of the radius. This is found by beginning at the left hand, and subtracting each figure from 9, but the last figure from 10.

ANT'INOUS, in Astronomy, a part of the constellation aquila, or the eagle.

AN'ÍIOCHIAN Sect, or Academy, a name given to the fifth academy or branch of academics. It took its name from being founded by Antiochus, a philosopher contemporary with Cicero; and it succeeded the Philonian academy. Though Antiochus was really a stoic, and only nominally an academic.

ANTIOCHIAN epocha, a method of computing time from the proclamation of liberty granted to the city of Antioch, about the time of the battle of Pharsalia.

ANTIPARALLELS, in Geometry, are those lines which make equal angles with two other lines, but contrary ways; that is, calling the former pair the 1st and $2 \mathrm{~d}$ lines, and the latter pair the $3 \mathrm{~d}$ and 4 th Iines, if the angle made by the $1 \mathrm{st}$ and $3 \mathrm{~d}$ lines be equal to the angle made by the $2 \mathrm{~d}$ and $4 \mathrm{th}$, and contrariwise the angle made by the 1st and 4 th equal to the angle made by the $2 d$ and $3 \mathrm{~d}$; then each pair of lines are antiparallels with respect to the other, viz, the 1 st and $2 \mathrm{~d}$, and the $3 \mathrm{~d}$ and 4 th. Thus, if $\Lambda \mathrm{B}$ and $\mathrm{AC}$ be any two lines, and $\mathrm{FC}$ and $\mathrm{FE}$ be two others, cutting them so,

that the angle $B$ is equal to the angle $E$,

and the angle $c$ is equal to the angle $D$;

then $\mathrm{EC}$ and $\mathrm{DE}$ are antiparallels with respect to $\mathrm{AB}$ and AC; also these latter are antiparallels with regard to the two former.-See also Su BCONTRARY.

It is a property of these lines, that each pair cuts the other into proportional segments, taking them alternately,

viz, $\triangle \mathrm{B}: \mathrm{AC}:: \mathrm{AE}: \mathrm{AD}:: \mathrm{DE}: \mathrm{BC}$, and $\mathrm{FE}: \mathrm{FC}:: \mathrm{FB}: \mathrm{FD}:: \mathrm{DB}: \mathrm{EC}$.

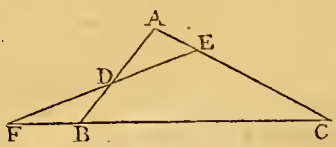

ANTIPODES, in Geography, are the inhabitants of two places on the earth which lie diametrically opposite to each other, or that walk feet to feet; that is, if a line be continued down from our feet, quite through the centre of the earth, till it arrive at the surface on the other side, it will fall on the feet of our Antipodes, and vicc versa. Antipodes arc 180 degrees distant froin each other every way, on the surface of the globe; they have equal latitudes, the one north and the other south, but they differ by 180 degrees of longitude: they have therefore the same climates or degrecs of beat and cold, with the same scasons and length of days and nights; but all of these at contrary times, it boing day to the one, when it is night to the other, summer to the one when it is winter to the other, \&c: they have also the same horizon, the one being as far distant on the one side, as the other on the opposite, and therefore when the sun, \&c, rises to the one, it sets to the other. The Antipodes to London are a part a little south of New 'Zealaud.

It has been said that Plato first started the notion of 
Antipodes, and gave them the name; which is likely enough, as he conceived that the earth was of a globular figure. But there have been many disputes on this point, the fathers of the church having greatiy opposed it, especially Lactantius and Augustine, who pretended to laugh at it, and were greatly perplexed to think how men and trees should hang pendulous in the air with their feet uppermost, as they thought must be the case in the other hemisphere.

ANTISCIANS, or ANTISCII, in Geography, are people who dwell in the opposite hemispheres of the earth, as to worth and south, and whose shadows at noon fall in contrary directions. This term is more general than antœci, with which it is often confounded. The Antiscians stand contradistinguished from Periscians.

\section{ANTECI, see ANTECians.}

APERTURE, -in Optics, is the hole next the objectglass of a telescope or microscope, through which the light and the image of the object come into the tube, and are thence conveyed to the eye. Aperture is also understood of that part of the object-glass itself which covers the former, and which is left pervious to the rays.

A great deal depends on having a just aperture.-To find it experimentally : apply several circles of dark paper, of various sizes, on the face of the glass, from the breadth of a straw, to such as leave only a small hole in the glass; and with each of these, separately, view some distinct ubjects, as the moon, stars, \& $c$; then that aperture is to be chosen through which they appear the most distinctly.

Huygens first found the nse of apertures to conduce much to the perfection of telescopes; and he found by experience (Dioptr. prop. 56) that the best aperture for an object-glass, for example of 30 feet, is to be determined by this proportion, as 30 to 3 , so is the square root of 30 times the distance of the focus of any lens, to its proper aperture: and that the focal distances of the eyc-glasses are proportional to the apertures, And M. Auzont says he found, by experience, that the apertures of telescopes ought to be nearly in the subduplicate ratio of their lengths. It has also been found by experience that ob. ject-glasses will admit of greater apertures, if the tubes be blacked within side, and their.passagc furnished with wooden rings.

It is to be noted, that the greater or less aperture of an object-glass, does not increase or diminish the visible area of the object; all that is effected by this, is the admittance of more or fewer rays, and consequently the appearance of the object more or less bright. But the largeness of the aperture or focal distance, causes the irregularity of its refractions. Ilence, in viewing Venus through a tclescope, a much less aperture is to be used than for the moon, or Jupiter, or Saturn, because her light is so bright and glaring. And this circumstance somewhat invalidates and disturbs Auzout's proportion, as is shown by Dr. Hook, Philos. Trans. No. 4.

A perture in Hydraulics. See Hydraulics.

APHELION, or A PIIELIU m, in Astronomy, that point in the orbit of the earth, or a planet, in which it is at the greatest distance from the sun. Which is the point $A$ (in the fig. to the art. ANOMALY) or extremity of the transverse axis, of the elliptic orbit, farthest from the focus s, where the sun is placed; and diametrically opposite to the peribelion $\mathrm{B}$, or nearer extremity of the same axis. In the
Ptolemaic system, or in the supposition that the sun mores about the earth, the aphelion becomes the apogee.

The times of the aphelia of the primary planets, may be known by their apparent diameter appearing the smallest, and also by their moving slowest in a given time: and the sane of the moon's apogee. Calculations and methods of finding them have been given by many astronomers, as Riccioli, Almag. Nov. lib. 7, sect. 2 and 3; Wolfius, Elem. Astron. $\$ 659$; Dr. Halley, Philos. Trans. No. 128 ; Sir I. Newton, Princip. lib. 3, prop. 14; Dr. James Gregory, Astron. lib. 3, prop. 14; Keil, Astron. Lect.; De la Lande, Memoires de l'Acad. 1755, 1757, 1766, and in his Astron. liv. 22; also in the writings of MM. Evles, D'Alembert, Clairaut, \&c, on attraction; Vince's Astron.; vol. i, p. 133; and Gregory's Astron. p. 220.

The aphelia of the planets are not fixed; for their mutual actions on one another keep those points of their orbits in a continual motion, which is greater or less in the different planets. 'This motion is made in consequentia, or according to the order of the signs; and Sir I. Newton shows that it is in the sesquiplicate ratio of the distance of the planet from the sun, that is, as the square root of the cube of the distance.

The quantities of this motion, as well as the place of the aphelion for a given time, are variously given by different authors. Kepler states them, for the year 1700, as in the following table.

\begin{tabular}{|c|c|c|c|c|c|c|}
\hline Planets. & \multicolumn{4}{|c|}{ Aphelions. } & Annu: & Motion. \\
\hline Mercury & 20 & $8^{0}$ & $25^{\prime}$ & $30^{\prime \prime}$ & $1^{\prime}$ & $45^{\prime \prime}$ \\
\hline Venus & mw & 3 & $2+$ & 27 & 1 & 18 \\
\hline Mars & $m$ & 0 & 51 & 29 & 1 & 7 \\
\hline Jupiter & $\approx$ & 8 & 10 & 40 & 0 & 47 \\
\hline Saturn & $\hat{f}$ & 28 & 3 & 48 & 1 & 10 \\
\hline The Earth & & $s$ & 25 & 30 & & \\
\hline
\end{tabular}

By De la Hirc they are given as follows, for the same

\begin{tabular}{|c|c|c|c|c|c|c|}
\hline Planets. & & Apl. & elion. & & Annua & Mation. \\
\hline Mercury & is & $13^{0}$ & $3^{\prime}$ & $40^{\prime \prime}$ & $1^{\prime}$ & $39^{\prime \prime}$ \\
\hline Venus & w & 6 & 56 & 10 & 1 & 26 \\
\hline Mars & $m$ & 0 - & 35 & 25 & 1 & 7 \\
\hline Jupiter & $\bumpeq$ & 10 & 17 & 14 & 1 & 34 \\
\hline Saturn & 1 & 29 & 14 & 41 & 1 & 22 \\
\hline
\end{tabular}

And Lalande states them as follows, for the year 1750 .

\begin{tabular}{|c|c|c|c|c|c|c|}
\hline Planets. & \multicolumn{3}{|c|}{ Aphelion. } & \multicolumn{3}{|c|}{ Secular Motion. } \\
\hline Mercury & $s^{s}$ & $13^{\circ}$ & $33^{\prime}$ & & & $40^{\prime}$ \\
\hline Venus & 10 & 8 & 13 & 4 & & \\
\hline Mar: & 5 & 1 & 28 & 1 & & 40 \\
\hline fupi & 6 & 10 & 22 & 1 & & 2 \\
\hline & 8 & 29 & 53 & 2 & & 20 \\
\hline [he Earth & 9 & 8 & 38 & 1 & & \\
\hline
\end{tabular}

And for the year 1800 , they are thus:

\begin{tabular}{l|rrrr|rrr}
\multicolumn{1}{c|}{ Planets. } & \multicolumn{4}{c|}{ A phelion. } & \multicolumn{3}{|c}{ Secular Motion. } \\
Mercury & $8^{\prime}$ & $14^{0}$ & $20^{\prime}$ & $50^{\prime \prime}$ & $1^{0}$ & $33^{\prime}$ & $45^{\prime \prime}$ \\
Venus & 10 & 7 & 59 & 1 & 1 & 21 & 0 \\
Mars & 5 & 2 & 24 & 4 & 1 & 51 & 40 \\
Jupiter & 6 & 11 & 8 & 20 & 1 & 34 & 33 \\
Saturn & 8 & 29 & 4 & 11 & 1 & 50 & 7 \\
Herschel & 11 & 16 & 30 & 31 & 1 & 29 & 2
\end{tabular}

APHELLAN, the name of a bright star in the constellation Gemini.

APHRODISIUS, in Chronology, denotes the eleventb month in the Bithynian year, commencing on the 25 tb July in ours.

APIAN or Aprian (Petar), called in Grir 
newitz, a celebrated astronomer and mathematician, was born at Leisnig or Leipsick in Misnia, 1495, and became professor of mathematics at Ingolstadt, in 1524, where he died in the year 1552 , at 57 years of age.

Apian wrote treatises. on many of the mathematical sciences, and greatly improved them; more especially astroumy and astrology, which in that age were much the same thing; also geometry, geography, arithmetic, \&c. He particularly enriched astronomy with many instruments, and observations of eclipses, comets, \&c. His principal work was the Astronomicum Cæsareum, published in folio at Ingolstadt in 1540, and which contains a number of interesting observations, with the descriptions and divisions of instruments. In this work he predicts eclipses, and constructs the figures of them in plano. In the $2 \mathrm{~d}$ part of the work, or the Meteoroscopium Planum, he gives the description of the most accurate astronomical quadrant, and its uses. To it are added observations of five different comets, viz, in the years $1531,1532,1533$, 1538, and 1539; where he first shows that the tails of comets are always projected in a direction from the sun.

Apian also wrote a treatise on Cosmography, or Geographical Instruction, with various inathematical instruments. This work Vossius says he published in 1524, and that Gemma Frisius republished it in 1540 . But Weidler says he wrote it only in 1530 , and that Gemma Frisius published it at Antwerp in 1.550 and 1584, with observations of many eclipses. The truth may be, that perhaps all these editions were published.

In 1533 he made, at Norimberg, a curious instrument, which from its figure he called Folium Populi; which, by the sun's rays, showed the hour in all parts of the earth, and even the unequal hours of the Jews.

In 1534 he published his Inscriptiones Orbis.

In 1540, his Instrumentum Sinuum, sive Primi Mobilis, with 100 problems.

Beside these, Apian was the author of many other works: among which may be mentioned the Fphemerides from the year 1534 to 1570: Books upon Shadows: Arithmetical Centilogues: Books upon Arithmetic, with the Rule of Coss (Algebra) demonstrated: On Gauging: Almanacs, with astrological directions: A book on Conjunctions: Ptolemy, with very correct figures, dcawn in a quadrangular form: Ptolemy's works in Greek: Books of Eclipses: the works of Azoph, a very ancient astrologer : the works of Gebre: the Perspective of Vitello: of Critical Days, and of the Rainbow: a new Astronomical and Geometrical Radius, with various uses of Sines and Chords: Universal Astrolabe of Numbers: Maps of the World, and of particular countries: \&c, \&c.

Apinn left a son, who many years afterwards taught mathematics ut Ingolstadt, and at Tubinga. Tyche has preserved (Progymn. p. 643) his letter to the Landgrave of Hesse, in which he gives an opinion on the new star in Cassiopeia, of the year 1572 .

One of the comets observed by Apian, viz, that of 1532 , had its elements nearly the same as of one observed $128 \frac{1}{4}$ years after, viz, in 1661 , by Hevelius and other astronomers; and hence Dr. Halley judged that they were the same comet, and that therefore it might be expected to appear again in the beginniug of the year 1789 . But it was not found that it returned at this period, though the astronomers then looked anxiously for it; and it is doubtful whether the disappointment might be owing to it passing unobscrved, or to any errors in the observations of Apian, or to its period being disturbed and greatly altered by the actions of the superior planets, \&c.

APIS, musca, the Bee, or Fly, in Astronomy, one of the southern constellations, containing 4 stars.

APOGEE, Apogaum, in Astronomy, that point in the orbit of the sun, moon, \&c, which is farthest distant from the earth. It is at the extremity of the line of the apses: and the point opposite to it is called the perigee, where the distance from the earth is the least.

The ancient astronomers, considering the earth as the centre of the system, chiefly regarded the apogee and perigee: but the moderns, placing the sun in the centre, change these terms for the aphelion and perihelion.The apogee of the sun, is the same thing as the aphelion of the earth; and the perigee of the sun is the same as the perilielion of the earth.

The manner of finding the apogee of the sun or moon, is shown by Ricciolus, Almag. Nov. lib. 3, cap. 24; by Wolfius in Elem. Astr. $\S 618$; by Cassini, De la Hire, and many others: see also Memoires de l'Academie, the Philos. Trans. vol. 5, 47, \&.c.

The apogee and perigee, or the higher and lower apses, would be two fixed points, if the moon, or planet, were only acted on by the single body about which they revolve in an elliptic orbit. But, by the compound actions of the other planets, those points become changed, as if the orbit itself had a motion about its lower focus. The moon, in particular, has her regular orbit about the earth disturbed, and that in an irregular manner, according to their different positions with regard to the earth.

The quantity of motion in the apogee may be found by comparing two observations of it made at a great distance. of time ; converting the difference into minutes, and dividing them by the number of years elapsed between the two observations; the quotient gives the annual motion of the apogee. I'hus, from an observation made by Hipparchus in the year before Christ 140, by which the sun's apogee was found $5^{\circ} 30^{\prime}$ of $\mathrm{\Pi}$; and another mate by Ricciolus, in the year 1646 , by which it was found $70^{\circ} 26^{\prime}$ of of ; the amnual motion of the apogee is found to be $1^{\prime} 2^{\prime \prime}$. And the annual motion of the moon's apogee is about $1^{5} 10^{0} 39^{\prime} 52^{\prime \prime}$.

But the moon's apogee moves unequably. When she is in the syzygy with the sun, it moves forwards; but in the quadratures, backwards; and these progressions and regressions are not equable, but it goes forward slower when the moon is in the quadratures, or perhaps goes retrograde; and when the moon is in the syzygy, it goes forward the fastest of all.-See the articies APIILL10N, Apsis, and LAPLACE; also Newton's Theory of the Moon, for more upon this subject, and his Princip. lib. i. pr. $43,44,45$.

APOLLODORUS, a celebrated architect, under Trajan and Adrian, was born at Damascus, and flourished about the year of Christ 100 . He had the direction of the stone bridge which 'Trajan ordered to be built over the Danube in the year 104 , which was estcemed the most magnificent of all the works of that emperor. Adrian, one day as Trajan was discoursing with this architect on the buildings be had raised at Rome, would neods give his judgment, in which he showed that he knew nothing of the matter. Apollorlorus turned upon him bluntly, and sail to bim, Go paint Citruls, for you are very ignorant of the subject we are talking on. Adrian at this time boasted of lis painting Citruls well. This was the 


\section{A P O}

first step towards the ruin of Apollodorus; a slip which he was so far from attempting to retrieve, that he even added a new offence, and that too after Adrian was advanced to the empire, on the following occasion: Adrian sent to bim the plan of a temple of Venus; and though he asked his opinion, yet to show that he had no seed of him, and that he did not mean to be directed by $\mathrm{jt}$, the temple was already built. A pollodorus wrote his opinion very freely, and remarked such essential faults in it, as the emperor could neither deny nor remedy. He showed that it was neither high enough nor large enough; that the statues in it were disproportioned to its bulk : for, said he, if the gorldesses should have a mind to rise and ho out, they could not do it. This put Adrian into a great passion, and prompted him to the destruction of Apollodorus. He banished him at first; then unter the pretext of certain supposed crimes, of which he had him accused, he at last put lim to death.

A P'OLLONICS, of Perga, a city in Pamphylia, was a celebrated geometrician, who flourished in the reign of Ptolemy Euergetes, about 240 years before Christ; being about 160 years after Euclid, and 49 years later than $\mathrm{Ar}$ chịmedes. He studied a long time in Alexandria under the disciples of Euclid; and afterwards he composed several curious and ingenious geometrical works, of which only his books of Conic Sections al'e now extant, and even these are not perfect. For it appears from the author's dedicatory epistle to Eudemus, a gesmetrician in Pergamus, that this work consisted of $S$ books; only 7 of which however have come down to us.

From the Collections of Pappus, and the Commentaries of Eutocius, it appears that Apollonius was the author of various pieces in geometry, on account of which be acquired the title of the Great Geometrician. His Conics was however the principal of them. Some have thought that Apollonius appropriated the writings and discoveries of Archimedes; Heraclius, who wrote the life of Archimedes, affirms it; though. Eutocius endeavours to refute him. But though it should be allowed a groundless supposition, that Archimedes was the first who wrote upon conics, yet as his treatise on that subject was greatly esteemed, it is highly probable that Aprollonius would avail himself of the writings of that author, as well as others who had gone before him; and, upon the whole, he has the honour of explaining a difficult subject better than had been done before; laving made several improvements, buth in Archimcdes's problems, and in Euclid. His work was doubtless the most perfect. of the kind among tle ancients, and in some respects even among the moderns. Before Apollonius, it had been customary, is we are informed by Eutocius, for the writers on conics to require three different kinds of cones to cut the diffewit sections from, viz, the parabola from a right-angled cone, the ellipse from an acute, and the hyperbola from an obtuse cone; because they always supposed the sections made by a plane cutting the cones to be perpendicular to the side of them : but Apollonius cut his sections all froin any one cone, by only varying the inclination or position of the cutting plane; an improvement lhat hats been followed by all other nuthors since his time. but that Arclimedes was acquainted with the same manner of cutting any cone, is sufficiently proverl, against Futocius, P'appus, and others, by Guido Ulsaldus, in the bceginning of his Commentary on the 21 book of Archimedes's. liquiponderantes, published at Pisa in 1588.

Vol., I.
$A$ P O

The first four books of Apollonius's Conics only liave conse down to us in their original Greek language; but the next three, the 5th, 6 th, and 7 th, in an Arabic version; and the 8th not at all. These have been commented on, translated, and published by various authors. Pappus, in his Mathematical Collections, has left some account of his various works, with notes and lcmmas upon them, and particularly on the conics. And Eutocius wrote a regular elaborate commentary on the propositions of several of the books.

The first four books were badly translated by Joan. Baptista Memmius. But a better translation of them in Latin was made by Commandine, and published at Bononia in 1566 ; - Vossius mentions an edition of the $\mathrm{Co}$ nics in 1650 ; the 5 th, 6 th, and 7 th books being recovered by Golius.-Claude Richard, professor of mathematics in the imperial college of his order at Madrid, in the year 1632, explained, in his public lectures, the first four books of Apollonius, which were printed at Antwerp in 1655 , in folio.- The Grand Duke Furdinand the $2 \mathrm{~d}$, and his brother Prince Leopold de Medicis, employed a professor of the Oriental languages at Rome to translate the 5 th, 6 th, and 7 th books into Latin. These were published at Florence in 1661, by Borelli, with his uwn notes, who also maintains that these books are the genuine production of Apollonius, by many strong authuritics, against Mydorgius and others, who suspected that these three books were not the real production of Apollonius.

As to the Sth, some mention is made of it in a work of Golius's, where he states that it had not been translated into Arabic; because it was wanting in the Greek copies from which the Arabians translated the others. But the learned Mersinne, in the preface to $\Lambda$ pollonius"s Conics, printed in his Synopsis of the Mathematics, quotes the Arabic philosupher A ben Nedin for a work of his about the ycar 400 of Mahomet, in which is part of that Sth book, and who asserts that all, the books of Apollonius are extant in his language, and even more than are enumerated by Pappus; and Vossius says he has read the same; De Scieutiis Nathematicis, pa. 55.-A neat edition of the first four books in Latin was published by Dr. Barrow, in 4 to, at London in 1675.-A mannificent edition of all the 8 books was published in folio, by Dr. Halley, at Oxford in 1710; together with the Lemmas of Pappus, and the Commentaries of Eutocius. The first four in Greek and Latin, but the latter four in Latin only, the 8 th book being restored by himself.-The other writings of Apollonius, mentioned by Pappus, are,

1. The Section of a Ratio, or Proportional Sections, two books.

2. The Section of a Space, in two books.

3 . Determinate Section, in two books.

4. The Tangencies, in two books.

5. The Inclinations, in two books.

6 The Plane Loci, in two broks.

The contents of all these are mentioned by Pappus, and many lemmas are delivered relative to them; but none, or very little of these books themselves have descendert down to the moderns. From the account however thait has been given of their contents, many restorations hasc been made of these works, by the moderin mathematiciaris, as follow: viz,

Vieta, A pollonius Gallus. The Tangencies. l'aris, 1600 , in 4 to. 
Sincllius, Apollonius Batavus.

Lugd. 1601, 4 to.

Snellius, Sectio Rationis et Spatii. 1607.

Ghetaldus, Apollonius Redivivus. The Inclinations.

Venice, $160.7,4$ to.

Ghetriluz, Supplement to the Apollonius Redivivus.

Tangencies. $160 \bar{t}$.

Ghetaldus, Apollonius Redivivus, lib. 2. 1613.

Alex. Anderson, Supplem. Apol. Redivivi. Inclin. Paris,

1612,4 to

Alex. Anderson, Pro Zetetico Apolloniani problematis a se jam pridem edito in Supplemento Apollonii Redivivi. Paris, 1615, 4to.

Schooten, Loca Plana restituta. Lug. Bat. 1656.

Fermat, Luca Plana, 2 lib. Tolos. 1679, folio.

Halley, Apol. de Sectione Rationis libri duo ex Arabico

MS. Latine versi duo restituti. Oxon. 1706, 8vo.

Sinson, Loca Plana, libri duo. Glasg. 1749, 4to.

Simson, Sectio Determinat. Glasg. 1776, 4to.

Horsley, A pol. Inclinat. libri duo. Oxon: 1770, 4 to.

Lawson, The Tangencies, in two books. Lond. 1771,4 to.

Lawson, Determinate Section, two books. Lond. 1772, 4 to.

Wales, Determinate Section, two books. Lond. 1772,4 to:

P. Gianini, Sectio Determinat. Parma, 1773, 4to.

Burrow, The Inclinations. Lond, 1779, 4to.

APONO (Peter de) a learned astronomer and philosopher, was born at.Apono near Padua, about the year 1250. He described the Astrolabium Planum, by which were shown the equations of the celestial houses, for any hour and minute, and for any part of the world : it was published at Venice in 1502 . He acquired the name of the Conciliator, on account of a book of his, in which be reconciles the writings of the ancient philosophers and pbysicians; the book was published at Venice in 1483. He resided at Padua, where, from his practising medicine, and his skill in astronomy, he fell under the suspicion of magic. He died in 1316 , at 66 years of age.

APOPHYGL, in Architecture, is a concave part or ring of a column, lying above or below the flat meinber; and it owes its origin to the ring by which the ends of wooden columns were hooped, to prevent them from splitting.

APOTONE, the remainder or difference between two lines or quantities which are only commensurable in power. Such is the difference between 1 and $\sqrt{ } 2$, or the difference between the side of the square and its diagonal.

The term is used by Euclid; and a pretty full explanation of such quantities is giv' $n$ in the tenth book of his F.lemonts, where he distinguishes six kinds of apotomes, and shows how to find them all geometrically.

Apotone Prima, is when the greater term is rational, and the difference of the squares of the two is a square number: is the difference $3-\sqrt{ } 5$.

A porwas: Secunda, is when the less number is rational, and the square root of the difference of the squares of the two terms, has to the greater term, a ratio expressible in numbers; such is $\sqrt{ } 18-4$, because the differcnce of the squarrs 18 and 16 is 2 , and $\sqrt{ } 2$ is to $\sqrt{ } 18$ as $\sqrt{ } 1$ to $\sqrt{ } 9$ or as 1 i. 3

A polus a Fertin, is when both the terms are irrational, and, as in the second, the square root of the difference of their rynares, has to the greater term, a rational ratio: ats $\sqrt{ } 2+-\sqrt{ }$ IS ; for the ditlerence of their squares 24 and is is 6 , and $\sqrt{ }^{6}$ is to $\sqrt{ } 24$ as $\sqrt{ } 1$ to $\sqrt{ } 4$, or als 1 to 2 .

\section{A P P}

Apotom E 2uarta, is when the greater term is a rational number, and the square root of the difference of the squares of the two terms has not a rational ratio to it : as $4-$ $\sqrt{ } 3$, where the difference of the squares 16 and 3 is 13 , and $\sqrt{ } 13$ has not a ratio in numbers to 4 .

A pritome 2uinta, is when the, less ferm is a rational number, and the square root of the difference of the squares of the two has not a rational ratio to the greater: a $\sqrt{ } 6-2$, where the difference of the squares 6 and 4 is 2 , and $\sqrt{2}$ to $\sqrt{ } 6$ or $\sqrt{ } 1$ to $\sqrt{3}$ or 1 to $\sqrt{ } 3$ is not 8 rational ratio.

А ротом $\mathrm{E}$ Sexta, is where both terms are irrational, and the square root of the difference of their squares has not a rational ratio to the greater; as $\sqrt{ } 6-\sqrt{ } 2$; where the difference of the squares 6 and 2 is 4 , and $\sqrt{ } 4$ to $\sqrt{ } 6$ or 2 to $\sqrt{ } 6$, is not a rational ratio.

The doctrine of apotomes, in lines, as dicliviered by Euclid in the tenth book, is a very curious subject, and has always been much admired and cultivated by all mathematicians who have rightly understood this part of the elemcits; so that Peter Ramus has greatly exposed his judgment by censuring that book: And the tirst algebraical writers in Europe commonly employed a considerable portion of their works on an algebraical expusition of it, which led them to the doctrine of surd quantities; as Lucas de Burgo, Cardan, Tartalea, Stitelius, Peletarius, \&c, \&c. See also Pappus, lib. 4, prop. 3, and the introduc. to lib. 7. And Dr. Wallis's tlgubra, pa. 109.

A Pотомe, in Music, is the difference betwern a greater and less semitone, bein

APPARENT, that which is visible, or evident to the eye, or the understanding.

APPARENT Conjunction of the planets, is when a right line, supposed to be drawn through their centres, passes through the eye of the spectator, and not through the centre of the earth.-And, in gineral, the apparent conjunction of any objects, is when they appear or are placid in the same right line with the cye.

ApPa Rint ltitude Dlameter, Distance, Horizom, MIagnitude, MIotion, Place, Time, \&c. See the rrspective substantives, for the quantuty and measure of it.

The apparent state of things, is commonly very different from their real state, either as to distance, figure, magnitude, position, \&rc, \&rc. Thus,

A P PA RENT Diameter or Magnilude, as for 'sample of the heavenly bodies, is not the real length of the diameter, but the angle which they subtind at the "ye, wr under which they appear. And hence, the angle, or apparent extent, diminishing with the increased distance of the object, a very small object, as $\mathrm{AB}$, may have the same apparent diameter as a very large on $\mathbf{F G}$; and inded the objects have all the same apparent diнmeter, that are contained in the same angle, reg. And it these are paralicl, the real magnitudes are directly proportional to their distances.

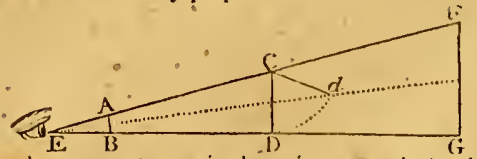

But the apparent magnitude valic's, not only by the distance, hut also by the position of it. So, if the object co bre changud frum the durect posituon, to the oblique one cd, its apparent mignitude would then be only the angle ced, instead of the angle ceD. 


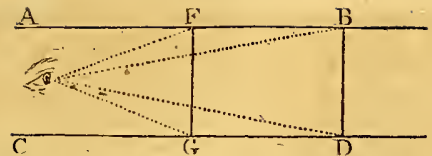

If the eye $\mathrm{E}$ be placed between two parallels $\mathrm{AB}, \mathrm{CD}$, these parallels will appear to converge, or come nearer and nearer to each other, the farther they are continued out, till at last they will appear to coincide in that point where the sight terminates, which will happen when the optic arigle BED becomes equal to about one minute of a degree, the smallest angle under which a common object is distinctly visible.-Also the apparent magnitudes of the same object FG or BD, seen at different distances, that is the angles $F E G, B E D$, are in a less ratio than the reciprocal ratio of the distances, or the distance increases in a greater ratio than the angle or apparent magnitude diminishes. But when the object is very remote, or the optic angle is very small, as one degree or thereabouts, the angle then varies nearly as the distance reciprocally.

But though the optic angle be the usual or sensible measure of the apparent magnitude of an object, yet habit, and the frequent ('xperience of looking at distant objects, by which we know that they are larger than they appear, has so far prevailed on the imagination and judgment, as to cause this too to have some share in our estimation of apparent magnitudes; so that these will be judged to be more than in the ratio of the optic angles.

The apparent magnitude of the same object, at the same distance, is different to different persons, and different animals, and even to the same person, when viewed in different lights; all which may be occasioned by the different magnitudes of the cye, causing the optic angle to differ as that is greater or less: and since, in the same person, the more light there comes from an object, the less is the pupil of the eye, looking at that object; therefore the optic angle will also be less, and consequently the apparent magnitude of the object. Every one must have experienced the truth of this, by looking at another person in a room, and afterwarls abroad in the sunshine, when he always appears snraller than in a room where the light is less. So also, objects elevated in the air, having more light coming from them than when they are upon the ground, or near it, may appear less in the former case than in the latter; thus the ball of the cross on the top of St. Paul's church, which is 6 feet in diameter, appears less than an object of the same diameter seen at the same distance below, near the ground. And this is, by some, supposed to be the reason why the sun and moon appear so much larger when seen in the horizon, where their beams are weak, than when they are more elevated, and their light stronger.

This apparent variation in the magnitude of the heavenly bodies, which is mentioned under the article optice angle, is a very singular phenomenon, which has engaged the attention of many eminent philosophers; and various attempts have accordingly been made to explain the cause from which it arises; the most satisfactory of which appears to be the following.

In judging of the maguitude of a body, we do not estimate it from the angle only under which we perceive it, but also from its rlistance, and this distance is estimated by a comparison with that of other objects. Now there is nothing betwcen us and the moon, when she is near the acuith, liom which to draw that comparisou, and there- fore, deceived by the absence of intermediate bodies, we involuntarily conclude that she is nearer to us than when we ubserve her rising or setting in the horizon, beyond hills, valleys, forests, and other objects, which we know to be at a considerable distance; and hence it happens, that secing this, and the other beavenly bodies, always under the same angle, and conceiving them to be sometimes near and sometimes more remote, our senses are deceived, and we attribute to them, alternately, a greater and less magnitude.

That this is the cause of the phanomenon is demonstrable from the following circumstance, that the illusion ceases as soon as we lose sight of other objects. To prove this, it will be sufficient to observe any of the hearenly bodies through a tube, or through a small hole pierced in a card; in either, case, they will appear no larger in the horizon than in the zenitl, because the smallness of the aperture prevents us from seeing any other object than that on which the observation is made, and consequently all means of comparison are destroyed.'

Again, if the eye be placed in a rare medium, and view an object through a denser, as glass or wates, having plane surfaces; the object will appear larger than it really is: and contrariwise, smaller. And hence it is that fishes, and other objects, seen in the water, by an eye in the air, always appear larger than they are.-In like manner, an object will appear larger when viewed through a globe of glass or water, or any transparent convex spherical segment; and, on the contrary, it will appear smaller when viewed through a concave of glass or water.-See MaGnitude, Apparent; also Robius's Tracts, vol. 2, p. 225, \&c.

Apparent Distance, is that distance which we judge an object to be from us, when seen afar off. This is conmonly very different from the true distance; because we are apt to think that all very remote objects, whose parts cannot well be distinguished, and which have no othervisible objects acar them, are at the same distance from us; though perhaps they may be thousands or millions of miles off; as in the cases of the sun and moon and stars. The apparent distances of objects are also greatly altered by the refraction of the mediun through which they are seen.

APPARENT Figure, is the figure or shape which air object is scen under when viewed at a distance; and is often very different from the true figure. For a straight line, viewed at a distance, may appear but as a point; a surface, as a line; and a solid, as a surface. Also these may appear of different magnitudes, and the surface and solis of different figures, according to their situation with respect to the eye : thus, the arc of a circle may appeal" at straight line; a square, a trapezium, or even a triangle; a circle, an ellipsis; angular magnitudes, round; and : sphere, a circle. Also all objects have a tendency to roundness and smoothness, or appear less angular, as their. distance is greater: fol; as the distance is increased, thic smaller angles and asperities first disappear, by subtencling a less angle than one minute; after these, the next larger disappear, for the same reason; and so on continually, as the distance is more and more increased; the object seeming still more and more round and smooth. So, a triangle, or square, at a great distance, shows only as a round speck; atsd the edge of the moon appears round and regular to the eye, notwithstanding the hills and valleys on her surfice. And bence it is also, that 
near objects, as a range of lamps, or such like, seen at a great distance, appear to be contiguous, and to form one uniform continued magnitude, by the intervals between them disappearing, from the smallness of the angtes subtended by them.

APrAlient Motion, is either that motion which we perccive in a distant bocly that moves, the cye at the same time being cither in motion or at rest; or that motion which an ubject at rest seens to have, while the eye jtself only is in motion.

The motions of bodies at a great distance, though really moving equally, or passing over equal spaces in equal times, may appear to be very unequal and irregular to the eye', which can only judge of them by the mutation of the angle at the eye. And motions, to be equally visible, or appear cqual, must be'directly proportional to the distances of the objects moving. Again, very swift motions, as those of the luminaries, may not appear to be any 'motions at' all, but like that of the hour hand of a clock, on account of the great distance of the objects: and this will always happen, when the space actually passed over in one second of time, is less than about the 14000 th part of its distance from the eye; for the hour band of a clock, and the stars about the earth, move at the rate of 15 seconds of a degree in one second of time, which is only the 13751 part of the radius or distance from the eye. On the other hand, it is possible for the motion of a body to be so swift, as not to appear any motion at all; as when through the whole space it describes there constantly appcars a continued surface or solid, as it were generated by the motion of the object, as when any thing is whirled very swiftly round, describing a ring, \&c.

Also, the more oblique the eye is to the line which a distant body moves in, the more will the apparent motion differ from the true one. So, if a body revolve with an equable motion in the circumference of the circle $\mathbf{A B C D}$ \&c, and the eye be at $\mathbf{E}$ in the plane of the circle; as the body moves from $A$ to $B$ and $c$, it scems to move slower and slower along the line $A \mathrm{~L} K$, till when the body arrives at $\mathrm{c}$, it appears at rest at $\mathrm{K}$; then while it really moves from $\mathrm{c}$ by $\mathrm{D}$ to $\mathbf{F}$, it appears to move quicker and quicker from $\kappa$ by $L$ to $A$, where its motion is quickest of all; after this it appears to move slower and slower from $A$ to $N$, while the body moves from $v$ to $n$ : there becoming stationary again, it appears to return from $\mathrm{N}$ to $\mathrm{A}$ in the straight line, while its real motion is from

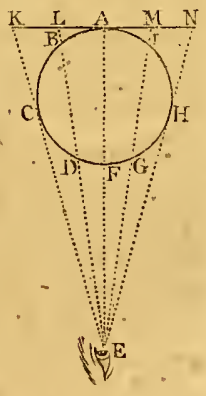
$\boldsymbol{H}$ by 1 to $A$ in the circle. And thus it appears to move in the line $\mathrm{kN}$, by a motion continually varying between the least, or nothing, at the extremes $K$ and $N$, and the greatest of all at the middle point $A$. Or, if the inotion be referred to the concave side of the circle, instend of the line $\mathrm{KN}$, the appearances will be the same.

If au eyc move directly forwards from $\mathrm{E}$ to $\mathrm{O}, \& \mathbf{c}$; any remote object at rest at $P$, will appear to move the contrary way, or from $P$ to 2 , with the same velocity. But if the object I move the same way, and

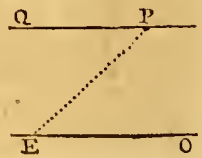

with the same velocity as the eye; it will secm to stand still. If the object have a less vilocity than the eye, it will appear to move back towards $\mathbf{Q}$ with the difiterence of the velocities; and if it move taster than the eyc, it will appear to move forwards from $\Omega$, with the same aith rence of the velocities. And so likewise when the object $P$ inoves contrary to the motion of the eye, it appears to move backwards with the sum of the motions of the two. And. the truth of all this is experienced by persons sitting in a boat moving on a river, or in a wheel-carriage when running fast, and viewing houses or trees, \&zc, on the shro or side of the road, or other boats or wheel-carriages also in motion.

APraRent Place of an olject, in Optics, is that in which it appeais, when seen in or thruugh glass, water, or other refracting mediums; which is commonly different from the true place. So, if an object be secu in or through glass, or water, either plane or concave, it will appear nearer to the eye than its true place; but when seen through a convex glass, it appears mele remote.

A PPA RENT Place of the Image of an olject, in Catoptrics, is that where the inage of an object made by the reflection of a speculum appears to be; and the optical writers, from Euclid downwards, give it as a general rule, that this is where the reflected rajs mect the perpendicular to the speculum drawn from the object: so that if the speculum be a plane, the apparent place of the image will be at the same distance. behind the speculum, as the eye is before it; if convex, it will appear behind the glass ncarer to the same; but if concave, it will appear before the speculum. Aul yet in some cases there are exceptions to this rulc, as is shown by Kepler in lis Paralipomena in Vitellionem, prop. 18. See also Wollius Catoptr. $\$ 51,188,233,234$.

Apparent Place of a Planet, \&c, in Astronomy, is that point in the surface of the sphere of the fixed stars, where the contre of the luminary appears from the surface of the earth.

APPARITION, in Astronoiny, denotes a star's or other "luminary's becoming visible, which before was hid. So, the heliacal rising, is rather an apparition than a proper rising.

APPEARANCE, in Perspective, is the representation or projection of a figure, body, or the like object, on the perspective plane.-The appearance of an objective right line, is always a right line. Sce Pensrectrve.-Having given the appearance of an opake body, and of a luminary, to find the appearance of the shadow; see SIr a Dow.

ApPearances, in Astronomy, $\mathcal{E}$, are more usually called phanomena and phases.-In Optics, the term direct appearance is used for the vicw or sight of any objcct by direct rays; without either refraction or reflection.

APPLiCATL, Applicata, Ordinate Appicite, in Geometry, is a right line drawn to a curve, and bisected by its diameter: 'This is otherwise called an ORDINATE; which see.

Applicate Number. See Concrete.

ApPI.rcation, the act of applying one thing to ano: ther, by approaching or bringing them nenrer together. So a longer space is measured by the contlnual application of a less; as a foot or yard by an inch, S.C. And motion is determincd by a successive application of any thing to different parts of space.

APPLICATION is sometimes uscd, both in arithmetic and geometry, for the rule or operation of division, or what is similar to it in geometry. Thus, 20 applicel to, 
or divided by 4 , gives 5 . And a rectangle $a b$, applied to a line $c$, gives the 4 th proportional $\frac{a b}{c}$, or another line which, with the given line $c$, will contain another rectangle which shall be equal to the forner rectangle $a b$. And this is the sense in which Euclid uses the term, lib. 6 , pr. 28.

Application, in Geometry, is also used for the act or suppusition of putting or placing one figure upon another, to find wherher they be equal or unequal; which seems to be the primary way in which the mind first acquires both the islea and proof of equality. And in this way Juclid, and other geometricians, demonstrate sume of the first or leading properties in geometry. Thus, if two triangles lave two sides in the one triangle equal to two side's in the other, and also the angle included by the same sides cqual to each other; then are the two triangles equal in all respects: for by conceiving the one triangle placed on, or applied to the othere, it is proved that they coincilie or rexactly agree in all their parts. And the same happens if, of two triangles, one side and the two adjacent angles of the one triangle, are equal, respectively, to one sitle and the two corresponding angles of the other. Thus also it may be proved that the diameter of a circle divides it into two equal parts, as also that the diagonal of a square or parallelogram bisects or divides it into two equal parts.

APPLICATION of one science to another, as of algebra to geometry, is said of the use made of the principles and properties of the one, for augmenting and perfecting the other. Indeed all arts and sciknces mutually receive aid from each other. But the application here meant, is of a inore cxpress and limited nature; as will appear by what follows.

Applicatron of Algebra or of Analysis to Geometry. The first and principal applicatious of algebra, were to arithmetical questions and computations, as being the first and most useful science in all the concerns of buman life. Afterwards algebra was applied to geometry and all the other sciences in their turn. The application of algebra to geometry, is of two kinds; that which regards the plane or common geometry, and that which respects the higher geometry, or the nature and properties of curve lines.

The first of these, or the application of algebra to common geometry, is concerned in the algebraical solution of geometrical problems, and finding out theorems in geometrical figures, by means of algebraical investigations or demonstrations. This kind of application bas been made from the time of the carliest writers on algebra, as Diophantus, the Indians and Arabians, Lucas de Burgo, Cardan, Tartalea, \&c, \&cc, down to the present times. Some of the best precepts and exercises of this kind of application, are to be met with in Newton's Universal Arithmetic, and in Simpson's Algebra and Select Exercises. (ieometrical problems are commonly resolved more directly and easily by algebra than by the geometrical noalysis, especially by young beginners; but then the synthesis, or construction and demonstration, is most elegant as derluced from the latter method. Now it com-. monly happens, that the algebraical solution succeeds best in such problems as respect the sides and other lines in geometrical figures; while, on the contrary, those problems in which angles are concerned, are best efiected by the gcometrical analysis. Nowton gives these, among inany other remarks on this brunch. Having any problem puoposed: compare together the quantities concerued in it ; and, making no difference between the known and unknown quantities, consider how they depend upon, or are related to, one another; that it may be perceived what quantities, if they are assumed, will, by proceeding synthetically, give the rest, and that in the simplest manner. And in this comparison, the geometrical figure is to be feigned and constructed at random, as if all the parts were actually known or given; and any other lines drawn that may appear to conduce to the easier and simpler solution of the problem. Having considered the method of computation, and drawn out the scheme, names are then to be given to the quantities entering into the computation, that is, to some few of them, both known and unknown; from which the rest may most naturally and simply be derived or expressed, by means of the geometrical properties of figures, till an equation be obtained, by which the value of the unknown quantity may be clerived by the ordinary methods of reduction of equations, when only one unknown quantity is in the notation; or till as many equations are obtained as there are unknown letters in the same.

For example, suppose it were required to inscribe a square in a given triangle. Let $\mathrm{ABC}$ be the given triangle; and suppose $\mathrm{DEFG}$ to be the required square; also draw the perpendicular $\mathrm{B} P$ of the triangle, which will be given, as well asall the sides of $i t$. Then, con-

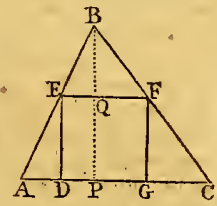
sidering that the triangles BAC, BEF are similar, it will be proper to make the nolation as follows, viz, making the base $\mathrm{AC}=b$, the perpendicular $\mathrm{EP}=p$, and the side of the square $\mathrm{DE}$ or $\mathrm{EF}=x$. Hence then $\mathrm{BQ}=\mathrm{BP}-\mathbf{E D}$ $=p-x ;$ consequently, by the proportionality of the parts of those two similar triangles, viz, BP : AC: : BQ: $\mathbf{E F}$, it is $p: b:: p-x: x$; then, multiply extremes and means \&c, there arises $p x=b p-b x$, or $b x+p x=b p$, and $x=\frac{l p}{b+p}$, the side of the square sought; that is, a fourth proportional to the base and perpendicular, and the sum of the two, taking this sum for the first term, or $\mathrm{AC}+\mathrm{DP}: \mathrm{BP}:: \mathrm{AC}: \mathrm{EF}$.

Erumip. 2. In a right-angled triangle, having given the perimeter, or sum of all the sides, and the perpendicular let fall from the riglit angle on the hypotheinuse, to determine the sides of the triangle.

Let $\triangle B C$ represent the triangle, and в D the given perpendicular. Also put $\mathrm{sD}=p$, A $\mathrm{B}$ $=x, \mathrm{BC}=y, \mathrm{Ac}=z$, and the sum of the sides $=s$. Now then we have three unknown quantities, and therefore we must

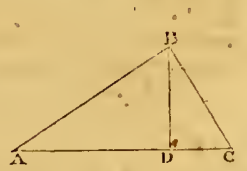
establish three equations before it will be possible to proceed in the solution, which are as follows;

$$
\begin{aligned}
& x^{2}+y^{2}=z^{2} \text {. Euclid prop. 47, book } 1 \text {. } \\
& x y=p z \text {. Euclid prop. s, beok } 4 . \text {. } \\
& x+y+z=s \text {. By the question. }
\end{aligned}
$$

From the first and second equation we find $x^{2}+2 x y+$ $y^{2}=z^{3}+2 z p$, or $x+y=\sqrt{ }\left(z^{2}+2 p z\right) ;$ but $x+y=s$ - z, by the third equation; therefore, $\sqrt{ }\left(z^{2}+2 p z\right)=s-z$, or by squaring buth sides and transposing $(2 p+2 s) z=$ $s^{2}$, hence $z=\frac{s^{2}}{2 p+2 s}$. Having thus found the value of $z$, we may procecrl to find $x$ and $y$, and consider $z$ as a 
known quantity, only substituting in the final expressions its value determined as above. This may be done by means of any two of the original equations, but the first and second are the most proper for this purpose; from them we determinc at once $x^{2}+2 x y+y^{2}=z^{2}+2 p z$, or $x+y=\sqrt{ }\left(z^{2}+2 p z\right) ; x^{2}-2 x y+y^{2}=z^{2}-2 p z$, or $x-y$ $=\sqrt{ }\left(z^{2}-2 p z\right)$; hence $x=\frac{1}{2} \sqrt{ }\left(z^{2}+2 p z\right)+\frac{1}{2} \sqrt{ }\left(z^{2}-2 p z\right)$, and $y=\frac{1}{2} \sqrt{ }\left(z^{2}+2 p z\right)-\frac{1}{2} \sqrt{ }\left(z^{2}-2 p z\right)$; and now reestablishing the value of $z$ as determined above, the sides of the triangle will be as follows:

$$
\begin{aligned}
& \mathrm{AB}=\frac{s}{2} \sqrt{ }\left[\left(\frac{\frac{\pi}{2} s}{p+s}\right)+\frac{p}{p+s}\right]+\frac{s}{2} \sqrt{ }\left[\left(\frac{\frac{\mathrm{T}}{2} s}{p+s}\right)-\frac{p}{p+s}\right] ; \\
& \mathrm{BC}=\frac{s}{2} \sqrt{ }\left[\left(\frac{\frac{\mathrm{T}}{2} s}{p+s}\right)+\frac{p}{p+s}\right]-\frac{s}{2} \sqrt{ }\left[\left(\frac{\frac{\mathrm{T}}{2} s}{p+s}\right)-\frac{p}{p+s}\right] ; \\
& \mathrm{AC}=\frac{\frac{\mathrm{T}}{2} s^{2}}{p+s}
\end{aligned}
$$

The other branch of the application of algebra to geometry, was introduced by Descartes, in his Geometry, which is the new or higher gcometry, and relates to preperties of curve lines. In this branch, the nature of the curve is expressed or denoted by an algebraic equation, which is.thus derived: A line is conceived to be drawn, as the diameter or some other principal line about the curve; and on any indefinite points of this, other lincs are erected perpendicularly, which are called ordinates, while the parts of the first line cut off by them, are called abscisses. Then, calling any absciss $x$, and its corresponding ordinate $y$, by means of the known nature, or relations of the other lines in the curve, an equation is derived, involving $x$ and $y$, with other given quantities in it. Hence, as $x$ and $y$ are common to every point in the primary line, that equation, so derived, will belong to every position or value of the absciss and ordinate, and so is properly considered as expressing the nature of the curve in all points of it ; and is commonly called the equation of the curve.

In this way it is found that any curve line has a peculiar form of equation belonging to it, and which is different from that of every other curve, either as to the number of the terms, the powers of the unknown letters $x$ and $y$, or the signs or coefficients of tbe terns of the equation. Thus, if the curve line нк be a circle, of which $\mathrm{HI}$ is part of the diameter, and IK a perpendicular ordinate: then putting $\mathrm{H} \mathbf{I}=x, \mathrm{I} \mathbf{K}=y$, and $p=$ the

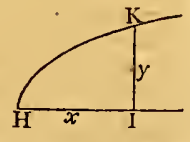
diameter, the equation of the circle will be $p x-x^{2}=y^{2}$. But if $\boldsymbol{н ~} \mathrm{K}$ be an ellipse, an hyperbola, or parabola, the equation of the curve will be different, and for all the four curves, will be respectively as follows, viz,

$$
\begin{aligned}
& \text { for the circle - }-p x-x^{2}=y^{2}, \\
& \text { for the ellipse - } p x-\frac{p}{t} x^{2}=y^{2}, \\
& \text { for the hyperbola }-p x+\frac{p}{t} x^{2}=\dot{y}^{2}, \\
& \text { for the parabola }-p x--=y^{2} \text {, }
\end{aligned}
$$

where $t$ is the transverse axis, and $p$ its parameter. "Aind, in like manner for other curves. Sce A Bsciss.

This way of expressing the nature of curve lines, by algebraic equations, has given occasion to the greatest improvement and extension of the geonctry of curve lines; for thus, all the properties of algebraic equations, and their roots, are transferred and added to curves, whose bsejsses and ordinates have similar properties. Indeed the bencfit of this sort of application is mutual and reciprocal, the known propertics of equations being transferred to the curves that they represent; and, on the contrary, the known properties of curves are transferred to their iepresentative equations. Sèe Curves.

Application of Geometry to Algebra. Besides the use and application of the higher geometry, for ubtaining the nature and roots of equations, and for finding the values of those roots by the geometrical construction of curve lines, even common geornetry may be made subservient to the purposes of algebra. Thus, to take a very plain and simple instance, if it were required to square the binomial $a+b$; by forming a square, as in the annexed figure, whose side is equal to $a+b$, or the two lines or parts.added together denoted by the letters $a$ and $b$; and then drawing two lines parallel to the sides, from the points where the two parts join, it will be immediately perceived that the whole square of the compound quantity $a+b$, is equal to the squares of both the parts, together with two rectangles under the two parts, or $a^{2}$ and $b^{2}$ and $2 a b$, that is the square of $a+b$ is equal to $a^{2}+b^{2}+$ $2 a b$, as derived from, a geonetrical

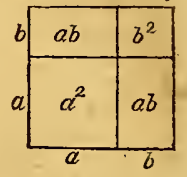
figure or construction. And in this very manner it was, that the Arabians, and the early European writers on algebra, derived and demonstrated the common rule for resolving compound quadratic equations. - And this also, in a similar way, it was, that Turtalea and Cardan derived and demonstrated all the rules for the solution of cubic equations, using cubes and parallelopipedons instead of squares and rectangles. And many other instances might be given of the use and application of geometry in algebra.

ApPlication of Algebra and Geometry to Mechanics. This is founded on the same principles as the application of algebra to geometry. It consists principally in representing by equations the curves described by bodies in motion, by deternining the equation between the spaces which the bodies describe, when actuated by any fortes, and the times employed in describing them, \& $c$. A familiar instance also of the application of gcometry to mechanics, may be seen at the article Acc eler a tion, where the perpendiculars of triangles represent the times, the bases the velocities, and the areas the spaces described by bodies in motion; a method first given by Galilco. In short, as velocities, times, forces, spaces, \&c, may be represented by lines and geometrical figur's; and as these again may be treated algebraically; it is evident how the principles and properties, of both algebra and geometry, may be applied to mechanics, and indeed to all the other branches of mixed mathenratics.

A p Plication of Mechanics to Geometry. This consists chiefly in the use that is sometimes made of the centre of gravity of figures, for determining the contents of solids described by those figures. This is called the centrobaric method.

Aprication of Geometry and Astronomy to Geography. This consists chicfly in three articles. 1st, In determining the figure of the globe we inhabit, by means of geometrical and astronomical operations. 2d, ln determining the positions of places, by observations of latitudes and longitudes. 3d, In detcrmining, by geometrical operations, the position of such places as are not far distant from one another.

Geomrtry and $\Lambda$ stronomy are also of great use in $\mathrm{Na}$ vigation. 
Application of Geometry and Algebra to Plysics or Natural Philosophy. This application we owe to Newton, whose philosopliy may therefore be called the geometrical or mashematical philosophy; and on this application are founded all the physico-mathematical sciences. Here a single observation or experiment will often produce a whole science: so, when we know, as we do by experience, that the rays of light, in reflecting, make the angle of inciclence equal to the angle of reflection; we therice deduce the whole science of catoptrics: for, that expcriment once admitted, catoptrics becomes a science purely geumetrical, since it is reduced to the-comparison of angles, and lines given in position. And the same in many other sciences.

APPLICATION of one thing to another, in general, is employed to denote the use that is made of the former, to understand or to perfect the latter. Thus, the applicatiun of the cycloid to pendulums, means the use made of the cycluidal curve for improving the doctrine and use of pendulums.

APPLY. This term is used two different ways, in geometry.

1st, It signifies to transfer or place a given line, cither in a circle or some other figure, so that the extremities of the line shall be in the perimeter of the figure.

$2 \mathrm{l}, \mathrm{lt}$ is also used to express division in geometry, or to find one limension of a rectangle, when the area and the other dimension are given. As the area $a b$ applied to the line $c$, is $\frac{a b}{c}$.

APPROACH, the curve of equable approach: This was a problem first proposed by Leibnitz, namely, to find a. curve, down which a body descending by the force of gravity, shall make equal approaches to the horizon in equal portions of time. It has been found by Bernoulli and others, that the curve is the second cubical parabola, placed with its vertex uppermost, and which the descending bociy must enter with a certain determinate velocity. -Varignon rendered the question general for any law of gravity, by which a body may approach towards a given point by equal spaces in equal times. And Maupertuis ilso resolved the problem in the case of a body descending in a medium which resists as the square of the velocity. See 11 ist. de l'Acad, des Sciences for 1699 and 1730.

Method of Approacies, a nane given by' Dr. Wallis, in his Alyctura, to a inethod of resolving eertain problems relating (1) square numbers, \&c. T'lis is done by first assigning certain limits to the quantities required, and then approaching nearer and nearer, till a coincidence is obtained.- In this sense, the method of Trial-and-error, or donble rule of lialse Position, may be considered as a mcthou of approaches.

APPliO ACHLS, in Fortification, the several works made by the besiegers, for advancing or getting nearer to a fortrens or plicc besieged. Such as the trenches, mines, saps, lodgments, batteries, galleries, ('paulments, \&c.

A PPRUACи Es, or Lines of A PPROACn, are particularly used for trenche's dug in the ground, and the earth thrown up on the side towards the place besieged; under the defence or sholter of which, the besiegers may approach withesil loss, (1) raise batteries, and plant guns, \&c, to batter ut.- l'he lines of approach are commonly carried on, in a zigzag firm, parallel to the oppessite faces of the brsieged work, or nearly so, that they maty not be cnsilarled by the guns from the enemy. And they are also connected by parallels or lines of communication.-The besieged commonly make counter-approaches, to interrupt and defcat the approaches of the besiegers.

The ancients made their approaches towards the place besiegerl, much after the same manner as the moderns. Folard show's, that they had their trenches, their parallels, saps, \&c; which, though usually thought of modern invention, it appears, have been practised long before, by the Grecks, Romans, Asiatics, \& c.

APPROXIMATION, a continual approacl, still nearer and nearer, to a root or any quantity sought.-Methods of continual approximation for the square roots and cube roots of numbers, have been employed by algebraists and arithmeticians, froin Lucas de Burgo down to the present time. And the later iwriters have given various approximations, not only for the roots of higher powers, or all simple equations, but for the roots of all sorts of compound equations whatever: especially Newton, Wallis, Raphson, Halley, De Lagny, Lagrange, \&c, \&c; all of them forming a kind of infinite series, either expressed or understood, converging nearer and nearer to the quantity sought, according to the nature of the process.

It is evident that if a number proposed be not a true square, that no exact square root of it can be found, explicable by rational numbers, whether integers or fractions: therefore, in such cases, we must be cuntent with approximations, or coming continually nearer and nearer to the truth. In like manner, for the cubic and other roots, when the proposed quantities are not exact cubes, or other powers.

The most easy and general mode of approximation, for any power above the square, is perhaps by the rule of Double Position, or, what is sometimes called, the Method of Trial-and-error; which see under its own name. And among all the rules for finding the roots of pure powers, of which there are many, 1 believe the best is that which was discovered by myself, and given in the first volume of my Mathematical Tracts, (printed in 1786) in point of ease, both of execution and for remembering it. Which is thiș: if $\mathrm{N}$ denute any number, out of which is to be extracted the root whose index is denoted by $r$, and if $n$ be the nearest root first taken; then will $\frac{(r+1) N+(r-1) u^{r}}{(r-1) N+(r+1) u^{r}} \times n$ be the required root of $\mathrm{N}$ very nearly; or as $r-1$ times the given number added to $r+i$ times the nearest powcr, is to $r+1$ times the given number adderl to $r-1$ times the nearest puwer, so is the assumed root $n$, to the required root, very nearly. Then this last value of the root, so found, if one still nearer is wanted, is to be used for $n$ in the same thcorem, to repeat the operation with it. And so on, repeating the operation as oft'n as nc'cessary. Which theor'm includes all the rational formula of Halley and De Lagny.

lor example, suppose it were required to double the cube, or to find the cube root of the number 2 . Here $r=3$; consequently $r+1=4$, and $r-1=2$; and thercfore the gencral theorem becomes $\frac{4 \mathrm{~N}+2 n^{3}}{2 \mathrm{~N}+4 n^{3}} \times n$ or $\frac{2 \mathrm{~N}+n^{3}}{\mathrm{~N}+2 n^{3}} \times n$ for the cube root of $\mathrm{N}$; or as $\mathrm{N}+2 n^{3}: \mathrm{N}^{2}$ $+n^{3}:: n:$ the root sought nearly. Now, in this case, $\mathrm{N}=2$, and therefore the nearest root $n$ is 1 , and its cube $n^{3}=1$ also: lience $\mathrm{N}+2 n^{3}=2+2=4$, and $2 \mathrm{~N}+n^{3}$ $=4+1=5 ;$ therefore, as $4: 5:: 1: \frac{5}{4}$ or $1 \frac{1}{4}=1 \cdot 25$ tho first approximation. Again, taking $r=\frac{s}{4}$, and conse- 


\section{A P P}

quently $r^{3}=\frac{125}{64} ;$

$$
\begin{aligned}
& \text { bence } \mathrm{N}+2 n^{3}=2+\frac{250}{64}=\frac{378}{64}, \\
& \text { and } 2 \mathrm{~N}+n^{3}=4+\frac{25}{64}=\frac{881}{34},
\end{aligned}
$$

therefore as $378: 381$, or as $120: 127^{\circ}:: \frac{5}{4}: \frac{63}{50} \frac{5}{4}=$ $1 \cdot 259921$, which is the cube root of 2 , true in all the tigures. And by taking $\frac{635}{50}$ for a new value of $n$, and repeating the process again, a great many more figures may be found.

Of the Roots of Equations by Approxination.-Stevinus and Vieta gave mothods for tinding values, always nearer and nearer, of the roots of equations. And Oughtred and others pursued and improsed the same. These however were very tedious and imperfect, and required a different process for eviry degre of equatinss. But Newton introduced, not only gencral methods for expressing radical quantities by approximating infinite series, but also for the roots of all sorts of compound cquations whatever, which are both easy and expeditious, and which will be more particularly described under their respective articles. His method of approximating, is in substance this: First take a value of the root as near as may bc, by trials, either greater or less; then assuming another letter to dcnote the unknown'difference betwcen this and the true value, substitute into the equation the sum or, difference of the approximate root and this assumed letter, instead of the unknown letter or root of the equation, which will produce a new equation having only the assumed small difference for its root or unknown letter; and find, from this equation, a near value of this small assunied quantity. Assume then another letter for the small difference between this last value and the true one, and substitute the sum or difference of them into the last equation, by which will arise a third equation, involving the second assumed quantity; whose value is found as before. Proceeding thus as far as we please, all the near values, connected together by their proper signs, will form a series approaching still nearer and neare to the true root of the first or proposed equation. The approximate values of the several small assumed differences, may be found in different ways: Newton's method is this: As the quautity solight is small, its higher powers decrease more and more, and therefore neglecting then will not lead to any great error. He therefore neglects all the terms having in them the $2 d$ and higher powers, leaving only the 1st power and the absolute known term; from which simple equation be always finds the value of the assumed unknown letter nearly, in a vory simple and easy manner. Halley's method of doing the saun thing, was to neglect all the terins above the square or $2 d$ power, and then to find the root of the remaining quadratic equation; which would indeed be a nearer ralue of the assumed letter than Newton's was, but then it is inuch more troublesome to perform.- Raphison bats another winy, which is a little varierl from that of Newton's agail, which is this: Ifaring found a near value of the first assumed small yuantity or difference, by this he corrects the first approximation to the root of the proposed equation; ind then, assuming another letter for the next, or smaller difference, he introduces it into the original equation in the simme way as befere. And thus he proceeds, from one correction to anotluer, employing always the first proposed equation to finct them, instend of the successive new equations used by Newton.

For example, let it be repuired to find the ront of the "quation $x^{2}-5 x=31$, or $x^{2}-5 x-31=0$ :- - Fere the soot $\dot{x}$, it is eviclent, is nearly $=8$; for $x$ theretore take $8+z$, and substitute $\&+z$ for $x$ in the given equation
A P P

"and the terms will be thus;

$$
\begin{gathered}
x^{2}=64+16 z+z^{2} \\
-5 x=-40-5 z \\
-31=-31 \\
\text { the sum is }-7+11 z+z^{2}=0
\end{gathered}
$$

Then, rejecting $\tilde{i}^{2}$, it is $11 z-\tau=0$, and $:=\frac{7}{11}=6363$, $\& c$, or $=6$ nearly.

Assume now $z=6+y$ : then

$$
\begin{aligned}
z^{2} & =36+1 \cdot 2 y+y^{4} \\
11 z & =6 \cdot 6+11 y \\
-7 & =-7
\end{aligned}
$$

the sum $-04+12 \cdot 2 y+y^{2}=0$,

$$
\text { where } y \div \frac{01-1}{12 \cdot 2}=003278 \text { inearly. }
$$

Honce then, collexing all the assumed difierences, with their signs, it is found that $x=8+z+y=\mathrm{s}+6+$ $\cdot 003278=8 \cdot 603278$ the root of the equation required, by Newton's method.

The same by Ravhsou's wryy.

First $x=\mathrm{S}-\mathrm{-1} \cdot z$;

then $x^{2}=64+16 z+z^{3}$

$-5 x=-40-5 z$

$-31=-31$

the sum $-7+11 z+z^{2}=0$ :

hence $z={ }_{1}^{2}=6$ nearly, as belure,

and $x=8+z=8 \cdot 6$ nearly.

Assmue it $x=8 \cdot 6+y$;

then $x^{2}=73 \cdot 96+17 \cdot 2 y+y^{3}$.

$-5 x=-43 \quad-5 y$

$-31=-31$

the sum $-04+12 \cdot 2 y+y^{3}=0$;

hence $y=\frac{\cdot 04}{12 \cdot 2}=\cdot 003278$ nearly,

and $x=8.6+y=8 \cdot 60327 \mathrm{~s}$, as before.

And in the same manner Newton performs the approximation for the roots of literal equations, that is, equiltions having literal coefficients; so the root of this cquation

$$
\begin{aligned}
& y^{3}+a x y+a^{2} y-x^{3}-2 a^{3}=0, \text { is } \\
& y=a-\frac{x}{4}+\frac{x^{2}}{64 a}+\frac{131 x^{3}}{512 a^{4}}+\frac{509 x^{4}}{16384 a^{3}} \text { \& } .
\end{aligned}
$$

See also a memoir on this method by the Marquis de Courtivron, in the Menoires de l'Academie for 1744 .

Other, Methods of A Pproximation. Besides the luregoing general roethods, other particular ways of approximating, tor various purposes, have been given by many different pérsons.-As for example, methods of approximating, by series, to the ronts of cubic equations belonging to the irreducible case, by Nicole in the same Memóores de l'àceademic, by M. Clairaut in his Algibrit, and by myself in the Philos, "Irrans. for 17 So. Sir also scre. ral parts of Simpson's works, or my 'l'racts vol. 1, and my Contse of Mathematics, vol. 1 ; or the treatise on numerical equations liy Lagrange. Also the methods of intinite stries by Widlis, fiewton, Gregner, Mercator, S.c, may be considered as approximations, in quadratures, and other branches of the mashematics, many instances of which may be seen in W'allis's Algebrit, and other books: -Likenise the methon of exhatusfions of the ancients, by which Archimedes and ofluers bave :yproximated to the quatratuse and rectification of the circle, dec, which was performerl by continually bisecting lae sides of polygons, both inscribed in at circle athd circumscribed about it; by which means the sum of the sides of the like polygons approach continually wearer and nearer together, and the circumference of the circle is nearly a mean betwern the two sums. "Sre atso liquations. 
APPULSE, in Astronomy, means the near approach of two luminaries to each other, so as to be seen, for instance, within the same telescope.

The appulses of the planets to the fixed stars have always been very useful to astronomers, as serving to fix and determine the places of the former. The ancients, wanting an easy method of comparing the planets with the ecliptic, which is not visible, had scarce any other way of fixing their situations, but by observing their track among the fixed stars, and marking their appulses to some of those visible points. See Hist. Acad. Scienc. for 1710 , pa. 417. And Philos. Trans. No. 369; where Dr. Halley has given a method for determining the places of the planets, by observing their near appulses to the fixed stars. See also Philos. Trans. No. 76 , pa. 361, and Mem. Acad. Scienc. for 1708 , where Flamsteed and De la Hire have given observations of the moon's appulses to the Pleiades. See also Flamsteed's Historia Colestis, where a multitude of observations of appulses, or small distances, of the moon and planets, from the fixed stars, are recorded. And Dr. Halley has published a map or planisphere of the starry zodiac, in which are accurately laid down all the stars to which the moon's appulse has ever becn observed in any part of the world. Sce Philos. Trans. No. 369; or my A bridg. vol. 6 , pa. 530 .

APRIL, the 4th month of the year according to the common computation, and the $2 \mathrm{~d}$ from the verial equinox.-The word is derived from Aprilis, of aperio, Iopen; because the earth, in this month, begins to open her bosom for the production of vegetables.-In this month the sun travels through part of the signs Aries and Taurus.

APRON, in Gunnery, a piece of thin or sheet lead, used to cover the vent or touch-hole of a cannon.

APSES, or APSIDEs, in Astronomy, are the two points in the orbits of planets, where they are at their grcatest and least distance from the sun or the earth. The point at the greatest distance being called the higher apsis, and that at the nearest distance the lower apsis. Also the ligher apsis is more particularly called the aphelion, or the apogee; and the lower apsis, the perihelion, or the perigee. The diameter which joins these two points, is called the line of the apses or of the apsides; it passes through the centre of the orbit of the planct, and the centre of the sun or the earth; and in the modern astronomy this line makes the longer or transverse axis of the elliptical orbit of the planet. In this line is counted the excentricity of the orbit; being the distance between the centre of the orbit and the focus, where is placed the sun or the earth.

The foregoing definitions suppose the lines of the greatest and lenst distances to lie in the same straight line; which is not always precisely the case; as they are sometimes out of a right line, making an angle greater or less than 180 degrees, and the difference from 180 degrces is called the motion of the line of the apses: when the angle is less than 180 degrees, the motion of the apses is said to be contrary to the order of the signs; on the other band, when the angle exceeds 180 degrees, the motion is according to the order of the signs.

Different means have been employed to determine the motion of the apses. Dr. Keil explains, in his Astronomy, the method used by the ancients, who supposed the orbits of the planets to be perfectly circular, and the sun out of the centre. But since it has been discovered that they lescribe elliptic orbits, various other methods have been Hevised for determining it. Dr. Halley has given onc, VoI.. I. which supposes to be known only the time of the planct's revolution, or periodic time. Seth Ward has also given a determination from three different observations of a planet, in any three places of its orbit: but his method being founded on an hypothesis not strictly true, Euler has given one much more exact in vol. 7 of the Petersburg Commentaries. See various ways explained in the Astronorny of Keil and Monnier.

Newton has also given, in the Pirncipia, an excellent method for determining the same motion, on the supposition that the orbits of the planets differ but little from circles, which is the case nearly. That great philosopher shows, that if the sun be immoveable, and all the planets gravitate towards him in the inverse ratio of the squares of their distances, then the apses will be fixed, or their motion nothing; that is, the lines of greatcst and least distance will form one right line, and the apses will be directly opposite, or at 180 degrees distance from each other. But, because of the mutual tendency of the planets towards each other, their gravitation towards the sun is not precisely in that ratio; and hence it happens, that the apses are not always exactly in a right line with the sun. And Newton bas given a very elegant method of determining their motion, on the supposition that we know the force which is thereby added to the gravitation of the planet towards the sun, and that this additional force is always in that dircction. Dr. Chas. Walmesley has a treatise on the Theorie du Mouvamens des Apsides, in 8 vo.

\section{APSIS. See Apses.}

APUS or A pous, Avis Indica, in Astronomy, a constellation of the southern hemisphere, situated near the south pole, between the triangulum australe and the chameleon, and supposed to represent the bird of paradise. Also supposed to be one of the birds named Apodes, as having no feet.

The number of stars contained in this constellation are, 11 in the British Catalogue, in Bayer's Maps 12, and a still greater number in La Caille's Catalogue; the principal star being but of the 4th or 5th order of magnitude. See Coelum Australe Stelliferum, and the Memoires de l'Acad. for 1752 , pa. 569 .

AQUARIUS, in Astronomy, one of the celestial constellations, being the eleventh sign in the zodiac, reckoning from Aries, and is marked by the character $\approx \pi$, represcnting part of a stream of water, issuing from the vessel of Aquarius, or the water-pourer. This sign also gives name to the eleventh part of the ecliptic, through which the sun moves in part of the months of January and February.

The poets feign that Aquarius was Gauymede, whom Jupiter ravished under the shape of an eagle, and carried away into Heaven to serve as a cup-bearer, instead of IIebe and Vulcan; whence the name. Others hold, that the sign was thus called, bccause that when it appears in the horizon, the weather commonly proves rainy.

The stars in the cunstellation Aquarius are, in Ptolemy's Catalogue 45 , in Tycho's 41 , in Hevelius's 47 , and in Flamsteed's 108. See the article Constellation; also Cata Logú.

AQUEDUCT, or $\triangle Q U$ \&DUCT, that is to say, ductus aquæ, a conduit of water, is a construction of stone or timber built on uneven ground, to preserve the level of water, and convey it, by a canal, from one place to another.-Some aqueducts are under ground, being conducted through hills, \&c; and others are raised above ground, and supportest on arches, to conduct the water over valleys, \&c. 
The Romans were very magnificent in their aqueducts; having some that extended a hundred miles, or more. Frontinus, a man of consular dignity, who had the direction of the aqueducts under the emperor Nerva, speaks of nime that emptied themselves through $13,59+$ pipes, of an inch diameter. And it is observed by Vigenere, that in the space of 24 hours, Rome received from these aqueducts not less than 500,000 hogsbeads of water. The chicf aqueducts now in being, are these: 1st, that of the Aqua Virginia, repaired by pope Paul Iv; $2 d$, the Aqua Felice, constructed by pope Sixius $v$, and is called from the name he assumed before he was exalted to the papal throne; $3 \mathrm{~d}$, the Aqua Paulina, repaired by pope Paul v, in the year 1611 ; and 4 thly, the aqueduct built by Louis $x I v$, near Maintenon, to convey the river Bure to Versailles, which is perhaps the largest in the world; being 7000 fathoms long, elevated 2560 fathoms in height, and containing 242 arcades. See Philos. Trans. for 1685, No. 171 ; or my Alridg. vol. 3, pa. 167 and 231.

AQUEOUS HUMOU $\mathrm{R}$, or the watry humour of the eyt, is the first or outermost, and the rarest of the three bumours of the eye. It is transparent and colourless, like water; and it fills up the space that lies between the cornea tunica, and the crystalline humour.

AQUILA, the Eagle, or the Vulture as it is sometimes called, is a constellation of the northern bemisphere, usually joined with Antinous. It is one of the 48 old constellations; according to the division of which Hipparchus marle his Catalogue of the Fixed Stars, and which are described by Ptolemy. The number of stars in Aquila, and those near it, now in the later-formed constellation Antinous, amount to 15 .in Ptolemy's Catalogue, to 19 in Tycho's, to 42 in that of Hevelius, and to 71 in Flamsteed's. But jn Aquila alone, Tycho counts only 12 stars, and Hevelius 23 ; the principal star being Lucida Aquila, and is between the 1 st and $2 \mathrm{~d}$ magnitude. The Greeks, as usual, relate various fables of this constellation, to make the science appear as of their own invention.

ARA, the Altar, one of the 43 old constellations, mentioned by the ancient astronomers, and is situated in the southern hemisphere; containing only 7 stars in Ptolemy's Catalogue, and 9 in that of Flamstecd; none of which exceed the 4 th magnitude.

ARATUS, celebrated for his Greek poem entitled $\Phi \alpha_{\text {s- }}$ vóuava, the Phenomena, flourished about the 127 th Olympiad, or sear 300 years before Christ, while Ptolemy Philadelphus reigned in Egypt. Being educated under Dionysius Heracleotes, a Stoic philosopher, he espoused the principles of that sect, and became physician to Antigonus Gonatus, the son of Demetrius Poliorcetes, king of Macedon. The Phenomena of Aratus gives him a title to the character of an astronomer, as well as a poct. In this work hedescribes the nature and motion of the stars, and shows their various dispositions and relations; he describes the figures of the constellations, their situations in the sphere, the origin of the names which they bear in Greece and in Egypt, the fables which have given rise to them, the rising and setting of the stars, and he indicates the manner of knowing the constellations by their respective situations.

The poem of Aratus has been commented on and translated by many authors: of whom, among the ancients, were Cicero, Germanicus Cesar, and Festus $\Lambda$ vicnus, who made Latin translations of it; a part of the former of which is still extant. Aratus inust have been much cs- teemed by the ancients, since we find so great a numbcr of scholiasts and commentators on him ; among whom are Aristarchus of Samos, the Arystylli the geometricians, Apollonius, the Evancti, Crates, Numenius the grammarian, Pyrrhus of Magnesia, Thales. Zeno, and many ot hers, as may be seen in Vossius, p. 156: Sujdas ascribes several other works to Aratus. Virgil, in his Georgics, has translated or imitated many passages from this author: Ovid speaks of him with admiration, as well as many others of the poets. And St. Paul has quoted a passage from him; which is in his speech to the Athenians (Acts xvii. 28.) where he tells them that some of their own poets have said, "For we are also his offspring," these words being the heginning of the $5 \mathrm{th}$, line of the Phenomena of Aratus.

His modern editors are as follow : Henry Stephens published his poem at Paris in 1566, in his collection of the poets, in folio. Grotius published an edition of the Phenomena at Leyden in quarto, 1600, in Greek and Latin, with the fragments of Cicero's version, and the translations of Germanicus and Avienus; all which the editor has illustrated with curious notes. Also at neat and correct edition of Aratus was published at Oxford, 1672, in 8vo. with the Scholia.

ARAOMETER, see Areometer.

ARC, or ARCH; which see.

ARCADE, in Architecture, denotes an opening in the wall of a building formed by an arch.

ARC-BOUTANT, is a kind of arched buttress, formed of a flat arch, or part of an arch, abutting against the feet. or sides of another arch or vault, to support them and prevent them from bursting or giving way.

ARCH, Arc, Arcus, in Geometry, a part of any curre line; as, of a circle, or ellipsis, or the like.

It is by means of circular ares, or arches, that all angles are measured; the arc being described from the angular point as a centre. For this purpose, every circle is supposed to be divided into 360 degrees, or equal parts; and an arch, or the angle jt subtends and measures, is estimated according to the number of those degrees it contains: thus, an arc, or angle, is said to be of 30 or 80 , or 100 degrees. Of late years the French mathematicians have divided the circle into 400 degrees, or the quadrant into 100 , called the centigrade division. Circular arcs are also of great use in finding of fluents.

Concentric Arcs, are such as have the same centre.

Equal Ancs, are such arcs, of the same circle, or of equal circles, as contain the same number of degrces. These have also equal chords, sines, tangents, \&.c.

Similar Arcs, of unequal circles, \&c, are such as contain the same number of legrces, or that are the like part or parts of their respective whole circles. Hence, in concentric circles, any two radii cut off, or intercept, similar arcs $\mathrm{M}$ and or.-Sinilar arcs are proportional to the radii $\mathrm{L}, \mathrm{LO}$, or to the whole circumferences.-Similar ares of other like curves, are also like parts of the

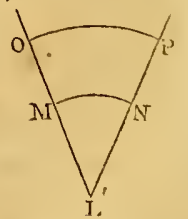
wholes, or determined by like parts similarly posited.

Of the Length of Circular $\Lambda$ ncs. The lengths of circular arcs, as found and expressed in various ways, may be secn in my large Trcatise on Mensuration, pa. 88 et seq. 4th edition: some of which are as follow. The radius of a circle being 1 ; and of any arc $a$, if the tangent be $t$, the sine $s$, 
the cosine $c$, and the versed sine $v$ : then the arc $a$ will be truly expressed by several series, as follow, viz, the are $a=t-\frac{x}{3} t^{3}+\frac{x}{5} t^{5}-\frac{x}{7} t^{7}+\frac{1}{9} t^{9} \& c$. $a=\frac{s}{c}-\frac{1}{3} \cdot \frac{s^{3}}{c^{3}}+\frac{1}{5} \cdot \frac{s^{5}}{c^{5}}-\frac{1}{7} \cdot \frac{s^{7}}{c^{7}} \& c$. $a=s+\frac{1}{2.3} s^{3}+\frac{1.3}{2.4 .5} s^{5}+\frac{1.3 .5}{2.4 .6 .7} s^{7} \& \mathrm{c}$. $a=\sqrt{ } 2 v \times\left(1+\frac{1}{2.3} \cdot \frac{v}{2}+\frac{1.3}{2.4 .5} \cdot \frac{v^{3}}{2^{2}}+\frac{1.3 .5}{2.4 .6 .7} \cdot \frac{v^{3}}{2^{3}} \& c\right.$. $a=\frac{3 \cdot 14159 \& c}{180} d=\cdot 01745329 \& c \times d$; where $d$ denotes the number of degrees in the given arc. Also $a=\frac{8 c-c}{3}$ nearly; where $\mathrm{c}$ is the chord of the arc, and $c$ the chord of half the arc; whatever the radius is.

To find the length of the arc of any curve. Put $x=$ the absciss, $y=$ the ordinate, of the arrc $z$, of any curve whatever. Put $\dot{z}=\sqrt{ }\left(\dot{x}^{2}+\dot{y}^{2}\right)$; then, by means of the equation of the curve, find the value of $\dot{x}$ in terms of $\dot{y}$, or of $\dot{y}$ in terms of $\dot{x}$, and substitute that value instead of it in the above expression $\dot{z}=\sqrt{ }\left(\dot{x}^{2}+\dot{y}^{2}\right)$; hence, taking the fluents, they will give the length of the $\operatorname{arc} z$, in the terms of $x$ or $y$.

A Rc, in Astronomy. Of this, there are various kinds. Thus, the latitude, elevation of the pole, and the declination, are measured by an arc of the meridian; and the longitude, by an arc of a parallel circle, \&c.

Diumal ARc of the sun, is part of a circle parallel to the equator, degcribed by the sun in his course from his rising to the setting. And his Nocturnal Arc is of the same kind; excepting that it is described from setting to rising.

A r c of Progression, or Direction, is an arch of the ecliptic, which a planet seems to pass over, when its motion is direct, or according to the order of the signs.

Anc of Retrogradation, is an arch of the ecliptic, described while a planet is retrograde, or moves contrary to the order of the signs.

ARC between the Centres, in eclipses, is an arc passing from the centre of the earth's shadow, perpendicular to the moon's orbit, meeting her centre at the middle of an eclipse.-If the aggregate of this arc and the apparent semidiameter of the moon, be equal to the semi-diameter of the shadow, the eclipse will be total for an instant, or without any duration; and if that sum be less than the radius of the shadow, the eclipse will be total, with some duration; but if greater, the eclipse will be only partial.

A Rc of Vision, is that which measures the sun's depth below the horizon, when a star, before hid by his rays, begins to appear again.- The quantity of this arc is not always the same, but varies with the latitude, declination, right ascension, or descension, and distance, of any planet or star. Ricciol. Almag. vol. 1, pa. 42. However, the following numbers will serve nearly for the stars and planets. TABLE exhibiting the Arc of Vision of the PLANETS and

\begin{tabular}{|c|c|c|c|c|c|c|c|c|}
\hline \multicolumn{5}{|c|}{ PLANETS. } & \multicolumn{4}{|c|}{$\begin{array}{l}\text { FIXED STARS. } \\
\text { Magnitude. }\end{array}$} \\
\hline Mercury & $\therefore$ & - & $10^{\circ}$ & $0^{\prime}$ & 1 & - & - & \\
\hline Venus: & - & - & 5 & 0 & 2 & - & - & - \\
\hline Mars - & - & - & 113 & 30 & 3 & - & - & - \\
\hline Jupiter & $-\quad-$ & - & 10 . & 0 & 4 & - & - & - \\
\hline Saturn & & - & & 0 & $\begin{array}{l}5 \\
6\end{array}$ & - & - & - \\
\hline
\end{tabular}

A ren, in Architecture, is a concave structure, raised or turned upon a mould, called the centering, in form of the arcls of a curve, and serving as the inward support of some superstructure. Sir Henry Wotton says, An arch is nothing but a narrow or contracted vault; and a vault is a dilated arch.

Arches are used in large intercolumniations of spacious buildings; in porticocs, both within and without temples; in public halls, as ceilings, the courts of palaces, cloisters, theatres, and amphitheatres. They are also used to cover the cellars in the foundations of houses, and powder magazines; also as buttresses and counter-forts, to support large walls laid deep in the earth; for triumphal arches, gates, windows, \&c; and, above all, for the foundations of bridges and aqueducts. And they are supported by piers, butments, imposts, \&c.

Arches are of several kinds, and are commonly denominated from the figure or curve of them; as circular, elliptical, cycloidal, catenarian, \&c, according as their curve is in the form of a circle, ellipse, cycloid, catenary, \&c.

There are also other denominations of circular arches, according to the different parts of a circle, or manner of placing them. Thus,

Semicircular Arches, which are those that make an exact semicircle, having their centre in the middle of the span or chord of the arch; called also by the French builders, perfect arches, and arches en plein centre. The arches of Westminster Bridge are semicircular.

Scheme Arcines, or skene, are those which are less than semicircles, and are consequently flatter arches, containing 120 , or 90 , or 60 , degrees, \&c. They are also called imperfect and diminished arçhes.

Ancr Ls of the third and fourth point, or Gothic arches; or, as the Italians call them, di terzo and quarto acuto, because they always meet in an acute angle at top. Thcse consist of two excentric circular arcs, meeting in an angle above, and are drawn from the division of the chord into three or four more parts at pleasure. Of this kind are many of the arches in churches and other old Gothic buildings.

Elliptical Arcues, usually consist of semi-ellipses. These were formerly much used instead of mantle-trees in chimneys; and are still employed, from their bold and beautiful appearance, for many purposes, and particularly for the arches of a bridge, like that at Black-Friars, both for their strength, beauty, convenience, and cheapness.

Straight ARCHes, are those which have their upper and under edges parallel straight lines, instearl of curves. These are chicfly used over doors and windows; and have their cnds and joints usually pointing towards one common centre.

Arcn is particularly used for the space between the two piers of a bridge, intended for the passage of the water, boats, \&c.

ARC $\mathrm{Af}$ oquitibration, is that which is in equilibrium in all its parts, having no tendency to break in one part more than in another, and which is therefore safer and stronger than any other figure. Every particular figure of the extrados, or upper side of the wall above an arch, requires a peculiar curve for the under side of the arch itself, to form an arch of equilibration, so that the incumbent pressure on every part may be proportional to the strength or resistance there. When the arch is equally thick throughout, a case that can hardly ever happen, then the catenarian curve is the arch of equilibration; but in no other case: and therefore it is a great mistake in some authors to suppose that this curve is the best figure for arches in all cases; when in reality it is commonly the $\mathrm{T} 2$ 


\section{A R C}

wnrst. This subject is fully treated in my Collection of 'Tracts, tract 1, on the Principles of Bridges, pr. xi, exam. 2, where the proper intrados is investigated for every extraclos, so as to form an arch of equilibration in all cases whatever. It there appears that, when the upper side of wall is a straight horizontal line, us in the anncxed figure,

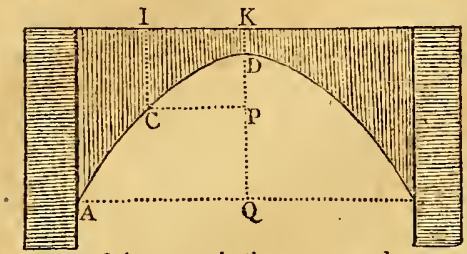

the equation of the curve is thus expressed,

$$
\text { K } \mathrm{L} \text { or } \mathrm{PC}=y=h \times \frac{\log . \text { of } \frac{w+\sqrt{\left(w^{2}-a^{2}\right)}}{a}}{\log . \text { of } \frac{c+\sqrt{\left(c^{2}-a^{2}\right)}}{a}} \text {; }
$$

where $k=\mathrm{AQ}, w=\mathrm{c} 5, a=\mathrm{DK}, c=\mathrm{KQ}$.

$$
\text { Or } \mathrm{cr}=w=\frac{a^{2}+n^{2}}{2 n} ; n=\text { the number whose log. is }
$$

$c y+A$, and $A=\log$. of $a$, and $c=\frac{1}{h} \times \log$. of $\frac{c+\sqrt{ }\left(c^{2}-a^{3}\right)}{a}$.

And hence, when $a, h, c$, are any given numbers, a table is formed for the corresponding values of $w$ and $y$, by which the curve is constructed for any particular occasion. Tlıus supposing $a$ or $\mathrm{DK}=6, h$ or $\mathrm{AQ}=50$, and $c$ or $\mathrm{KQ}=46$; then the corresponding values of $\mathrm{K} I$ and $1 \mathrm{c}$, or horizontal and vertical lines, will be as in this table.

\begin{tabular}{|c|c|c|c|c|c|}
\hline $\begin{array}{c}\text { Value of } \\
\text { KI }\end{array}$ & $\begin{array}{l}\text { Value of } \\
\text { Ic. }\end{array}$ & $\begin{array}{l}\text { Value of } \\
\text { Kr. }\end{array}$ & $\begin{array}{l}\text { Value of } \\
\text { IC. }\end{array}$ & $\begin{array}{c}\text { Value of } \\
\text { Kr. }\end{array}$ & $\begin{array}{l}\text { Value of } \\
\text { Ic. }\end{array}$ \\
\hline 0 & $6 \cdot 000$ & 21 & 10.381 & 36 & 21.774 \\
\hline 2 & 6.035 & 22 & 10.858 & 37 & $22 \cdot 948$ \\
\hline 4 & $6 \cdot 144$ & 23 & $11: 368$ & 38 & $24: 190$ \\
\hline 6 & 6.324 & 24 & 11.911 & 39 & 25.505 \\
\hline 8 & $6 \cdot 580$ & 25 & $12^{\circ} 489$ & 40 & $26 \cdot 894$ \\
\hline 10 & $6 \cdot 914$ & 26 & $13 \cdot 106$ & 41 & $28 \cdot 364$ \\
\hline 12 & $7 \cdot 330$ & 27 & $13^{\circ} 761$ & 42 & $29 \cdot 919$ \\
\hline 13 & 7.571 & 28 & $14^{\circ} 457$ & 43 & 31.563 \\
\hline 14 & $7 \cdot 834$ & 29 & $15 * 196$ & 44 & $33 \cdot 299$ \\
\hline 15 & $8 \cdot 120$ & 30 & 15980 & 45 & $35 \cdot 135$ \\
\hline 16 & 8.430 & 31 & $16 \cdot 811$ & 46 & $37 \cdot 075$ \\
\hline 17 & 8.766 & 32 & $17 \cdot 693$ & 47 & $39 \cdot 126$ \\
\hline 18 & $9 \cdot 168$ & 33 & $18 \cdot 627$ & 48 & $41 \cdot 293$ \\
\hline 19 & $9 \cdot 517$ & 34 & $19 \cdot 617$ & 49 & 43.581 \\
\hline 20 & 9.934 & 35 & 20.665 & 50 & $46^{\circ} 000$ \\
\hline
\end{tabular}

Table for constructing the Curve of Equilibration.

See my Tracts, vol. $j$, art. 1 .

The doctrine and use of arches are neatly delivered by sir Henry Wotton, though he is not always mathematically accurate in the priuciples. He says; 1st, All matter, unless impeded, tends to the centre of the earth in a perpendicular line. 2d, All solid materials, as bricks, stones, \& $c$, in their ordinary rectangular form, if laid in numbers, one by the side of another, in a level row, and their extreme ones sustained between two supporters; those in the middle will necessarily sink, even by their own gravity, much more if forced down by any superincumbent weight. 'To make them stand, therefore, either their figure or their position must be altered.-3d, Stones, or other materials, briug figured cuneatim, or wedge-like, broader above than below, and laid in a level row, with their two extremes supported as in the last article, and pointing all to the same centre; none of them can sink, till the supporters or butments give way, becuuse they want room in that situation to descend perpendicularly. But this is a weak structure; because the supporters are subject to too much inpulsion, especially where the line is long; for which reason the form of straight arches is seldom used, excepting over doors and windows, where the line is short, and the side walls strong. In order to fortify the work, thercfore, we nust change not unly the figure of the materials, but also their position.-4th, If the materials be shaped wedge-like, and be disposed in form of an arch, and pointing to some centre; in this case, neither the pieces of the said arcla can sink downwards, for want of room to descend perpendicularly; nor can the supporters or butments suffer much violence, as in the preceding flat form: for the convexity will always make the incumbent rather rest upon the supporters, than thrust or push them outwards. His reasoning, however, afterwards, on the effect of circular and other arches, is not accurate, as be attends only to the side pressure, without considering the effect of different vertical pressures.

The chief properties of arches of different curves may be seen in the $2 d$ sect. of my Tract on the Prunciples of Bridges, above quoted. It there appears that none, except the mechanical curve of the arch of equilibration, can admit of a horizontal line at top: that this arch is of a form both graceful and convenient, as it may be made higher or lower at pleasure, with the same span or opening: that all other arches require extrados that are curved, more or less, either upwards or downwards : of these, the elliptical arch approaches the nearest to that of equilibration, for equality of strength and corvenience; and it is also the best form for most bridges, as it can be male of any height to the same span, its hanches being at the same time sufficiently elevated above the water, even when it is very flat at top: elliptical arches also look bolder and lighter, are more uniformly strong, and much cheaper than most others, as they require less materials and labour. Of the other curves, the cycloidal arch is next in quality to the elliptical one, for those properties, and lastly the circle. As to the others, the parabola, hyperbola, and catenary, the'y are quite inadmissible in bridges that consist of spveral arches; but may, in some cases, be employed for a bridge of one single arch, which may be intended to rise very high, as in such cases they are not murch loaded at the hanches.

See also Bringe, and MAGAZINE.

Arch Mural. See Mura L Arch.

ARCHER, or Sagittarius, one of the constellations of the northern hemispluere, and one of the twelve signs of the zodiac, placed between the Scorpion and Capricorn. Sce SAgITTARIUS.

ARCHIMEDES, one of the most celebrated mathematicians among the ancients, who flomislsed about 28.9 years before Christ, being about 110 years later than liuclid. He was born at Syracuse in Sicily, anci was related to Hiero, according to Plutarch, the then king of that city, by his father. In his youth, he devoted himself to the study of geometry; and in his maturer years, he travelied into Egypt, whither the Grecks usually resorted in the pursuit of science. After un absence of several years, which he spent in the socicty of Conon and other eminent men, and during which time he gave very promising indications of his future fanc, he returned to his own country; and 
then continued his studies with the greatest assiduity. Such indeed were the ardour and intenseness of his application to mathematical science, that he prosecuted his studies to the neglect both of food and sleep, and improved the minutest circumstance that occurred into an occasion of making very important and useful discoveries. The mathematical genius of Archimedes set bim with such distinguished excellence in the view of the world, as rendered him both the honour of his own age, and the admiration of posterity. He was indeed the prince of the ancient mathematicians, being to them what Newton is to the moderns, to whom in his genius and character he bears a very near resemblance. He was frequently lost in a kind of reverie, so as to appear hardly sensible; he would study for days and nights together, neglecting his food ; and Plutarch tells us that he used to be carried to the baths by force. Many particulars of his life, and works, mathematical and mechanical, are recorded by several of the ancients, as Polybius, Livy, Plutarch, Pappus, \&c. He was equally skilled in all the sciences, astronomy, geometry, mechanics, hydrostatics, optics, \&c, in all of which he excelled, and made many and great discoveries.

Archimedes, it is said, marle a sphere of glass, of a most surprising contrivance and workmanship, exhibiting the motions of the heavenly bodies in a very pleasing manner. Claudian has an epigram on this invention, which has been thus translated :

When in a glass's narrow space confin'd,

Jove saw the fabric of th' almighty mind,

He smil'd, and said, Can mortals' art alone,

Our heavenly labours mimic with their own?

The Siracusan's brittle work contains

Th' eternal law, that through all nature reigns.

Fran'd by his art, see stars unnumber'd burn,

And in their courses rolling orbs return :

His sun thruugh various signs describes the year;

And every month his mimic mons appear.

Our rival's laws his litzle planets bind,

And rule their motions with a human mind.

Salmoneus could our thunder imitate,

But Archimedes can a werld create.

Many wonderful stories are told of his discoveries, and of his very powerful and curious machines, \&c. Hliero ouce admiring them, Archimedes replied, these cffects are nothing, "But give me," said he, "some other place to fix a unachine on, and I will move the carth." He fell upon a curious device for discovering the deceit which had been practised by a workman, employed by the king to make a golden crown. Hiero, having a mind to make an offering to the gods of a golden croww, agreed for one of great value, and weighed out the gold to the artificer. After some time be brought the crown home of the full weight ; but it was afterwards discovered or suspected that a part of the gold had been stolen, and the like weight of silver substituted in its stead. Hicro, being angry at this imposition, desired Archimedes to take it into consideration, how such a fraud might be certainly discovered. While engaged in the solution of this difficulty, he happened to go into the bath; where observing that a quantity of water uverflowed, equal to the bulk of his body, it presently occurred to him, that Hiero's question might be answered by a like method: upon which he lraped out, and ran bome-

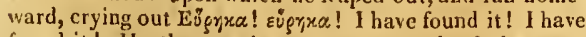
found it! He then made two masses, each of the same weight as the crown, one of gold and the other of silver: this done, he filled a vessel to the brim with water, and put the silver mass into it, upon which a quantity of water overflowed equal to the bulk of the mass; then taking the mass of silver out he filled up the vessel again, measuring the water exactly, which he put in; this showed him what measure of water answered to a certain quantity of silver. Ile then tricd the gold in like manmer, and found that it caused a less quantity of water to overflow, the gold being less in bulk than the silver, though of the same weight. He then filled the vessel a third time, and putting in the crown itself, he found that it caused more water to overflow than the golden mass of the same weight, but less than the silver one; so that, finding its bulk between the two masses of gold and silver, and that in certain known proportions, he hence computed the real quantities of gold and silver in the crown, and so manifestly discovered the fraud.

Archimedes also contrived many machines for useful and beneficial purposes; among these, engines for launching large ships; screw pumps, for exhausting the water out of slips, marshes, or overflowed lands, as Egypt, \&c, which they would do from any depth.

But he became most famous by his curious contrivances, by which the city of Syracuse was so long defended, when besieged by the Roman consul Marcellus; showering upon the enemy sometimes long darts, and stones of vast. weight, and in great quantities; at other times lifting their ships up into the air, that had come near the walls, and dashing them to picces by letting them fall down again; nor could they find their safety in removing out of the reach of his cranes and levers, for there he contrived to fire them with the rays of the sun reflected from burning glasses.

However, notwithstanding all his art, Syracuse was at length taken by storm, or by corrupting the guards. "What gave Marcellus the greatest concern," says Plutarch, "was the unhappy fate of Archimedes, who was at that time in his museum; and his mind, as well as his eyes, so fixed and intent upon some geometrical figures, that he neither heard the noise and hurry of the Romans, nor perceived the city to be taken. In this depth of study and contemplation, a soldier came suddenly upon him, and commanded him to follow him to Marcellus; which he refusing to do, till he had finished his problem, the soldier, in a rage, drew his sword, and ran him througl]." Jjivy says he was slain by a soldier, not knowing who he was, while he was drawing schemes in the dust: that Marcellus was grieved at his death, and took care of his funeral; and made his name a protection and honour to those who could claim a relationship to him. His death it seems happened about the $142 \mathrm{~d}$ or $143 \mathrm{~d}$ Olympiad, or 210 years before the birth of Christ. According to Tzetzes he lived 75 years.

When Cicero was questor for Sicily, he discovered the tomb of $\Lambda$ rchimedes, all overgrown with bushes and brambles; which be caused to be cleared, and the place set in order. There was a sphere and cylinder cut upon it, with an inscription, but the latter part of the verses quite worn out.

Many of the works of this great man are still extant, though the greatest part of them are lost. The pieces remaining are as follow: 1. Two books on the sphere and Cylinder:-2. The Dimension of the Circle, or proportion between the diameter and the circumference.-3. Of Spiral lines. - 4. Of Conoids and Spheroids. - 5. Of Equiponderants, or Centres of Gravity.-6. The Quadrature of the 1'arabola.-7. Of Bodies floating on Fluids, -8 . Lemmata. -9 . Of the Number of the Sands. 


\section{A R C}

[

Among the works of Archimedes which are lost, may be reckoned the descriptions of the following inventions, which may be gathered from himself and other ancient authors. 1. His account of the method which he employed to discover the mixture of gold and silver in the crown, mentioned by Vitruvius. - 2. His description of the Cochleon, or engine to draw water out of places where it is stagnated, still in use under the name of Archimedes's Screw. Athenæus, speaking of the prodigious ship built by the order of Hiero, says, that Archimedes invented the cochleon, by means of which the bold, notwithstanding its depth, could be drained by one man. And Diodorus Siculus says, that he contrived this machine to drain Egypt, and that by a wonderful mechanism it would exhaust the water from any depth.-3. The Helix, by means of whicb, Athenæus informs us, he launched Hieru's great ship.4. The Trispaston, which, according to Tzetzes and Oribasius, could draw the most stupendous weights. -5 . The macbines, which, according to Polybius, Livy, and Plutarch, he used in the defence of Syracuse against Marcellus, consisting of Tormenta, Balistæ, Catapults, Sagittarii, Scorpions, Cranes, \&c.--6. His Burning Glasses, with which be set fire to the Roman galleys. - 7. His Pneumatic and Hydrostatic engines, concerning which subjects he wrote some books, according to Tzetzes, Pappus, and Tertullian.-8. His Sphere, which exhibited the celestial motions. And probably many others.

A whole volume might be written on the curious methods and inventions of Archimedes, that appear in his mathematical writings now extant only. He was the first who squared a curvilineal space ; unless Hypocrates must be excepted, on account of his lunes. In his time the conic sections were admitted into geometry, and he applied himself closely to the measuring of them, as well as other figures. Accordingly, he determined the relations of spheres, spheroids, and conoids, to cylinders and cones; and the relations of parabolas to rectilineal planes, whose quadratures had long before been determined by Euclid. He has left us also his attempts upon the circle: he proved that a circle is equal to a right-angled triangle, whose base is equal to the circumference, and its altitude equal to the radius; and consequently, that its area is equal to the rectangle of half the diameter and half the circumference; thus reducing the quadrature of the circle to the determination of the ratio between the diameter and circumference; which determination however has never yet been douse. Being disappointed of the exact quadrature of the circle, for want of the rectification of its circumference, which all his methods would not effect, he proceeded to assign a useful approximation to it: this he effected by the numeral calculation of the perimeters of the inscribed and circumscribed polygons; from which calculation it appears, that the perimeter of the circumscribed regular polygon of 192 sides, is to the diameter, in a less ratio than that of $3 \frac{1}{7}$ or $3 \frac{1}{7} \frac{0}{8}$ to 1 ; and that the perimeter of the inscribed polygon of 96 sides, is to the diameter, in a greater ratio than that of $3 \frac{1}{7}^{\circ}$ to 1 ; consequently, that the ratio of the circuniference to the diameter, lies between these two ratios. Now the first ratio, of $3 \frac{1}{7}$ to 1 , reduced to whole numbers, gives that of 22 to 7 , for $3 \frac{1}{7}: 1:: 22: 7$; which therefore is nearly the ratio of the circumference to the diameter. From this ratio between the circumference and the diameter, Archimedes computed the approximate area of the circle, and he found that it is to the square of the diameter, as 11 is to 14.

\section{A R C}

He determined also the relation between the circle and the ellipse, with that of their similar parts. And it is probable that he also attempted the hypertola; but it is not to be expected that he met with any success, since approximations to its area are all that can be given by the rarious methods that have since been invented.

Besides these figures, he determined the measures of the spiral, described by a point moving uniformly along a right line, the line at the same time revolving with a uniform angular motion; determining the proportion of its area to that of the circumscribed circle, as also the proportion of their sectors.

Throughout the whole works of this great man we every where perceive the deepest design, and the finest invention. He seems to have been, with Euclid, exceedingly careful of admitting into his demonstrations nothing but principles perfectly geometrical and unexceptionable: and though his most general method of demonstrating the relations of curved figures to straight ones, be by inscribing polygons in them; yet to determine those relations, he does not increase the number, and diminish the magnitude, of the sides of the polygon ad infinitum, but from this plain fundamental principle, allowed in Euclid's Elements, (viz, that any quantity may be so often multiplied, or added to itself, as that the result shall exceed any proposed finite quantity of the same kind,) be proves that to deny his figures to have the proposed relations, would involve an absurdity. And when he demonstrated many geometrical properties, particularly in the parabola, by means of certain progressions of numbers, whose terms are similar to the inscribed figures; this was still done without considering such series as continued ad infinitum, and then collecting or summing up the terns of such infinite series.

There have been various editions of the existing writings of Archimedes. The whole of these works, together with the Commentary of Eutocius, were found in their original Greek language, on the taking of Constantinople, whence they were brought into Italy; and bere they were found by that excellent mathemntician John Nuller, otherwise called Regiomontanus, who brought them into Germany: where they were, with that Commentary, published long afterwards, viz, in 1544, at Basil, being most beautifully printed in folio, both in Greek and Latin, by Hervagius, under the care of Thomas Gechaufi Venatorius.-A Latin translation was published at Paris 1557, by Pascalius Hamellius.-A nother edition of the whole, in Greek and Latin, was published at Paris 1615 , in folio, by David Rivaltus, illustrated with new demonstritions and commentaries: a life of the author is prefixed; and at the end of the volume is added some account, by way of restoration, of our author's other works, which harc been lost; viz, The Crown of Hiero; the Cochleon or Water Screw; the Helicon, a kind of endless screw; the Trispaston, consisting of a combination of wheels and axles; the Machines employed in the detence of Syracuse; the Burning Speculum; the Machines mored by Air and Water; and the Material Sphere.-In 1675, Dr. Isaac Barrow published a neat edition of the works, in Latin, at London, in 4 to ; illustrated, and succinctly demonstrated in a new method.-But the most complete of any, is the magnificent edition, in folio, lately printed at the Clarendon press, Oxford, 1792. This edition was prepared ready for the press by the learned Joseph Torelli, of Verona, with his preface, and in that state presented to 
the University of Oxford. The Latin translation is a new one. We find here also Eutocius's Commentary on some of the-pieces, and notes on the whole. An account of the life and writings of Tirelli is prefixed, by Clemens Sibiliati. And at the end a large appendix is alded, in two parts; the first being a Commentary on Archimedes's paper on Bodies that float on Fluids, by the Rev. Abram Robertson of Christ-Church C'ullege, who had the care of this edition; and the latter is a large collection of various readings in the manuscript works of Archimedes, found in the library of the late king of France, and of another at Florence, as collated with the Basil edition above mentioned.

There are al -o extant other editions of certain parts of the works of Archimedes. Thus, Commandine published, in 4 to, at Bologna-1565, the two books concerning Bodies that foat on Fluids, with a Commentary. Commandine published also a translation of the Arenarius. And Borelli publisbed, in folio, at Florence, 1661 , Archimedes's Líber Assumptorum, translated into Latin from an Arabic manuscript copy. This is accompanied with the like translation, from the Arabic, of the $5 \mathrm{th}, 6 \mathrm{th}$, and 7 th books of Apollonius's Conics. Mr. G. Anderson published (in Svo. Lond. 1784) an English translation of the Arenarius of Archimedes, with learned and ingenious notes and illustrations. Dr. Wallis published a translation of the Arenarius. Lastly, the translation of Torelli's work into the French language, by $F$. Peyrard, in 2 vols, 8vo, at Paris, in 18-, and 1808. And there may be other editions beside the above; but these are all that I liave seen, or know of.

Archimedes's Screw. See Screw of Archimedes.

Archinedes's Burning-glass. See Burning-glass.

ARCHITECT, a person skillcd in architecture, or the art of building; who forms plans and designs for edifices, conducts the work, and directs the various artificers employed in it.

The most celebrated architects are, Vitruvius, Palladio, Scamozzi, Serlio, Vignola, Barbaro, Cataneo, Alberti, Viola, Inigo Jones, De Lorme, Perrault, S. Le Clerc, Sir Christopher Wren, the Earl of Burlington, \&c.

ARCHITECTURE, Archilectura, the art of planning and building or erecting any edifice, so as properly to answer the end proposed, for solidity, conveniency, and beauty; whether houses, temples, churches, bridges, halls, theatres, \&c, \&c.-Architecture is divided into civil, military, and naval or maine.

Civit AkCHITECTURE, is the art of designing and erecting edifices of every kind for the uses of civil life in every capacity; as churches, palaces, private houses, \&c ; and it has been divided into five orders or manners of building, under the names of the Tuscan, Doric, Ionic, Corinthian, and Composite.

There were many authors on architecture among the Greeks and Romans, before Vitruvius; but he is the first whose work is entire and extant. IJe lived in the reigns of Julius Cxesar and Augustus, and coruposed a complete system of architecture, in ten books, which he dedicated to this prince. The principal authors on architecture since Vitruvius, are Philander, Barbarus, Salmasius, Baldus, Atberti, Gauricus, Demoniosius, Perrault, De l'Orme, Rivius, Wotton, Serlio, Palladio, Sitrada, Vignola, Scanozzi, Dicussart, Catanei, Freard, De Cambray, Blondel, Goldinan, Sturmy, Wolfius, De liosi, Desgodetz, Baratteri, Mayer, Gulielumus, Ware, \&c, \&c. See also AnсHттест.

Milizary A Rcnitectu E, otherwise more usually called
Fortification, is the art of strengthening and fortifying places, to secure them from the insults or attacks of enemies, and the violence of arms; by erecting forts, castles, and other fortresses, with ramparts, bastions, \&c.-The anthors who have chiefly excelled in this art, are Coehorn, Pagan, Vauban, Scheiter, Blondel, and Montalembert.

Naval ARch I tecture, or Slip-building, is the art of constructing ships, galleys, and other vessels proper to float on the water.

ARCHITRAVE, is that part of a column which bears immediately on the capital. It is the lowest member of the entablature, and is supposed to represent the principal beam in timber buildings, in which it is sometimes called the Reason-piece, or Master-piece. Also, in chimneys it is called the Mantle-piece; and the Hyperthyron over the jaumbs of doors, or lintels of windows.

Architrave Cornice. See Cornice.

ARCHTRAVE doors, are those which have all architrave on the jaumbs, and over the door; on the cal' piece if straight; or on the arcb, if the top be curved.

A RCH ITR A VE windows, of timber, are usually an ogee raised out of the solid timber, with a list over it: though sometimes the monldings are struck, and laid on; and sometimes they are cut in brick.

ARCHIVAULT or ARCHIVOLT, the contour of an arch; a band or frame adorned with mouldings, running over the faces of the voussoirs or arch-stones, and bearing upon the imposts.

ARCHYTAS, of Tarentum, a celebrated mathematician, cosmographer, and Pythagorean philosopher, whon Horace calls

\section{Mensorem.}

He flourished about 400 years before Clirist; and was the master of Plato, Eudoxus, and Philolaus. He gave a method of finding two mean proportionals between two given lines, and thence the duplication of the cube, by means of the conic sections. His skill in mechanics was such, that he was said to be the juventor of the crane and the screw; and he made a wooden pigeon that could fly about, when it was once set off, but it could not rise again of itself, after it rested. He wrote several works, though none of then are now extant, particularly a treatise $\pi \varepsilon \rho \dot{\delta} \tau \tilde{8}$ ПTarròs, De Universo, cited by Simplicius in Aristot. Categ. It is said he invented the ten categories. He ac. quired great reputation both in his legislative and military capacity; having commanded an army seven times without ever being defented. He was at last shipwrecked, and drowned in the Adriatic sea.

ARCIIC Circle, is a lesser circle of the sphere, parallel to the equator, and passing through the north pole of the ecliptic, or distant from the north or arctic pole, by a quantity equal to the obliquity of the ecliptic, which was formerly cstimated at $23^{\circ} 30^{\prime}$, but its mean quantity is now $23^{\circ} 2 \mathrm{~s}^{\prime}$ nearly. 'This, and its opposite, the antarctic circle, are also called the polar circle's, where the longest day and longest night are 24, hours, and within all the space of these circles, at one time of the year, the sun never sets, and at the opposite season he never rises for some days, more or less according as the place is nearer the pole.

Arctic Pole, the north pole of the world, and so called from áprros, ursa, the bear, from its proximity to the constellation of that name.

ARCTURUS, a fixed star of the first magnitude, between the thighs, of the constellation Bootes. So called 


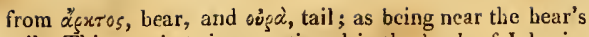
tail. This star is twice mentioned in the book of Job, viz, ix. 9 , and xxxviii. 32 , by the name $A$ ish, if the translation be right; and by many of the ancients under its Greek name Arcturus. The Greeks gave the fabulous history of this star, or constellation, to this purport: Calisto, who was afterwards, in form of the great bear, raised up into a constellation, they tell us, brought forth a son to Jupiter, whom they called Arcas. That Lyacon, when Jupiter afterwards came to visit him, cut the boy in pieces, and served him up at table. Jupiter, in revenge, as well as by way of punishment, called down lightning to consume the palace, and turned the monarch into a wolf. The limbs of the boy were gathered up, to which the gorl gave life again, and he was taken and educated by some of the people. His mother, who was all this time a bear in the woods, fell in his way: he chased her, ignorant of the fact, and, to avoid him, she threw herself into the temple of Jupiter: he followed her thither to destroy her; and this being death by the laws of the country, Jupiter took them both up into heaven, to prevent the punishment, making her the constcllation of the great bear, and converting the youth into this single star behind her.

Dr. Hornsby, the Savilian Professor of Astronomy, concludes that Arcturus is the nearest star to our system visible in the northern hemisphere, because the variation of its place, in consequence of a proper motion of its own, is more remarkable than that of any other of the stars; and by comparing a variety of observations respecting both the quantity and direction of the motion of this star, he infers, that the obliquity of the ecliptic slecreases at the rate of $j \delta^{\prime \prime}$. in 100 ycars; a quantity which ncarly corresponds to the mean of the computations framed by Euler, Lalande, and Laplace, on the principles of attraction. Philos. Trans, vol. 63.

AREA, in general, denotes any plain surface to walk upon ; and derived from arere, to be dry.

AREA, in Architecture, denotes the space or site of ground on which an edifice stands. It is also used for inner courts, and such like portions of ground.

AREA, in Gcometry, denotes the superficial content of any figure. The areas of figures are estimated in squares and parts of squares. Thus, suppose a rectangle EFG $H$ have its length $\mathrm{EH}$ equal to 4 inches, or feet, or yards, \&c, and its breadth EF cqual to 3 ; its area will then be 3 times 4 , or 12 squares, each side of which is respectively one inch, or foot, or yard, \&cc. The areas of other particular figures may be seen under their respectivenames.

The areas of all similar figures, are in the duplicate ratio, or as the squares of their like sides, or of any like linear dimensions. $-\mathrm{Al}$ so the law by which the planets move round the surn, is regulated by the areas described by a linc connecting the sun and planct; that is, the time in which the planet describes, or passes over, any arc of its elliptic orbit, is proportional to the clliptic area clescribed in that time by the said line, or the sector contained by the said arc and two radii drawn from its extremities to the focus in which the sun is placed.

\section{Area, in Optics. Sce FinLd.}

ARENARIUS, the name of a book of Archimedes, in which he demonstrated, that not only the sands of the carth, but even a grcater quantity of particles than could be containcd in the immense sphere of the fixed stars, might be expressed by numbers, in a way by him invented and described. This notation proceeds by certain geometrical progressions; and in denoting and producing certain very distant terms of the progression, he here first of any one makes usc of a property similar to that of logarithms, viz, adding the indices of the terms to find the index of their product. See Archinedes.

AREOMETER, Ar זOMETRUm, an instrument to measure the density or gravity of fluids.

The areometer, or water-poise, is commonly made of glass; consisting of a round hollow ball, which terminates in a long stender neck, hermetically sealed at top; having first as much runining mercury put isto it, as will serve to balance or keep it swimming in an rect position. The stem, or neck, is divided into degres or parts, which arc numbered, to show, by the ilepth of its descent inte any liquor, the lightness or density of it : for that fluid is heaviest in which it sinks least, and lightest in which it sinks deepest.

Another instrument of this kind is described by Homberg of Paris, in the Memoirs of the Acad of Sciences for the year 1699; also in the Philos.jTrans. $\mathrm{N}^{5}$ 262, where a table of numbers is given, expressing the density of various fluids, as determined by this instrument buth in summer and winter. By this table it appears that the density, or specific gravity of quiclisilver and distilled water, in the two seasons, were as follow, viz,

$\begin{array}{lll}\text { in summer as - } & 13.61 \text { to } 1 \text {, } \\ \text { in winter as } & - & 13.53 \text { to } 1 \text {; } \\ \text { me medium of these two is as } & 13.57 \text { to } 1 \text {. }\end{array}$

and the medium of these two is as 13.57 to 1 .

Sce the Philos. Trans, vol. 36 , or my $\Lambda$ bridg. vol. 7 , for the description and use of another new areometer. Sec also HY DROAETER.

AREOMETRY, the science of measuring the gravity and density of fluids. See the Philos. Trans. vol. 68, for an essay on areometry, \&c. Also my Abridgement, vol. 14.

AREOSTYLE, in Architecture, a sort of intercolumnation in which the columus were placed at a great distance from one another.

ARGETENAR, a star of the fourth-magnitude, in the flexure of the constellation Eridanus.

ARGO NAVIS, or the ship, is a constellation of fixed stars, in the southern hemisphere, being one of the 48 old constcllations. 'The number of stars in this constellation, are, in Ptolemy's catalogue 45 , in 'Tyclıo Brahe's 11 , in Flamsteed's 6 t.

The Greeks tell us, that this was the noted ship in which the Argonauts performed that celebrated expedition, which bas been so famous in all their history.

ARGUMENT, in Astronomy, is an arch given, by which another arch is found in some preportion toit. Hence, ARGUMENT of Inclination, or ARGUMENT of Latitude, of any planct, is an arc of a planet's orbit, intercepted between the ascending node, and the place of the planet from the sun, numbered according to the succession of the signs.

Menstrual Argunent of Latilude, is the distance of the moon's true place from the sun's true place.-By this is found the quantity of the real obscuration in eclipses, or how many digits are darkened in any place.

Annual ARGuMENT of the moon's apogee, or simply, Annual Argument, is the distance of the sun's place froin the place of the inoon's apogec; that is, the arc of the ccliptic comprised between those two places. 
ARIES, or the Ram, in Astronomy, one of the constellations of the northern hemisphere, being the first of tbe old twelve signs of the zodiac, and marked $\boldsymbol{r}$ in imitation of a ram's head. It gives name to a twelfth part of the ecliptic, which the sun enters commonly about the 20th of March.- The stars of this constellation in Ptolemy's catalogue are 18, in 'Tycho Brahe's 21 , in Hevelius's 27 , and in Flamsteed's 66: but they are mostly very small, orily one being of the $2 \mathrm{~d}$ magnitude, two of the $3 \mathrm{~d}$ magnitude, and all the rest smaller.

The fabulous account of this constellation, as given by the Greeks, is to this effict. That Nephele gave Phryxus, her son, a ram, which bore a golden fletce, as a guard against the greatest dargers. Juno, the step-mother both of him and Helle, laid designs against their lives; but Phryxus, remembering the admonition of his mother, took his sister with him, and getting upon the back of the ram, they were carried to the sea. The ram plunged in, and the youth was carried over; but Helle dropping off, was drowned, and so gave name to the Hellespont. When he arrived in Colchis, Eeta the king received him kindly; and, sacrificing the ram to Jupiter, dedicated the fleece to the god; which was afterwards carried off by Jason. The animal itself, they say, Jupiter snatched up into the heavens, and made of it the constellation Aries. They have nther fables also to account for its origin. But it is most probable that the inventors of this sign, placed it there as the father of those animals which are brought forth about the time the sun approaches to that part of the heavens, and so marking the beginning of spring.

An I És also denotes a battering ram; being a military engine with an iron head, much used by the ancients, to batter and beat down the walls of places besieged. Sce - Ram, and Battering Ram.

ARISTARCHUS, a celebrated Greek philosopher and astronomer, was a native of the city of Samos; but of what date is not exactly known; it must have been however before the time of Archimedes, as some parts of his writings and opinions are cited by that anthor, viz, in his Arenarius; he probably flourished about 420 years belore Christ. He held the opinion of Pythagoras as to the system of the world, but whether before or after him, is uncertain, teaching that the sun and stars were fixed in the heavens, and that the earth moved in a circle about the sun, at the same time that it revolved about its own centre or axis. He taught alsn, that the annual orbit of the earth, compared with the distance of the fixed stars, is but as a pnint. On this head A rchimedes says;" A Aristarchus, the Salnian, confuting the notions of astrologers, lajd down certain positions, from which it follows, that the world is much larger than is generally imagined; for he lays it down, that the fixed stars and the sun are immoveable; and that the earth is carried round the sun in the circumference of a circle. On which account, though he might not suffer persecution and imprisonment like Galileo, yet he did not escape censure for his supposed impiety ; for it is said Cleantlius was of opinion, that Greece ought to have tried Aristarchus for irreligion, for endeavouring to preserve the regular appearance of the heavenly bodies, by supposing that the heavens themselves stood sill; but that the earrh revolved in an oblique circle, and at the same time turned round its own axis.

Aristarchus invented a peculiar kind of sun-dials, mentioned by Vitruvius. There is extunt of his works only a treatise on the magnitude and distance of the sun and

VOL. 1 . moon: this was translated into Latin, and commented on by Commandine, who first published it 'with Pappos's Lxplanations, in 1572 . Dr. Wallis afterwards published it in Greek, with Commandine's Latin version, in 1688, and which he inserted agtin in the $3 \mathrm{~d}$ volume of his Mathematical Works, printed in folio at Oxford, 1699. The piece was animadverted on by Mr. Foster, in his Miscellanies. There is another piece which has gone under the name of Aristarchus, on the Mundane Systeni, its parts, and motions, published in Latin by Roberval, and by Mersenne, in his Mathematical Synopsis. But this piece is censured, by Menagius (in Ding. Laert.), and Descartes, in his Epistles, as a fictitious picce of Roberval's, and not the genuine work of Aristarchus.

ARISTO'ELIAN, something that relates to the philosopher Aristotle. Thus we say, an Aristutelian dogma, the Aristotelian schiool, \&c.

Aristutelian Philosophy, the philosophy taught by Aristotle, and maintained by his followers. It is otherwise called the Peripatetic Philosophy, from their practice of teaching while they were walking.-The principles of Aristutle's philosophy, the learned agree; are chiefly laid down in the four books de Cœlo. Instead of the more ancient systems, he introduced Matter, Form, and Privation, as the principles of all things; but it does not seem that he derived much benefit from them in natural philosophy. And his doctrines are, for the most part, so obscurely expressed, that it has not yet been satisfactorily ascertained, what were his sentiments on some of the most important subjects. He attempted to refute the Pythagorean doctrine concerning the two-fold motion of the earth; and pretended to demonstrate, that the matter of the heaveus is ungenerated, incorruptible, and not subject to any alteration: and he supposed that the stars were carried round the earth in solid orbs.

ARISTOTELIANS, a sect of philosophers, so called, from their leader Aristotle, and are otherwise called Peripatetics.-The Aristotelians and their dogmata prevailed for a long while, in the schools and universities; even in spite of all the efforts of the Cartesians, Newtonians, and other corpuscularians. But the systems of the latter have at length gainerl the ascendency; and the Newtonian philosuphy in particular is now very generally received.

ARISTO'lLE, a Grecian philosopher, the son of Nicomachus, physician to Amyntas king of Macedonia, was born 384 years before Christ, at Stagira, a town of Macedonia, or, as others say, of Thrace; from which he is also called the Stagirite. Not succeeding in the profession of arms, to which it seems he first applied himself, he turned his views to philosophy, and at 17 years of age entered himself a disciple of Plato, and attended in the academy till the death of that philosopher. Aristotle then retired to Atarna, where the prince Hermias gave him his daughter to wife. Repairing afterwards to the court of king Plilıp, he became preceptor to his son, Alexander the Great, whose education he attended for the space of 8 years; and by the magnificent encouragement of this prince he was afterwards enabled to procure all sorts of animals, from the inspection of which to write their history. On his quitting Macedon, he settles at Athens, where he established his school, having the Lyceum assigned him, by the magistrates, for the place of his instruction or disputation; where he became the head and founder of the sect called after his name, as also Peripatetics, from the circumstance of his giving instructions 


\section{A R I \\ [ 146$]$ \\ A R I}

while watking. But being here accused of impicty by Eurymedon, priest of Ceres, and fearing the fate of Socrates, he retired to Chalcis, where he died at 63 years of age, and 322 years before Christ. Some say that be poisoneit himself, others that he died of a colic, and others agnin pretend that he threw himself into the sea, for grief that he could not discover the cause of the flux and reflux of the waters. Laertius, in his life of Aristotle, estimates his books at the number of 4000; of which however scarce 20 have come down tu us : these may be comprised under five heads; the first, relating to poetry and rhetoric; the second, to logics; the third, to ethics and politics; the fourth, to physics; and the fifth, to metaphysics. In the schools, Aristotle has been called the philosoplser, and the prince of philosophers. Indeed, such was the veneration paid to him, that his opinion was allowed to stand on a level with reason itself: nor was any appcal from it admitted, the parties, in every dispute, being obliged to show, that their conclusions were no less conformable to the doctrine of A ristotle than to truth.

Aristotle's Mechanics were published in Greek and Latin, at Paris, in 4to, 1599, by Henry Monanthol, physician, and royal professor of the Mathematical arts, with a large commentary in Latin. This is a very short tract, and consists chiefly in explaining the reasons or causes of certain motions, and machines, and natural effects; some being true, and others erroneous. In explaining many physical phenomena, he takes occasion to advert to the propertics of the simple mechanical powers, the lever, the balance, the statera, the wheel-and-axle, the wedge, the screw, and the pulley; of which, most or all, he observes, may be referred to the lever, by means of which most mechanical effects are produced.-The form of the work, is by way of answcring certain queries, and explaining natural effects, and seeming contraries or paradoxes: as,

1. Of a circular line which, without any breadth, has both convexity and concavity.

2. How two things, the one moving the other, may nove contrary ways at the same time; as when one wheel turns another, by means of teeth on their circumferences, while the one turns from right to left, the other turns from left to right.

3. In a balance, because a greater line revolves circularly quicker than a less, therefore the larger balances are more exact than the smaller.

4. That two lines moved parallelwise, in different directions, with velocities having always the same ratio, hat we their intersection always in the diameter or diagonal of the parallelogram having its sides in the directions of the motions. Or, one line moved parallelwise, while a point moves along the line, with a motion in a constant ratio to that of the line; the point moves always in the diagonal of the said parallelogram. And this is the same as the celebrated theorem of the parallelogram of motions, or of forces. But if the motions be not al ways in the same ratio, then the intersections will not be in the diagonal of a parallelogram.

5. On the variable motions, to cause the intersections to describe the periphery of a circle.

ti. On the affections and properties of scales or balances.

7 . On the cause of the power of the lever.-That the weight and power are reciprocally proportional to the arms of the lever.

8. Hence the reason why the oars are the most effica- cious of those rowers who are seated in the middle of the ship.

9. On the cause of the power of the rudder, in turning the ship.

10. On the cause of the power of the sail-yard in a ship. As the yard is placed higher up, on the mast, so much quicker will the ship be moved, with the same wind and the same sail; because the lever (or distance up the mast) is so much the longer.

11. On shifting the sails and rudder when turning the corners and windings of a river.

12. Why round or circular figures are more moveable.

13. Why the greatest circles are the most moveable.

14. Why balista without weight are more easily moved.

15. Why a heavy or loaded carriage is easier than a light or running one.

16. Why a stone is thrown farther from a sling, than when thrown by the hand.

17. On the wheel-and-axle, and tooth-and-pinion work.

18. On the breaking of wood. Why a stick is more edsily broken over the knee, when the extremities are held by the hands, than when held nearer the middle.

19. On the rounciness of pebbles, or why those about the sea-shore are round; viz, from the continual rolling by the motion of the waters.

20. Why wood is weaker as it is longer.

21. The cause of the power of the wedge, is chiefly owing to percussion.

22. Of the pulley or little wheel.

23. Why an ax by striking divides; but by pressing not: because in the former way it acts as a thiu wedge.

24. Of the Statera, or Steclyard.

25. Of the Dentiduco, or Toothdrawer.

26. Of the Nutcracker.

27. Why, in a Rhombus, both the extreme points do not describe or pass over equal right lines. 'That is, either extreme point moving along an adjacent side, while that side is carried by an equal parallel motion, then the point describes the adjacent diagonal of the rliombus. But the diagonal connecting the acute anyles of the rhombus, being greater than the other which connect the obtuse angular points; therefore the motion of the acute angular point, is su much greater than that of the other.

28. Why a greater circle revolves equally with a less, when posited about the same centre.

29. Of Beds. - Why the sides of a bed are made in a donble ratio; the one being 6 feet, or little more; the other only 3 . And why the cordings are not extendesl diam gonal wise. Or that they be made in such magnitude as to correspond with the bodies. For thus the sides will be double, as 4 cubits in length, and 2 in breadth.

30. Why it is more difficult to support a long stick at the extremity of the arm, than in the middle of it.

31. Why it is more difficult to support, at the extremity of the arm, a very long weight, than a short unc.

32. Why a tolleno for drawing water, acts better as it works with a heavy weight at the end of the cross bur.

33. Why, when two men carry a weight on a pole between them, they are not equally pressed when it is not in the middle between them.

34. Why those who rise up from a seat, put themselves into a particular position; viz, putting their feet backwards, and bending their body forward.

35. Why a moving body is more casily moved, than a quicscent one 
36. Why a lath or rod projected loses of its motion.This, and the former cause, Aristotle seems not to understand.

37. Why any thing proceeds not on in the direction of its motion, but relaxes, when impelled in the contrary direction. As, when its weight causing it to descend downwards, is greater than the impulsive force can impel it upwards.

3s. Why any things, either small or large, when projected, do not go far.

39. Why a light body, floating in a vortex of water, goes into the middle of the vortex.

AlITHMETIC, is the art and science of numbers; or, that part of mathematics which considers their powers and properties, and teaches how to compute or calculate tru$\mathrm{ly}$, with ease and expedition. It is by some authors also defined the science of discrete quantity. Arithmetic consists chiefly in the four principal rules or operations of Addition, Subtraction, Multiplication, and Division; to which may perhaps be added involution and evolution, or raising of powers und extraction of roots. But besides these, for the facilitating and expediting of computations, mercantile, astronomical, \& cc, many other useful rules have been contrived, which are applications of the former, such as, the rules of proportion, progression, alligation, false position, fellowship, interest, barter, rebate, equation of payments, reduction, tare and tret, \&c ; besides the doctrine of the curious and abstract properties of numbers.

Very little is known of the origin and invention of arithretic. In fact it must have commenced with mankind, or as soon as they began to bold any sort of commerce together; and must have undergone continual improvements, as occasion was given by the extension of commerce, and by the discovery and cultivation of other sciences. It is therefore very probable that the art has been greatly indebted to the Phœnicians or Tyrians; and indeed Proclus, in his commentary on the first book of Euclid, says, that the Phonicians; by reason of their traffic and commerce, were accounted the first inventors of Arithmetic. From Asia the art passed into Egypt, whither it was carried by Abraham, according to the opinion of Josephus. Here it was greatly cultivated and improved; insomuch that a considerable part of the Egyptian philosophy and theology seems to bave turned altogether upon numbers. Hence those wonders related by them about unity, trinity, with the numbers 4, 7,9 , \&c. In effect, Kircher, in his Oedip. Ėgypt. shows, that the Egyptians explained every thing by numbers; Pythagoras himself affirming, that the nature of numbers pervades the whole'universe; and that the knowledge of numbers is the knowledgc of the Deity.

From Egypt arithmetic was transmitted to the Greeks, by mcans of Pythagoras and other travellers; among whom it was greatly cultivated and improved, as appears by the writings of Euclid, Archimedes, and others: with these improvements it passed to the Romans, and from them it bas descenderl to us.

The nature of the arithmetic however that is now in use, is very different from that above alluded to ; this art laving undergone a total alteration by the introduction of the Arabic or Indian notation, about 8 or 9 hundred years since, into Europe: so that nothing now remains of use from the Greeks, but the theory and alstract properties of numbers, which have no dependence on the peculiar nature of any particular scale or rnode of notation. 'That used by the Hebrews, Greeks, and Romans, was chiefly by means of the letters of their alphabets. The Greeks, particularly, had two different methods; the first of these was much the same with the Roman notation, which is sufficiently well knows, being still in common use with us, to denote dates, chapters and sections of books, \&c. Afterwards they had a better method, in which the first nine letters of their alphabet represented the first numbers, from one to nine, and the next nine letters represented any number of tens, from one to nine, that is, 10, 20,30, \&c, to 90 . Any number of hundreds they expressed by other letters, supplying what they wanted with some other marks or characters : and in this order they went on, using the same letters again, with some different marks, to express thousands, tens of thousands, hundreds of thousands, \& c : in which it is evident that they approached very near to the more perfect decuple scale of progression used by the Arabians, and who acknowledge that they had received it from the Indians. Archimedes also invented another peculiar scale and notation of his own, which he employed in his Arenarius, to compute the number of the sands. Ptolemy is supposed to have invented the sexagesimal division of numbers, with its peculiar notation and operations : a mode of computation still used in astronomy, \&c, for the subdivisions of the degrees of circles. Those notations bowever were ill adapted to the practical operations of arithmetic: and hence it is that the art advanced but very little in this part; for, setting aside Euclid, who has given many plain and useful properties of numbers in his Elements, and Archimedes, in his Arenarius, they mostly consist in dry and tedious distinctions and divisions of numbers; as appears from the treatises of Nicomachus, supposed to be written in the $3 \mathrm{~d}$ century of Rome, and published at Paris in 1538; as also that of Boethius, written at Rome in the 6 th century. A compendium of the ancient arithmetic, written in Greek, by Psellus, in the 9th century, was published in Latin by Xylander, in 1556. A similar work was written soon after in Greek by Jodocus Willichius; and a more ample work of the same kind was written by Jordanus, in the year 1200, and published with a comment by Faber Stapulensis in 1480 .

Since the introduction of the Indian notaion into Europe, about the 10 th century, arithmetic has greatly clanged its form, the whole algorithm, or practical operations with numbers, being quite altered, as the notation required; and the authors of arithmetic have gradually become more and more numerous. This method was brought into Spain by the Moors or Saracens; whither the learned men from all parts of Europe repaired, to learn the arts and sciences of them. This, Dr. Wallis proves, began about the year 1000; particularly that one Gerbert, a monk, afterwards pope Sylvester the 2nd, who dicd in the year 1003, brought this art from Spain into France, long before the date of his death: and that it was known in Britain before the year 1150, where it was brought into common use before 1250, as appcars by the treatise of arithmetic of Johannes de Sacro Bosco, or Halifax, who died about 1256. Since that time, the principal writers on this art have been, Barlaam, Lucas de Burgo, Tonstall, Aventinus, Purbacb, Cardan, Scheubclius, Tartalia, Faber, Stifelius, Regius, Recorde, Ramus, Maurolycus, Hemischius, Peletarius, Stevinus, Xylander, Kersey, Snellius, Tacquet, Clavius, Metius, Germa Frisius, Buteo, Ursinus, Romanus, Napier, Cerulen, Wingate, Kepler, Briggs, Ulacq, Oughtred, Cruger, Van Schooten, Wallis, Dee, Newton, 
Morland, Moore, Jeake, Ward, Hatton, Malcolm, \&c, \&c; the particular inventions or excellencies of whom, will be noticed under the articles of the several species or kinds of arithmetic bere following, whieh may be included under these beads, viz, Theoretical, Practical, Instrumental, Logarithmical, Numerous, Speciuus, Universal, Common or Decadal, Fractional, Radical or of Surds, Decinial, Duodeeimal, Sexagesimal, Dynamical or Binary, Tetractycal, Political, \&c.

Theoretical ARITHMRTIC, is the science of the properties, relations, \&c, of numbers, abstractedly considered ; with the reasons and demonstrations of the several rules. Such is that contained in the $7 \mathrm{th}, 8 \mathrm{th}$, and 9 th books of Euelid's Elements; the Logistics of Barlaam the monk, published in Latin by J. Chambers, in 1600; the Summa Arithmetica of Lucas de Burgo, printed 1494, who gives the several divisions of numbers from Nienmachus, and their properties from Euclid, with the algorithm, both in integers, fractions, extraction of roots, \&c; Malcolm's New System of Arithmetic, Theoretical and Practical, in 1730 , in which the subject is very completely treated, in all its branches, \&c.

Praetical Arith метцc, is the art or practice of numbering or computing; that is, from certain numbers given, to find others which shall have any proposesl relation to the former. As, having the two numbers 4 and 6 given ; to find their sum, which is 10; or their differenee, which is 2 ; or their product, 24 ; or their quotient, $1 \frac{1}{2}$; or a third proportional to them, which is 9; \&c.-Lucas de Burgo's works contain the whole practiee of arithmetic, then used, as well as the theory. Tunstall gave a neat practical treatise of Arithmetic in 1526; as did Stifelius, in 1544, both on the practical and other parts. Tartalea gave an entire body of practical arithmetic, which was printed at Venice in 1556, consisting of two parts; the former, the application of arithmetic to eivil uses; the latter, the grounds of algebra. And most of the authors in the list before enumerated, joined the practiee of arithmetic with the theory.

Binary or Dyadic: ArITHM ETJc, is that in which only two figures are used, viz, 1 and 0 . See BINARY.-Leibnitz and De Lagny both invented an arithmetic of this sort, about the same time: and Dangicourt, in the Miscel. Berol. gives a specimen of the use of it in arithmetical progressions; where he shows, that the laws of progression may be more easily discovered by it, than by any other method where more characters are used.

Common or Vulgar ARITHMETIC, is that which is concerning integers and vulgar fractions.

Decimal or Decadal Anithmetic, is that which is performed by a series of ten characters or fignres, the progression being ten-fold, or from 1 to 10 's, 100's, \&c; which includes both integers and decimal fractions, in the common scale of numbers; and the characters used are the ten $\Lambda$ rabic or Indian figures $0,1,2,3,4,5,6,7,8,9$. ' $i$ 'his methorl of arithmetic was not known to the Greeks and Ronians; but was borrowed from the Moors while they possessed a great part of Spain, and who aeknowledge that it came to them from the Indians. It is probable that this method took its origin from the ten fingers of the fands, whieh were used in computations before arithmetic was brought into an art. The Easteru missionaries assure us, that to this day the Indians are very expert at computing on their fingers, without any use of pen and ink. And it is asserted, that the P'eruvians, who perform all computations by the different arrangements of grains of maizc, outdo any European, both for certainty and dispatch, with all his rules.

Duodecimal ARITHAETic, is that which proceeds from 12 to 12 , or by a continual subdivision according to 12 . This is greatly used by most artificers, in squaring or ealculating the quantity of their work; as Bricklayers, Carpenters, J'ainters, Tilers, \&c.

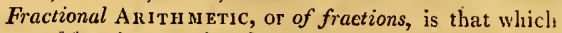
treats of fraetions, both vulgar and decimal.

Harmonical Arith m etic, is so much of the doctrine of numbers, as relates to making the comparisons, reductions, \&c, of musieal intervals.

A R ITH METIC of Infinites, is the method of summing up a series of numbers, of which the number of terms is infinite. This method was first invented by Dr. Wallis, as appears by bis treatise on that subject; where he shows its uses in geometry, in finding the areas of superfieies, the contents of solids, \&c. But the method of fluxions, which is a kind of universal arithmetic of infinites, performs all these more easily; as well as a great many other things, which the former cannot reach.

Instrumental ARtTHMETIC, is that in which the eommon rules are performed by instruments, or some sort of tangible or palpable substance. Such are the methods of computing by the ten fingers and the grains of maize, by the Last-Indians and Peruvians, above-mentioned; by the A bacus or Shwanpan of the Chinese; the several sorts of scales and sliding rules; Napier's bones or rods; the arithmetical machine of Pascal, and others; Sir Samuel Morland's instrument, described in 1666 ; that of Leibnitz, dcscribed in the Miscell. Berol,; that of Polenus, published in the Venetian Miscellany, 1709; and that of Dr. Saundersm, of Cambridge, described in the introduction to his Algebra.

Integral ARITHMETIC, or of integers, is that which respects integers, or whole numbers.

Literal ArITn metic, or Algebra, is that which is Ferformed by letters, which represent any numbers indefinitely.

Logarithmical ARITHM ETIC is perlormed by the tables of logarithms. These were invented by baron Napier; and the best trearise on the subject, is Brigg's Arithmetica Logarithmica, 1624.

Logistical A ritumetic. See Logistical.

Numerous or Numeral ARITnuETsc, is that which teaehes the ealculus of numbers, or of abstract ruantities; and is performed by the common numeral or Arabic characters.

Political Aritimetic, is the application of arithmetic to politieal subjects; such as, the strength and revenues of nations, the number of people, births, burials, \&c. See Politrica a Arithmetic. To this bead may also be referred the doctrine of Chances, Gaming, \&c.

AntTHMEIC of Radicals, Rationals, and Irrationals. See Radical, \&c.

Aritumetic of Sines. See Sines.

Sexngesimal or Sexagenary ARITH M E'uc, is that which proceeds by sisties; or the doctrine of sixigesimal fractions: a method which, it is supposed, was juvented by Ptolemy, in the $2 d$ century; at least they were used by lim. In this notation, the integral numbers from 1 to 59 were expressed in the eommon way, by the alphabetical letters: then sixty was called a Sexagena Prima, ant mark. esl with a dash to the character 1 , thus $1^{\prime}$; twice sixty, or 120 , thus 11 ; and so on to 59 times 60 , or 3540 , which is Lıx'. Agrain, 60 times 60 , or 3600 , was called Sexugetua 


\section{A R l}

\section{[}

Secunda, and marked with two dashes, thus I"; twice 3600 , thus $11^{\prime \prime}$; and ten times 3600 , thus $x^{\prime \prime} ; \&$. . And in this way the notation was continued to any length. But when a number less than sixty was to be joined with any of the sexagesimal integers, their proper expression was annexed without the dash: thus 4 times 60 and 25 , is $1 v^{\prime} \times x v$; the sum of twice 60 and 10 times 3600 and 15 , is $x^{\prime \prime} 11^{\prime} x v$. So near did the inventor of this method approach to the Arabic notation : instead of the sexagesimal progression, be had only to substitute decimal; and to make the signs of numbers, from 1 to 9 , simple characters, and to introduce another clraracter, which should signify nothing by itself, but serving only to fill up places.-The Sexagenæ Integrorum were soon laid aside, in ordinary calculations, after the introduction of the Arabic notation; but the sexagesinal fractions continued till the inventio:s of decimals, and indeed are still used in the subdivisions of the degrees of circular arcs and angles; except by the French, who now divide their circles into $400^{\circ}$; and were it not for the difficulty in overcoming the prejudices for old established customs, this method would doubtless be generally introduced, as it is much better in practice than that in crmmon use.

Sam. Reyber has invented a kind of sexagenal rods, in imitation of Napier's bones, by means of which the sexagesimal arithmetic is easily performed.

A good treatise on the Greek or Sexagenary Arithmetic has lately (1SOS) been given by M. Delambre, in F. Peyrard's French translation of the works of Archimedes; and a very good account is given of the same in the Edinburgh Review, vol. 18, pa. 185 .

Specious ARITH METIC, is that which gives the calculus of quantities is designed by the letters of the alphabet: a method which was more generally introduced into algebra, by Virta; being the same as literal arithmetic, or algebra.-Dr. Wallis has joined the numeral with the literal calculus; by which means he has demonstrated the rules for fractiuns, proportions, extraction of roots, \&c ; of which a compendiun is given by limself, under the title of Elementa Arithmeticæ; in the year 1698.

Tabular AR1TH ETic, is that in which the operations of multiplication, division, $\& c$, are perforined by means of tables calculated for that purpose : such as those of Herwart, in 1610; and my tables of powers and products, published by order of the Commissioners of Longitude, in 1781 .

Tetractic Arith metic, is that in which unly the four characters $0,1,2,3$, are used. A treatise of this kind of arithmetic is extant, by Erhard or Echard Weigel. But both this and binary arithmetic are little better than c11riosities, especially with regard to practice; as all numbers are much more compendiously and conveniently expressed by the common decuple scale.

Vulgar, or Common ARITHMETIC, is that which relates to integers and vilgal fractions.

Universal Aиттиметіс, is the name given by Newton tu the science of algebra; of which he left at Cambridge an excellent treatise, bcing the text-book drawn up for the use of his lectures, while he was professor of mathematics in that university.

ARITHMLI'ICAL, something relating to, or after the manner of arithuetic.

Arithatical Complement, of a logarithm, is what the lugarithm wants of $10.00000 \& \mathrm{c}$; and the easiest way to find it is, beginning at the left land, to subtract every figure from 9, and the last from 10. So, the arithmetical
A R I

complement of $8 \cdot 2501396$ is $1 \cdot 7498604 .-$ It is commonly used in trigonometrical calculations, when the first term of a proportion is nut radius; in that case, adding all together, the logarithms of the $3 \mathrm{~d}, 2 \mathrm{~d}$, and arithmetical complement of the 1st term.

Aritimetical Instrunénts, or Machines, are instruments for performing arithmetical computations; such as Napier's bones, scales, sliding rules, Pascal's machine, \&c.

Arith metical Mean, or Medium, is the middle term of three quantities in arithmetical progression; and is always equal to hatf the sum of the extremes. So, an arithmetical mean between 3 and 7 , is 5 ; and between $a$ and $b$, is $\frac{1}{2} a+\frac{1}{2} b$.

Also, an arithmetical mean among any number of quantities, is uften found by adding the quanrities all together, and then dividing the sum by the number of the terms. So, of the six numbers $1,3,4,6,7,9$, the sum is 30 , which divided by 6 , gives 5 , for the mean or medium among then all.

Arith metical Progression, is a series of three or more quantities that have all the same commoll difference: as $3,5,7, \& c$, which have the common difference 2; and $a$, $a+d, a+2 d \& c$, which have all the same difference $d$.

In an arithmetical progression, the chief properties are these: 1 st, The sum of any two terms, is equal to the suin of every uther two that are takell at equal distances from the two former, and equal to double the middle term when there is one equally distant between those two: so, in the series $0,1,2,3,4,5,6,8 \mathrm{c}, 0+6=1+5=2+4=$ twice 3 or $6 .-2 \mathrm{~d}$, The sum of all the terms of any arithmetical progression, is equal to the sum of as many terms of which each is the arithmetical mean between the extremes; or equal to half the sum of the extremes multiplied by the number of terms: so, the sum of these ten terms $0,1,2,3,4,5,6,7,8,9$, is $\frac{0+9}{2} \times 10$, or $9 \times 5$, which is 45 : and the reason of this will appear by inverting the terms, setting them under the former terms, and adding each two togetber, which will make double the same series;

$$
\begin{aligned}
\text { thus } & 0,1,2,3,4,5,6,7,8,9, \\
\text { inverted } & 9,8,7,6,5,4,3,2,1,0, \\
\text { sums } & 9,9,9,9,9,9,9,9,9,9 ;
\end{aligned}
$$

where the double series being the same number of 9 's, or sum of the extremes, the single series must be the half of that sum.-3d, The last, or any term, of such a series, is equal to the first term, with the product added of the common difference multiplied by I less than the number of terms, when the series ascends or increases; or the same product subtracted when the series descends or decreases: so, of the series $1,2,3,4, \& c$, whose common difference is 1 , the 50 th term is $1+1 \times 49$, or $1+49$, that is 50 ; and of the serics $50,49,48, \& c$, the 50 th term is $50-$ $1 \times 49$, or $50-49$, which is 1 . Also, if

$a$ denote the least term, $z$ the greatest term, $d$ the common difference, $n$ the number of the terms,

and $s$ the sum of them all;

then the principal properties are expressed by thesc equations, viz, $\quad z=a+d(n-1)$,

$$
\begin{aligned}
& a=z-d(n-1), \\
& s=(a+z) \frac{1}{2} n, \\
& s=\left[z-\frac{1}{2} d(n-1)\right] n, \\
& s=\left[a+\frac{1}{2} d(n-1)\right] n .
\end{aligned}
$$




\section{A R M}

$[150$

Also, when the first term $a$, is 0 or nothing; the theorems become $\quad z=d(n-1), \quad$ and $s=\frac{\pi}{2} z n$.

Arithatetical Proportion, is when the difference between two terms, is equal to the difference between two other terms. So, the four terms, $2,4,10,12$, are in arithmetical proportion, because the difference between 2 and 4 , which is 2 , is equal to the difference between 10 and 12.-The principal property, besides the above, and which indeed depends upon it, is this, that the sum of the first and last, is equal to the sum of the two means : so 2 +12 , or the sum of 2 and 12 , is equal to $4+10$, or the sum of 4 and 10 , which is 14 .

Arithmetical Ratio, is the same as the difference of any two ternis: so, the arithmetical ratio of the series $2,4,6,8$, is 2 ; and the arithmetical ratio of $a$ and $b$, is $a-b$.

ARtTin metical Scales, a name given by M. Buffon, in the Memoirs of the Acad. for 1741, to different progressions of numbers, according to which, arithmetical computations might be made. It bas already been remarked above, that our common decuple scale of numbers was probably derived from the number of fingers on the two hands, by means of which the earliest and most natural mode of computation was performed; and that other scales of numbers, formed in a similar way, but of a different numher of characters, have been devised; such as the binary and tetractic scales of arithmetic. In the memoir above cited, Buffon gives a short and simple method to find, at once, the manner of writing down a number given in any scale of numbers whatever; with remarks on different scales. The general effect of any number of characters, different from ten, is, that by a smaller number of characters, any given number would require more places of figures to express or denote it by, but then arithmetical calculations, by multiplication and division, would be easier, as the small numbers $2,3,4, \& \mathrm{c}$, are easier to use than the larger $7,8,9$; and by employing more than ten characters, though any given number would be expressed by fewer of them, yet the calculations in arithmetic would be more difficult, as by the larger numbers $11,12,13, \& \mathrm{c}$. It is therefore concluded, on the whole, that the ordinary decuple scale is a good convenient medium among them all, the numbers expressed being tolerably short and compendious, and no single character representing too large a number. The same might also be said, and perhaps more, of a duodecimal scale, by twelve characters, which would express all numbers in a more compendious way than the decuple one, and yet no single character would represent a number too large to compute by; as is confirmed by the now common practice of extending the multiplication table, in school books, to 12 numbers or dimensions, each way, instead of 10 ; and every person is taught, with sufficient ease, to multiply and divide by 11 and 12 , as easily as by $S$ or 9 or 10 . Another convenience might be added, namely, that the number 12 admitting of more submultiples than the number 10 , there would be fewer expressions of interminate fractions in that way than in decimals. So that on all accounts, it is very probable that the duodecimal would be the best of any scale of numbers whatever.

A it TH metical Triangle. SeeTriangle Arithmetical.

ARMED. $\Lambda$ magnet or loadstone is said to be armed, when it is capped, cased, or set in iron or steel; to make it take up a greater weight; and also rcadily to distinguish its polcs.

\section{A $\mathbf{R} \mathrm{M}$}

It is surprising, that a little iron fastened to the poles of a magnet, should so greatly improve its power, as to make it even 150 times stronger, or more, than it is naturally, or when unarmed. The effect however, it seems, is not uniform; but that some magnets, by arming, gain nuch more, and others much less, than one would expect; and that some magnets even lose some of their efficacy by arming. In general however, the thickness of the iron armour ought to be nearly proportioned to the natural strength of the magnet; giving thick irons to a strong magnet, and to the weaker ones thinner: so that a magnet may easily be over-loaded.

The usual armour of a load-stone, in form of a rightangled parallelopipedon, consists of two thin pieces of iron or steel, of a square figure, and of a thickness proportioned to the goodness of the stone; the proper thickness being found by trials; always filing it thinner and thinner, till the effect be found to be the greatest possible.The armour of a spherical load-stone, consists of two steel shells, fastened together by a joint, and covering a good part of the convexity of the stone. This also is to be fled away, till the effect is found to be the greatest.

Kircher, in his book De Magnete, says, that the best way to arm a luad-stone, is to drill a hole through the stone, from pole to pole, in which is to be placed a steel rod of a moderate length: this rod, he asserts, will take up more weight at the end, than the stone itself when armed in the common way. And Gassendus and Cabæus prescribe the same method of arming. But Muschenbroek found, by repeated trials, that the usual armour, already mentioned, is preferable to Kircher's; and he gires the following directions for preparing it. When, by means of steel filings and a small needle, the poles of a magnet have beell discovered, he directs that the adjacent parts should be rubbed or ground into parallel planes, without shortening the polar axis; and the magnet may be afterwards shaped into the figure of a cube or parallelopipedon, or any other figure that may be more convenient. Plates of the softest iron are then prepared, of the same length and breadth with the whole polar'sides of the magnet: the thickness of which plates, so as that they may admit and convey the greatest quantity of the magnetic virtue, is to be previously determined by experiment, in a manner which he prescribes for the purpose. A thicker piece of iron is to be annexed at right angles to these plates, which is called pes armaturæ, the font or base of the armour: then the plates, nicely smoothed and polished, are to be firmly attached to cach of the polar sides, while the thicker part or base is brought into close contact with the lower part of the magnet. In this way, he says, almost all the magnetic virtue issuing from the poles, enters into the armour, is directed to the base, and condensed by means of its roundness, so as to sustain the greatest weight of iron. Phys. Exper. and Geom. Dissert. 1729, pa. 131.

ARMILLARY Sphere, a name given to the artificial spliere, composed of a number of circles of metal, wood, or paper, which represent the several circles of the system of the world, put together in their natural order. It serves to assist the imagination to conceive the disposition of the heavens, and the motion of the celestial bodies.

This sphere is represented at Plate 2, Fig. 6 , where $P$ and $Q$ represent the poles of the world, $A D$ the equator, EL the ecliptic and zodiac, PAGD the meridian, or the solstitial colure, $\mathrm{T}$ the earth, $\mathrm{FG}$ the tropic of cancer, $\mathrm{nT}$ the tropic of capricorn, $M N$ the arctic circle, ov the ant- 

A S C
[ 151$]$
A S C

arctic, $N$ and o the poles of the ecliptic, and RS the horizon.

The Armillary sphere constructed by Dr. Long, in Pembroke-hall, Cambridge, was 18 feet in diameter; and will contain more than 30 persons sitting within $\mathrm{jt}$, to view, as from a centre, the representation of the celestial spheres. The lower part of the sphere, which is not visible to England, is cut off; and the whole apparatus is so contrived, that it may be turned round with as little labour as is employed to wind up a common jack. See also Mr. Ferguson's sphere, in his Lectures, p. 194.

AnMillan y Trigonometer, an instrument first contrived by Mr. Mungo Murray, and improved by Mr. Ferguson, consisting of five semicircles; viz, meridian, vertical circle, horizon, hour circle, and equator; so adapted to each other by joints and hinges, and so divided and graduated, as to serve for expeditiously resolving many problems in astronomy, dialling, and spherical trigonometry. The drawing, description, and method of using it, may be seen in Ferguson's Tracts, pa. 80, \&c.

AR'TILLERY, the heavy equipage of war; comprehending all sorts of large fire-arms, with their appurtenances; as cannon, mortars, howitzers, carronades, balls, shells, petards, musquets, carbines, \&c ; being what is otherwise called Ordnance. The term is also applied to the larger instruments of war used by the ancients, as the catapult, balista, battering-ram, \&c.

The term Artillery, or Royal Artillery, is also applied to the persons employed in that service; as also to the art or science itself; and formerly it was used for what is otherwise called Pyrotechnia, or the art of fire-works, with the apparatus and instruments belonging to the same.

There have been many authors on the subject of artillery; the principal of which are, Bucherius, Braunius, Tartalea, Collado, Sardi, Uiano, Hanzelet, Digges, Moretti, Simienowitz, Mieth, l'Avelour, Manesson, Mallet, St. Julien; and the later authors, of still more consequence, are Belidor, St. Reny, le Blond, Valiere, Morogue, Puget, Coudray, Robins, Muller, Euler, Antoni, Tignola, Scheele; to which may be added the extensive and accurate experiments published in the $2 \mathrm{~d}$ and $3 \mathrm{~d}$ volumes of my Tracts, and in the Philos. Trans, for 1778.

Park of ARTiLLERY, is that place in a camp which is set apart for the artillery, or large fire-arms.

Traile or Train of ARTILLERY, a number of pieces of ordnance, mounted on carriages, with all their furniture tit for marching. 'To this commonly belong mortars, cannon, balls, shells, \&c.- There are trains of artillery in most of the royal magazines; as in the Tower, at Portsmouth, Plymouth, \&c, but, above all, at Woolwich, from whence the ships commonly receive their orduance, and where they are all completely proved before they are received into the public service.

The officers and men of the artillery were formerly called also the Train of Artillery, but are now called the Royal Regiment of Artillery; consisting at present of ten battalions, and several troops of Horse or Cavalry Artillery.

ASCENDING, in Astronomy, a term used to denote any star, or degree, or other point of the heavens, rising above the horizon.

Ascending Latitude, is the latitude of a planet when going towards the north.

ASCENDING Node, is that point of a planet's orbit where it crosses the ecliptic, in proceeding northward. It is otherwise called the Northeru Node, and is denoted by this character 8 , representing a node, or knot, with the larger part upwards; and the same character reversed is used to denote the opposite, or Descending Node 8 .

AscendING Signs, are such as are upon their ascent, or rise, from the nadir or lowest point of the heavens, towards the zenith, or highest point.

ASCENSION-DAY, otherwise called Holy Thursday, is a festival of the church, held 10 days before Whit-sunday, in memory of our Saviour's Ascension.

ASCENSION, in Astronomy, is either Rightor Oblique.

Right Ascinsion of the sun, or of a star, is that degree of the equinoctial, accounted from the begiming of Aries, which rises with them, in a right sphere-OOr, Right Ascension, is that point of the equinoctial, counted as before, which comes to the meridian with the sun or star, or other point of the beavens. And the reason of thus referring it to the meridian, is, because this is always at right angles to the equinoctial; whereas the horizon is so only in a right or direct sphere.-The right ascension stands opposed to the right descension; and is similar to the longitude of places on the earth. All the fixed stars, \&c, which have the same right ascension, that is, which are at the same distance from the first point of Aries, or, which comes to the same thing, which are in the same meridian, rise at the same time in a right sphere, that is, to the pcople who live at the equator. And if they be not in the same meridian, the difference between their times of rising, or of coming to the meridian of any place, is the precise difference of their right ascension.-But, in an oblique sphere, where the horizon cuts all the metidians obliquely, different points of the same meridian never rise or set together: so that two or more stars on the same meridian, or having the same right ascension, never rise or set at the same time in an oblique sphere; and the more oblique the sphere is, the greater is the interval of time between them.

To find the right ascension of the sun, stars, \&c, by trigonometry, say, As radius is to the cosine of the sun's greatest declination, or obliquity of the ecliptic, so is the tangent of the sun's or star's longitude, to the tangent. of the right ascension.

Right Ascension of the Mid-heaven, often used by astronomers, especially in calculating eclipses by means of the nonagesimal degree, is the right ascension of that point of the equator which is in the meridian; and it is equal to the sum of the sun's right ascension and the horary angle or true time reduced to degrees, or to the sum of the mean longitude and mean time.

Oblique Ascension, is an arch of the equator intercepted between the first point of Aries, and that point of the equator which rises together with the star, \&c, in an oblique sphere.-The oblique ascension is counted from west to east; and is greater or less, according to the various obliquity of the sphere.-To find the oblique ascension of the sun, see AsCENSInNAL and GLOBL.

The Arch of Oblique Ascension, is an arch of the horizon intercepted between the begiuning of Arits, and the point of the equator which rises with a star or planet, in an oblique spbere; and it varies with the latitude of the place.

Refraction of Ascension and Descension. See REFRACTION.

ASCENSIONAL Difrerence, is the difference between the right and oblique ascension of the same point on the surface of the spherc. Or it is the time the sun rises or scts belorc or after 6 o'clock. 

A S C
$\left[\begin{array}{lll}152 & \end{array}\right]$
A S P

To find the ascensional difference, having given the sun's declination and the latitude of the place, say, As radins is to the tangent of the latitude, so is the tangent of the sun's declination, to the sine of the ascensional difference sought. The sun's ascensional difference converted into time, shows how much he rises before, or sets after, 6 o'clock. When the sun has north declination, the right ascension is greater than the oblique; but the contrary, when the sun has south declination; and the difference, in either case, is the ascensional difference.

ASCENT, the motion of a body from below tending upwards; or the continual recess of a body from the earth, or from some other centre of force. And it is opposed to descent, or motion downwards.

An acclivity is also sometimes called an ascent, as a declivity is called a descent.

The Peripatetics attributed the spontaneous ascent of bodies to a principle of levity, inherent in them. But the moderns have demonstrated that there is no such thing as spontaneous levity; and they show, that whatever ascends, does so by virtue of some external impulse or extrusion. Thus it is that smoke, and other rare bodies, ascend in the atmosphere; and oil, light woods, \&cc, in,water; not by any inherent principle of levity, but superior gravity, or pressure of the medium in which they ascend and float.

The ascent of light bodies in heavy mediums, is produced after the same manner as the ascent of the lighter scale of a balancc. It is not that such scale has an internal principle, by which it immediately tends upwards; but it is impelled upwards by the preponderancy of the other scale; the excess of the weight in the one having the same effect, by augmenting its impetus downwards, as so mnch real levity in the other: because the tendencies mutually oppose each other, and action and reaction are always equal.-See this farther illustrated under the articles Fluid, and Specific Gravity.

Ascrn t of Bodies on Inclined Planes. See the doctrine and laws of them under Inclined Prane.

AsCENT of Fluid's, is particularly understood of their rising above their own level, between the surfaces of nearly contiguons bodies, or in slender capillary glass tubes, or in vessels filled with sand, asbes, or the like porous substances. Which is an effect that takes place as well in vacuo, as in the open air, and in bent, as well as straight tubes. Indeed some fluids ascend swifter than others, as spirit of wine, and oil of turpentine; and some rise after a different manner from others. The phenomenon, with its causes, \& c, in the instance of capillary tubes, will be treated more at large under CAPILLARY Tube.

As to planes: Two smooth polisbed plates of glass, metal, stouc, or other matter, being placed almost contiguous, have the effect of several capillary tubes, and the fluid rises in them accordingly : the same may be said of at vessel filled with sand, \&c; the various small interstices of which form, as it were, it kind of capillary tubes. So that the same principle accounts for the appearance in them all. And to the same cause may be ascribed the ascent of the sap in vegetables. And on this subject Sir Isaac Newton says, "If a large pipe of glass be filied with sifted ashes, well pressed together, and one end dipped into stagnant water, the fiuid will ascend slowly in the ashes, so as in the space of a week or fortnight, to reach the height of 30 or 40 inches above the stagnant water. This ascent is wholly owing to the action of those particles of the ashes which are on the surface of the elevated water; those witbin the water attracting as much downwards as npwards : it follows, that the action of such particles is very strong; though being less dense and close than those of glass, their action is not equal to that of glass, which keeps quicksilver suspended to the height of 60 or 70 inches, and therefore acts with a force which would kcep water suspended to the height of above 60 feet. By the sane principle, a sponge sucks in water, and the glands in the budies of animals, according to their several natures and dispositions, imbibe varions juices from the blood." Optics, pa. 367 .

Again, if a drop of water, oil, or other fluid, be dropped upon a glass plane, perpendicular to the horizon, so as to stand without brcaking, or running off; and another plane touching it at one end, be gradually inclined towards the former, till it touch the drop; then will the drop break and move along towards the touching end of the planes; and it will move the faster in proportion as it proceeds farther, because the distance between the planes is constantly diminishing. And after the same manner, the drop may be brought to any part of the planes, either upward $\mathrm{m}$ downward, or sideways, by altering the angle of inclination.

Lastly, if the same planes be so placed, as that two of their sides meet, and form a small angle, the other two being only kept apart by the interposition of some thin body; and thns immerged in a fluid, tinged with some colour to render it visible; the fuid will ascend between the planes, and that the highest where the planes are nearest; so as to form a curve line which is found to be a true hyperbola, of which onc of the asymptotes is the line of the fluid, the orher being a line drawn along the toucling sides.

And the physical cause of all these plucnomena, is the same power of attraction.

Ascent of Vapour. Sce Cloud and VAPOUR.

Ascent, in Astronomy, \&c. See $A$ scension.

Ascri, are those inhabitants of the globe, who, at certain times of the year, have no shadow. Such are the inhabitants of the torrid zone, who twice a year having the sun at noon in their zenith, have then no shadow.

ASELLI, two fixed stars of the furth magnitude, in the constellation Cancer.

ASH-WEDnesDar, the first day of Lent, supposed to bave been so called from a custom in the church, of sprinkling ashes that day on the heads of penitents then admitted to penance.

ASPECT, is the situation of the stars and planets in respect of each other. Or; in Astrolngy, it denotes a certain configuration and mutual relation between the planets, arising from their situations in the zodiac, by which it is supposed that their powers are mutually either increased or diminished, as they happen to agree or disagree in their active or passive qualities. Though such configurations may be varied and combined a thousand ways, y't only a few of them are considered. Hence, Wolfius merre accurately defines aspect to be, the inceting of luminous rays emitted from two planets to the carth, either posited in the same right line, or including an angle which is an aliquot part, or some number of aliquot parts, of four right angles, or of 360 degrees.

.'The doctrine of aspects was introduced by the astrologers, as the foundation of their predictions. And hence Kepler defines aspect to be, an angle formed by the rays of two planets mecting on the cartl, capable of exciting some natural power or influence. 


\section{A S S}

The ancients reckoned five aspects, viz, conjunction, sexfile, quartile, trine, and opposition.

Conjunction is denoted by this character $\delta$, and is when the planets are in the same sign and degree, or have the same longitude.

Sextile is denoted by $*$, and is when the planets are distant by the 6 th part of a circle, or 2 signs, or 60 degrees.

Quartile is denoted by $\square$, and is when the planets are distant $\frac{\pi}{4}$ of the circle, or 90 degrees, or 3 signs.

Trine is denoted by $\Delta$, and is when the planets are distant by $\frac{1}{3}$ of the circle, or 4 signs, or 120 degrees.

Opposition is denoted by 8 , and is when the planets are in opposite points of the circle, or differ by $\frac{\pi}{2}$ the circle, or 6 signs, or 180 degrees of longitude.

Or their characters and distances are as in this following tablet.

\begin{tabular}{|c|c|c|c|}
\hline \multirow{2}{*}{\multicolumn{2}{|c|}{$\begin{array}{l}\text { NAME. } \\
\text { Conjunction }\end{array}$}} & & \\
\hline & & CH ARACTER, & $\begin{array}{l}\text { DISTANCE. } \\
00=0^{s}\end{array}$ \\
\hline Sextile - & - & $\begin{array}{l}0 \\
*\end{array}$ & $60=2$ \\
\hline Quartile - & - & a & $90=3$ \\
\hline Trine - & - & $\Delta$ & $120=4$ \\
\hline Opposition & - & 8 & $180=6$ \\
\hline
\end{tabular}

These intervals are reckoned according to the longitudes of the planets; so that the aspects are the same, whether the planct be in the ecliptic or out of it.

$\Lambda$ SPERITY, signifies the inequality or roughness of the surface of any body; by which some parts of it are more promiuent than the rest, so as to hinder the hand, \&c, from passing over it with ease and freedom; and thus producing what is called friction.-Asperity, or roughness, stands opposed to smoothness, erenness, \&c.

According to the relations of Vermausen, the blind man so celebrated for distinguishing colours by the touch, it seems that every colour hes its peculiar degree and kind of asperity. He makes black the roughest, as it is the darkest of colours; but the others are not smoother in proportion as they are lighter; that is, the roughest do not always reflect the least light: for, according to him, yellow is two degrees rougher than blue, and as much smoother than green. See Colours.

ASSIG NABLE Mugnitude, is used for any finite magnitude that can be expressed or denoted. And,

Assignable Rutio, for any expressible ratio.

ASSUMLPION, a teast celebrated in the Romish church, in honour of the miraculous ascent of the Holy Virgin, as they describe it, body and soul, into heaven. It is kept on the 15th of Angust.

ASSURANCE on Lives, a compact by which security is granted for the payment of a certain sum of money on the expiration of the life on which the policy is given, in consideration of such a previous payment made to the assurer as is accounted a sufficient compensation for the loss and hazard to which he exposes limself:

The sum at which this compensation should be valued, repends principally on these tivo circumstances, viz. 1st, On the rate of interest given for the use of money; and 2, , On the probability of the duration of the life assured, and the values of annuities. For, 1st, If the interest of money be high, the value of the insurance will be proportionally low, and the contrary; because the ligher the rate of interest, the less will be the present value which amounts to a certain proposed sum in any given time. Also, if the probability of the duration of life be bigh, the value of the assurance will again be proportionably low, VOL. I.

and the contrary; because the longer the time is, the less will be the principal which will amount to any assigned sum. Thus, if it be required to know the premium or present value, to be given for 100 pounds to be reccived at the end of any time, as suppose 10 years; then, if the interest of money be at the rate of 5 per cent. the answer, or present premium, would be $61 l 7 s 10 d$; but at 4 per cent. it would be $67 l 11 \mathrm{~s} 1 \mathrm{~d}$; and at 3 per cent. it ivould amount to $74 l 8 s 2 d$. Again, suppose it were required to assure $100 \mathrm{l}$ on a life, for any time, for instance 1 yeär; that is, let $100 \mathrm{l}$ be supposed to be payable a year hence, provided a life of a given age fails in that time: here it is evident that, whatever be the rate of interest, the less the probability of the life failing within the year, the less the risk is, and the less the premium ought to be. In effect, the rate of interrest being 5 per cent, if it were sure that the life would fail in that year, the value of the assurance would be the same as the present value of $100 l$. payable at the end of the year, which is $95 l 4 s 9 d$.

But, if it be an equal chance whether the life does or does not fail in the year, in which case the probability of failing is $\frac{1}{2}$; then the value of the assurance will be but half the former value, or $47 l 12 s 4 \frac{\pi}{2} d$. Or if the odds against its failing be as 2 to 1 , that is, if one person out of every 3 die at the age of the proposed life, the probability of dying being only $\frac{1}{3}$, the value of the assurance will be $\frac{\mathrm{r}}{3}$ of the first value, or $31 \mathrm{l} 14 \mathrm{~s} 11 \mathrm{~d}$. And if the odds be 19 to 1 , or one person die out of 20 , of that age, the probability of dying will be $\frac{x}{20}$, and the value of the assurance will also be $\frac{1}{20}$ of the first value, or $4 l 15 s 3 d$ nearly. Lastly, if only one person die out of 50 at the given age, the probability of dying will be $\frac{r}{50}$, and the value of the assurance will be accordingly only $\frac{1}{50}$ of the first sum, or $1 l 18 s 1 d$ : the interest of money being all along considered as after the rate of 5 per cent, - Now, according to Dr. Halley's table of observations, one person dies out of 3 , at the age of 87 ; one in 20 at the age of 64 ; and one in 50 at the age of 39 : It follows, therefore, that the value of the assurance of $100 l$. for one year, on a life aged 87 , is $31 l 14 s 11 d$; on a life aged 64 , it is $4 l 15 s 3 d$; and on a life aged 39 , it is $l l 18 s$ ld: reckoning interest at 5 per cent. But if interest were rated at 3 per cent. these values would be $32 l 7 s 3 d$, and $4 l 17 s 1 d$, and $1 l 18 s 10 d$.

When a life is assured for any number of years; the premium or value may be paid, either in one single present payment; in consequence of which the sum assured will become payable without any farther compensation, whenever, within the given term, the life shall happen to drop: or the value may be paid in annual payments, to be continued till the failure of the life, should that happen within the tern; or, if not, till the determination of the term. And the determination of the value of assurance, $i$ ts all cases, is to be made out from the rules for computing annuities on lives; the principal writers on which are Halley, De Moivre, Simpson, Smart, Kersscboom, De Parcieux, Price, Morgan, Maseres, and Baily. See also LifE ANnuties, Peversion, \&c.

Assurances may be made either on single lives; as above explained; or they may be made on any number of joint lives, or on the longest of any lives; that is, an assurer may bind himself to pay any sums at the extinction of any joint lives, or the longest of any lives, or at the extinction of any one or two of any number of lives. Tliere are further assurances on survivorships; by which is meant an obligation, for the value received, to pay a given sum 
or annuity, provided a given life shall survive any other given life or lives. For which see Survivonsurp.

The principal offices for making these insurances, in England, are the London and the Koyal Exchange Assurance Offices; the Amicable Society, incorporated for a perpetual Assurance Office; the Society for equitable Assurances on Lives and Survivorships; the Westminster Society for granting Arnuities and insuring Money on Lives; the Phœnix fire-office; the Westminster fire-office; the Life-assurance Society, for widows and female relations; the British fire-office; the Pelican life-insurance; the Laudable Society, for widows; the Imperial Insurance Company; the Globe ; the Albion; the Rock; the Provident Institution; the Philanthropic; the Hope; the County fire-office; the Eagle; the London Life Association; and the Atlas.

Most of these offices, besides various extensive companies in the country, make also insurances on houses, goods, ships, \&c, from fire and damages, \&c. These articles of assurances are commonly divided into 3 classes : first, common assurances, which are made at $2 s$ per cent. per annum; $2 \mathrm{~d}$, hazardous assurances, at $3 s$; and $3 \mathrm{dly}$, doubly hazardous, at $5 s$ per cent. per annum: and the mode of classification may be learned from the proposals of any of the companies. Besides these rates, a duty of $1 s 6 d$ on every $100 l$ was imposed in 1782 , which was increased in 1797 to $2 s$ per cent, and in $180+$ to $2 s 6 d$ per cent. From the produce of this duty, an estimate has been formed of the total amount of property assured from fire in Great Britain, which appears to have been at several periods nearly as follows:

$$
\begin{aligned}
& \text { In } 1785 \text { - }-\ldots \\
& 1789-- \text { - - - } 142 \\
& 1793 \text { - - - - } 167 \\
& 1797 \text { - - - - - } 184 \\
& 1801 \text { - - - - . - } 223 \\
& 1806 \text { - - - - - } 260
\end{aligned}
$$

\begin{tabular}{|c|c|c|c|c|c|c|c|c|c|}
\hline \multicolumn{10}{|c|}{$\begin{array}{l}\text { Table of Premiums for assuring the Sum of one hundred } \\
\text { Pounds upon the Life of any healthy Person, from the } \\
\text { Age of Eight to Sisty-seven. }\end{array}$} \\
\hline Age. & \multicolumn{3}{|c|}{ For 1 Year. } & \multicolumn{3}{|c|}{$\mid \begin{array}{lll}\text { For } 7 & \text { Years at an } \\
\text { annual } & \text { Payment of }\end{array}$} & \multicolumn{3}{|c|}{$\begin{array}{l}\text { For the whole Life } \\
\text { at an aniual Pay- } \\
\text { ment of }\end{array}$} \\
\hline & $l$ & & & $l$ & $s$ & & l & $s$ & \\
\hline 8 to 14 & 0 & 17 & 9 & 1 & 1 & 5 & 1 & 17 & 7 \\
\hline 15 & 0 & 17 & 11 & ] & 2 & $1 \mathrm{I}$ & 1 & 18 & 7 \\
\hline 10 & 0 & 19 & 2 & 1 & 4. & 7 & 1 & 19 & 8 \\
\hline 17 & 1 & 1 & 2 & I & 6 & 1 & 2 & 0 & 8 \\
\hline 18 & I & 3 & 3 & 1 & 7 & 5 & 2 & 1 & 8 \\
\hline 19 & 1 & 5 & 0 & 1 & 8 & 6 & 2 & 2 & 8 \\
\hline 20 & 1 & 7 & 3 & 1 & 9 & 5 & 2 & 3 & 7 \\
\hline 21 & 1 & 8 & 10 & 1 & 10 & 1 & 2 & 4 & 6 \\
\hline 22 & 1 & 9 & 3 & 1 & 10 & 6 & 2 & 5 & 4 \\
\hline 23 & 1 & 9 & 8 & 1 & 11 & 0 & 2 & 0 & 3 \\
\hline 24 & 1 & 10 & 2 & 1 & 11 & 6 & 2 & 7 & 1 \\
\hline 25 & 1 & 10 & 7 & 1 & 12 & 1 & 2 & 8 & 1 \\
\hline 26 & 1 & 11 & 1 & 1 & 12 & 7 & 2 & 9 & 1 \\
\hline 27 & 1 & 11 & 7 & 1 & 13 & 2 & 2 & 10 & 1 \\
\hline 28 & 1 & 12 & 1 & 1 & 13 & 9 & 2 & II & 1 \\
\hline 29 & 1 & 12 & 8 & 1 & 14 & 4 & 2 & 12 & 3 \\
\hline 30 & 1 & 13 & 3 & 1 & 14 & 11 & 2 & I. 3 & 5 \\
\hline 31 & 1 & 13 & y & 1 & 15 & 7 & 2 & 14 & 7 \\
\hline
\end{tabular}

As to the assurances on lives, the premium or rates of

\begin{tabular}{|c|c|c|c|c|c|c|c|c|c|}
\hline \multicolumn{10}{|c|}{ The Table Continued. } \\
\hline \multirow[t]{2}{*}{ Age. } & \multicolumn{3}{|c|}{ For 1 Year. } & \multicolumn{3}{|c|}{$\begin{array}{l}\text { For } 7 \text { Years at an } \\
\text { annnul Payment of }\end{array}$} & \multicolumn{3}{|c|}{$\begin{array}{c}\text { For whe whole Life } \\
\text { at an annual Pay- } \\
\text { ment of }\end{array}$} \\
\hline & $\iota$ & $s$ & & $l$ & & & $l$ & $s$ & \\
\hline 32 & 1 & 14 & 4 & 1 & 16 & 3 & 2 & 1.5 & 9 \\
\hline 33 & 1 & 15 & 0 & 1 & ] 6 & 10 & 2 & 17 & -1 \\
\hline 34 & 1 & 15 & 8 & 1 & 17 & 8 & 2 & $1 \mathrm{~s}^{\prime}$ & 5 \\
\hline 35 & 1 & 16 & 4 & 1 & 18 & 10 & 2 & 19 & 10 \\
\hline 36 & 1 & 17 & 0 & 1 & 19 & 7 & 3 & 1 & 4 \\
\hline 37 & 1 & 17 & 9 & 2 & 0 & 8 & 3 & 2 & 10 \\
\hline 38 & 1 & 18 & 6 & 2 & 1 & 9 & 3 & 4 & 6 \\
\hline 39 & I & 19 & 3 & 2 & 12 & 11 & 3 & 6 & 2 \\
\hline 40 & 2 & 0 & 8 & 2 & 4 & 1 & 3 & 7 & II \\
\hline $4 \mathrm{I}$ & 2 & 2 & 0 & 2 & 5 & 4 & 3 & 9 & 9 \\
\hline 42 & 2 & 3 & 6 & 2 & 6 & 6 & 3 & 11 & $s$ \\
\hline 4.3 & 2 & 4 & 6 & 2 & 7 & 9 & 3 & 13 & 8 \\
\hline 44 & 2 & 5 & 6 & 2 & 9 & 2 & 3 & 15 & 9 \\
\hline 4.5 & 2 & 6 & $s$ & 2 & 10 & 10 & 3 & 17 & 11 \\
\hline 46 & 2 & 7 & 10 & 2 & 12 & 6 & 4 & 0 & 2 \\
\hline 47 & 2 & 9 & 0 & 2 & 14 & 4 & 4 & 2 & 7 \\
\hline 48 & 2 & 10 & 3 & 2 & 16 & 4 & 4 & 5 & 1 \\
\hline 49 & 2 & 12 & 3 & 2 & 18 & ci & 4 & 7 & 10 \\
\hline 50 & 2 & 15 & 1 & 3 & 0 & 8 & 4 & 10 & 8 \\
\hline 51 & 2 & 17 & 4 & 3 & 2 & 8 & 4 & 13 & 6 \\
\hline 52 & 2 & 19 & 1 & 3 & 4 & 9 & 4 & 10 & 5 \\
\hline 53 & 3 & 1 & 0 & 3 & 7 & 0 & 4 & 19 & 7 \\
\hline 54 & 3 & 3 & 0 & 3 & 9 & 5 & 5 & 2 & 10 \\
\hline 55 & 3 & 5 & 0 & 3 & 12 & 0 & 5 & 6 & 4 \\
\hline 56 & 3 & 7 & 3 & 3 & 14 & 8 & 5 & 10 & i \\
\hline 57 & 3 & 9 & 8 & 3 & 17 & 6 & 5 & 14 & 0 \\
\hline 58 & 3 & 12 & 3 & 4 & 0 & 6 & 5 & 18 & 2 \\
\hline 59 & 3 & 15 & 1 & 4 & 3. & 8 & 6 & 2 & 8 \\
\hline 60 & 3 & 18 & 1 & 4 & 7 & 1 & 6 & 7 & 4 \\
\hline 61 & 4 & 1 & 5 & 4 & 10 & 11 & $\sigma$ & 12 & 4 \\
\hline 62 & 4 & 3 & 11 & 4 & 15 & 0 & 6 & 17 & 9 \\
\hline 63 & 4 & 7 & 8 & 4 & 19 & 8 & 7 & 3 & 7 \\
\hline 64 & 4 & 10 & 9 & 5 & 4 & 10 & 7 & 9 & 10 \\
\hline 65 & 4 & 15 & 2 & 5 & 10 & 10 & 7 & 10 & 9 \\
\hline 66 & 5 & 0 & 1 & 5 & 17 & 7 & 8 & 4 & 1 \\
\hline 67 & 5 & 5 & 6 & 6 & 5 & 2 & 8 & 12 & 1 \\
\hline
\end{tabular}
the different companies are nearly alike; as indeed they must be if founded on correct principles, and equally advantageous to the public; and the following tables of the rates are nearly the same in all.

\begin{tabular}{|c|c|c|c|c|c|c|c|}
\hline \multicolumn{2}{|c|}{ Ages. } & \multirow{2}{*}{\multicolumn{3}{|c|}{ Premium. }} & \multirow{2}{*}{\multicolumn{3}{|c|}{$\begin{array}{l}\text { Annuity equival, ne to } \\
\text { 10ol, ro be paid from the } \\
\text { Death of the Life assured, } \\
\text { during the R R mainder of } \\
\text { lhe other life. }\end{array}$}} \\
\hline $\begin{array}{l}\text { Life to be } \\
\text { assured. }\end{array}$ & $\begin{array}{l}\text { Life against } \\
\text { which the } \\
\text { Assurance is }\end{array}$ & & & & & & \\
\hline \multirow{9}{*}{ J0 } & & $t$ & $s$ & & I & & \\
\hline & 10 & I & 8 & 6 & 5 & 14 & 0 \\
\hline & 20 & I & 9 & 1 & 6 & 1.4 & 10 \\
\hline & 30 & 1 & 8 & 3 & 7 & 14 & 11 \\
\hline & 40 & 1 & 7 & 8 & 9 & 5 & 6 \\
\hline & 50) & I & 6 & 11 & 11 & 13 & 0 \\
\hline & 60 & I & 6 & 0 & 15 & 13 & 5 \\
\hline & 70 & 1 & 4 & 11 & 23 & 13 & 0 \\
\hline & 80 & I & 3 & 4 & 40 & 10 & 8 \\
\hline \multirow[t]{8}{*}{20} & 10 & 1 & 16 & 6 & 5 & 6 & 11 \\
\hline & 20 & 1 & 17 & 0 & 6 & t. & 1 \\
\hline & 30 & 1 & 15 & 9 & 7 & 0 & 6 \\
\hline & 40 & I & 14 & 8 & 8 & 4 & 11 \\
\hline & 50 & I & 13 & 6 & 10 & 1 & 9 \\
\hline & 60 & I & 12 & 1 & 13 & $\theta$ & 7 \\
\hline & 70 & 1 & 10 & 6 & is & 12 & $s$ \\
\hline & sò & 1 & 8 & 3 & 30 & $y$ & $i^{\prime}$ \\
\hline
\end{tabular}

A T'able of annual Preniums payable during the joint Continuance of two lives for assuring one hundred Pounds, or an equivalent Annuity on the Contingency of one Life's surviving the other. 


\section{A S S}

\begin{tabular}{|c|c|c|c|c|c|c|c|}
\hline \multicolumn{8}{|c|}{ The Table continued. } \\
\hline \multicolumn{2}{|c|}{ Ages. } & \multirow{2}{*}{\multicolumn{3}{|c|}{ Premium. }} & \multirow{2}{*}{\multicolumn{3}{|c|}{$\begin{array}{l}\text { Annuity equivalent to } \\
1 \text { ool, to be paid fiom the } \\
\text { Death of the Life assured, } \\
\text { during the Remainder of } \\
\text { the other Life. }\end{array}$}} \\
\hline $\begin{array}{l}\text { Life to be } \\
\text { assured. }\end{array}$ & $\left|\begin{array}{c}\text { Lite against } \\
\text { which tlie } \\
\text { Assurance is } \\
\text { to be made. }\end{array}\right|$ & & & & & & \\
\hline \multirow[b]{2}{*}{30} & & $l$ & $s$ & $d$ & $l$ & $s$ & $d$ \\
\hline & 10 & 2 & -5 & & 5 & 5 & 8 \\
\hline \multirow[t]{7}{*}{4} & 20 & 2 & 6 & & 6 & 2 & 9 \\
\hline & 30 & 2 & 4. & & 6 & 19 & 6 \\
\hline & 40 & 2 & 2 & 9 & 8 & 3 & 8 \\
\hline & 50 & 2 & 0 & 11 & 10 & 0 & 6 \\
\hline & 60 & 1 & 18 & 10 & 13 & 0 & 0 \\
\hline & 70 & 1 & 16 & 7 & 18 & 12 & 10 \\
\hline & 80 & 1 & 13 & 9 & 30 & 9 & 3 \\
\hline \multirow{8}{*}{40} & 10 & 2 & 19 & 2 & 5 & 3 & 6 \\
\hline & 20 & 2 & 19 & 10 & 5 & 19 & 9 \\
\hline & 30 & 2 & 18 & 2 & 6 & 16 & 8 \\
\hline & 40 & 2 & 15 & 11 & 8 & 1 & 0 \\
\hline & 50 & 2 & 12 & 10 & 9 & 16 & 6 \\
\hline & 60 & 2 & 9 & 4 & 12 & 14 & 3 \\
\hline & 70 & 2 & 5 & 11 & 18 & 5 & 6 \\
\hline & 80 & 2 & 1 & 10 & 29 & 19 & I0 \\
\hline \multirow[t]{8}{*}{50} & 10 & 4. & 0. & 11 & 5 & 1 & 4 \\
\hline & 20 & 4. & 1 & 10 & 5 & 16 & 2 \\
\hline & 30 & 4. & 0 & 1 & 6 & 12 & 2 \\
\hline & 40 & 3 & 17 & 10 & 7 & 16 & 9 \\
\hline & 50 & .3 & 13 & 10, & 9 & 12 & 8 \\
\hline & 60 & 3 & 7 & 7 & 12 & 6 & 8 \\
\hline & 70 & 3 & 1 & 6 & 17 & 11 & 5 \\
\hline & so & 2 & 15 & 0 & 28 & 12 & 6 \\
\hline \multirow{8}{*}{60} & 10 & 5 & 16 & 9 & 4 & 19 & 3 \\
\hline & 20 & 5 & 18 & 1 & 5 & 12 & 10 \\
\hline & 30 & 5 & .16 & 3 & 6 & 7 & 7 \\
\hline & 40 & 5 & 14 & 0 & 7 & 10 & 10 \\
\hline & 50 & 5 & 10 & 7 & 9 & 8 & 0 \\
\hline & 60 & 5 & 2 & 4 & 12 & 5 & 6 \\
\hline & 70 & 4 & 9 & 10 & 17 & 5 & 8 \\
\hline & 80 & 3 & 17 & 11 & 27 & 19 & 10 \\
\hline \multirow[t]{8}{*}{67} & 10 & 8 & 1 & 0 & 4 & 17 & 8 \\
\hline & 20 & 8 & 2 & 9 & 5 & 10 & 5 \\
\hline & 30 & 8 & 0 & 10 & 6 & 4 & 0 \\
\hline & 40 & 7 & 18 & 7 & 7 & 5 & 5 \\
\hline & 50 & 7 & 15 & 6 & 9 & 0 & 6 \\
\hline & 60 & 7 & 8 & 8 & 12 & 0 & 3 \\
\hline & 70 & 6 & 10 & 8 & 17 & 1 & 8 \\
\hline & 80 & .5 & 8 & 9 & 27 & 5 & 11 \\
\hline
\end{tabular}

A Table of anual Premiums payable during the Continuance of two joint Lives for assuring one bundred ['ounds, to be paid when either of the lives shall drop.

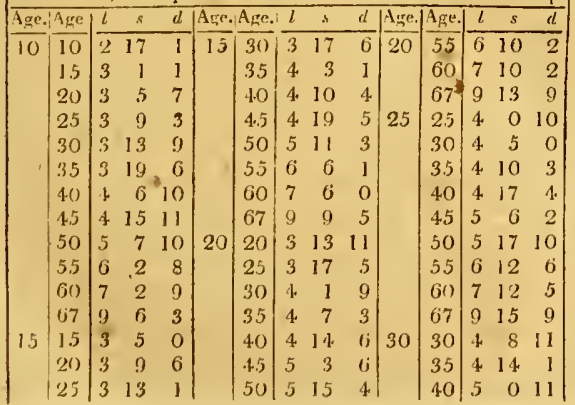

\begin{tabular}{|c|c|c|c|c|c|c|c|c|c|c|c|c|c|c|}
\hline \multicolumn{15}{|c|}{ The T'able continued. } \\
\hline Age. & Age. & $l$ & $s$ & $d$ & Age. & Age. & 1 & $s$ & $d$ & Age. & Age. & $t$ & $s$ & \\
\hline \multirow[t]{5}{*}{30} & $\overline{45}$ & 5 & & 6 & 35 & 67 & 10 & 1 & 2 & 45 & $\overline{67}$ & 10 & II & \\
\hline & 50 & 6 & & 0 & 40 & 40 & 5 & $1 \mathrm{I}$ & & 50 & 50 & & 7 & \\
\hline & 55 & 6 & 15 & 5 & & 45 & 5 & 19 & 9 & & 55 & 8 & 0 & \\
\hline & 60 & 7 & 15 & 0 & & 50 & 6 & 10 & b. & & 60 & 8 & 18 & \\
\hline & 67 & 9 & 18 & 1 & & 55 & 7 & 4 & 5 & & 67 & 10 & 18 & 10 \\
\hline \multirow[t]{6}{*}{35} & 35 & 4 & 19 & 0 & & 60 & 8 & 3 & & 55 & 55 & 8 & 12 & 2 \\
\hline & 40 & 5 & 5 & 6 & & 67 & 10 & 5 & 6 & & 60 & 9 & 9 & \\
\hline & 45 & 5 & 13 & 10 & 4.5 & 45 & 6 & 7 & 4 & & 67 & 11 & 8 & \\
\hline & 50 & 6 & 5 & 0 & & 50 & 6 & 17 & & 60 & 60 & 10 & 4 & \\
\hline & 55 & & 19 & 2 & & 55 & 7 & 11 & 0 & & 67 & 12 & 2 & \\
\hline & 60 & & 18 & 6 & & 60 & 8 & 9 & & 67 & 67 & 13 & 15 & \\
\hline
\end{tabular}

From the above specimen of premiums, the reader will easily judge of the proportional premium for any intermediate age.

ASTERISM, the same with constellation, or a collcction of many stars, which are usually represented on globes by some particular image or figure, to distinguish the stars which compose this constellation from those of others.

ASTREA, a name given by some to the sign Virgo, by others called Erigone, and sonetimes Isis. The poets feign that Justice quitted beaven to reside on earth, in the golden age; but, growing weary of the iniquities of mankind, she left the earth, and returned to heaven, placing herself in that part of the zodiac called Virgo, where she became a constellation of stars, and from her orb still looks down on the ways of men. Ovid. Metam. lib; i. ver. 149.

ASTRAGAL, in Architecture, a small round moulding, which encompasses the top of the fust or shaft of a column, like a ring or bracelet. The shaft always terminates at top with an astragal, and at bottom with a fillet, which in this place is called ozia.

AstragAL, in Gunnery, is a kind of ring or moulding on a piece of ordnance, at about half a foot distance from the muzzle or mouth; scrving as an ornament to the gun, as the former does to a column.

ASTRAL, something belonging to or depending on the -stars.

Astral Year, or Sidereal Year. See Year.

ASTRODICTICUM, an astronomical instrument invented by $M$. Weighel, by means of which many persons shall be able to view the same star at the same time.

ASTROLABE, from $\alpha \sigma \tau \gamma \rho$, star, and $\lambda \alpha \mu[\alpha \nu \omega$, I take ; alluding to its use in taking, or observing, the stars. The Arabians call it in their tongue astharlab; a word formed by corruption from the common Greek name.

This name was originally used for a system or assemblage of the several circles of the sphere, in their proper order and situation with respect to each other. And the ancient instruments were much the same as our armillary spheres.

The first and most celebrated of this kind, was that of Hipparchus, which he made at Alexandria, the capital of Egypt, and lodged in a secure place, where it served for divers astrommical operations. Ptolemy made the same use of it: but as the instrument had several inconveniences, he contrived to cliange its figure, though perfectly natural, and agreeable to the doctrine of the sphere; and to reduce the whole astrolabe to a plain surface, to which he gave the name of the Planisphere. Hence,

Astrulabe is used among the moderns for a Planisphere, or a sterengraphic projection of the circles of the sphere on the plane of one of the great circles; which is usually cither the plane of the equinoctial, the eye being then placed in the pole of the world; or that of the meri- 
dian, the eye being supposed in the point of intersection of the equinoctial and horizon; or on that of the horizon.

The Astrolabe has been treated at large by Stoffler, Gemma Frisjus; Clavius, and our poet Chaucer, who wrote a long and learned tract upon it, and indeed on the whole practice of astronony, as the art stood in his day. For a farther account of the nature and kinds of $i t$, see the article Planisphere.

Astrolabe, or Sea Astrolabf, more particularly denotes an instrument chiefly used for taking altitudes at sea; "as the altitude of the pole, the sun, or the stars.

The common Astrolabe, represented Plate 2, fig. 7, consists of a large brass ring, about 15 inches in diameter, whose limb, or a convenient part of it, is divided into degrees and minutes. It is fitted with a moveable label or index, which turns upon the centre, and carrics two sights; and having a small ring, at $A$, to hang it by in time of observation.

To make use of the Astrolabe in taking altitudes; suspend it by the ring $A$, and turn it to the sun, \& $\mathrm{c}$, so as that the rays may pass freely through both the sights $\mathrm{F}$ and $\mathrm{G}$; then will the label cut or point out the altitude on the divided limb. There are many other uses of the Astrolabe; of which Clavius, Henrion, and others have written very largely.

The Astrolabe, though now come into disuse, is by many esteemed equal to any other instrument for taking the altitude at sea; especially between the tropics, where the sun comes near the zenith.

ASTROLOGICAL, something relating to Astrology.

ASTROLOGY, the pretended art of foretelling future events, from the positions, aspccts, and influences of the heavenly bodies. The word is compounded of $\alpha \sigma \tau \% \rho$, star, and noyos, discourse; whence, in the literal sense of the term, astrology should signify no more than the doctrine or science of the stars: this indeed was its original acceptation, and constituted the ancient astrology; which consisted formerly of both the branches now called astronomy and astrology, under the name of the latter only; and for the sake of making judiciary predictions it was, that astronomical observations, properly so called, were chiefly made by the ancients. And though the two branclies be now perfectly separated, and that of astrology almost universally rejected by men of real learning, this has but lately been the case, as their union subsisted, in some degree, from Prolemy till Kepler, who had a strong bias towards the ancient astrology.

Astrology may be divicled into two branches, natural and judiciary:

To Natural Astrologr belongs the predicting of natural effects; such as the changes of weather, winds, storms, hurricancs, thunder, floods, carthquakes, \&c. But this art properly belongs to Pliysiology, or Natural Plitosuphy; and is only to be deduced, à posteriori, from phenomena and observations. And for this kind of astrology it is that Mr. Boyle makes an apology in his History of the Air. Its foundation and merits may be gatbered from what is saicl under the articles Air, AtmosPuERE, and WeATHER.

Judicial, or Judiciary As trozog y, which is what is commonly and properly called astrology, is that which professes to foretel moral cvents, or such as have a dependence on the free will and agency of man; as if they were produced or directed by the stars.

The professors of this bind of astrolugy inaintain,
"That the heavens are one great volume ur book, wherein God has written the history of the world; and in which every man may read his own fortuue, and the transactions of his time. The art," say they, "had its rise from the same people as astronomy itself: while the ancient Assyrians, whose serene unclouded sky fayoured their celestial observations, were intent on tracing the paths and periods of the heavenly bodies, they discovered a constant, settled relation of analogy, between them and things below; and hence were led to conclude thcse to be the Parcæ, the $\mathrm{D}_{\text {- }}$ stinies so much talked of, which preside at our births, and dispose of our future fate."

"The laws therefore of this relation being ascertained by a series of observations, and the share each planet has therein; by knowing the precise time of any person's nativity, they were enabled, from their knowledge in astronomy, to erect a scheme or boroscope of the situation of the planets, at that point of time; and hence, by considering their degrees of power and influence, and how each was either strengthened or tempered by some other, to compute what must be the result thereof."

Judicial Astrology, it is commonly said, was invented in Chaldæa, and from thence transmitted to the Egyptians, Greeks, and Romans; though some insist that it was of Egyptian origin, and ascribe the invention to Chan. But it is to the Arabs that we owe it. At Rome the people were so infatuated with it, that the astrologers, or, as they were then called, the nathematicians, maintained their ground in spite of all the edicts of the emperors to expel them out of the city. . See Genfruliacr.

Among the Indians, the bramins, who introduced and practised this art in the East, have hereby made themselves the arbiters of good and evil hours, which gives them great authority: they are consulted as oracles; and they have taken care always to sell their answers at good rates.

The same superstition has prevailed in more modern ages and nations. The French historians remark, that in the time of queen Catharine de Medicis astrology was so much in vogue, that the most inconsiderable thing was not to be done without consulting the stars. And in the roigns of king Henry In and iv of France, the predictions of astrologers were the common theme of the court conversation. And this predominant humour in that court was well rallied by Barclay, in his Argenis, lib. 2, on occasion of an astrologer, who had undertaken to instruct king Henry in the erent of a war which was then threatened by the faction of the Guises.

History of Astrology. - Nothing conlel be more vain, ridiculous, and absurd, than the pretended science of astrology; yet nothing, perhaps, could possibly have led men to a more diligent inquiry into, and finally to a knowledge of, the laws that regulate the motions of the heavenly bodies. Thus it has been said, thit astrology was the daughter of ignorance, and the mother of astronomy; and certainly, if it was not the offspring of passive ignorance, it must be acknowledged to have derived its origin from the natural vanity and credulity of the human mind, aided perhaps by the coincidence of some accidental circumstances, such as an eclipse, the appearance of a comet, or other celestial phenomenou, at the time of any remarkable calamity, the cleath of a prince, or the defat of an army; which being once observed, nothing more was necrssary, in this infant state of the reasoning faculty of man, to lead him to a belief that all great events were connected witl the motion of the heuvenly borlies, that the death of princes 
was always announced by some celcstial phenomenon; and finally, that every man had his destiny written in the heavens, in which might be read all the physical and political events of his life.

Whether or 1 ot, astronomical observations were made, before they were thought necessary to astrological predictions, we bave now no means of determining; but at all events, we know that many were made with no other view, and in consequence of which the knowledge of astronomy was advanced, if it did not derive its immediate origin from astrology. It is also probable, that to the same frivolous motive, wc are indebted for the prescrvation of so many of the learned works of Grecce, which have been handed down to us. For, as a celebrated author justly observes, "What conld be the charms of truth, purely geometrical, to a people sunk for so many ages in the deepest abyss of ignorance and superstition:" but that arithmetic, geometry and even optics, such as it was, were the necessary steps to astronomy, and this again was the preliminary to every astrological prediction? In this respect astrology, if not the mother of astronomy, was at least its nurse, and the preserver of many of the other sciences: for which reason its name may still be preserved from that oblivion which, considered as a science, it so justly merits. At what time, or in what country this pretended science first originated, are alike unknown; or whether it may not have been invented, neglected, and revived, in diflerent countrics, at different periods, according as the sciences were in their infancy, plenitude, or decline. Indecd the latter seems the most probable, as there were different modes of drawing horoscopes in different watious; which scems to indicate that they had not all obtained it from one and the same original. However this may be, we find its traces in the ancient records and manuscripts of every nation, where those records are to be found; in India, China, Chaldea, Grecce, Egypt, Arabia, and Europe. In short, in every country where the sciences have been introduced, the fontsteps of astrology are likewise to be discovered.

In India the pretenderl knowledge of astrology, or the art of divination, remained entirely with the bramins, who were too cunning to promulgate its mysteries, being aware that it would be the most probable means of detecting the imposition, and their interest was too much involved in the deception, for them not to be anxious to keep the secret to themselves: it was not only in their hands a source of emolument and riches, but likewise a convenient instrument for securing the reverence of the people. They not only professed a knowledge of judicial astrology, but also as it regarded incteorology; publishing yearly an almanac or panjamgam, in which every wcek, and hour, both of day and night, were set aside for performing certain avocations in agriculture and other concerns of life: they annually prescuted to the prince a prediction of events for the following year; and such as were found to deccive three times were condemned to silence, but the others who were fortunate in their predictions, enjoyed every distinction that was due to such profound knowledge. The Chinese had, and still continue to have, their astrologers; and many records, both of jurlicial, and natural or meteornlogical astrology of very great anticpuity, still remain. The following is a remarkable instance of the estimation in which astrolugy was held in China, and of the lrutal barbarity and ignorance of their cmperor Tsin-chi-hoang, who in the year 246 , after having re-united under one he'id all the little statcs, into which the cmpire had been divided by intestine broils, and presuming that the sword was sufficient to secure what it had alrearly acquired, he ordered every book to be burnt, except those relating to agriculture, medicine, and astrology ; three sciences which, it thus appears, he thought equally serviceable to the nation; whilc all those relating to history and pure astronomy were destroyed in the barbarous conflagration. But of all the professors of this doctrine, nonc appear to have been so celebrated as the Chaldeans, even the name of Chaldean and Astrologer became as it were synonymous; and according to their records it has been practiscd among them for thousands of ages: even taking their years for days, whicb, according to some modern authors, is what they are supposed to represent, thicy anount to more than 2000 years before the birth of Christ. Zoroaster is said to have been the founder of astrology among the Chaldeans; he was followed by Hostanes, from whom the Greeks and Egyptians are supposed to have received this doctrine. Belezes, another noted Chaldean astrologer, became famous for having predicted to Arbases the victory that he afterwards gained over Sardanapalus, a prediction by no means difficult, when we consider the tyranny and debauchery of the latter, which caused him to be abhorresl and despised, both by his troops and his nation. But thus it frequently happened, that the prediction of an event, which required only a little foresight and knowledge of mankind to know nearly to a certainty the result, was the mcans of raising men to celebrity, and they were generally too cunning to undecrive the wondering multitude. As to the Greeks, it dues not appear that they were so much infatuated with the belief of judicial astrology, as the nations we have alrearly mentioned; but to what is called meteurolugical astrology, they paid particular attention; various predictions of tbis kind having beer given in the Greek calendars by Eudoxus, Callippus, Aratus, Hipparchus, and other authors of eminence. It is a long time after this, that we find some account of the destiny of particular mein pretended to be predicted from an inspection of the stars. We must not however omit to mention, that there are two works on the subject of jullicial astrolngy under the name of P'tolemy; but it appears there is some reason to suspect that they are not his works, and for the honour of the author of the Almagest, it is to be hoped they are not.

Next to the Chaldeans, the Egyptians were cortainly. the most celebrated astrologers; their belief in this doctrine being carricd to the highest degree of enthusiasm : all that we suppose to depend upon our will, or on our determination, they belicved to be connected with the mntion of the stars, and that our destinies are writtrn in the hearens, wherein may be read our thoughts, inclinations, constitutions, and every event of our lives.

Till about the commencement of the Cliristian æra, or a little before, astrology was circumscribed to the East, and to Egypt; but about this time it invaded the Roman empire, and Rome itself was inundated with the professors of this imaginary science. It, was in vain that the scnate issued decress against them, under the name of Chaldeans, astrologers, or mathematicians, they having arrogated to themselves the latter appellation; thus degrading one of the noblest sciences, by associating it with their ridiculous doetrine: but notwithstanding these decrees, they still maintaincl themselves, through the support which they experienced from individuals, and some cven gained great cclebrity for their pretended knowledge and ability, as $\mathrm{Ni}$ gidius Ligilus 'Trassylus, the astrologer to Tiberius; Tit- 
runtius Firmanus, the friend both of Cicero and Varro; and particularly the poet Manilius, whose poen entitled Astronomicon sice Matheseos, lib. viii, published in 1551, with some Greek and Arabian works of the same kind, is a sort of course of judicial astrology, having scarcely any thing in it relating to pure astronomy. Nigidius became celebrated for predicting to Octavius, father of Augustus, that the infant would be one day master of Rome. Trâssylus was the constant attendant of Tiberius; and all those persons whom the latter had reason, or thought that he had reason to fear, he caused their horoscope to be drawn, and if, as it frequently happened, their stars announced them to be ambitious, or that they had any pretensions to the empire, they were put to death: such was the effect of this most abominable, and disgusting doctrine; and who knows how many may tlius have inuocently suffered death, from such predictions, through the tyranny, ignorance, and superstition of princes, and the rulers of nations? Under these circumstances, astrologers could not fail of having a complete ascendency over the people; as it must have been very dangerous to offend those, who could make the stars say what they pleased, to gain the favour of their prince, or to gratify their own private revenge. It should be observed that this art was not always limited to predicting events from certain periods of time being given, but they performed also the converse of the proposition; that is, by knowing the events, they pretended to discover the time; this indeed was an easier problem, at least they were not so likely to be belied by the event, as frequently happened in other cases. Thus Varro having demanded of Firmanus the time of the birth of Romulus, from the events recorded of his life, the astrologer discovered that he was conceived the first year of the second Olympiad, about 3 o'clock of the 23d day of the Egyptian month Chæac, that is, the $23 \mathrm{~d}$ of December, and that he was born under the influence of the sun, the 21 st of September following; and noreover, that Rome was founded by him on the 9th of April, when the moon was in the sign libra: hence also he determined the fate of the city; for cities and towns had their horoscopes as well as men; such was the pitch to which this most ridiculous science was carried.

The Arabs are another people who made a considerable figure in the history of judicial astrology. All the knowledge they had of astronomy was made entirely subservient to that doctrine, and their history is filled with traits on this subject; a circumstance which proves their great predilection for this pretended science.

The number of Arabian authors, who have written on astrology, would form a long catalogue; but we shall only montion some of the most celebrated. Such were Mesalah and Alchindi, both Jews; and Ali-ben-roduan, who was so much admired by Cardin ; Aleabitius, whose work was published in 1502 in quarto, and afterwarls in folio in 1.503 and 1512 ; Altarabius; Alboacen; Aliaben Ragel; Albubator; Almansor; and a great number of otbers, worthy of the obscurity in which they rare placed at this day. Jacob Alchindi the Jew enjoyed great crlebrity under the reign of Almanson; but the Arabs, jealous of the favour which he experienced, accused him of magic, which led to a violent dispute between him and a mosulan doctor, till at last they defied each other's art. 'This being put to the test, these two learned professors each drew a circle abust himself, to which they atributed a magical power' and thus posited, the doctor, by way of challeuge, wrote two words on a picce of paper which he sealed up and delivered to the calif, and defied Alchindi to divine what they were: this was no easy task for Jacob; but still he cunluingly applied himself to his books and instruments; and then, with all the importance of self-consequence, answered, that the first of these words was a plant, and the other an animal; the calif having opened the paper, he, and all present, were struck with astonishment to find the words assa, mousa. This lucky guess gare Jacob such a decided advantage oyer his confounderl adversary, that Albumazar, one of the doctor's pupils, was so much hurt at the disgrace of his master, that hiding a dagger under his cloak, he was going to kill Alchindi; when the latter, no doubt apprised of his design, called out with a stern voice, "You come to slay me; quit this design and the poniard that you carry, and I will teach you astronony." Astonished at these words he let fall the dagger, and became one of Alchindi's most zealous disciples, and afterwards one of the most celebrated astrologers. He wrote a treatise on the conjunction of the planets, in order to read, as it were, the destiny of the earth; to find the time of its origin, and the end of the world. He predicted the fates of the different religions; the Christian religion was to subsist for 1460 years, that of Mahomet 544 years; but all these predictions, like inost others of a similar nature, have been contradicted by facts.

In 1179 , the Arabian astrologers predicted for the year 1186 , a dreadful inundation, and such terrible tempests, that would owerthrow every thing in their course, in consequence of a conjunction of five planets, and a great eclipse of the sun. During seven years alarm was spread in every quarter; but at length the time arrived, and nothing extraordinary having been observed, it gave decidedly the lie both to the prediction and the art; but notwithstanding this, the people still continued to pay the same reverence to the science and its professors as formerly, nothing being sufficient to open the eyes of the infatuated multitude.

The Europeans having received their first notions of astronomy from the Arabians, it was to be expected that they would partake also of their superstitious belief in astrology. Indeed in all those obscure ages which preceded the regeneration of the arts, none seemed to doubt of the influence of the stars on the destiny of man, and most of those who studied or cultivated astronomy, had no other object in vicw, than its application to astrological predictions.

But what must be our opinion of the church of Rome, when we find one of its cardinals so much infaluated with his belief in this doctrine, that he actually drew the horoscope of Jesus Christ, from a pretended knowledge that he professed to have of the moment of his birth; and this being what may be called predicting after the event, he failed not to find written in the heavens all the circumstances of his life and death. And the manner in which he ('ndeavoured to defend himself from the charge of impicty alleged against hitn was equally as ridiculous is the ussertiop itsell:

Among the most celebrated astrologers uf Europe, ve find many whose works on other subjects hare dom honour to themselro's and to their country, and it is to be wished that they had confined their labours only to these subjects. Such were liegiomontauns, Camerarius, Cardan, Argoli, Morin, Tycho Brahe, and his patron and friend count Rantzau; to these we may add, Guide 


\section{$\triangle \mathrm{S} \mathrm{T}$}

Botanus de Forli, who was the author of six treatises on Astrology; Stoffler, who published for many years an ephemeris containing astrolugical predictions. In his almanas for 1499, he annolinced for the year 1514 a terrible inundation throughout Europe, on account of the conjunction of the superior planets in the sign pisces: but this, as also a simitar one by Virdungus, was, when the time arrived, most completely belied by the events, for these years were rather more dry than usual. These ephemerids were similar to our present Moore's almanac, which to the disgrace of the country still carries on this species of deception, and which, notwithstanding its many and palpable contradictions, is at this day regarded as the oracle of wisdom among the greater part of our peasantry.

But perhaps none of those above cited, except Tycho Brahe, was so enthusiastically devoted to astrology as Cardan. He even, in imitation of the Roman cardinal, attempted from the configuration of the hearens to draw the horoscope of Christ; and many other of his notions were equally absurd and ridicutous. He firmly believed in the influence of the stars on the fates and destinics of kingdoms. Thus Butler says :

"Cardan believ'd great states depend

Upon the tip o' th' bear's tail's end;

That as she whisk'd it t'wards the sun,

Strow'd mighty empires up anıl down."

He drew his own horoscope; and it has been said that be even starved himsclf, in order that the time of his death should not give the lie to his doctrine; but this is not true, for he died before the time that his stars had predicted for that event. Junctinus is another who has written largely on this subject; he was astrologer to Catharine de Medicis, at the same time that he was almoner to the duke of Anjou; and a column was erected to him in the Halle au Blé; but he died at last in indigent circumstances at Lyons; au event which he did not probably foresce notwithstanding his astrological skill. To these we may farther aud the fimous John Dee, who acted in the capacity of astrologer to queen Elizabeth, by whom he was consulted as to the time propitious for her coronation; and his friend and companion Kelly, another of the heroes that Butler has introduced in that character,

"Kelly did all his fates upon,"

The devil's looking-glass, a stone."

From which we may conclude, that he dialt a little in the magical way, beside his more common occupation of astrology. But cotuit Rantzau, and his ally TychoBrahe, were perihaps the most zealous defenders of astrology; the latter having written largely in dcfence of the truth of this scicnce, the tendency of which was to show its connexion with the christian religion, and the impiety in doubting of the truth of its loctrine. Even Kepler was in some measure addicted to astrulngy, as appears from several of his letters to his friends, where he is serionsly discussing the best method of drawing an lioroscope: lie was not however so violent and dariug as Stoffer, and Cardan; making hardy and dangerous predictions, which dishonoured both the art and artist.

Morin, who was born in 1583, was the last astrologer of any note, and whose attempts to defend the doctrine of astrology may be considered as the last ctforts of this expiring art; he was an outrageous opponent to the Cupernican systern, with respect to the motion of the carth, and is said to have laboured 30 yeats at his Astrologin Gullica, and predicted the death of his at versary the learued Gas- sendi, a number of times; but the latter seemed to rise from under his frequent afflictions, in order to give the lie to Morin's doctrine. He cven forctold the death of Louis xuII, who never was in better health than at the time announced by Morin for his death: finally, his works and his predictions being gencrally contradicted by the events, they were despised and ridiculed by all his contemporaries who had any reputation in the sciences, and his favourite ductrine seemed to lose ground, as the truth of the modern system of astronomy became more generally acknowledged. That a man of this description, an astrological bigot, should so strongly combat the idea of the motion of the ear.th, is by 1 o means surprising; because the old system of astronomy was much more favourable to the dactrine of astrology than the modern. Indeed it was to this system having finally prevailed over the obstacles that ignorance and superstition had raised against it, that was the principal cause of plunging astrology into that contempt it so justly merited. For as soon as it was known that the earth was not the ccntre.of the universe; that it was only one of the little satellites of the sun; that instead of the sun and stars turning about the earth, it turned on its uwn axis to receive the light and heat of that lnminary; and finally, that the fixed stars are placed at an immense distance, 'that even startles the imagination;how could it for a moment be admitted, that all this vast machine was subordinate to this little globe of earth, and be designed only to preside over the destinies of its inhabitants!

Morin died in 1656 , since which time astrology has been declining; and at this day there is not a man of scicnce in Europe who is not fully aware of the absurdity of this pretended doctrine.

But in the East, astrology still preserves its credit; and astronomy is only cultivated there, so far as it is thouglit necessary for astrological purposes; for the Turks can no more be persuaded that the Europeans study astronomy without belicring in astrology, than imagine that wa can examine the inscriptions and ruins of antiquity, without scarching for treasure.

It may be thought that too much has been said on this subject, that eren its name ought not to be introduced into books of science; and certainly, were it not for the bene fit that astronomy loas derived from it, and the respectability of many author's who, tinfurtunately for thicir memoirs, have been its admirers and supporters, it would long before this have been lost in total ublivion, and even its name would not have been remembered by philosophers. But those circumstances cannot fail of giving it some intercst when we are contemplating the progress of human knowledge, and when we reflect, that without this superstitous stimulus, we might still have remained ignorant of those grand and sublime laws, that regulate the motion of the heavenly bodies, and preserve unimpaircet, the universal harmony of our jlanetary system.

ASTROMETL OROLOCiJA, the art of forctelling the weather, and its changes, from the aspects and configurations of the moon and planets: a species of astrology distinguished by some under the denomination of meteurological astrology.

ASTILONONICAI, something relating to Astronomy. Astronomical Calendar, Characters, Columi, Horizon, Hours, Month, 2uadrant, Ring-Dial, Sector, Tables, Telescope, Time, I'ear. Ser the several substantives.

Astrofomical Observetions. Of these thete are rem 


\section{A S T}

[ 160

cords, or mention, in almost all ages. It is said that the Chinese have observations for a course of many thousand ycars. But of these, as well as those of the Indians, we have yet had but little benefit. But the observations of most of the other ancients, as Babylonians, Greeks, \&c. among which those of Hipparchus make a principal figure, are carefully preserved by Ptolemy, in his Almagest.

About the year 880 , Albategni, a Saracen, applied himsclf to the making of observations; in which he was followed by others of the same nation, as well as Persians and Tartars; among whom were Nassir-Eddin-Ettusi, Arzachel, who also constructed a table of sines, and Ulug Bcigh. In 1457 Regiomontanus undertook the province at Nuremberg; and his disciples, J. Werner and Ber. Walther, continued the same from 1475 to 1504 . Their observations were published together in $1544 .-\ln 1509, \mathrm{Co}-$ pernicus, and after him the landgrave of Hesse, with his assistants Rothman and Byrge; observed; and after them Tycho Brahe, assisted by the celebrated Kepler, from 1582 to 1601 . All the foregoing observations, together with Tycho's apparatus of instruments, are coutained in the Historia Colestis, published in 1672 , by order of the emperor Ferdinand.-In 1651, was published at Bononia, by Ricciolus, Almagestum Novum, being a complete body of ancient and modern observations, which he so named after the work of the same nature by Ptolemy.-Soon after, Hcvelius, with a magnificent and well-contrived apparatus of instruments, described in his Machina Coelestis, began a course of observations. It has becn objected to him, that he only used plain sights, and could never be brought to take the advantage of telescopic ones; which occasioned Dr. Hooke to write animadversions on Hevelius's instruments, printed in 1674 , in which he too rashly despises them, on account of their inaccuracy: but Dr. Halley, who at the instance of the Royal Society went over to Dantzick in the year 1679 , to jnspect his instruments, approved of their justness, as well as of the observations made with them. See Sighis.-Our two countrymen Jer. Horrox and Will. Crabtree, are celebrated for their observations from the year 1635 to 1645 , who first observed the transit of Venus over the sun in the year 1639.-They were followed by Flamsteed, Cassini the father, and son, Halley, de la Hire, Roemer, and Kirchius.-We have also now had published the accurate observations of Dr. Bradley and of Dr. Maskelyne; as also those of the French and other obscrvatories, with the observations of many ingenious private astronomers, which are to be found in the Transactions and Memoirs of the various Philosophical Societies.-There have been also observations of many other eminent astronumers; as, Galileo, Huygens, and our countryman I Iarriot, whose very interesting observations have lately.becn brought to light by the earl of Egremont, and count Bruhl, though they have not been thought fit to be published. Other publications of celestial observations, are those of Cassini, La Caille, Monnier, Lalande, Herschel, \&c.-See farther under Celestial Observations, C八taLogue, ObSERvatory, \&c.

Astronomical Place of a star or planet, is its lomgitude, or place in the ecliptic, reckoned from the beginning of aries, in consequentia, or according to the order of the signs.

ASTRONOMICALS; a name used by some writers for sexagesinal fractions; on account of their usc in astronomical calculations.

ASTIRONOMICUS Radius. Sce Ra dius.
A S T

ASTRONOMY, the doctrine of the hearens, and their phenomena. Astronomy is properly a mixed mathematical science, by which we become acquainted with the celestial bodies, their motions, periods, eclipses, magnitudes, distances, and other phenomena. Some, however, understand the term astronomy in a more extensive sensc, as comprising in it the theory of the universe, with the primary laws of nature: in which sense it seems to be rather a branch of physics than of mathematics.

History of Astronomy.-The invention of astronomy has been variously given, and ascribed to several persons, several nations, and sereral ages. Indeed it is probable that mankind never existed without some knowledge of astronomy anongst them. For, besides the motives of mere curiosity, which are sufficient of themselves to have excited men to a contemplation of the glorious and varying celestial canopy, it is obvious that some parts of the science answer such essential purposes to mankind, as to make the cultivation of it a matter of indispensible necessity.-Accordingly we find traces of it in various nations, and rccords of $\mathrm{jts}$ cultivation by the earliest inhabitants. Josephus informs us, that the sons of Seth employed themselves in the study of astronomy, and that they engraved their observations on two pillars, one of brick and the other of stone, in order that they might be preserved from the destruction wbich Adam had forctold should come upon the earth. He also relates that "A brahain gave lectures in astronomy and arithmetic to the Egyptians," "which," he says, " they knew nothing of, till Abraham brought them from Chaldea to Egypt, and from thence they passed to the Greeks." Berosus also observes, " that Abraham was a great and just nan, and famous for" his celestial observations." The same ruthor ascribes to the antediluvians a knowledge of the astronomical cycle of 600 ycars, which may be supposed to mean the period in which the sun and moon came again into the same situstion with regard to the nodes, apogee of the moon, \& $\mathrm{c}$. "This period," says Cas'sini, " of which we find no intimation in any mouument of any other nation, is the most accurate of the kind that ever was invented : for it brings out the solar year more exactly than that of Hipparchus and Ptolemy; and the lunar month within about one sccond of what is determined by modern astronomers." If the antediluvians had such a perjod of 600 years, they must have known the motions of the sun and moon more exactly than their descendants kniew them some ages after the flood.

The accounts however which we have of these matters, anterior to the flood, do not carry with them the degree of credibility that would justify a more particular mention of them; nor would a more copious detail conduct us to any certain conclusion of the age or country where the science of astronomy first originated, or by whom it was first methodised and extended. Whatever credibility these uncertain testimonies of the ancients may pessess; certain it is that most authors are agreed that the commencement of the science originated either in Chaldea, Egypt, or lndia; all of which nations pretended to a very high antiquity, and equally claimed the homour of producing the first cultivators of this science. The Chaldeans in particular boasted of their temple, and cnormous tower of $\mathrm{BC}$ lus, which has been thought by some to have bech an as. tronomical observatory; and no less of thcir famous astronomer and philosopluer Zoroaster, whom they placed 500 years before the destruction of Troy. While the Egyjtians, with similar ostentation, vaunted of their Col- 
leges of priests, which were the depits of every species of knowledge; and of the monument of Osymandyas, in which tradition asserts, there was a golden circle of 365 cubits in circuinference, and one cubit thick, divided into 365 equal parts according to the days of the year, and containing the heliacal risings and settings of the stars for each day, \&ic.

It is indeed evident that both Chaldea and Egypt were countries very proper for astronomical observations, on account of the extended flatness of the country, and the purity and serenity of the air. The tower of Belus, or of Babel, being of a great height, was probably an astronomical observatory; and the lofty pyramids of Egypt, whatever they were originally designed for, might perhaps answer the same purpose; at least, they show the skill of this people in practical astronomy, as they are all placed with their four fronts exactly facing the cardinal points of the compass. For it is hardly possible, that they should lave placed them in that striking position, and with such a degree of exactness, without some particular reason; and this being the case, it will follow, that they were acquainted with the correct method of drawing a meridian line, which is a matter of more difficulty than is generally supposed: it bcing well known that Tycho Brahé, the most able astronomer of his time, committed an error of several minutes in tracing that of the observatory of Uranisburg.

The Chaldeans certainly began to make observations soon after the confusion of languages, as appears from the observations found there on the taking of Babylon by Alcxander; and probably much earlier. It hence appears that they had determined, with tolerable exactness, the length buth of a periodical and synodical month. They lrad also discovered, that the motion of the moon was not uniform; and they even attempted to assign those parts of the orbit in which the motion is quicker or slower. We are also assured by Ptolemy that they were not unacquainted with the motion of the moon's apogee and nodes, the latter of which they supposed made a complete revolution in $6585 \frac{1}{3}$ days, or a little more than is years, and that it contained 223 complete lunations, which period is called the Chalelean Saros. From Hipparchus, the same author also gives us several observations of Iunar.eclipses made at Babylon above 720 years before Christ. And Aristutle informs us, that they had many occultations of the planets and fixed stars by the moon; a circumstance which lcd them to conceive that fclipses of the sun were to be attributed to the same cause. They had also no inconsiclerables share in arranging the stars into constellations. Nor had even those eccentric bodies the comets escaperl their observatinn: for both Diodorus Siculus and Appollinus Mynticus, Seneca informs us, accounted these to le permatuent bodies, itaving statcil revolutions as well as the planets, but in much more extensive orbits: although others of them were of opinion, that the comets were only meteors raised very ligh in the air, which, blazing for a while, disappear when the matter of which they consist is consumed or dispersed. The art of dialling wis also practised among then long before the Greeks wre acquainterl with that science. Herodotus says, that the Cirecks were made acquainted with the use of the l'ole and Gromm through the medium of the Babylonians; the latter of which scems to have been the inost ancient mutrunicut made use of in astronomy.

The Egyptiıns, it appears from variuus circumstances, I, 1.. I. laid claim to nearly the same critical pretensions in the knowledge of astronomy as the Chaldcans. : Herodotus ascribes their knowledge in the science to Sesostris; probably not the same whom Newton makes contemporary with Solomoil, as they were acquainted with astronomy at least many hundred ycars before that æra. We learn. from the testimony of some ancient authors, many particulars relative to the state of their knowledge in this science; such as, that they believed the figure of the earth was spherical; that the moon was eclipsed by passing through the eartl?'s shadow, though it does not ecrlainly appear that they had any knowledge of the true system of the universe; that they attempted to moasure the magnitude of the earth and sun, though their methods of ascertaining the latter were very erroneous; and that they even $p$ retended to foretel the appearance of comets.

Diodorus Siculus also informs us that the Egyptians discovered that the planets had sometimes a direct, and sumetimes a retrograde motion, and at others were stationary: that they made the sun move in a circle inclined to the equator, and in a contrary direction to the diurnal motion. The idca of dedicating the seven days of the week to the planets, is also ascribed to the ligyptians. We are also furthir informed by Mr. E. Barnard, that these people discovered that the stars had an annual motion of $50^{\prime \prime} 9^{\prime \prime \prime} 4.5^{\prime \prime \prime \prime}$ in a year (Phil. 'I'rans. No. 158): and by Macrobius that the Egyptians made the planets revolve about the sun in the same order as is ascribed to them by us : but it does not appear at what time the planets were first discovered to be of a different nature from the fixerl stars nor by whom. They were also acquainted, as is gencrally supposed, with several other branches of this science, as indeed were the Chaldeans; which will appear from the first notions they entertained of the system of the world, and the agreement which has been found among several ancient inetsures of the circumference of the earth.

Many other nations, it is true, have claimed the honour of the original cultivation of this science; but among the various observations of the ancients which have be'en transmitted to us, none appear of greater antiquity than those of the Chinese. The most remarkable of these is a conjunction of tive of the plasets which, according to their annals, is saicl to hase taken place in the reign of their empcror Tchcun-hiu, a bout 2500 years before Christ. They also mention an eclipse of the sun, which happerred in the constellation Scorpio about the year 2150 of the same xra; and which is saicl to have proved fatal to twis Chincse astronomers, of the names of $\mathrm{Ho}$ and $\mathrm{Hi}$, who were condenuned to death by the emperor Tchong-kang, on account of their omisting, through negligence and intoxication, to announce the precise time at which it arrived. And from these data, apparently well attested, several eminent astronomers have endeavoured to discover whether these events could have possibly happened about the time here inentioned; but the subject is attendeet with too many difficulties $t o$ afford any satisfactory result.

Mlost of the accounts which we have of the Chinese astronomers have come from the Jesuit missionaric's. They inform us, that the Chincse were originally taught the science of astronomy by their first emperor Fo-bi, who is supposed by some to be the same with Noah. He alsa culistructed astronomical tables, and gave the figures of the heavenly bodics. In the reign of the emperor Iloang-ti, about 2ij9t years before Christ, Yuchi likewise obsersed the pole-star and the censtellations circumscribing it. 110 $\mathrm{Y}$ 
alsu constructed a sphere with several fixed and moveatie circles; and was the author of an instrument by wuch lye could find the four cardinal points, without any relerence to the heavens; which was probably the same as a compass. Nearly about this time the cycle of 60 years was also established, and it is asserted by F. Gaubril, that for more than a century A. C. the Chine'se had discovered the obliquity of the ecliptic, with the theory of eclipses: and that they were, long before that, acquainted with the true length of the solar year, the method of observing meridian altitudes of the sun by the shadow of a gnomon, and of deducing from thence his declination, and the height of the pole. The same missionary also says, that the Chinese have yet remaining some books of astronomy, which were written about 200 years before Christ; from which it appears, that the Chinese had known the daily motion of the sun and moon, and the times of the revolutions of the planets, many years before that period.

Du Halde likewise infurms us, that Tcheou-cong, the most'skilful astronomer that ever China produced, lived more than a thousand ycars before Christ; that he passed whole nights in observing the celestial bodies, and arranging them into constellations, \&c. At present, however, the state of astronomy is but very low in that country, although it be cultivated at Peking, by public authority, in like manner as in most' of the capital cities of Europe.

The inhabitants of Japan, of Siam, and of the Mogul's empire, have also been acquainted with astronomy from time iminemorial ; and the celebrated observatory at Benares, is a monument both of the ingenuity of the people, and of their skill in that science.

According to Porphyry, astronomy must have been of very ancient standing in the east. He informs us that, when Babylon was taken by Alexander, there were brought from thence celestial observations for the space of 1903 years; which therefore must have commenced within 115 years after the flood, or within 15 years after the building of Babel.-Epigenes, according to Pliny, affirmed that the Babylonians had observations of 720 years engraven on bricks. - Again, Achilles Tatius ascribes the invention of actronomy to the F.gyptians; and adds, that their knowledge of that science was engraven on pillars, and by that means transmitted to posterity.

M. Bailly, in his elaborate bistory of ancient and modern astronomy, endeavours to trace the origin of this science among the Chaldeans, Egyptians, Persians, Indians and Chinese, to a very early period. And thence he maintains, that it was cultivated in Egypt and Chaldea 2800 year's before Christ; in Persia, 3209; in India, 3101; and in China, 2952 years before that xra. He also apprehends, that astronomy had been studied even long before these distant periods, and that we are only to date its revival from thence.

In investigating the antiquity and progress of astronomy anong the Indians, M. Bailly examines and compares four difficrent sets of astrunomical tables of the Indian philosophers, namely that of the Siamese, explained by M. Cassini in 1689; that brought from India by M. le Gentil of the Acarlemy of Sciences; and two other manuscript tables, found among the papers of the late M. de Lisle; all of which be found to agrce very accurately, and referring to the merillian of benares, above mentioned. It appears that the fundanontal epuch of the Indian astronomy, is a conjunction of the sun and moon, which took place at the allazing distance of 3102 years before $C h r^{\mathrm{j}} \mathrm{st}$ : and $\mathrm{M}$.
A S T

Bailly informs us that, by our most accurate astronomical tables, such a conjunction did really happen at that time. He further observes that, at present, the Indians calculate eclipses by the mean motions of the sun and moon ubserved 5000 years since; and that their accuracy, with regard to the solar motion, far exceeds that of the bost Grecian astronomers. They had also settled the lunar nortions by computing the space through which that luminary had passed in 1,600,984 days, or a little more than 4383 ycars. M. Bailly also informs us, that they make use of the cycle of 19 years, the same as that ascribed by the Greeks to Meton; that their theory of the planets is much better than Ptolemy's, as they do not suppose the earth in the centre of the celestial motions, and believe that Venus and Mercury move round the sun; and that their astronomy agrees with the most modern discoveries as to the decrease of the obliquity of the ecliptic, the acceleration of the motion of the equinoctial points, \&c.

In the $2 d$ vol. of the transactions of the Royal Society of Edinburgh, is also a learned and ingenious dissertation on the astronomy of the Brabmins of India, by Mr. Professor Playfair ; in which the great accuracy and high antiquity of the science, among them, is reduced to the greatest probability. It hence appears, that their tables and rules of computation have peculiar reference to an epoch, and to observations, 3 or 4 thousand years before Christ; and many other instances are there adduced, of their critical knowledge in the other mathematical sciences, employed in their precipts and calculations.

Astronomy, it seems too, was not unknown to the Americans; though in their division of time, they made use only of the solar, and not of the lunar motions. And that the Mexicans, in particular, had a strange predilection for the number 13, by means of which they regulated almost every thing: their shortest periorls consisted of 13 days; thcir cycle of 13 months, each containing 20 days; and their century of 4 periods, of 13 years each: and this excessive veneration for the number 13 , arose, according to Siguenza, from its being the number of their greater gods. And it is very remarkable, that the Abbe Clavigero asserts it as a fact, that, having discovered the excess of a few hours in the solar above the lunar year, they made use of intercalary days, to bring them to an equality, as established by Julius Cæesar in the Roman Calendar; but with this difference, that, instead of one day every 4 years, they interposed 13 days every 52 years, which produces the same effect.

This science however fell into great decay with the Egyptians, and in the time of the emperor Augustus, it was cutirely extinct among them.

from Clialdea and ligypt the science of astronomy passed into Placnicia, which this prople applied to the purposes of narigation, steering their course by the north polar star; and hence they became masters of the sea, and of almost all the commerce in the world.

'The Greeks, it is probable, derived their astronomical knowledge chiefly from the ligyptians and Phomiciank, by means of sercral of their countrymen who visited thene nations, for the purpose of learuing the different sciences. Newton supposes that most of the constellations were invented about the time of the $A$ rgonatic expedition; but it is more probable that they were, at least the greater part of them, of a much older date, and derived from other nations, though clothed in fables of their own invention or application. Several of the consteltations are 
mentioned by Hesiod and Homer, the two most ancient writers among the Greeks, and who lived about 870 years before Christ. Their knowledge in this science; however, was greatly improved by Thales the Milesian, and other Greeks, who travelled into Egypt, and brought from thence the chief principles of the science. Thales was born about 640 years before Christ; and he, first of all among the Greeks, observed the stars, the solstices, the eclipses of the sun and moon, and predicted the same. And the same was farther cultivated and extended by his successors Anaximander, Anaximanes, and Anaxagoras; but most especially by Pythagoras, who was born 577 years before Christ, and having resided for several years in Egypt, \&cc, brought from thence the learning of these people, taught the same in Greece and Italy, and founded the sect of the Pythagoreans. He taught that the sun was in the contre of the universe; that the earth was round, and people had antipodes; also that the moon reflected the rays of the sun, and was inhabited like the earth; that comets were a kind of wandering stars, disappearing in the further parts of their orbits; that the white colour of the milky-way was owing to the united brightness of a great multitude of small stars; and he supposed that the distances of the moon and planets from the earth, were in certain harmonic proportions to one another.

Philolaus, a Pythagorean, who flourished about 450 years before Christ, asserted the annual motion of the earth about the sun. He was persecuted for propagating this opinion, and obliged to fly; and it is a circumstance worthy of remark, that Galileo lost his liberty for maintaining the same. Not long after, the diurnal motion of the earth on her own axis, was taught by Hicetas, a Syracusan. A bout the same time flourished at Athens, Meton and Euctemon, where they observed the summer solstice 432 years before Christ, and observed the risings and settings of the stars, and what seasons they answered to. Meton also invented the cycle of 19 ycars, which still bears his name.

The next astronomer was Eucloxus the Cnidian, and scholar of Plato, who lived about 370 years before Christ, and was accounted oue of the most skilful astronomers and geometricians of antiquity, being esteemed the inventor of many of the propositions in Euclid's Elements, and having introduced geometry into the science of astronomy. He travelled into Asia, Africa, Sicily, and Italy, for improvements in astronomy; and we are informed by Pliny, that he determined the annual year to contain 365 days 6 hours, and also the periodical times of the planets, and made other important observations and discoveries.

Calippus flourished soon after Eudoxus, and his celestial sphere is mentioned by Aristotle; but he is better known by his invented period of 76 , containing 4 corrected Metunic periods, and which commenced at the summer solstice in the year 330 before Christ. About his time the knowledge of the Pythagorean system was carried into Italy, Gaul, and Egypt, Ly certain colonies of Greeks.

However, the introduction of astronomy into Greece is represeuted by Vitruvins in a manner somew hat different. He maintains, that Berosus, a Babylonian, brought it immediately from Babylon itself, and opened an astronomical school in the isle of Cos. And Pliny says, that in consideration of his wonderful predictions, the Athenians erected him a statue in the gymnasium, with a gilded tongue. But if this Berosus be the same with the author of the Chaldaic histories, he must have lived before Alexander.
After the death of this conqueror, the sciences flourished chiefly in Egypt, under the auspices of Ptolemy Philadelphus and bis successors. Ile founded a schoul there, which continued to be the grand seminary of learning, till the invasion of the Saracens in the year of Christ 650 . From the founding of that school, the science of astronomy advanced considerably. Aristarchus, about 270 years before Christ, strenuously asserted the Pythagorean system, and gave a method of determining the sun's distance by the dichotomy of the moon.- Eratosthenes, who was born at C'yrene in the year 271 before Christ,' measured the circumference of the earth by means of a gnomon; and being invited to Alexandria, from Athens, by Ptolemy Euergetes, and made kceper of the royal lihrary there, lie set up for that prince those-armillary spheres, which Hipparchus and Ptoleny the astronomer afterwards employed so successfully in observing the heavens. He also determined the distance between the tropics to be $\frac{1}{8} \frac{1}{3}$ of the whole meridian circle, which makes the obliquity of the ecliptic in his time to be $23^{0} 51 \frac{1}{3}^{\prime}$.- The celebrated Archimedes likewise cultivated astronomy, as well as geometry and mechanics: he detcrmined the distances of the planets from one another, and constructed a kind of planetarium or orrery, to represent the phenomena and motions of the heavenly bodies.

The next ancient astronomer that deserves our most grateful acknowledgements, for the number of his correct observations, as well as the important conclusions he derived from them, is Hipparchus. This celebrated astronomer was the first who cultivated all the branches of this science; his first labours were directed to the obliquity of the ecliptic, observed by Eratosthenes, which he found to be perfectly correct; and this was afterwards confirmed by Ptoleny. He next attempted to determine the length of the tropical year; a thing which had not been done by his predecessors, as he conceived, with that degree of accuracy as the importance of it deserved: this he did by observing the interval of the return of the sun to the same tropic or equinox: he imagined that if he could get two corresponding observations, after a great number of revolutions, the error would be proportionally diminished; which was the incthod employed by future astrunomers, in deternining the mean motions of all the planets; a discovery of very high importance in this science. He compared the observation of a solstice by Aristarchus, with one made by hinself, at the interval of 145 years, and found that this bappened half a day sooner than it ought to have done, if the year consisted of $365 \frac{1}{4}$ days, as the Greeks believed before him. Thus then he determined the tropical year to be $365^{\mathrm{t}} 5^{\mathrm{h}} 55^{\mathrm{m}} 12^{\mathrm{t}}$, being nearly $4 \frac{x}{2}$ minutes too great. He constructed the first solar tables, of which any mention is made in the history of astronomy; and discovered the excentricity of the sun's orbit: he also consiclered the motion of the moon, and attempted to estinate the exact time of her revoliztion, by a comparison of ancient cclipses ; he likewise discovered the excentricity of her orbit, as indeed he did that of all the planets : he found that the moon's motion was not uniform, that she moved slower in the apogee of her orbit than in the perigce, and that there was a motion of anticipation of her nodes: be collected the accounts of the Egyptiaus and Chaldeans relative to eclipses; and calculated all that were to happen for 600 yoars to come: It is also to this celebrated astrononer that we are indebted lor the discovery of the priticiple of the e'put$\mathrm{Y}_{2}$ 
tion of time, and the equally important one of the precession of the equinoxes: he found that the stars changed their declinations, but not their latitudes, and on which account he referred them to the ecliptic. He also knew that an eclipse of the sun varied on different parts of the earth; and thus discovered that the moon had a parallax; and this suggested to him a method of determining the sun's distance from the earth more accuratcly than any of his predecessors had done; he also enriched geography. with many disceveries, and was the first writer that we are acquainted with on spherical trigonometry; but bis chicf work is a catalogue which he made of the fixed stars, to the number of 1022 , with their longitudes, latitudes, and apparent magnitudes; which, with most of his other observations, are preserved by Ptolemy in his Almage'st.

After this period it may be remarked, that little progress was inade in this science from the time of the lastmentioned astronomer to that of Ptolemy, who was born at Ptolemais in Egypt, in the first century of christianity, and who inade the greatest part of his observations at the celcbrated school of Alexandria in that country. Profiting by those of Hipparchus, and other ancient astronomers, be formed a system of his own, which, though erroneous, was followed for many ages by all nations. He compiled a great work, called the Almagest, a name given jt by the Arabs: but which was originally entitled $\mathrm{M} \varepsilon \gamma \dot{a}-$ $\lambda . \eta \sum_{i} \dot{v} \tau \alpha \xi i s$, or Great Construction. From its containing the observations and cullections of Hipparchus, 'Timocharis, and others his predecessors in astronomy, it will ever be valuable to the professors of that science. Thus did the.Alexandrian school continue to be enriclied, and to gain celebrity in science, for more than five centuries; when (as has been observed) an event the most calamitous, and the most disgraceful to its perpetrators, laid in ruins its valuable library, and consumed in one hour the labours of all the most celebrated astronomers of that period. But it deserves to be remarked, that the very people who destroyed those admirable treasures of learning and invention, became afterwards the protectors of science and litcrature, and cultivated astronomy with the gritat'st ardour.

Geography likewise is much indebted to Ptolemy for his meritorious services in collecting all the determinations of the latitudes and longitudes of places then known, and for this fundanental principle of the methods of projections for constructing geographical charts; which was scarcely known before his time. The Almagest was preserved from the lanentable configration of the Alexandrine library by the Saracens, and translated out of Greck into Arabic in the year 827 , and from thence into Latin in 1230. Th: Greek origiual was not known in Europe till the beginning of the 15 th centuly, when it was brought from Constantinople, then taken by the 'Turks, by Goorge, a monk of Trabezond, by whom it was trinslated into Latin; and various other editions bave since alpeared.

During the long period from the year soo till the beginning of the 14th century, the western parts of Europe were involved in the utmost ignorance and barbarily, while the Arabians, profiting by the books they had prescreed from the week of the Alexandrine library, cultivated and improved all the sciences, and particularly that of astronomy, in which they had many able professors and authors. The caliph $\Lambda$ ] if ansur first introkluced a taste for the scicnces into his empire. Ilis grandson $\Lambda$ I Mamon, who ascoubled the throne in 814 , was a great cn- courager and improver of the sciences, and especially of astronomy. Having constructed proper instruments, he made many observations; determined the obliquity of the ecliptic to be $23^{\circ} 35^{\prime}$; and under his auspices a degree of tile circle of the carth was measurcd a second time in the plain of Singar, on the border of the Red Sea. About the same time Alferganus wrote elements of astronony; and the science was from heuce greatly cultivated by the $\Delta$ rabians, but principally by Albategnius, who flourished about tbe year 880 , and who greatly reformed astrunomy, by comparing his own observations with those of Ptoleny: hence he computed the motion of the sun's apogee from Ptolemy's time to his own; settled the precession of the equinnxes at one degree in 70 ytars; and fixed the obliquity of the ecliptic at $23^{\circ} 35^{\prime}$. The tables which he composed, for the ineridian of Aracta, were long estcemed by the Arabians. After his time, though the Saracens bad many eminent astronomers, several centuries elapsed without producing any very valuable observations, excepting those of some eclipses observed by Ebn Younis, astronomer to the caliph of Egypt, by means of which the cuantity of the moon's acceleration since that time may be cietermined.

Other emiment Arabian astronomers, were, Arzachel a Mnor of Spain, who observed the oblicuity of the ecliptic: he also improved trigonometry by constructing tables of sines, instead of cliords of arches, clividing the diameter into 300 equal parts. And Alliazen, his contemporary, who wrote upon the twilight, the height of the clouds, the phenomenon of the horizontal moor, and who first showed the importance of the theory of refractions in astronoiny.

Ulug Beg, grandson of the ceichrated Tartar prince Tamerlane, was a great proficient in practical astronomy; he bad very large instruments, particularly a quadrane of about iso feet high, with which be made gorid observations. From these he determined the latitude of Sancrcand, his capital, to be $39^{\circ} 37^{\prime} 23^{\prime \prime}$; and composel astronomical tables for the meridian. of the sane so exact, that they differ very little from those constructed afterwards by Tycho Brahé; but his principal work was his Cata. logue of the fixed stars, made also from his own obscriaticins in the year 1437 .

During this period, almost all Europo was immersed in gross ignorance. But the settlement of the Moors in Spein introduced the sciences into listope; from which time they have continued to improve, and to be communicated from one people to another, to the present time, when astronomy, and all the sciences, have arrived at a very en,jnent decree of perfection. The emperor Frederick it, about 1230 , first began to cncoturage liarning; restoring some decayed universitio's, and founding a new one in Vienna: he also causcd the works of Aristotle, and J'tulemy's Alnagest, to be translated into Latin; and from the translation of this wouk wo ma date the revival of astromomy iu Europe. Two ycars after this, Iolon de Sacro Bosco, that is, of Halifas, likewise compiled, from l'tolemy, Albategnius, Alferganus, and other Arabic astromomers, his work De Spliara, which was held in the gratest estimation for 300 ycars after, and was honoured with commentaries by Clavius and other learned men. In 1240, Alphonso, king of C'ustile, not only cultivated astronomy himself, but grealy cncouraged others; and by the assistance of several learned men he corrected the tables of l'tolemy, and composed those which were denomi- 
nated from him the Alphonsine Tables. About the same time also, Roger Bacon, an English monk, wrote several tracts relative to astronomy, particularly of the lunar aspects, the solar rays, and the places of the fixed stars. And, about the year 1270, Vitello, a Polander, composed a treatise on optics, in which he showed the use of refractions in astronomy. Also our poet Chaucer, who was born in 1328, wrote a large and learned work on the Astrolabie, as he calls it, and indeed on practical astronomy in general, detailing all the precepts very particularly and orderly.

Little other improvement was made in astronomy till the time of Purbach, who was born in 1423. He composed new tables of sines for every 10 minutes, making the radius 60 , with four ciphers annexed. He constructcd spheres and globes, and wròte several astronomical tracts; as, a commentary on Ptolemy's Almagest ; some treatises on Arithmetic and Dialling, with tables for various climates; new tables of the fixed stars reduced to the middle of that century; and he corrected the tables of the planets, making new equations to then where the Alphonsine tables were erroneous. In his solar tables, he placed the sun's apogee in the beginning of Cancer; but retained the ubliquity of the ecliptic $23^{0} 33 \frac{x^{\prime}}{2}$, as determined by the latest observations. He also obscrved some (clipses, made new tables for computing them, and had just finished a theory of the planets, when he died, in 1462 , being only 39 years of age.

Purbach was succeded in his astronomical and mathematical labours by lis pupil and fricnd, John Mluller, commonly called Regiomontanus, from Montergio, or Koningsherg; a town of Franconia, where he was born. He completed the epitome of Pulemy's Almagest, which Purbach har begun; and after the death of his frient, was invited to liome, where he alsu made many astronomical observations. Being returncd to Nuremberg in $147 \mathrm{l}$, by the encouragement of a wealihy citizen named Bernard Walther, he made sereral instruments for astrunomical observations, among which was an armillary astrolabe, like that used in Alexandria by Hipparchus and Ptolemy, with which he made many obserrations, using also a good cluck, which was then but a tate invention. Ile also made cphemerides for 30 years to come, showing the lunations, eclips:s, \&c; and, the art of printing baving then bern lately inventect, he printed the works of many of the most celcbrated ancient astronomers. If wrote the Theory of the Planets and Comets, and a treatise on triangles, still in repute $f r$ several good theorem; computing the table of sines for every single minute, to the radius 1000000 , and introducing the use of taugents also into trigonometry. After his deaih, which happened at Rome in 1476 , being only 40 years of anc, Walther collected his papers, and contiwued the astronomical observations till his own death also. 'The olss rvations of both were collected by order of the seuste of Nuremberg, and published there in $15+4$ by John Schoner: they were also afterwards published in 1618 by Sin.llius, at the end of the observations made by the Landgrave of llesse; and lastly with those of 'lycho Brahe in 1666 .

Walther was succederl, as astronomer at Nuremberg, by Juhn Werner, a clergyman. He observed the motion of the conet in 1500; aud wrote scveral tracts on geometry, astronemy, and geography in a masterly manner; the uiost remarkible of which, are those concesning the mo- tion of the Sth sphere, or of the fixed stars: in this tract, by comparing his own observations, made in 151\}, witl those of Ptolemy, Alphonsus, and others; he showed that the motion of the fixed stars, since called the precession of the equinoxes, is $1^{0} 10^{\prime}$ in 100 years. He made also the first star of Aries $26^{\circ}$ distant from the equinoctial point, and the obliquity af the ecliptic only $23^{\circ} 2 \mathrm{~S}^{\prime}$. He also constructed a planctarium, representing the celestial motions according to the Ptolemaic hypothesis; and published a translation of Ptolemy's Geography, with a commentary, in which he first proposed the method of finding the longitude at sea by observing the moon's distance from the fixed stars; now so successfully practised for that purpose. Weruer died in 1528 , at 60 years of age.

The next astronomer of cminence after the celebrated characters just mentioned, was Nicholas Copernicus, who was born at 'Thorn, in Prussia, January 19th, 1472. He is peculiarly deserving of notice, for having restores the old Pytlagorean system. of the workd, which had continued to be universally neglectèd from the time of Ptolc$\mathrm{my}$, and which is now so universally received throughout all the civilised countries of Euroje. After much time spent in meditation on the various sy'stems, and an att'ntive examination of all the hypotbese's of his predccessors, he found that a circular motion of the planets about the earth was insufficient to solve the phenomena; and he could not admit the revolution of these bodies about an inaginary centre, which the doctrine of epicycles inculcated. Ile therelore sought for a happier expedient, and was suon led $t$ ( ) the establishment of his own systen, by the opinions which he found had bien suggested by other astronomers. He learnt that Philolaus had placed the san in the centre, and that Nicetas had given the carth a rotation about its axis; the previous knowledge of which was, donbtloss, of the greatest utility in facilitating his laudable enquiries. The notion of placing the most glorious luminary in the beavens in the centre, could not but strike him with delight, it being so natural an idea; but, to bring it forward as the basis of a system, supported by such new and demonstrative arguments in its linour, was a circumstance of much greater moment. Spraking of its merits, h" says, "By lang observations, I discovered that if the motions of the planets be compared with that of the earth, and be estimated according to the tines in which they perrorm their revolutions, not only therr several apprarances will follow from this hypothesis, but it will so connect the order of the planets, their urbits, magnitudes, and clistunces, and even the apparent motion of the fixed stal's, that it with be impossible to remove one of these bodies out of its place without disordering the rest, and even the whole frame of the universe." llaving forancd the rudiments of lis system, he applied himself with the greatest assiduity to the improvement of it, by making numerous observations, and comparing them with those of his predecessors. By these he construcied new table's, which, with the true system of the univers, were given in his great work of astronomy, entitled Astronomial lnstaurata. But, in conseguence of the dread ol persecution lrom religions bigots, and violent opposition from others und'r the denonination of astronomers, the publication of this valuable porfiomance was protracted, and the printing of it delayed till the year 1543 , when Schower and Osiander undeitook the care of it, and the work came out under the title of Revolutiones Orbium Coelestium, 
a copy of which Copernicus received a few hours before his dissulution, which happened on the $23 \mathrm{~d}$ of May, 1543, in the 71 st year of his age.

After the death of Copernicus, the science and practice of astronomy were greatly impruved by many otlier persons, as Schuner, Nonius, Appian, Gemma Frisius, Rothman, Byrgius, the Landgrave of Hesse, \&c.-Schoner reformed and explained the calendar, improved the methods of ruaking celestial observations, and published a treatise on cosmography ; but he dicd four years after Copernicus.-Nonius, or Nunez, wrote several works on mathematics, astronomy and navigation, and inventerl some useful and more accurate instruments than formerly; one of these was the astronomical quadrant, on which he divided the degrees into minutes by a number of concentric circles; the first of which was divided into 90 equal parts or degrees, the second into 89 , the third into $\$ 8$, and so on, to 46 ; so that, the index of the quadrant always falling upon or near one of the divisions, the minutes would be known by an easy computation. Nunez wrote also a tract on the Twilight; and a neat treatise on Algebra; besides other pieces. - The chief work of Appian, the Cæsarean Astronomy, was published at Ingoldstat in 1540 ; in which he shows, how to observe the places of the stars and planets by the astrolabe ; to resolve astronomical problems by certain instruments ; to predict eclipses, and to describe the figures of them; and the method of dividing and using an astronomical quadrant: at the end are added observations of 5 comets, une of which has been supposed the same with that observed by Hevelius, and if so, it ought to have returned again in the year 1789 ;-but it was not observed thell. Gemma Frisius wrote a commentary on Appian's Cosmography, accompanied with many observations of. eclipses: he also invented the astronomical ring, and several other instruments, useful in taking obsẹvations at sea; and was also the first who recommended a time-keeper for determining the longitude at sea.-Rheticus gave up his professorship of mathematics at Wittemberg, that he might attend the astronomical lectures of Copernicus; and, for improving astronomical calculations, he began a very extensive work, being a table of sines, tangents, and secants, to a very large radius, and to every 10 seconds, or $\frac{1}{6}$ of a minute; which was completed by his pupil Valentine Otho, and published in 1594.

About the year 1561, William Iv, Landgrave of Hesse Cassel, applied himself to the study of astronomy, having furnished hiruself with the best instruments that could then be made: with these he made a great number of observations, which were published by Snellius in 1618 , and which were preferred by Hevelius to those of Tycho Brahé. From these observations he formed a catalogue of 400 stars, with their latitudes and longitudes, adapted to the beginning of the year 1593 .

The next astronomer of note was Tycho Brahe, a noble Dane, who, from his assiduous attention to this science, became one of the greatest observers that ever 'xisted: he began his observations about the same time with the Landgrave of Hesse, above mentioned, and observed the great conjunction of Jupiter and Saturn; but finding the usual instruments very inaccurate, he constructed many others, much larger and exacter, with which he applied himself diligently to observe the celestial plicuomena. In 1571 he discovered a new star in the chair of Cassiopeia ; which induced him, like Hipparchus on a similar occasion, to make a new catalogue of the stars; which he did to the number of $77 \%$, and a dapted theil places to the year 1600 . In the year 1576 , by favour of the king of Denmark, he built his new observatory, called Uraniburg, on the small island Huenna, opposite to Copenliagen, and which he very amply furnisheil with many large instruments, some of them so divided as to show single minutes, and in others the arch might be read off to 10 seconds. One quadrant was divided according to the method invented by Nonius, that is, by 47 concentric circles; but most of them were divided by diagonals, a method of division invented by a Mr. Richard Chanceler, an Englishman, Tycho employed his time at Uraniburg to the best advantage, till the death of the king; when, falling into discredit, be was obliged to remove to Holstein; and he afterwards found meaus of introducing himsclf to the Emperor Rodulph, with whom he continued at Prague till the time of his death in 1601 . It is well known that Tycho was the inventor of a system of astronomy, a kind of semi-Ptolemaic, which he vainly endeavoured to establish instead of the Copernican or true system. His works, however, which are very numerous, sliow that he was a inan of great abilities; and his discoveries, together with those of Purbach and Regiomontanus, were collected and published together in 1621 , by Lorigomontanus, the favourite disciple of Tycho.

While Tycho resided at Prague with the emperor, he prevailed on Kepler, who had formerly been his disciple and assistant at Holstein, and from his conduct and abilities had rendered himself dear to him, to leave the university of Glatz, and to come to him, which he did with his family and library in 1600: but Tycho dying in 1601, Kepler enjoyed during his life the title of mathematician to the Emperor, who ordered him to finish the tables of Tycho Brabé, which he did accordingly, and publisbed them in 1627 under the title of Rodolphine. He died about the year 1630 at Ratisbon, where he was soliciting the arrears of his pensiom. From his own observations, and those of Tycho, Kepler discovered several of the true laws of nature, by which the motions of the celestial bodies are regulated. He discovered that all the planets revolved about the sun, not in circular, but in elliptical orbits, having the sun in one of the foci of the ellipse; that their motions were not equable, but varying, quicker or slower, as they were near to the sun or farther from him; but that this motion was so regulated, that the areas described by the variable line drawn from the planet to the sun, are equal in equal times, aud always proportional to the times of describing them. He also discuscred, by trials, that the cubes of the distances of the planets from the sun, were in the same proportion as the squares of their periodical times of revolution. By observations also on comets, be concluded that they moved in very excentric orbits, and crossing those of the planets in all directions, but which he could not then determine. These liscoveries, and many others which are to be found in his writings, but of which no traces are di-coverable in the annals of antiquity, will lor ever immortalize his fame, and render his history interesting to the most distint ages.

In Kepler's time there were many uther gool proficients in astronomy; as Ldward Wright, Baron Napicr, John Bayer, \&c. Wright masle stveral good mericlional observations of the sun, with a quadrant of 6 teet 1adius, iu the 
years 1594, 1595, and 1596; from which he greatly improved the theory of the sun's motion, and computed more accurately his declination than any person had done before. In 1599 he published also an excellent work entitled "Certain Errors in Navigation discovered and de. tected," containing a method which has commonly, though erroneously, been ascribed to Mercator.-To Napier we owe some excellent theorems and improvements in spherics, besides the ever-memorable invention of logarithms, one of the most useful ever made in the art of numbering, and of the greatest use in all the other mathematical sciences.-Bayer, a German, published his Uranometria, being a complete celestial atlas, or the figures of all the constellations visible in Europe, with the stars marked on them, and the stars atso accompanied by names, or theletters of the Greek atphabet; a contrivance by which tlıe stars may easily be referred to with distinctness and prescision.

The next astronomer, whose labours deserve to be particularly recorded, is Galileo, who was born at Florence in the year 1564 . Hê discovered the laws by which bodies falling freely are accelerated, and meditated deeply on their motions in general. From the valuable discoveries be made in this branch of physico-mechanical sciences; Newton and Huygens were aiterwards enablerl to derive the most complete and satisfactory theories of the planetary motions. Being informed of the invention of the telescope, an ever-memorable instrument, and to which we are indebted for the most brilliant discoveries, and all the accuracy to which the science of astronomy has attained, he, by duly considering the principles of refraction, constructed one himself of far supcrior power, by which he discovered the inequalities of the moon's surface, the satellites of Jupiter, and the ring of Saturn; also spots on the sun, by which he determined the revolution of that luminary about its axis: he also found that Venus exhibited the same phases as the moon; and discovered that nebulæ and the galaxy were full of sinall stars. He was also a warm defender of the Copernican system, for which he was cruelly persecuted by a congregation of cardinals, who made him abjure, upon his knees, in the most soleinn manner, a truth which nature and his own conviction had shown to be infallible. He was afterwards imprisoned in the inquisition at Flornce, where he was born; and died in the year 1642 , leaving behind him many works which testify bis great skill in the sciences.

The history of astronomy, about this period, also offers us many names of very respectable observers; among whom may be noticed Mercator, Manrolycus, Homelius, and Stevin, abroad; and in England, Thomas and Leonard Digges, John Dee, Robert Flood, Harriot, \&c; the latter of whom, who had hitherto only bcen distinguished as an algebraist, made nearly the same discoveries as Galileo, aud at as early a period, if not more so, as appears by his papers not yet printed, in the possession of the Earl of Egremont. After whom, Mr. Horrox, a young astronomer of great talents, made considerable discoveries and improvements in this science. In 1633 he discovered that the planet Venus would pass over the sun's disc on the 24 th of November 1639, an event which he announced only to his friend Mr. Crabtree; and these two were the only persons in the world that observed this transit, which was also the first time it had ever been seen by human ryes. Mr. Horrox made also many other nseful obser-vations, and had cven formed a new theory of the moon, taken notice of by Newton; but lis early death, in the beginning of the year 1640 , put a stop to his useful and valuable labours.

The next discoveries to Kepler's and Galileo's, were those of Huygens. This celebrated genius was the first who applied pendulums to clocks, a discovery as brilliant and advantageous as ever was presented to astronomy. He was also the first who found that the singular appearances of Saturn are produced by a ring by which the planet is surrounded; and from his great assiduity in observing it, he was led to the discovery of one of its satellites. To this memorable man, also, we are indebted for sonce of the most useful theorems on centrifugal forces; and, had he had an idea of combining them with his enquiries into the developement of curves and the laws of Kepler, Newton had certainly not been the first to announce to the world the universal law of gravitation; but these are the things in which discoveries generally consist.

Next flourished Hevelius, Burgomaster of Dantzic, who. furnished an excellent observatory in his own house, wherc he observed the spots and phases of the moon, from which observations he compiled his Selenographia; and published an account of his apparatus in his work entitled Machina Carlestis, a book now vory scarce, as most of the copies were accidentally burnt, with the whole house and apparatus, in 1679 . Hevelius died in 1688 , aged 76 .

About this time the celebrated Dr. Hooke invented other instruments with telescopic sights, and ccnsured those of Hevelius, which occasioned a sharp dispute between them ; to settle which, the celebrated Dr. Halley was.sent over to Hevelius to examine his instruments. The two astronomers made several observations together, very much to their satisfaction, and among them was one of an occultation of Jupiter by the moon, when they determined the diameter of the latter to be $30^{\prime} 33^{\prime \prime}$.

Before the middle of the 17 th century the construction of telescopes had been greatly improved, particularly by Huygens and Fontaua. The former constructed one of 123 feet, with which he long observed the moon and planets, and discovered that Saturn was encompassed with a ring. With telescopes, also, of 200 and 300 feet focus, Cassini saw five satellites of Saturn, with his zones or belts, and likewise the shadows of Jupiter's satellites passing over his body. In 1666 Azout applied a micrometer to telescopes, to measure the diameters of the planets and other small distance's in the heavens; but an instrument of this kind had been invented before, by Mr. Gascoigne, though it was but little known abroad.

'To obviate the difficulties of the great lengths of refracting telescopes and the aberration of the rays, it is said that Mersene first started the idca of making telescopes of reflectors, instead of lenses, in a letter to Descartes; and in 1663 James Gregory of Aberdeen showed how such a telescope might be constructed. After some time spent. also by Newton, on the construction of both sorts of telescupes, he discovered the great inconvenience which arises to refractors from the different refrangibility of the rays of light, and for which he could not then find a remedy; and therefore, pursuing the other kind, in the year 1672 he presented to the Royal Society two reflectors, which were constructed with spherical speculums, as he could not plucure other figures. The inconveniences, however, arising from the different refrangibility of the rays of light, have since been fully obviated by the ingenious Ml. Dollond. Towards the latter part of the $17 \mathrm{th}$, and beginning of the 18 th century,practical astronomy, it scems, 
rather languished. But at the same time the speculative part was carried to the highest perfection by the immortal Newton in his Principia, and by the Astronomy of David Gregurs.

Suon after this, however, great improvements of astronomical instruments began to take place, particularly in Britain. MIr. Graham, a celebrated mechanic and watchmaker, not only improved clocks and watch work, but also carried the accuracy of astronomical instruments to a surprising degres. He constructed the old 8 feet mural arch at the Royal Observatory, Greenwich, and a small equaturial sector for making ubservations out of the meridian; but he is chiefly to be noticed for contriving the zenith sector of 24 feet radius, and afterwards one of $12 \frac{1}{2}$ fect, with which Dr. Bralley discovered the aberration of the fixed stars. The relecting telescope of Gregory and Newton was also greatly improved hy Mr. Hadley, who presented a very poweriul instrument of that kind to the Royal Society in 1719. The same gentleman has also immortalized his memory by the invention of the reflecting quadrant or sector, now called by his name, which he presented to the society in 1731 , and which is now used so universally at sea, espccially where nice observations.are required. It appears, however, that an instrument similar to this in its principles had been invented by Newton; and a description with a drawing of it given by him to Dr. Ilalley, when he was preparing for his voyage in 1701 , to discover the variation of the necdle. It has also been asserted, that a Mr. Godfrey, of Pliladelphia in Amcricā, made the same discovery, and the first instrument of this kind. About the middle of this century, the constructing and dividing of large astronomical instruinents were carried to great perfection by Mr. John Bird; and re. flecting telescopes were not less improved by Mr. Short, who also first executed the divided object-glass micrometer, which had been proposed and described by $M$. Louville and others. Mr. Dollond also brought refracting telescopes to the greatest perfection, by means of his acromatic glasses; and, lately, the discoveries of Herschel are owing to the amazing powers of reflectors of his own construction.

Thus the astronomical improvements in the 1 sth century have been chiefly owing to the foregoing inventions and improvements in the instruments, and to the establishment of regular observatories in Eingland, France, and other parts of Europe. Roemer, a celebrated Danish astronomer, first made use of a meridional telescope; and, by observing the eclipses of Jupiter's satellites, he first discovered the progressive motion of light, concerning which he read a dissertation before the Academy of Scicuces in Paris in 1675.-Mr. Flanstec d was appointed the first Astronomer Royal at Greenwich in $\mathbf{1 6 7 5}$. He obscrved; for 44 years, all the celestial phenomena, of the sun, monn, planets, and fixed stars, of all which he gave r: improved theory and tables, viz, a catalogue of 3000 stars with their places, to the year 1689 ; also new solar rables, and a theory of the moon according to Horrox; likewisc, in Sir Joulas Moore's Systen of Mathematics, he gave a curious tract on the doctrine of the sphere, showing how, geometrically, to construct eclipse's of the sun and moon, as well as occultations of the fixed stars by the moon. And it was on his tables that Halley's tables and Newton's theory of the moon were also constructed. Cassini, also, the first lirench Astronomer Ruyal, very much distinguished himself, making many observations on the sun, moon, planets, and comets, and greatly improved the elements of their motions. He also erected the gnomon, and drew the celebrated meridian line in the church of Petronia at Bologna.

In $1719 \mathrm{Mr}$. Flamsteed was succeeded by Dr. Halley, as Astronomer Royal at Greenwich. The Doctor had been sent, at the early age of 21 , to the island of St. He lena, to observe the sonthern stars, and make a cataloguc of them, which was published in 1679 . In 1705 he published his Synopsis Astronomic Cometica, in which he ven. tured to predict the return of a comet in 1755 or 17.59 He was the first who discovered the acceleration of the moon, and he gave a very ingenious method for finding her parallax by three observed phascs of a solar eclipse. $\mathrm{He}$ also published, in the Philosophical Transactions, many learned papers, and among them some that were concerning the use that might be made of the next transit of Venus in determining the distance of the sun from the earth. He composed tables of the sun, moon, and all the planets, which are still in great repute; with which he compared the observations he made of the moon at Greenwich, amounting to near 1500 , and noted the differences. He recommenderl the metbod of detcrmining the longitude by the moon's distances from the sun and certain fixed stars; a method which had before been noticed, and which has since been carried into execution, more particularly at the instance of the late Astronomer Royal, Dr. Maskelync.

About this time a dispute arose concerning the figure of the earth. Sir Isaac Newton had determined, from a consideration of the laws of gravity, and the diurnal motion of the earth, that the figure of it was an oblate spheroid, or flatted at the poles; but Cassini had determined, from the measures of Picart, that the figure was an oblong spheroid, or lengthened at the poles. To settle this dispute, it was resolved, under Lewis $x v$, to measure 2 degrees of the meridian; one near the equator, and the other as near the pole as possible. For this purpose, the Royal Academy of Sciences sent to Lapland Mless. Maupertuis, Clairault, Camus, and Le Monier, \&c; and, on the southcru expedition were sent Mess. Godin, Condamine, and Bouguer, to whom the king of Spain joined Don Gcorge Juan and Don Antonio de Ullna. These sct out in 1735 , and returned at different times in 1744,1745 , and $17+6$; but the former party, who set out only in 1736 , returned the year following, having both fulfilled their commissions. Picart's mensure was also revised by Cassini and De la Cailic, which, after his errors were correcteil, was found to agree very well with the olber two; and the result of the whole served to confirm the letermination of the figure before laid down by Newton. On the southern expedition, it was found that the attraction of the great monntains of Peru bud a sensible eftict on the plamb-line of one of their largest instruments, deffecting it 7 of 8 seconds from the true perpendicular.

On the death of 1)r. Halley, in 1742, he was succceded by Dr. Bradley as dstronomer Royal at Grenwich. The: accuracy of his observations enabled him to detect the smaller inequalities in the motions of the planets and fised stars. The consequence of this accuracy was, the discovery of the aberration of light, the nutation of the carth's axis, and a much greater degree of perfiection in the lunar tables. Ile also observel the placrs, and computcd the 
elements of the comets which appeared in the years 1723 , 1736,1743 , and 1757 . He made new and accurate tables of the motions of Jupiter's satellites; and from a multitude of observations of the luminaries, he constructed a most accurate table of refractions, with a gencral rule for computing them. Also, with a very large transit instrument, and a new mural quadrant of $\mathrm{S}$ feet radius, constructed by $\mathrm{Mr}$. Bird in 1750 , he made an inmense number of observations, for settling the places of all the stars in the British catalogue, together with near 1500 places of the moon, the greater part of which he compared with Mayer's tables. Dr. Bradley died in 1762 .

In the year 1760 all the learned societies in Europe made preparations for observing the transit of Venus over the sun, which had been predicted by Dr. Halley more than 80 years before, with the use that might be made of it ir determining the sun's parallax, and the distances of the planets from the sun. The same exertions were also repeated, to observe the transit in $\mathbf{1 7 6 9}$, by sending observers to different parts of the world, for the more convenience in observing. From the whole, Mr. Short computed that the sun's parallax was nearly $8 \frac{3}{5}$ seconds, and consequently the distance of the sun fron the cartl about 24114 of the earth's diameters, or 96 millions of miles.

Dr. Bradley was suiccecded, in 1762, in his office of Astronomer Royal, by Mr. Bliss, Savilian professor of astrono$\mathrm{my}$; who being in a declining state of health, did not long elljoy it. But, dying in 1765 , was succeeded by Dr. MaskeJyne, the late Astronomer Royal, who discharged the duties of that office with the greatest honour to himself, and benefit to the science. In January 1761 this gentleman was sent by the Royal Society, at a very early age, to the 1sland of St. Helena, to observe the transit of Venus over the sun, and the parallax of the star Sirius. The first of these objects partly failed, by clouds preventing the sight of the $2 \mathrm{~d}$ internal contact; and the $2 \mathrm{~d}$ also, owing to Mr. Short having suspended the plumb-line by a loop from the neck of the central pin. However, our astronomer indemnified himself by many other valuable observations: Thus, at St. Helena, he observed the tides; the liorary parallaxes of the moon; and the going of a clock, to find, by comparison with its previous motion which had been ubserved in England, the difference of gravity at the two places : aivo, in going out and returning, he practiseci the method of finding the longitude by the lunar distances taken with a Hadley's quadrant, makiug out rules for the use of seamen, and taught the method to the officers on board the ship; which he afterwards explained in a letter to the sceretary of the Royal Society, inscrted in the Philos. Trans. for the year 1762 , and still more fully afterwards, in the British Mariner's Guide, which be published in the year 1763 . He returned from St. Helena in the spring of 1762 , after a stay there of 10 months; and i) September 1763 sailed for the island of Barbadoes, to setile the longitude of the place, and to compare Mr. Ilarrison's watch with the time there, when this gentleman shouki bring it out: ansther object was also to try $\mathrm{Mr}$. Irwin's marine chair, which he did in his way out. While at Parbadoes, he also made many other observations; and anong them, several relating to the moon's horary parallaxes, not yet publislied. Returning to England in the latter part of the year 1764 , he was alppointid in 1765 to succeed $\mathrm{Mr}$. Bliss as $\Lambda$ stronomcr Rayal, and immediately recommended to the Board of Lisngitude the lunar method

VoL. I. of finding the longitude, and proposed to them the project of a Nautical Almanac, to be caiculated and published io facilitate that method: this they agreed to, and the first volume was published for 1767 , and which continued tull his death under his direction, to the great benefit of navigation and universal commerce.

A multitude of other useful writings by this gentleman are inserted iu the volumes of the Philos. Trins.; and partictularly, in consequence of a proposal mate by him to the Royal Society, the noble project was formed of measuring very accurately the effect of some mountain on the plumb-line, in deflecting it from the perpendicular; and the mountain of Schihallien, in Scotland, having been found the inost convenient in this island for the purpose, at the request of the Society he went into Scotland to conduct the business, which be performed in the most accurate manner; showing that the sum of the defictions on the two opposite sides was about $11 \frac{3}{5}$ seconds of a degrue; and proving, to the satisfaction of the world, the universal attraction of all matter: from the data resulting from which measures I have computed the mean density of the whole matter in the earth, and have found it to be nearly 5 times that of common water. Besides many learned and valuable papers in the Philosophical Transactions, the most assiduous exertions in the disties of the observatory, as abundantly appears by the curious and voluminous observations which he has given to the public, tho world is particularly obliged to his endeavours with the Board of Longitude, for the publication of the Nautical Ephemeris, and the method of observing the longitude, by the distances of the moon and stars, now adopted by all nations, and by which the practice of navigation has been brought to the greatest feifection. Dr. Maskclyne died the 9th of February 1811, and has been succeeded in his office by Mr. John Pond.

The discuveries of Dr. Herschel form a new ara in astronomy. He first, in 1781 , began with observations on the periodical star in Collo Ceti, and a new methord of measuring the lunar mountains, none of which he made more than half a mile in height : and, having constructed tclescopes vastly more powerful than any former ones, he proceeded to other observations, concerning which he has had several papers printed in the Philosophical Transactions; as, On the rotation of the planets round their axes; On the parallax of the fixed stars; Catalogues of double, triple, \&c stars; On the proper motion of the sun and solar system; On the remarkable appeerances of the solar regions of the planet Mars, \&c, \&c; and, above all, his discovery of a new primary planet, on the 13 th of March 1781, which he called the Georgian planet; though at first named the planet Ilerscled by the French and toreign astronomers, but lately, and inore generally, Uranus; by which, and its six satellites, he has greatly cularged the bounds of the solar system, this new planet being more than twice as far from the sur as the planet Saturtu.

M. Piazzi, astronomer royal at Palermo, on Jan. 1, 1801, the first day of the 19th century, discorered another planet, moving in an intermediate orbit between Mars and Jupiter. It had lone beell conjectured by Maclaurin, Bax. ter, Lambert, Bode, Zach, and others, that there was it primary planet within this space; and the discovery has answered to some of these conjectures in a very extraordinary manner. This planet is now distingutshed by the name Ceres; though it would pethaps be betrer to call ahis, and all the new planets, by the names of then 
respective discoverers. Another new and small planet was discovered by Dr. Olbers of Bremen, on March 28, 1802 : it is called Pallas, and is distant from Mars nearly the same as Ceres, their orbits indeed intersecting each other. -Juno is also another very small planet, in the same interval, discovered by M. Harding, at Lilienthal, on Sept. 1, 1804; and is a little farther distant than Ceres and Pallas. A fourth of these small planetary bodies was discovered by Dr. Olbers the 29th of March, 1807, which is denoted by the name of Vesta. And indeed it is highly probable that, as astronomical instruments are improved, new discoveries of this kind will be made, and our system be enlarged by the discovery of planets making their excursions even beyond the orbit of the planet Uranus, or Herschel, as well as some within the orbit of Mercury.

In the labours of scientific men on physical astronomy, the most distinguished names after Newton, are Maclaurin, Clairaut, Simpson, Euler, Walmesley, Dalembert, Frisi, Lagrange, Landen, and Laplace. Simpson, in his tracts, directed his attention to the theory of the moon. He showed that the effects of such forces as are proportional to the cosine of an arc $z$, are explicable by means of the cosines of that arc and of its multiples; and thus determined a very important point : for, since it hence appears, that no terms enter into the equation of the moon's orbit, but what by a regular increase and decrease do, after a certain time, return to their former values; it is therefore evident that the mean motion, and the greatest quantities of the several equations, receive no change from gravity. Frisi, in tis Cosmographia, applied a similar mode of reasonipg to the variations in the obliquity of the ecliptic; which be thus showed to be confined within certain limits. Laplace was conducted farther: in the general movement of a system of bodies, such as is actually exemplified in nature, every thing is in motion; not only every body, but the plane or position of every orbit. The mutual actions of the plancts change the positions of the planes in which they revolve; being perpetualiy made to depart by a small quantity, from side to side, each from that plane in which it would go on continually, if their mutual actions were to cease. The calculus of Lajlace, in the first book of his Mecanique Celeste, shows that the inclinations of these orbits, in the planctary system, are stable, or that the planes of the orbits oscillate a little, to and fro, on each side of a fixed and immoveable plane. This plane is shown to be one, on which, if every one of the bodies of the system be projected by a perpendicular let fall upon it, and if the mass of each body be inultiplied into the area described in a given time by its projection on the said plane; the sum of all these products shall be a maximum.

The celebrated problem of the three bodies, engaged in succession the attention of many eminent philosophers. The problem is this: Having given the masses of thrce bodies, projected from three points given in position, with velocities given it their quantity and dircetion; and supposing the bodies to gravitate to one alsother, with forces that are as their masses directly, and the squares of their distance's inversely; to find the lines described by those bodies, and their position at any given instant. Or, the problem may be rendcred universal, by assuming any number of bodies above three. To resolve the problem generally, according to cither of these cnunciations, exceeds the pewers even of the most refined analysis: but, under the conditiol: presented by nature, much lias been done by Chairas Dalcmbert, Lulcr, and oshers. Clairaut was the first who deduced, from his solution of the problem, a complete set of lunar tables, far more accurate than any former ones. Their accuracy, however, was exceeded by another set produced by 'Tobias Mayer of Guttingen, and founded on a comparison of Euler's solution with correct observations. The problem of finding the longitude at sea, which was now understood to depend so much on the esactness with which the moon's place could be computed, gave much additional value to these researches, and established a very elose connexion between the conclusions of theory and the art of navigation. Mayer's tables were rewarded by the Board of Longitude in England; and Euler's, at the suggestion of Turgot, by the Board of Longitude in France.

Thus the lunar theory was brought to a high degree of accuracy; the tables constructed by means of it giving the moon's place true to $\frac{x}{2}$ a minute. Yet there remained still one inequality in the moor's motion, for which the principles of gravitation seemed to afford no account; this was what is known by the name of the moon's acceleration. Various attempts were made to explain that phenomenon by Halley, and others : it was at last satisfactorily elucidated by Laplace, who thus gave the finishing toucls to the theory of the moon, nearly a century after it had been propounded in the first edition of the Principia. See ACcELERATION of the Monn.

That branch of the theory of disturbing forces which relates to the action of the primary planets on one another, was successfully cultivated between 1740 and 1780 . In the course of these researches, the change in the obliquity of the ecliptic came first to be perfectly recognized, and ascribed to the action of the plancts, on the earth. Euler proved, independently of Frisi, that the change in this obliquity is periodical; that it is not a constant diminution, but a slow and small oscillation, never exceeding $2^{\circ}$ altogetber on both sides of a mean quantity, by which it alternately increases and diminishes in the course of periods, which are not all of the same length, but by which, in the course of ages, a compensation ultimatcly takes place.

Lagrange, struck with the circumstance that the calculus had never given any inequalities but such as were periodical, applied himself to the investigation of a general question, from which he found, by a method peculiar to hinself, and independent of any approximation, that the inequalities produced by the mutual action of the plancts, inust in effect be all periodical; that such changes are confined within narrow limits; that none of the planets ever has been, nor ever can be a comet, moving in a very excentric orhit; but that the planetary system oscillates as it were round a medium state, from which it never deviates far: that amid all the changes which arise from the mutual actions of the planets, two things remain perpetually the same, viz, the length of the greater axis of the cllipse. described by the planet, and its periodical time round the sun; or, which is the same thing, the mean distance of each planet from the sun, and jts mcan motion, botli rcmain constant. The plane of the orbit varies, the species of the ellipse and its excentricity change, but never, by any means whatever, the greatcr axis of the cllipse, or the time of the entire revolution of the planct. 'The discovery of this grent principle, which may be considered as the bul wark that secures the stability of our system, and excludes all access to confusion and disorder, nust render the name of Lagrange for ever memorable in science, and ever lievered by those who delighte in the contemplation of whit- 


\section{A S T}

ever is excellent and sublime. After Newton's discovery of the elliptic orbits of the planets from gravitation, La. grange's discovery of their periodical inequalities, is doubtless the noblest truth in physical astronomy; and, in respect of the doctrine of final causes, it may be regarded as the greatest of all.

Nearly allied to this truth, is the following theorem, resulting from one of Laplace's investigations: If the mass of each planet be multiplied into the square of the excentricity of its orbit, and this product into the square root of the axis of the same orbit, the sum of all these quantities, when added together, will remain for ever the same: this sum is of a constant magnitude, "which the mutual action of the planets cannot change. Hence, no one of the excentricities can ever increase to a greater magnitude: for, as the mass of each planet is given, as well as the axis of its orbit, the square of the excentricity in each is multiplied into a given coefficient, and the sum of all the products so formed is incapable of change. Hence, therefore, we have an "her general property, indicating the stability of our. system within narrow limits. Yet it does not follow that this permanency is necessary and unavoidable, under every possible constitution of the planetary orbits: for, if the planets did not all move the same way; if their orbits were not all nearly circular; if their excentricities were not small; or if the law of planetary deflection were different; the permanency of the preceding quantity could not obtain. Such permanency depends on conditions which are not necessary in thenselves, and is therefore an argument of design in the construction of the universe.

The subject of the tides liad its true theory sketched by Newton, but was not completed till long after his time. The state of neither mechanical nor mathematical scicnce was such in his time as would enable any one to deternine the motions of a fluid, acted on by three gravitations, and having besides a rotatory motion. The dissertations of D. Bernoulli, Euler, and Maclaurin, which shared the prize with that of P. Cavalleri on the principle of vortices, were excellent: but the first man who felt himself in possession of all the principles required in this arduous investigation, was Laplace, who, in the years 1775,1779 , and 1790 , communicated to the Academy of Sciences a scries of memoirs on this subject, which he has united and cxtended in the 4th book of the Mecanique Celeste. See 'TıDes; as also Edinb. Review, N²2.-The precession of the equinoxes is anotber interesting subject, to which the attention of Frisi, Walmesley, Simpson, Dalembert, Landen, Milner, M. Young, and Robertson, have been successively directed.-See the articles, Сомets, Eclipses, Gravitation, Moon, Planets, Satellites, Sun, Solar Ststes, Tides, and the several planets Mercury, Vevus, \&c, \&c.

\section{On the Solar System.}

'The most conspicuous of all the celestial bodies is the suli, that magnificent luminary necupying the centre of the system which comprehends our earih, with a variety of other iprimary and secondary plancts, and a still greater number of comets.-The sun is a body nearly spherical, whose diameter is about 883,250 English niles, nearly 4 times the distance of the moon from the earth. Situated nearly at the centre of the system of the planets, it exerts nn theen all a remarkable influence: it heats them, it enlighters them, and enchains then in their clliptical orbits, by virtue of a force varying inversely as the squares of the distances, and directly as the masses.

The sun agrees with the fixed stars in the property of emitting light continually, and in retaining constantly its relative situation, with very little variation: it is probable also that these bodies have many other properties in common. The sun is therefore considered as a fixed star comparatively near us: and from the same analogy it is inferred that the stars are possessed of gravitation, and of the other general properties of matter; they are supposed to emit heat as well as light; and it has with reason been conjectured, that they serve to cherish the inhabitants of a multitude of planetary bodies revolving about them.

The sun, like many other stars, has probably a progressive motion, which, from a comparison of the apparent motions of a great number of the stars, is supposed to be directed towards the constellation Hercules. It is beyond all question that many of the stars have motions peculiar to themselves, and it is not certain that any of them are without such motions: it is therefore highly probable that the sun may have such motion; and indeed Dr. Herschel has confirmed this conjecture by arguments that are almost demonstrative.

Besides this progressive motion, the stin is subject to a small change of place, dependent on the situations of the planetary bodies; which was long inferred from theory only, but which has been actually demonstrated by 'modern observations. Supposing all the planets to be in conjunction, or nearly in the same direction from the sun, the common centre of inertia of the system is then at the distance of about a diameter of the sun from his centre, or half a diameter without his surface: and since the centre of inertia of the whole system must be undisturbed by any reciprocal actions of the bodies composing it, the sun must describe an irregular orbit round this centre, his greatest distance from it being equal to his owu diameter.

The sun revolves on his axis in 25 days 10 hours, with respect to the fixed stars; this axis is directed towards a point about lialf way between the pole-star and Lyra, the plane of the rotation being inclined a little more than 7 degrees to the plane of the ecliptic. The direction of this inotion is from west to east; and all the rotations of the different bodies which compose the solar system, as far as they have been ascertained, are in the same direction.

The time, and direction of the sun's rotation is ascertained by the change in the situation of the spots, which are often visible on his disc; and which some astronomers suppose to be elevations, but others, apparently with better reason, to be excavations or deficiencies in the luminous matter covering the sun's surface. These poirts are often observed to appear and disappear, and they are in the mean time liable to great variations; but they are generally found about the middle parts of the sun's surface. Lalande imagined that they were parts of the solid body of the sun, which by some agitations of the luminous ocean, with which be conceived the sun to be surrounded, are left nearly or entirely bare. Dr. Wilson and Dr. Herschel are disposed to consider this ocean as consisting, rather of a flame, than of a liquid substance; and Dr. Herschel attributes the spots to the emission of an aëriform fluid, not yet in combustion, which displaces the general lumino:s atmosphere, and which is afterward to scrve as fuel for supporting the process: hence he supposes the appearance of copious spots to be indicative of the approach of wariz $\angle 2$ 
seasons on the surface of the earth, and he has attempted to maintain this opinion by historical evidence. Whatever be the cause of these spots, by their often continuing during the whole time of the rotation, they have given occasion, by observations, to determine the duration of that period, as above mentioned.

The planetary system comprises at least 30 bodies, without including comets. Among these 30 bodies, the sun is the only one which is really phosphoric, or that shines with a lustre which is, properly speaking, its own. All the others are opake; that is, they intercept the luminous fluid, and are only visible by the reflected light. 11 of these bear the name of primary plunets; the other 18 are known under that of satellites or secondary planets.

The planets perform their revolutions abnut the sun in elliptical curves, differing but little from circles, and of which the centre of the sun (or rather the common centre of inertia of the whole system) occupies one of the foci. Commencing with that which is nearest the sun, they have the following disposition: Mercury, Venus, Terra or the Earth, Mars, Ceres or Piazzi, Pallas or Olbers ${ }^{1}$, Juno or Harding, Vesta or Olbers", Jupiter, Saturn, and Uranus or Herschel. See plate 31 .

Each of the planetary orbits is in a plane which passes through the sun's centre. The earth's orbit is named the ecliptic; which is conceived as indefinitely prolonged on all sides; and astronomers observe the situations of the planes of the other orbits by referring them to this.

All the planets move in their orbits from west to east, perform. their revolutions each in equal times, and in an orbit always at the same mean distance from the sun. The velocities in their orbits are variable, being quicker in the parts nearer the sun, and proportionally slower when farther off, in such sort that equal areas are described by the radius vector, or line connecting the sun and the planet in its motion.

Mercury and Venus are nearer the sun than the earth is; on which account they are called inferior or interior planets. Those which are farther from the sun than the earth, are called superior or exterior planets.

The inferior planets can never be in opposition to the sun (see Orposition); but they will be found twice in conjunction with that luminary during the course of their sidereal revolution: first, when they come betwcen the sun aud the earth, making the inferior conjunction; and again, when the sun is between the planet and the earth, making the superior conjunctinn. See Conjunctron.

The inferior planets present diffurent phases, when they are viewed with telescopes: these appearances are more perceptible for Venus than for Mercury, and they depend on the proner motion of those plancts. If this motion be combined with that of the earth in its orbit, which is effrected more slowly by reason of its greater distance from the sun, new appearances will arise, such as the direct motion of those planets in the inferior conjunction, and their retrograle motion in the superior conjunction. See RETUOGRADE.

The orbits of the superior planets aje above or include that of the earth; at the sanc: time that the velocity of she earth is greater thin those of the superior planets. Hence it results that the earth, in its notion, passe's beisvern chese planets and the sun; which catuses them to a)pear in opposition to that tuminary. In the opposition they lave at inotion apparently retromgude; but it is direct in the conjunction, as that of Venus and Mercury is in their superior conjunctions.

Some of the planets, as the Earth, Jupiter, Saturn, and Uranus, have satellites or moons, which revolve round those primaries, as these do about the sun. Thus, the earth ha1 moon or satellite, Jupiter 4 , Saturn 7 , and Uranus 6 . See Moon and Satriltres.

With respect to these satellites or secondary planets, tlie following remarkable circumstance takes place. Our moon, with the 4 satellites of Jupiter, and 1 of Saturn's, are found by observation to turn about an axis, in the same time as they respectively revolve about their primaries. And though it has not yet been ascertained from observations whether the same be true for the other satellites, of Saturn, and those of Uranus; yet, from the uniformity which obviously. pervades the system, it is concluded that the same is true for all the secondaries.

The planet Saturn is encompassed with a thin flat ring, or, as it is now found, with two rings, lying one within the other edgewise towards the planet, and detached from it, having their planes passing through the centre of Saturn. If a circular annulus be cut out of a card, and divided into two parts by a concentric circle, leaving the inner breadth about 3 times that of the outer; and a ball be then put within, of such a size, that the space between the ball and the annulus may be a little larger than the breadth of the annulus, a representation will thus be obtained of Saturn and his two rings. That side next the sun is bright, like the body of the planet. The rings revolve in their own plane; and, not being of a regular figure, their centre of inertia is at a small distance from the centre of Saturn. AI. Laplace computed the time of their rotation to be $10^{\mathrm{h}} 33^{\mathrm{m}}$ $36^{\mathrm{s}}$; nearly agreeing with the time found by Dr. Herschel from observation.

If the mean distances, either of the planets or of their satellites, be compared with the duration of their sidereal revolutions, it will be easy to trace the beautiful relation discovered by Kepler; namely, that while several bodics turn about the same point, the squares of the periodical times are respectively as the cubes of their mean distances from that point: and, by combining this law with the theory of Huygens, viz, that when the squares of the periorlical times, of several bodics circulating about the same point, are respectively as the cubes of the distances from that point, the central forces which animate them, are in the inverse ratio of the squares of the same distances; it will be easy to discover the law of gravitation; and, as it were, to unveil the mechanism of the planetary system.

"The following tables exhibit a bricf view of the solar system, according to the latest and correct account.

I. Duration of the Sidereal Revolutions of the Planets, or of their Periodic Times.

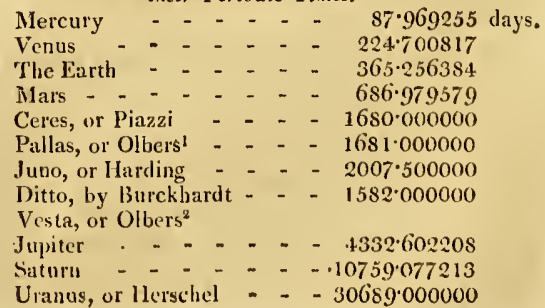


11. Greater Semiaxes of the Planetary Orbits, or their Mean Distances from the Sun, that of the Earth (swhich is about 95 millions of miles) being denoted by unity.

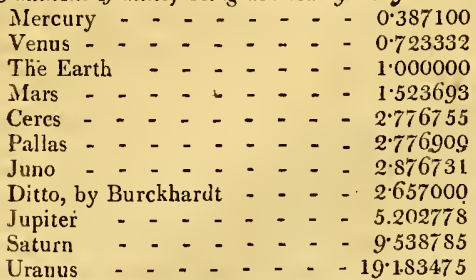

111. Relations of Excentricity to the Greater Semiaxis 1.

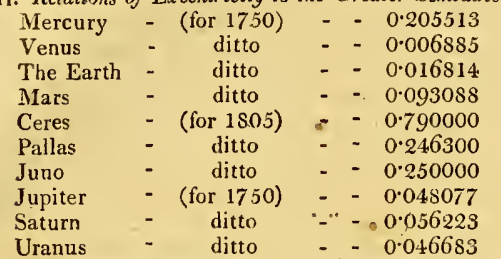

IV. Inclination of Orbits to the Ecliptic.

\begin{tabular}{|c|c|c|c|}
\hline Mercury & $-\quad($ for 1750$)$ & -- & $-7 \cdot 0001)$ \\
\hline Venus & ditto & - & $3 \cdot 3930$ \\
\hline The Earth & ditto & - & 0.0000 \\
\hline Mars & ditto & - & $1 \cdot 8501$ \\
\hline Ceres & (for 1805) & - & $-10 \cdot 6167$ \\
\hline Pallas & ditto & -. & -33.7500 \\
\hline Juno & ditto & - & $-21 \cdot 0000$ \\
\hline Jupiter & (for 1750$)$ & - & -1.3172 \\
\hline Saturn & ditto & - & - $\quad 2.4986$ \\
\hline Uranus & ditto & - & - 0.7706 \\
\hline
\end{tabular}

V. Dianeters of the Planets, that of the Earth (7914 English miles) being assumed as Unity.

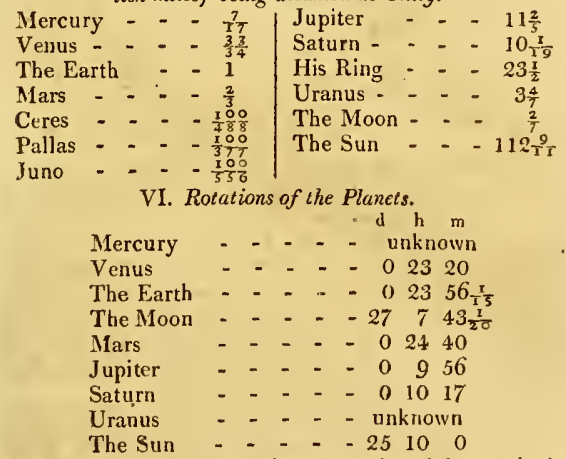

The masses of the planets have been found from principles as follow: The forces that urge two bodies, moving circularly, are in a ratio composed of the masses, the distances from the centre, and the inverse square of the periodic times (see CENTRAL Forces): whence it results, that the gravity of one of the satellites towards its planet, is to that of the earth towards the sun, as the mean distance of the satellite from the centre of its planet, divided by the square of its periodic tine, is to the mean distance of the earth from the sun, divided by the square of its periodic time : or, expressing these gravitating tendencies by $\mathrm{G}, g$, the mcan distances by $\mathrm{R}, r$, the periodic times by $\mathrm{T}, t$, it is $\mathrm{G}: g:: \frac{\mathrm{R}}{\mathrm{T}^{2}}: \frac{r}{t^{2}}$. But, denoting by $\mathrm{M}$ the mass of the sun, and by $m$, that of the planet about which the satellite revolves, by the nature of gravitation it is G.: $g:: \frac{M}{\mathrm{R}^{2}}: \frac{m}{r^{2}}$; therefore $\frac{\mathrm{R}}{\mathrm{T}^{2}}: \frac{r}{t^{2}}:: \frac{\mathrm{M}}{\mathrm{R}^{2}}: \frac{m}{r^{2}} ;$ consequently $\frac{\mathrm{R}^{3}}{\mathrm{~T}^{2}}: \frac{r^{3}}{t^{2}}:: M: m$.

By applying this result to the planets which have satellites, it is easy to find the value of their masses; for, we know the radii of the orbits of the satellites, as well as the length of their sidereal revolutions, or their periodic times. Therefore, taking the cubes of the radii of these orbits, and dividing them successively by the squares of the periodic times, the quotients will give the values of the masses of the bodies, about which the satellites revolve.

As to the planets which have not satellites, Laplace has deduced the values of the masses of Venus and hiars, from the secular diminution of the obliquity of the ecliptic, and the acceleration of the moon's mean motion. The mass of Mercury was inferred from its volume, supposing the densities of that planet and the earth reciprocally as their mean distances from the sun. Mecanique Celeste, tom.3, p. 64. Exposition du Système du Monde, ed. 2, p, 193. Thus was deduced the following table.

VII. Masses of the Planets, that of the Sun being taken for Unity.

$$
\begin{aligned}
& \text { Mercury - - - - } \frac{1}{2025810} \\
& \text { Venus - - - - } \frac{1}{383130} \\
& \text { The Earth - - - } \frac{1}{329620} \\
& \text { Mars - - - - } \frac{1}{3846082} \\
& \text { Jupiter - - - - } \frac{1}{1067} \\
& \text { Saturn - - - - - } \frac{1}{3359} \\
& \text { Uranus - - - - } \frac{1}{19504} \\
& \text { The Moon - - - - } \frac{1}{22578970}
\end{aligned}
$$

\begin{tabular}{|c|c|c|c|c|c|c|c|}
\hline & - & & & & - & & $\begin{array}{l}\text { ter } 1 \\
912\end{array}$ \\
\hline & - & & & & - & 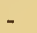 & - \\
\hline & - & & & & - & - & \\
\hline & . & - & & & - & & \\
\hline & - & - & & & - & & \\
\hline & - & - & & & & & \\
\hline & - & - & & & - & 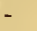 & \\
\hline & & - & & & & & \\
\hline & & & & 1.0000 & & & $1 \cdot 26$ \\
\hline
\end{tabular}

The densities of bodies being in the direct ratio of their masses, and the inversc ratio of the volumes; and in bodies nearly spherical, the volumes being as the cubes of their radii; it results that the densities are then as the masses divided by the cubes of the radii. By this process the following numbers are found for the mean densities of the planets, that of the sun being assumed as unity; viz,

Where the first column of numbers denotes the proportion of the densities of the planets to the sun considered as unity. Then multiplying these by $1 \cdot 2692=\frac{5}{3 \cdot 9: 192}$, we 
obtain the last column of numbers, denoting the densities of the planets in respect of that of water 1 . See my Tracts, vol. 2 , p. 64,66 .

Besides the bodies which revolve completely round the sun, within the limits of our observation, there are otbers, of which we only conclude from analogy that they perform such revolutions. These are the comets; they generally appear åttended by a nebulous light, either surrounding then as a coma, or stretched out to a cousiderable length as a tail; and they sometimes seem to consist of such light only. Their orbits are so excentric, that in their remoter situations the comets are no longer visible to us, though at other times they approach much nearer to the sun than any of the planets; for the comet of 1680 , when at its perihelion, was at the distance of only $\frac{\pi}{6}$ of the sun's diameter from his surface. Their tails are often of great extent, appearing as a faint light, directed aliways nearly opposite to the sun. It is quite uncertain of what substance they consist; and it is difficult to determine which of the conjectures concerning them is the least improbable. For further particulars, see Coner.

Lists and historical accounts of the principal writings and authors on astronomy are contained in Weidler's History of Astronomy, which is brought down to the year 1737. There is also Bailly's excellent History of astronomy, ancient and modern. For this purpose, consult also the following authors, viz, Adam, Vossius, Bayle, Chauffepié, Costard, Perraut, the chronological table of Riccioli, and that of Sherburn, at the end of his edition of Manilius; also the first volume of Lalande's astronomy. The more modern, and popular books on elementary astronomy, are very numerous, and well known : as those of Ferguson, Long, Emerson, Vince, Leadbetter, Brent, Keil, Whiston, Wing, Street; Gregory, \&c, \&c.

ASTROSCOPE, a kind of astronomical instrument, composed of two cones, on the surface of which are delineated the constellations, with their stars, by means of which these may easily be known in the heavens. The astroscope was invented by William Shukhard, professor of mathematics at Tubingen, nn which he published a treatise in 1698.

ASTROSCOPIA, the art of observing and examining the stars, by means of tclescopes; and other instruments, to discover their nature and properties.

ASTROTHEMATA, the places or positions of the stars, in an astrological scheme of the heavens.

ASTROTIIESIA, is used by some for a constellation or collection of stars in the heavens.

ASTRUM, or Astron, a constellation, or assemblage of stars; in which sense it is distinguished from Aster, which denotes a single star. Some apply the term, in a more particular sense, to Sirius the Great Dog, or rather to the large bright star in his mouth.

ASYMMETRY, the want of proportion, otherwise called Inconmensurability, or the relation of two quantities which have no common measure; as between 1 and $\sqrt{ } 2$, or the side and diagonal of a square.

ASYMP'TOTE, is properly a right line, which approaches continually nearer and nearer to some curve, whose asymptote it is said to be, in such à manner, that when they are both indefinitely produced, they are nealer together thin by any assiguable finite distance; or it niay be considered its a tangent to the curve, when conceived to be produced (1) an infinite distance. Tivo curves are also said to be asynututical, when they thus continually approach inde-

\section{A S Y}

finitely to a coincidence: thus, two parabolas, placed with their axes in the same right line, are asymptotes to each other.

Of lines of the second kind, or curves of the first kind, viz. the conic sections, only the hyperbola has asymptotes, which are two in number. All curves of the second kind have at least one asymptote; but they may have three. Those of the third kind may have 4 asymptotes, those of the fourth kind 5 ; and so on for every order of curves whatever; viz, that they have as many asymptotes as the equations expressing them have dimensions, and no more. The conchoid, cissoid, and logarithmic curve, though not reputed geometrical curves, have each one asymptote. And the branch or leg of a curve that. has an asymptote, is said to be of the hyperbolic kind.

The nature of asymptotes will be easily conceived from the instance of that of the conchoid. Thus, if $A \mathrm{BC}$ \& $\mathrm{C}$ be part of a conchoid, and the line $\mathrm{MN}$ be so drawn, that the parts $\mathrm{F} \mathrm{B}, \mathrm{GC}, \mathrm{HD}$, $\mathrm{x}$, \& $\mathrm{c}$, of right lines, drawn from the pole $\mathbf{P}$,

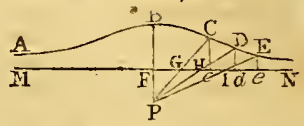
to the curve $\mathrm{SCD}$, be equal to each other; then will the line $M N$ be the aymptote of the curve: because the perpendicular $\mathrm{c} c$ is shorter than $\mathrm{r} \mathrm{B}$, and $\mathrm{v} d$ than $\mathrm{c} c$, \& $\mathrm{c}$; so that the two lines continually approach; yet the points $x e$ \&c can never coincide.

Asymptotes of the Hyperbola are thus described. Suppose a right line DE drawn to touch the curve in any point $A$, and equal to the conjugate de of the diameter $A C B$ drawn to that point $A$, viz, $A D$ or $A \mathrm{E}$ equal to the semiconjugate $\mathrm{c} d$ or $c e$; then the two lines CDF, CEH, drawn from the centre $\mathbf{C}$, through the points $\mathbf{D}$ and $\mathbf{E}$, are the two asymptotes of the curve.
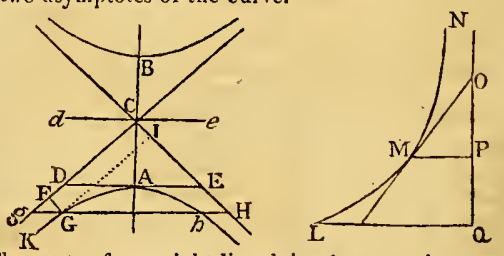

The parts of any right line, lying between the curve of the common hyperbola and its asymptotes, are equal to one another on both sides, that is, $g \mathrm{G}=h \mathrm{H}$. In like manner, in hyperbolas of the second kind, if there be drawn any right line cutting both the curve and its three asymptotes in three points, the sum of the two parts of that right line extended in the same direction from any two of the asymp. totes to two points of the curve, is equal to the third part which extends in the contrary direction from the third asymptote to the third point of the curve.

If $A G K$ be an hyperbola of any kind, whose nature, with regard to the curve and asymptote, is expressed by this general equation $x^{m} y^{n}=a \times a^{m+n}$, where $\boldsymbol{x}$ is $=\mathbf{C F}$, and $y=$ FG drawn any where parallel to the other asymptote cII; and the parallelogram crGs be completed: 'Then $m-n$ is to $n$, as this parallelogran cras is to the liyperbolic space $\mathrm{FGK}$, contained under the determinate line $\mathrm{F} / \mathrm{i}$, with the asymptote $\mathrm{F} K$ and the curve $\mathrm{c} K$, both indefinitcly continued towards $k$. So that, if $m$ be greater than $n$, the said asymptotic space is finite and quadrable : but when $m=n$, as in the cominon or conic lyperbola, them $m-n$ 
$=0$, the ratio of that space to the said parallelogram, is as $n$ to $0:$ that is, the hyperbolic space is infinitely great, in respect of the finite parallelogram : and when $m$ is less than $n$, then, $m-n$ being negative, the asymptotic space is to the determinate parallelogram, as a positive number is to a negative one, and is what Dr. Wallis calls more than infinite.

Asymptote of the Logarithmic CuRve. If LMN be the logarithmic curve, QON an asymptote, $L Q$ and $M P$ ordinates, Mo a tangent, and Po the subtangent, which in this curve is a constant quantity. Then the indeterminate space $L M N Q$ is equal to $L Q \times P O$, the rectangle under the ordinate $L Q$ and the constant subtangent Po; and the solid generated by the rotation of that curve space about the asymptote $\mathrm{N} Q$, is equal to half the cylinder, whose altitude is the said constant subtangent Po, and the radius of its base is LQ.

AsYMPTOTES, by some mathematicians, are distinguished into various orders. The asymptote is said to be of the first order, when it coincides with the base of the curvilinear figure: of the $2 \mathrm{~d}$ order, when it is a right line parallel th the base : of the $3 \mathrm{~d}$ order, when it is a right line oblique to the base : of the 4 th order, when it is the common parabola, having its axis perpendicular to the base: and, in general, of the $n+2$ oreler, when it is a parabola whose ordinate is always as the $n$ power of the base. The asymptute is oblique. to the base, when the ratio of the first fluxion of the ordinate to the fluxinn of the base, approaches to an assignable ratio, as its limit; but it is parallel to the base, or coincides with it, when this limit is not assignable.

The doctrine and determination of the asymptotes of curves, is a curious part of the higher geometry. Fontenelle has given several theorems relating to thi, subject, in his Geometrie de l'Infini. See also Stirling's Linece Tertii Ordinis, prop.vi, where the subject of asymptotes is learncelly treatel ; and Cramer's Introduction a' l'amalyse des lignes courbes, art. $14 \pi \& s e q$. for an excellent theory of asymptotes of genmetrical curves and their branches. Likewise Maclaurin's Algebra and bis Fluxions, bouk i, chap. 10, where he has carefully avoided the inodern paradoxes concerning infinites and infinitesimals. But the easiest way of determining asymptotes, it seems, is by consilering them as tangents to the curves at an infinite distance from the beginning of the absciss; that is, when the absciss $x$ isinfinite in the cquation of the curve, and in the proportion of $\dot{x}$ to $\dot{y}$, or in that of the subtangent to the ordinate.

The areas bounded by curves and their asymptotes, tlough indefinitely extended, have sometimes limits to which they may approach indefinitely near: and this happens in hyperbolas of all kinds, except the first or Apollo-o nian, and in the logarithmic curve; as was observed above. But in the common hyperbola, and many other curves, the asymptutical area has no such limit, but is infinitely great.-Solids, too, generated by hyperbolic areas, revolving about their asymptotes, have sometimes their limits; and sometimes they may be produced till they exceed any given solid.-Also the surface of such snlid, when supposcd to be infinitely produced, is either finite or infinite, according as the area of the generating figure is finite or infinite.

ATLANTIDLS, a name given to the Pleiades, or scven stars, sometimes also called Vergilix. They were thus called, as being supposed by the pocts to have been the daughters either of Atlas or his brother Hesperus, who were translated to beaven.

ATMOSPHERE, is that invisible elastic fluid which surrounds the earth to an unknown height, and partakes of all its motions. - It received its uame from the Greeks, in consequence of the vapours which are continually mixing with it.

Neither the properties nor the composition of the atmosphere seem to have occupied much of the attention of the ancients. Aristotle considered it as one of the four elements, situated between the regions of water and firc, and mingled with two exhalations, the dry and the inoist; the former of which he considered the cause of thunder, lightning, and wind; while the second produced rain, hail, and snow. The ancients in general seemed to have considered the blue colour of the sky as essential to the atmosphere; and several of their philosophers believed that air was the constituent principle of other bodies, or at least that air and otber bodies are mutually convertible into each other. But these opinions floated merely in a state of vague conjecture, till the matter was explained by the sagacity of Hales, and of those philosophers who followed the career that he had opened to them.

It was not till the time of Lord Bacon, who first taught mankind to investigate natural phenomena correctly, that the nature of the atmosphere began to be elucidated with precision. Galileo introduced the study, by showing that the air had heaviness, a point which was soon after investigated completely by his pupil Torricelli, Pascal, \&c. Many otber of its, mechanical properties were afterwards discnvered by Boyle, Mariotte, Hooke, Newton, Derham, \&ce but as these belong to the subject of Pneumatics, a more particular mention of them shall be made in the articles which they respectively constitute.

The opinions of the ancient chemists, concerning the component parts of the atmosphere, are too vague to merit any particular notice. Boyle however, and his contemporaries, put it beyond doubt, that the atmnsphere. contained. two distinct substances, viz, an elastic fluid distinguished by the name of air; and water in the state of vapour. Besides these two bodies, it was supposed also that the atmosphere contained a great variety of other substances, which were continually mixing with it, from the eartb, and which often altered its properties, and rendered it noxious or fatal. Since the discovery however of carbonic acid gas; by Dr. Black, it bas been ascertained that this elastic fluid always constitutes a part of the atmosphere. The constituent parts of the atmosphere, therefore, are,-1. air; 2. water; 3. carbonic acid gas; 4. unknown bodies.

That part of the composition of the atmosphere called air, has not till very lately been perfectly recognised : it seems to liave been used at first to denote the atmosphere in general, and was considered for many ages merely as a simple, homogeneous, and elementary fluid; but philosophers afterwards restricted it to the elastic fluid which constitutes the greatest and most important part of the atmosphere, excluding water and otlier forcign bodies, which are occasionally found mixed with it. For many years, all permanently elastic fluids were considered as air, from whatever combinations they were extricated, and supposed to possess exactly the same properties as the air of the atmosphere; and it was not till the discoveries of Cavendish and Priestley had determined the peculiar properties of a 
varicty of elastic tuids, that philosophers became sensible that there existed various species of them. In consequence of this discovery, the word air became generic, and was applied by Priestley, and the British and Swedish philosophers in general, to all permanently elastic fluids; while the air of the atmosphere was distinguished by the epithets of cominon or atınospheric air: but Macquer thought proper to apply the term gas to all permanently elastic fuids except common or atmospheric air, and to confine the term air to this last fuid, an acceptation of the word which is now universally adopted by all philosophers: the word air therefore now denotes common or atmospheric air, and all other permanently elastic fluids are denominated gases.

For the knowledge of the component parts of air, we are indebted to the labours of tliose philosophers in whose hands chemistry advanced with such rapidity, during the last 40 years of the 18 th century. The first step was made by Dr. Priestley in 1774, by the discovery of oxygen gas. This gas, according to the prevailing notions of the time, he considered as air totally deprived of phlogiston. Hence he considered common air as oxygen gas combined with an indefinite portion of phlogiston, varying in purity according to that portion; being also the purer the smaller the quantity of phlogiston it contained. Schecle also analysed the air, and found, from a process distinct from Priestley, that it was composed of two different elastic fluids, similar to those discovered by Priestley : the oxygen gas of Priestley he called empyreal air, and azotic gas he denominated foul air. Lavoisier was also conducted to the same conclusion by another different process; and thus was atmospheric air confirmed to be a cumpound of oxygen and azotic gas.

This point being established, it next became a question of considerable consequence to determine the proportion of these two ingredients in any given bulk of air, and to ascertain whether that proportion is in every case the same: accordingly the must eninent philosophers engaged themsolves in this deternination. Mr. Cavendish, by improving the eudiometer employed by Pricstley and Fontana, was enabled to ascertain correctly the constituents of air (See Eudom ETER): he found that, by mixing together oxygen gas and azote in various proportions, he should at last be able.tu determine the absolute quantity of oxygen in air: for since azotic gas, one of the component parts of that Huid, cannot be scparated by any substance with which chemists are acquainted; the analysis of air can only be attempted by exposing it to the action of those bodies which have the property of absorbing its oxygen. By these bodies, the oxygen gas is separated, and the azote left behind, and the proportion of oxygen may be ascertained by the dininution of bulk; which being once known, it is easy to ascertain the proportion of azntic gas, and thus to determine the relative quantity of the component parts of air. Therefore, to find the absolute quantity of oxygen in air, he mixed together oxygen gas and a\%ote in various proportions, and at last lound that a mixture of 5 measures of the purest oxyen which he could procure, with 19 measures of a\%ote, was just as much diminished by nitrous gas, as the same butk of atmospheric air. Hence he concluded that air is composed of 5 parts of oxys(+1) and 19 of azote, of every 2.4 measures of atmospheric air; which gives for its compositon per cent. 79.16 azote, and 20 s.t of axygen; or very nearly 21 per cent. of oxygen gas. The trials mate by others, (1) discover the true quantity of uxjgen in air, liave all ended in the same result as that obtained by $\mathrm{Mr}$. Cavendish, whenever the experiment was made with precision. They all indicate that common air consists very nearly of 21 parts of oxygen, and 79 of azote; and prove that in whatever place or altitude, above the surlace of the earth, the experiment is made, the same proportion of its constituents is always manifested, let the state of the atnosphere be what it may. Gay Lussac examined air brought from an altitude of 6000 feet in a balloon, and found its constituents precisely the same as the air at the earth's surface.

The next ingredient in the composition of the atmosphere, which claims our particular notice, is water; for since it is now fully confirmed that the atmosphere always contains some portion of this fluid, it deserves to be explained in what state this comparatively heavier fluid exists in the air, and the quantity which a given bulk of the atmosphere contains precisely, if possible, made known. With respect to the state in which water exists in air, two opinions have been formed, each of which has been supported by the most eminent philosophers. 1. Water may be dissolved in air in the same manner as a salt is held in solution by water. 2. It may be mixed with air in the state of steam or vapour, after having been converted iuto vapour. The first of these opinions was hinted at by Dr. Hooke, in his Miçographia, and afterwards proposed by Dr. Halley; but it was much more developed by M. Le Roy, of Montpclier, in 1751. Dr. Hamilton of Dublin also made known the same discoveries about the same time. The phenomena in general coincide remarkably well with this theory. The quantity of water which air is capable of holding in solution, is increased by every augmentation of temperature, and diminished by cold, which is precisely analogous to almost all otber solvents. These analogies, and several others which will easily suggest themselves to the reader, have induced by far the greater number of philosophers to adopt this opinion.

The second theory, namely that water exists in a state of vapour, is strongly confirmed by the experiments of Saussure, and the reasoning of Dr. Black: but it is to Mr. Dalton that we are indebted for the most precise infurmation on this subject, and the perfect establishment of its reality. He demonstrated that the water existing in air possesses precisely the same degree of elasticity that it dors when in the state of vapour, in a vacuum, at the same temperature; from which it follows irresistibly that it exists in air not in the state of water, but of an elastic fuid or vapour.

'The tirst who attained any thing like ptecision, in attompting to measure the quantity of water in the atmosphere, was Saussure. This ingenious philosopher has shown in his Hygrometrical Essays, that an English cubic foot of air, when saturated with water, at the tcmperature of $66^{\circ}$, contains only about 8 grains troy of that liquid, or about $\frac{1}{6}$ th ol its weight. But the experiments of Mr. Ditton were susceptible of far greater precision, and reflect much credit on his talents as a philesopher. His results are these: -1 . That the quantity of vapour in the at mosphere is virriable. 2. 'Ihat in the turrid zone its force of clasticity vaties from 0.6 to une inch of mercury. 3. That in Britatu it seldom amounts to $0 .(i$, but is often as great as 0.5 chring summer. 4. 'l'hat in winter it is freruently as low is 0.1 of an inch of mercury. From these facts, the absolute quantity of water containcd in the atmosphere, at any given 
time, might be correctly ascertained, proviled we were certain that the density and elasticity of vapours follow the same law as that of gases, as is extremely likely to be the case. If so, the vapour will vary from $\frac{1}{60}$ th to $\frac{x}{300}$ th part of the atmosphere. Dalton supposes that the medium quantity of vapour held in solution at once in the atmosphere, may amount to about $\frac{1}{70}$ th of its bulk. See Manchester Memoirs, vol. 3.

With regard to the next constituent part of the atmosphere, carbonic acid gas, it will be first proper to remark, that its existence was first determined by Dr. Black, immediately after he had ascertained the nature of that peculiar fluid. The exposure of a pure alkali, or alkaline earth, to the atmosphere, having been observed to be the cause of a gradual conversion of it into a carbonate, by the absorption of carbonic acid gas, rendered unavoidable the inference (as soon as the difference between a pure alkali and its carbonate had been ascertained to depend upon that acid) that carbonic acid gas existed in the atmosphere. This fact has not only been proved for the atmosphere near the surface of the earth, but established for the greatest heights accessible to man. Saussure found it at the top of Mount Blanc, the highest point of the old continent; which is covered with eternal snow, and not exposed to the influence of regetables or animals. Humbolt has more lately ascertrined the existence of this gas in air brought by M. Garnerin, from an aerial excursion into the atmosphere not Iess than 4280 feet above the surface of the earth; which is a fact sufficient to prove that the presence of carbonic acid in air does not depend on its vicinity to the earth.

The difficulty of separating this gas from air, has rendered it no easy matter to determine, with accuracy, the relative quantity of it in a given bulk of air. From the experiments of Humbolt it appears to vary from 005 to 01 . Mr. Dalton's experiments give the quantity much smaller; he concludes that air contains only $\frac{r}{\sqrt{460}}$ th of it bulk of carbonic acid gas. Hence, and from the results of other experiments, it is inferred, that the bulk of carbonic acid in air, does not exceed much the $x_{10}$ th th part of the atmosphere.

From all, which we conclude, that the average proportion of cach of these three distinct elastic fluids, forming chiefly the whole body of the atmosphere, is, -

$$
\begin{aligned}
& 98.9 \text { Air. } \\
& 1.0 \text { Vapour } \\
& 0.1 \text { Carbonic acid. } \\
& \hline 100.0
\end{aligned}
$$

It is indeed true that other bodies, besides the three elastic fluids just mentioned, have been suspected in the atmosphere; as, for instance, hydrogen gas, and carburetted hydrogen; but as no satisfactory accounts concerning their quantitics have yet been ascertained, or that would justify any particular notice of them in this place, we will therefore content ourselves by barely mentioning them, and refer our rearlers for the little information we have been able to collect respecting them, to their proper articles. The electric matter, ind other substances raised from bodies on the earth's surface, by effuvia, exhalations, \&c, are always distinguishable; and, being exposed to the continual action of the sun, give rise to innumerable operations, sublimations, separations, compositions, digestions, fermentations, putrefactions, \&c. Thomson's Chem. vol. 4.

Electricity of the Aтmosphere. The subject of atmospherical electricity has engaged the attention of nany VOL. I. very eminent philosophers; since it is well known that, besides those large quantities of the electrical matter, with which the clouds are generally charged in a thunder storm, the atmosphere is never wholly destitute of the electric fluid. A person electrified negatively may satisfy himself of this by extending his arm in the open air, and presenting a long sharp needle with its point upwards; for the electric matter collected from the remoter air will appear luminous as it converges to the point of the needle. Mr. Canton's balls are likewise an excellent contrivance for the same purpose, and may be made use of, not only for determining the electricity of the atmosphere in general, but the positive or negative quality of it. ' According to this ingenious philosopher, desiccated atmospheric air, when heated, becomes negatively electric; and when cooled, the electricity is of the positive kind, even when the air is not permitted to expand or-contract; and the expansion or contraction of atmospheric air occasions changes in its electrical state.

Beccaria informs us, that the electricity of the atmosphere is always positive during the day, and in dry weather; but always negative when a bright or serene atmosphere succeeds dark and moist weather: that the quantity of it increases after the rising of the sun, and during his progress; and that its augmentation is the more considerable as the moisture of the air diminishes; but it decreases in the evening. In days equally dry, the degree of electricity at noon was proportional to the degree of heat; and in a serene atmosphere with little wind, a considerable quantity of the electrical matter commonly arose after sun-set during the precipitation of the dew. Thick fogs were observed, during their ascent into dry air, to carry with them a considerable quantity of the electric matter. And the electricity was always stronger when his rods were higher, and the strings which were extended and insulated in the open air were longer. See his Essay on Atmospheric Electricity annexed to the English translation of his Artificial Electricity.

Mr. Cavallo, from numerous experiments and observations, infers, that there is in the atmosphere at all times a quantity of electric matter:- - that the electricity of the atmosphere and of fogs is always positive :-that in general the strongest electricity is observable in thick fogs, and also in frosty weather; and the weakest when it is cloudy and warm, and rain approaches; - that it does not seen to be less by night than by day; and that it is stronger in places more elevated, than in those which are lower. See his complete Treatise on Electricity, vol. 2. p. 42. edit. 4.

Among the celebrated men who have engaged in the cultivation of this science, few have cnjoyed the advantages of observing the phenomena attending atmospherical electricity of MI. Saussure, or indecd who have possessed a more extensive acquaintance with meteorology in general, for enabling him to illustrate these phenomena by apposite observations. He confirms the fact, noticed by others, and previously known, that aerial electricity varies according to the situation, being generally strongest in elevated and insulated places, and not observable under trees, in streets, houses, \& c. But it is not so much the height as the situation of the places which determines the degree of electricity: for the projecting angle of a high hill, he says, will often exhlbit a stronger electricity than the plain at the top of the hill, as there are fewer points of the former to deprive the air of its electricity. The intensity of the atmospherical electricity is also subject to a great variety $2 \mathrm{~A}$ 
of changes, of which some depend on obvious circumstances, while otbers are altogether inexplicable; they are sometimes so rapid, says Saussure, as to preclude all possibility of noting them down. When rain falls, without a storm, these changes are not so sudden; but with respect to the intensity of the electric force, they are excessively irregular; wbile the quality of it is more constant. Rain or snow almost al ways gives positive electricity. In cloudy weather, without rains or storms, the electricity generally follows the same laws as in serene weather. Its intensity is most conmonly diminished by strong wipds, which blend the different strata of the atmosphere, cause them to subside towards the ground, and thus distribute the electricity uniformly between the earth and the air. The various modifications of electricity in the atmosphere, are observed with the greatest advantage in serene weather. M. Saussure found in winter, and in such weather, that the electricity was generally weakest in the evening, when the dew had fallen, and so continued till sun-rise; afterwards its intensity augmented by degrees, sometimes sooner and sometimes later; but usually before noon it attained a certain maximum, from which it again declined till the fall of the dew, when it would be sometimes stronger than it had been during the whole day; after which, it would again gradually decrease during the whole night; but it was never quite destroyed in weather perfectly serene. Hence it may be inferred, that atmospherical electricity, like the waters of the ocean, is subject to a fux and reflux; which produce an increase and diminution twice in 24 hours. The same author also remarks, after numerous experiments made on evaporation, that this which appeared to be the vehicle that conveys electric matter into the atmosphere, from silver and china always produced negative electricity; and from iron and copper generally positive electricity: and bence it may be inferred, that electricity is positive with those bodies that are capable of decomposing water, or of being decomposed themselves by their contact with the water; and negative with all those that are not at all decomposed or altered.

As to the producing causes or sources of atmospherical electricity, we may observe in general, that they may be reduced to four, viz, friction, evaporation, heat and cold, and condensation and expansion: and with respect to the changes and modifications to which the atmospherical electricity is continually subject, they may be attributed to the operation of the various causes that produce them, and to the chemical processes that are constantly carried on by means of the various ingredients that compose the atmosphere. For further observations on this subject, consult the authors from whence these particulars were chiefly taken; also Mr. Read's very ingenious work, entitled a Suınmary View of the Spontaneous Electricity of the Earth and Atmosphere. See also the articles $H_{A} \mathrm{~J}_{\mathrm{L}}, \mathrm{RA} \mathrm{IN}_{\mathrm{N}}, \mathrm{S}_{\mathrm{N}}$ ow, LIG ITNING, and those of other meteors.

Figure of the ATMOSPHFRE.-As the atmosphere envelops all parts of the surface of our globe, it is evident, if they botb continued at rest, and were not endowed with a diurnal motion about their common axis, that the atmosphere would be exactly globular, according to the laws of gravity; for all the parts of the surface of a fluid in a stitte of rest, must be equally removed from its ceutre. But as the earth and the ambient parts of the atmosphere revolve uniformly together about theiraxis, the different parts of both have a centrifugal force, the tendency of which is unore considerable, and that of the centripetal less, as the parts are inore remote from the axis; and hence the figure of the atmosphere must become an oblate spheroid; since the parts that correspond to the equator are farther removed from the axis, than the parts which correspond to the poles. Besides, the figure of the atmosphere must, on another account, represent a flattened spheroid, namcly because the sun strikes more directly the air which encompasses the equator, and is comprehended between the two tropics, than that which pertains to the polar regions; whence it follows, that the mass of air, or part of the atmosphere, adjoining to the poles, being less beated, cannot expand so mucl, nor reach so high. Yet, notwithstanding, as the same force which contributes to elevate the air, diminishes its pressure on the surface of the earth, higher columns of it about the equatorial parts, all other circumstances being the same, may not be heavier than those about the poles.

In the Transactions of the Royal Irish Academy for $1788, \mathrm{Mr}$. Kirwan has an ingenious dissertation on the figure, height, weight, \&c, of the atmosphere. He obserres that, in the natural state of the atmosphere, that is, when the barometer would every where, at the level of the seas stand at 30 inches, the weight of the atmosphere, at the surface of the sea, must be equal all over the globe; and in order to produce this equality, as the weight proceeds from its density and height, it must be lowest where the density is greatest, and lighest where the density is least; that is, highest at the equator and lowest at the poles, with several intermediate gradations.

Though the equatorial air however be less dense to a certain height than the polar, yet at,some greater heights it must evidently be more dense: for since an equatorial and polar column are equal in total wejght or mass, the lower part of the equatorial column, being more expanded by heat $\& c$ than that of the polar, must have less mass, and therefore a proportionably greater 'part of its mass must be found in its superior section; so that the lower extremity of the superior section of the equatorial column is more compressed, and consequently denser, than the corresponding part of the polar column. The same thing is to be understood also of the extra-tropical columns with respect to each other, where differences of heat prevail.

Hence, in the highest regions of the atmosphere, the denser equatorial air, not being supported by the collateral extra-tropical columns, gradually flows over, and expands itself towards the north and south.

These superior tides consist chiefly of inflammable air, as it is much lighter than any other, and is genernted iu great plenty between the tropics; it furnishes the matter of the aurora borealis and australis, by whose combustion it is destroyed, otherwise its quantity would in time bccome too great, and the weight of the atmosphere annually increased; but its combustion is the primary source of the greatest perturbations of the atmosphere. See an ingenious work on the Atmosphere by H. Robertson, M. D. in 2 vols. 8vo, 1808 .

Weight or Pressure of the $\Lambda$ Tmospuens.-That the mass of the atmospliere has weight, ant consequently presses upon bodies exposed to its action, is a fact which has been asserted by almost all philosoghers, both ancient and inodern. But it was only by mons of the experiments made with pumps and the barometrical tube, by Galileo and Torricelli, that we have been convinced of its reality, and enabled to ascertain the precise measure and quantity of that pressure. Thus, it is found that the pres- 
sure of the atmosphere sustains a column of quicksilver, in the tube of the barometer, of about 30 inches in height; it therefore follows, that the whole pressure of the atmosphere is equal to the weight of a column of quicksilver, of an equal base, and 30 inches height: and because a cubical inch of quicksilver is found to weigh nearly half a pound avoirdupois, therefore the whole 30 inches, or the weight of the atmosphere on every square inch of surface, is almost equal to 15 pounds. Again, it has been found that the pressure of the atmosphere balances', in the case of pumps \&c, a column of water of about $34 \frac{r}{2}$ feet high ; and, the cubical foot of water weighing just 1000 ounces, or $62 \frac{\mathrm{r}}{2}$ pounds, $34 \frac{\mathrm{r}}{2}$ times $62 \frac{\mathrm{r}}{2}$, or $2158 \mathrm{lb}$, will be the weight of the column of water, or of the atmosphere on a base of one square foot; and consequently the 144th part of this, or $15 \mathrm{lb}$ nearly, is the weight of the atmosphere on a square inch; the same as before. Hence Mr. Cotes computed that the pressure of this ambient fluid on the whole surface of the earth, is equivalent to that of a globe of lead of 60 miles in diameter. And hence also it appears, that the pressure upon the human body must be very considerable; for as cvery square inch of surface sustains a pressure of 15 pounds, every square foot will sustain 14.times as much, or 2160 pounds; then, if the whole surface of a man's body be supposed to contain 15 square feet, which is pretty near the truth, he must sustain 15 times 2160 , or 324.00 pounds, that is nearly $14 \frac{\mathrm{T}}{2}$ tons wcight, for his ordinary load. By this enormous pressure we should undoubtedly be crushed in a moment, if all parts of our bodies were not filled cither with air or some other elastic fluid, the spring of which is just sufficient to counterbalance the weight of the atmosphere. But whatever this fluid may be, it is certain that it is just able to counteract the weight of the atmosphere, and no more: for, if any considerable pressure be superadded to that of the air, as by going into deep water, or the like, it is alway's severely felt, at least when the change is made suddenly; and if, on the other hand, the pressure of the atmosphere be taken off from any part of the human body, as the hand for instance, when put over an open receiver, from which the air is afterwards extracted, the weight of the external atmosphere then prevails, and we imagine the hand strongly sucked down into the glass.

The difference in the weight of the air which our bodies sustain at one time more than another, is also very considerable; this difference in the same situation arises from changes in the state of the atmosphere, and it most frequently takes place in countries at some distance from the equator; and as the barometer varies at times from 28 to 31 inches, or about one tenth of the whole quantity, it follows that this difference amounts to about a ton and a half on the whole body of a man, which he therefore may sustain at one time more than at another. On the increase of this natural weight, the weather is commonly fine, and we feel ourselves in better spirits and more alert and active; but, on the contrary, when the weight of the air diminishes, the weather is generally moist and foggy, and people frel a listlessness and inactivity about them. And hence it is no wonder that persons suffer very much in their health from such changes in the atmosphere, especially when they take place very suddenly, for it is to this circumstance chiefly that a sensation of uneasiness and indisposition is to be attributed; thus, when the variations of the barometer and atmosphere are sudden and great, we fecl the alteration and its effect on our bodies and spi- rits very much; but when a clange of this kind is gradual, we are scarcety sensible of it, owing, undoubtedly, to the power with which the body is naturally endowed, of accommodating itself to this alteration in the state of the air, as well as to the change of many other circumstances of life, the body requiring a certain interval of time to effect the alteration in its state, proper to that of the air \& $c$. Thus, in going up to the tops of mountains, where the pressure of the atmosphere is diminished two or three times inore than on the plain below, little or no inconvenience is felt from the rarity of the air, if it be not mixed with other noxious vapours \& $\mathrm{c}$; because that, in the as. cent the body has had sufficient time to accommodate itself gradually to the slow variation in the state of the atmosphere: but, when a person ascends with a balloon very rapidly to a great height in the atmosphere, he feels a difficulty in breathing and an uneasiness of body; and the same is also true of an animal when inclosed in a receiver, and the air suddenly drawn or pumped out of it. So likewise, on the condensation of the air, we feel little or no change in ourselves, except when the alteration occurs suddenly, as in very rapid changes in the weather, and in descending to great depths in a diving-bell, \&c. I have often heard the late unfortunate Mr. Spalding speak of his experience on this point: he always found it absolutely necessary to descend with the belt very slowly, and that only from one depth to another, resting a while at each depth before he began to descend farther: he first descended slowly for about 5 or 6 fathom, and then stopped a while; he felt an uneasiness in his head and ears, which increased more and more as be descended, till he was obliged to stop at the deptb above mentioned, where the density of the air was nearly doubled; having remained there a while, he felt his ears give a sudden crack, and after that he was soon relieved from any uneasiness in that part, and it seemed as if the density of the air was not altered. He then descended 30 fect more, with the same precaution and similar sensations, being again relieved in the same nannier, after remaining a while stationary at the next stage of his descent, where the density of the air was tripled. And thus he continued proceeding to a great depth $_{2}$ always with the same circumstances, repeated at every 5 or 6 fathoms, and adding the pressure of one more atmosphere at every period of the progress.

It is not easy to assign the true reason for the variations that happen in the gravity of the atmosphere in the same place. In places within the tropics, where these variations are not very considerable, the chief cause seems to be the lieat of the sun, as the barometcr constantly sinks about half an inch every day, and rises to its former station again in the night. But in the temperate zones the barometer ranges from 28 to near 31 inches, showing, by its various altitudes, the changes that are about to take place in the weather. If we could know, therefore, the causes by which the weather is influenced; we should also know those by which the gravity of the atmosphere is affected. These may perhaps be reduced to inmediate oncs, viz, an emission of latent heat from the vapour contained in the atmosphere, or of electric fluid from the same, or from the earth; as it is observed that they both produce the same effect with the solar beat in the tropical climates, viz, to rarefy the air, by mixing with it, or setting loose a lighter fluid, which did not betore act in such large proportion in any particular place.

Temperature of the Ara OSPI E RE.-To no part of natural $2 \mathrm{~A} Q$ 
and experimental philosophy, has the attention of learned men been directed with greater assiduity, than that which resnects the various states of the atmosphere in regard to temperature: many reasons have been adduced, as well as theories proposed, to account fur these plienomena, and numerous experiments made to ascertain the precise degrees of variation, at different seasons, and in different situations, both at sea and land. Among the writers who have rendered themselves conspicuous in these investigations, are Dr. Hales, M. Bouguer, Mariotte, Halley, De Luc, Saussure, Mairan, Raymond, Playfair, Kirwan, and Darwin. They all agree, that the principal alterationsin the state of the atmosphere are effected by means of the sun's heat, and that arising from the condensation of vapour: it was from the former fact that the ancient philosophers of Greece and Rome concluded (though too abruptly) that the torrid zone under a vertical sun, and the frigid zone where its rays fall very obliquely, were uninhabitable: time corrected this mistake, and presented new plienomena, which it has been found difficult to explain. The hottest days are frequently felt in the coldest climates; and the greatest cold, as well as perpetual snow, are found in countries bordering on, or even immediately under, the equator. Dr. Halley has indeed proved, that abstracting from the intervention of fogs, mists, and mountains of ice, the hottest weather might in summer take place even under the poles; the duration of the sun's light more than compensating for the obliquity of its direction, (see HЕAT); but as many physical causes obstruct the activity of the solar rays in these and other regions, it was still necessary to recur to some other cause. At length M. de Mairan (Mem. Acad. Paris, 1719 and 1767) discovered, that the rigotir or intensity of the cold in winter, is tempered by the heat imparted to the atmosphere by the earth itself; which heat, probably possessed from its origin, is preserved and renewed by the iucessant influences of the sun, to which one half of its surface is constantly exposed: hence it must follow, that the temperature of the atmosphere depends on the capacity of the earth to receive and retain heat, and for communicating it to the surrounding medium. But as the enrth is composed of land and water, it should be considered that the capacities of these constituent parts, for receiving both heat and cold, are very different. Land, particularly when dry, receives heat from the sun's rays very readily, but transmits it through its own substance to great depths very slowly; and on the other hand water, by reason of its transparency, receives heat very slowly, but diffuses what it receives more readily. The experiments of Dr. Hales, and those of other plilosophers, to ascertain the degrees of heat of the earth at different deptlss, and at various times of the year, appear to confirm the idea that the surface of the carth is much beated during the summer, but that the heat descends very slowly, a grcat part of it being communicated to the air; and that during the winter, the earth gives out to the air the heat which it had recoived during the sumyner; also that wet summers must be succeded by cold winters. Mariotte, also obtained nearly the same results: he found that the carth is gradually heated during the summer, and as gradually cooled during the winter months; and that at the distance of a few fert under the surface, it is constantly warmer than the external air; and the excess was found to remain till April, when the surface is again heated by the sun's rays, and slowly transmits its heat downwards. Hence it apleat's, that at the distance of about 80 or 90 feet below the surface, provided there be a communication with the external air, or at a less depth if there be no such communication, the temperature of the earth admits of very slight variation, and generally approaches to the mean annual heat. From which it has been inferred, that the temperature of spring is nearly the same as the annual temperature, and varies very little. 'These facts tend to prove very satisfactorily, that the heat of the earth does not increase as we descend below its surface, but at the greatest depths it is nearly the same as the mean annual temperature of the latitude. We have observed, that land is capable of receiving heat and cold much more readily than water. To which purpose Dr. Raymond found, in the neighbourhood of Marseilles, land frequently heated to $160^{\circ}$; but he never observed that the sea was hotter than $77^{\circ}$; and in winter he frequently observed the earth cooled down to $14^{\circ}$ or $15^{\circ}$, but the sea never lower than $44^{\circ}$ or $45^{\circ}$ : Mem. de la Societ. de Med. de Paris, an. 1778, p.70. From these facts therefore, it must follow, that the atmosphere which lies over the sea should maintain a more uniform tcmperature, than that over the land; and this is found to be the fact; nor is it difficult to give a satisfactory explication of it. During summer, the temperature of the sea on its surface is constantly diminished by the process of evaporation; and in the winter, when the superficial water is cooled, it descends by its augmented gravity to the bottom, and its place is occupied by water of a higher temperature. Thus alternate changes of heavier and lighter air proceed, and the winter elapses before the atmosphere has chiminished the temperature of the water below a certain degree. Betwcen the mean annual temperature of the atmosphere over the ocean, and that of countries situated ut a considerable distance from it, there is a very perceptible dificrence. As the sea is never heated to the same degree as the land, the mean temperature of summer over the sea may be considered as lower than that over the land. In winter, when the force of the sun's rays is weakened, the sca imparts its beat to the atmosphere much more readily than the carth; therefore the mean temperature on sea, at this season, is higher than on land; and in cold countric's, this difierence in the evolution of heat is so very considerable, that it more than counterbalances the difference which talics place in summer; insomuch that, in high latitudes, the mean annual temperature at sea ought to execed that on land.

In latitudes distant from the equator, islands a re warmer than continents, because they participate more of the tomperature of the sca. Countries that lie southward of any sea, are warmer than those that have the sea to the south of them, at least in cur hemisphere, because the winds that should cool them in winter are tempered by passing. to them from that sea; and those that are northward of the sea, are cooled in summer by the breezes that issue from it; but a northern or southern bearing of the sea renders. a country warmer than if it layeither to the east or west. Tracts of land which ape covered with ures and luxuriant regetables, are much colder than those which have less surface of regetable matter; for though living vegetabl's alter their temyerature slowly and with difliculty, yet the evaporution from their numerous surfaces is much greatcr than from the same space of lamil uncovered with vegetables; aud besides, when they are tall and close, as. forests, they cxclude the sun's rays, and shelier the winter suows from the wind and stm. From some cxperiments of $\mathrm{Mr}$. 
Williauns (Philad. Trans, vol. 2, p.150), it appears that forests discharge one third more vapour into the atmospliere, than thesame space of ground would do if actually covered with water. From this reasoning, it seems that woody countries are much colder than those that are open and cultivated. With regard to the leat afiorded from the condensation of vapour, we need not expatiate very considerably, since it is well known that vapour contains a great quantity of sucl matter. This heat produces no other effect but that of making it assume an aerial expanded form, until the vapour is condensed into a liquid; and during this condensation, a quantity of sensible heat is let loose, which warms the surrounding atmosphere. This condensation is frequently occasioned by the attraction of an electrical cloud; and hence proceeds the sultriness which we often experience before rain.

The following is a view of the annual temperature of different places according to the order of their latitudes.

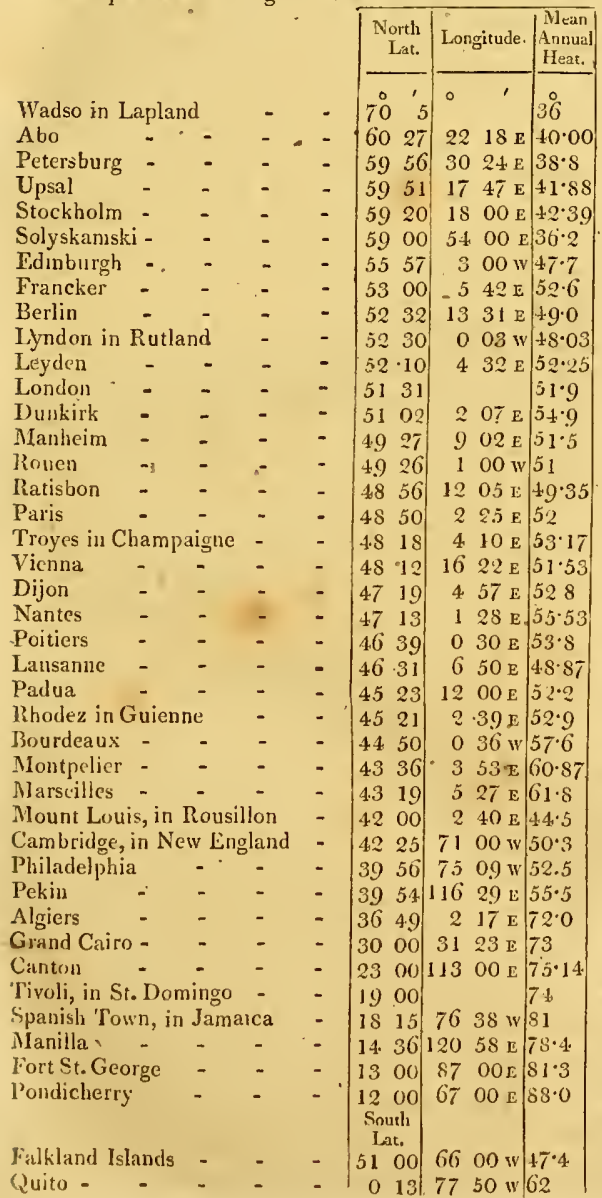

As to the effect of elevation on the temperature of the atmosphere, we may observe, that as heat is propagaterl through the atmosplaere chiefly by communication and contact with the earth, lofty moinntains of limited surface cannot warm it to any considerable degrec, as they receive the sun's rays more ubliquely, and communicating less with the common mass of the earth, are less heated than plains. Hence it happens that the steejest mountains are always the coldest. Before M. Bouguer demonstrated and laid open the true causes of the diminution of heat on the tops of mountains, many hypotheses were assumed to account for this phenomenon; but this cclebrated philosopher has shown, that there is no necessity for recurring to any dubious suppositions for an explication of the fact, as will evidently appear from his account of what was experienced on the mountains of Peru. He says, "It was proper, in order to explain this subject, to consider the short duration of the sun's rays, which cannot strike the different sides of mountains but for a' few hours, and even this not always. A horizontal plain, when the sun is clear, is exposed at mid-day to the perpendicular and undiminished action of these rays, while they fall but obliquely on a plain uot much inclined, or on the sides of a high pile of steep rocks. But let us conceive for a moinent an insulated point, half the lieight of the atmosphere, at a distance from all mountains, as wel] as froni the clouds which float in the air. The more a medium is tramsparent, the less heat it ought to receive by the immediate action of the sun. The free passage which a very transparent body allow's to the rays of light, shows that its small particles are hardly touched by them. Indeed, what impression could they make on it when they pass through almost without obstruction? Light, when it consists of parallel rays, does not, by passing through a foot of free atmospheric air near the earth, lose an hundred-thousandtl part of its force. From this we may judge how few rays are weakenced, or can act upon this fluid, in their pussage through a stratum of the diameter, not of an inch or line, but of a particle; yct the subtilty or transparency are still greater at greater heights, as was obvious in the Cordilleras when. we looked at distant objects. Lastly, the grosser air is heated below by the contact or neighbourhood of bodies of greater density than itself, which it surrounds and on which it rests; and the heat may be communicated by small degrces to a certain distance. The inferior part of the atmosphere by this means contracts daily a very considerable degree of heat, and may reccive it in proportion to its. density or bulk; but it is evident that she same thing cassnot happen at the distance of a league and a half or two leagues above the surface of the earth, though the light may be there soncthing more actire. 'The air and wind, therefore, it this height, must be extremely cold, and colder in proportion to its elevation."

Saussure adopts this theory, and adsls the following fact, to prove that the force of the sun's rays, considered abstractedly and independently of any extrinsic source of cold, is no less powerful on mountains than on plains, viz, that the power of burning lenses and mirrors is the same at all leights. For ascertaining this fact, he procured a burning glass so wrak in its iffect, that at Geneva it would just set fire to tinder. This glass svas carried to the summit of Mount Saleve, 3000 fiet high, and it there produced the same effect, and with greater ease. Hence he concluded that the principal source of cold on the tops of mountains is their bcing yerpetwally surrounded by an 

A $\mathrm{T} M$
[ 182
A $\mathrm{T}$ M

atmosphere which cannut be much heated by the rays of the sun, on account of its transparency, or by their reflection from the earth, by reason of its distance; but he wished also to know whether the direct solar rays had the same power on the top of a high mountain as on the plain below, while the body on which they acted was placed in such a manner as to be unaffected by the surrounding air. With this view be instituted a set of experiments, from which he deduced the following conclusions, viz, that a difference of 777 toises in lieight, diminishes the heat which the rays of the sun are able to communicate to a body exposed to the external air, $14^{\circ}$ of the thermometer; that it diminishes the heat of a body partially exposed only $6^{\circ}$; and that it augments by $1^{0}$ the heat of a third body completely defended from the air. Hence it appears, that the atmosphere counteracts the operations of the solar rays in producing heat, by a power which is excrted at all distances, from the surface to the higher regions.

Dr. Darvin, from his experiments and observations, infers, that there is good reason for concluding that in all circumstances where air is mechanically expanded, it becomes capable of attracting the fluid matter of heat from other bodies in contact with it. Now, as the vast region of air which surrounds our globe is perpetually moving along its surface, rising up the sides of mountains, and descending jito the valleys; as it passes along, it must be perpetually varying the degree of heat, according to the elevation of the country it traverses: for, in rising to the summits of mountains, it becomes expanded, having so much of the pressure of the superincumbent atmosphere taken away; and when thus expanded, it attracts or absorbs heat from the mountains in contiguity with it; and, when it descends into the valleys, and is compressed into less compass, it again gives out the heat it has acquired to the bodies it comes in contact with. The same thing must happen to the superior regions of the atmospluere, which are regions of perpetual frost, as has lately been evinced by the aerial navigators. When large districts of air, fiom the lower parts of the atmosphere, are raised two or three niles high, they become so much expanded by the great diminution of the pressure over them, and thence become so cold, that hail or snow is produced by the precipitation of the vapour: and as there is, in these high regions of the atmosphere, nothing else for the expanded air to acquire heat from after it luas parted with its vapour, the same degree of cold continues, till the air, on descending to the earth, acquires its former. state of condensation and of warmiti. The Aniles, almost under the line, rests its base on burning sands: about its middle beight is a most pleasant and temperate climate covering an extensive plain, on which is built the city of Quito; while its forehead is encircled with eternal smow, perhaps coeval with the mountain.. Yet, according to the accounts of Don Ulloa, these three discordant climates seldom encroach much on each other's territories. The hot winds below, if they ascend, become cooled by their expansion; and hence they cannot affect the snow upon the summit; and the cold winds that sweep the summit become condeused as they descend, and of a moderate temperature before they reach the fertile plains of Quito.

Many other experiments to this purpose were also made by M. Pictet of G(nc'vi, Dl: Wilson of Glasgow, and Mr. James Six; but as out limits will not permit us to give observations of this kind in detail, we beg to reler our readers to the works containing them; viz, the 76 th vol. of the Phil. Trans, for Mr. James Six's, and the same 'Trans. for 1780 and 1781 , for Dr. Wilson's: also see Rees's new edit: of his Cyclopedia, art. Atmosphere.

Height and Density of the Atmospnere. Various attempts have been made to ascertain the height to which the atmosphere extends above the earth. These commenced soon after it was discovered, by mears of the Torricellian tube, that air is endued with weight and pressure. And had not the air an elastic power, but were every where of the same density, frum the surface of the earth to the extreme limit of the atmosphere, like water, which is equally dense at all depths, it would be a very easy matter to determine its beight from its density and the column of mercury which it would counterbalance in the barometrical tube: for, it having been observed that the weight of the atmosphiere is equivalent to a column of about 30 inches or $2 \frac{1}{2}$ feet of quicksilier, and the density of the former to that of the latter, ncarly as 1 to 11040 ; therefore the height of the uniform atmosphere would be 11040 times $2 \frac{\pi}{2}$ feet, that is 27600 feet, or little more than 5 miles and a quarter. But the air, by its elastic quality, expands and contracts; and it being found by repeated experiments in most nations of Europe, that the spaces it occupies, when compressed by different weights, are reciprocally proportional to those weights; or, that the more air is pressed, so much the less space it takes up; it follows that the air in the upper regions of the atmosphere must become confinually more and more rare, as the height of it increases, and thus, according to that law, must necessarily be extended to an indefinite height. Now, if we suppose the height of the whole to be divided into innumerable equal parts, the quantity of each part will be as its density; and the weight of the whole incumbent atmosplere being alsb as its density; it follows, that the wejght of the incumbent air is every where as the quantity contained in the subjacent part; which causes a-difference between the weights of each two contiguous strata of air. But, by a theorem in the doctrine of proportion, when a magnitude is continually diminished by the like part of itself, and the remainders the same, they will express a series of continued quantities decreasing in geometrical progression : therefore if, according to the supposition, the altitude of the air, by the addition of new parts into which it is divided, do continually increase in arithmetical progression, its density will be dininished, or, which is the same thing, its gravity will be decreased, in continued geometrical progression. And hence, again, it appears that, according to the hypothesis of the density being always proportional to the compressing force, the height of the atmosphere must necessarily be extersded indefinitely. And, farther, as an arithmetical series adapted to a geometrical one, is analogous to the logarithms of the said geometrical one; it follows iherefore that the altitudes are proportional to the logarithms of the densities or weights of air; and that any height taken from the earth's surface, which is the difference of two altitudes to the top of the atmosphere, is proportional to the difference of the logarithms of the two delisities there, or to the logarithm of the ratio of those densities, or their corresponding compressing forces, as measured by the two heights of the barometer there. 'Ihis law was first observed and demonstrated by Dr. Halley, from the sature of the byperbola; and afterwards by Dr. James Gregory, by means of the logarithmic curve. Sec Philos. 'I'rans. $N^{*} 181$, or my Abrilg. vol. 3, p. 300 , and Greg. Astron. lib. v, prop. 3. 
It is now easy, fiom the foregoing property, and two or three experiments, or barometrical observations, made at known altitudes, to deduce a general rule to determine the absolute height answering to any density, or the density answering to any given altitude above the earth. And accordinaly, calculations have been made upon this plan by many philosophers, particularly by the Freneh; but it having been found that the barometrical observations did not correspond with the altitudes as measured geometrically, it was suspeeted that the upper parts of the atmospherical regions were not subject to the same laws with the lower ones, in regard to the density and elasticity. And indeed, when it is considered that the atmosphere is a heterogeneous mass of particles of all sorts of matter, some elastic, and others not, it is not improbable but this may be the case, at least in the reginns very high in the atmosphere, which it is likely may more copiously abound with the electrical fluid. Be this however as it may, it has lately been discovered that the law above given, holds very well for all such altitudes as are within our reach, or as far as to the tops of the highest nountains on the earth, when a eorrection is made for the difference of the heat or temperature of the air only; as was fully evinced by M. de Luc, in a long series of observations, in which he deternined the altitudes of hills, both by the barometer and by geometrical measurement, from which he deduced a practical rule for determining the allowance to be made for the difference of temperature. See lis Treatise on the Modifications of the Atmosphere. Similar rules have also been deduced from aecurate experiments, by Sir George Slivekburgh and Gcneral Roy, both coneurring to show, that such a rule for the altitudes and densities holds true for all heights that are accessible to us, when the elasticity of the air is corrected on account of its density: and the result of their experiments showed that the difference of the lugarithms of the heights of the mercury in the barometer, at two stations, when multiplied by 10000 , is equal to the altitude in English fathoms, of the one place above the other; that is, when the temperature of the air is about 31 or 32 degrees of Fahrenheit's thermometer; and a eertain quantity more or less, aecording as the actual temperature is different froin that degree.

But it may here be shown that the same rule ean be deduced independent of such a train of experiments as those above, and that from a single observation of the density of the air at the surface of the earth alone. Thus, let D denote the density of the air at one place, and $d$ the density at the other; both measured by the column of mercury in the barometrical tube: then the difference of altitude between the two places, will be proportional to the log. of $\mathrm{D}-$ the $\log$. of $d$, or to the $\log$. of $\frac{\mathrm{D}}{d}$. But as this formula expresses only the relation between different altitudes, and not the absolute quantity of them, assume some indeterminate but constant quantity $h$, which multiplied into the expression $\log . \frac{D}{d}$, may be equal to the real differenee of altitude $a$, that is $a=h \times \log$. of $\frac{\mathrm{D}}{d}$. Then, to determine the value of the general quantity $h$, let us take a case in which we know the altitude $a$ which eorresponds to a known density $d$; as for instanee, $a=1$ foot, or 1 ineh, or some such small altitude: then because the density $\mathrm{D}$ may be measured by the pressure of the whole atmosphere, or the uniform column of 27600 fect, when the temperature is $55^{\circ}$; therefore 27600 feet will denote the density $\mathrm{D}$ at the lower place, and 27599 the less density $d$ at 1 toot above it; consequently $1=\bar{h} \times \log$. of $\frac{27600}{27599}$, which is nearly $=h \times \frac{43429448}{27600}$ or $\frac{1}{63551}$ by the nature of logarithms; and hence we find $h=63551$ feet; which gives us this formula for any altitude $a$ in general, viz, $a=63551 \times \log$. of $\frac{1}{d}$, or $\bar{a}=63551 \times \log$. of $\frac{\mathrm{m}}{\mathrm{m}}$ feet, or $10592 \times \log$. of $\frac{\mathrm{MI}}{\mathrm{m}} \mathrm{fa}-$ thoms; where $\mathrm{M}$ denotes the column of mercury in the tube at the lower place, and $m$ that at the upper. This formula is adapted to the mean temperazure of the air $55^{\circ}$ : but it has been found, by the experiments of Sir Geo. Shuckburgh and General Roy, that for every degree of the thermometer, different from $55^{\circ}$, the altitude $a$ uill vary by its 435 th part ; henee, if we would ebange the factor $h$ from 10592 to 10000 , beeause the difference 592 is nearly the $18 \mathrm{th}$ part of the whole factor 10592 , or sufficiently so for all practical purposes, and 18 the 24 th part of 435 ; therefore the change of temperature, answering to the change of the factor $h$, is $24^{\circ}$, which reduces the $55^{\circ}$ to $31^{0}$. So that, $a=10000 \times \log$. of $\frac{M}{m}$ fathoms, is the easiest expression for the altitude, and answers to the temperature of $31^{\circ}$, or very nearly the freezing point: and for every degree above that, the result must be increased by so inany times its 435 tl part, and diminished when below it.

From this theorem it follows, that, at the height of $3 \frac{\mathrm{r}}{2}$ miles, the density of the atmosphere is nearly 2 times rarer than it is at the surface of the earth; at the beight of 7 miles, 4 times rarer; and so on, according to the following table:

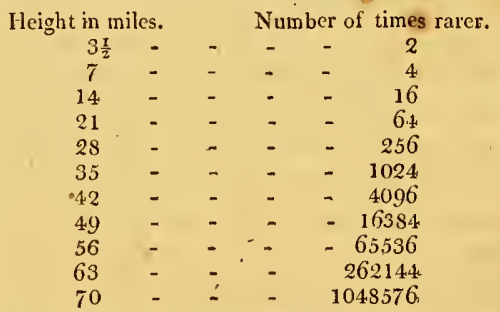

And, by pursuing the calculations in this table; it might be easily shown, that a eubic inch of the air we breathe would be so much rarefied at the height of 500 miles, that it would fill a sphere equal in diameter to the orbit of 'sáturn.

Henee we may perceive how very soon the air becomes so extremely rare and light, as to be utterly impcreeptible to all experience; and that hence, if all the planets have such atmospheres as our earth, they will, at the distances of the planets from one another, be soextremely attenuated, as to give no sensible resistanee to the planets in their motion round the sun, for many, perhaps liundreds or thousands of ages to come. Even at the lieight of 50 miles, it is so rare as to have no sensible effeet on the rays of light: for it was found by Kepler, and Lahire after him, who computed the height of the sensible atmosphere from the duration of 'twilight, and from the magnitude of the terrestrial shadow in lunar eclipses, that the effect of the atmosphere to reflect and intercept the light of the sun, is 
only sensible to the altitude of between 40 and 50 miles: and at that altitude we may collect, from what bas been already said, that the air is above 10000 times rarer than at the surface of the earth. It is well known that the twilight begins and ends when the centre of the sun is about 18 degrees below the horizon, or only $17^{0} 27^{\prime}$, by súbtracting $33^{\prime}$ for refraction, which raises the sun so much higher than he would be. And a ray coming from the sun in that position, and entering the eartb's atmosphere, is refracted and bent into a curve line in passing through it to the eye. M.Lahire took great pains to demonstrate, that, supposing the density of the atmosphere proportional to its weight, this curve is a cycloid; he also says, that if the ray be a tangent to the atmosphere, at the point of impact, the diameter of its generating circle will be the height of the atmosphere; and that this diameter increases, till at'last, when the rays are perpendicular, it becomes infinite, or the circle degenerates into a right line, This reasoning supposes that the refracting surface of the atmosphere is a plane; but since it, is in reality a curve, he observes that these cycloids become in fact epicycloids. Herman however detected the crror of M. Lahire, and showed that this curve is infinitely extended, and lias an asymptote. And it is observed by Dr. Brook Taylor, in his Method. Increm. pa. $168, \& c$, that this curve is one of the most intricate and perplexed that can well be proposed. The same ingenious author also computes, that the refractive power of the air is to the force of gravity at the surface of the earth, as 320 millions to 1 .

The extreme rarity of the atmosphere at considerable distances from the earth's surface, such as those of 40 or 50 miles, bounding the production of twilight, bas much perplexed philosophers in accounting for meteors which, whatever be their origin, whether electrical or otherwise, are observed at a much greater elevation than that to which the refractive power of the atmosphere extends. A very rcmarkable one of this kind was observed by Dr. Halley in the month of March 1719 , the altitude of which he computed at between 69 and $73 \frac{1}{2}$ English miles; its diameter 2800 yards, or more than a mile and a half; and its velocity about 350 miles in a minute. Others, apparently of the same kind, but whose altitude and velocity were still greater, have been observed; particularly that very remarkable one, of August 18 th $17 \mathrm{~S} 3$, whose distance from the earth could not be less than 90 miles, and its diameter at least as large as the former, while its velocity was certainly not less than 1000 miles in a minute. Now, from analogy of reasoning, it scems very probable, that the metcors which appear at such great leights in the air, are not essentially different from those which are seen on or near the surface of the earth. The difficulty with regard to the former is, that at the great heights above-mentioned, the atmospliere ought not, according to computation, to have any density sufficient to support flame, or to propagate sound; and yet such metcors are commonly succeeded by one explosion or more, and it is said are evert sometimes accompanied with a lissing noise as they pass over our heads. The metcor of 1719 was not only very bright, sceming for a short time to turn night into day, but was attended with an explosion that was heard over the whole island of Britain, causing a violent concussion in the atmosphere, and scciming toshake the earth itself: and yet, in the regions in which this meteor moved, the air ought to have bren 300 thousand times rarer than the air we breathe, or 1000 timcs rarer than the vacuum commonly made by a good air-pump. Dr. IIalley offers a conjecture, indeed, that the vast magnitude of such bodies might compensate for the thinness of the medium in which they moved. But appearances of this kind are, by otbers, attributed to electricity; though the circumstances of them cannot well be reconciled to that causc; for the meteors move with various legrees of velocity; and though the electrical fire easily pervades the vacuum of an airpump, yet it does not in that case appear in bright welldefined sparks, as in the open air, but rather in long streams resembling the aurora borealis; and from some late experiments il has been concluded that the electric fluid cannot even penetrate, or be apparent in a perfect vacuum.

Of the Refractive and Reflective Power of the AтmoSPHERE. It has been observed above, that the atmosphere has a refractive power, by which the rays of light are bent from the right-lined direction, as in the case of the twilight; and many other experiments manifest the same virtue, which is the cause of various phenomena. Alhazen, the A rabian, who lived about the year 1100 , seems to have been more inquisitive into the nat ure of refraction than former writers. But neither Alhazen, nor his follower Vitellio were acquainted with its just quantity, nor was it known to any tolcrable degree of exactness, before 'Tycho Brahé, with his accustomed diligence, settled this point. This cclebrated philosopher however did not discover, nor indeed did Kepler, in what manner the rays of light were refracted by the atmosphere. Tycho thought the refraction was chiefly caused by dense vapours, very near the earth's surface: while Kepler placed the cause wholly at the top of the atmosphere, which he thought was uniformly dense; and thence he determined its altitude to be little more than that of the highest mountains. But the true constitution of the density of the atmosphere, deduced afterwards from the Torricellian experiment, afforded a juster idea of these refractions, especially after it appeared, by a repetition of $\mathrm{Mr}$. Lowthorp's experiment, that the refractive power of the air is proportional to its deusity. By this variation in the density and refractive power of the air, a ray of light, in passing through the atmosphere, is continually refracted at every point, and thereby made to describe a curve, and not a straight line, as it would have done were there no atmosphere, or were its density uniform.

The atmosphere has also a reflective power; ' and this power is the mcans by which objects are enlightened so uniformly on all sides. The want of this power would occasion a strange alteration in the appearance of things; the shadows of which would be so very dark, and their sides enlightened by the sun so very bright, that probably we could see no more of them than their bright halyes; so that for a view of the other halves, we must turn them half round; or if immoveable, must wait till the sun could come round upon them. Such a pellucid unreflective atmosphere would indeed have been very commodious for astronomical observations on the course of the sun and planets among the fixed stars, visible by day as well as by night; but then such a sudden transition trom darkncss to light, and from light to darkness, immediately on the rising and setting of the sun, without any twilight, and everr on turning to or from the sun at noon day, would have been very inconvenient and offensive to our eyes. Ilowever, though the atmosplere be greatly assistant in the illumination of objects, yct it must also be observed that it stops a great deal of light. By M. Bouguer's 'x- 
periments, it seems that the light of the moon is often 2000 times weaker in the horizon, than at the altitude of $66 \mathrm{de}-$ grees; and that the proportion of her light at the altitudes of 66 and 19 degrees, is about 3 to 2 ; and the lights of the sun must bear the same proportion to each other at those heights; which Bouguer made choice of, as being the meridian heights of the sun, at the summer and winter solstices, in the latitude of Croisic in France. Smith's Optics, Rem. 95.-For the atmosphere of the sun, noon, and planets, see the respective articles.

A twospli Er E of Solid or Consistent Bodies, is a kind of sphere formed by the effluvia, or minute corpuscles, cmitted from them. Mr. Boyle endeavours to show, that all bodics, even the hardest and most coherent, as gems, \&c, have their atmospheres.

АтмOSPHERE, in Elcctricity, denotes that medium which is conceived to be diffused over the surface, as is generally supposed, of elcctrified bodies, and to some distance a round them, and consisting of effluvia issuing from them; by whicb, other bodies immerged in it become cndued with an electricity contrary to that of the body to which the atmosphere belongs. This was first noticed at a very carly period in the history of this science by Otto Guericke, and afterwards by the academicians del Cimento, who contrived to render the electric atmosphere visible, by means of smoke attracted by and uniting itself with a piece of -amber, and gently rising from it, but vanishing as the amber cooled. Dr. Franklin exhibited this clectric atmosphere with greater advantage, by dropping rosin on hot iron plates held under electrificd bodies, from which the smoke arose and encompassed the bodies, giving them a very beautiful appearance. But the theory of electric atmospheres was not well explained and understood for a considerable time; and the investigation led to many curious experiments and observations. The cxpcriments of Mr. Canton and Dr. Franklin prepared the way for the conclusion that was afterwards drawn from them by Mess. Wilcke and Epinus; though they retained the common opinion of electric atmospheres, and endeavoured to explain the phenomena by that means. The conclusion was, that the electric fluid, when there is a redundancy of it in any body, repels the clectric fluid in any other body, when they are brought within the sphere of each other's infuence, and drives it into the remote parts of the body, or quite out of it, if there be any outlet for that purpose.

By atmosphere, Epinus says, no more is to be understuod than the sphere of action belonging to any body, or the ncighbouring air electrified by it. Sig. Beccaria unites in the same opinion, that clectrified bodies have no other atmosphere than the electricity communicated to the neigh. bouring air, and which goes with the air, and not with the electrified bodics. AIr. Canton also, having relinquished the opinion that electrical atroospheres were composed of c|fluvia from excited or clectrified bodies, maintained that they only result from an alteration in the state of the electric fluid contained in it, or belonging to the air surrounding these bodies to a certain distance; for instance, that excited glass repels the electric fluid from it, and consequently beyorid that distance makes it more dense; whereas excited wax attracts the electric fluid existing in the air nearer to it, making it rarer than it was before. In the course of experiments that were performed on this occasion, Mess. Wilcke and Epinus succeeded in charging a Ilate of air, by suspending large boards of wood covered with tin, with the that sides parallel to one another, and at

VuL. 1. some inclies asunder: for they found, on electrifying one of the boards positively, that the other was always negative; and a sbock was produced by forming a communication betwcen the upper and lower plates. Beccaria has largely considered the subject of electric atmospheres, in his Aitificial Electricity, p. 179 \&c, Eng. edit. Sce also Dr. Priestley's Hist. of Electricity, vol. ii. scct. 5 ; and Cavallo's Electricity, p.241.

Atmospliere, Mragnetic, \&c, is understood of the spherc within which the virtue of the magnet, \&c, acts.

Atmospheric Stones. See Aeroliths.

Atmospheric Tides, certain periodical changes of the atmosphere, similar, in some respects, to those of the ocean, and produced, in a great measure, by the same causes.

There are twokinds of motion in the atmosphere which come under this denomination; the first is occasioned by the joint influence of the sun and moon on the, body of air with which we are surrounded, in the same manner as they act upon the mass of water, and cause the flux and reflux of the ocean; the second is produced by the heat of the sun alone, which exerting upon the air its well known power of rarefaction and expansion, gires rise to those perpetual clanges in the atmósphere, which follow him in his course, though such changes are not altingether observable or appreciable by us. The former of these are termed by the Abbé Mann attraction tides, and the latter heat tides. The tides of attraction, like those of the ocean, and from the like grounds, have at the.same time; at two opposite ends of the globe, projecting parts, and these lie almost in that line which iniglit be drawn from the centre of the earth to that of the moon. The heat tides, on the other hand, can take place only on one point of the globo, that is, in the point to which the sun is vertical. Their projecting parts will be dirceted towards that luminary, and nearly follow its movements.

Bacon, and the most eminent philosophers who have since written on the wind, unanimously observe, that the periods of the year most exposed to it are the two equinuxes; that storms are most frequent at the times of new and full moon, and particularly those which happen near the equinoxes; that at periods otherwise calm, a small breeze alway's take's place at high water; and that a small movement in the atmosphere is each time perceived a little after noon and midnight.

For the investigation of this subject, the abbe lays down the following principles. 1. The elasticity of fluids is in the inverse ratio of their density. 2. The force or elasticity of the air expands and contracts itself in the direct ratio of the wcigbt with which it is loaded, and diffuses itself in the inverse ratio of the force with which it is compressed. 3. The air is rarefied, or diffuses itself, in the direct ratio of the quantity of heat which acts upon it. 4. The air, as well as all fluids in general, has a tendency to put itself in equilibrium, and does not rest till it has obtained it.

Our limits preclude the possibility of following tho abbé in the application of thesc principles to the various phenomena of the subject in question; we therefore sccomnend to the attention of our readers the whole of his observations on the flux and reflux of the atmosphere, inscrted from the Brussels Trans. in Tillocli's Phil. Mlag. vol. 5 . In the 7 th vol. of that work is a paper Ots a periodical Variation of the Barometer, \&c, by lụke Howard, escp. to which we also refer; and conclude by usticing the ubservations made by llumbolt near the equatur: "I have $2 \mathrm{~B}$ 
read," says he, "in the Transactions of the Bengal Society, that the barometer rises and falls there regularly every 24 hours. Here, in South America, its motion is more astonishing: there are four atmospherical tides every 24 hours, which depend only on the attraction of the sun; the mercury falls from 9 o'clock in the morning till 4 in the afternoon; it rises from 4 till 11 o'clock; it falls from 11 till half past 4 in the morning; and reascends from that time 'till 9 o'clock; neither winds, storms, nor earthquakes have any influence on this motion." See farther Laplace, Exposition du Système du Monde, liv. 4, ch.12.

ATOM, a particle of matter indivisible on account of its solidity, hardness, and inpevetrability; which preclude all division, and leave no vacancy for the admission of any foreign force to separate it into parts. As atoms are the first matter, it is necessary they should be indissolvable, that they may be incorruptible. Newton adds, It is also required that they be immutable, that the world may continue in the same state, and bodies be of the same nature now as formerly.

ATOMiCAL Philosopiy, or the doctrine of atoms, a system which accounted for the origin and formation of things, from the hypothesis that atoms arc endued with weight and motion. This philosophy was first taught by Moschus, some time before the Trojan war; but it was most cultivated by Epicurus; whence it is called the Epicurean philosophy.

aTTRaCTiON, or Attractive Power, a general term used to denote the cause, power, or principle, by which all bodies mutually tend towards each other, and cohere, till separated by some other power. The laws, phenomena, \&c, of attraction, form the chief subject of Newton's philosophy, being the principal agent of nature, in almost all her wonderful operations.

The principle of attraction, in the Newtonian sense of it, appears to have been first surmised by Coperricus. "As for gravity," says he, "I consider it as nothing more than a certain natural appetence (appetentia) which the Creator has impressed upon all the parts of matter, in order to their uniting or coalescing into a globular form, for their better preservation; and it is probable that the same power is also inherent in the sun and moon, and plinets, that those bodies may constautly retain that round form in which we see them." De Revol. O. b. Ceelest. lib. i, cap.9. Kepler calls gravity a corporeal and mutual afiection between similar bodies, in order to their union. Ast. Nov. in Introd. And he pronounced more positively, that no bodies whatever were absolutely light, but only relatively so; and consequently that all matter was subjected to the power and law of gravitation. Ibid.

The first in this country who adopted the notion of attraction was Dr. Gilbert, in his book De Mlagnete; and the next was the celebrated Lord Bacon, in bis Nov. Organ. lib. ii, aphor. $30,45,48$. Sylv. cent. i, exp. 33; also in his treatise De Motu, particularly under the articles of the 9 th and the 13th kinds of Motion. In France it was received by Fermat and Roberval; and in Italy by Galilco and Borelli.

But before Newton none appear to have bad clearer ideas of the doctrine of universal gravitation, or to bave approached so near the application of it to the laws of nature, as Dr. Hooke. This celebrated man, in his work entitled "An Attempt to prove the Motion of the Earth," seems to have embraced it in nearly the whole: of its generality. The hypothesis on which the system of the world is there explained, is grounded on the three following principles: 1. That all the celestial bodies have not only an attraction or gravitation towards their proper centres, but that they mutually attract each other within their splsere of activity. 2. That all bodies which have a simple and direct motion, continue to move in a direct line, if some force which operates without ceasing does not constrain them to describe a circle, an ellipse, or some other nore complicated curve. 3 . That attraction is so much the more powerful, as the' attracting bodics are nearer to each other. Newton makes use of the word attraction, in common with the school philosophers, but at the same time very studiously distinguishes between the ideas. The ancient attraction was conceived to be a kind of quality inherent in certain bollies themselves, and arising from their particular or spceific forms. But the Newtonian attraction is a more indefinite principle; denoting not any particular kind or mode of action; nor the physical cause of such action; but only.a general tendency, a conatus accedendi, to whatever cause, physical or metaphysical, such effect be owing; whether to a power inherent in the bodies themselves, or to the impulsc of an external agent. Accordingly, that author remarks, in his Philos. Nat. Prin. Math. " that he uses the words attraction, impulse, and propension to the centre, indifferently; and cautions the reader not to imagine that by attraction he exprosses the modus of the action, or its efficient cause, as if there were any proper powers in the centres, which in reality are only mathematical points ; or as if centres could attract." Lib. 1, p. 5. So, he "considers centripetal powers as attractions; though, physically speaking, it were perhaps more just to call them impulses. 16. p. 14\%. He adds, "that what he calls attraction may possibly be iffected by impulse, though not a comnion or corporeal impulse, or after some other manner unknown to us." Optics, p. 322.

Attraction, if considered as a quality arising from the specific forms of bodies, ought, together with sympathy, antipathy, and the whole tribe of occult qualities, to be exploded. But when these are set aside, there will remain innumerable phenomena of nature, and particularly the gravity or weight of bodies, or their tendency to a centre, that argue a principle of action scemingly distinct from impulse; where, at least, there is no sciusible impulsion concerned. Nay, what is more, this action, in some respects, differs from all impulsion we know of; impulse being always found to act in proportion to the surfaces of bodies; whereas gravity acts according to their solicl content, and consequently it must arise from some cause that penetrates or pervades the whole substance of them. This unknown principle, (we mean in respect of its cause, for its phenomena and effects are most obvious, with all its species and modifications, ) is called attraction; being a general name, under which nay be ranged all mutual tendencies, where no physical impulse appears, and which consceluently camot be accounted for on any known laws of nature.

And hence arise divers particular kinds of attraction; as Gravity, Magnetism, likctricity, \&c, which are so many different principles, acting by diflerent laws; and only agrecing in this, that we do not perceive any physical causes of them : but that, as to our senses, they may renliy urise from some power or efficacy in such bodies, by which they are enabled to act even upon distant bocke's; though our reason absolutely disallows of any such action. 
Attraction may be divided, with respect to the law it nuserves, into two kinds.

1. That which extends to a sensible distance, As the attraction of gravity, whicl is found in all bodies; and the attractions of magnetism and electricity, found only in particular bodies. The several laws and phenomena of each, see under their respective articles.

The attraction of gravity, called also among mathematicians the centripetal force, is one of the greatest and most universal principles in nature. We see and feel it operate on bodies ncar the earth, and find by observation that the same power (i. e. a power which acts in the same manner, and by the same rules, viz, always proportionally to the quantities of matter, and inversely as the squares of the distances) does also obtain in the moon, and the other planets, both primary and sccondary, as well as in the comets; and even that this is the very power by which they are all retained in their orbits, szc. And hence, as gravity is found in all the bodies which come under our observation, it is easily inferred, by one of the established rules of philosophising, that it obtains in all others. And since it is found to be proportional to the quantity of matter in any body, it must exist in every particle of it : and hence it is proved that every particle in nature attracts every other particle.

From this attraction arises all the motion, and consequently all the mutation, in the universe. By this heavy bodies descend, and light ones are made to ascend: by this also projectiles are directed, vapours and exhalations rise, and rain, \&c, fall: also from the same cause rivers glide, the océan swells, the air presses, \&c. In short, the motions and forces arising from this principle, constitute the subject of that extensive branch of mathematics, called meclianics or statics, with the parts or appendages of it, as hydrostatics, pneumatics, hydraulics, \&c.

2. That which does not extend to sensible distances. Such is found to obtain in the minute particles of which bodies are composed, attracting each other at or extremely near the point of contact, with forces often much superior to that of gravity, butwhich at any distance decrease much faster than the power of gravity. This power is generally called the at:raction of cohesion, as being that by which the atoms or insensible particles of bodies are united into sensible masses.

This latter kind of attraction claims Sir Isaac Newton for its discoverer; as the former does for its improver. The laws of motion, percussion, \&c, in sensible bollies, under varjous circumstances, as falling, projected, \&c, ascertained by the later plitosophers, do not reach those more recluse, intestine inotions in the component particles of the same bodics, on which the clianges of the texture, colour, properties, \&xc, depend. So that our philosnphy, if it were only founded on the principle of gravitation, and even carried as far as this would lead us, would still.be deficient.

But besides the common laws of sensible masses, the minute parts they are composed of are found subject to some others, which have but lately been noticed, and are even yet inperfectly known. Newton himsell; to whose happy penetration we owe the hint, is satisfied with establishing that there are such motions in the minima natura, and that thoy flow from certain powers or forces, not reducible to any of those in the great world. Ile shows that, by virtue of these powers, "the sinall particles act on one another even at a distance; and that many of the phenomena of nature result from this action. Sensible budies, we have already observed, act on one another several ways; and as we thus perceive the tenor and course of nature, it appears highly probable that there may be other powers of the like kind; nature being always uniform and consistent with herself. Those just mentioned, reach to sensible distances, and by that means bave been observed by vulgar eyes; but there may be others which reach to such small distances as have hitherto escaped observation; and it is probable electricity may reach to such distances, even without being excited by friction."

The great author just mentioned proceeds to confirm the reality of these suspicions by a great number of phenomena and experiments, which plainly argue such powers and actions between the particles, for example of salts and water, oil of vitriol and water, aquafortis and iron, spirit of vitriol and saltpetre, and most other chemical operations. He also shows, that these powers, \&c, are unequally strong between different bodies; stronger, for instance, between the particles of salt of tartar and those of aquafortis than those of silver, between aquafortis and lapis calaninaris thas iron, between iron and copjer than silver or mercury. Thus spirit of vitriol acts on water, but more on iron or copper, \&c. And the other experiments are innumerable which countenance the existence of such principle of attraction in the particles of matter.

These actions, by virtue of which the particles of the bodies above-mentioned tend towards each other, the author calls by a general indefinite name, attraction; a name equally applicable to all actions by which distant bodies. tend towards each other, whether by impulse, or by any. other more latent power: and lience he accounts for an infinity of phenomicna which would be otherwise inexplicable from the principle of gravity.

"Thus," adds Newton, "will nature be found very conformable to herselt, and very simple; perfurming all the great motions of the heavenly bodies by the attraction of gravity, which interccdes those bodies, and alnust all the sinall ones and their constituent parts, by some other attractive power diffused through their particles. Without such principles, there could have been no such thing as motion; and withunt the continuance of it, motion would soon perish, there being otleerwise a great decrease or diminution of it, which is only supplied by these active principles."

It need not be said how unjust it was, in the generality of forcigu philosophers, to declare against a principle which furnishes so beautiful a view, for no other reason but be cause they could not conceive how one borly should act on another at a distance. It is indeed true, that philosophy allows of no action but what is by immediate contact and impulsion; for how can a body excrt any active power where it does not exist? Yet we see effects, without perceiving any such impulse; and where effects are observed, there must exist causes, whether we perceive them or not. But we may contemplate sucli effects, without entering into the consideration of the causes, as indeed it seems the business of a philosopher to do: for to exclude a number of phenomena which we see, would be to leave a great chasm in the history of nature; and to argue about actions which we do not see, would be to raise castles in the air. It follows therefore, that the phenomena of attraction are matter of physical consideration, and as such entitled to a share in the system of physics; but that their causes will only become so when they become sensible, that is when they appear to be the effect of sonie other higher causes; 
for a cause is no otherwise seen than as itsclf is an effect, so that the first cause must needs be always invisible; we are therefore at liberty to suppose the causes of attractions what we please, without any injury to the effects. The illustrinus author himself seems to be a little indeternined as to the causes; inclining sometimes to attribute gravity to the action of an inmaterial cause (Optics, p. 343, \&c.) and sometimes to that of a material one, Ib. p. 325.

In his philosophy, the research into causes is the last thing, and never comes under consideration till the laws and phenomena of the effect be settled; it being to these phenomena that the cause is to be accommodatid. The cause even of any, the grossest and most sensible action, is not adequately known. "How impulse or percussion itself produces its effects, that is, how motion is communicated from one body to another, or by what agency it is continued after it is communicated, confounds the deepest philosophers. Yet jmpulse is received not only into philosophy, but into mathematics; and accordiugly the laws and phenomena of its eflects make the chief part of common mechanics.

The other species of attraction, therefore, in which no impulse is observable, when their phenomena are sufficiently ascertained, have the same title to be promoted from physical to mathematical consideration; and this without any previous inquiry into their causes, to which our conceptions may not be proportionate.

Our great philosopher, then, far from adulterating science with any thing foreign or metaphysical, as many have reproached him with doing, has the glory of having thrown every thing of this kind out of his system, and of having opened a new source of more sublime nechanics, which, duly cultivated, might be of far greater,extent than all the mechanics yet known: it is hence therefore that we must expect to learn the manner of the cbanges, productions, generations, corruptions, \&c, of natural things; 'with all that scene of wonders opened to us by the operations of chemistry.

Some of our own countrymen have prosecuted the discovery with laudable zeal. Dr. Kieil particularly has endeavoured to deduce some of the laws of this new action, and applied them in resolving several of the more general phenomena of matter, as cohesion, fluidity, elasticity, softness, fermentation, coagulation, \&c: and Dr. Freind, seconding his endeavours, has made a larther application of the sarve principles, to account for almost all the phenomena that chemistry presents. So that some philosophers are inclined to think that the new mechanics should seen already raised to a complete science, and that nothing now can occur but what we have an immediate solution of, from the principles of attractive forces.

But this seems a little too precipitate: a principle so fertile should have been further explained; its particular laws, limits, \&c, more industriously detected and laid down, before we had proceeded to the application. Attraction in the gross is so complex a thing, that it may solve a thousand different phenomena alike. The notion is but one degree more simple and precise than action itself; and, till its properties be more fully ascertained, it w ri better to apply it less, and study it more. It may he addea, that some of Newton's followers have been clatiged with falling into that error which he industriousIy avoided, viz, of considering attraction as a cause or active property in bodics, not merely as a phenomenon or ethect.
Certain attempts have lately been made to assign the probable or possible cause of gravitation, but too absurd to merit more particular notice.

For the laws, properties, \&c, of the different kinds of attraction, see their particular articles, CoHesion, GraVітY, MAG NTIS M, \&c.

Attraction, Centre of. See Centre of Attraction.

Atrraction of Mountains. This is but a late discovery, and must be esteemed a complete confirmation of the Newtonian theory of universal gravitation. According to that system, an attractive power is excrted, not only between those large masses of matter the sun and planets, but likewise between all comparatively smaller bodies, and even between the smallest particles that compose them. Agreeably to this hypothesis, a lieavy body, which gravitates or tends toward the centre of the earth, ought likewise, though in a less degree, to be attracted and to tend laterally towards a neighbouring mountain on the earth's surface. So that, a plumb-line, for instance, of a quadrant, hanging near such a mountain, ought to be drawn a little aside, from the perpendicular direction, in consequence of the attractive power of the quantity of matter, of which it is composed, acting in a direction different from that exerted by the. whole mass of matter in the earth, and with a proportionally inferior degree of force.

Though Sir Isaac Newton had long ago hinted at an experiment of this kind, and had remarked, that "a mountain of an hemispherical figure, 3 miles high, and 6 broad, would not by its attraction draw the plumb-line $2 \mathrm{mi}$ nutes out of the perpendicular ;" yet no attempt to ascertain this matter by actual experiment was made, till about the year 173s; when the Frenich-academicians, particularly Messrs. Bouguer and Condamine, who were sent to Peru to measure a degree of the meridian at the equator, attempted to discover the attractive power of Chimboraço, a mountain in the province of Quito. According to their observations, made under unfavourable circumstances, that mountain exerted an attraction equal to $\mathrm{S}^{\prime \prime}$. Though this experiment was not perbaps sufficient to prove satisfactorily even the reality of an attraction, much less the precise quantity of it; yet it does not appear that any steps had been since taken to repeat it.

In the year $\mathbf{1 7 7 2}$, the astronomer royal, Dr. Maskely ne, made a proposal for this purpose to the Royal Society: and in 1774, he was deputed to make the trial, accompanied with proper assistants, and provided with accurate instruments. The mountain Schehallicn, situated nearly in the centre of Scotland, was chosen as the most proper then found in this island. The observations were made by observing the meridian zenith distances of some fixed stars near the zenith, by means of a zenith sector of 10 feet radius; first on the south, and then on the north side of the hill, the greatest length of which was considerably extended in the east and west direction.

It is plain, that if the mass of matter in the hill should exert any sensible attraction, it would cause the plumbline of the sector, through which the observer viewed a star in the meridian, to deviate from its perpendicular situation, and would attract it contrarywise at the two opposite stations, thus doubling the effect. On the south side the plummet would be drawn to the nortliward, by the attractive power of the hill placed on the north side of it; and on the north side, a contrary and equal deflection of the plummet would take place, by the like attraction of the liill, now to the soutleward of it. The apparent zenilh di- 
stances of the stars must be affected contrarywise; those being increased at the one station, which were diminished at the other; and the correspondent quantities of the deflection of the.plumb-line must give the observer the sum of the contrary attractions of the hill, acting on the plummet at the two stations; and the half of which will of course indicate the attractive power of the hill.

The various operations requisite for this experiment lasted about 4 months; and from them it appears, that the sum of the two contrary attractions of the hill, in the two temporary observatories, which were successively fixed half way up the hill (where however the effect of the attraction would not be the greatest), was found to be $11^{\prime \prime} \cdot 6$; the half of which therefore, or $5^{\prime \prime} \cdot \mathrm{s}$, is the mean attraction of the mountain, at that height.

The inferences drawn from these experiments may be reduced to the following:

1. It appears that the mountain Scheballien exerts a sensible attraction ; therefore, by the rules of philosophising, we are to conclude, that cvery mountain, and indeed every particle of the earth, is endued with the same property, in proportion to its quantity of matter.

2. The law of the variation of this force, in the inverse ratio of the squares of the distances, as laid down by Sir Isaac Newton, is also confirmed by this experiment. For, if the force of attraction of the hill, had been only to that of the earth, as the matter in the hill is to that in the earth, and had not been greatly increased by the near approach to its centre, the attraction of it must have been wholly insensible. But now, by only supposing the mean density of the earth to be double of that in the hill, which seems very probable from other considerations, the attraction of the hill will be reconciled to the general law of the variation of attraction, in the inverse duplicate ratio of the distances, as deduced by Sir Isaac Newton from the comparison of the motion of the heavenly bodies, with the force of gravity at the surface of the earth; and the analogy of nature will be preserved.

3. We may now therefore be allowed to admit this law, and to acknowlerge, that the mean density of the earth is about double of that at the surface; and consequently that the density of the internal parts of the earth, is much greater than near the surface. Hence also, the whole quantity of matter in the cartls, will be at least as great again, as if it had been all composed of matter of the same density with that at the surface; or will be about 4 or 5 times as great as if it were all composed of water. 'This conclusion is totally contrary to the hypothesis of some naturalists, who suppose the carth to be only a large hollow shell of matter; supporting itsclf from the property of an arch, with an immense vacuity in the midst of it: But, were that the case, the attraction of mountains, and even smaller inequalities in the earth's surface, would be very great, contrary to experiment, and would affect the measures of the degrees of the meridian much more than we find they do; and the variation of gravity, in different lititudes, in going from the equator to the poles, as found by pendulums, would not be near so regular as experiments have shown it to be.

4. As mountains are, by these experiments, found capable of producing sensible deflections in the plunh-lines of astrononical iustruments; it becomes a matter of great inportance, in the mensuration of meridional degrees, either to choose places where the irregular attractions of the elevated parts may be small; or where, by their situation, they may compensate or counteract each other's effects. Sce Philos. Trans, vol. 65; or my Aluridg. vol. 13, p. 702.

Besides what is abuve related of this grand experiment, and its results, as it was necessary, so a great deal more was done, than what yet appears by that account. For, though the experiment showed the quantity of deviation in the piumb-line, from its due position, to be $5^{\prime \prime} \cdot 8$, and thereby proved the fact of the universal attraction or gravitation of matter, yet this was all that it proved; for as to the mean density of the whole matter in the earth, as above noticed, much more remained to be done, what is there said having been partly from anticipation by a near guess, or rather from the results of accurate calculations which I was at that very time employed in making, and which results I was in the habit of communicating to Dr. Maskelyne, while he was composing his account of the experiment. In fact, to deduce from the experiment the real mean density of the earth, was, from the first projecting of the experiment, considered as a very important part of the business; and in order to this, it was necessary to procure a very minute and correct survey and measurement of the mountain, from which to calculate theoretically the effect of the attraction of every particle of matter contained in its composition. Accordingly, Dr. Maskelyne, with his assistants, made accurate neasurements of the hill, with numerous sections, both borizontal and vertical; by means of which, all exact model of it could be made, and the distance and position of every point in it could be ascertained. This survey Dr. M. brought to the committee of the R. Soc. along with the observations of the plumb-line before mentioned; but he declined undertaking the laborions calculations of the attractions of the matter in the hill, which were necessary to show the real mean density of the earth ; ealculations which would probably occupy four times as much labour and time as had been employed in making the experiment and survey.

Accordingly, at the request of the Royal Society, I undertook this arduous task, and, after about a year's labour, delivered to that body an account of my computations and results, which gave universal satisfaction; which account was printed in the $6 \mathrm{sth}$ volume of the Philos. Trans. It has also been printed at large in the $2 \mathrm{~d}$ volume of my Tracts, where it is proved that the mean density of the whole earth is near 5 times the density of water, or almost double the density of the rocks near the earth's surface.

AT"IRITION, the striking or rubbing of bodies against one another, so as to throw off some of their superficial particles: such as the grinding and polishing of bodies. Or simply the act of rubbing: as when amber and other electric bodies are rubbed, to make them attract, or emit their electric force.

ATWOOD (GEoRGE) F. R. S. a celebrated mathematician and philosopher, was educated at Westminster school; after which he studied at Trinity college, Cambridge, where he was afterwards for some time a tutor, and many year's fellow of that college. He was very celebrated also for his courses of kectures, on several branches of experimental philosophy, which he read in the university, with neat illustrations by means of a large and excellent colfection of instruments; 'sevcral of which were of his own contrivance; particularly a very ingenious one, exbibiting the theories respecting the rectilinear motion of bodies which are acted on by constant forces. Of these lectures, he published a very neat Syllabus; also, in 1784, he published, in one large volume in sro, A Treatise on the Rec- 
tilinear Motion and Rotation of Bodies; a learned and ingenious work, but not treated in the most simple and perspicuous manner. Mr. A. communicated some learned and ingenious papers to the Royal Society, which were printed in their Transactions : viz, 1. On the Mensuration of a certinin Angle. 2.On the Times of Vibration of Watch Balances. 3. 'The Theory of Floating Bodies. 4. The Stability of Ships: all of them in some degree liable to the like imputation with the foregoing; as well as the two Tracts on the subject of Arches and Bridges, in 1801 and 1804; to him apparently a new study; as nıst of the properties which occurred to him, though common and well known to other writers, he unfurtunately fancied to be new discoveries. The whole composition being indeed strongly indicative of the feeble state of body and mind, in which $\mathrm{Mr}$. A. had lingered during some years before his death; which happened in 1807, at 62 years of age.The late minister, Mr. Pitt, having been one of Mr. A's auditors at Cambridge, gave him, in 1784, it is said, a sinecure place of $500 l$. a-year, which he enjoyed during the remainder of his life. It is also said, that the same minister employed him in financial calculations for the public service, in the latter years of his life, till such time as the declining state of his health rendered him incapable of severe application.

Avant-Foss, or Ditch of the Counterscarp, in Fortification, is a wet ditch surrounding the counterscarp, on the outer side, next to the country, at the foot of the glacis. It would not be proper to have such a ditch if it could be laid dry, as it would then serve as a lodgment for the enemy.

AVENPACE, a philosopher among the Saracens that invaded Spain, who flourished in the 12th century, and was a follower of Aristotle. He wrote a commentary on Euclid, as well as philosophical and theological epistles. He was also intimately conversant with the Peripatetic philosophy, and applied it to the illustration of the Islamic system of theology, and to the explanation of the Koran; and on this account he was suspected of heresy, and thrown into prison at Corduba. It is said that be was poisoned at $\mathrm{Fez}$, in the year of the Hegira $533, \mathrm{~A} . \mathrm{D}$. 1138 ; or according to others, $525, \mathrm{~A} . \mathrm{D} .1130$.

Averroes, or Aben-Roes, a very subtile Arabian philosopher, who flourished about the end of the 11th century, when the Moors had possession of part of Spain. He was the son of the high priest and chief judge of Corduba or Cordova in Spain; but educated in the university of Morocco, where he was professor, and where he lied in 1206, having there studied natural philosophy, medicine, mathematics, law, and divinity. After the death of his father, he enjoyed his posts in Spain, to which was afterward added that of judge of Morocco and Mauritania, where having settled deputies, he returned to his duty in Spain. Notwithstanding le was very rich, and bad a large income, his liberality to men of letters in nocessity, whether they were his friends or his enemies, kept him always in debt. He was afterwards deprived of these means of emolument, and thrown into prison for heresy, by the vile instigations of his enemies; but the oppressions of the judge who succeeded him, caused him to be restored to his former employments.

This philosopher has been highly celebrated for his personal virtues. Such was his temperance, that he partook only once a day of the plainest food. In his application, he was unremitting and indefatigable; allowing himself no other recreation in the course of the day than the change of several literary occupations, for those of poetry or history, and spending whole nights in study: In the exercise of forbearance, meekness, and self-command, he was signally exemplary. When a servant employed by an enemy intruded upon him in one of his public lectures, and whispered some abusive language, Averroes, with perfect self-possession, turned round to him, and said, "Well, well;" and proceeded with his business. This servant waited on him next day to implore his pardon for the insult he had offered him. "God forgive thee," said Averroes, "thou hast publicly shown me to be a patient man; and as for thine injury, it is not worthy of notice." $\mathrm{He}$ then gave him money, and dismissed him with this admonition ; "What thou hast done to me, do not to another." He was extremely fond of the works of Aristotle, and wrote commentarles upon them; whence he was styled the Commentator, by way of eminence. He also wrote many other pieces; among which is a work on the Whole Art of Physic; an Epitome of Ptolemy's Almagest, which Vossius dates, about the year 1149 ; also a Treatise of Astrology, which was translated into Hebrew by R. Jacob Ben Samson, and said to be extant in the French regal library. He likewise wrote several poems, and many ama. tory verses, but these last he destroyed when lie grew old. His other poems are lost, except a small piece, in which he says, "that when he was young, he acted against his reason; but that when he was in years, be followed its dictates;" upon which he utters this wish, "Would to God I had been born old, and that in nyy youth I had been in a state of perfection!" As to religion, his opinions were, that Christianity is absurd; Judaism, the religion of children; Mahometanism, the religion of swine.

AVICENA, Avicenne, or Avicenes, an eminent writer, who has been accounted the prince of Arabian philosophers and physicians. He was born at Assena, near Bokhara, in 978 ; and died at Hamadan in 1036, being 58 years of age.

The first years of Avicena were employed on the study of the Belles Lettres and the Koran, and at ten years of age he was perfect master of the bidden senses of that book. Then applying to those of logic, philosophy, and mathematics, be made a rapid progress. After studying under a master the first principles of logic, and the first 5 or 6 propositions of Euclid's elements, he became disgusted with the slow manner of the schools, applied himself alone, and soon accomplished all the rest by the help of the commentators only.

Possessed with an extreme avidity to be acquainted with all the sciences, he studied medicine also. Persuaded that this art consists as much in practice as in theory, he sought all opportunities of seeing the sick; and alterwards confessed that he had learnt more from such experience than from all the books he had read. Being now in his 16 th year, and already celebrated for being the light of his age, he determined to resume his studies of philosophy, which medicine, \&c, had made lim for some time neglect: he spent a year and a half in this painful industry, without ever slecping a whole night together. At the age of 21 , he conceived the bold design of incorporating, in one work, all the objects of human knowledge; which he carried into execution in an Encyclopedia of 20 volumes, to which he gave the title of the Utility of Utilities.

Many wonderful stories are related of his skill in medicine, and the cures which be performed. Several princes 


\section{A U R}

had been taken dangerously ill, and Avicenes was the only one that could discover the cause of their disorders and the mode of curing them. His reputation increased daily, and all the princes of the East desired to retain him in their families, and in fact he passed through several of them. But the irregularities of his conduct sometimes lost him their favour, and threw him into great distresses. His excesses in pleasures, and his infirmities, made a poet say, who. wrote his epitaph, that the profound study of philosophy had not taugbt him good morals, nor that of medicine the art of preserving his own health.

After his death however, he enjoyed so great a reputation, that till the 12th century Lis works of philosophy and medicine were preferred to those of all his predecessors. Even in Europe his works were the only writings in vogue in the schools. They were very numerous, and various, the titles of which are as follow: 1 . Of the Utility and Advantage of the Sciences, in 20 books. -2 . Of lnnocence and Crininality, 2 books.-3. Of Health and Remedies, 18 books. -4. On the.Means of preserving Health; 3 books. -5. Canons of Physic, 14 books. -6. On Astronomical Observations, 1 book.-7. On Mathematical Sciences.-8. Of Theorems, or Mathematical and Theological Demonstrutions, 1 book.-9. On the Arabic Language, and its Properties, 10 books. - 10. On the Last Judgment. -11. On the Origin of the Soul, and the Resurrection of Bodies.-12. On the end we should propose to ourselves in Harangues and Philosophical Argumentations.-13. Demonstration of the Collateral Lines in the Sphere.-14. Abridgment of Euclid.-15. On Finity and lnfinity.-16. On Pliysics and Metapbysics.-17. Un Animals and Vegetables, \&c.-18. Encyclopedia, 20 volumes.

AUGUST, the sth month of the year, containing 31 days. In the ancient Roman calendar it was called sextilis, as being the 6th month from March, with which their year began; but changed to its present name by the emperor Augustus, who gave it his own name on account of his having obtained many victories and honours in that month.

AVOIRDUPOIS Weiglt, a weight used in England for weighing all the larger and coarser kinds of goods; as groceries, cheese, butter, flesh, wool, salt, hops, \&c, and all metals except gold and silver. Avoirdupois weight is lihus divided, viz.
16 ir. or drams make
1 ounce, marked $o z$.
$16 \mathrm{oz}$.
1 pound, - - lb.
$112 \mathrm{lb}$.
1 hundred weight, $c$ wt.
$20 \mathrm{cwt}$.
1 ton - - ton.

The Avoirrlupois ounce is less than the Troy ounce, in the proportion of 700 to 768 , but the Avoirdupois pound is greater than the Troy pound in the proportion of 700 to 576 ;

for $1 \mathrm{lb} \mathrm{Avoird}$ is $=7000$ grains Troy,

but $1 \mathrm{lb}$ Troy is $=5760$ grains Troy,

also $\mathrm{J}$ oz Avoird. is $=437 \frac{1}{2}$ grains Troy,

and $1 \mathrm{oz}$ 'Troy is $=480$ grains Troy.

AUliGA, the Waggoner, a constellation in the northern bemisplere, consisting of 14. stas in Ptolemy's catalogue; but in 'Tycho's, 27; in Hevelius's, 40; and in the Britunnic catalogue, 66 .

This is one of the 48 old asterisms, mentioned by all the most ancient astronomers. It is represented by the figure of an old man, in posture somewhat like sitting, with a goat and her kids in his left hand, and a bridle in his right. AURORA, the morning twilight; or that fuint light

\section{A U R}

which appears in the morning when the sun is within 18 degrees of the horizon.

AURORA BOREALIS, NoRthern Light, or Sireamers; a kind of meteor appearing in the northern part of the heavens, mostly in the winter season, and in frosty weather. It is usually of a reddish colour, inclining to yellow, and sends out frequent coruscations of pale light, which seem to rise from the horizon in a pyramidical undulating form, and shooting with great velucity up to the zenith. It appears often in form of an arch, which is partly bright, and partly dark, but generally transparent. And the matter of it is not found to have any effect on the rays of light, which pass freely through it. Dr. Hamil. ton observes, that he could plainly discern the smallest speck in the Pleiades through the density of those clouds which formed part of the Aurora borealis in 1763 , without the least diminution of $i t s$ splendour, or increase of twinkling. Philos. Essays, p. 106.

Sometimes it produces an Iris. Hence M. Godin judges, that most of the extraordinary meteors and phenomena in the skies, related as prodigies by historians, as battles and the like, may probably enough be reduced to the class of Auroræ Boreales. Hist. Acad. R. Scienc. for 1762, p. 405.

This kind of meteor never appears near the equator; but it seems is frequent enough towards the south pole, like as towards the north, having been observed there by voyagers. See Philos. Trans. No. 461, and vol. 54; also Forster's account of his voyage round the world with Captain Cook, where be describes their appearance as observed for several nights together, in sharp frosty weather, which was much the same as those observed in the north, excepting that they were of a lighter colour.

In the Shetland Isles these phenomena are the constant attendants of clear evenings, and afford great relief to the inhabitants 'among the gloom of the long winter nights: they are called there the merry dancers. The same kind of appearances are also seen in the northern latitudes of Sweden and Lapland, where they are particularly beautiful, and afford light to travellers during the whole night. In Hudson's Bay the aurora borealis diffiuses a variegated splendour, which is said to cqual that of the full moon. In the north-eastern parts of Siberia, according to the description of Gmelin (Reise durch Siberien, vol. 3, p. 135) cited and translated by Dr. Blagden (Phil. Trans, vol. 74, pa. 228) those northern lights are observed to begin with single bright pillars, rising in the north, and almost at the same time in the north-east, which, gradually increasing, comprehend a large'space of the beavens, rush. about from place to place with incredible velocity, and finally almost cover the whole sky up to the zenith, producing an appearance as if a vast tent were expanded in the heavens, glittering with gold, rubies, and sapphire. A more beautiful spectacle cannot be painted; but whoever should see such a northern light for the first time, could not behold it without terror. For, however grand the illumination may appear, it is attended, as I have learnt from the relation of many persons, with as much hissing, cracking, and tunult, as if the largest fire-works were playing off. To describe what they then bear, they make use of the expression "spolochi, chodjat," that is, the raging heat is passing. The hunters who pursue the white and blue foxes in the confines of the icy sea, are often overtaken in their courscs by these northern lights; their dogs are tlyen so much frightened, that they will not move, but lic obstinately on the ground till the noise has passed. Commonly clear and 
calm weather follows this kind af meteor. I have heard this account, not from one person only, but confirmed by the uniform testimony of many, who have spent part of several years in these very northern regions, and inhabited different countries, from the Yenisei to the Lena; so that no doubt of its truth can remain. This seems, indeed, to be the real birth-place of the aurora borealis.

It scems that metcors of this kind have appeared sometimes more frequently than others. They were so rave in England, or else so little regarded, that none are recorded in our annals since that remarkable one of Nov. J 4,1574 , till the surprising aurora borealis of Narch 6,1716 , which appeared for three nigbts successively, but by far more strongly on the first; except that five small ones were observed in the year 1707 and 1708 . Hence it would seem, that the air, or earth, or both, are not at all times disposed to produce this phenomenon.

The extent of these appearances is also very great : that in March 1716 was visible from the west of Ireland to the confines of Russia and the east of Poland, extending at least near 30 -degrees of longitude, and from about the 50th degree in latitude, over almost all the north of Europe; and in all places, at the same time, it cxhibited the like wondrous appearances. Father Boscovich has determined the height of an aurora borealis, which was observed by the Marquis of Polini the 16th of December 1737, to have been 825 miles high; and Bergman, from a mean of 30 computations, makes the average height of the aurora borealis amount to 70 Swedish, or 469 English miles. Bnt Euler supposes the height to be several thousands of miles; and Mairan also assigns to them a yery elevated region.

Many attempts have been made to determine the cause of this phenomenon. Dr. Halley imagines that the watery vapours, or effluvia, exccedingly rarefied by subtcrraneous firc, and tinged with sulphureous streams, which many naturalists have supposed to be the cause of earthquakes, may also be the cause of this appearance; or that it is produced by a kind of subtile matter, freely pervading the pores of the earth, and which, entering into it nearcr the southern poie, passes out again with a similar force into the ether, at the same distance from the northern. This subtile matter, by becoming more dense, or having its velucity increased, may perhaps be capable of producing a small dcgree of light, after the manner of effluvia from electric bodies, which, by a strong and quick friction, emit light in the dark, to which sort of light this seems to have a great affinity. Philos. Trans. No. 347. Sce also Mr. Cotes's dcscription of this phenomenon, and his method of explaining $\mathrm{jt}$, by streans emitted from the heterogeneous and fermenting vapours of the atmosphere, in Smith's Optics, p. 69; or my Philos. Trans. Abr. vol. 5, p. 477.

'The celebrated M. de Mairan, in an express treatise on the Aurora Borealis, published in 1731, supposes its cause to be the zodiacal light, which, according to him, is no other than the sun's atmosphere: this light happening, on some occasious, to mect the upper parts of our atmosphere about the limits where universal gravity begins to act more forcibly towards the earth than towards the sim, falls into our air to a greater or less depth, as its specific glavity is greater or less, compared with the air through which it passes. See Tract. Phys, ct llist. de l'Aurore Boreale. Suite des Memoires de l'Acad. R. des Scien. 1731. Also Jhilos. Trans. No.433.

However, Euler is of a different opinion: he does not think that the cause of the aurora borealis is owing to the zodiacal light, as M. de Mairan supposes, but to particles of our atmosphere, dricen beyond its limits by the impulse of the solar light. And on this supposition he endeavours to account for the phenomena observed concerning this light. He supposes the zodiacal light and the tails of comets to be owing to a similar cause.

But from the period that the identity of lightning and the electric matter was determined, philosophers bave been naturally led to seck for the explication of aërial meteors in the principles of electricity; and there is now no doubt but most of them, and especially the aurora borealis, are electrical phenomena. Besides the more obvious and known appearances which constitute a resemblance bctween this meteor and the electric matter by which lightning is produced, it has been observed that the aurora occasions a very sensible fluctuation in the magnctic needle; and that when it has extended lower than usual in the atmosphere, the flashes have been attended with various sounds of rushing and hissing, especially in Russia and the other more northern parts of Europe, as noticed by Sig. Beccaria and M. Messier. Mr. Canton, soon afier he had obtained electricity from the clouds, offered a conjecture, that the aurora is occasioned by the flashing of electric fire from positive towards negative clouds at a great distance, through the upper part of the atmosphere, wherc the resistance is least: and he supposes that the aurora which happens at the time when the magnctic ncelle is disturbed by the heat of the earth, is the electricity of the heated air above it; and this appears chiefly in the northern regions, as the alteration in the heat of the air in those parts is the greatest. Nor is this hypothesis improbable, when it is considered that electricity is the cause of thunder and lightning; that it has been extracted from the air at the time of the aurora borealis; that the inhabitants of the northern countries observe it remarkably strong when a sudden thaw succeeds very cold severe weather; and that the tourmalin is known to emit and absorb the electric fluid only by the increase or diminution of its heat. Positive and negative electricity in the air, with a proper quantity of moisture to serve as a conductor, will account for this and other meteors, sometimes seen in a screne sky. Mr. Canton has since contrived to exhibit this meteor by means of the 'l'uricellian vacuum, in a glass tube about 3 feet long, and sealed hermerically. When one end of the tube is held in the hand, and the other applied to the conductor, the whole tube is illuminated from end to end, and continues luminous without interruption for a considerable time after it has been removed from the conductor. Ii, after this, it be drawu through the hand eithej way, the light will be remarkably intense through the whole length of the tube; and though a great part of the electricity be discharged by this operation, it still flashes at intervals, when held only at one extremity, and kept quite still; but if at the same time it be grasped by the other hand in a ditferent place, strong flashes of light dart fiom one end to the other; and these continue 24 hours or more, without a fresh excitation. Sig. Beccaria conjectures that there is a constant and regular circulation of the electric fluid from north to south; and he thinks that the aturori borealis may be this electric matter pertorming its circulation in such a state of the atmosphere as renders it visible, or approaching nearer than u-tal to the earth: thuugh probably this is not the mode of its operation, as the metcor is obscrved in the southen bemisphere with the same appearances as in the northern. Dr. Franklin 


\section{A U R}

$[193$

supposes, that the electric fire discharged into the polar regions, from many leagues of vaporised air raised from the ocean between the tropics, accounts for the aurora borealis; and that it appears first, where it is first in mution, namely, in the most northern part; and the appearance proceeds southward, though the fire really moves in the opposite direction. Franklin's Exper. and Obs. 1769, pa. 49. Philos. Trans. vol. 48, pa. 358, 784; Ib. vol. 51, pa. 403. Lettere dell' Ellettricismo, pa. 269; or Priestley's Hist. of Electricity. See also an ingenious solution of this phtnomenon, on the same principles, by Dr. Hamilton, in his Philos. Essays. Mr. Kirwan (in the Transactions of the Royal Irish Academy, ann. 17 88), has some ingenious remarks on the aurora borealis et australis. He gives his reasons for supposing the rarefaction of the atmospliere in the polar regions to proceed from them, and these from a combustion of inflammable air caused by electricity. He observes, that after an aurora borealis the barometer cominonly falls, and high winds from the south generally follow.

To the same purpose it is observed by Mr. Wynn (Phil. Trans, vol. 73), that the appearance of an aurora borealis is a sure indication of a hard gale of wind from the south or suuth-west. This occurred without fail in 23 instances; and he thinks that the splendor of these phenomena will enable the observer to form some judgement concerning the ensuing tempest. If the aurora is bright, the gale will come on within 24 hours, but will be of no long duration: if the light be faint and dull, the gale will be less violent, and longer in gathering, but will continue longer. His observations were made in the English Channel, where such winds are very dangerous; and, by attending to the aurora, he says, that he often escaped, when others were nearly shipwrecked. Observations of this kind would serve to lessen the dangers of navigation.

MI. Libes has suggested a new theory, in his Nouv. Dict. de Physique, whicl, it secms, is adopted by most of the northern philosophers. In his opinion, electric light is not the cause of the aurora borealis; nor has electricity itself any further influence on their existence, than as it fixes the aëriform substances, whose combination occasions the meteor. This philosopher's theory is founded on the following principles:-

I. If we excite the electric spark in a mixture of azotic and oxygen gas, there will result nitric acid, or nitrons acid, or nitrous gas, according to the rclation that subsists bètween the gases which compose the mixture.

2. Nitric acid, when exposed to the sun, assumes more colour and volatility. M. Libes placed a receiver over a salver containing nitric acid, and exposed to the action of the solar rays. Some minutes after, the acid appeared coloured, and the receiver filled with red and volatile vapours, which were sustained in it a long while, and diffused a light similar to that of the aurora borealis.

3. In flasks which contain nitrous acid, a ruddy and volatile vapour is always perceived above the vapour.

4. Nitrous gas, in contact with atmospheric air, exhales ruddy vapours, which fly off into the atmosphere.

5. The hydrogen, which is disengaged from the earth's surface, rises, till it occupies, in the higher regions of the atmosplicre, al place deterinined by its specific gravity.

6. The solar heat has very little activity in the polar reginas.

These several principles rest on observations and experiments made with great exactness, and most of them too VoL. l.
A U T

well known to necd being here described. Now it is plain, from a simple combination of these facts, 1 st, that the production of hydrogen must be next to nothing in the polar regions; $2 \mathrm{dly}$, that the ligher regions of the polar atmosphere contain wery little if any hydrogen; 3uly, that whenever there is a re-establishment of equilibrium of the electric fluid in the polar atmosphere, this fluid can only find in its passage a mixture of azot and oxygen; thly, that the electric spark ought to fix and combine these gascous substances; $5 \mathrm{thl}_{\mathrm{l}} \mathrm{y}$, that from this combination must result a production of nitrous acid, or of nitric acid, or of nitrous gas, according to the relation subsisting between the oxygen and azot, that constitute the nixture; 6thly, that the production of either of those acids, or of the gis, will give birth to red and volatile vapours, whose elevation in the atmosphere will form the meteor known by the name of the aurora borealis. After removing some general abjections to these preliminary notions, M. Libes then applies them to the phenomena as generally observed of that meteor.

AURUM FULminans, a preparation from gold, which bcing thrown into the fire, it explodes with a violent noise like thunder. The matter is produced by dissulving yold in aqua regia, and precipitating the solution by oil of turtar per deliquium, or volatile spirit of sal ammoniac. The powder being washed in warm water, and dried to the consistence of a paste, is afterwards formed into small grains of the size of hempseed.

It is inflammable, not only by fire, but also by a gentle warmth; and gives a report much louder than that of gunpowder. A single grain laid on the point of a knife, and lighted at a candlc, explodes with a greater report than a nusquet : and a scruple of this powder, it is said, acts more loudly than balf a pound of gunpowder; and yet it is said that, by mixture, it does not increase the elastic force of fired gunpowder. Dr. Black attributes the increase of weight, and also the explosive property of this powder, to adhering fixable air.-This is a very dangerous preparation, and should be used with great caution.

AUSTRAL, the same with Southern. Thus, Austral signs, are the last 6 signs of the zodiac; and are so called because they are on the south side of the equinoctial.

aUSTRALIS Corona. Sce Corona Australis.

Australis Piscis, the Southern Fish, is a constellation of the southern hemisphere. See Piscis Australis.

AUSTRIACA Sy Der A, in Astronomy, a name ascribed by Maupertuis to the spots in the sun, as supposing them to be small stars between the sun and us.

AUTOLYCUS, a Greek mathematician and astronomer, of Pitane, in Kolia, who flourished thout 320 year before Christ. He was preceptor in mathematics to $\mathrm{Mr}$ cesilaus, who was also a disciple of Theophrastus, the successor of Aristotle. That he was eminent in mathematical learning, appears from two of his works that are cxtant; viz, a treatise On the Aloveable Sphere, published by Dasypodius, in Greek and Latin, 8vo, at Strasburg, in 1572; and in a Latin translation in the Synopsis Mathematica of Mersennus, published in 4to, at Paris, in 1642; and also a treatise On the Rising and Setting of the Stars, cdited with the former work by Dasypodius. Diog. Lacrt. Vit. Arcesil. Fabr. Bib. Grxc. tom. 2, p. 89. Montucla Hist. Mathem. 1 tom. p. 192.

AUTOMATON, a scemingly self-moving machine; ; one so coustructed, by incans of wcights, levers, pulleys, springs, \&c, as to move for a considerable time as if it $2 \mathrm{C}$ 
were endued with animal life. And according to this description, clocks, watches, and all machines of that kind are automatons.

It is said, that Archytas of Tarentum, 400 years before Christ, made a wooden pigeon that could fly; that Archimedes also made such-like automatons; that Regiomontanus made a wooden eagle that flew forth from the city, met the emperor, saluted him, and returned; also, that be made an iron fly, which flew out of his hand at a feast, and returned again after flying about the room; that Dr. Hooke made the model of a flying chariot, capable of supporting itself in the air. Many other surprising automatons we have been eye-witnesses of, in the present age: thus, we have seen figures that could write, and perform many other actions in imitation of animals: M. Vaucanson made a figure that played on the flute; the same gentleman also made a duck, which was capable of eating; drinking, and imitating exactly the voice of a natural one; and, what is still inore surprising, the food $j$ swallowed was evacuated in a digested state, or considerably altered on the principles of solution; also the wings, viscera, and bones, were formed so as strongly to resemble those of a living duck; and the actions of eating and drinking showed the strongest resemblance, even to the muddling the water with its bill. M. Le Droz of la Chaux de Fonds, in the province of Neufchatel, has also executed some very curious pieces of mechanism; one was a clock, presented to the king of Spain, which had, among other curiosities, a slicep that imitated the bleating of a natural one; and a dog watching a basket of fruit, that barked and snarled when any one offered to take it away; besides a variety of human figures, exhibiting motions truly surprising. But all these seem to be inferior to M. Kempell's chess-player, which may truly be considered as the greatest masterpiece in mechanics that ever appeared in the world. "So it certainly would have been," said my esteemed friend, Thos. Collinson, esq. (nephew of the late ingenious Peter Collinson, esq, F. R. S.) had its scientific movements depended merely on mechanism. "Being slightly acquainted with M. Kempell when he exhibited his chess-playing figure in London, I called on him about five years since at his house at Vienna (another gentleman and myself being then on a tour on the continent). The baron (for I think he is such) showed me some working models which he had lately made-among them an improvement on Arkwright's cotton-mill, and also one which he thought an improvement on Boulton and Watt's last steam-engine. I asked him after a piece of speaking mechanism which he had shown me when in London. It spoke as before, and I gave the same word as I gave when I first saw it, Exploitation, which it distinctly pronounced with the French accent. But I particularly noticed, that not a word passed about the chess-player; and of course I did not ask to see it. In the progress of the tour I came to Dresden, where, becoming acquainted with Mr. liden, our envoy there, by means of a lettel given me by lis brother, lord Auckland, who was ambassador when I was at Madrid, he obligingly accompanied me in seeing several things worthy of attention. And he introduced iny companion and nyself to a gentleman of rank and talents, named dosceph Freidrick Freylyere, who seems completely to have discovered the vitality and soul of the chess-playing figure. This gentleman courteously presenterl me with a trentise he had published, dated at Dresàcr, Sept. 30, 1789, explaining its principles, accompanied with curious plates neatly coloured. This treatise is in the German language; and $\mathbf{I}$ hope soon to get a translation of it. A weli-taughit boy, very thin and small of his age (sufficiently so that he could be concealed in a drawer almost immediately under the chess-board,) agitated the whole. Even after this abatement of its being strictly an automaton, much ingenuity remains to the contriver.-This discovery at Dresden accounts for the silence about it at Vienna; for 1 understand, by Mr. Eden, that Mr. Freyliere had sent a copy to Baron Kempell: though he seems unwilling to acknowledge that Mr. F. has completely analysed the whole.

"I know that long and uninteresting letters are formidable things to inen who know the value of time and science; but as this happens to be upon the subject, forgive me for adding one very admirable piece of mechanisin to those you have touched upon. When at Geueva, I called upon Droz, son of the original Droz of la Cliaux de Fonds, where I also was. He showed me an oval gold snuff-box, about (if I recollect right) 4 inches and a half long, by 3 inches broad, and about an inch and a half thick. It was double, having an horizontal partition; so that it may be considered as one box placed on another, with a lid, of course, to each box: one contained snuff-in the other, as soon as the lid was opened, there rose up a very small bird, of green enamelled gold, sitting on a gold stand. Inmediately this minute curiosity wagged its tail, shook its. wings, opencd its bill of white enamelled gold, and pourcd forth, minute as it was (being only three quarters of an inch from the beak to the extremity of the tail) such a clear melodious song, as would have filled a room of 20 or 30 feet square with its harmony. Droz agreed to mcet me at Florence; and we visited the Abbé Fonvana together. He afterwards joined me at Rome, and exhibited his bird to the pope and the cardinals in the Vatican palace, to the admiration, I may say, to the astonishment, of all who saw and heard it."

Another extract from a second letter on the same subject, by Mr. Collinson, is as follows: "Permit me to spcak of another automaton of Droz's, which scveral years since be exhibited in England; and which, from my personal acquaintance, I had a commodious opportunity of particularly examining. It was a figure of a man, I think, the size of life. It held in its hand a metal style; a card of Dutch vellum being laid under it. A spring was touched, which released the internal clock-work from its stop, when the figure immediately began to draw. Mr. Droz, happcning once to be sent for in a great hurry to wait upon some considerable personage at the west end of the town, left me in possession of the keys, which opened the recesses of all his machincry. He opened the drawingmaster himself, wound it up, explained its leading parts, and faught me how to make it obcy my requirings, as it had nbeyed lis own: Droz then went away. After the first card was finished, the figure rested. I put a scrond; and so on, to five separate cards, all different subjects; but five or six was the extent of its delineating powers. The first card contained, I may ruly say, elegant portrats and likenesses of the king and queen, facing cach other; and it was curious to observe with what precision the figure lifted up his pencil, in the transition of it from one point of the Iraft to another, without making the lcast slur whatever: for instance, in passing from the forchearl to the eye, nuse, and chin; or from the waving curls of the hair to the car, \&c. 1 have the cards now by me, \&c, \&.c." 
To the preceding account of such surprising accounts of automata, may be added, that we have lately seen exhibited at Spring:Gardens, in London, by a Mr. Maliardet, similar and other such mechanical contrivances; viz, the singing-bird, and the figure of a young man that wrote many words and lines, and drew very neatly several figures as above described. There were also several others, equally curious; as a tumbling figure, which performed surprising evolutions about a horizontal wire; also a spider resembling a living one, and which crept all over the table; and the musical lady, being one that much resembled life, by its motions and respirations, \&ce, and naturally performing some fine pieces on the piano-forte, \&c.

AU'TUMN, the third season of the year, when the harvest and fruits are gathered in. This begins at the descending equinox, which, in the northern hemisphere, is when the sun enters the sign Libra, or about the $22 \mathrm{~d}$ day of September; and it ends, when winter commences, about the same day in December.

A U TUMNa, something belonging to autumn. Thus,

Autuanal Equinox, the time when the sun enters the descending point of the ecliptic, where it crosses the equinoctial; and is so called, because the nights and days are then equal.

Autumal Point, the point of the ecliptic answering to the autumnal equinox.

A U T M M a L Signs, are the signs Libra, Scorpio, Sagittary, through which the sun passes during the autumn.

AUZOUT, or Azout, (Adria N), a French mathematician of the 17 th century, and one of the earliest members of the Academy of Sciences at Paris, was born at Rouen, and died in 1693 . Some have ascribed to him the honour of having invented the micrometer; but he is more justly entitled to the praise of having contributed to the improvement of it, in pursuance of the ideas suggested by $M$. Huygens, and the Marquis of Malvasia. He published a treatise on this subject in 1667 , which was afterwards inserted in the Memoirs of the Academy for 1693, tom. 6 . Auzout was also concerned with M. Picard in the important discovery of the method of applying the telescupe to the quadrant, which has been highly useful to astronomers. He published An Ephemeris of the Comet of 1665 ; also, A Letter to the Abbé Charles on the Observations of Campani, in 1665; and some Remarks on the Machise of Hooke, mentioned in the article Automaton. These tivo last pieces are also contained in the 6th volume of the Memoirs of the Academy. Montucla, Hist. Mathem. tom. 2, p. 569-572.

AXIOM, a self-evident truth, or a proposition immediately assented to, when the terms of it are properly understood. Such as, that the whole is grcater than its part; that a thing cannot be and not be at the same time; and that any whole is equal to all its parts taken together.

Some axioms are in effect, strictly speaking, no other than identical propositions. Thus, to say that all right angles are equal to each other, is as much as to say, all right angles are right angles; such equality being implied in the very definition, or the very name or term itself.

Axiom is also an established principle in some art or science. Thus, it is an axiom in physics, that nature does nothing in vain; that effects are proportional to their causes; \&c. It is an axiom in geometry, that two things equal to the same thing, are also equal to each other; that if to two equal things equals be added, the sums will be equal. And it is an axiom in optics, that the augle of in- cidence is equal to the angle of reflection. In this sense also the general laws of motion are called axioms; as, that all motion is rectilinear, that action and reaction are equal, \&c.

These particular axioms, it may be observed, do not bowever immediately arise from any first notions or ideas, but are deduced from certain hyputheses; this is particularly obscrved in physical matters, wherein, as several experiments contribute to make one hypothesis, so several hypotheses contribute to one axiom.

The axioms of Euclid are general propositions; and so are the axioms of the Newtonian philosophy; but these two kinds of axioms have very different origins. The former appear true upon a bare contemplation of our ideas; whereas the latter are the result of the most laborious induction; and therefore " ought not," as Lord Bacon says, "to be admitted upon conjecture or even upon the authority of the learned; but as they are the general principles and grounds of all learning, they should be canvassed and examined with the most scrupulous attention."

AXE or AXIS, in Geometry, the straight line in a plane figure, about which it revolves, to produce or generate a solid. Thus, if a semicircle be moved round its diameter at rest, it will generate a sphere, whose axis is that diameter. And if a right-angled triangle be turned about its perpendicular at rest, it will describe a cone, whose axis is that perpendicular.

AxIs is yet more generally used for a right line conceived to be drawn from the vertex of a figure to the middle of the base. So the

Axis of a circle or sphere, is any line drawn through the centre, and terminated at the circumference, on both sides.

Axis of a cone, is the line from the vertex to the centre of the base.

AxIs of a cylinder, is the line from the centre of the one end to that of the other.

AxIs of a conic section, is the line from the principal vertex, or vertices, perpendicular to the tangent at that point. The ellipsis and hyperbola have each two axes, which are finite and perpendicular to each other; but the parabola has only one, and that infinite in length.

Transverse Axis, in the Ellipse and Hyperbola, is the diameter passing through the two foci, and the two principal vertices of the figure. In the hyperbola it is the shortest diameter, but in the ellipse it is the longest.

Conjugate Axıs, or Second Axis, in the Ellipse and $\mathrm{Hy}_{y}$ perbola, is the diameter passing through the centre, and perpendicular to the transverse axis; and is the shortest of all the diameters, as the transverse axis is the longest.

Axis, of a curve line, is still more generally used for that diameter which has its ordinates at right angles to it, when that is possible. For, like as in the conic sections, any diameter bisects all its parallel ordinates, making the two parts of them on both sides of it equal; and that diameter which has such ordinates perpendicular to it, is an Axis: So, in curves of the second order, if there be any two parallel lines each meeting the curve in three points: the right line which cuts these two parallels so, that the sum of the two parts on one side of the cutting line, between it and the curve, is equal to the third part terminated by the curve on the other side, then the said line will in like manner cut all other parallels to the former two lines, viz, so that, of every one of them, the sum of the two parts, or ordinates, on one side, will be equal to the $2 \mathrm{C} 2$ 


\section{A X I}

$[196$

third part or ordinate on the other side. Such cutting line then is a dianeter; and rhat diameter whose parallel ordinates are at right angles to $\mathrm{it}$, when possible, is an Axis. And the same for other curves of still higher orders. Newton, Enumeratio Lincarum Tertii Urdinis, sect. 2 , art. 1 .

Axis, in Astronemy. As the AxIs of the world, is an imaginary riglit line conceived to pass through the centre of the earth, aud terminating at each end in the surface of the mundane sphere. About this line, as an axis, the sphere, in the Ptolemaic system, is supposed daily to revolve.

Axis of the Earth, is the line connecting its two poles, and about which the earth performs its diurnal rotation, from west to east. This is a part of the axis of the world, and always remains parallel to itself during the motion of the earth in its orbit about the sun, and perpendicular to the plane of the equator.

Axis of a Planet, is the line passing through its centre, and about which the planet revolves.-The Sun, Earth, Moon, Jupiter, Mars, and Venus, it is known from observation, move about their several axes; and the like mo:ion is easily inferred of the other three, Mercury, Saturn, and the Georgian planet.

Axis of the Horizon, Equator, Ecliptic, Zodiac, \&c, are right lines passing through the centres of those circles, perpendicular to their planes.

Axis of a Magnet, or Magnetical Axis, is a line passing through the middle of a magnet, lengthwișe; in such manner, that however the magnet be divided, provided the division be made according to a plane passing through that line, the magnet will then be cut into two loadstones. And the extremities of such lines are called the poles of the stone.

Axis, in Mechanics.-The axis of a balance, is the line upon which it moves or turns.

Axis of Rotation, of any solid, is' the line about which the body really revolves, when it is put in motion. The impulse given to a homogeneous spliere, in a direction which does not pass through its centre, will cause it to revolve constantly round the diameter, which is perpendicular to a plane passing through its centre, and the line of direction of the impressed force. New forces acting on all its parts, and of which the result passes through its centre, will not change the parallelism of its axis of rotation. Thus it is that the axis of the earth remains alway.s nearly parallel to itself; in its revolution round the sun, without its being necessary to suppose, with Copernicus, an anuual motion of the poles of the earth round those of the ccliptic. If the body possess a certain figure, its axis of rotation may change every instant. The determination of these changes, whatever may be the forcesacting on the bodies, is one of the most interesting prublems of mechanics, respecting hard bodies, on account of its connexion with the precession of the equinoxes, and the libration of the moon. The solution of this problem has led to a curious and very useful result, namely, that in all bodies there exist three axes perpendicular to each other, round which the body may turn uniformiy when not solicited by exturnal forces. On this account these axes are properly called the three principal axes of rotation.

A.sis of Oscillation, is a line parallel to the horizon, passing through the centre, about which a pesdulum vibrates, and perpendicular to the plane in which it oscillates.

\section{A $\times 1$}

Axis in Peritrochio, or Whect and axle, is one of the five mechanical powers, or simple machines; contrived chiefly for the raising of weights to a considerable height, as water from a well, \&c. This unachine consists of a circle $\mathbf{A B}$, concentric with the base of a cylinder, and moveable together with it about its axis $\mathrm{CD}$. This cylinder is called the axis; and the circle, the peritruchium; and the radii, or spokes, which are sometimes fitted immediately into

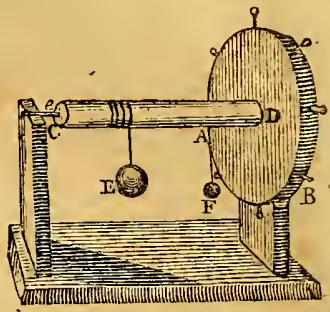
the cylinder, without any circle, the scytalæ. About the axis winds a rope, or chain, by means of which great weights are raised by turning the wheel.-The axis in peritrochio takes place in the motion of every machine, in which a circle may be conceived as described about a fixed axis, concentric with the plane of a cylinder about which it is placed; as in Crane-wheels, Mill-wheels, Capstans, \&c.-The chief properties of the Axis-in-peritrochio, are as follow :

1. If the power $F$ applied in the direction a $\mathrm{F}$ a tangent to the circumference, or perpendicular to the spoke, be to a weight $\mathrm{E}$, as the radius of the axis $\mathrm{c} e$ is to the radius of the wheel $A D$, or the length of the spoke; tlie power will just sustain the weight; that is, the power and the weight will be in equilibrio, when they are in the reciprocal proportion of their distance from the centre.

2. When the wheel moves, with the power and weight; the velocities, of their motion, and the spaces passed over by them, will be both in the same proportion as above, namely, directly proportional to their distances from the centre, and reciprocally proportional to their own weights when they are in equilibrio.

3. A power and a weight being given, to construct an axis-in-peritrochio, by which it shall be sustained and raised. Let the axis be taken large enough to support the weight and power without breaking: then, as the weight is to the power, so make the radius of the wheel to the radius of the axis. Hence, if the power be very small in respect of the weight, the radius of the wheel will be vastly great. For example, suppose the weight 4050 , and the power 50 ; then the radius of the wheel will be 81 times that of the axis; which would be a very inconvenient size. But this inconvenience is obviated by increasing the number of the wheels and axes; making one to turn another, by means of teeth and pinions. And to find the effect of a number of wheels and axes, thus turning one another, multiply together, all the radii of the axes, and all the radii of the wheels, and then it will be, as the product of the former is to the product of the latter, so is the power to the weight. So, if there be 4 wheels and axes, the radius of each axis being 1 foot, and the radius of each wheel 3 feet; then the contiuual product of all the wheels is $3 \times 3 \times 3 \times 3$ or 81 feet, and that of the axis only 1 ; therefore the effect is as $\$ 1$ to 1 , or the weight is 81 times the power. And, on the contrary, if it be required to find the diameter of each of four equal wheels, by which a weight of $4050 \mathrm{lb}$ shall be balanced by a power of $50 \mathrm{lb}$, the diameter of each axis being 1 foot: dividing 4050 by 50 , the quotient is 81 , extract the 4 th root of 81 , or twice 
the square root, and it will give 3 , for the dianieter of the four wheels songht.

Axrs of a vessel, is that quiescent right line passing through the middle of it, perpendicular to its base, and equally distant from its sides.

Axis, in Optics.-Optic axis, or visual axis, is a ray passing through the centre of the eye, or falling perpendicularly on the eye.

Axis of a lens, or glass, is the axis of the solid of which the lens is a segment. Or the axis of a glass, is the line joining the two vertices or middle points of the two opposite surfaces of the glass.

Axıs of Incidence, in Dioptrics, is the line pasiing through the point of incidence, perpendicularly to the refracting surface.

Axis of Refraction, is the line continued from the point of incidence or refraction, perpendicularly to the refracting surface, along the farther medium.

AZIMUTH, of the sun, or star, \&c, is an arch of the horizon, jntercepted between the meridian of the place, and the azimuth or vertical circle passing through the sun or star; and is equal to the angle at the zenith formed by the said meridian and vertical circle. Or it is the complement to the eastern or wcstern amplitude.-The azimuth is thus found by trigonometry;

As radius is to the tangent of the latitude,

So is the tangent of the altitude of the sun or star,

To the cosine of the azimuth from the south, at the time of the equinox.

Azıм Uт, magnetical, an arch of the horizon contained between the magnetical meridian, and the azimuth or vertical circle of the object; or jts apparent distance from the north or south point of the compass. This is found by observing the sun, or star, \&c, with an azimuth compass, when it is 10 or 15 degrees high, either before or after noon.

Azinuti Compass, an instrument for finding either the magnetical azimuth or amplitude of a celestial object. The description and use of this instrument, see under the article Conpass.

Azrmuth DiaL, a dial whose stile or gnomon is perpendicular to the plane of the horizon.

AzIMUTu, or Vertical Circles, are grcat circles of the sphere-intersecting each other in the zenith and nadir, and cutting the horizon at right angles.-These azimuths are represented by the rhumbs on common sea charts; and on the globe by the quadrant of altitude, when screwed in the zenith. On these azimuths is counted the height of the sun, or stars, \&c, when out of the meridian.

AZOTIC GAS, the same as the phlogisticated air of Scheele and Priestley, or the mephitic air of Lavoisier, or the nitrogen gas of Chaptal, and sume other French chemists. This gas was discovered in 1772 , by Dr. Rutherford of Edinburgh ; and many of its properties were ascertained by Cavendish, Kirwan, and Priestley. It is nearly of the same specific gravity as common air, or perhaps a very little heavier, being indeed only atmospheric air deprived of its oxygen; and hence its noxious quality of presently extinguishing flame and animal life; as in unused wells or cellars, and close places of stagnant air.

\section{B.}

\section{B A C}

$\mathbf{B}^{\mathrm{A}}$ ACK-STAFF, an instrument formerly used for taking the sun's altitude at sea; being so called because the back of the observer was turned towards the sun when he made the observation. It was sometimes called Davis's quadrant, from its inventor Captain John Davis, a Welchman, and a celcbrated navigator, who produced it about the year 1590 .

This instrument consists of two concentric arches of box-wood, and three vanes : the arch of the longer radius

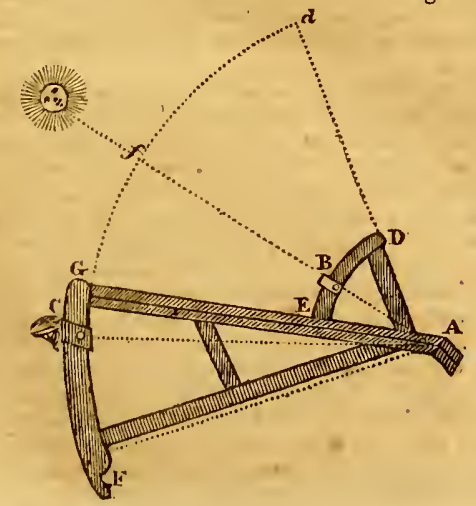

\section{B A C}

is of 30 degrees, and the other of 60 degrees, making between them 90 dcgrees, or a quadrant: also the vane $A$ at the centre is called the horizon-vane, that on the arch of $60^{\circ}$ at $\mathrm{B}$ the shade-vane, and that on the other arch at c the sight-vane.

To use the Back-Staff.-The shade-vane is to be set upon the 60 arch, at an even degree with some latitude, less by 10 or 15 degrees than the complement of the sun's altitude is judged to be; also the horizon-vane being put on at $\boldsymbol{A}$, and the sight-vane on the 30 arch $F G$, the observer then turns bis back to the sun, lifts up the instrument, and looks through the sight-vane, raising or falling the quadrant, till the shadow of the upper edge of the shade-vane fall on the upper edge of the slit in the horizon-vane; and then if he can see the horizon through the said slit, the observation is exact, and the vanes are rightly adjusted: But if the sea appear instead of the horizon, the sight-vane must be moved downward towards F; or if the sky appear, it must be moved upward towards $G$; thus trying till it comes right : the observer next examines how many degrees and minutes are cut by that edge of the sight-vane that answers to the sight-hole, and to them he adds the degrees cut by the upper edge of the shade vane; the sum of which is the sun's distance from the zenith, or the complement of his altitude; that is, of his upper limb when the superior end of the shade-vane is used in the ubservation, or of his lower limb when the lower part of that vane is used; therefore in the former case add 16 minutes, the sun's semidiameter, and subtract. 
16 minutes in the latter case, to give the zenith distance or cu-altitude of the sun's centre.

Mr. Flamsteed contrived a glass lens, or double convex, to be placed in the middle of the shade-vane, which throws a small bright spot on the slit of the horizon-vane, instead of the shade; which is a great improvement, if the glass be truly made; for by this means, the instrument may be used in hazy weather, and a much more accurate observation made at all times.

BACON (Rogen), a celebrated English monk of the Franciscan order, was born near Ilchester in Somersetshire, in the year 1214. He commenced his studies at Oxford; whence he removed to the university of Paris, which at that time was esteemed the centre of learning; and where it appears he made such progress in the sciences, that be was esteemed the glory of that university, and was there greatly caressed by several of his countrymen, particularly by Robert Groothead or Grouthead, afterwards bishop of Lincoln, his great friend and patron. Having taken the degree of doctor of divinity, he returned to England in 1240, and took the habit of the Franciscan order, being then about 26 years of age; but according to some he became a monk before he left France. He now pursued his favourite study of experimental philosophy with unremitting ardour and assiduity. In this pursuit, in experiments, he informs us he spent, in the course of 20 years, no less than $2000 l$. in the purchase of instruments and scarce books, an amazing sum in those days, but which was generously furnished him by some of the heads of the university, to enable him the better to pursue his noble researches. By such extruordinary talents and rapid progress in the sciences, which in that ignorant age were so little known to the rest of mankind, while they raised the admiration of the more intelligent few, could not fail to excite the envy of his illiterate fraternity, whose malice against him was the more inflamed by the freedom with which he treated the clergy in his writings, in which he spared neither their ignorance nor their immorality: these therefore found no difficulty in possessing the vulgar with the notion of Bacon's communication with evil spirifs. Under this pretence he was restrained from reading lectures to the young students in the university, and at length so closely confined as to be debarred from all intercourse with his firiends, and from receiving a necessary supply of food. These efforts of malevolence and cruelty, whatever might have been the real or pretended causes from which they originated, could not however deprive this great man of the esteem and respect to which his distinguished talents and character entitled him. Such was the high opinion entertained by the cardinal bishop of Sabina, who was the pope's legate in England, of his genius and merit, that he requested from him a complete copy of all his works. As he was restrained, by the prohibition of his own fraternity, from communicating any of his works to any person whatever, he at first declined complying with the cardinal's request; but as soon as he heard that the cardinal-legate was raised to the pontifical dignity, under the name of Clement $1 \mathrm{v}$, he signified to him by letter his readiness to perform what his holiness had desired; and the pope assured him of protection against any interference of his own order. Bacon immediately began to collect, arrange, and improve the pieces he had already written: and having digested them into one volume, under the title of "Opus Majus" (the greater work, he sent it to the pope in the year 1267 , by a special messenger, whose name was John of Paris, and who was his own favourite disciple. This John of Paris was a poor boy, of promising talents, taken by Bacon under his tuition, in order to try by experience the efficacy of his peculiar mode of instruction; and as the result of it he observes, "that there was no room to conceive any high notions of the perfection of human wisdom, when it was possible in a year's time to teach a young man all that, with the utmost industry and application, a zealous inquirer after knowledge was able either to attain or discover in the space of 20 or even 40 years. The pope was so gratified with the present of this learned work, that it procured for Bacon extraordinary favour and encouragement in his studies.

With the life of the enlightened and liberal Clement Iv, terminated the tranquillity of this philosopher; for, under the pontificate of Nicholas III, and with the sanction of his authority, Jerom de Esculo, or de Ascoli, general of the Francisean order, prohibited the reading of his works, and sentenced him to imprisonment. The pretended cause of this severity has been sought by some writer's in certain tracts of Bacon on necromancy, astrology, and alcliemy; but the true reason was most probably that dread of innovation, which Bacon's improvements in science caused in the minds of bigoted or interested persons. Bacon continued in prison 10 years; but on the accession of Jerom de Esculo to the papal see, under the name of Nicholas Iv, he attempted to conciliate the favour of the pope, by presenting to him a treatise " $O n$ the means of avoiding the infirmities of old age." What effect this had on the pope does not appear; it did not at least produce an immediate discharge : however, towards the latter end of his reign, by the interposition of some noblemen, Bacon obtained his liberty; and afterwards spent the remainder of his life in the college of his order, where he died in the year 1294, at 80 years of age, and was buried in the Franciscan church. Such are the few particulars which the most diligent researches have been able to discover concerning the life of this very extraordinary man.

Bacon's printed works are, 1. Epistola Fratris Rogeri Baconis de Secretis Operibus Artis et Naturz, et de Nullitate Magice: Paris, 1542, in 4to. Basil, 1593, in svo. 2. Opus Majus; London, 1733, in fol.-published by Dr. Jebb. 3. Thesaurus Chemicus; Francf. 1603 and 1620 . These printed works of Bacon contain a number of essays, which have been considered as distinct books in the catalogue of his writings by Bale, Pitts, \&c; but there remain also in different libraries several manuscripts not yet published.

By an attentive perusal of his works, the reader is astonished to find that this great luminary of the 13th century was deeply skilled in all the arts and sciences, and in many of them had made the most important inventions and discoveries. He was, says Dr. Peter Shaw, beyond all comparison the greatcst man of his time, and he might perhaps stand in competition with the greatest that have appeared since. It is wonderful, consiclering the ignorant age in which he lived, how he came by such a depth of knowledge on all subjects. His writings are composed with that elegance, conciseness, and strength, and adorned with such just and exquisite observations on nature, that, among all the chemists, we do not know his cqual. In his chemical writings, he attempts to show how imperfect metals may be ripened into perfect ones; making, with Geber, mercury the common basis of all metals, and sulphur the cement. 
His other physical writings show no less genius and force of mind. In his, treatise Of the Secret Works of Art and Nature, he shows that a person perfectly acquainted with the manner observed by nature in her operations, would be able to rival, and even to surpass ber. In another piece, Of the Nullity of Magic, he shows with great sagacity and penetration, whence the notion of it sprang, and how weak all pretences to it are. From a pernsal of his works, adds the same author, we find Bacon was no stranger to many of the principal discoveries of the present and past ages. 'The ingredients and effects of gunpowder he was doubtless acquainted with; and thunder and lightning, he tells us, may be produced by art; for though sulphur, nitre, and charcoal, when separate have no sensible effect, yet when mixed together in due proportion, and closely confined, and fired, they yield a loud report. A more precise description of gunpowder cannot be given in words. He also mentions a sort of unextinguishable fire prepared by art; which shows he was not unacquainted with phosphorus: and that he had some notiou of the rarefaction of the air, and the structure of an air-pump, is past contradiction. He was the miracle, says Dr. Friend, of the age he lived in, and the greatest genius, perhaps, for mechanical knowledge, that ever appeared in the world since Archimedes. He appears also to have been master of the whole science of optics: he has accurately described the uses of readingglasses, and shown the way of making them. Dr. Friend adds, that he also describes the camera obscura, and all sorts of glasses, which magnify or diminish any object, or bring it nearer to the eye, or remove it farther off. Bacon says himself, that he had great numbers of burning-glasses: and that there were none ever in use among the Latins, till his friend Peter de Mahara Curia applied himself to the making of them. That the telescope was not unknown to him, appears from a passage where he says, that he was able to form glasses in such a manner, with respect to our sight and the objects, that the rays shall be refracted and reflected wherever we please, so that we may see a thing under what angle we think proper, either near or at a distance, and be able to read the smallest letters at an incredible distance, and to count the dust and sand from the greatness of the angle under which we see the objects; and also that we shall scarce see the greatest bodies near us, on account of the smallness of the angle under which we view them. His skill in astronomy was also very great: he discovered that error which occasioned the reformation of the calendar; one of the greatest efforts, according to Dr. Jebb, of human industry: and his plan for correcting it was followed by pope Gregory xinr, with this variation, that Bacon would have had the correction to begin from the birth of our Saviour, whereas Gregory's amendment reaches no higher than the Nicene council.

$\mathrm{B} \Lambda \mathrm{CON}$ (FRANCIS), baron of Verulam, viscount of St. Albans, and lord high chancellor of England under king James $\mathbf{r}$. He was born in 1560 , heing son of Sir Nicholas Bacon, lord keeper of the great seal in the reign of Queen Elizabeth, by Anne daughter of Sir Anthony Cook, eminent for ber skill in the Latin and Greek languages. He gave even in bis infuncy tokens of what he would one day become; and Queen Elizabeth had many times occasion to admire his wit and talents, and used to call him her young lord keeper. He studied the philosophy of Aristotle at Cambridge; where he made such progress in general literature, that at 16 years of age he had run through the whole circle of the liberal arts as they were then taught, and even began to perccive those imperfections in the reigning philosophy which he afterwards so effectually exposerl, and thence not only overcame that tyranny which checked the progress of true knowledge, but laid the foundation of that free and useful philosophy which has since opened a way to so many glorious discoveries. On his leaving the university, his father sent him to France; where, before he was 19 years of age, he wrote a general view of the state of Europe: but his father dying, he was obliged suddenly to return to England; where he applied limself to the study of the common law, at Gray's-inn. His merit at length raised him to the highest dignities in his profession, namely, those of attorney-general, and lord-high-chancellor. But being of an easy and liberal disposition, his servants took advantage of that temper, and of their situation under him, by accepting presents in the line of-his profession. Being abandoned by the king, he was tried by the house of lords, for bribery and corruption, and by them sentenced to pay a fine of $40,000 /$, and to remain prisoner in the Tower during the king's pleasure. 'The king however soon after remitted the fine and punishment: but his misfortunes had given him a distaste for public affairs, and be afterwards mostly lived a retired life, closely pursuing his philosophical studies and amusements, during which time he composed the greatest part of his English and Latin works. Though even in the midst of his honours and employments he forgot not his philosophy, but in 1620 published his great work Novum Organum. After some years spent in his philosophical retirement, he died in 1626 , being 66 years of age.

The chancellor Bacon is one of those extraordinary geniuses who have contributed most to the advancement of the sciences. He clearly perceived the imperfection of the school philosophy, and pointed out the only means of reforming it, by proceeding in the opposite way, from experiments to the discovery of the laws of nature. Addison has said of him, That he had the sound, distinct, comprehensive knowledge of Aristotle, with all the beautiful light graces and embellishments of Cicero. Mr. Walpole calls him the Prophet of Arts, which Newton was afterwards to develope; and adds, that his genius and his works will be universally admired as long as science exists. He did not yet, said another great man, understand nature, but he knew and pointed out all the ways that lead to her. He very early despised all that the universities called philosophy; and used every exertion in his power that they should not disgrace her by their quiddities, their horrors of a vacuum, their substantial forms, and such-like impertinencies.

He composed two works for perfecting the sciences. The former On the Dignity and Augmentation of the Sciences. In this he shows the state in which they then were, and points out what remains to be discovered for perfecting them; condemning the method employed by Aristotle, of reversing the natural order of things. He here also proposes his celebrated division of the sciences.

To remedy the faults of the common logic, Bacon composed his second work, the New Organ of Sciences, above mentioned. He here teaches a new logic, the chief end of which is to show how to make a good inference, as that of $\Lambda$ ristotle's is to make a syllogism. Bacon was 18 years in composing this work, and he always esteemed it as the chief of his compositions.

The pains which Bacon bestowed on the sciences in general, prevented him from making any considerable appli- 
cations to any one in particular: and as he knew that natural phitosuphy is the foundation of all the other sciences, he chicfly endeavoured to give perfection to it. He therefore proposed to establish a new system of physics, rejecting the doubtful principles of the ancients. For this purpose he took the resolution of composing every month a treatise on some branch of physics; and accordingly beyan with that of the winds; after which he gave that of heat; next that of motion; and lastly that of life and death. But as it was impossible that one man alone could so compose the whole circle of sciences with the same precision,-after having given these patterns, to serve as a model for those who might cboose to labour on his principles, he contented himself with tracing in a few words the design of four other tracts, and with furnishing the materials, in his Silva Silvarum, where he has amassed a vast number of experiments, to serve as a foundation for his new physics. In fact, no one before Bacon understood any thing of the experimental philosophy; and of all the pbysical experiments which have been made since his time, there is scarcely one that is not pointed out in his works.

This great precursor of philosophy was also an elegant writer, an historian, and a wit. His moral essays are valuable, but are formed more to instruct than to please. There are also many excellent things in his work On the Wisdom of the Ancients, in which he has moralized the fables which formed the theology of the Greeks and Romans. He wrote also The History of Henry the 7 th king of England, by wbich it appears that he was no less a great politician than a great philosopher.

Bacon had also some other writings, published at different times; the whole of which were collected together, and published at Frankfort, in the year 1665, in a large folio volume, with an introduction concerning his life and writings. Another edition of his works was also published at London in 1740; the enumeration of which is as below :

1. De Dignitate et Augmentis Scientiarum.

2. Novum Organum Scientiarum, sive Judicia vera de Interpretatione Naturæ; cum Parasceve ad Historiam Naturalem et Experimentalem.

3. Phanomena Universi, sive Historia Naturalis et Experimentalis de Ventis; Historia Densi et Rari; Historia Gravis et Levis; Historia Sympathiæ et Antipathiæ Rerum; Historia Sulphuris, Mercurii, et Salis; Historia Vita et Mortis; Historia Naturalis et Experimentalis de Forma Calidi ; De Mlotus, sive Virtutis activa variis speciebus; Ratio inveniendi causas Fluxus et Refluxus Maris ; \&c, \&c.

4. Silva Silvarum, sive Ilistoria Naturalis.

5. Novus Atlas.

6. Historia Regni Henrici vı Angliæ Regis.

7. Sermones Fideles, Ethici, Politici, Oeconomici.

8. De Sapientia Veterum.

BACULE, in Fortification, a kind of portcullis, or gate, made like a pit-fall, with a counterpoise, and supported by two great stakes. It is usually made before the corps de garde, near the gate of a place.

BACULOME'IRY, the art of measuring either accessible or inaccessible lines, by the help of baculi, staves, or rods. Schwenter has explained this art in his Geometria Practica; and the rules of it are delivered by Wolfus, in his Elcments: Ozanam also gives an illustration of the principles of baculometry.

BAILLY (JiAN SYLVAIN), a celebrated French astro- nomer, historiographer, and politician, was born at Paris the 15 th of September 1736 , and has tigured as one of the greatest men of the age, being a member of several academies, and an excellent scholar and writer. He enjoyed for several years the office of keeper of the king's pictures at Paris. About the year 1762 , the theory of Jupiter's satellites became a particular object of his inquiries; and, in the competition for this prize question, he had a formidable rival in La Grange, generally considered as one of the greatest mathematicians in Europe. The results of his investigations were afterwards collected into a treatise, and published in 1 vol. 4 to; also in 1766 , when it was preceded by a History of the Astronomy of these Satellites. In the Journal Encyclopédique for May and July 1773 , he addressed a letter to M. Bernoulli, astronomer royal at Berlin, on some discoveries relative to these satellites, which he had disputed. In 1768 he publislied the eulogy of Leibnitz, which obtained "the prize at the Academy of Berlin, where it was printed. In 1770 he printed at $\mathrm{Pa}$ ris, in 8 vo, the eulogies of Charles the $5 \mathrm{th}$, also of Lecaille, of Leibnitz, and of Corneille. This last had the second prize at the Academy of Rouen, while that of Moliere had the same honour at the French Academy.

M. Bailly was admitted into the Academy as Adjunct the 29th of January 1763, and as Associate the 14th of July 1770 . In 1771 he was a candidate, under the patronage of Buffon, for the office of Secretary; but the interest of Condorcet, and the influence of Dalembert, prevailed in favour of Condorcet. Of the Academic Francoise, he was chosen Secretary in 1754 ; and he was admitted in the following year into the Academy of Inscriptions and Belles Lettres, the only instance since Fontenelle of the same person being at once a member of all the three Academies. In 1775 came out at Paris, in 4to, his History of the Ancient Astronomy, in $1 \mathrm{vol}$ : in 1779 the History of Modern $\Lambda$ stronomy, in 2 vols: and in 1787 the History of the Indian and Oriental Astronomy, being the $2 \mathrm{~d}$ vol. of the Ancient Astronomy.

M. Bailly's memoirs, published in the volumes of the Academy, are as follow:

Memoir upon the Theory of the Comet of 1759.

Memoir upon the Epoques of the Moon's motions at the end of the 17 th century.

First, second, and third Memoirs on the Theory of Jupiter's Satellites; 1763.

Memoir on the Comet of 1762 ; vol. for 1763.

Astronomical Observations, made at Noston ; 17 (it.

On the Sun's Eclipse of the 1st of April, $176+4$.

On the Lougitude of Polling; $176+$.

Observations made at the Louvre from 1760 to $176 \mathrm{ft}$; 1765.

On the cause of the Variation of the Inclination of the Orbit of Jupiter's second Satcllite; 1765.

On the Motion of the Nodes, and on the Vatriation of the Inclination of Jupiter's Satellite's; 1766 .

On the Theory of Jupiter's Satellites, pubtished by M. Bailly, also according to the 'lables of their Mlotions and of those of 1upiter, published by ML. leaurat ; 1766 .

Observation.6 on the Opposition of the $\mathrm{S}_{\mathrm{un}}$ and Jupitcr; $176 \mathrm{~s}$.

On the Equation of Jupiter's Centre, and on some other Elements of the Theory of that Planet; 1768 .

On the 'Transit of Venus over the Stun, the $3 \mathrm{~d}$ of Iure 1769; and on the Solar Eclipse the the June the sause year : 1769 . 
In the beginning of the revolution in France, in 1789, M. Bailly took an active part in that business, and was so popular and generally esteemed, that he was chosen the first president of the States General, and of the National Assembly, and was afterwards for two years together the Mayor of Paris ; in both which offices he conducted himself with great spirit, and gave general satisfaction.

He soon afterward however experienced a sad reverse of fortune; being accused by the ruling party of favouring the king, he was condemned for incivism and for wanting to overturn the Republic; in consequence he died by the guillotine at Paris, November 12, 1793, at 57 years of age.

BAINBRIDGE (Јонг), an eminent physician, astronomer, and mathematician, was born in 1582 , at Ashby-de-la-Zouch, Leicestershire. He studied at Cambridge, and having taken his degrees of Bachelor and Master of Arts, he returned to Leicestershire, where for some years he kept a grammar-school, and at the same time practised physic ; employing his leisure hours in studying mathematics, especially astronomy, which had been his favourite science from his earliest years. By the advice of his friends, he removed to London, where he was admitted a fellow of the College of Physicians. His description of the comet which appeared in 1618, greatly raised his character, and procured him the acquaintance of Sir Henry Savile, who, in 1619, appointed bim his first professor of astronomy at $\mathrm{Oxford}$. On his removal to this university, he entered a master-commoner of Merton college; the master and fellows of which appointed him junior reader of Linacer's lecture in 1631, and superior reader in 1635 . At the age of 40 he began the study of Arabic, with an intention of publishing correct editions of the ancient astronomers. Before completing that work however he died, in the year 1643 , at 61 years of age.

Dr. Bainbridge wrote many works, but most of them have never been published; those that were published, were the three following, viz:

1. An Astronomical Description of the late Comet, from the 18 th of November 1618 , to the 16 th of December following; 4to, London, 1619.--This piece was only a specimen of a larger work, which the author intended to publish in Latin, under the title of Cometographia.

2. Procli Sphæra, Ptolemæi de Hypothesibus Planeta. rum liber singularis. To which he added Ptolemy's $\mathrm{Ca}$. non Regnorum. He collated these pieces with ancient inanuscripts, and gave a Latin version of them, illustrated with figures: printed in 4.to, 1620.

3. Canicularia. A treatise concerning the Dog-star, and the Canicular Days: published at Oxford in 1648, by Mr. Greaves, together with a demonstration of the heliacal rising of Sirius, the dog-star, for the parallel of Lower Egypt. Dr. Bainbridge also undertook this work at the request of Archbislop Usher, but he left it imperfect; being prevented by the breaking out of the civil war, or by death.

There were also sevcral dissertations of his prepared and committed to the press the year after his death, but the edition of them was never completed. The titles of them are as follow:

1st, Antiprognosticon, in quo Mavrirỹs Astrologicæ, Cœlestium Donorum, et Triplicitatum Commentis, magnisque Saturni et Jovis (cujusmodi anno 1623, et 1643, contigerunt, et vicesimo fere quoque deinceps anno, ratis

VOL. I. naturæ legibus recurrent) Conjunctionibus innixæ, Vanitas breviter detegitur.

2nd, De Meridionorum sive Longitudinum Differentiis irıveniendis Dissertatio.

$3 \mathrm{~d}$, De Stella Veneris Diatriba.

Besides the foregoing, there were several other tracts, never printed, but left by his will to Archbishop Usher; among whose manuscripts they are preserved in the library of the college of Dublin. Among which are the following: 1. A Theory of the Sun. 2. A Theory of the Moon. 3. A Discourse concerning the Quantity of the Year. 4. Two volumes of Astronomical Observations. 5. Nine or ten volumes of Miscellaneous Papers relating to Mathematical Subjects.

BAKER (ThомAs), a mathematician of some eminence, was born at llton in Somersetshire, in 1625 . He entered on his studies at $O x$ ford in 1640 , where he remained seven years. He was afterwards appointed vicar of Bishop's-Nymmet in Devonshire, where he lived astudious and retired life for many years, chiefly pursuing the mathematical sciences, of which he gave a proof of his critical knowledge, in the book he published, concerning the general construction of biquadratic equations, by a parabola and a circle; the title of whicl book at full length is, "The Geometrical Key; or the Gate of Equations unlocked: or a new Discovery of the Construction of all Equations, howsoever affected, not excceding the 4th degree, viz, of Linears, Quadratics, Cubics, Biquadratics, and the finding of all their Roots, as well False as True, without the use of Mesolabe, Trisection of Angles, without Reduction, Depression, or any other previous preparations, of equations by a circle, and any (and that one only) Parabole, \&c:" 1684, 4to, in English and Latin.

There is some account of this work in the Philos. Trans. an. 1684. And a little before his death, the Royal Society sent him some mathematical queries; to which be returned such satisfactory answers, as procured the present of a medal, with an inscription honourable and respectful. Mr. Baker died at Bishop's-Nymmet, 1690, in the 65th year of his age.

BAKER (HENRY), an ingenious and diligent naturalist, was born in London about the beginning of the 18 th century. He was trought up under an eminent bookseller, but being of a philosophical turn of mind, he quitted tbat line of business soon after the expiration of his apprenticeship, and engaged himself in teaching deaf and dumb persons to speak and write \&c, in which occupation, in the course of his life, he acquired a handsome fortune. For his amusement he cultivated various natural and philosophical sciences, particularly botany, natural history, and microscopical subjects, in which he especially excelled, having, in the year 1744, obtained the Royal Society's gold medal, for his miscroscopical experiments on the crystallizations and configurations of saline particles. He had also various papers published in the Philos. Trans. of the Royal Society, of which he was a worthy member, as well as of the Societies of Antiquaries, and of Arts. He was author of many pieces, on various subjects, the principal of which were, his Treatise on the Water Polype, and two Trcatises on the Microscope, viz, The Microscope made easy, and Employment for the Microscope, which have gone through several editions.

Mr. Baker married Sophia, youngest daughter of the celebrated Daniel Defoe, by whom he had two sons, one $2 \mathrm{D}$ 
of whom, Henry Erskine Baker, was the author of the first edit. of the little work called, The Companion to the Play-house. He terminated an houourable and useful life, at his apartments in the Strand, on the 25th of November 1774 , being then upwards of 70 years of age.

BAKER'S CENTRAL RULE, for the Construction of Equations; is a method of constructing all equations, not exceeding the 4th degree, by means of a given parabola and a circle, without any previous reduction of them, or first taking away their second term. See Centrat Rule.

BALANCE, one of the six simple powers in mechanics, clictly used in determining the equality or difference of weight in heavy hodies, and consequently their masses or quantities of matter.

The balance is of two kinds, the ancient and modern. The ancient or Roman balance, ealled also Statera Romana, or Steelyard, consists of a lever or beam, moveable on a centre, and suspended near one of its extremities. The bodics to be weighed are suspended from the shorter end, and their weight is shown by the division marked on the beam, where the power or constant weight, which is moveable along the lever, kecps the steclyard in equilibrio. This balance is still in common use for weighing heavy bodies.

The moderr balance, now commonly used, consists of a lever or beam suspended exactly at the middle, and having scales hung from the two extremities, to receive the wcights to be weighed.

In either case the lever is called the jugum or the beam, and its two halves on each side the axis, the brachia or arms; also the line on which the beam turns, or which divides it in two, is called the axis; which when considered with regard to the length of the brachia, is esteemed only a point, and called the centre of the balance, or centre of motion: the extremities where the weights are applied, are the points of application or suspension; the handle by which the balance is held, or by which the whole apparatus is suspended, is called trutina; and the slender part perpendicular to the beam, by which is determined either the equilibrium or preponderancy of bodies, is called the tongue of the balance.

From these descriptions we easily gather the characteristic distinction between the Roman balance and the common one, viz, that in the Roman balance, there is one constant weight used as a counterpoise, the point where it is suspended being varied; but, on the contrary, in the common balance or scales, the point of suspension remains the same, and the counterpoise is varied. The principle of both of them may be easily understood from the general properties of the lever, and the following observations. Sec LeVER.

The beam A BC, the principal part of the balance, is a lever of the first kind; but instead of resting on a fulcrum, it is suspended by a handle, \&c, fastened to its centre of motion $\mathrm{B}$ : and bence the mechanism of the balance dcpends on the same theorems as that of the lever. Consequently as the distance between the centre of motion and the place of the unknown weight, is to the distance between the same centre and the place of the known weight, so is the latter weight to the former. So that the unknown weight is discovered by means of the known one, and their distances from the common centre of motion; viz, if the distances from the centre be equal, then the two weights will be equal also, as in the common balunce; but if the distances be onequal, then the weights will also be unequal, and in the very same proportion, alternately, the less weight having so mucb the grater distance, as in the steelyard.

\section{The Common Bulance or Scales.}

The two brachia $A \mathrm{~B}, \mathrm{sc}$, should be exactly equal in length, and in weight also when their scales $\mathrm{D}$ and $\mathrm{E}$ are fixed on their ends; the beam should hang exactly level or horizontal in the case of an equipoise; for which purpose the centre of gravity of the whole should tall a little below the centre

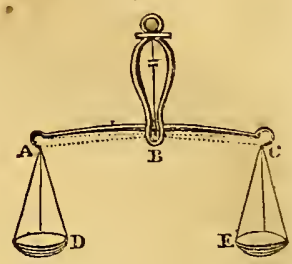
of motion, and but a little, that the balance be sufficiently sensible to the least variation of weight: the friction on the centre should also be as small as possible.

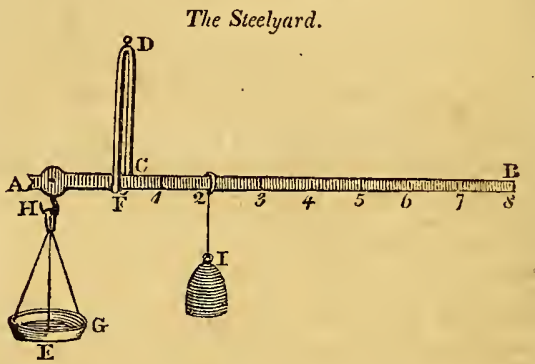

Having taken a proper bar of steel $\mathrm{A} \mathrm{B}$, tapering at the longer end, and very strong at the other, suspend it by a centre $\mathrm{c}$ near the shorter or thicher end, so that it may exactly balance itself in equilibrio, and prepare a constant weight 1 to weigh with: then hang on any weight, as one pound for instance, at the shorter arm, and slide the constant weight backwards and forwards upon the longer arm, till it be just in equilibrio with the former; and there make a notch and number 1 , for the place of 1 pound: take off the 1lb, and hang a two ponnd weight in its stead at the shorter arm; then slide the copstant weight back on the longer arm, till the whole come again into a state of equilibrium, making a notch at the place of the constant weight and the number 2, for the place of 216 . Proceed in the same manner for all other weights, $3,4,5$, sc: as also for the intermediate halves and quarters, $\delta \mathrm{c}$, if $\mathrm{jt}$ be necessary; always suspending the variable weights at the end of the shorter arm, shifting the constant wight so as to balance them, and marking and numbering the places on the longer arm where the constant weight always makes a counterpoise. The use of the stcelynird is hence very evident: the thing whose weight is required being suspended by a hook at the short end, move the constant weight backwards and forwards on the longer arm, till the beam is balanced horizontally: then look what notels the constant weight is placed at, and its number will show the weight of the body that was required. $\mathrm{nc}$ is the handle and tongue; $F$ the centre of motion; Eci a scale sometimes hung on at the end by the hook $n$. 
B A L

The Bent-Lever Balance.

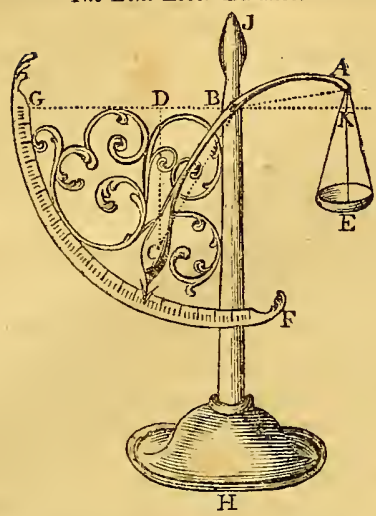

This instrument operates by a fixed weight, $c$, increasing in power as it ascends along the arc FG of a circle, and pointing by an index to the number or division of the arc which denotes the weight of any body put into the scale at E. And thus one constant weight serves to weigh all others, by only varying the position of the arms of the balance, instead of varying the places or points of suspension in the arms themselves.

The Deceitful, or False Balance. This operates in the same manner as the steelyard, and cheats or deceives by having one arm a little longer than the other; though the deception is not perceived, because the shorter arm is made somewhat heavier, so as to compensate for its shortness, by which means the beam of the balance, when no weights are in the scales, hangs horizontal in cquilibrio. The consequence of this construction is, that any commodity put in the scale of the longer arm, requires a greater weight in the other scale to balance it ; and so the body is fallaciously accounted heavier than it really is. But the trick will casily be detected by making the body and the weight change places, removing them to the opposite scales, when the weiglt will imnediately be scen to preponderate.

The true weight of any thing may however be found out by means of the false balance, in this manner: viz, Weigh the body first in the one scale, next in the other; then take a mean proportional between the two difierent weights thus indicated, and that will be the true weight rcquired. Thus, suppose the two false weights be 7 and $5 \frac{x}{7}$; the product of these two is 36 , the square root of which, 6 , is the true weight. Or, balf the sum of the two false weights, 7 and $5 \frac{1}{7}$, which in this case is $6_{\frac{1}{4}}$, will commonly be very near the true weight, though always a very little too much.

Assay-Balance. This is a very nice balance, used in determining the exact weights of very small bodies. Its structure is but little different from the common sort ; except that it is made of the best and hardest steel, and made to turn with the smallest weight. For an account of several balances, which have been constructed by different persons for delicate experiments, consult the 66 th vol. of the Phil. Trans., also the same work for 17.99 , part 2. See also Nicholson's Chemistry, chap. 6, and Parkinson's System of Mechanics.

\section{B A L}

Hydrostatical Balance. This is an instrument for determining the specific gravity of bodies. See HYDRosi ATICAL; and SPECIFIC Gravity.

BaLANCE, in Astronomy, the same as Libra.

BALANCE of a Clock or Watch, is that part which, by its motion, regulates and determines the beats. The circular part of it is called the Rim, and its spindle the Verge; there belong to it also two pallets or nuts, that play in the fangs of the crown-wheel: in pocket-watches, that strong stud in which the lower pivot of the verge plays, and in the middle of whicb one pivot of the crown-wheel runs, is called the Potence; the wrought piece which covers the balance, and in which the upper pivot of the balance plays, is the cock; and the small spring in the new pocket-watches is called the Regulator.

BaLance of Forces, in Mechanics. See Compound MTotion.

BALCONY, a projecture in the front of a bouse, or other building, commonly supported by pillars or consoles, and encompassed by a ballustrade.

BALL, any spherical, globular, or round body.

$\mathrm{BALL}_{\mathrm{A}}$, in the military art, signifies all sorts of bullets for fire-arms, from the pistol up to the largest cannon. Cannon balls are made of cast-iron; but the musket and pistol-balls of lead, as these are both heavier under the same bulk, and do not furrow the barrels of the pieces.

BALL of a Pendulum, is the weight at the bottom of it; and is sometimes, especially in shorter pendulums, callcd the Bob.

BALLS of Fire, in Meteorology. See Fin E Balls.

BA L LS, in Electricity, invented by Mr. Canton, are two pieces of cork, or pith of elder-tree, nicely turned in a lathe to the size of a small pea, and suspended by very delicate threads. They are used as electrometers, and are of excellent use to discover small degrees of electricity; and to observe its changes from positive to negative, or the reverse; as also to estimate the force of a shock before the discliarge, so that the operator shall always be able to tell very nearly beforehand what the explosion will be, by knowing how high he has charged his jars.

BALLISTA, a military engine much used by the ancients for throwing stones, darts, and javelins; it somewhat resembled our cross-bows, but much larger and stronger. The word is Latin, signifying a cross-bow; but is derived from the Greek $\beta a \lambda \lambda \omega$, to shoot, or throw.

$V$ egetius informs us, that the ballista discharged darts with such violence and rapidity, that nothing could resist their force: and Athenzus adds, that Agistratus made one of little more than 2 feet in length, that slot darts 500 paces. Authors have often confounded the ballista with the catapulta, attributing to the one what belongs to the other. According to Vitruvius, the ballista was made after divers manners, though all were used for the same purpose: one surt was framed with levers and bars; another with pulleys; some with a crane; and others again with a toothed wheel. Marcellinus describes the ballista thus: A round iron cylinder is fastened between two planks, from which reaches a hollow square beam placed cross-wise, fastened with cords, to which are added screws. At one end of this stands the engineer, who puts a wooden shaft, or arrow, with a large head, into the cavity of the beam; this done, two men bend the engine, by drawing some wheels; when the top of the head is drawn to the utmost end of the cords, the shaft is driven out of the ballista, \&c.

The ballista is ranked by the ancients among the sling $2 \mathrm{D} 2$ 
kind, and its structure and effect reduced to the principles of that instrument; whence it is called by Hero and others; Funda, and Fundibulus. Gunther calls it Balearica machina, as a sling peculiar to the Balearic islands.-Perrault, in his notes on Vitruvius, givés a new contrivance of a like engine for throwing bombs without powder.

Fig. 1, Plate v, represents the ballista used in sieges, according to Folard: where 2,2 , denote the base of the ballista; 3,4 , upright beams; 5,6 , transverse beams; 7,7 , the two capitals in the upper transverse beam, (the lower transverse beam has also two similar capitals, which cannot be seen in this transverse figure); 9,9 , two posts or supports for strengthening the transverse beams; 10, 10, two skains of cords fastened to the capitals; 11, 11, two arms inserted between the two strands, or parts of the skains; 12 , a cord fastened to the two arms; 13 , darts which are shot by the ballista; 14, 14, curves in the upright beams, and in the concavity of which cushions are fastened, in order to break the force of the arms, which strike against them with great force when the dart is discharged: 16 , the arbor of the machine, in which a straight groove or canal is formed to receive the darts, in order to their being shot by the ballista; 17 , the nut of the trigger, 18 , the roll or windlass, about which the cord is wound; 19 , a hook, by which the cord is drawn towards the centre, and the ballista cocked; 20, a stage or table on which the arbor is in part sustained.

Ba Llista, in Practical Geometry, the same as the geometrical cross, called also Jacob's Staff. See Cross-Staff.

BALLISTIC PEN DULUM, an ingenious machine invented by Benjamin Robins, for ascertaining the velocity of military projectiles, and consequently the force of fired gunpowder. It consists of a large block of wood, annexed to the end of a strong iron stem, having a cross steel axis at the other end, placed horizontally, about which the whole vibrates together like the pendulum of a clock. This machine being at rest, a piece of ordnance is pointed straight towards the wooden block, or ball of this pendulum, and then discharged: the consequence is this; the ball discharged from the gun strikes and enters the block, and causes the pendulum to vibrate more or less according to the velocity of the projectile, or the force of the blow; and by observing the extent of the vibration, the force of that blow becomes known, or the greatest velocity with which the block is moved out of its place, and consequently the velocity of the projectile itself which struck the blow and urged the pendulum.

A more minute and particular description may be seen in iny Tracts, vols. $2 \& 3$, where are given the various rules for using it, and for computing the velocities, with a multitude of accurate experiments performed with cannon balls, by means of which the most useful and important conclusions have been deduced in military projectiles and the nature of physics. In those Tracts are detailed at great length many experiments of the same kind, by discharging cannon balls at various distances from the block; from which have resulted the discovery of a complete series of the resistances of the air to balls passing through it with all degrees of velocity, from 0 up to 2000 fect in a second of time.

Other writers on this subject are Euler, Antoni, Le Roy, Darcy, \&c. See also Robins's Mathematical Tracts.

BALLISTICS, is used by some for projectiles, or the art of throwing heavy bodies. Mersenne has published a treatise on the projection of bodies, under this title.
BALLOON, or BALLON, in a general sense signifies any spherical hollow body. Thus, with chemists, it denotes a round short-necked vessel, used to receive what is distilled by means of fire: in architecture, a ball or globe on the top of a pillar, \&c: and among engineers, a kind of bomb made of pasteboard, and played off in fireworks, in imitation of a real iron biomb-shell.

Air-Balloon. See Aerostation.

BALLUSTER, a small kind of column or pillar, used for ballustrades.

BALLUSTRADE, a series or row of ballusters, joined by a rail; serving for a rest to the arms, or as a fence or inclosure to balconies, altars, staircases, \&c.

BAND, in Architecture, denotes any flat low menber, or moulding, that is broad, but not very deep. The word lace sometimes means the same thing.

BANQUET, or BANQUETTE, in Fortification, a little foot-bank, or elevation of earth, forming a path along the inside of a parapet, for the soldiers to stand upon to dis. cover the counterscarp, or to fire on the enemy in the moat, or in the covert way. It is commonly about 3 feet wide, and a foot and a half high,

BARITONO, in Music, a voice of low pitch between a tenor and bass.

BARLOWE (WILLIA II), an eminent mathematician and divine, of the 16th century. He was born in Pembrokeshire, his father (Wiiliam Barlowe) being then bishop of St. David's. In 1560 he was entered commoner of Baliol college, Oxford; and in 1564, having taken a degree in arts, he left the university, and went to sea; but in what capacity is uncertain: however lie thence acquired considerable knowledge in the art of navigation, as his writings afterwards showed. About the year 1573 , he entered into orders, and became prebendary of Winchester, and rector of Easton, near that city. In 1588 he was made prebendary of Litchfield, which he exchanged for the office of treasurer of that church. He afterwards was appointed chaplain to prince Henry, eldest son of king James the First; and, in 1614, archdeacon of Salisbury. Barlowe was especially remarkable for having been the first writer on the nature and properties of the loadstone, 20 years before Gilbert published his book on that subject. He was the first who made the inclinatory instrument transparent, and to be used with a glass on both sides. It was Barlowe also who suspended it in a compass-box, where, with 2 ounces weight, it was made fit for use at sea. He also found out the difference between iron and steel, and thcir tempers for magnetical uses. He likewise discovered the proper way of touching magnetical needles; and of piecing and cementing of loadstones; and also why a loadstone, being double-capped, must take up so great a weight.

Barlowe died in the year 1625.-His works are as follow :

1. The Navigator's Supply, containing many things of principal importance belonging to Navigation, and use of diverse Instruments framed chiefly for that purpose. Lond. 1597,4 to ; dedicated to Robert earl of Lssex.

2. Magnetical Advertiscment, or Diverse pertinent $\mathrm{Ob}-$ servations and improved Experiments concerning the nature and properties of the Loadstone. Lond. 1616, 4to.

3. A Brief Discovery of the idle Animadversions of Mark Ridley, M. D. upon a treatise entitled Magnetical Advertisements. Lond, 1618, 4to.

In the first of these picces, Barlowe gave a demonstration of Wright's or Mercator's division of the meridinn 
line, as communicated by a friend; observing that "This manner of carde has been publiquely extant in print these thirtie yeares at least [he should have said $2 S$ only], but a cloude (as it were) and thicke miste of ignorance doth keepe it hitherto concealed: And so much the more, because some who were reckoned for men of good knowledge, have by glauncing speeches (but never by any one reason of moment) gone about what they could to disgrace it."

. This work of Barlowe's contains descriptions of several instruments for the use of Navigation, the principal of which is an Azimuth Compass, with two upright sights; and as the author was very curious in making experiments on the loadstone, he treats well and fully upon the SeaCompass. He also treated still farther on the same instrument in his second work, the Magnetical Advertisement.

BAROMETER, an instrument for measuring the weight or pressure of the atmosphere; and by that means the variations in the state of the air, foretelling the changes in the weather, and measuring heights or depths, \&c.

This instrument is founded on what is called the Torricellian experiment, related below, and commonly consists of a cylindrical glass tube, open at one end; which being first filled with quicksilver, and then inverted with the open end downwards into a bason of the same, the mercury descends. in the tube till it remains at about the beight of 29 or 30 inches, according to the weight or pressure of the atmosphere at the time, which is just equal to the weight of that column of the quicksilver. Hence it follows that, if by any means the pressure of the air be altered, it will be indicated by the rising or falling of the mercury in the tube; or if the barometer be carried to a higher station, the quicksilver will descend in the cylinder, but when carried to a lower place, it will rise higher in the tube, according to the differencc of elevation between the two places.

History of the Burameter.-About the beginning of the 17 th century, when the doctrine of a plenum was in vogue, it was a common opinion among philosophers, that the ascent of water in pumps was owing to what they called nature's abhorrence of a vacuum; and that in consequence fuids might be raised by suction to any height whatever. But an accident having discovered, that water could not be raised in a pump unless the sucker reached to within 33 fect of the water in the well, it was conjectured by Galikeo, who flourished about that time, that there might be some other cause of the ascent of water in pumps, or at least that the effect of this abhorrence was limited to the finite beight of 33 feet. Being unable to satisfy hiuself on this head, he recommended the consideration of the difficulty to Torricelli, who had been his disciple. After some time Torricelli fell upon the suspicion that the pressure of the atmosphere was the cause of the ascent of water in pumps; that a column of water 33 feet higl was a just counterpoise to a column of air, of the same base, and which extended up to the top of the atmosphere; and that this was the true reason why the water did not follow the sucker any farther. And this conjecture was soon after confirmed by various experiments. Torricelli considered, that if a column of water 33 feet high were a counterpoise to a whole column of the atmospherc, then a column of mercury of about 2 feet and a half high would also be a counterpoise to it, since quicksilver is nearly 14 times heavicr than water, and so the 14 th part of the height, or 2 feet and a half, very nearly, would be as heavy as the column of water. This reasoning was soon verificd; for baviug filled a glass tube with quicksilver, and inverted it into a bason of the same, the mercury presently descended till its height, above that in the bason, was about two fict and a half, just as he had estimated. And this is what has, from him, been called the Torricellian experiment.

The new opinion, with this confirmation of it, was readily acquiesced in by most of the philosophers, who repeated the experiment in various ways. Others however still adhered to the old doctrinc, and raised several pretended objections against the new one; such as that there was a film or imperceptible rope of mercury, extended through the upper part of the tube, which retained the column of mercury, and kept it from falling into that in the bason. This and other objections were however soon overcome by additional confirmations of the true doctrine, particularly by varying the elevation of the place. It was hinted by Descartes and Pascal, that if the mercury be sustained in the tube by the pressure of the atmosphere, by carrying it to a higher situation, it would descend lower in the tube, having a shorter column of the atmosphere to sustain it, and vice versa. In consequence of which, Pascal engaged his brother-in-law, M. Perier, to try that experiment for him, being more conveniently situated for that purpose than be was at Paris. This he accordingly executed, by observing the height of the quicksilver in the tube, first at the bottom of the Puy de Dome, a mountain in Auvergne, and then at several stations, or different altitudes, in ascending, by which it was found that the mercury fell lower and lower all the way to the top of the mountain; by this means confirming the truth of the doctrine relating to the universal pressure of the atmosphere, and the consequentsuspension of the mercury in the tube of the barometer. Thus, by the united endeavours of Torricelli, Descartes, Pascal, Mersenne, Huygens, and others, the cause of the suspension of the quicksilver in the tube of the barometer becaine pretty generally established.

It was some time however after this general consent, before it was known that the pressure of the air was various at different times, in the same place. This could not bowever remain long unknown, as the frequent measuring of the column of mercury, must soon show its variations in altitude; and experience and observation would presently show that those variations in the mercurial column, were always succecded by ccrtain changes in the weather, as rain, wind, frosts, \&c. Hence this instrument snon came into use as the means of foretclling the changes of the weather; and on this account it obtained the mame of the weather-glass, as it did that of barometer from its being the measure of the weight or pressure of the air. We may now proceed to take a view of its various forms and uses.

The Common Barometer. This is represented at fig. 1, plate iv, in the same form as it was invented by Torricelli. A B is a glass tube, of $\frac{x}{4}$, or $\frac{x}{3}$, or $\frac{x}{2}$ inch wide, the more the better, and about 34 inclics long, being hermetically sealed at the top $A$, and the open end $\mathbf{B}$ immersed in a bason of quicksilver $\mathrm{cD}$, which is also the better the wider it is. To fill this, or any other barometer; take a clean new glass tube, of the dimensions above named, and pour into it well purified quicksilver, with a small funnel, either of glass or paper, in a finc continued stream, till it wants about half an inch or an inch of being full; then stopping it close with the finger, invert it slowly, and the air in the empty part will ascend gradually to the other end, collecting in its ascent such other sinall air-bubbles as unavoidably get into the tube among the incrcury, when 
filling it with the funnel: and thus continue to invert it several times, turning the two ends alternately upwards, till all the air-bubbles are collected, and brought up to the open end of the tube, or till the part filled shall appear, without speck, like a fine polished steel rod. "This done, pour in more quicksilver, to fill the empty part entirely, and so exclude all air from the tube: then, stopping the orifice again with the finger, invert the tube, and immerse the finger and end, thus stopped, into a bason of like purified quicksilver; in this position withdraw the finger, so shall the mercury descend in the tube to some place, as $E$, between $2 S$ and 31 inches above that in the bason at $F$, these being the limits between which it always stands in this country on the common surface of the earth. Then measure, from the surface of the quicksilver in the bason at $F, 28$ inches to $G$, and 31 inches to $\mathrm{F}$, dividing the space between them into inches and tenths, which are marked on a scale placed against the side of the tube; and the tenths are subdivided into hundredth parts of an inch by a sliding index carrying a vernier or nonius. (See art. Vernier.) These 3 inches, between 28 and 31 , so divided, will answer for all the ordinary purposes of a stationary or chamber barometer; but for experiments on altitudes and depths, it is proper to have the divisions carried on" somewhat higher, and a great deal lower down. In the proper filling and otherwise fitting up of the barometer, several circumstances are to be carefully noted; as, that the bore of the tube be pretty wide, to allow the freer motion of the quicksilver, without being impeded by an adhesion to the sides; that the bason below it be also tolerably large, at least 10 times larger in diameter than that of the tube, in order that the surface of the mercury at $F$ may not sensibly rise or fall with that in the tube; for the numbers marked in the scale annexed to the tube, denote their distance from a certain fixed point, and therefore cannot truly indicate the height of the column above the mercury in the vessel, unless its surface coincides with this point, and be immoveable; also, that the bottom of the tube be cut off rather obliquely, that when it rests on the buttom of the bason there may be a free passage for the quicksilver; and that, to have the quicksilver very pure, it is best to boil it in the tube, in order to expel the air from it, which may be done by the following simple process. Having procured a tube of $2 \frac{\pi}{2}$ lines or 3 lines in bore, and about half a line in thickness, fill it witl melcury within' two iuches of the top, and hold it with the sealed end lowest, in an inclined position, over a chafingdish of burning charcoal, presenting first the scalcd end to the fire, and moving it obliquely over the chafing-olish. As the mercury is lieated, the air-bubbles will assume an appearance like so many studs on the inner surface of the tube, and graclually rushing into one another, ascend towards the ligher parts of the tube, which arc not heated; here they are condensed and almost disappear, and after repeated emigrations they acquire a bulk by their union, which enables them at length to escape. When the mercury boils, its parts strike against each other, and ngainst the sides of the tube, wh such violence, that a person unaccustoned to this operation is ready to appreliend their force to be sufficient to break the tube. The mercury is thus freed front all heterogencous particles contained in it, together with their surrounding atmospheres, and the air which lines the inside of the tube, which cannot be expelled in any other way, is discharged; when this last-mentioned stratum of air is thus expelled, the tube may be afterwards emptied, and filled even with cold mercury, and will be found nearly as free from the air as before: the mercury in the tube thus preparcd, will rise higher than in those of the common sort, and the barometers will more nearly correspond with ench other, whereas there will be a difierence of 6 or 8 fines in the ascent of the mercury in the common barometers. When this operation is completed, the mercury generally remains suspended at the top, and will not descend to its proper level without shaking the tube to bring it down. The tubes, which should not be chosen lessthan 3 feet long, may now be filled to their proper length. This barometer is commonly fitted up in a neat mahogany case, together with a thermometer and hygrometer, as represented in plate 4 , fig. 13.

As the scale of variation is but small, being included within 2 inches in the conmon barometer, several contrivances have been devised to cularge the scale, or to render the motion of the quicksilver more sensible.

Descartes first suggested a uncthod of increasing the sensibility, which was executed by Huygens. This was effected by making the barometrical tube end in a pretty large cylindrical vessel at top, into which was inserted also the lower or open end of a much finer tube than the former, which was partly filled with water, to give little obstruction by its weight to the motion of the mercury, while it moved through a considerable space of the very fine tube by a small variation of the mercury below it, and so rendered the small changes in the state of the air very sensible. But the inconvenience was this, that the air contained in the water gradually disengaged itself, and escaped through into the vacuum in the top of the small tube, till it was collected in a body there, and by its elasticity preventing the free rise of the fluids in the tubes, spoiled the instrument as a true barometer. And this, it may be observed by-the-bye, is the reason why a water barometer cannotsucceed. This barometer is here represented in fig. 2, where $C D$ is the vessel, in which are united the upper or small water tube $\mathrm{A} c$, with the lower or mercurial one $\mathrm{cB}$.

'T'o remedy this inconvenience, Huygens thought of placing the mercury at top, and the water at bottom, which he thus contrived. ADG (fig. 3) is a bent tube hermetically sealed at $\Lambda$, but open at $G$, of about one line in $\mathrm{di}$ ameter, and passing through the two equal cylindrical vessels BC, EF, which are about 20 juches apart, and of 15 lines diameter, their length being 10. The mercury being put iuto the tube, will stand betwcen the middle of the vessels $\mathrm{EF}$ and $\mathrm{BC}$, the remaining space to $\mathrm{A}$ being roid both of air and mercury. Lastly, common water, tiuged with a 6 th part of aqua regia, to prevent its freezing, is poured into the tube $F G$, till it rises a toot above the morcury in DE. To prevent the water from evaporating, a drop of oil of sweet almonds fluats on the top of it. 13ut the column of water will be sensibly affected by heat and cold, and consequently the accuracy of the instrument will be spoiled, for which renson other contrivances have been made, as below.

The Horizontal "1. Rectangular Barometer, fig. 4 , was invented by J. Bernoulli and Cassini; where $\Lambda$ is is a pretty wide cylindrical part at the top of the tube, which tube is bent at right angles at $c$, the lower part of it $\mathrm{cn}$ bcing turned into the horizontal direction, and closed above at $\Lambda$, but open at the lower end $D$, where the nercury cannot run out, being opposed by the pressure of the atmosphere. 'This and the foregoing contrivance of Iluygens 
are founded on a theorem in hydrostatics, viz, that fluids of the same base, press according to their perpendicular altitude, not according to the quantity of their matter; so that the same pressure of the atmosphere sustains the quicksilver that fills the tube $\mathrm{ACD}$, and the cistern B, as would support the mercury in the tube alone. Hence, having fixed upon the size of the scale, as suppose the extent of 12 incbes, instead of the 3 , in the common barometer from 28 to $3 t$, that is 4 times as long; then the area of a section of the cylinder $A \mathrm{~B}$ must be 4 times that of the tube, and consequently its diameter double, since the areas of circles are as the squares of their diameters: then for every natural variation of an inch in the cylinder $\mathrm{AB}$, there will be a variation of 4 inches $\mathrm{in}$ the tube $\mathrm{CD}$.But on account of the attrition of the mercury against the sides of the glass, and the great nomentum from the quick motion in $\mathbf{C D}$, the quicksilver is apt to break, and the rise and fall is no longer equable; and besides, the mercury is apt to be thrown ont of the orifice at $D$ by sudden inotions of the machine.

The Diagonal Barometer of Sir Samuel Moreland, fig. 5, is another method of enlarging the natural scale of 3 inches perpendicular, or $\mathrm{CD}$, by extending it to any length $\mathrm{BC}$ in an oblique direction. 'This is liable in some degree to the same inconvenience, from friction and breaking, as the horizontal one: and hence it is found that the diagonal part $\mathrm{BC}$ cammot properly be bent from the perpendicular more than in an angle of $45^{0}$, which only increases the scale nearly in the proportion of 7 to 5 .

Dr. Honke's Wheel Burameter, fig. 6. This was invented about $166 \mathrm{~s}$, and is meant to render the alterations in the air more sensible. Here the barometer-tule has a large ball $\mathrm{AB}$ at top, and is bent up at the lower or open end, where an iron ball $\mathrm{G}$ floats on the top of the mercury in the tube, to which is connected another ball it by a cord, hanging frely over a pulley, turning an index $\mathrm{K} \mathrm{L}$ about its centre. When the mercury rises in the part rg, it raises the hall, and the other ball descends and turns the pulley with the inclex round a graduated circle from $\mathrm{N}$ towards $M$ and $\mathbf{P}$; and the contrary way when the quicksilver and the ball sink in the bent part of the tube. Hence the scale is easily enlarged 10 or 12 fold, being increased in proportion of the axis of the pulley to the length of the index $\mathrm{KL}$. But then the friction of the pulley and axis is some obstruction to the free motion of the quicksilver. Contrivances to lessen the friction \&c, may also be seen in the Pliilos. Trans. vol. 52, art.29, and vol. 60, art. 10.

The Steclyard Burometer, for so that may be called which is represented by fig. $T$, which enlarges the scale in the proportion of the shorter to the Yonger arm of the steelyard. $A B$ is the barometer tube, closed at $A$ and open at $\mathrm{B}$, immersed in a cylindrical glass cistern $\mathrm{CD}$, which is but very little wider than the tube $\mathrm{AB}$ is. The barometer tube is suspended to the shorter arm of an index like a steelyard, moving on the fulcrum $\mathrm{E}$, and the extremity of its longest arm pointing to the divisions of a graduated arch, with which index the tube is nearly in equilibrio. When the pressure of the atmosphere is lessened, the mercury descends out of the tube into the cistern, which raises the tube and the shorter arm of the index, and consequently the extremity of the longer moves downwards, and passers over a part of the graduated arch. And on the contrary this moves upwards when the pressure of the atmospliere increases.

The Pendant Barometcr, fig. 8, was iuvented by M.Amon- tons, in 1695. It consists of a singte conical tube $\mathrm{AB}$, hung up by a thread, the larger or open end downivards, and having no vessel or cistern, because the conical figure supplies that, and the column of mercury sustained is always equal to that in the common barometer tube; which is effected thus: when the pressure of the air is less, the mercury sinks-down to a lower and wider part of the tube, and consequently the altitude of its column will be less; and on the contrary, by a greater pressure of the atmosphere, the mercury is forced up to a higher and narrower part, till the length of the column $\mathrm{CD}$ be equal to that in the tube of the common barometer.--The inconvenience of this barometer is, that as the bore must be made very small, to prevent the mercury from falling out by an accidental shake, the friction and adhesion to the sides of the tube prevent the free motion of the mercury.

Mr. Rouning's Compound Barometers. This gentleman has several contrivances for enlarging the scale, and that in any proportion whatever. One of these is described in the Philos: Trans. No. 427 , and also in his Nat. Philos. part 2; and another in the same part,. which is here represented at fig. 9. $\mathrm{ABC}$ is a compound tube, hermetically sealed at $A$, and open at $\mathrm{C}$; empty from $\mathrm{A}$ to $\mathrm{D}$, filled with mercury from thence to $B$, and from hence to $E$ with water. Hence by varying the proportions of the two tubes $A F$ and $\mathrm{FC}$, the scale of variation may be changed in any degree.

The Marine Barometer. This was first invented by Dr. Hooke, to be used at sea, being contrived so as not to be affected or injured by the motion of the ship. His contrivance consisted of a double thermometer, or a couple of tubes half filled with spirit of wine; the one sealed at both ends, with a quantity of air included; the other sealed at one end only. The tormer of these is aflected only by the warmth of the air; but the other is affected both by the exierual warmth and by the variable pressure of the atmosphere. Hence, considering the spirit thermometer as a standard, the excess of the rise or fall of the other above it will show the increase or decrease of the pressure of the atmosphere. 'This instrument is described by Dr. Halley, in the Philos. Trans. No. 269, where he says of it, "I had one of these baroneters with me in my late southeru voyage, and it never failed to prognosticate and give early notice of all the bad weather we had, so that I depended thereon, and made provision accordingly; and from my own experience I conclude, that a more useful contrivance hath not for this long time been offered for the benefit of navigation." - Dr. Hooke also contrived another barometer, similar to that of Mr. Rowning above described. The Rev. J. Wilson has also clescribed a method of insuring the sensibility of the barometer ad libitum. See Nich. Journal, No. 9, new series.

Mr. Nairne, an ingenious artist in London, has lately invented a new kind of Marine Barometer; which differs from the common barometer by having the lower part of the tube, for about 2 feet in length, made very small, to check the vibrations of the mercury, which would otherwise arise from the motions of the ship. This is also assisted by being fixed in gimbals, by a part which subjects it to be the least affected by such inotions.

Another sort of Marine Barometer has also been invented by M. Passemente, an ingenious artist at Paris. This contrivance consists only in twisting the midlle of the tube into a spiral of two revolutions; by which contrivance the impulses which the mercury receives from the 
motions of the ship are destroyed, by being transmitted in contrary directions.

Messrs. W. and S. Jones of London, have also constructed two excellent Marine Barometers, which seem to te well adapted to the purposes intended.

The Stat ical Baroscope, or Burometer, of Mr. Boyle, \&c. This consists of a large glass bubble, blown very thin, and then balanced by a small brass weight. Hence these two bodies being of unequal bulk, the larger will be very much affected by a change of the density of the medium, but the less not at all that is perceptible: so that, when the atmosphere becomes deuser, the ball loses more of its weight, and the brass weight preponderates; and contrariwise when the air grows lighter.

Mr. Caszuell's Baroscope, or Barometer. 'This is described in the Philos. Trans. vol. 24, and seems to be the most sensible and exact of any. It is thus described: Suppose A BCD (Fig. 10) a bucket of water, wherein is placed the baroscope xrezyosm, which consists of a body xrsm, and a tube ezyo, which are both concave cylinders, made of tin, or rather glass, and communicating with each other. The bottom of the tube $z y$ has a leaden weight to sink it, so that the top of the body may just swim even with the surface of the water by the addition of some grain weights on the top. When the instrument is forced with its mouth downwards, the water ascends into the tube to the height $y u$. Tu the top is added a stuall concave cylinder, or pipe, to keep the instrument from sinking down to the bottom: $m d$ is a wire: and $m S$, de are two threads oblique to the surface of the water, which perform the office of diagonals: for while the instrument sinks more or less by an alteration in the gravity of the air, there where the surface of the water cuts the thread is formed a small bubble, which ascends up the thread while the mercury of the common baroscope ascends, and vice versa.

It appears from a calculation which the author makes, that this instrument shows the alterations in the air 1200 times more accurately than the common barometer. He observes, that the bubble is seldom known to stand still even for a ininute; that a small blust of wind, which cannot be heard in a chamber, will sensibly make it sink; and that a cloud passing over it always makes it descend, \&c.

While some have been increasing the sensibility of the barometer by enlarging the variations, others have cndeavoured to make it more convenient by reducing the length of the tube. M. Ainontons, in 1688 , first proposed this alteration in the structure of barometers, by joining several tubes to one another, alternately filled with mercury and with air, or some other fluid; and the number of these tubes may be increased at pleasure: but the contrivance is perhaps more ingenious than useful.

$M$. Mairan's reduced Barometer, which is only 3 inches long, serves the purpose of a manometer, in showing the dilatations of the air in the receiver of an air-pump; and instruments of this kind are now commonly applied to this use.

The Portable Barometer, is so contrived that it may be carried from one place to another without being disordered. The end of the tube is ticd up in a leathern bag not quite full of mircury; which being pressed by the air, forces the mercury into the tube, and keeps it suspended at its proper height. 'This bag is usually inclosed in a box, through the bottom of which passes a screw, by means of which the mercury may be forced up to the top of the tube, and prevented from breaking it by dashing against the top when the instrument is removed from one siation to auother. It seems Mr. Patrick first made a coutrivance of this kind: but the portable barometer has received various improvements since; and the most complete of this kind has been described by M. De Luc, iu his Recherches, vol. 2 , pa. $5 \& \mathrm{c}$, together with the apparatus belonging tw it, the method of construction and use, and the advantages attending it. Improvements have also been suggestcd by Sir George Shuckburgh, and Col. Roy, which have been carried into execution, with farther inprovements also, by Mr. Ramsden, and other ingenious artists in London.

Sir Henry Englefield has likewise much simplified the portable barometer, reducing $i$ ts weight to less than a pound and a half. See Nicholson's Journal, No. 55 ; Phjlos. Mag. No: 117 ; or Retrospect of Discoveries, No. 13.

Fig. 11 represents this instrument, as inclosed in its mahogany case by means of three metallic rings aaa. This case is a hollow cone, so shaped within as to contain steadily the body of the barometer, and is divided into three branches from $b$ to $c$, forming three legs or supports for the instrument when observations are inaking, and sustaining it at the part $d$ of the case, as it appears in Fig. 12 , by an improved kind of gimbals, in which its own weight renders it suffi iently steady at any time.

A thermometer is always attached to the instrument, as a necessary appendage to $\mathrm{it}$, being fastened to the body at $h$, and sunk into the surface of the frame, to preserve it from injury; the degrees of this thermometer are marked on two scales, one on each side of it, viz, the scale of Fahrenheit, and that of Reaumur; the freezing point uf the former being at 32, and of the latter at 0 . Also on the right-hand side of these two scales there is a third, called a scale of correction, placed oppositely to that of Fahrenheit, with the word add and subtract marked, which shows the necessary correction of the observed altitude of the mercury at any given temperature of the air, indicated by the thermometer.

The Common Chamber Weatherglass, is also usually fitted up in a ncat mahogany frame, and other embellishments, to make it an ornamental piece of furniture. It consists of the common tube barometer, with a thermometer by the side of it, and an hygrometer at the top, as exhibited in fig. 13.

To the foregoing may be added a new sort of Barometer, or Weather Instrument by the Sound of a Wire. This is mentioned by M. Lazowski in his tour through Switzerland: it is as yet but in an imperfect state, and was lately discovered there by accident. It seems that a clergyman, though near-sighted, often amused himself with firing at a mark, and contrived to stretch a wire so as to draw the mark to him to see how he had aimed. He observed that the wire sometimes sounded as if it ribrated like a musical cliord; and that after such soundings, a change always ensued in the state of the atmosphere; from whence he came to predict rain or finc weather. On making farther experiments, it was found that the sounds were most distiuct when extended in the plane of the meridian. And according to the weather which was to follow, it was found that the sounds were more or less soft, or more or less continued; also fine weather, it is said, was announced by the tones of counter-tenor, and rain by those of bass. It has been said that M. Volta mounted 15 chords in this way at Pavia, to bring this method to some precision, but no accounts have yet appeared of the success of his obscrvations. 
Humbolt, the celebrated traveller, contrived a portable barometer, composed of several parts, which could be easily put together, and which should correct itself at each observation, like an astronomical instrument. In this barometer the tube is distinct from the scale; and when this breaks, its place can be supplied by another, even on the top of a mountain.

The Phenomena and Observations of the Barometer.-The phenomena of the barometer are various; but authors are not yet agreed upon the causes of them; nor is the use of it, as a weather-glass, yet perfectly ascertained, though daily observations and experience lead us still nearer to precision. Mr. Boyle observes, that the phenomena of the barometer are so precarious, that it is exceedingly difficult to form any certain general rules concerning the rise and fall of the mercury. Even that rule fails which scems to hold the most generally, viz, that the mercury is low in high winds. The best rules however that have been deduced by several authors are as follow :

Dr. Halley's Rules for judging of the Weather.

1. In calm weather, when the air is inclined to rain, the mercury is commonly low.

2. In serene, good, and settled weather, the mcrcury is generally high.

3. Upon very great winds, though they be not accompanied with rain, the mercury sinks lowest of all, according to the point of the compass the wind blows from.

4. The greatest heights of the mercury are found upon easterly or north-easterly winds, other circumstances alike. - The above four observations made by Dr. Halley in England appear to be most universal, as they were found by Mr. Melander to lat. $39^{\circ}$, and by M. de Luc to lat. $46^{\circ}$.

5. In calm frosty weather, the mercury commonly stands high.

6. After very great storms of wind, when the mercury has been very low, it generally rises again very fast.

7. The more northerly places have greater alterations of the barometer than the morc southerly, near the equator.

8. Within the tropics, and near them, there is little or no variation of the barometer, in all weathers. For instance, at St. Helena, it is little or nothing, at Jamaica but 3-10ths of an incl, and at Naples the variation seldom exceeds an inch; whereas in England it amounts to 2 inches and a half, and at Petersburgh to $3 \frac{1}{3}$ nearly.

Dr. Beal, who followed the opinion of M. Pascal, observes that, creteris paribus, the mercury is higher in cold weather than in warm: and in the morning and evening usually higher than at mid-day.-That in settled and fair weather, the mercury is higher than either a little before or after, or in the rain; and that it commonly descends lower after rain than it was before it. And he ascribes these effects to the vapours with which the air is charged in the former case, and which are dispersed by the falling rain in the latter. If it chance to rise higher after rain, $i t$ is usually followed by a settled serenity. And that there are often great changes in the air, without any perceptible alteration in the barometcr.

Mr. Patricl's Rules for judging of the Weather.-These are esteemed the best of any general rules hitherto made.

1. The rising of the mercury presages, in general, fair weather; and its falling, foul weather, as rain, snow, high winds, and storms.

2 . In very hot weather, the falling of the mercury indicates thunder.

VOL. I.
3. In winter, the rising forebodes frost: and in frosty weather, if the mercury fall 3 or 4 divisions, there will certainly follow a thaw. But in a continued frost, if the mercury rises, it will certainly snow.

4. When foul weather happens soon after the falling of the mercury, expect but little of it; and on the contrary, expect but little fair weather when it proves fair shortly after the mercury has risen.

5. In foul weather, when the mercury rises much and high, and so continues for 2 or 3 days before the foul weather is quite over, then expect a continuance of fair weather to follow.

6 . In fair weather, when the mercury falls much and low, and thus continues for 2 or 3 days before the rain comes; then expect a great deal of wet, and probably high winds.

7. The unsettled motion of the mercury, denotes uncertain and changeable weather.

8. You are not so strictly to observe the words engraved on the plates, as the mercury's rising and falling; though in general it will agree with them. For if it stands at much rain, and then rises up to changeable, it presages fair weather; though not to continue so long as if the mercury had risen higher. And so, on the contrary, if the mercury stood at fair, and falls to changeable, it presages foul weather; though not so much of it as if it had sunk lower.

- Upon these rules of Mr. Patrick, the following remarks are made by Mr. Rowning; who also observes, that it is not so much the absolute height of the mercury in the tube that indicates the weather, as its motion up and down: wherefore, to pass a right judgment of what weather is to be expected, we onght to know whether the mercury is actually rising or falling; to which end the following rules are of use.

1. If the surface of the mercury be convex, standing higher in the middle of the tube than at the sides, it is a sign that the mercury is then rising.

2. But if the surface be concave, or hollow in the middle, it is then sinking. And,

3. If it be plain, or rather a little convex, the mercury is stationary: for mercury being put into a glass tube, especially a small one, naturally has its surface a little convex, because the particles of mercury attract one another more forcibly than they are attracted by glass. Farther,

4. If the glass be small, shake the tube; then if the air be grown heavier, the mercury will rise about half a loth of an inch higher than it stood before; but it it be grown lighter, it will sink as much. And, it may be added, in the wheel or circular barometer, tap the instrument gently with the finger, and the index will visibly start forwards or backwards according to the tendency of the fluid to rise or fall at that time. This proceeds from the mercury's adhering to the sides of the tube, which prevents the free motion of it till it be discugagerl by the shock: and therefore when an observation is to be made with such a tube, it ought to be first shaken; for sometimcs the mercury will not vary of its own accord, till the weather is present which it ought to have indicated.

To the foregoing rules may be added the following additional ones, deduced from later and more close observation of the motions of the barometer, and the consequent changes in the air in this country.

1. In winter, spring, and autumn, the sudden falling of the mercury, and that for a large space, denotes ligh winds and storms; but in summer it presages licavy $2 \mathrm{E}$ 
showers, and thunder : and it always sinks lowest of all for great winds, though not accompanied with rain; though it falls more for wind and rain together than for either of thein alone. Also, if, after rain, the wind change into any. part of the north, with a clear and dry sky, and the mercury rise, it is a certain indication of fair weather.

2. After very great storms of wind, when the mercury has been low, it commonly rises again very fast. In settled fair and dry weather, except the barometer sink much, expect but little rain; for its small sinking then, is only for a little wind, or a fetw drops of rain; and the mercury soon rises again to its former station. In a wet season, suppose in hay-time and harvest, the smallest sinking of the mercury must be regarded; for when the constitution of the air is much inclined to showers, a little sinking in the barometer then denotes more rain, as it never then stands very high. And, if in such a season it rises suddenly, very fast, and high, expect not fair weather more than a day or two, but rather that the mercury, will fall again very soon, and an immediate rain follow: the slow gradual rising, and keeping on to do so for 2 or 3 days, are most to be depended on for a week's fair weather. And the unsettled state of the quicksilver always denotes uncertain and changeable weather, especially when the mercury stands any where about the word changeable on the scale.

3. The greatest heights of the mercury, in this country, are found upon easterly and north-easterly winds; and it may often rain or snow, the wind being in these points, and the barometer sinks little or none, or it may even be in a rising state, the effect of those winds counteracting. But the mercury sinks for wind, as well as rain, in all the other points of the compass; but rises as the wind shifts about to the north or the east, or between those two points: but if the barometer should sink with the wind in that quarter, expect it soon to change from thence; or clse, should the fall of the mercury be much, a heavy rain is then likely to ensue, as it sometimes happens.

\section{Cause of the Phenomena of the Barometer.}

To account for the foregoing phenomena of the barometer, many hypotheses have been framed, which may be reduced to two general heads, viz, mechanical and chemi$\mathrm{cal}$. The chief writers on these causes, are Pascal, Beal, Wallis, Garcin, Garden, Lister, Halley, Garsten, Lahire, Mariotte, Le Cat, Woodward, Leibnitz, De Mairan, Hamberger, 1). Bernoulli, Muschenbroek, Chambers, De Luc, Black, \&c : and an account of most of their hypotheses may be seen at large.in M. De Luc's" Recherches sur les Modifications de l'Atmosphere, vol. 1, chap. 3; see also the Philus. Trans. and various other works on this subject. It may suffice to notice here slightly a few of the principal of them.

Dr. Lister accounts for the changes of the barometer from the alterations by heat and cold in the mercury itself; contracting by cold, and expanding by heat. But this, it is now well known, is quite insufficient to account for the whole of the effect.

The changes in the weight or pressure of the atmosphere must therefore he regarded as the principal cause of those in the barometcr. But then, the difficulty will be to assign the catuse of that cause, or whence arise: those alterations that take plice in the atmosplere, which are somctimes so great is to alter its pressure by the toth part of the whole quantity. It is probable that the winds, as driven about in difficrent dircctions, bave a great share in then; vapours and exhalations, rising from the earth, may also bave some share; and some perhaps the flux and reflux occasioned in the air by the moon; as well as some chemical causes operating between the different particles of matter.

Dr. Halley thinks the winds and exhalations sufficient; and on this principle gives a theory, the substance of which may be comprised in what follows:

1st, That the winds must alter the weight of the air in any particular country; and this, either by bringing together a greater quantity of air, and so loading the atmusphere of any place; which will be the case as often as two winds blow from opposite parts, at the same time, towards the same point: or by sweeping away sume part of the air, and giving room for the atmosphere to expand itself; which will happen when two winds blow opposite ways from the same point at the same time: or lastly, by cutting off tlie perpendicular pressure of the air; which is the case when a single wind blows briskly any way; it being found by experience, that a strong blast of wind, even maic by art, will resder the atmosphere lighter; and hence the mercury in a tube below it, as well as in others more distant, will considerably subside. See Philos. Trans. No. 292.

$2 \mathrm{dly}$, That the cold nitrous particles, and even the air itself condensed in the northern regions, and driven elsewhere, must load the atmosphere, and increase its pressure.

3dly, That heavy dry exhalations from the eaith must increase the weight of the atmosphere, as well as its elastic force; as we find the specific gravity of menstruums increased by dissolved salts and metals.

4thly, That the air being rendered heavier by these and the like causes, is thence better able to support the valpours; which being also intimatcly mixed with it, make the weather serene and fair. Again, the air bring made ligliter from the contrary causes, it bccomes unable to support the vapours with which it is replete; these therefore precipitating, are collected into clouds, the particles of which in their progress unite into drops of rain.

Hence, he infers, it is evident that the same causes which increase the weight of the air, and render it more able to support the mercury in the barometer, do likewise produce a serene sky, and a dry scason; and that the same causes which render the air lighter, and less able to support the mercury, do also generate clouds and rain.

But these principles, though well adapted to many of the particular cases of the barometer, scem however to fall short of some of the principal and inost obvious ones, besides being lisble to several objections.

For first, If the wind were the sole agent in effecting these alterations, we should have no sensible alterations without a sensible wind, nor any wind without some alteration of the barometer; both which are contrary to $\mathrm{ex}$ perience.

2 dly, If two winds be supposed blowing from the same place, viz, London, opposite ways, viz, N. F. and s. W. there will be two others blowing from opposite points, riz, N. W. and 5. E. to the same place; which two last will balance tho first, and bring as much air towards the point as the others swept lrom it. This follows from the laws of fluiclity.

$3 \mathrm{dly}$, If the wind were the sole agent, the siterations in the height of the mercury would only te relative, or topical; there would be still the same quantity supported at several places taken collcctively; thus, what a tube at 
London lost, another at Paris, or at Pisa, or at Zurich, would gain. But we find the very contrary true in fact; for, from all the observations hitherto made, the barometers in several parts of the globe rise and fall together; so that it must be some alteration in the absolute weight of the atmosphere that accounts for the rise and fall of the mercury.

Lastly, setting aside these popular phenomena, the mercury's fall hefore, and rise after rain, seem to be inexplicable on the ground of this hypothesis: for suppose two contrary winds sweeping the air from over London; we know that few if any of the winds reach above a mile high; all therefore that they can do, will be to cut off a certain part of the column of air over London: if the consequence of this be the fall of the mercury, yet there is no apparent reason for the rain's following it. The vapours indeed may be let lower, but it will only be till they come into an air of the same specific gravity with themselves; and there they will be suspended as before.

Leibnitz accounted for the fall of the mercury before rain on another principle, viz, That as a body specifically lighter than a fluid, while it is sustained by it, adds more weight to that fluid than when, by being reduced in bulk, it becomes specifically heavier, and Jescends; so the vapour, after it is reduced into the form of clouds, and descends, adds less weight to the air than it did before; and hence the mercury sinks in the tube.-But here, granting that the drops of rain formed from the vapours always increasing in size as they fall lower, were continually accelerated also in their motion, and, so the air suffer a continued loss of their weight as they descend; it may however be objected, that by the descent of the mercury the rain is foretold a much longer time before it comes, than the vapour can be supposed to take up in falling: that many times, and in different places, there falls a great deal of rain, witbout any sinking of the mercury at all; as also that there often happens a fall of the mercury, without any rain ensuing: and that sumetimes the mercury will surdenly sink, in a short space of time, half an inch or more, which answers to 7 inches of rain, or about one third of the whole quantity falling in the whole year.

M. De Luc supposes that the changes observed in the pressure of the atmosphere, are chiefly produced by the greater or less quantity of vapours floating in it: others have attributed them to the same cause, but have given a different explanation of it. His opinion is, that vapours diminish the specific gravity, and consequently the absolute weight of those columns of the atmosphere into which they are received, and which, notwithstanding this admixture, still remain of the same height with adjoining columns that consise of pure or dry air. He afterwards vindicates and more fully explains this theory, and applies it to the solution of the principal phenomena of the barometer, as depending on the varying density and weight of the atmosphere.

Dr. James Hutton, in his Theory of Rain, printed in the Transactions of the Royal Society of Edinburgh, vol. 1, gives ingenious and plausible reasons for thinking that the diminution of the weight of the atmosphere by the fall of sain, is not the cause of the descent of the mercury in the barometer tuhe; but that the principal, if not the only cause, arises from the commotions in the atmosphere, which are chicfly produced by sudden changes of beat and cold in the air. "The barometer," he observes, "is an instrument necessarily connected with motions in the at- mosphere; but it is not equally affected with every motion in that fluid body. The barometer is chiefly affected by those motions by which there are produced accumulations and abstractions of this fluid, in places or regions of sufficient extent to affect the pressure of the atmosphere on the surface of the earth. But, as every commotion in the atmosphere may, under proper conditions, be a cause of rain, and as the want of commotion in the atmosphere is naturally a cause of fair weather, this instrument may be made of great importance for the purpose of meteorological observations, though not in the certain and more simple manner in which it has been, with the increase of science, so successfully applied to the measuring of heights." See RaIN.

In the Encyclopædia Britannica there is another theory of the changes in the barometer, as depending on the heat in the atmosphere, not as producing commotions there, but as altering the specific gravity of the air by the changes of heat and cold. The principles of this theory are, -1 st, That vapour is formed by an intimate union between the elements of fire and water, in consequence of which the fire or beat is so totally enveloped, and its action so perfectly suspended by the aqueous particles, that it not only loses its properties of burning and of giving light, but becomes incapable of affecting the most sensible thermometer; in which case it is said by Dr. Black to be in a latent state: and 2d, That if the atmosphere be affected by any unusual degree of heat, it thence becomes incapable of supporting so long a column of mercury as before; for which reason the barometer sinks.

From these axions it would follow, that as vapour is formed by the union of fire with water, whether by attraction or a solution of the water in the fire, the vapour cannot be condensed till this union, attraction, or solution, be at an end. Flerice the beginning of the condensation of the vapuur, or the first signs of approaching rain, must be the separation of the fire which is latent in the vapour. In the beginning, this may be either slow and partial, or it may be sudden and violent: in the first case, the rain will come on slowly, and fall after a considerable time; but in the other, it will accumulate very quickly, and in a great quantity. But Dr. Black bas proved, that when fire quits its latent state, however long it may have lain dormant and insensible, it always reassumes its proper qualities, and atfects the thermometer just the same as if it had never been absorbed. The consequence of this must be, that in proportion as the latent heat is discharged from the vapour, those parts of the atinosphere into which it is discharged must be sensibly affected by it; and in proportion to the heat communicated to those parts, they will become specifically lighter, and the mercury will sink of course.

In the Memoirs of the Literary Society of Manchester, vol. 4 , is also a curious paper on this subject, viz. Meteorological Observations made on different Parts of the Western Coast of Great Britain : arranged by T. Garnett, M. D. 'This paper is composed of materials furnished by several observers; those of Mr. Copland, surgeon at Dumfries, are of special importance. This gentleman is of opinion that the changes of the barometer indicate approaching hot and cold weather, with much more certainty than dry and wet. "Every remarkable elevation of the barometer," he says, "where it is of any duration, is followed by very warm or dry weather, and moderate as to wind, or by all of them: but heat seens to have most inflience $2 \mathfrak{E} 2$ 
and connexion; and when it is deficient, the continuance of the other two will be longer and more r'emarkable; therefore the calculation must be in a compound ratio of the excess and deficiency of the heat, and of the dryness of the weather in comparison of the medium of the season; and with regard to the want of strong wind, it appears to be intimately connected with the last, as they show that no precipitation is going on in any of the neighbouring regions."

In his 14th and 15th remarks, he had said,

' $14 \mathrm{th}$, That the barometer being lower, and continuing so longer than what can be accounted for by immediate falls, or stormy weather, indicates the approach of very cold weather for the season; and also, cold weather, though dry, is always accompanied by a low barometer, till near its termination.'

' 15 th, That warm weather is always preceded and mostly accompanied by a high barometer; and the rising of the barometer in the time of broken or cold weather, is a sign of the approach of warmer weather; and also if the wind is in any of the cold points, a sudden rise of the barometer indicates the approach of a southerly wind, which in winter generally brings rain with it.'

In the two following remarks, Mr. Copland had explained certain phenomena from a principle similar to that on which Dr. Darwin has so much insisted: Botanic Garden, I. notes p. 79, \&c.

"That the falling of the barometer may proceed from a decomposition of the atmosphere occurring around or near that part of the globe where we are placed, which will occasion the electricity of the atmosphere to be repelled upwards in fine lambent portions; or driven downwards or upwards in more compacted balls of fire; or lastly, to be carried along with the rain, \&c, in an imperceptible manner to the surface of the earth: the precipitation of the watery parts generally very soon takes place, which diminishes the real gravity of the atmosphere, and also by the decomposition of some of the more active parts, the air loses part of that elastic and repulsive power which it so eminently possesseci, and will therefore press with less force on the mercury of the barometer than before, by which means a fall ensues.

- That the cause of the currents of air, or winds, may also be this way accounted for: and in very severe storms, where great decompositions of the atmosphere take place, this is particularly evident, such as generally occur in one or more of the West-India islands at one time, a great loss of real gravity, together with a considerable diminution of the spring of the air immediately ensues; hence a current commences, first in that direction whence the air has most gravity, or is most disposed to undergo such a change; but it being soon relieved of its superior weight or spring on that side, by the decomposition going on as fast as the wind arrives on the island, it immediately veers to another point, which then rushes in mostly with an increase of force; thus it goes on till it has blown more than half way round the points of the compass during the continuation of the hurricane. For in this manner the IVest-India phenomena, as well as the alterntion of the wind during henvy rains in this country, can only be properly accounted for.' See rematk No. 4.

Mr. C.'s 4th aplorism is, "That the heaviest rains, when of long continuance, generally begin with the wind blowing easterly, when it gradually veers round to the south; and that the rain does not then begin to cease tilt the wind has got to the west, or rather a little to the northward of it, when, it may be added, it commonly blows witb some violence.'

Many other observations on the barometer, the weather, \&c, may be seen in various parts of the Philos. Trans. And for some curious papers on the same, and other subjects connected with the barometer, see the Gentleman's Magazine for 1789 , p. 317 ; also Greu's Journal of Nat. Philos. printed at Leipzig 1792, for the influence of the sun and moon on the barometer. See also a very elaborate paper on this.subject in vol. 2 , p. 49 , \&c, of the Royal Irish Trans. by Mr. Kirwan.

The Burometer applied to the Measuring of Altitudes.

The secondary character of the barometer, namely, as an instrument for measuring accessible heights or depths, was first proposed by Pascal and Descartes, as bas been before observed; and succeeding philosoplers bave been at great pains to ascertain the proportion between the fall of the barometer and the height to which it is carried; as Halley, Mariotte, Maraldi, Scheuchzer, J. Cassini, D. Bernoulli, Horrebow, Bouguer, Shuckburgh, Roy, and more especially by De Luc, who has given a critical and historical detail of most of the attempts that have at different times been made for applying the motion of the mercury in the barometer to the measurement of accessible heights. And for this purpose serves the portable barometer, before described, (fig. 11 and 12, plate 4,) which should be made with all possible accuracy. Various rules have been given by the writers on this subject, for computing the height ascended from the given fall of the mercury in the tube of the barometer, the most accurate of which was that of Dr. Halley, till it was rendered much more'so by the indefatigable researches of De Luc, by introducing into it the corrections of the columns of mercury and air, on account of heat. Other corrections and modifications of the same may be seen inserted under the article ATMOSPHERE, where the most correct rule is deduced from one single cxperiment only.

The Rule for computing Altitudes, is this,

Viz, $10000 \times \log$. of $\frac{M}{m}$ is the altitude in fathoms, in the temperature of $31^{\circ}$ : and for every degree of the thermometer above that, the result must be increased by so many times its 435 th part, and diminished when below it: in which theorem $\mathrm{M}$ denotes the length of the column of mercury in the barometer tube at the bottom, and $m$ that at the top of the hill, or other eminence; which lengths may be expressed in auy one and the same sort of measures, whether feet, or inches, or tenths, \&c, and cither English, or French, or of any other nation; but the result in this case is ałways in fathoms, of 6 English fect cach.

And the Prccepts, in words, for the practice of measuring altitudes by the barometer, are these following:

1st, Observe the height of the barometer at the bottom of any height or depth, proposed to be measural; together with the temperature of the mercury by means of the thermometer attached to the barometer, and also the temperature of the air in the shade by another thermometer which is detached from the barometer.

2dly, Let the same thing be done also at the top of the said height or depth, and as near the time of the former as possible. And let those altitudes of mercury be reluced to the same temperature, if it be thought necessary, by correcting either the one or the other, viz, augnenting the lecight of the mercury in the colder temperature, or dimi- 


\section{B A R}

nishing that in the warmer, by its 9600 th part for every degree of difference between the two; and the altitudes of mercury so corrected, are those which are denoted by $\mathrm{s}$ and $m$ in the algebraic formula above.

$3 \mathrm{dly}$, Take out the common logarith ms of the two heights of mercury, so corrected, and subtract the less from the greater, cutting off from the right hand side of the remainder three places for decimals; so shall those on the left be fathoms in whole numbers, the tables of logarithms being understuod to be such as have 7 places of decimals.

4.thly, Correct the number last found, for the difference of the temperature of the air, as follows: viz, Take half the sum of the two temperatures of the air, shown by the detached thermometers, for the mean one; and for every degree which this differs from the standard temperature of $31^{\circ}$, take so many times the 435 th part of the fathoms above found, and add them if the mean temperature be more than $31^{\circ}$, but subtract them if it be below $31^{\circ}$; so shall the sum or difference be the true altitude in fathoms, or being multiplied by 6 , it will give the true altitude in English feet.

Example 1. Let the state of the barometers and thermometers be as follows, to find the altitude: viz,

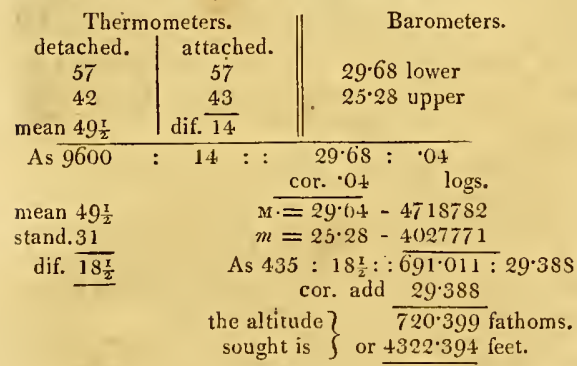

Example 2. To find the altitude of a hill, when the state of the barometer and thermometer, as observed at the bottom and summit of the same are as follows: viz,

Thermometers.

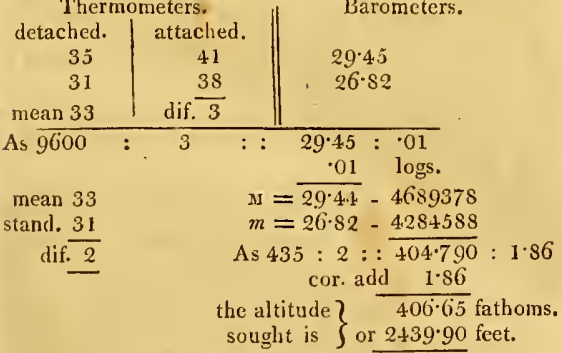

See this rule investigated under the article PNEUMATICs, at the end.

N. B. The mean height of the barometer in London, on an average of two observations in every day of the year, kept at the house of the Royal Society, for many years past, is 29.88; the medium temperature, or height of the thermometer, according to the same, being $58^{\circ}$. But the medium height at the surface of the sea, according to Sir Geo. Shuckburgh (Philos. Trans. 1777, p. 586) is 30.0 t inches, the heat of the barometer being $55^{\circ}$, and of the air

\section{B A R}

$62^{0}$. See a learned paper in vol. 1 of the Trans. of the R. Soc. of Edinburgh, " on the Causes which affect the Accuracy of Barometrical Measurements; by John Playfair, A. M. F. R. S. Edin. and Professur of Mathematics in the University of Edinburgh ;" also, another by Dr. Damen, late Professor of Mathematics and Philosophy in the University of Leyden, entitled "Dissertatio Physica et Mathematica de MIontium Altitudine Barometro Metienda. Accedit Refractionis Astrononica Theoria," in Svo, at the Hague, 1783. Also other corrections and modifications that have been given by several philosophers, as Robison, Trembley, Englefield, Prony, Laplace, \&c.

We shall now conclude this subject by an account of the height of some of the most remarkable mountains, \&c, on the earth, above the surface of the ocean, in feet.

Mount Puy de Dome, in Auvergne, the first mountain measured by the barometer, - $\}$

Mount Blanc

Monte Rosa

Aiguille d'Argentine - $\}$ Alps

Monastery of St. Bernard

Mount Cenis

Pic de los Reyes )

$\left.\begin{array}{ll}\text { Pic du Medi } & - \\ \text { Pic d'Ossano } & -\end{array}\right\}$

Canegou

Lake of Geneva

Mount Fina -

Nount Vesuvius

Mount Hecla, in Iceland

Snowden

Ben Moir

Ben Laurs

Ben Gloe

Shihallien

Ben Lomond

Tinto

Table-Hill, Cape of Good Hope,

Gondar city, in Abyssinia,

Source of the Nile

Pic of Teneriffe

Chimboraçou -

Cayambourou

Antisana

Pichinha -

City of Quito -

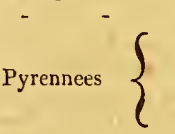

5088

15662

- 15084

- $13 \pm 02$

- 7944

- $\quad$ y212

- 9300

- 11700

- 8544

- 1232

- 10954

- 3938

- 4587

- 3555

- $\quad 3723$

- $\quad 3858$

- 3472

- 3461

- 3180

- 2342

- 3454

- $8+40$

- 8082

- $14026^{\circ}$

- 19595

- 19391

- 19290

- 15670

- 9977

Caspian Sea below the Ocean - - $\quad 306$

BAROSCOPE, a machine for showing the alterations in the weight or pressure of the atmosphere. See BaroneTER.

BARREL, an English vessel or cask, containing 36 gallons of beer-measure, or 32 gallons of ale-measure. The barrel of beer, vinegar, or of liquor preparing for vinegar, ought to contain 34 gallons, according to the standard of the ale quart.

BARMEL, in clock-work, is the cylinder about which the spring is wrapped.

BARRICADE, or BARRICADO, a military term for a fence, or retrenchment, hastily made with vessels, or baskets of earth, carts, trees, stakes, or the like, to preserve an army from the shot or assault of an enemy.

BARRIER, a kind of fence made at a passage, retrenchment, gate, or such likc, to stop it up against an encmy.

BARROW (IsAAC), a very eminent mathematician and divine of the 17 th century, was born at London in Octo- 
ber, 1630 , being the son of Thomas Barrow, then a linendraper of that city, but descended from an ancient family in Suffolk. He was first placed at the Charter-house school for two or three years; where his behaviour afforded but little hopes of suceess in the profession of a scholar, being much addicted to fighting, and promoting it among his school-fellows; but on removal to Felsted in Essex, his disposition took a different turn, and be applied himself with great assiduity to his studies, in which he made very rapid advancement. He was first admitted a pensioner of Peter-house in Cambridge; but when he came to join the university in Feb. 164.5, he was entered at Trinity-college. In 1647 he was chosen a scholar of the house, and subscribed the engagement; but afterwards repenting what he had done, he went back and struck out his name from the list. At this period he began to apply himself with great diligence to the study of all parts of literatire, especially natural philosophy. Ile afterwards turned his attention to the profession of physic, and made considerable progress in anatomy, botany, and chemistry : be next studied divinity; then ehronology, astronomy, geometry, and the other branches of the mathematics; with what suceess, his writings afterwards most eminently showed. In $16+9$ he was chosen fellow of his eollege.

When Dr. Duport resigned the ehair of Greek professor, he recommended his pupil Mr. Barrow for his successor, who, in his probationary exercise, showed himself equal to the character that had been given of him by this gentleman; but being suspected of favouring Arminianism, be was not preferred. This disappointment, it appears, determined him to quit the college, and visit foreign countries; but sach were his. finances, that he was obliged to dispose of his books, to enable him to execute that design.

He left England in June 1655, and visited France, Italy, Turkey, \&c. At several places, in the course of this tour, he met with much kindness and liberal assistance from the English ambassadors, \&c, which enabled him to benefit the more from it, by protracting his stay, and prolonging his journey. He spent more than a year in Turkey, and returned to England by way of Venice, Germany, and Holland, in 1659. At Constantinople he read over the works of St. Chrysostom, once bishop of that see, whom be preferred to all the other fathers.

On his return bome Barrow was episcopally ordained by bishop Brownrig; and in 1660, he was chosen to the Greek professorship at Cambridge. In 1662 be was elected professor of geometry in Gresham-college; in which station he not only discharged his own duty, but supplied also the absence of Dr. Pope the astrunomical professor. Among his lectures, some were on the projection of the sphere and perspective, which are lost; but his Latin oration, previous to his lectures, is still extant. About this time Mr. Barrow was offered a good living; but the condition annexed, of teaching the patron's son, made him refuse it, as thinking it too like a simoniacal contract. On the 20th of May 1663 he was elected a fellow of the Royal Society, in the first choice made by the council after their charter. The same year the executors of Mr. Lucas having, according to his appointment, founded a mathematical lecture at Cambridge, they selected Mr. Barrow for the first professor; ind though his two professorships were not incompatible with each other, he ehose to resign that of Gresham-college, which be did May the 20th, 1664; and at the same time his Greek professorship also. In 1669 be resigned the mathematical chair to his learned friend Mr. Isaac Newton, having detcrmined to quit the study of mathematics altogether for that of divinity. On leaving his professorship, he had only his fellowship of Trinity-college, till his uncle gave him a small sinccure in Wales; and Dr. Seth Ward, bishop of Salisbury, cunferred on him a prebendary in his church. In the year 1670 he was created doctor in divinity by mandate; and, on the promotion of Dr. Pearson, master of Trinity-college, to the see of Chester, he was appointed to succeed him by the king's patent, bearing date the 13th of Feb. 1672; on which occasion the king was pleased to say, "he had given it to the best scholar in England." In this his naajesty did not speak from report, but from his own knowledge; the doctor being then his chaplain, he used often to converse with him, and, in his humorous way, would call him an " unfair preacher," because he exhausted every subject, and left no rnom for others to come after him. In 1675 he was chosen vice-chancellor of the university; and he omitted no endeavours for the good of that society, nor in the line of his profession as a divine, for the promotion of piety and virtue: but his useful labours were abruptly terminated by a fever on the 4th of May, 1677, in the 47 th year of his age. He was interred in Westminster Abbey, where a monument, adorned with his bust, was soon after creeted by the contribution of bis friends.

Dr. Barrow's works are very numerous, and indeed various-inathematical, theological, poetical, \&c, and such as do honour to the English nation. They are principally as follow:

1. Euclidis Elementa. Cantab. 1655, in 8vo.

2. Euclidis Data. Cantab. 1657 , in 8 vo.

3. Leetiones Opticæ xviii. Lond. 1669, 4 to.

4. Lectiones Geometrica xiii. Lond. 1670, 4to.

5. Archimedis Opera, A pollonilConicorum libri iv, Theodosii Sphericorum lib. iii; nova methodo illustrata, et suceinete demonstrata. Lond. 1675 , in 4 to.

The following were published after his decease, viz:

6. Lectio, in qua thcoremata Archimedis de sphrera et cylindro per methodum indivisibilium investigata, ac breviter investigata, exhibentur. Lond. $1678,12 \mathrm{mo}$.

7. Mathematicæ Lectiones habitæ in scholis publicis academiæ Cantabrigiensis, an. 1664, 5, 6, \&c. Lond.1683.

8. All his Englisin works, in 3 volumes. Lond. 1683, folio.-These are all theological, and were published by Dr. John Tillotson.

9. Isaaci Barrow Opuscula; viz, Determinationes, Conciones ad Clerum, Orationes, Poemata, \&c, volumen quartum Lond. 1687, folio.

Dr. Barrow left also several curious papers on mathematical subjects, written in his own band, which were communicated by Mr. Jones to the author of "The Lives of the Gresham Professors," a partieular account of which may bc seen in that book, in the Life of Barrow.

Several of his works have. been translated into English, and published: as the Elements and Data of Euclid; the Gcometrical Lectures; the Mathematical Lectures; and accounts of some of them were also given in several volumes of the Philos. Trans.

It is remarkable, that Dr. Barrow's method of drawing tangents to eurves is exactly similar to that by the method of fluxions, the diflerence being only in the notation: viz, he considered the elemental triangle, formed by the distance and the difference of two indefinitely near ordinates, with the element of the curve, as similar to the triangle formed by the ordisate, the tangent, and the subtangent; 
which thence he found by this proportion: As the difference of the ordinates is to their distance, so is the ordinate to the subtangent.

Dr. Barrow must ever be esteemed, in all the subjects which exercised his pen, a person of the clearest perception, the finest fancy, the soundest judgment, the profoundest thought, and the closest and most nervous reasoning. "The name of Dr. Barrow (says the learned MIr. Granger) will ever be illustrious for a strength of mind and a compass of knowledge that did honour to his country. He was unrivaled in mathematical learning, and especially in the sublime geometry; in which he has been excelled only by his successor Newton. The same genius that seemed to be born only to bring bidden truths to light, and to rise to the beights or descend to the depths of science, would sometimes amuse itself in the flowery paths of poetry, and compose verses both in Greek and Latin. He at length gave bimself up entirely to divinity; and particularly to the most useful part of it, that which has a tendency to make inen wiser and better."

Several good anecdotes are told of Barrow, as well of his great integrity, as of his wit, and bold intrepid spirit and strength of body. His eurly attaclıment to fighting when a boy is some indication of the latter; to which may be added the two following anecdotes: In his voyage between Leghorn and Smyrna, the ship was attacked by an Algerine pirate, which, after a stout resistance, they compelled to sheer off, Barrow keeping his post at the gun to the last. And when Dr. Pope in their conversation asked him, "Why he did not go down into the hold, and leave the defence of the ship to those to whom it did belong ?" He replied, "It concerned no man more than myself: I would rather have lost my life, than to bave fallen into the hands of those merciiess infidels."

There is another anecdote told of him, which showed not only his intrepidity, but an uncommon goodness of disposition, in circumstances where an ordinary share of it would have been probably extinguished. Being once on a visit at a gentleman's house in the country, where a large mastiff was kept, as he was going out one morning before day (for he wats a very early riser) the fierce animal that used to be chained up during the day, aud let loose at night for the security of the house, perceiving a strange person in the garden at that unusual time, set upon him with great fury. The doctor canght him by the throat, grappled with bim, and throwing him down, lay upon him: once he thought of killing him; but he altered his resolution, on recullecting that this would be unjust, since the. log did only his duty, and he himself was in fault for rambling out of his room before it was light. At length. he called out so lond, that he was heard by some of the family, who came presently out, and released the doctor and the $\operatorname{dog}$ from their disagreeable situation.

BARS, in Music, are the spaces quite through any composition, scparated by upright lines drawn across the five borizontal lines, each of which either contains the same number of notes of the same kind, or so many other notes as will make up a like interval of time; for all the bars, in any picce, must be of the same leugth, and played in the sanc time.

BARS, in Architecture, are long slender pieces of wood, or iron, used to keep things securc, and at their proper assigned distances.

BARTEl, or Truck, is the exchanging of one commodity for another; and forns a rule in the commercial part of arithmetic, by which the commodities are properly calculated and equalled, by computing first the value of the commodity which is given, and then the quantity of the other which will be adequate to the same sum.

Example. How much wine at $70 l$ per pipe must be given in barter for 500 loads of timber at $2 l 5 s$ per load?

First fiad the value of the commodity whose quantity is given; thus,

$$
\begin{array}{l|l}
s & \\
5 & \frac{2}{1000} \\
\frac{125}{125}
\end{array} .
$$

$\overline{1125}$ the value of the timber: hence, to find what quantity of wine at $70 l$ per pipe will amount to this sum, say, as $70 l: 1$ pipe : $: 1125 l: 16$ pipes 9 gallons the quantity sought.

BARTHOLINE(ERASMUs), a mathematician and philosopher, in the latter part of the 17th century, who died in 1698 , at 73 years of age. He was successively professor of mathematics and philosophy at Copenhagen; and attained the rank of counsellor of state. He published De Cometis, an. 1664 et 1665 , Opusculum ex observationibus Hafniæ habitis adornatum. Hafnix, 4to. Also, Experimenta Crystalli Islandici, 1670, in 4to; and De Aere Hafniensi, 1679 , in 8 vo.

BASE, or BAsis, in Chemistry, is a term applied by the old chemists to designate those substances of a fixed, inert, passive nature, which combined with, and were acted on, by more volatile or active menstrua. Thus, the alkalies, earths, and metallic oxyds, which form compound salts, by uniting with acids, were called the bases of these salts. Modern chemists, though they maintain, that in every combination the nisus or force of affinity between two ingredients is mutual and equal, have yet retained the term for the sake of precision, to express either species or families of salts which differ with regard to the acid, but agree as to the alkali, earth, or metallic oxyd which they contain. Thus, salts with a base of potash, include all those species which are formed by the combination of the various acids with the particular alkali, potash. Again, salts with an alkaline base comprehend the three families of salts with bases of potash, soda, or ammonia, as distinguished from the otler salts with earthy or metallic bases.

The utility, therefore, of this mode of expression is evident; for though the compound salts are usually divided into genera according to their acids, as sulphats, nitrats, muriats, \& c, jet it is often desirable to arrange them according to their other element or base, for which the Lavoisierian nomencląure has not particularly provided.

The term base is also used on other occasions as a method of denoting species; as when we say sulphuric acid is composed of oxygen united with the base of sulphur; the vegetable acids, of oxygen and a compound base of hydrogen and carbon. Sometimes also the word base is applied in a more indefinite manner, as in the expressions, phosphat of lime is the base of animal bone, azot is the base of muscular fibre, where it means merely the characteristic or principal part.

BASE, BAs1s, in Architecture, denotes the lower part of a column or pedestal. The base of columns is differently formed in different orders: Thus, the Tuscan base consists only of a single tore, besides the plinth: the Doric has an astragal more than the Tuscan : the Ionic has a large torc over two slender scutias, separated by two 
astragals: the Corinthian has two tores, two scotias, and two astragals: the Composite has an astragal less than the Corinthian: the Attic base has two tores and a scotia, and is proper for either the Ionic or Composite columns.

Bas $\mathrm{s}$, in Geometry, the lowest side of any figure. Any side of a figure may be considered as its base, according to the position in which it may be conceived as standing; but commonly it is understood of the lowest side: as the base: of a triangle, of a cone, cylinder, \&c.

BASE LINE, in Perspective, denotes the common section of the picture and the geometrical plane.

BASE RING, of a cannon, is the great ring next behind the vent or touch-liole.

Base, Alternate. See Alternate.

BASEMENT, in Architecture, a continued base, extended a considerable length, as about a house, a room, or other piece of building.

BASILIC, in the ancient Architecture, was a large hall, or court of judicature, where the magistrates sat to administer justice.

BASILICA, or Basizicus, the same as Regulus, or Cor Leonis, being a fixed star of the first magnitude in the constellation of Leo.

BASILISK, in the older artillery, was a large piece of ordnance so called from its resemblance to the supposed serpent of that name. It threw an iron ball of 200 pounds weight; and was in great repute in the time of Solyman emperor of the Turks, in the wars of Hungary; but it is now grown - out of use in most parts of Europe. Paulus Jovius relates the terrible slaughter made in a Spanish ship by a single ball from one of these basilisks; after passing througl the beams and planks in the ship's head, it killed upwards of 30 men. And Maffeus speaks of basilisks made of brass, each of which required 100 yoke of oxen to draw them.-More modern writers also give the name basilisk to a much smaller and sizeable piece of ordnance, made of 15 feet long by the Dutch, but of only 10 by the French, and carrying a ball of 48 pounds. The largest size of cannon now used by the English, are the 32pounders.

BASIS, in Geometry, the same as BAsE.

BASS, in Music, that part of a concert which is the most heard, which consists of the gravest, deepest, and longest sounds: it is played on the largest pipes or strings of common instruments; or on instruments larger than ordinary for the purpose; by some it is esteerned the basis and principal part in music, and by others as scarcely necessary in some tunes.

BASSANTIN (JAMES), a Scotch astronomer of the 16 th century, born in the reign of James the 4 th of Scotland. He was a son of the Laird of Bassantin in the Merse. After funisling his education at the university of Glasgow, he travelled through Germany and ltaly, and then settled in the university of Paris, where he taught mathematics with great applause. Jlaving acquired some property in this employment, he returned to Scotland in 1562 , where he died 6 ycars after.

From his writings it appears he was no inconsiderable astronomer, for the age in which he lived; but, according to the fashion of the times, lie was greatly addicted to judicial astrology. It was doubtless to our author that Sir James Melvil allucles in his Memoirs, when he says that his brother Sir Robert, when he was using his endeavours to reconcile the two queens Elizabeth and Mary, met with one Bassantin, a man learned in the high sciences, who told him, "that all his travels would be in rain; for," said he, "they will never meet together; and next, there will never be any thing but dissembling and secret hatred for a while, and at length captivity and utter wreck to our queen from England." He added, "that the kingdom of England at length shall fall, of right, to the crown of Scotland: but it shall cost many bloody battles; and the Spanjards shall be helpers, and take a part to themselves for their labour." A prediction in which Bassantin partly guessed right, whicl it is likely he was enabled to do from a judicious consideration of probable circumstances and appearances.

Bassantin's works are,

1. Astronomia Jacobi Bassantini Scoti, opus absolutissimum, \&c; ter, edit. Latine et Gallice. Genev. 1599. fol. This is the title given it by Tornœsius, who translated it into Latin from the French, in which language it was first published.

2. Paraphrase de l'Astrolabe, avec une amplification de l'usage d'Astrolabe. Lyons 1555. Paris 1617 , 8vo.

3. Mathematica Genethliaca.

4. Arithmetica.

5. Musica secundum Platonem.

6. De Mathesi in Genere.

BASTION, in the modern fortification, a large mass of earth at the angles of a work, connecting the curtains to each other; and answers to the bulwark of the ancients. It is formed by two faces, two flanks, and two demigorges: The two faces form the saliant angle, or angle of the bastion; the two flanks form with the faces, the epaules or shoulders; and the union of the other two ends of the flanks with the curtains forms the two angles of the flanks.

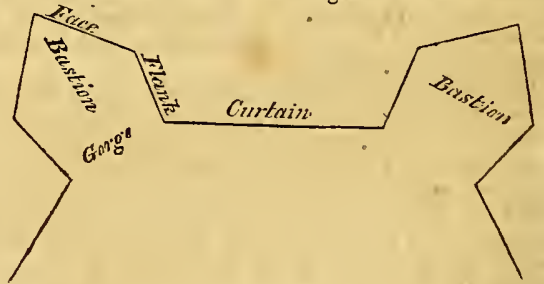

The first introduction of bastions into fortification, is not precisely defined. However, they were well known soon after the year 1500; for Tartalea gives a plan of Turin, which had been completely fortified for some time with four bastions, in his Quesiti et Inventioni diverse, published in 1546 .

If the angle of the bastion be less than $60^{\circ}$, it will be too small to give room for guns; and besicles so acute as to be easily beaten down by the enemy's guns : to which may be added, that it will either render the line of defence too long, or the flanks too short; it must therefore be more than $60^{\circ}$; but whether or no it should be a right angle, some intermediate angle between 60 and $90^{\circ}$, or even whether or no it should exceed $90^{\circ}$, is still disputed, though those are generally preferred which are not much less than $90^{\circ}$, and not exceeding 120 or $130^{\circ}$. Hence it follows that a triangle can never be fortified; because either some or all of the angles will be either $60^{\circ}$, or less than 60 .

Bastions are of various kinds; solid, void, regular, Sc.

Solid Bastrons, are those that are entirely filled up with earth to the height of the rampart, without any roid space towards the centre. 
Void or Hollow Bast yos, has the rampart and parapet ranging only round the flanks and spaces, so that a void space is left within towards the celltre, where the ground is so low that if the rampait be taken, no retrenchment can be made in the centre, but what will lie under the fire of the besieged.

Regular BAstion, is that which has its due proportion of faces, flanks, and gorges.

Deformed or Irregular $\mathrm{BAST} 1 \mathrm{ON}$, is when the irregularity of the lines and angles throws the bastion out of shape; as when it wants one of the demigorges, one side of the interior polygon being too short, \&c.

Demi Bastion, or Half Basion, also otherwise called an Epaulment, has but one face and flank.

Double Bastron, is when one bastion is raised within, and upon the plane of another bastion.

Flat Bastron, is one built in the middle of the curtain, when it is ton long to be defended by the usual bastions at the extremities.

Composed BAstion, is when the twosides of the interior polygon are very unequal, which makes the gorges also unequal.

Out BAstron, is that which has a re-entering angle at the point, and is sometimes called a. BAstion with a Tencille, whose point is cut off, making an angle inwards, and two points outwards. 'This is used wben the saliant angle would be too sharp, or when water or some other impediment prevents it from being carried out to its full extent.

BAS'TON, or BATOoN; in Architecture, a moulding in the base of a column, called also a Tore, or Torus.

BATTARDEAU, or COFFERDAM, in bridge-building, is a case of piling, \&c, without a bottom, fixed in the bed of the river, by which to lay the bottom dry for a space large enough to build the pier on. When it is fixed, its sides reaching above the surface of the river, the water is drawn out by pumps, \&c, and it is in like manner kept dry till the pier is built up; and then the materials of the cofferdam are drawn up. See my Tracts, vol, i, p. 100.

BATTEN, a name given by workmen to a scantling or piece of wooden stuff, about an inch thick, and from 2 to 4 inches broad; of a considexable but indeterminatelength.

BATTERING, the attackiıg a place, work, or the like, with heavy artillery.

BAtTERING-R $\Lambda$ M, a military engine used for beating down walls before the invention of gunpowder and the modern artillery. It was no other than a long heavy beam of timber, armed with an iron head, something like the head of a ram. 'This being pushed violently with constant successive blows against a wall, gradually shakes it with a vibratory motion, till the stones are disjointed and the wall falls down. There were several kinds of battering-rams : the first was rude and plain, which the soldiers carricd in their arms by main force, and so struck the head of it against the wall. The second was slung by a rope about the middle to another beam lying across upon a couple of posts; which was the kind described by Joscphus as used at the siege of Jerusalem. A third sort was covered with a shell or screen of boards, to defend the men from the stones and darts of the besieged upon the walls, and thence called Testudo Arictária. And Felibien describes a fourth sort of battering-rain, which ran upon whcels; and was the most perfect and effectual of them all.

Vitruvius affirms, that the battering-ram was first invented by the Carthaginians, while they laid siege to Cadiz; yet Pliny assures us, that the ram was invented or used at

VoL. I. the siege of Troy; and that it was this that gave occasion to the fable of the wooden-horse. Thie ram so used by the Carthaginians was of the most simple construction, being the one first mentioned. Pephasmenos, a Tyrian, afterwards contrived to suspend it with ropes; and lastly, Polydus, the Thessalian, mounted it on wheels, at the siege of Byzantium, under Philip of Macedon. It has been suspected that the walls of Jericho, mentioned in the Book of Joshua, were beaten down with this instrument; the ram's horns there mentioned, and by means of which they were overthrown, being no other than the horns of the batteringram.

Plutarch relates, that Marc Anthony, in the Parthian war, made use of a ram 80 feet long: and Vitruvius affirms that they were sometimes 106, and even 120 fect long; which must have given an immense force to this engine. The ram required 100 soldiers to work and manage it at one time; who being exhausted, another century relieved them; by which means it was kept playing continually. See fig. 2 , plate $v$, which represents the battering-ram suspended in its open frame; in which 3 denotes the form of the head, fastened to the enormous beam 2 , by three or four bands (4) of iron, of about four feet in breadth. At the extremity of each of these bands was an iron chain (5), one end of which was fastened to a hook $(6)$, and to the last link at the other extremity was firmly bound a cable, which ran along the whole length of the beam to the end of the ram 7 , where these cables were bound together as fast as possible with small ropes. To the end of these cables was likewise fastened another, that consisted of several strong cords platted together to a certain length, and then running single (8), at each of which were placed several men, to balance and work the machine. 10 is the chain or cable by whicl the ram was hung to the cross beam (11), fixed on the top of the frame; and 12 is the base of the machine.

The unsuspended ram differed from this only in the manner of working it; as it moved on small whecls upon another large beam, instead of being slung by a chain or cable.

Mr. Attwood, in his Mechanics, p. 265, has compared the effects of a battering-ram to that of a 24-poundier; 'the metal extremity of the ram, or the head of it, he supposes to be of the same magnitude as the shot: "In order," says he, "that the ball may have the same effect in making a breach in a wall, the weight of the ram must exceed that of the ball in the proportion of about $1700^{\text {: }}$ to the square of the velocity with which this engine could be made to impinge agaiust a wall, expressed in feet; if this may beestimated at about 10 fcet in a sccond, the proportion of the weights will be that of about 2890000 to 100 , or 25900 to $1:$ the weight of the battering-ram must therefore be equal to 346 ton. In this case the batteringram and the cannon-ball, moving with the velocities of 10 feet and 1700 feet respectively in a second, would have the same effect in penetrating the substance of an opposcd obstacle; but it is probable that the weight of the ram never amounted to so much as is above described, and consequently the effects of the cannon-ball to cut dowr walls by making a breach in them, must exceed those of the ancient battering-rams: but the momentum of these, or the impetus whereby they comnunicated a sliock to the whole building, was far greater than the utmost force of cannon-balls; for if the weight of the battering-ram were no more than 170 times greater than that of a cannon$2 \mathrm{~F}$ 


\section{B A r}

[ 218

ball, eacis moving with its respective velucity; the momenta of both would be equal : but as it is certain, that the weight of these ancient machines was far more than 170 times our heaviest cannon-ball, ; it follows that their momentum or impetus, to shatie or overturn walls, \&c, was tar superior to that which is exerted by modern artifiery. And since the strength of fortifications will in general be proportioned to the means which can be used for their demolition, the military walls of the moderns have been constructed with less attention with regard to their solidity and massy weight, than the ancients thought a necessary defence against the puwer of the ram; that sort of cohesive firmness of texture which resists the penetration of bodies being now more necessary than in ancient times: but it is manifest that even now, solidity or weight in fortifications also is of material consequence, to the effectual construction of a wall or battery."

BATTERY, in the Military Art, a place raised to plant cannon upon, to play with more advantage on the enemy. It consists of an epaulment or a breast-work, of about 8 feet high, and 18 or 20 feet thick.

In all batteries, the open spaces through which the muzzles of the cannon are pointed, are called Embrazures, and the distances between the embrasures, Merlons. The guns are placed on a platform composed of plinks \&c, ascending a little from the parapet, to check the recoil, and that the gun may be the easier brought back again to the parapet: they are placed from 12 to 16 fiet distant from one another, that the parapet may be strong, and the gunners have room to work.

Mortar BatTeries differ from the others, in that the slope of the parapet is inwards, and is without embrasures, the shells being fired quite over the parapet, commonly at an angle of 4,5 degrees elevation.

Open BATTERY, is nothing more than a numher of cannon, commonly field-pieces, ranged in a row abreast of one another, perhaps on some small natural elevation of the ground, or an artificial bank a little raised for the purpose.

Covered or Masked BATTERY, is when the cannon and gunners are covered by a bank or breast-work, commonly male of brush-wood, faggots, and earth, called a fascine battery.

Sunk or Buried Batt ERy, is when its platform is sunk, or let down into the groúnd, so that trenches must be cut into the earth opposite the muzzles of the guns, to scrve as embrasures to fire through. This is mostly used on the first making of approaches in besieging and battering a place.

Ricochet BATtrRy, so called by its inventor M. Vauban, and first used at the siege of Aeth in 1697. It is a method of firing with a very small quantity of powder, and a little elevation, so as just to fre over the parapet; and then the shot will roll along the opposite rampart, dismounting the cannon, and driving or destroying the troops. In a siege, they are generally placed at about 300 feet before the first parallel, perpendicular to the faces produced which they are to enfilade. Ricochet practiee is not confined to cannon alone; small mortars and howitzers may effectually be used for the same purpose. They. are of singular use in action to enfilade the enemy's ranks; for when the men perceive the shells rolling about with their fuscs burning, expecting them to burst every moment, the bravestamong them will not have courage to wait their approach, and face the havoc of their explosion.

\section{B A $\mathrm{T}$}

Cross Batreries, are two batteries playing athwart each other upon the same object, forming an angle there, and battering to more effect, because what one battery siakes, the other brats down.

Battiny d'Enfilade, is nine that scours or sweeps the whole length of a straight line.

BatтRкy en Echarpe, is one that plays ubliquely.

Battery de Reverse, or Murdering Battery, is one that plays upon the enemy's back.

Camerade or Joint B.a TTERY, is when several guns play upon one place at the same time.

BatTerY, in Electricity, is a combination of coated surfaces of glass, commonly jars, so connected together that they may be charged at once, and discharged by a common conductor. Mir. Gralath, a German electician, first contrived to increase the shock by charging screral phitls at the same time.-Dr. Franklin, having analyzed the Leyden phial, and found that it lost at one surface the electric fire received at the other, constructed a battery of eleren large panes of sash window glass, coated on both sides, and so connected that the whole might be charged together, and with the same labour as one single pane; then by bringing all the giving sides into contact with one wire, and all the receiving sides with another, he contrived to unite the furce of all the plates, and by that means to discbarge them at once.-Dr. Prirstley describes a still more complete battery. This consists of $6+$ jars, each 10 inches long, and $2 \frac{1}{2}$ inches in diameter, all coated within an inch and a half of the top, forming in the whole about 31 square feet of coated surface. A piece of very fine wire is also twisted about the lower end of the wire of cach jar, to touch the inside coating in several places; and it is put throngh a pretty large piece of cork, within the jar, to prevent any part of it from touching the side, by which a spontaneous discharge might be made. Each wire is turned round so as to make a loop at the upper end; and through these loops passes a considerably large brass rod with knobs, each rod serving for one row of the jars; and these rods are made to communicate together by a thick chain laid over them, or as many of them as may be wanted. The jars stand in a box, the bottom of which is covered with a tin plate; and a bent wire touching the plate passes through the box, and appcars on the outside. To this wire is fastencd any conductor designed to communicate with the outside of the battery; and the discharge is made by bringing the brass knob into contact with any of the knobs of the battery. When at very great force is required, the size or number of the jars must be increased, or two or more batteries may be used.-But the lurgest and most powerful battery of all, is that employed by Dr. Van Marum, to the great electrical machine, constructed for Teyler's museum at Haarlem. This grand battery consists of a great number of jars coated as above, to the amount of about 130 square feet; and the effects of it, which are truly astonishing, are related by Dr. Yan Marum in his description of this machine, and of the experiments made with it, at Haarlem $3785, \mathrm{Nc}$. Sre also Franklin's Exper. and Observ. and Priestley's History of Electricity.

BATTERY or PJLE, in Galvanism, is an apparatus cmployed for accumulating the electricity of galranism, which is produced by the mutual agencies of certain metallic and carbonaceous substances, and peculiar fluids. It was invented by the celebrated Volta, from whose labours the new science of galvanism has derived many advantages, 
and much improvement. Mr. Henry, in his valuable Epitome of Chemistry, gives the following easy directions for the construction of this pile: "Procure at a brazier's or coppersmith's, 30,40 , or 50 pieces of zinc, or speltre, cast in sand, of the size of half-crowns or shillings, but rather thicker. A corresponding number of halfcrowns or shillings will also be required, according to the sizes of the pieces of zinc that may be employed. Lct an equal number of pieces of woollen cloth be cut, of a circular shape to correspond with the pieces of zinc, and steep these in a strong solution of common salt in water. Then dispose the three substances alternately, in the following order; silver, zinc, moistened cloth; silver, zinc, \&c ; till a sufficient number of these triplicates, not less than 20 or 30 , have been thus arranged, the silver terminating the pile at top. In order to facilitate the touching of the bottom piece of silver, it may be well to put under it a slip of tinfoil, or Dutch leaf, which may project a few inches. Next Iet the hands be moistened with salt and water; and on toliching the picce of tinfoil with one hand, the uppermost piece of silver with the other, a shock will pass through the arms, which will be strong in proportion to the number of pieces of ainc \&c employed." Of late, copper has been used instead of silver, on account of its being cheaper; and solutions of muriate of ammonia (salammoniac), of nitrous acid and of muriatic acid, have been substituted for the solution of common salt with increased effect. Any two metallic substances which are perfect conductors of electricity will answer the purpose, on condition that the interposed fluid is capable of oxy. dating at least one of them.

Various forms of this battery have been adopted by different philosophers; but none have been brought into such general use as the galvatic trough invented by Mr. Cruicshank, of Woolwich. "It consists of a box of baked wood, in which plates of copper and zinc, or of silver and zinc, soldered together at their edges, are cemented in such a manner as to leave a number of water-tight cells corresponding to the number of the series." The common voltaic pile, on account of the loss of moisture, generally" loses its electrical action in a few days, and this cannot be renewed without the trouble of reconstruction; but by Mr. Cruicshank's contrivance, which becomes active on merely filliug the cells with the proper saline fluids, and freeing it when necessary from oxyd'by muriatic acid, greater permanency is secured, much trouble is prevented, and nuch time saved.

A very powerful galvanic pile is that of M. Pcpys, junior; for a description of which the reader may consult the Phil. Mag. No. 57. See also Galvan ism.

BA'TTLEMENTS, in Architecture, are notches or indentures in the top of a wall or other building, like embrasures, to look through.

$\mathrm{B} \wedge \mathrm{Y}$, in Geography, denotes a small gulf, or an arm of the sea stretching up into the land; being larger in the middle within, than at its entrance, called the mouth of the bay.

BAYER (Joux), a German lawyer and astronomer, flourished the latter part of the 16 th and begiuning of the 17 th century, but in what particular year or place he was born, is not known; his name however will be ever memorable in the annals of astronomy, on account of that great and excellent work which he first published in the year 1603 , under the title of Uranometria, being a completc celestial atlas, or large folio charts of all the con- stellations, with a nomenclature collected from the different tables of astronomy, ancient and modern, together with the useful invention of denoting the stars in every constellation by the letters of the Greek alphabet, in their order, and according to the order of their magnitude in each constellation. By means of these marks, the stars of the heavens may, with as great facility, be distinguished and referred to, as the several places of the earth are by means of geographical tables; and as a proof of the usefulness of this method, our celestial globes and atlasses have ever since retained it; and hence its general use through all the literary world. Astronomers, in speaking of any star in the constellation, denote it by saying it is marked by Bayer, $\alpha$, or $\beta$, or $\gamma, \&$ c.

Bayer lived many years after the first publication of this work, which he greatly improved and augmented by his constant attention to the study of the stars. At length, in the year 1627, it was republished under a new title, viz, Coelum Stellatum Christianum, or the Christian Stellated Heaven, or the Starry Hcavens Christianized : for in this work, the heathen names and characters, or figures of the constellations are rejected, and others, taken from the Scriptures, inserted in their stead, to circumscribe the respective constellations. This was the project of one $\mathrm{Ju}$ lius Schiller, a civilian of the same place. But this attempt was too great an innovation, to find success, or a general reception, which might occasion great confusion. And, we even find in the latter editions of this work, that the ancient figures and llames were restored again; at least so I find them in two editions, of the years 1654 , and $166 \mathrm{l}$, which are now before me.

BAYLY (IVM.), a respcctable astronomer and mathematician, was born at Bishop-Carions in Wiltshire, 1737, where the early part of his youth was enployed in the line of his father, on a small farm in that parish; till accident threw in his way books and assistance, by which means he became qualificd for an usher in a school. He afterwards passed a fow years as assistant with Dr. Maskclyne, the astronomer royal at Greenwich; by whose recommendation Mr. B. in 1769, was sent out by the Royal Society to the North Cape, to observe the transit of Vcnus of that ycar; of which his account was published in the Philos. Trans. In $1772 \mathrm{Mr}$. B. as one of the astronomers, saikd with Capt. Cook, on his second royage of discovery in the southern hemisphere; and again, in 1776 , in the third and last voyage, which unfortunately terminated the useful life of that brave and excellent commander. On the decease of Mr. Witchell, the first master in the Royal Naral Academy at Portsmouth, in 1785, Mr. B. was appointed his successor, which situation he held, with much credit to bimself, and benefit to the institution, until the now establishment of the Royal Naval College, in 1807; when Mr. B. was allowed to retire on a handsome pellsion, which he enjoyed till the time of his death, which happened at Portsea the latter part of 1810 , at 73 years of age.

BE.AD, in Architecture, is a round moulding, carred in short embossments, like beads in necklaces; and sometimes an astragal is thus carved. There is also a sort of plain bead often set on the edge of each fascia of an architrave; as also sometimes on the lining board of a doorcase, the upper cdge of skirting boards, \&c.

BEA $\mathrm{D}$, in Assaying, the small ball or mass of pure metal scparated from the scoria, and seen distinct and pure in the middle of the coppel while in the fire. 
BEAM, in Architecture, the largest piece of wood in a building; being laid across the walls, and serving to support the principal rafters of the roof. Sercral ingenious authors have considered the force or strength of beams, as supporting their own weight and any other additional weight; particularly Varignon, and Parent in the Memoir. Acad. R. Scien, an. 1708, and Mr. Fmerson, on the Strength and Stress of Timber, in bis Mechanics. Mr.Parent makes the proportion of the depth to the breadth of a rectangular beam to be as 7 to 5 when it is strongest.

The proportions of beams near London, are fixed by statute, as follows: a beam 15 feet long must be, at least, 7 inches on one side its square or end, and 5 on the other; if 16 feet long, the ends must be 8 inches by 6 ; if 17 feet long, 10 inches by $6:$ in country places they, are usually made stronger. Sir $\mathrm{H}$. Wotton advises these to be of the strongest and most durable timber. Sume of the best authors have examined the force or strength of beams, and brought their resistance to a precise calculation; and the chief results of their investigations are the following:

1. The strengths of two beams, of the same wood, and of different dimensions, are to each other, as the products of their sections multiplied by the distances or heights of their centres of gravity from the base, or underside, and divided by the lengths.

2. When the lengths are equal, the strengths are as the sections multiplied by the distances of the centres of gravity above the base.

3. When the sections are equal, the strengths are as the heights of their centres of gravity divided by their lengths.

4 . When the heights of the centres of gravity are equal, the strengths are as their sections divided by their lengths.

5. A rectangular beam with its narrower face upwards, is stronger than with its broader face, in proportion as the broader face is to the narrower.

6. A rectangular beam will bear a greater weight when it is fixed so, that its diagonal is in a vertical position, than when it is placed flat on one of its sides.

7. The strength of a cylindric beam, is to that of the circuniscribed square one, as their sections, or as the area of a circle is to its circumscribed square, that is, as 7854 to 1 , or as 11 to 14 .

8. The strongest rectangular beam which can be cut out of a cylindrical tree, is that whose breadth is to its depth, as 1 to $\sqrt{ } 2$, or very nearly as 5 to 7 .

9. The strength of a triangular beam when laid flat on its base, is double the strength of it when laid the reverse way, or with the base uppermost.

10. The stress upon a beam, to break it, by a weight laid upon the middle, is to the stress when laid nearer one end than the other, as the square of half the length, is to the product of the distances of the weight from each end. Therefore a beam will sustain the least weight in the middle, but gradually more and more towards one end.

11. When a beam is laid in an oblique position, its strength is to the strength in a horizontal position, as radius is to the cosine of the inclination.

12. A bcam supported near both ends on two props, will sustain twice as much when the ends beyond the props are kept from rising, as when it rests loosely on the props. Sec Carpentry.

13. When a bar or beam is sustaining alyy very great compression endways, it is much easier broken by any transverse strain. Several experiments have been made on this kind of strain: a piecc of white marble, for in- stance, of $\frac{1}{4}$ an inch square, and 3 inclics between the props, bore $38 \mathrm{lbs}$; but when compressed endways with $300 l b s$, it broke with $14 \frac{1}{2}$. The difference is much more remarkable in timber and soft bodies, but it is considerable in all.

14. As the strength of the same kind of wood varies very much, it is impossible ever to come to an exact knowledgc of the just proportion between the strengths of different kinds. Tlius much however may be said, with regard to the two kinds commonly used for beams, \&c, namely oak and fir, that the former is stronger than the latter, nearly in the proportion of 9 to 8 .

Beans of a ship, are the large, main, cross timbers, stretched from side to side, to support the decks, and kicp the sides of the slip from falling together.

BEAM of a balance, is the horizontal piece of wood or iron supported on a pivot in the middle, and at the extremities of which the two scales are suspended, for weighing any thing.

Bram-Compass, an instrument consisting of a wooden or brass square beam, having sliding sockets carrying steel or pencil points; and are used for describing large circles, the radii of which are beyond the extent of the common compasses.

$\mathrm{BE} A \mathrm{R}$, in Astronomy, a name given to two constellations, called the greater and the lesser bear, or UR a ma. jor and minor. The pole star is in the tail of the little bear, and is within less than 2 degrees of the north pole. See URSA, major and minor.

BEARD, of $॥$ Comet, the rays which it emits in the direction in which it moves; as distinguished from the tail, or the rays emitted or left behind it as it moves along, being always in a direction from the sun.

BEARER, in Architecture, any subsidiary or intermediate support in aid of the principal supports, as the small joists or brackets which bear a gutter or the covering board of a cornice; the piers and blockings under the joints of a ground-floor; or the joists, \&-c, which bear any thing independent of or unconinected with the building; as the bcarers of a cistern, of a vat, of a platform.

BEARING, in Geography and Navigation, the situation of one place from another, with regard to the points of the compass; or an arch of the horizon between the ineridian of a place and a line drawn through this and another place, or the angle formed by a line drawn through the two placesand their meridians. - The bearings of places on the ground are usually determined by the magnetic needle.

BEATS, in a Clock or Watch, are the strokes made by the fangs or pallets of the spindle of the balance; or of the pads in a royal pendulum. For the number and use of the beats, see Derham's Artificial Clock Maker, pa. 14 and seq.

$\mathrm{BEAT}$, in Music, is a grace marked thus ", or thus $\chi$. Its etlect is just the contrary of a transient shake in rapid movements, where it can neither be prepared nor turhed.

BEAUNE (FLORIMONE), counsellor or presidial of Blois, was born in 1601, and died in 1651. He illustrated with notes the most diflicult passages in the geonictry of Descartes, all the mysteries of which he understood. Indeed that great man had such a respect for Bcaune, that in several of his letters he declares, that he relied more on his learning and approbation, than on those of all tho other mathematicians then in France.-Beaune proposed a problem which gave rise to the inverse method of tangents. 


\section{B E 1}

[ 2

BECCARIA (JAMES BARTIIOLOM Ew), a celebrated mathematician and philosopher of the 17 th century, was born at Bononia, in 1682, and received the early part of his education among the Jesuits. Turning his attention to thestudy of natural philosophy, he soon becaine distinguished for the variety and depth of his knowledge in physics and in mathematics, of which he was made public professor, and in conjunction with Morgagni, and other celebrated characters at Bononia, assister in forming an academy there for teaching mathematics, natural history, chemistry, anatomy, and medicine. He was a frequent correspondent with the Royal Society of London, of which he was made an honorary member. Among other communications from Beccaria, which appear in the Phil. Trans. are his "Observations on the Weather," "On the Ignis Fatuus," and "On the power some persons have enjoyed of supporting life for a great length of time without food." This was afterwards published at Padua, under the title of "De jejuniis longis Dissertatio," folio 1748 . He died Jan. 1766, being 84 years of age. Among his publications are the following:

1. Dissertatio Meteorologica Medica, in qua aeris temperies et morbi Bononiæ grassantes annis 1729 et sequenti describuntur.

2. De quamplurimis phosphoris nunc primum detectis Commentarius, in 4 to, 1744 at Bonon.

3." Scriptura Medico-legalis, 1749 .

Besides numerous other compositions, the titles of which may be seen in Gen. Biog. and Hal. Bib. Ansat.

BECCARIA (GIAMBATtista), an eminent philosopher, and a monk of Ecoles-Pies, was a native of MIendovi, in Piedmont, and became a professor of mathematics and philosophy, first at Palermo, and next at Rome. He afterwards uccupied the chair of experimental philosophy at Turin, and was appointed preccptor to the princes of Sardinia. His chief works are:

1. Dell' Elettricismo Artificiale c Naturale, in 4.to, 17.53, and of which an English translation was published also in 4 to, 1776 .

2. Lettere dell' Elettricismo, folio 1758.

3. Gralus Taurinensis, 1774 , in 4 to, being an account of his measuremont of a degree of the meridian near Turin, which he carefully executed between the years 1760 and 1764 .

He also published Essays on the Cause of Storms and Tempests on the meridian of Turin, \&c. This philosopher, no less respected for his virtues than his knowledge, died at Turin in un advanced age, May 22, 1781 .

BED, of a Great Gun, a plank laid between the checks of the carriage, on the middle transum, for the breech of the gun to rest upon.

BFD, or Stool, of a mortar, a thick and strong planking on which a mortar is placed, hollowed a little to receive the breech and trunions.

BED-MOULDING, in Architecture, a term used by wnrkmen for those member's in a cornice, which are placed below the coronet, or crown. It ustally consists of these four members, an ogee, a list, a large boultine, and ahother list under the coronet.

BEES, in Naval Architecture, denote pieccs of elm plank bolted to the outward cnds of bowsprits.

BEIGHTON (HENแ ), ₹. R.s., was a respectable mathematician and mechanist, being well skilled in machinery, and followed the profession of a civil-engincer, being indeed the most eninent one of his time. IIe was the
B E L

intimate friend of Dr. Desaguliers, who received from him many valuable communications in mechanics, engines, \& c, some of which may be seen in several parts of the $2 \mathrm{~d}$ vol. of the Doctor's Course of Experimental Philosophy, particularly at pp. $431,437,449,46 \mathrm{t}, 472,497,534,539$, \&c. He communicated to the Royal Society also a curious letter, containing a Description of the Water-Works at London Bridge; printed in the Philos. Trans. vol. 37, p. 5; or my Abridg. vol. 7 , p. 442.

It is probable that Mr. B. died in 1743 or 1744 , as it appears he conducted the Laclies' Diary for the Stationers' Company, from 1714 to 1744 inclusively; discharging that trust with such satisfaction to the company, that they permitted his widow to enjoy it for many years afterwards, by employing a deputy to compile that useful annual little book. In this almanac, for the year 1721, Mr. B. inserted a curious table of calculations on the steam-engine.

BELIDOR (BERNaRD Forest DE), an engineer in the service of France, but born in Catalonia in 169s. He was professor in the new school of artillery at la Fere, where he published his course of mathematics for the use of the artillery and engincers. He was the first who seriously considered the quantity of gunpowder proper for charges, and reduced it to 2-3ds the quantity. He was named Associate in the Academy of Sciences in 1751 ; and died Sept. 8,1761 , at 63 years of age.

His works that have been published, are :

1. Sommaire d'un Cours d'Arclitecture militäire, civile, et hydraulique, in $121 \mathrm{no}, 1720$.

2. Nouveau Cours de Mathematiques, \&c. in 4to, 1725.

3. La Science des Inénieurs, in 4 to, 1729 .

4. Le Bombardier Francuis, in 4.to, 1734.

5. Architecture Hydraulique, 4 vols. in 4 to, 1737.

6. Dictionnaire portatif de l'Ingénieur, in $8 v o$.

7. Traité des Fortifications, 4 vols. in 4 to.

Besides several picces inserted in the volumes of the Memoirs of the Academy of Scionces, for the years 1737 , 1750,1753 , and 1756 .

BELLATRIX, in Astronomy, a ruddy, glittering stär of the 2d inagnitude, in the left shoulder of Orion. Its name is from the Latin bellum, as being anciently supposed to have great influence in kindling wars, and forming warriors.

BELLOWS, a machine so contrived as to exspirc and inspire the air by turns, by enlarging and contractiug its capacity. This machine is used in chambers and kitchens, in forges, furnaces, and founderies, to blow up the fire: it serves also for organs and other pneumatic instruments, to give them a proper supply of air. All these are of various constructions according to their different purposes; but in general they are composed of two flat boards, sometimes of an oval, sometimes of a triangular. figure: two or more hoops, bent according to the figure of the boards, are placed between them; a piece of leather, broad in the middle, and narrow at both ends, is nailed on the edges of the boards, which it thus unites together; as also in the hoops which separate the boards, that the lcather may the easier open and fold again: a tube of iron, brass, or copper, is fistened to the undermost board, and there is avalve within that covers the hole in the underboard, to kcep the air from escaping.

BELTS, Fascice, in Astronomy, two zones or girdles surrounding the planet Jupiter, brighter than any of the rest of his body, and terminated by parallel lines. They are obscrved however to be sometimes broader and some- 
times narrower, and not always occupying exactly the same part of the disc. Jupiter's belts were first observed and described by Huygens, in his Syst. Saturn. Dark spots have often been observed on the belts of Jupiter; and M. Cassini ubserved a pernanent one on the northern side of the most southern belt, by which he determined the length of Jupiter's days, or the time in which the planet revolves upon its axis, which is $9 \mathrm{~h} .56 \mathrm{~m}$. Some astronomers suppose that these belts are seas, which alternately cover and leave bare large tracts of the planet's surface: and that the spots are gulfs in those seas, which are sometimes dry and sometimes full. But Azout conceived that the spots are protuberances of the belts; and others again are of opinion that the transparent and moveable spots are the shadows of Jupiter's satellites.

Cassini also speaks of the belts of Saturn; being three dark, straight, parallel bands, or fascice, on the disc of that planet. But it does not appear that Saturn's belts adhere to his body, as those of Jupiter du; but rather that they are large dark rings surrounding the planet at a distance. Some imagine that they are clouds in the atmosphere of Saturn, though it would seem that the middlemost is the shadow of his ring.

BENDING, the reducing a body to a curved or crooked form. The bending of boards, planks, \&c, is effected by meaus of heat, whether by boiling or otherwise, by which their fibres are so relaxed that they may be bent into any figure. Bernoulli has a discourse on the bending of springs, or elastic boelies. And Amontons gives several experiments concerning the bending of ropes. The friction of a rope bent or wound about an immoveable cylinder, is suficient, with a very small power, to sustain very great weights.

BERENICE's Hair; see Coma Berenices.

BERKELEY (GEORGE), the virtuous and lcarned bishop of Cloyne in Ireland, was born in that kingdom, at Kilcrin, the 12 th of Harch $16 \mathrm{st}$. After receiving the first part of his education at Kilkenny school, he was admitted a pensioner of Trinity College, Dublin, at 15 years old; and chosen fellow of that college in 1707 .

The first public proof he gave of his literary abilities was in his Arithmetica absque Algebra aut Euclide demonstrata: which from the preface it appears he wrote before he was 20 years old, though he did not publish it till 1707 . It is followed by a Mathematical Miscellany; containing observations and theorems inscribed to his pupil Samucl Molineux.

In 1709 came out the Theory of Vision; which of all his works appears to do the greatest honour to his sagacity; being the first attempt that ever was made to distinguisli the immediate and natural objects of sight, from the conclusions we have been accustomed from infancy to draw from them. The boundary is here traced out between the ideas of sight and touch; and it is shown, that though habit has so connected these two classes of idcas in the inind, that they are not without a strong effort to be scparated from each other, yet originally they have no such connexion: insomuch, that a person born blind, and suddenly madè to see, would at first be utterly' unable to tell how any object that affected his sight would aliect his tooch; and particularly would not from sight receise any idea of distance, or external space, but would imagine all objects to be in his eyc, or rather in his mind.

Next to his Theory of Vision, in the year 1710 appeared The Principles of IIuman Knowledge; and in 1713 Dialugues between Hylas and Philonous: the object of both which pieces is, to prove that the commonly received notion of the existence of matter, is false; that sensible material objects, as they are called, are not external to the mind, but exist in it, and are nothing more than impressions made upon it by the immediate act of God, according to certain rules called laws of nature.

For acuteness of purts and beauty of imagination, few anthors can vie with Berkeley: his writings excited universal admiration, and his company was courted even by men of opposite parties, who also concurred in recommending him. For Stecle he wrote several papers in the Guardian, and at his house became acquainted with Pope, with whom he always lived in friendship. Swift recommended him to the celcbrated earl of Peterborough, who being appointed ambassador to the king of Sicily and the Italian States, took Berkeley with him as chaplain and secretary in 1713 , with whom he returned to England the year following.

His hopes of preferment expiring with the fall of queen Anne's ministry, he some time after embraced an offer made him by Ashe, bishop of Clogher, of accompanying his son in a tour through Europe. At Paris he visited the illustrious Malebranche, whom he found in his cell cooking in a small pipkin a medicine for an inflammation of the lungs, with which he was afflicted; and as they engaged in a conversation on Berkeley's system, Malebranche in the heat of disputation, raised his voice so high, and indulged the natural impetuosity of his temper to such a degree, that he brought on an increase of his disorder, which carfied him off a few days after. During four years' absence from England, he not only visited those places which fell within the grand tour, but some that are less frequented, and with great industry collected materials for a natural history of those parts, but which were unfortunately lost in his passage to Naples. He arrived at London in 1721 ; and being much concerned for the miseries of the nation, brought on by the disastrous Suuth-sea scheme of 1720, he published the same year An Essay towards preventing the ruin of Great Britain : reprinted in bis Miscellaneous Tracts.

The path leading to the highest company being now open, Pope introduced him to Lord Burlington, by whom he was recommended to the Duke of Grafton, then appointed lord-lieutenant of Ireland, who took Berkeley over as one of his chaplains in 1721 . The latter part of this year he obtained the dregrees of bachclor and doctor in divinity: and the year following he had a viry unexpected increase of fortune from the death of Mis. Vanhonirigh, the celebrated Vanessa, to whom he had been introdeced by Swift: this lady had intended Swift for her heir; but perceiving herodf to be slighted by him, she lelt her fortune, of soool, between her two executors, of whoin Berkeley was one. In 1724 he was promoted to the deanery of Derry, worth $1100 l$ a year.

In 1725 he published A ['roposal for converting the savage Americans to Chrisianity, by a college to be crected in the Summer lslec, otherwise called lhe lskes of Bermuda, which has also been repriuted in his Mliscrllaneous Tracts. The proposal was well received, at least by the king; and he oltained a charter line tisunding the collige, with a parliamentary grant of $20,000 /$ towards carrying it into execution: hut he could never get the moncy, it being otherwise 'mployed by the ministry: so that after two years' stay in America on this business, he was obliged to roturn, and the scheme consequently expired. 


\section{B E R}

In 1732 be publisherl The Minute Philosopher, in 2 volumes 8 vo, against Freethinkers. In 1733 he was made bishop of Cloyne; and might bave been remored in 17.5 , by Lord Clesterfield, to Clogher; but declined it. He resided constantly at Cloyne, where be faithfully discharged all the offices of a good bishop, yet continued his studies with unabated attention.

About this time he engaged in a controversy with the mathematicians, which excited the highest interest in the literary world; the occasion of it was this: Mr. Addison had given the bishop an account of the belaviour of their common friend, Dr. Garth, in his last illness, which was equally unpleasing to both these advocates of revealed religion. For when Addison went to see the doctor, and began to discourse, with him seriously about another world, "Surely, Addison," repliesl he, "I have good reason not to believe those trifles, since nyy friend Dr. Halley, who has dealt so much in demonstration, lus assured me, that the doctrines of Christianity are incomprehensible, and the religion itself an imposture." 'The bishop therefore took up arms against Halley, and addresser to him, as to an Infidel Mathematician, a discourse called The Analyst; with a view of showing that mysteries in faith were unjustly objected to by mathematicians, who he thought admitted others much greater, and even falsehoods in science, of which he endeavoured to prove that the doetrine of Fluxions furnished a clear example. This attack gave uccasinn to Rubins's Discourse concerning the Methar of Fluxions, to Maclaurin's Flusions, and to other smalker works on the same subject; but the direct answers to the Analyst were made by a person under the name of Philalethes Cantabrigiensis, but commonly supposed to be Dr. Jurin, whose first piece was, Geometry no Friend to Infidelity, 1734. To this the bishop replied in A Defence of Freethinking in Mathematics; witb an Appendix concerning Mr. Walton's Vindication, 1735; which drew a second answer the same year from Philalethes, under the title of The Minute Mathematician, or The Freethinker no just Thinker, 1735. Other writings in this controversy, besides those before mentioned, were,

1. A Vindication of Newton's Principles of Fluxions against the objections contained in the Analyst, by J. Walton, Dublin, 1735.

2. The Catechism of the Author of the Minute Philosopher fully answered, by J.Walton, Dublin, 1735 .

3. Reasons for not replying to Mr. Walton's Full Answer, in a letter to P. T. P. by the author of the Minute Philosopher, Dublin, 1735 .

4. An Introduction to the Doctrine of Fluxions, and Defence of the Mathematicians against the objections of the author of the Analyst, \&c. Lond. 1736 .

5. A new Treatise of Fluxions; with answers to the principal objections in the Analyst, by James Smith, A. M. Lond. 1737.

6. Mr. Robins's Discourse of Newton's Methods of Fluxions, and of Prime and Ultimate Ratios, 1735.

7. Mr. Robins's Account of the preceding Discourse, in the Repub. of Letters, fir October 1735.

8. Philalethes's Considerations upon some passages contained in two letters to the author of the Analyst \&c, in Repub. of Letters, Novemb. 1735.

9. Mr. Rubins's Review of some of the principal objections that have been made to the doctrine of Fluxions \& $c$, Repub. of Letters for Decem. 1735.
B E $\mathbf{R}$

10. Philalethes's Reply to ditto, in the Repub. of Letters, Jan. 1736.

11. Mr. Robins's Dissertation, showing that the account of the doctrines of Fluxions \& $\mathrm{c}$, is agreeable to the real sense and meaning of their great Inventor; \&c, Repub. of Letters, April 1736.

12. Philalethes's Considerations upon ditto, in Repub. of Letters, July 1736.

13. Mr. Robins's Remarks on ditto, in Repub. of Letters, Aug. 1736.

14. Mr. Robins's Remainder of ditto, in an Appendix to the Repub. of Letters, Sept. 1736. 15. Philalethes's Observations upon ditto, in an Appendix
to the Repub. of Letters, Nov. 1736 .

16. Mr. Robins's Advertisement in Repub. of Letters, Decemb. 1736 .

17. Philalethes's Reply to ditto, in an Appendix to the Repub. of Letters for Decem. 1736.

18. Some Observations on the Appendix to the Repub. of Letters for Decem. 1736, by Dr. Pemberton, in the Works of the Learned for Feb. 1737. With some smaller pieces in the-same.

In 1736 Bishop Berkeley published "The Querist, a discourse addre'ssed to magistrates, occasioned by the enormous licence and irreligion of the times;" and many other things afterward of a smaller kind. In 1744 appeared his celebrated and curious book, "Siris; a Chain of Philosophical Reflections and Inquiries concerning the virtues of Tar-water." It received a second impression, with additions and emendations, in 1747 ; and was followed by Farther Thoughts on 'Tar-water, in 1752 . In July the same year he removed, with his lady and family, to Oxford, partly to superintend the education of a son, but chicfly to indulge the passion for learned retirement, which had always strongly possessed him. He would have resigned his bishopric for a canonry or headship at O. ford; but it was not permitted him. Here he lived highly respected, and collected and printed the same year all his smaller pieces in 8 vo. But this happiness did not long eontinue, being suddenly cut off by a palsy of the heart, Jan. 14, 1753 , in the 69 th year of his age, while listening to a sermon that his lady was reading to him.

The excellence of Berkeley's moral character is fully attested by his writings: he was certainly a very amiable as well as a very learned man; and it is thonght that Pope scarcely said too much, when he ascribed

"To Berkeley every virtue under heaven."

BERME, in Fortification, a small space of ground, 4 or 5 feet wide, left without the rampart, between it and the side of the moat, to receive the earth that rolls down from the rampart, and prevent its 'falling into and filling up the ditch.-Sometimes, for greater sccurity, the berme is pallisacloed.

BERNARD (Dr. EDwaRD), a learned Euglish astronomer, critic, and linguist, was born at Perry St. Paul, near Towcester, the $2 \mathrm{~d}$ of May 1638 , and educated at Merchant-Taylor's scloool, whence he was removed in 1655 to St. John's college, Oxforl. Having laid in a gond fund of classical learning at school, in the Greek and Latin languages, he applied himself very diligently at the university to the study of history, the Eastern languages, and mathematics under the celebrated Dr. Wallis. In 1668 he went to Leyden, to consult some Oriental manuscripts left to that university by Joseph Scaliger and Levin Warter, and espe. 
cially' the 5th, 6th, and 7 th books of Apollonius Pergæus's Conics, brought from the East by the celebrated Golius. These he transcribed and brought to Oxford, with a view of publishing them, but was obliged to relinquish that design for want of proper encouragement. This however was afterwards carried into effect by Dr. Halley in 1710 , with the addition of the Sth book, which he supplied by his own ingenuity and industry.

On his return to Oxford, he examined and collated the most valuable manuscripts in the Bodleian library. In 1669, the celebrated Christopher Wren, Savilian professor of astronomy at Oxford, having been appointed surveyor-general of his majesty's works, and being much detained at London by thisemployment, obtained leave to name a deputy at Oxford. He accordingly fixed on Mr. Bernard, which engaged the latter in a more particular application to the study of astronomy. But in 1673 he was appointed to the professorship bimself, which Wren was obliged to resign, as, by the statutes of the founder, Sir Henry Saville, the professors are not allowed to hold any other office cither ecclesiastical or civil. About this time a scheme was set on foot at Oxford for collecting and publishing the works of the ancient mathematicians. Mr. Bernard, who had first formed the project, collected all the old books published on that subject since the invention of printing, together with all the manuscripts he could get from the Bodleian and Savilian libraries, which he arranged in order of time, and according to the matter they contained; of these he drew up a synopsis; and as a specimen he published a few sheets of Euclid, containing the Greek text, and a Latin version, with Proclus's commentary in Greek and Latin, and learned scholia and corollaries. The synopsis itself was published by Dr. Smith, under the title of Veterum Mathernaticorum Gracorun, Latinorum, et Arabum, Synopsis.

Mr. Bernard undertook also an edition of the Parva Syntaxis Alexandrina; in which, besides Euclid, are contained the small treatises of Theodosius, Menelaus, Aristarchus, and Hipsicles; but it never was published.

In $\mathbf{1 6 7 6}$ he was sent to France, as tutor to the dukes of Grufton and Northumberland, sons to king Cliarles the $2 d$ by the duchess of Cleveland, who then lived with their mother at Paris: but the simplicity of his manners not suiting the gaiety of the duchess's family, he returned about a year after to Oxford, and pursued his studies with his usual ardour and great success. In 1691, being presented to the rectory of Brightwel! in Berkshire, he quitted his professorship at Oxford, in which he was succeded by David Gregory, professor of mathematics at Edinburgh.

In the year 1696 , he undertook a voyage to Holland, to attend the sale of Golius's manuscripts, as he had once before done at the sale of Heiusius's library; and soon after his return to Englum, he fell into a languishing consumption, which put an end to his life the 12 th of January 1696 , in the 58 th year ol his age.

Besides his works already mentioned, he was author of many other compositions. 1le composed tables of the longitudes, latitudes, right-ascensions, \&c, of the fixed stars: and wrote Observations on the Obliquity of the Ecliptic; and other pieces inserted in the Philosophical Transactions. lliwrote also,

1. A 'Treatise of the Ancient Weights and Measures.

2. Chronologix Samaritanx Synopsis, in two tables.

3. Testimenies of the Ancients concerning the Greck Version of the Ohl 'l'estament by the Serenty.
And several other learned works. Besides a great number.of valuable manuscripts left at his death.

BERNARD (Dr. JAMEs), professor of philosophy and mathematics, and minister of the Walloon church at Leyden, was born September the 1st 1658, at Nions in Dauphiné. Having studied at Geneva, lue returned to France in 1679 , and was chosen minister of Venterol, a village in 1)auphiné; but some time after lie was removed to the church of Vinsobres in the same province. To avoid the persecutions against the protestants in France, lie went into Holland, where he was appointed one of the pensionary ministers of Ganda. He here published several political and historical works. And in 1699 he began the Nouvelles de la Republique des Lettres, which continued till December 1710. In 1705 he was chosen minister of the Walloon church at Leyden; and about the same time, Mr. de Volder, professor of philosophy and mathematics at Leyden, having resigned, Mir. Bernard was appointed his successor; upon which occasion the university also presented him with the degrees of doctor of philosophy and master of arts. In 1716 he published a supplement to Moreri's dictionary in 2 volumes folio. The same year he resumed his Nouvelles de la Republique des Lettres; which he continued till his death, which happened the 27 th of April 1718, in the 60th year of his age.

BERNOULLI (JAM $\mathrm{MS}$ ), a celebrated philosopher and mathematician, born at Basil the 27 th of December 1654 . Having taken his degrees in that university, he applied himself to divinity agreeably to the entreatics of his father, but against his own inclination, which led bim to astronomy and mathematics. He gave very early proofs of his genius for these sciences, and soon became a geometrician, without a preceptor, and almost without books; for if one by chance fell into his hands, he was obliged to conceal it, to avoid the displeasure of his father. This situation induced him to choose for lis device, Phacton driving the chariot of the sum, with these words, Inizto patre sidera verso, "I traverse the stars against my father's will :" alluding particularly to astronomy, to which lie then chiefly applied hinself.

In $1676^{\circ}$ he began his travels. When he was at Geneva, he fell upion a method to teach a young girl to write who had been blind from her infancy. At Bourdeaux he composed universal gnomonic tables; but they were never published. He returned from France to his own country in 1680. About this time there appeared a comet, the return of which he foretold; he wrote also a sinall treatise upon it. Soon after this he went into Holland, where lie applied himself to the study of the new philosopliy. Having visited Flanters and Bribant, he passed over to England; where he formed an acquaintance with the most eminent men in science, and attonded their philosophical meetings. He returned to his native country again in 1682 ; and exhibited at Basil a course of ixperiments in natural philosophy and mechanics, which consisted of a variety of new discoveries. The stame year he published his lissay on a new Syst'm of Comets; and the year following, his Disscrtation on the Weight of the Air. About this time loibuitz having published, in the Acla Eruditorum at Leipsic, some c'ssays on his new Calculus Diflerentialis, but conceraling the art and methorl of it, Bormoulli and his brother John soon discovered, lrom what they saw, its beatity amb extent: this induced them to endeavour to unravel the secret; which they did with such sticcess, that 
Leibnitz declared that the invention belonged to them as much as to himself.

In $1687 \mathrm{James}$. Bernoulli succeeded to the professorship of inathematics at Basil; a trust which he discharged with great applause; and his reputation drew many foreigners from all parts to attend his lectures. In 1699 he was adnitted a foreign member of the Academy of Sciences of Paris; $;$ and in 1701 the same honour was also conferred ot him by the Academy of Berlin: in both of which he published several ingenious compositions, about the years $1502,-3$, and-4. He wrote likewise several pieces in the Acta Eruditorum of Leipsic, and in the Journal des Sçavans. His intense application to study brought on him the gout, and by degrees a slow fever, which terminated his life the 16th of August 1705, in the 51st year of his age - Archimedes having found out the proportion of a sphere and its circumscribing cylinder, ordered them to be engraven on his monument: in imiration thercof Bernoulli appointed that a logarithmic spiral curve should be inscribed on his tomb, with these words, Eadem mutata resurgo; in allusion to the hopes of the resurrection, which are in some measure represented by the properties of that curve, which he had the honour to discover.

James Bernoulli had an excellent genius for invention and elegant simplicity, as well as.a close application. He was eminently skilled in all the branches of the mathematics, and contributel much to the promotion of the new analysis, infinite series, \&c. Hc carried to a great extent the theory of the quidrature of the parabola; the geometry of curve lines, of spirals, of cycloids, and epicycloids.

His works, that had been published, were collected, and printed in 2 volumes 4 to, at Geneva in 1744. At the time of his death he wras occupied on a great work entitled De Arte Conjectandi, which was published in 4 to in 1713 . It contains an elegant and most excellent introduction to Infinite Series, \&c. This posthumous piece is omitted in the collection of his works above mentioned.

BERNOULLI (JоH N), the brother of James, last mentioned, and a celebrated mathematician, was boru at Basil the 7 th of August 1667. His father intended him for trade; but his own inclination was at first for the belleslettres, which however, like his brother, he forsook for that of mathematics. He laboured with his brother to discover the methorl used by Leibnitz in his cssays on the Differential Calculus, and gave the first principles of the Integral Calculus. Our author, with Messieurs Huygens and Leibnitz, was the first who gave the solution of the problen proposed by James Bernoulli, concerning the catenary, or curve formed by a chain suspended by its two extremities,

John Bernoulli had the degree of doctor of physic at Basil, and two years afterward was named professor of mathematics in the university of Groningen. It was here that he discovered the mercurial plosphorus or luninous barometer, occusioned by triction of mercury against glass in a vacuum; and where he also resolved the problem proposed by his brother concerning lsoperiinetricals.

On the death of James, the professor at Basil, our author returned to his native country, against the pressing invitations of the magistrates of Utrecht to come to that city, and of the university of Groningen, who also wished to retain him. The Academic Senate of Basil soon appointed thim to succeed bis brother, without assembling competitors, and contrary to the established practice: an appointsus which he held during his wholc life.

VOL. I.
In 1714 was published his treatise on the management of ships; and in 1730, his memoir on the elliptical figure of the planets which gaiued the prize of the Academy of Sciences at Paris. The same academy also divided the prize, for their question concerning the inclination of the planetary orbits, between our author and his son Danicl.

John Bernoulli was a member of most of the academies of Europe, and received as a foreign associate of that of Paris in 1699. After a long life spent in constant study and the improvement of all the braiches of the mathematics, he died full of honours the 1st of January 1748 , in the 81 st year of his age. Of five sons which he had, three pursued the same study with himself. One died before him ; the two others, Nicholas and Daniel, he lived to see become eminent and respected in mathematical science.

The writings of this great man were dispersed through the periodical memoirs of several academies, as well as in many separate treatises. And the whole of them were carefully collected and published at Lausanne and Geneva, 1742 , in 4 volumes, 4 to.

BERNOULLI (JoHN), son of the preceding, was born at Basil, Jan. 17, 1695, and died at Petersburgh, July 26, 1726. He was licentiate of law, professor of law at Berne; and afterwards professor of nathematics at Petersburg, and member of the Institute of Bologna.

BERNOULLI (NICHOLAS), nepbew of the two first nained, was professor of mathematics at Padua, afterwards of logic, and then of law at Basil. He was member of the Academy of Scionces and Belles-Letters at Berlin, and also of the Royal Society of London, and of the Institute of Bologna ; was born at Basil; Oct. 10, 1687, where he also died the 29th of Nov. in the year 1759 .

BERNOULLI (DANIEL), a celebrated physician and philosupher, son of John, and nephew of James, was born at Groningen Feb. the 9th, 1700, where his father was then professor of mathematics. He was first designed for trade, but his genius led him to other pursuits. He passed some time in Italy; and at 24 years of age be declined the honour offered him of becoming president of an academy intended to have been established at Genoa. He spent several years with great credit at Petersburg; and in $1733^{\circ}$ relurned to Basil, where his father was then professor of mathematics; and here our author successively filled the chair of physic, of nat ural and of speculative philosophy.

In his work Exercitationes Malhematica, 1724, he took the only title he then had, viz, "Son of Jubn Bernoulli," and never would suffer any other to be added to it. This work was published in ltaly, while be was there on his travels; and it classed him in the rank of inventors. His work entitled Hydrodynamica, was published in 4to at Argentoratum or Strasbourg; in 1738.

Daniel Bernoulli wrote a multitucle of other pieces, which have all heen published either in the Mem. of the Acad. of Sciences at Paris, or in those of other Academics. He gained and divided ten prizes from the Academy of Sciences at Paris, which were contended for by the most illustrious inathematicians in Europe. The only person who has had similar success is Euler, his countryman, disciple, rival, and friend. His first prize he gained at 24 years of age. In 1734 lie divided that with his father rispecting the inclination of the orbits of the planets; which hurt the family union; for the father considered the contest itself as a want of respect; and the son did not sufficiently conceal that he thought (what was really the case) $2 \mathrm{G}$ 
his own piece better than bis father's. And besides, he declared for Newton, against whom his father had contended all his life. In 1740 our author divided the prize, "On the Tides of the Sea," with Euler and Maclaurin. The Academy at the sane time rewarded a fulth piece, the chief merit of which was that of being Cartesian; but this was the last public act of adoration paid by the Academy to the authority of the author of the Vortices, which it had already obeyed too long. In 1748 Daniel Bernoulli succeeded his father John in the Academy of Sciences, who had succeeded his brother James; this place, since its first erection in 1699 , having never been without a Bernoulli to fill $\mathrm{it}$.

Our author was extremely respected at Basil; and to bow to Daniel Bernoulli, when theymet him in the strcets, was one of the first lessons which every father gave to his cbild. He was a man of great simplicity and modesty of manners. He used to tell two little adventures, which he said had given him more pleasure than all the other honours he had received. Travelling with a learned stranger, who, being pleased with his conversation, asked his nane; "I am Daniel Bernoulli," answered he with great modesty; "and I," said the stranger (who thought he meant to laugh at him) " am Isaac Newton." Another time, having to dine with him the celebrated Koenig, the mathematician, who boasted, with sume degree of self-complacency, of a difficult problem he had resolved with much trouble, Bernoulli went on doing the honours of his table, and when they went to drink coffee he presented' Koenig with a solution of the problem more elegant than bis own.

After a long, useful, and honourable life, Danicl Bernoulli died the 17 th of March, 1782 , in the $83 \mathrm{~d}$ year of his age.

BERNOULLI (JAMES), another mathematical branch of the foregoing celebrated family. He was born at Basil in October 1759; being the son of John Bernoulli, and grandson of the first John Bernoulli, before mentioned, and the nephew of Daniel Bernoulli last noticed. Our author's clder brother John, who still Jives at Berlin, is also well known in the republic of science, particularly for his astronomical labours.

The gentleman to whom this article relates, was educated, as most of his relations had been, for the profession of the law : but his genius led him very early into the study of mathematics; and at 20 years of age he read public lectures on experimental philosophy in the university of Basil, for his uncle Daniel Bernoulli, whom he hoped to have succeeded as professor. Being disappointed in this view, he resolved to leave his native place, and to scek his fortune elsewhere; hence he accepted the uffice of secretary to Count Breuner, the emperor's envoy to the republic of Venice; and in this city he remained till the year 1786 , when, on the recommendation of his countrymau, M. Fuss, he was invited to Petersburg to succeed M. Lexell in the academy there, where he continued till his death, which happened the $3 \mathrm{~d}$ of July 1789 , at not quite 30 years of age, and when he had been married only two months, to the youngest daughter of John Albert Euler, the son of the celebrated Leonard Euler.

BETELGEUSE, a fixed star of the first magnitude in the right shoulder of Orion.

BEVIS (JolN, M. D.), an able astronomer, was born October 31, 1695, near Old Sarum in IViltslire. Ile was entered at Christ-Church College, Oxford, where he applied himself diligently, not only to the study of physic, for which he was designed, but to other sciences, particularly astronomy and optics, in which he became a considerable proficient. After taking the degrce of $\mathrm{N}$. D. he left the university, and devoted himself almost entirely to pursuits connected with astronomy. In $173 \mathrm{~s}$ he made an excellent collection of astronomical instruments for a utw observatory, which he had erected at Nevington Green, in Middlesex. Here he became an indefatigable observer, often observing the transits of 160 stars in one night.About $\mathbf{1 7 4 5}$ the doctor undertook the laborious task of arranging a work called Uranographia Britannica, or als Exact View of the Heavens, on 52 plates, similar to Bayer's work representing the constellations, \&c. The plates, which would have done great honour as well to the nation as Dr. Bevis, were engraven but never published, the doctor having consigned the management of them to a person who bccame a bankrupt. Dr. Bevis was the real author of a great many works which have been well received by the public, but which his modesty prevented bim from taking the merit of. It is to him we are indebted for the publication of Dr. Halley's Astronomical Tables, after they had been printed more than 20 years: having supplied some auxiliary tables, and the precepts for using them, he favoured the public with the whole in the year 1749 . In Mr. Simpson's Essays, p. 10, are delivered practical rules for finding the aberration, which were drawn up and given him by Dr. Bevis, with examples of the correction applied to several stars, which he bad carefully observed with proper justruments; by which he proved, first of any one, that the phenomena are as conformable in right ascension, as Dr. Bradley, who made this great discovery, found they were in declination. Several pieces by the doctor were inserted in the few numbers that were published of a work called the Mathematical Magazıne, by Messrs. Whitchell and Moss; particularly a curious paper on the satellites of Venus, and several sheets of a new matbematical dictionary. Dr. Bevis enriched the Philosophical 'Transactions with 27 valuable papers, from vol. 40 to vol. 59 inclusive: The only pieces which appeared separately with his name, besides thesc papers in the Transactions, were two pamphlets, the one entitled The Satellite's Sliding Rule, for determining the imnersions and emersions of Jupiter's four satellites. The other was An Experimental Enquiry concerning the Contents, Qualitics, and Medicinal Virtues, of the. Two Mineral Waters lately discovered at BagniggeWells, near London, 1760 . He was the first who appropriated the term achromatic to the curious telescopes invented by Mr. Dollond. On the death of AIr. Bliss in 1765 , Dr. Bevis was a candidate with Dr. Maskelyne for the honourable and important situation of Astronomer Royal. Soon after which, he removed from Newington to the Temple, London, where be followed his occupation as a physician, and continued to make astronomical observations. Here he died, Nov. 6,1771 , at 76 years of age; his death having been occasioned by a fall he received a short time before, in going too hastily from his instrument to the clock when observiug the sun's meridian altitude.

BEZOUT (STEPnEN), a celebrated l'rench mathematician, Member of the Acadenies of Scicnces and the Marinc, and Examiner of the Guards of the Marine and of the Elèves of Artillery, was born at Nemours the 31st of March, 1730. In the course of his studies he inct with soine books of geometry, which gave him in taste for that science; nnd the Eloges of lontenelle, which showed him the honours tbat attomicd talents and the love of the'sci- 
ences. His father in vain opposed the strong attachment of young Bezout to the mathematical sciences. April 8, 1758 , he was named adjoint-mechanician in the French Academy of Sciences; having before that sent thein two ingenious inemoirs on the integral calculus, and given other proofs of his proficiency in the sciences. In 1763 he was named to the new office of Examiner to the Marine, and appointed to compose a Coursc of Mathematics for their use; and in 1768, on the death of M.Camus, he succeeded as Examiner of the Artillery Elèves.

Bezout fixed his attention more particularly to the resolution of algebraic equations; and he first-found out the sulution of a particular class of equations of all degrees. This method, different from all former ones, was general for the cubic and biquadratic equations, and just became particular at those of the fifth degree. At this work of finding the roots of equations, our author láboured from 1762 till 1779 , when he published it. He composed two courses of mathematics; the one for the Marine, the other firr the Artillery. The foundation of these two works was the same; the applications only being difierent, according to the two different objects: thesc courses have every where been held in great estimation. In his office of Examiner, he discharged the dutics with great attention, care, and tenderness. A trait of his justice and zeal is rcmarkable in the following instance: During an examination which he held at Toulon, he was told that two of the pupils could not be present, being confined by the small-pox; he himself had never had that disease, and he was greatly afraid of it; but as he knew that if he did not see these two young inen, it would much impede their advancement, he ventured therefore to their bed-sides, to examiue them, and was happy to find them so deserving the hazard he had made for their benefit.

M. Bezout lived thus several years, beloved of his family and friends, and respected by all, enjoying the fruits and the credit of his labours. But the trouble and fatigues of his offices, with some personal chagrins, had reduced his strength and constitution; he.was attacked by a malignant fever, of which he died Sept. $27,17 \mathrm{S3}$, in the 54th ycar of his age, regretted by bis family, his friends, the young students, and by all his acquaintance in general.

The books published by him wcre:

1. Course of Mathematics for the use of the Marine, with a 'Treatise on Navigation; 6 vols, in 8 vo. Paris, 1764 .

2. Course of Mathematics for the Corps of Artillery; 4 vols, in 8 vo, 1770 .

3. General Theory of Algebraic Equations, 1779.

His papers printed in the volumes of the Memoirs of the Academy of Sciences, are:

1. On curves whose rectification depends on a given quantity, in the vol. for 1758 .

2. On scveral classes of equations that admit of an algebraic solution, 1762 .

3. First vol, of a course of mathematics, 1764 .

4. On certain equations, \& c. 1764 .

5. General resolution of all equations, 1765 .

6. Second vol. of a course of mathematics, 1765 .

7. Third vol. of the same, ' 1766 .

8. Fourth vol. of the same, 1767 .

9.' Integration of differentials, \&c. vol. 3, Sav. Etr.

10. Experiments on cold, 1777 .

BIANCHINI (FRANCIS), a very learned Italian philosopher and mathematician of the 17 th century, was born at Verona the 13th of December, 1662. He was highly esteemed by the learned, and was a member of several academics; and was even the founder of that at.Verona, called the Academy of Aletofili, or Lovers of Truth. He went to Rome in 1684; and was made librarian to Cardinal Ottoboni, who was afterwards Pope by the name of Alexander the 8 th. He entered into the church, and became canon of St. Mary de la Rotondo, and afterward of St: Lawrence in Damaso.

Bianchini was author of several learned and ingenious dissertations. In 1697 he published La Istoria universale provata con monumenti, et figurata con simboli de gli antichi. In 1701 Pope Clement the 11 th named him secretary of the conferences for the reformation of the calendar; in which situation he published in $1703, \mathrm{De}$ Calendario et Cyclo Cæsaris, ac de Canone Paschali sancti-Hypoliti, Mattyris, Disserțationes duæ; Bianchini was emplojed likcwise to construct the large gnomon in the church of the Cliartreux at Rome, on which he published an ample dissertation entitled, De Nummo et Gnomone Clementino. The research concerning the parallax and the spots of $\mathrm{Ve}$ nus occupied him a long time; but his most remarkable discovery is that of the parallelism of the axis of Venus in her orbit. He was employed 8 years in preparing neans for tracing a meridian line through the wholc extent of Italy, but he never commenced this undertaking. He was adnitted a foreign Associate in the Paris Academy of Sciences, in 1706; and had many astronomical dissertations inserted in thicir Memoirs, particularly in those of the years $1702,1703,1704,1706,1707,1708,1713$, and 1718 . -Bianchini died the $2 \mathrm{~d}$ of March, 1729 , in the 67 th year of his age.

BILLION, in Numeration, the same as a million millions; as much as to say bismillion or bimillion, or by further contraction, billion.

BILLY (JAMES DE), of Compeigne, a Jesuit, died in 1679 at Dijon, aged 77 . He was a respectable mathematician, and published sone good works in that science; as,

1. Nova Geometriæ Clavis Algebra. Paris, 1643 , in 4to.

2. Tabulæ Lorloiceæ de Doctrina Eclipseon. Divionæ, 1656 , in 4 to.

3. De Propartione Harmonica Tractatus. Paris, 1658, in s.to.

4. Diophantis Geometria, ex Græco versa, in qua per rationes Euclidianas efficiuntur Problemata, quæ ille Rationibus Algebraicis enodavit. Paris, 1660, in 4to.

5. Opus Astronomicum. In which he explained the hypotheses and notions of the stars, and every thing relating to the theory and practice of astronomy. Dijon, 1661, in 4 to.

6. Crisis Astronomica, de Motu Cometarum. In which he refuted the rectilinear hypothesis, and discovered a new system of comets. Dijon, 1666 , in 8 vo.

7. Diophantes Redivivus. Lyons, 1670 , in 8 vo.

8. Doctrinæ Analyticæ inventum novum. Contained in the Commentaries on Diophantus, composed by M. Fermat. Toulouse, 1671 , in folio.

- BIMEDIAL Line, is the sum of two medials. Euclid reckons two of these bimedials, in pr. 38 and 39 , lib. $x$; the first is when the rectangle is rational, which is contained by the two medial lines whose sum makes the bimedial; and the second when that rectangle is a medial, or contained under two lines that are commensurable only in power.

BINARY Number, that which is composed of two units. $2 \mathrm{G} 2$ 
Bryany Aritlmetic, that in which two figures or characters, yiz. 1 and 0 , only are used; the cipher multiplying exery thing by two, as in the common arithmetic by 10 : thus, $l$ is one, 10 is 2,11 is 3,100 is 4,101 is 5,110 is 6 , 111 is 7,1000 is 8,1001 is 9,1010 is ten; being founded on the same principles as common arithmetic. This kind of arithmetic was invented by Leibnitz, who pretended that it is better adapted than the common arithmetic, for discovering certain properties of numbers, and for constructing tables; but he does not venture to recommend it for ordinary use, on account of the great number of places of figures requisite to express all numbers, even very small ones. Jos. PeJican, of Prague, has more largely explaincd the principles and practice of the binary arithmetic, in a book entitled Arithmeticus Perfectus, qui tria uumerare nescit: 1712 . And De Lagni proposed a new system of logarithms, on the plan of the binary arithmetic; which he finds shortcr, and more easy and natural than the common ones.

BINOCLE, or BrNocular Telescope, is one by which an object is viewed with both eyes at the same time. It consists of two tubes, each furnished with glasses, of the same power, by which means it has been said to show objects larger and more clearly than a monocular or single telcscope; though this is probably only an illusion, occasioned by the stronger impression which two equal images, alike illuminated, make upon the eyes: they are however more embarrassing than useful. This telescope has been chiefly treated of by the fathers Reita and Cherubin of Orleans. - There are also nicroscopes of the same kind, though but little used, being subject to the same inconveniences as the telescopes.

BINOMIAL, a quantity consisting of two terms or members connected by the sign $\%$ or - , viz, plus or minus; as $a+b$, or $3 a-2 c$, or $a^{2}+b$, or $x^{2}-2 \sqrt{ } c$, \&c ; denoting the suin or the difference of the two terms. When either term is negative, the hinomial is sometimes called a residual, and by Euclid an apotome. The term binomial was first introduced by Lucas de Burgo. See his Arith. carta 120, edit. 1494 .

Brinomial Line, or Surd, is that in which at least one of the parts is a surd. Euclid enumerates six kinds of binomial lines or surds, in the 10th book of his Elements, which are exactly similar to the 6 residuals or apotomes there treated of also, and of which an account is gjven under the art. Apotòn, which see. Those apotomes become binomials by only changing the sign of the latter. term from minus to plus, which therefore are as below.

\section{Euclid's 6 Binomial Lines. \\ 1st binomial $3+\sqrt{ } 5$, \\ $2 d$ binomial $\sqrt{ } 18+4$, . \\ 3d binomial $\sqrt{ } 24+\sqrt{ } 18$, \\ 4 th binomial $++\sqrt{ } 3$, \\ 5 th binomial $\sqrt{ } 6+2$, \\ 6 th binomial $\sqrt{ } 6+\sqrt{ } 2$.}

To extract the Square Root of a Binomial, as of $a+\sqrt{ } b$, or $\sqrt{ } c+\sqrt{ } b .-V$ arious rules have been given for this purpose. The first is that of Lucals de Burgo, in his Summa de Arith. \&ce, which is this: When one part, as a, is rational, divide it into two parts such, that their prorluct may be equal to $\frac{1}{4}$ of the number under the radical $b$; then sliall the sum of the roots of those parts be the root of the binnmial sought: or their difference is the root when the quantity is residual; that is, if $e+c=a$, and $c e=\frac{x}{4} b$; then is $\sqrt{ } c+\sqrt{ } e=\sqrt{ }(a+\sqrt{ } b)$ the root sought. For example, let the binomial be $23+\sqrt{ } / 44$; then the parts of 23 are 16 and 7 , and their product is 112 , which is $\frac{x}{4}$ th of 448 ; therefore the sum of their roots $4+\sqrt{ } 7$ is the root sought of $23+\sqrt{ } 448$.

Lucas de Burgo gives also another rulc for the same extractions, which is this: The given binomial being, for example, $\sqrt{ } c+\sqrt{ } b$, its root will be $\sqrt{ }\left[\frac{x}{2} \sqrt{ } c+\frac{\pi}{2} \sqrt{ }(c-b)\right]$ $+\sqrt{ }\left[\frac{x}{2} \sqrt{ } c-\frac{1}{2} \sqrt{ }(c-b)\right]$. - So, in the foregoing example, $23+\sqrt{ } 448$, here $\sqrt{ } c=23$, and $\sqrt{ } b=\sqrt{ } 448$; hence $\frac{\mathrm{x}}{2} \sqrt{ } c=11 \frac{1}{2}$, and $\frac{x}{2} \sqrt{ }(c-b)=\frac{x}{2} \sqrt{ }\left(23^{2}-448\right)=$ $\frac{x}{2} \sqrt{ } 81=4 \frac{\pi}{2}$;

theref. $\sqrt{ }\left[\frac{1}{2} \sqrt{ } c+\frac{x}{2} \sqrt{ }(c-b)\right]=\sqrt{ }\left(11 \frac{1}{2}+4 \frac{x}{2}\right)=16=4$, and $\sqrt{ }\left[\frac{1}{2} \sqrt{ } c-\frac{1}{2} \sqrt{ }(c-b)\right]=\sqrt{ }\left(11 \frac{1}{2}-4 \frac{1}{2}\right)=\sqrt{ } 7$; conseq. $4+\sqrt{ } 7$ is the root sought, as before.

Again, if the binomial be $\sqrt{ } 18+\sqrt{10}$; here $c=18$, and $b=10$; theref. $\frac{1}{2} \sqrt{ } c=\frac{1}{2} \sqrt{ } 18=\frac{3}{2} \sqrt{ } 2$, and $\frac{1}{2} \sqrt{ }(c-b)=$ $\frac{x}{2} \sqrt{ } 8=\sqrt{ } 2$; hence,

$\sqrt{ }\left[\frac{1}{2} \sqrt{ } c+\frac{1}{2} \sqrt{ }(c-b)\right]=\sqrt{ }\left(\frac{3}{2} \sqrt{ } 2+\sqrt{ } 2\right)=\sqrt{ } \frac{5}{2} \sqrt{ } 2=$ $\sqrt[4]{\frac{25}{2}}$, and

$\sqrt{ }\left[\frac{x}{2} \sqrt{ } c-\frac{1}{2} \sqrt{ }(c-b)\right]=\sqrt{ }\left(\frac{3}{2} \sqrt{ } 2-\sqrt{ } 2\right)=\sqrt{ } \frac{x}{2} \sqrt{ } 2=\sqrt[4]{\frac{x}{2}} ;$ cousequently, $\sqrt{\frac{25}{2}}+\sqrt[4]{\frac{1}{2}}$, or $\frac{\sqrt{5}+1}{\sqrt{ } / 2}$ is the root of $\sqrt{ } 18+$ $\sqrt{ } 10$ sought. This latter rule bas been used by all authors, down to the present time.

To extract the Cubic and other higher Roots of a Binonial. This is useful in resolving cubic and higher equations, and was introduced with the resolution of those equations by Turtalea and Cardan. 'The rules for-such extractions are in great measure tentative; some of the principal ones of which are the following.

Tartaleu's Rule for the Cube Root of a Binomial $p+q$. This rule is given in his 9 th book of Miscellaneous Questions, 'quest. 40 ; being made out from either of the terms, $p$ or $q$, of the binomial, taken singly, in this manner: Separate either term, as $p$, into two such parts that one of them may be a cubic number, and the other part divisible by 3 without a remainder; then the cube root of the said cubic part will be one term of the root, and the other term will be the square root of the quotient arising from dividing the aforesaid third part by the first term just found. Thus if $p$ be divided into $r^{3}+3 s$, then the root is $r+\sqrt{ } \frac{s}{r}$. For example, to extract the cube root of $\sqrt{ } 10 s+10$. Suppose the part 10 to be taken: this separates into the parts 1 and 9 , the former of which is a cube, and the latter divisible by 3 ; that is $r^{3}=1$, and $3 s=9$; hence $r=1$, and $s=3$; consequently $r+\sqrt{ } \frac{s}{r}=1+\sqrt{ } 3$ is the cube root of $\sqrt{ } 108+10$ sought. Again, to use the other term $\sqrt{ } 108$ : this divides into $\sqrt{ } 27+\sqrt[V]{27}$, of which the former is a cube, and the latter divisible by 3 ; that is, $r^{3}=$ $\sqrt{ } 27$, and $3 s=\sqrt{ } 27$; therefore $r=\sqrt{ } 3$, and $s=\frac{5}{3} \sqrt{ } 27$ $=\sqrt{ } 3$ also: hence $r+\sqrt{ } \frac{s}{r}=\sqrt{ } 3+\sqrt{ } \frac{\sqrt{ } 3}{\sqrt{ } 3}=\sqrt{ } 3+1$ the cube root, the same as before.

Bombell's Rule for the Cube Root of the Binominl $a+$ $\sqrt{ }-b$. First find $\sqrt[3]{ }\left(a^{2}+b\right)$; then, by trials, search out a number $c$, and a square root $\sqrt{ } d$, such that the sum of their squares $c^{2}+d$ nuay be $=3 \sqrt[3]{ }(a+b)$, and also $c^{3}-$ $3 c l=a ;$ then shall $c+\sqrt{ }-d$ be the cube root of $a+$ $\sqrt{ }-b$ sought. For example, to find the cube root of $2+$ $\sqrt{ }-121:$ here $\sqrt[3]{ }\left(a^{2}+b\right)=\mathfrak{V} 125=5$; then taking $c=$ 2 , and $d=1$, it is $c^{2}+d=5=\sqrt[3]{\left(a^{2}+b\right)}$ and $c^{3}-3 c d$ 
$=8-6=2=a$, as it ought ; therefore $2+\sqrt{ }-1$ is the cube root of $2+\sqrt{ }-121$. - Bombetli gave also a rule for the cube root of the binomial $a+\sqrt{ } l$, but it is good for nothing.

Albert Girard's Rule for the Cube Root of a Einomial. This is given in his Invention Nouvelle en l'Algèbre, and is explained by him thus: Let $7 \dot{2}+\sqrt{ } 5120$ be the given binomial whose cube root is sought.

The square of 72 the greater term is 5184 . and of the less term is 5120 their difference 64 its cube root 4 ,

which 4 must be the difference between the squares of the two terms of the root sought; and as the rational part 72 of the given binomial is the "greater term, therefore the rational part of the required root will be the greater part also; conscquently the root sought must be one of the binominals here set in the margin, where the difference of the squares of the terms is always 4 , as required; and to find out the true one, proceed thus:

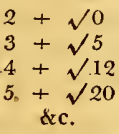
The first, $2+\sqrt{ } 0$ must be rejected, because one term of it is 0 or nothing; also because 5 exceeds the cube root of 72 , or $\sqrt{ } 20$ exceeds the cube root of $\sqrt{ } 5120$, therefore 5 . $+\sqrt{ } 20$, and all after it must be rejected likewise; so that the root must.te either $3+\sqrt{ } 5$ or $4+\sqrt{ } 12$, if the given quantity has a binomial root: to know which of these is to be taken, it must be considered that the rational term of the root must measure the rational term given; and also the irrational term of the root must mcasure the irrational tcrni given; then, on examination it is found that both -3 and 4 measure or divide the 72 without a remainder, but that only the $\sqrt{5}$, and not $\sqrt{ } 12$, measures $\sqrt{ } 5120$; consequently none but $3+\sqrt{ } 5$ can be the cube root of the given quantity $72+\sqrt{ } 5120$; which is found to answer, by cubing the said root $3+\sqrt{ } 5$.

Dr. Wallis's Rule for the Cule Root of the Binomial $a \pm$ $m \sqrt{ } b$ or $a \pm m \sqrt{ }-b$. In these forms the greatest rational part $m$ is extracted out of the radical part, leaving only $b$ the least radical part possible under the radical sign. IIc first observes that if the given quantity have a binomial root, it must be of this form $c \pm n \sqrt{ } b$, with the same radical $b$. Then to find the value of $c$ and $n$, he raises this root to the 3d power, which gives $c^{3}+3 c n^{2} b \pm \overline{3 c^{2} n+n^{3} b} \cdot \sqrt{ } b$, which must $b c=a \pm m \sqrt{ } b$, the given quantity; hence putting the rational part of the one quantity equal to that of the other, and also the radical part of the one equal to that of the other, gives $c^{3}+3 c n^{2} b=a$, and $3 c^{2} n+n^{3} b=m$. Then assuming several values of $n$, from the last equation he finds the value of $c$; hence if these values of $c$ and $n$, substituted in the first equation, make it obtain, they are right; but if not, another value of $n$ is assumed, and so on, till the first cquation hold true. And it is to be noted that $n$ is always an integer, or else the half of an integer. For example, if the cube root of $135 \pm \sqrt{ } 1825$ be required, or $135 \pm 78$ $\checkmark 3$; here $a=135, m=78$, and $l=3$; hence $3 c^{2} n+$ $n^{3} b=m$ is $3 c^{2} n+3 n^{3}=78$, or $c^{2} n+n^{3}=26$; then assuming $n=1$, this last equation becomes $c^{2}+1=26$, from which $c$ is found $=5$; which values of $c$ and $n$ being substituted in the first equation $c^{3}+3 c n^{2} b=a$, nake $5^{3}+$ $3.5 .3=170$, but ought to be 135 , showing that $c$ is too great, and consequently $n$ taken too little. Let $n$ therefore ice assumed $=2$, so shall $2 c^{2}+8=26$, and $c=3$; and the first equation becomes $3^{3}+3 \cdot 3 \cdot 2^{2} \cdot 3=27.5=135$ $=a$ as it ought, which shows that the true value of $n$ is 2 , and that of $c$ is 3 ; hence then the cube root of $135 \pm 78$ $\sqrt{ } 3$ or $c \pm ? \sqrt{ } b$ is $3 \pm 2 \sqrt{ } 3$ or $3 \pm \sqrt{ } 12$. And in like manner is the process instituted when the number in the radical is negative, as the cube root of $81 \pm 30 \sqrt{ }-3$, which is $\frac{9}{2} \pm \frac{1}{2} \sqrt{ }-3$.

Another rule for extracting the cube, root of an inaginary binomial was also given by Demoivre, at the end of Saunderson's Algebra, by means of the trisection of an arc or angle.,

Sir I. Newton's Rule for any Root of a Binqmial $a \pm b$. In his Universal Arith. is given a rule for the square root of a binomial, which is the same as the $2 \mathrm{~d}$ by Lucas de Burgo, before given ; and also a general rule for any root of a binomial, which $I$ have not met with elsewlicre; it is this: Of the given quantity $a \pm b$, let $a$ be the greater term, and $c$ the index of the root to be extracted. Seek the least number $n$ whose power $n^{6}$ can be divided by $a a-b b$ without a remainder, and let the quotient be $q$; Compute $c /((a$ $+b) \sqrt{ } q$ ) in the nearest integer number, which call $r$; divide $a \sqrt{ } q$ by its greatest rational divisor, calling the quotient $s:$ and let the nearest integer number above $\frac{r+\frac{n}{2 s}}{2 s}$ be $t$ : so shall $\frac{t s \pm \sqrt{ }\left(t^{2} s^{2}-n\right)}{\sqrt[3]{q}}$ bc the root sought, if the extraction of it be possible. And this rule is demonstrated by Gravesande in his commentaly on Newton's Arithmetic. Many numeral examples, illustrating this rule, are given in Gravesande's Algebra, abovementioned, pa. 160, as also in Newton's Univers. Arith. pa. 53, $2 \mathrm{~d}$ edit. and in Maclaurin's Algebra, pa. 118. Other rules may be found in Schooten's Commentary on the Geometry of Descartes, and clsewhere.

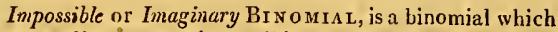
has one of its terms an impossible or an imaginary quantity: as $a+\sqrt{ }-b$.

In the foregoing article are given several rules for the roots of Binomials. Dr. Maskelyne, the late Astronomer Royal, has also given a method of finding any power of an Impossible Binomial, by another like Binomial. This rule is given in his Introduction prefixed to Taylor's Tables of Logarithms, pa. 56 ; and is as follows.

The logarithms of $a$ and $b$ being given, it is required to find the power of the Inpossible Binomial $a \pm \sqrt{ }-b^{2}$. whose index is $\frac{m}{n}$, that is, to find $\left(a \pm \sqrt{ }-b^{2}\right)^{\frac{m}{n}}$ by another Impossible Binomial; and thence the value of $(a+$ $\left.\sqrt{ }-b^{2}\right)^{\frac{m}{n}}+\left(a-\sqrt{ }-b^{2}\right)^{\frac{m}{n}}$, which is always possible, whether $a$ or $b$ be the greater of the two.

Solution. Put $\frac{b}{a}=$ tang. $z$. Then

$\left(a \pm \sqrt{ }-b^{2}\right)^{\frac{m}{n}}=\left(a^{2}+b^{2}\right)^{\frac{m}{2 n}} \times\left(\cos \cdot \frac{m}{n} z \pm \sqrt{ }-\sin \cdot \frac{{ }^{2} m}{n} z\right)$ Hence $\left(a+\sqrt{ }-b^{2}\right)^{\frac{m}{n}}+\left(a-\sqrt{ }-b^{2}\right)^{\frac{m}{a}}=\left(a^{2}+b^{2}\right)^{\frac{m^{2}}{2 a}} \times$ $2 \cos \cdot \frac{m}{n} z=(a \times \sec \cdot z)^{\frac{m}{n}} \times 2 \operatorname{cosin} \cdot \frac{m}{n} z=(b \times \operatorname{cosec} . z)^{\frac{m}{n}}$ $\times 2 \operatorname{cosin} \cdot \frac{m}{n} z$, where the first or second of these two last expressions is to bc used, according as $z$ is an cxtreme or mean arc; or rather, because $\frac{b}{a}$ is not only the tangent of $z$, but also of $z+360^{\circ}, z+720^{\circ}$, \&c; therefore the factor in the answer will have several values, viz, 
$2 \cos \frac{m}{n} z ; 2 \cos \cdot \frac{m}{n}\left(z+360^{\circ}\right) ; 2 \cos \frac{m}{n}\left(z+720^{\circ}\right) ; \& \mathrm{c} ;$ the number of which, if $m$ and $n$ be whole numbers, and the fraction $\frac{m}{n}$ be in its least terms, will be equal to the denominator $n$; otherwise infinite.

By Logarithms. Put log. $b+10-\log . a=\log \cdot \tan . z$. Then log. $\left[\left(a+\sqrt{ }-b^{2}\right)^{\frac{m}{n}}+\left(a-\sqrt{ }-b^{2}\right)^{\frac{m}{n}}\right]=\frac{m}{n} \times(1 . a$ $+10-1 \cdot \cos . z)+1.2+1 \cdot \cos \cdot \frac{m}{n} z-10=\frac{m}{n} \times(1 . b+$ $10-1 . \sin . z)+1.2+1 \cdot \cos \cdot \frac{m}{n} z-10$; where the first or second expression is to be used, according as $z$ is an extreme or mean arc. Moreover by taking successively, $\mathrm{i}$. cos. $\frac{m}{n} z ; 1 \cdot \cos \cdot \frac{m}{n}\left(z+360^{\circ}\right) ; 1 . \cos \cdot \frac{m}{n}\left(z+720^{\circ}\right) ; \quad \& \mathrm{c}$, there will arise several distinct answers to the question, agreeably to the remark made above.

Binomiat. Curve, is a curve whose ordinate is expressed by a binomial quantity; as the curve whose ordinate is $x^{a} \times \overleftarrow{b+d x}$ c). Stirling, Method. Diff. pa. 58 .

Brivomial Theorem, is used to denote the celebrated theorem given by Sir I. Newton for raising a binomial to any power, or for extracting any root of it by an approximating infinite series. It was known by Stifelius, and others, about the beginning of the 16 th century, how to raise the integral powers, not barely by a continued multiplication of the binomial given, but also by a table of numbers formed by Stifelius, which showed by inspection the coefficients of the terms of any power of the binomial, contained within the limits of the table; but still they could not independent of a table, and of any of the lower powers, raise any power of a binomial at once, by determining its têms one from another only, viz, the $2 \mathrm{~d}$ term from the 1 st, the $3 \mathrm{~d}$ from the $2 \mathrm{~d}$, and so on as far as we please, by a general rule; and much less could they extract general algebraic roots in infinite series by any rule whatever.

For though the nature and construction of that table, which is composed of figurate numbers, was so early known, and employed in raising powers, and extracting roots; yet it was only by raising the numbers one from another by continual additions, and then taking them from the table when wanted; till Mr. Briggs first pointed out the way of raising any line in the table by itself, without any of the preceding lines; and thus teaching to raise the terms of any power of a binomial, independent of any of the other powers; and so gave the substance of the binomial theorem in words, wanting only the algebraic notation in symbols; as is shown at large at pa. 78 of the historical introduction to my Mathematical Tables. Whatever was known however of this matter, related only to pure or integral powers, no one before Newton having thought of extracting roots by infinite serics. He happily discovered that, by considering powers and roots in a continued series, roots being a's powers having fractional exponents, the same binomial series would equally serve for them all, whether the index should be fractional or itstegral, or whether the series be finite or infinite. The truth of this method however was long known only by trial in particular cases, and by induction from analogy; nor does it appear that even Newton himself ever attempted any direct proof of it: however, various demonstrations of the theorem have since been given by the more modern mathematicians, some of which are by means of the doctrine of fluxions, and others, more legally, from the pure principles of algebra only: for a full account of which, see pa. 228, \&c, of my Mathematical Tracts, vol. 1.

This theorem was first discovered by Sir I. Newton in 1669 , and sent in a letter of June 13,1676 , to $\mathrm{Mr}$. Oldenburg, Secretary to the Royal Society, to be by him communicated to Mr. Leibnitz; and. it. was in this form: $(p+p q)^{\frac{m}{n}}=p^{\frac{m}{n}}+\frac{m}{n} a q+\frac{n-n}{2 n} b q+\frac{m-2 n}{3 n} c q+\& c:$ where $p+p q$ signifies the quantity whose root, or power, or root of any power, is to be found; $p$ being the first term of that quantity; $q$ the quotient of all the rest of the terms divided. by that first term; and $\frac{m}{n}$ the numeral index of the power or root of the quantity $p+p q$, whether it be integral or fractional, positive or negative; and lastly $a, b, c, d, \& c$, are assumed to denote the several terms in their order as they are found, viz, $a=$ the first term $p^{\frac{m}{a}}, b=$ the $2 \mathrm{~d}$ term $\frac{m}{n} u q, c=$ the third term $\frac{m-n}{2 n} b q$, and soon. As Newton's general notation of indices was not commonly known, he takes this occasion to explain it; and then he gives many examples of the application of tbis theorem, one of which is the following.

Ex. 1. To find the value of $\sqrt{ }\left(c^{2}+x^{2}\right)$ or $\left(c^{2}+x^{2}\right)^{\frac{\pi}{3}}$, that is, to extract the square root of $c^{2}+x^{2}$ in an infinite series. Here $p=c^{2}, q=\frac{x^{2}}{c^{2}}, m=1$, and $n=2$; therefore $a=p^{\frac{m}{n}}=(c c)^{\frac{x}{2}}=c, b=\frac{m}{n} a q=\frac{x^{3}}{2 c}, c=\frac{m-n}{2 n} b_{q}=\frac{-x^{4}}{8 c^{2}}$, \&c; therefore the root sought is $c+\frac{x^{2}}{2 c}-\frac{x^{4}}{8 c^{3}}+\frac{x^{6}}{16 c^{5}}-\frac{5 x^{8}}{125 c^{5}}$ $+\& c$.

A variety of other examples are also given in the same place, by which it is shown that the theorem is of universal application to all sorts of quantitics whatever.-This theorem is sometimes represented in other forms, as $p^{\frac{m}{n}} \times: 1+\frac{m}{n} q+\frac{m}{n} \cdot \frac{m-n}{2 n} q^{2}+{ }_{n}^{m} \cdot \frac{m-n}{2 n} \cdot \frac{m-2 n}{3 n} q^{3}$ $+\& c$; which comes to the same thing. Or thus $p^{\frac{m}{u}}+$ $\frac{m}{n} p^{\frac{m-n}{n r}}+\frac{m}{n} \cdot \frac{m-n}{2 n} p^{\frac{m-2 n}{u r^{3}}}$ \&c; where the binomial is $(p+r)^{\frac{m}{n}}$.

In another letter to Mr. Oldenburg, of Oct. 24, 1676 , Newton explains the train of reasoning by which be obtained the said theorem, as follows: "In the beginning of my mathematical studies, when $I$ was perusing the works of the celebrated Dr. Wallis ' (sce bis Arith. of Infinites, prop. 118 , and 121 , also his Algebra, chap. 82), and considering the series by the interpolation of which he exhibits the area of the circle and hyperbola ; for instance, in this scries of curves, whose common base or axis is $x$, and the ordinates respectively $(1-x x)^{\frac{0}{2}},(1-x x)^{\frac{3}{2}}$, $(1-x x)^{\frac{2}{2}},(1-x x)^{\frac{3}{3}},(1-x x)^{\frac{4}{2}}, \& x$; I perceived that if the areas of the alteruate curves, which are

$$
\begin{aligned}
& x-\frac{1}{3} x^{3} \\
& x-\frac{2}{3} x^{3}+\frac{2}{5} x^{5}, \\
& x-\frac{3}{3} x^{3}+\frac{3}{5} x^{5}-\frac{1}{7} x^{7},
\end{aligned}
$$

\&c; could be interpolated, we should be able to obtain the areas of the intermediate ones; the first of which, $(1-x x)^{\frac{x}{2}}$, is the aren of the circle: now in order to this, it appeared that in all the series the first term was $x$; that the $2 d$ terms $\frac{0}{3} x^{3}, \frac{3}{3} x^{3}, \frac{2}{3} x^{3}, \frac{3}{3} x^{3}$, \&c, were in arithmetical 
progression; and consequently that the first two terms of all the series to be interpolated would be

$$
x-\frac{\frac{1}{2} x^{3}}{3}, \quad x-\frac{\frac{3}{2} x^{3}}{3}, \quad x-\frac{\frac{6}{2} x^{3}}{3}, \text { \&c. }
$$

"Now for the interpolation of the rest, I considered that the denominators, $1,3,5,7$, \&c, were in arithmetical progression; and that therefore only the numeral coefficients of the numerators were to be investigated. But these in the alternate areas, which are given, were the same with the figures of which the several powers of 11 consist; viz, of $11^{0}, 11^{\mathrm{I}}, 11^{2}, 11^{3}$, \&c ; that is,

the first 1,
the second 1,1
the third $1,2,1$
the fourth $1,3,3,1$
the fifth $1,4,6,4,1$
\& c.

"I enquired therefore how, in these series, the rest of the terms may be derived from the first two being given; and $I$ found that by putting $m$ for the $2 \mathrm{~d}$ figure or term, the rest would be produced by the continued multiplication of the terms of this series,

$\frac{m-0}{1} \times \frac{m-1}{2} \times \frac{m-2}{3} \times \frac{m-3}{4} \times \frac{m-4}{5} \&$ c.

" lor instance, if the $2 \mathrm{~d}$ term $m=4$; then shall 4. $\times \frac{m-1}{2}$, or 6 , be the $3 \mathrm{~d}$ term; and $6 \times \frac{m-2}{3}$, or 4 , the 4 th term; and. $4 \times \frac{m-3}{4}$ or 1 , the 5 th term; and $1 \times \frac{m-4}{5}$, or 0 , the 6 th; which shows that in this. case the series terminates.

" This rule therefore I applied to the series to be interpolated. And since, in the series for the circle, the $2 \mathrm{~d}$ term was $\frac{\frac{1}{2} x^{3}}{3}$ I put $m=\frac{x}{2}$, which produced the terms $\frac{x}{2} \times \frac{\frac{\pi}{2}-1}{2}$ or $-\frac{1}{8} ;-\frac{1}{8} \times \frac{\frac{1}{2}-2}{3}$ or $+\frac{1}{16} ;+\frac{1}{16} \times \frac{\frac{r}{2}-3}{4}$ or $-\frac{5}{128}$; and so on ad infinitum. And bence $I$ found that the required area of the circular segment is $x-\frac{\frac{7}{2} x^{3}}{3}-\frac{\frac{3}{8} x^{6}}{5}-\frac{\frac{3}{15} x^{7}}{7}-\frac{\frac{3}{12 x^{9}} x^{9}}{9}-8 \mathrm{c}$.

" And in the same manner might be produced the interpolated areas of the other curves: as also the area of the byperbola and the other alternates in thisseries $(1+x x)^{\frac{0}{2}},(1+x x)^{\frac{1}{2}},(1+x x)^{\frac{2}{2}},(1+x x)^{\frac{3}{2}}$, \&c. And in the same way also may other series be interpolated, and that too if they should be taken at the distance of two or more terms.

"T'his was the way then in which I first entered upon these speculations; which I should not have remembered, but that in turning over my papers a few wceks since, I chanced to turn my eyes on those relating to this matter.

"Having proceeled so far, I considered that the terms $(1-x x)^{\frac{0}{2}},(1-x x)^{\frac{2}{2}},(1-x x)^{\frac{4}{2}},(1-x x)^{\frac{6}{2}}, \& \mathrm{c}$, that is, 1

$$
\begin{aligned}
& 1-x^{2} \\
& 1-2 x^{2}+x^{4}
\end{aligned}
$$

$1-3 x^{2}+3 x^{4}-x^{0}, \& c$, might be interpolated in the same manner as the areas generated by them: and for this, nothing more was required but to omit the denominators $1,3,5,7, \& c$, in the terms expressing the areas; that is, the cocfficients of the terms of the quantity to be interpolated $(1-x x)^{\frac{1}{2}}$, or $(1-x x)^{\frac{3}{2}}$, or generally $\left(1-x_{x}\right)^{+\infty}$, will be produced by the continued multiplication of the terms of this series

$m \times \frac{m-I}{2} \times \frac{m-2}{3} \times \frac{m-3}{4} \& c$.

"Thus, for example, there would be found

$(1-x x)^{\frac{1}{2}}=1-\frac{1}{2} x^{2}-\frac{1}{8} x^{4}-\frac{1}{16} x^{6}$ \&c.

$(1-x x)^{\frac{3}{2}}=1-\frac{3}{2} x^{2}+\frac{3}{8} x^{4}+\frac{1}{10} x^{6} \& \mathrm{c}$.

$(1-x x)^{\frac{x}{3}}=1-\frac{x}{3} x^{2}-\frac{x}{9} x^{4}-\frac{5}{85} x^{6} \& \mathrm{c}$.

"Thus then $I$ discovered a general method of reducing radical quantities into infinite series, by the theorem which I sent in the beginning of the former letter, before I knew the same by the extraction of roots.

"But having discovered that way, this other could not long remain unknown: for to prove the truth of those operations, I multiplied

$1-\frac{1}{2} x^{2}-\frac{1}{8} x^{4}-\frac{5}{16} x^{6} \& c$, by itself, and the product was $1-x^{2}$, all the rest of the terms vanishing after these, in infinitum. In like manner,

$1-\frac{x}{3} x^{2}-\frac{\pi}{9} x^{4}-\frac{5}{8} x x^{6} \& c$, twice multiplied by itself, produced $1-x^{2}$. But as this was a certain proof of those conclusions, so I was naturally led to try conversely whether these series, which were thus known to be the roots of the quantity $1-x^{2}$, could not be extracted out of it after the manner of arithmetic; and upon trial I found it to succeed. The process for the square root is here set down

$$
\begin{aligned}
& \frac{1-x^{2}\left(1-\frac{1}{2} x^{2}-\frac{1}{8} x^{4}-\frac{1}{16} x^{6}\right. \text { \&c. }}{0-x^{2}} \\
& \frac{-x^{2}+\frac{1}{4} x^{4}}{-\frac{1}{4} x^{4}} \\
& \frac{-\frac{1}{4} x^{4}+\frac{1}{8} x^{6}+\frac{1}{64} x^{8}}{-\frac{1}{8} x^{6}-\frac{1}{64} x^{8}}
\end{aligned}
$$

"These methads being found, I laid aside the other way by interpolation of series, and used these operations only as a more genuine foundation. Neither was I ignorant of the reduction by division, which is so much easier." See Collins's Commercium Epistolicum.

And this is all the account that Newton gives of the invention of this theorem, wbich is engraved on his manument in Westminster Abbey, as one of his greatest discoverics.

Francis Maseres, Esq. (Cursitor Baron of theExchequer) has communicated the following observations on the Binomial theorem, and its demonstration; viz, About the year 1666 the celcbrated Sir Isaac Newton discovered that, if $m$ were put for any whole number whatsoever, the coefficients of the terms of the $m$ th power of $1+x$ would be

$$
1, \frac{m}{1}, \frac{m}{1} \cdot \frac{m-1}{2}, \frac{m}{1} \cdot \frac{m-1}{2} \cdot \frac{m-2}{3}, \text { \&c ; }
$$

till we come to the term $\frac{m-(m-1)}{m}$, which will be the last term. But by what particular means he discorered this proposition, he has not told us, nor has he even attempted to give a demonstration of it. Dr. John Wallis, of U.xford, informs us (in his Algebra, chap. 85, pa. 319) that he had endeavoured to find this manner of generating these coefficients one from another, but without success; and he was greatly delighted with the discovery, when he found that Mr. Newton had made it. But he likesvise has omitted to give a demonstration of it, as well as Sir Isaac Newton; and probably he did not know how to demonstrate it. 
Newton, after having discovered this rule for generating the coefficients of the powers of $1+x$ when the indices of those powers were whole numbers, conjectured that the same might possibly obtain also when the indices were fractions. He therefore resolved to try whether it would or not, by applying it to such inclices in a few easy instances, and particularly to those of $\frac{\pi}{2}$ and $\frac{1}{3}$, which, if the rule served in the case of fractional indices, would enable him to find series equal to the values of $(1+x)^{\frac{1}{2}}$ and $(1+x)^{\frac{1}{3}}$, or the square-root and the cube-root of the binomial quantity $1+x$. And, when he bad in this manner obtained a series for $(1+x)^{\frac{x}{2}}$, which he suspected to be equal to $(1+x)^{\frac{1}{2}}$, ir the square root of $1+x$, he multiplied the said series into itself, and found that the product was $1+x$; and when he had discovered in like manner a series for $(1+x)^{\frac{\pi}{3}}$ he multiplied the same twice into itself, and found that the product was $1+x$; and thence he concluded that the former series was really equal to the square-root of $1+x$, and that the latter series was really equal to its cube-root. And from these and a few more such trials, in which he found the rule to answer, he concluded universally that the rule was always true, whether the index $m$ stood for a whole number or a fraction of any kind, as $\frac{1}{2}, \frac{1}{3}, \frac{2}{3}, \frac{3}{2}, \frac{5}{9}, \frac{9}{5}$, or, in general, $\frac{p}{q}$.

After the discovery of this rule by Sir Isaac Newton, and the publication of it by Dr. Wallis, in his Algebra, chap. 85 , in the year 1685 , (which I believe was the first time it was published to the world at large, though it was inserted in Sir Isaac Newton's first letter to Mr. Oldenburg, the secretary to the Royal Society, dated June 13, 1676 , and the said letter was shown to Mr. Leibnitz, and probably to some other of the learned mathematicians of that time) it remained for some years without a demonstration, either in the case of integral powers or of roots. At last however it was demonstrated in the case of integral powers by means of the properties of the figurate numbers, by that learned, sagacious, and accurate mathematician Mr. James Bernoulli, in the $3 d$ chapter of the $2 d$ part of his excellent treatise De Arte Conjectandi, or, On the Art of forming reasonable Conjectures concerning Events that depend on Chance; which appears to me to be by far the best-written treatise on the doctrine of Chances that has yet been puhlished, though M. Demoivre's book on the same subject may have carried the doctrine something further. This treatise of James Bernoulli's was not published till 8 years after his death, which happened in August 1705 ; but there is reason to think that it was composed in the latter 5 or 6 years of the preceding century, and even that some parts of it, or some of the propositions inserted therein, had been discovered by the author in the years $1689,1690,1691$, and 1692 . For the first part of his very curious tract, entitled, Positiones Arithmetica de Seriebus Infinitis, was published at Basil or Basle in Switzerland in the year 1689 ; and the second part of the said Positiones (in the 19th Position of which those propertics of the figurate numbers from which the binomial theorem may be deduced, are set down) was published at the same place in the year 1692 . But the demonstrations of those properties of the figurate numbers, and of the binomial theorem, which depends upon them, were never I believe communicated to the public till the year 1713 , when the zuthor's posthumous treatise Dc Arte Conjectandi mado its appearance. These demonstrations are founded on clear and simple principles, and afiord as much satisfaction as can well be expected on this subject. But the full display and explanation of these principles, and the deduction of the said properties of the figurate numbers, and ultimately of the Binomial theorem, from them, is a matter of considerable length. It will therefore not be improper to give a shorter proof of the truth of this important theorem, that shall not require a previous knowledge of the properties of the figurate numbers, but yet shall be equally conclusive with that which is derived from those properties. Now this may be done in the manner following.

Let us suppose that the coefficients of the terms of the first six powers of the Binomial quantity $1+x$ have been found, upon trial, to be such as would be produced by the general expressions

$$
1, \frac{m}{1}, \frac{m}{1} \frac{m-1}{2}, \frac{m}{1} \cdot \frac{m-1}{2} \cdot \frac{m-2}{3}, \& c,
$$

by substituting in them first 1 , then 2 , then 3 , then 4 , then 5 , and lastly 6 instead of $m$. This may easily be tried by first raising $1+x$ to the 6 th power, by repeated multiplications in the common way, and afterwards finding the terms of the powers by means of the said general expressions above ; which will be found to produce the very same terms as arose from the multiplications. After these trials we shall be sure that those general expressions are the true values of the coefficients of the powers of $1+x$ at least in the said first six powers. And it will therefore only remain to be proved that, since the rule is true in the said first six powers, it will also be true in the next following, or the 7 th power, and consequently in the 8 th, 9 th, and 10th powcrs, and in all higher powers whatsoever.

Now, If the coefficients of the 1st, 2d, 3d, 4th, and other following terms of $1+\left.x\right|^{n}$ be denoted by the letters $a, b, c, d$, \&c, respectively, it is evident from the nature of multiplication, that the coefficients of the Ist, $2 \mathrm{~d}, 3 \mathrm{~d}$, 4.th, and other following terms of the next higher power of $1+x$, to wit, $1+x)^{m+1}$ will be equal to $a, a+b$, $b+c, c+d, \& c$, respectively, or to the sums of every two contiguous coefficients of the terms of the preceding series which is $=\overline{1+x}^{m}$. This will appear from the operation of multiplication, which is as follows :

$$
\begin{aligned}
& a+b x+c x^{2}+d x^{3}+e x^{4}+\& c \\
& \frac{1+x}{a+b x+c x^{2}+d x^{3}+e x^{4}+\& c} \\
& +a x+b x^{2}+c x^{3}+d x^{4}+\& c . \\
& \text { if } \overline{1+x} \text { be equal to the series } \\
& a+b x+c x^{2}+d x^{3}+e x^{4}+\& c
\end{aligned}
$$

Thercfore, if $\left.\overline{1+x}\right|^{n}$ be equal to the series

then $\widetilde{1+x}^{m+1}$ will be equal to the series

$$
a+\overline{a+b} \cdot x+\overline{b+c} \cdot x^{2}+\overline{c+d} \cdot x^{3}+8 \mathrm{c} .
$$

Now lat $n$ be $=m+1$. We shall then bave to prove that, if the cocfficients $a, b, c, d$, \&c, be respectively cqual to $1, \frac{m}{1}, \frac{m}{1}, \frac{m-1}{2}, \frac{m}{3} \cdot \frac{m-1}{2} \cdot \frac{m-2}{3}, \& c$.

the cocfficients $a, a+b, b+c, \& c$, will be respectively cqual to $1, \frac{n}{1}, \frac{n}{1} \cdot \frac{n-1}{2}, \frac{n}{1} \cdot \frac{n-1}{2} \cdot \frac{n-2}{3}$, \&c.

And to do which, there is nothing more requised than to collect together every two terms of the former of the:e two series, and then substitute into these sums, $n$ instead 


\section{B I $Q$}

of $m+1$, when there will immediately come out the terms of the latter series, as above, viz,

$$
\widetilde{1+\left.x\right|^{n}}=1+\frac{n}{1} x+\frac{n}{1} \cdot \frac{n-1}{2} x^{2}+\& \text { c. Q. E. D. }
$$

For several other demonstrations of the Binomial Theorem, see my new Tracts, vol. 1, pa. 228, \&c.

BIPARTIENT, is a number that divides another into two equal parts without a relnainder. So 2 is a bipartient to 4 , and 5 a bipartient to 10 .

BIPAPTITION, is a division into two equal parts.

BIQUADRATE, or BiQua Dratic Pozer, is the squared square, or 4th power of any number or quantity. Thus 16 is the biquadrate or 4 th power of 2 , or it is the square of 4 which is the $2 \mathrm{~d}$ power of 2 .

Biquadratic Root, of any quantity; is the square root of the square root, or the 4 th root of that quantity. So the biquadratic root of 16 is 2 , and the biquadratic root of 81 is 3 .

BIQUADLATIC Equation, is that which rises" to 4 dimensions, or in wlich the unknown quantity rises to the 4th power; as $x^{4}+a x^{3}+b x^{2}+c x+d=0$.

Any biquadratic equation may be conceived to be generated or produced from the continual multiplication of four simple equations,

as $(x-p) \times(x-q) \times(x-r) \times(x-s)=0$;

or from that of two quadratic equations,

as $\left(x^{2}+p x+q\right) \times\left(x^{2}+r x+s\right)=0$;

or, lastly, from that of a cubic and a simple equation,

as $(x-p) \times\left(x^{3}+q x^{2}+r x+s\right)=0$ : which was the invention of Harriot. And on the contrary, a biquadratic equation may be resolved into four simple equations, or into two quadratics, or into a cubic and a simple equation, having all the same roots with it.

1. Ferrari's Method for Biquadratic Equations.

The first resolution of a biquadratic equation was given in Cardan's Algebra, chap. 39, being the invention of his pupil and friend Lewis Ferrari, about the year 1540. This is effected by means of a cubic equation, and is indeed a method of depressing the biquadratic equation to a cubic, which Cardas demonstrates, and applies in a great variety of examples. The principle is very general, and cousists in making one side of the equ:tion a square, by help of some multiples or parts of its own terms and an assumed unknown quantity; which it is always casy to do; and then the other side is made to be a square also, by assuming the product of its 1 st and $3 \mathrm{~d}$ terms equal to the square of hatf the $2 \mathrm{~d}$ term; for it consists only of three terms, or three different denoninations of the original letter; then this equality will determine the value of the assumed quantity by a cubic equation: other circumstances depend on the arise's judgment. But the method will be farther explained by the following examples, extracted from Cardan's bouk.

Ex. 1. Given $x^{4}-6 x^{8}+36=60 x$, to be resolved. Add $6 x^{2}$ to both sides of the equation, so shall $x^{4}+12 x^{2}+36$ or $\left(x^{2}+6\right)^{2}=6 x^{2}+60 x$. Assume $y$, and add $\left(x^{2}+6\right) 2 y+y^{2}$ to both sides, then is $\left(x^{2}+6\right)^{2}+2 y\left(x^{2}+6\right)+y^{2}=6 x^{2}+60 x+2 y\left(x^{3}+6\right)$ $+y^{2}$, or $\left(x^{2}+6+y\right)^{2}=(6+2 y) x^{2}+60 x+12 y$ $+y^{2}$.

Make now the $1 \mathrm{st} \times 3 \mathrm{~d}$ term $=\frac{\mathrm{r}}{4}$ sq. $2 \mathrm{~d}$, this gives $(6+2 y) \cdot\left(12 y+y^{2}\right) x^{2}=900 x^{2}$, or $y^{3}+15 y^{2}+36 y=450$; and hence

$y=\sqrt[3]{ }\left(287 \frac{1}{2}+\sqrt{ } 80449 \frac{x}{4}\right)+\sqrt[3]{ }\left(287 \frac{1}{2}-\sqrt{ } 80449 \frac{1}{4}\right)-5$. From which $x$ may be found by a quadratic cquation.

VoL. I.

\section{B I $\mathrm{Q}$}

For, because $(6+2 y) \cdot(12 y+y) \cdot x^{2}=900 x^{2}$, $(6+2 y) \cdot x^{2}+60 x+12 y+y^{2}$, becomes a square, whose root is evidently equal to $x \sqrt{ }(6+2 y)+\sqrt{ }\left(12 y+y^{2}\right)$; and since $\left(x^{2}+6+y\right)^{2}=(6+2 y) x^{2}+60 x+12 y+y^{2}$, their roots are also equal, that is $x^{2}+6+y=x \sqrt{ }(6$ $+2 y)+\sqrt{ }\left(12 y+y^{2}\right)$, a quadratic equation.

Ex. 2. Given $x^{4}=x+2$.

Before applying Ferrari's method to this example, Cardan resolves it by another way as follows: subtract 1 , from both sides of the equation, then is $x^{4}-1=x+1$; divide by $x+1$, then is $x^{3}-x^{2}+x-1=1$, or $x^{3}+x$ $=x^{2}+2 ;$ and hence $x=\sqrt[3]{ }\left(\sqrt{ } \frac{2241}{2916}+\frac{47}{54}\right)-\sqrt[3]{ }\left(\sqrt{ } \frac{2241}{2916}\right.$ $\left.-\frac{47}{54}\right)+\frac{1}{3}$.

But to resolve it by Ferrari's rule:

Because $x^{4}=x+2$; therefore $x^{4}+2 y x^{2}+y^{2}$ or $\left(x^{2}+y\right)^{2}=2 y x^{2}+x+\left(2+y^{2}\right)$; bence $2 y^{3}+4 y=\frac{x}{4}$, or $y^{3}+2 y=\frac{1}{8}$; and the root is $y^{\prime}=\sqrt[3]{ }\left(\sqrt{ } \frac{2075}{6912}+\frac{1}{10}\right)-\sqrt[3]{ }\left(\sqrt{ } \frac{2075}{6912}-\frac{1}{16}\right)$ :

by means of which $x$ is found by a quadratic equation.

Ex. 3. Given $x^{4}+32 x^{2}+16=48 x$. Add 240 , then $x^{4}+32 x^{2}+256$ or $\left(x^{2}+16\right)^{2}=48 x+240$; complete square again, then

$\left(x^{2}+16\right)^{2}+2 y \cdot\left(x^{2}+16\right)+y^{2}=2 y x^{2}+4 \cdot 8 x+\left(y^{2}\right.$ $+32 y+240)$; make the last side a sq. by the rule, which gives $y^{3}+32 y^{2}+240 y=24 \times 12=288$.

Put now $z=y+10 \frac{z}{3}$, and the last transforms to $z^{3}=101 \frac{1}{3} z+420 \frac{16}{2} ;$; then the value of $z$ found from this, gives the value of $y$, and hence the value of $x$ as before.

\section{Descartes's Rule for Biquadratic Eouations.}

Another solution was given of biquadratic equations by Descartes, in the $3 \mathrm{~d}$ book of his Geometry. In this solution he resolved the given biquadratic equation into two quadratics, by means of a cubic equation, in this manner: First, let the $2 d$ term or $3 d$ power be taken away out of the equation, after which it will stand thus, $x^{4}+p x^{2}+q x+r=0$. Find $y$ in this cubic equation $\left.y^{6}+2 p y^{4}+4 r\right\} p^{2}-q^{2}=0$; and theu $x$ in these $\left\{x^{2}-y x+\frac{x}{2} y^{n}+\frac{x}{2} p+\frac{q}{2 y}=0\right.$ two quadra- $\left\{x^{2}+y x+\frac{x}{2} y^{2}+\frac{x}{2} p-\frac{q}{2 y}=0\right.$, so shall these values of $x$ be the roots of the given bijtadratic equation.

Ex: Let the equ. be $x^{4}-17 x^{2}-20 x-6=0$.

Here $p=-17, q=-20$, and $r=-6$; and the cubic equ. is $x^{6}-3+y^{4}+313 y^{2}-400=0$, the root of which is $y^{2}=16$, or $y=4$; hence the two qua- $\left\{x^{2}-4 x-3=0\right.$ dratics are - $\left\{x^{2}+4 x+2=0\right.$, the four roots of which are $2 \pm \sqrt{ } 7$ and $-2 \pm \sqrt{ } 2$.

\section{Euler's Method for Biquadratic Equations.}

The celebrated Leonard Eulcr has also given, in the 6 th volume of the Petersburg Ancient Conmentaries, for the year 1738, an ingenious and general method for resolving equations of all degrees, by means of the equation of the next lower degree, and among them that of the biquadratic cquation by means of the cubic; and this last was also given more at large in his treatise of Algebra, translated frum the German into French in 1774 , in 2 volumes svo. The method is this: Let $x^{4}-a x^{2}-b x-c=0$, be the $2 \mathrm{H}$ 
given biquadratic equation, wanting the $2 d$ term. Take $\hat{f}=\frac{\pi}{2} a, g=\frac{1}{16} a a+\frac{\pi}{4} c$, and $h=\frac{1}{64} \vec{b} b$; with which values of $f, g, h$, form the cubic equation $z^{3}-f z^{2}+g z-h=0$. Then find the three roots of this cubic equation, and let them be called $p, q, r$. So shall the four roots of the proposed biquadratic be these following, viz,

When $\frac{1}{8} b$ is positive :

1st. $\sqrt{ } p+\sqrt{ } q+\sqrt{ } r$

2l. $\sqrt{ } p+\sqrt{ } q-\sqrt{ } r$

3d. $\quad \sqrt{ } p-\sqrt{ } q+\sqrt{ } / r$

When $\frac{1}{8} b$ is negative:

4th. $\sqrt{ } p-\sqrt{ } q-\sqrt{ } r \mid-\sqrt{ } p-\sqrt{ } q-\sqrt{ } r$.

$\boldsymbol{E} x$. Let the equ. be $x^{4}-25 x^{2}+60 x-36=0$.

Here $a=25, b=-60$, and $c=36$;

therefore $f=\frac{25}{2}, g=\frac{625}{16}+9=\frac{769}{16}$, and $h=\frac{225}{4}$.

Corsequently the cubic equation will be

$$
z^{3}-\frac{25}{2} z^{2}+\frac{769}{16} z-\frac{225}{4}=0 .
$$

The three roots of which are

$z=\frac{9}{4}=p$, and $z=4=q$, and $z=\frac{2.5}{4}=r ;$ and the roots of these are $\sqrt{ } p=\frac{3}{2}, \sqrt{ } q=2$ or $\frac{4}{2}, \sqrt{ } r=\frac{5}{2}$.

Hence, as the value of $\frac{1}{8} b$ is negative, the four roots are

1st. $x=\frac{3}{2}+\frac{4}{2}-\frac{5}{2}=1$,
2d. $x=\frac{3}{2}-\frac{4}{2}+\frac{5}{2}=2$,
3 d. $x=-\frac{3}{2}+\frac{4}{2}+\frac{5}{2}=3$,
4th. $x=-\frac{3}{2}-\frac{4}{2}-\frac{5}{2}=-6$.

4. Simpson's Rule for Biquadratic Equations.

Mr. Simpson gave a general rule for the solution of biquadratic equations, having all its terms, in the $2 \mathrm{~d}$ edit. of his Algebra, pa. 150, published in 1755 , in which the given equation is also resolved by means of a cubic equation, as well as the two former ways; and it is investigated on the principle, that the given equation is equal to the difference between two squares; being indeed a kind of generalization of Ferrari's method.

Thus, he supposes the given equation, viz, $x^{4}+p x^{3}+q x^{2}+r x+s=\left(x^{2}+\frac{1}{2} p x+A\right)^{2}-(\text { B } x+C)^{2} ;$ then from a comparison of the like terms, the values of the assumed letters are found, and the final equation becomes $A^{3}-\frac{1}{2} q A^{2}+k A-\frac{1}{2} l=0$,

where $k=\frac{3}{4} p r-s$, and $l=\frac{x}{4} r^{2}+s \cdot\left(\frac{x}{4} p^{2}-q\right)$.

The value of $A$ being found in this cubic equation, those of $\mathrm{B}$ and $\mathrm{C}$ will be had from their general values, viz, $\mathrm{B}=\sqrt{ }\left(2 \mathrm{~A}+\frac{\mathrm{T}}{4} p^{2}-q\right)$, and $\mathrm{c}=\frac{p_{\mathrm{A}}-r}{2 \mathrm{~B}}$. Hence, finally, the root $x$ will be obtained from the assumed cquation $\left(x^{2}+\frac{1}{2} p x+A\right)^{2}-(B x+c)^{2}=0$, or $x^{2}+\frac{1}{2} p x+A=$ $\pm \mathbf{B} x \pm \mathbf{c}$ (which always is the case when the value of $x$ is taken such, that the given biquadratic becomes equal to nothing), in four several values.

Ex. Given the equ. $x^{4}-6 x^{3}-58 x^{2}-114 x-11=0$. Here $p=-6, q=-58, r=-114$, and $s=-11$, whence

$k$ or $\frac{\pi}{4} p r-s=182, l$ or $\frac{T}{4} r^{2}+s \cdot\left(\frac{x}{4} p^{2}-q\right)=2512$; and therefore the cubic equation becomes

$\Lambda^{3}+29 \Lambda^{2}+182 \Lambda-1256=0$,

the root of which is $A=4$.

Hence then $\mathrm{B}$ or $\sqrt{2 \Lambda+\frac{7}{7} p^{2}-q}=\sqrt{ } 75$, and $\mathrm{c}$ or $\frac{p \mathrm{~A}-r}{2 \mathrm{~B}}=\sqrt{ } 27$ :

then the quadratic equation becomes

$x^{2}-3 x+4= \pm x \sqrt{ } 75 \pm \sqrt{ } 27$; which solved gives $x=1 \frac{\pi}{2} \pm 2 \frac{x}{2} \sqrt{ } 3+\sqrt{ }\left(17 \pm 11 \frac{1}{2} \sqrt{ } 3\right)$, or $=1 \frac{1}{2} \pm 2 \frac{t}{2} \sqrt{ } 3-\sqrt{ }\left(17 \pm 11 \frac{1}{2} \sqrt{ } 3\right)$, exhibiting all the 4 roots of the given equation according to the rariations of the signs.

Mr. Simpson here subjoins an observation which is tolally erroneous, viz, that "The value of $A$, in this equation, wilt be commensurate and rational (and therefore the casier to be discorered), not only when all the roots of the given cquation are commensurate, but when they are irrational and even impossible; as will appear from the example subjoincd." It must indeed appear singular that he should have adduced such a reason for the proof of a proposition without any foundation; and it is wonderful that he fell upon no examples that refuted it, as the instances in which it holds true, are so few, in comparison with the number of those in which it fails.

By niethods similar to most of the foregoing ones, Dr. Waring gave very general in restigations of such rules for biquadratics, in his Meditationes Algebraicæ, published in 1770 .

Note. In any biquadratic equation having all its terms, if $\frac{3}{8}$ of the square of the coefficient of the $2 \mathrm{dterm}$ be greater than the product of the coefficients of the 1st and $3 \mathrm{~d}$ terms, or $\frac{3}{8}$ of the square of the coefficient of the 4 th term be greater than the product of the coefficients of the $3 \mathrm{~d}$ and 5 th terms, or $\frac{4}{9}$ of the square of the coefficients of the $3 d$ term greater than the product of the coefficients of the $2 \mathrm{~d}$ and 4 th terms; then all the roots of that equation will be real and unequal; but if either of the said parts of those squares be less than either of those products, the equation will have imaginary roots.

For the construction of biquadratic equations, sec Construction. See also Descartes's Geometry, with the Commentaries of Schooten and others; Baker's Geometrical Key; Slusius's Mcsolabium; l'Hnspital's Conic Sections; Wolfius's Elcmenta Matheseos; \&c.

Biqua dratic Parabola, a curve of the 3 d order, having two infinite legs, and expressed by one of these threc equations, viz,

$a^{3} x=y^{4}$, as in fig. 1 ,

$a^{3} x=y^{4}-a^{2} y^{2}$, as in fig. 2 ,

$a^{3} x=y^{4}-(a+b) \cdot y^{3}-a b y^{2}$, as in fig. 3 ;

where $x=A \mathrm{P}$ the absciss, $y=\mathrm{PQ}$ the ordinate, $b=\Lambda \mathrm{B}$, $\mathrm{c}=\mathrm{AC}$, and $a=$ a certain given quantity.
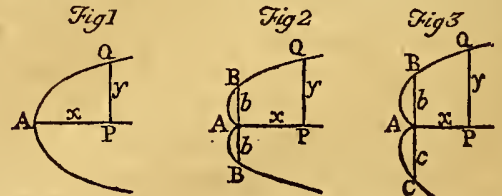

But the most general equation of this curve is the following, which belongs to fig. 4 , viz,

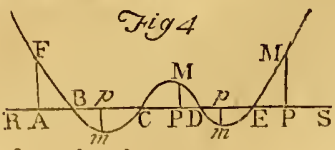

$a y=x^{4}+b x^{3}+c x^{2}+d x+c$;

where $x=\wedge p$ or $\Lambda \mathrm{p}$ the absciss, and $-y$ or $+y$ is the ordinate $p m$ or $p M$, also $a, b, c, d, e$, are constant quantities; the beginning of the absciss being at any point $\mathrm{A}$ in the indefinite line $a p$.

But if the beginning of the absciss $A$ be at the point where this line intersects the curve, as in fig. 5 , then the 

B I S
[ 235$]$
B L A

nature of the curve will be defined by this equation $a \times$ $p m=\Lambda p \times$ в $p \times c p \times \mathrm{D} p$, wherever the point $p$ is taken in the infinite line $\mathrm{RS}$.
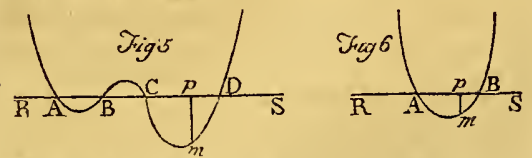

When the curve has no serpentine part, as fig. 6 , the equation is more simple, being in this case barely $a \times p m$ $=A p^{2} \times p B^{2}$. See Curve-Lines, and Geometrical Lines.

BIQUINTILE, an aspect of the planets when the distance between them is 144 degrees, or twice the 5 th part of 360 degrees.

BISECTION, or Bissection, the division of a quantity into two equal parts, otherwise called bipartition.

BISSEXTILE, or Leap-year, a year consisting of 366 days, and happening cvery 4 th year, in consequence of the addition of 1 day to the month of February, when that month consists of 29 days. And this is done to compensate for the 6 hours which the sun takes up nearly in his course, more than the 365 days commonly allowed for it in other years.

The day thus added was by Julius Cæsar appointed to be the day before the 24th of February, which among the Romans was the 6 th of the calends, and which on this occasion was reckoned twice; whence it was called the Bissextile.

By the statute De anno bissextile, $21 \mathrm{Hen} .3 \mathrm{~d}$, to prevent misunderstandings, the intercalary day and that next before it are to be accounted as one day.

To find what year of the period any given year is ; divide the given year by 4 ; then if 0 remains, it is leap-year; but if any thing remain, the given year is so many after leap-year.

But the astronomers concerned in reforming the calendar in 1582, by order of Pope Gregory the 13th, observing that in 4 years the bissextile added 4.4 minutes more than the sun spent in returning to the same puint of the ecliptic; and computing that in 133 years these supernumerary minutes would form a day; to prevent any changes being thus insensibly introduced into the scasons, directed, that in the course of 400 years there should be three sextiles retrenched; so that every centesimal year (which is a leapyear according to the Julian account), is a common year in the Gregorian account, unless the number of centuries can be divided by 4 without a remainder. So 1600 and 2000 are bissextile; but 1700,1800 , and 1900 are common years.

The Gregorian computation has been reccived in most foreign countries ever since the reformation of the calendar in 1582; excepting some northern countries, as Russia, \&c. And by act of parliament, passed in 1751 , it commenced in all the dominions under the crcwn of Great Britain in the year following; it being ordered by that act that the natural day next following the $2 \mathrm{~d}$ of September, should be accounted the 14th; omitting the intermediate 11 days of the common calendar. The supernumerary disy, in leap-years, being added at the end of the month of February, and called the 29th of that month.

The centesimal year 1800 having been a common year, instead of a bissextile, from that time there are now 12 days difference between the old and new style, and so will continue till the year 1900, when the difference between will be one day more, and so on, at every centesimal year which is not exactly divisible by 4 .

$\mathrm{BLACK}$, a colour so called, or rather a privation of all colour. This, it appears, arises from such a peculiar texture and situation of the superficial parts of a black body, that they absorb all or most of the rays of light, reflecting little or none to the eye; and hence it happens that black bodies, thus imbibing the rays, are always found to be hotter than those of a lighter colour. Dr. Franklin observes that black clothes heat more, and dry snoner in the sun than white clothes; that therefore black is a bad colour for clothes in hot chimates; but a fit colour for the linings of ladies'summer hats; and that a chinmey painted black, when exposed to the sun, will draw more strongly. Franklin's Experin. \&c.-Dr. Watson, the present bishop of Landaff, covered the bulb of a tbermometer with a black coating of lndian ink, and the consequence was that the mercury rose 10 degrees higher. Philos. Trans. vol. 63, pa. 40 ; and the same thing has since been evinced by the experiments of Mr. Leslie and other philosoplers.-And a virtuoso of some credit assured Mr. Boyle, that in a hot climate by blacking the shells of eggs, and exposing them to the sun, he had scen them thus well roasted.

BLACKNESS, the quality of a black body, as to colour; arising from its stifling or absorbing the rays of light, and reflecting little or none. In which sense it stands directly opposed to whiteness; which consists in such a texture of parts, as indifferently reflects all the rays thrown upon it, of whatever colour they may be.

Descartes, it appears, first rightly distinguished these causes of black and white, though he might be mistaken with respect to the general nature of light and colours.Sir Isaac Newton shows, in his Optics, that to produce black colours, the corpuscles must be smaller than for exhibiting those other colours; because, where the sizes of the component particles of a body are greater, the light reflected is too much for constituting this colour; but when they are somewhat smaller than is requisite to reflcct the white, and very faint blue of the first order, they will reflect so small a portion of light, as to appear intensely black; and yet they may perhaps reflect it variously to and fro within then so long, till it be stifled and lost.

And hence it appears, why fire, or putrefaction, by dividing the particles or substances, turn them black; why small quantities of black substances impart their colours very freely, and intensely, to other substances, to which they are applied; the minute particles of these, on account of their very great number, easily overspreading the gross particles of others. Hence it also appears, why glass ground very elaborately on a copper-plate with sand, till it be well polished, makes the sand, with what is rubbed off from thecopper and glass, become very black; also why blacks commonly incline a little towards a blueish colour; as may be seen by illuminating white paper with light refecteri from black substances, when the paper usually appears of a blueish white; the reason of which is, that black borrlers on the obscure blue of the first order of colours, and therefore refects more rays of that colour than of any other; and lastly, why black substances sooner than others become hot in the sun's light, and burn; an eflect which may proceed partly from the multitude of refractivis in a little space, and partly from the easy commotion among such minute particles.

BLAGRAVE (JoHN), an eminent mathenatician, who 2112 
flourished in the 16 th and 17 th centuries. He was the second son of John Blagrave, of Bulmarsh-court, near Sunning in Berkshire, descended from an ancient family in that country, From a grammar-school at heading he was sent to St. John's-college in Oxford, where he applied himself chiefly to the study of mathematics. From hence he retired to his patrimunial seat of Southcote-lodge, near Reading, where he spent the rest of his life, in a retired manner, without marrying, that he might have more leisure to pursue his favourite studies; which he did with great application and success. After a life thus spent in study, and in acts of benevolence to all around him, he died in the year 1611; and was buried at Keading in the church of St. Lawrence, where a sumptuous monument was erected to his memory.

He left the bulk of his fortune to the posterity of his three brothers, which were very numerous. There have been mentioned various acts of his beneficence in private life, for the encouragement of learning, the reward of merit, and the relief of distress. Some of these were the result of a quaint, humorous disposition, discovered chiefly in his legacies. One of these was 10 pounds left to be annually disposed of in the following manner: On Good-friday, the churchwardens of each of the three parishes of Reading send to the town-hall " one virtuous maid who has lived five years with her master;" there, in the presence of the magistrates, these three virtuous maids throw dice for the 10 pounds. The year following the two losers are returned with a fresh one, and again the third year, till each has had three chances. He also left an annuity to 80 poor widows, who should attend annually on Good-friday also, and hear a sermun, for the preaching of which he left 10 shillings to the minister. He took care also for the maintenance of his servants, rewarding their diligence and fidelity, and providing amply for their support. Thus it appears he was not more remarkable for his scientific knowledge, than for his generosity and philanthropy. His works are,

1. A Mathematical Jewel. Lond. 1585 , folio.

2. Of the Making and Use of the Familiar-Staft. Lond. 1590 , tho.

3. Astrolabium Uranicum Generale. Lond. 1596, 4to. 4. The Art of Dyalling. Lond. 1609 , 4to.

BLAIR (ЈоHN), an eminent chronologist, was born and educated at Edinburgh. Afterward, coming to London, he was for some time usher of a school in Hedge-lame. In 1754 he first published The Chronology and Histury of the World, from the Creation to the year of Christ 1753 , illustrated in 56 tabl's. In 1755 he was elected a fellow of the Royal Society, and in 1761 of the Suciety of Antiquaries. In 1756 he published a $2 \mathrm{~d}$ edition of his Chronological Talsles, and in 1768 an improved edition of the same, with the addition of 14. Maps of Ancient and Modern Geugraphy, for illustrating the Tables of Chronolegy and History; to which is prefixed a Dissertation on the Progress of Geoggraphy. In 1757 he was appointed chaplain to the Princess Dowager of Wate's, and Mathematical Tutur to the 1)uke of York; whom he attended in 17 (i3 in a tour to the continent, from which they returned the year after. Dr. Bhair has successively several good cluturch preferments: as a prebendal stall at W'estminster, the vicarage of Ilinckley, and the rectory of Burton Cougeles in Lincohnshire, all in 176t; the vicarage of St. Brile's in London, in $177 \mathrm{t}$, in exchange fir that of llinckley; the rectury of St. John the Evangelist in Westminster, in 1776 , in exchange for the vicarage of St. Bride's; in the same year the rectory of Horton near Colebrooke, Bucks. Dr. Blair died the 24th of June, 1782.

BLIND, an epithet applied to an animal deprived of the use of eyes; ur one from whom light, colours, and all the glorious objects of the visible creation, are interccpted by some natural or accidental cause.

BLINDNESS; a privation of the sense of sight. The ordinary causes of blinduess are, some external violence, viciuus conformation, growth of a cataract, gutta serena, small-pox, \&c; or a decay of the optic nerve; an instance of which we have in the Academy of Sciences, where, on opening the eye of a person long blind, it was found that the optic nerve was extremely shrunk and decayed, and without any medulla in it. 'The more extraordinary causes of blindness are malignant stenches, puisonous juices dropped into the eye, baneful vermin, long confinement in the dark, \&c.

We find various compensations for blindness, or substitutes for the use of eyes, in the wonderful sagacity of many blind persons, related by Zaphnius in his Uculus Artificialis, and others. In some the defect has bern supplied by a most excellent gift of memory; others by a delicate nose or the sense of sinelling; others by a very nice ear; and others again by an exquisite touch, or sense of feeling, which they bave possessed in great perfection.

Some have been able to perform many sorts of curious works in the nicest and most dexterous manner. Aldrovandus speaks of a sculptor, who liad become blind at 20 years of age, and yet 10 years afterwards he made a perfect marble statue of Cusmo II de Medicis, and another uf clay like pope Urban viry. Barthulin speaks of a blind sculptor in Denmark, who, by mere touch, clistinguished perfectly well evcry kind of wood, and even colours; and father Grimaldi relates an instance of the same kind; besives the blind organist, lately living in Paris, who it was saisl did the same thing. What seems more extraortinary still, we are told by authors of good report, of a blind guide, who used to conduct the merchants through the sands and deserts of Arabia; and a not less marrcllous instance is now existing in this country, in owe John Metcalf, near Manchester, who became quite blind at a very early age; and yet passed many years of his life as a waggoner, and occasionally, as a guide in different roads during the might, or when the paths were covered with snow; and, what is stranger still, his present occupation is that of surveyor and projector of highways in difficult and mountainotis parts, particularly about Buxton, and the Peak in Derbyshire.

There are also many instances of blind men who have been highly distinguisbed for their mental and literary talents, not to speak of the poets Homer, Milton, (ssian, \&e; of these we have a remarkable instance in the late Dr. Sanderson, professor of mathematics in the unisessity of Cambridge, and in Dr. Ilenry Moyes, public hecturer in phitosophy, who both of them lost their sight by the-smallpox at an age before they had any recollection; hese men were well thilled in all branches of the matho matice philosophy, optics, sce, which they tateht with the preatest reputation; bo sides the nomunu of lane which the lormer has left behind him in his mathematical and phitosophical works. Another instance of these sery cerratratinary characters is likewise eriuced in the prestit day, in Mr. John Gough of Niddleshow, near Kendal, who, though blind trom his infancy, has neverthetess acquired not only a very extensive knowledge in almust all the 
branches of the mathematics, but also in botany and chemistry.

The effects of a sudden recovery of sight in such as have been born blind, are also very remarkable. Devoid of the experience of distance and figtre arising frorn sight, they are liable to the greatest mistakes in this respect; so much that it has been said they could not distinguish by the mere sight between a cube and a globe, without first touching them. Mr. Boyle nentions a gentleman that was blind, who having been restored to sight at 18 years of age, was nearly distracted with joy. See Boyle's works abridg. vol.1, p. 4. See also a remarkable case of this kind in the Tatler, No. 55, vol. 1. And the gentleman couched by Mr. Cheselden had no ideas of colour, shape, or distance: though he knew the colours asunder in a good light during his blind state, yet when he saw them after he had been couched, the faint ideas he had of them before, were not sufficient for discriminating them afterwards. As to distance, his ideas were so deficient, that he thought all the objects he saw touched his eyes, as what he felt did his skin; and it was a considerable time before be could remember which was the dog and which the cat, though often infurmed, without feeling then.

BLI $\backslash D S$, or BLINDES, in Fortification, a kind of defence usilally made of oziers or branches interwoven, and laid across between two rows of stakes, about a man's height, and 4, or 5 feet asunder. They are used particularly at the heads of trenches, when these are extended in front towards the glacis, serving to defend the workmen, and prevent the enemy from overlooking them.

BLOCKADE, is the blocking up of a place by posting troops in all the avenues and roads leading to it, to prevent farther supplies of men and provisions to the besieged, and thus starving them out, without forming any regular siege or atticks.

BLONDEL (Francis), a celebrated French mathematician and military engineer, was born at Ribemond in Picarly in 1617. While he was yet but young, he was chosen Regius Professor of Mathemutics and Architecture at Paris. Not long after he was appointed governor to Lewis-Henry de Lomenix, Count de Brienne, whom he accompanied in his travels from 1652 to 1655 , of which he published an uccount. He enjoyed many honourable employments, both in the navy and army; and was intrusted with the management of several negociations with foreign princes. He arrived at the dignity of marshal de camp, and counsellor of state, and had the hohour to be appointed mathematical preceptor to the dauphin. He was a member of the Royal Academy of Sciences, director of the Academy of Architecture, and lecturer to the Royal, College, in all which he supported his character with dignity and applause. Blondrl was no less versed in the knowledge of the belles lettres than in the mathematical sciences, as appears by the comparison he published between Pindar and Horace. He dierl at Paris the 22d of February 1686, in the 60 th year of his age. 1 lis chirf mathematical works were,

1. Cours d'Architecture. P'aris, 1675 , in folio.

2. Resolution de's Quatre l'rincipaux Problemes d'Architecture. Paris, 1676 , in folio.

3. Histoiredn Calcudrier Romain. Paris, 1682 , in 4 to.

4. Cours de Mathematique's. P’aris, $16 \mathrm{~s} 3$, in 4 to.

5. L'Art de jetter de's l3ombes. La llaye, 1655 , in tito.

Besides $\Lambda$ New Mlcthod of fortifying l'laces, and other works.
Blondel had also many ingenious pieces inserted in the Memoirs of the French $\Lambda$ cademy of Sciences, particularly in the year 1666 .

BLOW, in a general sense, denotes a stroke given either with the hand, a wcapon, or an instrument. The efleet of a blow is estimated like the force of percussion, and expressed also by the velocity of the body multiplied by its weight.

BLOWING of a Fire-arm, is when the vent or touchhole is run or gullied, and becomes wide, so that the powder will flame out.

BLUE, one of the seven primitive colours of the rays of light, into which they are divided when refracted through a glass prism. See Newton's Optics, \&c. See also CuroMATICS.

BLUENESS, that quality of a body, as to colour, from which it is called blue; depending on such a size and texture of the parts that compose the surface of a body, as disposes them to reflect only the blue or azure rays of light to the eye.

The blueness of the sky is thus accounted for by De la Hire, after Da Vinci; viz, that a black bocly viewed through a thin white one, gives the sensation of blue, like the immense-expanse viewed through the air illuminated and whitened by the sun. For the same reason he says it is, that soot mised with white, makes a blue; for that white bodies, being always somewhat transparent, when mixing with a black behind, give the perception of blue. From the same principle too he accounts for the bluencss of the veins on the surface of the skin, though the blood they are filled with be a deep red.

In the same manner was the blue colour of the sky accounted for by many other of the earlier writcrs, as Fromondus, Funccius, Otto Guericke, and many others, with several of the more modern writers, Wolfus, Muschenbroek, \& c. But in the explication of this phenomenon by Newtun, he observes, that all the vapours, when they begin to condense and coalesce into natural particles, become first of such a magnitude as to reflect the azure rays, before they can constitute clouds of any other colour. This being therefore the first colour they begin to reflect, nust be that of the finest and most transparent skies, in which the vapours are not yet arrived at a grossness sufficient to reflect other colours.

Bouguer however ascribes this blueness of the sky to the constitution of the air jtseli, being of such a nature that these fainter-coloured rays are incapable of making their way through any considerable tract of it. And as to the blue shadows which were first ubsurved by Butfon in the year 1742 , he accounts for them by the acrial colour of the atmosphere, which enlightens these shadows, and in which the blue rays prevail; wbile the red rays are not reflected so soon, but pass on to the remoter regions of the atmosphere. Aurd the Abbe Mazeas accounts for the phenomenon of blue shadows by the diminution of light; observing that, of two shadows which were cast upon a white wall from an opaque body, illuminated by the moon and by a candle at the same time, that from the candle was somewhat red, while the other from the moon was blue. See Newton's Optics, pa. 228 ; Buuguer 'l'raté d'Optique pa. 36 ; ; lidinb. Ess. vol. 2, pa.75; or Priestley's Hist. of Vision \&ce, pa. 436 .

BLUNDERBUSS, in the Military Art, a short kind of fire-arm, with a large bore, contrived to carry a number of pistol or musket bullets at once. The blunderbuss is proper 
to do execution in a crowd, or to make good any narrow passage.

BLUNDEVILLE (Thomas), of Newton Flotman, a writer on navigation near the end of the 16 th century. In his Briefe Description of Universal Mappes and Cardes, first printed in 1589 , he gives an account of Gerrard Mercator's universal map, observing that Bernard Putean of Bruges had published in 1579, one altogether like it. And though Blundeville is so particular, as to set down numbers expressing the distances between each parallel of latitude in those maps, yet he seems to slight them, by saying, that no better rules than those given by Ptolemy can be devised: but, in the after editions of his book, Blundeville omitted the commendation he had given of Ptolemy's method of delineating an.universal map. But Blundeville's chief work seens to be his Exercises, written in 1593, the 4th edit. in 1613, 4to, 800 pages, containing treatises of Arithmetic; tables of Sines, Tangents, and Secants; Cosmographic; Description and Ise of the Globes, Mappes, Cardes, Astrolabe, Navigation, \&c.

$\mathrm{BOB}$ of a Pendulum, the same as the ball or weight; "hich see.

BODY, in Geometry, is a figure conceived to be extended in all directions, or what is usually said to consist of length, breadth, and thickness; being otherwise called a Solid. A body is conceived to be formed or generated by the motion of a surface; in the same manner as a surface is by the motion of a line, and a line by the motion of a point.-Similar bodies, or solids, are in proportion to each other, as the cubes of their like sides, or linear dimensions.

BoDr, in Physics, or Natural Pbilosophy, is a solid, extended, palpable substance; of itself merely passive, or rather being tenacious either of motion or rest, but capable of any sort of motion or figure.

Body is composed, according to the Peripatetics, of matter, form, and privation; according to the Epicureans and Corpuscularians, of an assemblage of hooked, heavy atoms; according to the Cartesians, of a certain extension; and zccording to the Newtonians, of a system or association of solid, massy, hard, impenetrable, moveable particles, ranged or disposed in a certain manner; from which arise bodies of various kinds, and distinguished by particular names: these elementary or component particles of bodies, they assert, must be perfectly hard, so as never to wear or break in pieces; which, Newton observes, is necessary, in order to the world's persisting in the same state, and bodies continuing of the same nature and texture in several ages.

BoDY of a plece of Ordnance, the part contained between the centre of the trunnions and the cascabel. This should always be more fortified or stronger than the rest, to sustain the first or greatest effort of the powder. See $\mathrm{C}_{\mathrm{A} \text { N }}$ on.

Bony of the Place, in Fortification, dewotes either the buildings inclosed, or more generally the inclosure itself. Thus to construct the body of the place, is to fortify or inclose the place with bastions and curtains.

BODY of a I'ump, the thickest part of the barrel or pipe of a pump, within which the piston moves.

Boviss, Regular or Platonic, are those which have all their sides, angtes, and planes, similar and equal. Of these there are only 5 ; vie,

the Tetraedron, contained by 4 equilateral triangles; the Hexaedron or cube, by 6 squares;

the Uctaedruis, by 8 triangles;

the Dodecaedron, by 12 pentagons; and

the leositedron, by 20 triangles.
To form the five Regular Bodies.

Let the annexed figures be exactly drawn on pasteboard, or stiff paper, and cut out by the extreme or bounding lines: then cut the others, or internal lines, only half through, so that the parts may be turned up by them, and then glued or otherwise fastened together with paste, sealing-wax, \&c; so shall they form the respective body marked with the corresponding number; viz, $\mathrm{N}^{0} 1$ the tetraedron, $\mathrm{x}^{0} 2$ the hexaedron or cube, $\mathrm{N}^{0} 3$ the octaedron, $\mathrm{N}^{0}+$ the dodecacdron, and $\mathrm{N}^{0} 5$ the icosaedron.
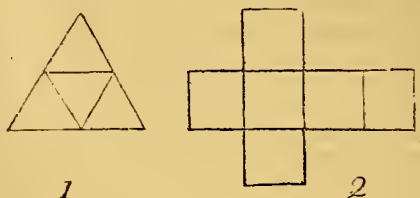

1
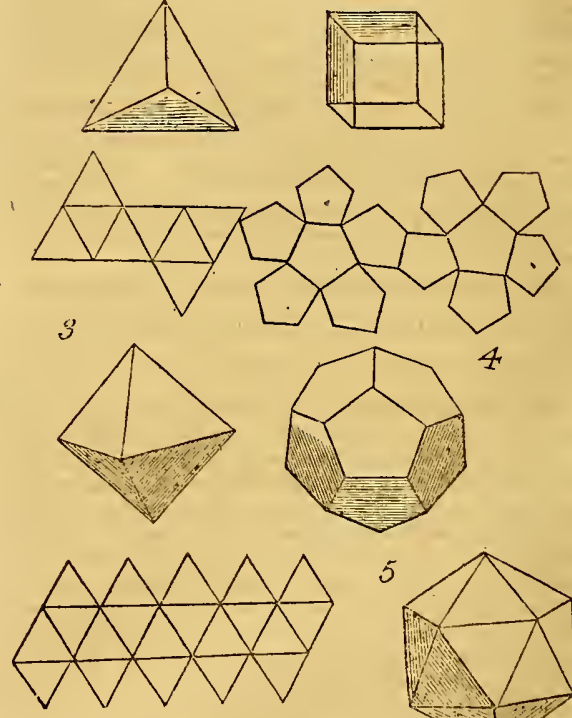

4

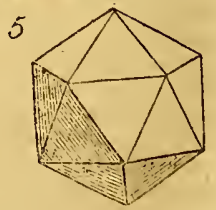

To find the Superficies or Solidity of the Regular Bodies.

1. Multiply the proper tabular area (taken from the following table) by the square of the linear edge of the solid, for the superficies.

2. Multiply the tabular solidity by the cube of the linear edge, for the solid content.

\begin{tabular}{|c|c|c|c|}
\hline \multicolumn{4}{|c|}{$\begin{array}{c}\text { Table of the Surfices and Solidities of the live liegulat } \\
\text { Bodies, the linear edge being } 1 .\end{array}$} \\
\hline No,of Faces & Names & Surfaces. & solidities \\
\hline 4 & Tetrikedron & $1 \cdot 73205$ & $0 . \overline{1785}$ \\
\hline 6 & Hexacdron & $6 \cdot 00000$ & 1.00000 \\
\hline 8 & Octacelron & $3+46+10$ & $0 .+71+0$ \\
\hline 12 & Dodecaedron & $20 \cdot 6.4573$ & 7.66312 \\
\hline 20 & lcosacklron & s.660025 & 2.18169 \\
\hline
\end{tabular}


For more particular properties, sce each respective word. See also my large Mensuration, pa. 185, edit. 4.

These bodies were called Platonic, because they were said to have been invented, or first treated of, by Plato, who conceived certain mysteries annexed to them.

Bodies, Descent of. See Acceleration.

BOETHIUS, or BOETIUS, (А.м.т.s.), a Roman philosopher and mathematician, was descended of a patrician family, and born at Padua in the 5 th century. He studied at Athens, where he adopted the opinions of Aristotle; and, returning to Rome, was raised to the rank of senator and patrician, and even to the consulate, in 487 . On the entry of Theodoric, king of the Goths into Rome, 500 , Boethius pronounced his panegyric, in the name of the senate. He was consul in 510 and 511 ; but, in 523, having remonstrated against the violence of Theodoric, he was accused to that prince of having conspired with the emperor Justin against the Goths; and, being seized with his father-in-law Symeuachus, he was conducted to Padua, and there beheaded by order of Theodoric, in 524, after 6 months' inprisonment.--Besides some books on mystical divinity, Boethius wrote 5 books on the Consolation of Philosuphy, said to have bcen composed to soften the rigour of his confinement ; a work wlich has been translated into most other languages, particularly into AngloSaxon, by the illustrious Alfred. It seems that in his time they had the use of the Arabian arithmetic, with their ten numeral characiers, which he employed, and from which our present numerals have been derived by several successive variations.

BOFFRAND (GERMAIN), a celebrated French architect and engineer, was born at Nantes in Bretagne, in 1667. He was brought up under Harduin Mansarad, who trusted lim with conducting his greatest works. Boffrand was admitted into the French Acallemy of Architccture in 1709. Many German princes chose him for their architect, and raised considerable edifices on his plans. His manner of building approached that of Paliadio; and there was much of grandeur in all his designs. As engineer and inspector-general of bridges and higlwways, he directed and constructed a number of canals, sluices, bridges, and other mechanical works. He published a curious and useful book, containing the general prisciples of his art; with an account of the plans, profiles, and elevations of the principal works which he executed in France and other countries. Boffrand died at Paris in 1755, dean of the Academy of Architecture, first congineer and inspector-general of the bridges and highways, and architect and administrator of the general hospital.

BOLLING, or EBullition, the bubbling up of any fluid by the application of heat. This is, in general, occasioned by the discharge of an elastic vapour through the fluid, which is then said to boil; and the same appearance is observed, whether it be that of common air, fixed air, or steam, \&ec. It is proved by Dr. Hamilton of Dublin, in his lissay on the ascent of vapour, that the boiling of water is occasioned by the lowermost particles of it being heated and rareficl into vapour, or steam ; in consequence of this climinution of their specific gravity, they ascend through the surrounding heavier fluid with great velocity, agitating and throwing up the body of water in their ascent, and giving it the tumultuous motion called boiling.

That this is occasioned by clastic steam, and not by particles of fire or air, as some have imagined, is easily proved by the following simple experiment: Take a common drinking-glass filled with hot water, and invert it into a vessel of the same; then, as soun as the water in the vessel begins to böil, large hubbles will be seen to ascend in the glass, by which the water in it will be clisplaced, and there will soon be a continued bubbling from under its edge: but if the glass be then drawn up, so that its mouth may just touch the water, and a cloth wetted in cold water be applied to the outside, the elastic stean within it will be instantly condensed, on which the water will ascend so as nearly to fill it again. Some small parts of air \& c, that may happen to. be lodged in the fluid, may also perhaps be expelled, as well as the rarefied stean. And this is particularly recommended as a method of purifying quicksilver, for more accurately making barometers and thermometers. If a vessel cuntaining water be placed over a steady fire, the water will grow continually hotter, till it reaches the limit of boiling; after which, the regular accessions of heat are wholly speut in converting it intosteam, or the steam carries off the heat as fast as it is generated by the fire. The water therefore remains at the same degree of temperature, however fiercely it may boil; the only difference is, that with a strurg fire it sooner boils, and more quickly evaporates.' Hence the reason why a vessel full of water, and plunged into the centre of a larger one, which is likewise filled with that fluid, barely acquires the boiling heat, but will never actually boil.

We commonly annex the idea of a certain very great degree of heat to the builing of liquids, though often without reason; for different liquids boil with different degrees of heat; and any one given liquid also, under different pressures of the atmospliere. Thus, a vessel of tar being set over the fire till it boils, it is said a person may then put his hand into it without injury: and by putting water under the receiver of an air-pump, and applying the flame of a candle or lamp under it, it will be found, by gradually exhausting the receiver, that the water is made to boil with less and less degrees of heat : and without applying any heat at all, the water, or even the moistureabout the bottum or edlges of the receiver, will rise in an elastic vapour up into it, when the exhaustion is nearly completed.

Spirit of wine buils still sooner in vacuo than water. And Dr. Freind gires at table of the different times required to make several fluids boil by the same heat. See also Philos. Trans. N0 122.

Every particular liquid has a fixed point of the thermometer, at which boiling takes place, and this is called the boiling point of the liquid. Thus, water begins to boil when heated to 212 degrees of Fahrenheit's scale. A strong heat makes it boil more rapidly, but roes not increase its tefnperature: a fact which wasfirst observed by Dr. Hooke. The annexed table shows the boiling puint of a number of liquids, under the common pressure of 30 inches of the barometer; for the heat and boiling point are found to depend on the degree of pressure upon the liquid. When the pressure is diminished, the liquid boils at a lower temperature; when it is incrcased, a ligher temperature is neces. sary to produce cbullition. From the expcriments of Professor Robinson, it appears that, in a

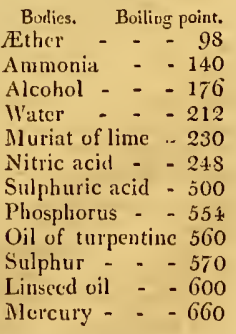
vacuum, all liquids boil at about $1+5^{\circ}$ lower than in open 
air, under a pressure of 30 inchcs of mercury: therefore water would boil in vacuo at $67^{\circ}$ and alcohol at $34^{\circ}$. In Papin's digester, the temperature of water may be raised to 300 or 400 degrees, without ebullition; but the moment that the pressure is removed, the boiling commences with the greatest violence.

The mix ture of various salts with water affects its boiling point considerably; as appears from Mr. Achard's experiments recorded in the Berlin Memoirs for 1785 . He found that a saturated solution of muriat of soda raised the boiling point 10.35 degrees; one of muriat of ammonia raised it 9.79 degrees; one of carbonat of potass raised it $11 \cdot 2$ degrees; while a small quantity of borax lowered it 1.35 degrees; ditto of sulphat of magnesia lowered it 2.47 degrees; and sulphat of lime in any proportion lowered it 2.02 degrees.

BOMB, in Artillery, a shell or hollow ball of cast-iron, having a large vent, by which it is filled with gun-powder, and which is fitted with a fuze or bollow plug to give fire by, when thrown out of a mortar, \&c. About the time when the shell arrives at the intended place, the composition in the pipe of the fuze sets fire to the powder in the shell, which blows it in pieces, to the great annoyance of the enemy, by killing the people, or firing the houses, \&c. They are now commonly called Shells simply, in the English artillery.

These shells, or bombs, are of various sizes, from that of 17 or 18 inches diameter downwards. But these very large ones are not used by the English, that of 13 inches diameter being the greatest size now employed by them; the weight, dimensions, and other circumstances of the 13-inch shells, and of those downwards, are as in the following table.

\begin{tabular}{|c|c|c|c|}
\hline $\begin{array}{l}\text { Diameter of } \\
\text { the Shell. }\end{array}$ & $\begin{array}{l}\text { Weight of } \\
\text { the Shell. }\end{array}$ & $\begin{array}{l}\text { Powder to } \\
\text { fill them. }\end{array}$ & $\begin{array}{l}\text { Powder to burst } \\
\text { them into most } \\
\text { pieces. }\end{array}$ \\
\hline 13 inch & $\begin{array}{l}\text { lbs. } \\
195\end{array}$ & $\begin{array}{cc}\text { tb. } & \text { oz. } \\
9 & 4 \frac{x}{2}\end{array}$ & $\begin{array}{cc}\text { lb. } & \text { oz } \\
7 & 8\end{array}$ \\
\hline 10 & 89 & $4 \quad 14 \frac{\pi}{2}$ & 3 \\
\hline 8 & 46 & $2 \quad 3 \frac{\pi}{2}$ & 0 \\
\hline $5 \frac{4}{6}$ Royal & $14 \frac{1}{2}$ & $1 \frac{\pi}{2}$ & $0 \quad 14$ \\
\hline $4 \frac{3}{3}$ Cohorn & $7 \frac{2}{2}$ & 08 & $0 \quad 7$ \\
\hline
\end{tabular}

Mr. Muller gives the following proportion for all shells. Dividing the diameter of the mortar into 30 equal parts, then the other dimensions, in 30ths of that diameter, will be thus:

Diametcr of the bore, or mortar - - 30

Diameter of the shell - - - - $\quad$ - $29 \frac{1}{2}$

Diameter of the bollow sphere - - $\quad-21$

Thickness of metal at the fuze hole - - - $3 \frac{x}{2}$

Thickness at the opposite part - $\quad$ - 5

Diameter of the fuzo hole - - - - 4

Wright of sheil empty - - - - $\quad x_{10}^{x} d$

Weight of powcler to fill it - . - - $\frac{2}{473} d$ where $d$ denotes the cube of the diameter of the bore in inches.-But shells have also lately been made of the same thickness of metal throughout.

In general the windage, or difference between the diameter of the shell and mortar, is $\frac{r}{6}$ of the latter; also the diameter of the hollow part of the shell is $T^{7}$ of the same.

Bumbs are thrown out of mortars, or howitzers; but they may also be thrown out of cannon; and a very small sort are thrown by the hand, which are called granados: and the Venetians at the siege of Candia, when the Turks had possessed themselves of the ditch, used large bombs without any piece of ordnance, barely rolling them down upon the enemy along a plank set aslope, with ledges on the sides to keep the bomb directly forward.

Mr. Blondel, in his Art de jetter des Bombes, says the first bombs were those thrown into the city of Watchtendonch in Guelderland, in 1588 ; and they are described by our countryman Lucar, in his book on Artillery published this same year 1588; though it is pretended by others that they were practised near a century beiore, namely at the siege of Naples in 1495. They only came into common use, however, in 1634, and then only in the Dutch and Spanish armics. It is said that one Malthus, an English engineer, was sent for from Holland by Lewis the $14 \mathrm{th}$, who used them for him with much success, particularly at the siege of Cohoure in 1642.

The art of throwing bombs, or shells, constitutes one of the chief branches of Gunnery, founded on the theory of projectiles, and the quantities and laws of the force of gunpowder. And the principal writers on this art are Mess. Blondel, Guisuée, De Ressons, De La Hire, ¿c.

Bомь-Снest, is a kind of chest usually filled with bombs, and sometimes only with gunpowder, placed under ground, to be blown up into the air with those who stand upon it; being set on fire by means of a saucissee fastened at one end. But they are now much out of use.

BOMBARD, an ancient piece of ordnance, nuw out of use. It was very short and thick, with a large mouth; some of which threw balls of 300 pounds weight, requiring the use of cranes to load them. The Bombard is by some called basilisk, and by the Dutch donderbus.

To воMBARD, is to attack by throwing of bombs, or shells.

BOMBARDIER, a person employed in throwing bombs or shells. He adjusts the fuze, and loads and fires themortar.

BUND (HENRY), was an intelliwent mathematician, and a teacher of navigation at Ratcliff, near London, in the carly part of the 17 th century. In the course of his nautical concerns he interested himself much about the magnetical needle, its variation and inclination. The variation of the magnetic needle was first observed by John Moir, then by Edn. Guuter, next by Ilen. Gallibrand, and then by our author Bond. In the year 1657 there was no variation at London: before that time it had been eastwards, as observed by several of those persons, but gradually decreasing, or the needle moving westwards, till it pointed due north in that year 1657; ever since it bas been travelling westwards, but at unequal rates, till now, in 1812 , it has arrived at above $24^{\circ}$ west variation, and appears to be nearly stationary, after which it will prubably return eastward again; according to which rate of motion, the magnetic needle, or pole, appears to perform an entire circular revolution in about 620 years. Besides the changenble variation east and west, the ncedle was also found to have a changeable jnclination, or dip below the horizon; and on this last change it was that Mr. Bond founded $h$ is method of finding the longitude; whit however has failed, as might be expected. On this method he published a book, called The Longitude found, printed at Lonton 1676 , in 4 to. He here states, as his prediction, after many boasting andempirical pretensions, what would be the latitude, and longitude, and the inclination of the needle, in muny of the most noted places on the earth, in the year 1676 ; also another table of the latitude of the magnetic pole, answering to cvery 5 minutes in the inclination of the needle. He also had iuscrted in the Philos. 
Trans. No. 40, of the year 1668 , or pa. 282 , vol. 1, of my Abridgment, a table of the needle's variation at London, for every year from 1668 to 1716 , when he states it at $9^{0} 17 \frac{1}{2}^{\prime}$.

Dr. Birch states that Sir Charles Cavendish, brother to William, marquis, and afterwards duke of Newcastle, in an original letter of his from Antwerp, Nov, 13, 1648, to Mr. John Pell, then professor of matbematics at Breda, mentions Mr. Bond as an old mathematician at Lendorr, a humble man, who speaks, says he, very meanly of himself, and "yet be found an easy and short demonstration of that proposition concerning spherical triangles, which Mr. Oughtred demonstrated first, who told me, Mr. Bond's demonstration was shorter." Sir Charles adds, that Mr. Bond was in hopes of finding the longitude by the loadstone; and his treatise on that subject, entitled "The Longitude found," was printed at London 1676 , in 4 to.

Bond's scheme for the longitude was soon opposed, a book bcing published at London in 1678 , called " The Longitude not found," written by one Bcckborrow. And indeed as Bond's hypothesis did not in anywise answer its author's sanguine expectations, Dr. Halley also undertook this affair ; and from a multitude of observations concluded, that the magnetic needle was influenced by four poles. His speculations on this subject are in the Philos. Trans. No. 148 and 195. But this wonderful phenomenon seems to have hitherto eluded all researches. However, Dr. Halley, in 1700 , published a general map, on which were delineated curve lines, showing the patlıs where the magnetic ncedle had the same variation. The positions of these curves will indeed continually suffer alterations, requiring to be corrected from time to time, as they have since been for the years $174+$ and 1756 , by two ingenious persons, William Mountaine and James Dodson; the latter of whoun died not long after he had been chosen, for his merit, mathematical master at Christ's Hospital, in London.

RONES, Napier's. See Napler.

BONING, ir Surveying and Levelling, \&c, is the placing thrce or more rods or poles, all of the same length, in or upon the ground, in such a manner, that the tops of them be all in one continued straight line, whether it be horizontal or inclined, so that the eye can look along the tops of them all, from one end of the line to the other.

BONNET, in Fortification, a small work of two faces, having only a parapet, with two rows of palisadoes at about 10 or 12 feet distance. It is commonly placed before the salliant angle of the counterscarp, and having a communication with the covered way, by means of a trench cut through the glacis, and palisadoes on each side.

Bonnet à P'être, or Priest's Cap, is an outwork, baving three salliant angles at the head, besides two inwards. It differs from the double tenaille only in this, that its sides, instead of being parallel, become narrower, or closer, at the grrge, and opening at the front; from whence it is called Queue d'aronde, or swallow's tail.

BOO'TES, a constellation of the northern hemisphere, and one of the 48 old ones; having 23 stars in Ptolemy's catalngue, 28 in 'Tycho's, 34 in Bayer's, 52 in Hevelius's, and 54 in Flansteed's; of which one, in the skirt of the coat, is of the first magnitude, and called Arcturus.

Bootes is represcnted as a man in the posture of walking; his right hand grasping a club, and his left extended upwards, and holding the cord of the two dogs which scem barking at the Great Bear.

This constellation is called by various other names; as Vol. I.
Arcas, Arctophylax, Arcturns-Minor, Bubulcus, Bubulus, Canis-Latrans, Clamator, Icarus, Lycaon, Philometus, Plaustri-Custos, Plorans, Thegnis, and Vociferator ; by Hesychius it is called Orion, and by the Arabs, Aramech, or Archamech. Schiller, instead of Bootes, makes the figure of St. Sylvester; Schickhard, that of Nimrod; and Weigelius, the three Swedish crowns. See Wolf. Lex
Math. p. 266 .

BORDA (CharLES), a celebrated French mathematician and philosopher, died in 1799 , in the 64 th year of his age; in whom the National Institute, and the Parisian Board of Lorgitude, lost one of their most learned and active members. Borda very early gave proofs of his eminent mathematical talents; as appears by his many excellent tracts inserted in the Memoirs of the Royal Academy of Sciences, on a variety of abstruse subjects; on bydraulics, on the resistance of fuids, ou water-whcels, on pumps, on the projection of military sbells, and their resistarice by the air, \&c.

In 1771 and $177^{2}$, by order of the king, he accompanied La Crenne and Pingré, on a literary voyage to variuus coasts of Europe, Africa, and America, for improving the science of geography, and for trying several new nautical instruments, time-pieces, and methods of finding the longitude. The three travellers afterwards published conjointly, in 2 vols 4 to 1778 , an account of the fruits of their numerous researches; in which Borda's share was certainly not the smallest. The results of this expedition are also recorded in the Memoirs of the Academy for the year 1773 .

In the year 1787 , he published his much-esteemed Description and Use of the Circle of Reflection; in which he revived and recommended the use of the specular circles, which had been proposed in 1756 by Tobias Mayer. Borda was the founder of the schools of naval architecture in France: he first conceived the project, aud formed the plan' of instruction, with the regulations for those seminaries. By his exertions too, a uniformity in the building of the ships was introduced, according to the principles of Euler, by which improvement, an equality of sailing was effected in all the ships of the royal navy. Hence, the form of the French ships, buing constructed on mathematical principles, is clearly preferable to that of the ships of the other naval powers; being the most advantageons, and the best adapted for fast sailing, and for mancuvring.

Borda also brought into use Mayer's old method of measuring terrestrial arigles, applied it to astronomical obscrvations, and, fur that purposse, invented a new construction of circles, with double moveable telescopes, which have bcen used in the new measurement of degrees in France. He was also the inventor of the ingenious mensuration rod, with which the now station-lines were measured; and he had the chief share in the reform of wcights and meastres; of which he was so zealous a promoter, that he printed, at his own expense, Tables of Sines, \&c, on the decimal system. In 1792 he determined, with au accuracy perhaps never before attained, the lengtb of the pendulum vibrating seconds at Paris. In 1797, we find lis name in the list of candidates for the office of Director of the French Republic,

BURE, of a gun, or other piece of ordnance, is the chase, cylinder, or hollow part of the piece.

BOREAL Signs, are the first six signs of the Zorliac, or those on the northern side of the equinoctial; viz, the signs $r$ arics, $\gamma$ taurus, II gemini, go cancer, $\Omega \mathrm{lco}, \boldsymbol{p}_{2}$ virgo. 
BOrealis, Aurora. See Auror a Boteulís.

BORELLI (Joн A ALPHonso), a celebrated philosopher and mathematician, born at Naples the 28th of Jan. 1608. He was professor of philosophy and mathematics in some of the most celebrated universities of. 1taly, particularly at Florence and Pisa, where he became highly in favour with the princes of the house of Medicis. But having been concerned in the revolt of Messina, be was obliged to retire to Rome, where be spent the remainder of his life under the protection of Christina qucen of Sweden, who honoured him with her friendship, and by her liberality towards him softened the rigour of his hard fortune. He continuell two years in the convent of the regular clergy of St. Pantaleon, called the Pious Schools, where heinstructed the youth in the mathenatical sciences. And this study he prosecuted with great diligence for many years afterward, as appears by his correspondence with several ingenious mathematicians of his time, and the frequent mention that has been made of him by others, who have endeavoured to do justice to his memory. He wrote a letter to Mr. John Collins; in which he discovers his great desire and endeavours to promote the improvement of those sciences: he also speaks of his correspondence with, and great affection fur, Mr. Henry Oldenburg, Secretary of the Royal Society; of Dr. Wallis ; of the then late learhed Mr. Boyle, and lamented the loss sustained by his death to the commonivealth of learning. Mr. Baxter, in his Enquiry into the Nature of the Human Soul, makes frequent use of our author's book De Motu AnimaJiuim, and tells us, that he was the first who discovered that the forcc exêrted within thie body prodigiously exceeds the weight to be moved without, or that nature empioys an immense power to move a small weight. He acknowledges, however, that Dr. James Keil had shown that Borelli was misfaken in his calculation of the force of the muscle of the heart; but that he nèvertheless ranks him with the most authentic writers; and says he is seldom mistaken: and, having remarked, that it is so far from being true that great things are brought about by small poiver's, that, on the contrary, a stupendous power is manifest in the most ordinary operntions of nature, he observes that the ingenious Borelli first observed this in animal motion; and that Dr. Stephen Hales, by a course of experiments in his Vegetable Statics, had shown the same in the force of the ascending sap in vegetables. And this circunistance, that nature employs a vcry great power, or force, in the animal frame, to move a small iveight, we may here obscrve, is owing to the levers in the animal frame being all necessarily of the third kind, the power acting very near the centre of motion, and the weight at a comparatively great distance from it.

After a course of uncéasing labnurs, Borelli died at Pantaleon of a pleurisy, the 31 st of December 1679 , at 72 years of age.

Beside several books 'on physical subjects, Borelli published the following mathematical ones: viz,

1. Apollonii Pergæi Conicorum Lib. 5, 6, and 7. Floren. 1661 , fol.

2. Theoriz Medicorum Planetarun ex causis physicis deductæ. Flor. 1666, 4 to.

3. De Vi Percussionis. Bölogna, 1667, 4to.-This piece was reprinted, with bis colebrated treatise De Motu $\Lambda_{n i}$ malium, and that other De Motionibus Naturalibus, in 1686 .

4. Euclides Restitutus, \&c. Pisa, 1668, 4to.
5. Osservatione intorno alla vistu ineguali degli Occi-This piece was inserted in the Journal of Rome, for the yeas 1669.

6. De Notionibus Naturalibus de Gravitate pendentibus. Regio Julio, 1670,4 to.

7. Meteorologia Aetnea, \&c. Regio Julio, 1670, 4to.

8. Osservatione dell' Ecclissi Lunare, 11 Gennaro 1675. -Inserted in the Journal of Rome 1675 , p. 34.

9. Elementa Conica Apollonii Pergæi, et Archimedis Operi, nova et breviori methodo demonstrata.-Printed at Rome in 1679 , in $12 \mathrm{mo}$, at the end of the $3 \mathrm{~d}$ edition of his Euclides Restitutus.

10. De Motu Animalium. Pars prima in 1680, and Pars altern in 1681,4 to.- Thesc were reprinted at Leyden 1685 , revised and purged from many errors; with the addition of Jolnn Bernoulli's Mathematical Meditations concerring the Motion of the Muscles.

11. At Leyden, 1686 , in 4 to, a more correct and accurate edition, revised by J. Broen, M. D. of Leyden, of his two pieces, De Vi Percussionis, et De Motionibus de Gravitate pendentibus.

BORELLI (PETER), who fourished about the middle of the 16 th century, appears to have been a native of Middlcburg, capital of the island of Walcheren and all Zealand; and was coumsellor and physician in ordinary to his most Christian majesty. He wrote two treatises on the telescope and microscope, printed at the Hague, in 1655 . and 1650. The former is a very full and particular treatise on the history of the invention of those very uscful instruments; which, by a train of strong evidence he ascribes to Zacluariah Jansen, a spectacle-rnaher of Miduleburg, in. 1590. He gives a particular account of the pretensions. that have been ascribed to all the other persons that have been named as the inventors; the whole forming a very. interesting history of the invention. T'he chief body of evidence it seems he received from a $\mathrm{Wm}$. Borelli, of the sane. city, some time ambassador in Fingland; but whether any. relation of Peter's does not appear.

The first volume consists of two parts: the former treating of the invention jiself; and the latter of the fabric, construction, and uses of the instruments. The second volume contains the account and appearances of onc hundred minute objects, as viewed by the microscope. The volumes are both neatly printed in 4to, by Adrian Vlacq, at the Hague.

BOSCOVICH (Roger Josepr), an eminent mathematician and philosopher, was born May 11 th, 1711 , at Ragusa. He studied Latin grammar in the schools which were taught by the Jesuits in his native city, until 1725 , when, in consistence with a maxim of the Jesuits to send. their most eminent pupils to Rome for the completion of their education, he was removed to that city. After this be soon acquired very great reputation for lis eminent attainments in divinity and science: at three successive periods be became professor of mathematics and astronomy at Rome, at Pavia, and at Milan. When the order of Jesuits was suppressed he was invited to Paris, and received the place of clirector of the optical instruments of the marine. Previous to this however he had been employed, in conjunction with Majer, in measuring a clegree of the meridian in Italy, and correcting the maps of the Papal estate. Ile published, in 1755, an interesting account of the cxpedition in which these objects were effected. Ile had also been employed in adjusting a disagreeable affair between the republic of Lucca and the regency of 'Tuscany, and in a 
similar business between the republic of Ragusa and the court of Great Britain, which brought him to London, where he soon bećame acquainted with the most eminent of the English philusophers. During his stay he was elected a fellow of the Ruyal Sociéty, and he dedicated to that learned body his Poem on Eclipses, which contains a neat compendium of astronomy. He remained at Paris 10 years, where he was much respected; but, being a foreigner, his celebrity was envied: this; together with the irreligion which then prevailed among many of the French philosophers, was disagreeable to him, so that he obtained leave of absence to revisit his friends in Italy. The first place he resided at for any considerable length of time in Italy, was Bassano, where he published his Opuscules, in five volumes 4 to, composed in Latin, Italian, and French; and containing a variety of elegant and ingenious disquisitions connected with astronomical and optical science. From Bassano our author went to Rome, and thence to Milan, where he took up his abode; being in the neighbourhood of his favourite observatory at Brera. Here he continued to enjoy the pleasures of study, and occasionally the society of many respected friends. His unwillingness to leave Italy, and at the same time a solicitude to avoid the charge of ingratitude from the French nation, occasioned him great perplexity of mind, which was followed by deep melancholy, a disordered imagination, and at length direct madness. During the heat of delirium, he frequently exclaimed that be would die poor and inglorious. He bad indeed some short lucid moments, and once there were hopes of his recovery; but he soon relapsed, and an imposthume, breaking in his breast, put an end to his mortal life the 13th of Feb. 1787 , in his 76 th year. He was interred decently, but without pomp, in the parochial church of S. Maria Pedrone. "Such was the exit," says Fabroni, " of this sublime genius, whom: Rome honoured as her master; whoin all Italy regarded as her ornament; and to whom Greece would have erected a statue, had she for want of space been obliged even to throw down some of her heroes." The works of this memorable man are the following:-1. Elements of Mathematics, with a Treatise on Conic Sections. 2. His many Dissertations published during his professorship in the Roman College. 3. His Account of the Survey of the Pope's Estate. 4. A curious and elegant Poem on Solar and Lunar Eclipses. 5. The five volumes published at Bassano. 6. His Hydronamical Pjeces. 7. A Theury of Natural Philosophy.

BOUGUER (PETER), a celebrated French mathematician, was born at Croisic, in Lower Bretagne, the 10 th of Fcbruary 1698. He was the son of John Bouguer, Professor Royal of Hydrography, a tolerably good mathematician, and author of A complete Treatise on Navigation. Young Bouguer was accustomed to learn mathematics from his father, from the time he was able to spcak, and thus became a proficient in those scieuces while he was yet a child. He was sent very early to the Jesuits' college at Vannes, where he had the lonour to instruct lis regent in the rnathematics, at eleven years of age.

Two years after this be had a public contest with a professor of mathematics, on a proposition which the latter had advanced erroneously; and he triumphed over him; on which the professor, unable to bear the disgrace, left the country.

"I'wo years after this, whell young Bouguer had not yet finished hịs studies, he lost his father; whom lye was ap. pointed to succeed in his office of hydrographer, after a public examination of his qualifications; being then only 15 years of age; an occupation which he discharged with great respect and dignity at that early age.

At the age of 28 in 1727 he obtained the prize proposed by the Açademy of Sciences, for the best way of masting of ships. This first sụccess of Bouguer was soon after followed by tivo others of the same kind; he sucessively gained the prizes of 1729 and 1731 ; the former, for the best manner of observing at sea the height of the stars; and the latter, for the most advantageous way of observing the declination of the-magnetic needle, or the variation of the compass.

In 1729, he gave an Optical Essay upon the Gradation of Light; a subject quite new, in which he examines the intensity of light, and determines its degrees of diminution in passing through difierent pellucid mediums, and particularly that of the sun in traversing the earth's atmosphere. Mairan gave an extract of this first essay in the Journal des Savans, in 1730.

During this year, 1730, he was removed from the port of Croisic to that of Havre, which brought him into a nearer connection with the Academy of Sciences, in which he obtained, in 1731, the place of associate geometrician, vacant by the promotion of Maupertuis to that of pensioner; and in 1735 he was promoted to the office of pensioner-astronomer. The same year he was likewise sent on the commission to South America, along with Messieurs Godin, Condamine, and Jeussicu, to determine the measure of the degrees of the meridian, and the figure of the earth. In this painful and troublesome búsiness, of 10 years' duration, chiefly among the lofty Cordelier mountains, our author determined many other new circumstances, besides the inain object of the voyage; such as the expansion and contraction of metals and other substances, by the sudden and alternate changes of heat and cold among those mountains; observations on the refraction of the atmosphere from the tops of the same, with the singular phenomenon of the sudden increase of the refraction, when the star can be observed below the line of the level; the layss of density of the air at different heights, from observations made at different points of these enormous mountains; a determination that the mountains have an effect on a plummet, though he did not assign the exact quantity of it; a method of estimating the errors conmitted by navigators in determining their course; a new construction of the log for measuring a ship's way; with several other useful improvements.

Other inventions of Bouguer, made upon different occasions, were as follow: The heliometer, being a telescope with two object glasses, affording a good method of measuring the diameters of the larger planets with ease and exactness: his rescarches on the figure in which two lines or two long ranges of parallel trees apjear : his experiments on the famous reciprocation of the pendulum : and those upon the manner of measuring the force of light: \&c, \&.c.

The close application which Bouguer made to study, undermined his heal $\mathrm{h}$, and terminated his life the 15th of August 1758, at 60 years of age,-His chief works, that have bcen published, are,

1. The Figure of the Earth, determined by the observitions made in South America; 1749, in 4.10 .

2. Treatise on Navigation and Pilotage; Paris, 175\%, 212 
in 4 to. This work has been abridged by M. La Caille, in 1 volume, 8vo, 1768 .

3. Treatise on Ships, their Construction and Motions; in $4.0,1756$.

4. Optical Treatise on the Gradation of Light; first in 1729; then at now edition in 1760 , in 4 to.

His papers that were inserted in the Memoirs of the Academy, are very numerous and important: as, in the Memoirs for 1720, On the Comparison of the force of the solar and lunar light with that of candles.-1731, Observations on the curvilinear motion of bodies in mediums. 1732, Un the new curves called the Lines of Pursuit.1733, To determire the species of conoid, to be constructed on a given base which is exposed to the action of a fluid, so that the impulse may be the least possible.-Determination of the orbit of comets.- 1734, Comparison of the two laws which the carth and the other planets must observe under the figure which gravity causes them to take.On the curve lines proper to form the arches in domes. 1735, Observations on the equinoxes.-On the length of the pendulum. -1796 , On the length of the pendulum in the torrid zone.-On the manner of determining the figure of the earth by the measure of the degrees of latitude and longitude. -1739 , On the astronomical refractions in the torrid zonc-Observations on the lunar eclipse of the $s$ th of September 1757, made at Quito._-1744, Short account of the voyage to Pern, by the members of the Royal Academy of Sciences, to measure the degrees of the meridian near the equator, and from thence to determine the figure of the earth.-1745, Experiments made at Quito, and divers other places in the torrid zone, on the expansion and contraction of metals by heat and cold.-On the probletn of the masting of ships.-1746, Treatise $m$ ships, their structure and motions.-On the impulse of Auids upon the fore parts of pyramidoids baving their base a trapezium.-Continuation of the short account giren in 1744, of the voyage to Peru for measuring the earlh. -1747 , On a new construction of the $\log$, and other instruments for measuring the run of a ship.-1748, On the diameters of the larger planets. The new instrument called a Heliometer, used for determining them; with observations of the sun.-Observation on the eclipsc of the moon the 8 th of August 1748.-1749, Second meinoir on astronomical refractions, observed in the torrid zone, with remarks on the manner of constructing the tables of them,-Figure of the earth determined by $\mathbf{M} M$. Bouguer and Condamine, with an abridgment of the expedition to Peru. - 1750, Observation of the lunar eclipse of the 13 th of December $1750 .-1751$, On the form of bodies most proper to be assumed for their revolution, when they are urged at one of their extremities, or any other point.-On the moon's parallax, with the estimation of the changes caused in the parallaxes by the figure of the earth.-Observation of the Iunar eclipse, the $2 \mathrm{~d}$ of December $1751 .-1752$, On the operations made by seamen, called Corrections.-1753, Olservation of the passage of Mercury over the sun, the 6 th of May 1753.- On the dilatations of the air in the atmosphere.-New treatise of navigation, containing the theory and practice of pilotage, or working of ships. 1754, Operations, \&ce, for distinguishing, anong the different determinations of the degree of the mericlian neat Paris, that which ought to be preferred.-On the direction which the string of a plummet takes. - Solution of the chief problems in the working of ships. - 1755 , On the ap- parent magnitude of objects.-Second memoir on the chief problems in the working of ships. - 1757, Account of the treatise on the working of ships.-On the means of measuring the light.-1758, His Eulogy.

In the volumes of the prizes given by the acadeny, are the following pieces, by Bonguer:

In vol. 1, on the masting of ships.-Vol. 2, On the method of exactly observing at sea the height of the stars; and the variation of the compass. Also on the cause of the inclination of the planets' orbits.

BOULTINE, in Architecture, a convex moulding, of a quarter of a circle, and placed next below the plinth in the Tuscan and Doric capital.

BOURNE (WM.), was a useful and ingenious writer on navigation about the middle of the 16 th century. In 1567 was published what he calls "Rules of Navigation," as appears from his Almanac, printed in 1571 . And in 1577 was published his "Regiment for the Sea," designed as a supplement to Cortes, whom he often yuotes. Besides many things cominon with others, Bourne gives a table of the places and declinations of 32 principal stars, by which to find the latitude and hour; as also a larger tiric-table than that published by Leonard Digges, in 1556. He show's, by considering the irregularities in the momm's motion, the errors of the sailors in finding her age by the epact; as also in their determining the hour from observing on what point of the compass the sun and inoon appeared. He advises, in sailing towards high latitucles, to keep the reckoning by the globe; as there the plane chart errs most. He despairs of our ever being able to find the longitude by any instrument, unless the variation of the compass shoulat be catised by some such attractive point as Cortes hail imagined. Though of this he donbts: and as he had shown how to find the variation of the compass at alt times, he advises to keep an account of the observations, as useful to discover the place of a ship: which advice the famous Simon Stevin prosecuted at large, in a treatike published at Leyden in 1599, entitled "Portuum investigandorum Ratio Metaphrasto Hugone Grotio;" the substance of which was the same year printed at London, in English, by Edw. Wright, entitled " The Haven-finding Art."

But the most remarkable thing in this ancient tract is, the describing of the way by which our sailors estimated the rate a ship made in her course, by an instrument called the Log; an instrument so named from the wooden $\operatorname{lng}$ that floats in the water, while the time is reckoned during which the line fastened to it is veering out. The origin of this device is not known; and it secms no further mention was inade of it till 1607, in an East-India voyage, published by Purchas; but from that time its name occurs in other royages, among bis collections. And benceforward it was often noticed, by foreigners as well as our own authors; as by Gunter in 1623, Snellius in 1624, Metius in 1631, Oughtred in 1633, Herigone in 16.34, Saltonstall in 1636 , Norwood in 1637, Foumier in 1643; and inderd by most succeding writers on navigation of every country. And it still continues to be in use as at tirst, though attempts have often been made to improve it, and other contrivances proposed to stipply its place. Many of these have succceded in quiet water, but proved useless in a troubled sca. See our article Log.

$\Lambda$ following edition of this book was revised by the author; among the additions to which, he enlarges on the account of the log-line; and at the cud subjoins a "Hy. 
drographical Discourse touching the five sereral Passages into Cathay." Bourne published other tracts also; as, one entitled "Inventions or Devises;" where he describes a method, by wheel-work, of measuring the veloeity of a ship at sea; an artifice which he attributes to one Humfrey Cole.

BOW, an offensive weapon made of wood, horn, steel, or other elastic matter, by which arrows are thrown with great force. This instrument was of very general use among the ancients, and is still found among all savage nations unacquainted with the use of fire-arms. There are two species of the Bow, the Long and the Cross Bow.

The Long Bow is simply a bow, or a rod, with a string fastened to each end of it, to the middle of which the end of an arrow being applied, and then drawn by the hand, on suddenly quitting the hold, the bow returns by means of its elasticity, and impels the arrow from the string with great violence. The old English archers were famous for the long bow, by means of which they gained many victories in France and other countries.

The Cross Bow, called also Arbalest or Arlalet, is a bow strung and set in a shaft of wood, and furnished with a trigger; serving to throw bullets, darts, and large arrows, \&c: The ancients lad large machines for throwing many arrows at once, called Arbalets, or Balistx.

The force of a bow may be calculated on this principle, that its spring, i.e. the power by which it rentores itself to its natural position, is always proportional to the space or distance it is bent or removed from it.

Bow, a mathematical instrument formerly used at sea for taking the sun's altitude. It consisted of a large arch divided into 90 degrees, fixed on a staff, and furnished with three vanes, viz, a side vane, a sight vane, and another called the horizon vane.

Bow-Compass, an instrument for drawing arches of very large circles, for which the common compasses are too small. It consists of a beanit of wood or brass, with three long screws that govern or bend a lath of wood or steel to any arch.

BOX AND NEEDLE, the small compass of a theodolite, circumferentor, or plain-table.

BOYAU, in Fortification, a ditch covered by a parapet, and serving as a communication between two trenches. It runs parallel to the works of the bodly of the place, and serves as a tine of contravallation, both to hinder the sallies of the besieged, and to secure the miners. When it is a particular cut running from the trenches, to cover some spot of ground, it is drawn so as not to be enfiladed or scoured by the enemy's shot.

BOYLE (RosErT), one of the greatest philosophers, as well as best men, that any cuuntry has ever produced, was the $7 t_{1}$ son and the 14 th child of Richard earl of Cork, and was born at Lismore in the province of Munster in Ireland, the 25th of January', 1026-7; the very year of the death of the learnest Lord Bacon, whose plans of experimental philosophy our author afterwards so ably secondel. While very young, he was instructed in his father's house to read and write, and to speak French and Latin. In 1635 , when only 8 years old, he was sent over to England to be educated at kiton school. Here he soon discovered an extranrdinary force of understanding, with a disposition to cultivate and improve it to the utmost.

After remaining at Eton betwcen 3 and 4 years, his father sent our author and his brother Francis, in 1638, on their travels upon the continent. They passed through France to Geneva, where they settled for some time to pur- sue their studies : here our author resumed his acquaintance with the elements of the mathematics, which he had commenced at Eton when 10 years old, on account of an illness which prevented his other usual studies.

In the autumn of $16+1$ he quitted Geneva, and travelled through Switzerland and Italy to Venice, whence be returned again to Florence, where he spent the winter, studying the Italian language and history, and also the works of the celebrated astronomer Galilco, who died in a village near this city during Mr. Boyle's residence here.

About the end of March $16+2$, lie set out from Florence, visited Rome and other places in Italy, then returned to the south of France. At Marseilles, in May 164,2, Mr. Boyle received letters from his father, which infornsed him that the rebellion had broken out in Irekand, and with what difficulty he had procured $250 l$ then remitted to help him and his brother home. This remittance bowever never reached them, and they were obliged to return to Geneva with their governor Mr. Marcombes, who contrived on his own credit, and by selling some jewels, to raise money enough to bring them to Englanil, where they arrived in 164.4. On their arrival they foum that their father was dead, and had left our author the manor of Stalbridge in England, with some other conside rable estates in Ireland.

From this time Mr. Boyte's chict residence, for some years at least, was at bis manor of Stalbridge, whence he made occasional excursions to Oxford, London, \&c ; applying himself with great industry to various kinds of studies, but especially to philosophy and chemistry; and seizing every opportunity of cultivating the acquaintance of the most learned men of his time. He was one of the members of that small but learned body of men, in 1645 , who, when all academical studies were interrupted by the civil wars, secreted themselves; and held private meetings, first in London, afterwards at Oxford, to cultivate subjects of natural knowledge on that plan of experiment which Lord Bacon bad delineated. They styled themselves then The Philosophic College; Lut after the restoration, when they were incorporated, and distinguished openly, they took the name of the Royal Socicty.

In the summer of 1654 he retired to settle at Oxford, the Philosophical Society being removed from London to that place, that he might enjoy the conversation of the other learned members, his friends, who had retired thither, such as Wilkins, Wallis, Ward, Willis, Wren, \&c. It was during his residence here that he improved that admirable engine the air-pump; and by numerous experiments was enabled to discover several qualities of the air, so as to lay a foundation for a complete thwory. He declared against the philosophy of Aristotle, as having in it more of words thals things; promising much, but performing little; and giving the inventions of men for indubitable proofs, instead of building on observation and experiment. He was also so zealous for this true method of learning by experiment, and so careful about it, that though the Cartesian philosophy then made a great interest in the world, yet be could never be persuaded to read the works of Descartes, for fear he should be amused and led away by plausible accounts of things founded on conjecture, and merely hypothetical. But philosophy, and inquiries into nature, though they engaged his attention decply, did not occupy him entirely; as he still continued to pursue critical and theological studies. He had offers of preferinent to enter into holy orilers, by the government, after the restoration. But he declined the offer, choosing ratlicr to pursue his 
studies as a layınan, in such a manner as might be most effectual for the support of religion; and began to communicate to the world the fruits of these studies. These productions were very numerous and important, as well as various: the principal of which, as well as of some other memorable occurrences of his life, werc nearly in the following order :

In 1660 came out, 1. New experiments, physico-mechanical, touching the spring of the air and its effects.2. Seraphic love; or some motives and incentives to the love of God, pathetically discoursed of in a letter to a friend. A work which it has becn said was owing to his courtship of a lady, the daughter of Cary earl of Monmouth; though oul author was never married.-3. Certitin physiological essays and other tracts, in 1661.-4. Sceptical chemist, $166 \%$; reprinted about the year 1679 , with the addition of divers experiments and notes on the producibleness of chemical principles.

In the ycar 1663 , the Royal Society being incorporated by king Charles the 2d, Mr. Boylc was named one of the council; and as he might justly be reckoned among the founders of that lcarned brdy, so he continued one of the most useful and industrious of its members during the whole coursc of his life. His next publications were, 5 . Considerations touching the usefulness of experimiental natural philosophy, 1663.-6. Experiments and considerations upon colours; to which was added a letter, containing Observations on a diamond tlat shines in the dark, 1663. This treatise is full of curious and uscful remarks on the hitherto unexplained doctrine of light and colours; in which he shows great judgment, accuracy, and penetration; and which may be said to have led the way for Newton, who made such brilliant discoveries in that branch of physics.-7. Considerations on the style of the holy Scriptures, 1663. This was an extract from a larger work, entitled An cssay on Scripture; which was afterwards published by Sir Peter Pett, a fricnd of Mr. Boyle's.

In 1664 he was elected into the company of the royal miners; and was all this year occupied in prosecuting various good designs, which was probably the reason that he did not publish any works in this ycar. Soon after came out, 8. Occasional reflections, upon several subjects, 1665. This piece exposed our author to the censurc of the celebrated Dean Swift, who, to ridicule these discourses, wrote A Pious Meditation upon a Broomstick, in the Style of the honourable Mr. Boyle.-9. New experiments and obscrvations upon cold, 1665.-10. Hydrostatical paradoxes made out by new expcriments, for the most part physical and easy, 1666.-11. The origin of forms and qualitics, according to the corpuscular philosophy, 1666.-Both in this and the former year, our author communicated to his friend Mr. Oldenburg, then sccretary to the Royal Society, several curious and excellent short pieces of his own, on a great variety of subjects, and others transmitted to him by his learned friends, which are printed in the Philosophical Transactions.

In the year $1668 \mathrm{Mr}$. Boyle resolved to settle in London for life; and for that purpose he removed to the house of his sister, the Lady Ranclagh, in Pall-Mall. This removal was to the great benefit of the learned in general, and particularly of the Royal Society, to whom he gave great and continual assistance, as abuulantly appears by the scvera! pieces communicated to them from time to time, and printed in their Transactions. To avoid improper waste of time, he had set hours in the day appointed for' receiv- ing sucl persons as wanted to consult bim, either for their own assistance, or to communicate new discoveries to him : and he besides kept up an extensive correspondence witl the most learned men in Europe; so that it is wonderfuI how he could bring out so many new works as he did. His next publications were, 12. A continuation of new experiments touching the weight and spring of the air; to which is added, $\mathbf{A}$ discourse of the atmosphere of consistent bodies, 1669.-13. Tracts about the cosmical qualities of things; cosmical suspicions; the temperature of the subterraneous regions; the bottom of the sea; to which is prefixed an introduction to the history of particular qualities, $1669 .-1+$. Considerations on the usefulness of experimental and natural philosophy, the $2 \mathrm{~d}$ pait, 1671.-15. A collection of tracts on several useful and important points of practical philosophy, 1671.-16. An essay on the origin and virtues of gems, 1672.-17. A collection of tracts on the relation between flame and air; and several other uscful and curious subjects, 1672; besides furnishing, in this and the former year, a number of short dissertations on various topics, addressed to the Royal Society, and inserted in their Transactions.-18. Essays on the strange subtilty, great efficacy, and determinate nature, of effuvia; with a variety of experiments on other subjects, 1673.-19. The excellency of theology compared with philosophy, 1673. This discourse was written in the year 1665 , while our author, to avoid the great plague which then raged in London, was forced to go from place to place in the country, having little or no opportunity of consulting his books. -20 . A collection of tracts on the saltness of the sea, the moisture of the air, the natural and preternatural state of bodies; to which is prefixed a dialogue concerning cold, 1674,-21. A collection of tracts containing suspicions about hidden qualities of the air; with an appendix touching celestial mag. nets; animadversions on Mr. Hobbes's problem conccrning a vacuum; a discourse of the cause of attraction and suction, 1674.-22. Some considerations about the reasonableness of reason and religion; by ' $\mathrm{T}$. $\mathrm{E}$. (the final letters of his names). To which is annexed a discourse about the possibility of the resurrection; by Mr. Bnyle, 1675. The same year several papers communicated to the Royal Society, among which were two upon quicksilver growing hot with gold,-23. Experiments and notes about the mechanical origin or production of particular qualities, in several discourscs on a great variety of subjects, and among the rest on electricity, 1676.-He then communicated to Mr. Hook a short memorial of some observations madc on an artificial substance that shines without any preceding illustration; publisherl by Honk in his Lectiones Cutlerianæ.-24. Historical account of a degradation of gold made by an anti-clixir.-25. Acrial noctiluca; or some new phenomena, and a process of a factitious self-shining substance, 1680 . This year the Royal Society, as a proof of the just sense of his great worth, and of the constant and particular services which through a course of many years he had done them, made choice of lim for their president; but he being extremely, and, as he says, pcculiarly tender in point of oaths, declined that houour.-26. Discourse of things above renson; inquiring, whether a philosopher should admit any such, $1681 .-27$. New cxperiments and observntions on the icy noctifuca; to which is addeda chemical parados, grounded on new experiments, making it probable that chemical principles are transmutable, so that out of one 
of then others may be produced, 1682.-28. A continuation of new experiments, physico-mechanical, touching the spring and weight of the air, and their effects, 1682.29. A short letter to Dr. Beale, in relation to the making of fresh water out of salt, 1683.-30. Memoirs for the natural histury of human blood, especially the spirit of that liquor, 1684.-31. Experiments and considerations about the porosity of bodies, 1684.-32. Short memoirs for the natural experimental history of mineral waters, \&c, 1685.33. An essay on the great effects of even languid and unheeded motion, \&c, 1683.-34. Of the reconcileableness of specific medicines to the corpuscular philosophy, \&c, 1685.-35. Of the high veneration man's intellect owes to God, peculiarly for bis wisdom and power, 1685.-36. Free inquiry into the vulgarly received notion of nature, 1686.-37. The martyrdom of Theodora and Didymia, 1687 ; a work he had drawn up in his youth.-38. A disquisition about the final causes of natural things, and about vitiated light, $168 \mathrm{~s}$.

Mr. Boyle's bealth declining very fast, he abridged grcatly his time given to conversations and communications with other persons, that he might have more time to prepare for the press some others of his papers, before lis death, which were as fullow: -39 . Medicina Hydrostatica, \&c, 1690.-40. The Christian virtuoso, \&c, 1690.--41. Experimenta et Observationcs Physicre, \&c, 1691; which is the last work that he published.

Mr. Boyle died on the last day of December of the same year, 1691, in the 65th year of his age, and was buried in St. Martin's church in the Fields, Westminster; his funeral sermon being preached by Dr. Gilbert Burnet, bishop of Salisbury; in which he displayed the excellent qualities of our author, with many circumstances of his life, \&c. But as the limits of this work will not allow us to fullow the bishop in the copious and cloquent account he has given of this great man's abilities, we must coutent ourselves with adding the following short eulogium by the celebrated physician, philosopher, and chemist, Dr. Bocrhave; who, after having declared Lord Bacon to be the father of experimental philosopliy, asserts, that, "Mr. Boyle, the ornament of his age and country, succeeded to the genius and inquiries of the great chancellor Verulam. Which," says lie, "of. Mr. Boyle's writings shall I recommend? All of them. To him we owe the secrets of fire, air, water, animals, vegetables, fossils :. so that from his works may be derluced the whole system of natural knowledge."

Mr. Boyle left also several papers behind him, which have been published since his death. Beautiful editions of all his works have been printed at London, in 5 volumes. folio, and 6 volumes 4 to. Dr. Shaw also published in 3 volumes 4 to, the same works " abridged, methodized, and disposed under the general beads of Physics, Statics, Pneumatics, Natural History, Chemistry, and Medicine;" to which he has prefixed a short catalogue of the philosophical writings, according to the order of time when they were first published, as follows:

Physico-mechanical experiments on the spring and weight of the air - $\quad-166$

The Sceptical Chemist _ - $\quad 1661$

Physiological Essays $\quad$ - $\quad$ - 1662

History of Colours - $\quad 1663$

Usefinlness of Experimental Philosophy - 1663

History of Cold . - - $\quad 1665$

Ilistorical Paradoxes $\quad$ - $\quad$ - $1666^{\circ}$

Origin of Forms and Qualitics - 1666
Cosmical Qualities - $\quad 1670$

The admirable Rarefaction of the Air - 1670

The Origin and Virtues of Gems - 1672

The Relation betwixt Flame and Air : 1672

Effluviums - $\quad$ - $\quad$ - 1673

Saltness of the Sea - - 1674

Hidden Qualities of the $\Lambda \mathrm{ir}$ - - 1674

The Excellence, \&c, of the Mechanical Hypothesis

Considerations on the Resurrection

Particular Qualicies

Acrial Noctiluca

Icy Noctiluca

Things above Reason

Natural History of Human Bloorl

Porosity of Bodics

Natural History of Mineral Waters

Specific Medicjnes

The High Veneration due to God

Languid Motion

Tlie Notion of. Nature

Final Causes

Medicina. Hydrostatica

The Christian Virtuoso

Experimenta et Observationes Physicæ

Natural History of the Air

Medicinal Experiments

-

-

BRACE, in Architecture, denotes a piece of timber framed in with bevel-joints; scrving to keep the building from swerving either way.

BRACHMINS, or BRACHMANS, a branch of the ancient Gymnosophists, or philosophers of India, remarkable for the severity of their lives and manners.

BRACKE', in Building, denotes a kind of wooden stay, in form of a knee or shoulder, serving for the support of shelves, \&c, or a cramping-iron, which scrves as a stay in timber-work.

BRADLEY (DR. JAMES), a celebrated English astronomer, the third son of William Bradley, was born at Sherborne in Gloucestershire in the year 1692. He was fitted for the university at Northleach, in the same county, at the boarding-school of Mr. Iles and Mr. Brice. From thence ho was sent to Oxford, and admitted a commoner of Baliol collego, March 15, 1710 ; where he took the degree of bachelor the 14 th of.October 1714 , and of master of arts the 21 st of January, 1716 . His friends intending him for the church, his studies were regulated with that view; and as soon as he was of a proper age to receive holy orders, the bishop of Hereford, who had entertained great esteem for him, gave him the living of Bridstow, and soon after he was inducted to that of Landewy. Welfrey in Pembrokeshire.

He was nephew to Mr. Pound, a gentleman well known in the learned world, by many excellent astronomical and, other observatiuns, and who would have enriched it much. more, if the journals of his voyages had not been burnt at: Pudor Condor, when the place was set on fire, and the English who were settled there cruelly massacred, Mr.. Pound himself very narrowly escaping with his life. With, this gentleman, at Wanstead, Mr. Bradley passed all the time that he could spare from the duties of his function,. being then sufieciently acquainted with the mathematics to improve by Mr. Pound's conversation. It may casily be imagincel that the example and conversition of this gentleman did not render Bradlcy inore fond of his profession, to, 
which he had before no great attachmont: he continued however as yet to fulfil the duties of it, though at this time he bad made such observations as laid the foundation of those discoveries which afterwards distinguished him as one of the greatest astronomers of lis age. These observations gained him the notice and friendship of the lord-chancelfor Macclesfield, Mr. Newton, afterwards Sir lsaac, Mr. Halley, and many other members of the Royal Society into which he was soon after elected a membér.

Soon after, the chair of Savilian professor of astronomy at $O x$ ford became vacant, by the death of the celebrated Dr. John Keil ; and Mr. Bradley was elected to succeed him on the 3 ist of October, 1721 , at 29 years of age : his colleagne being Mr. Halley, who was professor of geometry on the same foundation. On this appointment Mr. Bradley resigned his church livings, and applied himself wholly is the study of his favourite science. In the course of his observations, which were innumerable, he discovered and settled the laws of the alterations of the fixed stars, from the progressive motion of light, combined with the earth's annual motion about the sun, and the nutation of the earth's axis, arising from the unequal attraction of the sun and moon on the different parts of the earth. The former of these effects is called the Aberration of the fixed stars, the theory of which he published in 1727 ; and the latter the Nutation of the earth's axis, the theory of which appeared in 1747 , deduced from 20 ycars' assiduous observations, by whicb he communicated to the world two of the finest discoveries in modern astronomy, making a memorable epnch in the history of that science. See ABERRATION and Nutation.

In 1730 Bradley succeeded Mr. Whiteside, as lecturer in astronomy and experimental philosophy in the Museum at Oxford; which was a considerable emolument to him, and which he enjoyed till within a year or two of his death, when the ill state of his health induced him to resign it.

Our author always preserved the esteem and friendship of Dr. Halley; who, being worn out by age and infirmities, thought he could not do better for the service of astrono$\mathrm{my}$, than procure for Mr. Bralley the place of regius professor of astronomy at Greenwich, which he himself had many years possessed with the greatest reputation. With this view be wrote many letters, desiring Mr. Bradley's permission to apply for a grant of the reversion of it to him, and even offered to resign it in his favour, if it should be thought necessary; but Dr. Halley died before be could accomplish this kind object. Bradley however obtained the place, by the interest of lord Macclesfield, who was afterwards president of the Royal Society; and upon this appointment the university of Oxford conferred on him a diploma.

The appointment of astronomer royal at Greenwich, which was dated the $3 \mathrm{~d}$ of February, 1741-2, placed our author in his proper sphere; and he pursued his observations with unwearied diligence. However numerous the collection of astronomical instruments at that observatory, it was impossible that sisch an observer as Dr. Bradley should not desire to increase them, as well to answer those particular views, as in general to make observations with greater exactness. In the year $17+8$, therefore, he took the (1)portunity of the visit of the Royal Society to the observatory, annually made to examine the instruments and receive the professor's observations for the year, to represent so strongly the necessity of repairing the old instrunents and providing new ones, that the society thought proper to make application to the king, who was pleased to order 1000 pounds for that purpose. This sum was laid out under the direction of Bradley, who, with the assistance of the late celebrated Mr. Grabam and Mr. Bird, furnished the observatory with as complete a collection of astronomical instruments, as the most skilful and diligent observer could desire. Our author being thus furnished with such assistance, pursued his observations with great assiduity during the rest of his life; an immense number of which was found after his death, in 13 folio volumes, and were presented to the university of Oxford in 1776 , on condition of their printing and publishing them.

During Dr. Bradley's residence at the Royal Observatory, the livitig of the church at Greenwich became vacant, and was oftered to him: upon his refusing to accept it, from a conscientious scruple, " that the duty of a pastor was incompatible with bis other studies and necessary engagements," the king was pleased to grant him a pension of $250 l$ over and above the astronomer's original salary (100l) from the Board of Ordnance, "in consideration (as the sign manual, dated the 15 th Feb. 1752 , expresses it) of his great skill and knowledge in the several branches of astronomy and other parts of the mathematics, which have proved so useful to the trade and navigation of this kingdom ;" a pension which has been regularly continued to the astronomers royal ever since.

A bout 1748 , our author became entitled to bishop Crew's benefaction of $30 l$ a year, to the lecture reader in experimental philosophy at Oxford. He was elected a member of the academy of Sciences at Berlin in 1747 ; of that at Paris, in 1748; of that at Petersburg, in 1754; and of that at Bologna, in $175 \%$. He was married in the year 1744, but never had more than one child, a daughter.

By his unremitting application to study and observations, he becanze afflicted, for near two years before his death, with a violent oppression on his spirits, which interrupted his useful labours. 'This distress arnse chiefly from an apprehension that he should outlive his rational faculties; but this so much dreaded evil never came upon him. In June 1762 he was seized with a suppression of urine, occasioned by an inflammation in the reins, which terminated his existence the 13th of July following. His death bappened at Chalford in Gloucestershire, in the 70 th year of his age; and he was interred at Minchinhampton in the same county.

As to the cliaracter of Dr. Bradley, he was remarkable for a placid and gentle modesty, very uncommon in persons of an active temper and robust constitution. Though he was a good speaker, and possessed the rare but happy art of expressing his ideas with the utmost precision and clearness, yet no man was a greater lover of silence, for he never spoke but when he thought it absolutcly nocessary. Nor was he more inclined to write than to speak, as he has published very little : he had a natural diffidence, which made him always afraid that his works might injure his character; so that he suppressed many which might have been worthy of publication.

His papers, which have been inserted in the Plilos. T'rans. are,

1. Observations on the Comet of 1723, vol. 33, p.41.2. 'The Longitude of Lisbon and of the Fort of New York from IVansted and London determined by the Eclipse of the First Satellite of Jupiter, vol. 34, p. 85.-3. An Account of a new discovered Motion of the lixed Stars, wol. 35, p. 637.-4. On the Going of Clocks with Isoch rouical 
Pendulums, vol. 38, p. 302.-5. Observations on the Comet of $1736-7$, vol. 40, p. 111. -6. On the Apparent Motion of the Fixed Stars, vol. 45, p. 1.-7. On the Occultation of Venus by the Moon, the 15th of April, 1751, vol. 46 , p. 201,-8. On the Comet of 1757 , vol. 50 , p. 408 . -9. Directions for Using the Common Micrometer, vol. 62, p. 46.

BRADWARDIN (THomas), a skilful mathematician and divine, was born at Hartfield in Sussex, about the close of the 13 th century. He was educated at Merton-college, Oxford, where he took the degree of doctor of divinity. It has been said he was professor of divinity at Oxford; that he was chancellor of the diocese of London, and confessor to Edward the 3d, whom he constantly attended during his war with France. After his return from the field, he was made prebendary of Lincoln, and afterward archbishop of Canterbury. He died at Lambeth in the year 1349 , forty days after bis consecration. His works are, 1. De Causa Dei, printed in London, 16is, published by J. II. Savil.,-2. De Geometria Speculativa, \&c. Paris, 1495,15 12, 1530. - 3. De Arithmetica Practica. Paris, 1502, 1512 -4. De Proportionibus. Paris, 1495. Venice, 1505, folio.-5. De Quadratatura Circuli. Paris, 1495, fol.

BRAHE' (TYCHo), a celebrated astronomer, descended from a noble family, originally of Sweden, but settled in Denmark, was born the 14th of December 1546, at Knudstorp in the county of Schonen, ncar Helsimbourg. Hc was taught Latin when 7 years old, and studied 5 years under private tutors. His father dying while he was very young, his uncle, George Brahé, having no children, adopted him, and sent him, in 1559, to study philosophy and rhctoric at Copenhagen. The great eclipse of the sun, on the 21st of August 1650, happening at the precise time the astronomers had foretold, he began to consider astronomy as something divinc; and purchasing the tables of Stadius, he gained some notion of the theory of the planets. In 1562 he was sent by his uncle to Lcipsic to study the law, where his acquirements gave manifest indications of extraordinary abilities. His natural inclination bowever was to the study of the heavens, to which he applied himself so assiduously, that, notwithstanding the care of his tutor to keep bim close to the study of the law, he made use of evcry means in his power for improving his knowledge of astronomy; he purchased with his pocket-money whatever books he could meet with on this subject, and read them with great attention, procuring assistance in difficult cases from Bartholomew Scultens, his private tutor; and having procured a small celestial globe, he took opportunities, when his tutor was in bed, and when the weather was clear, to examine the constellations in the heavens, to learn their names from the globe, and their motions from observation.

After a course of 3 years study at Leipsic, his uncle dying, he returned home in 1565 . In this year a difler-. ence arising between Brahé and a Danish nobleman, they fought, and our author lost part of his nose cut off by a blow in the contest. This defect he so artfully supplied with one made of precious metal and wax, that it was not perceivable. Alsout this time he began to apply himself to chemistry, proposing nothing less than to obtain the philosopher's stone. But becoming greatly disgusted to sce the liberal arts despised, and finding his own relations and friends uneasy that he applied himself to astronomy, as thinking it a study unsuitable to a person of his quality,

Voz. I. he went to Wirtemberg in 1566 , from which the breaking out of the plague soon occasioned his removal to Rostock, and in 1569 to Augsburg, where he was visited by Peter Ramus, then professor of astronomy at Paris, and who greatly admired his uncommon shill in this science.

In 1571 he returned to Denmark; and was favoured by his maternal uncle, Steno Billes, a lover of learning, with a convenient place at his castle of Herritzvad near Knudstorp, for making his observations, and building a laboratory. And here it was he discovered, in 1573, a new star in the constellation Cassiopeia. But soon after, his marrying a country girl, beneath his rank, occasioned so violent a quarrel between him and his relations, that the king was obliged to interpose to reconcile them.

In 1574 , by the king's command, he read lectures at Copenhagen on the theory of the planets. The year following he began his travels through Germany, and proceeded as far as Venice. He then resolved to remove his family, and settle at Basil ; but Frederick the 2d, king of Denmark, being informed of his design, and unwilling to lnse a man who was capable of doing so much honiour to his country, he promised to enable him to pursue his studies, and bestowed on him for life the island of Huen in the Sound, and engaged that an observatory and laboratory should be built for him, with a supply of money for carrying on his designs : accordingly the first stone of the observatory was laid the 8 th of August 1576, under the name of Uranibourg. The king also gave him a pension of 2000 crowns out of his treasury, a fee in Norway, and a canonry of Roshild, which amounted to 1000 more. This situation he enjoyed about 20 years, pursuing his observations and studies with great industry: here he kept always in his house ten or twclve young men, who assisted him in bis observations, and whom he instructed in astronomy and mathematics. And it was also at this place that he reccived a visit from James vr, king of Scotland, afterwards James I of England, having come to Denmark to espouse Anne, daughter of Frederick II. This king made our author some noble presents, and wrote a copy of Latin verses in his praise.

Brahe's tranquillity however in this happy situation was at length fatally interrupted. Soon after the death of King Frederick, by the aspersions of envious and malevolent ministers, he was deprived of his pension, fee, and canonry, in 15.96. Being thus rendered incapable of supporting the expenses of his establishment, he quitted his favourite Uranibourg, and withdrew to Copenhagen, with some of his instruments, and continued his astronomical observations and chemical experiments in that city, till the same malevolence procured from the new king, Charles Iv, an order for him to discontinue thens. This induced him to fall upon means of being introduced to the emperor Rodolphus, who was fond of mechanism and chemical expcriments: and to smooth the way to an interview, Tycho now published his book, Astronomia instaurata Mechanica, adorned with figures, and dedicated it to the emperor. That prince received him at Prague with great civility and respect; gave him a magnificent house, till he could procure one for him more fit for astronomical observations; he also assigned him a pension of 3000 crowns; and promised him a fec for himself and his descendants. Here then he settled in the latter part of 1598, with his sons and sclolars, and anong them the celebrated Kepler, who had joincd him. But he did nut $2 \mathrm{~K}$ 
long enjoy this happy situation; for, about 3 years after, he died, on the 24th of October 1601, of a retention of urine, in the 55 th year of his age, and was interred in a magnificent manner in the principal church at Prague, where a noble monument was erected to his memory; leaving, besides his wife, two sons and four daughters. On the approach of death, he enjoined his sons to take care that none of his works should be lost; exhorted the studerits to attend closely to their exercises; and recommended to Kepler the finishing of the liodolphine tables, which he had constructed for regulating the motion of the plaucts.

Brahe's skill in astronomy is universally hnown; and he is famed for being the inventor of a new system of the planets, which he encieavoured, though without success, to establish on the ruins of that of Copernicus. He was very credulous with regard to judicial astrology and presages. If he met an old woman when he went out of doors, or a hare upon the road on a journey, he would turn back immediately, being persualed that it was a bad omen: also, when he lived at Uranibourg, he kept in his house a madman, whon he generally placed at his feet at table; for as lie imagined that every thing spoken by mad persons presaged something, he carefully observed all that this man said; and because it sometimes proved true, he fancied it might always be depended on. He was of a very itritable disposition : a mere trifle would put him in a passion; and against persons of the first rank, whom he thuught his enemies, he openly discovered his resentment. He was very apt to rally others, but highly provoked when the same liberty was taken with himself,-The principal part of his writings, according to Gassendus, are,

1. An account of the New Star, which appearcd Nov. 11 th, 1572, in Cassiopeia; Copenh. 1573, in 4to.-2. An Oration concerning the Matliematical Sciences, pronounced in the university of Copenhagen, in the year 1574: published by Conrad Aslac, of Bergen in Norway. -3. A treatise on the Comet of the year 1577, immediately after it disappcared. Nine years afterwards he revised it, and added a 10 th chapter; printed at Uranibourg, 1559.-4. Another treatise on the New Phenomena of the heavens. In the first part of which he treats of the Restitution, as he calls it, of the sun, and of the fixed stars. And in the $2 d$ part, of a New Star, which had then made its appearance.-5. A collection of Astronumical Epistles: printed in 4to, at Uranibourg in 1596; at Nuremberg in 1602; and at Frankfort in 1610 . It was dedicated to Maurice Landgrave of Hesse; because there is in it a considerable number of letters of the Landgrave William his father, and of Christopher Rothmann, the mathematician of that prince, to Tycho, and of Tycho to them.-6. The Mecltanical Principles of $\Lambda$ stronomy restored: Wandesburg, 1598, in folio.- 7 . $\mathbf{A n}$ Answer to the Letter of a certain Scotchman, concerning the comet, in the year 1577 . - S. On the composition of an Elixir for the plague; addressed to the emperor Rodolphus.9. An Elegy on his Exile: Rostock, 1614, 4to.-10. The Rodolphine Tables; which he had not finished when he died; but were revised, and published by kepler, as 'Tycho lad desired.-11. An Accurate Enumeration of the Fixed Stars: addressed to the emperor Rodolphus.-12. A complete Catalogue of 1000 of the fixed Stars; which Kepler has inserted in the Rodolpine 'Tables.-13. Historu Coelestis; or a History of the Heavens; in two parts. 'The lst contains the Observations he had made at Urani- bourg, in 16 books; the latter contains the Observations made at Wandesburg, Wittemberg, Prague, \&c.; in 4 books.-14. Is an Epistle to Caster Pucer; printed at Copenhagen 1668 .

BRANCKER, or BRANKER (ThомAs), an eminent mathematician of the 17th century, son of 'Thomas Brancker some time bachelor of arts in Exeter-college, Oxford, was born in Devonshire in 1636, and was admittcd butler of the said college, Nos. 8,1652 , in the 17 th year of his age. In 1655 , June the 15 th, he took the degree of bachelor of arts, and was elected probationary-fellow the 30 th of the same month. In 1658, April the $22 \mathrm{~d}$, lie took the degree of master of arts, and became a preacher ; but after the Restoration, refusing to conform to the ceremonies of the church of England, he quitted his fellowslip in 1662, and retired to Chester: but not long after, he became reconciled to the service of the church, took orders from a bishop, and was made a minister of Whitegate. He liad however, for some time, enjoyed great opportunity and leisure for pursuing the bent of his genius in the mathematical sciences; and his skill both in the mathematics and chemistry procured him the favour of Lord Brereton, who gave hini the rectory of Tilston. He was afterward chosen master of the well-endowed school at Macclesficld, in that county, where he spent the few remaining years of his life, which was terminated by a short illness in 1676 , at 40 ycars of age; and was interred in the church at Macclesfield.

Brancker wrote a piece on the Doctrine of the Sphere, in Latin, which was published at Oxford in 1662; and in 1668 he published at London, in 4 to, a translation of Rhonius's Algebra, with the title of $\Lambda_{n}$ Introduction to Algebra; which treatise having been cominunicated to Dr. Juhn Pell, he received from him some assistance towards improving it; which he generously acknowledges in a letter to Mr. John Collins; with whoni, and some other gonthemen, proficients in this science, he continued a corlcspondence during his life.

BREACH, in Fortification, a gap or opening made in any part of the works of the town, by the camon or mines of the besiegers, with intent to storn or attack the place.

BREAKING Ground, in Military Affairs, is beginning the works for carrying on the siege of a place; more especially the commencement of digging trenches, or approaclies.

BREAST-WOR $\mathrm{K}$, in the Nilitary Art, is an elevation of earth thrown up around a fortified place or post, to conceal or protect the garrison, and whicls is at the sime time so strong that the enemy's shot cannot picrce through it. Sec Para PET.

BREECH of a Gun, the hinder part, from the cascabel to the lower part of the bore.

BREREWOOD (LDw ARD), a learned mathematician and antiquary, was the son of Robert Brercwood, a reputable tradesman, who was three times mayor of Chester. Our author was born in that city in 1565 , where he was educated in gratnniar crudition at the free-school ; and was afterward admitted, in 1581, of Brazen-nose college, Oxforl, where lie soon acquired the chancter of a hard student; as he has shown in his commentanies on Aristotle's Ethics, which he produced abour the age of 21 .

In the year 1596 he wals chosen the first professor of astronomy in Gresham-college, being one of the two who, at the desire of the electors, were rocommented to them 
by the university of Oxford. He loved retirement, and entirely devoted himself to the pursuit of knowledge. And though he never published any thing himself, yet he was very communicative, and ready to impart what he knew, to others, either in conversation or in writing. His retired situation at Gresham-college being agreeable, it did not appear that he had any other views, but continued there the remainder of his life, which was terminated hy a fever the 4 th of November 1613, at 48 years of age, in the midst of his pursuits, and before he had taken proper care to collect and digest his learned labours; which however were not lost; being reduced to order, and published after his death. These were little or nothing mathematical, being of a miscellaneous nature, on the several subjects of Weights, Money, Languages, Religion, Logic, the Sabbath, Meteors, the Eye, Ethics, \&c.

BRIDGE, a work of carpentry or masonry, built over a river, canal, or the like, for the convenience of passing from one side to the other; and may be considered as a road over water, supported by one or more arches, and these again supported by proper piers or butments. Besides these essential parts, may be added the paving at top, the banquet, or raised footway, on each side, leaving a sufficient breadth in the middle for horses and carriages, also the parapet wall either with or without a balustrade, or other ornamental and useful parts. The breadtb of a bridge for a great city should be such, as to allow an easy passage for three carriages and two borsemen abreast in the middle way, and for 3 foot passengers in the same manner on each banquet: but for other smaller bridges, a less breadth may suffice.

Bridges"are commonly very difficult to execute, on account of the inconvenience of laying foundations and walling under water. The earliest rules and instructions for building of bridges are given by Alberti, in his Archit. 1, 8. Other rules were afterwarits laid down by Palladio, Serlio, and Scamozzi, which are collected by Blondel, in his Cours d'Archit. pa. 629, \&c. The best of these rules were also given by Goldman, Baukhurst, and in Hawkesmonr's History of London Bridge. M. Gautier has a considerable volume expressly on bridges, ancient and modern. See also Rinu's Short Principles for the Architecture of Stone Bridges; as also Emerson, M Iuller, Labelye, and ny own Principles of Bridges, in the 1st vol. of my Tracts, besides neat accounts in the various new Encyclopredias, as well as several French authors.

The conditions required in a bridge are, That it be well designed, commodious, durable, and suitably decorated. It should be of such a height as to be quite convenient for the passage over it, and yet easily admitting through its arches the vessels that navigate under it, and also the water, cven at high tides and floods; the neglect of this precept has caused the destruction of many bridges. Bridges wre commonly continued in a straight direction perpendicular to the stream; though some think they should be tulade rather convex towards the stream, the better to resist floods, \&c. And briclges of this sort have been executed in some places, as Pont St. Esprit near Lynns. Again, a bridge should, not be made in too narrow a part of a navigable river, or one that is subject to tides or floods: because the breadth being still more contracted by the piers, this will increase the deptb, velocity, and fall of the water under the arches, and endanger the whole bridge and navigation. There ought to be an uncven uumber of arches, or an even number of piers; both that the middle of the stream or chief current may flow freely without the interruption of a pier; and that the two halves of the bridge, by gradually rising from the ends to the middle, may there meet in the highest and largest arch; and also, that by being open it: the middle, the eye in viewing it may have a better command and look directly through there. When the niddle and ends are of different heights, their difference however ought not to be great in proportion to the length, that the ascent and descent may be easy; and in that case alsu it is more beautiful to make the top in one continued curve than two straight lines forming an angle in the middle. Bridges should rather be of few and large arches, than of many smaller ones, if the height and situation will possibly admit of it ; for this will leave passage for the water and navigation, and be a great saving in materials and labour, as there will be fewer piers and centres, and the arches \&c will require less materials; a remarkable instance of which appears in the diference between the bridges of Westminster and Blackfriars, the expense of the former being more than double the latter.

For the proper execution of a bridge, and making an estimate of the expense $\mathbb{E} x$, it is necessary to have threc plans, three sections, and an elevation. 'Ihe three plans are so many horizontal sections, viz, 1st a plan of the foundation under the piers, with the particular circum-' stances attending it, whether of gratings, planks, pilek, $\& c$; the $2 d$ is the plan of the piers and arches; and the $3 \mathrm{~d}$ is the plan of the superstructure, with the paved road and banquet. The three sections are vertical ones; the 1 st of then a longitudinal section from end to end of the bridge, and through the middle of the breadth; the $2 \mathrm{~d}$ a transverse one, or across it, and through the summit of an arch; and the $3 \mathrm{~d}$ also across, but taken upon a pier. The elevation is an orthographic projection of one side or face of the bridge, or its appearance as viewed at a distance, showing the exterior aspect of the materials, with the manner in which they are disposed, \&c.

For the figure of the arches, some prefer the semicircle, though perhaps without any just reason; others the elliptical form, as having many advantages over the semicircular; and some talk of the catenarian arch, thougin its pretended advantages are only chimerical; but the arch of equilibration is the only perfect one, so as to be equally strong in every part: see my Principles of Bridges. The picrs are of divers thickness, according to the figure, span, and height of the arches; as may be seen in the work above mentioned.

With the Romans, the repairing and building of bridges were committed to the priests, thence named Pontifices; next to the censors, or curators of the roads; but at last the emperors took the care of the briclges to themselves. Thus the Pons Janiculensis was built of marbie by Antoninus Pius; the Pons Cestius was restored by Gurdian; and Arian built a new one, which was called after his own name. In the middle age, bridge-building was reckoned among the acts of religion; and, towards the end of the 12 th century, St. Benezet founcled a regular order of hospitallers, vider the name Pontifices, or bridge-builder's, whose office was to assist travellers, by making brilges, settling ferries, and receiving strangers into hospitals, or houses, built on the banks of rivers. We real of an hospital of this kind at $\Lambda$ vignon, where the hospitallers $r e$ sided under the direction of their first superior St. Benezet : and the Jesuit Raynaldus has a treatisc on St. Johu the bridge-builder.

$2 \mathrm{~K} 2$ 
Among the bridges of antiquity, that built by Trajan over the Danube is the most'magnificent. It was demolished by his next successor Adrian, the ruins of which are still to be seen in the middle of the Danube, near the city Warhel in Hungary. It had 20 piers, of square stone, each of which was 150 feet high above the foundation, 60 feet in breadth, and 170 feet distant from one another, which is the span or width of the arches; so that the whole length of the bridge was mure than 1530 yards, or one mile nearly.

In France, the Pont de Garde is a very bold structure; the piers being only 13 feet thick, yet serving to support an immense weight of a triplicate arcade, and joining two mountains. It consists of three bridges, one over another; the uppermost of which is an aqueduct, which carries water to Nismes.

The aqueduct bridgc of Alcantara, near the city of Lisbon, which was finislied in 1732 , is also a very magnificent piece of workmanahip; it consists of 35 arches, the largest of which is $22 T$ feet ligh, and $108 \frac{1}{2}$ feet wide.

The bridge of Avignon, which was finished in the year 1188 , consists of 18 arches, and measures 1340 paces, or about 1000 yards in lengrth.

Over the several canals at Venice are laid nearly 500 bridges of different sizes; the greater number of them are of stone. The chief of these, called the Rialto, is celebrated as a master-piece of art : it consists of one flat and bold arch, nearly 100 feet span, and only 23 feet high above the water; it was built in 1591 from a design of Michael Angelo. The breadth of the bridge, which is 43 feet, is divided by two rows of shops into three narrow streets, that in the middle being the widest; and there is in the centre an open arch-way, by which the three streets communicate with one another. At each end of the Rialto is an ascent of 56 steps; the view from its summit is very lively and magnificent. The whole interior of the shops and the bridge is of marble. The foundation extends 90 feet, and rests upon $12000 \mathrm{elm}$ piles. This structure cost the republic 250,000 ducats. Poulet also mentions a bridge of a single arch, in the city of MIunster in Bothnia, much nobler than that of the Rialto at Venice. Yet these are nothing to a bridge in China, built, from one mountain to another, consisting only of a single arch, 4.00 cubits long, and 500 cubits high, whence it is called the flying bridge; a figure of which is given in the Philos. Trans. Kircher also speaks of a bridge in the same country 360 perches long without any arch, but supported by 300 pillars.

There are many bridges of considerable note in our own country. The triangular bridge at Crowland in Lincolnshire, is esteemed the most ancient Gothic structure remaining entire in the kingdom; and was erected about the year 860 .

London bridge is on the old Gothic structure, with 20 small locks or arches, each of about 20 feet wide; but there are now only is open, two having lately been thrown into one in the centre, and another on oke side is concealed or covered up. It is 900 feet long, $60 \mathrm{high}$, and 74 wide; the piers are from 25 to 34 feet broad, with starlings projecting at the ends: so that the greatest waterway, wlicn the tide is above the starlings, was 450 feet, scarce half the brealth of the river; and below the starlings, the water-way was reduced to 194 feet, before the late opening of the centre. London bridge was first built with timber between the years 993 and 1016 ; and it was repaired, or rather rebuilt with timber in 1163 . The store bridge was begun in 1176 , and finished in 1209. It is probable there were no houses on this bridge for upwards of 200 years; since we read of a tilt and tournament held on it in 1395. Houses it appears were erected on it afterwards; but being found of great ineonvenience and nuisance, they were removed in 1758 , and the avenues to it also enlarged, and the whole made more commodious; the two iniddle arches were then thrown into one, by rcmoving the pier from between them ; the whole amounting to above $50,000 l$.

There were other bridges in England built in the old manner of London bridge; as the bridge at Rocbester, which is 550 feet long, and has 11 arches; also the late bridge at Newcastle-upon-'Tyne, which was broken down by a great flood in the year 1771 , for want of a sufficient quantity of water-way through the arches.

The longest bridge in England is that over the Trent at Burton, built in the 12th century, of squared free-stone, and is strong and lofty; it contains 34 arches, and the whole length is 1545 feet. But this falls far short of the wooden bridge over the Drave, which according to $\mathrm{D}_{1 \mathrm{r}}$. Brown is at least 5 miles long.

But one of the inost singular bridges in Europe is that built over the Taaf in Glamorganshire, by William Edwards, a poor country mason, in the year 1756 . This remarkable bridge consists of but one stupendous arcly, which, though only 8 feet broad, and 35 feet high, is no less than 140 span, being part of a circle of 175 feet dir ameter.

There is also a remarkable bridge of one arch, built at

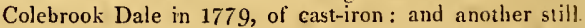
larger of the same metal, raised over the river Wear, at Sunderland, the arch being of 240 feet span. And since that time several other iron bridges have been erected in various places, accounts and drawings of which may be seen in my T'racts before noticed.

Of moderu bridges, perhaps the two finest in Europe are those of Westminster and Blackfriars over the river Thames at London. The former is 1220 foct long, and 44 feet wide, having a commodious broad foot-path on. each side for passengers. It consists of 13 large and two small arches, all semicircular, with 14 intermediate piers. The arches all spring from about 2 feet above lowwater mark; the middle arch is 76 feet wide, and the others on each side decrease regularly by 4 feet at a time. The two middle piers are cach 17 feet thick at the springing of the arches; and the others decrease equally on each side by ove foot at a time; every pier terminating with a saliant right angle against either stream. 'This bridge is built of the best materials, and in a neat and elegant taste, but the arches are too small for the quantity of masonry contained in it. This bridge was begun in $173 \mathrm{~S}$, and opened in 1750; the whole sum of money granted and paid for the erection of it, with the purchase of houses to take down, and widening the avenues, \& 4 , amounted to389,500l.

Blackfriars bridge, nearly opposite the centre of the city of London, was begun in 1760 , and completed in 10 years. and three quarters; it is an excecling light and elegant structure, but the materials unfortunately do $130 \mathrm{scem}$ to be of the best sort, as many of the arch stones are decaying. It consists of 9 large, clegant, elliptical arches; the centre arch being 100 feet wide, and those on each side decreasing in a regular gradation to the smallest, at each extremity, which is 70 feet wide. The brcadth of the 
bridge is 42 feet, and the length from wharf to wharf 995 . The upper surface is a portion of a very large circle, which forms an elegant figure, and is of convenicnt passage over it. The whole expense was $150,840 l$.

Wooden BRIDGES. The simplest case of these edifices, is that in which the road-way is laid over beams placed horizontally, and supported at each end by piers or posts. This method however is deficient in strength and width of opening: it is therefore recessary, in atl works of any magnitude, to apply the principles of trussing, as used in roofs and arches. Wooden bridges of this kind are stiff frames of carpentry, in which, by a proper disposition, beams are put so as to stand instead of solid bodies, as large as the spaces which the beams enclose; and thus two or three more of these are sct in abutment with each other, like mighty arch stones.

Palladio has given several very elegant designs of wooden bridges; one of which is that over the river Cismoine. 'The river where this bridge is erected, is 100 feet wide; this wilth is divided into six equal parts; and at the end of each part, excepting at the banks, which are strengthened with pilasters of stone, the beams are placed that form the breadth of the bridge, upon which, a little space being left at their ends, were placed other beams lengthwise, which form the sides. Over these, directly upon the first, the colonelli (king-posts) were disposed on each side; these king-posts arc connected to the beams which furm the breadth of the bridge, by means of irons passing through the projecting ends of the beams, and bolied and pinned through both.

Mr. Coxe, in the first volume of his Travels, has slightly described a very singular bridge at Wittingen in SwitzerIand; the construction of which is quite simple. The span is 230 feet, and it rises only 5 . The arches approach to a catenarian form, built up of 7 courses of solid lngs of oak, in lengths of 12 or 14 feet, and 16 inches or more in thickness. These are all picked of a natural shape, suited to the intended curve; so that the wood is no where cut across the grain to trim it into shape. 'These logs are laid above each other, so that their abutting joints are al ternate like those of a brick wall; or, in the language of the workmen, they break joint. It is indeed a wooden structure simply built up by laying the picces upon each other, taking care to make the abutting joints as close as possible. They are not fastened together by pins or bolts, but held united by iron straps, which surround them at the distance of 5 feet from each other, where they are fastencd by bolts and keys. The two arches being erected and well butted against the rock on each side, were freed from their supports, and allowed to settle. They are so placed that the intended road intersects them about the misldle of their height. The road-way is supported by cross-joints which rest on a long horizontal summer-beam; and this is connected with the arches on each side by uprights holted into them. The whole is covered with a roof which projects over the arches on each side to def(nd them from the weather. Three of the spaces between these uprights have struts or braces, which give the upper work a sort of trussing in that part. This bridge is of a strength much more than adequate to support any load that can be laid upon it; though it is manifest, by the attempts to truss the ends, that it was the contrivance of a person ignorant of principle. It was the work of one Ulrich Grubenhamm, of I'uffen, in the canton of Appenzcl, a carpenter without education, but celebrated for scveral works of the same kind.

At Schaffhausen, in Switzerland, where the Rline flows with great rapidity, several stone bridges had been destroyed, when in 1754 Grubenhamm offered to throw a wooden bridge of a single arch across the river, which is nearly 390 feet wide. The magistrates, however, required that it should consist of two arches, and that he should for that purpose employ the middle pier of the last stone bridge, which would divide the new one into two unequal arche's, of $t 7 \approx$ and $t 93$ feet span. The carpenter did so, but contrived to leave it a matter of doubt whether the bridge is at all supported by the middle pier. It was erected on a plan nearly similar to the Wittingen bridge, at the expense of a bout $8000 l$ sterling. Travellers inform us, that it shook if a man passed over it; yet waggons heavily laden also went over it without danger. We are sorry to add that this curious bridge was burnt by the French when they evacuated Schaffhausen in April 1799.

Iron BRIDGES, are those in which iron is the chief material used. Iron brilges are the exclusive invention of. British artists. The first that has been erected on a large scale, is that over the river Severn at Colebrook Dale, in. Shropshire. This luridge is composed of 5 ribs, and 'each rib of 3 concentric arcs, connected together by radiating pieces. The interior arc forms a complete semicircle, but the others extend only to the cills under the road-svay. These arcs pass through an upright frame of iron at each end, which serves as a guide; and the small space in the haunches between the frames and the outer are is fitted in with a ring of about 7 . feet diameter. Upon the top of the ribs are laid Gast-iron plates, which sustain the roadway. The arch of this bridge is 100 feet 6 inches in span; the interior ring is cast in two pieces, each piece being about 70 feet in length. It was constructed in the year 1779 , by Mr. Abraham Darby, iron-master at Colebrook Dide, and must be consilered as a very bold effort in the first instance of adopting a new material. The total weight of the metal is $378 \frac{1}{2}$ tuns.

'The sccond iron bridge of which the particulars have conic to nur knowledge was that designed by Mr. Thomas Paine, author of many political works. It was constructed by Messrs. Walker at Rotherham, and was brought to Londun and set up in a bowling-grecn at Paddington, where it was exhibited for the first time. After which it was intended to have been sent to America; but Mr. Paine not being able to defray the expense, the manufacturers took it back, and the malleable iron was afterwards worked up in the construction of the bridge at Wearmouth.

The thircl iron brilge of importance erecterl in Great Britain, was that over the river Wear at Bisbop Wearmouth, near Sunderland, the chief projector of which was Rowland Burton, esq. M. P. It consists of a single arch, whose span is 236 fert; and as the springing stones at cach sisle project 2 feet, the whole opening is 240 feet. The arch is the seument of a circle of about $44+4$ feet diameter, its versed sine is 3.4 fect, and the whole leight from low water about 100 fert, admitting vessels of from 2 to 300 tons burthen to pas under it without striking their masts. A series of 105 blocks form a. rib, and 6 of these ribs compose the brealth of the bridge. The spandrels, or the spaces between the arch and the road-way, are fillex up with cast-iron circles which tonch the outer circumfercnce of the arch, and at the same time support the 
road-way, thus gradually diminishing from the abutments towards the centre of the bridge. There are also diagonal iron bars, which are laid on the tops of the ribs and extended to the abutments, to keep the ribs from twisting. Tlie superstructure is a strong frame of timber planked over to support the carriage-road, which is composed of marl, lime-stone, and gravel, with a cement of tar and chalk immediately upon the planks, to preserve them. The whole width of the bridge is 32 feet. The abutments are masses of almost solid masonry, 24 feet in thickness, 42 in breadth at bottom, and 37 at top. The south pier is founded on the solid roek, and rises from about 22 feet above the bed of the river. On the north side the ground was not so favourable, so that it was necessary to carry the foundation 10 feet below the bed. The weight of the iron in this extraordinary fabrie amounts to 260 tons; 46 of these are malleable, and 214 cast. The entire expense was $27,000 l$.

Many other iron bridges have since been constructed or proposed by different persons, and in various situations, which are exhibited and described in the first volume of my Tracts.

There are various other kinds of bridgcs, besides these already mencioned, such as hanging or pendant bridges, draw-bridges, flying bridges, \&c, \&c, and even watural bridges, of which kind a most wonderful one is described by Mr. Jefferson, in his State of Virginia; and another 'described by Don Ulloa, in the province of Angariz, in South America. Sec also ARCH.

BRIGGS (HEN RY), one of the greatest mathematicians in the 16 th and 17 th centuries, was born at Warleywood, near Halifax in Yorkshire, in 1556 . From a grammarschool in that country he was sent to St. Joln's-college, Cambridge, in 1579; where after taking both the degrees in arts, he was chosen fellow of lis college in 1588. He applied himself chiefly to the study of mathematics, in which he so greatly excelled, that in 1592 he was made examiner and lecturer in that faculty; and soon after, reader of the physical lecture, founded by Dr. Linacer.

On the settlement of Gresham-college, in London, he was chosen the first professor of geometry there, in $\mathbf{1 5 9 6 .}$ Soon after this, he constructed a table, for finding the latitude, from the variation of the magnetic needle being given. In the year 1609 he contracted an acquaintance with the learned Mr. James Usher, afterwards archbishop of Armagh, which was continued many years after by letters, two of Mr. Briggs being still extant in the collection of Usher's letters that were published: in the former of these, dated August 1601, he writes, among other things, that he was engaged in the subject of eclipses; and in the latter, dated the 10 th of March 1615 , that be was wholly taken up and employed about the noble invention of logarithms, which had come out the year before, and in the improvement of which he had afterwarels so great a concern. For Briggs immediatelyapplied himself to the study and improvement of them; expounding them also to his auditors in his lectures at Gresham-college. In these lectures he proposed the alteration of the scale of logarithms, from the byperbolic form which Lord Napier bad given them, to that in which 1 should be the logarithm of the ratio of 10 to 1 ; and soon after he wrote to I.ord Napier to make tise same proposal to himsclf. In the year 1616 Briggs made a visit to Napicr at Edinburgh, to confer with him on this change; and the next year be did the same also. In these conferences, the alterations were agreed. upon accordingly, and on Briggs's return from his second visit, in 1617 , he published the first chiliad, or 1000 of his logarithms. Sce the Introduction to my Logarithms, for an ample history of these transactions.

In 1619 he was made the first Savilian professor of geometry at Oxford; and resigned the professorship of Gresham-college the 25th of July 1620. At Oxford be settled bimself at Merton-college, where he continued a most laborious and studious life, employed partly in the duties of his office as geometry lecturer, and partly in the computation of the logarithms, and in other useful works. In the year 1622 he published a small tract on the "North-west passage to the South Seas, through the continent of Virginia and Hudsun's Bay ;" the reason of which was probably, that he was then a member of the company trading to Virginia His next performance was his great and elaborate work, the Arithmetica Logarithmica in folio, printed at London in 1624; a stupendous work for so short a time! containing the logarithms of 30 thousand natural numbers, to 14 places of figures besides the index. Briggs lived also to complete a table of logarithınic sines and tangents for the 100 th part of every degree to 14 places of figures, besides the index; with a table of natural sines for the same 100th parts to 15 places, and the tangents and secants for the same to ten places; with the description and construction of the whole. These tables were printed at Gouda in 1631 , under the care of Adrian Vlacq, and published in 1633 , under the title of Trigonometria Britannica. In the construction of these two works, on the logarithms of numbers, and of sincs and tangents, our author, besides extreme labour and application, manifests the highest power of. genius and invention; as we here, for the first time, meet with several of the most important discoveries in the mathematics, and what have hitherto been considered as of much later invention; sueb as the Binomial Theorem; the Differential Method and Construction of Tables by Differences; the Interpolation by Differences; with Angular Sections, and several other ingenious compositions; a particular account of which may be seen in the Introduction to my Mathematical Tables.

This truly great man terminated his useful life the 26 th of January 1630, and was buried in the choir of the chapel of Merton-college. As to his character, he was not less esteemed for his great sincerity and otber eminent virtues, than for his excellent skill in mathematics. Doctor Smith gives hiı the character of a man of great probity; easy of access to all; free from arrogance, moroseness, envy, ambition, and avarice; a contemner of riches, and contented in bis own situation; prefirring a studious retirement to all the splendid circumstances of life. The lrarned Mr. Thomas Gataker, who attended his lectures when he was reader of mathenatics at Canbriclge, represents him as highly esteemed by all persuns skilled in mathematics, both at home and abroad; and says, that desiring him once to give his judgment concerning judicial astrology, his answer was, "that he conceived it to be a mere system of groundless conceits." Oughtred calls him the mirror of the ag', for his excellent skill in geometry. And one of his successors at Gresham-crillege, the learned Dr. Isaac Barrow, in his oration there upon his admission, has drawn his cliaracter more fully; celebrating his sreat abilities, skill, and industry, particularly in perfect- 
ing the invention of logarithms, which, without his care and pains, might have continued an imperfect and useless design.

His writings were more important than numerous: some of them were published by other persons: the list of the principal part of them is as follows:

1. A Table to find the Height of the Pole; the Magnetical Declination being given. This was published in Mr. Thomas Blundeville's Theoriques of the Seven Planets; London 1602, 4 to.

2. Tables for the Improvement of Navigation. These consist of, A table of declination to every minute of the ecliptic, in degrees, minutes, and seconds: $A$ table of the sun's prosthaphaereses: A table of equations of the sun's ephemerides: A table of the sun's declination: Tables to find the beight of the pole in any latitude, from the height of the pole star. These tables are printed in the $2 \mathrm{~d}$ edition of Edward Wright's treatise, entitled, Certain Errors in Navigation detected and corrected; London 1610, 4to.

3. A Description of an Instrumental Table to find the Part Proportional, devised by Mr. Edward Wright. This is subjoined to Napier's table of logarithms, translated into English by Nr. Wright, and after his death published by Briggs, with a preface of his own; London, 1616 and $1618,12 \mathrm{mo}$.

4. Logarithmorum Chiljas Prima. Lond. 1617, svo.

5. Lucubrationes et Anmotationes in Opera Posthuma J. Neperi; Edinb. 1619, 4to.

6. Euclidis Elementorum vi libri priores \&c. Lond. 1620 , folio. This was printed without lis name to it.

7. A treatise of the North-west Passage to the South Sea \&c. By H. B., Lond. 1622,4 to. This was reprinzed in Purchas's Pilgrins, vol. 3, p. 852.

8. A rithmetica Logarithmica, \&c. Lond. 1624, folio.

9. Trigonometria Britannica, \&c. Goudie 1633, folio.

10. Two letters to Archbishop Uslier.

11. Mathenzatica ab Antiquis minus Cognita.-This is a summary account of the most observable invention of modern mathematicians, communicated by Mr. Briggs to Dr. Geurge IJakewill, and published by him in his Apologie; Lond. folio.

Besides these publications, Driggs wrote some other pieces, that bave not been printed: as,

(1.) Commentaries on the Geometry of Peter Ramus.

(2.) Duæ Epistolæ ad celeberrimum virum Chr. Sever. Longomontanum. One of these letters contained some remarks on a treatise of Longomontanus, about squaring the circle; and the other a detence of arithmetical geometry.

(3.) Animadverses Geornetrica: 4to.

(4.) De codem Argumento: 4.to.- These two were in the possession of the late: Mr. Joneg. 'They both contain a great variety of geometrical propositions, conecrning the properties of many figures, with several arithmetical computations, relating to the circle, angular sections, \&c. The two following were also in posscission of Mr. Jones:

(5.) A treatise of Common Arithmetic; folio.

(6.) A letter to Mr. Clarke of Gravesend, dated 25 Fel. 1606 ; with which he sends lim the description of a ruler called Bedwell's ruler, with directions lıow to use it.

BRIGGS (WILLIAM), an emincut physician in the latter part of the 17 th century, was born at Norwich, for which town his father was four times member of parliament. He studied at the university of Cambridge; and afterwards travelled into France, where he attended the lectures of the famous anatomist Vienssens, at Nontpelier. On lis raturn he published his Ophthalmographia, in 1676 . The year following he was made doctor of medicine at Cambridge, and soon after fellow of the College of Physicians at London. In 1682 lie resigned his fellowship to his brother: and the same year his 'Theory of $\mathrm{Vi}$ sion was published by Hooke. The ensuing year he sent to the Royal Society a continuation of that discourse, which was publisherl in their Transactions; and the same year he was appointed physician to St. 'Thomas's Hospital. In 1684 he communicated to the Royal Society two remarkable cases relating to vision, which were likewise printed in their Transactions; and in 1685 be published a Latin version of his 'Theory of Vision, at the desire of Mr. Newton, afterwards Sir lsaac, then professor of mathematics at Cambrilge, with a recommendatory epistle from him prefixed to it. He was afterwards made physician in ordinary to King William, and continued in great esteem for his skill in his profession till his death, which happened the 4 th of September 1704.

BRIGGs's Logarithms, that species of them in which 1 is the lugarithm of the ratio of 10 to 1 , or the logarithm of 10 . See Logatritims.

BROKEN Number, the same as Fraction; which sce.

Broken Ray, or Ray of Refraction, in Dioptrics, is the line into which an incident ray is refracted or broken, in crossing the second medium.

BROUNCKER, or BROUNKER (WILL1AM), Lord Viscount, of Castle I,yons in Ireland, son of Sir William Brounker, afterwards made viscount in 164.5 , was born about the year 1620 . He very early discovered a genius for mathematics, in which he afterwards became very eminent. He was made doctor of physic at Oxford, June 23,1646 . In 1657 and 1658 , he was engaged in a correspondence by letters on tnathematical subjects with Dr. John Wallis, who published them in his Comnercium Epistulicum, printed 1658, at Oxford. Brounker was one of the nobility and gentry who signed the remarkable dectaration concerning hing Charles 11, published in April 1660.

Afrer the Restoration, Lord Brounker was made chancellor and keeper of the grent seal to the queen, also one of the commissioners of the navy, and naster of St. Katherine's hospital near the Tower of London. He was one of those great men who first formed the Royal Society, of which he was by the charter appointed the first president in 1662: which office he held, with great advantage to the society, and honour to limself, till the anniversary election, Nov. 30,1677, when he was succeeded by SijJoseph Williamson. He died at his house in St. James'sstreet, Westminster, the 5 th of April 1684; and was succeeded in his title by his younger brother Harry, who died in January 1687.

Lord Brounker had several papers inserted in the Philosophical 'Transactions, the chicf of which were, 1. Experiments concerning the Recoiling of Guns.-2. A Series for the Quadrature of the Hyperbola; which was the first series of the kind on that subject.-3. Several of his letters to Archbishop Usher were also printed in Usher's Letters; as well as some to Dr: Wallis, in his Commercium Epistolicum, above mentioned; particularly an approximation to the circular area by means of continued fractions, of which he was the first inventor.

BROWN (Sir WILLJA Ai), a noted physician and miscellancous writer of the 18th century. lle first settled 
at Lynn in Norfolk, where he published a translation of Dr. Gregory's Elements of Catoptrics and Dioptrics; to which he added, 1. A Method for finding the Foci of all Specula and Lenses universally; as also Magnifying or Lessening a given object by a given Speculum or Lens, in any assigned proportion.-2. A Solution of those Problems which Dr. Gregory has left undemonstrated.-3. A particular account of Nicroscopes and Telescopes, from Mr. Huygens; with the discoveries made by Catoptrics and Dioptrics.

Having acquired a competence by his profession, he removed to Queen's-square, Ormond street, London, where he resided till his death, in $\mathbf{1 7 7 4}$, at $\$ 2$ years of age; leaving by his will two prize-medals to be annually contended for by the Cambridge poets.

Sir William Brown it seems was a very facetious man; and a great number of his lively essays, both in prose and verse, were printed and circulated among his friends. The active part taken by him in the contest with the licentiates, in 1768 , occasioned his being introduced by Mr. Foote in lis Devil upon Two Sticks.-Upon Foote's exact representation of him with his identical wig and coat, tall figure, and glass stiffly applicd to his eye, he sent him a complimentary card for hatving so happily represented him; but as he had forgot the muff, he sent him his own.-This goodnatured way of resenting disarmed Foote completely.He used to frequent the annual ball at the ladies' boarding-school, Queen-square, merely as a neighbour, a goodnatured man, and a lover of youthful company. A dignitary of the church being there one day to see his daughter dance, and finding this upright figure stationed in the room, told him he believed he was Hermippus Redivivus who lived anhelitu puellarum.- IVhen be lived at Lymn, a pamphlet was written against him; which he nailed up against his house-door.-At the age of 80 , on St. Luke's day 1771, be came to Batson's coffee-house in his laced coat and band, and fringed white gloves, to introduce himself to Mr. Crosby, then lord mayor. A gentleman present observing that he looked very well, he replied, he had neither wife nor debts.

BRUIN (JOHN DE), a respectable mathematician and philosopher, was born at Gorcum in 1620; and having pursued a course of philosophy at Leyden, and prosecuted his studies at Bois-le-Duc and Utrecht, he removed to Leyden, where he taught matheniatics. He afterwards became professor at Utrecht, and besides his official duties made dissertations in private, and read lectures on Grotius's treatise De Jure Belli ct Pacis. He was distinguished by his attachment to experimental philosophy, and by his observations in astronomy. Mle published dissertations De vi altrice; De corporum gravitate ct levitate; De cognitione Dej naturali; De lucis causa et origine, \&c. He had a dispute with Isaac Yossius, to whom he wrote a letter printed at Amsterdam in 1663, wherein he criticiscs Vossius's book De matura et proprietate lucis; and strenuously inaintains the hypothesis of Descartes. He died in 1675 , after be had been professor 23 years.

BULLIALD (ISMAEL), an eminent astronomer and mathematician, was born it Laon in the Isle of France in 1605. He travelled in his youth, for the sake of improvement, and gave very early proofs of his antronomical genius. Riccioli styled him, $\Lambda$ stronomus profunda indaginis. Ile first published his dissertation cntitled, PhiloLaus, sive de vero Systemate Mundi; or his true system of the world, according to Philolaus, an ancient philosopher and astronomer. Afterward, in the year 1645, he set forth his Astronomia Philolaica, grounded upon the hypothesis of the earth's motion, and the elliptical orbit described by the planet's motion about a cone. To which he added tables entitled, Tabula Philolaica: a work which Riccioli says ought to be attentively read by all students of astronomy.-He considered also the hypothesis or approximation of Bishop Ward, and found it not to agree with the planet Mars; and he showed, in his defence of the Philolaic astronomy against the bishop, that fiom four observations made by Tycho on the planet Mars, that planet in the first and third quarters of the mean anomaly, was more forward than it ought to be according to Ward's hypothesis; but in the $2 \mathrm{~d}$ and 4 th quadrant of the same, the planet was not so far advanced as that hypothesis required. He therefore set about a correction of the bishop's hypothesis, and made it answer more exactly to the orbits of the planets, which were most excentric, and introduced what is called, by Street in his Caroline Tables, the Variation: these tables were calculated from this correction of Bulliald's, and exceeded all others in exactness that had before been published. This correction is, in the judgment of Dr. Gregory, a very happy one, if it be not set above its due place; and be accounted no more than a correction of an approximation to the true system: for by this means we are enabled to gather the coequate anomaly a priori and that directly from the means, and the observations are well enough answered at the same time; which, in Mercator's opinion, no one bad effected before.-It is remarkable that the ellipsis which he has chosen for a planet's motion, is such a one as, if cut out of a cone, will have the axis of the cone passing through one of its foci, viz, that next the aphelion.

In 1657 was published his treatise De Lineis Spiralibus, Exerc. Geom. et Astron. Paris, 4 to. In 1682 came out at Paris, in folio, his large work entitled, Opus norum ad Arithmeticam Infinitorum; a work which is a cliffuse amplification of Dr. Wallis's Arithmetic of Infinites, and which Wallis treats of particularly in the Soth chapter of his Historical Treatise of Algebra. He wrote also two Admonitions to $A$ stronomers ; the first, concerning a new star in the neck of the Whale, appearing at some times, and disappearing at others; the second, concerning a nebulous star in the northern part of Andromeda's girdle, which had not been noticed by any of the ancients. This star also appeared and disappeared by turns. And as these phenomena were new and surprising, he strongly recommended the observing them to all that might be curious in astronomy.

BURGO (LUcas Pacroll DE), a cclebrated author on arithmetic and algebra, who flourished in the 15 th century; and whose works on those subjects were the first that we have in print. See an account of them in the article Algebra.

BURNING, the action of fire on some pabulum or fuel by which its minute parts are put into a violent motion, and some of them, assuming the nature of fire, fly off in orbem, while the rest are dissipated in vapour, or reduced to ashes.

BURNı⿻-Glass, or Burning-Mirror, a machine by which the sun's rays are collected into a point; and by that means their force and effect are extremely heightened, so as to burn objects placed in it.

Burning-glasses are of two kinds, convex and concave. 
The convex ones are lenses, which, acting according to the laws of refraction, incline the rays of light towards the axis, and unite them in a point or focus. The concave ones are mirrors or reflectors, whether made of polished metal or silvered glass, and which acting by the laws of reflection, throw the rays back into a point or focus before the glass.

The use of burning-glasses, it appears, is very ancient, many of the old authors recording some effects of them. Diodorus Siculus, Lucian, Dion, Zonaras, Galen, Anthemius, Eustatius, Tzetzes, and others, relate that by means of them Archimedes set fire to the Roman fleet at the siege of Syracuse. Tzetzes is so particular in his account of this matter, that his description suggested to Kircher the method by which it was probably accomplished. That author says that “Archimedes set fire to Marcellus's navy by means of a burning-glass composed of small square mirrors, moving every way upon binges; which, when placed in the sun's rays, directed them upon the Poman fleet, so as to reduce it to ashes at the distance of a bowshot." And the burning power of reflectors is mentioned in Euclid's Optics, theor. 31. Again, A ristophanes, in his comedy of The Clouds, introduces Socrates as examining Strepsiades about a method he had discovered of getting clear of his deots. He replies, that " he thought of making use of a burning-glass which he had hitherto used in kindling his fire; for should they bring a writ against me, I'll immediately place my glass in the sun at some little distance from it, and set it on fire." Pliny and Lactantius have also spoken of glasses that burn by refraction. The former calls them balls or globes of crystal or glass, which being exposed to the sun, transmit a heat sufficient to set fire to cloth, or corrode the dead flesh of those patients who stand in need of caustics; aud the latter, after Clemens Alexandrinus, observes that fire may be kindled by interposing glasses filled with water between the sun and the buject, so as to transmit the rays to it.

Among the ancients, the most celebrated burning-mirrors were those of Archimedes and Proclus; by the former was burnt the fleet of Marcellus, as above mentioned; and by the latter, the navy of Vitellius, besicging Byzantium, according to Zonaras was burnt to ashes.

Among the noderns, the most remarkable burningglasses, arc those of Magine, of 20 inches diameter; of Sepatala of Milan, near 2 inches diameter, and which burnt at the distance of 15 feet; of Settala of Villette; of Tschirnhausen, of Buffon, of Trudaine, and of Parker.

Villette, a French artist at Lyous, made a large mirror, wibich was bought by Tavernier, and presented to the king of Prussia ; a second, bought by the king of Denmark; a third, presented to the Royal Academy by the king of France; and a fourth came to England, and was publicly shown. This mirror is 47 inches wide, being a segment of a sphere of 76 inches radius; so that its focus is about 38 inches from the vertex ; and its substance is a composition of tin, copper, and tin-glass. Some of its effects were as follow:

A silver sixpence melted in - _ - $\quad$ - $7 \frac{\mathrm{r}}{2}$

1 George the 1st's halfpenny in - $\quad$ - $\quad$ - 16 and runs with a liole in - $\quad-\quad-34$

Tin molts in

Cast iron in

Slate in

A fossil shell calcines in

Kiv.. I.
Piece of Pompey's Pillar vitrifies, the black part in 50 sec. the white part in $\mathbf{5 4}$

Copper ore in - - $\quad$ - $\quad$ - $\quad$ - $\quad$ - $\quad-8$

Bone calcines in 4, and vitrifies iu - - $\quad-33$

An emerald melts into a substance like a torquois stone; a diamond weighing 4 grains loses $\frac{7}{8}$ of its weight; the asbestus vitrifies, as all other bodies will do if kept long enough in the focus; but when once vitrified, the mirror can go no further with them. Philos. 'Trans. vol. 4, p. 198.

Tschirnhausen's reflecting mirrors produced equally surprising eficcts; as they may be seen described in the Acta Erudit. for 1687 , p. 52. And other persons have made very good ones of wood, straw, paper, ice, and other substances capable of taking a proper form and polisi.

Every lens, whether convex, plano-convex, or convexoconvex, collects the sun's rays, dispersed over its convexity into a point by refraction; and it is therefore a burning-glass. 'The most considerable of this kind is that made by Tschirnhausen, and described in the same Acta Erudit. The diameters of his lenses are from 3 to 4 fcet, having the focus at the distance of 12 feet, and its diameters an inch and a half. To make the fucus more vivid, the rays are collected a second time, by a second lens parallel to the first, and placed at such a distance that the diameter of the cone of rays formed by the first lens is equal to the diameter of the second; so that it receives then all, and the focus is reduced from an inch and a half to half the quantity, and consequently its force is quadrupled. 'This glass vitrifies tiles, slate's, purnice-stones, \& $c$, in a moment. It melts sulphur, pitch, and all rosins, under water; the ashes of vegetables, woods, and other matters, are transmuted into glass, and every thing applied to its focus is either melted, changed into a calx, or into fumes. The author observes that it succeeds best when the matter applied is laid on a hard charcoal well burnt. But though the force of the solar ray's be thus found so surprising, yet the rays of the full moon, collected by the same burning-glass, do not show the least increase of heat.

Sir Isaac Newton presented a burning-glass to the Roya) Society, consisting of 7 concave glasses, so placed, that all their foci join in one physical point. Each glass is about $11 \frac{1}{2}$ inches diametcr: six of them are placed contiguous to, and round the 7 th, forning a kind of spherical segment, whose subtense is about $34 \frac{1}{2}$ inches: the common focus is about $22 \frac{\pi}{2}$ inches distant, and about an inch in diameter. This glass vitrifics brick or tile in 1 second, and melts gold in 30 seconds.

M. Buffon also made a varicty of very powerful buraing-glasses, both as mirrors and as lenses; but at length concluded with one which is probably of the same nature with that of $A$ rchimedes, and consisted of 400 mirrors reflecting their rays all to onc point, and with which he could melt lead and tin at the distance of 140 feet; and with others he consumed substances at the distance of 210 feet. See Philos. Trans, vol. 44; Buffon's Hist. Nat. Suppl. vol. 1; or Montucla's Hist. des Math. vol. 1, p. 246.

It appears there is no solid substance capable of resisting the efficacy of modern burning-glasses; though water, \&c, are not afiected by thein at all. Thưs, Messrs. Macquer and Baumé have succeeded in melting small portions of platina by means of a concave glass 22 inches diameter, and 28 inches focus, though this metal is not fusible by the strongest fires that can be cxcited in furnaces, or sustained by any chemical appuratus. Yct it was long since obscrved, by the Academicians del Cimento, that spirit-of- 
wine could not be fired by any burning-glass which they used; and notwithstanding the great improvements these instruments have since received, MI. Nollet has not been able, by the most powerful burning-mirrors, to set fire to any inflammable liquors whatever.

However, a large burning-lens, for fusing and vitrifying such substances as resist the fires of furnaces, and especially for the application of heat in vacuo, and in certain other circumstances in which heat cannot be applied by other means, has long been a desideratum with persons concerned in philosophical experiments : and this it appears is now in a great neasure accomplished by Mr. Parker, an ingenious glass-manufacturer in Fleet-street, London. His lens is niade of flint-glass, and is 3 feet in diameter; but when fixed in its frame, exposes a surface of 32 inches in the clear; the length of the focus is 6 feet 8 inches, and its diameter one inch. The rays from this large lens are received and transmitted through a smaller, of 13 inches diameter in the clear within the frame, its focal length 29 inches, and diameter of its focus 3-8ths of an inch: so that this second lens increases the power of the former more than 7 times, or as the square of 8 to the square of 3 .

From a great number of experiments made with this lens, the following are selected to serve as specimens of its powers :

Substances Fused.

\begin{tabular}{|c|c|c|c|c|c|c|c|}
\hline \multicolumn{3}{|c|}{ seristances tesede. } & & & & \multicolumn{2}{|c|}{ in Sec. in Grs. } \\
\hline Scoria of wroug & ght iron & & - & - & - & 2 & 12 \\
\hline Common slate & - & - & - & - & - & 2 & 10 \\
\hline Silver, pure & - & - & - & - & - & 3 & 20 \\
\hline Platina, pure & - & - & - & - & - & 3 & 10 \\
\hline Nickell - & - & - & - & - & - & 3 & 16 \\
\hline Cast iron, a cul & & - & - & - & - & 3 & 10 \\
\hline Kearsh - & - & - & - & - & - & 3 & 10 \\
\hline Gold, pure & - & - & - & - & - & 4 & 20 \\
\hline Crystal pebble & - & - & - & - & - & 6 & 7 \\
\hline Cank, or terra & ponder & rosa & - & - & - & 7 & 10 \\
\hline Lava - & - & - & - & - & - & 7 & 10 \\
\hline Asbestos - & - & - & - & - & - & 10 & 10 \\
\hline Bar iron, a cub & & - & - & - & - & 12 & 10 \\
\hline Steel, a cube & - & - & - & - & - & 12 & 10 \\
\hline Garnet - & - & - & - & - & - & 17 & 10 \\
\hline Copper, pure & - & - & - & - & - & 20 & 33 \\
\hline Onyx - & - & - & - & - & - & 20 & 10 \\
\hline Zeolites - & - & - & - & - & - & 23 & 10 \\
\hline Pumice-stone & - & - & - & - & - & 24 & 10 \\
\hline Oriental emera & & - & - & - & - & 25 & 2 \\
\hline Jasper - & - & - & - & - & - & 25 & 10 \\
\hline White agate & - & - & - & - & - & 30 & 10 \\
\hline Flint, oriental & - & - & - & - & - & 30 & 10 \\
\hline Topaz, or chry & ysolite & - & - & - & - & 45 & 3 \\
\hline Common limes & stone & - & - & - & - & 55 & 10 \\
\hline Whitc rhomboi & idal spe & & - & - & - & 60 & 10 \\
\hline Volcánic clay & - & - & - & - & - & 60 & 10 \\
\hline Cornish moorst & tone & - & - & - & - & 60 & 10 \\
\hline Rough cornclia & & - & - & - & - & 75 & 10 \\
\hline Rotten-stonc & - & $\therefore$ & - & - & - & so & 10 \\
\hline
\end{tabular}

BurNING Zone, or Torrid Zone, the space within $23 \frac{\pi}{2}$ degrees of the equator, both north and south.

BURROIVS (WILLIAM), a writer on the subject of navigation in the 16 th century. His "Discourse of the Variation of the Compass or Magnetic Necdle," is subjoined to Norman's "Newe Atractive." Burrows had been a famous navigator, having used the sea from 15 years of age, and for his merit was promoted to be controller of the navy by Queen Elizabeth. He shows bow to determine the variation several ways, setting down many observations of it, made by an azimuth compass of Norman's invention, but improved by himself. He demonstrates the falsehood of the rules used to find the latitudes by the guard-stars. He particularizes many errors in the then sea-charts, occasioned by the neglect of the variation; addiug, "But of these coasts (towards the north) and of the inwarde partes of the countries of Russia, Muscovia, $\delta c$, I have made a perfect plat and description, by myne owne experience in sundrie voiages and travailes, bothe by sea and lande, to and fro in these partes, which $I$ gave to her majestie in anno 1578." And lastly, he justly blames Coignet's instrument, called a Nautical Hemisphere; but speaks too severely against the writers on navigation.

BUSHEL, a measure of capacity for dry goods; as grain, pulse, fruits, \&c, containing 4 pecks, or 8 gallons, or $\frac{1}{8}$ of a quarter. By act of parliament, made in 1697 , it was ordained that " Every round bushel with a plain and even bottom, being made $18 \frac{x}{2}$ inches wide throughout, and 8 inches deep, shall be esteemed a legal IVinchester bushel, according to the standard in his majesty's cxchequer." Now a bushel being thus made will contain $2150.42 \mathrm{cu}-$ bic inches, and consequently the corn gallon contains only $268 \frac{4}{5}$ cubic inches. - The weight of a bushel of good corn, or pulse, $\& c$, is commonly accounted to be as follows; viz, of

$\begin{array}{llll}\text { Wheat - } & \text { - } & 60 \mathrm{lb} \text {. avoirdupois. } \\ \text { Rye - } & \text { - } & 56 \\ \text { Barley - } & - & 50 \\ \text { Beer, or Big } & - & 44 \\ \text { Oats - } & - & 40 \\ \text { Pease - } & - & 60 \\ \text { Beans - } & - & 60 \\ \text { Potatoes (beaped) } & 56\end{array}$

But some experienced persons say that the bushel of good wheat weighs and produces as below; viz,

$\begin{array}{llll}\text { Bushel of wheat weighs - } & - & 63 \\ \text { Yiclds of meal } & - & - & 62 \\ \text { Loss in grinding } & - & - & -\end{array}$

$\begin{array}{lllll}\text { Loss in grinding } & - & - & - & 1 \\ \text { Bran in the meal } & - & - & - & 6\end{array}$

BUTMENTS, are those supporters, or props, by which the feet of arches or the extremities of bridges are supported; and should be made very strong and firm.

BUTTRESS, is an arch, or a mass of masonry, serving to support the sides of a building, wall, or the like, on the outside. See Arcu, and Arcu-Bov'sint. 


\section{c}

C A L

C AISSON, in Architecture, a kind of chest or flat-bottomed vessel, in which the pier of a bridge is built, then sunk to the bottom of the water, and the sides loosenel and taken off from the bottom, by a contrivance for that purpose; the bottom of the caisson being left under the pier as a foundation to it. The caisson is kept afloat till the pier is built above the height of low-water mark; and for that purpose, its sides are either made of more than that beight at first, or else gradually raised to it as it sinks by the weight of the work, so as always to keep its top above water. Mr. Labelye tells us, that the caissons in which he built some of the piers of Westminster bridge, contained 150 loud of fir timber, of 40 cubic feet each, and that it was of more tonnage or capacity than a 40-gun ship of war.

CAISSON, in Military Affairs, is sometimes used for a chest; and in particular for a lomb or shell chest, and is used as a superficial inine, or fourneau. This is done by filling a chest either with gunpowder and loaded shells, or else with shells alone, and burying it in a spot where an enemy, besieging a place, is expected to come, and then firing it by a train to destroy the men.

CALANIUS Pastoralis, in Music, the Shepherd's Pipe; an ancient musical instrument, the origin of the flute, at first made of a straw or reed.

CALCULATION, the act of computing several sums, by adding, subtracting, multiplying, dividing, \&cc. From calculus, in allusion to the practice of the ancients, who used calculi, or little stones, in making computations, in taking suffrages, and in kecping accounts, \&c; as we now use counters, figures, \&c. Calculation is more particularly used to signify the computations in astronomy, trigonometry, \&ce, for making tables of astronomy, of logarithms, ephemerides, finding the times of eclipses, and such like.

CALCULATOR, a person who makes or performs calculations. - lt is also the name of a machine constructed by Mr. Ferguson, in the shape of an orrery, for exhibiting the motions of the earth and moon, and resolving a variety of astronomical problems.' See his Astron, 4to, p.' 265 , or $8 \mathrm{vo}$, p. 393.

CALCULATORES were anciently accountants who reckoned their sums by calculi, or little stones, or counters. - In ancient canons too we find a sort of diviners or enchanters, censured under the detiomination of Calculatores, probably so called from their calculating nativities.

CÁLCULUS denotes primarily a small stone, pebble, or counter, used by the ancients in making calculations or computations, taking of suffrages, playing at tables, and the like.

CALCULUS denotes now a certain way of performing mathematical investigations and resolutions. Thus, we sity the Arithmetical or Numcral Calculus, the Algebraical Calculus, the Differential Calculus, the Exponential Cirlculus, the Fluxional Calculus, the Integral Calculus, the Literal or Symbolical Calculus, \&c; for which, see each respective word.

Arithmetical or Numeral Calculus, is the method of
C $\perp$ L

performing arithmetical computations by numbers. See ArtThMETIC, and Number.

Algebraical, Literal, or Symbolical CA Lcu Lus, is the method of performing algebraical calculations by letters or other symbols. See ALgEBRA.

Differential $\mathrm{C}_{A} \mathrm{LCULUS}$, is the arithmetic of the indefinitely small differences of variable quantities; a mode of computation much used by foreign mathematicians, and introduced by Leibnitz, as similar to Newton's method of Fluxions. See Differential \&c.

Exponential Cancubus, is the applying the fluxional or differential methods to exponential quantities; such as $a^{x}$, or $x^{x}$, or $a y^{x}, \& c$. See Exponential.

Fluxional $\mathrm{C}_{\mathrm{A}} \mathrm{LCU} \mathrm{Cus}$, is the method of fluxions, invented by Newton. See Fluxions.

Integral CaLcolus, or Summatorius, is a method of integrating, or summing up differential quantities; and is similar to the finding of fluents. See INTEGRAL and Fiuent.

CALCU Lus Literalis, or Literal Calculus, is the same with algebra, or specious arithmetic, so called from its using the letters of the alpbabet; in contradistinction from numeral arithmetic, in which figures are used.

CALENDAR, or KALENDAR, a distribution of time as accommodated to the uses of life; or an almanac, or table, containing the order of days, weeks, months, feasts, \&c, accurring in the course of the year: it is so called from the word Calendæ, which among the Romans denoted the first days of every month, and anciently was written in large characters at the head of each month. See ALm ANAC, C Alen DS, Month, Time, Year, \&c.

In calendars, the days were originally divided into octoades, or eights; but afterwards, in imitation of the Jews and Orientals, they were divided into hebdomades, or sevens, for what we now call a week : which custom, Scaliger observes, was not in use among the Romans till after the time of 'Theodosius.

Divers calendars are established in different countries, according to the different forms of the year, and distributions of time: as the Persian, the Roman, the Jewish, the Julian, the Gregorlan, \&c, calendars.-The ancient Ruman calendar is given by Ricciolus, Struvius, Danet, and others; in which we perceive the order and number of the Roman holy-days and work-days.-The Jewish calendar was fixed by Rabbi Hillel, about the year 360 ; from which time the days of their year may be reduced to those of the Julian calendar.- The three Christian calendars are given by Wolfius in his Elemerts of Chronology; as also the Jewish and Mohamedan calendars. Other writers on the calendars are Vieta, Clavius, Scaliger, Blondel, \&rc.

The Roman $\mathrm{C}_{\mathrm{ALEN}} \mathrm{DAR}$ was first formed by lomulus, who distributed time into several periods for the use of his followers and people under his command. He divided the year into 10 montlss, of 304 days; begiming on the first of March, and ending with December.

Numa rcformed the calendar of Romulus. Headded the montlss of January and February, making it to com- 
mence on the first of January, and to consist of 355 days. But as this was evidently deficient of the true year, he ordered an intercalation of 45 days to be made every 4 years, in this manner, viz, Every 2 years an additional month of 22 days, between February and March; and at the end of each two years more, another month of 23 days; the month thus interposed, being called Marcedonius, or the intercalary February.

Julius Cæsar, with the aid of Sosigenes, a celebrated astronomer of those times, furtber reformed the Roman calendar, whence arose the Julian calendar, and the Julian or old style. Finding that the sun performed his annual course in $36 j$ days and a quarter nearly, lie divided the year into 365 days, but every 4 th year 366 days, adding a day to the $23 \mathrm{~d}$ of February, which being the 6 th of the calends, and thus reckoned twice, gave occasion to the name Bissextile, or what we also call leap-year.

This calendar was further reformed by order of the pope, Gregory xill; from whence arose the term Gregorian calendar and style, or what is now called the new style, which is now observed by almost all nations in Europe. The year of Julius was too long by nearly 11 minutes, which amounts to about 3 days in 400 years; the pope therefore, by the advice of Clavius and Ciaconius, ordained that there should be omitted a day in every 3 centuries out of 4; so that every century, which would otherwise be a bissextile year, is made to be only a common year, excepting only such centuries as are exactly divisible by 4 , which happens once in 4 centuries. See Bissextile. This reformation of the calendar, or the new style, as we call it, commenced in the countries under the popish influence, on the 4 th of October 1582, when 10 days were omitted at once, which had been over-run since the time of the council of Nice, in the year 325, by the surplus of 11 minutes each year. But in England it only commenced in 1752 , when 11 days were onitted at once, the $3 \mathrm{~d}$ of September being accounted the 14th that year; as the surplus minutes had then amounted to 11 days. And now, since the jear 1800 , another day has been addel on account of the odd minutes; so that now our new style differs from the old by 12 days.

Juliun Christian CALENDAR, is that in which the days of the week are dicternsined by the letters $A, B, C, D, E, F, G$, by means of the sular cycle; and the new and full moons, particularly the paschal full moon, with the feast of Easter, and the other moveable feasts depending on it, by means of golden numbers, or lunar cycles, rightly disposed through the Julian year. See Cyche, and Golden Num ber.

In this calendar, it is supposed that the verual equinox is fixed to the 21st day of March; ; and that the golden numbers, or cycles of 19 years, constantly indicate the places of the new and full moons; though both are erroneous; and from hence arose a great irregularity in the time of Easter.

Gregorian CALENDAR, is that which, by means of epacts, rightly disprosed through the several months, determines the new and full moons, with the time of Easter, and the moveable feasts depending on it, in the Gregorian year. This differs therefore from the Julian calendar, both in the form of the year, and in as much as epacts are substituted instead of golden numbers. Sce ЕРАст.

Though the Gregorian calendar be more accurate than the Julian, yet it is not without imperfections, as Scaliger and Calvisius have fully shown; nor docs it appear pos- sible to devise any one that shall be quite perfect. I ft the Reformed Calendar, and that which is ordered to be observed in England, by act of parliament made the 24th. of George II, come very near to the point of accuracy: For, by that act it is ordered that "Easter-day, $\mathrm{cn}$ which the rest depend, is always the first Sunday atier the full moon, which happens upon, or next after the 21 st day of March; and if the full-moon bappens on a Sunday, Easterday is the Sunday after."

Reformed, or Corrected, CALEN a R is that which, rejecting all the apparatus of golden numbers, epacts, and dominical letters, determines the equinox, and the paschal full moon, with the moveable feasts depending on it, by computation from astronomical tables. This calendat was introduced amoug the protestant states of Germany in the year 1700 , when 11 days were omitted in the month of February, to make the corrected style agree with the Gregorian. This alteration in the form of the year, they admitted for a time; in expectation that, the true quantity of the tropical year being at length more accurately determined by observation, the Romanists wóld agree with them on some more convenient intercalation.

French New CALENDAR, is quite of a different form of calendar from all others. It commenced in France on the 22d of September 1792 , soon after the change of government. At the time of printing this (viz, in July 1794), tt does not certainly appear whether this new calendar will be made permanent or not; but merely as a curiosity in the science of chronology, a very brief notice of it inay here be added, as follows.

The year, in this caleudar, commences at midnight the beginning of that day in which fall the true autumal equinox for the observatory of Paris. The year is divided ints 12 equal months, of 30 days eacb; after which 5 supplementary days are added, to complete the 365 days ot the ordinary year: these 5 days do not belong to any'month. Each month is divided into three decade's of 10 days each; distinguished by 1 st, $2 \mathrm{~d}$, and $3 \mathrm{~d}$ decade. All these are named according to the order of the natural numbers, viz, the $1 \mathrm{st}, 2 \mathrm{~d}, 3 \mathrm{~d}, \& \mathrm{c}$, month, or day of the decade, or of the supplensentary days. The years which receive an intercalary day, when the position of the equinox requires it, which we call embolismic or bissextile, they call olympic; and the period of four years, ending with an ulympic year, is called an olympiacle; the intercalary day being placed alter the ordinary five supplementary days, and making the last day of the olympic year. Lach day, from midnight to midnight, is divided into 10 parts, each part into 10 others, and so on to the last measurable portion of time.

In this calendar the months and days have new names. The first three months of the year, of which the antumu is composed, take their etymology, the first from the vintage which takes place from September to October, and is called Vendemaire; the sccond, Brumaire, from the mists and low fogs, which show as it were the transudation of nature from Uctober to Novenıber: the third, Frimaire, from the various colds which are generally felt from Nuvember to December: The three winter montis tahe their etymology, the first, Nivose, from the snow which whitens the earth from December to January; the second, Pluviose, from the rains which usually fill in greater abundance from January to February; the third, Ventose, from the wind which dries the eartli from February to March. The three spring months take thcir ctymology, the first, Ger- 
minal, from the fermentation and developement of the sap from March to April ; the second, Floreal, from the blorring of the flowers from April to May; the third. Prairial, from the smiling fecundity of the meadow crops from May to June. Lastly, the three summer months take their etymology, the first, Messidor, from the appearance of the waving ears of corn and the golden harvests which cover the fields from June to July; the second, Thermidor, from the heat, at once solar and terrestrial, which inflames the air from July to August; the third, Fructiclor, from the fruits gilt and ripened by the sun from August to September. Thus, the whole 12 months are,

$\begin{array}{ll}\quad \text { Autuin. } & \text { Spring. } \\ \text { Vendemaire } & \text { Germinal } \\ \text { Brumaire } & \text { Floreal } \\ \text { Frimaire. } & \text { Prairial. } \\ \text { Winter. } & \text { SummEr. } \\ \text { Nivose } & \text { Messidor } \\ \text { Pluviose } & \text { Thermidor } \\ \text { Ventose. } & \text { Fructidor. }\end{array}$

From these denominations it follows, that by the mere pronunciation of the name of the month, every one rcadily perceives three things and all their relations, viz, the kind of season, the temperature, and the state of vegetation : for instance, in the word germinal, his imagination will easily conceive, by the termination of the word, that the spring commesces; by the construction of the word, that the elementary agents are busied; and by the signification of the word, that the buds unfold themselves.

As to the names of the days of the week, or decade of 10 days each, which they have adopted instead of severi, as these bear the stamp of judicial astrology and heathen mythology, they are simply called from the first ten numbers; thus,

$\begin{array}{ll}\text { Primidi } & \text { Sextidi } \\ \text { Duodi } & \text { Septidi } \\ \text { Tridi } & \text { Octidi } \\ \text { Quartidi } & \text { Nonidi } \\ \text { Quintidi } & \text { Decadi. }\end{array}$

In the almanac, or annual calendar, instead of the multitucle of saints, one for each day in the year, as in the popish calendars, they annex to every day the name of some animal, or utensil, or work, or fruit, or flower, or veretablé, \&cc, appropriate and most proper to the times. This calendar however was changed for the old one again, with the change of the form of government. For the calendars of the Greeks, Egyptians, Jews, Syrians, Persians, Mahomedans, Ethiopians, \&c, see the article $\mathbf{Y}_{\mathbf{E A}} \mathbf{R}$.

Calendar Months, the solar months as they stand in the calendar, viz, January 31 days, \&c. The number of days in each month may ue suggested to the memory by the following canou:

"Thirty days liath September,

April, June, and November;

February has twenty-eight alone,

And all the rest have thirty-one."

Astronomical CALEN DAR, au instrument engraven upon copper-plates, printed on paper, and pasted on board, with a brass slider which carrics a hair, and shows by inspection the sun's meridian altitude, right ascension, declina-tion, rising, sctting, amplitude, \&c, to a greater exactness than can be shown by the common globes.

CALENDS, Calenda, in the Roman Chronology, denoted the first days of each month; being so named from

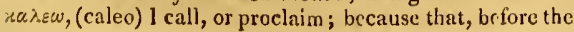

publication of the Roman Fasti, and counting their montlis by the motion of the monn, a priest was appointed to observe the tirst appearance of the new moon; who, having seen ber, gave notice to the president of the sacrifices to offer one; and calling the people together, he proclaimed unto them how they should reckon the days until the nones; pronouncing the word Caleo 5 times if the nones should happen on the 5 th day, or seven times if they happened on the 7 th day of the month.

The calends were reckoned backwards, or in a retrograde order: thus, for example, the first of May being the calcnds of May, the last or 30th day of April was the pridie calendarum, or $2 \mathrm{~d}$ of the calends of May; the 29th of April, the $3 \mathrm{~d}$ of the calends, or before the calends: and so back to the 13 th, where the ides commence; which are likewise numbered bacl: wards to the 5 th, where the nones begin; andalso reckoned after the same manner to the first day of the month, which is the calends of April.

Hence comes this rule to find the day of the calends answering to any day of the month, viz, Cousider how many days of the montl are yet remaining after the day proposed, and to that number add 2, for the number of or from the calends. For example, suppose it were the $23 \mathrm{~d}$ day of April, it would then be the 9th of the calends of May: for April containing 30 days, from which 23 being taken, there remains 7 ; to which 2 being added, makes the sum 9. And the reuson for this addition of the constant uumber 2 , is because the last day of the month is called the $2 \mathrm{~d}$ of the calends of the month following.

CALIBER, or CALIPER, is the thickness or diameter of a round body, particularly the bore or width of a piece of ordnance, or that of its ball.

Caliber Compusses, or Ca laper Campasses, or simple Calipers, a sort of compasses made with bowed or arched legs, the better to take the diameter of any round body; as the diameters of balls, or the bores of guns; or the diameter, and even the length of casks, and such like. The best sort of calipers usually contain the following articles, viz, 1st, the measure of convex diameters in inches \&c; $2 \mathrm{~d}$, of concave diameters; $3 \mathrm{~d}$, the weight of iron shot of given diameters; 4th, the weight of iron shot for given gun bores; 5 th, the rlegrees of a semicircle; 6 th, the proportion of troy and avoirdupois weight; 7 th, the proportion of English and French feet and pounds weight; 8 th, factors used in circular and spherical figures; 9th, tables of the specific gravities and weights of bodies; 10 th, tables of the quantity of powder necessary for the proof and service of brass and iron guns; 11 th, rules for conputing the number of shot or shells in a complete pile; $12 \mathrm{th}$, rules for the fall or descent of beavy bodies; $13 \mathrm{th}$, rules for the raising of water; 14th, rules for firing artillery and mortars; 15 th, a line of inclses; 16 th, logarithmic scales of numbers, sines, versed sincs, and tangents; 17 th, a sectoral line of equal parts, or the line of lines; $18 \mathrm{th}$, a sectoral line of planes and superficies; and 19th, a sectoral line of sulids.

CAlippic, Period, in Chronology, a period of 76 years, continually recurring; at every repetition of which, it was supposed, by its inventor Calippus, an Athenian istronomer, that the mean new and full moons would always return to the same day and hour.

About a century before, the golden number or cycle of 19 years had been invented by Meton, which Calippus finding to contain 19 of Nabonassar's year, 4 days and $\frac{33}{45} \frac{1}{9}$, to avoid fractions he quadrupleal it, and so produced, 
his period of 76 years, or 4 times 19 ; after which he supposed all the lunations \& c would regularly return to the same hour. But neither is this exact, as it brings them too late by a whole day in 225 years.

CALORIC, in the new Chemical Nomenclature, is that supposed material substance which possesses the power of exciting the feeling of heat in the animal body; or it is the nuatter and the cause of heat.

The general properties of caloric are the following: If it be considered as material, it must be a substance of extreme subtlety and tenuity, entirely imponderable by human instruments, derived jmmediately from the sun together with light, and like liglit obeying certain and similar laws of reflection and refraction, and also elicited by cumbustion and by various other irstances of chemical action, percussion or collision, friction, the electric spark, galvanism, \&c: when uncombined, moving with immense velocity in every direction, and constantly tending to an equiIibrium; penetrating all bodies in nature with more or less facility, and thereby enlarging their dimensions, without increasing their weight: when combined, probably entering into chemical union with other bodies, and thereby constituting the different states of liquid, vapour, or gas: and lastly, when applied sudelenly to the animal body, occasioning the sensations of warmth, heat, or, in greater intensity, burning. See the articles LIGHT and HEAT.

Caloric, then, is a word used to denote that substance by which the phenomena of heat are produced; and the sume as was formerly termed igneous fluid, malter of heat, and other analogous demominations. There are perhaps few subjects respecting which a more remarkable versatility of general opinion has been evinced, than with regard to the existence or non-existence of this principle. Are the physical effects of heat produced by the operation of a material fluid, sui generis? or is heat merely an affection, consisting in internal vibrations and collisions of its particles, or in some other mode of corpuscular action, of which we are ignorant, and is there consequently no such thing as caloric? These are the questions that have divided the opinions of many of the ablest philosophers of both ancient and moderu times; but though difierent opinions still prevail on this subject, yet the former of these hypotheses seems to be most generally received. Among the ancients who supposed the materiality of heat, may be reckoned Zeno and Cicero: the latter, in his book De Natura Deorum, says expressly, that heat is combined with water, as its liquefaction sufficiently proves; and that it cannot frceze nor congeal into snow or hoar-frost, without suffering that heat to escape, \&c, \&c: which is very nearly the idea that we have of it at the present day; and indeed this appear's to have been the most prevailing opinion till the time of Lord Bacon, who, in his work De Forma Calidi, considers heat as the effect of an intestine motion, or mutual collision of the particles of the body lieated; an expansive undulatory motion in the minute particles of the body, by which they tend with some rapidity towards the circumference, and at the same time incline a little upwards; which idea was also with some modifications adopted by Descartes, Newton, Boyle, and the other mechanical philosophers of the succeding age. The general opinion however among chemists, was still in favour of the materiality of heat; and as cliemistry became more generally known, so this opinion gained strongth, till Count Rumford by some experiments secmed to show, that heat was imponderable, and capable of being produced, ad infinitum, from a finite quantity of matter, which again threw some doubt on this question; and though these results appear, in some legree, to have been confuted by subsequent experiments, yet is the matter at present undecided, and will probably always be involved in some obscurity. We caunot here enter into the merits of the particular arguments that have been advanced on both sides of this intricate and interesting inquiry; but we shall enumerate a few of the principal experiments that have been made on this subject, and shall then leave the reader to draw his own conclusion, as to the inateriality or immateriality of heat, or the cause of heat; or, in other words, of the existence or non-existence of that substance which is defined by the term Caloric.

It is known, that when water freezes, a portion of heat is given out by it during the congelation, which is so considerable, that if we were to conceive it to be transmitted to, and imbibed by an equal quantity of water at the temperature of $32^{\circ}$ of Fahrenheit's thermometer, the latter would be heated to no less than $140^{\circ}$, or to $172^{\circ}$; if therefore heat were a ponderable substance, it might be imagined that a given quantity of water would become lighter when frozen in a vessel hermetically sealed. This circumstance therefore induced sume philosophers to ascertain, if possible, the ponderability of caloric, and thus to decide the question on which so many opinions had been formed. Of the experiments made for this purpose, that of $\mathrm{Dr}$. Fordyce seems to have been conducted with the greatest caution. He took a glass globe, three inches in diameter, with a short neck, and weighing 451 grains; poured into it 1700 grains of water from the New River, London, and then seiled it hermetically. The whole of which then weighed $2150 \frac{\mathrm{x}}{\mathrm{T} 2}$ grains, at the temperature of $32^{\circ}$ : this was then put for 20 minutes into a frcezing mixture of snow and salt, till some of it was frozen, and was then taken out, and wiped perfcetly dry with a linen cloth, and next with a clean dry piece of washed leather; and being then immediately weighed, it was found to be $\frac{r}{6}$ th of a grain heavier than before: this was repeated five times, at each time more of the water was frozen, ind more wcight gained; and when the whole was frozen, it was found to have gained $\frac{3}{16}$ ths of a grain; but the temperature of the globe, and contained ice, by having been kept in the frozen mixture, had been reduced to 120 of Fahreuheit's thermometer: and on allowing it to rise to $32^{\circ}$, it was found to weigh $\frac{1}{16}$ th of a grain more than when the water was fluid. The beam in this experiment was so adjusted that it would mark $\frac{\mathrm{T}}{\mathrm{T} \delta}$ of a grain.

By this result therefore it appears that water by freezing, an operation in which it evolves caloric, becomes heavier, and that of course the addition of caloric to a body renders it absolutely lighter; which if arlmitted goes to prove, that caloric counteracts the efiect of gravitation, as it does that of contiguous attraction or affinity. 'This cxperiment was repeated by Morveau and Chaussier, and with the same result, the water being heavier after it had been frozen in glass resscls hermetically sealed, than it was before when in its fluid state.

This subject soun attracted the attention of Lavoisicr; and bis experiments, which were published in the Memoirs of the French $A$ cademy, led him to conclude that the wright of borlies is not at all altered by heating or cooling them, and consequently that caloric produces no sensible change in the weight of bodies. Count Rumford also made similar experiments, and was at first led to the same 
conclusion as Dr. Fordyce; but afterwards, by varying the experiments, be had reason to suppose that he had been mistaken in the first instance, and he finally came to the same conclusion as Lavoisier. Hence then we may conclude from these experiments, and others of a simiIar nature which have been made on this subject, that if caloric be pessessed of weight, it exists at least in such a state of rarity, that its gravity cannot be ascertained by experiment. But Count Rumford went still further, and endeavoured to prove that an inexhaustible supply of heat may be produced from a finite quantity of matter; and that therefore heat must be an effect arising from some species of corpuscular action annong the constituent particles of the body, agreeably to the notions of Bacon. This experiment was conducted in the following manner. The Count caused a cylinder of brass to be turned $7 \frac{3}{4}$ inches in diameter, and 9.8 inches long, wbich was bored like a cannon with a caliber 3.7 inches in diameter, and $7 \cdot 2$ juches deep; so that the bottom was 2.6 inches in thickness. The hollow cylinder contained $385 \frac{3}{4}$ eubic inches of brass, and weighed $113.13 \mathrm{lbs}$ avoirdupois. By means of the engine used for boring cannon in the arsenal of Munich, a blunt borer, or flat piece of hardened steel, 4 inclies long, 0.63 inch thick, and $3 \frac{x}{2}$ inches wide, was kept with one of its extrenities, whose area was about $2 \frac{\mathrm{I}}{3}$ square inclies, pressed against the bottom of this hollow cylinder on the inside, with a force of about $10,000 \mathrm{lbs}$ avoirdupois, while the latter was turned about its axis, with a velocity of 32 revolutions in a minute. In one experiment, the cylinder was covered on the outside with a coating of thick flannel, to prevent the access of heat from the atmospbere; and in another, the borcr was made to work through a collar of leatbers, so as to prevent access of air also to the interior of the bore; and in a thircl, the whole cylinder was immersed in water, the borcr still working through a collar of leathers, so as to prevent the access of the water to the interior of the bore; again, in a fourth, the collar of leathers was removed, and the water had access to the bottom of the interior of the bore where the friction took place. The result was, that in all these cases beat was generated by the friction in sufficient quantity to cause about $26 \frac{x}{2}$ lbs of icc-cold water to boil in two hours and a half; or at about the same rate as that at which it would have been produced by 9 large wax candles. 'The capacity of brass for heat, or its power of producing it by friction, did not appear to be diminish$\mathrm{cd}$, and it seemed that this generation of heat would have gone on for ever, if the friction had been continued, or that the source of it was inexhaustible. Now any thing which en insulated body, or a system of bodies, can continue to supply without limitation, cannot possibly be a inaterial substance; and hence the conclusion was, that heat must be an effect arising from some species of corpuscular action among the constituent particles of the body.

Some otber experiments of M. Pictet, Mr. Davy, and other celebrated chemists, seem to be in favour of the conclusions drawn by Count liumford from those above stated; but there are others, that appear to contradict this principle, some of which were made by the Count himself.

If heat arise only from the corpuscular motion of the particles of the body, it would follow that no heat could be produced, but in those cases where motion could be communicated; whence again heat could not be derived through a vacuum. But Count Rumford, hy a very satis- factory experiment, has shown that heat may be communicated through a Torricellian vacuum, in which there could be nothing to communjeate or propagate motion; for though such a vacuum may be not quite complete, yet the matter of it must be in such a state of rarity, as to prevent us from supposing for a moment, that it is capable of exciting motion in the particles, of the body placed in it; and consequently, that the conclusion drawn from the boring experiment was inaceurate: and a recent discovery made by Dr. Herschel, has at last nearly put an end to the dispute; by demonstrating that caloric is not a property of matter, but a peculiar substance; or at least that we hare the same reason for considering it as a substance, as we have for believing light to be material.

Dr. Herschel had been enployed in making observations on the sun by means of telescopes; and to prevent the inconvenience arising from the heat, he used coloured glasses; but these glasses, when thry were deep enough coloured to intercept the light, very soon cracked and broke to pieces; which circunistance induced him to examine the heating power of the different coloured rays. He therefore made eacb of them in its turn fall upon the bulb of a thermometer, near which two other thernometers were placed to serve as a standard; and the number of degrees that the thermometer exposed to the coloured ray rose above the other two thermometers, indicated the heating power of that ray. He thus found, that the most refrangible rays loave the least heating power; this power gradually increasing as the refrangibility diminishes. The heating power of the green, violet, and red rays, as determined by these experiments are expressed by Dr. Herschel, by the annexed numbers.

$$
\begin{aligned}
& \text { Violet }=16 \\
& \text { Green }=22 \cdot 4 \\
& \text { Red }=55
\end{aligned}
$$

It struck Dr. Herschel as very remarkable, that the illominating power and the heating power of the solar rays should follow such different laws: the first existing in greatest perfection in the middle of the spectrum, and djminishing as we approach either extremity; but the second constantlyincreasing from the violet end, and being greatest at the red end. This led him to suspect, that probably the beating power did not'stop at the termination of the visible spectrum, but is continued beyond it; he therefore placed the thermometer completely without the boundary of the red ray, but still in the line of the spectrum; and it rose still higher than when exposed to the red ray : and on shifting the thermometer still further it contiuned to rise; the rise not reaching its maximum till the thermometer was half an inch beyond the visible spectrum: when shifted still further, it sank a little, but the power of heating did not cease at the distance of an inch and a half trom the real ray.

These experiments were afterwards repeated and fully confirmed by Sir Henry Englefield, in the presence of some very goorl judges. The apparatus was very different from that of Dr. Herschel, being contrived on purpose to obviate certain objections which had been made to the conclusion drawn by that celcbrated philosopher; and the bulbs of the thermometer's were mostly blackened. The following are the results of these experiments.

Thermometer in the blue ray rose in $3^{\mathrm{m}}$ from $55^{\circ}$ to $56^{\circ}$

green - $-3-54=58$
yellow $-3=56-62$
full rud $-2 \frac{3}{2}-56=72$
confines of res $2 \frac{1}{2}=58=73 \frac{3}{2}$
the visiblc light $2 \frac{1}{2}-61-79$




\section{C $\triangle \mathrm{L}$}

[ 264

The thermometer with its bulb blackened, rose much more when placed in the same circumstances, than the thermometers whose bulb was either naked, or whitened with paint; as is shown in the following table:

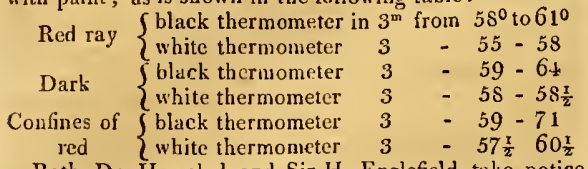

Both Dr. Herschel and Sir H. Engleficld take notice of a faint blush of red, of a seni-eval form, visible when the rays beyond the end of the spectrum were collected by a lens:

From these experiments it follows, that there are rays emitted from the sun which produce heat, but have not the power of illuminating; and that these are the rays that produce the greatest quantity of licat: and consequently caloric is emitted from the sun in rays, and the rays of caloric are not the same with the rays of light.

We have thus enumerated many of the principal experiments that have bcén made, in order to prove the materiality or immateriality of heat; and without insisting further on this subject, on which probably the present state of knowledge is insufficient for furming a decided opinion. It seems however that, under all the circumstances attending this inquiry, we ought rather to consider it as a material substance; because of the two theories, that which supposes it to be so is by far the most intelligible, the most agreeable to the analogy of nature, and the least exceptionable; and we shall accordingly regard it as an elastic fluid, sui generis, capable of pervading, with various degrees of facility, all the solid bodies with which we are acquainted, and of being imbibed and retained by theur in different proportions, according to their respective degrees of specific attraction, or capacity for it.

It will easily be conceivecl, that from the elasticity and power of pervading other substances, which we have attributed to this fluid, it inust mecessarily follow that when a body is by any means charged with a larger quantity of it than is proportional to its mass and capacity, when compared with thuse of other bodies in its vicinity; the surplus will be communicated to those other bodies, until the density of the fluid in every body in the system becomes equal; in like manner as a quantity of air thrown into one of a number of ressels conmunicating with each other, will pass from it to the others, until it becomes distributed among them all, in proportion to their respective capacities; or till it becones of equal density in them all: and this state of density or compression of the caloric is called its temiperature.

The celerity and facility with which this fuid pervades different substances, are however extremely various, as may be shown by the following experiment. Take a small cylindrical earticn or wooden vessel, between three and four inches in diameter, and insert into it, through boles drilled in its bottom for that purpose, several wires about $\frac{\pi}{8}$ th of an inch in dianeter, and 6 or 7 inches long, of equal size in every respect, but of different metuls, as gold, silver, copper, iron, lirass, and zinc; and also two small rods, one of glass and the other of woorl, of equal dimensions with the wire; so that they may each of them project about $\frac{1}{2}$ an incl on the inside of the vessel; the other parts leeing on the outsidc, and forming a kind of stand for it. Dip a portion of the lower cxtrcmities of
C A L

these wires into meliel wax, so that they may becóme equally coated with it; and when the wax has cooled, ill the vessel with a hrated fluid, as boiling water, or melted lead, and observe the intervals between the time of filling it, and the melting of the wax; wbich intervals, will indicate the relative conducting power of the substances.

We shall now conclude this article by a slight investigation of the effects arising from the condensation or rarefaction of this fluid in bodies: by the former their temperature is elevated, or they are heated; and by the latter it is depressed, or they become cooled.

In our investigation of the most universal operations in nature, we generally find that the more immediate instruments employed for the production of the effects which we perceive, are two counteracting powers, by whose co-operation, and mutual counterpoise, the necessary equilibrium is preserved, and the purposes of their creation effected, with greater regularity thau would probably result from the enployment of a single agent. Thus, gravity is, as we know, counteracted by inertia; and the effects of cohesion, a no less universal principle than gravity, are in like manner restrained and inudified by the agency of caloric: and as without inertia, all the celestial bodies would be drawn together intu some one part of extended space; so without heat, all the matter in the universe would become a congealed and concrete mass; and fluidity and organization, vegetation, and life, would have no existence. Sulicls become first expanded, and then liquified, by its influence, and liquids assume the aëriform state. It is essential to the idea of a solid body, that it should in a certain degree resist buth compression and dilatation; that is, that 1 s particles should repel each other when brought nearer together, and attract each other when further separated, within certain limits; and it is incontrovertible that they can only remain in a quiescent state, by the equilibrium of these opposite forces. The latter of these constitutes cohesion, and the existence of the former is probably attributable to the substance of which we are now speaking. But be this as it may, it is at least a fact, that the repulsive encrgies of these corpliscles are increased, and the sphere of action enlarged by its influence, for the body becomes expanded; that is, this equilibrium no longer takes place, unless its molecula are removed further from each other than when it was at a lower temperature. Elevate its temperature still further, and the body fuses; that is, the attractive power of its particles becomes annililated, or wholly counteracted, and th'y are only kept together, like a quantity of slot, by the action of gravity, operating independently on each. Increase still the action of heat on the body, and the splsere of repulsion of its particles, or the distance to which this power operates, again becones much further extended, perliaps infinitely so, and is thercfore sufficient to overpower the action of gravity, and the body becomes resolved into an elastic fuid. We know of no power that is capable of resisting this universal sulvent: gold itself may be expanded into vapour by the action of heat; and as these eflects are always producible by the same cause, su is it at least probable, that the converse of this is also true; that whenever a substance is formed in a stuc of fuidity, it is attributable solvly to this cause, and that the abstraction of the caloric, by which it is held in solution, would rgain reduce it to a solid. Morcury is, we know, capuble of congelation; and the atmosphere itselt would prubably become a conerete bolly, if we conld deprive it of all, or the greater part of its hedt. 
Hence then we arrive at a very distinctive and appropriate definition of caloric; that it is that substance, or affection of matter, which is the cause of fluidity, in all or the greater part of the bodies that we meet with in that state.

For a fuller and more detailed account of caloric and its various properties, see MIurray's or 'Thomson's Chemistry; and for the ancient opinions on this subject sec HEA T.

CAMBER-BEAM, a piece of timber cut arch-wise, or with an obtuse angle in the middle. They are commonly used in platforms, as for church-roofs, and other occasions where long timbers are wanted to lie at a small slope. A camber-beam is much stronger than another of the same dimensions; for being laid with the hollow side downwards, and having good butments at the ends, they serve for a kind of arcl..

CAMELEON, one of the constellations of the southern hemisphere, near the south pole, and invisible in our latitude. There are ten stars marked in this constellation in Sharpe's catalogue.

CAMELOPARDALUS, a new constellation of the northern hemisphere, formed by Hevelius, consisting of 32 stars first observed by him. It is situated between Cepheus, Cassiopeia, Perseus, the Two Bears, and Draco; and it contains 58 stars in the British catalogue.

CAMERA Eolia, a name given by Kirclier to a contrivance for blowing the fire, for the fusion of ores, without bellows. This is effected by means of water falling through a funnel into a close vessel, which sends from it so much air or vapour, as continually blows the fire. See Hooke's Philos. Coll. No. 3, pa. 80.

Camera Lucida, a contrivance of Dr. Hooke to make the image of any thing appear on a wall in a light room, either by day or night. See Philos. Trans. No. 38, pa.741. Another camera lucida has lately been introduced by William Hyde Wollaston, M. D. secretary of the Royal Society.

Camera Obscura, or Dark Chamber, an optical machine or apparatus, representing an artificial eye, by which the images of external objects, received through a double convex glass, are shown distinctly, and in their native colours, on a white ground placed within the machine, in the focus of the glass. The first invention of the camera obscura is ascribed to John Baptista Porta. See his Magia Naturalis, lib. 17, cap. 6 , where he largely describes the effects of it. See also the end of Gravesande's Perspective, and other optical writers, for the construction and uses of various sorts of camera obscuras.

This machine serves for many useful and entertaining purposes. For example, it is very useful in explaining the naturc of vision, representing a kind of artificial cye: it exhibits very diverting spectacles; showing images perfectly like their objects, clothed in their natural colours, but more intense and vivid, and at the same time accompanied with all their motions; an advantage which no art can imitate: and by this instrument, a person unacquainted with painting, or drawing, may delineate objects with the greatest accuracy of drawing or colouring.

Theory of the Camera Obscura. The theory and principle of this instrument may be thus explained. If any object $\triangle \mathrm{B}$ radiate through a sinall aperture $\mathrm{L}$, upon a white ground opposite to it, within a darkened room, or box, \&c ; the inage of the ubject will be painted on that ground in an inverted position. For, by the smallncss of the aperture, the rays from the object will cruss each other

VOL.1.

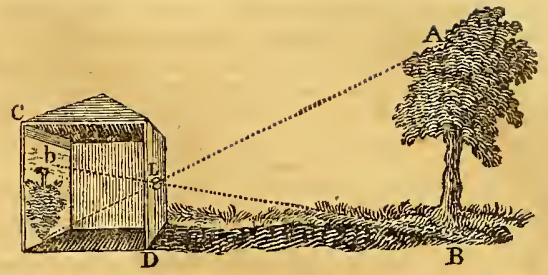

there, the inage of the point $\mathrm{A}$ being at $a$, and that of $\mathrm{B}$ at $b$; so that the whole object $\mathrm{AB}$ will appear inverted, as at $a b$. And as the corresponding rays make equal angles on both sides of the aperture, if the ground be parallel to the object, their heights will be to each other directly as their distances from the aperture.

Construction of a Camera Obscura, in which the images of external objects shall be represented distinctly, and in their genuine colours, either in an inverted or an erect situation. 1st, Darken a chamber that has one of its windows looking towards a place containing various objects to be viewed, leaving only a small aperture open in one shutter. $2 \mathrm{~d}$, In this aperture fit a proper lens, either plano-convex, or convex on both sides; the convexity forming a small portion of a large sphere. But note, that if the aperture be made very small, as of the size of a pea, the objects will be represented even without any lens at all. 3d, At a proper distance, to be determined by trials, stretch a paper or white cloth, unless there be a white wall at that distance, to receive the images of the objects: or the best way is to have some plaster of Paris cast on a convex mould, so as to form a smooth concave surface, and of a curvature and size adapted to the lens, to be placed occasionally at the proper distance. $4 \mathrm{~h}$, If it be desired to have the objects appear erect, instead of inverted, this may be done either by placing a concave lens between the centre and the focus of the first lens; or by reflecting the image from a plane speculum inclined to the horizon in an angle of 4.5 degrees; or by having two lenses included in a draw-tube, instead of one.

That the images be clear and distinct, it is necessary that the objects be illuminated by the sun's light shining upon them from the opposite quarter: so that, in a western prospect, the images will be best scen in a foremoon, an eastern prospect the afternoon, and a northern prospect about noon; a southern aspect is the least eligible of any. But the best way of any is, if the lens be fixed in a proper frame, on the top of a building, and inade to move easily round in all dircctions, by a handle extended to the person who manages the instrument; the images being then thrown down into a dark room immediately below it, on a horizontal plaster of Paris ground a little concave: for thus a view of all the objects quite around may easily be taken in the space of a few minutes; as in the case of the excellent camera obscura placed on the top of the Royal Observatory at Grcenwich.

The objects will he scen brighter, if the spectator first wait a few minutes in, the dark. Care should also be taken, that no light escape through any chinks; and that the ground be not too much illuminated. It may further be observed, that the greater distance there is between the aperture and the ground, the larger the images will be; but then at the same time the brightness is weakened more and more with the increase of distance. 
To construct a Porlable Camera Obscura. 1st, Provide a small box or chest of dry wood, about 10 inches broad, and 2 feet long or more, according to the size of the lenses. $2 d$, In one side of it, as BD, fit a sliding tube EF with two Ienses; or, to have the image at a less distance from the tube, with three lenses, convex on both sides; the diameter of the two outer ones to be about 7 inches, but that of the inner to be less, as $4 \frac{3}{4}$ or 5 inches. $3 \mathrm{~d}$, At a proper distance, within the box, set up perpendicularly an oiled paper GH, so that images thrown upon it may appear on the other side. 4th, In the opposite side, at I, make a round hole, for a person to look conveniently through with both eyes. Then if the tube be turned towards the objects, and the lenses be placed by trials at the proper distance, by sliding the tube in and out, the objects will be seen delineated on the paper, erect as before.

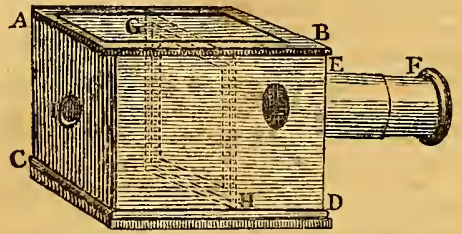

The machine may be better accommodated for drawing, by placing a nirror to pass from $\mathrm{G}$ to $\mathrm{c}$; for this will reflect the image upon a rough glass plane, or an oiled paper, placed lorizontally at AB ; and a copy of it may there be sketched with a black-lead pencil.

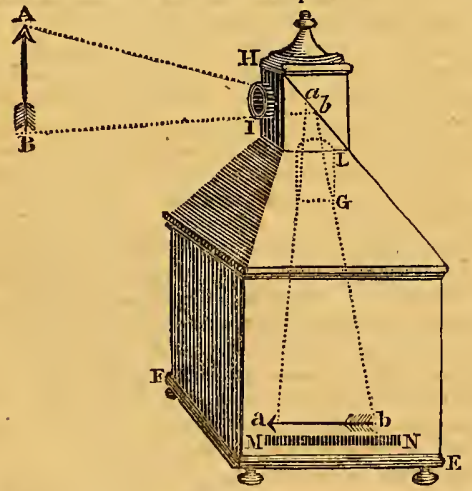

Another Portable Camera Obscura is thus made. 1st, On the top of a box or chest raise a little turret $\mathrm{H}$, open towards the object $\mathrm{AB}$. 2d, Behind the aperture, incline a small mirror $a b$ at an angle of 45 degrees, to reficet the rays $\mathrm{A} a$ and $\mathrm{E} b$ upon a lens $\mathrm{G}$ convex on both sides, and included in a tube GL. Or the lens may be fixed in the aperture. $3 \mathrm{~d}$, At the distance of the focus of this lens place a table, or board $\mathrm{Er}$, covered with a white praper, to receive the image $a b$. Lastly, In $M N$ make an oblong aperture to look through; arid an opening may also be made in the side of the box, for the convenience of drawing.

This sort of camera is casily changed into a show-box, for viewing prints, \&c: placing the print at the bottom of the box, with its upper part inwards, where it is enlightened through the front, left open for this purpose, either by day or candle-light; and the print may be viewed through the aperture in $31 \mathrm{I}$.-A varicty of contrivances for this purpose may be seen described in Harris's Optics, b. ii. sect. 4.-Mr. Storer has also procured a patent for an instrument of this kind, which he calls a delineator; being formed of two double convex lenses and a plane mirror, fitted into a proper box. One lens is placed close to the mirror, making with it an angle of 45 degrees; the other being placed at right angles to the former, and fixed in a moveable tube. If the moveable leus be directed towards the object whicb is to be viewed or copied, and moved ncarer to or farther from the mirror, till the image is distinctly furmed on a greyed glass, laid upon that surface of the upper lens which is next the eye, it will be found more sharp and vivid than those formed in the common instruments; because the image is received so near the upper lens. And by increasing the diameter and currature of the lenses, the effect will be much heightened.

CAMPANI (MaTTHEW), an eminent Italian optician. born in the diocese of Spoletto, in the 17 th century, but resided chiefly at Rome, where he was very eminent for his mcchanical contrivances of optical and philosophical instruments, and for several books on such subjects ; particularly relating to pendulums, or tinepieces, for the longitude; also to spectacles, and to telescopes, of which it is said he made the very best and largest of his time. It is said it was by his telescopes that the first Cassini made his best observations and chief discoveries in the heavens; and they still show at Paris, among the astronomical antiquities, several of Campani's object-glasses, of 8,10 , and 12 inches diameter. He was curate at Rome, and much respected.

CAMPANI (JUSEPH), younger brother and pupil to Matthew, above mentioned, was also very skilful in mechanics and pliysic, and executed his brother's plans with great ingenuity. They were both living in 1678 .

CAMUS (CIIARLES-STepileN-Lewis), a celebrated French mathematician, examiner of the Roy'al Schools of Artillery and Engineers, secretary and professor of the Royal Acalemy of Architceture, hunorary member of that of the Marine, and fellow of the Royal Socicty of London, was horn at Cressy en Brie, the 25th of August 1699. His early ingenuity in mechanics and his nwn entreaties, induced his parents to send him to study at a college in Paris, at 10 years of age; where in the space of two jears his progress was so great, that he was able to give lessous in mathematics, and thus to defray his own expenses at the college, without any further charge to lis parents. By the assistance of the celebrated Varignon, young Camus sonn ran through the course of the higice mathe matics, and acquired a name among the lcarned. He made himself more particularly known to the Academy of Sciences in 1727 , by his memoir on the subject of the prize which they had proposed for that year, viz, "To determine the most advantageous way of masting ships;" in consequence of which he was numed that year Adjoint-Mechanician to the Acrldemy; and in 1730 he was appointed professor of architecture. In less than three years after, he was honoured with the secretaryship of the same; and the $1 S$ th of $A$ pril 1733, he obtained the degree of Associate in the Academy, where he distinguished himsolf greatly by his memoirs on living forces, or bodies in motion acted on by forces, on the figure of the teetls of whecls and pinions, on pump work, and several other ingenious memoirs.

In 1736 he was sent, in company with Messicurs Clai- 
raut, Maupertuis, and Monnier, on the celebrated expedition to measure a degree at the north polar circle; in which he rendered himself highly useful, wot only as a mathematician, but also as a mechanician and an artist, branches for which he had a remarkable talent.

In 1741 Camus had the honour to be appointed Pensioner-Geometrician in the Academy; and tbe same year he invented a gauging-rod and sliding-rule, proper at once to gauge all sorts of casks, and to calculate their contents. About the year 1747 he was named Examiner of the Schools of Artillery and Engineers: and, in 1756, one of the eight mathematicians appointed to examine by a new measurement, the base which had formerly been measured by Picard, between Viltejuifve and Juvisi; an operation in which his ingenuity and exactness were of great utility. In $1765 \mathrm{M}$. Camus was elected a fellow of the Royal Society of London; and died the 4th of May 1768, in the 69 th year of his age; being succeeded by the celebrated d'Alembert in his office of Geometrician in the French Academy; and leaving behind bim a great number of manuscript treatises on various branches of the matbematics.

The works published by M. Camus are:

1. Course of Mathematics for the use of the Engineers, 4. vols. in Svo.

2. Elements of Mecbanics.

3. Elements of Arithmetic.

And his memoirs in the volumes of the Academy are:

1. Of Accelerated Motions by living forces: vol. for 1728.

2. Solution of a Geomet. Prob. of M. Cramer: 1732.

3. On the figure of the teeth and pinions in clocks: 1733 .

4. On the action of a musket-ball, piercing a pretty thick piece of wood, without communicating any considerable velocity to it: 1738 .

5. On the best manner of employing buckets for raising water: 1739 .

6. A Problem in Statics: 1740 .

7. On an Instrument for gauging of vessels: 1741 .

8. On the Standard of the Eil Measure: 1746 .

9. On the tangents of Points common to several branches of the same curve: 1747 .

10. On the operations in measuring the distance between the centres of the pyramids of Villejuifve and Juvisi, to discover the best measure of the degrce about Paris: 1754 .

11. On the Masting of Ships: Prize tom. 2.

12. The Manner of working Oars: Mach. tom. 2.

13. A Machine for moving many Colters at once: Mach. tom. 2.

CANAL, in general, denotes a long, round, hollow instrument, through which a fluid matter may be conveyed. In which sense, it amounts to the same as what is otherwise called a pipe, tube, channel, \&c. Thus the canal of an aqueduct, is the part through whicls the water passes; which, in the ancient works of this kind, is lincd with a coat of mastic of a peculiar composition.

CANAL more particularly denotes a kind of artificial river, often furnished with locks and sluices, and sustained by banks or mounds. They are contrived for divers purposes: some for forming a communication between one place and another; as the canals between Bruges and Ghent, or between Brussels and Antworp: others for the decoration of a garden, or house of pleasure; as the canals of Versailles, Fontainbleau,. St. James's Park, \&c: and others are made for draining wet and marshy lands; which last, however, are more properly callcd watcr-gangs, drains, ditches, \&ec.
It is needless to enumerate the many advantages arising from canals and artificial navigations. Their utility is now so apparent, that most nations in Europe give the highest encouragement to undertakings of this kind, wherever they are practicable. Nor did their advantagies escape the observation of the ancients; for in the earliest accounts of society we read of attempts to cut through large isthmuses, to make communications by water, either between one sea and another, or between different nations, or distant parts of the same nation, where land-carriage was tedious and expensive.

Egypt is full of canals, dug to.receive and distribute the waters of the Nile, at the time of its inundation. They are dry the rest of the year, except the Canal of Joseph, and four or five others, which may be ranked as considerable rivers. There were also subterraneous canals, or tunnels, dug by an ancient king of Egypt, by which those lakes, forned by the inundations of the Nile, were conveyed into the Mediterranean Sea.

A new canal for conveying the waters of the Nile from Ethiopia into the Red Sea without passing into Fgypt, was projected by Albuquerque, viceroy of India for the Portuguese, to render Egypt barren and unprofitable to the Turks. And MI. Gaildereau attributes the frequency of the plague in Egypt, of late days, to the decay or stopping up of the canals; which happened upon the Turks becoming masters of the country.

Herodotus relates, that the Cnidians, a people of Coria, in Asia Minor, designed to cut through the isthmus which joins that peniusula to the continent; but were superstitious enough to give up the undertaking, because it was interdicted by an oracle.

Several kings of Egypt attempted to join the Red Sea to the Mediterrinean; a project which Cleopatra was very fond of. This canal was begun, according to Herodotus, by Necus son of Psanımeticus, who desisted from the attempt on au answer from the oracle, after having lost 120 thousand men in the enterprise. It was resumed and completed by Darius son of Hystaspes, or, according to Diodorus and Strabo, by Ptolemy Philadelphus; who relate that Darius relinquished the work on a representation made to bim by unskilful engineers, that the Red Sea, being higher than the land of Egypt, would overflow and drown the whole country. It was wide cnough for two galleys to pass abreast, and its length was four days sailing. Diodorus adds, that it was also called Ptolemy's river; that this prince built a city at its mouth on the Red Sea, which he called Arsinoë, from the name of his favourite sister; and that the canal might be cither opened or shut, as occasion required. Diod. Sic. lib. 1; Strabo, Geog. lib. 17; Herod. lib. 2. Soliman the 2 d, emperor of the Turks, employed 50 thousand men in this great work; which was completed under the caliphate of Omar, about the year 635 ; but it was afterwards allowed to fall into neglect and disrepair; so that it is now difficult to discover any traces of it. Hist. Acad. Scienc. ann. 1703, pa. 110.

Botb the Grceks and Romans intended to make a canal across the Isthmus of Corinth, which joins the Morea and Achaia, for a navigable passage by the Ionian Sea into the Archipelago. Demetrius, Julius Casar, Caligula, and Nero, made several unsuccessful efforts to open this passage. But as the ancients were entirely ignurant of the use of water-locks, their whole attention was employed in making level cuts, which is probably the chief reason why they so often failcd in their attempts. Charlemague formsed $2 \mathrm{ML}, 2$ 
a design of joining the Rhine and the Danube, to make a communication between the Ocean and the Black Sea, by a canal from the river Almutz which discharges itself into the Danube, to the Reditz, which falls into the Maine, which last falls inio the Rhine near Mayence or Mentz: for this purpose he employed a prodigious number of workmen; but he met with so many obstacles from different quarters, that he was obliged to relinquish the attempt.

In China there is scarce a town or village without the advantage either of an arm of the sea, a navigable river, or a canal, by which means navigation is rendered so commoll, that there are almost as many people on the water as the land. The great canal of China is one of the wonders of art, extending from north to south quite across the empire, from Pekin to Canton, a distance of $\$ 25$ miles, and was made upwards of 900 yedrs ago. Its breadth and depth are sufficient to carry barks of considerable burthen, which are managed by sails and masts, as well as towed by hand. On this canal it seems the emperor employs near ten thousand ships. It passes through, or by, 41 large cities; there are in it 75 vast locks and sluices, to keep up the water, and pass the ships where the ground will not admit of sufficient depth of channel, besides several thousand draw- and other bridges. Indeed, F. Magaillane assures us, there are passages by canals from one end of China to the other, for the length of 600 French leagues : and that a person may go from one end of the empire to the other, either by canals or rivers, except a single day's journey by land in crossing a mountain.

The French at present have many fine canals. That of Briere, otherwise called the Canal of Burgundy, was begun under Henry rv, and finished under the direction of Cardinal Richelieu in the reign of Louis xrr.- This canal makes a communication between the Loire and the Seine, and so to Paris. It extends 11 French great leagues from Bricre to Montargis, and has 42 locks upon it.

The Canal of Orleans was begun in 1675 , for establishing a communication also between the Seine and the Loire. It is consiclerably shorter than that of Briere, and has only 20 sluices.

The Canal of Bourbon was but lately undertaken: its design is to make a communication from the river Oisc to Paris.

But the greatest and most useful work of this kind, is the junction of the Ocean with the Mediterranean by the Canal of Languedoc, called also the Canal of the Two Seas. It was proposed in the reigns of Francis I, and Henry iv, and was begun and finished under Louis XIv; having been planned by Francis Riquet in the year 1666 , and finished before his death, which happened in 1680 . It begins with a large reservoir 4000 paces in circumference, and 24 feet deep, which receives many springs from the mountain Noire. The canal is about 200 niles in length, extending fiom Narbonne to'Tholouse, being supplied by a number of rivulets in the way, and furnished with 104 locks or sluices, of about 8 feet rise each. In some places it is carried over bridges and aquelucts of vast height, which give passage underneath to other rivers; and in some places it is cut through solid rocks for a mile together.

'The new canal of the lake Ladoga, cut from Volhowa to the Neva, by which a communication is made between the Baltic, or rather Ocean, and the Caspian Sea, was begun by the czar l'cter the 1st, in 1719: by means of which the English and Dutch merehandise is easily colt- veyed into Persia, without being obliged to double the Cape of Good Hope. There was a former canal of communication between the Ladoga lake and the river Wolga, by which timber and other goods had been brought from Persia to Petersburg; but the navigation of it was so dangerous, that a new one was undertiken.

The Spaniards have several times had in view the digging a canal through the Isthmus of Darien, between North and South America, from Panama to Nombre de Dios, to make a ready communication between the $A$ t] antic and the South Sea, and thus afford a straight passage to China and the East Indies.

In the Dutch, Austrian, and French Netherlands, there is a great number of canals: that from Bruges to Osterd carries vessels of 200 tons. But it would be an endless task to describe the numberless canals in Holland, Germany, Russia, \&c. We will therefore only take a view of those in our own country.

In England, that ancient canal from the river Nyne, a little below Peterborough, to the river Witham, three inilcs below Lincoln, called by the modern inhabitants Caerdike, may be ranked among the nonuments of the Roman grandeur, though it is now most of it filled up. Morton will have it made under the emperor Domitian. Urns and medals have been discovered on the banks of this canal. which appear to confirm that opinion. Yet some authors take it to be a Danish work. It was 40 miles in length ; and, so far as appears from the ruins, must have been very broad and deep. Notwithstanding that early beginning, it is but very lately that canals have been revised in this country. They are now however bccome very numerous, particularly in the counties of York, Lincoln, and Cheshire. Mlost of the counties between the mouth of the Thames and the Bristol Channel are connected, either by natural or artificial navigations; those upon the Thames and Isis reaching within 20 miles of those upon the Severn.

The canal for supplying London with water hy means of the New River, was projected and begun by Mr. Edward Wright, author of the celebrated treatise on Navigation, about the year 1608 ; but finished by Mr. (afierwards Sir Hugh) Middleton, five years after. This canal commonces near Ware, in Hertfordshire, and takes a course of 60 miles before it reaches the cistern at Islington, which supplies several water-pipes that convey it to the city and parts adjacent. In some places it is 30 feet deep, and in others it is conveyed over a valley between two hills, by means of a trough supported by wooden arches, and rising above 23 fect in height.

The Duke of Bridgewater's Canal, projected and executed under the direction of Mr. Brindley, was begun about the year 1759. It was first designed only for conveying coals to Manchester, from a mine in the duke's cstate; but hats since been applicd to many other useful purpescs of inland navigation. This canal begins at a place callecl Worsley Mill, 7 miles from Manchester, where a bason is made capable of holding all the boats, and a great body of water which serve's as at reservoir or head to the natrigattion. The canal russ through a hill by a subtcrameous passage, large cnough for admitting long flat-bottomed boats, which are towed by a rail on each hand, mear three quiturers of a mile, to the coal-works. There the passage divides into two channels, one of which goes off 300 yards. to the right, and the other as many to the left; and both may be continued at pleasure. 'The passage is in some. 
places cut through the solid rock, and in others arched over with brick; and air-funnels, some of which are near 37 yards perpendicular, are cut, at certain distances, through the rock to the top of the hill. The arch at its entrance is about 6 feet wide, and about 5 feet high from the surface of the water; but widens within, so that in some places the boats may pass one anotber, and at the pits it is $\mathbf{1 0}$ feet wide. When the boats are loaded arid brought out of the bason, five or six of them are linked together, and drawn along the cainal by a single horse, and thus reaching Manchester in a course of 9 miles. It is broad enough for two barges to pass or go abreast; and on one side there is a good road for the passage of the people, and the borses or mules employed in the work. Tine canal is raised over public roads by means of arches; andit passes over the navigable river Irwell near 50 feet above it; so that large vessels in full sail pass under the canal, while the duke's barges are at the same time passing over them. This canal joins that which passes from the river Mersey towards the Trent, taking in the whole a course of 34 miles.

The Lancaster Canal begins near Kendal, and terminates near Eccleston, comprebending the distance of $72 \frac{1}{2}$ miles.

The canal from Liverposl to Leed is $108 \frac{1}{3}$ miles in length: that from Leeds to Selby, $23 \frac{\pi}{4}$ miles; from Chichester to Middlewich, $26 \frac{3}{4}$ miles; from the Trent to the Mersey, 88 miles; from the Trent to the Severn, $46 \frac{x}{2}$ miles. The Birningbam Canal joins this near Wolverlampton, and is $24 \frac{\pi}{4}$ miles: the Droitwich Canal is $5 \frac{\pi}{2}$ miles: the Coventry Canal, commencing near Lichfield, and joining that of the Trent, is $36 \frac{\pi}{4}$ miles: the Oxford Canal breaks off from this, and is 82 miles: the Chesterfield Canal joins the Trent near Gainsborough, and is 4.1 miles.

A communication is now formed, by means of this inland navigation, between Kendal and London, by way of Oxford; between Liverpool and Hull, by the way of Lceds; and between the Bristol Channel and the Humber, by the junction formed between the Trent and the Severn. Other schemes have been projected, which the present spirit of improvement will probably soon carry into exicution, of opening a communication between the German and Irish seas, so as to reduce a hazardous navigation of more than 800 miles by sea, into a little more than 150 miles by linct, or inland navigation; and also of joining the Isis with the Severn; besides many other canals in various parts of England.

In Scotland, a navigable canal between the Forth and Clyde, which divides that country into two parts, was meditated upon more than a century since, for transports and small ships of war. It was again projected in the ycar 1722 , and a survey marle; but isothing more was done till 1761 , when the then Lord Napier, at his own expense, had a survey, plan, and estimate, made on a small scale. In 1764, the trustees for fisherie's, \& c, in Scotlancl, procured another survey, plan, and estimate, of a canal 5 leet deep, which was to cost 79,000 pounds. In 1766 , a sulıscription was obtained by a number of the most pespectable merchants in Glasgow, for making a canal 4 fret deep and 24 feet in breadth; but when the bill was nearly obtained in parliament, it was given up on account of the smailness of the scale, and a new subscription set on foot for a canal 7 feet deep, estimated at 150,000 pounds. This obtained the sanction of parliament; and the work was begun in 1768 , by Mr. Sineaton the engineer. The extreme length of the canal from the Forth to the Clyde is 35 niles, begimning at the mouth of the Carron, and ending at Dalmure
Burnfoot on the Clyde, 6 miles below Glasgow, rising and falling 160 feet by means of 39 locks, 20 on the east side of the summit, and 19 on the west, as the tide does not ebb so low in the Clyde as in the Forth by 9 feet; and it was deepened to upwards of 8 feet. This canal was finished a few years since, after having expericnced some interruptions and delays, for want of resources, and is esteemed the greatest work of the kind in this island. Vessels drawing 8 feet water, with 19 feet in the beam and 73 feet in lengtb, pass with ease; and the whole enterprise displays the art of man in a high degree. To supply the canal with water was of itself a very great work. There is one reservoir of 50 acres 24 feet deep, and another of 70 acres 22 feet deep, in whicb many rivers and springs terminate, which it is expected will afford sufficient supply of water at all times.

\section{The Practice of Canal Digging and Inland Navigations.}

The particular operations necessary for making artiticial navigations depend upon a variety of circumstances. The situation of the ground; the vicinity or connexion of it with rivers; the ease or difficulty with which a proper quantity of water can be obtained: these and many other circumstances necessarily produce great variation in the structure of artificial navigations, and augment or diminish the labour and expense of executing them. When the ground is naturally level, and unconnected with rivers, the execution is easy, and the navigation is not liable to be disturbed by floods: but when the ground rises and lalls, and cannot be reduced to a level, artificial methods of raising and lowering vessels must be employed; which also vary according to circumstances.

Sometimes a kind of temporary sluices are employed, to raise boats over falls or shoals in rivers, by a very simple operation. 'I'wo pillars of mason-work, with grooves, are fixed, one on each bank of the river, at some distance below the' shoal. 'The boat having passed these pillars, strong planks are let down across the river hy pulleys into the grooves; by which neans the water is dammed up to a proper hoight for allowing the boat to pass up the rivey over the shoal.

The Dutch and Flemings at this day sometimes, when obstructed by cascades, form an inclined plane or rollingbridge upon dry land, along which their ves'els are drawn from the river below the cascade, into the river above it. This, it is said, was the only metbod employesl by the anciunts, and is still sometimes used by the Chinese. 'I'hese rolling-bridges consist of a number of cylindrical roller's which turn easily on pivots. And a mill is commonly built near; so that the same machinery may serve the double purpose of working the mill and drawing up vesscls.

But in the present improved state of inland navigation, these falls and shoals are commonly surmounted by means of what are called locks or sluices. A lock is a bason placed lengthwise in a river or canal, lined with walls of masonry on each side, and terminated by two gates placed across the canal, where there is a cascade or natural fall of the country; and so constructerl, that the bason being filled with water by an upper sluice to the level of the waters above, a vessel may ascend through the upper gate; or the water in the lock being reduced to the level of the water at the bottom of the cascade, the vessel may descend through the lower gate: for when the waters are brought to a level on either side, the gate on that side may be casily. opened.

Lut as the lower gate is strained in proportion to the 
depth of water it supports, when the perpendicular height of the water exceeds 12 or 13 feet, it becomes necessary to bave more locks than one. Thus, if the fall be 16 feet, two locks are required, each of $\delta$ feet fall; and if the fall be $\mathbf{2 5}$ feet, three locks are necessary, each baving 8 feet 4 inches fall. - It is evident that the side-walls of locks should be made very strong: and where the natural foundation is bad, they should be founded on piles and platforms of wood. They should likewise slope outwards, in order to resist the pressure of the earth from behind.

To illustrate this by representations: Plate 6 , fig. 1 , is a perspective view of part of a canal, with several locks, \& $c$; the vessel $\mathrm{L}$ being within the lock AC.-Fig. 2 is an elevation or upright section along the canal ; the vessel $\mathrm{L}$. about to enter.-Fig. 3, a like section of a lock full of water; the vessel $t$ being raised to a level with the water in the superior canal.- - And fig. 4 is the plan or ground section of a lock: where $L$ is a vessel in the inferior canal; $\mathrm{C}$, the under gate; $A$, the upper gate; $\mathrm{GH}$, a subterraneous passage for letting water from the superior canal run into the lock; and $\mathrm{KF}$, a subterraneous passage for water from the lock to the interior canal.

$\mathbf{X}$ and $\mathbf{Y}$ (fig, 1) are the two flood-gates, each of which consists of two leaves, pressing against one another, so as to form an obtuse angle, the better to resist the pressure of the water. The first $(x)$,prevents the water of the superior canal from falling into the lock; and the second $(Y)$ dams up and sustains the water in the lock. These floodgates ought to be very strong, and to turn freely on their hinges. They should also be made very tight and close, that as little water as possible may be lost. And, to make them open and shut with ease, each leaf is furnished with a long lever $\mathrm{A} b, \mathrm{~A} b ; \mathrm{c} b, \mathrm{c} b$.

By the subterraneous passage $\mathbf{G H}$ (fig. 2, 3, 4) which descends obliquely, by opening the sluice $G$, the water is let down from the superior canal $\mathrm{D}$, in to the lock, where it is stopped and retained by the gate $\mathbf{c}$ when shut, till the water in the lock bc on a level with the water in the superior canal D; as represented in fig. 3 . When, on the other hand, the water contained by the lock is to be let out, the passage $\mathrm{GH}$ must be shut, by letting down the sluice $G$; the gate $A$ inust also be shut, and the passage KF opened by raising the sluice $k$. A frec passage being thus given to the water, it descends through $\mathrm{KF}$, into the inferior canal, until the water in the lock be on a level with the water in the inferior canal $\mathrm{B}$; as represented in fig. 2 .

Now suppose it be required to raise the vessel $L$ (fig. 2) from the inferior canal $B$ to the superior one $D$. If the lock be full of water, the sluice $\mathrm{r}$ inust be shut, as als? the gate $A$, and the sluice $K$ opened, so that the water in the lock may run out till it becomes level with the water in the inferior canal $B$. When the water in the lock is on a level with the water at $\mathrm{B}$, the leaves of the gate $\mathrm{c}$ are opened by the levers $\mathrm{ch}$, which is easily performed, the water on each side of the gate being in equilibrio; the vessel then sails into the lock. After this, the gate $\mathbf{c}$ and the sluice $k$ are shut, and the sluice $\mathrm{G}$ opened, $\mathrm{j}$ order to fill the lock, till the water in the lock, and consequently the vessel, be upon a level with the water in the superior canal $\mathrm{D}$; as is reprecented in fig. 3. The gate $\mathbf{A}$ is then opened, and the vessel passes into the canal $D$.

Again, let it be repuired to make a vessel descend from the canal $\mathrm{D}$ into the inferior canat $\mathrm{x}$. If the lock be empty as in fig. 2 , the gate $\mathrm{c}$ and sivice $K$ must be shut, and the upper sluice $G$ opened, so that the water in the lock may rise to a level with the water in the upper canal D. Then, opening the gate $A$, the vessel will pass through into the lock. This done, shut the gate $A$ and the sluice $\mathrm{G}$; then open the sluice $\mathrm{K}$, till the water in the lock be on a level with the water in the inferior canal; this done, the gate $\mathbf{c}$ is opened, and the vessel proceeds into the caual $B$, as was required.

Other contrivances bave lately been made for raising and lowering the vessels between the under and upper canal.

CANCER, the Crab, one of the twelve signs of the zodiac, usually drawn on the globe in the form of a crab, and in books of astronomy denoted by a character resembling the number sixty-nine, turned sideways, thus go. $^{\circ}$

The number of stars in the sign Cancer, Ptolemy makes 13, Tycho 15, Bayer and Hevelius 29, and Flamsteed 83.

Tropic of CANCER, a little circle of the splicre parallel to the equinoctial, and passing through the beginning of tlie sign Cancer.

CANDLEMAS, or the Purification, a feast of the church, held on the $2 \mathrm{~d}$ of February, in memory of the purification of the Virgin; taking its name of Candlemas, either from the number of lighted candles used by the Romish church in the processions of this day, or because that the church then consecrated candles for the whole year.

CANES Venatici, the Hounds, or the Greyliounds, one of the new constellations of the northern bemisphere, which Hevelius has formed out of the unformed stars of the old catalogues. These two dogs are further distinguished by the names of Asterion and Chara. They contain 23 stars according to Hevelius, but 25 in the British catalogue.

CANICULAR, a name given, by many of the carlier astronomers to the constellation which we call the Lesser Dog, and Canis Minor, but some Procyon and Antecanis. See Can is Minor.

It is also used for one of the stars of the constellation Canis Major; called also simply the Dog-star ; and by the Greeks $\Sigma$ eipro5, Sirius. It is situated in the mouth of the constellation, and is the largest and brightest of all the stars in the beavens. From the heliacal rising of this star, that is, its cmersion from the sun's rays, which now happens with us about the 11th of August, the ancients reckoned their Dies Caniculares, or Dog-days.

The Egyptians and Ethiopians began their year at the heliacal rising of Canicula ; reckoning to its rise again the next year, which is called the Annus Canarius.

CANICULAR Days, or Dog-days, denote a certain number of days, befure and after the heliacal rising of canicula, or the dng-star, in the morning. The ancients imagined that this rising of the star occasioned the sultry weather usually felt in the latter part of the summer, or dog-days; with all the distempers of that sickly season: Homer's II. lib. 5, v, 10, and Virgil's En. Jib. 10, v. 270. Some authors say, from Hippocrates and Pliny, that the day this star first rises in the morning, the sea boils, wine turns sour, dogs begin to grow mad, the bile increases and irritates, and all animals grow languid; also that the diseases it usually occasions in men, are burning fevers, dysenteries, and phrensics. The Romans sacrificed a brown dog every year to Canicula at his rising, to appease its ragc. All this however arose from the groundless idea that the dog-star, so rising, was the occasion of the extreme heat and the diseases of that season: for the rising of the star not only varies, in any one year, as the latitude varies, but is always later and later every year in all latitudes; so that in time the star may, by the same 
rule, be charged with bringing frost and snow, when he rises in winter.

The dog-days were commonly reckoned about 40 , viz, 20 days before and 20 days after the heliacal rising; and almanac-makers have usually set down the dog-days in their almanacs to the clianging time of the star's rising, by which means they had at length fallen considerably after the hottest time of the year, till of late we have observed an alteration of them in the almanacs, and very properly, from July 3 to August 11. For, by the dogdays, the ancients ineant to express the hottest time of the year, which is commonly during the month of July, about which time the dog-star rose heliacally in the time of the most ancient astronomers that we know of : but the precession of the equinoxes has carrier this heliacal rising into a much later and cooler part of the year; and because Hesion tells us that the hot time of the year ends on the 50 th day after the summer solstice, which brings us to about August 10 or 1.1, the above alteration appears to be very proper.

CANicular Year, denotes the Egyptian natural year, which was computed from one heliacal rising of Canicula to the next. This ycar was also called annus canurius, and annus cynicus; and by the Lgyptians themsclves the Sethic year, from Seth, by which name they called Sirius. Some call it also the heliacal year. This year consisted ordinarily of 365 days, and every 4th year of 366 ; by which means it was accommodated to the civil year, like the Julian account. And the reason why they chose this star, in preference to others, to compute their time by, was not only the superior brightness of that star, but because in Egypt its heliacal rising was a time of very singular note, as coinciding with the greatest augmentation of the Nile, the reputed father of Egypt. Ephestion adds, that from the aspect of canicula, its colour, \&c, the Egyptians drew prognostics concerning the rise of the Nile; and according to Florus, predicted the future state of the year. So it is no wonder that the first rising of this star was observed with great attention. Bainbrigge, Canicul. cap. 4, p. 26.

CAN1S Major, the Great Dog, a constellation of the southern liemisphere, below the feet of Urion, and one of the old 4.8 constellations. The Greeks, as usual, have many fables of their own about the exaltation of the dog into the skies; but the origin of this constellation, as well as its other name Sirius, lies more probably anong the ligyptians, who carefully watched the rising of this star, and by it judged of the swelling of the Nile, calling the star the sentinel and watch of the year; and hence, according to their manner of hieroglyphic writing, reprosented it under the figure of a dog. 'They also called the Nile Siris; and hence their Osiris.

The stars in this constellation, Ptolemy makes 29; Tyclio however observed only 13, and Hevelius 21; but in Flamsteed's catalogue they are 31 .

Canss Minor, a constellation of the northern hemisphere, just below Gemini, and is one of the 48 old constellations. The Greeks fabled that this is one of Orion's hounds; bot the Egyptians were most probably the inventors of this constellation, and they may have given it this figure to express a little dog, or watchful creature, going before, as conductor of the larger, or rising before it: and hence the Latins have called it Antecanis, the star before the dog.

'The stars in this constellation are, in Ptolemy's cata- logue 2, the principal of which is the star Procyon; in 'Tycho's, 5; in Hevelius's, 13; and in Flamsteed's, 14.

CANNON, in Military Affairs, a long round hollow engine, made of iron or brass, \&c, for throwing balls, \&c, by means of gunpowder. The length is distinguished into three parts; the first reinforce, the second reinforce, and the chase: the inside hollow where the charge is lodged, being also called the chase, or bore. But for the several parts and members of a cannon, see AstrAGAL, BAsEring, Bore, Brejch, Cascabel, Chase, Muzzle, Ogee, Reinfurce-ring, Trunnions, \&c. Sue also Gun, and GunNery.

Cannon were first niade of several bars of iron adapted to each other lengthways, and hooped together with strong iron rings. They were employed in throwing stones and metal of several hundred weight. Others were made of thin sheets of iron rolled up, and hooped: and on emergencies they have been made of leather, with plates of iron or copper. They are now constituted of cast-iron or brass; being cast solid, and the tube bored out of the middle by means of a very strong machine for that purpose. When or by whom caunon were first invented is uncertain.

Larrey makes brass cannow the invention of J. Owen; and asserts that the first known in England, were in 1535; and further that iron cannon were first cast here in 1547 . He acknowledged that canion were known before; and remarks that at the battle of Cressy, in 134.6, there were 5 pieces of cannon in the linglish army, which were the first ever scen in France. Mezeray also observes, that King Edward struck terror in to the French army, by 5 or 6 pieces of cannon; it being the first time they had met such thunlering machines.

In the list of aids raised for the redemption of King John

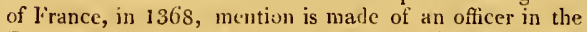
French army called Master of the King's Cannon, and of his providing 4 large cannon for the garrison of Harfleur. But Father Danicl, in bis Life of Philip of Valois, produces a proof from the records of the chamber of accounts at Paris, that canmon and gumpowder were used in the year 1338. And Du-Cange even finds mention of the same. engines in Froissart, and other French historians, some time earlicr.

The Germans carry the invention of cannon farther back, and ascribe it to Albertus Magnus, a IJominican. monk, about the year 1250. But Isaac Tossius finds cannon in China upwaris of 1700 years ago ; being used by the emperor Kit'y, in the year of Christ 85 . The ancients too, of Furope and Asia, had thrir fiery tubes, or Cannæ, which being loaded with pitch, stones, and iron: balls, were exploded with a vehement noise, smoke, and great effect.

Cannon were formerly made of a very great length, which rendered then exceedingly heavy, and their use very troublesome and confrned. But it has lately been found by expriment, that there is very little added to the force of: the ball by a great length of the camnon; and therefore they have very properly been much reduced both in their length and weight, and rendered easily manageable on all occasions. They were formerly distinguished by many hard and terible names, but are now only named from the weight of their ball; as a 6 potinder, a 12 pounder, a 24 pouncler, or a 36 pounder, which is the lurgest size now. used by the English for battering.

CANON, in Algebra, Aribmetic, Geometry, \&c, is a gencral rule for resolving all cases of a like nature with the: 
present inquiry. Thus the last step of every equation is a canon, and if turned into words, becomes a rule to resolve all cases or questions of the same kind with that proposed:

Tables of sines, tangents, \&c, whether natural or artificial, are also called canons.

Canon, in Ancient Music, is a method of determining the intervals of musical notes. Ptolemy, rejecting the Aristoxenian way of measuring the intervals in music by the magnitude of a tone, formed by the difference between a diapente and a diatessaron, thought that they should be distinguished by the ratios which the sounds terminating those intervals bear to one another, when considered according to their degree of acutenesṡ or gravity; which, hefore Airstoxenus, was the old Pythagorean method. ' He therefore made the diapason consist in a double ratio; the diapente consist in a sesquial terate; the diatessaron, in a sesquitertian; and the tone itself, in a sesquioctave; and all the other intervals, according to the proportion of the sounds that terminate them : therefore, taking the canon, for a determinate line of any lengtl, he shows in what manner it must be cut, to represent the respective intervals: and this method answers exactly to experiments in the different lengths of musical chords. From this canon, Ptolemy and his followers have been called Canonici; as those of Aristoxenus were called Musici.

CANON, in Modern Music, is a short composition of two or more parts, in which one leads and the other follows; and is a fugue so bound up, or restrained, that the following part or parts must precisely repeat the same notes, with the same degrees rising or falling, wlich were expressed by the, leading part; it is therefore tied to so strict a rule, that it is called canon.

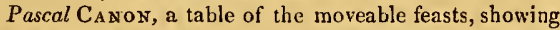
the day of Easter, and the other feasts depending on it, for a cycle or period of 19 years. It is said that the Pascal Canon was the calculation of Euscbius of Casarea, and that it was made by order of the council of Nice.

CANOPUS, a name given by some of the old astronomers to a star under the $2 d$ bend of Eridanus. These writers say that the river in the heavens is not the Eridanus, but the Nile, and that this star commemorates an island made by that river, which was called by the same name.

Canopus is also the name of a bright star of the first magnitude in the rudder of Argo, one of the southern constellations. Its situation, as given by several authors, at different times, is as follows:

\begin{tabular}{|c|c|c|c|c|c|c|}
\hline $\begin{array}{c}\text { Aut } \\
\text { F. Th }\end{array}$ & Dites & Longit. & Lat. & Rt. Ascen. & $\begin{array}{l}\text { Declin. } \\
52^{\circ} 31^{\prime}\end{array}$ & So. \\
\hline F. No & 1697 & & & $93 \quad 54$ & $52 \quad 29$ & \\
\hline Dr. Halley & 1700 & 10 & 72 & & & \\
\hline F. Feuille & Mar. 1709 & & & & 30 & 4 \\
\hline
\end{tabular}

C.ANTALIVERS, in Architecture, are the same with modillions, except that the former are plain, and the latter carved. They are both a kind of cartouses, set at equal distances under the corona of the cornice of a building.

CANTON (JoIN), an ingenious natural philosopher, was born at Stroud, in Gloucestershire, in 1718 ; and was placed, when young, under the care of Mr. Davis, an able mathematician of that place, with whom he learnt the rudiments of arithmetic, \&c. He next proceeded to higher parts of the mathematics, and particularly to algebra and astronomy, in which he had made a considerable progress, when his father took him from school and set him to learn his own business, which was that of a broad-cloth weaver.
This circumstance did not check his zeal for acquiring knowledge. All his leisure time was devoted to the assiduous cultivation of science; and he had now acquired a sufficient knowledge of astronomy to calculate lunar eclipses and other phenomena, and to construct varions kinds of sun-dials, which be did at times when he ought to have slept, and without the knowledge of his father; who feared that the prosecution of such studies inight injure bis health. It was during this discouragement, and at these hous's, that he computcd, and cut upon stone, with no better an instrument than'a common knife, the lines of a large upright sun-dial, on which, beside the hour of the day, were shown the sun's risfng, his place in the ecliptic, and some other particulars. When this was finished and made known to his father, he permitted it to be placed against the front of his house, where it excited the admiration of several neighbouring gentlemen, and introduced young Canton to their acquaintance, which was followed by the offer of the use of their libraries. In the library of one of these gentlemen he found Martin's Philosophical Grammar, which was the first book that gave him a taste for natural philosophy. In the possession of another gentleman he first saw a pair of globes; a circumstance that afforded him great pleasure, from the great case with which he could resolve those problems he had hitherto been accustomed to compute.

Among other persons with whom our author becane acquainted in early life, was Dr. Hewry Miles of Tooting; who perceiving that he possessed abilities too promising to be confined within the narrow limits of a country town, prevailed on his father to permit him to come to London. Accordingly he arrived at the metropolis the 4th of March 1737 , and resided with Dr. Miles at Tooting till the 6 th of May following; when he articled himself, for the term of 5 years, as an assistant to MIr. Samuel Watkins, master of an acadomy in Spital Square. In this situation his ingenuity, diligence, and prudence, were so distinguished, that on the expiration of his servitude in May 1742, he was taken into partmership with Mr. Watkins for 3 years; which gentleman he afterwards succceded in the school, and there continued during his whole life.

Towards the end of 174,5 , electricity reccived a great improvement by the discovery of the famous Leyden phial. This event turned the thoughts of most of the philosophers of Europe to that branch of natural philosophy; and Canton, who was one of the first to repeat and to pursue the experiment, found his endeavours rewarded by many discoveries.-Towards the end of 1749 , he was engaged with his friend, the late ingenious Benjamin Robins, in making experiments to determine the height to which rockets may be made to ascend, and at what distance their light may be seen.-In 1750 was read at the Royal Society Mr. Canton's " Method of making Artificial Magnets, without the use of, and yet far superior to, any natural ones." 'This paper procured him the honour of being elected a member of the Society, and the present of their gold medal. The same year he was complimented with the degrce of M. A. by the university of Aberdeen. And in 1751 he was chosen one of the council of the Royal Socicty.

In 1752 , our philosopher was so fortunate as to be the first person in England who, by attracting the electric fire from the clouds during a thunder-storm, verificd 1)r. Franklin's hypothesis of the similarity of lightning and clectricity. Next year his paper entitled "Elcctrical Ex- 
periments, with an atteinpt to account for their scveral phenomena," was read at the Royal Society; in which Mr. Canton mentions his having discovered, by many experiments, that some clouds were in a positive, and some in a negative state of electricity: a discovery which was also made by Dr. Franklin in Anerica much about the same time. This circumstance, together with our author's constant defence of the doctor's bypothesis, induced that excellent philosopher, on his arrivil in England, to pay Mr. Canton a visit, and gave rise to a friendship which ever after continued between them.- In the Laöies' Diary for 1756 , our author answered the prize query that had been proposed in the preceding year, concerning the meteor called shooting-stars. The solution, though only signed A. м. was so satisfactory to his friend, the excellent mathematician Mr. Thomas Simpson, who then conducted that ingenious and useful little work, that he sent $\mathrm{Mr}$. Canton the prizc, accompanied with a note, in which he said be was sure that he was not mistaken in the author of it, as no correspondent besides him, could have given that ansiver.-Our philosopher's next communication to the public was a letter in the Gentleman's Magazine for September 1759 , on the electrical properties of the tourmalin, in which the laws of that wonderful stone are laid down in a very concise and elegant manner. On the 13th of December in the same year, was read at the Royal Society, An attempt to account for the Regular Diurnal Variation of the Horizontal Magnetic Needle; and also for its Irregular Variation at the time of an Aurora Borealis. A complete year's observations of the diurnal variations of the needle are annexed to the paper.- - Nov. 5,1761 , our' author communicated to the Royal Suciety an account of the Transit of Venus of the 6 th of June that year, observed in Spital-square; and in 1762 a letter addressed by him to Dr. Franklin was read, containiug some remarks on Mr. Delaval's electrical experiments. Un the 16 th of Dec. the same year, another curious addition was made by him to philosophical knowledge, in a paper entitled Experiments to prove that Water is not Incompressible. And on Nov. 8, the year following, were read before the Society, his farther Experiments and Observations on the Compressibility of Water, and some other Fluids. These experiments are a complete refutation of the famous Florentine experiment, which so many plilosophers bave mentioned as a proof of the incompressibility of water. For this communication he received the Society's gold medal.

In 1768 our author communicated to the Society, An easy methed of making a phosphorus that will imbibe and emit light like the Bolognian Stone; with experiments and observations. When he first showed to Dr. Franklin the instantancous light acquired by some of this phosphorus from the wear discharge of an clectrified bottle, the desctor immediately exclaimed," And God said, Let there be light, and there was light."

The Dean and Chapter of St. Paul's having, in a letter, dated March 6, 1769, requested the opinion of the Ringal Society relative to the best method of fixing electrical conductors to preserve that eathedral from iujury by lightning, Mr. Canton was one of the comunitlec appointed to take the letter into consideration, and to reprort their opinion thereon. The gentlemen who joined hisn in this business were, Mr. Delaval, Mr. liranklin, Dr. Watson, and Mr. Wilson, Their report was made on the sth of June following; and the noote recommended by them has been Vol. I. carried into execution. Our author's last communication to the Royal Society, was a paper read Dec. 21, 1769, containing Experiments to prove that the Luminousness of the Sea arises from the Putrefaction of its animal Substances.

Besides the papers above mentioned, Mr. Canton wrote a number of others, both in the earlier and the latter purts of his life, which appeared in several publications, and particularly in the Gentleman's Magazine.-He died of a dropsy, the 22d of March, 1772, in the 54th year of his age.

CAPACITY, is the solid content of any body. Also our hollow measures for corn, beer, wine, \&c, are called measures of capacity.

Capacity, in the Modern Doctrine of Heat, significs the proportional capability of a given quantity of any substance to absorb and retain caloric; or that disposition or property by which various bodics respectively require more or less of this fluid to superinduce any giren temperature in a given mass. Sce Cazoric and Tlimperature.

CAPE, or Promontory, is any high land runuing out with a point into the sea; as Cape Verde, Cape Horn, the Cape of Good Hope, \&c.

CAPELLA, a bright star of the first magnitude, in the left shoulder of Auriga.

CAPJLLARY Tubes, in Physics, are very minute pipes, whose canals are exceedingly narrow; being so called from their resemblance to a hair in smallness. Their usual diameter may be from $\frac{x}{20}$ to $\frac{x}{50}$ of an inch: though Dr. Hooke assures us that he drew tubes in the flame of a lamp much smaller, and resembling a spider's thread.

The Ascent of Water, \&c, in capillary tubes, is a noted phenomenon in philosophy. 'Take sereral small glass tu bes, of different diameters, and open at both ends; immerse them a little way in water, and the fluid will be seen to stand higher in the tubes than the surface of the water without, and higher as the tube is smaller, almost in the reciprocal ratio of the diameter of the tube; and that buth in open air and in vacuo. The greatest height to which Dr. Hooke ever observed the water to stand, in the smallest tubes, was 21 inches above the surface in the vessel.

From this property, of a higher ascent in smaller tubss, a neat experiment exhibits a pleasing appearance in this manner: Scleet a number of these glass tubes all different in their diameter, from the very smallest to the largest size; fix these against a small piece of board, all parallel and near to cach other, with their lower ends all on a level, and so as that the tubes may succeed each other in a regular gradation, from the smallest to the largest; then dip the whole machine into water with the lower ends egually immorsed, and it will be seen that, by the fluid ascending to ditterent lueights in the tubes, the tops of the columns will appear to form a regular curve line, being highest in the smallest tirbe, and lowest in the largest; and if the water be a little tinged with rochineal, or ink, \&e, it wilt runder the effect more visible and pleasing.-A more pleasing variety of this experiment is made in the following manner: Take two equal square picees of perlectly flat glass, of any convenient size, 6 or 8 inches, fur instance, on cach side; cunnect two of their equal edges close togcther by any means, but separatc their opposite edges a little from each other, by fixing a small bit of chip, \&c, between then, so as to give the two the appearanee of a very thin werlge, which witl represent, and have the aflect of, $2 \mathrm{~N}$ 
an intinite number of contigunus tubes as disposed paralIs! to the said closer and opened (d) ges; for then, on dipping the bottoms in the columel water, this will be seen to ascend up between the two planes, highest on the side next their closed edges, but gradually lower towards the upposite sides, the top of the fluid forming a regular hyperbolic curve line, to which the closed eages and the bottom are scen as the asymptotes.

This ascent and suspension of the water in the tube, is by 1)r. Jurin, Mr. Hauksbee, and other philosophers, ascribed to the attraction of the periphery of the concave surface of the tabe, to $u$ hich the upper surface of the water is contiguous and adheres.

This does not however happen uniformly the same in all fluids, some standing higher than others; and in quicksilver the contrary takes place, as that fluid stands lower within the tube than its surface in the ressel, and the lower as the tube is smaller. See Philos. Trans. No. 355, \&.c, or Cote's Hydr. and Pneum. Lect. p. 265. But these effects suppose that the tubes are used in the clean state in which they are naturally presentcd; for, by certain precautions, mercury may be shown to have a like elevation above the level. And if the interior of these tubes be coated over with greasy matter, such as oil or tallow, the usual effect will not take place, as the fluid will retain its level.

Anstber phenomenon of these tubes is, that sucb of them as would only naturally discharge water by drops, when electrified, yield a continued and accelerated stream; and the acceleration is proportional to the smallness of the tube: indeed, the effect of electricity is so considerable, that it produces a continued stream from a very small tube, out of which the water had not before been able to drop. Priestley's Hist. Electr. 8vo, vol. 1, p. 17 1, ed. $3 d$.

The celebrated philosopher, Laplace, has written pretty fully on the theory of this capillary attraction, confirming the opinion of Jurin and Hauksbee, \&c, above mentioned, and explaining it by mathematical calculations, in the Supplement to the ioth book of his Celestial Mechanics, on Capillary Attraction. On this subject, see also $\mathrm{Dr}$. Young's paper on the cohesion of fluids, in the Philos. Trans. for 1805, or in his Natural Philos, vol. 2; or Ciregory's Mechanics, vol. 1, p. 393; or, translation of liaü's Nat. Philos. vol. 1, p. 158; Nicholson's Journal, Nos. 74, 76 , N. S.; or Retrospect of Philosophical Discoveries, No. 10.

Among the various methods of deternining the diameter of a uniform capillary tube, the following appears the best and most accurate. Pour into the tube a certain quantity of mercury, whose weight in troy yrains is $w$, and weasure carefully the length, $l$, of the tube which it occupies, then is the diameter, $d=0192523 \sqrt{ } \frac{w}{l}$. For the specific gravity of inercury being 13568 , a cubic inch weighs 3435.16 grains: hence, $1: d^{2} l \times 785395:: 3435 \cdot 16$ grains: $w:$. Whence, multiplying extremes and menns, \&rc, we get $d=\sqrt{\frac{w}{3.35 \cdot 1(1 \times 785398 \times l}}=0192523 \sqrt{ } \frac{w}{l}$.

CAPIT $\Lambda$ L, in Architecture, the uppermost part of a rolumn or pilaster, serving as a heal or crowning to it, being placid immediately over the sluaft, and under the mitablature. It is made differently in the different orders, and is that indeed which chiefly distinguishes the orders themselves.
CAP1TAL of a Bastion, is an imaginary line dividing any work into two equil and similar parts; or a line drawn from the angle of the polygon to the point of the bustion, or from the point of the bastion to the middle of the gorge:

CAPONilite, or Caponsiere, in Fortification, is a passage made from one work to anuther, of 10 or 12 feet wide, and about five feet deep, covered on cach side by a parapet, terminating in a glacis or slope: sonetimes it is covered with planks wnet earth.

CAPRA, or the She-gout, a name given to the star Cae pella, on the left shoulder of A uriga; and sometimes to the constellation Cappricorn. Some again represent Capra as a constellation in the northern hemisphere, consisting of 3 stars, conjprised between the 45 th and 55 th degree of latitude. 'The poets fable her to be Ansalthea's goat, which suckled Jupiter in his infancy.

CAPRICORN, the Gout, a southern constellation, and the loth sign of the zodiac, as also one of the 48 original constellations receired by the Greeks from the Egyptrans. The figure of this sign is drawn as having the fore part of a goat; but the hinder part of a fish; and sometimes simply under the form of a goat. In writing, it is denoted by a character representing the crooked horns of a goat's head, thus $r p$.

'The stars in this constellation, in Ptolemy's and Tycho's catalogue, are 28 ; in that of Hevelius, 29 ; though it is to be remarked that one of those in the tail, of the 6th magnitude, marked the 27 th in 'Tycho's book, was lost in Hevelius's time. Flamsteed gives $\mathbf{5 1}$ stars to this sign.

Tropic of Capricon n, a suall circle of the sphere, parallel to the equator, passing through the beginning of $\mathrm{Ca}$ pricorn, or the winter solstice, or the point of the sun's grcatest south declination.

CAPSTAN, a large massy column shaped like a truncated cone; being set upright on the deck of a ship, and turned by levers or bars, passing through holes in its upper extremity. The capstan is a kind of perpetual lever, or an axis-in-peritrochio, which, by means of a strong rope or cable paseed round serves to raise very great weights; such as to hoist sails, to weigh the anchor's, to draw the vessels on shorc, and hoist them up to be refitted, \&c.

CAPUT DuAConıs, or dragon's bead, a name given by some to a fixed star of the first magnitude, in the heas of the constelation Draco.

CARAC'T, or CARAT, a name given to the weight which expresses the degree of goodness or fineness of gold. The whole quanity of mital is considered as consisting of 24 parts, which ar. the carats; so that the carat is the 24th part of the whole: this carat is divided into 4 equal parts, called grains of a carat, and the grains into halves and yuarters.

Whient gold is purified to the utmost degree possible, so that it luses no more by further trials, it is considered as quite pure, and satid to be 24 carats fine; if it lose 1 carat, or $1-24$ th in purifying, it was of 23 carats fine; and if it lose 2 carats, it was 22 carats tine; and so on.

CARC $4.5 S$, is a hollow case formed of ribs of iron, and coverel wer with pitclied cloth, \&c, about the size ui tomb-shells; or somctimes male all of iron except two or three boles for the fire to blaze through. 'These were filled with various combustibles, to fire houses, when thrown out of mortars into besieged places.

CARCAVI (PETEu DE), was bornat Lyons, but in what 
yrar is not known. He was counsellor to the Parliament of Toulouse, afterwards counsellor to the grand council, and keeper of the king's library. He was appointed geometrician to the French Academy of Sciences in 1666, and died at Paris in 16st. There are extant some letters of bis, printed among these of Des Cartes.

CARDAN (IIIERONYMUS, or JEROM), one of the most extraordinary geniuses of his age, was bom at Pavia, in Italy, Sept. 24, 1501. At 4 years old he was carricd to Mlilan, bis father being an adrocate and physician in that city: at the age of 20 he entered the university of the same eity, and prosecuted his studies with great success. 1n 1524 he went to Padua, and the same year was admitted to the degree of master of arts, and the year following, that of doctor of physic. He married about the year 1531 ; and vecume professur of mathematics at Milan in 1533. In 15.39 he was admitted a member of the college of physiciains at Milan; in 1543 he read public lectures in medicine there, and the same at Pavia the year following; but in consequence of non-payment of his salary, he discontinued them, and returned to Milan.

In 1552 be went into Scotland, having been sent for by the archbishop of St. Andrew's, to cure him of an afflicting asthma, after trying the physicians of the king of France and of the emperor of Germany, without receiving any benefit. He began to recover from the day that Cardan prescribed for him: our author returned at the end of 6 weeks and 3 days, leaving him prescriptions which in two years wronght a complete cure. On this visit Cardan passed through London, and calculated King Edward's nativity; being famons for his pretensions in astrology, as well as his skill in mathematics and medicine. Returning to Milan, after 4 months absence, he remained there till the beginning of Oct. 1552 ; and then went to Pavia, from whence be was invited to Bologna in 1562 . He taught in this last city till the year 1570 ; at which time he was thrown into prison; but some months after lie was sent home to his own house. He quitted Bologna in 1571; and went to Rome, where he lived for some time without any public employment. He was however admitted a member of the college of physicians, and received a pension from the Pope, till the time of his death, which happened at Rome on the 21 st of September 1575 .

Cardan, at the same time that he was one of the greatest geniuses and most learned men of his age, in all the sciences, was one of the most eccentric and fickle in conduct of alt men that cver lived; despising every good principlc and opinion, and without one friend in the world. The same capriciousness that was remarkable in his outward conduct, is also observable in the composition of his numerous and claborate works. In many of his treatises the reader is stopped almost every moment by the obscurity of his text, or by digressions from the point in hand. In his arithmetical writings there are several discourses on the motions of the planets, on the creation, on the Tower of Babel, and such like. And the apology which he made for these frequent digressions is, that he might by that means enlarge and fill up his book, his hargain with the bookseller being at so much per sheet; and that he worked as much for his daily support as for fame. The Lyons edition of his works, printed in 1663 , consists of no less than 10 volumes folio.

In fact, when we consider the transcendent qualities of Cardan's mind, 'it cannot be denied that hc cultivated it with every species of knowledge, and made a greater progress in philosophy, in the medical art, in astronomy, in mathematics, and the other sciences, than the most prost of his contemporaries who had applied thicinselves but to oncr of those sciences. In pitricular, he was perhaps the best algebraist of his time, a science in which he made great improvements; and his labours in cubic equations cispecially have rendered his name inmostal, the rules for $\mathrm{r}$ solving them having ever since borne his name, and are likely to do so as long as this science exists, although he received the first knowledge of them from another persons the account of which, and his disputes with Tartalea, have been given at large under the article ALGE B RA.

Scaliger afforns, that Cardan, having by astrology predicted and fixed the tinse of his death, abstained from all food, in order that his prediction might be verified, and that his continuance to live might not discredit his art. It is further remarkable, that Cardan's father also died in this manner, in the year 1524 , having abstained from sustenance for nine days. Our author, too, informs us that his father had white eyes, and could see in the night-time.

CARDINAL Points, in Geography, are the cast, west, north, and south points of the horizon.

Cardinal Points of the Heavens, or of a Natizity, are the rising and setting of the sun, the zenith and nadir.

Cardinal Signs, are those at the four quarters, or the equinoxes and solstices, viz, the signs Aries, Libra, Cancer, and Capricorn.

Cardinal Winds, are those that blow from the four cardinal points, viz, the east, west, north, and south winds.

CARDIOIDE, the name of a curve so called by Castilliani.-But it was first treated of by Koersma, and by Carré. See Philos. Trans. 1741, and Memoires de l'Acad. 1705.

The Cardioide is thus generated. $\mathrm{A} \mathrm{P} \mathrm{P}$ is a circle, and A B its diameter. Through one extremity $\Delta$ of the diameter drav any num. ber of lines A PQ, cutting the circle in $P$; upon these set off always $P Q$ equal to the diameter $A B$; so shall the points $Q$ be always in the curve of the cardioide.

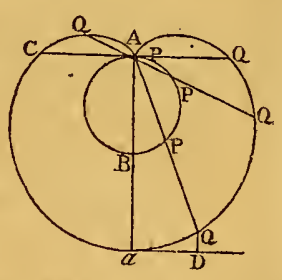

From this generation of the curve, its chief propertics are evident, viz, that,

everywhere $\mathbf{P} \Omega=\Lambda \mathrm{B}$,

$C Q$, or $Q Q$ is $=A a$ or $2 A B$,

$\triangle Q=A B \pm A P$,

$\mathbf{P}$ always bisects $\mathbf{Q Q}$.

The cardioide is an algebraical curve, and the equation expressing its nature is this: putting

$a=\mathrm{AB}$ the diameter,

$x=a \mathrm{D}$ perp. to $a \mathrm{~B}$,

$y=\mathrm{DQ}$ perp. to $a \mathrm{D}$; then is

$\left.\begin{array}{rl}y^{1}-6 u y^{3} & +2 x^{2} y^{2}-6 a x^{2} y+x^{4} \\ & +12 a^{2} y^{2}-8 a^{3} y+3 a^{2} x^{2}\end{array}\right\}=0$

which is the equation of the curve.

Many propertics of the cardioide may be seen in the places above cited.

CARRE' (LEW1s), an ingenious mathematician and philusopher, was born in the year 1663 , in the province of Brie in France. His father, a substantial farmer, intended bim for the church. But, after going through the usual course of education for that purpose, and having an utter aversion to it, he refused to enter upon that function; by $2 \mathrm{~N}_{2}$ 
which he incurred his father's displeasure. 1lis resources being thus cut off, he was obliged to quit the university, and lonk iuto the world for some employment. In this exigency he had the good-fortune to be engaged as an amanuensis by the celebrated Father Malebranche; by which he found himself at once transported from the mazes of scholastic darkness, to the source of the most brilliant and enlightened philosophy. Under this great master he studied mathematics and the most sublime motaphysics. After seven years spent in this excellent school, M. Carré found it necessary, in order to procure himself some less precarious establishment, to teach mathematics and philosophy in Paris; but especially that philosoplyy which, on account of its tendency to impiove our morals, he valued more than all the mathematics in the world. And accordingly his greatest care was to make geometry serve as an introduction to his well-beloved metaphysics.

Most of Carrés pupils were of the fair sex. The first of these, who soon porceived that his language was rather the reverse of elegant and correct, told him pleasantly that, as an acknowledgment for the pains he took to teach her philosophy, she would teach him French; and he ever after owned that her lessons were of great service to him. In general he seemed to set more value on the genius of women than that of men.

M. Carré, although he gave the preference to the metaphysics, did not neglect mathematics; and while he taught both, he took care to make himself acquainted with all the new discoveries in the latter. This was all that his constant attendance on his pupils would allow him to do, till the year 1697, when M. Varignon, so remarkable for his extreme scrupulousness in the choice of his elèves, took M. Carre to him in that station. Soon after, viz, in the y'ear 1700 , our autlior, thinking himself bound to do something that might render him worthy of that title, published the first complete work on the Integral Calcalus, under the title of "A metliod of measuring Surfaces and Solids, and finding their Centres of Gravity, Percussion, and Oscillation." He afterwards discovered some errors in the work, and was candid enough to own and correct thern in a subsequent cdition.

In a short time $M$. Carré became associate, and at length one of the pensioners of the Academy. And as this was a sufficient establishment for one, who knew so ivell how to kecp his desires within just bounds, lye gave himself up entirely to study; and as he enjoyed the appointment of Mechanician, lie applied himself more particularly to mechanics. He took also a survey of every branch relating to music; such as the doctrine of sounds, the description of musical instruments; though he despised the practice of music, as a mere sensual pleasure. Some sketches of his ingenuity and industry in this way may be seen in the Memoirs of the Frencli Academy of Sciences. Ile also composed some treatises on other branches of natural philosophy, and some on mathematical subjects; all which he bequerathed to that jllustrious borly; thougl it does not appear that any of them have jet been published. It is not unlikely that he was linbecel from putting the last hand to them by a train of discrelcrs proceeding from a bad digestion, which, after liarassing him during the course of five or six years, at length brought him to the grave in 1711 , at 48 years of ane.

His, memoirs printed in the velumes of the Academy, with the yoars of the volumes, arc as below.
1. Rectification of Curve Lines by Tangents : 1701 .

2. Solution of a Prob. propused to Geometricians, \&c. 1701.

3. Reflections on the Table of Equations : 1701.

4. On'the Cau'se of the Refraction of Light: 1702.

5. Why the Tides are always augmenting from Brest to. St. Malo, and diminishing aloug the cousts of Normandly: 1702 .

6. Number and Names of Musical Instruments : 1702.

7. On the Vinegar which causes small stones to roll upon an inclined plane: 1703.

8. On the Rectification \&c, of the Caustics by reflection: 1703 .

9. Method for the Rectification of Curves: 1704.

10. Observations on the Prodiction of Sound: 1704.

11. On a Curve formed from a Circle: 1705 .

12. On the Refraction of Ausket-balls in wuter, and ol: the Resistance of that fluid : 1705 .

13. Experiments on Capillary Tubes: 1705.

14. On the Proportion of Pipes to have a determinate quantity of water: 1705.

15. On the Laws of Alotion: 1706.

10. On the Properties of Pendulums; with some new properties of the Parabola: 1707 .

17. On the Proportion of Cylinders that their sounds, may form the musical chords: 1709 .

18. On the Elasticity of the Air: 1710.

19. On Catoptrics: 1710.

20. On the Monochord: in the Machines, tom. 1, with some other pieces, not mathematical.

CARRIAGE, of a Cannon, is the machine upon which it is mounted ; serving to point or direct it for firing, and to convey it from place to place.

Wheel CAnRiage, one that is mounted and mored about upon wheels. Horses draw in general, to most advantage, when the direction of their draft is parallel to the ground, or rather a little upwards. A carriage also goes casiest when the centre of gravity is placed very high; since, when once put in motion, it continues it with rery little labour to the horses.

CARTES (RENE DES), one of the mosi eninent philosophers and mathematicians of the 17 th century, or indeed of any age whatever. He was descended if an ancient noble tamily in Touraine in France, benng a younger son of a counsellor in the parliament of Remnes, and was born March 31, 1596. His, father gave him a liberal coducation, and the more so as he observed in him the appearance of a promising gcnius, using to call lim the Philusoples, on account of lis insatiable curiosity in asking the reasons of every thing that he did not understand.

He soon made a remarkable progress in his stuclics, and from bis infancy showed a decided taste for nutural and philosophical knowledge. Disgusted with the jargon of an absurd philosophy, he found unly in mathenatics the certainty with which he was charmed. Jle gave himself up chiefly to this science, and thenec he derived the most solid and indisputed part of his remwn. lint this philosopber, who lawght others to think, who broke the yoke of amiquity, and re-cetablished reason on her throne, this very Dos Cartes, was himscli led astray by his imagination. lit us respect his errors; for never did an ordinary man fall into the like. Ilis edifice of Vurlices, like she phibosophy of Aristulle, bas been temolisled by the cfluets of his serccessors; thengh his system concerning the nature of animals, in whichtie saw no principle superior to mecha- 
nism, still divides the literary world. But if we do not always find truth in the works of Des Cartes, we are at no Joss to trace evident marks of genius. That philosopher was a profound thinker, and spent his life in solitude. In vain did Cardinal Richelieu, in the name of the king, offer him important posts: he preferred lis retirement to the slavery of honours. Yet, yiclding to the urgent solicitations of Qucen Christina of Sweden, who wished to see and converse with him, he repaired to Stockholin. But the hours of their interviews were not regulated by the habits of Des Cartes, nor congenial to his nature. That great man, born with a weak constitution, which was rendered still more delicate by his custom of meditating in bed, conld not long sustain the task of rising every morning at five o'clock, in the winter rigours of a northern climate, to repair to the library of Christina. A defuction on the lungs terminated his life on the 11th of February, 1650 . In 1667 , his corpse was conveyed to France, and is now deposited in the Pantheon at Paris.

"Nature," says Voltaire, "had favoured Des Cartes with a strong and clear imagination, whence he became a very singular person, both in private life and in his manner of reasoning. This imagination could not be concealed even in his philosophical writings, which are cvery where adorned with very brilliant ingenious metaphors. Naturc had almost made hin a poett; and indeed he wrote a picce of poetry for the entertainment of Christina, quecn of Sweden, which however was suppressed in honour of his memory. He extended the limits of geometry as far beyond the place where be found them, as Newton did after him; and first taught the method of expressing curves by cquations. He applied this gcometrical and inventive genius to dioptrics, which when treated by him became a new art; and if he was mistaken in some things, the reason is, that a man who discovers a new tract of land, cannot at once know all the properties of the soil. Those who come after him, and make these lands fruitful, are at least obliged to him for the discovery." Voltaire acknowledges, that there are innumcrable errors in the rest of Des Cartes's works: but adds, that genmetry was a guide which he himself had in some measure formed, and which would have safely conducted him through the several paths of natural philosophy: yet he had at last abandoned this guide, and gave entirely into the humour of framing hypotheses; and then philosophy was no more than an ingenious romance, fit only to amuse the ignorant.

It lias been pretty generally acknowledged, that he borrowed his improvements in algebra from Harriot's Artis Analytice Praxis; which is highly probable, as he was in Jingland about the time when llatriot's book was published, and follows the manner of Harriot, cxcept in the melhod of uoting the powers. On this liead the following anecdote is related by Dr. Pell, in Wallis's Alyebra, pa. 198. Sir Charles Cavendish, then resiclent in Paris, discoursing there with .I. Roherval, concerning Des Cartes' Gcometry, then lately published: I admire, said lhoberval, that nucthod in De's Cartes, of placing all the terms of the equatinn on one side, making the whole equal to nothing, and how he felt upon it. The renson why you admire it, said Sir Clyarles, is because you are a lirenchman; for if you were an linglishunan, you would not admire it. Why so? asked Roberval. liecause, replied Sir Charles, we in England know whence he had it; namely, from Harriot's Al. ge bra. What book is that? says Roberval; I never saw ji. Nicxt time you come to my chamber, a aid Sir Charles,
I will show it to you. Which a while after he did: and on perusal of it, Roberval exclaimed with admiration, $\boldsymbol{I l}$ l'a vu! il l'a vu! He had seen it! he had seen it! finding all that in Harriot which he had before admired in Des Cartes, and not doubting but that Des Cartes had it from thence. Sce also Montucla's History of Mathematics.

'The real improvement of Des Cartes in algebra and genmetry, I have particularly treated of under the article $\mathrm{AL}_{\mathrm{L}}$ GEBIIA; and his philosophical doctrines are displayed in the article Cantesian Plilosophy, licre following. He was never married, but had onc natural daughter, who died when she was five years old. There liave becn several editions of his works, and commentaries upon them; particularly those of Schooten on his Geometry.

CARTESIAN Plilosophy, or Cartesianism, the system of philosoplyy adianeed by Des Cartes, and maintained by his followers, the Cartesians.

The Cartesian philosophy is founded on two great principles, the one metaphysical, the other physical. The metaphysical one is this: I think, therefore I an, or I exist : the physical principle is, that nothing exists but substances. Substance he makes of two kinds; the one a substance that thinks, the other a substance extended: so that actual thought and actual cxtension make the essence of substance.

The essence of matter heing thus fixed in extension, Des Cartes concludes that there is no vacuum, nor any possibility of it in nature; but that the universe is absolutely full: by this principle, mere space is quite $\mathrm{cx}-$ cluded; for extension being implied in the idea of space, niatter is so too.

Des Cartes defines motion to be the translation of a body from the neighbourlood of others that are in contact with $\mathrm{jt}$, and considered as at rest, to the neighbourhood of other bodies: by which he destroys the clistinction between motion that is absolute or real, and that which is relative or apparent. He maintains that the same quantity of motion is always preserved in the universe, because God must be supposed to act in the most constant and immutable manner. And hence also he deduces his three liws of motion. See Motron.

On these principles, Des Cartes explains mechanically how the world was formed, and how the present phenomena of nature came to arise. He supposes that God created. matter of an indefinite extension, which he separated into small cubic portions or masses, full of angles: that he impressed two motions on this inatter; the one, by which each part revolved about its own centre; and-another, by which an assemblage, or system of them, turned round a common centre. From whence arose as many different vortices, or eddies, as there were different masses of-matter, thus moving about common centres.

The consequence of these motions in each vortex, according to Des Cartes, is as follows: The prarts of matter could not thus move and revolve among one another, without having their angles gradually broken; and this continual friction of parts and angles must produse thrce clements: the first of these, an infuitely fine dust, formed of the angles brnken off; the second, the spheres remaining, after all the angular parts are thus removed; and those particles not yet rendered smooth and spherical, but still retaining some of their angles, and hamous parts, form the third element.

Now the first or subtilest element, according to the laws. of motion, must occupy the centre of eacls system, or vore- 
tex, by reason of the smallness of its parts: and this is the matter which constitutes the sun, with the fixed stars above, and the fire below. The second element, made up of spheres, forms the atmosphere, and all the matter between the enrth and the fixed stars; in such sort, that the largest spheres are always next tbe circuniference of the vortex, and the smallest next $i$ ts centre. The third element, formed of the irregular particles, is the matter that composes the eartb, and all lerrestrial bodies, together with comets, spots in the sun, \&c.

He accounts for the gravity of terrestrial bodies from the centrifugal force of the ather revolving-round the earth: and on the same general principles he pretends, to explain the phenomena of the magnet, and to account for all the other operations in nature.

The Cartesian system has received many alterations and amendments; ingenious men, for full a century, employing their talents in reforming one part, and new-modelling another; but it is now generally acknowledged that the foundation was faulty, and the superstructure erroneous; so that the fabric, though allowed to be. a work of genius, is abandoned to neglect and ruin, and is pointed at as a memorial of philosophical presumption.

CARTOUCHE, in the Military Art, a case of wood, about 3 inches thick at the bottom, girt with marlin, holding about 400 musket balls, besides 6 or 8 balls of iron of a pound weight, to be fired from howitzers for the defence of a pass, \&c.

CARTRIDGE, in the Military Art, the charge or load of a fire-arm, wrapped up in a thick paper, pasteboard, or parchment, to be the more readily charged, or conveyed into the piece. Cartridges of cannon and mortars are usually in cases of pasteboard, or tin, sometimes of wood half a foot long; taking up the place of the bullet in the picce to whose caliber the diameter is proportioned. Those of muskets, pistols, and smull-arms, only contain the charge of powder, with a tall wrapped up in thick paper.

Flannel cartridges are now preferred, because they do not retain the fire, and are therefore less liable to accidents in loading. A parchment cap is marle to cover them, in order to prevent the dust of powder from passing through them, which is taken off before they are put into the piece.

CartRIDGE-Box, a case of wood or turned iron, cuvered with l'ather, holding a dozen musket-cartridges. It is worn upon a belt, and hangs a little lower than the right pocket-hole.

CARY (RовеRT), a larned English chronologer and divine, was born at Cockington, in the county of Devon, about the ycar 1615. He took his degrees in arts, and L L. D. in Oxford. After returning from his travels he was presented to the rectory of Portlemouth, near Kingsbridge in Devonshirc: but not long after he was drawn over by the presbyterian ministers to their party, and chosen moderator in that part of the second division of the courity of Devon, which was appointed to meet at Kingsbridge. And yet, on the restoration of Charles the $2 \mathrm{~d}$, he was one of the first to congratulate that prince on his return, and soon atter was preferred to the archdeaconry of Exeter; but from which he was however some time atterward ejected. The remainder of his days he spent at his rectory at Portlemouth, and clicd in 1688 , at 73 years of age.-Ile pub. lished Palaelogia Chronica, a chromological account of ancient time, in three parts: 1 , Didactical; 2, Apodidactical; 3, Canonical : in 1677.
CASATI (PAi L), a learned Jesuit, born at Placentia in 1617. He entered early among the Jesuits; and after having taught mathematics and livinity at Rome, he was sent into Sweden to Queen Christina, whom he prevailed on to embrace the popish religion. His writings are as follow :

1. Vacuum Proscriptum.-2. Terra Machinis mota.3. Mechanicorum, libri octo.-4. De Igne Disscrtationes. -5. De Angelis Disputatio Theolog.-6. Hydrostaticæ Dissertationes.- - T. Opticæ Disputationes. It is remarkable that he wrote this treatise on optics at 88 years of age, and after he was blind. He was also author of several books in the Italiau language.

CASCABEL, the knob or button of metal behind the "brcech of a cannon, serving as a kind of handle by which to elevate and direct the piece; to which some adid the fillet and ogees as far as the base-ring.

CASEMATE, or CAzEMATE, in Fortification, a kind of vault or arch, of stone-work, in that part of the Hank. of a bastion next the curtain; serving as a batlery, to defend the face of the opposite bastion, and the moat or ditch.

It is now seldom used, because the batteries of the enemy are apt to bury the artillery of the casemate in the ruins of the vault: beside, the great smoke made by the disclrarge of the canuon, renders it intolerable to the men. So that, instead of the ancient covered casemates, later engincers have contrived open ones, only guarded by a parapet, \&c.

Casemate, or Casenent, in Architecture, a hollow moulding, which some architects make $\frac{x}{6}$ of a circle, and others $\frac{\pi}{4}$. Casement is also used for a sinall moveable window, usually within a larger, bcing made open to turu on hinges.

CASERNS, or CAzER s, in Fortification, small roouns, or buts, erected between the ramparts and the houses of fortified towns, or even on the ramparts themselves; 10 serve as lodgings for the suldiers on immediate duty, to ease the garrison.

CASE-SHot, or CANNנSTER-SIIot, are a number of small balls put into a round tin cannister, and so shót out of great guns. These liave superseded, and been substituted instead of, the grape-shot, which have been laid aside.

CASSINI (JonN-Domıмc), an eminent astronumer, was born of noble parents, at a town in Piedmont in Italy, June 8, 1625. Atter laying a proper foundation in lis studies at bome, he was sent to continue them in a college of Jesuits at Genoa. He had an uncommon turn for Latin poetry, which he exercised so very early, that some of his poems were published when he was but 11 years old. At length lie met with books of astronomy, which he read with great eagerness, Pursuing the bent of his inclinations in this way, he soon acquired a very extcnsict knowledge of the subject, and in 1650 the senate of Bologna invited him to be their public mathematical professor. Cassini was but 25 years of age when he went to Bologia, where he taught mathematics, and made cetestial observations with great care and assiduity.- - 11 1652 a comet appeared, which lie observed with great accuricy; and he discovered that comets were not bodics accidentally generated in the atmosphere, as had been supposed, but of the same nature, and probably governed by the same laws, as the planets. 'The same your he resolved an astronomical problem, which Kepler and Bulliald had given up as insolvable; viz, to determine geonetrically the apogece and excentricity of a planet, trom its true and neas place.- 
In 1653 , when a church in Bologna was repaired and enlarged, he obtained leave of the senate to correct and settle a meridian line, which had tren drawn by an astronomer in 1575 . - In 1657 he attended, as an assistant, a nobleman, who wan scnt to Rome to compose some differences, which had arisen between Bologna and Ferrara, from the inundations of the Po; and he showed so much skill and judgment in the management of the affair, that in 1663 the pope's brother appiinted him inspector general of the fortifications of the castle of Urbino: and he had afterwards committed to him the care of all the rivers in the ecclesiastical state.

Meauwhile he did not neglect his astronomical studies, but cultivated them with great care. He made several discoveries relative to the planets Mars and Venus, particularly the revolution of Mars about his axis: but the point he had chiefly in vicw, was to settle an accurate theory of Jupiter's satellites; which, after much labour and ohservation, he happily effected, and published it at Rome, among other astronomical pieces, in 1666 .

Picard, the French astronomer, getting Cassinj's tables of Jupiter's satellites, found them so very exact, that he conceived the highest opinion of his skill; and from that time his fame increased so fast in France, that the govern= ment desired to have him a member of the Academy. Cassini however could not quit his station without leave of his superiors ; and therefore the king, Lewis the 14th, requested of the pope and the senate of Bulogna, that Cassini might be permitted to come into France. Leave was granted for 6 ycars; and he came to Paris in the beginning of 1669 , where he was immediately made the king's astronomer. When this term of 6 years was nearly expired, the pope and the senate of Bologna insisted on his return, on pain of forfeiting his revenues and emoluments, which lad bitherto been faithfully remitted to him: but the minister Colbert notwithstandive prevailed on hin to stay, and he was naturalized in 1673 ; the year in which he was married.

The Royal Ohservatory of Paris had been finished some rime. The uccasion of its being built was this: In 1638, the culcbrated Mersenne was the chief institutor and promoter of a suciety, where several ingenious and learned men met together to talk upon plyysical and astronomical subjects; among whom were Gassendi, Descartes, Monmort, Thevenot, Bulliald, our countryman Hobbes, \& c: and this society was kept up by a succession of learned men for many years. At length the government considering that a number of such men, acting in a body, would succeed much better in the promotion of science, than if they acted separately, each in his particular'art or province, established under the direction of Colbert, in 1666 , the Royal Acarlemy of Sciences: and for the advancement of astronomy in particular, erected the Royal Observatory at Paris, and furnished it with all kinds of instruments shat were necessary to make obscrvations. The foundation of this noble pile was laid in 1667 , and the building completed in 1670. Of this obscrvatory, Cassini was appointed the first inhabiter; which be took possession of in Sept. 1671. In 1672 he endeavoured to determine the parallax of. Mars and the sun: and in 1677 be proved that the diurmal rotation of Jupiter about his axis was performed in 9 hours 58 minutes, from the motion of a spot in one of his larger belts: also in 1684 be discovered four satellites of Saturn, besides that which Iluygens ljad observel. In 1693 he published a ncw cdition of his Tables. of Jupiter's Satellites, corrected by later observations. In 1695 he took a journey to Bologna, to examine the meridian line, which he had fixed there in 1655; and be showed, in the presence of eminent mathematicians, that it had not varied in the least, during that 40 years. In 1700 he continued the meridian line through France, which Picard had begun, to the very southern limits of that country.

Our author resided at the royal observatory for more than 40 years, making many excellent and useful discoveries. The titles of his publications occupy nearly 5 pages in the closely printed index of Rozier, and consequently far exceed our bounds even to enumerate in this place. He liad the misfortune in his latter years to be deprived of his sight; and he ended a useful and honourable life September 14th 1712, at the age of $\$ 7$ ycars.

CASSINI (JAMES), a celebrated French astronomer, and niember of the several Academies of Sciences of France, England, Prussia, and Bologna, was borm at Parlz, Feb. 18, 1677 , being the younger son of John-Dominic Cassini, above mentioned, whom he succeeded as astronomer at the royal observatory, the elder son baving lost his life at the battle of La Hogue.

After his first studies in his father's house, in which it is not to be supposed that mathematics and astronorny were neglected, he was sent to study philosophy at the Mazarine college, where the celebrated Varignon was then professor of mathematics; from whose assistance young Cassini profited so well, that at 15 years of age he supported a mathematical thesis with great honour. At the age of 17 be was admitted a member of the Acarlemy of Sciences; and the same year he accompanied his father in his journey to Italy, where he assisted him in the verification of the meridian at Bologna, and other measurements. On his return he made other similar operations in a journey into Holland, where he discovered some errors in the measure of the earth by Sisell, the result of which was communicated to the Academy in 1702 . He also visited England in 1696, where he was made a member of the Royal Society. - In 1712 he succeeded his father as astronomer royal at the observatory.-In 1717 he gave to the Academy his rusearches on the distance of the fixed stars, in which he showed that the whole annual orbit, of near 200 millions of miles diameter, is but as a point in comparison of this distance. The same year be communicated also his discoveries concerning the inclination of the orbits of the satellites in general, and especially of those of Saturn's satellites and ring.-In 1725 he undertook to determine the cause of the moon's libration.

In 1732 an important question in astronomy cxercised the ingenuity of our author. His father had determined, by his observations, that the planet Venus madc one complete revolution about her axis in 23 hours : and M. Bianchini had published a work in 1729 , in which he settled the period of the same revolution at 24 days 8 hours. From an examination of Bianchini's obscrvations, which were upon the spots in Venus, he discovered that he liad intermitted his observations for the duration of 3 hours, from which cause lie had probably mistaken new spots for the old ones, and so had becn led into the mistake. He soon afterwarls determined the nature and quiantity of the accrleration of the motion of Jupiter, at half a second per ycar, and of that of the retardation of Saturn at two minutes. per year; that these quantities would go on increasing for 2000 years, and then would decrease again.-In 


\section{$C \perp S$}

[ 280

1740 he published his $\Lambda$ stronomical Tables; and his Elements of Astronomy; both of which are very extensive and accurate works.

Though astronomy was the principal object of our author's consideration, he did not confine himself absolutely to that branch, but made occasional excursions into other fields. We owe also to him, for example, Experiments on Electricity, or the light produced by friction; Experimerts on the recoil of fre-arms; Rescarches on the rise of the mercury in the barometer at different heights above the level of the sea; Reflections on the perfecting of burning-glasses; and other memoirs.

The French Academy had properly judged that one of its most important objects, was the measurement of the earth. In 1669 Picard measured a little more than a degree of latitude to the north of Paris; but as that extent appeared ton small from which to conclude the whole circumference with sufficient accuracy, it was resolved to continue that measurement on the neridian of Paris to the north and south, through the whole extent of the country. Accordingly, in $1683, M$. Lahire continued that on the north side of Paris, and the older Cassini that on the south side. The latter was assisted in 1700 in the continuation of this operation by his son James. The same work was farther continned by the same Academicians; and finally the part left unfinished by Lahire in the north, was finished in 1718 by our anthor, with the late Maraldi, and Lahire the younger.

These operations produced a considerable degree of precision. It appeared also, from this measured extent of 6 degrees, that the degrees were of different lengths in different parts of the meridian; and in such sort that our author concluded, in the volume published for 1718, that they decreased more and more towards the pole, and that therefore the figure of the earth was that of an oblong spheroid, having its axis longer than the equatorial diameter. He also measured the perpendicular to the same meridian, and compared the measured distance with the differences of longitude, as before determined by the eclipses of Jupiter's satellites; whence he inferred, that the length of the degrees of longitude was smaller than it wonld be on a sphere, and that therefore again the figure of the earth was an oblong speroid; contrary to the determination of Newton by the theory of gravity. In consequence of these assertions of our author, the French government sent two different classes of mathematicians, the one to measure a degree at the equator, and the other at the polar circle; and the comparison of the whole deterinined the figure to be an oblate spheroid, contrary to Cassini's determination.

After a long and laborious life, our author James Cassini lost his life by a fall in April 1756, in the soth year of his agc, and was succecled in the Acalemy and Observatory by his second son Ccsar-François de Thury. He published $A$ Treatise on the Magnitude and Figure of the Earth; as also The Elements or Theory of the Planets, with tables; besides a great number of papers in the Memoirs of the Academy, from the year 1699 to 1755 .

CASSINI de Tiuut: (Cesar-Firançors), a celebrated French astronomer, dircctor of the obscriatory, and member of most of the learned socicties of Lurnpe, was born at Paris June $t 7,1714$, being the second son of James Cassini, whose occupations and talents our author inherited and supported with great honour. IIe received his first lessons in astronomy and mathematics from MM. Ma- raldi and Camus; and his progress was so great, that at 10 years of age he was able to calculate the phases of the total eclipse of the sun of $172 \%$. At the age of 18 he accompanied his fatber in his twojourneys undertaken for drawing the perpendicular to the observatory meridian, from Strasbourg to Brest. From that time a general cliart of France was devised; for which purpose it was necessary to traverse the country by several lines, parallel and perpendicular to the meridian of Paris, and our author was intrusted with this business. He did not content himself with the measure of a degree by Picard; suspecting even that the measures which bad been taken by his father and grandfather were not exempt from some errors the imperfections of their instruments at least would be liable to, he again undertook to measure the meridian at Paris, by means of a new series of triangles, of a smaller number, and more advantageously disposed. This great work was published in 1740 , with a chart showing the new meridian of Paris, by two different series of triangles, passing along the seacoasts to Bayonne, traversing the fromtiers of Spain to the Mediterranean and Antibes, and thence along the castern limits of France to Dunkirk, with parallel and perpendicular lines described at the distance of 6000 toises from one another, from side to side of the country.-In 1735 he had been received into the academy as adjoint supernumerary at 21 years of age.

A tour which our author made in Flanders, in company with the king, about 174.1, gave rise to the particular chart of France, at the instance of the king. Cassini published different works relative to these charts, and a great number of the sheets of the charts themselves.

In 1761 Cassini undertook an expedition into Germany, for the purpose of continuing to Vienna the perpendicular of the Paris meridian; to unite the triangles of the chart of France with the points taken in Germany; to prepare the means of extending into this country the same plan as in Erance; and thus to establish successively for all Europe a most uscful uniformity. Our author was at Vienna the 6th of June 1761, the day of the transit of Venus over the sun, of which he observed as much as the state of the weather would permit lim to do, and published the account thereof in his Voyage en Allcmagne.

Finally, M. Cassini, always meditating the perfection of his grand design, proposed at the peace between England and France in 1783 , the joining of certain points taken upon the English const with those which had becn detcrmined on the coast of France, and thus to connect the general chart of the latter with that of the British isles, like as he had before united it with those of Flanders and Germany. The proposal was favourably receired by the English government, and presently carried into effect, under the direction of the Royal Socicty, the exceution being committed to the late Gencral Roy; after whose icath the business was for some time suspended; hut it was afterwards revived under the auspices of the Duke of Richmond, Master-General of the Ordnance, and the execution committed to the care of Col. Edward Williams and Captain (now Col.) William Mudge, both respectable ofticers of the Artillery, and Mr. Isaac Dalby, who had before accompanicd and assisted Gencral koy; from whose united skill and zeal the happiest prosecution of this business was to be exjected; as it has since been most ably completed by Col. Mudge, now Licut. Governor of the Royal Military Academy; where as i cadet be received hos mathematical education under the direction of the author of this Dictionary. 


\section{A S}

The volumes of the Memoirs of the French Academy for the years between 1735 and 1770 contain a prodigious number of papers by Cassini. They consist chiefly of astronomical observations and questions; among which are, Researches concerning the parallax of the sun, the moon, Mars, and Venus; On astronomical refractions, and the effect caused in their quantity and laws by the weather; Numerous observations on the obliquity of the ecliptic, and on the law of its variations. In short, he cultivated astronomy for 50 years, a period the most important for that science that ever elapsed, for the magnitude and variety of objects, in which he commonly, sustained a corisiderable share.

M. Cassini was of a very strong and vigorous constitution, which carried him through the many laborious operations in gengraphy and astronomy which he conducted. An habitual retention of urine however rendered the last 12 years of his life very painful and distressing, till it was at length terminated by the smali-pox the 4 th of Scptember 1784 , in the 71 st year of his agc; being succeeded in the academy, and as director of the observatory, by his only son Count John-Dominic Cassini; who is the 4 th in order by direct descent in that honourable station.

CASSIOPE1A, one of the 48 old constellations, placed near Ccpheus, not far from the north pole. The Greeks probably received this figure, as they did the rest, from the Egyptians, and in their fables added it to the family in the neighbouring part of the heavens, making her the wife of Cepheus, and mother of Andromeda. They pretend that she was placed in this situation, to behold the destruction of her favourite daughter Andromeda, who is chained just by her on the shore, to be devoured; and this as a punishment for her pride and vanity in presuming to stand the comparison of beauty with the Nereids.

In the year 1572 there burst out all at once in this constellation a new star, which at first surpassed Jupiter himself in magnitude and brightness; but it diminished by degrees, till it quite disappeared at the end of 18 months. This star alarmed all the astronomers of that age, many of whom wrote dissertations upon it; among the rest $\mathrm{T}_{y}$ cho Brahé, Kepler, Maurolycus, Lycetus, Gramineus, and others. Beza, the Landgrave of Hesse, Rosa, and some more, wrote to prove it a comet, and the same that appeared to the Magi at the birth of Christ, and that it came to declare his second coming: these were answered by Tycho.

The stars in the constellation of Cassiopeia, are in PtoIemy's Catalogue 13, in Hevelius's 37, in Tycho's 16 , and in Flamsteed's 55.

CASTELLI (Benedict A в вате), an eminent mathematician, was born of an ancienr and noble family at Brescia, in the year 1577 . In 1595 he entered into a monastery of the order of St. Benedict, in his native city. He was for some time a disciple of Galileo at Florence, and assisted him in his astronomical observations, and afterwards maintained a regular correspondence with him. Under his name, the apology of Galileo against the censures of Leudovico delle Colombe and Vincont di Grazia appeared, thrugh it was principally written by Galit co himself. From 1615 to 1625 he occupied the mathenratical chair at Pisa. ln 1625 Castelli was invited to Rome by Pope Urban vin, and made mathematical professor in the colicge Gella Sapirnza. 'Thr subject of his particular attention, and in the investigation of which he chisfly excelted, wats the motion of watter and in 1628 he published two works upon it,

YoL. I. which gained much reputation; viz, The Mensuration of running waters, and Geometrical Demonstrations of the mensuration of running waters. These have been lately inserted in the collection of the author's works on similar topics, printed at Florence, with other trcatises on the laguna of Venice, on the improvement of the Pontine, Bolognese, Ferrarese, and Romagnese marshes, \&c. Guglielmini, though in other things he impugns Castelli, allows him the honour of having first applied geometry to the motion of water; and Montucla (Hist. Math. t. 2, p. 201) calls him "The creator of a new part of hydraulics." He died at Rome in 1644 .

CASTOR, a moiety of the constellation Gemini ; called also Apollo. Also a star in this constellation, whose latitude, for the year 1700, according to Hevclius, was $10^{\circ} 4^{\prime} 20^{\prime \prime}$ north; and its longitude og $16^{\circ} 4^{\prime} 14^{\prime \prime}$.

CASTOR and Pollux. See Gemini.

CASTOR and PoLlux, in Metcorology, is a fiery meteor, which at sea appears sometimes adhering to a part of the ship, in the form of a ball, or even several balls. When one is seen alone, it is properly called Helena; but two are called Castor and Pollux, and sometimes Tyndaridz.

By the Spaniards, Castor and Pollux are called San Elmo; by the French, St. Elme, St. Nicholas, St. Clare, St. Helene; by the Italians, Hermo; and by the Dutch, Vree Vuuren.

The meteor Castor and Pollux, it is commonly thought, denotes a cessation of the storm, and a future calm; as it is rarely seen till the tempest is nearly spent. But Helena alone portends ill weather, and denotes the severest part of the storm yet behind.

When the metcor adheres to the masts, yards, \&c, it is concluded, from the air not having motion enough to dissipate this flame, that a profound calm is at hand; but if it flutter about, that it denotes a storm.

CASTRAMETATION, the art, or act, of encamping an arniy.

CATACAUSTICS, or Catacaustic Curves, in the Higher Geometry, and in Optics, are the species of caustic curves formed by reflection. These curves are generated after the following manncr: If there be an infinite number of rays $\mathrm{AB}, \mathrm{AC}$, $A D, \& c$, procecding from the radiating point $A$, and reflected at any given curve $\mathrm{BCDH}$, so that the angles of incidence be still equal to the angles of reflection; then the curve BEG, to which the reflected rays B 1, CE, DF, \& c, are everywhere tangents, as at the points $I, E, F, \& c$, is the catacaustic, or caustic-by-reflection.

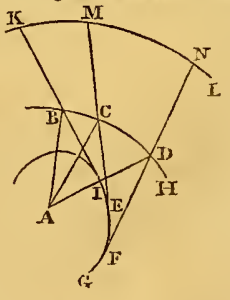

Or it is the same thing as saying, that a caustic curve is that formed by joining the points of concurrence of the sevrral reflected rays.

Some propertics of these curves are as follow. If thic reflected ray $1 \mathrm{~B}$ be produced to $\mathrm{K}$, so that $\mathrm{AB}=\mathrm{BK}$, and the curve $\mathrm{K} L$ be the evolute of the caustic $B E G$, beginning at the point $\mathrm{K}$; then the portion of the caustic $\mathrm{BE}$ is $=(A C-A B)+(C E-B 1)$, that is, the difference of the two incident rays added to the difference of the two reflected rays.

When the given curve $\mathbf{B C n}$ is a geometrical one, the caustic will be so too, and will alsvays be rectifiable. 'The 20 


\section{$\mathrm{C} \wedge \mathrm{T}$}

[ 282

caustic of the circle is a cycloid, or epicycloid, formed'by the revolution of a circle upon a circle.

Thus, A BD being a semicircle, exposed to parallel rays; then those rays which fall near the axis св will be reflected to $\mathbf{r}$, the middle point of $\mathrm{BC}$; and those which fall at $A$, as they touch the curve only, will not be re-

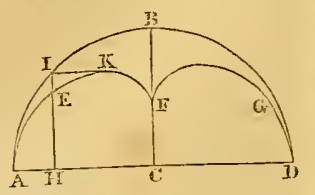

flected at all; but any intermediate ray $\mathrm{H} \mathrm{X}$ will be reflected to a point $\mathrm{K}$, somewhere between $\mathrm{A}$ and $\mathrm{F}$. And since every different incident ray will have a different focal point, therefore those various focal points will form a curve line $A E F$ in one quadrant, and FGD in the other, being the cycloid above mentioned. The caustic of the common cycloid, when the rays are parallel to its axis, is also a common cycloid, described by the revolution of a circle on the same base. The caustic of the logarithmic spiral, is the sanse curve.

The catacaustic may be beautifully exhibited experimentally by exposing the inside of a smooth bowl, or glass, to the sun-beams, or strong candle-light; for then this curve $A$ ETGD will appear plainly delineated on any white surface placed horizontally within the same, as on the surface of milk contained in the bowl.

The principal writers on the caustics are, l'Hôspital, Carré, \&cc. See Memoires de l'Acad. an. 1666 \& 1703.

CATACOUSTICS, or Cataphonics, is the science of reflected sounds; or that part of acoustics which treats of the properties of echoes.

Catacoustics, in Military Language, are écoutes or small galleries, from distance to distance, in front of the glacis of a fortified place, all of which communicate with a gallery that is carried parallel to the covert-way. The besieged make use of them in going to meet the enemy's miners, and interrupt their progress.

CATADIOPTRICAL Telescope, the same as Reflecting Telescope; which see.

CATALOGUE of the Stars, is a list of the fixed stars, disposed according to some order, in their several constellations; with the longitudes, latitudes, right ascensions, \&c, of each.

Catalogues of the stars have usually been disposed, either as collected into certain figures called constellations, or according to their right ascensinns, that is the order of their passing over the meridian. All the catalogues, from the most ancient down to Flamstecd's inclusively, were of the first of these forms, or in constellations; but most of the others that have since appeared are of the Iatter form, as being much more convenicnt for most purposes. Indeed one has lately been constructed in which the stars are disposed in classes according to zones or degrees of polar distance.

Hipparchus of Rlıodes first undertook to make a catalogue of the stars, about 128 yeatrs before Christ; in which he made use of the observations of Timocharis and Aristyllus, for about 14.0 years before him. Ptolemy retained Hipparcluss's catalogue, containing 1026 fixed stars in 48 constellations, though he limself made abundance of observations, with a view to a new catalogue, an. Dom. 140 Albategni, a Syrian, brought the same down to his own time, viz, about the year of Christ 880 . Anno 14.37, Ulugh Beigh, or Begr, king of Parthia and India, formed a new catalogue of 1022 fixed stars, or according to some 1016 ; since translated out of Persian into Iatin by Dr. Hyde, in 1665. The tbird person who made a catalogue of stars from his own observations was Tycho Brahé, who determined the places of 777 stars, for the end of the year 1600 ; which Kepler, from other observations of Tycho, afterwards increased to the number of 1000 in the Rudulphine tables; adding those of Ptolemy and other authors, omitted by Tycho; so that his catalogue amounts to abore 1160. A bout the same time, William landgrave of Hesse, with his mathematicians Byrgius and Rothman, determined the places of 400 stars from new observations, rectifying them for the year 1593 ; which Hevelius prefers to those of Tycho. Ricciolus, in his Astronomia Reformata, determined the places of 101 stars for the year 1700 , from his own observations: for the rest, he tollowed Tycho's catalogue; altering it where he thought proper. Anno $1667 \mathrm{Dr}$. Halley, in the island of St. Helena, observed 350 of the southern stars, not visible in our horizon. The same labour was repented by Father Nuel in 1710, who published a new catalogue of the same stars constructed for the year 1687. Also De la Caille, at the Cape of Good Hope, made accurate observations of about 10 thousand stars near the south pole, in the years 1751 and 1752 ; the catalogue of which was published in the Memoirs of the French Academy of Sciences for the year $\mathbf{1 7 5 2}$, and in some of his own works, as more particularly nuticed below.

Bayer, in his Uranometria, published in 1603 a catalogue of 1160 stars, compiled chictly from Ptolemy and Tycho, in which every star is marked with some letter of the Greek alphabet ; the brightest or principal star in any constellation being denoted by the first letter of the alphabet, the next star in order by the second letter; and so on; and when the number of stars exceeds the Greck alphabet, the remaining stars are marked by the letters of the Roman alphabet; which letters are preserved by Flamsteed in his catalogue, and also by Senex on his globes, and indeed by most astronomers since that time.

In 1673 , the celebrated John Ilevelius, of Dantzic, published, in his Machina Cœlestis, a catalogue of 1888 stars, of which 1553 were observed by himself; and their places set down for the end of the year 1660 . But this catalogue, as it stands in Flamsteed's Historia Ceelestis of 1725, contains only 1520 stars.

The inost complete catalogue ever given from the labours of one man, was the Britannic catalogue, compiled from the observations of the accurate and indefatigable Mr. Flamsteed, the first Royal Astronomer at Gremwich; who for a long series of years devoted himsclf wholly to that employment. As there was nothing wanting, cither in the olserver or appraratus, this may be consiclered as a perfect work, so far as it goes. It is however to be regretted that the edition did not pass through his own hands : that now cxtant was published by authority, but without the author's consent, and contains 2734 stars. Another edition was published in 1725 , pursuant to his testament, containing 3000 stars, with ibeir places adapted to the begiming of the year 168.9 ; to which is added $\mathrm{Mr}$. Sharp's catalogie of the southern stars not visible in our hemisphere, set down for the year 1726. Sec vol. 3 of his Historia Coelestis, in which are printed the catalogues of l'tolemy, Uluglı Beigh,'l'ycho, the P'rince of l lesse, and Hevelius; with an accuunt of each of them in the I'rolegomena. 
The first catalogue, we believe, that was printed in the new or second form, according to the order of the right ascensions, is that of Lacaille, given in his Ephemerides for the 10 years between 1755 and 1765 , and printed in 1755. It contains the right ascensions and declinations of $307 \mathrm{stars}$, which are rectified to the beginning of the year 1750.-In 1757, Lacaille published his Astronomiæ Fundamenta, containing a catalogue of the right ascensions and declinations of 398 stars, Jikewise adapled to the beginning of $1750 .-$ And in 1763 , the year after his death, was published the Cœlum Australe Stelliferum of the same author; containing a catalogue of the places of 1942 stars, all situated to the southward of the tropic of Capricorn, and observed by him while he was at the Cape of Good Hope, in 1751 and 1752 ; their places being also suited to the beginning of $1750 .-$ In the same year was published his Ephemerides for the 10 years between 1765 and 1775 ; in the introduction to which are given the places of 515 zodiacal stars, all deduced from the observations of the same author; and adjusted to the beginning of the year 1765 .

In the Nautical Almanac for 1773 , is given a catalogue of $387 \mathrm{stars}$, in right ascension, declination, longitude, and latitude, derived from the observations of the late celebrated Dr. Bradley, and adapted to the beginning of the year 1760. This small catalogue, and the results of about 1200 observations of the moon, are all that the public have yet seen of the multiplied labours of this most accurate and indefatigableobserver, though he has now (1814) been dead upwards of 52 years.

In 1775 was published a thin volume entitled' Opera Inedita, containing several papers of the late Tobias Mayer, and among them a catalogue of the right ascensions and declinations of 998 stars, which may be occulted by the moon and planets; the places being adjusted to the beginning of the year 1756 .

At the end of the first volume of Astronomical Observations made at the Royal Obscrvatory at Greenwich, published in 1776 , Dr. Maskelyne, the late Astronomer Royal, gave a catalogue of the places of 34 principal stars, in right ascension and north-polar distance, adapted to the beginning of the year 1770 . These being the result of several years' repeated observations, made with the utmost care and the best instruments, may be presumed to be exceedingly accurate.

In 1782 , M. Bode, of Berlin, publisherl a very extensive catalogue of 5058 of the fixed stars, collected from the observations of Flamsteed, Bradley, II cvelius, Mayer, Lacaille, Messier, Monnier, D'Arquier, and other astronomers; all rectified to the beginning of the year 1780 ; and accompanied with a Celestial $\Lambda$ tlas, or sets of maps of the constellations, engraved in a most delicate and beautiful manner.

To these may be added, $\bar{D}$ r. Herschel's catalogue of double stars, printed in the Philos. Trans. for 1782 and 1783; Messicr's nebulæ and clusters of stars, published in the Connoissance des Temps for 17 84; and Herschel's catalogue of the same kind, given in the Philos. Trans. for 1786 , and other years.

In 1789 , the Rev. Francis Wollaston published A Specimen of a General Astronomical Catalogue, in Zones of North-polar Distance, and arlapted to Jan. 1, 1790. These stars are collected from all the catalogues beforementioned, from that of llevelius downwards. This work contains five distinct catalogues; viz, Dr. Mlaskelyne's new catalogue of 36 principal stars; a general catalogue of all the stars, in zones of north-polar distance; an index to the general catalogue; a catalogue of all the stars, in the order in which they pass the mcridian; and a catalogue of zodiacal stars, in longitude and latitude.

In 1792, Dr. Zach published at Gotha, Tabulæ Motuum Solis, to which is annexed a new catalogue of the principal fixed stars, from his own observations made in the years $1787,1788,1789,1790$. This catalogue contains the right ascensions and declinations of 381 principal stars, adapted to the beginning of the year 1800 .

The catalogues of Dr. Bradley, Lacaille, Zach, and Mayer, are published by Professor Vince, in the second volume of his Astronomy. M. Lalande has published a new catalogue of more than 12,000 stars, in the volumes of the Connoissance des Temps, from the year 7 (1799) to the year 12. Almost all these are stars which had not becn before observed. M. C. Vidal has lately communicated to the Lyceum of Toulouse a catalogue of 888 austral stars, from the 5 th to the 7 th magnitude inclusively: every star has been observed three times, and all are reduced to a mean position, regard being had to the effect of refraction, the aberration of light, and the rotation of the earth's axis. The mean position of all these stars has been calculated to a common period, viz. Jan. 31,1798 ; the equation and precession of the equinoxes being previously allowed for. The place of M. Vidal's observations was Mirepoix, a situation admirably suited to his purpose, by the serenity of its atmosphere, and the excellence of the instruments with which the observatory is furnished, and commanding nearly six degrees of the heavens southwards more than Paris. On this account $\mathrm{C}$. Lalande and his nephew C. François Lalande, in their grand work of completing a catalogue of 48,000 stars, engaged M. Vidal to form a catalogue of the austral stars, which he has executed with great success and admirable precision. From the history of astronomy for 1800 by Jerome de Lalande, it appears that M. F. Lalande has terminated the labour commenced August 5, 1789, and determined the places of 50,000 stars, from the pole to 2 or 3 degrees below the tropic of Capricorn. In 1800, S. Piazzi, of Palermo, published his large catalogue, of 5500 stars.

CATAPULT, Catapulta, a military engine, much used by the ancients for throwing huge stones, and sometimes large darts and javelins, 12,15 , or even 18 feet long, on the enemy. It is sometimes confounded with the Balista, which is more peculiarly adapted for throwing stones; some authors making them the same, and others different.

The catapulta, which it is said was invented by the Syrians, consisted of two large timbers, like masts of ships, placed against each other, and bent by an engine for this purpose; these being suddenly unbent again by the stroke of a hammer, threw the javelins with prodigious force. lts structure and the manner of working it are described hy Vitruvius; and a figure of it is also given by Perrault. M. Folard asserts that the catapult made infinitely more disorder in the ranks, than our cannon charged with iron halls. Sce Vitruv. Archit. lib. x. cap. 15 and 18; and Perr. notes on the same; also Rivius, p. 597.

Josephus takes notice of the surprising effects of these engines, and says, that the stones thrown out of them, of a hundred wcight or more, beat down the battlements, knocked the angles off the towers, and would level a whole 
filc of men from one end to the other, if the phalanx were ever so deep.

See plate $V$, fig. 3 and 4 , for two forms of the catapult, the one for throwing darts and javelins, the other for stones.

CATENARY, a curve line which a chain, cord, or such like, forms itself into, by hanging frcely from two points of suspension, whether these be in the same horizontal line or not; as the curve BAc, formed by a heavy flexible line suspended by any two points $\mathrm{C}$ and $\mathrm{B}$. It is otherwise called the elastic curve.

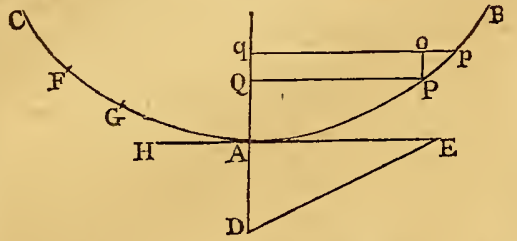

The nature of this curve was sought after by Galileo, who thought it was the same with the parabola; but though Jungius detected this mistake, its true nature was not discovered till the year 1691 , in consequence of M. John Bernoulli having published it as a problem in the Acta Eruditorum, to the mathematicians of Europe. In 1697 Dr. David Gregory published an investigation of the properties before discovered by Bcrnoulli and Leibnitz; in which he asserts that an inverted catenary is the best figure for an arch of a bridge, \&c. See Philos. Trans. abr. vol. 4 ; also Bernoulli Opera, vol. 1. pa. 48, and vol. 3, pa. 491; and Cotes's Harmon. Mersur. pa. 108.

The catenary is a curve of the mechanical kind, and cannot be expressed by a finite algebraical equation, in simple terms of its absciss and ordinate; but is easily expressed by means of fluxions: thus, if $A Q$ be its axis perpendicular to the horizon, and $P Q$ an ordinate parallel to the same, or perp. to $\mathrm{AQ}$; also $p q$ another ordinate indefinitely near the former, and po parallel to $\mathrm{AQ}$ : then, $a$ being some givell or constant quantity, the fundamental property of the curve is this, viz, $\mathrm{Po}:$ op $:: \mathrm{AP}: a$, or $\dot{x}: \dot{y}:: z: a$, that is, the fluxion of the axis, is to the fluxion of the ordinate, as the length of the curve, is to the given quantity $a$; where $x=\mathrm{AQ}, y=\mathrm{PQ}$, and $z=\mathrm{AP}$. This, and the other propertics of the curve, will plainly appear from the following considerations : First, supposing the curve hung up by its two points $\mathbf{B}$ and c, against a perpendicular or upright wall : then, every lower part of the curve being kept in its position by the tension of that which is immediately above it, the lower parts of the curve will retain the same position unvaried, by whatever points it is suspended above; thus, if it were fixed to the wall by the point $\mathrm{F}$, or $\mathrm{G}, \mathrm{\& c}$, the whole curve $c A \mathrm{~B}$ will remain just as it was; for the tensions at $\mathbf{F}$ and $G$ have the same effect on the other parts of the curve, as when it is fixed by those points: and hence it follows that the tension of the curve at the point $\Lambda$, in the horizontal direction, is a constant quantity, whether the two legs or branches of the curve, on both sides of it, be longer or shorter; which coustant tension at $A$ let be denoted by the quantity $a$.

Now, because any portion of the curve, as $\Delta \mathrm{P}$, is kept in its position by three forces, viz, the tensions at its cxtremitics $A$ and $P$, and its own wcight, of which the tension at $A$ acts in the direction $A 1$ or po, and the tension at 5 acts in the direction of $\mathrm{PP}$, and the weight of the line acts in the perpendicular direction oP ; that is, the three forces which retain the curve $A P$ in its position, act in the directions of the sides of the elementary triangle $o p P$; but, by the principles of mechanics, any three forces, keeping a body in equilibrio, are proportional to the three sides of a triangle drawn in the directions in which those forces act; therefore it follows, that the forces keeping $A P$ in its position, viz, the tension at $A$, the tension at $P$, and the wcight of $A P$, are respectively as $O p, p P$, and $O P$,

that is, as $\dot{y}, \dot{z}$, and $\dot{x}$.

But the tension at $A$ is the constant quantity $a$, and the weight of the uniform curve $A P$ may be espounded by its length $z$; therefore it follows that $\dot{x}: \dot{y}:: z: a$; which was to be proved.

Also from this last proportion, by proper analogy, or similar combinations of the terms, there arises this other property, $\dot{x}: \sqrt{ }(\dot{x} \dot{x}+\dot{y})$ or $\dot{z}:: z: \sqrt{ }(a a+z z)$, or $z \dot{z}=$ $\dot{x} \sqrt{ }(a a+z z)$, or $\dot{x}=\sqrt{ } \frac{z \dot{z}}{\left(a^{2}+z^{3}\right)} ;$ and the fluents of these give $x=\sqrt{ }\left(a^{2}+z^{2}\right)$. But, at the vertex of the curve, where $x=0$, and $z=0$, this becomes $0=\downarrow\left(a^{2}+0\right)=a$; and therefore, by correction, the true equation of the fllents is $x=\sqrt{ }\left(a^{2}+z^{2}\right)-a$, or $a+x=\sqrt{ }\left(a^{2}+z^{2}\right)$ : and bence also $z=\sqrt{ }\left[(a+x)^{2}-a^{2}\right]=\sqrt{ }\left(2 a x+x^{2}\right)$, and $a=\frac{z^{2}-x^{2}}{2 x}$ : either of which is the equation of the curve, in terms of the arc and its absciss; from this it appears that $a+x$ is the bypothenuse of a right-angled triangle, whose two legs are $a$ and $z$. So that, if in QA and $1 \mathrm{~A}$ produced, there be taken $\mathrm{AD}=a$, and $\mathrm{AE}=$ the curve $z$ or Al; then will the hypothenuse $\mathrm{DE}$ be $=a+x$ or DQ. And hence, any two of these three, $a, x, z$, being given, the third is given also.

Again, from the first simple property, viz, $\dot{x}: \dot{y}:: z: a$, or $a \dot{x}=z \dot{y}$, by substituting the value of $z$ above found, it becomes $a \dot{x}=\dot{y} \sqrt{ }\left(2 a x+x^{2}\right)$, or $\dot{y}=\frac{a \dot{x}}{\sqrt{\left(2 a x+x^{2}\right)}}$; and the fluent of this equation is $y=2 a \times$ hyp. $\log$. of $[\sqrt{ } x+$ $\sqrt{ }(2 a+x)]$. But at the vertex of the curve, where $x=0$ and $y=0$, this becomes $0=2 a \times$ hyp. $\log$. of $\sqrt{ } 2 a$; therefore the correct fluent is $y=2 a \times$ hyp. $\log$. of $\frac{\sqrt{ } x+\sqrt{ }(2 a+x)}{\sqrt{ } 2 a}$; an equation to the curve also, in terms of $x$ and $y$, but not in simple algebraic terms. This last equation however may be brought to much simpler terms in different ways; as first, by squaring the logarithmic quantity and dividing its coefficient by 2 , then $y=a \times$ hyp. $\log$. of $\frac{a+x+\sqrt{ }}{a} \frac{\left(2 a x+x^{2}\right)}{a}=a \times$ hyp. $\log . \frac{a+x+z}{a}$; and 2 dly, by multiplying both numcrator and denominator by $\sqrt{ }(2 a+x)-\sqrt{ } x$, then squaring the prorluct, and dividing the coefficient by 2 , which gives $y=a \times$ hyp. $\log$. $\frac{z+x}{\sqrt{\left(z^{2}-x^{2}\right)}}=a \times \operatorname{lyp} \cdot \log \cdot \frac{(E \times x)^{2}}{z^{2}-x^{3}}=a \times \operatorname{lyy} p \cdot \log \cdot \frac{z+x}{z-x^{0}}$.

The catenary is the crolute to the equitangential curve.

CATHLTUS, in Geometry, a name by which the perpendicular leg of a right-angled triangle is sometimes called ; or it is in general any tinc or radius filling perpendicularly on another line or surface.

Catuetus of Incidence, in Catoptrics, is a right line drawn from a radiant point, or point of incidence, perpendicular to the reflecting line, or plane of the speculum.

Catruetus of Refiction, or of the Eye, a right line drawn from the cyc, perpendicular to the plane of reflection.

Catnetus, in Architceture, denotes the axis of a co- 
lumn, irc. In the Ionic capital, it signifies a line passing. perpendicularly through the eye or centre of the volute.

CATOPTRICS, the science of reflex vision; or that part of optics which explains the laws and properties of light reflected from mirrors, or specula.

The first treatise extant on catoptrics, is that which was composed by Euclid: this was published in Latin in 1604 by John Pena; it is also contained in Herigon's Course of Mathematics, and in Gregory's edition of Euclid's works; though it is suspected by some that this piece was not the work of that great geometrician, notwithstanding it is ascribed to him by Proclus in lib. 2, and by Marinus in nis Preface to Euclid's Data. Alhazen, an Arabian autbor, composed a large volume of optics about the year 1100 , in which he treats pretty fully of catoptrics: and after him Vitello, a Polish writer, composed another about the year 1270. Tacquct, in his Optics, has very well demonstrated the chief propositions of plane and spherical speculums. And the same is very ably done by Dr. Barrow in his Optical Lectures. There are also Trabe's Catoptrics, David Gregory's Elements of Catoptrics, Wolfuus's Elements of Catoptrics, and those of Dr. Smith, contained in his learned and very elahorate Treatise on Optics, and many others of less note.

As this subject is treated under the general term Optics, the less need be said of it here. The whole doctrine of catoptrics depends on this simple principle, that the angle of incidence is equal to the angle of reflection; that is, that the angle in which a ray of light falls upon any surface, called the angle of incidence, is equal to the angle in which it quits it, when refiected from it, called the angle of reflection; though it is sometimes defined that the angles of incidence and reflection, are those which the incident and reflected rays make, not with the reflecting surface itself, but with a perpendicular to that surface, at the point of incidence, which are the complements to the others: but it matters not by what name these angles are called, as to the truth and principles of the science; since, if the angles are equal, their complements are also equal. This principle of the equality between the angles of incidence and reflection, is inere matter of experience, being a phenomenon that has always been observed to take place, in evcry case that has fallen under observation, as near at least as mechanical measurcments can ascertain; and hence it is inferrel that it is a universal law of nature, and to be considered as matter of fact in all cases. Thus, let $\mathrm{AC}$ be an incident ray, falling on the reflecting surface $\mathrm{DE}$, and $\mathrm{CB}$ the reflected ray, also co perpendicular to $\mathrm{DE}$; then is the an-

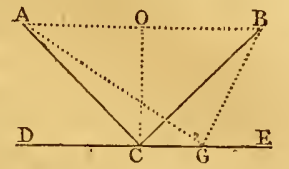
gle $\mathrm{ACD}=\mathrm{BCE}$, or the angle $\mathrm{ACO}=\mathrm{BCO}$.

Of this law in nature, viz, the equality between the angles cfincidence and reflection, it is remarkable, that in this way, the length or route $\mathrm{AC}+\mathrm{CB}$, in a ray passing from any point $A$ to another given point $B$, by being reflected from any surface $D E$, is the shortest possible, namely $A C+C B$ is shorter than the suin $A G+G B$, of any other two lines inflected at the line $D E$; and hence also the passage of the ray from $A$ to $B$ is performed in a shorter time than if it had passed by any other way.

From this simple pripciple, and the common properties of linear geometry, the chief phenomena of catoptrics are easily deduced, and are as follows:

1. Rays of light reflected from a plane surface, have the same inclination to each other after reflection as they had before it. Thus, the rays $A C, A I, A K$, issuing from the radiant point $A$, and $\mathrm{rC}-$ flected by the surface $D E$ into the lines $\mathrm{CF}, \mathrm{IL}, \mathrm{KM}$; these

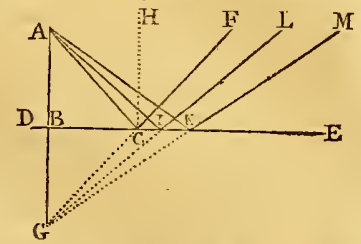
latter lines will have tlie same inclination to each other as the former AC, AT, AK, have. For draw ABG perpendicular to $\mathrm{DE}$, and produce $\mathrm{FC}, \mathrm{L} 1$, MK backwards to meet this perpendicular, so shall they all meet in the same point $G$, and $A B$ will in every case be equal to $\mathrm{B} D$ : for the incident $\angle A C B$ is equal to the reflected $\angle F C E$, which is equal to the opposite angle $\mathrm{BCG}$; so that the two triangles $\mathrm{ABC}$, $\mathrm{GBC}$, have the angles at C cqual, as also the right angles at B equal, and consequently the $3 \mathrm{~d}$ angles at $\mathrm{A}$ and $\mathrm{G}$ equal; and having also the side $\mathbf{B C}$ common, they are cqual in all respects, and $\mathbf{A B}=\mathbf{B G}$; and the same for the other rays: consequently the angles $B G C, B G I, B G K$, are respectively equal to the angles $B \mathrm{AC}, \mathrm{BAl}, \mathrm{BAK}$; that is, the reflected rays have the same inclinations as the incident ones have.

2. Hence it is that the image of an object, seen by reflection from a plain mirror, appears to proceed from a place $\mathrm{G}$ as far beyond, or on the other side of the reflecting plane $\mathrm{DE}$, as the object $\mathrm{A}$ itsclf is before the plane. This is when the incident rays diverge from some point as $A$.

But if the case be reversed, and $\mathrm{FC}, \mathrm{Lr}, \mathrm{MK}$, be considered as incident rays, issuing from points, $F, L, M$, and converging to some point $\mathrm{f}$ beyond the reflecting plane; then $\mathrm{CA}, 1 \mathrm{~A}, \mathrm{KA}$ will become the reflected rays, and they. will converge to the point $A$ as firr before the plane as the point $\mathrm{G}$ is beyond it.

So that universally, when the incident rays diverge from a point $\Lambda$, the reflected rays will also diverge from a point $G$; and when the incident rays converge towards a point $G$, the reflected ones will also converge to a point $A$; and in both cases these two points arc at cqual clistances on the opposite sides of the reflecting plane $\mathrm{DE}$.

3. Parallel rays reflected from a concave spherical surface, converge after reflection. For, let A F, CD, Es be three parallel rayss falting on the concave surface ${ }^{\prime} B$, whose centre is $\mathrm{c}$. To the centre draw the perpendiculars $\mathrm{FC}$ and $\mathrm{BC}$; also draw FM making the reflected angle, CFM, equal to the incident angle $\mathrm{Cr} A$; and in like manner BM to make the angle $\mathrm{CBM}=$ the angle $C B E ;$ sn shal the rays $A$ I and $\mathrm{EI}$, be reflected in. to the converging rays $F M$ and $B$ M. As to the

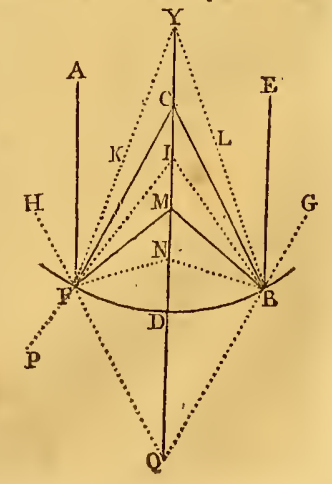


ray $\mathrm{CD}$, being perpendicular to the surface, it is reflected back again in the same line $D C$.

4. Converging rays falling on the concave surface are made to converge more. Thus, let $\mathbf{G} B$ and $I F$ be the incident rays : then because tse incident angle $\mathbf{H} F C$ is larger than the angle $A F C$, therefore the equal reflected angle $\mathrm{NFC}$ is greater than the reflected angle $\mathrm{MFC}$, and so the point $\mathrm{N}$ is below the point $\mathrm{M}$, or the line $\mathrm{IN}$ below the lime $\mathrm{PN}$; in like manmer $\mathrm{BN}$ is below $\mathrm{BM}$; that is, the reflected rays $\mathrm{FN}$ and $\mathrm{BN}$ are more converging in this case, than $E M$ and $B M$ in the other.

5. The focus to which all parallel rays, falling near the vertex $D$, are reflected, is in the middle of the radius $M$. For, because the $\angle M \mathrm{rC}=\angle A F C$, which is = the alternate $\angle \mathrm{FCN}$, therefore the sides opposite these angles are also equal, namely the side $\mathrm{FM}=\mathrm{CM}$; consequently, when the point $F$ is very near the vertex $D$, then the sum $\mathrm{CM}+\mathrm{Mr}$ is nearly $=\mathrm{CD}$, and cousequently $\mathrm{CM}$ nearly $=$ $M D$, or the focus of the parallel rays is nearly in the middle of the radius. But the focus of other reflected rays is either above or below that of the parallel rays; namely, below when the incident rays are converging, and above when they arc diverging; as is evident by inspection; thus, $\mathrm{N}$ the reflected focus of the converging rays $\mathrm{GB}$ and $\mathrm{HF}$, is below $M$; I that of the diverging rays $Y \mathbf{B}$ and $Y \mathbf{F}$, is above $M$.

6 . Incident and reflected rays are reciprocal, or so that if the reflected rays be returned back, or considered as incident ones, they will be reflected back into what were before their incident rays. And hence it follows that diverging rays, after reflection from a concave spherical surface, become either parallel or less diverging than before. Thus, the incident rays $M F$ and $M B$ are reflected into the parallel rays $\mathrm{FA}$ and $\mathrm{BE}$, and the rays $\mathrm{NF}$ and $\mathrm{NB}$ are reflected into $\mathrm{FH}$ and $\mathrm{BG}$, which are less diverging; also the rays $I F$ and $I E$ are reflected into $F K$ and $B L$, which converge.-And hence all the phenomena of concave mirrors will be evident.

7. Rays reflected from a convex speculum, become quite contrary to those reflected fron a concave one; so that the parallel rays become diverging, and the diverging rays become still more diverging; also converging rays will become either diverging, or parallel, or else less converging. Thus BDF being a spherical surface, whose centre is $\mathrm{C}$, produce the radii $\mathrm{CBV}$ and CFT which are perpendicular to the surface; then it is evident that the parallel rays $\Lambda \mathrm{F}$ and r. $\mathrm{B}$ will be reflected into the diverging ones $\mathrm{FK}$ and $B \mathrm{~L}$; and the diverging rays $Y \mathrm{~B}$ and $\mathrm{Yr}$ become $\mathrm{BO}$ and $\mathrm{FP}$ which are more diverging; also the converging

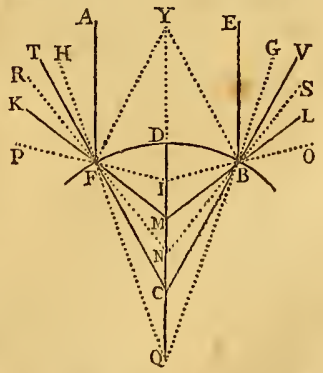

rays $1 \mathrm{~F}$ and $\mathrm{G} \mathbf{B}$ become $\mathrm{F} \Omega$ and $\mathrm{BS}$ which diverge, or else $\mathrm{KF}$ and $\mathrm{LB}$ become $\mathrm{FA}$ and $\mathrm{BE}$ which are parallel, or else lastly $\mathrm{PF}$ and on become $\mathrm{FY}$ and $\mathrm{BY}$ which are converging.

8. Hence, as in the concave speculum, so also in the convex one, of parallet incident rays $\Lambda \mathrm{F}$ and $\mathrm{Em}$, the innginary locus $M$ of their reflected rays $\mathrm{F} K$ and $\mathrm{BL}$, is in the middle of the radius when the speculum is a small segment of a sphere: but the reflected imaginary focus of other rays is either above or below the middle point $\mathrm{M}$, viz $\mathrm{N}$ being that of the converging rays $\mathrm{GB}$ and $\mathrm{H} F$, below $M$; but $\mathrm{I}$, that of the diverging rays $\mathrm{YB}$ and $\mathrm{r} F$, above $M$.

9. When the speculum is the small seginent of a sphere, either convex or concave, and the incident rays either converging or diverging, the distances of the foci, or points of concurrence, of the incident rays, and of the reflected rays, from the vertex of the speculum, are directly proportional to the distances of the same from the centre of it; that is, YD: ID : : YC: $I C$,

and $Q D: N D:: Q C: N C$.

For because the radius $\mathrm{CF}$, or the same produced, bisects the angle YFr in the concave speculum, or the external angle YFP in the convex one, therefore YF: IF : : YC: IC; but when $v$ is very near to $D$, then $Y F$ and IF become nearly $\mathrm{YD}$ and ID; consequently $\mathrm{YD}:$ ID : : YC: IC.

In like manner, because $\mathrm{c} F$ bisects the angle QFN in the convex, or its external angle NFF in the concave speculum, therefore $\mathrm{QF}: \mathrm{FN}:: \mathrm{QC}: \mathrm{CN}$; but when $\mathrm{F}$ is very near to $D$, then $Q F$ and $F N$ become nearly $Q D$ and $N D$; and therefore QD : ND : : \&C: NC.

For example, suppose it were required to find the focal distance of diverging rays incident on a convex surface, the radius of the sphere being 5 inches, and the distance of the radiant point from the surface 20 inches. Here then are given $\mathrm{YD}=20$, and $\mathrm{CD}=5$, to find $\mathrm{ID}:$ then the theorem in numbers is YD : ID : : YC : IC, $20: 10:: 25: 5-10$ a permutation $20: 25::$ ID : $5-$ ID, and by composition $45: 20:: 5:$ ID $=\frac{100}{45}=\frac{20}{9}=$ $2 \frac{2}{9}$ the focal distance sought.

And if it should happen in any case, that the value of ID in the calculation comes out a negative quantity, the focal distance must then be taken on the contrary side of the surface.

From the foregoing principles may be deduced and collected the following practical maxims, for plane and spherical mirrors, viz,

\section{In a Plane Mirror.}

(1.) The image will appear as far behind the mirror, as the object is before it.

(2.) The image will appear of the same size, and in the same position as the object.

(3.) Any plain mirror will reflect the image of an object of twice its own length and breadth.

\section{In a Spherical Concex Mirror.}

(1.) The image will always appear behind the mirror, or within the sphere.

(2.) The image will be in the same position, but less than the object.

(3.) The image will be curved, but not spherical, like the mirror.

(4.) Parallel rays falling on this mirror, will have the image at half the distance of the centre from the mirror.

(5.) In converging rays, the distance of the object must be eyual to half the distance of the centre, to make the image appear behind the mirror.

(6.) Diverging rays will have their image at less than half the distance of the centre.

III. In a Spherical Concave MTirror.

(1.) Parallel rays have their focus, or the image, at half the distance of the centre. 
(2.) In the centre of the sphere the image appears of the same dimensions as the object.

(3.) Converging rays form an image before the mirror.

(4.) In diverging rays, if the object be at less than balf the distance of the centre, the image will be behind the mirror, erect, curved, and magnified; but if the distance of the object be greater, the image will be before the mirror, inverted and diminished.

(5.) The solar rays, being parallel, will be collected in a focus at half the distance of its centre, where their heat will be augmented in proportion as the surface of the mirror exceeds that of the focal spot.

(6.) If a Iuminous body be placed in the focus of a concave mirror, its rays, being reflected in parallel lines, will strongly enlighten a space of the same dimensions with the mirror, at a great distance. If the luminous object be placed nearer than the focus, its rays will diverge, and so enlighten a larger space, but not so strongly. And on this principle it is that reverberators are constructed.

Catoptric Dial, a dial that exhibits objects by reflected rays. See Reflecting Dial.

Catoptric Telescope, a telescope that exbibits objects by reffection. See Reflecting Té escope.

Ca toptric Cistula, a machine, or apparatus, by which small bodies are represented extremely large, and near ones extrensely wide, and diffused through a vast space; with otber very pleasing phenomena, by means of mirrors, disposed by the laws of catoptrics in the concavity of a kind of chest.

There are various kinds of these machines, accommodated to the various intentions of the artificer: some multiply the objects, some magnify, some deform them, \&c. The structure of one or two of them will suffice to show how many more may be made.

To make a Catoptric Cistula to represent several different scenes of oljjects, when viewed through different holes.
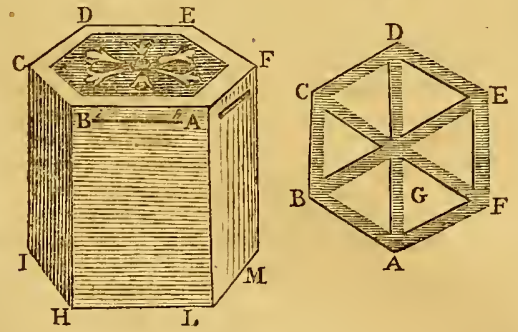

Provide a polygonal cistula, or box, like the mult-angular prism $A B C D E F$, and divide its cavity by diagonal planes $A D, B E, C F$, intersecting in the centre, into as many triangular cells as the chest has sides. Line those diagonal partitions with plain mirrors; and in the sides of the box make round holes, through which the eye may look within the cells of it. These holes are to be covered with plain glasses, ground withinside, but not polished, to prevent the objects in the cells fiom appearing too distinctly. In each cell are to be placed the different objects whose images are to be exhibiter; then covering up the top of the chest with a thin transparent membrane, or parchment, to admit the light, the machine is complete.

For, from the laws of rellection, it fullows, that the images of objects, placed within the augles of mirrors, are multiplied, and appear some more remote than others; by which the objects in one cell will appear to take up more room than is contained in the whole box. Therefore by looking through one hole only, the objects in one cell will be seen, but those multiplied, and diffused through a space much larger than the whole box. Thus every bole will afford a new scene; and according to the different angles the mirrors make with each other, the representations will be different : if they be at an angle greater than a right one, the images will be monstrous, \&c.

To make a Catoptric Cistula to represent the objects within it prodigiously multiplied, and diffused through a rast space.

Make a polygonous cistula or box, as before, but without dividing the inner cavity into any apartments, or cells; line the insides $C B H 1$, BHLA, ALMF, \& $\mathrm{c}$, with plane mirrors, and at the boles pare off the tin anci quicksilver, that the eye may look through; place any object in the bottom $\mathrm{Mi}$, as a bird in a cage, \&c.

Now by looking throngh the aperture $l i$, each object placed at the bottom will be seen vastly multiplied, and the images removed at equal distances from one another, like a great multitude of birds, or a large aviary.

CAYALIER, in Fortification, a mount of earth raised in ra fortress higher than the other works, on which to place camnofi. \&c for scouring the field, or opposing a commanding work. Cavaliers are of different shapes; and are bordered with a parapet, to cover the cannon mounted upon them; their situation is also various, eitber in the curtain, the bastion, or the gorge. The cavalier is sometimes called a double bastion, and its use is to overlook the enemy's batteries, and to scour their trenches.

CAVAlieiri (Bunaventura), a celebrated Italian mathematician of the 17 th century. He was born at Milan in 1598, and became a friar of the order of the Jesuati of St. Jerome. Cavalieri was a disciple of Galileo, and the friend of Torricelli. He was professor of mathematics at Bologna: where several of his books were published, and where he died in the year 1647. His works that have been published, as far as I can discover, are as follow :

1. Directorium Generale Uranometricum; 4to, Bononia, 1632.- In this work the author treats of Trigonometry; and Logarithms, their construction, uses, and applications. The work includes also tables of logarithms of common numbers; with trigonometrical tables of natural sines, and logarithmic sines, tangents, secants and versed sines.

2. Lo Spechio Ustorio overo Trattato delle Settioni Coniche: 4to, Bologna, 1632.-An ingenious treatise of conic sections.

3. Geometria Indivisibilibus continuorum nova quadam ratione promota: 4.to, Bononiæ, 1635 ; and a $2 \mathrm{~d}$ edition in 1653.-This is a curious original work in geometry, in which the author conceives the geometrical figures as resolved into their very sinall clements, or as made up of an infinite number of infinitely small parts, and on account of which he passes in Italy for the inventor of the infinitesimal calculus.

4. Trigonometria Plana et Sphærica, Linetris, et Logarithmica: 4 to, Bononire, 1643 .- A very neat and ingenious treatisc on Trigonometry; with the tables of sines, tangents, and secants, both natural and logarithmic.

5. Excreitationes Gcometrice Sex; 4to, Bunonix, 1647. This work contains Excrcises on the incthod of Indivisi- 
bles; Answers to the abjections of Guldini; The use of Indivisibles in cossic powers or algebra, and in considerations concerning gravity; with a miscellaneous collection of problems.

CAVALLO (TIBERIUS), F. R. s, was born in the city of Naples the 30th of March 1749 . His father, who was an eminent physician, died when Tiberius was scarcely 11 years of age. His relations continued to give him a liberal education; and as he was intended for a commercial life, with that view they sent him to England. He arrived in London in 1771 , and continued in this country during the rest of his life. Here his inclination led him to pursue scientific studies, the rudiments of which he had received at the university of Naples, and he attained to a considerable rank in several of the more fashionable and popular physical sciences. In March 1799, he was elected member of the Royal Academy of Sciences of Naples; and in December the same year he was elected home member of the Royul Society of London; where he died the 26 th of December 1809 , at 60 years of age.

Besides several ingenious papers inserted in the Philos. Trans., Mr. C. published separate and very interesting volumes on various popular and useful subjects: as, A Complete Treatise of Electricity in 'Theory and Practice, 1 vol. Svo. 1777 (enlarged to 3 vols. in 1793).-An Essay on the Theory and Practice of Medical Electricity, 1 vol. Svo. 1780.-A Treatise on the Nature and Properties of Air, and other Permanently Elastic Fluids, with an Introduction to Chemistry, 1 vol. 4to, 1781.-The History and Practice of Aërostation; 1 vol. $8 v 0,1785$.- Mineralogical Tables; folio, (accompanied with an svo explanatory pamphlet) 1785.-A Treatise on Magnetism, in Theory and Practice; 1 vol. 8vo, 1787.-Description and Use of the Telescopical mother-of-pearl Micrometer; 8vo, 1793. -An Essay on the Medicinal Properties of Factitious Airs, with an Appendix on the nature of Blood; 1 vol. 8 vo, 1798, many of which have gone through several editions.

CAUDA Capricorni, a fixed star of the th magnitude, in the tail of Capricorn; called also by the Arabs, Dineb Algedi; and marked $\gamma$ by Bayer.

CaUDA Ceti, a fixed star of the $3 \mathrm{~d}$ magnitude; called a)so by the Arabs, Dineb Kaetos; marked $\beta$ by Baycr.

CAUDA Cygni, a fixed star of the $2 \mathrm{~d}$ magnitude in the Swan's tail ; called by the Arabs, Dineb,Adigcge, or Eldegiagich; and marked $\alpha$ by Bayer.

Cauva Delphini, a fixed star of the $3 \mathrm{~d}$ magnitude, in the tail of the Dolphin; marked $\varepsilon$ by Bayer.

CA U D A Draconis, or Dragon's tail, the moon's southern or descending node.

CaUna Leonis, a fixed star of the first magnitude in the Lion's tail; called also by the Arabs, Dineb lileced; and marked $\beta$ by Bayer. It is called also Lucida Cauda.

Carpa Ursa Mujoris, a fixed stir of the 3 d magnitude, in the (ip of the Grent Bear's tail; called also by the Arabs, Alalioth, and Benenath; aud marked $\gamma$ by Bayer.

Ca un A Urse Minoris, a fixed star of the 3 d magnitude, at the end of the Lesser Bear's tril; called also the Pule Star, and by the Arabs, Alrukabah; and marked $\alpha$ by lBayer.

CAVENDISII (HENiy), F. z.s. son of the late Lord Charles Cavendish, was born ()ct. 10, 1731 , and died lieb. 24, 1810 , consequently in lis 79th year. Mr. C. lived and died a bachelor, devoting the whole hours and attentions of his long life most assiduously in cultivating the sciences, mathematical, physical, chemical, Sc, in all which he excelled in a very eminent degree. His genius and labours were rewarded with the most brilliant discoveries and improvements in the various branches of science; the records of which are chicfly to be fund in the volumes of the Philos. Trans. He had stuclied, and rendered himself familiatly conversant with, every part of Nowton's philosophy; the principles of which be applied, 40 years ago, to an investigation of the laws on which the phenomena of electricity depend. Pursuing the same research, on the occasion of the first experiments with the torpedo, he gave a satisfactory explanation of the remarkable powers of electrical fishes; pointing out that distinction between conmon and animal elcetricity, which has since been amply confirmed by the brilliant discoveries in $\mathrm{Gal}$ vanism. Having very early turned his attention to pnenmatic chemistry, in $1766^{\circ}$ he ascertained the extreme levity of inflanmable air, now called hydrogen gas; a discovery on which are founded many curious experiments, particularly that of aërial navigation. In the same line of science, he made the important discovery of the composition of water, by the mnion of two airs; which laid the fuundation of the modern system of chemistry, which rests principally on this fact, and that of the decompusition of water, announced soon atterwards by M. Lavoisier.

As the purity of atmospherical air had been a subject of controversy, Mr. C. contrived essential improvements in the method of performing experiments witl the cudiometer; by means of which lie was the first who showed, that the proportion of pure air, in the atmosphere, is nearly the same in all open places. The other, and much larger portion of our atmosphere, he sagaciously conjectured to be the basis of the acid of nitre; an opinion which he soon brought to the test, by an ingenious and laborious experiment, completely proving its truth; whence this air has now very generally obtained the name of nitrogen. So many and great discoveries spread his fame throughout Europe; and he was universally considered as one of the greatest philosophers of the age.

Among the labours of his latter days, was the nice and difficult experiment, by a mode and instrument contrived by the late Rev. John Michell, by which he determined the mran density of the earth, in a result not widely different from what was obtained in the more perfect way by the attraction of mountains. This density forms an element of consequence in delicate calculations of astronomy, as well as in geological inquiries. Fven in the last year of his life, he proposed and described improrements in the manner of dividing large astronomical instruments, which promise very great advantages.

These pursuits, together with reading, of various kinds, by which he acquired a deep insight into almost every topic of general knowledge, formed the whole occupation of his life, and indeed constifuted his sole amusement. The love of trutb alone was sufficient to till his mind. From lis attachment to sucls occupations, and the constant resource be found in them, iogetier with a shyness and diffidence natural to his disposition, his habits had, from early life, beren seciuded. Ilis manners were mildhis mind firm - bis nature benevoleme and complicent. He was liberal, without being profuse; and charitable, withollt ostentation.

CAYE'T"TO, a bollow member or mouldine, containing a quadrant of a circle, and having an cllect just contrary to that of a quarter round. It is used as an ormament in cornices. 
CAULICOLES, Cauliculi, in Architecture, are those 8 lisser branches or stalks in the Corinthian capital, which spring out from the four greater principal caules or stalks.

CAUSE, Physical, that which produces a sconsible corporeal eflect. Thus, the sun is a physical cause of ligbt. Others define a physical cause to be that which produces its eflects by a physical virtuc, or natural influence.

CAUSTIC Cunve, in the higher geometry, is a curve formed by the concourse ur coincidence of the rays of light, when either refected, or refracted, from some other curve.

Every curve therefore has its twofold caustic: accordingly, caustics are divided into catacaustics, and diacaustics; the one formed by reflection, the angle of reflection being equal to that of incidence; the other by refraction. Sce the two articles Catacaustics and Diacaustics.

To pursue the doctrine of caustics, as it relates to optics, consult Haüy's Philosophy, vol. 2 ; and as a braisch of the doctrine of curves, consult the writers on fluxions, as Hays, or Hodgson, or Stone, \&r.

CAZEMATE. See Casemate.

CAZERN. See Casern.

CEGINUS, a fixed star of the $3 \mathrm{~d}$ magnitude, in the left sloulder of Boötes; and marked $\gamma$ by Bayer.

CELERITY, is the velocity ar swiftness of a body in motion; or that affection of a body in motion by which it can pass over a certain space in a certain time.

CELESTIAL G lobe, \&c. See Globe, \&c.

CELLARIUS (CHRISTOPII E ), a learned geographer and historiographer of the 17 th century. He was born in 1638, at Smalcalde in Franconia, where his father was minister. Our author was successively rector of the colleges at Weymar, Zeits, and Mersbourg, and professor of eloquence and history in the university founded by the king of Prussia at Hall in 1693, where he composed the greatest part of his works.

His great application to study hastened the infirmities of old age; for it has been said, he would spend whole days and nights together over his books, without any attention to his health, or even the calls of nature. He died in 1707 , at 69 years of age.

Cellarius was author of a great number of books, on various subjects; but those for which he has a place here, are his geographical works, which are as follow:

1. Notitia Orbis Antiqui, 2 vols, in 4to; and is estcemed the best work extant on the ancient geography.

2. Atlas Colestis; in folio.

3. Historia Antiqua, 2 vols, in $12 \mathrm{n} \mathrm{o}$; being an abridgement of universal history.

CENTAURUS, the Centaur, one of the 48 old constellatious, being a southern one, and is in form half man and half horse. It is fabled by the Greeks that it was Chiron the Centaur, who was the tutor of Achilles and Esculapius. The stars of this constellation are, in Ptolemy's catalogue 37 , in Tycho's 4 , and in the Britannic catalogue, with Sharpe's appendix, 35 .

Center, and Centering. Sec Centre, and CenTRING.

CENTESM, the 100th part of any thing.

CLNTIGRADE, the division into grades or degreos by hundredth parts, called also Centesimals, contracted into Centemes, ou still morc contracted Cents.

The North-American states have thcir money (dollars), divided into hundredth parts or cents. And the French nation have lately divided al! their money, weights, and

Vol. I. measures, decimally; which is allowed to be the best way. They have even divided the circular quadrant into cents, for trigonometrical uses, on the centigrade way, having 100 in the quadrant, while we have 90 : therefore the magnitude of their degree, is to that of ours, as 9 to 10 ; but the number of their degrees to the number of ours, as 10 to 9 : hence, to change French degrees into English, diminish them by one-tenth, or multiply them by 9 , and cut off one figure for a decimal: and to find the number of French degrees answering to a given number of English, annex a cipher to the latter, then divide them by 9 .

The French have also their centigrade thermometer, \&c. In that instiument they divide the distance between the freezing and boiling points into 100 , which we divide into 180; therefore the length of their thermometrical degree, is to the length of ours, as 180 to 100 , or as 18 to 10 , or 9 to 5 ; and the number of theirs to the number of ours, as 10 to 18 , or 5 to 9 ; therefore, to change French thermoneter degrees into English, multiply the furmer by 9 ; and divide by $5:$ so, to find the linglish degrees answering to 15 French, the 5 th of 15 is 3 , then $3 \times 9=27$ are the English degrees answering to 15 Frencls : but as the French freezing point is at 0 , and ours at 32 , therefore $27+32=$ 59 is the point or degree on the English thermometer, answering to that of 15 on the French.

CENTRAL, something relating to a centre. Thus we say central eclipse, central forces, central rule, \&c.

Centrat Eclipse, is when the centres of the luminaries exactly coincide, and fall directly in a line with the eye.

CENTRAL. Forces, are forces having a tendency directly towards or from some point or centre; or forces which cause a moving body to tend towards, or recede from, the centre of motion. And accordingly they are divided into two kinds, in respect to their different relations to the centre, and hence are called centripetal, and centrifugal.

The doctrine of central forces constitutes a considerable branch of the Newtonian philosophy, and has been greatly cultivated by mathematicians, on account of its extensive use in the theory of gravity, and other branches of physico-mathematical science.

In this doctrine, it is supposed that matter is equally indifferent to motion or rest, or rather that it always resists any attempt to change its state; or that a body at rest never moves itself, and that a body in motion never of jtself changes either the velocity or the direction of its motion; but that every motion would continue uniformly, and its direction rectilinear, unless some external force or: resistance affected it or should act upon it. Hence, wheat a body at rest always tends to move, or when the velocity of any rectilinear motion is continually accelerated or retarded, or when the direction of a motion is continually changed, and a curve line is thereby described, it is supposed that these circumstances proceed from the influence of some power that acts incessantly; which power may be measured, in the first case, by the pressure of the quiescent body against the obstacle which prevents it from moving, or by the velocity gained or lost in the secoud case, or by the flexure of the curve described in the $3 \mathrm{~d}$ case; due regard being had to the time in which these effects are produced, and other circumstances, according to the principles of mechanics. Now the power or force of gravity produces effects of each of these kinds, which fall under our constant observation near the surface of the earth; for the same power which renders bodies beavy, 
while they are at rest, accelerates their motion when they descend perpendicularly; or retards them when they ascend; and bends the track of their motion into a curve line, when they are projected in a direction oblique to that of their gravity. But we can judge of the forces or powers that act on the celestial bodies by effects of the last hind only. And hence it is, that the doctrine of central forces is of so much use in the theory of the planetary motions.

Sir 1. Newton bas treated of central forces in lib, 1 , sec. 2, of his Principia, and has demunstrated this fundamental theorem of central forces, viz, that the areas which revolving bodies describe by radii drawn to an immoveable centre, lie in the same immoveable planes, and are proportional to the times in which they are described. Prop. 1.

It is remarked by a laté eminent mathematician, that this law, which was originally observed by Kepler, is the only general principle in the doctrine of centripetal forces; but since this law, as Newton himself has proved, cannot hold in cases where a body has a tendency to any other than one and the same point, there seems to be wanting some law that may serve to explain the motions of the moon and satellites which gravitate towards two different centres: the law he lays down for this purpose is, that when a budy is urged by two forces tending constantly to two fixed points, it will describe, by lines drawn from the two fixed points, equal solids in equal times, about the line joining those fixed points. See Machin, on the Laws of the Moon's Motion, in the Postscript. This short treatise is published at the end of the English translation of Sir Isaac Newton's Principia. See also a demonstration of this law by Mr. William Jones, in the Philos. Trans. vol. 59. Very learned tracts have also been since given, when the motion respects, not tivo only, but sereral centres, by many ingenious authors, and practical rules deduced from them for computing the places \& $c$ of planets and satellites; as by Lagrange, Laplace, Waring, \&c, \&c. See Berlin Memoirs; those of the Academy of Sciences at Paris; and the Philos. Trans. of London.

M. Demoivre gave elegant general theorems relating to central forces, in the Philos. Trans. and in his Miscel. Analyt. 231.-Let $M P Q$ be any given curve, in which a body moves: let $\mathbf{P}$ be the place of the body at any time; $s$ the centre of force, or the point to which the central force acting on

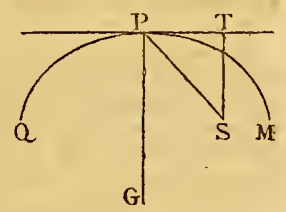

the body is always directed; $P G$ the radius of curvature at the point P; and ST perpendicular to the tangent PT; then will the centripetal force be every where proportional to the quantity $\frac{\mathrm{sP}}{\mathrm{GP} \times \mathrm{ST^{2 }}}$. Vid. ut supra.

M. Varignon has also given two general thcorems on this subject in the Memoirs of the Acad. an. 1700, 1701; and has shown their application to the motions of the planets. See also the sanie Memoirs, an. 1706, 1710.

Mr. Maclaurin lias also treated the subject of central forces very ably and fully, in his Treatise on Fluxions, art. 4.16 to 498 ; where he gives a great variety of expressions for theso forces, and several elegant methods of investigating rhem.

Laws of Cextra . Forces.

1. 'The following is a very clear alul comprehensive rule, for which we are obliged to the Marquis de l'Hopital:
Suppose a body of any determinate weight to revolve uniformly about a centre, with any given velocity; find from what height it must have fallen, by the force of gravity, to acquire that velocity; then, as the radius of the circle it describes, is to double that height, so is its weight to its centrifugal force. Su that, if $b$ be the body, or its weight or quantity of matter, $v$ its velocity, and $r$ the radius of the circle described, alsn $g=16 \frac{1}{1^{2}}$ feet; then, by the laws of falling bodies we have $4 g^{2}: v^{2}:: g: \frac{v^{2}}{4 g}$ the beight due to the velucity $v$; and by rule $r: \frac{v^{2}}{2 g}:: b: \frac{6 v^{2}}{2 g r}=f$ the centrifugal force. Hence, if the centrifugal force be equal to the gravity, the velocity is equal to that acquired by falling through half the radius.

2. The central force of a body moving in the periphery of a circle, is as the-versed sine $A M$ of the indefinitely small arc $A \mathrm{E}$; or it is as the square of that arc $\mathrm{A} E$ direct$l y$, and as the diameter $A B$ inversely. For AM is the space through whicli the body is drawn from the tangent in the given time, and $2 \mathrm{ABI}$ is the proper measure of the intensity of the central force. But, AE being very

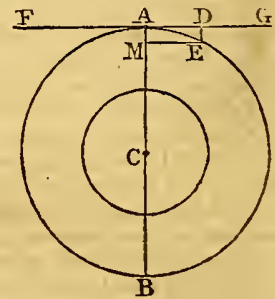
small, and therefore nearly equal to its chord, we shall have by the nature of the circle

$A B: A E:: A E: A M=\frac{A E^{2}}{A B}$.

3. If two bodies revolve uniformly in different circles; their central forces are in the duplicate ratio of their velocities directly, and the diameters or radii of the circles inversely; that is, $\mathrm{F}: f:: \frac{\mathrm{v}^{2}}{\mathrm{D}}: \frac{v^{2}}{d}:: \frac{v^{2}}{\mathrm{n}}: \frac{v^{2}}{\mathrm{x}}$.

For the force, by the last article, is as $\frac{A E^{3}}{A B}$ or $\frac{A E^{2}}{D}$; and the velocity $v$ is as the space $A \mathrm{E}$ uniformly described.

4. And hence, if the radii or diameters be reciprocally in the duplicate ratio of the velocities, the central forces will be reciprocally in the duplicate ratio of the radii, or directly as the 4 th power of the velucities; that is, if $\mathrm{v}^{2}: v^{2}:: r: \mathrm{R}$, then $\mathrm{F}: f:: r^{2}: \mathrm{R}^{2}:: \mathrm{v}^{4}: v^{4}$.

5 . The central forces are as the diameters of the circles directly, and squares of the periodic times inversely. For if $c$ be the circumference described in the time $t$, with the velocity $v$; then the space $c=t v$, or $v=\frac{c}{l}$; hence, using this value of $v$ in the $3 \mathrm{~d}$ rule, it becomes $\mathbf{E}: \boldsymbol{f}:: \frac{\mathrm{c}^{3}}{\mathrm{DT}^{3}}: \frac{c^{2}}{d l^{3}}:: \frac{\mathrm{D}}{\mathrm{T}^{2}}: \frac{d}{t^{3}}:: \frac{\mathrm{R}}{\mathrm{T}^{2}}: \frac{r}{l^{2}}$; since the diameter is as the circumference.

6 . If two bodies, revolving in different circles, be acted on by the same central force; the periolic times are in the subduplicate ratio of the diameters or radii of the circles; for when $\mathrm{F}=f$, then $\frac{\mathrm{D}}{\mathrm{T}^{2}}=\frac{d}{t^{2}}$, and $\mathrm{D}: d:: \mathrm{T}^{2}: t^{2}$, or $\mathrm{T}: t:: \sqrt{ } \mathrm{D}: \sqrt{ } d:: \sqrt{ } \boldsymbol{} \mathrm{x}: \sqrt{ } r$.

7. If the velocities be reciprocally as the distances from the centre, the central forces will be reciprocally as the cubes of the same distances, or dircctly as the cubes of the velocities. That is, if $\mathrm{v}: v:: r: \mathrm{R}$, then is $\mathrm{F}: f:: r^{3}:$ $\mathrm{R}^{3}:: v^{3}: v^{3}$. For $\mathrm{v}: f:: \frac{v^{3}}{\mathrm{R}}: \frac{v^{3}}{r}:: \frac{r^{2}}{\mathrm{R}}: \frac{\mathrm{n}^{2}}{r}:: r^{3}: \mathrm{n}^{3}::$ $v^{3}: \tau^{3}$. 


\section{E N}

8 . If the velocitics be reciprocally in the subduplicate ratio of the central distances, the squares of the times will be as the cubes of the distances: thus, if $\mathrm{v}^{2}: v^{2}:: r: \mathrm{R}$, then is $\mathrm{T}^{2}: t^{2}:: \mathrm{R}^{3}: r^{3}$. For by $3 \mathrm{~d}$ and 5 th $\frac{\mathrm{V}^{2}}{\mathrm{R}}: \frac{v^{3}}{r}:: \frac{\mathrm{R}}{\mathrm{T}^{2}}: \frac{r}{t^{2}}$, and $\frac{\mathrm{v}^{3} \times r}{v^{2} \times \mathrm{R}}=\frac{r^{3}}{\mathrm{R}^{2}}$ (by this) $=\frac{\mathrm{R} \times t^{2}}{r \times \mathrm{T}^{3}}$, and hence $\mathrm{T}^{2}: t^{2}:: \mathrm{R}^{3}: r^{3}$.

9. Therefore, if the forces be reciprocally as the squares of the central distances, the squares of the periodic times will be as the cubes of the distances; or when $F: f:: r^{2}: \mathrm{R}^{2}$, then is $\mathrm{T}^{2}: i^{2}:: \mathbf{\mathbf { n }}^{3}: r^{3}$. For by 5 th, $\mathbf{F}: f:: \frac{\mathbf{R}}{\mathrm{T}^{3}}: \frac{r}{t}$, and by this $\mathrm{F}: f:: r^{2}: \mathrm{R}^{2}$, theref. $\frac{\mathrm{R}}{\mathrm{T}^{2}}: \frac{r}{t^{3}}:: r^{2}: \mathrm{R}^{2}$, and hence $\mathrm{T}^{2}: t^{2}:: \mathrm{R}^{3}: r^{3}$.

Exam. From this, and some of the foregoing theorems, may be deduced the velocity and periodic time of a body revolving in a circle, at any given distance from the earth's centre, by means of its own gravity. Put $g=16_{\mathrm{T}^{2}}^{\frac{1}{2}}$ feet, the space described by gravity, at the surface, in the first second of time, viz, = A $\mathrm{Ax}$ in the foregoing fig. and by rule 2 ; then putting $r=$ the radius $\mathrm{AC}$; it is $\mathrm{AE}=\sqrt{ }(\mathrm{AB} \times$ $A M)=\sqrt{ } 2 g r$ the velocity in a circle at its surface, in one second of time; and bence, putting $c=3.14159 \& c$, the circumference of the earth being $2 c r=25,000$ miles, or $132,000,000$ feet, it will be $\sqrt{ } 2 g r: 2 c r:: 1^{\prime \prime}: c \sqrt{ } \frac{2 r}{g}=$ 5078 seconds nearly, or $1^{\text {h }} 24^{\mathrm{m}} 38^{\text {, }}$, the periodic time at the circumference: Also the velocity there, or $\sqrt{ } 2 g r$ is $=$ 26000 feet per second nearly. Then, since the force of gravity varies in the inverse duplicate ratio of the distance, by rules 8 and 9 , it is $\sqrt{ } \mathrm{R}: \sqrt{ } r:: v$ or $26000: 26000$ $\sqrt{ } \frac{r}{R}=\mathrm{v}$ the velocity of a body revolving about the earth at the distance $\mathrm{R}$; and $\sqrt{ } r^{3}: \sqrt{ } \mathrm{R}^{3}:: t$ or $5078^{\prime \prime}: 5078$ $\sqrt{\frac{\pi^{3}}{r^{3}}}=\mathrm{T}$ the time of revolution in the same. So if, for instance, it be the moon revolving about the earth at the distance of 60 semidiameters; then $\mathrm{R}=60 \mathrm{r}$, and the above expressions become $\mathrm{v}=26000 \sqrt{ } \frac{\mathrm{r}}{60}=3357$ feet per second, or $38 \frac{x}{7}$ miles per minute, for the velocity of the moon in her orbit; and $\mathrm{T}=5078 \sqrt{\frac{\mathrm{n}^{3}}{\gamma^{3}}}=2360051$ seconds, or $27 \frac{3}{10}$ days nearly, for the periodic time of the moon in her orbit at that distance.

Thus also the ratio of the forces of gravitation of the moon towards the sun and earth may be estimated. For, 1 year or $365 \frac{\pi}{4}$ days being the periodic time of the earth and moon about the sun, and $27 \frac{3}{10}$ days the periodic time of the moon about the earth, also 60 being the distance of the moon frnm the earth, and 23920 the distance from the sun, in semidiameters of the earth, by art. 5 it is

$\frac{60}{27 \cdot 3^{2}}: \frac{23920}{365 \cdot 25^{3}}: ; f$ or $1: \frac{23902}{60} \times \frac{27 \cdot 3^{2}}{365 \cdot 25^{2}}=2 \frac{2}{9}$; that is, the proportion of the moon's gravitation towards the sun, is to that towards the earth, as $2 \frac{2}{9}$ to 1 nearly.

Again, we may hence compute the centrifugal force of a body at the equator, arising from the earth's rotation. For, the periodic time when the centrifugal force is equal to the force of gravity, it has been shown above, is 5078 seconds, and 23 hours 56 minutes, or 86160 seconds, is the period of the earth's rotation on its axis: therefore, by art. 5 , as $86160^{2}: 5078^{3}:: 1: \frac{7}{2} \frac{7}{8}$, the centrifugal force required, which therefore is the 289 th part of gravity at the earth's surface. Simpson's Flux. pra. 24.0, \& c.
C F N

By similar means we may determine the centrifugal force of bodies in different latitudes; for as the earth turns round its axis, it is evident that those bodies on the surface of it, which lie nearer to the axis, or, which is the same thing, lie nearer to the poles, describe circles smaller than those which lic nearer to the equator, though they are all performed in the same interval of time, viz, $23^{\mathrm{h}} 56^{\mathrm{m}}$. Hence the periodical times being equal, the central forces are as the radii of the circles (5); and since, in different latitudes, we may use the following proportion; viz, as the radius is to the co-sine of a given latitude, so is the centrifugal force of bodies situated at the equator, to the centrifugal force of bodies at that given latitude. Now as the co-sines decrease in length the nearer they approach the poles, so the tendency of bodies to fly off from the surface of the earth is greatest at the equator, but diminishes in approaching towards the poles; and hence we perceive why the earth has been found, by means of unquestionable measu rements and other observations, to be an oblate spheroid, whose polar diameter is the shortest. This circumstance furnishes a convincing evidence of the earth's daily rotation about its axis.

For another example, suppose $\mathrm{A}$ to be a ball of 1 ounce, which is whirled about the centre $c$, so as to describe the circle $A \& B$, each revolution being made in half a second; and the length of the cord AC equal to 2 feet. Here $t=\frac{x}{2}$, $r=2$, and it having been found above that $c \sqrt{ } \frac{2 R}{g}=T$ is the periodic time at the circumference of the earth when the centrifugal force is equal to gravity; hence then, by art. 5 , as $\frac{\mathrm{R}}{\mathrm{T}^{2}}: \frac{r}{t^{2}}:: \mathrm{F}$ or $1: f$, which proportion becomes $\frac{g}{2 c^{3}}: \frac{r}{t^{3}}:: 1: \frac{2 c^{2} r}{g t^{2}}=\frac{16 c^{2}}{g}=\frac{16 \times 3 \cdot 1416^{2}}{16 \frac{1}{12}}=9 \cdot 819=$ the centrifugal force, or that by which the string is stretched, viz, nearly 10 ounces, or 10 times the weight of the ball

Lastly, suppose the string and ball to be suspended trom a point $D$, and describes in its motion a conical surface $A D B$; then putting $\mathrm{DC}=a, \mathrm{AC}=r$, and $\mathrm{AD}$ $=h$; and putting $\mathrm{F}=1$ the force of gravity as before; then will the body $A$ be affected by three forces, viz, gravity acting parallel to $\mathrm{DC}$, a centrifugal force in the direction $\mathrm{C}_{A}$, and the ten-

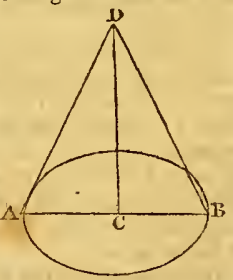
sion of the string, or force by which it is stretched, in the direction DA: hence these three powers will be as the three sides of the triangle $\Delta \mathrm{DC}$ respectively, and therefore as $C D$ or $a: A \mathrm{D}$ or $h:: 1: \frac{h}{a}$ the tension of the string as compared with the weight of the body. Also DC or $a: \mathrm{AC}$ or $r:: 1: \frac{2 c^{2} r}{3 t^{3}}$ the general expression for the centrifugal force above found; hence, $g t^{2}=2 a c^{2}$ and consequently $t=c \sqrt{ } \frac{2 a}{g}=1 \cdot 108 \sqrt{ } a=$ the periodic time.

10. When the force by which a body is urged towards a point is not always the same, but is either increased or decreased as some power of the distance; several currcs will then arise according to that power. If the force decrease as the squares of the distances increase, the body will describe an ellipsis, and the force is directed towards one of its foci; so that in every revolution the body once $2 \mathrm{P} 2$ 

C E N
[ 292$]$

approaches towards it, and once receles from it: also the excentricity of the ellipse is greater or less, according to the projectile force; and the curve may sornetimes become. a circle, when the excentricity is nothing; the body may also describe the other two conic sections, the parabola and hyperbola, which do not return into themselves, by süp posing the velocity greater in certain proportions. Also if the force increase in the simple ratio as the distance increases, the body will still describe an ellipse; but the force will in this case be directed to the centre of the ellipse; and the body, in each revolution, will twice approach towards it, and again twice recede from that point. For the investigations of these and other particulars, consult Simpson's Fluxions, vol. 1, sect. 12, and Gregory's Mechan. vol. 1 , chap. 3 , bk. 2 .

The general doctrine of central forces is called most into application in investigating the orbits of the planets as urged towards the eentral bady the sun: and, considered in that vies, the following deduction of the princjpal laws is offered.

(1). When a body, urged by a centripetal force, moves in any curve; then, in every point of the curve, the force is in a ratio, compounded of the direct ratio of the body's distance from the centre of force, and the inverse ratio of the cube of the perpendicular on the tangent to the same point of the curve, drawn into the radius of curvature of the same point.

(2). Hence, if the body move in a circle, by a force tending to a point in the circumference, the force will be everywhere in the inverse ratio of the 5 th power of the body's distance from that point.

(3). When a body moves in a conic section, the force urging it towards the focus is reciprocally proportional to the square of the distance.

(4). Hence, if a body move in an ellipsis, by a centripetal force tending to its centre, that force will be directly as the distance.

(5). Hencer results an easy construction, fer determining the radius of curvature in any conic section. Thus, from the given point $A$, draw $A$ B through the focus $F$, and $A R$ perpendicular to the curve at $A$, cutting the axis $\mathrm{v} C$ in $\mathrm{C}$; at $\mathrm{C}$ erect $\mathrm{CB}$ perpendicular to $A C$, and draw BR perpendicular $A B$; then $R A$ is the radius of curvature

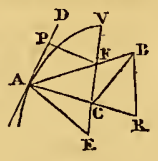
at the point $A$.

In the parabola, the construction becomes a little simpler. For, since $\mathbf{A} \mathbf{F}=\mathbf{F C}$, and $\mathrm{ACB}$ a right angle, therefore $F$ is the centre of a circle passing through $A, C, B$, and $A F=F B$. Hence, if $A F$ be produced till $F B=A F$, and B R be drawn perpendicular to $\mathrm{A} B$, meeting the perpendicular $\Lambda \mathbf{R}$; then $\mathbf{R}$ will be the centre of curvation of the parabola.

Hence, in all the conic sections, $\triangle \mathrm{C}: \mathrm{AB}:: \mathrm{AB}: \triangle \mathrm{R}$ the radius of curvature; which therefore is a $3 \mathrm{~d}$ proportional to $\Lambda \mathrm{C}$ and $\Lambda \mathrm{B}$. And when the point $\Lambda$ coincides with the vertex $v$, then $\Lambda \mathrm{C}: A \mathrm{~B}$ becomes the ratio of the two semiaxes; that is, the radius of curvature at the vertex, or extremity of the axis, is equal to a $3 \mathrm{~d}$ proportional to the two semiaxes, and is therefore equal to the semiparuncter of the axis.

Further, the ralii of curvature at the extremities of the transverse and conjugate axes, are to each other reciprocally as the cubes of the axes. For, let $a$ denote the for- mer semiaxis, and $c$ the latter: then the radius of curvature to the former is $\frac{c^{3}}{a}$, and to the latter is $\frac{a^{2}}{c}$; and $\frac{c^{2}}{a}: \frac{a^{2}}{c}:: c^{3}: a^{3}$.

(6). When a body moves in a parabola, by a ceritripetal force tending to the focus; then the velocity of the body in the parabola, is to the velocity of a body describing a circle at the same distance, everywhere as $\sqrt{ } 2$ to 1 . But the velocity of a body in an ellipsis, is to the velocity of a body moving in a circle at the same distance, in a less ratio than that of $\sqrt{ } \approx$ to 1 . And the velocity in an hyperbola, is to the velocity in a circle, in a greater ratio than that of $\sqrt{ } 2$ to 1 .- If the body move in the nautical spiral, its velocity is everywhere equal to the velocity of a body describing a circle at the same distance.

(7). Supposing a body is projected in a given direction, with a given velocity, and that it is urged by a force which is always reciprocally as the square of the distance; reguired to determine the curve in which the body will move.-lt is plain, in the first place, that the path of the body will be one of the conic sections. Let the body be projected in the right line $\Lambda_{\mathrm{n}}$, with the given velocity $\mathrm{c}$ : and since the absolute quantity of the centripetal force is known, there will thence be given the velocity with which a body can describe a circle at the distance $A F$, by the same force: for it is equal to that acquired by a body in falling through $\frac{I}{2} A x$, while urged constantly by the same force. I.et that velocity be $c$. Erect Ac perpendicular to $A D$, in which take $A R$ a 4 th proportional to $c^{2}, C^{2}, \frac{A F^{2}}{F P}$, where $\mathbf{F}$ is a perpendicular on $A D$; then $A R$ will be the radius of curvature at $A$. Draw RB perpendicular fo $\Lambda \boldsymbol{v}$, and $\mathrm{BC}$ perpendicular to $\mathrm{AR}$; then drawing $\mathrm{FC}$, it will give the position of the axis. Make the angle $\mathrm{E} A \mathrm{C}$ equal to the angle FAC. Thon, if $A \mathrm{E}$ be parallel to $\mathrm{FC}$, the figure in which the body moves will be a parabola. But if it meet the axis $\mathrm{FC}$ in $\mathrm{E}$; and if the points $\mathrm{F}$ and $\mathrm{E}$ fall on the same side of the point $c$, the figure will be an hyperbola; but if $F$ and $E$ fail on contrary sides, the figure will be an ellipsis. Hence, with the foci $\mathrm{E}$ and $\mathrm{F}$, and the axis $A F \pm A \mathrm{E}$, the section may be described in which the body will move.

For further particulars on central forces, the reader may consult Newton's Principia, Varignon in the Memoirs of the French Academy, 1700, 1701; Maclaurin's Fluxions; Simpson's Fluxions; Gregory's Mechanics, vol. 1 ; Dawson's Essay on the inverse problem of central forces, in the Manchester Memoirs, or in Leybouru's Mathematical Reposifory, Nos. 4 and 5, N. s.; and Waring's paper on central forces, Philos. Trans. vol. $7 \mathrm{~S}$, or my $\mathrm{Abridg-}$ ment, v. 16 , p. 384 .

Central Rule, is a rulc or method discovered by Mr. Thomas Baker, rector of Nympton in Devonshire, which he published in his Geometrical Key, in the ycar 1684 , for determining the centre of a circle which shall cut a given parabola in as many points as a given equation, to be constructed, has real roots; which he has applled with good success in the construction of all equations as far as the 4 th power inclusive.

The Central Rule is chiefly founded on this property of the parabola; that if a line be inscribed in the curve perpendicular to any diameser, the rectarglo of the segments of this line, is equal to the rectangle of the intercepted part of the diancter and the parameter of the axis. 
The Central Rule has the advantage over the methods of constructing equations by Descartes and Delatteres, which are liable to the trouble of preparing the equations by taking away the second term; whereas Baker's method effects the same thing without any previous preparation whatever. Sce Philos. Trans. N0 157.

CENTRE, or Centel, in a general sense, signifies a point equally remote from the extremes of a line, plane, or solid ; or a middle point dividing them so that some certain effects are equal on all sides of it.

CEN Tre of Attraction, or Gravitation, is the point to which bodies tend by gravity; or that point to which a revolving planet or comet is impelled or attracted, by the force or impetus of gravity.

CEN TRE, in Architècture. This term is üsed to denote a frame of timber, constructed for the purpose of supporting the stones or bricks forming an arch or vault, during the erection. Thus the centre serves as in foundation for the arch to be built upon, which at the completion of the work is struck or taken down, and then the arch will support itself by the reciprocal pressure of its parts.

Centre of a Bustion, is a point in the middle of the gorge, where the capital line commences, and which is usually at the angle of the inner polygon of the figure. Or it is the point where the two adjacent curtains produced intersect each other.

Centre of a Circle, is the point in the middle of a circle, or circular figure, from which all lines drawn to the circumference are equal.

Centre of a Conic Saction, is the middle point of any diameter, or the point in which all the diamoters intersect and bisect one another.

In the ellipse the centre iswrithin the figure; but in the hyperbola it is without, or between the conjugate hyperbolas; and in the parabola it is at an infinite distance from the vertex.

CENTRE of Conversion, in Mcchanics, a term first used by M. Parent, and may be thus conceived: Suppose a stick laid on stagnant water, and then drawn by a thread fastened to it, so that the thread always makes the same angle with the stick, either a right angle or any other; then it will be found that the stick will turn on one point of it, which will be immoveable; and this point is termed the centre of conversion.

This effect arises from the resistance of the fluid to the stick partly immersed in it. And if, instead of the body thus floating on a fluid, the same be conceived to be laid on the surface of another body; then the resistance of this plane to the stick will still have the same effect, and will determine the same centre of conversion, And this resistance is precisely what is called friction, so prejudicial to the effects of machines.

11. Parent has determined this centre in some certain cases, with much laborious calculation. When the thread is fastened to the extremity of the stick, he found that the distance of the centre from this extremity would be nearly $\frac{1}{2} \frac{3}{8}$ of the whole length. But when it is a surface or a solid, there will be some change in the place of this centre, according to the nature of the figure. Sce $M \mathrm{em}$, of the Acad. of Sciences, vol. 1, p. 191.

C'ENTкE of a Curve, of the higher kind, is the point where two dianeters meet.-When all the dianeters meet in the same point, it is called, by Sir Isaac Newton, the general centre.

CENTRE of a Dial, is the point where its gnomon or stile, which is placed parallel to the axis of the carth, mcets the plane of the dial; and from this all the hourlines are drawn, in stich dials as have centres, viz, all except that whose plane is parallel to the axis of the earth where there ean be no centre; in which case all the hourlines will be parallel to the stile, and to one another, the centre being as it were at an infinite distance.

Centre of Equilibrium, is the same with respect to bodies immersed in a fluid, as the centre of gravity is to bodies in free space; being a certain point, upon which if the body or bodies be suspended, they will rest in any position. To determine this centre, see Emerson's Mechan. prop. 92, p. 134 .

Cent R E of Friction, is that point in the base of a body on which it revolves, into which if the wloole surface of the base, and the mass of the body were collected, and made to revolve about the centre of the base of the given body, the angular velocity destroyed by its friction would be equal to the angular velocity destroyed in the given body by its friction in the same time.-Sce Vince on the Motion of Bodies affected by friction, in the Philos. Trans. 1785 .

Centre of Gravity, is that point about which all the parts of a body do in any situation exactly balance each other. Hence, by means of this property, if the body be supported or suspended by this point, the body will rest in any position into which it is put; as also, that if a plane pass through the same point, the segments on each side will equiponderate, neither of them being able to inove the other.

The centre of gravity of a body is not al ways within the body itself: thus the centre of gravity of a ring is not in the substance of a ring, but in the axis of its circumscribing cylinder: and the centre of gravity of a hollow staff, or of a bone, is not in the matter of which it is constituted, but somewhere in its imaginary axis. Every body however has a centre of gravity, and so has every system of bodies.

If a lieavy borly be sustained by two or more forces, their directions must meet either at the centre of gravity of that body, or in the vertical line which passes through it.

When a body stands upon a plane, if a rertical line passing through the centre of gravity fall within the base on which the body stands, it will not fall over; but if that vertical line passes without the base, the body will fall, unless it be prevented by a prop or cord. Where the vertical line falls upon the extremity of the base, the body may stand, but the equilibrium may be disturbed by a very trifling force; and the ncarer this line passes to any adge of the base, the more easily the body is thrown over; the nearer it falls to the middle of the base, the more firmly the body stinds.

The whole gravity, or the whole matter, of a body, may be conceived united in the centre of gravity; and in demonstrations it is usual to conccive all the matters as really colleeted in that point.

Through the centre of gravity passes a right line, called the diameter of gravity; and thercfure the intersection of two such dianeters determines the centre of gravity. Also the plane upon which the centre of gravity is placed, is called the plane of gravity; so that the eommon interscetion of two such planes determines the diancter of gravity.

In homogeneal bodies, which may be divided lengthways into similar and equal parts, the centre of gravity is the same with the centre of magnitude. II'nce therefore the centre of gravity of a line is in the middle point of it, or that point which bisects the line. Also the ecntre of gravity of a parallelogram, or cylinder, or any prism 
whatever, is in the nidllie point of the axis. And the centre of gravity of a circle or any regular figure, is the same as the centre of magnitude.

Also, if a line can be so drawn as to divide a plane into cqual and similar parts, that line will be a diameter of gravity, or will pass through the centre of gravity; and it is the same as the axis oi the plane. Thus the line drawn from the vertes and perpendicular to the base of an isosccles triangle, is a diameter of gravity; and thus also the axis of an ellipse, or a parabola, \&c, is a diameter of gravity. The centre of gravity of a segment or arc of a circle, is in the radius or line perpendicularly bisecting its chord or base.

Likewise, it a plane divide a solid in the same manner, making the parts on brith sides of it perfectly cqual and similar in all respects, it will be a plane of gravity, or will pass through the centre of gravity. Thus, as the intersection of two such planes determincs the diameter of gravity, the centre of gravity of a right cone, or spherical segment, or conoid, \&c, will be in the axis of the same.

The centre of gravity of a ship, is always before the point, which is the middle of her absolute length : for the fore-part, being of greater capacity than the after-part, must conseguently have greater weight; and therefore it carries the centre of gravity forwards in proportion to its greater weight (which in large ships is from 50 to 80 tons), and also to the interval between every centre of gravity of each particular part both forward and aft. When a ship is at sea and loaded, the centre of gravity may well be supposed not to change, unless the cargo be moved. But experience shows that the fore or after-part of the bottom of a ship plunges and labours more and more, in proportion as the wind acts with more or less force on the sails; because ships are generally not masted according to the point velique; so that a ship, which has the centre of the effort of her sails ill-placed, draws al ways more water forward or aft when the impulse of the wind upon her sails is very powerful, than when she is at ease under her burthen. From the centre of gravity of the floating line of a ship let a perpendicular be raised, and continued till it be intersected by the direction of the impulse of the water on the bows, in sailing directly before the wind; and where these two lines cut each other, that point is the point velique, and where the centre of effort of all the sails should be placed.

Common Centre of Gravity of two or more badies, or the different parts of the same body, is such a point as that, if it be suspended or supported, the system of bodies will cquiponderate, and rest in any position. Thus, the point of suspension in a conmon balance beam, or stcelyard, is the centre of gravity of the same.

When any number of bodies move in right lines with uniform motions, their common centre of gravity moves also in a right line with a uniform motion; and the sum of their motions, estimated in any given direction, is precisely the same as if all the borlies in one mass were carried on with the direction and motion of their common centre of gravity. Nor is the centre of gravity of any number of bodies affected by their collision or actions on each otber. For further particulars on the motion of the centre of gravity of any number of bodies, see Emerson's Micchan. Props. 48, 49, \&c.

\section{Laws and Determination of the Centre of Gratity.}

1. 'The centre of gravity of two 'qual bodies or masses is equally distant from their respective contres. For these are as two equal weights suspended at equal distances from the point of suspension; in which case they will equiponderate, and rest in any position.

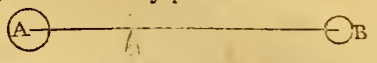

2. If the centres of gravity of two bodics, $A$ and $B$, be connected by the right line $\mathrm{AB}$, the distances $\mathrm{AC}$ and $\mathrm{BC}$, from the common centre of gravity $c$, are reciprocally as the weights or bodies $A$ and $\mathrm{B}$; that is, $A C: B C:: B: A$.

See this demonstrated under the article LEVER.

Hence, if the weights of the bodies $A$ and $B$ be equal, their common centre of gravity $\mathrm{c}$ will be in tlie middle of the right line $A \mathrm{~B}$, as in the forcgoing article. Also since $\mathrm{A}: \mathrm{B}:: \mathrm{BC}: \mathrm{AC}$, therefore $\mathrm{A} \times \mathrm{AC}=\mathrm{B} \times \mathrm{EC}$; whence it appears that the powers of equiponderating bodies are to be estimated by the product of the mass multiplied by the distance from the centre of gravity; which product is usually called the momentum of the weights.

Further, from the foregoing propertion, by composition it will be $\mathrm{A}+\mathrm{B}: \mathrm{A}:: \mathrm{AB}: \mathrm{BC}$, or $\mathrm{A}+\mathrm{B}: \mathrm{B}:: \mathrm{AB}: \mathrm{AC}$. So that the common centre of gravity $\mathrm{C}$ of two hodies will be found, if the product of one weight by the whole distance betwcen the two, be divided by the sum of the two weights. Suppose, for example, that $A=12$ pounds, $\mathbf{B}=4 \mathrm{lb}$, and $\mathbf{A} \mathbf{B}=36$ inches : then $16: 12:: 36: 27=$ Bc, and consequently $\mathrm{AC}=9$, the two distances from the common centre of gravity.

$$
\text { V A P B S }
$$

3. The common Centre of Gravity of tliree or more given bodies or points $\mathrm{A}, \mathrm{B}, \mathrm{C}, \mathrm{D}, \& \mathbf{C}$, will be thus determincd.If the given bodies lie all in the same straight line $A \mathrm{D}$; by the last article, find $P$ the centre of gravity of the two $A$ and $\mathbf{B}$, and $\mathbf{Q}$ the centre of gravity of $\mathbf{C}$ and $\mathrm{D}$; then, considering $P$ as the place of a body equal to the sum of $A$ and $B$, and $Q$ as the place of another body cqual to both $C$ and $D$, find $s$ the common centre of gravity of these two sums, viz, A + B collected in $\mathbf{P}$, and $\mathrm{C}+\mathrm{D}$ united in $\mathrm{Q}$; so shall $\mathrm{s}$ be the common centre of gravity of all the four bodies, A, B, C, D. And the same for any other number of bodies, always considering the sum of any number of them as united or placed in their common centre of gravity, when found.

Othervise, thus. Take the distances of the given bodies from some fixed point as $\mathrm{v}$, calling the distance $\mathrm{vA}=a$, $\mathrm{v} \mathrm{B}=b, \mathrm{vc}=c, \mathrm{v} \mathrm{D}=d$, and the distance of the centre of gravity vs $=x$; then $\mathrm{s}_{\mathrm{A}}=x-a, \mathrm{sB}=x-b, \mathrm{sc}=$ $c-x$, sD $=d-x$, and by the nature of the lever $\mathrm{A} \cdot(x-a)+\mathrm{B} \cdot(x-b)=\mathrm{C}(c-x)+\mathrm{D}(d-x)$ hence $\mathrm{A} x+\mathrm{B} x+\mathrm{c} x+\mathrm{D} x=\mathrm{A} a+\mathrm{B} b+\mathrm{C} c+\mathrm{Dd}$, and $x=\frac{A a+B b+c c+D d}{A+B+C+D}=v s$ the distance sought; which therefore is equal to the sum of all the momenta, divided by the sum of all the weights or bodies.

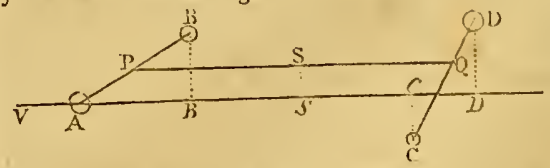

Or thes. When the bodies are not in the same straight line, connect them with the lines $\mathrm{A} B, \mathrm{CD}$; then, as before, find $P$ the common centre of $A$ and $B$, and $Q$ the common ccutre of $\mathrm{C}$ and $\mathrm{D}$; then, conceiving $\mathrm{A}$ and $\mathrm{B}$ united in $\mathrm{P}$, 
and $\mathrm{C}$ and $\mathrm{D}$ united in $\mathrm{Q}$, find $\mathrm{s}$ the common centre of $\mathrm{P}$ and $Q$, which will be the centre of gravity of the whole.

Or the bodies may be all reduced to any line $V A B, \& c$, drawn in any direction whatever, by perpendiculars $\mathrm{B} B$, $c c$, \& c, let fall thereon from the centres of gravity of those bodies; and then the common centre $s$ in this line, as before, will be at the same distance from $\mathrm{v}$ as the true centre $\mathrm{s}$ is; and consequently the perpendicular fron $s$ will pass through $s$ the real centre.

4. From the foregoing general expression, viz,

$x=\frac{A a+B b+c c+\& c}{A+B+c+\& c}$, for the centre of gravity of any system of bodies, may be derived a general method for finding that centre in any body; for $\boldsymbol{A}, \dot{\mathrm{x}}, \mathrm{c}, \delta \mathrm{c}$, may be considered as the elementary parts of any body, whose sum or mass is $\mathrm{M}=\mathrm{A}+\mathrm{B}+\mathrm{c} \& \mathrm{c}$, and $\mathrm{A} a, \mathrm{~B} b, \mathrm{Cc}, \& \mathrm{c}$, are the several momenta of all these parts, viz, the product of each part multiplied by its distance from the fixed point $\mathrm{v}$. Hence then, in any body, find a general expression for the sum of the momenta, and divide it by the content of the body, so shall the quotient be the distance of the centre of gravity from the vcrtex, or from any other fixed point, from which the momenta are estimated.

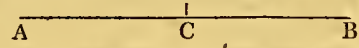

5. Thus, in a right line $\mathrm{AB}$, all the particles which compose it may be considered as so many very small weights, each equal to $\dot{x}$, which is therefore the fluxion of the weights, or of the line denoted by $x$. So that the small weight $\dot{x}$ multiplied by its distance from $\mathrm{A}$, viz $x$, is $x \dot{x}$ the momentum of that weight $\dot{x}$; that is, $x \dot{x}$ is the fluxion of all the momenta in the line $\mathrm{A} \mathrm{B}$ or $x$; and therefore its fluent $\frac{x}{2} x^{2}$ is the sum of all those momenta; which being divided by $x$ the sum of all the weights, gives $\frac{\pi}{2} x$ or $\frac{I}{2} A \mathrm{~B}$ for the distance of the centre of gravity $\mathrm{C}$ from the point $\mathrm{A}$; that is, the centre $\mathrm{C}$ is in the nidddle of the line $\mathrm{AB}$.

6. Also in the parallelogram, whose axis or length $\mathrm{A} \mathrm{B}=x$, and its breadth $\mathrm{DE}=b$; drawing de parallel and indefinitely near $\mathrm{DE}$, the arcola $d \mathrm{DE} e=$ $b \dot{x}$ will be the fluxion of all the weights, which multiplied by its distance $x$ from the point $A$, gives $b x \dot{x}$ for the fluxion of all the mo-

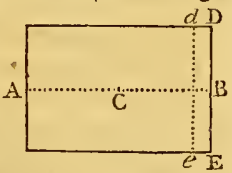
menta, and consequently the flient $\frac{x}{2} b x^{2}$ is the sum of all those momenta themselves; which being divided by $b x$ the sum of all the weights, give $\frac{\pi}{2} x=\frac{1}{2} \mathrm{AB}$ for the distance of the centre $\mathrm{C}$ from the extremity at $\Lambda$, and is therefore in the middle of the axis, as is known from other principles.

And the process and conclusion will be exactly the same for a cylinder, or any prism whatever, making $b$ to denote the area of the cnd or of a transverse section of the body.

7. In a triangle $\mathrm{ABC}$; the line $\mathrm{AD}$ drawn from one angle to bisect the opposite side, will be a diameter of gravity, or will pass through the centre of gravity: for if that line be supported, or conceivr.d to be laid upon the edge of something, the two halves of the triangle on both sides of that line will just balance one

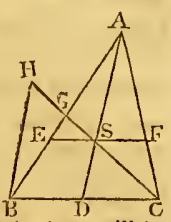
another, since all the parallels $x y \& c$ to the base will be bisected, as wcll as the base itself, and so the two halves of rach line will just balance each other. 'Therefore, putting the basc $\mathrm{BC}=b$, and the axis or bisccting line $\mathrm{AD}=a$, the variable part $\mathrm{AS}=x$; then, by similar triangles $\triangle \mathrm{D}$ : $\mathrm{BC}:: \mathrm{AS}: \mathrm{EF}$, that is $a: b:: x: \frac{l: x}{a}=\mathrm{EF} ;$ which, as a weight, multiplicd by $\dot{x}$, gives $\frac{\operatorname{lix} \dot{x}}{a}$ for the fluxion of the weights; and this again rnultiplied by $\hat{x}=\mathrm{As}$, the distance from $A$, gives $\frac{b x^{2} \dot{x}}{a}$ for the fluxion of the momenta; the fluent of which, or $\frac{b x^{3}}{3 a}$ divided by $\frac{l x^{2}}{2 a}$ the fluent for the weights, gires $\frac{2}{3} x=\frac{2}{3} \Lambda \mathrm{S}$ for the distance of the centre of gravity from the vertex $A$ in the triangle $A \mathrm{EF}$; and whel $x=A \mathrm{D}$, then $\frac{2}{3} \mathrm{AD}$ is the distance of the centre of gravity of the triangle $A \mathrm{BC}$.

The same otherwise, without Fluxions. Since a line drawn from any angle to the middle of the opposite side passes through the centre of gravity, therefore rhe intersection of any two of sucls lines, will be that centre: thus then the centre of gravity is in the line $\mathrm{AD}$; and it is also in the line $C G$ bisccting $A B$; it is therefore in their intersection $\mathrm{s}$. Now to determine the distance of $\mathrm{s}$ from any angle, as $A$, produce $C G$ to meet $B \mathrm{H}$ parallel to $A S$ in $H$; then the two triangles $A G S, B G H$ are mutually equal and similar; for the opposite angles at $\mathbf{G}$ are equal, as are the alternate angles at $\mathrm{H}$ and $\mathrm{S}$, and at $\mathrm{A}$ and $\mathrm{B}$, also the side $A G=B G$; therefore the other sides BI, $A S$ are equal. But the triangles cDs, СвH are similar, and the side $\mathrm{CB}=2 \mathrm{CD}$, therefore $\mathrm{BH}$ or its equal $\mathrm{AS}=2 \mathrm{DS}$, that is $\mathrm{AS}=\frac{2}{3} \mathrm{AD}$, the same as was found before. And in like manner cs $=\frac{2}{3} \mathrm{CG}$.

8. In a trapezium. Divide the figure into two triangles by the diagonal $\Delta \mathrm{C}$, and find the centres of gravity $\mathrm{E}$ and $\mathrm{F}$ of these triangles; join $\mathrm{Er}$, and find the common centre $G$ of these two by this proportion, $\triangle \mathrm{BC}: \triangle \mathrm{DC}:: \mathrm{FG}: \mathrm{EG}$, or $A B C D: \triangle D C:: E F: E G$.

In like manner, for any other

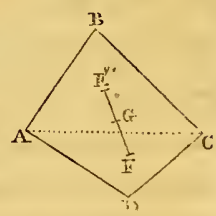
figure, whatever be the number of sides, divide it into several triangles, and find the centre of gravity of each; then connect two centres together, and find their common centre as above; do the same with this and the centre of a third, and so on, always connecting the last found common centre to another centre, till the whole are included in this process; so shall the last common centre be that which is required.

9. In the Purabola вас. Fut $\triangle \mathrm{D}=x, \mathrm{si}=\dot{\mathrm{D}}=$, and the parameter $=p$. Then, by the nature of the figure, $p x=y^{2}$, and $2 y=2 \sqrt{ } p x$; hence $2 \dot{x} \overrightarrow{ } p x$ is the fluxion of the mass, and $2 x \dot{x} \sqrt{p} x$ is the fluxion of the momentum; then the fluent of the latter divided by that of the former, or $\frac{4}{5} x^{2} \sqrt{ } p x$ divided by $\frac{4}{3} x \sqrt{ } p x$, gives $\frac{3}{5} x=\frac{3}{5} A \mathrm{D}$, for $A G$, the distance of the centre of gravity $G$, from the vertex $A$ of the parabola.

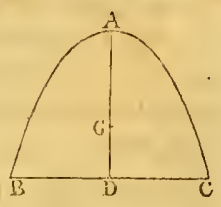

10. In the Circular Arc $\mathrm{A} \mathrm{B} \mathrm{D}$, considered as a physical line having gravity. $1 \mathrm{l}$ is manifest that the centre of-gravity $\mathrm{G}$ of the arc, will be somewhere in the axis, or iniddle radius $\mathrm{BC}$, $\mathrm{c}$ being

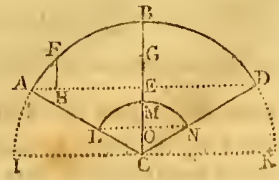


the centre of the eircle, which is considered as the point of suspension. Suppose $r$ inciefinitely near to $\alpha$, and FHparallel to $\mathrm{BC}$. Put the ratius $\mathrm{BCOr} \mathrm{AC}=r$, the semiarc $\mathrm{AB}=z$, and the semichord $\Lambda \mathrm{E}=x ;$ the $n$ is $\Lambda \mu=\dot{x}$, and $\Lambda \mathrm{F}=\dot{z}$ thefluxion of the mass; therefore $c L x \dot{z}$ is the fluxion of the momentum. But, by similar triangles, Ac or $r: \mathrm{cE}:: \mathrm{AF}$ or $\dot{z}:$ AH or $\dot{x}$, therefore $r \dot{x}=\mathrm{cE} \times \dot{z}$, and so $r \dot{x}$ is also the fluxion of the momentum; the fluent of which is $r x$, and this divided by $z$ the whole weight, gives $\frac{\tau x}{\approx}=\frac{A C \times A E}{A B}=$ $\frac{A C \times A E D}{A B D}=C G$ the distance of the centre of gravity from the centre $c$ of the circle; being a 4 th proportional to the given arc, its chord, and the radius of the circle.

Hence, when the arc becomes the semicircle Iвк, the above expression becomes $\frac{\mathrm{IC}^{2}}{1 \mathrm{~B}}$ or $\frac{r^{2}}{1.3708 r}=\frac{r}{1.5708}=$ - $6366 r$, viz a third proportional to a quadrant and the radius.

11. In the Circular Sector A BDc. Here also the centre of gravity will be in the axis or middle radius BC. Now with any smaller radius deseribe the concenitric arc $L M N$, and put the radius $\mathrm{AC}$ or $\mathbf{B C}=r$, the $\operatorname{arC} \mathbf{A} \mathbf{B} \mathbf{D}=a$, its chord $\mathrm{AED}=c$, and the variable radius $\mathrm{CL}$ or $\mathrm{CM}=y$; then as $r: y:: a: \frac{a y}{r}=$ the arc $\mathrm{LMN}$, and $r: y:: c: \frac{c y}{r}$ $=$ the chord LoN; also, by the last article, the distance of the centre of gravity of the arc LMN is $\frac{\mathrm{CM} \times \text { LON }}{\text { LMN }}=$ $\frac{\mathrm{CM} \times \mathrm{AED}}{\mathrm{ABD}}=\frac{c y}{a}$; hence the arc IMN or $\frac{a y}{r}$ multiplied by $\dot{y}$ gives $\frac{a y \dot{y}}{r}$ the fluxion of all the weights or mass, and this multiplied by $\frac{c y}{g}$ the distance of the common centre of gravity, gives $\frac{c y^{2} \dot{y}}{r}$ the fluxion of the momentum; the fluent of which, viz $\frac{c y^{3}}{3 r}$ divided by $\frac{a y^{2}}{2 r}$, the fuent of the mass, gives $\frac{2 r y}{3 a}$ for the distance of the centre of gravity. of the sector CLMN from the centre $\mathrm{c}$; and when $y=r$, it becomes $\frac{2 c r^{\prime}}{3 a}=\mathrm{cG}$ for that of the sector $\mathrm{C} \triangle \mathrm{BD}$ proposed; being $\frac{2}{3}$ of a 4 th proportional to the arc of the sector, its chord, and the radius of the circle.

Hence, when the sector becomes a semicircle, the last expression becomes $\frac{4 r^{2}}{3 a}=\frac{21 \mathrm{c}^{2}}{3 \mathrm{iB}}$ or $\frac{2}{3}$ of a $3 \mathrm{~d}$ proportional to a quadrantal arc and the radius. Or it is equal to $\frac{s r}{B p}=-4244 r$ from the centre $\mathrm{c}$; where $p=3 \cdot 1416$. So that the distance of the centre of gravity of the sector, is just equal to $\frac{2}{3}$ of that of its arc.

12. In the Cone $\Lambda \mathrm{DL}$. Putting $a=\mathrm{Dc}, l=$ area of the base $\wedge \mathrm{EB}$, and $x=D c^{2}$ any variable altitude; then as $a^{2}: x^{2}:: b$ : $\frac{b x}{a^{2}}=$ area $a e b$; hence the fluxion of the mass is $\frac{k x^{2} \dot{x}}{a^{2}}$ whose fluent, or the solid, is $\frac{l x^{3}}{3 a^{2}}$; and the fluxion of the momentum is $\frac{l x^{3} \dot{x}}{a^{2}}$, whose fuent is $\frac{b x^{4}}{4 a^{2}}$; then this fluent divided by the former fluent, the whole weight or mass, gives $3 x$ or $-\frac{3}{4} D E$ for the distance of the centre of gravity of the cone $\mathrm{D} a b$, or $\frac{3}{4} \mathrm{bc}$ for that of the cone DAB below the rertex $\mathrm{D}$. And the same is the distance in any other pyramid. So that all pyramids of the same altitude, have the same contre of gravity.

13. General Scholium to the foregoing articles, for the place of the centre of gravity in geometrical figures.

(1). The centre of gravity of every geometrical figure, lies in its axis or diameter, being that line which is drawn from the midlle of the base of one end, to the middle of the opposite end, or to the opposite angle or vertex; because this line bisects all the ordinates or sections parallel to the said

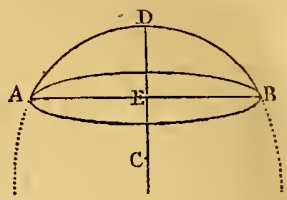

base or ends. And the distance of the centre of gravity, as measured on that axis, either from the base or froin the vertex, is for the several figures as below.

(2). The centre of gravity is in the middle of that axis, or lengtl, or equally distant from both ends, or coinciding with the centre of magnitude in all regular figures, or such as have all the said parallel sections equal; such as right lines, all parallelograms and prisms, all regular plane polygons and solid polyhedrons, also the circle and spherc.

(3). In a triangle and paraboloid, it is $\frac{1}{3}$ of the axis from the base, or $\frac{2}{3}$ from the vertex.

(4). In a parabola it is $\frac{2}{5}$ from the base, or $\frac{3}{5}$ from the vertex.

(5). In a circular arc, it is from the centre of the circle, a 4 th proportional to the arc, its chord, and the radius of the circle, viz, $\frac{c r}{a}$.

(6). In a circular sector, the distance from the centre is, $\frac{2 c r}{a c}$ or $\frac{2}{3}$ of the said 4 th proportional to the arc, chord, ant radius.

(7). In a semicircle the distance from the centre is $\frac{4 r^{3}}{3 a}$, or $\frac{4}{3}$ of a third proportional to the arc and radius.

(8). In a circular segment, the distance from the centre is $\frac{c^{3}}{12 \text { area }}$ or $\frac{x}{\sqrt{2}}$ of the cube of the chord divided by the area of the segment, or $\frac{x}{I^{2}}$ of a 4 th proportional to the said area, the square of the chord, and the same chord.

(9). In any pyramid or cone, it is $\frac{1}{4}$ from the base, or $\frac{3}{4}$ from the vertex.

(10). In the hemisphere, or hemispheroid, $j$ is $\frac{3}{8}$ from the base, or $\frac{5}{8}$ from the vertex.

(11). In the segment of a sphere, or of a spheroid, the distance from the base is $\frac{2 d-h}{6 d-1 h} h$; where $h$ denotes the height of the seginent, and $d$ the whole diameter or axis.

(12). In the segment of an lyyperbolic conoid, the di stance from the base is $\frac{2 d+h}{6 d+4 h} h$; where $h$ and $d$ are the samo as above, viz, the height of the segment and the whole diameter or axis.

(13). In any figure, composed of two parts, or for any two bodies: find the centre of gravity of each of the two bodies, or two parts, and connect these two centres by a right line, in which line will lie the common centre of gravity of the compound body; and the distance of that centre from the ccutre of each body, or each part, will be 


\section{E N}

[ 297

regulated by this proportion, viz, that each distance is reciprocally proportional to the mass or weight, or content of each body; or, as the sum of the contents of the two bodies, or of the whole compound mass, is to the whole distance of the tivo centres, so is each particular body or part, to the distance from the centre of the other body or part.

14. To determine the Centre of Gravity in any Body meclanically. Lay the body on the edge of any thing, as a triangular prism, or such like, moving it backwards and forwards till the parts on both sides are in equilibrio; then is that line just in, or under the centre of gravity. Balance it again in another position, to find another line passing through the centre of gravity; then the intersection of these two lines will be the centre required.

The same may be done by laying the body on an horizontal table, as near the edge as possible without its falling, and that in two positions, as lengthwise and breadthwise: then the common intersection of the two lines contiguous to the edge, will be its centre of gravity. Or it may be done by placing the body on the point of a style, $\& \mathrm{c}$, till it rest in equilibrio. It was by this method that Borelli found that the centre of gravity in a human body is between the nates and pubis; so that the whole gravity of the body is collected into the place of the genitals; an instance of the wisdom of the Creator, in placing the membrum virile in the part which is the most convenient for copulation.

The same otherwise: thus, Hang the body up by any point; then a plumb-line hung over the same point, will pass through the centre of gravity; because that centre will always descend to the lowest point when the body comes to rest, which it cannot do except when it falls in the plumb-line. Therefore, marking that line upon it, and suspending the body by another point, with the plummet, to find another such line, the interscetion of the two will give the centre of gravity.

Or thus. Hang the body by two strings from the same tack, but fixed to different points of the body; then a plummet, hung by the same tack, will fall on the centre of gravity.

In the 4th volume of the New Acts of the Academy of Petersburg, is the demonstration of a very general theorein concerning centres of gravity, by MI. Lhuilier; a particular example only of the general proposition will be.as follows: Let $A, B, C$, be the centres of gravity of three bodies; $a, b, c$ their respective masses, and $Q$ their common centre of gravity. Let right lines $Q A, Q B, Q C$, be drawn from the common centre to that of each body, and the lat ter be connected by right lines $\triangle B, A C$, and $B C$; then $\mathrm{QA}^{2} \times a+\mathrm{QB}^{2} \times b+\mathrm{QC}^{2} \times c=$

$$
A \mathrm{~B}^{2} \times \frac{a b}{a+b+c}+\mathrm{AC}^{2} \times \frac{a c}{a+b+c}+\mathrm{BC}^{2} \times \frac{b c}{a+b+c} \text {. }
$$

Uses of the Centre of Gravity. This point is of the greatest use in mechanics, and many injportant concerns in life; because the place of that centre is to be considered as the place of the body itself, in computing mechanical effects; as in the oblique pressures of hodies, banks of earth, arches of bridges, and such like.

The same centre is even useful in finding the superficial and solid contents of bodies; for it is a general rule, that the superficies or solid generated by the rotation of a line or plane about any axis, is always equal to the product of thes said line or plane strawn into the circumference or path described by the ccntre of gravity. For example, it

V'OL. I.
C E N

was found above at art. 11, that in a semicircle, the distance of the centre of gravity from the centre of the circle is $\frac{4 r}{3 p}$; and therefore the path of that centre, or the circumference described by it while the semicircle revolves about its diameter, is $\frac{8}{3} r$; also the area of the semicircle is $\frac{1}{2} p r^{2}$; hence the product of the two is $\frac{4}{3} \mathrm{pr}^{3}$; which, it is well known, is equal to the solidity of the sphere generated by the revolution of the semicircle.

And hence also is obtained another method of finding mathematically the centre of gravity of a line or plane, from the contents of the superficies or solid generated by it. For if the generated superficies or solid be divided by the generating line or plane, the quotient will be the circumference described by the centre of gravity; and consequently this divided by $2 p$ gives tlie radius, or distance of that centre from the axis of rotation. For example, in the semicircle, whose area is $\frac{1}{2} p r^{2}$, and the content of the sphere generated by it $\frac{4}{3} \mathrm{pr}^{3}$; here the latter divided by the former is $\frac{8}{3} r$, and this divided by $2 p$ gives $\frac{4 r}{3 p}$ for the distance of the centre of gravity from the axis, or from the centre of the semicircle, the same as before. The property last mentioned, relative to the relation between the centre of gravity and the figure generated by the revolution of any line or plane, is mentioned by Pappus, in the preface to his 7 th book; and Father Guldini has more fully demonstrated it in his $2 \mathrm{~d}$ and $3 \mathrm{~d}$ books on the Centre of Gravity.

The principal writers on the centre of gravity are Archimedes, Pappus, Guldini, Wallis, Casatus, Carré, Hays, Wolfius, \&c. See also my Mensuration, $p .382$, 4th edit.

CENTRE of Gyration, is that point in which if the whole mass be collected, the same angular velocity will be generated in the same time, by a given force acting at any place, as in the body or system itself. This point differs from the centre of oscillation, inasmuch as in this latter case the motion of the body is produced by the gravity of its own particles, but in the case of the céntre of gyration the body is put in motion by some other force acting at one place only.

To determine the Centre of Gyration, in any body, or system of bodies composed of the parts A, B, C, \& c, moving about the point $\mathrm{s}$, when urged by a force $f$ acting at any point $\mathrm{P}$. See the following figure. Let $\mathrm{R}$ be that centre; then, by mechanics, the angular velocity generated in the system by the force $f$, is as $\frac{f . \mathrm{sp}}{\mathrm{A} \cdot \mathrm{sA}^{2}+\mathrm{B} \cdot \mathrm{su}^{2}+\mathrm{c} \cdot \mathrm{sc}^{2} \& \mathrm{c}}$, and, by the same, the angular velocity of the inatter placed all in the point $\mathrm{R}$, is $\frac{f . \mathrm{sp}}{(\mathrm{A}+\mathrm{B}+\mathrm{c} \& \mathrm{c}) \times \mathrm{sR}^{3}}$; then since these two are to be equal, their equation will give $\mathrm{s} R$ $=\sqrt{A \cdot S A^{2}+B \cdot S B^{2}+c \cdot s c^{2} \& C}$, for the distance of the centre of gyration sought, below the axis of motion.

Now because the quantity $\mathbf{A} \cdot \mathbf{S} \Lambda^{2}+\mathbf{B} \cdot \mathbf{S B ^ { 2 }}+\& \mathbf{c}$ is $=$ sc. so. $b$, where $\mathrm{c}$ is the centre of gravity, o the centre of oscillation, and $b$ the whole body or sum of $A, B, C, \& C$; therefore it follows that $\mathrm{sR}^{2}=\mathrm{sG}$. so; that is, the distance of the centre of gyration, is a mean proportional betwcen those of gravity and oscillation.

And hence also, if $p$ denote any particle of a body, placed at the distance $d$ from the axis of motion; then is $\mathrm{s} \Omega=\frac{\text { sum of all the } p d^{2}}{\text { body } b}$; whence the point $\mathrm{R}$ may be determined in bodies by means of Fluxions. 
The distance of the centre of gyration from the point or axis of motion, being a mean proportional between the distances of the centres of gravity and oscillation from the same axis. Hence, when any two of these distances is known, the third may readily be determined.

The distance $s \mathrm{R}$ of the centre of gyration from the axis of roration in several bodies will be as follows :

1. In a right line, or thin cylinder, revolving about the end, $\mathrm{SR}=$ lengrh $\times \sqrt{\frac{1}{3}}$.

2. The plane of a circle, or cylinder, revolving about the axis, $\mathbf{S R}=$ radius $\times \sqrt{ } \frac{x}{2}$.

3. The periphery of a circle, about the diameter, $\mathrm{sR}=$ radius $\times \sqrt{\frac{I}{2}}$.

4. A wheel with a very thin rim, revolving about its axle, $\mathbf{S} \mathbf{R}=$ radius.

5. The plane of a circle, about the diameter, $s R=\frac{I}{2}$ radius.

6. The surface of a sphere, about the diameter, $\mathrm{sR}=$ radius $\times \sqrt{ } \frac{2}{3}$.

7. A globe, about the diameter, $s \mathbf{R}=$ radius $\times \sqrt{ } \frac{2}{5}$.

8. A cone, about the axis, $\mathrm{sR}=$ radius $\times \sqrt{\mathrm{I}^{\circ}}$.

CENTRE of Inertion. See CENTRE of Gravity.

CENTRE of Magnitude, is the point which is equally distant from all the similar cxtcrnal parts of a body. This is the same as the centre of gravity it bomogeneal bodics that can be cut into like and equal parts according to their length, as in a cylinder or any otber prism.

CEnTRE of Motion, is the point about which any body, or system of bodies, moves, in a revolving motion.

Centre of Motion of a ship, is the point upon which a vessel oscillates or rolls, when put in motion.

CENTRE of Oscillation, is that point in the axis or line of suspension of a vibrating body, or system of bodies, in which if the whole matter or weight be collected, the vibrations will still be performed in the same time, and with the same angular velocity, as before. Hence, in a compound pendulum, its distance from the point of suspension is equal to the length of a simple pendulum whose oscillations are isochronal with those of the compound une.

Mr. Huygens, in his Horologium Oscillatorium, first showed how to find the centre of oscillation. At the beginning of his discourse on this subject, he says, that Mersennus first proposed the problem to him while he was yet very young, requiring him to resolve it in the cases of sectors of circles suspended by their angles, and by the middle of their bases, both when they oscillate sideways and flatways; as also for triangles and the scgments of circles, either suspended from their vertex or the middle of their bases. But, continucs he, not having immediately discovered any thing that would open a passage into this business, I was repulsed at first setting out, and stopped from a farther prosecution of the subject; till being farther incited to it in adjusting the motion of the pendulums of my clock, 1 surmounted all difficulties, going far beyond Descartes, Fabry, and others, who had done the thing in a few of the most casy cases only, without any sufficient demonstration; and sulving not only the problems proposed by Mersenne, but many others that were much more difficult, and showing a general way of determining this centre, in lines, superficies, and solids.

In the Leipsic Acts for 1691 and 1714, this doctrine is handled by the two Bernoullis: and the same is also done by Herman, in his treatise De Motu Corporum Solidorum et Fluidorum.

It may also be seen in treatises on the Inverse Method of Fluxions, where it is introciuced as ope of the rxamples of that methosl. See Haris, Canké, Wolfues, Simpsox, Fluxions, \&c.

To determine the Centre of Oscillation, in any Compond M ass or Body MN, or of any System of Bodirs A, B, C, SC.

Let ar be the plane of vibration, to which plane conceive all the matter to be reduced by letting fall perpendiculars to this plane from every particle in the body; i supposition which will not alter the vibration of the body, because the particles are still at the same distance from the axis of motion. Let 0 be the centre of oscillation, and $G$ the centre of gravity; through the axis $\mathrm{s}$ draw sGo, and the horizontal

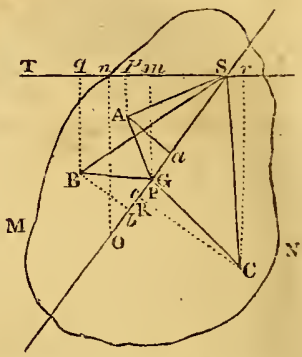

line $S T$; then from every particle $A, B, c, \& c$, let fall perpendiculars $\mathrm{A} a$ and $\mathrm{A} p, \mathrm{~B} b$ and $\mathrm{B} q, \mathrm{c} c$ and $c r, \& c$, to these two lines; and join $\mathrm{SA}, \mathrm{SB}, \mathrm{SC}$; also draw $\mathrm{G} m$ and on perpendicular to $\mathrm{ST}$.

Now the furces of the weights $A, B, c$, to turn the body about the axis, are A.sp, B.s $q,-\mathrm{c} . \mathrm{s} r$; and, by mechanics, the forces opposing or resisting that motion are $A \cdot S A^{2}, B \cdot S B^{2}, c . S C^{2}$; therefore the angular velocity generated by the whole system is $\frac{A \cdot s p+B \cdot s q-c \cdot s r}{A \cdot s^{2}+B \cdot s s^{2}+c \cdot s c^{2}}$. In like manner, the angular velncity which any body or particle $p$, situated in $\propto$, generates in the system, by its weight, is $\frac{p . s n}{p \cdot \mathrm{so}^{2}}$, or $\frac{\mathrm{sn}}{\mathrm{so}^{2}}$, or $\frac{\mathrm{sm}}{\mathrm{sG} \cdot \mathrm{s} \mathrm{s}^{2}}$, because of the similar triangles $\mathrm{sG} m$, son. But, by the conditions of the problem, the timeand angular velucity are alike in both these cases; therefure these two expressions must be equal to each other, that is, $\frac{\mathrm{s} m}{\mathrm{sg} \cdot \mathrm{so}}=\frac{\mathrm{A} \cdot \mathrm{sp}+\mathrm{B} \cdot \mathrm{s} q-\mathrm{c} \cdot \mathrm{s} r}{\mathrm{~A} \cdot \mathrm{sA}^{2}+\mathrm{B} \cdot \mathrm{sB}^{2}+\mathrm{C} \cdot \mathrm{sc}^{2}}$, and consequently so $=\frac{s m}{s \mathrm{G}} \times \frac{A \cdot \mathrm{sA}^{2}+\mathrm{B} \cdot \mathrm{ss}^{2}+\mathrm{c} \cdot \mathrm{sc}^{2}}{\mathrm{~A} \cdot \mathrm{s} p+\mathrm{B} \cdot \mathrm{s} q-\mathrm{s} \cdot \mathrm{s} r}$. Again, by mechanics, the sum of the forces $\Lambda . s p+B . s q-c . s \ddot{r}$ is equal $(A+B+C) s m$ the force of the same matter collected all into its centre of gravity $G$; therefore

so $=\frac{A \cdot s^{2}+B \cdot s B^{2}+c \cdot s c^{2}}{(A+B+c) \cdot s G}$, which is the distance of the centre of oscillation o below the axis of suspension.

Farther, because it was found under the article Centre of Gravity, that $(\mathrm{A}+\mathrm{B}+\mathrm{c}) \cdot \mathrm{s} \mathrm{s}=\mathrm{A} \cdot \mathrm{s} a+\mathrm{B} \cdot \mathrm{s} b+\mathrm{C} \cdot \mathrm{s} c$, thercfore $s o=\frac{A \cdot s \Lambda^{2}+B \cdot s B^{2}+c \cdot s c^{2}}{A \cdot s a+B \cdot s b+c \cdot s c}$ is the same distance of the centre of oscillation; where any of the products A. $s a$, B.$s b$, \&c are to be considered negative when the points $a, b, \& \mathrm{c}$ lie above the point $\mathrm{s}$, or where the axis passes through.

$\Lambda$ gain, because, by Eucl. 11.12 and 13, $\mathrm{SA}^{2}=\mathrm{SG}^{2}+\mathrm{GA}^{2}-2 \mathrm{SG} \cdot \mathrm{G} \|$,

and $\mathrm{SB}^{2}=\mathrm{SG}^{2}+\mathrm{GB}^{2}+2 \mathrm{SG} \cdot \mathrm{Gb}$,

and $\mathrm{Sc}^{2}=\mathrm{SG}^{2}+\mathrm{Gc}^{2}+2 \mathrm{sc}$. Gr, Sc ;

and since by Mechanics, the sum of the last terms is nothing, namely-2sa. $\mathrm{G} a+2 \mathrm{sc} \cdot \mathrm{G} b+2 \mathrm{sG} \cdot \mathrm{Gc} \& \mathrm{c}=0$; therefore the sum of the others, or $\mathrm{A} \cdot \mathrm{SA}^{2}+\mathrm{B} \cdot \mathrm{SB}^{2} \& \mathrm{C}$

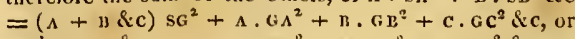
$=b \cdot \mathrm{sG}^{2}+\Lambda \cdot \theta \Lambda^{2}+\mathrm{B} \cdot \mathrm{G} \mathrm{A}^{2}+\mathrm{C} \cdot \mathrm{Gc}^{2} \& \mathrm{c}$; where $b$ denotes the body, or sum $A+B+C \& C$ of all 
the parts: this value then being substituted in the nume$r$ ator- of the $2 \mathrm{~d}$ value of so above-found, it becomes

$\mathrm{so}=\frac{b \cdot S G^{3}+A \cdot G \mathrm{~A}^{3}+\mathrm{B} \cdot \mathrm{Gn}^{2} \& \mathrm{c}}{b \cdot \mathrm{SG}}$, or

$\mathrm{SO}=\mathrm{SG}+\frac{\mathrm{A} \cdot \mathrm{GA}^{2}+\mathrm{B} \cdot \mathrm{GB}^{2} \& \mathrm{CC}}{b \cdot \mathrm{SG}}$;

From which it appears that the centre of oscillation is always below the centre of gravity, and that the difference

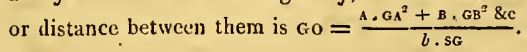

It follows also from hence, that $\mathrm{SG}$. Go = $\frac{A \cdot G A^{2}+B \cdot G B^{2} S c c}{\text { the body } b}$; that is, the rectangle $\mathrm{SG} . \mathrm{Go}$ is always the same constant quantity; wherever the point of suspension $\mathrm{S}$ is placed, since the point $\mathrm{G}$ and the bodies $\Lambda, \mathrm{B}, \& \mathrm{c}$, are constant. Or Go is always reciprocally as $\mathbf{S G}$, that is $\mathrm{Go}$ is less as $\mathrm{SG}$ is greater; and th points $\mathrm{G}$ and $o$ coincide when $S G$ is infinite; but when $s$ coincides with $G$, then Go is infinite, or o is at an infinite distance.

To find the Centre of Oscillation by means of Fluxions. From the premises is derived this general method for the centre of oscillation, viz, let $x$ be the abscissa of an oscillating body, and $y$ its corresponding ordinate or section; then will the distance so of the centre of oscillation below the axis of suspension $\mathrm{s}$, be equal to the fluent of $y x^{2} \dot{x}$ divided by the fluent of $y x \dot{x}$. So that, if from the nature or equation of any given figure, the value of $y$ be expressed in terms of $x$, or otherwise, and substituted in these two fluxions; then the fluents being taken, and the one divided by the other, the quotient will be the distance to the centre of oscillation in terms of the absciss $x$.

But when the body is suspended by a very fine thread of a given length $a$, then the fluent of $(a+x)^{2} \cdot y x$ divided by the fluent of $(a+x) y \dot{x}$ gives the distance of the same centre of oscillation below the point of suspension.

Ex. For example, in a right line, or rectangle or cylinder or any other prism, whose constant section is $y$, or the constant quantity $a$; then $y x^{2} \dot{x}$ is $a x^{2} \dot{x}$, whose fluent is $\frac{x}{3} a x^{3}$; also $y \cdot \dot{x}$ is $a x \dot{x}$, whose fluent is $\frac{x}{2} a x^{2} ;$ and the quotient of the former $\frac{1}{3} a x^{3}$ divided by the latter $\frac{x}{2} a x^{2}$, is $\frac{2}{3} x$ for the distance of the centre of oscillation below the vertex in any such figure, namely having every where the same breadth or scction, that is, at two-thirds of its length. - In like manner the centre of oscillation is found for various figures, vibrating flatways, and are as they are expressed below, viz,

Nature of the Figure.

Isosceles triangle

Common Parabola

Any Parabola

When suspended by Vertex. $\frac{3}{4}$ of its altitude $\frac{5}{7}$ of its altitude $\frac{2 m+1}{3 m+1} \times$ its altitude.

As to figures moved laterally or sideways, or edgeways, that is about an axis perpendicular to the plane of the figure, the finding the centre of oscillation is somewhat difficult; because all the parts of the weight in the same horizontsl plane, on account of their unequal distances from the point of suspension, do not move with the same velucity; as is shown by Huygens, in his Horol. Oscil. He found, in this case, the distance of the centre of oscillation below the axis, viz,

In a circle,

In a rectangle, susp. by one angle, In a parabola, susp. by its vertex, The same susp. by inid. of base,

Iu a sector of a circle
In a cone

In a sphere

the radius, and $g=a+r$ the rad. added to the length of the thread.

See also Simpson's Fluxions, art. 183 \&c.

To find the Centre of Oscillation Mechanically or Experimentally. Make the body oscillate about its point of suspension; and hang up also a simple pendulum of such a length that it may vibrate or just keep time with the other body : then the length of the simple pendulum is equal to the distance of the centre of oscillation of the body below the point of suspension.

Or it will be still better found thus: Suspend the body very freely by the given point, and make it vibrate in small arcs, counting the vibrations it makes in any portion of time, as a minute, by a good stop-watch; and let that number of oscillations made in a minute be called $n$ : then shall the distance of the centre of oscillation be

so $=\frac{140350}{n n}$ inches. For, the length of the penduluin vibrating seconds, or 60 times in a minute, being $39 \frac{1}{8}$ inches, and the lengths of pendulums being reciprocally as the square of the number of vibrations made in the same time, therefore $n^{2}: 60^{2}:: 39 \frac{x}{8}: \frac{140850}{n n}$, the length of the pendulum which vibrates $n$ times in a minute, or the distance of the centre of oscillation below the axis of motion.

Centre of Percussion, in a moving body, is that point where the percussion or stroke is the greatest, in which the whole percutient force of the body is supposed to be collected; or about which the impetus of the parts is balanced on every side, so that it may be stopped by an immoveable obstacle at this point, and rest on it, without acting on the centre of suspension.

1. When the percutient body revolves about a fixed point, the centre of percussion is the same with the centre of oscillation; and is determined in the same manner, viz, by eonsidering the impetus of the parts as so many weights applied to an inflexible right line void of gravity; namely, by dividing the sum of the products of the furces of the parts multiplied hy their distances from the point of suspension, by the sum of the forces. And therefore what has been abose showit of the centre of oscillation, will hold also of the centre of percussion, when the borly revolves about a fixed point. For instance, that the centre of percussion in a cylinder is at $\frac{2}{3}$ of its lengtls from the point of suspension, or that a stick of a cylindrical figure, supposing the centre of motion at the hand, will strike the greatest blow at a point about two-thirds of its length from the hand.See the investigation at large in my Course of Mathematics, p. 4.4.

2. But when the body moves with a parallel motion, or all its parts with the same celerity, then the centre of per-' cussion is the same as the centre of gravity; for the mumenta are the products of the weights and celerities; and to multiply equiponderating bodies by the sime velocity, is the same thing as to take equimultiple's; but the rquimultiples of equiponderating bodies do also equiponderate; therefore equivalent momenta are disposed about the centre of gravity, consequently in this case the two centres coincide, and what is showiz of the one will loold in lie other.

Centre of Percussion, in at lluid, is the satne ats out of it. 2 (?) 
Cent R $\varepsilon$ of a Parallelogram, the point in which its diagonals intersect.

\section{Centre of Position. Sce Centre of Gravity.}

CEN T RE of Pressure, of a fluid against a plane, is that point agaiust which a force being applied, equal to the whole pressure, but in a contrary direction, it will keep the surface at rest. It is evident that, on a plane parallel to the horizon, or on any plane where the pressure is uniform, the centre of pressure coincides with the centre of gravity of the plane. But for other cases, it will be found by the following method.

\section{To find the Centreoof Pressure of any Plane Surface.}

Let $\triangle \mathrm{BCD}$ be the surface of the fluid, $\mathrm{vw}$, the plane; which being produced, let $\mathbf{c} D$ beits intersection with the surface, $P$ the centre of pressure, and $\mathrm{G}$ the centre of gravity; and conceive the whole plane to be divided into an indefinite number of extremely small parts of which one is $x$ : draw $\mathrm{PQ}, \mathrm{G} g, x v$,

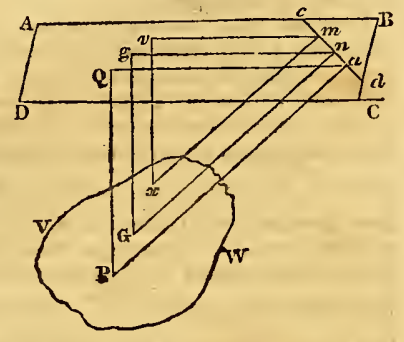
perpendicular to the surface, and $\mathbf{P} a, \mathrm{G} n, x m$ perpendicular to $c d$; and join $Q a, g n, v m$, then it is manifest that the triangles $\mathrm{P} Q a, \mathrm{Gg} n, x v m$ are similar. Now, by the principles of hydrostatics, the pressure on $x$, perpendicular to $\mathrm{vw}$, is as $x \times x v$; and by mechanics, its effect to turn the planc about $c d$, is as $x \times x o \times x m$; but $\mathrm{G} n: \mathrm{Gg}:: x m: x 0$ $=x m \times \frac{\mathrm{Gg}}{\mathrm{G} x}$; hence the effect of the pressure at $x$, to turn the plane about $c d$, is as $x \times x m^{2} \times \frac{G g}{G n}$; therefore the whole effect is as the sum of all the $x \times x m^{2} \times \frac{G g}{G n}$. But if $\mathrm{A}=$ the area of $\mathrm{vw}$, the whole quantity of pressure on $\mathrm{v} w=\Lambda$ $x \mathrm{Gg}$; therefore the effect of the pressure at $P$, to turn the plane about $c d$, is as $\mathrm{A} \times \mathrm{eg} \times \mathrm{P} a$. Hence $\mathrm{A} \times \mathrm{Gg} \times \mathrm{P} a=$ sum of all the $x \times x m^{2} \times \frac{G x}{G / 2}$; consequently

$$
\mathrm{P} a=\frac{\text { sum of all the } x \times a \mathrm{~m}^{3} \times \frac{\mathrm{G} n}{\mathrm{Gg}}}{\mathrm{A} \times \mathrm{G} n} \text {. }
$$

Hence it appears, that $P$ is at the same distance from $c d$ as the centre of percussion is, $c d$ being the axis of suspension. They do not however in general coincide or lie in the same line, that is, in the line $n \mathrm{G}$; for the efficacy of the pressure at $x$, to turn the plane about $n \mathrm{G}$, is as $x \times x v \times$ $m n$ (or since $x o$ variesas $x m$ ), as $x \times x m \times m n$; but the sum of all the $x \times x m \times m n$, is not generally $=0$, thercfore the whole pressure will not necessitrily balance itself upon the line $\mathrm{G} n$. The situation of the line $\mathrm{P}(\boldsymbol{t}$ must be determined by making the sum of all the $x \times x m \times m n=0$, which in any particular case inay be done by a fluxional process. It is not therefore true, in general, that the centres of pressure and percussion are the samc point.

Cextre of is Regular Polygon, or Regular Body, is the same as that of the inscribed, or circumscribed circle or spluere.

CENTRE of Rotation, is that point about which a body, w therwise at liberty, revolves or weuds to revolve when it is acted upon unequally at different poists, or by a force the direction of which does not pass through its centre of gravity. M. J. Bernoulli was the first who published any thing on this subject; and he first found the point about which a body at rest would begin to revolve when struck by another body, and he called this point the centre of spontaneous rotation, to distinguish it from the centre of forced rotation. He observed however that D. Bernoulli liad discovered the same; he has also mentioned the curve described by that point in the progressive motion of the boriy, and has described a method of inquiry by which the velocity of the bodics may be found after the stroke. Mr. Vince has treated this subject much at large, and with great perspicuity, in the Philos. Trass. vol. 70, for 1780 , p. $546,8 \mathrm{~s}$.

Centre of a Spleere, is the same as that of its generating semicircle, or the middle point of the sphere, from whence all right lines drawn to the superficies, are equal.

CENTRE of Sipontaneous Rotation, is that point which remains at rest the instant a body is struck, or about which the body begins to move. The term was first used by Jolın Bernoulli to distinguish this centre from the centre of forced rotation, or that about which a body is compelled to rcvolve, such for instance as the point of suspension of a pendulum. See Clintre of Rotation.

Centurne of on Optic Gilass, the grinding it so as that the thickest part be cxactly in the middle.

Cassini the younger has a discourse expressly on the necessity of well centring the object glass of a large telescope, that is, of grinding it so as that the centre may fall exactly in the axis of the telescope. Mem. Acad. 1710.

Indeed, one of the greatest difficulties in grinding large optic glasses is, that in figures, so little convex, the least difference will throw the centre 2 or 3 inches out of the middle. And yet Dr. Hooke remarks, that though it were better the thickest part of a long object glass were exactly in the middle, yet it may be a very good one when it is an inch or two out of it. Philos. Trans, No. 4.

CENTRING, or CENTERING, in Building, denotes the frame of timber by which the brick or stone work of arched vaulting is supported during its erection, and from which it receives its form and curvature. For their description and properties, sce the Principles of Bridges, in my vew Tracts, vol. 1, p. 105.

CENTRIFUGAL Machine, a curious machine invented by a Mr. Erskine, for raising water by means of a centrifugal force, combined with the pressure of the atmosphere. This machine consists of a large tube of copper, \&c, in the form of a cross, placed perpendicularly in the water, and resting at the bottom on a pivot. $\Delta t$ the upper part of the tube is a horizontal toothed wheel, which touches the teeth of another in a vertical position; so that by the aid of a double winch the whole nachine is moved round with very great velocity. Near the bottom of the perpendicular part of the tube is a valve opening upwards; and near the two extremities, but on the contrary sides of the arms, or cross part of the tube, are two other valves opening outwards. These two valves are kept shut by means of springs, till the machine is put in motion, when the centrifugal velocity of the water forces them open, and clischarges itself into a cistern or reservoir placed there for that purpose. On the upper part of the arm are two holes, which are closed by pieces that screw into the metal of the tube. Before the macline can work, these holes must be opened, and water poured in through them, till the whole tube be full; by these means all the air is forced out of the machine, and, 


\section{E N}

the water supported in the tube by means of the valve at the bottom. The tabe being thus filled with water, and the holes closed by their screw caps, it is turned round by the winch, when the water in the arms of the tube acquires a centrifugal force, opens the valves near the extremities of the arms, and flics out with a velocity nearly equal to that of the extremities of the said arms.

Centrifugat Force, is that by which a body revolving about a centre, or about another body, endeavours to recede from it. And

CENTRIPETAL Force, is that by which a moving body is perpetually urged towards a centre, and made to revolve in a curve instead of a right line.

Hence, when a body revolves in a circle, these two forces, riz, the centrifugal and centripetal; are equal and contrary to each other, since neither of them gains upon the other, the body being in a manner equally balanced by them. But when, in revolving, the body recedes farther from the centre, then the centrifugal exceeds the centripetal force; as in a body revolving from the lower to the higher apsis, in an ellipse, and respecting the focus as the centre. And when the revolving body approaches nearer to the centre, the centrifugal is less than the centripetal force; as while the body mores from the farther to the nearer extremity of the transver'se axis of the cllipse.

It is onc of the established laws of nature, that all motion is of itself rectilinear, and that the moving body never recedes from its first right line, till some new impulse be superadded in a different direction; after that new impulse the motion becomes compounded, but it is still rectilinear, though not in the same line or direction as before. To move in a curve it must receive a new impulse in a different direction every moment, a curve not being reducible to any number of finite right lines. If then a body, continually drawn towards a centre, be projected in a line that does not pass through that centre, it will describe a curve; in every point of which, as $\Lambda$, it will endeavour to recede from the curve, and proceed in the tangent $\mathbf{A D}$; and if nothing bindered, it would actually proceed in it; so as in the same time in which it describes the arch AE, it would re-

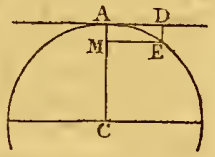
cede the length of the line $\mathrm{DE}$, perpendicular to $\mathrm{AD}$, by its centrifugal force: or, being projected in the direction $\mathrm{AD}$, but continually drawn out of its direction into a curve by a centripetal force, so as to fall below the line of direction by the perpendicular space $\mathrm{DE}$; then the centrifugal or centripetal force is as this line of deviation $\mathrm{DE}$, supposing the arch A E indefinitely small.

The doctrine of centrifugal forces was first mentioned by Huygens, at the end of his Horologium Oscillatorium, published in 1673 , and demonstrated in the volume of his Posthumous Works ; as also by Guido Grando, who gave a few easy cases in bodics revolving in the circumference of circles. But Newton, in his Principia, was the first who fully prosecuted this doctrine; at least as far as regards the conic sections. After him there have been several other writers on this subject; as Leibnitz, Varignon, in the Mem. de l'Acad., Keil, in the l'hilos. Trans. and in his Physics, Bernoulli, Herman, Cotes, in his IJarmonia Mensurarnm, Maclaurin, in his Gcometrica Organica, and in his Fluxions, and Euler, in his book de Motu, where he considers the curves described by a body acted on by centripetal forces tending to several fixed points.
C E R

Sce also the article Central Forces, where this doctrine is more fully explained.

CENTROBARYCO, the same as centre of gravity.

Centrobaryc Method, is a method of determining the quantity of a surface or solid, by means of the generating line or plane, and its centre of gravity. The doctrine is chiefly comprised in this theorem : Every figure, whether superficial or solid, generated by the motion of a line or plane, is equal to the product of the generating magnitude and the path of its centre of gravity, ar the line described by its centre of gravity.

See more of this subject in the art. Centre of Gravity. CENTRUM, in Geom. Mechanics, \&c. See Centre. Centrum Plonicum, in Acoustics, is the place where the speaker stands in polysyllabical and articulate echoes.

CеNтиUM Phonocampticum, is the place or object that returns the voice in an echo.

CEPHEUS, a constellation of the northern hemisphere, being one of the 48 old asterisins. The Greeks fable that Cepheus was a king of Ethiopia, and the father of Andromeda, the princess who was delirered up to be destroyed by a sea monster, from which she was rescued by Persens. The stars of this constellation, in Ptolemy's catalogue, are. 13 , in Tycho's 11, in Hevelius's 51 , and in the Britannic catalogue 35.

CERBERUS, one of the new constellations, formed by Hevelius out of the unformed stars, and added to the 48 old asterisms. It contains only 4 stars, which are enumerated under Hercules in the Britannic catalogue.

CERES, or Piazzi, in Astronomy, a new primary planet intermediate between the orbits of Mars and Jupiter, was discovered on the Ist of January 1801, by $M$. Piazzi, astronomer royal at Palermo in Sicily, who also gave it this name.

M. Piazzi in a brief account he has published of the discovery of this' planet states, that having been engaged for nine years in rectifying the positions of the stars as collected in the catalogues of various astronomers, he was searching on the first of January $180 t$, among many others, for the 87 th in the catalogue of the zodiacal stars of the Abbe de la Caille, when he observed that this star was preceded by another, which according to his usual custom he wished to observe also; especially as it did not interrupt the principal observation. Its light was somewhat faint, and its colour resembled that of Jupiter; but like many others, which, in regard to their magnitude, are usually placed in the 8 th class. At that time no derubt arose respecting the nature of it; but on the evening of the $2 \mathrm{~d}$, having repeated his ouservations, and finding that they did not correspond either in time or zenith distance, he suspected that some error had been committed in his observations on the preceding day. He then began to cntertain some idea that it might perhaps be a new planet. In the cvening of the $3 \mathrm{~d}$ his conjecture was confirmed, and he assured hinself it was not a fixed star. However before he would speak of it, he waited till the evening of the 4 th, in which he had the satisfaction of finding that it had moved according to the same laws which it had observed on the preceding days. At this time the motion was retrograde; but on the loth of January it becane direct. Ile continued to observe the planet till the 13th of February, when he was obliged by illness to discontinue his observations. M. Piazzi then transmitted accounts of his ouservations to sevcral celebrated astronomers, in order that they might calculate the orbit of the new plaset, 


\section{E U}

and trace out its progress in the heavens: but it eluded every search that was made for it, until December 7 th, when it was re-discovered by the assiduous Dr. Zach of Saxe-Gotha; and soun after it was observed by Dr. Olbers at Bremen; by Mechain, at Paris ; by the royal astromomor at Greenwich; by Dr. Herschel, at Slough; and by various other persons both in England and Scotland.

Elements of its Theory according to Burckhardt.

Inclination of orbit

$\theta^{3} \quad 10^{0} \quad 37^{\prime}$

Place of the ascending node - 2216

Epoch of $1801 \quad-\quad$ - 21719

Place of the aphelion $\quad-\quad \begin{array}{llll}10 & 26 & 9\end{array}$

Passage of the aphelion Jan. 1, 1801 - 8 hours

Excentricity of orbit - $\quad$ - 0.0784

Mean distance - $\quad$ - $\quad 2.7677$

Time of revolution - - - 4.606 years.

From the observations of Dr. Herschel it is probable that the diameter of this planet, is much less than that of the moon; it appeared under a disc of less than $2^{\prime \prime}$. Yet, sinall as is this planet, M. Schröeter suspects it has two satellites. It has been expected for nearly a century, that such a discovery as this of M. Piazzi, would be made by some diligent astronomer. Maclaurin, Lambert, Bode, Zach, Capel Lofft, and others have supported the conjecture. And six astronomers assembled at Lilienthal, in September 1800, resolved to establish a society of 24 practical astronomers, dispersed throughout different parts of Europe, for the express purpose of searching out this planet between Mars and Jupiter: they elected Schröeter as their president, and Dr. Zach was chosen their secretary. Accordingly, the consequence has been, that no less than four new planets have been discovered, all nearly agreeing in size, period, and situation; viz, the above one by Piazzi, one by Harding of Lilienthal, and two by $\mathrm{Ol}-$ bers of Bremen, all of which have received names of different heathen divinities : but it may be remarked on this occasion, that it would be a more fit practice to call the several new planets after the names of their respective discoverers, as Hcrschel, Piazzi, Olbers, \&c.

CETUS, the Whale, a southern constellation, and one of the 48 old asterisms. The Greeks pretend that it was the sea monster sent by Neptune to devour Andromeda, but waskilled byPerseus. - In the neck of the whale is a remarkable star, Collo Ceti, which appears and disappears periodically, or rather becumes brighter and fainter by tums, arising it is supposed from the alternate turnings of its bright and rark sides towards us, as it revolves upon its axis, or else from the flatness of its form. The period of its changes is about 312 days. Bulliald in Phil. Trans. vol. 2, Hevelius, ibid. vol. 6, Herschel, ibid. vol. 70, Marald. in Mlem. Acad. 1,19 .

'I'he stal's in the constellation Cetus, in Ptolemy's catalogue, are 22, in 'Tycho's 21, in Hevelius's 4.5, and in the Britanic catalogue 97.

CEULlEN, or KEUIEN (Ludolpn VAN), a noted Dutch professor of mathematics, who flowrished in the latter part of the 16 th century. Ludolph was surnaned Van Ceulen, because he was from Cologne, which is called Ceulen or Keulen in Dutch. He was author of several picess, both in arithmetic and geometry, in the Dutch language, some of which were translated into Latin by Willebrord Sucll, as mentioned under his life. Ceulen was chiefly rematiable for his approximation to the circumference of the circte, to 36 places of figures, which he ordered to be engraved on his tombstone.
CHAIN, in Surveying, is a lineal measure, consisting of a certain number of iron links, usually 100 ; serving to take the dimensions of fields \& 8 .

At every 10 th link is usually fastened a small brass plate, with a figure engraven upon it, or else cut into different shapes, to show how many links it is from one end of the chain.

Chains are of various kinds and lengths; as

1. A chain of 100 feet long, each link one foot, is used for measuring of large distances only, when no regard is had to acres \&c, in the superficial content.

2. A chain of one pole or 16 feet and a half in length ; is particularly useful in measuring and laying out gardens and orchards, or the like, by the pole or rod-measure.

3. A chain of 4 poles, or $66^{\circ}$ fcet, or 22 yards, in length, called Gunter's chain, and is peculiarly adapted to the business of land-surveying, hecause 10 square chains just make an English acre of land; so that the dimensions being taken in these chains, they are readily reduced to acres by dividing by 10 , or barely cutting off the last figure from the square chains. But it is still better in practice to proceed thus, viz, count the dimensions, not in chains, but all in links; then the contents are in square links; and five figures being cut off for decimals, the rest are acres; that is four figures to bring the square links to square chains, and one more to bring the square chains to acres.

In this chain, the links are eacb 7 inches and $\frac{92}{50}$, or $7 \cdot 92$ inches in length, which is very nearly $\frac{2}{3}$ of a foot. And hence any number of chains or links are casily reduced to feet or inches, or the contrary: the best way of doing which is this: multiply the number of links by 66 , then cut off two figures for decimals, and the rest are feet : or multiply links by 22 for yards, cutting off two figures.

Crs Rule, called also Rule of Exchange, Rule of Reduction, and Rule of Equation, is a mode of arbitration very useful in the higher operations of exchunge, as well as in the arbitration of bullion, specie, and merchandisc. Foreign merchants, it secms, are very expert in their application of this rule to commercial computations; and that it is, in a great measure, to this accomplishment that their acknowledged superiority in the practice of exclange may be attributed. See Arbitration, also. Dr. Kelly's Cambist.

CHALDER, or CHALDRON, of Coals, an English dry measure of capacity consisting of 36 bushels heaped up.

The chaldron of coals is accounted to wcigh about 28 cwt. or 3136 pounds.-On ship-board, 21 chaldrons of coals are allowed to the scorc.

By act of parliament, a Newcastle chaldion is to weigh $52 \frac{1}{2} \mathrm{cwt}$, or 3 waggons of $17 \frac{1}{2} \mathrm{cwt}$. or 6 carts of $8 \frac{3}{4} \mathrm{cwt}$. each, making $52 \frac{r}{2} \mathrm{cwt}$. to the chaldron. The statute London chaldron is to consist of 36 bushels heaped up, eacl bushel to contain a IVinchester bushel and one quart, and to be $19 \frac{1}{2}$ inches diameter externally. Now it lias been found by repeated trials that 15 London chaldrons are equal to 8 Newcastle chaldrons, which, reckoning $52 \frac{2}{2}$ cwt. to the latter, gives $28 \mathrm{cwt}$. to the former, or $3136 \mathrm{lbs}$ to the London chaldron.

This 1 find ncarly confirmed by experiment. I weighed one peck of coals, which amounted to "2 13 lbs. Then 4 times this gives 87 lb for the weight of the bushel; and $36^{\circ}$ tiines the bushel gives 3132 lbs for the chaldron; to which if the weight of the odd quart be adcled, or 316 nearly,. it gives $3135 \mathrm{lb}$ for the weight of the chaldron, which is only 1 pound short of that by statute. 
CHAMBER of a Mortar, or Cannon, is a cell or cavity at the bottom of the bore, to receive the cluarge of powder. It is not found by experience that chambers have any stusible effect on the velocity of the shot, unless in the largest ordnance, as mortars or very large cannon. Neither is it found that the form of them is very material; a small cylinder is as goorl as any; though mathematical speculations may show a preference of one form over another. But in practice, the ehief point to be observed, is. to have the chamber of a size just to contain the charge of powder, and no more, that the ball may lie close to the charge; and that its entrance may point exactly to the centre of the ball.

CHAMBERS (EPHRAIN), author of the dictionary of sciences called the Cyclopædia. He was born at Milton, in the county of Westmoreland, where be received the common education for qualifying a youth for trade and commerce. When at a proper age, he was apprenticed to Mr. Senex the globe-maker, a business which is connected with literature, especially with geography and astronomy. It was during Mr. Chambers's residence with this skilful artist, that he acquired that taste for literature which accompanied him through life, and directed all his pursuits. At this time, he formed the design of his grand work; the Cyclopædia; some of the first articles of which were written behind the counter. To bave leisure to pursue this work, he quitted Mr. Senex, and took chambers at Gray's Inn, where he chiefly resided during the rest of his life. The first edition of the Cyclopædia, which was the result of many years intense application, appeared in 1728 , in 2 vols folio. The reputation that Mr. Chambers acquired by the execution of this work, procured him the honour of being elected r. R.s. Nov. 6,.1729. In less than ten years time, a second edition became necessary; which accordingly was printed, with corrections and additions, in 1738; and this was followed by a third edition the very next year.

Though the Cyclopredia was the chief business of Mr. Chambers's life, and may he regarded as almost the sole foundation of his fame, his attention was not wholly confined to this undertaking. He was concerned in a periodical publication called The L.jterary Magazine, which was begun in 1735 . In this work lie wrote a variety of articles; particularly a review of Morgan's Moral Philosopher. He was also concerned with Mr. John Martyn, professor of botany at Cambridge, in preparing for the press a translation and abridgment of the Philosophical History and Memoirs of the Royal Academy of Sciences at Paris; which work was not published till 1742 , some time after our author's decease, in 5 volumes 8 vo. Mr. Chambers was also author of the translation of the Jesuit's Perspective, from the French, in 4to; which has gone through several editions. He was also joint editor, with Dr. Shaw, of a translation of Boerhaave's Institutiones Chemix, under the title of A New Method of Chcmistry; including the 'Theory and Practice of that Art, \&c, published jointly by $\mathrm{P}$. Shaw, M. B. and E. Chambers, gent. with additional notes and sculptures, Lond. 4 to, 1727 : and it afterwards went through several editions, with augmentations. Mr. C. was also the editor of the Abridgment of Lord Bacon's Philosophical Works, in 3 vols 4to, 1739; and of those of Mr. Buyle, 3 vols 4to, 1725 . Besides which, he was the author of 3 Chemical Lectures, read in London in 1731 and 1732, and Scarborough 1733, for the improvement of arts, trades, and natusal philosophy, 8vo, $2 \mathrm{~d}$ edit. 1725.
Subsequent to which, he published Essays for the improvemcnt of arts, manufactures, and commerce, by means of chemistry, 2d edit. improved by P. Shaw, fellow of the college of physicians, and physician in ordinary to his majesty.

Mr. Chambers's close and unremitting attention to his studies at length impaired his health, and obliged him occasionally to take a country lodging, but without inuch benefit; he afterwards visited the south of France, but still with little effect; be therefore returned to England, where he soon after died, at Islington, Mlay 15, 17.40, and was buried in Westminster Abbey.

After our author's death, two more editions of his $\mathrm{Cy}$ clopædia were published. The proprietors afterwards procured a supplement to be compiled, by Mr. Scott and Dr. Hill, which extended to two volumes more; and the whole has since been reduced into one alphabet in 4 volumes, by Dr. Rees, forming a very valuable body of the sciences. And the same is now extending in a new edition, in $4 t 0$, under the title of Encyclopædia.

CHAMBRANLE, the border, frame, or ornament of stone or wood, surrounding the three sides of doors, windows, and chimneys. This is different in the different orders: when it is plain, and withont mouldings, it is called simply and properly, band, case, or frume. In an ordinary door, it is commonly termed door-case; in a window, the window-frame.

'The chambranle consists of three parts; the two sides, called ascendants; and the top, called the traverse or supercilium.

CHAMFER, or CHAMFERET, an ornament, in architecture, consisting of, half a scutia ; being a kind of small furrow or gutter on a column.

CHAMFERING, is used for cutting the edge or the end of any thing bevel, or aslope.

CHANCE, the Doctrine and Laws of, are the same as those of Expectation, or Probability, \&c ; which sec. Chances, in play, consist of the number of ways by which events may happen. Thus, if a halfpenny, or other piece of money, be tossed up, there are two events, or chances, or sides that may turn up, namely, one chance for turning up a head, and onc for the contrary; that is, it is an equal chance to throw a head or not. And in throwing a common die, which bas 6 faces, there are in all 6 chances, that is one chance for throwing an ace or any other single point, and 5 chances against it; or it is 5 to $I$ that such assigned point does not come up.

The term chance is applied to events, to denote that they happen without any necessary, foreknown, or intending cause; or it is used to denote the bare possibility of an event when nothing is known either to prevent or hinder it: thus, when we say a thing happens by chance, we really mean no more than that its cause is unknown to us; not, as some vajnly imagine, that chance itself can be the cause of any thing.

Doctrine of Chaxces, History of. This subject, which is no less useful than curious, does not appear to have engaged the attention of mathematicians in former times so much as its importance required. Until the beginning of the last century little is to be found in any of their writings concerning this subject. Of the few pro blems which they had been accustomed to investigate, they withheld the solutions both from the public and from each other; and they seem to have considered the doctrinc of chances rather as an exercise for their ingenuity, 
than as capable of being applied to any useful purpose. Before Mr. Huygens published his book De Ratiociniis in Ludo Alex, no person had treated the subject methodically; and, with the exception of Messrs. Pascal and Fermat, who had solved a few problems of no great importance or difficulty, he appear's to have been the first who attempted either to give rules for the solutions of any question, or to lay down the principles from which those solutions might be deduced. To this work succeeded a small anonymous tract, On the Laws of Chance, which was published in London in 1692, and a French publication of not much larger size, entitled L'Analyse des Jeux de Hazard, written by M. Monmort, and published in the year 1708. In this latter work, the author having chiefiy insisted on the same mode of reasoning with Huygens, in the solution of his problems, Mr. Demoivre (who considered such reasoning as neither genuine nor natural) was induced in his celebrated work on the Doctrine of Chances (which was first published in 1717), to adopt a plainer and less exceptionable mode, in which he has proceeded from the most simple to the most complicated cases; so that by the variety of his problems, as well as by the improvements and additions which he made in two subsequent editions, he had rendered his ,work one of the best and most copions that has ever been written on the subject. In the year $1740, \mathrm{Mr}$. Thomas Simpson published a small treatise on The Nature and Laws of Chance, which, like his other publications, is not only clear and concise, but contains some problems, whose solutions had either never been attempted, or at least never before communicated to the public. Prior however to the two lastmentioned publications, a posthumous work of Mr. James Bernoulli was published in the year 1713 , entitled De Arte Conjectandi, containing an explanation of Huygens's tract, and the solution of a great variety of other problems, deduced from the general principles of combination. The second part of this valuable work has lately been translated into English by Mr. Baron Maseres, with notes and commentaries. In the year 1765 , and at other times, $M$. Dalembert, in his Opuscules \&c, wrote different essays on the doctrine of chances; and not long ago M. Condorcet published a small treatise on the same subject. In addition to these, which are the principal publications on the subject, may be noticed a small tract, De Mensura Sortis, given by Demoivre in his Miscellanea Analytica, and some other papers written by him, by Messrs. Bernoulli, Euler, and others, in the Acts of Leipsic, the Journial des Scavans, the Philosophical Transictions, \&cc, among which may be particularly mentioned an Essay on the Method of calculating the exact Probability of all Conclusions founded on Induction, and a Supplement to that Essay; the one preserved from the papers of the late Rev. Mr. Bayes, and communicated with an appendix, by 1)r. Price, to the Royal Society in the year 1762; the other cliefly written by Dr. Price, and communicated in the following year. These tracts contain the investigation of a problem, the converse of which had formerly exercised the ingenuity of Bernoulli, Demoivre, and Simpson. Indeel both the problem and its converse may justly be considered not only as the most difficult, but as the most important that can be proposed on the subject; having (as Dr. Price well observes) "no less an object in view thin to show what reason we have for believing that there are in the constitution of things, fixed laws, according to which events happen; and that therefore, the frame of the world must be the effect of the wisdom and power of an intelligent cause; and thus to confirm the argument taken from final causes for the existence of the Deity." ReEs.

For the doctrine itself consult Demoivre, T. Simpson, and a work on the same subject lately published by the celebrated Laplace. See also CredibiLitr, GaniNg, Expectation, and Probability.

CHANDELIERS, in Fortification, a kind of wooden parapet, consisting of upright timbers supporting others laid across the tops of them, 6 feet high, and fortified with fascines, \&cc. They are used to cover the workmen in approaches, galleries, and mines. And they differ from blinds only in this, that the former serve to cover the men before, and the latter over hend.

CHANGES, the permutations or variations of any number of things, with regard to their position, order, \&c ; as how many changes may be rung on any number of bells, or how many different ways any number of persons may be placed, or how many several variations may be made of any number of letters, or any other things prom posed to be varied.

To find out such numbers of changes, multiply continually together all the terms in a series of arithmetical progression, whose first term and common difference are each unity or 1, and the last term the number of things proposed to be varied, thus $1 \times 2 \times 3 \times 4 \times 5 \& c$, till the last number be the proposed number of things. For let there be two things as $a$ and $b$. Then since they may be placed either thus $a b$ or $b a$, it is obvious that the number of changes is $1 \times 2$ or 2 . If three things, as $a, b, c$ be supposed ; they will admit of 6 variations as in the margin; for each of the three may be combined three different ways with each combination of the other two; thus $a$ may be first combined with $b c$ and then with $c b$, and hence the number of changes arises, viz, $1 \times 2 \times$ $3=6$.

\begin{tabular}{lll}
$a$ & $b$ & $c$ \\
$b$ & $a$ & $c$ \\
$b$ & $c$ & $a$ \\
\hline$a$ & $c$ & $b$ \\
$c$ & $a$ & $b$ \\
$c$ & $b$ & $a$
\end{tabular}

And if there be 4 things, each of them may be combined 4 ways with each combination of the other three, that is 4. times 6 ways, or $1 \times 2 \times 3 \times 4=24$ ways.

In like manner, the combinations

of 5 things are $1 \times 2 \times 3 \times 4 \times 5=120$

of 6 things are $1 \times 2 \times 3 \times 4 \times 5 \times 6=720$

\&c.

So that if it be proposed to assign how many dificrent ways a company of 6 persons may be placcd, at table for instance, the answer will be 720 ways. Also the number of changes that can be rung on 7 bells, is $1 \times 2 \times 3 \times 4$ $\times 5 \times 6 \times 7$ or $720 \times 7=5040$,

CHAPITERS, the crowns or upper parts of a pillar or column.

ClHAPPE (JEAN D'AUTerocrie), a French astronomer, was born at Mauriac, in Auvergne, March 2, 1728. A taste for drawing and mathematics appeared in him at a very early period; and he owed to Dom. Germain a knowledge of the first elements of mathematics and astronomy. M. Cassini, after assuring himself of the genius of this young man, undertonk the cultisation of it. 1le employed him on the map of lyance, and the translation of Halley's tables, to which he made consislerable additions. The king cliargot him in 1753 with drawing the plan of the county of Bitclue, in Lorraine, all the elements of which he determined gcogruphically. IJe occupied him- 
self greatly with the two comets of 1760 ; and the fruit of bis labour was his Elementary treatise on the theory of those comets, enriched with observations on the zodiacal light, and on the aurora borealis. Soun after he went to Tobolsk, in Siberia, to observe the transit of Venus over the sun; a journey which greatly impaired his health. After two years absence be returned to France in 1762 , where he occupied himself for some time in arranging the great number of observations he had then made. Mr. Chappe also went to observe the next transit of Venus, viz, that of 1769 , at California, on the west side of North America, where he died of an cpidemic disease, the 1st of August 1769. He had been nanied adjunct astronomer to the Academy the 17 th of January 1759.

The published works of M. Chappe, are,

1. The Astronomical Tables of Dr. Halley; with observations and additions: in 8 vo, 1754 .

2. Voyage to California to observe the transit of Venus over the sun, June 3, 1769: in 4to, 1772.

3. He had a cousiderable number of papers inserted in the Memoirs of the Academy, for the years 1760,1761 , $1764,1765,1766,1767$, and 1768 ; chiefly relating to astronomical matters.

CHAPTREL, the same with Impost.

CHARACTERISTIC, of a Logarithm, the same as Index, or Exponent. This term was first used by Briggs in the 4 th section of his Arithmetica Logarithmica, where he treats particularly of it; meaning by it, the integral or first part of a logarithm towards the left hand, which expresses 1 less than the integer places or figures in the number answering to that logarithm, or how far the first figure of this number is removed from the place of units; namely, that 0 is the charactcristic of all numbers from 1 to 10 ; and 1 the characteristic of all those from 10 to 100 ; and 2 the characteristic of all those from 100 to 1000 ; and so on

CHARACTERS, are certain marks used by astronomers, mathematicians, \&c, to denote certain things, whether for the sake of brevity, or perspicuity, in their operations.

\section{Astronomical Characters.}

\section{Planets, \&c.}

The twelve Signs or Constellations of the adiac.

D The Moon

$\oplus$ The Earth

$\checkmark$ Mercury

\% Venus

\& Mars

2f Jupiter

h Saturn

H Herschel, or the Georgian Planet

8 Ascending Node

8 Descending Node $r$ Aries, the Ram

$\checkmark$ Taurus, the Bull

II Gemini, the Twins

Go Cancer, the $\mathrm{Crab}_{\mathrm{r}}$

$\Omega$ Leo, the Lion

m Virgo, the Maid

$\bumpeq$ Libra, the Balance

$m$ Scorpio, the Scorpion

I Sagittary, the Archer

bo Capricorn, the Goat

m Aquarius, the Water-bearer

\& Coljunction

8 Opposition

* Sextile

o Quartile

$\Delta$ T'rine

The Aspects, Time, Motion, \&c.

- Degrees

- Minutes or Primes

"Seconds, \&c.

A, M. Ante merid. or m. morn.

P. M. Post merid, or a. aftern.

h, m, s, Hours, min. scc.

2. Matuematical \& c. Characters.

Numerical Churacters used by different Nations.

The most common numerical characters, are those Vou. I. called Arabic or Indian, viz, 1, 2, 3, 4, 5, 6, 7, 8, 9, with 0 for cipher or nothing.

The Roman numeral characters are seven, viz, I one, $v$ five, $x$ ten, $I$ fifty, $c$ a hundred, $\mathrm{D}$ or 10 five hundred, $M$ or $D A$ or $\mathrm{C} 10$ a thousand. Other combinations are as in the following synopsis of the Roman Notation

$$
\begin{aligned}
1 & =\mathrm{I} \\
2 & =\mathrm{II}: \text { As often as any character is repeated, } \\
3 & =\mathrm{II} \text { so many times its value is repeated. } \\
4 & =\mathrm{III} \text {. or } \mathrm{I} \mathrm{v}: \text { A less character before a greater } \\
5 & =\mathrm{V} \text { diminishes its value. } \\
6 & =\mathrm{VI}: \text { A less character after a greater increases } \\
7 & =\mathrm{V} 1 \mathrm{i} \text { its value. } \\
8 & =\mathrm{VIII} \\
9 & =\mathrm{IX} \\
10 & =\mathrm{X} \\
50 & =\mathrm{L} \\
100 & =\mathrm{C} \\
500 & =\mathrm{D} \text { or } 10: \text { For every } 0 \text { added, this becomes }
\end{aligned}
$$
10 times as many.

$1000=\mathrm{m}$ or $\mathrm{cr} \mathrm{0}:$ For every $\mathrm{c}$ and $\mathrm{o}$, set one at eachi $2000=\mathrm{NM} \quad$ end, it becomes 10 times as much.

$5000=100$ or $\overrightarrow{\mathrm{v}}:$ A line over any number increases

$6000=\overline{v I}$ it 1000 fold.

$10000=\bar{x}$ or cciso

$50000=1300$

$60000=\overline{\mathrm{Lx}}$ or $\operatorname{ccc} 1000$

$100000=\overline{\mathrm{c}}$ or $\operatorname{ccc} 1000$

$1000000=\bar{M}$ or ccccisjo

$2000000=\overrightarrow{M M}, \& c$.

Greek Numerals.

The Greeks had three ways of expressing numbers. First, For every single letter, according to its place in the alphabet, was denoted a number from $\alpha 1$ to $\omega 24$; in which manner the books of Homer's Jlias are distinguished. Secondly, Another way was by dividing the alphabet into (1st) 8 units, a 1, $\beta 2$, \&c; (2nd) 8 tens, $110, \times 20$, $\& c$; $(3 d) 8$ hundreds, $\rho 100, \sigma 200, \& c$ : and thousands, they expressed by, a point or accent under a letter, as $\alpha$ $1000, \beta 2000$, \&c. Thirdly, By six capital letters, thus,

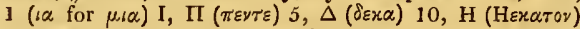
$100, \mathrm{X}(\chi i \lambda, \alpha) 1000, \mathrm{M}(\mu v \varepsilon i \alpha) 10000$ : and when the letter $I I$ inclosed any of these, except $I$, it showed that the inclosed letter was 5 times its own valuc, as $\overline{\Delta \mid} 50, \overline{\mid \mathrm{H}} 500$, $|\overline{\mathrm{X}}| 5000, \overline{\mathrm{M}} \mid 50000$.

Hebrew Numerals.

The Hebrew alphabet was divided into nine units, as $\ll 1,22, \& c$; nine tens, as 110,220 ; nine hundreds, as p 100, 7 200, \&ce, 7 500, מ 600, †700, ๆ 800 , צ 900. Thousands were sometimes expressed by the units prefixed to hundreds, as אדר 1534, \&c. and even to tens, as vi 1070 , \&c, But more commonly thousands were ex-

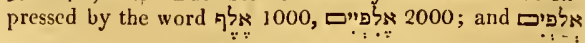
with the other numerals prefixed to signify the number of thousands, as שישל 3000 , \& c.

\section{Characters used in Arithmetic and Algebra.}

The first letters of the alphabet, $a, b, c, \delta c$, denote given quantities; and the last letters, $z, y, x, \& c$, represent such as are unknown or sought. Stifelius first used the capitals $\mathrm{A}, \mathrm{B}, \mathrm{c}, \& \mathrm{c}$, for the unk nown or required quantitics After that, Vieta employed the capital vowels $A$, 2 IR 
$E, x, o, U, Y$, for the unknown or required yuantities, and the consonants B, C, D, \& $c$, for known or gisen numbers. llarriot changed Vieta's capitals into the small letters, viz, $a, e, i, b, u$, fir unknown, and $b, c, d$, sc, for known quantities. And Descartes changed Harrioi's vowels for the latter letters $z, y, x, \& \mathrm{c}$, and the consonants for the leading letters, $a, b, c, d$, \&c.

Newton denotes the several orders of fuxions of variable quantities by as many points over the latter letters; as, of $x, y, z ; \quad$ as $\dot{x}, \dot{y}, \dot{z}$ are the 1 st fuxions,

$\ddot{x}, \ddot{y}, \ddot{z}$ are the $2 d$ fluxions,

$\dot{\dot{x}}, \dot{\hat{y}}, \dot{\ddot{z}}$ are the $3 \mathrm{~d}$ fluxions.

And Leibnitz denotes the differentials of the same quantitics by prefixing $d$ to cach of them, thus $d x, d y, d z$.

Powers of quantities are denoted by placing the index or exponent after them, towards the upper part; thus $a^{2}$ is the 2d power, $a^{3}$ the third power, and $a^{\mathrm{n}}$ the $n$ power of a. Diophantus marked the powers by their initials, thus $\delta^{v}, x^{v}, \delta \delta^{v}, \delta x^{v}, x x^{v}, \& c$, for dynamis, cubus, dynamodynamis, \&c, or the 2d, 3d, 4th, \&c, powers; and the same method has been practised by several of the early writers, since the introduction of algebra into Europe: but the first of them, as Paciolus, Cardan, \&ce, used no mark for powers, but the words themselves. Stifel, and uthers about his time, used the initials or abbreviations $2,3, \pi, 33$, $\& c$, of res or coss, zenzus, cubus, zenzizenzus, \&c, barbarous corruptions of the Italian cosa, census, cubo, censicensus, \& c. But he used also numeral exponents, both positive and negative, to the general characters or roots $A$, B, C, \& c. Bombelli made use of a half circle thus $\smile$ as a general character for the unknown or quantity required to be found in any question, and the several powers of it he denoted by figures set above it; thus $\mathbf{x}^{2}, 2,3$, are the 1 st, $2 \mathrm{~d}, 3 \mathrm{~d}$ powers of $\smile$; which powers he called dignities. Stevinus used a whole circle for the same unknown quantity, with the numeral index within it, and that both integral and fractional; thus (10), (1), (2); ; (3), are the 0,1 , 2,3 powers of the general quantity $O$; also (2), (3), (4), he uses as the square root, cube root, 4 th root of the same; and $(2)$, the cule root of the square,

and $(\theta)$, the square root of the cube, and so on.

And these fractional exponents were adopted and farther practised by his commentator Albert Girard. So that Stevinus ought to be esteemed the first person who rendered general the notation of all powers and roots in the same way, the former by integral, and the latter by fractionil exponents. Harriot denoted his powers by a repetition of the letters; thus $a$, aa, aan, \&c. And Descartes, instead of this, set the numeral index at the upper part of the letters, as at present, thus $a, a^{2}, a^{3}, \& c$; though, I am informed, by such as have seen Harriot's postlumous paper's, that he also there makes use of exponents.

The character $\checkmark$ is the sign of radicality, or of a root, bcing derived from the initial $\mathrm{n}$ or $r$, which was used at first by Paciolus, Cardan, \&c. This character $\sqrt{ }, 1$ find first used by Stifel, in 1544, and by Robert Recorde in 1557. The bare character $\sqrt{ }$ denotes the square root only; but at first they prefixed the initial of the name after it, to denoto the several roots: as $\sqrt{ } q$ the quadrate or square root, $\sqrt{ } c$ the cube root, \&c. But the numeral indices of the rout were prefixed by AJhert Girard, exactly the same as they are use'd at present, viz, $\sqrt[2]{ }, \sqrt[3]{ }, \sqrt[4]{ }$, the $2 \mathrm{~d}, 3 \mathrm{~d}$, or sth root.

The cbaracter + represents addition, and a positive quantity. At first the word itself was used, plus, piu, or the initial $p$. by Paciolus, Cardan, Tartalca, \&c. And the character + for addition occurs in Stitclius.

- is the character of subtraction, and denotes also a negative quantity; which also first occurs in the same author Stifelius. Hefore that, the word minus, mene, or the initial $m$. was uscl. Other characters have also been sometimes uscd by other authors, for aldition and subtraction; but they are now obsolete.

$x$ is the character of inultiplication, and was introduced by Oughired.

$\div$ representing division, was introduced by Dr. Pull. Division is also denoted like a fraction, thus $\frac{\Omega}{b}$ or $\frac{6}{3}=2$.

$=$ denotes equality, and was used by Rubert Recorde. Descartes employs $x$ for the same purpose.

The character : : for proportionality, or equality of ratios, was introduced by Oughtred; as was afso the mark $\because$ - continued proportion.

$>$ for greater, and < for less, were used by Harriot. And $\neg$ and $\neg$ were used by Oughtred for the same purposes.

Dr. Pell used $Q$ for involution, and $l w$ for evolution.

in denotes a general difference between any two quantities, and was used by Dr. Wallis.

The Parenthesis ( ), as a vinculum, was invented by Albert Girard, and used in such expressions as these, $\sqrt[3]{(72}+\sqrt{ } 5120)$, and $\mathrm{B}(\mathrm{B} q+\mathrm{c} q)$, both for universal roots, and multiplication, \&c.

The straiglt-lined vinculum, , was used by Vieta for the same purpose; thus $\overline{\mathrm{A}-\mathrm{B}}$ in $\overline{\mathrm{B}+\mathrm{C}}$.
ㅁ A Squarc
$\triangle$ A Triangle
$\square$ A Rectangle
$O$ or $\odot$ A Circle

CHARGE, in Electricity, in a strict sense, imports the accumulation of the electric matter on one surface of an electric, as the Leyden phial, a pane of glass, \&r, while an equal quantity passes off from the opposite surface. Or, more generally, clectrics are sairl to be charged, when the cquilibrium of the electric matter on the opposite surface is destroyed, by communicating one kind of electricity on one side, and the contrary kind to the opposite side: nor can the equilibrium be restored till a communication be made by means of conducting substances between the two opposite surfaces: and when this is donc, the elcetric is said to be discharged. The charge properly refers to one side, in contradistinction from the other; since the whole quantity in the electric is the same before and after the process of charging; and the operation canuot succed, unless what is gained on one side is lost by the other, by means of conductors applied to it, and communicating either with the earth, or with a sulficicut number of nonelectrics. To facilitate the communication of electricity to an electric plate, \&c, the opposite surfaces are coated with some conducting substance, usually with tin-foil, within some distanec of the edge; in consequence of which the electricity communicated to one part of the coating is readily diflused through all parts of the surface of the electric in contact with it; and a discharge is eatsily made by forming a communication with any conductor from one coating to the other. If the opposite coatings approach 
too near each other, the electric matter forces a passage from one surface to the other before the charge is complete. And some kinds of glass have the property of conducting the electricity over the surface, so that they are altugether unfit for the operation of charging and discharging. If indeed the charge be too high, and the glass plate or phial too thin, the attraction between the two opposite electricities forcesa passage through theglass, making a spontaneous discharge, and the glass becomes unfit for farther use. See Conductors, Elecrutcs, Leyden Pinia L, \&c.

CHARGE, in Gunnery, the load of a piece of ordnance, or the quantity of powder and ball, or shot, with which it is prepared for execution.

The charge of powder, for proving guns, is equal to the weight of the ball; but for service, it is $\frac{1}{2}$ or $\frac{1}{3}$ the weight of the ball, or still less; and indeed in most cases of service, the quantity of powder used is too great for the intended exccution. In the British navy, the allowance for 32pounders is but $\frac{7}{16}$ of the weight of the ball. For it is probable that, if the powder in all ship guns was reduced to $\frac{x}{3}$ the weight of the ball, or even less, it would be a considerable advantage, not only by saving ammunition, but by kecping the guns cooler and quieter, and at the same time more effectually injuring the vesscls of the enemy. With the present allowance of powder, the guns are heated, and their tackle and furniture strained; and all this only to render the ball less efficacious; for a ball which can but just pass through a piece of timber, and in the passage loses almost all its motion, is found to rend and fracture it much more, than when it passes through with considerable velocity. See Rohins's Tracts, vol.1, pa. 2.90, 2.91; and my own Tracts, vols. 2 and 3.

Again, the same author observes, that the charge is not to be determined by the greatest velocity that may be produced; but that it should be such a quantity of powder as will produce the least velocity necessary for the purpose in view; and if the windage be moderate, no field-picce should ever be loaded with more than $\frac{1}{6}$, or at the utmost $\frac{x}{5}$ of the weight of its ball in powder; nor should the charge of any battering piece exceed $\frac{1}{3}$ of the weight of its bullet. Ib. pa. 266.

Different charges of powder, with the same weight of ball, produce difierent velocities in the ball, which are in the subduplicate ratio of the weights of powder; and when the weight of powder is the same, and the ball varied, the velocity produced is in the reciprocal subduplicate ratio of the weight of the ball: which is agrecable both to theory and practice. See my paper on Gunpowder in the Philos. Trans. 1778 , pa. 50 ; and my Tracts, vols. 2 and 3.

But this is on a supposition that the gun is of an indefinite length; whereas, on account of the limited length of guns, there is some variation from this law in practice, as well as in theory; in consequenee of which it appears that the velucity of the ball increases with the charge only to a certain point, which is peculiar to each gun, where the velocity is the greatest; and that by farther increasing the charge, the velocity gradually liminishes, till the bore is quite full of powder. By an easy fluxionary process it is found that, calling the length of the bore of the gun $b$, the length of the charge prisducing the greatest velocity, ought to be $\frac{b}{2 \cdot 718281828}$, or about $\frac{3}{8}$ of the length of the bore; where 2718281828 is the number whose hyp. lug. is 1. But, for scveral reasons, in practice the length of the charge producing the greatest velocity, falls short of that above mentioned, and the nore so as the gun is longer. From many experiments I have found the length of the charge producing the greatest velocity, in guns of various lengths of bore, from 15 to 40 calibers, as follows:

\begin{tabular}{|c|c|c|}
\hline $\begin{array}{l}\text { Length of Bore } \\
\text { in Calibers. }\end{array}$ & & $\begin{array}{l}\text { Length of Charge } \\
\text { fur greatest Veluc. }\end{array}$ \\
\hline 15 & - & $-\quad 3$ \\
\hline 20 & - & $\frac{3}{r^{2}}$ \\
\hline 30 & - & $\begin{array}{l}12 \\
\frac{3}{16}\end{array}$ \\
\hline 40 & - & $\frac{3}{20}$. \\
\hline
\end{tabular}

CHARLES'S WAIN, a name by which some of the astronomical writers, in our own language, have called Ursa Major, or the great bear; though some writers say the lesser bear. Indeed both of the two bears have been called waggons or wains, and by the Latins, who have followed the Arabians, two biers, Feretrum majus et minus.

CHART, or SEA-CY A RT, a hydrograpbical or sea map, for the use of navigators; being a projection of some part of the sea in plano, showing the sea coasts, rocks, sands, bearings, \&cc. Fournier ascribes the invention of sea-charts to Henry son of John king of Portugal. These charts are of various kinds, the Plain chart, Mercator's or IVright's chart, the Globular chart, \&c.

In the construction of cliarts, great care should be taken that the several parts of them preserve their position to one another, in the same order as on the earth; and it is probable that the finding out of proper methods to do this, gave rise to the various modes of projection.

There are many ways of constructing maps and charts; but they depend chiefly on two principles. First, by considering the earth as a large extended flat surface; and the charts made on this supposition are usually called Plain Charts. Sccondly, by considering the earth as a sphere; and the charts constructed on this principle are sometimes called Globular Charts, or Mercator's Charts, or Reduced Charts, or Projected Charts.

Plain Charts lave the meridians, as well as the parallels of latitude, drawn parallel to each other, and the degrees of longitude and latitude every where equal to those at the equator. And therefore such charts must be deficient in several respects. For, 1 st, Since in reality all the meridians meet in the pole's, it is absurd to represent them, especially in large charts, by parallel right lines. $2 \mathrm{dly}$, As plain charts show the degrees of the several parallels as equal to those of the equator, therefore the distance of places lying east and west, must be represented much larger than they really are. And 3 dly, In a plain chart, while the same rhumb is kept, the ressel appears to sail on a great circle, which is not the case. Y et plain charts made for a small extent, as a few degrees in length and breadth, may be tolerably exact, especially for any part within the turrid zone; and even a plain chart made for the whole of this zone will differ but little from the truth.

Mercator's Chart, like the plain charts, has the meridians represented by parallel right lines, and the degrees of the parallels or longitude, every where equal to those at the equator; so that they are increased more and more, above their natural size, as they approach towards the pole; but then the degrees of the meridians, or of latitude, are increased in the same proportion at the same part; so that the same proportion is preserved between them as on the globe itself. 'This chart has its name from that of the author, Girard Mercator, who first proposed it for use in the year 1556, and made the first charts of this kind; though 
they were not altogether on true or exact principles, nor does it appear that he perfectly understood them. Neither Indeed was the thought originally his own, viz, of lengthening the degrees of the meridian in some proportion, for this was hinted by Ptolemy near two thousand years'ago. It was not perfected however till Mr. Wright first demonstrated the same about the year 15.90 , and showed a ready way of constructing this chart, by enlarging the meridian line by the continual addition of the secants. See his Correction of Errors in Navigation, published in 1599.

Globular Chart, is a projection so called from the conformity it bears to the globe itself; and was proposed by Messrs. Senex, Wilson, and Harris. This is a meridional projection, in which the parallels are equidistant circles, having the pole for their common centre, and the meridians curvilinear and inclined, so as all to meet in the pole, or common centre of the parallels. By which means the several parts of the earth have their proper proportion of magnitude, distance, and situation, nearly the same as on the globe itself; which renders it a good method for geographical maps.

Selenographic Charts, are particular descriptions of the appearances, spots, and maculæ of the moon.

Topographic Charts, are draughts of some small parts only of the earth, or of some particular place, without regard to its relative situation, as London, York, \&c.

For the Construction of Charts, see GEograPHY, Maps, Projection, \&c.

CHASE, of a Gun, is its bore or cylinder.

CHAULNES (ThE DUKE DE), a peer of France, remarkable as an astronomer and mathematician, was born at Paris, Dec. 30, 1714.-At an early period he discovered a singular taste and genius for the sciences; and in the tumults of armies and camps, he cultivated mathematics, astronomy, mechanics, \&c. He was named honorary academician the 27 th of February 1743, and few members were more punctual in attending the meetings of that body; where he often brought different constructions and corrections of instruments of astronomy, of dioptrics, and achromatic telescopes. These researches were followed with a new parallactic machine, more solicl and convenient than those that were in use; together with many reflections on the manuer of applying the micrometer to those telescopes, and of measuring exactly the value of the parts of that instrument. The Duke of Chaulnes proposed many other works of the same kind, whon death surprised him the $23 \mathrm{~d}$ Sept. 1769 .

He had several papers published in the volumes of Memoirs of the Academy of Sciences, as follow :

1. Observations on some Experiments in the 4th part of the $2 \mathrm{~d}$ book of Newton's Optics : an. 1755.-2. Observations on the Platform for dividing mathematical instruments : $1765 .-3$. Determination of the distance of Arcturus from the sun's limb, at the summer solstice: 1765 . -4 . On some means of perfecting astronomical instruments : $1765 .-5$. On some experiments relative to dioptrics : $1767 .-6$. The art of dividling inathematical instruments : $1768-7$. Observations on the Transit of Venus, June $3,1769 .-8$. New method of dividing mathematical abd astronomical instruments.

CHAUSE TIRAPPES, or Caltrops, or Crowsfeet, are iron instruments of spikes about 4 inches long, made like a star, in such a manner that whichever way they fall, one point stunds always upwards, like a nail. They arc usually thrown and scattered into moats and breaches, to gall the horses' feet, and stop the hasty approaches of the enemy.

CHAZELLES (Join-Mattinew), a French mathematician and engineer, was born at Lyons in 1657 , and educated there in the college of Jesuits, from whence he removed to Paris in 1675 . He first became acquainter with $\mathrm{Du}$ Hamel, secretary to the Academy of Sciences, and through him with Cassini, who employed him with himsclf at the Observatory, where Chazelles greatly improved himself, and also assisted Cassini in the measurement of the southern part of the meridian of France. Having, in 1684, instructed the Duke of Montemar in the mathematical sciences, this nobleman procured him the appointment of hydrography-professor to the galleys of Marseilles. In discharging the duties of this department, he made numerous geometrical and astronomical observations, from which he drew a new map of the coast of Provence. - He also performed many otber services in that department, and as an engincer, along with the armies and nawal expeditions. To make observations in geograply and astronomy, he undertook also a voyage to the Levant, and among other things he measured the pyramids of Egypt, and found the four sides of the largest of them exactly to face the four cardinal points of the compass. He made a report of his voyage, on his return, to the Academy of Sciences, upon which he was named a nember of their body in 1695, and had many papers inserted in the vom lumes of their Memoirs, from 1693 to 1708 . Chazelles died at Marseilles the 16 th of January 1710 .

CHEMIN des Ronds, in Fortification, the way of the rounds, or a space between the rampart and the low para. pet under it, for the rounds to go about it.

CHEMISE, a wall that lines a bastion, or ditch, or the like, for its greater support and strength.

CHEVAL de Frise, pl. Cheraux de Frise, or Friseland Horse, so called because it was first used in that country. It consists of a joist or piece of timber, about a foot in diameter, and 10 or 12 long, pierced and transversed with a great number of wooden spikes of 5 or 6 feet in length, and armed or pointed with iron. It is also called turmpike, or tourniquet, and is chiefly used to stop a breach, defend a passage, or make a retrenchment to stop the cavalry.

CHEVRET'L', in Artillery, an engine to raise guns or mortars into their carriage. It is formed of two pieces of wood of about 4 feet long, standing upright upon a third, which is square. The uprights are about a foot asunder, and pierced with holes exactly opposite to one another, to receive a bolt of iron, which is put in either higher or lower at pleasure, to serve as a support to a handspike by which the gun is raised up.

CHEYNE (GEORGE), a British physician and muthematician, was born in Scutland, 1671, and clucated at Edinburgh under Dr. Pitcairn. He passed his youth in close study and great abstemiousness; but on coming to London, at 30 years of age, he suddenly changed his whole manner of living; which had such an eflect on lis constitution, that his body grew to an immense size, weighing it is said +48 pounds. From this load of oppression however he was afterwards in a great measure rclieved, by means of a milk and vegetable diet, which reduced his weight to about one-third of what it had been, and restored him to a goot state of heulth; by which his life was prolonged to the $72 \mathrm{~d}$ year of his age.

Dr. Cheyne was author of various medical and other tracts, and of a treatise on the Inverse method of Flux- 
ions, under the title of Fluxionum Methodus Inversa; sive quantitatum fluentium leges generaliores: in 4to, 1703. Upon this book Demoivre wrote some animadversions in an 8 vo vol. 1704; which were replied to by Cheyne in 1705 . Besides this work, he published Philosophical Principles of Religion, natural and revealed. Also the English Malady, or a Treatise on Nervous Diseases.

CHILIAD, an assemblage of several things ranged by thousands. It was particularly applied to tables of logarithms, because they were at first divided into thousands. Thus, in the year $1624, \mathrm{Mr}$. Briggs published a table of logarithms for 20 chiliads of absolute numbers; afterward, he published 10 chiliads more; and lastly, one more; making in all 31 chiliads.

CHILIAEDRON, a solid figure having a thousand faces.

CHILIAGON, a regular plane figure of a thousand sides and angles.

CHORD, a right line connecting the two extremes of an arch; so called from its resemblance to the chord or string of a bow; as $A B$, which is common to the two parts or arches $A D B, A E B$ that make up the whole circle. The chords have several properties:

1. The chord is bisected by a perpendicular cF drawn to it from the centre.-2. Chords of equal arcs, in the same or equal circles,

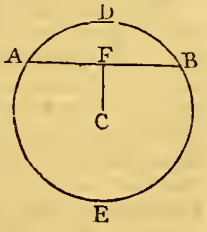
are equal to each other.-3. Unequal chords have to one another a less ratio than that of their arcs. -4 . The chord of an alc, is a mean proportional between the diameter and the versed sine of that arc.

Scale or Line of Chords. See Plane Scale.

CHORD, or CORD, in Music, denotes the string or line by whose vibrations the sensation of sound is excited; and by whose divisions the several degrees of tune are determined. Chords of gold wire in harpsichords, yield a sound almost twice as strong as those of brass; while chords of steel impart a feebler sound than those of brass.

To divide a Chord $\mathrm{AB}$ in the most simple manner, so as to exhibit all the original concords.

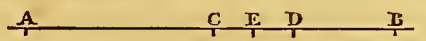

Divide the given line into two equal parts at $\mathrm{c}$; then subdivide the part $C B$ into two equal parts at $D$, and again the part $\mathrm{CD}$ into two equal parts at E. Here $\mathrm{AC}$ to $\mathrm{AB}$ is an octave; $A C$ to $A D$ a fifth; $A D$ to $A B$ a fourth; $A C$ to $A E$ a greater third, and $A E$ to $A D$ a less third; $A$ E to $\mathbf{E B}$ a greater sixth, and AE to $A B$ a less sixth. Malcolm's Treatise of Music, ch. 6. sec. 3. See Movochord.

To find the number of Vibrations made by a Musical Chord or String in a given time; having given its weight, length, and tension. Let $l$ be the length of the chord in feet, 1 its weight, or rather a small weight fixed to the middle and equal to that of the whole chord, and $w$ the tension, or weight by which the chord is stretched. Then shall the time of one vibration be expressed by $\frac{\pi}{7} \sqrt{\frac{l}{32 \frac{1}{6} w}}$, and consequently $\frac{7}{\pi} \sqrt{ } \frac{32 \frac{1}{l} w}{l}$ will be the number of vibrations per sccond.

For example, suppose $v=28800$, or the tension equal to 28800 times the weight of the chord, and the length of it 3 feet; then the last theorem gives 35 t nearly for the number of vibrations made in each second of time.-But if $w$ were 14,400 , there would be made but 250 vibrations per second; and if $w$ were only $28 s$, there would be no more than $35 \frac{16}{4}$ vibrations per second. See my Tracts, vol. 3 , prob. $21 \& 22$, pa. 246 .

On the supposition that chords or strings are perfectly elastic, the following proportions have been deduced mathematically. 1. If the length of the chord and the tending force be given, the inflecting force will be nearly as the space through which the chord is bent. 2. If the length of the string and the space through which it is bent be given, the inflecting force is as the tension. 3 . If the tending force, and the space through which the chord is bent be given, the infiecting force is inversely as the length of the chord. 4. If the diameter of the chord, and the tension be given, the times of vibration are as the length of the chord : on which principle the monochord is founded. 5 . If the tension and length of the chord be given, the time of vibration is as the diameter of the chord. 6 . If the diameter, and length of the chord be given, the time of vibration is inversely as the square root of the tension.

CHORD, in Music, is also used for the union of two or more sounds uttered at the same time, and forming together a complete harmony.

Chords are divided into perfect and imperfect. In the perfect chord we have the third, fifth, and octave of the fundamental sound: they are also subdivided into major and minor, according as the thirds which enter into their composition are flat or sharp. Imperfect chords are those in which the sixth, instcad of the fifth, prevails; and in general all those whose lowest are not their fundamental sounds.

Chords are again divided into consonances and dissonances. The consonances are the perfect chord, and its derivatives. Every other chord is a dissonance. A table of both, accorling to the system of M. Rameau, may be scen in Rousseau's Murical Dictionary, vol. 1, pa. 27.

CHROMATIC, a species of music which proceeds by semitones and minor thirds. The word is derived from the Greek $\chi f w \mu \alpha$, which signifies colour, and perhaps the shade or intermediate shades of colour, which mingle and connect colours, like as the small intervals in this scale. easily slide or run into each other.

Boethius and Zarlin ascribe' the invention of the chromatic genus to 'Timotheus, a Milesian, ir the time of Alexander the Great. The Spartans banished it their city on account of its softness. The character of this genus, according to Aristides Quintillianus, was swcetness and pathos.

CHROMATICS, is that part of optics which explains the several properties of the colours of light, and of natural bodies.

Before the time of Newton, the notions concerning colour were very vague and extravagant. The Pythagoreans. called colour the superficies of bodies: Plato said that it was a flame issuing from them: According to Zeno, it is the first configuration of matter: And Aristotle suid it was. that which made bodies actually transparent. Descartes. accounted colour a modification of light, and he imagined that the difference of colour proceeds from the prevalence of the direct or rotatory motion of the particles of light. Grimaldi, Dechales, and many others, conjectured that the differences of colour depended on the quick or slow vibrations of a certain elastic medium pervading the universe. Rohault conceived, that the different colours weremade by the rays of light entering the eye at different angles with respect to the optic axis. And Dr. Hooke ima- 
gined that colour is caused by the sensation of the oblique or uncren pulse of light; which being capable of but two varieties, he concluded there could be nu more than two primary colours.

Sir I. Nevton, in the ycar 1666, brgan to investigate this subject; when finding that the coloured image of the sun, formed by a glass prisim, was of an oblong, and not of a circular form, as, according to the laws of equal refraction, it wught to be, he conjectured, that light is not bomogeneal; hut that it consists of rays of different colours, and endued with divers degrecs of retrangibility. And, from a further prosecution of his experiments, he concluded that the different colours of bodies arise from their reflecting this or that kind of rays most copiously. This method of accounting for the different colours of bodies soon became generally adopted, and still continues to be the most prevailing opinion. It is hence agreed that the light of the sun, which to us appears white and perfectly homngeneal, is composed of no less than seven different colours, viz. red, orange, yellow, green, blue, purple, and violet or indign: that a body which appears of a red colour, has the property of reflecting the red rays more profusely than the rest; and so of the other colours, the orange, yellow, green, \&c: also that a body which appears black, instead of reflecting, absorbs all or the most part of the rays that fall upon it; while, on the contrary, a body which appears white, reflects the greatest part of all the rays indiscriminately, without separating them one from another.

The foundation of a rational theory of colours being thus laid, the next inquiry was, by what peculiar mechanism, in the structure of each particular body, it was rendered capable of reflecting one kind of rays more than another ; and this is attributed, by Sir I. Newton, to the density of these bodies. Dr. Hooke had remarked, that thin transparent substances, particularly soap-ivater blown into bubbles, exhibited various colours, according to their thinness; and yet, when they have a considerable degrce of thickness, they appear colourless. And Sir Isaac himsclf had also obscrved, on compressing two prisms hard togcther, in order to make their sides (which happened to be rather convex) to touch one another, that in the place of contact they were both perfectly transparent, as if they had been but one continued piece of glass : but round the point of cuntact, where the glasses were a little separated from each other, rings of different colours appeared. And when he afterwards, further to clucidate this matter, employed two convex glasses of telescopes, pressing their convex sides upon each other, he observed several series of circles or rings of such colours, diflerent, and of various intensities, according to their distance from the common central pellucid point of contact.

As the colours were thus found to vary according to the different distances between the glass plates, Sir Isaac conceived that they proceeded from the different thickness of the plate of air intercepted between the glasses; this plate of air being, by the mere circumstance of thinness or thickness, disposed to reflect or transmit the rays of this or that particular colour. Hence he concluded, that the colours of all natural bodies depend on their density, or the inagnitude of their component particles: and hence also he constructed a table, in which the thickness of a plate necessary to reflect any particular colour, was expressed in millionth parts of an inch.

From a great varicty of such experiments, and observa- tions tpon them, our author deduced his theory of colours. And from which it appears that crery substance in nature is transparent, provided it be made sufficiently thin; as gold, the densest sulestance we know of, when reduced into thin leaves, transmits a blueish-green light through it. If we suppose any body therefore, as anld for instance, to be divided into a vast number of plates, so thin as $t$ ' $)$ be almost perfectly transparent; it is evident that all, or the greatest part of the rays, will pass through the upper plates, and when they lose their force will be reflected from the under unes. They will then have the same number of plates to pass through which they had before penetrated; and thus, according to the number of those plates through which they are obliged to pass, the object appears of this or that colour, just as the rings of culours appeared different in this experiment of the two plates, according to their distance from one another, or the thickness of the plate of air between them.

This theory of colours has been illustrated and confirmed by various experiments, made by other philosophers. Mr. E. II. Delaval produced similar effects by the intusion of flowers of different colours, and also by the intimate inixture of the various metals with glass, when they are reduçed to very fine parts ; the denser metals imparting to the glass the less refrangible colours, and the lighter ones those colours that are mure easily refrangible. $D r$. Priestley and Mr. Canton also, by laying very thin leaves or slips of the metals upon glass, ivory, wood, or metal, and passing an electrical stroke through them, found that the same effect ivas produced, viz, that the substrated was tinged with different colours, according to the distance from the point of explosion.

However, the Abbé Mazeas and M. du Tour contended, that the colours between the glasses are not to be ascribed to the thin stratum of air, since they cyually produced them by rubbing and pressing together two flat glasses, which cohered so strongly that it required the greatest force to move or side tbem over each other. See Priestley's IIistory of Vision.

Of Newton's 8th Exper. in the 2d Book of Optics.

The event of this experinent, which has been contradicted by repetitions of the sume by other philosophers, having been the occasion of much controversy; and relatiug to a material part of the doctrine of chromatics, it will not be improper here to give an account of what has passed concerning it. Newton found, that when light, by contrary refractions through different mediums, is so corrected, that it cmerges in lines parallel to the incident rilys, it continues ever after to be white: But that if the emergent rays be inclined to the incident ones, the whiteness of the cmerging light will, by degrees, in passing on from the place of energence, become tinged at its edges with colours. And these laws he inferred from experiments made by refracting light witls prisms of glass, placed within a prismatic vessel of water.

By theorems deduced from this experiment lie infers, that the refraction of the rays of every sort, out of any medium into air, are known by having the refraction of the rays of any one sort: and also, that the refraction out of one medium into another is found, whenever we have the refractions out of them both, into any third medium.

Now the same experiment, having been since performed by other persons, sund turning out contrary to what is stated above, has vecasioncel rather free reflections to be thrown on Newton concerning it; but which however have 
been very satisfactorily obviated by Mr. Peter Dollond, in a pramphlet ou this subject; as we shall show below.

In the first place then, M. Klingenstierna, a Swedish philosopher, having in the year 1755 considered the controversy between Euler and Mr. John Dollond, ralative to the reliaction of light, formed a theorem of his own, from geometrical reasoning, by which he was induced to belite that the result of Newtou's experiment could not be as he has represented; except when the angles of the refracting mediums are small. Sce the paper on this matter by Klingenstierna in the pamphlet above cited by Mr. Peter Dollonil.

This paper of Klingenstierna being communicated to Mr. John Dollond by Mr. Mallet, to whom it was sent for that purpose, made Dollond entertain doubts concerning Newton's report. of the result of his experiment, and determined him to have recourse to experiments of his own, which be accordingly made in the year 1757 , as follows.

Having cemented two glass planes tugether by their edges, so as to form a prismatic vessel, when closed at the ends or bases; and the edge being turned downward, he placed it in at glass prism with one of its edges upward, filling up the vacancy with clear water; so that the refraction of the prism was contrived to be contrary to that of the water, in order that a ray of light, transmitted through both these refracting mediums, might be affected by the difference only between the two refractions. As he found the water to refract more or less than the glass prism, he diminished or augmented the angle between the glass plates, till the two contrary refractions becane equal, which he discovered by viewing an object through this double prism. For when it appeared neither rajed nor depressed, he concluded that the refractions were equal, and that the emergent ray's were parallet to the incident ones.

Now, according to the prevailing opinion, be observes, that the object ought to have appeared throngh this double prism in its natural colour; for if the difference of refrangibility had been in all respects equal, in the two equal refractions, they would have rectificd each other. But this experiment fully proved the fallacy of the received opinion, by showing that the divergency of the light by the glass prism, was almost double of that by the water; for the image of the object; though not at all refracted, was yet as much infected witl prismatic colours, as if it had been seen through a glass wedge only, having its angle near 30 degrees.

This experiment is the very same with that of Newton's above-mentioned, notwithstanding the result was so strikingly different. Mr. Dollond plainly saw however, that if the refracting angle of the water-vessel could have admitted of a sufficient increase, the divergency of the coloured rays would have been greatly diminished, or entirely rectified; and that there would bave been a very great refraction without colour, as he had already proruced a great discolouring without refraction: but the inconveniency of so large an angle as that of the prismatic vessel must have been, to bring the light to an equal divergency with that of the glass prism, whose angle was about 60 degrees, rendered it necessary to try sone experiments of the same kind with smaller angles.

Accordingly he procured a wedge of plate-glass, whose angle was only 9 degrees; and, using it under the same circumstances, be increased the angle of the water-wedge, in which it was placed, till. the divergency of the light by the water was equal to that by the glass; that is, till the image of the object, though considerably refracted by the excess of the refraction of the water, appeared nevertheless quite free from any colours proceeding from the different refrangibility of the light.

Many conjectures were made as to the cause of so great a difference in the results of the same cxperiment; but none that gave any great satisfaction; till it was afterwards shown to be probably owing to the nature of the glass then used by Newton. This conjecture is made by Mr. Peter Dollond, son of Juhn, the inventor of the achromatic telescope, in a pamphlet by him published in defence of his lather's invention, against the misrepresentatious of some persons who have unjustly attempted to gire the invention to other philosophers, who themselves never imagined that they had any just claim to it. After a full and satisfactory vindication of his father, $\mathrm{Mr}$. P. Dollond then adds,

"I now come to a more agrecable part of this paper, which is, to endeavour to reconcile the different results of the $S$ th experiment of the $2 \mathrm{~d}$ part of the 1st book of Newton's Optics, as related by himself, and as it was fuund by Dollond, when he tried the same experiment, in the year 1757. Newtun observes, that light, as often as by contrary refractions it is so corrected, that it emergeth in lines parallel to the incident, continues ever after to be white. Now Dollond says, when he tried the same experiment, and made the mean refraction of the water equal to that of the glass prism, so that the light emerged in lines parallel to the incident, he found the divergency of the light by the glass prism to be neitrly double to what it was by the water prism. The light appeared to be so evidently coloured, that it was directly said by some persons who were present, that if Newton had actually made the experiment, be must have perceived it to have been so. Yet who could for a moment loubt the veracity of Newton? or feel indifferent in vindicating if possible the assertion of such a character? Many conjectures were therefore made by different persons. Mr. Murdoch in particular gave a paper to the Royal Society in defence of. Newton; but it was such as very little tended to clear up the matter. Philos. Trans, vol. 53, p. 192.-Some have supposed that Newton made use of water strongly impregnated with saccharum saturni, because he mentions sometimes using such water, to increase the refraction, when he used water prisins instead of glass prisms. Newton's Opt. p. 62.-And others lave thought that be tried the experiment.with so strong a persuasion in his own mind, that the divergency of the colours was always in the same proportion to the mean refraction, in all sorts of refracting mediums, that he did not attend so much to that experiment as he ought to have done, or as he usually did. None of these conjectures having appeared at all satisfactury, I have therefore endeavoured to discover the true cause of the diflerence, and thereby show, how the experimeut may be made to agree with Newton's description of it, and to get rid of those doubts, which have listherto remained to be cleared up.

"It is wcll known, that in Newton's time the Englisl, were not the most famous for making optical instruments : telescopes, opera-glasses, \&c, were imported from Italy in great numbers, and particularly from Venice; where they manufactured a kind of glass which was much moro proper for optical purposes than any made in England at 
that time. The glass made at Venice was nearly of the same refractive quality as our own crown-glass, but of a much better colour, being sufficiently clear and transparent for the purpose of prisms. It is probable that Newton's prisms were made with this kind of glass; and it appears to be the more so, because he mentions the specific gravity of common glass to be to water as 2.58 to 1 , Newton's Opt. p. 247, which nearly answers to the specific gravity we find the Venetian glass generally to have. Having a very thick plate of this kind of glass, which was presented to me about 25 years ago by the late Professor Allemand, of Leyden, and which he then informed me had been made many years; 1 cut a piece from this plate of glass to form a prism, which I conceived would be similar to those made use of by Newton himself. I have tried the Newtonian experiment with this prism, and find it answers so nearly to what Newton relates, that the difference which remains may very easily be supposed to arise from any little difference which may and does often happen in the same kind of glass made at the same place at different times. Now the glass prism made use of by Dollond to try the same experiment, was made of English flint-glass, the specific gravity of which I have never known to be less than 3.22 . This difference in the densities of the prisms, used by Newton and Dollond, was sufficient to cause all the difference which appeared to the two experimenters in trying the same experiment.

"From this it appears, that Newton was accurate in this experiment as in all others, and that his not having discovered that which was discovered by Dollond so many years afterwards, was owing entirely to accident; for if his prism had been made of glass of a greater or less density, he would certainly have then made the discovery, and refructing telescopes would not have remained so long in their original imperfect state." See Achromatic, and TeleSCOPE.

Mr. Delaval's experiment on the coiours of opaque bodies. Besides the experiments of this gentleman, before-mentioned, on the colours of transparent bodies, he has also published an account of some made upon the permanent colours of opaque substances, the discovery of which must be of the utmost consequence in the arts of colour-making and dyeing.

The changes of colour in permanently coloured bodies, our author observes, are produced by the same laws that take place in transparent colourless substances; and the cxpcriments by which they are investigated consist chiefly of various methods of uniting the colouring particles into larger masses, or dividing them into smaller ones. Sir Isaac Newton made his experiments chiefly on transparent substances; and in the few places where he treats of others, lie acknowledges his want of experiments. He makes the following remark however on those bodies which reflect one kind of light and transmit another, viz, that if these glasses or liquors were so thick and massy that no light could get through them, he questioned whether they would not, like other opatue bodies, appear of one and the same colour in all positious of the eje; though he could not yet affirm it from exprience. Indeed it was the opinion of this great philosopher, that all coloured matter reflects the rays of light, some reflecting the more refrangible rays inost copiously, and others those that are less so; and that this is at once the true and only reason of these colours. lle was likewise of opinion that opaque bodies reflect the light from their anterior surface, by some power of the body evenly diffused over and external thereto. With respect to transparent coloured bodies, he thus expresses himself: "A transparent body which looks of any colour by transmitted light, may also appear of the same colour by reflected light; the light of that colour being reflected by the farther surface of that body, or by the air beyoni it : and then the reflected colour will be diminished, and perhaps cease, by making the body very thick, and pitching $j$ on the back-side, to diminish the reflection of its farther surface, so that the light reflected from the tingeing particles may predominate. In such cases the colour of the reffected light will be apt to vary from that of the light transmitted."

To investigate the truth of these opinions, Mr. Delaval entered upon a course of experinents with transparent coloured liquors and glasses, as well as with opaque and semitransparent bodies. And from these experiments he discovered several remarkable properties of the colouring matter; particularly, that in transparent coloured substances it does not reflect any light; and when, by intercepting the light which was transmitted, it is hindered frum passing through such substances, they do not vary from their former colour to any other, but become entirely black.

This incapacity of the colouring particles of transparent bodies to reflect light, being deduced from very numerous experiments, may therefore be taken as a general law. It will appear the more extensive, if it be considered that, for the most part, the tingeing particles of liquors, or other transparent substances, are extracted from opaque bodies; that these bodies owe their colours to those particles, in the same manner as the transparent substances; and that by the loss of them they are also deprived of their colvurs.

Notwithstanding these and many other experiments, tlie theory of colours is not yet determined with certainty; and it must be acknowledged that very strong objections might be brought against every hypothesis on this subject that has been invented. The discoveries of Newton however are sufficient to justify the following A phorisns.

Aphorism 1. All the colours in nature arise from the rays of liglit.

2. There are seven primary colours, namely red, orange, yellow, green, blue, indigo, and violet.

3. Every ray of light may be scparated into these seven primary colours.

4. The rays of light, in passing through the same medium, have dificrent degrees of refrangibility.

5. The difference in the colours of light arises from its different refrangibility: tbat which is the least refrangible producing red; and that which is the most refrangible, violet.

6. By compounding any two of the primary, as red and yellow, or yellow and blue, the internediate colour, orange or green, maty be produccd.

7. The colours of bodies arise from their dispositions to reflect one sort of rays, and to absorb the others: those that roflect the least refrangible rays appenring red; and those that reflect the most rofrangible, violet.

8. Such bodics as reflect two or more sorts of rays, appear of various colours.

9. The whiteness of bodies arises from their disposition to reflect all the rays of light promiscuously. 
10. The blackness of bodies proceeds from their incapacity to reflect any of the rays of light. - And from their thus absorbing all the rays of light that are thrown upon them, it arises, that black bodies when exposed to the sun, become hot sooner than all others.

of the Diatonic Scale of Colours. - Newton, in the course of his investigations of the properties of light, discovered that the lengths of the spaces occupied in the spectrum by the seven primary colours, exactly correspond to the lengths of chords that sound the seven notes in the diatonic scale of music: which is made evident by the following experiment.

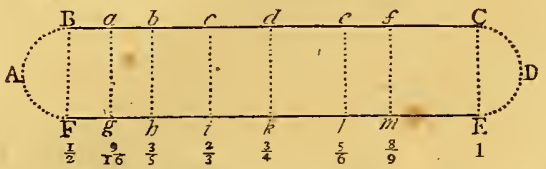

On a paper, in a dark chamber, let a ray of light be largely refracted into the spectrum $\triangle \mathrm{BCDEF}$, marking upon it the precise boundaries of the several colours, as $a, b, c$, $\& c$; and across the spectrum draw the perpendicular lines $a_{5}, b h, \& c$. Then it will be found that the spaces, by which the several colours are bounded, viz, Bagr containing the red, ablg containing the orange, bcih containing the yellow, \&c, will be in exact proportion to the divisions of a musical chord for the notes of an octave; that is, as the intervals of these numbers $1, \frac{8}{9}, \frac{5}{6}, \frac{3}{4}, \frac{2}{3}, \frac{3}{5}, \frac{9}{16}, \frac{x}{2}$.

CHRONOLOGICAL, belonging to chronology.

Cirronological Characters, are characters by which times are distinguished. Of these, some are natural, or astronomical; others are artificial or historical. The natural characters are such as depend on the motions of the stars or luminaries; as eclipses, solstices, equinoxes, the different aspects of planets, \&c. And the artificial characters are those that have been invented and established by men; as the solar cycle, the lunar cycle, \&c. Historical Chronological Characters are those supported by the testimonies of historians, when they fix the dates of certain events to certain periods.

CHRONOLOGY, the art of measuring and distinguishing time; with the doctrine of dates, epochs, eras, \&c. The measurement of time in the most early periods, was by means of the seasons, or the revolutions of the sun and monu. Thesuccession of Iuno's prirstesses at Argos served Hellanicus for the regulation of his narrative; while Ephorus reckoned his matters by generations. Even in the histories of Herodotus and Thucydides, there are no regular dates for the events recorded; nor were there any endeavours to establish a fixed era until the time of Ptolemy Philadelphus, who attempted it by comparing and correcting the dates of the olympiads, the kings of Sparta, and the succession of the priestesses of Juno at Argos. Eratosthenes and A pollodorus digested the events related by them, according to the succession of the olympiads and of the Spartan kings.

The chronology of the Latins is still more uncertain. The records of the Romans were destroyed by the Gauls; and Fabius Pictor, the most ancient of their historians, was obliged to borrow the chief part of his information from the Greks. In other European nations the chronoJogy is still more imperfect, and of a later date: and even in modern times a considerable degree of confusion and inaccuracy lias arisen, from the want of attention in the VOL. I. historians to ascertain the dates and epocbs with precision.

Hence it is evident, how necessary a proper system of chronology must be for the right understanding of history, and also bow difficult it must be to establish such a system. For this purpose, however, several learned men have spent much time, particularly Julius Africanus, Eusebius of Crsarea, George Cyncelle, John of Antioch, Dennis, Petau, Clavius, Calvisius, Scaliger, Vieta, Newton, Usher, Simson, Marsham, Helvicus, Vossius, Strauchius, Blair, Playfair, and Hales.

A system of this kind is founded, 1st, On Astronomical observations, especially of the eclipses of the sun and moon, combined with calculations of the years and eras of different nations. 2d, The testimonies of credible authors. $3 \mathrm{~d}$, Such epochs in history as are so well attested and dctermined, that they have never been controverted. 4 th, Ancient medals, coins, monuments, and inscriptions.

The nost obvious division of time, is derived from the apparent or real revolutions of the luminaries, the sun and moon. Thus, the apparent revolution of the sun, or the real rotation of the earth on her axis causing the sun to appear to rise and set, constitutes the vicissitudes of day and night, which nust be evident to the most barbarous and ignorant nations. The moon, by her revolution about the earth, and her changes, as naturally and obviously forms months; while the great annual course of the sun through the several constellations of the zodiac, points out the larger division of the year.

The Day is divided into hours, minutes, \&c; wbile the month is divided into weeks, and the year into months, baving particular names, and a certain number of days.See a particular account of each of these under the respective words.

Besides the natural divisions of time arising immediately from the revolutions of the heavenly bodies, there are others which are formed from some of the less obvious consequences of these revolutions, and are called Cycles, or circles. The most remarkable of these are, 1, The Solar Cycle, or cycle of the sun, a period or revolution of 28 years, in which time the day's of the months return to the same days of the week, the sun's place to the same signs and degrees of the ecliptic on the same months and days, and the leap-years begin the same course over again with respect to the days of the week on which the days of the months fall. 2, 'The Lunar Cycle, or cycle of the moon, commonly called the Golden Number, is a revolution of 19 ycars; in which time the conjunctions, oppositions, and other aspects of the moon, are on the same days of the months as they were 19 years beforc, and within an bour and a balf of the time of the day.

The Indiction, or Roman Indiction, is a period of 15 years, used unly by the Romans for indicating the times of certain payments made by the subjects to the republic.

The Cycle of Easter, called also the Dionysian Period, is a revolution of 532 years, and is produced by multiplying the solar cycle 28 , by the lunar cycle 19 .

The Julian Period, is a revolution of 7980 years, and is produced from the continual multiplication of the three numbers $28,19,15$, of the three former cycles, viz, the solar, lunar, and incliction.

As there are certain fixed points in the lieavens, from which astronomers begin thcir computations, so there are certain points of time, from which historians begin to reckon; and these points or roots of time are called liras 
or Epochs. The most remarkable of these are, those of the Creation, the Greek Olympiads, the building of Rome, the era of Nabonnassar, the death of Alexander, the birth of Christ, the Arabian Hegira, or flight of Mahomet, and the Persian Jesdegird. All of which, with some others of less note, have their beginnings fixed by chronologers to the years of the Julian period, to the age of the world, and to the years before and after the birth of Christ.

The testimony of authors is the second principal part of historical chronology. Though no man has a right to be considered as infallible, it would however be making a very unfair judgment of mankind, to treat them all as dupes or impostors; and it would be an injury offered to public integrity, to doubt the veracity of authors universally esteemed, and facts that are truly worthy of belief. When the historian is allowed to be completely able to judge of an event, and to bave no intent of decciving by his relation, his testimony cannot be refused.

The Epochs form the $3 \mathrm{~d}$ principal part of chronology; being those fixed points in history that have never been contested, and of which there cannot reasonably be any doubt. Notwithstanding that chronologers fix upon the events which are to serve as epochs, in a manner quite arbitrary; yet this is of little consequence, provided the dates of these epochs agree, and that there is no contradiction in the facts themselves.

Medals, Monuments, and Inscriptions, form the last of the four principal parts of chronology; and this study is but of very modern date, scarcely more than 200 years having elapsed sincc close application has been made to the study of these. To the celebrated Spanheim 'we owe the greatest obligations, for the progress that is made in this method; and it is by the aid of medals that M. Vaillant has composed his judicious history of the kings of Syria, from the time of Alexander the Great to that of Pompey. Nor have they been of less service in elucidating all ancient history, especially that of the Romans; and even sometimes that of the middle ages.

Besides the foregoing general account, there are some few systems of chronology which may deserve'some more particular notice, as follows.

Sacred Chronology. There have been various systems relating to sacred clironology; which is not to be wondered at, as the three chief copies of the Bible give a very different account of the first ages of the world. For while the IIebrew text reckons about 4000 years from the creation to the birth of Christ, and to the flond 1656 years; tbe Samaritan makes the former much longer, though it reckons from the creation to the flood only 1307 years; and the Septuagint removes the creation of the world to 6000 years beforc Christ, and 2250 years betore the flood. NIany attempts have been made to reconcile these differences; though none of them are quite satisfactory. Walton and Vossius give the preference to the account of the Septuagint; while others have defended the Hebrew text. Sec an abstract of the different opinious of learned men on this subject, in Strauchius's Brev. Chron. translated by Sault, p. 166 and 176 .

The Chinese Chronology. No nation has boasted morc of its antiquity than the Chinese : but though they be allowed to trace their origin as far back as the deluge, they have few if any authentic records of their history for so long a period as 500 years before the Christian era. This indecd may be owing to the general destruction of ancient remains by the tyrant Tsin-chi-hoang, in the ycar 213 , or some say 246 , before Christ. From a chronology of the Chinese bistory (for which we are obliged to an illtistrious Tartar who was viceroy of Canton in the year 1724, and of which a Latin translation was published at Rome in 1730), we learn that the most remote epoch of the Chinese chronology does nut surpass the first year of Guel-liewang, or $42+$ years before our rulgar era. And this opinion is confirmed by the practice of two of the most approved listorians of China, who admit nothing into their histories previous to this period.

The Chinese, in their computation, make use of a cycle of 60 years, called kia-tse, from the name given to the first year of it, which serves as the basis of their whole chronology. Every year of this cycle is marked with two letters which distinguish it from the others; and all the times of the emperors, for upwards of 2000 years, have names in listory common to them with the corresponding years of the cycle. Philos. Trans. Abridg. vol. 9, pa. $3+3$.

According to M. Freret, in his Essays, the Chinese date the epocha of $\mathrm{Yao}$, one of their first emperors, about the year 2145, or 2057, before Christ; and they reckon that their first astrononical observations, and the composition of their calendar, preceded Yao 150 years : whence it is inferred that the era of their astronomical observations coincides with that of the Chaldeans. But later authors date the rise and progress of the sciences in China from the grand dynasty of 'Tcheou, abont 1200 years before the Christian era, and show that all historical relations of events prior to the reign of $\mathrm{Yao}$ are fabulous. Mem. de l'Histoire des Sciences, \&c. Clinois. vol. 1, Paris 1776.

The Hindu Chronology. The Hindus employ, in their chronology, certain large periods, called Yoogs or Yergs. These are 4 in number, the last of which, called cali yug, or kalee yug, is only now attended to, and hegan 3101 before the Christian era. It comprehends within. it the following, viz,

The era of Bikramajit, which began 3045 years before Christ, .... Salabân, ..... 78 years after Christ, ..... Bengal, not strictly Hindu, cycle of 60 years : the present began 1807 of Christ.

Their solar-sidereal year contains $365^{\mathrm{d}} 6^{\mathrm{h}} 12^{\mathrm{m}} 30^{\mathrm{s}}$, and their tropical year $365^{\mathrm{d}} 5^{\mathrm{h}} 50^{\mathrm{m}} 35^{\mathrm{s}}$. They divide the zodiac into 28 lutur, and into 12 solar constellations or signs; and their astronomical year commences when the sum arrives at the first point of Aries. The length of their months is determined by the time employed by the sun in passing through each sign, and they are accordingly longer in the apogec, and shorter in the perigce; that which corresponds with the higher apsis being $31^{\mathrm{d}} 14^{\mathrm{h}} 39^{\mathrm{m}}$, and that with the lower $29^{\mathrm{d}} 8^{\mathrm{H}} 21^{\mathrm{m}}$. For civil purposes, they count the days by the age of the moon that happens to fall within each month, and often from the simple phase of the monn.

Their fasts and festivals, like those of the Jews and Christians, being regulated by the lunar revolutions, they therefore cimploy a lunar year. This they make to consist of 12 months, of 30 lunar days, or eacli semi-lunation of 15 days, which are somewhat shorter than the natural day. The commencement of the solat clay is counted from sunrise, and the interval till the next sun-rise, is divided into 60 equal parts, each equal to 24 of our minutes. The subdivisions also follow the sexagesimal scalc.

The days of the week are denominated from the planets, as with the Europeans, and their arrangencut is the same also, proceeding from the sun and moon, to Mars, Mercury, Jupiter, Venus, and Saturn. Friday, or Venus's 
day, appears as the first of the week in their calculations, and probably because the cali yug began on that day; but in common, the week is considered by the Hindus as beginning with Sunday. From the view of their astronomy and chronology, they appear to be quite astrological; and they further appear to be the parents or originals of those of the Europeans and other nations.

For more particular information, see the Philos. Trans. Abridg. vol. 16, p. 742, and vol. 17, p. 250.

Babylonian, Egyptian, and Chaldean Annals. These, M. Gibert has attempted to reduce to our chronology, in a letter published at Amsterdam in 1743. He begins with showing, by the authorities of Macrobius, Eudoxus, Varro, Diodorus Siculus, Pliny, Plutarch, St. Augustin, \&c, that by a year, the ancients meant the revolution of any planet in the heavens; so that it might consist sometimes of only one day. Thus, according to him, the solar day was the astronomical year of the Chaldeans; and so the boasted period of 473,000 years, assigned to their observations, is reduced to 1297 years 9 months; the number of years which, according to Eusebius, elapsed from the first discoveries of Atlas in astronomy, in the 384t, year of Abraham, to the march of Alexander into Asia in the year 1682 of the same era. And the 17,000 years added by Berosus to the observations of the Chaldeans, reduced iu the same manner, will give 46 years and 6 or 7 months; being the exact interval between Alexander's march, and the first year of the 123d Olympiad, or the time to which Berosus carried his bistory.

Epigenius ascribes 720,000 years to the observations preserved at Babylon; but these, according to M. Gibert's system, amount only to 1971 years 3 months; which differ from Callisthenes's period of 1903 years, allotted to the same observations, only by 68 years, the period elapsed from the taking of Babylon by Alexander, which terminated the latter account, and to the time of Ptolemy Philadelphus, to which Epigenius extended his account.

The Newtonian Principles of Chronology.-Sir Isaac Newton has shown, that the chronology of ancient kingdoms is involved in the greatest uncertainty: that the Europeans in particular had no chronology before the Persian empire, which commenced 536 years before the birth of Christ, when Cyrus conquered Darius the Mede : that the antiquities of the Greeks are full of fables, because their writings were in verse only, till the conquest of Asia by Cyrus the Persian; about which time prose was introduced by Pherecides Syrius and Cadmus Milesins. After this time several of the Greek historians introduced the computation by generations. The chronology of the Latins was still more uncertain : their old records having been burnt by the Gauls 120 years after the expulsion of their kings, or 64 years before the death of Alexander the Great, answering to 388 before the birth of Christ. The chromologers of Gaul, Spain, Germany, Scythia, Sweden, Britain, and Ireland, are of a still later date. For Scythia, beyond the Danube, had no letters till Ulphilas, their bishop, formed them, about the year 276 . Germany had none till it received them from the western empire of the Latins, aluout the year 400 . The lluns had none in the days of Procopius, about the year 526. And Sweden and Norway received them still later.

Newton, after a general account of the olscurity and defects of the ancient chrowology, observes that, though many, of the ancients computed by successions and gene- rations, yet the Egyptians, Greeks, and Latins, reckoned the reigns of kings equal to generations of men, and three of them to a hundred, and sometimes to 120 years; and this was the foundation of their technical chronology. He then proceeds from the ordinary course of nature, and a detail of historical facts, to show the difference between reigns and generations; and that, though a gencration from father to son may at an average be reckoned about 33 years, or three of them equal to 100 years, yet, when they are taken by the eldest sons, three of them cannot be estimated at more than about 75 or 80 years; and the reigns of kings are still shorter; so that 18 or 20 years may be allowed a just medium. Sir Isaac then fixes on four remarkable periods, viz, the return of the Heraclidæ into the Peloponnesus, the taking of 'Troy, the Argonautic expedition, and the return of Sesostris into Egypt, after his wars in Thrace; and he settles the epoch of each by the true value of a generation. To instance only his estimate of that of the Argonautic expedition: Having fixed the return of the Heraclida to about the 159 th year after the death of Sulomon, and the destruction of Troy to about the 76 th year after the same period, he observes, that Hercules the Argonaut was the father of Hyllus, the father of Clerdius, the father of Aristomachus, and the father of A ristodemus, who conducted the Heraclidæ into Peloponnesus; so that reckoning by the chief of the family, their return was four generations later than the Argonautic expedition, which therefore happened about 43 years after the death of Solomon. This is further confirmed by another argument: Assculapius and Hercules were Ärgonauts: Hippocrates was the 18 th inclusively from the former by the father's side, and the 19 th from the latter by the muther's side: now, allowing 28 or 30 years to each of them, the 17 intervals by the father, and the 18 intervals by the mother, will on a medium give 507 years; and these, reckoning back from the commencement of the $\mathrm{P}_{\mathrm{C}}$ loponnesian war, or the 431 st year before Christ, when Hippocrates began to flourish, will place the Argonautic expedition in the $43 \mathrm{~d}$ year after the death of Solomon, or 937 years before Christ.

The other kind of reasoning, by which Newton endeavours to establish this epoch, is purely astronomical. The sphere was formed by Chiron and Musæus, for the use of the Argonautic expedition, as is plainly shown by several of the asterisms referring to that event: and at the time of the expedition the cardinal points of the equinoxes and solstices were placed in the middle of the constellations Aries, Cancer, Chelæ, and Capricorn. This point is established by Newton from the consideration of the ancient Greek calendar, which consisted of 12 tunar months, and each month of 30 days, which required an intercalary month. Of course this luni-solar year with the intercalary month, began sometimes a week or two before or after the cquinox or solsticc; and hence the first astronomers were led to the before-mentioned disposition of the equinoxes and solstices: and that this was really the case, is confirmed by the testimonies of Eudoxus, Aratus, and Hipparchus. Upon these principles Sir Isaac proceeds to argue in the following manner. In the end of the year 1689 the star called the Prima Arietis, was in $r 28^{\circ} 51^{\prime}$, with north latitude $7^{\circ} 8^{\prime} 5 \mathrm{~S}^{\prime \prime}$; and the star called the Ultima caude Arietis was in $819^{\circ} 3^{\prime} 42^{\prime \prime}$, with north latitude $2^{0} 34^{\prime} 5^{\prime \prime}$; consequently the equinoctial colure at this time cut the ecliptic in $\gamma 6^{0} 4.4^{\prime}$; and by this he reckons that the equinox had then gone back $36^{\circ} 44^{\prime}$ since iS 2 


\section{C $\mathrm{H} \mathrm{R}$}

[ 310

the time of the Argonautic expedition. But it recedes $50^{\prime}$ in a year, or $1^{\circ}$ in 72 years, and consequently $36^{\circ} 44^{\prime}$ in 2645 years; and this, counted backwaris from the beginning of 1690 , will place this expedition about 25 years after the death of Solomon. But as there is no necessity for allowing that the middle of the constellations, according to the general account of the ancients, should be precisely the middle between the prima Arietis and ultima Caudæ, our author proceeds to "examine what were those stars through which Eudoxus made the colures to pass in the primitive sphere, and in this way to fix the position of the cardinal points." Now from the mean of five places he finds, tlat the great circle, which in the primitive sphere, described by Euduxus, or which at the time of the Argonautic expedition was the equinoctial colure, did in the end of 1689 cut, the ecliptic in $86^{\circ} 29^{\prime} 15^{\prime \prime}$. In the same manner our author determines that the mean place of the solstitial colure is $\Omega 6^{\circ} 28^{\prime} 46^{\prime \prime}$, and as it is at right angles with the other, he cuncludes that it is rightly drawn. And bence he infers that the cardinal points, in the interval between that expedition and the year 1689 , have receded from those colures-1s $6^{\circ} 29^{\prime}$; which, allowing 72 years to a degree, amounts to 2627 years; and these counted backwards, as above, will place the Argonautic expedition 4.3 years after the death of Solomon. Our author has, by other methods also of a similar nature, established this epoch, and reduced the age of the world 500 years.

This elaborate system has not escaped censure; Mess. Freret and Souciet having both attacked it, and on much the same ground: but the former has confounded reigns and generations, which are carefully distinguished in this system. The astronomical objections of buth have been answered by Sir Isaac Newton himself, and by Dr. Halley. Philos. Trans. abr. vul. 7, pa. 172, 191 ; Newton's Chronol. ch. 1.

CHRONOMLTER, is any instrument or machine used in measuring time; sucl as dials, clocks, watches, \&c.

The term is however more particularly used for a kind of clock, so contrived as tu measure a small portion of time, even to the $16 \mathrm{th}$, or the 40 th part of a sccond; one of this latter kind I have seen, made by an ingenious artist; but it could not be stopped to the loth part of the proposed degree of accuracy. There is a description of one also in Desagulier's I'xperimental Philosophy, invented by the late ingenious Mr. George Graham; which inight be of great use for measuring small portions of time in astronomical observations, the time of the fall of bodies, the velocity of running waters, \&c. But long intervals of time cannot be measured by it with sufficient exactness, unless its pendulum be made to vibrate in a cycloid; for otherwise it is liable to err considerably, as is the case of all clocks with short pendulums that swing in large arches of the circle.

Various other contrivances, besides clocks, have been used for measuring time for some particular purposes. See a musical chronometer described in Malcolm's Treatise of Music, pa.407. The excullent watches that have lately been made, for the purpose of finding the longitude, by the ingenious artists, Harrison, Arnold, Mudge, Emery, llardy, \&c, are sometimes called chronometers, also timekeepers.

CHIRONOSCOPE, a word sometimes used for a pendulum, or machine, to measure time.

CIILYSTALLINE. SeC CHYSTALLINE.
C I R

CINA, or Sin A, in Architecture, a inember, or noulding, called also ogee, and cimatium.

ClNCTURE, in Architecture; is a ring or list around the shaft of a column, at its tup and buttom.

CINTRE, in Building, the same as Centre; which see. CIPHER, or Cypher, one of the numeral charactess, or figures, tlius formed 0 . The word comes from the Hebrew saphar, to number.

The cipher of itself signifies nothing, or implics a privation of value; but when combined with other numeral characters, it alters their value in a tenfold proportion, for every cipher so annexed; viz, when sct after a figure in common integral arithmetic, it increases its value in that proportion, though it has no effect when placed bcfore or to the left-hand side of such figures; but on the contrary, in decinal arithmetic, it decreases their value in that proportion when set before the figures, yet makes no alteration in their value when placed after them.

Thus, 5 is five,

but 50 is fifty,

and $500^{\circ}$ is five hundred;

whereas 05 , or $005, \& \mathrm{c}$, is still but 5 or five.

Also $\cdot 5$ is five tenths,

but 05 is five hundredths,

and .005 is five thousand his;

whereas $\cdot 50$, or $\cdot 500$, \& $\mathrm{c}$, is still but 5 or five tinths.

The invention and use of the cipher, as in the common arithmetic and notation of numbers, is one of the happiest devices that can be imagined; and is ascribed to the Indians, by the Arabians, by whom it was made known to Europe at the revival of literature.

CIRCLE, a plane figure, bounded by a curve line which returns into itself, called its circumfirence, and which is cvery wbere equally distant from a certain point within, called its centre. 'The circumference or perijhery itseli is sometimes called the circle, but impioperly, as that name denotes the space contained within the circumference.-A circle is described with a pair of compasses, fixing one foot in the centre, and turning the other round to trace out the circumference.

The circumference of every circle is supposed to be divided into 360 equal parts, called degrees, and marked '; each degree into 60 minutes or primes, marked'; 'ach uninute into 60 seconds, marked "; and so on. So $24^{\circ} 12^{\prime}$ $15^{\prime \prime} 20^{\prime \prime \prime}$, is 24 degrees 12 minutes 15 seconds and 20 thirds.

Circles have many curious propertics, some of the most important of which are the following.

1. The circle is the most capacious of all plain figures, or contains the greatest area within the same perimeter, or has the least perimeter about thesame area; being the limit and last of all regular polygons, having the number of $i$ ts sides infinite.

2. The area of a circle is always less than the area of any regular polygon circumscribed about it, and its circumference always less than the perimeter of the polygon. But on the other hand, its area is always grenter than that of its inscribed polygon, and $i$ s circumterence greater than the perimeter of the said inscribed polygon. How:ever, the area and perimeter of the circle ippronch always nearer and nearer to those of the two polygons, as the number of the sides of these is greater ; the circle being always limited between the two polygons.

3. The area of a circle is cqual to that of a triangle whose base is cqual to the circumference, and perpendi- 
ular equal to the radius. And therefore the area of the circle is found by multiplying half the circumference by half the diameter, or the whole circumference by the whole diameter, and taking the 4th part of the product; as demonstrated by Archimedes.

4. Circles, like other similar plane figures, are to one another, as the squares of their diameters. And the area of the circle is to the square of the diameter, as 11 to 14 nearly, as proved by Archimedes; or as $785+$ to 1 more nearly; or still more nearly as

$7853981633,9744830961,5660845819,8757210492$, $9234984377,6455243736,1480769541,0157155224$,

$9657008706,3355292669,9553702162,8318076661$,

$7734611+$ to 1 ;

as it has been found by modern mathematicians.

In Wallis's Arithmetic of Infinites are contained the first infinite series for expressing the ratio of a circle to the square of its diameter: viz,

1 st, The circle is to the square of its diameter, as 1 to $\frac{3 \times 3 \times 5 \times 5 \times 7 \times 78 \mathrm{cc}}{2 \times 4 \times 4 \times 6 \times 6 \times 8 \& \mathrm{c}}$, $\}$ discovered by Wallis or 1 to $\left.\frac{9}{8} \times \frac{25}{24} \times \frac{49}{48} \& \mathrm{c},\right\}$ himself; oras 1 to $1+\frac{1}{9}+$, by Ld. Brounker; $2+\frac{9}{2+\frac{25}{2+-\frac{49}{2+8 c}}}$

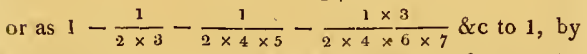
Sir I. Newton; or as $1-\frac{x}{3}+\frac{x}{5}-\frac{1}{7}+\frac{x}{4}-\frac{1}{T} \&$ is to 1 , by Gregory and Leibnitz; and a great many other forms of series have been invented also by other authors to express the same ratio between the circle and circumscribed square.

5. The circumferences of circles are one to another, as their diameters, or radii. Hence, as the areas of circles are proportional to the rectangles of their radii and circumferences; the quadrature of the circle will therefore be effected by the rectification of its circumference; that is, if the true length of the circumference could be found, the true area could be determined also. But while several mathematicians have endeavoured to investigate the true area and circunference, others have even doubted of the possibility of the same. Of this latter opinion is Dr. Isaac Barrow: towards the end of his 15 th mathematical lecture he says, he is of opinion, that the radius and circumference of a circle, are lines of such a nature, as to be not nnly incommensurable in length and square, but even in length, square, cube, biquadrate, and all other powers to infinity: for, cuntinucs he, the side of the inscribed square is incommensurable to the radius, and the square of the side of the inscribed octagon is incommensurable to the square of the radius; and consequently the square of the octagonal perimeter is also incommensurable to the square of the radius: and thus the ambits of all regular polygons, inscribed in a circle, may have their, superior powers incommensurate with the co-ordinate powers of the radius; whence the last polygon, that is, the circle itself, appears to lave its periphery incommensurate with the radius; which, if true, will put a final stop to the quadrature of the circle, since the ratio of the circumference to the radius is altogether inexplicable from the nature of the thing, and consequently the problem requiring the explication of such a ratio, is inpossible to be solved, or rather it requires that for its solution which is impossible to be apprehended. He concludes by observing that this great mystery cannot be explained in a few words: but if time and opportunity had permitted, he would have enckeavoured to produce many things for the elucidation and confirmation of this conjecture. Newton also, in book 1 of his Principia, has attcrnpted to demonstrate the impossibility of the general quadrature of oval figures, by the description of a spiral, and likewise the impussibility of determining, by a finite equation, the intersections of that oval and spiral, which must be the case, if the oval be quadrable. And several other authors bave attempted to demonstrate the impossibility of the general quadrature of the circle by any means whatever. On the other hand, many mathensaticians not only believe in the possibility of the quadrature of the circle, but some have even professed to have discovered the same, and have published to the world their pretended discoveries: of which no one has rendered himself more conspicuous than our country man Mr. Hobbes, though a great scholar, and of excrlient understanding in uther matters. Sec Quadrature.

The approximate quadrature of the circle however, or the determination of the ratio between the diameter and the circumference, is what the mathematicians of all ages bave successfully attempted, and with different degrees of accuracy, according to the inproved state of science. Archimedes, in his book de Dimensione Circuli, first gave a near value of that ratio in small numbers, being that of 7 to 22 , which are still used as very convenient numbers for this purpose in common measurements. 'Other and nearer ratios have since been successively assigned, but in larger numbers,

$$
\begin{aligned}
& \text { as } 106 \text { to } 333, \\
& \text { or } 113 \text { to } 355, \\
& \text { or } 1702 \text { to } 5347, \\
& \text { or } 1815 \text { to } 5702,8 c \text {, }
\end{aligned}
$$

which are each more accurate than the foregoing: and several of these same ratios, as well as others, have lately been found in the nathematical books of the Hindus, accounts of which may be seen in my Tracts, vol. 2, pa.158, \&c. Vieta, it his Universalium lnspectionum ad Canonum Mathematicum, published 1579 , by means of the inscribed. and circumscribed polygons of 393216 sides, has carriel the ratio to ten places of figures, showing thluat if the diameter of a circle be $1000 \& \mathrm{c}$, the circumlerence will be greater than $314,159,265,35$,

but le:s than $314,159,265,37$.

And Van Colen, in his book De Circulo et Adscriptis, has, by the same means, carried that ratio to 36 places of figures; which were also recomputed and confirmed by Willehrord Snell. After these, that indefatigable computer Mr. Abraham Sharp extended the ratio to 72 places of figures, in a paper, published about the year 1706, by means of the series of Dr. Halley, from the tangent of an arc of 30 degrees. And the ingenious Mr. Machin also carried the same to a hundred places, by other series, depending on the differences of arcs whose tangents have certain relations to one another. See this method explaincd in my Mensuration, pa. 89, 4t hedit.; or my new Tracts, vol. 1, pa. 266. And, finally, M. De Lagny, in the Memoires de l' $A$ cad. 1719 , by means of the tangent of the arc of 30 degrees, has extended the same ratio to the astonishing length of 128 places of figures; finding, that, if thie. diameter be 1000 \&ce, the circumference will be 
$31415,92653,58979,32384,62643,38327,95028$, $84197,16939,93751,05820,97494,45923,07816$, $40628,62089,98628,03482,53421,17067,98214$, $80865,13272,30664,70938,146+$ or $447-$

From such methods as the foregoing, a variety of series have been discovered for the length of the circumference of a circle, such as the following, viz, lf the diameter be 1 , the circumference $c$ will be variously expressed thus, $c=4 \times\left(1-\frac{1}{3}+\frac{1}{5}-\frac{1}{7}+\frac{1}{9}-\frac{1}{11}+\frac{1}{13}-\frac{1}{15} \& c\right)$, $c=\sqrt{ } 8 \times\left(1+\frac{1}{3}-\frac{1}{5}-\frac{1}{7}+\frac{1}{9}+\frac{1}{11}-\frac{1}{13}-\frac{1}{15}, \& c\right)$, $c=\sqrt{12} \times\left(1-\frac{1}{3.3}+\frac{1}{5.3^{3}}-\frac{1}{7.3^{3}}+\frac{1}{9.3^{4}} \& c\right)$, $c=8 \times\left(\frac{1}{1.1 .3}+\frac{1}{1.3 .5}-\frac{1}{3.5 .7}+\frac{1}{5.7 .9}-\frac{1}{7.9 .11} \& \mathrm{c}\right)$, $c=8 \times\left(\frac{2}{3}-\frac{1}{5}-\frac{1}{4.7}-\frac{1.3}{4.6 .9}-\frac{1.3 .5}{4.6 .8 .11} \& \mathrm{c}\right)$, $c=4 \sqrt{ } 2 \times\left(\frac{2}{3}-\frac{1}{5.2}-\frac{1}{4.7 .2^{2}}-\frac{1.3}{4.6 .9 .2^{3}}-\frac{1.3 .5}{4.6 .8 .11 .2^{4}} \& c\right)$, $c=4 \times\left(1-\frac{1}{2.3}-\frac{1}{2.4 .5}-\frac{1.3}{2.4 .6 .7}-\frac{1.3 .5}{2.4 .6 .8 .9} \& c\right)$.

And many other series might here be added. See my Mensuration in several places; also my paper on such series in the Philos. Trans. 1776, or my new Tracts, vol. 1 , pa. 268; Euler's Introductio in Analysin Infinitorum; and many other authors.

6 . Some of the more remarkable properties of the circle are as follow:

If two lines $A B, C D$ cut the circle, and intersect within $i t$, the angle of intersection $\mathrm{E}$ is measured by half the sum of the intercepted arcs $\mathrm{AC}, \mathrm{DB}$.

But if the lines intersect without the circle, the angle $\mathrm{E}$ is measured by half the difference of the intercepted arcs Ac, D B.

7. The angle at the centre of a circle is double the angle at the circumference, standing on the same arc; and all angles in the same segment are cqual. Also the angle at the centre is measured by the arc it stands upon, and the angle at the circumference by half the same'arc.

8. If the cliords $\mathrm{FG}$, III intersect each other at right angles, the sums of the opposite arcs are cqual; viz, F $\mathbf{H}+$ $\mathrm{Gl}=\mathrm{FI}+\mathrm{GH}$.

9. If one side no of a trapezium inscribed in a circle be produced out, the external angle Lor, will be equal to the internal opposite angle $\mathrm{M}$.

10. Any angle, as res, formed by a tangent $Q R$ and cliord $Q \mathrm{~s}$, is measured by lialf the arc of the chord $Q$ s, and is equal to any ungle $\mathrm{T}$ in the alternate segment QTS.
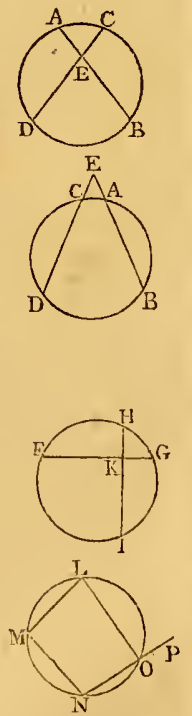

'11. If $\mathrm{v} w$ be a diameter, and $\mathrm{X} z \mathrm{x}$ a chord perpendicular to it; then is $\mathrm{xz}$ or $\mathrm{z} \mathrm{x}$ a mean proportional between the segments $\mathbf{v z}, \mathbf{z w}$. So that if $d$ denote the diameter $\mathrm{vw}, x$ the absciss $\mathrm{vz}$, and $y$ the ordinate $\mathrm{zx}$; then is $y^{2}=(d-x) \times x$ or $=d x-x^{2} ;$ which is called the equation of the circle.

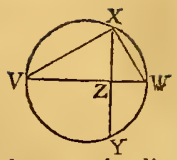

The chord $\mathrm{vx}$ is a mean proportional between the diameter $\mathrm{vw}$ and the versed sine $\mathrm{v} z$; and the chord $\mathrm{wx}$ is a mean proportional betwcen the diameter and the versert sine $\mathrm{wz}$; also each versed sine is proportional to the square of the corresponding chord; viz, $\mathbf{v z}: \mathrm{wz}:: \mathrm{vx}^{2}: \mathrm{wx}^{2}$.

12. If the diameter $P Q$ be divided into two parts at any point $\mathrm{R}$; and if $\mathrm{RS}$ be drawn perpendicular to $\mathrm{PQ}$, also $\mathbf{R T}$ applied equal to the radius, and $\mathrm{TR}$ produced to the circumference at $\mathrm{v}$ : Then, between the two segments $P R, R Q$, $\mathrm{IT}$ is the arithmetical mean,

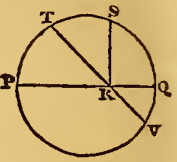
Rs is the geometrical mean,

$\mathrm{RV}$ is the harmonial mean.

13. When two lines cut the circle, whether they intersect within the circle, or without it, as in the two figures to article 6 , the segments between the common intersection and the two points where each line cuts the curve, are reciprocally proportional, and their rectangles are equal; viz, $\mathbf{E A}: E C:: \mathrm{ED}: \mathrm{EB}$, Or $E A \times E B=E C \times E D$.

14. In a trapezium inscribed in a circle, the rectangle of the two diagonals is equal to the sum of the two rectangles contained by each pair of opposite sides; viz, $\mathrm{AC} \times \mathrm{BD}=\mathrm{A} \mathrm{B} \times$ $D C+A D \times B C$.

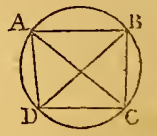

15. If any chords EF, EG, drawn from the saine point $\mathrm{E}$ in the circumference, be cut by any other line HI, the rectangles will be all equal which are contained by each chord and the part intercepted by this line, viz, $\mathrm{EF} \times$ $\mathrm{EI}=\mathrm{EG} \times \mathrm{EII}=\mathrm{EK}^{2}$.

16. In a circle whose centre is $\mathrm{N}$ and radius $\mathrm{No}$, if two points $\mathrm{s}, \mathrm{r}$, in the radius produced, be so taken that the three, $\mathrm{NM}, \mathrm{NO}, \mathrm{NP}$, be in continued proportion; then if from the points $M$ and $P$ lincs be drawn to any, or cvery

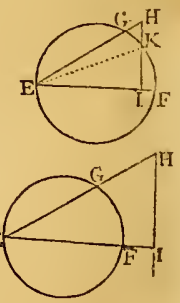
point in the circumference, as $Q$; these lines will be always

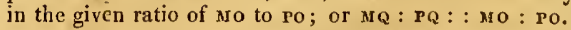

17. If $\mathrm{vw}$ be two points in the diancter, equidistant from the centre $T$; and if two lines be drawn from these to any point $\mathbf{x}$ in the circumference; the sum of theirsquares will be equal to the sum of the squares of the segments of the diameter made by either point; viz, $v^{2}+w x^{2}=$

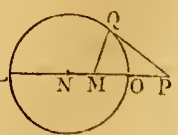
$\mathrm{RV}^{2}+\mathrm{vs}^{2}$, or $=1 \mathrm{w}^{2}+\mathrm{Ws}^{2}$, or $=2 \mathrm{RT}^{2}+2 \mathrm{VT}^{2}$.

18. If a line $F E$ perpendicular to the dianeter $A \mathrm{~s}$, meet any other chord $\mathrm{CD}$ in the point $\mathrm{E}$; then is $A F \times \quad \mathrm{R}$ $=\mathrm{CE} \times \mathrm{ED}+\mathrm{EF}^{2}$. 

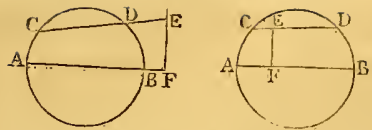

19. If upon the diameter $\mathrm{GH}$ of $\mathrm{a}$ circle there be formed a rectangle GIIKI, whose breadth $\mathrm{GI}$ or $\mathrm{HK}$ is equal to $\mathrm{GL}$ or H L, the chord of a quadrant, or side of the inscribed square; then if from $\mathrm{I}$ and $\mathrm{K}$ lines be drawn to any point $M$ in the circle $G M H$, they will

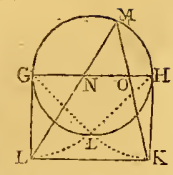
cut the diameter $\mathrm{GI}$ in such a manner that $\mathrm{GO}^{2}+\mathrm{HN}^{2}$ $=\mathrm{GH}^{2}$.

20. If the arcs $P Q, Q R, R S, \& c$, be equal, and there be drawn the chords $P Q, P R, P S, P T, \& c$, then it will be $P Q: P I:: P R: P Q+P S:$ : PS : PI + PT : : PT: PS + PV, \&c.

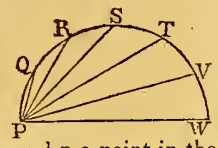

21. The centre of a circle being $o$, and $P$ a point in the radius, or in the radius produced; if the circumference be divided into as many equal parts $\mathrm{AB}, \mathrm{BC}, \mathrm{CD}, \& \mathrm{C}$, as there are units in $2 n$, and lines be drawn from $\mathrm{x}$ to all the points of division; ther shall the continual product of all
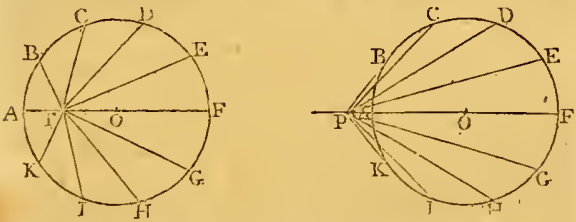

the alternate lines, viz, PA $\times$ PC $\times$ PE \& $\&$ be $=r^{\mathrm{n}}-x^{\mathrm{n}}$ when $\mathrm{P}$ is within the circle, or $=x^{\mathrm{n}}-r^{\mathrm{n}}$ when $\mathrm{P}$ is without the circle; and the product of the rest of the lines, viz $\mathrm{PB} \times$ PD $\times$ PF \& $\mathrm{C}=r^{\mathrm{n}}+x^{\mathrm{n}}$ : where $r=A 0$ the radius, and $x=\mathrm{op}$ the distance of $\mathrm{P}$ from the centre.

22. To divide a circle into any number of equal parts, by means of concentric circles.ADB being half the given circle, divide its radius $\mathrm{Ac}$ into the like number of parts; at the puints, of division erect the perpen-

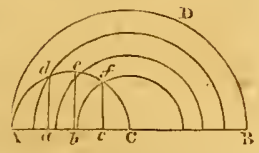
dicular $a d, b e, c f$, meeting the semicircle $\wedge$ def $\mathrm{c}$ in the points $d, e, f$, \&c. Lastly, from the centre $c$, describe circles through the points $d, e, f, \& c$, and these will $\mathrm{di}-$ vicle the space in the manner required, either all equal, or in any ratios as the radius $\mathrm{AC}$ is first divided into. Sce my Tracts, vol.3, p.378, \&c.

23. A circle may thus be divided into any number of parts that shall be equal to one another both in area and perimeter. Divide the diameter $Q R$ into the same number of equal parts at the points $s, T, v, \& c$; then, on one side of the diameter describe semicircles on the diamcters Qs, QT, Qv, and on the other side of it describe semicircles on RV, RT, RS; so shall the parts 17 ,

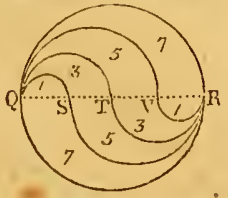
$35,53,71$, be all equal, both in area and perimeter. See my Tracts, vol. 1, p. 25t.

24. To describe a Circle either about or within a given
Regular Polygon. Bisect two of its angles, or two of its sides, with perpendiculars, and the intersection of the bisecting lines will, in either case, be the centre of the circles.

Parallel, or Concentric $\mathrm{C}_{1 \mathrm{R}} \mathrm{CL} \mathrm{s}$, are such as are equally distant from each other in every point of their peripheries; or that have the same centre. As, on the other hand, those are called the excentric circles, that have not the same point for their centres.

The 2uadrature of the $\mathrm{C}_{1 \mathrm{RCLE}}$, is the manner of describing, or assigning, a square, whose surface shall be perfectly equal to that of a circle. This problem has exercised the geometricians of all ages, but it is now generally abandoned as a problem impossible to be effected, by most persons that have any just claim to that rank, Descartes insists on the impossibility of it, for this reason, that a right line and a circle being of different uatures, there can be no strict proportion between them; which is not satisfactory. Dr. Barrow shows the strongest probability of the same thing; and not only that the diameter and circumference themselves, but that all powers of them to infinity, are incommensurate.

The Emperor Charles v offered a reivard of 100,000 crowns to any person who should resolve this celebrated problem: and the States of Holland also proposed a reward for the same thing. See QUADRATURE.

\section{Cincles of the Higher Orders,} are curves in which

$\mathrm{W} \mathrm{Y}^{\mathrm{m}}: \mathrm{YZ}^{\mathrm{m}}:: \mathrm{YZ}: \mathrm{YX}$,

or $\mathrm{WY}^{\mathrm{m}}: \mathrm{YZ}^{\mathrm{m}}:: \mathrm{Y}^{\mathrm{n}}: \mathrm{YX}^{\mathrm{n}}$.

When $m$ and $n$ are each equal

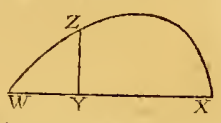

to 1 , then $W Y: Y Z:: Y Z: Y x$, which is the property of the common circle.

Put $\mathrm{WY}=x, \mathrm{Yz}=y, \mathrm{w} \mathrm{X}=a$; then is $\mathrm{Yx}=a-x$, and the above proportions become

$$
\begin{gathered}
x^{m}: y^{m}:: y:(a-x) \text { or } y^{m+1}=x^{m} \cdot(a-x) \text { and } \\
x^{m}: y^{m}:: y^{n}:(a-x)^{n} \text {, or } y^{m+x}=x^{m} \cdot(a-x)^{n} \text {, the }
\end{gathered}
$$
equations to curves of this kind.

Curves difined by this equation will be ovals when $m$ is an odd number. 'l'hus suppose $m=1$, then the equation becomes $y^{2}=x \cdot(a-x)$, or $a x-x^{2}$, the equation to the common circle. And if $m=3$, the equation is $y^{2}=x^{3} .(a-x)$ or $a x^{3}-x^{4}$, which denotes a curve of the form $A B$, the first fig. below.
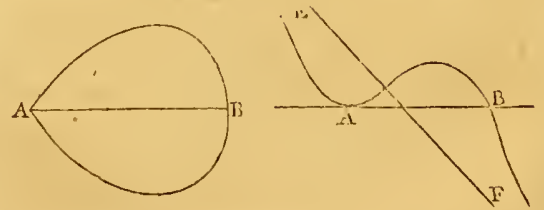

But when $m$ denotes an cven number, the curve will have two infinite legs. So if $m=2$, the cquation becomes $y^{3}=x^{2}:(a-x)$, or $a x^{2}-x^{3}$, for a circle of the $2 \mathrm{~d}$ order, and which defines one of Ncwton's defective hyperbolas, being his 37 th species of curves, whose asymptote is the right line EF, making an angle of 40 degrees with the absciss $A B$, as in the $2 \mathrm{~d}$ fig.

C1rcu of Curvature, or circle of cqui-curvature, is that circle which has the same curvature with a given curve at a certain point; or that circle whose radius is equal to the radius of curvature of the given curve at that point. 
A circular arc is the only curve line that is equally cursed in every point. In all other curve lines, such as the arc of an ellipse, or a parabola, or an hyperbola, or a cycloid, the curvature is different in different points, and the degree of currature in any point is estimated by the curvature of a circle which is said to have the same curvature as the proposed curve line in that point; by which is understood the circle which, having the tangent of the proposed curve in the said point for its tangent, approaclies so nearly to the proposed curve that no other circle whatever can be drawn between it and that curve.

This circle is also said to osculate the curve in the said point, and is therefore often called the osculating circle, as well as the circle of equal curvature with the curve in that point. And the radius of this circle is called the radius of curvature of the proposed curve in the said point; also its centre is called the centre of curvature.

Now there are some curve lines so very highly curved in particular points, that every circle, of how small a radius soever, having the tangent to the curve in one of those points for its tangent, will pass without the curve, or between the curve and its tangent. This, for example, is the case with the curve of a cycluid in the two points contignous to its base, as also with the cissoid at its vertex. And in such points the curvature of these curves is said to be infinite, because it is greater than the curvature of any circle, how small soever. Also the radius of the circle of curvature in such points is nothing; the length of that radius being always inversely or reciprocally as the degree of curvature at any point.

The theory of these circles of equal curvature with curves in particular points was first cultivated by Apollonius in his Conic Sections : and it has since been carried much farther by several great mathematicians of modern times; particularly by Mr. Huygens in his doctrine of Evolute Curves and Curves of Evolution, and by the great Sir Isaac Newton. See Curvature.

Cincles of the Sphere, are such as cut the mundane sphere, and bave their circumference in its surface.

These circles are either fixed or moveable.

The latter are those whose peripheries are in the moveable or revolving surface; and which therefore move or turn with it; as the meridians, \&c. The former, having their periphery in the immoveable surface, do not revolve; as the ecliptic, equator, and its parallels.

'The circles of the sphere are either great or lesser circles.

A Great Circle of the Sphere, is that which divides it into two equal parts or hemispheres, having the same centre and diameter with it. As the horizon, meridian, equator, ecliptic, the colures, and the azimuths.

A Lesser Circle of the Sphere, divides the sphere into two unequal parts, having neither the same centre nor diancter with the sphere; its diameter being only some chord of the sphere less than its axis. Such are the parallels of latitude, \&c.

Cincles of Alitude, or Alnucantars, are lesser circles parallel to the horizon, having their common pole in the zenith, and still diminishing as they approach it. They are so called from their use, which is to show the altitude of a star above the horizon.

Cincres of Declination, are great circles intersecting each other in the poles of the world.

Culcle of Dissipation, in Optics. See Dissipation.

Diurnal C'ncess, are parallels to the equinoctial, supposed to be described by the several stars, and other points of the heavens, in their apparent diurnal rotation abous the earth.

Circle Equant, in the Ptolemaic Astronomy, is a circle described on the centre of the equant. Its chicf use is, to find the variation of the first inequality.

CircLes of Excursion, are lesser circles parallel to the ecliptic, and at such a distance from it, that the cxcu rsions of the planets towards the poles of the ecliptic, may be included within them; being usually fixed at about 10 d çgrees.

It may here be observed, that all the circles of the sphere, described above, are transferred from the beavens to the earth: and thence come to have a place in geography as well as in astronomy: all the point of each circle being conceived as let fall perpendicularly on the surface of the terrestrial globe, and thus tracing out circles perfectly similar to them. So, the tertestrial equator is a circle conceived precisely under the equinoctial line, which is in the heavens; and so of the rest.

Horary Ci RCLES, in Dialling, are the lines which slow the hours on dials. These are straight lines on the dials, but called circles as being the projections of the meridians.

Horary $\mathrm{C}_{1 \mathrm{RCL}}$, or Hour Circle, on the globe, is a small brazen circle fixed to the north pole, divided into 24 hours, and furnished with an index to point them out, thereby showing the difference of meridians in time, and serving for the solution of many problems, on the artificial globes.

CincLe of Illumination, is that imaginary circle on the surface of the earth, which separates the illuminated side or hemisphere of the earth from the dark side : and all lines passing from the sun to the earth, being physically parallel, are therefore perpendicular to the plane of this circle.

Circles of Latitude, or Secondaries of the Ecliptic, are great circles perpendicular to the plane of the ecliptic, intersecting each other in its poles, and passing through every star and planet, \&c.-These are so called, because they serve to measure the latitude of the stars, which is an arch of one of these circles, intersected between the stir and the ecliptic.

Cincles of Longitude, are lesser circles parallel to the ecliptic, diminishing nore and more as they recede from it, or as they appronch the pole of that circle.

They are so called, because the longitudes of the stars are reckoned upon them.

Cincles of Perpetual Apparition, one of the lesser circles parallel to the equator, described by the most northern point of the horizon, as the sphere revolves round by its diurnal motion.-All the stars included within this circle, arc continually above the horizon, and consequently never set.

Circle of Perpetual Occultation, is another lesser circle at a like distance from the equator, but on the other side of it, being described by the most southern point of the horizon, and contains all those stars which never appear in our hemisplere, or which never rise.

All other stars, being contained between these two circles, do alternately rise and set, at certain moments of the diurnal rotation.

Polar Cincles, are immoveable circles, parallel to the equator, and at such a distance from the pole as is cyual to the greatest declination of the ecliptic, which is now nearly $23^{\circ} 28^{\prime}$. 'That next the northern pole is called the arctic, and that next the sonthem one the antarctic.

Circles of Position, are circles passing through the common interscetions of the horizon and meridian, and 
through any degree of the ecliptic, or the centre of any star, or other point in the heavens; and are used for finding the situation or position of any star. These are usually 6 in number, cutting the equinoctial into 12 equal parts.

CIRCUIT, Electrical, denotes the course of the electric fluid from the charged surface of an clectric body, to the opposite surface, into which the discharge is made. Some electricians at first apprehended, that the same particles of the electric fluid that were thrown on one side of the charged glass, actually made the whole circuit of the intervening conductors, and arrived at the opposite side: whereas Dr. Franklin's theory only requires, that the redundancy of electric matter on the charged surface should pass into those bodies which form that part of the circuit which is contiguous to it, driving forward that portion of the fluid which they naturally possess; and that the deficiency of the exhausted surface should be supplied by the neighbouring conductors, which form the last part of the circuit. On this supposition, a vibrating motion is successively communicated through the whole length of the circuit.

Many attempts were made, both in France and England, at an early period in the practice of electricity, to ascertain the distance to which the electric shock might be carried, and the velocity of its motion. 'The French philosophers, at different times, caused it to pass through circuits of 900 and even 2000 toises, or about 2 English miles and a half; and they discharged the Leyden phial through a bason of water, whose surface was equal to one acre. M. Monier found that the fluid, in passing through an iron wire of 950 toises in length, did not spend a quarter of a second; and that its motion was instantaneous through a wire of 1319 feet. In 1747, Dr. Watson, and other English philosophers, after many experiments of a similar kind, conveyed the electric matter through a circuit of 4 miles; and, from two several trials, they concluded that its passage is instantaneous. By all which doubtless is meant, that its motion is too rapid to be measured. Priestley's Hist. of Lilect. vol. 1, p. 128, 8vo edit.

CIRCULAR, appertaining to a circle; as a circular form, circular motion, \&c.

Circula I Instruments of Reflection, or Multiplying Circles, are such circles as, in a small size, by means of reflection, procure at pleasure all the advantages of the size of large instruments, as well as diminishing the errors of division.-Circular instruments are in fact considerable improvements on Hadley's octant, and the marine sextant, devised for the same purposes. We shall herc describe the progress of these instruments under the contrivances of Tobias Mayer, Chevalier Borda, and Captain Mendoza.

Mr. Mayer proposed to complete the limb of the sextant to a whole circle, with the horizon glass moveable round the centre, and with an additional index, which may be called the horizon index, to distinguish it from the centre index, to which the centre glass is at tached. This instrument is represented in plate 7, fig. 8 ; and the manner of using it is as follow's: After the index $A$ is set at o (the beginning of the divisions) the two glasses are rendered parallel, as in using Hadley's quadrant, by moving the horizon index $B$, till the horizon of the sea (or the sun or any other object), or its direct image, and the doubly reflected image of the same, seen through the telescope, coincide. After fixing the horizon index in that position, the centre index $\Delta$ is to be moved, in order to mcasure the VOL. I. distance of the two objects $s$ and $L$ (suppose the sun and moon), by bringing into contact the double reflected image of the sun with the direct image of the moon, seen through the telescope. The centre image will then be at $\mathrm{M}$, and the arch on might give, as in the sextant, the angular distance required; but the construction of the circle renders it easy, in this position, to effect again the parallelism of the glasses, and to make another observation of the contact, in the like manuer as from 0 ; which operation will bring the centre index to $\mathrm{N}$. The index will then give oy, or double the distance; which divided by 2 , reduces the errors of the instrument to one half. And it is obvious, that by successive repetitions of the same process, triple, quadruple, \&c, the distance may be obtained, and the said error further reduced, in the inverse ratio of the multiplication of the distance, to any degree of approximation desired.

The method of rendering the glasses parallel, by means of the horizon of the sea, is not acculate, on account of the indistinctness of the images; and when the sun is used for that purpose, the observation becomes fatiguing to the eye. T'be repetition of that observation remained therefore a considerable inconvenience attached to this circle. The author himself seems to bave been aware of it, as be proposed to provide the instrument with a diagonal ruler, fixed on one of the indexes, so that the other index should touch it when the glasses were parallel: but an adjustment of this nature must be subject to great errors, and never was adopted in practice. The chevalier Borda, wishing to remove that imperfection, contrived to render the parallelism of the glasses unnecessary, by substituting the observation of the angular distance of the two objects, for that of the coincidences of the images of the same object. This constitutes the second great improvement of the reflecting circle.

In Borda's circle, the telescope is fixed at some distance from the centre, and the horizon glass is carried near the border of the instrument, as in plate 7, fig. 9. By this arrangement, the rays of light can arrive at the centre glass, both from the hcavenly bodies, situated to the right of the horizon index, as $\mathrm{s}^{\prime}$, and from those situated to the left, as $\mathrm{s}$. Thus, if the glasses are parallel to one another, when the centre index is at $o$, it is obvious that there are two ways of making the observations. While the direct image of the moon $L$ is scen through the telescope, the angular distance to the sun, if at $\mathrm{s}$, may be measured by moving the centre index to $m$, in order to produce the contact; or, if the sun be at $s^{\prime}$, the same operation may be performed, by using the contrary motion to $m^{\prime}$. The first kind of observation, M. Borda calts observation to the left; and the second observation to the right. Suppose now, that (the horizon index being fixed in the same position) the distance from $L$ to $S$ is observed to the left, by bringing into contact the double reflected inage of $s$ with the image of $L$, seen without reflection; Jet us then turn the instrument round, keeping it in the same plane, so as to have the direct image of s through the telescope, and thus make an observation of the sime distance to the right; the position of the centre index being in the first observation at $m$, and in the sccond at $m^{\prime}$, it is clear, that if o be the point where the parallelism of the glasses takes place, $0 m$ will be equal to o $m^{\prime}$, and that the arch $m m^{\prime}$, determined by the two positims, will give double the distance--lt will be more convenient to have the centre index at 0 , when the first obscrvation is made, $2 \mathrm{I}^{\circ}$ 
in order to take the double distance at one reading, after the second observation. For this purpose, the first part of the process may be inverted, by previously fixing the centre index at the beginning of the divisions, and moving the horizon index $\mathrm{I}$ towards o, instead of moving the centre index A to $m$, or towards $\boldsymbol{H}$.

The last kind of observation, in which the incident ray, which produces the first image on the centre glass, may be conceired to run double the angular distance, passing in its way over the line of collimation, has by $M$. Borda been called the crossed observation. - The same process nuay be repeated, by fixing alternately one of the indexes, and moving the other, and continuing successive sets of observations; each set of two crossed observations, one to the right and another to the left. The angle given by the instrument, will be equal to double the angular distance multiplied by the number of sets observed.

Whether a circle is used simply, as Mayer proposed it, or in Borda's method, its peculiar advantages chiefly depend on the multiplication of the distance sought. Capt. Mendoza has therefore turned his attention to the improvement of this principle, and has accordingly contrived the following construction: In the crossed observations made with Borda's circle, the indexes move alternately through an arch which, jn the divisions, is equal to double the distance: for example, the centre index comes, in the first crossed observation, from $m$ to $m^{\prime}$; in the third crossed observation, from $\dot{m}^{\prime}$ to $m^{\prime \prime}$, \&c ; and the horizon index, in the $2 \mathrm{~d}$ crossed observation, to $h^{\prime}$; in the 4 th crossed observation to $h^{\prime \prime}, \& c$; and by cach of the two indexes may be found the same multiple of the distance sought. Let us now place the nonius in a circle moving round the centre, over, or adjacent to, the usual limb which contains the divisions: then it will easily be conceived that, by attaching that circle, which M. Mendoza calls the flying nonius, alternately to each of the indexes, it will serve as nonius for both; and that, after any number of obscrvations, it will give the compound motion of the two indexes. Thus, after the first observation, the flying nonius will, at each crossed observation, advance double the distance over the divisions, while each separate nonius, fixed on the indexes, requires a set of two observations to produce the same effect in Borda's circle. The peculiar property of the instrument being that of giving double the distance, it was thought proper to divide the circle into 360 degrees, and not into 720 according to the nature of the sextant. Thus, after a crossed observation, the reading of the nonius will, without reduction, exhibit the measure of the simple distance. Mendoza has also extended the nonius rousd the circumference, so that, by the coincidence of two divisions, the number of degrees will appear on the limb, and that of the minutes and seconds on the flying nonius. See Philos. Trans. 1801.

For more on the nature of circular instruments of reflection, see Montucla, Histoire des Mathematiques, tom.3, p. 527 : for the computation of the probable extent of the errors to which they are liable, sce Swanberg, Exposition des opérations faites en Lapponic \&c, p. 29; and for a catalogue of papers \&c, connected with such instruments, consult Dr. T. loung's Natural Philos. vol. 2, p. 350.-For Sir Howard Douglas's Reflecting Circle or Semicircle, sec the article Refuecting in this Dictionary.

Circulak Lines, a name givell by some authors to such straight lines as are divided by mcans of the divisions made in the arch of a circle. Such as the Sines, Tangents, Secants, \&rc.

CIRcular Numbers, are such as have their powera ending in the roots themselves. As the number $j$, whose square is 25 , and its cube $125, \& c$.

Napier's Circular Parts, are five parts of a right-angled or a quadrantal spherical triangle; they are the two legs, the complement of the hypothenuse, and, the complements of the two oblique angles.

Concerning these circular parts, Napier gave a general rule in his Logarithmorum Canonis Descriptio, which is this: "The rectangle under the radius and the sine of the middle part, is equal to the rectangle under the tangents of the adjacent parts, and to the rectangle under the cosines of the opposite parts. The right angle or quadrastal si:le being neglected, the two sides and the complements of the other three natural parts are called the circular parts; as they follow each other as it were in a circular order. Of these, any one being fixed upon as the middle part, those next it are the adjacent, and those farthest from it the opposite parts." Lord Buchan's Life of $\mathrm{Na}$ pier, pa. 98 .

This excellent, though very concise rule, including all the particular rules for the solution of right-angled spherical triangles, may be readily applied to those that are oblique, by letting fall a perpendicular, excepting the two cases in which there are given either the three sides, or the three angles. -And for thesc a similar expedient has been devised by Lord Buchan and Dr. Minto. M. Pingre, in the Memoires de Mathematique et de Physique for the year 1756 , reduces the solution of all the cases of spherical triangles to four analogies. These four analogies are in fact, under another form, Napier's rule of the circular parts, and his second or fundamental theorem, with its application to the supplemental triangle. Though it would be no difficult matter to get by heart the four analogics of M. Pingre, yet there are few persons blessed with a menory capable of retaining them for any considerable time. For this reason, the rule for the circular parts ouglit to be kept under its present form. If the reader attends to the circumstance of the sccond letters of the words tangents and cosines being the same with the first of the words adjacent and opposite, he will find it almost impossible to forget $i t$. And the rule for the solution of the two cases of spherical triangles, for which the former alone is insufficient, may be thus expressed: Of the circular parts of an oblique spherical triangle, the rectangle under the tangents of hali the sum and half the difference of the segments at the middle part (formed by a perpendicular drawn from an angle to the opposite side), is equal to the rectangle under the tangents of half the sum and half the difference of the opposite parts. By the circular parts of an oblique spherical triangle, are meant its three sides and the supplements of its three angles. Any of these six being assumed as a middle part, the opposite parts are those two of the same denomination with it; that is, if the misdle part is one of the sides, the opposite parts are the other two, and if the middle part is the supplenent of one of the angles, the opposite parts are the supplements of the other two.-Since every plane triannle maty be considered as described on the surfice of a splere of an intinite radius, these two rules inay be applied to plane triangles, provided the middle part be restricted to a side.

"'Thus it appears thrt two simple rules suffice for the solutiou of all the possible cuses ol plane and spherical 


\section{I R}

[ 3

triangles. These rules, from their neatness and the manner in which they are expressed, cannot fail of engraving themselves deeply on the memory of every one who is a little sersed in trigonometry. It is a circumstance worthy of notice, that a person of a very weak memory may carry the whole art of trigonometny in his head." Napier's Life, pa. 102.

Cincular Sailing, is that performed in the arch of a great circle.-It is chicfly on account of the shortest distance that this method of sailing has been proposed; and - for the most part it is advantageous for a ship to reach her port by the shortest course.

As the solutions of the cases in Mercator's sailing are performed by plane triangles; so the cases in great-circle sailing are resolved by the solution of spherical triangles. But, after all, the several cases in this kind of sailing serve rather for exercises in the solution of spherical triangles, than for any real use towards the navigating of a ship.

Circula r Spots, are made on pieces of metal by large electrical explosions. See Philos. Trans. vol. 5s, pa. 68; or my Abridgment, vol. 12, p. 510 ; also Priestley's Hist. of Electricity, vol. 2, sect. 9 , edit. 8 vo.

These beautiful spots, produced by the morlerate charge of a large battery, discharged between two smooth surfaces of metals, or semi-metals, lying at a small distance from each other, consist of one ceutral spot, and several concentric circles, which are more or less distinct, and more or fewer in number, as the metal on which they are marked is more easy or difficult of fusion, and as a greater or less force is employed. They are composed of dots or cavities, which indicate a real fusion. If the explosion of a battery, issuing from a pointed body, be repeatedly taken on the plain surface of a.piece of metal near the point, or be received from the surface on a point, the metal will be marked with a spot, consisting of all the prismatic colours disposed in circles, and formed by scales of the metal separated by the force of the explosion.

Circulan Velocity, a term in Astronomy, signifying that velocity of a planet, or revolving body, which is measured by the arch of a circle.

Circulating Decinals, called also recurring or re. peating decimals, are those in which a figure or several figures are continually repeated. They are distinguished into single and multiple, and these again into pure and mixed.

A pure single circulate, is that in which onc figure only is repeated; as " $222 \mathrm{\& c}$, and is marked thus $\cdot \dot{2}$.

A pure multiple circulate, is that in which several figures are continually repeated; as 2323 '23 \&c, marked $\cdot \dot{2} \dot{3}$; and 524524 \&c, marked $\cdot 52 \dot{4}$.

A mixed single circulate, is that which consists of a terd minate part, and a single repeating figure; as $4: 222 \& \mathrm{c}$, or $4: \dot{2}$. And

A nixed multiple circulate, is that which contains a terminate part with several repeating figures; as $45 \cdot \dot{5} \dot{4}$.

That part of the circulate which repcats, is called the repetend: and the whole repetend, supposed infinitely contisued, is cqual to a vulgar fraction, whose numerator is the repeating number, or figures, and its denominator the same number of nines : so $\cdot \dot{2}$ is $=\frac{2}{9}$; and $\ddot{2}$ is $=$ $\frac{23}{59} ;$ and $\cdot 52 \dot{4}$ is $=\frac{5}{9} \frac{24}{5}$.

It seems it was Dr. Wallis who first distinctly considered, or treated of infinite circulating decimals, as he himself informs us in his Treatise of Infinites. Since his time many other authors have treated on this part of arithmetic; the principal of these, however, to whom the art is mostly indebted, are Messrs. Brown, Cunn, Martin, Emerson, Malcolm, Donn, and Henry Clarke, in whose writings the nature and practice of this brauch of arithmetic may be fully seen, especially in the last-mentioned ingenious a uthor.

CIRCUMFERENCE, in a general sense, denotes the line or liues bounding any figure. But it is commonly used in a more limited sense, to denote the curve line which bounds a circle, and which is otherwise called the periphery; the boundary of a right-lined figure being expressed by the term perimeter.

The circumference of a circle is every where equidistant from the centre. And the circumferences of different circles are to one another as thcir radii or diameters, or the ratio of the diameter to the circumference is the same in every circle, which is nearly as 7 to 22 , as it was found by Archimedes, or, more nearly, as 1 to 3.1416. Under the article Circle may be seen various other approximations to that ratio, one of which is carried to 128 places of figures, viz, by M. De Lagny.

The circumference of every circle is supposed to be divided into 360 equal parts, called degrees.-Any part of a circumference is called an arc or arch; and a right line drawn from one end of an arc to the other, is called its chord.-The angle at the circumference is equal to half the angle at the centre, standing on the same arc; and thercfore it is measured by the half of that arc.

CIRCUMFERENTOR, a particular instrument used by surveyors for taking angles. It consists of a brass circle and index all of one piece; the diameter of the circle is commonly about 7 inches; the index about 14 inches long, and an inch and a half broad. On the circle is a card or compass, divided into 360 degrces; the meridian line of which answers to the middle of the breadth of the index. On the limb, or circumference of the circle, is soldered a brass ring; which, with another fitted with a glass, forms a kind of box for the needle, which is suspended on a pivot in the centre of the circle. There are also two sights to screw on, and slide up and down the index, as also a spangle and socket screwed on the underside of the circle, to receive the head of the three-legged staff.

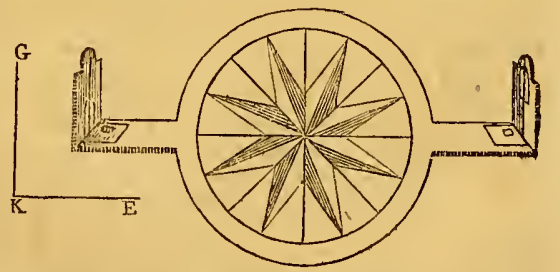

To take, or obserce, the 2uantity of in Angle by the Circumferentor. The angle proposed being EKG; place the instrument at $\mathrm{k}$, with the flower-de-luce of the card towards you; then direct the sights to $\mathrm{E}$, and observe what degrees are cut by the south end of the necdle, which let be 295 ; then, turning the instrument about on its stand, direct the sights to $G$, noting again what degrees are cut by the south end of the needle, which suppose are 213 . This done, subtract the less number from the greater, viz, $2 \mathrm{~T} 2$ 
213 from 2950 , and the remainder, or 82 degrees, is the cuantity of the angle $\mathrm{EKG}$ sought.

CIRCUMIGYRATION, is the revolving motion of any body about a ceutre; as of the planets about the sun, \&c.

CIRCUM-Pou a Stars, are those stars which, by reason of their vicinity to the pole, move round it, without setting.

CIRCUMSCRIBED Figure, is a figure which is drawn about another, so that all its sides or planes touch the latter or inscribed figure.

The area and perimeter of every polygon that can be circumscribed about a circle, are greater than those of the circle; and the area and perimeter of every inscribed polygon, are less than those of the circle; but they approach alvays nearer to equality as the number of sides is greater. And on these principles Archimedes, and some otber authors since his time, attempted the quadrature of the circle; which is nothing else, in effect, but the measuring the area or capacity of a circle.

Circumscribed Hyperbola, is one of Newton's hyperbolas of the $2 \mathrm{~d}$ order, that cuts its asymptotes, and contains the parts cut off within its own space.

CIRCUMVALLATION, or Line of Circumvallation, in the Art of War, is a trench, bordered with a parapet, thrown up around the besieger's camp, as a security against any army that may attempt to relieve the place, as well as to prevent desertion.

CIRCUMVOLUTION, in Architecture, the torus of the spiral line of the Ionic order.

CISSOID, is a curve line of the second order, invented by Diocles for the purpose of finding two continued mean proportionals between two other given lines. The generation or description of this curve is as follows :

On the extremity $B$ of the diameter $A \mathrm{~B}$ of the circle $\mathrm{AOB}$, erect the indefinite perpendicular $\mathrm{CBD}$, to which from the other extremity a draw several lines, cutting the circle in $\mathrm{r}, \mathrm{o}, \mathrm{N}, \& \mathrm{c}$; and on these lines set off the corresponding equal distances, viz, $\mathrm{HM}=A \mathrm{r}, \mathrm{FO}=\mathrm{AO}$, and $\mathrm{CL}$ $=A N$, \&c; then the curve line drawn through all the points $M, 0, L$, $\mathbb{E c}$, is the cissoid of Diocles, who was an ancient Greek geometrician.

This curve is, by Newton, reckoned among the defective hyperbolas, being the $42 \mathrm{~d}$ species in his Enumeratio-Linearum Tertii Ordinis. And in his Appendix de Equationum Constructione Lincari, at the end of his Arithmetica Universalis, he gives another elegant method of describing this curve by the continual motion of a square ruler. Otlier me-

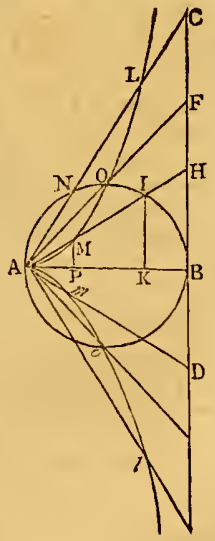
thods have also becn devised by different authors for the same thing.

The Properties of the Cissoid are the following:

1. 'The curve has two infinite legs 4 moL, amol meeting in a cusp $A$, and tending towards the indefinite line $\mathrm{CBD}$, which is their common asymptate.

2. 'The curve passes thruugh o and $o$, points in the circle equally distant from $A$ and $\mathrm{b}$; or it bisccts cach seinicircle.
3. Letting fall perpendiculars $3 \mathrm{P}$, I $\mathrm{K}$ from any corresponding points $\mathrm{I}$, $\mathbf{M}$; then is $\Lambda \mathrm{H}=\mathbf{B K}$, and $\mathbf{A M}=\mathbf{H} \mathbf{I}$, because $\mathrm{AI}=\mathbf{M} \mathbf{H}$.

4. AP : $\mathbf{P B}:: \mathrm{MP}^{2}: \mathrm{AP}^{2}$. So that, if the diameter $\mathrm{AB}$ $\mathrm{bc}=a$, the absciss $\mathrm{AP}=x$, and the ordinate $\mathrm{PM}=y$; then is $x: a-x:: y^{2}: x^{2}$, or $x^{3}=(a-x) \times y^{2}$, which is the equation of the curve.

5. Sir Isaac Newton, in his last letter to M. Leibuitz, has shown how to find a right line equal to one of the legs of this curve, by means of the hypeibola; but he there suppressed the investigation, which however may be scen in his Fluxions.

6 . The whole infinitely long cissoidal space, contained betwcen the infinite asymptote $\mathrm{CBD}$, and the curves Lonol \&c, of the cissoid, is cqual to triple the generating circle АОВОА.- Sce more of this curve in Dr. Wallis's Works, vol. 1, pir. 545.

CIVIL Day. See Day.

Crvil Month. See Month.

Civil Year, is the legal year, or annual account of time which every government appoints to be used within its own dominions. It is so called in contradistinction from the natural year, which is measured exactly by the revolution of the heavenly bodies.

CLAIRAUT (ALEXIS-ClAUDE), a celebrated French mathematician and academician, was born at Paris the 13th of May 1713, and died the 17 th of May 1765 , at 52 years of age. His father, a teacher of mathematics at Paris, was his sole instructor; and such was the progress of our author under him, that at the early age of 4 years, he could read and write. The letters of the alphabet he taught him in the figures of Euclid's Elements. At 9 years of age he studied Guisnée's Application of Algebra to Geometry; at 10 De l'Hôpital's Conie Sections; and between 12 and 13 he read a memoir to the Acadeny of Sciences concerning 4 new geometrical curves of his own invention. About the same time he laid the first foundation of his work upon curves that have a double curvature, which he finished in 1729 , at 16 years of age. He was named Adjoint-Mechanician to the Academy in 1731, at the age of 18, Associate in 1733, and Pensioner in 1738. During his connexion with the Academy, he had a great number of learned and ingenious communications inserted in their Memoirs, besides several other works which he published separately; the list of which is as follows:

1. On Curves of a Double Curvature; in 1730,4 to.

2. Elements of Geometry; 1741, svo.

3. Theory of the Figure of the Earth; 1743 , Sro.

4. Elements of Algebra; 1746 , Svo.

5. Tables of the Mloon; 1754, 8vo.

His papers inserted in the Memoirs of the Academy are too numerous to be particularized here; but they may be found from the year 1727, for almost every year till 1762; being upon a variety of subjects, astronomical, mathematical, optical, \&c.

CLARKE (DR. SA MEL), a celebrated English divine, philosopher, and metaphysician, was born at Norwich the 11 th of Oct. 1675 . He was the son of Fdward Clarke, esq, alderman of that city, and for several yenrs one of its representatives in parliament. He received instruction in classical learning at the free-school of that town; and in 1691 removed thence to Caius-college, Cambridge, where his shining abilities soon began to display themselves.Though the philosophy of Descartes was at that time the 
established system of the university, yet Clarke's sagacity soun discovcred its imperfections, and led him to the belief of one more solid and rational, viz, the new system of Newton: this he soon mastered; and in order to his first degree of arts, performed a public exercise in the schools upon a question taken from it. He greatly contributed to the establishment of the Newtonian philosophy by an excellent translation of Rohault's Physics, with notes, which he finished before he was 22 years of age, a book which had been for some time the system used in the university, and founded on Cartesian principles. This was first published in the year 1697, and it soon after went through several other editions, all with improvements.

Mr. Whiston relates that, in that year, 1697, while he was chaplain to Dr. Moore, bishop of Norwich, he met with young Clarke, then wholly tinknown to him, at a coffee-house in that city, where they entered into a conversation about the Cartesian philosophy, particularly Rohault's Physics, which Clarke's tutor, as he tells ns, had put him upon translating. "The result of this conversation was," says Whiston, "that I was greatly surprised that so young a man as Clarke then was, should know so much of those subline discoveries, which were then almost a secret to all but a few particular mathematicians. Nor did I remember (continues he) above one or two at the most, whom I bad then met with, that appeared to know so much of that philosophy as Mr. Clarke."

He afterwards turned his thoughts to diviuity; and laving taken holy orders, in 1698 he succeeded Mr. Whiston as chaplain to Dr. Moore, bishop of Norwich, who was ever after his constant friend and patron. Soon after he published some theological treatises; and-Bishop Moore gave him the rectory of Drayton, near Norwich, with a lectureship in that city. In 1704 , he was appointed to preach Boyle's lecture; and the subject he chose was, The Being and Attributes of God. He succeeded so well in this, and gave so much satisfaction, that he was appointed to preach the same lecture the next year, when he chose for his subject, The Evidences of Natural and Revealed Religion. These sermons were first printed in two volumes, in 1705 and 1706 , and contained some remarks on such objections that had been made by Hobbes and Spinoza, and other opposers of natural and revealed religion. In the 6 th edition was added, A Discourse concerning the Connexion of the Prophecies of the Old Testament, and the application of them to Christ.

About this time, Mr. Whiston informs us, he discovered that Mr. Clarke (having read much of the primitive writers) began to suspect that the Athanasian doctrine of the Trinity was not the doctrine of those early ages; auch it was particularly remarked of him, that he never read the Athanasian Creed at his parish church.

In the midst of his theological labours, Mr. Clarke found time to show his regard to mathematical and philosophical researches, with his exact knowledge and skill in them. And his natural affection and capacity for these studies were not a little improved by the frieniship of Newton; at whose request he translated his Optics into Latin in 1706. With this version Sir lsaac was so highly pleased, that he presented him with the sum of $500 \mathrm{l}$ or $100 \mathrm{l}$ to each of his 5 children.

The same year, also, Bishop Moore procured for him the rectory of St. Bennett's, Paul's Wharf, in London; and soon after carried him to court, and recommended him to the favour of Queen Anne. She appointed lim one of her chaplains in ordinary; and alsu presented him to the rectory of St. James's, Westminster, when it became vacant in 1709. On this occasion he took the degree of $D$. D. when the public excrcise which he performed for it at Cambridge was highly admired.

In 1715 and 1716 , Dr. Clarke had a dispute with the celebrated Leibnitz, concerning the principles of natural philosophy and religion; and a collection of the papers which passed between them was published in 1717 . This work was addressed to Queen Carcline, then princess of Wales, who was pleased to have the controversy pass through her hands. It related chiefly to the subjects of liberty and necessity.

About the year 1718 he was presented by the lord Lechmere to the mastership of Wigston's hospital in Leicestershire. In 1724 and 1725 he published 18 sermons, preached on several occasions. In 1727 , on the death of Newton, he had the offer of succeeding him as Master of the Mint, a place worth from 12 to 15 lundred a year; but to this secular preferment he could not reconcile himself, and therefore absolutely refused it. In 1728 was published, A Letter from Dr. Clarke to Mr. Benjamin Hoadley, occasioned by the Controversy relating to the Proportion of Velocity and Force of Bodies in Motion, which was printed in the Ptilosophical Transactions, No. 401. Dr. Clarke died the 17 th of May, 1729 , in the 54 th year of his age.

Queen Caroline took great pleasure in the doctor's conversation and friendship; seldom a week passed in which slie did not receive some proof of the greatness of his genius and the force of his understanding.

As to the character of Dr. Clarke, he is represented as possessing one of the most amiable and affectionate dispositions, remarkably free and easy in his conversation, cheerful and even playful in tis manner. Bishop Hare says of him, "He was a man who had all the good qualities that could meet together to recommend him. He was possessed of all the parts of learning that are valuable in a clergyman, in a degree that few possess any single one. He bad joined to a good skill in the three learnesl languages a great compass of the best philosophy and mathematics, as appears by his Latin works; and his Eriglish ones are such a proof of his own piety and of his knowledge in divinity, and have done so much service to religion, as would make any other man, that was not under a suspicion of heresy, secure of the friendship of all good churclimen, especially the clergy.-Much more may be seen, related in his praise, by Bishop Hoadley, Dr. Sykcs, and Mr. Whiston, in their Memoirs of his life.

CLAVIUS (Cnirstophen), a German Jesuit, was born at Bamberg in Germany, in 1537. He became a very studious mathematician and elaborate writer; his works, when collected, and closely printed, making 5 large folio volumes, being a complete body or course of mathematics. They are mostly elementary, with commentaries on Euclid and others, possessing very little of invention of his own. His talents and writings have been variously spoken of, and it inust be acknowledged that they are heavy and elaborate. He was invited to Rome, to assist, with other learned mcn, in the reformation of the calendar, by Pope Gregory, of which he afterwards undertook a defence against Scaliger, Vieta, and others, who attacked it. Hc died at Rome the 6 th of February, 1612, at 75 years of age, after 
more than 50 years close application to the mathematical sciences.

CLEF, or CLIF F, in Music, a mark at the beginuing of the lines of a song, which shows the tone or key in which the picce is to begin. Or, it is a letter marked on any line, which explains and poins out the names of all the rest.

Anciently, every line had a letter marked for a clef; but now a letter on one line suffices; since by this all the rest are known, reckoning up and down, in the order of the letters.

It is called the clef, or key, because that thereby are known the names of all the other lines and spaces; and consequently the quantity of every degree or interval. But because every note in the octave is called a key, though, in another sense, this letter marked is called peculiarly the signed clef; since, being written on any line, it not only signs and marks that one, but it also explains all the rest. By clef, therefore, for distinction sake, is meant that letter, signed on a line, which explains the rest; and by key, the principal note of a song, in which the melody closes.

There are three of these signed clefs, $c, f, g$. The clef of the highest part in a song, called treble, or alt, is $g$, set on the second line, rcckoning upwards. The clef of the bass, or the lowest part, is $f$, on the 4 th line upwards. For all the other mean parts, the clef is $c$, sometimes on one, and at others on another line. Indeed, some that are really mean parts, are sometimes set with the $g$ clef. It must however be observed, that the ordinary signatures of clefs bear little resemblance to those letters. Mr. Malcolm is of opinion that it would be well if the letters themselves were used. Kepler takes great pains to show, that the common signatures are only corruptions of the letters they represent.

The clefs are always taken fifths to one another. So the clef $f$ bcing lowest, $c$ is a fifth above it, and $g$ a fifth above $c$.

When the place of the clef is changed, which is not frcquent in the mean clef, it is with a design to make the system comprehend as many notes of the song as possible, and so to have the fewer notes above or below it. So that, if there be many lines above the clef, and few below it, this purpose is answered by placing the clef in the first or second line; but if there be many notes below the elef, it is placed lower in the system. In effect, according to the relation of the other nots to the clef note, the particular system is taken differently in the scale, the clef line making one in all the variety.

But-still, in whatever line of the particular system any clef 'is found, it must be understood to belong to the same of the general system, and likewise to be the same individual note or sound in the scale. By this constant relation of clefs, we learn how to compare the several particular systems of the severul parts, and to know how they communicate in the scale, that is, which lines are unison and which are not; for it is not to be supposed that each part has certain bounds, within which another may never enter-some notes of the treble, for example, must be lower than some of the mean parts, or even of the bass. 'Thercfore, to join together in to one system all the parts of a composition writt'n separately, the note of each particular part must be placed at the same distances above and below the proper clef, as they stand in the separate system: and because all the notes that are consonant, or heard together, must stand directly over each other, that the notes belong- ing to each part may be distinctly ascertained, they may. be made with such differences as shall not confound or alter their significations with respect to time, but only show their relation to this or that part. Thus we shall discover iu what manner the parts change and pass through one another; and which, in every note, is highest, lowest, or unison.

It must here be observed, that for the performance of any single piece, the clef only serves for explaining the intervals in the lincs and spaces: so that it need not be regarded what part of any greater system it is; but the first note may be taken as high or low as we please. For as the proper use of the scale is not to limit the abrolute degree of tone, so the proper use of the signed clef is not to bound the pitch at which the first note of any part is to be taken, but to determine the tune of the rest, with respect to the first; and considering all the parts together, to determine the relation of their several notes by the relations of their clefs in the scale: thus, their pitch of tune being determined in a certain note of one part, the other notes of that part are determined by the constant relations of the letters of the scale, and the notes of other parts by the relations of their clefs.

In effect, for performing any single part, the clef note may be taken in an octave, that is, at any note of the same name; provided we do not go too bigh or too low for finding the rest of the notes of a song. But in a concert of several parts, all the clefs must be taken, not only in the relations, but also in the places of the system abovementioned, that every part may be comprehended in it.

The natural and artificial note expressed by the same letter, as $c$ and $c$, are both set on the same line or space. When there is nu charactcr of flat or sharp, at the beginning with the clef, all the notes are natural; and if in any particular place the artificial note be required, it is denoted by the sign of a flat or sharp, set on the line a space before that note.

If a sharp or flat be placed at the broginning in any line or space with the clef, all the notes on that line or space are artificial ones; that is, are to be taken a semitone higher or lower than they would be without such sign. And the same affects all their octaves above and below, thongh they be not marked so. In the course of the song, if the natural note be sometimes required, it is signified by the character $b$.

CLEPSIDRA, a kind of water-clock, or an hour-glass serving to measure time by the fall of a certain quantity, commonly out of one vessel jinto another.-There have been also clepsydra made with quicksilver; and the term is also used for hour-glasses of sand.

By this instrunent the Egyptians measured thrir time and the course of the sun. N!lso Tycho Brabé, in modern times, made use of it to measure the motion of the stars, \&c; and Dudley used the same contrivance in making all his maritime observations.

The use of clepsydrax is very ancient. They were probably invented in Ligypt under the l'tolemies; though some authors ascribe the invention of them to the Greeks, and others to the Romans. Pliny informs us, that Scipio Nasica, about 150 ycars.betore Clurist, gave the first hint for the construction of them: and Pancirollus las particularly described them. According to his account, the clepsydra was a vessel made of glass, with a small hole in the bottom, edged with gold: in the upper part of this ressel 
a line was drawn, and marked with the 12 hours: the vessel was filled with water, and a cork with a pin fixed in it floated on the surface, pointing to the first hour; and as the water sunk in the vessel by issuing out of the small hole, the pin indicated the other hours as it descended. The same kind of instruments were also used by the Hindus, for mcasuring time; and from the invention might come into all the countries westward from them. See my Tracts, vol. 2, p. 178.

Clepsydræ were chiefly used in the winter; as sun-dials served for the summer. They had however two great defects; the one, that the water ran out more or less easily, as the air was more or less dense; the other, that the water flowed more rapidly at the beginning, than towards the conclusion when its quantity and pressure were much decreased. Amontons has invented a clepsydra which, it is said, is free from both these inconveniences; and the same effect is produced by one described by Mr. Hamilton, in the Philos. Trans. vol. 44, pa. 17 1, or my Abridg. vol. 9, pa. 236. Varignon also, in the Memoires de l'Acad. 1699 , delivers a geperal geometrical method of making clepsydræ, or water-clocks, with any kind of vessels, and with any given orifices for the water to run through.

Vitruvius, in lib. 9 of his Architecture, treats of these instruments; and Pliny in chap. 60 , lib. 7 , says that Scipio Nasica was the first who measured time at Rome by clepsydrx, or water-clocks. Gesner, in his Pandects, pa. 91, gives several contrivances for these instruments. Solomon de Caus also treats on this subject in his Reasons of Moving Forces, \&c. So also does Ozanam, in his Mathematical Recreations, in which is contained a Treatise on Elementary Clocks, translated from the Italian of Dominique Martinelli. There is likewise a treatise on HourGlasses by Arcangelo Maria Radi, called Nova Scienza de Horologi Polvere. See also the Technica Curiosa of Gasper Schottus; and Amontons Remarques et Experiences Physiques sur la Construction d'une nouvelle Clepsydre, excmpte des défauts des autres.

CLERC (Joms LE), a celebrated writer and universal scholar, was born at Geneva in 1657. After passing through the usual course of study at Geneva, he went to France in 1678; but returning the year after, he took holy orders. In 1682 Le Clerc visited England, to learn the language : but the smoky air of London not agreeing with his constitution, he snon returned to Holland, where he settled; and was appointed professor of philosophy, polite literature, and the Hebrew tongue, in the school at Amsterdam. Here he long continued to read lectures; for which purpose he drew up and published his Logic, Ontology, Pneumatology, and Natural Philosophy. He published also Ars Critica; a Commentary on the Old Testament; a Compendium of Universal History ; an Ecclesiastical History of the first two centuries; a French translation of the New Testament, and other works. In 1686 , he began, jointly with M. de la Crose, his Bibliotheque Universelle et Historique, in imitation of other literary journals; which was continued to the year 1693 , making 26 volumes. In 1703 he commenced his Bibliotheque Choisie, and continucd it to 1714 , when he began another work on the same plan, called Bibliotheque Anci(nne it Moderne, which he continued to the year 1728 ; all of them justiy esteemed excellent stores of useful knowledge. 1le published also, in 1713 , a small treatise on practical Geomitry, in 2 vols. small svo, called Pratique de la Geometrie, sur le papier et sur le terrain. In 1728 he was seized with a palsy and fever; and after spending the last six years of his life with little or no understanding, he died in 1736 , at 79 years of age.

CLIFF, in Music, the same as Clef, which see.

CLIMACTERIC, a critical year in a person's life.

According to some, this is every 7 th year : but others allow it only to those years produced by multiplying 7 by the odd numbers $3,5, \tau, 9$. These years, observe they, bring with them some remarkable change with respect to health, life, or fortune : the grand climacteric is the $63 \mathrm{~d}$ year; but some ald also the 81 st to it : the other remarkable climacterics are the 7 th, 21 st, 35 th, 49 th, and 56 th.

CLIMATE, or Clime, in Geography, a part of the surface of the earth bounded by two lesser circles parallel to the equator; and of such a breadth, as that the longest day in the parallel nearer the pole exceeds the longest day in that next the equator, by some certain space, as half an hour, or an hour, or a month.

The beginning of a climate, is a parallel circle in which the day is the shortest; and the encl of the climate, is that in which the day is the longest. The climates therefore are reckoned from the equator to the pole; and are so many zones or bands, terminated by lines parallel to the equator : though, in strictness, there are several climates, or different degrees of light or temperature, in the breadth of one zone. Each climate only differs from its contiguous ones, in that the longest day in summer is longer or shorter, by half an hour, for instance, in the one place than in the other.

As the climates commence at the equator, at the beginning of the first climate, that is at the equator, the day is just 12 hours long; but at the end of it, or at the beginning of the $2 d$ climate, the longest day is 12 hours and a half long; and at the end of the $2 d$, or begiuning of the $3 \mathrm{~d}$ climate, the longest day is 13 hours long; and so of the rest, as far as the polar circles, where the hour climates terminate, and month climates conmence. And as an hour climate is a space comprised between two parallels of the equator, in the first of which the longest day exceeds that in the latter by an hour; so the month climate is a space contained between two circles parallel to the polar circles, and having its longest day longer or shorter than that of its contigunus one, by a month, or 30 days. But some anthors, as Riccioli, make the longest day of the contiguous climates to differ by half hours, to about the latitude of 45 degrees; then to differ by an hour, or sometimes 2 hours, to the polar circle; and after that by a month each. Sue tables of climates in Varenius, chap. 25, prop. 13.

The ancients, who confined the climates to what they thought the habitable parts of the carth, reckoned only seven, the middles of which they made to pass through some remarkable places; as the 1st through Moroe, the 2d through Sienna, the Sil through Alexandria, the 4th through. Rhodes, the 5 th through Rome, the 6th through Pontus, and the 7 th through the mouth of the Borysthenes. But the moderns, who have sailed farther towards the poles, make 30 climates on cach sille.

Vulgarly the term climate is bestowed on any country or region differing from another either in respect of the seasons, the quality of the soil, or even the manners of the inhabitants; without any regard to the length of the. longest day. $\Lambda$ bulfedat, an Arabic author, distinguishes 
the first kind of climates by the term Real Climates, and the latter by that of Apparent Climates.

CLOCK, a machine now constructed in such a nanner, and so regulated by the uniform motion of a pendulum, as to measure time, and all its subdivisions, with great exactness. Before the invention of the pendulum, a balance, not unlike the tly of a kitchen-jack, was used instead of it. - They were at first called nocturnal dials, to distinguish then trom sun-dials, which showed the bour by the shadow of the sun.

The invention of clocks with wheels is ascribed to $\mathrm{Pa}-$ cificus, archdeacon of Verona, in the 9 th century, on the credit of an epitaph quoted by Ughelli, and borrowed by him from Panvinius. Others attribute the invention to Buethius, about the year 510 .

Mr. Derham however reduces clock-work to a much older date; ranking Archimedes's sphere, made 200 years before Christ, as mentioned by Claudian, and that of Posidonius, mentioned by Cicero, among machines of this kind: not that either their form or use were the saine with those of modern mechanics; but that they had their motion from some hidden weights, or springs, with wheels or pulleys, or some such clock-work principle.

In the Disquisitiones Monasticæ of Benedictus Haëften, published in the year 1644, we are informed, that clocks were invented by Silvester IV, a monk of his order, about the year 998,' as Dithmarus and Bozius have also shown; for before that time, they had nothing but sundials and clepsydræ for pointing out the hour.-Conrade Gesner, in his Epitome, pa. 604, says, that Richard Wallingford, an English abbot of St. Albans, who flourished in the year 1326, made a wonderful clock by a most excellent art, the like of which could not be produced by all Europe-Moreri, under the word Horologe du Palais, observes, that Charles v, called the wise king of France, ordered at Paris the first large clock to be made by Henry de Vie, whom he sent for from Germany, and set it upon the tower of his palace, in the year 1372 . -John Froissart, in his Histoire et Chronique, vol. 2, ch. 28, says, the duke of Bourgogne lad a clock, which sounded the hour, taken away from the city of Courtray, in the year 1382: and the same thing is said by $\mathrm{Wm} . \mathrm{Pa}-$ radin, in his Annales de Bourgogne.

Clock-makers were first introduced into England in 1368, when Edward 111 granted a licence for three artists to come over from Delft in Holland, and practise their occupation in this country.

The water-clocks, or clepsydræ, and sun-dials, have both a much better claim to antiquity. The French annals mention one of the former kind, sent by Aarnn king of Persia, to Charlemagne, about the year 807 , which it would seem bore some resemblance to the modern clocks : it was of brass, and showed the hours by 12 little balls of the same metal, which at the end of each hour fell upon a bell, and made a sound. There were also figures of 12 cavaliers, which at the end of each hour came out through certain apertures, or windows, in the side of the clock, and shut them again, \& $x$.

The invention of pendulum clocks is owing to the happy industry of the 17 th century; and the honour of that discuvery is disputed betwcen Galileo and Huygens. The latter, who wrote an excelleut volume on the subject, declares it was first put in practice in the year 1657, and the description of it printed in 1658. Becher, De Nova
Temporis dimetiendi Theoria, anno 1680 , contends for Galileo; and relates, though at second-hand, the whole history of the invention; adding that one Trefler, clockmaker to the father of the then grand duke of Tuscany, made the first pendulum clock at Florence, under the direction of Galileo Galilei, a pattern of which was brought to Holland. And the Academy del Cimento expressly observes, that the application of the pendulum to the movement of a clock was first proposed by Galileo, and put in practice by his son Vicenzo Galilei, in 1649. But whoever may have becn the inventor, it is certain that the invention never flourished till it came into the hands of Huygens, who insists, that if ever Galileo thought of sueh a thing, he never brought it to any degree of perfection. The first pendulum clock made in England was in the year 1662 , by one Fromantil, a Dutchman.

Among the modern clocks, those of Strasburg and Lyons are very eminent for the richness of their furniture, and the variety of their motions and figures. In the former, a cock claps his wings, and proclaims the hour : the angel opens a dour, and salutes the Virgin, \&c. In the latter, two horsemen encounter, and beat the hour upon each other: a door opens, and there appears on the theatre the Virgin, with Jesus in her arms; the Magi, with their retinue, marching in order, and presenting their gifts; two trumpeters sounding all the while to proclaim the procession.

These however are far excelled by two that have lately been made by English artists, as a present from the EastIndia company to the einperor of China. These two clocks are in the form of chariots, in each of which a lady is placed, in a fine attitude, leaning her right hand on a part of the chariot, under which appears a clock of curious workmanship, little larger than a shilling, that strikes and repeats, and goes for eight days. On the lady's finger sits a bird, fincly.modelled, and set with diamonds and rubies, with its wings expanded in a flying posture, and actually flutters for a considerable time, on toucling a diamond button below it; the body of the bird, in which are contained part of the wheels that animate it as it were, is less than the 16 th part of an inch. The lady holds in her left-hand a golden tube little thicker than a large pin, on the top of which is a small round box, to which is fixed a circular ornament not larger than a sixpence, set with diamonds, which goes round in three hours in a constant regular motion. Over the lady's bead is a double unbrella, supported by a small futed pillar not thicker than a quill, and under the larger of which a bell is fixed at a considerable distance from the clock, with which it secnss not to have any connexion; but from which a communication is secretly conveyed to a hammer, that regularly strikes the hour, and repeats the same at pleasure, by touching a diamend button fixed to the clock below. At the feet of the lady is a golden dog; before which, from the point of the chariot, are two birds fixed on spiral springs, the wings and feathers of which are set with stones of various colours, and they appear as if tying away with the chariot, which, from another secret motion, is contrived to run in any direction, either straight or circular, \&e; while a boy, that lays hold of the chariot behind, appears also to push it forwards. Above the umbrella are flowers and ornanents of precious stones; and it terminate's with a flying dragon set in the same manner. The whole is of gold, most curiously executed, and embellished with rubies and pearls. 
The ingenious Dr. Franklin contrived a clock to show the hours, minutes, and seconds, with only three wheels and two pirions in the whole movement. The dial-plate hat the hours engraven on it in spiral spaces along two diameters of a circle, containing four times 60 minutes. The index goes round in four liours, and points out the minutes from any hour by which it has passed to the next following hour. The snall hand, in an arch at top, goes round once in a minute, and shows the seconds. The clock is wound up by a line going over a pulley, on the axis of the great wheel, like a common 30-hour clock. Many of these very simple machines have since been constructed, that measure time excecdingly well. This clock is subject, however, to the inconvenience of requiring frequent winding, by drawing up the weight; as also to some uncertainty as to the particular hour sliown by the index.

Mr. Ferguson proposed to remedy these inconveniences by another construction, which is described in his Select Exercises, pa. 4. This clock will go a week without winding, and always shows the precise hour; but, as Mr. Ferguson acknowledges, it has two disadvantages which do not belong to Dr. Franklin's clock: when the minute hand is adjusted, the hour plate must also be set right, by means of a pin: and the smallness of the teeth in the pendulum wheel will cause the pendulum ball to describe but small arcs in its vibrations; and therefore the momentum of the ball will be less, and the times of the vibrations will be more affected by any unequal impulse of the pendulum wheel on the pallets. Besices, the weight of the flat ring, on which the seconds are engraven, will load the pivots of the axis" of the pendulum wheel with a great deal of friction, which ought by all possible means to be avoided. To remedy this inconvenience, the seconds plate inight be omitted:

Mr. Ferguson also contrived a clock, showing the apparent diurnal motions of the sun and moon, the age and phases of the moon, with the time of her coming to the meridian, and the times of high and low water; and all this by laving only two wherls and a pinion addled to the common inovement. See his Selcct Exercises before mentioned. In this clock the figure of the sun serves as an hour index, by going round the dial-plate in 24 hours; and a figure of the moon goes round in $24 \mathrm{~h} .50 \frac{\mathrm{x}}{2} \mathrm{~min}$. the time of her going round in the heavens from any meridian to the same meridian again. A clock of this kind was adapted by Mr. Forgusen to the movement of an old watch. See also a description and drawing of an astronomical clock, showing the apparcnt daily motions of the sun, moon, and stars, with the times of their rising, soutlling, and setting; the places of the sun and moon in the ccliptic, and the age and phases of the moon for every day in the year, in tlie same book, p. 19.

There have been several treatises upon clocks; the principal of which are the following: Hieronymus Cardan, de Varietate Rerum, libri 17.-Conrade Dasypodius, Descriptio Horologii Astronomici Argentinensis in summa Templi erecti-Guido Pancirollus, Antiqua deperdita et nova reperta.-L'Usage du Cadran, ou de l'Horloge Physique Universelle, par Galilée, Mathematicien du Duc de Florence.-Oughtred's Opuscula Mathematica.-Huygens's Horologium Oscillatorium.-Pendule perpetuelle, par l'Abbé de Hautcfeuille.-J. J. Becheri Theoria et Expericntia de nova Temporis dimeticndi Ratione et Horologiorum Constructione._-Clark's Oughtredus explicatus, ubi de Constructione Horologiorum,-Horological VoL. I.
Disquisitions. - Huygens's Posthumous Works.-Sully's Regle Artificielle du Temps, \&c.-Serviere's Recueil d'(iuvrages Curieux.-Derham's Artificial Clock-maker.-Camus's Traités des Forces Mouvantes. - Alexandre's Traité Général des Horologies.-Also 'Treatises and Principles of Clock-making, by Hatton, Cuming, \&c, \&c.

CLOUD, a visible collection of vapours, suspended in the atmosphere.

Though it be generally allowed that the clouds are formed from the aqueous vapours, which before were so closely united with the atmosphere as to be invisible; it is however not easy to accuunt for the long continuance of some very opaque clouds without dissolving; or to assign the reason why the vapours, when they have once begun to condense, do not continue to do so till they at last fall to the ground in the form of rain or snow, \&c. It is now known, that a separation of the latent heat from the water, of which vapour is composed, is attended with a condensation of that vapour in some degree; in such case, it will first appear as a smoke, mist, or fog; which, if interposed between the sun and earth, will form a cloud; and the same causes continuing to operate, the cloud will produce rain or snow. It is however abundantly evident that some other cause, besides mere heat or cold, is concerned in the formation of clouds, and the condensation of atmospherical vapours. This cause is esteemed in a great measurc the electrical fluid: indeed electricity is now so generally admitted as an agent in all the great operations of nature, that it is no wonder to find the formation of clouds attributed to it; and this has accordingly been given by Beccaria as the cause of the formation of all clouds whatsoever, whether of thunder, rain, hail, or snow.

But whether the clouds are produced, that is, the atmospheric vapours rendered visible, by means of clectricity or not, it is certain that they do often contain the electric fluid in prodigious quantitics, and many terrible and destructive accidents have heen occasioned by clouds very highly electrified. The most cxtraordinary instance of this kind perhaps on record happened in the island of Java, in the East-Indies, in August, 1772 . On the 11th of that month, at midnight, a bright cloud was observed covering a mountain in the district called Cheribou, and several reports like those of a gun were heard at the same time. The people who dwelt on the upper part of the mountain not being able to fly fast enough, a great part of the cloud, eight or nine miles in circumference, detached itself under them, and was seen at a distance, rising and falling like the waves of the sea, and emitting globes of fire soluminous, that the night became as bright as day. The effects of it were astonishing; every thing was destroyed for 20 miles round; the houses were demolished; plantations were buried in the earth; and 2140 people lost their lives, besides 1500 head of cattle, and a vast number of horses, goats, \&c. Another remarkable instance of the dreadful effects of electric clouds, which happened at Malta the 29th of October 1757 , is also related in Brydone's Tour through Malta.

The height of the clouds is not usually great; the summits of high mountains being commonly quitc free from thern, as many travellers have experienced iu passing these mountains. It is found that the most highly clectrified clouds descend lowest, their height being often not more than 7 or 800 yards above the carth's surface; and sometimes thunder-clouds appear actually to touch the ground $2 \mathrm{U}$ 
with one of their edges; but the generality of clouds are suspended at the height of a inile, or little more, above the varth.

The motions of the clouds, though often directed by the wind, are not always so, especially when thunder is about to ensue. In this case they are seen to move very slowly, or even to appear quite stationary fọ some time. The reason of this probably is, that they are impelled by two opposite streams of air nearly of equal strength; and in such cases it appears that both the aerial currents ascend to a considerable height ; for Messis. Charles and Robert, while endeavouring to avoid a thunder-cloud, in one of their aerial voyages with a balloon, could find no alteration in the course of the curreut, though they ascendet to the height of 4000 feet above the earth. In some cases the motions of the clouds evidently depend on their clectricity, independent of any current of air whatever. Thus, in a calm and warm day, small clouds are often seen meeting each other in opposite directions, and setting out from such short distances, that it cannot be supposed that any opposite winds are the cause. Such clouds, when they meet, instead of forming a larger one, become much smaller, and sometimes entirely vanish; a circumstance most probably owing to the discharge of opposite electricities into each other. And this serves also to throw some light on the true cause of the formation of clouds; for if two clouds, the one electrified positively, and the other negatively, destroy each other on contact, it follows that any quantity of vapour suspended in the atmosphere, will, while it retains its natural quantity of electricity, remain invisible, but becomes a cloud when clectrified either plus or minus.

The shapes of the clouds arealso probably owing to their electricity; for in those seasons in which a great commotion has been excited in the atmospherical electricity, the clouds are seen assuming strange and whinsical forms, that are continually varying. This, as well as the meeting of small clouds in the air, and vanishing upon contaet, is a sure indication of thunder.

The uses of the clouds are evident, as from them proceeds the rain that fructifies the earth, and without which, according to the present state of mature, the whole surface of the earth would soon become a mere desert. They are also useful as a screen interposed between the earth and the scorching rays of the sul, which are often so powerful as to destroy the grass and other tender vegetables. In the more secret operations of nature also, where the electric fluid is concerned, the clouds bear a principal share; and chiefly serve as a medium for conveying that fluidfrum the atmosphere into the earth, and from the earth into the atmosphere: in doing which, when electrified to a great degree, they sometimes produce very terrible effects; an instance of which is related above. See the articles A $\mathrm{r}$ MOSPHERICAL ELECTRICITY, Eva PORATION, RAIN, \&c.

CLOUTS, in Artillery; are thin plates of iron nailed on that part of the axle-trce of a gun-carriage which comes through the nave, and through which the linspin goes.

CLUVIER, or Cluviuus, (P\|iLjP), a celebrated gengrapher, was born at Dantyic iı 1580. After an edu- cation at home, he travelled into Poland, Germany, and the Netherlands, to improve himself in the knowledge of the law. But, when at Leyden, Joseph Scaliger persuaded him to give way to his genius for geography. In pursuance of this advice, Cluvier visited the grealest part of the European states. He was well skilled in many lan- guages, speaking ten with facility, viz, Grcek, Latin, Gexman, French, English, Dutch, Italian, Hungarian, Polish, and Bohemian. On his return to Leyden, he taught there with great applause; and died in 1623 , being only 43 years of age, justly esteened the first geographer who had put his researches in order, and reduced them to certain principles. He was author of several ingenious works in geography, viz:

1. De Tribus Rheni Alveis.

2. Germania Anticua.

3. Italia Antiqua, Sicilia, Sardinia, et Corsica.

4. Introductio in Universam Geographiam.

COASTING, is that part of Navigation in which the places are not far asunder, so that a ship may sail in sight of land, or within soundings, between them.

COCHLEA, one of the five Mechanical powers, otherwise called the Screw ; being so named from the resen-blance a screw bears to the spiral shell of a snail, in Latin Cochlea. See Screw, and Mechan ical Pozvers.

COCK of a Dial, the pin, stile, or gnomon.

COEFFICIENTS, in Algebra, are numbers, or given quantities, usually prefixed to letters, or unknown quantities, by which it is supposed they are multiplied; and so, with such letters, or quantities, making a product, or coefficient production; whence the name.

Thus, in $3 a$ the coefficient is 3 , in $b x$ it is $b$, and in $c x^{2}$ it is $c$. If a quantity have no number prefixed, unity or 1 is understood; as $a$ is the same as $1 a$, and $b c$ the same as 1bc. The name coefficient was first given by Vieta.

In any equation so reduced as that its highest power or term has 1 for its coefficient; then the coefficient of the $2 \mathrm{~d}$ term is equal to the sum of all the roots, buth positive and negative ; so that if the $2 \mathrm{~d}$ term is wanting in an equation, the sum of the positive roots of that equation is equal to the sum of the negative roots, as they mutually balance and cancel each nther. Also the coefficient of the 3d term of an equation is equal to the sum of all the rectangles arising by the multiplication of every two of the roots, how many ways soever they can be combined by twos; as once in the quadratic, 3 times in the cubic, 6 times in the biquadratic equation, \&c. And the coefticient of the 4 th term of an equation, is the sum of all the solids made by the continual multiplication of every three of the roots, how often soever such a ternary can be had; as once in a cubic, 4 times in a biquadratic, 10 times in an equation of 5 dimensions, \&c. And thus it goes on infinitely.

Confricients of the same Order, is a term sometimes used for the coefficients prefixed to the same unknown quantities, in different equations.

$$
\text { Thus in the equations }\left\{\begin{array}{l}
a x+b y+c z=m, \\
d x+c y+f z=n, \\
g x+h y+k z=p,
\end{array}\right.
$$

the coefficients $a, d, g$, are of the same order, being the coefficients of the same letter $x$; also $b, c, h$, are of the same order, being the cocfficients of $y$; and so on.

Opposile Coefricients, such as are taken each from a different equation, and from a diflerent order of cocfficients. Thus, in the foregoing equations, $a, e, k$, or $a, h, f$, or $d, b, k$, \&c, are opposite coeflicionts.

CINLlistial. Sec Cezestual.

COFFli, in Architecture, a square depressure or sinking, ils cach interval between the modillions of the Corinthian cornice; usually filled up with a rose; sometimes with a ponesranate, or other enrichment. 
Coffer, in Fortification, denotes a hollow lodgment, athwart a dry moat, 6 or 7 fect deep, and 16 or 18 broad. The upper part of $i t$ is made of picces of timber, raised 2 feet above the level of the moat; the elevation baving hurdles laden with earth for its covering, and serving as a parapet with embrazures.

The coffer is nearly the same with the caponiere, excepting that this last is sometimes made beyond the counterscarp on the glacis, and the coffer always in the moat, taking up its whole breadth, which the caponiere docs not. It differs from the traverse and gallery, in that these are made by the besiegers, and the coffer by the besieged.

The besieged commonly malie use of coffers to repulse the besiegers, when they endeavour to pass the ditch. And, on the other hand, the besiegers, to save themselves from the fire of these coffers, throw up the earth on that side towards the coffer.

CoFFER-Dams, or Batardeaur, in Bridge-building, are inclosures formed for laying the foundation of piers, and for other works iu water, to exclude the surrounding water, and so prevent it from interrupting the workmen.

These inclosures are sometimes single, and sometimes double, with clay rammed between them; sometimes they are made with piles driven close together, and sometimes the piles are notched or dove-tailed into one another; but the most usual method is, to drive piles with grooves in them, at the distance of five or six feet from each other, and then boards are let down between them, after which the water is pumped out.

COGGESHALL'S Sliding-Rule, an instrument used in Gauging, and so called from its inventor. See the description and use under SLIDING-Rule.

COHESION, in Natural Philosophy, as distinguished from adhesion, is that species of attraction which, uniting particle to particle, retains together the component parts of the same mass or kind. Or it is that action or power by which the homogeneous particles of bodies are kept attached to each other.

This power was first considered by Newton as one of the properties essential to all matter, and the cause of all that variety observed in the texture of different terrestrial bodies. He does not attempt to investigate the nature of this power, but wisely contents himself with calling it a mutual attraction peculiar to the particles of matter. His words are as follow: "The particles of all hard homogeneous bodies, which touch one another, cohere with a great force; to account for which, some philosophers have recourse to a kind of hooked atoms, which in effect is nothing else but to beg the thing in question. Others imagine that the particles of bodies are connected by rest, i.e. in effect by nothing at all; and others by conspiring motions, i. e. by a relative rest among thenselves. For - myself, it rather appears to me, that the particles of bodics cohere by an attractive force, whereby they tend mutually toward each other: which forci, in the very point of contact, is very great; at little distances is less, and at a little farther distance is quite insensible."

It is uncertain in what proportion this force decreases as the distance increases: Desaguliers conjectures, from some pheriomena, that it decrease's as the biquadratic or 4 th power of the distance, so that at twice the distance it acts 16 times more weakly, \&c.

"Now if compound bodies he so hard, as by experience we find some of them to be, and yet have a great many hidden pores within them, and consist of parts only laid together; no doubt those simple particles which have no pores within them, and which were never divided into parts, must be vastly harder. For such hard particles, gathered into a mass, cannot possibly touch in more than a few points; and therefore much less force is required to sever them, than to break a solid particle, whose parts touch throughout all their surfaces, without any intermediate pores or interstices. But how such hard particles, only laid together, and touching only in a few points, should come to cohere so firmly, as in fact we find they do, is inconceivable; unless there be some cause, whereby they are attracted and pressed together. Now the smallest particles of matter may cohere by the strongest attractions, an constitute larger, whosea ttracting force is feebler: and again, many of these larger particles cohering, may constitute others still larger, whose attractive force is still weaker; and so on for several successions, till the progression end in the biggest particle, on which the operations in chemistry, and the colours of natural bodies depend; and which by cohering compose bodies of a sensible magnitude."

Again, the opinion maintained by many, is that which is so strongly defended by J. Bernuulli, De Gravitate IEtheris; who attributes the cohesion of the parts of matter to the uniform pressure of the atmosphere: confirming this opinion by the known experiment of two polished marble planes, which cohere very stongly in the open air, but easily drop asunder in an exhausted recejver. However, if two plates of this kind be smeared with oil, to fill up the pores in their surfaces, and prevent the lodgment of air, and onc of them be gently rubbed upon the other, they will adhere so strongly, even when suspended in an exhausted receiver, that the weight of the lower plate will not be able to separate it from the upper one. And though this theory might serve tolerably well to explain the cohesion of compositions, or greater collections of matter ; yet it falls far short of accounting for that first cohesion of the atoms, or primitive corpuscules, of which the particles of hard bodies are composed; for a botly does not fall in pieces in the exhausted receiver.

Again, some philosophers have positively asserted, that the powers, or means, are immaterial, by which matter coleres; and, in consequence of this supposition, they have so refined upon attractions and repulsions, that their systems appear but little short of scepticism, or denying the existence of matter altogether. A system of this kind is adopted by Dr. Priestley, from Messrs. Boscovich and Michell, to solve some difficulties concerning the Newtonian dactrine of light. See his History of Vision, vol. 1, pa. 392. "The easiest method," says Priestley, " of solving all difficulties, is to adopt the hypothesis of MIr. Boscovich, who supposes that inatter is not impenetrable, as has becn perhaps universally taken for granted; but that it consists of physical points only, endued with powers of attraction and repulsion in the same manner as solicl matter is generally supposed to be: provided therefore that any body moves with a sufficient degree of velocity, or has a sufficient momentum to overcome any powers of repulsion that it may meet with, it will find no difficulty in making its way through any body whatever; for nothing clse will penetrate each other but powers, such as we know do in fact exist in the same place, and counterbalance or over-rule each other. The most obvious difficulty, and indeed almost the only one, that attends this hypothesis, as it supposes the mutual penetrability of $\approx \mathrm{U} \approx$ 


\section{$\mathrm{C} \mathrm{OH}$}

[ 339

matter, arises from the idea of the nature of matter, and the perplexity wo neet with in attempting to force two bodies into the same place. But it is demonstrable, that the first obstruction arises from no actual contact of matter, but from mere powers of repulsion. This difficulty we can overcome; and having got within one sphere of repulsion, we fancy that we are now impeded by the solid matter itself. But the very same is the opinion of the generality of mankind with respect to the first obstruction. Why, therefore, may not the next be another sphere of repulsion, which may only require a greater force than we can apply to make it yield without disordering the arrangement of the constituent particles; but which may be overcome by a body moving with the astonishing ve. locity of light."

Other philosophers have supposed that the powers, both of gravitation and cohesion, are material; and that they are only different actions of the ethereal fuid, or elementary fire. In proof of this doctrine, they allege the experiment with the Magdeburg hemispheres, as they are called. The pressure of the atmosphere, we know, is, in this case, capable of producing a very strong cohesion; and if there be in nature any fluid nore penetrating, as well as more powerful in its effects, than the air we breathe, it is possible that what is called the attraction of cohesion may in some measure be an effect of the action of that fluid. Such a fluid as this is the element of fire. Its activity is such as to penetrate all bodies whatever : and in the state in which it is commonly called fire, it acts according to the quantity of solid matter contained in the body. In this state it is capable of dissolving the strongest cohesions observed in nature. Fire, therefore, being able to dissolve cohesions, must also be capable of causing them, provided its power be exerted for that purpose, which possibly it may be, when we consider its various modes or appearances, viz, as fire or heat, in which state it consumes, destroys, and dissolves ; or as light, when it seems deprived of that destructive power; and as the electric fluid, when it attracts, repels, and moves bodies, in a great variety of ways. In the Philos. Trans. for 1777 this hypothesis is noticed, and in some measure adopted by Mr. Henley. "Some gentlemen (he observes) have supposed that the electric matter is the cause of the cohesion of the particles of bodies. If the electric matter be, as I suspect, a real elementary fire inherent in all bodies, that opinion may probably be well founded; and perhaps the soldering of metals, and the cementation of iron, by fire, may be considered as strong proofs of the truth of their hypothesis."

But whatever the cause of cohesion may be, its effects are evident and certain. The different degrees thereof, constitute bodies of different forms and properties. Thus, Newton observes, the particles of fluids, which do not cohere too strongly, and are small enough to render them susceptible of those agitations which keep liquors in a fluor, are most easily separated and rarcfied into vapour, and make what the chemists call volatile bodies; being rarefied with an easy heat, and again conclensed with a moderate cold. Those that have grosser particles, and so are less susceptible of agitation, or coliere by a stronger attraction, are not separable without a greater degree of heat; and some of them not without fermentation; and these constitute what the chemists call fixed bodies.

Air, in its fixed state, possesses the interstices of sulic
$\mathrm{C} \mathrm{O} \mathrm{H}$

substances, and probably serves as a bond of union to their constituent parts; for when these parts are separated, the air is discharged, and recovers its elasticity. And this kind of attraction is evinced by a variety of faniliar experiments : as, by the unios of two contiguous drops of mercury; by the mutual approach of two pieces of cork. floating near each other in a bason of water; by the adhesion of two leaden balls, whose surfaces are scraped and joined together with a gentle twist, which is so considerable, that, if the surfaces are about a quarter of an inch in diameter, they will not be separated by a weight of $100 \mathrm{lb}$. by the ascent of oil or water between two glass planes, $s$ ) as to form the hyperbolic curve, when they are made to touch on one side, and kept scparate at a small distance on the other; by the depression of mercury, and by the rise of water in capillary tubes, and on the sides' of glasy vessels; also in sugar, sponge, and all porous substances And where this cohesive attraction ends, a power of tepulsion begins.

To determine the force of Coliesion, in a variety of different substances, many experiments bave bcen made, and particularly by Professor Muschenbroek. The adhesion of polished planes, about two inches in dianeter, heated in boiling water, and smeared with grease, required the fols lowing weights to separate them:

$\begin{array}{ccccc}\text { Plane of Glass } & & \text { Cold Grease } & & \text { Hot Grease } \\ \text { Brass } & - & 130 \mathrm{lb} & - & 300 \mathrm{lb} \\ \text { Copper } & - & 150 & - & 800 \\ \text { Marble } & - & 225 & - & 850 \\ \text { Silver } & - & 150 & - & 600 \\ \text { Iron } & - & 300 & - & 950\end{array}$

But when the brass planes were inade to adhere by other sorts of matter, the results were as in the following table :

$\begin{array}{lccc}\text { With Water } & - & - & 1202 \\ \text { Oil } & - & - & 1 \mathrm{~S} \\ \text { Venice Turpentine } & - & 24 \\ \text { Tallow Candle } & - & - & 800 \\ \text { Rosin } & - & - & 850 \\ \text { Pitch } & - & - & 1400\end{array}$

In estimating the Absolute Cohesion of solid pieces of bodies, he applied weights to separate them according to their length : his pieces of wood were long square parallelopipedons, each side of which was 26 of an inch, or abont $\frac{x}{4}$, and they were drawn asunder by the following weights :

$\begin{array}{lccc}\text { Fir } & - & - & 600 \mathrm{lb} \\ \text { Elm } & - & - & 950 \\ \text { Alder } & - & - & 1000 \\ \text { Linden-tree } & - & - & 1000 \\ \text { Oak } & - & - & 1150 \\ \text { Beech } & - & - & 1250 \\ \text { Ash } & - & - & 1250\end{array}$

He tried also wires of metal, 1-10th of a Rhinland inch in diameter: the metals and weights were as follows:
Of Lead
Tin
Copper
$29 \frac{\pi}{4}$
$-\quad 40 \frac{\pi}{4}$
$-\quad 299 \frac{7}{4}$
Yollow Brass
Silver
Iron
Gold
-
$29 \frac{\pi}{4}$
360
370
450
500

He then tried the Relative Cohesion, or the force with which bodics resist in action applied to them in a direction 
perpendicular to their length. For this purpose he fixed pieces of wood by one end into a square hole in a metal plate, and hung weights towards the other end, till they broke at the hole: the weights and distances from the hole are exhibited in the following table:

$\begin{array}{lllll} & & \text { Distance } & & \text { Weight } \\ \text { Pine } & - & 9 \frac{\pi}{2} \text { inc. } & - & 36 \frac{\mathrm{I}}{2} \mathrm{oz} . \\ \text { Fir } & - & 9 & - & 40 \\ \text { Beech } & - & 7 & - & 56 \frac{\mathrm{I}}{2} \\ \text { Elm } & - & 9 & - & 44 \\ \text { Oak } & - & 8 \frac{x}{2} & - & 4 \mathrm{~S} \\ \text { Alder } & - & 9 \frac{1}{4} & - & 48\end{array}$

See his Elem. Nat. Philos. cap. 19.

The inquisitive reader may further consult Ritter on Cohesion; Gilbert's Journal, iv, 1 ; Benzenberg on Cohesion, xvi, 76 ; Fontana on Solidity and Fluidity, Soc. Ital. i, 89 ; and Dr. T. Young on the Cohesion of Fluids, in the Philos. Trans. 1805, or in the $2 \mathrm{~d}$ vol. of his Nat. Philos.

COIGNET ( native of Antwerp, who flourished in the latter part of the 16th century, and died in 1623 . He published a work on navigation in the Elemish language; which was afterwards enlarged, and published in French, under the title of "Instruction nouvelle des Points plus excellents et necessaires touchant l'Art de Naviger." This served as a supplement to Medina's book on the same subject, whose mistakes are well exposed by Coignet. In treating of the sun's declination, he took notice of the gradual decrease in the obliquity of the ecliptic, a point long disputed, but now settled from the theory of attraction, as well as long observation. He also described the cross-staff with three transverse pieces, which he mentioned as being then in common use among mariners; but he preferred that of Gemma Frisius. He mentioned likewise some instruments of his own invention, which however are uow quite disused. As the old sea-table, above noticed, erred more and more in advancing towards the poles, he set down another, to be used by such as sailed beyond the 60th degree of latitude. At the end of the book is delivered a method of sailing on a parallel of latitude, by means of a ring dial, and a 24-hour glass; on which the author very much values himself.

COLD, the privation of heat, or the opposite to it.

As it is supposed that heat consists in al particular motion of the parts of a hot body, hence the nature of cold, which is its opposite, is deduced ; for it is found that cold extinguislies, or rather abates heat; hence it would seem to follow, that thuse bodies are cold, which check and restrain the motion of the particles in which heat consists.

In general, cold contracts most bodies, and heat expands tluem: though there are some instances to the coutrary, especially in the extreme cases or states of these qualities of borlies. Thus, though iron, in common with other bodies, expand with heat, yet, when melted, it is always found to expand in cooling again. So also, though water always is found to expand gradually as it is heated, and to contract as it cools, yet in the act of freezing, it suddenly expands again, and that with a most enormous force, capable of rending rocks, or bursting the very thick shells of metal, \&c. A computation of the force of freezing water has been made by the Florentine Academicians, from the bursting of a very strong brass globe or shell, by freezing water in it; when, from the known thickness and tenacity of the metal, it was found that the expansive power of a spherule of water only one inch in diameter, was sufficient to overcome a resistance of more than 27,000 pounds, or 13 tons and a half. See also experiments on bursting thick iron mortar-shells by freezing water in them, by Major Edward Williams of the Royal Artillery, in the Edinb. Philos. Trans. vol.2.

Such a prodigious power of expansion, almost double that of the most powerful stearm-engines, and exerted in so small a mass, seemingly by the force of cold, was thought a very powerful argument in favour of those who supposed that cold, like heat, is a positive substance. Dr. Black's discovery of latent heal, however, has now afforded a very easy and natural explication of this phenomenon. He has shown, that, in the act of congelation, water is not cooled more than it was before, but rather becomes warmer : that as much heat is discharged, and passes from a latent to a sensible state, as, had it been applied to water in its fluid state, would have heated it to $135^{\circ}$. In this process, the expansion is oćcasioned by a great number of minute bubbles suddenly produced. Formerly these were conjectured to be cold in the abstract ; and to be so subtile, that, insinuating themselves into the substances of the fluid, they augmented its bulk, at the saine time that, by impeding the motion of its particles on cach other, they changed it from a fluid to a solid. But Dr. Black shows that these are only air extricated during the congelation; and to the extrication of which he ascribes the great expansive force exerted by freezing water. The only question therefore now rcmaining, is, by what means this air comes to be extricated, and to take up more room than it naturally does in the fluid. To this it may be answered, that perhaps part of the heat which is discharged from the freezing water, combines with the air in its unclastic state, and, by restoring its elasticity, gives it that extraordinary force, as is seen also in the case of air suddenly extricated in the explasion of gunpowder.

Cold also usually tends to make bodies electric, which are not so naturally, and to increase the electric properties of such as are so. And it is farther found, that all substances do not transmit cold with equal facility; but that the best conductors of electricity, viz, metals, are also the best conductors of cold. It may farther be added, that when cold has been carried to such an extremity as to render any body an electric, it then ceases to conduct the cold so well as before. This is exemplified in the practice of the Laplanders and Siberians; where, to exclude the extreme cold of the winters from their habitations the more effectually, and to admit at the same time a small portion of light, they cut pieces of ice, which in the winter season inust always be electric in those countries, and place them in their windows; which they find to be much more eflictual in keeping out the cold, than any other substance.

Cold is the destroyer of all vegetable life, when increased. to an excessive degree. It is found that many garden plants and flowers, which seem to be very stout and hardy, go off at a little increase of cold beyond the ordinary standard. And in severe winters, nature has provided tlie best natural defence for the corn-fields and gardeus, by a covering of snow, which preserves such parts green and liealthy as are under it, while such as are not covered by it are either killed or greatly injured.

Dr. Clarke is of opinion, that cold is owing to certain nitrous, and other saline particles, endued with particular 
figures proper to produce such effects. Hence, sal-ammoniac, saltpetre, or salt of urine, and many other volatile and alkalizate salts, mixed with water, very much increase its degree of cold. In the Pbilos. Trans. number 274 , M. Geoffroy relates some remarkable experiments with regard to the production of cold. Four onisces of sal-animoniac dissolved in a pint of water, made his thermorneter descend 2 inches and $\frac{3}{4}$ in less than 15 miuutes. An ounce of the same salt put into 4 or 5 ounces of distillerl water, made the thermometer descend 2 inches and $\frac{x}{4}$. Half an ounce of sal-ammoniac mixed with 3 ounces of spirit of nitre, made the thermometer descend 2 inches and $\frac{5}{12}$; but, on using spirit of vitriol instead of nitre, it sunk 2 inches and $\frac{\mathrm{r}}{2}$. In this last experiment it was remarked, that the vapours raised from the mixture had a considerable degrce of heat, though the liquid itself was so extremely cold. Four ounces of saltpetre mixed with a pint of water, sank the thermometer an inch and $\frac{x}{4}$; but a like quantity of sea salt sank it only $\frac{x}{6}$ of an inch. Acius'always produced heat, even common salt with its own spirit. Volatile alkaline salts produced cold in proportion to their purity, but fixed alkalies heat.

But a far greater degree of cold produced by the mixture of salts and aqueous fluids, was that shown by Homberg; who gives the following receipt for making the experiment: Take a pound of corrosive sublimate, and as much sal-anmoniac; powder them separately, and mix the powders well; put the mixture into a phial, pouring upon it a pint and a half of distilled vinegar, shaking all well together. This composition becomes so cold, that it can scarce be held in the hand in summer; and it happened, as MI. Homberg was making the experiment, that the matter froze. The same thing once happened to $M$. Geoffroy, in making an experiment with sal-ammoniac and water, but it never was in his power to make it succeed a second time.

If, instead of making these experiments with fluid water, it be taken in its congealed state of ice, or rather snow, degrees of cold will be produced greatly superior to any that have yet been mentioned. A mixture of snow and common salt sinks a Fahrenheit's thermometer to 0 ; potashes and pounded ice sank it $S$ degrees farther; two affusions of spirit of salt on pounded ice, sank it $14 \frac{x}{2}$ below 0 ; and by repeated affusions of spirit of nitre M. Fahrenheit sark it to $40^{\circ}$ below 0 . This is the ultimate degree of cold which the mercurial thermometer will measure; for the mercury itself begins then to congeal; and therefore recourse must afterwards be had to spirit of wine, naphtha, or some other fluid that will not congeal. The greatest degree of cold hitherto produced by artificial means, has been $80^{\circ}$ below 0 ; which was done at lludson's Bay by means of snow and vitriolic acid, the thermometer standing naturally at $20^{\circ}$ below 0 . Indecd greater degrees of cold than this have been supposed: Mr. Martin, in his Treatise on Heat, relates, that at Kirchga in Siberia, the mercurial thernometer sunk to $115^{\circ}$ below 0 ; and Professor Braun at Petersburg, when he made the first experiment of congealing quicksilwer, fixed the point of congelation at $350^{\circ}$ below 0 ; but from later experiments it has been more accurately determined, that $40^{\circ}$ below 0 is the freezing point of quicksilver.

The most renarkable experiment however was made by Mr. Walker of Oxford, with spirit of nitre poured rin Glauber's salt, the effect of which was fouml to be similar to that of the same spirit poured on ice or snow; alw the addition of sal-ammoniac rendered the cold still more intense. The proportions of these ingredients recommended by Mr. Walker, are concentrated nitrous acid two parts by weight, water one part; of this mixture, cooled to the temperature of the atmosphere, 18 ounces; of Glauber's salt, a pound and a half avoirdupoise; and of sal-ammoniac, 12 ounces. On adding the Glauber's salt to the nitrous acid, the thermometer fell $52^{\circ}$, viz, from 50 to -2 ; and on the addition of the sal-ammoniac it fell to -9 . Thus Mr. Walker was able to freeze quicksilver without either ice or snow, when the thermoneter stood at $45^{\circ}$; viz, by putting the ingredients in 4 different pans, and inclosing these within each other.

Excessive degrees of cold also occur naturally in many parts of the globe in the winter season.

Though the thernometer in this country seldom descends so low as 0 , yet in the winter of $17 \mathrm{So}$, Mir. Wilson of Glasgow observed, that a thermometer laid on the snow sunk to $25^{\circ}$ below 0 ; and Mr. Derham, in the year 1708 , observed in England, that the mercury stood within onetenth of au inch of its station when plunged into a mixture of snow and salt. At Petersburg, in 1732, the thermometer stond at $28^{\circ}$ below 0 ; and when the French academicians wintered near the polar circle, they observed the thermometer at $33^{\circ}$ below 0 ; and in the Asiatic and American continents still greater degrees of cold are often obscrved. Lately, Professor Leslie, of Edinburgh, has frozen water, merely by evaporation in the exhausted receiver of an air-pump; and, it is said, he can freeze mercury in the same way. Mercury has also bcen frozen by Dr. Marcet, by the evaporation of ether. See Nicholson's Journal, v. 34, pa. 119; also the article FR E EZING in this Dictionary. Further, a paper of Dr. Alarcet's was read at the Royal Society, July 8, 1813 , on the intense degree of cold produced by the sulphuret of carbon. From the author's experiments it appears that this liquor is the most evaporable of all known fluids, or at least to produce, by its evaporation, the most intense degree of cold. If the bulb of a spirit-thermometer, closely enveloped in fine Aamnel, or cotton wool, be moistened with the fluid, its temperature falls to about 0 ; but if the thermometer be exposed to the effect of a vacuum, by being enclosed in the recciver of a good air-pump, it sinks to $-80^{\circ}$, in a minute or two. The congelation of mercury in glass tubes may therefore be nost quickly and easily performed by this process at all seasons, and under any atmospheric temperature.

In the last bulletin of the retreat of the French from Moscow, in November and December 1812, the cold, there stated at 16 and $1 \mathrm{~s}$ below freezing, is perhaps to be understood as being on the scale of Reaumur. The thermometer most in use through Lngland is Fabrenheit's; and upon that scale the cold above mentioned by the French rould be from 8 to 11 below zero; a degree in England very rarely known, but common enough amidst the horrors of Russia; where, in 1773 , the Empress Catharine made the famous experiment on the ordnauce shell, of the largest diameter, $1 \mathrm{~s}$ inches. The shell was filled with water, in her presence, as she states, and in less than an hour the whole was so frozen as to burst, and with no small explosion !"Avec beaucoup de fracas!" Some few years before, there had been another curious experiment by Prince. Orlorf, who, in a foundation for a massy brick coach gateway to his court-yard, in a soil so loose and wet, that pile's must have been driven, filled so many corresponding cuvities with water, on which, when turned by the frost into 
columns, he raised the projected supcrstructure! And at that time (1773) it had lasted four years, and so might be likely to last for ever! The words of the report are, "Une parte cochere voutée en briques, et très solide! Elle existe dcpuis quatre ans; et elle existera, je crois, jusqu'a ce qu'on l'abatte!".

'The effects of these extreme degrecs of cold are very surprising. Trees are burst, rucks rent, and rivers and lakes frozen several fect deep: metallic substances blister the skiu, like red-hot iron: the air, when drawn in by breathing, hurts the lungs, and excites a crugh: even the effects al fire in a great measure secms to ceasc; and it is obscrved, that though metals are kept for a considerable time before a strong fire, they will still freeze water when thrown upon them. When the French mathematicians wintered at Tornea in Lapland, the external air, when suddenly admitted into their room's, converted the moisture of the air into whirls of snow ; their breasts seemed to be rent when they breathed it, and the contact thereof was intolerable to their bodics; and the spiric of wine, which had not been highly rectified, burst some of their thermometers by the congelation of the aqueous part.

Extreme cold often proves fatal to animals in those countries where the winters are very severe; thus 7000 Swedes perished at once in attempting to pass the mountains which divide Norway from Sweden. But it is not necessary that the cold, in order to destroy human life, should be so very intense as has just been mentioned; it is only requisite to be a little below $32^{\circ}$ of Fahrenheit, or the freezing point, accompanied with snow ar liail, from which shelter cannot be obtained. 'The snow which falls upon the clothes, or the uncovered parts of the body, then inelts, and by a continual cvaporation carries off the animal heat to such a degree, that a sufficient yuantity is not left for the support of life. In such cases, the person first feels himself extremely chill and uncasy; he turns listless, unwilling to walk or use exercise to kecp himself warm, and at last turns drowsy, sits down to refresh himself with slecp, but awakes no morc. See FreEzıx .

A striking illustration of these effects of cold is related by captain Cook, in an occurrence which took place during a botanical excursion of Sir Juseph Banks and Dr. Solander among the hills of Terra del Fuego. The party, consisting of 11 persons, were overtaken by darkness, and obliged to spend the night on the bills, during extreme cold. Dr. Solander, who had more than ouce crossed the mountains which divide Sweden from Norway, well knew that extreme cold, especially whet joined with fatigue, produces a torpor and sleepiness that are almost irresistible; he therefore conjured the company to keep moving, whatever pains it might cost them, and whatever relief they inight be promised by an inclination to rest: "Whoever sits down," said he, "will sleep; and whocver slecps, will wake no more." Thus at once admonisherl and alarmed, they set forward; but whilc they wcre still upon the naked rock, and before they had got anong the bushes, the cold became suddenly so intense, as to produce the effect that had been most dreaded; Dr. Solander himself was the first who foumil the inclination, against which he had warned others, irresistible, and insisted upon being suffered to lie down. Mr. Banks entreated and renonstrated in vain; duwn he lay upon the ground, though it was covered with stow; and it was with great difficulty thit his friend kept bin from slceping. Richmond also, one of the black scrvants, began to linger, having suffered from the cold in the same manner as the Doctor. Mr. Banks therefore sent 5 of the company forward to get a fire ready at the first convenient place they could find; and himself, with 4 others, remained with the Doctor and Richmond, whom partlyby persuasion and entreaty, and partly by force, they brought in; but when they harl got through the greater part of the birch and swamp, they both declared they could go no farther. Mr. llanks had recourse again to entreaty and expostulation, but they produced no effect. When Richmond was told that if he did not go on he would in a short time be frozen to death, he answered that be desired nothing but to lie down and die. The Doctor did not so explicitly renounce his life; be said he was willing to go on, but that he must first take sume sleep, though he bad before told the company that to sleep was to perish. Mr. Banks and the rest found it impossible to carry them, and there being no remedy, they were both suffered to sit down, being partly supported by the bushes; and in a few minutes they fell into a profound sleep. Soon after some of the people who had been sent forwarl returned with the welcome news, that a fire was kindled about a quarter of a mile farther on the way. Mr. Banks then endeavoured to wake Dr. Solander, and happily succeedcd; but, though be had not slept five minutes, he liad almnst lost the use of his limbs, and the muscles were so shrunk, that his shoes fell from his feet; - he consented to go forward with such assistance as could be given him; but no attempts to relieve poor Richmond wcre successful. He, together with another black left with bim, died. Several others began to lose their sensibility, having been expased to the cold and snow near an hour and a half, but the fire recovered them. See Captain Cook's first Voyage; also Rees's Cyclopædia, art. Cold.

COLLECTOR, in Electricity, is a small appendage to thic prime conductor of the electrical machine, and generally consisting of pointed wires, affixed to that end of the prime conductor which stands contiguous to the glass globe, or cylinder, or other electric of the machine. Its office is to receive the electricity, whether positive or negative, from the excited electric, much more rearlily than the blunt end of the prime conductor would be able to receive it, without that appendage.

COLLEGE (Royal MifTary), was instituted in 1799, under the direction of 12 commissioners, a governor, and professor of mathematics. It consists of two departments; the senior, established first at High Wycombe, and the junior at Great Marlow, in Buckinghamshire, each under the care of a commandant, superintendant, and adjutant, and under the instruction of a number of able masters in different branches of science. But in the year 1813 the sehior department has been removed to Faruham, and the junior to Sandhurst, near Bagshot, Surrey. The pupils in the senior departinent are young officers who have received their commissions; thase in the junior department are gentlemen cadets, amounting to ahout 3 or 400 in number, are cducated and fitted for commissions in the army, each cadet payilg a certain annual suin, towards the expense of his ellucation and maintenance, \& $c$.

College, Naral, the new name of the Natial Academy at Portsmouth. Sce ACADEMY.

COLLIMATION, Line of, in a telesenpe, is a line passing through the intersection of those wires that are fixed in the focus of the object-glass, and the centre of the sime glass.

COLLINS (Joux), an eminent accountant und mathe- 
Inatician, was born at Wood Eaton near Oxford, March 5, 1624. Lie received his education from his father, a dissenting minister, and at 16 years of age was put apprentice to a bookseller at Oxford ; hut soon after the commencement of the civil wars he became a clerk of the Prince of Wales's kitchen. In this situation be was under the superintendence of Mr. Marr, a good niathematician, and famous for the dials with which he adorned the king's gardens. From hence he travelled abroad, to prosecute his favouritc study; and on his return he took upon him the profession of an accountant, and published, in the year 1632 , a large work entitled, An Introduction to Merchants Acconsts; which was followed by several other publications on different branches of accounts. In $165 \mathrm{~s}$, he pullished a treatise called The Sector on a Quadrant; containing the description and use of four several quadrants, each accommodated to the making of sun-dials, \&c; to which he afterwards added an appendix concerning reflected dialling, from a glass placed reclining.- In 1659 , he published bis Geometrical Dialling; and the same year also bis Mariner's Plain Scale new plained,-Collins now became a Fellow of the Royal Society in London, to which he inade various communications; particularly some ingenious chronological rules for the calendar, printed in the Philos. Trans. number 46, for April 1669: also a curious dissertation concerning the resolution of equations in numbers, printed also in the Trans. for March 1671: an elegant construction of the curious problem, Having given the mutual distances of three objects in a plane, with the angles made by them at a fourth place in that plane, to find the distance of this place from each of the three former, vol. 6, pa. 2093: and thoughts about some defects in algebra, vil. 14 , pa. 375 .

Collins wrote also several commercial tracts, highly acceptable to the public; viz, A Plea for bringing over Irish cattle, and keeping out the fish caught by forcigners:For the promotion of the Einglish fishery:-For the working of the Tin-mines:-A discourse on Salt and Fishery. He was frequently consulted in nice and critical cases of accounts, of commerce, and engincering. On one of these occasions, being appointed to inspect the ground for cutting a canal or river between the lsis and the $A$ von, he contracted a disorder by drinking cider when he was too warm, which ended in his death the 10th of November 1683 , at 59 years of age.

Mr. Collins was a very useful man to the sciences, keeping up a constant correspondence with the most learned men, both at home and abroad, and promoting the publication of many valuable works, which, but for him, ivould never have been introducel to the public; particularly Dr. Barrow's optical and geometrical Lectures; his abridgment of the Works of Archimedes, Apollonius, and 'Theodosius; Branker's transiattion of Rhronius's Algebra, with Dr. Pell's additions, \&c ; which were procured by his fiequent solicitations.

After a considerable time had elapsed, his papers were all delivered into the hands of the learned and ingenious Arr. William Jones, F. sr. s, among which were found manuscripts, on mathematical subjects, of Briggs, Oughtred, Barrow, Newton, Pell, and niany others. From a variety of letters from these, and many other celcbrated mathematicians, it appears that Collins spared neither pains nor expense to procure what tended to promote real scicnce: and even many of the late discoveries in physical know. ledge are indebted to hin for their inprovement; for while he excited some to make known every now and useful invention, he employed other's to improve them. Sometimes he was pecutiarly useful, by showing where the defect was in any useful branch of science, pointing out the difficultits attending the inquiry, and at other times settirg forth the advantages, and kceping up a spirit and warm desire for improscment. Mr. Collins was also as it wcre the register of all the new improvements made in the mathematical sciences; the magazine to which the curious had frequent recourse: in so much that he acquired the appellation of the English Mersennus. If some of his corresponcients had not olliged bim to conceal their communications, there could have becn no dispute about the priority of the invention of a method of analysis, the honour of which evidently belongs to Newton; as appears undeniably from the papers printed in the Commercium Epistolicum D. Joannis Collins et aliorum de Analysi promota; jussu Societatis Regix in lucem editum, 1712 ; a work that was composed from the letters in the possession of our author.

Collins's' 2uadrant. See Quadrant.

COLLISION, is the percussion or striking of bodies against each other. Striking bodies are considered either as elastic, or non-elastic. They may also be either both in motion, or one of them in motion and the other at rest. When non-elastic bodies strike, they adhere together as one mass; which, after collision, either remains at rest, or moves forward as one body. But when elastic bodies strike, they always separate after the stroke. 'The principal theorems relating to the collision of bodies, are the following:

-1. If any body impinge or act obliquely on a plane surface; the force or energy of the stroke, or action, is as the sine of the angle of incidence. Or the force upon the surface, is to the same when acting perpeudicularly, as the siue of incidence is to radius.

2. If one body act on another, in any direction, and by any kind of force; the action of that force on the second body is made only in a direction perpendicular to the surface on which it acts.

3. If the plane, acted on, be not absolutely fixed, it will move, after the stroke, in the direction perpendicular to its surface.

4. If a body $\Lambda$ strike another body $\mathrm{B}$, which is eitherat rest, or else in motion, either towards $A$ or from it; then the momenta, or quantities of motion, of the two bodirs, estimated in any one direction, will be the very same after the stroke that they were before it.

$$
\begin{array}{lll}
A & B & C
\end{array}
$$

Thus, first, il $A$ with a momcntum of 10 , strike $B$ at rest, and communicate to it a momentum of 4 , in the sirction AB. Then there will remain in a conly a momentum of 6 in that direction: which together with the momentum of 3 , viz 4 , makes up still the same momentum betwen them as before- - But it is were in motion before the stroke, with a momentum of 5 , in the same direction, and reccive from $A$ an additional momentum of $2:$ then the motion of $A$ after the stroke will be 8 , and that of $\mathrm{B}, \bar{T}$; which together make 15 , the same as 10 and 5 , the motions before the stroke.-Lastly, if the borlies move in opposite directions, and meet eacl other, namely $A$ with a motion of 10 , and $n$, of 5 ; and $A$ communicate to $B$ a motion of 6 in the direction $A$ in of its motion: then, before the stroke, the whole motion from both, in thedirection $\triangle \mathrm{B}$, is $10-5$, or 


\section{O L}

5 : but after the stroke the motion of $A$ is 4 in the direction $\mathbf{A B}$, and the motion of $\mathbf{B}$ is $6-5$, or 1 , in the same direction $A B$; therefore the sum $4+1$, or 5 , is still the same motion from both as it was before.

5. If a hard and fixed plane be struck either by a soft or a hard unelastic body; the body will adhere to it. But if the plane be struck by a perfectly elastic body, it will rebound from it with the same velocity with which it struck the plane.

6. The effect of the blow of the elastic body, upon the plane, is double to that of the non-elastic one; the velocity and mass being the same in both.

7. Hence, non-elastic bodies lose, by their collision, but half the motion that is lost by elastic bodies; the masses and velocities being equal.

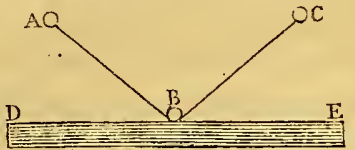

8. If an elastic body $A$ impinge upon a firm plane $D E$ at the point $\mathrm{B}$, it will rebound from it in an angle equal to that in which it struck it; or the angle of incidence will be equal to the angle of reflection: namely, the angle $A B D$ $=\mathrm{c} \mathrm{B}$.

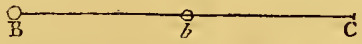

9. If the non-elastic body $B$; moving with the velocity $\mathrm{v}$ in the direction $\mathrm{B} b$, and the body $b$ with the velocity $v$, strike each other, the direction of the motion being in the line BC; then they will move after the stroke with a common velocity, wbich will be more or less according as, before the stroke, $b$ moved towards $\mathrm{B}$, or from $\mathrm{B}$, or was at rest; and that common velocity, in each of these cases, will be as follows: viz,

$\frac{\mathrm{ev}+b y}{\mathrm{~B}+b}$ when $b$ moved from $\mathrm{B}$,

$\frac{\mathrm{Bv}-l v}{\mathbf{B}+b}$ when $b$ moved towards $\mathbf{B}$,

$\frac{\mathrm{BV}}{\mathrm{B}+b}$ when $b$ was at rest.

For example, if the bodies or weights, B and $b$, be $5 \mathrm{lb}$ and $3 \mathrm{lb}$; and their velocities $\mathrm{v}$ and $v, 60$ feet and 40 feet per second; then 300 and 120 will be their momenta B V and $b v$, and $8=\mathrm{B}+b$ the sum of the weights. Consequently the common velocity after the stroke, in the three cases above-mentioned, will be thus, viz,

$\frac{300+120}{8}=\frac{420}{8}$ or $52 \frac{1}{2}$ in the first case,

$\frac{300-120}{8}=\frac{180}{8}$ or $22 \frac{1}{2}$ in the second case,

$\frac{300}{8}$ - - or $37 \frac{1}{2}$ in the third case.

10. If two perfectly clastic bodies impinge on each other; their relative velocity is the same both before and after the impulse; that is, they will recede from each other with the sune velocity with which they approached and met.

It is not meant lowever by this theorem, that each body will have the very same velocity after the impulse as it had bcfore; for that will be varied according to the relation of the masses of the two bodies; but that the velocity of the one, after the stroke, will be so much increased, and the wher decreased, as to have the same difference as before,

Vox. I. in one and the same direction. So, if the elastic body $\mathrm{E}$ move with the velocity $\mathrm{v}$, and overtake the elastic body $b$, moving the same way, with the velocity $v$; then their relative velocity, or that with which they strike, is only $v-v$; and it is with this same velocity that they separare from each other after the stroke: but if they meet each other, or the body $b$ move contrary to the body $\mathrm{B}$; then they meet and strike with the velocity $r+v$, and it is with the same velocity that they separate again, and recede from each other after the stroke : in like manuer, they would separate with the velocity $\mathrm{v}$ of $\mathrm{B}$, if $b$ were at rest before the stroke. Also the sum of the velocities of the one body, is equal to the sum of the others. But whether they nove forwards or backwards after the impulse, and with what particular velocities, are circumstances tbat depend on the various masses and velocities of the bodies before the stroke, and are as specified in the next theorem.

11. If the two elastic bodies $B$ and $b$ move directly towards each other, or directly from each other, the former with the velocity $\mathrm{v}$, and the latter with the velocity $v$; then, after their meeting and impulse, the respectjve velocities of $\mathbf{B}$ and $b$ in the direction $\mathbf{B C}$, in the three cases of motion, will be as follow: viz,

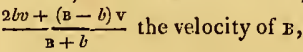
$\frac{2 \mathrm{BV}-(\mathrm{B}-b) v}{\mathrm{~B}+b}$ the velocity of $b$ when the bodies both moved towards $\mathrm{c}$ before the stroke; and

$$
\begin{aligned}
& \frac{-2 b v+(B-b) v}{B+b} \text { the velocity of } B, \\
& \frac{2 B+(B-b) v}{B+b} \text { the velocity of } b,
\end{aligned}
$$

when в moved towards $\mathbf{c}$, and $\boldsymbol{b}$ towards в before the stroke; also,

$\frac{\mathrm{B}-b}{\mathrm{~B}+b} \times \mathrm{v}$ the velocity of $\mathrm{B}$;

$\frac{2 B}{B+b} \times \vee$ the velocity of $b$,

when $b$ was at rest before the stroke.

12. The motions of bodies after impact, that strike each other obliquely, are thus deternined.

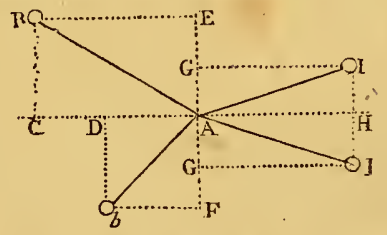

Let the two bodies $\mathbf{B}, b$, move in the oblique diréctions $B A, b_{A}$, and strike each other at $A$, with velucities which are in proportion to the lines $\mathrm{B} A, b_{\Lambda}$. Let $\mathrm{CAH}_{\mathrm{A}}$ represent the plane in which the bodies touch in the point of concourse; to which draw the perpendiculars $\mathbf{B C}, b_{\mathbf{D}}$, and complete the rectangles CE, DF. Now the motion in BA is resolved into the two $\mathrm{BC}, \mathrm{CA}_{\mathrm{A}}$; and the motion in $\boldsymbol{b}_{\mathrm{A}}$ is re. solved into the two $b \mathrm{D}, \mathrm{DA}$; of which the antecedents $\mathrm{BC}$, $b \mathrm{D}$ are the velocities with which they directly meet, and the consequents $\mathbf{C A}_{A}, \mathbf{D A}$ are parallel, and therefore by these the bodics do not impinge on each othej, and consequently the motions according to these directions will not be changed by the impulse; so that the volocities with which the bodics mect, are as $\mathrm{EC}$ or $\mathrm{E} A$, and $b \mathrm{D}$ or $\mathrm{FA}$. $2 \mathrm{X}$ 
The motgons thereforc of the bodies $\mathrm{B}, b$, directly striking each other with the celerities EA, FA, will be determined by art. 11 or 9 , according as the bodies are elastic or nonclastic; which being done, let AG be the velocity, so determined, of one of them, as $A$; and since there remains also in the same body a force of moving in the direction parallel to $\mathrm{BE}$, with a velocity as $\mathrm{BE}$, make $\mathrm{AH}$ equal to $B E$, and complete the rectangle $G n$ : then the two motions in $A \mathrm{~A}$ and $\mathrm{AG}$, or $\mathrm{H} \mathrm{I}$, are compounded into the diagonal $A \mathrm{I}$, which therefore will be the path and celerity of the body в after the stroke. And after the same manner is the motion of the other body $b$ determined after the inpact.

13. The state of the common centre of gravity of bodies is not affected by the collision or other actions of those bodies on each other. That is, if it were at rest before their collision, so will it be also at rest after collision: and if it were moving in any direction, and with any velocity, before collision; it will do the very șame after collision.

See more on this subject under the article Percussion.

COLONNADE, a peristyle of a circular figure; or a series of columns disposed in a circle, and insulated withinside.

COLOUR, a property inherent in light, by which, according to the various sizes of its parts, or from some other cause, it excites different vibrations in the optic nerve; which, propayated to the sensorium, affect the mind with different sensations. See the doctrine of colours fully explained under Chromatics. See also O P'T ICs, ACH RoMatic, and TELESCOPE.

COLSON (Joir), a respectable mathematician, who flourished about the beginning of the 18th century, and who appears to have been a Cambridge scholar. - IVe first notice him as author of some papers- in the Philos. Trans. viz, one in vol. 25 (an. 1707) on The Universal Resolution of Cubic and Biquadratic Equations; .a second in vol. 34, on Negative-Affirmative Aritlumetic; and 3dly, in vol. 39, on the Construction and Use of Spherical Maps. He was also author of sevcral separate works; as, The British Hemisphere, being a map of a new contrivance, in the form of a half globe, of about 15 inches in diameter, but comprehending the whole known surface of the habitable eartl, the city of London being the centre or vertex of the map. In 1736 he published a translation from the Latin of Sir Isaac Newton's Metbod of Fluxions and Infinite Series, with a large comment on the whole work, consisting of annotations, illustrations, and supplements. At the date of this publication Mr. Colson was in the situation of inaster of Sir' Joseph Williamson's free mathematical school at Rocliester, and a F. R.s. Three years after this we find he succeeded Mr. Sanderson as Lucasian Professor of Mathematics at Cambridge, a situation which he held for 20 years, after which he was succeederl, in 1759 , by Dr. Waring; though Mr. Colson lived till Dec. 20, 1760. The character of our author seems to have been chiefly that of minute precision and patient laborious industry. His paper on the solution of cubic and biquarlatic cquations above-mentioned, is founded icaily on the idea of the solution by Descartes, by assuming the biquadratic as equal to the product of two quadratics with incleterminate coefficients. The construction of the equations also, by means of the circle and parabola, is in imitation of the like constructions of Descartes in his Geometry, and of $\mathrm{Mr}$. Baker in his Geometrical Key. Mr. Colson, it secms, was so well pleased with the Analytical Institutions of the Sig- nora Agnesi, that he made an entire translation of that lady's work, from the ltalian, the copy of which was found anong his papers, and published at the expense of $\mathrm{Mr}$. Baron Maseres in 2 volumes 4 to.

COLUMBA Noachi, Noah's Dove, a sinall corstellatiou in the southern hemisphere, consisting of 10 stars.

COLUMIN, in Architecture, a round pillar, made to support or adorn a builtling.

The column is the principal part of an architectonical order, and is composed of three parts, the base, the shaft, and the capital; each of which is subdivided into a number of lesser parts, called members, or mouldings.

Columns are different according to the different orders in which they are used; and also according to their matter, construction, form, disposition, and purpose. The proportion of the length of each to its diameter, and the diminution of the diameter upwards, are diversly stated by different authors. The medium of them is 1:early as follows :

The Tuscan is the simplest and shortest of all: its height $3 \frac{x}{2}$ diameters, or 7 modules; and it diminishes $\frac{x}{4}$ part of its diameter.

The Duric is more delicate, and adorned with flutings; its height $7 \frac{1}{2}$ or $S$ diameters.

The Ionic is more delicate still, being 9 diameters long. It is distinguished from the rest by the volutes, or curled scrolls in its capital, and by its base, which is peculiar to it.

The Corinthian is the richest and most delicate of all the columns, being 10 diameters in lengtb, and adorned with two rows of leaves, and stalks or stems, from whence spring out small volutes.

The Composite column is also 10 diameters long, its capital adorned with rows of leaves like the Corinthian, and with angular volutes like the Ionic.

COLURES, are two great circles imagined to intersect at right angles in the poles of the world, and to pass, the one through the equinoctial points Aries and Libra, and the other through the solstitial points Cancer and Capricorn; whence they are called the Equinoctial and Solstitial Colures. By thus dividing the ecliptic into four cqual parts, they mark the four seasons, or quarters of the year.

It is a matter of dispute over what part of the back of Aries the equinoctial colure passed in the time of Hlipparchus. Newton, in his Chronology, takes it to have been over the middle of this constcllation. Souciet insists that it passed over the dodecatemorion of Aries, or midway between the rump and first of the tail. There are some observations in the Plilos. Trans. number 466 , concerning the position of this colure in the ancient sphere, from a draught of the constellation Aries, in the Aratia published at Leyden and Ainsterdam in 1652 , which appears to confirm Newton's opinion; but the antiquity and authority of the original draught may still be questioned.

COMA BERENICEs, Berenice's Hair, a modern constellation of the northern hemispher'; compased of unformed stars between the Lion's tail and Bootes.

It is said that this constellation was formed by Conon, an astrononier, to console the quicen of P'tolemy Euergetes, for the loss of a lock of her hair, which was stolen out of the temple of Venus, where she had derlicated it on account of a victory obtained by her husbind.

The stars in this constellation are, in Tycho's catalogue 14, in Hevelius's 21 , and in the Britamnic catalogue +3.

COMBINATIONS, denote the alternations or varia- 
tions of any number of quantities, letters, sounds, or the like, in all possible ways. Merseune gives the combinations of all the notes and sounds in music, as far as 64 ; the sum of which amounts to a number expressed by 90 places of figures. And the number of possible combinations of the 24 letters of the alphabet, taken first two by two, then three by three, and so on, according to Prestet's calculation, amounits to $1391724288887252999+25128493402200$.

Father Truchet, in Mem. de l'Acad. shows, that two square pieces, each divided diagonally into two colours, may be arranged and combined 64 different ways, so as to form as many different kinds of chequer-work : a circumstance worthy the attention of masons, paviours, \&c.

Doctrine of Cominations.

1. Having given any number of things, with the number in each combination; to find the number of combinations.

1. When only two are combined together.

One thing admits of no combination.

Two, $a$ and $b$, admit of one only, viz, $a b$.

Three, $a, b, c$, admit of three, viz, $a b, a c, b c$.

Four admit of six, viz, $a b ; a c, a d, b c, b d, c d$.

Five admit of ten, viz, $a b, a c, a d, a e, b c, b d, b e, c d$, ce, de.

Whence it appears that the numbers of combinations, of two and two only, proceed according to the triangular numbers $1,3,6,10,15,21$, \&c, which are produced by the continual addition of the arithmetical series $0,1,2$, $3,4,5, \& c$. So that if $n$ be the number of things, then the general formula for expressing the sum of all their combinations by twos, will be $\frac{n \cdot(n-1)}{1 \cdot 2}$.

Thus, if $n=2$; this becomes $\frac{2 \cdot 1}{2}=1$.

$$
\begin{aligned}
& \text { If } n=3 ; \text { it is }{ }^{3.2}{ }_{2}-\quad-\quad=3 . \\
& \text { If } n=4 ; \text { it is } \frac{4.3}{2} \quad-\quad-\quad=6.8 \mathrm{c} .
\end{aligned}
$$

2. When three are combined together; then

Three things admit of one order, ahc.

Four admit of 4 ; viz, abc, abd, acd, bcd.

Five admit of 10 ; viz, abc, abd, abe, acd, ace, ade, bcd, $b c e, b d e, c d e$. And so on according to the first pyranidal numbers 1, 4, 10, 20, \&c, which are formed by the continual addition of the former, or triangular numbers 1,3 , $6,10, \&$ c. And the general formula for any number $n$ of combinations, taken by threes, is, $\frac{n}{1} \times \frac{n-1}{2} \times \frac{n-2}{3}$.

$$
\begin{aligned}
\text { So, if } n & =3 ; \text { it is } \frac{3 \cdot 2 \cdot 1}{1 \cdot 2 \cdot 3}=1 . \\
\text { If } n & =4 ; \text { it is } \frac{4 \cdot 3 \cdot 2}{6}=4 . \\
\text { If } n & =5 ; \text { it is } \frac{5 \cdot 4 \cdot 3}{6}=10.8 \mathrm{c} \text {. }
\end{aligned}
$$

Proceeding thus, it is found that a general formula for any number $n$ of things, combined by $m$ at each tinc, is $s=\frac{n}{3} \times \frac{n-1}{2} \times \frac{n-2}{3} \times \frac{n-3}{4} \& c_{2}$ continued to $m$ factors, or terms, or till the last factor in the denominator be $m$.

So, in 6 things, combined by 4 's, the number of combinations is $\frac{6 \cdot 5 \cdot 4 \cdot 3}{1 \cdot 2 \cdot 3 \cdot 4}=15$.

3. By adding all these series together, their sum will be the whole number of possible combinations of $n$ things combined both by twos, by threes, by fours, \&c. And as the said series are evidently the coefficients of the power $n$ of a binomial, wanting only the first two 1 and $n$; there- fore the said sum, or whole number of all such combinations, will be

$(1+1)^{n}-n-1$, or $2^{n}-n-1$. Thus if the number of things be 5 ; then $2^{5}-5-1=32-6=26$.

II. To find the number of Changes and Alterations which any number of quantities can unaergo, when combined in all possible varieties of ways, with themselves and each other, both as to the things themselves, and the Order or Position of them.

One thing admits but of one order or position.

Two things may be varied four ways; thus, $a a, a b$, $b \dot{a}, b b$.

Three quantities, taken by twos, may be varjed nine ways; thus, $a a, a b, a c, b a, c a, b b, b c, c b, c c$.

In like manuer four things, taken by twos, nay be varied, $4^{2}$ or 16 ways; and 5 things, by twos, $5^{2}$ or 25 ways; and, in general, $n$ things, taken by twos, may be changed or varied $n^{2}$ different ways.

For the same reason, when taken by threes, the changes will be $n^{5}$; and when taken by fours, they will be $n^{4}$; and

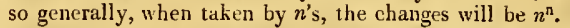

Hence, then, adding all these together, the whole inumber of changes, or combinations in $n$ things, taken both by 2's, by 3's, by 4's, \&c, to ' $n$ 's, will be the sum of the geometrical series, $n+n^{2}+n^{3}+n^{4} \cdots \cdots n^{n}$, which sum is $=$ $\frac{n^{n}-1}{n-1} \times n$.

For example, if the number of things $n$ be 4 ; this gives $\frac{4^{2}-1}{4-1} \times 4=\frac{255}{3} \times 4=840$.

And if $n$ be 24, the number of letters in the alphabet; the theorem gives

$\frac{24^{24}-1}{24-1} \times 24=\left(24^{24}-1\right) \times \frac{24}{23}=$

1391724288887252999425128493402200 . In so many different ways, therefore, may the 24 letters of the alphabet be varied or combined anong themselves, or so many different words may be made out of them.

COMBUST, or Com Busron, is said of a planet when it is in conjunction with the sun, or not distant from it above half their disc. But according to Argol, a planet is Combust, or in Combustion, when it is within $8 \frac{x_{2}}{2}$ of the sun.

COMBUSTION, in Chemical and Natural Philosophy, a tern denoting the decomposition of certain substances accompanied by light and heat.

When a stone or a brick is heated, it undergoès no change, except an augmentation of temperature; and when left to itself it soon cools again, and becomes as at first. But with combustible bodies the case is very different. When heated to a certain degree in the open air, they suddenly become much botter of themselves; continue for a considerable time intensely hot, sending out a copious streain of caloric and light to the surrounding bodies. This emission after a certain period begins to diminish, and at last ceases altogetber. The combustible has now untergone a complete change; it is converted into a substance possessing very difierent propertics, and no longer capable of combustion. Thus, when charcoal is kept for some time at the temperature of about $800^{\circ}$, it kindles, becomes intensely hot, and continues to emit light and caloric firr a long time. When the emission ceases, the charcoal has all disappeared, except an inconsiderable residuum of ashes; being almost entirely converted into carbonic acid gas, which makes its escape, unless the experiment be conducted in proper vessels. If it be collected, it is found to excecd greatly in weight the whole of the charcoal consumed. $2 \mathrm{X}$ ? 
Previous to the $17 \mathrm{th}$ century, the theory of combustion was but little understood or attended to. Since that period the labours of Bacon and Boyle, and Hooke and Nayow, together with those of Stahl and Lavoisier, have thrown much light on the subject, particularly, the theory of Lavoisier, which is by far the most rational that have yet been offered to the public. He corrects the errors of his predecessors, and has advanced before them one very important stcp; but many new steps are still wanting to render his theory of combustion complete. It explains indeed, in a satisfactory manner, why during the process of combustion the burning body gradually wastes away; but it gives no explanation of the constant emission of heat and light, though a circumstance as worthy of attention as the wasting of the body.

Dr. Thomson, admitting the truth and accuracy of the Lavoiserian theory as far as it proceeds, divides the bodics which occupy the attention of chemists into:-1. Combustibles; 2. Supporters of combustion; and 3 . lncombustibles.

The combustibles, or those bodies which in common language are said to burn, may be divided into, 1 . Simple combustibles; 2. Compound combustibles; and 3. Combustible oxides. Simple combustibles are, sulphur, phosphorus, carbon, hydrogen, and all the metals, except perhaps gold, silver, and mercury. Compound combustibles consist of compounds formed by the simple combustibles uniting together two and two; and combustible oxides are composed of one or more simple combustibles combined with a dose of oxygen. These oxides may be arranged under two heads: viz, those containing only a single base combined with oxygen may therefore be termed simple combustible oxides; and those containing more than one base may therefore be termed compound combustible oxides. The simple combustible oxides are only four in number: namely, oxide of sulphur, oxide of phosphorus, charcoal, and carbonic oxide gas. $\Lambda l l$ the simple combustible oxides are by combustion converted into acids. The compound combustible oxides include by far the greater number of combustible bodies; for almost all the animal and vegetable substances belong to them, and the double base is usually carbon and hydrogen.

The supporters of combustion are a set of bodies which are not of themselves, strictly speaking, capable of undergoing combustion, but which are absolutely necessary for the process. All the supporters known at present are six; viz,-1. Oxygen gas; 2. Air; 3. Gaseous oxide of azote; 4. Nitrous gas; 5 . Nitric acid; and 6. Oxy-muriatic acid. There are other substances to which the etuthor gives the name of purtial supporters; but all supporters contain one common principle, namely, oxygen.

The incombustible bodies are neither capable of undergoing combustion themsclves, nor of supporting the combustion of bodies that are. Of course they are not immediately connected with combustion; but they are noticed here because some of the alkalies and earths which belong to this class possess certain propertics in common with combustibles, and are capable of exhibiting phenomena somewhat analogous to combustion; phenomena which Dr. Thomson describes under the title of semi-combustion.

From these observations it is obvious, that in cvery case of combustion there inust be present a combustible and a supporter; and Lavoisier ascertaincd beyond a doubt, that, during the process, the combustible alsays unites with the oxygen of the supporter. This new compound 1)r. 'I'thom- son calls a product of combustion; and maintains that every such product is either water, ur an acid, or a metallic oxide. He admits indeed that other bodies sometimes make their appearance during combustion; but affirms that these, on examination, will be found neither to be products, nor to have urdergone combustion. See Nicholson's Journal, vol. 2, 2d series; also 1)r. Thomson's Chemistry.

COMLT, a beav'n]y body in the planetary rogion, appearing suddenly, and again disappearing; and during the time of its appearance moving in a proper, though rery excentric orbit, like a planet.

Comets are vulgarly called Blazing Sturs, and have this to distinguish them from other stars, that they are usually attended with a long train of light, tending always opposite to the sun, and being of a fainter lustre, the fartber it is from the body of the comet. And hence arises a popular division of comcts, into three kinds, viz, bearded, tailed, and hairy comets; though in reality, this division rather relates to the scieral circumstances of the same cumet, than to the phenomena of several. Thus, when the comet is eastward of the sum, and moves from him, it is said to be bearded, because the light precedes it in the manner of a beard: when the comet is westward of the sun, and sets after him, it is said to be tailed, because the train of light folluws it in the manner of a tail: and lastly, when the sun and comet are diametrically opposite, the earth being between them, the train is hid behind the body of the comet, excepting the extrenities, which, being broader than the body of the comet, appear as it were around it, like a border of hair, or coma, from which it is called hairy, and a comct. But there bave been comets whose disc was as clear, round, and well defined, as that of Jupiter, withont either tail, beard, or coma.

Of the Nature of Comets.-Philosophers and astronomers, of all ages, have been much divided in their opinions as to the nature of comets. Their strange appearance has always bcen matter of terror to the vulgar, who have uniformly considered them as cvil omens, and forcrunners of war, pestilence, \&c. Diodorus Siculus and Apollinus Myndius, in Seneca, inform us, that many of the Chaldeans held them to be lasting bodies, baving stated revolutions as wcll as the planets, but in orbits vastly more extensive; on which account they are only visible while near the earth, but disappear again when they go into the higher regions. Others were of opinion, that the comets were only meteors raised very high in the air, which blaze for a while, and disappear when the matter of which they consist is consumed or dispersed.

Some of the Grceks, before Aristotle, supposed that a comet was a vast heap or assemblage of very small stars mecting together, by reason of the inequality of their motions, and so uniting into a visible mass, by the union of all their small lights; which must again disappear, as those stars separated, and cach procecded in its course. Pythagoras, however, accounted them a kind of planets or wandering stars, disappen ring in the superior parts of their orbits, and becoming visible only in the lower parts of them.

But Aristotle held, that comets were only' a kind of transient fires, or metcors, consisting of exlatations raised to the upper region of the air, and there set on fire; far below the course of the moon.

Sineca, who lived in the first century, and who had scen two or three comets himself, plainly intimates that he thought them above the moon; aud argues strongly against 
those who supposed them to be meteors, or maintained other absurd opinions cnncęrning them ; declaring his belief that they were not fires suddenly kindled, but the cternal productions of nature. He points out also the only way to arrive at a certainty on this subject, viz, by collecting a number of observations concerning their appearance, in order to discover whether they return periodically or not. "For this purpose," he observes, "one age is not sufficient; but the time will come when the nature of comets and their magnitudes will be demonstrated, and the routes they take, so different from the planets, explained. Posterity will then wonder, that the preceding ages should be ignorant of matters so plain and easy to be known."

For a long time this prediction of Seneca seemed not likêly to be fulfilled; and Tycho Brahé was the first among the moderns, who restored the comets to their true rank in the creation; for after diligently observing the comet of 1577 , and finding that it had no sensible diurnal parallax, he assigned it its true place in the planetary regions. See his book De Cometa anni 1577 .

Bcfore this, however, there were various opinions concerning them; In the dark and superstitinus ages, comets were held to be forerunners of every kind of calamity, and it was supposed they had different degrees of malignity, according to the shape they assumed; whence also they were differently denominated. Thus, it was said that some were bearded, others hairy; that some represented a beam, sword, or spear; others a target, \&c; whereas modern astronomers acknowledge only one species of comets, and account for their different appearances from their different situations with respect to the sun and the earth.

Kepler, in other respects a very great genius, indulged the most extravagant conjectures, not only concerning comets, but the whole system of nature in general. ,The planets he imagined were huge animals swimming round the sun; and the comets monstrous and uncommon animals gencrated in the celestial spaces.

A still inore ridiculous opinion, if possible, was that of John Bodin, a learned Frenchman in the 16th century; who maintained that comets "are spirits, which baving lived on the earth innumerable ages, and bcing at last arrived on the confines of death, celebrate their last triumph, or are recalled to the firmament like shining stars! 'This is followed by famine, plague, \&cc, because the cities and people destroy the governors and chiefs who appease the wrath of God." - Others again have denied even the existence of comets, and maintained that they were nnly false appearances, occasioned by the refraction or reflection of light.

Hevelius, from a great number of observations, proposed it as his opinioll, that the comets, like the solar macula or spots, are formed or condensed out of the grosser exhalatiuns of his body; in which he differs-but little from the opinıon of Kepler.

Janes Bernoulli, in his Systema Cometarum, imagined that comets were no other than the satellites of some very distant planet, which was itself invisible to us on account of its distance, as werc also the satellites unless when in a certain part of their orbits.

Descartes advances another opinion: He conjectures that, comets are only stars, formerly fixed, like the rest, in the beavens; but which becoming gradually covered svith maculæ or spots, and at length wholly deprived of their light, cannot keep their places, but are carried off by the vortices of the circumjacent stars; and in proportion to their magnitude and solidity, moved in such a manner, as to be brought nearer the orh of Saturn; and thus coming within reach of the sun's light, rendered visible.

But the absurdity of all these hypotheses now abundantly appears from the observed phenomena of comets, and from the doctrine of Newton, which is as follows:

The comets, he says, are compact, solid, fixed, and durable bodies; in fact a kind of planets, whicls move in very oblique and excentric orbits, every way with the greatest freedom; persevering in their motions, even against the course and direction of the planets: and their tail is a very thin and slender vapour, emitted by the head or nucleus of the comet, ignited or heated by the sun. This theory of the comets at once solves their principal phenomena, which are as beluw.

The Principal Plienomena of the Comets.

1. First then, those comets which more according to the order of the signs, do all, a little before they disappear, either advance slower than usual, or clse go rctrograde, if the earth be between them and the sun; but more swiftly, if the earth be placed in a contrary part. On the other hand, those which proceed contrary to the order of the signs, move more swiftly than usual, if the earth be between them and the sun; and more slowly, or else retrograde, when the earth is in a contrary part.-For since this course is not amnng the fixed stars, but among the planets; as the motion of the earth either conspires with them, or goes against them; their appearance, with respect to the earth, must be changed; and, like the planets, they must sometimes appear to move swifter, sometimes slower, and sometimes ret lograde.

2. So long as their velucity is increased, they nearly move in great circles; but towards the end of their course, they deviate from those circles; and when the earth proceeds one way, they go the contrary way. Because, in the end of their course, when they recede almost directly from the sun, that part of the apparent motion which arises from the parallax, must bear a greater proportion to the whole apparent motion.

3. The comets move in cllipses, having one of their fo$c i$ in the centre of the sun; and by radii drawn to the sun, describe areas proportional to the times. Because. they do not wander precariously from one fictitious vortex to another; but, making a part of the solar system, return perpetually, and run a constant mund. Hence, their elliptic orbits being very long and excentric, they become invisible when in that part which is most remote from the sun. And from the curvity of the paths of cumets, Newton concludes, that when they disappear, they are.much beyond the orbit of Jupiter; and that in their perihelion they frequently descend within the orbits of Mars and the inferior plancts.

4. The light of their nuclei, or hodies, increases as they reccde from the eartls towards the sun; and on the contrary, it decreases as they recede from the sun. Because, as they are in the regions of the planets, their access tom wards the sun bears a considerable proportion to theip whole distance.

5. Their tails appear the largest and brightest, immediately after their transit through the region of the sun, or ifter their perihelion. Because then, their lieads bring the most heated, will emit the most vapours,-From lie light of the nucleus we infer their vicinity to the sarth, 
and that they are by no means in the region of the fixed stars, as some have imagined; since, in that case, their heads would be no more illuminated by the sun, than the planets are by the fixed stars.

6. The tails always decline from a just opposition to the sun towards those parts which the nuclei or bodies pass over, in their progress through their orbits. Because all smoke, or vapour, emitted from a body in motion, tends obliquely upwards, still receding from that part towards which the smoking body proceeds.

7. This declination, cateris paribus, is the smallest when the nuclei approach nearest the sun: and it is also less near the nucleus, or head, than towards the extremity of the tail. Because the vapour ascends more swiftly near the haad of the comet, than in the higher extremity of its tail; and aisu when the comet is nearer the sun, than when itois farther off.

S. 'The tails a re somewhat brighter, and more distinctly defined in their convex, than in their concare part. Because the vapour in the convex part, which goes first, being somewhat nearer and denser reflects the light more copiously.

9. The tails always appear broader at their upper extremity, than near the centre of the comet. Because the vapour in a free space continually rarefies and dilates.

10. The tails are always transparent, and the smallest stars appear through them; because they consist of infinitely thin vapour.

The Phases of Comets.-The nuclei, which are also called the heads, and bodies, of comets, viewed through a telescope, exlibit faces very different from those of the fixed stars or planets. They are liable to apparent alterations, which Newton ascribes to changes in the atmosphere of comets : and this opinion was confirmed by observations. of the comet in 1744; Hist: Acal. Scienc. 1744. Sturmius says that, observing the cumet of 1680 with a telescope; it appeared like a coal dimly glowing, or a rude mass of matter illuminated with a dusky fumid light, less sensible towards the extremes than in the middle; whereas a star appears with a round disc, and a vivid light.

Of the comet of 1661 , Hevelius observes, that its body was of a yellowish colour, very bright and conspicuous, but without any glittering light: in the middle was a dense ruddy nucleus, almost equal to Jupiter, encompassed by a much fainter, thimer matter. February 5 th, its head was somewhat larger and brighter, and of a gold colour; but jts light more dusky than that of the star's: and here the nucleus appeared divided into seyeral parts. Feb, 6 th, the disc was lessened; the parts of the nucleus still existed, though less than before: one of them, on the lower part of the disc, on the left, much denser and brighter than the rest; its body round, and representing a very lucid small star: the nucleus still encompassed with another kincl of matter. l'eb. Ioth, the head somewhat more obscure, and the nucleus more confused, but brighter at top than buttom. Feb. 13 th, the head diminished nuch both in size and splendor. March 2d, its roundness a litthe impaired, and its edges lacerated, \&c. March 2Stb, very palc, and exceeding thin; its matter much dispersed; and $n$ o distinct nucleus at at appearing.

Veigelius; who saw the comet of 1664, as also the moon, and a small cloud in the horizon illuminated by the sum at the same time, ubservid, that through the telescope the moon appeared of a continued luminous surface: but the comet very different; being exactly like the small cloud.
And from these observations it was that Hevelius formed his opinion, that comets are like macula or spots formed out of the solar exhalations.

Of the Magnitude of Comets.-The estimatrs that have been given of the magnitude of comets by Tycho Brahé, Hevelius, and other astronomers, are not very accurate; as it does not appear that they distinguished between the nucleus and the surrounding atmosphere. Thus Tycho computes that the true diameter of the comet in 1577 , was in proportion to the diameter of the earth, as 3 to 14; and Hevelius made the diameter of the comet of 1652 , to that of the earth, as 52 to 100 . But the diameter of the atmosphere is often 10 or 15 times as great as that of the nucleus : the former, in the comet of 1682 , was measured by Flamsteed, and found to be 2 , when the diameter of the nucleus alone was unly 11 or $12^{\prime \prime}$. Though some comets, estimated by a comparison of their distance and apparent magnitude, have been reckoned much larger than the moon, and even equal to some of the primary planets. The diameter of that of 1744 , when at the distance of the sun from us, measured about 1', which makes its diameter about three times that of the earth: at auother time the diameter of its nucleus was mearly equal to that of the planet Jupiter.

Of the Tails of Comets.-There have been various conjectures about the nature of the tails of comets, the principal of which are those of Newton, and his followers. Newton shows that the atmospheres of comets will furnish vapour sufficient to form their tails. This he argues from their wonderful rarefaction in our air at a distance from the earth; which is such that a cubic inch of common air, expanded to the rarity of that at the distance of half the earth's dianeter, or 4000 miles, would fill a space larger than the whole region of the stars. Since then the coma, or atmosphere of a comet, is ten times higher than the surface of the nucleus, from the centre; the tail, ascending still much higher, must necessarily be immensely rare: so that it is no wonder the stars are visible through it.

Now the ascent of vapours into the tail of the comet, he supposes to be occasioned by the rarefaction of the matter of the atmosphere at the time of the periliclion. Smoke, it is observed, ascends the chimmey by the impulse of the air in which it floats; and air, rarefied by licat, ascends by the diminution of its specific gravity, carrying up the smoke along with it: in the same manuer then it may be supposed that the tail of a comet is raised by the sun.

The tails therefore thus produced in the peribelions of comets, will go off along with their head, into remote regions; and either return from thence, together with the comets, after a long series of years; or rather be there lost, and vanish by little and little; and the comet be lutit bare; till at its returm, descending towards the sun, some short tails are again gradually produced from the head: which afterwards, in the perihelion, descending jinto the sun's atmosphere, will be immensely increased.

Newton farther observes, that the vajours, when thus dilated, rarefied, and diffused through all the celestial regions, may probably, by means of their own gravity, be gradually attriced down to the planets, and become intermingled with their utmospheres. He adds that this intermixture may be uscful and necessary for the conservation of the water and moisture of the planets, dried up or consumed in rarious ways. And I stuspect, adds our atithor, that the spirit, which makes the timest, subtilest, and 
best part our air, and which is absolutely requisite for the life and being of all things, is derived principally from the comets.-On this principle there may appear to be some foundation for the popular opinion of presages from comets; since the tail of a comet thus intermingled with our atmosphere, may produce changes very sensible in ani$\mathrm{mal}$ and vegrtable bodies.

It may here be addled, that another use which Newton conjectures comets may be designed to serve, is that of recruiting the sun with fresh fuel, and repairing the consunption of his light by the streams continually sent forth in every direction from that luminary. In support of this conjecture he observes, that comets in their perihelion may suffer a diminution of their projectile force, by the resistance of the solar atmosphere; so that by degrees their gravitation towards the sun may be so far increased, as to precipitate their fall into his body.

Various opinjons have been entertained respecting the generation of the tails of comets. Apian, Tycho Brahé, and some others, think they are produced by the sun's rays transmitted through the nucleus ot the comet, which they supposed was transparent, and there refracted as in a glass lens, so as to furm a beam of light behind the comet. Descartes accounted for the phenomenon of the tail by the refraction of light from the head of the comet to the spectator's eye. Mairan supposes that the tails are furmed from the luminous matter composing the sun's atmosphere : and M. De Lalande combines this bypothesis with that of Newton recited above. But Mr. Rowning, not satisfied with Newton's opinion, accounts for the tails of comets in the following mainer: lt is well known, says he, that when the sun's light pasues through the atmosphere of any body, as the carth, that which passes on one side, is by the refiaction made to converge towards that which passes on the opposite side; and this convergency is not wholly effected either at the entrance of the light into the atmosphere, or at its egression from it; but begiuning at its entrance, it increases in every point of its progress: It is also agreed, that the atmospheres of the comets are very large and dense: he therefore supposes that by such time as the light of the sun has passed through a considerable part of the atmosphere of the comet, the rays are so far refracted towards each other, that they then begin sensibly to illuminate it, or rather the vapours floating in it; and so render that part they have yet to pass through, visible to us: and that this portion of the atmosphere of a comet thus illuminated, appears to us in form of a beam of the sun's light, and passes under the denomination of a comet's tail. Rowning's Nat. Philos. part 4. chap. 11.

M. Eulcr, Mem. Berlin, tom. 2, pa. 117, thinks there is a great affinity between the tails of comcts, the zodiacal light, and the aurora borcalis, and that the common cause of all of them, is the action of the sun's light on the atmospheres of the comets, of the sun, and of the earth. He supposes that the impulse of the rays of light on the atmosphere of a comet, may drive sume of the finer particles of that atmosphere lar beyond its limits; and that this force of impulse combined with that of gravity towards the comet, would produce a tail, which would always be in opposition to the sun, if the comet did not move. But the motion of the comet in its orbit, and about an axis, must vary the position and figure of the tail, giving it a curvature, and deviation from a line join- ing the centres of the sun and comet; and that this deviation will be greater, as the orbit of the comet has the greater curvature, and as the motion of the comet is more rapid. It may even happen, that the velocity of the coInet, in its perihelion, may be so great, that the force of the sun's rays may produce a new tail, before the old one can follow; in which case the comet might have two or more trils. The possibility of this is confirmed by the comet of 1744 , which was observed to have several tails while it was in its perihelion.

Dr. Hamilton urges several objections against the Newtonian hyputbesis; and concludes that the tail of a comet is formed of matter which has not the power of refracting or reflecting the rays of light; but that it is a lucid or self-shining substance: and from its similarity to the aurora borealis, that it is produced by the same cause, and is properly an electrical phenumenon. Dr. Halley likewise was doubtless inclined to this hypothesis, when he said, that the streams of light in an aurora borealis so much resembled the long tails of comets, that at first sight they might well be taken for such: and that this light scems to have a greater affinity to that which the effiuvia of electric bodies emit in the dark. Philos. Trans. No. 347. Hamilton's Philos. Essays, pa. 91.

The Motion of Comets.-If it be supposed that the paths of comets are perfectly parabolical, as some have imagined, it will follow that, being impelled towards the sun by a centripetal force, they descend as from spaces infinitely distant; and that by their falls they acquire such a velocity as will carry thein off again into the remotest regions, never more to return. But the frequency of their appearance, and their degree of velocity, which does not exceed what they might acquire by their gravity towards the sun, seem to put it past doubt that they move like the planets, in elliptic orbits, though excedingly excentric; and so return again after very long periods.

The apparent velocity of the comet of 1472 , as observed by Regiomontanus, was such as to carry it through $40^{\circ}$ of a great circle in 24 hours: and it was observed that the comet of 1770 moved through more than $45^{\circ}$ in the last 25 hours.

- Respecting the return of comets there have been different opinions. Newton, Flamsteed, Halley, and other English astronomers, appear quite satisfied of the return of these bodics. Cassini and some of the French think it highly probable; but De la Hire and nthers oppose it. Those on the affirmative side suppose that the comets describe orbits prodigiously excentric, insomuch that we can observe them only in a very smiall part of their revolution: out of this, they are lost in the immensity of space; hid not only from our eyes, but our telescopes: that small part of their orbit next us passing sometimes vithin those of all the inferior plancts.

Cassini gives the following reasons in favour of the return of comets. 1. It is found that they move a considerable time in the arcl of a great circle, when referred to the fixed stars, that is, a circle whose plane passes through the centre of the earth; deviating but a little from it chiefly towards the end of their appearance; a deviation however common to them with the planets.-2. Comets, as well as planets, appear to move so much the faster as thcy are nearer the carth; and when they are at equal distances from thrir perigee, their velocities are nearly the same. By subtracting fom their inotion the 
apparent inequality of velocity occasioned by their different distance from the earth, their equal motion might be found: but we should not still be certain that this is their true motion; because they might have considerable inequalities, not distinguishable in that small part of their orbit visible to us. It is rather probable that their real motion, as well as that of the planets, is unequal in itself; and hence we have a reason why the observation made during the appearance of a comet, cánnot give the just period of its revolution. - 3 . There are no two different planets whose orbits cut the ecliptic in the same angie, and whose nodes are in the same points of the ecliptic, and having the same apparent velocity in their perigee: consequently, two comets seen at different times, yet agreeing in all those three circumstances, can only be one and the same comet. Not that this exact agreement, in rhese circumstances, is absolutely necessary to determine their identity: for the moon herself is irregular in all of them, so that it appears there may be cases in which the same comet, at different periods of revolution, may disagree in these points.

As to the objections against the return of comets, the principal is that "of the unfrequency of their appearance, with regard to the number of revolutions assigned to them. In 1702 there was a comet, or rather the tail of one, seen at Rome, which M. Cassini takes to be the same with that olserved by Aristotle, and again lately in the year 1668 ; which would imply a period of 34 years: Now, it may appear strange that a star.which has so short a revolution, and of consequence such frequent returns, should be so seldom seen. Again, in April of the same year 1702, a comet was observed by Messrs. Bianchini and Maraldi, which the latter supposed was the same with that of 1664 , both on account of its motion, velocity, and direction. M. de la Hire thought it had some relation to another he had observed in 1698 , which Cassini refers to that of 1652 ; which would make it a period of 43 months, and the number of revolutions between 1652 and 1692,14 : now, it is hard to suppose, that in this age, when the beavens are so uarrowly watched, a star should make 14 revolutions unperceived; especially such a star as this, which inight appear above a month together; and consequently be often disengaged from the crepuscula. For this reason M. Cassini is very reserved in maintaining the hypothesis of the return of comets, and only proposes those for planets where the motions are easy and simple, and are solved without straining, or allowing any irregularities.

M. de la Hire proposes one general difficulty against the whole system of the return of comets, which would seem to prevent any cothet from returning as a planet; which is this; that by the disposition necessarily given to their courses, they ouglit to appear as small at first as at last ; and always increase till they arrive at their nearest proximity to the earth; or if they should chance not to be observed, as soon as they are capable of being seen, it is yet hardly possible but they must often show themselves before they have arrived at their full magnitude and brightness: but, adds he, none were ever yet observed till they had arrived at it. However, the appearance of a comet in the month of Octuber 1723, while at a great distance, so as to be too small and dim to be viewed without a telescope, as well as the observations of several others since, may serve to remove this obstacle, and set the com ets still on the same footing with the planets.

It is a conjecture of Newton, that as those planets which are nearest to the sun, and revolve in the least orbits, are the smallest; 'so among the comets, such as in their perihelion come nearest the sun, are the smallest, and revolve in the least orbits.

Of the Writings and Lists of Comets.

There have been many writings on the subject of comets, beside the notices of historians as to the appearance of certain particular ones.

Regiomontanus first showed how to find the magnitudes of comets, their distance from the earth, and their true place in the heavens. His 16 problems De Cometæ Magnituline, Longitudine, ac Loco, are to be found in a book published in the year 1544, with the title of Scripta Joannis Regiomontans.

Peter A pian observed and wrote on the comets of 1631 , 1632 , \&c. Other writers are Tycho Brahé, in his Progymnasmata Astronomix Instauratæ.-Kepler, of the comet in the year 1607, and de Cometis Libelli tres.-Ricciolus, in his Almagestum Novum, published 1651, enumerates 154 comets cited by historians down to the year 1618. - Hevelius's Prodromus Cometicus, containing the history of the comet of the year 1664. Also his Cometographia.-Lubienietz, in a large folio work expressly on this subject, published 1667 , extracts, with immense labour, from the passages of all historians, an account of 4.15 comets, ending with that of 1665 .-Dr. Hooke, in his Posthumous works.-M. Cassini's little Tract of Comets. -Sturmius's Dissertatio de Cometarum Natura.-Newton, in his Principia, lib. 3 ; wlso first assigned their proper orbits, and by calculatious compared the observations of the great comet of 1680 with his theory.-Dr. Halley, in his Synopsis Cometica, in the Pbilos. Trans. No.218, $\& \mathrm{c}$; who computed the elements and orbits of 24 comets, and who first ventured to predict the return of one in 17.59, which happened accordingiy. - De Lalande, Théurie des Comètes, 1759 ; also, in his Astronomie, vol. 3 Clairant, Théorie du mouvement des Comètes, 1760.D'Alembert, Opuscules Mathématiques, vol. 2, pa. 97.M. Albert Euler, 1762.-Séjour, Essai sur les Conètes, 177 5. - Besides Boscovich, De la Grange; De la Place, Frisi, Lexel, Barker, Hancocks, Cole, with many others. -And M. Pringre's Cométographie, in 2 vols. 4to, 1784 ; in which is contained the most ample list of such comets as have been well observed, and their elements coniputed, to the number of $6 \%$. And accounts of a very few more that have been observed since that time, may be seen in the $\mathrm{Mlem}$. de l'Acad. and in the Plilos. Trans. A very ingenious and ample work on comets is also published by Sir Henry Englefield, entitled, On the determinations of the Orbits of Comets.

'The whole list of comets that have been noticed, on record, amount to upwards of 500 , but the following is a complete list of all that have been properly observed, and their elements computed, the mean distance of the carth from the sun being supposed 100,000 . 
C O M $\left[\begin{array}{lll}345 & 3\end{array} \quad\right.$ C O M

TABLE OF THE ELEMENTS OF COMETS.

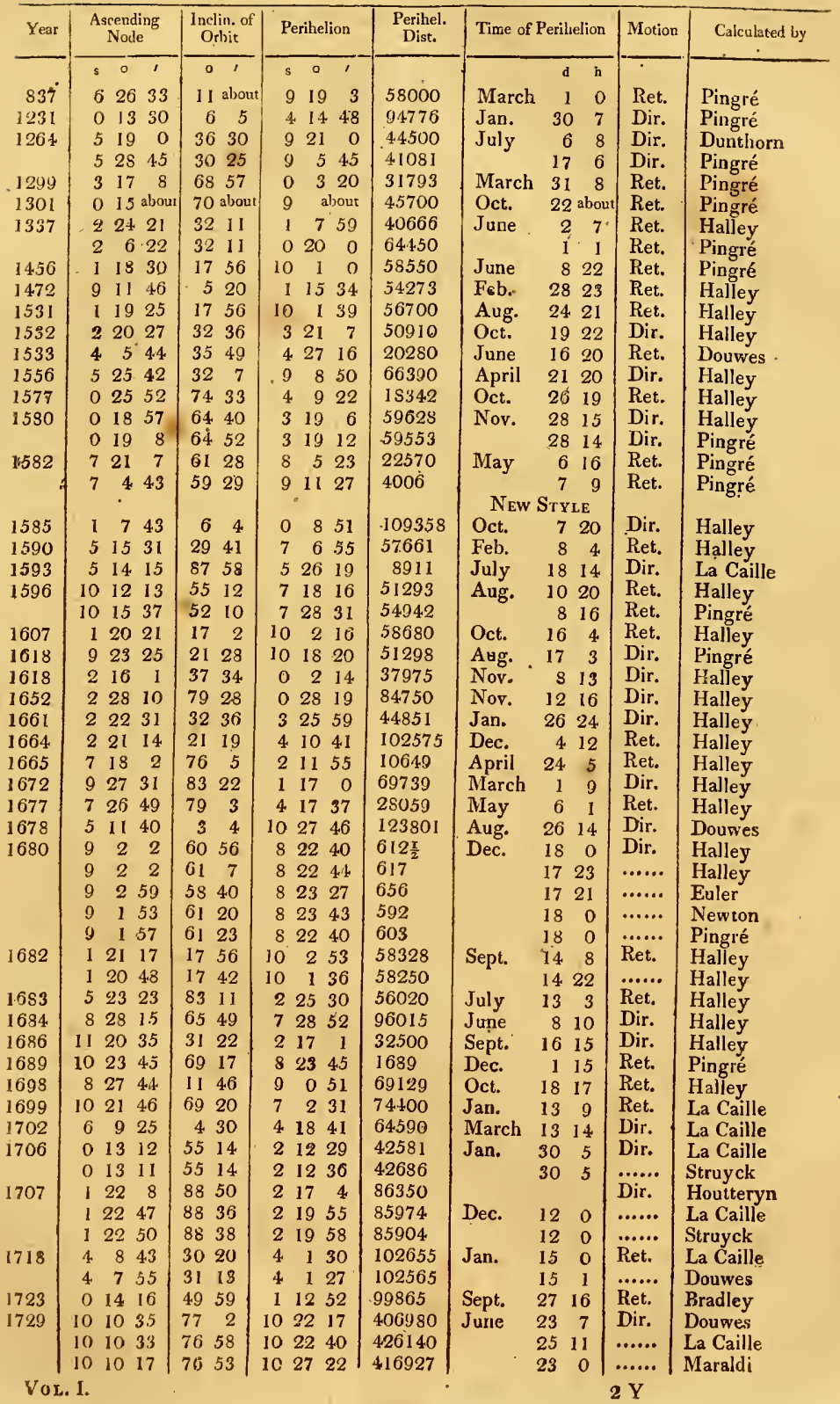




\begin{tabular}{|c|c|c|c|c|c|c|c|c|c|c|c|c|c|c|}
\hline \multirow{2}{*}{ Year } & \multicolumn{3}{|c|}{$\begin{array}{l}\text { Ascending } \\
\text { Node }\end{array}$} & \multicolumn{2}{|c|}{$\begin{array}{c}\text { Inclin. of } \\
\text { Orbit }\end{array}$} & \multicolumn{3}{|c|}{ Perihelion } & \multirow[t]{2}{*}{$\begin{array}{l}\text { Perihel. } \\
\text { Dist. }\end{array}$} & \multicolumn{3}{|c|}{ Time of Perihelion } & \multirow[t]{2}{*}{ Motion } & \multirow[t]{2}{*}{ Calculated by } \\
\hline & s & 。 & , & & ' & s & 0 & $\therefore$ & & & d & h & & \\
\hline \multirow[t]{2}{*}{1729} & 10 & 10 & 52 & 77 & 19 & 10 & 16 & 27 & 394927 & May & 221 & II & ....... & Kies \\
\hline & 10 & 10 & 33 & 77 & 1 & 10 & 22 & 37 & & & 25 & 9 & $\ldots . .$. & De I'Isle \\
\hline 1737 & 7 & 16 & 22 & 18 & 21 & 10 & 25 & 55 & 22282 & Jall. & 30 & 9 & Dir. & Bradley ${ }^{\circ}$ \\
\hline 1739 & & 27 & 18 & 55 & 53 & 3 & 12 & 34 & 67160 & June & 17 & 11 & Ret. & Zanotti \\
\hline & & 27 & 25 & 55 & 43 & 3 & 12 & 39 & 67358 & & 17 & 10 & $\ldots \ldots$. & La Caille \\
\hline 1742 & 6 & 5 & 35 & 67 & 4 & 7 & . 7 & 33 & 76555 & Feb. & 8 & 5 & Ret. & \\
\hline & 6 & 5 & 33 & & & 7 & 7 & 32 & 76550 & & 8 & 4 & ...... & Le Monnier \\
\hline & 6 & 5 & 38 & 66 & 59 & 7 & 7 & 35 & 76568 & & 8 & 5 & ....... & La Caille \\
\hline & 6 & 5 & 4.3 & 66 & 52 & 7 & 7 & 39 & 76530 & & 8 & 8 & ...... & Zanotti \\
\hline & 6 & $16^{\circ}$ & 9 & 56 & 35 & 7 & 16 & 42 & 73766 & & 15 & 22 & $\ldots \ldots$ & Euler \\
\hline & 6 & 9 & 32 & $6 \mathrm{I}$ & 44 & 7 & 10 & 49 & 73668 & Feb. & 7 & 4 & Ret. & Euler \\
\hline & 6 & 5 & 47 & 68 & 14 & 7 & 7 & 33 & 76890 & & 7 & 22 & ....... & Wrigt \\
\hline & 6 & 5 & 29 & 67 & 11 & 7 & 7 & 26 & 766 & & 8 & 5 & ...... & senberg \\
\hline & 6 & 5 & 42 & 66 & 51 & 7 & 7 & 38 & & & 8 & 7 & $\ldots \ldots$ & \\
\hline 1743 & 2 & 8 & 11 & 2 & 16 & 3 & 2 & 58 & 83811 & Jan. & 10 & 21 & Dir. & \\
\hline & 2 & 8 & 21 & 2 & 20 & 3 & 2 & 42 & 835 & & 10 & 21 & ...... & \\
\hline 1743 & 0 & 5 & 16 & 4.5 & 48 & 8 & 6 & 34 & 57 & Sept. & 20 & 21 & Ret. & Klinkenberg \\
\hline & 1 & 15 & 45 & 47 & 9 & 6 & 17 & 13 & 22206 & March & I & 8 & Dir. & nd Blis \\
\hline & ] & 15 & 47 & 47 & 4 & 6 & 17 & 6 & 22322 . & & I & 8 & ...... & \\
\hline & 1 & 15 & 46 & 47 & 5 & 6 & 17 & 10 & 222 & March & 1 & 8 & Dir. & $\mathrm{La}$ \\
\hline & 1 & 15 & $5 \mathrm{I}$ & 47 & 18 & 6 & 17 & 18 & 22156 & & 1 & 8 & ....... & \\
\hline & 1 & 16 & 5 & 47 & 50 & 6 & 17 & 19 & 22192 & - & 1 & 9 & ...... & \\
\hline & 1 & 15 & 46 & 47 & 11 & 6 & 17 & 12 & 222 & & 1 & 8 & ...... & $\mathrm{Eul}$ \\
\hline & & .15 & 48 & 47 & 8 & 6 & 17 & 13 & 2222 & & 1 & 8 & ....... & \\
\hline & 1 & 15 & 4.9 & 4.7 & 18 & 6 & 17 & 15 & $22200^{\circ}$ & & 1 & 8 & ...... & nberg \\
\hline & 1 & 15 & 4.9 & 47 & 14 & 6 & 17 & 16 & 22176 & & I & 9 & $\ldots \ldots$ & \\
\hline 1747 & & 26 & 58 & 77 & 57 & 9 & 10 & 6 & 2293 & Feb. & 28 & 12 & Ret. & \\
\hline & & 27 & 19 & 79 & 6 & 9 & 7 & 2 & 2198 & March & 3 & 10 & ...... & \\
\hline & & 27 & 19 & 79 & 6 & 9 & 7 & 2 & 219851 & & 3 & 7 & ....... & \\
\hline 1748 & 7 & 22 & 52 & 85 & 27 & 7 & 5 & I & 84066 & April & 28 & 20 & Ret. & Mar \\
\hline & 7 & 22 & 46 & 85 & 35 & 7 & 4 & 39. & 84150 & & 29 &. $\mathrm{I}$ & ...... & nnier \\
\hline & 7 & 22 & 52 & 85 & 28 & 7 & 5 & $23^{\circ}$ & 840 & & 28 & 19 & $\ldots \ldots$ & \\
\hline 1748 & 1 & 4 & 40 & 56 & 59 & 9 & 6 & 9 & 65525 & June & 18 & 2 & Dir. & \\
\hline 1757 & 7 & 4 & 13 & 12 & 50 & 4 & 2 & 58 & & Oct. & $2 \mathrm{I}$ & 8 & Dir. & \\
\hline & 7 & 4 & 6 & 12 & 39 & 4 & 2 & 39 & 3390 & & 21 & 10 & ...... & \\
\hline & 7 & 4 & 4 & 12 & 48 & 4 & 2 & 49 & 33797 & & 21 & 10 & ...... & \\
\hline$\cdot$ & 7 & 4 & 7 & 12 & 41 & 4 & 2 & 36 & 339 & & 21 & 9 & ....... & \\
\hline 1758 & 7 & 20 & 50 & 68 & 19 & 8 & 27 & 38 & 21535 & June & 11 & 3 & Dir. & \\
\hline & 1 & 23 & 48 & 17 & 38 & 10 & 3 & 14 & 58255 & March & 12 & 14 & Ret. & \\
\hline & & 23 & 46 & 17 & 40 & 10 & 3 & $s$ & & & 12 & 14 & ....... & \\
\hline & 1 & 23 & 49 & 17 & 35 & 10 & 3 & 16 & 5836 & & 12 & 13 & ...... & \\
\hline & & 23 & 49 & 17 & 38 & 10 & 3 & 16 & 58380 & & 12 & $13 \frac{\pi}{2}$ & ...... & ille \\
\hline & & 23 & $49^{\circ}$ & 17 & 39 & 10 & 3 & 16 & 583 & & 12 & $13 \frac{2}{3}$ & $\ldots \ldots$ & aille \\
\hline & 1 & 23 & 46 & 17 & 40 & 10 & 3 & 19 & 5828 & & 12 & $13^{2}$ & ....... & berg: \\
\hline & I & 24 & 7 & 17 & 29 & 10 & 1 & 0 & 597 & & 13 & 10 & $\ldots$ & berg \\
\hline & & 23 & 45 & 17 & 41 & 10 & 3 & 23 & 58234 & & 12 & 13 & $\ldots \ldots$ & \\
\hline 1759 & 4 & 19 & 40 & 79 & 7 & 1 & 23 & 34 & $\$ 0139$ & Nov. & 27 & 0 & Dir. & Ping \\
\hline & & 19 & 39 & 78 & 59 & & 23 & 24. & & & 27 & 2 & ...... & \\
\hline & 4 & 19 & 40 & 79 & 3 & & 23 & 38 & 802 & & 27 & I & & \\
\hline 1759 & & 19 & 51 & & 52 & 4 & 18 & 25 & & Dec. & 16 & 21 & Ret. & \\
\hline & & 19 & 20 & & 42 & & 19 & 4 & & & 16 & 13 & Ret. & \\
\hline 1762 & 11 & 18 & 56 & 85 & 22 & & 15 & 22 & 101415 & May & 29 & 0 & Dir. & \\
\hline & II & 19 & 20 & 84 & 45 & & 15 & 15 & & & 28 & 15 & & \\
\hline & 11 & 18 & 58 & 85 & 12 & & 14 & 24 & & - & 29 & 2 & & \\
\hline & 11 & 18 & 35 & 85 & 40 & & 13 & 43 & & & $2 \mathrm{~S}$ & 2 & .... & kenberç \\
\hline & 11 & 19 & 2 & & 53 & & 14 & 30 & 100986 & & 28 & 7 & & \\
\hline 1763 & II & 26 & 23 & 72 & 41 & & 24 & 52 & & No & 1 & 20 & Dir. & \\
\hline 1764 & 4 & 0 & 5 & 52 & 254 & & 15 & 15 & & & 12 & 14 & Ret. & \\
\hline 3766 & & 34 & 11 & 40 & 50 & & 23 & 15 & 505 & Feb. & 17 & 9 & Ret. & \\
\hline 1766 & & 214 & 23 & 11 & 8 & & 2 & 18 & & & 22 & 21 & Dir. & \\
\hline 1769 & & 525 & 1 & 40 & 38 & & 24 & 6 & 12376 & Oct. & 7 & 13 & Dir. & Lalande \\
\hline & & 525 & 2 & 40 & 43 & & 24 & 14 & 12287 & & 7 & & & \\
\hline & 5 & 25 & 3 & 40 & 47 & & 24 & 11 & 12258 & & 7 & 13 & & Cassin, jun. \\
\hline
\end{tabular}




\begin{tabular}{|c|c|c|c|c|c|c|c|c|c|c|c|c|c|c|c|}
\hline \multirow{3}{*}{$\begin{array}{r}\text { Year } \\
769\end{array}$} & \multicolumn{3}{|c|}{$\begin{array}{l}\text { Ascending } \\
\text { Node }\end{array}$} & \multicolumn{2}{|c|}{$\begin{array}{l}\text { Inclin. of } \\
\text { Oibit }\end{array}$} & \multicolumn{3}{|c|}{ Perihelion } & \multirow[t]{2}{*}{$\begin{array}{c}\text { Perihel. } \\
\text { Dist. }\end{array}$} & \multicolumn{3}{|c|}{ Time of Perihelion } & \multirow[t]{2}{*}{ Motion } & \multicolumn{2}{|l|}{ Calculated by } \\
\hline & $s$ & 0 & ' & 0 & 1 & & 0 & 1 & & & d & $\mathrm{h}$ & & & \\
\hline & 5 & 25 & 7 & 40 & 49 & 4 & 24 & 11 & 12272 & & 7 & 14 & $\ldots \ldots$ & Prosperin & \\
\hline & 5 & 25 & 3 & 40 & 41 & 4 & 24 & 9 & 12289 & & 7 & 14 & ..... & Audiffrédi & \\
\hline & 5 & 25 & 11 & 41 & I & 4 & 24 & 33 & 12100 & & 7 & 12 & ..... & Slop & \\
\hline & 5 & 19 & 4.1 & 29 & 41 & 4 & 13 & 15 & 15880 & & 16 & 10 & $\ldots \ldots$ & Zanotti & \\
\hline & 5 & 25 & 5 & 40 & 41 & 4 & 24 & 7 & 12308 & & 7 & 14 & $\ldots \ldots$ & Asclépi & \\
\hline & 5 & 24 & 42 & 41 & 28 & 4 & 25 & 46 & 11640 & & 7 & 11 & ...... & Lambert & \\
\hline & 5 & 25 & 14 & 40 & 43 & 4 & 24 & 22 & 12280 & & 7 & 18 & $\ldots \ldots$ & Widder & \\
\hline & 5 & 25 & 3 & 40 & 50 & 4 & 24 & 16 & 12264 & Oct. & 7 & 15 & Dir. & Euler & \\
\hline & 5 & 25. & 5 & 40 & 50 & 4 & 24 & 11 & 12269 & & 7 & 16 & ...... & Lexell & \\
\hline & 5 & 25 & 6 & 40 & 4.7 & 4 & 24 & 16 & 12274 & & 7 & I 6 & $\ldots \ldots$ & Pingré & \\
\hline \multirow[t]{11}{*}{1770} & 4 & 16 & 39 & 1 & 44 & 11 & 26 & 7 & 62959 & Aug. & 9 & 0 & Dir. & Pingré & \\
\hline & 4 & 13 & 39 & I & 41 & 11 & 25 & 5 & 65800 & & 10 & 22 & ....... & Pingré & \\
\hline & 4 & 15 & 29 & 1 & 47 & 11 & 26 & 7 & 62955 & & 9 & 0 & ...... & Prosperin & \\
\hline & 4 & 15 & 4 & 14 & 4.5 & 11 & 22 & 51 & 64456 & & 8 & 9 & $\ldots \ldots$ & Prosperin & \\
\hline & 4 & 14 & 30 & I 2 & 23 & 0 & 7 & 14 & 71717 & & 25 & 2 & $\ldots \ldots$ & Prosperin & \\
\hline & 4 & 12 & 56 & 1 & 46 & 11 & 29 & 45 & 64946 & & 12 & $2 \mathrm{I}$ & ....... & Widder & \\
\hline & 4 & 12. & 0 & $1:$ & 34 & 11 & 26 & 16 & 67438 & Aug. & 13 & 13 & Dir. & Lexell & \\
\hline & 4 & 12 & 17 & $1:$ & 35 & 11 & 26 & 26 & 67689 & & 14 & 0 & $\ldots \ldots$ & Pingré & \\
\hline & 4 & 16 & 14 & 14 & 45 & 11 & 26 & I 3 & 62872 & & 9 & I & ...... & Slop & \\
\hline & 4 & 12 & 0 & 15 & 55 & I1 & 25 & 57 & 63100 & & 9 & 4 & $\ldots \ldots$ & Lambert & \\
\hline & 4 & 14 & 22 & 1 & 49 & 11 & 20 & 19 & 62758 & & 8 & 19 & $\ldots \ldots$ & Rittenhouse & \\
\hline 1770 & 3 & 18 & 42 & 312 & 26 & 6 & 28 & 23 & 52824 & Nov. & 22 & 6 & Ret. & Pingré & \\
\hline 1771 & 0 & 27 & 51 & 111 & 15 & 3 & 13 & 28 & 90576 & April & 18 & 22 & Dir. & Pingré & \\
\hline & 0 & 27 & 50 & 111 & 17 & 3 & 13 & 48 & 90188 & & 19 & 1 & $\ldots \ldots$ & Prosperin & \\
\hline 1772 & 8 & 12 & 43 & 19 & 0 & 3 & 18 & 6 & 101814 & Feb. & 18 & 21 & Dir. & Lalande & \\
\hline 1773 & 4 & I & I 6 & 61 & 25 & 2 & 15 & 36 & 113390 & Sept. & 5 & 11 & Dir. & Pingré & \\
\hline & 4 & 3 & 15 & 62 & 36 & 2 & $2 \mathrm{I}$ & 40 & 123800 & 8 & 2 & 12 & ...... & Lambert & \\
\hline & 4 & 3 & 35 & 623 & 36 & 2 & 20 & 43 & 121550 & - & 2 & 19 & ...... & Scultz & \\
\hline & 4 & 1 & 12 & $6 I 2$ & 21 & 2 & 15 & I 6 & $113010^{\circ}$ & & 5 & 6 & ..... & Lexell & \\
\hline & 4 & 1 & 5 & 611 & 13 & 2 & 14 & 58 & 112530 & 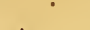 & 5 & 9 & & Pingré & \\
\hline 1774 & 6 & 0 & 57 & 824 & 4.8 & 10 & 16 & 28 & 142525 & Aug. & 14 & 4 & Dir. & De Saron & \\
\hline & 6 & 0 & 50 & 824 & 49 & 10 & 16 & 48 & 142525 & & 14 & 18 & $\ldots \ldots$ & De Saron & \\
\hline & 6 & 1 & 22 & 822 & 21 & 10 & I7 & 26 & $142600^{\circ}$ & & 15 & 5 & $\ldots \ldots$ & Boscovich & \\
\hline & 6 & 0 & 50 & 83 & 0 & 10 & 17 & 22 & 142860 & & 15 & $1 I^{\circ}$ & i...... & Mechain & \\
\hline 1779 & 0 & 25 & 3 & 322 & 26 & 2 & 27 & 14 & 71322 & Jan. & 4 & 3 & Dir. & De Saron & \\
\hline & 0 & 25 & 6 & 322 & 24 & 2 & 27 & 13. & 71313 & & 4 & 2 & (...... & Mechain & \\
\hline & 0 & 25 & 4 & 322 & 26 & 2 & 27 & 14 & 71319 & & 4 & 2 & $\because \ldots .$. & D'Angos & \\
\hline 1780 & 4 & 4 & 0 & 535 & 56 & 8 & 6 & 30 & 9781 & Sept. & 30 & 20 & Ret. & Lexell & \\
\hline & 4 & 4 & 30 & 531 & 15 & 8 & 6 & 19 & 10047 & & 30 & 16 & ....... & Lexell & \\
\hline & 4 & 4 & 9 & 534 & 48 & 8 & 6 & 21 & 9926 & & 30 & 18 & ;..... & Mechain & \\
\hline 1781 & 2 & 25 & 13 & 51 & 16 & 5 & 22 & 17 & 1027558 & March & 13 & 0 & Dir. & Boscovich & \\
\hline & & & & & & 5 & 28 & 13 & 944040 & Jan. & 27 & 6 & m..... & La Place & \\
\hline 1781 & 2 & 23 & 1 & 814 & 43 & 7 & 29 & 11 & 77586 & July & 7 & 5 & Dir. & Mechain & \\
\hline 1781 & 2 & 17 & 23 & 271 & 13 & 0 & 16 & 3 & 96101 & Nov. & 29 & 13 & Ret. & Mechain & \\
\hline 1783 & 1 & 24 & 14 & 53 & 9 & I & 1.5 & 25 & 156530 & Nov. & 15 & 6 & Dir. & Mechain & \\
\hline 1784 & I & 26 & 50 & 51 & 9 & 2 & 20 & 44 & 70786 & Jan. & 21 & 5 & Ret. & Mechain & \\
\hline 1784 & 2 & 26 & 52 & 475 & 55 & 10 & 28 & 55 & 65053 & April & 9 & 21 & ...... & Chev. d'Angos & \\
\hline 1785 & 8 & 24 & 12 & 70 & 14 & 3 & 19 & 52 & 114340 & -Jan. & 27 & 8 & Dir. & Mechain & \\
\hline 1785 & 2 & 4 & 34 & 875 & 32 & 9 & 27 & 30) & 42730 & April & 8 & 9 & Ret. & Mechain & \\
\hline 1786 & 6 & 14 & 23 & 505 & 54 & 5 & 9 & 26 & 41010 & July & 7 & 22 & Dir. & Mechain & \\
\hline 1787 & 3 & 16 & 52 & 48 & 16 & 0 & 7 & 44 & 34891 & May & 10 & 20 & Ret. & P. de Saron & \\
\hline 1788 & 5 & 7 & 11 & 122 & 28 & 3 & 9 & 8 & 106301 & Nov. & 10 & 7 & & Mechain & - \\
\hline 1788 & 11 & 21 & 42 & 645 & 53 & 0 & 23 & 12 & 76691 & & 20 & 9 & Dir. & Mechain & \\
\hline 1790 & 5 & 26 & 12 & 315 & 54 & 2 & 0 & 15 & 75810 & Jan. & 15 & 5 & Ret. & De Saron & \\
\hline 1790 & 8 & 27 & 9 & $56 \cdot 5$ & 58 & 3 & 21 & 45 & 106328 & & 28 & 8 & Dir. & Mechain & \\
\hline 1790 & 1 & 3 & 11 & 635 & 52 & 9 & -3 & 4.3 & 79796 & May & 21 & 6 & Ret. & Lalande & \\
\hline 1792 & 6 & 10 & 46 & 39 & 47 & I & 6 & 30 & 129300 & Jan. & 13 & 14 & Dir. & & \\
\hline 1795 & 11 & 29 & 11 & 24 & 17 & 5 & 13 & 37 & 22700 & Dec. & 14 & 23 & ...... & Zach & \\
\hline & 11 & 13 & 2.3 & 20 & 3 & 5 & 7 & 37 & 25800 & & 15 & 15 & ...... & Bouvard & \\
\hline & 0 & $l$ & 7 & $2+4$ & 42 & 5 & 15 & 34 & 21500 & & 14 & 19 & & Prosperin & \\
\hline 1796 & 0 & 17 & 2 & 645 & 55 & 6 & 12 & 44 & 157800 & April & 2 & 20 & Ret. & Olbers & \\
\hline 1797 & 10 & 29 & 17 & 50 & $30 j$ & 1 & 19 & 35 & 52545 & July & 9 & 3 & & Bouvard & \\
\hline & 4 & 2 & 9 & 435 & 52 & 3 & 11 & 59 & 48476 & April & 4 & 12 & $\begin{array}{l}\text { Dir. } \\
2 \mathrm{Y} 2\end{array}$ & Burckhardt & \\
\hline
\end{tabular}


C O M $[348] \quad$ C O M

\begin{tabular}{|c|c|c|c|c|c|c|c|c|c|c|c|c|}
\hline \multirow[t]{2}{*}{ Year } & \multicolumn{3}{|c|}{$\begin{array}{l}\text { Ascending } \\
\text { Node }\end{array}$} & $\begin{array}{c}\text { Inclin. of } \\
\text { Orbit }\end{array}$ & \multicolumn{2}{|c|}{ Perihelion } & \multirow[t]{2}{*}{$\begin{array}{l}\text { Perihel. } \\
\text { Dist. }\end{array}$} & \multicolumn{3}{|c|}{ Time of Perihelion } & \multirow[t]{2}{*}{ Motion } & \multirow[t]{2}{*}{ Calculated by } \\
\hline & $\mathrm{s}$ & $\circ$ & 1 & 01 & $s \quad 0$ & ' & & & & h & & \\
\hline 1798 & 8 & 9 & 31 & 4223 & 14 & 30 & 77968 & Dec. & 31 & 13 & & Burckhardt \\
\hline \multirow[t]{3}{*}{1799} & 3 & 9 & 32 & 5056 & 0 & 39 & & Sept. & 7 & 6 & Ret. & \\
\hline & 3 & & 27 & $50 \quad 57$ & 0 & 39 & 84018 & & 7 & 6 & ...... & Vo \\
\hline & 3 & 9 & 34 & 5052 & 03 & 36 & & & 7 & 4 & ....... & irdt \\
\hline 1799 & 10 & 26 & 49 & 772 & 610 & 20 & 625 & Dec & 25 & 22 & Ret. & \\
\hline 180 & ] & 14 & $2 S$ & 2120 & 63 & 49 & 261 & $\mathrm{Au}$ & 8 & 13 & ......' & hardt \\
\hline 1803 & 10 & 10 & 17 & 570 & 112 & 8 & & Sep & 9 & 21 & Dir. & ain \\
\hline \multirow[t]{2}{*}{1804} & 5 & 26 & 48 & 5629 & 428 & 4.5 & & & 13 & 14 & ....... & Gauss \\
\hline & 5 & 26 & 50 & 5644 & 4. $2 S$ & 54 & & $\mathrm{Fe}$ & 13 & 16 & $\ldots \ldots$ & \\
\hline 8 & 11 & 15 & 7 & 1558 & 4. 29 & 0 & & No & 18 & 0 & Dir. & \\
\hline 1805 & 8 & 10 & 35. & 1625 & $\begin{array}{ll}3 & 19\end{array}$ & 24 & & & 31 & 6 & ....... & Legendre \\
\hline 1807 & 8 & 26 & 46 & 6311 & $\begin{array}{ll}9 & 0\end{array}$ & 56 & 64648 & Sept. & 18 & 8 & $\ldots \ldots$ & Olbers \\
\hline
\end{tabular}

M. Facio has suggested, that some of the comets have their nodes so very near the annual orbit of the earth, that if the earth should happen to be found in that part next the node at the time of a comet's passing by ; as the apparent motion of the comet will be immensely swift, so its parallax will become very sensible; and its proportion to that of the sun will be given: whence, such transits of comets will afford the best means of determining the distance between the earth and sun.

The comet of 1472 , for instance, had a parallax above 20 times greater than the sun's; and if that of 1618 had come down in the beginning of March to its descending node, it would have been much nearer the earth, and its parallax much more notable. But hitherto none has threatened the earth with a nearer appulse than that of 1680: for Dr. Halley finds, by calculation, that Nov. 11 th, at $1 \mathrm{~h} .6 \mathrm{~min}$. afternoon, that comet was not more than one semidiameter of the earth to the northward of the earth's path; at which time had the earth been in that part of its orbit, the comet would have had a parallax equal to that of the moon.-What might have been the consequence of so near an appulse, a contact, or lastly, a shock of these bodies? Mr. Whiston says, a deluge!

To determine the Place and Course of a Comet.-Observe the distance of the comet from two fixed stars, whose longitudes and latitudes are known: then with the distances thus found, calculate the place of the comet by spherical trigonometry.

Longomontanus shows an easy method of finding and tracing out the places of a comet mechanically, which is, to find two stars in the sanie line with the comet, by stretching a thread before the eye over all the three; then do the same by two other stars and the comet: this done, take a celestial globe, or a planisphere, and draw a line upon it first through the former two stars, and then through the latter two; so shall the intersection of the two lines be the place of the comet at that time. If this be repeated from time to timc, and all the points of interséction connected, it will show the path of the comet in the heavens.

The last comet which has been observed, was that of 1807. It was distinctly seen by the naked eye for many werks in September and October. Its elements are as recorded above; to which it may be added that its diameter, arcording to Dr. IIerschel, is $\mathbf{5 3 8}$ miles.

The following observations on this comet have also been published by MI. Burckhardt, of the Institute, on Sept. 22, 1807. "The comet approached nearest the sun on the 12 th of September, at 10 o'clock in the evening; it was then distant 39 millions of leagues from the sun, and 54 from the

earth. Since that time the distance of the comet from the sun has increased, but its distance from the earth will diminish till the middle of October, when its least distance will exceed 41 millions of "leagues. It is not probable that the comet will become more visible, as it loses somewhat more of its light by receding from the sun, than it gains by approaching nearer the earth. In general, the orbit of this comet is such, that it can never approach very near the earth. The body of the comet appears to be separated from the luminous matter, which surrounds it in the shape of a parabola. This phenomenon, which has not been perceived in other comets, has been ascertained by the observations of all the astronomers in Paris, and without donbt will not escape the notice of other astronomers. It does not follow, however, that the body of the comet is wholly separated from the luminous appearance, as the space that seems empty may be filled up by particles less luminous than the rest."

COMETARIUM, a machine adapted to give a representation of the revolution of a comet about the sun. It is so contrived as, by clliptical wheels, to show the unequal motion of a comet in every part of its orbit. The comet is represented by a small brass ball, carried by a radius vector, or wire, in an elliptical grovve about the sun in one of its foci; and the years of its period are shown by an index moving with an equable motion over a graduated silvered circle. See a particular description, with a figure of it, in Fergusson's Astron. 8vo. pa. 400.

COMMANDINE (FREDERICK), a celebrated mathematician and linguist, was born at Urbino in Italy, in 1509 ; and died in 1575 , at 66 years of age. He was famous for his learning and knowledge in the sciences. To a great depth, and just taste in the mathematics, he joined a critical skill in the Greck language, a happy conjunction that well qualified him for translating and expounding the writings of the Greek mathematicians. And accordingly, with a most laudable zeal and industry, he translated and published several of their works, to which no former writer had done that good office. On which account, Francis Moria, duke of Urbino, who was very conversant in thuse sciences, proved a very aficctionate patron to him. He is greatly applauded by Bianchanus, and other writers; and he justly deserved their encomiums.

Of his own works he published the following:

1. Commentarius in Planisphariun Ptolemei: 1558, in 4 to.

2. De Centro Gravitatis Solid. Fonon. 1565, in 4.0.

3. Horologiorun Descriptio: Rom. 1562 , in 4 to.

He translated and illustrated with notes, the following 
works, most of them beautifully printed in 4 to, by the celcbrated printer Aldus:

1. Archimedis Circuli Dimensio; de Lineis Spiralibus; Quadratura Parabolæ; de Conoidibus et Spharoidibus; de Arenæ Numero: 1558.

2. Ptolemæi Planisphærium; et Planisph. Jordani : 1558.

3. Ptolemæi Analemma: 1562.

4. Archimedis de iis que vehuntur in aqua: 1565 .

5. Apollonii Pergæi Conicorum libri quatuor, una cum Pappi Alexandrini Lemmatibus, et Commentariis Eutocii Ascalonitæ, \&c: 1566.

6. Machometes Bagdadinus de Superficierum Divisionibus: 1570 .

7. Elementa Euclidis: 1572.

8. Aristarchus de Magnitudinibus et Distantiis Solis et Lunæ: 1572.

9. Heronis Alexandrini Spiritualium liber: 1583.

10. Pappi Alexandrini. Collectiones Mathem. 1588.

COMMANDING Grqund, in Fortification, an eminence or rising ground, overlooking any post or strong place.This is of three sorts; 1st, A Front Commanding Ground, or a height opposite to the face of the post, which batters upon its front: 2dly, A Reverse Comınanding Ground, or an eminence that can play upon the rear or back of the post : 3dly, An Enfilade Commanding Ground, or an eminence in flank which can, with its shot, scour the whole length of a straight line.

COMMENSURABLE 2uantities, or Magnitudes, are such as have some common aliquot part, or which may be measured or divided, without a remainder, by one and the same measure or divisor, called their common measure. Thus, a foot and a yard are commensurable, because there is a third quantity that will measure each, viz, an inch; which is 12 times contained in the foot, and 36 times in the yard,-Commensurables are to each other, as one rational whole number is to another; but incommensurables are not so: and therefore the ratio of commensurables is rational, but that of incommensurables, irrational; hence also the exponent of the ratio of commensurables is a rational number.

COMMENSURABLE Numbers, whether intégers, or fractions, or surds, are such as have some other number, which will measure or divide them exactly, or without a remainder. Thus, 6 and 8 are commensurable, because 2 measures or divilles them both. And $\frac{2}{3}$ and $\frac{3}{4}$, or $\frac{8}{12}$ and $\frac{9}{12}$ are commensurable fractions, because the fraction $\frac{1}{6}$ or $\frac{1}{\frac{1}{12}}, \& \mathrm{c}$, will measure them both; and in this sense, all fractions may be said to be commensurabie. Also the surds $2 \sqrt{ } 2$ and $3 \sqrt{ } 2$ are commensurable, being measured by $\sqrt{ } 2$, or being to each, other as 2 to 3 .

Commensurabe in P'ower. Euclid says, right lines are commensurable in power, when their squares are measured by one and the same space or superficies.

COMMON, is applied to an angle, line, measure, or the like, that belongs to two or more figures or other things. As a conmon angle, a common side, a common base, a common measure, \& $\mathrm{c}$.

Common Mrasure, or divisor, is that which measures two or more numbers without a remainder. So of 8 and 12 , a common measure is 2, and so is 4 .

The greatest Conmon Measure is the greatest number that can measure two other uumbers. So of 8 and 12, the greatest common measure is 4 .

To find the great est common nicasure of two numbers. Di- vide the greater term by the less; then divide the divisor by the remainder, if there be any; and so on continually, always dividing the last divisor by the last remainder, till nothing remains; then is the last divisor the greatest consmon measure sought.

Thus, to find the greatest common measure of 816 and 1488.

$$
\begin{aligned}
& \text { 816) } 1488(1 \\
& \left.\frac{816}{672}\right) 816(1 \\
& \left.\frac{672}{144}\right) 672(4 \\
& \left.\frac{576}{96}\right) 144(1 \\
& \text { The common measure, } \left.\frac{.96}{48}\right) 96(2 \\
& 96
\end{aligned}
$$

Therefore 48 is the greatest common measure of 816 and 1488, thus:
$48) 816(17$
$\frac{48}{336}$
48) $14.88(31$
144
48
48

The common measure is useful in fractions, to reduce a fraction to its least terms, by dividing those that are given by their greatest common measure. So $\frac{816}{x+88}$ reduces to $\frac{1}{3} \frac{7}{1}$, by dividing 816 and 1488 both by their yreatest common measure 48 .

COMMUNICATION of Motion, that act of a moving body, by which it gives motion, or transfers its motion to another body. Father Mlallebranche considers the communication of motion as something metaphysical ; that is, as not necessarily arising from any physical principles, or any properties of bodies, but flowing from the immediate agency of God.

The communication of motion results from, and is an evidence of the impenetrability and inertia of matter, as such; unless we admit the hypothesis of the penetrability of matter, advanced by Boscovich and Michell, and ascribe to the power of repulsion those effects which have been usually ascribed to its solidity and actual resistance.

Newton shows that action and reaction are equal and opposite; so that one body striking or acting against another, and thence causing a change in its motion, does itself undergo the very same change in its own motion, the contrary way. And lience, a moving boly striking directly another at rest, it loses just as much of its motion it it communicates to the other. For the laws and quantity of motion so communicated, eitler in elastic or non-elastic bodies, see COLLISION.

COMIUTA'TION, Angle of, is the distance between the sun's true place seen from the earth, and the place of a planct reduced to the ecliptic: which therefore is found by taking the difference between the sun's longitude and the heliocentric longitude of the planet.

COMPANY, Rule of, or Rule of Fellowship, in Arithmetic, is a rule by which are cletermined the true sharcs of profit or loss, due to the several partners, or associates, in any enterprise, or trade, in due proportion to the stock contributed by each, and the time it was employed. To do which properly, see the Rule of Fellouslipp.

COMPARTMEN', a design composed of serral dif 
ferent figures, disposed with symmetry; to adorn a parterre, a cieling, pannel of joinery, or the like.

COMPARTITION, the useful and graceful distribution of the whole ground-plot of an edifice, into rooms of office, and of reception, or entertainment.

COMPASS, or Mariner's Compass, is an instrument used at sea by mariners to direct and ascertain the course of their ships. It consists of a circular brass box, which contains a paper card with the 32 points of the compass, or winds, fixed on a magnetic needle that always turns to the north, excepting a small deviation, which is variable at different places, and at the same place at different times. See Variation of the Compass.

The needle with the card turns on an upright pin fixed in the centre of the box. To the middle of the needle is fixed a brass conical socket or cap, by which the card hanging on the pin turns freely round the centre.

The top of the box is covered with a glass, to prevent the wind from disturbing the motion of the card. The whole is inclosed in another box of wood, where it is suspended by gimbals, or brass hoops, to keep the card in a horizontal position during the various motions of the ship. The whole ought to be so placed in the ship, that the middle section of the box, parallel to its sides, may be parallel to the middle section of the ship along its kecl.

The invention of the compass is aseriber by some writers to Flavio Gioia, or Flavio of Malphi, about the year 1302; and hence it is that the territory of Principato, the part of the kingdom of Naples, where he was born, has a compass for its arms. He divided his compass only into 8 points. Others are of opinion that the invention is due to the Chinese; and Gilbert, in his book De Magnete, affirms that Marcus Paulus, a Venetian, making a journey to China, brought back the invention with him in 1260 . What strengthens this conjecture is, that at first they used the compass, in the same manner as the Chinese still do, viz, letting it float on a small piece of cork, insteitd of suspending it on a pivot. It is also arlded, that their emperor Chiningus, a celebrated astrologer, had a knowledge of it 1120 years before Christ. The Chincse divide their compass only into 24 points. But Ludi Vertomanus affirms, that when he was in the East Indies, about the year 1500, he saw a pilot of a ship direct his course by a compass, fastened and framed as those now commonly used. And Barlow, in his book called The Navigator's Supply, anno 1597, informs us, that in a personal conference with two East Indians, they affirmed, that instead of our compass, they use a magnetical needle of 6 inches, and longer, upon a pin in a dish of white China earth, filled witb water; in the bottom of which they have two cross lines for the 4 principal winds, the rest of the divisions being left to the skill of their pilots. Also in the same book he observes, that the Portuguese, in their first discovery of the East Inclies, got a pilot of Mahinde, who brought them from thence in 33 days, within sight of Calicut.

But Fauchette relates sone verses of Guoyot de Provence, who lived in France about the year 1200, which seem to make mention of the compass under the name of Marinette, or Mariner's Stone; which show it was used in lirance near 100 years before either the Malfite or Venetian one. The French even lay claim to the invention, from the fleur de tis witl which most people distinguish the north point of the carcl. With as much reason Dr. Wallis ascribes it to the linglish, from its nane Compass, by which name most nations call it, and which he observes is used in many parts of England to signify a circle.

The mariner's compass long continued very rude and imperfect, but at length received great improvement from the invention and experiments of Dr. Knight, who discovered the useful practice of making artificial magnets; and the further emendations of Mr. Smeaton, and Mr. MI'Culloch, by which the needles are larger and stronger than former$\mathrm{ly}$, and instead of swinging in gimbals, the compass is supported in its very centre upon a prop, and the centres of motion, gravity, and magnetism, are brought almost all to the same point.

After the discovery of that most useful property of the magnet, or loadstone, viz, its giving a polarity to bardened iron or steel, the compass was many years in use before it was known in anywise to deviate from the poles of the world. About the midclle of the 16 th century, so confident were soine persons that the needile invariably pointed due north, that they treated with contempt the notion of the variation, which about that time began to be suspected. However, careful observations soon discovered that in England, and its neighbourhood, the needle pointed to the castward of the true north line; and the quantity of this deviation being known, mariners became as well satisfied as if the compass had none; because the true course could readily be obtained by making allowance for the true variation.

From succecding observations it was afterwards discovered, that the deviation of the needle from the north was not a constant quantity, but that it gradually diminished, and at last, namely, about the year 1657 , it was found that the needle pointed due north at London, and has ever since been going to the westward, till now the variation is upwards of two points of the compass: indeed it was $22^{\circ}$ $41^{\prime}$ about the middle of the year 1781 , as appears by the Philos. Trans. pa. 225, for that year, and is probably now somewhat more, which it would be of consequence to know. So that in any one place it may be suspected the varia-. tion has a lind of libratory motion, traversing through the notth, to unknown limits eastward and westward. But the settling of this point must be left to time and future experiments. Since the above was printed in the former edition of this work, the magnetic observations have been partially resumed by the Royal Society; in consequence, the variation of the needle was there found to be (in September 1811) $24^{\circ} 14^{\prime}$ west, and the dip or inclination $70^{\circ} 32 \frac{1}{2}^{\prime}$. See Variation, also Inclination, and Dip. Also for a further (keseription of different compasses and their uses, see that useful book, Robertson's Navigation, vol. 2, ra231 .

The Azimuth Con pass difiers from the common sea compass in this, that the circumference of the card, or box is divided into degres; and there is fitted to the box an index with two sights, which are upright pieces of biass, placed diametrically opposite to each other, having a slit down the middle of them, through which the sun or star is to be viewed at the time of observation.

The Use of the Azimuth Compass, is to take the bearing of any celestial object, when it is in, or above the horizon, that from the magnetical azimuth or amplitude, the variation of the neelle may be known. See Azrutru, and AMPI,ITUDE.

The figure of the compass card, with the names of the 32 points or winds, are as in fig. 5 , plate 8 ; where other 
compasses are also exhibited. See also a new one in the Supplement to Cavallo's Treatise on Magnetism.

As there are 32 whole points quite around the circle, which contains 360 degrees, therefore each point of the compass contains the $32 \mathrm{~d}$ part of 360 , that is $11 \frac{\mathrm{x}}{4}$ degrees, or $11^{\circ} 15^{\prime}$; consequently the half point is $5^{\circ} 37^{\prime} 30^{\prime \prime}$, and the quarter point $2^{\circ} 48^{\prime} 45^{\prime \prime}$.

The points of the compass are otherwise called rhumbs; and the numbers of degrees, minutes, and seconds, made by every quarter point with the meridian, are exbibited in the following table:

A TA вLE of Rhumbs, showing the Degrees, Minutes, and Seconds, that every Point and 2uarter-point of the Compass makes with the MTeridian.

\begin{tabular}{|c|c|c|c|c|c|c|c|c|}
\hline \multicolumn{2}{|c|}{ North } & Pts.qr & \multicolumn{3}{|c|}{01} & Pts.qr. & \multicolumn{2}{|c|}{ Sunth } \\
\hline \multirow{7}{*}{$N$ by $E$} & \multirow{7}{*}{$\mathrm{NbW}$} & & & 48 & $\overline{45}$ & & \multirow{7}{*}{ S b E } & \multirow{7}{*}{ S b W } \\
\hline & & 0 & 5 & 37 & 30 & 0 & & \\
\hline & & 0 & 8 & 26 & 15 & 03 & & \\
\hline & & 1 & 11 & 15 & 0 & 10 & & \\
\hline & & 1 & 14 & 3 & 45 & 1 & & \\
\hline & & & 16 & 52 & 30 & I & & \\
\hline & & 1 & 19 & 41 & 15 & 1 & & \\
\hline \multirow[t]{4}{*}{ NNE } & N NW & 2 & 22 & 30 & 0 & 2 & SSE & SSW \\
\hline & & 2 & 25 & 18 & 45 & 2 & & \\
\hline & $\therefore$ & 2 & 28 & 7 & 30 & 2 & & \\
\hline & TTS & 2 & 30 & 56 & 15 & 2 & & \\
\hline \multirow[t]{4}{*}{ NEbN } & NW b $\mathrm{N}$ & 3 & 33 & 45 & 0 & 3 & SE b S & SW b S \\
\hline & & 3 & 36 & 33 & 45 & 3 & & 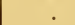 \\
\hline & & 3 & 39 & 22 & 30 & 3 & & \\
\hline & & 3 & 42 & JI & 15 & 3 & & \\
\hline \multirow[t]{4}{*}{ NE } & NW & 4 & 45 & 0 & 0 & 4 & SE & SW \\
\hline & & 4 & 47 & 48 & 45 & 4 & & \\
\hline & & 4 & so & 37 & 30 & 4 & & \\
\hline & & 4 & 5.3 & 26 & 15 & 4 & & \\
\hline \multirow[t]{4}{*}{ NEb E } & NW b W & 5 & 56 & 15 & -0 & 5 & SE b E & SW b IV \\
\hline & & 5 & 59 & 3 & 45 & 5 & & \\
\hline & & 5 & 61 & 52 & 30 & 5 & & \\
\hline & & 5 & 64 & 41 & 15 & 5 & & \\
\hline \multirow[t]{4}{*}{ ENE } & WNIV & 6 & 67 & 30 & 0 & 6 & ESE & WSW \\
\hline & & 6 & 70 & 18 & $45^{\prime}$ & 6 & & \\
\hline & & 6 & 73 & 7 & 30 & 6 & & \\
\hline & & 6 & 75 & 56 & 15 & 6 & & Wh $\mathrm{s}$ \\
\hline \multirow[t]{3}{*}{$\mathrm{E} b \mathrm{~N}$} & W b $N$ & 7. & 78 & 45 & 0 & 7. & E b $\mathrm{S}$ & Wh s \\
\hline & & 7 & $\begin{array}{l}81 \\
84\end{array}$ & $\begin{array}{l}33 \\
22\end{array}$ & $\begin{array}{l}45 \\
30\end{array}$ & $\begin{array}{l}7 \\
7\end{array}$ & & \\
\hline & & 7 & 87 & 31 & 15 & 7 & & \\
\hline Eqst & West & 80 & $0 \mid 90$ & 0 & 0 & 80 & Eist & West \\
\hline
\end{tabular}

Compass Dials, are small dials, fitted in boxes, for the pocket, to show the hour of the day by the direction of the needle that indicates how to place them right, by turning the dial about till the cuck or stile stand directly over the needle. But these can never be very exact, hecause of the variation of the needle itself; unless that variation be allowed for, in making and placing the instrument.

COMPASSES, or Pair of Compasses, a mathematical instrument for describing circles, measuring and dividing lines or figures, \&c.

The common compasses consist of two sharp-pointed branches or legs of iron, steel, brass, or other metal, joined together at the top by a rivet, about which they move as on a centre. Those compasses are of the best kind in which the pin or axle, on which the joint turns, is made of steel, and also balf the joint itself, as the opposite metals wear more equally: the points should also be made of hard steel, wcll polished; and the joint should open and shut with a smooth, casy, and uniform motion. In some compasses, the points are both fixed; but in others, one is made to take out occasionally, and a drawing-pen, or pencil, put in its place. There are in use compasses of various kinds and contrivances, adapted to the various purposes they are intended for ; as,

Compasses of Three Legs, or Triangular Compasses; the construction of which is like that of the common compasses, with the addition of a thind leg or point, which bas a motion every way. Their use is to take three points at once, and so to form triangles; and lay down three positions of a map to be copied at once.

Beam Conpasses, consist of a long straight beam or bar, carrying two brass cursors; one of these being fised at one end, the otber sliding along the beam, with a screw to fasten it on occasionally. To the cursors nay be screwed points of any kind, whether steel, pencils, or the like.. To the fixed cursor is sometimes applied an adjusting or micrometer screw, by which an extent is obtained to very great nicety. The beam compasses are used to draw large circles, to take great extents, or the like.

Bow Compasses, or Bows, arc a small sort of compasses, that shut up in a hoop, which serres for a handle. Their use is to describe arcs or circumferences with a very small radius.

Caliber Compasses. See Ca liber.

Clockmakers Conpasses, are jointed like the common compasses; with a quadrant or bow, like the spring compasses, only of different use, serving here to keep the instrument firm at any opening. They are made very strong, with the points of-their legs of well-tempered steel, as being used to draw or cut lines in pasteboard, or copper, \&c.

Cylindrical and Spherical Compasses, consist of four branches, joined in a centre, two of them being circular and two flat, a little bent at the ends. The use of them is to take the diameter, thickness, or caliber of round or cy lindrical bodies; as caunons, balls, pipes, \&c.

There are also splecrical compasses, differing in nothing. from the common ones, but that their legs are arched; serving to take the diameters of round bodies.

Another sort of compasses also has lately been invented for measuring the dianeter of round bodics, as balls, \&c, which consist of two flat pieces of metal set at right angles on a straight bar or beam of the same; the one piece being fixed, and the otber sliding thereon, so far as just to receive the round body brtween them; and then its diameter, or distance between the two pieces, is shown by the divisions marked on the beam.

Elliptical Compasses, are used to drav ellipses or ovals of any kind. The instrument consists of a beam $\Lambda \mathrm{B}$ (Plate 8, fig. 6,) about a foot long, bearing three cursors; to one of which may be screwed points of any kind; and to the bottom of the other two are riveted two slirling dove-tails, adjusted in grooves made in the cross branches of the beam. The dove-tails having a motion every way, by turning about the long branch, they go backward and forward along the cross; so that when the beam has gone half-way round, one of these will have moved the whole length of one of the branches; and when the beam has gone quite round, the same dove-tail has gone back the whole length of the branch. The same is understood of the other dove-tail.

Note, the distance between the twoslicling dove-tails, is the distance between the two foci of the ellipse; so that by changing that distance, the ellipse will be more circular 
or slevider. Under the ends of the branclies of the cross, are placed four steel points to keep it fast.

The use of this compass is easy: by turning round the long branch, the pen, pencil, or other points will draw the ellipse required.

Its figure shows both its use and construction.

German Conpasses have their legs a little bent outwards, near the top; so that when sliut, the points only meet.

Hair Compasses are so contrived withinside by a small adjusting screw to one of the legs, as to take an extent to a hair's breadtb.

Proportional Compasses are those whose joint lies, not at the end of the legs, but between the points terminating each leg. These are either sinple, or compound. In the former kind the centre, or place of the joint is fixed; so that one pair of these serves only for one proportion.

Compound Proportional CoMPASses have the joint or centre moveable. They consist of two parts or sides of brass, which lie upon each other so exactly as to appear but one when they are shut. These sides easily open, and move about the centre, which is itself moveable in a hollow canal cut through the greatest part of their length. To this centre on each side is fixed a sliding piece, of a small length, with a fine line drawn thereon serving as an index, to be set against other lines or divisions placed upon the compasses on both sides. These lines are, $1, \mathrm{~A}$ line of lines; 2, a line of supcrficies, areas, or planes, the numbers on which answer to the squares of those on the line of lines; 3 , a line of solids, the numbers on which answer to the cubes of those on the line of lines; 4 , a line of circles, or rather of polygons to be inscribed in circles. These lines are all unequally divided, the first three from 1 to 20 , and the last from 6 to 20 . The use of the first is to divide a line into any number of equal parts; by the $2 \mathrm{~d}$ and $3 \mathrm{~d}$ are found the sides of like planes or solids in any givell proportion; and by the 4th, circles are divided into any number of equal parts, or any polygons inscribed in them. See Plate 8 , fig. 7.

Spring Compasses, or Dividers, are made of hardened steel, with an arched head, which by its spring opens the legs; the opening being directed by a circular screw fastencd to one of the legs, let through the other, and worked with a nut.

Trisecting CoMpasses, for the trisecting of angles mechanically, for which purpose they were invented by $M$. Tarragon. The instrument consists of two central rules, and an arch of a circle of 120 degrees, immoveable, with its radius: the radius is fastened with one of the central rules, like the two legs of a sector, that the central rule may be carried through all the points of the circumference of the arch. The radius and rule should be as thin as possible; and the rule fastened to the radius should be hammered cold, to be more elastic; and the breadth of the other central rule inust be triple the breadth of the radius : in this rule also is a groove, with a dove-tail fastened on it, for its motion; there must also be a hole in the centre of each rulc.

Turn-up ConPassus, a late contrivance to save the trouble of changing the points; the body is like the common comprasses; and towards the bottom of the legs withoutside, are added two other points, besides the usual ones; the one carrying a drawing-pen point, and the other a pret-crayon; both being adjusted to turu up, to be used or not, as occasion may require.
COMPLEMENT in general, is what is wanting, or necessary, to complete sume certain quantity or thing. As, the

CoMplement of an arch or angle, as of $90^{\circ}$ or a quadrant, is what any given arch or angle wants of it; so the complement of $50^{\circ}$ is $40^{\circ}$, and the complement of $100^{\circ}$ is $-10^{\circ}$, a negative quantity. - The complement to $180^{\circ}$ is usually called the supplement, to distinguish it from the complement to $90^{\circ}$, properly so called. - The sine of the complement of an arc, is contracted into the word cosine; the tangent of the complement, into cotangent ; Sc.

Arithmetical CompexmeN T, is what a number or logarithm wants of unity or 1 with some number of ciphers. It is best found, by beginning at the left-hand side, and subtracting every figtire from 9, cxcept the last, or righthand figure, which must be subtracted from 10 .

So, the arithmetical comp. of the log. 9.5329714 , by subtracting from 9's, \&c, is $0^{\circ} 46702 s 6$.

The arithmetical complements are mucli used in operations by logarithms, to change subtractions into additions, which are more conveniently performed, especially when there are more than one of them in the operation.

CUMPLEMEXT, in Astronomy, is used for the distance of - a star from the zenith; or the arc contained between the zenith and the place of a star which is above the horizon. It is the same as the complement of the altitude, or co-altitude, or the zenith distance.

Complement of the Course, in Navigation, is the quantity which the course wants of $90^{\circ}$, or 8 points, viz, a quarter of the compass.

CoMplement of the Curtain, in Fortification, is that part of the anterior side of the curtain, which makes the demigorge.

Con PLEM EN Ts of the Line of Defence, is the remainder of that line, after the angle of the flank is takell away.

Complements of a Parallelogram, or in a Parallelogram. are the two lesser parallelograms, made by drawing two right lines parallel to each side of the given parallelogram, through the same point in the diagonal. So $P$ and $Q$ are the complements in the parallelogram $\triangle \mathrm{BCD}$.

In every case, these complements are always equal, viz, the parallelo-

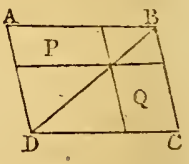
$\operatorname{gram} \mathrm{P}=\mathrm{Q}$.

Compleanent of Life, a term much used, in the doctrine of Life Annuities, by De Moivre, and, according to him, it denotes the number of years which a given life wants of 86 , this being the age which he considered as the ntmost probable extent of life. So 56 is the complement of 30 , and 30 is the complement of 56 .

That author supposed an equal annual decrement of life through all its stages, till the age of 86 . Thus, if there be 56 persons living at 30 ycars of age, it is supposed that one will die every year, till they be all dead in 56 years. This hypothesis in some cases is near the truth, for the value of single lives. Demoive had another hypothesis, that of life decreasing in geometrical progression, for calculating the value of joint lives; but more inaccurate than the former. See Life Annuitus; De Moivre's 'Treatise on Annuitics, pa. 83 ; Simpson's Life A1ınuities; Price on Reversionary Payments, pa. 2; and Baily's Life Annuities.

COMPOSITE Nunber, is one that is compounded of, 
or made up by the multiplication of two other numbers, greater than 1, or which can be measured by some other number greater than 1. As 12, which is composed, or compounded of 2 and 6 , or 3 and 4 , viz, by multiplying together 2 and 6 , or 3 and 4 , both products making the same number 12; which therefore is a composite number. Composites are opposed to prime numbers, or primes, which cannot be exactly measured by any other number except unity; or be produced by multiplying together two other factors.

Composite Numbers between themselves, are the same with commensurable numbers, or such as have a common measure or factor; as 15 and -12 , whicls have the common term 3. The doctrine of Prime and Composite numbers is pretty fully trcated in the 7 th and Sth books of Euclid's Elemenis.

Composite Numbers, in musical calculations, are such as are composed by the multiplication of the prime integers $2,3,5$. Alf primes larger than these, or composite numbers into which such cnter, are not musical numbers, but are irrational, or surd, in a musical sense.

Composite Order, in Architecture, is the last of the five orders of columns; and is so catled, because its capital is composed out of those of the other orders. Thus, it borrows a quarter-round from the Tuscan and Doric; a double row of leaves from the Corinthian; and volutes from the Ionic. Its cornice has single modillions, or dentils; and its column is 10 diameters in height. This order is also called the Roman order, and Italic order, as having been inveuted by the Romans, like as the other orders are denominated from the people among whom they had their rise.

COMPOSITION, is a species of reasoning by which we procecd from things that are known and given, step by step, till we arrive at such as were before unknown or required; viz, proceeding upon principles self-evident, on definitions, postulates, and axioms, with a previously demonstrated series of propositions, step by step, till it gives a clear knowledge of the thing to be known or demonstrated. Conposition, otherwise called the synthetical method, is opposed to Resolution, or the analytical method, and is chictly used by the ancients, Euclid, Apollonius, \&c. Sce Pappus; also the term Axalrsis.

CoM P.OSITiox of forces, or of motion, is the union or assemblage of several forces or motions that are oblique to one another, into an equivalent one in another direction.

1. When several forees or motions are united, that act in the same line of direction, the combined force or motion will be in the same line of direction also. But when oblique forces are united, the compounded force takes a new direction, diflerent from both, and is either a right line or a curve, according to the nature of the forces compounded.

2. If two compounding motions be both equable ones, whether equal to each other or not, the line of the compound motion will be a straight oue. Thus, if the one equable in the direction $\mathrm{AB}$ be sufficient to carry a body over the space $A B$ in any time, and the other equable motion sufficient to pass over $\mathrm{AC}$ in the same time; then by the compound motion, or both acting on the body together, it would in the same time pass over the diagonal $A D$ of the parallelogram $A$ B DC. For because the motions are uniform, VOL. I. any spaces $A b, \mathbf{A} c$ passed over in the same time, are proportional to the velocities, or to $A B$ and $A C$; and consequently all the points $\Delta, d, d, \mathrm{D}$, of the path are in the same right line.

3. And though the compounding motions be not equable, but variable, either accelerated or retarded, provided they do but vary in a similar manner, the compounded motion will still be in a straight line. Thus, suppose for instance, that the motions both vary in such a manner, as that the spaces passed over in the same time, whether they be equal to each other or not, are both as the same power $n$ of the time; then $A \mathrm{~B}^{\mathrm{n}}: A \mathrm{C}^{\mathrm{n}}:: A \mathrm{~b}^{\mathrm{n}}: A \mathrm{C}^{\mathrm{n}}$, and hence $A B: A C:: A b: A c$, and therefore, as before, $A d d D$ is still a right line.

4. But if the compounding motions be not similar to each other, as when the one is equable and the other variable, or when they are varied in a dissimilar manner, then the compounded motion is in some curve line: so, if the motion in the one direction $\mathrm{Er}$ be in a less proportion, with respect to the time, than that in the direction $\mathrm{EG}$ is, then the path will be a curve line $E / h \mathbf{H}$ conves towards EF; but if the motion in EF be in a greater proportion than that in EG, then the path of the compound motion will be a curve EiH concave towards EF: that is, in general, the curvilineal path is convex towards that direction in which the motion is in the less proportion to the time. Hence, for a particular instance, if the mo-

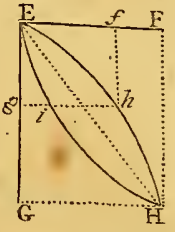
tion in the direction $\mathrm{EF}$ be a motion of projection, which is an cquable motion, and the motion in the direction EG that arising from gravity, which is a uniformly accelerated motion, or in proportion to the squares of the times; then is $\mathrm{EG}$ as $\mathrm{GH}^{2}$, and $\mathrm{Eg}$ as $\mathrm{g} h^{2}$, that is $\mathrm{EG}: \mathrm{Eg}:: \mathrm{GH}^{2}: g h^{2}$, which is the property of the parabola; and therefore the path Elini of any body projected, is the common parabola.

5. If there be three furces united, or acting against the same point $\mathbf{A}$ at the same time, viz, the force or weight $\mathbf{B}$ in the direction $\mathrm{AB}$, and the forces or tensions in the directions $A C, \triangle D$; and if these three forces mutually balance each other so as to keep the common point $A$ in equilibrio, then are these forces directly proportional to the respective sides of a triangle, formed by drawing lines parallel to the directions of these forces; or indeed perpendicular to those directions, or making any one and the same angle with them. So that, if $\mathrm{BE}$ be drawn parallel, for instance, to $\mathrm{AD}$, and meet $C_{A}$ produced in $\mathrm{E}$, forming the triangle $A \mathrm{BE}$, then are the three forces in the directions $A B, A C, A D$, respectively proportional to the sides $\mathrm{AB}, \mathrm{AE}, \mathrm{BE}$.

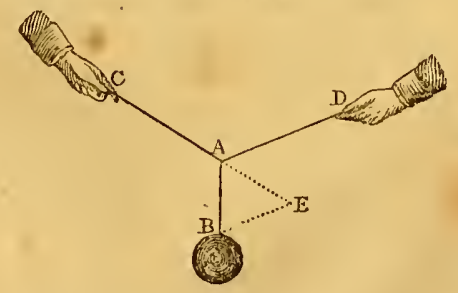

And this theorem, with its corollaries, Dr. Keil observes, is the foundation of all the new mechanics of M. Varignon; $2 \mathrm{Z}$ 
by help of which may the force of the muscles be computed, and most of tise mechanic theorems in Borelli, De Motu Animalium, may immediately be deduced. And hence also may any number of forces be compounded together into one only. Sec Collisiox, Composition of Forces, \&c.

Composition of Numbers and Quantities. Sec ComBINATION.

Composition of Proportion, according to the 15 th definition of the 5 th book of Euclid's Elements, is when, of 4 proportionals, the sum of the 1 st and $2 d$ is to the $2 d$, as the sum of the $3 \mathrm{~d}$ and 4 th is to the 4 th:

$\checkmark$ as if it be $a: b:: c: d$, then by composition $a+b: b:: c+d: d$. Or, in numbers, if $2: 4:: 9: 18$, then by composition $6: 4:: 27: 1$ s.

Composition of Ratios, is the adding of ratios together; which is performed by multiplying together their corresponding terms, viz, the untecedents together, and the consequents together, for the antecedent and consequent of the compounded ratio; like as the addition of logarithms is the same thing as the multiplication of their corresponding numbers. Or, if the terms of the ratios be placed fraction-wise, then the addition or composition of the ratios is performed by multiplying the fractions together.

Thus, the ratio of $a: b$, or of $2: 4$, added to the ratio of $c: d$, or of $6: s$, makes the ratio of $a c: b d$, or of $12: 32$; and so the ratio of $a c$ to $b d$ is said to be componnded of the ratios of $a$ to $b$, and $c$ to $d$. So likewise, if it were required to compound together the three ratios, viz, of $a$ to $b, c$ to $d$, and $e$ to $f$; then $\frac{a}{b} \times \frac{c}{d} \times \frac{e}{f}=\frac{a c e}{v d f}$ are the terms of the compound ratio; or the ratio of ace to $b d f$ is compounded or made up of the ratios of $a$ to $b, c$ to $d$, and $e$ to $f$.

Hence, if the consequent of each ratio be the same as the antecedent of the preceding ratio, then is the ratio of the first term to the last, compounded, or made up of all the other ratios, viz, the ratio of $a$ to $e$, equal to the sum of all the ratios of $a$ to $b$, of $b$ to $c$, of $c$ to $d$, and of $d$ to $c$; for $\frac{a}{b} \times \frac{b}{c} \times \frac{c}{d} \times \frac{d}{c}=\frac{a}{e}$ the terms or exponents of the compounded ratio.

Hence also, in a series of continual proportionals, the ratio of the first term to the third is double of the ratio of the first to the second,

and the ratio of the 1 st to the 4 th is triple of it, and the ratio of the Ist to the 5 th is quadruple of $i t$, and so on; that is, the exponents are double, triple, quadruple, \&c, of the first exponent: as in the series $1, a, a^{2}$, $a^{3}, a^{4}, 8 \mathrm{c}$; where the ratio 1 to $a^{2}$ is double, of 1 to $a^{3}$ triple, \& c c, of the ratio of 1 to $a$; or the exponent of $a^{2}$, $a^{3}, a^{4}, \& c$, double, triple, quadruph', \&c, of $a$.

COMPOUND InTEREST, called also linterest upon Interest, is that which, is reckoned not only upon the principal, but on the interest itself forborn, which thus becomes a sort of secondary principal.

If $r$ be the amount of 1 pound for 1 yeal, that is the sum of the principal and interest together for one ycar; then is $r^{2}$ the amount for 2 years,

and $r^{3}$ the amount for 3 years,

and in general $r^{t}$ the amount for $t$ years; that is $r^{t}$ is the sum or total amount of all the principals and interests together of $1 l$ fur the whole time or number of years $t$; con-

sequentiy, if $p$ be any other principal sum, forborn for $t$ yeas, then its amount in that time at compound interest, is $a=m^{t}$.

The Rule therefore in words is this: to one pound add its interest for one year, or balf year, or for the first time at which the interest is reckoned; raise the sum $r$ to the power denoted by the time or number of terms; then this power multiplied by the principal, or first sum lent, will produce the whole amount.

For example, To find how much $50 \mathrm{l}$ will amount to in 5 years at 5 per cent. per annum, compuund interest.Here the interest of $1 l$ for 1 year is $\cdot 05$, and theretore $r^{*}=1.05 \quad ;$ hence the 5 th power of it for 5 years, is $r^{5}=1.27628$ \& ;

multiply this by $p$ or -50

gives the amount $p^{\text {t }}$ or $63.814 l$

or $63 l 16 s 3 \frac{x}{4} d$ for the amount sought.

If the compound interest, or amount of any sum, be required for the parts of a year, it may be determined as follows:

1. When the time is any aliquot part of a year.

Rule. Find the amount of $1 l$ for 1 year us before, and that root of it which is denoted by the aliquot part will be the amount of $1 l$ for that time; which multiplied by the principal, will le the amount of the given sum required.

2. When the time is not an aliquot part of a year.

Rule. 1. Reduce the time into days, and the 365 th root of the amount of $1 l$ for 1 year, is the amount of the same sum for one day. 2. Raise this amount to that power whose index is equal to the number of days, and it will be the amount of $1 l$ for the given time. 3. Multiply this amount by the principal, and it will be the amount of the given sum required.

Note. The readiest way of extracting the roots, especially bigh ones, is by logarithms, thus: Divide the lugarithm of the rate, or amount of 11 , for a year, by the denominator of the given aliquot part, and the quotient will be the logarithm of the root sought.

But Compound Interest for any given number of whole years, is best computed by means of such a table as the following, being the amounts of $l l$ for any number of years, and at several rates of compound interest.

As an example of the use of this table, suppose it were required to find the amount of $250 l$ for 35 years at 4 per cent. compound interest.

In the column of 4 per cent, and line of 35 years, which is inuliplied by the principal gives -
or -
which is the amount sought. -

$$
\begin{aligned}
& \text { - 3.94609, } \\
& \text { - } \frac{250}{956 \cdot 52.50} \\
& \text { - gscil los } 5 \frac{1}{4} d \text {, }
\end{aligned}
$$

Note. By a bare impection of this table, it appears how many years are requirel tor any sum of money to double itself, at any rate of compound interest; riz, by looking in the columus when the amount becomes the number 2 . So it is tound that at the several rates the respective times requisite for loubling any sum, are nearly thus; viz,
Rate $3 \quad 3 \frac{5}{2}+4 \frac{1}{3} \quad 5 \quad 6$
Yicars $23 \frac{\pi}{2} \quad 20 \frac{\pi}{4} \quad 17 \frac{3}{4} \quad 15 \frac{3}{4} \quad 14 \frac{8}{4} \quad 12$ 
$T_{\mathrm{ABLE}}$ of the Amount of $1 /$ at Compound Interest for many Years and several Rates of Interest.

\begin{tabular}{|c|c|c|c|c|c|c|}
\hline 5 & $\begin{array}{l}\text { at } 3 \text { per } \\
\text { cent. }\end{array}$ & $\begin{array}{l}3 \frac{1}{2} \text { pur } \\
\text { cent. }\end{array}$ & $\begin{array}{l}4 \text { y } \\
\text { cent. }\end{array}$ & $\begin{array}{l}4 \frac{1}{2} \text { per } \\
\text { cent. }\end{array}$ & $\begin{array}{l}5 \text { per } \\
\text { cent. }\end{array}$ & $\begin{array}{l}6 \text { per } \\
\text { cent. }\end{array}$ \\
\hline 1 & 1.03000 & 1.03500 & 1.04000 & $1 \cdot 04500$ & 1.05000 & 106600 \\
\hline 2 & & & 0 & 1.09203 & & \\
\hline 3 & & & & $1 \cdot 14117$ & & \\
\hline 4 & & 4752 & & I.19252 & & \\
\hline 5 & $1 \cdot 15927$ & 3769 & $1 \cdot 21663$ & $1 \cdot 24618$ & $1 \cdot 27628$ & 3823 \\
\hline 6 & $I \cdot 19405$ & J.22926 & $\mathrm{I} \cdot 26532$ & 1.30226 & 34010 & 1. \\
\hline 7 & & & & $1 \cdot 36086$ & & \\
\hline 8 & & 31681 & 36857 & 1.42210 & & \\
\hline 9 & & $1 \cdot 36290$ & $1 \cdot 423$ & & & \\
\hline 10. & & 060 & $1 \cdot 48024$ & $1 \cdot 55297$ & 890 & \\
\hline II & $1 \cdot 38$ & & & $1 \cdot 62285$ & & \\
\hline 12 & & & & & & \\
\hline 13 & & & 97 & .77220 & & \\
\hline [4 & & & & & & \\
\hline 15 & & & & $1 \cdot 93528$ & & \\
\hline 16 & & & 87298 & 237 & & \\
\hline 17 & & & & & & \\
\hline 18 & & & & & & \\
\hline 18 & & & & & & \\
\hline 20 & & 79 & $2 \cdot 19$ & & & \\
\hline 21 & & & 27877 & & 96 & \\
\hline 22 & & & & & & \\
\hline 23 & & & & & & \\
\hline 24 & & & & & & \\
\hline 25 & & & & & & \\
\hline 26 & & & & & & \\
\hline 27 & & & & & & \\
\hline 28 & & & & & & \\
\hline 29 & & & & & & \\
\hline 30 & & & & & & \\
\hline 31 & & & $3 \cdot 37313$ & & & \\
\hline 32 & & & & & & \\
\hline 33 & & & & & & \\
\hline 34 & & & & & & \\
\hline 35 & & & & & & \\
\hline 36 & & & & & & \\
\hline 37 & & & & & & \\
\hline 38 & & & & & & \\
\hline 39 & & & & & & \\
\hline 40 & & & & & & \\
\hline 41 & & & & & & \\
\hline 42 & & & & & & \\
\hline 43 & & & & & & \\
\hline 44 & & & & & & \\
\hline 45 & & & & & & \\
\hline 46 & & & & & & \\
\hline 4 & & & & & & \\
\hline 48 & & & & & & \\
\hline 49 & & & & & & \\
\hline 00 & & & & & 6740 & 18.42015 \\
\hline
\end{tabular}

COMPOUND Motion, that motion which is the effect of several conspiring powers or forces, viz, such forces as are not directly opposite to each other: as when the radius of a circle is considered as revolving about a centre, and at the same time a point moving along it ; which produces a kind of a spiral for the path of the point. And hence it is easily perceived, that all curvilinear motion is compound, or the effect of two or more forces ; though every compound motion is not curvilinear.

It is a popular theorem in Mechanics, that in uniform compound motions, the velocity produced by the conspiring powers or forces, is to that of either of the two compounding powers separately, as the diagonal of a parallelogram, according to the direction of whose sides they act separately, is to cither of the sides. Sec Cov Position of Molion, and CoLlusion.

CoMPound Numbers, those conposed of the multipli- cation of two or more numbers: as 12 , composed of 3 times 4. See Composite.

Compound Pendulum, that which consists of several weights constantly kecping the same distance, both from each other, and from the centre about which they oscillate.

Compound Quantities, in Algebra, are such as are connected together by the signs + or - . Thus, $a+b$, or $a-c+d$, or $a a-2 a$, are compound quantities. Compound quantities are distinguished into binomials, trinomials, quadrinomials, \& $c$, according to the number of terms in them; viz, the binomial having two terms; the trinomial, three; the quadrinomial, 4.; \&c. Also, those that have inore than two terms, are called by the general naine of multinomials, as also polynomials.

Compound Ratio, is that which is made by adding two or more ratios together; viz, by multiplying all their antecedents together for the antecedent, and all the consequents together for the consequent of the compound ratio. So 6 to 72 is a ratio compounded of the ratios 2 to 6 , and 3 to 12 ; because $\frac{6}{72}=\frac{2}{6} \times \frac{3}{12}$ : also $a b$ to $c d$ is a ratio compounded of the ratio of $a$ to $c$, and $b$ to $d$; for $\frac{a b}{c d}=\frac{a}{c} \times \frac{b}{d}$. See Cumposition of Ratios.

COMPRESSION, the act of pressing, or squeczing something, so as to bring its parts nearer together, and inake it occupy less space. Compression differs from condensation as the cause from the effect, compression being the action of any force on a body, witlout regarding its effects; whereas condensation denotes the state of a body that is actually reduced into a less bulk, and is an effect of compression, though it may be effected also by other means. Nevirtheless, compression and condensation are often confounded.

Pumps, which the ancients imagined to act by suction, do in reality act by compression; the piston, in working in the narrow pipe, compresses the inclosed air, so as to enable it, by the force of its increased elasticity, to raise the valve, and make its escape; on which, the balance being destroyed, the pressure of the atmosphere on the stagnant surface, forces up the water in the pipe, thus evacuated of its air.

It was long thought that water was not compressible into less bulk: and it was belicved, till lately, that after the air had been purged out of it, no art or violence was able to press it into less space. In an experiment made by the Academy del Cimento, water, when violently squeezed, made its way through the fine pores of a globe of gold, rather than yield to the compression.

But the ingenious Mr. Canton, attentively considering this experiment, found that it was not sufficiently accurate to justify the conclusion which had always been drawn from it; since the Florentine philosuphers had no method of determining that the alteration of figure in their globe of gold, occasioned such a diminution of its interual capacity, as was exactly equal to the quantity of water forced into its pores. To bring this matter therefore to a more accurate and decisive trial, he procurel a small glass tube of about two feet long, with a ball at one end, of an inch and a quarter in diameter. Having filled the ball and part of the tube with mercury, and brought it exactly to the heat of $50^{\circ}$ of Fahrenleit's thermometer, he marked the place where the mercury stood in the mibe, 
which was about six inches and a half above the ball; he then raised the mercury by heat to the top of the tube, and there sealed the tube bermetically; then on reducing the mercury to the same degree of heat as before, it stood in the tube $\frac{32}{10}$ of an inch higher than the mark. The same experiment was repeated with water exhausted of air, instead of mercury, and the water stood in the tube $\frac{43}{100}$ of an inch above the mark. Since the weight of the atmosphere on the outside of the ball, without any counterbalance from within, will compress the ball, and equally raise both the mercury and water, it appears that the water expands $\frac{I x}{100}$ of an inch more than the mercury by renoving the weight of the atmosphere. Having thus determined that water is really compressible, he proceeded to estimate the degree of condensation corresponding to any given weight. For this purpose he prepared another ball, with a tube joined thereto; and finding that the mercury in $\frac{23}{T_{0}}$ of an inch of the tube was the hundred thousandth part of that contained in the ball, he divided the tube accordingly. He then filled the ball and part of the tube with water extrausted of air; and leaving the tube open, placed this apparatus under the receiver of an airpump, and observed the degree of expansion of the water answcring to any degree of rarefaction of the air; and again by putting it into the glass receiver of a condensing engine, he noted the degree of condensation of the water corresponding to any degree of condensation of the air. He thus found, by repeated trials, that, in a tempcrature of $50^{\circ}$, and.when the mercury has been at its mean beight in the barometer, the water expands one part in 21740 ; and is as much compressed by the weight of an additional atmosphere; or the compression of water by twice the weight of the atmosphere, is one part in 10870 of its whole bulk. Should it be objected, that the compressibility of the water was owing to any air which it might be supposed to contain, he answers, that more air would make it more compressible; he therefore let into the ball a bubble of air, and found that the water was not more compressed by the same weight than before.

In some further experiments of the same kind, Mr. Canton determined that water is more compressible in winter than in summer; but he observed the contrary in spirit of wine, and oil of olives. The following table was formed, when the barometer was at 29 inches and a half, and the thermoniter at 50 degrees.

\begin{tabular}{lrrrr} 
Compression of & \multicolumn{3}{c}{ Millionih pts. } & Spec. grav. \\
Spirit of wine & - & 66 & - & 846 \\
Oil of olives & - & 48 & - & 918 \\
Rain water & - & 46 & - & 1000 \\
Sia water & - & 40 & - & 1028 \\
Mercury & - & 3 & - & 13595
\end{tabular}

He infers that these fluids are not only compressible, but elastic; and that the compressions of them, by the same weight, are not in the inverse ratin of their densitics, or specific gravities, as might be supposed. Phil.Trans, vol.lii. 1762 , art. 103 ; and vol. liv. 1764 , art. 47 .

The compression of the air, by its own weight, is surprisingly great : but the air may be still farther compressr.d by art. See Elasticily of $\Lambda$ r. This immense compression and dilatation, Newton obscrves, cannot be accounted for in any other way, but by a repelling force, with which the particles of air are endued; by virtue of which, when at liberty, they mutually recede from each other.
He also further informs us, that this repelling power is stronger and more sensible in air, than in other bodies; because air is generated out of very fixed bodies, but not without great difficulty, and by the help of fermentation: now those particles always recede from each other with the greatest violence, and are compressed with the greatest dificulty, which, when contiguous, cohere the most strongly. See Air; Atriaction, Confsion, Dilatation, and REPULSION.

COMPUTATION, the manner of accounting and estimating tíme, weights, measures, moncy, \&c. See $\mathrm{C}_{\mathrm{AL}}$ CULATION, which it is also used for.

CONCAVE, an appellation used in speaking of the inner surface of hollow bodlies, more especially of spherical or circular ones.

Concave glasses, lenses, and mirrors, have either one side or both sides concave.

The property of all concare linses is, that the rays of light, in passing through then, are deflected, or made to recede from one another; as in convex lenses they are inflccted towards each other; and that the more as the concavity or convexity has a smaller radius. Hence paralled rays, as those of the sun, by passing through a cuncave lens, become diverging; diverging rays are made to diverge more; and converging rays are made either to converge less, or to become parallel, or go cut diverging. And hence it is, that objects viewed through concave lense's, appear diminished; and the more so, as they are portions of less splieres. See Lers.

Concave mirrors have the contrary effect to lenses: they reflect the rays which fall on them, so as to make thein approach more to; or recede from each other, than before, according to the sittiation of the object; and that the more as the concavity is greater, or as the radius of concarity is less. Hence it is that concave mirrors magnify objects that are presented to tliem; and that in a greater proportion, as they are portions of smaller spheres. And hence also concave mirrors bave the effect of burning glasses. See Mirror, and Burning-Giass.

CUNCAVITY, that side of a figure or body which is hollow.-An arch of a curve lias its concavity turned all the same way, when the right lines that join any two of its points are all on the same side of the arch.

Archimedes, intending to include in bis definition such lines as have rectilinear parts, says, a line has its concavity turned one way, when the right lines that join any two of its points, are either all on une side of it, or while some fall upon the line itself, none fall on the opposite side. Archim. de Splixer et Cyl. Def. 2; and Mitclaurin's Fluxions, art. 150 .

When two lines that have their concavity turned the same way, have the same extremes, and the one includes the other, or has its concavity towards it, the perimeter of that which includes, is greater than that which is included. Archim. ib. ax. 2.

CONCENTRIC, having the same centre. It is opposed to excentric, or having different centres. The word is chiefly used in speaking of rousd bodies and figures, such as circular, and clliptic ones; but it may also be used for polygons that are drawn paralled to each oller, from the same centre.-Nonius's method of graduating instruments consists in describing with the same qualrat 45 concentric arches, dividing the outermost into 90 equal parts, the next into 89 , and so on. 


\section{O N}

CONCHOID, or CoNciltLes, the name of a curve invented by Nicomedes. It was much used by the ancients in the construction of solid problems, as appears by what Pappus says. - It is thus constructed: $\mathrm{AP}$ and $\mathrm{BD}$ being two lines intcrsecting at right angles; from $P$ draw a number of other lines PFDE, \& $\mathrm{C}$, on which take always $\mathrm{DE}=\mathrm{DF}$ $=\mathrm{AB}$ or $\mathrm{BC}$; so shall the curve line drawn through all the points $E, E, E$, be the first conchoid, or that of Nicomedes; and the curve drawn through all the other points $\mathrm{F}, \dot{\mathrm{F}}, \mathrm{F}$, is called the second conchoid; though in reality, they are both but parts of the same curve, having the saine pole $P$, and four infinite legs, to which the line $\mathrm{DBD}$ is a common asymptote.
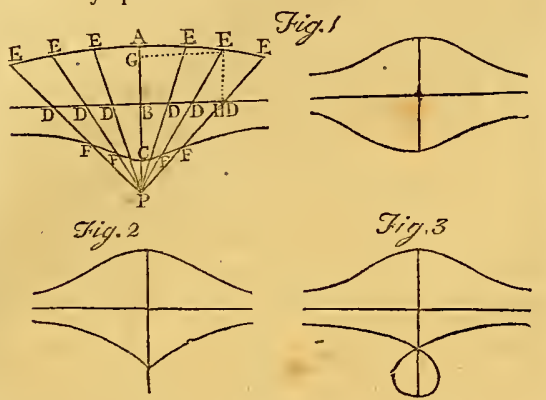

The fixed point $P$ is called the pole or centre of the conchoid. And the line BD is called the directrix.

To determine the equation of the curve: put $\mathrm{AB}=\mathrm{BC}$ $=\mathrm{D} \mathrm{i}=\mathrm{DF}=a, \mathrm{~PB}=b, \mathrm{BG}=\mathrm{EH}=x$, and $\mathrm{GE}=\mathrm{BH}$ $=y$; then the equation to the first conchoid will be $x^{2} \times$ $(b+x)^{2}+x^{2} y^{2}=a^{2} \times(b+x)^{2}$, or $x^{4}+2 b x^{3}+b^{2} x^{2}+$ $x^{2} y^{2}=a^{2} b^{2}+2 a^{2} b x+a^{2} x^{2} ;$ and, changingonly the sign of $x$, as being negative in the other curve, the cquation to the $2 \mathrm{~d}$ conchoid will be $x^{2} \times(b-x)^{2}+x^{2} y^{2}=a^{2} \times$ $(b-x)^{2}$ or $x^{4}-2 b x^{3}+b^{2} x^{2}+x^{2} y^{2}=a^{2} b^{2}-2 a^{2} b x+$ $a^{2} x^{2}$.

Of the whole conchoid, expressed by these two equations, or rather one equation only, with different signs, there are three cases or species; as first,

when BC is less than BP, the conchoid will be as in fig. 1; when $\mathrm{BC}$ is equal to $\mathrm{BP}$, the conchoid will be as in fig. 2; and when BC is greater than $\mathbf{B P}$, the conchoid will be as in fig. 3.

Newton approves of the use of the conchoid for trisecting angles, or finding two mean proportionals, or fur constructing other solid problems. Thus, in the Lincar Construction of equations, towards the end of his Universal Arithmetic, he says, "The ancients at first endeavoured in vain at the trisection of an anglc, and the finding of two mean proportionals by a right line and a circle. Afterwards they begin to consider several other lines, as the conchoid, the cissnid, and the conic sections, and by some of these to solve thesc problems." Again, "Either therefore the trochoid is not to be admitted at all into geometry, or else, in the construction of problems, it is to be preferred to all lines of a more difficult description: and there is the same reason for other curves; for which reaon we approve of the trisections of an angle by a conchuid, which Archimerles in his Lemmas, and Pappus in his Collections, have preferred to the inventions of all others in this case; because we ought either to cxclude
$\mathrm{C} \mathrm{O} \mathrm{N}$

all lines, besides the circle and right line, out of geometry, or admit them according to the simplicity of their descriptions, in which case the conchoid yields to none, except the circle." Lastly, "That is arithmetically more simple which is detcrmined by the more simple equations, but that is geometrically more simple which is determined by the more simple drawing of lines;' and in geometry, that ought to be reckoned best which is geometrically most simple: wherefore I ought not to be blamed, if, with that prince of mathematicians, Archimedes, and other ancients, 1 make use of the concboid for the construction of solid problems."

CONCRETE Numbers are those that are applied to express or denote any particular subject; as 3 men, 2 pounds, $\& c$. Whereas, if nothing be connected with a number, it is taken abstractedly or universally: thus, 4 signifies only an aggregate of 4 units, without any regard to a particular subject, whether men or pounds, or any thing else.

CONCURRING, or CONGRUBNT Figures, in Geometry, are such as, being laid upon one another, do exactly correspond to, and cover one anotber, and consequently must be equal among themselves. Thus, triangles having two sides and the contained angle equal, each to each, are congruent or equal to each other in all respects.

CONDAMINE (CHARLLS MARIE DE LA), a French mathematician, and chevalier of the order of St. Lazare. He was born at Paris in 1701; and began his career in the military service; but, soon yielding to bis inclination for ratural curiosities, he travelled over the East. Afterwards, in $1735,1736, \& \mathrm{c}$, he was employed, along with M. Godin, Bouguer, and otber mathematicians, to measure the meridian in Peru, near Quito; from which he returned by the river Amazon, through vast dangers and difficulties, as may be seen in the account he published of the voyage, which was not finished under the course of ten years. After in some degree recovering his health he travelled through Italy and England, \&c, and died in 1774 . Condamine was admitted into the Academy of Sciences in 1730, the French Academy in 1760 , and was also a member of several foreign academies. The published works of Condamine are,

1. Voyage dans l'Amerique Meridionale, 1745, svo.

2. Figure de la Terre, 1749 , in 4 to.

3. Voyage a l'Equateur, avec le Supplement, et l'Histoire des Piramides de Quito, 1751 and 1752,2 vols. 4 to.

4. Three Memoirs on Ineculation, and Letters on tbe saine subject.

Besides numerous scientific articles in most of the voJumes of Memoirs of the Academy of Sciences, from the year 1731 to 1768 inclusively.

CONDENSATION, is the compressing or reducing a body into a less bulk or space; by which means it is rendered more dense and compact. Compression and Condensation (though sometimes used the one for the other) differ in this; viz, that the former denotes a diminution of bulk occasioned by the application of external force; whereas, the latter expresses the same cfect when produced without that external application, as is the case with most bodies in cooling. But the word Condensation has been more commonly used for denoting the conversion of vapour into water, or of vapours in general into liquids.

Notwithstanding this definition, it is to be remarked, that in every case of condensation, or of the contraction of a borly into a narrower space, the effect is produced by 
the cscape of something; and in bodies which appear to be the simpiest in nature, the contraction which they uncergo in cooling, is occusioned by the escape of the caloric, which the present_state of philosophical knowledge reckons among the clementary substances. But the reverse of this is not always true; viz, bolies are not always cuntracted in their dimensious when the caloric escapes from them; and such has been found to be the case with water, with iron, and with some othcr substances in certain temperatures.

It is howerer evident that condensation in general consists in bringing the parts closer to each other, and increasing their contact, whatever nay be their form or state of previous existence, or the means by which it is effected: in opposition to rarefaction, which renders the body lighter and looser, by setting the parts farther asunder, and diminishing their contact, and of consequence their cohesion.

Air condenses, either by cold, or by pressure, but much inore by the latter; and most of all by chemical process. Water condenses also under the same circumstances of action; but it suddenly expands by congelation: indeed almost all matter, both solids and fluids, has the same property of condensation by those means. See Compression. Soalso vapour is condensed, or converted into water, by distillation, or naturally in the clouds. The way in which vapour commonly condenses, is by the escape of the caloric. On touching it, the vapour parts with this heat: and on doing so, it immediately loses the proper characteristics of vapour, and becomes watcr. But though this be the most common and usual way in which vapour in general is condensed, yet nature certainly proceeds after another manner; since we often observe the vapours most plentifully condensed when the weather is really warmer than at other times.-Most bodies are susceptible of three successive states of existence; namely, the solid, the liquid, and the elastic or vaporous; and all these are effected by the introduction of caloric. During every one of those states, a different degree of condensation is produced by the intermediate gradations of temperature, viz, such as are not quite sufficient to induce a different state of existence in the body concerned. Thus, a quantity of the vapour of water, which at the temperature of $242^{\circ}$ (Fahrenheit's thermometer), and under the mean gravity of the atmosphere, occupies the space of 3600 cubic inches; if it be gradually cooled until the temperature becomes equal to about $212^{\circ}$, its bulk will be contracted so as to occupy the half of the space it did before, viz, about 1800 cubic inches. If the temperature be lowered below $212^{\circ}$, the vapour will be condensed into liquid water, which will occupy the space of not more than a single cubic inch. If the cooling be continued, the water will be contracted in its bulk, but not very regularly (that is, the decrements of bulk will not be exactly proportional to the decrements of beat) until the temperature descends to about $42^{\circ}$. Below that degree the water, instead of contracting its bulk, is expanded by further cooling; viz, by a further abstraction of caloric. This is a very remarkable property of water, on which some interesting phenoincua of nature are depending. The water, though expanding below the temperature of $42^{\circ}$, still continues fluid, as far as about the temperature of $32^{\circ}$; but below this last-mentioned point, by further cooling it becomes a solid, namely, ice; and in this form water occupies a grcater space than it did in a liquid state.

Since the enlargement of the bulk of water commences at about the temperature of $42^{\circ}$, which is ten degrees above the point of melting ice, commonly called the freezing point, which is $32^{\circ}$, it is evident that the particles of water begin to arrange themselves in a particular order long before the freezing talies place. And, by following the ana$\operatorname{logy}$, it may be supposed that in erery state of existence, the particles of bodies have, more or less, a tendency to arrange themselves in a certain order; to which tendency the irregularities in the condensations of bodies are probably to be attributed.

CONDENSER, a pneumatic engine, or syringe, by which an extraordinary quantity of air may be forced into a given space; so that frequently ten atmospberes, or ten times as much air as the space naturally contains, without the engine, may be thrown in by means of it, and its egress prevented by valves properly disposed.

The condenser is made either of metal or glass, and either in a cylindrical or g]obular form, into which the air is thrown with an injecting syringe. The receiver, or vessel containing the condensed air, should be made very strong, to bear the force of the air's elasticity thus increased; for which reason it is commonly made of brass. When glass is used, it will not bear so great a condensation of air; but then the experiment will be more entertaining, as the effect may be viewed of the condensed air upon any subject put therein.

CONDITION, EQuations of, in the Integral Calculus, are certain equations of this form, $\frac{\dot{\mathrm{A}}}{\dot{y}}=\frac{\dot{\mathrm{B}}}{\dot{x}}$, useful in ascertaining whethur a proposed fluxion will admit of tinite integration, or a finite fluent. This method was given by M. Clairaut, in the Memoirs of the Academy of Sciences for the year 1740, and may be explained in this inauner. When a given fluxional equation is very complex, it is often difficult to determine whether it be integrable or not ; the object of this method is then to ascertain that point. In order to which, Clairaut demonstrates, that if there be a fluxional equation of the first order, expressed generally by these two terms $\Delta \dot{x}+\nabla \dot{y}$, where $\Delta$ denotes all the terms or quantities that multiply $\dot{x}$, and $\mathrm{B}$ all those that multiply $j$, being fluxions of $x$ and $y$; and if in such equation, there be taken the fluxion of $\mathrm{A}$, making $y$ only variable; next in like manner the fluxion of $B$, making $x$ only variable; then if that fluxion of a when divided by $\dot{y}$, give the same result as the fluxion of $\mathrm{B}$ when divided by $\dot{x}$, the proposed equation will be integrated. Suppose, for instance, the general proposed quantity be $a x^{\mathrm{m}} y^{\mathrm{n}}$ : its fluxion is am $x^{\mathrm{m}-1} y^{\mathrm{n}} \dot{x}+a n y^{\mathrm{n}-1} x^{\mathrm{m}} y^{\text {. Here then }} \mathrm{A}$ is $a m x^{m-1} y^{n}$, and $B$ is any $y^{n-1} x^{m}$. Now, by taking the fluxions of these two quantities again, making only $y$ variable in $A$, and $x$ only in $B$, striking out $\dot{y}$ in the former, and $\dot{x}$ from the latter, there results the same quantity amm $x^{\mathrm{m}-1} y^{\mathrm{n}-2}$. This rule is expressed more briefly by these kinds of symbols: if the fluxion of $A \dot{x}+13 \dot{y}$ be intcgrable, then must $\frac{\dot{i}}{\dot{y}}=\frac{\dot{B}}{\dot{x}}$, that is, the fluxion of $A$, making only $y$ variable, and dividing by $\dot{y}$, will be equal to the fluxion of $\mathrm{B}$, making only $x$ variable, and dividiug by $\dot{x}$.

Clairaut shows, in like manner, that in a fluxional cx. pression from three variables, $x, y, z$, thus expressed, $\dot{x} \dot{x}+3 \dot{y}+c \dot{z}$, in order that it may be integrable, these three following equations must be true, $\frac{\dot{i}}{\dot{y}}=\frac{\dot{B}}{\dot{x}}$, and $\frac{\dot{i}}{\dot{z}}=\frac{\dot{\mathrm{c}}}{\dot{x}}$, and $\frac{\dot{i}}{\dot{z}}=\frac{\dot{c}}{y}$; whence it is sufficiently suggested that if there 
be a fluxional expression from four variables, it must be satisficd by six such equations, viz, as many as are the combinations of 4 things by 2 at a time; and so on for more complex cases. And this is what is meant by Equations of Condition.

For example, suppose the fuxional expression $\dot{x} \sqrt{ } y+$ $\frac{x \dot{y}}{2 \sqrt{y}}-a \dot{y} \dot{y}^{3} x-\frac{a y \dot{x}}{3 x^{2}}$ be proposed, which is sufficiently comples not to be easily judged whether it be intcgrable or not. Here, taking all the terms that multiply $\dot{x}$, they give $\sqrt{ } y-\frac{a y}{8 r_{\frac{2}{3}}^{2}}=\mathrm{A}$, and all the tcrms that multiply $\dot{j}$ gire $\frac{x}{2 \sqrt{y}}-a \sqrt[3]{x}=\mathrm{E}$. Then taking the fluxion of $\mathrm{A}$, making only $y$ variable, and suppressing $\dot{y}$, gives $\frac{1}{2 \sqrt{y}}-\frac{a}{3 x^{2}}$ : again, taking the fluxion of $\mathrm{B}$, making only $x$ variable, and dividing by $\dot{x}$, gives also $\frac{1}{2 \sqrt{y}}-\frac{a}{3 x_{\frac{2}{3}}}$, the same as the former; that is, $\frac{\dot{y}}{\dot{y}}=\frac{\dot{B}}{\dot{x}}$, and consequently the proposed expression is quadrable.

Hence the integration is easy and evident, for it is only to integrate either $\boldsymbol{A}^{\dot{x}}$ making $y$ constant, or в $\dot{y}$ making $\dot{x}$; that is either $\dot{x} \sqrt{ } y-\frac{a y \dot{x}}{3 x_{\frac{2}{3}}^{2}}$ or $\frac{x y}{2 \sqrt{ } y}-a \dot{y} \sqrt[3]{x}$, both of which give, by their integration, the same quantity $x \sqrt{ } y-a y$ $\sqrt[3]{x}$, which is therefore the fluent of the proposed fluxion $\dot{x} \sqrt{y}+\frac{x \dot{y}}{\sqrt[y]{y}}-a \dot{3} \sqrt[3]{ } x-\frac{a y \dot{x}}{3 x_{\frac{3}{3}}^{3}}$.

CONDORCET (John-Anthony Nicholas de CARITAT, Marquis of), member of the Institute of Bologna, of the Academies of Turin, Berlin, Stockholm, Upsal, Philadelphia, Petersburg, Padua, \&c, and secretary to the Academy of Sciences at Paris, was born at Ribemont in Picardie, the 17 th of September 17 13. His early attachment to the sciences, and progress in them, soon rendered him a conspicuous character in the commonwealth of letters. He was received as a member of the Academy of Sciences at 25 years of age, namely, in March 1769 , as Adjunct-Mechanician; afterwards, be became Associate in 1770 , Adjunct-Secretary in 1773, and sole Secretary soon after, which he enjoyed till his death, or till the dissolution of the Academy by the Convention.

Condorcet soon becaine an author, and that in the most sublime branches of mathematics. Ile published his Essais d'Analyse in several parts; the first part in 1765 , at 22 years of age; the second, in 1767 ; and the third, in 1768. Thise works are chiefly on the Integral Calculus, or the finding of Fluents, and make one volume 4 to.

Ile published the Eloges of the Academicians or members of the Acadeny of Sciences, from the year 1666 till 1700 , in several volumes. He wrote also sinilar Eloges of the Acarlemicians who died during the time that he discharged the important office of Secretary to the Academy; as well as the very useful histories of the difierent branches of science commonly prefixed to the volumes of Memoirs, till the volume for the year 1783 , when it is to be lamented that so useful a part of the plan of the Academy was discontinued.

Ilis other memoirs contained in the volumes of the Acadesuy, are the following.

1. 'I'ract on the Integral Calculus; 1765 .

2. On the problem of Three IBodies; 1767 .

3. Observations on the Integral Calculus; 1767 .

4. On the Nature of Infinite Series; on the Extent of the Solutions which thry give; and on a new method of Approximation for Differential Equations of all Orders; 1769 .

5. On Equations for Finite Differences; 1770.

6. On Equations for Partial Differenees; 1770.

7. On Differential Equations; 1770.

8. Additions to the foregoing Tracts; 1770 .

9. On the Determination of Arbitrary Functions which enter the Integrals of Equations to Partial Differences; 1771.

10. Reflexions on the Methods of Approximation hitherto known for Differential Equations; 1771.

11. Theorem concerning Quadratures; 1771.

12. Inquiry concerning the Integral Calculus; 1772.

13. Calculation of Probabilities, part 1 and $2 ; 1781$.

14. Continuation of the same, part $3 ; 1782$.

15. Ditto, part $4 ; 1783$.

16. Ditto, part $5 ; 1784$.

Condorcet had the character of being a very worthy honest man, and a respectable author, though perhaps not a first-rate one, and produced an excellent set of Eloges of the deceased Academicians, during the time of his secretaryship. A late French political writer has observed of him, that he laboured to succeed to the literary throne of d'Alembert, but that he cannot be ranked anong illustrious authors; that his works have neither animation nor depth, and that his style is dull and dry; that some bold attacks on religion and declamations against despotism have chiefly given a degree of fame to his writings.

On the breaking out of the troubles in France, our author took a decided part on the side of the people, and steadily maintained the cause he had espoused amid all the shocks and intrigues of contending parties; till, under the tyranny of Robespierre, he was driven from the convention, being one of those members proscribed on the 31 st of May 1793, and he died March 28, 1794. The manner of his death is thus described by the public prints of that time. He was obliged to conceal himself with the greatest care for the purpose of avoiding the fate of Brissot and the other deputies who were executed. He did not however attempt to quit Paris, but concealed himself in the housc of a female, who, though she knew him only by name, did not hesitate to risk her own life for the purpose of prescrving that of Condorcet. In her house he remained till the month of March 1794, when it was rumoured that a domiciliary visit was to be made, which obliged lim to leave Paris. Though he had neither pass. port nor civic carcl, he escaped through the barrier, and arrived at the plain of Mont Rouge, where he expected to find an asylum in the country-house of an intimate friend. Unfortunately this friend had set out for Paris, where he was to remain for three days.-During all this period, Condorcet wandered about the fields and in the woods, not daring to enter an inn on account of not having a civic card. Half dead with hunger, fatigue, and fear, and scarcely able to walk on account of a, wound in his foot, he passed the night under a tree.

At length his friend returned, and received him with great cordiality; but as it was deemed imprudent that lie should enter the house in the day-time, he returned to the woods till night. In this short interval between morning and night his caution forsook him, and he went to an inn at Clamars, where be ordered an omlette. His torn clothes, his dirty cap, his meagre and pale countenance, and the grecdiness with which he devoured the omlette, fixed the attention of the persons in the inn, aniong whom was a member of the revolutionary committe of Cla- 
mars. 'Ihis man conceiving him to be Condorcet, who had effected his escape from the Bicetre, asked him whither he was going, and whether he had a passport. The confused manner in which he replicd to these questions, induced the member to orler him to be conveyed before the committee, who, after an examination, sent him to the district of Boury la Reine. He was there inierrogated again; and the unsatisfactory answers which he gave, determined the directors of the district to send him to prison on the succeeding day.-During the night lie was confined in a kind of dungeon. On the next morning, when his keeper entered with some bread and water for him, he found him stretched on the ground without any signs of life.

On inspecting the body, the immediate cause of his death could not be discovered, but it was conjectured that he had poisoned himself. Condorcet indeed always carried a dose of poison in his pocket; and be said to the friend who was to have received him into his house, that he had been often tempted to make use of it, but that the idea of a wife and daughter, whom he loved tenderly, restrained him. During the time that he was conccaled at Paris, he wrote a history of the Progress of the Human $\mathrm{Mind}$, in two volumes.

CONDUCTOR, in Electricity, a term first introduced in this science by Dr. Desaguliers, and used to denote those substances which are capable of receiving and transmitting electricity; in opposition to electrics, in which the matter or virtue of electricity may be excited and accumulated, or retained. The former are also called nonelectrics, and the latter non-conductors. And all bodies are ranked under one or other of these two classes, though none of them are perfect electrics, nor perfect conductors, so as wholly to retain, or freely and without resistance to transmit the electric fluid.

To the class of conductors, belong all metals and semimetals, ores, and all fluids (except air and oils), together with the substances containing tbem, the effluvia of flaming bodies, ice (unless very hard frozen), and snow, most saline and stony substances, charcoals, of which the best are those that have been exposed to the greatest heat, smoke, and the vapour of hot water.

It seems probable that the electric fluid passes through the substance, and not merely over the surfaces of metallic conductors; because, if a wire of any kind of metal be covered with some electric substance, as resin, scalingwax, \&c, and a jar be discharged through it, the charge will be conducted quite as well as without the electric coating.

Some philosophers have even alleged, that electricity will pervade a vacuum, and be transmitted through it a)niost as freely as through the substance of the best conductor: but Mr. Walsh found, that the electric spark or shock would no more pass through a perfect vacuum, than through a piece of solid glass. In other instances however, when the vacuum has been made witl all possible care, the experiment has not succecled.

It may also be observed, that many of the forementioned substances are capable of heing electrified, and that their conducting power may be destroyed and recovered by different processes: for eximple, green wood is a conductor; but baked, it becomes a non-conductor; again its conducting power is restored by charring it; and lastly it is destroyed by reducing this to ashes.

Again, many electric substances, as glass, resin, air, \&c, become conductors by being made very hot: however, air heated by glass must be excepted.

See, on this subject, Priestley's Hist. of Electricity, vol. 1 ; Franklin's Letters \& c, p.. 96 and 262, edit. 1769; Cavallo's Complete 'Treat. of Electr. chap. 2 ; Henley's Exper. and Obser. in Electr. also Philos. Trans. vol. 67, p. 122; and clsewhere in the difierent volumes of the 'Transactions.

Prime Conductor, is an insulated fonductor, so connected with the electrical machine, as to receive the electricity imnediately from the excited electric. Mr. Grer first employed metallic conductors in this way, in 1734; and these were several pieces of metal suspended on silken strings, which he charged with electuicity. M. Du Fay fastened to the end of an iron bar, which he used as his prime conductor, a bundle of linen threads, to which, he applied the excited tube; but these were afterwards changed for small wircs suspended from a common gun-barrel, or other metallic rod.

In the present advanced state of the science, this part of the electrical apparatus bas been considerably improved. The prime conductor is made of hollow brass, and usually of a cylindrical form. Care should be taken, that it be perfectly smooth and round, without points and sharp edges. The ends of the conductor are spherical; and it is neccssary, that the part most remote from the electric should be made round and much Jarger than the rest, the better to prevent the electric matter from escaping, which it always endeavours most to do at the greatest distance from the electric: and the other cnd also should be furmished with several pointed wircs or needles, either suspended from, or fixed to an open metallic ring, and pointing to the globe, or cylinder, or plate, to collect the electricity. It is liest supported by pillars of solid glass, covered with sealing-wax or good parmish. Prime conductors of a large size are usually made of pasteboard, covered with tiu-foil or gilt paper; these being useful for throwing off a longer and denser spark than those of a smaller size: they should terninate in a smaller knob or obtuse edge, at which the sparks should be solicited. Mr. Nairne prepared a conductor 6 feet in length, and 1 foot in diameter, from which he drew clectrical sparks at the distance of 16,17 , or 18 inches; and Dr. Van Marum still far exceeded this, with a coniluctor of 8 inches dianeter, and upwards of 20 feet long, formed of diflerent picces, and applied to the large electrical machine in Teyler's Museum at Haerlem, the most powerful machine of the kind ever yet constructed. But the size of the conductor is always limited by that of the electric, there being a maximum which the size of the former should not exceed; for it may be so large, that the dissipation of the electricity from its surface may be greater than that which the electric is capable of supplying.

Dr. Priestley recommends a prime conductor of polished copper, in the form of a pear, supported by a pillar and a firm basis of baked wood: this receires its fire by a long arched wire of solt brass, which may be easily bent, and raised or lowered to the globe: it is terminated by an open ring, in which some sharp-pointed wires ate suspended. in the body of this conductor are holes for the insertion of metalline rods. This, he observes, collects the fire perfectly well, and retains it coually cvery where. Sce Philosoph. Transact. vol. 64, art. 7 ; and IIist. Elcct. vol. 2, $\S \mathcal{Q}$.

Mr. Ienley has contrived a new hind of prime conduc - 
tar, which, from its use, is called the luminous conductor. It consists of a glass iube 18 inches long, and 2 inches diametcr. The tube is furnished at both ends with brass caps and ferrules about 2 inches long, cemented and made air-1ight, and terminated by brass balls. In one of these caps is drilled a small hole, which is covered by a strong valve, and serves for exhausting the tube of its air. Within the tube at each end there is a knobbed wire, projecting to the distance of 2 inches and a half from the brass caps. To one of the balls is annexed a fine-pointed wire for receiving and collecting the electricity, and to the other a wire with a knob or ball for discharring it. 'The conduc. tor, thus prepared, is supported on pillar's of sealing-wax or glass. besides the common purposes of a prime conductor to an elcctrical machine, this apparatus serves to exhibit and ascertain the direction of the electric matter in its passage through it. See a figure of this conductor in the Philus. Trans. with a description of experiments, \&c, with it, vol. 64 , pa. 403 .

Cosuuctors of Lightning, are pointed metallic rods, fixed to the upper parts of buildings, to secure them from strokes of lightning. ' These were invented and proposed by Dr. Franklin for this purpose, soon after the identity of electricity and lightning was ascertained; and they exhibit a very important and useful application of modern discoveries in this science. This ingenious philosopher, having found that pointed bodies are bctter fitted for receiving and throwing off the electric fire, than such as are terminated by blunt enels or flat surfaces, and that metals are the readiest and best conductors, soon discovered that lightning and clectricity rescmbled each other in this and other distinguishing properties : he therefore recommended a pointed metalline rod, to be raised some feet above the highest part of a building, and to be continued down into the ground, or the nearest watcr. The lightning, shruld it ever come within a certain distance of this rod or wire, would be attracted by it, and pass through it, preferably to any other part of the building, and be conveyed into the earth or water, and there dissipated, without doing any damage to the building. Many facts have occurred to evince the utility of this simple and apparently trifing apparatus. And yct some clectricians, of whom Mr. Wilson was the chief, have objected to the pointerl termination of this conductor; preferring rather a blunt end: because, they pretend, a point invites the electricity from the clouds, and attracts it at a greater distance than a blunt conductor. Philos.. Trans. vol. 54, pa. 234; vol. 63, pa. 49 ; and vol. $6 s$, pa. 232.

This subject has indeed been very accurately examined and disçussed; and pointed conductors are almost universally, and for the best reasons, recommerided as the most proper and eligible. A sharp-pointed conductor, as it attracts the electric matter of a cloud at a greater distance than the other, draws it off gradually : and by conveying it away gently, and in a continued stream, prevents an accumulation and a stroke; whercas a conductor with a blunt termination recives the whole discharge of a cloud at once, and is much more likely to be exploded, whenever a cloud comes within a striking distance. To this may be added experience; for buildings gnarded by either natural or artificial conductors terminating in a point, have very seldom been struck by lightning; but others, having flat or blunt terminatious, have often bcen struck and damaged by it.

The best conductor for this purpose, is a rod of iron, VOL. I. or rather of copper, as being a better conductor of clectricity, and less liable to rust, about 3 quatters of an inch thick, whicb is either to be fastened to the walls of a building by wooden cramps, or supportcd by wooden posts, at the distance of a foot or two from the wall; though less may do: its upper end should terminate in a pyramidal form, with a sharp point and cdges; and, when made of iron, gilt or painted near the top, or else pointed with copper; and be elevated 5 or 6 feet above the highest part of the building, or chimneys, to which it may be tastened. The lower end should be driven 5 or 6 feet into the ground, and directed from the foundations of the building, or continued till it communicates with the nearest water: and if this part be made of lead, it will be less apt to decay. When the conductor is formed of different pieces of metal, care should be taken that they are well joined : and it is farther recommended, that a communication be made from the conductor by plates of lead, 8 or 10 inches broad, with the lead on the ridges and gutters, and with the pipes that carry down the rain, which should be continued to the bottom of the building, and be made to communicate either with water or moist carth, or with the main pipe which supplies the house with water. If the building be large, two, three, or more conductors should be applied to different parts of it, in proportion to its extent. Pbilos. Trans. vol. 64 , pa. 403.

Chains have been used as conductors for preserving ships; but as the electric matter does not pass readily through the linis composing them, copper wires, a little thicker than a goose-quill, have been preferred, and are now generally used. They should reach 2 or 3 feet above the highest mast, and be continued down in any convenient direction, so as always to touch the sca water. Philos. Trans. vol, 52, pa. 633. See also Franklin's Letters \&c, 1769, pa. 65, 124, 479, \&c; and Cavallo's Electr. ch. 9 .

For the Construction and management of Electrical Kites, and Conductors or Machines for drawing electricity from the clouds, sce Priestley's Ilist. of Electr. vol. ?, pa. 103 , edit. 1775 .

CONE, a kind of round pyramid, or a solid body having a circle for its base, and its sides formed by right lines drawn from the circumference of the base to a point at top, being the vertex or apex of the cone.

Euclid defines a conc to be a solid figure, whose base is a circle, and is produced by the entire revolution of a right-angled triangle about its perpendicular leg, called the axis of the conc. If this leg, or axis, be greater than the base of the triangle, or radius of the circular base of the cone, then the cone is acute-angled, that is, the angle at its vertex is an acute angle; but if the axis be less than the radius of the basc, it is an obtuse-angled cone; and if they be equal, it is a right-angled cone.

But Euclid's definition only extends to a riglit cone, that is, to a conc whose axis is perpendicular or at right-angles to its base; and not to oblique ones, in which the axis is inclined to the same, the general definition, or description of which may be this: If a line va continually pass through the point $v$, turning on that point as a joint, and the lower part of it be carried round the circumference $\mathrm{ABC}$ of a circle; then the space inclosed between that circle

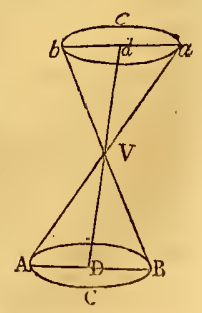




\section{$\mathrm{CON}$}

and the path of the line, is a cone. The circle $\triangle \mathrm{BC}$ is the base of the cune; $v$ is its vertex; and the line vD, from the vertex to the centre of the base, is the axis of the cone. Also the other part of the revolving line, produced above $\mathrm{v}$, will describe another cone $\mathrm{v} a c b$, called the opposite cone, and having the same common axis produced $\mathrm{ov} d$, and vertex $\mathrm{v}$.

Properties of the CONE. - 1 . The area or sulface of every right cone, exclusive of $i t s$ base, is equal to a triangle whose base is the periphery, and its height the slant side of the cone. Or, the curve superficies of a right cone, is to the area of its circular base, as the slant side is to the radius of the base. And therefore, the same curve surface of the cone is equal to the sector of a circle whose radius is the slant side, and its arch equal to the circumference of the base of the cone.

2. Every cone, whether right or oblique, is equal to one-third part of a cylinder of equal base and altitude; and therefore the solid content is found by multiplying the base by the altitude, and taking $\frac{1}{3}$ of the product; and hence also all cones of the same or equat base and altitude, are equal.

3. Though the solidity of an oblique cone be obtained in the same manuer as that of a right one, it is otherwise with regard to the surface, since this cannot be reduced to the measure of a sector of a circle, because all the lines drawn from the vertex to the base are not equal. Sce a Memoir on this subject, by M. Euler, in the Nouv. NIem. de Petersburg, vol, 1. Dr. Barrow has demonstrated, in his Lectiones Geometrica, that the solidity of a cone with an elliptic base, forming part of a right cone, is equal to the product of its surface by a third part of one of the perpendiculars drawn from the point in which the axis of the right cone intersects the ellipse; and that it is also equal to $\frac{x}{3}$ of the beight of the cone multiplied by the elliptic base : consequently that the perpendicular is to the hejght of the cone, as the elliptic base is to the curve surface. For the curve surface of all the oblique parts of a cone, see my Mensur. pa. 174, \&c.

4. To find the Curve Surface of the Frustum of a Cone. Multiply half the sum of the circumferences of the two ends, by the slant side, or distance between these circumferences.

5. For the Solidity of the Frustum of a Cone, add into one sum the areas of the two ends and the mean proportional between them, multiply that sum by the perpendicular height, and $\frac{x}{3}$ of the product will be the solidity. See also my Mensuration, pa. 136, \&c.

6 . The Centre of Gravity of a cone is $\frac{3}{4}$ of the axis distant from the vertex.

Cones of the Higher Kinds, are those whose bases are circles of the higher kinds; and are generated, like the common cone, by conceiving a line turning on a point or vertex on bigh, and revolving round the circle of the higher kind.

Cone of Rays, in Optics, includes all the several rays which fall from any point of a radiant object, on the surface of a glass.

Double Cone, or Spindle, in Mechanics, is a solid formed of two equal cones joined at their bases. If this be laid on the lower part of two rulers, making an angle with each other, and clevated in a certain degree above the horizontal plane, the cones will roll up towards the superior ends, and appear to ascend, though in reality its centre of gravity descends perpendicularly lower.
CONFIGURATION, the exterior surface or shape that bounds bodies, and gives then their particular figure.

CONFUSED Vision. See V1sion.

CONGLLATION, or FreEzing, the act of fixing the fuidity of any liquid, by cold, or the application of cold bodies: in which it differs from coagulation, which is produced by other causes. See FrEezing, Frost, and ICE.

CONGRUITY, in Gcometiy, is applied to lines and figures, which exactly' correspond when laid over each other; as haying the same terms, or bounds. It is assumed, as an axiom, that those things are equal and similar, between which there is a congruity. Euclid, and most geometricians after him; demonstrate great part of their elements from the principle of congruity: though Leibnitz and Wolfius substitute the notion of Similitude instead of that of congruity.

CONIC SECTIONs, are the figures made by cutting a cone by a plane.

2. According to the different positions of the cutting plane, there arse five different figures or scctions, viz, a Triangle, a Circlc, an E.llipse, a Parabola, and an Hyperbola: the last three of which only are peculiarly called conic sections.

3. If the cutting plane pass through the vertex of the cone, and any part of the base, the section will evidently be a Triangle; as $\mathrm{vAB}$.

4. If the plane cut the cone parallel to the base, or make no angle with it, the section will be a Circle, as $A B D$.

5. The section $\mathbf{D A} \mathbf{B}$ is an Ellipse, when the cone is cut obliquely through both sides, or when the plane is inclined to the base in a less angle than the side of the cone is.

6. The section is a Parabola, when the cone is cut by a plane parallel to the side, or when the cutting plane and the side of the cone make equal angles with the base.
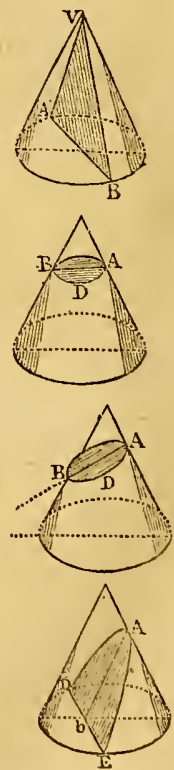

7. The scction is an Iyperbola, when the culting plane makes a greater angle with the base than the side of the cone does. And if the plane be continued to cut the opposite cone, this latter section is called the opposite hyperbola to the former; as $d B c$. 
C O N

8. The Vertices of any section, are the points where the cutting plaue meets the oppasite sides of the cone, or the sides of the vertical triangular section; as $A$ and B.--Hence, the ellipse and the opposite hyperbolas have each two vertices; but the parabola only one; unless we consider the other as at an infinite distance.

9. The Axis, or Transverse Diameter, of a conic section, is the line or distance $\mathbf{A} \mathbf{B}$ between the vertices. - Hence the axis of a parabola is infinite in length.

Ellipse:

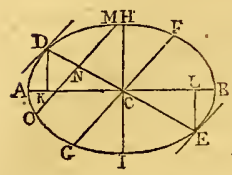

Oppos. Hyperb.
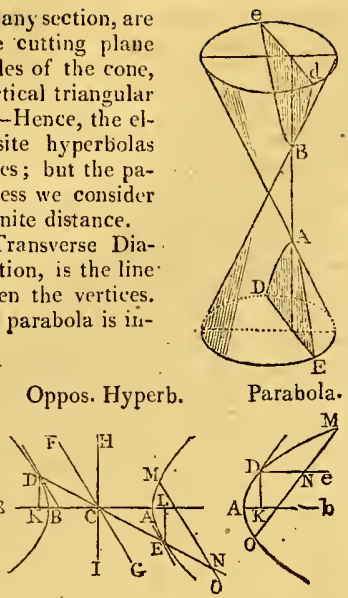

Parabola.

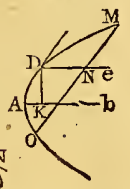

10. The Centre $c$, is the middle point of the axis. Hence the centre of a parabola is infinitely distant from the vertex. And of an ellipse, the axis and centre lie within the curve; but of an hyperbola, without.

11. A.Diameter is any right line, as A B or $\mathbf{D E}$, drawn through the centre, and terminated on each side by the curve : and the extremities of the diameter, or its intersections with the curve, are its Vertices.-Hence all the diameters of a parabola are parallel to the axis, and infinite in length; because drawn through the centre, a point at an infinite distance. And hence also every diameter of the ellipse and hyperbola has two vertices; but of the parabola, ouly one; unless we consider the other as at an infinite distance.

12. 'The Conjugate to any liameter, is the line drawn through the centre, and parallel to the tangen of the curve at the vertex of the diameter. So FG, parallel to the tangent at $\mathrm{D}$, is the conjugate to $\mathrm{DE}$; and $\mathrm{HI}$, parallel to the tangent at $A$, is the conjugate to $A B$.- Hence the conjugate 111 , of the axis $A B$, is perpendicular to the same; but the conjugates of other diameters are oblique to them.

13. An Ordinate to any diameter, is a line parallel to its conjugate, or to the tangent at its vertex, and terminated by the diameter and curve. SO DK and EL are ordinates to the axis $\mathrm{AB}$; and $\mathrm{MN}$ and $\mathrm{No}$ ordinates to the diameter DE.-Hence the ordinates of the axis are also perpendicular to it ; but of other diamcters, the ordinates are oblique to them.

14. An Absciss is any part of a diameter, contained between its vertex and an ordinate to it; as $\mathrm{AK}$ or $\mathrm{s} \mathrm{K}$, and DN or EN. - Hence, in the ellipse and hyperbola, every ordinate has two abscisses; but in the parabola, only one; the other vertex of the diameter being infinitely distant.

15. The Parameter of any diameter, is a third propor"tional to that cliameter and its conjugate, in the ellipse and hyperbola, or to any absciss and its ordinate in the parabola.

16. The Focus is the point in the axis where the ordinate is equal to half the parameter : as $K$ and $L$, where $D K$ or EL is equal to the semiparameter.-Hence, the ellipse and hyperbola bave each two foci; but the parabola only

one--The foci, or burning points, were so called, because all rays are united or reflected into one of them, which pro. ceed from the other focus, and are reflected from the curve.

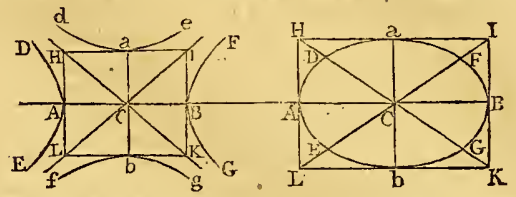

17. If $\mathrm{DAE}, \mathrm{FBG}$, be two opposite hyperbolas, having $A B$ for their first or transverse axis, and $a b$ for their second or conjugate axis; and if dae, $f b y$ be two other opposite hyperbolas, having the same axes, but in the contrary order, viz, $a b$ their first axis, and $\mathrm{A} \mathrm{B}$ their second; then these two latter curves $\mathrm{dae}, \mathrm{fbg}$, are called the Conjugate $\mathrm{Hy}$ perbolas to the former tivo, DAE, FBG; and each pair of opposite curves mutually conjugate to the other.

18. And if tangents be drawn to the four vertices of the curves, or extremities of the axis, forming the inscribed rectangle HIK L; the diagonals HCK and ICL, of this rectangle, are called the Asymptotes of the curves.

19. Scholium. The rectangle inscribed between the four conjugate hyperbolas, is similar to a rectangle circumscribed atout an ellipse, by drawing tangents, in like manner, to the four extremities of the two axes; also the asymptotes or diagonals in the liyperbola, are analogous to those in the ellipse, curtting this curve in similar points, and making the pair of equal conjugate diameters. Moreover, the whole figure, formed by the four hyperbolas, is as it were, an ellipse turned inside out, cut open at the extremities $D, E, T, G$, of the said equal conjugate diameters, and those four points drawn out to an infinite distance, the curvature being turned the contrary way, but the' axes, and the rectangle passing through their extremities, remaining fixed, or unaltered.

Further if there be placed or conjoined four cones, in such a manner, as to have all their vertices meet in a common point, also all their axes in the same plane, and their sides touching and coinciding through their whole length, in two lines crossing in the said common vertex point; then if these four cones be all cut by one plane, parallel to the common plase of their four axes, the section will exhibit a very beautiful appearance of four hyperbolic sections, of which each pair of opposite ones are equals, and each pair has also the resemblance of what are usually called the conjugates to the other pair; but are not really so, except only when the four cones are all equal; in which case the four hyperbolic sections are all equalalso.

From the foregoing definitions are easily derived the following general corollaries to the sections.

$$
\text { Ellipse. Hyperbola. Parabola. }
$$

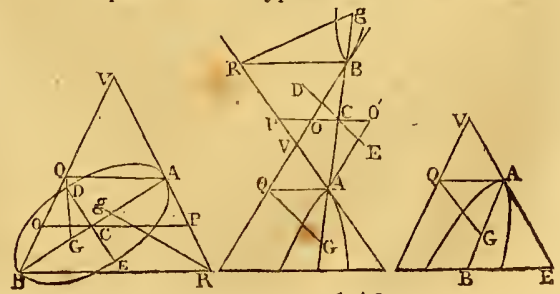


20. Corol. 1. In the ellipse, the semiconjugate axis, $C D$, or $C E$, is a mean pruportional between $C O$ and $C P$, the parts of the diameter op of a circular section of the cone, drawn through the centre $c$ of the ellipse, and parallel to the base of the conc. For DE is a ducuble ordinate in this circle, being perpendicular to $O P$ as well as to $A \mathrm{~B}$.

21. In like manner, in the hyperbola, the length of the semiconjugate axis, $\mathrm{CD}$ or $\mathrm{CE}$, is a mean proportional between co and $C P$, slrawn parallel to the base, and meeting the sides of the cone in o and $P$. Ol, if $A O^{\prime}$ be drawn parallel to the side $\mathrm{v} B$, and meet $P C$ produced in $o^{\prime}$, making $\mathrm{Co}^{\prime}=\mathrm{Co}$; and on this diameter ó $\mathrm{P}$ a circle be drawn parallel to the base: then the semiconjugate $C D$ or $C E$ will be an ordinate of this circle; being perpendicular to o' $P$ as well as to $\mathrm{AB}$.

$\mathrm{Or}$, in both figures, the whole conjugate axis $\mathrm{DE}$ is a mean proportional between $Q A$ and $B R$, parallel to the base of the cone. See my Conic Sections, pa. 6.

In the parabula, both the transverse and conjugate are infinite; for $A B$ and $6 \mathrm{R}$ are both intinite.

22. Corol. 2. ln all the sections, $A$ G will be equal to the parameter of the axis, if QG be drawn making the angle $A Q G$ equal to the angle $B A \varkappa$. In like manner $B g$ will be equal to the same parameter, if $\mathrm{Rg}$ be drawn to make the angle $\mathrm{BRg}=$ the augle $\mathbf{A} \mathbf{B Q}$.

23. Corol. 3. Ilence the upper hyperbolic section, or section of the opposite cone, is equal and similar to the lower one. For the two sections have the same transverse or first axis $\Lambda \mathrm{x}$, and the sane conjugate or second axis $\mathrm{DE}$, which is the mean proportional between $A Q$ and $B R$; and they have also equal parameters AG, Bg. So that the two opposite sections make, as it were, but the two opposite ends of one entire section or hyperbola, the two being every where inutually equal and similar. Like the two halves of an ellipse, with their ends turned the contrary way.

24. Corol. 4. And hence, though both the transverse and conjugate axis in the parabola be infinite, yet the former is infiuitely greater than the latter, or has an infinite ratio to it. For the transverse has the same ratio to the conjugate, as the conjugate has to the parameter, that is, as an infinite to a finite quantity, which is an infinite ratio.

The peculiar properties of each particular curve, will be best referred to the particularwords Ellips E, HypenBola, Parabola; and therefore it will only be proper here to notice a few of the properties that are common to all the conic sections.

\section{Some olher General Properties.}

25. From the foregoing definitions, \&c, it appears, that the conic sections are in themsclves a system of rcgular curves, naturally allied to each other; and that one is changed into another perpetually, when it is either increased, or dimiuished, in infinitum. Thus, the curvature of a circle being ever so little increased or diminished, passes into an cllipse; and again, the centre of the ellipse going off infinitcly, and the curvature being thercby diminisbed, is changed into a parabola; and lastly, the curvature of a parabola being cver so little changed, tlıere ariseth the first of the hyperbolas; the innumcrable species of which will all of them arise orderly by a gradual diminution of the curvature; till this quite vanishing, the last hyperbola ends in a right line. Hence it is manifest, that every regular curvature, like that of a circle, from the circle itself to a right line, is a conical curvature, and is distinguished witl its peculiarname, according to the divers degrees of that curvature.

26. That all dianeters in a circle and ellipse interscet one another in the centre of the figure within the section: that in the parabola they are all parallel among themselves, and to the axis: but in the hyperbolit, they intersect one another, without the figure, in the common centre of the opposite and conjugate sections.

27 . In the circle, the latus rectum, or parameter, is double the distance from the vertex to the tocus, which is also the centre. But in ellipses, the parameters are in all proportions to that distance, between the double and quadruple, according to their different species. While, in the parabola, the parameter is just quadruple that distance. And, lastly, in liyperbolas the parameters are in all proportions beyond the quadruple, according to their various kinds.

28. The first general property of the conic sections, with regard to the abscisses and ordinates of any diameter, is, that the rectungles of the abscisses are to each other, as the squares of their corresponding ordinates. Or, which is the same thing, that the square of any diameter is to the square of its conjugate, as the rectangle of two abscisses of that diameter, to the square of the ordinate which divides them. That is, in all the figures,

the rect, $\triangle \mathrm{C}, \mathrm{CB}:$ rect, $\mathrm{AE} \cdot \mathrm{EB}: \mathrm{CD}^{2}: \mathrm{EF}^{2}$ :

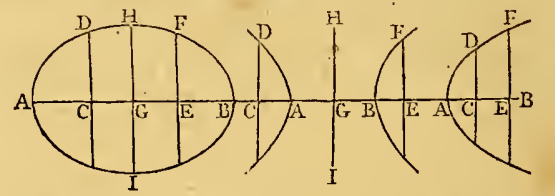

But as in the parabola the infinites $\mathbf{C} \mathbf{B}$ and $\mathbf{E B}$ are in a ratio of cquality, for this curve the same property becomes AC : AE : : CD $^{\prime 2}: \mathrm{EF}^{2}$, that is, in the parabola, the abscisscs are as the squares of their ordinates.

$\mathrm{Or}$, when one of the ordinates is the semiconjugate $\mathrm{GII}$, dividing the diameter equally in the centre, the same gencral property becomes,

$A G \cdot G B$ or $A G^{2}: A C \cdot C B:: \mathrm{GJ}^{2} \cdot \mathrm{CD}^{2}$;

or $\triangle B^{2}: 111^{2}:: A C \cdot C B: C^{2}$.

29. Hunce is derived the equation of the curves of the conic sections ; thus, puting the diameter $\mathrm{A} \mathrm{B}=d$, its conjugate $\mathrm{m}_{\mathrm{L}}=c$, absciss $\mathrm{AC}=x$, and its ordinate $\mathrm{CD}=y$; then is the other absciss $\mathbf{c B}=d-x$ in the ellipse, or $d+x$ in the hyperbola, or $d$ in the parabola; and hence the last analogy above, becomes $d^{2}: c^{2}:: x(d \mp x)$ or $d x \mp x^{2}: y^{2}$, or $d^{\prime \prime} y^{2}=c^{2}\left(d x \mp x^{2}\right)$ is the general equation for all the conic sections; and, in particular it is

$d^{2} y^{2}=c^{2}\left(d x-x^{2}\right)$ in the cllipse,

$d^{2} y^{2}=c^{2}\left(d x+x^{2}\right)$ in the hyperbola, and

$d^{2} y^{2}=c^{2} d x$, or $d y^{2}=c^{2} x$ in the parabola: Or the three equations may be otherwise expressed thus:

$y^{2}=\frac{c^{2}}{d^{2}}\left(d x-x^{2}\right)$ in the ellipse,

$y^{2}=\frac{c^{2}}{d^{2}}\left(d x+x^{2}\right)$ in the hyperbola, and

$y^{2}=\frac{c^{2}}{d} t$ or $=p x$ in the parabola, where the parameter

$p=\frac{c^{2}}{d}$ the third proportional to the diameter and its conjugate, by the definition of it.

$\Lambda$ ud from this one general proposition alone, which is 
easily derived from the section in the solid cone itself, together with the definitions only, as laid down above, all the other properties of all the sections may easily be desived, without any further reference to the cone, and without mechanical descriptions of the curves in plano; as is done in my Trentise on Conic Sections, for the use of the Royal Mil. Acad.; in which also all the similar propositious in the ellipse and hyperbola are carried on word for word in them buth.

'The wore ancient mathematicians, before the time of Apollonius Pergæus, admitted only the right cone into their geometry, and they supposed the section of it to be made by a plane perpendicular to one of its sides; and as the vertical angle of a right cone may be either right, tcute, or obtuse, the same method of cutting these several cones, viz, by a plane perpendicular to one side, produced all the three conic sections. The parabola was called the scetion of a right-angled cone; the ellipse, the section of the acute-angled cone; and the hyperbola, the section of the obtuse-angled cone. But Apollowius, who, on account of his writings on this subject, obtained the appellation of Magnus Geometra, the Great Geometrician, observed, that these threesections might be obtained in every cone, both oblique and right, and that they depended on the different inclinations of the plane of the section to the cone itself Apollon. Con. Halley's edit, lib. 1, p. 9.

Instead of considering these curves as sections cut from the solid cone, which is the true genuine way of all the ancients, and of the most elegant writers among the moderns, Descartes aud some others of the moderns have given arbitrary constructions of curves on a plane, from which constructions they have demonstrated the properties of these, and have afterwards proved that some principal property of them belongs to such curves or sections as are cut from a cone; and hence it is inferred by them that those curves, so described on a plane, are the same with the conic sections.

The doctrine of the conic sections is of great use in physical and geometrical astronony, as well as in the physico-mathematical sciences. It has been much cultivated by both ancient and modern gcometricians, who have left many good treatiges on the subject. The most ancient of these is that of Apollonius Pergæus himself, containing 8 books, the first 4 of which have often been published; but Dr. Halley's edition has all the eight. Pappus, in his Colleet. Mathern. lib. 7, informs us, that the first four of these were written by Euclid, though perfected by Apollonius, who added. the other 4 to them. Among the moderns, the chief writers are Mydorgius de Sectionibus Conicis; Gregory St. Vincent's Quadratura Circuli et Sectionum Coni ; Lahire de Sectionibus Conicis; Trevigar Elem. Section. Con.; De IVitt's Elementa Curvarum; Dr. IVallis's Conic Sections; De l'Hospital's Anal. Treat. of Conic Sections; Dr. Simson's Section. Con.; Milne's Elementa Section. Conicarum; Boscoviclı's excellent treatise; Muller's Conic Sections; Stcel's Conic Sections; Emer'son's Conic Sections; Dr. Hamilton's elegant treatise; my own treatise, above cited; and my friend Dr. Abram Robertson of Oxforl has also published a curious work on this subject, containing not only a treatise on the science, but a history of the writings relating to it.

CONICS, that part of the higher geometry, or geometry of curves, which considers the cone, and the several curve lines arising from the sections of it.
CONJUGATE Axis, or Diameter, in the Conic Sections, is the axis, or a diameter parallel to a tangent to the curve at the vertex of another axis, or diameter, to which that is a conjugate. Indeed the two are inutually conjugates to each other, and each is parallel to the tangent at the vertex of the other.

Conjugat t H renbol a s, also called Adjacent Hyperbolas, are such as have the same axes, but in the contrary order; the first or principal axis of the one being the $2 \mathrm{~d}$ axis of theother, and the $2 \mathrm{~d}$ axis of the former, the 1st axis of the latter. See art. 17 of Conic Sections.

CONJUNCTION, in Astronomy, is the meeting of the stars and planets in the same point or place in the heavens; and is either true or apparent.

True Conjunction is when the line drawn through the centres of the two stars passes also through the centre of the earth. - And Apparent $\mathrm{CoNJUNcTION}$ is when that line does not pass through the earth's centre, but having only the same apparent longiturle.

CONOID, is a figure resembling a cone, except that the slant sides from the base to the vertex are not straight lines as in the cone, but curved. It is generated by the revolution of a conic section about its axis; and it is therefore threefold, answering to the three sections of the cone, viz, the Elliptical Conoid, or spheroid, the Hyperbolic Conoid, and the I'arabolic Conoid.

If a conoid be cut by a plane in any position, the section will be of the figure of some one of the cunic sections; and all parallel sections, of the same conoid, are like and similar figures. When the section of the solid returns into itself, it is an ellipse; which is always the case in the sections of the spheroid, except when it is perpendicular to the axis; which position is also to be excepted in the other solids, the section being always a circle in that position. In the parabolic conoid, the section is always an ellipse, except when it is parallel to the axis, when it is a parabola. And in the hyperbolic conoid, the section is an ellipse, when its axis makes with the axis of the solid, an angle greater than that made by the said axe of the solid and the asymptote of the generating hyperbola; the section being an hyperbola in all other cases, but when those angles are equal, in which case it is a parabola.

Bat when the section is parallel to the fixed axis, it is of the same kind with, and similar to the generating plane itself; that is, the section parallel to the axis, in the spheroid, is an ellipse similar to the generating ellipse; in the parabolic conoid it is a parabola, similar to the generating one; and in the hyperbolic conoid, it is an hyperbola similar to the generating one.

The section through the axis, which is the generating plane, is, in the spheroid the greatest of the parallel sections, but in the hyperboloid it is the least, and in the paraboloid those parallel sections are all equal.

-The analogy of the sections of the hyperboloid to those of the cone, are very remarkable, all the three conic sections being formed by cutting an hyperboloid in the same pusitions as the cone is cut. Thus, let an hyperbola and its asymptote revolve together about the transverse axis, the former describing an hyperboloid, and the latter a cone circumseribing it: then let it be supposed that they are both cut by one plane in any position; so shall the two sections belike, similar, and concentric figures; that is, if the plane cut both the sides of each, the sections will lse concentric and similar cllipse's; but if the cutting planc be parallel to the asymptote, or to the side of the conc, the 
sections will be parabolas; and in all other positions, the sections will be similar and concentric hyperbolas.

And this analogy of the scctions will not appear strange, when it is considered that y cone is a specjes of the hyperboloid; or a triangle a species of the hyperbola, the axes being infinitely small. See my Mensuration, prop. 1, part 3, sect 4, pa. 20I ; or my Tracts, vol. 2, pa. 137.

CONON (of $S_{A}$ yos), a respectable mathematician and philosopher, who flourished about the 130thulympiad, being a contemporary and friend of Archimedes, to whom Conon communicated his writings, and sent him some problems, which Archimedes reccived with approbation, observing they ought to be published while Conon was living, for he compreliends them with ease, and can give a proper demonstration of them.

At another time he laments the loss of Conon, thus admiring his genius: "How many theorems in geometry," says he, "which at first seemed jmpossible, would in time have been brought to perfection! Alas! Conon, though he invented many, with which he enriched gcometry, had not time to perfect them, but left many in the dark, being prevented by death." He had an uncornmon skill in mathematics, joined to an extraordinary patience and application. This is farther confirmed by a letter sent to Archimedes by a friend of Conon's. "Having heard of $\mathrm{Co}$ non's death, with whose friendship $\rfloor$ was honoured, and with whom you kept an intimate correspondence; as he was thoroughly versed in geometry, I greatly lament the loss of a sincere friend, and a person of surprising knowledge in mathematics. I then determined to send to you, as 1 had before done to him, a theorem in geometry, hitherto observed by no one."

Conon had some disputes with Nicoteles, who wrote against him, and treated him with too much contempt. Apollonius confesses it; though he acknowledges that Conon was not fortunate in his demonstritions.

Our author invented a kind of volute, or spiral, different from that of Dinostratus; but because Archimedes explained the properties of it more clearly, the name of the inventor was forgotten, and $j$ was bence called Archimedes's rolute or spiral.

CONSECTARY, or Corollary, a consequence deduced from some foregoing principles.

CONEQUENT, is the latter of the two terms of a ratio; or that to which the antecedent is reforred and compared. Thus, in the ratio $a: b$, or $a$ to $b,-$ the latter term $b$ is the consequent, and $a$ is the antecedent.

CONSISTINT Bodies, is a term nuch used by $\mathrm{Mr}$. Buyle, for such as are usually called firm, or fixed bodies; in opposition to tuid ones.

CONSOLE, in Architecture, is an ornament cut upon the key of an arch, baving a projecture or jetting, and occasionally scrving to support small cornices, busts, and bases.

CONSONANCE, in Music, is commonly used in the same sense with concord, viz, for the union or agreement of two sounds produced at the same time, the one grave, the other acute, which is compounded together by such a proportion of each, as proves agrecable to the car.

An unison is the first consonance, an eighth is the $2 \mathrm{~d}$, it fifth is the $3 \mathrm{~d}$; and then follow the fourth, with the third and sixtlis, major and minor.

CONSTANT Quantities are such as remain invariably the same, while others increase or decrease. Thus, the diameter of a circle is-a constant quantity; for it remains the same while the abscisses and ordinates, or the sines, tangents, \&c, are variable. These are sometimes called given, or invariable, or permanent quantities; and in algebra it is now usual to represent them by the leading letters of the alpbabet, $a, b, c, \& z c$; while the variable ones are denoted by the last letters, $z, y, x$, \&c.

CONSTELLATIONS, certain imaginary finures of birds, beasts, fishes, and other animals in the heavens, within which are arranged certain stars. These assemblages are also sometimes called asterisms.

The ancients portioned out the firmament into several parts, or constellatious ; reducing a certain number of stars under the representation of certain images, to assist the imagination and memory, to conceive or retain their number, order, and disposition, or even to distinguish the virtues they attributed to them.

The division of the heavens into constellations is very ancient; being known to the most early authors, whether sacred or profane. In the Book of Job the names of some of them are mentioned; witness that sublime expostulation, "Canst thou restrain the sweet influence of the Pleiades, or loosen the bands of Oriun ?" And the same may be observed of the oldest among the heathen writers, Hesiod and Homer.

The ancients in their division took in only the visible firmament, or so much as came under their notice, as visible to the nalied eye. The first or earliest of these, is contained jn the catalogue of Ptolemy, given in the $7 \mathrm{th}$ book of his Almagest, prepared, as he assures us, from his own observations, compared with those of Hipparchus, and the other ancient astronomers. In this cataloguc Ptolemy has formed 48 constellations. Of these, 12 are about the ccliptic, commonly called the 12-signs; 21 to the north of it; and 15 to the south. The northern constellations arc, the Little Bear, the Great Bear, the Dragon, Cepheus, Buotes, the Northern Crown, Hercules, the Harp, the Swan, Cassiopeia, Perscus, Auriga, Ophiucus or Serpentary, the Serpent, the Arrow, the Eagle, the Dolphin, the Horse, Pegasus, Andromeda, and the Triangle.

The constellations about the ecliptic are Aries, Taurus, Gemini, Cancer, Leo, Virgo, Libra, Scorpio, Sagittarjus, Capricorn, Aquarius, and Pisces: or according to the English names, the Ram, the Bull, the Tivins, the Crab, the Lion, the Virgin, the Balance, the Scorpion, the Archer, the Goat, the IVater-bearer, ami the Fishes.

The Southern constellations ar'e, the Whale, Orion, the Ericlanus, the Ilare, the Great Dug, the Little Dog, the Ship, the Hydra, the Cup. the Raven, the Centaur, the Wolf, the Altar, the Southern Crown, and the Soutbern Fish.

The other stars not comprebended under these constellations, yet visible to the naked eye, the ancients called Informes, or Sporades, some of which the modern astronomers have since reduecd into new tigures, or constellattions. But on the moleris celestial globes, the consteliations are made to include all the unformed stars. Ptolemy has set down the longrtude and latituce of all these stars to about the year of Christ 137, amounting to the number of 1022 , vi\%,

in the northern constellations

in the zodiacal constellations

in the southern constellations

in all of Ptolemy's catalogue 360 - $\quad 346$

$-\overline{1022}$

Among the moderu astronomers, Tycho Brulie is the 
first who determined, with exactness, and in consequence of his own observations, the long. and lat. of the fixed stars, ont of whıch lie formed 45 constellations; of these, 43 were of the old ones described by Ptolemy, to which Tycho added the Coma Berenices, and Antinous; but he omits 5 of the old southern eonstellations, viz, the Centaur, the Wolf, the Altar, the Southern Crown, and Southern Fish; which be could not observe, because of the high northern latitucle of Uranibourg.

After Tycho, Bayer gave the figures of 60 constellations, very exactly represented, and with tables annexed, having acleded to the 48 old ones of Ptolemy the following 12 about the south pole; viz, the Peacock, the Toucan, the Crune, the Phœnix, the Dorado, the Flying Fisb, the Hydra, the Chameleon, the Bce, the Bird of Paradise, the Triangle, and the Indian. Besides accurately distinguishing the relative size and situation of every star, Bayer marks the stars in cach constellation with the letters of the Greek and lioman alphabets, setting the first letter $\alpha$ to the first or principal star in each constellation, $\beta$ to the $2 \mathrm{~d}$ in order, $\gamma$ to the $3 \mathrm{~d}$, and so on; a very useful method of noting and describing the stars, which bas been used by all astronomers since, and who have farther enlarged this method, by adding the ordinal numbers $1,2,3$, \& c , to the other stars discovered siuce his time, when any constellation contains more than can be murked by the two alphabets. The number and order of the stars, as mentioned ly Bayer, are,

$\begin{array}{lcrr}\text { of the Ist magnitude } & - & - & 17 \\ \text { of the 2d magnitude } & - & - & 63 \\ \text { of the 3d magnitude } & - & - & 196 \\ \text { of the 4th magnitude } & - & - & 415 \\ \text { of the 5th magnitude } & - & - & 348 \\ \text { of the 6th magnitude } & - & - & 341 \\ \text { of the unformed stars } & - & - & 326 \\ & \text { in all } & - & \mathbf{1 7 0 6}\end{array}$

After Bayer, a catalogue, with new constellations, was published by Schiller, in 1627, in a work entitled Cœlum Stellatum Christianum, the Christian Starry Heaven, in which he substitutes, very improperly, other figures of the constellations, and names, taken from the sacred scriptures, instead of the old ones.

In the year 1065, Riccioli published his Astronomy Reformed, containing a catalogue of the stars in 62 constellations, viz, the 60 of Bayer, with the Coma Berenices and Antinous of Tycho. He distributes the stars in all the constellations into four classes. In the first of these classes are contained those stars determined by his own observations, and those of Grimaldi. In the second are those stars which had been ascertained by Tycho Brahé and Kepler. In the third are the stars determined by Hipparchus and Ptolemy. And the 4 th class consists of those of the southern hemisphere discovered by navigators, who have ascertained their places in a more or less accurate manner; in which he has marked the longitudes and latitudes for the year 1700 , the period to which he has rerluced all his observations. This catalogue was followed by a number of celestial schemes and maps of the heavens, published in 1673 by Pardies, who has represented very carefully all the constellations, with the stars they contain. After this, Vitalis published a catalogue of the fixed stars in his Tables of the Prinum Mobile, in which their longitudes and latitudes, with the right ascensions and declinations are set down for the year $167 j$.

Some time after this, Royer published maps of the bea- vens, reduced into 4 tables, with a catalogue of the fixed stars for the year 1700. To the stars marked by Bayer, he adds a number of stars not before seen, with others taken from the tables of Riccioli, and not mentioned by Bayer: he also formed, out of the unformed stars, eleven other constellations; five of which are to the north, and are called the Giraffe, the River Jordan, the River Tigris, the Sceptre, and the Flower-de-luce; with 6 on the south part, wlich are the Dove, the Unicorlu, the Cross, the Great Cloud, the Little Cloud, and the Rhomboide. To this work Royer has joined the catalogue of the southern stars observed by Dr. Halley at the island of St. Helena.

Hevelius has also improved on the labours of those who went before him, and collected togetlier several stars of the before unformed class into some new constellations. These are, the Unicorn, the Camelopardalis, described by Bartschius, the Sextant of Urania, the Dogs, the Little Lion, the Lynx, the Fox and Goose, the Sobieski's Crown, the Lizard, the Little Triangle, and the Cerberus; to which Gregory has added the Ring and the Armilla. Some of these new constellations however answer to those of Royer, as the Camelopardal to the Giraffe, the Dogs to the River Jordan, and the Fox to the River 'Tigris. The latitudes and longitudes are added for the year 1700 .

Finally, Flamsteed has given a catalogue of the fixed stars, not only much more correct, but much larger than any of those that had appeared before his time. He has set down the longitude, latitude, right ascension, and polar distance of 2934 stars, as they were at the beginning of 1690, all determined from his own observations. He distinguishes all the stars into-seven classes, or orders of magnitude, distinguishing those of Bayer by his lctters, and marking their variation in right ascension, for showing their situation in the succeeding years. See the term $\mathrm{CA}_{A-}$ T A LOGUE.

This catalogue was followed by an Atlas Colestis, published at London in the year 1729 , describing, in several schemes, the figures of the constellations seen in our hemisphere, with the exact position of the fixed stars, with respect to the circles of the spherc, as resulting from the last catalogue corrected by Flamsteed. And still later observations, made with farther improved telescopes, have greatly enlarged the number and accuracy of the stars; but the number of the constellations remains the same as above described, except that an attempt was afterwards made by Dr. Hill to add to the list 14 new ones, formed out of more of the clusters of unformed stars.

Besicle the literal marks of the stars introduced by Bayer, it is uswill also to distinguish them by that part of the constellation in which they are placed; and many of them again have their peculiar names; as Arcturus, between the knees of Bootes; Gemina, or Lucida, in the Corona Septentrionalis, or Northern Crown; Palilitim, or Aldebaran, in the Bull's eye, Pleiades in his neck, and Hyades in his forchead; Castor and Pollux in the heads of Gemini; Capella, with the Hœdi in the shoulder of Auriga; Regulus, or Cor Leonis, the Lion's Heart; Spica Virginis in the hand, and Vindemiatrix in the shoulder of Virgo; Antares or Cor Scorpionis, the Scorpion's Heart; Fomalhaut, in the mouth of Piscis Australis, or Southern Fish; Regel, in the foot of Orion; Sirius, in the mouth of Canis Major, the Grcat Dog; Procyon, in the back of $\mathrm{Ca}$ nis Minor, the Little Dog; and the Pole Star, the last in the tail of Ursa AIinor, the Little Bear.

'The Greek and Roman poets, from the aucient theology, 
give wild and romantic fables about the origin of the constellations, probably derived from the bieroglyphics of the Egyptians, and transınitted, with some alterations, from them to the Grecks, who probably obscured them greatly with their own tables. See Hyginus's Poeticon Astron.; Riccioli $\Lambda$ magest. lii. 6, cap. 3,4, 5; Shelburne's Notes upon Manilius; Bailly's Ancient Astronomy; and Gebelin's Monde Primitif, vol. 4: from the whole of which it appears probable, that the invention of the signs of the zodiac, and probably of most of the other constellations of the sphere, is to be ascribed to some very ancient nation, inhabiting the northern temiperate zone, probably what is now called Tartary, or the parts to the northward of Persia and China; and from thence transmitted through China, India, Babylon, Arabia, Egypt, Greece, \&c.

It is a very probable conjecture, that the figures of the signs in the zodiac are descriptive of the seasons of the year, or months, in the sun's path: thus, the first sign Aries, denotes, that about the time when the sun enters that part of the ecliptic, the lambs begin to follow the shcep; that on the sun's approach to the $2 \mathrm{~d}$ constellation, Taurus, the Bull, is about the time of the cows bringing forth their young. The third sign, now Gemini, was originally two kids, and signified the time of the goats bringing forth their young, which are usually two at a birth, while the former, the sheep and cow, commonly produce only one. The 4 th sign, Cancer, the Crab, an animal that goes sideways and backwards, was placed at the northern solstice, the point where the sun begins to return back again from the north to the southward. The 5 th sign, Leo, the Lion, as being a very furious animal, was thought to denote the beat and fury of the burning sun, when he has left Cancer, and entered the next sign Leo. The succeeding constellation, the $6 \mathrm{th}$ in order, reccived the sun at the time of the ripening of corn and the approach of harvest; which was aptly expressed by one of the female reapers, with an ear of corn in her hand; viz, Virgo, the maid. Libra, or the Balance, which is the following sign, evidently denotes the equality of days and nights, that takes place at that season: and Scorpio, the next in order, denotes the time of gathering in the fruits of the earth, which being generally an unhealthy season, is represented by this venomous animal, extending his long claws, threatening the mischief that is likely to follow. The fall of the leaf was the season of the ancient hunting; for which reason the constellation Sagittary represents a buntsman with his arrows and his club, the weapons of destruction for the creatures he pursues. The reason of the Goat's being chosen to mark the southen solstice, when the sun has attained his extreme limit in that quarter, and begins to return and mount again to the northward, is obvious 'nough; the character of that animal being, that it is mostly found climbing, and ascending some mountain, as it browzes. There yet remain two of the signs of the zodiac to be considered with regard to their origin, viz, Aquarius and Pisces. As to the former, it is to be considered that the winter is a wet and uncomfortable season; this therefore was expressed by Aquarius, the figure of a man pouring out water from an urn. The last of the zodiacal constellations was Pisces, a couple of fishes, tied together, that bad been caught: 'The lesson was, the severe season is over, your flocks do not yet yield their store; but the seas and rivers are open, and there you may take fish in abundance.

Through a vain and blind zcal, rather than from any love for the science, some persons lave been induced to alter either the figures of the constellations, or their names. Thus, venerable Bede, instead of the profane names ancl figures of the twclie zodiacal constellations, substituted those of the 12 apostles; which example was followed by schiller, who completed the reformation, and gave Scripture names to all the constellations in the heavens. Thus, Aries, or the Ram, was changed into P'ter; Taurus, or the Bull, into St. Andrew; Andrnmeda, into the Sepulchre of Christ; Lyra, into the Manger of Christ; Hercules, into the Magi coming from the East; the Great Dog, into David; and so on. And Weigel, professor of mathematics in the university of Jena, made a new order of constellations; changing the firmament into a Coelum Heraldicum; and introducing the arms of all the princes in Europe, by way of constellations. Thus Ursa Major, the Great Bcar, he transformed into the elephant of the kingdom of Denmark; the Swan, into the Ruta with swords of the House of Sasony; Ophiucbus, into the Cruss of Cologne; the Triangle, into Compasses, which he calls the Symbol of Artificers; and the Pleiades into the Abacus Pythagoricus, which he calls that of merchants; \&c.

But the more judicious among astronomers never approved of these innovations; as they only tend to int10duce confusion into the science. The old constellations are thcrefore still retained; both because better could not be substituted, and also to kcep up the greater correspondence and uniformity betwcen the old astronomy and the new. See Catalogut.

CONSTRUCTION, in Geometry, the art or manner of drawing or describing figures, schemes, the lines of a prublem, or such like.

Construction of Equations, in Algebra, is the finding the roots or unknown quantities of an equation, by geometrical construction of right lines or curves; or the reducing given rquations into geometrical figures; which is effected by lines or curves according to the order or rank of the equation.

The roots of any equation may be determined, that is, the equation may be constructed, by the intersections of is straight line with another line or curve of the same dimensions as the equation to be constructed : for the roots of the cquation are the ordinat's of the curve at the points of intersection with the right line; and it is well known that a curve may be cut by a right line in as many points as its dimensions amount to. 'Thus, then, a simple equation will be construeted by the interection of one right line with another: a'yuadratic equation, or an affected equation of the $2 \mathrm{~d}$ rank, by the intersections of a 1 ight line with a circle, or any of the conic scctions, which are all lines of the $2 \mathrm{~d}$ order; and which may be cut, by the right line, in two points, thereby giving the two routs of the guadratic cquation. A cubic cquation may he constructed by the intersection of the right line with a line of the 3el order: and so on.

But if, instead of the right line, some other line of a higher order be used; then the ad line, whese intersections with the former are to determine the juots of the equation, may be taken as many dimensions lower, as the former is taken higher. And, in general, an equation of any degree will be constructed by the intersections of 2 wo lines whose timensions, multiplied tugether, produce the dimension of the given equation. Thus, the intersections of a circle with the conic sections, or of these with each other, will construct biquadratic cquations, or those of 
the 4 th power, because $2 \times 2=4$; and the intersections of the circle or conic sections with a line of the $3 \mathrm{~d}$ order, will construct equations of the 5 th and 6 th power; and so on.-For example,

To construct a Simple Equation. This is done by resolving the given simple equation into a proportion, or finding a third or fourth proportional, \&c. Thus, 1. If the equation be $a x=b c$; then $a: b:: c: x=\frac{b c}{a}$, the fourth proportional to $a, b, c$.

2. If $a x=b^{2}:$ then $a: b:: b: x=\frac{b^{2}}{a}$, a third proportional to $a$ and $b$.

3. If $a x=b^{2}-c^{2}$; then, since $b^{2}-c^{2}=(b+c)$ $\times(b-c)$, it will be $a: b+c:: b-c: x=\frac{b+c \times b-c \text {, }}{a}$ a fourth proportional to $a, b+c$ and $b-c$.

4. If $a x=b^{2}+c^{2}$; then construct the right-angled triangle $\mathrm{ABC}$, whose base is $b$, and perpendicular is $c$, so shall the square of the hypothenuse be $b^{2}+c^{2}$, which call $h^{2}$; then the equation is $a x=h^{2}$, and $x=\frac{h^{2}}{a}$, a third proportional to $a$ and $h$.

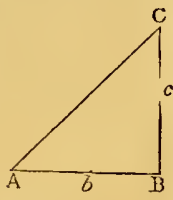

To consiruct a 2uadratic Equation.

1. If it be a simple quadratic, it may be reduced to this form $x^{2}=a b$; and hence $a: x:: x: b$, or $x=\sqrt{ } a b$ a mean proportional between $a$ and $b$. Therefore upon a straight line take $\mathrm{AB}=a$, and $\mathrm{BC}=b$; then upon the diameter Ac describe a semicircle, and raise the perpendicular $\mathrm{BD}$ to

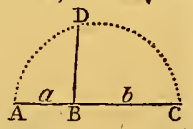
meet it in $\mathrm{D}$; so shall $\mathbf{B D}$ be $=x$ the mean proportional sought between $\mathbf{A B}$ and $\mathbf{E C}$, or between $a$ and $b$.

2. If the quadratic be affected, let it first be $x^{2}+$ $2 a x=b^{2}$; then form the right-angled triangle $A \mathrm{BC}$, whose base $\mathbf{A B}$ is $a$, and perpendicular $\mathbf{B C}$ is $b$; and with the centre $A$ and radius $A C$ describe the semicircle DCE; so shall $\mathrm{DB}$ and $\mathrm{BE}$ be the two roots of the given quadratic equation $x^{2}+2 a x=b^{2}$

3 . If the quadratic be $x^{2}-2 a x=b^{2}$, then the construction will be the very same as of the preceding one $x^{2}+2 a x=b^{2}$.
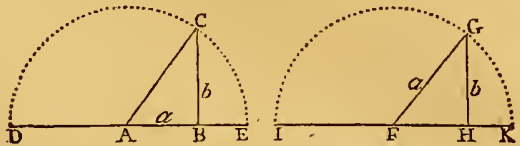

4. But if the form be $2 a x-x^{2}=b^{2}$ : form a rightangled triangle whose hypothenuse $\mathrm{FG}$ is $a$, and perpendicular $\mathrm{G} I \mathrm{II}$ is $b$; then with the radius $\mathrm{FG}$ and centre $\mathrm{F}$ describe a semicircle $I G K$; so shall $I I$ and $I \mathbf{K}$ be the two roots of the given equation $2 a x-x^{2}=b^{2}$, or $x^{2}-2 a x$ $=-b^{2}$. See Maclaurin's Algebra, part 3, cap. 2, and Simpson's Algebra, pa. 267.

To construct Cubic and Biquadratic Equations,-These aro constructed by the intersections of two conic sections; for the equation will rise to 4 dimensions, by which are determined the ordinates from the 4 points in which these conic sections may cut one another; and the conic sections may be assumed in such a manner, as to make this VOL. I. equation coincide with any proposed biquadratic : so that the ordinates from these 4 intersections will be equal to the roots of the proposed biquadratic. When one of the intersections of the conic section falls upon the axis, then one of the ordinates vanishes, und the equation, by which these ordinates are determined, will then be of 3 dimensions only, or a cubic; to which any proposed cubic equation may be accommodated. So that the three remaining ordinates will be the roots of that proposed cubic. The conic sections for this purpose should be such as are most easily described; the circle may be one, and the parabola is usually assumed for the other.

Vieta, in his Canonica Recensione Effectionum Geometricarum, and Ghetaldus, in his Opus Posthumum de Resolutione et Compositione Mathematica, as also Descartes, in bis Geometria, have shown how to construct simple and quadratic equations. Descartes has also shown how to construct cubic and biquadratic equations, by the intersection of a circle and a parabola: And the same bais been done more generally by Baker, in his Clavis Geometrica, or Geometrical Key. But the genuine foundation of all these constructions was first laid and explained by Slusius in his Mesolabium, part 2. This doctrine is also pretty well handled by Lahire, in a small treatise, called La Construction des Equations Analytiques, annexed to his Conic Sections. Newton, at the end of his Algebra, has given the construction of cubic and biquadratic equations mechanically; as also by the conchoid and cissoid, as well as the conic sections. See also Dr. Halley's Construction of Cubic and Biquadratic Equations; Colson's, in the Philos. Trans.; the Marquis de l'Hospital's 'Traité Analytique des Sections Coniques; Maclaurin's $\Lambda$ Igebra, part 3 , c. 3 , \&c.

CONTAC'T, the relative state of two things that touch each other, but without cutting or entering; or whose surfaces join to each other without any interstice.

The contact of curve lines or surfaces, with either straight or curved ones, is only in points; and yet these points have different proportions to one another, as is shown by Mr. Robartes, in the Philos. Trans. vol. 27, pa. 470 ; or my Abr. vol. 5, pa. 678 . Because few or no surfaces are capable of touching in all points, and the cohesion of bodies is in proportion to their contact, those bodies will adhere fastest together, that are capable of the greatest contact.

Angle of Contact, is the opening between a curve line and a tangent to it, particularly the circle and its tangent; as the angle formed at $\mathrm{A}$ between $\mathrm{B} A$ and $\mathrm{AC}$, at the point of contact $A$. It is demonstrated by Euclid, that the line $\mathrm{CA}$ standing perpendicular on the radius $\mathrm{DA}$, touches the

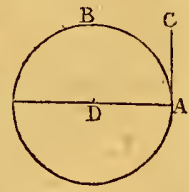
circle only in one point: and that no right line can be drawn between the tangent and the circle. Hence, the angle of contact is less than any rectilinear angle; and the angle of the semicircle between the radius $\mathbf{D A}$ and the arch $A B$, is greater than any rectilinear acute ang]e. 'This seeming paradox of Euclid has exercised the wits' of mathematicians: it was the subject of a long controversy between Peletarius and Clavius; the former of whom maintained that the angle of contact is heterogeneous to a rectilinear one, as a line is to a surface; the latter maintaincd the contrary. Dr. Wallis has a formal treatise on the 
angle of contact, and of the semicircle; where, with other great mathematicians, he approves of the opinion of Peletarius.

CONTENT, a turm often used fur the measurement of bodies and surfaces, whether solid or superficial; or the. capacity of a ressel and the arca of a space; being the quantity either of matter or space included within certain bounds or limits.

CONTJGUITY, the rclation of bodies touching one another.

CONTIGUOUS, a relative term, understood of things so disposed, that their surfaces touch each other.

Conriguous Angles, are such as have one leg or side common to each angle; and are otherwise called adjacent angles; in contradistinction to those made by continuing their legs through the point of contact, which are called opposite or vertical angles.

CONTINENT, a terra firma, main-]and, or a large extent of country, not interrupted by seas : so called, in opposition to island, peninsula, \&c.

Our earth is usually divided into two grand continents, the old and the new : the old continent comprehonds Europe, Asia, and Africa; the new continent comprehends North and South America. Since the discovery of New Holland and New South Wales, it is a doubt with many whether to call that vast country an island or a continent.

CONTINGENT Line, the same with tangent line in Dialling, being the intersection of the planes of the dial and equinoctial, and at right angles to the substilar line.

CONTINUAL, or Continued Proportionals, ate a series of three or more quantities compared together, so that the ratio is the same between every two adjacent terms, viz. between the 1 st and $2 \mathrm{~d}$, the $2 \mathrm{~d}$ and $3 \mathrm{~d}$, the $3 \mathrm{~d}$ and 4th, \&c. As $1,2,4,8,16$, \& c , where the terms continually increase in a double ratio; or $12,4,4, \frac{4}{9}$, where the terms decrease in a triple ratio. A series of continual or continued proportionals, is otherwise called a Progression.

Continuen Fractions. Sce Fractions.

ConT1 v E D Quatity, or Body, is that whose parts are joined and united together.

Continued Proportion, is that in which the consequent of the first ratio is the same with the antecedent of the sccond; as in these, $3: 6:: 6: 12$. See Contruat Proportion.

On the contrary, if the consequent of the first ratio be different from the antecedent of the sccond, the proportion is called Discrete: as $3: 6:: 4: 8$.

CONTR $\triangle$ CTION, in Arithmetic, is the shortening or contracting certain operations, as in Multiplication, Division, Extraction of the Square Ront, \& $x$ : the method for which see under the respective heads.

CONTRA-Harmonical Proportion, that relation of three terms, in which the difference of the first and second is to the difference of the $2 \mathrm{~d}$ and $3 \mathrm{~d}$, as the $3 \mathrm{~d}$ is to the first.' Thus, for instance, 3,5 , and 6 , are numbers contra-harmonically proportional; for $2: 1:: 6: 3$.

CONTRA-MUR R, in Fortification, is a little wall built before another partition wall, to strengthen it, so that it may receive no damage from the adjacent buildings.

CON'TATE-W WEEL, is that wheel in watches which is next to the crown, whose tècth and hoop lie comtrary to those of the other whels; from whence comes its name.

('ON'TRA VALLA'TiON, Line of, in Fortification, is a trencb, guarded with a parapet; being made by the be- siegers, between them and the place besieged, to secure themselyes on that side, and stop the sallies of the garrison. It is made beyond the reach of musket-shot from the town; sometimes going quite around it, and sometimes not, as occasion may require. The besiegers lie between the lines of circumvallation and contravallation: but it is now seldom used.

CONVERGING Curves. Sce Curve.

Cunverging, or Convergent Limes, in Geometry, are those that continually approximate, or whose distance becomes continually less and less the farther they are continued, till they meet: in opposition to divergent lines, whose distance becomes continually greater.

Lines that converge the one way, diverge the other.

Converging Rays, in Optics, are such as incline towards one another in their passage, and in Dioptrics, are those rays which, in their passage out of one medim into another of a different density, ale refracted towards une another; so that, if far enough continued, they will meet in a point or focus.

CoNvERG 1 NG Series, a series of terms or quantities, that always decrease the further they proceed, or which tend to a certain magnitude or limit: in opposition to diverging series, or such as become larger and larger continually. -See SFries.

CONVERSE. A proposition is said to be the converse of another, when, after drawing a conclusion from something first supposed, we return again, by making a suppo sition of what had before becn concIuded, and draw trom thence as a conclusion what before was male the supposition. Thus, when it is supposed that the tivo sides of a triangle are equal, and thence demonstrate or conclude that the two angles opposite to those sides are equal also; then the converse is to suppose that the two angles of a triangle are equal, and thence to prove or conclude that the sides opposite to those angles are also equal.

CONVERSION, or CON VERTEND.o, is when there are four proportionals, and it is inferred, that the first is to its excess above the $2 \mathrm{~d}$, as the $3 \mathrm{~d}$ to its excess above the $4 \mathrm{th}$ : according to Euclid, lib. 5, def. 17.

Thus, if it be - $s: 6:: 4: 3$, then convertendo, or by conversion, - $8: 2:: 4: 1$. Or if there be - - - $a: b:: c: d$, then convertendo, or by conversion, $a: a-b:: c: c-d$. CONVEX, round or curved and protuberant outwards, as the outside of a globular body.

Convex Lens, Mirror, \&c. See Lens, Mraror, \&c.

CONVEXITY, the exterior or outward surface of a convex or round body.

COORDINATES, in the Higher Geometry, is the generill $t \in r m$ used, when the absciss and ordinates of in curve are considered connectedly, whether they are at right angles with each other or not; and the nature of a curve is determined by the equation between its coordinates.

COPERNICAN, something relating to Copernicus. As, thie

Copernican Sphere. Sec Spinene.

CoPER ICAN System, is that system of the worle, in which it is supposed that the sun is at rest in the centre, and the earth and planets all moring around him in their own orbits. Ilere it is supposed, that the heavens and stars are at rest; and the diurnal motion which they appear to have, from east to west, is imputsil to the cindli's diurnal motion from west to east. 
This system was maintained by many of the ancients; particularly Ecphantus, Scleucus, A ristarchus, Philolaus, Cleanthes Samius, Nicetas, Heraclides Ponticus, Plato, and Pythagora; from the last of whom it was ancicntly called the Pythagoric, or Pythagorean System. The same system was also held by Archimedes, in his book of the number of the Grains of Sand; but after him it became neglected, and even forgotten, for many ages; till about 300 ycars since, when Copernicus revived it; from whom it took the new name of the Cupernican System. See the next article.

COPERNICUS '(NichoLAs), an emineut astronomer, was born at Thorn in Prussia, January 19, 1+73. He was instructed in the Latin and Greek languages at home; and afterward sent to Cracow, where lie studied philosophy, mathematics, and medicine: though his genius was naturally turned to mathematics, which he chiefly studied, and pursued through all its various branches.

He set out for ltaly at 23 years of age; stopping at Bologna, that he might converse with the celebrated astronomer of that place, Dominic Maria, whom he assisted for some time in making his observations. From hence he passed to Rome, where he was presently considered as not inferior to the famous Regiomontanus. Here he soon acquired so great a reputation, that he was chosen professor of mathematics, which he taught there for a long time with the greatest applause; and here also he made some astronomical ubservations about the year 1500 .

Afterward, returning to his own country, he began to apply his fund of obscrvations and mathematical knowledge, to correcting the system of astronomy which then prevailed. He sct about collecting all the books that had been written by philosophers and astronomers, and to examine all the various hypotheses they had invented for the solution of the celestial phenomena ; to try if a more symmetrical order and constitution of the parts of the world could not be discovered, and a more just and exquisite harmony in its motions established, than what the astronomers of those times so generally admitted. But of all their hypotheses, none pleased him so well as the Pythagorean, which made the sun to be the ccutre of the system, and supposed the earth to move both round the sun, and also round its own axis. He thought he discerned much beautiful order and proportion in this; and that all the embarrassment and perplexity, from epicycles and excentrics, which attended the Ptolemaic hypotheses, would here be entirely removed.

He began to consider this systcm, and to write upon it, when he was about 35 years of age. He carefully contensplated the phenomena, made mathematical calculations, examined the observations of the ancients, and made new ones of his own; till, after more than 20 years chiefly spent in this inanner, he brought his scheme to perfection, establishing that system of the world which goes by his name, and is now universally received by all philosophers.

'This system however was at first cunsidered as a most dangerous heresy, and his work had long been finished and perfected, before he could be prevailed on to give it to the world, notwithstanding being strongly urged to it by his friends. At length, yiclding to their entreaties, it was printed, and he had but just received a perfect copy, when he died the 24th of May 1543, at 70 years of age; by which it is probable he was happily relieved from the violent lanatical persecutions of the churcl, which were
$C \cap R$

but too likely to follow the publication of his astronn. mical opinions; and which indeed was afterwards the fute of Galileo, for adopting and defending them. This work of Copernicus, first printed at Norimberg in folio, 1543 , and of which there have been. other editions since, is intitled De Revolutionibus Orbium Cœlestium, being a large bocly of astronomy, in 6 books.

When Rheticus, the disciple of our author, returned out of Prussia, he brought with him a tract of Copernicus, on plane and splerical trigonometry, which he had printed at Norimberg, and which contained a table of sines. It was afterward printed at the end of the first book of the Revolutions. An edition of our author's great work was also published in 4to at Amsterdam in 1617 , under the title of Astronomia Instaurata, illustrated with notes by Nicolas Muler of Groningeñ.

COPERNICUS, the nane of an astronomical instrument, invented by Whiston, to show the motion and phenomena of the planets, both primary and sccondary. It is founded on the Copernican system, and thercfore called by his name.

COPPER, is a malleable and ductile metal, of a palered colour, with a tinge of yellow. It is soluble with effervescence in nitrous acid, to which it gives a sky-blue colour: on the addition of liquid ammonia, a green precipitate is thrown down, which is resoluble in an excess of ammonia, forming a very rich purplish-blue fluid, and from this, if slightly supersaturated with muriatic acid, the copper is again precipitable in its metallic form, by a bar of clean iron. See Aikin's Chemical Dictionary, Article Copper.

COR CAROLI, Charles's Heart, an extra-constellated star of the $2 \mathrm{~d}$ magnitude in the northern hemisphere, between the Coma Berenices and Ursa Major; so called by Sir Charles Scarbórongh, in honour of King Charles I.

Cor Hydre, the Hydra's Heart, a star of the $2 d$ magnitude, in the. Heart of the constellation Hydra.

Con Lsonis, Lion's Heart, or Regulus, a star of the first magnitude in the constellation Leo.

Con Scorpi. Sec Axtares.

CORBEILS, in Fortification, are little baskets about a foot and a half high, $\delta$ inches broad at the bottom, and 12 at the top; which being fillcd with earth, are set against one another on the parapet, or elsewhere, leaving certain port-holes, from whence to fire under cover upois the enemy.

CORBEL, in Architecture, the representation of a basket, sometimes seen on the heads of caryatides.

CORBEL, or ConBIL, is also used, in Building, for a short piece of timber placed in a wall, with its end projecting out 6 or 8 inches, as occasion serves, in the manner of a shouldering-piece.

CORBET, the same as CORBEL.

CORDON, in Fortification, a row of stones jutting out between the rampart and the basis of the parapt, like the tore of a column. The cordon ranges round the whole fortress, and serves to join the rampart, which is aslope, and the parapet, which is perpendicular, more agreeably together. In fortifications raised of earth, this space is filled up with pointed stakes instead of a cordon.

CORDS, in Music, are the sounds produced by an instrument or the voice.

CORIDOR, or CoRridon, in Fortification, is thic covert-way lying cutirely round the whole compass of the $3 \mathrm{~B} 2$ 
works of a place, between the outside of the moat and the pullisadoes, being about 20 yards broad.

CORIDOR is also used, in Architecture, for a gallery, or long aisle, around a building, leading to several chambers at a distance from each other, sometimes wholly inclosed, and sometimes open on one side.

CORINTHIAN Order, of Architecture, is the 4th in order, or the 5th and last according to Scamozzi and Le Clerc. This order was invented by an Athenian architect, and is the richest and most delicate of them all; its capital is adorned with rows of leaves, and of $\mathrm{S}$ volutas, which support the abacus. The height of its column is 10 diameters, and its cornice is supported by modillions.

CORNEA Tunica, the second cont of the eye; so called from its substance resembling the horn of a lantern. This is situated in the fore-part; and is surrounded by the sclerotica. It has a greater convexity than the rest of the eye, and is a portion of a small sphere, or rather spheroid, and consolidates the whole eye.

CORNICE, CorNicne, or Cornish, the third and uppermost part of the entablature of a column, or the uppermost ornament of any wainscoting, \&c.

COROLLARY, or CONSECTARY, a consequence drawn from some proposition or principles already advanced or demonstrated, witbout the aid of any other proposition : thus from this theorem, That a triangle which has two equal sides, has also two equal angles, this consequence may be drawn, That a triangle which hath the three sides equal, has also its three angles equal. - What is now called a corollary, was by the earlier geometers named a Porism.

CORONA, Crown or Crowning, in Architecture, the flat and most advanced part of the cornice; so called because it crowns the cornice and entablature: by the workmen it is called the drip, as serving by its projecture to screen the rest of the building from the rain.

CORONA, in Optics, a luminous circle, usually coloured, whicl is seen round the sun, moon, or largest planets. See HaLo.

Coros a Borealis, or Septentrionalis, the Northern Crown or Gurland, a constellation of the uorthern hemisphere, being one of the 48 old ones. It contains 8 stars according to the catalogue of Ptolemy, Tycho, and Hevelius; but according to the Britannic Catalogue, 21.

Corova Australis, or Meridionalis, the Southern Crown, a constellation of the southern hemisphere, whose stars in Ptolemy's catalogue are 13 ; in the British catalogue, 12.

CORPUSCLE, the diminutive of corpus, used to express the minute parts, or particles, that constitute natural bodies; meaning much the same as atoms. Newton shows a method of determining the sizes of the corpuscles of bodies, from their colours.

CORPUSCULAR Attraction, denotes that power by which the minute component particles of bodies are united, and adhere to each other. It may be distinguished into attraction of aggregation, viz, that power by which the homogeneous particles of bodies are united; and attraction of affinity or of composition, viz, that power by which the heterogentous particles of bodies are united.

Corpuscular Plilosophy, that scheme or system of physics, in which the phenomena of bodics are accounted for, from the motion, rest, position, \&cc, of the corpuscles or atoms of which bodies consist.

'The Corpuscular philosoply, which now fourishes un- der the name of the mechanical philosophy, is very ancient. Leucippus and Democritus taught it in Greece; from them Epicurus received it, and improved it; and from him it was called the Epicurean Philosophy.

Leucippus, it is sajd, reccived it from one Mochus, a Phenician physiologist, before the time of the Trojan war, and the-first who philosophized about atoms: which Moclus is, according to the opinion of some, the Moses of the Scriptures.

After Epicurus, the corpuscular philosophy gave way to the peripatetic, which became the popular system. Tlus, instead of atoms, were introduced specific and substantial forms, qualities, sympathies, \&c, which amused the world, till Gassendus, Charleton, Descartes, Boyle, Newton, and others, retrieved the corpuscularian bypothesis; which is now become tbe basis of the mechanical and experimental philosophy.

Boyle reduces the principles of the corpuscular philosophy to the 4 following heads.

1. That there is but one universal kind of matter, which is an extended, impenetrable, and divisible substance, common to all bodies, and capable of all forns.-On this bead, Newton finely remarks thus: "All things considered, it appears probable to me, that God in the beginning created matter in solid, hard, impenetrable, moreable particles; of such sizes and figures, and with such other properties, as most conduced to the end for which he formed them : and that these prinitive particles, being solids, are incomparably harder than any of the sensible porous bodies compounded of them; even so hard as never to wear, or break in picces : no other power being able to divide what God made one in the first creation. While these corpuscles remain entire, they may compose bodies of one and the same nature and texture in all ages: but should they wear away, or break in pieces, the nature of things depending on them would be changed: water and earth, composed of old worn particles, of fragments of particles, would not be of the same nature and texture now, with water and earth composed of entire particles at the beginning. And therefore, that nature may be lasting, the changes of corporeal things are to be placed only in the various separations, and new associations, of these permanent corpuscles."

2. That this matter, in order to form the vast variety of natural bodies, must have motion in some, or all its assignable parts; and that this motion was given to matter by God, the creator of all things; and has all manner of directions and tendencies.- "These corpuscls (says Newton) have not only a vis inertiæ, accompanied with such passive laws of motion as naturally result from that force; but also are moved by certain active principles; such as that of gravity, and that which causes fermentation, and the cohesion of bodies."

3. That matter must also be actually divided into parts; and each of these primitive particles, frugments, or atoms of matter, must have its proper magnitude, figure, and shape.

4. That these differently sized and shaped particles, have different orders, positions, situations, and postures, from whence all the variety of compound bodies arises.

CORRECTION of a Fluent. Sce Fluent.

CORRIDOR. Sce Coridor.

CORTES (MARTIN), a Spanish author on Navigation, who flourished in the mildle of the 16 th century. In 1551 


\section{$\mathrm{C} O \mathrm{~S}$}

was published at Seville, his book entitled, Breve de la Sphera y de la Arte de Nauegar con nueuos Instrumentos y Reglas; which he says he composcd at Cadiz in 1545 . In the dedication to the emperor, Cortes boasts that he was the first who had reduced navigation into a Compendium, enlarging much on what he had performed. He briefly and clearly exposed the errors of plane-charts, and seemed to reflect on what had been said against the variation of the compass by Medina, when he advised the mariner, rather to be guided by experience, than to mind subtle reasonings. Besides, he endeavoured to account for this variation, in imagining the needle to be influenced by a magnetic pole (which he called the Point Attractive) different from that of the world; a notion which has bcen farther prosecuted by others. - A translation of Cortes's book by Richard Eden, was, on the rccommendation of that great navigator Steven Burrough, and the encouragement of the Society for making discoveries at sea, published at London in 1561 .

CORVUS, the Raven, a southern constellation, fabled by the Greeks, as taken up to heaven by Apollo, to whom it tattled that the beautiful maid Coronis, the daughter of Phlegeos, and mother of Esculapius by Apollo, played the deity false with Ischys, under a tree upon which the animal happened to be perched. The stars in this constellation, in Ptolemy's and Tycho's catalogues, are 7 ; but in the Britannic catalogue, 9 .

COSECANT, COSINE, COTANGENT, COVERSED SINE, are the secant, sinc, tangent, and versed sine of the complement of an arch or angle; $\boldsymbol{C o}$ being, in this case, a contraction of the word complement, and was first introduced by Gunter.

COSIICAL Aspecr, among astrologers, is the aspect of a planct with respect to the earth.

Cosmical Rising, or Setting, is said of a star when it rises or sets at the same time when the sun rises.

But, according to Kepler, to rise or set cosmically, is only simply to rise or set, that is, to ascend above, or dcscend below, the horizon; as much as to say, to risc or set to the world.

COSMOGONY, the science of the formation of the universe; as distinguished from cosmography, which is the science of the parts of the universe, supposing it formed, and in the state as we behold it; and from cosmology, which reasons on the actual arid permanent state of the world as it now is; whereas cosmogony reasons on the variable state of the world at the timc of its formation.

COSMOGRAPHY, the description of the world; or the art that tcaches the construction, figure, disposition, and relation of all the parts of the world, with the manner of representing them on a plane. It consists chiefly of two parts; viz, Astronomy, which shows the structure of the heavens, with the disposition of the stars; and Geography, which shows those of the earth.

CUSMOLOGY, the science of the world in general.

COSS, Rule of, meant the same as Algebra, by which name it was for some time called, when first introduced into Europe through the Italians, who named it Regola de cosa, the Rule of the thing; the unknown quantity, or that which was required in any question, being called cosa, the thing; the terms whence we have Coss, and Cossic numbers, \&c.

COSTARD (GEoRge), was born at Shrewsbury 1710 , and educated at Warlham-college, Oxford, of which he became a fellow and a tutor; he died at his vicarage at
C O T

Twickenham, Jan. 10, 1782, in the $72 \mathrm{~d}$ year of his age. $\mathrm{Mr}$. Costard was a man of great learning; dceply read in divinity and astronomy ; well versed in the Greek, and a perfect master of the Hebrew and other oriental languages. - His private character was amiable, and his correspondence with the literati was extensive both at home and abroad. He had a great veneration for the Greeks and their literature; and was generally skilled in the learning of most of the ancient nations. Besicles various publications on divinity, \&c, he was author of the History of Astronomy, 4to, 1767 , with its application to geography, history, and chronology. His communications to the Royal Society, which are chiefly on astronomical subjects, are contained in vols. $43,44,48$ and 68 of the Philosophical Transactions.

COTES (ROGER), a very eminent mathematician, philosopher, and astronomer, was born July 10, 1682, at Burbach in Leicestershire, where his father Robert was rector. He was first placed at Leicester school; where, at 12 years of -age, he dliscovered a strong inclination to the mathenuatics. This being observed by his uncle, the Rev. Mr. John Smith, he gave him all the encouragement he could; and prevailed on his father to send him for some time to his house in Lincolnshire, that he might assist him in those studies: and here he laid the foundation of that deep and extensive knowledge in that science, for which he was afterwards so deservedly famous. Hc was hence removed to St. Paul's school, London, where he made a great progress in classical lcarning; and yet he found so much leisure as to support a constant correspondence with his uncle, not only in mathematics, but also in metaphysics, philosophy, and divinity. His next remove was to Trinity-college, Cambridge, where he took his degrees, and became fellow.

$\mathrm{Jan} .1706$, he was appointed professor of astronomy and experimental philosophy, on the foundation of Dr. Thomas Plume, archdeacon of Rochester; being the first that enjoyed that office, to which he wis unanimously chosen, on account of his bigh reputation and merits. He entered into orders in 1713; and the same year, at the desire of Dr. Bentley, he published at Canbridgc the second edition of Newton's M1athematica Principia ; inserting all the improvements which the author had made to that time. To this edition he prefixed an excellent preface, in which he pointed out the true method of philosophizing, showing the foundation on which the Newtonian philosophy was raised, and refuting the objections of the Cartesians and all other philosophers against it.

The publication of this edition of Newton's Principia added greatly to his reputation; nor was the high opinion the public now conceived of him in the least diminished, but rather much increased, by scveral productions of his own, which afterward appeared. He gave in the Philos. Transactions, two papers, viz, 1, Logometria, in vol. 29; and a Description of the great fiery, meteor that was seen March 6, 1716, in vol. 31 .

This extraordinary genius in the mathematics died, to the great regret of the university, and all the lovers of the sciences, June 5,1716 , in the very prime of his life, being not quite 34 years of age.

Mr. Cotes left behind him some very ingenious, and indeed admirable tracts, part of which, with the Logometria

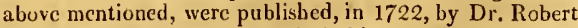
Smith, his cousin and successor in his professorship, afterwards inaster of 'Trinity-college, under the title of Har- 
monia Mensurarum, which contains a number of very ingenious and learned works: See the Introduction to my Logarithms. He wrote also a Compendium of Arithmetic; on the Resolution of Equations; on Dioptrics; and on the Nature of Curves. Besides these piecrs, he drew up, in the time of his lectures, a course of Hydrostatical and Pueunatical Lectures, in English, which were published also by Dr. Smith in $8 v 0,1737$, and are beld in great estimation.

So high an opinion had Sir Isaac Newton of our author's genius, that he used to say," If Cotes had lived, we might have known something."

COTESIAN theorem, in Geometry, an appellation used for an clegant property of the circle discovered by Mr. Cotes. The theorem is this: If the factors of the binomial $a^{\mathbf{c}} \neq x^{\mathbf{c}}$ be required, the index $c$ being an integer number. With the centre $o$, and radius $A O=a$, describe a circle,

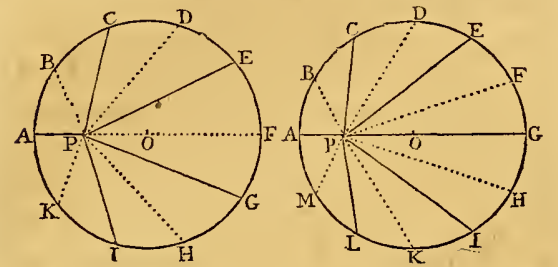

and divide its circumference into as many equal parts as there are units in $2 c$, at the points $\Lambda, \mathrm{B}, \mathrm{C}, \mathrm{D}, \& \mathrm{C}$; then in the radius, produced if necessary, take $O P=x$, and from the point $\mathbf{P}$, to all the points of division in the circumference, draw the lines $\mathrm{PA}, \mathrm{PB}, \mathrm{PC}, \& \mathrm{c}$; so shall these lines takeu alternately, be the factors sought; viz,

$\mathrm{PB} \times \mathrm{PD} \times \mathrm{PF} \& \mathrm{C}=a^{\mathrm{c}}+x^{\mathrm{c}}$, and

$\mathrm{PA} \times \mathrm{PC} \times \mathrm{PE} \& \mathrm{c}=a^{\mathrm{c}} \sim x^{\mathrm{c}}, \mathrm{viz}, a^{\mathrm{c}}-x^{\mathrm{c}}$ or $x^{\mathrm{c}}-a^{\mathrm{c}}$, according as the point $P$ is within or without the circle. For instance, if $c=5$, divide the circumf. into 10 equal parts, and the point $\mathrm{P}$ being within the circle, then will $O A^{5}+O P^{5}=B P \times D P \times F P \times H P \times K P$, and $O A^{5}-O P^{5}=A P \times C P \times E P \times G P \times I P$.

In like manncr, if $c=6$, baving divided the circumference into 12 equal parts, then :vill

$0 \Lambda^{6}+O P^{6}=\mathrm{BP} \times \mathrm{DP} \times \mathrm{FP} \times \mathrm{HP} \times \mathrm{KP} \times M \mathrm{P}$, $O A^{6}-O P^{6}=A P \times C P \times E P \times G P \times I P \times L P$.

The demonstration of this theorem may be seen in Dr. Pemberton's Epist. de Cotesii inventis. See also Dr. Smith's Theoremata Logometrica and Trigonometrica, adkced to Cotes's Harm. Mens. pa. 114; De Moivre Miscel. Analyt. pa. 17; Waring's Letter to Dr. Powell, pa. 39, and Simpson's Essays, p. 113.

By means of this theorem, the acute and elegant author was enabled to make a farther progress in the inverse method of Fluxions, than had been done before. But in the application of his discovery there still remained a limitation, which was renoved by Demoivre. Vide ut supra.

COVERT-IVAY, in Fortification, a space of ground lercl with the adjoining country, on the outer edge of the ditch, ranging quite round all the works. This is otherwise called the Corridor, and has a parapet with its banquette and glacis, which form the beight of the parapet. It is sometimes also called the Connterscarp, because it is on the edge of the scarp.-One of the greatest difficultics in a siege, is to make a lodgment on the covert-ivay; because it is usual for the besieged to palisade it along the middle, and undermine it on all sides.

COVING, in Building, is when houses are built projecting over the ground plot, and the turned projecture formed into an arch.

Corng Cornice, is one that has a large casemate or hollow in it.

COUNT-IYHEEL, is a wheel in the striking part of :t clock, moving round once in 12 or 24 hours. It it solnetimes called the locking-wheel, because it has usually 11 notches in it at unequal distances from one another, to make the clock strike.

COUNTER-APPROACnEs, in Fortification, lines or trenches nade by the besieged, where they come out to attack the lines of the besiegers in form.

Counter-BAtTerY, a batiery raised to play on another, to dismount the guns, \& $c$.

Counter-Breast-work, the same as Fausse-Braye.

Counter-Forts, Buttresses, or Spurs, are pillars of masonry serving to prop or sustain walls, or terraces, subject to bulge, or be thrown down.

Counter-Fugue, in Music, is when fugues proceed contrary to one another.

Counter-Guand, in Fortification, a work commonly serving to cover a bastion. It is consposed of two faces, forming a salient angle before the flanked angle of a bastion.

Counter-Harmonical. See Contra-Harmonical. COUNTER-MINE, a subterraneous passage, made by the besieged, in search of the enemy's mine, to give air to it, to take away the powder; or by any other means to irustrate the effect of it.

Counter-Part, a term in Music, only denoting that one part is opposite to another: so, the bass and treble are counter-parts to cach other.

Cownter-Pornt, in Music, the art of composing harmony; or disposing and concerting several parts so tugether, as that they may make an agreeable whole.

COU NTER-Porse, any thing serving to weigh against another; particularly a piece of metal, usually of brass or iron, making an appendage to the Roman statera, or steelyard. It is contrived to slide along the beam; and from the division at which it keeps the balance in equilibrio, the weight of the body is determined. It is sometimes called the Pear, on account of its figure; and Mass, by reason of its weight. Rope-dancers make use of a pole by way of counterpoise, to kecp their bodies in equilibrio.

COUNTERSCARP, is that side of the ditch that is next the country; or properly the talus that supports the earth of the covert-way: though by this word is often understood the whole covert-way, with its parapet and glacis. And so it must be understood when it is said, The enemy lodged themselves on the counterscarp.

COUNTER-SWALLOWS-TAIL, is an outwork in Fortification, in form of a single tenaille, wider towards the place, or at the gorge, than at the head, or next the country.

COUNTER-TENOR, one of the mean or middle parts of music; so called, as being ofposite to the tenor.

COURSE, in Navigation, the point of the compass, or borizon, which a ship steers on; or the angle which the rhumb-line on which it sails makes with the meridian; being sometimes reckoned in degreses, and sometines in points of the compass.

When a ship saik e'ither due north or south, she sails on a meridian, makes no departure, and her distance ansl dif- 
ference of latitude are the same. When she sails duc east or west, her course makes right-angles with the meridian, and she sails either upon the equator, or a parallet to it; in which case she makes nu difference of latitude, but her distance and departure are the same. But when the ship sails between the cardinal points, on a course making always the same oblique "angle with the meridians, her path is then the loxodromic curve, being a spiral cutting all the meridians in the same angle, and terminating in the pole.

Hence arises a kind of geographical, or nautical paradox; at least to those who have but a limited knowledge of those subjects; which is this. If we suppose two places having different latitudes and longitudes, and that a person leaves one of those places on a certain point of the compass, bearing dircetly towards the other; and having arrived there, leaves this last place upon the dirfet opposite point of the compass to that on which he first set out, it will be found on his pursuing this course, that he will Jever return again to the place whence he first departed; but will leave it more or less on the right or left, according to the relative situations of the two places.

The cause of this deviation from tris original track is however easily explained : for in the first case, as the traveller is supposed to go dircctly from one place to the other, he does not continue, in any two successive instances, to pursue the same point of the compass; whereas on his return, he is considered as constantly observing the same point, and hence arises the deviation in question.

For the same reason, if a vessel depart from a port situated in north latitude, and constantly pursue the same north-easterly, or north-westerly course, it will continually approach towards the north pole in a spiral traced on the terrestrial surface, but it will never arrive there, however far the voyage may be pursued. And exactly the same thing will take place with respect to the south polc, if the course be directed towards the south-east, or south-west.

COURTAIN. See Curtin.

CRAB, in Mechanics, an engine used for mounting guns on their carriages. See Gin.

Cra B, ón ship-board, is a wonden pillar, having its lower end let down through the ship's decks, and resting on a sockct, like the capstan: in its upper end are three or four holes, at different heights, through the middle of it, one above another, to receive long bar's, against which men act by pusling or thrusting. - It is employed to wind-in the cable, and for other purposes requiring a great mechanical power.

The Crab with three claws is used to launch ships, and to heave them into the dock, or off the key.

CIABTREE (IVM.), an ingenious English astronomer, who resided at Broughton near Manchester in Lancashire, in the carly part of the 17 th century. He was the friend of the congenial young astronomer Horrox, near Liverpool, with whom he carried on a learned astronomical currespondence for the several years $1636,1637,1638,1639$, 1640 ; by which means he was very instrumental in encouraging $\mathrm{Mr}$. Iorrox to prosecute his use ful astronomical sbservations and writings. A collection of those of $\mathrm{Mr}$. Crabtree, mostly for the same years as those above, was published by I)r. Wallic, along with the works of his liend IIorrox, in onevol. 4to, 1672. A long and learned letter of his to Mr. Gascoigne (the inventor of the Micrometer), on the nature and appearances of the solar spots, in the Philos. Trans. vol. 27, and in my Abridg. vol. 5, pa. (i26.

CIRAlG (Rev. Jous), F.u.s, a respectable Scotch di- vine and mathematician, who it scems settled some time at Cambridge, but was afterwards vicar of Gillingham, in Dorsetshire, whence he commonly addressed his letters to the Royal Society. In 1685 he had a dispute with John Bernoulli, on the quadrature of curve lines and curvilinear figures. He had also anither difference with that great mathematician on an algebraic equation; but afterwards finding himself wrong, he candidly acknowledyed it in the Philosophical 'Transactions. He wrote several good picces on mathematical subjects, partly published separately, and several published in the Philos. Trans. as also the translation of them from the Latin in my Abridgement, where a catalogue of them may be scen, in the index at the end of the 1 sth or last volume; being, 1. Quadratures of curves geometrically irrational.-2. Quadrature of the logarithmic curve.-3. On the curve of quickest descent.4. On the solid of least resistance.-5. Method of determining the quadrature of figures.-6. Sulution of Bernoulli's problem on curves. -7 . On the length of curve lines. -8 . Nethod of making logaritlıms. -9 . On the head of a monstrous calf.

And his separate publications are, 1. Methodus figurarum quadraturas, $\& c$, anno 1685 , 4to.-2. De quadraturis et locis, \&c, an. 1693, 4to.-3. De calculo fluentium, et de Optica analytica, \&c, an. 1718,4 to.-4. Thelogide Christianæ principia mathematica, 1699 , and afterwards printed at Lcipsic, with a learned preface. The object of this curious tract, is to apply mathematical calculation to the credibility of the Gospel history; and on this principle he maintains that the Christian religion inust end, accurding to the doctrine of chances, annodom. 3150 , at which time Christ will make his second appearance. This work was answered by the abbé Hauteville, in a piece entitled, The Christian religion proved by facts.

CRAMllR (GABRieL), an eminent Swls mathematician, was horn at Geneva in 1704, and died 1752. He was made professor of mathematics at 19 years of age, and was author of several good works; as, bis Theory of Curve Lines, 1750, 4to; he simplified the art of reducing the equations of a problem to the smallest number possible. To him the world owes the useful labour of collecting and publishing the works of James and John Bernoulli. He possessed a fund of kunwledge in physics and the belles lettres, and rendered himself famous throughout Europe by his progress and skill in mathematics.

CRANE, a machine used in building, and in commerce, for raising large stones and other weights.

Perrault, in his notes on Vitruvius, makes the crane the same with the corvas, or raven, of the ancients.

'The modern crane consists of' several members or pjeces, the principal of which is a strong upright beam, or arbor, firmly fixed in the ground, and sustained by eight arms, coming from the extremities ol four pieces of wood laid across, through the middle ol which the foot of the beun passes. Abont the miclalle of the arbor the arms ineet, and are mortised into it: its top' cuds in an iron pirot, on which is borne a transverse piece, advaricing out to a good distance like a crane's neck; whenee the name, The micldle and extremities of this are again sustained by arms from the middle of the anbor: and over it passes a rope, or chain, to one end of which the weight is fixed; whe other is wound about the spindle of a whel, which, turned, draws the rope, and that heaves up the weight; to be afterwards applied to any side or quatert, by the mobility ot the transverse piece on the pivot. 
Several improvements of this useful machine are mentioned in De-saguliers's Exper. Philos. pa. 178 \& seq. particularly how to prevent the inconveniences arising from sudden jerks, as well as to increase its force by using a double axis in peritrochio, and two handles.

The Crane is of two kinds; in the first kind, called the rat-tailcd crane, the whole machine, with the load, turns upon a strong axis: in the second kind, the vibbet alone moves on its axis. See Desaguliers, as above, for a particular account of the different cranes, and of the gradual improvenents they have received. See also the Supplement to Ferguson's Lectures, pa. 3, \&c; or Philos. Trans. vol. 54, pa. 24, for a description of a nęw and safe crane, with funr different power's adapted to different weights.

Crane is the name of a soutbern constellation. See Grus.

CrANE is also a popular name for a syphon.

CRANK, a contrivance in machines, in manner of an elbow, only of-a square form; projecting out from an axis, or spindle; and serving, by its rotation, to raise and fall the pistons of engines for raising water, or the like.

CRATER, the Cup, a constellation in the southern bemisphere; whose stars, in Ptolemy's catalogue, are 7 ; in Tycho's, 8; in Hevelius's, 10; and in the Britannic catalogue, 31.

CREEK, a part of a haven, where any thing is landed from the sea. It is also said to be a shore or bank on which the water beats, runuing in a small channel from any part of the sea.

CREPUSCULUM, Twilight; the time from the first dawn or appearance of the morning, to the rising of the sun; and again, between the setting of the sun, and the last remains of day.

The Crepusculum, or twilight, it is supposed, usually begins and ends when the sun is about 18 degrees below the burizon; for then the stars of the 6th magnitude disappear in the morning, and appear in the evening. It is of longer duration in the solstices than in the equinoxes, but it is longer in an oblique sphere, than in a right one; because, in those cases the sun, by the obliquity of his path, is longer in ascending through is degrees of altitude.

Twilight is occasioned by the sun's rays refracted in our atmosphere, and reflected from the particles of it to the eye. For let $A$ be the place of an observer on the earth $\triangle D L, A B$ the scnsible' horizon, meeting in s the circle $C B M$ bounding that part of the atmosphere which is capable of refracting and reflecting light to the eye. It is plain that when the sun is under this horizon, no direct rays can come to the eye at

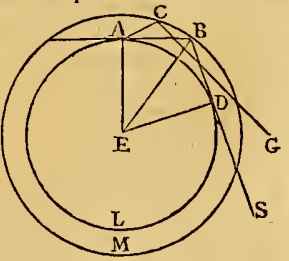
$A$ : hot the sun being in the refracted line $\mathrm{cG}$, the particle $c$ will be illuminated by the direct rays of the sun; and that particle may reflect those rays to $A$, where they enter the eyc of the spectator. And thus the sun's light illuminating an innumerable multitude of particles, may be all reflected to the spectator at $A$.- From $B$ draw $B D$ touching the circle $A D L$ in $D$; and let the sun be in the line $\mathrm{Bn}$ at $\mathrm{s}$ : Then the ray $\mathrm{s} B$ will be reflected into $\mathrm{B} \boldsymbol{A}$, und will enter the eye, because the angie of incidence $\mathrm{DBE}$ is equal to the angle of reflection $\triangle \mathrm{BE}$ : And this will be the first ray that reaches the eye in the morning, when the dawning begins; or the last that falls upon the eye at night, when the twilight ends: for when the sun goes lower down, the particles at $\mathrm{B}$ can be no longer illuminated.

Kepler indeed assigns another cause of the crepusculum, viz, the luninous matter or atmosphere about the sun; which, arising near the horizon, in a circular figure, exhibits the crepusculum; in no wise, he thinks, owing to the refraction of the atmosphere.-'The sun's luminous atmosphere indeed, though neither the sole nor principal cause of twilight, may lengthen its duration, by illuminating our air, when the sun is too low to reach it with his own light. Gregor. Astr. lib. 2, prop. \&.

The depression of the sun below the horizon, at the beginning of the morning, or end of the evcning twikght, is determined in the same manner as the arch of vision; viz, by observing the moment when the air first begins to sbine in the morning, or ceases to shine in the evening; then finding the sun's place for that moment, and thence the time till bis rising in the horizon, or from bis setting in it in the evening. It is now generally agreed that this depth is about 18 degrećs upon an average.-Albazen found it to be $19^{0}$; Tycho, $17^{0}$; Rothmann, 240 ; Stevenius, $18^{0}$; Cassini, $15^{\circ}$; Riccioli, in the equinox in the morning $16^{\circ}$, in the evening $20^{\circ} 30^{\prime}$; in the summer solstice in the morning $21^{\circ} 25^{\prime}$, in the winter solstice in the morning $17^{\circ} 25^{\prime}$.

Nor is this difference among the detcrminations of astronomers to be wondered at; the cause of the crepusculum being inconstant : for; if the exhalations in the atmosphere be either more copious, or ligher, than ordinary; the morning twilight will begin sooner, and the evening hold longer than ordinary: for the more copious the exhalations are, the more rays will they reffect, consequently the more will they shine; and the higher they are, the sooner will they be illuminated by the sun. On this account too, the evening twilight is longer than the morning, at the same time of the year in the same place. To this it may be added, that in a denser air the refraction is greater; and that not only the brightness of the atmosphere is variable, but also its height from the earth: and therefore the twilight is longer in loot weather than in cold, in summer than in winter, and also in hot countries than in cold, other circumstances being the same. But the chief differences are owing to the different situations of places on the carth, or to the difference of the sun's place in the licavens. Thus, the twilight is longest in a parallcl sphere, and shortest in a right sphere, and longer to places in an oblique sphere, in proportion as they are nearer to one of the poles; a circumstance which affords relief to the inhabitants of the more northern countries, in their long winter nights. And the twilights are longest in all places of north latitude, when the sun is in the tropic of cancer; and to those in south latitude, when he is in the tropic of capricorn. The time of the shortest twilight is also different in difierent latitudes; in England, it is about the beginning of October and of March, when the sun is in the signs $\bumpeq$ and $x$. For the method of determining it by trigononetry, see Gregor. Astron. lib. 2, prob. 41. See also Robertson's Nitvigation, book 5 , prob. 12.-Hence, from what has been said, it appears that when the difference between the sun's declination and the depth of the equator is less than $18^{\circ}$, so that the suin does not descend more than $18^{\circ}$ below the ho'rizon; the crepusculum will continue the whole night, as is the case in England from about the $22 \mathrm{~d}$ of May to the $22 \mathrm{~d}$ of July.

Givin the latitude of the place, and the sun's declination; 
to find the beginning of the morning, and end of the exening twilight. - In the ablique-angled spherical triangle $\mathrm{zPS}$, are given $\mathrm{zP}$ the colatitude, PS the codeclination, and $\mathrm{zs}=108^{\circ}$, being the sum of $90^{\circ}$ the quadrant and $18^{\circ}$ the depression at the extremity of the twilight. Then, by spherical trigonometry, calculate the angle $z$ Ps the hourangle from noon; which changed into time, at the rate of $15^{\circ}$ for an hour, gives the time from noon at the beginning or end of twilight. See Robertson, ubi supra.

Of the Height of the sensible Atmosphere, as determined from the duration of twilight, see Keil's Astron. Lect. lect. 20, pa. 235, ed. 1721 ; or Long's Astron. vol. 1, pa. 260; where it is determined that the height at which the atmosphere is dense enough to reflect the rays of light, is about 42 miles.

CRESCENT, the new moon, which, as it begins to recede from the sun, shows a small rim of light, terminating in lorns or points, which increase till it becomes full, and round in the opposition.

The term is sometimes also used for the same figure of the moon in her wane, or decrease, but improperly; both because the horns are then turned towards the west, and because the figure is on the decrease; the crcscent properly signifying increase, from cresco, $I$ grow.

\section{CRONICAL. See Acronical.}

CRONOS, a name given to Saturn by some of the old astronomical writers.

CROSIER, four stars, in form of a cross; by the help of which, those that sail in the southern bemisphere find the antarctic pole.

CROSS, in Surveying, is an instrument consisting of a brass circle, divided into 4 cqual parts, by two lines crossing each other in the centre. At each extremity of these lines is fixed a perpendicular sight, with snall holes below each slit, for the better discuvering of distant objects. The cross is mounted on a staff, or stand, to fix it in the gruund, and is very useful for measuring small picces of land, and taking oftisets, \&c.

Ex. Suppose it be required to survey the field $\triangle B C D E$ with the Cross. Measure along the diagonal line $\Lambda \mathrm{c}$, and $\mathrm{ob}$ serve, with the Cross, when you are perpendicularly opposite to the corners, as at $\mathrm{F}$, $G$, If, and from thence measure the perpendiculars $\mathrm{FE}$, GB, HD. When you think you

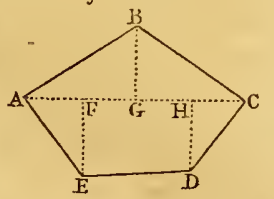
arc nicarly opposite a corner, set up the cross, with one of the bars or cross lines in the direction $\Lambda \mathrm{C}$; then look through the sights of the other cross bar for the corner, as $\mathrm{e}$; if it be secn through them, the cross is fixed in the right place; if not, take it up and move it backward or forward in the line $\Lambda \mathrm{c}$, till the point $\mathrm{B}$ be seen through thuse sights; and then you have the true place of the perpendicular.

Invention of the Cross, Inventio Crucis, an ancient feast, which is still retained in our calendar, and solemnized on the $3 \mathrm{~d}$ of May, in memory of the finding of the true cross of Christ, it is said, decp in the ground, on Mount Calvary, by St. Ifelena, the mother of Constantine; where she erected a church for the preservation of part of it: the

Vol. I. rest being brought to Rome, and deposited in the church of the Holy Cross of Jerusalem.

Exaltation of the Cross, an ancient feast, held on the 14th of September, in memory of this, that Heraclitus restored to Mlount Calvary the true cross, in 642, which had been carried off, 14 years before, by Cosrues king of Persia, on his taking Jerusalem from the emperor Phocas. This feast is still retained in our calendar, on Sept. 14, under the name of Holy Rood, or Holy Cross.

Cross-Multiplication, a method used chiefly by artificers in multiplying feet and inches by feet and inches, or the like; so called, because the factors are multiplied crusswise, thus:

\begin{tabular}{ccc}
$9 \mathrm{f}$ & $10 \mathrm{inc}$ \\
6 & 8 & \\
\hline 59 & 0 & \\
6 & 6 & 8 \\
\hline 65 & 6 & 8
\end{tabular}

Cross-Staff, or Fore-Staff, was a nautical instrument of box, or pear-tree, consisting of a square staff, of about 3 feet long, having each of its faces divided like a line of tangents, and having 4 cross pieces of unequal lengths to fit on to the staff, the halves of these being as the radii to the tangent lines on the faces of the staff.-The instrument was used in taking the altitudes of the celestial bodies at sea. It seems to have been pretty ancient, and was described by $J_{0 h n}$ Werner of Nuremberg, in his Annotations on the 1st book of Ptolemy's Geography, printed in 1514 , recommending it for observing the distance between the moon and some star, for the purpose of determining he longitude. Sce Fore-Staff.

CROUSAZ (Join Peter de), a learned philosopher and mathematician, was born at Lausanne in Switzerland, April 13, 1663; where he died in 1748 , at 85 years of age. Having made great progress in mathematics and the philosophy of Descartes, he travelled into Geneva, Holland, and France. He was successively professor in several universities; and at length was chosen governor to Prince Frederick of Hesse-Cassel, nephew to the king of Sweden.

Crousaz was author of many works, in various branches ; belles-lettres, logic, philosophy, divinity, \&c, \&c; but the most estecmed of them are, 1 . His Logic; the best edition of which is that of 1741 , in 6 vols. 8 vo. -2 . A Treatise on Beauty.-3. A Treatise on Education, 2 vols, 12 no.-4. A Treatise on the Human Understanding. - 5, ieveral Treatises on Philusophy and Mathenatics; as a Treatise on Motion, \&c, with several papers inserted in the Menoirs of the French Academy of Sciences.

CROW, in Mechanics, an iron lever, made with a sharp point at one end, and two claws at the other; being used in heaving and purchasing great weights, \& $c$.

CrOWN, in Astronomy, a name given to two constellations, the southern and the northern.

Cnown, in Geometry, a plane ring included between two parallel or concentric peripheries of unequal circles.

The area of this is obtained, by multiplying its breadth by the length of a middle periphery, which is an arithmetical mean between the two peripheries that bound it; or by multiplying half the sum of the circumferences liy half the difference of the diameters; or lastly, by multiplying the sum of the diameters by the difference of the diameters, and this last product by $\cdot 7854$. See my Mensuration, pa. 110,4 th. edit.

Crown-Post, is a post in some buildings standing upright in the middlc, betwcen two principal rafters; and from which proceed struts or braces to the middlc of each $3 \mathrm{C}$ 
rafter. It is utherwise called a King-post; or King's-piece, or Joggle-piece.

Crow $\mathrm{x}$-IVlieel, of a Watch, is the upper whecl next the balance, or that which drives the balance.

Crow $\mathrm{x}$-Work, in Fortification, is an out-work running into the ticld; designed to ke'p wfithe enemy, gain some bill, or advantagerus prist, and corce the other works of the place. It con-ists of two demi-bastions at the extremities, and an entire bastion in the middle, with curtains.

CROw NED Horn-ivork, is a horn-work with a crownwork before it.

CRYSTALLINE Humour, is a thick compact humour of the eye, in form of a flattish convex lens, placed in the middle of the eye, and serving to make that refraction of the rays of light whicb is necessary to have them meet in the retina, and form an image there, by which vision may be performed.

Crystalline Heatens, in the Old Astrenomy, two orbs imagined between the primum mobile and the firmament, in the Ptolemaic system, which supposed the heavens solid, and only susceptible of a single motion.

King Alphonsus of Arragon, it is said, introduced the Crystallines, to explain what was called the motion of trepidation, or titubation.-The first Crystalline, according to Regiomontanus, \&c, serves to account for the slow motion of the fixed stars; by which they advance a degree in about 70 years, according to the order of the signs, or from west to east; which occasions a precession of the equinox. The $2 d$ serves to account for the motion of libration, or trepidation; by which the celestial sphere librates from one pole towards the other, causing a difference in the sun's greatest declination.

CTESIBIUS, or CTESE RES, a mathematician of Alexan. dria, who was contemporary with Ptolemy king of Egypt, in the 165 th olympiad, about 120 years before Christ. He was possessed of a very great strength of imagination, which placed him at once in a situation much above that which his birth otherwise entitled lim to expect, being the son of a barber in that city. His memory is particularly cherished as the inventor of the pump. He also invented an hydraulic organ, though the circumstances that led to the discovery were purely accidental. On lowering a mirror in his father's shop, he observed that the counterpoise, which was included in a cylinder, produced a sound, by driving the air before it; and on examining the phenomenon more strictly, he conceived the idea of making an instrument, in which sounds should be produced by means of the action of water drivirg the air before it, which invention was afterwards carried into effect by the emperor Nero. Ctesibins was the inventor likewise of the clepsydra, or water-clock, by means of water falling upon a wheel, or a train of whecls, which were turned by it. The wheels communicated their motion to a small wooden image, which, by being gradually raised, pointel with an index to the proper hours, that were engraved on a column near the machinc. 'This invention, it is highly prubable, led to that ol hour-glasses, or sand-glasses, for measuring time, which secms an imitation of the clepsydra. Ctesilius was also author of a treatise," Georlesia, or the Art of dividing and measuring loodies," which is said to be preserved in the library of the Vatican.

Ctesinius' P'ump. See 'Pump.

CUBATURE, or CUBATion, of a soljel, is the measu- ring the space contained in it, or finding the solid content of it, or finding a cube equal to it.

The cubature regards the content of a body, as the quadrature dees the superficies or arca of a figure.

CUBE, a regular solid body, inclosed by six cqual sides or laces, which are squares. $-A$ die is a small cube. It is also called a hexaeciron, because of its six sides, and is the $2 \mathrm{~d}$ of the five Platonic or Regular bodies?

The cube is supposed to be generated by the motion of a square plane, atorgg a line equal and perpendicular to one of its sides.

To desc ${ }_{1}$. ve a licte, or Net, for forming a cube, or wití which it may be covered. See Body (remular).

To determine the Surface and Solidity of a Cube.-Multiply one side by itself, which will give one square or face; then this multiplied by 6 , the number of faces, will gise the whole surface. Also multiply one side, twice by itoclt, that is, cube it, which will give the solid content.

Duplication of $a$ Cube. See Duplication.

Cubes, or Cubic Numbers, are formed by nultip] $y^{-}$ ing any numbers twice by themselves. So the cubes of $1,2,3,4,5,6, \& \mathrm{c}$.

are $1,8,27,64,125,216$, \&c.

The third differences of the cubes of the natural numbers are ail equal to each other, being the constant number 6 . For, let $m^{3}, n^{3}, p^{3}$, be any three adjacent cubes in the natural series ats above, that is, whowe roots $m, n, p$, have the common difference 1 ; then because

$n=m+1$, theref. $n^{3}=m^{3}+3 m^{2}+3 m+1$,

$p=n+1$, theref. $p^{3}=n^{3}+3 n^{2}+3 n+1$; so that the difierence between the 1 st and $2 \mathrm{~d}$, and between the $2 \mathrm{~d}$ and $3 d$ cubes, are

$\left.\begin{array}{l}n^{3}-m^{3}=3 m^{2}+3 m+1, \\ p^{3}-n^{3}=3 n^{2}+3 n+1,\end{array}\right\}$ the 1 st differences;

and the dif. of these differences, is

$3\left(n^{2}-m^{2}\right)+3(n-m)=3(n+m) \cdot(n-m)+3$ $(n-m)$, or since $n-m=1$, it becomes $3(n+m)+3$ $=3(n+m+1)=6(m+1)$, the $2 \mathrm{~d}$ difference.

In like manner the next $2 \mathrm{~d}$ dif. is $6(n+1)$ : hence the dif. of these two $2 \mathrm{~d}$ diffs. is $6(n-m)=6$, which is therefore the constant $3 \mathrm{~d}$ difference of all the series of cubes. And hence the series of cubes will be formed by arldition only, viz, adding always the $3 \mathrm{~d}$ dif. 6 to find the column or series of $2 \mathrm{~d}$ difi's, then these addied always for the 1st difis, and lastly these always added for the cubes thenselves, as below:

3d Difs.
6
6
6
6
6
6
6
6

$2 d$ Difs.
6
12
18
24
30
36
42
48

\begin{tabular}{c|c}
1 st Difs. & Cubes. \\
1 & 0 \\
7 & 1 \\
19 & 8 \\
37 & 27 \\
61 & 64 \\
$91^{-}$ & 125 \\
127 & 216 \\
169 & 343
\end{tabular}

Peletarius, among various speculations concerning square and cubic numbers, slows ibat the continual sums of the cubic numbers, whose ronts are $1,2,3, \& \mathrm{c}$, form the series of squares whose roots are $1,3,6,10,15,21$, sc.
Thus,
$1=1=1^{2}$
$1+8=9=3^{2}$,
$1+8+27=36=6^{2}$,
$1+8+27+64=100=10^{2}$, \& e. 
Or, in gencral, $\mathrm{I}^{3}+2^{3}+3^{3}+4^{3} \&$ c., to $n^{3}=(1+2+3$ $+4 \ldots n)^{2}$, whose root is $\frac{1}{2} n \cdot(n+1)$.

It is also a pretty property, that any number, and the cube of it, being divided by 6 , leave the same remainder; the series of remainders being $0,1,2,3,4,5$, continually repeated. Or that the differences between the numbers and their cubes, divided by 6 , leave always 0 remaining; and the quotients, with their successive differences, form the several orders of figurate numbers. Thus,

\begin{tabular}{|c|c|c|c|c|c|}
\hline Num. & Cubes. & Difs. & Qunt. & 1 Dif. & 2 Dif. \\
\hline 1 & 1 & 0 & 0 & 0 & 0 \\
2 & 8 & 6 & 1 & 1 & 1 \\
3 & 27 & 24 & 4 & 3 & 2 \\
4 & 64 & 60 & 10 & 6 & 3 \\
5 & 125 & 120 & 20 & 10 & 4 \\
6 & 216 & 210 & 35 & 15 & 5 \\
7 & 343 & 336 & 56 & 21 & 6 \\
\hline
\end{tabular}

See a large table of cubes and squares, with their roots, $\& \mathrm{c}$, in the first rolume of my Tracts, at the end.

The Cube of a Binomial, is equal to the cubes of the two parts or mcmbers, together with triple of the two parallelopipedons under each part and the square of the other; viz, $(a+b)^{3}=a^{3}+3 a^{2} b+3 a b^{2}+b^{3}$. And hence the common method of extracting the cube root.

$\mathrm{Cu}$ в гс Equations, are those in which the unknown quantities rise to three dimensions; as $x^{3}=a$, or $x^{3}+a x^{2}=b$, or $x^{3}+a x^{2}+b x=c, \& \mathbf{c}$.

All cubic equations may be reduced to this forn, $x^{3}+p x=q$; viz, by taking away the $2 \mathrm{~d}$ term, when there is one; which is done by substituting, in the given equation $z-\frac{x}{3} a$ for $x$.

All cubic equations have three roots; which are either all real, or else one only is real, and the other two imaginary; for all roots become imaginary by pairs.

But the nature of the roots, as to real and imaginary, is known partly from the sign of the co-efficient $p$, and partly from the relation between $p$, and $g$ : for the equation bas always two imaginary roots when $p$ is positive; it luas also two imaginary ronts when $p$ is negative, provided $\left(\frac{x}{3} p\right)^{3}$ be less $\left(\frac{x}{2} q\right)^{2}$, or $4 p^{3}$ less than $27 q^{2}$; otherwise the roots are all real; namely, whenever $p$ is negative, and $4 p^{3}$ either equal to, or greater than $27 q^{2}$.

Every cubic equation of the above form, viz, wanting the $2 \mathrm{~d}$ tern, has both positive and negative ronts, and the greatest root is always equal to the sum of the two less roots; viz, either one positive root equal to the sum of the two negative ones, or else one negative root equal to the sum of two smaller and positive ones. And the sign of the greatest, or single root, is positive or negative, accurding as $q$ is positive or negative when it stands on the right-liand side of the equation, thus $x^{3}+p x=q$; and the two smaller roots have always the contrary sign to $q$.

So that, in general, the sign of $p$ determines the nature of the roots, as to real and imaginary; and the sign of $q$ determines the affection of the roots, as to positive and negative. See my Tract on Cubic Equations in the Philos. 'Trans. for 1780, and in my new Tracts, vol. 2, tract 28.

To find the Values of the Roots of Cubic Equations. Having reduced the equation to this form $x^{3}+p x=q$, its root may be found in varions ways: the first of these, is that which is called Cardan's Rule, by whom it was first published, but invented by Ferreus and Turtalea. Sec $A_{L}$ -
GEBra. The rule is this: Put $a=\frac{x}{3} p$, and $b=\frac{x}{2} q$; then by Cardan's method the root is

$x=\sqrt[3]{ }\left[b+\sqrt{ }\left(b^{2}+a^{3}\right)\right]+\sqrt[3]{ }\left[b-\sqrt{ }\left(b^{2}+a^{3}\right)\right] ;$ or if there be put $s=\sqrt[3]{ }\left[b+\sqrt{ }\left(b^{2}+a^{3}\right)\right]$, and $d=\sqrt[3]{ }\left[b-\sqrt{ }\left(b^{2}+a^{3}\right)\right]$, then $s+d=x$, the 1st or Cardan's root,

also $-\frac{s+d}{2}+\frac{s-d}{2} \sqrt{ }-3$ is the $2 \mathrm{~d}$ root,

and $-\frac{s+d}{2}-\frac{s-d}{2} \sqrt{ }-3$ is the $3 \mathrm{~d}$ root.

Now the first of these, or Cardan's root, is always a real root, though it is not always the greatest root, as it has been commonly mistaken for. And yet this rule always exhibits the root in the form of an imaginary quantity when the equation has no imaginary roots at all; but in the form of a real quantity when the equation has two imaginary roots. See the reason of this explained in my Tract above cited, art. 47. As to the other two roots, viz, $-\frac{s+d}{2} \pm \frac{s-d}{2} \sqrt{ }-3$, though, in their general form they have an imaginary appearance; yet, by substituting certain particular numbers, they come out in a real form in all such cases as they ought to be so.

But, after the first root is found by Cardan's rule, the other two roots may be found, or exhibited, in several other different ways; some of which are as follow :

Let $r$. denote the 1st, or Cardan's root,

and $v$ and $w$ the other two roots:

then is $v+v=-r$, and $v w r=q$;

and the resolution of these two equations will give the other two roots $v$ and $w$, by a quadratic equation.

Or resolve the quadratic equation $x^{2}+r x+r^{2}+p=0$, and its two roots will be those sought.

Or the same two roots will be

either $-\frac{1}{2} r+\frac{1}{2} \sqrt{ }\left(-p-\frac{3 q}{r}\right)$ and $-\frac{1}{2} r-\frac{1}{2} \sqrt{ }\left(-p-\frac{3 q}{r}\right)$, or $\quad-\frac{1}{2} r+\frac{1}{2} \sqrt{ }\left(r^{2}-\frac{4 q}{r}\right)$ and $-\frac{1}{2} r-\frac{1}{2} v^{\prime}\left(r^{2}-\frac{4 q}{r}\right)$, or $-\frac{x}{2} r+\frac{\pi}{2} \sqrt{ }\left(1-\frac{4 q}{q-p r}\right)$ and $-\frac{1}{2} r-\frac{x}{2} r \sqrt{ }\left(1-\frac{4 q}{q-p r}\right)$.

$E x$. 1. If the equation be $x^{3}-15 x=4$ : here $p=$ $-15, q=4, a=-5, b=2$; hence $\sqrt{ }\left(b^{2}+a^{3}\right)=$ $\sqrt{ }(t-125)=\sqrt{ }-121=11 \sqrt{ }-1, s=\sqrt[3]{ }(2+\sqrt{ }-121)$ $=2+\sqrt{ }-1$, and $d=\sqrt[3]{ }(2-\sqrt{ }-121)=2-\sqrt{ }-1$ : therefore $r=c+d=4$, the 1st root; and $-\frac{s+d}{2} \pm \frac{s-d}{2} \sqrt{ }-3=-2 \mp \sqrt{ } 3$, the other two roots.

$E x$. 2. If $x^{3}-6 x=4$ : here $a=-2$, and $b=2$; therefore $\sqrt{ }\left(b^{2}+a^{3}\right)=\sqrt{ }(4-8)=\sqrt{ }-4=2 \sqrt{ }-1$, $s=\sqrt[3]{ }(2+2 \sqrt{ }-1)=-1+\sqrt{ }-1$, and $d=\sqrt[3]{ }(2-2$ $\sqrt{ }-1)=-1-\sqrt{ }-1$ : hence then $r=s+d=-2$, the first root; and $1 \pm \sqrt{ } 3$ the other two roots.

Ex. 3. If $x^{3}+18 x=6$ : here $a=6$, and $b=3$; then $\sqrt{ }\left(b^{2}+a^{3}\right)=\sqrt{ }(9+216)=\sqrt{ } 225=15, s=\sqrt[3]{ }(3+15)$ $=\sqrt[3]{18}$, and $d=\sqrt[3]{ }(3-15)=\sqrt[3]{ }-12=-\sqrt[3]{12}$ : therefore $r=s+d=\sqrt[3]{18}-\sqrt[3]{12}=\cdot 331313$, the 1st root, and $-\frac{\sqrt[3]{18-\sqrt[V]{ } 12}}{2} \pm \frac{\sqrt[3]{18}+\sqrt[V]{ } 12}{2} \sqrt{ }-3$ are the two other roots.

2. Another method for the ronts of the equation $x^{3}+p x=q$, is by means of infinite series, as shown at art. 68 , and seq. of my Tract above cited; whence it appears that the roots are exhibited in various forms of series as fullow: viz,

$$
2 \sqrt[3]{b} \times\left(1-\frac{2 c^{2}}{3 \cdot 6 b^{4}}-\frac{2 \cdot 5 \cdot 8 c^{4}}{3 \cdot 6 \cdot 9 \cdot 12 b^{4}} \& \mathrm{c}\right)
$$

for the 1st root, and 


\section{U B}

$-\sqrt[3]{b \times}\left(1-\frac{2 c^{2}}{3 \cdot 6 b^{2}}-\frac{2 \cdot 5 \cdot 8 c^{4}}{3 \cdot 6 \cdot 9 \cdot 12 b^{3}} \& c\right)$
$\pm \frac{c \sqrt{ }-3}{\sqrt[3]{b^{2}}} \times\left(\frac{1}{3}+\frac{2 \cdot 5 c^{3}}{3 \cdot 6 \cdot 9 b^{2}}+\frac{2 \cdot 5 \cdot 8 \cdot 11 c^{2}}{3 \cdot 6 \cdot 9 \cdot 12 \cdot 15 b^{4}} \& c\right)$

for the two other roots: where $a=\frac{1}{3} p, b=\frac{\pi}{2} q$, and $e=$ $\sqrt{ }\left(b^{2}+a^{3}\right)$. And similar series, for the solution of eubic eçuations, were also employed by M. Lesbnitz of Hanover, in the year 1677. Various other series for the same purpose may also be seen in my Tract, so often before cited.

3. A third method for the roots of cubic equations, is by angular sections, and the table of sines. It was first hinted by Bombelli, in hıs Algebra, that angles are trisected by the resolution of cubic equations. Afterwards Vieta gave the resolution of eubies, and the higher equations, by angular sections. Next, Albert Girard, in his Invention Nouvelle en l'Algebre, shows how to resolve the irieducible case in cubics by a table of sines : and he also constructs the same, or fiuds the roots, by the interseetion of the hyperbola and circle. Halley and Demoivre also gave rules and examples of the same kind of resolutions by a table of sines. And, lastly, Mr. Anthony Thacker invented, and Mr. William Brown computed, a large set of similar tables, for resolving affected quadratic and cubic equations, with their application to the resolution of biquadratic ones. See IRreducible Case. Also the resolution of these cases, by the tabies of sines, tangents,

$$
\begin{aligned}
& \text { 1st. } x^{3}+p x-q=0 \ldots \ldots=\sqrt[3]{ }\left[\frac{q}{2}+\sqrt{ }\left(\frac{q^{2}}{4}+\frac{p^{3}}{27}\right)\right]-\sqrt[3]{ }\left[-\frac{q}{2}+\sqrt{ }\left(\frac{q^{3}}{4}+\frac{p^{3}}{27}\right)\right] \text {, } \\
& \text { 2d. } x^{3}+p x+p=0 \ldots x=\sqrt[3]{ }\left[-\frac{q}{2}+\sqrt{ }\left(\frac{q^{2}}{4}+\frac{p^{3}}{27}\right)\right]-\sqrt[3]{ }\left[\frac{q}{2}+\sqrt{ }\left(\frac{q^{3}}{4}+\frac{p^{3}}{27}\right)\right] \text {, } \\
& \text { 3d. } x^{3}-p x-q=0 \ldots x=\sqrt[3]{ }\left[\frac{q}{2}+\sqrt{ }\left(\frac{q^{3}}{4}-\frac{p^{3}}{27}\right)\right]+\sqrt[3]{ }\left[\frac{q}{2}-\sqrt{ }\left(\frac{q^{2}}{4}-\frac{p^{3}}{27}\right)\right] \text {, } \\
& \text { 4th. } x^{3}-p x+q=0 \ldots . . . . .-x=-\sqrt[3]{ }\left[\frac{q}{2}-\sqrt{ }\left(\frac{q^{2}}{4}-\frac{p^{3}}{27}\right)\right]-\sqrt[3]{ }\left[\frac{q}{2}+\sqrt{ }\left(\frac{q^{2}}{4}-\frac{p^{3}}{27}\right)\right] \text {. }
\end{aligned}
$$

The roots of the first and second forms are negatives of each other; and those of the third and fourth are also negatives of each other. The first and second forms have ouly one root each. The third and fourth forms have also only one root each, when the quadratic surd $\sqrt{ }\left(\frac{q^{2}}{4}-\frac{p^{3}}{27}\right)$ is possible; but have three roots each when that surd is impossible.

The roots of all the four forms may, in all cases, be easily computed as follows :

Forms 1st and $2 d$. Put $\frac{q}{2} \times\left(\frac{3}{p}\right)^{\frac{3}{2}}=$ tang. $z$; and $\sqrt[3]{ }\left[\tan .\left(45^{\circ}-\frac{1}{2} z\right)\right]=\tan . u$. Then $x= \pm \sqrt{ } \frac{4 p}{a} \times \cot$. $2 u$; where the upper sign belongs to the first form, and the lower sign to the second form.

Forms $3 d$ and $4 t h$. Put $\frac{2}{q} \times\left(\frac{p}{3}\right)^{\frac{2}{3}}$ if less than unity, otherwise its reciprocal $\frac{q}{2} \times\left(\frac{3}{4}\right)^{\frac{3}{2}}=\cos . z$. Then,

Case 1st. $\frac{2}{q} \times\left(\frac{p}{3}\right)^{2}<$ unity. Put $\sqrt[3]{ }\left[\tan .\left(45^{0}-\frac{1}{2} z\right)\right]$ $=\tan . u$. Then $x= \pm \sqrt{ } \frac{A P}{3} \times$ cosec. $2 u$; where the upper sign belongs to the third form, and the lower sign to the fourth form.

Casc $2 d . \frac{2}{q} \times\left(\frac{p}{3}\right)^{\frac{3}{2}}>$ unity. Then $x$ luas three values in each form, viz, $x= \pm \sqrt{ } \frac{4 p}{3} \times \cos \cdot \frac{z}{3}=\mp \sqrt{ } \frac{4 p}{3} \times \cos$. $\left(60^{\circ}-\frac{z}{3}\right)=\mp \sqrt{ } \frac{4 p}{3} \times \cos .\left(60^{0}+\frac{z}{3}\right) ;$ where the upper signs belong to the third form, and the lower signs to the fourth form.

\section{By Logarithms.}

Forms 1st and $2 d$. $\quad$ Log. $\frac{q}{2}+10-\frac{3}{2} \times \log \cdot \frac{p}{3}=\log$.

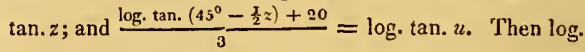
$x=\frac{1}{2} \log \cdot \frac{4 p}{3}+\log$. cot. $2 u-10$; and $x$ will be affirmative in the first form, and negative in the second form.

Forms $3 d$ and 4 th. $\frac{3}{2} \times \log \cdot \frac{p}{3}+10-\log \cdot \frac{g}{2}$ being less than 10 (which is case first) or $\log \frac{q}{2}+10-\frac{3}{2} \times \log$. $\frac{p}{3}$ boing less than 10 (which is case $\left.2 \mathrm{~d}\right)=\log . \cos . \approx$.

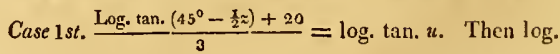
$x=\frac{1}{2} \log \cdot \frac{4 p}{3}+10-\log \cdot \sin .2 u$; and $x$ will be affirmative in the third form, and negative in the 4 th form.

Case $2 d$. Here $x$ has three values.

1st. Log. $\pm x=\frac{1}{2} \log \cdot \frac{4 p}{3}+\log \cdot \cos \cdot \frac{z}{3}-10$,

2d. Log. $\mp x=\frac{1}{2} \log \frac{4 p}{3}+\log \cdot \cos \cdot\left(60^{\circ}-\frac{z}{3}\right)$,

3d. Log. $\mp x=\frac{1}{2} \log \cdot \frac{4 p}{3}+\log \cdot \cos \cdot\left(60^{0}+\frac{z}{3}\right)-10$; where the upper signs belong to the third form, and the lower sigus to the fourth form; that is, the first value of $x$ in the third form is positive, and its second and third 
values negative; and the first value of $x$ in the fourth form is negative, and its second and third values affirmative."

See also Ir reducible Case.

For the cunstruction of cubic equations, see ConSTRUCTION.

Cc B1C Foot, of any thing, is so much of it as is contained in a culse whose side is one foot.

Cu в IC Hyperbola, is a figure espressed by the equation $x y^{2}=u$, having two asymptotes, and consisting of two hyperbolas, lying in the adjoining angles of the asymptotes, and not in the opposite angles, like the Apollonian byperbola; being otherwise called by Newton, in his Enumeratio Linearum Tertii Ordinis, an hyperbolismus of a parabola; and is the 65 th species of thuse lines according to him.

Cu вic Numbers. Sce Cubes.
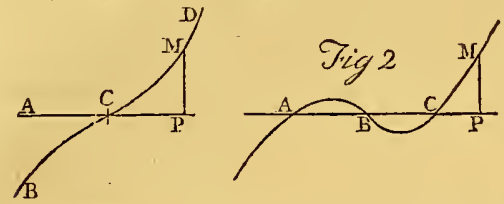

Cu Bic Parabola, a curve, as всD, of the $2 \mathrm{~d}$ order, having two infinite legs $\mathrm{CB}, \mathrm{CD}$, tending contrary ways. And if the absciss, $\mathbf{A} \mathbf{P}$ or $x$, touch the curve in $\mathbf{C}$, the relation between the absciss and ordinate, $v i z, \mathrm{~A} P=x$, and $\mathrm{PM}=y$, is expressed by the cquation $y=a x^{3}+b x^{2}+c x+d$; or when $a$ coincides with $\mathrm{c}$, by the equation $y=a x^{3}$, which is the simplest form of the equation of this curve.

If the right line AP (fig. 2) cut the cubical parabola in three points $A, B, C$; and from any point $P$ there be drawn the right line or ordinate $P M$, cutting the curve in one point M only: then will PM be always as the solid AP $\times$ BP X CP ; which is an essential property of this curve.

And hence it is easy to construct a cubic equation, as $x^{3}+a^{2} x=b^{3}$, by the intersection of this curve and a right line. See the Construction of a cubic equation by means of the cubic parabola and a right line, by Dr. Wallis, in his Aluebra: As also the Construction of equations of 6 dimensions, by means of the cubic parabola and a circle, by Dr. Halley, in a lecture formerly read at Oxford.

The curve of this purabola cannot be rectified, not even by means of the conic sections. But a circle may be found equal to the Curve Surface, generated by the rotation of the curve $\Lambda M$ about the tangent AP to the principal ver$\operatorname{tex} A$. Let $M N$ be an ordinate, and $M T$ a tangent at the point $M$; and let $P M$ be parallel to $A \mathrm{~N}$. Divide $M \mathrm{~N}$ in the point $\mathrm{o}$, so that Mo be to oN as TM to MN. Then a mean proportional between TM $+O N$ and $\frac{x}{3}$ of $A N$ will be the radius of a circle, whose area is equal to the superficies described by that rotation, viz, of AM about $\mathbf{A P}$.

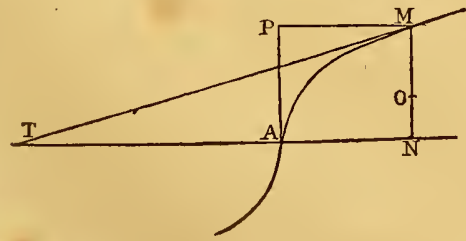

The Arca of a Culic Parabola is 3 of its circumscribing parallelogram.
CuBic Root, of any number, or quantity, is such a quantity as being cubed, or twice multiplied by itself, shall produce that which was given: So, the cubic root of 8 is 2 , because $2^{3}$ or $2 \times 2 \times 2$ is equal to 8 .

The common method of extracting the cube root, founded on the property given abuve, viz, $(a+b)^{3}=a^{3}$ $+3 a^{2} b+3 a b^{2}+b^{3}$, is found in every bouk of common arithmetic, and is as old at least as Lucas de Burgo, where it is first met with in print. Other methods for the cube root may be seen under the article Exтnaction of Roots, particularly this one, viz, the cube root of $n$, or $\sqrt[3]{n}=$ $\frac{2 n+a^{3}}{n+2 a^{3}} \times a$, very nearly, or $2 a^{3}+n: 2 n+a^{3}:: a: \sqrt[3]{n}$ the cube root of $n$ nearly; where $n$ is any number given whose cube root is sought, and $a^{3}$ is the nearest complete cube to $n$, whether greater or less.

For example, Suppose it were proposed to double the cube, or, which comes to the same thing, to extract the cube root of the number 2 . Here the nearest cube is 1 , whose cube root is 1 also, that is, $a^{3}=1$, and $a=1$, also $n=2$; therefore

$2 a^{3}+n \quad 2 n+a^{3} \quad a$

$4: 5:: 1: \frac{5}{4}=1 \cdot 25=\sqrt[3]{2}$ nearly.

But, for a nearer value, assume now $a=\frac{5}{4}$, or $a^{3}=\frac{125}{64}$; then is $2 a^{3}+n=\frac{250}{64}+2=\frac{3}{64}$, and $2 n+a^{3}=4+$ $\frac{325}{64}=\frac{381}{64}$; hence $3 \frac{64}{37}: 381:: \frac{5}{4}: \frac{635}{504}$ or $1 \cdot 25.9921=$ $\sqrt[3]{2}$, or the cube root of 2 , which is true in the last place of decimals - And this is the sinplest and easiest method for the cube root of any number. See its investigation in my new Tracts, vol. 1, pa. 210.

Every number or quantity has three cubic roots, one that is real, and two imaginary: So, the cube root of 1 is either 1 , or $-\frac{1+\sqrt{ }-3}{2}$, or $-\frac{1-\sqrt{ }-3}{2}$; and if $r$ be the real root of any cule $r^{3}$, the two imaginary cubic roots of it will be $-\frac{1+\sqrt{ }-3}{2} r$, and $-\frac{1-\sqrt{ }-3}{2} r$ : for any one of these being cubed, gives the same cube $r^{3}$.

Cuning of a Solid. See Cubature.

Cuвo-cuвe, the 6 th power.

CURo-CuBo-CuBE, the 9 th power.

CUBIT, a measure equal to the distance from the elbow of a man to the extremity of his fingers; and commonly estimated at half a yard, or 18 inches.

CULMINATION, the passage of a star or planet over the meridian, or that point of its orbit which it is in at its greatest altitude. Hence a star is said to culminate, when it passes the meridian.

To find the time of a Star's culminating. Estimate the time nearly; and find the right ascension, both of the sun and star, corrected for this estimated time; then the difference between these right ascensions, converted into solar time, at the rate of 15 degrees to the hour, gives the time of southing. See an example of this calculation every ycar in White's Ephemeris, pa. 45.

CULVERIN, was the name of a piece of ordnance; but is now disused.

\section{CUNETTE. See CuvETtE.}

CUNEUS. See WEDGE.

CUNITIA $\left(M_{\Lambda \mathrm{R} 1 \mathrm{~A}}\right)$, a lady of Silesia, who was famous for her extensive knowledge in many branches of learning, but more particularly in mathematics and astronomy, on which she wrote several ingenious treatises; particularly one entitled Urania Propitia, printed in 1650, in Latiu and German, and dedicated to lierdinand the $3 d$, emperor 
of Germany. In this work are contained nstronomical tables of grat ease and accutacy, founded on kepler's hypothesis. Liut notwithstanding her merit shines with sucls peculiar lustre as to reflect honour on her ses, histury dues not infurm us of the time of her birth or death.

CURlRENT, a stream or thux of water in any direction. The Setting of the current, is that point of the compass towards which the waters run; and the Drift of a current is the rate it runs an hour.

Currents in the sea, are either natural and general, as arising from the diurnal rotation of the earth on its axis, or the tisles, \&ic; or accidental and particular, caused by the waters being driven against promuntorics, or into gulphs and streights; whence they are furced back, and thus disturb the natural tiux of the sea.

The currents are so violent under the equator, where the motion of the eartls is greatest, that they hurry vessels very speedily from Africa to America, but absolutely prevent their return the same way: so that ships are forced to run as far as the 40 th or 45 th degrec of north latitude, to fall into the return of the current again, to bring them home to Europe. It is shown by Governor Pownal, that this current performs a continual circulation, setting out from the Guinea coast in Africa, for example, thence crossing straight over the Atlantic Ocean, and so setting into the Gulph of Mexico by the south side of it; then sweeping round by the bottom of the Gulph, it issues out by the north side of $i t$, and thence takes a direction northeasterly alung the coast of North America, till it arrive near Newfoundland, where it is turned by a whirling motion backward across the Atlantic again, upon the coasts of Europe, and thence southward to tlic coast of Africa, from which it commenced.

In the streights of Gibraltar, the currents set in by the srutb side, sweep along the coast of Africa to Fgypt, by Palestine, and return by the northern side, or luropean coasts, and issue out again by the northern side of the streights. In St. George's Channel too they usually set eastward. The great violence and danger of the sea in the Streights of Magcllan, is attributed to two contrary currents setting in, one from the south and the other from the north sea.

Currents are of some consideration in the art of navigation, as a ship is either accelerated or retarded by them in her course, according as the set is with or against the slip's mution. If the ship sail along the direction of a current, it is evident that the velocity of the current must be added to that of the vessel as observed by the log, because in this estimation the water is supposed to be at rest; but if her course be dircelly against the current, it must for the same reason be subtracted: and if she sail athwart it, her motion will be compounded with that of the current; and her velocity augmented or retarded, according to the angle of her direction with it; that is, she will proceed in the diagonal of the parallelogram formed according to the two lines of direction, and will describe or pass over that diagonal in the same time in which she would liave described either of the sides, by the separate velocities.

For suppose a s de be a parallelogram, the diagonal of which is a 1). Now if the wind alone would drive the ship from $A$ to $\mathrm{B}$, in the sime time as the current alone would drive it from $\wedge$ to $\mathrm{c}$ : then, as the wind neitlier helps nor hinders the ship from coming towards the line $c$, the current will bring it there in the same time as i f the wind did not act: and as the curront neither belps nor hinders the slip from com:ng towards the line $\mathbb{B} D$, the wind will bring it there in the same time as if the current dia not act: therefrie the ship must, at the end of that timc, be found in both those lines, that is, in their musting $\mathrm{D}$ : consequently the ship must have passed from $A$ (n $\mathrm{D}$ in the diagonal AD. See Couposituon of Forces.

See the Sailing in Currents largely exemplified in Robertson's Navigation, vol. 2, book 7 , sect. 8 .

CuRrents, Under, are thuse currents which run at a certain depth below the surface of the water, and in a contrary direction. Dr. Smith, in the 14th vosl. of the Philosophical Transactions, relates an experimerit made in the Baltic Sound, which was communicated to him by an able seaman, who was present at the time it was made. Being at that place in one of his majesty's trigates, they went with their pinnace into the mid-stream, where they were carricd away violently by the current: But having sunk a large basket by means of iron shot placed in it, and having at the same time a rope affixed to it, the motion of the boat, which was then drifting with the current, was checked; and by sinking the basket still lower and lower, the motion of the boat was more and more retarded, until it was finally driven in a contrary direction to that of the upper current, which last did not appear to extend to a greater depth than that of four or five fathoms; and as the upper current was stronger, the nearer it was to the surface; so the under current appeared to be more rapid, as the depth increased. Dr. Smith derives another argument in farour of an under current at the Straits, from the ossing between the north and south Foreland, where it runs tide and half tide; viz, it is either ebb or flood in that part of the Downs, three hours before it is so off at sea; a certain sign that, though the tide or flood runs above, the tide of ebb must run below, that is, close to the ground; and on the contrary, when it is r.bb tide above, it will be flowing below.

\section{CurRents of Air. See Wind.}

CURSOR, a small picce of brass \&c, that slides; as, the piece in an equinoctial ring dial that slides to the day of the nouth; or the little ruler or label of brass sliding in a groove along the middle of another label, representing the horizon in the analemma; or the point that slides along the beam compass; \&c.

CURTATE Distance, is the distance of a planet's place from the sun, reduced to the ecliptic; or, the interval between the sun and that point where a perpendicular, let fall from the planet, mocts the ecliptic.

CURTATION, the interval between a planet's distance from the sun, and the curtate distance. From the foregoing article it is easy to find the curtate distance; whence the manner of constructing tables of curtation is obvious; the quantity of inclination, reduction, and curtation of a planet, depending on the argument of latitude. Kepler, in his Rodolphine Tables, reduces the tables of them all into one, under the title of 'labulas Latitudinaria.

CUR'TlN, Cuntas, or Countine, in Fortification, that part of a wall or rampart that joins two bastions, or lying between the flank of one and that of another.-The curtain is usually bordered with a parapet 5 feet high; behind which the soldiors stand to fire upon the covertway, and into the moat.

CULV A'TURE of a Line, is its bending, or flexure; by which it becomes a currc, of any peculiar form and pro- 
perties. Thus, the nature of the curvature of a circle is such, as that every point in the periphery is equally distant from a point within, called the centre; and so the curvaunre of the same circle is every where the same; but the curvature in all other curves is continually varying. - The curvature of a circle is so much the more, as its radius is less, being alway's reciprocally as the radius, for the curvature of a circle is its deviation from the tangent in a very small arc; and this deviation is equal to the versed sine of the arc, which is reciprocally as the ritdius. And, in like manner, the curvature of other curves is measured by the reciprocal of the radius of a circle having the same degree of curvature as itself, at some certain point.

Every curve recedes from its tangent by its curvature, the measure of which is the same as that of the angle of contact formed by the curve and tangent. Now the same tangent $A B$ is common to an infinite number of circles, or other curves, all touching it and each other in the same point of contact c. So that any curve DCE may be toucled by an infinites number of different circles at the same

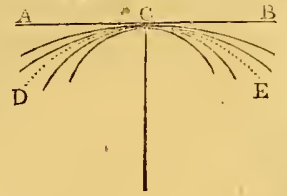

point $\mathbf{c}$; and some of these circles fall wholly within it, being more curved, or having a greater curvature than that curve; while others fall without it near the point of contact, or between the curve and tangent at that point, and being thus less deflected from the tangent than the curve is, they have a less degree of curvature there. Consequently there is cne, of this infinite number of circles; which neither falls below it nor above it, but, being equally deflected from the tangent, coincides with it most intimately of all the circles; and the radius of this circle is called the Radius of Curvature of the curve; also the circle itself is called the Circle of Curvature, or the Osculatory circle of that curve, because $\mathrm{jt}$ touches it so closely that no other circle can be drawn between it and the curve.

As a curve is separated from its tangent by its flexure or curvature, so it is separated from the osculatory circle by the increase ur decrease of its curvature; and as its curvature is greater or less, according as it is more or less deflected from the tangent, so the variation of curvature is greater or less, according as it is more or less separated fiom the circle of curvature.

It appears however, from the demonstration of geometricians, that circles may touch curvelines in such a manner, that there may be indefinite degrees of more or less intimate contact between the curve and its osculatory circle; and that a conic section may be described that shall have the same curvature with a given line at ir given point, and the same variation of curvature, or a contact of the same kind with the circle of curvaturc.

If we conceive-tho: tangent of any proposed curve to be a base, and that a new line or curve be describer, whose ordinate, on the same base or absciss, is a $3 \mathrm{~d}$ proportional to the ordinute and base of the first; this new curve will determine the chord of the circle of curvature, by its intersection with the ordinate at the point of contact; and it will also measure the variation of curvature, by means of the tangent of the angle in which it cuts that circle: the less this angle is, the closer is the contact of the curve and circle of curvature; and of this contact there may be indefinite degrees.
For example, let EMH be any curve, to which ET is a tangent at the point $\mathrm{E}$; then let there be always taken $\mathrm{MT}: \mathrm{ET}:: \mathbf{E T}: \mathbf{T K}$, and through all the points $\mathbf{k}$ draw the curve $\mathbf{\text { B }} \boldsymbol{F}$; then from the point of contuct $\mathrm{E}$ draw $\mathrm{EB}$ parallel to the ordinate $\mathrm{T} \kappa$, meeting the last curve in $\mathrm{B}$; and finally, describe a circle ERQB through the point $B$ and touching $\mathrm{ET}$ in $\mathrm{E}$; and it shall be the osculatory circle to the given curve $\mathrm{R}$ M. And the contact of EM and ER is always the closer, the less the angle $K B Q$ is. See Maclaurin's Fluxions, art. 366 .

Hence it follows, that the contact of the curve Exil and its osculatory circle, is closest, when the curve $\mathbf{B K}$ touclies the arch $\mathrm{BQ}$ in $\mathrm{B}$, the angle $\mathrm{B} \mathbf{E T}$ being given; and it is farthest from this, or most open, when $B \mathrm{~K}$ touches the right line $\mathrm{BE}$ in $\mathrm{B}$.

Hence also there may be indefinite degrees of more and more intimate contact between a circle and a curve. The first degree is when the same right line touches them both in the same point; and a contact of this sort may take place between any circle and any arch of a curve. The $2 \mathrm{~d}$ is when the curve $\mathrm{EMH}$ and circle ERB have the same curvature, and the tangents of the curve $\mathrm{BK} \mathrm{E}$ and circle $\mathrm{BQE}$ intersect each other at $\mathrm{B}$ in any asnignable angle. The contact of the curve EM and circle of curvature Ele at $\mathbf{E}$, is of the $3 \mathrm{~d}$ degree, or order, and their osculation is of the $2 d$, when the curve $\mathbf{B K F}$ touches the circle $\mathbf{B Q E}$ at $\mathrm{n}$, but so as not to have the same curvature with it. The contact is of the $4 \mathrm{th}$ degree, or order, and their osculation of the $3 \mathrm{~d}$, when the curve $\mathrm{B}$ if $\mathrm{F}$ has the same curvatare with the circle $\mathrm{BQE}$ at $\mathrm{B}$, but so as that their contact is only of the $2 \mathrm{~d}$ degree. And this gradation of more and more intimate contact, or of approximation towards coincidence, may be continued indefinitely, the contact of EM and Eu at $\mathrm{E}$ being always of an order two degrees closer than that of $B K$ and $B Q$ at $B$. There is also an indefinite variety comprehended under each order: thus, when EM and Ex have the same curvature, the angle formed by the tangents of $\mathrm{B} K$ and $\mathrm{BQ}$ admits of indefinite variety, and the contact of $\mathrm{EM}$ and $\mathrm{ER}$ is the closer the less that angle is. And when that angle is of the same magnitude, the contact of EM and ER is the closer, the greater the circle of curvature is. When $\mathrm{BK}$ and $\mathrm{BQ}$ touch at $\mathrm{B}$, they may touch on the sume or on different sides of their common tangent; and the angle of contact $\mathrm{KBQ}$ mat achit of the same variety with the angle of contact MER; but as there is seldom occasion for considering those higher degrees of more intimate contact of the curve r.M 11 and circle of curvature E. 12 , Maclaurin calls the contact or osculation of the same kind, when, the chord $E$ b aud angle BET being given, the angle contained by the tangents of $\mathrm{BK}$ and $\mathbf{B Q}$ is of the same magnitude.

When the curvature of EMH increases from $\mathrm{E}$ towards $\mathrm{H}$, and consequently corresponds to that of a circle gradually less and less, the arch E. falls within $\mathrm{E} R$, the arch of the osculatory circle, and $\mathbf{B K}$ is within $\mathrm{BQ}$. The contrary happens when the curvature of En decrcase's from $\mathbf{E}$ towards II, and consequently corresponds to that of a circle which is gradually grcater and greater, the arch $\mathrm{B}$ II falls without $\mathrm{ER}$, and $\mathrm{BK}$ is without $\mathrm{BQ}$. And according as the curvature of EM varies more or less, it is more or less unlike to the uniform curvature of a circle; 
the arch of the curve EMu separates more or less from the arch of the osculatory circle $\mathrm{ERB}$, and the angle contained by the tangents of $\mathrm{BKF}$ and $\mathrm{BQE}$ at $\mathrm{B}$, is greater or less. Thus the quality of curvature, as it is called by Newton, depends on the angle contained by the tangents of $\mathrm{BK}$ and $B Q$ at $B$; and the measure of the inequability or variation of curvature, is as the tangent of this angle, the radius being given, and the angle $\mathbf{B E T}$ being a right one.

The radii of curvature of similar arcs in similar figures, are in the same ratio as any homologous lines of these figures; and the variation of curvature is the same. See Mlaclaurin, art. 370 .

When the proposed curve $\mathrm{E}$ мH is a conic section, the new line BKF is also a conic section; and it is a right line when EмH is a parabola, to the axis of which the ordinates $\mathrm{TK}$ are parallel. $\mathrm{BKF}$ is also a right line when EM1 is an hyperbola, to one asymptote of which the ordinate $\mathrm{TK}$ is parallel.

When the ordinate $\mathrm{EB}$, at the point of contact $\mathrm{E}$, instead of meeting the new curve $\mathrm{BK}$, is an asymptote to it, the curvature of EM is less than in any circle; and this is the case in which it is said to be infinitely small, or that the radius of curvature is infinitely great. And of this kind is the curvature at the points of contrary flexure in lines of the $3 \mathrm{~d}$ order.

When the curve $\mathrm{B} K$ passes through the point of contact $E$; the curvature is greater than in any circle, or the radius of curvature vanishes; and in this case the curvature is said to be infinitely great. Of this kind is the curvature at the cusps of the lines of the $3 \mathrm{~d}$ order.

As to the degree of curvature in lines of the $3 \mathrm{~d}$ and higher orders, see Maclaurin, art. 379 ; also art. 380 , when the proposed curve is mechanical.

As curves which pass through the same point have the same tangent when the first fluxions of the ordinates are equal, so they have the same curvature when the $2 \mathrm{~d}$ fluxions of the ordinate are likewise equal; and half the chord of the osculatory circle that is intercepted between the points where it intersects the ordinate, is a $3 \mathrm{~d}$ proportional to the right lines that incasure the $2 \mathrm{~d}$ fluxion of the ordinate and first fluxion of the curve, the base being supposed to flow uniformly. When a ray revolving about a given point, and terminated by the curve, becomes perpencticular to it, the first fluxion of the radius vanishes; and if its $2 \mathrm{~d}$ fluxion vanish at the same time, that point must be the centre of curvature. 'The same may be said, when the angular motion of the ray about that point is equal to the angular motion of the tangent of the curve; as the angular motion of the radius of a circle about its centre is always equal to the angular motion of the tangent of the circle. Hence the various properties of the circle may suggest several thoręms for determining the centre of curvature.

See art 396 of the said book, also the following, concerning the curvature of lines that are described by means of riglit lines revolving about given polcs, or of angles that either revolve about such poles, or are carried along fixed lines.

It is to be observed that, as when a right line intersects an arc of a curve in two points, if by varying the position of that line the two intersections unite in one point, it then becomes the tangent of the arc; so when a circle touches a curve in one point, and interssets it in another, if, by varying the centre, this intersection join the point of contact, the circle has then the closest contact with the arc, and be- comes the circle of curvature; but it still continues to intersect the curve at the same point where it touches it, that is, where the same right line is their common tangent, unless another intersection join that point at the same time. In general, the circle of curvature intersects the curve at the point of osculation, only when the number of the successive orders of fuxions of the radius of curvature, that vanish when this radius comes to the point of osculation, is an even number.

It lias bcen supposed by some, that two points of contact, or four intersections of the curve and circle of curvature, must join to form an osculation. But Mr. James Bernoulli justly insisted, that the coalition of one point of contact and one intersection, or of three intersections, "as sufficient. In which case, and in general, when an odd number of intersections only join each other, the point where they coincide continues to be an intersection of the curve and circle of curvature, as well as a point of their mutual contact and osculation. See Maclaurin's Flux. art. 4.93 .
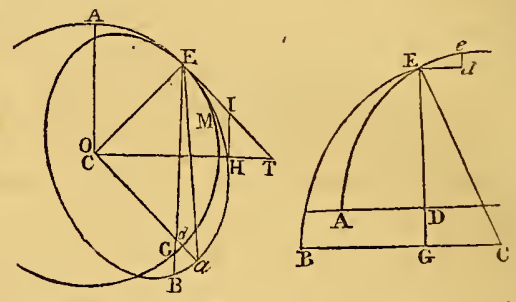

From these principles may be determined the circle of curvature at any point of a conic section. Thus, suppose A EMHG be any corric section, to the point $E$ of which the circle or radius of curvature is to be found. Let ET be a tangent at E, and draw EG B and the tangent $\mathrm{H} 1$ parallel to the chords of the circle of curvature; then take EB to EG as $\mathrm{El}^{2}$ to $\mathrm{II}^{2}$; or, when the section has a centre $\mathrm{o}$, as in the ellipse and hyperbola, as the square of the semi-dianeter $o a$ parallel to $\mathbf{E T}$, is to the square of the semi-diameter on parallel to EB; and a circle EB described on the chord в в that touches ET, will be the circle of curvature sought.

When BET is a right angle, or $\mathrm{EB}$ is the diameter of the circle of curvature, EG will be the axis of the conic section, and $\mathrm{EB}$ will be the parameter of this axis; also when the point $G$, where the conic section cuts $E B$, and the poilut $B$, are on the same side of $E$, then $k M G$ will be an ellipsis, and ra the greater or less axis, according as $\mathrm{EG}$ is greater or less than вв.

The propositions relating to the curvature of the conic sections, commonly given by authors, follow without much difficulty from this construetion.

1. When the chord of curvature, thus found, passes throngh the centre of the conic section, it will then be equal to the parameter of the dimmeter that passes through the point of contact.

2. The square of the semi-diameter $o a$, is to the rectangle of half the transverse and hali the conjugate axis, as the radius of curvature $c x$ is to oa. And therefore the cube of the scmi-diancter oa, parallel to the tangent ET, is equal to the solid contained by the radius of curvature $\mathrm{CE}$, and the rectangle of the two semiaxes.

3. The perpendicular to either axis bisects the angle 
made by the chord of curvature, and the common tangent of the conic section and circle of curvature.

4. The chord of the osculatory circle that passes through the focus, the diameter conjugate to that which passes through the point of coutact, and the transverse axis of the figure, are in continued proportion.

5. When the section is an ellipse, if the circle of curvature at $\mathbf{E}$ meet $o a$ in $d$, the square of $\mathbf{E} d$ will be equal to twice the square of $0 a$. Hence $\mathrm{E} d: 0 a:: \sqrt{ } 2: 1$. Which gives an easy method of determining the circle of curvature to any point $\mathrm{E}$, when the semi-diamcter $\mathrm{o} a$ is given in magnitude and position.

Several other properties of the circle of curvature, and methods of determining it, when the section is given; or vice versa, of determining the section when the circle of curvature is given; may be seen in Maciaurin's Flux. art. 375. See also the Appendix to Maclaurin's Algebra, sect. 1.

To determine the Radius and Circle of Curvature by the Method of Fluxions. Let AEe be any curve, concave toward its axis AD; draw an ordinate DE to the point $\mathbf{E}$ where the curvature is required to be found; and suppose Ec perpendicular to the curve, and equal to the radius of the circle в $\mathrm{E} e$ of curvature sought ; lastly, draw $\mathbf{E} d$ parallel to $A D$, and $d c$ parallel and indefinitely near to $D E$; thereby making $\mathrm{E} d$ the fluxion or increment of the absciss $A D$, also de the fluxion of the ordinate $\mathrm{DE}$, and $\mathrm{E} e$ that of the curve AE. Now put $x=A \mathrm{D}, y=\mathrm{DE}, z=\mathrm{AE}$, and $r=\mathbf{C E}$ the radius of curvature; then is $\mathrm{E} d=\dot{x}$, de $=\dot{y}$, and $\mathrm{E} e=\dot{z}$.

Now, by sim. tri. the 3 lines or

are respectively as the three therefore and the fluxion of this last equation is

$-\mathrm{E} d, \quad d e, \mathrm{Ee}$,
$-\quad \dot{x}, \dot{y}, \dot{z} ;$
$-\mathrm{GE}, \dot{\mathrm{G}}, \mathrm{cE} ;$
$-\mathrm{GC} \cdot \dot{x}=\mathrm{GE} \dot{y} ;$

or because $\dot{\mathrm{GC}}=-\dot{\mathrm{GC}} \cdot \ddot{x}+\mathrm{GC}, \dot{x}=\mathrm{G} \dot{\mathrm{E}} \cdot \ddot{y}+\mathrm{GE} \dot{y}$, $\mathrm{GC} \cdot \ddot{x}-\dot{\mathrm{EG}} \cdot \dot{x}=\mathrm{GE} \cdot \dot{y}+\dot{\mathrm{GE}} \cdot \dot{y}$.

But, since the two curves $A E$ and $B E$ have the same curvature at the point $\mathrm{E}$, their abscisses and ordinates have the samefluxions at that point, that is $\mathrm{e} d$ or $\dot{x}$ is the fluxion both of $A D$ and $B G$, and de or $j$ is the fluxion both of DE and GE. In the above equation therefore substitute $\dot{x}$ for $\dot{B G}$, and $\dot{y}$ for $\dot{\mathrm{E}} \mathrm{E}$, and it becomes

or $\quad \mathrm{GC} \cdot \vec{x}-\dot{x} \dot{x}=\mathrm{GE} \cdot \ddot{y}+\dot{y} \dot{y}$,
$\mathrm{GC} \cdot \ddot{x}-\mathrm{GE} \cdot \ddot{y}=\dot{x}^{2}+\dot{y}^{2}$ or $\dot{z}^{2}$. Now mult. the three terms of this equation respectively by these three quantities, $\frac{\dot{y}}{G \mathbf{C}}, \frac{\dot{x}}{\mathbf{G E}}, \frac{\dot{z}}{\mathbf{C E}}$, which are equal, and it bccomes $\ddot{y} \ddot{x}-\ddot{x} \ddot{y}=\frac{\dot{z}^{3}}{\mathrm{cE}}$, or $\frac{\dot{z}^{3}}{r}$; and hence $r=\frac{\dot{z}^{3}}{\dot{y} \ddot{x}-\dot{x} \ddot{y}}$, which is the geveral value of the radius of curvature for all curves whatever, in terms of the fluxions of the absciss and ordinate.

Farther, as in any case cither $x$ or $y$ may be supposed to flow equably, that is, either $\dot{x}$ or $\dot{y}$ constant quantities, or $\ddot{x}$ or $\ddot{y}=$ to nothing, by this supposition either of the terms in the denominator of the value of $r$ may be made to vanish. So that when $\dot{x}$ is constant, the value of $r$ is $\frac{\dot{z}^{3}}{-\dot{x} \ddot{j}}$, but $=\frac{\dot{x}^{3}}{\dot{y} \ddot{x}}$ when $\dot{y}$ is constant.

For example, suppose it were required to find the radius or circle of curvature to any point of a parabola, its vertex being $A$, and axis AD.-Now the equation of the curve is $a x=y^{2}$; hence $a \dot{x}=2 y \dot{y}$, and $a \ddot{x}=2 \dot{y}^{2}$, supposing $j$ constant; hence $r$ or

VOL.I. $\frac{\dot{z}^{3}}{\dot{y} \ddot{x}}$ or $\frac{\left(\dot{x}^{2}+\dot{y}^{2}\right) \frac{3}{2}}{\dot{y} \ddot{x}}$ is $\frac{\left(a^{2}+4 y^{a}\right)^{3}}{2}$ or $\frac{(a+4 x)^{\frac{3}{2}}}{2 \sqrt{a}}$, the general value of the radius of curvature for any point $\mathrm{E}$, the ordinate to which cuts of the absciss A D $=x$.

Hence, when $x$ or the absciss is nothing, the last expression becomes barely $\frac{a^{\frac{3}{2}}}{2 \sqrt{a}}=\frac{x}{2} a=r$, for the radius of curvature at the vertex of the parabola; that is, the diameter of the circle of curvature at the vertex of a parabola, is equal to $a$ the parameter of the axis.

See CrRcle of Curvature.

Variation of Curvature. See Variation.

Double Curvature, is used for the curvature of a line, which twists so that all the parts of it do not lie in the same plane. Such as the rhumb-line, or loxodromic curve.

CURVE, a line whose several parts proceed bowing, or tend different ways; in opposition to a straight line, all whose parts have the same course or direction.

The doctrine of curves, and of the figures and solids generated from them, constitute what is called the higher geometry.

In a curve, the line $\mathrm{AD}$, which bisects all the parallel lines $\mathrm{MN}$, is called a Diameter; and the point $A$, where the diameter meets the curve, is called the Vertex : if A $\mathrm{D}$ bisect all the parallels at right angles, it is called the Axis. The parallel lines $\mathrm{M} \mathrm{N}$ are called Ordi-

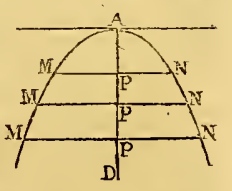
natesfor Applicates; and their halves, PM, or PN, Semi-erdinates. The portion of thediameter $\mathbf{A} \mathbf{P}$, between the vertex, or any other fixed point, and an ordinate, is called the Ab+ sciss: also the concourse of all the diameters, if they meet all in one point, is the Centre. This definition of the diameter, as bisecting the parallel ordinates, respects only the conic sections, or such curves as are cut only in two points by the ordinates; but in the lines of the $3 \mathrm{~d}$ order, which may be cut in three points by the ordinates, then the diameter is that line which cuts the ordinates so, that the sum of the two parts that lie on one side of it, shall be equal to the part on the other side: and so on for curves of higher orders, the sum of the parts of the ordinates on one side of the diameter, being al ways equal to the sum of the parts on the other side of it.

Curve lines are distinguished into Algebraical or Geometrical, and Transcendental or Mechanical.

Algebraical or Geometrical CuRves, are those in which the relation of the abscisses $A P$, to the ordinates PM, can be expressed by a common algebraic equation.

And Transcendental or Mechanical Curves, are such as cannot be so defined or expressed by an algebraical equation. See Transcendental Curve.

Thus, suppose, for instance, the curve be the circle; and that the radius $\mathrm{AC}=r$, the absciss $A \mathrm{P}=x$, and the ordinate $\mathrm{FM}=y$; then, because the nature of the circle is such, that the rectangle $A P \times P B$ is always $=\mathrm{P}_{\mathrm{N}} \mathrm{z}^{2}$, therefore the equation is $x \cdot(2 r-x)=y^{2}$, or $2 r x-x^{2}=y^{2}$, defining this

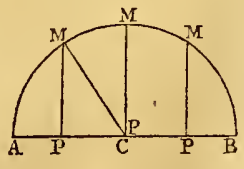
curve, which is therefore an algebraical or geometrical line. Or, suppose $\mathrm{Cr}=x$; then is $\mathrm{CM}^{2}-\mathrm{Cr}^{2}=\mathrm{PN}$, that is $r^{2}-x^{2}=y^{3}$; which is another form of the equation of tlie curve. 
The doctrine of curre lines in general, as expressed by algebraical equations, was first introduced by Descartes, wbo called algebraical curves geometrical ones; as admitting none else into the construction of problems, nor consequently into geometry. But Newton, and after him Leibnitz and Wolfius, are of another opinion; and think, that in the construction of a problem, one curve is not to be preferred to another for its being defined by a more simple equation, but for its bcing more easily described.

Algebraical or geometrical lines are best distinguished into orders according to the number of dimensions of the equation expressing the relation between its ordinates and abscisses, or, which is the same thing, according to the number of points in which they may be cut by a right line. And curves of the same kind or order, are those whose equations rise to the same dimension. Hence, of the first order, there is the right line only; of the $2 d$ order of lines, or the first order of curves, are the circle and conic sections, being 4 species only, viz, $d x-x^{2}=y^{2}$ the circle, $\frac{c^{2}}{d^{2}} \cdot\left(d x-x^{2}\right)=y^{2}$ the ellipse, $\frac{c^{2}}{d^{2}} \cdot\left(d x+x^{2}\right)=y^{2}$ the hyperbola, and $d x=y^{2}$ the parabola; the lines of the $3 \mathrm{~d}$ order, or curves of the $2 \mathrm{~d}$ order, are expressed by an equation of the $3 \mathrm{~d}$ degree, having three roots; and so on. Of these lines of the $3 \mathrm{~d}$ order, Newton wrote an express treatise, under the title of Enumeratio Linearum Tertii Ordinis, showing their distinctive characters and properties, to the number of 72 different species of curves: •but Mlr. Stirling afterwards added four more to that number; and Mr. Nic. Bernoulli and Mr. Stone added two more.

Curves of the $2 d$ and other higher kinds, New ton observes, have parts and properties similar to those of the 1st kind : Thus, as the conic sections have diameters and axes; the lines bisected by these are ordinates; and the intersection of the curve and diameter, the vertex: so, in curves of the $2 \mathrm{~d}$ kind, any two parallel right lines being drawn to meet the curve in 3 points; a right line cutting these parallels so, as that the sum of the two parts between the secant and the curve on one side, is equal to the 3d part terminated by the curve on the other side, will cut, in the same manner, all other right lines parallel to these, and that meet the curve in three points, that is, so as that the sum of the two parts on one side, will still be equal to the $3 \mathrm{~d}$ part on the other side. These three parts therefore thus equal, unay be called Ordinates, or Applicates; the cutting line, the Dianseter; and where it cuts the ordinates at right angles, the Axis; the intersection of the diameter and the curve, the Vertex; and the concourse of two diameters, the Centre; also the concuurse of all the diameters, the Common or General Centre.

Again, as an hyperbola of the first kind has two asymptotes; that of the $2 \mathrm{~d}$ has 3 ; that of the $3 \mathrm{~d}$ has 4 ; $\mathrm{kc}$ : and as the parts of any right line between the conic byperbola and its two asymptotes, are equal or cither side; so, in hyperbolas of the $2 \mathrm{~d}$ kind, any right line cutting the curve and its three asymptotes in three points; the sum of the two parts of that right line, extended from any two asymptotes, the same way, to two points of the curve, will be equal to the $3 \mathrm{~d}$ part extended from the $3 \mathrm{~d}$ asymptote, the contrary way, to the $3 \mathrm{~d}$ point of the curve.

Again, as in the conic sections that are not parabolical, the square of an ordinate, i. e. the rectangle of the ordinates drawn on the contrary sides of the diameter, is to the rectangle of the parts of the diameter terminated at the vertices of an ellipse or hyperbola, in the same proportion as a given line called the latus rectum, is to that part of the diameter which lies between the vertices, and called the latus transversum; so, in curves of the $2 \mathrm{~d}$ kind, not parabolical, the product under three ordinates, is to the product under the three parts of the diameter cut off at the ordinates and the three vertices of the figure, in a given ratio: in which, if there be taken three right lines situated at the three parts of the diamcter between the vertices of the figure, each to each; then these three right lines may be called the Latera Recta of the figure; and the parts of the diameter between the vertices, the Latera Transversa.

And, as in a conic parabola, which has only one vertex to one and the same diameter, the rectangle under the ordinates is equal to the rectangle under the part of the diameter cut off at the ordinates and vertex, and a given right line called the latus rectum: so, in curves of the $2 \mathrm{~d}$ kind, which lave only two vertices to the same diameter, the product under three ordinates, is equal to the product under two parts of the diameter cut off at the ordinates and the two vertices, and a giren right line, which may therefore be called the Latus Transiersum.

Further, as in the conic sections, where two parallels, terminated on each side by a curve, are cut by two other parallels terminated on each side by a curve, the 1st by the $3 \mathrm{~d}$, and the $2 \mathrm{~d}$ by the 4 th; the reciangle of the parts of the 1st is to the rectangle of the parts of the $3 \mathrm{~d}$, as that of the $2 \mathrm{~d}$ is to that of the 4 th; so, when four such right lines occur in a curve of the $2 d$ kind, each in three points; the product of the parts of the 1st, will be to that of the parts of the $3 \mathrm{~d}$, as that of the $2 \mathrm{~d}$ to that of the $4 \mathrm{th}$.

Lastly, the legs of curves, both of the 1st, 2d, and higher kinds, are either of the parabolic or hyperbolic kind: an hyperbolic leg being that which approaches infinitely towards some asymptote; and a parabolic one, that which has no asymptote.

These legs are best distinguished by their tangents; for, if the point of contact go off to an infinite distance, the tangent of the hyperbolic ley will coincide with the asymptote; and that of the parabolic leg, recede infinitely, and vanish. Therefore the asymptote of any leg is found, by seeking the tangent of that leg to a point infinitely distant; and the dircction of an infinite leg is found, by seeking the position of a right line parallel to the tangent, where the point of contact is infinitely remote, for this line tends that way towards which the infinite leg is directed.

\section{Reduction of Curves of the $2 d$ lind.}

Newton reduces all curves of the $2 d$ kind to four cases of equations, expressing the relation between the ordinate and absciss, viz,

in the 1st case, $x y^{2}+e y=a x^{3}+b x^{2}+c x+d$;

in the 2d, - $x y=a x^{3}+b x^{2}+c x+d$;

in the $3 d, \quad-\quad y^{2}=a x^{3}+b x^{2}+c x+d$

in the 4th, - $y=a x^{3}+b x^{2}+c x+d$.

See Newton's Enumeratio, sect. 3 ; and Stirling's Linex, \&c, p. 83.

Enumeration of the Curves of the $2 d$ kind.

Under these four cases, the author brings a great number of different forms of curves, to which he gives different names. An lyperbola lying wholly within the angle of the asymptotes, like a conic hyperbola, he calls an Inscribed Hyperbola; that which cuts the asymptotes, and contains the parts cut of within its own periphery, a 
Circumscribed Hyperbola; that which has one of its infinite legs inscribed and the other circumscribed, he calls Ambigenal ; that whose legs tend towards each other, and are directed the same way, Converging; that where they tend contrary ways, Diverging; that where they are convex different ways, Cross-legged; that applied to its asymptote with a concave vertex, and diverging legs, Conchoidal; that which cuts its asyınptote with contrary flexures, and is produced each way into contrary legs, Anguineous, or Snake-like; that which cuts its conjugate across, Cruciform; that which returning around cuts itself, Nodated; that whose parts concur in the angle of contact, and there terminate, Cuspidated; that whose conjugate is oval, and infinitely small, i. e. a point, Pointed; that which, from the impossibility of two roots, is without either oval, node, cusp, or point, Pure. And in the same manner he denominates a parabola Converging, Diverging, Cruciform, \&c. Also when the number of hyperbolic legs exceeds that of the conic hyperbola, that is more than two, he calls the hyperbola Redundant.

Under those 4 cases the author enumerates $\mathbf{7 2}$ different curves: of these, 9 are redundant hyperbolas, without diameters, having three asymptotes including a triangle ; the first consisting of three hyperbolas, one inscribed, another circumscribed, and the third ambigenal, with an oval; the $2 \mathrm{~d}$, nodated; the $3 \mathrm{~d}$, cuspidated; the 4 th, pointed; the 5 th and 6 th, pure; the 7 th and 8 th, cruciform; the 9 th or last, anguineal. There are 12 redundant hyperbolas, having only one diameter: the 1st, oval; the $2 \mathrm{~d}$, nodated; the $3 \mathrm{~d}$, cuspidated ; the $4 \mathrm{th}$, pointed; 5 th, $6 \mathrm{th}$, 7 th, and 8 th, pure; the 9th and 10th, cruciform; the 11 th and 12th, conchoidal. And to this class Stirling adds 2 more. There are 2 redundant hyperbolas, with three diameters. There are 9 redundant hyperbolas, with three asymptotes converging to a common point; the 1st being formed of the 5th and 6th redundant parabolas, whose asymptotes include a triangle; the $2 \mathrm{~d}$ formed of the 7 th and 8 th ; the $3 \mathrm{~d}$ and 4 th, of the 9 th ; the 5 th. is formed of the 5 th and 7 th of the redundant hyperbolas, with one diameter; the 6 th, of the 6 th and 7 th ; the 7 th, of the 8 th and 9 th; the 8 th, of the 10 th and 11 th; the 9 th, of the 12th and 13th : all which conversions are effected, by diminishing the triangle comprehended between the asymptotes, till it vanish into a point.

Six are defective parabolas, having no diameters: the $1 \mathrm{st}$, oval; the $2 \mathrm{~d}$, nodated; the $3 \mathrm{~d}$, cuspidated; the $4 \mathrm{th}$, pointed; the 5 th, pure; \& 8 .

Seven are defective hyperbolas, having diameters: the 1 st and $2 \mathrm{~d}$, conchoidal, with an oval; the $3 \mathrm{~d}$, nodated; the 4 th, cuspidated, which is the cissoid of the ancients; the 5 th and 6 th, pointed; the 7 th, pure.

Seven are parabolic hyperbolas, having diameters : the Ist, oval ; the $2 \mathrm{~d}$, nodated; the $3 \mathrm{~d}$, cuspidated ; the $4 \mathrm{th}$, pointed; the 5 th, pure ; the 6 th, cruciform; the 7 th, anguineous.

Four are parabolic hyperbolas; four are hyperbolisms of the byperbola : three, hyperbolas of the ellipsis : two, hyperbolisms of the parabola.

Six are diverging parabolas; the 1 st, a trident; the $2 \mathrm{~d}$, oval; the $3 \mathrm{~d}$, nodated; the $4 \mathrm{th}$, pointed; the 5 th, cuspidated (which is Neil's parabola, usually called tlse semicubical parabola); the 6th, purc.

Lastly, one, commonly called the cubical parabola.

Mr. Stirling and Mr. Stone have shown that this enu- meration is imperfect, the former having added four new species of curves to the number, and the latter two, or rather these two were first noticed by Mr. Nic. Bernoulli. Also Mr. Murdoch and Mr. Geo. Sanderson have found some new,species; though some persons dispute the reality of them. See the Genesis Curvarum per Umbras, and the Ladies' Diary 1788 and 1789 , the prize question.

Organical Description of Curves.-Sir Isaac Newton shows that curves may be generated by shadows. He says, if upon an infinite plane, illuminated from a lucid point, the shadows of figures be projected; the shadows of the conic sections will always be conic sections; those of the curves of the $2 d$ kind, will always be curves of the $2 d$ kind; those of the curves of the $3 \mathrm{~d}$ kind, will always be curves of the $3 \mathrm{~d}$ kind; and so on ad infinitum.

And, like as the projected shadow of a circle generates all the conic sections, so the 5 diverging parabolas, by their shadows, will generate and exhibit all the rest of the curves of the $2 \mathrm{~d}$ kind : and thus some of the most simple curves of the other kinds may be found, which will form, by their shadows upon a plane, projected from a lucid point, all the other curves of the same kind. And in the French Memoirs may be seen a demonstration of this projection, with a specimen of a few of the curves of the $2 \mathrm{~d}$ order, which may be generated by a plane cutting a solid formed from the motion of an infinite right line along a diverging parabola, having an oval, always passing through a given or fixed point above the plane of that parabola. The above method of Newton has also heen pursued and illustrated with great elegance by Mr. Murdoch, in his treatise entitled Newtoni Genesis Curvarum per Umbras, seu Perspectivæ Universalis Elementa.

Mr. Maclaurin, in his Geometria Organica, shows how to describe several of the species of curves of the $2 \mathrm{~d}$ order, especially those having a double point, by the motion of right lines and angles: but a good commodious description by a continued motion of those curves which have no double point, is ranked by Newton among the most diffcult problems. Newton gives also other methods of description, by lines or angles revolving above given poles; and Mr. Brackenridge has given a general method of describing curves, by the intersection of right lines moving about points in a given plane. See Philos. Trans. No. 437, or my Abr. vol. 8, pa. 5; and some particular cases are demonstrated in his Exerc. Geometrica de Curvarum Descriptione.

CUR VEs above the $2 d$ Order. The number of species in the bigher orders of curves increase amazingly, those of the $3 \mathrm{~d}$ order only it is thought amounting to some thousands, all comprehended under the following ten particular cquations,

$$
\begin{aligned}
& \text { viz, 1. } y^{4}+f x^{2} y^{3}+g x y^{3}+h x^{2} y+i y^{2}+h x y+l y, \\
& \text { or 2. } y^{4}+f x y^{2}+g x^{2} y+h x y^{2}+i x y+k y, \\
& \text { or 3. } x^{2} y^{2}+f y^{3}+g x^{2} y+h y^{3}+k y, \\
& \text { or 4. } x^{2} y^{2}+f y^{3}+g y^{2}+h x y+i y, \\
& \text { or 5. } y^{3}+f x y^{2}+g x^{2} y+h y, \\
& \text { or } 6 \cdot y^{3}+f x y^{2}+g x y+h y \\
& =a x^{4}+b x^{3}+c x^{2}+d x+e ; \\
& \text { or 7. } y^{4}+e x^{3} y+f x y^{3}+g x y^{2}+h y^{2}+i x y+k y, \\
& \text { or } x^{3} y+e x y^{3}+f x^{2} y+g y^{2}+l x y+i y, \\
& \text { or 9. } x^{3} y+e y^{3}+f x y^{2}+g x y+h y, \\
& \text { or } 10 . x^{3} y+e y^{3}+f y^{2}+g x y+h y, \\
& =a x^{3}+b x^{2}+c x+d .
\end{aligned}
$$

Those who wish to sce how far this doctrinc has beell $3 \mathrm{D} 2$ 


\section{U R}

[ 388

advanced, with regard to curves of the higher orders, as well as those of the 1 st and $2 \mathrm{~d}$ orders, may consult $\mathrm{Mr}$. Maclaurin's Geometria Organica, and Brackenridge's Exerc. Geom.

All geometrical lines of the odd orders, viz, the $3 \mathrm{~d}, 5$ th, 7 th, \&c, have at least one leg running on infinitely; because all equations of the odd dimensions have at least one real root. But vast numbers of the lines of the even orders are only ovals; among which there are several having very pretty figures, some being like single hearts, some double ones, some resembling fiddles, and others again single knots, double kisots, \&.c.

Two geometrical lines of any order will cut each other in as many points as are denoted by the product of the two numbers expressing those orders.

The theory of curves forms a considerable branch of the mathematical sciences. Those who are curious of advancing beyond the knowledge of the circle and the conic sections, and to consider geometrical curves of a higher nature, and in a general view, will do well to study Cramer's Introduction à l'Analyse des Lignes Courbes Algebraiques, which the learned and ingenious author composed for the use of beginners. There is an excellent posthumous piece too of Maclaurin's, printed as an Appendix to his Algebra, and entitled De Linearum Gcometricarum Proprietatibus Generalibus. The same author, at a very early age, gave a remarkable specimen of his genius and knowledge in his Geometria Organica; and be carried these speculations farther afterwards, as may be seen in the theorems he has given in the Philos. Trans. See Abr, vol. 6. Other writings on this subject, besides the Treatises on the Conic Sections, are Archimedes de Spiralibus; Descartes Geometria; Dr. Barrow's Lectiones Geometricæ; Newton's Enumeratio Linearum 'Tertii Ordinis; Stirling's Illustratio 'Tractatûs Newtoni de Lineis Tertii Ordinis; Maclaurin's Geometria Organica; Brackenridge's Descriptio Linearum Curvarum; M. de Gua's Usages de l'Analyse de Descartes; besides many other Tracts on Curves in the Memoirs of several Academies \&c.

Use of Cunves in the Construction of Equations. One great use of curves in Geometry is, by means of their intersections, to give the solution of problems. Sce ConSTRUCTION.

Suppose, ex. gr. it were required to construct the following equation of 9 dimensions,

$x^{9}+b x^{7}+c x^{6}+d x^{5}+c x^{4}+(m+f) x^{3}+g x^{2}+h x+k=0:$ assume the equation to a cubic parabola $x^{3}=y$; then, by writing $y$ for $x^{3}$, the given equation will become $y^{3}+b x y^{2}+c y^{2}+d x^{2} y+e x y+m y+f x^{3}+g x^{2}+b x+k=0$ an equation to another curve of the $2 \mathrm{~d}$ kind, where $m$ or $f$ may be assumed $=0$ or any thing else: and by the descriptions and intersections of these curves will be given the roots of the equation to be constructed. It is suffcient to describe the cubic parabola once. When the equation to be constructed, by omitting the two last terms $h x$ and $k$, is reduced to 7 dimensions; the other curve, by expunging $m$, will have the double point in the beginning of the absciss, and may be casily described as above: If it be reluced to 6 dimensions, by omitting the last three terms, $g x^{2}+h x+k$; the other curve, by expunging $f$, will become a conic section. And if, by omitting the last three terms, the equation be reduced to three dimensions, we shall fall upon Wallis's covstruction by the cubic paratiola and right line.
C U R

Rectification, Inflection, 2uadrature, \&c. of Curves. See the respective terms.

Curve of a Double Curvature, is a curve of which all its parts are not in the same plane.

A curve which can be traced upon a curve surface, and not upon a plane, is called a curre of double curvature. These kinds of curves may be considered as generated by the motion of a point upon a curve surface, provided the duration of its motion be not constantly in the same plane: for, except in the latter case, it is evident that the line so described is curved in two ways; that is, it partakes of the curvature of the surface, and of the continual and successive deflections of the describing point. Or, we may consider those curves as generated by the motion of at variable ordinate along any curve line regarded as an absciss.

Curves of double curvature may also be formed by two curve surfaces penetrating each other; as is the case when a sphere is penetrated by a right cylinder, if the axis of the latter do not pass through the centre of the former.

In the rectification of those curves, they may be considered as projected, first, upon some plane, whose position is known; by which means a plane curve will be obtained, which being considered as an absciss, the rectification of the original curve may be deduced from the usual methods employed for the rectification of plane curves.

Clairaut has published an ingenious treatise on curves of a double curvature. See his Recherches sur les Courbes à Double Courbure. Euler has also treated on this subject in the Appendix to his Analysis Infinitorum, vol. 2, pa. 323. $\Lambda$ s likewise Lacroix in his Calcul Differentiel $\& c$, and other modern writers.

Fumily of Cunves, is an assemblage of several curves of different kinds, all defined by the same equation of an indeterininate degree; but differently, according to the diversity of their kind. Fo example, Suppose an equation of an indeterminate degree, $a^{m-1} x=y^{\mathrm{m}}$ : if $m=2$, then will $a x=y^{2}$; if $m=3$, then will $a^{2} x=y^{3}$; if $m=4$, then is $a^{3} x=y^{4}$, \&c: all which curves are said to be of the same family or tribe.

The equations by which the families of curves are defined, are not to be confounderl with the transcendental ones: for thougl, with regard to the whole fumily, they be of an indeterminate degree, yet with respect to estch several curve of the family, they are leterminate; whereas transcendental equations are of an indefinite degree with respect to the same curve.

All algebraical curves therefore compose a certain family, consisting of innumerable others, each of which comprehends infinite kinds. For the rquations by which curves are defined involve only products, either of powers of the abscisses and ordinates by constant coefficients; or of powers of the abscisses by powers of the ordinates; or of constant, pure, and simple quantities, by onc another. Morcover, every equation to a curve may luave 0 for one member or side of it; for eximple $\iota x=y^{2}$, by transposition becomes $a x-y^{2}=0$. 'Therefore the equation for all algebraic curves will be

$$
\left.\begin{array}{rl}
a y^{m} & +b \cdot r y^{m-1} \\
+f y^{m-1} & +k x^{2} y^{m-2} \delta \cdot c \cdot y^{m-2} \\
& +\eta y^{m-2}
\end{array}\right\}=0 .
$$

Catacaustic, and Diacuustic Curves. Sce Catac'auSTIC, and DiACAUSTiC. 
Exponential CURVE, is that which is defined by an exponential equation, as $a x^{z}=y$, \&c.

Curves by the Light, or Cov r Bes a la Lumiere, a name given to certain curves by M. Kurdwanowski, a Polish gentleman. He observed that any line, straight or curved, exposed to the action of a luminous point, received the light differently in its different parts, according to their distance from the light. These different effects of the light upon each point of the line, may be represented by the ordinates of some curve, which will vary precisely with these effects. Priestley's Hist. of Vision, pa. 752.

Logarithmic Curvb. See Loganithic Curve.

Curve Reflectoire, so called because it is the appearance of the plane bottom of a bason covered with water, to an eye perpendicularly over it. In this position, the bottom of the bason will appear to rise upwards, from the centre outwards; but the curvature will be less and less, and at last the surface of the water will be an asymptote to it. M. Mairan, who first conceived this idea from the phenomena of light, found also several kinds of these curves; and he gives a geometrical deduction of their properties, showing their analogy to caustics by refraction. Mem. Ac. 1740; Priestley's Hist. of Vision, pa. 752.

Radical Curves, a name given by some authors to curves of the spiral kind, whose ordinates, if they may be so called, do all terminate in the centre of the including circle, and appear like so many radii of that circle: whence the name.

Regular CuRves, are such as have their curvature turning regularly and continually the same way; in opposition to such as bend contrary ways, by having points of contrary flexure, which are called irregular curves.

Characteristic Triangle of a CURVE, is the differential or elementary right-angled triangle whose three sides are, the fluxions of the absciss, ordinate, and curve; the fluxion of the curve being the hypothenuse. So, if $p q$ be parallel to, and inde-finitely near to the ordinate $\mathrm{PQ}$, and $\mathrm{Q} r$ parallel to the absciss $A \mathrm{P}$; then $Q r$ is the fuxion of the absciss $A \mathrm{P}$, and $g r$ the fluxion of the ordinate $\mathrm{PQ}$, and $\mathrm{Qq}$ the

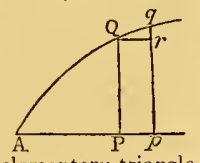
fluxion of the curve $A Q$; hence the elementary triangle Qqr is the characteristic triangle of the curre $A Q$; and the three sides are $\dot{x}, \dot{y}, \dot{z}$; in which $\dot{x}^{2}+\dot{y}^{2}=\dot{z}^{2}$.

CURVILINEAR Angle, Figure, Superficies, \&c, are such as are formed or bounded by curves; in opposition to rectilinear ones, which are formed by straight lines or planes.

CUSP, in Astronomy, is used to express the points or horns of the moon, or other luminary.

Cusp, in the Higher Geometry, is used for the point or corner formed by tivo parts of a curve meeting and terminating there. Siee CURvE.

Cuspidated Hyperbola, \&e. Sec Curve.

CUT-Bastion. See Bastion.

CUVETTE, or CUNETTE, in Fortification, is a kind of ditch within a ditch, being a pretty deep trench, about four fathoms broad, sunk and running along the middle of the great dry ditch, to hold water; serving both to keep off the enemy, and prevent him from mining.

CYCLE, a certain period or series of numbers proceeding orderly from first to last, then returning again to the first, and so circulating perpetually.

Cycles have chiefly arisen from the incommensurability of the periods of revolutions of the earth and cclestial bo- dies to one another. The apparent revolution of the sus about the earth has been arbitrarily divided into 24 hours, whicls is the basis or foundation of all our mensuration of time, whether days, years, \&r. But neither the annual motion of the sun, nor that of the other heavenly bodies, can be measured exactly, and without any remainder, by hours, or their multiples. That of the sun, for example, is 365 days 5 hours 49 minutes nearly; that of the moon, 29 days 12 hours 44 minutes nearly.

Hence, in order to find equivalent cxpressions for these fractions in whole numbers, and yet in numbers which only express days and years, cycles have been invented; which, comprehending several revolutions of thesame body, replace it, after a certain number of years, in the same points of the heaven whence it first departed; or, which is the same thing, in the same place of the civil calendar.

There are various cycles; as, the cycle of indiction, the cycle of the moon, the cycle of the sun, \&c.

CYCLE of Indiction, is a series of 15 years, returning constantly the same as the other cycles; and it commenced from the third year before Chist; whence it happens that if 3 be added to any given year of Christ, and the sum be divided by 15 , what remains is the year of the indiction. See In Diction.

CYCLE of the Moon, or the Lunar Cycle, is a period of 19 years; in which time the new and full moons return to the same day of the Julian year. See Calippic.

This cycle is also called the Metonic period or cycle, from its inventor Mcton, the Athenian; and also the Golden Number, from its excellent use in the calendar: though, properly speaking, the golden number is rather the particular number which slows the year of the lunar cycle, which any given year is in. This cycle of the moon ouly holds true for 310 ? ycars: for, though the new moons do return to the same day after 1.9 years; yet not to the same time of the day, but near an hour and a half soouer; all crror which in $310_{\frac{7}{0}}^{7}$ years amounts to an entire day. Yet those employ ed in reforming the calendar made their calculations on a supposition that the lunations return preciscly from 19 years to 19 years, for ever.

The use of this cycle, in the ancient calendar, is to show the new moon of cach ycar, and the time of Easter. In the new one, it only serves to find the Kjacts; which show, in either calendar, that the new moon falls 11 days too late.

As the Orientals began the use of this cycle at the time of the Council of Nice in 325, they assumed, that the first year of the cycle the paschal new moon fell on the 13 th of March; on which account the lunar cycle 3 . fell on the 1 st of January in the third year.

The Occidentals, on the contrary, placed the number 1 to the 1 st of January, which occasioned a considerable difference in the time of Easter. Hence, Dionysius Exiguns, on framing a new calendar, persuaded the Christians of the west to salve the difference, and come juto the practice of the church of Alesandria.

To find the Year of the Lunar Cycle, is to find the golden number. See Gouden-Number.

Cy cue of the Sun, or Solar Cycle, is a period or revolum. tion of 28 years; teginning with 1 , and ending with 28 ; which elapsed, the Dominical or Sunday-letters, and those that express the other feasts, \&c, return into their former place, and proceed in the same order as before. The days of the month return again to the same days of the week.; the suns place to the same sigus and degrees of the ecliptic on the same months and days, so as not to differ one. 
degree in a hundred years; and the leap-years begin the same course with respect to the days of the week on which the days of the month fall.

This is called the cycle of the sun, or the solar cycle, not from any regard to the sun's course, which has no concern in it; but from Sunday, anciently called dies solis, the sun's day; as the dominical or sunday letter is chiefly sought for from this revolution.

The reformation of the calendar under Pope Gregory the 13th, occasioned a considerable alteration of this cycle : In the Gregorian calenclar, the solar cycle is not constant and perpetual; because every 4 th secular year is common; whereas, in the Julian, it is bissextile. The epoch, or beginning of the solar cycle, both Julian and Gregorian, is the 9th year before Christ. And therefore,

To find the Cycle of the Sun for any given year: add 9 to the number given, and divide the sum by 28 ; the remainder will be the number of the cycle, and the-quotient the number of revolutions since Christ. If there be no remainder, it will be the 28 th or last year of the cycle.

CYCLE of the Sun, and Sunday Letters, from the Year 1800 to the Year 1900.

\begin{tabular}{ll|ll|ll|ll|ll|ll|ll}
\hline 1 & $\mathrm{ED}$ & 5 & $\mathrm{GF}$ & 9 & $\mathrm{BA}$ & 13 & $\mathrm{DC}$ & 17 & $\mathrm{FE}$ & 21 & $\mathrm{AG}$ & 25 & $\mathrm{CB}$ \\
2 & $\mathrm{C}$ & 6 & $\mathrm{E}$ & 10 & $\mathrm{G}$ & 14 & $\mathrm{~B}$ & 18 & $\mathrm{D}$ & 22 & $\mathrm{~F}$ & 26 & $\mathrm{~A}$ \\
3 & $\mathrm{~B}$ & 7 & $\mathrm{D}$ & 11 & $\mathrm{~F}$ & 15 & $\mathrm{~A}$ & 19 & $\mathrm{C}$ & 23 & $\mathrm{E}$ & 27 & $\mathrm{G}$ \\
4 & $\mathrm{~A}$ & 6 & $\mathrm{C}$ & 12 & $\mathrm{E}$ & 16 & $\mathrm{G}$ & 20 & $\mathrm{~B}$ & 24 & $\mathrm{D}$ & $2 \mathrm{~F}$ & $\mathrm{~F}$
\end{tabular}

Great Pascal CrcLe, is another name for the Victorian or Dionysian Period. Which see.

CYCLOID, or Troсног, a mechanical or transcendental curve, which is thus generated : Suppose a wheel, or a circle, $A \mathrm{E}$, to roll along a straight line $\mathrm{AB}$, beginning at the point $A$, and ending at $\mathrm{B}$, where it bas completed just one revolution, thus measuring out a right line $A B$ exactly equal to the circumference of the generating circle $A \mathrm{x}$, while a nail or point $\mathrm{A}$ in the circumference of the wheel, or circle, traces out or describes a curvilineal path $A D B$; then this curve $A D B$ is the cycloid, or trochoid.

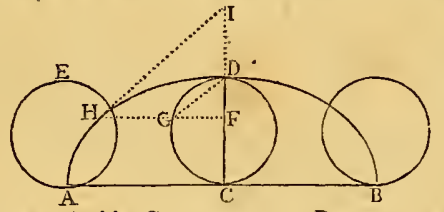

Schooten, in his Commentary on Descartes, says that Descartes first conceived the notion of this elegant curve, and after him it was first published by Father Mersenne, in the year 1615. But Torricelli, in the Appendix de Dimensione Cycloidis, at the end of his treatise De Dimensione Parabolix, published 1644, says that this curve was considered and named a cycloid, by his predecessors, and particularly by Galilen about 45 years before, i. e. about 1599. And Dr. Wallis shows that it is of a much older standing, having been known to Bovilli about the year 1500 , and even considered by Cardinal Cusanus much earlier, viz, before the year 14.51. Plilos. Trams. Abr. vol. 4 , pa. 169 . It would seem however that Torricilli's was the first regular treatise on the Cycloid; though several particular propertie's of it might be known prior to his work. Ile first showed, that the cycloidal space is equal to triple the gencrating circle, (though Pascal contends that Roberval showed this): also that the solid generated by the rotation of that spacc about its base, is to the cir- cumscribing cylinder, as 5 to 8 : about the tangent parallel to the base, as 7 to 8 : about the tangent parallel to the axis, as 3 to 4 : \&c.

Honoratus Fabri, in his Synopsis Geom. has a short treatise on the cycloid, containing demonstrations of the above, and many other theorcms concerning the centres of gravity of the cycloidal space, \&c; which hc says befound out before the year 1658 .

From the preface to Dr. Wallis's treatise on the Cycloid we learn, that, in the year 1658 , M. Pascal publicly proposed at Paris, under the name of Dettonville, the two following problems as a challenge, to be solved by the ma* thematicians of Europe, with a reward of 20 pistoles for the solution: viz, to find the area of any segment of the cycloid, cut off by a right line parallel to the base; also the content of the solid generated by the rotation of the same about the axis, and about the base of that segment. This challenge set the Doctor upon writing that treatise on the cycloid, which is a much better and more complete piece than had been given before upon this curve. He there gives the curve surfaces of the solids generated by the rotation of the cycloidal space about its axis, and about its base, with determinations of the centres of gravity, \&c. He here likewise asserts that Sir Christopher Wren, in 1658 , was the first who found a right line equal to the curve of the cycloid; and Mr. Huygens, in bis Horolog. Oscillat. says that he himself was the first iuventor of the segment of a cycloidal space, cut off by a right line parallel to the base at the distance of $\frac{I}{4}$ the axis of the curve from the centre, being equal to a rectilinear space, viz, to a regular hexagon inscribed in the gemerating circle; the demonstration of which may be seen in Wallis's treatise.

Several other authors bave treated of the cycloid: as Pascal, in his treatise, under, the name of Dettonville: Schooten in his Commentary on Descartes's Geometry, near the end of the $2 \mathrm{~d}$ book; M. Reinau, in his Analyse Démontrée, tom. 2, pa. 595: also Newton, Leibnitz, Lalouberc, Roberval, Descartes, Wren, Fabri, the Bernoulli's, Lahire, Cotes, Emerson, \&c, \&c.

Properties of the CrclorD.-The circle A E, by whose revolution the cycloid is traced out, is called the Generating Circle; the line $A \mathrm{~B}$, which is equal to the circumference of the circle, is the Base of the cycloid; and the perpendicular De on the middle of the base, is its Axis. The properties of the cycioid are among the most beautiful and useful of all curve lines: some of the most remarkable of which are as follow:

1. The circ. arc $D G=G H$ parallel to $A B$.

2. The scmicircumf. $\mathrm{DGC}=$ the semihase $\mathrm{AC}$.

3. The arc $\mathrm{DH}=$ double the chord $\mathrm{DG}$.

4. The arc $\mathrm{DA}=$ double the diamcter $\mathrm{DC}$.

5. The tang. 11 is parallel to the chord DG.

6 . The space $\triangle \mathrm{DB} \Delta=$ triple the circle $A \mathrm{E}$.

7. The spacc $A \mathrm{DGCA}=$ the same circle $A \mathrm{E}$, \& $\mathrm{C}$.

8. $\Lambda$ body falls through any arc $\mathrm{KL}$ of a cycloid reversed, in the same time, whether that arc bc great or small; that is, from any point $\mathrm{L}$, to the lowest point $\kappa$, which is the vertex reversed; and that time is to the time of falling perpendi-

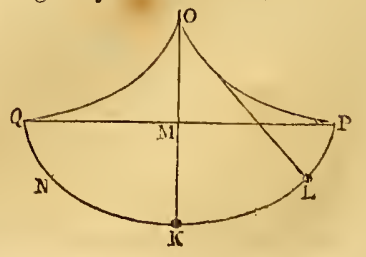


C Y

cularly through the axis $\mathrm{M} \mathrm{K}$, as the semicircumference of a circle is to its diameter, or as 3.1416 to 2. And hence it follows that, if a pendulum be made to "vibrate in the arc $L K N$ of a cycloid, all the vibrations will be performed in the same time.

9. The evolute of a cycloid, is another equal cycloid. So that if two equal semicycloids or, $O Q$, be joined at $o$, so that $0 \mathrm{~m}$ be equal to $\mathrm{m}$ the diameter of the generating circle, and the string of a pendulum hung up at $o$, having its length equal to on or to the curve or ; then, by plying the string round the curve oP, to which it is equal, and the ball being let go, it will describe and vibrate in the other cycloid $\mathbf{P K Q}$. We must not however conclude from this, that a body so vibrating will really perform all its vibrations in equal times, for this is only true in theory, that is, by supposing the string by which the vibrating body is suspended to be void of gravity. For in estimating the centre of oscillation of any body, regard must be had to this circumstance, and in the final expression the distance from the point of suspension is found to enter, together with the distance of the centre of oscillation of the string, and consequently this last centre of oscillation is constantly varying; which will prevent the body from performing all its vibrations in the same time: but the greater the weiglit of the body is, when compared with that of the string, the nearer will these times approach to an equality.

10. The cycloid is the curve of swiftest descent: or a heavy body will fall from one given point to another, through the arc of a cycloid passing by those two points, in a less time, than if it passed along any other curve, or even along a right line joining those two points.

John Bernoulli $u$ as the first who proposed to inathematicians this problem under the title of the Brachystoc/zronon, that is, to find the curve of swiftest descent, or that curve, along which a heary body would fall from one point to another, not in the same vertical line, in the least time. And solutions were given to it by his brother James Bernoulli, Leibnitz, Hôpital, and Newton. See Gregory's Mechanics, art. 277, vol. 1 : also the Works of James and John Bernoulli, for many other curious properties concerning the descents in cycloids, \&c.

Cycroids are also either Curtate or Prolate.

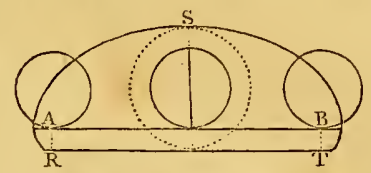

Cycroin, Curtate, or contracted, is the path described by some point without the circle, while the circumference rolls along a straight line; and a

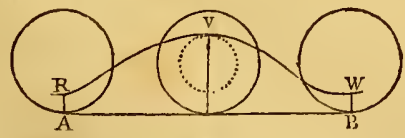

Cyclos n, Prolute, or Inflected, is in like manner the path of some point taken within the generating circle.

'Thus, if, while the circle rolls along the line $\mathrm{Als}$, the point $\mathrm{x}$ be taken without the circle, it will sescribe or trace out the curtate or contracted cycloid iss; bit the point being taken within the circle, it will describe the prolate or inflected cycloid $\mathrm{n} v \mathrm{w}$.
C I L

These two curves were both noticed by Torricelli and Schooten, and more fully treated of by Wallis, in his T'reatise on the Cycloid, printed at Oxford in 1659; where he shows that these have properties similar to the first or primary cycloid; only the last of them is a curve having a point of inflection, and the other crossing itself, and forming a node.

By continuing the motion of the wheel, or circle, so as to describe a right line equal to the generating circumference several times repeated, there will be produced as many repetitions of the cycloids, which so united together will appear as in these figures following:

\section{Curtate Cycloid.}

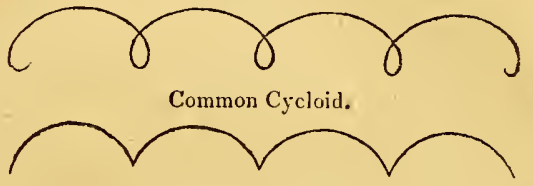

Inflected Cycloid.

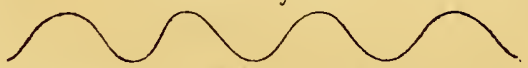

CYCLOPADIA. See ENCYCLOP EDIA.

CYGNUS, the Swan, a constellation of the northern hemisphere, being one of the 48 old ones, and fabled by the Greeks to be the swan, under the form of which Jupiter deccived Leda or Nemesis, from which embrace sprung the beautous Helen. The stars in the constellation Cygnus, in Ptulemy's catalogue are 19, in Tycho's 18, in Hevelius's 47 , and in the Britannic catalogue 81 .

CYLINDER, a solid having two equal circular ends, and every plane section parallel to the ends a circle equal to them also.-The cylinder may be conceived to be thus generated :

Suppose two parallel circles $\triangle \mathrm{B}, \mathrm{CD}$, and a right line carried continually round them, always parallel to itself; this line will describe the curve surface of a cylinder, A R DC; of which the two parallel

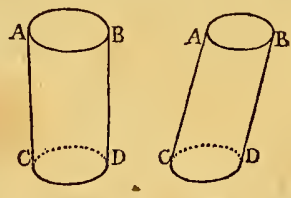
circles $\triangle B$ and $C D$ lorm the two ends. When the line or side is perpendicular to the ends, the cylinder is a right or perpendicular one; otherwise it is oblique.

Or the right cylinder may be conceived to be generated by the rotation of a rectangle about one of its sides. The Axis of the cylinder is the line connecting the centres of its two parallel circular ends; and is cqual to the altitude of the cylinder when this is a right one, but exceeds the altitude in the oblique cylinder, in the propurtion of radius to the sine of the angle of its inclination to the base.

The convex surface of -a cylinder is equal to the product of the axis and the circumference of its base.--The solidity of a cylinder is equal to the area of its base multiplied by its perpendicular altitude.

Cylinders of equal bases and altitudes, are equal.

Cylinders are to each other, as the product of their bases and altitudes. And equal cylinders have their bases reciprocally as their altitudes.

A cylinder is to its inscribed sphere, or spheroid, as 3 to 2: and to its inscribed cone as 3 to 1 . 
The oblique plane sections of a cylinder, are cllipses; but all the sections parallel to the ends, are circles.

For the surfaces and solidities of the ungulas or oblique slices of a cylinder, see my Mensuration, p. 161, 4th edit.

CYLINDRICAL, pertaining to a cylinder.

CYLINDROID, a solid resembling the figure of a cylinder; but differing from it as having ellipses for its ends or bases, insteal of circles in the cylinder.

In the cylindroid, the solidity and curve superficies are found the same way as those of the cylinder ; viz, by muItiplying the circumference of the base by the length or axis, for the surface; and the area of the base by the altitude, for the solidity.

CYMA'TIUM, Cimatiun, or Cima, in Architecture, a member, or moulding of the cornice, whose profile is waved; i. e. concave at top, and convex at bottom.

CYPHER, or nought. See CipHRR.

CYSSOID. See Crssord.

\section{A L}

D ADO, that part in the middle of the pedestal of a column \&c, between its base and cornice.

DAILY, in Astronomy. See Diurnal.

DALEMBERT, an eminent French mathematician and philosopher, and one of the brightest ornaments of the 18 th century. He was perpetual secretary to the French Academy of Sciences, and a member of most of the philosophical academies and societies of Europe.

Dalcmbert was born at Paris, the 16 th of November 17 17. He derived the name of John le Rond from that of the church near which, after his birth, he was exposed as a foundling. But his father, informed of this circumstance, listening to the voice of nature and duty, took measures for the proper education of his child, and for his future subsistence in a state of ease and independence. His mother was a lady of rank, the celebrated Mademoiselle Tencin, sister to Cardinal Tencin, archbishop of Lyons.

He received his first education among the Jansenists, in the college of the four nations, where he gave early signs of genius and capacity. On quitting the college, finding himself alone, and unconnected in the world, he sought an asylum in the house of his nurse. He hoped that his fortune, though not ample, would enlarge the subsistence, and better the condition of her family, which was the only one that be could consider as his own. It was here therefore that he fixed his residence, resolving to apply himself entirely to the study of geometry.-And here he lived, during the space of 40 years, witli the greatest simplicity, discovering the augmentation of his means only by increasing displays of his beneficence, concealing his growing reputation and celebrity from these honest people, and making their plain and uncouth manners the subject of good-natured pleasantry and philosophical observation. His good nurse perccived his ardent activity; heard him mentioned as the writer of many books; but never took it into her head that he was a great man, and rather belield him with a kind of compassion. "You will never," said she to him one daly, "be any thing but a philosopherand what is a philosopher?-a fool, who toils and plagues limself all his life, that people may talk of him when he is dead."

In the year 174.1 he was admitted a member of the $\Lambda$ cademy of Sciences; for which distinguished literary promotion, at so early an age (24), he had prepared the way by correcting the errors of a celebrated work (The Analyse Demontrée of lieyncau), which was estecmed classical in l'rance in the lirk: of analytics. He after-

\section{A L}

wards set himself to examine, with close attention and assiduity, what must be the motion and path of a body, which passes from one fluid into another denser fluid, in a direction oblique to the surface between the two fluids. Every onc knows the phenomenon which happens in this case, and amuses children, under the denomination of ducks and drakes; but it was Dalembert who first explained it in a satisfactory and philosophical manner.

Two years after his election to a place in the Academy;, he published his Treatise on Dynamics. The new principle developed in this treatise, consisted in establishing an equality, at each instant, between the changes that the motion of a body lias undergone, and the forces or powers which have been employed to produce them: or, to express the same thing otherwise, in separating into two parts the action of the moving powers, and considering the one as producing alone the motion of the body, in the second instant, and the other as employed to destroy that which it had in the first. So early as the year 1744, Dalembert had applied this principle to the theory of the equilibrium, and the motion of fluids: and all the problems before resolved in physics, became in some measure its corollaries. The discovery of this new principle was followed by that of a new calculus, the first cssays of which were published in a Discourse on the General Theory of the Winds, to which the prize-medal was adjudged by the Academy of Berlin in the year 1746 , which proved a new and brilliant addition to the fame of Dalembert. This ncw calculus of Partial Difterences he applied, the year following, to the problcm of vibrating cliords, the resolution of which, as well as the thcory of the oscillations of the air and the propagation of sound, had been but imperfectly given by the mathematicians who preceled him; and these were his masters or his rivals.

In the year 1749 he furnished a method of applying his principle to the motion of any body of a given figure. He also resolved the problem of the precession of the equinoxes; determining its quantity, and explaining the phenomenon of the mutation of the terrestrial axis discovered by Dr. Bradley.

In 1752 , Dalembert published a treatise on the Resistance of Fluids, to which he gave the modest title of al Essay; though it contains a multitude of original idens and new observations. About the same time lie published, in the Memoirs of the Academy of Berlin, Rescarches concerning the Integral Calculus, which is greatly indebted to him for the ralpid progress it has since mide.

Our author's eulogist ascribes to envy, detraction, \&c, 
all the opposition and censure that Dalembert met with on account of the celebrated Encyclopédie, or Dictionary of Arts and Sciences, in conjunction with Diderot. None surely will refuse the well-deserved tribute of applanse to the eminent displays of genius, judgment, and true literary taste, with which Dafembert has enriched that great work. Among others, the Preliminary Discourse be has prefixed to it, concerning the rise, progress, connesions, and affnities of all the branches of human knowledge, is perhaps one of the most capital productions the philosophy of the age can boast of.

Some time after this, Dalembert published his Philosophical, Historical, and Philological Miscellanies. These were followed by the Memoirs of Christina queen of Sweden; in which he showed that he was acquainted with the natural rights of mankind, and was bold enough to assert them. His Essay on the Intercourse of Men of Letters with Persons high in Rank and Office, wounded the former to the quick, as it exposed to the eyes of the public the ignominy of those servile cbains, which they feared to shake off, or were proud to wear. Dalembert gave elegant specimens of his literary abilities in his translations of some select pieces of Tacitus. But these occupations did not divert him from his mathematical studies: for about the same time he enriched the Encyclopédie with a multitude of excellent articles in that line, and composed his Researches on several Important Points of the System of the World, in which he carried to a higher degree of perfection the solution of the problem concerning the perturbations of the planets, that had several years before been presented to the Academy.

In 1759 he published his Elements of Philosophy : a work much cxtolled, as remarkable for its precision and perspicuity. The resentment that was kindled (and the disputes that followed it) by the article Geneva, inserted in the Encyclopédie, are well known. Dalembert did not leave this field of controversy with flying colours. It was on this occasion that Frederick king of Prussia offered him an honourable asylum at his court, and the office of president of his Academy : but the king was not offended at Dalembert's refusal of these distinctions, though he cultivated an intimate friendship with him during the rest of his life. He had refused, some time before this, a proposal made by the empress of Russia to entrust him with the education of the Grand Duke;-a proposal accompanied with all the flattering offers that could tempt a man, ambitious of tilles, or desirous of making an ample fortune: but the objects of his ambition were tranquillity and study.

In the year 1765 , he published his Dissertation on the Destruction of the Jesuits. This picce drew upon him a swarm of adversaries, who only confirmed the merit of his work by their mamer of attacking it. Besiles the works already mentioned, he published 9 volumes of memoirs and treatises, under the title of Opuscules; in which he has resolved a multitude of problems relating to astronony, - mathematics, and natural philosoplyy; of which his panegyrist, Condorcet, gives a particular account, more especially of those which exhibit new subjects, or new methods of investigation. Ile published also Elements of Music; and rendered, at length, the system of Rameau intelligible: but he did not think the mathenatical theory of the sonorous body sufficient to accuunt for the rules of that art.

In the year 1772 he was chosen secretary to the French VoL. 1.
Academy of Sciences. He formed, soon after this preferment, the design of writing the lives of all the deceased academicians, from 1700 to 1772 ; and in the space of three ycars he executed this design, by-composing 70 eulogies. Dalembert died on the 29 th of October 1783 , being nearly 66 years of age.

As it may be curious and uscful to have in one view an entire list of Dalembert's writings, 1 shall here insert a catalogue of them, from Rozier's Nouvelle Table des Articles contenus daus les volumes de l'Academie Royale des Sciences de Paris, \&c, as follows:

Traité de Dynamique, in 4to, Paris, 1743 . The $2 \mathrm{~d}$ ed. in 1758.-Traité de l'Equilibre et du Mouvement des Fluides. Paris, 1744; and the $2 d$ edit. in 1770.

Reflexions sur la Cause Générale des Vents; which gained the prize at Berlin in 1746 ; and was printed at Paris in $\mathbf{1 7 4 7}$, in 4 to.-Recherches sur la Précession des Equinoxes, et sur la Nutation de l'Axe de la Terre dans le Système Newtonicn. Paris, 1749, in 4to.-Essais d'ime Nouvelle Théorie du Mouvement des Fluides. Paris, 1752, in 4to.-Recherches sur differens Points importans du Système du Monde. Paris, 1754 and 1756, 3 vols. in 4 to. -Elemens de Philosophic, 1759.

Opuscules Mathématiques, ou Memoires sur différens Sujets de Géonétrie, de Méchaniques, d'Optiques, et d'Astronomie. Paris, 9 vol. in 4 to; 1761 to 1773.

Elémens de Musique, théorique et pratique, suivant les Principes de M. Rameau, eclairés, développés, et simplifiés. 1 vol. in 8vo. à Lyon.-De la Destruction des Jesuites, 1765.-In the Memoirs of the Academy of Paris are the following pieces, by Dalembert: viz, Précis de Dynamique, 1743, Hist. 164.-Précis de l'Equilibre et de Mouvement des Fluides, 1744, Hist. 55.-Methode générale pour déterminer les Orbites et les Mouvements de toutes les Planètes, cu ayant égard d̀ leur action mutuelle, 1745, p. 365.-Précis des Rélexions sur la Cause Générale des Vents, 1750, Hist. 41.-Précis des Recherches sur la Précession des Equinoxes, et sur la Nutation de l'Axe de la Terre dans le Système Newtonien, 1750 , Hist. 134.-Essai d'une Nouvelle Théorie sur la Résistance des Fluides, 1752, Hist. 116.-Précis des Essais d'une Nouvelle Théorie de la Résistance des Fluides, 1753, Hist. 289.-P'récis des Recherches sur les differens Points importans du Système du Monde, 1754, Hist. 125.-Recherches sur la Précession des Equinoxes, et sur la Nutation de l'Axe de la Terre, dans l'Hypothese de la Dissimilitude des Méridiens, 1754, p. 413, Hist. 116.-Reponse à uf́ Article du Mémoire de M. l'Abbé de la Caille, sur la Théorie du Soleil, 1757, p. 145, Hist. 118.-Addition à ce Mémoire, 1757 , p. 567 , Hist. 118.

Précis des Opuscules Mathénatiques, 1761, Hist. 86.

Précis du troisième volume des Opuscules Mathématiques, 1764, Hist. 92.-Nouvelles Recherches sur les Verres Optiques, pour servir de stite à la théorie qui en à été donnée dans le volume $3^{\mathrm{e}}$ des Opuscules Matlématiques. Premier Mlémoire, 1764, p. 75, Flist. 175. - Nouvelles Rccherches sur les Verres Optiques, pour servir de suite'i la théorie qui en a été donnée dans le troisième volume des Opuscules Mathématiques. Second Mlémoize, 1765 , p. 53,-Observations sur les Lunettes $\Lambda$ chromaliques, 1765, p. 53, Hist. 119.- Suite des Recherches sur les Verres Optiques. Troisième Mémoire, 1767, p. 43, Hist. 153.-Reclierches sur le Calcul Intégral, 1767, p. 573.Accident arrive par l'Explosion d'une Mcule d'Emonleur, $176 \mathrm{~s}$, Hist. 31.-P'récis des Opuscules de Mathematiques, $3 \mathrm{E}$ 
$4^{e}$ et $5^{e}$ volumes. Leur Analyse, 1768, Hist. 83.-Recherches sur les Mouvemeus de l'Axe d'une Planete quelconque dans l'hypothese de la Dissimilitude des Méridi(nes, $176 \mathrm{~s}$, p. 1, Hist. 95.-Suite des Recherches sur les Mouvemens, \&c, 1768, p. 332, Hist. 95.-Recherches sur 1. Calcul lntégral, 1769, p. 73.-ALémoire sur les Principes cie la Mech. 1769 , p. 278 . And in the Memoirs of the Academy of Berlin, are the following pieces, by our anthor :- viz, Recherches sur le Calcul Intégral, premiere partie, 1746. - Solution de quelques Problemes d'Astronomie, 1747.-Recherches sur la courbe que forme une Ccrde Tendue, mise en Vibration, 1747.- Suite des recherches sur le Calcul Intégral, 1748.-Lettre à $\mathrm{M}$. de Maupertuis, 1749.-Addition aux recherches sur la courbu que forme une Corde Tendue mise en Vibration, 1750.- - Addition aux recherches sur le Calcul Intégral, 1750.- Lettre à MI. le professeur Formey, 1755.-Extr. de differ. lettres à M. dé la Grange, 1763.-Sur les Tautochrones, 1765 . -Extr. de differ, lettres à $\mathrm{M}$. de la Grange, 1769. Also in the Memoirs of Turin are, Differentes Lettres à M. de la. Grange, en 1764 et 1765 , tom. 3 of these Memoirs.-Recherches sur differens sujets de Math. t. 4 .

DARCY (Count), an ingenious philosopher and mathenatician, was born in Ireland in 1725; but his friends being attuched to the Stuart family, he was sent to France, at 14 years of age, where he spent the rest of his life. Being put under the care of the celebrated Clairaut, he improved so rapidly in the mathematics, that at 17 years of age he gave a new solution of the problem concerning the curre of equal pressure in a resisting medium. This was followed the year after by a determination of the curve described by a heavy body, sliding by its own weight along a moveable plane, at the same time that the pressure of the body causes a horizontal motion in the plane. Darcy served in the war of 1744 , and was taken prisoner by the English : and yet, during the course of the war he gave two memoirs to the Acadenay the first of these contained a geucral principle in mechanics, that of the preservation of the rotatory motion; a principle which he again brought forward in 1750 , by the name of the principle of the preservation of action.

In 1760 , Darcy published An Essay on Artillery, containing some curious experiments on the charges of gunpowder, \&c, \&c, and improvements on those of the inyenious Robins; which kind of experiments our author carried on vccasionally to the end of his life.

In 1765 , he published his Memoir on the Duration of the Sensation of Sight, the most ingenious of his works: the result of these researches was, that a body may sometimes pass by our eyes without being scen, or marking its presence, otherwise than by weakening the brightness of the object it covers.

All Darcy's works bear the character which results from the union of genius and philosophy; but as he measured every thing on the largest scale, and required extreme accuracy in experiment, neither his time, fortune, nor avocations, allowed him to execute more than a very small part of what he projected. In his disposition, lię was amiable, spirited, lively, and a lover of independence, a passion to which he nobly sacrificed, even in the millst of literary socicty.-IIe died of a cholera morbus in 1779 , at 54 years of age.

Darcy was admitted a member of the French Acadeny in 1749 , and was made peusioner-geonctrician in 1770 .
- His essays, printed in the Memoirs of the Academy of Sciences, are various and very ingenious, and are contained in the volumes for the years $17+2,1747,1749$ s $1750,1751,1752,1753,1754,1758,1759,1760,1765$, and in tom. 1 of the Savans Etrangers.

DARK Chamber. See CAM BRA Obscura.

DARK Tint, a portable camera obscura, made somewhat like a desk, and fitted with optic glasses, to take prospects of landscapes, buildings, \& c.

DATA, in Mathematics, denote certain things or quantities, supposed to be given or known, from which other quantities are discovered that were unknown, or sought. A problem or question usually consists of two parts, Data and Quæsita, Given and Sought. Euclid has an express. and cxcellent treatise of Data; in which he uses the word for such spaces, lines, angles, \&c, as are given; or to which others can be found equal.

Euclid's Data is the first in order of the books that have been written by the ancient geometricians, to facilitate and promote the method of resolution or analysis. In general, a thing is said to be given, which is either actually exhibited, or that can be found, that is, which is either known by hypothesis, or that can be demonstrated to be known: and the propositions in the book of Enclid's Data show what things can be found or known, from those, that by bypothesis are already given: so that in the analysis or investigation of a problem, from those things that are laid down as given it is demonstrated, by the help of these propositions, that other things are given, and from these last that others are given, and so on, till it is demonstrated that that which was proposed to be found in the problem is given; and when this is done, the problem is solved; and its composition is made and derived from the compositions of the Data which were employed in the analysis. And thus the Data of Euclid are of the most general and necessary use in the solution of problems of every kind.

Mlarinus, at the end of his Preface to the Data, is mistaken in asserting that Euclid has not used the synthetical, but the analytical method in delivering them: for, though in the analysis of a theorem, the thing to be demonstrated is assumed in the analysis ; yet, in the demonstrations of the Data, the thing to be demonstrated, which is, that something is given, is never once assumed in the demonstration; from which it is manifest that every one of then is demonstrated synthetically : though indeed if a proposition of the Data be turned into a problem, the demonstration of the proposition becomes the analysis of the problem. See Simson's edition of Euclid's Data, which is estcemed the best.

DAVIS (CAPT. JoHN), a celebrated navigator, who flourished in the latter part of the 16 th century. IIe gave name to the straits which be discovered; and great expectations were formed from his skill and long experience. In 1594 he published a small treatise, entitlid, The Seaman's Secrets; a piece written with brevity, and was esteemed in its time, an 8th edition being printed in 1657 ; so that it scems to bave supplanted Cortes'. Davis treats of plane sailing, calling it llorizontal; and gives the form for keeping a sea-reckoning. He shows also how to sail by the globe, and boasts of what he intended to do ; much commending great circle sailing, without describing it ; as also what he calts Paradoxal, that is, by a projection on the plane of the equator with spiral rhumbs, promising to publish a chart for that purpose. But above all he extols 


\section{A Y}

[ 39

the use of calculations in the cases of uavigation, and states his intention to treat on that subject.

At the end of the book is given the figure of a staff of his contrivance, to make a back-observation, which it appears he was very vain of, and which seems to have been some time in use; but was afterwards supplanted by one of a inore commodious form, called Davis's Quadrant, as if it were also of his invention, though perhaps only because a back-observation was inade by both instruments, like as the quadrant itself was at first called Staff, and Back-staff.

DAvis's 2uadrant, the common sea quadrant, or backstaff. See Back-STafF. See also Robertson's Navigation, book 9 , sect. 7 .

DAY, a division of time arising from the appearance and disappearance of the sun.

$D_{A Y}$ is either natural or artificial.

Artificial $\mathrm{DAY}_{\mathrm{Y}}$ is that which is generally meant by the word Day, and is the time of its being light, or the time while the sun is above the horizon. Though sometimes the twilight is included in the term day-light; in opposition to night or darkness, being the time from the end of twilight to the beginning of day-light.

Natural $\mathrm{DA}_{\mathrm{A}} \mathrm{Y}$ is the portion of time in which the sun performs one, revolution round the earth; or rather the time in which the earth makes a rotation on its axis. And this is either astronomical or civil.

Astronomical $\mathrm{D}_{\mathrm{A}} \mathrm{Y}$ begins at noon, or when the sun's centre is on the meridian, and is counted 24 hours to the following noon.

Civil DAY is the time allotted for day in civil purposes, and begins differently in different nations, but still including ane whole rotation of the earth on its axis; beginning either at sun-rise, sun-set, noon, or miduight.

1st, At sun-rising, among the ancient Babylonians, Persians, Syrians, and most other eastern nations, with the present inhabitants of the Balearic islands, the Greeks, \&c. $2 \mathrm{dly}$, At sun-setting, among the ancient Athenians and Jews, with the Austrians, Bohemians, Narcomanni, Silesians, modern Italians, and Chinese. 3dly, At noon, with dstronomers, and the ancient Umbri anrl Arabians. And 4thly, at misluight, ansong the ancient Egyptians and Romans, with the modern English, French, Dutcb, Germans, Spaniards, and Portuguese.

The day is divided into hours; and a certain number of days makes a wcek, a month, or a year.

The different length of the natural day in difierent climates, has been matter of controversy, viz, whether the natural days be all equally long throughout the year; and if not, what their difficrence is? A professor of mathematícs at Seville, in the Philos. Trans. vol. 10, pa. 425, asserts, from a continued series of observations for three years, that they are all equal. But Mr. Flamsteed, in the 6ame Trans. pa. 429 , refutes the opinion; and shows that a day, when the surn is in the equinoctial, is shorter than when he is in the tropies, by 4.0 seconds; and that 14 tropical days are longer than so many equinoctial ones, by 10 minutes. This inequality ol" the days depends upon two different principles: the one, the excentricity of the earth's orbit; the other, the obliquity of the ecliptic with regard to the equitor, which is the measure of time. As these two cause's happen to be differently combined, the length of the day is varied. Sue EQu ATion of time.

I A.Y Sidereal, is the time in which any star appears to revolve from tho meridiau, to the meridian again, which is $23^{\mathrm{h}} 56^{\prime} 4^{\prime \prime} \mathrm{T}^{-}$of mean solar time; being the time in which the earth makes one complete revolution on its axis. See Si DeR EA L.

DAY's-Work, in Navigation, denotes the reckoning or account of the ship's course, during 24 hours, or between noon and noon.

DEAD BEAT, a peculiar kind of scapement for clock and watch work, invented by Mr. Mudge for correcting the motion of pendulums \&c. See Sca PEn ENT.

Dead Reckoning, in Navigation, the judgment or estimation which is made of the place where a ship is, without any observation of the heavenly bocties; being generally performed by keeping an uccount of her way by the $\log$, in knowing the course she has been steered by the compass, and by combining and rectifying all the allowances for drift, lee-ssay, \&c, according to the known trim of the ship. This reckoning however is al ways to be corrected, as often as any good observation can be obtained.

DECAGON, a plane gcometrical figure of ten sides and ten angles. Whell all the sides and angles are equal, it is a regular decagon, and may be inscribed in a circle; otherwise, not.

If the radius of a circle, or the side of the inscribed liexagon, be dividerl in extreme and meall ratio, the greater segment will be the side of a decagon inscribed in the same circle. And therefore, as the side of the decagon is to the radius, so is the radius to the sum of the two. Whence, if the radius of the circle be $r$, the side of the inscribed decagon will be $(\sqrt{ } 5-1) \times \frac{1}{2} r$.

If the side of a regular decagon be 1 , its area will be $\frac{5}{2} \sqrt{ }(5+2 \sqrt{ } 5)=7 \cdot 6942088$; therelore as 1 is to $7 \cdot 69+2088$, so is the square of the side of any regular decagon, to the area of the same: consequently, if $s$ be the side of such a decagon, its area will be equal to $7 \cdot 69420 s 8 s^{2}$. See Regular Figure.

To inscribe a decagon in a circle geometrically. See my Mensuration, prob. 35, pa. 20, 4th edit.

DECEMBER, the last month of the year; in which the sun enters the tropic of Capricorn, making the winter solstice. In the time of Romulus, December was the loth month; whence the name, viz, from decem, ten; for the Ronans began their year ia March, from which December is the 10th month. The month of December was under the protection of Vesta. Romulus assigned it 30 days; Numa reduced it to 29; which Julius Cæsar increased to 31. At the latter part of this month they had the Juveniles Ludi, and the country-people kept the feast of the goddess Vacuna in the fields, having then gathered in thcir fruits, and sown their corn; whence it seems is derived our popular festival called Harvest-home.

DECHaLES (Claud-Francis-Milliet), an excellent mathematician, mechanist, and astronomer, was born at Chambery, the capital of Savoy, in 1611. He chiefly excelled in a just knowledge of the mathematical and mechanical sciences: not that he was bent upon new discoveries, or happy in making them; as his talent rather lay in explaining those sciences with ease and accuracy; which perhaps rendered him equally uscful and deserving of esteem. Indeed it was generally allowed that he made the best use of the productions of other men, and that he drew the several parts of the mathematical sciences together with great judgment and perspicuity. His probity wasnot inferior to his learning; and these two qualitics made him generally admired and belored at l'aris, where $3 \mathrm{E} 2$ 

D E C
[ 396 ]
D E C

for four years together he read public mathematicạl lectures in the college of Clermont.-From hence he removed to Marseilles, where he taught the art of navigation and the practical' mathematical sciences.- - He afterwards became professor of nathematics in the university of Turin, where he died March 28, 1678 , at 67 years of age.

Among other works which do honour to his memory, are, 1. An edition of Euclid's Elements; in which he has omitted the less important propositions, and explained the uses of those he has retained.-2. A Discourse on Fortification; and another on Navigation.-3. These performances, with some others, were collected in 3 volumes folio, under the titie of Mundus Mathematicus, being indeed a complete course of mathematics. And the sanie was afterward much enlarged, and published at Lyons, 1690 , in 4 large volumes, folio.

DECINALS, any thing proceeding by tens; as Decinal arithmetic, Decimal fractions, Decimal scales, \&c.

Decrinat Arithmetic, in a general sense, may be considered as the common arithinctical computation in use, in which the decimal scale of numbers is used, or in which the places of the figures change their value in a tenfold proportion, being 10 times as much for every place more towards the left hand, or 10 times less for cvery place removed towards the right hand; the places being supposed indefinitely continued, both to the right and left. In this sense, the word includes both the arithmetic of integers, and decimal fractions. In a more restrained sense, however, it means only

Decimal Fractions, which are fractions whose denominator is always a 1 with some number of ciphers annexed, on the number of which depend the value of the fraction, but the numerator may be any number whatever; as $\frac{1}{10}, \frac{3}{108}, \frac{7}{1080}, \& c$.

As the denominator of a decimal is always one of the numbers $10,100,1000$, \&c , the inconvenience of writing these denominators down may be avoided, by placing a proper distinction before the figures of the numerator only, to distinguish them from integers, for the value of each place of figures will be known in decimals, as well as in integers, by their distance from the 1st or unit's place of integers, having similar names at equal distances, as appears by the following scale of places, both in decimals and integers :

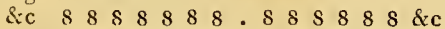

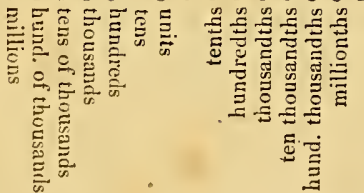

The mark of clistinction for decimals, called the separatrix, has been various at difterent times, according to the fancy of different althors; sometimes a semiparenthesis, or a semicrotchet, or a perpendicular bar, or the same with a line drawn under the figure's, or simply this line itself, Sc ; but it is usual now to write either a comma or a full point near the bottom of the figures; I place the point sear the upper part of the figures, in which $I$ follow the practice of Sir l. Newton; a method which prevents the sparatrix fiom being confounded with mere marks of punctuition, and which method is now used by the most correct inathethaticians.
In setting down a decimal fraction without its denominator, the numerator must consist of as many places as there are ciphers in the denominator; and if it hats not so many figures, the defect must be supplied by prefixing ciphers: thus $\frac{3}{16}$ is $\cdot 3$; and $\frac{1}{10}{ }^{4}$ is $\cdot 14$; and $\frac{14}{1000}$ is $\cdot 014$; and ${ }_{\text {To }}^{3}$ is ${ }^{\circ} 003$; \& $c$.

As ciphers on the right-hand side of integers increase their value decimally, or in a tenfold proportion, as 2,20 , 200 , \&c; so, when set on the left-haud of decimal fractions, they decrease the value decimally, or in a tenfold proportion, as $\cdot 2, \cdot 02, \cdot 002$, \&.c. But ciphers set on the other sides of these numbers, make no alteration in their value, neither of increase nor decrease, viz, on the lifthand of integers, or on the right-hand of decimals; so 2 , or 02 , or $002, \& \mathrm{c}$, are all the same; as are also $\cdot 2$, or $\cdot 20$, or $\cdot 200$, sic.

Decimal fractions may be considered as having been introduced by Regiomontanus, about the year $1+64$, viz, when he transformed the tables of sines from a sexagesimal to a decimal scale. They were also used by Ramus, in his Arithmetic, written in 1550; and befure his time by our countrymen Buckley and Recorde. But it was Stevinus, who first wrote an express treatise on decimals, viz, about the year 15\$2, in La Practique d'Arithmetique; since which time, this has conmonly made a part in most treatises on arithmetic.

To reduce any Vulgar fraction, or parts of any thing, as suppose $\frac{3}{8}$, to a decimal traction of the same value; add ciphers at pleasure to the numerator, and divide by the denominator: thus,

$$
\text { S) } \begin{aligned}
& 3.000 \\
& .375=\frac{3}{8}
\end{aligned}
$$

and therefore 375 or $\frac{37}{1000}$ is a decimal of the same value with the proposed vulgar fraction $\frac{3}{8}$.

Some vulgar fractions can never be reduced into decimals without defect; as $\frac{x}{3}$, which by division is $\cdot 33333 \mathrm{\&}-\mathrm{c}$ infinitely. Such numbers are very properly called circulating decimals, and repetends, because of the continual return of the same figures. Sec Repetends and $\mathrm{C}_{1 \mathrm{neu}}$ LATES.

The common arithmeticul operations are performed the same way in slecimals, as they are in integers; regard heing had only to the particular notation, to distinguish the fractional from the integral part of a sum. Thus, in Addition and Subtraction of decimals, all figures of the same place or denomination are set directly uneler each other, the separatrix, or decimal points, forming a straight column. The operation of addition, or subtraction, is then performed the same as in integers. In Multiplication of decimals set down the numbers, and multiply them as in integers; aisd point off from the product as many places for decimals as there are decimals in both factors; prefixing ciphers if necessary. In Division, set down the numbers and divide also as in integers; making as many decimals in the quotient, as those in the dividend excced those in the divisor. Examples are numerous and common in most books of arithmetic.

Decuand Scales, are any seales divided decimally, or by tens.

DECLINA'ION, in Astronony, is the distance of the sun, star, planet, \&c, from the equinoctial, cither northward or southward; being the same with latitude in geograpliy, or distance from the equator.

Declination is rither real or appareut, according as the real or apparent place of the point or object is cunsidered. 
The declination of any point $s$ is an arch of the meridian SE, contained between the given point and the equinoctial EQ. The declination of a star $\& c$, is found by knowing or observing the latitude of the place, i. e. the height of the pole, and then the meridian altitode of the star, \&c; hence the difference between the co-latitude and the altitude of the star \&c, is the declination, viz, the difference betwcen En the co-latitude, and st the altitude, is $x \mathrm{~s}$ the

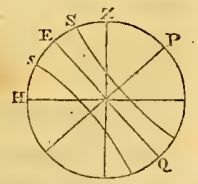
declination. For example, 'Tycho found at Uranibourg the meridian altitude of Cauda Leonis, viz, $n \mathrm{~s}=50^{\circ} 59^{\circ} 00^{\prime \prime}$ the co-latitude is - - - TIE $=34 \quad 545$ remains the declin. north - - ES $=16 \quad 53 \quad 15$

To find the Sun's Declination at any time; having given his place in the ecliptic; the rule is, as radius is to the sine of the sun's longitude, so is the sine of the greatest declination, or obliquity of the ecliptic, to his present declination sought.

In constructing tables of declination of the sun, planets, and stars, regard should be had to refraction, abcrration, ustation, and parallax.

By"comparing ancient observations with the modern, it appears that the declination of the fixed stars is variable; and that differently in different stars; for in some it increases, and in others decreases, and that in different quantities.

Circles of DeCLiNation, are great circles of the sphere passing through the poles of the world, on which the declination is measured; and consequently are the same as meridians in geography.

Parallax of DeCLination, is an arch of the circle of dcclination, by which the parallax in altitude increases or diminishes the declination of a star.

Purallels of DecLination, are lesser circles parallel to the equinoctial. The tropic of Cancer is a parallel of declination at $23^{\circ} 28^{\prime}$ distance from the equinoctial northward; and the tropic of Capricorn is the parallel of declination as far distant southward.

Refraction of the DECLINATION, an arch of the circle of declination, by which the declination of a star is increased or diminished by means of the refraction.

Declination of the Compass, or Needle, is its deviation from the true meridian. See Variation.

Declination of a Vertical Plane, or Wall, in Dialling, is an arcls of the horizon, comprehended either between the plane and the prime vertical, when it is counted from the cast or west; or between the plane and the meridian, if it be reckoned from the north or south.

DECLINATOR, or DECLINATORY, an instrument in dialiing, by which the declination, inclination, and reclination of pianc's are determined.

DECLINERS, or DECLINIEg Dials, are those which cut obliquely, either the plane of the prime vertical, or the plane of the horizon.

The use of declining vertical dials is very frequent; because the erect walls of houses, on which ditals are commonly dratun, mostly decline from the cardinal points. But incliners and recliners are very rare.

DLCLIVIT'Y, a sloping or oblique descent.

D]CILALNT, fiqual, of lifc. Sce Conplement of $L$ ife.

DHCRFMENTS are the small parts by which a variable and decreasing quantity becomes less and less. I'he indefinitely small decrements are proportional to the Huxions, which in this case are negative. Sce Fuxions: also $I_{N-}$ CREMENTS.

DECUPLE, a term of relation or proportion in arithmetic, implying a tenfold change or scale of variation, or one thing 10 times as much as another.

DECUSSATION, a term in Geometry and Optics, signifying the crossing of any two lines or rays \& $\mathrm{c}$ : or the action itself of crossing.-The rays of light decussate in the crystalline, before they reach the retina.- Many of the lines of the 3d order decussate themselves. See Newton's Enimeratio, \&c.

DEE (Jurr), a famous mathematician and astrologer, was born at London 1527. In 1542 he was sent to St. John's-college, Cambridge. After five years close application to study, chiefly in the mathematical and astronomical sciences, he went over to Holland, to visit some mathematicians on the Continent; whence, after a year's absence, he returned to Cambridge, and was there elected one of the Fellows of Trinity-college, then first erected by King Henry the 8 th. In 1548 he left England a second time, his stay at home being rendered uneasy to him, by the suspicions that were cntertained of his being a conjurer, arising chiefly from his application to astronomy, and from some of his mechanical inventions.

He now visited the university of Louvain ; where he was much caressed, and visited by several persons of high rank. After two years he went into France, and read lectures, in the college of Rheims, on Euclid's Elements. In 1551 , he returned to England, and was introduced to King Edward, who assigned him a pension of 100 crowns, which he afterward relinquished tor the rectory of $U_{p}$ tonon-Severn. But soon after the accession of Queen Mary, holding some correspondence witb ber sister likizateth, he was accused of practising against the queen's life by enchantment : on which account he suffered a tedious confinement, and was several times examined; till, in the ycar 1555 , he obtained lis liberty by an order of council.

When Queen Elizabeth ascended the throne, Dee was consulted concerning a propitious day for the coronation: on which occasion he was introduced to the queen, who made him great promises, which werc but ill performed. In 1564 , he made another voyage to the continent, to present a bouk which he had dedicated to the Emperor Mlaximilian. He returned to England the same ycar; but in 1571 we fund him in Lorrain; where, being dangerously ill, the queen sent over two physicians to his relief. IIaving once more returned to his native country, he settled at Mortlake in Surrey, where he continued his studies with much ardour, and collected a great library of printed books and masuscripts, with a number of instruments; most of which were alterwards destroyed by the mob, as belonging to one who dealt with the devil.

In 1578 , the queen being inuch indisposed, Mr. Dee was sent abroad to consult with German physicians and philosophers (astrologers no doubt) on the occasion; though some have said she employed him as a spy; probably be acted in a double capacity. We next find him again in England, where he was soon after employed in a more rational service. The queen, desirous to be informed concerning ber titic to those countries which had been discovered by Englishmen, ordered Dee to cousult the attcient records, and to furnish her with proper geographical descriptions. Accordingly, in a short time, he presented to the quem at lichmond, two large rolls, in which the discovered countries were geographically described aus 
historically illustrated. His next employment was the reformation of the calendar, on which subject be wrote a rational and learned treatise, preserved in the Ashmolean library at Oxtord.

Hitherto the extravagancies of bur eccentrical philosopher seem to have been tempered with a tolerable proportión of reason and science; but henceforward he is to be considered as a mere necromancer and credulous alchymist. In the year 1581 lie became acquainted with one Edward Kelly, by whose assistance he performed dip̣ers incantations, and maintained a frequent imaginary intercourse with spirits and angels; one of whom made him a present of a black speculum (a polished piece of cannelcoal), in which these appeared to him as often as he had occasion for them, answering his questions, \&c. Hence Butler says,

\section{"Kelly did all his feats upon}

The devil's looking-glass, a stone."

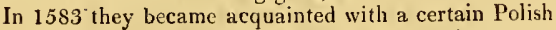
nobleman, then in England, named Albert Laski, a person equally addicted to the same ridiculous pursuits: he was so charmed with Dee and Kelly, that he persuaded them to accompany him to his native country; by whose means they were introduced to Rodolph king of Bohemia; who, though a credulous man, was soon disgusted with their nonsense. They were afterwards introduced to the king of Poland, but with no better success. Soon after this they were entertained at the castle of a rich Bohemian nobleman, where they lived for some time in grcat affluence; owving, as they asserted, to their art of transmutation by means of a certain powder in thepossession of Kelly.

Dee, now quarrelling with his companion, quitted Bohemia, and returned to England, where he was once more graciously received by the queen; who, in 1595 , made him warden of Manchester college, in which town he resided several years. In 1604, he returned to his house at Mortlake, whice lie died in 1608 , at 81 years of age; leaving a large family and many works behind him.

T'he bocks that were printed and published by Dee, are, 1.,Propoelumata Aphoristica, \&c. in 1558, in 12mo.2. Monas Hieroglyphica ad Regem Romanorum Maximilianum: 1564.-3. Épistola ad eximium ducis Urbini mathematicum, Fredericum Ciymmandinum, prefixa libelJo Machometi Bagdadini de superticierum Divisionibus \&c; 1570.-4. 'The British Monarchy, otherwise called, The Petty Nary Royal; 1576.-5. Preface Mathematical to the English Euclid, published by Henry Billingsley, 1570: certainly a very curious and elaborate composition, and where he says, many more arts are wholly invented by name, definition, property, and use, than either the Grecian or Roman mathematicians have left to our knowledge. -6 . Divers and many annotations and inventions dispersed and added after the 10th book of English Euclid; $1570 .-7$. Epistola prefixa Ephemeridibus Joannis Feldi a 1557, cuirationem declaraverat Fplemerides conseribendi.-3. ParallaticaCommentationis Paxeosque $\mathrm{Nu}$ cleus guidam ; 1573.

This catalogue of Dee's printed and published works is to be found in his Compendious Rehearsal \&c, as well as in his letter to Abp. Whitgift: and from the same places might be transcribed more than 4.0 titles of books unpublished, which be had written.

DEF liNCi, in Sieges, is used for any thing that serves to preserve or sereen the soldiers, or the place. So the perapels, tlanks, cuscmates, ravelins, and out-works, that cover the place, are called the defences, or covers of the place : and when the cannon have beaten down or ruined these works, so that the men cannot fight under cover, the defences of the place are said to be demolished.

Line of DEFENCE, is that which flanks a bastion, being drawn from the flank opposite to it. The line of defence should not exceed a musket shot, i. e. 120 fathoms : indeed Mclder allows 130, Scheiter 140, Vauban and Pagan 150 .

Line of DEFLNCE, greater; or fichant, is a line drawn frum the point of the bastion to the concourse of the opposite flank and curtin.

Line of DeFEnce, lesser, or rasant, or flanquant, is the face of the bastion continued to the curtin.

DEFERENT, or DEFERENs, in the Ancient Astronomy, an inaginary eircle, which, as it were, carries about the body of a planet, and is the same with the excentric; being invented to account for the excentricity, perigee, and apogee of the planets.

DEFICIENT Hyperbola, is a curve baving only one asymptote, though two hyperbolic legs runniug out infinitely by the side of the asymptote, but contrary ways. See CuRVE.

This name was given to the curves by Newton, in Lis Enumeratio Linearum certii Ordinis. There are 6 different species of them, wbich have no diameters, expressed by the cquation $x y+c y=-a x^{3}+b x^{2}+c x+d$, the term $a x^{3}$ being negative. When the equation $a x^{4}=b x^{3}+c x^{2}$ $+d x+\frac{5}{4} e e$ has all its roots real and unequal, the curve has an oval joined to it. When the two middle roots are equal, the oval joins to the legs, which then cut one another in shape of a noose. When three roots are equal, the nodus is changed into a very acute cusp or point. When, of three roots with the same sign, the two greatest are equal, the oval vanishes into a point. When any two roots are imaginary, there is only a pure serpentine hyperbola, without any oval, decussation, cusp, or conjugate point; and when the terms $b$ and $d$ are wanting, it is of the 6 th species.

There are also 7 diflerent species of these curves, having each one diameter, expressed by the above equation when the term $e y$ is wanting : according to the various conditions of the roots of the equation $a x^{3}=b x^{2}+c x+d$, as to their reality, equality, their having the same signs, or two of them being imaginary.

Deficient Numbers, are those whose aliquot parts added together, make a sum less than the whole number: as 8 , whose parts $1,2,4$, make only 7 ; or the number 16 , whose parts $1,2,4,8$ make only 15 .

DEFILE, in Fortification, a narrow line or pussage through which troops can pass only in file, making a smail front, so that the enemy nay easily stop their masch, and charge them with the more advantage, as the front and rear cannot come tu the relief of one another.

DEFINITION, an enumeration, or specitication of the chief simple ideas of which a compound idea consists, in order to ascertain or explain its nature and character. Definitions are of two kinds; the one nominal, or of the name; the other real, or of the thing.

Nominal DEFIRITION, is an enumcration of such known characters as are sufficient for distinguishing any proposed thing trom otbers; as is that of a square, when it is snid that it is a quadrilateral, equilateral, rectangular figure.

Real Derinitros, al distinct notion, explaining the genesis of a thing: that is, how the thing is made or clone: 
as is this definition of a circle, viz, that it is a figure described by the motion of a right line about a fixed point.

DEFLECTION, the turning any thing aside from its former course, by some adventitious or external cause.The word is often applied to the tendency of a ship from her true course, by reason of currents, \&c, which turn her out of her right way:

DEFLECTION of the Rays of Light, is a property which Dr. Hooke observed in 1675 . He found it different both from reflection and refraction; and that it was made perpendicularly towards the surface of the opacous body. 'I'his is the same property which Newton calls inflection; and by others it is called diffraction.

DEGREE, in Algebra, is used in speaking of equations, when they are said to be of such a degree, according to the lighest power of the unknown quantity. If the index of that power le 2 , the equation is of the $2 \mathrm{~d}$ degree; if $\mathrm{a}$, it is of the $3 \mathrm{~d}$ degree, and so on.

DEGREE, in Geometry or Trigonometry, is the 360 th part of the circumference of any circle; for every circle is considered as divided into 360 parts, called degrees; which are marked by a small ${ }^{\circ}$ near the top of the figure; thus $45^{\circ}$ is 45 degrees. The degree is subdivided into 60 smaller parts, called minutes, meaning first minutes; the minute into 60 others called seconds; the second into 60 thirds; \&c. Thus $45^{\circ} 12^{\prime} 20^{\prime \prime}$ must be rearl 45 degrees, 12 minutes, 20 seconds.

The magnitude or quantity of angles is reckoned in degrees; for because of the uniform curvature of a circle in all its parts, cqual angles at the centre are subtended by equal ares, and by similar ares in peripheries of different diameters; and an angle is said to be of so many degrees, as are contained in the arc of any circle comprehended between the legs of the angle, and having the angular point for its centre. Thus we say an angle of $90^{\circ}$, or of $45^{\circ} 24^{\prime}$, or of $12^{\circ} 20^{\prime} 30^{\prime \prime}$. It is also usual to say, such at star is so many degrees above the horizon, or declines so many degrees from the equator; or such a town is situate in so many degrees of latitude or longitude.-A sign of the ecliptic, or zodiac, contains 30 degrees.

The division of the circle into 360 degrees is usually ascribed to the Egyptians, probably from the circle of the sun's annual course, or according to their number of days in the year, allotting a degree to each day. It is a convenient number too, as admitting of a great many aliquot parts, as $2,3,4,5,6,8,9$, \&c. The sexagesimal subdivision, however, has often been condemned as improper, by many eminent mathematicians, as Stevinus, Oughtred, Wallis, Briggs, Gellibrand, Newton, \&c; who arvise a decimal division instead of it, or else that of centesms; as the degrec into 100 parts, and each of these into 100 parts again, and so on. Stevinus even holds, that this division of the circle which he contends for, obtained in the wise age, in sæculn sapienti. Stev. Cosmog. lib. 1, def. 6. And several large tables of sines \&c bave been constructed according to that plan, and published, by Briggs, Newton, and others. And I inyself have carried the idea still much farther,' in a memoir published in the Philos. 'T'rans. of 1783 , containing a proposal for a new division of the quadrant, viz, into equal decimal parts of the radins; by which means the degrees or divisions of the arch would be the real lengths of the arcs, in terms of the radius: and $\mathbf{I}$ have since computed those lengths of the arcs, with their sines, \&c, to a great extent and accuracy. Sec my new Tracts, vol. 2, pa. 122. The French mathematicians have lately calculated their new Trigonometrical Tables on an entire decimal division of arcs; viz, first dividing the quadrant into 100 degrees, and these intu decimals of degrees. But it appears by Mr. Crabtree's letter to Mr. Gascoinue (inventor of the micrometer) Aug. 7, 1640, that both these gentlemen, as well as thcir mutual friend Mr. Horrox, had projected those tables by a complete decimal division, the whole arch being divided into $100,000,000$ parts. See Philos. 'Trans. vol. 27, pa. 230; or iny Abridg. vol. 5 , pa. 631 .

DEGRE of Latitude, is the space or distance on the meridian through which an observer must move, to vary lis latitude by one degree, or to increase or diminish the distance of a star from the zenith by one degree; and whicb, on the supposition of the perfect sphericity of the earth, is the 360th part of the meridian.

The quantity of a degree of a meridian, or other great circle, on the surface of the earth, is variously determined by different observers; and the methods made use of are also variozis.

Eratosthenes, 250 years before Christ, first determined the measure of a degree of the meridian, between Alexandria and Syene on the borders of Ethiopia, by measuring the distance between those places, and comparing it with the difference of a star's zenith distances in the same places; and found it to be $694 \frac{4}{9}$ stadia.

Posidonius, in the time of Pompey the Great, by means of the different altitudes of a star near the horizon, taken at different places under the same meridium, compared in like manner with the distance between those places, determined the length of a degree to be only 600 stadia.

Ptolemy fixes the degree at $6 \mathrm{~S}_{\frac{2}{3}} \mathrm{Ar}$ rabic milcs, counting $7 \frac{I}{2}$ stadia to a mile. The Arabs themselves, who made a computation of the diameter of the earth, by measuring the distance of two places under the same meridian, in the plains of Sennar, by order of Almamon, make it only 56 miles. Kepler determined the dianıter of the earth by the distance of two mountains, and makes a degree 13 Gorman miles; but his method is far from being accurate. Snell, by seeking the diamcter of the earth from the distance between two parallels of the equator, finds the quantity of a degree,

by one method 57064. Puris toises, or 342384 feet; by another meth. 57057 - toises, or 34.2342 fiet.

The mean between which two numbers, M. Picard found by mensuration, in 1669 , from Aniens to Malvoisin, to be the most accurate, and he makes the quantity of a degree 57060 toises, or 342360 fect. However, M. Cassini, at the king's command, in the year 1700 , repeated the same labour, and measuring the space of ' $6{ }^{\circ} 18^{\prime}$, from the observatory at Paris, along the mericlian, to the city of Collioure in Roussillon, that the greatness of the interval might diminish the error, found the length of the degree equal to 57292 toises, or 343742 Paris feet, amounting to 365184. English feet.

And this account nearly agrees with that of our countryman Norwood, who, about the year 1635 , measured the distance between London and York, and found that distance to be 905751 English feet; the difference of latitude being $202 s^{\prime}$; hence, he determined the quantity of one degree at 3671.96 English fect, or 57300 Paris toises, or 69 miles, 288 yards. Sec Newt. Princ. Phil. prop. 19; and Hist. Acad. Scienc. anno 1700, pa. 153.

M. Cassini, the son, completed the work of measuring the whole arc of the meridian through France, in 1718 ; 
to perform which, he divided the meridian of France into two arcs, which he measured separately.

The one from Paris to Collioure gave him 57097 toises; the other from Paris to Dunkirk - _ '56960; and the whole arc from Dunkirk to Collioure 57060 , the same as Picard's.

M. Muschenbrock, in 1700, resolved to correct the errors of Snell, and found by particular observations, that the degree between Alcmaer and Bergen-op-zoom contained 57033 toises.

Messieurs Maupertuis, Clairant, Camus, Monnier, and Outhier of France, were sent on a northern expedition, and began their operations, assisted by M. Celsus, an eminent astronomer of Sweden, in .Swedish Lapland, in July 1736 , and finished them by the end of May following. They obtained the measure of that degree, whose middle point was in lat. $66^{\circ} 20^{\prime}$ north, and found it 57439 toises, when reduced to the level of the sea. About the same time another company of philosophers was sent to South America, viz, Messicurs Godin, Bonguer, and Condamine of France, with whom were Dou Jorge Juan, and Don Antonio de Ulloa of Spain. They left Europe in 1735 , and began their operatious in the province of Quito in Peru, abont October 1736, and frished them, after many interruptions, about 8 years after. The Spanish gentlemen published a separate account, and assigned for the measure of a degree of the meridian at the cquator 56768 toises. M. Bouguer makes it 56753 toises, when reduced to the level of the sea; and M. Condamine states it at 56749 toises.

M. Lacaille, being at the Cape of Good Hope in 1752, found the length of a degree of the meridian in lat. $33^{\prime \prime} 18^{\prime} 30^{\prime \prime}$ south, to be 57037 toises; but in 1755 , father Boscovich found the length of a degrce in lat. $43^{\circ}$ north to be 56972 toises, as measured between Rome and Rimini in Italy. In the year 1740, Messrs. Cassini and Lacaille again examined the former measures in lrance, and, after making all the necessary corrections, found the measure of a degree, whose niddle point is in lat. $49^{\circ} 22^{\prime}$ north, to be 57074 toises; and in the lat. of $45^{\circ}$, it was 57050 toises.

In $1764, \mathrm{~F}$. Beccaria completed the measurement of a portion of the meridian near Turin; from which it is deduced that the length of a degree, whose middle lat. is $44^{\circ} 44^{\prime}$ north, is 57024 Paris toises.

At Vienna, 3 degrees of the meridian werc measured; and the'medium, for the latitude of $47^{\circ} 40^{\prime}$ nortl may be taken at 57091 Paris toises. See an account of this measurement, by father Joseph Liesganig, in the Philos. Trans. 1768 , pa. 15 .

In the same vol.there is also an account of the measurement of a part of the mericlian in Maryland and Pcnsylvania, North America, 1766, by Messis. Mason aud Dixon; from which it appears that the length of a degree whose middle point is $39^{\circ} 12^{\prime}$ north, is 363763 English feet, or $56904 \frac{\pi}{2}$ Paris toises.

From the trigonometrical survey of England and Wales, completed in 1802 , under the direction of Licut.-col. Mudge, four degrees of the meridian were mcisured; the mean latitude of which was $51^{\circ} 29^{\prime} 54 \frac{x^{\prime \prime}}{2}$, and the mean l'ngth of a degree $69 \cdot 1457$ English miles.

The remeasurement of a degrec in Lapland, as a correction of the former French operations, was performed during the years 1801,1802 , and 1803 , by the Swedish mathematicians, Ofverboom, Swanberg, IIolinquist, and
Polander; and from the account of their operations, published by $\mathrm{M}$. Swanberg, the length of the degree in lat. $66^{\circ} 20^{\prime} 10^{\prime \prime}$, is $69^{\circ} 2689$ English miles. From a comparison of this result, with those from the measurements taken in Peru, the East Indies, and France, M. Swanberg deduces a mean of $\frac{\pi}{3}{ }_{3}$ for the ellipticity, and $3 y 63.26$ miles for the equatorial radius of the earth.

In 1803 Colonel Lambton also measured a degree in the East Indies, north lat. $12^{\circ} 32^{\prime}$, which he made $68 \cdot 7445$ miles. But the latcst measurement of this nature is that of the meridian, comprised between Barcelona in Spain and the Belearic isles. This meridian had before been measured by Messrs. Mechain and Delambre, from Dunkirk to Barcclona, and the two French mathematicians Biot and Arago, with the Spanish commissioners Chaix and Roderiguez, were appointed to continue the line to the small island of Formentera. The lat. of this island was found to be $38^{\circ} 40^{\prime}$, that of Dunkirk $51^{\circ} 5^{\prime}$; and the distance between the two places 1374438.73 metres: hence a degree is equal to $68 \cdot 769$ English miles; and its middle latitu'de $44^{0} 052 \frac{I}{2}^{\prime}$.

The following table, collected from the precerling accounts, shows at one view the several lengths of a degrce, as measured in various parts of the earth, with the line of its measurement, the courtry in which it was measured, the names of the measurers, and the lat. of the mirldle point :

\begin{tabular}{|c|c|c|c|c|c|}
\hline Date. & Lat & tude. & $\begin{array}{l}\text { Length, } \\
\text { miles. }\end{array}$ & Mleasurers. & Countries. \\
\hline 1525 & $49^{\circ}$ & $20^{\prime} \mathrm{N}$. & $68 \cdot 763$ & M. Furnel & France. \\
\hline 1620 & 52 & $4 \mathrm{~N}$. & $66 \cdot 91$ & Snell & Holland \\
\hline 1685 & 53 & $15 \mathrm{~N}$. & $69 \cdot 545$ & Norwood & England \\
\hline 1644 & & & 75.066 & Riccioli & Italy , \\
\hline 1669 & 49 & $22 \mathrm{~N}$. & $68 \cdot 9 \pm 5$ & Picard & France \\
\hline 1718 & 49 & $22 \mathrm{~N}$. & $69 \cdot 119$ & Cass̄ini & France \\
\hline 1737 & 66 & $20 \mathrm{~N}$. & $69 \cdot 403$ & Maupertuis & Lapland \\
\hline 1740 & 49 & $22 \mathrm{~N}$. & $69 \cdot 121$ & Cassini \& & France. \\
\hline 1740 & 45 & ON. & $69^{\circ} 092$ & $\int$ Lacaille & France \\
\hline 1744 & 0 & 0 & $68 \cdot 751$ & Juan \&c. & Peru \\
\hline 1744 & 0 & 0 & $68 \cdot 732$ & Bouguer & Peru \\
\hline 1744 & 0 & 0 & $68 \cdot 713$ & Condamine & Peru \\
\hline 1752 & 33 & $18 \mathrm{~s}$. & 69.076 & Lacaille & Cape of G.H. \\
\hline 1755 & 43 & ON. & 68.998 & Boscovich & Italy \\
\hline 1764 & 44 & $44 \mathrm{~N}$. & $69 \cdot 061$ & Beccaria & Italy \\
\hline 1766 & 47 & $40 \mathrm{~N}$ & $69 \cdot 142$ & Leisgauig & Germany \\
\hline $176 \mathrm{~s}$ & 39 & $12 \mathrm{~N}$ & 68.893 & Mason \& D. & America \\
\hline 1802 & 51 & $30 \mathrm{~N}$. & $69 \cdot 146$ & Mudge & England \\
\hline 1803 & 66 & $20 \mathrm{~N}$. & $69 \cdot 292$ & Swanberg & Lapland \\
\hline 1803 & 12 & $32 \mathrm{~N}$. & 68.743 & Lambton & India \\
\hline 1808 & 44 & $52 \mathrm{~N}$. & $68 \cdot 769$ & Biot and $\mathrm{Ar}$. & France \\
\hline
\end{tabular}

The difference between Swanberg's and Maupertuis' result, taken in the same place, was owing it secms to the latter having omisted to allow for the difierence of level in the measurement base, above the sea.-On the whole, it seems the length of the iniddle degree, or $4.5^{\circ}$ of lat. may be accounted $69 y_{T}^{\prime}$ miles.

The inctbod of obtaining the length of a degree of the terrestrial meridian, is to measure a certain distance upon it by a series of triangles, whose angles may be found by observation, connected with a base, the length of which may be taken by an actual survey, or otherwise; and to observe the different altitudes of some star at the two extremities of that distance, which gives the difference of latitude between them: then, by proportion, as this diflerence of latitude is to one degree, so is the measured length 
to the length of one degree of the meridian sought; which method was first practised by Eratostlienes, in Egypt. See Earth, Geography, and the beginning of this article; also Dr. T. Young's Philos. vol. 2, for a list of writings on this subject.

It appears that the meridional degrees of an ellipsnid increase from the equator to the pole very nearly as the square of the sine of the latitude. And the length of the degree at any point, is to the length at the equator, accurately as the cube of a line drawn parallel to the plumb line, from a point in the axis equidistant from the centre with the equator, and terminating in a point of the plane of the equator, to the cube of the line drawn from this point to the true pole. Or, if $e$ be the ellipticity, aind $s$ the sine of the latitude, the length of the degree will vary as $(1+(2 e+e e) x x)^{\frac{3}{2}}$.

DEGREE of Longitude, is the space between two meridians that make an angle of $1^{\circ}$ with each other at the poles; the quantity or length of which is variable, according to the latitude, being every where as the cosine of the latitude; viz, as the cosine of one lat. is to the cósine of another, so is the length of a degree in the former lat. to that in the latter; and from this theorem is computed the following Table of the length of a degree of long. in different latitudes, supposing the earth to be a perfect sphere.

\begin{tabular}{|c|c|c|c|c|c|}
\hline $\begin{array}{l}\text { Degr. } \\
\text { lat. }\end{array}$ & $\begin{array}{c}\text { English } \\
\text { miles. }\end{array}$ & $\begin{array}{l}\text { Degr. } \\
\text { lat. }\end{array}$ & $\begin{array}{l}\text { English } \\
\text { miles. }\end{array}$ & $\begin{array}{l}\text { Degr. } \\
\text { lat. }\end{array}$ & $\begin{array}{l}\text { English } \\
\text { miles. }\end{array}$ \\
\hline 0 & $69 \cdot 07$ & 31 & $59 \cdot 13$ & 61 & \\
\hline 1 & & 32 & & & \\
\hline 2 & & 3 & & & \\
\hline 3 & & 34 & & & \\
\hline 4 & & 35 & & 65 & $29 \cdot I 5$ \\
\hline 5 & & 36 & & & \\
\hline 6 & & 37 & & & \\
\hline 7 & & 38 & & 68 & 25.85 \\
\hline 8 & & 39 & & 69 & \\
\hline 9 & & 40 & & 70 & \\
\hline 10 & & 41 & & & \\
\hline 11 & & 42 & & 72 & \\
\hline 12 & & 43 & & 73 & \\
\hline 13 & & 44 & & 74 & \\
\hline 14 & & 4.5 & & & \\
\hline 15 & & 46 & & 76 & \\
\hline 10 & & 4 & & 77 & \\
\hline 17 & & 4 & & 78 & \\
\hline 18 & & 49 & & 79 & \\
\hline 15 & & 50 & & & \\
\hline 20 & & 51 & & 81 & \\
\hline 21 & & 52 & & 8 & \\
\hline 22 & & 5 & & 8 & \\
\hline 23 & & 54 & & 8. & \\
\hline 24 & & & & s & \\
\hline 25 & & 5 & & 8 & \\
\hline 26 & & 57 & & 87 & (6) $]$ \\
\hline 27 & & 58 & & 85 & $2+1$ \\
\hline 29 & & 5 & & B & \\
\hline 2 & & 6 & 34.50 & 90 & 0 \\
\hline & & & & & \\
\hline
\end{tabular}

Note, This tahle is computed on the supposition that the length of the degrees of the equator are equal to those of the meridian at the medium latitude of $45^{\circ}$, which length is $69 x^{\frac{x}{5}}$ linglish miles nearly.

But, taking the earth as a spheroid, the degree of longitude may be found, in any given latitude $l$, by saying, VOL. I.
Ist. As the equatorial diameter is to the polar, so is tang. $\left(90^{\circ}-l\right)$, to tang. of an ungle $a$ : then $2 \mathrm{~d}$. As radius, to sine of $a$, so is the length of a degree of the equator to tha length of a degree on the parallel of the given latitude.

DEINCLINERS, or DEINCIINING Dials, are such as both decline and incline, or recline, at the same time. Suppose, for instance, a plane cutting the prime vertical circle at an angle of $30^{\circ}$, and the horizontal plane at an angle of $24^{\circ}$, the latitude of the place being $52^{\circ}$; a dial drawn on this plane, is called a deincliner.

DELIACAL Problem, a celebrated problem among the ancients, concerning the duplication of the cube.

DELISLE. See LISTE (DE).

DELPHINUS, the Dolphin, a constellation of the northern hemisphere; whose stars, in Ptolemy's catalogue, are 10; in Tycho's the same; in Hevelius's 14; and in Flamsteed's 18.

DEMETRIUS, a Cyuic philosopher, and disciple of Apollonius Thyancus, in the age of Caligula. That emperor wishing to gain the philosopher to his interest by a large present, he refused it with indignation, saying, "If Caligula wishes to bribe me, let him send me his crown." Vespasian was displeased with his insolence, and banished him to an island. The cynic derided the punishment, and bitterly inveighed against the emperor. Demetrius lived to a very great age. And Seneca observes, that "nature had brought him forth to show mankind that an exalted genius can live securely without being corrupted by the vice of the surrounding world."

DEMI-Bastion, in Fortification, that which has only one face and one flank.

Demi-Cannon, and Demi-Culverin, names of certain species of cannon, now no longer used.

DEм T-Cross, an instrument used by the Dutch to take the altitude of the sun or a star at sea ; instead of which we use the cross-staff, or fore-staff.

DEMI-Gorge, is half the gorge or entrance into the bastion; not taken directly from angle to angle, where the bastion joins to the curtin, but from the angle of the flank to the centre of the bastion; or the angle the two curtins would make, were they thus protracted to meet in the bastion.

DE M I - Lune, or Half-moon, an outwork consisting of two faces, and two little flanks. It is often built before the angle of a bastion, and sometimes also before the curtin; though now it is very seldom used.

DEMIOCRITUS, one of the greatest philosophers of antiquity, was born at Abdera, a town of Thrace, about the SOth olympiarl, or about 400 years before Christ. Il is father, says Valerius Maximus, was able to entertain the army of Xerxes; and Diogenes Lacrtius adds, upon the testimony of Herodotus, that the king, in requital, presented him with some Mlagi and Chaldeans. From these, it seems, Democritus received the first part of his education; and from them, while yet a boy, he learned theology and astronomy. He next applied to Leucippus, from whom he learned the system of atoms and a vacuum. llis father dying, he and his two brothers divided his effects. Democritus made choice of that part which consisted in money, as being, though the least share, the most convenient for travelling; and it is said that his portion amounted to more than 100 talcuts, which is near 20 thousand pounds sterling. Ilis extraordinary inclination for knowledge and the sciences, induced him to travel into all parts of the world where lic might find learned men. 
He went to visit the priests of Egypt, from whom he learned genmetry: he cunsulted the Chaldean and Persian philosophers; and it is said that he penetrated even into India and Ethiopia, to confer with the Gymnosophists. In these travels he wasted his substance; after which, at his return he was obliged for sume time to he maintained by his brother. Settling bimself at Abdera, be there governed in the most absolute manner, by virtue of his consummate wisdom. The magistrates of that city made him a present of 500 talents, and erected statues to him, even in his lifetime; but being naturally more inclined to contemplation than delighted with public honours and employments, he withdrew into solitude and retirement.

Deniocritus always laughed at human life, as a continued farce, which made the pcople think he was mad; on which they sent for Hippocrates to cure him: but that celebrated physician having discoursed with the philosopher, told the people that he had a great vencration for Democritus; and that, in his opinion, those who esteemed themselves the most healthy, were the most distempered. It is said, though with little probability, that Dcmocritus put out his own eyes, that he might meditate more profoundly on philosophical subjects. He died, according to Diogenes Laertius, in the 361 st year before the Christian cra, at 109 ycars of age. He was the author of many books, which are lost ; from which Epicurus borrowed his philosophy.

DEMOIVRE (AвRAHAM), a celebrated mathematician, of French original, but who spent most of his life in lingland. He was born at Vitri in Champagne, 1667. On account of the revocation of the edict of Nantes, in 1685, he determined, with many others, to take shelter in England; where he perfected his mathematical studies, the foundation of which he had laid in his own country. A mediocrity of fortune obliged him to employ his talent in this way in giving lessons, and rcading public lectures, for his better support : in the latter part of lis life too, he chicfly subsisted by giving answers to questions in chances, play, annuities, \&c, and it is said most of these responses were delivered at a coffee-house in St. Martin's-lane, where he spent must of his time. The Principin Mathematica of Newton, which chance is said to have thrown in his way, soon convinced Demoivre how little he had anvanced in the science he professel. This induced him to redouble his application; which was attended by a considerable degree of success; and he soon became connected with, and colebrated among, the first-rate mathematicians. His eminence and abilities in this line, opened bim an entrance into the Royal Society of London, and into the Academies of Berlin and Paris. By the former his merit was so wcll known and esteemed, that they judged hin a fit person to decide the famous contest between Newton and Leibnitz, concerning the invention of Fluxions.

Demoivre died at London Nov, 1754, at 87 years of age. 'The Pbilosophical Transactions of London contain several of his papers, and all of them interesting, viz. in the volunies $19,20,22,23,25,27,29,30,32,40,41,43$.

He published also some very respectable works, viz,

1. Misccllanca Analytica, de Sericbus et Quadraturis \&c; 1730 , in 4 to. But perhays he has been more generally known by his

2. Ioctrine of Chances; or, Mcthod of Calculating the Probabilitics of Events at Play. This work was first printed, 1718 , in 4 to, and dedicated to Sir Isaic Newton: it was reprinted in 1738 , with grcat alterations and jmprovenents; and a third edition was afterwards printed.

3. Annuities on Lives; first printed 1724 , in $8 v 0 .-$ In 174.2 the ingenious Thomas Simpson (then only 33 years of age) published his Doctrine of Annuities and lieversions; in which be paid some handsome compliments to our anthor. Notuillsstanding which, Demoivre presintly brought out a second edition of his Annuities, in the preface to which he passed some harsh reffctions upon Simpson. To these the latter gavc a handsome and effectual answer, 1743, in An Appendix, containing some Remarks on a late book on the same subject, with answers to some personal and malignant misrepresentations, in the preface thereof. At the end of this answer, Mr.Simpson concludes, "Lastly, I appeal to all mankind, whether, in his treatment of me, he has not discovered an air of self-sufficiency, ill-nature and inveteracy, unbecoming a gentlcman." Here it would scem the controversy dropped: Mr. Demoivre published the $3 \mathrm{~d}$ cdition of his book in 1750 , without any farther notice of Simpson, but omitted the offensire reflectious that had been in the preface.

DENONSTRATION, a certain or convincing proof of some proposition: such as the demonstrations of the propositions in Euclid's Elements. 'The method of demonstrating in mathematics, is the same with that of drawing conclusions from principles in logic. Indeed, the demonstrations of mathematicians ate no other than series of enthymemes ; every thing is concluded by force of syllogism, only omitting the premiscs, which either occur of their own accord, or are recollected by means of quotations.

DENDROMETER, an instrument lately invented by Messrs. Duncombe and Whittel; so called, from its use in measuring trees.

DENEB, an Arabic term, signifying tail; used by astronomers as a name lo some of the fixed stars, but especialiy for the bright star in the Lion's tail.

DENOMINATOR, of a Fraction, is the number or quantity placed bclow the line, which shows the whole integer, or into how many parts it is supposed to be divided by the fraction; as that which gives denomination or name to the parts of the fraction. Thus, in the fraction $\frac{5}{i^{2}}$ (five-twelfits) the number 12 is the denominator, and shows that the integer is here lisvided into 12 parts, or thet it consists of 12 of those parts of which the numerator contains 5. Also $b$ is the denominator of the fraction $\frac{a}{b}$.

DENOMINator of a Ratio, is the quotient arising from the division of the antecedent by the consequent. 'Thus, 6 is the denominator of the ratio 30 to 5 , because $30 \mathrm{di}$ vided by 5 gives 6 . It is otherwise called the exponent of the ratio.

DE:NSITY, that property of bodies by which they contain a certain quantity of matter, under a certain bulk or magnitude. Accordingly, a body that contains more matter than another, under the same bulk, is said to be denser than the other, and that in proportion to the quantity of matter; or if the quantity of matter be the sume, but under a less bulk, it is said to be denser, and so much the more so as the bulk is less. Su that, in gencral, the density is directly proportional to the mass or quantity of matter, and reciprocally or inversely proportional to the bulk or mangnitude under which it is contained.

The quantities of matter in bodies, or at least the proportions of them, are known by their gravity or weight; every equal purticle of matter being endowed with an equat 
gravity, it is inferred that cqual masses or quantities of matter have an equal weight or gravity; and unequal masses have proportionally uncqual weights. So that, when body, or mass, nr quantity of matter is spoken of, we are to understand their weight or gravity.

From the furegoing gencral propurtion of the density of bodlies, viz, that it is as the mass directly, and as the bulk inversely, may be inferred the proportion of the masses, or of the magnitudes; viz, that the mass or quantity of natter is in the compound ratio of the bulk and density; and that the bulk or magnitude is as the mass directly, and the density inversely. Hence, if $B, b$ be two bodies, or masses, or weights;

and $\mathrm{D}, d$ their respective densities;

also $\mathrm{N}, m$ their magnitudes, or bulks:

Then the theorems above are thus expressed,

viz, $\mathrm{D} \propto \frac{\mathrm{B}}{\mathrm{M}}$, and $\mathrm{B} \propto \mathrm{DM}$, and $\mathrm{M} \propto \frac{\mathrm{B}}{\mathrm{D}}$;

or $\mathrm{D}: d:: \frac{\mathrm{B}}{\mathrm{M}}: \frac{b}{m}$, and $\mathrm{B}: b:: \mathrm{DM}: d m, \& \mathrm{c}$;

or $\frac{\mathrm{D}}{d}=\frac{\mathrm{B} m}{l \mathrm{M}}$, and $\frac{\mathrm{B}}{b}=\frac{\mathrm{DM}}{d m}$, and $\frac{\mathrm{M}}{m}=\frac{\mathrm{B} d}{b \mathrm{D}}$.

No body is absolutely or perfectly dense; or no space is perfectly full of matter, so as to have no vacuity or interstices; on the contrary, it is the opinion of Newton, that even the densest bodies, as gold, \&cc, contain but a small portion of matter, and a very great portion of vacuity; or that it contains a great deal more of pores or empty space, than of real sulstance.

It bas been observed above, that the relative density of bodies may be known by their weigbt or gravity; and hence the most general way of knowing those densities, is by actually weighing an equal bulk or magnitude of the bodies, whetber solid or fluid; if solid, by shaping them to the same figure and dimensions; if fluid, by filling the same vessel witl them, and weighing it.

For thids, there are also other methods of finding their density: as 1st, by making an equilibrium between them in tubes that communicate; for, the diameters of the tubes bcing equal, and the weights or quantities of matter also equal, the densities will be inversely as the altitudes of the liquids in them, that is inversely as the bulk.

$2 \mathrm{dly}$, The densities of fluids are also compared together by immerging a solid in them; for if the solid be lighter than the liquids, the part immerged by its own wcight will be inversely as the density of the fluid; or if it be heavier, and sink in the liquids, by weighing it in them; then the weights lust by the body will be directly proportional to the densities of the fluids.

DENSITY of the Air, is a property that has much engaged the attention of the later philosophers, since the discovery of the Torricellian experiment, and the air-pump. By means of the barometer it is demonstrated, that the air is of the same density at all places at the same distance from the level of the sea, provided the temperature, or degree of heat, be the same. Also the density of the air always increases in proportion to the compression, or the compressing forces. And hence the lower parts of the atmosphere are always denser than the upper: yet the density of the lower air is not exactly proportional to the weight of the atmosphere, by reason of heat and cold, which make considerable alterations as to rarity and density; so that the barometer mcasures the elasticity of the air, rather than its density. If the height of the barometer be considered as the incasure buth of the density and elas- ticity of the air, when the thermometer is $4 t 31^{\circ}$, and $b$ be any other height of the barometer, when the thermometel is at $t$ degrees; then in this case, $b$ is the measure of the elasticity, and $\frac{466-t}{435} b$ is the measure of the density of the air.

DENSITY of the Planets. In homogeneous, unequal, spherical bodies, the gravities on their surfaces are as their diameters when the densities are equal, or the gravities are as the densities when the bulks are equal; therefore, in spheres of unequal magnitude and density, the gravity is in the compound ratio of the diameters and densities, or the densities are as the gravities divided by the diameters. Knowing therefore the diameters of the planets by observation and comparison, and the gravities at their surface by means of the revolution of the satellites, the relation of their densities becomes known. And as I have frund the mean density of the earth to be nearly 5 times thát of water (iny new Tracts, vol. 2, Tract 27); hence the densities of the planets, with respect to water, become known, and are as below:

$\begin{array}{lccc}\text { Water } & - & - & \text { Densities. } \\ \text { The Sun } & - & - & 1 \\ \text { Mercury } & - & - & 1 \frac{4}{1} \\ \text { Venus } & - & - & 6 \frac{1}{6} \\ \text { The Earth } & - & - & 5 \\ \text { Mars } & - & - & 3 \frac{5}{3} \\ \text { The Moon } & - & - & 3 \frac{5}{\frac{5}{1}} \\ \text { Jupitcr } & - & - & 1 \frac{1}{6} \\ \text { Saturn } & - & - & 0 \frac{5}{4} \\ \text { Herschel } & - & - & 1 \frac{1}{\frac{1}{6}}\end{array}$

As it is not likely that any of these bodies are homogeneal, the densities here determincd are supposed to be the mean densities, or such as the bodies would have if they were homogeneal, and of the same mass of matter and magnitude.

DENTICLES, of DENTILS, are ornaments in a cornice, cut after the manner of teeth. These arc mostly affected in the Ionic and Corinthian orders; and of late also in the Doric. The square member on which they are cut, is called the Denticule.

DEPARTURE, in Navigation, is the easting or westing of a ship, with regard to the meridian she departed or sailed from. Or, it is the difference in lungitude, either east or west, between the present meridian the ship is under, and that where the last reckoning or observation was made. This departure, any where but under the equator, must be accounted according to the number of miles in a degree proper to the parallel the ship is in.-The Departure, in Plane and Mercator's Sailing, is always represented by the base of a right-angled plane triangle, where the course is the angle opposite to it, and the distance sailed is the hypothenuse, the perpendicular or other leg being the difference of latitude. And then the theorem for finding it, is always this: As radius is to the sine of the course, so is the distance sailed, to the departure.

DEPHLOGISTICATED Air. See OXYgEN.

DEPRESSION of Equations, in Algebra, is the reducing them to lower degrees; as, from biquadratics to cubies, or from cubics to quadratics. It is only in snme certain cases that this can be performed 3 as 1st, When thiere can be found a compound binonial measure, or divisor of the given equation, of which one term is the unknown letter or quantity; then the other part of the $3 \mathrm{~F} 2$ 


\section{E P}

divisor is one root of the equation, and the whole equation being divided by the divisor, the quotient is the equation depressed one degree lower. So, tor example, if the equation be $x^{3}-6 x^{2}+11 x-6=0$; and it be discovered that $x$ is $=2$, then $x-2$ will be the divisor, by which dividing the given equation, it is depressed to the quadratic $x^{2}-4 x+3=0$, the two roots of which are 1 and 3 . So that all the three roots of the given cubic equation, are, 1 , 2, 3.-Or, if the given equation were $x^{3}-b x^{2}-a^{2} x+a^{2}$ $b=0$, and it be found that $b$ is one root of it, or that $x-b$ is a divisor; then making the division, the quotient, $x^{2}-$ $a^{2}=0$, is the depressed equation, the two roots of which are $x=+a$ or $-a$ : so that the three roots are $+a,-a,+b$.

$2 d$. If an equation contain equal roots, these may be found, and the equation reduced as many degrees or dimensions lower, as there are equal roots. For if the original equation $x^{\mathrm{n}}-a x^{\mathrm{n}-1}+b x^{\mathrm{n}-2}-\& \mathrm{c}=0$, have $m$ equal roots, then $n x^{n-1}-(n-1) a x^{n-2}+(n-2) b x^{n-3}-\& \mathrm{c}=0$, has $m-1$ of those roots, as may be readily shown. Hence, when there are two equal roots, the two equations have a common measure of this form $(x-r)^{\mathrm{m}-1},(r$ being one of the roots, ) which may be obtained in the usual way, and thus $m$ roots of the original equation may be known. Then divide this equation by $(x-r)^{\mathrm{m}}$, and the resulting equation, of $n-m$ dimensions, contains the other roots.

Thus, let the cubic $x^{3}-a x^{2}+b x-c=0$, have two equal roots; then $3 x^{2}-2 a x+b=0$ has one of them; and the two equations have a common measure, which is a simple equation: the quantities $3 x^{3}-3 a x^{2}+3 b x-3 c$, and $3 x^{2}-2 a x+b$, have also the same commun measure, which being found, we have $\frac{1}{3}\left(6 b-2 a^{2}\right) x-\frac{1}{3}(b c-a b)$ for a divisor of the equation $x^{3}-a x^{2}+b x-c=0$; this divisor being also put $=0$, gives $x=\frac{b c-a b}{6 b-2 a^{2}}$. Thus two roots of the equation are discovered; and, since $a$ is the sum of all the ruots, the $3 \mathrm{~d}$ root is the difference between $a$ and the sum of the two equal roots.

If two roots of the equation be of the form $+a,-a$, differing only in their signs; change the signs of the roots, and the resulting equation has two roots, $-a,+a$; thus we have two equations with a common measure, $x^{2}-a^{2}$, which may be found, and the equation depressed, as in the former case.

To be more particular:-There bring only certain cases in which this depression can be effected, viz, ist. When there is any known relation between two or more of the roots; and, 2 dly. When the equation is reciprocal, that is, when each of the terms of the equation, which are equally distant from the first and last terms, have equal co-efficients; under which form the relation of each pair of roots becomesknown.

With regard to the first, it seldom happens that any known relation subsists between the roots of an equation if the roots themselves are unknown, except it be that of equality, we shall thorefore only consider it under this point of view; namely, when two or more of the roots are cqual, having either like, or contrary signs; referring the reader, for further information on this subject, to Lacroix' Complément des Elémens d'Algebre, art. 38 ; and to Waring's Meditationes Algeb. cap. 3.

1. When an equation has two equal roots, with contrary signs, it may be depressed two degrees.

Lit there be given the equation

$x^{5}-p x^{4}-q x^{3}+r x^{2}+s x-t=0$, which we are supposed to know contains two equal roots with contrary signs.
D E P

This being the case, it is evident that we shall not alter the value of the equation by writing $-x$ instead of $x$; which gives us, $-x^{5}-p x^{4}+q x^{3}+r x^{2}-s x-t=0$, Or $\quad x^{5}+p x^{4}-q x^{3}-r x^{2}+s x+t=0$, Also $x^{5}-p x^{4}-q x^{3}+r x^{2}+s x-t=0$, being the given equation. Now these two equations have a common measure, which, being obtained by the usual method, will be found to involve the $2 d$ power of $x$; whence the equal roots may be determined, and the whole equation being divided by this commun measure, will give us the depressed equation. We may also discuver the value of the equal roots in equations, not exceeding the 5 th degree by the addition and subtraction of the above equations, for by the former we obtain

$2 x^{5}-2 q x^{3}+2 s x=0$, or $x^{4}-q x^{2}+s=0$, and by the latter $-2 p x^{4}+2 r x^{2}-a t=0$, or $x^{4}-\frac{r}{p} x^{2}+\frac{t}{p}=0$.

Therefore $-q x^{2}+s=-\frac{r}{p}-x^{2}+\frac{t}{p} ;$ whence we have $x= \pm \sqrt{ }\left(\frac{t-p s}{r-p q}\right)$, which are the two equal roots, and consequently $x^{2}-\left(\frac{t-p s}{r-p q}\right)=0$ is a divisor of the origilial equation, which nay thus be depressed to a culse, containing the other three roots.

Suppose, for example, our giren equation had been $x^{5}-3 x^{4}-17 x^{3}+27 x^{2}+52 x-60=0$.

Then $p=3, q=17, r=27, s=52, t=60$;

And $x= \pm \sqrt{ }\left(\frac{t-p s}{r-p q}\right)= \pm 2$; the whole equation therefore being divided by $x^{2}-4=0$, gives $x^{3}-3 x^{3}-$ $3 x+15=0$, which is a cubic equation, in which are contained the other three roots.

And exactly in the same manner may any other equation of this kind be depressed two degrees.

2. When two or more of the-roots are equal, having like signs, the equation may be depressed as many degrees as there are equal roots.

Let $x^{3}-p x^{2}+q x-r=0$, be an equation having two equal roots with like signs, and let $a$ and $b$ represent those roots; then we shall have the two following identical equations: $a^{3}-p a^{2}+q a-r=0, b^{3}-p b^{2}+q b-r=0$, whence we draw $\left(a^{3}-b^{3}\right)-p\left(a^{2}-b^{2}\right)+q(a-b)=0$, and dividing this by $a-b$, we obtain $\left(a^{2}+a b+b^{2}\right)-p$ $(a+b)+q=0 ;$ or, since $a=b, 3 a^{2}-2 p a+q=0 ;$ which has a common divisor with the original equation, and writing $x$ again instead of $a$, this divisor will be readily obtained by the rule for finding the greatest common measure of two quantities, which in the present case is $\frac{6 q-2 p^{2}}{3} x-\frac{9 r-p q}{3}=0$, whence $x=\frac{9 r-p q}{6 q-2 p^{2}}$.

Thus, two of the ronts are discovered, and consequently. the equation may be depressed two degrees as before, by dividing the whole of it by $\left(x-\frac{9 r-p q}{6 q-2 p^{2}}\right)^{2}$.

But in the present case, as the original equation was only of three dimensions, and $p$ being cqual to the sum of all its roots, from the nature of cquations; we have

$x=p-\frac{9 r-\eta \eta}{3 q-p^{2}}$, for the third root.

Suppose, for example, the proposed cquation had been $x^{3}-4 x^{2}+5 x-2=0$ : here $p=4, q=5, r=2$; aul one of the equal roots is $\frac{9 r-\eta \eta}{d q-2 p^{2}}=1$; the third root therefore is $p-2=t-2=2$, and the three roots sought are 1,1 , and 2 . 


\section{E P}

We should have obtained exactly the same result, by considering that an equation having two equal roots, is necessarily divisible by a factor of the form $x^{2}-2 a x+a^{2}$, where $a$ represents one of the equal roots: and if three of the roots are equal, the equation has a divisor of the form $x^{3}-3 a x^{2}+3 a^{2} x-a^{5}$, and so oll, for any number of equal roots : that is, an equation may always be depressed as many degrees as there are equal roots contained in it.

3 Reciprocal equations may be depressed to others of half the original dimension, when the highest power of the unknown quantity is even. Let the given reciprocal equation be $x^{4}+p x^{3}+q x^{2}+p x+1=0$; and dividing this by $x^{4}$, we obtain $1+p_{x}^{1}+g \frac{1}{x^{2}}+p^{\frac{1}{x^{3}}}+\frac{1}{x^{4}}=0$; whence it is evident, that if $a$ be one of the roots of this equation, $\frac{1}{a}$ is also a root; and in the same manner if $b$ be one of its roots, then $\frac{1}{b}$ is the other: and so on, for equations of higher dimensions.

We may therefore cousider the given equation as generated from the product $(x+a) \cdot\left(x+\frac{1}{a}\right) \cdot(x+b) \cdot\left(x+\frac{1}{b}\right)$, or from the quadratic factors

$$
\left(a^{2}+x\left(a+\frac{1}{u}\right)+1\right) \cdot\left(x^{2}+x\left(b+\frac{1}{b}\right)+1\right),
$$

or making $a+\frac{1}{a}=m$, and $b+\frac{1}{b}=n$, the above becomes $\left(x^{2}+m x+1\right) \cdot\left(x^{2}+n x+1\right)=$ $x^{4}+(n+n) x^{3}+(2+m n) x^{2}+(m+n) x+1=0$, and equating the coefficients we bave $m+n=p, n t n=$ $q-2$, whence $m-n=\sqrt{ }\left(p^{2}-4 q+8\right), m=\frac{1}{2} p+$ $\frac{1}{2} \sqrt{ }\left(p^{2}-4 q+8\right), n=\frac{1}{2} p-\frac{1}{2} \sqrt{ }\left(p^{2}-4 q+8\right)$.

Thus $n$ and $n$ are found by a quadratic, that is, by an equation of half the original dimension. As to $a$ and $b$; those are now readily determined: for since $a+\frac{1}{a}=m$, we have $a=\frac{1}{2} m \pm \sqrt{ }\left(\frac{\pi}{4} m^{2}-1\right)$, and since $b+\frac{1}{b}=n$, we derive $b=\frac{1}{2} n \pm \sqrt{ }\left(\frac{1}{4} n^{2}-1\right)$. Suppose, for example, our equation was $x^{4}+5 \frac{5}{5} x^{3}+10 \frac{1}{3} x^{2}+5 \frac{5}{6} x+1=0$.

Here $p=5 \frac{5}{6}$, and $q=10 \frac{1}{3}$,

whence $n=\frac{1}{2} p+\frac{1}{2} \sqrt{ }\left(p^{2}-4 q+8\right)=3 \frac{1}{3}$,

and $n=\frac{\pi}{2} p-\frac{1}{2} \sqrt{ }\left(p^{2}-4 q+8\right)=2 \frac{1}{2}$;

therefore $a=\frac{1}{2} m \pm \sqrt{ }\left(\frac{1}{4} m^{2}-1\right)=3$ or $\frac{1}{3}$,

and $b=\frac{1}{2} n \pm \sqrt{ }\left(\frac{x}{4} n^{2}-1\right)=2$ or $\frac{\pi}{2}$

consequently, the four roots are $3, \frac{r}{3}, 2$, and $\frac{1}{2}$.

And in the same manner may any other equation of this kind be depressed to half its original dimension.

When the highest power of the unknown quantity is uncven, then +1 or -1 is a root of the equation, as is evidant from inspection; it may therefore be divided by $x \pm$ $\mathrm{l}=0$; which will depress it one degree, still leaving it reciprocal, after which, it may be reduced to half that dimension, by the method above explained. Let $x^{5}+p x^{4}+$ $g x^{3}+q x^{2}+p x+1=0$, be the given equation, then +1 is one of its roots; dividingit therefore by $x-1=0$, we obtain $x^{4}+(p+1) x^{3}+(p+q+1) x^{2}+(p+1) x+1=0$, which is still reciprocal, and may therefore be depressed tua quadratic by the method already employed. MI. Gauss, in his Disquisitiones Arithmetica, which is mentioned under the article Algebra, has shown that every equation of two terms, of which the exponent is in prime number, may be rationally decomposed into other eugutions, the degrees of which are indicated by the prime factors of the given exponent minus 1 , by means of which he has been able to
D E $R$

express the side of a regular 17 -sided polygon inscribed in a circle, by a quadratic equation; which was before thought impossible. This curious piece of analysis has been translated into French under the title of Recherches Arithmetiques, and is highly deserving of an attentive perusal.

Depression of the Pole. So many degrees \&c as you sail or travel from the pole towards the equator, so many it is said you depress the pole, because it becomes so much lower, or nearer the borizon.

Depression of a Star, or of the Sun, is its distance below the horizon; and is measuresl by an arc of a vertical circle, intercepted between the horjzon and the place of the star.

Depression of the Visible Horizon, or Dipof the Hori$z o n$, denotes its sinking or dipping below the true horizontal plane, by the observer's eye being raised above the surface of the sea ; in consequence of which, the observed altitude of an object is by so much too great. Thus, the eye being at $E$, the beight is $\mathrm{AE}$ above the surface of the earth, whose centre is $\mathrm{C}$; then $\mathrm{E} \mathrm{H}$ is the real horizon, and $\mathrm{E} h$ the visible one, below the former by the angle

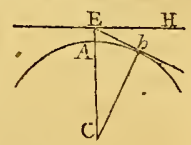
$M E h$, by reason of the elevation ' $A \mathrm{E}$ of the eyc.

To compute the Depression or Dip of the Horizon.

In the right-angled triangle $\mathrm{cE} h$, are given $\mathrm{c} h$ the earth's radius $=21000000$ feet, and the hypothenuse $\mathrm{CE}=$ the radius increased by the height $A \mathrm{l}$ : of the eye; to find the angle $\mathrm{c}$ which is $=$ the angle $n \mathrm{I} h$, or the depression sought; viz, as $\mathrm{c} / \mathrm{:}: \mathrm{CE}:$ : radius : sccant $\angle \mathrm{C}$, or, as $\mathrm{ce}: \mathrm{c} h$ : : radius : cosine $\angle \mathrm{c}$.

By either of these theorems are computed the numbers in the following table, which shows the depression or dip of the horizon of the sca, for different heights of the eye, from 1 foot to 100 fect.

\begin{tabular}{|c|c|c|c|c|c|}
\hline $\begin{array}{l}\text { Height } \\
\text { of the } \\
\text { eye }\end{array}$ & $\begin{array}{l}\text { Dip of } \\
\text { the } \\
\text { horizon }\end{array}$ & $\begin{array}{c}\text { Height } \\
\text { of the } \\
\text { eye }\end{array}$ & $\begin{array}{l}\text { Dip of } \\
\text { the } \\
\text { Lorizon }\end{array}$ & $\mid \begin{array}{c}\text { Height } \\
\text { of the } \\
\text { eye }\end{array}$ & $\begin{array}{l}\text { Dip of } \\
\text { the } \\
\text { horizon }\end{array}$ \\
\hline feet & , " & fcet & 11 & feet & ' " \\
\hline 1 & 057 & 13 & 326 & 26 & 452 \\
\hline 2 & 121 & 14 & 334 & 28 & 53 \\
\hline 3 & 139 & 15 & 342 & 30 & 514 \\
\hline 4 & 155 & $10^{\circ}$ & $\begin{array}{ll}3 & 49\end{array}$ & 35 & 539 \\
\hline 5 & 28 & 17 & 356 & 40 & 62 \\
\hline 6 & 220 & 18 & 43 & 45 & 62.4 \\
\hline 7 & 231 & 19 & 410 & 50 & 644 \\
\hline 8 & 242 & 20 & 416 & 60 & 723 \\
\hline 9 & 252 & 21 & 4. 22 & 70 & 759 \\
\hline 10 & $\begin{array}{ll}3 & 1\end{array}$ & 22 & 428 & 80 & 832 \\
\hline 11 & 310 & 23 & 434 & 90 & 93 \\
\hline 12 & 318 & 24 & 4.0 & 100 & 933 \\
\hline
\end{tabular}

Sce Robertson's Navigation, book 9 appendix; and Tables requisite to be used with the Nautical liphemeris, pa. 1. See also Leveluteg.

DEPTH, the opposite of Height, and one of the dimensions of bodies, or of space. See Height, Altitune, ELEVATION, EC.

DERHAM (Dr. WILLIAM), an eminent English philosopher and divine, was born at Stowton, near Worcester, 1657, and educated in Trinity-college, Oxford. In 1682, he was presented to the vicarage of Wargrave in Berkshire; and, in 1689 , to the valuable rectory of $\mathrm{Up}_{\mathrm{p}}$ - 
minster in Essex; which, lying at a convenient distance from Lo!ton, afforded him an opportunity of conversing and corresponding with the principal literary men of the nation. Applying himself there with great eagcrness to the pursuit of his studies in natural and experimental philosophy, he soon became a distinguished and useful member of the Royal Society, whose Philosophical Transactions contain a great variety of curious and raluable pieces, the fruits of his laudable industry, in all or most of the volumes, from the 20th to the 39 th, both inclusive; the principal of which are :

1. Experiments on Pendulums in vacuo.

2. Of an instrument for finding the Meridian.

3. Experim. and Observat. on the Motion of Suund.

4. On the Migration of Birds.

5. On the Spots in the Sun, from 1703 to 1711 .

6. Observations on the Nurthern Lights, Oct. 8,1726 , and Oct. 13, 1728 .

7. Tables of the Eclipses of Jupiter's Satellites.

8. Difference of Time in the meridian of diff. places.

9. Of the meteor called Ignis Fatuus.

10. The History of the Death Watch.

11. Meteorological Diaries for several years.

In his younger days he published his Artificial Clockmaker, a very useful little work, which has gone through several editions. In $1711,1712,1714$, he preached those sermons at Boyle's lecture, which he afterwards digested under the well-known titles of Physico-Theology and Astro-Theology, or Demonstrations of the being and attributes of God, from his works of creation, and a survey of the heavens.

ln 1716 he was made a canon of Windsor, being at that time chaplain to the prince of Wales; and in 1730 he received, from the university of Oxford, the degree of Doctor of Divinity. He revised the Miscellanea Curiosa, in 3 vols. Svo, containing many curious papers of Dr. Halley and scveral other ingenious philosophers. To him also the world is indebted for the publication of the Philosophical Experiments of the late eminent Dr. Hooke, and other ingenious men of his time; as well as notes and illustrations of several other works.

Dr. Derham was very well skilled in medical as well as in physical knowledge; and was constantly a physician to the bodies as well as the souls of his parishioners. This great and good man, after spending his life in the most agreeable and improving study of nature, and the diligent and pious discharge of his duty, died at Upminster in 1735 , at 78 years of age.

DERIVATIONS (Calcul des), a newi species of calculation, which may be considered as an important discovery in analysis.-This method was first published by M. Arbugast, professor of mathematics at Strasburgh, in 1800, and has since been translated into French; but the first idea of this method appears to have been formed by the celebrated Dr. Waring, and is alluded to by him in the conclusion of his Meditationes Analytica.

As the name of this method imports, it depends on the principle of deriving one term of a series from the foregoing ones; and by means of a peculiar notation, this derivation is found to be very simple and commodious. Here, instrad of symbols being made use of to represent quantities, they are employed to represent certain opcrations that are performed upon them. Thus let $\mathrm{r}(a+x)$ be any function of the binomial $a+x$, this function may be expanded into a series proceeding according to the powers of $x$, as $\mathrm{F}(a+x)=a+b x+\frac{c}{1 \cdot 2} x^{2}+\frac{d}{1 \cdot 2 \cdot 3} x^{3} \& c$; whence it will be seen that $a=\mathrm{F} a$, that $b$ is derived from $a$, or from $\mathbf{r} a ; c$ from $b ; d$ from $c, \& c$, by the same law: therefore if we conceive the manner in which we derive $b$ from $a$, in the same manner we derive $c$ from $b, d$ from $c$, \&c. Then representing by $D$ the operation to be performed on $\mathrm{F} a$, to produce $b$, we shall have $b=\mathrm{DF} a$, $c=\mathrm{D}^{2} \mathrm{~F} a, d=\mathrm{D}^{3} \mathrm{~F} a$, \& $c$; so that the above series is expressed thus, $\mathrm{F}(a+x)=\mathrm{F} a+\frac{\mathrm{DF} a}{1} x+\frac{\mathrm{D}^{2} \mathrm{~F} a}{1.2} x^{2}+$ $\frac{\mathrm{D}^{3} \mathrm{~F} a}{1.2 .3} x^{3} \& \mathrm{c}$.

For farther particulars relating to this important branch of analysis, the riader is referred to the work of M. Arbogast, entitled Des Calcul des Derivations; also to Mr. Woodhouse's Analytical Calculations.

DESAGULIERS (JoHn Theophilus), an eminent experimental philosopher, was the sun of the Rev. John Desaguliers, a French Protestant refugee, and born at Rochelle in $\mathrm{J} 683$. His father brought hin to England an infant; and having taught him the classics himself, be sent him at a proper age to Christ-church college, Oxford; where in 1702 be succeeded Dr. Keil in reading lectures on experimental philosophy at Hart-Hall. In 1712 lee married, and settled in London, when he first of any irtroduced the reading of lectures in experimental philosophy into the metropolis, which he continued during the rest of his life with the greatest applause, having several times the honour of reading his lectures before the king and royal family. In 1714 he was elected F.R.s, and proved a very useful momber, as appears from the great number of bis papers that are printed in their Philos. Trans. on the subjects of optics, mechanics, and meteorology. The magnificent Duke of Clandos made Dr. Desaguliers his chaplain, and presented him to the living of Edgware, near bis seat at Cannons; and he became afterwards chaplain to Frederick prince of Wales; but in the latter part of his life, he removed to lodgings over the Great Piazza in Covent Garden, where he carried on his lectures with great success till the time of his death in 1749 , at 66 years of age.

He was a member of several forcign academies, and corresponding member of the Royal Academy of Sciences at Paris; from which academy he obtained the prize, proposed by them for the best account of electricity. He communicated a multitude of curious and valuable papers to the Royal Society, from the year 1714 to 1743 , or from vol. 29 to vol. 42 .

Besides those numerous communications, be published a valuable Course of Experimental Plilosophy, 1734, in 2 large vols. 4to.; and gave an edition of David Gregory's Elements of Catoptrics and Dioptrics, with an Appendix on Reflecting Telescopes, 8vo, 1735. This appendix contains some Original Letters that passed between Sir Isaac Newton and Mr. James Gregory, relating to those trlescopes. He gave also an Englisb translition of Gravesande's Natural Philosuphy, in 2 large volumes in 4 to, 1747. A son of Dr. D. was long an officer in the corps of Royal Artillery, was a great fitvourite with his majesty, and died at an advanced age, colonel of one of the then lour regiments or battalions of that corps, about the year $\mathbf{1 7 7 5}$.

Recollecting all these fivourable circumstances; that Dr. D. was much noticed by the learned and the great, and posscssed two church livings, we cannot account for 
the circumstance of his dying in obscurity and indigence: yet such it would seem was the case; at least, if we may credit the poet Cawthorne, who, speaking of the neglected Desagullers, asks,

". How he who taught two gracious kings to view

All Boyle ennobled, and all Bacon knew,

Died in a cell, without a friend to save,

Without a guinea, and without a grave."

DESCARITS. See Cartes.

DESCENDING, a going or moving from above, downwards.-There are ascending and descending stars, and ascending and descending degrees, \&c.

Descenning Latitude, is the latitude of a planet in its return from the nodes to the equator.

DESCENSION, in Astronomy, is either Right, or $O$ blique.

Right Descenston, is a point, or arch, of the equator, which descends with a star, or sign, below the horizon, in a right sphere; and

Oblique Descension, is a point, or arch, of the cquator, which descends at the same time with a star, or sign, below the horizon, in an oblique sphere.

Descensions, both right and oblique, are counted from the first point of Aries, or the vernal intersection, according to the order of the signs, i. e. from west to east. And, as they are unequal, when it happens that they answer to equal arcs of the ecliptic, as for example to the 12 signs of the zodiac, it follows, that sometimes a greater part of the equator rises or descends with a sign, in which case the sign is said to ascend or descend rightly: and sometimes again, a less part of the equator rises or sets with the same sign, in which case it is said to ascend or descend obliquely. See Ascension.

Refraction of the Descension. See Refraction.

Descensional Difference, is the difference between the right and oblique descension of the same star, or point of the heavens.

Descent, or Fall, in mechanics, \&zc, is the motion, or tendency, of a body towards the centre of the earth, either directly or obliquely. Bodies may be considered either as freely descending, as in a vacuum, or as resisted by some external force, as an opposing bridy, or a fluid medium, \&c. 1st, If the body $b$ descend freely, and perpendiculitrly, by the force of gravity; then the motive force urging it downwards, is equal to its whole weight $b$; and the guantity of matter being $b$ also, the accelerative force will be $\frac{b}{b}$ or 1 .

$2 \mathrm{dly}$, If the body $b$, descending, be opposed by some mechanical power, suppose a wedge or inclined plane, that is, instead of pursuing the perpendicular line of gravity, it is made to descend in a sloping direction down the inclined plane: then if the natural sine of the angle the plane makes with the horizon be $s$, the motive force urging the body down the plane will be $b s$; and therefure the accelerative force is $\frac{t s}{b}$ or $s$; which is less than in the furmer case in the proportion of $s$ to 1 .

3 dlly, $\mathrm{A}$ body immersed in a fluid, loses as much of its weight, as is equal to the weight of a like bulk of the fluid; and when descending, it loses the same, besides the obstruction arising from the cohesion of the parts of the medium, and the opposing force of the particles struck, which last produces in greater or less resistance, according to the velocity of the motion. But, the weight of the body being $b$, and that of a like bulk of the fluid medium $m$, the motive force urging the body to descend, is only $b-m$; that is, the body only fallo by the excess of its weight above that of an equal bulk of the fluid.-Hence, the power that sustains a body in a medium, is equal to the excess of the absolute weight of the body above an equal bulk of the medium. Thus, a piece of copper weighing $47 \frac{1}{3} \mathrm{lb}$, loses $5 \frac{1}{3} \mathrm{lb}$. of its weight in water: and therefore a power of $42 \mathrm{lb}$, will sustain it in the water.

4thly, If two bodies have the same specific gravity, the less the bulk of the descending body is, the more of its gravity does it lose, and the slower does it descend, in the same medium. For, though the proportion of the specific gravity of the body to that of the fluid be still the same, whether the bulk be greater or less, yet the smaller the body, the more the surlace is, in proportion to the mass; and the more the surface, the inore the resistance of the parts of the fluid, in proportion.

5 thly, If the specific gravities of two bodies be different; that which has the greatest specific gravity will descend with greater velocity in the air, or resisting medium, than the other body. Thus, a ball of lead descends swifter than wood or cork, because it loses less of its weight, though in a vacuum they both fall equally swift.

The cause of this descent, or tendency downwards, has been greatly controverted. 'Two opposite hypotheses have been advanced; the one, that it proceeds from an internal principle, and the other frum an external one: the first is maintained by the Peripatetics, Epicureans, and the Newtonians; and the latter by the Cartesians and Gassendists. See also Acceleration.

\section{Laws of DEsCEN T of Bodies.}

Ist, Heavy bodies, in an unresisting medium, fall with an uniformly accelerated motion. For, it is the nature of all constant and uniform forces, such as that of gravity at the same distance from the centre of the earth, to generate or produce equal additions of velocity in cqual times. So that, if in one second of time there be produced a certain velocity, in 2 seconds there will be double that velocity, in 3 seconds triple, and so on, the degree or quantity of velocity being always proportional to the length of the time.

$2 d$, The space descended by an uniform gravity, in any time, is just the half of the space that might be uniformly described in the same time by the last acquired velocity at the end of that time, if uniformly continued. For, as the velocity increases uniformly ir an arithmetic progression, the whole space descended by the variable velocity, will be equal to the space that would be described with the middle velocity uniformly continued for the sanie time; and this again will he only half the space that would be described with the last velocity, also uniformly continued for the same time, bccause the last velocity is double of the middle velocity, being produced in a double time.

$3 d$, The spaces descended by an uniform gravity, in different times, are proportional to the squares of the times, or to the squares of the velocities. For the whole space descended in any time, consists of the stums of all the particular spaces, or velucitics, which are in arithmetical progression; but the sum of such an arithmetical progression, beginning at 0 , and having the last term and the number of terms the same quantity, is equal to balf 
the square of the last term, or of the number of terms; tberefore the whole sums are as the squares of the times, or of the velocitics.

This theory of the-descents by gravity was first discovered and taught by Galileo, who afterwards confirmed the same by experiments; which have often been repeated in various ways by many other persons since his time, as Grimaldi, Riccioli, Huygens, Newton, and many others, all confirming the same laws.

The experiments of Grimaldi and Riccioli were made by dropping a number of balls, of half a pound weight, from the tops of several toivers, and measuring the times of falling by a pendulum. Ricciol. Almag. Nov. tom. 1, lib. 2, cap. 21, prop. 4. An abstract of their experiments is exhibited here below:

\begin{tabular}{|c|cc|c|c|}
\hline $\begin{array}{c}\text { Vibrations } \\
\text { of the } \\
\text { pendulum }\end{array}$ & The time & $\begin{array}{c}\text { Space at the } \\
\text { end of the } \\
\text { time }\end{array}$ & $\begin{array}{c}\text { Space } \\
\text { descended } \\
\text { each time }\end{array}$ \\
\hline & "'l' & Rom. feet & $\begin{array}{c}\text { Rom. feet } \\
10\end{array}$ \\
5 & 0 & 50 & 10 & 30 \\
10 & 1 & 40 & 40 & 50 \\
15 & 2 & 30 & 90 & 70 \\
20 & 3 & 20 & 160 & 90 \\
25 & 4 & 10 & 250 & 15 \\
\hline 6 & 1 & 0 & 15 & 15 \\
12 & 2 & 0 & 60 & 45 \\
18 & 3 & 0 & 135 & 75 \\
24 & 4 & 0 & 240 & 105 \\
\hline
\end{tabular}

The space descended by a heavy body in any given time, being determined by experiment, is sufficient, in connexion with the preceding theorems, for determining every inquiry concerning the times, velocities, and spaces descended, depending on a uniform force of gravity. From many accurate experiments made in England, it has been found that a heavy body descends freely through 16 feet 1 inch, or $16 \frac{\mathrm{T}}{12}$ feet, in the first second of time; and consequently, by theorem 2 , the velocity gained at the end of 1 second, is $32 \frac{1}{6}$ feet per second. Hence, by the same, and theorem 3 , the velocity gained in any uther time $t$ is $32 \frac{1}{6} t$, and the space descended is $16_{\frac{1}{12}} t^{2}$. So that, if $v$ denote the velocity, and sthe space due to the time $t$, and there be put $g=16_{\frac{1}{12}}$; then is

$$
\begin{aligned}
& v=2 g t=2 \sqrt{ } g 8=\frac{2 s}{t} . \\
& s=g t^{2}=\frac{v^{2}}{4 g}=\frac{1}{2} t v . \\
& t=\frac{v}{2 g}=\sqrt{ } \frac{s}{g}=\frac{2 s}{v} .
\end{aligned}
$$

'The experiments with pendulums give also the same space for the descent of a heavy body in a second of time. Thus, in the latitude of London, it is found by experiment, that the length of a pendulum vibrating seconds is ncarly $39 \frac{x}{8}$ inches; and it being known that the time of one vibration of any pendulum, is to the time in which a heavy body will fall through lalf the length of the pendulum, as the circumference of a cirele is to its diameter, therefore as $3 \cdot 1416: 1:: 1: \frac{1}{3 \cdot 1416}$ which is the time of descending through $19 \frac{9}{16}$ inches, or hatf the length of . the penduluin; und the spaces being as the squares of the times, we hase as $\frac{1}{3.1416}: 1^{2}:: 19 \frac{9}{16}: 19 \frac{9}{16} \times 3.1416^{2}$ $=193$ inches, or 16 feet 1 inch, which therefore is the space a heavy body will descend through in one second; the very same as before.

4 th, For any other constant force, instead of the perpendicular free descent by gravity, find by experiment, or otherwisc, the space descended in one second by that force, and substitute that instead of $16 \frac{\pi}{\frac{\pi}{12}}$ for the value of $g$ in these formula: or, if the proportion of the force to the force of gravity be known, let the value of $g$ be altered in the same proportion, and the same formula will still hold good. So, if the descent be on an inclined plase, making, for instance, an angle of $30^{\circ}$ with the horizon; then, the force of desceut upon the plane being always as the sine of the angle it makes with the horizon, which in the present case will be as the sine of $30^{\circ}$, that is, as $\frac{I}{2}$ the radius; therefore the, value of $g$ will be but balf the former, $8 \frac{1}{2} 4$, in all the foregoing formulæ.

$\mathrm{Or}$, if one body descending perpendicularly draw another after it, by means of a cord sliding over a pulley; then it will be, as the sum of the two budies is to the descending body, so is $16 \frac{1}{T^{2}}$ to the value of $g$ in this case; which value of it being used in the said formula, they will still hold good. And in like manner for any other constant forces whatever.

5 th, The time of the oblique descent down any chord of a circle, drawn cither from the uppermost point or lowermost point of the circle, is equal to the perpendicular descent through the diameter of the circle.

$6 \mathrm{th}$, The descent, or vibration, through all arcs of the same cycloid are equal, whether great or small.

7 th, But the descent, or vibration, through unequal arcs of a circle, are unequal; the times being greater in the greater arcs, and less in the less.

8 th, For Descents by Forces that are variable, see Forces, \&c. See also Inclined Plane, Cyclori, PENDULUM, \&C.

Line of Siviftest DEscenr, is that which a body, falling by the action of gravity, describes in the shortest time possible, from one given point to another. And this line, it is proved by phitosophers, is the are of a cycloid, when the one point is not perpendicularly over the other. See Crcloto.

DESCRIBENT, a term in Geometry, signifying a line or superficies, by the motion of which a-superficies or solid is described.

DETENTS, in a clock, are those stops which, by being lifted up or let down, lock and unlock the clock in striking.

DeтеNт-Wheel, or Hoop-Whed, that wheel in a clock which bas a hoop almost round it, in which there is a vacancy, where the clock locks.

DETERMIINATE Number. See Nunber.

Deterana Te Problem, is that which has but one solution, or a certain limited number of solutions; in contradistinction to an indeterminate problem, which admits of infinite solutions. Such, for instance, is the problem, To form an isoscrles triangle on a giren line, so that each of the angles at the base shall be double of that at the vertex; which has only one solution: or this, 'I'o find an isosceles triangle whose area and perimeter are given; which arhitis of two solutions. But if it had only been required to describe an isosceles triangle on a given base, then it is evident that an infinite number of such triangles might have been constructed, and therefore, the problem would have been an indeterminate one. 
Determinate Section, the name of a Tract, or General Problem, written by the ancient geometrician Apollonius. None of this work has come down to us, excepting some extracts, and an account of it by Pappus, in the preface to the 7 th book of his Mathematical Collections. He there says that the general problem was, "To cut an infinite right line in one point so, that, of the segments contained between the point of section sought, and given points in the said line, either the square on one of them, or the rectangle contained by two of them, may have a given ratio, either to the rectangle contained by one of them and a given lire, or to the rectangle contained by two of them."

Pappus further informs us, that this Tract of Apollonius was divided into two books; that the first book contained 6 problems, and the second 3 ; that the 6 problems of the first book contained 16 epitagmas, or cases, respecting the dispositions of the points; and the second book 9 . Further, that of the epitagmas of the 6 problems of the first book, 4 were maxima, and one a minimum : that the maxima are at the $2 \mathrm{~d}$ epitagma of the $2 \mathrm{~d}$ problem, at the $3 \mathrm{~d}$ of the $4 \mathrm{th}$, the $3 \mathrm{~d}$ of the 5 th, and the $3 \mathrm{~d}$ of the $6 \mathrm{th}$; but that the minimum was at the $3 \mathrm{~d}$ epitagma of the $3 \mathrm{~d}$ problem. Also, that the second book contained three determinations; of which the $3 \mathrm{~d}$ epitagma of the 1 st problem, and the $3 \mathrm{~d}$ of the $2 \mathrm{~d}$ were minima, and the $3 \mathrm{~d}$ of the $3 \mathrm{~d}$ a maximum. Moreover, that the first book had 27 lemmas, and the $2 \mathrm{~d}$ book 24 ; and lastly, that both books contained 83 theorems.

From such account of the contents of this Tract, and the lemmas, also given by Pappus, several persons have attempted to restore, or recompose what they thought might be nearly the form of Apollonius's tract, or the subject of each problem, case, determination, \&c; ainong whom are, Snellius, an eminent Dutch mathematician of the 17 th century; a translation of whose work was published in English by Mr. John Lawson, in 1772, together with a new restoration of the whole work by his friend Mr. William Wales. A more complete restoration is given in Dr. Simson's posthumous works, in 1776 ; as also by P. Giannini, at Parma, 1773.

DEviation, in Astronomy. See Nutation.

DEVIATION of a falling body from ils perpendicular direction. When the notion of the revolution of the earth about its axis was revived by Copernicus, various objections were started against it by the adherents of the old. system; and among others, it was asscrted that, if the earth did really turn on its own axis, it would have followed as a natural consequence, that a stone or other heavy body, being let fall from an eminence, as the top of a tower, must have struck the earth at a consilerable distance from its foot towards the west; for as the motion of the earth was from west to east, they conceived that it must necessarily have passed through a space towards the cast, greater or less, according to the height of the tower, or to the time the body was in falling, before the latter reacherd the ground; and as, from experiments, this was found not to be the case, they considered it as an unanswerable objection to the Copernican system. But as soon as the laws of motion became better known, and the composition of forces was properly understood, it was found that, contrary to the opinions of those objectors, irsstead of the stone falling considerably to the westward of the tower, it ought to fall a little to the east of it, which is what ixperiments have since confirmed to be the case. VoL. I.
And this is what is now generally understood by the deviation of a falling body.

This phenomenon is the natural consequence of the first law of motion, namely, that every body has a tendency to preserve its state, whether it be in motion or at rest. Thus, a bullet let fall from the topmast of a ship, will fall in a direction parallel to the mast, whatever may be the velocity of the vessel, and to those on board it will have the appearance of a perpendicular descent, notwithstanding its real path is that of the curve of a parabola, the same as if it had been projectèd from a state of rest with a velo. city equal to that of the ship.

With respect to a body falling from the top of a tower, the only difference is that, by reason of the revolution of the earth, the bigher a body is elevated, the greater will be its velocity, because it describes a greater circle in the same time; therefore the body having previously to its fall acquired a greater velocity, from west to east, than the foot of the tower has, it will naturally be carried a little towards the east. Thus the same circumstance that was considered as an unanswerable objection to the modern system of astronomy, may be now regarded as an additional evidence of its truth.

This fact is confirmed by experiment. M. Guglielmini was the first who drew the attention of philosophers to this subject. On causing bodies to fall from a height of 241 feet, he found a deviation of 8 lines to the east from the vertical. M. Benzenberg, professor of physics and astronomy at Dusseldorp, made 28 experiments with balls well turned and polished, which were dropped from a height of 262 French feet, and which at a medium produced 5 lines of deviation to the east: the theory gives $4 \cdot 6$ lines. And the last experiments made at Bologna, by Guglielmini, give nearly the same results. Yet so many are the causes of inaccuracy in the experiments, and so small the deviation required by the theory, that we hesitate in laying much stress on the results of these philosopliers. Of the truth of the theory, however, there can be no doubt: we may therefore here notice MI. Laplace's ultimate theorem. Let $h$ denote the height from which the body falls, $g=$ the space fallen by gravity in the first sccond, $a=$ the angle of the earth's rotation in the same time, at the rate of $360^{\circ} \div 0.99727$ in a day, and $c=$ cosine of the latitude of the place, also $d$ the deviation towards the east: then is the deviation $d=\frac{2}{3} a c h \sqrt{ } \frac{h}{g}$. (Bulletin des Sciences, $N^{0} 75$.) - In this theorem, the value of the angle $a$ is $15^{\prime \prime} \cdot 0411$, or nearly $15^{\prime \prime}$; and as a small arc is nearly equal to its sine or tangent, therefore the value of $a$ is the sine of $15^{\prime \prime}$ to radius 1 ; that is, $a=\cdot 000072725$, the $\log$. of which is 5.8616661 , which will be useful in computing by the above theorem.

DEW, a thin light insensible mist, or rain, ascending with a slow motion, and falling while the sun is below the horizon. 'To us it appears to differ from rain, as less from more. Its origin and matter are doubtless from the vapours and exhalations that rise from the earth and water. See Exmalation. Some define it a vapour liquefied, and let fall in drops. M. Huet, in one of his letters, asserts that dew does not fall, but rises; and others have adopted the same opinion.

II. du Fay made several experiments, first with glasses, then with pieces of cloth stretched horizontally at different lieights; and he found that the lower bodies, with their under surfaces, were wetted before those that were placed $3 \mathrm{G}$ 
higher, or their upper surfaces. And Du Fay and Muschenbroek both found, that different substances, and even different colours, receive the dew differently, and some little or not at all.

From the principles laid down under the article EvaPORATION, the several phenomena of dews are easily accounted for. Such as, for instance, that dews are more copious in the spring, than in the other seasons of the year; there being then a greater stock of vapour in readiness, than at other times, by reason of the sinall expense of it in the winter's cold and frost. Hence it is too, that Egypt, and some other hot countries, abound with dews throughout all the heats of summer; for the air there being too hot to constipate the vapours in the day-time, they never gather into clouds; and hence they have no rain: but in climates that are cxcessively hot, the nights are rcmarkably cold; so that the vapours raised after sun-set, are readily condensed into dews.

It is natural to conclude, from the different substances which are combined with dew, that it must be either salutary or injurious, both to plants and animals.

It is not easy to ascertain the quantity of dew that rises every night, or in the whole year, because of the winds which disperse it, the rains which carry it down, and other inconveniences:- but it is known that it rises in greater abundance after rain than after dry weather, and in warm countries than in cold ones. There are some places in which dew is observed only to ascend, and not to fall : and others again in which it is carried upwards in greater plenty than downwards, being dispersed by the winds.

Dr. Hales made some experiments, to determine the quantity of dew that falls in the night; and for this purpose, on the 15th of August, at 7 in the evening, he filled two glazed earthen pans with moist earth; the dimensions of the paus being, 3 inches decp, and 12 inches diameter: and he observes, that the moister the earth, the more dew falls on it in a night; and that more than a double quantity of dew falls on a surface of water, than on an equal surface of moist earth. These pans increased in weight, by the night's dew, 180 grains; and decreased in weight, by the evaporation of the day, 1 oz 282 grs : so that 540 grains more are evaporated from the earth every 24 hours in summer, than the dew that falls in the night; i. e. in 21 days near 26 ounces from a circular area of a foot diameter. Now if 180 grains of dew, falling in one night on such an arca, which is equal to 113 square inches, be equally spread on the surface, its depth will be the 159th part of an inch. He likcwise found that the depth of dew in a winter's night was the 90th part of an inch. If therefore we allow 159 nights for the extent of the summer's dew, it will in that time amount to one inch in depth; and reckoning the remaining 206 uights for the extent of the winter's dew, it will produce $2 \cdot 28$ inches depth; and the dew of the whole year will amount to 3.28 inches depth. But the quantity which evaporated in a fair summer's day from the same surface, being $1 \mathrm{oz}$ and 282 grs, gives the toth part of an inch deep for eraporation, which is 4 times as much as fell at night. Dr. Hales observes, that the evaporation of a winter's day is wearly the same as in a summer's clay; the carth's greater moisture in winter compensating for the sun's grcater heat in summer. Ilales's Vegetable Statics, vol, 1, p. 52 of the the dit. See Evaponation.

Signor Beccaria made several experiments to demonstrate the existence of the electricity that is produced by dew. He observes in general, that such electricity took place in clear and dry weather, during which no strong wind prevailed; and that it depends on the quantity of the dew, as the electricity of the rain depends on the quantity of the rain. He sometimes found that it hegan before sun-set; at other times not till 11 o'clock at night. Artificial Electricity, Appendix, letter 3.

DE WIT (Jonn), the famous Dutch pensionary, was born at Dort, in 1625; where he prosecuted his studies so diligently, that at 23 years of age he published Elementa Curvarum Linearum, one of the deepest books in mathematics at that time. After taking his degrees, and travelling, he, in 1650, became pensionary of Durt, and distinguished himself very early in the management of public affairs, which soon after raised lim to the rank of pensionary of Holland. After rendering the grcatest benefits to his country in many important instances, and serving it in several bigh capacities, with the greatest ability, diligence, and integrity, by some intrigues of a party, it is said, he and his brother were thrown into prison, from whicnce they were dragged by the mob, and butchered with the most cruel and savage barbarity.

DIACAUSTIC Curve, or the Cuustic by Refraclion, is a species of caustic curves, the genesis of which is in the following manner. Imagine an infinite number of rays $B A$, BM, BD, \& , issuing from the same luminous point $\mathrm{B}$, refracted to or from the perpendicular ac, by the given curve $A M D$; and so that $\mathrm{CE}$, the sines of the angles of incidence $C M E$, be always to $\mathrm{CG}$ the sines of the refracted angles $\mathrm{CMG}_{\mathrm{G}}$, in a given ratio; then the curve $1 \mathrm{FN}$ that touches all the refracted rays $\mathrm{AH}, \mathrm{MF}, \mathrm{DN}, \& \mathrm{c}$, is called the Diacaustic, or Caustic by Refraction.

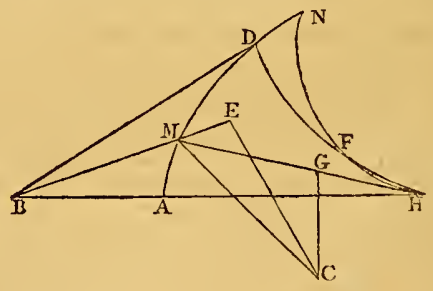

- DIACOUSTICS, or Drapionics, the consideration of the propertics of sound refracted in passing through different mediums; that is, out of a denser into a more subtile, or out of a more subtile into a denser medium. Sce Sound.

DI $\Lambda$ GONAL, is a right line drawn across a figure, from one angle to another; and is sometimes called a diameter. It is used chiefly in quadrilateral figures, viz, in pirallelograms and trapcziums.

1. Every diagonal, as a $\mathrm{c}$, divides a parallelogram into two equal parts or triangles $A B C, A D C$.

2. Two diagonals, $A c, \mathrm{BD}$, drawn in a parallelogram, bisect cach other; as in the point $\mathbf{E}$.

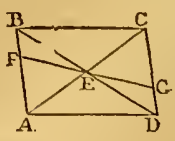

3. Any line, as $\mathrm{FG}$, drawn through the midclle of the diagonal of a parallelogram, is bisected by it at the point $\mathrm{E}$; and it divides the parallelogram into two equal parts, BYGC and A F'GD.

4. The diagonal of a stuare is incommensurable with its side. 
5. The complements about the diagonal of any paral!clogram are equal to each other.

6 . In any parallelogram, the suin of the squares of the four sides is equal to the sum of the squares of the two diagonals.

7. In any trapezium, the sum of the squares of the four sides is equal to the sum of the squares of the two diagonals, together with 4 times the square of the distance between the middle points of the diagonals.

8. In any trapezium, the sum of the squares of the two diagonals, is double the sum of the squares of two lines bisecting the tivo pairs of opposite sides.

9. In any quadrilateral iuscribed in a circle, the rectangle of the two diagonals is equal to the sum of the two rectangles under the two pairs of opposite sides.

Diagonal Scale. See Scales.

DIAGRAM, is a scheme for the explanation or demonstration of any figure, or of its properties.

DIAL, or SUN-DIAL, an instrument for measuring time by means of the sun's shadow. Or, it is a draught or description of certain lines on the surface of a body, so that the shadow of a style, or ray of the sun through a hole, should touch certain marks at certain hours.

Sun-Dials are doubtless of great antiquity. But the first upon record is, it scems, the dial of Ahaz, who began to reign 400 years before Alexander, and within 12 years of the building of Rome: it is mentioned in Isaiah, chap. 38 , ver. 8 .

Several of the ancients are spoken of, as the constructors of dials; as Anaximenes Milesius, Thales, \&rc. Vitruvius mentions one made by the ancient Chaldee historian Berosus, on a reclining plane, almost parallel to the equator. Aristarchus Samius invented the hemispherical dial. And there were at the same time some spherical ones, with a needle for a gnomon. The discus of Aristarchus was an horizontal dial, with its rim raised up all around, to prevent the shadow from stretching too far.

It was late before the Romans became acquainted with dials. The first sun-dial at Rome was set up by Papyrius Cursor, about the 460 th year of the city; before which time, Pliny says, there is no inention of any account of time but by the sun's rising and setting: the first dial was set up near the temple of Quirinus; but being inaccurate, about 30 years after, another was brought out of Sicily by the consul M. Valerius Messala, which he placed on a pillar near the Rostrum; but neither did this show time truly, because not made for that latitude; and, after using it 99 years, Martius Philippus set up another more exact.

The diversity of sun-dials arises from the different situation of the planes, and from the different figure of the surfaces on which they are described; whence they become denominated Equinoctial, Horizontal, Vertical, Polar, Direct, Erect, Declining, Inclining, Reclining, $\mathrm{Cy}$ lindrical, \&c. For the gencral principles of their construction, see Dialling.

Dials are distinguished into Primary and Sccondary.

Primary LiaLs, are such as are drawn either on the plane of the horizon, and thence called horizontal dials; or perpendicular to it, and called vertical dials; or else drawn on the polar and equinoctial planes, though ncither horizontal nor vertical.

Secondary DiALs, are all those that are drawn on the planes of other circles, besides those last mentioned; or those which either decline, incline, recline, or deincline.

Each of these again is divided into several others, as:
Equinoctial $\mathrm{D}_{\mathrm{IAL}}$, is that which is described on an equinoctial plane, or one parallel to it.

Horizontal D $1 \mathrm{~L}$, is described on an horizontal plane, or a plane parallel to the horizon.-This dial shows the hours from sun-rise to sun-set.

South Dia , or an Erect, Direct South Dial, is that described on the surface of the prime vertical circle facing towards the south.-This dial shows the time from 6 in the morning till 6 at night.

North D $14 \mathrm{~L}$, or an Eiect, Direct North Dial, is that which is described on the surface of the prime vertical pointing northward. This dial only shows the hours before 6 in the morning, and after 6 in the evening.

East DiA L, or Erect, Direct East Dial, is that drawn on the plane of the meridian, directly to the east.-This can only show the hours tin 12 o'clock.

West Dial, or Erect, Direct West Dial, is that described on the western side of the meridian.-This can only show the hours after noon. Consequently this, and the last preceding one, will show all the hours of the day between them.

Polar Dra $\mathrm{L}$, is that which is described on a plane passing through the poles of the world, and the east and west points of the horizon. It is of two kinds; the first directed towards the zenith, and called the upper; the latter, down towards the nadir, called the lower. The polar dial therefore is inclined to the horizon in an angle equal to the elevation of the pole.-The upper polar dial shows the hours from 6 in the morning till 6 at night, and the lower one shows the hours before 6 in the morning, and after 6 in the evening, viz, from sun-rise and till sun-set.

Declining DIaLs, are erect or vertical dials which decline from any of the cardinal points; or they are such as cut either the plane of the prime verical, or of the hori$z o n$, at oblique angles.

Dcclining dials are of very frequent use; as the walls of houses, on which dials are mostly drawn, commonly deviate from the cardinal points.

Of declining dials there are several kinds, which are denominated from the cardinal points which they are nearest to; as decliners from the south, and from the north, and even from the zenith.

Inclined DraLs, are such as are drawn on planes not erect, but inclining, or leaning forward towards the soutl, or southern side of the horizon, in an angle, either greater or less than the equinoctial plane.

Reclining Dials, are those drawn on planes not erect, but reclined, or leaning backwards from the zenith towards the north, in an angle greater or less than the polar plane.

Deinclined Dra as, are sucl as both decline and incline, or recline.-These last three kinds of dials are very rare.

Di a Ls without Centres, are those whose hour lines converge so slowly, that the centre, or point of their concoursc, cannot be expressed on the given plane.

2uadrantal Dial. See Horodicticul QUa drant.

Reflecting Dial. Sce Reflecting Dial.

Cylindrical Dra L, is one drawn on the curve surface of a cylinder. This may first be drawn on a paper plane, and then pasted round a cylinder of wood, \&c. It will show the time of the day, the sun's place in the ecliptic, and his altitude at any time of observation.

There are also Portable Dra Ls, or on a Card, and Utiiversul Drals on a Plain Cross, Sc.

Refracted DiaLs, are such as show the hour by means of some refracting transparent fluid. 
Ring DiaL, is a small portable dial, consisting of a brass ring or rim, about 2 inches in diameter, and onethird of an inch in breadth. In a point of this rim there is a hole, through which the sun-beams pass, and form a bright speck in the concavity of the opposite semi-circle, which gives the hour of the day in the divisions marked within it.

When the bole is fixed, the dial only shows true about the time of the equinox. But to have it perform throughout the whole year, the hole is made moveable, the signs of the zodiac, or the days of the month, being marked on the convex side of the ring; hence, in using it, the moveable hole is set to the day of the month, or the degree of the zodiac the sun is in; then suspending the dial by the little ring, turn it towards the sun, and bis rays through the hole will show the hour on the divisions within side.

Universal, or Astronomical Ring-Dra L, is a ring dial which shows the hour of the day in any part of the earth; whereas the former is confined to a certain latitude. Its figure see represented below.

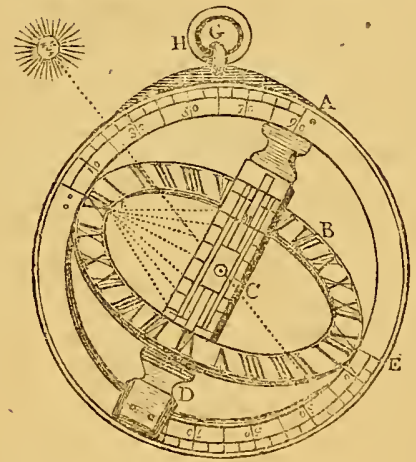

It consists of two rings or flat circles, from 2 to 6 inches in diameter, and of a proportionable breadth \&c. The outward ring $\mathrm{A}$ represents the meridian of any place you are at, and contains two divisions of 90 degrees each, dianetrically opposite to each other, the one serving from the equator to the north pole, the other to the south pole. The inner ring represents the equator, and turns exactly within the outer, by means of two pivots in each ring at the hour of 12.

Across the two circles is placed a thin reglet or bridge, with a cursor $\mathrm{c}$, sliding along the middle of the bridge, and having a small hole for the sun to shine through. The middle of this bridge is conceived as the axis of the world, and the extremities as the poles: on the one side are drawn the signs of the zodiac, and on the other the days of the nonth. On the edge of the meridian a picce slides, to which is fitted a small ring to suspend the instrument by.

In this dial, the divisions on the axis are the tangents of the angles of the sun's declination, adapted to the semidiameter of the equator as rarlius, and placed on either side of the centre: but instead of laying them down from a line of tangents, a scale of equal parts maly be made, of which 1000 shall answer exactly to the length of the semiaxis, from the centre to the insicle of the equinoctial ring; and then $43 \pm$ of these parts may be laid down from the centre towards each end, which will limit all divisions on the axis, because 434 is the natural tangent of $23^{\circ} 28^{\circ}$. And thus, by a nonius fixed to the sliding piece, and taking the sun's declination from an ephemeris, and the tangent of that declination from the table of natural tangents, the slider may be always set true within 2 minutes of a degree. This scale of 434 equal parts might be placed directly against the $23^{\circ} 28^{\prime}$ of the sun's declination, on the axis, instead of the sun's place, which is there of little use. By this means the slider might be set in the usual way, to the day of the month, for common use ; or to the natural tangent of the declination, when great accuracy is required.

To use this Dial: Place the line $a$ (on the midrle of the sliding picce) over the degrce of latitude of the place, as for instance $51 \frac{1}{2}$ degrees for London: put the line which crosses the hole of the cursor to the degree of the sign, or day of the month. Open the instrument so as that the two rings be at right angles to each other, and suspend it by the ring $\mathrm{H}$, that the axis of the dial, represented by the middle of the bridge, may be parallel to the axis of the earth. Then turn the flat side of the bridge towards the sun, so that his rays, striking through the small hole in the middle of the cursor, may fall exactly on a line drawn round the middle of the concave surface of the inner ring ; in which case the bright spot shows the hour of the day in the said concave surface of the ring.

Nocturnal or Night-DI A L, is that which shows the hour of the night, by the light, or shadow projected from the moon or stars.

Lunar or Moon Dials may be either purposely described and adapted to the moon's motion; or the hour may be found on a sun-dial by the moon shining upon it, thus: Observe the hour which the shadow of the index points at by moon-light; find the days of the moon's age in the calendar, and take 3-4ths of that number, for the hours to be added to the time shown by the shadow, to give the hour of the night. The reason of which is, that the moun comes to the same horary circle later than the sun by about three quarters of an hour every day; and at the time of new moon the solar and lunar hours coincide.

Dral Planes, are the plane superficies on which the hour lines of dials are drawn.

Tide Dial. See Tide Dial.

DIALLING, the art of drawing dials on any kind of surface, whether plane or curyed.

Dialling is wholly founded on the first motion of the heavenly bodics, and chicfly the sun; or rather on the diurnal rotation of the earth : so that the clements of spherics, and spherical trigonometry, should be understood, before a person attempts to enter upon the doctrine of dialling.

The principles of dialling may be aptly declucel from, and illustrated by, the phenomena of a hollow or transparent sphere, as of glass. Thus, suppose $а$ гс to represent the carth as transparent; and 'its equator as divided into 24 equal parts, by so many meridian semicircles $a$, $b, c, d, e$, sce, one of which is the geographical meridian of any given place, as London, which it is supposed is at the point $a$; and if the bour of 12 were marked at the equator, both on that meridian and the opposite one, and all the rest of the hours in order on the other mericlians, those meridians would be the henr circles of London: be. cause, as the sun appears to move round the carth, which is in the centre of the visible heavens, in 24 hours, he will pass from one meridian to another in one hour. Then, if 
the sphere had an opake axis, as PEp, terminating in the poles $P$ and $p$, the shadow of the axis, which is in the

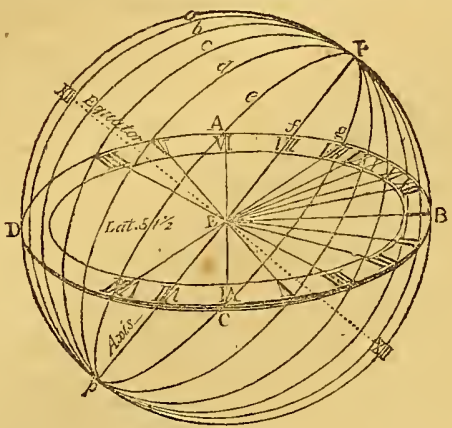

same plane with the sun and witl each meridian, would fall upon every particular meridian and hour, when, the sun came to the plane of the opposite meridian, and would consequently show the time at London, and at all other places on the sane meridian. If this sphere were cut through the niddle by a solid plane $A \mathrm{BCD}$ in the rational horizon of London, one half of the axis $\mathrm{EP}$ would be above the plane, and the other half below it; and if straight lines were drawn from the centre of the plane to those points where its circumference is cut by the hour circles of the sphere, those lines would be the hour lines of an horizontal dial for London; for the shadow of the axis would fall upon each particuliar hour line of the dial, when it fell upon the like bour circle of the sphere.

It the plane which cuts the sphere be upright, as AFCG, touching the given place, for example, London, at $\mathbf{F}$, and

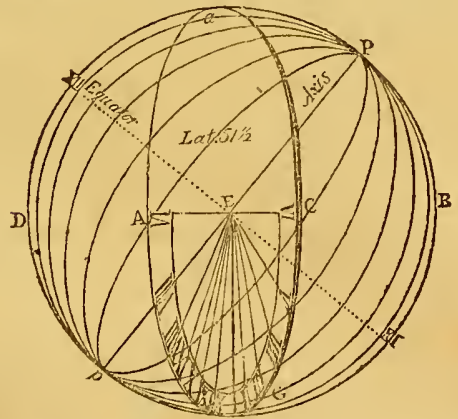

directly opposite to the necridian of London, it will then bscome the plane of an ercet direct suuth dial; and if right lines be drasw from its centre $E$, to those points of its circumference where the hour circles of the sphere cut it, these will be the hour lines of a vertical or direct south dial for London, to which the hours are to be sct in the figure, contrary to thuse on an horizontil dial; and the lower half $x p$ of the axis will cast a sluadow on the hour of the day in this dial, at the same time that it would fall upon the like hour circle of the sphere, if the dial plane was not in the way.
If the plane, still facing the meridian, be made to incline, or recline, any number of degrees, the hour circles of the spherc will continue to cut the cdge of the plane in those points to which the hour lines must be drawn straight from the centre; and the axis of the sphere will cast a shadow on these lines at the respective hours. The like will still hold, if the plane be made to decline by any number of degrees from the meridian towards the east or west; provided the declination be less than 90 degrees, or the reclination be less than the co-latitude of the place; and the axis of the sphere will be the guomon: otherwise, the axis will have no elevation above the plane of the dial, and cannot be a gnomon.

Thus it appears that the plane of every dial represents the plane of some great circle on the earth, and the guomon the earth's axis; the vertex of a right gnomon the centre of the earth or visible heavens; and the plane of the dial is just as far from this centre as from the vertex of the stile. The earth itself, compared with its distance from the sun, is considered as a point; and therefore, if a small sphere of glass be placed upon any part of the earth's surface, so that its axis be parallel to the axis of the earth, and the suhere have such lines upon it, and such planes within it, as above described; it will show the hours of the day as tiuly as if it were placed at the earth's centre, and the shell of the earth were as transparent as glass. Ferguson, lect. 10.

The principal writers on Dials, and Dialling, are the following: Vitruvius, in his Aichitecture, cap. 4 and 7 , lib. 9: Sebastian Munster, his Horolographia: John Dryander de Horologiorum varia Compositione : Conrade Gesner's Pandectæ: Andrew Schoner's Gnomonica: Fred. Commandine de Horologiorum I escriptione: Joan. Bapt. Benedictus de Gnomonum Umbrarumque Solarium Usu : Joannes Georgins Schomberg, Exegesis Fundameutorum Gnomonicorum : Solomon de Caus, Traité des Horologes Solaires: Joan. Bapt. Trolta, Praxis Horologioruın: Desargues, Maniere Universelle pour poser l'Essieu et placer les Heures et autres Choses aux Cadrans Solaires: Ath. Kircher, Ars nagna Lucis et Umbræ: Hallum, Explicatio Horologii in lIorto Regio Londini: Tractatus Horologiorum Joannis Mark: Clavius, Gnomonices de Horologiis; in which he demonstrates both the theory and the operations after the rigid manner of the ancient inathematicians: Dechales, Ozanam, and Scbottus, gave much easier treatises on this subject; as did also Wolfius in his Elementa: M. Picard gave a new method of making large dials, by calculating the lour lines; and M. Lahire, in his Dialling, printed in 1683, gave a geometrical method of drawing hour lines from certain points, determined by observation. Everhard Walper, iñ 1625, published his Dialling, in which he lays down a method of drawing the primaty dials on very simple principles; and the same principles are also described at length by Sebastian Mluuster, in his Rudimenta Mathematica, publisbed in 1651. In 1672, Sturmius published a new edition of Walper's Dialling, with the addition of a whole secord part, concerning inclining and doclining dials, \&c. In 1708 , the same work was re-published, with Sturmius's additions, and likevise a 4 th part, containing Picard's and Lahire's methods of drawing large dials, which forms much the best and ablest work on the subject. Paterson, Michael, and Muller, have each written on Dialling, in the German language: Coetsius, in his Horologiographia Plana, printed in 1689: 
Gauppen, in his Gnomonica Mechanica: Leybourn, in his Dialling: Bion, in his Use of Mathematical Instruments: WVells, in his Art of Shadows. There is also a treatise by M. Deparceux, 1740. Mr. Ferguson has also written on the same subject, in bis Lectures on Mechanics; besides Emerson, in his Dialling; and Mr. W. Jones, in his Instrumental Dialling. See also my Recreations, vol. 3.

Unizersal Dialling Cylinder, is represented in fig. 2, plate $i x$, where $A \mathrm{BCD}$ is a glass $c y$ lindrical tube, closed at both ends with brass plates, in the centres of which a wire or axis EFG is fixed. The tube is either fixed to an horizontal board $\mathrm{I}$, so that its axis may make an angle with the board equal to that which the earth's axis makes with the horizon of any given place, being thus parallel to the axis of the earth; or it may be made to move on a joint, and clevated for any particular latitude. The 2.4 hour lines are drawn with a diamond on the outside of the glass, equidistant from each other, and parallel to the axis. The xil next $B$ stands for midnight, and the xil next the board $\boldsymbol{H}$ for noon. When the axis of this instrument is elevated according to the latitude, and the board set level, with the line $H \mathrm{~N}$ in the plane of the meridian, and the end towards the north; the axis EFG, when the sun shises on the instrument, will scrve as a gnomon or stile, and cast a sladow on the hour of the day among the parallel hour lines. As the plate AD at the top is parallel to the equator, and the axis EFG perpendicular to it, right lines arawn from the centre to the extremities of the parallels will be the hour lines of an equinoctial dial, and the axis will be the stile. An horizontal plate ef put down into the tube, with lines drawn from the centre to the several parallels, cutting its edge, will be an borizontal dial for the given latitude; and a vertical plate $g c$, fronting the meridian, and touching the tube with its edge, with lines drawn from its centre to the parallels, will be a vertical south dial: the axis of the instrument serving in both cases for the stile of the dial: and if a plate be placed within the tube, so as to decline, incline, or recline, by any given number of degrees, and lines be drawn, as above, a declining, inclining. or reclining dial will be formed for the given latitude. If the axis with the several plates fixed to it be drawn out of the tube, and set up in sunshine in the same position as they were in the tube, $A D$ will be an equjnoctial dial, of an horizontal dial, and $g c$ a vertical soutb dial; and the time of the day will be shown by the axis EFG. If the cylinder were wood, instead of glass, and the parallel lines drawn upon it in the same manner, it would serve to facilitate the operation of inaking these several dials. The upper plate with lines drawn to the several intersections of the parallels, which appears obliquely in fig. 2, would be an equinoctial dial as in fig. 3, and the axis perpendicular to it be its stile. An horizontal dial for the latitude of the elevation of the axis might be made, by drawing out the axis and cutting the cylinder, as at efgh, parallel to the horizontal board in ; the section would be clliptic as in fig. 4. A circle migbt be described on the contre, and lines drawn to the divisions of the dlipse would be the hour lines; and the wire put in its place again, as $\varepsilon$, would be the stile. If this cylinder were cut by a plane perpendieular to the horizontal brard 11 , or to the line sir $N$, beginning at $g$, the plane of the section would be elliptical as in fig. 5 , and lines drawn to the points of intersection of the parallels on its edge would be the hour lines of a vertical direct south dial, which might be made of any shape, either circular or square, and $\mathrm{F}$ the axis of the cylinder would be its stile. Thus also inclining, declining, or reclining dials might be easily constructed, for any given latitude. Ferguson, ubi supra.

DIALL:NG Globe, is an instrument made of brass, or wood, with a plane fitted to the horizon, and an index: particularly contrived to draw all sorts of dials, and to give a clear exhibition of the principles of the art.

Dialling Lines, or Scales, are graduated lines, placed on rules or the edges of quadrants, and other instruments, to expedite the construction of dials. The principal of these lines are, 1. A scale of six hours, which is only a double tangent, or two lines of tangents each of 4.5 degrees, joined together in the middle, and equal to the whole line of sines, with the declination set against the meridian altitudes in the latitude of Lumkn, suppose, or any place for which it is made: the radius of which line of sines is equal to the dialling scale of six hours. 2. A line of latitudes, which is fitted to the hour scale, and is made by this canon: as the radius is to the chord of 90 degrees; so are the tangents of each respective degree of the line of latitudes, to the tangents of other arcs: and then the natural sines of those arcs are the numbers, which, taken from a diagonal scale of equal parts, will graduate the divisions of the line of latitude to any radius. The line of hours and latitudes is generally for pricking down all dials with centres. For the method of constructing these scales, see Sc A LE.

DiaLLING Sphere, is an instrument made of brass, witl several semicircles sliding over each other, on a moveable horizon, to demonstrate the nature of the doctrine of spherical triangles, and to give a true idea of the drawing of dials on all kinds of planes.

DIAMETER, of a Circle, is a right line passing through the centre, and terminated at the circumference on both sides.

The diameter divides the circumference, and the area of the circle, into two equal parts. And half the diameter, or the semi-diamcter, is called the radius.

For the proportion between the diameter and the circumfercuce of a circle, sce CirCLF, and Circumference.

Dinmeter of a Conic Section, or Transerse Diameter, is a right-line passing through the centre of the section, or the middle of the axis.-The diameter bisects all ordinates, or lines drawn parallel to the tangent at its vertex. See Conic Sections.

Conjugate Diameter, is a diameter, in Conic Sections, parallel to the ordinates of another diameter, called the transverse; or parallel to the tangent at the vortex of this other.

DIA NIETER, of any Curve, is a right line which rlivides two other parallel right lines, in such manner that, in each of them, all the segments or ordinates on one side, between the diameter and different points of the curve, are equal to all those on the other side, accorling to Newton's definition of a Diameter.

But some define a cliameter to be that line, whether right or curved, which bisects all the parallels drawn from one point to another of a curve. So that in this way every curve will have a diameter; and hence the curves of the $2 \mathrm{~d}$ order have, all of them, either a right-lined diameter, or else the curves of some one of the conic sections for diameters. And many geometrical curves of the higher orters, my also have for dianeters, curves of more inferior orders. 
Diameter of Grevity, is a right line passing through the centre of gravity.

Diameter, in Astronomy. The diameters of the heavenly bodies are either apparent, i. e. such as they appear to the eye; or real, i. e. such as they are in themselve's.

The apparent diameters are best measured with a micrometer, and are estimated by the measure of the angle they subtend at the eye. These are different in different circumstances and parts of the orbits, or according to the various distances of the luminary; being in the inverse ratio of the distance.

The sun's vertical diameter is found by taking the height of the upper and lower edge of his disk, when he is in the meridian, or near it; correcting the altitude of each edge on account of refraction and parallax; then the difference between the true altitudes of the two, is the true apparent diameter sought. Or the apparent diameter may be determined by observing, with a good clock, the time which the sun's disc takes in passing over the meridian: and here, when the sun is in or near the equator, the following proportion may be used; viz, as the time between the sun's leaving the meridian and returning to it agtin, is to 360 degrees, so is the time of the sun's passing over the meridian, to the number of minutes and seconds of a degree contained in his apparent diameter: but when the sun is in a parallel at some distance from the equator, his diameter measures a greater number of minutes and seconds in that parallel than it would do in a great circle, and takes up proportionally more time in passing over the meridian; in which case we have this proportion: as radius is to the cosine of the sun's declination, so is the time of the sun's passing the meridian reduced to minutes and seconds of a degree, to the arc of a great circle which measures the sun's apparent horizontal diameter. See Transit.

The sun's apparent diameter may also be taken by the projection of lis image in a dark ronm. There are several ways of finding the apparent dianeters of the planets; but the nost certuin mechod is that with the micrometer. In these diameters considerable errors were made by the more ancient astronomers; but, according to the best of the modern observations, those diameters are as below: viz, the surn's diameter is found to vary from $32^{\prime} 38^{\prime \prime} 6$ in January, to $31^{\prime} 33^{\prime \prime} 8$ in July: the apparent diameter of the moon varies from about $29^{\prime} 28^{\prime \prime}$ to $33^{\prime} 36^{\prime \prime}$; her real diameter being about 2180 miles. 'The apparent diameters of the planets, when at their respective mean distances from the earth, are as follow:

$$
\begin{aligned}
& \text { Mercury, Venus, Mars, Jupiter, Sarurn, Herschel, } \\
& 11^{\prime \prime} \quad 58^{\prime \prime} \quad 27^{\prime \prime} \quad 39^{\prime \prime} \quad 18^{\prime \prime} 3^{\prime \prime} 5 . l^{\prime \prime \prime} .
\end{aligned}
$$

And from these apparent diameters, with the respective distances from the barth, the real diameters of the sun and planets have been determined in Euglish miles as below:

Mercury, Vonu, Mars, Jupiter, Saturu, Hersehcl,' Sun, $\begin{array}{lllllll}3224 & 7687 & 4189 & 89170 & 790+2 & 35112 & 883246 .\end{array}$

Observations on the planets Herschel, Saturn, Jupiter, Mars, and the Farth, prove that there is a sensible diffeence between their equatorial and polar diameters; and it is probatle that there is a like diffierence between the diameters of the other planets, but this has not yet been determined by observation. 'T'he mean apparcnt diameters of the planets, as seen firom the sun, have been thus given:

Mercury, Venus, Earth, Mars, Japiecr, Saturn, Hesschel,

$20^{\prime \prime} \quad 30^{\prime \prime} \quad 17^{\prime \prime} \quad 10^{\prime \prime} \quad 37^{\prime \prime} \quad 16^{\prime \prime} 4^{\prime \prime}$.

Diamete of a Column, is its thickness just above the base. From this the module is taken, which measures all the other parts of the column.

Diamerer of the Diminution, is that taken at the top of the shaft.

Diam ETEn of the Swelling, is that taken at the heiglit of one third from the base.

DIAPASON, a musical interval, otherwise called an octave, or eighth; so called, because it contains all the possible diversities of sound. If the lengths of two strings be to each other as 1 to 2, the tensions being equal, their tones will produce an octave.

DIAPENTE, is the perfect fiftl, or second of the concords, making an octave with the Diatessaron. The lengths of tho chórds are as 3 to 2 .

DIAPHANOUS Body or Medium, one that is translucent, or through which the rays of light easily pass; as water, air, glass, talc, fine porcelain, \&c. Sce TransPARENCY.

DIAPHONICS, is sometimes used for tbe science of refracterl sound, as it passes through different inediums.

DIASTYLE, a sort of edifice, in which the pillar's stand at such a distance from one another, that three diameters of their thickness are allowed for the intereolumniation.

DIATESSARON, is the perfect tourth; or a musical interval, consisting of one greater tone, one lesser, and une greater semitone. The lengths of strings to sound the diatessaron, are as 3 to 4 .

DIATONIC, a term signifying the ordinary solt of music, which procecds by tones or degrees, both ascending and descending. It contains or admits only the greater and lesser tone, and the greater semitone.

DIESIS, a division of a tone, less than a semitone; or an interval consisting of a lesser, or imperfect semitone. The Diesis is the smallest and softest change, or infexion, of the voice inaginable. It is also called a feint, and is expressed by a St. Andrew's cross, or salticr.

DIFFERENCE, is the excess by which one magnitude or quantity exceeds another. When a less quantity is subtracted from a greater, the remainder is otherwise called difference.

\section{Ascensional Difference. See Ascenstonat.}

Difference of Latitude, in Geography, \&ic, is an arch of the meridian included between the parallels of latitude in which any two places lie. When the latitudes are both north or both south, the less lat. taken from the greater leaves the difference of latitude; but when the one lat. is north, the other south, their sum shows the dif. of lat.

DiFFERENCE of Longitude, of two places, is au arch of the equator contained between the meridians of those two places, or the measure of the angle formed by their meridians. When the longitudes are both east or both west, the one taken from the other leaves the dif. of longitude; but when the one is east and the other west, they are then ta be added together for the dif. of lungitude.

DIFFERENTIAL, an indefinitely small quantity, part, or difference. By some, the Differential is considered as infinitely small, or less than any assignable quantity; and also as of the same import as fluxion. It is called a Differential, or Differential Quantity, because often considered as the difference betwcen two quantities; and as such it is the foundation of the Differential Calculus. Newton used the term Moment in a like sense, as being the momentary increase or decrease of a variable quintity. $\mathbf{~ I C}$. Lcibnitz and others call it also an infinitesimal. 


\section{1) I IF}

Differential, of the $1 s t, 2 d, 3 d, \& c$, degree. See DIFFERENTIO-DIFFERENTIAL.

Dufferential Calculus, is a method of differencing quantities. See Dufferential Metlood, Calculus, and Finjions.

Differentio-Differential Calculus, is a method of differencing differentiai quantities. As the sign of a differential is the letter $d$ prefixed to the quantity, as $d x$ the differential of $x$; so that of a diflerential of $d x$ is $d d x$, and the differential of $d d x$ is $d d d x$, \&zc; similar to the fluxions $\dot{x}, \ddot{x}, \ddot{x}$, Sc. Thus we have degrees of differentials. The differential of an ordinary quantity, is a differential of the first order or degree, as $d x$; that of the $2 \mathrm{~d}$ degree is $d d x$; that of the $3 d$ degree is $d d d x$, \&xc. The rules for differentials, are the very same as those for fluxions. See FLuXions.

Differinta L, in Logaritums. Kepler calls the logarithms of tangents, Difierentiales; which we usually call artificial tangents.

Differentral Equation, is an equation involving or containing differcntial quantities; as the equation $3 x^{2} d x-2 a x d x+a y d x+a x d y=0$. Some mathematicians, as Stirling, \&c, have also applied the term differential equation in another sense, to certain equations defining the nature of serics.

DIFFERENTIAL Method, a methud of finding quantities by means of theil successive differences. This method is of very general use and application, but especially in the construction of tables of numbers, and the summation of series, \&c. It was first uscd, and the rules of it lajd down, by Briggs, in his Construction of Logarithms and other Numbers, much the same as they were afterwards taught by Cotes, in his Constructio Tabularum per Differentias; as is shown in the Introduction to my Logs. pa. 69 et seq. See Briggs's Arithmetica Logarithmica, cap. 12 and 13, and his Trigonometria Britannica.

The method was next treated in another form by Newton, in the 5 th Lemma of the $3 \mathrm{~d}$ book of his Principia, and in his Methodus Differentialis, published by Jones in 1711, with the other tracts of Newton. 'This author here treats it as a method of describing a curve of the parabolic kind, through any given number of points. He distinguishes two cases of this problem; the first, when the ordinates drawn from the given points to any line given in position, are at equal distances from one another; and the $2 \mathrm{~d}$, when these ordinates are not at equal distances. He has given a solution of both cases, at first without demonstration, which was afterwards supplied by himself aud others: see his Methodus Differentialis above mentioned; and Stirling's Explanation of the Newtonian Differential Method, in the Philos. Trans. No 362 ; Cotes, De. Methodo Differentiali Newtoniana, published with his Ilarmonia Mensurarum; llerman's Phoronomia; and Le Seur and Jacquier, in their Commentary on Newton's Principia. It may be observed, that the muthods there demonstrated, by some of these authors, extend to the description of any algebraic curve through a given number of points, which Newton, writing to Leibnitz, mentions as a problem of the greatest use.

By this mothod, some terms of a series being given, and conceived as placed at given intervals, any intermediate term may be found; which therufore gives a method for interpolations. Briggs's $\Lambda$ rith. Log. ubi supra ; Newton, Metl. Differ. prop. 5; Stirling, Methodus Differentialis.
Thus also may any curvilineas figure be squared nearly, having some few of its ordinates. Newton, ibid. prop. 6 ; Cotes De Metbod. Differ.; Simpson's Míathematical Dissert. pa. 115. And thus may mathematical tables be constructed by interpolations: Briggs, ibid. Cotes Canunotechnia. The successive differences of the ordinates of parabolic curves, becoming ultimately cqual, and the intermediate ordinate required being determined by these differences of the ordinates, is the reason for the name Differential Method.

To be a little more particular.-The first case of Newton's problem amounts to this: A series of numbers, placed at equal intervals, being given, to find any intermediate-number of that series, when its interval or distance from the first term of the series is given. Subtract each term of the scries from the next following term, and call the remainders first differences; then subtract in like manner each of these differences from the next following one, calling these remainders $2 \mathrm{~d}$ differences; again, subtract each $2 \mathrm{~d}$ difference from the next following, for the $3 \mathrm{~d}$ differences; and so on: then if $\mathrm{A}$ be the 1 st term of the series, $d^{\prime}$ the filst of the 1st difierences,

$d^{\prime \prime}$ the first of the $2 \mathrm{~d}$ difierences,

$d^{\prime \prime \prime}$ the first of the $3 \mathrm{~d}$ difierences, $8 \mathrm{xc}$;

and if $x$ be the interval or distance between the first term of theseries and any term sought, $T$, thatis, let the number of terms from a to $\mathrm{T}$, both included, be $\triangle x+1$; then will the term sought, $\mathrm{T}$, be $=$

$\mathrm{A}+\frac{x}{3} d^{\prime}+\frac{x}{2} \cdot \frac{x-1}{2} d^{\prime \prime}+\frac{x}{1} \cdot \frac{x-1}{2} \cdot \frac{x-2}{3} d^{\prime \prime \prime}$, Scc.

Hence, if the differences of any order become equal, that is, if any of the diffs. $d^{\prime \prime}, d^{\prime \prime \prime}$, \&c, become $=0$, the above series will give a finite expression for $T$, the term sought; it being fevident, that the series must terminate when any of the diffs. $d^{\prime \prime}, d^{\prime \prime \prime}, \& c$, become $=0$.

It is also evident that the co-efficients $\frac{x}{1}, \frac{x}{1}, \frac{x-1}{2}$, \&c of the difierences, are the same as the terms of the binomial theorem.

For Example, Suppose it were rcquired to find the $\log$. tangent of $5^{\prime} 1^{\prime \prime} 12^{\prime \prime \prime} 24^{\prime \prime \prime \prime}$, or $5^{\prime} 1^{\prime \prime} \frac{62}{300}$, or $5^{\prime} 1^{\prime \prime} \cdot 2066$, \&c.

Take out the log. tangents to several minutes and seconds, and take their first 'and second differences, as below :

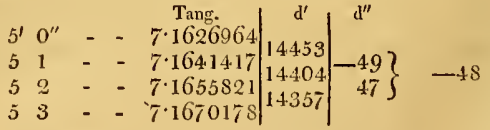

IIcre $\Lambda=7 \cdot 16414.17 ; x=\frac{62}{3} \frac{2}{0} ; d^{\prime}=14404 ;$;nd the mean second difference $d^{\prime \prime}=-4.8$. Hence

$$
\begin{aligned}
& \text { A - . . . } 7 \cdot 16+1417 \\
& x d^{\prime} \ldots \ldots . . .2977 \\
& \frac{x}{1} \cdot \frac{x-1}{1} d^{\prime \prime}--4
\end{aligned}
$$

Theref. the tang. of $5^{\prime} 1^{\prime \prime} 12^{\prime \prime \prime} 24^{\prime \prime \prime \prime}$ is $\overline{7 \cdot 16 \cdot 14398}$

Hence may be deduced a method of finding the sums of the terms of such a series, calling its terms $A, B, c D$, Sc. For, conceive a n:w series having its 1 st term $=0$, its $2 \mathrm{~d}=A$, its $3 \mathrm{~d}=\mathrm{A}+\mathrm{B}$, its $4 \cdot \mathrm{l}_{1}=A+\mathrm{n}+\mathrm{c}$, its 5 th $=\mathrm{A}+\mathrm{B}+\mathrm{C}+\mathrm{D}$, and so on; then it is plain that assigning one tern of this series, is fiuding the sum of all the terms $\Lambda, n, c, D, \alpha c$. Now since these terms are the difliences of the sums, $0, A, A+B, A+B+C, \& C$; 
and as some of the differences of $A, B, C, \& c$, are $=0$ by supposition; it follows that some of the differences of the sums will be $=0$; and since in the series $A+\frac{x}{1} d^{\prime}+$ $\frac{x}{1} \cdot \frac{x-1}{2} d^{\prime \prime} \& \mathrm{c}$, by which a term was assigned, $\mathrm{A}$ represented the 1 st term; $d^{\prime}$ the 1 st of the 1st differences, and $x$ the interval between the first term and the last; we are to write 0 instead of A, A instead of $d^{\prime}, d^{\prime}$ instcad of $d^{\prime \prime}, d^{\prime \prime}$ instead of $d^{\prime \prime \prime}, \& \mathrm{c}$, also $x+1$ instead of $x$; which being done, the series expressing the sums will be $0+$ $\frac{x+1}{1} \mathrm{~A}+\frac{x+1}{1} \cdot \frac{x}{2} d^{\prime}+\frac{x+1}{1} \cdot \frac{x}{2} \cdot \frac{x-1}{3} d^{\prime \prime}$, \&c. Or, if the real number of terms of the lines be called $z$, that is, if $z=x+1$, or $x=z-1$, the sum of the series will be $A z+\frac{z}{1} \cdot \frac{z-1}{2} d^{\prime}+\frac{z}{1} \cdot \frac{z-1}{2} \cdot \frac{z-2}{3} d^{\prime \prime}$ \&c. See De Moivre's Doct. of Chances, pa. 59,60; or his Miscel. Analyt. pa. 15 ; ; or Simpson's Essays, pa. 95.

For Exumple. To find the sum of six terms of the series of squares $1+4+9+16+25+36$, of the natural numbers.

$\begin{array}{cccc}\text { Terms } & d^{\prime} & d^{\prime \prime} & d^{\prime \prime \prime} \\ 1 & 3 & 2 & \\ 4 & 5 & 2 & 0 \\ 9 & 7 & 2 & 0 \\ 16 & 9 & 2 & \\ 25 & 9 & & \end{array}$

Here $\mathrm{A}=1, d^{\prime}=3, d^{\prime \prime}=2, d^{\prime \prime \prime} \& \mathrm{c}=0$, and $z=6$; therefore the sum is $6+\frac{6}{T} \cdot \frac{5}{2} \cdot 3+\frac{6}{3} \cdot \frac{5}{2} \cdot \frac{4}{3} \cdot 2=6+45+$ $40=91$ the sum required, viz, of $1+4+9+16+25$ +36 .

A variety of examples may be seen in the places above cited, or in Stirling's Methodus Differentialis, \&c.

As to the Differential method, it may be observed, that though Newton and some others have treated it as a method of describing an algebraic curve, at least of the parabolic kind, through any number of given points; yet the consideration of curves is not at all essential to it, though it may help the imagination. The description of a parabolic curve through given points, is the same problem as the finding of quantities from their given differences, which may always be done by algebra, by the resolution of simple equations. See Stirling's Method. Differ. pa. 97. This ingenious author has treated very fully of the differential method, and shown its use in the solution of some difficult problems. See also SER IES.

Differentral Scale, in Algebra, is used for the scale of relation subtracted from unity. Sie Recurring SERIES.

DIFFRACTION, a term first used by Grimaldi, to denote that property of the rays of light, which others have called Inflection; the discovery of which is attributed by some to Grimaldi, and by others to Dr. Hooke.

DIGBY (Sir KENELM), a celebrated linglish philosupher, was born at Gothurst in Buckinghamshire, 1603. He was the descendant of an ancient fanily: his greatgrandfather, with six of his brothers, fought valiantly at Busivorth-field on the side of Henry 7 th, agaipst Richard the 3d. Ilis father, Sir Everard, engaged in the gurpowder plot against Janes the 1st, for which he was bcheaded; but the son was restored to his estate, and had afterwards several appointments under King Charles the 1st, who granted him letters of reprisal against the Venetians, from whom he took several prizes with a small fleet which he commanded. He fought the Venetians near the VoL.l. port of Scanderoon, and bravely made his way through them with his booty.

In the beginning of the civil wars, he exerted himself greatly in the king's cause, and was afterwards imprisoned, by order of the parliament; but was set at liberty in 1643 . He afterwards compounded for his estate; but being banished from England, he retired to France, and was sent on two embassies to Pope Innocent the 10th, from the queen, widow to Charles the 1st, whose chancellor he then was. On the restoration of Charles the $2 d$, he returned to London; where he died in 1665 , at 62 years of age.

Digby was a great lover of learning, and translated several authors into English, as well as published several works of his own; as, 1 . Observatious upon Dr. Brown's Religio Medici, 1643.-2. Observations un part of Spenser's Fairy Queen, 1644.-3. A Treatise of the Nature of Bodies, 1644.-4. A Treatise declaring the Operations and Nature of Man's Soul, out of which the Immortality of reasonable souls is evinced: works that discover great penetration and extensive knowledge.

He applied much to chemistry ; and found out several useful medicines, which he distributed with a liberal hand. He particularly distinguished himself by his sympathetic powder for the cure of wounds at a distance; his discourse concerning which made great noise for a while. He held several conferences with Descartes, about the nature of the soul, and the principles of things. At the beginning of the Royal Society, he became a distinguished member, being one of the first council. And he had at his own house regular levees or meetings of learned men, to improve themselves in knowledge, by conversing with one another.

This eminent person was, for the early pregnancy of his talents, and his great proficiency in learning, compared to the celebrated Picus de Mirandola, who was one of the wonders of human nature. Yet his knowledge, though various and extensive, probably appeared greater than it really was; as he had all the powers of elocution and address to recommend it. He knew how to shine in a circle, either of ladies or philosophers; and was as much attended to when he spoke on the nust trivial sabjects, as when he spoke on the most important. It has been said that one of the princes of It:]y, who had no child, was desirous that his princess should bring him a son by Sir Kenelm, whom he esteemed a just model of perfection.

DIGGES (LEONARD), an ingenious mathematician in the 16 th century, was descended from an ancient family, and born at Digges-court in the parish of Barham in Kent ; but in what year is not known; and died about the year 1574. He was educated for some time at Oxford, where he laid a good foundation of learning; but retiring from thence, he prosecuted his studies, and became an excellent mathematician, a skilful architect, and an expert surveyor of land, \&ce. He composed several books : as, 1. Tectonicum: bricfly showing the exact Measuring, and speedy Reckoning of all manner of Lands, Squares, Tinber, Stones, Steeples, \& c ; 1556, 4to.; this was augmented and published again by his son Thomas Digges, in 1592 ; and also reprinted in 1647.-2. A Geometrical Practical Treatise, named Pantometria, in three books, which ho lelt in manuscript; but after his death, his son supplied such parts of it as were obscure and imperfect, and published it in 15.91, folio; subjoining, "A Discourse Geometrical of the five regular and Platonic bodies, containing 311 
sundry theoretical and practical propositions, arising by mutual conference of these solids, Inscription, Circumscription, and Transformation."-3. Prugnostication Everlasting of right good effect: or Choice Rules to judge the weather by the Sun, Moon, and Stars, \&c ; in 4to, 1555, 1556 , and 1564: corrected and augmented by his son, with divers general tables, and many compendious rules, in 4 to, 1592.

DIGGES (Thомas), only son of Leonard Digges, after a liberal education from his tenderest years, went and studied for some time at Oxford; and by the improvements be made there, and the subsequent instructions of his learued father, became one of the best mathematicians of his age. When Queen Elizabeth sent some forces to assist the oppressed inhabitants of the Netherlands, Mr. Digges was appointed muster-master-general of them; by which he became well skilled in military affairs; as his writings afterwards showed. He died in 1595 .

Mr. Digges, beside revising, correcting, and enlarging some pieces of his father's, already mentioned, wrote and published the following learned works himself: viz, 1. Alæ sive Scalæ Mathematicæ: or Mathematical Wings or Ladders, 1573, 4to. A book which contains several demonstrations for fiuding the parallaxes of any comet, or other celestial body, with a correction of the errors in the use of the radius astronomicus. -2. An Arithmetical Military Treatise, containing so much of Arithmetic as is necessary towards military discipline, 1579,4 to -3 . A Geometrical Treatise, named Stratioticos, requisite for the perfection of Soldiers, 1579,4 to. This was begun by his father, but finished by himself. They were both reprinted together in 1590, with several additions and amendments, under this title: "Au Arithmetical Warlike Treatise, named Stratioticos, compendiously teaching the Science of Numbers, as well in Fractions as Integers, and so much of the Rules and Equations. Algebraical, and Art of Numbers Cossical, as are requisite for the profession of a souldier. Together with the Moderne militaire discipline, offices, lawes, and orders in every well-governed campe and armie, inviolably to be observed." At the end of this work there are two pieces; the first, "A bricfe and true report of the proceedings of the Earle of Leycester, for the reliefe of the towne of Slace, from his arrival at Vlishing, about the end of June 1587, untill the surrendrie thereof 26 Julii next ensuing. Whereby it shall plainelie appear, his excellencie was not in anie fault for the losse of that towne :" the second, " A briefe discourse what orders were best for repulsing of foraine forces, if at any time they should invade us by sea in Kent, or elsewherc." $\rightarrow$. A perfect Description of the Celestial Orbs, according to the most ancient doctrine of the Pythagoreans, \&c. This was placed at the end of his father's "Prognostication Everlasting, \&c." printed in 1592, 4.to. - 5. A humble Motive for Association to maintain the religion established, $1601,8 v 0$. To which is added, his Letter to the same purpose to the archbishops and bishops of England.-6. England's Defence: or, A Treatise conccrning Invasion. 'This is a tract of the same nature with that printed at the end of his Stratioticos, and called, A briefe Discourse, \&c. It was written in 1599, but not published till 1686.-7. A Letter printed before Dr. John Dee's Parallatica Commentationis praxcosque nuclens quidan, 1573,4 to.-Beside these, and his Nova Corpora, he left several methematical treatiscs ready for the press; which, by reason of law- suits and other avocations, he was hindered from publishing.

If our author was great in himself, he was not less so in his sun, Sir Dudley Digges, so celebrated as a politician and elegant writer; and also in his grandson Dudley.

DIGIT, in Arithmetic, one of the ten characters 0 , $1,2,3,4,5,6,7,8,9$, by means of which all numbers are expressed.

Digit, in Astronomy, is the measure by which the part of the luminaries in eclipses is estimated, being the 12th part of the diameter of the luminary. Thus, an eclipse is said to be of 10 digits, when 10 parts out of 12 of the diameter are in the eclipsed part; when the whole of the luminary is exactly covered, the digits eclipsed are just 12 ; and when the luminary is more than covered, as often happens in lunar eclipses, then more than 12 digits are said to be eclipsed: Thus, if the diameter or breadth of the earth's shadow, where the moon passes through, be equal to one diameter and a half of the moon, then $18^{\sigma}$ or digits are said to be eclipsed. These digits are by Wolfius, and some others, called Digiti Ecliptici.

DILATATION, a motion of the parts of a body by which it expands, or opens itself, so as to occupy a greater space.-Many authors confound dilatation with rarefaction; but the more accurate writers distinguish between them; defining dilatation to be the expansion of a body into a greater bulk, by its own elastic power; and rarefaction, the like expansion produced by means of heat.

The moderns have observed, that bodies which, after being compressed, and again left at liberty, restore themselves perfectly, do endeavour to dilate themselves with the same force by which they are compressed; and accordingly they sustain a force, and raise a weight equal to that with which they are compressed.

Again, bodies, in dilating by their elastic power, excrt a greater fouce at the beginning of their dilatation, than towards the end; as being at first more compressed; and the greater the compression, the greater the elastic power and endeavour to dilate. So that these three, the compressing power, the compression, and the elastic power, are always equal.

Finally, the mution by which compressed borlies restore themselves, is usually accelerated : thus, when compressed air begins to restore itself, and dilate into a greater space, it is still compressed ; and consequently a new impetus is still impressed upon it, from the dilatative cause; and the former remaining, with the increase of the cause, the effect, that is the motion and velocity, must be increased also. Indeerl it may happen, that where the compression is only partial, the motion of dilatation shall not be accelcrated, but retarded; as is evident in the compression of a spunge, soft bread, gauze, \&c.

DIMENSION, the extension of a body, considered as measurable. Hence, as we conceive a body extended, and measurable in length, breadth, and depth, dimension is considered as threefold, viz, length, breudth, and thickness. So a line has one dimension only, viz, length; a superficies two, length and breadth; and a body or solic has thrce, viz, length, breadth, and thickness.

Dinension is also particularly used with regard to the powers of quantities in equations. Thus, in a simple equation $x=a+b$, the unknown quantity is only of one dimension; in a quadratic equation, $x^{2}=a^{2}+b^{2}$, it is of two dimensions; in a cubic $x^{3}=a^{3}+b^{3}$, it is of three dimensions; and so on. 
DIMINISHED Angle, in Fortification. See Angle.

DIMINUTION, in Music, is the abating something of the full value or quantity of any note.

Diminution, in Architecture, is a contraction of the upper part of a column, by which its diameter is made less than that of the lower part.

DINOCRATES, a celebrated ancient architect of $\mathrm{Ma}$ cedonia, of whom several extraordinary things are related. $\mathrm{He}$ was taken, by Alexander the Great, into Egypt, where he employed him in marking out and building the city of Alexandria : here he formed a design, in which Mount Athos was to be laid out into the form of a man, in whose left hand were to be represented the walls of a great city; and all the rivers of the mount flowing into his right, and from thence into the sea. Another memorable instance of Dinocrates's architectonic skill, is his restoring and building, in a more august and magniticent manner than before, the celebrated temple of Diana at Ephesus, after Herostratus, for the sake of imınortalizing his name, had destroyed it by fire. A third instance, more extraordinary and wonderful than either of the former, is related by Pliny in his Natural History; who says he had formed a scheme for building the dome of the temple of Arsinoë at Alexandria of loadstone, iu order that her image made entirely of iron should hang in the middle of it, as if it were in the air; but the king's death, and his own, prevented the execution or attempt of this project.

DIOCLES, a Greek mathematician of some celebrity, but more particularly remembered for his invention of the curve called the cyssoid or the Cissoid of Diocles. See C1ssoID.

DIONYSIUS, the Periegetic, an ancient geographer and poet. Pliny says, he was a native of the Persian Alexandria, afterwards called Antioch, and at last Charrax; that lie was sent by Augustus, to survey the eastern part of the earth. Dionysius wrote a great number of pieces, enumerated by Suidas and his commentator Eustathius: but his Periegesis, or Survey of the World, is the only one now extant; which may be well esteemed one of the most exact systems of ancient geography, since Pliny himself proposed it as his pattern.

DIONYSIAN, or Victorian Period. See Period.

DIOPHAN'TINE Problems, are certain questions relating to square and cubic numbers, and to right-angled triangles, \&c; the nature of which were first and chiefly treated by Diophantus, in his Arithmetic, or rather A]gebra.

In these questions, it is chiefly intended to find commensurable numbers to answer indeterminate problems; which often bring out an infinite number of incommensurable quantities. For example, If it be proposed to find a right-angled triangle, whose three sides $x, y, z$ are expressed by rational numbers; from the nature of the figure it is known that $x^{2}+y^{2}=z^{2}$, where $z$ denotes the hypothenuse. Now it is plain that $x$ and $y$ may also be so taken, that $z$ shall be irrational; for if $x=1$, and $y=2$, then is $z=\sqrt{ } 5$.

Now the art of resolving such problems, consists in ordering the unknown quantity or quantities, in such a manner, that the square or higher power may vanish out of the squation, and then by means of the unk nown quantity in its first dimension, the equation may be resolved without having recourse to incommensurables. For example, in the "quation above, $x^{2}+y^{2}=z^{2}$, suppose $z=x+u$, then is $x^{2}+y^{2}=x^{2}+2 x u+u^{2}$, out of which equation $x^{2}$ vanishes, and then it is $y^{2}=2 x u+u^{2}$, which gives $x=\frac{y^{2}-u^{3}}{2 u}$ : hence, assuming $y$ and $u$ equal to any numbers at pleasure, the three sides of the triangle will be $y, \frac{y^{2}-u^{2}}{2 u}$, and $\frac{y^{2}+u^{z}}{2 u}$, or out of fractions, $2 u y$, and $y^{2}-u^{2}$, and $y^{2}+u^{2}$, which are all rational whenever $y$ and $u$ are rational. For example, if $y=2$, and $u=1$, then $y^{2}-u^{2}=3$, and $2 u y=4$, and $y^{2}+u^{2}=5$. It is evident that this problem admits of infinite numbers of solutions, as $y$ or $u$ may be assumed any numbers at pleasure.

But, in questions of this kind, very few directions can be given, as the method of solution must be left, in a great measure, to the skill and ingenuity employed in the substitution \& c, for unknown quantities. Thus, a person acquainted with this branch of algebra, would have inmediately substituted in the above equation,

$$
\begin{aligned}
& x=v^{2}-u^{2}, \text { or } x^{2}=v^{4}-2 v^{2} u^{2}+u^{4}, \\
& \text { and } y=2 v u, \text { or } y^{2}=4 v^{2} u^{2}, \\
& \text { hence } z=v^{2}+u^{2} \text {, or } z^{2}=v^{4}+2 v^{2} u^{2}+u^{2} .
\end{aligned}
$$
where $v$ and $u$ may be taken at pleasure.

Or if the equation had been $x^{2}-y^{2}=z^{2}$. Then by making $x^{2}=\left(v^{2}+u^{2}\right)^{3}$, and $y^{2}=4 v^{2} u^{2}$, we should bave $z^{2}=\left(v^{2}-u^{2}\right)^{2}:$ where $v$ and $u$ may be taken any num. bers whatever, as before.

$E x$. 2. To find the general values of $x$ and $y$, such, that $x^{2}+y$, and $x^{2}-y$, may be both complete squares. $\mathrm{Or}$, which is the same thing, to find three square numbers in arithmetical progression.

First, put $\left.x^{2}+y=w^{2}\right\}$

$$
\left.\begin{array}{l}
\text { put } x^{2}+y=w^{2} \\
\text { and } x^{2}-y=z^{2}
\end{array}\right\} \text { Then } 2 x^{2}=w^{2}+z^{2} \text {, }
$$

or $2 x^{2}-w^{2}=z^{2}$. Now make $x=w+m$, or $2 x^{2}=2 w^{2}$ $+4 w m+2 m^{2}$ : hence $z^{2}=w^{2}+4 w m+2 m^{2}$, which must be a complete square; therefore substitute $z=w+r$, and we obtain $w^{2}+4 v m+2 m^{2}=v v^{2}+2 v r+r^{2}$,

or $(4 m-2 r) w=r^{2}-2 m^{2}$; hence we have $w=\frac{r^{3}-2 m^{3}}{4 m-2 r}$; $w+m=x=\frac{r^{3}-2 r m+2 m^{3}}{4 m-2 r} ; w+r=z=\frac{-r^{2}+4 r m-2 m^{3}}{4 m-2 r}$.

$\mathrm{Or}$, since the denominators are equal, we may make $w=r^{2}-2 m^{2}, x=r^{2}-2 r m+2 m^{2}$, and $z=-r^{2}+4 r m$ $-2 m^{2}$; where $r$ and $m$ may be assumed at pleasure, cither integral or fractional. In the first values of $w, x$, and $z$; by assuming $r=6$, and $m=4$, we have $w=1, x=5$, and $z=7$; and therefore

1,25 , and 49 , the three square numbers required. Now some of those general expressions being once obtained, they may be frequently introduced to abreviate the operations in more intricate questions. For an example of which we will propose the following problem.

Ex. 3. To find the values of $x$ and $y$, such, that unity being adderl to each of them, as also to their sum, and difference, the four numbers thus arising shall be square numbers.

$$
\text { First substitute }\left\{\begin{array}{r}
x+1=l^{2} \\
y+1=n^{2} \\
x+y+1=p^{2} \\
x-y+1=s^{2} .
\end{array}\right.
$$

Iere, we see that the thrce squares $s^{2}, l^{2}$, and $p^{2}$, are in arithmetical progression, their common difference being $y$.

The question therefore is reduced to the finding of three square numbers in arithmetical progression, whose common difference +1 may be a square; therefore from the foregoing example let $s^{2}=\left(r^{2}-2 m^{2}\right)^{2}, l^{2}=\left(r^{2}-2 m m+\right.$ $\left.2 m^{2}\right)^{2}, p^{2}=\left(-r^{2}+4 r m-2 m^{2}\right)^{2}$, which we know are in arithmetical progression, their common difference being $4 r^{3} m-12 r^{2} m^{2}+8 r m^{1}=y$; and we have to ind $4 r^{3} m$. 3 II 2 
$-12 r^{2} m^{2}+8 r m^{3}+1$ a square. Substitute its ront $=1$ $+4 r m^{3}$, and we thus obtain $16 r^{2} m^{6}=4 r^{3} m-12 r^{2} m^{2}$; or dividing by $4 r^{2} m$, we have $r=4 m^{5}+3 m$ : where $m$ may be assumed at pleasure. By taking $m=1$, we have $r=7$, also $p^{2}=529$, and $l^{2}=1369$; again $l^{2}-1=1368=x$, and $l^{2}-p^{2}=840=y$, which are the two numbers sought,

$$
\text { the four squares being }\left\{\begin{array}{r}
1368+1=37^{2} \\
840+1=29^{2} \\
1368+840+1=47^{2} \\
1368-840+1=23^{2} \text {. }
\end{array}\right.
$$

The operations in the solution of Diophantine problems may also be frequently simplified, from certain known properties of numbers, when they are included under peculiar forms, particularly when the answer is required to be given in integers; many ingenious specimens of which may be seen in the solution of Diophantine problems in the New Series of the Mathematical Repository, by Mr. Leybourn, of the Royal Military College.

Another method, which is frequently adopted to considerable advantage, is by means of the development of certain algebraical functions; an example of which will be seen in the following problem.

Ex.4. To find a number, from which two given squares being severally subtracted, each of the remainders may be a square.

Theorem. Let $a^{2}$ and $b^{2}$ represent the given squares. Resolve $\frac{\pi}{2} a-\frac{1}{2} b$ into any tivo unequal factors $m$ and $m^{\prime}$; and $I a-\frac{1}{2} b$ into any two unequal factors $n$ and $n^{\prime}:$ then will $\left(m^{2}+n^{2}\right) \cdot\left(m^{\prime 2}+n^{\prime 2}\right)$ be the number required.

Demonstration. For $\left(m^{2}+n^{2}\right) \cdot\left(m^{\prime 2}+n^{\prime 2}\right)$

$=\left\{\begin{array}{r}\left(m m^{\prime}+n n^{\prime}\right)^{2}+\left(m n^{\prime}-n m^{\prime}\right)^{2} \\ \operatorname{or}\left(m m^{\prime}-n n^{\prime}\right)^{2}+\left(m n^{\prime}+n m^{\prime}\right)^{2},\end{array}\right.$

as is immediately seen by the development of the above formula. And since $\frac{1}{2} a+\frac{1}{2} b=m n^{\prime}$, and $\frac{1}{2} a-\frac{1}{2} b=n n^{\prime}$, therefore $\left.a=m m^{\prime}+n n^{\prime}\right\}$

and $\left.b=m x^{\prime}-n n^{\prime}\right\}$ Consequently, the square of each being taking from the above product, will leave a square remainder.

Suppose, for example, 4 and 36 were the two squares, then $\frac{6+2}{2}=4.1$, and $\frac{6-2}{2}=2.1$, therefore $\left(4^{2}+1^{2}\right)$. $\left(2^{2}+1^{2}\right)=85$ the number sought; for $85-4=81=9^{2}$
and $85-36=49=7^{2}$.

For further information on this interesting branch of algebra, the reader may consult Fermat, Bachet, Ozanam, Kersey, Saunderson, and Euler, particularly the latter, who in the $2 \mathrm{~d}$ vol, of his Algebra has carried this subject to a considerable length, which, with the additions by $\mathrm{La}$ Grange, may be consilered as one of the most conplete pieces of Diophantine algebra. But with regard to the Theory of Numbers, which is intimately connected with the above subject, the best works are Le Gendre's Essai sur la Theorie des Nombres; and the Recherches arithmetiques par ML. Gauss.

DIOPHAN'TUS, a celebrated mathematician of Alexandria, who has commonly been reputed to be the inventor of algebra; but notwithstanding his is the earliest work extant on that science, there appears to be no reason for supposing that he was the inventor of it: indecd the work itscif is almost it proof to the contrary; for though he dues not mention any other authors in the course of his bouk, yet lie evidently treats the subject as one alrearly known. It is not certain when Diophantus lived. Some have placed him before Christ, and some alter, in the reigns of Nero and the Antoinines; but all with equal uncertainty.
It seems he is the same Diophantus who wrote the Canon Astronomicus, wbich Suidas says was commented on by the celebrated Hypatia, daughter of Theon of Alexandria. His reputation must have been very high among the ancients, since they ranked him with Pythagnras and Euclid in mathematical learning. Bachet, in his notes upon the 5th book De Arithmeticis, has collected, from Dioplantus's epitaph in the Anthologia, the following circumsiances of his life; namely, that he was married when be was 33 years old, and had a son born 5 years after; that this son died when he was 42 years of age, and that his father did not survive him above 4 years; from which it appears, that Diophantus lived to the age of 84 years.

This epitaph as translated by Bachet is as Jollows:

Hic Diophantus habet tumulum, qui tempora vitæ Illiuss mutâ denotat arte tibi

Egit sextantem juvenis, lanugine malas Vestire hinc copit parte duodecina

Septante uxori post hæc sociatur, et anno Formosus quinto nascitur indè puer

Semissem ætatis postquam attigit ille paterna, Infelix subitâ morte peremptus obit

Quatuor æstates genitor lugere superstes Cogitur; hinc annos illius assequere.

Diophantus wrote 13 books of Arithmetic, or Algebra, which Regiomontanus, in his preface to Alfraganus, tells us, are still preserved in manuscript in the Vatican library: indeed we are informed by Diophantus himself that his work consisted of 13 books, viz, at the end of his address to Dionysius, placed at the beginning of the work, and hence Regiomontanus might be led to say the 13 books were in that library; but no more than 6 whole books, with part of a seventh, have ever been publislied; and I am of opinion there are no more in being; indeed Bombelli, in the preface to his Algebra, written 1572, says there were but 6 of the books them in the library, and that he and another person were about a translation of them.

Those 6 books, with the imperfect 7 th, were first published at Basil by Xylander in 1575 , but in a Latiu version only, with the Greck scholia of Maximus Planudes on the first two books, and observations of his own; the same books were afterwards publishod in Greck and Latin at Paris in 1621, by Bacliet, an ingenious and learned Frenchman, who made a new Latin translation of the work, and enriclied it with learned commentarics; and al.though he did not entirely neglect the notes of Xylander in his edition, yet lie treated the scholiast Planucies with the utmost contempt. He scems to intimate, in what he says upon the 28 tli question of the $2 \mathrm{~d}$ book, that the 6 books which we have of Diophantlis, may be nothing more than a collection made by soms novice, of such propositions as he judged proper, out of the whole 13 : but Fabricius thinks there is no just ground for such a supposition. This work was afterwards (1670) revised and republished by Fermat, who added many excellent note's in addition to those of Bachet.

DlOPJER, or Droptra, the same with the index or alhidade of an astrolabe, or other such instrument.

Droptua was an instrument invented by llipparchus, which served for several uses; as, to level water-courses; to take the height of towers, or places at a distance; to determine the places, magnitudes, and distances of the planets, \&c.

DIOPTRICS, called also Annclastics, is the doctrinc of refracted vision; or that part of optics whicli explains the 
effects of light as refracted by passing through different mediums, as air, water, glass, \&cc, and especially lens’s.

Dioptrics is one of the most uscful and pleasant of all the human sciences; as it brings the remotest objects near hand, enlarging the smallest objects so as to show their minute parts, and even giving sight to the blind; and all this by the simple means of the attractive power in glass and water, causing the rays of light in their passage through them to alter their course according to the different substances of the medium; whence it happens that the objects seen through thern, do, in appearance, alter their magnitude, distance, and situation.

The ancients have treated of direct and reflected vision; but what we have of refracted vision, is very imperfect. J. Baptista Porta wrote a treatise on retraction, in 9 books, but without any great improvement. Kepler was the first who succreded in any great degree, on this subject ; having demonstrated the properties of sphericallenses very accurately, in a treatise first published anno 1611 . After Kepler, Galileo gave sonewhat of this doctrine in his Letters; as also an Lixamination of the Preface of Johannes Pena upon Euclid's Oplics, concerning the use of optics in astronomy. Descartes also wrote a treatise on Dioptrics, commonly annexed to his Priticiples of Philosophy, which is one of his best works; where the true manner of vision is more distinctly explaincd than by any former writer, and in which is contained the true law of refraction, which was first discovered by Sncll, though the name of the inventor is suppressed: here are also laid down the properties of elliptical and hyperbolical lenses, with the practice of grinding glasses. Dr. Barrow has treated on Dioptrics in a very eleyant manner, though rather too briefly, in his Optical Lectures, read at Cambridge. Húygens's Dioptrics, is also an excellent work of its kind. Molyneux's Dioptrics is a work rather heavy and dull. Beside these, there are scveral other treatises on Dioptrics, such as Hartsolier's lissai de Dioptrique. Cherubiu's Dioptrique Oculaire, et La Vision Parfaite. David Gregory's lilements of Dioptrics. 'Traber's Nervus Opticus. Zahn's Oculus Artificialis Teledioptricus. Dr. Smith's Optics, which is a complete work of its kind. Wolfius's Dioptrics, contained in his Elementa Mathěseos Universalis. But especially the Treatise on Optics, and the Optical Lectures of Newton, in whose experinents are contained far more discoveries than in all the former writers. Lastly, this science was perfected by Dollond's discorery of the achromatic glasses, by which the colours are obviated in refracting telescopes.

The laws of Dioptrics aro delivered under the article REFuATron, LENS, \&c; and the application of them in the construction of telescopes, miscruscopes, and other dioptrical instruments, under the articles TELESCope and IItcroscopr.

DIP of the Horizon. Sec DEPREssion.

Dipping-Needle, or Inclinatory Needle, a magnetical needle, so hung, as that, instrat of playing horizontally, atsd pointing out nortl and south, one end dips, or incline's to the horizon, and the other points to a certisin degree of elevation above it.

The inventor if the dipping-needle was Robert Norman, a compass-maker at Ratclific, about the ycar 1580 : this is not only testiged by his wwn account, in his New Attractive, but also by Dr. Gilbert, Mr. William Burrowes, Mr. Henry loond, and other writers of that time, or soon after it. T'he occision of the discovery he him- self relates, viz, that it being his custom to finish, and hang the needles of his compasses, before he touchet thin, he always found that, immediately after the touch, the 'north point would dip or decline downward, pointing in a direction under the horizon; so that, to balance the needle again, he was always obliged to put a piece of wax on the south end, as a counterpoise. The constancy of this effect led him, at length, to observe the precise quantity of the dip, or to measure the greatest angle which the needle would make with the horizon. This, in the year 1576 , he fóund at London was $71^{\circ} 50^{\prime}$. It is not quite certain whether the dip varies, as well as the horizontal direction, in the same place. Mr. Graham made a great many experiments with the dipping-needle in 1723 , and found the dip between 74 and 75 degrees. Mr. Nairne, in 1772 , found it somewhat above $72^{\circ}$. And by many observations inade since that time at the Royal Society, the medium quantity is $72^{\circ} \frac{x}{2}$. Indeed, in 1812 it is only stated at $70^{\circ} 32^{\prime}$ at the Royal Society. The trifling difference between the first observations of Norman, aud the last of Mr. Naime and the Royal Society, lead to the opinion that the dip is unalterable; and yet it may be difficult to account for the great difference between these and Mr. Graham's numbers, considering the well-known accuracy of that ingenious gentleman. Philos. Trans. vol. 45, pa. 279 , vol. 62 , pa. 476 , vol. $69,70,71$. Sec atso the description of a new dipping-needle, by Dr. Lorimer, in the Philosophical Transactions 1775 ; and in the supplement to Cavallo's Treatise on Magnetism.

It is certain however, from many experiments and observations, that the dip is different in different latitudes; and that it increases in going northward. It appears from a table of observations made with a marine dipping-needle of Mr. Nairne's, in a voyage towards the north pole, is 1773 , that

in Iatitude $60^{\circ} 1 \mathrm{~S}^{\prime}$ the dip was $75^{\circ} \mathrm{O}^{\prime}$, in latitude 7045 the dip was 7752 , in latitude 8012 the dip was 8152 , and

in latitude 8027 the dip was $822 \frac{1}{2}$.

Sce Phipps's Voyage, pa. 12:. See also the Observations of Mr. Hutchins, made in Hudson's Bay and Straits, Philos. Trans. vol. 65, pa. 129.

Burrowes, Gilbert, Ridley, Bond, Ec, endeavonred to apply this discovery of the dip to the finding of the latitude; and Bond, going still farther, first of any proposed finding the longitude by it; but for want of observations. and experiments, he could not go any length. $M_{1}$. Whiston, being furnished with the further observations of Colond Windham, Dr. Halley, Mr. Pound, Mr. Cunningham, M. Noel, M. Feuille, and his own, made great improvements in the cloctrine and use of the dipping-needle, brought it to more certain rules, and cndeavoured in good earncst to find the longitude by it. For this purpose, he observes, 1st, That the true tendency of the north or south: end of every nagnetic needle, is not to that point of the horizon, to which the horizontal needle points, but towards another, directly under it, in the same vertical, and in different degrees under it, in different ages, and at difficrent places. 2dly, That the power by which the horizontal. needle is governed, and all our navigation usually directed, it is proved is only one quarter of the power by which the dipping-necdle is moved; which should render the larter. far the nore effectual and accurate instrument. 3dly, That a dipping-needle of a toot long will plainly show an alteration of the angle of inclination, in these parts of the 


\section{I R}

world, in half a quarter of a degree, or $7 \frac{x}{2}$ geographical miles; and a needle of 4 feet, in 2 or 3 miles; i. c. supposing these distances taken along, or near a meridian. 4 thly, A dipping-needle, 4 feet long, in these parts of the world, will show an equal alteration along a parallel, as auother of a foot long will show along a meridian; i. e. that will, with equal exactness, show the longitude, as this the latitude.

This depends on the position of the lines of equal dip, in these parts of the world, which are found to lie about 14 or 15 degrees from the parallels. Hence he argues, that as we can have needles of $5,6,7,8$, or more feet long, which will move with strength sufficient for exact observation; and since microscopes may be applied for viewing the smallest divisions of degrees on the limb of the instrument, it is evident, that the longitude at land may thus be found to less than 4 miles.

And as there have been many observations made at sea with the same instrument by Noel, Feuille, \&c, which have determined the dip usually within a degree, sometimes within $\frac{1}{2}$ or $\frac{1}{3}$ of a degree, and this with small needles, of 5 or 6 , or at the most 9 inches long; it is inferred, that the longituche may be found, even at sea, to less than half a quarter of a degree. This premised, the observation itself follows.

To find the Longitude or Latitude by the Dipping-Needle. -If the lines of equal dip, below the horizon, be drawn on maps, or sea-charts, from good observations, it will be easy, from the longitude known, to find the latitude; and from the latitude knowm, to find the longitude either at sea or land.-Suppose, for example, a person travelling or sailiug along the meridian of London, should find that the angle of dip, with a needle of one foot, was $75^{\circ}$; the chart will show that this meridian, and the line of dip, meet in the latitude of $53^{\circ} 11^{\prime}$; which is therefore the latitude sought.-Or if be be travelling or sailing along the parallel of London, i. e. in $51^{\circ} 31^{\prime}$ north latitude, and find the angle of $\operatorname{dip} 74^{\circ}$; then this parallel, and the line of this dip, will meet on the map in $1^{\circ} 46^{\prime}$ of cast longitude from London; which therefore is the longitude sought.

Many other persons have uselessly endeavoured to determine the longitude of places fros the dip of the magnetic needle. One among this number, was Mandilo, a Genois pilot, who contrived a small weight for measuring the degree of inclination of the needle; and though nothing could be much more absurd, he continued in his opinion to the last, and died under the persuasion that he had truly discovered a method of determining the longitude, and that justice had not been done him, nor his merits fairly appreciated.

DIRECT, in Arithmetic, is when the proportion of any terms, or quantities, is in the natural or direct order in which they stand; being the opposite to inverse, which considers the proportion in the inverted order of the terms, So, $3: 4:: 6: 8$ directly; or $3: 4:: 8: 6$ inversely.

Rule of Three DuREcr, is when both pairs of terms are in direct proportion.

Dinfecr, in Astronomy. $\Lambda$ planet is said to be direct, or its motion direct, when it goes forward by its proper motion in the zodiac, according to the succession or orler of the signs; or when it appears so to do, to an observer standing on the earth. Whereas it is said to be, or to move, retrograde, when it appears to go the contrary way, or backward; and to be stationary, when it seems not to move either way.
D I $\mathrm{R}$

Direct Dials. See Dial and Dialling.

Dinect, in Optics. Direct vision is that performed by direct rays; in contradistinction to vision by refracted, or reflected rays. Direct vision is the subject of optics, which prescribes the laws and rules of it.

Direct Rays, are those which pass on in right lines from the object to the eye, without being turned out of their rectilinear direction by any intervening body, either opaque or pellucid; or without bcing either reflected or refracted.

Direct Sphere. See Rigut Sphere.

DIRECTION, in Astronomy, the motion and other phenomena of a planet, when direct.

Line of DrRecrios, in Gunnery, is the direct line in which a piece is pointed. Sometimes a line of direction is marked on the upper side of the gun, by a small notch or slit, or knob, in the base and muzzle rings: but, unless the two wheels of the carriage stand equally high, this line will be fallacious; for which reason gunners commonly find a new line of direction every time, by means of a plummet.

Line of Druector, in Mechanics, denotes the line in which a body moves, or endeavours to proceed.

1 Angle of Direcrion, is that comprehended between the lines of direction of two conspiring powers.

Quantity of DiREcTION, is used for the probluct arising from multiplying the velocity of the common centre of gravity, in a system of bodies, by the sum of their masses. In the collision of borlies, the quantity of direction is the sane both betore and after the impulse.

DIRECTION of the Loadstone, that property by which the magnet, or a needle touched by it, always presents one of its ends towards one of the poles of the world, and the opposite end to the other pole. This is also called the polarity of the magnet or needle. The attractive property of the magnet was kuown long before its directive; and the directive long before the inclinatory.

Number of Direction, is the number of days that Septuagesima Sunday falls after the 17 th of January. Sco NUMBER.

DIRECTLY, in Geometry: We say two lines lie directly against each other, when they are parts of the same right line : also quantities are said to be directly proportional, when the proportion is according to the order of the terms; in contradistinction to inversely, or reciprocally proportional, which is taking the proportion contrary to the order of the terms.

In Mechanics, a body is said to strike or impinge directly against another body, when the stroke is in a direction perpendicular to the surface at the point of impact. And a sphere, in particular, strikes directly against another, when the line of direction passes through both their centres.

1)IRECTRIX, a particular right line, perpendicular to the axis of a conic section, and much referesl to by the authors who demomstrate the properties of those curves from the description of them in plano. Thus, the indefinite right line $A$ is is the directrix, and is so placed, that if any point $\mathrm{r}$ be assumed without it, and while the line $\mathrm{D}$ revolves about $r$ as a centre, a point n moves in it in such a manuer, that its distance from $\mathrm{F}$ shall always be to $\mathrm{CD}$, its distance from the line $\mathrm{AB}$, in a constant

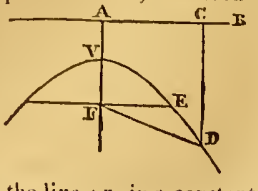
. 


\section{D $1 \mathrm{~S}$}

ratio; then the curve $\mathrm{V} D$, described hy the point $\mathrm{D}$, is a conic section. And the curve is an ellipse, or a parabola, or an hyperbola, according as $\mathrm{FD}$ is less than, equal to, or greater than, $\mathrm{CD}$, or $\mathrm{FV}$ than $\mathrm{VA}$. The constant ratio of $\mathrm{FD}$ to $\mathrm{CD}$, or of $\mathrm{FV}$ to $\mathrm{VA}$, is called the determining ratio. In the ellipse, the distance $A \nabla$ of the directrix from the vertex, is greater than $v F$ the distance of the focus from the vertex; in the parabola $A V$ is equal to $\mathrm{VF}$; but.in the hyperbola $A v$ is less than $v F$.

Directrix, in a Parabola, a line perpendicular to the axis produced, at the distance of the focus without the vcrtex; as shown above.

DIRIGENT, a term expressing the line of motion, along which a describent line, or surface, is carried in the genesis of any plane or solid figure.

Thus, if the line A B move parallel to itself, and along the line $\mathrm{AD}$, so that the point $A$ always keeps in the line $A D$, and the point $B$ in the line $\mathrm{BC}$; a parallelogram $\mathrm{ABCD}$ will be formed; of which the line $A B$ is the describent, and the line $A D$ the

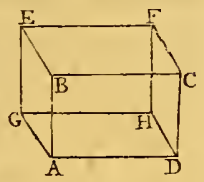
dirigent. So also, if the surface $A B E G$ be supposed carried along the line $\mathrm{AD}$, in a position always parallel to itself at its first situation, the solid AF will be formed; where the surface $A \mathrm{E}$ is the describent, and the line $A D$ is the dirigent.

DISC, or Disk, the body or face of the sun or moon; such as it appears to us; for though they be really spherical bodies, they are apparently circular planes.-The diameter of the disc is considered as divided into 12 equal parts, called digits; by means of which it is, that the magnitude of an eclipse is measured, or estimated.-In a total eclipse of either of those luminaries, the whole disc is obscured, or darkenerl; in a partial eclipse, only part of them.

Illuminated Disc of the Earth. Sce CrRCLE of Illuminution.

Disc, in Optics, the magnitude of a telescope glass, or the widtl of its aperture, whatever its figure be, whether a plane, convex, meniscus, or the like.

DISCHARGER, or Discinarging Rod, in Electricity, consists of a bandle of glass or baked wood, $A$, and two bent metal rods, $\mathrm{B} \mathrm{B}$, terminating in poiists, and capable of being screwed into the knobs DD, which move by a joint $c$, fixed to the

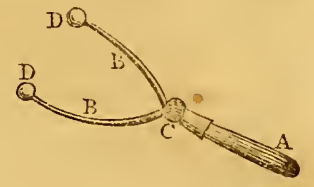
handle $A$. 'Thus it may be used either with the points or balls, as occasion requires; and by being made moveable on a joint, it may be applied to larger or smaller jars at pleasure. By bringing one of these knobs or points to one coaterl side of a charged electric, and the other to the other side, or to any conductor connected with it, the communication is completed between the two sides, and the electric is rlischarged.-For the description and use of a Universal Discharger, by Mr. IIenly, with which many curious experiments may be performed, see Cavallo's Electricity, pa. 164.

DISCORD, the relation of twn sounds which are always, and of themselves disagreeable, whether applied in succession or consonance
423 D D I S

DISCOUNT, or Rebate, is used for an allowance made on a bill, or any other debt not yet become due, in consideration of making present payment of the bill or debt.

Among merchants and traders, it is usual to allow a sum for discount that is equal to the interest of the debt, calculated for the time till it bccomes due: but this is not the correct or just sum; for, as the true value of the discount is equal to the difference between the debt and its present worth, it is equal only to the interest of that present wortb, instead of the interest on the whole debt. And therefore the rule for finding the true discount is this;

As the amount of $100 l$, for the given rate and time :

Is to the given sum or debt : :

So is the interest of $100 l$, for the given rate and time :

To the discount of the debt.

So that, if $p$ be the principal or debt, $r$ the rate of interest per cent, and $t$ the time;

then as $100+r t: p:: r t: \frac{p r t}{160+r t}$, which is the true discount. Hence also $p-\frac{p r t}{100+r t} \frac{100 p}{100+r t}$ is the present worth, or sum to be received.

For Ex. Suppose it be required to find the discount of $250 l$, for five months, at the rate of 5 per cent per annum interest. Here $p=250, r=5$, and $t=\frac{5}{12}$ or 5 months; then $\frac{p r t}{100+r t}=\frac{250 \times 5 \times \frac{4}{12}}{100+5 \times \frac{4}{12}}=\frac{250 \times 25}{1.200+25}=\frac{250}{49}=5 l 2 s \frac{2}{49}$ the discount sought.

A Table of Discounts may be scen in Smart's Tables of Interest, the use of which makes calculations of discount very easy.

Discousx is also the name of a rule in books of Arithmetic, by which calculations of Discount are made.

DISCRETE, or Disju ncr, Proportion, is that in which the ratio between two or more pairs of numbers is the same, and yet the proportion is not continued, so that the ratio may be the sane between the consequent of one pair and the antecedent of the next pair.

Thus, if the numbers or proportion $6: 8:: 3: 4$ be considered : the ratio of 6 to 8 is the same as that of 3 to 4 , and therefore these four numbers are proportional: but it is only discretely or disjunctly, and not continued; for 8 to 3 is not the same ratio as the former; that is, the proportion is broken off between 8 and 3 , and not continued throughout, as it is in these following four numbers, which are called continual proportionals, viz, $3: 6:: 12: 24$.

Discrete 2uantity, is such as is not continued and joined together. Such for instance is any number; for. its parts, being distinct units, cannot be-united into one continuum ; for in a continuum there are uo actual determinate parts before division, but they are potentially infinite : so that it is usually and truly said that continued quantity is divisible in infinitum.

DISDIAPASON, in Music, a compound concord, in the quadruple ratio of 4 to 1 , being a fifteenth or double cighth, and is produced when the voice goes from the first tone to the fifteenth.

DISJUNCT Proportion. See Discrete Proportion.

DISK. See Disc.

DISPART, a term in Gunuery, used for a mark set upon the muzzle-ring of a piecc of ordnance, of such height, that a sight-line taken from the top of the base ring near the vent or touch-hole, to the top of the dispart near the muzzle, may be parallel to the axis of the con- 


\section{I S}

[ 424$]$

cave cylinder; for which reason it is evident, that the height of the dispart is equal to the difference between the radii of the piece at the base and muzzle-rings, or to half the difference of the diameters there. Hence comes the common method of disparting the gun, which is this: Take, with the calipers, the two diameters, viz, of the base ring and the place where the dispart is to stand, subtract the less froin the greater, and take half the difference, which will be the length of the dispart; this is commonly cut to that length from a small bit of wood, and so fixed upright in its place with a bit of wax or pitch.

DISPLRSION, in Dioptrics, is the divergency of refracted rays of light.

Point of Dispension, is a point from which refracted rays begin to diverge, when their refraction renders them divergent.- It is called point of dispersion, in opposition to the point of concourse, or point in which converging rays concur after refraction. But it is more usual to call the latter the focus, and the former the virtual focus.

Dispensjox of Light, occasioned by the refrangibility of the rays, or the nature of the refracting mediam. See ABERRATION, and INFLECTION.

Dr. Wollaston's mode of examining refractive and dispersice powers is described in Philos. Trans. for 1802 , or Nicholsun's Journ. vol. 4, pa. 89 . Extensive tables of refractive and dispersive powers are given in Dr. T. Young's Nat. Philos. vol.2, pa. 296, 299.

DISSIPATION, in Physics, a gradual, slow, insensible loss or consumption of the minute parts of a body; or, more properly, the flux by which they fly off and are lost. See EirfuUvia.

Circle of Dissipation or Aberration, in Optics, denotes that circular space on the retina of the eye, which is occupied by the rays of each pencil in indistinct vision: thus, if the distance of the object, or the constitution of the eye, be such, that the image falls beyond the retina, as when objects are too near; or before the retina, when thé rays have not a sufficient divergency; the rays of a pencil, instead of being collected into a central point, will be dissipated over this circular space: and, all other circumstancs being alike, this circle will be greater or less, according to the distances from the retina of the foci of refracted rays. But this circle causes no perceptible difference in the distinctness of vision, unless it exceed a certain magnitude: as soon as that is the case, we begin to perceive an indistinctness, which increases as that circle increases, till at length the object is lost in confusion. This circle is also greater or less, according to the greater or less magnitude of the visible object; and though it be not easy to assign the diameter of the said circle, it seems very probable that vision continues distinct for alt such distances, or so long as these circles, or the pencils of light from them, do not touch one another on the retina; and the indistinctness begins when the said circles begin to interfere. It has been often observed, that a precise union of the respective rays on the retina, is not necessary to distinct vision; but the first author whon ascertained the fact beyond all doubt, was Dr. Jurin. See a variety of observations and experiments on this subject, in his Essay on Distinct and Indistinct Vision, in Smitl's Optics, Appendix. In the Philos. Trans. for 1789 , pa. 256, is an excellent paper on this subject by Dr. Maskelyne; in which be computes the cliameter of the circtu of clissipation it . 002667 of an incli, making it answer to an external angle of $15^{\circ}$, which he shows is very compatible with distinct vision. See also Mloos, and Vision.

Radius of Dissipation, is the radius of the circle of dissipation.

DlSSOLVENT, something that dissolves; i. e. divides, and reduces a body into its smallest parts.

DISSOLUTION, is a separation of the structure of a body, into small or minute parts. According to Newton, and others, this is effected by certain powerful attractions.

DISSONANCE, or Discond, is a false consonance or concord; being produced by the mixture or meeting of two sounds which are disagreeable to the ear.

DISTANCE, properly speaking, denotes the shortest line between two points, objects, \& c.

Distance, in Astronomy, as of the sun, planets, comets, \&c. The Real Distances are found from the parallaxes of the planets, \&c. See Paraliax, Plane't, and Transit. The distance of the earth from the sun has been determined at 95 millions of miles, by the late transits of Venus: and from this one real distance, and the several relative distances, by analogy are found all the other real distances, as in the table below.

'The Proportional or Relative Distances of the planets are very well deduced from the theory of gravity: for Kepler has long since discovered, and Newton has demonstrated, that the squares of their periodical times are proportional to the cubes of their distances. Kepler's Epit. Astron. lib. 4 ; Newton's Principia, lib. 3, phæn. 4 ; and Gregory's Astron. book 1, prop. 40. If therefore the mean distance of the earth from the sun be assumed, or supposed 100000 , we shall then, from the foregoing analogy, and the known periodical times, obtain the relative distances of the other planets: thus,

The Periodical Revolutions in Days and Parts. Mercury, Venus, Eartl, Mars, Jupiter, Saturn, Herschel, $87 \frac{23}{2} 4^{3} \quad 224 \frac{17}{24} \quad 365 \frac{1}{4} 686 \frac{23}{24} 4332 \frac{1}{2} \quad 10759 \frac{7}{24} 30445$. Relative Distance of the Plane/s.

\begin{tabular}{|c|c|c|c|c|c|}
\hline - & Kiepler. & Cassini. & Halley. & $\begin{array}{l}\text { De la } \\
\text { Lande. }\end{array}$ & $\begin{array}{l}\text { Log. of those } \\
\text { distances. }\end{array}$ \\
\hline Mercury & $38800^{\circ}$ & 35506 & 38710 & 35710 & $9 \overline{5878221}$ \\
\hline Venus & 72413 & 72340 & 72333 & 72333 & $y \cdot S 593379$ \\
\hline Earth & 100000 & 100000 & 100000 & 100000 & 10.0000000 \\
\hline Mars & 152350 & 152573 & 152369 & 152369 & $10 \cdot 1828975$ \\
\hline Vesta & $-\cdots$ & $\ldots$ & $-\cdots$ & 235513 & \\
\hline Juno & & & --- & $266+00$ & \\
\hline Pallas & & -- & -- & 276500 & ono- \\
\hline Ceres & $\cdots$ & $\ldots-$ & --- & 276700 & \\
\hline Jupiter & 520000 & 520290 & 520098 & 520279 & $10 * 7162364$ \\
\hline Saturn & 951000 & 954180 & $95+007$ & 954072 & $10 \cdot 9795813$ \\
\hline $\left.\begin{array}{l}\text { Uranus or } \\
\text { Herschel }\end{array}\right\}$ & & & & 1908180 & 11.2806193 \\
\hline
\end{tabular}

And from these relative distances, the real distance of any planet is easily found, that of the earth being known, by the following proportion, namely; as $100000: 95000000$, or as 1 to 950 , so is the relative distance in the table, to the real distance required.

Hence we find the real distances expressed in eren millions to be,

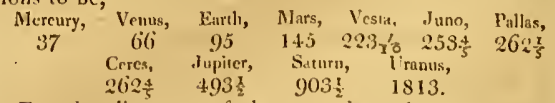

For the distances of the secondary planets from the centres of their respective primaries, see SATELIITES. 
As to that of the fixed stars, as having no sensible parallax, little more can be done than-to guess at it.

Dist a N E of the Sun fiom the Moon's node, or apogee, is an arch of the ecliptic, intercepted between the sun's true place and the moon's node, or apogee. See None.

Curtate Distance. See CuRtate.

Distance of the Bastions, in Fortification, is the side of the exterior polygon.

Accessible Dis T A N ces, in Geometry, are measured with the chain, decempeda or ten-foot rod, or the like.

Inaccessible Distances, are found by taking bearings to them, from the two extremities of a line whose length is given. Various ways of performing this may be seen in my Treatise on Mensuration, sect. 3, on Heights and Distances.

Dista yce, in Geography, is the arch of a great circle intercepted between two places.

To find the distance of two places, $A$ and B, far remote from each other. Assume two stations, $c$ and $D$, from which both the places $A$ and $B$ may be seen; and there, with a theodolite, observe the quantity of the angles $A C D$, $B C D, A D C, B D C$, and measure any distance as $A C$. Then, in the triangle $\triangle C D$, there are given the angles $A C D$, $A D C$, and the side $A C$; to find the side

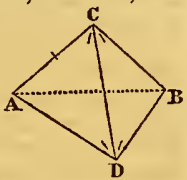
CD.--Next, in the triangle $B C D$, there are given the angles $\mathrm{BCD}, \mathrm{BDC}$, and the side $\mathrm{CD}$; to find the side BC.-Lastly, in the triangle $A B C$, there are given the angle $A C B$, and the sides $A C$, , $B$; to find the side $A B$, which is the distance sought. And in these operations, the triangles may be computed either as plane triangles, or as spherical ones, as the case may require, or according to the magnitude of the distances.

The DIsTANCE of a remote object may also be found from its height. This admits of several cases, according as the distances are large or small, \&c. 1st, Suppose that from the top of a tower at $A$, whose height $A \mathrm{~B}$ is 120 feet, there be taken the angle $\mathrm{BAC}=33^{\circ}$, and the angle $\mathrm{BAD}=64^{\circ} \frac{\mathrm{I}}{2}$, to two trees, or other objects, $\mathrm{C}, \mathrm{D}$; to find the distance betwcen them $\mathbf{C D}$, and the distance of each from the bottom of the tower at $\mathrm{B}$.
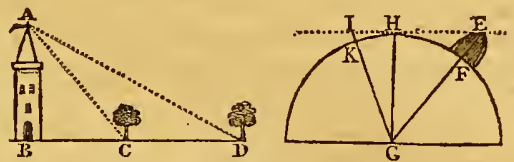

First, rad. : tang. $\angle \mathrm{B} \boldsymbol{\mathrm { A }} \mathrm{D}:: \mathrm{AB}: \mathrm{B} \mathbf{D}=251 \cdot 585$, next, rad. : tang. $\angle \mathrm{BAC}:: \mathbf{A} \mathrm{B}: \mathbf{B C}=77 \cdot 929$, their difference is the distance $\quad \mathrm{CD}=173.656$.

2d, Suppose it be required to find the distance to which an object can be seen, by knowing its altitude; ex. gr. the Peak of Teneriffe, whose height is said to be 3 miles above the level of the sea, supposing the circumference of the earth 25,000 miles, or the diameter 7958 miles. Let FG be the radius $=3979, \mathrm{EF}=3$ the height of the mountain, and $E j$ a tungent to the earth at the point $H$, which is the farthest point to which the top of the mountain $\mathrm{E}$ can be seen. Herc, in the right-angled triangle $\mathbf{E G} H$, are given the hypothenuse $\mathrm{E} \mathbf{G}=3982$, and the $\log \mathrm{G} \mathbf{I}=3979$; to find the other $\operatorname{leg} \| \mathrm{E}=154 \cdot \frac{\mathrm{T}}{2}$ miles = the distance sought nearly. Or, rather, as EG : 6 Ir : : rad. : cosin. $\angle \mathrm{G}=2^{0} 13^{\prime} \frac{\pi}{2}$; then as $360^{\circ}: 2^{0} 13^{\prime} \frac{\mathrm{x}}{2}:: 25,000: 154 \frac{\mathrm{T}}{2}$ miles $=$ the arch of dist, nf sought, the same as before.

Vol. I.
$3 \mathrm{~d}$, If the eye, instead of being in the borizon at $\mathrm{r}$, were elevated above it at $I$, any known height, as suppose $26+$ fcet, or $\frac{1}{20}$ th of a mile, as on the top of a high eminence; then the mountain can be secn much farther off along the line $\mathbf{I}$, , and the distance will be the two tangents $I H$ and $H E$, or rather the two ares $K \boldsymbol{H}$ and Hr. Hence, as above, as $1 \mathrm{G}: \mathrm{GH}::$ rad. : cosin. $\angle \mathbf{I G H}=17^{\prime 2}$; then as $360^{\circ}: 17^{\prime} \frac{2}{7}:: 25,000: 20$ miles $=$ the arc $\mathrm{kH}:$ this added to the former arc $\mathrm{HF}=154 \frac{\mathrm{T}}{2}$, makes the whole arc $\mathrm{kF}=174 \frac{\mathrm{T}}{2}$ miles, for the whole distance to which the top of the mountain can be seer in this case.

Apparent Dista x CE, in Optics, that distance which we judge an object is placed at when seen afar off. This is usually very different from the true distance; because we are apt to think that all very remote objects, whose parts cannot well be distinguished, and which have no other object in view near them, are at the same distance from us, though perhaps the one is thousands of miles nearer than the other, as is the case with regard to the sun and moon.

M. Lahire enumerates five circumstances, which assist us in judging of the distance of objects; viz, their apparent magnitude, the strength of the colouring, the direction of the two eyes, the parallax of the objects, and the distinctness of their small parts. On the contrary, Dr. Smith maintains, that we judge of distance principally, or solely, by the apparent magnitude of objects; and concludes universally, that the apparent distance of an object seen in a glass, is to its apparent distance seen by the naked eye, as the apparent magnitude to the naked eye is to its apparent magnitude in the glass. But it was long since observed by Alhazen, that we do not judge of distance merely by the angle under which objects are seen; and Mr. Robins clearly shows that Dr. Smith's hypothesis is contrary to fact, in the most common and simple cases. Thus, if a double convex glass be held upright before some luminous object, as a candle, there will be seen two images, one crect, and the other inverted; the first is made simply by reflexion from the nearest surface; the second by reflexion from the farther surface, the rays undergoing a re fraction from the first surface both before and after the reflexion. If this glass lias not too short a focal distance, when it is held near the object, the inverted image will appear larger than the other, and also nearer; but if the glass be carried off from the object, though the eye remain as near to it as before, the inverted image will be diminished so much faster than the other, that at length it will appear much less than it, but still nearer. Herc, says Mr. Robins, two images of the same object are seen under one view, and their apparent distances immediately compared; and it is evident that those distances have no necessary connexion with the apparent magnitude. This experiment may be made still more convincing, by sticking a piece of paper on the midlle of the lens, and viewing it through a short tube. He observes further, that the apparent magnitude of very distant objects is neither determined by the magnitude of the angle only under which they are seen, nor is the exact proportion of that angle compared with their true distance, but is compounded also with a deception concerning that distance; so that if we had no idea of difference in the distance of objects, each would appear in magnitude proportional to the augle under which $\mathrm{jt}$ was scen; and if our supposition of the distance were always just, our idea of their niagnitude would be unvaried, in all distances; but in proportion as we err in our conception of their distance, the greater angle sug3 I 
gests a greater magnitude. By not attending to this compound effect, Mr. Robins apprehends that Dr. Smith was led into his mistake.

Dr. Porterfield has made severa] remarks on the five methods of judging concerning the distance of objects above recited from $M$. Lahire; and he has also added to them one more, viz, the conformation of each eye. See Circle of Dissipation. This, he says, can be of no use to us, with respect to objects that are placed without the limits of distinct vision. But the greater or less confusion with which the object appears, as it is more or less removed from those limits, will assist the mind in judging of its distance; and the more confused it appears, the farther we shall imagine it to be from us. However, this confusion has its limits; for when an object is placed at a certain distance from the eye, to which the breadth of the pupil bears no sensible proportion, the rays proceeding from a point in the object may be considered as parallel; in which case, the picture on the retina will not be sensibly more confused, though the object be removed to a much greater distance. The most universal, and often the most sure means of judging of the distance of objects, he says, is the angle made by the optic axes: our two eyes are like two different stations, by the assistance of which, distances are taken; and this is the reason why those persons who have lost the sight of one eye so frequently miss their mark in pouring liquor into a glass, snuffing a candle, and such other actions as require that the distance be exactly distinguished. With respect to the method of judging by the apparent magnitude of objects, he observes that this can only serve when we are otherwise acquainted with their real magnitude. Thus he accounts for the deception to which we are liable in estimating distances, by any extraordinary inagnitudes that terminate them; as, in travelling towards a large city, castle, or cathedral, we fancy they are nearer than they really are. Hence also, animals and small objects seen in a valley contiguous to large mountains, or on the top of a mountain or high building, appear exceedingly small. Dr. Jurin accounts for the last-recited phcnomenon, by observing that we have no distinct idea of distance in that oblique dircction, and therefore judge of them mercly by their pictures on the eyc.

Dr. Porterfield observes, with respect to the strength of colouring, that if we are assured they are of a similar colour, and one appears more bright and liveiy than the other; we judge that the brighter object is the nearer. When the small parts of objects appear confused, or do not appear at all, we judge that they are at a great distance, and vice versa; brcause the inage of any object, or part of an object, diminishes as the distance of it increases. Finally, we judge of the distance of objects by the number of intervening bodies, by which it is divided into separate and distinct parts; and the more this is the case, the greater will the distance appear. Thus distances upon uneven surfaces appear less than upon a plane, because the inequalities do not appear, and the whole apparent distance is diminished by the parts that do not appear in it; and thus the banks of a river appear contiguous as viewed at a distance, when the river is low and not seen. Accidens de la Vue, pa. 358. Smith's Optics, vol. 1, pil. 52, and Rem. pa. 51. Robins's Tracts; vol. 2, pa. 230, 247, 251. Porterficla on the Eye, vol. 1, pa. 105, vol. 2, pa. 387. See Priestley's Hist, of Vision, pa. 205, and pa. 689.
Distance, in Navigation, is the number of miles or leagues that a ship has sailed from any point or place. See SA I LING.

Line of Distance, in Perspective, is a right line drawn from the eye to the principal point: as the line or, drawn between the eye at $o$, and the principal point $F$; which being perpendicular to the plane, $P Q$ is therefore the distance of the eye from that plane.

Point of Distance, in Perspective, is a point in the horizontal line at the same distance from the principal point, as the eye is from the same. Such are the points $P$ and $Q$, in the horizontal line $P Q$, whose distance from the principal point $\mathrm{F}$, is equal to that of the eye from the same point.

DISTINCT Base, in Optics, is that distance from the pole of a convex glass, at which objects, beheld through it, appear distinct and well defined : so that the distinct base is the same with what is otherwise called the focus. The distinct base is caused by the collection of the rays procceding from a single point in the object, into a single point in the representation: and therefore concave glasses, which do not unite, but scatter and dissipate the rays, can have no real distinct base.

Distinct Vision. See VIsIon.

DITCH, in Fortification, called also Foss, and Moat, is a trench dug round the rampart, or wall of a fortified place, between the scarp and counterscarp.

Ditches are either dry, or wet, that is having water in them; both of which have their particular advantages. The earth dug out of the ditch serves to raise the rampart. The ditch in front should be of such breadth as that tall trees may not leach over it, being from 12 to 24 fathoms wide, and 7 or 8 feet deep. The ditches on the sides are made smaller; but the most general rule is perhaps, that the dimensions of the ditch should be such as that the earth dug out may be sufficient to build the rampart of a proper magnitude. The space sometimes left betwecrs the rampart and ditch, being about 6 or 8 feet, is called the berm, or list, serving to pass and repass, and to prevent the earth from rolling into the ditch.

DITONE, in Music, an interval comprehending two tones, a greater and a less. The ratio of the soumets that form the ditone, is 4 to 5 ; and that of the semi-ditone, 5 to 6 .

DITTON (IUMPFREY), an cminent mathematician, was born at Salisbury, May 29, 1675. Being an only son, and his father observing in him an extriordinary good capacity, determined to cultivate it with a good education. For this purpose he placed him in a reputable private academy; on quitting of which, at the desire of his father, though against his own inclination, he engagerl in the profession of divinity, and began to exercise his function at Tumbridge in the county of Kent, where he continucd to preach some years; during whicl time he married a lady of that place.

But a weak constitution, and the dcath of his father, induced Mr. Ditton to quit that profession; aud at the persuasion of 1)r. Harris and $\mathrm{Mr}$. Whiston, both eminent mathematicians, he engaged in the study of mathematics, a science to which he had always a strong inclination. In the prosecution of this science, he was nuch encouraged by the success and applause he reccived; being greatly esteemed by the chicl professors of it, and particularly by 
Sir Isaac Newton, by whose interest and recommendation he was elected master of the new Mathematical School in Christ's Hospital; where he continued till his death, which happened in 1715 , in the 40 th year of his age, much regretted by the philosophical world, who expected many useful and ingenious discoveries from his assiduity, learning, and penetrating genius.

Mr. Ditton published several mathematical and other tracts, as below.-1. Of the Tangents of Curves, \&c. Fhilos. Trans. vol. 23.

2. A Treatise on Spherical Catoptrics, published in the Philos. Trans. for 1705; from whence it was copied and reprinted in the Acta Eruditorum, 1707, and also in the Memoirs of the Academy of Sciences at Paris.

3. General Laws of Nature and Motion; 8vo, 1705. Wolfius mentions this work, and says that it illustrates and renders easy the writings of Galileo, Huygens, and the Principia of Newton. It is also noticed by La Roche, in the Memoires de Literature, vol. 8, pa. 46 .

4. An Iustitution of Fluxions, containing the first Principles, Operatious, and Applications, of that admirable Method, as invented by Sir lsaac Newton; 8vo, 1706 . This work, with additions and alterations, was again published by Mr. John Clarke, in the year 1726 .

5. In 1709 he published the Synopsis Algebraica of John Alexander, with many additions and corrections.

6. His Treatise on Perspective was published in 1712 . In this work he explained the principles of that art mathematically; and besides teaching the methods then generally practised, gave the first hints of the new method afterward enlarged upon and improved by Dr. Brook Taylor; and which was published in the year 1715 .

7. In 1714, Mr. Ditton published several pieces, both theological and mathematical; particularly his Discourse on the Resurrection of Jesus Christ ; and The New Law of Fluids, or a Discourse concerning the Ascent of Liquids, in exact Geometrical Figures, between two nearly contiguous Surfaces. To this was annexed a tract, to demonstrate the impossibility of thinking or perception being the result of any combination of the parts of matter and motion: a subject which was much agitatel about that time. To this work also was added an advertiscment from him and Mr. Whiston, concerning a method for discovering the longitude, which it seems they had published about half a year before. This attempt probably cost our author his life; for though it was approved and cuuntenanced by Sir Isaac Newton, before it was presented to the Board of Longitude, and the method has since been successfully put in practice, in finding the longitude between Paris and Vienıa, yet that board determined against it : so that the disappointment, together with some public ridicule (particularly in some verses written by Dean Swift), affecterl his health, that he died the ensuing year, 1715 .

In an account of Mr. Ditton, prefixed to the German translation of his Discourse on the Resurrection, it is sair that he had publislied, in his own name only, another method for finding the longitude; but this Mr. Whiston denied. However, Raphacl Levi, a learned Jew, who had studied under Leibnitz, informed the German cditor, that he well knew that Ditton and Leibuitz had corresponded on the subject; and that Diton had sent to Leibnitz a delineation of a machine he had invented for that purpose; which was a piece of mechanism constructed with many wheels like a clock, and which Leibnitz highly al]proved of for land use; but doubted whether it would answer on ship-board, on account of the motion of the ship.

\section{DIVERGENT Point. See Virtual Focus.}

Divergent, or Diverging Lines, in Geometry, are those whose distance is continually increasing. - Lines which diverge one way, converge the other.

DIVERGENT, or DIVERGING, in Optics, is particularly applied to rays which, issuing from a radiant point, or having, in their passage, undergone a refraction, or reflexion, do continually recede farther from each other.

In this sense the word is opposed to convergent, which implies that the rays approach each other, or that they tend to a centre, where they intersect, and, being continued, go on liverging. Indeed all intersecting rays, or lines, diverge both ways from the centre, or point of intersection.

Concave glasses render the rays diverging; and convex ones, couverging.-Concave mirrors make the rays converge; and convex ones, diverge. - It is demonstrated in Optics, that as the diameter of a pretty large pupil does not exceed $\frac{x}{5}$ of a digit; diverging rays, flowing from a radiant point, will enter the pupil as parallel, to all intents and purposes, if the distance of the radiant from the eye amount to 40,000 feet. See Focus, Lrght, and Vision.

Diverging Hyperbola, is one whose legs turn their convexities toward each other, and run out contrary ways. See Hy perbola.

Diverging Parabola. See Diverging Parabola.

Divergang Series, is a series whose terms always become larger the farther they are continued.

DIVIDEND, in Arithmetic, is the number given to be divided by some other number, called the divisor. Or it is the number given to be divided, or separated, into a certain number of equal parts, viz. as many as the divisor contains units; and the number of such equal parts is called the quotient. Or, more generally, the dividend contains the divisor, as many times as the quotient contains unity.The dividend is the numerator of a fraction, whose denominator is the divisor, and the quotient is the value of the fraction. Thus, $\frac{8}{2}=4$, and $\frac{3}{4}=75$.

DIVING. The art, or act of descending under water, to considerable depths, and remaining there for a certain time. The uses of diving are very considerable, particularly in the fishing for pearls, corals, sponges, \&c.

Various methods have been proposed, and engines contrived, to render the business of diving more safe and easy. The grcat point in all these, is to furnish the diver with fresh air, without which he must either make but a short stay, or perish. Those who dive for sponges in the Mediterranean, help themselves by carrying down sponges dipt in oil in their mouths; but considering the small quantity of air that can be contained in the pores of a sponge, and how much that little will be contracted by the pressure of the incumbent water, it is evident that such a supply callnot be very useful; since it is found by experiment, that a gallon of air included in a bladder, and by a pipe reciprocally inspired and expired by the lungs, becomes unfit for respiration in little more than one minute; for though its elasticity be but little altered in passing the lungs, yet it loses its vivifying spirit, and is rendered unfit for the support of animal life. Dr. Halley assures us, a naked diver, without a sponge, cannot remain above two minutes inclosed in water; nor much longer with one, without suffocating; nor, without long practice, near so long: per3 I 2 
sons unaccustomed to diving generally begin to be suffocated in about half a minute. Besides, if the depth be considerable, the pressure of the water on the vessels makes the eyes blood-shotten, and frequently occasions a spitting of blood: hence, where there has been occasion to continue long at the bottom, some have contrived double flexible pipes, to circulate air down into a cavity inclosing the diver, as with armour, both to furnish air, and to bear off the pressure of the water, and give leave to his breast to dilate upon inspiration; the fresh aix being forced down one of the pipes with bellows, and returning by the other, not unlike to an artery and vein.

But this method is impracticable when the depth exceeds three fatlonss; the water embracing the bare limbs so closely, as to obstruct the circulation of the blood in them; and withal pressing so strongly on all the junctures where the armour is made tight with leather; that if there be the least defect in any of them, the water rushes in, and instantly fills the whole engine, to the great danger of the diver's life.

Diving-Bell, is a machine contrived to remedy all these inconveniencies. In this the diver is safely conveyed to any reasonable depth, and may stay more or less time under the water, as the bell is greater or less; and it is most conveniently made in form of a truncated cone, the smaller base being closed, and the larger open; being weighed with lead, and so suspended, that it may sink full of air, with its open basis downward, and as near as may be in a situation parallel to the borizon, so as to close with the surface of the water all at once.

The diver sitting under this machine, sinks down with the included air to the depth desired; and if the cavity of the vessel can contain a ton of water, a single man may remain a full hour, without much inconvenience, at five or six fathoms deep; but the lower he goes, still the more the included air contracts itself, according to the weight of the water that compresses it; so that at thirty-three fect deep, the bell becomes half-full of water; the pressure of the incumbent fluid being then equal to that of the atmosphere; and at all other depths, the space occupied by the compressed air in the upper part of its capacity, is to the space filled with water, as thirty-three feet to the depth of the surface of the water in the bell below its common surface; and this condensed air, being taken in with the breath, soon insinuates itself into all the cavities of the body, and has no ill effect, provided the bell be permitted to descend so slowly as to allow time for that purpose.

One inconvenience that attends it, is found in the cars, within which there are cavities which open only outwards, and that by pores so snall, as not to give admission even to the air itself, unless they be dilated and distended by a considerable force; hence, on the first descent of the bell, a pressure begins to be felt on each ear, which by degrees becomes painful, till the force overcoming the obstacle, that constringes these pores, yields to the pressure, and letting some condensed air slip in, presently ease ensues; the bell descending lower, the pain is renewed, and afterwards it is again eased in the same manner. But the greatest inconvenience of this engine is, that the water entering it, contracts the bulk of air into so small a compass, that it soon heats, and becomes unfit for respiration: so that there is a necessity for its being drawn up to recruit it; besites the uncomfortable situation of the diver, who is almost covered with water.

To obviate the difficulties of the diving-bell, Dr. Halley, to whom we owe the preceding account, contrived some further apparatus, by which not only to recruit and refresh the air from time to time, but also to keep the water wholly out of it at any depth; which he efiected after the following manner:-His diving-bell (plate is, fig. 6) was of wood, 3 feet wide at top, 5 feet at bottom, and 8 feet high, containing about 63 cubic feet in its concavity, coated externally with lead so heavy, that it would sink empty; a particular weight being distributed about its bottom $\mathrm{R}$, to make it descend perpendicularly, and no otherwise. In the top was fixed a meniscus glass $\mathrm{D}$, concave downwards, like a window, to let in light from above; with a cock, as at $B$, to let out the hot air ; and a circular seat, as at $\mathbf{L} M$, for the divers to sit on: and below, about a yard under the bell, was a stage suspended from it by three ropes, each charged with a hundred weight, to keep it steady, and for the divers to stand upon to do their business. The machine was suspended from the mast of a ship by a sprit, which was secured by stays to the mast-head, and was di-rected by braces to carry it overboard clear of the side of the vessel, and to bring it in again.

To supply air to this bell when under water, he had two barrels, as c, holding 36 gallons each, cased with lead, so as to sink empty, each having a bung-hole at bottom, to let in the water as they descended, and let it out again as they were drawn up. In the top of the barrels was another hole, to which was fixed a leathern pipe, or hose, well prepared with bees wax and oil, long enough to hang below the bung-hole; being kept down by a weight for that purpose; so that the air driven to the upper part of the barrel by the influx of the water, in the descent, could not escape up this pipe, unless the lower end were lifted up.

These air-barrels were fitted with tackle, to make them rise and fall alternately, like two buckets; being directed in their descent by lines fastened to the uuder cdge of the bell: so that they came readily to the hand of a man placed on the stage, to receive them; who taking up the ends of the pipes, as soon as they came above the surface of the water in the barrels, all the air included in the upper part of it was blown forcibly into the bell; the water taking its place.

One barrel thus received, and emptied; on a signal given, it was drawn up, and at the same time the other let down; by which altemate succession, fresh air was furnished so plentifully, that the learned Doctor himseif was one of five, who were all together in nine or ten fathorns deep of water for above an hour and a half, without the last inconvenience; the whole cavity of the bell boing perfectly dry.

All the precaution he observed, was, to be let down gradually about 12 fiet at a time, and then to strp, and divive out the water that had entered, by taking in three or lour barrels of fresh air, before he descended farther. And, being arrived at the depth intended, he let out as much of the hot air that had been breathed, as each barrel would replace with cold, by means of the coek $\mathrm{n}$, at the top of the bell, through whose aperture, though very small, the air would rush with so much violence, as to make the surface of the sea boil.

Thus he found any thing could be done that was requirer to be done underneath; and by raking off the stage, lie could, for a space as wide as the circuit of the bell, lay the bottom of the sen so far dry as not to be over shoes in. water. Besides, by the glass wintow so much light was. 
transmitted, that when the sea was clear, and especially when the sun shone, he could see perfectly well to write or read, much more to fasten or lay hold of any thing under him that was to be taken up; aud by the return of the air-barrel he often sent up orders written with an iron pen on a plate of lead, directing how he would be moved from place to place. At uther times, when the water was troubled and thick, it would be as dark as night below; but in such cases he was able to keep a candle burning in the bell.

Dr. Halley observes, that they were subject to one inconvenience in this bell; they felt at first a small pain in their ears, as if the end of a tubacco-pipe were thrust into them; but after a little while there was a small puff of air, with a little noise, and they were easy.

This he supposes to be occasioned by the condensed air shutting up a valve leading from some cavity in the ear, full of common air; but when the condensed air pressed harder, it forced the valve to yield, and filled every cavity. One of the divers, in order to prevent this pressure, stopped his ear with a pledget of paper; which was pushed in so far, that a surgeon foupd considerable difficulty in extracting it.

The same author intimates, that by an additional contrivance he has found it practicable for a diver to go out of the bell to a good distance from it; the air being conveyed to lim in a continued stream by small flexible pipes, which serve him as a clue to direct him back again to the bell. For this purpose, one end of these pipes, kept open against the pressure of the sea by a small spiral wire, and made tight without by painted leather and sheep's guts drawn over it, being open, was fastened in the bell, as at $P$, to receive air, and the other end was fixed to a leaden cap on the man's head, reaching down below his shoulders, open at bottom, to serve him as a little brll, full of air, for him to breathe at his work, which would keep out the water from him, when at the level of the great bell, because the density of the air in both was the same: but when he stooped down lower than the level of the great bell, he shut the cock $\mathrm{F}$, to cut off the communication between the two. Phil. Trans. abr, vol, vi. pa. 258, 522.

The air in this bell would serve him for a minute or two; and he might instantly change it, by raising himself above the great bell, and opening the cock $F$. The diver was furnished with a girlle of large leaden weights, and clogs of lead for the feet, whicl, with the weight of the leaden cap, kept him firm on the ground; he was also well clothed with thick fannels, which being first made wet, and then warmed in the bell by the heat of his body, kept off the chill of the cold water for a considerable time, when he was out of the bell.

Mr. Martin Triewald, F. R. S, and military architect to the king of Sweden, contrived to construct a diving-bell on a smaller scale, and at a less expense, than that of Dr. Halley, and yet capable of answering the same purposes. This bell, $\mathrm{AB}$ (fig. 7.) sinks with leaden weights $\mathrm{DD}$, suspended from the bottom of it. It is made of copper, and tinned all over on the inside; and it is illuminated by three strong convex lenses GGG, defended by the copper lids $11+1 \mathrm{t}$. The iron plate $\mathrm{E}$ serves the diver to stand upon, when he is at work; and this is suspended by the chains $\backslash F F$, at such a distance from the botiom of the bell, that when he stands upright, his herd is just above the water within it, where lie has theadvantuge of air more proper for respiration, than when he is much higher up; but as there is occasion for the diver to be wholly in the bell, and consequently his head in the upper part of it, Mr. Triewald also contrived, that even there, after he has breathed the hot air as long as he can, by means of a spiral copper tube placed close to the inside of the bell, he may draw the cooler and fresher air from the lowermost parts; for which purpose a flexible leather pipe, about two fect long, is fixed to the upper end of the tube at $b$; and to the other end of the pipe is fastened an ivory mouth-piece, for the diver to hold in his mouth, by which to respire the air from below. We shall only remark, that as air rendered effete by respiration is somewhat heavier than common air, it must naturally subside in the bell; but it may probably be restored by the agitation of the sca-water, and thus become more fit for respiration. See Fixed Air, Phil. Trans. abr. vol. viii. pa. 634. Or Desaguliers's Exper. Phil, vol. ii. pa.220, \&c. Several other persons bave practised with diving-macbines, on the same principles.

The famuus Corn. Drebell had- an expedient in some respects superior even to the diving-bell, if what is related of it be true. He contrived not only a vessel to be rowed under water, but also a liquor to be carried in the vessel, which supplied the place of fresh air. The vessel was made for King James the 1st, carrying 12 rowers, besides the passengers. It was tried in the river Thames; and one of the persons in that submarine navigation, then living, told it one, from whom Mr. Boyle had the relation. As to the liquor, Mr. Boyle assures us, he discovered by a physician, who married Drebell's daughter, that it was used from time to time, whon the air in that submarine boat was clogged by the brcath of the company, and rendered unfit for respiration: at which time, by unstopping the vessel full of this liquor, he conld spcedily restore to the troubled air such a proportion of vital parts, as would make it serve again a good while. The secret of this liquor Drebell wulld never disclose to above one person, who himself assured Mr. Boyle what it was. Boyle's Exp. Phys. Mech. of the Spring of the Air.

We have had many projects of diving-machines, and. diving-ships of various kinds, which have proved abortive.

Diving-Bladder, a term used by Borclli for a machine which he contrived for diving under water to great depths, with considerable facility, which he prefers to the common diving-bell. The vesica, or bladder, as it is usually called, is of brass or copper, and about two feet in diameter, which is to contain the diver's head; and this is fixed to a goat's skin habit, exactly fitted to the shape of the body of the person. Within this vesica there are pipes, by means of which a circulation of air is contrived; and the person carries an air-pump by his side, by means of which he may make himself leavier or lighter, as the fishes. do, by contracting or dilating their air-blaclder: by this means, the objections all other living-machines are liable to are obviated, and particularly that of the air; the moisture by which it is clogged in respiration, and by which it is rendered unfit for the same use again, being liere taken from it by its circulation through the pipes, to the sides of which it adheres, and leaves the air as free as before. Borclli Opera Posthuma.

DIVINI (Eustacmo), an ingenious Italian artist, very eminent for his telescopes, though, it seems, inferior to Campani. He wrote a book (pullished at the Hague 1660 , in 4 to) against the discovery of Saturn's ring, made: 
by the celebrated Huygens, contesting the truth of it; to which the latter wrote a reply. Divini was living in 1663; though Moreri thinks be died about that time.

DIVISIBILITY, a property in quantity, body, or extension, by which it becomes separable into parts; either actually, or at least mentally. Such divisibility is infinite, if not actually, at least potentially; as no part can be conceived so small, but another may be conceived stili smaller; for every part of matter must have some finite extension, and that extension may be bisected, or otherwise divided; for the same reason, these parts may be divided again, and so on without end.

It is not here contended for the possibility of an actual division in infinitum: it is only asserted that however small a body is, it may be still farther divided; which it is presuned may be called a division in infinitum, because what has no limits, is called infinite. 'The infinite, or indetinite divisibility of mathematical quantity is thus proved, and illustrated by inathematicians: Suppose a line $A D$ perpendicular to $\mathrm{BF}$; and another as $\mathrm{GH}$ also perpendicular to the same $\mathrm{BF}$; with the centres $c, c, c, \& c$, and distances $\mathbf{C A}, \mathbf{C A}, \& \mathbf{c}$, describe circles cutting the line $\mathrm{GH}$ in the points $c$, $e, \& c$. Now, the greater the radius $\mathrm{AC}$ is, the less is the part $e \mathrm{G}$; but the radius may be augmented in infmi-

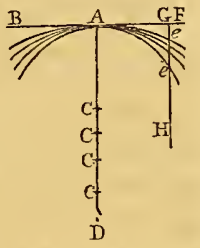
tum, and therefore the part, $e_{G}$ may be diminished in the same manner; and yet it can never be reduced to nothing, because the circle can never coincide with the right line BF. Consequently the parts of any magnitude may be diminished in infinitum.

All that is supposed, in strict geometry, concerning the divisibijity of magnitude, amounts to no more, than that a given magnitude may be conceived as divided into a number of parts, equal to any given or proposed number. It is true, that there are no such things as parts infinitely small; yet the subtilty of the particles of several bodies is such, that they far surpass our conception; and there are innumerable instances in nature of such parts actually separated from one another.

Several instances of this are given by Mr. Boyle. He speaks of a silken thread 300 yards long, that weighed but two grains and a half. He measured leaf-gold, and found by weighing it, that 50 square inches weighed but one grain : if the length of an inch be divided into 200 parts, the eye nay distinguish them all; therefore in one square inch there are 40,000 visible parts; and in one grain of it there are two milions of such parts; which visible parts no one will deny are still farther divisible.

Again, an ounce weight of silver may be gilt over with 8 grains of gold, which may be afterwards drawn into a wire 13,000 fect long, and stil] be all covered with the same gilding.

In odloriferous bodies a still greater subtilty of parts is perceived, and even such as are actually separated from one another: several bodies scarce lose any sensible part of their weight in a long time, and yet continually fill a very large space with odoriferous particles. Dr. Keil, in his Vera Physica, lect. 5, has calculated the maguitude of a particle of assatoetida, which will be the 57 $\overline{10000000000000000}^{t h}$ part of a cubic inch. And in the same Lecturc, he shows that the particles of the blood in animalculæ, observed in fluids by means of microscopes, must be less than that part of a cubic inch which is expressed by a fraction whose numerator is 8 , and denominator unity witb 30 ciphers after it.

The particles of light, if light consist of real particles, furnish another surprising instance of the minuteness of some parts of matter. A small lighted candle placed on a plain, will be visible two miles, and consequently its light fills a sphere of 4 miles diameter, bcfore it has lost any sensible part of its weight. Now, as the force of any hody is directly in proportion to its quantity of matter multiplied by its velocity; and since it is demonstrated that the velocity of the particics of light is at least a million of times greater than the velocity of a caunon-hall, it is plain, tbat if a million of these particles were round, and of the size of a small grain of sand, we durst no more open oui eyes to the light, than expose them to sand shot puint-blank from a cannon.

By help of microscopes, such objects as would otherwise escape our sight, appear very large: there are some small animals scarce visible with the best microscopes; and yet these have all the parts necessary for life, as bluod, and other fluids. How wonderful then must the subtilty of the parts be, which make up such fluids! Whence is deducible the following theorem:

Any particle of matter, however small, and any finite space, however large, being given; it is possible for that small particle of matter to be diffused through that space, and to fill it so as that there shall be no pore in it, whose diameter shall exceed any given line; as is demonstrated by Dr. Keil, Introduct, ad Ver. Phys.

DIVISIBLE, the facuity or quality of being capable of being divided.

DIVISION, is one of the four principal rules of arithmetic, being that by which we find how often one quantity is contained in another; so that division is in reality only a compendious method of subtraction; its effect being to take one number from another as often as possible; that is, as often as it is contained in it. There are therefore three numbers concerned in division: 1st, That which is given to be divided, called the dividend; $2 \mathrm{~d}$, That by which the dividend is to be divided, called the divisor; $3 d$, That which expresses how often the divisor is contained in the dividend; or the number resulting from the division of the dividend by the divisor, called the quotient.

There are various ways of performing division, one called the English, another the Flemish, unother the Italian, another the Spanish, another the German, and another the Indian way, all equally just, as finding the quotient with the same certainty, and only differing in the manner of arranging and disposing the numbers.

There is also division in integers, division in fractions, and division in species, or algebra, \&c.

Division is performed by secking how often the divisor is contained in the dividend; and when the latter consists of a greater number of figures than the former, the dividend must be taken into parts, beginning on the left, and procccding to the right, and secking how often the divisor is found in each of those parts; after the manner as taught in all books of arithmetic, as well as various contractions adapted to particular cases: such as, 1 st, when the divisor has any number of ciphers at the end of it, they are cut off, with the same number of figures from the end of the dividend, and then the work is performed withont them both, annexing only the figures last cut off, to the lust re- 
mainder; $2 \mathrm{~d}$, when the divisor is equal to the product of several single digits, it is easier to divide successively by those digits, instead of the divisor at once; $3 \mathrm{~d}$, when it is required to continue a quotient to a great many places of figures, as in decimals, a very expeditious method of performing it, is as follows: Suppose it were required to divide 1 by 29 , to a great many places of decimals. Adding ciphers to the 1, first divide 10000 by 29 in the common way, till the remainder become a single figure, and annex the fractioual supplement to complete the quotient, which gives $\frac{1}{29}=0.03448 \frac{8}{29}$ : next multiply each of these by the numerator 8 , so shall $\frac{8}{2} \frac{9}{9}=0 \cdot 27584 \frac{5}{2} \frac{4}{9}$ or rather $0.27586 \frac{6}{2}$; which figures substituted instead of the fraction $\frac{8}{29}$ in the first value of $\frac{1}{29}$, it becomes $\frac{x}{29}=$ $0.0344827586 \frac{6}{29}$ : again, multiply both of these by the last numerator 6 , and it will be $\frac{6}{29}=0.2068965517 \frac{7}{2} \frac{9}{9}$; which figures substituted for $\frac{6}{29}$ in the last-found value of $\frac{1}{29}$, it becomes $\frac{1}{29}=0.03448275862068965517 \frac{7}{29}$ : and again, multiplying these by the numerator 7 , gives $\frac{7}{2}=0.2413793103+482758620_{2}^{10} \frac{0}{9}$; which figures substituted instead of $\frac{7}{29}$ in the last-found value of $\frac{r}{29}$, it becomes $\frac{1}{2}=$

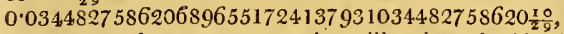
and so on; where every operation will at least double the number of figures before found by the last one.

Proof of Division. In every example of division, unity is always in the same proportion to the divisor, as, the quotient is to the dividend; and therefore the product of the divisor and quotient is equal to the product of 1 and the dividend, that is, the dividend itself. Hence, to prove division, multiply the divisor by the quotient, to the product add the remainder, and the sum will be equal to the divideud when the work is right; if not, there is a mistake.

Division, in Vulgar Fractions, is performed by dividing the numerators by each other, and the denominators by each other, if they will exactly divide; but if not, then the dividend is multiplied by the reciprocal of the divisor, that is, having its terms inverted; for, taking the reciprocal of any quantity, converts it from a divisor to a multiplier, and from a muliplier to a divisor. For $\mathrm{Ex}, \frac{15}{16} \div$ by $\frac{5}{8}$ gives $\frac{3}{2}$, by dividing the numerators and denominators; but $\frac{15}{1} \frac{5}{6} \div$ by $\frac{4}{3}$ is the same as $\frac{13}{16} \times \frac{3}{4}$, which is $=\frac{45}{64}$. Where $x$ is the sign of multiplication, and the character $\div$ is the mark of division. Or division is also denoted like a vulgar fraction; so 3 divided by 2 , is $\frac{3}{2}$.

Drvision, in Decimal Fractions, is performed the same way as in integers, regard being had to the number of decimals, viz, making as many in the quotient as those of the dividend exceed those in the divisor.

But in this case we sometimes make use of a contracted method, whicls it may not be amiss to explain. Thus, when there are many tigures in the divisor, or when only a certain number of decimals are necessary to be retained in the quotient; then take only as many of the left-hand figures of the divisor as will excee? the number of figures, both integers and decimals, to be retained in the quotient by unity, and find how many times they are contained in the first figures of the dividend as usual.

Let each remainder form a new dividend ; and for every such dividend, leave ont one figure more on the right-hand side of the divisur, observing to carry for the increase of the figures cut off as follow: namely, 1 , from 5 to 14 ; 2 , from 15 to $24 ; 3$, from 25 to 34 , \&c.

liut when there are not so many figures in the divisor as are required to be in the quotient, then the operation must be begun with all the figures, continuing it the same as in the common rule, till the number of figures in the divisor excced those remaining to be found in the quotient by unity, after which begin the contraction. See the following example, where 5 figures are to be retained in the quotient.

$92 \cdot 4.103,5) 2508 \cdot 92806(27 \cdot 149$
660721
13849
4608
912
80

Drvision, in Algebra, is performed like that of common numbers, either making a fraction of the dividend and divisor, and cancelling or dividing by the terms or parts that are common to both; or else dividing after the manner of long division, when the quantities are compound ones. Thus,

$a b$ divided by $a$, gives $b$ for the quotient :

and $12 a b$ divided by $4 b$, gives $3 a$ for the quotient :

$16 a b c^{2}$ divided by $8 a c$, gives $2 b c$ :

$a$ divided by $3 b$, gives $\frac{a}{3 b}$ :

$15 a b c^{3}$ divided by $12 b c^{2}$, gives $\frac{15 a b c^{3}}{12 b c^{2}}=\frac{5 a c}{4}$ : and $a^{2}-b^{2}$ by $a+b$, gives $a-b$; thus,

$$
\begin{aligned}
&a+b) a^{2}-b^{2}(a-b \\
& \frac{a^{2}+a b}{-a b-b^{2}} \\
&-a b-b^{2}:
\end{aligned}
$$

again, to divide $x^{6}-8 x^{4}-124 x^{2}-64$ by $x^{2}-16$;

$$
\begin{aligned}
& \left.x^{2}-16\right) x^{6}-8 x^{4}-124 x^{2}-64\left(x^{4}+8 x^{2}+4\right. \\
& \frac{x^{6}-16 x^{4}}{8 x^{4}-124 x^{2}} \\
& \frac{8 x^{4}-128 x^{2}}{4 x^{2}-64} \\
& 4 x^{3}-64
\end{aligned}
$$

In some cases, the quotient will run out to an infinite series; and then, after continuing it to any certain number of terms, it is usual to annex, by way of a fraction, the remainder with the divisor set under it.-It is to be noted that, in dividing any terms by one another, if the signs be both alike, either both plus or both minus, the sign of the quotient will be plus; but when the signs are different, the one plus and the other minus, the sign of the quotient is minus.

Division by Logurithens." See Logarithis.

Division of Mlathematical Instruments. See GrapuaTrON, and Mu RaL Arc or \&uadrant.

Drvision in Music, is the dividing the interval of an octave into a number of lesser intervals.

Division by Napier's Bones. See Napier's Bones.

Division of Powers, is performed by subtracting their exponents. Thus, $a^{5} \div a^{4}$ is $=a^{2}$; and $4 a^{\frac{3}{4}} b^{2} \div 2 a^{\frac{1}{2}} b^{\frac{4}{3}}$ is $=2 a^{\frac{1}{4}} b^{\frac{2}{3}}$.

Division of Proportion, is comparing the difference between the antecedent and consequent, with either of them. Thus,

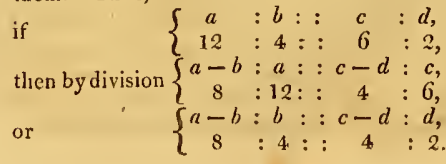


Divisor, is the dividing number; or that which shows how many parts the dividend is to be divided into.

Divisors of a Number, are those numbers by which it is exactly divisible; thus, $1,2,3,4,6$ and 12 , are divisors of the number 12 , because 12 may be divided by each of those numbers, without leaving a remainder. But if we take a prime number, as for example 13, we shall find that it has only two divisors; namely, 1 and 13, or unity and itself, which is indeed what constitutes a prime number.

In composite numbers, it is frequently necessary to know what those numbers are by which they are divisible, the sum of all those divisors, how many such divisors belong to a given number, their forms, and other particulars, which find their application in various branches of analysis. In order to enter into these particulars, it is necessary that we know all the prime divisors of a number, which are found by dividing it by all those prime numbers, less than the square root of itself, by which it is divisible; repeating the division by each, as often as it can be done without leaving a remainder: by which means every number $\mathrm{N}$ is reduced to the following form, $\mathrm{N}=$ $a^{\mathrm{m}} b^{\mathrm{n}} c^{\mathrm{p}} d^{\mathrm{q}} \& \mathrm{c}$; where $a, b, c \& c$ are the prime divisors of $\mathrm{N}$, and $m, n, p \& \mathrm{c}$ the powers of those divisors, or the number of times that $\mathrm{N}$ is divisible by $a, b, c \& c$.

This reduction being made, it is evident from inspection only, that by the developement of the formula $\left(1+a+a^{2} \ldots \ldots a^{\mathrm{m}}\right) \cdot\left(1+b+b^{2} \ldots b^{\mathrm{n}}\right) \cdot(1+c+$ $\left.c^{2} \ldots c^{\mathrm{P}}\right) \& c$, we shall obtain every possible combination, that can be formed with $m-a s, n-b s, p-c s \& c$, and consequently, every divisor of $\mathrm{N}$ is contained in the result obtained from this developenent, and which will therefore express the sum of all the divisors of the given number $\mathrm{N}$.

Suppose, for example, the sum of all the divisors of 360 were required.

Here $360=2^{4} \cdot 3^{2} \cdot 5^{2}$, that is $a=2, b=3$, and $c=5$; also $m=4, n=2$, and $p=2$ : therefore $\left(1+2+2^{2}\right.$ $\left.+2^{3}+2^{4}\right) \cdot\left(1+3+3^{2}\right) \cdot\left(1+5+5^{2}\right)=31.13 \cdot 31$ $=12+93$, which is the sum of all the divisors of 360 . But if, instead of 360 , the sum of the divisors of 361 had bech required, we should have found it equal only to 362 , because 361 is a prime. From which circumstance we should be led to conclude, that no order could be observed between the sum of the divisors of the consecutive numbers; but Euler has proved in his Introduction to the Analysis Infinitorum, tom. 1, p. 355, that these sums follow a certain law, after the manner of a recurring series.

We may also from our first formula find the number of divisors of any given number, which is evidently expressed by $(n+1) \cdot(n+1) \cdot(p+1)$. \&c. Suppose, for example, it were required to find how many divisors belong to the number 1800 .

First $1800=2^{3} \cdot 3^{2} \cdot 5^{2}$; that is, $m=3, n=2$, and $p=2:$ therefore $(3+1) \cdot(2+1) \cdot(2+1) \cdot=36$, the number of divisors of 1500 . Hence again, we may readliy fincl a number that shall have any given number of divisors. 'Thus, let it be required to find a number that. shall have 20 divisors. Hore, it is only necessary to resolve 20 into any number of factors, as $20=4.5$; subtract 1 from each of those factors, and make $m=3, n=4$; then will $a^{\mathrm{m}}, b^{\mathrm{a}}$ be the number sought ; where $a$ and $b$ may be taken any prime numbers at pleasurc. If $a=3$, and $b=2$, we have $27.16=432$, for the number required.
Various other properties of divisors might be here enumerated, but we shall only mention a few of those which have been most successfully employed in the Theory of Numbers.

1. If $a$ be a prime number, and $x$ not divisible by $a$, then is $a$ a divisor of the formula $\left(x^{3-1}-1\right)$.

2. If $a$ be a prime number, it is a divisor of the formula $(1.2 .3---a-1)+1$.

3. If $a$ be a prime number, it is a divisor of the formula $\left(1^{2} \cdot 2^{2} \cdot 3^{2} \cdot-\left(\frac{a-1}{2}\right)^{2}\right) \pm 1$, the upper sign baving place when $a$ is of the form $4 n+1$; and the under one when $a$ is of the form $4 n-1$.

4. The divisors of every number comprised under any of the forms $p^{2}+n^{2}, p^{2}+2 n^{2}, p^{2}-2 n^{2}$, are always of the same form, as the numbers that they divide. That is, the sum of two squares can only be divided by numbers that are also the sum of two squares, \&c. It being alivays understond that $p$ and $n$ are prime to each otlier.

5. Every prime number is a divisor of the formula $p^{2}+n^{2}+1$

6. Every odd number, except 5 and its inultiples, is a divisor of any repetend digit; and the number of digits necessary to form the dividend, never excecds the number expressed by the divisor.

7. If the sum of the digits of any number is divisible by 3 or 9 , the number itself is divisible by the same number 3 or 9 .

8. If the sum of the digits in the even places, are equal to the sum of those in the odd places, in any number, that number is divisible by 11 .

9. If the $n$ right-hand digits of any number be divisible by $2^{n}$, the whole number is divisible by $2^{n}$.

The rcader is referred for further information on this subject, to Euler's Introduction to the Analysis Infinitorum, also to the $2 \mathrm{~d}$ vol. of his Algebra; to Waring's Meditationes Algebraica; Le Gendre's Essai sur la Theoric des Nombres; and to the Recherches Arithinetiques, par M. Gauss. See also Numbers in this Dictionary.

DIURNAL, something relating to the day; in opposition to Nocturnal, relating to the night.

Diurnal Arch, is the arch described by the sun, moon, or stars, between their rising and setting.

Diurnat Circle, is the apparent circle described by the sun, moon, or stars, in consequence of the rotation of the earth.

Diurnal Motion of a Planet, is so many degrees and minutes \& $\mathrm{C}$ as any plauet moves in 24 hours.

The Drurnal Motion of the Earth, is its rolation round its axis, the duration of which constitutes the natural day.

Diurnal Parullax. Sec Parallax.

DIURNax is also used in speaking of what belongs to the nycthemeron, or natural day of 24 hours: in which sense it is opposed to annual, menstrual, \&c. The diurnal phenomena of the heaveuly bodies are solved from the diurnal revolution of the carth; that is, from the rotation of the earth round its own axis in 24 lours; which rotation is cquable, and from west to east, about an axis whose inclination to the ecliptic is now $66^{\circ} 32^{\prime}$. Now since the earth is an opaque boly, that small part of $i$ s surface which comes at the same time under the confined view of the spectator, though really spherical, seoms to be extended like a plane: and the eyc, taking an entire view of the heavens, they secm to form a concave splierical superficies, concentric with the carth, or ruther with tho 
eye, which the said plane of the earth's superficies divides into two equal parts, the one of which is visible, but the other, because of the earth's opacity, is hid from the view.

And as the earth. revolves about its axis, the spectator, together with the said plane he stands upon, called his horizon, dividing the visible from the invisible hemisphere of the heavens, is carried round the same way, viz, toivards the east : hence it is, that the sun and stars, placed towards the east, being before hid, now become visible, the horizon as it were sinking below them; and the stars \& $\mathrm{c}$ towards the west are covered or hid, and become invisible, the horizon being elevated above them: so that the former stars, to the spectator, who reckons the place he stands on as immoveahle, appear to ascend above the horizon, or rise; and the latter to descend below the horizon, or set.

Since the earth, with the horizon of the spectator, continues to move always towards the same parts, and about the same axis equally; all bodies, and all phemomena, that do not partake of the said motion, (that is, all such things as are entirely separate from the earth,) seem to move in the same time uniformly, but towards the opposite parts, or from east to west: and every one of these objects, according to sense, describes the circumference of a circle, whose plane is perpendicular to the axis of the earth. And becausc all these circles, together with the visible objects describing them, appear to be in the concave spherical supcrficics of the heavens, every visible object seems to describe a grater or less circle, according to its greater or less distance from the poles, or extremities of the earth's axis produced; the middle circle bctween these poles, called the equator, being consequently the greatest.

DUDECAGON, a regular polygon of 12 equal sides and angles. If the side of a dodecagon be 1 , its area will be equal to 3 times the tang. of $75^{\circ}=3 \times(2+\sqrt{ } 3)=$ 11.1961524 nearly; and, the areas of plane figures being as the squares of their sides, therefore $11 \cdot 1961524$ multiplied by the sqquare of the side of any dodecagon, will give its area. See my Mensuration, pa, $85,4 t h$ edit.

To inscribe a Dodecugon in a given Circle. Carry the radius 6 times round the circumference, which will divide it into 6 equal parts, or will make a hexagon; then bisect each of those parts, which will divide the whole into 12 parts, for the dodecagon. See also other methods of describing the same figure in my Mensuration, pa. 16 , Rrc. See Puly gon.

DODECAHEDRON, one of the Platonic bodies, or five regular solids, being contained under a surface composed of twelve equal and regular pentagons.

To form a Dodecahedron. See Regular Body.

If the side, or linear edge, of a dodecahedron be $s$, its surface will be $15 s^{2} \sqrt{ }\left(1+\frac{2}{5} \sqrt{ } 5\right)=20.64 .57788 s^{2}$, and its solidity $\frac{5}{2} s^{3} \sqrt{ }(4 \cdot 7+2 \cdot 1 \sqrt{ } 5)=7 \cdot 66311896 s^{3}$.

If the radius of the sphere that circumscribes a dodecahedron be $r$, then is

its side or linear edge $=(\sqrt{ } 15-\sqrt{ } 3) \times \frac{7}{3} r$;

its superficies - $=10 r^{2} \sqrt{ }\left(2-\frac{2}{5} \sqrt{ } 5\right)$;

and its solidity - $=\frac{20 r^{3}}{3} \sqrt{ } \frac{3+\sqrt{ } 5}{30}$.

The side of a dodecahedron inscribed in a sphere, is (qual to the greater part of the side of a cube inscribed in the same sphere, cut in extrene and mean proportion.-If a line be cut according to extreme and mean ration, and the kess segment be take'l) for the side of a doVUL. I. decahedron, the greater segment will be the side of a cube inscribed in the same sphere.-The side of the cube is equal to the right line which subtends the angle of a pentagon of the dodecahedron, inscribed in the same sphere. See Polyuedron; also my Mensur. pa. 188, \&c.

DODSON (JAMS), was an ingenious and very industrious inathematician, as well as author of several useful books; but we are acquainted with very few particulars of his life. He was some time master of the Royal Mathematical Schoul in Christ's Hospital, London. His publications chicfly were, 1 . The Antilogarithmic Canou, folio, 1742.-2. The Calculator, a collection of useful tables, large 8vo, 1747.-3. Mathematical Repository, being a collection of analytical questions and solutions, in 3 vols. $12 \mathrm{mo}$. an. $1748,1753,1755$.

-DOG, a name common to two constellations, called the Great and Little Dog; but more usually Canis Major, and Canis Minor.

DOLLOND (JoHs), the celebrated optician, was born in Spitalfields, in June, 1706: his parents were French protestants, who quittcd Normandy at the revocation of the edict of Nantz, in 1685.

The first years of Mr. Dollond's life were employed at the loom; but, being of a very studious and philosophic turn of mind, his leisure hours were engiged in mathematical pursuits; and though by the death of his father, which happened in his infancy, his education gave way to the necessities of his family, yet at the age of fifteen, before he bad an opportunity of seeing works of science or elcmentary treatises, he amused himself by constructing sun-dials, drawing geometrical scbemes, and solving problems. Under the pressure of a close application to business for the support of his family, he found time, by abridging the hours of his rest, to extend his mathematical knowledge, and made a considerable proficiency in optics and astronomy, to wlich be now principally devoted his attention, having in the earlier stages of his lite prepured hinself for the higher parts of those subjects, by a correct knowledge of algebra and geometry. He also acquired a very respectable knowledge of the Latin and Greek languages.

He designed his eldest son, Peter Dollond, for the sane business with.himself; and for several years they carricd on their manufacture together in Spitalfields; but the employment neither suited the expectations nor disposition of the son, who, having received much information on mathematical and philosophical subjects, from the instruction of his father, and observing the great value which was set upon his father's knowledge in the thory of optics by professional men, determined to apply that knowledge to the benefit of himself and bis family ; and accordingly, under the directions of his father, commenced optician. Success attended every effort; and in the year 1752 , John Dollond, embracing the opportunity of pursuing a profession congenial with his onind, joined his son, and in consequence of his theoretical knowledge, soon became a proficient in the practical parts of optics.

His first attention was directed to improve the combination of the eye-glasses of refracting telescopes; and having succeeded in his system of four eyc-glasses, he procecded one step further, and produced telescopes furnished with five eyc-glasses, which considerably surpassed the former; and of which he gave a particular account in a paper presented to the Royal Society, and which was printed in the Philos. 'Trans. vol. 48, pa. 103.

$3 \mathrm{~K}$ 
Soon after this he made a very useful improvement in Mr. Savery's micrometer : for, instead of employing two entire object-glasses, as Mr. Savery and M. Bouguer had done, he used only one glass cut into two equal parts, one of them sliding or moving laterally by the other. This was considered to be a great improvement, as the micrometer could now be applied to the reflecting telescope with nuch advantage, and which Mr. James Short immediately did. An account of the same was given to the Royal Society, in a paper, which was afterwards printed in the Philos. Trans. vol. 48 , pa. 178.

Mr. Dollond's celebrity in optics now became universal; and the friendship and protection of the most eminent men of science flattered and encouraged his pursuits. Surrounded by these enlightened men, in a state of mind prepared for the severest investigation of philosophic truths, and in circumstances favourable to liberal inquiry; Mr. Dollond engaged in the discussion of a subject, which at that time not only interested this country, but all Europe. Sir Isaac Newton had declared, in his Trentise on Optics, page 112, "That all refracting substances divcrged the prismatic colours in a constant proportion to their mean refraction ;" and drew this conclusion, "that refraction could not be produced without colour " and consequently, " that no improvement could be expected in the refracting telescope." No one dloubted the accuracy with which Sir Isaac Newton had made the experiment; yet some men, particularly M. Euler and others, were of opinion that the conclusion, which Newton had drawn from it, went too far, and maintained, that in very small angles refraction might be obtained withont colour. Mr. Dollond was not of that opinion, but defended Newton's doctrine with much learning and ingenuity, as may be seen by a reference to the letters which passed between Euler and Dollond on that occasion, and which were published in the Philos. Trans. vol. 48, pa. 287 , and contended that, "If the result of the experiment had been as described by Sir lsaac Newton, there could not be refraction without colour."

A mind constituted like Mr. Dollond's could not remain satisfied with arguing in this manner from an $\mathrm{ex}$ periment made by another, but determined to try it himself: and accordingly, in the year 1757 , he began the examination; and, to use his own words, with "a resolute perseverance," continued during that year, and a great part of the next, to bestow his whole mind on the subject, until in the month of Junc, 1758, he found, after a complete course of experiments, the result to be very different from that which he expected, and from that which Sir Isaac Newton had related. He discovered " the difference in the dispersion of the colours of light, when the mean rays are equally refracted by different mediuns." The discovery was complete, and he immediately irew from it this practical conclusion, "That the object-glasses of refracting telescopes were capable of being made without being affected by the different refrangibility of the rays of light." His account of this experiinent, ansl of others connected with it, was given to the lioyal Society, and printed in their Transactions, vol. 50, pa. 733 , and he was presented, in the same year, by that learned body, with Sir Godfrey Copley's medal, as a reward of his merit, and a memorial of the discovery, though not at that time a member of the Socicty.

This discovery no way affected the points in dispute between Euler and Dollond, respecting the doctrine ad- vanced by Sir Isaac Newton. A new principle was in a manner found out, which had no part in their former reasonings, and it was reserved for the accuracy of Dollond to have the honour of making a discovery which had eluded the observation of Newton. This new principle being now established, he was soon able to construct object-glasses, in which the different refrangibility of the rays of light was corrected, and the name of achromatic given to them by the late Dr. Bevis, on account of their being free from the prismatic colours.

As usually happens on such occasions, no sooner was the achromatic telescope made public, than the rivalship of foreigners, and the jealousy of philosophers at home, led them to doubt of its reality; and Euler himself, in his paper read before the Academy of Sciences at Berlin, in the year 1764, says, "I am not ashamed frankly to avow, that the first accounts which were published of it, appeared so suspicious; and even so contrary to the best established principles, that I could not prevail upon myself to give credit to them;" and he adds, "I should never have submitted to the proofs which Mr. Dollond produced to support this strange phenomenon, if $M$. Clairaut, who must at first bave been equalty surprised at it, had not most positively assured me, that Dollond's experiments were but too well founded." And when the fact conld no longer be disputed, they endeavoured to find a prior inventor, to whom it might be ascribed, and several conjecturers were honoured with the title of discoverers.

In the beginning of the year 1761 , Mr. Dollond was elected Fellow of the Royal Society, and appointed opticiall to. his majesty, but did not live to enjoy those honours long; for on the 30th of November, in the same year, as he was reading a new publication by M. Clairaut, on the theory of the moon, and on which he had been intently engaged for several hours, he was seized with apoplexy, which rendered him immediately spcechless, and occasioned his deatb in a few hours afterwards.

Besides Mr. Peter Dollond, whom we have had occasion to mention in the course of this memoir, his family, at his death, consisted of three daughters and a son, who, possessing the name of his father, and, we may add, a portion of the family abilities, carries on the optical business, in partnership with his elder brother. Sce also the articles Achromatic and Texescope.

DOME, is a round, vaulted, or arched roof, of a church, hall, pavilion, vestibule, stair-case, \&c, by way of crowning, or acroter.-In plate 7 , is represented the plan and clevation of a dome constructed without ccntring, by Mr. S. Bunce; viz, fig. 1 the plan, and fig. 2 the elevation. The first course consists of the stones marked 1, 1, 1, \&ce, of different sizes, the large onc's exactly twice the height of the small ones, placed alternately, and forming intervals to receive the stones marked $2,2,2$. The other courses are continued in the same manner, accorrling to the order of the figures to the top.

It is evident, from the converging or wedge-like form of the intervals, that the stones they receive can only be inserted from the nutside, and camot fall through: therefore the whole dome may be built without centring or temporary support. To break the upright joints, the stones may be cut of the form inarked in fig. 3 ; and those marked 16,17 , \&c, near the key-stones, may be enlarged as at fig. 4 .

For the mathematical theory of Domes, see my new Tracts, vol. 1; pa. 63, \&.c. 
DOMINICAL Letter, otherwise called the SurDAY Letter, is one of these first seven letters of the alphabet, A BCDEFG, used in almanacs, \& c, to mark or denote the Sundays throughout the year. The reason for using seven letters, is because that is the number of days in a week; and the method of using them is this: the first letter $A$ is set opposite the Ist day of the year, the $2 d$ letter в opposite the $2 \mathrm{~d}$ day of the year, the $3 \mathrm{~d}$ letter c opposite the 3d day of the year, and so on through the seven letters; after which they are repeated over and over again, all the way to the end of the year, the letter $A$ denoting the 8 th day, the letter в the 9 th, \&c. Then, whichever of the letters so placed, falls opposite the first Sunday in the year, the same letter, it is evident, will fall opposite every future Sunday tbroughout the year, because the number of the letters is the same as the number of days in the week, being both $\gamma$ in number; that is, in common ycars; but in leap-years, an interruption of the order takes place in them. For, on account of the intercalary day, either the letters must be thrust out of their places for the whole year afterwards, so as that the letter, fur example, which answers to the 1st of March, shall likewise answer to the $2 \mathrm{~d}, \& \mathrm{c}$; or else the intercalary day must be denoted by the same letter as the preceding one. This latter expedient was judged to be the best, and accordingly all the Sundays in the year after the intercalary day have another Dominical letter.

The Dominical letters were introduced into the calendar by the primitive Christians, instead of the nundinal letters in the Roman calendar; and in this manner were those seven letters set opposite the days of the year, to denote the days in the wcek, in most of our common almanacs, till the year $17 \%$, when the initial letters of the days of the wcek were generally introduced instead of them, excepting the Sunday letter itself, which is still retained.

From the foregoing account it follows, that,

Ist, As the common year consists of 365 days, or 52 weeks, and one day over; the letters go one day backwards every common year: so that in such a ycar, if the beginning or first day fall on a Sunday, the next juar it will fall on a Saturday, the next on a Friday, and so on. Consequently, if $\mathrm{G}$ be the Domınical letter for the present year, $F$ will be that for the next year; and so on in a retrograde order.

$2 \mathrm{~d}$, As the bissextile or leap-year consists of 360 days, or 52 weeks, and 2 days over, the beginning of the year next after bissextile goes back 2 days. Whence, if in the beginning of the bissextile year the Dominical letter were $\mathrm{G}$, that of the following year will be $\mathrm{E}$.

$3 d$, Since in leap-years the intercalary day falls on the 24th of February, in which case the 24th and 25th days are considered as one day, and denoted by the same letter; after the 24th day of February the Dominical letter goes back one place: thus, if in the beginning of the year the Dominical letter be 6 , it will afterwards change to the letter $\mathbf{F}$ for the remaining Sundays of the year. With us, however, this day is now added at the end of February, and from thence it is that the change takes place.

4th, As every fourth year is bissextile, or leap-year, and as the number of letters is 7 ; the same order of Dominical letters only returns in 4 times 7 , or 28 y'ears; which, without the interruption of bissextiles, would return in 7 years.
5 th, Hence the invention of the solar cycle of 28 years; on the expiration of which the Dominical letters are restured successively to the same days of the month, or the same order of the letters returns.

To find the Dominical Letter of any given year. Find the cycle of the sun for that year, as directed under Cycle; and the Dominical letter is found corresponding to it. When there are two letters, the proposed year is bissextile; the former of them serving till the end of February, and the latter for the rest of the year.

The Dominical letter for any year of the present century may be found thus :

To the number of years since the date 1800 , add their fourth part, plus 2; and divide the sum by 7 , then subtract the remainder from 7 , and the last number will be that answering to the Dominical letter; that is, if the remainder be 1 , it answers to $A, 2$ to $B, \& c$, and 0 to $G$.

Suppose, for example, we wanted the Dominical letter for the year 1833 .

Here $33+8+2=43$, being divided by 7 , leaves a remainder 1 , which taken from 7 , gives 6 ; answering to $F$, which therefore is the Dominical letter for that year.

When the year is bissextile, that is, when the date of it is divisible by 4 , then the letter obtained from this rule is that used from February to the end of the ycar, and the Sunday letter from the beginning of the year, to the end of February, will be that, next in order in the alphabet, unless the former is $\mathrm{G}$, in which case the lattcr will be $\mathbf{A}$.

By the reformation of the calendar under Pope Gregory the 13th, the oidder of the Dominical letters was again disturbed in the Gregorian year; for the year 1582, which had $\mathrm{G}$ for its letter at the beginning, by retrenching 10 days after the 4 th of October, came to have $c$ : by which means the Dominical letter of the ancient $\mathbf{J u}$ lian calendar is 4 places before that of the Gregorian, the letter $\mathrm{A}$ in the former answering to $\mathrm{D}$ in the latter.

DONJON. Sce Dungeon.

DONN (BENJAMIN), a respectable mathematician, was born at Bideford, in Devonshire, in $17 \approx 9$. He kept a school in that town for some years, and while there, made a complete survey and map of the county, for which he received a premium of $100 l$. from the society for promoting arts and commerce. He also published his Mathematical Essays in 8vo, which bad a favourable reception, and procured him the office of keeper of the library at Bristol; where he also kept a flourishing academy for some years. In 1771 he printed an Epitome of Natural and Experimental P'tilosophy, 12mo; and in 1774, a work, entitled, The British MIariner's Assistant, being a collection of tables for nautical purposes. In 1796 he was appointed to the sinecurc office of master of mechanics to the king. Besides the books above mentioned, he published also trentises on Arithmetic, Bookkceping, Geometry, and Trigonometry. Mr. Donn died in 1798 , leaving behind him the character of an ingenious and worthy man.

DORADO, a southern constellation, not visible in our latitude; called also Xiphias, or the Sword-fish. Its stars, in Sharp's catalogue, are 6.

DORIC Order of Architecture, is the second of the five orders, being placed betwcen the Tuscan and the Ionic. The Doric scems the most natural, and best proportioned, of all the orders; all its parts being founded on the natural position of solid bodic's; for which reason it is most proper to be used in great. and massy luuildings, $3 \mathrm{~K} 2$ 
D R A

as the outside of churches and public places. The Doric order has no ornaments on its base nor its capital. Its column is $\mathbf{S}$ diameters high, and its frieze is divided between triglyphs and metopes.

DORMLR, or DURMaNT, in Architecture, denotes a window made in the roof of a building, or above the entablature; being raised upon the rafters.

DOUBLE Horizontal Dial, one with a double gnomon, the one pointing out the hour on the outer circle, the other, on the stereugraphic projection drawn upon it. This dial finds the meridian, the hour, the sun's place, rising, setting, \&c, and many other problems of the globe.

Dou b Le Point, in the Higher Geometry, is a point which is common to two parts or legs, or branches of some curve of the $2 \mathrm{~d}$ or higher order: such as, an infinitely small oval, or a cusp, or the cruciform intersection, \&c, of such curves. See Newton's Lnumeratio Linearum, \&c, de Curvarum Punctis Duplicibus.

Dovble Slars. See StaR.

DOUBLER of Electricity, is an instrument capable of augmenting a very small quantity of electricity, so as to render it more than sufficiently manifest, by means of an electrometer, or even capable of affording sparks.

The invention of this instrument is due to the Rev. Abraham Bennet; the description of which is contained in the second part of the 77 th volume of the Philosophical Transactions; and in the same Transactions, for the year $17 \mathrm{~S} 8$, Mr. Cavallo has given an account, at considerable length, of his experiments and observations on this instrument, as improved by himself, and also in its original form; from which he concludes, "that the invention of the douhler is very ingenious, but its use cannot be depended on ;" that is to say, for ascertaining small quantities of electricity, because though no electricity be communicated to the plates of the instruments, yet by the usual process of doubling, a certain quantity of it will be produced. For the description of a very ingenious instrument for this purpose, by Mr. Nicholson, see Philosophical Transactions for the year 1788 .

Doubling a Cape, or Point of Land, in Navigation, signifies the coming up with it, passing by it, and leaving it behind the ship. The Portuguese pretend that they first doubled the Cape of Good Hope, under their admiral Vasquez de Gama; but there are accounts in history, particularly in Herodotus, that the Egyptians, Carthaginians, \&cc, had done the same long before them.

DOUCINE, in Architecture, is an ornament on the highest part of the cornice, or a moulding cut in the figure of a wave, half convex, and half concave.

DRACHM, or DRAM, is the name of a small weight used with us, and is of two kinds, viz, the 8th part of an ounce in Apothecaries' weight, and the 16 th part of an ousce in Avoirdupois weight.

DRACO, the DRAGON, a constcllation of the northern hemisphere, whose origin is variously fabled by the Greeks; some of them representing it as the Dragon which guarded the Hesperian fruit, or golden apples, but being killed by Hercules, Juno, as a reward for its faithful services, took it up to heaven, and so formed this constcllation: while others say, that in the war of the giants, this D) ragon was brought into the combat, and opposed to Minerva, when the goddess taking the Dragon in her hand, threw him, I wisted as he was, up to the skies, and fixed him to the axis of the beavens, before he had time to unwinl his concortions.
D U C

The stars in this constellation are, according to Ptor lemy, 31; according to Tycho, 32; Hevelius, 40; Bayer, 33 ; and Flamsteed, so.

DRAGON, in Astronomy. See Draco.

Dragon's Head, and Tail, are the nodes of the planets, but more particularly of the moon, being the puints in which the ecliptic is intersected by her orbit, in an angle of about $5^{\circ} 18^{\prime}$. One of these points is northward, the moon then beginning to have north latitude; and the other southward, where she cominences south latitude; the former point being represented by the knot 8 for the head, and the other by the same reversed, or 8 fir the tail. And near these points it is that all eclipses of the sun and moon happen. These intersections are not always in the same two points of the ecliptic, but shift by a retrograde motion, at the rote of $s^{\prime} 11^{\prime \prime}$ per day, and completing their circle in 18 years 225 days.

DraGoN-Beams, in Architecture, are two strong braces or struts, standing under a breast-summer, and meeting in an angle on the shoulder of the king-piece.

DRAM. See DrachM.

DliAUGHT-Conpasses, those provided with several moveable points, to draw fine draughts in architecture, \&cc. See Compass.

DRALGHT-Hooks, are large hooks of iron, fixed on the cheeks of a gun-carriage, two on each side, one near the trunnion hole, and the other at the train.

DRAW-Bridge, a bridge made after the manner of a floor, to be drawn up, or let down, as occasion requires, before the gate of a town or castle.

DRIFT, Sноот, or ThRUst of an Arch, is the push or force it exerts.

DrIFT, in Navigation, denotes the angle which the line of a ship's motion makes with the nearest meridian, when she drives with her side to the wind and waves, and is not governcd by the power of the helm ; and also the distance which the ship drives on that line, so called only in a storm.

DRIP, in Architecture. See Larmier.

DUCTILITY, a property of certain bodies, by which they are capable of being beaten, pressed, drawn, or stretched forth, without breaking; or by which they are capable of great alterations in their figure and dimensions, and of gaining in one way as they lose in anotber.

Such are metals, which, being urged by the hammer, gain in length and breadth what they lose in thickness; or, being drawn into wire through holes in iron, becomelonger as they are made more slender. Such also are gums, glues, resins, and some other bodies; which, though not malleable, may yet be demominated ductile, in as much as, when softened by water, fire, or some other menstruum, they may be drawn into threads.

Some bodies are ductile both when thry are hot and cold, and in all circumstances: such are metals, and especially gold and silser; other bodies are ductile only when they have a certain degree of heat; such as glass, and wax, and such like substances : others again areductile only when cold, and brittle when hot; as some kinds of iron, viz, those called by workmen redshort, as also brass, and sume inctallic alloys.

'The catise of ductility is very obscure; as depending much on hardness, a quality whose nature we know very little about. It is true, it is usual to account for hardness from the force of attraction between the particles of the hard body; and lor ductility, from the particles of the 
ductile body being as it were jointed, and cutangled with each other. But without dwelling on any fanciful hypotheses about ductility, we may amuse ourselves with some truly amazing circumstances and phenomena of it, in the instances of gold, glass, and spiders' webs. Ubserving, however, that the ductility of metals decreases in the following order : gold, silver, cupper, iron, tin, lead.

Ductility of Gold. One of the properties of gold is, to be the most ductile of all bodies; of which the goldbeaters and gold-wire-irawers, furnish us with abundant proof. Fa. Mersenne, M. Robault, Dr. Halley, \&c, have made computations of it : but they trusted to the reports of the workmen. M. Reaumur, in the Memoires de l'Academie Royale des Sciences, an. 1713, took a surer way; he made the experiment himself. A single grain of gold, he found, even in the common gold leat, used in most of " our gildings, is extended into 36 and a half square inches; and an ounce of gold, which, in form of a cube, has not half an incl for its side, is beaten under the hanmer into a surface of 146 and a half square feet; an extent almost double to what could be done in former times. In Fa. Mersemne's time, it was deemed prodigious, that an ounce of gold should form 1600 leaves; which, together, only made a surface of 105 square feet.

But the distension of gold under the hanmer (however considerable), is nothing to that which it undergoes in the drawing-iron. There are gold leaves, in some parts scarce the $\frac{1}{360000}$ part of an incli thick; hut $\frac{1}{30000}$ part of an inch is a considerable thickness, in comparison of that of the gold spun.on silk in our gold thread. To conceive this prodigious ductility, it is necessary to have some idea of the manner in which the wire-drawers proceed. The wire, and thread that we commonly call gold thread, \&c, (which is only silver wire gilt, or covered over with gold) js drawn from a large ingot of silver, usually about thirty pounds weight. This they form into a cylinder, or roll, about an inch and a half in diameter, and twenty-two inches long, and cover it with the leaves prepared by the gold-beater, laying one over another, till the cover is a deal thicker than that in our urdinary gilding; and yet, even then, it is very thin; as will be easily conceived from the quantity of gold that is used for gilding the thirty pounds of silver, which seldom exceeds two ounces; and, frequently, little more than one. In effect, the full thickness of the gold on the ingot rarely exceeds $\frac{x}{400}$ or $\frac{x}{5} \frac{x}{0}$ part; and somctimes not Tơ part if an inch.

The ingot is then successively drawn through the boles of several irons, euch smaller than the other, till it be as fine as, or funer than, a hair. livery new hole lessens its diameter; but it gains in length what it loses in thickness; and of course increases in surface: yet the gold still covers it; it follows the silver in all its extension, and never leaves the minutest part bare, not even to the microscope. How inconcrivably therefore must it be attenuated while the ingot is drawn into a thread, whose rlianeter is 9000 times less than that of the ingot. M. lieaumur, by exact weighing, and rigorous calculation, found, that one ounce of the thread was 3232 feet long; and the whole ingot 1163520 feet. Paris measure, or 96 Firench league's; cqual to 1264400 English feet, or 240 miles English; an extent which far surpasses what $F_{a}$. Mersenne, Furetière, Dr. Halley, \&c, cver thought of.

Mersenne salys, that half an ounce of the thread is 100 tarises or fathoms long; on which calculation, an ounce would only be 1200 feet: whereas M. Reaumur finds it 3232. Dr. Halley makes 6 feet of the wire one grain in weight, and one grain of the gold 98 yards; and consequently the ten-thousandth part of a grain, above one third of an inch. The diameter of the wire he found one- $186 \mathrm{th}$ part of au incls; and the thickness of the gold one-154500th part of an ilicl. But this too comes short of M. Reaumur; for, on this principle, the ounce of wire would only be 2680 feet.

But the ingot is not even in this case extended to its full length. The greatest part of our gold thread is spun, or wound on silk; and, before it is spun, they flat it, by passing it between two rolls, or wheels of exceedingly well polished steel ; which wheels, in flatting it, lengthen it by above one-seventh. So that oúr 240 miles are thus extended to 274 . Now, the breadth of these laminze, or plates, M. Reaunur finds, is only one- 8 th of a line, or one-96th of an inch; and their thickness one-3072d. The ounce of gold, then, is here extended to a surface of 1190 square feet; whereas, the utmost the gold-beaters can do, we have obscrved, is to extend it to 146 square feet. From M. Reaumur's calculus, it is found to be one-175000th of a line, or one-2100000th of an inch; which is scarce one13 th of the thickness that Dr. Halley supposed.

But he adds, that, from this supposition the thickness of the gold is every where equal, which is no ways probable; for in beating the gold leaves, whatever care they can bestow, it is impossible to extend them equally. This we easily find, by the greater opacity of some parts than others; for, where the leaf is thickest, it will gild the wire the thickest ; and Reaumur, by computing what the thickness of the gold must be where it is thinnest, finds it only one-3150000th part of an inch. Yet even this is not the utmost ductility of gold ; for, instead of two ounces of gold to the ingot, which we have here computed upon, a single one might have been used; and then the thickness of the gold, in the thimnest places, would only have been the 6300000 th part of an inch.

And still by only pressing them more between the flatter's wheels, they are exteniled to double the breadth and proportionably in length. So that their thickness, at last, will be reduced to one-thirteen or fourteen millionth part of an inch.

Yet, with this amazing thinness of the gold, it is still a perfect cover for the silver: the best eye, or even the best microscope, cannot discover the least chasm, or discontinuity. There is not an aperture to admit alcohol of wine, the subtilest fluid in nature, or even light itself; unless it be owing to cracks occasioned by repeated strokes of the hammer. To which we may add, that if a piece of this gold thread, or gold plate, be laid to dissolve in aquafortis, the silver will be all excavated, or eaten out, and the gold left entire, in little tubules.

It should be observed, that gold, when it has been struck for some time by a hammer, or violently compressed, as by gold wire-drawers, becomes more hard, elastic and stiff, and less ductile, so that it is apt to be cracked or torn : the same thing happens to the other metals by percussion and compression; but ductility and tractability may be restored to metals in that state, by annealing them, or making them red hot. Gold, however, seemsto be more affected by percussion aud annealing, than any otlier metals.

As to the Ductility of soft bodics, it is not yet carried. 
to that pitch. We must not be surprised that, among the ductile bodies of this class, the first place is given to the most brittle of all others, - glass.

Ductility of Glass. It is known that, when well penetrated with the heat of the fire, the workmen can figure and manage glass like soft wax; but what is most remarkable, it may be drawn or spun out into threads exceedingly fine and long. Our ordinary spinners do not form their threads of silk, flax, or the like, with half the ease and expedition, as the glass-spinners do threads of this brittle matter. Some of them that are used in plumes for children's heads, and divers other works, are much finer than any hair, and which bend and wave like it with every wind.

Nothing is more simple and easy than the method of making them : there are two workmen employed; the first holds one end of a piece of glass over the flame of a lamp; and, when the heat has softeued it, a second operator applies a glass hook to the metal thus in fusion; and, withdrawing the hook again, it brings with it a thread of glass, which still adheres to the ruass: then, fitting bis hook on the circumference of a wheel about two feet and a half in diameter, he turns the wheel'as fast as he pleases; which, drawing out the thread, winds it on its rim; till, after a certain number of revolutions, it is covered with a skein of glass thread.

The mass in fusion over the lamp diminishes insensibly : being wound out, as it were, like a pelotoon, or clue of silk, upon the wheel; and the parts, as they recede from the flame, cooling, become more coherent to those next to them; and this by degrees: the parts nearest the fire are always the least coherent, and of course must give way to the effort the rest make to draw thein towards the wheel. The circumference of these threads is usually a flat oval, being 3 or 4 times as broad as thick: some of them seem scarce larger than the thread of a silk-wvorm, and are surprisingly flexible. If the two ends of such threads be knotted together, they may be drawn and bent, till the aperture, or space in the middle of the knot, doth not excced one-4th of a line, or one-4sth of an inch diameter.

Hence M. Reaumur advances, that the flexibility of glass increases in proportion to the fineness of the threads; and that, probably, had we but the art of drawing threads as fine as a spider's web, we might weave stuffs and cloths of them for wear. Accordingly, he made some experiments this way, and found he could make threads fine enough, viz, as fine, in his judgment, as spiders' thread, but he could never make them long enough to do any thing with them.

Ductility of Spiders' Webs. See Weв.

DUNGEON, DoN J $\cap \mathrm{N}$, in Fortification, the highest part of a castle built after the ancient mode; serving as a watchtower, or place of observation; and also for the retreat of a garrison, in case of necessity, so that they may capitulate with greater advantage.

DUNN (SAMULL), was a native of Crediton, in Devonshire, where he kept a mathematical school for several years; but afterwarcls removed to Chelsea, where he long kept an academy. He was a person well skilled in nautical calculations, and was a good practical astronomer, being employed as mathematical examiner for the EastIndia service. 13esides several papers of his inserted in the Philos. Trans, he was also the author of some separate works on practical branches of mathematics; and he publislied a folio Atlas, which has been held in some estimation. Mr. Dunn died in good circumstances, and bequeathed an estate of about $30 l$. a year, to support a mathematical school in his native town, the first master to which was appointed in $\mathbf{1 7 9 3}$.

NUODECIMALS, a kind of multiplication in arithinetic, by which artificers square their dimensions, that is, multiply them together, to obtain the contents of their works, in square feet, inches, and parts. The method is, to multiply the feet and inches in the one dimension, by each of those in the other separately, in so many lines, the like manes set under each other, feet und'r feet, and inches under inches, and then adding their like parts together, as here annexed; first multiplying $14 \mathrm{f}$. 9inc. by the tf. and then by the 6inc. observing to carry 1 for every 12 , from one denomimation to the other. Or, instead of multiplying by the 6 inches, or half a foot, it is better to take halt the upper line, or divide 14f. 9 inc. 4.6

590

$7 \quad 4 \frac{1}{2}$

it by 2 . The sum of the products is $66 \mathrm{f} .4 \frac{\mathrm{T}}{2} \mathrm{inc}$. for the content. But it is to be noted, that though the 66 are square feet, yet the $4 \frac{1}{2}$ inc, are not square inches, but $4 \frac{x}{2}$ parts, in 12, of a square foot, or $4 \frac{\mathrm{r}}{2}$ twelfth-parts of a foot, that is, $4 \frac{r}{2}$ parallelograms, each of 1 foot long and 1 inch broad. See the article Duodecimal ArItı aric; as also Cross Multiplication, and most books on Aritimetic or Mlensuration.

DUPLE, or DoU BLF Ratio, is that in which the antecedent is double tlie consequent; or where the exponent of the ratio is 2. Thus, 6 to 3 is in a duple ratio.

Sub-D UPLE Ratio, is that in which the conscquent is double the antecedent; or in which the exponent of the ratio is $\frac{\pi}{2}$. As in 3 to 6 , which is in subduple ratio.

DUPLICATE Ratio, is the square of a ratio, or the ratio of the squares of two quantities. Thus, the duplicate ratio of $a$ to $b$, is the ratio of $a^{2}$ to $b^{2}$, or of the square of $a$ to the square of $b$.- In a series of geometrical proportionals, the 1 st term is to the $3 \mathrm{~d}$, in a duplicate ratio of the 1 st to the $2 \mathrm{~d}$, or as the square of the first to the square of the $2 \mathrm{~d}$ : Thus, in the geometricals $2,4,8,16$, \& $\mathrm{x}$, the ratio of 2 to 8 , is the duplicate of that of 2 to 4 , or as the square of 2 to the square of 4 , that is, as 4 to 16 . So that duplicate ratio. is the ratio of the squares, as triplicate ratio is the ratio of the cubes, \&c.

DUPLICATION, is the doubling of a quantity, or multiplying it by 2 , or adding it to itself.

DUPLICATION of a Gube, is finding out the side of a cube that shall be double in solidity to a given cube: which is a celebrated problem, much cultivated by the ancient geometricians, a bout 2000 years ago.

It was first proposed by the oracle of Apollo at Delphos; which, being consulted about the manner of stopping a plague then raging at Athens, returned for answer, that the plague should cease when Apollo's altar, which was cubical, should be doubled. Upon this, they applied themselves in good earnest, to seck the duplicature of the cule, which from thence was called the Delian problem.

This problem cannot be cfiected geometrically, as it requires the solution of a cubic equation, or requires the finding of two mean proportionals, viz, between the side of the given cube and the double of the same, the first of which two mean proportionals is the side of the double cube, us was first observed by llippocrates of Chios. For, 
let $a$ be the side of the given cube, and $z$ the side of the double cube sought; then it is $z^{3}=2 a^{3}$, or $a^{2}: z^{2}:: z$ : $2 a$; so that, if $a$ and $z$ be the 1st and $2 d$ terms of a set of continued proportionals, then $a^{2}: z^{2}$ is the ratio of the square of the 1 st to the square of the $2 \mathrm{~d}$, which, it is known, is the same as the ratio of the 1 st term to the $3 \mathrm{~d}$, or of the $2 d$ to the $4 t h$, that is of $z$ to $2 a$; therefore, $z$ being the $2 \mathrm{~d}$ term, $2 a$ will be the 4 th. So that $z$, the side of the cube sought, is the $2 \mathrm{sl}$ of four terms in continued proportion, the 1 st and 4th being $a$ and $2 a$, that is, the side of the double cube is the first of two mean proportionals between $a$ and $2 a$.

Eutocius, in his Commentaries on Arcnimedes, gives several ways of performing this by the inesolabe. In Pappus tos are found three different ways; the first according to Archimedes, the second according to Hero, and the $3 \mathrm{~d}$ by an instrument invented by Pappus, which-gives all the proportions required. The Sieur de Comiers has also published a demonstration of the same problem, by means of a compass with three legs. But all these methods are only mechanical. See Valerius Maximus, lib. 8; also Eutocius's Comm. on lib. 2. Archimedes de Sphæra et Cylindro; and Pappus, lib. 3, prop.5, and lib.4, prop. 22.

DURER (ALBERT), descended of an Hungarian family, but born at Nuremberg, in 1471 , was one of the best engravers, painters, and practical geometricians of his age. He was at the same time a man of letters and a philosopher; and he was an intimate friend of Erasmus, who revised some of the pieces which he published. He was also a man of business, and for many years the leading magistrate of Nuremberg.

Though not the inventor, he was one of the first and greatest improvers of the art of engraving. He was however the inventor of cutting in wood, which he devised and practised in great perfection, using this way for expedition, as he bad a multitude of designs to execute; and as his work was usually done in the most exquisite manner, lis pieces took him up much time. For in many of those prints which he exccuted on copper, the engraving is clegant to a high degree. His Hell-Scene in particular, engraven in the year 1513 , is as bighly finished a print as ever was engraved, and as happily executed. In his wooden prints too it is surprising to see so much meaning in so early a master. In fact, Durer was a man of a very cxtensive genius. His pictures were excellent; as well as his prints, which were very numerous. They were much admircl, from the first, and soon bought up; which put his wife, who was another Xantippe, on urging him to spend more time upon engraving than he was inclined to do: for he was rich; and chose rather to practise his art as an amuscment, than as a business. He died at $\mathrm{Nu}-$ remberg, in 1528, at 57 ycars of age.

Albert Durer wrote several books, in the German language, which were translated into Latin by other persons, and published after his death : viz,
1. His book upon the rules of painting, entitled, De Symmetria Partium in rectis formis Humanorun Corporum, is one of them: printed in folio, at Nuremberg, in 1532, and at Paris in 1557. An Italian version also was published at Venice, in 1591.

2. Institutiones Geometricæ; Paris 1532.

3. De Urbibus, Arcibus, Castellisque condendis et muniendis; Paris 1531.

4. De Varietate Figurarum, et Flexuris Partium, et Gestibus Imaginum; Nuremberg 1534.

The figures in these books, which are from wooden plates, are very numerous, and most admirably well executed, indeed far beyond any thing of the kind done in our own days. Some of them also are of a very large size, as much as 16 inches in length, and of a proportional breadth, which being exquisitely worked, must have cost great labour. His geometry is chiefly of the practical kind, consisting of the most curious descriptions, inscriptions, and circumscriptions of geometrical lines, planes, and solids. We here meet, for the first time, with the plane figures, which folded up make the five regular or platonic bodies, as well as that curious construction of a pentagon, being the last method in prob. 23 of my Mensuration.

DYE, in Architecture, the trunk of the pedestal, or that part between the base and the cornice, being so called, because it is often made in the form of a dye or cube.

DYNAMICS, is the science of moving powers; more particularly of the motion of bodies that mutually act on each other. See Mecuinics, Motion, Communicatron of Motion, Oscrillation, Percussion, \&c; also my Course of Mathematics, vol. 2; hesides the Mecanique Philosophique of Prony, the Mecanique Analytique of Lagrange, and the Mecanique Celeste of Laplace.

DYNOMETER is the name of an instrument intended for measuring the muscular strength of men and other animals. Mr. Graham, many years ago, invented an instrument for this purpose, which was afterwards improved by Dr. Desaguliers; but it was still too bulky and limited in its operations, to be of much use. The defect of this machine induced M. Leroy, of the Academy of Sciences at Paris, to construct another on more simple principles, and which was certainly more useful for the purpose it was designed for, than Mr. Graham's. The principle of this instrument was not much unlike that of the common spring steelyard, and perhaps it would have been still better, had its principles been exactly the same; for with this last-mentioned instrument, it is evident that the power of an animal may be as accurately ascertained as the weight of a body, and nothing more simple can well be devised.

DYPTERE, or DI PTERE, was a kind of temple, encompassed round with a double row of columns; and the pseudo-diptere, or false diptere, was the same, only this was encompassed with a single row of columns, instead of a double one. 


\section{E A R}

$\mathbf{E}$ AGLE, Aquila, is a constellation of the northern hemisphere, having its right wing contiguous to the equinoctial. For the stars in this constellation, see Aquila. EARTH, Terra, in Natural Philosophy, one of the four elements of the ancients; for, according to their system, all bodies were considered as resolvable into four simple elements, or constituent parts, namely, air, fire, water, and earth. The two former were, avowedly almost unknown; most liquids were supposed to be modifications of the third, and the solid particles of bodies were attributed to the last. Earth in this extended sense was defined, a simple, dry, and cold substance; and as such, an ingredient that entered into the composition of all natural bodies. Modern chemists, though they have retained the term in a much more restricted sense, have yet included under it a.sufficiently heterogeneous assemblage of bodies, many of which may be more properly considered as belonging to the class of alkalies.

The earths at present known, are nine in number; namely, Silex, Zircon, Alumine, Glycine, Yttria, Bary tes, Strontian, Lime, and Magnesia. And when they are purified by art from all foreign inixture, they agree in the following properties: 1 . They are of a snow-white colour. 2. They are infusible by a very intense beat. 3. They are not reducible to the metallic state by being heated in contact with combustible matter. Of those nine earths, however, the last four have all the properties of alkalies; whence they are sometimes called alkaline earths; and many chemists are of opinion that the term earth ought only to be applied to the five former, by which arrange. ment the two classes of earths and alkalies would admit of distinct chemical defnitions, which at present is wanted.

EARTH, in Gcography, the terraqueous globe or ball which we inhabit, consisting of land and sea.

Figure of the EARTr. The ancients had various opinions as to the figure of the earth; some, as Anaximander and Leucippus, held it cylindrical, or in form of a drum; but the principal opinion was, that it was flat; that the visible horizon was the bounds of the earth, and the ocean the bounds of the horizon; that the heavens and earth above this ocean constituted the universe; and that all beneath the ocean was Hades: and of this same opinion were also some of the Christian fathers; as Lactantius, St. Augustine, \&c. See Lactan. lib. 3, cap. 24; St. Aug. lib. 16, de Civitate Dei; $A$ ristot. de Cœlo, lib. 2 , cap. 13.

Such of the ancients however as understood any thing of astronomy, and especially the doctrine of eclipses, must have been acquainted with the round figure of the earth; as the ancient Babylonian astronomers, who had calculated eclipses long before the time of Alexander, and Thales the Grecian, who predicted an cclipse of the sun.

That the exterior of the carth is round, or rotund, is manifest to the most common perception, in the case of a ship sailing either from the land, or towards it; for when a person stands upon the shore, and sees a ship sail from the land, out to sea; at first he loses sight of the hull and lower parts of the ship, next the rigging and middle parts, and lastly of the tops of the masts themselves, in every

\section{$E A R$}

case the rotundity of the sea between the ship and the eye being very visible: the contrary happens when a ship sails towards us; we first see the tops of the masts appear just over the rotundity of the sea ; next we perceise the rigging, and lastly the luull of the ship itself: all which is véll illustrated by the following figure.

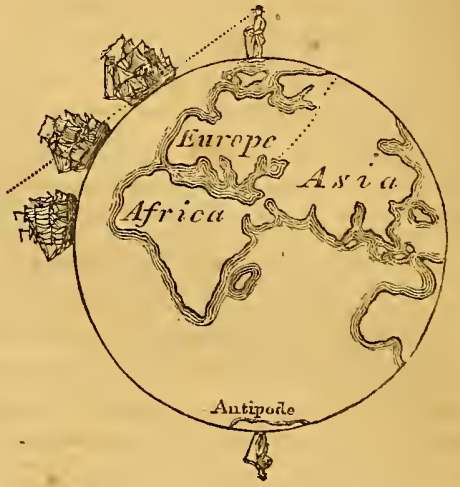

The round figure of the earth is also evident from the eclipses of the sun and moon; for in all eclipses of the moon, which are caused by the moon passing through the earth's shadow, that shadow always appears circular upon the face of the moon, whatever way it be projected, whether east, west, north, or south, and however its diameter vary, according to the greater or less distance from the earth. Hence it follows, that the shadow of the earth, in all situations, is really conical ; and conseguently the body that projects it, i. c. the earth, is at least nearly spherical.

The spherical figure of the carth is also evinced from the rising and setting of the sun, moon, and stars; all which happen sooner to those who live to the cast, and later to those living westwardly; and that more or less so, according to the distance.

So also, in sailing to the northward, the north pole and northern stars become more elevated, and the suuth pole and southern stars more depressed; the elevation northerly increasing equally with the depression southerly; and each of them proportionably to the distance; and the same thing happens in going to the southward; that is, in this case the south pole is elevated, and the north pole depressed. Besides, the oblique ascensions, descensions, emersions, and amplitudes of the rising and sctting of the sun and stars in every latitude, are agreeable to the supposition of the earth's being of a spherical form : all which could not happen if it was of any other figure.

But the most convincing proof of the spherical figure of the earth, and which bids defiance to every objection, is, that many celcbrated aavigators have sailed round it, proceeding in a cortain direction, and by constantly pursuing the saine course, have finally arrivel at the port 
whence they departed. The first who suceeded in this daring attempt, was Ferd. Magellan, a Portugucse, in the ycar 1519, and who completed his tour in 1124 days. In the year 1557 Francis Drake performed the same in 1056 days : in the year 1586 , Sir Tho. Cavendish made the same voyage in 777 days; Simon Cordes, of Rotterdam, in the year 1590; and in the year 1598, Oliver Noort, a IIollander, in 1077 days; Van Schouten, in the ycar 1615, in 749 days; Jac. Heremites and Joh. Huygens, in the year 1623, in 802 days: and many others have since performed the same navigation, particularly Anson. Bougainville, and Cook; sometimes sailing round by the eastward, sumetimes to the westward, till at length they arrived again in Europe, whence they set out; and in the conrse of their voyage, observed that all the phenomena, botb of the heavens and earth, corresponded to, and evinced this spherieal figure.

The globular figure of the earth is also inferred from the operation of levelling, in which it is found necessary to make an allowance for the difference between the apparent and the true level.

The natural cause of this sphericity of the globe is, according to Sir Isaac Newton, the great principle of attraction, which the Creator has stamped on all the matter in the universe; and by which all bodies, and all the parts of boties, mutually attract one another.-And the same is the cause of the sphericity of the drops of rain, quicksilver, \&c.

What the earth loses of its sphericity by mountains and valleys, is nothing considerable; the highest eminence being scarce equivalent to the minutest protuberance on the surface of an orange. Its difference from a perfect sphere, however, is more considerable in another respect, by which it approaches nearly to the shape of an orange, or to an oblate spheroid, being a little flatted at the poles, and raised about the equatorial parts, so that the axis from pole to pole is less than the equatorial diameter. What gave the first occasion to the discovery of this figure of the earth, was the observations of some French and English philusophers in the East Indies, and other parts, who found that pendulums performed their vibrations slower, the nearer they came to the equator; whence it follows, that the velocity of the descent of bodies by gravity, is less in countries nearer to the equator ; and consequently that those parts are farther removed from the centre of the earth, or from the common centre of gravity. See the History of the Royal Academy of Sciences, by Du Hamel, p. 110, 156, 206; and Hist. de l'Acad. Roy. 1700 and 1701 . This circumstance put Huygens and Newton upon finding out the cause, which they attributed to the revolution of the earth about its axis. If the earth were in a fluid state, its rotation round its axis would necessarily make it put on such a figure, because the centrifugal furce being greatest towards the equator, the fluid would there rise and swell most; and that its figure really should be so now, seems necessary, to keep the sea in the equinoctial regions from overflowing the earth about those parts. See this curious subject well handled by Huygens, in his discourse De Causil Gravitatis, pa. 154, where he states the ratio of the polar diameter to that of the equator, as 577 to 578 . And Newton, in his Principia, first published in 1686 , demonstrates, from the theory of gravity, that the figure of the earth must be that of an oblate splieroid generated by the rotation of an ellipse about its shortest diameter, provided VoL. 1. all the parts of the earth were of an uniform density throughout, and that the proportion of the polar to the equatorial diameter of the earth, would be that of 689 to 692 , or nearly that of 229 to 230 , or as $\$ 9956522$ to $\mathrm{J}$.

This proportion of the two diameters was ealculated by Newton in the following manner. Having found that the centrifugal force at the equator is $\frac{x}{2} \frac{x}{9}$ th of gravity, he assumes, as an hypothesis, that the axis of the earth is to the diameter of the equator as 100 to 101 , and thence determines what must be the centrifugal force at the equator to give the earth such a form, and finds it to be ${ }_{5}^{4}{ }_{5}$ ths of gravity : then, by the rule of proportion, if a centrifugal force equal to $\frac{4}{505}$ ths of gravity would make the earth higher at the equator than at the poles by $\frac{\mathrm{I}}{\mathrm{x} \delta} \mathrm{t}$ th of the whole height at the poles, a centrifugal force that is the $\frac{7}{89}$ th of gravity will make it higher by a proportional cxcess, which by calculation is $\frac{1}{2} \frac{1}{2}$ th of the height at the poles; and thus he discovered that the diameter at the equator is to the diameter at the poles, or the axis, as 230 to 229. But this computation supposes the carth to be every where of a uniform density; whereas if the earth is more dense near the centre, then bodies at the poles will be more attracted by this additional matter being nearer; and therefore the excess of the semi-diameter of the equator above the semi-axis, will be different. According to this proportion between the two diameters, Newton farther computes, from the different measures of a degrce, that the equatorial diameter will exceed the polar, by $34 \frac{2}{5}$ miles.

Nevertheless, Messrs. Cassini, both father and son, the one in 1701 , and the other in 1713 , attempted to prove, in the Memoirs of the Royal Academy of Sciences, that the earth was an oblong spheroid; and in 1718, M. Cassini again undertook, from observations, to show that, on the contrary, the longest diameter passes through the poles; which gave occasion for John Bernoulli, in his Essai d'une Nouvelle Physique Celeste, printed at Paris in 1735, to triumph over the British philosopher, apprehending that these observations would invalidate what Newton had demonstrated. And in 1720 , M. De Mairan advanced arguments, supposed to be strengthenet by geometrical de:inonstrations, lurther to confirm the assertions of Cassini. But in 1735 two companies of mathematicians wcre employed, one for a northern, and another for a southern expedition, the result of whose observations and measure. ment plainly proved that the earth was flatted at the poles; and so it has been considered ever since. See DFG RE E.

The proportion of the equatorial diameter to the polar, as stated by the gentlemen employed on the northern expedition for measuring a degree of the meridian, is as 1 to 0.9891 ; by the Spanish mathematicians as 266 to 265 , or as 1 to 0.99624 ; by $M$. Bouguer as 179 to 178 , or as 1 to 0.99441 .

As to all conclusions however deduced from the length of pendulums in different places, it is to be observed that they proceed upon the supposition of the uniform density of the earth, which is a very improbable circumstance; as justly observed by Dr. Horslcy in his letter to Captain Phipps: "You finish your article," he concludes, "relating to the pendulum with saying, "that these observations give a figure of the earth nearer to Sir Isaac Newton's computation, than any others that have hitherto been made;' and then you state the several figures given, as you imagine, by former observations, and by your own. Now it is very true, that if the meridians be eillipses, or if the figure of the earth be that of a spheroid generated is L 
Ly the revolution of an ellipsis, turning on its shorter axis, the particular figure, or the cllipticity of the generating cllipsis, $u$ hich your observations give, is nearer to what Sir loa: Newton says it should be, if tise globe were lomogeneous, than any that can be clerised frum former observations. But yet it is not what you imagine. Taking the gain of the pendulum in latitude $79^{\circ} 50^{\prime}$ exactly as you state it, the difierence between the equaturial and the polar diameter, is about as much less than the Newtonian computation makes it, and the hypothesis of hon:ogeneity would require, as you reckon it to be grater. The proportion of 212 to 211 sliould indced, according to your obscrvations, be the proportion of the force that acts upon the pendulum at the poles, to the force acting upon it at the equator. But this is by no pneans the same with the proportion of the equatorial diameier to the polar. If the globe were homog(ncous, the equatorial diameter would exceed the polar by $\frac{-1}{2} \frac{r}{3}$ ol the length of the latter: and the polar force would also cxceed the equatorial by the like part. But if the diference between the polar and equatorial force be greater than $\frac{\pi}{2} \frac{\pi}{3}$, (which may be the case in an heterngeneous globe, and scems to tec the case in ours,) then the difierence of the diameters should, according to thcory, be less than $\frac{1}{2} \frac{1}{3} \delta$, and vice versa.

"I I confess this is by no means obvious at first sight; so far otherwise, that the mistake, which you bave fallen into, was once very general. Many of the best mathematicians were misled by too implicit a reliance upon the authority of Newton, who had certainly confined his investigations to the homogeneous splieroid, and had thought about the heterogencous only in a loose and general way. The late Mr. Clairaut was the first who set the matter right, in bis elegant and subtle treatise on the figure of the earth. That work has now been many years in the hands of mathematicians, among whom 1 imagine there are none, who have considered the subject attentively, that do not acquiesce in the author's conclusions.

"In the second part of that treatise, it is proved, that putting $P$ for the polar force, $\Pi$ for the equatorial, $\delta$ for the true ellipticity of the earth's figure, and $\varepsilon$ for the ellipticity of the homogeneous spheriod, then

$\frac{p-\Pi}{\Pi}=2 \varepsilon-\delta:$ therefore $\delta=2 \varepsilon-\frac{P-\Pi}{\Pi}$;

and therefore, according to your observation, $\delta=\frac{x}{2} \frac{1}{3}$. This is the just conclusion from your observafions of the pendulum, taking it for granted, that the meridians are ellipses: which is an hypothesis, upon which all the reasonings of theory have hitherto proceeded. But plausible as it may seem, I must say, that there is much reason from experiment to call it in question. If it were true, the increment of the force which actuates the pendulum, us we approach the poles, should be as the square of the sine of the latitude: or, which is the same thing, the clecrement, as we approach the equator, should be as the square of the cosine of the latitude. But whoever takes the pains to conipare together such of the observations of the pendulum in different latitudes, as seem to have been made with the greatest care, will find that the increments and decrements do by no means follow these proportions; and in those which 1 have cxamined, 1 find a regularity in the deviation which little resembles the mere error of observation. The unavoidable conclusion is, that the true figure of the meridians is not elliptical. If the meridians are not ellipses, the difference of the diameters may indeed, or it may not, be proportional to the difference between the polar and the muatorial force; but it is quite an un- certainty, what relation subsists between the one quantity and the other; our whole theory, except so far as it relates to the homogeneous spheroid, is built upon false assumptions, and there is no saying what figure of the earth any "bservations of the pendulum give."

He then lays dowi the following table, which shows the difierent results of observations made in different latitudes; in which the first three columns contain the names of the several observer's, the places of observation, and the latitude of each; the 4 th column shows the quantity of $\mathrm{P}-\Pi$ in such parts as $\Pi$ is 100000 , as deduced from comparing the length of the pendulum at each place of observation, with the length of the equatorial pendulum as determined by $\mathrm{Ml}$. Bouguer, upon the supposition that the increments and decrements of force, as the latitude is increased or lowered, observe the proportion which theory assigns. Only the $2 \mathrm{~d}$ and the last value of $\mathrm{P}-\Pi$ are concluded from comparisons with the pendulum at Greeswich and at London, not at ihe cquator. '1 he 5 th column shows the value of $\delta$ corresponding to every value of $\mathrm{P}-\Pi$, according to Clairaut's theorem :

\begin{tabular}{|c|c|c|c|c|c|}
\hline Glservers. & Places. & Lati & ades. & $\mathbf{P}-\Pi$. & $\delta$. \\
\hline Bonguer & Equ & & $0^{\prime}$ & & \\
\hline Bouguer & Porto Bello & 9 & 34 & $741 \cdot 5$ & $7 \frac{1}{84}$ \\
\hline Green & Otaheitee & 17 & 29 & $563 \cdot 2$ & $3 \frac{1}{2} \frac{1}{6}$ \\
\hline Bouguer & San Domingo & 18 & 27 & $591^{\circ} 0$ & $3 \frac{7}{5}$ \\
\hline $\left.\begin{array}{c}\text { Abbé de la } \\
\text { Caille }\end{array}\right\}$ & $\left.\begin{array}{l}\text { Cape of } \\
\text { Good Hope }\end{array}\right\}$ & 33 & 55 & $731 \cdot 5$ & \\
\hline$-\quad-\overline{-}$ & Paris & 48 & 50 & $585 \cdot 1$ & $3 \frac{x}{5} x$ \\
\hline $\left.\begin{array}{c}\text { The Acade- } \\
\text { micians }\end{array}\right\}$ & Peillo & 66 & 48 & $565 \cdot 9$ & $3 \frac{1}{2} 9$ \\
\hline Capt. Phipps & - & 7.9 & 50 & $471 \cdot 2$ & $\frac{I}{2} \leq T$ \\
\hline
\end{tabular}

"By this table it appears, that the observations in the middle parts of the globe, setting aside the single one at the Cape, are as consistent as could reasonably be expected; and they represent the ellipticity of the earth at about $\frac{1}{4} \bar{c}$. But when we come within 10 degresof the equator, it should seem that the force of gravity suddenly becomes much less, and within the like distance of the poles much greater than it could be in such a splseroid."

The following problem, communicated by Dr. Leatherland to Dr. Penberton, and publisbed by Mr. Robertson, serves for finding the proportion betwcen the axis and the equatorial diameter, from measures taken of a degree of the meridian in two different latitudes, supposing the earth an oblate spheroid.

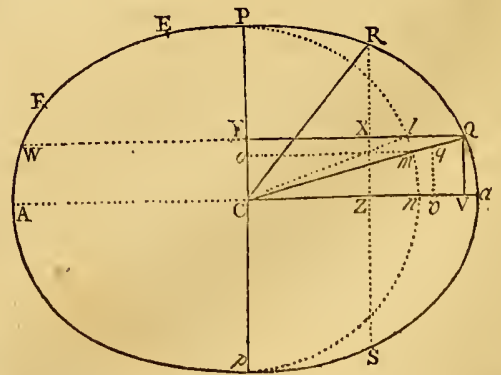

Let $\wedge$ Pap be an ellipse, representing a section of the eartl through the axis $r p$; the equatorial diameter, or 
the greater axis of the ellipse, being $A a$; let $\mathrm{E}$ and $\mathrm{F}$ be two places where the measure of a degree has been taken; these measures are proportional to the radii of curvature in the ellipse at those places; and if $\mathrm{CQ}, \mathrm{CR}$ be conjugates. to the diameters whose vertices are $\mathrm{E}$ and $\mathrm{F}, \mathrm{CQ}$ will be to $\mathrm{Cr}$ in the subtriplicate ratio of the radins of curvature at $\mathrm{E}$, to that at F, by Cor. 1, prop. 4. part 6 of Milnes's Conic Sections, and therefore in a given ratio to one another; also the angles QCP, $\mathrm{RCP}$ are the latitudes of $\mathrm{E}$ and $\mathrm{F}$; so that, drawing $Q \mathrm{v}$ parallel to $\mathrm{P} p$, and $Q \times \mathrm{Y} w$ to $\mathrm{A}$, these angles being given, as well as the ratio of $\mathrm{c} \Omega$ to $\mathrm{cR}$, the rectilinear figure crQXY is given in species; and the ratio of $\mathrm{rc}^{2}-\mathrm{zC}^{2}(=\mathrm{QX} \times \mathrm{Xw})$ to $\mathrm{Rz}^{2}-\mathrm{Qv}^{2}(=\mathrm{RX} \times$ $\mathrm{xs}$ ) is given, which is the ratio of $\mathrm{CA}^{2}$ to $\mathrm{CP}^{2}$; therefore the ratio of $\mathrm{CA}$ to $\mathrm{CP}$ is given.

Hence, if the sine and cosine of the grater latitude, be each augmented in the subtriplicate ratio of the measure of the degree in the greater latitude, to that in the lesser, then the difference of the squares of the augmented sine, and the sine of the lesser latitude, will be to the difference of the squares of the cosine of the lesser latitude and the augmented cosine, in the duplicate ratio of the equatorial to the polar diameter. For $\mathrm{c} q$, being taken in $\mathrm{CQ}$ equal to $\mathrm{Cr}$, and $q v$ drawn parallel to $Q \mathrm{v}, \mathrm{C} v$ and $v q, \mathrm{cz}$ and $\mathrm{z} \mathrm{R}$ will be the signs and cosines of the respective latitudes, to the same radius; and $\mathrm{cv}, \mathrm{V} Q$ will be the augmentations of $\mathrm{c} v$ and $\mathrm{c} q$, in the ratio named.

Hence, to find the ratio between the two axes of the earth, let $\mathrm{E}$ denote the greater, and $\mathrm{F}$ the lcsser of the two latitudes, in and $\mathrm{N}$ the respective measures taken in each; and let $\mathbf{P}$ denote

$\sqrt[3]{\frac{X}{N}}:$ then $\sqrt{ } \frac{\cos ^{3} F-p^{2} \times \cos ^{2} E}{p^{2}+\sin ^{2} E-\sin ^{2} F}$ is $=\frac{\text { lesser axis }}{\text { greater axis }}$.

It also appears by the above probiem, that when one of the degrees measured, is at the equator, the cosine of the latitude of the other being augmented in the subtriplicate ratio of the regrees, the tangent of the latitude will be to the tangent answering to the augmented cosine, in the ratio of the greater axjs to the less. For supposing $\mathrm{E}$ the place out of the equator; then if the semi-circle plimnp be described, and $l \mathrm{c}$ joined, and mo drawn parallel to $a \mathrm{c}$ : co is the cosine of the latitude to the raclius $C P$, and $C Y$ that cosine augmented in the ratio before-named; $\mathrm{YQ}$ being to $\mathrm{Yl}$, that is $\mathrm{C} \iota$ to $\mathrm{C} n$ or $\mathrm{CP}$, as the tangent of the angle $\mathrm{YCQ}$, the latitude of the point $\mathrm{E}$, to the tangent of the angle $\mathrm{y} c$, belonging to the augmented cosine. 'Thus, if n represent the measure in at latitude denoted by $\mathrm{E}$, and $\mathrm{N}$ the measure at the equator, let $\mathrm{A}$ denote an angle whose measure is cos. $\mathrm{E} \times \sqrt[3]{\frac{\mathrm{M}}{\mathrm{N}}}$. Then $\frac{\operatorname{tan.} \mathrm{A}}{\tan . \mathrm{E}}$ is $=\frac{\text { lesser axis }}{\text { greateraxis }}$.

But $\mathrm{m}$, or the length of a degree, obtained by actual mensuration in diflerent latitudes, is known from the followiug table:

\begin{tabular}{|c|c|c|c|}
\hline \multicolumn{2}{|l|}{ Names. } & - Iatit. & Value of $\mathrm{s}$. \\
\hline Swanberg and & soc. & $66^{\circ} 20^{\prime}$ & $\mathrm{u}=57209$ \\
\hline Cassini and $\}$ & - & $49 \quad 22$ & $M=57074$ \\
\hline La Caille $\}$ & - & 4500 & $\mathrm{M}=57050$ \\
\hline Boscovich & - & $43 \quad 00$ & $\mathrm{x}=56972$ \\
\hline De la Caille - & - & $33 \quad 18$ & $M=57037$ \\
\hline $\begin{array}{l}\text { Bouguer and } \\
\text { measur. at es }\end{array}$ & tor $\}$ & 00 & $M=56757$ \\
\hline
\end{tabular}

Now, by comparing each of the first five with the 6 th or last, there are obtained 5 results, each showing the relation of the axes or dianeters; the arithmetical means of all of which gives that ratio as 1 to 0.996747 , which is the ratio of 308 to 307 . But if the last of the four results be omitted, the medium among the other four give's the ratio of 1 to 0.996876 , or of 320 to 319 , which is probably nearest the truth.

Now the magnitude, as well as the figure of the earth, that is the polar and equatorial diameters, may be deduced from the foregoing problem. For, as half the latus rectum of the greater axis $\mathrm{A} a$ is the radius of curvature at $A$, it is given in magnitude from the degree measured there, and thence the axes themselves are given. 'Thus, the circular arc whose length is equal to the radius being $57 \cdot 29578$ degrees, if this number be multiplied by 56757 toises, the measure of a degree at the equator, the product will be the radius of curvature there, or balf the latus rectum of the greater axis; and this is to half the lesser axis in the ratio of the less axis to the greater, that is, as 319 to 320 ; whence the two axes are 6524252 and 6514704 toises, or 7901 and 7926 English miles; and the difference between the two axes about 25 miles. See Robertson's Navigation, vol. 2, pa. 206 \&c. See also Suite des Mem. de l'Acad. 1718, pa.24.7; and Maclaurin's Fluxions, vol.2, book 1, ch. 14. Also the later measurements of the French Institute, of Swanberg, of Colonel Mudge, \&c, \&c.

And nearly the same ratio is deduced from the lengths of pendulums vibrating in the same time, in different latitucies; provided it be allowed that the meridians are real ellipses, or the earth a true spheroid, which however can only take place in the case of an uniform gravity in all parts of the earth.

Thus, in the new Petersburg Acts, for the years 1788 and 1789 , are accounts and calculations of experiments relative to this subject, by $\mathbf{M}$. Krafft. These experiments were made at different times, and in various parts of the Russian empire. This gentleman has collected and compared them, and drawn the proper conclusions from them: thus he infers that the length $\boldsymbol{x}$ of a pendulum that swings seconds in any given latitude $\lambda$, and in a temperature of 10 degrees of Reaumur's thermomiter, may be determined by this equation: $x=439 \cdot 178+2 \cdot 321$ sine $^{2} \lambda$, línes of a French foot, or $x=39^{\circ} 0045+0.206 \operatorname{sine}^{2} \lambda$, in English inches, in the temperature of 53 of Fahrenheit's thermometer.

This expression nearly agrees, not only with all the experiments made on the pendulum in Russia, but also with those of Mr. Graham in England, and those of Mr. Lyons in $79^{\circ} 50^{\prime}$ north latitude, where he found its leugth to be $431.3 \mathrm{~S}$ lines. It also shows the augmentation of gravity from the equator to the parallel of a given latitude $\lambda$ : for, putting $g$ for the gravity under the equator, $G$ for that under the pole, and $y$ for that under the latitude $\lambda$, M. Krafft finds $y=\left(1+0.005284 .8 \sin ^{2} \lambda\right) g$; and there-

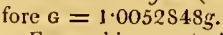

From this proportion of gravity under different latitudes, the sane author infers that, in case the earth is a bomogeneous ellipsoid, its oblateness must be $\frac{1}{T} \mathrm{~T}$; instead of $\frac{x}{2} \frac{\pi}{3}$, which ought to be the result of this hypothesis: but on the supposition that the earth is a heterogeneous ellipsoid, he finds its oblatencss, as deduced from these experiments, to be $\frac{1}{2} \frac{7}{7}$; which agrees with that resulting from the measurement of some of the degrees of the meridian nearly. This confirms an observation of MI. Láplace, that, if the hypothesis of the earth's homogeneity be given 
up, then theory, the measurement of degrees of latitude, and experiments with the pendulum, all agree in their result with respect to the oblateness of the earth. See Memoires de l'Acad. 1783, pa. 17, and lis later works.

In the Philos. Trans. for 1791, pa. 236, Mr. Dalby has given some calculations on measured degrees of the meridian; whence he infers, that those degrees measured in middle latitudes, will answer nearly to an ellipsoid whose ares are in the ratio assigned by Newton, viz, that of 230 to 229. And as to the deviations of some of the others, viz, towards the poles and equator, he thinks they are caused by the errors in the observed celestial arcs.

On the supposition that the earth's figure is that of an oblate spheroid, it has been stown, 1st, 'That a degree of the earth's equator is the first of two mean proportionals, between the last and first degrees of latitude. $2 \mathrm{tl}$, If the equatorial diameter be to the polar axis, as 180 to 179 , the lat. in which the degree of lat. is equal to a degree of the equator, will be $54^{\circ} 48^{\prime} 24^{\prime \prime}$. If the proportion be 230 to 229 , the lat comes out $54^{\circ} 45^{\prime} 31^{\prime \prime}$. 3d, If the proportion be 180 to 179 , the lat. where the degree of the meridian is equal to a degree of a circle whose diameter is equal to the earth's axis, will be $35^{\circ} 20^{\prime} 30^{\prime \prime}$; assuming the proportion 230 to 229 , that lat. will be $35^{\circ} 19^{\prime} 25^{\prime \prime}$. (Horsley's Tracts, p. 394, \&c.)

M. Swanberg obscrves, that occultations of the fixed stars by the moon is another method by which the figure of the earth may be determined : and $M$. Treisnecker, after comparing a great number of these uccultations, concludes from them that the ellipticity of the earth is $\frac{1}{3} \frac{1}{2}$.

The exceltent mathematician and astronomer Laplace, in his Mécanique Céleste, has calculated the ellipticity of she earth, from the effect of precession and nutation, to be $\frac{\pi}{3 \frac{1}{3}}$; which is nearly ait arithmetical mean among those obtained from occultations of the stars, and the vibrations of the pendulum. The theory of the moon also gives $\frac{x}{305}$ for the ellipticity.

The - preceding ellipticities of the earth, expressed in parts of its equatorial diameter, with their authors, and the principles fiom which they were derived, may be exhibited in one view, as follows:

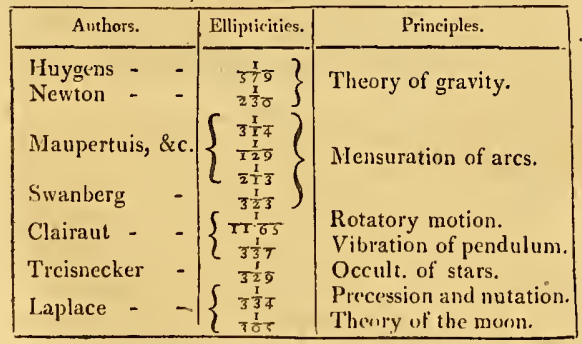

'The variety in the preceding results presents an interesting circumstance, as it indicates something in the figure and conformation of the earth, which is not yet understood, and naturally gives rise to the followisg im portant and intricate questions, to which all the accumulated knowledge of the present time's bas not been able to abtain complete solutions. Is the earth a spheroid of rotation? Are the northern and southern hemispheses cqualand similar to each ofher? What is the ratio of an arc of the mericlian, measured in a given latitude, to the whole meridian? It is perhaps no improbable conjecture, that the effects of precession and nutation, with the vibrations of the pendulum, will be found among the most accurate means of determining, at least, the general outlines of our globe.

Tacquet draws some pleasing inferences, in the form of paradoxes, from the ruund figure of the earth; as, $1 \mathrm{st}$, That if any part of the surface of the earth were quite plane, a man could so more walk upright upon it, than on the side of a mountain. 2d, That the traveller's head goes a greater space than his feet; and a horseman than a footman; as moving in a greater circle. $3 \mathrm{~d}$, That a vessel, full of water, being raised perpendicularly, some of the water will be continually flowing out, yet the vessel still remain full; and on the contrary, if a vessel of water be let perpendicularly down, though nothing flow out, yet it will cease to be full: consequently there is more water contained in the same vessel at the foot of a mountain, than on the top ; because the surface of the water is formed into a segment of a smaller sphere below than above. Tacq. Astron. lib. 1, cap. 2.

Changes of the EARTH. Mr. Boyle suspects that there are great, though slow internal changes, in the mass of the earth. He draws his arguments from the varieties observed in the change of the magnetic needle, and from the observed changes in the temperature of climates. But as to the latter, there is reason to suspect that he could not have diaries of the weather sufficient to direct his judgement. Boyle's Works abr. vol. 1, pa. 292, \&c.

Magnetism of the LARTH. The notion of the magnetism of the earth was started by Gilbert; and Boyle supposes magnetic offluvia moving from one pole to the other. Sice his Works abr. vol. 1, pa. 285,290

Dr. Knight also thinks that the earth may be considered as a great loadstone, whose magnetical parts are disposed in a very irregular manuer; and that the south pole of the earth is analogous to the north pole in maguets, that is, the pole by which the magnetical stream enters. See MAGNET.

He observes also, that all the phenomena attending the direction of the needle, in different parts of the eartl, in a grcat neasure correspond with what happens to a needle, when placed on a large terrella; if we make allowances for the different dispositions of the magnetical parts, witl respect to each other, and consider the south pole of the carth as a north pole with regard to magnetism. The earth might become magnetical by the iron ores it contains, for all iron ores are capable of magnetism; and that a great part of the earth consists of iron is rendered probable by its great mclium specific gravity, being wearly 5 times that of water. It is true, the globe might notwithstanding have remained unmagnetical, unless somo cause had existed capable of making that repcllent matter producing magnetism move in a stream through the earth.

Now the doctor thinks that such a cause does exist. For if the earth revolves round the sun in an ellipsis, and the south pole of the carth is directed towards the stm, at the time of its descent towirds it, a stream of repellent matter will thenec be made to enter at the south pole, and issue out it the north. And he suggests, that the earth bring in its perilulion in winter may be one reasun why magnetism is strunger in this season than in summer. The cause here assigned for the earth's magnetism must continue, and perhaps improve it, from year to year. 
Hence the doctor thinks it probable, that the earth's magnetism has been improving ever since the creation, and that this may be one reason why the use of the compass was not discovered sooner. See Dr. Kinight's attempt to demonstrate, that all the phenomena in nature may be explained by Attraction and Repulsion, prop. 87 .

Magnitude and Constitution of the EARTn. This las been variously determined by different authors, both ancient and modern. The usual way has been, to measure the length of one degree of the muridian, and multiply it by 360 , for the whole circumference. See DEgreE. Diogenes Laertius informs us, that Anaximander, a scholar of Thales, who lived about 550 years before the birth of Christ, was the first who gave an account of the circumference of the sea and land; and it seems his measure was used by succceding mathematicians, till the time of Eratosthenes. Aristotle, at the end of lib. 2 De Ccelo, says, the mathematicians who have attempted to measure the circuit of the earth, make it 40000 stadiums; which it is thought is the number determined by Anaximander.

Eratosthenes, who lived about 200 years before Christ, was the next who undertook this business; which, as Cleomedes relates, he performed by taking the sun's zenith distances, and mcasuring the distance between two places under the same meridian; by which he deducel for the whole circuit about 250000 stadiums, which Pliny states at 31500 Roman miles, reckoning each at $1000^{\circ}$ paces. But this measure was considered to be false by many of the ancient mathematicians, and particularly by Hipparchus, who lived 100 years afterwards, and who added 25000 stadiums to the circuit of Eratosthenes.

Possidonius, in the time of Cicero and Pompey the Great, next measured the earth, viz, by means of the altitudes of a star, and measuring a part of a meridian; from which he concluded that the circumference was 240000 stalliums, according to Cleomedes, but only 180000 according to Strabo.

Ptolemy, in his Gengraphy, says that Marinus, a celebrated geographer, attempted something of the same kind; and in lib. 1, cap. 3, he nentions that he himsulf had tried to perform the same thing in a manner different from any others before his time, which was by means of places under different meridians: but he does not say how much he made the number; for he still made use of the number 180000 , which had been before employed.

Snellius relates, from the Arabian geographer Abelfedea, who lived about the year 1300 , that about the year S00, Aluaimon, au Arabian king, having collected together some skilful mathematicians, commanded them to find out the circumference of the earth. Accorlingly they made choice of the fields of Mesopotamia, where they measured under the same meridian from north to south, till the prole was depressed one degree lower: which measure they found equal to 56 miles, or $56 \frac{\mathrm{x}}{2}$ : so that according to them the circuit of the earth is $20160 \mathrm{or}$ 20340 miles.

It was in long time after this, before any more attempts of this kinl ivere made. At length, however, Snell, above mentioned, professor of mathematics at Leyden, about the year 1620, with great skill and labour, by measuring large distances between tivo parallels, found one degree equal to 28500 perches, each of which is 12 Rhinland feet, amounting to 19 Dutch miles, and so the whole periphery 6840 miles; a mile being, according to him,
1500 perches, or 18000 Rhinland feet. See his treatise called Eratosthenes Batavus.

The next that undertook this measurement, was Richard Norwood, who in the year 1635 , by measuring the distance from London to York with a chain, and taking the sun's meridian altitude, June 11 th old style, with a sextant of about 5 feet radius, found a degree contained 367200 feet, or 69 miles and a half and 14 poles; and thence the circumfeirnce of a great circle of the earth is a little more than 25036 miles, and the diameter a little more than 7966 miles. See the particulars of this meam surement in his Seaman's Practice.

The measurement of the earth by Snell, though very ingenious and troublesome, and much more accurate than any of the ancients, being still thought by some French mathematicians liable to certain small errors, the business was renewed, alter Snell's manner, by Picard and other mathematicians, by the king's orler ; using a quadrant of $3 \frac{x}{6}$ French feet radius; and they thus found a degree contained 342360 French feet. See Picard's treatise, La Mesure de la Terre.

M. Cassini the younger, in the year 1700, by the king's command also, renewed the business with a quadrant of 10 feet radius, for taking the latitude, and another of $3 \frac{1}{8}$ feet for taking the angles of the triangles; and found a degree, from his calculation, contained 57292 toises, or almost $699_{2}^{\perp}$ English miles.

Several other measurements have been taken, the best of which are the following, with the corresponding mean semidiameter annexed, in English miles.

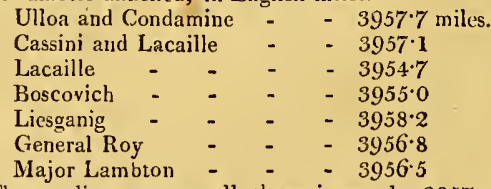

The nedium among all these is nearly 3957 miles, which may be taken for the mean semidiameter of th earth. And supposing the solar parallacic angle to be $8^{\prime \prime} \cdot 6$, it will be, as $\sin .8^{\prime \prime} \cdot 6$ : radius : : $3957: 95$ millions of miles pearly, the carth's distance from the sun.

Hence the mean circumference is 24.862 miles, which gives $69_{\frac{1}{16}}^{\frac{1}{6}}$ miles for the medium length of a degree of a great circle.

See the results of many other measurements under the article DEGREE, and from the preceding part of the present article. From the mean of all which, the following dimensions may be taken as near the truth:

the circumference - $\quad 4862$ miles,
the diameter - $\quad 27914$ miles,
the superficies - 196757900 square miles,
the solidity - 259523200000 cubic miles.

Also the seas and unknown parts of the earth, by a measurement of the best maps, contain 160522026 square miles, the inhabited parts 38922180 ; of which Europe contains 44.56065; Asia, 10768823; Africa, 9654807; and America, 14.110874.

The terraqueous globe has two motions, besides that on which the precession of the equinoxes depends; the one diurnal around its own axis in the space of 24 hours, which constitutes the natural day; the other annual, about the sun, in an elliptical orbit or track, in 365 days 6 hours, constituting the year. From the former arise the divcrsities of day and night; and from the latter, 
the vicissitudes of scasuns, spring, summer, sutumn, and winter.

The terraqueous globe is distinguished into thrce parts or regions, viz, Ist, The external part or crust, being that from which vigetables spring and animals are nursed. 2d, The middle, or intermediate part, which is possessed by fossils, extending further than human labour ever yet penetrated. $3 \mathrm{~d}$, the internal or central part, which is utterly unknown to us, though by many anthors supposed of a magnetic nature; by others, a mass or sphere of firc; by others, an abyss or collection of watcrs, surrounded by the strata of earth; and by others, a hollow, empty space, inhabited by animals, who have their sun, moon, planets, and other conveniences within tbe same. But others divide the body of the globe into two parts, viz, the external part, called the cortex, including the internal, which they call the nucleus, being of a different nature from the former, and possessed by fire, water, or more likely by a considerable portion of metals, and probably mostly iron, as it has been found, by my calculation, that the mean density of the whole earth is near double the density of common stone. See my determination of it, Philos. Trans. 177 S, pa. $7 \mathrm{S1}$; or my Tracts, vol. 1 , tract 26.

The extermal part of the globe either exhibits inequalitics, as mouutains and valleys ; or it is plane and level; or hollowed into channels, fissures, \&c, where rivers, lakes, and seas, flow in all directions. These inequalities in the face of the earth most naturalists suppose bave arisen from a rupture or subversion of it, by the force either of the subterrancous fires or waters. The earth, in its natural and orininal state, it has been supposed by Descartes, and after him, Burnet, Steno, Woodward, Whiston, and others, was perfectly round, smooth, and equable; and they account for its present rude and irregular form, principilly from the great rleluge. See DELuge.

In the external, or cortical part of the earth, there appear various strata, supposed the sediments of several floods; the waters of which, being replete with matters of divers kjnds, as they dried up, or oozed through, deposited these differeut matters, which in time hardencel into strata of stone, sand, coal, clay, \&c.

I)r. Woodward has consiclered the circumsiances of these strata with great attuntion, viz, their order, number, and situation with respect to the horizon, depth, intersections, fissures, colour, consistence, \&c. He ascribes the origin and formation of then all, to the great flood, \&c. At that terrible revolution he supposes that all sorts of terrestrial bodies had been dissolved and mixed with the waters, forming all together a chaos or confused mass. This mass of terrestrial particles, intermixed with water, he conccives was at length precipitated to the bottom; and that generally according to the order of graviry, the heaviest sinking first, and the lightest afterwards; by which means the strata were formed of which the earth consists; which, attaining their solidity and hardness by legrees, have continued so ever since. 'Thu'se sediments, he further concludes, were at first all parallel and concentrical; and the surface of the eartl formed of them, perfectly smooth and regular; but that in course of time, clivers changes arising, from earthquakes, volcanoes, \&c, the order and regularity of the strata was disturbed and broken, and the surface of the earth by such means brought to the irregular form in which it now appears.

M. De Buffon surmises that the earth, as well as the other plauets, are parts struck off from the body of the sun by the collision of comets; and that when the earth assumed its form, it was in a state of liquefaction by fire. But this cannet be granted; for if they were struck of from the bodly of the sun, they would move in orbits that would always pass through the sun, instead of having the sun lor their fucus, or centre, as they are now found to have; so that having been struck off they would fall down into the sun again, terminating their career as it were after one revolution only.

Besides these, various other hypotheses have been advanced, all rof them however more or fess liable to objections; as must always be the case with conclusions drawn from uncertain data. We may however particularize Dr. James Hutton's treatise, on this subject, as one deserving the most attention, and from which much useful information may be derived.

EAnth, in Astronomy, is one of the primary planets, according to the system of Copernicus, or Pythagoras; its astronomical character or mark bcing $\Theta$ : but according to the Ptolemaic hypothesis, it is the centre of the systen. For, whether the earth morc or remain at rest, that is, whether it be fixed in the centre, baving the sun, the heavens and stars moving round it from east to west; or, these buing at rest, whether the earth only moves from west to east, is the great article that distinguishes the Ptolemaic system from the Copernican.

Motion of the Eג RTH. It has already been observed that, besides the small motion of the earth which causes the precession of the equinoses, the earth has two grcat and independent motions; viz, the one by which it turus round its own ax is, in the space of 24 hours nearly, which causes the continual succession of day and night; and the other an absolute motion of its whole mass in a large orbit about the sun, having that luminary for its centre, so that its axis keeps always parallel to itself, inclined in the same angle to $\mathrm{jts}$ jath, and by that means causing the vicissitudes of seasons, spring, summer, autumn, and winter.

It is indeed true that, as to sense, the earth appears to be fixed in the centre, with.the sun, stars and heavens moving round it every dity; and such, doubtless would be considered as the true nature of the motions in the first early ages of mankind, as they are still by the rude and unlearned. But to a thinking and leamed mind, the contrary will soon appear.

Indeed there are traces of the knowledge of these motions in the carliest age of the sciences. Cicero, in his Tusc. Quæst. says that Nicetas of Syracuse first discovered that the eartl had a diurnal motion, by which it revolved round its axis every 24 hours; and Plutitech, de Placit. Philosoph. inforns us that Philolaus discovered its annual motion round the sun; also Aristarchus, abuut 100 years after Philolaus, alludes to the notion of the earth in stronger and clearer terms, as we are assured by Archimedes, in his Arenarius. And the same, we are further assured, was the opinion and inct rine of l'y thatgoras. But the religious opinions of the heathen world prevented this doctrine from being more cultivated. For, Aristarchus bcing accused of arcrilege by Cleauthes for moving Vesta and the tutelar deitic's of the universe out of their places, the philosophers were obliged to dissemble, and seem to relinquist so prerilous a position.

Many ages aftewards, Nic. Cusausus revived the ancient system, in his Doct. de Pignorant. and asserted the motion of the earth: but the doctrine gained very little ground till the time of Copernicus, who showed its great 
utility and advantages in astronomy; and who had immediately all the philosophers and astronomers on bis side, that clared to ditfer from the crowd, and were not afraid of ecclesiastical censure, which was not less dangerous under the Christian dispensation, than if had been under that of the heathen. For, because eertain parts of Scripture nake mention of the stability of the carth, and of the motion of the sum, as the rising and setting, \&c, the fathers of the church thought their religion reguired that they should defend, with all its puwer, what they conceired to -be its doctrines, and to censure and punish every attempt at innovation on those points. There is now howerer no doubt that in such instances the expressions are only to be considered as accominodated to appearances, and the vulgar notions of things.

By the diurnal rotation of the curth on its axis, the same phenomena must take place as if it had no such motion, that is, as if the sun and stars revolved about it. For, turning round from west to cast, causes the sun and all the visible heavens to have the appearance of moving the contrary way, or from east to west, as we daily sce them. So that, when in its rotation it has brought the sun or a star to appear just in the horizon in the east, they are then said to be rising; and as the earth continues to revolve more and more towards the cast, other stars seem to rise and advance westwards, passing the meridian of the observer, when they are due south from him, and at their greatest altituḑe above his horizon; after which, by a continuance of the same motions, viz, of the earth's rotation eastwards, and the luminaries' appareut counter mution westwards, these decline from the meridian, or south point, towards the west, where being arrived, they are said to set and descend below it; and so on continum ally from day to day; thus making it day while the sun is above the horizon, and night while he is below it.

While the earth is thus revolving on its ixis, it is at the same time carried by its proper motlon in its orbit round the sun, as one of the planets, namely, between the orbits of Vents and Mars, having the orbits of Venus and Mercury within its own, or between it and the sun, in the centre, and those of Mars, Jupiter, Saturn, \&c, without or above it; which are therefore called superior planets, and the others the inferior ones; which is called the ammal motion of the earth, because it is performed in a year, of 365 days 6 hours nearly; or rather 365 days 5 hours 49 minutes, from either equinux or solstice to the same again, which completes the tropical year; but from any fixed star to the same again, as seen from the sun, in 365 days 6 hours 9 minutes, which is calted the sidereal year. 'The figure of this orbit is elliptical, having the sun in one focus, the mean distance being about 95 millions of miles, which is upon the supposition that the sun's parallax is about $\mathrm{s}^{\prime \prime} \frac{2}{3}$, or the angle under which the earth's semi-diancter would appear to an observer placed in the sun: and the excenltricity of the orbit, or distance of the sun, in the focus, from the centre of this elliptic orbit, is about $\frac{x}{6}$ th of the snean distance.

Now this annual motion is performed in such a manner, that the earth's anis is cvery where parallel, or in the same direction in every part of the orbit; by which means it happens, that at one time of the year the sun enliglitens more of the north polar parts, and at the opposite season of the year more of the southern parts, thus showing all the varictics of seasons, spring, sunmer, autumn and winter; which may be illustrated in the following manner:
Let the caudle 1 (tig. 1, plate $\mathrm{x}$ ) represent the sun, about which the earth $\mathrm{E}$, or $\mathrm{F}$, \& $\&$, is moved in its elliptical orbit $A B C D$, or ecliptic, and cutting the cquator $a b c d$ in the nodes $\mathrm{E}$ and $\mathrm{G}$ : then, suspending the terrella by its north pole, and moving it, so suspended, round the ecliptic, its axis will always be parallel to its first position, and the various scasons will be represented at the different parts of the path. 'Thus, when the earth is at $\sigma$ or $F$, the enliglitened half of it includes the south pole, and leaves the nurrh pole in larkness, making our winter; at $G$ it is spring, and the two poles are equally illuminated, and the

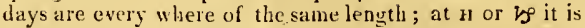
our sumner, the north polar parts being in the illuminated hemisphere, and the southern in the dark one; lastly, at $\mathrm{E}$ it is autumn, the poles being cqually illuminated again, and the days of equal length every where.

EA RTI, its 2untity of Matter, Density, and Attractive Power. Though the retative densities of the earth and most of the otljer planets have been known a considerable time, it is but very lately that we have come to the knowledge of the absolute gravity or density of the whole mass of the earth. This I have calculated and deduced from the obscrvations made by Dr. Maskelyne, (late Astrononer Royal,) at the mountain Schchallien, in the years $1774,-5$, and -6 . 'The attraction of that mountain on a plummet, being observed on both sides of it, and its mass being computed from a number of sections in all directions, and consisting of stone; these data being then compared with the known attraction and magnitude of the earth, gave by proportion its mean density, which is to that of water nearly as 5 to 1 , and to common stone as 2 to 1 : from which very considerable mean density, it nay be presumed that the internal parts contain some great quantities of metals.

From the density, thus found, its quantity of inatter becomes known, being equal to the product of its density by its magnitude. From various experiments too, we know that its attractive force, at the surface, is such, that bodics fall there through a space of $16_{\frac{1}{2}}^{\frac{1}{2}}$ feet in the first second of time: whence the force at any other place, either within or without it, becomes known; for rhe force at any part within it (supposing it every where uniform) is directly as its distance from the centre; but the force of any part without it, reciprocaliy as the square of its distance from the centre.

EAST', one of the cardinal points of the horizon, or of the compass, being the middle point of it between north and south, on that sicle where the sun rises, or the point in which it is intersected on that side by the prime vertical.

EASTER, a feast of the church, held in memory of our Saviour's resurrection. This feast has been annually celebrated ever since the time of the apostles, and is one of the most considerable fe'stivals in the C'rristian calendar; being that which regulates and determines the times of all the other moveable feasts.

The rule for the celebrition of caster, fixed by the council of Nice, in the year 325 , is, that it be held on the surulay which falls upon, or next after, the full inoon which happens next after the 21 st of March; that is, the sunday which falls upon, or next after the first full inoon after the verial equinox. The retson of which decree was, that the Christians might avoid celcbrating their easter at the same time with the Jewish passover, which, according to the institution of Moses, was held the very day of the full moun. 
To find Easter according to the Old, or Julian Style.

In the annexed table, find the golden number, with the day of the paschal full moon, and the sunday letter annexed; compare this letter with the dominical letter of the given year, that it may appear how. many days are to be added to the day of the paschal full moon, to give easter-day.

For Example. In the year 1715 , the dominical letter is $\mathrm{B}$, and the golden number is 6 , opposite to which stands April 10 for the day of the paschal full moon ; opposite to which is the sunday letter $\mathrm{B}$, which happening to be the same with that of the given year, that day is a sunday; and therefore easter will fall 7 days after, viz, on the 17th of April.

But in this computation, the vernal eyuinox is supposed fixed to the 21 st of March; and the cycle of 19 years, or golden numbers, is supposed to point out the places of the new and full moons exactly; both which suppositions are erroneous: so that the Julian easter never happens at its due time,

\begin{tabular}{|r|r|r|}
\hline $\begin{array}{r}\text { Gold. } \\
\text { Nut. }\end{array}$ & $\begin{array}{r}\text { Paschal full } \\
\text { moon. }\end{array}$ & $\begin{array}{c}\text { Dom. } \\
\text { letter }\end{array}$ \\
\hline 16 & March 21 & C \\
5 & 22 & D \\
13 & 23 & E \\
2 & 24 & F \\
& 25 & G \\
10 & 26 & A \\
& 27 & B \\
18 & 28 & C \\
7 & 29 & D \\
& 30 & E \\
15 & 31 & F \\
4 & April & G \\
& 2 & A \\
12 & 3 & B \\
1 & 4 & C \\
& 5 & D \\
9 & 6 & E \\
17 & 7 & F \\
6 & S & G \\
& 9 & A \\
14 & 10 & E \\
3 & 11 & C \\
11 & 12 & D \\
19 & 13 & E \\
8 & 14 & F \\
& 15 & G \\
& 16 & A \\
\hline & 17 & B \\
& 18 & C \\
\hline
\end{tabular}
unless by accident. For instance, in the above example the vernal equinox falls on the 10th of March, eleven days before the rule supposes it; and the paschal full moon on the 7 th of April, or 3 days earlier than was supposed: and therefore easter-day should be held on the 10th of April, instead of the 17 th.

This error had grown to such a height, that Pope Gregory the 13th thought it necessary to correct it; and accordingly, in the year 1582, by the advice of Aloysius $\mathrm{Li}$ lius and others, he ordered 10 days to be thrown out of October, to bring the vernal equinox back again to the 21 st of March : and hence arise the terms Gregorian calendar, Gregorian year, \&c.

This correction however did not entirely remove the error; for the equinoxes and solstices still anticipate $2 \mathrm{~S}^{\prime}$ $20^{\prime \prime}$ in every 100 Gregorian years; but the difference is so inconsiderable as not to amount to a whole day, or 24 hours, in less than 5082 Gregorian years.

The Gregorian, or New Style, was not introduced into England till the year 1752, when eleven days were thrown out, viz, between the $3 \mathrm{~d}$ and 14 th of September, the error amounting then to that quantity.

To find Easter according to the New or Gregorian Style, till the Year 1900 exclusive.

Look for the golden number of the year in the first co-, lumn of the table, against which stands the day of the paschal full moon; thenlook in the $3 \mathrm{~d}$ columu for the sunday letter, next after the day of the full moon, and the day of the month stanling against that sunday letter is caster-day. When the full moon happens on a sunday, then the next sunday after is easter-day.
For Example. For the year 1813, the golden number is 9 ; against which stands April the 15 th, and the next sunday letter, which is c, below that, stands opposite April 18; which is therefore the easter-day for the year 1813 .

Though the Gregorian calendar be much preferable to the Julian, it is yet not without its defects. It cannot, tor instance, keep the equinox always fixed on the 21 st of March, but it will vary between the 19 th and the 23d. To which we may add, that the full moon happening on the 20th of March, might sometimes be paschal; yet it is not allowed as such in the Gregorian computation; as on the contrary, the full moon of the 22d of March may be allowed for paschal, which it is not. Scaliger and Calvisius have also pointed out other inaccuracies in this calendar. An excellent paper on this subject by the Earl of Macclesticld, may be seen in the Philos. Trans. vol. 40 , pa. 417 ; or my A bridg. vol. x, pa. 33.

Easter Term. See Term.

EBBING and FLowING of

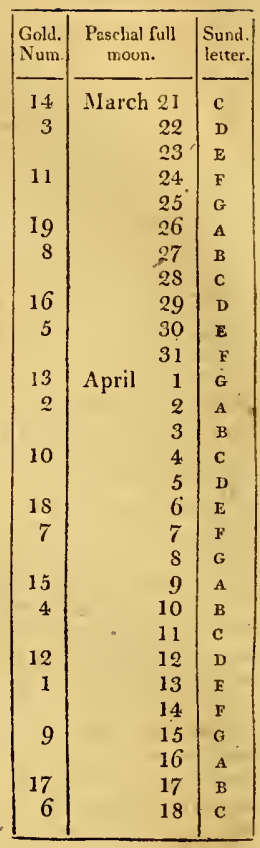
the Sea. See Tines.

ECCENTRIC. See Excentric.

ECCENTRICITY. See Excentricity.

ECHO, a sound reflected, or reverberated from some body, and thence returned or repeated to the ear.-For an echo to be heard, the ear must be in the line of reflection; that the person who made the sound, may hear the ccho, it is necessary he should be in a perpendicular to the place which reflects it; and for a multiple or tautological echo, it is necessary that there should be a number of walls and vaults, rocks and cavities, either placed behind each other, or fronting each other. Those murmurs in the air, that are occasioned by the discharge of great guns, \&c, are a kind of indefinite echoes, and are produced from the vaporous particles suspended in the atmosphere, which resist the undulations of sound, and reverberate them to the ear.

There can be no echo, unless the direct and reflex sounds follow one another at a sufficient distance of time; for if the reflex sound arrive at the ear before the inpression of the direct sound ceases, it will not be doubled, but only rendered more intense. Now if we allow that 9 or 10 syllables can be pronounced in a second, in order to preserve the sounds articulate and distinct, there should be about the 9th part of a second between the times of their appulse to the ear; or, as sound fies about 1142 feet in a second, the said difference should be $\frac{x}{9}$ of 1142 , or 127 feet; and therefore every syllable will be reflected to the ear at the distance of about 70 feet from the reflecting body ; but as, in the ordinary way of speaking, 3 or 4 syllables only are uttercel in a second, the speaker, that he 


\section{$\mathrm{E} \mathrm{CH}$}

may have the ccho returned as soon as they are expressed, should stand about 500 feet from the reflecting body; and so in proportion for any other number of syllables. Mersenne allwws for a monosyllable the distance of 69 feet; Mortun, 90 feet; for at clissyllable 105 fieet, a trisyllable 160 feet, a tetrasyllable: 18 \& feet, and a pentasyllable 204 feet. Nat. Hist. Northampton, cap. 5 , pa. 358 .

From what has been said, it follows lhat echoes may be appiied for measuring inaccessible distances. Thus, Mr. Derham, standing on the banks of the Thames, opposite to Woolwich, observed that the echo of a single sound was reflected back from the houses in 3 seconds; consequently the sum of the direct and reflex rays must have been 1142 $\times 3=3426$ feet, and the half of it, $1 \pi 13$ feet, the breadth of the river in that place.

It also follows, that the echoing body being re moved farther off, it reflects more of the sound than when nearer; which is the reason why some echocs repeat but one syllable, or one word, and some many. Of these, some are tonical, which only return a voice when modnlated into some particular nusical tone; and others polysyllabical. That fine echo in Woodstock park, Dr. Plot assures us, in the day-time will returm very distinctly 17 syllables, and in the night 20. " Nat. Hist. Oxf. cap. 1, pa. 7 .

Echoing bodies may be so contrived, and placed, as that reflecting the sound from one to the other, a mulriple echo, or many echoes, shall arise.-At Rosneath, near Glasgow, in Scotland, there is an echo that repeats a tune played with a trumpet three timcs completely and distinctly.At the sepulchre of Metella, wife of Crassus, there was an echo, which repeated what a man said five times.-Some authors mention a tower at Cyzicus, where the echo is repeated seren times, and one at Brussels, that answers 15 times.

But the finest echo we read of, is that mentionea" by Barthius, in his notes on Statius's Thebais, lib. 6, ver. 30, which repeated the words a man uttered 17 times. This was on the banks of the Naha, betwcen Coblentz and Bingen. And whereas, in common echoes, the repetition is not heard till some time after hearing the words spolicti, or the notessung; in this, the person who speaks, or sings, is scarce heard at all; but the repetition very clcarly, and always in surprising varieties; the echo seeming sometimes to approach nearer, and sometimes farther off; sometimes the voice is heard very distinetly, and sometimes scarce at all: one person hears only one voice, and another several; one hears the echo on the right, and the other on the left, \&c.

Addison, and other travellers in Italy, mention an ccho at Simonetta palace, near Milan, still more cxtraordinary, returning the sound of a pistol 56 times. The echo is heard behin:l the house, which has two wings; the pistol is discharged from a window in one of these wings, the sound is returned from a dead wall in the other wing, and heard from a window in the back-front. See Addis. Travers, pa. 32; Misson. Voyag. d'ltal. tom. 2, pa. 196; Philos. Trans. $N^{\circ} 480$, pa. 220.

Again, a multiple echo may be made, by so placing the choing bodies, at unequal distances, as that they may reflect all one way, and not one on the other; by which means, a manifold successive sound will be heard; one clap of the hands like many; one ha like a laughtter; one single word like many of the same tone and accent; and so one musical jnstrument like many of the same kind, imjtating each other.

VOL. I.
E C L

Lastly, Echoing bodies may be so contrived, that from any one given sound, they shall produce many echoes, different both as to tone and intension. By which means a musical room may be so contrived, that not only one instrument playing in it shall seem many of the same sort and size, but cren a concert of difierent ones; this may be contrived by placing certain echoing bodies so, as that any nute played, shall be returned by them in $3 \mathrm{ds}$, 5 ths, and Sths.

Ecuo is also used for the place where the repetition of the sound is produced, or heard. This is cither natural or artificial. The place where the speaker stands is called the centrum phonicuin; and the object or place that returns the voice, the centrum phonocampticum.

Ecuo, in Architecture, is applied to certain vaults and arches, mostly of elliptical or parabolical figures; used to redouble sounds, and produce artificial cchoes.-The method of making them is taught by $F$. Blancani, in his Echometria, at the ent of his book on the Sphere.

One of the finest echoes in England, is that commonly called the whispering-gallery, in the dome of St. Paul's, London; and another in Gloucester cathedral. see WnisPERING-Places.

Vitruvius tells us, that in divers parts of Greece and Italy there were brazen vessels, artfully ranged under the seats of the thearres, to render the sound of the actors' voiccs more clear, and make a kind of echo; by which-means, every one of the prodigious multitude of persons, present at those spectacles, might hear with ease and pleasure.

ECLJPSAREON, an instrument invented by Mr. Ferguson for showing the phenomena of eclipses; as to their time, quantity, duration, progress, \&c. Ferguson's Astron., or Pbilos. Trans. vol. 48 , pa. 520 .

ECLIPSE, a privation of the light of one of the luminaries, by the interposition of some opaque body, cither between it and the eye, or between it and the sun. The ancients had terrible ideas of eclipses; supposing them presages of some dreadful events. Plutarch assures us, that at Rome it was not allowed to talk publicly of any natural causes of eclipses; the popular opinion running so strongly in favour of their supernatural production, at least those of the moon; for as to those of the sun, they had some idea that they were caused by the interposition of the moon between us and the sun; but were at a loss for a body to interpose between us and the moon, which they. thought must be the way, if the eclipses of the n:oon were produced by natural causes. They therefore nade a great noise with brazen instruments, and set up loud shouts, during eclipses of the moon; thinking by that means to ease her in labour: whence Juvenil, speaking of a talkative woman, says, " Una laboranti poterit succurrere lunæ." Others attributed the cclipse of the moon to the arts of magicians, who, by their enchantments, flucked her out of heaven, and made lier skim over the grass.

The natives of Mexico kecp fast during the time of eclipses; and particularly their women, who beat and ab use themselves, drawing blood from their arms, \&c; imagining the moon bas been wounded by the sun, in some quarrel between them.

The Chinese have an idea that cclipses are occasioned by great dragons, who are ready to devour the sun and moon; and thercfore when they perceive an eclipse, they rattle drums and brass kettles, till they think the monster, terrified by the noise, lets go his prey.

The superstitious notions entertained of eclipses, have $3 \mathrm{M}$ 
buen sometimes of considerable advantage, as was the case with Christopher Columbus, the discoverer of America, who, being driven on the island of Jamaica in the year 1493 , and distressed for want of provisions, was refused relief by the natives; but having threatened them with a plague, at the same time foretelling an eclipse as a token of it, which happened according to his prediction, the barbarians were so terrified, that they strove who should be the first in bringing him supplies, throwing them at his feet, and imploring lis forgiveness. But the most striking circumstance of this kind was the prediction of an eclipse of the sun by Thales, the great father of astronomy, which happened, according to Mayer's calculation, on the 17 th of May, fos year's before Christ, at the moment that Cyaxares king of the Medes, and Aliathos king of the Lydians, were preparing for battle; but alarmed at the appearance of the eclipse, they immediately laid down their arıns, and negociated a peace: and thus ignorance, which is often so injurious to the happiness of man, may, in this instance, be said to have largely contributed towards it.

Duration of an EcLIPSE, is the time of its continuance, or the time between the immersion and emersion.

Immersion, or Incidence, of an ECLIPSE, is the numment when the eclipse begins, or when part of the sun, moon, or planet tirst begins to be obseured.

Emersion, or Expurgation, of an Ecusps, is the time when the eclipsed luminary begins to je-appear, or emerge out of the shadow.

Quantity of an EcLIPse, is the part of the luminary eclipsed. To determine this part, it is usual to divide the diameter of the luminary into 12 equal portions, called digits; whence the eclipse is said to be of so many digits, according to the number of them contained in that part of the diameter which is eclipsed or obscured.

Eclipses are divided, with respect to the luminary eclipsed, into Eclipses of the sun, of the moon, and of the satellites; and with respect to the circumstances, into total, partial, annular, central, \&c.

Animular EcLIPSE, is when the whole is eclipsed, except a ring, or annulus, which appears round the border or edge.

Central Ecurse, is one in which the centres of the two Jumiaries and the earth come into the same right line.

Parlial Ec LIPse, is when only a part of the luminary is clipsed. And a

Total ECLIPse, is that in which the whole luminary is darkened.

EcLIPSE of the MIon, is a privation of the light of the noon, occasioned by the interposition of the body of the earth directly between the sun and moon, and so intercepting the sun's rays that they cannot arrive at the moon, to illuminate her. Or, the obscuration of the moon may be considered as a section of the earth's conical shadow, by the rnoon passing through some part of it.

The manner of this celips" is represented in this figure, where $s$ is thesum, $E$ the carth, and $M$ or $\mathrm{m}$ the moon.

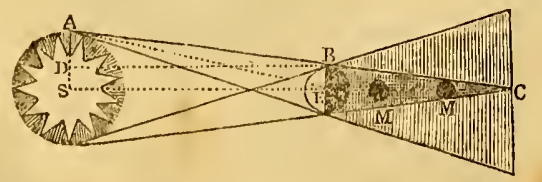

l.unar eclipses only happen at the time of full moon; hecause it is only at that time the earth is betwcen the sun and moon: nor do they happen every full moon, because of the obliquity of the moon's path with respect to the sun's, or more properly the eartb's; but only in such full moons as happen either at the intersection of those two paths, called the moon's nodes, or very near them; viz, when the moon's latitude, or distance between the centres of the earth and moon, is less than the sum of the apparent semi-diameters of the moon and the earth's shadow.

The chief Circumstances in Lunar Eclipses are the. following:-1. All lunar eclipses are universal, or visible in all parts of the earth which have the moon above their horizon; and are every where of the same magnitude, with the same beginning and end.-2. In all lunar eclipses, the eastern side is what first immerges and emerges again, i.e. the left-hand side of the moon as we look towards ber from the north; for the proper motion of the moon being swifter than that of the earth's shadow, the moon approaches it from the west, overtakes and passes through it with the moon's east side foremost, leaving the shadow behind, to the westward.-3. Total eclipses, and those of the longest duration, happen in the very nodes of the ecliptic; because the section of the rartl's shadow, then falling on the moon, is considerably larger than her disc. There may, however, be total eclipses witbin a small distance of the nodes; but their duration is the less as they are farther from it ; till they become only partial ones, and at last, none at all.-4. The moon, even in the middle of an eclipse, has usually a faint appearance of light, resembling tarnished copper; which Gassendus, Ricciolus, Kepler, \&c, attribute to the light of the sun, refracted by the earth's atmosphere, and so transmitted thither-Lastly. She becomes sensibly more dim and pale, before entering into the real shadow; owing $t$ ) a penumbra which surrounds that shadow to some distance.

Astronomy of Lunar Ecripses, or the method of calculating their times, places, magnitudes, and other phenomena. The Ist preliminary is to find the length of the earth's conical shadow; which may be found either from the distance between the earth and sum, and the proportion of their diameters, or from the angle of the sun's apparent magnitude at the time. Thus, suppose the semiaxis of the earth's orbit 95,000000 miles, and the excentricity of the orbit 1,377000 miles, making the greatest distance 96,377000 miles, or 24194 semidianneter's of the earth; and the sun's scmidiameter being to the earth's, as 112 to 1 ; then as $A D: B E:: D B: E C$, that is, $111: 1::$ $2+194: 21 \mathrm{~S}$ semidiancters of the earth $=$ ac the length of the carth's shadow. Otherwise, suppose the angle AEs, or the sun's apparent semidiameter be $15^{\prime} 56^{\prime \prime}$, and the angle $B A E$, or the sun's parallax $8 \cdot 6^{\prime \prime}$, then is their difference, or the angle $A C E=15^{\prime} 47^{\circ} 4^{\prime \prime}$; hence, as tang. $15^{\prime} 47^{\circ} 4^{\prime \prime}$ : radius : : BE or $t: 218$ nearly $=\mathrm{ck}$, the same distance as before. Hence, as the moon's least distance from the earth is scarce 56 semidiameters, and the greatest not more than 64 , the moon, when in opposition to the sun, in or near the nodes, will fall into the earth's shadow, and will be eclipsed, as the length of the sliadow is almost 4 times the moon's distance.

2. To find the apparent semidiancter of the earth's shadow, in the place where the mown passes through it, at any given time. Add together the sun and moon's parallaxes, and from the sum subtract the apparent semidiameter of the sun; so shall the remander be the apparent semidiameter of the shandow at the place of the moon's passage. For example, the 28 th of $A$ pril 1790 , at midnight, the 


\section{E C L}

moon's parallax is $61^{\prime} 9^{\prime \prime}$, to which add $s \cdot 6^{\prime \prime}$ or $9^{\prime \prime}$, for the sun's parallax, from the sum $61^{\prime} 1 \mathrm{~S}^{\prime \prime}$ take $15^{\prime} 56^{\prime \prime}$, the sun's apparent semidiameter, and the remaincler $4 j^{\prime} 22^{\prime \prime}$ is the scmidiameter of the shadow at the place where the moon passes through at that time. But some omit the sun's parallas, as of no consequence; and increase the apparent semidiameter of the shadow by one whole minute, for the shadow of the atmosphere; which would give the semidiameter of the shadow, in the case above, $46^{\prime \prime} 13^{\prime \prime}$.

3. There must also be had, the true distance of the moon from the node, at the mean opposition; and the true time of the opposition, with the true place of the sun and moon, reduced to the ecliptic; also the moon's true latitude at the time of the true opposition; the angle of the moon's way with the ecliptic, and the true horary motions of the sun and moon: from which all the circumstances of her cclipse may be computed by common arith. metic and trigonometry.

To Construct an Eclipse of the Moon.-Let Ew be a part of the ecliptic, and $c$ the centre of the earth's shadow, through which draw perpendicular to $\mathrm{EW}$, the line $\mathrm{CN}$ towards the north, if the moon have north latitude at the time of the eclipse, or cs southward, if she bave south latitude. Make the angle NCD equai to the angle of the moon's way with the ecliptic, which may be always taken at $5^{\circ} 35^{\prime}$, on an average, withont sensible error; and bisect this angle by the right line cF; in which line it is that the true equal time of opposition of the sun and moon falls, as given by the tables.

From a convenient scale of equal parts, representing minutes of a degree, take the moon's latitude at the true time of full moon, and set it from $\mathrm{c}$ to $\mathrm{G}$, on the line $\mathrm{Cr}$; and througlt the point $G$, at right anglis to $\mathrm{CD}$, draw the right line HKGLI for the path of the moon's centre. Then is $\mathbf{L}$ the point in the earth's shadow, where the moon's

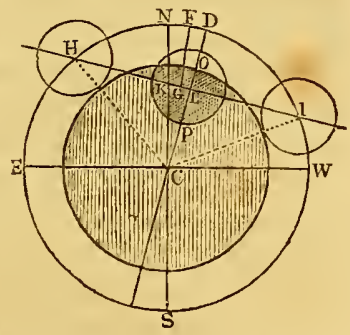

centre is at the middle of the cclipse; $G$ the point where her centre is at the tabular time of her being full; and $\mathrm{K}$ the point where her centre is at the instant of her ecliptic opposition: also I the moon's centre at the moment of immersion, and $x$ lier centre at the end of the eclipse.

With the moon's semidiameter as a radius, and the points $r, L, I I$, as centres, describe circles for the moon at the beginning, middle, and end of the eclipse.

Finally, the length of the line of path II, measured on the same scale, will scrve to determine the duration of the eclipse, viz, by saying, As the moon's horary motion from the sun is to In: : 1 hour or 60 nin. to the whole turation of the eclipse.

To Compute a Lunar Ecursse. This will be very casy from the foregoing construction. For, 1 ,, in the triangle CGL, right-angled at $\mathrm{L}$, there are given the hypothenuse $\mathrm{c}_{\mathrm{r}}=$ the moon's latitude at the time of full moon, and the angle $\mathrm{GCL}=$ the half of $5^{\circ} 35^{\prime}$; to find the legs $\mathrm{CL}$ and LG.-2d, In the right-angled triangle $(31$, or $\operatorname{Cr} L$, are gives the leg CL, and hypothersuse csi or $\mathrm{C}$, the sum of
E C L.

the semidiameters of the moon and the carth's shadow; to find Lin or LI, half the difference of the sun's and moon's motions during the time of the eclipse.--3d, As the difference of the horary motions of the luminaries is to one hour, or 60 min. : : H $\mathrm{L}$ to the semiduration of the eclipse, and :: $G L$ to the difference between the opposition and middle of the eclipse; this last therefore taken from the time of fult moon, gives the time of the middle of the eclipse; from which subtracting the time in $L I$, or seniduration before found, gives the beginning of the eciipse; or add the same, and it gives the end of it.-Lastly, from cothe semidiameter of the shadow, take $\mathbf{C L}$, and there remains LO ; to which add LP, the moon's semidiameter, when necessary, the sum of which is op the quantity eclipsed.

Note, When the moon's distance from the node exceeds $12^{\circ}$, there can be no eclipse of the moon; or, more accurately, the limit is from $10 \frac{1}{2}$ to $12 \frac{\mathrm{r}}{3} \mathrm{O}$ degrees, according to the distances of the sun, earth, and moon.

Ectipse of the Sun, is an occultation of the sun's body, occasioned by the interposition of the moon between the earth and sun. On which account it is by some considered as an eclipse of the carth, since the light of the sun is hid from it by the moon, whose shadow involves a part of the eartb.

The manner of a solar eclipse is represented in this figure; where $s$ is the sun, $m$ the monn, and $\mathrm{CD}$ the carth,

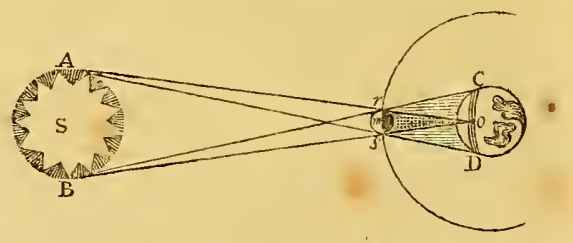

rmso the moon's conical sharlow, travelling over a part of the earth CoD, and making a complete eclipse to all the inhabitants residing in that track, but no where else; excepting that for a large space around it there is a fainter shade, included withiu all the space $r \mathrm{CD} s$, which is called the Penumbra.

Hence, solar eclipses happen when the moon is in conjunction with the sun, or at the new moon, and also in the wodes or near them, the limit being about 17 degrees on each side of it; and such eclipses only happen when the latitude of the moon, viewed from the earth, is less than the sum of the apparent semicliameters of the sun and moon; becausc the moon's way is oblique to the ecliptic, or sun's path, making an angle of nearly $5^{\circ} 35^{\prime}$ with it.

In the nodes, when the moon has no visible latitude, the occultation is total; and with some continuance, when the disc of the moon in perigee appears greater than that of the sun in apogee, and its shadow is extended beyond the surface of the earth; and without continuance at small distances when the cusp, or point of the moon's shadow, barely touches the earth. Leistly, out of the nodes, but near them, the cclipses are partial.

The other circumstances of solar cclipses are, 1. That none of them are nniversal; that is, none of them are scen throughout the whole hemisphere which the sun is then above; the moon's disc being too small and much too near the carth, to bide the sun from its whole disc. Communly the moon's dark shadow covers only a spot on the carth's surfice, about 180 mill's broad when the sun's 3 M 2 
E C L $\left[452^{\circ}\right]$ distance is greatest, and the moon's least. But her partial shadow, or penumbra, may then cover a circular space of 4900 miles in diameter, within which the sun is more or luss eclipsed, as the places are nearer to or farther from the centre of this shadow. In this ease the axis of the shade passes through the centre of the earth, or the new moon happens exactly in the node, and then it is evident that the section of the shadow is circular; but in every other case the conical shadow is cut obliquely by the surface of the eartb, and the section is an oval, and very nearly a true ellipsis.

2. Nor does the eclipse appear the same in all parts of the earth, where it is seen; but when in one place it is total, in another it is only partial. Further, when the moon appears much less than the sun, as is chiefly the case when she is in apogee and the sun in perigee, the vertex of the Junar shadow is then too short to reach the earth; for thongh the moon be in a central conjunction with the sun at this time, yet she is not large cnough to cover his whole disc, but lets his limb appear like a lucid ring or bracelet, and so causes an annular eclipse.

3. A solar eclipse does not happen at the same time, in all places where it is seen; but appears more early to the western parts, and later to the eastern; as the motion of the moon, and consequently of her shadow, is from west to east.

4. In most solar celipses the moon's disc is covered with a faint light; which is attributed to the refiexion of the light from the illuminated part of the earth.

Lastly, In total eclipses of the sun, the moon's limb is seen surrounded by a pale circle of light; which some astronomers consider as an indication of a lumar atmosphere; but others as the atmosphere of the sun, because it has been observed to more equally with the sun, and not with the moon; and besides, it is generally believed that the moon is without any atmosphere, unless it be one that is very small, and very rare; which latter supposition may now be considered as a fact well established, being confirmed by a vast number of attentive ubservations.

To determine the Bounds of $a$ Solar EcLipse. If the moon's parallax were inconsiderable, the bounds of a solar eclipse would be determined in the same manner as those of a lunar; but as it has a sensible parallax, the method of proceeding is a litule diffierent, viz.

1. Add together the apparent semidianeters of the luminaries, both in apage and perigee; which gives $33^{\prime} 6^{\prime \prime}$ for the greatest sum of them, and $3 \alpha^{\prime} 31^{\prime \prime}$ for the least sum.

2. Since the parallax diminishes the northern latitude and augnent; the sututhern, therefore let the greatest parallax in latitude be alded to the former sums, and also subtracted from them: ibus in each case there will be had the true latitude, bryond which there ean be no eclipse. This latitule being given, the moon's dintance from the nodes, beyoud which eclipses cannot lappen, is tound as for a lunar celipse. This limit is nearly between $16 \frac{1}{2}$ and $18 \frac{1}{3}$ degrees distance from the nocies.

To find the Digits eclipsed. Add the apparent scmidiameters of the luminaries into one sum; from which subtract the moon's apparent latitude; the remainder is the scruples, or parts of the diametor eclipsed. Then say, $A$ s the semidiameter of the sun is to the scruples eclipsed; so are $G$ rligits reduced into scruples, viz, 360 seruples or ninutes, to the rigits de eclipsed.

Tis determme the Duration of a Solter Fichass.. Find the horary motion of the moon liom the sun, lor one hour before the conjunction, and another lour after : then say, As the former horary motion is to the seconds in an hour, so are the scruples of half duration (found as in a lunar eclipse) to the time of inmersion; and as the latter horary motion is to the same seconds, so are the same scruples of balf duration to the time of emersion. Lastly, Adding the times of inmersion and emersion together, the aggregate is the total duration.

The moon's apparent diameter when largest, exceeds the sun's when least, only by $2^{\prime}$ of a degree; and in the grestest solar eclipse that can happen at any time and place, the total darkness cannot continue any longer than whilst the moon is moring through $\mathrm{tl}$ is $2^{\prime}$ from the sun in her orbit, which is about a minutes of time: for the motion of the shadow on the earth's disc is cuual to the moon's motion from the sun, which on account of the earth's rotation on its axis in the same direction, or eastwarl, is about $30 \frac{1}{2}$ minutes of a degree every hour, at a mean rate; but so much of the moon's orbit is equal to $30 \frac{1}{2}$ degrces, of a great circle on the earth, because the circumference of the mon's orbit is about 60 times the circumference of the earth; and therefore the moon's sharlow goes $30 \frac{1}{2}$ degrees, or 1830 geographical miles in au hour, or $30 \frac{1}{3}$ miles in a minute.

To delermine the Beginning, Middle, and End, of a Solar Eclipse. From the moon's latitude, for the time of conjunction, find the arch GL. (last fig. but one), oI the distance of the greatest obscurity. Then say, As the horary motion of the moon from the sun, before the conjunction, is to 1 hour; so is the distance of the gratest darkness, to the interval of time between the greatest darkuess and the conjunction. Subtract this interval, in the 1st and 3d quarter of the anomaly, from the time of the conjuiction; and in the other quarters, add it to the same; then the result will be the time of the greatest darkness. Lastly, from the tine of the greatest darkness subtract the time of incidence, and add it to the time of emersion; the difference in the first case will be the beginning; and the sum, in the latter case, the end of the eclipse.

To Calculate Ecripses of the Sun. 1. Find the mean new moon, and thence the true one; with the place of the luminaries for the apparent time of the truc one-2. For the apparent time of the true new moon, compute the apparent time of the new moon observed.-3. For the apparent time of the new moon seen, compute the latitude seren-4. Thence determine the digits eclipsed.-5. Find the tinies of the greatest darkness, immersion, and emersion.-6. Thence determine the beginning and ending of the eclipse.

From the foregoing problems, it is eviclent that all the trouble and fatigue of the calculus arises from the parallases of longitude and latitude, without which, the calculation of solar eclipses would be the same with that of lunar ones.

See the Construction and Calculation of Eclipses by Flanstecel, in Sir Jonas Muore's System of Mafbematics, and in Fereusun's Astronomy, \&c.

In the Philos. Trans. $\mathrm{N}^{0}-1.61$, is a contrivance to represent solar iclipses, by means of the terrestrial globe, by N1. Secruer, professin of mathematics at Gottingen. And Mr. Fergusun lined a terrestrial ghobe, so as to show the time, quantity, furation, and progress of solar celipses, at any place of the carth where they are risible; which he calls the Ficlipsalreon. Ila bas also given a large catalogue of uncient and modern celipses, including those re- 
corder in history, from 721 years before Christ, to A. D. 1485; also computed eclipses from 1485 to 1700 , and all the eclipses visible in Eurupe from 1700 to 1800 . Sue his Astron.

The Number of EcLipess, of both luminaries, in any year, cannot be less than two, nor more than seven; the most usual number is 4 , and it is rare to have more than 6. The reason of which limitation is obvious; because the sun passes by both the nodes but once in a year, unless he pass by one of them in the beginning of the year; in which case he will pass by the same again a little bufore the end of the year; because the nodes move hackwards $19 \frac{\mathrm{I}}{3}$ degrees every ycar, so that the sun will come to either of tbem $\mathbf{1 7 3}$ days after the other: if either node be within $17^{\circ}$ of the sun at the tinse of new moon, the sun. will be eclipsed; and at the subsequent opposition, the moon will be eclipsed in the other node, and come round to the next conjunction before the former node be $17^{\circ}$ beyond the sun, and eclipse him again. When three eclipses happen about either node, the like number commonlyr haypens about the opposite one ; as the sun comes to it in 173 days afterward, and 6 lunations contain only 4 days more. Thus there may be two eclipses of the sun, and one of the moon, about each of the nodes. But when the moon changes in either of the nodes, she cannot be near enough the other node, at the next full, to be celipsed; and in 6 lunar months afterward she will change near the other node; in which case there cannot be more than two cclipses in a year, both of the sun.

Feriod of Ecrupses, is the period of time in which the same celipses return again; and as the nodes move backwards $19 \frac{x}{3}$ degrees every year, they must shift through evcry point of the ecliptic in 18 years and 225 days; and this would be the regular period of their return, if any complete number of lunations were finished without a fraction; but this is not the case. However, in 223 mean lunations, after the sun, moon, and nodes bave been once in a line of conjunction, they roturn so nearly to the same state acain, is that the same node which was in conjunction with the sun and moon at the beginning of the first of these lunations, will be within $28^{\prime} 12^{\prime \prime}$ of the line of conjunction with the sun and moon ugain, when the last of these lunations is completed; and in this period there will be a regular return of eclipses for many ages. To the mean time of any soliar or lunar eclipse, by adding this period, or is Julian yenrs 11 dilys 7 hours +3 minutes 20 seconds, when the last dily of Feliruary in leap-years is 4 rimes included, (1) a day less when it occurs 5 times, we shall have the mean time of the return of the same eclipse. In an interval of 6890 inean lumations, containing 557 years 21 days 18 hours 30 minutes 11 seconds, the sun and node meet so nearly, is to be distant only 11 seconds.

The Use of Ect.1PSEs. In astronomy, eclipses of the moon deterinine the splocipal figure of the carth ; they also show that the sun is larger than the earth, and the carth than the moon. Eclipses also, that are similar in all circumstances, and that happen at considerable intervals of time, serve 10 ascertain the period of the monn's motion. In gergraphy, eclipse's discover the longitude of ditferent places; for which purpose those of the moon are the more useful, because they are more often visible, and the same lunar celipses is of equal magnitude and duration at all places where it is secn. In chronology, both solar and lumar celipses serve to determine exactly the time of ally jast exen!.
The following is a list of all the solar cclipses visible in this country till the year 2000 ; the times adapted to Leicestershire, the middle of England.

A Table of all the SoLAR ECLu PSEs that will be visible till the Yerr 2000.

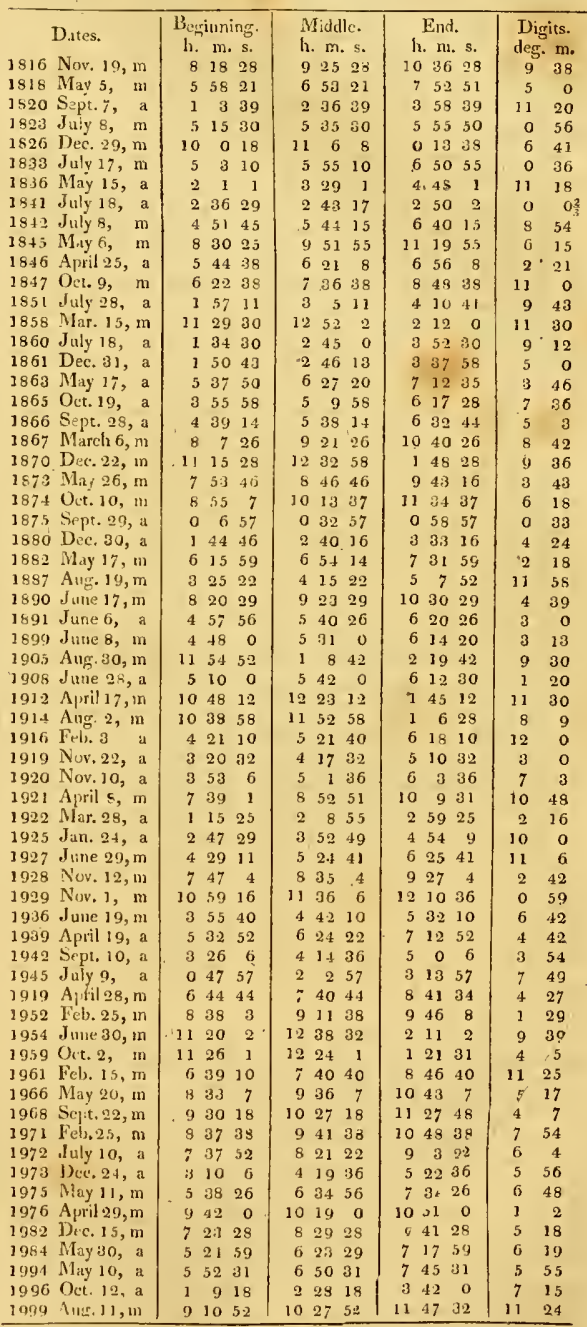

EcL1PS lis of the Satellites. See Satrenites of Jupiter. -'The pritucipal circumstinces here observed are, 1. That the satcllites of .lupiter undergo two or three kinds of edlipses; the first of which are proper, being such as happen when Jupiter's borly is directly interposcel betwe'n then and the sun; which eclipses bappen almost every day, Various authors have given tables for computing 
eclipses of the satellites of Jupiter; as Flamsteed, Cassini, Wargentin, \&c.

The second sort are occultations, rather than observations; when the satellites, coming too near the body of Jupiter, are lost in his light; which Riccioli calls occidere zeusiace, setting jovially. In which case, the nearest or first satellite exhibits a third kind of eclipse; being observed like a round macula, or dark spot, transiting the disc of Jupiter, with a motion contrary to that of the sitellite; like as the moon's shadow, projected on the carth, will appear to do, to the lunar inhabitants.

The eclipses of Jupiter's satellites furnish very good means of finding the longitude at sea. Those especially of the first satellite are much surer than the eclipses of the moon, and they also happen much oftener : the manner of applying them is also very easy. In the Philos. Trans. for $1786, \mathrm{Mr}$. Pigott gave a preferable method by the lunar transits. See LoNGITUDE.

ECLIPTIC, in Astronomy, a great circle of the sphere conceived to pass through the middle of the zodiac. It is sometimes called the via solis, or sun's path, being the track which he appears to describe among the fixed stars; though more properly it is the apparent path of the earth, as viewed from the sun, and thence called the heliocentric circle of the earth. It is called the ecliptic, because all the eclipses of the sun or moon happen when the moon crosses it, or is nearly in one of those two parts of her orbit where it crosses the ecliptic, which points are called the moon's nodes.

Upon the ecliptic are marked and counted the $12 \mathrm{cc}-$ lestial signs, Aries, Taurus, Gemini, \&c ; and also the longitude of the planets and stars. Its situation is oblique with respect to the equator, which it cuts in two opposite points, viz, the beginning of Arics and Libra, being directly opposite to each other, and called the equinoxes, making one half of the ecliptic to the north, and the otber halt on the south side of the equator; the two extreme points of it, to the north and south, which are opposite to each other, and at a quadrant distance from the equinoctial points both ways, are called the solstices, or solstitial points, or also the two tropies, which are at the beginning of Cancer and Capricorn, being at the farthest distance of any points of it from the equator, which distance is the measure of the sun's grcatest declination, and is the same with the obliquity of the ecliptic, or the angle it makes with the equator.

This obliquity of the ecliptic is not permanent, but is coniinually varying, by the ecliptic approaching nearer and narer to a parallelism with the equator, at the rate at present of $42^{\prime \prime} \cdot 6$ in 100 years, as is deduced from ancient and indern observations compared together ; and as the mean obliquity of the ecliptic was $23^{\circ} 28^{\prime}$ about the end of the year 1788 , or beginning of 1789 , by adding $-4,26$ of a second for each preceding year, or subtracting the same for each collowing year, the mean obliquity will be found nearly, for any year either before or since that period. 'The quantity however of this change is variously' stated by different authors. Hipparchus, al most two thousand years since, observed the obliquity of the celiptic, and found it about $23^{\circ} 51^{\prime}$; and all succceding astronomers, to the present time, having observed the same, have found it always less and less; being now rather uniler $23^{\circ} 28^{\prime}$; a difference of abont $23^{\prime}$ in 1950 years; which gives a mediun of $70^{\prime \prime}$ in 100 years. There is no doubt, however, that the diminution is variable.
It is now well known that this change in the obliquily of the ecliptic, is wholly owing to the actions of the planets upon the earth, and especially the planets Venus and Ju* piter, but chiefly the former. See Lagrange's excellent paper on this subject in the Memoirs of the French Acadeiny for 1774 ; Cassini's in 1778 ; and Lalande's Astron. vol. 3, art. 2737. According to Lagrange, who proceeds upon theory, the annual change of obliquity is variable, and has its limits: about 2000 years ago, he thinks it was after the rate of about $3 \mathrm{~s}^{\prime \prime}$ in 100 years; that it is now, and will be for 400 years to come, $56^{\prime \prime}$ per century; but 2000 years hence, $49^{\prime \prime}$ per century. According to Cassini, who computes from observations of the obliquity between the years 1739 and 1778 , the annual cluange at present is $60^{\prime \prime}$ or $l^{\prime}$ in 100 years. But according to Lalande, the diminution is at the rate of $42^{\prime \prime} \cdot 6$ per century ; while Dr. Maskelyne makes it $50^{\prime \prime}$ in the same time. But later observations and the calculations of Laplace, give now $52^{\prime \prime} \cdot 1$ for the mean secular variation.

Besides the diminution of the obliquity of the ecliptic, at the above rate, which arises from a change of the ecliptic itself, it is subject to two periodical inequalities, the onc produced by the unequal force of the sun in causing the precession of the equinoxes, and the other depending on the nutation of the earth's axis. See the Explanation and Use of Dr. Miaskelyne's Tables and Observations, pa. vi, where he shows how to calculate those inequalities; and also, from his own observations, determines the mean obliquity of the ecliptic to the beginning of the year 1769 , to be $23^{\circ} 28^{\prime} 9^{\prime \prime} \cdot 7$.

To find the Obliquity of the Ecliptic, or the greatest declination of the sun:-About the time of the summer solstice observe very carefully the sun's zenith distance for several days together; then the difference between this distance and the latitude of the place, will be the obliquity sought, when the sun and equator are both on one side of the place of observation; but their sum will be the obliquity when they are on different sides of it. Or, it may be found by observing the meridian altitude, or zenith distance, of the sun's centre, on the days of the sunmer and winter solstice; then the difference of the two will be the distance between the tropics, the half of which will be the obliquity sought.

By the same method too, the declination of the sun from the equator for any other day may be found; and thus a table of his declination for every day in the year might be constructed. Thus also the dectination of the stars might be found.

The observations of astronomers of all ages, on the obliquity of the celiptic, have becn collected together; and although some of them may not be quite accurate, yet they sufficiently show the gradual and continual decrease of the obliquity from the times of the earliest observations down to the present time. The chief of those olscrvations may be seen in the following table; where the frist column contains the name of the observer, or author; the 2d, the year before or al ter Christ; and the 3d, the obliquity of the ccliptic for the time.

Sec Ptolem. $\Lambda \mathrm{m}$. lib. 1, cap. 10 ; Riccioli $\Lambda$ mm. vol. 1 , lib. 3, cap. 27 ; litumstecd, Proleg. Ilist. Col. vol. 3; Philos. 'T'rans. $\mathrm{N}^{\circ} 163$; ibid. vol. 63 , pt. 1 ; Long's Astron. vol. 1, cap. 16; Menuirs of the Acad. an. 1716, 1734, $1762,1767,177$ 4, 1778 ; $\Lambda$ cta Lrud. Lipsix, 1719 ; Naut. Alm. 1779; Maskelyne's Observ. Explan. pa.vi ; \&.c. Sir. LAPLACE. 
Table exhibiting tite Degree of Obliquity of the Eclipic at various Times, as determined fiom ancient and modern Observations.

\begin{tabular}{|c|c|c|c|c|c|c|c|}
\hline \multicolumn{5}{|c|}{ Authors' Names. } & \multirow{2}{*}{$\mid \begin{array}{c}\text { Years before } \\
\text { Christ. } \\
324\end{array}$} & \multicolumn{2}{|c|}{ Obliquity. } \\
\hline Pytheas & - & - & - & - & & 2349 & $9 \stackrel{23}{2}$ \\
\hline \multicolumn{5}{|c|}{ Eratosthenes and Hipparclius } & 230 \& 140 & 2351 & 120 \\
\hline Ptolemy & - & - & - & - & 140 & 2348 & $8 \quad 4.5$ \\
\hline Almahmon & - & - & - & - & 832 & 2335 & \\
\hline Albategniu: & & - & - & - & 880 & 2335 & \\
\hline Thebat & - & - & - & - & 911 & $23 \quad 33$ & 330 \\
\hline \multirow{2}{*}{\multicolumn{5}{|c|}{$\begin{array}{l}\text { Abul Wasi and Hamed } \\
\text { Persian Tables in Chrysococea }\end{array}$}} & 999 & 2335 & \\
\hline & & & & & 1004 & 2335 & \\
\hline Albatrunius & & - & - & - & 1007 & 2335 & \\
\hline Arzachel & - & - & - & - & 1104 & 2333 & 330 \\
\hline Almæon & - & - & - & - & 1140 & 2333 & 330 \\
\hline \multicolumn{3}{|c|}{ Choja Nassir Oddin } & - & - & 1290 & 2330 & \\
\hline \multicolumn{3}{|c|}{ Prophatius the Jew } & - & - & 1300 & 2332 & \\
\hline Ebn Shattir & & - - & - & - & 1363 & 2331 & \\
\hline \multicolumn{4}{|c|}{ Purbach and Regiomontanus } & - & 1460 & 2330 & \\
\hline Ulugh Beig & & - & - & - & 1463 & 2330 & $0 \quad 17$ \\
\hline Walther & & - & - & - & 1476 & 2330 & \\
\hline \multicolumn{5}{|c|}{ Ditto corrected by refraction, \&c. } & $\ldots \ldots$ & 2329 & 98 \\
\hline Werner & - & & - & - & 1510 & 2328 & 830 \\
\hline Copernicus & & - & - & - & 1525 & 2328 & 24 \\
\hline Egnatio Da & anti & - & - & - & 1570 & 2329 & \\
\hline Prince of $\mathrm{H}$ & lesse & - & - & - & 1570 & 2331 & \\
\hline \multicolumn{3}{|c|}{ Rothmann and Byrge } & - & - & 1570 & 2330 & 020 \\
\hline 'Tycho Bral & & - & - & - & 1584 & 2331 & 130 \\
\hline \multicolumn{2}{|c|}{ Ditto corrected } & - & - & - & ........ & 2329 & \\
\hline Wright & - & - & - & - & 1594 & 2330 & \\
\hline Kepler & - & - & - & - & 1627 & 2330 & 030 \\
\hline Gassendus & - & - & - & - & 16.30 & 2331 & 1 \\
\hline Ricciolus & - & - & - & - & 1646 & 2330 & 020 \\
\hline \multicolumn{2}{|c|}{ Ditto corrected } & - & - & - & 1655 & $23 \quad 29$ & \\
\hline Hevelius & - & - & - & - & 1653 & 2330 & 020 \\
\hline \multicolumn{2}{|c|}{ Ditto corrected } & - & - & - & 1661 & 2328 & 852 \\
\hline Cassini & - & - & - & - & 1655 & 2329 & $9 \quad 15$ \\
\hline \multicolumn{2}{|c|}{ Montons, correct } & ed, \& & c & - & 1660 & 2329 & 93 \\
\hline \multicolumn{3}{|c|}{ Richer corrected } & - & - & 1672 & 2328 & 852 \\
\hline La Hire & - & - & - & - & 1686 & 2329 & \\
\hline \multicolumn{2}{|c|}{ Ditto corrected } & $=$ & - & - & ....... & 2329 & y 28 \\
\hline \multicolumn{2}{|c|}{ Flamsteed } & - & - & - & 1690 & 2329 & \\
\hline \multicolumn{2}{|l|}{ Bianchini } & - & - & - & 1703 & 2323 & 325 \\
\hline Roemer & - & - & - & - & 1706 & 2328 & 84.1 \\
\hline Louville & - & - & - & - & 1715 & 2328 & 824 \\
\hline Godin & - & - & - & - & 1730 & 2328 & 820 \\
\hline Bradley & - & - & - & - & 1750 & 2328 & 818 \\
\hline Mayer & - & - & - & - & 1756 & 2329 & 816 \\
\hline Maskelyne & & - & - & - & 1769 & 2329 & 810 \\
\hline Hornsby & - & - & - & - & 1772 & 2328 & 88 \\
\hline Nautical Al & Imanac & & - & - & 1779 & 2329 & 87 \\
\hline Ditto & - & - & - & - & 1800 & $23 \quad 27$ & 750 \\
\hline Dr. Maskel & lyne"s & ubsert & vations & & 1800 & 2327 & $56 \cdot 6$ \\
\hline Piazzi & 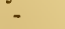 & - & - & - & 1800 & 2327 & $756 \cdot 3$ \\
\hline Mr. Pund & - & - & - & - & 1800 & $23 \quad 27$ & $756 \cdot 5$ \\
\hline Delambre & with , & reprat & ing ci & ircle? & & & \\
\hline $\begin{array}{l}\text { of Borda, } \\
\text { dred obse }\end{array}$ & servatic & & & & 1800 & 2327 & 757 \\
\hline English ani & d) Fren & ach ast & romon & & 1812 & 2327 & 742 \\
\hline
\end{tabular}

Table of the Obliquity of the Ecliptic for Forty Conturies, with its Secular Variation.

\begin{tabular}{|c|c|c|c|c|c|}
\hline & & \multicolumn{3}{|c|}{ Mean Otsliquity. } & Secular Variation. \\
\hline \multirow[t]{10}{*}{ A. C. } & 900 & $23^{\circ}$ & $50^{\prime}$ & $26^{\prime \prime} \cdot 2$ & \\
\hline & 800 & 23 & 49 & $39 \cdot 6$ & $46^{\prime \prime} \cdot 9$ \\
\hline & 700 & 23 & 48 & $52 \cdot 7$ & $47 \cdot 2$ \\
\hline & 600 & 23 & 48 & $5 \cdot 5$ & $47 \cdot 5$ \\
\hline & 500 & 23 & 47 & $18 \cdot 0$ & $47 \cdot 9$ \\
\hline & 400 & 23 & 46 & $30 \cdot 1$ & $48 \cdot 2$ \\
\hline & 300 & 23 & 45 & $4.1 \cdot 9$ & $48 \cdot 5$ \\
\hline & 200 & 23 & 44 & $53 \cdot 4$ & $48 \cdot 9$ \\
\hline & 100 & 23 & 44 & $4 \cdot 5$ & $49 \cdot 1$ \\
\hline & 0 & 23 & 4.3 & $15 \cdot 4$ & $49 \cdot 3$ \\
\hline \multirow[t]{30}{*}{ A. D. } & 100 & 23 & 42 & $26 \cdot 1$ & $49 \cdot 7$ \\
\hline & 200 & 23 & 41 & $36 \cdot 4$ & $49 \cdot 9$ \\
\hline & 300 & 23 & 40 & $46 \cdot 5$ & $50 \cdot 1$ \\
\hline & 400 & 23 & 39 & $56 \cdot 4$ & $50 \cdot 3$ \\
\hline & 500 & 23 & 39 & $6 \cdot 1$ & $50 \cdot 5$ \\
\hline & 600 & 23 & 38 & $15 \cdot 6$ & $50 \cdot 8$ \\
\hline & 700 & 23 & 37 & $24 \cdot 8$ & $51 \cdot 0$ \\
\hline & 800 & 23 & 36 & $33 \cdot 8$ & $51 \cdot 1$ \\
\hline & 900 & 23 & 35 & $42 \cdot 7$ & $51 \cdot 3$ \\
\hline & 1000 & 23 & 34 & $51 \cdot 4$ & $5] \cdot 4$ \\
\hline & 1100 & 23 & 34 & 0.0 & $51 \cdot 5$ \\
\hline & 1200 & 23 & 33 & $8 \cdot 5$ & $51 \cdot 7$ \\
\hline & 1300 & 23 & 32 & $16 \cdot 8$ & $51 \cdot 8$ \\
\hline & 1400 & 23 & 31 & $25 \cdot 0$ & $51 \cdot 9$ \\
\hline & 1500 & 23 & 30 & $33 \cdot 1$ & $52 \cdot 0$ \\
\hline & 1600 & 23 & 29 & $41 \cdot 1$ & $52 \cdot 0$ \\
\hline & 1700 & 23 & 28 & $49 \cdot 1$ & $52 \cdot 1$ \\
\hline & 1800 & 23 & 27 & $57 \cdot 0$ & $52 \cdot 1$ \\
\hline & 1900 & 23 & 27 & $5 \cdot 0$ & $52 \cdot 1$ \\
\hline & 2000 & 23 & 26 & $12 \cdot 8$ & $52 \cdot 2$ \\
\hline & 2100 & 23 & 25 & $20 \cdot 6$ & $52 \cdot 3$ \\
\hline & 2200 & 23 & 24 & $28 \cdot 3$ & $52 \cdot 2$ \\
\hline & 2300 & -23 & 23 & $36 \cdot 1$ & $52 \cdot 2$ \\
\hline & 2400 & 23 & 22 & $43 \cdot 9$ & $52 \cdot 2$ \\
\hline & 2500 & 23 & 21 & $51 \cdot 7$ & $52 \cdot 2$ \\
\hline & 2600 & 23 & 20 & $59 \cdot 5$ & $52 \cdot 1$ \\
\hline & 2700 & 23 & 20 & $7 \cdot 4$ & $52 \cdot 1$ \\
\hline & 2800 & 23 & 19 & $15 \cdot 3$ & $52 \cdot 1$ \\
\hline & 2900 & 23 & 18 & $23 \cdot 3$ & $52 \cdot 0$ \\
\hline & 3000 & 23 & 17 & $31 \cdot 3$ & $52 \cdot 0$ \\
\hline
\end{tabular}

According to an ancient tradition of the Egyptians, mentioned by Herodotus, the ecliptic had formerly been perpendicular to the equator: they were led into this notion by observing, for a long series of years, that the obliquity was continually diminishing; or, which amounts to the same thing, that the ecliptic was continually approaching to the equator. Hence they tonk occasion to suspect that those two circles, in the begimning, had been as far off each other as possible, that is, perpendicular to each other. Diodorus Siculus relatcs, that the Chaldeans reckoned 403,000 years from their first observations to the time of Alexander's entering Babylon. This enormous account may have some foundation, on the supposition that the Chaldeans deduced it from the diminution of the obliquity of the ecliptic at the rate of a minute in 100 years. M. de Louville, taking the obliquity, such as it must have been at the time of Alexander's cntrance into 


\section{E L A}

[ 450

Babylon, and going back to the time when the ecliptic, at that rate, must have been perpendicular to the equator, actually finds $402,9+2$ Egyptian or Chaldean years; which is only 58 years short of that epocha. Indced there is no way so probable of accounting for the fabulous antiquity of the Egyptians, Chaldeans, \&c, as from the supposition of loug periods of very slow celestial motions, a small part of which they had observed, and from which they calculated the beginning of the period, making the world and their own nation to commence together. $\mathrm{Or}^{*}$ perhaps they sometimes counted months or days for years.

ECLi pTic, in Geography, a great circle on the terrestrial globe, in the plane of, or directly under, the celestial ecliptic.

Ecliptic, Eclipticus, something belonging to the ecliptic, or to eclipses; as ecliptic conjunction, opposition, \&c.

EcLiptic Bounds, or Limits, are the greatest distances from the nodes at which the sun or moon can be eclipsed; namely, near 18 degrees for the sun, and 12 degrees for the moon.

Ecriptic Digits, Digiti ecliptici. See Digits.

Poles of the EcLIPTIC, are the two opposite points of the sphere which are each every where equally distant from the ecliptic, viz, $90^{\circ}$. The distance of the poles of the ecliptic from the poles of the equator, or of the world, is always equal to the varying distance of the obliquity of the ecliptic, which for the year 1800 was, according to Dr. Maskelyne, $23^{\circ} 27^{\prime} 56^{\prime \prime} \cdot 6$.

Reduction to the Ecliptic. See Reduction.

EFFECT. The result or consequence of the application of a cause, or agent, on some subject. It is one of the great axioms in philosophy, that effects are always proportional to the powcrs of their adequate causes.

EFFECTION, denotes the geometrical construction of a proposition. The term is also used in reference to problems and practices; which, when they are deducible from, or founded on some general propositions, are called the geometrical effection of them.

EFFERVESCENCE, is popularly used for a light ebullition, or a brisk intestine motion, produced in a liquor by the first action of heat, with any remarkable separation of $i$ ts parts.

EFFICIENT Cause, is that which produces an effect. See CAUSE and EFFECT.

EFFicisins, in $\Lambda$ rithmetic, are the numbers given for an operation of multiplication, and are otherwise called the factors. Hence the term coefficients in algebra, which are the numbers prefixed to, or that inultiply the letters or algebraic quantities.

EFFLUVIUM, a flux or exhalation of minute particles from any boxly; or an cmanation of subtile corpuscles from a mixed, sensible body, by a kind of motion of transpiration.

ELASTIC, an appellation given to all bodies endoved with the property of elasticity or springiness.

Elastic Body, is that which changes its figure, and yiclds to any impulse or pressure, but endeavours by its own nature and force to, restore the same again; or, it is a body of such a mature that, when compressed or colldensed, it will make an effort to sct itself at liberty, and to repel the body that constrained it: such, for instance, as a bow, or a sword-blade, \&c, which are casily bent, but presently return to their former figure and extension. All bodies partake of this property in some degree, though
E L A

perhaps none are perfectly clastic, as none are found to restore themselres with a force equal to that with which they are compressed.

The principal plienomena observable in elastic bodics, are, 1. That an elastic bocly (i. e. a body perfictly elastic, if any such there be) (nduavours to restore itself with the same torce with which it is pressed or bent. ?. An elastic body exerts its force cyua!ly towards all sides; thuugh the effect is chiefly found on that side where the resistance is weakest; as is evident in the case of a gun explocting a ball, a bow shooting out an arrow, \&c.-3. Elatstic bodies, in whatever manner lhey are struck or impelled, are inflected and rebound after the same manner: thus a bell yields the same musical sound, in whatever manner, or on whatever side it be struck; the same of a tense or musical chord; and a body rebounds from a plane in the same angle in which it meets or strikes it, making the angle of incidence equal to the angle of reficction, whether the intensity of the stroke be great or small.-4. 1 perfectly fluid body cannot be elastic, if it be allowed that its parts cannot be compressed.-5. A body perfectly sulid, cannot be elastic; because, having no porrs, it is incapable of being compressed.- - i. 'The clastic propertics of bodies seem to differ, according to their greater or less density or compactness, though not in an cqual degrce: thus, metals are rendered more compact and elastic by being limmered: tempered steel is much more elastic than soft steel; and the density of the former is to that of the latter as 7809 to 7738 . Cold condenses solid bodics, and renders then more elastic; while heat, that relaxes them, has the opposite effect : but, on the contrary, air, and other elastic fluids, are expanded by heat, and rendered more clastic.-For the laws of motion and percussion in clastic bodies, see Motion, and Pencussion.

Litastic Curve. See Catenaria.

Elastic Fluids. See Air, Electricity, Gas, ElasTIC VA pours, Sic.

Elastic Gum. The same as Caovtcinouc, or Indian Rubber.

Exastic Vapours, or Fluids, are such as may be compressed mechanically into a less space, and which resume their former state when the compressing force is withdrawn; such as atmospheric air, and all the acrial fluids, with all kinds of fumes raised by means of heat, whether from solid or fluid bodies.

Of these, some remain elastic only while a considerable degree of heat is applicd to them, or to the substance which produces them; while others continue elastic in every degree of cold that has yet been obscried. Of the former kind, are the vapours of water, spirit of wine, mercury, sal-ammoniac, and all kinds of sublimable salis : of the latter, those of spirit of salt, mixtures of vitriolic acid and iron, nitrous acid, and various other metals, and in short the several species of aerial fuids indiscriminately.

The elastic force with which any one of these fluids is endowed, has not yet been calculated, as being ultimately greater than any ohstacle we can put in its way'. 'l'hus, on compressing atmospheric air, we find that for some litile time at first it easily yiclis to any force ajplicd; but at every succeding moment the resistance becomes stronger, and a greater and greater force must be applied, to compress it further. As the compression goes on, the resach containing the air becomes hot; but no power whatever has yet becn able in any digree to destroy the elasticity of 

E L A
$[457$
E L E

the contained fluid; for, upon removing the pressure, it is always found to occupy the very same space that it did before. The case is the same with the steam of water, to which a sufficient heat is applied to keep it from condensing into water again.

Elasticity, or Elastic Force, that property of bodies by which they restore themselves to their former figure, after any external pressure.

The cause or principle of this important property, elasticity, is variously accounted for. The Cartesians ascribe it to a subtile matter making an effort to pass through pores that are too narrow for it. Thus, say they, in bending or compressing a hard clastic body, as a bow, for instance, its parts recede from each other on the convex side, and approach on the concave one: consequently the pores are contracted or straitened on the concave side; and, if they were before round, are now perhaps oval : so that the materia subtilis, or matter of the second element, endeavouring to pass out of the pores thus straitened, must make an effort, at the snme time, to restore the budy to the state it was in when the pores were cylindrical,i.e. before the bow was bent : and in this consists its elasticity.

Other later philosophers account for clasticity much after the sane manner as the Cartesians; with this difference only, that instear of the subtile matter of the Cartesians, these substitute ether, or a fine cthereal medium that pervades all bodies.

Others, setting aside the precarious notion of a materia subtilis, account for elasticity from the great law of nature, attraction, or the cause of the cohesion of the parts of solid and firm bodies. Thus, say they, when a hard body is struck or bent, so that the component parts are moved a little from each other, but not quite disjointed or broken off, or scparated so far as to be out of the power of that attracting force by which they cohere; they must, on removing the external violence, spring back to their former natural state.

Others again resolve elasticity into the pressure of the atmosphere : for a violent tension, or compressiun. though not so great as to separate the constituent particles of bodies far enough to let in any foreign matter, must jet occasion many little vacuola between the scparated surfaces; so that on the removal of the force they will close again by the pressure of the acrial fluid upon the external parts.

Lastly, Others attribute the elasticity of all hard bodies to the power of resilition in the air included withirr them; and so make the elastic force of the air the principle of elasticity in all other bodies. See Desaguliers's Exper. Philos. vol. 2, pa. 38, \&c.

The Elasticity of Fluids is accounted for from their particles being ail endowed with a centrifugal force; whence Sir Isaac Newton demonstrates, prop. 23, lib. 2, that particles, which naturally avoid or fly off from one another by such forces as are reciprocally proportional to the distances of their centres, will compose an elastic fluid, whose density is proportional to its compression ; and vice versa, if any fluid be composed of particles that $\mathrm{Ay}$ off or a void one another, and have its density proportional to its compression, then the centrifugal forces of those particles will be reciprocally proportional to the distances of their centres.

Elasticity of the Air is the force with which that element endeavours to expand, and with which it does actually dilate itself, on removing the force that compresses it. The elasticity or spring of the air was first Vol. 1. discovered by Lord Bacon, and farther established by $\mathrm{Ga}$ lileo. Its existence is thus proved by the latter philosopher: An extraordinary quantity of air being intruded, by means of a syringe, into a loollow ball or shell of glass or metal, till such time as the ball, with its accession of air, weigh considerably more in the balance than it did before; then, opening the mouth of the ball, the air rushes out, till the ball sink to its former weight. Hence he inferred, that just as much air had issued out, as compressed air had been compressed in. Air therefore returus to its former degree of expansion, on removing the force that compressed or resisted its expansion; and consequently it is endowed with an elastic force. It may be added, that as the air is found to rush out in every situation or direction of the orifice, the elastic force acts every way, or in every direction alike. See AIR, and ATMoSPHERE.

The cause of elasticity in air has been ustrally ascribed to a repulsion between its particles; but what is the cause of that repulsion? The term repulsion, like that of attraction, requires to be defined; and probably it will be found in most cases to be the effect of the action of some other fluid. Thus, it is found that the elasticity of the atmosphere is very considerably affected by heat. Supposing a quantity of air beated to such a degree as to raise Fahrenbeit's thermometer to 212 , it will then occupy a considerable space; but if it be cooled again to such is degree, as to sink the thermometer to 0 , it will shrink up to less than half the former bulk. The quantity of repulsive power therefore acquired by the air, while passing from one of these states to the other, is evidently owing to the heat added to it, or taken away from it. Nor does there seem to be any reasun to suppose, that the quantity. of elasticity or repulsive power it still possesses, is owing to any other cause than the fire contained in it. The supposition that repulsion is a primary cause, independent of all others, has given rise to many erroneous theories, and very much enbarrassed philosophers in accounting for the phenomena of elasticity.

The elasticity of the air is not only proportional to its density, but is always equal to the force which compresses it, because these two forces exactly balance each other. This elasticity, in the atmospheric air, is measured by the height of the barometer at any time, allowing for its heat or temperature, after this rate, viz, the 434th part for each degree of Fahrenheit's thermometer, above or below some mean temperature, as $55^{\circ}$; for by that part of the whole it is that air expands or contracts, or else increases or decreases in its elasticity, for eacls degree of the thermometer. Sir Geo. Shuckburgh, in the Philos. Trans. for 1777,. pa. 561.

ELECTIONs, or Choice, signify the several different ways of taking any number of things proposed, either separately, or as combined in pairs, in threes, in fours, \&c; not as to the order, but only as to the number and variety of them. Thus, of the things $a, b, c, d, e, \& \mathrm{c}$, the elections of

one thing are $(a) \quad 1=,2^{1}-1$, two things are $(a, b, a b) 3=2^{2}-1$, three thingsare $(a, b, c, a b, a c, b c, a b c) 7=2^{3}-1, \& c$; and of any nuinber, $n$, all the elections are $2^{n}-1$; that is, one less than the power of 2 whose exponent is $n$, the number of single things to be chosen, either separately or in combination. Sec Combination.

ELECTRIC, in Physics, is a term applicd to thuse sub$3 \mathrm{~N}$ 
stances in which the electric fluid can be excited, and accumulated, without transmitting it; and which are therefore called non-conductors. They are also called original Electrics, and Electrics per se. The word is derived from $r \lambda a \varkappa \% \rho \%$, amber, one of the most observable non-conductors, and the only one known to the ancients. To this class also belong glass, and all vitrifications, even of metals; all precious stones, of whicl the most transparent are the best; resins, and resinous compositions; sulphur, and baked wood; all biruminous substances, wax, silk, and cotton; all dry animal substances, as feathers, wool, hair, \&c ; also paper, white sugar, and sugar-candy; likewise aic, oils, chocolate, calces of metals and semimetals, the ashes of animal and vegetable substances, the rust of metals, all dry vegetable substances, and stones, of which the hardest are the best.

substances of this kind may be excited, so as to exhibit the electric appearances of attracting and repelling light bodies, emitting a spark of light, atteuded with a snapping noise, and yielding a current of air, the sensation of which rescmbles that of a spider's web drawn over the face, \&c, and a small like that of phosphorus; and this exciting may be cither produced by friction, or by heating and cooling, or by muting, and pouring one meited substance into another.-The term is peculiarly applied to the electric, viz, the globe, or cylinder, \&c, used in electrical machines, for collecting the fluid by friction.

Elecruic a t. Air 'Thermometer, an instrument contrived by Mr. Kinnersley of Philadelphia, and used in determining the effects of the electrical explosion upon air; a description of which may be seen in. Franklin's Letters, \&c, pa. 389,4 to, 1769.

Biectrical Apparatus, consists of glass tubes, about 3 feet long, and an inch and a half in diameter, one of which should be closed at one end, and furnished at the other end with a brass cap and stop-cock, to rarefy or condense the inclosed air; sticks of sealing-wax, or tubes of rough glass, or glass tubes covered with sealing-wax, or cylinders of baked wood for producing the negative elecricity; with proper rubbers, as black oiled silk, with analgam upon it for the former, and soft new flamnel, or hare-skins, or cat-skins, tanned with the hair on, for the latter; coated jars, or plates of glass, either single, or combined in a battery, for accumulating electricity; unetal rods, as dischargers; an electrical machine; electroneters, and insulated stools, supported by pillars of glass, covered with sealing-wax, or baked wood, varnished or boiled in linseed oil.

Elecrtical Aimosplere, is a stream or mass of the electric fluid which surromus an excited or electrificd body, to a certain distance.

Electrical Bralls. See Batis, and Electrometer.

Electrica L Battery, consists of a number of coated jars, placed near each other in a convenient manner. These being charged, or electrified, and connected with each other, are then suddenly expleded or discharged, with a prodigions iflect. See lBatTERy.

ELECTRICAL Bells, are a set of bells mounted in a peculiar manner, so as to be rung by means of the action of the electric fluid. Fig. 10, plate vii, represents a set of bells of this kind; when the clapper is suspented from the fly bcd, the axis of which resis in a small hole on the top of the glass pillar ef; the upper part of the axis moves lireely on, and is supported by a hole in the brass piece $g s$, and bells of different toues are placed round the board
hIK. This apparatus being placed near the cylinder, the prime conductor being removed, will, when the machine is in action, be put in motion, the clapper of which striking the bells in succession, produces an agreeable harmony.

ELEct RIC Experiments, are certain experiments made by means of an electric macbine and apparatus; sometimes in order to discover the nature and propertics of the electric fluid, and at others for the amuscment of the operator or by-standers. But, in a work like the present, it is impossible to give any thing like a complete set of experiments: we have however selccted the following; and must refer the reader, for others, to Adams's, Cavallo's, and Cuthbertson's works on this subject.

Experiment 1. Put the machine in action, connect the cushion by a chain with the ground, and those bodies which communicate with the positive conductor will be electrificd positively. Connect the positive conductor with the earth by a chaiu, take off the chain from the cushion, and those bodies which communicate with the cushion, or negarive conductor, will be electrificd negatively.

Exper. 2. Connect the positive conductor by a chain with the table; turn the cylinder, and the cushion will be found to be negatively electrified. Take the chain of from the positive conductor, and both will exhibit signs of electricity; but any clectrified body, which is attracted by the one, will be repelled by the other. If they are brought sufficiently near to each other, sparks will pass between them, and they will act on each other stronger than on any other bodies. If they are connected together, the electricity of the one will destroy that of the other; for though the fluid seems to proceed from the cushion to the conductor, the two, when thus conjoined, will exhibit no signs of electricity, because the fluid is continually circulating from one to the other, and is therefore kept always. in the same state.

We see, by this experiment, that electric appearances are produced both in the electric which is excited, and the substance by which it is excited, provided that substance be insulated; but their electric powers are directly reverse of each other, and may be distinguished by opposite effects.

Exper. 3. If the cushion and the conductor are both insulated, it is observed, that the less electric fuid.is obtained, the more perfect the insulation is made.

'the moisture, which is at all times floating in the air, together with the small points, from which it is impossible totally to free the cushion, do not permit it to be perfectly insulated, so as to aftord no supply of electric matter to the cushion. If the air, and other parts of the apparatus, are very dry, little or no electricity will be produced under the above-mentioned circumstances. From this experiment it is inferred, that the electric powers do not exist in the electrics themsclves, but are prosluced from the earth by the excitation of electrics; or that the electric matter on the prime conductor is not produced by the firiction of the cylinder against the cusbion, but is collocted by that operation from it, and from those bodies which are comnecter with $\mathrm{it}$.

Exper. 4. 'Iouch a pair of insulated pith balls with an excited glass tube, they will become electritied, and will separate from each other; the balls are electrified positively, and are therefore attracted by excited wax, and repelled by excited glass. As those light substances, which possess the same clectric power, repel cach other; 


\section{E L F}

we can easily discover whether they are electrified positively or negatively, by presenting an excited stick of sealing-wax or glass to them. If they are attracted by the glass, they are negatively, if repelied by it, they are positively electrified; on the contrary, if repelled by the excited wax, they are negative, if attracteo, positive.

In ascertaining the nature of the electric powers, we must avoid bringing the bodies to be tried near each other suddenly; or one with a strong electricity near another which is weakly so; as it may render the experiment doubiful, by attracting, and not repelling the light body.

Exper. 5. Insulate a long metallic rod, suspend a pair of pith balls from each end of it, place one of the encis at about two inches from the prime conductor, the other end as far from it as possible, electrify the conductor, and the electric fluid in the rod will be driven to that end which is furthest from the conductor; so that one end will be electrified negatively, the other cnd positively, as will be seen by the balls.

Exper. 6. Fix the end of a wire, having featliers attached to it, in the small hole which is at the end of the prime conductor; turn the cylinder, and the feathers, which are connected with the wire by linen threads, will separate from each other; the fibrous and downy parts will become turgid, and expand in a pleasing nanner, and in a variety of directions.

Prescnt a mitallic point, or the finger, or any other conducting substance, to the featliers, the downy parts thereof will immediately collapse, the divergence of the feathers will cease, and they will approach each other, and cling round the non-electric body.

The feathers separate from cach other, and tend towards unclectrified bodies, from the effort made by the electricity which is communicated to then to diffuse itself, and the resistance it meets with from the air.

Exper. 7. Fix tlie end of the wire into the hole at the end of the conductor, as before, having now two pith balls attached, instead of the feathers; put the machine in action, and the two small balls will recede from cach other. Bring a conducting substance within the sphere of their action, and they will fly towards it; touch the conductor with a non-electric, and they will immediately come together.

The balls do not always diverge so much as might be expected, from the action of their atmospheres, bccausc thicy are influenced by that of the conductor.

Ihe balls, or feathers, will separate, \&c, in the same manner, if they are annexed to a negative conductor.

Exper. 8. Present a fine thread towards an electrified conluctor ; when it is at a proper distance, it will fly towards, and stick to the conluctor, and convey the electric fuid lrom it to the band; remove the thread to a small distance fro:n the conductor, and it will fly backwards and forwards with great velocity and in a very pleasing mamner: present the same thead towards one tlat bangs from the conductor, they will attract and join each other. ling a non-electric body, as a brass ball, near these threads, the ball will repel that held by the hand, and attract that which is fixed to the conductor: the upper thread renders the brass ball negative, and therefore goes uwards it; while the under thread, which is also negative, is repelled. Let the ball he brought near the lower part of the under one, and it will be attracted by it. The junction of the threads arises from the effort the elcctric Auid makes to diffuse itself through them.
E L E

Exper. 9. Place a square piece of leaf brass or silver on the under plate, hold this parallel to the upper one, at about 5 or 6 inches from it; turn the machine, and the leaf will then rise up into a vertical situation, and remain between the two plates, without touching either of them. Present a metal point towards the leaf, and it will immediately fall down. Place a brass ball at the end of the conductor, and when the leaf of brass is suspended between the plate and ball, move the plate round the ball, and the leuf will also move round without touching eitber plate or ball.

Exper. 10. Place two wircs directly under, and parallel, to each other; suspend one from the conductor, let the other communicate with the table; then a light image placed between these, will, when the conductor is electrified, appear like a kind of electrical rope-dancer.

Exper. 11. Put a pointed wire into one of the holes which are at the end of the conductor, hold a gliss tumbler over the point, covering it with your hands, which serve as a tcmporary coating; then electrify the con, ductor, and turn the tumbler round, that the whole interior surface may receive the fluid from the point; place a few pith balls on the table, and cover them with this glass tumbler, the balls will immediately begin to leap up and down as if they were animated, and will continue to move for a considerable time.

Exper. 12. Connect one end of a chain with the outside of a charged jar, and let the other end lie on the table, place the end of another piece of chain at about one quarter of an inch distant from the former; then set a decanter of water on these separated ends, and on making the discharge through the chain, the water will appear perfectly and beautifully luminous.

Exper. 13. Let any person stand on an insulated stool, and connect himself by a wire or chain with the prime conductor, and he will then exhibit the same appearances which are observed in the conductor, and will attract liglit bodies, give the spark, \&c, and thus afiord a pleasing mole of diversifying every expcriment. It is however absolutely necessary, to the complete success of this experiment, that no part of the clothes touch the floor, table, \&c, and that the glass feet be carefully dried; a sheet of dry brown paper placed under the stool will be found of considerable service, by rendering the insulation nore complete.

If the insulated person lav his hands on the clothes of one that is not so, especially if they are woollen, they will both feel as it wcre many pins pricking them, as long as the cylinder is in motion.

Exper. 14. Place the biass ball of a coated jar in contact with the prinse cunductor, while the outside comnunicates with the table, twin the cylinder, and the bottle wiil in a little time be charged. Now to discharge the jar, that is to restore it to its natural state, bring one end of a conducting substance in contact with the ourside coating, and let the other be brought near the knob of the jar which communicates with the insite coatjing, and a strong explosion will take place, the clectric light will be visible, and the report very loud. 'Charge the jur again, then touch the ontsile coating with one band, and the knob with the other, by which means the bottle will be discharged, and a sudden peculiar sensation will be perceived, which is called the clectric shock. 'Mlie slooch, when it is taken in this manner, generally aficets the wrists, clbows, and breast; and when it is strong it re$3 \mathrm{~N} q$ 
sembles a universal blow. This peculiar sensation is probably owing to the two-fuld and instantaneous action of the electric fiuid, which enters and goes out of the body, and the various parts through which it passes, at one and the same instiut. It has been also observed, that nature has appointed a certain modification of the electric fluid in all terrestrial bodies, which we violate in our experiments; when this violation is small, the puwers of nature operate in a gentle manner, to rectify the disorder we have introduced; but when the deviation is considerable, the natural powers restore the oriuginal constitution with extreme violence.

If several persons join hands, and the first touches the outside of a charged jar, and the last the knob, the bottle will be discharged, and they will all feel the shock at the same instant; but the greater the number of persons are that take a shock, the weaker are its effects.

The force of the shock is in proportion to the quantity of coated surfaces, the thinness of the glass, and the power of the machine; or the effect of the Leyden phial is increased in proportion as we destroy the equilibrium on the surfaces.

Exper. 15. A cork ball, or an artificial spider, made of burnt cork with legs of linen thread, suspended by silk, will play between the knobs of two bottles, one of which is charged positively, the other negatively, and will in a little time discharge them.

Exper. 16. A ball suspended on silk, and placed between two brass balls, one proceeding from the outside, and the other from the inside of a Leyden jar, when the bottle is charged, will fly from one knob to the other ; and by thus conveying the fuid from the inside to the outside of the bottle, will soon discharge it.

Exper. 17. Take a glass tube, round which, at small, but equal distances from each other, pieces of tin-foil are pasted in a spiral form from end to end; this tube is inclosed in a larger one, fitted with brass caps at each end, which are connected with the tin-foil of the inner tube. Hold one end in the hand, and apply the other near enough to the prime conductor to take sparks from it, a bcautiful and lucid spot will then be seen at each separation of the tin-foil; which multiply, as it were, the spark taken from the conductor; for if there was no break in the tin-foil, the electric fluid would pass off unperceived.

Exper. 18. To take the electric spark with a metal point, screw a pointed brass wire into one end of a spiral tube, and present it to the conductor while the machine is in action; then a strong spark will pass between the conductor and the point.

Exper. 19. Take a clean dry glass tube, of about a quarter of an inch bore, and insert a pointed wire in this tube, keep the pointed end at some distance from the end of the tube, at the same time let the other end be connected with the ground, bring the former towards the prime conductor, and strong zig-zag sparks, attended with a peculiar noise, will pass between the conductor and the point.

Exper. 20. The Luminous Word. This experiment is exactly on the same principles as Experiment 17. The word is formed by the sinall separations nade in the tinfoil, which is pasted on a piece of glass, that is fixed in a frame of baked wood, having a brass ball at one end. To make the experiment, hold the frame in the hand, and present the ball to the conductor, the spark reccived on this will be communicated to the tin-foil, and follow it in all its windings, till it arrives at the hook, whence it is conveyed to the ground by a chain : and thus the lucid appearance at each break exhibits a word in characters of fire.

Electrical Fluid, is a fine rare fluid which issues from, and surrounds, electrified bodies.

Electrical Kite, an instrument contrived by Dr. Franklin, to verify lis hypothesis of the identity of elictricity and lightning. It consisted of a large thin silk handkerchief, extended and fastened at the four corners to two slender strips of cedar, and accommodated with a tail, loop, and string, so as to rise in the air like a paper kite. To the top of the upright stick of the cross-yas fixed a very shary-poiuted wire, rising a foot or more above the wood; and to the end of the twine, next the hand, a silk ribband was tied. From a key suspended at the junction of the twine and silk, when the kite is raised during a thunder-storm, a phial may be charged, ant electric fire collected, as is usually done by means of an excited glass tube or globe. Philos. Trans. vol. 47, pa.565, or Franklin's Letters, pa. 111 and 112 .- Kites made of paper, covered with varnish, or with well boiled linseed oil, to preserve them from the rain, with a stick and cane bow, like the common ones used by boys, will answer the purpose extremely well, and are very useful in determining the electricity of the atmosphere. See ConDUCTOR.

Electrucal Machine, a piece of mechanism in which an electric boly, such as glass, sulphur, resin, \&c, is subjected to friction, in order to excite in it that power which is commonly known under the name of electricity.

It would be useless to trace this machine through all its various forms and improvements, which the ingenuity and industry of philosophers have given to it; we shall therefore limit ourselves to describing and exhibiting some of the most approved constructions, which may be enumerated as follows.

Description of the most useful Electrical Machines.

Fig. 1, plate xi, represents Dr. Priestley's machine, which is a very extensively useful one, described in his History of Electricity; in which $g$ is the globe, or electric; $f$ the rubber; in the two pillars $d, d$, of baked wood, are several'holes to receive the spindles of different globes or cylinders, several of which may be put on together, to increase the electricity: $k l m$ is the prime conductor, being a copper tube, supported on a stand of glass or baked wood.

Dr. Watson's machine, for using several globes at once, to accumulate a great quantity of electricity, is represented fig. 2 .

Fig. 3 represents a very portable electrical machine invented by Mr. Read, and improved by Mr. Lane. A is the glass cylinder, moved vertically by means of the pulley at the lower end of the axis, the pulley being turned by the large wheel B parallel to the table: there are also several pulleys, of different sizes, either of which may be used, accurding as the motion is required to be quicker or slower. The conductor $\mathrm{c}$ is furnsished with points to collect the Auid, and is screwed to the wire of a coated jar $\mathrm{D}$. 'The figure shows also the manner of applying Mr. Lanc's clectrometer to this machine.

Electrical machines lave of late jear's undergone some very essential alterations and inprovenents; both from the suggestions of private electricians, and the inventions of Messrs. Adams, Nairue, and Jones, instrument-makcrs in London; some of which are as follow: 
Fig. 4 represents a very convenient machine for practice. The frame of this machine consists of the bottom board $\triangle \triangle C D$; which, when the machine is used, must be fastened to the table by two metal cramps. EF are two round pillars, of baked wood, which support the cylinder $\mathrm{G}$ by the axles of the brass or wonden caps $n$, turned sometimes by á simple winch I, and sometimes by a pulley and wheel, as in the next fig. The rubber is fixed to a glass pillar $к$, which is fastened to a wooden basis $\mathrm{L}$ at the buttoin. The conductor $\mathrm{N}$ is usually made of brass or tin japanned, and is insulated by a glass pillar, screwed into a wooden basis or foot, most conveniently placed parallel to the cylinder.

Fig. 5 represent's an electrical machine, with a conductor in the slape of a $r$; and an improved medical apparatus, where it is neccssary to give the shock in the arms, or any other particular part of the body.

Fig. 6 shows Mr. Nairne's patent machine for medical purposes. Its glass cylinder is about 7 inches in diameter, and 12 long, with two confluctors parallel to it. The rubber is fastened to the conductor $\mathrm{R}$; and consists of a cushion of leather stuffed, having a piece of silk glued to its under part. The conductors are made of tin, and covered with black lacker, each of them containing a large coated glass jar, and likewise a smaller one, or a coated tube, which are visible when the caps $\mathrm{N} N$ are removed. To each conductor is fixed a kıob o, for the occasional suspension of a chain to produce positive or negative electricity. That part of the winch $c$ which acts as a lever in turning the cylinder, is of glass. Thus every part of the machine is insulated, the cylinder itself and its brass caps not excepted; by which means very little of the electricity is dissipated, and hence of course the effects are likely. to be the more powerful: the inventor has also adapted to this machine some flexible conducting joints, a discharging electrometer, \&c, for the practice of medical electricity.

The large electrical machine placed in Teyler's Museum at Harlem, was constructed by Mr. John Cuthbertson, an English instrument-maker; and it has, for the electric, two glass plates of 65 inches diameter, made of French glass, as this is found to produce the most electricity next to English fint glass, which could not be made of a sufficient size: these plates are set on the same horizontal axis, at the distance of $7 \frac{1}{2}$ inches, and are excited by 8 rubbers, each $15 \frac{x}{2}$ inches long; and both silles of the plates are covered with a resinous substance to the distance of $16 \frac{\mathrm{r}}{2}$ inches from the centre, both to strengthen the plates, and to prevent any eleetricity from being carried off by the axis. Its battery of jars contains 225 square feet of coated surface, and its effects are astonishingly great; as a proof of which we mention the following particulars: The sensation commonly called the spider's web, when this machine is in action, is felt by the by-standers at the distance of 8 feet from the prime conductor. A thread 6 feet long, suspended perpendicularly, was sensibly attracted by the prime conductor at the distance of 38 feet, and a pointed wire presented to the prime conductor appeared luminous even at the distance of 28 fect. A single spark from the prime conductor melted a considerable length of gold leaf. And a Leyrlen phial, containing about a square foot of coated surface, was fully charged by about half a revolution of the plates, so as to discharge itself; and by repeated trials it was found that, in one minute's time, this phial discharged itself 76,78 , and frequently even 80 times in a minute. Having thus described the electrical machines of the most upproved construction, it only remains to add some general and practical directions necessary to be observed for the preservation and proper management of them, in making experiments.

Moisture and dust, but particularly the former, being detrimental to the power of an electrical machine, it becomes necessary to guard against both, as much as may be practicable; hence when not actually in use, the machine should be kept in a dry and clean place, and at least the glass part of it should not be suffered to remain dirty and soiled. If it has been long neglected, the operator, in order to render it ready for use, must, in the first place, remove the rubber; he must then place the machine at a moderate distance from the fire, so as to render every part of it perfectly dry, but not too warm. This done, and the dust removed, the glass part must be repeatedly rubbed with a clean and warm handkerchief, or towel; the rubber likewise must be cleaned, removing all the old amalgam that may have adhered to it. The glass plate, or cylinder, in its rotation frequently contracts some dark spots ur concretions on its surface, which tend to diminish its power, and which must therefore be removed. Previously to replacing the rubber, the following operation generally contributes towards increasing the excitation. It consists in touching the cylinder with the bottom of a tallow candle, in streaks parallel to the axis of the cylinder, then rub the cylinder again with a dry warm linen clotb, taking care that this cloth be not very old, for in that case it is apt to leave filaments about the glass and about the rest of the machine. This done, the rubber is fixed in its place, and its supporter being properly adjusted as to pressure, as also a chain suspended from the rubber, connecting it with the floor of the room, then the machine is ready for operation, except the application of the amalgam, in which the following directions nust be observed.

Formerly the amalgam was spread upon the rubber; but experience has shown that it is much better for it to be fixed clean in its place, and then to apply the amalgam upon a picce of leather, to the surface of the cylinder, while this is revolving in its usual direction; for by this means the revolution of the plate or cylinder will carry from the leather a sufficient quantity of the amalgam, and will deposit it upon the rubber. 'The leather with the amalgam need not be applied longer than the cylinder makes 10 or 12 revolutions, moving the leatluer at the same time, backward and forward, from one end of the cylinder to the other, in order that the amalgam may be equally distributed. Now if the cylinder be turned, and a hand or the ends of the fingers be presented to it, a crackling noise, which is accompanied with luminous brushes and dense sparks of the electric fire, will be observed, particularly in a dark room, which appearances indicate that the machine is in good action; then, the prime conductor being situated in its proper place, you may proceed to perform the experiments. During the performance of which, the electrified part of the machine is apt to attract dust from all quarters, which the operator must guard against as much as possible, by frequently wiping those parts with à clean and perfectly dry cloth, or silk handkerchief.

The amalgam which we have mentioned above is generally coinposed of 1 part of zinc, and $4 \mathrm{or} 5$ parts of quicksilver, which are amalganated in the following manner:

Let the quicksilver be heated to about the degree of boiling water; then, the zinc being already melted in a crucible, pour the quicksilver first into a wooden box, and immediately after the melted zinc. Then slut the box, 
and shake it for about half a minute. After this you must wait till the amalgam is quite cold, or nearly so, when you may mix some grease with it by trituration. If the incled zine be poured into the quicksilver when cold, a very small portion of the former will be amalgamated, the r'st remaining in lumps of different sizes.

The above is the method recommended by M. Cavallo, but Mr. Cuthbertson gives the following directions for preparing amalgam. Take one part of tin and zinc, melt them in a crucible, and pour them on two parts of mercury, which put into a wooden box made for the purpose; and shake the box till the metals are cold. The amalgam is then to be pulverised in a metal mortar to a very fine powder, and afterwards mixed with a sufficient quantity of logs-lard, to make it into a paste.

\section{Électural Plial. See Leyden Phial.}

Electrica L Rubber. See Electrical Apparatus, and Electrical Machine.

ELEC Tric.1 Shock, is the sudden explosion between the opposite sides of a charged electric; s:) called because if the discharge be made through the body of an animal, it occasions a sudden motion by the contraction of the muscles through which it passes, accompanied with a disagreeable seusation. 'The force of this shock is proportioned to the quantity of coated surface, the thinness of the glass, and the power of the machine by which it is charged. Its velocity is almost instantaneous, and it bas not been found to take up the least sensible time in passing to the greatest distances.

It has however been observed that the electrical shock is weakened by being communicated through several persons in contact with, one another. Indeed it is obstructed in its passage, even through the best conductors, as it will prefer a shurt passage through the air to a long one through the most perfect conductors; and if the circuit be interrupted, either by electrics, or very imperfect conductors of a moderate thickness, the shock will rend them in its passage, disperse them in every direction, and exhibit the appearance of a sudden expansion of the air about the centre of the shock. A strong shock made to pass through or over the belly of a muscle, forces it to contract; and sent through a small animal body, deprives it instantly of life, and bastens putrefaction. It gives polarity to magnetic needles, reverses their poles, and produces effects precisely similar, though inferior in degree, to those of lightning.

Electrical Star. Sce Star.

ELECTRICITY, or Fiectric $\Delta \mathrm{L}$ Force, is that power or property, which was first observed in amber, the lyneurium, or tourmalin, and which sealing-wax, glass, and a variety of other substances, called clectrics, are now known to possess, of attracting light bodies, when excited by heat or friction; and which is also capable of being communcated in particular circumstances to other bodies.

ELECTRiCity also denotes the science, or that part of natural philosophy, which proposes to investigate the nature and effects of this pawer. From $\gamma_{\gamma} \lambda \varepsilon r \tau e 0 \nu$, the Greek name for amber, is derived the term electricity, which is now very extensively applied, not only to the power of attracting light bodies inherent in amber, but to other similar powers, and their various effects, in whatever bodies they reside, or to whatever bodies they may be communicated. Muschenbroek and Apinus have observed a considerable analogy, in a variety of particulars, between the powers of elcctricity and magnetism; and they have nlso pointed out many iustances in which they differ.
History of ELECTRICITY.-The property which amber pussesses of attracting light bodies, was observed by Thates of Mliletus, 600 years before Christ, who concluded from henee that it was animated. But the first person who expressly mentioned this substance, was Theophrastus, about 300 years before Christ. The attractive property of amber is also occasionally noticed by Pliny, and other naturalists, particularly Gassendus, Sir Kenelm Digby, and Sir Thos. Brown. But it was generally apprehended that this quality was peculiar to amber and jet, and perhaps agate, till Dr. Gilbert, a native of Colchester, and a plysician in London, published his treatise De Magnete, in the year 1600 . Dr. Gilbert made many considerable experiments and discoveries, considering the then infant state of the science. He enlarged the list both of electrics, and of the bodies on which they act; and remarked, that a dry air was most favourable to electrical appearances, while a meist air almost anuihilates the electric virtue: he also observed the conical figure assumed by electrified drops of water. He considered electrical attraction separatcly from repulsion, which he thuught had no place in electricity, as a phenomenon similar to the attraction of cohesion, and tie imagined, that electrics were brought into contact with the bodies on which they act by their effluvia, excited by friction.

The ingenious Mr. Boyle added to the catalogue of electric substances; buc he thought that glass possessed this power in a very low degree: he found; that the electricity of all bodies, in which it might be excited, was increased by wiping and warming them before they were rubbed; that an excited electric was acted on by other bodies as strongly as it acted upon them: that diamonds rubbed aguinst any kind of stuff, emitted light in the dark; and that feathers would cling to the fingers, and to other substances, after they had been attracted by electrics. He accounted fur electrical attraction, by supposing a glutinous effluvia emitted from electrics, which laid hold of small bodies, in its way, and carried them back to the body from which it proceeded.

Otto Guericke, the celebrated inventor of the air-pump, lived about the same time. 'This ingenious philosophe' discovered, by means of a globe of sulphur, that a body once attracted by an electric, was next repelled, and that it continued in this state of repulsion till it was touched by sume other body; he also observed the sound and light produced lyy the excitation of his globe; and that bodies immerged in electrical atmospheres are themselves electrified, with an electricity opposite to that of the atmosphere.

The light emittel by clectrical bodies was, soon after, observed to much greater advantage by $\mathrm{Dr}$. Wall, who ascribes the light to the electrical property which they possess; and he suggests a similarity between the cifects of electricity and lightuing.

Sir Isaac Newton was not inattentive to this subject: he observed that excitcd glass attracted light bodies on the sicte opposite to that on which it was rubbed; and he ascribed the action of electric bodies to an chastic tluid, which freely penetrates glass, and the emission of it to the viluratory motions of the parts of excited bodies.

Mr. llawksbee wrote on this subject in the year 1709 , when a new ara commenced in the history of this science. He first took notice of the great elcctrical power of glass, and the light proceeding from it; though others hat be:fore observed the light proceeding from other electrified 

E L E
[ 463 ]
E L E

substances: he also noted the noise cceasioned by it, with a variety of phenomena relating to electrical attraction and repulsion. He first introduced a glass globe into the electrical apparatus, to which circumstance many of his important cliscoveries may be attributed.

After his time there was an interval of near 20 years in the progress of this science, till Mr. Stephen Grey established a new wa in the bistory of electricity. To him we owe the important discovery of communicating the pover of native electrics to other bodies, in which it cannot be excited, by supporting them on silken lines, hair lines, cakes of resin or glass; aud also a more accurate distinction than had hitherto been made between electrics and non-electrics: he likewise showed the effect of electricity on water much inore obviously than Gilbert had done in the infancy of this science.

The experiments of Mr. Grey were repeated by M. du Fay, member of the Academy of Sciences at Paris, to which he added many new experiments and discoveries of his ow I. He observed, that electrical operations are obstructed by great heat, as well as by a moist air; that all bodies, both solicl and fluid, would rcceive electricity, when placed on warm or dry glass, or sealing-wax; that those bodics which are naturally the least electric, have the greatest degree of electricity communicated to them by the approach of the excited tube. He transmitted the electric virtue through a distance of 1256 feet; and tirst observed the electric spark from a living body, suspended on silken lines, and noted scveral circumstances attending it. M. du Fay also established a principle, first suggested by Otto Guericke, that electric bodies attract all those that are not so, and repel them as soon as they are become electric, by the vicinity or contact of the electric body. He likewise inferred from other experiments, that there were two kinds of electricity; one of which he called the vitreous, belonging to glass, rock crystal, \&rc; and the other resinous, as that of amber, gum-lac, \&ce, which are distinguished by their repelling those of the same kind, and attracting cach other. He farther observed, that communicated electricity had the same property as the excited; and that electric substances attract the dew more than conductors.

Mr. Grey, resuming his experiments in 1734, suspended several pieces of metal on silken lines, and found that by rlectrifying them they gave sparks; which was the origin of metallic conductors: and on this occasion be discovered a cune or pencil of electric light, such as is now known to issue from an electrified point. And from other experiments he coscluded that the electric power seems to be of the same nature with that of thunder and lightning.

Dr. Desaguliers succceded Mr. Grey in the prosecution of this branch of philosophy. The accuunt of his first experiments is dated in 1739 , and to him we owe those technical teruns of conductors or non-electrics, and electrics per se; he also first ranked pure air among the electrics per se, and supposed its electricity to be of the vitreous kind.

After the year 1742, in which Dr. Desaguliers concluded his experiments, the subject was taken up and pursued in Germany: the globe was substituted for the tube, which hat been used ever since the time of Hawksbee, and a cushion was soon after used as a rubber, instead of the hand; it was also about this time that cylinders bewan to be used instead of the globes; and some of the German electricians inade use of many globes at the same time. By thus increasing the electrical power, they were the first who slicceded in scting fire to inflammable sub- stances: which was first done by Dr. Ludolf, in the beginning of the year 1744 , who, with sparks excited by the friction of a glass tube, kindled the ethereal spirit of Frobenius. Winkler did the same by a spark from his own finger, by which he kindled French brandy, and otlier spirits, after previously heating them. Mr. Gralath firced the smoke of a candle just blown out, and so lighted it again; and Mr. Boze fired gun-powder, by means of its inflammable vapour. It was also at this time Ludolf the younger demonstrated, that the luminous barometer w'as made perfectly electrical by the motion of the quicksilver. The electrical star and electrical bells were also of German invention.

From this period Dr. Watson considerably distinguished himself in the pursuit of this science; he fired a variety of substances by the electrical spark, and first discovered that they are capable of being fired by the repulsive power of electricity. In the year 1745 , the accumulation of the electrical power in glass, by means of the Leyden phial, was first discovered. See Lex Den Plial: and for the method practised about this time, of measuring the distance to which the electrical shock may be conveyed, see Electrical Circuit. Dr. Watson discovered that the glass tubes and globes do not contain the electric matter in themselves, but only serve as first-movers or determiners, as he expresses it, of that power; which was also confirmed towards the end of 1746 , by Mr. Benjamin Wilson, who made the same discovery, that the electric fluid does not come from the globe, but from the earth, and other nonelectric bodies about the apparatus. Dr. Wátson also discovered what Dr. Franklin observed about the same time in America, and called the plus and minus electricity. He likewise shored that the electric matter passed through the substance of the metal of communication, and not mercly over the surface. The history of medical electricity commencesl in the year 1747 ; but we are under the necessity of omitting many experiments, and conclusions drawn from them, by Mr. Wilson, Mr. Smeaton, and Dr. Miles in England, and by the Abbé Nollet, with regard to the effect of elcctricity on the evaporation of fuids, on solids, and on animal and other organized bodics, in France.

While the philosophers of Europe were busily employed in clectrical experiments and pursuits, those of Amcrica, and Dr. Franklin in particular, were equally industrious, and no less successful. 1lis discoveries and observations in electricity were comnumicated in several letters to a friend; the first of which is dated in 174.7 , and the last in 1754 ; the particulars of his system may be seen under the articles, Theory of Lisectricity, Ley dex Phial, Points, Cirarging, Conductors, Electimics, \&c.

The similarity between electricity and lightning had been suggested by sereral writers: and Dr. Franklin first proposed a method of bringing the matter to the test of experiment, by raising an electrical kite; by which means be succecded in collecting electrical fire from the clouds, in 1752 , one month after the same theory had had been verified in France, and without knowing what had bcen done there: and to him we owe the practical application of this discovery, in securing buildings from the damage of lightning, by erecting metallic conductors. Sue CoNDUCTORS, and LIGITNING.

In the subscquent period of the history of electricity, Mr. Canton in England, and Signior Beccaria in Italy, acquired distinguished reputation. 'They both discovered, 
independently of each other, that air is capable of receiving electricity by communication, and of retaining it when received. Mr. Canton also, towards the latter end of the year 1753, pursued a series of experiments, which proved that the appearances of positive and negative electricity, which had hitherto been deemed essential and unchangeable properties of different substances, as of glass and scaling-wax for instance, depend on the surface of the electrics, and that of the rubber. This hypothesis, verified by $1 \mathrm{u}$ merous cxperiments, occasioned a controversy between Mr. Canton and Mr. Delaval, who still maintained that these different powers depended entirely on the substances themselves. About this time also some curious experiments were performcd by four of the principal electricians of that period, viz. Dr. Franklin, and Messrs. Canton, Vilcke, and Epinus, to ascertain the nature of electric atmospheres; the result of which see under that article. The theory of two electric fluids, always co-existent and counteracting each other, though not absolutely independent, was maintained by a course of experiments on silk stockings of different colours, communicated to the Royal Society by Mr. Symmer, in the year 1759 , and which were farther pursued by Mr. Cigna of Turin, who published an account of them in the Memoirs of the Academy at Turin for the year 1765 .

Many instances occur in the history of the science about this time, of the astonishing force of the clectric shock, in melting wires, and producing other similar effects: but the most remarkable is an experiment of $S$. Beccaria, in which he thus revivified metals. Several experiments were also made by Dr. Watson, Mr. Smeaton, Mr. Canton, and others, on the passage of the electric fluid through a vacuum, and its luminous appearance, and on the power possessed by certain substances of retaining the light communicated to them by an electric explosion. Mr. Canton, S. Beccaria, and others, made many experiments to identify electricity and lightning, to ascertain the state of the atmosphere at different times, and to explain the various phenomena of the aurora borealis, water-spouts, hurricanes, \&c. on the principles of this science.

Those who are desirous of farther information with respect to the history of electrical experiments and discoveries, may consult Dr. Priestiey's History and Present State of Electricity. This author however, is not merely an historian : his work contains many original experiments and discoveries made by himself. He ascertained the conducting power of charcoal and of hot glass, the electricity of fixed and inflanmable air, and of oil; the difforence between new and old glass, with respect to the diffusion of electricity over its surface; the lateral explosion in electrical discharges; a new method of fixing circulal-coloured spots on the surfaces of metals, and the most probable difference between electrics and conductors, ac. The science is also greatly indebted to many other persons, cither for their experiments and improvements in it, or for treatises and other writings upon it ; as Mr. Henley, to whom we owe several curious experiments and observations on the electrical and conducting quality of different substances, as chocolate, vapour, \&c, with the reason of the difference between them; the fusion of platina; the nature of the electric fluid, and its course in at discharge; the method of estimating the quantity of it in electrical bodies by an electrometer; the infuence of points ; \&c, \&c. Also Mcssrs. Van Maruin, Van Swinden, Ferguson, Cavallo, Lord Mahon, Nairne, \&c, \&c, for their several treatises on the subject of electricity, any of which may be consulted with adrantage for the experiments and principles of the science.

Medical Electricsty. It is natural to imagine that a power of such efficacy as that of electricity would soon be applied to medical purposes; especially, since it has been found iuvariably to increase the sensible perspiration, to quicken the circulation of the blood, and to promote the glandular secretion : accordingly, many instances accur in the latter period of the history of this science, in which it has been applied with considerable advantage and success. And among the variety of cases in which it has been tried, there are none where it has been found prejudicial, except those of pregnancy and the vencreal disease. In must disorders, in which it has been used with perseverance, it has given at least a temporary and partial relief, and in many it has effected a complete cure. $\mathrm{Nu}$ merous instances of this kind may be seen in the Philos. Trans., and the writings on this science by Messrs. Lovet, Westley, Firguson, Cavallo, \&c, \&c.

Theory of ELECTRICITY. It is hardly necessary to recite the ancient hypotheses on this subject; such as that of the sympathetic powicr of the Peripatetics; that of unctuous effuria emitted by excited bodies, and returning to them again, adopted by Gilbert, Gassendus, Sir Kenelm Digby, \&c; or that of the Cartesians, who ascribed electricity to the globules of the first elements, discliarged through the pores of the rubbed substance, and in their return carrying with them those light bodies, in whose pores they were entangled: these hypotheses were framed in the infancy, not only of this science, but of philosophy in general, and luve long since been deservedly exploded. In the more advanced state of electricity there have been two principal theories, each of which has had its advocates. The onc, is that of two distinct electric fluids, repulsive with respect to themselves, and attractive of one another, adopted by M. du Fay, on discovering the two opposite species of electricity, viz, the vitreous and resinous, and since new-modelled by Mr. Symmer. It is supposed that these two fluids are equally attracted by all bodies, and exist in intimate union in their pores; and that in this state they exhibit no mark of their cxistence. But that the friction of an electric by a rubber separates these fluids, and causes the vitreous elcetricity of the rubber to pass to the ciectric, and then to the prime conductor of a machine, while the resinous elcctricity of the conductor and clectric is conveyed to the rubber: and thus the quality of the electric fluid, possessed by the conductor and the rubber, is changed, while the quantity remains the same in each. In this state of separation, the two clectric fluids will exert their respective powers; and any number of bodies charged with either of then will repel cach other, attract those bodies that have less of each particular fuid than themselves, and be still more attracted by bodies that are wholly destitute of it, or that are overcharged with the contrary. According to this theory, the electric spark makes a double current; one fluid passing to an electrified conductor from any substance presented to it, while the same quantity of the other thid passes from it; and when each body recoives its untural quantity of both fluids, the balance of the two powers is restored, and both bodies are unelectrified. For a farther account of the explicatiot of some of the principal phenomena of electricity by this theory, sce Dr. Priestley's History, vol. $2, \S 3$. 


\section{E L E}

The other theory is commonly distinguished under the denomination of positize and negative electricity, being first suggested by Dr. Watson, but digested, illustrated, and confirmed by Dr. Franklin; and since that it has been known by the appellation of the Franklinian hypothesis. It is here supposed that all the phenomena of electricity depend on one fluid, sui generis, extremely subtile and elastic, dispersed through the pores of all bodies, by which the particles of it are as strungly attracted as they are repelled by one another. When bodies possess their natnral share of this fluid, or such a quantity as they can retain by their non-attraction, it is then said they are in an unelectrified state; but when the equilibrium is clisturbed, and they either acquire an additional quantity from other bodies, or lose part of their own natural share by communication to other bodies, they exhibit electrical appearances. In the former case it is said they are electrified positively, or plus; and in the other negatirely, or minus. This electric fluid, it is supposed, moves with great ease in those bodies that are called conductors, but with extreme difficulty and slowness in the pores of electrics; whence it happens, that all electrics are impermeable to it. It is further supposed that electrics contain always an equal quantity of this fluid, so that there can be nu surcharge or increase on one side, without a proportionate decrease or loss on the other, and vice versa; and as the electric does not admit the passage of the fluid through its pores, there will be an accumulation on one side, and a corresponding deficiency on the other. Thus when both sides are connected together by proper conductors, the equilibrium will be restored by the rushing of the reduudant fluid from the overcharged surface to the exhausted one. Thus also, if an electric be rubbed by a conducting substance, the electricity is only conveyed from one to the other, the one giving what the other receives; and if one be electrified positively, the other will be electrified negatively, unless the loss be supplied by other bodies connected with it, as in the case of the clectric and insulated rubber of a machine. 'This theory serves likewise to illustrate the other pienomena and operations in the scicnce of electricity. Thus, bodies differently electrified will naturally attract each other, till they mutually give and receive an equal quantity of the electric fluid, and the equilibrium is restored between then. Beccaria supposes, that this effect is produced by the electric matter making a vacuum in its passage, and the contiguous air afterwards collapsing, and so pushing the bodies together.

'l'he influence of points, in drawing or throwing off the electric fluid, depends on the less resistance it finds to $\mathrm{cm}$ ter or pass off through fewer particles than through a greater number, whose resistance is united in flat or round surfaces. The clectric light is supposed to be part of the electric lluid, which appears when it is properly agitated; and the sound of an explosion is produced by vibrations, occisioned by the air's being displaced by the chectric fluid, and agaiu suddenly collapsing.

As to the nature of the clectric fluid, philosophers have entertained very diflerent sentiments: some, and among them Mr. Wilvon, have supposed that it is the same with the ether of Sil Isatac Newton, to which the phenomena of attraction and repulsion are ascribed; while the light, smetl, and other sensible qualities of it, are referred to the grosser partictes of bodies, driven from them by the forcible actuon of this ether; and other appearances are ex-

VOL. 1,
E L E

plained by means of a subtile medium diffused over the surfaces of all bodies, and resisting the entrance and exit of the ether; which medium, it is supposed, is the same with the electric fluid, and is more rare on the surfaces of conductors, and more dense and resisting on those of clectrics: but Dr. Priestley remarks that, though they may possess some common properties, they have uthers cosentially distinct; the ether is repelled by all other matter, whereas the electric fluid is strongly. attracted by it. Others have had recourse to the element of fire; and from the supposed iclentity of fire and the electric finid, as well as from the sinilarity of some of their effects, the latter has been usually called tlie electric fire: but mose electricians have supposed that it is a fluid sui generis. Mr. Cavendish has published an attempt to deduce and explain some of the principal phenomena of electricity in a mathematical and systematic mauner, from the nature of this ftuid, considered as composed of particles that repel each other, and attract the particles of all other matter, with a force inversely as some less power of the dstance than the cube, while the particles of all other matter repel each other, and attract those uf the electric fuid, according to the same law. Philos. Trans, vol. 61 , pa. 581 -667. And a similar hypothesis and method of reasuning was also proposed by M. Epinus, in his Tentamen Theoria Electricitatis et Mlagnetismi.

Dr. Priestley concludes, from experiments which lie made, that the electric matter either is phlogiston, or con:tains it, since he found that both produced similar effects. And Mr. Henley also apprehends, that the electric fluid is a modification of that element, which, in its quieseent state, is called phlogiston; in its first active state, electricity; and whon violently agitated, fire. Perhaps we may be allowed to cularge our views, and consiler the sun as the fountain of the electric fluid, and the zodiasal light, the tails of comets, the aurora borealis, lightning, and artificial electricity; as its various and not very dissimilar modifications. On this subject, see Priestley's Hist. of Llectr. vol. 2, part $3, \S 1,2,3$; Wilson's Lissay turards an Explication of the Phenomena of Electricity, \&c; Wilson and Huadley's Obs. \&c, pa. 55, 1759; Freke's Lissity on the Cause of Electricity, $17+6$; Priesticy on Air, vol. 3 , pa. 1\$6,274, \&e; Philus. Trans vol. 67, pa. 129; liales's Letters, on the same subject; and Cavallo's Lilectricity.

ELECTROMETLR, is an instrument that measures the quantity, and deternines the quality of electricity, in any electrified bokly. Previous to the invention of instruments of this kind, Mr. Canton estimated the quantity of slictricity in a charged phial, by presenting the phial with one liand to an insulated conductor, and giving it a spark, which he touk off with the other; proceeding in this manner till the plial was discharged, whom he decrmined the height of the clatrge by the number of spanks. Ekectormeters ąre of vartious kind : an 1, the single thread ; "?, the cork or pith balls; 3 , the qundrant; and de, the discharging electrometer, de.

The lst, or most simple. electrometer, is a linen threat? called by 1)r. Desagulicis, the thered of trial; which, being brought nealr an electrified budy, is attracted by $\|$ : but this deses lintle more than determine whether the borly is in any degres dectrificd or not; withont determining with any precision its quantity, much less the quality of it. 'The Abbe Nollet used two threads, showing the degres of electricity by the anghe of their disergency exhe bited in their shatow un a boatal placed behind them.

30 
Yr. Canton's electrometer consisted of two balls of cork, or pith of elder, about the size of a small pea, suspended by fine linen threads, about 6 inches long, which may be wetted in a weak solution of salt. (Sce fig. 7.) If the box containing these balls be insulated, by placing it on a drinking-glass, \&c, and an excited smooth glass tule be brought near them, they will first be attracted by it, and then be both repelled, from the glass, and from each other; but if excited wax be applied to them, they will gradually approach and come together; and vice versa. This apparatus will also serve to determine the electricity of the clouds and air, by holding them at a sufficient distance from buildings, trees, \&c; for if the electricity of the clouds or air be positive, their mutual repulsion will increase by the approach of excited glass, or decrease by the approach of amber or sealing-wax; and on the contrary, if it be negative, their repulsion will be diminished by the former, and increased by the latter. Sce Philos. Transact. vol. 48, part 1 and 2, for an account of Mr. Canton's curious experiments with this apparatus. If two balls of this kind be annexed to a prime conductor, they will serve to determine both the degree and quality of its electrification, by their mutual repulsion and divergency.

The Discharging Electrometer (fig. 3, plate xi.) was invented by $\mathrm{M} \mathrm{r}$. Lane. It consists of brass work $\mathrm{G}$, the lower part of which is enclosed in the pillar $\mathbf{F}$, made of bakcd wood, and boiled in linseed oil, and bored cylindrically about two-thirds of its length; the brass work is fixed to the pillar by the screw $\mathrm{n}$, moveable in the groove $I$; and through the same is made to pass a steel screw $x$, to the end of which, and opposite to $\mathrm{K}$, which is a polished hemispherical piece of brass, attached to the prime conductor, is fixed a batl of brass $\mathrm{m}$, well polished; and to this screw is annexed a circular plate o, divided into 12 equal parts. The use of this electrometer is to discharge a jar $\mathrm{D}$, or any battery connected with the conductor, without a discharging rod, and to give shocks successively of the same degice of strength; on which account it is very fit for medical purposes. Now, if a person holds a wire fastened to the screw $H$ in one hand, and another wire fixed to $E$, which is a loop of brass wire passing from the frame of the machine to a tin-plate, on which the phial D stands, be will perceive no shock, while $\mathrm{K}$ and $\mathrm{M}$ are in contact; and the degrec of the explosion, as well as the quantity of electricity accumulated in the phial, will be regulated by the distance between $K$ and $M$. Philos. Trans. vol. 57, pa. 451 . - Mr. Henley much improved Mr. Lane's electrometer, by taking away the screw, the double-milled nut, and the sharp-edged graduated plate, and adding other things in their stead. Mr. Henley's discharger of this kind has two tubes, one sliding within the other, to lengthen and accommodate it to larger apparatus.

The Quadrant Electrometer of Mr. Henley, consists of a stem, terminating at its lower (nd with a brass ferrule and screw, for fastening it on any occasion; and its upber part ends in a ball. Near the top is fixed a graduated semicircle of ivory, on the centre of which the index, being a very light rod with a cork ball at its extremity, reaching to the brass ferrule of the stem, is made to turn on a pin in the brass piece, so as to keep near the graduated limb of the semicircle. When the electrometer is not electrified, the index hangs parallel to the stem; but as soon as it begins to be electrified, the inclex, repelled by the stem, begins to move along the graduated edge of the semicircle, and so mark the degree to which the conductor is electrified, or the height to which the clrarge of any jar or battery is advanced. (See fig. 8.)

Ii. Cavallo also contrived several ingenious electrometers, for different uses; as may be seen in his Treatise on Eleetricity, pa. 370, \&c, and in the Philos, Trans. vol.67, pa. 4.8 and 399.

ELECIROPHOR, or FLECTROPIORUS, an instrument for showing perpetual electricity; which was invented by $M$. Volta, of Como, near Milan, in Italy. The machine consists of two platès, fig. 9, one of which $\mathrm{B}$ is a circular plate of glass, covered on either side with some resinous clectric, and the other $A$ is a plate of brass, or a circular board, coated with tin-foil, and furnished with a glass handle s, which may be screwed into its centre by means of a sockct. Now, if the plate B be excited by rubbing it witb new white flannel, and the plate $A$ be applied to its coated side, a finger, or any other conductor, will reccive a spark on touching this plate; and if the plate $\mathrm{A}$ be then scparated, by means of the handle 1 , it will be found strongly clectrified, with an electricity contrary to that of the plate $\mathbf{B}$. Then replacing the plate $A$, touching it with the finger, and scparating it again, it will be found electrificd as before, and give a spark to any conductor, attended with a snapping noise; and by this means a coated phial may be charged. The same phenomena may repeatedly be exhibited, without any renewed excitation of the electric plate $B$; the electric power of $B$ having continned for several days, and cren weeks, after excitation; though there is no reason to imagine that it is perpetual.

M. Cavallo prepares this machine by coating the glass plate with sealing-wax; and the late Mr. Adams, philosophical-instrument-maker, prepared them with plates formed from a composition of Iwo parts of shcli-lac, and one of Venice turpentine, without any glass. The action of this plate depends on a principle discovered and illustrated by the experiments of Franklin, Canton, Wilcke, and IEpinus, viz, that an excited clectric repels the electricity of another body, brought within its sphere of action, and gives it a contrary electricity. Thus the plate $\boldsymbol{A}$, touched by a conductor, while in contact with the plate $\mathrm{B}$, which is negatively electrified, it will acquire an additional quantity of the electric fluid from the conductor; bnt if it were in contact with a plate electrified positively, it would part with its clectricity to the conductor connected with it. See an account of several curious experiments with this machine, by Mr. Henley, M. Cavallo, and Dr. Ingenhousz, in the Philos. Traus. vol. 66, pa. 513; rol. 67, pa. 116 and 389 ; and vol. 68 , pa. 1027 and 1049 .

ELEMENTARY, something that relates to the principles or elements of bodies, or sciences; as Elementary Air, Fire, Geometry, Music, \&c.

ELEMENTS, the first principles of which all bodies and things are composed. These are supposed few in number, unchangcable, and by their combinations producing that extensive variety of objects to be met with in the works of nature. Democritus stands at the heal of the elcmentary philosophers, in which he is followed by Epicurus, aus many otbers after them, of the Ipicurean and corpuscular philosophers.

Among those who consider matter, or the elements, as corruptible, some assert that there is only one clement in nature, while others maintain that there are several. Of the former, the principalare Heraclitus, who considers tire 


\section{E L E}

as this element; Anaximenes asserts that it is air; Thales Milesius says water, and Hesiod eartl. Hesiod is folluweil by Bernardin, Telesius; and Thales by many of the chemists.

Among those who admit several corruptible elements, the principal are the Peripatetics; who, after their leader Aristotle, contend for futir elcments, viz, fire, air, water, and earth. Aristotle tuok the notion frum Hippocrates; Hippocrates from Pythagoras; and Pythagoras from Ocellus Lucanus, who it seems was the first author of it, or from the orientals.

The nodern chcmists, however, dn not acknowledge any particular number of elements, but freely admit into this class all substances that have not been decomposed. An element is therefore merely one of the last results of chemical analysis; and in denominating substances elementary or simple, we consider them as such only with regard to the present state of chemical science. It is not probable any one of the simplest bodies with which we are acquainted, is really and essentially elementary, and it is at best needless to enter into an enquiry in which there are no facts to rest on for support.

ELEMENTS, a term also used for the first grounds and principles of arts and sciences; as the Elements of geometry, Elements of mathematies, \&c. So Fuclicl's Elements, or simply the Llements, as they were anciently and peculiarly named, denotes the treatise on the chicf properties of geometrical figures by that author.

The ELEMENTS of mathematics have been delivered by several authors in their courses, systems, \&c. The first work of this kind is that of Herigon, in Latin and French, and published in 1634 , in 5 tomes; which contains Euclid's Elements and Data, Apollonius, Theodosius, \&c; with: the modern elements of arithmetic, algebra, trigonometry, architecture, geography, navigation, optics, spherics, astronomy, music, perspective, \&c. The work is remarkable for this, that a kind of real and universal characters are used throughout; so that the demonstrations may be understood by such as only remember the characters, without any dependence ou language or words at all.

Since Herigon, the elements of the several parts of mathematics have been also delivered by many others; particularly the Jesuit Schottus, in his Cursus Mathematicus, in 1674; De Chales, in his Cursus, $167+$; Sir Jonas Noorc, in his New System of Mathematics, in 1681; Ozanam, in his Cours de Mathematique, in 1699; Jones, in his Synopsis Palmariorum Matheseos, in 1706; and many others, but above all, Christ. Wolfius, or Wolf, in luis Elementa Matheseos Universæ, in 2 vols 4 , the 1st published in 1713 , and the $2 \mathrm{~d}$ in 1715 ; a very excellent work of the kind. Another edition of the work was published at Geneva, in 5 vols 4 to, of the several dates $1732,1733,1735,1738$, and 1741 .

The Ezerrex's of Euclid, as they were the first, so they still continue to be the best systen of geometry yet extant. They are contained in $\mathbf{1 5}$ books, of which numerous editions and commentaries have been published. Proclus wrote a cominentary on this work, and Orontius Fineus gave a printed edition of the first 6 buoks, in 1530, with notes, to explain Euclid's sense. Peletarius did the same in 1557. Nic. Tartaglia also, about the same time, madie a comment on all the 15 books, with the addition of malyy things of his own. And the same was also done by Billingsley in 1570; and by Flussates Candalla, a
E L E

French nobleman, in the year 1578 , with consicterible additions as to the comparison and iuscriptions of solid bodies; which work was afterwards republished with a prolix commentary, by Clavius. But the first who translated them into Latin, while they were yet unknown to the European nations, was Adhelard, or Adelard, a learued Englishman, who performed this task in the 12 th century. Commandine also gave a good edition of this work, and in $1703 \mathrm{Dr}$. Gregóry published an edition of the whole works of Euclid, in Greek and Latin. But it would be endless to notice all the editions of this celebrated performance that have been given; some of the best of these however are those of Tacquet, Ozanam, Whiston, Stone, Playfair, Ingram, and more particularly that of Dr. Rob. Simson, of Glasgow.

Beside these there are several other writers on geometry, who bave not followed Euclid strictly either in form or arrangement; as it is thought by some, that in the present state of marhematical knowledge, a system of geometry less tedious and prolix that Euclid's would be a considerable acquisition.

ELEMENTs, in the Higher or Sublime Geometry, are the infinitely small parts, or differentials, of a right line, curve, surface, or solid.

ErEuENT, in Astronomy, are those principles deduced from astronomical observations and calculations, and such fundamental numbers, as are employed in the construction of tables exhibiting the planetary motions. Thus, the elements of the theory of the sun, or rather of the earth, show its mean motion and excentricity, with the motion of the iphelia. And the elements of the theory of the moon, relate to lier mean motion, that of the nude and apogee, the excentricity, the inclination of her orbit to the plane of the ecliptic; \&c. For the particulars of which see the respective names above mentioned.

FLEVATION, the height or altitude of any object.

Exevation, in Architecture, denotes a draught or description of the principal front or side of a building; called also its upright or orthography.

Exte ation, in Astronomy and Geography, is various; as elevation of the equator, of the pole, of a star, \&c.

Elevation of the Equator, is the height of the equator above the horizon; or an arc of the meridian intercepted between the equator and the horizon of the place.-The elevation of the equator and of the pole together always make up a guadrant; the one being the complement of the other. Therefore, the elevation of the pole being found, and subtracted from $90^{\circ}$, leaves the elevation of the equator.

ExEvatios of the Pole, is its height above the horizon; or that arc of the meridian which is comprehended between the equator and the horizon of the place. The elevation of the pole is always equal to the latitude of the place; that is, the arc of the meridian intercepted between the pole and the horizon, is every where equal to the arc of the same meridian intercepted between the equator and the zenith. Thus the north pole is elevated $51^{\circ} 31^{\prime}$ above the horizon of London; and the distance, or number of degrees, is the same between London and the equator; so that London is also in $51^{\circ} 31^{\prime}$ of north latitude.

ELEVATion of a Star, or of any other point in the sphere, is the angular height above the horizon; or an arc of the vertical circle intercepted between the star and 302 
E L L

the horizon. The meridian altitude of any such point, or its altitude when in the meridian, is the greatest possible.

VIE A TIOS of a Cannon, or Mortar, is the angle which the bore or the axis of the picce makes with the horizuntal plane.

Angle of ELEVATIOX, is the angle which any line of direction makes above a borizontal line.

Encvatiox is also used by some writers ou Perspecive, fol the scenography, or perspective representation wt the whole body or building.

ELIAINATION, in Algebra, is that operation by "hich any number $n$ of equations, containing $n$ unknown quantities, being given, we find one equation which inwhes only one urknown quantity; so that by resolving this equation, we obtain the value of that unknown quantity, and retracing our steps back again, we obtain ilso the values of all the other quantities, which, in the course of the operation, are found to have certain relations with, or dependence on each other. See ExterMIINATIO N.

ELLIPSE, or ELLIPSis, is one of the conic sections, popularly called an oval: it was first called an Ellipse or Ellipsis by Apollonius, the first and principal author on the conic sections, hecause in this figure the squares of the ordinates are less than, or defective of, the rectangles under the parameters and abscisses.

This figure is differently defined by different authors; either from some of its properties, or from nuechanical construction, or fiom the section of a cone, which is the best and most natural way. Thus;

Fig. 1.

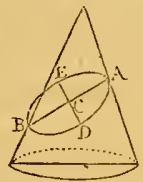

Fig. 2.

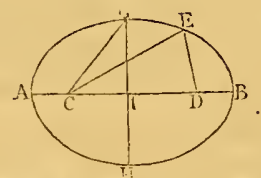

1. An ellipse is a plane figure made by cutting a cone by a plane obliquely through the opposite sides of it; or so as that the plane makes a less angle with the base than the side of the cone makes with it; as $A$ BD fig. 1 . The line AB connecting the upper and lower points of the section, is the transverse axis; the middle of it $\mathrm{c}$, is the centre; and the perpendicular to it DCE, through the centre, is the conjugate axis. The parameter, or latus rectum, is a $3 \mathrm{~d}$ proportional to the transverse and conjugate axes; and the foci are two points in the transverse axis, at such equal distances from the centre, that the duuble ordinates passing through those points, and perpendicular to the transverse, are equal to the parameter.

2. The ellipse is also variously described from some of its properties; the principal of which are as follow: That if two lines be drawn from two certain points $c$ and $D$ in the axis, fig. 2, to any point $\mathrm{E}$ in the circumference, the sum of those two lines $\mathbf{C E}$ and $\mathrm{nE}$, will be every where equal to the same constant quantity, viz, to the axis $\mathrm{A} B$. Fig. 3.
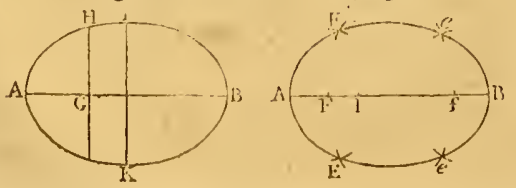

E L L

And, secondly, that the rectangle $A G \times G B$ (fig. 3) of the abscisses, tending contrary ways, is to $\mathrm{GII}^{\circ}$ the square of the ordinate, as $\mathrm{A} \mathrm{B}^{2}$ to $\mathrm{IK}^{2}$, the square of the transverse axis to the square of the conjugate, or, which is the same thing, as the transverse axis is to the parameter; beside various other properties.

3. The ellipse is aiso rariously described from mechanical constructions, which also depend on some of its peculiar properties. 1st, If in the axis $\mathrm{A} \mathrm{B}$, there be taken any point $I$ (fig. 4); and with the radii $A I, B 1$, and cenrres $F$ and $f$, the two foci, arcs be described, these arcs will intersect in certain points $\mathrm{E}, \mathrm{E}, c, e$, which will be in the curve or circumference of the figure: and thus several points I being taken in the axis $\mathrm{AB}$, as many more points, $\mathrm{E}, e, \& \mathrm{c}$, will be found then the curve line drawn through all these points $\mathrm{E} ; c$, will be an ellipse. And if there be taken a thread of the exact lengtl of the transverse axis $A B$, and the ends of the thread be fixed by pins in the two foci $F$ and $f$ (fig. 5); then moving a pen or pencil within the thread, so as to keep it always strutched, it will describe an ellipse. Or it may be described from the property of the directrix.

Fig. 5 .

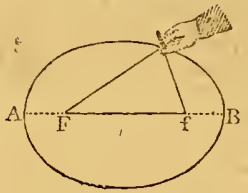
To construct an Exirpse. There are many other ways of describing or constructing an ellipse, besides those already mentioned.

1st. If upon the given transverse axis there be described a circle AGB (fig.6), to which there be drawn any ordinate $D G$, and $D E$ be taken a 4 th proportional to the transverse, the conjugate, and the ordinate $D G$; then $E$ is a point in the curve. Or if the circle $a g b$ be described on the conjugate axis $a b$, to which any ordinate $d g$ is drawn, in which taking $d_{\mathrm{E}}$ in like manner a 4 th proportional to the conjugate, the transverse, and ordinate $d g$, then shall $\mathbf{E}$ be a point in the curve. Or, having described the two circles, and drawn the common radius cgG cutting them in $\mathrm{G}$ and $g$; and then $d g \mathrm{E}$ being drawn parallel to the transverse, and DeE parallel to the conjugate, the intersection $E$ of these two lines will be in the curve of the ellipse. And thus several points $\mathrm{E}$ being found, the curve may be drawn through them all with a steady hand.

Fig. 7 .
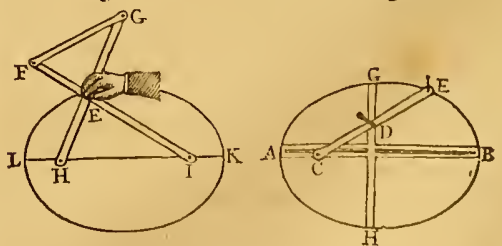

2. Or take three rulers, of which the two gir and $y x$ (fig. 7 ) are of the length of the transverse axis LK, and the third ra equal to 111 the distance between the foci; then conuccting these rulers so as to be moveable about the foci $s$ and $I$, and about the points $F$ and $G$, their in- 
E L L .

tersection $\mathrm{E}$ will always be in the curve of the ellipse; so that by moving the rulers about the joints, with a pencil passed through the slits made in them, it will trace out the ellipse $\mathrm{L} \mathrm{K}$.
Fig. 9.

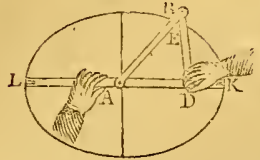

Fig. 10.

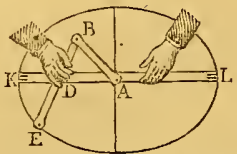

3. If one end $A$ of any two equal rulers $A B, D B$, (fig. 9 and 10), which are moveable about the point $B$, like a carpenter's joint-rule, be fastened to the ruler $\mathrm{LK}$, so as to be moveable ahout the point $A$; and if the end $D$ of the ruler $D B$ be drawn along the side of the ruler $I, \mathbf{K}$, then any point $\mathrm{x}$, taken in the side of the ruler $\mathrm{DB}$, will describe an ellipse, whose centre is $\mathrm{A}$, conjugate axis $2 \mathrm{DE}$, and transterse axis $2 \mathrm{AB}+2 \mathrm{BE}$.

Another method of description is by the elliptical compass. See that article, below.

The most Remarkable Properties of the Eltipsc, are as follow:-1. The rectangles under the abscisses are proportional to the squares of their ordinates; or as the square of any axis, or any diameter, is to the square of its conjugate, so is the rectangle under two abscisses of the former, to the square of their ordinate parallel to the latter; or again, as any dianeter is to its parameter, so is the said rectangle under two abscisses of that diameter, to the square of their ordinate. So that if $d$ be any diameter, $c$ its conjugate, $p$ its parameter, so that $p=c^{2} \div d, x$ the one absciss, $d=x$ the uther, and $y$ the ordinate; then, as $d^{2}: c^{2}:: x(d-x): y^{2}$, or $d^{2} y^{2}=c^{2} x(d-x)$; or as $d: p:: x(d-x): y^{2}$, or $d y^{2}=p x(d-x)$.

From either of which equations, called the equation of the curve, any one of the quantities may be found, when the other three are given.

2. The sum of tivo lines drawn from the foci to meet in any point of the curve, is always equal to the transverse axis; that is, $C E+D E=A B$, in the $2 \mathrm{~d}$ fig. Consequently the line $c \mathrm{G}$ slrawn from the focus to the end of the conjugate axis, is equal to $\Lambda \mathrm{I}$ the semi-transverse.

3. If from any point of the curve, there be drawn an ordinate to either axis, and also a tangent meeting the axis produced; then half that axis will be a meau proportional

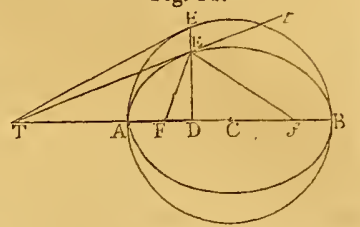
between the distances from the centre to the two points of intersection; viz, $\mathrm{cA}$ a mean proportional between $\mathrm{CD}$ and $\mathrm{CT}$; and $\mathrm{crnsequently}$ all the tangents TE, TE, meet in the same yoint of the axis produced, whicl are drawn from the extremilies $E, E$, of the common ordinates $D E$, $D E$, of all ellipses described on the same axis $\triangle \mathrm{B}$.

4. Two line's ctrawn from the foci to any point of the curve, make equal angles with the tangent at that point: that is, the $\angle \mathrm{FET}=\angle f \mathrm{~T}=$. And hence a line drawn to bisect the angle $\mathrm{F} F f$, will be perpendicular to the tangent and curve at the point $\mathrm{t}$.

5. All parallelograins that are formed by tangents drawn
E L L

through the cxtremities of any diameter and its conjugate, and thus circumscribed about an ellipse, are equal to each other; and every such parallelogram is cqual to the rectangle of the two axes.

6 . The sum of the squares of every pair of conjugate diameters, is equal to the same constant quantity, viz, the sum of the squares of the two axes.

7. If a circle be described upon either axis, and from any point in that axis an ordinate be drawn both to the circle and to the ellipsis; then will the ordinate of the circle be to the ordinate, of the ellipse, as that axis is to the other axis: viz,

A $\mathrm{B}: a b::$ DG : DE, (in the 6th fig.)

and $a b: A$ B : : $d g: d \mathbf{E}$.

And in the same proportion is the area of the circle to the arca of, the ellipse, or any corresponding segments $A D G$, ADE. Also the area of the ellipse is a mean proportional betwcen the areas of the inscribed and circumscribed circles. Hence therefore,

8. To find the area of an Ellipse. Multiply the two axes together, and that product by $\cdot 7854$, for the area. Or,

9. To find the area of any segment $\mathrm{ADE}$. Find the area of the corresponding segment $A D G$ of a circle on the same diameter $\mathrm{AB}$; then say, as the axis $\mathrm{AB}$ : its conj. $a b::$ circ. seg. ADG : elliptic seg. ADE.

10. To find the length of the whole circumference of the Ellipse. Multiply the circumference of the circumscribing circle by the sum of the series

$1-\frac{d}{2^{2}}-\frac{3 d^{2}}{2^{2} \cdot 4^{2}}-\frac{3^{2} \cdot 5 d^{3}}{2^{7} \cdot 4^{2} \cdot 6^{2}}-\frac{3^{2} \cdot 5^{2} \cdot 7 d^{3}}{2^{2} \cdot 4^{2} \cdot 6^{2} \cdot 8^{2}} \& \mathrm{c}$, for the circumference; where $d$ is the difference between an unit and the square of the less axis divided by the square of the greater.

See another series, p. 178, vol. 4, Elinb. Philos. Trans.

Or, for a near approximation, take the circumference of the circle whose diancter is an arithmetical mean between the two axes, or half their sum: that is,

$\frac{x}{2}(\ell+c) \times 3.1416=$ the perimeter nearly; being about the 200th part too little; where $t$ denotes the transverse, and $c$ the conjugate axis.

Or, again, take the circumference of the circle, the square of whose diameter is half the sum of, or an arithmetical mean between, the squares of the two axes:

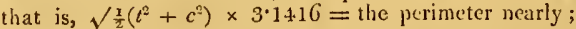
being about the 200 th part too great.

Hence combining these two approximating rules together, the periphery of the ellipse will be very nearly equal to half their sum, or equal to

$\frac{r}{2}\left[\frac{1}{2}(t+c)+\sqrt{ } \frac{1}{2}\left(t^{2}+c^{2}\right)\right] \times 3.1416$, within about the 30000 th part of the truth.

For the length of any particular arc, and many other parts about the ellipse, sce my Munsuration, pa. 212, \&ic, 4th edit. See also my Conic Sections, for many other propertics of the ellipse, especially such as are common to the hyperbola also, or to the conic sections in general.

Infinite lizipses. See Elutptord.

ELLIPSOID, is an elliptical spheroid, being the solid generated by the revolution of an ellipse about either axis. See SPHERoId.

ELLIPTIC or ElLi PTICAL, something relating to an ellipse.

Elciptic Compasses, or Elliprical Compass, is an instrument for describing ellipses at one revolution of the index. It consists of a cross $\triangle \mathrm{BC}$ II (fig. S.) with grooves in it, and an index $\mathrm{CE}$, sliding in dovetail grooves; by which motion the end 1 describes the curve of an ellipse. 

E. $M$

Eluiptical, Conoid, is sometimes used for the spheroid, or ellipsoid.

El ziptica L Dial, an instrument usually made of brass, with a joint to fold together, and the gnomon to fall flat, for the convenience of the pocket. By this instrument are found the meridian, the hour of the day, the rising and setting of the sun, \& $\mathrm{c}$.

ELLIPTICITY, of the terrestrial spheroid, is the ratio, or the difference, between the two semiaxes. 1t is usually expressed in terms of the greater, or radius of the equator, that is, by what fractional part this exceeds the axis or polar diameter. 'The quantity of this ellipticity has been variously assigned by different mathematiciaus. Sir Isaac Newton, supposing the earth of uniform dersity, gave $\frac{1}{2} \frac{1}{3}$ for the ellipticity; Boscovich, from a inean of several admeasurem ints, stated it tht $\frac{1}{24} \overline{8}$; Lalancle at $\frac{1}{3} \frac{1}{3}$; Laplace, $\frac{1}{3 \frac{1}{2}}$; Sajour, $\frac{1}{3 \frac{1}{8}}$; Carouge, $\frac{1}{30}$; Kraft, $\frac{1}{297}$; and Playfair, from a theorem of Clairaut, applied to the heterogeneous spheroid, states it at $\frac{x_{3}}{4}$. Setting aside those which are deduced from the hypothesis of uniform density, $\frac{1}{2} \sigma$ may be admitted as the most probable value of the ellipticity. Under the article EARTH, see other proportions, and the manner of deducing them.

ELLiptoid, an infinite or indefinite ellipse, defined by the indefinite equation $a y^{\mathrm{m}+\mathrm{n}}=b x^{\mathrm{m}}(a-x)^{\mathrm{n}}$, where $m$ or $n$ are greater than $I$ : for when they are each $I$, it denotes the common cllipse. 'There are several kinds or degrees of elliptoids, which receive their denominations from the cxponent $m+n$ of the ordinate $y$. As the cubical clliptoid, expressed by $a y^{3}=b x^{2}(a-x)$; the biquadratic, or sursolid $a y^{4}=b x^{2}(a-x)^{2} ; \& c$.

ELONGATION, in Astronomy, the distance of a planet from the sun, with respect to the earth; or the angle formed by two lines drawn from the earth, the one to the sun, and the other to the planet; or the arc measuring that angle: or it is the difference between the sun's place and the geocentric place of the planet.

The greatest elongation, is the greatest distance to which the planets recede from the sun, on either side. This is chiefly considered in the inferior planets, Venus and Mercury; the greatest elongation of Venus bcing about $47 \frac{\mathrm{J}}{2}$ degrees, and of Mercury only about $27 \frac{\mathrm{x}}{2} \mathrm{de-}$ grees; which is the reason that this planet is so rarely secn, being usually lost in the sun's beams.

EMBEl:-Days, are certain day's observed by the cliurch at four different seasons of the year; viz, the wednesday, friday, and saturday next after Quadragesima sunday, or the 1st sunday in Lent; after Whit-sunday; after Holyrood, or Holycross, the 14 th day of September; and after St. Lucy, the 13th day of December. The name, it scems, is derived from embers, or ashes, which it is supposed the primitive Christians strewed on their heads at these solemn fasts.

EMBER-Weeks, are those weeks in which the emberdays fall. These ember-woeks are now chicfly noticed on account of the ordination of priests and deacons; because the canon appeints the sundays next alter the ember-weeks for the solemn times of ordination; though the bishops, if they please, may ordain on any sumbly or holiday.

EMBOL.IMLAN, and EMBoLisne, Interculary, is chicfly used in speaking of the additional months inserted by chronologists to form the lunar cycle of 19 years. The 19 solar years, consist of 6939 days and 18 hours, and the 19 lunar years of only 6726 days, so that it was found necessary to intercalate or insert 7 lunar months, contain- ing 209 days; which, with the to Lissextile dags happen. ing in the lunar cycle, make 213 days, and the whole 6939 days, the same as the 19 solar years, which make the lunar cycle.

In the course of 19 years there are 228 common moons, and 7 emloolismic moons, which are distributed in this manner, riz, the $3 \mathrm{~d}, 6 \mathrm{th}, 9 \mathrm{th}, 11 \mathrm{th}, 14 \mathrm{th}, 17 \mathrm{th}$, and $19 \mathrm{th}$ years, are embolismic, and so coniain 384 days cach. And this was the method of computing time among the Greeks; though they did not strictly observe it, as it seems the Jews did. And the method of the Greeks was followed by the Romans till the time of Julius Casar. The embolismic months, like other lunar months, sometimes contain 30 rlays, and sometimes only 29 days.

The Embolismic Epacts are those between 19 and 29 ; which are so called, because, with the addition of the cpact 11 , they exceed the number 30 : or ratber, because the year's which have these cpacts, are embolismic; having13 moons each, the 13th being the embolismic.

EMBOLISMUS, in Chronology, signifies intercalation. As the Greeks used the lunar year, which contains only 354 days; in order that they might bring it to the solar year, of 365 days, they had an cmbolism every two or three years, when they added a 13th lunar month.

EMBRASURE, in Architecture, an enlargement of the aperture or opening of a door, or winlow, within-side the wall, sloping back inwards, to gire the greater play for the opening of the door, casenent, \&c, or to take in the more light.

Embrasures, in Fortification, are the apertures or holes through which the camnon are pointed, whether in casemates, batteries, or in the parapets of walls, In the navy, these are called port-holes. The embrasures are placed 12 or 15 fcet apart from each otlier; being made sloping or opening outwards, from 6 to 9 feet wide on the outside of the wall, and from 2 to 3 within, to allow the gun to traverse from side to side. Their base is about $2 \frac{\pi}{2}$ or 3 feet above the platform on the inside of the wall, but sloping down outwards, so as to be only about $1 \frac{1}{2}$ above it on the outside; in order that the muzzle may be occasionally depressed, when it is necessary to point the gun in that dircction.

EMERGENT Year, in Chronology, is the epoch, from which any people begin to compute their time or dates. So, our emergent yrar is sometimes the your of the creation, but more usually the year of the bith of Christ. The Jews used that of the Deluge, or the Fixodus, \&c. The emergent year of the Greeks, was the beginning of the Olympic games; while that of the Romans was the date of the Building of their City.

EMERSION, in Astronomy, is the re-appearance of the sun, moon, or other planet, atter having been eclipsed, or hid by the interposition of the moon, carth, or other body.

The immersions and cmersions of Jupiter's first sitellite, are particularly useful lor funding the longitudes of places; the immersions being observed from Jupiter's conjunction with the sun, till his opposition; and the emersions from the opposition till the conjunction. But within 15 day's of the conjunction, both before and after it, tiey cannot be observed, the planet and his satellites being then lost in the sun's light.

EnEmsion is also used when a star, after being hid by the sun, begins to re-appear, and emerges out of his rays.

Minutes or Scruples of ENE $\mathrm{ES} \perp \mathrm{ON}$, au arc of the nuov's orbit, which her centre passes over, from the time she 
begins to emerge out of the earth's shaduw, to the end of the eclipse.

Emersion, in Physics, the rising of any solid above the surface of a fluid that is specifically heavier than the solid, into which it had bcen violently immerged, or pushed. It is one of the known laws of hydrostatics, that a lighter solid, being forced down into a heavier fluid, immediately endeatvours to emerge; and that with a force equal to the excess of the weight of a quantity of the fluid above that of an equal bulk of the solid. Thus, if the body be immerged in a thuid of double its specific gravity, it will emerge again, till half its bulk be above the surface of the fluid.

EMLRSON (WrLLAM), a lite eunent mathematician, was burn in June 1701, at Hurworth, a village about three miles south of Darlington, on the borders of the county of Durham; at least it is certain that he resided here from his childhood. His lather Dudley Emerson was a schoolmaster, and a tolerable proficient in the mathematics; and without his books and instructions, probably his son's genius, though eminently fitted for mathematical sturlies, might never have been unfolded : bnt besides his father's instructions, our author was assisted in the learned languages by a young clergyman, then curate of Hurworth, who was boarded at his father's house. Peing satisfied with a moderate compctence left him by his parents, he devoted himself to a studious retirenent, which he thus closely pursued, in the same place, through the course of a long life, being mostly very healthy, till towards the latter part of his days, whon he was much afflicted with ibestone. Towards the close of the year 1781 , being sensible of his approaching dissolution, he disposed of the whole of his mathematical library to a bookseller at York; and on May the $20 \mathrm{th}, 1782$, his lingering and painful disorder put an end to his life at his native village, being near 81 years of age.

Mr. Emerson, in his person, was rather short, but strong and well made, with an open countenance and ruddy complexion. He was of a singular turn in his behaviour, Iress, and conversation; his inanner and appearance being rather plain and uncourtly: he had strong natural mental parts, and coulu discourse sensibly on any subject, but was always positive and impatient of contradiction. Hc spent his whole life in close study, and writing books, from the profits of which, he redeemed his little patrimony from some original incumbrance. In his dress he was as singular as in every thing else. He possessed commonly but one suit of clothes at a time, and those very old in their appearance. He seldom used a waistcoat; and his coat be wore open before, except the lower button; and his shirt quite the reverse of one in common use, the hind side turned foremost, to cover his breast, -and buttoned close at the collar behind. He also had a kind of rustycoloured wig, without a crooked hair in it, which probably liarl never been tortured with a comb frum the time of its being made; and the bat he wore had served him the greater part of his life, of which he gradually lessencd the tlaps, bit by bit, as it lost its clasticity, till little or nothing but the crown remained.

He sometimes walked up to London when he had any book to be published, revising sheet by sheet himsclf :--for trusting no eye but lis own, was always a favourite maxim with him. In mechanical subjects, he always tried the propositions practically, making all the difterent parts himsclf on a small scale; so that his house was filled with all kinds of meclianical instruments, together or disjointed.
He would frequently stand up to bis middle in water while fishing; a diversion he was very fond of. He used to study incessantly for some time, and then for relaxation take a ramble to some pot-alehouse, where he could get any body to drink with and talk to. He was a married man, but without children; and his wife used to spin on an oldfashioned wheel, of his own making, a drawing of which is given in his Mechanics.

Mr. Emerson, from his strong vigorous mind and close application, had acquired a deep knowledge of all the branches of muthematics and physics, on every part of which he wrote good treatises, though in a rather antiquated style and manner. He was not remarkable, however, for genius or discoveries of his own, as his works show hardly any traces of original invention. He was well skilled in the science of music, the theory of sounds, and the various scales both ancient and modern; but he was a very poor performer, though he cuuld make and repair some instruments, and sometimes went, about the country tuning harpsichords, as well as cleaning clocks.

The following is a list of Mr. Emerson's works; all of them printed in Svo, excepting his Mechanics and his Increments in 4to, and his Navigation in $12 \mathrm{mo}$. 1. The Doctrine of Fluxions.-2. The Projection of the Sphere, orthographic, stereographic, and gnomonical. -3 . The Elcments of Trigonometry.-4. The Principles of Mechanics. -5. A Treatise of Nivigation.-6. A Treatise on Arithmetic. -7. A Treatise on Geometry,-8. A Treatise of Algebra, in 2 books.- 9 . The Method of Increments.10. Arithmetic of Infinites, and the Conic Sections, with other Curve Lines. - 11. Elements of Optics and Perspcctive-12. Astronomy.-13. Mechanics, with Centripetal and Centrifugal Forces.-14. Mathematica! Principles of Geograpby, Navigation, and Dialling.-15. Commentary on the Principia, with the Defence of Newton.-16. Miscellanies.

Besides these, Mr. Imerson was a frequent mathematical correspondent in the Ladies' Diary, the Palladium, \&c, in the fictitious names of Mcrones, Nichol Dixon, and other fanciful appellations. In the same works, sometimes a riddle or a question in rhyme, showed that he was not destitute of poetical talents.

EMINENTIAL Equation, a term used by some algebraists, in the investigation of the areas of curvilineal figures, for a kind of assumed equation that contains another equation eminently, the latter being a particular case of the former. Hayes's Flux. pa. 97.

ENCEINTL, a French term, in Fortification, signifying the whole inclosure, circumference, or compass, of a fortified place, whether built with stonc or brick, or only nade of earth, and wheth'r with or without bastions, \&c.

ENCYCLOPEDIA, the circle or chain of arts and sciences; sometimes also written Cyclopadia.

Numerous are the encyclopædias, or general dictionaries that have been published; but our business is only with those which treat chicfly on the mathematical or philosophical sciences. The first dictionary of this kind, it appears, was that of Dasypodius, printed in 1573 , under the title of $\Lambda \varepsilon \xi_{\text {s }}$ เov, seu Dictionarium mathematicarum, in quo definitiones et divisiones continentur scientiarum mathematicarum, arthmetic, \&c, M. Courado Dasyporlio authore; a very cxtraordinary work for the time of its publication, and of which a now edition was printed at Strasburg, in 1579.-In 1632 canc out the work J.H. Alstelii Encyclopedia, in 2 vols. folio : an claborate perlurmance; 
which was followed in $165 \pi$, by Erhardi Wegalii Idea Encyclopædia Mathematico-philosophica; a work not superlatively interesting, even for that period. Of a similar kind is the Lexicon Matb. Astron. Geomet. of Vatalis, printed at Paris in 1668, Svo; as also the Dictionnaire Mathematique of Ozanam, printed in 1 vol. 4 to 1691.

As to the works of Ger. J. Vussius (1650), and of Beughem ( $691 \frac{1}{2}$, they are jittle more than catalogues of mathematical books.

The next important step was made by Dr. Harris, in his Lexicon Technicum, published, the 1st vol. in 1704, the $2 \mathrm{~d}$ in 1710. This is the earliest English work that assumes to good purpose the systematic form of a general dictionary, and attempts to allot to each article its comparative portion in the scale of science. The author possessed very considerable general knowledge; but his attainments as a mathematician and philosopher were most conspicuous. To the 5 th edit. in 1736 , a supplement was added by the booksellers, but of minor importance.-Various dictionaries, comprising more or less of scientific materials, were published between the times of Harris's Lexicon and Chambers's Cyclopærlia ; such, for instance, as the Great Dictionary of the French Academy, the Dictionary of the Jesuits of Trevoux, the Chemical Dictionary of Johnson, the Medical ones of Blancharil and Castellas, the Nathematical Dictionaries of Stone and Wolfius, the Sea Dictionary of Nainwaring, the Dictionary of the Bible by Calmet, the Lexicon Philosophicum of Chauvin, a work of importance, especially in the mathematics and philosophy of the ancients; the Lexicon of J. Burkard Monkens, in, 1715; Jablonski's Lexicon in 1721; and Collier's great 1-listorical Dictionary, begun in 1694, and finished in 1727.

The first edition of Chambers's Cyclopredia came out in 1727,2 vols. folio; of which the public demand occasioned a $2 \mathrm{~d}$ edit. in 1738 , a $3 \mathrm{~d}$ in $173 \mathrm{y}$, a 4 th in 1741 , and it 5 th in 1746 . This unprecedented success induced the preprietors, at a great expense, to engage G. L. Scott abd Dr. IIill to prepare a Supplement to the 6th edition, which was accordingly published in 2 additional volumes. 'The $7 t_{1}$ calition, completed in 1786 in four thick folio volumes, removed the disadvantage of the donble alphabets, by incorporating them into one, by the labour and skill of that able cditor Dr. Abr. liees.

From th is periorl, dictionarics devoted to arts and sciences, as welt in general dictionaries, increased very rapidly, both it number and importance; of which a few of the clief may be noticed : as, the Medical Dictionaries of Motherby, Quincy, lames, 'Turton, and the lidinburgh Dictionary ; the Chemical Dictionarics of Macquer, Nicholson, and the Aikins; the Dictionaries of Gusseme and Risches, on Numismatology ; those of Miller, Martyn, and Dickson, on Gardening; those of Burn, Cunningham, anel Jacob, (1) Law: the Marine Dictionaries of Chapman and Falconcr; the Builder's Mligazine, and liclihien's Dictionary of 'Architerture; Jombert's Dictionatire de l'Engenieur et de l'Arillewr, and James's Military l)ictionary'; Pilkington's Dictionary of Painters; Mintimer's and l'ostlethwate's Dicuonaries of 'Trade aud Commerce; Rousseatu's, Iloyle's, alm Busby's Dictionaries of MI unic ; our own MIathematical and Philosophical Dictionary; and the Dictionmaire de I'hysique of M. Lilses. 'The diligence exerted by the authers of these, and a lew other dictionatries appropriated to sepiarate branches of science, and by the editors of encyclepadias, has operated reciprocally to improve buth; and hesec it has happened, that many of such dic- tionaries, during the last 50 years, tave grcatly contributed to the improvement and diffusion of buman knowledge.

The labours of the continental encyclopedists, during this period, are also of much importance. Among the works of the Germans may be noticed, the Universal Lexicon of Ludwig, in 1732-1750, in 64 volumes; Oekononische Encyclopädia, by Krünitz, in 1773; the Encyclopälia der Historischen, P’hilosophiscen, und Mathematischen W'issenchaften, by Büsch, in 1775 and 1795; Klïgel's Fncyclopädic aller Mathematischen Wissenchaften ihre Geschichte und Litteratur, by Rosenthal, in 1790. To these may be added, the Swedish Encyclopædia, by Giorwell, in 1785; and the Encyclopedia Italiana ovvero Bibliotheca universale della umane cognizioni, at Naples in 1788 .

Our neighbours the French also have the Dictionnaire universel de Mathematique et de Physique, by M. Saverien, in 1753; the Encyclopedie, ou Dictionnaire raisonné des Sciences, des Arts, et les Metiers, by Diderot, Dalembert, \&c, in 1754-1757; the Dictionnaire Portatif, in 1760; the Dictionnaire de Physique Portatif, in 1763; the new edition of the Encyclopedie, by Diderot, \&c, in 39 vols. in 1778, 1779; and the Ency clopedie Methoclique, by Dalembert, Bossut, Condorcet, Lalande, \&:c, which commenced in 1785 , and consists of scparate alpliabets, or dictionaries, for the several arts and sciences.

We must now glance rapidly at the labours of British encyclopxdists since the time of Chambers; passing over the productions of Owen, Procter, Castieau, Hall, Howard, and Kendal, with a mere notice of their names; the only one of which that we do not feel desirous to forget is Owen. We must however mention, with commendation, the Dictionary of Arts and Scicnces, published in 1766 , by Crowder, in 3 vols. 4 to ; the editors of which were, the Rev. J. Scott, 'Trinity-college, Cambridge; Mr. Chas. Green, assistant at the royal observatory; Mr. J. Meades; and Mr. lialconer, the unfortunate author of the Shipwreck.

The work last mentioned, though respectable, is far inferior to Chambers, who has however now had some formiclable rivals in Britain; viz, the Encyclopædia Britunnica, published in Edimburgh, first we believe in 766 , in 4 vols. 4 to, and afterwards in 10 vols. This work scems also to have been the first that attempted the imnovation of incorporating systems or treatises, along with the usual articles in the alphabetical arrangement. The $3 \mathrm{~d}$ edition of this Encyclopadia, superintended by Dr. Gleig, was finished in 1800 ; the whole with a supplement, making 20 large volumes. It contains, besides the general matters treated in Chambers's work, the adclitional subjects of biography, history, and geography. It is a work, in many respects, of considerable excellence: it comnonly explains the principles and practices, in the various arts and sciences, with great correctness and perspicuity. Many of the treatises it contains, were drawn up by some of the most rminent Scotch professors, which do them gratt credit.-The linglish Encyclopedia was conpleted in 10 vols. 410 , in 1803. The names of its conductors are not mentioned; but they are commonly understond to have been 1). Aikin and Mr. Ifoulston. Its general plan is much like that of the lincyclopeedia Britamnicn, but much less imbued with orivinis misterly compositions.

Such is the general difiusion of linowledge in this country, and so prevalent is the desire to possess a library as it were in one work, that beside's two general dictionaries 


\section{E N T}

[ 473

just finished, no less than six others are now in course of publication, viz, The Encyclopædia Londinensis; a new edition of the Encyclopæedia Perthensis; Brewster's Edinburgh Encyclopædia; the Pantologia, or New Cyclopædia, by Mr. Good, Dr. O. Gregory, and Mr. Bosworth, including all English words, besides all sciences and human knowledge; to be comprised in 10 or 12 volumes; also a new edition of the Encyclopædia Britannica, and Dr. Rees's New Cyclopædia, both to be extended to several more volumes.

ENDECAGON, a plane geometrical figure of eleven sides and angles, otherwise called undecagon. If each side of this figure be 1 , its area will be $9.3656399=\frac{11}{4}$ of the tang. of $73 \frac{7}{15}$ degrees, to the radius 1 . See my MIensuration, pa. $85, \&$ c, 4th edit. See also Regular Figure.

ENFILADE, a French term in Fortification, applied to those trenches, and other lines, that are ranged in a right Iine, and so may be scoured or swept by the cannon lengthways, or in the direction of the line.

To ENFILADE, is to sweep lengthways by the firing of cannon, \&c.

A Battery'd'EnFilaDE, is that where the cannon sweep a right line.

$A$ Post, or Command d'EnfiLADE, is a height from which a whole line may be swept at once.

ENGINE, in Mechanics, a compound machine, consisting of several simple ones, as wheels, screws, levers, or the like, combined together, in order to lift, cast, or sustain a weight, or produce some other considerable effect, so as to save either force or time. There are numberless kinds of engines; of which some are for war, as the Balista, Catapulta, Scorpion, Aries or Ram, \&c; others for the arts of peace, as mills, cranes, presses, clocks, watches, \& c, \&c.

ENGINEER is applied to a contriver or maker of any kind of useful engines or machines; or who is particularly skilled or employed in them. And he is denominated either a civil or military engineer, according as the objects of his profession respect civil or military purposes.

A militaly engineer should be an expert mathematician and draughtsman, and particularly versed in fortification and gunnery, being the person officially employed to direct the operations both for attacking and defending works. When at a siege the engineers have narrowly surveyed the place, they are to make their report to the general, or commander, by acquainting him which part they judge the weakest, and where approaches may be made with most success. It is their business also to draw the lines of circumvallation and contravallation; also to mark out the trenches, places of arms, batteries, and lodgments, and in general to direct the workmen in all such operations.

ENIIARMONIC, the last of the three kinds of music. It abounds in dicses, or the least sensible divisions of a tone. See Philos. Trans. No. 481 ; also Wallis's Appendix to Ptolem. pa. 165, 166.

ENNEADECATERIS, in Chronology, a cycle or period of 19 solar years, being the same as the golden num"ber and lunar cycle, or cycle of the moon; which see; as also Lim BoLISMic.

ENNEAGON, a plane geometrical figure of 9 sides and angles; and is otherwise callerd a nonagon. If each side of this figure be 1 , its area will be $6.1818242=\frac{9}{4}$ of the tang. of 70 degrees, to the radius 1 . See my Mensuration, pir. 85, 4th edit. Sec also the article RegulA Figure.

EN'TABLATURE, in Architecture, is that part of the VuL. I.
E P A

order of a column which is over the capital, comprehend. ing the architrave, frize, and cornice.

ENTABLATURE, or ENTABLEMENT, is sometimes also used for the last row of stones on the top of the wall of a building, on which the timber and covering rest ; sometimes also called the drip, because it projects a little, to throw the water off.

ENVELOPE, in Fortification, is a mound of earth, sometimes raised in the ditch of a place, and sometimes beyond it, being either in form of a single parapet, or of a small parapet bordered with a parapet. These envelopes are made only to cover weak parts with single lines, without advancing towards the field, which cannot be done without works that require a great deal of room, such as horn-works, half-moons, \&c. Envelopes are sometimes called Sillons, Contregards, Conserves, Lunettes, \&r.

ENUMERA'TlON, numbering or counting. Sir Isaac Newton wrote a very ingenious treatise on the enumeration of the lines of the $3 \mathrm{~d}$ order.

EOLIPILE. See FolipiLe.

EPACT, in Chronology, the excess of the solar month above the lunar synodical month; or of the solar year above the lunar year of 12 synodical months; or of several solar months above as many synodical months; or of several solar years above as many periods each consisting of 12 synodical months.

The epacts therefore are either annual or menstrual.

Menstrual Eрастs, are the excesses of the civil calendar month above the lunar month. Suppose, for example, it were new moon on the 1st day of January: then since the month of January contains 31 days,

And the lunar month - $\quad-29^{\mathrm{ds}} 12^{\mathrm{b}} 44^{\mathrm{m}} \cdot 3^{\mathrm{s}}$; The menstrual epact is - $\quad-\quad \begin{array}{lllll}11 & 15 & 57\end{array}$

Annual Epicts, are the excesses of the solar year above the lunar. Hence,

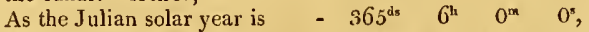
And the Julian lunar year - $354 \quad 8 \quad 4 \mathrm{~S} \quad 3 \mathrm{~S}$, The annual epact will be $\quad-\quad \begin{array}{llll}1 & 21 & 11 & 22,\end{array}$ that is, almost 11 days. Consequently. the epact of 2 years, is 22 days; of 3 years, 33 days; or rather 3 , since 30 days make an embolismic, or intercalary month; therefore, adding still 11 , the cpact of 4 years is 14 days; and so of the rest as in the following table, where it may be observed, that they do not become 30 , or 0 again, till the 19 th year; so that at the 20 th year the epact is 11 again; and herice the cycle of epacts expires with the golden number, or lunar cycle of 19 years, and begins with the same again.

\begin{tabular}{|c|c|c|c|c|c|}
\hline $\begin{array}{l}\text { Golden } \\
\text { Numb. }\end{array}$ & Epacts. & $\begin{array}{l}\text { Golden } \\
\text { Numb. }\end{array}$ & Epacts. & $\begin{array}{l}\text { Golden } \\
\text { Numb. }\end{array}$ & Epacts. \\
\hline I & 11 & VIII & 28 & $\mathrm{xV}$ & 15 \\
\hline II & 22 & $\mathrm{IX}$ & 9 & XVI & 26 \\
\hline III & 3 & $\mathrm{x}$ & 20 & XV1I & $s$ \\
\hline IV & 1.4 & $\mathrm{xI}$ & 1 & xvili & 19 \\
\hline $\mathrm{v}$ & 25 & X11 & 12 & XIX & $30\}$ \\
\hline vI & 6 & xilI & 23 & & or 0$\}$ \\
\hline VII & 17 & xiv & 4 & & \\
\hline
\end{tabular}

$\Lambda$ gain, as the new moons are the same, or fall on the same day, every 19 years, so the difference between the solar and lunar years is the same évery 19 years. And because the said difference is alivays to be added to the lunar year, to arljust or make it equal to the solar year; therefore the said difference respectively belonging to each yeal $3 \mathrm{P}^{3}$. 
of the moon's cyclc, is called the epact of the said year, that is, the number to be added to the said year, to make it equal to the solar year; and upon this mutual relation between the cycle of the noon and the cycle of the epacts, is founded this

Rule for finding the Julian Epact, belonging to any year of the Moon's Cycle.-Multiply the golden number, or the given year of the moon's cycle, by 11 , and the product will be the epact, if it be less than 30 ; but if it exceed 30 , then reject as many 30 's as the product contains, and the remainder will be the epact.

Rule to find the Gregorian Epact.-1st, The difference between the Julian and Gregorian years being equal to the difference between the solar and lunar year, or 11 days, therefore the Gregorian epact for any year is the same with the Julian epact for the preceding year; and hence the Gregorian epact will be fuund, by subtracting 1 from the golden number, multiplying the remainder by 11 , and rejecting the 30's; which rule will serve till the year 1900; but after that year, the Gregorian cpact will be found by this rule: Divide the centuries of the given year by 4 ; multiply the remainder by 17 ; and to this product add 43 times the quotient, as also the number 86 , and divide the whole sum by 25 , reserving the quotient : next multiply the golden number by 11 , and from the product subtract the reserved quotient; so shall the remainder, after rejecting all the $30^{\prime}$ 's contained in it, be the epact sought.

The following table contains the golden numbers, with their corresponding epacts, till the year 1900 .

Table of Gregorian Epacts.

\begin{tabular}{|l|r||l|r||l|r|}
\hline $\begin{array}{l}\text { Golden } \\
\text { Numb. }\end{array}$ & Epacts. & $\begin{array}{l}\text { Goiden } \\
\text { Numb. }\end{array}$ & Epacts. & $\begin{array}{l}\text { Golden } \\
\text { Numb. }\end{array}$ & Epacts. \\
\hline I & 0 & VIII & 17 & XV & 4 \\
II & II & IX & 28 & XV I & 15 \\
III & 22 & X & 9 & X VII & $20^{\circ}$ \\
IV & 3 & XI & 20 & X V III & 7 \\
V & 14 & XII & 1 & XIX & 18 \\
VI & 25 & XIII & 12 & I & 0 \\
vII & 6 & XIV & 23 & & \\
\hline
\end{tabular}

On the subjcct of epacts, see Wolfius's Elementa Chromologiæ; apud Opera, tom. 4, pa. 133; also Philos. Trans. vol. 46 , pa. 417 , or No. 4.95 , art. 5 .

EPAULE, or EsPAULE, in Fortification, the shoulder of the bastion, or the angle made by the face and flank, otherwise called the angle of the epaule.

EPAULEMENT, in Fortification, a side-work hastily raised, to cover the cannon or the men; and is made either of carth thrown up, or bags filled with carth or sand, or of gabions, or fascines, \&c; of which latter sort are commonly constructed the epaulements of the places of arms for the cavalry behind the trenches.

EPA ULEMENT, is also used for a demi-bastion, consisting of a face and flank, placel at the point of a hornwork or crown-work: and a little flank added to the sides. of horn-works, to defend them when they are too long: also for the redoubts made on a right line, to fortify it. And lastly, for an orillen, or mass of earth almost square, faced and lined with a wall, and designed to cover the cannon of at casemate.

EPHINMERIS, EPHenerrdes, tables calculated by astronomers, showing the present state of the heavens for ('very day at noon; that is, the places in which all the plancts are found at that time; differing but little from an astronomical almanac. It is from such tables as these that the eclipses, conjunctions, and aspects of the planets are computed; ; as also horoscopes, or cclestial schemes, constructed, \&c.

The following list of authors of published ephemerides has been chiefly collected from Weidler's History of Astronomy, in 4 to. $7+1$.

\begin{tabular}{|c|c|c|c|c|c|}
\hline Regiomonta & from & $n 1475$ to 1506 & Beaulieu & from & $1703: 01714$ \\
\hline Angelas & - & $1494-1500$ & Manfredi & - & $1715-1725$ \\
\hline Stoefler & - & $1494-155]$ & & & $1725-1750$ \\
\hline Sclıoner & 一 & 1533 & Ghisler & 一 & $1721-1740$ \\
\hline Gauricus & 一 & $1534-1551$ & & - & $1739-1756$ \\
\hline Rheticus & - & 1550 & Connoissance de & & \\
\hline $\begin{array}{l}\text { Simi } \\
\text { Mizald }\end{array}$ & 二 & $\begin{array}{l}1554-1568 \\
1555-1557\end{array}$ & $\begin{array}{l}\text { Temps, by } \\
\text { Picard }\end{array}$ & - & \\
\hline $\begin{array}{l}\text { Mizald } \\
\text { Pitstus }\end{array}$ & Z & $\begin{array}{l}1555-1557 \\
1552-1562\end{array}$ & $\begin{array}{l}\text { Picard } \\
\text { Lefebvre }\end{array}$ & 二 & $\begin{array}{l}1679-1695 \\
1656-1701\end{array}$ \\
\hline $\begin{array}{l}\text { Pitatus } \\
\text { Stadius }\end{array}$ & 二 & $\begin{array}{l}1552-1562 \\
1554-1576\end{array}$ & $\begin{array}{l}\text { Letebvre } \\
\text { Lieutaud }\end{array}$ & $=$ & $1701=1729$ \\
\hline Corelti & 一 & $1558-1577$ & Godin & 一 & $1729-1735$ \\
\hline Leovitius & - & $1556-1606$ & Maraldi & 一 & $1735-1760$ \\
\hline Moletius & - & $1564-1584$ & Lalande & 一 & $1760-1775$ \\
\hline Magrious & - & $1581-1630$ & Jeaurat & - & $1775-1788$ \\
\hline Scala & 一 & $1589-1600$ & Mechain & - & $1788-1794$ \\
\hline Everhart & - & $1590-1615$ & Commission & 一 & 1795 \\
\hline Origan & 一 & $1595-1655$ & Board of Lon- $\}$ & & נ 796, \&c, \&c. \\
\hline Kepler & - & $1617-1620$ & Eitude $S$ & & \\
\hline rgoli & 一 & & des Mlouve. & & \\
\hline Durret & - & $1637-1649$ & mens Celes- & & \\
\hline & & $3648-3700$ & tes, by & & \\
\hline Inntebrun & 一 & $1641-1660$ & Desplaces & 一 & $1715-1725$ \\
\hline Wing & - & $1652-1672$ & & 一 & $1725-1735$ \\
\hline Malvasia & 一 & $1662-1667$ & & 一 & $1735-1744$ \\
\hline Palati & 一 & $1664-1670$ & Lacaille & - & $1745-1755$ \\
\hline Hecker' & 一 & $1666-1680$ & & - & $1755-1765$ \\
\hline Gadbury & - & $1672-1701$ & & - & $1765-1775$ \\
\hline Mozzavachis & 一 & $1675-1684$ & Lalande & 一 & $1775-1784$ \\
\hline & & $1702-1720$ & & - & $1785-1792$ \\
\hline & & $1681-1692$ & Nautical Alma- & & \\
\hline & 一 & $1693-1729$ & & & \\
\hline inius & - & $1701-1703$ & Maskelyne & 一 & $1767-1817$ \\
\hline & - & $3701-1702$ & & & \\
\hline
\end{tabular}

There are now published such ephemerides by the $A$ cademies of several other natinns; but that which is in most. estecm for its accuracy and nse in finding the longitude, is the Nautical Almanac, or Astronomical Fphemeris, published in England by the Board of Longitude, under the direction of the astronomer royal, which commenced with the year 1767 , by Dr. Maskelyne: besides many other. smaller annual cphcinerides, by Whitc, Partridge, Parker, and many morc.

EPICHARMUS, an ancient poet and philosopher, born in Sicily, was a scholar of Pythagoras, and fourished. in the time of Hicro, in whose reign it is said he introduced comedy at Syracuse. He also wrote treatises on philosophy and medicine; but none of his works have been preserved. He died at 90 years of agc, according to Lacrtius, who has preserved four verses inscribed on his statue.

EPICURUS, a celcbrated ancient philusopher, was born at Gargettium in $A$ ttica, in the 10gth Olympiad, of about 340 years before Christ. He settled at $\lambda$ thens in a fine garden he had bought; where he lived with his friends in much tranquillity, and cducated a great number of clisciples; who lived all in common with their master. Ilis scliool was never divided, but his doctrine was followed as an oracle; and the respect which his disciples paid to his memory is aumirable; his birtli-day being kept even in Pliny's time, and the month he was brom in was obscrverl as a continued festival ; his picture was also displayed in numberless places. He wrote a great many books, and valued himself upan making no quotations. 1 le raised the atomical system to great reputation, though he 


\section{E P I}

[ 475

was not the inventor of $j$, but only made some change in that of Democritus. As to his doctrine concerning the supreme good or happiness, it was very liable to be misrepresented, and some ill effects proceeded from thence, which discredited his sect, though undeservedly. He was charged with perverting the worship of the gods, and inciting men to debauchery. But he did not forget himself on this occasion : he published his opinions to the whole world; wrote some books of devotion; recommended the veneration of the gods, sobriety, and chastity, living in an exemplary manner, and conformably to the rules of philosophical wisdom and frugality. Gassendus has given us all that he could collect from the ancients concerning the person and doctrine of this philosopher; by whom we are informed that he died of a suppression of urine, at the age of 72 years.

Epicurear Philosoply, the doctrine, or system of philosopliy, maintained by Epicurus and his followers; which consisted of three parts; canonical, physical, and ethical. The first respected the canons or rules of judging; in which soundness and simplicity of sense, assisted by some natural reflections, chiefly formed his art. His search after truth proceeded only by the senses; to the evidence of which he gave so great a certainty, that he considered them as an infallible rule of truth, and termed them the first natural light of mankind. In the $2 \mathrm{~d}$ part of his philosophy he laid down atoms, space, and gravity, as the first principles of all things. He asserted the existence of a God, whom he accounted a blessed immortal being, but who did not concern himself with human affairs.

As to his ethics, he made the supreme good of man to consist in pleasure, and consequently supreme evil in pain. Nature itself, says he, teaches us this truth; and prompts us from our birth to procure whatever gives us pleasure, and to avoid that which gives us pain. To this end he proposes a remedy against the sharpness of pain, which is to divert the mind from it, by turning our whole attention upon the p.easures we have formerly enjoyed. He held that the wise man must be happy, as long as he is wise: pain, by not depriving him of his wisdom, cannot deprive him of his happiness: from which it would seem that his pleasure consisted rather in intellectural than in sensual enjoyments: though this is a point strongly contested : probably in both.

EPICUREANS, the sect of philosophers holding or following the principles and doctrine of Epicurus. As the nature of the pleasure, in which the chicf happiness of man is supposed to be seated, is a great problem in the morals of Epicurus, there hence arise two kinds of Epicureans, the rigid and the remiss: the first were those who understood Epicurus's notion of pleasure in the best sense, and placed all their happiness in the pure pleasurcs of the mind, arising from the practice of virtue: while the loose or remiss Epicureans, taking the words of that philosopher in a gross sense, placed all their nappiness in bodily pleasures or debauchery.

EPICYCLE, in the ancient Astronomy, a little circle having its centre in the circumference of a greater one: or a small orb or sphere, which being fixed in the deferent of a planet, is carried along with it, and yet, by its own peculiar motion, carries the planet fastened to it round its proper centre.-It was by means of epicycles that Ptolemy and his followers solved the various phenomena of the planets, but more cspecially their stations and retrogradations.
E P I

EPICYCLOID, is a curve generated by the revolution of a point of the periphery of a circle, which rolls along or upon the circumference of another circle, either on the convex or concave side of it. When a circle rolls along a straight line, a point in its circumference describes the curve calked a cycloid. But if, instead of the right line, the circle roll along the circumference of another circle, either equal to the former or not, then the curve described by any point in its circuinference is what is called the epicycloid.

If the generating circle roll along the convexity of the circumference, the curve is called an upper, or exterior epicycloid; but if along the concavity, it is called a lower, or interior epicycloid. Also the circle that revolves is called the generant; and the arc of that circle along which it revolves, is called the base of the epicycloid.

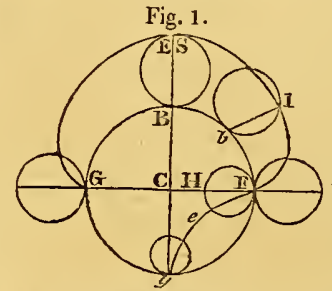

Fig. 2.

Thus, in the above figure, GEIF is the exterior epicycloid, and ger the interior epicycloid, to the former of which EB is the generant, and GBF the base, and to the latter the circle FII is the generant, having the arc GF for its base.

The honour of the invention of this curve is due to $M$. Roemer, a celebrated Danish astronomer, who did not treat of it as a purely geometrical speculation; but as one of great utility, being, as he thought, the form which ought to be given to the teeth of wheels, in machinery; in order to diminish the friction of them against each other, and to render the action of the powers more equal. This idea occurred to M. Roemer while he was in Paris about the year 1674 , notwithstanding M. Lahire, in 1694, published a work upon this subject, and seems to lay claim to the invention; but he was justly confuted by Lcibnitz, who was in Paris during the visit of the Danish mathematician Roemer, and knew both of the invention and its application to mechanical purposes. Sir Isaac Newton and John Bernoulli have each considered the nature and properties of this curve, which are both numerous and interesting; but our limits will only admit the insertion of some of those which are more particularly deserving of attention. It is a remarkable property of circular epicycloids, that they are sometimes gcometrical, though the common cycloid, which is much more simple in appearance, as a right is inore simple than a curve, is always inechanical, or transcendental. The cases where the epicycloid is geometrical, are those in which there is a numerical ratio between the circumferences of the circle which forms the base, and the generant; for where there is no rational ratio between them, the curve becomes mechanical.

It is also a remarkable property of epicycloids, whether they be geometrical or tianscendental, that they are always rectifiable, at least in the case wliere the describing point is taken in the circumference of the generating 3 P \& 

E P I

circle. Thus, the curve of the epicycloid GLF, is to 4 times the diameter of the generating circle $\mathrm{в} \mathrm{L}$, as the sum of the diameters of the two circles, is to that of the base. But if the epicycloid is inferior, as Feg, then instead of the sum of the diameters we must take their difference.

It may also be observed, as a curious circumstance, that when the diameter of the generant is equal to half that of the circle forming the base, as in fig. 2 ; then the epicycloid degenerates into a right line, describing the diameter of the latter circle. We may also further remark, that when the describing point is taken either within or without the generant, the length of the epicycloid is equal to the circumference of an ellipse, which is easily constructed.

As to the areas of epicycloids, they are determined from the following proportion. As the radius of the circle forming the base, is to 3 times that radius plus twice that of the generant; so is the circular segment $b \mathrm{r}$, to the epicycloidical space $b_{\mathrm{IF}}$; or, so is the whole area of the generant, to the entire area of the epicycloid FEGB.

Dr. Halley has also given a general proposition for the measuring of all cycloids and epicycloids: whicb is this, the area of a cychoid, or epicycloid, either primary, or contracted, or prolate, is to the area of the generating circle; and also the areas of the parts generated in those curves, to the areas of analogous segments of the circle; as the sum of double the velocity of the centre and the velocity of the circular motion, is to this velocity of the circular motion. See the Demonstr. in the Philos. Trans. No. 218, or my Abridg. vol.4. pa. 47.

Spherical Epicycloids are formed by a point of the revolving circle, when its plane makes a constant angle with the plane of the circle on which it revolves. Messrs. Bernoulli, Maupertuis, Nicole, and Clairaut, have demonstrated several properties of these epicycloids, in Hist. Acad. Sci. for 1732 .

Epicycloids. Parabolic, Elliptic, \&c.

If a parabola roll upon another equal to it; its focus will describe a right line perpendicular to the axis of the quiescent parabola: also the vertex of the rolling parabola will describe the cissoid of Diocles; and any other point of it will describe some one of Newton's defective hyperbolas, having a double point in the like point of the quiescent parabola.

In like manner, if an ellipse revolve upon another ellipse, equal and similar to it, its focus will describe a circle, whose centre is in the other focus, and consequently the radius is equal to the axis of the ellipsis; and any other point in the plane of the ellipse will describe a line of the 4 , th order.

The same may be said atso of an hyperbola, revolving upon another, equal and similar to it; for one of the foci will describe a circle, having its centre in the other focus, and the radius will be the principal axis of the hyperbola; and any other point of the liyperbola will describe a line. of the 4 th order.

Concerning these lines, see Newton's Principia, lib. I; also Lahire's Memoires de Mathematique \&c, where he shows the nature of this line, and its use in mechanics; see also Maclaurin's Geometria Organica; the Philos. Trans. No.217, pa.113; and No.218, pa. 125.

EPIPHANI, a Christian festival, otherwise called the Manifestation of Christ to the Gentiles, observed on the 6 th of January, in bonour of the appearance of Christ to the three magi or wise men, who came to adore him and bring him presents.

EPISTYLE, in the ancient Architecture, a term used by the Greeks for what we call the architrave, viz, a massire stone, or a piece of wood, laid 'immediately over the capital of a column.

EPOCH, or Еросна, a term or fixed point of time, whence the succeeding years are numbered or reckoned. Different nations make use of different epochs. The Christians chiefly use the epoch of the nativity or incarnation of Jesus Christ; the Mahometans, that of the Hegira; the Jews, that of the Creation of the World, or that of the Deluge; the ancient Greeks, that of the Olympiads; the Romans, that of the Building of their City; the ancient Persians and Assyrians, that of Nabınassar ; $\mathcal{S} \cdot \mathcal{C}$.

The doctrine and use of epochs is of very great extent in chronology. To reduce the years of one epoch to those of another, i. e. to find what year of one corresponds to a given year of another; a period of years has been invented, which, commencing before all the known epochs, is, as it were, a common receptacle of them all, called the Julian Period. To this period all the epochs are reduced; that is, the year of this period when each epoch commences, is so determined; that, adding the given year of one epoch to the year of the period corresponding with its rise, and from the sum subtracting the year of the same period corresponding to the other epoch, the remainder is the year of the required epoch.

Epocir of Christ, is the common epoch throughout Europe, commencing at the supposed time of his nativity, December 25 ; or rather, according to the usual account, from lis circumcision, or the 1st of January. The author of this epoch was an abbot of Rome, one Dionysius Exiguus, a Scythian, about the year 507 or 527. Dionysius began his account from the conception or incamation, usually called the Annunciation, or LadyDay; which method obtained in the dominions of Great Britain till the year 1752 , before which time the Dionysian was the same as the English epoch: but in that year the Gregorian calendar having been admitted by act of parliament, they now reckon from the first of January, as in the other parts of Europe, except in the court of Rome, where the epoch of the lncamation still obtains. for the date of their bulls. 
A TABLE of the Years of the most remarkable Epoctis or Eras and Events.

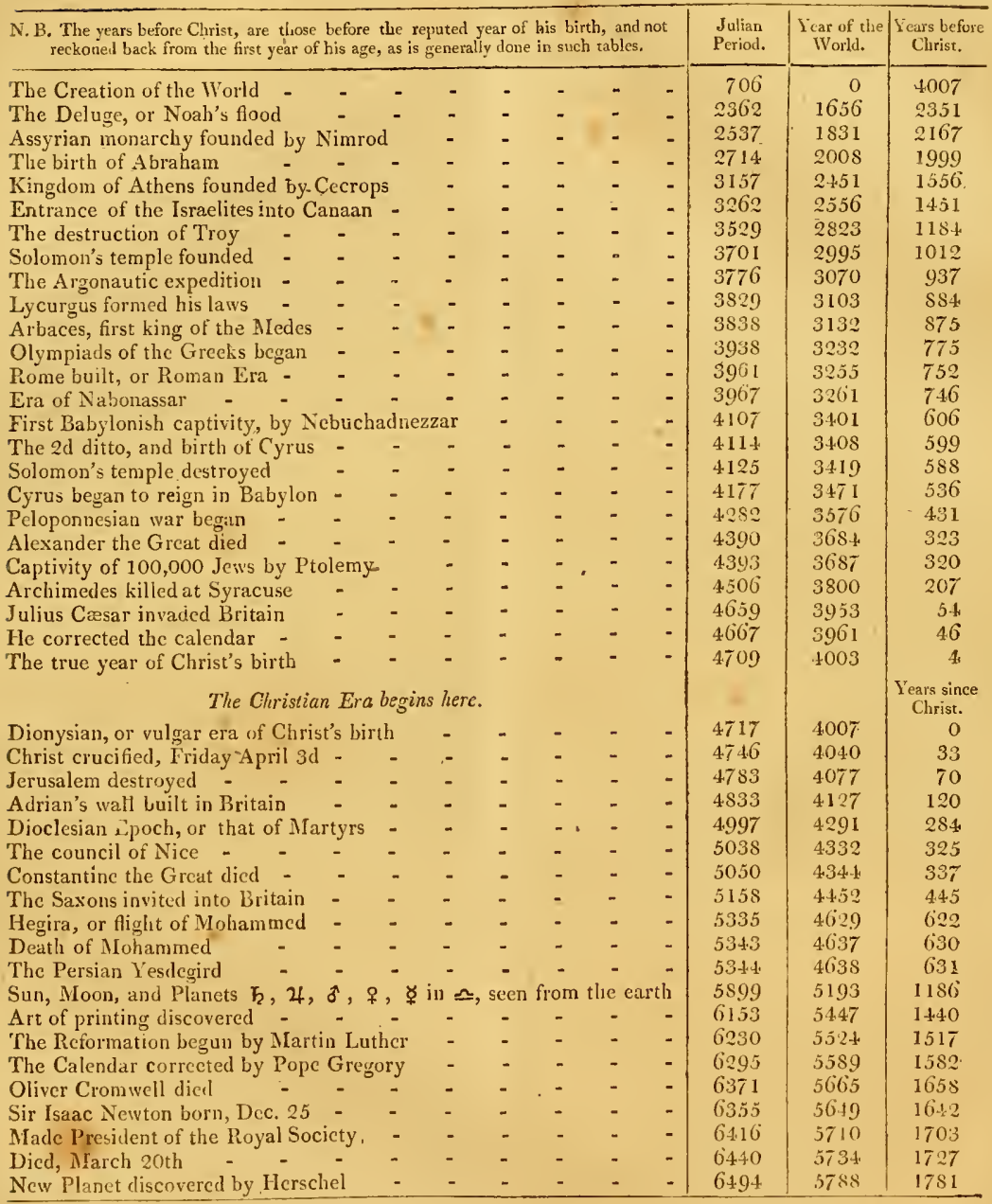

EPROUVETTE, in Gunnery, is a machine for showing the strength or quality of gunpowder. Eprouvettes bave been made of various kinds and shapes. The best is that described in my Tracts, vol. 3, pa..153.

EQUABLI: Motion, Celerity, Velocity, \&c, is that which is uniform, or without alteration, or by which equal spaces are passed over in equal times. Hence, the spaces, passed over in cquable motions, are proportional to the timcs. So that if a body pass over 20 feet in 1 sceond of time, it will pass over $40 \mathrm{fect}$ in 2 seconds, and so on.

LQUABLY Accelerated or Retarded, \&c, is when the mo- tion or change is increased or decreased by cqual quantities or degrees in equal tines.

EQUAL, a term of relation between different things, but of the same kind, magnitude, quantity, or quality.Wolfius defines equals to he those things that may be substituted for each other, without any alteration of their quantity.- It is an axiom in mathematics \&ce, that two things which are equal to the same thing, are also equal to each other. And if equals be equally altered, by equal addition, subtraction, multiplication, division, \&ce, the results will be also equal. 


\section{$E Q U$}

EQUar Circles, are those whose dianeters are equal.

EQUa $A$ Angles, are those whose sides are equally inclined, or which are measured by similar arcs of circles.

EQUaL Lines, are lines of the same length.

EqUal Plane Figures, are those whose areas are equal; whether the figures be of the same form or not.

EQU AL Solids, are such as are of the same space, capacity, or solid content; whether they be of the same kind or not.

EQU a L Curvatures, are such as have the same or equal radii of curvature.

EQUAL Ratios, are those whose terms are in the same proportion.

Equal, in Optics, is said of things that are seen uncler equal angles.

EQUALITY, the exact agreement of two things in respect to their quantity. Those figures are equal which may occupy the same space, or may be conceived to possess the same space, by the flexion or transposition of their parts. See a learned discourse upon this subject, by Dr. Barrow, in his 11th and 12th Mathematical Lectures.

EQUALITY, in Algebra, the relation or comparison between two quautities that are really or effectually equal. See EQUATION.-Equality, in Algebra, is usually denoted by two equal parallel lines, as $=$ : thus $2+3=5$, i. e. 2 plus 3 , are equal to 5 . This character $=$ was first introduced by Robert Recorde. Descaries, and some others after him, use the mark $\propto$ instead of it: as $2+3 \propto 5$.

EQUALITT, in Astronomy. Circle of EQUALITy, or the Equant. See Crrcre, and Equant.

Ratio or Proporition of EQUaLITY, is that between two equal numbers or, quantities.

Proportion of EQUALITY evenly ranged, or ex squo ordinaia, is that in which two terms, in a rank or series, are proportional to as many terms in another series, compared to each other in the same order, i. e. the first of one rank to the first of another, the $2 \mathrm{~d}$ to the $2 \mathrm{~d}$, \& $\mathrm{x}$.

Proportion of LQUality evenly disturbed, called also $e x$ aquo perturbata, is that in which more than two terms of one rank, are proportional to as many terms of another, compared to each other in a different and interrupted order; viz, the 1st of one rank to the $2 \mathrm{~d}$ of another, the $2 \mathrm{~d}$ to the $3 d$, \& 8 .

EQUANT, or ÆQUANT, in Astronomy, a circle formerly conceived by astronomers, in the plane of the deferent, or excentric; for regulating and adjusting certain motions of the planets, and reducing them more easily to a calculus: but in the modern astronomy they are not used.

- EQUATED Anomaly. See ANona Ly.

Equated Bodies. On Gunter's Sector are sometimes placed two lines, answering to one another, and called the lines of equated bodies; being situated between the lines of superficies and solids, and are marked with the letters $D, I, C, S, O, T$, to signify dodecahedron, icosahedron, cube, sphere, octahedron, and tetrahedron.

The uses of these lines are, 1st, When the diameter of the sphere is given, to find the sides of the five regular bodies, each equal to that sphere; 2l, From the side of any one of those bodies being given, to find the diameter of the sphere, and the sides of the other bodies, which shall be each equal to the first given body. So that when the spbere is given, take its diameter, and apply it on the sector in the points $\mathrm{s}$, s; but when one of the otber five bodies is given, apply its side over in its proper points;
E Q U

then the parallels taken from between the points of the other bodies, or sphere, will be the sides or diameter, equal severally to the sphere or body first given.

EQUATION, in Algebra, an expression of equality between two different quantities; or two quantities, whether simple or compound, with the mark of equality between then : as $2+3=7-2$; or $2 \times 3=6$; or $5 \times 3$ $=30 \div 2$; or $a+b=c$; or $x^{2}+a x=b$; \&c. When the two sides of the equation are the same, the expression becomes an identity, as $5=5$, or $a=a, \& c$. Sometimes the quantities are placed all on one side, and made equal to 0 , or nothing, on the other side; as $5-5=0$, or $a-b$ $=0:$ which is no more than setting down the difference of two equal quantities equal to nothing.-The character or sign usually employed to denote an equation, is =, which is placed between the two equal quantities, called the two sides of the equation.

The Terms of an EQUATIon, are the several quantities or parts of which it is composed. Thus, of the equation $a+b=c$, the terms are $a, b$, and $c$ : and the tenor or inport of the expression is, that some quantity represented by $c$, is equal to two others represented by $a$ and $b$.

Equations are either simple or affected or compound.

A Simple EQUation is that which has only one power of the unknown quantity: as $a+x=3 b$, or $a x^{2}=b c$, or $a^{3}+2 x^{3}=5 b$, \&c; where $x$ denotes the unknown quantity, and the other letters known ones.

An Affected, or Adfected, or Compound EQu a Tion, contains two or more different powers: as $x^{2}+a x=b$, or $x^{3}-4 x^{2}+3 x=25$, \& $c$

Again, equations are denominated from the highest power contained in them; as quadratic, cubic, biquadratic, \&c. Thus,

A Quadratic EQUation, is that in which the unlinown quantity rises to two dimensions, or to the square or $2 \mathrm{~d}$ power: as $x^{2}+20 x=200$, or $x^{2}-a x=b$.

A Cubic Equation, is that in which the unknown quantity is of three dimensions, or rises to the $3 \mathrm{~d}$ power: as $x^{3}=25$, or $x^{3}-2 x^{2}=27$, or $x^{3}-a x^{2}+b x=c$.

A Biquadratic Equation, is that in which the unknown quantity is of 4 dimensions, or rises to the 4 th or biquadratic power : as $x^{4}=25$, or $x^{4}-20 x=10$, or $x^{4}+a x^{3}$ $+b x^{2}+c x=d$.

And so on for other higher orders of equations.

The Root of an EQUation, is the value of the unknown letter or quantity contained in it. And this value being substituted in the terms of the equation instead of that letter or quantity, will cause both sides to vanish, or will make the one side exactly equal to the other. So the root of the equation $3 x+18=24$, is 2 ; for by using 2 for $x$, it becomes $3 \times 2+18=6+18=24$.

Every equation has as many roots as it has dimensions, or as it contains units in the index of the highest power, when the powers are all reduced to integral exponents. So the simple equation of the 1st power, has only one root; but the quadratic has 2 , the cubic 3 , the biquatdratic 4, \&c. Thus the two roots of this equation $x^{2}-4 x=-3$ are 1 and 3 ; for either of these substituted for $x$ makes $x^{2}-4 x=-3$. Also the three roots of $x^{3}-4 x^{2}-11 x=-30$, or $x^{3}-4 x^{2}-11 x+30=0$, are 2,5 , and -3 ; as will appear by substituting each of these insteal of $x$ in the equation, by which means it will always be found to be equal to 0 . And so ol others.

On the Relation betaveen the Roots of Liquations, and the 
Coefficients of their Terms.-In cvery equation, when the terms are ranged in order according to the order of the powers, the greater before the less; the first term or highest power being freed of its coefficient, by dividing all the terms of the equation by it, and all brought to one side, and made equal to nothing on the other side, then it will appear in this form,

$$
x^{\mathrm{n}}+a x^{\mathrm{n}-1}+b x^{\mathrm{n}-2}+c x^{\mathrm{n}-3} \ldots \ldots=0 ;
$$

and the relations between the roots and coefficients, are as follow:

1st, The coefficient $a$ of the $2 \mathrm{~d}$ tcrm, is equal to the sum of all the roots.

$2 \mathrm{~d}$, The coefficient $b$ of the $3 \mathrm{~d}$ term, is equal to the sum of all the products of the roots that can be made by multiplying every two of them together.

$3 \mathrm{~d}$, The coefficients $c, d, e, \& c$, of the following terms, are respectively equal to the sum of the products of the roots made by multiplying every three together, or every four together, or every five together, \&c, the signs of all the roots being changed, as will be seen when we treat of the Generation of Equations.

The roots of equations are positive or negative, and real or imaginary. Thus, the two roots of the equation $x^{2}-4 x=-3$, are 1 and 3 , real and both positive; but the roots of the equation $x^{3}-4 x^{2}-11 x=-30$, are 2 , 5 , and -3 , viz, real, two positive and one negative; and the roots of the equation $x^{3}+9 x=10$, are 1 and $-\frac{r}{2} \pm \frac{r}{2}$ $\sqrt{ }-39$, one real and positive, and two imaginary.

On the Generation of Equations.-This is only the multiplying of certain assumed simple equations together, to produce compound ones, with intert to show the nature of these; a method which was invented by Harriot, and is as follows: Suppose $x$ to denote the unknown quantity of any equation, and let the roots of that equation, or the values of $x$, be, $a, b, c, d$, \&c; that is $x=a$, and $x=b$, and $x=c, \& c$; or $x-a=0$, and $x-b=0$, and $x-c=0$, $\& c$; then multiply these last equations together, thus,

$x-a=0$

$x-b=0$

$\left.\overline{x^{2}-a} \frac{a}{b}\right\} x+a b=0$

$x-c \cdot \cdot=0$

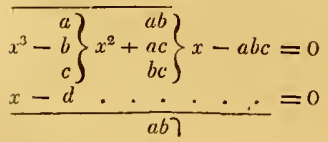

$\left.\left.\left.x^{4}-\begin{array}{l}a \\ b \\ c \\ d\end{array}\right\} x^{3}-\begin{array}{r}a c \\ b c \\ a d \\ b c \\ d c\end{array}\right\} x^{2}-\begin{array}{r}a b c \\ a b d \\ a c d \\ d b c\end{array}\right\} x+a b c d=0$

Now the roots of these equations are $a, b, c, d, \& c$; and it is obvious that the sum of all the roots is the coefficient of the $2 \mathrm{~d}$ term, the sum of all the products of every two is the cocflicient of the $3 \mathrm{~d}$ term, the sum of all the products of every three that of the 4 th term, and so on, to the last term, which is the continual product of all the roots; and the same law may always be observed, whatever be the dimension or degree of the equation.

On the Reduction of EQUarions. - T'his is to transform or change them to their simplest and most commodious form, in order to prepare them for finding or extracting their roots. . It is always most convenient to arrange them according to the powers of the unknown ketter, the highest power being placed first on the left hand, and that term to have only +1 for its coefficient; also all the terms containing the unknown letter to be on one side of the equation, and the absolute known term only on the other side.

Now this reduction chiefly respects the first term, or that which contains the highest power of the unknown quantity; and the general rule for reducing it is, to consider in what manner it is involved or connected with other quantities, and then perform the cuunter or opposite relation or operation; for every operation is undone or counteracted by the reverse of it; as addition by subtraction, multiplication by division, involution by evolution, \&c: then bring all the unknown terms to one side, and the known term to the other side, changing the signs, from + to - , or from - to + , of those terms which are changed from one side to the other; and lastly divide by the coefficient of the first torm, with its sign.

Thus, for example, if $5 x-12=3 x+4$ :

$$
\text { then is } 5 x-3 x=12+4 \text {, or } 2 x=16 \text {; }
$$
and so $x=\frac{16}{2}=8$.

See other cxamples in my Course of Mathematics, vol. 1.

On Extracting or Finding the Roots of EQuations.

This is finding the value or values of the unknown letter in an equation, the rules for which are various, according to the degree of the equation.

1. For the Root of a Simple EQUa tion.

Having reduced the equation as above, by bringing the unknown terms to one side, and the known ones to the other, freeing the former from radicals and fractions, by their counter operations, and lastly dividing by the coefficients of the unknown quantity, the value of it is then found: as in the example of reduction above given.

2. For the Roots of Quadrutic EQUATIONs.

These are usually found by what is called completing the square; which consists in squaring half the cocflicient of the $2 \mathrm{~d}$ term, and adding it to both sides of the equation; for then the unknowu side is a complete sujuare of a binomial, and the other side consists only of known quantities. 'Therefore, extracting the root on both sides, the root of the first side will be a binomial, one part of which is the unknown letter, and the other a known quantity, and the root of the other sicle is taken either + or -, since the square of either of these is the same given quantity: lastly, bringing over the known part of the binomial root to the other side, with a contrary sign, it will give the two roots or values of the unknown letter sought. Sce examples in my Course, vol. 1.

\section{For the Rools of Cubic EQuations.}

A Cubic EQuation is that in which the unknown letter. ascends to the $3 \mathrm{~d}$ power; as $x^{3}+a^{3}=b^{3}$, or $x^{3}+a x^{2}+$ $b x=c$. The $2 \mathrm{~d}$ term of every cubic equation being taken away, (see Transrormatron, those equations may all ${ }^{-}$ be reduced to this form, $x^{3}+a x=b$; and the general value of one root is $x=\sqrt[3]{ }\left[\frac{1}{2} b+\sqrt{ }\left(\frac{1}{4} b^{2}+\frac{1}{27} a^{3}\right)\right]+$

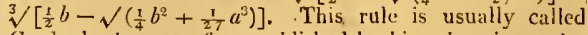
Cardan's, because first published by bim, but it was invented both by Scipio Ferreus, and Nich. Tartalea, by the latter of whom it was communicated to Cardan. See the article $A$ I. EBRA.

When the $2 \mathrm{~d}$ term is negative, or the equation of this form, $x^{3}-a x= \pm b$, the radical $\sqrt{ }\left(\frac{1}{4} b^{2}+\frac{t}{2} 7 a^{3}\right)$ bccomes $\sqrt{ }\left(\frac{x}{4} b^{2}-\frac{1}{27} a^{3}\right)$, which will be imaginary or impossible when $\frac{x}{2} a^{3}$ is greater than $\frac{1}{4} b^{2}$, for $\sqrt{ }\left(\frac{x}{4} b^{2}-\frac{1}{2} a^{3}\right)$, 


\section{E Q U}

[ 480

will then be the square root of a negative quantity, which is impossible: and yet, in this case, the root $x$ is a real quantity; though algebraists have never been able to find a real finite general expression for it. And this is what is generally called the Irreducible Case.

This case may indeed be resolved by the trisection of an arc or angle; or by any of the usual methods of converging; or by general expressions in infinite serics. See Saunderson's Aigebra, pa. 713; Philos. Trans. vol. 18, pa. 136, and vol. 70, pa.415, or iny Abridg. vol. 3, pa. 396 ; vol. 5 , pa. $33 \pm$, and vol. 15 , pa. 139 . See also the article Cu в Iс Equations.

Mr. Cotes observes, in lis Logometria, p. 29, that the solution of all cubic equations depends either on the trisection of a ratio, or of an angle: which method is explained in Saunder'son's Algeb. pa. 718.

Biquadratic EQCatrons, or those that are of 4 dimensions, are resolved after various methods. The first rule was given by Lewis Ferrari, the companion of Cardan, which is one of the best. A second method was given by Descartes, and another by Mr. Simpson and Dr. Waring. For the explanation of which, see BIQU ADRATic Equations. Equations of the Higher Degrees or Orders.

There is no general rule to express algebraically the roots of equations above those of the 4 th degree; and therefore methods of approximation are here made use of, which, though not accurately, are yet practically true.

Some of these excel in ease and simplicity, and others in quickness of converging. Among these may be reckoned first, Double Position, or Trial-and-Error, both in respect of ease and universality, as it applies in the simplest manner to all kinds of equations whatever, not excepting even exponential ones, radical expressions of cver so complex a form, expressions of logarithms, of arches by the sines or tangents, of arcs of curves by the abscisses, or any other fluents, or roots of fluxional equations. For an explanation of this and other methods of converging to the roots of equations, by Halley, Newton, Raphson, \&c, \&c, sec ApProximation, and CONVERGING; and my Course of Mathematics, vol. 1.

Besides the methods above adverted to, there have been some others, given in the Memoirs of several Academies, and elsewhere. As, by M. Daniel Bernoulli, in the Acta Petropolitana, tom. 3, pa. 92 ; by Euler, in the same, vol. 6 , New Scries, and tom. 5, pa. 63 and 82 ; by Mr. Thos. Simpson, in his Essays, pa. 82 ; in his Dissertations, pa. 102; in his Algebra, pa. 158; and in his Select Exercises, pa. 215.

Construction of Equations. See Construction.

Depression of Eevations. See Demression.

Elimination in EQUations. Sec Elimination.

Transformation of EQUaTions. See TransFonnaTION.

Alsolute Equation. See Absolute.

Alfected, or Affected EQuation. See AfFected.

Differential EQUAT1ON, is the equation of difierences or fluxions.

RQUATIONS of Condition. Sce Condition, Equations of.

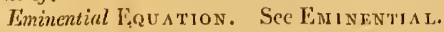

Exponential Equ $\operatorname{cton}$, one in which the exponents of the powers are variable or unknown quantities. Sec Ex$P()$ NENTIAL.

Fluential lieuntron, is the equation of the fluents.

Fluxional Lev $\Delta$ TuoN, is the equation of the fluxions.
$E Q U$

Limits of the Roots of an Equation. See Limits.

Literal EQUATION, is a general equation expressed in letters, as contradistinguished from a

Numeral EQUATron, one expressed in numbers.

Reciprocal Equation. See Reciprocal.

Transcendental Equation.- See Transcendertal,

EQuation of Payments, in Arithmetic, is the finding a time when, if a sum of money be paid, which is equal to the sum of several others due at different times, no loss will be sustained by either party.-The rule usually given for this purpose, in the cummon books of arithmetic, is this: Multiply each payment by the time it is due at, then divide the sun of the products by the sum of the payments, for the equated time. For example, If $20 l$. be due at 2 months, and $40 l$. at 4 months, to find, as an equivalent, when the whole $60 l$. may be paid at once. Here then $20 \times 2+40 \times 4=40+160=200$, is the sum of the products; and $20+40=60$, is the sum of the payments; hence $200 \div 60=3 \frac{\pi}{3}$ is the equated time, for making the whole paynient at once.

This method, it is obvious, cannot be quite correct when the two payments, as here, are unequal, because no account of the interest on the two sums is considered. When this is allowedfor, Mr. Nlalcolm, in his Arithmetic, has given a correct rule for two payments at simple interest, which is this: If $p$ denote the first payment, and $t$ the time till it is due; also $\mathrm{P}=$ another payment, and $\mathbf{T}=$ its time; and if $r$ denote one year's interest of it ; next, putting $a=\mathrm{T}+t+\frac{\mathrm{P}+p}{p r}$, and $c=\mathrm{T} t+\frac{\mathrm{PT}+p t}{p r}$; then $\frac{1}{2} a-\frac{T}{2} \sqrt{ }\left(a^{2}-4 c\right)$ is the equated time for the two payments. And here if the formcr example be taken, it gives $p=20, t=2, \mathrm{P}=40, \mathrm{x}=4$, and $r=\frac{\mathrm{r}}{2 \delta}$, for $\mathrm{a}$ rate of 5 per cent. Hence $a=66$, and $c=208$; then $\frac{\pi}{2}$ $a-\frac{1}{2} \sqrt{ }\left(a^{2}-4 c\right)=3.3436$ is the true equated time, allowing simple interest, being very nearly equal to the former, which was 3.3333 \&c. And if compound interest were allowed, the result would be a small matter different still.

When three or more payments are concerned, then Malcolm's method recommended, of first equating for two payments, and next for the result and a $3 \mathrm{~d}$ payment, and so on, is not strictly just. But in all such cases, to obtain a true answer, Malcolm's general principle ought to be used, viz, making the interests of the sums that are kept later than they are duc, equal to the disconnts of those that are paid before they are due. The resolution of the result ing equation will, indeçl, require some knowledge in algebra. But for ordinary use, the common rnle will bring ont answers sufficiently near the truth.

Equation of a Curve, is an equation showing the nature of a curve by cxpressing the relation between any absciss and its correrponding ordinate, or else the relation of their fluxions, ixc. Thus, the equation to the circle, is $a x-x^{2}=y^{2}$, where $a$ is its diameter, $x$ any absciss, or part of that diameter, and $y$ the ordinite at that point of the diameter; that is, whatever absciss is denoted by $x$, the square of its corresponding ordinate will be $a x-x^{2}$. In like manner the equation

of the ellipse is ${ }_{a}^{p}\left(a x-x^{2}\right)=y^{2}$,

of the hyperboli is $\frac{p}{a}(a x+x)=y^{2}$,

of the parabola is - $-p x=y^{2}$,

Where $a$ is an axis, and $p$ the parancter. 
And in like manner for any other curves. Sec Absciss.

This method of expressing the nature of curves by algebraical equations, was first introduced by Descartes, who, by thus connecting together the two sciences of algebra and geonetry, made them mutually assist each. other, and so laid the foundation of the great improvements that have been made in every branch of them since that time. See Descartes's Geometry; also Newton's Lines of the 3d Order, and many other similar works on curve lines, by several authors. See Curve.

Equation, in Astronomy, as Anmual Equation, is either of the mean motion of the sun and moon, or of the moon's apogee and nodes.

The annual equation of the sun's centre being given, the other three correspondent annual equations will be also given, and therefore a table of the first will serve for them all. Thus, if the annual equation of the sun's centre, taken from such a table for any time, be called $s$; and if $\frac{\pi}{x} s=A$, and $\frac{\mathrm{I}}{6} s=\mathrm{B}$; then will the other annual equations for that time be as follow:

$A+\frac{I}{3} \mathrm{~A}=m$, that of the moon's mean motion; and $\mathrm{B}+\frac{\mathrm{T}}{3} \mathrm{~B}=a$, that of the moon's apogee;

and $\frac{\mathrm{I}}{2} \mathrm{~B}-\frac{\mathrm{I}}{\mathrm{T}} \mathrm{O} \mathrm{B}=n$, that of her nodes.

And bere it must be observed, that when $s$, or the equation of the sun's centre, is additive; then $m$ is negative, $a$ is positive, and $n$ is negative. But on the contrary, when $s$ is negative or subductive; then $m$ is positive, a negative, and $n$ positive.

There is also an Equation of the moon's mean motion, depending on the situation of her apogee in respect of the sun; which is greatest when the moon's apogee is in an octant with the sun, and is nothing at all when it is in the quadratures or syzygies. This equation when greatest, and the sun in perigee, is $3^{\prime} 56^{\prime \prime}$. But it is never above $3^{\prime} 34^{\prime \prime}$ when the sun is in apogee. At other distances of the sun from the earth, this equation when greatest, is reciprocally as the cube of that distance. But when the monn's apogee is any where out of the octants, this equation becomes commonly less, and is at the same distance between the earth and sun, as the sine of double the distance of the moon's apogee from the next auadrature or syzygy, is to radius. This is to be added to the moon's motion while her apogeo passes from a quadrature with the sun to a syzygy; but is to be subtracted from it, while the apogee moves from the syzygy to the quadrature.

There is another Equation of the moon's motion, which depends on the aspect of the nodes of the moon's orbit with respect to the sun: and this is greatest when her nodes are in octants to the sun, and quite vanishes when they come to their quadratures or syzygics. This equation is proportional to the sine of double the distance of the node from the next syzygy or quadrature; and at the greatest is only $47^{\prime \prime}$. This must be added to the moon's mean motion while the nodes are passing from the syzygies with the sun to their quadratures; but subtracted while they pass from the quadratures to the syzygies. From the sun's true place subtract the equated mean motion of the lunar apogee, as was shown above, and the remainder will be the annual argument of the said apogee; from which the excentricity of the moon and the $2 \mathrm{~d}$ equation of her apogee may be compared. See Theory of the Moos's motions, \&c.

EQUation of the Centre, callerl also Prosthapheresis, and Total Prosthapheresis, is the difference between the true and mean place of a planet, or the angle made by the lines of the true and mean place; or, whicb amounts to the VOL. I. same, between the mean and equated anomaly. The greatest equation of the centre may be obtained by find ing the sun's lougitude at the times when he is near his mean distances, for then the difference will give the true motion for that interval of time: next find the sun's mean motion for the same interval of time; and then half the difference between the true and mean motions will show the greatest equation of the centre.

For example, by observations made at the Royal Observatory at Grcenwich, it appears that at the following mean times the sun's longitudes were as expressed below, viz, Mean times.

1769 Oct. 1 at $23^{\mathrm{h}} 49^{\mathrm{m}} 12^{\mathrm{s}} \quad-\quad-6^{\mathrm{s}} 9^{\circ} 32^{\prime} 0 \cdot 6^{\prime 7}$ 1770 Mar. 29 at 0 4 50 - - - 085027.5 dif. of time $17 \mathrm{~S}^{\mathrm{d}} 01538$; true dif. lon. $\begin{array}{llll}5 & 29 & 18 & 27\end{array}$ tropical year $=365^{\mathrm{d}} 5^{\mathrm{h}} 4 \mathrm{~S}^{\mathrm{m}} 42^{\mathrm{s}}=365.2421527$; observed interval $=178 \quad 0 \quad 1538=178^{\circ} 0108565$ : then $365 \cdot 2421527: 178 \cdot 01085648:: 360^{\circ}: 175 \cdot 455948$ or $175^{\circ} 27^{\prime} 21^{\prime \prime}$ the mean motion.

Therefore - $175^{\circ} 2 \overline{7}^{\prime} 21^{\prime \prime}$ of mean motion,

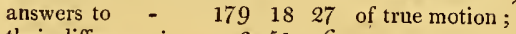
their difference is $\quad 3516$ and its half - 15533 is the greatest equation of the centre according to these observations.

To find the Equation of the Centre, which is Kepler's problem, and requires a very troublesome operation, especially in the more excentric orbits. The method of performing this, has been shown by Newton, Gregory, Keil, Machin, Lacaille, and others, by methods little differing from one another; which consist principally in finding a certain intermediate angle, called the excentric anomaly; having known the mean anamoly, and the dimensions of the sun's orbit. The mean anomaly is easily found, by determining the exact time when the sun is in the aphelion, and using the following proportion, viz, As the time of a tropical revolution, or solar year, Is to the interval between the aphelion and given time, So is 360 degrees, to the degrees of the mean anomaly. Or it may be found by taking the sun's mean motion at the given time out of tables.

To find the Excentric Anomaly, say,

As the aphelion distance,

Is th the perihclion distance;

So is the tangent of half the mean anomaly,

To the tangewt of an arc.

Which arc added to half the mean anomaly, gives the excentric anomaly. Then,

To find the True Anomaly, say,

As the square root of the aphelion distance,

Is to the square root of the perihelion distance;

So is the tangent of half the excentric anomaly,

To the tangent of half the true anomaly.

Then, the difference between the true and mean anomaly, gives the equation of the centre, sought. Which is subtractive, from the aphelion to the perihelion, or in the first 6 signs of anomaly; and additive, from the perihelion to the aphelion, or in the last 6 signs of anomaly; and hence called Prosthapheresis.

By this problem a table may easily be formed. When the equations of the centre for cvery degree of the first 6 signs of mean anomaly are found, ihey will serve also for the degrees of the last 6 signs, because equal anomalies are at equal distances on both sides of their apses. 'Therefore set these equations orderly to theirsigns and degrees $3 \mathrm{Q}$ 
of anomaly; the first 6 being reekoned from the top of the table downwards, and signed subtract; the last 6 , for which the same equations serve, in a contrary order, being reckoned from the bottom upwards, and marked add. Let also the difference between every adjacent two equations, called tabular differences, be set in another column. Hence, from these equations of the centre, augmented or diminished by the proportional parts of their respective tabular differences, for any given minutes and seconds, may easily be deduced equations of the centre to any mean anomaly proposed. See Robertson's Elem. of $\mathrm{Na}$ vig, book 5 , pa. $286,290,295$, and 308 , where such a table of equations is given.

Euler has particularly considered this subject, in the Mem. de l'Acad. de Berlin, tom. 2, pa. 225 et seq. where he resolves the following problems :

1. To find the true and mean anomaly corresponding to the planet's mean distance from the sun; that is, where the planet is in the extremity of the conjugate axis of its orbit.-2. The excentricity of a planet being given, to find the excentric anomaly corresponding to the greatest equation.-3. The excentricity being given, to find the mean anomaly corresponding to the greatest equation.4. From the same data, to find the true anomaly corresponding to this equation.-5. From the same data, to find the greatest equation.-6. The greatest equation being given, to find the excentricity.

Euler observes, that this problem is very difficult, and that it can only be resolved by approximation and tentatively, in the manner he mentions : but if the excentricity be not great, it may then be found directly from the greatest equation. 'Thus, if the greatest equation be $=m$, and the excentricity $=n$; then is

$m=2 n+\frac{11}{2^{4} \cdot 3} n^{3}+\frac{599}{2^{6} \cdot 3 \cdot 5 \cdot 7} n^{5}+\& c$; and by reversion $n=\frac{1}{2} m-\frac{11}{2^{4} .3} m^{3}-\frac{578}{2^{10} .3 .5} m^{5}-\& c$. Where the greatest equation $m$ must be expressed in parts of the radius, which, may be done by reducing the angle $m$ into seconds, aud adding 4.6855749 to the $\log$. of the resulting number, which will be the log. of the number $m$. The mean anomaly to which this greatest equation corresponds, will be $x=90^{\circ}+\frac{5}{8} m-\frac{5}{2 \cdot \cdot^{9} 3} m^{3}-\frac{1}{29^{9}} m^{5}-\& x$. Whence,

if $90^{\circ}$ be added to $\frac{5}{8}$ of the greatest equation, the sum will be the mean anomaly sufficiently exact.

Euler subjoins a table, by which may be found the greatest equations, with the mean and excentric anomalies corresponding to these greatest equations for every 100th part of unity, which he supposes equal to the greatest excentricity, or when the transverse and distance of the foci become infinite. The last column of the table gives also the logarithm of that distance of the planet from the sum where its equation is greatest. By means of this table, any excentricity being given, the corresponding greatest equation will be found by interpolation. But the principal use of the table is to determine the excentricity when the greatest equation is known; and without this help Euler thinks the problen cannot be resolved.

EQuation of Time; denotes the difference between mean and apparent time, or the reduction of the apparent unequal time, or motion of the sun or a planet, to cipal and mean time, or motion; or the equation of time is the difference between the sun's mean motion, and his right ascension. Apparent time is that which takes its begin. ning ron the patsange of the sun's centre over the meri- dian of any place; and had the sun no motion in the ecliptic, or was his motion reduced to the equator or in right ascension uniform, he would always return to the meridian after equal intervals of time. But his apparent motion in the ecliptic being continually varying, and his motion in right ascension being rendered further unequal on account of the obliquity of the ecliptic to the equator, it follows from these causes that the intervals of his return to the meridian become unequal, and the sun will gradually come too slow or too soon to the meridian for an equable motion, such as that of clocks and watches ought to be; and this retardation or acceleration of the sun's coming to the mericlian, is called the equation of time.

Now, computing the celestial motions according to equal time, it is necessary to turn that time back again into apparent time, that they may correspond to observation: on the contrary, any phenomenon being observed, the apparent time of it must be converted into equal time, to bave it correspond with the times marked in the astronomical tables.

The equation of time is nothing at four different times in the year, at which time the whole mean and unequal motions exactly agree; viz, about the 15 th of April, the 15 th of June, the 31st of August, and the 24th of Deccmber: but at all other times the sun is eitluer too fast or too slow for mear, equal, or clock time, by a certain number of minutes and seconds, which at the greatest is $16^{n}$ $14^{\prime \prime}$, and happens about the 1st of November; cvery other day throughout the year having a certain quantity of this difference belonging to it; which however is not exactly the same every year, but only every 4th year; for which reason it is necessary to have 4 tables of this equation, viz, one for each of the four years in the period of leap-ycars. Instead of these, we shall jnsert here, one general equation of time, according to the place of the sun, in every point of the ecliptic: where it is to be observed, that the sign of the ecliptic is placed at the tops of the columns, and the particular degree of the sun's place, in each sign, in. the first and last columns; and in the angle of meeting in. all the other columns, is the equation of time, in minutes and seconds, when the sun has any particular longitude: supposing the obliquity of the ecliptic $23^{\circ} 2 \mathrm{~s}^{\prime}$, and the sun's apogee in $9^{\circ}$ of .

The equations with the sign + , are to be added to, and those with the sign - are to be subtracted from, the -apparent time, to give the mean time. The preceding sign, whether it be + or - , at the top of any column, belongs to all the numbers or equations in that column till the sign changes; after which, the remainder of the column takes the contrary sign.

The equation answering to any point of longitude between one degree and another, or any number of minutes or parts of a degree, is to be found by proportion in the usual way, viz, as $1^{\circ}$ or $60^{\prime}$, is to that nus aber of minutes, so is the whole difierence in the equation, from the given whole degree of longitude to the next degree, to the proportional part of it answering to the given number of minutes.

See Tables of the Equation of Time computed for every year, in the Nautical $A$ lmanac, by a method proposed and illustrated by Dr. Masholyne, late astromomer royal, viz, by taking the difference between the sun's true right ascension and his mean longitude, corrected by the equation of the equinoxes in right ascension, and turning it into fime at the rate of 1 minute of time to 15 . of right ascension. Plulos. 'Trans. vol. 5.1, pa. 336. 
TABLE of the Equation of Time, for every Degree of the Sun's Longitude.

\begin{tabular}{|c|c|c|c|c|c|c|c|c|c|c|c|c|c|c|c|c|c|c|c|c|c|c|c|c|c|}
\hline שִ & $\begin{array}{r}r \\
\text { r }\end{array}$ & $\begin{array}{l}0 \\
s\end{array}$ & $\begin{array}{l}\gamma \\
\mathrm{m}\end{array}$ & $\begin{array}{l}1 \\
s\end{array}$ & & $\begin{array}{l}2 \\
s\end{array}$ & & $\begin{array}{l}3 \\
\mathrm{~s}\end{array}$ & & $\begin{array}{l}4 \\
\text { s }\end{array}$ & & $\begin{array}{l}5 \\
\mathrm{~s}\end{array}$ & & $\begin{array}{l}6 \\
8\end{array}$ & $\begin{array}{l}m \\
m\end{array}$ & $\begin{array}{l}7 \\
\text { s }\end{array}$ & $\frac{f}{m}$ & $\begin{array}{c}8 \\
s\end{array}$ & $\begin{array}{l}\text { bo } \\
\text { m }\end{array}$ & $\begin{array}{l}9 \\
\mathrm{~s}\end{array}$ & $\stackrel{m}{m}$ & $\begin{array}{r}10 \\
\mathrm{~s}\end{array}$ & & $\begin{array}{c}11 \\
s\end{array}$ & \\
\hline 0 & $7+$ & & $1-$ & & & $-5 I$ & & -13 & $5+$ & -57 & $2+$ & 20 & $7-$ & & $15-$ & -31 & $13-$ & -33 & $1-$ & 11 & $11+$ & -28 & $14+$ & & \\
\hline 1 & 7 & 17 & 1 & 23 & 3 & 47 & 1 & 26 & 5 & 59 & 2 & 4 & 7 & 58 & 15 & 39 & 13 & 17 & 0 & 42 & 11 & 45 & 14 & 13 & 1 \\
\hline 2 & 6 & 58 & I & 36 & 3 & 41 & 1 & 40 & 6 & 0 & 1 & 48 & S & 19 & 15 & 46 & 13 & 0 & 0 & 1.2 & 12 & 1 & 14 & 6 & 2 \\
\hline 3 & 6 & 39 & 1 & 48 & 3 & 37 & 1 & 53 & 6 & 1 & 1 & 31 & 8 & 40 & 15 & 52 & 12 & 42 & & & 12 & 17 & 13 & 59 & 3 \\
\hline 4 & 6 & 20 & 2 & 0 & 3 & 32 & 2 & 7 & 6 & 1 & 1 & 14 & 9 & 1 & 15 & 57 & 12 & 23 & 0 & 46 & 12 & 32 & 13 & 51 & 4 \\
\hline 5 & 6 & 1 & 2 & 11 & 3 & 26 & 2 & 20 & 6 & 0 & 0 & 56 & 9 & 21 & 16 & 2 & 12 & 4 & 1 & 16 & 12 & 46 & 13 & 43 & 5 \\
\hline 6 & 5 & 42 & 2 & 22 & 3 & 19 & 2 & 33 & 5 & 59 & 0 & 38 & 9 & 41 & 16 & 6 & 11 & 44 & 1 & 45 & 12 & 59 & 13 & 34 & 0 \\
\hline 7 & 5 & 24 & 2 & 32 & 3 & 12 & 2 & 45 & 5 & 57 & 0 & 20 & 10 & 1 & 16 & 9 & 11 & 23 & 2 & 14 & 13 & 12 & 13 & 24 & 7 \\
\hline 8 & 5 & 5 & 2 & 42 & 3 & 4 & 2 & 58 & 5 & 54. & 0 & 1 & 10 & 20 & 16 & 11 & II & 1 & 2 & 43 & 13 & 24 & 13 & 14 & 8 \\
\hline 9 & 4 & 47 & 2 & 51 & 2 & 56 & 3 & 11 & 5 & 51 & $0-$ & 18 & 10 & 39 & 16 & 13 & 10 & 39 & 3 & 11 & 13 & 35 & 13 & 3 & 9 \\
\hline 10 & 4 & 28 & 3 & 0 & 2 & 47 & 3 & 23 & 5 & 47 & 0 & 37 & 10 & 57 & 16 & 13 & 10 & 16 & 3 & 39 & 13 & 45 & 12 & 51 & 10 \\
\hline 11 & 4 & 9 & 3 & 8 & 2 & 38 & 3 & 35 & 5 & 42 & 0 & .57 & 11 & 15 & 16 & 13 & 9 & 53 & 4 & 7 & 13 & 54 & 12 & 39 & 11 \\
\hline 12 & 3 & 50 & 3 & 16 & 2 & 29 & 3 & 46 & 5 & 37 & 1 & 17 & 11 & 33 & 16 & 12 & 9 & 29 & 4 & 3.5 & 14. & 2 & 12 & 27 & 12 \\
\hline 13 & 3 & 32 & 3 & 23 & 2 & 19 & 3 & 58 & 5 & 31 & 1 & 38 & 11 & 51 & 16 & 10 & 9 & 5 & 5 & 2 & 14 & 9 & 12 & 14 & 13 \\
\hline 14 & 3 & 13 & 3 & 30 & 2 & 8 & 4 & 9 & 5 & 24 & 1 & 58 & 12 & 8 & 16 & 7 & 8 & 40 & 5 & 29 & 14 & 16 & 12 & 0 & 14 \\
\hline 15 & 2 & 55 & 3 & 36 & 1 & 57 & 4 & 19 & 5 & 17 & 2 & 19 & 12 & 25 & 16 & 4 & 8 & 14 & 5 & 56 & 14 & 22 & 11 & 46 & 15 \\
\hline 16 & 2 & 37 & 3 & 41 & I & 46 & 4 & 29 & 5 & 9 & 2 & 40 & 12 & 41 & 16 & 0 & 7 & 48 & 6 & 22 & 14 & 27 & 11 & 31 & 16 \\
\hline 17 & 2 & 19 & 3 & 46 & 1 & 35 & 4 & 39 & 5 & 1 & 3 & 1 & 12 & 57 & 15 & 55 & 7 & 22 & 6 & 48 & 14 & 31 & 11 & 16 & 17 \\
\hline 18 & 2 & 1 & 3 & 50 & 1 & 23 & 4 & 48 & 4. & 52 & 3 & 22 & 13 & 12 & 15 & 49 & 6 & .55 & 7 & 13 & 14 & 35 & 11 & 1 & 18 \\
\hline 19 & 1 & 43 & 3 & 5.3 & I & II & 4 & 57 & 4 & 43 & 3 & 44 & 13 & 27 & 1.5 & 42 & 6 & 28 & 7 & 37 & 14 & 38 & 10 & 4.6 & 19 \\
\hline 20 & 1 & 26 & 3 & 56 & 0 & 59 & 5 & 5 & 4 & 33 & 4 & 5 & 13 & 42 & 15 & 35 & 6 & 0 & 8 & 1 & I 4 & 40 & 10 & 30 & 20 \\
\hline 21 & 1 & 9 & 3 & 58 & 0 & 46 & 5 & $13^{\prime}$ & 4 & 22 & 4 & 26 & 13 & 56 & 15 & 26 & 5 & 32 & 8 & 24 & 14 & 41 & 10 & 14 & 21 \\
\hline 22 & 0 & 52 & 4 & 0 & 0 & 34 & 5 & 20 & 4 & 11 & 4 & 47 & 14 & 9 & 15 & 17 & 5 & 4 & 8 & 47 & 14 & 42 & 9 & 58 & 22 \\
\hline 23 & 0 & 36 & 4 & 1 & 0 & 21 & 5 & 27 & 3 & 59 & 5 & 9 & 14 & 21 & 15 & 7 & 4 & 36 & 9 & 9 & 14 & 41 & 9 & 41 & 23 \\
\hline 24 & 0 & 20 & 4 & 1 & 0 & 8 & 5 & 33 & 3 & 46 & 5 & 30 & 14 & 33 & 14 & 56 & 4 & 8 & 9 & 31 & 14 & 40 & 9 & 24 & 24 \\
\hline 25 & 0 & & 4 & 1 & & +5 & 5 & 39 & 3 & 33 & 5 & 52 & 14 & 44 & 14 & 44 & 3 & 39 & 9 & 53 & 14 & 39 & 9 & 6 & 25 \\
\hline 26 & $0-$ & & 4 & 0 & c & 19 & 5 & 44 & 3 & 19 & 6 & 13 & 14 & 53 & 14 & 31 & 3 & 10 & 10 & 14 & 14 & 37 & 8 & 48 & 26 \\
\hline 27 & 0 & & 3 & 59 & 0 & 31 & 5 & 48 & 3 & 4 & 6 & 35 & 15 & 5 & 14 & 17 & 2 & 41 & 10 & 34 & 14 & 34 & 8 & 30 & 27 \\
\hline 28 & 0 & 40 & 3 & 57 & 0 & 46 & 5 & 52 & 2 & 50 & 6 & 56 & 15 & 14 & 1 & 3 & 2 & 11 & 10 & 53 & 14 & 30 & 8 & 12 & 28 \\
\hline 29 & 0 & 53 & 3 & 54 & c & 59 & 5 & 55 & 2 & 35 & 7 & 17 & 15 & 23 & 13 & 48 & 1 & 41 & 11 & 11 & 14 & 25 & 7 & 54 & 29 \\
\hline 30 & 1 & 9 & 3 & 51 & 1 & 13 & 5 & 57 & 2 & 20 & 7 & 28 & 15 & 31 & 13 & 33 & 1 & 11 & 11 & 28 & 14 & 19 & 17 & 36 & 30 \\
\hline
\end{tabular}

EQUATOR, in Geography, a great circle of the earth, The circle in the heavens conceived directly over the equaequally distant from its two poles, and dividing it into tor, is the equinoctial. Sce EQUINoctial. The greatest two equal parts, or hemispheres, the northern and south- height of the equator above the horizon, is equal to the ern. The equator is sometimes simply called the line. latitude of the place.

TaBle for turning Degrees and Minutes into Time, and the Contrary.

\begin{tabular}{|c|c|c|c|c|c|c|c|c|c|c|c|c|c|c|c|c|c|}
\hline & H. & D. & H. M. & D. 1 & H. м. & D. & H. M. & D. & H. М. & D. & H. M. & D. & M. & D. & H. I & D. & H. $\mathrm{N}$ \\
\hline & M. s. & м. & & M. & $\mathrm{M}$ & M. & M. S. & M. & M. $s$. & M. & M. $s$. & M. & M. $s$. & M. & M. 8 . & M. & M. S \\
\hline 1 & 4 & 21 & $2+$ & & 44 & & & 81 & 24 & 01 & 44 & 121 & & & 24 & 61 & 0 \\
\hline & & & & & & & & & & & & & & & & & \\
\hline & $\begin{array}{ll}0 & 12\end{array}$ & 3 & 32 & 3 & 252 & & 412 & 83 & $\begin{array}{ll}5 & 32\end{array}$ & & & 12.3 & $\begin{array}{|ll|}8 & 12 \\
\end{array}$ & & & 63 & \\
\hline & $\begin{array}{ll}0 & 16\end{array}$ & 24 & & 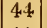 & 256 & & $4 \quad 16$ & 8 & $\begin{array}{ll}5 & 36\end{array}$ & & 56 & 24. & $\left|\begin{array}{ll}8 & 16\end{array}\right|$ & & $\begin{array}{ll}9 & 36\end{array}$ & 64 & 10 \\
\hline & 20 & 2 & 40 & 4.5 & & & 4. 20 & & 540 & & & 125 & & & & & \\
\hline 6 & 24 & & 4 & & & & 424 & & 544 & & & & & & & & \\
\hline 7 & & & & 47 & & & 428 & 87 & 548 & & & 27 & & & & 67 & 11 \\
\hline & 32 & 28 & 52 & 48 & 12 & & 32 & 88 & $5 \quad 52$ & & $\begin{array}{ll}7 & 12\end{array}$ & $12 \mathrm{~s}$ & & & 52 & 168 & 1 \\
\hline & 0 & 29 & 5 & & 10 & & & & 556 & & 71 & & & & 56 & & \\
\hline 10 & 0 & $3 c$ & & & 3 & & & & & & & 30 & & & 10 & 170 & 1 \\
\hline 1 & & & & & $\begin{array}{ll}3 & 24 \\
0\end{array}$ & & 4. & 9 I & & & 724 & 131 & & 151 & 10 & 171 & 11 \\
\hline 12. & 48 & 32 & & & 328 & & 4 & 9 & & & 72 & 132 & & & 10 & 72 & 1 \\
\hline 13 & $\begin{array}{lll}0 & 52\end{array}$ & 33 & 12 & 5 & $\begin{array}{ll}3 & 32\end{array}$ & & 4 & 93 & (b) 12 & & 32 & & & & $\begin{array}{ll}10 & 12\end{array}$ & 173 & 3 \\
\hline $1+$ & 056 & & & & $\begin{array}{ll}3 & 36\end{array}$ & 7 & 56 & & 61 & & 736 & 134 & , & 154 & 1016 & 174 & \\
\hline 15 & & 3 & 2 & 5 & 340 & 7 & & 9 & 62 & & 74 & 135 & & & 1020 & 175 & 1 \\
\hline 16 & & 36 & 24 & 56 & $\begin{array}{ll}3 & 4\end{array}$ & 7 & & 9 & $\begin{array}{lll}6 & 24\end{array}$ & & 74 & 36 & & & 10 & 176 & 1 \\
\hline 17 & I & 3 & & 5 & $\begin{array}{ll}3 & 4.8\end{array}$ & 7 & & & & & 74 & & & & $10 \quad 28$ & 177 & \\
\hline & 112 & & 2 & & & & 512 & & 63 & & 75 & & & & 1032 & 178 & 11 \\
\hline 19 & 16 & $3 !$ & 23 & $5 !$ & 356 & 7 & $5 \quad 16$ & 99 & 636 & & 756 & 139 & 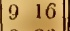 & 150 & 10 & 179 & 1150 \\
\hline & & & & & & & & & & & & & & & $10+$ & & \\
\hline
\end{tabular}


As one whole revolution of the earth, or of the $360^{\circ}$ of the equator, is performed in 24 hours, which is at the rate of $15^{\circ}$ per hour; hence the number of degrees of the equator, answering to any other given time, or the time answering to any given number of degrees of the equator, will be easily found by proportion, viz,

\section{as $1^{\mathrm{h}}: 15^{\circ}$ : : any time : its degrees,}

or as $15^{\circ}: 1^{\mathrm{h}}:$ : any degrees : their time.

And thus is computed the foregoing table for turning time into degrees of the equator, and the contrary.

EQUATORIAL, Universal, or Portable ObservaTORY, is an instrument intended to answer a number of useful purposes in practical astronomy, independently of any particular observatory. It may be employed in any steady room or place, and it performs most of the useful problems in the science of astronomy. The following is the description of one lately invented by Mr. Ramsden, and named the Universal Equatorial, the description of which was given by the Hon. Stewart Mackenzie.

The principal parts of this instrument (fig. 2, plate viii.) are, 1st, The azimuth or horizontal circle $A$, which represents the horizon of the place, and moves on a long axis $\mathrm{B}$, called the vertical axis. $2 \mathrm{~d}$, The equatorial or hour-circle $c$, representing the equator, placed at jight angles to the polar axis $\mathrm{D}$, or the axis of the earth, upon which it moves. 3d, The semicircle of declination $\mathbf{E}$, on which the telescope is placed, and moving on the axis of declination, or the axis of motion of the line of collimation $F$; which circles are measured and divided as in the following table :

\begin{tabular}{|c|c|c|c|c|c|}
\hline $\begin{array}{l}\text { Measures of the se- } \\
\text { veral circles and } \\
\text { divisions on them }\end{array}$ & 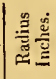 & $\left|\begin{array}{ll}\text { Limb di- } \\
\text { vided to }\end{array}\right|$ & $\left|\begin{array}{c}\text { Non. of } \\
30 \text { gives } \\
\text { seconds. }\end{array}\right|$ & $\left|\begin{array}{l}\text { Divid, on } \\
\text { limb into } \\
\text { pts. of Inc }\end{array}\right|$ & $\begin{array}{l}\text { Divided by } \\
\text { Non. into } \\
\text { pts. of Inc. }\end{array}$ \\
\hline $\left.\begin{array}{c}\text { Azimuth or } \\
\text { horizontal } \\
\text { circle }\end{array}\right\}$ & $5 \cdot 1$ & $15^{\prime}$ & $30^{\prime \prime}$ & 4.5 th & 1350th \\
\hline $\left.\begin{array}{c}\text { Equatorial } \\
\text { or hour } \\
\text { circie }\end{array}\right\}$ & $5 \cdot 1$ & $\begin{array}{c}15^{\prime} \\
\text { or } \mathrm{tm} . \\
\text { in time }\end{array}$ & $\begin{array}{r}30^{\prime \prime} \\
2^{\prime \prime}\end{array}$ & 45 th & 1350th \\
\hline $\left.\begin{array}{l}\text { Vertical se- } \\
\text { micircle for } \\
\text { declination } \\
\text { orlatitude. }\end{array}\right\}$ & $5^{\circ}$ & $15^{\prime}$ & $30^{\prime \prime}$ & $42 d$ & 1260 th \\
\hline
\end{tabular}

4th, The telescope, is an achromatic refractor with a triple objert-glass, whose focal distance is 17 inches, and its aperture $2 \cdot 45$ inc., and it is furnished with 6 difierent eye-tubes; so that its magnifying powers extend from 4.4 to 168 . The telescope in this equatorial may be brought parallel to the polar axis, as in the figure, so as to point to the pole-star in any part of its diurnal revolution; and thus it has been observed near noon, when the sun has shone very bright. 5th, The apparatis for correcting the error in altitude occasioned by refraction, which is applied to the end of the telescope next the eye-glass, and consists of a slide $\mathrm{G}$ moving in a groove or dove-tail, and carrying the several cye-tubes of the telescope, on which slide there is an index corresponding to five small divisions engraved or the dove-tail; a very sinall circle, called the refiaction circle 11 , noveable by a linger-screw at the extremity of the and of the telescope next the eyc-ghtiss; which circle is divided into halt minutes, one whole revolution of it teing equal to $3^{\prime} 18^{\prime \prime}$, and by its motion it raises the centre: of the cross hairs on a circle of altitude; and also at quadrane 1 of $1 \frac{1}{2}$ inc, 1 adius, with divisions on each side, one expressing the degree of altitude of the object viewed, and the other expressing the minutes and seconds of the error occasioned by refraction, corresponding to that degree of altitude. To this quadrant is connected a small round level $k$, which is adjusted partly by the pinion that turns the whole of this apparatus, and partly by the index of the quadrant; for which purpose the refraction circle is set to the same minute $\delta c$, which the index points to on the limb of the quadrant; and if the minute \&c, given by the quadrant, exceed the $3^{\prime} 1 \mathrm{~s}^{\prime \prime}$ contained in oncentire revolution of the refraction circle, this must be set to the excess above one or more of its entire revolutions; then the centre of the cross bairs will appear to be raised on a circle of altitude to the additional height which the error of refraction will occusion at that altitude.

The principal adjustment in this instrument, is that of making the line of collimation to describe a portion of an hour-circle in the licavens : in order to which, the azinuth circle must be truly leve! the line of collimation, or some corresponding line represented by the small brass rod $M$ parallel to it, must be perpendicular to the axis of its own proper motion; and this last axis must be perpendicular to the polar axis. On the brass rod a there is occasionally placed a banging level $\mathrm{N}$, the use of which will appear from the following directions :

The azimuth circle may be made level by turning the instrument till one of the levels be parallel to an imaginary line joining two of the feet screws; then adjust that level with these two feet screws; turn the circle $180^{\circ}$, or half round; and if the bubble be not then right, correct half the error by the screw belonging to the level, and the other half error by the two foot screws, repeating this operation till the bubble come right; then turn the circle $90^{\circ}$ from the two former positions, and set the bubble right again, if it be wrong, by the foot screw at the cud of the level; when this is done, adjusit the other level by its own screw, and the azimuth circle will be truly level. The hanging level must then be fixed to the brass rod by two hooks of equal length, and made truly parallel to it: for which purpose, the polar ax is must be made perpendicular or nearly perpendicular to the horizon; then adjust the level by the pinion of the declination semicircle : reverse the level, and if it be wrong, correct halt the crror by a small stecl screw that lies under one end of the level, and the other half error by the piniou uf the declination-scmicircle, repeating the operation till the bubble be right in both positions. To make the brass rod, on which the level is suspended, at right angles to the axis of motion of the telescope, or line of collimation, make the polar axis horizontal, or nearly so; set the declination semicircle to $0^{\circ}$, and turn the hour-circle till the bubble be properly arljusted; then turn the declinationcircle to $90^{\circ}$; adjust the bubble by raising or depressing the polar axis (first by land till it be nearly right, afterwards tighten with an ivory key the socket which runs on the arch with the polar axis, and then apply the same ivory kry to the adjusting serew at the ('nd of the said arch till the bubble be properly adjusted); then turu the reclination-circle to the opposite $90^{\circ}$; and if the lerel be not then right, correct halt the error by the afores:isl adjusting screw at the end of the arch, and the other half error by the two screws that raise or depress the end of the brass rod. The polar axis remaining nearly horizontal as before, and the declimation-semicircle at $0^{\circ}$, adjust the 
bubble by the hour-circle; then turn the declinationsemicircle to $90^{\circ}$, and adjust the bubble by raising or depressing the polar axis; then turn the hour-circle 12 hours; and if the bubble be wrong, correct half the error by the polar axis, and the other half error by the two pair of capstan screws at the feet of the two supports on one side of the axis of motion of the telescope; and thus this axis will be at right angles to the polar axis. The next adjustment, is to make the centre of the cross hairs remain on the same object, while the eyc-tube is turned quite round by the pinion of the refraction apparatus: in order to perform this, set the index on the slicle to the first division on the dove-tail; and set the division marked $18^{\prime \prime}$ on the refraction-circle to its index; then look through the telescope, and with the pinion turn the eye-tube quite round; then if the centre of the bairs do not remain on the same spot during that revolution, it must be corrected by the four small screws, 2 and 2 at a time, which will be found upon unscrewing the nearest end of the eye-tube that contains the first eye-glass; repeating this correction till the centre of the hairs remain on the spot looked at during a whole revolution. 'To make the line of collimation parallel to the brass rod on which the level bangs, set the polar axis horizontal, and the declination-circle to $90^{\circ}$, adjust the level by the polar axis; look through the telescope on some distant horizonta] object, covered by the centre of the cross hairs: then invert the telescope, which is done by turning the hour-circle half round; and if the centre of the cross bairs do not cover the same object as before, currect half the error by means of the upper and lower of the 4 small screws at the eye-end of the large tube of the telescope; this correction will give a second object now covered by the centre of the hair : which must be adopted instead of the first object; then invert the telescope as before; and if the second object be not covered by the centre of the hairs, correct half the elror by the same two sclews as were used before: this correction will give a third object, now covered by. the centre of the hairs, which must be adopted instead of the second object; repeat this operation till no error remains; then set the hour-circle exactly to 12 hours, the declination-circle remaining at $90^{\circ}$ as before; and if the centre of the cross hairs do not cover the last object fixed on, set it to that object by the two lemaining small screws at the eye-end of the large tube, and then the line of collimation will be parallel to the brass rod. For rectifying the nonius of the declination and equatorial circles, depress the telescope as many, degrees \& $\mathrm{c}$ below $0^{\circ}$ or \& on the declination-senicircle, as are equal to the complement of the latitude; then clevate the polar axis till the bubble be horizontal; and thus the equatorial circle will be elevated to the co-latiturle of the place: set this circle to 6 hours; and adjust the level by the pinion of the declination-circle; now turn the equatorial circle exactly 12 hours from the last pusition; and if the level be not right, correct one laif of the error by the equatorial circle, and the other half by the dechination-circle: then turn the equatorial circle back again exactly 12 hours from the last position; and if the level be still ivrong, repeat the correction as before, till it be right, when turned to either position: which being done, set the nonius of the equatorial circle exactly to 6 hours, and the nonius of the declination-ciscle exactly to $0^{\circ}$.

The principal uses of this erpuatorial are,

1st, To find the meridian by one observation only: For which purpose, elevate the equatorial circle to the co-latitude of the place, and set the declination-semicircle to the sun's declination for the day and hour of the day required; then move the azimuth and hour-circles both at the same time, either in the same or in contrary direction, till you bring the centre of the cross hairs in the telescope exactly to cover the centre of the sun; then, the index of the hour-circle will give the apparent or solar tine at the instant of observation; and thus the time is known, though the sun be at a distance from the meridian; after which turn the hour-circle till the index points precisely to 12 o'clock, and lower the telescope to the horizon, in order to observe some point there in the centre of the glass; and that point is the meridian mark, found by one observation only. The best time for this operation is 3 hours before, or 3 hours after, 12 at noon.

2d, To point the telescope to a star, though not on the meridian, in full duy-light. - Having clevated the equatorial circle to the co-latitude of the place, and set the declination-semicircle to the star's declination, move the index of the hour-circle till it points to the precise tinse at which the star is then distant from the meridian, found in the tables of the right ascension of the stars, and the star will then appear in the glass.

Besides these uses, peculiar to this instrument, it may also be applied to all the purposes to which the principal astronomical instruments are applicd; such as a transit instrument, a quadrant, and an equal-altitude instrument, sc.

See the description and drawing of an equatorial telescope, or portable observatory, jnvented by Mr. Short, in the Philos. Trans. No. 493, or vol. 46 , pa. 242 ; and another by Mr. Nairne, vol. 61, pa. 107 ; also the description by Sir Geo. Shuckburgh, in the Philos. Trans. vol. \$3, pa. 67 ; or my Abridg. vol. 17, pa. 299.

EQUIANGULAR Figure, is one that has all its angles equal among themselves; as the square, and all regular polygons. An equilateral figure inscribed in a circle, is always equiangular. But an equiangular figure inscribed in a circle, is not always equilateral, except when it has an odd number of sides: If the sicles be of an even numbér, then the'y may either lie all equal, or else half of them will be equal to each other, and the other half to each otber, the equals being placed alternately. See the demonstration in iny Mathematical Misecklany, pa. 272.

FQUIANGULAR, is also said of any two tigures of the same kind, when each angle of the one is equal to a corresponding angle in the other, whether each figure, separately considered in itselt, be an equiangular figure or not, that is, hitving all its angles oqual to each other. Thus, two triangles are equiangular to cach other, if, $t x . g r$. one angle in each be of $30^{\circ}$, a second angle in each of $50^{\circ}$, and the third angle of each equal to 100 degrees.- Iiquiangular triangles have not-their like sides necessarily equal, but only proportional to each other; but such triangles being always similar to each other.

EQUICRURAL Triangle, is one that has two of its sides. equal to each other; but is more usually called an Isosceles triangle.

EQULCULUS, EQUULEUS, or EQUUS Minor, a constellation of the northern hemisphere. See EeuUueus.

EQUIDIFFERENT, are such things as have equal differences, or alithmetically proportional. It the terms liave all the same difference, viz, the 1 st and $2 \mathrm{~d}$, the $2 \mathrm{~d}$. and $3 d$, the $3 d$ and 4 th, \&c, they are said to be continually, 


\section{$\mathrm{E} Q \mathrm{U}$}

equidifferent; as the uumbers $3,6,9,12, \& \mathrm{c}$, where the common difference is 3 . But if the several different couplets only have the same difierence, as the 1 st and $2 \mathrm{~d}$, the $3 \mathrm{~d}$ and 4 th, the 5th and 6 th, \&zc, they are said to be discretely equidifferent; as the terms 3 and 6,7 and 10,9 and 12 , \&c. See AnIthuetical Progression and Proportion.

EQUILATERAL Figure, is one that has all its sides equal to each other. Such as the square, and all regular polygons, or a triangle that has all its angles equal. Sce Equiangular.

Equilaterat Hyperbola, is that which has the two axes equal to each other, and every pair of conjugate diameters also equal to each other. The asymptotes also are at right angles to each other, and make each balf a right angle with either axis. Also, such an hyperbola is equal to its conjugate hyperbola, as well as to its opposite hyperbola; so that all the four conjugate hyperbolas are mutually equal to each other.

Moreover, as the $3 \mathrm{~d}$ proportional to the two axes is the parameter; therefore, in such a figure, the parameter and two ascs are all three equal to one another. Hence, as the general equation to hyperbolas is $y^{2}=\frac{p}{t}\left(t x+x^{2}\right)$ or $=$ $\frac{c^{3}}{t^{3}}\left(t x+x^{2}\right)$, where $t$ is the transverse axis, $c$ the conjugate, $p$ the parameter, $x$ the absciss, and $y$ the ordinate; then making $t, c$, and $p$ all equal, the equation, for the equilateral hyperbola, becomes $y^{2}=t x+x^{2}$; differing from the equation of the circle only in the sign of the term $x^{2}$, which in the circle is $t x-x^{2}$, when $t$ is taken to represent the diameter of the circle.

EQUILIBRIUM, is an equality between two equal forces acting in opposite directions; so that they mutually balance each other ; like the two equal arms, or scales, of á balance, \&c.

EQuILIBRIUn, in solid bodies, forms a considerable part of the science of Statics. And Equilibrium of fluids, a considerable part of the doctrine of Hydrostatics.

EQUIMULTIPLES, the products of quantities equally multiplied.

Thus $3 a$ and $3 b$ are equimultiples of $a$ and $b$;

and $m c$ and $m d$ are equimultiples of $c$ and $d$.

Equimultiples of any quantities, have the same ratio as the quantities themselves. Thus $a: b:: 3 a: 3 b:: m a: m b$.

EQUINOCTIAL, $a^{\circ}$ great circle in the heavens under which the equator moves in its rliurnal motion. The poles of this circle are the poles of the world, and it divides the sphere in to two equal parts, the northern and southerm. It cuts the horizon of any place, in the east and west points; and at the meridian $\mathrm{its}$ elevation above the horizon is equal to the co-latitude of the place. It has also various other properties; as,

1. When the sun is in this circle, the days and nights in all parts of the earth are equal; because he then rises due east, and sets due west. Ilence it has the name equinoctial. All stars which are under this circle, or have no decination, also rise duc east, and set due west.-2. All pcople living under this circle, or upon the equator, or line, have their days and nights at all times equal to cach other. - 3 . From this circle, on the globe, is counted, upon the meridian, the declination in the heavens, and the latitude on the (arth._4. Upon the equinoctial, or cquator, is counted the longitude, making in all $360^{\circ}$, that is, $180^{\circ}$ east, and $180^{\circ}$ west, -5 . And as the time of one whole
$\mathrm{E} Q \mathrm{U}$

revolution is divided into 24 hours; therefore 1 hour answers to $15^{\circ}$, or the 24 th part of $360^{\circ}$. Hence,

$1^{\circ}$ of longitude answers to $4 \mathrm{~min}$. of time,

$15^{\prime}$ - - - - - - to 1 min. of time,

$1^{\prime}$ - - - - - - to 4 sec. of time, \&c.

6. The shadows of oljects situated under this circle are cast to the southward one half of the year, and to the northward during the other half; and twice in a year, viz, at the time. of the equinoxes, the sun at noon casts no shadow, being exactly in their zenith.

EQuin octin L Colure, is the great circle passing through the poles of the world and the equinoctial points, or first points of Aries and Libra.

Equinoctial Dial, is one whose plane is parallel to the equinoctial. Its properties or principles are:

1. 'The hour-lines are all equally distant from each other, viz, $15^{\circ}$; and the style is a straight pin, or wire, set up in the centre of the circle, perpendicular to the plane of the dial.

2. The sun shines upon the upper part of this dial-plaue from the 21 st of March to the $22 \mathrm{~d}$ of September, and on the under part the other half of the year.

Some of these dials are made of brass, \&c; and set up in a frane, to be elevated to any given latitude.

EQuin octial Points, are the two opposite points where the ecliptic and equinoctial cross each other; the one point being in the beginning of Aries, and called the vernal point, or vernal equinox; and the other in the beginning of Libra, and called the autumnal point, or autumnal equinox.-It is found by observation, that the equinoctial points, and all the other points of the ecliptic, are continually moving backwards, or in antecedentia, i. e. westwards. 'This retrograde motion of the equinoctial points, arises from the Prccession of the equinoxes, and is made at the rate of $50 \frac{x}{4}$ seconds every year nearly. Sec PRECession, also the preface to the Nautical Almanac for the year 1797 .

EQUINOXES, the times when the sun enters the equinoctial points; that is, about the 21 st of March and $22 \mathrm{~d}$ of September: the former being the vernal or spring equinox, and the latter the autumnal equinox.-As the sun's motion is unequal, being sometimes quicker and sometimes slower, it hence happens that there are about $\mathbf{s}$ days more from the vernal to the autumnal equinox, or while the sun is on the northern side of the equator, than while he is in moving through the southern signs, from the autumnal to the verual equinox, or on the southern side of the equator. According to the observations of M.Cassini, the sun is $186^{\mathrm{d}} 14^{\mathrm{h}} 53^{\mathrm{m}}$ in the northern signs,

and only $178 \quad 1456$ in the southern signs,

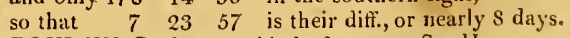
EQUINUS Barbatus, a kind of comet. See lippeus.

EQUITANG ISNTIAL Curve, is one that was first constructed by Mr. J. Perks, the tangent of which is always equal to a constant line. It is the involute of the catenaria; and is also the curve of traction to a straight line. - For the various properties of this curve, its mechanical construction, its relation to the catenaria, and its use in dividing the meridiun in Mcrcator's projection, we refer to the Philos. 'Trans. No. 34.5: séc also Brougham's paper on Porisms, in the Philos. Trans. vol. ss; or my Abridg. vol. 18 , pa. 354, for other curious properties of this curve.

LQUULEUS, Equicurus, and Eques Minor, Equi scetio, the Horse's Head, one of the 48 old constellations, in the northern licmispherc. Its stars in Ptolcmy's cata- 
ogue are 4, in Tycho's 4, in Hevelius's 6, and in Flamsteed's 10.

ERATOSTHENES, a learned Greek philosopher of Cyrene, and librarian of Alexandria under Ptolemy Euergetes, king of Egypt. He was learned in all the sciences, and first discovered a method of measuring the earth's magnitude; whence he was called the Cosmographer and Surveyor of the Universe. He gave a mechanical solution to the famous problem of doubling the cube. And he gave a method of finding prime numbers, in arithmetic, commonly called Eratosthenes' Sive, a learned account of which is given by Dr. Horsley, in the Philos. Trans. of 1772 ; or in my Abridgment, vol. 13, pa. 314. Some of his works remain, which were printed at Oxford, in Greek, 1672, svo. Eratosthenes died about 194 before the year Christ, at 81 years of age.

ERECT Vision. See Vision.

ERECr Dials, such as stand perpendicular to the horizon, and are of various kinds; as erect direct, when they face exactly one of the four cardinal points, east, west, north, south ; and erect declining, when they decline from the cardinal points. See Dial.

To EneCT a P'erpendicular, is a popular problem in practical geometry, and denotes to raise a perpendicular from a given line, \& $x$, as distinguished from demitting or letting fall a perpendicular on a line, \&c, from some point out of it. See Perpendicular.

ERIDANUS, the River, a constellation of the southern hemisphere, and one of the 48 old asterisms. The stars in this constellation, in Ptolemy's catalogue, are 34 , in Tycho's 19, and in the British catalogue 84 .

ERRATIC, an epithet applied to the planets, which are called erratic or wandering stars, in contradistinction to the fixed stars.

ESC $\triangle$ LADE, or ScALADF, a furious attack on a wall or a rampart; carried on with ladders, to pass the ditch, or mount the rampart; without procceding in form, breaking ground, or carrying on regular works to secure the men.

ESPAUle, or Epaule. See Erauje.

ESPLANADE, in Fortification, called also Glacis, a part which serves as a parapet to the counterscarp, or covert way; being a declivity or slope of earth, commencing from the top of the counterscarp, and losing itself insensibly in the level of the champaign.

ESPLANADE means also the ground which bas been levelled from the glacis of the counterscarp, to the first houses; or the vacaut space between the works and the houses of the town. The term is also applied, in the general, to any piece of ground that is male fut or level, and which before had some eminence that incommoded the place.

ESTJVAL Occident, Orient, ar Solstice. See OcciDENT, ORIENT, SOLSTICE.

FSTU $\Lambda$ RY, an arm of the sea; a frith; or the mouth of a lake or river in which the tide reciprocates.

ETHER, an element more fine and subtile than air; or air refined and sublimed; or the matter of the highest regions above.

EтиER, in Chemistry, a certain very light, volatile, fragrant, inflammable liquor, procured by distillation, by heat, from a mixture of alcohol, and sulphuric or some other acid, in equal quantities.

'The specific gravity of ether, is from 0.725 to 0.632 , according to its different slegrees of purity. It appears a limpid and colourless liquor, of a hot pungent taste, highly fragrant, and so volatile that it can hardly be poured from one vessel to another without much loss by evaporation, the purest portion of it assuming the state of elastic fluid.

When exposed to a cold of $-46^{\circ}$, ther freezes and crystallizes. In moderate temperatures it is not affected by oxygen gas or common air; but when kindled in a state of vapour, it burns rapidly with a fine white flame, and leaves behind a trace of charcoal. In the state of vapour it detonates with cominon air and oxygen gas.

EVAPORATION, the act of dissipating the humidity of a body in fumes or vapour; differing from exhalation, which is properly a dispersion of dry particles issuing from a body.-Evaporation is usually produced by heat, and by the change of air: thus, common salt is formed by evaporating all the humidity from the brine or salt water; which evaporation is either performed by the heat of the sun, as in the salt-works on the sea-coast, Sc ; or by means of fire, as at the salt-springs, \&c: and it is well known how useful a brisk wind is in drying wet clothes, or the surface of the ground; while in a calm, still atmosphere, they dry extremely slow.

But, though evaporation be generally considered as an effect of the heat and motion of the air, yet M. Gauteron, in the Memoires de l'Acad. des Scienc. an. 1705, shows, that a quite opposite cause may have the same effect, and that fluids lose more of their parts in the severest frost than when the air is moderately warı : thus, in the great frost of the year 5708 , he found that the greater the cold, the more considerable the evaporation; and that ice itself lost full as much as the warmer liquors that did not freeze.

There are indeed few subjects of philosophical investigation that have occasioned a greater variety of opinion than the theory of evaporation, or of the ascent of water in such a fluid as air, between 8 and 9 hundred times lighter than itself, to different heights, according to the different densities or states of the atmosphere; in which case it must be specifically lighter than the air through which it ascends. The Cartesians account for it by supposing, that by the action of the sun upon the water, small particles of the water are formed into hollow spheres and filled with the materia subtilis, which renders them specificallylighter than the ambient air, so that they are buoyed up by it.

Dr. Nieuwentyt, in his Religious Philosopher, cont. 19, and several others, have alleged, that the sun emits particles of fire, which adhere to those of water, and form molecula, or small bodies, lighter than an equal bulk of air, which consequently ascend till they come to a height where the air is of the same specific gravity with themselves; and that these particles being separated from the fire with which they are incorporated, coalesce and descend in dew or rain.

Dr. Halley has advanced another bypothesis, which has been more generally reccived: he imagined, that by the aetion of the sun on the surface of the water, the aqueous particles are formed into hollow spherules, that are filled with a finer air highly rarefied, so as to become specifically lighter than the external air. Philos. Trans. No. 192, or my Abridg. vol. 3, pa. 427.

Dr. Desaguliers, dissatisfied with these hypotheses, proposes another in the Philos. Trans. No. 407, or Abridg. vol. 7, pa. 323. See also his Course of Experimental Philosophy, vol, 2, pa. 336. He supposcs that lseat acts. 
more powerfully on water than on common air; that the same degree of heat which rarefies air two-thirds, will rarefy water nearly 14,000 times; and that a very small degree of heat will raise a steam or vapour from water, even in winter, while it condenses the air; and thus the particles of water are converted into vapour by being made to repel each other strongly, and, deriving clectricity from the particles of air to which they arc contiguous, are repelled by them and by each other, so as to form a fluid which, being lighter than the air, rises in it, according to their relative gravities. The particles of this vapour retain their repellent force for a considerable time, till, by some diminution of the density ol the air in which they float, they are precipitated downwards, and brought within the sphere of each other's attraction of cohesion, and so join again into drops of water.

Many objections have been urged against this opinion; as, by Mr. Clare, in his Treatise of the Motion of Fluids, pa. 294; and by Mr. Rowning in his System of Philosophy, part 2, diss. 6 ; to which Dr. Hamilton has added the two following, viz, That if heat were the only cause of evaporation, water would evaporate faster in a warm close room, than when exposed in a colder place, where there is a constant current of air; which is contrary to experience; and that the evaporation of water is so far from depending on its bejug rarefied by heat, that it is carried on even while water is condensed by the coldness of the air, till it freezes; and since it evaporates even when frozen into hard ice, it must also evaporate in all the lesser degrees of cold. And therefore heat does not seem to be the principal, much less the only cause of evaporation.

Others have more successfully accounted for the phenomena of evaporation on another priuciple, viz, that of solution; having shown, from a variety of experiments, that what we call evaporation, is nothing more than a gradual solution of water in air, produced and supported by the same means, viz, attraction, heat, and motion, by which other solutions are effected.

It seems however that the Abbé Nollet first started this opinion, in his Leçons de Plyysique' Experimentale, published in 1743, without pursuing it much further: he there only conjectures that the air of the atmosphere may serve as a solvent or sponge, with regard to the bodies that encompass it, and receive into its pores the vapours and exhalations that are detached from the masses to which they belong in a fluid state; and lıe accounts for their ascent on the same principles with the ascent of liquors in capillary tubes. On his hypothesis, the condensation of the ajr contributes; like the squeczing of a sponge, to their descent.

Dr. Franklin, in a paper of Philosophical and Meteorological Observations, Conjectures, and Suppositions, delivered to the Royal Society about the year 1747, and read in 1756 , suggested a similar hypothesis: he observes, that air and water mutually attract each other; and hence he concludes, that water will dissolve in air, as salt in water; every particle of air assuming one or more particle of water; and when too much is added, it precipitates in rain. But as there is not the same contiguity botween the particles of air as there is between those of water, the solution of water in air is not carried on without a motion of the air, so as to cause a fresh accession of dry particles. A small degree of heat so weakens the coliesion of the particles of water, that those on the surface casily quit it, and adhere to the particles of air: a greater degree of heat is necessary to break the cohesion between water and ajr; for its particles being by heat repelled to a greater distance from each other, they thereby more easily keep the particles of water, that are annexed to them, from running into cohesions that would obsiruct, refract, or reflect the heat: and hence it happens that when we breathe in warm air, though the same quantity of moisture may be taken up from the lungs as when we breathe in cold air, yet that moisture is not so visible. On which principles he accounts for the production and different appearances of fogs, mists, and clouds. And be ards, that if the particles of water bring with them electrical fire when they attach themselves to air, the repulsion between the particles of water electrified, joins with the natural repulsion of the air to force its particles to a greater distance, so that the air being more dilated, it rises and carries the water up with it; which mutual repulsion of the particles of air is increased by a mixture of common fire in the particles of water. When air, loaded with surrounding particles of water, is compressed by adverse winds, or by being driven against mountains, $\& \mathrm{c}$, or condensed by taking away the fire that assisted it in expanding, then the particles will approach one another, and the air with its water will descend as a dew; or if the water surrounding one particle of air come in contact with the water surrounding another, they will coalesce and form a drop, producing rain; and since it is a well-known fact, that vapour is a good conductor of electricity, as well as of common fre, it is reasonable to conclude with Mr. Henley, that evaporation is one great cause of the clouds becoming at times surcharged with this fluid. Philos. Trans. vol. 67, pa. 134. See also vol. 55 , pa. 18,2; or Franklin's Letters and Papers on Philosophical Subjects, pa. 42, \&c, and pa. 182 , ed. 1769 .

M.le Roi, of the Academy of Sciences at Paris, has also advanced the same opinion, and supported it by a variety of facts and observations in the Memoirs for the year 1751. He shows, that water undergoes in the ajr a real dissolution, forming with it a transparent mixture, and possessing the same properties with the solutions of most salts in water; and that the two principal causes which promote the solution of water in the air, are beat and wind; that the hotter the air is, within a certain limit, the more water it will dissolve; and that at a certain degree of heat the air will be saturated with water; and by determining at different times the degree of the air's saturation, he estimates the influence of those causes on which the quantity depends that is suspended in the air in a state of solution. Accordingly, the air, heated by evaporating substances to which it is contiguous, becomes more rare and light, rises and gives way to a denser air; and, by being thus removed, contributes to accelerate the evaporation. The fixed air contained in the internal parts of evaporating bodies, put into action by heat, seems also to increase their evaporation. The wind is another cause of the increase of evaporation, chiefly by changing and renewing the' air which immediately encompasses the evaporating substances; and from the consideration of these two cause's combined, it appears why the quantity of vapour raised in the night is less than that raised in the day, since the air is then both less heated and less agitated. To the objection urged against this hypothesis, on account of the evaporation of water in a vacuum, this ingenious writer replies, that the water itself contaius a great quantity of air, which gradually disengages itself, und causes the craporation; and that it is impossible that a space containing water which eviporates should remain perlectly free from air. To this 
objection Dr. Dobson of Liverpool replics, that though air appears, by unquestionable experiments, to be a chemical solvent of water, and, as such, is to be considered as one cause of its evaporation; heat is another cause, acting without the intervention of air, and producing a copious evaporation in an exhausted receiver; agreeably to an experiment of Dr. Irving, who says, that in an exhausted receiver water rises in vapour more copiously at $180^{\circ}$ of Fahrenheit's thermometer, than in the open air at $212^{\circ}$, its boiling point. Dr. Dobson further adds, that water may exist in air in three different states; in a state of perfect solution, when the air will be clear, dry, and heavy, and its powers of solution still active; in a state of beginning precipitation, when it becomes moist and foggy, its powers of solution are diminished, and it becomes lighter in proportion as its water is deposited ; and also, when it is completely precipitated, whicb may happen either by a slower process, when the dissolved water falls in a drizzling rain, or by a more sudden process, when it descends in brisk showers. Philos. Trans. vol. 67, pa. 257 ; and Phipps's Voyage towards the Nortl Pole, pa. 211.

Dr. Hamilton, professor of philosophy in the university of Dublin, transmitted to the Royal Society in 1765 , a long dissertation on the nature of evaporation, in which he proposes and establishes this theory of solution; and though other writers had been prior in their conjectures, and even in their reassoning on this subject, Dr. Hamilton assures us, that he has not represented any thing as new which he was conscious had ever been proposed by any one before him, even as a conjecture. Dr. Hamilton having evinced the agreement between solution and evaporation, concludes, that evaporation is nothing more than a gradual solution of water in air, produced and promoted by attraction, heat, and motion, just as other solutions are effected.

In order to account for the ascent of aqueous vapours into the atmosphere, this ingenious writer observes, that the lower beds of the air being pressed by the weight of the upper beds against the surface of the water, and continually rubbing upon it by its motion, attracts and dissolvcs those particles with which it is in contact, and separates them from the rest of the water. And since the cause of solution in this case is the stronger attraction of the particles of water towards the air than tuwards each other, those that are already dissolved and taken up, will be still further raised by the attraction of the dry air that lies over them, and thus they will diffuse themselves, rising gra. dually higher and higher, and so leave the lower strata of air not so much saturated but that it will still be able to dissolve and take up fresh particles of water; which process is greatly promoted by the motion of the wind. When the vapours are thus raised and carried by the winds into the higher and colder parts of the atmosphere, some of them will coalesce into small particles, which slightly attracting each other, and being intermixed with air, will form clouds; and these clouds will float at different heights, according to the quantity of vapour borne up, and the degree of heat in the upper parts of the atmosphere: and thus clouds are generally higher in summer than in winter. When the clouds are much increased by a continual addition of vapours, and their particles are driven close tugether by the force of the winds, they will run into drops heavy enough to fall down in ruin. If the clouds be frozen brfore their particles are gathered into drops, small picces of them, being condensed auxl made VOL. I. heavier by the cold, fall down in thin flakes of snow. When the particles are formed into drops before they are frozen, they become hailstones. When the air is replete with vapours, and a cold breeze springs up, which checks the solution of them, clonds are formed in the lower parts of the atmosphere, and compose a mist or fog, which usually bappens in a cold morning, and is dispersed when the sun has warmed the air, and made it capable of dissolving these watery particles. Southerly winds commonly bring rain, because, being warm and replete with aqueous vapours, they are cooled by coming into a coldcr climate; and therefore they part with some of them, which precipitate in rain: whereas northerly winds, being cold, and acquiring additional heat by coming into a warmer climate, are ready to dissolve and receive more vapour tban they before contained; and therefore are commonly attended with fair weather.

Changes of the air, with respect to its density and rarity, as well as its heat and cold, will produce contrary effects in the solution of water, and the consequent ascent or fall of vapours. Several experiments have been made which prove that air, when rarefied, cannot keep so much water dissolved in it as it does in a more condensed state; and therefore when the atmosphere is saturated with water, and changes from a denser to a rarer state, the high and colder parts of it will let go some of the water before it is dissolved, forming new clouds, and disposing them to fall down in rain: but a change from a rarer to a denser state will stop the precipitation of the water, and enable the air to dissolve, either in whole or in part, some of those clouds that were formed before, and render their particles less apt to run into drops and fall down in rain : on this account, we generally find that the rarefied and condensed states of the atmosphere are respectively attended with rain or fair weather. See more on this subject in the Philos. Trans, vol. 55, pa. 146; or Hamilton's Philosophical Essays, pa. 33.

Dr. Halley, before mentioned, furnished several experiments on the evaporation of water; the result of which is contained in the following articles: 1 . That water salted to about the same degree as sea-water, and exposed to a heat equal to that of a summer's day, dicl, from a circular surface of about 8 inches diameter, evaporate at the rate of 6 ounces in 24 hours: whence by a calculus he finds that, in such circumstances, the water cvaporates $1-10$ th of an inch deep in 12 hours : which quantity, he observes, will be found abundantly sufficient to furnish all the rains, springs, dews, \&c. By this expcriment, every 10 square inches of surface of the water, yield in vapour, per diem, a cubic inch of water : and each square foot half a wine pint ; every space of 4 fect square, a gallon; a mile square, 6914 tons; and a square degree, of 69 English miles, will evaporate 33 millions of tons a day; and thus the whole Mediterranean, which is computed to contain 160 square degrees, will yield at least 5280 millions of tons each day. Philos. Trans. No. 189; or my Abridg. vol. 3, pa. $387 .-2$. A surface of $\&$ square inches, evaporated purely by the natural warmth of the weather, without either wind or sun, in the course of a whole year, 16292 grains of water, or 64 cubic inches; consequently, the deptl of water thus evaporated in one year, amounts to 8 inches. But this being too little to answer the experiments of the French, who found that it rained 19 inclies of water in one year at Paris ; or those of Mr. Townley, who found the annual quantity of rain in Lancashire above 40 inches; $3 \mathrm{R}$ 


\section{E U C}

he therefore concludes, that the sun and wind contribute more to evaporation than any internal heat or agitation of the water. In effect, Dr. Halley fixes the annual evaporation of London at 4.8 inches ; and Dr. Dobson states the same for Liverpool at $36 \frac{3}{4}$ inches. Philos. Trans. vol. 67, p 252.

3. The effect of the wind is very considerable, on a double account ; for the same observations sbow a very singular quality in the vapours of water, viz, that of adhering to the surface that exhaled them, which they clothe as it were with a flecce of vaporous air ; which once investing the vapour, it afterwards rises in much less quantity. Whence the quantity of water lost in 2.4 hours, when the air is very still, was very small, in proportion to what went off when there was a brisk gale of wind abroad to dissipate the fleece, and make room for the emission of vapour; and this, even though the experiment was made in a plice as secure from the wind as could be contrived. Moreover, this fleece of water, hanging to the surface of waters in still weather, is the occasion of very singular appearances, by the refraction of the vapours differing from and exceeding that of common air; whence evcry thing appears more lofy, or raised above its usual level, as houses, steeples, ships, \&c.

4. The same experiments show that the evaporation in May, June, July, and August, which are nearly equal, are about three times as great as those in the montlis of November, Decomber, January, and February. Philos. Trans. No. 212, or my Abridg. vol. 3, pa. 658.

Dr. Brownrigg, in his Art of making common salt, pa. 189 , fixes the evaporation of some parts of England at 73.8 inches during the months of May, June, July, and August; and the evaporation of the whole year at more than 140 inches. But the evaporation of the four summer months at Liverpool, on a medium of 4 years, was found to be only 18.88 inches. Also Dr. Hales calculates the greatest ennual evaporation from the surface of the earth in England-at 6.66 inches; and therefore the annual evaporation from a surface of water, is to the annual evaporation from the surface of the earth at Liverpool, nearly as 6 to 1. Philos. Trans, vol, 67, ubi supra.

In the Transactions of the American Philosophical Society, rol. 3, pa. 125, there is an ingenious paper on evaporation, by 1)r. Wistar: where he shows that evaporation arises when the moist body is warmer than the medium it is inclosed in : and, on the contrary, it acquires moisture from the air, the colder the body is. This carrying off, and acquiring of moisture, it is shown, is by the passage of heat out of the body, or into it.

EUCLID, of Megara, a celebrated philosopher and logician; he was a disciple of Socrates, and fourished about 400 years before Christ. The Athenians having prohibited the Megarians from entering their city on pain of death, this philosopher disguised himself in women's clothes to attend the lectures of Socrates. $\Lambda$ fter the death of Socrates, Plato and other philosophers went to '́uclid at Mcgara, to shelter themselves from the tyrants who governed Athens. Euclid admitted but one chief good; which he at different times called God, or the Spirit, or Prosvidence.

'Tlis philosopher las, by sonc, been confounded with Euclid of Alexandria, the prince of geometricians; but independently of the differcint subject of their writings, it is well known that Euclid of Mceara flourished at least a century before Euclid of Alexandria was born:

EucLID, the celebrated mathematician, according to the account of Pappus and Proclus, was born at Alexandria, in Egypt, where be flourished and tanght mathematics with great applause, under the rcign of Ptolemy Lagos, about 280 years before Christ. Some Arabian historians, however, inform us, that he was born at Tyre, that his father's name was Naucrates, an inhabitant of Damas,The particular place of his nativity appears, therefore, to be uncertain; but whether or not Alexandria had the ho. nour of giving birth to this celebrated mathematician, all historians agree that he finurished and taught mathematics there, at the time abovementioned; which city, from that period to the conquest of it by the Saracens, seems to have been the residence, if not the birth-place, of all the most eminent mathematicians of that tine. Euclid reduced into regularity and order all the fundamental principles of pure mathematics, which had been delivered down by Thaless, Pythagoras, Eudoxus, and other mathematicians before him, and added many others of his own : on which account it is said he was the first who reduced arithmetic and geometry into the form of a science. He likewise applied himself to the study of mixed mathematics, particularly to astronomy and optics.

His works, as we learn frum Pappus and Proclus, are the Elemients, Data, Introduction to Harmony, Phenomena, Optics, Catoprics, a Treatise on the Division of Superficies, Porisms, Loci ad Superficiem, lallacies, and. Four books of Conics. 'The most celebrated of these, is the first work, the Elements of Geometry; of which there have been numberless"editions, in all languages; and a fine edition of all his works, now extant, was printed in 1703 , by. David Gregnry, Savilian professor of astronomy at Oxford.

The Elements, as commonly published, consist of 15 books, of which the last two, it is suspected, are not Euclid's, but a comment of Hypsicles of Alexandria, who lived 200 years after him. They are divided into three parts, viz, the Contemplation of Superficies, Numbers, and Solids: the first 4 books treat of planes only; the 5 th of the proportions of magnitudes in general; the 6th of the proportion of plane figures; the $7 \mathrm{th}$, sth, and $9 \mathrm{th}$, give us the flindamental properties of number's; the 10th contains the theory of commensurable and incommensurable lines and spaces; the 11 th, 12 th, $13 \mathrm{th}, 14 \mathrm{th}$, and 15 th, treat of the doctrine of sulids.

There is no doubt hut, before Euclid, Elements of Genmetry were compiled by Hippocrates ot Chios, Eudoxus, Leon, and many others, mentioned by Proclus in the beginning of his second book; for he affirms that Euclid ne'wordered many things in the lilements of Eudoxus, cumpleted many things in those of 'Theatetus, and besides strengthened such propositions as before were 100 slightly, or but superficially established, with the most firm and convincing demonstrations.

History is silent as to the time of Euclid's death, or his age. But pappus represents him as a person of al cou1teous and agrceable behaviour, and in great esterm with Ptolemy Lagos, king of Egyjt; who one day ashing him, whether there was not any shorter way of cuming at geometry than by his Elements, Euclid is said to have answered, "that there zuas no royal road to geometry."

EUDIOMEIER, an instrument for deternining the purity of the air, or the quantity of pure and dephlogisticated or vital air coutained in it, chiefly by means of its. diminution on a mixture with nitrous air.

Instruments of this kind were first made, in consequenco of the experiments and discoveries of Dr. P'ricstley, for de- 
termining the salubrity of different kinds of air. That writer having discovered, that when nitrous air is mixed with any other air, their original bulk is diminished; and that the diminution is nearly, if not exactly, in proportion to its salubrity; he was hence led to adopt nitrous air as a true test of the purity of respirable air ; and nothing more seemed to be necessary but an easy, expeditious, and accurate method of estimating the degree of diminution in different cases; and for this purpose, the eudiometer was contrived; of which several kinds have been invented, the principal of which are the following.

I. 'The eudiometer originally used by Dr. Pricstley is a divided glass tube, into which, after having filled it with common water, and inverted it into the same, one measure or more of common air, and an equal quantity of nitrous air, are introduced by means of a small phial, which is called the measure; and thus the diminution of the volumc of the mixture, which is seen at once by means of the graduations of the tube, instantly discovers the purity of the air required.

II. The discovery of Dr. Priestley was announced to the public in the year 1772; and several persous, both at home and abroad, presently availed themselves of it, by framing other more accurate instruments. The first of these was contrived by M. Landriani; a description of which is given in the 6th volume of Rosier's Journal, for the year 1775 . It consists of a glass tube, fitted, by grinding, to a cylindrical vessel, to which are joined two glass cocks and a small bason; the whole being fixed to a wooden frame. In this instrument quicksilver is used instead of water; though that is attended with an inconvenience, because the nitrous air acts upon the metal, and rendcrs the experiment ambiguous.

III. In 1777, Mr. Mageltan published an account of three eudiometers invented by himself, consisting of glass vessels of rather difficult construction, and troublesome to use. M. Cavallo observes, that the construction of all the three is founded on a supposition, that the mixture of nitrous and atmospherical air, having continued for sonc time to diminish, afterwards increases again; which it seems is a mistake: nor do they give accurate or uniform results, in experiments made with nitrous and common air of precisely the same quality.

IV. A preferable and very accurate method of discovering the purity of the air by means of a cudiometer, is recommended by M. Fontana. The instrument is originally nothing more than a divided glass tube, though the inventor afterwards added to it a complicated apparatus, perhaps of little or no use. The first simple eudiometer consisted only of a glass tube, uniformly cylindrical, about 18 inches long, and $3-4$ ths of an incli diameter within-side, the outside being marked with a diamond at such distances as are exactly filled by equal measures of elastic fluids : and when any parts of these divisions are required, the edge of a ruler, divided into inches and smaller parts, is held against the tube, so that the first livision of the ruler may coincide with one of the marks on the tube. The nitrous and atmospherical air are introduced into this tubc, in order to be diminished, and thence the purity of the atmospheric air ascertained.

V. M. Saussure of Geneva also invented a eudiometer, which he thinks is more exact than any of those before described; the apparatus of which is as follows: 1. A cylindrical glass bottle, with a ground stopple, containing about $5 \frac{1}{2}$ ounces of air, which serves as a receiver for mix- ing the tivo airs._- A small glass phial, to serve as a incasure, and is about one-third the size of the receiver.3. A small pair of scales that may weigh very exactly.4. Several glass bottles, for containing the nitrous or other air to be used, and which may supply the place of the recipient when broken. The method of using this instrument is as follows:-The receiver is to be filled with water, closed exactly with its glass stopper, wiped dry on the outside, and then weighed very accurately. Being then immerged in a vessel of water, and held with the mouth downwards, the stopple is removed, and, by means of a funnel, two measures of common and one of nitrous air are introduced into it, one after another: these diminish as soon as they come into contact; in consequence of which the water enters the recipient in proportionable quantity. After being stopped and well shaken, to promote the diminution, the receiver is to be opened again under water; then stopped and shaken again, and so on for three times successively, after which the bottle is stopped for the last time under water, then taken out, wiped very clean and dry, and exactly weighed as before. It is plain that now, the bottle being filled partly with elastic fluid and partly with water, it must be lighter than when quite full of water; and the difference between those two weights, shows nearly what quantity of water would fill the space occupied by the diminished elastic fluid. But, in making experiments with airs of different degrees of purity, the said difference will be grcater when the diminution is less, or when the air is less pure, and vice versa; by whicb means the comparative purity between two different kinds of air is determined.

VI. But as this method, notwithstanding the encomiuris bestowed on it by the inventor, is subject to several errors and inconveniences; to remedy all these, another instrument was invented by M. Cavallo; the description of which, being long, may be seen in his Treatise on the Nature and Properties of Air, pa. 344.

Other constructions of the eudiometer have also been given by Mr. Cavendish and Mr. Schcele. For further information, seè Magellan's Letter to Dr. Priestley, containing the Description of a Glass Apparatus, \&zc and of New Eudiometers, \&c, 1777, pa. 15, \&c; Priestley's Exp. and Obs. on Air, vol. 3, preface and appentix; the methods of Dr. Ingenhousz in Philos. Trans. vol. 66, art. 15; see also the Philos. Trans. vol. 73 ; and Cavallo's Treatise on Air, pa. 274, 315, 316, 317, 328, 340, 344, and 348 .

EUDOXUS, of Cnidus, a city of Caria in Asia Ninor, flourished about 370 years before Christ. He learned geometry from Archytas, and afterwards travclled into Egypt to learn astronomy and othes' sciences. There he and Plato studied together, as Laertius informs us, for the space of 13 years; and afterwards came to $A$ thens, fraught with all sorts of knowledge, which they liad learned from the priests. Here Eudoxus opened a school; which he supported with so much glory and renown, that cven Plato, though his friend, is said to have envied him. Eudosus composed Elements of Geometry, from which Euclid liberally borrowed, as mentioned by Proclus. Cicero calls Eudoxus the greatest astronomer that bad ever lived: and Petronius says, lie spent the latter part of his life upon the top of a very high mountain, that he might contesplate the stars and the heavens with more convenience and less interruption: and we learn from 'Strabo, that there were some remains of his observatory at C'nidus to be seen cren in his time. He died in the $53 \mathrm{~J}$ year of his age. 
EVECTION, is used by some astronomers for the libration of the moon; being an inequality in her motion, by which, at or near the quadratures, she is not in a line drawn tbrough the centre of the earth to the sun, as she is at the syzygies, or conjunction and opposition, but makes an angle with that line of about $2^{\circ} 5 \mathbf{l}^{\prime}$. The motion of the moon about lier axis only is equable, which rotation is performed exactly in the same time as she revolves about the earth; for which reason it is that she turns always the same face towards the earth nearly, and would do so exactly were it not that her menstrual motion about the earth, in an elliptic orbit, is not equable; on which account the moon, seen from the earth, appears to liberate a little upon her axis, sometimes from east to west, and sometimes from west to east; or some parts in the eastern limb of the moon go backwards and forwards a small space, and some that were conspicuous, are bid, and then appear again.

The term Evection is used by some astronomers to denote that equation of the moon's motion, which is proportional to the sine of double the distance of the moon from the sun, diminished by the moon's aoomaly: this equation is not yet accurately determined; some state it at $1^{\circ} 30^{\prime}$, others at $l^{\circ} 16^{\prime}$, \& 8 c. It is the greatest of all the moon's equations, except the equation of the centre.

EVEN Number, is that which can be divided into two equal whole numbers; such as the series of alternate numbers, $2,4,6,8,10, \& \mathrm{c}$, and is therefore properly expressed by the form $2 n$.

EVENLY Even Number, is that which can be twice divided by 2 , and therefore is expressed by the form $4 n$.

EvenLy Odd Number, is that which can he only divided by 2 once, the quotient being an odd number, and is therefore of the form $4 n+2=2(m+1)$, that is, the quotient is of the form $2 n+1$, which is therefore necessarily an odd number.

EVERARD's Sliding Rule, a particnlar sort of rule invented by Mr. Thomas Everard, for the purpose of gauging. See SuIding Rule.

EULER (LEONARD), one of the most celebrated mathematicians of the 18 th, or perhaps of any other century. He was a nutive of Basil, and was born April 15, 1707 . The years of his infancy were passed at Richen, where lis father was minister. He, was afterwards sent to the university of Basil; and as his memory was astonishingly retentive, and his application regular, he performed his academical tasks with great rapidity; and all the time that he saved by this, was consecratell to the study of mathematics, which soon became his favourite science. The carly progress he made in this branch of study, added fresh ardour to bis application; by which he likewise obtained a distinguishing mark of the attention and esteem of prof 'ssor John Bernoulli, who was thetu one of the most eminent mathematicians in Europe.

In $1723, \mathrm{M}$. Euler took his degree as master of arts; and delivered on that occasion a Latin discourse, in which he drew a comparison between the philosophy of Newton and the Cartesian system, which was received with the greatest applause. At his father's desire, he next applied himself to the study of the logy and the oriental languages: and though these studies were foreign to his predominant plopensity, his success was considerable even in this respect: howeverr, with his lather's conscnt, he alterwards returned to mathematics is his principal object. In continuing to a vait himself of the counsels and instructions of
M. Bernoulli, be contracted an intimate friendship with his two sons Nicholas and Daniel; and it was chiefly in consequence of these connexions that he afterwards became the principal ornament of the philosophical world.

'The project of erecting an academy at Petersburg, which had been formed by Peter the Great, was executed by Catharine the 1st; and the two young Bernoullis being invited to Petersburg in 1725 , promised Euler, who was desirous of following them, that they would use their endeavours to procure for him an advantageous settlement in that city. In the mean time, by their advice, he made close application to the study of philosoplay, to which he made happy applications of his mathematical knowledge, in a dissertation on the nature and propagation of sound, and an answer to a prize question colıcerning the nuasting of ships; to which the Academy of Sciences adjugded the accessit, or second rank, in the year 1727 . From this latter discourse, and other circumstances, it appears that Eúler had very early embarked in the curious and useful study of naval architecture, which he afterward cnriched with so many valuable discoveries. The study of mathematics and philosophy however did not solely engage his attention, as be in the mean time attended the medical and botanical lectures of the professors at Basil.

Euler's merit would have given him an easy admission to honourable preforment either in the magistracy or university of his native city, if both civil and academical honours had not been there distributed by lot. This being against him in a certain promotion, he left his country, set.out for Petersburg, and was made joint professor with his countrymen Jlermann and Daniel Bernoulli, in the university of that city.

At bis first setting out in his new career, he enriched the academical collection with many memoirs, which excited a noble emulation between him and the Bernoullis; an emulation that always continued, without either degenerating into a selfish jealousy, or producing the least alteration in their friendship. It was at this time that be carried to new degrees of perfection the integral calculus, invented the calculation by sioes, reduced analytical operations to a greater simplicity, and thus was enabled to throw new light on all the parts of mathenatical science.

In 1730 , Euler was promoted to the professorship of natural philosophy; and in 1733 he succeeded his friend D. Bernoulli in the mathematical clair. In 1735 , a problem was proposed by the academy, which required expedition, and for the calculation of which some cminent mathematicians had icmanded the space of some months. The problem was undertaken by Euler, who completed the calculation in three days, to the great astonishment of the academy: but the violent and laborious efforts that he made to accomplish it, threw hin into a fiver, that cndangered his life, and deprived him of the use of his right eyc, which afterwards brought on a total blindiness.

The Academy of Sciences at Paris, which in 1738 lad adjucged the prize to his memoir Concerning the Nature and Properties of Fire, proposed for the year 1740 the im. portant subject of the 'Tides of the Sea ; al problem whose solution comprehended the theory of the solar system, and required the most arduous calculations. Éuler's solution of this question was adjudged a master-piece of analysis and geometry; and it was more honourable for him to share the academical prize with such illustrious competitors as Maclaurin and Danie! Bernoulli, than to have carried it away from rivals of less magnitude: Scldom, if 
ever, did such a brilliant competition adorn the annals of the academy; and perhaps no subject, proposed by that learned body, was ever treated with such force of reasouing and accuracy of investigation, as that which here displayed the philosophical powers of this extraordinary triumvirate.

In the year 1741, Eulcr was invited to Berlin to direct and assist the academy that was there rising into fame. On this occasion he enriched the last volume of the Miscellanies (Melanges) of Berlin with five memoirs, which form an eminent, perhaps the principal, figure in that collection. These were followed, with amazing rapidity, by a great number of important researches, which are dispersed through the memoirs of the Prussian academy; a volume of which has been regularly published every year since its establishinent in 1744. "The labours of Euler will appear ınore particularly astonishing, when it is considered, that while he was cnriching the academy of Berlin with a profusion of memoirs, on the deepest parts of mathematical science, contained always under some new points of view, and often réplete witl sublime truths, and sometimes discoveries of great importance; he still continued his philosophical contributions to the Petersburg aca demy, whose inemoirs display the marvellous fecundity of his genius.

It was with great difficulty that this extraordinary man, in 1766, obtained permission fiom the king of Prussia to return to Petersburg, where he wished to pass the remainder of his days; but soon after his return, which was graciously rewarded by the munificence of Catharine the $2 \mathrm{~d}$, he was seized with a violent disorder, which ended in the total loss of his sight: a cataract, formed in his left eye, which had been essentially damaged by the loss of the other.eye, and too close an application to study, deprived him entirely of the use of that organ. It was in this distressing situation that he dictated to his servant, a tailor's apprentice, who was absolutely devoid of mathematical knowledge, his Elements of Algebra; which by their intrinsic merit in point of perspicuity and method, and the unhappy circumstances in which they were composed, have equally excited the wonder and applause of the learned. This work, though purely clementary, plainly exhibits the proofs of an inventive genius; and it is perhaps here alone that we mect witl a complete theory of the Diophantine analysis.

About this time M. Euler was honoured by the Academy of Sciences at Paris with the place of one of the foreign members of that learned body; after which, the academical prize was adjudged to thrce of his memoirs, Concerning the lnequalities in the Motions of the Planets. The two prize questions proposed by the same Academy for 1770 and 1772 were designed to obtain from the labours of astronomers a more-perfect Theory of the MIoon. M. Euler, assistel by his cldest son, was a competitor for these prizes, and obtained them both. In this last memoir, he reserved for further consideration several incqualities of the mon's motion, which he could not determine in his first theory, on account of the complicated calculations in which the method lo then employed had engaged him. He atterwards revised his whole theory, with the assistancc of his son and Messrs. Krafit and Lexcll; and pursued his researches till he had constructed the new tables, which appeared, together with the great work, in 1772 . Instead of comfining himself, as before, to the fruitless integration of three differential equations of the seconsl de- gree, which are furnished by mathematical principles, he reduced them to the three ordinates, which deternine the place of the moon: and he divided into classes all the inequalities of that planet, as far as ihey depend either on the elongation of the sun and moon, or on the cxcentricity, or the parallax, or the inclination of the lunar orbit. All these means of investigation, employed with such art and dexterity as could only be expected from a genius of the first order, were attended with the greatest success; and it is impossible to observe without admiration, such immense calculations on the orre hand, and on the other the ingenious methods employed by this great man to abridge them, and to facilitate their application to the real motion of the moon. But this admiration will become astonishment, when wc consider at what period and in what circumstances all this was effected. It was when our author was totally blind, and consequently obliged to arrange all his computations by the sole powers of his memory and his genius: it was when he was embarrassed in his domestic affairs by a dreadful fire, that had consumed great part of his substance, and forced him to quit a ruined house, every conner of which was known to lim by habit, which in some measnre supplied the want of sight. It was in these circumstances that Euler composed a work which alone was sufficient to render his name inmortal.

Sorne time after this, the famous oculist Wentzell, by couching the cataract, restored sight to our author; but the joy produced by this operation was of short duration. Sorne instances of negligence, on the part of his surgeons, and his own impatience to use an organ, whose cure was not completely finished, deprived him a second time and for ever of his sight : a relapse which was also accompanied with tormenting pain. With the assistance of his sons, however, and of Messrs. Kraftt and Lexell, he continued his labours: neither the infirmities of old age, the loss of his sight, nor the acuteness of the pain, could quell the ardour of his genius. He had engaged to furnish the Academy of Petersburg with as many meinoirs as would be sufficient to complete its acts for 20 years after his death. In the space of 7 years he transmitted to the Academy above 70 memoirs, and above 200 more, left behind him, were revised and completed by a friend. Such of these memoirs as were of ancient ditte were separated from the rest, and form a collection that was published in the year 1783, under the title of Analytical Works.

The gencral knowledge of our author was more extensive than could well be expected, in one who had pursued, with such unremitting ardour, mathenatics and astronomy as his favourite studies. He had made a very consiglerable progress in medical, botanical, and chemical science. What was still more extraordinary, he was an excellent scholar, and possesserl in a high degrec what is generally called erudition. He had attentively read the most emin'nt writers of ancient Rome; the civil and literary history of all ages and all nations was familiar to him ; and foreigners, who were only acquainted with his works, were "astonished to find in the conversation of a man, whose long life scemed solcly occupied in mathenatical and physical researches and tiscoveries, such an extensive acquaintance with the inost interesting branclies of literature. In this respect, no doubt, he was much indebted to a very uncommon menory, which scemed to ritain every idea that was conveycd to it, either from reading or from meditation. He could repeat the Encid of Virgil, from the beginning to the end, without hesitation, and indi- 


\section{E V O}

E 49

cate the first and last line of every page of the edition he used.

Several attacks of a vertigo, in the beginning of September 1783 , which did not prevent his computing the motions of the aerostatic globes, were however the forerunners of his mild passage out of this life. While he was amusing himself at tea with one of his grand-children, he was struck with an apoplexy, which terminated his jliustrious career at $76^{\circ}$ years of age.

M. Euler's constitution was uncommonly strong and vigorous. His liealth was good; and the evening of his long life was calm and serene, sweetened by the fame that follows genius, the public esteem and respect that are never with held from exemplary virtue, and several domestic comforts, which he was capable of feeling, and therefore deserved to enjoy.

The catalogue of bis works has been printed in 50 pages, 14 of which contain the manuscript works. - The printed ones consist of works published separately, and works to be found in the memoirs of several Academies, riz, in 38 volumes of the Petersburg Acts (from 6 to 10 papers in each volume); - in several volumes of the Paris Acts; in 26 volumes of the Berlin Acts (about 5 papers to each volume); -in the Acta Eruditorum, in 2 volumes; - in the Miscellanea Taurinensia; -in vol. 9 of the Society of Ulyssingue ; - in the Ephemerides of Berlin;-in the Memoires de la Société Economique for 1766 ; - and in the Philos. Trans. by seven memoirs, from vol, 44 to vol. 62.

EVOLVENT, in the Higher Geometry, a term used by some writers for the involute, or curve resulting from the evolution of a curve, in contradistinction to that evolute, or curve supposed to be opended or evolved. See EvoLUTE, and IN VOLUTE.

EVOLUTE, in the Higher Geometry, a curve first proposed by M. Iluygens, and since much studied by many eminent mathematicians. It is any curve supposed to be evolved or opened, by having a thread wrapped close upon it, fastened at one end, and beginning to evolve or unwind the thread from the other end, keeping stretched the part evolved, or wound off; then this end of the thread will describe another curve called the Involute. Or the same involute is described in the contrary way, by wrapping the thread upon the evolute, keeping it always stretched.

Thus, if EFGl be any curve, and AE either a part of the curve, or a right line; then if a thread be wound close upon the curve from $A$ to $H$, where it is fixed, and then be unwound from $A$; the curve $\triangle \mathbf{E F G H}$, from which it is evolved, is called the evolute; and the other curve $\triangle B C D$, described by the end of the thread, as it evolves or unwinds, is the involute. Or, if the thread $\mathrm{H} \mathrm{D}$, fixed at $\mathrm{H}$, be wound or wrapped upon the evolute nGFEA, keeping it always stretched, as at the several positions of it $\mathrm{HD}$, $G C, F B, E A$, the extremity will describe the involute curve DCBA.
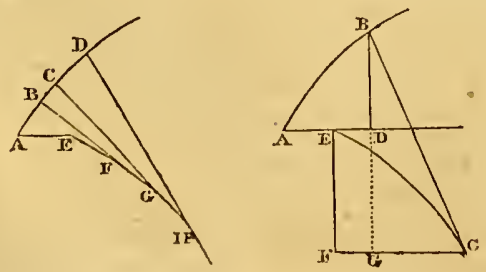

\section{E V O}

From this description it appears, 1. That the parts of the thread at any positions, as $\mathrm{EA}, \mathrm{EF}, \mathrm{GC}, \mathrm{nD}, \mathrm{Bc}$, art: radii of curvature, or osculatory radji, of the involute curve, at the points $\mathrm{A}, \mathrm{B}, \mathrm{C}, \mathrm{D}$.

2. The same parts of the thread are also equal to the corresponding lengths AE, AEP, ALFG, \& $\mathrm{C}$, of the evolute; that is,

$$
\begin{aligned}
& \mathrm{AE}=\mathrm{AE} \text { is the rad. of curvature to the puint } \mathrm{A}, \\
& \mathrm{BF}=\mathrm{AE} \\
& \mathrm{CG}=\mathrm{AG}
\end{aligned}
$$

3. Any radius of curvature $\mathbf{B} F$, is perpendicular to the involute at the point $B$, and is a tangent to the evolute curve at the point $F,-4$. The evolute is the locus of the centre of curvature of the involute curve.

The finding the radii of evolutes, is a matter of great importance in the higher speculations of geometry; and is even sometimes useful in practice; as is shown by Huygens, the inventor of this theory, in applying it to the pendulum. Horol. Oscil. part 3. For the doctrine of the oscula of evolutes we are indebted to M. Leibnitz, who first showed the use of evolutes in the measuring of curva tures.

To find the Evolute and Involute Curves, the one frota the other.-For this purpose, put

$z=A B$ the involute curve,

$x=A D$ the absciss of the involute,

$y=\mathrm{DB}$ its ordinate,

$r=\mathrm{BC}$ its radius of curvature,

$v=\mathrm{EF}$ the absciss of the evolute,

$u=\mathrm{rc}$ its ordinate, and

$a=\mathrm{AE}$ a given line (fig. 2 above).

Then, by the nature of the radius of curvature, it is $r=\frac{\dot{z}^{3}}{y \ddot{x}-x y}=\mathrm{BC}=\mathrm{AE}+\mathrm{EC}$; also by sim. triangles,

$\dot{z}: \dot{x}:: r: \mathrm{GB}=\frac{r \dot{x}}{\dot{\tilde{x}}}=\frac{\dot{x} \dot{z}^{2}}{j \ddot{x}-x \ddot{y}}$,

$\dot{z}: \dot{y}:: r: \mathrm{Gc}=\frac{\dot{y} \dot{y}}{\dot{z}}=\frac{\dot{x} \dot{z}^{2}}{y \ddot{x}-\dot{x} \dot{y}}$.

Hence $\mathrm{EF}=\mathrm{GB}-\mathrm{DB}=\frac{\dot{x} \dot{z}^{3}}{y \ddot{x}-\dot{x} \ddot{y}}-y=v$; and

$\mathrm{FC}=\mathrm{AD}-\mathrm{AE}+\mathrm{GC}=x-a+\frac{j \dot{z}^{2}}{j \ddot{x}-\dot{x} \dot{y}}=u$; which are

the values of the absciss and ordinate of the evolute curve vc; which therefore may be found when the involute is given.

On the other hand, if $v$ and $u$, or the evolute be given: then, puttiug the given curve $\mathrm{Ec}=s$; since $\mathrm{CB}=\Lambda \mathrm{E}+\mathrm{Ec}$, or $r=a+s$, this gives $r$ the radius of curvature. Also, by similar triangles, there result these proportions, viz, $\dot{s}: \dot{v}:: r: \frac{r \dot{v}}{\dot{s}}=\frac{a+s}{\dot{s}} \dot{v}=\mathrm{GB}, \mathrm{s}$

$s: \dot{u}:: r: \frac{\dot{r u}}{\dot{s}}=\frac{a+s}{s} \dot{u}=\mathrm{GC}$; therefore

$\mathrm{AD}=\mathrm{A} \mathrm{E}+\mathrm{EC}-\mathrm{GC}=a+u-\frac{n+s}{\dot{j}} \dot{u}=x$,

and $\mathrm{DB}=\mathrm{Gr}-\mathrm{EF}=\frac{n+s}{\dot{s}} \dot{v}-x=y$; which are the absciss and ordinate of the involute curve, and which may therefore be found, when the evolute is given. It may also be observed that $\dot{s}^{2}=\dot{v}^{2}+\dot{u}^{2}$, and $\dot{z}^{2}=\dot{x}^{2}+\dot{y}^{2}$. And cither of the quintities $x, y$, may be supposed to flow equably, in which case the respective second fluxion $\ddot{x}$ or $\ddot{y}$ will be nothing, and the corresponding torm in the denominator $j \ddot{x}-\ddot{x} y$ will vanish, leaving only the other 


\section{E V O}

term in it; which will have the effect of rendering the operation simpler.

For Example. If it be required to find the evolution $\mathbf{x c}$ when the given involute $\mathrm{A} \mathrm{B}$ is the cornmon parabola, whose equation is $p x=y^{2}$, the parameter being $p$.

Here $y=\sqrt{ } p x, \dot{y}=\frac{1}{2} \dot{x} \sqrt{ } \frac{p}{x}$, and $\dot{y}=\frac{-\dot{x}^{3}}{4 x^{2}} \sqrt{ } \frac{p}{x}$, making $\ddot{x}=0$. 'Then, to find first $\mathrm{AE}$ the radius of curvature of the parabola $\mathrm{AB}$ at the vertex, when $\bar{x}=0$, the gencral value of the radius of curvature above given becomes

$r=\frac{\dot{z}^{3}}{-\dot{x} \ddot{y}}=\frac{\left(\dot{x}^{2}+\dot{y}^{2}\right)^{\frac{3}{2}}}{-x \ddot{y}}=$ (by substituting the value of $\dot{y}$ and $\ddot{y} \&$ c) $\frac{(p+4 x)^{\frac{3}{2}}}{2 \sqrt{p}}$, which is the gencral value of $r$ or в , the radius of curvature, for any value of $x$ or $A D$; and when $x$ or $\mathrm{AD}$ is $=0$ or nothing, the value of $r$, or $\mathrm{AE}$, becomes then $a=\frac{p^{\frac{3}{2}}}{2 \sqrt{p}}=\frac{1}{2} p$ only; or half the parameter of the axis is the radius of curvature at the vertex of the parabola.

Again, in the general values of $v$ and $u$ ahove given, by substituting the values of $\dot{y}, \ddot{y}$, and $\dot{z}$, also 0 for $\ddot{x}$, and $\frac{1}{2} p$ for $a$; those quantities bccome

$v=\frac{\dot{z}^{2}}{-}-y=\frac{\dot{x}^{2}+\dot{y}^{2}}{-\dot{y}}-y=\frac{4 x^{\frac{3}{2}}}{p_{2}^{\frac{1}{2}}}=4 x \sqrt{ } \frac{x}{p} ;$ and

$u=x-a+\frac{y z^{2}}{-\dot{x} y}=3 x+\frac{1}{2} p-a=3 r$.

Hence then, comparing the values of $x^{n}$ in both of these, we have $x^{3}=\frac{1}{16} p v^{2}=\frac{1}{27} u^{2}$, and conseq. $27 p v^{2} \doteq 16 u^{3}$; which is the equation between the absciss and ordinate of the evolute curve Ec, showing it to be the semicubical parabola.

In like manner the evolute to any other curve is found. The evolute to the common cycloid, is an equal cycloid; a property first demonstrated by Huygens, and which be used as a contrivance to make a pendulum vibrate in the curve of a cycloid. See his Ilorolng. Oscil. Sec also, on the subject of evolute and involute curres, the Fluxions of Newton, Maclaurin, Simpson, De l'Hôpital, \&: ; Wolf. Elem. Math. tom. 1, \&c, \&c.

MI. Varignon has applied the doctrine of the radius of the evolute, to that of central forces; so that having the radius of the crolute of any curve, there may be found the value of the central force of a body; which, moving in that curve, is found in the same point where that radius terminates; or reciprocally, having the central force given, the radius of the evolute may be determined. Hist. de l'Acarl. an. 1706.

The variation of curvature of the line described by the evolution of a curre, is measured by the ratio of the radius of curvature of the evolute, to the radius of curvature of the line Jescribed by the evolution. Sce Maclaurin's Hlux, art. 402, prop. 36 .

Imperfect EvouUte, a name given by M. Reaumur to a uew kisd of evolute. Mathematicians had hitherto only considered the perpendiculars let fall from the involute on the cotivex side of the evolute: but if other lines not perpendicular be drawn upon the same peints, provided they be all drawn under the same angle, the effect will still be the same; that is, the oblique lines will all inter. sect in the curve, and by their interscetions form the infi- nitcly small sides of a new curve, to which they would be so many tangents. - Such a curve is a kind of evolute, and has its radii; but it is an imperfect one, since the radii are not perpendicular to the first curve, or involute. Hist. de l'Acad. \&c, an. 1709.

EVOLUTION, in Arithmetic and Algebra, denotes the extraction of roots. In which sense it stands opposed to involution, which is the raising of powers.

Erolution, in Geometry, the opening, or unfolding of a curve, and making it describe an evolvent.

The equable evolution of the periphery of a circle, or other curve, is such a gradual approach of the circumference to rectitude, as that its parts do all concur, and equally evolve or unbend; so that the same line becomes successively a less arc of a reciprocally greater circle; till at last they change into a straight line. In the Philos. Trans. No. 260, a new quadratrix to the circle is found by this means, bcing the curve described by the equable cvolution of its periphery.

EURYTHMY, in Architecture, Painting, and Sculpture, is a kind of majesty, elegance, and easiness appearing in the composition of certain members or parts of a body, building, or painting, and resulting from the fine and exact proportions of them.

EUSTYLE, is the best manner of placing columins, with regard to their distance; which, according to Vitruvius, should be 4 modules, or $2 \frac{1}{4}$ diameters.

EUTOCIUS, a respectable Greek mathematician, of Ascalon, in Palestine, abunt the year of Christ 550. He was one of the most considerable mathematicians that flourished about the decline of the sciences among the Greeks, and had for his preceptor Isidorus, the principal architect of the church of St. Sophia at Constantinople. He is chiefly known however by bis commentaries on the works of the two ancient authors, Archimedes and Apollonius. Those two commentaries are both excellent compositions, to which we owe many useful observations in the bistory of the mathematics.

His commentaries on Apollonius are published in Halley's edition of the works of that author; and those on Archinuedes, first in the Basle edition, in Greek and, Latin, in 15.4, and since in some others, as the late Oxford exition, in 1792. Of these commentaries, those rank the highest, which illustrate Archimedes's work on the Sphere and Cylinder; in one of which we have a recital of the various nuethods practised by the ancients in the solution of the Delian problem, or that of doubling the cube. The others are of less value; though it cannot but be regretted that Eutocius did not pursue his plan of commenting on all the works of Archimedes, with the same attention and diligence which he employed in his remarks on the sphere and cylinder.

EXCENTRIC, is applied to such figures, circles, spheres, \&c, as have not the same centre; as opposed to conceniric, which lave the same centre.

Excentric, or Excentric Circle, in the ancient Ptolemaic Astronomy, was the very orbit of the planet itself which it was supposed to describe about the earth, and which was conceived excentric with it; called also the deferent. I3ut instead of these excentric circles round the earth, the planets are now known to describe elliptic. orbits about the sun; which accounts for all the irregularitics of thcir inutions, and their various distances from the: eat th, Scc, more justly and naturally. 


\section{E X C}

[ 496

Excentrac, or Excentric Circle, in the Modern Astronomy, is the circle described from the centre of the orbit of a planet, with half the greatest axis as a radius; or it is the circle that circumscribes the elliptic orbit of the planet; as the circle $\Lambda$ QB.

Excentric Anomaly, or Anomaly of the Centre, is an arc A\& of the excentric circle, interceptcd between the aphelion $\Lambda$, and the right line QH, drawn through the centre $P$ of the planet perpendicular to the line of the apses AB.

Excentric Equation, in the Astronomy of the ancients, is an

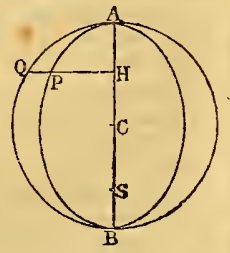
angle made by a line drawn from the centre of the earth, with another line drawn from the centre of the cxcentric, to the body or place of any planct. This is the same with the prosthapheresis; and is equal to the difference, accounted in an arch of the ecliptic, between the real and apparent place of the sun or planet. See EQUation of the Centre.

Excentric Place of a planet, in its orbit, is the heliocentric place, or that in which it appears as seen from the sun.

Excentric Place in the ecliptic, is the point of the ccliptic to which the planet is referred as viewed from the sun; and which coincides with the heliocentric longitude.

EXCENTRICITY, is the distance between the centres of two circles, or splieres, which have not the same centre.

Excentricity, in the Old Astronomy, is the distance between the centre of a planet and the centre of the earth. -That the planets have such an excentricity, is allowed on all sides, being cvickent from various circumstances; and particularly from this, that the planets at some times appear larger, and at other times less; which can only proceed from hence, that their orbits being excentric to the earth, in some parts of those orbits the planets are nearer to us, and in others more remote. And as to the excentricities of the sun and moon, it is thought they are sufficiently proved, both from eclipses, from the moon's greater and less parallax at the same distance from the zenith, and from the sun's continuing longer by 8 days in the northern hemisphere than in the southern one.

Excentricity, in the new Astronomy, is the distance cs between the sun $\mathrm{s}$ and the ccntre $\mathrm{c}$ of a planet's orbit; or the distance of the centre from the focus of the elliptic orbit; called also the Simple or Single Excentricity.

When the greatest equation of the centre is given, the excentricity of the earth's orbit may be found by the following proportion; viz,

As the diameter of a circle in degrees,

Is to the diameter in equal parts;

So is the greatest equation of the centre in degrees,

To the excentricity in equal parts. Thus,

Greatest equat. of the cent. $1^{\circ} 55^{\prime} 33^{\prime \prime}=1^{\circ} \cdot 9258333$ \& c. The diam. of a circ. beiug 1 , its circumf. is $3 \cdot 1415926$. Then $3 \cdot 1415926: 1:: 360^{\circ}: 114^{\circ} 5915609$ diam. in deg. And 114:5915609: 1::1.9258333:0.016806, the ex. Hence, by adding this to 1 , and subtracting it from 1, gives $1^{\circ} .016806=A$ s the aphelion distance, and 0.983194 $=$ вs the perilıelion distance.

See Robertson's Elem of Navig. book 5, pa. 286 .
E X C

Otherwise, thus : Since it is found that the sun's greatest apparent semi-diameter is to his least, as $32^{\prime}+3^{\prime \prime}$ to $31^{\prime} 38^{\prime \prime}$, or as $1963^{\prime \prime}$ to $1898^{\prime \prime}$; the sun's greatest distance from the earth will be to lis least, or AS to $\mathrm{SB}$, as 1963 to 1898 ; of which,

the half dif. is $32 \frac{x}{2}=\mathrm{cs}$,

and half sum $1930 \frac{1}{2}=\mathrm{CB}$; therefore,

as 1 y $30 \frac{1}{2}: 32 \frac{x}{2}:: 1:: 016835=c 9$ the excentricity, to the nean distance or semi-axis 1 ; which is nearly the same as before.

The excentricities of the orbits of the several planets, in parts of their own mean distances 1000 , and also in English miles, are as below, viz, the excentricity of the orbit of

\begin{tabular}{|c|c|c|c|c|}
\hline & & Parts. & & \\
\hline Mercury & & 210 & - & $7,730,000$ \\
\hline & & 7 & - & 482,000 \\
\hline & & 17 & - & \\
\hline & & 93 & & 13: \\
\hline & & 36 & - & \\
\hline & & 254 & - & 000 \\
\hline & 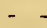 & $246^{\circ}$ & - & \\
\hline Cer & - & 78 & - & 000 \\
\hline & - & 48 & - & $23,760,000$ \\
\hline & - & 55 & - & $49,940,000$ \\
\hline & & $47^{\frac{1}{2}}$ & & $86,000,000$ \\
\hline
\end{tabular}

Or the excentricities of the orbits in parts of their own mean distances 1 , and the secular variations of the same, according to Laplace, are as bclow :

\begin{tabular}{|c|c|c|c|c|}
\hline Y) & & Excentr. & & $\begin{array}{c}\text { Sec. Variat. } \\
0.00000387\end{array}$ \\
\hline & & $0.0068529 \mathrm{~s}$ & - & 0.00006371 \\
\hline Earth & & 0.01683518 & - & 0.00004168 \\
\hline Mars & & 0.09313400 & - & 0.00009018 \\
\hline Jupiter & & 0.04817840 & - & 0.00015935 \\
\hline Saturn & & 0.05616830 & - & 0.00031240 \\
\hline $\begin{array}{l}\text { Herschel } \\
\text { or Uranus }\end{array}$ & & 0.04667030 & & 0.00002507 \\
\hline
\end{tabular}

Excentricities of the Orbits of the new or Telescopic Planets, are,

Piazzi, or Ceres (discovered 1801) - $\quad$ - 0.078349

Olbers, or Pallas ( - - 1802) - - 0.245354

Harding, or Juno ( - - 1804) - - 0.254944

Ditto, according to Burckhardt - 0.25096

Olbers 2d, or Vesta (discov. 1807) - - 0.093220

The excentricity of the moon's orbit is 0.0548553 , the greater semi-axis being 1 .

Double Excentricity, is the distance between the two foci of the elliptic orbit, and is equal to double the single excentricity above given.

EXCESS (Spherical) in Trigonometry, is the excess of the sum of the three angles, of any spherical triangle, above 2 right angles. Let, $A, B, c$, be the angles of a spherical triangle; and $a, b, c$, the sides opposite to the angles $A, \mathrm{~B}, \mathrm{C}$, respectively; $n=2$ right angles, and $r=$ the radius of the sphere; then the spherical cxcess $(\alpha)$ is ascertained by the following theorem, first given by Simon Lhuillier of Geneva: Tan. $\frac{1}{4} \mathrm{E}$, or $\tan \frac{1}{4}(\Lambda+n+c-n)=$ $\frac{1}{r} \sqrt{ }\left(\tan . \frac{a+b+c}{4} \cdot \tan \cdot \frac{a+b-c}{4} \cdot \tan \cdot \frac{a+c-b}{4} \cdot \tan \cdot \frac{b+c-a}{4}\right)$, or $=\frac{1}{r} \sqrt{ }\left(\tan \cdot \frac{s}{2} \cdot \tan \cdot \frac{s-a}{2} \cdot \tan \cdot \frac{s-b}{2} \cdot \tan ^{s-c}\right)$; where $s=\frac{1}{2}(a+b+c)$, half the sum of the sides. M. Puissant gives the following theorems for the excess: 
$\mathrm{E}=\left(\frac{\mathbf{R}^{\prime \prime}}{r r}\right) a^{2} \sin .2 \mathrm{C}+\left(\frac{\mathbf{R}^{\prime \prime}}{\pi r}\right) c^{2} \cdot \sin .2 \Lambda$, or

$\mathbf{E}=\left(\frac{\mathbf{R}^{\prime \prime}}{2 r^{3}}\right) b h$; where $b=$ the base, and $h=$ the height of

the triangle, also $\mathrm{R}^{\prime \prime}=$ the number of seconds contained in radius.

In a memoir on this by Burckhardt, in vol. 7 of the Hemoirs of the National Institute of France, it is shown that the same propertyequally applies to such small triangles on the spheroidal surface of the earth as occur in the usual geodotical operations; as no appreciable error will arise by considering such triangles as spherical ones, nor in any whose sides are small in comparison with a great circle of the sphere. It follows, says the author, that the small triangles traced on the surface of a spheroid may be calculated on the same principles as such triangles traced on the surface of a sphere; and both of them may be reduced to rectilinear triangles, by diminishing their angles, each by a quantity equal to one-third of the area of a similar triangle, described on a sphere whose radius is $=1$. - The solution of spheroidal triangles, whose sides are small with regard to the dimensions of the spheroid, depends therefore immediately on that of rectilinear triangles; not only when the spheroid is elliptical and of revolution, but when it is in any manner irregular, provided only that it differs but little from a sphere.

EXCH NGE, in Aritbmetic, is the bartering or exchanging the money of one place for that of another; or finding what quantity of the money of one place is equal to a given sum of another, according to a certain course of exchange.-The several operations in this rule are orly different applications of the Rule of Three: which may be seen in most books of aritbmetic.

Arbitration of ExснANGE, is the method of remitting to, and drawing upon, foreign places, in such a manner as shall turn out the most profitable.

Arbitration is either simple or compound.

Simple Arbitration respects three places only. Here, by comparing the par of arbitration between a first and second place, and between the lst and a $3 \mathrm{~d}$, the rate between the $2 \mathrm{~d}$ and $3 \mathrm{~d}$ is discovered; whence a person can judge how to remit or draw to the most advantage, and to determine what that advantage is.

Compound Arbitintion respects the cases in which the exchanges among three, four, or more places are concerned. A person who knows at what rate he can draw or remit directly, and also has advice of the course of exchange in foreign parts, may trace out a path for circulating his money, through more or fewer of such places, and also in such order, as to make a benefit of his skill and credit: and in this lies the great art of such negociations. See Hutton's Arithmetic 2 pa. 105 ; also the articles Anbitration, and the Cirain Rule.

EXCURSION, in Astronomy. See Elongatrun.

Circles of Excunsion. Sce C1RcLes.

EXEGESIS, or EXEGrica, in Algebra, is the finding, either in numbers or lines, the roots of the equation of a problem, according as the problem is either numeral or geometrical.

EXHALATION, a fumc or steam, issuing from a body, and diffusing itself in the atmosphers. The terms exhalation and vapour are often used indifierently; but the more accurate writers distinguish then, appropriating the term apour to the moist fumes raised from water and other Vis.. 1. liquid bodies; and the term exhalation to the dry ones emitted from solid bodics; as earth, minerals, \&c. In this sense, exhalations are dry and subtile curpuscles, or effluvia, loosened from hard terrestrial bodies, either by the heat of the sun, or the action of the air, or some other cause; being emitted upwards to a certain height in the atmosphere, where, mixing with the vapours, they help to constituteclouds, and return back in dews, mists, rains, \&c. Sir Isaac Newton thinks, that truc and permanent air is formed from the exhalations raised from the hardest aud most compact bodies.

EXHAUSTED Receiver, is a glass, or other vesscl, applied on the plate of an air-pump, to have the air extracted out of it by the action of the pump.-Things placed in such an exhausted receiver, are said to be in vacuo.

EXHAUSTIONS, or the Method of ExHAUSTIONs, is a mode of demonstration founded on the principle of exhausting a quantity by cortinually taking away cestain parts of it. The methor of exhaustions was frequently resorted to by the ancient matheinaticians; as iuclial, Archimedes, \&c. And it is founded on what Luclid ays in the 10 th book of his Elements; viz, that those quantides are equal, whose difference is less than any assignacte quantity. Or it may be otherwise expressed thus: two yuantities $A$ and $B$ are equal, when, if to or from on of them as $A$, any other quantity as $d$ be subtracted, however small it may be, the sum or rifference thus obtained is respectively greater or less than the other quantity $\mathrm{B}: \boldsymbol{d}$ being an indefinitively small quantity,

if $\mathbf{A}+d$ be greater than $\mathbf{B}$,

and $A-d$ less than $B$, then is $A$ equal to $\mathbf{B}$.

This principle is used in the 1st prop. of the 10th book, which imports, that if from the greater of two quantities be taken more than its half, and from the remainder more than its half, and so on; there will at length remain a quantity less than either of those proposed. On this foundation it is demonstrated, that if a regular polygon of infinite sides be inscribed in a circle, or circumscribed about it; that the space, which is the difference betwcen the circle and the polygon, will by degrees be quite exhausted, and the circle become ultimately equal to the polygon. And in this way it is that Archimedes demonstrates, that a circle is equal to a right-angled triangle, whose two sides about the right angle are equal, the one to the semidiameter, and the other to the perimeter of the circle. Prop. 1 De Dimensione Circuli.

On the method of exhaustions depends the method of indivisibles introduced by Cavalerius, which is but a shorter way of expressing the method of exhaustions; as also Wallis's Arithmetic of Infinites, which is a further improvement of the method of indivisibles; and hence also the inethods of increments, differentials, fluxions, and infuite series. Sec some account of the method of cxhaustions in Wallis's Algebra, chap. 73, and Ronayne's Algebra, part 3 , pa. 395.

EXPANSION, is the dilating, stretching, or spreading out of a body; whether from any extcrnal cause, as the cause of rarefaction, or from an internal cause, as clasticity. Bodies naturally expand by heat, and arc contracted by cold; and hence it happens that their dimensions and specific gravities are different in different temperatures and seasons of the year. Air compressed or condeused, as soon as the compressing or condensing force $3 \mathrm{~S}$ 
is rcmoved, expands itself by its elastic power to its former dimensions.

In some few cases indeed, bodies seem to expand as they cool, as water in the act of freezing: but this is owing to the extrication of a number of air bubbles from the fluid at a certain time; and is not at all a regular and gradual expansion like that of metals, \&c, by means of heat. Mr. Boyle, in his History of Cold, says that ice takes up a 12 h part more space than water; but by MajorWilliams's experiments on the force of freezing water, 1 have found it occupies only about the 17 th or 18 th part more space. Transact. of the R. Soc. of Edinb. vol. 2, pa. 28. In certain metals also, an expansion takes place wben they pass from a fluid to a solid state: but this too is not to be accounted any proper effect of cold, but of the arrangement of the parts of the metal in a certain manner; and is therefore to be considered as a kind of crystallization.

The expansion of different bodies by heat is very various; and many experiments upon it are to be met with in the volumes of the Philos. Trans. and in other places. In the 48th vol. in particular, Mr. Smeaton has given a table of the expansion of many different substances, as determined by experiment, from which the following particulars are extracted. Where it is to be noted, that the quantities of expansion which answer to 180 degrees of Fahrenheit's thermomcter, are expressed in ten-thousandth parts of an English inch, each substance being 1 foot or 12 inches in length.

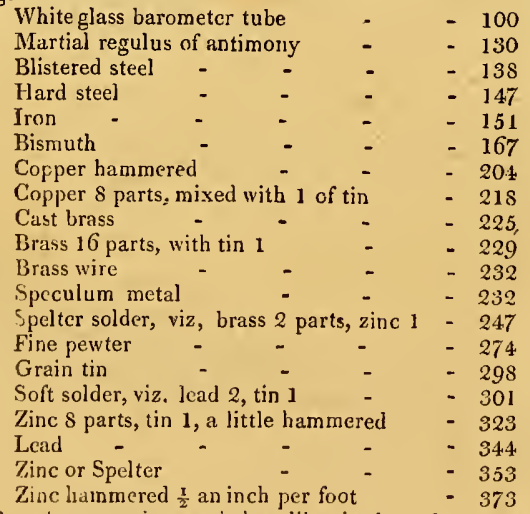

By other esperiments it has likewise been found that, tor each thermometrical degree of heat, mercury, water, and air, cxpand the following parts of their own bulk, viz,

Mercury the 9600 th?

Water the 6666 th $\}$ part of its bulk.

Air the 435 th

From the foregoing table it appears, that there is no general rule for the degree of expansion to which bodies are subject by the sime ingrec of heat, cither from their specific gravity or otherwise. 'Zinc, which is much lighter than lead, expands more with heat; while glass, which is lighter than either, expands much less; and copper, which is leavier than a mixture of brass and tin, expands less. It secms also that metals observe a proportion of expansion in a fluid state, quite diflerent from what they do in a solid one: for regulus of antimony secmed to con- tract in fixing, after having been melted, considerably more than zinc under similar circumstances.

But of all known substances, those of the aerrial kind expand most by an equal degree of heat; and in general the greater quantity of latent heat that any substance contains, the more easily is it expanded: yet even here no general law can be observed. It is indeed certain that the densest fluids ${ }_{2}$ such as mercury, oil of vitriol, \&c, are less expansible than water, spirit of wine, or ether. Which last is so easily expanded, that were it not for the pressure of the atmosphere it would be in a continual state of vapour. And indeed this is the case, in some measure, with perhaps all fluids; as it has been found, by experiments with the best air-pumps, that water, and other fluids, ascend in vapours the more as the cxhaustion is the more perfect; from which it would seem that wates would wholly rise in vapour, in any temperature, if the pressure of the atmosphere was entircly taken off.

After bodies are reducerl to a state of vapour, their cspansion seems to go on without ally limitation, in proportion to the degree of heat applice; though it may be impossible to say what would be the ultimate effecter ol that principle acting upon them in this manner. The force with which these vapours expand on the application of high degrees of heat is so great, that no body or force appears to be sufficient to counteract their effects

On this principle depends the construction of stean-engines, so much employed in various mechanical operations; likewise some hydraulic machines; and the instruments called manometers, which show the variation of gravity in the extcrnal atmosphere, by the expansion or cendensation of a small quantity of air confined in a proper vessel. On this principle also, perpetual movements might be constructed similar to those invented by Mr. Coxe, on the principle of the barometer. And a variety of other curious machines may be constructed on the principle of aërial expansion; an accouut of some of which is given under the articles Hydrostatics and PNEUMATICS.

The construction of thermometers depends also on the principle of the expansion of fuids. See'THERMOMETER. And for the effects of the different expansions of metals, in correcting the vibrations of pendulums, see PENDULUN.

The expansion of solid bodies is measured by an instrument called the pyroweter; and the force with which they expand is still greater than that of ae rial vapours; the flame of a small candle produces an expansion in a bar of iron capable of counteracting a weight of 500 pounds. The quantity of expansion, however, is so small, that it has never been applied to the movement of any mechanical engine.

EXPLCIATION, in the Doctrine of Chances, is applied to any contingent cvilit, on the happening of which, some benefit \& c, is expected. This is capable of being reduccel to the rules of computation: for a sum of money in expectation when a particular event happens, has a eleterminate value before that erent happens. 'Thus, if a person is to reccive any sum, as 10 . when an crent takes place which has an equal chance or probability of happening and failing, the value of the expectation is latf that sum, or 51.: but if there are 3 chances for failing, and only 1 for jts happening, or 1 chance mly in its favour out of all the 4 chances; then the probability of its happening is only 1 out of 4 , or $\frac{T}{4}$, and the value of the expectation is but $\frac{x}{4}$ 
of $10 l$. which is only $2 l .10 s$. or half the former sum. And in all cases, the value of the expectation of any sum is found by multiplying that șum by the fraction expressing the probability of obtaining it. So the value of the expectation on $100 l$. when there are 3 chances out of 5 for obtaining it, or when the probability of obtaining it is $\frac{3}{5}$, is $\frac{3}{5}$ of $100 \%$. which is $60 l$. And if $s$ be any sum expected on the happening of an event, $h$ the chances for that event happening, and $f$ the chances for its failling; then, there being $h$ chances out of $f+h$ for its happening, the probability will be

$\frac{h}{f+h}$, and the value of the expectation is $\frac{h s}{f+h}$. Suppose, for example, that a person is entitled to $100 l$. if, in casting a dic, he brings up an ace; and to $50 l$. if he brings up a six; but in all other cases he is to have nothing, to find the value of his expectation before the die is cast. In each case the number of chances, both for happening and failing, are 6 , but for the event taking place only 1 ; therefore $\frac{1}{6}$ of $100 l_{.}=16 l .13 s .4 d$. is the value of his expectation in the first case, and $\frac{x}{6}$ of $50 l .=8 l .6 s .8 d$. is its worth in the second case; consequently, their sum $25 l$. is the entire value of his expectation before he unclertakes to throw. See Simpson's or De Moivre's Doctrine of Chances.

Expectation of Life, in the Doctrine of Life Annuities, is the share, or number of years of life, which a person of a given age may, on an equality of chance, expect to enjoy. By the expectation or share of life, says Mr. Simpson (Select Exercises, pa. 273), is not here to be understood that particular period which a person hath an equal chance of surviving; this last being a different, and more simple consideration. The expectation of a life, to put it in the most familiar light, may be taken as the number of years at which the purchase of an annuity, granted upon it, without discount of money, ought to be valued. Which number of years will differ more or less from the period above-mentioned, according to the different degrees of mortality to which the several stages of life are incident. Thus it is much more than an equal chance, according to the table of the probability of the duration of life (pa. 254 ut supra), that an infant, just come into the world, arrives not to the age of 10 years; yet the expectation or share of life due to it, on an average, is near 20 years. The reason of which wide difference, is the great excess of the probability of mortality in the first tender years of life, above that respecting the inore mature and stronger ages. Indeed, if the numbers that die at every age were to be the same, the two quantities above specified wouId also be equal; but when the said numbers become continually less and less, the expectation must of consequence be the greater of the two.

-Mr. Simpson has given a table and rule for finding this expectation, pa. 255 and 273 as below.

lor example, if it be required to find the expectation or share of life, due to a, person of 30 years old. Opposite the given age in the first column of the table, stands $23 \cdot 6$ in the second col. for the years in the expectation sought. See l)e Moivre's Doctrine of Chances applicel to the valuation of annuities, pa. 288; or Dr. Price's Observations on Reversionary Payments, pa. 168,364, 374, \&c; or Philos. 'Traus. vol. 59, pa. 89 .
A Table of the Expectations of Life in London.

\begin{tabular}{|c|c|c|c|c|c|}
\hline Age. & Expectation. & Age. & Expectation. & Ağe. & Expectation. \\
\hline 1 & $27 \cdot 0$ & 28 & $24 \cdot 6$ & 55 & 14.2 \\
\hline 2 & $32 \cdot 0$ & 29 & $24 \cdot 1$ & 56 & $13 \cdot 8$ \\
\hline 3 & $34 \cdot 0$ & 30 & 236 & 57 & $13 \cdot 4$ \\
\hline 4 & $35 \cdot 6$ & 31 & $23 \cdot 1$ & 58 & $13 \cdot 1$ \\
\hline 5 & $36 \cdot 0$ & 32 & $22 \cdot 7$ & 59 & $12 \cdot 7$ \\
\hline .6 & $36 \cdot 0$ & 33 & $22 \cdot 3$ & 60 & $12 \cdot 4$ \\
\hline 7 & $35 \cdot 8$ & 34 & $2 l \cdot 9$ & 61 & 12.0 \\
\hline 8 & $35 \cdot 6$ & 35 & $21 \cdot 5$ & 62 & $11 \cdot 6$ \\
\hline 9 & $35 \cdot 2$ & 36 & $21 \cdot 1$ & 63 & $11 \cdot 2$ \\
\hline 10 & $34 \cdot 8$ & 37 & $20 \cdot 7$ & 64 & $10 \cdot 8$ \\
\hline 11 & $34 \cdot 3$ & 38 & $20 \cdot 3$ & 65 & $10 \cdot 5$ \\
\hline 12 & $33 \cdot 7$ & 39 & 19.9 & 66 & $10 \cdot 1$ \\
\hline 13 & $33 \cdot 1$ & 40 & $19 \cdot 6$ & 67 & $9 \cdot 8$ \\
\hline 14 & $32 \cdot 5$ & 41 & $19 \cdot 2$ & 68 & $9 \cdot 4$ \\
\hline 15 & $31 \cdot 9$ & 42 & 18.8 & 69 & $9 \cdot 1$ \\
\hline 16 & $31 \cdot 3$ & 43 & $18 \cdot 5$ & 70 & $8 \cdot 8$ \\
\hline 17 & $30 \cdot 7$ & 44 & $18 \cdot 1$ & 71 & $8 \cdot 4$ \\
\hline 18 & 30.1 & 45 & $17 \cdot \mathrm{s}$ & 72 & $8 \cdot 1$ \\
\hline 19 & 29.5 & 46 & $17 \cdot 4$ & 73 & $7 \cdot 8$ \\
\hline 20 & $28 \cdot 9$ & 47 & 17.0 & 74 & $7 \cdot 5$ \\
\hline 21 & $2 s \cdot 3$ & 48 & $16 \cdot 7$ & 75 & $7 \cdot 2$ \\
\hline 22 & $27 \cdot 7$ & 49 & $16 \cdot 3$ & 76 & 6.8 \\
\hline 23 & $27 \cdot 2$ & 50 & 16.0 & 77 & $.6 \cdot 4$ \\
\hline 24 & $26 \cdot 6$ & 51 & $15 \cdot 6$ & 78 & $6 \cdot 0$ \\
\hline 25 & $26 \cdot 1$ & 52 & $15 \cdot 2$ & 79 & $5 \cdot 5$ \\
\hline 26 & $25 \cdot 6$ & 53 & 14.9 & 80 & $5 \cdot 0$ \\
\hline 27 & $25 \cdot 1$ & 54 & 14.5 & & \\
\hline
\end{tabular}

EXPERIMENT, in Philosophy, a trial of the effect or result of certain applications and motions of natural bodies, in order to discover something of their laws and relations, \&c. The making of experiments is become a kind of art; and there are now many collections of them, mostly under the denomination of Courses of Experimental Philosophy. Sturmius made a curious collection of the principal discoveries and experiments of the 17 th century, under the title of Collegium Experimentale. Other courses of experiments have been published by Gravesande, Desaguliers, Helsham, Cotes, \&c.

EXPERIMENTAL Philosoply, is that which proceeds on experiments, or which deduces the laws of nature and the properties and powers of bodies, and their actions upon each other, from sensible experiments and observations. Experiments are of the utmost importance in philosophy; and the great advantages the modern physies have over the ancient, is chiefly owing to our making more experiments, and then founding our theories on the results thus obtained: whereas the method of the ancients was chiefly to begin with the assumed causes of things, and thence argue to the phenomena and effects; on the contrary, that of the moderns proceeds from experiments and observations, whence the properties and laws of natural things are deduced, aurl general theories are formed.

Several of the ancients indecd thought as highly of experiments as the moderns, and practised them also. Plato omits no nccasion of speaking of tho advantages of them ; and Aristotle's history of animals bears ample testimuny for him. Democritus's great employment was to make experinsents; and even Epicurus bimself owes part of his glory to the same cause.

Among the moderns, the making of experiments was clicfly begun by Friar Bacon, in the 13th century, whe

3 S 2 
spert a gict deal of money and labour in this pursuit. After him, the lord chancellor Bacon is considered as the founder of the present mode of philosophising by experiments. And his method has been prosecuted with laudable emulation by the Academy del Cimento, the Royal Society, the Royal Academy at Paris; by Mr. Boyle, and, above all, by Sir Isaac Newton, with many other illustrious names.

Indeed, the making of experiments, within the last century, is so much practisel, that nothing will pass in philosophy, but what is either founded on expcriments, or confirned by them; so that the new philosophy is almost wbolly experimental.

Yet there are some, even among the learned, who speak of experiments in a different manner, or perhaps rather of the abuse of them, and in derision of the pretenders to this practicc. Thus, though Dr. Keil allows that philosophy has received considerable advantages from those who make experiments; yet he complains of their disingenvity, in too often wresting and distorting their experiments and observations, to favour some darling theories they had espoused. Nay more, M. Hartsneker, in his Recueil de plusieurs Pieces de Physique, undertakes to show, that such as employ themselves in the making of experiments, are not properly philosophers, but as it were the labourers or operators of philosophers, that work under them, and for them, furnishing them with materials to build their systems and hypotheses on. And the learned M. Dacier, in the beginning of his discourse on Plato, at the head of his translation of the works of that philosopher, deals still more severely with the makers of experiments. He breaks out with a kind of indignation at a tribe of idly curious people, whose sole employment consists in making experiments on the gravity of the air, the equilibrium of fluids, the loadstone, \&c, and yet arrngate to themselves the noble title of philosophers.

EXYLOSION, a sudden and violent expansion of an elastic fluid, by which it instantly throws off any obstacle that bappens to be in the way, sometimes with astonishing force and rapidity, as the explosion of fired gunpowder, \&c.

Explosion differs from expansion, in this, that the latter is a gradual and cominued power, acting uniformly for soine certain time; whereas the former is always sudden, and only of momentary or immensurably short duration. The expansions of solid substances do not terminate in violent explosions, on account of their slowness of action, and the small space through which the expanding suhstance moves; though their strength may be equally great with that of the most active aerial fluids. Thus we find that though wedges of wood, when wetted, will cleave solid blocks of stone, they never throw them to any distance, as is the case with gunpowder. On the other band, it is seldom that the expansion of any elastic fuid bursts a solid substance without throwing the fragments of it to a considerable distance, with effects that are often very terrible.

The greater number of explosive substances are either aerial, or convertible into such, and raised into an elastic fluid. Thus gunpowder, whose essence scems to consist in common air fised in the nitre, or at least an air of similar elasticity, where it is condensed into a bulk many liundred times less than the natural state of the atmosphere; which air boing snddenly disengaged by the firing of the gunpowder, and the decomposition of its parts, it rapidly cxpands itsclf again with a force proportioned to the degree of its condensation when fixcl in the gunpowder, and so explodcs, and produces all those terrible effects that attend the explosion. The elastic fluid generated by fired gunpowder expands itself with a velocity of almost 10,000 feet per second, and with a force nearly 2000 times greater than the pressure of the atmosphere on the same base.

The electric explosions seem to be still much more strong and astonishing; as in the cases of lightning, earthquakes, and volcanoes; and even in the artificial electricity produced by the ordinary machines. The astonishing strength of clectric explosions, which is beyond all possible means of measuring it, manifests itself by the many tremendous effects we hear of fire-balls and lightning.

In cases where the electric matter acts like common fire, the force of the explosions, thongh very great, is capable of measurement, by comparing the distances to which bodies are thrown, with their weight. This is most evident in volcanoes, where the projections of the burning rocks and lava manifest the immensity of the power, at the same time that they aford a method of measuring it : and these explosions are owing to the extrication of aerial rapours, and their rarefaction by intense heat.

Nest in strength to the aerial vapours, are those of aqueous and other liquids. Very remarkable effects of these are observed in steam-engines; and there is one case from which it has been inferred that aqueons steum is even much stronger than firel gunpowder. This is when water is thrown upon melted copper: for here the explosion is so strong as almost to exceed imagination; and the most terrible accidents have happened, even from so slight a cause as onc of the workmen spitting in the furnace where copper was melting; arising probably from a sudden decomposition of the water. Explosions happen also from the application of water to other melted metals, though in a lower degree, when the fluid is applied in small quantities, and even to comınon fire itself, as cvery person's own experience must have informed him; and this secms to be occasioned by the sudden rarefaction of the water into steam. Examples of this kind often occur when workmen are fastening cramps of iron into stones; where there sometimes happens to be a little water in the bole into which the lead is poured; in this case the metal will fly out in such a manner as sometimes to burn then very severely. Terrible accidents of this kind sometimes occur in founderies, when large quantitics of melted metal is poured into wet or damp moulds. In these cases, the sudden expansion of the aqueous stcam has thrown out the metal with great violence; and if any decomposition has taken place at the same time, so as to convert the aqucous vapour into an acrial one, the explosinn must be still grcater.

To this last kind of explosion must be referred that which takes place on pouring cold water iuto boiling or burning oil or tallow, or in pouring the latter upon the former; the water however being always used in a small quantity.-Another remarkable kind of explosion is that produced by inflammable and dephlogisticated air, when inixed together, and set on fire; a circumstance that often lappens in coal-mines, \&c. 'This diflers from any of the cases before mentioned; for here is an absolute condensation rather than an expansion throughout the while of the operation; and could the airs be made to take fire throughout their whole substunce absolutely at the same instant, there would be no explosion, but only a sudden production of heat. 


\section{E X P}

Though explosions be sometimes very destructive, they are also of considerable use in life, as in removing obsta. cles that could scarcely be overcone by any mechanical power whatever. The principal of these are the blowingup of rocks, the scparating of stones in quarries, and other purposes of that kind. The destruction occasioned by them in times of war, and the machines formed upon the principle of explosion for the destruction of the human race, are well known; and if we cannot call these useful, they must be allowed at least to be necessary evils.

The effects of explosions, when violent, are felt at a considerable distance, by rcason of the concussions they give to the atmosphere. Sir $\mathrm{WWm}$. Hamilton relates, that at the explosions of Vesuvius, in 1767 , the doors and windows of the houses at Naples flew open if unbolted, and one door was burst open that had been locked, though at the elistance of 6 miles: and the explosion of a powdermagazine, or a powder-mill, it is well known, spreads destruction for many miles round; and even kills people by the mere concussion of the air. A curious cffect of them also is, that they electrify the air, and even glass windows, at a-considerable distance. This is always observable in firing the guns at the Tower of London: and some years ago, after an explosion of some powder mills near that city, many people were alarmed by a rattling and breaking of their china-ware. In this respect, however, the effects of electrical explosions are the most renarkable, though not in the uncommon way just mentioned; but it is certain that the influence of a flash of lightning is diffused for a great way round the place where the explosion happens, producing very perceptible changes both on the animal and vegetable creation.

EXPUNENT of a Pouter, in Arithmetic and Algebra, denotes the number or quantity expressing the degree or elevation of the power, or which shows how often a given power is to be divided by its root before it be brought down to unity or 1 . Thus, the exponent or inclex of a square number, or the $2 \mathrm{~d}$ power, is 2 ; of a cube 3 ; and so on; the square being a power of the $2 \mathrm{~d}$ degree; the cube, of a $3 \mathrm{~d}$, \&c. It is otherwise called the Index.

Exponents, as now used, are rather of modern invention. Diophantus, with the Arabian and the first European authors, denoted the powers of quantitics by subjoining an abbreviation of the name of the power; though with some variation, and difference from one another. The names of the powcrs, and the marks for denoting them, according to Diophantus, are as follow: viz,

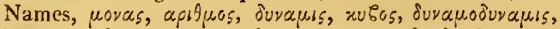
Marks, $\mu_{0}^{\circ},{ }_{5}, \delta u, x^{u}, \delta u \delta v, \& c$; which we now denote by $1, a, a^{2}, a^{3}, a^{4}, \& c$.

F. Lucas Paciolus, or De Burgo, lor the root, square, cube, \&c, uses the terms cosa, censo, cubo, relato (primo, secundo, tertio, \&c), or the abbreviations co. ce. $c u$.; and $\mathbf{R}$ for root or radicality.

Cardan used the Latin contractions of the names of the powers; and other contemporary, and cver, succceding anthors, especially the Germans, as Stifelius, Scheubelius, Pelitarins, \&c, used the like contractions, luut somewhat varied, thus:

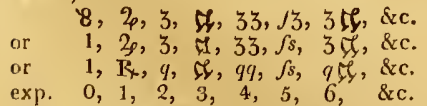

But besides that method of representation, the same authors also inade use of the numbers as in the last line here
E X P

above, and it was Stifelius who first called them exponents:

Bombelli, whose Algebra was published in 1579 , denotes the res, or unknown quantity, by this mark , and the powers by numeral exponents set over it, thus: 1,2 , 3 , Sc. And

Stevinus, who published his Arithmetic in 1585, and bis Algebra soon afterwards, makes use of a similar method, but instead of he uses a small circle $O$, within which he places the numeral exponent of the power; thus (), (1), (2), (3), \&c: and in this way le extends bis notation to fractional exponents, and even to radical ones;

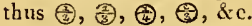

Vieta after this used words again to denote the powers. And Harriot denoted the powers by a repetition of the root; as $a, a a$, aut , for the $1 \mathrm{st}, 2 \mathrm{~d}$, and $3 \mathrm{~d}$ powers. Instead of which, Descartes again restored the numeral exponents, placing them after the root, when the power is high, in order to avoid a too frequent repetition of the same letter; as $a^{3} a^{4}, \& c$, the same as is used at present. Also Albert Girard, in 1629, used the exponents to roots, thus; $\sqrt{ }, \sqrt[2]{ }, \sqrt[3]{V}, 8 \mathrm{c}$.

The notation of powers and roots by the present mode of exponents, has introduced a new and general arithmetic of exponents or powers; for hence powers are multiplicd by only adding their exponents, divided by subtracting the exponents, raiscd to other powers, or roots of them extracted, by multiplying or dividing the exponent by the index of the power or root.-

$$
\begin{aligned}
& \text { So } a^{2} \times a^{3}=a^{5} \text {, and } a^{\frac{x}{2}} \times a^{\frac{x}{4}}=a^{\frac{3}{4}} ; \\
& a^{5} \div a^{3}=a^{2} \text {, and } a^{\frac{3}{4}} \div a^{\frac{1}{2}}=a^{\frac{1}{4}} \text {; } \\
& \text { the } 2 \mathrm{~d} \text { powcr of } a^{3} \text { is } a^{6} \text {, } \\
& \text { and the 3d root of } a^{6} \text { is } a^{2} \text {. }
\end{aligned}
$$

This algorithm of powers led the way to the invention of logarithms, which are only the indices or exponents of powers: and hence the addition and subtraction of logarithms, answer to the multiplication and division of numbers; while the raising of powers, and extracting of roots, is effected by multiplying the logarithm by the index of the power, or dividing the logarithm by the index of the root.

Exponent of a Rutio, is, by some, understood to be the quotient arising from the division of the antecedent of the ratio by the consequent: in which sense, the exponent of the ratio of 3 to 2 is $\frac{3}{2}$; and that of the ratio of 2 to 3 is $\frac{2}{3}$. But others, and those among the best mathematicians, understand logarithms as the exponents of ratios; in which sense they coincide with the idea of mensures of ratios, as de livered by Kepler, Hcrcator, Halley, Cotes, \&c.

EXPONENTIAL Calculus, the method of differencing, or finding the fuxions of exponential quantities, and of summing up those differences, nr finding their fluents. See Calculus, Fluxions, and Fuents.

Exponential Curve, is that whose nature is defined or expressed by an cxponential equation; as the curve denoted by $a^{x}=y$, or by $x^{x}=y$.

Exponential Equation, is one in which is contained an exponential quantity: as the cquation $a^{x}=b$, or $x^{x}$ $=a b$, \&c. Exponential equations are commonly best resolved by means of logarithms, viz, first taking the log. of the given equation : thus, by taking the ling. of the cquation $a^{x}=b$, it is $x \times \log$. of $a=\log$. of $b$; and hence $x=\log _{.} b \div \log _{.} a$.

Also, the log. of the cquation $x^{x}=a b$, is $x \times \log , x=$ 
log. $a b$; and then $x$ is easily found by trial-and-error, or the double rule of position.

ExpoNential Quantity, is that whose power is a variable quantity; as the expression $a^{x}$, or $x^{x}$. Exponential quantities are of several degrees, and orders, according to the number of exponents or powers, one above another. Thus,

$a^{\mathrm{x}}$ is an exponential of the 1 st order,

$a^{x}$, is one of the $2 \mathrm{~d}$ order,

$a^{z}$

$a^{x^{y}}$ is one of the $3 \mathrm{~d}$ order, and so on.

See Bernoulli Oper. tom. 1, pa. 182, \&c.

EXPRESSION, in Algebra, is any algebraical quantity, simple or compound : as the expression, $3 a$, or $2 a b$, or $\sqrt{ }\left(a^{2} \pm c^{2}\right)$. Sometimes also called a function.

EXTENSION, one of the common and essential properties of body; or that by which it possesses or takes up some part of universal space, called the place of that body. The extension of a body, is properly speaking in every direction whatever; but it is usual to consider it as extended only in length, breadth, and thickness.

EXTERIOR Polygon, or Talus, is the outer or circumscribing one. See PoLygon, and TaLus.

EXTEPMINATION, or Exterminating, in Algebra, is to take away certain unknown quantities from depending equations, so as finally to have only one equation containing one unknown quantity; whence its value may be determined, and by means of this, and the equations obtained in the extermination, all the other quantities become known.

The rules for performing this are various, and after all, much must be left to the ingenuity of the algebraist, as the application and combination of those particular rules, depend, in a great measure, on the forms and nature of the equations, out of which the unknown quantities are to be exterminated. The following however may be considered as forming principles of the operation.

1. Find the value of one of the unknown letters, in terms of the other quantities, in each of the given equations. Next put two of these values equal to each other; then one of these and a third, fourth, \&c; by which means one unknown quantity wil] be exterminated; and in the same way others may be taken away, till there remain only one equation asd one unknown quantity, the value of which may then be found by the common rules.

2. Find the value of one of the unknown letters in one of the equations; then substitute this value of $\mathrm{jt}$ in each of the other equations into which that letter enters, by which means it will be exterminated; and in the same manner we may take away all but one as before.

3. Multiply or divide the given equations by such numbers, that the coefficients of one of the unknown quantities in each of the equations may be equal to each other. Then by adding or subtracting them as the case requires, that unknown quantity will be taken awvay.

The following equations will serve to illustrate all these rules.

Given $a x+b y=n$, and $c x+d y=m$.

By the first method, $x=\frac{n-l y}{a}$; and $x=\frac{m-d y}{c}$;

therefore $\frac{n-m y}{a}=\frac{m-l y}{c}$, or $(a d-b c) y=a m-c n$, which is an equation containing only one unkmown quautity.

Sicond nucthod. Ilere $x=\frac{n-l y}{a}$; and substituting this value of $x$ in the second equation, we have

$$
\begin{aligned}
& \frac{c n-b_{c y}}{a}+d y=m \text {; which gives } \\
& (a d-b c) y=a m-c n \text {, as before. }
\end{aligned}
$$

Thirdmethod. By multiplying the first equation by $c$, and the second by $a$, we obtain

$$
a c x+b c y=c n \text {, }
$$

and $a c x+a d y=a m$; then by subtraction we have $(a d-b c)$ y $x \mathrm{ram}-\mathrm{cm}$, still the same result as before. See Hutton's Course, Simple Equat. prob. 1, pa. $22 \%$, \&c.

Many ingenious specimens of the different methods of extermination are given in Newton's Universal Arithmetic, and Maclaurin's Algebra. Sce also an excellent piece on this subject under the article Elimination in the, Encyclopedia Methodique.

EXTERNAL Angles, are the angles formed without a figure, by producing its sides out. In a triangle, any external angle is equal to the sum of both the two internal opposite angles taken together: and, $\mathrm{jn}$ any right-lined figure, the sum of all the external angles, is equal to 4 right angles, except in the case where one of them tends inwards. In which case, that angle must be taken from the sum of the $n$ thers to leave four right angles. See Hutton's Geom. Theor. 16 and 20.

EXTRA-Constellary Stars, such as are not properly included in any constellation.

EXTRA-Mundane Space, is the infinite, empty, void space, which is by some supposed to be extended beyond the bounds of the universe, and consequently in which there is really nothing existing.

EXTRACTION of Roots, is finding the roots of given numbers, quantities, or equations. The roots of quantities are denominated from their powers; as the square or $2 \mathrm{~d}$ root, the cubic or $3 \mathrm{~d}$ root, the biquadratic or 4 th root, the 5 th root, \&c; which are the roots of the $2 \mathrm{~d}, 3 \mathrm{~d}, 4 \mathrm{th}$, 5 th, \&c powers. The extraction of roots has always made a part of arithmetical calculation, at least as far back as the composition of powers has been known: for the composition of powers always led to their resolution, or extraction of roots, which is performed by the rules exactly reverse of the former. Thus, if any root be considered as consisting of two parts $a+x$, of which the former $a$ is known, and the latter $x$ unknown, then the square of this root is $a^{2}+2 a x+x^{2}$, which is its composition, and this indicated the resulution, or methor of discovering the unknown part $x$; for having subtracted the nearest square $a^{2}$ from the given quantity, there remains $2 a x+x^{2}$ or $(2 a+x) \times x$; thereforc divide this remainder by $2 a$, the double of the first member of the root, and the quotient will be nearly equal to $x$ the other member; then to sa add this quotient $x$, and multiply the sum $s a+x$ by $x$, and the product will make uptle remaning patt $2 a x+x^{2}$ of the given power.

The composition of the cubic or $3 d$ power next preschted itserf, which consists of these four terms $a^{3}+3 a^{2} x$ $+3 u x^{2}+x^{3} ;$ by 111 ans of which the cubc roots of numbers bave been exaracted; viz, by subtracting the nearest cube $a^{3}$ from the niven power, and dividing the remainder by $3 \iota^{\prime \prime}$, which grves $x$ nearly for the puotient; then completing the divisor up to $3 a^{2}+3 u x+x^{2}$, multiply it by $x$ fir the other part of the power to be subtracted. And this was the extent of the extraction of roots in the time 
of Lucas de Burgo, who, from 1470 to 1500 , wrote scveral pieces on arithmetic and algcbra, which were the first works of this kind printed in Eurupe.

It was not long huwever before the nature and composition of all the higher powers became known, and general tables of coefficients formed for raising them; the first of which is contained in Stifelius's Arithmetic, printed at Norimberg in 154.3, where he fully cxplains their use in extracting the roots of all powers whatever, by methods similar to those for the square and cube roots, as above described ; and thus completed the extraction of numerical roots, at least so far as that method of resolution extends. Since that time, however, many new modes of extraction have been devised, and the old method considerably improved.

The extraction of ronts of equations fullowed closely that of known numbers. In De Burgo's time they extracted the roots of quadratic cquations, the same way as at present. Ferreus, Tartalea, and Carilan extracted the roots of cubic equations, by general rukes. Soon alterwards the roots of higher enuations were extracted, at least in numbers, by approximation. And the late improvements in analytics have furnished general rules for extracting the roots, in infinite series, of all equations whatever. All which methods may be scen in most books of arithmetic and algebra; thercture it nay be sufficient to give here a short specimen of somc of the easiest rules for extracting the roots of quantities and equations.

\section{To Extract the Square Ront.}

Separate the given numbers into pcriods of two figures each, by setting a point over the place of units, another over the place of hundreds; and so on, over every second figure, both to the left hand in integers, and to the right hand in decimals.

Find the greatest square in the first period on the left hand, and set its root on the right hand of the given number, after the manner of a quotient figure in division.

Subtract the square, thus found, from the said period, and to the remainder annex the two figures of the next following period for a dividend. Duuble the root above found for a divisor, and find how often it is contained in the said dividend, exclusive of its right-hand figure, and set that quotient figure both in the quotient and in the divisor.

Multiply the whole augmented divisor by the last quotient figure, and subtract the product from the said dividend; bringing duwn to it the next period of the given number for a new dividend.

Repeat the same process over again; namely, find another new divisor, by doubling all the figures now found in the rnot; from which, and the last dividend, find the next figure of the not as befure, and so on through all the periorls to the last.

But the best way of doubling the root, to form the new divisur, is liy adding the last figure always to the last divisur; its apperers lrom the following examples.

When the root is to be carried into decimals, couplets of ciphers are: to bo added, insterd of figures, as far as may be necessary. In which case also a considerable abbreviation is made, alter the work has been carried on to half the number of figure's, or one more than balf, by continuing it to the other liall unly by the contracterl method of division; as in the fullowing example for the squarc root of 2 to cight decimals, or nine places of figures.
It may also not be amiss to add for the information of beginners, that when there are an odd number of decimals in the number whose root is to be extracted, they should be made even, by annexing a cipher, before the operation is begun. Sce the following examples.

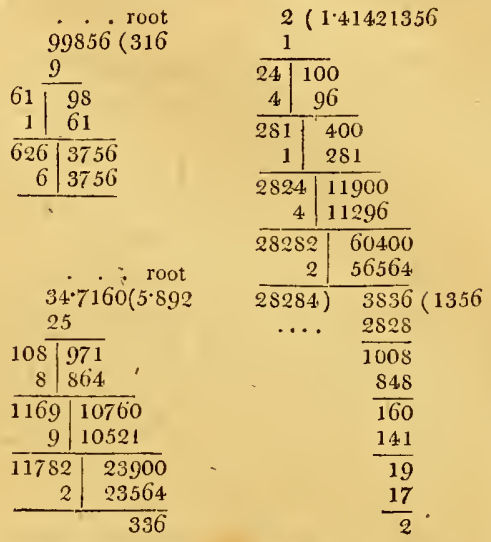

To extract the square root of a number by continued fractions, see Continued Fractions.

\section{To Extract the Cube Root.}

Divide the given number into periods of three figures each, by setting a point over the place of units, another over the place of thousands, and so on, over every third figure, to the left hand in integers, and to the right in decimals. Find the nearest less cube to the first period; and set its root in the quotient, subtracting the said cube from the first period; and to the remainder bring down the second period, which forms the resolvend.

To three times the square of the root, thus found, add thrce times the rout itself, setting this one place more to the right than the former, and call this sum the divisor. 'Then divicle tlie resolvend, wanting the last figure, by the divisor, for the next figure of the root, which annex to the former; and let this last figure be called $e$, and the part of the root before found $a$.

Add altogether these three products, namely, three times a square multiplied by $e$, thrice $a$ nultiplied by $e$ square, and $e$ cube, setting each of them one place mure to the right than the former, and call this sum the subtrahend; which should not exceed the resolvend; therefore if it do, make the last figure $e$ less, and repeal the operation for finding the subtraliend, till one be found less than the resolvend.

From the resolsend take the subtraturuel, and to the rcmainder join the next period of the given number for an new resolvend; to which form a new divisor from the whole root now found, and from thence another figure of the root as above directed, and so in, till all the figures are used. After whicls the operation may be cuntinued to any length by bringing always new periods of three ciphers each.

Suppose for cxample it were required to cxtract the cubc root of $48228 \cdot 544$. 


$$
\begin{aligned}
& 3 \times 3^{2}=27 \\
& 3 \times 3=\frac{48228.544(36.4 \text { root }}{279} \mid \frac{27}{21228} \text { resolvend } \\
& \text { Divisor } \\
& \begin{array}{c}
3 \times 3^{2} \times 6=162 \\
3 \times 3 \times 6^{2}=324 \\
6^{3}=216
\end{array} .
\end{aligned}
$$

$3 \times 36^{2}=3888 \mid 19656$ subtrahend$$
3 \times 36=\frac{108}{35988} \quad \frac{1572544}{\text { resolvend }}
$$

$$
\left.\begin{array}{rl}
3 \times 36^{4} \times 4 & =15552 \\
3 \times 36 \times 4^{2}= & 1728 \\
4^{3}= & 64
\end{array}\right\} \text { add }
$$

III. To Extract the Cube Root, or any other Root whatever.

This is easicst done by one general rule, that I have invented, and published in my new Tracts, vol. 1, pa. 210, which is to this effect: Let $\mathrm{N}$ be any number or power, whose $n$th root is to be extracted; and let $\mathrm{R}$ be the nearest rational root of $\mathrm{N}$, of the same kind, or $\mathrm{R}^{\mathrm{D}}$ the nearest rational power to $\mathrm{N}$, either greater or less than it; then shall the true root be very nearly equal to

$(n+1) \mathrm{N}+(n-1) \mathrm{R}^{\mathrm{n}}$

$\frac{(n+1) \mathrm{N}+(n-1) \mathrm{R}^{n}}{(n-1) \mathrm{N}+(n+1) \mathrm{R}^{\mathrm{n}}} \times \mathbf{R}$; which rule is general for any root whuse index is denoted by $n$. And by expoundiug $n$ successively by all the numbers $2,3,4,5$, \&c, this theorem will give the following particular rules for the several roots, viz, the

$$
\begin{aligned}
& 2 d \text { or squ. root, } \frac{3 N+R^{2}}{N+3 n^{2}} \times R \text {; } \\
& 3 d \text { or cube root, } \frac{4 N+2 R^{3}}{2 N+4 R^{3}} \times k \text {, or } \frac{2 N+R^{3}}{N+2 R^{3}} \times R \text {; } \\
& 4 \text { th root }-\frac{5 \mathrm{~N}+3 \mathrm{R}^{4}}{3 \mathrm{~N}+5 \mathrm{R}^{4}} \times \mathrm{R} \text {; } \\
& 5 \text { th root }-\frac{6 \mathrm{~N}+4 \mathrm{R}^{5}}{4 \mathrm{~N}+6 \mathrm{R}^{5}} \times \mathrm{R} \text {, or } \frac{3 \mathrm{~N}+2 \mathrm{R}^{5}}{2 \mathrm{~N}+3 \mathrm{R}^{5}} \times \mathrm{R} \text {; } \\
& 6 \text { th root }-\frac{7 N+5 R^{6}}{5 N+7 R^{6}} \times R \text {; } \\
& 7 \text { th root }-\frac{S N+6 R^{7}}{6 N+8 R^{7}} \times R \text {, or } \frac{4 N+3 R^{7}}{3 N+4 R^{7}} \times R \text {; } \\
& \text { \&c. \&c. }
\end{aligned}
$$

Or the theorem may be stated in the form of a proportion, thus:

as $(n-1) \mathrm{N}+(n+1) \mathrm{R}^{\mathrm{n}}:(n+1) \mathrm{N}+(n-1) \mathrm{R}^{\mathrm{n}}:: \mathrm{R}:$ the root sought very nearly.

Suppose for example it was required to find the cube root of the number 2 . IJere $\mathrm{N}=2, n=3$, and the ncarest power, and root are each 1.

$$
\begin{aligned}
\text { Hence } 2 \mathrm{~N}+\mathrm{n}^{3} & =4+1=5, \\
\text { and } \mathrm{N}+2 \mathrm{n}^{3} & =2+2=4 ;
\end{aligned}
$$

then $4: 5:: 1: \frac{5}{4}=1 \cdot 25$ the first approximation.

Again, taking $\mathrm{R}=\frac{5}{4}$, and consc' $] . \mathrm{R}^{3}=\frac{123}{64}$ :

$$
\begin{array}{r}
\text { Hence } 2 \mathrm{~N}+\mathrm{R}^{3}=4+\frac{12}{64}=\frac{381}{64}, \\
\text { and } \mathrm{N}+2 \mathrm{n}^{3}=2+\frac{250}{64}=\frac{378}{64}
\end{array}
$$

then $378: 381:: \frac{5}{4}: \frac{6}{5} 3 \frac{5}{4}=1.259921$, for the cube root of 2 , which is exact in the very last figure.

And agit:1 by taking $\frac{63}{50} \frac{5}{4}$ for the value of $\mathbf{R}$, a great miny mare dignres mat be found.

IV. To Extract the Roots of Algebraic Quantities.-This is done by the same rules, and in the same manner as for the roots of numbers in aritbmetic, as abore taught. Thus, to extract the square root of $4 a^{2}+12 a x+9 x^{2}$.

$$
\begin{array}{rl}
4 a^{2}+12 a x+9 x^{2}(2 a+3 x \text { the root } \\
4 a + 3 x \longdiv { a ^ { 2 } } \longdiv { 1 2 a x + 9 x ^ { 2 } } \\
3 x & 12 a x+9 x^{2} \\
\hline
\end{array}
$$

So also the root is carried out in an infinite series, in imitation of the like extraction of numbers in infinite decimals : thus, the syuare root of $a^{2}+x^{2}$ is

$$
\begin{aligned}
& a^{2}+x^{2}\left(a+\frac{x^{2}}{2 a}-\frac{x^{3}}{8 a^{3}}+\frac{x^{6}}{16 a^{5}} \& c .\right. \\
& \frac{\frac{a^{2}}{x^{2}} \mid x^{2}}{2 a+\frac{x^{2}}{2 a} \mid x^{2}+-\frac{x^{4}}{4 a^{2}}} \\
& 2 a+\frac{x^{3}}{a}-\frac{x^{4}}{8 a^{3}} \mid-\frac{x^{4}}{4 a^{3}} \\
& -\frac{x^{4}}{8 a^{3}}-\frac{x^{4}}{4 a^{2}}-\frac{x^{6}}{8 a^{4}}+\frac{x^{8}}{4 a^{6}} \text { \&c. }
\end{aligned}
$$

To extract the cube root of $a^{3}-x^{3}$ by the general rule in the $2 \mathrm{~d}$ article.-Here $\mathrm{N}=a^{3}-x^{3}, \mathrm{R}=a$, or $\mathrm{R}^{3}=a^{3}$; $\mathrm{N}+2 \mathrm{R}^{3}=3 a^{3}-x^{3}$, and $2 \mathrm{~N}+\mathrm{R}^{3}=3 a^{3}-2 x^{3}$; therefore, by the rule,

$8 a^{3}-x^{3}: 3 a^{3}-2 x^{3}:: a: a-\frac{x^{3}}{3 a^{3}}-\frac{x^{6}}{9 a^{5}}-\frac{x^{9}}{27 a^{7}} \& c$, which is the cube root of $c^{3}-x^{3}$ very nearly.

But these kinds of roots are best extracted by the $\mathrm{Bi}$ nomial Theorem; which see.

V. To Extract the Roots of Equations.-This is the same thing as to find the value of the unknown quantity in an equation; which is effected by various means, depending on the form of the equation, and the dimension of the highest power of the unknown quantity in it: for which, see the respective terms, EQuation, Root, QuaDRATic, Cubic, \&c.

The most general, as well as the most easy, method of extracting the roots of all the higher equatıons, is by double position, or trial-and-error; as it easily applies to allkinds of equations, however complex they may be, even logarithmic and cxponential ones. There are also several other good methods of approximating to the roots of equations, given by Newton, Halley, Raphson, De Moivre, Lagrange, \&c ; of which the most general is a rule for extracting the root of the following indefinite equation,

$$
\begin{aligned}
& \text { viz, } a z+b z^{2}+c z^{3}+d z^{4}+e z^{5} \& c \\
& \Rightarrow g y+b y^{2}+i y^{3}+k y^{4}+l y^{5} \& c,
\end{aligned}
$$

given by M. De Moivre in the Philos. Trans, vol. ?०, pa. 190, or my Abr. vol. 4, pa. 275.

EXTRADOS, the outside of an arch, or vault, \&c.

EXTREME-and-Mean Proportion, is when a line, or any quantity is so divided, as that the whole line is to the greater part, as that greuter part is to the less. Ilence, in any line so divided, the rectangle of the whole line and the less segment, is equal to the square of the greater segment. Fuclid shows how to divide a line in exremeand-mean ratio, in his Elements, book 2, prop. 11, to this effect: Let $A$ s be the given line; to which draw $A E$ perpendicular and equal to half $A B$; in lis produced take $\mathrm{EF}=\mathrm{n} \mathrm{n}$, so shall $\mathrm{A} \mathbf{F}$ be equal to the greater part; consequently, if $A \mathrm{G}$ be taken equal to $\Delta \mathrm{F}$, the line $\mathbf{A} \mathrm{B}$ will be divided in $\mathrm{g}$ as required. 

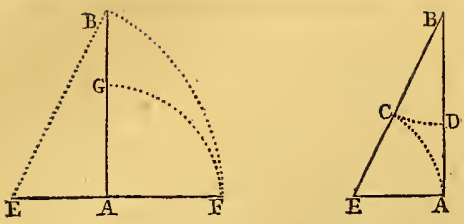

The same may be done otherwise thus:

As before, make $A E$ (fig. 2) perpendicular and $=\frac{1}{2} A B$; join $\mathrm{EB}$, on which take $\mathrm{EC}=\mathrm{EA}$, and then take $\mathrm{BD}=\mathrm{BC}$, so shall the line be divided in $\mathrm{D}$ as required.

No number can be divided into extreme and mean proportion, so that its two parts shall be rational; as is well demonstrated by Clavius, in his Commentary on the 9th book of Euclid's Elements; and the same thing will also appear from the following algebraical solution of the same problem: Let $a$ denote the whole line, and $x$ the greater part; then shall $a-x$ be the less part, and the rectangle of the whole and less part being put equal to the square of the greater part, gives this equation, $x^{2}=a(a-x)=a^{2}$ $-a x$; hence $x^{2}+a x=a^{2}$ and by completing the square, and extracting the root, $\& c$, there is at last obtained for the greater part $x=\frac{\sqrt{5}-1}{2} a$; conseq. $a-x=\frac{3-\sqrt{ } 5}{2} a$ is the less part. And as the square root of 5 , which cannot be exactly extracted, makes a portion of both these parts, it is manifest that neither of them can be obtained in rational numbers.

Euclid makes great use of this problem, in several parts of the 13th book of the Elements; and by means of it he constructs the 10th proposition of the $4 \mathrm{th}$ book, which is to construct an isosceles triangle having each angle at the base double the angle at the vertex.

EXTIREMLS Conjunct, and Extremes Disjunct, in Spherical Trigonometry, are in the former case the two circular parts that lie next the assumer middle part, and in the latter case they are the two that lie remote from the middle part. These were terms employed by lord $\mathrm{Na}$ pier, in his universal theorem for resolving all right-angled and quadrantal spherical triangles, and published in his Logarithmorum Canonis Descriptio, an. 1614. In this theorem, Napier condenses into one rule, in two parts, the rules for all the cases of right-angled spherical triangles, which had been separately demonstrated by Pitiscus, Lansbergius, Copernicus, Regiomontanus, and others. In this theorem, neglecting the right angle, the author calls the other five parts, circular parts, which are, the two legs about the right angle, and the complements of the other three, viz of the hypothenuse, and the two oblique angles. Then, taking any three of these five parts, one of them will be in the middle between the other two, and these two are the extremes conjunct when they are immediately adjacent to that middle part, or they are the extremes disjunct when they are each separated from the middle one by another part. Thus, the five parts being $\mathbf{A B}, \mathbf{A C}$, and the complements of $\mathrm{BC}$ and of the two angles $B$ and $c$ : then if the three parts $\mathrm{AB}$, and the complements of the angle $B$ and hypotheuuse $\mathbf{B C}$ be taken, these three are contiguous to each other, the angle sying in the to each other, the angle $\mathrm{B}$ lying in the
middle between the other two: there-
fore the complement of $\mathrm{B}$ is the middle part, and $\mathrm{AB}$ with

VOL. I. the complement of $\mathrm{BC}$ the extremes conjunct. But if the three sides be taken; $\mathrm{BC}$ is equally separated from the two legs $A B$ and $A C$, by the two angles $B$ and $C$; and therefore these two legs $A B$ and $A C$ are the extremes disjunct, and the complement of $\mathrm{BC}$ the middle part.-The author's rule for resolving each of these cases is in two parts, as beluw :

The rectangle contained by radius and the sine of the middle part, is equal to the rectangle of the tangents of the extremes conjunct, or equal to the rectangle of the sines of the extremes disjunct. Which rule comprehends all the cases that can happen in right-angled spherical triangles; in the application of which rule, the equal rectangles are divided into a proportion or analogy, so that the term sought may be the last of the four terms that are concerned, and consequently its corresponding term in the same rectangle must be the first of those terms.

EYE, the organ of sight, consisting of several parts, and of sucb forms as best to answer the purpuse for which it was designed. Vision or sight being cffected by a refraction of light through the humours of the eye to the bottom er farther internal part of $\mathrm{it}$, where the images of external objects are formed on a fine expansion of the optic nerve, called the retina, therefore the fore part of the eye must be of a convex figure, and of such a precise degree of convexity, as the particular refractive power of the several humours require, for forming the image of an object at a given focal distance, viz, the diameter of the eye. Hence we find,

1 st ; The external part of the eye-ball $\mathrm{cD}$ (Plate 2, fig. 8 ) is a strong pellucid substance, properly convex, and which, when dried, has some resemblance to a piece of transparent hol'n, for which reason it is called the cornea, or borny coat of the eye.

$2 \mathrm{dly}$; Immediately behind this coat there is a fine clear humour which, from its similarity to water, is called the aqueous or watery humour, and is contained in the space bctween $\mathrm{CD}$ and GFE.

3lly; In this space there is a nembrane or diaphragm, called the uvea, with a hole in the middle as at $\mathrm{F}$, called the pupil, of a muscular contexture for altering the dimensions of that hole, in order to adjust or admit a due quantity of light.

4thly; Immediately behind this diaphragm is placed a lenticular-formed substance $\mathrm{GE}$, of a considerable consistence, called from its transparency the crystalline humour. This is contained in a fine tunic called the choroides, and is suspended in the middle of the eye by a ring of muscular fibres called the ligamentum ciliure, as at $G$ and $E$; by which means it is moved a little nearer to, or farther from, the bottom of the eye, to alter the focal distance.

5thly; All the remaining interior part of the eye, constituting the great body of it, from $\mathrm{GHE}$ to $1 \mathrm{MK}$, is made up of a large quantity of a jelly-like substance, called the vitreous or glassy humour; though it resembles glass in nothing except its transparency; it being more like the white of an egg than any other thing.

6 thly; On one side of the backward part of the eye, as at $\mathrm{K}$, the optic nerve enters it from the brain, and is expanded over all the interior part of the eye to $G$ and $E$, the expansion being named the retina. On this delicate membrane, the image $1 \mathrm{M}$ of every external object $\mathrm{OB}$, is formed according to the optic laws of nature, in the following mamer.

Let $O B$ be any very distant object. Now a pencil of $3 \mathrm{~T}$ 
rays proceeding from any point $\mathrm{L}$, will fall on the cornca $\mathrm{CD}$, and be refracted by the aqueous humour under it, to a point in the axis of that pencil continued out. Also the radius of convexity of the cornca being nearly $\frac{\mathrm{r}}{3}$ of an inch; and the sine of incidence in air to that of refraction in the aqueous bumour, being nearly as 4 to 3 , supposing the rays parallel, or the object very far distant, the focal distance alter the first refraction, by the proper theorem (See Op'rics) $\frac{m r}{m-n}$, will be found $1 \frac{1}{3}$ inch from the cornea: $r$ being the radius $\frac{r}{3}$, and $m$ to $n$ as 4 to 3 , that is as the sine of the angle of incidence, to that of refraction.

The rays thus refracted by the cornea, fall converging on the crystalline humour, and tend to a point 1:228 inch belind it; also the radii of convexity in the said humour are $\frac{1}{3}$ and $\frac{1}{4}$ respectively; and the sine of incidence to that of refraction of the aqueous into the crystalline humour, having been found by experiments to be as 13 to 12 ; thereforc, by this theorem $\frac{m d r}{m d-u d+n r}$, the focal distance after refraction in the crystalline, will be 1.02 inch from tbe fore part of it: where $m=13, n=12$, $r=\frac{\mathrm{r}}{3}$, and $d=1.228$.

The rays now pass from the crystalline to the vitreous humour still in a converging state, and the sines of incidence and refraction being here as 12 to 13 , as found by experiment; and since the surface of the vitreous humour is concave which receives the rays, and is the same with the convexity of the hinder surface of the crystalline, the radius will be the same, viz $\frac{1}{4}$ of an inch. Therefore the focal distance after this third refraction will be found, by the same theorem $\frac{m d r}{n d-m d+n r}$, to be 6 tenths of an inch nearly, from the hinder part of the cornea: where $m=12$, $n=13, r=\frac{1}{4}$, and $d=\cdot 82$ : the thickness of the lens of the cornea being nearly $\frac{x}{5}$ of an inch.

Now expericnce shows that the distance of the retina in the back part of the cye, behind the cornea, is nearly equal to that focal distance; and therefore it follows that all ubjects at a great distance have their images formed on the retina in the bottom or hinder part of the eye, and thus is distinct vision produced by this wonderful organ.

When the distance of objects is nct very great, the focal distance, alter the last refraction in the vitreous humour, will be a little increased; and to do this we can move the crystalline a little noarer the cornea by means of the ligamentum ciliare, and thus on all occasions it may be: adjusted for a due ficcal distance for every distance of objects, excepting that which is less than 6 or 7 inches, in persons whose sight is perfect. But many are of opinion, that this is rficted by a power in the eye to alter the convexity of the crystalline humour as occasion requires; though this is rather doubtul.

By what has been silid it apprars, that rays of light flowing from every part of an object os, placed at a proper distance from the eye, will have an image 1 m thereby formed on the retina in the bottom of the cye; and since the rays $\mathrm{M}, \mathrm{m}$, which come from the extreme parts of the object, cross each other in the millde of the pupil, the position of the image in will be contrary to that of the olject, or inverted, ats in the case of at lens.

The apparent place of any part of an object is in the axis, and conjugate focus of that pencil of rays by which that part or point is formed in lhe image. 'Thus, on is the axis, and o the locus proper to the rays by which the point $\mathrm{M}$ in the image is made; therefore the sensation of the place of that part will be conceived in the mind to be at $\mathrm{o}$; in like manner the idea of place belonging to the point $\mathbf{I}$, will be referred in the axis $I B$, to the proper focus $\mathrm{B}$; therefore the apparent place of the whole image IM, will be conceived in the mind to occupy all the space between $O$ and $B$, and at the distance $A L$ from the cye.

Hence likewise appears the reason, why we see an object upright by means of an inverted image; for since the apparent place of every point $m$ will be in the axis so at $o$; and this axis crossing the axis of the cye $\mathrm{HL}$ in the pupil, it foliows, that the sensible place $o$ of that point will lie, without the eyc, on the contrary side of its axis, to that of the point in the eye; and since this is true of all other parts or points in the image, it is evident that the position of every part of the object will be on the contrary side of the axis to every corresponding part in the image, and thercfore the whole object on will have a contrary position to that of the image IM, or will appear upright.

If the convexity of the cornea $\mathrm{CD}$ happens not exactly to correspond to the diameter of the eye, considered as the natural focal distance, then the image will not be formed on the retina, and consequently no distinct vision can be effected in such an eye.

If the cornea be too convex, the focal distance in the eye will be less than its diameter, and the image will be formed short of the retina. Hence the reason why people having eyes thas formed are obliged to hold things very near them, to lengthen the focal distances; and also why they use concave glasses to counteract or remedy the excess of convexity, in order to vicw distant objects distinctly.

When the eye has less than a just degrce of convexity, or is too flat, as is generally the case with old people, by a natural deficiency of the aqueous humour, then the rays tend to a point or focus beyond the retina or bottom of the eye; and to supply this want of convexity in the cornea, we use convex lenses in those frames called spectacles, or visual glasses.

Since the rays of light $O A, B A$, which constitute the visual angle $O A B$, will, when they are intercepted by a lens, be refracted sooner to the axis; the said angle will thercby be enlarged, and the object of course become magnified; which is the reason why those lenses are called magnifiers, or reading-sylasses.

The dimensions, or magnitude, of an object os, are judged of by the quantity of the angle OAB which it subtends at the eye. For if the same object be placed at two different distances $\mathbf{L}$ and $\mathrm{N}$, the angles $0 \mathrm{AB}, 0 \mathrm{~A} b$, which in these two places it subtends at the eye, will be of diflerent magnitudes, and the lincal dimensions, viz, lengtl and breacth, will be at $\mathrm{N}$ and at $\mathrm{L}$, as the angle $0 \mathrm{~A} b$ is to the angle $о \mathrm{AB}$. But the surfaces of the objects will be as the squares of those angles, and the soliditics as the cubcs of thens.

It is found by experience, that two points $o, d$, in any object, will not be distinctly seen by the eyc, till they are near cunough to subtend an angle ox of one minute. And hence when objects, however large they maly be, are so remote as not to be secn under an angle of one minute, they cannot properly be said to have any apparent dimensions or magnitude at all : such is the case of the large bodies of the planets, comets, and fixed stars. But the science of optics has supplied means of enlarging this nutural small angle under which most distant objects appear, and thereby increasing their apparent magnitudes to a very 
surprising degree, in the instance of that noble instrument the telescope.

On the other hand, there are in creation an infinity of objects, of such small dimensions, that they will not subtend the requisite angle, if brought to the nearest limits of distinct vision, viz, 6,7 , or 8 inches from the eye, as found by experience; and therefore to render them visible at a very near distance, we have a variety of glasses, and instruments of different constructions, as microscopes, \& c, by which those minute objects appear many thousand times larger than to the naked eye; and thereby enrich the mind with discoveries of the sublimest nature, in regard to creative power, wistom, and economy. See Vision.

EYE-glciss, in Optical Instruments, is that which is applied to the cye bo using the instrument. This is usually a lens convex on both sides; but Eustachia Divini long since invented a microscope of this kind, the power of which he places very greatly above that of the common sort; and this chiefly icpending on the eye-glass, which was double, consisting of tro plano-convex glasses, so posited as to touch one another in the middle of their convex surface. 'l'bis instrument is much commended by Fabri in his Optics, as possessing this peculiar excellence, that it shows all the objects flat, and not crooked, and takes in a large area, though it magnifies very much.

Bull's EYli, a star of the first magnitude, in the eye of the constellation Taurus, the bull, and by the Arabs called Aldebaran.

\section{F.}

\section{F A C}

$\mathbf{F}$ ACE, or FAçaDE, in Architecture, is sometimes used for the front or outward part of a building, which immediately presents itself to the eye; or the side where the chief entrance is, or next the street, \& $\mathrm{c}$.

FACE, FACia, or FASCIa, also denotes a flat member, having a considerable breadth, and but a small projecture. Such are the bands of an architrave, larmier, \&c.

Faces of a Bastion, in Fortification, are the two foremost sides, reaching from the flanks to the outermost point of the bastion, where they meet, and form the saliant angle of the bastion. These are usually the first parts that are undermined, or beaten down; because they reach the farthest out, are the least flanked, and are therefore the weakest.

FACE of a Place, is the extent between the outermost points of two adjacent bastions; containing the curtain, the two flarks, and the two faces of those bastions that look towards each other. This is otherwise called the Tenaille of the place.

Fice Prolonged, is that part of a line of defence rasant, which is between the angle of the epaule or shoulder of a bastion and the curtain; or the line of a defence rasant diminished by the face of the bastion.

FACIA, in Aichitecture, See FACE, and FAscia.

FACTORS, in Multiplication, a name given to the two numbers that are multiplied together, viz, the multiplicand and multiplier; so called, because they are to facere productum, make or constitute the factum or product.

In algebra, we generally call all those quantities factors, the product of which constitute any algebraical expression: thus, $(a+b)$ and $(a-b)$ are the factors of the expression $a^{2}-b^{2}$; also $a, b, c, d$, are the factors of the quantity abcd. But in arithmetic these are commonly called divisors. Sue Divisons.

In the Dioplantine aralysis, and in the theory of numbers, it is frequently very advantageous to be able to resolve certain algebraical formulie into their respective factors, as also in the investigation of the nature of equations, and in short almost every brinch of algebra may sometimes derive peculiar advantagcs from such resolu-

\section{F A. C}

tion. Suppose, for example, we had to multiply $\frac{x^{2}-b^{1}}{x^{2}-2 l x+b^{2}}$ by $\frac{x-b}{x^{2}+b x}$. Now by resolving this into its factors it becomes $\frac{\left(x^{2}+b^{2}\right) \cdot(x+b) \cdot(x-b) \cdot(x-b)}{(x-b) \cdot(x-b) \cdot(x+b) \cdot x}=\frac{x^{2}+b^{2}}{x}$, by canceling the common factors; which from the common mode of operation would have been attended with conisiderable labour.

The limits of this work will not however admit of our entering upon this subject to any extent; we therefore refer the reader for farther information on this subject to Euler's Algebra, vol. 2, chapter 11; to the additions in the same vol. by Lagrange; to the Analysis Infinitorum; and to Legendre's Theorie des Nombres; where most of the following theorems are demonstrated in a very masterly manner.

$a-b$ is a factor of $a^{m}-b^{m}$ for all values of $m$.

$a+b---\quad-a^{\mathrm{m}}-b^{\mathrm{m}}$ when $m$ is even.

$a+b-\ldots-a^{\mathrm{m}}+b^{\mathrm{m}}$ when $m$ is odd.

$a-1-\quad-\quad-a^{m}-a^{n}$ for all values of $m$ and $n$,

$a+1-\ldots-a^{m}-a^{n}$ when $m-n$ is even.

$a+1-\ldots-a^{\mathrm{m}}+a^{\mathrm{n}}$ when $m-n$ is odd.

$a$ is a numer. factor of $r^{2}-r$ when $a$ is a prime number.

a - - - - $r^{\mathrm{n}-1}-1$ when $a$ is also prime to $r$.

a - - $(1 \cdot 2 \cdot 3-a-1)+1$ when $a$ is a prime.

The product of two factors, each of which is the sum of two squares, is itseli the sum of two squares.

That is, $\left(a^{2}+b^{2}\right) \cdot\left(c^{2}+d^{2}\right)=m^{2}+n^{2}$, also $\left(a^{2}+b^{2}+c^{2}+d^{2}\right) \cdot\left({ }^{2} a^{2}+b^{2}+c^{2}+d^{2}\right)=m^{2}+n^{2}+p^{2}+q^{2}$.

These two last properties were first demonstrated by Euler, in the Act. Petrop., where there are also many other ingenious papers on this subject.

Imaginar y FaCtors. See Imaginary.

FACTUM, the product of two quantities multiplied together. As, the factum of 3 and 4 is 12 ; and the factum of $2 a$ and $5 b$ is $10 a b$.

FACULA, in Astronmy, a name given by Scheiner, and others after him, to certain bright spots on the sun's disc, that appear more bright and lucid than the rest of his body.-Hevelius assures us that, on July 20, 1634, he observed a facula whose breadth was equal to a $3 \mathrm{~d}$ part 3 'I' 2 
of the sur's diameter. He says also that the macula often change into facula; but these seldom or never into maculæ. And some authors even contend that all the maculæ degenerate into faculæ before they quite disappear. Many authors, after Kircher and Scheiner, have represented the sun's bolly full of bright, fiery spots, which they conceive to be a kind of volcanos in the body of the sun: but Huygens, and others of the latest and best ubservers, finding that the best telescopes discover nothing of the matter, they therefore agree in exploding entircly the phenomena of lacula. All the fuundation he could see for the notion of facula, he says, was, that in the darkish clouds which frequently surround the macula, there are sometimes seen little points or sparks brighter than the rest: the cause of which is attributed by these authors to the tremulous agitation of the vapours near our earth; the same as sometimes shows a little unevenness in the circumference of the sun's disc when viewed through a telescope. Strictly therefore, the faculæ are not eructutions of fire and flame, but refractions of the sun's rays in the rarer exhalations, which, being cundensed near that shade, seem to exhibit a light grester than tbat of the sun.

FAHRENHEIT (GA BRIEz DA NIEL), by some writers said to be a native of Dantzic, and by others to be born at Hamburgh, in 1686. He was intended for mercantile affairs. But his inclination led him to the study of natural philosophy, and he applied particularly to the improvement of barometers and thermometers, constructing great numbers of them, which were much valued. He improved the thermometer, by substituting mercury instead of spirits of wine, and formed a new scale for the instrument, founded on accurate experiments, fising the freezing point of water at $32^{\circ}$, and that of boiling at 212. This was probably done about the year 17.20 ; as, in his Dissertation on Thermoneters, published 1724, be speaks of having used it some years, to ascertain M. Amonton's experiment, who asserted, that the heat of boiling water was constant. The English have generally adopted this scale, though the lirench preferred tbat of Reaumur, and very lately their own centigrade thermometer. Fahrenheit by some is said to have been living iu 1740 , while others say he died in 1736 .

FALCATED, one of the phases of the planets, vulgarly called horned. Astronomers say, the moon, or any planet, is falcated, when the enlightened part appears in the form of a crescent, like a sickle, or reaping-hook, which by the Latins is called fal.x. 'The moon is falcated while slue moves from the $3 d$ quarter to the conjunction, and so on from hence to the first quarter; the bright part appearing then like a cresc'nt, viz, during the first and last quarters. But during the $2 d$ and $3 d$ quarters, the light part appears gibbous, and the dark part falcated.

FALCON or FAUCON, and FALCONET or FAUCONET, certain old specics of cannon, long since disused.

FALL, the descent or natural motion of bodies towards the centre of the earth, \&c. Galileo first discovered the law of the acceleration of falling boclies; viz, that the spaces descruled from rest are as the squares of the times of descent; or, which comes to the same thing, that if the whole time of lalling be divided into any number of cqual parts, whatever space it falls through in the first part of the time, it will fall 3 times as far in the $2 \mathrm{~d}$ part of time, and 5 times as far in the $3 d$ portion of time, and so on, according to the uneven numbers $1,3,5,7$, \&c. See Acceleration, Descent, Gravity, \&ic.

FALSE-Brare, in Fortification. Sce FAusse-Bra yt: False Position, in Arithmetic. See Position.

FALsE Root, a name given by Cardan, to the negative routs of equations, and numbers. So the root of 9 may be either 3 or -3 , the former be calls the true, and the latter the false or fictitious root; also of this equation $x^{2}-x=6$, the two roots are 3 and -2 , the former true, aud the latter false.

FASCIA, in Architecture. Sce FACIA and FACE.

FASCI $Æ$, in Astronomy, are certain stripes or rows of bright parts, observed on the bodies of some of the planets, like swathes, bands, or belts; especially on the planet Jupiter. The fisciz, or belts of Jupiter, are more lucid than the rest of his disc, and are terminated by parallel lines. They are sometimes broader and sometimes narrower; and do not always possess the same part of the disc.

M. Huygens also observed a very large kind of fascia in Mars, in the year 1656 ; but it was darker than the rest of the disc, and occupied its middle part.

FASCINES, in Fortification, are faggots nude of the twigs and snall branches of trees and brush-wood, bound up in bundles; these, being mixed with earth, scrve to fill up ditches, and to make the parapets of trenches, batteries, \&c.

FATHOM, an English measure of the length of 6 fect or 2 yards.

\section{FATUUS Ignis. 'See I g is Fatuus.}

FAUCON, and FAUCONET, the same as Falcon and Falconet; the old names of certain species of ordnance ; which, as well as many others, are now no longer in use, as it has been for some time past the practice to denominate the several sizes of cannon from the weight of their ball, instead of calling them by those fanciful and unmeaning names.

FAUSSE-Braye, in Fortification, anelevation of earth, about three feet above the level ground; round the foot of the rampart on the outside, defended by a parapet about four or five fathoms distant from the upper parapet, which parts it from the berme, and the edge of the ditch. The fausse-braye is the same with what is otherwise called chemin des' rondes, and basse enceinte; its use being for the defence of the ditch.

FEBRUARY, the $2 \mathrm{~d}$ month of the year, containing 28 days for three years, and every fourth year 29 days. These years being called Bissextile, or Leap-years; and return every fourth year, except at the commencement of a new century, which are not bissextile, unless the number of the century be divisible by 4 ; so 1800 was not bissextile, nor will 1900 be 50 , but 2000 will, because 20 is clivisible by 4 . (See Bissextile.) In the first ages of Rome, February was the last month of the year, and preceded Jauuary, till the Decemviri made an order that February should be the $2 d$ month of the year, and come after January.

FELLOWSHIP, CoMPANY, or PARTNERSHP, is a rule in arithmetic, of great use in balancing accounts among merchants, and partners in trade, teaching how to assign to every one of them his due share of the gain or loss, in proportion to the stock lie has contributed, and the time it has been employed, or according to any other conditions. Or, more generally, it is a method of dividing a given number, or quantity, into any number of parts, that shall have any assigned ratios to onc another. 
And hence comes this general rule: Having alded into one sum the several numbers that express the proportions of the parts, it will be,

As the sum of the proportional numbers :

Is to the given quantity to be divided : :

So is each proportional number :

To the corresponding share of the given quantity.

For Example. Suppose it be required to divide the sumber 120 into three parts that shall be in proportion to each other as the numbers $1,2,3$.- Here 120 is the quantity to be divided, and 6 is the sum of the numbers 1,2 , and 3 , which express the proportions of the parts; therefore as

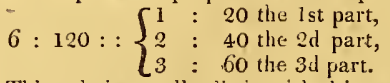

This rule is usually distinguished into two cases, one in which time is concerned, or in which the stocks of partners are continued for difterent times; and the other in which time is not considered; the latter being called Single Fellowship, and the former Double Fellowship.

Single Fellowship, or Fellowship vithout Time, is the case in which the times of continuance of the shares of partners are not considered, being all the same; and in this case, the rule, will be as above, viz,

As the whole stock of the partners :

Is to the whole gain or loss : :

So is each one's particular stock :

To his share of the gain or loss.

Example. Two partners, A and B, form a joint stock, of which A contributed $75 l$, and $\mathrm{B} 45 l$; with which they gain $30 l$ : how much of it must each person have?

As $120: 30::\left\{\begin{array}{l}75: 18 l .15 s .=A \\ 45: 11 \text {. } 5 s .=\text { s's share, }\end{array}\right.$

Double FELLowship, or FELLow'shIP with Time, is the case in which the times of the stocks continuing are considered, because they are not all the same. In this case, the shares of the gain or loss must be proportional, both to the several shares of the stock, and to the times of their continuance, and therefore proportional to the products of the two. Hence this rule: Multiply each particular share of the stock by the time of its continuance, and add all the products together into one sum; then say,

As the sum of the products :

Is to the whole gain or loss : :

So is each several product :

To the corresponding share of the gain or loss.

For Example. A had in company $50 l$. for 4 months, and в $60 l$. for 5 months; and their gain was $24 l$ : low must it be divided between them ?

$\begin{array}{rr}50 & 60 \\ \frac{4}{200} & \frac{5}{300} \\ 300 & \end{array}$

As $500: 24::\left\{\begin{array}{l}200: 9 l .12 s .=A ' s \text { share, } \\ 300: 14 l .8 s .=\text { B's share. }\end{array}\right.$

FERGUSON (JAMES), an ingenious experimental phi- losopher, mechanist, and astronomer, was horn in Banffshire, in Sectland, 1710, of very poor parents. At the very carliest age his extraordinary genius began to unfold itsclf. He first learned to read, by overhearing his father teach his clder brother: and he had made this acquisition before any one suspected it. He soon discovered a peculiar taste for mechauics, which first arose on secing his father use a lever. He pursucd his study to a considerable length, while he was yet very young; and made a watch in wood-work, from having once seen one. As he had at first no instructor, nor any belp from books, every thing he learned had all the merit of an original discovery; and such, with inexpressible joy, he believed it to be.

As soon as his age would permit, be went to service; in which he met with hardstips, that rendered his constitution feeble through life. While he was servant to a farmer (whose goodness he acknowledges in the modest and humble account of himself which be prefixed to one of his publications), he contemplated and learned to know the stars, while he tended the sheep; and began the study of astronomy, by laying down, from his own observations only, a celestial globe. His kind master, observing in hin these marks of ingenuity, procured him the countenance and assistance of some neighbouring gentlemen, through whose belp and instructions he gained farther knowledge, having by these means been taught arith metic, with some algcbra, and practical geometry. He had also obtained some notion of drawing, and being sent to Edinburgh, he there began to take portraits in miniature, at a small price; an employment by which he supported himself and family for several years, both in Scotland and England, while he was pursuing more serious studies. In London he first published some curious astronomical tables and calculations; ard afterwards gave public lectures in experimental philosophy, both in London and most of the country towns in England, with the highest marks of general approbation. He was elected a fellow of the Royal Society, and was excused the payment of the admission fee and the usual annual contributions, on account of his supposed inability to pay them. He cnjoyed from the king a pension of 50 pounds a year, besides other occasional presents, which he privately accepted and received from different guarters, till the time of his death; through which, and the fruits of his own labour's, he died worth aboutsix thousand pounds, which astonished all his friends, who had always entertained an idea of his great poverty. His death happened in 1776 , at 66 years of age, though he had the appearance of being nuch older.

Mr. Ferguson must be allowed to have been a very uncommon genius, especially in mechanical contrivances and executions, for he constructed many machines himself in a very neat manner. He had also a good taste in astronomy, as well as in matural and experimental philosophy, and was possessed of a happy manuer of explaining himself in an easy, clear, and familiar way. His general mathematical knowledge, however, was little or nothing. Of algebra he understood but little more than the notation; and he has often told me that he could never demonstrate one proposition in Euclid's Elements; his constant method being to satisfy himself, as to the truth of any problem, with a measurement by scale and compasses. He was a man of a very clear judgment in any thing that he professed, and of unwearied application to study: benevolent, meek, and innocent in lis manners as a child: humble, courteous, and communicative: instead of pedantry, philosophy scemed to produce in him only diffidence and urbanity. 'The list of Mr. Ferguson's public works, is as follows:

1. Astrononical Tables and Precepts, for calculating the true times of New aud Full Moous, \&ec; 1763.- 
2. Tables and Tracts, relative to several arts and sciences; $1767,-3$. An Easy Introduction to Astronomy, for Young Gentlemen and Ladies; $2 \mathrm{~d}$ edit. 1769.-4. Astronomy explained upon Sir Isaac Newton's Principles; 5 th edit. 1772.-5. Lectures on Select Subjects in Mechanics, Hydrostatics, Pneumatics, and Optics; 4th edit. 1772. -6. Select Mechanical Exercises; with a short Account of the Life of the author, by himself; $1773 .-7$. The Art of Drawing in Perspective nade easy; $1775 .-8$. An Introduction to Electricity; 1775.-9. Three Letters to the Rev. John Kennedy, on the Harvest Moon, 1763 and 1775 .

FERMAT (PETER), a celebrated mathematician and philosopher, and counsellor of the parliament of Toulouse, where he was born towards the commencement of the seventeenth century, or just before; and notwithstanding he tid not rnake the mathematics the whole, nor cren the principal part of his study, he yet obtained considerable celebrity for his knowlerge and discoveries in this science. He had also a perfect knowledge of the Greek and Latin languages, beside several of the modern languages of Europe, as the Italian, Spanish, and English. He also cultivated poetry, some of his works being still extant. At the same time he fulfilled the duties of his office as counsellor to the parliament of Toulouse, with assiduity and honour, enjoying the reputation of an cnlightened judge.

It was the custom of the time in which Fermat flourished, for the mathematicians of different countries to propose problems to each other for solution, and in this kind of correspondence we find him deeply engaged, particularly in theorems relating to certain properties of numbers, the demonstrations of which he generally suppressed, if, as is doubtful, he was in possession of them : how'ever this may be, many of them are even now without demonstrations, and several others which were left by him in this state, would perhaps still have remained so had it not been for the ingenuity and industry of Euler, Lagrange, aud some other more modern mathematicians.

Fermat had a violent disjute with Descartes on the subject of Maxima and Minima of quantities, of which he was the author; as well as on the doctrine of refraction; and though a compromise at length took place between them, they were never good friends after; but in this Des. cartes was most to blame, as Fermat seems to have much wisbed for a complete rcconciliation. He died in the beginning of the year 1665 . Besides his new edition of Diophantus, Fermat was author of 1. A Method for the Quadrature of all sorts of Parabolas, - 2. Another on Maxima and Minima: which serves not only for the determination of plane and solid problems; but also for drawing tangents to curve lines, finding the centres of gravity in solids, and the solution of questions concerning numbers: in short, a method very similar to the Fluxions of Newton.-3. An Introduction to Geometric Loci, plane and solid.-4. A Treatise on Spherical Tangencies: where he demonstrates in the solids, the same things as Vieta demonstrated in plines.-5. A Restoration of Apollonius's two books on Plane Loci.-6. A General Method for the dimension of Curve Lines. Besides a number of other smaller pieces, and many letters to learned men; several of which are to be found in his Opera Varia Mathematica, printed at T'oulouse, in folio, 1679.

FTIRMLNTATION, an intestine motion, arising spon- tameously among the small and insensible particles of a mixed body, thus producing a new disposition, and a difierent cumbination of those parts. Fermentation differs from dissolution, as the cause from its effect, the latter being only a result or effect of the former.

FESTOUN, in Architecture \&c, a decoration in form of a garland or cluster of flowers.

FICHANT Flank. See Flaix.

Fich a T T Line of Defence. See Fixed Line of Defence.

FIELD-Book, in Surveying, a bouk userl for setting down angles, distances, and other things, remarkable in taking surveys. The pages of the field-book may be conveniently divided into three columns. In the middle column are to be entered the angles taken at the several stations by the theodolite, with the distances measured from station to station. And the ofisets, taken with the offset-statf, on either side of the station line, are to be entered in the columns on either side of the middle column, according to their position, on the right or left, with respect to that line: also on the right or left of these are to be set down the names and characters of the objects, witb proper remarks, \&c. See a specimen in my Treatise on Mensuration; pa. 386 , ed. 4 th. See also SurVEYING.

Field-Fort. See Fortine.

Fi LLD-Pieces, are small cannon, usually carried along with an army in the field : such as, one-pounders, one-anda-balf, two-, three-, four-, six-, nine-, and 12-pounders; which, being light and small, are easily carried.

FIE L D-Siaff, is a staff carried by the gunners, in which they screw lighted matches, when they are on service; which is called arming the field-staffs. Sce Linstock.

Fiel of View, or of Vision, is the whole space or extent within which objects can be seen through an optical macline, or at one view of the eye without turning it.

The precise limits of this space are not easily ascertained, for the natural view of the eye. In looking at a small distance, we have an imperfect glimpse of objects through almost the extent of a bemisphere, or at least for above 60 degrees each way from the optic axis; but towards the extremity of this space, objects are very imperfectly seen; and the diameter of the field of distinct vision does not subtend an angle of more than 5 slegrees at most, so that the diameter of a distinct image on the retina is less than $\frac{6}{100}$ of $a n$ inch; but it is probably much less.

Field Works, in Fortification, are those that are thrown up by an army in besieging a fortress, or by the besieged to defend the place. Such are the fortifications of camps, highways, \&c.

FIFTH, in Music, one of the harmonical intervals or concords; called by the ancients diapente.

The fifth is the $3 \mathrm{~d}$ in order of the concords, and the ratio of the chords that produce it, is that of 3 to 2 . It is called fifth, because it contains five terms, or sounds, between its extrenes, and four degrees; so thit in the natural scale of music it comes in the 5 th place, or order, from the fundamental.-The imperfect, or defective filth, by the ancients called semidiapente, is less than the fifth by a mean semitone.

FIGU1RA'T': Numbers, are those numbers which are formed from the addition of all the leading terms of successive series, beginning first with that of the natural numbers, as in the following table; where the law of continuation is manifest. 


\section{F I G}

\begin{tabular}{c|ccc} 
A & $1,2,3,4,5,6$ \\
B & $1,3,6,10,15,21$ \\
C & $1,4,10,20,35,56$ \\
D & $1,5,15,35,70,126$
\end{tabular}

$n$.

$\frac{n \cdot(n+1)}{1 \cdot 2}$

$\frac{n:(n+1) \cdot(n+2)}{1 \cdot 2 \cdot 3}$.

$\frac{n \cdot(n+1) \cdot(n+2) \cdot(n+3)}{1 \cdot 2 \cdot 3 \cdot 4}$

Here, the first series $\mathrm{A}$ is that of the natural numbers, of whicl the general term is $n$; the second series $\mathrm{B}$, is that of triangular numbers, of which the general term is $\frac{n \cdot(n+1)}{1.2}$. If now from this general term, which is the $n$th term of the series $\mathrm{B}$, there be subtracted the preceding term of the same series, which is $\frac{(n-1) \cdot n}{1 \cdot 2}$, the remainder will be $n$, which is the $n$th term of the series $A$. Hence we shall form the $n$th term of the series $\mathrm{B}$, by adding to the $n-1$ term of the same series, the $n$th term of the series $A$.

The third series $\mathrm{c}$ is that of pyramiclical numbers, the general term of which $\frac{n \cdot(n+1) \cdot(n+2)}{1 \cdot 2 \cdot 3}$, and if from this term we subtract the preceding one $\frac{(n-1) \cdot n \cdot(n+1)}{1.2 \cdot 3}$, the difference will be $\frac{n \cdot(n+1)}{1 \cdot 2}$, which is the $n$th term of the series $\mathbf{B}$. Whence we may form the series $\mathrm{c}$ by means of the series $\mathrm{B}$, as this latter is formed from the series $A$. And exactly in the same manner the 4th series $D$, of which the general term is $\frac{n \cdot(n+1) \cdot(n+2) \cdot(n+3)}{1 \cdot 2 \cdot 3 \cdot 4}$, may be formed from the third $c$, and so on of others.

The general terms given above, as definitions, and from which the successive law of formation is deduced, contains the whole theory of figurate numbers, and presents, at one view, the demonstration of the general proportion mentioned by Fermat in his notes on Diaphantus, pas 16 , and which he considered as one of his principal discoveries.

So that the figurate numbers of any order may be found without compiting those of the preceding orders; which is done by taking the successive products of as many of the terms of the arithmeticals $1,2,3,4,5, \& c$, in their natural order, as there are units in the number which denominates the order of figurates required, and dividing those products always by the first product : thus, the triangular numbers are found by dividing the products $1 \times 2,2 \times 3,3 \times 4,4 \times 5$, \&c, each by the 1 st pr. $1 \times 2$; the first pyramids by dividing the products $1 \times 2 \times 3,2 \times 3 \times 4,3 \times 4 \times 5, \& c$, by the first $1 \times 2 \times 3$. And, in general, the figurate numbers of any order $n$, are found by substituting successively 1,2 , $3,4,5$, \&cc, instead of $x$ in this general expression $\frac{x \cdot x+1 \cdot x+2 \cdot x+3 . \& \mathrm{c}}{1 .}$; where the factors in the numerator and denominator are supposed to be multiplied together, and to be continued till the number in each be less by $l$ than that which expresses the order of the figurates required. Sce Maclaurin's Fluxions, art. 351 , in the notes; also Simpson's Algebra, pa. 213; or Malcolm's Arithmetic, pa. 396, where the subject of figurates is treated in a very extensive and perspicuous manner.

FIGURE, in general, denotes the surface or terminuting extrenes of a body.-All finite borlics have some ijgure, form, or shape; whence, figurability is reckoned among the essential properties of body, or matter : a body without figure, would be an infinite body.
F $] \mathrm{C}$

FIGUREs, in Architecture and Sculpture, denote representations of things made in solid matier; as statues, \& $\&$.

FrGUREs, in Arithmetic, are the numeral characters, by which numbers are expressed or written, as the ten digits, $1,2,3,4,5,6,7,8,9,0$. These are usually called the Arabic, and Indian figures, from which people it is supposed they were derived, being brought into Europe by the Moors of Spain, and into England about 1130, as Dr. Wallis conjectures: See his Algebra, pa. 9. However, from some ancient dates, supposed to consist wholly or in part of Arabian figures, some have concluded that these figures, originally Indian, were known and used in this country at least as early as the 10 th century. The oldest date discovered by Dr. Wallis, was on a chimneypiece, at Helmdon, in Northamptonshire, thus M133, that is 1133. Other dates discovered since, are 1090, at Colchester, in Essex; м16 or 1016, at Widgel-hall, near Buntingford, in Hertfordshire; 1011 on the north front of the parish church of Rumsey in Hampshire; and 975 over a gate-way at Worcester.

Dr. Ward, however, has urged several objections against the antiquity of these dates. As no example occurs of the use of such figures in any ancient manuscript, earlier than some copies of Johannes de Sacro Bosco, who died in 1256 , he thinks it strange that these figures shotld have been uscd by artificers so long before they appear in the writings of the learned; and he also disputes the fact. The Helmdon date, according to him, should be 1233 ; the Colchester date 14.90; that at Widgel-hall has in it no Arabic figures, the 1 and 6 being $I$ and $\mathrm{G}$, the initial letters of a name; and the date at Worcester consists, he supposes, of Roman numerals, being really nxv. See my Abridg. Philos. Trans. vol. 8, pa. 32, 39, \&c.

Mr. Gibbon observes (in his History of the Decline and Fall of the Roman Empire, vol.v. pa.321) that "under the reign of the caliph Waled, the Greek language and characters were excluded from the accounts of the public revenue. If this change was productive of the invention or familiar use of our present numerals, the Arabic characters or ciphers, as they are commonly styled, this regulation has promoted the most important discoveries of arithmetic, algebra, and the mathemaiical sciences."

On. the other liand it may be observed that, " according to a new, thougb probable notion, maintained by M. de Villaison (Anecdota Græca, tom. ii. pa. 152, 157), our ciphers are not of Indian or Arabic invention. They were used by the Greek and Latin arithmeticians long before the age of Boethius. After the extinction of science in the West, they were adopted in the Arabic versions from the original manuscripts, and restored to the Latins about the 11 th century."

FlGURE of the Earth. See Earth.

FIGU Re of ant Ficlipse, in Astronomy, denutes a representation on paper \&c, of the path or orbit of the sun or moon, during the time of the cclipse; with the different phases, the digits eclipsed, and the beginning, middle, and end of darkness, \&c.

Figure, or Delineation, of the full moon, such as, viewed through a telescope with two convex glasses, is of considerable use in observations of eclipses, and conjunctions of the moon with other luminarics. In this figure are usually represented the maculx or spots of the moon, marked by numbers; beginning with those that usually enter first within the shade at the time of the cclipses, atid also emerge the first. 
FIgvR E, in Conic Sections, according to Apollonius, is the rectangle contained under the latus-rectum and the transverse axis, in the ellipse and hyperbola.

Figure, in Fortification, is the plan of any fortified place; or the interior polygon, \&c. When the sides, and the angles, are all equal, it is called a regular figure; but whes unequal, an irregular one.

FIGURE, in Geometry, denotes a surface or space inclosed on all sides; and is either superficial or solid ; superficial when it is inclosed by lines, and solid when it is inclosed or bounded by surfaces.

Figures are either straight, curved, or mixed, according as their bounds are straight, or curved, or both. The exterior bounds of a figure, are called its sides; the lowest side, its base; and the angular point opposite the base, the vertex of the figure; also its height, is the distance of the vertex from the base, or the perpendicular let fall upon it from the vertex.

For Figures, equal, equiangular, equilateral, circumscribed, inscribed, plane, regular, irregular, similar, \&c ; see the respective adjectives.

Apparent Figure, in Opties, that figure, or shape, under which an object appears, when viewed at a distance. This is often very different from the true figure; for a straight line viewed at a distance may appear but as a point; a surface as a line; a solid as a surface; and a crooked figure as a straight one. Also, each of these may appear of different magnitudes, and some of them of different shapes, according to their situation with regard to the eye. Thus, an arch of a circle may appear a straight line; a square or parallelogram, a trapezium, or even a triangle; a circle, an ellipsis; angular magnitudes, round; a sphere, a circle; \&c.

Also any small light, as a candle, seen at a distance in the dark, will appear magnified, and farther off than it really is. Add to this, that when several objects are seen at a distance, uncler angles that are so small as to be insensible, as well as each of the angles subtended by any one of them, and that bext to it; then all these objects appear not only as contiguous, but as constituting and seeming but one continued magnitude.

FIgURE of the Sines, Cosines, Versed-sines, Tangents, or Secants, \&c, are figures marde by conceiving the circamference of a circle extended out in a right line, upon every point of which are erected perpendicular ordinates equal to the sines, cosines, \&c, of the corresponding arcs; and then drawing the curve line through the extremity of all these ordinates; which is called the figure of the sines, cosines, \&c.

It is probable that these figures took their rise from the circumstance of the extension of the meridian line by Edward Wright, who computed that line by collecting the successive sums of the secants, which is the same thing as the area of the figure of the secants, being made up of all the ordinates, or sccants, by the construction of the figure. And in imitation of this, the figures of the other lines have been invented; and by means of the figure of the secants, James Gregory showed how the logarithmic tangents may be constructed, in his kxcrcitationes Geoinctrice, 4to, 1668 .

Construction of the ligures of Sines, Cosines, \&c.

Let $A D$ B \& $($ fig. 1) be the circle, $A D$ an arc, DE its sinc, $\mathrm{CE}$ its cosine, $\Delta \mathrm{E}$ the versed-sine, $\Delta \mathrm{F}$ the tangent, G $B$ the cotangent, ex the secant, and $\mathrm{cn}$ the cosecant. Draw a right line aa equal to the whole circumfereuce

$\triangle D G B A$ of the circle, upon which lay off also the lengtlis of several arcs, as the arcs at every $10^{\circ}$, from 0 at $a$, 10 $360^{\circ}$ at the other end at $a$; upon these points draw perpendicular ordinates, upwards or downivards, according as the sine, cosine, $\mathbb{N c}$, is affirmative or negative in that part of the circle, and equal to them; then drawing a curve line through the extremities of all these ordinates, it will form the figure of the sines, cosines, versed-sines, tangents, cotangents, secants, and cosecants, as in the annexcd figures. Where it may be observed, that the following curves are the same, viz, those of the sines and cosines, those of the tangents and cotangents, and those of the secants and cosecants; except in the disposition of some of their parts.

Fig. 1.

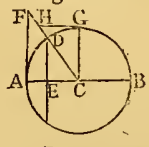

D

Cosines.

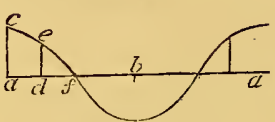

Tangents.

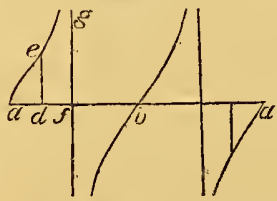

Secants.

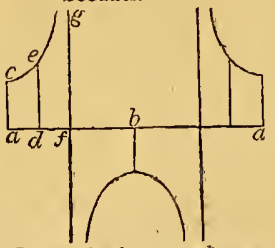

It may be known when any of these lines, viz, the sines, cosines, \&c, are affirmative or negative, i. e. to be set upwards or downwards, by observing the following general rules for those lines in the 1st, 2d, 3d, and 4th quadrants of the circle. T'he sines

The cosines

The taugents

The cotangents

The secants

The cosecants

And all the versed-sines are affirmative. in the 1st and $2 d$ are affrmative, in the $3 \mathrm{~d}$ and 4 th negative: in the 1 st and 4 th are affirmative, in the $2 \mathrm{~d}$ and $3 \mathrm{~d}$ negative: in the 1 st and $3 \mathrm{~d}$ are afirmative, in the 2l and 4th negutive; in the ist and $3 d$ are affirmative, in the 2d and 4 th negative: in the lst and 4 th are aflirmative, in the $2 \mathrm{~d}$ and $3 \mathrm{~d}$ newative : in the 1 st and $2 d$ are affirmntive, in the $3 \mathrm{~d}$ and 4 th negative: 
To find the Equation and Area, \&c, to each of these Curves. Draw any ordinate de; putting $r=A \mathrm{C}$ the radius of the given circle, $x=a d$ or $A D$ any absciss or arc, and $y=$ de its ordinate, which will be either the sine $\mathrm{DE}=s$, cosine $\mathrm{CE}=c$, versed-sine $\mathrm{A} \mathrm{E}=v$, tangent $\mathrm{Al}=t$, cotaugent $\mathrm{GH}=\tau$, secant $\mathrm{CF}=\mathrm{s}$, or cosecant $\mathrm{CH}=\sigma$, according to the nature of the particular construction. Now, from the property of the circle are obtaincd these following general equations, expressing the relations between the fluxions of a circular arc and its sine, or cosine, $S x$, viz,

$$
\begin{aligned}
\dot{x} & =\frac{\dot{r} s}{\sqrt{\left(r^{2}-s^{2}\right)}}=\frac{-\tau \dot{c}}{\sqrt{\left(r^{2}-c^{2}\right)}}=\frac{r v}{\sqrt{(2 r v-v)}}=\frac{r^{2} i}{r^{3}+t^{2}} \\
& =\frac{-r^{2} \tau}{r^{2}+\tau^{2}}=\frac{r^{2} \dot{s}}{s \sqrt{ }\left(s^{2}-r^{2}\right)}=\frac{-r^{2} \sigma^{2}}{\sigma \sqrt{ }\left(\sigma^{2}-\frac{1}{r^{2}}\right)} .
\end{aligned}
$$

And these also express the relation between the absciss and ordinate of the curves in question, each in the order in which it stands; where $x$ is the common absciss to all of them, and the respective ordinates are $s, c, v, t, \tau, s$, and $\sigma$. And hence the area $\& c$, of any of these curves may be found, as follows:

1. In the Figure of Sines,-Here $x=a d$, and $s=$ the ordinate de; and the equation of the curve, as above, is $\dot{x}=\frac{r \dot{ }}{\sqrt{\left(r^{2}-s^{3}\right)}}$. Hence $s \dot{x}=\frac{r \dot{s}}{\sqrt{\left(r^{2}-s^{2}\right)}}$ is the flusion of the area; the correct fluent of which is $r^{2} \mp r \sqrt{ }\left(r^{2}-s^{2}\right)$ $=r^{2}-r c=r v$ the rectangle of radius and vers. i. e. - or + as $s$ is increasing or decreasing; which is a general expression for the area ade in the figure of sines. When $s=0$, as at $a$ or $b$, this expression becomes 0 or $2 r^{2}$; that is 0 at $a$, and $2 r^{2}=$ thc area $a e b$; or $r^{2}=$ the area of $a f g$ when ad becomes a quadrant af.

2. In the F1Gune of Cosines.-Here $x=a d$ and $c=d e$; and the equation of the curve is $\dot{x}=$

$\frac{-r \dot{c}}{\sqrt{\left(r^{2}-c^{3}\right)}}$. Hence $c \dot{x}=\frac{-r c \dot{ }}{\sqrt{\left(r^{4}-c^{2}\right)}}$ is the fluxion of the area; and the fluent of this is $r \sqrt{ }\left(r^{2}-c^{2}\right)=r s$, the rectangle of radius and sine, for the general area adec. IVhen $s=r$, or $c=0$, this becomes $r^{2}=$ the area afc, whose absciss af is equal to a quadrant of the circumference; the same as in the figure of the sines, upon an equal absciss.

3. In the Figure of Versed-sines.-Here $x=a d$, and $v=d e$; and the equation of the curve is

$\dot{x}=\frac{\dot{v}}{\sqrt{(2 r v-v v)}}$. IInce $v \dot{x}=\frac{r v v}{\sqrt{ }(2 r v-v v)}=\frac{r \dot{v} \sqrt{ } v}{\sqrt{ }(2 r=v)}$ is the fluxion of the area; and the fucnt of this is $r x-r s$ $=r \times(A D-D E)$ for the area ade in the figure of versed sines. When $A D$ or $a d$ is a quadrant $A G$ or uf, this becomes $3.1416 \times \frac{1}{2} r^{2}-r^{2}=\cdot 570 s r^{2}$ for the area $a f g$. And when A D or ad is a semicircle $a b$, it becomes $3.116 r^{2}$ the arca $a b y=$ in the figure of versed-sines.

4. In the Figure of Tangents,-Herc $x=a d$, and $t=d e$; and the equation of the curve is $\dot{x}=$

$\frac{r^{2} i}{r^{2}+t^{2}}$. Hence the fluxion of the area is $t \dot{x}=\frac{r^{2} t}{r^{2}+t^{2}}$;

and the correct fluent of this is $\frac{1}{2} r^{2} \times$ hyp. $\log$. of $\frac{r^{2}+t^{2}}{r^{2}}=r^{2} \times$ hyp. log. of $\frac{\sqrt{ }\left(r^{2}+t^{2}\right)}{r}=r^{2} \times$ hyp. log. of $\frac{s}{r}$.

And hence: the figure of the tangents may be used for constructing the logarithmic secants; a property that was remarked by James Gregory at the end of his Exercit. Geonet.

When ad becomes a quadrant af, $t$ being then infinite, this becomes infunite for the area afg. And the same for the figure of cotangents, brgiming at $f$ instead of $a$.

VOL. I.
5. For the Figure of the Secants.-Here $x=a d$, andi $\mathrm{s}=d e$; and the equation of the curve is $\dot{x}=$ $\frac{r^{2} s^{0}}{s \sqrt{\left(s^{2}-r^{2}\right)}}$. Hence $s x=\frac{r^{2} s}{\sqrt{\left(s^{2}-r^{2}\right)}}$ is the fluxion of the area; the fluent of which is $r^{2} \times$ hyp. $\log$. of $\frac{s+\sqrt{ }\left(s^{2}-r^{2}\right)}{T}$ for the general arca ade. And when ad becomes the quadrant $a f$, this expression becomo in tinite for the area if $y$. The same process will serve for the figure of cosecants, beginning at $f$ instead of $a$.

Hence the meridional parts in Mercator's chart may be calculated for any latitude $\mathrm{A} D$ or $a d$ : Fur the merid. parts : are to the arc of latitude $A D::$ as the sum of the secants : to the sun of as many radii or: : as the area ade: to ad $x$ radius $a c$ or $A D \times A C$ in the first figure.

FILLET, in Architecture, any little square member or ornament used in crowning a larger moulding.

FIN IEUS (Orontius), in French, Finé, professor of mathematics in the. Royal Cullege of Paris, was the son of a physician, and was born at Briancon in Dauphine in 1494. Ile went young to Paris, where his friends procured him a place in the college of Navarre. He applied himself there to the study of philosophy and polite literature; but more especially to mathematics, in which he marle considerable progress, particularly in mechanics; for, having both a genius to invent instruments, and a skilful hand to execute them, he gained much reputation by the specimens he gave of his ingenuity.

Finæus first made himself publicly known by correcting and publishing Siliceus's Arithmetic, and the Margaria Philosophica. Heafterwards rcad private lectures in mathematics, and then taught that science publicly in the college of Gervais; from the reputation he thus gained, he was recommended to Francis the 1st, as a proper person to teach mathematics in the new college which that prince had founded at Paris. And here, tlough he spared no pains to improve his pupils, he yet found time to publish a great many books on many of the principal parts of the mathematics. But neither his genius, his labours, his inventions, nor the estrem which numberless persons showed him, could secure him from that fate which so often befalls mea of letters. He was obliged to struggle all his life-time with poverty; and when he died, left a numerous family decply in debt. However, as merit must always be estecmed in secret, though it seldom has the fortune to be rewarded openly; his children found friends who for their father's sake assisted his family. - He died in 1555 , at 61 yiars of age.

Like all other mathematicians and astronomers of those times, he was greatly addicted to astrology; and had the misfortune to be a long time imprisoned for having predicted some things, that were not acceptable to the court of France. He was also une of those, who vainly boasted of having found ont the quadrature of the circle, with the solution of the problem of two mean proportionals, and that of the triscction of an angle; but he was vigorously refuted by Nonius, as well as by Butron and Borclli, two of his own pupils.-Several of his pieces were published separately; as, his Practical Arithmetic in 1555, and Practical Geometry in 1544, both in 4to. And an edition of his whole works, translated into the Italian language, was published in 4to, at Venice, 1587 ; consisting of Arithmetic, Practical Geometry, Cosmograpliy, Astronomy, and Dialling.

FINITE, the property of any thing that is bounded $3 \mathrm{U}$ 


\section{F $1 \mathrm{~K}$}

or limited, either in its power, or extent, or duration, Sc ; as distinguished from the property of infinite, or without bounds.

FIRE, is that subtile invisible fluid by which bodies are made hot to the touch, and expanded or enlarged in bulk; by which fluids are rarefied into vapour; or solid bodies become fluid, and at last are either dissipated and carried off in vapour, or clse melted into glass. It seems also to be the chief agent in nature on which animal and vegetable life have an immediate dependence. Sce $\mathrm{CA}_{A}-$ LORIC and HEAT.

The disputes concerning fire, which long existed among philosophers, have now in a great measure subsided. Those celebrated philosophers of the 17 th century, Bacon, Boyle, and Newton, were of opinion, that fire was not a substance distinct from utber bodies, but that it entirely consisted in the riolent motion of the parts of any body, which was produced by the mechanical force of impulsion, or of attrition. So Buyle says, when a piece of iron becomes hot by hammering, "there is nothing to make it so, except the forcible motion of the hammer impressing a vehement and variously determined agitation on the small parts of the iron." And Bacon defines heat, which he makes synonymous with fire, an " expansive undulatory motion in the minute particles of a body, whereby they tend with some rapidity from a centre towardo a circumference, and at the same time a little upwards." And according to Newtoll, fire is a body heated so hot as to emit light copiously; for what else, says he, is red-hot iron, but fire? and what else is a fiery coal than red-hot wood? by which he suggests, that bodies which are not fire, may be changed and converted into it.

On the otber hand, the chemists strenuously contented that fire was a fluid of a certain kind, distinct from all others, and universally present throughout the whole globe. Boerbaave particularly maintained this doctrine ; and in support of it brought this argument, that flint and stcel would strike fire, and produce the same degrce of heat in Nova Zembla as they would do under the equator. Other arguments were drawn from the increased weight of metallic calces, which they thought proceeded from the fixing of the element of fire in the substance whose weight was thus increased. For a long time, however, the matter was most violently disputed ; but the mechanjcal philosophers at last prevailed through the deference paid to the principles of Newton, though he himself had scarcely taken any active part in the contest.

The experiments of Dr. Black, how ever, secmed to bring the dispute to a decision, and that in favour of the chemists, concerning what he called latent heat. From these discoveries it appears, that fire may exist in bodies in such a manner, as not to discover itself in any other way than by its action on the minute parts of the body; but that suddenly this action may be changed so, as no longer to be directed on the particles of the body itself, but upon ixternal objects; in which case we then perceive its action by our sense of feeling, or discover it by the thermometer, and call it heat, or sensible fire.

from this iliscovery, and others in electricity, it is now pretty generally allowed, that fire is a distinct fluid, capable of being transferred from one body to another. But when this was discovered, another question $n$ less perplexing arose, viz, what kind of a fluid it was; or whether it bears any analogy to those with which we are better acquainted. Now there are found two fluids, viz,
F I R

the solar light, and the electric natter, botb of which accasionally act as fire, and which therefore seem to indicate an original identity; and popularly the matter has been long since determined; the solar rays and the electric fluid having been indifferently accounted clementary fire. Some indeet have imagined both these fluids to be mere phlogiston itself, or at least containing a large portion of it; and Mr. Scheele went so far in this way as to form an bypothesis, which he endeavoured to support by experiments, that fire is composed of phlogiston and dephlogisticated air. But it is now ascertained beyond doubt, that the result of such a combination is not fire, but fixed air.

It was long since obserred by Newton, that heat was certainly conveyed by a medium more subtile than the common air ; for two thermometers, one included in the vacuum of an air-pump, the other placed in the open air, at an equal distance from the fire, would bocome equally hot in nearly the same time. This and other experiments show, that fire exists and acts where there is no other matter, and of consequence it is a fluid per se, independent of every terrestrial substance, without being generated or comprunded of any thing we are yet acquainted with. To determine the nature of the fluid, we have only to consider whether any other can be discovered "hich will pass through the perfect vacuum just mentioned, and act there as fire. Such a fluid is found in the solar ligh1, which is well known to act eren in vacuo as the most violent fire. The solar light will likewise act in the very same manner in the most intense cold; for M. de Saussure has found, that on the cold mountain-top the sun-beams are equally powerful as on the plain below, if not more so. It appears therefore, that the solar light will produce beat independent of any otber substance whatever; that is, where no other body is present, at least as far as we can judge, except the light itself, and the body to be acted on. We cannot therefore avoid concluding, that a certain modifcation of the solar light is the cause of heat, expansion, vapour, \&c, and answers to the rest of the characters given in the foregoing definition of fire, and that independent of any other substance whatever.

It is very probable 10o, that the electric matter is no other than the solar light absorbed by the earth, and thus becoming subject to new laws, and assuming many propertics apparently different from what it has $w$ hen it acts as light. Even in this case it manifests its identity with fire or light, viz, by producing a most intense heat where a large quantity of it passes through a small space. So that at any rate, the experiments which have already been made, and the proofs drawn from the phemment of nature, show such a strong affinity between the temients of fire, light, and electricity, that we may not only assert their iilentity on the most probable grounds, but lily it down as a position against which at present no argumi'tit of any weight has an existence.

Fine Arrow, is a small iron dart, furnished with springs and bars, and also a match impregnated with su! phur and powder, which is wound about its shaft. It is chictly used by priviteers and pirates to fire the sails of the enemy's ship, and for this purpose it is dincharged from a musketoon, or a swivel gun. The match being kinelled by the explosion, it communicates the llate to the sall against which it is directed, where the arrow fistens itself by means of its bars and springs. 'lhis weapon is poculiar to hot climates, particularly the West Indies; the sails being 
very dry, are quickly inflamed, and the fire is soon conveyed to the masts, rigging, and finally to the vessel itself.

Fr e-Balls, in Artillery, are certain balls composed of combustible matters, such as fine or mealed powder, sulphur, saltpetre, rosin, pitch, \&c. These are thrown into the enemy's works in the night-time, to discover where they are; or to set fire to houses, galleries, or blinds of the besiegers, \&c. They are sometimes armed with spikes or hooks of iron, that they may not roll off, but stick or hang where they are to take effcct.

Fin E-Bulls, or Fiery-Meteors, in Meteorology, a kind of Juminous bodies usually appearing at a great height above the earth, with a splendour surpassing that of the moon, and sometimes apparently as large. It has not been observed that they follow any regular course or motion, but, on the contrary, moving in all directions, and with very different degrees of celerity; frequently breaking into several smaller ones; sometimes making a strong hissing sound, sometimes bursting or vanishing with a loud report, and sometimes not.

These luminous appearances doubtless constitute one part of the ancient prodigies, blazing stars, or comets, which lust they sometimes resemble in being attended with a train; but more often they appear round. The first of these of which we have any accurate account, was observed by Dr. Halley, and some other philosophers at differentplaces, in the year 1719 , the height of which above the surface of the earth was computed at more than 70 miles. Many others bave been accurately observed since that time, and described by different philosophers. See French Memoirs, and Philos. Trans. vols. 30, 41, 42, 43, $46,47,48,51,53,54,63,74, \& c$. The velocities, directions, apprarances, and heights of all these were found to be very various; though the height of some of then was supposed above the limits assigned to our atmosphere, or where it loses its refractive power. The most remarkable phenomenon of this kind on record, appeared on the 18th of $\Lambda$ ugust 1783 , about 9 o'clock in the cvening. It was seen to the northward of Shetland, and took a south-casterly direction for an immense space, being observed as far as the southern provinces of France, and by some it was said to have been seen at Rome, passing over a space of 1000 miles in about half a minute of time, and at a very great height. During its course it appeared several times to change its shape; sometimes appearing in the form of one ball, sometimes of two or more; sometimes with a train, and sometimes without one.

There are divers opinions concerning the nature and origin of these meteors. The first thing that occurred to philosophers on this subject was, that they were burning bodies rising from the surface of the carth, and flying through the atmosphere with great rapidity. But this hypothesis was soon rejected, on considering that there was no power known by which such bodies could either be raised to a sufficient height, or projected with the velocity which they had by some means acyuired. The next hypothesis was, that, instead of one single body, they consist of a train of sulphureous vapours, extending an immense way through the atmosphere, and being kindled at one end, display the luminous appearances in question by the fire running from one extremity of the train to the other. l3ut it is not easy to conceive how such matters can exist and be disposed in such lines in so rare a part of the atmospluere, and even to burn there, in an almost perfect vacuun. For which reason this hypothesis was aban- doned, for another, which was, that those meteors are permanent solid bodies, not rising from the earth, but revolving round it in very excentric orbits, and thus in their perigeon moving with vast rapidity. But as the various appearances of one and the same meteur, to observers at different places, are not compatible with the idea of a single body so revolving, this hypothesis has also been given up in its turn. Again it has bren said, that these meteors are a kind of bodies that take fire as suon as they come within the atmosphere of the earth. But this cannot be supposed without implying a previous knowledge of the nature of these bodie's, which it is impossible we can have. Another hyporhesis is, that these fiery meteors are great bodies of electric matter, moving from one part of the heavens where, to our conccption, it is superabundant, to another where it is deficient: a conclusion attended with some probability, from the analogy wherved between electricity and the phenomena of these bodies: and hence they appear to be of the same origin with shooting-stars, lightuing, the aurora-borealis, \& $c$, being all referred to the same cause, viz, the electricity of the atmosphere. Sce AEROLiTE.

Fine-Engine, is a machine for extinguishing accidental fires by means of a stream or jet of water. The common squirting fire-engine consists of a lifting pump placed in a vessel of water, and wrought by two levers that act always together. During the stroke, the water raised by the piston of the pump spouts forcibly through a pipe joined to the pump-barrel, and made capable of any degree of elevation by means of a yielding leather pipe, or by a ball and socket turning every way, screwed on the top of the pump. The vessel containing the water is covered with a strainer, to prevent the mud, \&c, which is poured into it with the water, from choking the pump-work. Between the strokes of this engine the stream is discontinued, for want of an air-vessel. However, in some cases, engines of this construction have their use, becausi the stream, though interrupted, is much smarter than when the engine is made to throw water in a continued stream. See these engines particularly jescrubed in Desaguliers's Exper. Philos. vol. 2, pa. 505; or Martin's Philos.. Britan.vol. 2, pa. $69 ; 0$. G. Gregory's Mechanics. See also the figure of them, plate $x$. fig. 3 .

It appears that in the year 1675 , Sir Sam. Morland got, a patent for a powerful engine of this kind. This machine, by the strength of $8 \mathrm{men}$, would force water in a continued stream, from the river Thames, to the top of Windsor castle, and 60 feet higher, at the rate of 60 barrels an hour, which experiment was repeated several times, in the year 1681 , before the king, queen, and court; when his majesty presinted Morland with a medal, with his effigy set round with diamonds, and appointed him his master of mechanics, \&c.

F I r e-Lock, or Fusil, a small gun or musket, which fires with a flint and stcel; as distinguished from the old musket, or match-lock, which was fired with a match. The fire-lock is now in common use with the European armies, and carried by the foot-soldiers. It is usually about 3 feet 8 inches in the barrel; and, including the stock, 4 feet 8 inches, carrying a leaden bullet, of which 29 make 2]b. The diameter of the ball is ${ }^{\circ} 55$, and that of the barrel 56 parts of an incl. The time of the invention of fire-locks is uncertain; they were however used in 1690 .

Fire-Places, are contrivances for communicating heat $3 \mathrm{U}_{2}$ 
to rooms, and also for answering various purposes of art and manufacture.-The general properties of air and fire, on which their construction chiefly depends, are the following, viz, that air is rarefied by heat, and condensed by cold; or, in other words, the same quantity of air takes up more space when warm than when cold. $\Lambda$ ir rarefied and expanded by heat, is specifically lighter tlian it was before, and will rise in other air of greater density: so that a fire being made in any chimney, the air about and over the fire is rarefied by the heat, thence becomes lighter, and so rises in the funnel, and goes out at the top of the cbimney: the other air in the room, flowing towards the chimney, supplies its place, is then rareficd in its turn, and rises likewise; and the place of the air thus carried out of the room, is supplied by fresh air coming in through doors and windows, or, if they be shut, through every erevice with violence; or if the avenues to the room be so closed up, that little or no fresh supply of air can be obtained, the current up the funnel must flag; and the smoke, no longer driven up, float about in the room.

On these principles, various contrivances and kinds of fire-grates and stoves have been devised, from the old very open and wide chimney places, down to the present modish ones, which are much narrowed in the front, opening, by side and back jambs, and a low breast or mantle, besides the convenience of a flap, called a register, that covers the top of the fire-stove, but opening to any degree with a snall winch, which lifts the back part sloping upwards, and so throws the smoke freely up the funnel, admitting as little air to pass as you please; by which simple means the warm air is kept more in the room, while the very narrow and sloping orifice promotes the brisk ascent of the smoke, and yetprevents its return down again, for the same reason.

Another ingenious, but more complex, apparatus, called the Pennsylvania fire-place, was invented by Dr. Franklin, which keeps a room very warm hy a constant supply of fresl hot air, that passes into it through the stove itself. See the description in his Letters and Papers on Philosoplical Subjects.

Many other forms of fire-grates, often of whimsical figure's, have been recommended by different persous : but, after all, the common register-grate is far the best.

Fire-Pot, in the Military Art, is a small earthen pot, into which is put a grenade, filled with fine powder till the grenade be covered; the pot is then closed with a piece of parchment, and two pieces of match laid across and lighted. This pot being thrown where it is designed to do exccution, breaks and fires the powder, and this again fires the powder in the grenade, which ought to have no fuze, that its operation may be the quicker.

Rusant, or Razant $F_{1 R E}$, is a tire from the artillery and small arms, directed parallel to the horizon, or to those parts of the works of a place that are defended.

Running Frue, is when ranks of men fire one after another; or when the lines of an almy are drawn out to fire ou account ol' a victory; in which case cach squadron or battalion takes the fire from that on its right, from the right of the first line to the left, and from the left to the right of the sccond line, \&c. This is now commonly called a fen-de-joyc.

Iin E-Ships, in the navy, are vessels charged with combustible materials or artificial fire-works: which having the wind of an enemy's ship, grapple her, and set her on fire. Anderson, in lis History of Commerce, rol. 1, pa. 432, ascribes the invention to the English, in this instance, viz, some vessels being filled with combustible matter, and sent among the Spanish ships composing the Invincible Armada in 158s; and hence arose, it is said, the terrible invention of fire-ships. But Livy informs us, that the Rhodians had invented a kind of fire-ships, which were used in junction with the Roman fleet in their engagement with the Syrians, in the year' 190 before Christ : cauldrons of combustible and burning materials were hung out at their prows, so that none of the enemies' ships durst approach them : for these fell on the enemies' galleys, struck their beaks into them, and at the same time set them on fire. Livy, lib. 37, cap. 30.

Wild-Fıne, is a kind of artificial or fuctitious fire, that burns even under water, and that with greater violence than out of it. It is cumposed of sulphur, naphtha, pitch, gum, and bitumen, and it is only extinguishable by vinegar, mixed with sand and urine, or by covering it with raw hides. It is said its motion is contrary to that of natural fire, always following the direction in which it is throwu, whetber it be downwards, sideways, or otherwise.

The French call it Greek fire, or Feu Gregeois, because first used by the Greeks about the year 660, as is observed by the Jesuit Petavius, on the authority of Nicetas, Theophanes, Cedrenus, \&c. The inventor, according to the same author, was an engineer of Heliopolis, in Syria, named Callinicus, who first applied it in the sea-tight commanded by Constantine Pogonates, against the Saracens, nearCyzicus, in the Hcllespont; and with such effect, that he burnt the whole fleet, which had on board 30,000 men. But others refer it to a mucl older date, and ascribe the invention to Marcus Gracchus; an opinion that is supported by several passages, both in the Greek and Roman writers; which sbow that it was anciently used by each of these nations in their wars. See Scaliger against Cardan.

The successors of Constantine used it on sereral occasions, with great advantage : and it is said that thcy were able to keep the secret of the composition to themselves; so that no other nation knew it in the year 960 .

It is recorded by Chorier, in his Hist. de Dauph, that Hugh, king of Burgundy, demanding ships of the emperor Leo for the siege of Fresne, desired also the Greek fire.F. Daniel gives also a good description of the Greck fire, in his account of the siege of Damietta. under St. Louis. Every body, says he, was astonished with the Greek fire, which the Turks then prepared; and the secret of which is now lost. They threw it out of a kind of mortar, and sometimes shot it with an odd sort of cruss-bow, which was strosigly bent by means of a handle, or winch, with much greater force than the bare arm. That which was thrown from the mortar sometimes appeared in the air of the size of a tun, with a long tail, and a noise like that of thunder. The French, by degrees, got the secret of extinguishing it; in which they succeeded several tines.

After all, perhaps the invention of the wild-fire is to be ascribed to other nations, and to a still older date, and that it was the same as that used among the lndians in Alexander's invasion, when it was said they fought with thunder and lightning, or shot firc with a terrible noise.

Fine-Works, otherwise called Pyrotechula, are artificial fires, or preparations made of gumpowder, sulphur, and other inflamnuble and combustible ingredients, which are 
displayed at public rejoicings, and on other occasions. The principal of these are rockets, serpents, stars, hail, mines, bonıbs, garlands, letters, and other devices. The invention of fire-works is attributed, by M. Mahudel, to the Florentines and people of Sienna; who also discovered the method of adding to them decorations of statues, with fire issuing from their eyes and mouths.

FIRKIN, an English measure of capacity; being the 4th part of a barrel ; or containing 9 gallons.

FIRLO'T', a dry measure used in Scotland. The natfirlot contains $21 \frac{\mathrm{I}}{4}$ pints of that country, or about $85 \mathrm{ling}-$ lish piuss; and the barley-firlot, 31 standard pints. The wheat-firl t contains about 2211 cubic inches; and therefore exceeds the English bushel by 60 cubic inches, or almost an English quart.

FIRMAMENT, by some old astronomers, is the orb of the fixed stars, or the highest of all the beavens. But in Scripture and common language it is used for the middle regions, or the space or expanse appearing like an nich quite around or above us in the heavens. Many ancients and moderns also accounted the firmament a fluid matter; but thuse who gave it the name of firmsament must have taken it for a solud one.

FIRMNESS, is the consistence of a body; or that state in which its parts cohere, or are united together, so that the motion of one part induces a motion of the rest. And in this sense firmness stands opposed to fluidity. 'The firmness of bodics therefore depends on the connexion or cohesion of their particles; and the cause of cohesion the Newtonians hold to be an attractive force, inherent in bodies, which binds their small particles together; exerting itself unly at the points of contact, or extremely near them, and vanishing at greater distances.

FISSURES, in the History of the Earth, are ccrtain interruptions, mostly parallel to each other, that divide or separate its strata firom one another, in nearly horizontal directions; and the parts of the same stratum in nearly vertical directions.

FIXED Line of Defence, a line drawn along the face of the bastion, and terminating in the curtain.

Fixed Stgns of the Zodiac, according to some, are the four signs Taurus, Leo, Scorpio, Aquarius. They are so called because the sun passes them respectively in the middle of each quarter, when that season is more settled and fixed than under the signs which begin and end it.

Frxed Stars, are such as constantly retain the same position and distance with respect to cach other; by which they are contradistinguished from erratic or wandering stars, which are continually varying their situation and distance.-The fixed stars only are properly and absolutely called stars; the other celestial bodies falling under the denomination of planets or comets.

FIXITY, or Frxedness, the quality of a body which determines it fixed; or a property which cnables it to enilure the fire and other violent agents. A body may be said to be fixed in two respects: 1st, When on being exposed to the fire, or a corrosive menstrumm, its particles are indecd separated, and the body rendered fluid, but without being resolved into its first elements. The 2d, when the body sustains the active force of the fire or menstruums while its integral parts are not carried off in lumes. Each kind of fixity is the result of a strong or intimate cohesion between the particles.

FLAME, the subtlest and brightest part of the fucl, ascending above it in a pyramidal figure, and heated red- hot. Sir Isaac Newton defines flame as unly red-hot smoke, or the vapour of any substance raised fiom it by fire, and heated to such a degree as to emit light copiously. Is not flame, says be, a vapour, fume, or exhalation, heated red-hot ; that is, sn liot as to shine? For bodies do not flame without cmitting a copious fume; and this fume burns in the flame. 'The ignis fatuus is a vapour shining witbout heat; and is there not the sume difference between this yapour and flame, as between rotten wood shining without heat, and burning coals of fire? In distilling hot spirits, if the head of the still be taken off; the vapour which ascends will take fire at the flame of a candle, and turns into fame. Some bodies, heated by motion or fermentation, if the heat grow intense, fume copiously; and if the heat be great enough, the fumes will shine, and become flame. Metals in fusion do not flame, for want of a copious fume. All flaming bodies, as oil, tallow, wax, wood, fossil cual, pitch, sulphur, dxc, by burning, waste in smoke, which at first is lucid; but at a little distance from the body ceases to be so, and only continues hot. When tho flame is put out, the smoke is thick, and irequently smells strongly: but in the flame is loses its smeil; and, according to the nature of the fucl, the flame is of divers colours. That of sulphur and spirit ol wine is blue; that of copper opened with sublimate, green; that of tallow, ycllow; of camphire, whitc; \&c. Newton's Optics, pa. 318.

FLANISTEED (JoHN), an eminent English astronomer, being indeed the first astronomer-royal, for whose use the royal observatory was built at Greenwich, thence called Flamsteed House. He was born at Denby in Derbyshire the 19th of August 1646; and was educated at the free-school of Derby, where his father lived. At 14 . years of age he was afflicted with a severe illness, which rendered his constitution tender ever after, and prevented him then from going. to the university, for which he was intended. He ncvertheicss prosccuted his school education with the best effect; and, in 1662, on quitting the grammar-school, he pursued the natural bent of his genius, which led him to the study of astronomy, and closely pursued Sacrobosco's book De Sphæra, which f(ll in his way, and thus laid the foundation of all that mathematical and astronomical knowledge, for which he became afterward so justly celebrated. He next procured more modern books on the same subject, and among them Strcete's Astronomia Carolina, then latcly published, from which he learned to calculate eclipses and the planets' places. Some of these being shown to Mr. Halton, a consislerable mathematician, he lent lim Riccioli's Almagestum Novum, and Kepler's Tabulx Rudolphinx, from which he gained much information. In 1669, having calculated some remarkable eclipses of the moon, he sent them to lord Brouncker, president of the Royal Society, which were greatly approved by that learned body, and procured him a letter of thanks from Mr. Oldenlurg their secretary, and another from Mr. John Collins, with whom, and other lcarised men, Mr. Flansteed for a long time afterwards kept up a correspondence by letters on literary subjects. In 1670, his father, observing that he held correspondence with these ingenious persons, advised him to take a journey to London, to make himself personally acquainted with them; an offer which lic gladly embraced, and visited Mr. Oldenburg and Mr. Collins, who introduced him to Sir Jonas Moore, which proved the means of his greatest loonour and preferment. Ilc here got the knowledge and 
practice of astronomical instruments, as telescopes, micrometers, \&c: and on bis return, he calted at Cambridge, and visited Dr. Barrow, Newton, and other learned men there, and entered bimself a student of Jesus-college. In 1672 he made many celestial observations, which, witi calculations of appulses of the moon and planets io fised stars for the year following, he sent to Mr. Oldenburg, who published them in the Philos. Trans.

In 1673, Mr. Flamsteed wrute a small tract on the truc diameters of all the planets, when at their greatest and least distances from the earth; which he lent to Newton in 1685 , who made some use of it in the 4 th book of his Principia.-In 1674 he wrote an ephemcris, to show the falsity of astrology, and the ignorance of those who pretended to it: with calculations of the moon's rising and setting; also occultations and appulses of the moon and planets to the fixed stars. To which, at Sir Jonas Moore's request, be added a table of the moon's southings for that ycar; from which, and from Philips's theory of the tides, the high-waters being computed, he found the times come very near. In 1674 he also dreiv up an account of the tides, for the use of the king. Sir Jonas also showed the king, and the duke of York, some barometers and thermometers that Mr. Flamsteed bad given him, with the necessary rules for judging of the weather; and otherwise trok every opportunity of speaking favourably of Flamsteed to them, tili at length he brought him a warrant to be the king's astronomer, with a salary of $100 l$. per annum, to be paid out of the office of Ordnance, because Sir Jonas was then surveyur general of the Ordnance. This huwtver did not abate our author's propensity for holy orders, and he was accordingly ordained at Ely by bishop Gumning.

On the 10th of Angust 1675, the foundation of the royal observatory at Greenwich was laid; and during the building of it, Mr. Flamsteed's temporary observatory was in the queen's house, ivhere he made his observations of the appulses of the moon and planets to the fixed stars, and wrote his Doctrine of the Sphere, which was afterwards published by Sir Jonas Moore, in his System of the Nathematics.

About the year 1684 he was presented to the living of "Burslow in Surrey, which he held as long as he lived. Mr. Flamsteed was equally respected by the great men his contemporaries, and by those who succeeded him. Dr. Wotton, in his Reflections upon Ancient and Modern Learning, styles uur author one of the most accurate observers of the heavens, and say's he calculated tables of the cclipses of the several satcllites, which proved vcry useful to the astronomers. And Mr. Molyneaux, in bis Dioptrica Nova, speaks very highly of his abilities; and, in the admonition to the reader prefixed to the work, observes, that the geometrical method of calculating a ray's progress is quite new, and never before published; but for the first lint of it, says he, I must acknowledge myself obliged to my worthy friend Mr. Flamstced. 1le wrote several small tracts, and had many papers inserted in the Philosophical 'Transactions, in almosi every volume, from the th th the 29 th, which are too numerous to be particularly mentiuned in this place.

But his ereat work, and that which containerl the prioripal opprations of his life, was the Historia Cadestis Brirannica, published in 1725 , in 3 large folio volumes. 'Ihe first of which contains the observations of $\mathrm{Mr}$. William Gascoigne, the original inventor of the method of me.asuring angles in a telescope by means of screws, and the first who applied telescopical sights to astroromical instruments, in observations made at Middleton, near Leeds in Yorkshire, between the years 1638 and 1643 ; extracted from his lettro by Mr. Crabtree; with some of Mr. Crabtres's ob-ertations about the same time; and also those of 91r. Flamsted himself, made at Derby between the years 1670 and 1675 ; besides a mulcitude of curious observations, and nceessary tables to be used with them, made at the ruyal observatory, between the years 1675 and 1689 . - The $2 \mathrm{~d}$ volume cuntaus his observations, made with a mural arch of near 7 feet radius, and 140 degrees on the limb, of the meridional zenith distances of the fixed stárs, sun, moon, and planets, with their transits over the meridian; also observaticns of the diameters of the sun and moon, with their cclipses, and those of Jupiter's satellites, and variations of the compass, from 1689 to 1719 : also tables showing how to render the calculation of the places of the stars and planets easy and expeditious. To which are added, the moon's place at her oppositions, quadratures, \&ec; alo the plancts' places, derived from the observations.-The 3 d volume contains a catalogue of the ight-ascensions, polardistances, longitudes, and magnitudes of near 3000 fixed stars, with the cirresponding variations of the same. To this volume is prefixed a preface, at consilerable length, containing an account of all the astrenomical cheivations made before his time, with a description of the instruments employed; as also of his own observations and instruments; a new Latin version of Ptolemy's catalogue of 1026 fixed stars; and Ulegh-brig's places annexed on the Latin page, with the currections: a small catalogue of the Arabs: 'Tycbo Brahe's of about 780 fixed stars: the Lantigrave of Ilesse's of 386 : II'vetius's of 1534 : and a catalogue of sume of the southern fixed star nut visible in our hemisphere, calculated from the obscrvaticuns made by Dr. Halley at st. Helena, adapted to the year 1726 .

This work he prepared in a great measure fir the press, with much care and accuracy: but through a untural weakn'ss of constitution, and the adrance of age, he died of a strangury bifore he had completed it. December the 19th, 1719 , at 73 years of age ; leaving the care of finishing and publishing his work to his friend Mr. Hudgson. A less perfect edition of the Historia Calistis had before been published, but without his consent, viz, in 1712 , in one volume folio, containing his observations to the ycar 1705.

FLANK, in Fortification, is that part of the bastion reaching from the curtain to the face; and it defends the curtain, with the opposite face and flank.

Oblique or Second FunK, or FLaNK of the Curtain, is that part of the curtain from which the fice uf the oppusite bastion can be scen, being contained belween the lines rasant and fichant, or the grenter aud less lines of defence; or the part of the curtuin betwern the flank and the point where the fichant line of dofence terminates.

Cozered, Low, nr Retired FLANK, is the platiorm of the casemate, which lies hid in the bastion; and is otherwise called the orillon.

Fichsint $F_{\mathbf{L A N K}}$, is that from which a cannon playing, fires directly on the face of the opposite bastion.

Resant or Razant FLaNk, is the point from which the line of defence begins, from the conjunction of which with the curtain, the shot only raseth the face of the next bastion, which happens when the facc caunot be discovered but from the flank alonc. 
Simple FLANEs, are lines going from the angle of the shoulder to the curtain; the chief office of which is for the defence of the moat and place.

$F_{L A N K}$, is also a term of war, used by way of analogy for the side of a battalion, army, \&c, in contradistinction to the front and rear. So, to attack the enemy in flank, is to discover and fire upon them on one side.

FLANKED, is used of something that has flanks, or may be approached on the flank. As

Flavied Angle, or that formed by the two faces of the bastion, and forming its point or angle.

FLa KKed Line of Defence, Flanked Tenaille, \&c.

FLANKING, in general, is the discovering and firing upon the side of a place, body, batialion, \&c. To flank a place, or other work, is to dispose it in such a manner as that evcry part of it may be played upon both in front and rear.

FLAT Bastion, is that which is built on a right line, as on the midclle of the curtain, \&x.

FLETCHER ( $\triangle$ в RAнAM), an ingenious matliematician, was born at Little Broughton, in Cumberland, in 1714 , and brought up in his father's business, husbandry and tobaccu-pipe making. He learned to read and write by his own endeavours, and afterwards taught himself arithmetic, and gradually the several branches of mathematics, with botany, touching the medicinal qualities of herbs. At the age of 30 he turned schoolmaster, to which profession was added those of astrologer and country doctor; in which, by means of industry and oeconomy, the two handmaids of tortune, he was enabled to leave his family in possession of $4000 l$. at his death, which happened in 1793 , in the 79 th year of his age. Mr. Fletcher published, in 1752, an ingenious treatise, entitled The Universal Measurer, in one vol. 8 vo; which was afterwards (1762) much enlarged and improved, under the title of The Universal Measurer and Mechanic, being a general collection of exercises in most of the mathemarical sciences.

FLEXIBLE, is the property or quality of a body that may be bent.

FLEXION, the same as Flexure.

FLEXURE, or Flexion, is the bending or curving of a line or figure. When a line first bends one way, and then gradually changes to a bend the contrary way, the point where the two parts join, or where the hending changes to the other side, is called the point of inflexion, or of contrary flexure.

FLLE, or FLY, that part of the mariner's compass on which the 32 points of the wind are drawn, and over which the nesdle is placed, and fastened underneath.

FLOAT-Bourds, the boards fixed to the outer rim of undershot water-whecls, serving to receive the impulse of the stream, by which the wheel is carried round.-There may be too many of these buards on a whecl. It is thought to be the best rule, to have their distance asunder such, that each of them may come out of the water as soon as possible, after it has received and acted with its full impulse; or, which comes to the same thing, when the succeeding one is in a direction perpendicular to the surface of the water.

liloativg Bridge, is a bridge of boats, casks, \&c, covered witis planks, firmly bound together lor the passage of men, horses, or carriages, \&c.

FLOOD, a deluge or inundation of water.
FrOOD, is also tased in speaking of the tide, when it is rising or flowing up; in contradistinction to the ebb, which is when it is decreasing or running out.

FLOORING, in Carpentry, is commonly understood of the boarding of the floors. 'The measurement of flooring is estimated in squares, of 100 square feet each, or of 10 fect on each side every way; 10 times 10 being 100 . Hence, the length of the floor being multiplied by its breadth, in fect, and two figures cut off on the righthand, gives the squares, and fect, or decimals cut off. Thus, a floor being 22 feet long, and 16 wide; then $22 \times 16=352$ square feet, or 3.52 squares, the content.

FLULNT, or Floning Quantity, in the Doctrine of Fluxions, is the variable quantity which is consictered as increasing or decreasing ; or the flucnt of a given fluxion, is that quantity whose fluxion being taken, according to the rules of that calculus, shall be the same with the given flusion. See Fuvions.

Contemporary FLUENTS, are such as flow together or for the same time. And the same is to be understood of contemporary fluxions. - When contemporàry fluents are always equal, or in any constant ratio; then arso are their fluxions respectively either equal, or in that same constant ratio. That is, if $x=y$, then is $\dot{x}=\dot{y}$; or if $x: y:: n: 1$, then is $\dot{x}: \dot{y}:: n: 1$; or if $x=n y$, then is $\dot{x}=n \dot{y}$.

It is easy to find the fluxions to all the given forms of fluents; but, on the contrary, it is difficult to find the fluents of rnany given fluxions; and indeed there are numberless cases in which this cannot be effected, except it be by the quadrature and rectification of curve lines, or by Jogarithms, or infinite series.

This doctrine, first invented by Sir Isaac Newton, was carried by him to a considerable degree of perfection, at least as to the most frequent, and most useful forms of fluents; as may be seen in his Fluxions, and in his Quadrature of Curves. Maclaurin, in his Treatise of Fluxions, has made several inquiries into fluents, reducible to the rectification of the ellipse and hyperbola: and Dalembert has pursued the same subject, and carried it farther, in the Memuires de l'Acad, de Berlin, tom. 2. p. 200. To the celebrated M. Luler this doctrine is greatly indebted, in many parts of his various writings, as well as in the Institutio Calculi Integralis, in 3 vols 4to, Petr. 1768. The ingenious Mr. Cotes contributed very inuch to this doctrine, in his Harmonia Mensurarum, concerning the measures of ratios and anglcs, in a large collection of different forms of fluxions, with their corresponding fuents ; which was fapther prosecuted in the same way by Walmesley, in his Analyse des Mesure's des Rapportes et des Angles, a large vol. in 4 to, 1749 . Besid's many other authors who, by their ingenious labours, have greatly contributed to facilitate and extend the doctrine of fluents; as Emerson, Simpson, Lamlen, Waring, \&c, in this country ; l'llopital, and many other learned foreigners. Lastly, in $178 j$ was published at Viema, by M1. Paccassi, a German nobleman, Udhandlung uber cnic neuc Methode zu Integriren, being a mothod of integrating, or finding the fluents of given fluxions, by the ruk's of the direct method, or by taking again the fluxion of the given fluxion, or the $2 \mathrm{~d}$ fluxion of the fluent sought; and then making every flowing quantity, its fluxion, and $2 \mathrm{~d}$ fluxion, in geometrical progression; a method however, which, it seems, only holds true in the casiest cases or forms, whose 
fluents are easily had by the most common methods: and which nearly resembles the method employed by Clairaut, in treating what he called Equations of Condition.

As it is only in certain particular forms and cases that the fluents of given fluxions can be found; there being no method of pertorming this universally a priori, by a direct investigation, like finding the fluxion of a given fluent quantity; we can do little more than lay down a few rules for such forms of fluxions as are known, from the direct method, to belong to such and such kinds of fluents or flowing quantities: and these rules, it is evident, must chiefly consist in performing such operations as are the reverse of those by which the fluxions are found to given flowing quantities. The principal cases of which are as follow:

I. To find the Fluent of a simple fluxion; or that in which there is no variable quantity, and only one fluxional quantity. This is done by barely substituting the variable or flowing quantity instead of its fluxion, and is the result or reverse of the notation only. Thus,

The fluent of $a \dot{x}$ is $a x$.

The fluent of $a \dot{y}+2 \dot{y}$ is $a y+2 y$.

II. When any power of a flowing quantity is multiplied by the fluxion of the root. Then, having substituted, as befure, the flowing quantity for its fluxion, divide the result by the new index of the power. Or, which is the same thing, take out, or divide by, the fluxion of the root; add 1 to the index of the power; and divide by the index so increased.

So if the fluxion proposed be $-3 x^{5} \dot{x}$;

Strike out $\dot{x}$, then it is - - $3 x^{5}$;

ald 1 to the index, and it is $-3 x^{6}$;

divide by the index 6 , and it is - $\frac{3}{6} x^{6}$ or $\frac{1}{2} x^{6}$;

which is the fluent of the proposed fluxion $3 x^{5} \dot{x}$.

In like manner, the fluent

of $4 a x \dot{x}$ is $2 a x^{3}$;

of $3 x^{\frac{1}{2}} \dot{x}$ is $2 x^{\frac{3}{2}}$;

of $a x^{\mathrm{n}} \dot{x}$ is $\frac{a}{n+1} x^{\mathrm{n}}+{ }^{\mathrm{l}}$;

of $\frac{\dot{z}}{z^{3}}$ or $z^{-2} \dot{z}$ is $-z^{-1}$ or $\frac{-1}{z}$.

of $\left(a^{3}+z^{3}\right)^{4} z^{2} \dot{z}$ is $\frac{\mathrm{r}}{15}\left(a^{3}+z^{3}\right)^{5}$.

III. When the root under a vinculum is a compound quantity; and the index of the part or factor without the vinculum increased by 1 , is some multiple of that under the zinculum: Put a single variable letter for the compound root; and substitute its powers and fluxion instead of those, of the same value, in the given quantity; so will it be reduced to a simpler form, to which the preceding rule can then be applied.

Thus, if the given fluxion be $\dot{\mathrm{F}}=\left(a^{2}+x^{2}\right)^{\frac{2}{3}} x^{3} \dot{x}$; where 3 , the index of the quantity without the vinculum, increased by 1 , makes 4 , which is double of 2 , the exponent of $x^{2}$ within the same; therefore putting $z=a^{2}+x^{2}$, thence $x^{2}=z-a^{2}$, and its fluxion is $2 x \dot{x}=\dot{z}$; hence then $x^{3} \dot{x}=\frac{x}{2} x^{2} \dot{z}=\frac{x}{2} \dot{z}\left(z-a^{2}\right)$, and the given quantity $\dot{\boldsymbol{F}}$ or $\left(u^{2}+x^{2}\right)^{\frac{2}{3}} x^{3} \dot{x}$ is $=\frac{\pi}{2} z^{\frac{2}{3}} \dot{z}\left(z-u^{2}\right)$ or $=\frac{1}{2} z^{\frac{5}{3}} \dot{z}-$ $\frac{1}{2} l^{2} z^{\frac{2}{3}} \dot{z}$; and the fluent of each term gives

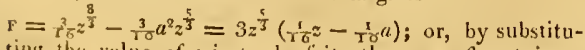
tiing the value of $z$ instead of it, the same flucnt is $3\left(a^{2}+x^{2}\right)^{\frac{5}{3}} \times\left(r^{\frac{1}{6}} x^{2}-\frac{3}{8} u^{2}\right)$, or $\frac{3}{r^{3}}\left(a^{2}+x^{2}\right)^{\frac{3}{3}} \cdot\left(x^{2}-\frac{3}{5} a^{2}\right)$.

IV. When there are sevcral terms involving two or morc variable quantities, having the fuxion of each multiplied by the other quantity or quantities: Take the fluent of each term, as if there was only one variable quantity in it, namely that whose fluxion is contained in it, supposing all the others to be constant in that term; then if the fuents of all the terms so found, be the very same quantity, that quantity will be the fluent of the whole.-l'hus, if the given fluxion be $\dot{x} y+x \dot{y}$. Then, the fluent of $\dot{x} y$ is $x y$, supposing $y$ constaut; and the fluent of $x y$ is alvo $x y$, when $x$ is constant ; therefore the common resulting quantity $x y$ is the required fluent of the given fluxion $x y+x \dot{y}$.

And, in like manner, the fluent of $\dot{x} y z+x \dot{y} z+x y \dot{z}$ is $x y z$.

V. When the given fluxional expression is in this form $\frac{\dot{x}_{y}-x \dot{y}}{y^{2}}$, viz, a fraction including two quantities, being the fuxion of the former drawn into the latter, minus the fluxion of the latter drawn into the former, and dizided by the square of the latter: then the fluent is the fraction $\frac{x}{y}$, or of the former quantity divided by the latter. That is,

The fluent of $\frac{\dot{x} y-x \dot{y}}{y^{2}}$ is $\frac{x}{y}$;

and the fluent of $\frac{2 x \dot{x} y^{2}-2 x^{2} y \dot{y}^{3}}{y^{4}}$ is $\frac{x^{3}}{y^{4}}$.

Though the examples of this case may be performed by the foregoing one. Thus the given fluxion

$$
\frac{\dot{x} y-x \dot{y}}{y^{2}} \text { rcduces to } \frac{\dot{x} y}{y^{3}}-\frac{x \dot{y}}{y^{2}} \text { or } \frac{\dot{x}}{y}-x \dot{y} y^{-2} \text {; }
$$

of which the fluent of $\frac{\dot{x}}{y}$ is $\frac{x}{y}$ when $y$ is constant; and the fluent of $x y y^{-2}$ is $+x y^{-1}$ or $\frac{x}{y}$ when $x$ is constant; and thercfore, by that case,

$\frac{x}{y}$ is the fluent of the whole $\frac{\dot{x} y-x \dot{y}}{y^{2}}$.

VI. When the fluxion of a quantity is dizided by the quantity itself: Then the fluent is equal to the hyperbolic logaritbm of that quantity; or, which is the same thing, the fluent is equal to $2.30258509 \& \mathrm{c}$, multiplied by the common log. of the same quantity.

So, the fluent

of $\frac{\dot{x}}{x}$ or $x^{-1} \dot{x}$ is the hyp. log. of $x$;

of $\frac{2 \dot{x}}{x}$ is $2 \times$ liyp. $\log$. of $x$, or $=$ h. l. of $x^{2}$;

of $\frac{a \dot{x}}{x}$ is $a \times$ h. l. of $x$, or h. 1. of $x^{2}$;

of $\frac{\dot{x}}{a+x}$ is the h. l. of $a+x$;

of $\frac{3 x^{2} \dot{x}}{a+x^{3}}$ is the h. l. of $a+x^{3}$.

VII. Many fluents may be found by the direct methoul of fluxions, thus: 'Take the fluxion again of the given fluxional expression, or the $2 \mathrm{l}$ fluxion of the fluent sought; into which substitute $\frac{\dot{x}^{2}}{x}$ for $\ddot{x}$, and $\frac{y^{2}}{y}$ for $\ddot{y}$, \&c, that is, makc $x, \dot{x}, \ddot{x}$, as also $y, \dot{y}, \ddot{y}, \mathcal{S} \cdot$, in continual proportion, or $x: \dot{x}:: \dot{x}: \ddot{x}$, and $y: y:: y: \ddot{y}$, \&c ; then divide the square of the gisen fluxional expression by the 2d fuxion, just found, and the quotient will be the fluent sought in many cases.

Or the same rule may be otherwise delirered thus: In the given fluxion $\dot{y}$, write $x$ for $\dot{x}, y$ for $\dot{y}$, \&c, and call the result $(i$, taking also the fluxion of this quantity, $\dot{\sigma}$; then 
make $\dot{G}: \dot{F}:: G: F$, so shall the 4 th proportional $F$ be the fluent as bofore. And this is the rule of M. Paccassi.

It may be proved whether this be the true fluent, by taking the fluxion of it again, which, if it agree with the proposed fluxion, will show that the fluent is right ; otherwise, it is not.

Thus, if it be proposed to find the fluent of $n x^{n^{-1}} \dot{x}$. Here $\dot{\mathrm{F}}=n x^{\mathrm{n}-1} \dot{x}$; write first $x$ for $\dot{x}$, and it is $n x^{\mathrm{n}^{-1}} x$ or $n x^{\mathrm{n}}=\mathrm{G}$; the fluxion of this is $\dot{\mathrm{G}}=n^{2} x^{\mathrm{n}-1} \dot{x}$; therefore $\dot{\mathrm{G}}: \dot{\mathrm{F}}:: \mathrm{G}: \mathrm{F}$ becomes $n^{2} x^{\mathrm{n}-\mathrm{x}} \dot{x}: n x^{\mathrm{n}-1} \dot{x}:: n x^{\mathrm{n}}: x^{\mathrm{n}}=\mathrm{F}$, the fluent sought.

Again, suppose it be proposed to find the fluent of $x y$ $+x \dot{y}$. Here $\dot{\mathrm{F}}=\dot{x} y+x \dot{y}$; then, writing $x$ for $\dot{x}$, and $y$ for $y$, it is $x y+x y$ or $2 x y=G$; the fluxion of which is $2 \dot{x} y+2 x \dot{y}=\dot{G} ;$ then $\dot{G}: \dot{F}:: \mathrm{G}: \mathrm{F}$ becomes $2 \dot{x} y+2 x \dot{y}$
$: \dot{x} y+x \dot{y}:: 2 x y: x y=\mathrm{F}$, the fluent sought. See also Condition, Equations of.

VIIl. To find Fluents by means of a table of forms of Fluxions and Fluents.

In the following table are contained the most usual forms of fluxions that occur in the practical solution of problems, with their corresponding fluents set opposite to them; by means of which, viz, comparing any proposed fluxion with the corresponding form here, the Auent of it will be found.

Where it is to be noted, that the logarithms in the said forms, are the hyperbolic ones, which are found by multiplying the common logs. by $2 \cdot 3025850929940$ \&c. Also the arcs whose sine, or tangent, \&c, are mentioned, have the radius 1 , and are those in the common tables of sines, tangents, \&c.-And the numbers $m, n$, \&c. are to be some quantities, as the forms fail when $n=0$, or $m=0$, \& c.

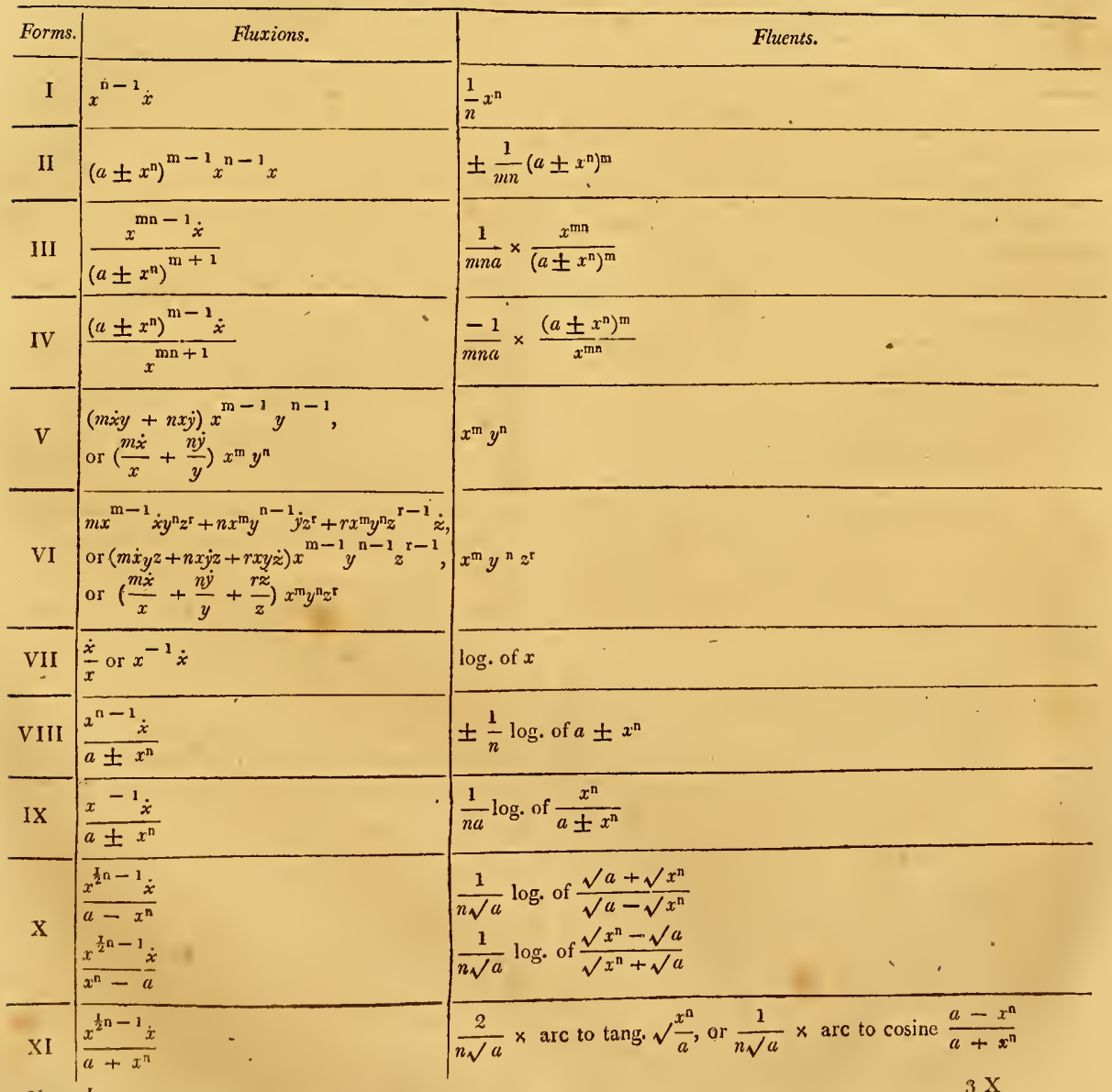

VOL.I. 


\begin{tabular}{|c|c|c|}
\hline Forms. & Fluxions. & Fluentsa \\
\hline XII & $\frac{x^{\frac{1}{2} \mathrm{n}-1} \dot{x}}{\sqrt{ }\left( \pm a+x^{\mathrm{n}}\right)}$ & $\frac{2}{n} \operatorname{Iog}$. of $\sqrt{ } x^{n}+\sqrt{ }\left( \pm a+x^{n}\right)$ \\
\hline $\mathrm{X}[\mathrm{II}$ & $\frac{x^{\frac{1}{2} n-1} x}{\sqrt{(}\left(u-x^{n}\right)}$ & $\frac{2}{n} \times$ arc to sin. $\sqrt{ } \frac{x^{n}}{a}$, or $\frac{1}{n} \times$ arc to vers. $\frac{2 x^{n}}{a}$ \\
\hline XIV & $\frac{x^{-1} x}{\sqrt{ }\left(a \pm x^{n}\right)}$ & $\frac{1}{n \sqrt{ } a} \log$. of $\frac{\sqrt{ } a-\sqrt{ }\left(a \pm x^{n}\right)}{\sqrt{ } a+\sqrt{ }\left(a \pm x^{3}\right)}$ \\
\hline $\mathrm{XV}$ & $\frac{x^{-1} \dot{x}}{\sqrt{\left(-a+x^{n}\right)}}$ & $\begin{array}{l}\frac{2}{n \sqrt{ } a} \times \text { arc to secant } \sqrt{\frac{x^{n}}{a}}, \text { or } \frac{2}{n \sqrt{ } a} \times \text { arc to cosine } \frac{a}{x^{n}} \\
\text { or } \frac{1}{n \sqrt{ } a} \times \text { arc to cosine } \frac{2 a-x^{n}}{x^{n}}\end{array}$ \\
\hline XVI & $\frac{\dot{x}}{\sqrt{ }\left(d x-x^{2}\right)}$ & $\frac{2}{d} \times$ arc to diam. $d$, and vers. $x$ \\
\hline XVII & $\begin{array}{l}\dot{x} \sqrt{ }\left(d x-x^{2}\right) \\
\dot{x} \sqrt{ }\left(u^{2}-x^{2}\right)\end{array}$ & $\begin{array}{l}\frac{1}{2} \text { circ. seg. to diam. } d \text { and vers. } x \\
\frac{1}{2} \text { circ. zone, rad. } a \text {, and height from centre } x\end{array}$ \\
\hline XVIII & $c^{\mathrm{nx}} \dot{x}$ & $\frac{c^{n x}}{n \log \cdot c}$ \\
\hline XIX & $\dot{x} y^{x} \log \cdot y+x y^{x-1} \dot{y}$ & $y^{x}$ \\
\hline $\mathrm{XX}$ & $\begin{array}{l}\left(a+x^{\mathrm{n}}\right)^{\mathrm{m}} x^{\mathrm{n}-1} \dot{x} \\
\text { Putting } s=m+r\end{array}$ & $\begin{array}{l}\frac{\left(a+x^{\mathrm{n}}\right)^{\mathrm{m}+1} x^{\mathrm{rn}-\mathrm{n}}}{n s} \times\left(\frac{1}{1}-\frac{r-1 \cdot a}{s-1 \cdot x^{\mathrm{n}}}+\frac{r-1 \cdot r-2 \cdot a^{2}}{6-1 \cdot s-2 \cdot x^{2 \mathrm{n}}}-\frac{r-1 \cdot r-2 \cdot r-3 \cdot a^{3}}{s-1 \cdot s-2 \cdot s-3 x^{3 \mathrm{n}}} 8 \mathrm{c}\right), \\
\mathrm{or} \frac{\left(a+x^{\mathrm{n}}\right)^{\mathrm{m}+1} x^{\mathrm{rn}}}{r n a} \times\left(\frac{1}{1}-\frac{s+1 \cdot x^{\mathrm{n}}}{r+1 \cdot a}+\frac{s+1 \cdot s+2 \cdot x^{2 \mathrm{n}}}{r+1 \cdot r+2 \cdot a^{2}}-\frac{s+1 \cdot s+2 \cdot s+3 \cdot x^{3 \mathrm{n}}}{r+1 \cdot r+2 \cdot r+3 \cdot a^{3}} s \mathrm{c}\right) .\end{array}$ \\
\hline
\end{tabular}

The Use of the foregoing Table of Forms of Fluxions and Fluents. - In the use of this table, it is to be observed, that the first column serves only to show the number of the form, as a mark of reference; in the $2 \mathrm{~d}$ column are the several forms of fluxions, which are of different kinds or classes; and in the $3 \mathrm{~d}$ or last column are the corresponding fluents.

The method of using the table is this. Having any fluxion given, whose fluent it is proposed to find: First, compare the given fluxion with the several forms of fluxions in the $2 \mathrm{~d}$ column of the table, till one of the forms be found that agrees with it; which is done by comparing the terns of the given fluxion with the like parts of the tabular fluxion, viz, the radical quantity of the one, with that of the other; 'and the exponents of the variable quantitics of each, both within and without the vinculum; all which, bcing found to agree or correspond, will give the particular values of the general quantities in the tabular form. Then substitute these particular values, for the same quantities in the general or tabular form of the fluent, and the result will be the particular fluent sought; after it is multiplied by any cocficient the proposed fluxion may have.

For Example. To find the flucnt of the fluxional expression $3 x^{3} \dot{x}$. This agrees with the first form; and by comparing the fluxions, it appears that $x=x$, and $n-1=\frac{3}{3}$, or $n=\frac{8}{3}$; which being substituted in the tabular fluent, or $\frac{1}{n} x^{n}$, gives, after multiplying by 3 the coefficient, $3 \times \frac{3}{8} x^{\frac{8}{3}}$ or $\frac{9}{8} x^{\frac{8}{3}}$ for the fluent sought.

Again, To find the fluent of $5 x^{2} \dot{x} \sqrt{ } c^{3}-x^{3}$, or $5 x^{2} \dot{x}$ $\left(c^{3}-x^{3}\right)^{\frac{\pi}{2}}$. This belongs to the $2 \mathrm{~d}$ form ; for $a=c^{3}$, and $-x^{n}=-x^{3}, n=3$ under the vinculum, and $m-1=\frac{x}{2}$, or $m=\frac{3}{2}$, also the exponent $n-1$ of $x^{\mathrm{n}-1}$ without the vinculum, by using 3 for $n$, is $n-1=2$, which agrees with $x^{2}$ in the fluxion given; and therefore all the parts of the form are found to answer. Then, substituting these values into the general fluent, $-\frac{1}{m n}\left(a-x^{\mathrm{n}}\right)^{\mathrm{m}}$, gives $-\frac{5}{3} \times \frac{2}{3}\left(c^{3}-x^{3}\right)^{\frac{3}{2}}=-\frac{x}{9}\left(c^{3}-x^{3}\right)^{\frac{3}{2}}$.

Thirdly, To find the fluent of $\frac{x^{0} \dot{x}}{1+x^{3}}$. This agrees with the sth form; where $\pm x^{n}=+x^{3}$ in the denominator, or $n=3$; and the numerator $x^{\mathrm{n}-\mathrm{x}}$ then becomes $x^{2}$, which agrees with the numerator in the given fluxion, also $a=1$. Hence then, by substituting in the general form of the fluent $\frac{x}{n}$ logarithm of $a+x^{n}$, it becomes ${ }_{3}^{x} \operatorname{logarithm}$ of $1+x^{3 !}$

IX. To find Fluents by means of Infinite Series.-When a finite form cannot be found to ugree with a proposed 
fluxion, it is then usual to throw it into an infinite series, either by division, or extraction of rnots, or by the binomial theorem, \&c ; after which, the fluents of all the terms are taken separately.

For Example. To find the fluent of $\frac{1-x}{1+x-x^{2}} \dot{x}$. Here, by dividing the numerator by the denominator, this becomes $\dot{x}-2 x \dot{x}+3 x^{2} \dot{x}-5 x^{3} \dot{x}+8 x^{4} \dot{x} \& \mathrm{c}$; and , the fluents of all the terms being taken, give $x-x^{2}+x^{3}-\frac{5}{4} x^{4}+\frac{8}{5} x^{5}$ $\& c$, for the fluent sought.

To Correct a FLU EN T.-The fluent of a given fluxion, found as above, sometimes wants a correction, to make it contemporary with that required by the problem under consideration, \&rc : for the fluent of any given fluxion, as $\dot{x}$, may be either $x$ (which is found by the rule) or it may be $x \pm c$, that is $x$ plus or minus some constant quantity $c$; because both $x$ and $x \pm c$ have the same fluxion $\dot{x}$ : and the finding of this constant quantity $c$, is called correcting the fluent. "Now this correction is to be determined from the nature of the problem in hand, by which means to know the relation which the fluent quantities have to each other at some certain point or time. Reduce therefore the general fuential equation, found by the rules above, to that point or time; then if the equation be true at that point, it is correct; but if not, it wants a correction, and the quantity of that correction is the difference betiveen the twa general sides of the equation when reduced to that particular state. Hence the general rule for the correction is this :

Connect the constant, but indeterminate, quantity $c$, with one side of the fluential equation, as determined by the foregoing rules; and, in this equation, substitute for the variable quantities such values as they are known to have at any particular state, place, or time; and then from that particular state of the equation find the value of $c$, the constant quantity of the correction.

Example. To find the correct fluent of $\dot{z}=a x^{3} \dot{x}$. First the general fluent of this is $z=\frac{\pi}{4} a x^{4}$, or $z=\frac{x}{4} a x^{4}+c$, taking in the correction $c$. -Now if it be known that $z$ and $x$ begin together, or that $z=0$, when $x=0$; then writing 0 both for $x$ and $z$, the general equation becomes $0=0+c$, or $c=0$; so that, the value of $c$ being 0 , the correct fluents are $z=\frac{r}{4} a x^{4}$.

But if when $z=0, x=b$, any known quantity; then substituting 0 for $z$, and $b$ for $x$, in the general equation, it becomes $0=\frac{1}{4} a b^{4}+c$, from which is found $c=-\frac{x}{4} a b^{4}$; and this being written for it in the gencral equation, this becomes $z=\frac{\pi}{4} a\left(x^{4}-b^{4}\right)$, for the correet, or contemporary fluents.

Or lastly, if it be knowu that when $z=d, x=b$; then substituting $d$ for $z$, and $b$ for $x$, in the general fluential equation $z=\frac{x}{4} a x^{4}+c$, it becomes $d=\frac{1}{4} a b^{4}+c$; and hence is deduced the value of the correction, viz, $c=d-\frac{1}{4} a b^{4}$; consequently, writing this value for $c$ in the general equation, it becomes $z=\frac{1}{4} a\left(x^{4}-b^{4}\right)+d$, for the correct equation of the fluents in this case.

And bence arises another easy and gencral way of correcting the fluents, which is this: In the general equation of the fluents, write the particular values of the quantities which they are known to have at any certain time; then subtract the sides of the resulting particular equation, from the corresponding sides of the general one, and the remainders will give the correct equation of the fluents songht. Su, as above,

the gigeneral equation being - . $z=a x^{4}$;

write $d$ for $z$, and $b$ for $x$, then $\quad d=a b^{4}$;

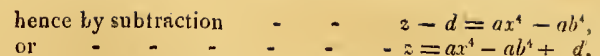
the correct fluents as before. - See other methods of finding o r transforming fluents, in my Course of Mathematics.

FLUID, or FLuid Body, according to Newton, is that whose parts yield to the smallest force impressed, and by yielding are easily moved among each otber; in whic sense it stands opposed to a solid. This is the definition of a perfect fluid: if the fluid require sone sensible force to move its parts, it is imperfect in proportion to that force; and such perhaps are all the fuids we know of in nature. See Fluidity.

That fluids have vacuities in their substance is evident, because certain bodies may be dissolved in them without increasing their bulk. Thus, water will dissolve a certain quantity of salt; after which it will receive a little sugar, and after that a little alum; and all this without increasing its first dimeusiuns. Which shows that the particles of these solids are so far separated as to become smaller than those of the fluid, and to be received and contained in the interstices between them.

Fluids are either elastic, such as air; or non-elastic', as water, mercury, \&ic. These latter occupy the same space, or are of the same bulk, under all pressures or forces; but the former dilate and expand themselves continually by taking of the external pressure from them; for which reason it is that the density and clasticity of such fluids, are proportional to the force or weight that compresses then. The doctrine and laws of fluids are of the greatest extent and importance in philosophy: the propertics of elastic fluids constituting the doctrine of Pneumatics; those of the non-elastic fluids, that of Hydrostatics; and the laws of their motions, Hydraulics. For which see these respective articles.-For the laws of the pressure and gravitation in fuids specifically heavier or lighter than the bodies immerged, see Speciric Gravity.-For the laws of the resistance of fluids, or the retardation of solid bodies moving in fluids, see Resistance.-For the ascent of fluids in capillary tubes; or between glass planes, \&c, see -Ascent, and Capillary Tubes.

FLUTES, or FLUTings, are certain channels or cavities cut along the shaft of a column, or pilaster.

FLUIDITY, that state or affection of bodies, which denominates or renders them fluisl ; or that property by which they yield to the smallest force impressed: in contradistinction to solidity or firmness. Fluidity is to be carefully distinguished from liquidity or humidity, which latter implies wetting or adhering. Thus, air, ether, mercury, and other melted metals, and even smoke and flame itself, are fluid bodies, but not liquid ones; while water, beer, milk, urine, \&ce, are both fluids and liquids at the same time.

The nature and causes of fluidity have been variously assigned. The Gassendists, and ancient corpuscularians, require only three conditions as necessary to it; viz, a smallness and smoothness of the particles of the body, vacuities between these particles, each of which being a spherical figure. The Cartesians, and after them Dr. Hooke, Mr. Boyle, \&c, besides these circumstances, require also a certain internal or intestine motion of the particles, as chiefly contributing to fluidity. Thus, Mr. Boyle, in his History of Fluidity, argues from various experiments: for cxample, a little dry powder of alabaster, or plaster of Paris, finely sifted, being put into a vessel over the fire, soon begins to boil like water; exhibiting all $3 \times$ a 
the motions and phenomena of a boiling liquor: it tumbles variuusly in great waves; will bear stirring with a stick or: Jaclle, without resisting; and if strongly stirred near the side of the vessel, its waves will apparently dash against it, thus resembling the boiling of water: yet it is all the while a dry parched powder.

The same is observed in sand; a dish of which being set on a drum-hcad, briskly beaten by the sticks, or on the upper stone of a mill, it in all respects emulates the properties of a fluirl body. A heavy body, ex. gr. will inmediately sink in it to the bottom, and a light one emerge to the top: each grain of sand has a constant vibratory and dancing motion; and if a hole be made in the side of the dish, the sand will spin out like watcr.

The Cartesians bring divers considerations to prove that the parts of fluids are in continual motion: as 1st, The change of solids into fluids, ex. gr, ice into water, and vice versa; the chief differcnce between the body in those two states consisting in this, that the parts, being fixed and at rest in the one, resist the touch; whereas in the other, being already in motion, they give way to the slightest impulse. 2dly, The effects of fluids, which commonly proceed from motion: such are the insinuation of fluids among the pores of bodies; the softening and dissolving hard bodies; the actions of corrosive menstruums; \&c: to which they add, that no solid can be brought to a state of fluidity, without the intervention of some moving or moveable body, as fire, air, or water. Air, these philosophers hold to be the first spring of the causes of fluidity, it being this that gives motion to fre and water, though itself receives its motion and action from the ether, or subtile medium.

But Boerbaave pleads strenuously that fire is the first mover, and the great cause of all fluidity in other bodies, as air, water, \&c: without this, he says, that the atmosphere itsclf would fix into one solid mass. And in like manner, Dr. Black, of Edinburgh, mentions fiuidity as an effect of heat. The different degrees of heat which are required to bring different bodies into a state of fluidity, he supposes may depend on some particulars in the misture and composition of the bodies themselves: which is rendered farther probable from considering that the natural state of bodic's in this respect is changed by certain mixtures; thus, when two metals are compounded, the mixture is commonly more fusible than either of them separately.

Newton's idea of the cause of fluidity is different: he considers it to be the great principle of attraction. The various intestine motion and agitation among the particles of fluid bodies, he tlinks is naturally accounted for, by supposing it a primary law of nature, that as all the particles of matter attract each other when within a certain distance; so at all greater distances, they avoid and fly from one another. For then, though their common gravity, together with the pressure of other bodies upon them, may keep them together in a mass, yet their continual cndeavour to avoid one another singly, and the andventitious inpulses of heat and light, or other external causes, may make the particle's of fluids continually move round each other, and so produce this quality. As thercfore the cause of cohesion of the parts of solid bodies appents to be their mutual attraction; so, en this principle, the chief causcoflluidity scems to be a contrary motion, inpressed on the partictes of Auids; by which they avoid and tly from eich other, as soon as they come at, and as long as the'y keep at, a certain distance. It is observed also in all fluids, that the direction of their pressure against the vessels which contain them, is in lines perpendicular to. the sides of such vessels; which property, being the necessary result of the spherical figure of the particles of any fluit, shows that the parts of all fluids are so, or of a figure very nearly approaching to it.

FLUX, in Hydrography, a regular and periodical motion of the sca, happening rwice in 24 hours and 45 minutes, nearly. The flux, or flow, is one of the motions of the tide: the other, by which the water sinks and retires, being called the reflux, or ebb. See Tide.

Betwren the flux and reflux there is a kind of rest or cessation, of about half an hour; during which time the water is at its greatest height, called higl-water.

The flux of the sea follows chielly the course of the moon; and is always highest and greatest at new and full moons, particularly near the time of the equinoxes. In some parts, as at Mount St. Michael, the water rises 18 or 20 feet, though in the open sea it never rises above a foot or two; and in sume places, as about the Morea, there is nu flux at all. It runs up some rivers above 120 miles : though up the river Thames it goes only about so, viz, near to Kingston in Surrey. Above London-bridge, the water flows + hours, and ebbs 8 ; and below the bridge, it flows 5 liours, and ebbs 7.

FLUXION, in the Newtonian analysis, denotes the rate or proportion at which a flowing or varying quantity increases its magnitude or quantity; and it is proportional to the magnitude by which the flowing quantity would be uniformly increased, in a given time, by the generating quantity continuing of the invariable magnitude it has at the moment of time for which the fluxion is taken: by which it stands contradistinguished from fluent, or the flowing quantity, which is gralualiy, and indefinitely increasing, after the manner of a space which a body in motion describes.

Mr. Simpson observes, that there is an advantage in thus considering fluxions, not as mere velocities of increase at a certain point, but as the magnitudes which would be uniformly generated in a given finite time: the imagination is not here confined to a single point, and the higher orders of fluxions are rendered nuch more casy and intelligible. And though Sir Isaac Newton elefines fluxions to be the velocities of motions, yct he has reconrse to the moments or increments, generated in equal particles of time, to determine those velocities, which he afterwards directs to expoutd by finite magnitudes of other kinds. As to the illustration of this definition, and the rules for finding the fluxions of all sorts of flowing quantities, see the following article, or the Method of Fluxions.

Mctlod of FuUxions, is the algorithm and analysis of fuxions and fluents, or flowing quantitics. Most forrigncrs define this as the method of differences or diftierentials, being the analysis of indefinitely small yuantities. But Newton, and other Finglish authous call these infinitcly small ynantities, moncents; considering them as the momentary increments of variable quantities; us of a line considered as generated by the flux or motion of a point, or of a surface generated by the flux of a line. Accordingly, the variable quantitien are called fuents, or fow ing quantities; and the methos of findung either the fluxion, or the fluent, the method of tluxions. But M. Leibnita considers the same infinitely small quantities as the differences, or differentials, of quantities; and the methor of finding those differences, be calls the Diflcrential Calculus. Besides 

F L U U
$\left[\begin{array}{ll}525 & 0\end{array}\right.$
F L U

this difference in the name, there is another in the notation. Newtun expresses the fluxion of a quantity, as of $x$, by a point placed over it, thus $\dot{x}$; while Leibnitz expresses his differential of the same $x$, by prefixing the initial letter $d$, as $d x$. And except in these circumstances, the two methods may be considered as the same.

The Method of Fluxions is probably one of the greatest, most subtle, and sublime discoveries of any age: it opens a new world to our view, and extends our knowledge, as it were, to infinity; carrying us beyond the bounds that seemed to have been prescribed to the humau mind, at least infinitely beyond those to which the ancient geometry was confined.

The history of this important discovery, recent as it is, is a little dark and embroiled. Two of the greatest men of the last age have both of them claimed the invention, Sir I. Newton, and M. Leibnitz; and nothing can be more glorious for the method itself, than the zeal with which the partisans of either side have asserted their title.

To exhibit a just view of this dispute; and of the pretensions of eacli party, we may here advert to the origin of the discovery, and mark where each claim commenced, and how it was supported.

The principles on which the Method of Fluxions is founded, or which conducted to it, had been laying, and gradually developing, from the beginning of the 17 th century, by Fermat, Napier, Barrow, Wallis, Slusius, \& c, who had methods of drawing tangents, of maxima and minima, of quadratures, \&c, in certain particular cases, as of rational quantities, on nearly the same principles. And it was not wonderful that such a genius as Newton should soon after raise those faint beginnings into a regular and general system of science, which be did about the year 1665 , or sooner.

The first time however that the method appeared in print, was in 1684 , when M. Leibnitz gave the rules of it in the Leipsic Acts of that year: but without the remonstrations. The two brothers however, John and James Bernoulli, being struck with this new method, applied themselves diligently to it, found out the demonstrations, and applied the calculus with great success.

But before this, M. Leibuitz had proposed his Differestial Method, viz, in a letter, dated Jan. 21,1677 , in which he exactly pursues Dr. Barrow's method of tangents, that had been published in 1670; and Newton communicated his method of drawing tangents to Mr. Collins, in a letter dated Dec. 10, 1672; which letter, together with another dated June 13,1676 , were sent to M. Leibnitz by Mr. Oldenburgh, in 1676 . So that there is a strong presumption that he might avail himself of the information contained in these letters, and other papers transmitted with them, and also in 1675 , before the publication of his own letter, containing the first hint of his differential method. Indeed it sufficiently appears that Newton had invented his method before the year 1669 , and ${ }^{\circ}$ that he actually made use of it in his Compendium of Analysis and Quadrature of Curves before that time. His attention seems to have been directed this way, even before the time of the plague which happened in London in 1665 and 1666 , when he was about 28 years of agc.

This is all that is heard of the method, till the year 1687, when Newton's great work the Principia appeared, which is almost wholly founded on the same calculus. The common opinion then was, that Newton and Leibnity had each invented it about the same time: and what seemed to confirm it was, that ncither of them made any mention of the other; and that, though they agreed in the substance of the thing, yet they differed in their ways of conceiving it, calling it by different names, and using different characters. However, foreigners having first learned the method throngh the medium of Leibnitz's publication, which spread the method through Europe, they were illsemsibly accustomed to consider him as the sole, or principal inventor, and became ever after strongly prejudiced in favour of his notation and mode of conceiving it.

The two greatauthors themselves, without any seeming cuncern, or dispute, as to the property of the invention, enjoyed the glorious prospect of the progresses continually making under their auspices, till the year 1699 , whicn the peace began to be disturbed.

M. Facio, in a treatise on the Line of Swiftest Deseent, declared, that he was obliged to own Newton as the first inventor of the differential calculus, and the first by many years; and that he left the world to judge, whether Leibnitz, the second inventor, had taken any thing from him. This precise distinction between first and second inventor, with the suspicion it insinuated, raised a controversy between M. Leibnitz, supported by the editors of the Leipsic Acts, and the English mathematicians, who declared for Newton. Sir Isaac himself nerer appeared on the scene; his glory was become that of the nation; and his adlierents, warm in the cause of their country, needed not his assistance to animate them.

Writings succeeded each other but slowly, on either side; probably in account of the distance of places; but the controversy grew still stronger and stronger: till at length 11. I.eibnitz, in the year 1711, complained to the Royal Society, that Dr. Keil had accused him of publishing the Method of Fluxions invented by Sir I. Newton, under other names and characters. He insisted that nobody knew better than Sir Isaac himself, that he had taken nothing from him; and requires that Dr. Keil should disavow the ill construction which might be put upon bis words.

The Society, thus appealed to as a judge, appointed a committee to examine all the old letters, papers, and documents, that had passed among the several mathematicians, relating to this subject; who, after a strict examination of all the evidence that could be procured, gave in their report as follows: "That M.Leibuitz was in London in 1673, and kept a correspondence with Mr. Collins by means of Mr. Oldenburgh, till Sept. 1676, when he returned from Paris to Hanover, by way of London and Amsterdam: that it did not appear that M. Leibnitz knew any thing of the differential calculus before bis letter of the 2 ist of June, 1677, which was a year after a copy of a letter, written by Newton in the year 1672, had been sent to Paris to be communicated to him, and above 4 years after Mr. Collins began to communicate that letter to his correspondents; in which the Method of Fluxions was sufficiently explained, to let. a man of his sagacity into the whole inatter : and that Sir I. Newton had even invented his method before the year 1669 , and consequently 15 years before M. Leibnitz had given any thing on the subject in the Lcipsic Acts." From which they concluded that Dr. Keil had not at all injured M1. Leibnitz in what he had said.

The Society printed this their determination, together with all the pieces and materials relating to it, under the 
titte of Commercium Epistolicum de Analysi Promota, Svo, Lonu'. 1712. This book was carcfully distributed through Europe, to vindicate the title of the English nation to the discovery; for Newton himself, as already hinted, never appeared in the affair : whether it was that he trusted his honour with his compatriots, who were zealous enough in the cause; or whether he felt himself even superior to the glory of it.

M. Luibnitz and his friends however could not show the same indifference: he was accused of a plagiarism; and the whole Commercium Epistulicum either expresses it in terms, or insinuates it. Soon after the publication therefore, a lonse shcet was printed at Paris, in behalf of MI. Leibnitz, then at Vienna. It is written with great zeal and spirit; and it boldly maintains that the Method of Fluxions bad not preceded the Method of Differences; and even insinuates that it might have arisen from it. The detail of the proofs however, on each side, would be too long, and could not be understood without a large comment, which must enter into the deepest geometry: M. Leibnitz had begun to work upon a Commercium Epistolicum, in opposition to that of the Royal Society; but he died before it was, completed.

A second edition of the Commercium Epistolicum was printed at London in 1722 ; when Newton, in the preface, account, and annotations, which were added to that edition, particularly auswered all the objections which Leibnitz and Bernoulli were able to make since the Com: mercium first appeared in 1712 ; and from the last edition of the Commercium, with the various original papers contained in it, it evidently appears that Newton had discovered his Method of Fluxions many years before the pretensions of Leibnitz. See also Raphson's History of Fluxious.

There are however, according to the opinion of some, strong presumptions in favour of Leibnitz; i. e. that he was no plagiary: for that Newton was at least the first inventor, is past all dispute; his glory is secure; the reasonable part, even among the foreigners, allow it : and the question is only, whether Leibnitz took it from him, or tell upon the same thing with him; for, in his theory of abstract notions, which he dedicated to the Royal Academy in 1671, before he had seen any thing of Neivton's, he already supposed infinitely small quantities, some greater than others; which is one of the great principles of his system. But, before prosecuting farther the history and improvements of this science, it will be proper to premise somewhat of the principles and practice of it, according to the idcas of the inventor.

\section{Principles of the Wethod of FLUNions.}

1. In the doctrine of fluxions, magnitudes or quantities, of all kinds, are considered, not as made up of a number of small parts, but as generated by continued motion, by means of which they increase or decrease : as at line by the motion of a point; a surface by the motion of a line; and a solid by the motion of a surface : which is no new principle in gcometry; having been used by Archimedes, \&c. So likewise, time may be considered ats represented by a line, increasing uniformly by the motion of a point. And quantitics of all kinds whatever, which are capable of increase and decrease, may thus be represented by linnes, surfaces, or solids, considered as gencrited by motion.

2. Any quantity, in this manner generated, and variable, is called a liluent, or a fluwing fuantity. And the rate or proportion according to which any flowing quantity increases, at any position or instant, is the fluxion of the said quantity, at that positiou or instant : and it is proportional to the magnitude by which the flowing quantity would be uniformly increased, in a given time, with the generating celerity uniformly continued during that time.

3. The small quantities thus generated or described, in any given small pertion of time, and by any continued motion, either uniform or variable, are called Increments.

4. Hence, if the motion of increase be uniform, by which increments are generated, the increments will in that case be proportional, or equal, to the measures of the fluxions : but if the motion of increase be accelerated, the increments so generated, in a given finite timc, will exceed the tluxion; and if it be a decreasing motion, the increment so generater, will be less than the fluxion. But if the time be indefinitely small, so that the motion be considered as uniform for that instant; then these nascent increments will always be proportional or equal to the fluxions, and may be substituted for them; in any calculation.

5. To illustrate these definitions: Suppose a point $m$ be conceived to move from the position $A$, and to generate a

$m$

P $p$

line $A P$, with a motion any-how regulated; and suppose the celerity of the point $m$, at any position $P$, to be such, as would, if from thence it should become, or continue, uniform, be sufficient to describe, or pass uniformly over, the distance $\mathrm{p} p$, in the given time allowed for the fluxion: then will the said line $p p$ represent the fluxion of the said fluent or flowing line $A \mathrm{P}$, at that position.

6. Again, suppose the right line mn to move, from the pasition $\mathrm{AB}$, continually parallel to itself, with any con-

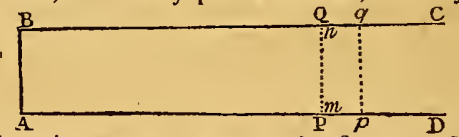

tinued motion, so as to generate the fluent, or flowing rectangle $A B Q P$, whilst the point $m$ describes the line $A P$; also let the distance $P p$ be taken, as above, to express the fluxion of the line or base $A P$; and complete the rectangle PQap. Then, in the same manner as $\mathrm{P} p$ is the fluxion of the line $\Delta P$, so the small parallelogram $P q$ is the fluxion of the flowing parallelogram, $A \&$; both these fluxions or increments being uniformly described in the sane time.

7. In like manner, if the solid $A E R P$ be conceired as generated by the plane PQR moving, from the position

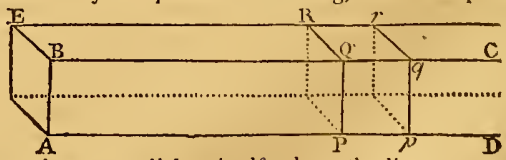

$\triangle \mathrm{BE}$, always parallel to itself, along the line $\triangle \mathrm{D}$; and if $P p$ denote the fluxion of the line $A P$. 'Th' $\mathrm{n}$, as the paralletogram $P q$, or $P p \times P Q$, expresses the liuxion of the flowing rectangle $A Q$, so likewise shall the fluxion of the variable solid or prism $A \mathrm{R}$ be expressed by the prism $\mathrm{rr}$, or Pp $\times$ the plane PR. And in both these last two cases, it appears that the fluxion of the generated rectangle, or prism, is equal to the product of the gencrant, whether 
line or plane, drawn into the fluxion of the line along which it moves.

s. Hitherto the generant, or generating line or plane, has been considered as of a constant or invariable magnitude; in which case the fluent, or quantity generated, is a parallelogram, or a prism, the former being described by the motion of a line, and the latter-by the motion of a plane. In like manner are other figures, whether plane or solid, conceived to be described, by the motion of a variable magnitude, whether it be a line or a planc. Thus, let a variable line $\mathbf{P Q}$ be carried with a parallel motion alung $\Delta P$, or while a point $P$ is carried along, and describes, the line AP, suppose another point $Q$ to be car-
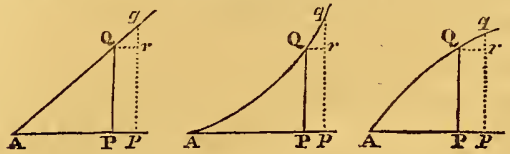

ried by a motion perpendicular to the former, and to describe the line $\mathbf{P Q}$ : let $p q$ be another position of $\mathbf{P Q}$, indefinitely near the formcr; and draw ar parallel to AP. Now in this case there are several fluents or flowing quantitics, with their respective fluxions : viz, the line or fluent $A P$, the fluxion of which is $\mathrm{P} p$, or $\mathrm{Q} r$; the line or fluent $\mathrm{PQ}$, the fluxion of which is $g r$; the curve, or oblique line $A Q$, described by the oblique motion of the point, the fluxion of which is $\mathrm{Q} q$; and lastly the surface $\mathrm{APQ}$, described by the variable line $P Q$, and the fluxion of which is the rectangle $P Q R P$, or $P Q \times P p$. And in the same manner may any solid be conceived to be described, by the motion of a variable plane parallel to itself, substituting the variable plane for the variable line; in which case, the fluxion of the solid, at any position, is represented by the variable plane, at that position, drawn into the fluxion of the line along which it is carried.

9. Hence then it follows gencrally, that the fluxion of any figure, whether plane or solid, at any position, is equal to the section of $i t$, at that position, drawn into the fluxion of the axis, or line along which the variable section is supposed to be perpendicularly carried; i. e. the fluxion of the figure $\triangle \mathrm{QP}$, is equal the plane $\mathrm{PQ} \times \mathrm{Pp}$ when that figure is a solid, or to the ordinate $P Q \times P P$ when the figure is a surface.

10. It also follows, from the same premises, that, in any curve, or oblique line, $A Q$, whase absciss is $\mathrm{AP}$, and ordinate is $P Q$, the fluxions of these three form a small rightangled plane triangle $Q q r$; for $Q r=P p$ is the fluxion of the absciss $A \mathrm{P}, q r$ the fluxion of the ordinate $\mathbf{P Q}$, and $\mathrm{Qq}$ the fluxion of the curve or right line $\Delta Q$. And consequently that, in any curve, the square of the fluxion of the curve, is equal to the sum of the squares of the fluxions of the absciss and ordinate, when these two lines are at right angles to cach other.

11. From the premises it also appears, that contemporaneous fluents, or quantities that flow or increase together, which are always in a constant ratio to each other, have their fluxions ulso in the same constant ratio at every position. For, let $\mathbf{A P}$ and $\mathbf{B Q}$ be two contemporaneous

\begin{tabular}{lllll}
\hline & $P$ & $P$ & $Q$
\end{tabular}

fluents, described in the same time by the motion of the points $\mathrm{P}$ and $\mathrm{Q}$, the contcmporaneous positions being $\mathrm{Q}, \mathrm{Q}$, and $p, q$; and let $\mathrm{AP}$ be to $\mathrm{BQ}$, or $\Lambda p$ to $\mathrm{B} q$, in the constant ratio of $n$ to 1 .

Then is - - - $A \mathrm{P}=n \times \mathrm{BQ}$, and - - - - $\mathrm{A} p=n \times \mathrm{B} q$; therefore by subtraction, - $\quad \mathbf{P} s=n \times \mathbf{Q} q$; that is, the fluxion $\mathrm{p} p$ : fluxion $\mathrm{Q} q:: n: 1$, the same as fluent $A P:$ fluent $B Q:: n: 1$; or the fluxions and fluents are in the same constant ratio.

But if the ratio of the fluents be variable, so will that of the fluxions be alsu, though not in the same variable ratio with the former, at every position.

The Nolation, \&c, in Fluxions.

12. To apply the foregoing principles to the determination of the fluxions of algebraic quantities, by means of which those of all other linds are determined, it will be necessary first to premise the notation used in this science, with some observations. As, first, that the final letters of the alphabet $z, y, x, w, \& c$, are used to denote variable or flowing quantities; and the initial letters $a, b, c, d$, \& $\mathrm{c}_{\text {, }}$ constant or invariable ones: thus, the variable base AP of the flowing rectangular figure $\mathrm{ABQP}$, at art. 6 , may be represented by $x$ : and the invariable altitude $\mathrm{PQ}$, by $a:$ also the variable basé or alsciss Ar, of the figures in art. 8 , may be represented by $x$; the variable ordinate $\mathrm{PQ}$, by $y$; and the variable curve or line $\mathrm{AQ}$, by $z$.

Secondly, that the fluxion of a quantity denoted by a single letter, is represented by the same letter with a point over it: Thus, the fluxion of $x$ is expressed by $\dot{x}$, that of $y$ by $\dot{y}$, and that of $z$ by $\dot{z}$. As to the fluxions of constant or invariable quantities, as of $a, b, c, \& \mathrm{c}$, they are equal to 0 or nothing, because they do not flow, or change their magnitude.

Thirdly, that the increments of variable or flowing quantities, are also denoted by the same letters with a small (') over them: So the increments of $x, y, z$, are $\dot{x}, y^{\prime}, z^{\prime}$.

13. From these notations, and the foregoing principles, the quantities and their fluxions, there considered, will be denoted as below.

In all the foregoing figures, put

the variable or flowing line - - $\quad-\quad \mathrm{AP}=x$, in art. 6 , the constant line $\quad-\quad-\quad-P Q=a$, in art. 8 , the variable ordinate - $\quad-P Q=y$, the variable curve or right line - $\quad-A Q=z$;

Then shall the scveral fluxions be thus represented, viz,

$\dot{x}=\mathrm{p} p$ the fluxion of the line AP,

$a \dot{x}=\mathrm{PQ} q \mathrm{p}$ the fluxion of $\mathrm{ABQP}$ in art. 6 ,

$y^{x}=\mathrm{PQrp}$ the fluxion of $\mathrm{APQ}$ in art. 8,

$\dot{z}=Q q=\sqrt{ }\left(\dot{x}^{2}+y^{2}\right)$ the fluxion of $\Delta \mathrm{Q}$,

and $a \dot{x}=\mathrm{pr}$ the flux. of the solid in art. 7 , if

$a$ denote the constant generating plane PQR.

Also $n x=\mathrm{B} Q$ in the figure to art. 11,

and $n \dot{x}=Q q$ the fluxion of the same.

14. The principles and notation being thus laid down, we may proceed to the practice and rules of this doctrine, which consists of two principal parts, called the direct and inverse method of fluxions; viz, the direct method, which consists in finding the fluxion of any proposed fluent or flowing quantity; and the iuverse method, which consists in finding the fluent of any proposed fluxion. As to the former of these two problems, it can always be determined, and that in finite algebraic terms; but the lattcr, or finding of fluents, only in some certain cases, except by menns of infinite series,-First then, of 


\section{The Direct Method of Fluxions.}

15. Th find the fluxion of the product or rectan. gle of two variable quantities; let $\Delta R Q F=x y$ be the flowing or varialble rectangle, generated by two lines $R Q$ and $P Q$ noving always perpendicular to each other, firm the positions $A \mathrm{P}$ and $\mathbf{A R}$; denoting the one by $x$, and the other by $y$; and

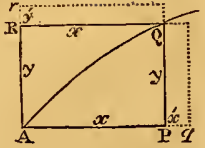
suppose $x$ and $y$ to be so reluted, that the curve $A Q$ may always pass through their intersection $Q$, or the opposite angle of the rectangle.

Now this rectangle consists of the two trilineal spaces $A P Q$, and $A R Q$, of which the fluxion of the former is $P Q \times$ $\mathbf{P} q$ or $\dot{x} y$, and that of the latter is RQ $\times \mathbf{R r}$ or $x \dot{y}$, by art. 8 ; therefore the sum of the two, $\dot{x} y+x \dot{y}$, is the fluxion of the whole rectangle $x y$ or ARQP.

The srme otherwise.-Let the sides of the rectanglc, $x$ and $y$, by flowing, becone $x+x^{\prime}$ and $y+y$ : then the product of the two, or $x y+x^{\prime} y+y y^{\prime}+x^{\prime} y^{\prime}$ will be the new or contemporaneous value of the flowing rectangle $\mathrm{PR}$ or $x y$; subtruct the one value from the other, and the remainder $x y^{\prime}+x^{\prime} y+x y^{\prime}$, will be the increment generated in the same time as $x^{\prime}$ or $y^{\prime}$; of which the last term $x^{\prime} y^{\prime}$ is nothing, or indefinitely small in respect of the other two terms, because $x^{\prime}$ and $y^{\prime}$ arc indefunitely small in respect of $x$ and $y$; which term being therefore omitted, there remains $x y+x y$ for the value of that increment: and hence, by substituting $x$ and $y$ for $x^{\prime}$ and $y^{\prime}$, to which they are proportional, there arises $x y+x y$ for the value of the fluxion of $x y$; the same às before.

17. Hence may be derived the fluxions of all powers and products, and of all other forms of algebraic quantities whatcver. And first for the continual products of any number of quantities, as $x y z$, or wxyz, or vuxyz, \&c. For $x y z$ put $q$ or $p z$, so that $p=x y$, and $x y z=p z=q$. Now, taking the fluxion of $y=p z$, by the last article, it is $\dot{q}=\dot{p} z+p \dot{z} ;$ but $p=x y$, and so $\dot{p}=\dot{x} y+x \dot{y}$ by the same article; stibstituting therefore these values of $p$ and $\phi$ instead of them, in the value of $\dot{q}$, this becomes $\dot{q}=x \dot{y} z+x \dot{y} z+x y \dot{z}$, the fluxion of $x y z$ required; which is therefore equal to the sum of the products arising from the fluxion of each letter or quantity multiplied by the product of the other two.

Again, to determine the fluxion of wxyz, the continual product of four variable quantitics; put this product, viz, wxy.x or $q w=r$, where $q=x y z$ as above; then, taking the fluxion by the last article, $\dot{r}=q \dot{w}+q \dot{w}$; and this, by substituting for $q$ and $\dot{q}$. their values as above, becomes

$\dot{r}=w x y z+w \dot{x y z}+w x y z+w x y z$, the fluxion of wxyz as required; consisting of the flusion of each quantity drawn into the products of the other three.

In the very same manner it is discovered that the fluxion of roxyz is

vivxyz + vwxyz + vwxyz + vwryz + vwxyz; and so on, for any number of quantities whatever; in which it is always found that there areas many terms as variable quantities in the proponed flucnt, and that these terms consist of the fluxion of each variable quantity multiplier by the product of all the rest of the quantities.

18. lirom hence is ansily derived the fluxion of any power of a variable quantity, as of $x^{2}$, or $x^{3}$, or $x^{4}$, \&c.
For, in the rectangle or product $x y$, if $x=y$, then is the product $x y=x x$ or $x^{2}$, and also $j$ ts fluxion $\dot{x} y+x \dot{y}=\dot{x} x+x \dot{x}$ or $2 x \dot{x}$, the fluxion $x^{2}$.

Again, if all the three $x, y, z$ be equal; then is the product of the three $x y z=x x x$ or $x^{3}$; and its fluxion $\dot{x} y z+x \dot{y} z+x y \dot{z}=\dot{x} x x+x \dot{x} x+x \dot{x} \dot{x}$ or $3 x^{2} \dot{x}$, the fluxion of $x^{3}$.

And in the same manner it will appear that the fluxion of $x^{4}$ is $=4 x^{3} \dot{x}$,

that of $x^{5}$ is $=5 x^{4} \dot{x}$,

that of $x^{n}$ is $=n x^{n-\cdot} \cdot \dot{x}$;

where $n$ is any positive whole number. That is, the flusion of any positive intcgral power, is equal to the exponent of the power $(n)$, multiplied by the next less power of the same quantity $\left(x^{n-x}\right)$, and by the fluxion of the root $(x)$.

19. Next, for the fluxion of any fractiou, as $\frac{x}{y}$, of one variable quantity divided by another ${ }^{\text {; }}$ put the proposed fraction $\frac{x}{y}=q$; then, multiplying by the denominator, $x=q y$; and taking the fiuxionis, $\dot{x}=\dot{q y}+q \dot{y}$, or $\dot{y} y=\dot{x}-q \dot{y} ;$ and, by division, $\dot{q}=\frac{\dot{x}}{y}-\frac{q \dot{y}}{y}=$ (by substituting the value of $\eta$, or $\left.\frac{x}{y}\right) \frac{\dot{x}}{y}-\frac{x \dot{y}}{y^{3}}=\frac{\dot{x} y-x \dot{y}}{y^{2}}$ the fluxion of $\frac{x}{y}$, as required. That is, the fluxion of any fraction, is equal to the fuxion of the numerator drawn jnto the denominator, minus the fluxion of the denominator drawn into the numerator, and the remainder divided by the square of the denominator.

20. Hence too is easily derived the fluxion of any negative integer power of a variable quantity, as of $x-n$ or $\frac{1}{x^{u}}$ which is the same thing. For here the numerator of the fraction is 1 , whose fluxion is nothing; and therefore, by the last article, the fluxion of such a fraction, or negative power, is barely equal to minus the fiuxion of the denominator, divided by the square of the said denominator. That is, the fluxion of $x^{-\mathrm{n}}$, or $\frac{1}{x^{\mathrm{n}}}$,

is $-\frac{n x^{n}-1}{x^{2 n}}$ or $-\frac{n \dot{x}}{x^{n}++^{1}}$ or $-n x^{-(n+1)} \dot{x}$; which is the same rule as before for integral powers.

$\mathrm{Or}$, the same thing is otherwise derived immediately from the fluxion of a rectangle or product, thus: put the proposed fraction, or quotient, $\frac{\mathrm{J}}{x^{\mathrm{n}}}=q$; then is $g \cdot x^{\mathrm{n}}=1$; and, taking the fluxions, $\dot{q} x^{n}+q n x^{n-1} \dot{x}=0$; hence $q x^{n}=-q n x^{n-1} \dot{x}$, and then dividing by $x^{n}$ we bave $q=-\frac{q n \dot{x}}{x^{x}}=$ (by substituting $\frac{1}{x^{u}}$ for $q$ ), $\frac{-n \dot{x}}{x^{\mathrm{u}}+1}$ or $-n x^{-n-1} \dot{x}$; the same as beforc.

21. Much in the same manner is obfained the fluxion of any surd, or fractional power of a fuent quantity, as of $x^{\frac{m}{n}}$ or $\sqrt[n]{x^{m}}$. For, putting the proposed quantity $x^{\frac{m}{11}}=q$, then, raising cach to the $n$ powcr, - - $x^{\mathrm{m}}=q^{\mathrm{n}}$; take the fluxions, - - - - $m x^{\mathrm{m}^{-x}} \dot{x}=n q^{\mathrm{n}-1} \dot{q}$; divide by $n q^{\mathrm{n}-1}, \dot{q}=\frac{m x^{\mathrm{m}-1} x}{n q^{n-1}}=\frac{m r^{\mathrm{m}}-{ }^{\prime} \dot{x}}{m x^{m-\frac{m}{n}}}=\frac{m}{n} x^{\frac{m}{u}-}{ }^{\prime} \dot{x}$ : which is still the same rule as before, for finding the fluxion of any power of a fluent quantity, and which is therefore general, whether the exponent be positive or negalive, integral or fractional. 
22. For the Fluxions of Logarithms: Let $A$ be the principal vertex of an hyperbola, having its asymptotes $\mathrm{CD}, \mathrm{CP}$, with the ordinates $\mathrm{DA}, \mathrm{BA}, \mathrm{PQ}$, \& $\mathrm{c}$, parallel to them. Then, from the nature of

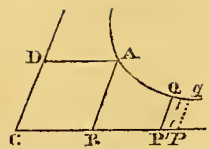
the hyperbola, and of logarithms, it is known that any space $A B P Q$ is the log. of the ratin of $C B$ to $C P$, to the modulus $\mathrm{ABCD}$. Now put $\mathrm{CB}=\mathrm{EA}=1$ the side of the square or rhombus $\mathrm{DB} ; m=$ the modnlus, or area of $\mathrm{DB}$, or sine of the angle $c$ to the ralius 1 ; also the abseiss $\mathrm{CP}=x$, and the ordinate $\mathrm{PQ}=y$. Then, by the mature of the hyperbola, $C P \times P Q$ is always equal to $\mathrm{DB}$, that is, $x y=m$; hence $y=\frac{m}{x}$, and the fluxion of the space, or $\dot{x} y$ is $\frac{m \dot{x}}{x}=\mathrm{PQqp}$ the fluxion of the $\log$. of $x$, to the modulus $m$. And in the ordinary hyp. logarithms the modulus $m$ being 1 , therefore $\frac{\dot{x}}{x}$ is the fluxion of the hyp. log. of $x$; which is therefore equal to the fluxion of the quantity, divided by the quantity itself. And the same might be shown in several other ways, independent of the figure of the hyperbola.

23. By meass of the fluxions of logarithms, are determined those of exponential quantities, i. e. quantities which have their exponent also a flowing or variable quantity. These exponentials are of two kinds, viz, when the root is a constant quantity, as $e^{\mathrm{x}}$; and when the root is variable, as $y^{\text {x }}$.

In the former case, put the proposed exponential $e^{x}=z$, a single variable quantity; then take the logarithm of each, so shall $\log$. of $z=x \cdot \log$. of $e$; take the fluxions of these, so shall $\frac{\dot{z}}{z}=\dot{x} \cdot \log$. of $e$; hence $\dot{z}=2 \dot{x} . \log$. of $e=e^{x} \dot{x}$. lng. of $e$, the fluxion of the proposed exponential $e^{x}$; and which therefore is equal to the said proposed quantity, drawn into the fluxion of the exponent, and also into the log. of the root.

24. And in the 2d case, put the exponential $y^{x}=z$; then the logarithms give log. $z=x \times \log . y$, and the fluxions give $\frac{\dot{z}}{z}=\dot{x} \cdot \log . y+x \cdot \frac{\dot{y}}{y}$; hence $\dot{z}=z \dot{x} \cdot \log$. $y+\frac{z x \dot{y}}{y}=$ (by substituting $y^{\mathrm{x}}$ for $z$ ) $y^{\mathrm{x}} \dot{x} \cdot \log \cdot y+x \dot{y}^{\mathrm{x}-\mathrm{x} \dot{y}}$, is the fluxion of the proposed exponential $y^{\mathrm{x}}$; which therefore consists of two terms, of which the one is the fluxion of the proposed quantity considering the exponent only as constant, and the other is the fluxion of the same quantity considering the root as constant.

Of Second, Third, \&c. Fluxions.-Having explained the manner of considering and determining the firstfluxions of flowing or variable quantities; it remains now to consider those of the higher orders, as $2 \mathrm{~d}, 3 \mathrm{~d}, 4$ th, $\& \mathrm{c}$, fluxions.

25 . If the rate or celerity with which any flowing quantity changes its magnitude, be constant, or the same, at revery position; then is the fluxion of it also constantly the same. But if the variation of magnitude be continually chanuing, either increasing or decreasing; then will there be a certain deyree of fluxion peculiar to every point or prsition; and the rate of variation or change in the fluxion, is called the fluxion of the fluxion, or the second fluxion of the given fluent quantity. In like manner, the variation or fluxion of this $2 \mathrm{~d}$ fluxion is called the third fuxion of the first proposed fluent quantity; and so on. And these orders of fluxions are denoted by the fluent let-

VOL. I. ter or quantity, with the corresponding number of points over it; viz, 2 points for the $2 d$ lluxion, 3 for the $3 \mathrm{~d}$ fluxion, 4 for the 4 th tluxion, and so on. So the ditferent orders of the Huxions of $x$, are $\ddot{x}, \ddot{x}, \ddot{x}, \& \mathrm{c}$; where each is the fluxion of the one next before it.

26. This description of the higher orders of fluxions may be illustrated by the three figures at the Sth article; where, if $x$ denote the absciss $A P$, and $y$ the ordinate $P Q$; and if the ordinate PQ or $y$ flow along the abseiss $A P$ or $x$, with an uniform motion; then the flusion of $x, \mathrm{viz}, x=\mathrm{p} p$ or $a r$ is a constant quantity, or $\ddot{x}=0$, in all the figures. Also, in fig. 1 , in which $\Delta \mathbb{Q}$ is a right line, $\dot{y}$ is $=r q$, or the fluxion of $\mathrm{PQ}$, is a constant quantity, or $\ddot{y}=0$; for, the angie $Q,=$ the angle $A$, being constant, $Q r$ is to $r q$, or $x$ to $y$, in a constant ratio. But in the $2 \mathrm{~d}$ figure, $r q$, or the fluxion of $\mathrm{PQ}$, continually increases more and more; and in fig. 3 it continually decreases more and more; and therefore in both these cases $y$ has a $2 \mathrm{~d}$ fluxion, being positive in fig. 2 , but negative in fig. 3 : and so on for the other orders of fluxions.

27. Thus if, for instance, the nature of the curve be such, that $x^{3}$ is everywhere equal to $a^{2} y$; then, taking the fluxions, it is $n^{2} \dot{y}=3 x^{2} \dot{x}$; and, considering $\dot{x}$ as a constant quantity, and taking the fluxions again, the equations of the several orders of fluxions will be as below; viz,

the 1 st fluxions $a^{2} \dot{y}=3 x^{2} \dot{x}$,

the $2 \mathrm{~d}$ fluxions $a^{2} \ddot{y}=6 x \vec{x}^{2}$,

the 3d fluxions $a^{2} \ddot{y}=6 \dot{x}^{3}$,

the 4th fluxions $a^{2} \ddot{y}=0$,

and all the higher fluxions $=0$ or nothing.

Also the ligher orders of fluxions are found in the same manner as the lower ones. Thus,

The 1st flux. of $y^{3}$ is - - $3 y^{2} y$;

its $2 \mathrm{~d}$ flux. or flux, of $3 y^{2}, 7$

considered as the rect- $\} 3 y^{2} y^{\prime \prime}+6 y y^{2}$;

angle of $3 y^{2}$ and $\dot{y}$, is - $\}$

$\left.\begin{array}{l}\text { and the flux. of this again, or } \\ \text { the } 3 \mathrm{~d} \text { fluxion of } y^{3}, \text { is }\end{array}\right\} 3 y^{2} \dot{y}+18 y \dot{y} \ddot{y}+6 \dot{y}^{3}$.

28. In the foregoing articles, it has been supposed that the fluents inerease; or that their fluxions are positive; but it often liappens that some fluents decrease, and that therefore their fluxions are negative: and whenever this is the case, the sign of the fluxion must be changed, or made contrary to that of the fluent. So, of the rectangle $x y$, when both $x$ and $y$ increase together, the fluxion is $x y+x y$ : but if one of them, as $y$, decrease, while the other, $x$, increases; then the fluxion of $y$ being $-\dot{y}$, the fluxion of $x y$ will in that case be $x y-x y$. 'T'his may be illustrated by the annexce rectangle $\mathrm{APQR}=x y$, supposed to be generated by the motion of the line $\mathrm{PQ}$ from $\mathrm{A}$ towards $\mathrm{C}$, and by the motion of the line $\mathrm{R} Q$ from $\mathrm{B}$ towards $\mathrm{A}$ : for, by the motion of $P Q$, from $A$ towards $\mathrm{c}$, the rectangle is increased, and its fluxion is $+\dot{x} y$; but by the motion of $R Q$, from $B$ towards $A$, the

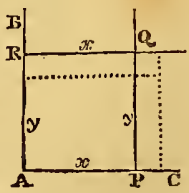
rectangle is decreased, and the fluxion of the decrease is $x \dot{y}$; therefore, taking the fluxion of the decrease from that of the increase, the fluxion of the rectangle $x y$, when $x$ increases and $y$ decreases, is $x y-x \dot{y}$.

For the Inverse Method, or the finding of fluents, sce FLUENT. And for the several applications of this science to Maxima and Minima, the drawing of TANGints, $\& c$, see the respective articles. 
Since the frrst incention of this calculus by Sir 1 . Newton, it bas reccived various improvements from many eminent authors, particularly John Bernoutli, who treated of the fluents belonging to the fluxions of exponential expressions; James Bernoulli, Craig, Cheyne, Cotes, Manfredi, Riccati, Tayior, Fagnanus, Clairaut, Dalembert, Euler, Maclaurin, Condorcet, Leseur and Jacquier, Bonganville, Walmesley, Lagrange, Emerson, Simpson, Landen, Waring, \&c. There are several other treaties also on the principles of Auxions, by Hayes, Newyentyt, L'Hôpital, Hodson, Rowe, Lyons, Dealtry, \&c, \&c, delivering the elements ol this science in an easy and familiar manner.

FLY, in Mechanics, a heavy weight applied to certain inachines, to regulate their motions, as in a jack; or to increase thir effect, as in the coining engine, \&c; by means of which the furce of the power is not only preserved, but equally distributed throughout the revolution.

The fly is cither like a cross with heavy weights at the ends of its arms; or like a heavy wheel at right-angles to the axis of motion. It may be applied to various kinds of engines, whether they be moved by men, borses, wind, or water; and it is of great use in those parts of an engine which have a quick circular motion, and where the power or resistance acts unequally in the different parts of a revolution. In this case the fly becomes a moderator, making the motion of revolution almost everywhere equal.

FLYERS, in Architecture, such stairs as go straght, and do not wind round, nor have the steps made tapering, but equally broad at both ends. Hence, if one flight do not rise to the top of the story, \&c, there is a bruact half pace, and then commonly another set of flyers.

FLYING, the progressive motion of a birl, or other winged animal, through the air. The parts of birds chiefly concerned in flyng, are the wings and tail : by the former, the bird sustains and wafts limself along; and by the lat ter be is assisted in ascending and descemling, to keep his body poised upright and steady. The wings are extended or stretched quite ont, and then struck forcibly downivards against the air, which by its resistance raises the bird upwards; then, to make another stroke, the wing, by means of its joints, readily closes in some degree, presenting the sharp edge of the pinion foremost to cut the air, and drawing the collapsed feathers after it like a flag, to diminish the resistance to the ascent as much as possible; the wing and feathers are then stretched out horizontally again, and another downward stroke is given, which raises the bird still higher; and so on as far as he pleases, or at least as far as the density of the air will sustain him; performing those motions of the wings very rapidly, that the flight may be the quicker.

Artificial Fin ing, is that att mpted by men, \&c, by the assistance of mechanics. The art of fying has been attempted by several persons in all ages. Firiar Bacon, about 500 years ago, not only asserts the possibility of Ayiug, but affirms that be himself knew how to make a machine with which a man might be able to convey bimself through the air tite a bird; and furtber adds, that it had been tried with success. Though the fict is to be doubt'd, if, at it was said, it consisted in the folluwing method; viz, in a couple of large thin hollow copper globes, exhausted of air ; which, being much lighter than air, would sustain a chair on which a person might sit. Jather lirancisco Lana, in lis Prodromo, proposes the samet thing, us his uwn iele'il. He computes, that a round vessel of plate-brass, 14 fect in diameter, weighing 3 ounces the square foot, will only wcigh 1848 ounces; whereas a quantity of air of the same bulk will weigh- near 2156 ounces; so that the globe will not only he sustained in the air, but will carry with it a wcight of 304 ounces; and by increasing the size of the glube, the thickness of the metal remaining the same, be adds, a vessel might be made to carry a much greater weight. But the fillacy is obrious: a globe of the dimensions he describes, as I)r. Hooke observes, would not sustain the pressure of the air, but be crushed inwards. Indeed it is not even probable that a globe can be made suficiently thin to float in the atmosphere after it is exhausted of air, and yet be strong enough to sustain the compressing force of the atmosphere. But for this purpose it seems that the globe should be filled with an air as clastic or strong as the atmosphere, and yet be rery much lighter; such as is used in the mongolfers and balloons; the former of which is filled with common air heated, so as to be more elastic, and l'ss heavy; and the latter with inflammahle air, which is as elastic as the common air, with only about one-tenth of its weight. And thus the idea of flying, or rather floating, in the air, bas been realized by the noderns, using bowever a dificrent kind of air. Sce Aerostation.

The same author describes a machine for flying, invented by the Sieur Besnier, a smith of Sable, in the county of Miain. See Philos. Collec. No. 1.

By the foreguing method however, at best, only a method of floating can be obtained, like is log floating in a current; but not of flying, which consists in moving through the air, independent of any current ; and which must be effected by something in the nature of wings. Attempts of this latter kind also have indeed been made by several persons; but it does not appear that any of them have been attended with such success is to induce the authors of those attempts to make them public. The philosophers in the reign of King Charles the $2 d$ were much employed on this subject; and the celebrated Bishup Wilkins ras so confident of success in it, that he says, he does not qnestion but in future age's it will be as usual to bear a man call for his wings, when lie is going a journey, as it is now to call for his boots.

The story of the flight of l)ædalus is well known.

Fureng Pinion, is part of a cluch hasing a tly or fitn with which to gather the air, ami so check the rapidity of the clock's motion, when the weight descends in the striking part.

FOCAL Distunce, the distance of the focus, which is sometimes understood as its distince from the vertex, as in the parabola; and sometimes its distance from the centre, as in the cltipse or hyperbola.

FOCUS, in Geometry and the Conic Sections, is ap. plicd to certain points in the ellipse, hyperbolia, and parabota, where the rays reflected from all parts of thesc curves concur or meet; that is, rilys issuing from a luminous point in the one focus, and falling on all $p$ ints of the curves, are rethe cted into the other focus, or mo the line directed to the other foecus, viz, into the other focus in the dlipse and parabola, and directly from it in the byperbula: which is the reason of the name locus, or burning-point. Hence, as the one focus of the parabola is at an intinite distance; and conscquently alt rays drawn from it, to any finite part of the curve about the vertes, are paraltel to one another; therefore, if rays from the sun, or any other object so distant that the rays may be considered as parallel, fall upon the curve of a parabolit 
or concave surface of a paraboloidal figure, those rays will all be reflected into its focus.

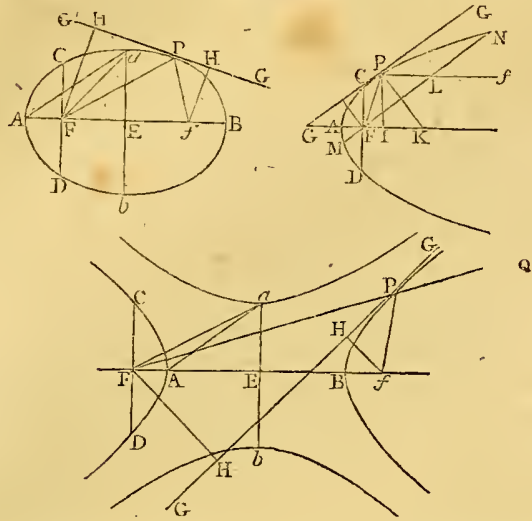

Thus, the rays of $f$, from the focus $f$, are reflected in the direction PF, into the other focus $\mathrm{F}$, in the ellipse and parabola, and form the focus $f$ into $\mathrm{PQ}$, in the hyperbola. -In all the three curves, the double ordinate $c D$ drawn through the focus $F$, is the parameter of the axis, or a $3 d$ proportional to $\mathrm{AB}$ and $a b$, the transverse and conjugate axes.- In the ellipse and parabola, the transverse axis is equal to the sum of the two lines $\mathbf{P F}+\mathrm{p} f$, drawn from the foci to any point $F$ in the curve; but in the hyperbola, the transverse is equal to the difference of those two lines. That is,

$\mathbf{A B}=\mathrm{PF}+\mathrm{P} f$ in the ellipse and parabola,

$\mathrm{AB}=\mathrm{PF}-\mathrm{P} f$ in the hyperbola.

In the ellipse and parabola, the square of the distance between the foci, is equal to the difference of the squares of the $t$ wo axes; and in the hyperbola, it is equal to the sum of their squares: that is

$\mathrm{F} f^{2}=\mathrm{AB}^{2}-a b^{2}$ in the ellipse and parabola.

$\mathbf{r} f^{2}=A \mathrm{~B}^{2}+a b^{2}$ in the hyperbola.

'Therefure the two semi-axes, with the distance of the focus from the centre, form always a rightit-angled triangle $\mathrm{F} A \mathrm{E}$, or $\mathrm{A} a \mathrm{E}$.

In all the curves, the conjugate semi-axis is a mean proportional between the distances of either focus from either end of the transverse axis : that is,

$$
\text { ÁF : } \mathbf{E} /:: \mathrm{E} a: F \mathrm{~B} \text {, }
$$

or $\mathrm{E} a^{2}=\mathrm{A} \mathbf{F}$. $\mathrm{FB}$.

If there be any tangent to these curves, and two lines dratis from the foci to the point of contact; these two lines will make equal angles with that tangent.

So, if $G P G$ touch the curve at $P$;

then is the angle $\mathrm{FPG}=\angle f \mathrm{PG}$.

If a line be drawn from either focus, perpendicularly upon a tangent; the distance of their intersection from the centre will be equal to the semi-transverse axis. So, if Fil or $f H$ be perpendicular to the tangent $\mathrm{PH}$; then is $\mathrm{E}_{\mathbf{H}}=\mathrm{E}_{\mathrm{A}}$ or $\mathrm{E}_{\mathrm{B}}$. Consequently, the circle described on the diameter AE, will pass through all the points $\mathrm{n}$, $\mathrm{II}$.

'The foregoing are the principal properties that are common to the foci of all the three conic sections. 'To which may be added the fullowing properties which are peculiar to the parabola : viz, In the parabula, the distance from $t$ e focus to the vertex, is equat to $\frac{t}{4}$ of the parameter, or half the ordinate at the focus: viz, $\mathrm{AF}=\frac{\mathrm{r}}{2} \mathrm{FC}$. Also, a line drawn from the focus to any point in the curve, is equal to the sum of the focal distance from the vertex and the absciss of the ordinate to that point : i. e. FP $=A F+$ $\mathrm{AI}=2 \mathrm{AF} \pm \mathrm{II}=\mathrm{CF} \pm \mathrm{FI}$. If from any point of a parabola there be drawn a tangent, and a perpendicular to it $\mathrm{PK}$, both to meet the axis produced; then the focus will be equally distant from the two intersections and the point of contact : i. e. $\mathrm{FG}=\mathrm{FP}=\mathrm{FK}$. Also the $\angle \mathrm{KPF}=\mathrm{KP} f$.

Hence also the subnormal $\mathrm{IK}$ is $=2 \mathrm{AF}=\mathrm{FC}$ the semiparameter.-The line drawn from the focus to any point of the curve, is equal to $\frac{\pi}{4}$ the parameter of the diameter to that point: i.e. FP $=\frac{1}{4}$ the parameter of the diameter $\mathrm{P} f$ - If an ordinate to any diameter pass through the focus, it will be equal to half its parameter; and its absciss equal to $\frac{1}{4}$ of the same parameter; or the absciss equal to half the ordinate: i.e. $\mathrm{PL}=\frac{\mathrm{T}}{4} \mathrm{MN}=\frac{\mathrm{T}}{2} \mathrm{LM}$ or $\frac{\mathrm{x}}{2} \mathrm{LN}$.

Focus, in Optics, is a point in which several rays meet, and are collected, after being either reflecred or refracted. It is so called, because the rays, being here brought together and united, their force and effect are increased, insomuch as to be able to burn; and therefore it is that bodies are placed in this point to be burnt, or to show the effect of burning-glasses, or mirrors.-It is to be observed, however, that in practice, the focus is not an absolute point, but a space of some small breadth, over which the rays are scattered; owing to the different nature and refrangibility of the rays of light, and to the imperfections in the figure of the lens, \&c. However, the smaller this space is, the better, or the nearer to perfection the instrument approaches. Huygens shows that the focus of a lens convex on both sides, has its breadth equal to $\frac{5}{8}$ of the thickness of the lens.

Virtual Focus, or Point of Divergence, so called by Mr. Molyneux, is the point from which rays tend, after refraction or reflection; being in this respect opposed to the ordinary focus, or point of concurrence, where rays are nmade to meet after refraction or reflection. Thus, the foci of an hyperbola are mutually virtual foci to each other; but, in an ellipse, they are common foci to each other; for the rays are refleeted from the other focus in the hyperbola, Lut towards it in the ellipse; as appears by the figures at the beginning of this article.

In Dioptrics, let $\triangle \mathbf{B C}$ be the concavity of a glass, whose centre is $D$, and axis $\mathrm{DE}$ : Let $\mathrm{FG}$ be a ray of light falling on the

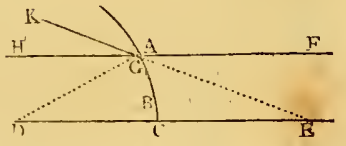
glass, parallel to the axis $D E$; this ray $F G$, after it has passed through the glass, at its emersion at $G$ will not proceed directly to $n$, but be refracted from the perpendicular $\mathrm{DG}$, and will become the ray $\mathrm{G} K$, which being produced to meet the axis in $\mathrm{E}$, this point $\mathrm{E}$ is the virtual focus, as the ray is refracted directly from this point.

\section{Rules for the Foes of Lenses and Mirrors.}

I. In Catopirics, or Lenses.

1. The focus of a convex glass, i.e. the point where parallel rays unite when transmitted through a convex glass whose surface is the segment of a sphere, is distant from the pole or vertex of the glass, nearly a cliameter and laalf of the convexity.-2. In a plano-convex glass, the focus of parallel rays is distant from the pole of the glass a diamctcr of the conrexity, if the sagment do not 
exceed 30 degrees. Or the rule in plano-convex glasses is, As $107: 193:$ : so is the radius of convexity : to the refracted ray taken to its concourse with the axis; which in glasses of large spheres is nearly equal to the distance of the focus taken in the axis.- 3 . In double-convex glasses of the same sphere, the focus is distant from the pole of the glass about the radius of the convexity, if the segment be but 30 degrees. But when the two convexities arc unequal, or segments of different spheres, then the rule is, As the sum of the radii of both convexities : is to the radius of either convexity alone : : so is double the radius of the other convexity : to the distance of the focus. But bere it un ust be observed, that the rays which fall nearer the axis of any glass, are not unitcd with it so near the pole of the glass as throse farther off : nor will the fucal distance be so great in a plano-convex glass, when the convex side is towards the object, as when the plane side is towards it. And hence it is truly concluded, that, in viewing any object by a plano-convex glass, the convex side should always be turned outwards; as also in burning by such a glass.

\section{For the Virtual Focus.}

1. In concave glasses, when a ray passes through air parallel to the axis, the virtual focus, by its first refraction, becomes at the distance of a diameter and a half of the concavity.-2. In plano-concave glasses; when the rays fall parallel to the axis, the virtual focus is distant from the glass, the diameter of the concavity.-3. In planoconcave glasses, as $107: 193:$ : so is the radius of the concavity : to the distance of the virtual focus.4. In double concaves of the same sphere, the virtual focus of parallel rays is at the distance of the radius of the concavity. But, whether the concavities be equal or unequal, the virtual focus, or point of dirergency of the parallel rays, is determined by this rule; As the sum of the radii of both concavities : is to the radius of either concavity : : so is double the radius of the other concavity : to the distance of the virtual focus.-5. In concave glasses, exposed to converging rays, if the point to which the incident ray converges, be farther distant from the glass than the virtual focus of parallel rays, the rule for finding the virtual focus of this ray, is this; As the difference between the distance of this point from the glass, and the distance of the virtual focus from the glass: is to the distance of the virtual focus : : so is the distance of this point of convergence from the glass : to the distance of the virtual focus of this converging ray.-6. In concave glasses, if the point to which the incident ray converges, be nearer to the glass than the virtual focus of parallel rays, the rulc to find where it crosses the axis, is this; As the excess of the virtual focus, above this point of convergency : is to the virtual focus : : so is the distance of this point of convergency from the glass : to the distance of the point where this ray crosscs the axis.

III. Practical Rules for finding the Foci of Glasses.

1. To find, by experiment, the focus of a convex spberical glass, being of a small sphere. Apply it to the end of a scale of inclies and decimal parts, and cxpose it to the sun; then on the scale may be seen the bright intersection of the rays measured out : or, fix it in the hole of a dark chamber; and where a white paper receives the distinct representation of clistant objects, there is the focus of the glass. - 2. For a glass of a pretty long focus, observe some distant object through it, and recede from the glass till thre eye perceives all in confusion, or the object begins to appear inverted; then the eye is in the focus.-
3. For a plano-convex glass: make it reflect the sun against the wall; then on the wall will be seen two sorts of light, a brighter within another more obscure: withdraw the glass from the wall, till the bright image be in its least dimensions ; then is the glass distant from the wall about a fourth part of its focal length. -4 . For a double convex : expose each side to the sun in like manner; and obscrve both the distances of the glass from the wall: then is the first distance abont half the radius of the convexity turned from the sun; and the second is about balf the radius of the other convexity. The radii of the two convexities being thus known, the focus is then found by this rule; As the sum of the radii of both convexities : is to the radius of either convexity $::$ so is double the radius of the other convexity : to the distance of the focus.

IV. To find the Foci of all Glasses Geometrically.

Dr. Halley has given a general method for finding the foci of spherical glasses of all kinds, both concave and convex; exposed to any kind of rays, cither parallel, converging, or diverging; as follows: To find the focus of any parcel of rays diverging from, or converging to, a given point in the axis of a spherical lens, and making the same angle with it; the ratio of the sines of refraction being given.

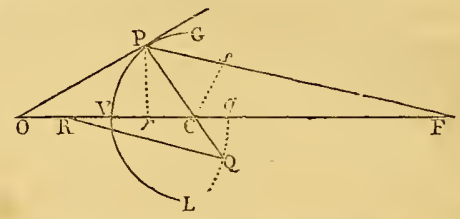

Suppose $\mathrm{GL}$ a lens; $\mathrm{P}$ a point in its surface; $\mathrm{V}$ its pole; $c$ the centre of the spherical segment; o the object, or point in the axis, to or from which the rays proceed; and OP a given ray: and suppose the ratio of refraction to be as $r$ to s. Then making $\mathrm{CR}$ to co, as $s$ to $r$ for the immersion of a ray, or as $r$ to $s$ for the emersion (i.e. as the sines of the angles in the medium which the ray enters, to the corresponding sines in the medium out of which it comes); and laying $\mathrm{cr}$ from $\mathrm{c}$ towards $\mathrm{o}$, the point $2 \mathrm{w}$ will be the same for all the rays of the point o. Lastly, drawing the radius $\mathrm{PC}$, continued if necessary; with the centre $\mathrm{R}$, and distance $\mathrm{OP}$, lescribe an arc intersecting $\mathbf{P C}$ in $\mathrm{Q}$. Then the line $Q R$, being drawn, it will be parallel to the reflected ray; and $\mathrm{PF}$, being drawn parallel to it, will intersect the axis in the point $\mathrm{F}$, the focus sought.-Or, say as $C Q: C P:: C R: C F$, which will be the distance of the focus from the centre of the sphere.-And from this general construction, he adverts to a number of particular simple cases.-Dr. Ilalley gave also a universal algebraical theorem to find the focus of all sorts of optic glasses, or lenses. See also the Philos. Trans. No. 205, or my Abr. vol. 3 , pa. 593.

\section{In Catopirics, or Foci by Refection.}

These are easily found for any known curve, from this principle, that the angle of incidence is always cqual to the angle of reflection. The same are also easily found by experiment, bring exposed to any object.

The increase of heat from collecting the sun's rays into a focus, has been found in many cases of burning-glasses, to be astonishingly great; the eftect being increased as the square of the diameter of the glass exceeds that of the focus. If, for instance, there be a burning-glass of 12 
inches diameter; this will collect or crowd together all the rays of the sun which fall upon the glass into the compass of about $\frac{\pi}{8}$ part of an inch : then, the areas of the two spaces being as the square of 12 to the square of $\frac{r}{8}$, or as the square of 96 to the square of 1 , that is, as 9216 to 1 ; it follows, that the heat in the focus will be 9216 times greater than the sun's common heat. And this will have an effect as great as the direct rays of the sun would have on a body placed at the 96 th part of the earth's distance from the sun; or the same as on a planet that should move round the sun at but a very little more than a diaineter of the sun's distance from him, or that would never appear farther from him than about 36 minutes.

Besides Dr. Halley's method for finding the foci, several other authors have written upon this subject; as Mr. Ditton, in his Fluxions; Dr. Gregory, in his Elements of Dioptrics; M. Carré, and Guisnée, in the Menoires de I'Acad.; Dr. Barrow and Sir I. Newton have also neat and elegant ways of fiuding geometrically the foci of spherical glasses; which may be seen in Barrow's Optical Lectures.

FOLIATE, a name given by some to a curve of the $2 \mathrm{~d}$ order, expressed by the equation $x^{3}+y^{3}=a x y$, being one species of defective hyperbolas, with one asymptote, and consisting of two infinite legs crossing each other, forming a kind of leaf. It is the $42 \mathrm{~d}$ species of Newton's Lines of the $3 \mathrm{~d}$ order.

FOLKLS (MARTIN), an English mathematician, philosopher, and antiquary, was born at Westminster, about 1690; and was greatly distinguished as a nember of the Royal Society in London, and of the Academy of Sciences at Paris. He was admitted into the former at 24 years of age; made one of their council two years after; named vice-president by Sir Isaac Newton himself; and, after Sir Hans Sloane, became president. Coins, ancient and modern, were a great object with him ; and his last production was a book upon the English Silver Coin, from the Conquest to his own times. He died at London in 1754 . Dr. Birch had drawn up materials for a life of Mr. Folkes, which are preserved at large in the Anecdotes of Bowyer, pa. 562. There are nuany memoirs of Mr. Folkes's in the Philos. Trans, from vol. 30 to vol. 46 , both inclusive; viz, 1. Account of an Aurora Borealis, vol. 30.-2. Of Licuwenhoek's curious Microscope, vol. 32.-3. On the Standard Measures in the Capitol at Rome, vol. 39.-4. Observations of three Mock-suns, vol. 40.-5. On the lieshwater Polypus, vol. 42.-6. On human bones petrified, vol. 43.-7. On a passage in Pliny's Natural History, vol. 44. - 8. On an Earthquake at London, vol, 46.-9. Ditto at Kensington, vol. 46.-10. Ditto at Newton, vol. 46 .

FOMAHAUT, or FonaLHAUT, a star of the first magnitude in the water of the constellation Aquarius, or in the mouth of the southern fish. Its latitude is $21^{\circ} 6^{\prime} 28^{\prime \prime}$ south, and mean longitude to the beginning of 1760 , $11^{5} 0^{\circ} 28^{\prime} 55^{\prime \prime}$.

FONTENELLE (BERNARD DE), a celebrated French author, was born at Roven in 1657 , and died in 1756 , having nearly attained his 100 th year. He was a universal genius: at a very early age he wrote several comedies and tragedies of considerable merit; and he did the same at a very advanced age. Voltaire declares him the most universal genius the age of Lewis the 14th produced; and compares him to lands situated in so happy a climate, as to produce all sorts of fruits. His last comedies, though they showed the elegance of Fontenclle, were, however, little fitted for the stage; he then also produced an Apology for the Vortices of Descartes; on which Voltaire says, "We must excuse his comedies, on account of his great age; and his Cartesian opinions, as they were those of his youth, when they were universally received all over Europe."

In his poetical performances and Dialogues of the $\bar{D}$ ead, the spirit of Voiture was discerned, though more extended and more philosophical. His Plurality of Worlds is a work singular in its kind: his design in this was, to present that part of philosophy to view in a gay and pleasing dress.

Fontenelle applied bimself also to mathematics and natural philosophy; in which he proved not less successful than he had been in polite literature. Having been appointed perpetual secretary to the Academy of Sciences, he discharged that trust above 40 years with universal applause: his History of the Academy often throws great light on their Memoirs, which are sometimes obscure; and it has been said, he was the first who introduced clegance into the sciences. The Eloges, which he pronnunced on the deceased inembers of the Academy, have this peculiar merit, that they excite a respect for the sciences as well as for the authors.

On the whole, Fontenelle must be considered as the great master of the new art of treating abstract sciences, in a manner that make their study at once easy and agreeable: nor are any of his works of other kinds void of nerit. All those talents which he possessed from nature, were assisted by a good knowledge of history and languages: and he perhaps surpasses all men of learning who have not had the gift of invention.

Besides lis poetical and theatrical works, with those of belles-lettres, \&c, he published Elemens de Geometrie de l'Infini, in 4to, 1727; also the Theorie des Tourbillons Cartesiens; and Discours Moraux et Philosophiques. All his different works were collected in eleven volumes $12 \mathrm{mo}$, under the title of CEuves Diverses.

FOOT, a measure of length, divided into 12 inches, and each inch supposed to contain 3 barley-corns in length. Geometricians divide the foot into 10 digits, and the digit into 10 lines, \&.c.

It seems this measure has been taken from the length of the human foot; but it is of different lengths in different countries. The Paris rogal foot is to the English foot, as 4263 to 4000 , or nearly 16 to 15 , and exceeds the English by $9 \frac{1}{2}$ lines. The ancient Roman foot of the Capitol consisted of 4 palms; equal to 11 inches and 7 . English; the Rhinland, or Leyden foot, used by the northern nations, is to the Roman foot, as 19 to 20 . For the proportions of the foot of severul nations, compared with the English, see the article Measure.

Square Foox, is a square whose side is 1 foot, or 12 inches, and consequently its area is 144 square inches.

Cubic Foot, is a cube whuse side is one foot, or 12 inches, and consequently it contains $12^{3}$ or 1728 cubic inches.

Foot-bank, or Foot-stcp, in Fortifi. See BANQU ETt E.

FORCE, Vis, or Power, in Mechanics, Philosoplyy, \&c, denotes the cause of the change in the state of a body, with respect to motion, rest, pressure, \&c; as well as its endeavour to oppose or resist any such change. Thus, whenever a body, which was at rest, begins to move; or when its motion is either not uniform, or not direct; the cause of this change in the state of the budy, is what is called Force, and is an external cause. Or, while a body remains in the same state, either of rest, or of uniform and rectilinear motion, the cause of its remaining in such state, 


\section{F O R}

is in the nature of the body, being an innate internal force, and is called its inertia.

Mechanical forces may be reduced to two sorts; one of a body at rest, the other of a body in motion.

The force, $\& c$, of a body at rest, is that which we conceive to be in it when lying on a plane, or hanging by a repe, or supported by a spring, \&cc; and this is called Pressure, Tension, Force, or Vis Mortua, Solicitatio, Conatus Movendi, Conamen, \&c ; which kind of force may be always measured by a weight, viz, the weight that susıains it. To this class of forces may also be referred cenrripetal and centrifugal forces, though they reside in a body ill motion; because these forces are homogeneous to weights, pressures, or tensions of any kind. The pressure, or force of gravity in any body, is proportional to the quantity of matter containcel in it.

The torce of a body in motion, is a power residing in that body, so long as itcontinues its motion; by means of which, it is able to remove those obstacles which are opposed to it ; or to lessen, destroy, or ovcrcome the force of any other moving body, which meets it in an opposite direction; or to surmount any the largest dead pressure or resistance, as tension, gravity, friction, \&c, for some time; but which will be lessened or destroyed by such resistance as lessens or destroys the motion of the body. This is called Vis Motrix, Moving Force, or Motive Force, and by some late writers $V$ is Viva, to distinguish it from the $V$ is Mortua before mentioned; and by these appellations, however different, the same thing is understood by all mathematicians; namely, that power which is inherent in a moving body, and which accompanies it in whole, or in part, as long as the body is in motion; so as to displace, or to withstand opposite moving forces, or by which it overcomes any dead resistance, \& $\&$, which force is otherwise called Percussion, or Percussive Force, or Momentum.

But concerning the measure of this kind of force, mathematicians have been divided into two parties. It is allowed on both sides, that the measure of this force depends partly on the mass of matter in the body, or its weight, and partly on the velocity of its motion; so that on any increase of either weight or velocity, the moving force bccomes greater. It is also agreed, that the velocity being given, or being the same in two moving bodies, their forces are in proportion to their masses or weights. But, when two bodies are rqual, and the velocities with which they move are different, they no longer agree about the measure of the moving force.

The Cartesians and Newtonians maintain, that, in this case, the moving force is in proportion simply as the relocity with which a body moves; so that with a double velocity it has a double force, \&c: But the l,eibnitians assert, that the moving force is proportional to the square of the velocity; so that, with a double velocity a quadruple force will be produced, \&c. Or, when the bodies are different, the former bold, that the momentum or moving force of bodies, is in the compound ratio of their weights and velocities: while the latter maintain, that it is in the compound ratio of the weights and squares of the velocitics.

Though Ieibnitz was the first who expressly asserted, that the force of a body in motion is as the square of its velocity, which was in a paper inserted in the Leipsic Acts for the year 1686 , yet it is thought that Iluygens led bim into that notion, by some demonstrations in the 4 th part of his book De IIorologio Oscillatorio, relating to the centre of oscillation, and by his dissertations, in answer to the objections of the abbot Catalan, one of which w ws published in 1684 . This eminent mathematician had o'emonstrated, that in the collision of two bodies that are perfectly elastic, the sum of the products of each body multiplied by the square of its velocity, was the same after the shock as before (though the same thing is true of the sums of the products of the bodies multiplied simply by their velocities). Now that proposition is so far general as to obtain in all collisions of bodies that are perfectly elastic: and it is also true, when bodies of a perfect e]asticity strike any immoveable obstacle, as well as when they strike one another; or when they are constrained by any power or resistance to move in directions different from those in which they impel one another. These considerations might have induced Huygens to lay it down as a general rule, that bodies constantly preserve their ascensional force, i.e. the product of their mass by the height to which their centre of gravity can ascend, which is as the square of the velocity; and ticrefore, in a given system of bodies, the sum of the squares of their velocitics will remain the same, and not be altered by the action of the bodies among themselves, nor against immovcable obstacles. Li.jbnitz's metaphysical system led him to think that the same quantity of action or force subsisted in the universe; and finding this impossible, if furce were estimatcd by the quantity of motion, he adopted Huygens's principle of the preservation of the ascensional force, and made it the measure of moving forces. But it is to be observed that Huygens's principle, above-mentioned, is general only when bodies are perfectly elastic: and in some other cases, which Maclaurin has endeavoured to distinguish: showing at the.same time that no useful conclusion in mechanics is affected by the disputes concerning the measure of the force of bodics in motion, which have been objected to mathematicians. Analyst, Query 9. See Maclaurin's Fluxious, vol.2, art. 533; Huygens, Oper. tom. 1, pa. 248; \&c.

Leibuitz's principle was adopted by sereral persons; as Wolfius, the Bernoullis, \&c. Mr. Dan. Bernoulli, in his Treatise, has assumed the preservation of the vis ascendens of Huygens, or, as olhers express it, the conservatio virium vivarum; and, in Bernoulli's own expression, æqualitas inter descensum actualem ascensumque potentialem, as an hypothesis of wouderful use in mechanics. But Dr. Jurin contends, that the conclusions drawn from this principle are oftener false than true. See De Conserwat. Virium Vivarum Dissert. Lond. 1744.

Catalan and Papin answered Leibuitz's paper published in 1686 ; and from that time the controversy became more general, and was carried on for several years by Leibnitz, John and Daniel Bernoulli, Poleni, Wolfius, Gravesande, Camus, Muschenbrosk, $\delta \mathrm{c}$, on one side; and Pemberton, Eames, Dusaguliers, Dr. S. Clark, M. de Mairan, Jurin, Maclaurin, Robins, \&e, on the other. Sec Act. Erud. 1686, 1690, 1691, 1695; Nouv, de la Rep. des Let. Sept. 1686, 1687, art.2; Comm. Epist. inter Leibn. et Bern. Ep. 24, p. 1+3; Discourses sur les Loix de li Comm. du Mouvement, Oper. tom. 3, \& Diss. de vera Notione Virium Vivarum, ib.; Act. Petropol. tom. 1, pa. 131, \&c ; Hydromanica, sect. 1 ; Herman, in Act. Petrop. tom. 1, p. 2, \&c; I'ulen. de Castellis; Wolf. in Act. Petrop. tom. 1, p. 217, \&cc, and in Cosmol. Gener.; Graves. in Journ. Lit, et Phys. Fheın. Math. 1742, lip. 2, cap. 2 and 3 ; Memoir. de l'Acad. des Ściences, 1725; 
Nuschenbr. Int. ad Phil. Nat. 1762, vol.1, p. 83 \&c; Pemb. \&c, in Phil. Trans. No. 371, 375, 376, 396, 400,401 , or my Abridg. vol. 6 , p. 570 \&c. Mairan in Mem. de l'Acad. des Sc. 1728 , Phil. Trans. No. 459, or Abridg. vol.7, p.637, Pliilos. Trans. vol. 43, p.423 \&c; Maciaurin's Acc. of Newton's Discoveries, p. 117 $\& c$, Flux. ubi supr. and Recucil des Pieces qui ont cmporté le Prix \&c. tom. 1; Desagul. Course Exp. Philos. vol.1, p.393 \&c, vol. 2, p. 49 \&c ; and Robins's Tracts, vol. 2, p. 135.

The nature and limits of this work will not admit of a full account of the arguments and experiments that have been urged on both sides of the question; but they may be found chiefly in the preceding references. A fcw of them however may be considered, as follows.

The defenders of Leibnitz's principle, besides the arguments above-mentioned, rufer to the spaces that bodies ascend to, when thrown upwards, or the penetrations of bodies let fall into soft wax, tallow, clay, snow, and other soft substances, which spaces are alway's as the squares of the velocities of the bodies. On the other hand, their opponents retort, that such spaces are not the measures of the force in question, which is rather percussive and momentary, as those above are passed over in unequal times, and are indeed the joint effect of the forces and times.

Desaguliers brings an argument from the familiar experiment of the balance, and the other simple mechanic powers, showing that the effect is in proportion to the velocity multiplied by the weight; for example, 4 pounds being placed at the distance of 6 inches from the centre of motion of a balance, and 2 pounds at the distance of 12 inches; these will have a vis viva if the balance be put into a. swinging motion. Now it appears that these forces are equal, because, with contrary directions, they soon destroy each other; and they are to each other in the simple ratio of the velocity multiplied by the mass, viz $4 \times 6=24$, and $2 \times 12=24$ also.

Mr. Robins, in his remarks on J. Bernoulli's treatise, entitled, Discours sur les Loix de la Conmunication du Nouvement, informs us, that Leibnitz adopted this opjnion throngh mistake; for though he maintained that the quantity of force is always the same in the universe, he endeavours to expuse the error of Descartes, who also asserted, that the quantity of motion is always the same; and in his discourse on this subject in the Acta Eruditorum for 1686 , he says that it is agreed on by the Cartesians, and all other philosophers and mathematicians, that there is the same furce requisite to raise a body of $t$ pound to the height of 4 yards, as to raise a body of 4 pounds to the height of 1 yard; but bong shown how much he was mistaken in taking that for the common opinion, which woukl, if allowed, prove the force of the body to be as the squitre of the velocity it mover with, he afterwards, rather than own hionself capable of such a mistake, endeavoured to defend it as true; since he found it was the necessary consequence of what he hat once asserted; and he maintainel, that the force of a bodly in motion was proportional to the height from which it must fall, to acquire that velucity; and the beinhts being as the square of the velocitics, the forces would be as the masses multiplied by them; whoreas, when a body disconds by its gravity, or is projected perpoulicularly upwards, its motion may be considered as the sum of the unilorm and contiunal impulses at the power of gravity, during its falling is the former case, and till they extinguish it in the latter.
Thus when a body is projected upwards with a double velocity, these uniform impulses must be continued for a double time, in order to destroy the motion of the body ; and hence it follows, that the body, by setting out with a double velocity, and ascending for a double time, must arise to a quadruple height, before its motion is exhausted. But this proves that a body with a double velocity moves with a double force, because it is produced or destroyed by the same uniform power continued for a double time, and not with a quadruple force, though it rises to a quadruple height; so that the error of Leibnitz consisted in his not considering the time, since the velucities alone are not the causes of the spaces described, but the times and the velocitics together; yet this is the fallaciou's argument on which he first built his new doctrine; and those which have been since much insisted on, and derived from the indentings or hollows produced in soft bodies by others falling into them, are much of the same kind. Robius's Tracts, vol.2, p.178.

But many of the experiments and reasonings, that have been urged on both sides of this controversy, bave been founded in the different senses applied to the term Force. The English and French plilosophers, by the word Force, mean the same thing as they do by momentum, motion, quantity of motion, percussion, or instantaneous pressure, which is measured by the mass drawn into the velocity, and may be known by its effect; and when they consider bodies as moving through a certain space, they allow for the time in which that space is described: whereas the Dutch, Italian, and German philosophers, who bave espoused the new opinion, mean by the word Force, or Force inherent in a body in motion, that which it is able to produce; or, in other words, the force is always measured by the whole effect produced by the body in motion, until its whole force be entirely communicated or destroyed, without any regard to the time employed in producing this total effect. Thus, say they, if a point runs through a. determinate space, and presses with a certain given force, or intensity of pressure, it will perform the same action whether it nove fast or slow, and therefore the time of the action in this case ought not to be regarded. Gravesande, Phys. El. Math. \$723-728.

Euler ubserves, with regard to this dispute concerning the measure of vivid force, or living force, as it is sonsetimes called, that we cannot absolutely ascribe any force to a borly in motion, whether we suppose this force proportional to the velocity, or to the square of the velocity: for the force exerted by a body, striking another at rest, is different from that which it exerts in striking the same bisdy in motion; so that this force cannot be ascribed to any body considered in itsc.lf, but only relatively to the other bodics it meets with. There is no force in a body absolutely considered, but its inertia, which is always the same, whether the bodly be in motion or at rest. But if this body be foreed by others to change irs state, its inertia then exerts itself as a force, properly so called, which is not absolutely determinable; because it depends on the changes that happen in the state of the body.

A second observation which has been male by some eninent writers, is, that the effect of a sloock of two or more bedics, is not produced in an instant, but requires a certain interval of time. Admitting this to be the case, the heterogeneity bctwecl the vis viva and mortua, or !iving and dead force, will vanish; since a pressure nay always be assigned, which in, the same time, lowever little, 
shall produce the same effect. If then the vis viva be nomogeneous io the vis mortua, and having a perfect measure and knowledge of the latter, we need require no other measure of the former than that which is derived from the vis mortua equivalent to it.

Now that the change in the state of two bodies, by their shock, does not happen in an instant, appears evidently from the experiments made on soft bodies : in these, percussion forns a small cavity, visible after the shock, if the bodies bave no elasticity. Such a cavity canmot cerlainly be made in an instant. And it the shock of soft bodies require a determinate time, we must certainly say as much of the harciest, though this time may be so small as to be beyond all our ideas. Neither can an instantaneous shock agroe with that constant law of nature, by virtue of which nothing is performed per saltum. But it is needless to insist farther upon this, since the duration of any shock may be determined from the most,certain principles.

The force of percussion, resulting from the pressures that bodies exert on each other, while the collision lasts, may be perfectly known, if these pressures be determined for every iustant of the shock. The mutual action of the budies begins the first moment of their contact; and is then least; after which this action increases, and becomes greatest when the reciprocal impressions are strongest. If the bodies have no elasticity, and the impressions they have received remain, the forces will then cease. But if the bodics be elastic, and the parts compressed restore themselves to their former state, then will the bodies continue to press each other till tbey separate. To comprebend therefore perfectly the force of percussion, it is requisite first to define the time the shock lasts, and then to assign the pressure corresponding to each instant of this time; and as the effect of pressures in changing the state of any body may be known, we may thence come at the true cause of the change of motion arising from collision. 'The force of percussion therefore is mo more than the operation of a variable pressure during a given time; and to measure this force, we must have regard to the time, and to the variations according to which the pressure increases and decreases.

Euler has given some calculations relative to these particulars; and he illustrates their tendency by this instance: Suppose that the hardness of the two bodies, $\mathbf{A}$ and $\mathbf{B}$, is equal; and such, that being pressed together with the furce of loolb, the impression made on each is of the

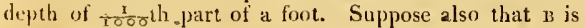
fixed, and that $\Lambda$ strikes it with the velocity of 100 feet in a second; then, according to this author, the greatest force of compression will be equivalent to $4.00 \mathrm{lb}$, and this force will produce in each of these bodies an impression equal ic $\frac{1}{2}$ of a foot; and the duration of the collision, that is, till the bodies arrive at their greatest compression, will be about zor of a second. M. Euler, in his calculations, supposes the hardness of a body to be proportional to the firce of pressure requisite to make a given impression on it; so that the force by which a given impression is made on a body, is in a compound ratio of the hardness of the body and of the quantity of the impression. But he observe's, that regard must be had to the magnitude of the bodies, as the same impression cannot be made on the least irudies as on the greatest, from the defect of space through which their component particles must be driven: he considers therefure only the least impressions, and supposes the bodies of such maginitudes, that the impressions with respect to them may be considered as nothing. What he supposes concerning the hardness of bodies, ncither implies elasticity nor the want of it, as elasticity only produces a restitution of figure and impression when the pressing force ceases; but this restitution need not be here considered. It is also supposed, that the bodies which strike each other, have plane and equal bases, by which they touch each other in the collision; so that the impression hereby made diminishes the length of each body. It is farther to be observed, that in M. Euler's calculations, bodies are supposed so constituted, that they may not only receive impressions from the forces pressing them, tut that a greater force is requisite to make a greater injpression. This excludes all bodies, fluid or solid, in which the same force may penetrate farther and farther, provided it has time, without ever being in equilibrio with the resistance: thus a body may continually penetrate farther into sott wax, though the force impeling it be not increased: in these, and the like cases, nothing is required but to surmount the first obstacles; which being once done, and the connexion of parts broken, the penetrating body always advances, meeting with the same obstacles as before, and destroying them by an equal force. But this celcbrated genius only considers the first obstacles which exist before any separation of parts, and which are doubtless such, that a greater impression requires a greater force. Indeed this chiefly takes place in elastic bodies; but it scems likewise to obtain in all bodies when the impressions made on them are small, and the contexture of their parts is not altered.

These things being premised, let the mass or weight of the body $A$ be cxpressed in gencral by $A$, and let its velocity before the shock be that which it might acquire by falling fron the height $a$. Further, let the hardness of $A$ lie expressed by $M$, and that of $B$ by $N$, and let the area of the base, on which the impression is made, be $c c$; then will the greatest compression be made with the force $\sqrt{ }\left(\frac{M N c c}{M+N} \times A a\right)$. Therefore, if the hardness of the two bodies, and the plane of their contact during the whole time of their collision be the same, this force will be as $\sqrt{ } \wedge a$, that is, as the square root of the vis viva of the striking body $A$. And as $\sqrt{ } a$ is proportional to the velocity of the body $A$, the force of percussion will be in a compound ratio of the velocity and of the subduplicate ratio of the mass of the body striking; so that in this case neither the Leibnitian nor the Cartesian propositions take place. But this force of percussion depends chiefly on the harduess of the bodies; the grreater this is, the greater will the force of percussion be. If $\mathrm{M}=\mathrm{N}$, this force will be as $\sqrt{ }(\mathrm{M} c c \times \mathbf{A l})$, that is, in a compound subduplicate ratio of the vis viva of the striking body, of the hardness, and of the plane of contact. But if $\mathrm{s}$, the hardness of one of the bodies, be infinite, the force of percussion will be as $\sqrt{ }(\mathrm{N} c c \times \Lambda a)$; at the sane time, if $n=N$, this force will be as $\sqrt{ }\left(\frac{1}{2} N c c \times \Delta a\right)$. Therefore, all otbet things being equal, the force of percussion, if the striking body be infinitely hard, will be to the force of pereussion when both the bodies are equally hard, as $\sqrt{ } 2$ to 1 .

Euler further deciuces from his calculation, that the impression received by the bodies $A$ and $B$ will be as follows; viz, as $\sqrt{\frac{N}{(M+N)} \times \frac{A a}{\times A c}}$ and $\sqrt{ } \frac{M \times A a}{(M+N) \times N c c}$ respectively. If therefore the hardness of $\Lambda$, that is $M$, be intinite, it will sulter no impression; whereas that on bi will exiend to the 
depth of $\sqrt{ } \frac{A a}{N c c}$. But if the hardness of the two bodies be the same, or $\mathrm{M}=\mathrm{N}$, they will each receive equal impressions of the depth $\sqrt{\frac{A a}{2 N C c}}$. So that the impression received by the body $B$ in this case, will be to the impression it receives in the former, as 1 to $\sqrt{ } 2$.

Euler bas likewise considered and computed the case where the striking body has its anterior surface convex, with which it strikes an inmoveable body whose surface is plane. He has also examined the case when both bodies are supposed immoveable; and from his formulæ he deduces the known laws of the collision of elastic and nonelastic bodies. He has also determined the greatest pressures the bodies receive in these cases; and likewise the impressions made on them. In particular he shows, that the impressions received by the body struck, or $\mathrm{B}$, if moveable, is to the impression received by the same body when fixed, as $\sqrt{ } \mathrm{B}$ to $\sqrt{ }(\mathrm{A}+\mathrm{B})$.

There are several curious, as well as useful observations, in Desaguliers's Experimental Philosophy, concerning the comparative forces of men and horses, and the best way of applying them. A horse draws with the greatest advantage when the line of direction is level with his breast; in such a situation, he is able to draw 200lb for $S$ hours a day, walking about $2 \frac{x}{2}$ miles an hour. But if the same horse be made to draw $240 \mathrm{lb}$, he can work only 6 hours a day, and cannot go quite so fast. On a carriage indeed, where friction alone is to be overcome, a middling horse will draw 1000lb. But the best way to try the force of a horse, is to make him draw up out of a well, over a single pulley or roller; and in that case, an ordinary horse will draw about $200 \mathrm{lb}$, as before observed.

It is found that 5 men are of equal force with one horse, and can with equal ease push round the horizontal beam of a mill, in a walk 40 feet wide; whereas 3 men will do it in a walk only 19 feet wide.

The worst way of applying the force of a horse is to make him carry or draw up hill : for if the hill be steep, 3 men will do more than a lorse, each man climbing up faster with a burden of 100 lb weight, than a horse that is loaded with S00lb: a difference which is owing to the position of the parts of the human body being better adapted to climb, than those of a horse.

On the other hand, the best way of applying the force of a horse, is the horizontal direction, in which a man can exert the least force: thus, a man that weighs $140 \mathrm{lb}$, when drawing a boat along by means of a rope coming over his shoulders, cannot draw above $27 \mathrm{lb}$, or exert above 1-7th part of the force of a horse employed to the same purpose; so that in this way the force of a horse is cqual to that of 7 men.

The best and rnost effectual posture in a man, is that of rowing; when he not only acts with more muscles at once for overcoming the resistance, than in any other position; but also as he pulls backwards, the weight of his body assists by way of lever. Sce Desaguliers's lixp. Philos. vol. 1, pa. 241, where several other observations are made relative to force acquired by certain positions of the body; from which that author accounts for most feats of strength and activity. Sce also a memoir on this subject by MI. Lahire, in the Mem. Roy. Acad. 1729; or in Desaguliers's Exp. \&c. pa. $267 \& \mathrm{c}$, who lias published a translation of part of it with remarks.

VoL. I.
Force is distinguished into motive and accelerative ur. retardive.

Motive For ce, otherwise called Momentum, or Force of Percussiun, is the absolute force of a body in motion, \&c; and is expressed by the product of the weight or mass of matter in the body multiplied by the velocity with which it moves. But

Accelerative Force, or Retardive Force, is that which respects the velocity of the motion only, accelerating or retarding it; and it is denoted by the quotient of the motive force divided by the mass or weight of the body. So, if $n$ denote the motive force, and $b$ the body, or its weight, and $f$ the accelerating or retarding force, then is $f$ as $\frac{m}{b}$.

Again, forces are either constant or variable.

Constant Forces are such as remain and act continually the same for some determinate time. Such, for example, is the force of gravity, which acts constantly the same upois a body while it continues at the same distance from the centre of the earth, or from the centre of force, wherever that may be. In the case of a constant force $\mathrm{F}$ acting upon a body $b$, for any time $t$, we have these following theorems; putting

$f=$ the constant accelerating force $=\mathrm{F} \div \mathrm{b}$,

$v=$ the velocity at the end of the time $t$,

$s=$ the space passed over in that time, by the constant action of that force on the body:

and $g=16_{T^{2}}$ feet, the space generated by gravity in 1 second, and calling the accelerating force of gravity 1 ; then is $s=\frac{\pi}{2} t o=g f t^{2}=\frac{v^{2}}{4 g f}$;

$$
\begin{aligned}
& v=2 g f t=\frac{2 s}{t}=\sqrt{ }+g f s ; \\
& t=\frac{v}{2 g f}=\frac{2 s}{v}=\sqrt{ } \frac{s}{g f} \\
& f=\frac{v}{2 g l}=\frac{s}{g l^{2}}=\frac{v^{2}}{4 g s} .
\end{aligned}
$$

Variable Forces are such as are continually changing in their cffect and intensity; such as the force of gravity at different distances from the centre of the earth, which decreases in proportion as the square of the distance increases. In variable forces, theorems similar to those above may be exhibited by using the fluxions of quantities, and afterwards taking the fluents of the given fluxional equations. And herein consists one of the great excellencies of the Newtonian or modern analysis, by which we are enabled to manage, and compute the cffects of all kinds of variable forces, whether accelerating or retarding. 'Thus, using the same notation as above for constant forces, viz. $f$ the accelerating force at any instant, $t$ the time a body has been in motion by the action of the variable force, $p$ the velocity generated in that time, s the space run uver in tliat time, and $g=16 \frac{r}{12}$ feet; then is

$$
\begin{aligned}
& \dot{s}=\frac{v \dot{v}}{2 g f}=v i ; \quad \dot{v}=\frac{2 g f \dot{s}}{v}=2 g f i ; \\
& i=\frac{\dot{s}}{v}=\frac{v}{2 g t} ; f=\frac{v \dot{v}}{2 g \dot{s}}=\frac{\dot{v}}{2 g i} .
\end{aligned}
$$

In these four theorems, the force $f$, though variable, is supposed to be constant for the indefinitely small time $i$; and they are to be used in all cases ol variable forces, is the former ones in constant forces; viz, from the circumstances of the problem under consideration, deduce a general expression for the value of the force $f$, at any indefinite time $t$; then substitute it in one of these theorems, $3 \mathrm{Z}$ 
which shall be proper to the casc in hand; and the equa. tion thence resulting will determine the corresponding va lues of the other quantities in the problem. It is also to be observed, that the foregoing theorcms equally hold good for the destruction of motion and rclocity, by mcans of retarding or resisting forces, as for the generation of the same by means of accelcrating forces. Many applications of these theorems may be seen in my Mathematics, vol. 2.

There are many other denominations and kinds of forces; such as attractive, central, centrifugal, \&c, \&c; for which see the respective words.

FORCES, the Equilibrium of, or the Composition and Resolution of, is the conspiring and opposing of two or more forces, so as to balance one another, or to hecp in equilibrio the body or point against which they, all mutually act.-This equilibrium may be of two, or of threc, or of any other number of forces. In the cise of two forces only, it is manifest that they must be both cqual in quantity, and in directions exactly opposite. For, if they be not directly opposite, the body or point of meeting must move towards one side; and if they be not equil, the greater will prevail over the less, and carry this along with it, with the difference of the two forces. Hence, in all the cases of cquilibrium and compositions of three or more forces, the practice must be to compose and reduce all the given forces into one, of a yiven quantity, and laving a given direction; for then taking another, of an ' qual quantity, and giving it the opposite direction, it will be in equilibrio with all the former given forces, so compounded together. - This will divide into two or three or any other number of forces, as below.

Parallelogram or Triangle of Fonc es, is when two oblique forces are given, to compound them, in order to find a third furce to balance them. So, if $\mathrm{i}$.

$A B, A C$ be the two given forces, acting at the common point $\mathrm{A}$ : shaw $\mathrm{BD}$ parallel and equal to $\mathrm{AC}$, and join $\mathrm{AD}$; then is $A D$ a force compounded of, and equiralent to, the two given forces $\mathrm{AB}, \mathrm{AC}$. Hence if, in AD produced, there be

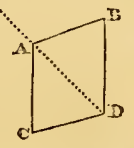
taken $\mathrm{AE}=\mathrm{AD}$; then $\mathrm{AE}$ will be the equivalent belancing force to $\mathrm{AD}$, and consequently to the two given forces $\mathrm{AB}$, $A c$. - From the circumstance of the two given forces $A B$ and $A C$, or $A B$ and $B D$, forming, with their equivalent compound $\triangle D$, a triangle $\triangle B D$, arises the phrase Triangle of Forces. And from the circumstance of a parallclugram $A E D C$ being formed by joining $\mathrm{CD}$, that the expression Parallelogram of Forces has arisan.-From the foregoing method of compounding two forces into one, will casily folluw the composition of any number whatever of forces into one; viz, first compound two together, then that and a $3 \mathrm{l}$, next that and a 4 th, and so on to the lant, whaterer their ntmber may be; which may be symmetrically callet the Polygon of Forces, as below.

Folygon of Forces, is the compounding tugether any number of forces into one. $A$ s, the four forces $A \mathrm{~A}, \mathrm{AC}, \mathrm{AD}, \mathrm{AE}$, all acting at the point $A$. Irom the extremity $n$ of the first, draw if provelled and cqual to the ?d Ac'; then rg parallel and cqual to the $3 d \mathrm{AD}$; last]y (in priballcl and orpual to the 4th Ar; then Aנr, being joinded, is the quantity and direction of a single force, which is compounded of, and equivalent to, the four first given forces; and consequently, taking a I oppositc and equal to $\mathrm{AH}$, then $\mathrm{AI}$, balancing $\mathrm{AII}$, will also balance all the four giren forces. For, by the principle of the Triangle of forces, $\mathrm{AB}$ and $\mathrm{AC}$, or $\mathrm{BF}$, are compounder into $\mathrm{AF}$; then $A F$ and $A D$, or $F G$, are compounded into $A G$; lastly $A G$ and $A E$, or $G H$, arc compounded into $A 11$; and so on, if there be ever so many forces.

ForCe of Running Water. This must be as the square of its relocity, or as the height from which a heavy borly must fall, to acquire that velocity; because the cfiect is first as the velocity of each particlc, and also as the number of them, which is as the velocity igain.

FORCER, in Mechanics, is properly a piston without a valie. For, by drawing up such a piston, the air is drawn uip, and the water follows; then pushing the piston down again, the water bcing prevented from descending by the lower valve, is forced up to any height above, by means of a side branch between the two.-See the ways of making these in Desnguliers's Exper. Philos. vol. 2, pa. 161 \&c. Sese :ilso Clase's Motion of Fluids, pa. 60.

Foncing Pump, one that acts, or raises wuter, by a forcing piston. See above.

FORELAND, or For ENEss, in Navigation, a point of land jutting out into the sea.

FORELAND, in Fortification, is a small picce of ground between the wall of a place and the moat; called also Berme and Liziere.

FORE-STAFF, an instrument formerly used at sea, for taking the altitudes of the heavenly bodics. It is so called, because the observer, in using it, turns his face forward or towards the object, in contradistinction to the back-staff, with which lie turns his back to the object. It is also called the cross-staff, because it consists of several pieces set acrosss a staff. See Cross-Staff.

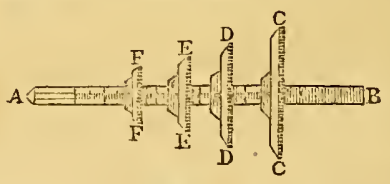

The forc-staff is formed of a straight square staff $A \mathrm{~F}$, of about 3 feet long, liaving each of its four sides graduated likic a line of tangents, and four crosscs, or vanes, $\mathrm{FF}, \mathrm{EE}$, D D, ec, sliding upon it, of unc qual lengths, the halves of which represent the radii to the lines of tangents on the clifierent sirles of the stafi. The first or shortest of these vanes, $\mathrm{rr}$, is called the ten cross, or ten vane, and belongs to the 10 scale, or that side of the instrument on which the divisions begin at 3 degrees, and end at 10 . The next longer cross, ee, is called the 30 cross, belonging to that side of the staff where the divisions begin at 10 digrees, and and at 30 , called the 30 scalc. Thic third vane on, is tcrmed the 60 rros", and belongs to that side where the divisions begin at 20 degrees, and end at 60 . 'The last or' longest vane ce, calied the co cross, belongs to the side where the dirisions begin at 30 degrees, and end at 90 .

'The chicf use of this intrument, is to take the height of the sun, and stars, or the distance between two stars: and the 10, 30,60, or 90 cross is to be used, according as the altitude is more or less; that is, if the altitude be less thin 10 degres, the 10 cross is in be: used; if above 10 , but less tlan 30 , the 30 cross is to be tused; and so on. 
To observe an Altitude with a Fore-staff. Apply the flat end of the staff to the eye, and slide one of the crosses backwards and forwards upon it, till over the upper end of the cross be just seen the centre of the sun or star, and over the under end the extreme horizun; then the degrees and minutes cut by the cross on the sirle of the staff proper to the vane in use, gives the altitude above the horizon.

In like manner, for the Distance betwoeen two luminaries; the staft being set to the eye, bring the cross just to subtend or cover that distance, by having the one luminary just at the one end of it, and the other luminary at the other end of it; and the degrees and minutes, in the distance, will be cut on the proper side of the staff, as before.

FORNIULA, a theorem or general rule, or expression, for resolving certain particular cases of some problem, \&rc. So ${ }^{I} s+\frac{1}{2} d$ is a general formula for the greater of two quantities whose sum is $s$ and difference $d$; and $\frac{T}{2} s-\frac{1}{2} d$ is the formula, or general value, for the less quanticy. Also $\sqrt{ }\left(d x-x^{2}\right)$ is the formula, or general value, of the ordinate to a circle, whose diameter is $d$, and absciss $x$.

FORT, a small castle or fortress; or a place of inconsiderable extent, fortified either by nature or art. The fort is usually encompassed with a moat, rampart, and parapet, to secure some high ground, or passage of a river; to make good or strengthei an advantageous post; or to fortify the lines and quarters of a siege.

Field Font, otherwise called Fortin, or Fortlet, and sometimes Sconce, is a small fort, built in haste, for the defence of a pass or post; but particularly constructed for the defence of a camp in the time of a siege, where the principal quarters are usually joined, or made to communicate with each other, by lines defended by fortins and redoubts. Their figure and size are various, according to the nature of the situation, and the importance of the service for which they are intended; but they are most commonly made square, each side about 100 toises, the perpendicular 10 , and the faces 25 ; the ditch about this fort may be 10 or 12 toises wide; the parapet is made of turf, and fraised, and the ditch pallisadoed when dry. There may be made a covert-way about this furt, or else a row of pallisades might be placed on the outside of the ditch. Some of these are fortified with bastions, and some with demi-bastions. -A fort differs from a citadel, as this last is crecter to command and guard some town; and from a redoubt, as it is closed on all sides, while the redoubt is open on one side.

Royal For T, is one whose line of defence is at least 26 fathoms long.

Stur FonT, is a sconce or redoubt, constituted by reentering and saliant angles, having commonly from five to eight points, and the sides fanking each other.

Forts are sometimes made triangular, only with half bastions; or of various other figures, regnlar or irregular, and sometimes in the form of a semicircle, especially when they are situated near a river, or the sea, as at the entrance of a harbour, for the convenience of firing at ships quite around them on that side. In the construction of all forts, it slould be remembered, that the figure of fewest sides and bastions, that can possibly answer the proposed defence, is always to be preferred; as works on such a plan are suoner executed, and with less expense; besides, fewer troops will serve, and they arc more readily brought together in case of necessity.

FORTIFICATION, called also Military Architecture, is the art of fortifying or strengthening a town or other place, by making certain works around it, to secure and defend it from the attacks of enemies. Fortification has doubtless been practised by all nations, and in all ages; being at first very rude and simple, and varying in its nature and manner, according to the mode of attack, and the weapons made use of. Thus, when villages and towns were first formed, it was found necessary, for the common safety, to encompass them with walls and ditches, to prevent any violence or sudden surprises from their neighbours. When offensive and missive weapons came to be used, walls were raised as a defence against the assailants, and look-holes or loop-holes made through the same to annoy the enemy, by shooting arrows, \&c, through them. But finding thât as soon as the enemy got close to the walls, they could no longer be seen or discomfited by the besieged, these added square towers along the wall, at proper distances from each other, so that all the intervening parts of the wall might be seen and defended from the adjacent sides of the towers. However, this manner of inclosing towns wits found to be rather imperfect, because there remained still the outer face of the tuwers which fronted the field, that could not be seen and defended from any other part. To remedy this imperfection, they next made the towcrs round instead of square, as seeming better adapted buth for strength to resist the battering engines, and for being defended from the other parts of the wall. Nevertheless, a small part of these towers still remained unseen, and incapable of being defended, for which reason they were again changed for square ones, as before, but with this difference, that now they presented an angle of the square outwards to the field, instead of a face or side; and thus such a disposition of the works was obtained, as that no part could be approached by the enerny without being seen and attacked.

Since the use of gunpowder, it has been found necessary to add thick ramparts of earth to the walls, and the towers bave been enlarged into bastions, as well as many other things added, that have given a new appearance to the whole art of defence, and the name of fortification, on account of the strength afforded by it, which was about the year 1500 , when the round towers were changed into bastions.

But notwithstanding all the improvements made in this art since the invention of gunpowder, that of attacking is still superior to it : the superiority of the besiegers' fire, together with the greater number of men, sooner or later compels the besieged to submit. A special advantage was addesl to the art of attacking by M. Vauban, at the siege of Ath, in the year 1697, viz, in the use of ricochet firing, or at a low elevation of the gun, by which the shot was made to run and roll a great way along the inside of the works, to the great annoyance of the besieged.

The chief authors who have treated of fortification, since it has been considered as a particular art, are the following, and mostly in the order of time: riz, La Treille, Alghisi, Marchi, Pasino, Ramelli, Cataneo, and Spcckle, who, as $\mathrm{Mr}$. Robins says, was one of the greatest geniuses that has applicd to this art: he was architect to the city of Strasburgh, and died in the year 1589: he published a treatise on fortification in the German language, which was reprinted at Leipsic in $\mathbf{3 7 3 6 . ~ A f t e r w a r d s , ~ E r r a r d , ~}$ who was engineer to Henry the Great of France; Stevinus, engineer to the prince of Orange; with Marolvis, the chevalier de Ville, Lorini, Cochorn, the count de Pagan, and the marshal de Vauban; which list two noble 
authors have contributed greatly to the perfection of the art; besides Scbeiter, Mallet, Belidor, Blondel, Muller, Montalambert, sc. Also a list of several works on the art of fortification may be added, as follows: viz. Melder's Praxis Fortificatoria: Les Fortifications du Comte de Pagan: L'Ingénicur Parfait du Sicur de Ville: Sturmy's Architectura Nlilitaris Hypothetical.: Blontel's Nouvelle Maniere de Fortifier les Places: the Abbé de đay's Veritable Maniere de Bien Fortifier: Vauban's Ingénieur François: Cochorn's Nouvelle Fortification tant pour un Terrain bas et humide, que sec et elevé : Alexander de Grotte's Fortification: Donatus Roselli's Fortification : Medrano's Ingénieur François: the Chevalier de St. JuIien's Architecture Militaire: Lansberg's Nouvelte Maniere de Fortifier les Places: an anonymous treatise in French, called Nouvelle Maniere de Fortifier les Places, tirée des Mlethodes du Chevalier de Ville, \&c: Ozanam's Traité de Fortification: Memoires de l'Artillerie de Surirey de St. Remy: Muller's treatises of Elementary and Practical Fortification: Riou's Elem. of Fortification, printed anonymously: and Montalambert's Fortification Perpendiculaire.

Maxims in Fortificatron. From the nature and circumstances of this art, certain general rules, or maxims, have been drawn, and laid down. These may indeel be multiplied to any extent, but the principal of them are the following: viz,

1. That the manner of fortifying should be accommodated to that of attacking. So that no one manner can be assured always to hold, unless it be assured that the manner of besieging is incapable of being altered. Also, to judge of the perfection of a fortification, the method of besieging at the time when it was built must be considered.

2. All the parts of a fortification should be equally strong on all sides, where there is equal danger; and they should be able to resist the most powerful machines used in besieging.

3. A fortification should be so contrived, as to be defended with the fewest men possible: which consideration, when well attended to, saves a great deal of expense.

4. That the defendants may be in the better condition, they must not be exposed to the enemies' artillery; but the aggressors must bc exposed to theirs.

5. All the parts of a fortification should be so disposed, as that they may defend each other. In order to this, every part ougbt to be flanked, i.e. seen sideways, capable of being seen and? defended from some other part ; so that there be no place where an enemy can lodge himself, either unseen, or under shclter.

6 . All the champaign around must lie open to the defendants; so that no hill or eninence be allowed, bchind which the enemy migbt shelter bimself from the guns of the fortification; or from which he might annoy them, with his own. Hence, the fortress is to command entirely the place surrounding it ; and consequently the outworks must all be lower than the body of the place.

7. No line of defence must exceed the point-blank inusket-shot, which is from 120 to 150 fathoms.

8 . The more acute the angle at the centre, the stronger is the place; as consisting of the more sides, and consequently more defensiblc.

9. All the defences should be as nearly dircet as possible.

10. The works that are most remote from the centre of the place, ought always to be open to those that are nearer.

These are the general laws and views of fortification. $\Lambda \mathrm{s}$ to the particular ones, or such as respect the several members' or parts of the work, they are given under those articles respectively.

Fortification is either theoretical or practical.

Theoretical For Tification, consists in tracing the plans and profiles of a work on paper, with scales and compasses; and in examining the systems proposed by different author:, to discover their advantages and defects.

Practical Fortification, consists in forming a project of a work according to the nature of the ground, and other necessary circumstances, tracing it on the ground, and executing the project, with all the military buildings; as magazines, storehouses, bridges, \&c.

Again, fortification is either defensive or offensive.

Defensive Fortirication, is the art of defending a town that is besicged, with all the advantages the fortification of it will admit. And

Offensine Fortification, is the same with the attack of a place, being the art of making and conducting all the different works in a siege, in order to gain possession of the place.

Fortification is also used for the place fortified; or the several works raised to defend and flank it, and keep off the enemy. All fortifications consist of lines and angles, which have names according to their various offices. The principal lines are those of circumvallation, of contravallation, of the capital, \&c. The principal angles are those of the centre, the flanking angle, flanked angle, angle of the epaule, \& $c$.

Fortifications are either durable or temporary.

Durable Fontrfication, is that which is built and intended to remain a long time. Such are the usual fortifications of cities, frontier places, \&c. And a

Temporary ForTIFICATION, is that which is erected on some emergent occasion, and only for a short time. Such are field-works, thrown up for the seizing and maintaining a post, or passage; those about camps, or in sieges; as circumvallations, contravallations, redoubts, trenches, batteries, \&c.

Again, fortifications are either regular or irregular.

Regular Fortification, is that in which the bastions are all equal; or which is built in a regular polygon, the sides and angles of which are usually about a musket-shot from each other. A regular fortification, having the parts all equal, has the advantage of being equally defensible; so that there are no weak places.

Irregular Fontification, is that in which the bastions are unequal, and unlike; or the sides and angles not all equal, and equidistant. In an irregular fortification, the defence and strength being unequal, it is necessary to rcduce the irregular shape of the ground, as ncar as may be, to a regular figure: i. e. by inscribing it in an oval, instead of a circle; so that one half may be similar and equal to the other balf.

Marine FonTIFICATION, is sometimes used for the art of raising works on the sea-coast, \&.c, to defend harbours against the attacks of shipping.- Sce a neat treatise on Marine Fortification, at the end of Robertson's Elements of Navigation.

There are many modes of fortification, \&c, that have been much esteemed and used; a small specimen of a comparative vicw of the principal of these, is represented in plate xii, viz, those of Count Pagam, and Mess. Vauban, Cochorn, Belidor, and Blondel; the explauation of which is as follows: 
F O R

A Half bastions.

\section{Pagan's System.}

B Ravelin and counterguard.

c Counterguards before the bastions.

D The ditch.

E The glacis.

$G$ The place of arms.

н Retired Aanks.

$a$ Line of defence.

\section{Vauban's System.}

$b$ Angle of the bastion, or flanked angle.

$c$ Angle of the shoulder.

$d$ Angle of the flank.

$e$ Saliant angle.

$f$ Face of the bastion.

$g$ The flank.

$h$ The curtain.

$i$ Tenailles.

$k$ Traverses in the covert way.

1 Concave Alanks.

\section{Coelion's System.}

2 The curtains.

3 Redoubts in the re-entering angles

4 Traverses.

5 Stone lodgments.

6 Round flanks.

7 Redoubt.

8 Coffers planked on the sides, and above covered overhead with a foot of earth.

I Cavaliers.

$$
\text { 4. Belidor's System. }
$$

₹ Rams-horns, or Tenailles.

L Retrenchments within the detached bastions.

м Circular curtain.

N The ravelin.

P Lunettes with retired batterics.

Q Redoubt.

R Detached redoubt.

$s$ An arrow.

T Small traverses.

$l$ Retired battery.

5. Blondel's System.

$m$ Lunettes.

n Ravelin, with retired bastion.

- Orillons.

Another, or new method of fortification has lately been proposed by M. Montalambert, called Fortification Perpendiculaire, because the faces of the works are made by a series of lines running zigzag perpendicular to each other.

Profile of a Fontivication, is a representation of a vertical section of a work; serving to show those dimensions which cannot be represented in plans, and are necessary in the building of a fortification. The names and dimensions of the principal parts are as follow (see fig. 8, p. ix), where the numbers or dimensions are all expressed in feet.

A B The level of the ground plane;

$\Lambda \mathrm{C}=27$;

$\mathrm{CD}=18$, and $\mathrm{CW}=16 \frac{\mathrm{I}}{3}$; also $\mathrm{DN}$ is parallel to $\mathrm{AB}$;

$\mathrm{DE}=30$;

$\mathrm{EF}=2, \mathrm{VG}=3, \mathrm{GII}=3, \mathrm{HI}=4 \frac{\mathrm{x}}{2}, \mathrm{IL}=1 \frac{1}{2}$;

$\mathrm{LK}=18, \mathrm{KM}=2 \frac{\mathrm{x}}{2}, \mathrm{NP}=36$, Nо $=5, \mathrm{PR}=7$;

rs $=1, \mathrm{ST}=12$ or 18, ov $=9, \mathrm{l} n=120$;

$m z=3, n u=3, m c=30, c d=2$;

$d e=3, e f=3, f=4 \frac{\pi}{2}, r g=120, l h=1$;

Aw The interior talus or slope of the rampart;
wE, or DE, The terre-plein of ditto;

rG The talus, (in the upper part, of the banquetie;

nI The interior side of the parapet ;

LM 'The upper part of ditto;

$\mathrm{N}$ 'The cordon of 1 foot radius;

NP The depth, and $P n$ the breadth of the ditcls;

$O Q$ Interior side of revetement;

NR The scarp or exterior side of ditto ;

ST The depth of the foundation;

yz Revetement of the parapet;

nu The counterscarp;

nc The covert-way;

ce Talus of the banquette;

ef The upper part of ditto;

fh Parapet of the covert-way;

hg The glacis.

Other sections are at fig. 2, pl. 14.

Fontified Place, a fortress, or fortification, i. e. a place well flanked, and sheitered with works.

Fortin, or Fortlet, a diminutive of the word Fort, meaning a small fort, or sconce, called also Field Fort.

Star Fortin, is that whose sides flank each other, \&c. FORTRESS, the same as Fort, or a Fortification.

FOSTER ( $\mathrm{S}_{A M U \mathrm{E}}$ ), an English mathematician, was born in Northamptonshire, and admitted a sizer at Emanuel-college Cambridge in 1616 . He took the degree of bachelor of arts in 1619, and of master in 1623. He applicd early to the mathematics, and attained a great proficiency in them, of which he gave the first specimen in 1624 , in a treatise on the use of the quadrant.

On the death of Mr. Gellibrand, our author was chosen to succeed him, in 1636, as astronomy-professor in Gresham-college, London. He quitted it again however the same year, though for what reason does not appear, and was succeeded by Mr. Mungo Murray, professor of philosophy at St. Andrews in Scotland. But this gentleman marrying, the professorship again became vacant, and Mr. Foster was re-clected in 1641 .

Mr. Foster was one of those gentlemen who held private meetings for cultivating philosophy and useful knowledge, which afterwards gave rise to the Royal Society. In 1646 , Dr. Wallis, who was one of those associating gentlemen, received from Mr. Foster a theorem De triangulo spherico, which he published in his Mechanics. Neither was it only in this branch of science that he excelled, but he was also well versed in the ancient languages; as appears from his revising and correcting the Lemmata of Archimedes, which had been translated into Latin from an Arabic manuscript by Mr. John Greavcs. He made also several curious observations on cclipses of the sun and moon, in various places; and was particularly noted for inventing, as well as improving, astronomical and other mathematical instruments. After a long declining state of health, he died at Greshamcollege in 1652 .

His printed works are as follow; of which the first two articles were published before his death, and the rest of them after it.

1. The Description and Use of a small Portable Quadrant, for the easy finding the Hour of Azinuth; 4 to, 1624 . Originally published at the end of Gunter's Description of the Cross-Staffe, as an appendix to it.

2. The Art of Dialling; 4.to, 1638. Reprinted, with additions, in 1675 .

3. Posthuma Fosteri ; by Wingate ; 4to, 1652.

4. Four Treatises of Dialling; 4to, 1654 .

5. Miscellanies, or Matliematical Lucubrations. Pub- 
lisked by John Twysden, with additions of his own; and an appendix by Leybourne; folio, 1659.

6. 'The Sector' altered, and other Scales added, \&c. Published by Leybourne in 1661, in 4to.

There have been two other persons of the same name, who bave published some mathematical pieces. The first was,

FOSTER (William), a disciple of Mr. Oughtred, and afterwards a teacher of the mathematics in London. He distinguished himself by a book, which he dedicated to Sir Kenelm Digby, entitled, The Circles of Proportion, and the Horizontal Instrument, \&c; 4to, 1633.-The otherwas,

FOSTER (MARK), who lived later in point of time than either of the other two, and published a treatise entitled, Arithmetical Trigonometry: being the Solution of all the usuál Cases in Plain Trigonometry by Common Arithmetic, without any tables wbatsoever. $12 \mathrm{mo}, 1690$.

FOUNDATION, that part of a building which is underground : or the mass which supports a building, and on which it stands : or it is the coffer or bed dug below the level of the ground, to raise a building on.

FOUNTAIN, in Philosophy, a spring or source of water rising out of the ground. See SPring.

Fountain, or Artificial Fountain, in Hydraulics, a unachine or contrivance by which water is violently spouted or thrown up ; called also a jet d'eau.

There are various kinds of artificial fountaius, but all formed by a pressure of one kind or other, on the water Sc, viz, either the pressure or weight of a head of water, or the pressure arising from the spring and elasticity of the air, \&c. When these are formed by the pressure of a head of water, or any other fluid of the same kind with the foutain, or jet, \&c, then will this spout up.nearly to the same height as that head, abating only a little for the resistance of the air, with that of the adjutage \&c, in the fluid's rushing through; but, when the tountain is produced by any other force than the pressure of a column of the same fluid with itself, it will rise to such a height as may be nearly equal to the altitude of a column of the same fluid whose pressure is equal to the given force that produces the fountain.

To Construct an Artificial Fountain, playing by the pressure of the water. This is to be effected by making a close comnexion betwcen a head or elevated piece of water, and the lower place, where the fountain is to play; which may be done in this manner: Having a head of water, naturnlly, or, for want of such, make an artificial one, raising the water by pumps, or other machinery : from this head convey the water in close pipes, in any dircetion, down to the place where the fountain is to play; and there let it issue through an adjutage or small hole, turned upwards, by which means it will spout up nearly as high as the liead of the water it comes from, as above mentioned.

'To Contruct an Artificial Founuin, playing by the spring or elusticity of the air. A vessci proper for a rescrvoir, as $A B$, fig. 4, plate $x$, is providerl eitler of netal, or glass, or the like, ending in a small meck $c$, at the top: through this neck is put it tube $c d$, till the lower cnd come near the botton of the ressel, this being about half full of water. The neck is so contrived, that a syringe, or condensing pipe, maty be screwed on the tube' by means of which a large quantity of air may be intrulled through the tube into the water, out of which it will disengaore itself, and cmerge into the vacant part of the vessel, aud lic over the surface of the water $C D$.
Now, the water in the ressel being thus pressed by the air, which is, for ex, double the density of the cxternal air ; and the elastic force of air being proportional to its density, or to its gravitating force, the effect wall be the same, as if the weight of the column of air, over the surface of the water, were double that of the column pressing in the tube; so that the water must be forced upwards through the tube, when the syringe is removed, with a force equal to the excess of pressure of the included air, above that of the external, that is, in this case, with a force equal to the pressure of an entire colums of the atmosphere; which being equal to the pressure of a column of 33 or 34 feet of water, it follows that the fountain will play to nearly 33 or 34 feet high.

These aerial or aquatic fountains may be applied in different ways, so as to exhibit various appearances; and from these alone arises the greatest part of artificial waterworks: even the engine for extinguishing fire, is a fountain playing by the force of confincd air.

A Fountain spouting the water in yarious directions. Suppose AB the vertical tube, or spout, in which the water rises, (fig. 5, pl. $\mathrm{x}$ ): into this let several other tubes be fitted; some horizontal, others oblique, or inclining, or reclining, \& $\&$, as at $\mathrm{E}, \mathrm{n}, \mathrm{L}, \mathrm{N}, \mathrm{p}, \& \mathrm{c}$. Then, as all water retains the direction of the aperture through which it comes, that issuing through $\Lambda$ will risc perpendiculary; and the rest will tend different ways, describing arches of different magnitudes.

Or tbus: Suppose the vertical tube a B (fig, 6) through which the water rises, to be stopped at the top, as in $\Lambda$; and, instead of pipes or cocks, let it be only perforated with small holes all around, or only half round its surface: then will the water spout out, in all directions, through the little holes, to different distances.

And hence, if the tube $A \mathrm{~B}$ be about the height of a man, and having a turn-cock at $\mathrm{c}$; on opening this cock, the spectators will be sprinkled unexpectedly with a shower.

FounTaIN playing by drauing the breath. Suppose A (fig. 8 , plate viii), a globe of glass, or metal, in which is fitted a tube $\mathrm{CD}$, having a small orifice in $\mathrm{C}$, and reaching almost to $D$, the bottom of the globe. Then if the air be sucked, or drawn with the mouth, out of the tube $C D$, and the orifice $\mathrm{c}$ be immediatcly immerged under cold water, the water will ascend through the tube into the sphere. Thus proceeding, by repeated exsuctions, till the ressel be above half full of water; then applying the mouth to $\mathrm{c}$, and blowing air into the tube, on removing the mouth, the water will spout forth.-Or, if the globe be put into hot water, the air being thus rarefied, will make the water spout as before.

And this kind of fountain is called Pila Heronis, of Hero's Ball, from the name of its inventor.

Fov N TAI, whose stream raises and plays a brass bull. Provide a hollow brass ball a (fig.9, pl.viii), made very thin, that its weight may not be too great for the force of the water; and let the tube BC, through which the water rises, be exactly perpendicular to the horizon. Then the ball, being laid in the bottom of the cup or basols $\mathrm{B}$, will be carried up by the stream, and sustained at a considerable height, playing a litle up and down.

FounTan which spouts z:ater in form of a shower. To the tube in which the watcr is to rise, fit a spherical or lenticular head an (fig. I, pl. siii) made of a plate of metal, and perforated at the top with a great number of small holes. The water rising violently towards $A B$, will be 
there divided into innumerable little threads, and afterwards broken, and dispersed into the finest draps.

FountaIN which spreads the water in form of a tablecloth. To the tube AB (6g. 2, pl. xiii) solder two sphierical scgments, c and D, almost touching each other, with a screw $\varepsilon$, to contract or amplify the interstice or chink, at pleasure. Some choose to make as smooth and eren cleft, in a spherical or lenticular head, fitted upen the tube. The water spouting through this chink or cleft will expand itsell like a cloth.- And thus, the fountain may be made to spout out in the figure of inen, or other animals.

Fountain, which, when it has done spouting, may be turned like an hour-glass. Provide two glasses, $\mathrm{A}$ and $\mathrm{x}$, (fig. 3, pl. xiii) to be so mucli the larger as the fountain is to pliey the longer, and placed at so much the greater distance from each other as the water is desired to spout the higher. Let $C D E$ bo a crooked tube, furnished in $E$ with a jet; and GH another bent tube, furnished with a jet in I: GF and $\mathrm{KL}$ are to be other lesser tubes, open at both ends, and seaching. near the bottom of the vessels $A$ and $B$, to which the tubes $\mathrm{CD}$ and $\mathrm{GH}$ are likewise to reach.

Now if the ressel $\mathrm{A}$ be filled with water, it will descend through the tubc CD; and it will spout up through the jet $\mathrm{E}$, by the pressure of the column of water $\mathrm{CD}$. But unless the pipe $\mathrm{G} F$ were open at $G$, to let the air run up to $F$, and press at the top of the surface of the water in the cavity $A$, the water could not run down and spout at $\mathrm{E}$. After its fall again, it will sink through the little tube $\mathrm{K} L$, into the vessel $\mathrm{B}$, and expel the air throngh the tube GI. At length, when all the water is cmptied out of the vessel $A$, by turning the machine upside-down, the vessel B will be the reservoir, and make the water spout up through the jet 1 , the pipe $\mathrm{KL}$ supplying $\mathrm{B}$ with air to $\mathrm{lct}$ the water deseend in the direction $\mathrm{G} \mathrm{HI}$.

Hence, if the vessels $\Delta$ and $\mathrm{B}$ contain just as much water as will be spouted up in an hour's time, we shall have a spouting clepsydra, or water-clock; which may be graduated or clivided into quarters, minutes, \&c.

Fountarn of Command. This depends on the same principles with those of the former : CAE (fig. 4, pl. xiii) is a vessel of water secured against the entrance of the air, except through the pipe $\mathrm{GF}$, when the cock $\mathrm{c}$, by which it is filled, is shut. There is another pipe EDH from the bottom of the water to the jut $\mathrm{B}$ in the bason $\mathrm{D} \mathrm{E}$; but this is stopped by the cock H. At the lowest part of the bason $\mathrm{DE}$, there is a small hole at $\mathrm{I}$, to let the water of the bason DB run into the bason $\mathrm{GII}$ under it ; there is also a small triangular hole or wotch, in the bottom of the pipe $\mathrm{FG}_{\text {, at }} \mathrm{G}$. Turn the cock $\mathrm{H}$, and the fountain will play for some time, then stop, then play again alternately for several times together. When those times of playing and stopping are known before-hand, you may command the fountain to play or siop; whence its name. The cause of this phenomenon is als follows: the water coming down the.pipe EDrrs, would not come out at $\mathbf{s}$, if the air $s s$, above the water, were not supplied as it dilated: now it is stipplicd by thic pipe $G r$, which takes it in at the notch $\Theta$, and delivers it out at $\mathbf{F}$; but after some time the water, which was spouted out at $\mathrm{B}$, falling down into the bason $\mathrm{Dn}$, rises ligh enough to come above the motch $\mathrm{c}$, which stops the passage of the air; so that the air ss, above the water in the vessel $\mathrm{C} A \mathrm{E}$, wanting a supply, cannot sufficiently press, and the fountain ceases playing.-But when the water of $\mathrm{Dn}$ has run down into the lower bason $\mathrm{c} n$, through the hole $\mathrm{r}$, till it falls below the top of the notch $\mathrm{G}$, the air runs up into the upper re: ceptacle, and supplies that at $s s$, and the fountain plays again. This is seen a little before-hand, by a skin of water on the notch $\mathrm{a}$, before the air firds a passige, and then ycu may command the fountain to play. It is evident that the hole 1 inust be less than the bole of the jet, or else all the water wosld run out into the lower bason, without rising high enough to stop the notch $\mathrm{G}$.

Fou NTAIN that begins to play upon the lighting of crindles, and ceases as they go out. Provide two cylindrical vessels, $A B$ and $C D$, (fig. 7 , pl. xiii,) connect them by tukes, opell at both ends, $K \mathrm{~L}, \mathrm{BE}$, \& $\mathrm{c}$, so that the air may descend uut of the higher into the lower : to the tubes solder candlesticks, H, \& c, and to the hollow cover of the lower vessel CE, fit a little tube or jet, FG, furnished with a cock (s, ain: reaching almost to the hottom of the vessel. In $\mathrm{c}$ let there be an iperture, furnished with a screw, by which water may be poured into $C D$. Then, on lighing the candles H, \&c, the air in the contiguous tubes becoming racefied by the heat, the water begins to spout througla $G \mathrm{~F}$.

By the same contrivance may a statue be marle to shed tears on the shining of the sun, or nn lighting a candle, \&c: all that is here requircd, being only to lay tubes from the cavity where the air is rarefied, to some other cavities placed near the eyes, full of water.

$A$ Founta ix by the Rarefuction of the Air, maly be made in the following manner: Let $A B$ and $C D$, fig. 5 , pl. xiii, be two pipes fixed to a brass head c, made to screw into a glass ressel $\mathrm{E}$, which having a little water in it, is inverted till the pipes are screwed on; then reverting it suddenly, so as to put $A$, the lower end of the spouting pipe $A B$, into a jar of water $A$, and the lower end of the descending pipe $C D$, into a receiving vessel $D$, the water will spout up from the jar $A$ into the tall glass vessel $\mathrm{E}$, from which it will go down at the mouth $c$, through $\mathrm{CD}$, into the vessel $\mathrm{n}$, till the water is wholly emptied out of $A$, making a fountain in $\mathbf{E}$, into $\mathbf{D}$. The reason of the play of the fountain is this: the pipe $C D$, being 2 feet 9 inches long, lets dewn a column of water, which rarefies the air 1-12th part in the vessel $\mathrm{E}$, wherc it presses against the water spouting at $B$ with $1-12$ th of the force by which the water is pushed up at the hole $A$, by the pressure of the common air en the water in the vessel $A$; so that the water spouts up into $\mathrm{E}$, when the air is rarefied $1-12$ th, with the difference of the pressure of the atmosphere, and the forcmentioned rarefied air; i.c. of 33 to $2 \frac{3}{4}$, or of 12 to 1. This wonld raise the water 2 feet 9 inches; but the length of the pipe $A$, of 9 inches, being deducted, the jet will only rise 2 fect. This, say's Desaguliers, may be called a syphon fountain, where $A B$ is the driving $\mathrm{I} g \mathrm{~g}$, and $C D$ the issuing leg.

Fou Nua of Jero of Alexandria, so called; because it was contriver by lita. In the second fountain above described, the air is compressed by a syringe; in this, (sce fig. 6 , pl. xiii) the air, being only compressed by the concealed fall of water, makcs a jet, which, aiter some continuance, is corsidered by the ignorant as a perpetual motion; because they imagine that the same water which fell from the jet rises again. The boxes $\mathrm{cr}$ and $\mathrm{nyx}$, being close, we see

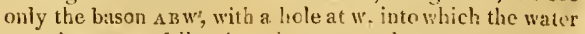
spouting at B falls; but that water does not come up again; for it runs rown thoush the pipe wx into the box DYx, whence it drives out the air, through the ascending pipe $\mathrm{r} z$, into the cavity of the box $\mathrm{CE}$, where, pressing upon the water that is in it, it forces it rout throngh the syenting-pipe $O B$, as long as there is any water in $C E$; so that this 
whole play is only while the water contained in CE, having spouted out, falls down through the pipe wx, or of the boxes CE. and DY above one another: the height of the water, measured from the bason $A B W$ to the surface of the water in the lower box Dxx, is always equal to the heigbt measured from the top of the jet to the surface of the water in the middle cavity at $\mathrm{CE}$. Now, since the surface $\mathrm{CE}$ is always falling, and the water in DY always rising, the height of the jet must continually decrease, till it is shorter by the deptl of the cavity $\mathrm{CE}$, which is emptying, added to the depth of the cavity $\mathrm{Dr}$, which is always filling; and when the jet is fallen so low, it immediately ceases. The air is represented by the points in this figure.

To prepare this fountain for playing, which should be done unobserved, pour in water at $w$, till the cavity $\mathbf{D X Y}$ is filled; then invert the fountain, and the water will run from the cavity DXY into the cavity $C E$, which may be known to be full, when the water runs out at $B$ held down. Set the fountain up again, and, to make it play, pour in about a pint of water into the bason $A B w$; and as soon as it has filled the pipe $w x$ it will begin to play, and continue as.long as there is any water in CE. You may then pour back the water left in the bason $A \mathrm{~B} w$, into any vessel, and invert the fountain, which being set upright again, will be inade to play, by putting back the water poured out into ABW; and so on as often as you please.

Spouting Foun TA1N, or Jet d'Eau, is any fountain whose water is darted forth impetuously through jets, or ajutages, and returns in form of rains, nets, folds, or the like.

Foun TAIN-Pen, is a pen contrived to contain a quantity of ink, and let it flow very gently, so as to supply the writer a long time without the necessity of taking fresh ink.

The fountain-pen, represented fig. s, pl. xiii, consists of divers pieces of metal, $F, G, 11$, the middle piece $F$ carrying the pen, which is screwed into the inside of a small pipe; and this again is soldered into another pipe of the same size as the lid $G$; in which lid is soldered a male screw, for screwing on the cover; as also for stopping a little hole at the place, and bindering the ink from passing through it : at the other end of the piece $F$ is a small pipe, on the outside of which may be screwed the top cover II. A portccraion goes in the cover, to be screwed into the last-mentioned pipe, to stop the end of the pipe into which the ink is to be poured by a funnel.

To use the p'n, the cover $\mathrm{G}$ must be taken off, and the pen a little shaken, to make the ink run more freely.

FOURTH, in Music, one of the harmonic intervals, or concords. It consists in the mixture of two sounds, which are in the ratio of 4, to 3 ; i. c. of two sounds produced by chords, whose lengths are to each other as 4 to.3.

FRACTION, or Broken Number, in Arithmetic and Atgebra, is a part, or some parts, of another number or quantity considered as a whole, but dirided into a certain number of parts; as 3-4.ths, which denotes 3 parts out of 4, of any quantity. Fractions are usually divided into vulgar, decimal, duodecimal, and sexagesimal. For the last three sorts, see the respective words.

Vulgar Fuacryons, called also simple Fractions, are usually denoted by two numbers, the one set under the other, with a small line between them : thus $\frac{3}{4}$ denotes the fraction three-fourths, or 3 parts out of 4 , of some whole considered as divided into 4 equal parts.

The lower number 4, is called the Denominator of the fraction, showing into how many parts the whole or inte- ger is divided; and the upper number 3 , is called the $\mathrm{Nu}$ merator, and shows how many of those equal parts are contained in the fraction. Hence it follows that as the numerator is to the denominator, so is the fraction itself, to the whole of which it is a fraction; or as the denominator is to the numerator, so is the whole or integer, to the fraction: thus, the integer being denoted by 1 , as $4: 3:: 1: \frac{3}{4}$ the fraction.-And hence there may be innumerable fractions all of the same value, as there may be innumerable quantities all in the same ratio, viz, of 4 to 3 ; such as 8 to 6 , or 12 to 9 , \&c. So that if the two terms of any fraction, i. e. the numerator and denominator, be either both multiplied or both divided by any number, the resulting fraction will still be of the same value: thus, $\frac{3}{4}$ or $\frac{6}{8}$ or $\frac{9}{12}$ or $\frac{12}{1} \frac{2}{6} \& c$, are all of the same value with each other.

Fractional expressions are usually distinguished into proper and improper, simple and compound, and mixt numbers.

$A$ Proper Fn actiox, is that whose numerator is less than the denominator; and consequently the fraction is less than the whole or integer; as $\frac{3}{4}$.

An Improper Fnactiox, is when the numerator is either equal to, or greater than, the denominator; and consequently the fraction either equal to, or greater than, the whole integer, as $\frac{4}{4}$, which is equal to the whole; or $\frac{5}{4}$, which is greater than the whole.

Simple Fractions, or Single Fractions, are such as consist of only one numerator, and one denominator; as $\frac{3}{4}$, or $\frac{5}{4}$, or $\frac{12}{2} \frac{2}{5}$.

Compound Fractroxs are fractions of fractions, and consist of several fractions, connected together by the word of : as $\frac{2}{3}$ of $\frac{3}{4}$, or $\frac{1}{2}$ of $\frac{2}{3}$, of $\frac{3}{4}$.

A Mixt Number consists of an integer and a fraction together: as $1 \frac{3}{4}$, or $12 \frac{2}{3}$.

The artihmetic of fractions consists in the reduction, addition, subtraction, multiplication, and division of them.

Reduction of $\mathrm{F}_{\mathrm{R}} \mathrm{A}$ CTIons is of several sorts; as 1 . To reduce a given whole number into a fraction of any given denominotor. Multiply the given integer by the proposed denominator, and the product will be the numerator. Thus, it is found that $3=\frac{\kappa}{2}$, and $5=\frac{20}{4}$.

If no denominator be given, or it be only proposed to express the integer fraction-wise, or like a fraction; set 1 beneath it, for its denominator. So $3=\frac{3}{1}$, and $5=\frac{5}{1}$, and $7=\frac{7}{1}$.

2. To reduce a givcn fraction to another fraction equal to it, that shall have a given denominator. MIultiply the numerator by the proposed denominator, and divide the product by the former denominator, then the quotient set over the proposed denominator will form the fraction required. Thus, if it be proposed to reduce $\frac{3}{4}$ to an equal fraction whose denominator shall be $S$; then $3 \times 8=24$, and $24 \div 4=6$ the numerator, so that $\frac{6}{8}$ is the fraction sought, being $=\frac{3}{4}$, and having 8 for its denominator.

3. To ablreviate, or reduce fractions to loter terms. Divide their terms, i.e. the numerator and denominator, by any number that will divide them without at renaineler, so shall the quotients be the corresponding torms of a new fraction, equal to the former, but in smaller numbers. In like manner abbreviate these new tr'ms ngain, and so on till there be no number grater than a that will divide them without a remainder, and then the fraction is said to be in its least terks. 'Tluus, to abbreviate $\frac{1}{0} 5$; first divide both terms by 5 , and the fraction becomes $T_{T}^{3}$; next divicle these by 3 , and it bccomes $\frac{1}{4}$ : so that $\frac{15}{60}=\frac{3}{x^{1}}=\frac{1}{4}$, in its least terms. 
To reduce a fraction at once to its lowest terms; divide woth numerator and denominator by their greatest common measure: to find which, see the article Common Measure.

It may further be observed, that a proper fraction is in its lowest terms, when the numerator and denominator are not both divisible by their difference, or by some submultiple of their difference, without a remainder.

4. Toreduce fractions to other equivalent ones of the same denominator. Multiply each numerator, separately taken, by all the denominators except its own, and the products will be the new numerators; then multiply all the denominators continually together, for the common denominator, to these numerators. Thus, $\frac{2}{3}$ and $\frac{4}{5}$ reduce to $\frac{10}{15}$ and $\frac{12}{1} \frac{2}{5} ;$ and $\frac{2}{3}, \frac{3}{4}$, and $\frac{4}{5}$ reduce to $\frac{4}{6} \frac{0}{6}, \frac{4}{6} 6$, and $\frac{48}{60}$.

5. To find the value of a fraction, in the known parts of its integer. Multiply always the numerator by the number of parts of the next inferior denomination, and divide the 16$) \overline{180}$ ( 11 s products by the denominator. Do the same with the remainders through all the denominations. So to find the value of $\frac{9}{16}$ of a pound sterling; multiply 9 by 20 for shillings, and dividing by 16 , gives 11 for the shillings; then multiply the remainder 4 by 12 pence, and dividing by 16 gives 3 for pence: so that $11 s .3 d$. is the value of $\frac{9}{Y b} l$.

$\frac{9}{20}$
$\frac{180}{16}(11 s$
$\frac{16}{20}$
$\frac{16}{4}$
$\frac{12}{48(3 d}$
48

6. To reduce a mixi number to an equivalent improper fraction. Multiply the integer by the denominator, and to the product add the numerator, for the new numerator, to be set over the same denominator as before. Thus $3 \frac{5}{8}$ becomes $\frac{29}{8}$.

7. To reduce an improper fraction to its equivalent whole or mixed number. Divide the numerator by the denominator; so shall the quotient be the integral part, and the remainder set over the denominator will form the fractional part of the equivalent mixed number. Thus $\frac{29}{8}$ reduces to $3 \frac{5}{8}$, and $\frac{32}{4}=8$.

S. To reduce a compound fraction to a simple one. Multiply all the numerators together for the numerator, and all the denominators together for the denominator, of the simple fraction sought. Thus, $\frac{1}{2}$ of $\frac{3}{4}=\frac{3}{5}$, and $\frac{2}{3}$ of $\frac{4}{5}$ of $\frac{7}{9}$ $=\frac{56}{13}$.

To reduce a vulgar fraction to a decimal. See DECIMALS. And for several other particulars concerning Reduction, as well as the other operations in fractions; see my Arithmetic.

Addition of Fractions. First reduce the fractions to their simplest form, and reduce them also to a common denominator, if their denominators are different; then asld all the numerators together, and set the sun over the common denominator, for the sum of all the fractions required.

Thus, $\frac{2}{5}+\frac{3}{5}=\frac{5}{5}=1$;

And $\frac{2}{7}+\frac{3}{4}=\frac{8}{28}+\frac{21}{28}=\frac{29}{28}=1 \frac{1}{28}$.

Subtraction of Fractions. Reduce the fractions the same as for addition; then subtract the one numerator from the other, and set the difierence over the common denominator.

$$
\begin{aligned}
& \text { So } \frac{7}{5}-\frac{3}{5}=\frac{4}{5} ; \\
& \text { And } \frac{7}{5}-\frac{4}{7}=\frac{4}{6} \frac{9}{3}-\frac{36}{6}=\frac{13}{6} \text {. }
\end{aligned}
$$

To Multiply Mnctions together. Reduce them all to the form of simple fractions, if they be not so; then multiply all the numerators together for the numerator, and all the denominators together for the denominator of the product sought.

VoL. 1.
Thus $\frac{2}{3} \times \frac{4}{5}=\frac{8}{15}$

And $\frac{3}{8} \times \frac{5}{6} \times \frac{7}{10}=\frac{105}{48}=\frac{21}{96}=\frac{7}{32}$.

To Divide Fractions. Divide the numerator by the numerator, and the denominator by the denominator, if they will exactly divide. Thus, $\frac{8}{15} \div \frac{4}{5}=\frac{2}{3}$.

But if they will not divide without a remainder, then multiply the dividend by the reciprocal, of the divisor, that is, by the fraction obtained by inverting or cluanging its terms. Thus, $\frac{8}{15} \div \frac{3}{4}=\frac{8}{15} \times \frac{4}{3}=\frac{32}{45}$.

Algebraic Fractions, or FRACTIONS in Species, are exactly similar to vulgar fractions, in numbers, and all the operations are perfurmed exactly in the same way; therefore the rules need not he repeated, and it may be sufficient here to set down a few examples to the foregoing rules. Thus,

1. The fraction $\frac{a a b}{b c}$ abbreviates to $\frac{a a}{c}$.

2. $\frac{6 a^{3}-9 a x^{2}}{6 a^{2}+3 a x}=\frac{2 a^{2}-3 x^{3}}{2 a+x}$, by dividing by $3 a$.

3. $\frac{a^{3}-a^{2} x+a x^{2}-x^{3}}{a^{3}-a x}=\frac{a^{3}+x^{2}}{a}$, by dividing by $a-x$.

See Common Measure.

4. $\frac{a}{b}$ and $\frac{c}{d}$ become $\frac{a d}{b d}$ and $\frac{b c}{b d}$, when reduced to a common denominator.

5. $\frac{a}{b}+\frac{c}{d}=\frac{a d+b c}{b d}$.

6. $\frac{a}{x}-\frac{b}{z}=\frac{a z-l x}{x z}$.

7. $\frac{a}{b} \times \frac{c}{d}=\frac{a c}{b d}$.

8. $\frac{a}{x} \div \frac{b}{z}=\frac{a}{x} \times \frac{z}{b}=\frac{a z}{b x}$.

Continued Fraction, is used for a fraction whose denominator is an integer with a fraction, which latter fraction has for its denominator an integer and a fraction, and the same for this last fraction again, and so on, to any extent, whether supposed to be infinitely continued, or broken off after any number of terms. Euler, Analys. Inf. vol. 1, p. 295.

$$
\begin{aligned}
& \text { or } \frac{x}{2}+\frac{x}{3}+\frac{x}{4} \text {, } \\
& \text { or } \frac{x}{2}+\frac{x}{3}+\frac{3}{4}+\frac{4}{5}+8 c \text {. }
\end{aligned}
$$

Or, using letters instead of numbers,

$$
\frac{1}{a}+\frac{1}{b}+\frac{1}{c}+\frac{1}{d}+\frac{1}{a}+\frac{A}{b}+\frac{\mathrm{c}}{c}+\frac{\mathrm{D}}{d}+\& \mathrm{c} \text {. }
$$

When these series are not far extended, it is not diffcult to collect them by common arithmetic.

Lord Brounker, it appears, was the first who considered continued fractions, or at least, who applied them to the quadrature of curves, in Wallis's Arith. Infin. prop. 191 , vol. 1, pa. $469 \& \mathrm{c}$, where this author explains the manner of forming them, giving several numeral examples, in approximating ratios, as well ás the general series

$$
\frac{a}{\alpha} \frac{b}{\beta} \frac{c}{\gamma} \frac{d}{\delta} \& \mathrm{c}, \text { as he denotes it. }
$$

Huygens also used it for the like purpose, viz, to approximate the ratios of large numbers, in his Descrip. Autom. 


\section{F R $\Lambda$}

Planet.in Oper. Reliq. pa. 173 \&c, edit. Amst. 1728. And a special treatise on Continued Fractions was given by Euler, in his Analys. Infm. vol, 1, pa. 295 \& c.

This subject is perlaps capable of much improvement, though it has been rather neglected, as very little use has been made of it, except, by those authors, in approximating to the value of fractions, and ratios, tbat are expressed in large numbers; besides a method of Goniometry by De Lagny, explained in the Introduction to my Logarithms, pa. 78 ; as also some use I have made of it in summing very slowly converging series, in my Tracts, vol. 1, pa. 203.

As to the reducing of common fractions, and ratios, that are expressed in large numbers, to continued fractions, it is no more than the common method of finding the greatest common measure of those two numbers, by dividing the greater by the less, and the last divisor always by the last remainder; for then the several quotients are the denominators of the fractions, the numerators being always 1 or unity. Thus, to find approximating values of the fraction $\frac{31415926535}{10000000000}$, or to the ratio of 314115926535 to 10000000000 , leing the ratio of the circumference of a circle to its diameter, by means of a continued fraction; or, to change the said common fraction to a continued fraction: Dividing the greater term always by the less, the same as to find the greatest common measure of the said numbers or terms, the several quotients will be 3,7 , $15,1,292,1,1, \& c$, which, after the first, will be the denominators, to the common numerator 1 ; and therefore the said fraction will be changed into this continued fraction,

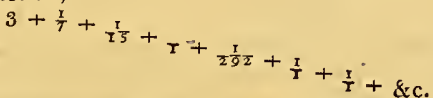

Hence, stopping at any part of these single fractions, one after another, will give several values of the proposed ratio, all successively nearer and nearer the truth, but alternately too great and too little. So, stopping at $\frac{r}{7}$, it is $3 \frac{1}{7}=\frac{22}{T}=3 \cdot 1428571$, too great, or 22 to 7 , the ratio of the circumference to the diameter as given by Archimedes. Again, leaving off at $\frac{T}{T 5}$, it is

$$
3 \frac{1}{7 \frac{1}{15}}=3 \frac{15}{106}=\frac{33}{106}=3.141509 \& \mathrm{c} \text {, too little. }
$$

But stopping at $\frac{x}{x}$,

it is $3 \frac{1}{7, \frac{1}{15}+\frac{1}{4}}=3 \frac{1}{7 \frac{1}{96}}=3 \frac{16}{113}=\frac{35}{1} \frac{5}{3}$ (the ratio of $\mathrm{Mc}$ tius) $=3.1415929 \& \mathrm{c}$, which is rather too great. And so on, always nearer and nearer, but alternately too great and too little.

And, in like manner is any algebraic fraction thrown into a continued fraction. As the fraction

$$
a \beta \gamma \delta+a \beta d+a c \delta
$$

$\overline{u \beta \gamma \gamma^{2}+u \beta d+a d^{2}+l \gamma \delta+b d}$, which being in like manner divided, the quotients are $\frac{a}{a}, \frac{\beta}{b}, \frac{\gamma}{c}, \frac{\delta}{d}$; which single fractions being considered as denominators to other fractions whose common numerator is 1 , these will be the reciprocals of the former, and so will become $\frac{a}{a}, \frac{b}{\beta}, \frac{c}{\gamma}, \frac{d}{\delta}$; and lience the proposed common fraction is equal to this terminate continucd,

$$
\frac{a}{a}+\frac{b}{+}+\frac{c}{\gamma}+\frac{i}{j} \text {. }
$$

F R A

On the other hand, any continued fraction being given, its equivalent common fraction will be found, by beginning at the last denominator, or lowest end of the given continued fraction, and gradually collecting the fractions backwards, till we arrive at the first, when the whole will thus be brought to one common fraction; as was done above in collecting the fractions

$$
\frac{x}{7}+\frac{x}{x \frac{x}{5}+\frac{x}{x}+\frac{x}{2} \bar{x}+\& c .}
$$

And in like manner the continued fraction

$$
\begin{aligned}
& \frac{a}{a}+\frac{b}{\beta}+\frac{c}{\gamma}+\frac{d}{\delta} \text { collects into the fraction } \\
& a \beta \gamma \delta+a \beta d+a c \delta
\end{aligned}
$$$$
\overline{a \beta \gamma \delta+a \beta d+a c \delta}+l y \delta+b u l^{l}
$$

When the given continued fraction is an infinite one, collect it successively, first one term, then two together, three together, \&c, till the sum is sufficiently exact. Or, if these collected sums be found to converge too slowly to the true value, having collected a few of the terms into successive sums, these being alternately too great and too little, the true value will be found as near as you please by the method of arithmetical means, explained in my Tracts, vol. 1, Tract 8, pa. 176 .

When the denominators of a continued fraction recur in any certain order, as in the following;

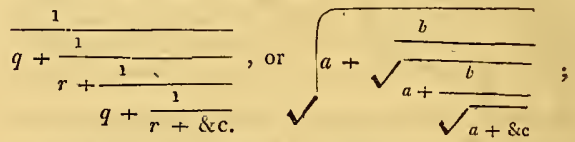

its value may be computed by making it first equal to $x$, and then solving the equation: thus, suppose the value of the first was required; then put the whole $=x$, and we get $\frac{1}{q+\frac{1}{r+x}}=x$, or $\frac{r+x}{q r+q x+1}=x$ : hence $r+x$ $=q r x+q x^{2}+x$, and $x^{2}+r x-\frac{r}{q}=0$, from which $x$, and consequently the value of the fraction, may be determined, by solving the quadratic.

In the same manner may the values of other quantities, which run on in infinitum, be found if the factors recur.

\section{Example. Required the value of}

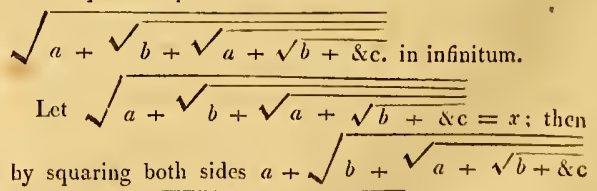
$=x^{2} ;$ and

$$
\sqrt{b+\sqrt{a+\sqrt{b+8 c}}}=x^{2}-a \text {; there- }
$$

fore $b+\sqrt{a+\sqrt{b+x c}}=x^{4}-2 a x^{2}+a^{2}$, or $b+x=x^{4}-2 a x^{2}+a^{2}$; hence $x^{4}-2 a x^{2}-x+a^{2}-$ $b=0$, whence the value of $x$ may be found.

If the letters be all equal, as

$$
\begin{aligned}
& \sqrt{a+\sqrt{a+\sqrt{a+\sqrt{a c c}}}} \text { Put it }=x \text {, then square } \\
& \text { the cquation, so sliall } x^{2}=a+
\end{aligned}
$$


$\sqrt{a+\sqrt{a+\sqrt{a+\sqrt{a \& c}}}}$ hence $x^{2}-a=$ $-\sqrt{a}$ $a+\sqrt{a+\sqrt{a \& c)}}=x$ the first value, because it reverts to the same quantity; and from this quadratic equation we find $x=\frac{1}{2}+\sqrt{ }\left(a+\frac{1}{2}\right)$. Again, to find the value of

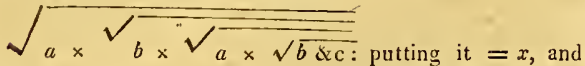
squaring, we have $x^{2}=a \sqrt{b \sqrt{a \sqrt{b \& c}}}$ or ${ }_{a}^{x^{2}}=$ $\sqrt{b \sqrt{a \sqrt{b \text { ac }}}}$; then this squared, and divided by $b$, gives $\frac{x^{*}}{a^{2} b}=(\sqrt{a \sqrt{b \sqrt{a \sqrt{b+c}})}}$ or $x^{3}=a^{2} b$, and $x=\sqrt[3]{a^{2} b}$.-If $b=a$, or the radicals be always the same letter $a$, then $x=\sqrt[3]{a^{3}}=a$.

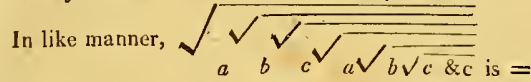
$\sqrt[7]{ } a^{4} b^{2} c$, or $=\sqrt[7]{ } a^{7}=a$, when $b$ and $c$ are taken $=a$.

Vanisting Fractions. Such fractions as have both their numerator and denominator vanish, or equal to 0 , at the same time, may be called Vanishing Fractions. We are not to conclude that such fractions are equal to nothing, or lave no value; for that they bave a certain determinate value, has been shown by the best mathematicians. The idea of such fractions as these, first originated in a very severe contest among some French mathematicians, in which Varignon and Rolle were the two chief opposite combatants, concerning the then new or ditierential calculus, of which the latter gentleman was a strenuous opponent. Among other arguments against it, he proposed an example of drawing a tangent to certain curves at the point where the two parts cross each other; and as the fractional expression for the subtangent, by that method, had both its numerator and denominator equal to 0 at the point proposed, Rolle considered it as an absurd expression, and as an argument against the method of solution itself. The seeming mystery however was soon explained, and first of all by John Bernoulli. See an account of this controversy in Montucla, Mist. Math. vol. 2, pa. 366 , Ist ed.

Since that tine, such kind of fractions have often been contemplated by mathematicians. As, by Maclaurin, in his fluxions, vol. 2, pa. 698: Saunderson, in his Algebra, vol. 2, art. 469: De Moivre, in Miscel. Anal. pa. 165: Emerson, in his Algebra, pa. 212: and by many others. The same fractions have also proved a stumbling-block to more mathematicians than one, and the cause of more violent controversies: witness that between Powell and Waring, at the time when Mr. Maseres was a competitor of the latter for the professorship at Cambridge. In the specimen of a work published on occasion of that competion, by Waring, was the fraction $\frac{p-p^{5}}{1-p}$, which he said became 4 when $p$ was $=1$. This was struck at by Powell, as absurd, becausc he said when $p=1$, then the iraction $\frac{p-p^{3}}{1-p}=\frac{1-1}{1-1}=\frac{0}{0}$. Waring replied that $\frac{p-p^{6}}{1-p}$ is $=p+p^{2}+p^{3}+p^{4}$ (by common division) $=1+1+1+1=4$, when $p$ is $=1$. See the contro- versial pamphlets that passed between those two gentlemen at that time.

There are several methots of finding the value of such fractions, that have been given by the gentlemen above quoted. The one is by considering the terms of the fraction as two variable quantities, continually decreasing, till they both vanish together; or finding the ultimate value of the ratio denoted by the fraction. In this way of considering the matter, it appears that, as the terms of the fraction are supposed to decrease till they vanish, or become only equal to their fluxions or their increments, the value of the fraction at that state, will be equal to the fluxion or increment of the numerator divided by that of the denominator. Hence then, taking the example $\frac{x-x^{3}}{1-x}$ when $x=1$; the fluxion of the numerator is $\dot{x}-5 x^{4} \dot{x}$, and of the denominator $-\dot{x}$; therefore

$\frac{\dot{x}-5 x^{2} \dot{x}}{-\dot{x}}=\frac{1-5 x^{4}}{-1}=\frac{5 x^{4}-1}{1}=5 x^{4}-1=5-1=4$, the value of the fraction $\frac{x-x^{5}}{1-x}$ when $x=1,-\mathrm{Or}$, thus, because $x=1$, therefore $\frac{x-x^{5}}{1-x}=\frac{1-x^{4}}{1-x}$; then the fluxion of the numerator, $-4 x^{3} \dot{x}$, divided by the fluxion of the denominator, or $-\dot{x}$, gives $4 x^{3}$ or 4 , the same as before.

Another method is by reducing the given cxpression to another, or simpler form, and then substituting the values of the letters. 'So in the above example $\frac{x-x^{5}}{1-x}$, or $\frac{1-x^{4}}{1-x}$, when $x=1$; divide the numerator by the denominator, and it becomes $1+x+x^{2}+x^{3}$, which when $x=1$, becomes 4 , for the given fraction, the same as beforcAgain, to find the value of $\frac{a^{\prime} \sqrt{ } u-x x}{a-\sqrt{ } a x}$ when $x$ is $=a$, in which case both the numerator and denominator become $=0$. Divide the numerator by the denominator, and the quoticnt is $\sqrt{ } a x+x+x \sqrt{\frac{x}{a}}$; which when $x=a$, becomes $a+a+a=3 a$, for the value of the fraction in that state of it.

Mr. Woodhouse, in his Principles of Analytical Calculation, has endeavoured to prove, that the method of expanding these fractions for estimating their values, is an arbitrary operation; that there is nothing contained in the significancy of such expressions which renders any such step necessary. He admits, that in the investigations of extension and of motion, we are necessitated, from the nature of the case, to compute a vanishing fraction by a process exactly similar to that just adverted to ; but contends the necessity of such operation when it is abstractedly proposed to find its value. For, says he; 'Propose the question separately, what does $\frac{x^{\mathrm{m}}-a^{\mathrm{m}}}{x^{n}-a^{\mathrm{n}}}$ become, when $x=a$ ? and the obvious answer to any mind undebauched with mathematical sophistry is $\frac{a^{\mathrm{m}}-a^{\mathrm{m}}}{a^{\mathrm{m}}-a^{\mathrm{n}}}=\frac{0}{0}$. A different result can be obtained by a different process, but why ought that process to be followed? No satisfactory answer can be given to this question, when it is abstractedly proposcul to find its value.'

In order to show somewhat the importance of this question, let us suppose that in the investigation of a problem, we obtained for the fluent corrected

$z=x^{\frac{5}{2}}-\left(x^{2}+b^{2}\right)^{\frac{2}{2}} \times \frac{x^{2}-a^{3}}{x-a}$, what would le the value 
of $z$ when $x=a$ ? It is evident that a case is bere presented, in which the computation of a vanishing fraction becomes an abstract proposition: but does it hence follow, that to expand the expression for effecting such computation is an arbitrary process? Surely not. The bare assertion that there is nothing contained in the significancy of the fraction which renders the expansion of the same necessary for the estimation of its value, is no proof whatever against the truth of the method; or that it is not a necessary step; since if it were true, that the fraction did not contain a value in this case, such truth would evidently appear in the result of that operation; that is neither more nor less than nothing would be expressed by the quotient. Now it is obvious that if $\frac{a^{2}-a^{a}}{a-a}$ has a value, we bave no other menns of determining it than by expanding the expression: for in the form $\frac{a^{2}-a^{2}}{a-a}$, we can derive nothing as a true and precise value of it. Let $\frac{a^{2}-a^{2}}{a-a}$ be put $=\frac{0}{0}$; it may be so, and we seceven in this case that the fraction may be equal to some finite quantity; that is to say, this expression does not preclude the possibility of its baving some definite value, though it affords no clue for computation in this particular state. Hence, since it must be granted, that a fraction and its equizalent whole number are in ull cases equal to cach other; it follows that if in any case a valuc be exhibited in one form, it is likewise contained in the other, and consequently must be given to that other; therefore, the equivalent whole number of $\frac{a^{2}-a^{2}}{a-a}=\frac{0}{0}$, having some definite value, $\frac{a^{2}-a^{2}}{a-a}$ itself, has also the same value. And hence the true value of $z$, in the case where $x=a$ is $a^{\frac{5}{2}}-\left(a^{2}+b^{2}\right)^{\frac{3}{2}} \times 2 a$, or $a \times\left[a \sqrt{ } a-2 \sqrt{ }\left(a^{2}+b^{2}\right)\right]$.

It is not therefore true that to expand the fractions to obtain its true value, is an arbitrary operation, but a process of necessity, rendered unavoidable by something contained in the significancy of the expression. 'To admit the necessity of expanding the fraction when its quantity is to be computed, in any investigation of extension or of motion; and to deny that necessity when it is abstractedly proposed to find its value, is confessing, that a fraction and its equivalent whole number are to be considered equal only in particular cases, which is absurd.

FRAISE, in Fortification, a kind of defence, consisting of pointed stakes, driven almost parallel to the horizon into the retrenchments of a camp, \&c, to ward off and prevent any approach or scalade.

FRANKLIN (Dr. BenJamin), one of the most celebrated philosophers and politicians of the 18 th century, was born at Boston in North America in the year 1706 , being the youngest of 13 , children. His father was a tallow-chandler in Boston, and young Franklin was taken from school at 10 years of age, to assist him in his business. In this situation he continued two years; but disliking that occupation, he was bound apprentice to an wiler brother, who was then a printer in Boston, but had learned that business in London, and who in the year 1721 began to print a newspaper, being the second ever published in America; the copies of which our author was sent to distribute, after having assisted in composing and printing it. On this occasion, our young philosopher enjoyed the sccret and singular pleasure of being the muchadnired author of many cssays in this paper; a circum- stance which he had the address to keep a secret even from his brother himself; and this when he was only 15 years of age. The frequent ill usage from his brother, induced our author to quit bis service, which he did, at the age of 17 , and went to New York. But not meeting cmployment bere, he went forward to Philadelphia, where be worked with a printer a short time; after which, at the instance of Sir Willliam Keith, governor of the province, he returned to Boston to solicit pecuniary assistance from his father, to set up a printing-house for himself at Philadelphia, on the promise of great encouragement from Sir William, \&c. His father, however, thought fit to refuse such aid, alleging that he was yet too young (18 years. old) to be intrusted with such a concern; so our author agair returned' to Pbiladelphia without it. Upon this, Sir William said he would advance the sum that might be necessary, and our young philosopher should go to England, and purchase all the types and materials, for which purpose he would give him letters of credit. He could never however get these letters; jet by dint of fair promises of their being sent on board the ship after him, he sailed for England, expecting these letters of credit were in the governor's packet, which he was to receive on its being opened. In this bowever he was cruelly deceived, and thus he was sent to London without either moncy; friends, or credit, at is years of age.

Under these circumstances his only resource was that of employment, which he shortly obtained, as a journeyman printer, first at a Mr. Palmer's, and afterwards with Mr. Watts, with whom he worked a consicierable time, and by whom he was greatly esteemed, being also treated with such kindness, that it was ever after most gratefully remembered by our philosopher.

After a stay of 18 months in London, he returned to Philadelphia, in 1726, with a merchant of that town, as his clerk, on a salary of 50 pounds a year. But bis master dying the year after, he again engaged to direct the printing business of the same person with whom he had worked before. After continuing with him the best part of a jear, Franklin, in partnership with another young man, at length set up a printing-house himself.

A short time previous to this, our author had gradually associated a number of persons, like himself, of a rational and philosophic turn of mind, and formesl them into a club or society, to hold meetings, to converse together for their mutual improvement in an kinds of uscful knowledge, which was in high repute for many ycars after. A mong many other useful regulations, they ayreed to bring such books is they had into one place, to form a common library. 'This resurece being found defective, at Franklin's persuasion they resolver to contribute a small sum monthly towards the purchase of books for their use from London. Thus their stock began to increase rapidly; and the inhabitants of Philadelphia, buing desirous of having a share in their literary knowledge, proposed that the books should be lent out on paying a small sum for the indulgence. Thus in a few years the society became rich, and possessed more books than were perhaps to be found in all the other colonies: the collection was advanced into a public library; and the other colonies, sensible of its advantages, began to form similar plans; whence originated the libraries at Boston, New lork, Charkstown, \&e ; that of Philadelphia being now not inferior to any in liurope.

About 1728 or 1729 , our author set up a newspaper, the second in Philadedphia, which prosed cery protitable, 
and otherwise useful, as affording an opportunity of making himself known as a political writer, by inserting several of his writings of that kind in it. In addition to his printing-house, he set up a shop to sell bocks and stationary; and in 1730, entering into a matrimonial engagement, his wife proved very useful in assisting to manage the shop, \&rc. He afterwards began to have some leisure, both for reading books, and writing them, of which be gave many specimens from time to time. In 1732 be began to publish Poor Richard's Almanac, which was continued for many years. It was always remarkable for the numerous and valuable concise maxims which it contained, for the œconomy of human life, all tending to exhort to industry and frugality: and in the almanac for the last year, all the maxims were collected in an address to the realler, entitled, The Way to IVealth. This has been translated into various languages, and inserted in different publications. It has also been printed on a large shect, proper to be framed, and hung up in conspicuous places in all houses, as it justly deserves to be. Mr. Franklin became gradually more known for his political talents, and in the year 1736 , he was appointed clerk to the General Assembly of Pennsylvania; and was re-elected by succeeding assemblies for several years, till he was chosen a representative for the city of Philadelphia ; and in 1737 he was appointed post-master of that city. In 1738 , he formed the first fire-company there, to extinguish and prevent fires and the burring of houses: an example which was soon followed by other persons, and in other places. And soon after, he suggested the plan of an association for insuring houses and ships from losses by fire, which was adopted; and the association continues to this day. In the year 1744, during a war between France and Great Britain, some French and Indians made iuroads on the frontier inhabitants of the province, who were unprovided for such an attack : the situation of the province was at this time truly alarming, bcing destitute of every means of defence. At this crisis Franklin stepped forth, and proposed to a meeting of the citizens of Philarlelphia, a plan of a voluntary association for the defence of the province. This was approved of, and signed by 1200 persons immediately. Copies of the same were circulated through the province; and in a short time the number of signers amounted to 10,000 . Franklin was chosen colonel of the Philadelphia regiment; but lie did not think proper to accept of the honour.

Pursuits of a different nature now occupied the greatest part of his attention for some years. Being always much addicted to the study of natural philosophy; and the discovery of the Jeyden experiment in electricity having rendered that science an object of general curiosity; Mr. Franklin applied bimself to it with great diligence, and soon began to distinguish himself very eminently in that way. He engaged in a course of electrical experiments with all the ardour and thirst for discovery which characterized the philosophers of thatday. By these, he was cnabled to make a number of important discoveries, and to propose theories to account for various phenomena; which have been generally adopted, and which will probably endure for ages. His observations he comnunicated, in a series of lettres, to his friend Mr. Collinson; the first of whicl is dated March 28, 1747. In these he makes known the power of points in drawing and throwing off the rlectric matter, which had hitherto escaped the notice of electricians. He also made the discovery of a plus and minus, or of a positive and ungative statc of electricity; from which be explained in a satisfactory manner the phenomena of the Leyden phial, first observed by Cuneus or Muschenbroeck, which had much perplexed philosophers. He showed that the bottle, when charged, contained no more elcctricity than before, but that as much was taken from one side as was thrown upon the other; and that, to discharge the same, it was only necessary to make a communication between the two sides, by which the equilibrium might be restored, and that then no signs of electricity would remain. He afterwards demonstrated by experiments, that the electricity did not reside in the coating, as had been supposed, but in the pores of the glass itself. After a phial was charged, he removed the coating, and found that upon applying a new coating the shock might still be received. In the year 1749 , he first suggested his idea of explaining the phenomena of thunder-gusts, and of the aurora borealis, upon electrical principles. He points out many particulars in which lightning and electricity agree; and likewise adduces many facts, and reasoning from facts, in support of his positions. In the same year he conceived the bold and grand idea of ascertaining the truth of his doctrine, by actually drawing down the forked lightning, by means of sharp-pointed iron rods, raised into the region of the clouds; whence he derived bis method of securing buildings and ships from being damaged by lightning. It was not until the summer, of 1752 that he was enabled to complete his grand discovery-the cxperiment of the electrical kite, which being raised up into the clouds, brought thence the electricity or lightning down to the earth; and M. D'Alibard made a like experiment about the same time in France, by following the track which Franklin bad before pointed out. The letters which he sent to Mr. Collinson, it is said, were refused a place anong the papers of the Royal Society of London; and Mlr. Collinson published them in a separate volume, under the title of New Experiments and Observations on Electricity, made at Philadelphia, in America; which were read with avidity, and soon translated into different languages. His theorics were at first opposed by several philosophers, and by the members of the Royal Society of London; but in 175.5 , when he returned to that city, they voted him the gold medal which is annually given to the person who presents the best paper on some interesting subject. He was a!so admitted a member of the society, and had the rlegree of doctor of laws conferred on him by different universities: but at this time, by reason of the war which broke out between Britain and France, he returned to Anerica, and interested himselt in the public aftairs of that country. Indeed he had clone this long before; for although philusophy was a principal object of Franklin's pursuit for several ycars, he did not confine himself to it alone. In the year 1747 he became a member of the General Assembly of Pennsylvania, as a burgess for the city of Philadelphia. Being a friend to the rights of man from his infancy, he soon distinguished himselfas a steady opponent of the unjust schenes of the proprictaries. He was looked up to as the head of the opposition; and to him have been attributed many of the spirited replics of the assembly, to the messages of the governors. Ilis influence in the body was very great. 'Ihis arose not from any superior powers of cloquence; he spoke but seldom, and he never was known to nake any thing like an claborate harangue. His speeches often consisted of a single sentence, or of a well-told story, the moral of which was always obviousiy to the point. He never attempted the flowery ficlds of 
oratory. His manner was plain and mild. His style in speaking wan, like that of his writings, simple, unadorned, and remarkably concise. With this plain manner, and his penetrating and solid judgment, he was áble to confound the most cloquent and subtle of his adversaries, to confirm the opinions of his friends, and to make converts of the unprejudiced who had opposed him. With a single observation, he has rendered of no avail a long and elegant discourse, and determined the fate of a question of importance.

In the year 1749 he proposed the plan of an academy, to be erected in the city of Philadelphia, as a foundation for posterity to (rect a seminary of learning, more extensive and suitable to future circumstances; and in the beginning of 1750 , three of the schools were opcued, namely, the Latin and Greck school, the Mathematical, and the English schools. This foundation soon after gave rise to another more extensive college, incorporated by charter May 27, 1755, which still subsists, and in a very flourishing condition. In 1752 , he wis instrumental in the establishment of the Pennsylvania Hospital, for the cure and relief of indigent invalids, which has proved of the greatest use to that class of persons. Having conducted himself $: 0$ well as postmaster of Philadelphia, he was, in 1753, appointed deputy postmaster-general for the whole British colonies.

These being inuch exposed to depredations on their frontier by the Indians and the French, at a meeting of commissioners from several of the provinces, Mr. Franklin proposed a plan for the general defence, to establish in the colonies a general government, to be administered by a president-general, appointed by the crown, and by a grand council, consisting of members chosen by the representatives of the different colonies; a plan which was unanimously agreed to by the commissioners present. The plan however harl a singular fate: it was disapproved of by the ministry of Great Britain, because it gave too much power to the representatives of the people; and it was rejected by every assembly, as giving to the president-general, who was to be the representative of the crown, an infuence greater than appeared to them proper, in a plan of government intmded for frecmen. Perbaps this rejection, on both sides, is the strongest proof that could be adduced of the excellence of the system, as suited to the situation of Great Britain and America at that time. It appears to have steered exactly in the middle, between the oppositc interests of both. Whether the adoption of this plan would bave prevented the separation of America from Great Britain, is a question which might afford much room for soeculation.

In the year 1755, General Braddock, with some regiinents of regular troops, and provincial levies, was sent to dispossess the French of the posts on which they had seized in the back settlenents. After the men were all ready, a difficntty occurred, which had nearly prevented the expethition. This was the want of waygons. Iranklin now stepped forwald, and with the assistance of his son, in a very short time procured 150. After the defeat of Braddock, our autbor introduced into the assembly a bill for organizing a militia, and has the desterity to get it parssed. In consecuence of this act a very respectuble militia was formed; and l'anklin was appointed colonel of a regiment in lhilidelplia, which consisted of $1200 \mathrm{men}$; in which capueity he acquitterl himself with much propricty, and was of singular service: though this inilitia was soon after disbanded by order of the English ministry.
In 1757 , he was sent to England, with a petition to the king and council, against the proprietaries, who refused to bear any share in the public expenses and assessments; which he got settled to the satisfaction of the state. After the coinpletion of this business, Franklin remained at the court of Great Britain for some time, as agent for the province of Pennsylvania; and also for those of Massachisetts, Maryland, and Georgia. Soon after this, he published his Canada pamphlet, in which he pointed out, in a very forcible manner, the advantages that would result from the conquest of this province from the Frepch. An expedition was accordingly planned and fitted out, and the command given to General Wolfe; the success of which is well known. He now divided his time indeed between phulosophy and politics, rendering many services to botb. While here, he invented the elegant musical instrument called the Harmonica, formed of glasses played on by the fingers. In the summer of 1762 he returned to America; on his passage to which he observed the singular effect produced by the agitation of a vessel, containing oil floating on water: the upper surface of the oil remaining sin ooth and undisturbed, while the water was agitated with the utmost commotion. On his return he received the thanks of the Assembly of Pennsylvania, which having annually elected him a member in his absence, he again took his seat in this body, and continued a steady defender of the liberties of the people.

In 1764 , by the intrigues of the proprietaries, Franklin lost his seat in the assembly, which he had possessed for 14 years; but was immediately appointed provincial agent to England, for which country he presently set out. In 1760 he was examined before the parliament relative to the stamp-act; which was soon after repealed. The same year he made a journey into Holland and Germany; and another in to France; being everywhere received with the greatest respect by the literati of all nations. In 1773 he attracted the public attention by a letter on the duel between Mr. Whately and Mr. Temple, concerning the publication of Governor Hutchinson's letters, declaring that he was the person who had discovered those letters. On the 29th of January next year, he was examined before the privy-council, on a petition he had presented long before, as agent for Massachusetts Bay, against Mr. Hutchinson: but this potition being disagreable to ministry, it was precipitately rejected, and Dr. Franklin was soon after removed from his office of postmaster-general for America. Finding now all efforts to restore harmony between Great Britain and her colonies useless, he returned to $A$ merica in 1775 ; just after the commencement of hostilities. Being named one of the delegates to the Contineutal Congress, he had a principal share in bringing about the revolution and declaration of independency on the part of the colonies. In 1776 he was deputed by Congress to Canada, to negociate with the people of that country, and to persuade them to throw off the British yoke; but the Canadians had been so much disgusted with the hot-headed real of the New Englanders, who had burnt some of their chapels, that they refused to listen to the proposals, though enforced by all the arguments Dr. Finklin could make use of. On his return to Plilarlelphia, Congress, sensible how much he was esteemed in france, sent lim thither to put a finishing hand to the prisate negrociations of Mr. Silas 1) (anc; and this important commission was readily accepted by the doctor, though then in the 7 st year of his age. 'Thic event is well hnown; a treaty of alliance and commerce was signed between France and America; and 
M. Le Roi asserts, that the doctor had a great share in the transaction, by strongly advising M. Maurepas not to lose a single moment, if he wished to secure the friendship of America, and to detach it from the mother-country. In 1777 he was regularly appointed plenipotentiary from Congress to the French court; but obtained leave of dismission in 1780. Having at length seen the full accomplistument of his wishes by the conclusion of the peace in 1783, which gave independency to America, he became desirous of revisiting his native country. He therefore requested to be recalled; and, after repeated solicitations, Mr. Jefferson was appointed in his stead. On the arrival of his successor, he repaired to Havre de Grace, and crossing the channel, landed at Newport in the Isle of Wight; whence, after a favourable passage, he arrived safely at Philadelphia in September 1785. He was received amidst the acclamations of a vast multitude, who flocked from all parts to see hím, and who conducted him in triumpl to his own house; where in a few days lie was visited by the members of the Congress and the principal inhabitunts of Philadelphia. He was afterward twice chosen president of the Assenubly of Philadelphia; but his increasing infirmities obliged him to ask permission to retire, and to spend the remainder of his life in tranquillity; which was granted, in 1788 . After this, the infirmities of age iucreased fast upon him; he became more and more afflicted with the gout and the stone, till the time of his death, which happened the $17 \mathrm{th}$ of April 1790 , about 11 at night, at 84 years of age; leaving one son, governor iVilliam Franklin, a zealous loyalist, who resided in London, on a pension from the English government, till his death in the year 1813 ; and a daughter, married to Mr. William Bache, merchant in Philadelphia.

Doctor Franklin was author of many tracts on electricity, and other branches of natural philosophy, as well as un politics, and miscellaneous subjects. He had also many papers inserted in the Philosophical Transactions, from vol. 47 to vol. 64 .

FREEZE, or FuIzE, in Architecture, a large fat member, being that part of the entablature of columns that separates the architrave from the curnice.

FREEZING, or Congclation, the fixing of a fluisl body into a firm or solid mass by the abstraction of its caloric : in which sense the term is applied to water when it freezes into ice; to metals wheu they resume their solid state, after being melted by heat; ol to glass, wax, pitch, tallow, \&cc, when they harden again after having been rendered fluid by leat. But it differs from crystallization, which is rather a separation of the particles of a solid from a fluid, in which it had been dissolved more by the moisture than the action of heat.

The process of congelation is always attended with the emission of heat or caloric, as is found by experiments on the freezing of water, wax, spermaceti, \&c; for in such cases it is always found, that a thermometer dipt into the fluid mass, keeps continually descending as this cools, till it arrive at a certain point, being the point of freezing, which is peculiar to each fluid, where it is rather stationary, and then rises for a little, while the congelation proceeds. Lut by what means it is that fluid bodies should thus be rentlered solid by cold, or loss of caloric; or fluid by the absorption of this substance; or what is introduced into the bodies by either of these principles, are matters which phitosophers luave never yet been able to discover, or to satisfy themselves upon. 'The following phenomena howerer are usually obscrved. Water, and some other fluids, suddenly dilate and expand in the act of freezing, so as to occuly a greater space in the furm of ice than before, in consequence of which it is that "ice is specifically lighter than the same fluid, and floats in it. And the uegree of expansion of water, in the state of ice, is by some authors computed at about $T_{T}^{\frac{1}{\delta}}$ of its volume. Oil however is an exception to this property, and quicksilver too, which slirinks and contracts still more after freezing. Boyle relates scveral experiments of vessels marle of metal, very thick and strong; in which, when filled with water, close stopped, and exposed to the cold, the water being expanded in freezing, and not finding either room or vent, burst the vessels. A strong barrel of a gutl, filled with water, and close stopped and frozen, was rent the whole lengtl. Huygens, to try the force with which water expands when frezing, filled a cannon with it, whose sides were an inch thick, and then closed up the mouth and vent, so that none cuuld escape; the whole being exposed to a strong freezing air, the water froze in about 12 hours, and burst the piece in two places. Mathematicians have. computed the force of the ice upon this occasion; alid they say, that such a force would raise a wright of $27 \% 20$ pounds. Lastly, Major Edward Williams, of the Royal Artillery, made many experiments on the force of freezing water at Quebec, in the years 1784 and 1785 . He filled all sizes of iron bomb-shells with this fluid, then plugged the fuze bole close up, and exposed them to the strong freczing air of the winter in that climate; sometimes driving in the iron plugs as liard as possible with a sledge hammer; and yet they were always thrown out by the sudden expansion of the ivater in the act of freezing, like a ball shot by gunpowder, sometimes to the distance of 4 or 500 feet, though they weighed nearly 3 pnunds; and when the plugs were screved in, or furvished with hooks or barls, to lay hold of the inside of the shell by, so that they could not possibly be forced out, in this case the shell was always split in two, though the thickuess of the metal of the shell was an inch and three quarters. It is further remarkable, that through the circular crack, round about the shells, where they burst, there stood out a thin film or sheet of ice, like a fin; and in the cases when the plugs were projected by freczing water, there suddenly jssued out from the fuze-hole, a bolt of ice, of the same diameter, and stond over it to the height sometimes of $S$ inches and a half. And hence we need not be surprised at the cffects of ice in destroying the substance of vegetables and trees, and even splitting rocks, when the frost is carried to excess. See other similar instances in the article Cond.

It is also observed that water loses of its weight by freezing, being found lighter after thawing again, tham before it was frozen. And indeed it evaporates almost as fast when truzen, as when it is fluid.

We likewise find it asserted, that water does not freeze in vacuo; requiring for thatypurpose the presence and contiguity of the air. But this circumstance is liable to some doubt, and it may be suspecter that the degree of cold has not been carricd far enough in these particular instances; as it is found that mercury in thermometers has even been frozen, thongh it requires a vastly greater degree of cold to frecze this Auid than water. Besides, the assertion lins been lately disprovul by the experiments of Profe'ssner Leslic and Dr. Marcet, \&c, who have frozen not water only, but even mercury, in the exhausted receiver, and that only by simple evaporation.

That water which has beeli boiled freezs more realily than that which has not been boiled; and that a slight 
commotion of the fleid disposes it to freeze more speedily; having sometimes been cooled several degrees below the freezing point, without congealing when kept quite still, but suddenly freezing into ice on the slightest agitation.

That the water, being covered over with a surface of oil of olives, does not freeze so readily as without it; and that nut oil absolutely preserves it under a strong frost, when olive oil would not.

That rectified spirit of wine, nut oil, and oil of turpentine, seldom freeze.

That the surface of the water, in freezing, appears all wrinkled; the wrinkles being sometimes in parallel lines, and sometimes like rays, proceeding from a centre to the circumference. See the articles Congelation, Cord, and $I_{C E}$.

Freezixg Mixture, a preparation for the artificial congelation of water, and other fluids. According to Mr. Boyle, all kinds of salts, whether alkaline or acid; and even all spirits, as spirit of wine, \& c ; as also sugar, and saccharum saturni, mixed with snow, are capable of freezing most fluids; and the same effect is produced, iu a very high degree, by a mixture of oil of vitriol, or spirit of nitre, with snow.

The same is also remarked by M. Homberg of equal quantities of corrosive sublimate, and sal ammoniac, with four times the quantity of distilled vinegar.

Boerhaave gives a method of producing artificial frost without either snow or ice: we must have for this purpose, at any season of the year, tbe coldest water that can be procured; this is to be mixed with a proper quantity of any salt (sal ammoniac will answer the intention best), at the rate of about 3 ounces to a quart of water. Another quart of water must be prepared in the same manner with the first; the salt, by being dissolved in each, will make the water much colder than it was before. The two quarts are then to be mixed together, and this will make them colder still. Two quarts more of water prepared and mixed in the same manner are to be inixed with these, which will increase the cold to a much higher degree in all. The whole of this operation is to be carricd on in a cold cellar; and a glass of common water is then to be placed in the vessel of the fluid thus artificially cooled, and it will be turned into ice in the space of 12 hours.

There is also a method of making artificial ice by means of snow, without any kind of salt. For this purpose fill a snall pewter dish with water, and upon that set a common pewter plate filled, but not heaped, with snow. Bring this simple apparatus near the fire, and stir the snow in the plate: the snow will dissolve, and the ice will be formed on the back of the plate, which was set in the dish of water.

M. Reaumur tried the effect of several salts, and examined the various degrees of cold by an ice thermometer, which being placed in the fluid to be frozen, showed very exactly the degree of cold by the descent of the spirit.

Nitre, or saltpetre, usually passes for a salt that may be very serviceable in these artificial congelations; but the experiments of this gentleman prove that this opinion is crroncons. The most perfectly refined saltpetre employed in the operation sunk the spirit in the thermometer only $3 \frac{x}{2}$ degrees below the fixed point. Less refined nitre depressed the thermometer lower, and gave a greater clegree of cold; owing to the common or sea-salt that it cuntains when less pure, which has a greater effect than the pure saltpetre itself.

Two parts of common salt being mixed with three parts of powdered icc in very hot weather, the spirit in the ther- mometer immediately descended 15 degrees, which is half a degree lower than it would have descended in the severest cold of our winters. M. Reaumur then tried the salts all round, determining with great regularity and exactness, what was the degree of cold occasioned by each in a given dose. Among the neutral salts, none produced a greater degree of cold than the common sea salt. Among the alkalies, sal ammoniac sunk the thermometer only to 13 degrees. Pot-ashes sunk it just as low as well-refined saltpetre.

For the common uses of the table, the ice is not required to be very hard, or such as is produced by long continuance of violent cold: it is rather desired to be like snow. Saltpetre, which is no very powerful agent in freèing, is therefore more fit for the, purpose than a more potent salt. It is not necessary that the congelation should be very suddenly made; but that it may retain its form as long as may be, when made, is of great importance. If it be desired to have ices very hard and firm, and very suddenly prepared, then sea salt is of all others most to be chosen for the operation. The ices thus made will be very hard, but they will soon run. Pot-ashes afford an ice of about the hardness that is usually required. This forms inceed very slowly, but then it will preserve a long time. And common wood-ashes will perform the business very rearly in the same manner as the pot-ashes; but for this purpose, the wood which is burnt, ought to be fresh.

The strong acid spirits of the neutral salts act much more powerfully in these congelations than the salts themselves, or indeed than any simple salt can do. Thus, spirit of nitre mixed with twice its quantity of powdered ice, immediately sinks the spirit in the thermometer to 19 degrees, or 4 degrees more than that obtained by means of sea salt, the most powerful of all the salts in making artificial cold. A much greater degree of cold may be given to this mixture, by piling it round with more ice mixed with sea salt. This gives a redoubled cold, and sinks the thermometer to 24 degrees. If this whole matter be covered with a fresh mixture of spirit of nitre and ice, a still greater degree of cold is produced, and so on; the cold being by this method of fresh additions to be increased almost without bounds: but it is to observed, that every addition gives a smaller increase than the former.

It is very rmarkable in the acid spirits, that though sea salt is so much more powerful than nitre in súbstance in producing cold, yet the spirit of nitre is much stronger than that of sea salt; and another not less wonderful phenomenon is, that spirit of wine, which is little else than liquid fire, has as powerful an etfect in congelations, or very nearly so, as the spirit of nitre itself.

The several liquid substances which produce cold, in the same manner as the dry salts on being mised with ice, are much more speedy in their action than the salts : because they immediately and much more intimately come into contact with the particles of the ice, than the salts can. Of this nature are spirit of nitre, spirit of wine, \&x. To produce the expected degree of cold, it is always necessary that the ice and the additive matter, whatever it may be, should both rush togother, and intimately uniting, form one clear fluid. It is hence that no new cold is prom duced with oil, which, though it melts the ice, yet cannot mix itself into a lomogeneous liquid with it, but must always remain floating on the surface of the water that is produced by the melting of the ice. Mcm. Acad. Scienc. Par. 1734.

It has been discovered, that fluids standing in a current of air, grow by this means much colder than beforc. Fah- 
renheit had long since observed, that a pond, which stands quite calm, often acquires a degree of cold much beyond what is sufficient for freezing, and yet no congelation ensued : but if a slight breath of air happens in such a case to glide over the surface of the water, it stiffens the whole in an instant. It has also been discovered, that all substances becone colder by the evaporation of the fluids they contain, or with which they are mixed. If both these methods therefore be practised on the same body at the same time, they will increase the cold to almost any degree of intenseness we please.

Most extraordinary instances of artificial freezing have since been made in Russia and at Hudson's Bay, and other parts, by which quicksilver was frozen into a solid mass of metal. This indeed had before been effected by the natural cold of the atmosphere alone, in Siberia. In the winter of 1733 , Professor Gmelin, with two other gentlemen of the Russian academy, were sent by Anne Ivanouna, the new empress, to explore and describe the different parts of her Asiatic dominions, with the communication of Asia and America. In the same season of the year 1734-5, M. Gmelin being at Yeneseisk in $58^{\circ} 30^{\prime}$ north lat. and $92^{\circ}$ long. east from Greenwich, first observed such a descent of the mercury, as must have been attended with congelation, being far brlow its freezing point, now fixed at -40 of Fahrenheit's thermometer. "Here," says he, "we first experienced the truth of what various travellers have related, with respect to the extreme cold of Siberia; for, about the middle of December, such severe weather set in, as we were sure had never been known in our time at Petersburg. The air seemed as if it were frozen, with the appearance of a fog, which did not suffer the smoke to ascend as it issued from the chimneys. 'Birds fell from the air as lead, and froze immediately, unless they were brought into a warm room. Whenever the door was opened, a fog suddenly formed round it. During the day, short as it was, parlelia and haloes about the sun were frequently seen; and in the night mock moons, and haloes about the moon. Finally, our thicrnometer, not subject to the same deception as the senses, left us no doubt of the excessive cold ; for the quicksilver in it was reduced, on the 5th of January, old style, to $-120^{\circ}$ of Fahrenheit's scale, lower than it had ever been observerl in nature."

The next instance of congelation occurred at Yakutsk, in $62^{\circ}$ north lat. and $1.50^{\circ}$ east longitude. The weather here was unusually mild for the climate, yet the thermoneter fell to $-72^{\circ}$; and one person informed the professor by a note, that the mercury in his barometer was frozen. He bastened immediately to his bouse to bebold such a surprising placumenon; but though he was witness to the fact, observing that the mercury did not continue in one column, but was divided in different places as into little cylinders, which appeared frozen, yet the prejudice he had entertained igainst the possibility of the congelation, would not allow him to believe it.

Another set of obsersations, in the course of which the mercury must frequently have boen congeated, were mate by $\mathrm{l}^{\text {'rofessor }} \mathrm{Gmelin}$ at Kirenga-furt, in $57 \frac{\pi}{2}$ rorth lat., and 108 east long.; his thermometer, at diflercht times, standing at $-108,-86,-100,-113$, and many other intermediate degrees; in the course of the winter of 1737-8. On the 27 th of November, after the thernometer had been standing lor two dlays at $-46^{\circ}$, he observed it sunk at noon to - ios. Suspecting some mistake, after be had noted down the observation, he iustiutly ran back, and

Vol. I. found it at -102 ; but ascending with such rapidity, that in the space of half an honr it had risen to $-19^{\circ}$. This phenomenon, which appeared so surprising, doubtless depended on the expansion of the mercury frozen in the bulb of the thermometer, and which, now nuelting, forced upwards the small thread in the stem. Similar appeárances were observed afterwards, when the thread of quicksilver in the thermometer was separated about 6 degrees.

A second instance, where a natural congelation of mercury has certainly been observed, is recorded in the Transactions of the Royal Academy of Sciences at Stockholm, as made by Mr. Andrew Hellant. The weather, in $\mathrm{J}_{\mathrm{a}-}$ nuary 1760 , was remarkably cold in Lapland; so that on the 5 th of that month, the thermometers fell to -76 , -128 , or lower; on the $23 \mathrm{~d}$ and following days they fell to $-58,-79,-92$, and below -238 entirely into the ball. This was observed at four different places in Lapland, situated between the 65 th and 78 th degrees of north lat. and the 21st and 2sth degrees of east longitude.

But the congelation of quicksilver, by an artificial freezing mixture, was first observed, and put beyond loutut, by Mr. Joseph Adam Braun, professor of philosophy at Petersburg. This gentlemran, wishing to try how many degrees of cold he could producc, availed himself of a good opportunity, which offered for that purpose, on the 14th of December 1759 , when the mercury in the thcrmometer stood in the natural cold at -34 , which it is now known is only 5 or 6 degrees above its point of congelation. Assisting this natural cold therefore, with a mixture prepared of aquafortis and pounded ice, his thermometer was sunk to -69 . Part of the quicksilver must now have been really congealed, but unexpected by him, and he only thought of pursuing his object of producing still greater degrees of cold ; and having expended all his pounded ice, he was obliged to use snow instead of it. With this fresh mixture the mercury sunk to $-100,-240$, and $-350^{\circ}$. Taking the thermongter out, he found it whole, but the quicksilver fixed, and it continued so for 12 minutes. On repeating the experiment, with anothè thermometer which had been graduated no lower than -220 , all the mercury sunk into the ball, and became solid as before, and did not re-ascend till after a still longer interval of time: Mr. Braun now suspected that the quicksilver was really frozen, and prepared for making a decisive experiment. This was accomplished on the 25 th of the same month, and the bulb of the thermometer broken as soon as the metal was congealed; when it appeared that the mercury was changed into a solid and sliuning metallic mass, which flatted and extended uncler the strokes a pestle, being rather less liard than lead, and yielding at dull sound lihe that metal. Mr. Epinus made similar experiments at the same time, amploying as well thermometers as tubes of a larger bore; in which last he remarked, that the quicksilver fell sensibly on being trozen, assuming a concave surtace, and also, that the congealed pices sunk in fluid mercury: also, in their furater experiments, they invantiably found that the mercury sunk lower when the whole of it was congealed, than if any part of it remained tluid: all showing that, contrary to water, mercury contracted in freczing. It was further observed, that the mercury when congenled looked like the most polished silver, and when beaten flat, it was casily cut with a penknife, like soft thin sheet lead.

'The fact being tlus established, and fuidity us) longer. to be considered as an essential property of yuicksilver, Mr. Braun communicated an account of bis experiments 4. B 
to the Petersburg academy, on the 6th of September 1760 ; of which a large extract was inserted in the Philos. Trans. vol.52, pa.156. He afterwards declared that he never suffered a winter to pass without repeating the experiment of freezing quicksilver, and never failed of success when the natural cold was of a sufficient strength for the purpose; and this degree of natural cold he supposes at -10 of Fahrenheit; though some commencement of the congelation might be perceived when the temperature of the air was as high as $\div 2$.

The results of all his experiments were, that with the abovementioned frigorific mixtures, and once with rectified spirits and snow, when the natural cold was at $-28^{\circ}$, he congealed the quicksilver, and discovered that it is a real metal, that melts with a very small degree of heat. How ever, not perceiving the necessary consequence of its great contraction in freezing, he always confounded its point of congelation, with that of its greatest contraction in freezing, and thus marked the former a great deal too low.

In the process of his observations, Mi. Braun found that double aquafortis was more effectual than spirit of nitre; but witb this simple spirit, which seldom brings the mercury lower than $-14 \mathrm{~S}$, this metal may le frozen in the following manner: Six glasses being filled with'snow as usual, and the thermometer placed in one of them, the spirit of nitre was poured upon it; when the mercury wonld fall no lower in this, the thermometer was removed to the second, and so on to the third and fourth, in which fourth immersion the mercury was congealed.

Mr. Epinus gives the following direction for using the fuming spirit of nitre : Take some of this spirit, cooled as much as possible, and put it into a wine-glass till it be about half full, filling it up with snow, and stirring them till the mixture become of the consistence of pap; by which means you obtain, almost in an instant, the necessary degree of cold for the freezing of quicksilver.

It is remarked by MIr. Braun, that by the mixture of snow and spirit of nitre, which congealed the mercury, be never was able to bring thermometers, filled with the most highly rectified spirit of wine, lower than -148 : so that the cold which will freeze mercury, will not frecze spirit of wine; and therefore spirit thermometers are the most fit to determine the degree of colleness in frigorific mixtures, till we can constrict solid metillic thermometers with sufficient accuracy. This author tried the effects of different fluids in bis frigorific compositions : he alivay's found that Glauber's spirit of nitre and double aquafortis were the most powerful; and from a number of experiments inade when the temperature of the air was between 21 and 28 of Fahrenheit, he concludes, that spirit of salt potred upon snow increased the natural cold $36^{\circ}$; spirit of sal ammoniac, 12; oil of vitriol, 42 ; Glauber's spirit of nitre, 70 ; aquafortis, 48 ; șimple spirit of nitre, 36; dulcified spirit of vitriol, 21; Hoffman's anodyne liquor, 38 ; spirit of hartshorn, 12 ; spirit of sulphur, 12; spirit of wine rectified, 24; camphorated spirit, 18; French brandy, 14; and several kinds, of wine increased the natural cold to 7,8 , or ? degrees.

The most remarkable congelation of mercury, by natural cold, that has ever been observed, was that related by Dr. P'eter Simon Pallas, who had been sent by the: empress: of liussia, with some other gentlemen, on an experdition similar to that of M. Gmelin. Bjeng at Krasnoyarsk in the year 1772 , in north lit. $56^{\circ} 30^{\prime}$, and east long. $93^{\circ}$, he had an opportunity of observing the pheno- menon we speak of. On the 6th and 7 th of December that year, says he, there happened the greatest cold I have ever experienced in Siberia : the air was calm at the time, and sceningly thickened; so that, though the sky was in other respects clear, the sun appeared as through a fog. I had only one small thermometer left, in which the scale went no lower than $-7^{\circ}$; and on the 6 th in the morning, I remarked that the quicksilver in the tube sunk into the ball, except some short columns which stuck fast to its surface. When the ball of the thermometer, as it hung in the open air, was tonched with the finger, the quicksilver rose'; and it could plainly be seen that the solid columns stuck and resisted a good while, and were at length pushed upward with a seening violence. He also placed upon the gallery, on the north side of his house, some quicksilver in an open bowl. Within an hour he observed the erlges and surface of it frozen solid; and some minutes afterward the whole was condensed by the natural cold into a soft mass very much like tin. While the inner part was still fluid, the frozen surface exhibited a great varety of branched wriukles; but in general it remained pretty smooth in freezing. 'The congealed mercury was more flexible than lead; but on being bent short, it was found more brittle than tin; and when bammered ont thin, it appeared somewhat granulated. When the hammer was not perfectly cooled, the quicksilver melted away under it in drops; and the same thing happened when the nietal was touched with the finger, by which also the finger was jmmediately benumbed. When the frozen mass was broken to pieces in the cold, the fragments adthered to each other and to the bowl in which they were placed. In the warm room it thawed on its surface gradually, by drops, like wax on the fire. Though the frost seemed to abate a little towards night, yet the congealed quicksilver remained unaltered, and the experiment with the thermometer could still be repeated. On the 7 th of December he had an opportunity of making the same observations all day; but some hours afte'r sunset, a porth-west wind sprung up, which raised the thermometer to $-46^{\circ}$, when the mass of quicksilver began to melt.

'The experiments of Mr. Braun were successfully repeated at Gottingen, in 17it, by Mr. John Frederick Blumenbach; being encouraged to this attempt by the excessive cold of the winter that year, especially the night of January the 11 th, when lie made the experiment, the thermometer standing at -10 in the open air. Mr. Blumenbach at 5 in the evening, put 3 drachms of quicksilver into a small sugar-glass, anil covered it with a misture of snow and Egyptian sal ammoniac, setting the glass out in the air upon a mixture also of sal ammoniac. At nne the next morning, the mercury was found frozen quite solid, and harel to the glass; and did not melt again till 7 or 8 the next morning. The colour of the frozen mercury was a dull pale white with a blucish casl, like zinc, very different from the natural appearance of quicksilwer.

By similar means, quicksilver was twice frozen by $\mathrm{Mr}$. Hutchins, governor of Albany-fort, in llurlson's-bay, in the months of January and February of 1775 . And the same was done on the 28 th of January 1776 , by Dr. Lambert bicker, secetury of liotterlam. The temperature of the atmosphere was then at $+2^{\circ}$; and the lowest it could reduce the thermometer by artiticial cold, was - $9+$; when, on breaking the glass, the mercury was found congealed.

In the beginuing of the year $17 \mathrm{SO}, \mathrm{M}$. Von Elterlein of 
Vytegra, a town of Russia, in lat. $61^{\circ}$ north, and long. $36^{\circ}$ east, froze quicksilver by natural cold. On the 4 th of January 1780 , the cold being increased to -34 that evening at Vytegra, he exposed to the open air 3 ounces of very pure quicksilver in a china teacup, covered with paper pierced full of holes. Next day, at 8 in the morning, he found it solid, and looking like a piece of cast lead, with a considerable depression in the middle. On attempting to loosen it in the cup, his knife raised shavings from it as if it had beenlead, which remained sticking up; and at length the metal separated from the bottom of the cup in one mass. He then took it in his liand to try if it would bend: it was stiff like glue, and broke into two pieces; but his fingers immediatcly lost all fecling, and coulr scarcely be restored in an hour and a lialf by rubbing them with snow. At $80^{\circ}$ clock the thermometer stood at -57 ; but balf after 9 it was risen to -40 ; and then the two picces of mercury which lay in the cup had lost so inuch of their liardness, that they could no longer be broken, or cut into shavings, but resembled a thick amalgam, which, though it became fluid when pressed by the fingers, immediately afterwards resumed the consistence of pap. With the thermometer at -39 , the quicksilver became fluirl. The cold was never less on the 5 th than -28 , and by 9 in the evening it had increased again to -33. This experiment seems to fix the freczing point of mercury at -40 of Fahrenheit's thermometer, or 40 below 0 ; which is $72^{\circ}$ below the freczing point of watcr.

During the winter of 1781 and $-82,-\mathrm{Mr}$. Ilutchins resumed the subject of freczing quicksilier by artificial cold, with such success, that from his experiments and those of M. Von Elterlein, last mentioned, the freezing point of mercury is now almost as well settled, viz, at -40 , as that of water is at +32 .

An instance of the natural congelation of quicksilver also occurred in Jemptland, one of the provinces of Siveden, on the 1st of January 1782; and lastly, on the 26 th of the same month, Mr. Hutchins obscrved the same effect of the cold at Hudson's-bay; when he found that at the point of its freezing a mercurial thermometer stuod at -40 , and a spirit thermometer at -30 .

On this subject, sce the article CoLD; see also the Philos. Trans.vol.51, pa. 672 ; vol. 52 , pa.156; vol. 66 , pa. 174 ; vol.73, pa. 303 and 325 ; vol.76, pa.24.1; vol. 77 , pa. 285 ; vol.78, pa. 43 ; and several others, particularly vol. 79, pa. 199, \&c, being experiments on the congclation of quicksilver in Frigland, by Mr. Richard Walker, where he proves that mercury may he frozen not only in England in summer, but even in the hottest climate, at any season of the year, and without the application of ice or suow.

About the ycar 1810, Professor Leslic, of Edinburgh, simply by evaporation, succceded in freezing watcr inclosed in an exhausterl receiver, to promote a quick evaporation. The water is placed over an open vessel, containing sulphuric acid, which absorbs the vapour as it exhales from the water; the whole being inclosed within the receiver of an air-pump, the water cools as the exhaustion proceeds; and is ultimately changed into ice. Mr. Leslie afterwards succcerled in freezing mercury by a similar process; viz, by investing the bulb of a mercurial thermometer with a thin coat of ice, and exposing this to the joint effect of cxhaustion and of sulphuric acid.

Dr. Marcet too has since cficcted the congelation of mercury with great facility and quickness, wimply by sub- stituting the evaporation of ether, instead of that of water, in the process. See the particulars in Nicholson's Journal, vol. 34, p. 119.

Dr.W. H. Wollaston has even succeeded in freezing water at a distance from the mixture. His method consists of a tube, with a ball at each end, bent at right angles: one of the balts contains a little water; and the remainder of the space is to be as perfect a vacuum as can be obtained. When this instrument has bcen successfully exhausted, if the empty ball be immersed in a freezing mixture of salt and snow, the watcr in the other ball, though at the distance of 2 or 3 feet, will be frozen solid in the course of a very few minutes. The vapour contained in the empty ball is condensed by the common operation of cold, and the vacuum produced by this coudensation gives opportunity for a fresh ouantity to arise from the opposice ball, with proportional reduction of its temperature. Sce Philos. Trans. $1 \$ 13$, pt. 1.

Frerzing Point, denotes the point or degree of cold, shown by a mercurial thermomiter, at which certain fluids begin to freeze, or, when frozen, at which they begin to thaw again. On Fahrenheit's thermometcr, this point is at +32 for water, and at -40 for quicksilver; these fluids congealing at those two points respectively. It would also be well if the freczing points for other fluids were ascertained, and the whole arranged in a table.

It may here be proper to remark, that though the freezing point of water be $32^{\circ}$, yet it may be cooled down in favourable circumstances consislcrably below that tempcrature, before it begins to shoot into crystals. Experiments were made on this subject by Mairan and Fahrenhcit; but it is to Sir Charles Blagden that we are indebted for the fullest investigation of it. He succecded in cooling water down to $21^{\circ}$ before it froze, by exposing it slowly to the action of frcezing mixtures. The experiment succeeds best when the water tried is well purged of its air. It ought also to be transparent; for opaque bodics floating thercin cause it to shoot into crystals when only a few degrces below the freczing point. When a piece of ice is thrown into water thus cooled, it causes it instantly to shoot out into crystals. The same effect is produced by throwing the liquid into a tremulons motion; but not by stirring it. It freezes also when cooled clown too suddenly.

FREEZING Rain, or Raining Ice, a very uncommon sort of shower which sometimes falls during winter. A remarkablc shower of this kind happened in December 1672, in the west of England: of wlich some accounts are given in the Philos. Transact. No. 90. This rain, as soon as it touched any thing above ground, as a bough, or the like, immedintcly settled into ice; and by cnlarging and multiplying the icicles, it broke all down with its weight. The rain that fell on the snow, immerliately congealed, without sinking in the snow at all. It made also an amazing destruction of trees, beyond any thing in all listory. " Had it concluded with some gust of wind," said a gentleman on the spot, "it might have been of terrible consequence. Having weighed the sprig of an ashtrec, the wood of which was just three quarters of is pound, the ice upon it amounted to 16 pounds. Some were frighted with the noise in the air ; till they discerned it was the clatter of icy boughs dashing against cach other." Dr. Beale observes, that there was no considerablo frost perceived on the ground during the whole of the shower; from which he concludes, that a frost may be very fierce and dangerous on the tops of some hills, while: $4 \mathrm{~B} 2$ 
in other places it kiceps at some fert above the ground; and may wander about very furiously in some places, and be remiss in others not far off. This rain was followed by glowing lieats, and a great forwardness of vegetation.

FRLNICLE (BERNARD), a celebrated French mathematician of the 17 th century. He was the contemporary and companion of Descartes, Fermat, and the other learned mathematicians of their time. He was admitted Geometrician of the French Academy in 1666; and died in 1675 . Frenicle had many papers inserted in the Ancient Memoirs of the Academy, of 1666 , particularly in vol. 5 of that collection, viz, 1. A method of resolving problems by Exclusions. -2. Treatise of right-angled Triangles in Numbers.-3. Short tract on Conbinations.-4. Tables of Magic Squares.-5. General method of making Tables of Magic Squares.

FRESCO, is a sort of painting which is made upon the plastering of walls before it is dry.

FRIABILITY, the quality of a body that is friable.

FRlABLE, a quality of bodies by which they are rendered tender and brittle, casily crumbled or reduced to powder between the fingers; their force of cohesion being such as easily exposes them to such solution. Such are pumice, and all calcined stones, burnt alum, \&c.

It is supposed that friability arises from hence, that the body consists wholly of dry parts irregularly combined, and which are readily separated, as having nothing unctuous or glutinous to bind them together.

FRICTION, the act of rubbing or grating the surfaces of bodies against or over each other, called also attrition. -The phenomena arising from the friction of divers bodies, under difierent-circumstances, are very numerous and considerable. Mr. Hawksbee gives a number of experiments of this kind ; particularly of the attrition or friction of glass, under various circuinstances; the result of which was, that it yiclded light, and became electrical. Indeed all bodies by friction are brought to conceive heat; many of them to emit liglit; particularly a cat's back, sugar, beaten sulphur, increury, sea-water, gold, copper, \&c, but above all diamonds, which when briskly rubbed against glass, gold, or the like, yield a light equal to that of a live coal when blowed by the bellows.

Furction, in Mechanics, denotes the resistance a moving body meets with from the surface on which it moves.

Friction arises from the roughness or asperity of the surface of the body moved on, and that of the body moving: for such surfaces consisting alternately of eminences and cavities, either the eminences of the one must be raised over those of the other, or they must be both broken and worn off: Lut neither can happen without motion, nor can motion be produced without a force impressed. Hence the force applied to move the bocly is cither wholly or partly spent on this effect; and consequently there arises a resistance, or friction, which will be greater as the eminences are greater, and the substatice the harder; and as the body, by continual rubbing, becomes more and more polished, the friction in consequence diminishes.

As the friction is less in a body that rolls, than when it slides, hence in machines, lest the friction should employ a great part of the power, care is to be taken that no part of the machine slidealong another, if it can be avoided; but rather that they roll, or turn upon each other, or at least slide as little as possible. With this view it may be proper to lay the axes of cylinders, not in a groove or concave matrix, as usual, but between two checks forming an angle, or on an horizontal support and between two upright pins; or best of all hetween little wheels, called triction wheels, moveable on their rispective axcs: for by this contrivance, the fiction is transferred from the circumterence of those wheels to their pivots, and is su much less as these are smaller. In like manner the ąt trition or ro sistance may be still farther diminisbed, by making the axis of those wheels rest upon other friction wheels that turn round with them. This was long since recommended by P. Casabus; and experience confirms the truth of it. Hence also it is, that a pulley moveable on its axis resists liss than if it were fixed, and the cord sliding over the circumference. And the same may be observed of the wheels of coaches, and other carriages. Indeed about 40 years ago friction balls or rollers were placed within the naves of carriage-whecls by some persuns, particularly a M r. Varlo; and lately Mr. Garnett had a patent for an improved manner of applying friction wheels to any axis, as of carriage blocks or pulleys, scale beams, \&c, in which the inclosed wheels or rollers are kept always at the same distance by connecting rods or bars.

From these priuciples, with the assistance of the higher geometry, Olaus Roemer determined the figure of the teeth of wheels, that should make the lcast resistance possible, which he found should be epicycloids. And the same was afterwards demonstrated by Lahire, and Camus.

II. Amontons by experiment attempted to settle a foundation for the precise calculation of the quantity of friction; which M. Parent endeavoured to confirı from reasoning and geometry. Amontons' principle is, that the friction of two bodies depends only on the wcight or force with which they press each other, being always more or less in proportion to that pressure; esteeming it a vulgar error, that the quantity of friction has any dependence on the cxtent of the surface that is rubbed, or that the friction increases with the surface; arguing that it will require the same weight to draw along upon a plane, a piece of wood on its narrow cdge, as on its broad and fat side; because, though on the broad side there be 4 times thenumber of touching particles, yet cach particle is pressed with but $\frac{x}{4}$ of the weight bearing on those of the narrow side; and since 4 times the number multiplied by $\frac{1}{4}$ of the weight is equal to $\frac{x}{4}$ of the number multiplied by 4 times the weight, it is plain that the eficct, that is, the resistance, is equal in both cases, and therefore requires the samc force to overcome it.

On the first proposal of this paradox, MI. Lahirc very properly had recourse to experiments, as the best test, had they been judiciously performed: such as they were, however, they succeded in favour of this system. He laid several pieces of unpolished wood on a rough table, whose sizes were unequal; and afterwards placed weights on them, so as to render them all equally heavy : and he found that the same precise force, or weight, applied to them by a small pulley, was required to put each in motion, notwitlstanding the inequality of the surfaces. The experiment succeeded in tlie same manner with picces of marble, on a marble table. After this, by reasoning, M. Lahire gave a physical solution of the effect. And M.Amontons settled a calculus of the value of friction, with the loss sustained by it in machines, on the foundation of this new principle. In wood, iron, lead, and brass, which are the chicf materials used in machines, he makes the resistance caused by friction to be nearly the same in all, when those materials are anointed with oil or fat: and the quantity of 
this resistance, independent of the magnitude of the surface, he finds nearly equal to a third part of the weight of the borly moved, or of the force with which the two borlies are presserl together. Others have asserted that if the surfaces be hard and well polished, the friction will be less than a third part of the wcight; but if the parts be soft or rugged, it will be much greater. It was farther observed, that in the cylinder moved on two small gudgeons, or on a small axis, the friction would be diminished in the sane proportion as the diameter of these gudgeons is less than the diameter of the cylinder; because in this case, the parts ou which the cylinder moves and rubs, will have less velocity than the power which moves it in the same proportion, which is in effect making the friction to be proportional to the velocity. So that, from the whole of their observations, this general proposition is deduced, viz, That the resistances arising from friction, are ro each other in a ratio compounded of the pressures of the rubbing parts, and the velocities of their motions. Principles which, it is now known from better experiments, are buth erroneous; notwithstanding the hypothesis of MI. Amontons has been adopted, and attempted to be confirmed by Camus, Desaguliers, and others.

M. Muschenbroek and the abbe Nollet, however, on the other hand, have concluded from experiments, that the friction of bodies depends on the magnitude of their surface, as well as on their weight. Though the former observes, that in small velocities the friction varies very nearly as the velocity, but that in great velocities the proportion increases faster: he has also attempted to prove, that by augmenting the weight of a hody, the friction rloes not always increase exactly in the same ratio. Introd. ad Phil. Nat. vol. 1, c. 9; and Lect. Phys. Exp. tom. 1, p. 241. Helsham and Ferguson, from the same kind of experiments, have endeavoured to prove, that the friction does not vary by changing the quantity of surface on which the body moves; and the latter of these asserts, that the friction increases very nearly as the velocity; and that by increas. ing the weight, the friction is also increased in the same ratio. Indeed there is scarce any subject of experiment, with regard to which, different persons have formed such varions conclusions. Of those who bave written on the theory, no one has established it altogether on true principles, till the experiments lately made by Mr. Professor Vince of Cambridge: Euler, whose theory is extremely elegant, and would have been quite satisfactory had his principles been founded on good experiments, supposes the friction to vary in proportion to the velocity of the body, and its pressure upon the plane; neither of which is true: and others, though they have justly imagined that friction is a uniformly retarding force, have yet retained the other supposition, and so rendered their solutions not at all applicable to the cases for which they werc intended.

For these reasons a new and ingenious set of experiments was successfully instituted by the Rev. Mr.Vince, Plumian Professor of Astronomy and Experimental Philosophy in the university of Cambridge, which are published in the 75 th vol. of the Philos. Trans. p.165. The object of these experiments was to determine, 1st, Whether friction be a uniformly retarding force.-2d, The quantity of friction. $-3 \mathrm{~d}$, Whether friction varies in proportion to the pressure or weight.-4th, Whether the friction be the same, on whichever of its surfaces a body moves.

The profcssor says, " The experiments were made with the utmost care and attention, and the several results agreed so very exactly with each other, that I do not scruple to pronuance thent to be conclusive."- "A plane was adjusted parallel to the horizon, at the extremity of which was placel a pulley, which cuuld be elevated or depressed in order to remder the string which connected the body and the moving force parallel to the plane or borizon. A scale accurately clivided was placed by the side of the pulley perpendicular to the horizon, by the side of which the moving force descended; on the scale also was placed a moveable stage, which could be adjusted to the space through which the moving furce descended in any given time, which time was measured by a well regulated pendulum clock vibrating seconds. Every thing being thus prepared, the following experiments were made to ascertain the law of friction. But let me first observe, that if friction be a uniform force, the difference betwcen it and the given force of the moving power must be also uniform, and therefore the moving body must descend with a uniformly accelerated velocity, and consequently the spaces described from the beginning of the motion must be: as the squares of the times, just as when there was no friction, only they will be diminished on account of the friction." Accordingly the experiments are then related, which are performed agreeably to these ideas, and from them are deduced these general conclusions, which may be considered as established and certain facts or maxims, viz,

1st, That friction is a uniformly retarding force in hard bodies; and is not subject to alteration by the velocity; except when the body is covered with cloth, woollen, \&c, in which case the friction increases a little with the velocity.

2 dly, Friction increases in a less ratio than the quantity of matter, or weight of the body. This increase however is different for the different bodies, mure or less; nor is it yet sufficiently known, for any one borly, what proportion the increase of friction bears to the increase of weight.

$3 \mathrm{dly}$, The smallest surface has the least friction; the weight being the same. But the ratio of the friction to the surface is not yet accurately known.

It is observed, that the professor's cxperiments consisted in detcrmining how far the sliding bodies would be drawn, in given times, by a weight laanging freely over a pulley. This method would both show him if the friction were a constant retarding force, and the other conclusions above stated. For, as the spaces described by any constant force, in given times, are as the squares of the times; and as the weight drawing the body is a constant force, if the friction, which acts in opposition to the weight, should also be a constant force, then their difference, or the force by which the body is urged, will also be constant, in which case the spaces described ought to be as the squares of the times; which happened accordingly in the experiments.

Mr. Vince adds some remarks likewise on the nature of the experiments which have been made by others. These, he observes, the authors " have instituted, to find what moving force would just put a body at rest, in motion: and they concluded from thence, that the accelerative force was then cqual to the friction; but it is mamifest, that any force which will put a body in motion must be greater than the force which opposes its motion, otherwise it could not overcome it; and hence, if there were no other objection than this, it is evident that the friction could not be very accurately obtained; but there is another objcction, which totally destroys the experiment, so far as it tends to show the quantity of friction, which is, the strong cohesion of the body to the plane when it lies 
at rest." This he confirms by several experiments, and then adds, "From these experiments therefore it appears, how very considerable the cohesion was, in proportion to the friction when the body was in motion: it being, in one case almost $\frac{x}{3}$, and in another it was found to be very nearly equal to the whole friction. All the conclusions therefore deduced from the experiments, which have been instituted to determine the friction from the force necessary to put a body in motion (and I have never seen any described but upon such a principle) lave manifestly been totally false; as such experiments only show the resistance which arises from the cohesion and friction conjointly." Philos. Trans. vol. 75 , pa. 165.

Emerson, in his Principles of Mechanics, deduces from expcriments the following remarks relating to the quantity of friction: When a cubic piece of soft wood, of 8 pounds veight, moves upon a sinuoth plane of soft wood, at the rate of 3 feet per second, its friction is about $\frac{1}{3}$ of the weight; but if the plane be rough, the friction is little less than half the weight: on the same supposition, when woth the pieces of wood are very smooth, the friction is about $\frac{1}{4}$ of the weight: the friction of soft wood on hasd, or of hard wood upon soft, is $\frac{1}{5}$ or $\frac{1}{6}$ of the weight; of hard wood upon hard wood, $\frac{1}{7}$ or $\frac{1}{8}$; of polished steel moving on steel or pewter, $\frac{1}{4}$; moving on copper or lead, $\frac{1}{5}$ of the weight. He observes in general, that metals of the same kind have more friction than those of different kinds; that lead makes much rcsistance; that iron or steel running in brass makes the least friction of any; and that metals oiled make the friction less than when polished, and twice as little as when unpolished. Desagulicrs also observes that, in M. Camus's experiments on small models of sledges in actual motion, there are more cases in which the friction is less than where it is more than $\frac{x}{3}$ of the weight. See a table, exhibiting the friction between various substances, formed from bis experinents in Desag. Exp. Philos. vol. 1, pa. I 93 \&c; also pa. 133 to 138 , and pa. 182 to 254 , and pa. 458 to 460 . On the subject of friction, see several vols. of the Philos. Trans. as vol. 1, pa. 206 ; vol. 34 , pa. 77 ; vol. 37, pa. 394 ; vol. 53 , pa. 139, \&c.

FRIDAY, the Gtl day of the week, so called from Friga, or Friya, a goddess worshipped by the Saxons on this day. It is a fast-day in the church of England, in memory of our Saviour's crucifixion, unless Christmas-day bappen to fall on Friday, which is always a festival.

Good FIIDAY, the Friday next before Easter, representing the day of our Saviour's crucifixion.

FRIGID zone, the space about ejther pole of the earth to which the sun rever rises for one whole day at least in their winter. These two zones extend to about $23 \frac{\mathrm{I}}{2} \mathrm{de}-$ grees every way from the pole considered as their centre.

FRIGORIFlC, in Plyssics, something belonging to, or that occasions cold.-Some philosophers, as Gassendus, and other corpuscularians, denying cold to be a mere privation, or absence of heat, contend that there are actual frigorific corpuscles or particles, as well as fiery ones: whence proceed cold and heat. But later philosophers allow of no other frigorific particles beside those nitrous salts, \&c, which float in the air in cold weather, and occasion freczing.

FRISI, or FRISIUS (Gємм A), appears to liave heen a native of the Flemish Netherlands, and flourished the beginning of the 16 th century. As Werner had proposed to find the longitude by observations on the moon; so
Gemma Frisi, in a tract entitled, De Principis Astionomix et Cosmographix, printcd at Antwerp 1530, advised keeping the time by means of small clocks or watclies, for the purpose, then, as he says, lately invented. He also contrived a new kind of cross-stafi, described in his tucatise De Radio Astronomico et Geometrico, printed at the same place $15+5$, and in lis additions to Puter Apian's Cosmography, gives the ligure of an instrument, lie calls a nautical quadrant, as very useful in navigation, promising to write largely on the subject : accordingly, in an edition he made in the year 1553, of his abovementuncd book, De Principijs Astrommire, \&c, he delivers several nautical axioms, as he calls them, which with some alterations were repeated by his son Cornelius G:mna, in the father's posthumous piece on the Universal Astrolabe, published in 1556.-Gemma Fisi died in 1555 , at 45 jeals of age.

FrlZE, Friezs, or FreEze, in Archilecture, a part of the entablature of columns, between the architrase and cornice.

FRONT, in Architecture, denotes the principal face or side of a building; or that presented to their chicf aspect or view.

Front, in Perspective, a projection or representation of the face, or forepart of an object, or of that part directly opposite to the eye, called also more usually $\mathrm{Or}$ thography.

FPON'l'lSPIECE, in Architecture, the portale, or principal face of a fine building,

FRONTON, in Architecture, an ornament among us more usually called Pediment.

FROST, such a state of the atmosphere as causes the congelation or freezing of water, or other fluids intu ice.

The nature and effects of frost in difierent countries, are. mentioned under the articlesCoNGELAT on, and F IREZ IN G. In the more nurthern parts of the world, even sulid bodies are affected by frost, though this is only or chiefly in consequence of the moisture they contain, which bcing frozen into ice, and so expanding as water is known to do when congeiled, it bursts and lacerates any tbing in which it is contained, as plants, trees, stones, and large rocks. Some fluids expand by frost, as water, which expands about roth part of its natural bulk, for wlich rcason ice tloats in water; but others again contract, as quicksilver, and hence frozen quicksilver sinks in the fluid metal.

Frost, being derived from the atmosphere, naturally proceeds from the upper parts of bodies downwards, as the water and the earth: so, the longer a frost is continued, the thicker the ice becomes upon the water in ponds, and the deeper into the earth the ground is frozen. In about 16 ur 17 days frost, Mr. Boyle found it had penetrated 14, juches jnto the ground. At Moscow, in a hard season, the frost will penctrate 2 feet deep in the ground; and Capt. James found it penetrated 10 feet deep in Charltonisland, and the water in the same island was frozen to the depth of 6 feet. Scheffer assures us, that in Sweden the frost pierces 2 cubits, or Swouish ells, into the earth, and turns what moisture is found there into a whitish substance, like ice; and standing waters $103 \mathrm{clls}$, or more. The same author also nuntions sudden crucks or rifts in the ice of the lakes of Swerlen, 9 or 10 foet deep, and many leagues long: the rupture being accompanied witl a noise not less loud than if many guns were discharged together. 13y such means, howcver, the fishes are furnished with air; so that they are rarely found dead.

'The several natural histories of frosts furuish vcry ex- 
traordinary effects of them. 'The trees are often scorched, and burnt up, as with the most excessive heat; and split or shattered. In the great frost in $\mathbf{1 6} 83$, the trunks of oak, ash, walnut, \&c, were miserably sulit and cleft, so that they might be seen through, and the cracks often attended with dreadful noises like the explosion of fire-arms. Philos. Traus. No. 105.

The close of the year $170 \mathrm{~s}$, and the beginning of 1709 , were remarkable, through the greatest part of Europe, for a severe frost. Dr. Derham says, it was the greatest in degree, if not the most universal, in the memory of man; extending through most parts of Europe, though scarcely felt in Scotland or Ireland.

In very cold countries, meat inay be preserved by the frost 6 or 7 months, and prove tolerably gond eating. See Capt.Middleton's observations made jil Hudson's-bay, in the Philos. Trans. No. 465 , sect. 2. In that climate the frost seems never out of the ground, it having been found hard frozen in the two summer months. Brandy and spirit of wine, placed in the open air, congeal to solid ice in 3 or 4 liours. Lakes and standing waters, not above 10 or 12 feet deep, are frozen to the ground in winter, and all their fish perish. But in rivers, wlsere the current of the tide is strong, the ice does not reach so deep, and the fish are preserved. Id. ib. Some remarkable instances of frost in Europe, and chiefly in England, are recorded as below: In the year

220 , Frost in Britain that lasted 5 months.

250 , 'The Thanes frozen 9 weeks.

291, Most rivers in Britain frozen 6 weeks.

359 , Severe frost in Scotland for 14 weeks.

508 , The rivers in Britain frozen for 2 month.

$55 \mathrm{~s}$, The Danube quite frozen over.

695 , Thames frozen 6 wceks; booths built on it.

759, Frost from Oct. 1 till Feb. 26, 760.

827, Frost in England for 9 weeks.

859 , Carriages used on the Adriatic sea.

gos, Most rivers in England frozen 2 montirs.

923, The 'Thames frozen 13 weeks.

987, Frost lasted 120 days: began Dcc. 22.

998, The Thames frozen 5 weeks.

1035, Severe frost on June 24: the corn and fruits destroyed.

1063, The Thames frozen 14 weeks.

1076, Frost in England from Nov. till April.

1114, Several woorlen bridges carried away by ice.

1205, Frost from Jan. 14 till March 22.

1407 , Frost that lasted 15 weeks.

1434, From Nov. 24 till Feb. 10. Thames frozen down to Gravesend.

1683, Frost for 13 wecks.

$170 \frac{8}{9}$, Severe frost for many weeks.

1715 , The same for many weeks.

1739, One for 9 weeks. Began Dec. 24. .

1742 , Severc frost for mally weeks.

1747, Severe frost in Russia.

1754, Severe one in lingland.

1760 , 'The same in Germany.

1776 , "The same in England.

1788, Thames frozen below bridge; booths on it.

1794 , Ilard frost of many weeks.

1813, Severe frost from the middle of December to the middle of March 1814. The Thames from Blackfriars to Lindon bridge, completely frozen over, booths and thousands of pcople upon it.
Hoar Frost, is the dew frozen or congealed, early in cold mornings; chiefly in autumn: though many Cartesians will have it formed of a cloud; and either congealed in the cloud, and so let fall; or ready to be congealed as soon as it arrives at the carth.

'This kind of frost, M. Regis observes, consists of an assemblage of small parcels of ice crystals; which are of various figures, according to the different disposition of the vapours, when met and condensed by the cold.

FRUSTUM, in Geometry, is the part of a solid next the base, left by cutting off the top, or segment, by a plane parallel to the base: as the frustum of a pyramid, of a cone, of a conoid, of a spheroid, or of a spluere, which is any part comprised between two parallel circular sections; and the middle frustum of a sphere, is that whose ends are cqual circles, having the centre of the sphere in the middle of it, and equally distant from both ends.

For the Solid Content of the Frustum of a cone, or of any pyramid, whatezer figure the base may have. Add into one sum, the areas of the two ends and the mean proportional between them; then $\frac{x}{3}$ of that sum will be a mean area, or the area of an equal prism, of the same altitude with the frustum; and consequently that mean area being multiplied by the height of the frustum, the product will be the solid content of it.

That is, if $\Lambda$ denote the area of the greater end, a that of the less, and $h$ the height;

then $(A+a+\sqrt{ } \Lambda a) \times \frac{1}{3} h$ is the solidity.

Other rules for pyramidal or conic frustums may be seen in my Mensuration, part 3.

The curve Surface of the zone or Frustum of a sphere, is had by multiplying the circumference of the sphere by the height of the frustum. Mensur. pa. 146 .

And the Solidity of the same Frustum is found, by adding together the squares of the radii of the two ends, and $\frac{r}{3}$ of the square of the height of the frustum, then multiplying the suin by the said height and by the number 1.5708 . That is $\left(\mathrm{R}^{2}+r^{2}+\frac{1}{3} / h^{2}\right) \times \frac{1}{2} p h$ is the solid content of the spheric fiustum, whose height is $h$, and the radii of its ends $\mathrm{R}$ and $r, p$ being $=3 \cdot 1416$. Mensur. pa. 152 .

For the frustums of spheroids, and conoids, either parabolic or hyperbolic, see Mensur, part 3. sect. 5, 6, 7. And in pa. 352 , \&c, are general theorems concerning the frustum of a sphere, cone, spheroid, or conoid, terminated by parallel planes, when compured with a cylinder of the same altitude, on a base equal to the middle section of the frustum made by a parallel plane. The difference between the frustum and the cylinder is always the same quantity, in ditferent parts of the same, or of similar solids, or whatever the magnitude of the two parallel ends may be; the inclination of those cnds to the axis, and the altitude of the frustum boing given; asd the said constant difference is $\frac{x}{4}$ part of a cone of the same altitude with the frustum, and the radius of its base is to that altitude, as the fixed axis is to the revolving axis of the frustum. Thus, if $\mathbf{B E C}$ be any conic section, or a right line, or a circle, whose axis, or a part of $i t$, is $A D$; $A B$ and $C D$ the extreme ordinates, $\mathrm{F} E$ the middle ordinate, $A \mathrm{~F}$ being $=\mathrm{rD}$; then taking, as $\Lambda \mathrm{D}$ to $\mathrm{DK}$, so is the whole

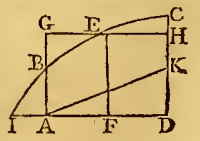
fixed axis, of which $\mathrm{AD}$ is a part, to its conjugate axis; and completing the parallelogram AGHD: then if the whole figure revolve about the axis $A \mathrm{D}$, the line BF.C will generate the frustum of the cone or conoid, according as it 
is a right line or a conic section, or it will generate the whole solid when A $\mathrm{B}$ vanishes, or $\mathbf{A}$ and $\mathbf{B}$ meet in the same point; likewise $\Lambda$ GH $\mathbf{D}$ will generate a cylinder, and ADK a cone: then is the 4 th part of this cone always equal to the difference between the said cylinder generated by AGHD, and the solid or frustum generated by $A B E C D$; having all the same altitude or axis $A D$.

In the parabolic conoid, this difference and the cone vanish, and the frustum, or whole conoid $A B E C D$, is always equal to the cylinder AGH D, of the same altitude.-In the sphere, or spheroid, the frustum A B ECD is less than the cylinder $A G H D$, by $\frac{\pi}{4}$ of the cone $A K D$. And, in the cone or hyperboloid, that frustum is greater than the cylinder, by $\frac{x}{4}$ of the said cone $A \mathrm{KD}$, which is similar to the other cone $\mathrm{IBCD}$.

These relations are likewise true, whether the ends of the frustum are perpendicular or oblique to the axis. And the same will obtain for the frustum of any pyramid, whether right or oblique; and such a frustum of a pyramid will exceed the prism, of the same altitude, and on the middle section of the frustum, by $\frac{1}{4}$ of the same cone.

It has been observed, that the difference, or $\frac{\pi}{4}$ of the cone A K D, is the same, or constant, when the altitude and inclination of the ends of the frustum remain the same. But when the inclination of the ends varies, the altitude being constant; then the said difference varies so as to be always reciprocally as the cube of the conjugate to the diameter aD. And when both the altitude and inclination of the ends vary, the diflerential cone is as the cube of the altitude directly, and the cube of the said conjugate diameter reciprocally: but if they vary so, as that the altitude is always reciprocally as that diameter, then the difference is a constant quantity.

Another general theoren for Frustums, is this. In the frustum of any solid, generated by the revolution of any conic section about its axis, if to the sum of the two ends be added 4 times the middle section, $\frac{1}{6}$ of the last sum will be a mean area, which being drawn into the altitude of the solid, will produce the content. That is $(\mathrm{AB}+\mathrm{DC}+$ $4 F E) \times \frac{x}{6} A D$ is. the content of $A B C D .-$ And this theorem is general for all frustums, as well as the complete solids, whether right or oblique to the axis, and not only of the solids generated from the circle or conic sections, but also of all pyramids, cones, and in short of any solid whose parallel sections are similar figures. - 'l'he same theorem also obtains for any parabolic area $\mathrm{ABECD}$, and is very nearly true for the area of any other curve whatever, or for the content of any other solid than those above montioned.

FUGUE, in music, is when the different parts of a musical composition follow each other, each repeating in order what the first had performed.

IULCRUM, or Prop, in Mechanics, is the fised point about which a lever \&c moves.

FULGURA'IING Plionphorus, a term used by some Engtish writers, to express a substance of the phosphorus kind. It was prepared both in a dry and liquid state, but the preparation it appears was not well known to any but the inventor of $\mathrm{it}$. This matter not only shonc in the dark in both states, but comnunicated its light to any thing it was rubbed on. When inclosed in a glass vessel well stopped, it would frequently lulgurate, or throw out little flashes of light, and sometimes fill the whole phinl with waves of fame. It loes not require laving its light recruited at the fire, or in the sunshine, like the phospliorus of the Bolognian stone, but of itself continues in a state of shining for several years together, and is seen as soon as exposed in the lark; the solid or dry matter always resembling a burning coal of fre, though not consuning itself. Philos. Trans. No. 13 t.

FULIGINOUS, an epithet applied to thick smoke or vapour replete with soot or orher crass matter.-In the first fusion of lead, there exhales a great deal of fuliginous vapour, which being retained and collected, makes what is called Litharge. And lampblack is what is gathered from the fuliginous vapours of pines, and other resinous wood, when burnt.

FULMINANT, FULMINANS, or FuLMINATiNg, a term applied to any thing thundering, or that makes a noise like thunder.

Aurum Futuinans. See Aurum.

Pulvis Fulaninas, is a composition of 3 parts of nitre, 2 parts of salt of tartar, and 1 of sulphur.-Both the aurum and pulvis fulminans produce their effect chiefly downwards; in which they differ from gun-powder, which acts in orbem, or all around, but principally upwards. When the composition is laid in brass ladles, and so set on fire, after fulmination, the ladles are often found perforated. It differs also from gunpowder in this, that it does not require to be confined, in order to fulminate, and it must be slowly and gradually heated. Some instants before explosion, a light blue flame appears on its surface, proceeding from the vapours beginning to kindle. No more fire or flame is perceived during the fulmination, being suffocated and extinguished by the quickness and violence of the commotion. Nor does the fulminating powder generally kindle the combustible bodies in contact with it, because the time of its infiamnation is too short.

Fulminating Damp. See Daip.

FULMINATION, or FuLGU RATION, a vehement noise or shock resembling thunder, caused by the sudden explosion and inflammation of divers preparations; as aurum fulminans, \&c, when set on fire.

FUNCTION, a term used in analytics, for an algebraical expression any how compounded of a certain letter or quantity with other quantities or numbers: and the expression is said to be a function of that letter or quantity. Thus $a-4 x$, or $a x+3 x^{2}$, or $2 x-a \sqrt{ }\left(a^{2}-x^{2}\right)$, or $x^{\mathrm{c}}$, or $e^{x}$, is each of them a function of the quantity $x$.

FuncTlons of Calculus, is that branch of analysis which treats of the doctrine of functions.

Landen, we believe, was the first who proposed to treat the method of thuxions as a branch of pure algebra, independently of any metaphysical considerations, or of such as are drawn lrom the prlnciples of motion. Soun after him, M. Lagrange endeavoured to establish the ámalytical principles in at memoir published in the Berlin Acts for the year 1772: and a farther developement of his ideas was presented iu 1796 , under the title of Theorie des Fonetions Analytiques, a work in which it is the object of the author to fis the primciples of the difierential calculus, independently of the consideration of infinicly smalt, or vanishing quantities, of limits, or of thowing quantitics. Another work also by M. Lagrang̣', entited Leçons sur le: Calcul des Fonctions, has sinee been published, which is meant to serve as a commentary and supplement to the first part of the 'Theorie des Fonctions $A$ natytiques, and offiers a course of anatysis on that branch of modern calculus which is commonly called Infinitesimal or 'Transcendental, and which, in the estimation ol this very distinguishol mathenutician, is alone properly the calculus of functions. 
The following extract will suffice to convey an idea, both of the nature of analytic functions, and of part of the notation employed by this author.

"The earlier analysts (says Lagrange) have only employed the word functions to design the different powers of the same quantity; it was afterwards extended in signification to every quantity formed it any nanner whatever from another quantity; and it is now generally adopted to denote that the value of a quantity depends, according to a given law, on one or many other given quantities.

"Under this point of view, algebra nust be regarled as the science of functions; and it is easy to perceive that the resolution of equations cousists, generally, in finding the values of the unknown quantities in determinate functions of kuown quantities. These functions represent, then, the different operations which must be performed on known quantities, to obtain the values of those which are sought, and they are, properly, only the ultimate result of the calculation.

"But, in algebra, we only consider the functions in as much as they result from arithmetical operations, generalized and transferred to the letters; while in the calculus of functions, properly speaking, we consider the functions which result from the algebraic operation of the deselopement in series, when we attribute to one or to several quantities of the function indeterminate augmentations.

"The developement of functions, viewed in a general manner, gives rise to derivative functions of different orders; and the algorithm of these functions once discovered, they may be considered in themselves and independent of the series whence they result. Thus, a given function being regarded as primitive, we may deduce, by simple and uniform rules, other functions which I call derivative; and when we have an equation between several variables, we may pass successirely to the derivative equations, and return again from these to the primitive equations. These transformations correspond to differentiations and integrations; but in the theory of functions they depend alone on operations purely algebraical, founded on the simple principles of the calculus.

"The derivative functions present themselves naturally in geometry, when we consider areas, taugents, radii of curvature, \& $c$; and in mechanics, when we cunsider velocities and forces. If we regard, for example, the area of a curve as a function of the abscissa, the ordinate is then the first derivative function, or prime function; the relation of the ordinate to the subtangent is expressed by the prime function of the ordinate, and of consequence by the second derivative function or second function of the area; the radius of the osculatory circle depends on two prime derivative functions of the ordinate, and so of others. In like manner, regarding the space run over as a function of the time, the velocity is then the prime function, and the accelerating force is the second function. It is not perhaps one of the least advantages of the calculus of functions, that it furnishes for these elements of the geometry of curves, and of mechanics, expressions as simple and intelligible as are the alyebraic expressions of powers and roots.

"When we consider a function with respect to one of the quantities which compose it, we make our abstraction of the value of that quantity, and consider it only as to the manner in which it enters the function; that is to say, in which it is combined with itself, and with the other quantities. Thus, the function is considered as remaining the sanc, while this quantity varies in any manner whatever,

VoL. I. provided that the other quantities with which it is blended remain constant: hence is introluced naturally, with respect to functions, the distinction of quantities into variable and constant quantities.

"In ordinary algebr"a, quantitics are simply distinguished into known and unknown, and it is customary to denote the former by the first letters of the alphabet, and the other by the latter. The application of algebra to the theory of curves first caused the distinction of the quantities which enter the equation of a curve into given ones, such as the axes, the parameters, \&c, and indeterminates, such as the co-ordinates. Afterwards these quantities became viewed under the more natural aspect of constant and variable quantities; and the consideration of functions leads us naturally to regard the quantities which compose them under the same point of view.

"We call therefore, simply, a function of one or of several quantities, every expression in which these quantities enter in any manner whatever, connected or not with other quantities considered as having given and invariable values, while the quantities of the functions are deemed capable of receiving all possible values.

"We ordinarily design the variable parts of functions by the final letter's of the alphabet $x, y, \& c$; and the constant parts by the initial letters $a, b, c, \& c$. And to denote a function of a single variable $x$, we merely place before that quantity the characteristic letter $f$ or $F$; but when we would design the function of a quantity already composed of that variable, as $x^{2}$, or $a+b x$, \& c , we include this quantity in a parenthesis. Thus $f x$ indicates a function of $x, f\left(x^{2}\right), f(a+b x), \& c$, denote functions of $x^{2}$ and of $a+b x$.

"To express a function of two independent variables as $x, y$, we write $f(x, y)$, and so of uthers.

"If $t(v)$ functions of two different variables, $x, y$, that is to say, the one of $x$, the other of $y$, are composed in the same manner, and with the same constant quantities; these functions will be similar, and may be designed in the same investigation by the same characteristic, thus $f x$, and $f y$, will be two similar functions which become identical when $x=y$. But if, while the two functions are composed in the same manner, the constant quantities which they contain are different, then we can no longer, generally speaking, represent them by the same characteristic in the course of the same calculation. Still, howeser, if the two functions differ only, for example, by the value of a constant quantity, which will be $a$ in the one, and $b$ in the other, we may yet design them by the same characteristic,representing them by $f(x, a)$, and $f(y, b)$, as similar functions of $x, a$, and of $y, b$. Thus, in this case, the quantities $a$ and $b$ enter likewise into the expression of the function; since, though they are constant quantities in each function, they may be regarded as variables from one function to the other."

For other particulars of the notation of the Calculus of Functions; and the doctrine itself, we must refer the reader to the works of Lagrange alrearly named, where he will find his curiosity for such rescarches amply satisfied. On the subject of functions, their divisions, transformations, explication by infinites, \&c, see also Euler's "Analys. Infinitorum," c. 1, where this branch of analysis is likewise fully treated. See also preface to Woorhouse's Principles of Analytical Calculation, for many sery proper remarks on the Theorie des Fonctions Analytiques of $M$. Lagrange. 
FUNDAMENTAL, something that serves as a base or foundation for anotber.

Fundamental, in Music. A fundamental sound is that which forms the lowest note of the cliord, and from which are deduced the barmonical relations of the rest; or it is that wbich serves for a key to the tone.

FUNDAMENTAL CHORD, is that which consists of the three fundamental consonances, the $3 \mathrm{~d}$, the 5 th, and the 8 th, or their inversions.

FUNDAMENTAL Bass, is that formed by the fundamental notes of every perfect chord that constitutes the harmony of the piece; so that under each chord it causes to be heard, or understood, the fundamental sound of that particular chord; that is to say, the sound from which it is derived by the rules of harmony. Whence we may see, that the fundamental bass can have no other contexture than that of a regular and fundamental succession, without whicb the procedure of the upper parts would be illegitimate.

FUNICULAR CuRve, the same as the Catenary; which see.

Funicular Polygon, is that formed by a chain hanging freely by its extremities.

FURLONG, an English long-measure, containing 660 feet, or 220 yards, or 40 polcs or perches, or the 8 th part of a mile.

FURNITURE, in Dialling, certain additional points and lines drawn on a dial, by way of ornament: such as the signs of the zodiac, length of days, parallels of declination, azimuths, points of the compass, meridians of chief cities, Babylonic, Jewish, or Italian hours, \&c.

FUSAROLE, in Architecture, a small round member cut in form of a collar, with oval beads, under the echinus, or quarter round, in the Doric, Ionic, and Compositc capitals.

FUSEE, or FUSY, in Watch-work, is that part resembling a low cone with its sides a little sunk or concave, which is drawn by the spring, and about which the chain or string is wound.

The spring of a watch is the first mover. It is rolled up in a cyliudrical box, against which it acts, and causes to turn round in unbending itself. The chain, which at one end is wound about the fusee, and at the other fastened to the spring-box, disengages itself from the fusee in proportion as the box is turned. And hence the motion of all the other parts of the spring-watch. The effort or action of the spring is continually diminishing from first to last; and unless that inequality were rectified, it would draw the chain with more force, and wind a greater quantity of it on the box, at one time than another; so that the movement would never kcep cqual time. To correct this irregularity of the spring, it was very happily contrived to have the spring applicd to the arms of levers, which are continually longer as the force of the spring is weaker : this foreign assistance, always increasing as it is most needed, maintains the action and effect of the spring in an cquality. It is for this reason then that the fusce is made tapering somcwhat conically, its radius at every point of the axis answering to the corresponding strength of the spring.

Now if the action of the spring diminished cqually, as the parallels to the base of a triangle do ; the cone, which is generated of a triangle, would be the precise figure required for the fusee; but it is certain that the weakening of the spring is not in that proportion; and therefore the fusee should not be exactly conical; and in fact experience shows that it should be a little hollowed about the middle, because the action of the spring is not there sufficiently diminished of itself.

M. Varignon has investigated the figure of the fusee, or the nature of the curve by whose revolution about its axis, shall be produced the solid whose figure the fusce is to have. This curve, it may easily be shown, is an hyperbola, whose asymptote is the axis of the fusee. Thus, let $\mathrm{DFE}$ be the curve of the fusee, its axis being $A B C$ : let $A D$ express the greatest strength of the spring when the watch is quite wound up, or when the spring

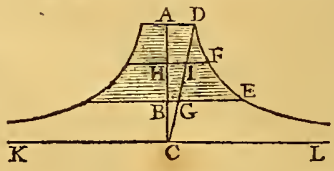
acts at $D$, and $B G$ the least strength when the watch is down, or when the spring acts at $\mathrm{E}$; so as that $\mathrm{BE}: \mathrm{AD}:$ : $A D: B G$, or $B E \times B G=\mathrm{AD}^{2}$; join $\mathrm{DG}$, producing it to meet the axis produced in $\mathrm{C}$; then slall III denote the strength of the spring acting at the corresponding point $\mathrm{F}$ of the fusce; and the nature of it must be such that the rectangle ${ }_{1} \times$ HF be cqual to a constant quantity, or HF must be reciprocally as $\mathrm{HI}$, Or $\mathrm{HI}: \mathrm{BG}:: \mathrm{BE}: \mathrm{NF}$; but because - $\quad-\quad$ - $A D, I I J, ~ B G$, are directly proportional to - . СA, сu, св, therefore these are reciprocally propor. to $\triangle \mathrm{D}$, II F, BE; and consequently the curve DFE is an hyperbola, whose centre is $c$, and asymptotes $A C$ and $\mathrm{KL}$ : so that the figure of the fusce is the solid generated by an equilateral hyperbola about its asymptotc. See also Martin's Mathem. Instit. vol. 2, pa.36.4.

Fusee, Fuse, or Fuze, in Artillery, is a wooden tap or tube used to set fire to the powder in a bomb-shell. The borc of this tube is filled with a composition, of sulphur one part, saltpetre 3 parts, and mealed powder 3,4 , or 5 parts. The tube is driven hard into the hole in the shell, having first cut it to the exact length, answering to the time of the intended flight of the shell, so that, the composition in the fuse catching fire by the discharge of the shell from the mortar, it just burns down to its lower end, and so sets fire to the porrder in the shell, thereby bursting it at the moment when it arrives at the end of its. range or fight.

FUST, in Architecture, the shaft of a column, or that part comprehended betwcen the base and the capitil, called also the Naked.

FUZE, or FuzeE. See FuseE. 


\section{G A G}

$\mathrm{G}$ $\triangle B J O N S$, in Fortification, are large cylindrical baskets, open at both ends, made of ozier twigs, of $3 \mathrm{or}$ 4 feet in diameter, and from 3 to 6 feet high. These, being filled with earth, are sometimes used as merlons for the batteries, and sometimes as a parapet for the lines of approach, when the attacks are carried on through a stony or rocky ground, and to advance them with extraordinary vigour. They serve also to make lodgments in some posts, and to secure other places from the shot of the enemy; who, on their part, endeavour to burn and destroy the gabions, by throwing lighted pitcli-faggots among them.

GABLE, or GABLE-end, of a house, is the upright triangular end, from the cornice or eaves to the top of its roof.

GAGE, in Hydrostatics, Pneumatics, \&ce, is an instrument for ascertaining measures of various kinds.

GAGE of the Air-pump, is adapted for showing the degree to which the air is rareficd, or the receiver is exhausted, at any time by the air-pump. This is either the common barometer-gage, both long and short, or the peargage, which at first was thought a great improvement, but afterwards it was discovered that its seeming accuracy was founded on a fallacy, which gave an erroneous indication of exhaustion. See A1R-pump.

GAGE of the Barometer, is a contrivance for estimating the exact degree of the rise and fall of the mercury in the tube of that instrument. It is wel] known that while the mercury rises in the tube, it sinks in the cistern, and vice versa ; and consequently, the divisions on the scale, fixed near the top of the tube, had their distance from the surface of the mercury in the cistern always various; from which there must often happen errors in determining the height of the mercury in the tube. To remedy this inconvenience, a line is cut upon a round piece of ivory, which is fixed near the cistern: this line is accurately placed at a given distance from the scale; for example, at 27 inches; and a small float of cork, with a cylindrical piece of ivory fixed to its upper surface, on which a line is cut at the exact distance of 2 inches from the under side of the cork, is left to play freely on the quicksilver, and the cylinder works in a groove made in the other piece. From this construction it appears, that if these marks are made to coincide, by raising or lowering the screw which acts on the quicksilver, then the divisions on the scale will express the true measure of the distance from the surface.

GAG E of the Condenser, is a glass tube of a particular construction, adapted to the condensing engine, and designed to show the exact density and quantity of the air containesl at any time in the conlenser. See Desaguliers's Exper. Philos. vol. 2, pa.394.

Sea GAsE, an instrument for sounding the depth of the sca. Several kinds of these have been invented, by Dr. Hales, Dr. Desaguliers, and others. Formerly, the machines for this purpose consisted of two bodics, the one specifically lighter, and the other specifically heavier, than the water, so joined tegether, that as soon as the heavy onc cane to the hottom, the lighter should get loose from it, and emerge; and the depth was to be estimated by the time the compound was in falling from the top to

\section{G A G}

the botton of the water, together with the time the lighter body was in rising, reckoned from the disappearing of the machine, till the emergent body was seen again. But no certain conclusion could be drawn from so precarious and incomplete an experiment.

But that invented by Drs. Hales and Desaguliers was of a more exact nature, depending on the pressure of the Aluid only. For as the pressure of fluids, in all directions, is the same at the same depth, a gage which discovers what the pressure is at the bottom of the sea, will show what the true depth of the sea is in that place, whether the time of the machine's descent be longer or shorter.

Dr. Hales, in his Vegetable Statics, describes his gage for estirnating the pressures made in opaque vessels; where hohey bcing poured over the surface of mercury in an open vessel, rises upon the surface of the mercury as it is pressed up into a tube whose lower orifice is immer'sed into the honey and mercury, and whose top is hermetically scaled. Now, as by the pressure, the air in the tube is condensed, and the mercury rises, so the mercury comes down again when the pressure is taken off, and would leave no mark of the beight to which it had risen; but the honey (or treacle, which does better) which is upon the mercury, sticking to the inside of the tube, leaves a mark, which shows the height to which it had risen, and consequently gives the quantity of pressure, and the height of the surface of the fluid.

Desaguliers's addition to this machine, consisted in a contrivance to carry it down to the bottom of the sea by means of a heavy wcight, which was immediately disengaged by striking the bottom, and the gage, made very light for the purpose, re-ascended to the top.

Dr. Ilales afterwards made more experiments of this kind, and proposed another sea gage for vast depths, which is described in the Philos. Trans. No, 4.05, and is to this effect. Suppose a pretty long tube of copper or iron, close at the upper end, to be let down into the sea, to any depth, the water will rise in the tube to a height bearing a certain proportion to the depth of the sea, to which the machine is sunk. And this proportion is as follows : 33 feet of sea water being nearly equal to the mean pressure of the atmosphere, therefore at 33 feet deep, the air in the tube will be compressed into half the length of the tube, or the water will rise and fill one half of the tube; in like manner, at 66 fi'et deep, the water will occupy $\frac{2}{3}$ of the tube; at 99 feet deep it will fill $\frac{3}{4}$ of the tube; at 132 feet deep it will fill $\frac{4}{5}$ of the tube; and so on. Hence therefore, by knowing the beight to which the water rises in the tube, there will be known the consequent depth of the sea.

But, in very great depths, the scale near the top of the tube would be so sinall, and the divisions so close, that there would be no accuracy in the experiment, unless the tube were of a very great length, and this again would render it both liable to be broken, and quite impracticable. To remedy this inconvenience, the Doctor made the following contrivance: To the bottom of the tube he screwed a large hollow globe of copper, with a small orifice, or a short pipe at bottom of the globe, to let in the $4 \mathrm{C}_{2}$ 


\section{G A L}

$[564$ water; by which means he had a very great quantity, of air, and the scale enlarged. See also Desagul. Exp. Phil. vol.2, pa. 224 and 241 .

Bucket Sea GAGE, is an instrument contrived also by Dr. Hales, to find the difierent degrees of coolness and saltness of the sea, at different depths. This gage consists of a common pail or bucket, with two heads; each of which has a round bole in the middle, about 4 jnches in diameter, covered with squarc valves opening upward. That these heads may both open and shut together, there is a sinall iron rod, having onc end fised to the upper side of the lower valve, and the other end to the lower side of the upper valve: so that as the bucket descends with its sinking weight into the sea, both the valves may open by the force of the water, which by that means has a free passage through the bucket. But when the bucket is drawn up, then both the valves shut by the force of the water at the upper end of the bucket; and consequently the bucket will be filled with the lowest sea water to which it has descended, and immediately the mercurial thermometer, fixed within it, is examined, to see the degree of temperature; and the degree of saltness is afterwards ascertained at leisure. Philos. Trans. No. 9, pa. 149, and No. 24, pa. 447.

Lord Charles Cavendish adapted a thermometer for the temperature of the sea water, at different depths. See Pbilos. Trans. vol. 50, pa. 300, and Phipps's Voyage towards the North Pole, pa. 142, \&c.

Aqueo-mercurial GAGE, is the hame of an apparatus contrived by Dr. Hales, and applied, in various forms, to the branches of trees, to determine the force with which they inbibe moisture. Vegetable Statics, vol. 1, ch. 2, pa. 84 .

Sliding GAG E, a tool used by mathematical-instrumentmakers, for measuring and setting off distances; consisting of a beam, tooth, sliding socket, and the shoulder of the socket.

Tide GAGE, an instrument used for determining the height of the tides, by Mr. Bayly, in the course of a voyage towards the south pole, \&c, in the Resolution and Adventure, in the years $1772,1773,1774$, and 1775 . This instrument consisted of a glass tube, whose internal diameter was 7-10ths of an inch, lashed fast to a 10-foot fir rod, divided into feet, inches, and parts; the rod being fastened to a strong post fixed firm and upright in the water. At the lower end of the tube was an excecding small aperture, through which the water was admitted. In consequence of this construction, the surface of the water in the tube was so little affected by the agitation of the sea, that its height was not altered the 10th part of an inch, when the swcll of the sea was 2 feet; and Mr. Bayly was certain, that with this instrument he could discern a difference of the loth of an inch in the height of the tide.

Water Gage. See Alitudde, and Hydrometer.

Wind $\mathrm{G}_{\Lambda \mathrm{GE}} \mathrm{E}$, an instrument for measuring the force of the wind upon any given surface. Several of these gages are of former invention; but there is one lately invented by Dr. Lind, which is described in the Philos. Trans. vol. 65. Sec several also under the article ANEMOM ETER.

GAGER. See GAUGRr.

GAGING. See GAUGING.

GAI.AXY, or Milky-Way, or Via Lactea, in Astronoiny, that long, whitish, luminous track, which appears to encompass the heavens like a swath, scarf, or girdle; and which is casily scen in a clear night, especially when the moon is not up. It is of a considerable, though unequal breadth; being also in some parts double, but in others single. The galaxy passes through many of the constellations in its circuit round the heavens, and keeps its exact place or position with respect to them.

There have been various strange and fabulous stories and opinions concerning the galaxy. The ancient poets, and even some of the philosophers, speak of it as the road or way by which the lieroes went to heaven. But the Egyptians called it the Way of Straw, from the story of its rising from burning straw, thrown behind the goddess Isis in her flight from the giant Typhon. While the Greeks, who affect to derive every thing in the heavens from some of their own fables, have two origins for it; the one, that Juno, without perceiring it, accidentally gave suck to Mercury when an infant, but that as soon as she turned her eyes upon him, she forced him from her, and as the nipple was drawn from his mouth, the milk streamed about for a moment and formed the galaxy. The other relates, that the infant Hercules being laid by the side of Juno when asleep, on waking she gave him the breast; but soon perceiving who it was, she threw him from her, and the heavens were marked by the wasted milk.

Other philosophers however gave it a different turn, and different origin : these esteemed it to be a tract of liquid fire, spread in this manner along the skies: and others again, supposing a celestial region beyond all that was visible, and imagining that fire, at some time let loose from thence, was to consume the world, made this a part of that celestial fire, and appealed to it as a presage of what would surely happen. This diffused brightness they considered as a crack in the vault or wall of heaven, and fancied this a glimmering of the celestial fire through it, and that there required nothing more than the undoing of this crack by some accident in nature, or by the will of the gods, to make the whole frame start, and let out the fire of destruction.

Aristotle makes the galaxy a kind of meteor, formed from a collection of vapours, drawn into that part by certain large stars disposed in the region of the heavens answering to it. Otleers, finding that the galasy was perceived in most parts of the globe, that it always corresponded to the same fixed stars, and that it was situated far above the lighest planets, set Aristotle's opinion aside, and placed the galaxy in the firmament or region of the fixed stars; thence concluding that it was nothing else but an assemblage of an infinite number of minute stars. And since the invention of telescopes, this opinion has been abundanily confirmed. For, by directing a good telescope to any part of the milky way, we perceive an innumerable multitude of very small stars, where before was only observed a confused whiteness, arising from the assemblage and union of their joint light; like as any thing powdered with fine white powder, at a distance we only obscrve the confused whiteness, but on examining it very near we perceive all the small particles of the powder scparately; as Milton firely expresses it,

" $\Lambda$ broad and ample road, whose dust is gold, And pavement stars, as stars to thec appear, Seen in the galaxy, that milky way, Which nightly, as a circling zone thou scest Powder'd with stars."

There are other similar appearances in the heavens; as the nubulx, or nebulous stars, and certain whitish parts about the south pole, called Magcllanic clouds, which are 
all of the same nature, appearing to be vast clusters of small stars when viewed through a telescope, which are too faint to affect the eye singly.

M. le Monnier however, not being able to discover more stars in this space, than in other parts of the heavens, disputes the opinion above recited, as to the reason of the whiteness, and supposes that this and the nebulous stars are occasioned by some other kind of matter. Inst. Ast. pa. 60. And Dr. Herschel has lately announced something of a similar opinion. See Philos. Trans. vul. 81, or my Abridg. vol. 17, pa. 18.

GALILLI (GALILEO), a most excellent philosopher, mathematician and astronomer, was born at Pisa in Italy, in 1564. From his infancy he had a strong propensity to philosophy and mathematics, which he indulged with sucl success that in 1592 he was chosen professur of mathematics at Padua. While he was professor there, visiting Venice, then famous for the art of glass-making, he heard that in Holland a glass had been invented, through which very distant objects were seen distinctly as if near at hand. This was sufficient for Galileo; his curiosity was raised, and his attention immediately engaged in considering what must be the form of such a glass, and the manner of making it. The result of his inquiry was the invention of the telescope, produced merely from this hint, without having seen the Dutch glass. All the discoveries he made in astronomy were easy and natural consequences of this invention, which opening a way, till then unknown, into the heavens, thence brought to light the finest discoveries. One of the first of these, was that of 4 of Jupiter's satellites, which he called the Medicean stars or planets, in honour of Cosmo the $2 \mathrm{~d}$, grand-duke of Tuscany, who was of that family. Cosmo sent lor our astronomer from Padua, and made him professor of mathematics at Pisa in $16 \mathrm{t} 1$; and soon after inviting him to Florence, gave hinı the office and title of principal philosopher and mathematiciau to his highness.

Our author had been but a few years at Florence, before the Inquisition began to be very troublesome concerning his opinions. Having obscrved some solar spots in 1612 , he printed that discovery the following year at Rome; in which, and in some other pieces, he ventured to assert the truth of the Copernican system, and brought several now arguments to confirm it. For these he was cited be. fore the Inquisition at llome, in 1615: after some inonths jmprisonmcnt, he was relcased, and sentence pronounced against him, that he should renounce his heretical opinions, ard not defend them by word or writing, or insinuate them into the minds of any persons. But having afterwards, in 1632, published at Florence his Dialogues of the two Grent Systems of the World, the Ptolemaic and Copernican, he was again summoned before the holy-office, and committed to the prison of that ccclesiastical court at Rome. The inquisitors convened in June that year; and in his presence pronounced sentence against him and his books, obliging him to abjure lis errors in the most solemn masner; committed him to the prison of their office during pleasure; aud enjoiner him, as a saving penance, for three years to come, to reneat once a week the seven penitential psalins; reserving to themselves, however, the power of moderating, cluanging, or taking away, altogether or in part, the said punishment and penance. On this sentence he was detained in prison till 1634; and his Dialogues of the System of tlie World were burnt at Rome.

Galileo lived ten years after this period; 7 of which were employed in making still further discoveries with his telescope. But by the continual application to that instrument, added to an injury of sight from the nocturnal air, his eycs grew gradually weaker, till he became totally blind in 1639. He bore this calamity with patience and resignation, worthy of a great philosopher. The loss neither broke his spirit, nor stopped the course of his studies. He supplied the defect by constant merlitation; by which means he prepared a large quantity of materials, and began to arrange them by dictating his jdeas; when, by a distemper of three months continuance, wasting away by degrees, he expired at Arcetri near Florence, in January 1642 , being the 78 th year of his age.

Galilco was in his person of small stature, though of a venerable aspect, and vigorous conslitution. His convrsation was affable and fiee, and full of pleasantry. He took great delight in architecture and painting, and designed extremely well. Heplayed exquisitely on the lute; and whenever he spent any time in the country, he took great pleasure in husbandry. His leaming was very extensive; and he possessed in a high degree a clearness and acuteness of wit. From the time of Archimedes, nothing had been done in mechanical geometry, till Galileo, who bcing possessed of an excellent judgment, and great skill in the most abtruse points of geometry, first extended the boundaries of that science, and began to reduce the resistance of solid bodies to its laws. Besides applying geometry to the doctrine of motion, by which philosophy became established on a sure foundation, he made surprising discoveries in the heavens, by means of his telescope. He made the evidence of the Copernican system more sensible, when he showed, from the phases of Venus, like to those of the moon, that Venus actually revolves about the sun. He proved the rotation of the sun on his axis, from many observations on the spots containcd on its surface; and thence the diurnal rotation of the earth became more credible. Thesatellites that attend Jupiter in his revolution about the sun, represented, in Jupiter's smaller system, a just image of the great solar system; and rendered it more easy to conccive how the moon might attend the carth, as a satellite, in her annual revolution. By discovering hills and cavities in the moon, and spots in the sun constantly varying, he showed that there was not so great a difference between the celestial bodies and the earth, as had been vainly imagined.

Our author likewise rendered no less service to science by treating, in a clear and geometrical manner, the doctrine of motion, which has justly been called the key of nature. The rational part of mcchanics had been so much neglected, that hardly any improvement was made in it for almost 2000 years. But Galilco las given us fully the theory of equable motious, and of such as are uniformly accelerated or retarded, and of these two compounded together. Hc, first of any, demonstrated that the spaces described by heavy bodies, from the beginning of their descent, are as the squares of the times; and that a body, projected in any direction not perpendicular to the hurizon, describes a parabola. These were the beginnings of the doctrine of the motion of heavy bodies, which has been since carried to so great a height by Newton. In geometry, he invented the cycloid, or trochoid; though the properties of this curve were afterwards chiclly demonstrated by his pupil Torricelli. He invented the simple pendulum, and made usc of it in his astronomical experiments : he had also thoughts of applying it to clocks; 
but did not execute that design: the glory of that invention was reserved for his son Vicenzio, who raade the experiment at Venice in 16.9; and Huygens afterwards carried the same to perfection. Our author was likewise projector of the machine, with which the Venetians render their Laguna fluid and navigable. He also discovered the gravity of the air, and endeavoured to compare it with that of water ; besides laying the foundation for several other inquiries in natural philosophy. In short, such was the character of this memorable genius, that he was not only esteemed and followed by philosophers, but was honoured by persons of the greatest distinction of all nations.

Galileo had scholars that were worthy of so great a master, by whorn the gravitation of the atmosphere was fully established, and its varying pressure accurately and conveniently measured, by the column of quicksilver of equal weight sustained thereby in the barometrical tube. The elasticity of the air, by which it perpetually endeavours to expand itself, and, while it admits of condensation, resists in proportion to its density, was a phenomenon of a new kind (the common fluids having no such property), and was of the utmost importance to philosophy. These principles opened a vast field of new and usetul knowledge, and explained a great variety of phenomena, which had been accounted for before that time in a very absurd manuer. It seemed as if the air, the fluid in which men lived from the beginning, had been then but first discovered. Philosophers were every where busy inquiring into its various properties and their effects: and valuable discoveries rewarded their industry. Of the great number who distinguished themselves on this occasion, may be mentioned Torricelli and Viviani in Italy, Pascal in Fl'unce, Otto Guerick in Germany, and Boyle in England.

Our author wrote a number of treatises, many of which were published in his life-time. Most of them were also collected after his death, and published by Mendessi in 2 vols. 4 to, under the title of L'Opere di Galileo Galilei Lynceo, in 1656. Some of these, with others of his pieces, were translated into English and published by Thomas Salisbury, in his Mathematical Collections, in 2 vols folio. $A$ volume also of his letters to several learned men, and solutions of several problems, were printed at Bologna in 4.to. His last disciple, Vincenzo Viviani, who proved a very eminent mathematician, methodized a work of his master's, and published it under this title, Quinto libro de gli Elementi d'Euclidi, \&c; at Florence in 1674, 4.to. Viviani published also other writings of Galilco, being extracts from his letters to a learned Frenchman, where he gives an account of the works which he intended to have p) ublished, and a passage from a letter of Galileo dated at Arcetri, Oct. 30, 1635, to John Camillo, a inathematician of Naples, concerning the angle of contact. Besides all these, he wrote many other pieces, which were unfortunately lost through his wife's devotion; who. solicited by her confessor, gave him leave to peruse her husband's manuscripts; of which he destroyed and took away as many as he said were unfit for publication.

GALLERY, in Architecture, a covered place in a building, much longer than broad; which is usually placed in the wings of the building, and serving to walk in as well as to place pictures. It denotes a small aisle, or walk, serving as a common passage to several rooms placed in a line, or row.

GALLERY, in Fortification, a covered walk, or passage, made across the ditch of a besieged town, with timbers fastened in the ground and covered over.

GALLON, an English measure of capacity, for things both liquid and dry, containing 2 pottles, or 4 quarts, or $S$ pints. But those pints and quarts, and consequently the gallon itself, are different, according to the quality of the things measured: the wine gallon, for instance, contains 231 cubic inches, and holds $81 \mathrm{~b}, 5 \frac{2}{3} \mathrm{oz}$, avoirdupois, of pure water; the beer and ale gallon contains 282 cubic inches, and holds $101 \mathrm{~h}, 3 \frac{\mathrm{I}}{5} \mathrm{oz}$ of water; and the gallon dry measure, for grain, meal, \&cc, contains $26 \mathrm{~S} \frac{4}{5}$ cubic inches, and holds $9 ! \mathrm{b}, 11 \frac{\mathrm{T}}{2} \mathrm{Oz}$ of water.

GALLOPER, in Artillery; the name of a carriage serving for the very small guns, and having shafts so as to be drawn without a limber.

GALVANISM, one of the most interesting branches of modern philosophy, which derives its name from its illustrious discoverer, Professor Galvani, late of Bologna: it comprises all those electrical phenomena arising from the chemical agency of certain metals with different fluids.

The following circumstance is said to have given rise to this very curious branch of philosophical inquiry. Professor Galvani had long been considered as one of the most able lecturers in the university to which he belonged; a character that he owed to his accurate knowledge of anatomy, chemistry, and other philosophical subjects: one day, while engaged in some experiments in his laboratory, a frog, which had been prepared for the purpose of making somp for his wife, who was sick, was laid on the same table where his electrical apparatus were placed; when one of his assistants in the experiments, by accident or design, brought the point of a scalpel near the crural nerves of the frog, lying not far from the conductor. In an instant the muscles of its limbs were agitated with strong convulsions. The experiment was repeated, the fact ascertained, and a great many ingenious experiments were put in execution, by which he investigated the law of nature, of which accident had thus given him a glimpse; and the success of his inquiries has immortalized his name.

His publication on the subject was printed for the institute at Bologna in 1791, cutitled, Aloysii Galvani de veribus Electricitatis in motu musculari Commentarius; which work immediately excited the attention of philosophers both in ltaly and in other countries; and the experiments were repeated and extended. Galvani, soon after he had ascertained the power of electricity on the limbs of dead frogs, and other animals, was led to a further discovery, that a similar effect might be produced without the assistance of any visible electrieal agent; that is, by the mere action of a metallic substanec; and after making a number of experiments on this head, he ascertained, that the convulsions were produced only when dissimilar metals were employed. These experiments, as before observed, were repeated by many philosophers ; none, however, added any thing new to what Galvani had discovered, excepting the celebrated Volta, of whom we shall lave frequent occasion to speak in the following piges : this great improvement took place in the year 1800 ; after which time, many other philosiphers pursued this subject with very great success and applause; and none with nore thain Professor" Davy, of the Royal lnstitution: Dr. Wollaston and Mr. Nicholson likenise have done themselve's considerable honotir, by the number and accuracy of their experiments, and the consequent developement of many new principles connected with this scicuce; and 
Mr. Wm. Cruickshank, a celebrated English chemist, by his invention of the galvanic trough, seems to have furnished the means of increasing the power- of the galvanic fluid to its greatest possible extent.

Having thus given a slight view of the discoverer and subsequent improvers of this interesting science, we shall proceed to explain, as much at length as uur limits will admit, the rature of its action, and the principal experiments that are necessary, in order to form a connected idea of $i$ ts several effects.

The discoveries of Galvani, as before observed, were made chiefly with dead frogs; the limbs of which, by means of electricity, acquired a tremulous motion, and generally a certain degree of extension; but this effect is likewise sensible, in a greater or less degree, on any other recently dead and skinned animal; though it is much more perceptible in cold-blooded creatures. The manner in which Dr. Galvani made his first experiments, was as follows: he skinned the legs of a frog recently dead, and left them attached to a small part of the spine, but separated from the rest of the body; and any other limb may be prepared in a similar manner; that is, the limb must be deprived of its integuments, and the nerve which belongs to it must be partly laid bare.

If the limbs thus prepared, for instance, the legs of a frog, be so situated, that a little electricity may pass through them, whether it be by the immediate cuntact of an clectrical body, or by the action of an electric atmosphere; as when the preparation is placed within a certain distance of an electrical machine, and a spark is taken from the prime conductor; the prepared legs will be instantly affected with a kind of spasmodic contraction, and sometimes so strong as to jump a considerable way.

The like movements may be produced in the prepared animal, without the aid of any apparent electrical agent. In an animal recently dead, detach one end of a nerve from the surrounding parts, taking care to cut it not too near its iusertion, into the muscle; remove the integuments from over the inuscles that depend on that nerve; then take a piece of metal, as a wire, and touch the nerve with one extremity of it, and the muscles with the other extremity; on doing whichr, the prepared limbs will nove in the same manner, as when electricity is made to pass through them. This, however, is not the most effectual way of forming the communication, though it will generally succeed; and the experiment will answer, whether the preparation be laid upon conductors, or on electrics. But if the communication between the nerve and the muscle be formed by nonconducting bodies, such as glass, wax, silk, \&cc, then ro motion will ensuc.

The conducting communication between the muscles and the nerve may consist of one or more pieces; and of the same, or, which is mucb better, of different borlies, connected together, as metals, water, a number of persons, and even of wood: but it must be obscrved, that the varioug bodies which form this circuit must be placed with full and perfect contact with each other, which is done by pressure, or by the interposition of water, \& $\mathrm{c}$. The less perfect conductors will answer only at first, when the prepared animal retains its vigour; but when the power begins to diminish, as it will after a few hours, then the more perfect conductors only will answer, and cven these will produce various effects.

'The most effectual way of producing thuse movenents, is by the application of two metals, of which silver and zinc seem to be rather the best; though silver and tin, or copper and zinc, and some other combinations, are not much inferior. If part of the nerve proceeding from a prepared limb be wrapped up in a piece of tinfoil, or only laid upon zinc; and a piece of silver laid with one end upon the bare muscle, and with the other on the abovementioned tin or zinc; the motion of the prepared limb will be very vigorous. The two metals may be so placed, as not to be in contact with the preparation; but in any part of the circuit; which may be completed by means of other conductor's, as water, \& c c.

In a similar manner, the action of the galvanic fluid may be made sensible on the human body. Thus, let a man lay a piece of metal, as zinc, upon his tongue; and a piece of some other metal, as silver, under his tongue; then by forming the communication betwecn these two metals, either by bringing their outer edges in contact, or by the interposition of some other piece of mctal, he will perceive a peculiar sensation, a kind of irritation, accompanied with a cool and subacid taste, not much unlike that which is produced by artificial clectricity. The sensation seems to be more distinct, when the inetals are of the usual temperature of the tongue; and on making the communication between the two pieces, the taste will be perceived more sensibly. The effect is rather more remarkable when the zinc touches the tongue in a smalt part, and the silver in a great portion of its surface, than when the contrary have place; that is, when the zinc touches more than the silver. Instead of the tongue, the two metals also may be placed in contact with the roof of the mouth, as far back as possible, and on completing the
communication, a similar effect will be produced.

Different persons are variously affectcel with this application of metals; with some, the sensation of taste is so slight, as scarcely to be perceptible; while with others it is very strong, and even disagreeable.

'The above experiment, which is one of the most simple that can be devised, was accidentally made about 60 years back, by a person of the name of Sulzer, who stated the fact in the following terins. If a piece of lead, and a similar. piece of silver, be laid together; and the edges of butli be brought in contact with the tongue, a taste is percerved similar to that of vitriol of irsn, at the same time that the metals applied separately produce no effect. The observer of this experiment does nut appear to have been at all surprised with its result: for at that time the doctrine of vibrations was employed to explain all natural phenomena; and he therefore conclucted, that some peculiar vibration took place on the application of the metals; and as every body was satisfied with this explanation, nothing further was thought of the experiment; and thus a prominent fact had slept in obscurity, from the time of Sultzer to the time of Galvani.

Having seen how the galvanic operation may be made sensible to the taste, by a similar application it may be made visible to the sight; thus, let a person in a dark place, put a piece of tinfoil on the bulb of one of his eyes, and a piece of silver, as a spoon, or the like, in his mouth; then on completing the communication between the spoon, and the tinfoil, a faint flash of white light will appear before his eyes: but this experiment may be performed in a more convenient manner, by placing a piece of ziuc between the upper lip and the gums, as high up as possible, and a silver piece of money on the tongue; for when the two metals are made to communicate, cither by the imme- 
diate contact of their edges, or by the interposition of good conducturs, the flash of light will be visible as before; but by continuing the contact of the two metals, the appearauce of light is not continued, it being only visible at the zoment the contact takes place; and sometimes, though but rarely, at the instant of separation: it may therefore be repeated at pleasure, by disjoining, and again connecting the two mctals.

For a considerable time after the first discovery made by Galrani, little more was known of its nature and its effects, than what is above stated; and it was doubtful, whether the convulsions in the animal's limbs, and the sensations produced upon the human body, were owing to an electrical property, peculiar to the animal parts, which it was thought might be conducted through the metals from one part to the other; or to a small quantity of electricity, which might be supplied by the metals themselves: Galvani himself was of the former opinion, and enthusiastic in the application of the ilectrical theory to animat economy; and particalarly when he found the metallic substances were capable of exciting muscular motion, he was confirmed in his opinion, that the inlserent electricity of the animal was transmitted from the nerve to the muscles by the metals employed.

Abunt this time, however, Signior Volta took up the subject, adopting a theory of a different nature; which, though not strictly truc, has contributed principally to its rapid advancement. Instead of supposing, as Galvani did, that the electricity belonged to the animal, he considered it as inherent in the different metals; which idca was truly fortunate for the cause of philosophy; for the very hypothesis of Galvani prevented him from deriving any farther knowledge of the science; because if the electricity belonged to the animal, no greater power was likcly to be produced, than what two picces of metal could effect; but on Volta's hýpothesis, since he conceived the action to be in the netals; it followed, or at least it was highly probable, that by increasing their number, thoir effect would also be increased in the same proportion.

He therefore repeated the experiments of Galvani, and found that when two pieces of metal of different linds were placed in different parts of an animal; at the same time that the metals were brought in contact, or were connecterl by a metallic arc; as often as the contact was made, convulsions were observed: lie also found that the greatest effect was produced, when the metals were zinc and silver; and when several pieces of metals were employed, having pieces of wet cloth between them, the effect appeared to be increased as the number of pairs; and hence his idea of the pile.

'This important cliscovery of accumulating the effects of this specius of electricity, was made by Volta in 1800 , and has hence been denominated the Voltaic pile. The apparatus first made by Volta, consisted of a number of pairs of zinc and silver plates, separated from each other by pieces of wet cloth, the arringement being as follows; viz, zinc, silver, wet clotls; zinc, silver, wet cloth, and so on: the silver plates were chicfly silver coins, the plates of zinc, and pieces of wet cloth being of the sanne size ; and he found the action of these combincd pairs, to be much more powerful when the pieces of cloth were moistrned with a solution of common salt, instead of pure water : a pile consisting of forty pairs, he found to possess the power of giving a pretty smart shock, similar to that of a small electric jar; and that this efiect took place, as often as a communication was made between each end of the pile; and as long as the pieces of cloth remained moist. An account of this discovery was communicated to the Royal Society, and published in the Philosuphical Transactions; but we do not hear of this celebrated philosopher making any farther discovery, after the invention of the pile, and ascertaining the nature and extent of its efiects on animals.

A Voltaic pile similar to that we have been describing, is represented, plate vii, fig. 6 ; it consists of picces of silver, about the size of half-crowns; pieces of zinc of an equal size; and pieces of cloth, or leather, or other bibulous substance, a little less in diameter than the metallic plates; these last being soaked in water, or any other proper fluid. These pieces are disposed in the order, silver, zinc, wet cloth; as indicated by the letters $s, z, w$; the pieces of cloth, or leather, must be well snaked in the fluid; but before they are applied, they should be squeezed, in order that the superfuous fluid may not run down the outside of the pile, or insinuate itself between the contiguous pieces of silver and ziuc. Those pieces of cluth, particularly if soaked in plain water, lose their moisture very soon, so that they can seldom last longer than for a day or two, after which the pile must be decomposed, the metallic pieces cleaned, thuse of cloth suaked again; and the whole arranged as before.

The three rods $\mathrm{R}$. $n$, $\mathrm{n}$, are of glass, or of balied wood, the piece o, slides freely up and down the rods, which serves to prevent the falling of the plates: but when such a pile is to be veiy powerful, viz, to comsist of many plates, the best metiod is to form two, thrce, or more piles; and to join them by pirces of metal, as $c, c$, fig. $\mathbf{5}$; where two pilis are joined togrether, so that $a$ is the regative extremity, and is is the other, or posizive cxtremity of the whole arrangement, or of the iwo piles, considered as one; that is, $a$ is a silver plate, and $b$ ihe zinc. It may also be observed, that copper may be substituted for the silver, without much decrense of the effect.

It was with piles similar to what we have been describing, but consisting of a greater or less number of plates that galvanic experiments were first made in this country by MIessrs. Yicholson and Carlisle, and many important chemical truths were developed by this means. At length Mr. Cruickshank, after having cmployed the Voltaic pile for various experiments; fell upon the Inappy idea of the galvanic trough. This consists of a box of baksd wood, of a width and depth agreeable to the size of the plates, and of convenient length for loanding about; the inside being furnished with grooves, passing across the side and botton, for the reception of the metallic plates, and ist such a distance, that when these are cementerl into the grooves, the trough may be diviled into a number of cells, about $\frac{3}{8}$ of an inch wide; cach of the plates being compound; viz, consisting of two plates, onc of zinc, and the other of silver or copper, soldered together; and the order in which they were placed was such, that all the zinc sides of the plates faced one nay, and the copper sides the other: in fact, the trough wis the pile placed horizontally, the cells being for the reception of a fluid, to answer the purpose of the pieces of nit cloth. 'The cementation of the metallic pieces ino the sides and bottom of the vessel, must be so accurate, as not to permit the fluid to pass from one cell to another ; the proper cement for this purpose being made, by melting together 5 parts of resin, 4 of bees-wax, and 2 parts of powdercel red ochre : 
two or more such batteries may be joined together, in order to increase the effect, as in the foregoing case of the double pile. See fig. 7 , pl. vii, which represcuts a single battery; with its communicating rods.

The action of all these batteries is the greatest, when they are first completed, or filled with the fluid; and it declines in proportion as the metal is oxidated, or the lluid loses its power: : therefore, after a certain time the fluid must be changed, and the metallic pieces cleaned, by removing the oxidated surface; which may be done, either by filing or by rubbing them with sand, or sand paper; or by immersing them for a short time in diluted muriatic acid, and then wiping them with a coarse cloth: the last method is to be preferred for the truugh; wiping them by means of a stick, which may be introduced between them, with a piece of cloth upon it.

Having thus describer the construction of the best and most simple galvanic arrangements, we shall proceed to state the effects of these combinations, in a popular manner; but omitting as much as possible those experiments that are purely of a chemical nature; as these are not only too numerous to beinserted in the space we have assigned for this article, but they are also incongruous wilt the plan of the present work; and we must therefore refer the curious reader, who wishes for particular information on this very interesting branch of philosophical inquiry, to the fuilowing works, viz, Rees's Cyclopoedia, and the professed chemical treatises, for more ample accounts.

The various powers of different simple galvanic circles may be ascertained, by applying them to such animal preparations as have their irritability more or less exhausterl. Thus, M. Volta in bis letter to Gren, says, "If you take a frog, the head of which has been cut off, and which has been deprived of all life, by thrusting a needle into the spiual marrow, and immerse it without skinning, or taking out the bowels, or any other preparation, into two glasses of water, the rump into ome and the legs into the other, it will be strongly agitated and violeutly convulsed, when you connect the water in both glasses, by a bow formed of very different metals, such as silver and lead; or, what is better, silver and zinc; but this will not be the case when the two metals are less different in regard to their powers, such as gold and silver, silver and copper, copper and iron, tin and lead; but what is more, the effect will be fully produced on this so little prepared frog, when you immerse in one of the two glasses, the end of a bow merely of tin or zinc; and into the other glass, the other end of this bow, which has been rubbed over with a little atkali: and you may perform the experiment still better with an iron bow, one end of which has been covered with a drop or thin coat of nitrous acid; but it will succeed beyond all expectation, when you take a silver bow, having a little sulphurate of potass adhering to its extremity."

It had long been asserted, that porter, and some other liquors, when drank out of pewter pots, had a different taste, from what they had when drank out of a glass or earthenware; but it was little expected, that this was a truth intimately connected with so interesting a branch of philosophy; though, now, there is no doubt of the fact. A similar, but more evident effect, may be made sensible by the following experiment. If a tin bason be filled with soap-suds, lime water, or a strong ley (which last is the best); and if you then lay bold of the bason with both hands, having first moistened them with pure water, and VOL. I. apply the tip of your tongue to the fluid in the bason, you will iminediately be sensible of an acid taste on your tongue, which is in contact with the alkaline liquor. This taste is very perceptible, aud for the moment pretty 'strong; but it is afterwards changed into a difierent one, less acid, but more saline and pungent, until at tast, it becomes alkaline, and sharp, in proportion as the fluid acts more upon the tongue.

In the foregoing part of this article, other experiments have been noticed, made by single combinations of different metals; we shall therefore now attend to those that are made by incans of the more powerful apparatus, such as we have already described; viz, the Voltaic pile; and the trough or battery; invented by Mr. Cruickshank. These powerful combinations not only convulse the prepared limbs of a fiog, or produce the appearance of a flash of light before the human eye, but they exhibit all the phenomena of electricity, in a very considerable degree: an arrangement of the kind we have described, will give the shock; it affects the electrometer, shows a luminous spark, accompanied with an audible report; it burns metallic, and other bodies; and continues in action for a very considerable time; viz, until the chemical action between the component parts of the battery be quite exhausted. Some of which effects are enumerated in the following paragraph.

If you take a Voltaic pile, consisting only of 20 repetitions of simple combinations, and touch with one hand one extremity of the pile, as at $b$, fig. 5 , and apply the other band to the other extremity, as at $a$; a very slight shock will be perceived, like that which is communicated by a Leyden phial weakly cliarged; and it will be scarcely felt beyond the fingers, or at most the wrists; but the shock is felt as often as the contact is renewed; and if you continue the hands in contact with the extremities $b$ and $a$, a slight but continued irritation will be perceived: and when the hand, or other part of the body, which touches the extremity of the battery, is excoriated or wounded, this sensation becomes disagreeable, and rather painful; but as the dry skin of the human body is seldom capable of conducting this shock, the touching fingers should be well moistened with water; and it will be still better to immerse a wire, that proceels from one extremity of the battery, in a bason of water, wherein may be plunged one hand; grasping, at the same time, with the otber hand well moistened, a large piece of netal, as a large silver spoon; by which means the shock will be felt more distinctly. Instead of one person, several may join hands, (which must be all well moistened,) and on completing the circuit, they will all feel the shock at the same time, as with an elcctrical discharge : but the strength of it is thus much diminished by passing through the several persons, as it is in general by passing through bodies that are not perfect conductors.

The shock of a battery, containing 50 or 60 pairs of combinations of silver or copper and zinc will be felt as ligh as the elbows ; and the combined effect of five or six such batteries will of course be much grcater, and such as few men would be willing to receive.

From the foregoing experiment it appears, that between the effects resulting from the common electrical machines, and those produced with the pile, there is this difference; that in the former, the effects are anuihilated at the instant of contact; while in the other, they remain as long as the 4. D 


\section{G A L}

[ 570

contact is continued. The pile once charged, becomes thus a reservoir of electricity, which, without the aid, and as it were without the knowledge, of the operafor, fills itself spontaneously; regains continually what is taken from it ; and would be inexhaustible, if the humid bodies, of which the pile is composed, could be prevented from losing their moisture.

If a wire, proceeding from one extremity of a pretty strong galvanic pile, be made to communicate with the inside coating of a common Leyden phial or electrical battery; and a wire which proceeds from the other extremity of the pile, be made to communicate with the outside coating of the same jar or battery ; the latter will become weakly, but almost instantaneously charged, in the same manner as would have been done with a few turns of the electrical machine; and with that charge you ma either give the shock, or effect an electrometer, \&c. In fact, every experiment convinces, more and more, that a galvanic battery produces a vast quantity of clectric fluid, which is but little condensed; and indeed it would be impossible to suppose that the electric fluid could proceed in a very condensed state, from an arrangement of bodies, which, whether more or less, are however all good conducturs of electricity; for if the fluid was much condensed at one extremity of the battery, and much rarefied at the other extremity, the condcrisation would soon be made through the pile itself: and indeed it is difficult to comprehend, why this compensation does not take place in all cases.

Having mentioned above, that the charge of a galvanic pile or battery may be communicated to a conimon electric battery, it is scarcely necessary to observe, that the same may be communicated to a condenser or a multiplier; and from hence to the electrometer; and if the pile consists of 200 repetitions, the electrometer will be affected by simple contact.

The spark or the discharge of an electric battery, when sent through thin inflammable bodies that are in contact with the common or oxygen air, sets them on fire, and consumes them with wonderful activity: it also fires gunpowder, liydrogen gas, phosphorus, and other combustible matter: it also renders red hot, fuses, and consumes, very slender metallic wires, and metallic leaves.

The method of employing the power of the battery for such purposes, is shown at fig. 7 , where $A B$ represents a powerful galvanic battery; $A C D F$ is a wire which conmunicates with the first plate of the battery A; BKIIIG is another wire, which communicates with the last plate at $B$; DE and in are two glass tubes, through which those wircs pass, and into which they are fastened sufficiently steady: these tubes serve to move the wires by; or if the operator applies his fingers to the midile part of those tubes, he may move the wires wherever he pleases, without the fear of recciving a shock, If the two extremitics $\mathbf{F}, \mathrm{G}$, are brought suffieiently near to each other, the spark will be seen between them; and it is between those extreinities, that the combustible substances, or inetallic leaf, is to be placed, in order to be fired or consumed; and in the figure is shown the situations of the wirc's, as they must be posited, in order to accomplish the firing of gunpowder, or the fusion of metals, \&c, \&c. $\Lambda$ battery, consisting of 200 pairs of metallic plates, viz, copper and zinc, rach five inches square, will melt 3 inches of very finc iron wire; and a platina wire about $r^{\frac{1}{3}}$ of an inch in diameter, may be melted into a globule by the same apparatus ; and more powerful effects will of course be produced from the combination of two or more such batteries.

Under the exhausted receiver of an air-pump, the galvanic battery acts less powcrfully than in the open air; but its eflect is increased in oxygen air. 'l'he flash of light that appears before the eye of lie cxperimenter, when the eje itself, or some other part not very remote frum it, is put in the circle of a galratic combination, does not appear much greater ivlisn a battery is employed, than when two plates are applied in the same manner; which experiment has been alreidy mentioned; but when the batrery is used, the sensation of a flash may be produced in various ways. If one hand or both be pliced in perfect contact with onc extremity of the bittery, and almost any part of the face be brought into contact with the other extremity of the battery; the flash will appear very distinctly, the operator bcing in the dark, or kecping his eyes shut. This flash appears very strong, when a wire, that proceeds from one extremity of the battery, is held between the teeth and rests on the tongue, while the other wire is held in the hand; and in this case, the lips and tongue are convulsed, the flash appears before the eycs, and a very pungent taste is perceived in the mouth.

If any part of the humum body forming part of the circuit of a galvanic lattery be kept some time in that situation, the irritation or numbness is more or less distinct, and more or less painful, according to the sensibility of the parts concerned.

The above are some of the most popular experiments that are inade by means of a galvanic battery; but by far the greater number of those that have been made have been directed to chemical purposes, and the results that have thus been obtained are truly curious and interesting: an extensive ficld has thus been opened for chemical research, too vast for human conception, by the introduction of this powerful agent; and more discoveries have through its means been made in one ycar than perbaps any one century can boast. But these experiments, as we before observed, do not properly belong to a work of this nature; we have therefore endeavoured, without introducing them, to give a familiar idea of the nature and power of this wonderful agent.

GARDLCAUT, or GuARD du CORD, in a watch, is that which stops the fusee, when wound up, and for that end is driven up by the spring. Some call it guard-cock; others guard du Gut.

GARRISON Guns, such as are mounted and used in a gartison, consistiug of the following weights, viz, the 42 , $32,24,18,12,9$, and 6 pounders; being made either of brass or iron.

Table of the Weiglt and Dimensions of Garrison Guns.

\begin{tabular}{|c|c|c|c|c|c|c|c|c|}
\hline \multicolumn{4}{|c|}{ Brass Garrison Gurs. } & \multicolumn{5}{|c|}{ Iron Garrison Guls. } \\
\hline Shot & Length & Wei & ght. & Shot & Len & $\operatorname{gil}$ & Weig & \\
\hline tb. & t. in. & Cir. g & . ib. & Ib. & & in. & Cw. qr & \\
\hline 42 & 100 & 6.8 & 0 & 32 & 9 & & 560 & 0 \\
\hline 32 & 92 & 40 & 18 & 24 & 9 & 8 & 480 & 0 \\
\hline 24 & 84 & 371 & 0 & 18 & 9 & 0 & 360 & 0 \\
\hline 18 & 76 & $27:$ & 0 & 12 & 7 & 8 & 240 & 0 \\
\hline 12 & 67 & $18 \approx$ & 0 & 9 & 7 & 0 & 180 & 0 \\
\hline 9 & 60 & 133 & 0 & 6 & 6 & 1 & 120 & 0 \\
\hline 6 & 53 & 9 & 0 & 4 & 5 & 4. & 80 & 0 \\
\hline
\end{tabular}


GAS. The term gas (with the exception of the common air we breathe) is used to signify any permanently elastic exhalation afforded in chemical processes. It was originally given by Van Helmont to the vapour of charcoal, the same with the fuid called fixed air; and some other factitious airs; and from him has been employed by modern philosophers as a general name for all the nuisls about which areology is couversant.

TABLE, showing the absolute weights and specific gravities of gases, and the quantity of cach absor bed by water. Temiperature $60^{\circ}$ Fallrenheit, Barometer 29.8 .

B. denotes Brisson; Cr. Cruickshánk; D. Davy; Dal. Dalton; Dei. Deiman ; H. Henry; K. Kirwan; S.Shuckburgh ; T. Thomson; Tls. Thonard; Tr. Tromsdorff.

\begin{tabular}{|c|c|c|c|c|c|c|c|c|c|c|c|c|c|}
\hline \multirow[t]{2}{*}{ - } & \multirow{2}{*}{\multicolumn{7}{|c|}{ Kind of Gas. }} & \multirow{2}{*}{$\begin{array}{l}\text { Weight of } \\
100 \text { cubic } \\
\text { inches in } \\
\text { grains. }\end{array}$} & \multicolumn{3}{|c|}{ Specific Gravity. } & \multirow{2}{*}{\multicolumn{2}{|c|}{$\begin{array}{l}\text { Number of cubic inches } \\
\text { absorbed by } 100 \text { inches of } \\
\text { water. }\end{array}$}} \\
\hline & & & & & & & & & \multirow{2}{*}{$\frac{\begin{array}{c}\text { Water, the } \\
\text { Standard at } 1000 .\end{array}}{3 .}$} & \multirow{2}{*}{$\begin{array}{c}\text { Air the } \\
\text { Standard. } \\
2425\end{array}$} & \multirow{2}{*}{$\frac{\text { Authority. }}{\text { D. }}$} & & \\
\hline Nitric acid & - & - & - & - & - & - & - & 76 & & & & 3300 & $\mathrm{~T}$ \\
\hline Sulphurous & - & - & - & - & - & - & - & $70 \cdot 215$ & $2 \cdot 75$ & 2240 & $\mathrm{~K}$. & & \\
\hline Vapour of ethe & & - & - & - & - & - & - & $62 \cdot 1^{*}$ & .......... & 2250 & Dal. & & \\
\hline Muriatic acid & & - & - & - & - & - & - & $59 \cdot 8$ & (......... & 1929 & $\mathrm{~K}$. & & \\
\hline Vapour of alco & oho & ol - & - & - & - & $=$ & - & $51 \cdot 5$ & .......... & 2100 & Dal. & & \\
\hline Nitrous oxide & & - & - & - & - & - $\quad-$ & - & $50 \cdot 1$ & 1.985 & 1615 & $\ldots \ldots$ & 86 & H. \\
\hline Carbonic acid & & $-\quad-$ & - & - & - & $-\quad-$ & - & $46 \cdot 5$ & $1 \cdot 84$ & 1500 & $\mathrm{~K}$. & 108 & H. \\
\hline Difto ditto & & - & - & - & - & - & $-\quad-$ & $45 \cdot 5$ & $1 \cdot 802$ & 1470 & D. & & \\
\hline Muriatic acid & - &.- & - & - & - & - & - & $44 \cdot 7$ & 1.765 & 1430 & B. & 51500 & T. \\
\hline Sulphuretted h & hydr & rogen & - & - & - & - & - & $38 \cdot 17$ & n....... & 1231 & T. & & \\
\hline Nitric oxide & - & $-\quad-$ & - & - & - & - & - & 37 & $1 \cdot 4.65$ & 1193 & $\mathrm{~K}$. & 5 & $\mathrm{H}$. \\
\hline Ditto ditto & - & $-\quad-$ & - & - & - & - & - & $34: 3$ & $1 \cdot 36$ & 1105 & D. & & \\
\hline Oxygen gas & - & $-\quad-$ & - & - & - & - & & $34 \cdot 74$ & $1 \cdot 39$ & 1127 & D. & & \\
\hline Sulphuretted h & $y d r$ & rogen & - & - & - & - & & $34 \cdot 286$ & $1 \cdot 36$ & 1142 & $\mathrm{~K}$. & 108 & H. \\
\hline Oxygen gas & $\therefore$ & $-\quad-$ & - & - & - & - & - & $3 \cdot 1$ & $1 \cdot 35$ & 1103 & K. & $3 \cdot 7$ & H. \\
\hline Atmuspheric a & air & $-\quad-$ & $=$ & - & - & - & & 31 & $1 \cdot 2279$ & 1000 & $S$. & & \\
\hline Azotic gas & - & $-\quad-$ & - & - & - & - & - & 30.535 & $1 \cdot 21$ & 985 & $\mathrm{~K}$. & $1 \cdot 53$ & H. \\
\hline Ditto - & - & - & - & - & - & - & - & $30 \cdot 4.5$ & $1 \cdot 20$ & 980 & D. & & \\
\hline Carbonic oxid & & $-\quad-$ & - & - & - & - & - & 30 & $1 \cdot 185$ & 967 & $\mathrm{Cr}$. & $2 \cdot 01$ & H. \\
\hline Olefiant gas & - & - & - & - & - & - & $=-$ & $28 \cdot 18$ & ......... & 905 & Dei. & 12.5 & Dal. \\
\hline Hydro carbure & et $\mathrm{fr}$ & rom st & agn & ant & wa & ter & - & $20 \cdot 66$ & ........ & 666 & Dal. & 1.40 & H. \\
\hline Ditto from coa & & $-\quad-$ & - & - & - & - & - & $20 \cdot 2$ & ......... & 650 & Dal. & 我 & \\
\hline Ditto from ethe & & - & - & - & - & - & - & 20 & ......... & 645 & Cr. & & \\
\hline Ammonia - & - & - & - & - & - &.- & - & $18 \cdot 16$ & 0.715 & 535 & K. & & \\
\hline Ditto - - - & $=$ & $-\quad-$ & - & - & - & - & - & 18 & $0.713=$ & 580 & D. & 47500 & D. ' \\
\hline Arsenicated by & dro & gen $\mathrm{g}$ & & - & - & - & - & .......... & 0.6499 & 529 & Tr. & & \\
\hline Hydro carburet & $t \mathrm{frc}$ & om alc & coh & & - & - & - & 16 & ......... & 516 & $\mathrm{Cr}$. & & \\
\hline Ditto from wat & ter & over is & gnit & & & rcoal & 1 & $14 \cdot 5$ & ........... & 468 & $\mathrm{Cr}$. & & \\
\hline Hydrogen gas & - & $-\quad-$ & - & - & - & - & - & $26 \cdot 13$ & $0 \cdot 1031$ & 84 & K. & $1 \cdot 6 I$ & H. \\
\hline Phosphuretted & hyo & drogen & & - & - & $\sim$ & - & $\ldots \ldots \ldots$ & .......... & $\ldots . .$. & ....... & $2 \cdot 14$ & H. \\
\hline
\end{tabular}

The following are the experiments made by professor Jacquin of Vienna on the different gases, as the vehicle of sounds. - A glass-bell being furnished with a metallic stopper cemented to a neck at the top; and in the bore of this cock within the glass, a small flute or pewter (etain) about six inches in length was fixed. The glass being then placed on the shelf of the pneumatic vessel, and filled witl any particular kind of gas, a bladder also filled with the same gas, and provided with a cock, was adapted to the external aperture of the cock belonging to the bellglass. In this disposition of the apparatus the flute was made to sound by gently pressing the bladder. Comparative experiments were also made with atmospheric air, oxygen, hydrogen, carbonic acid, and nitrous gas. The intensity of the sound did not vary; but when compared with that produced by atmospheric air, the oxygen gas afforded a sound hall' a tone lower; azotic gas, prepared by different methods, cunstantly gare a sound half a tone lower; hydrogen gas gave nine or eleven tones higher; carbunic acid gas gave one third lowcr; and nitrous gas also very nearly a third lower. A mixture of oxygen gas and azote, in the proportion contained in atmospheric air, afforded the tune of this last; that is to say, it was half at tone higher than each of the component parts alone. When the two gases were not uniformly mixed, the sound was abominably harsh. Chladni intends to give a fuller account of these interesting experiments. Joumal de Physique, vol. 4, N.S. p. 57.

GASCOIGNE, an ingenious young astronomer of Lancashire, who, about the year 1640 , nade many curious observations on some astronomical objects, and grẹatly improved that science, by the invention of the micrometer, and by applying telescopic sights to astronomical quadrants. See accounts of his inventions in the article Nicrometer. See also my Abridg. of the Plilos. Trans. vol. i, p. 169, 195, and vol. x, p. 369 .

Mr. Gracoigne was slain in the civil war's in the time of Charles $\mathrm{I}$.

GASSENDI (PETER), one of the most culebrated phi. losophers France has produccd, was brin at (hautersiex 
about 3 miles from Digne in Provence, in the year 1592. When a child, he took great delight in gazing at the moon and stars whenever they appeared: This pleasure often drew him into bye-places, that he might feast his eyes freely and undisturbed; by which means his parents bad him often to seck, with many anxious fears and apprehensions. In consequence of this promising disposition, he was sent to the best schools, to cultivate it with the instructions of the first masters. He profited so well by these aids, that he was invited to be professoj of rlietoric at Digne, belore he was quite 16 years of age. After filling this office three years, upon the death of his master at Aix, he was appointed to succeel him as professor of philosopliy. After a few years residence here, he composed his Paradoxical Exercitations; which coming to the hands of Nicholas Peiresc, that great patron of learning joined with Joseph Valter, prior of Valette, in promoting him; and, having entered into holy orders, he was first made canon of the church of Digne and doctor of divinity, and then warden or rector of the same church.

Our autlior's fondness for astronomy grew up with his years; and his reputation daily increasing, he was appointed the king's professor of mathematics at Paris in 1645. This institution being chiefly intended for. astronomy, Gassendi read lectures on that science to a crowded audience. However, he did not long enjoy this situation; for a dangerous cough and inflammation of the luugs obliged him, in $16+7$, to return to Digne for the benefit of his native air. Having thus, and by the intermission of his studies, recovered his health, he again returned to $\mathrm{Pa}$ ris in 1653; where, after first writing and publishing the lives of Tycho Brahé, Coperuicus, Purbach, and Regiomontanus, in 1654 he again renewed his astronomical labours, with the design of completing the system of the heavens. But while he was thus employed, too intensely for the feeble state of his liealth, he relapsed into his former disorder, under which, with the aid of too copious and numerous bleedings, by order of three physicians, he expired in the year 1655 , at 63 years of age.

Gassendi wrote against the metaphysical meditations of Descartes; and divided with that great man the philosophers of his time, alınost all of whom were either Cartesians or Gassendists. To his knowledge in philosophy and mathematics, he joined profound erudition and deep skill in the languages. He wrote, 1. Three volumes on Licurus's philosophy; and six otheis, which contain his own philosophy.-2. Astronomical Works.-3. The lives of Nicholas de Peiresc, Epicurus, Copernicus, Tycho Brahé, Purbach, and Regiomontanus.-4. Epistles, and other treatises. All his works were collected together, and printed at Lyons in 1658, in 6 volumes folin.

Gassendi was the first person wlso satw the transit of Nercury over the sun, viz, Nov. 7,1631 ; as Ilorrox first predicted'and showed the transit of Venus.-His library was large and valuable: $t$ (n) which he added an astronomical and plilosophical apparatus, which, for their accuracy and magnitude, were purchased by the emperor Ferdinand 1,1.-It appears by his letiers, printed in the 6th volume of his works, that he was olten consulted by the mont cclcbrated astronomers of his time, as Kepls's, Longomontenus, Snell, llevelius, Galiteo, Kirclser, Bulliald, and others: and he lias generally been esteemed one of thre founders of the reformed phitusophy, in opposition to the groundless hypotheses and empty subtleties of $\mathrm{Ari}$ stotle and the scluoolmen.
GATE, in Architecture, a large door leading or giving. entrance into a city, town, castle, palace, or other conside rable building.

Gate, in Forlification, is made of strong planks with iron bars to oppose an enemy. They are generally made in the middle of the curtain, whence they are seen and defended by the two fianlis of the bastions. They should be covered with a good ravelin, that they may not be seen or infiladed by the enemy. These gates belonging to a fortified place, are passages through the rampart, which may be shut and opened by means of doors and a portcnllis. They are either private or public.

Private Gates, are those passages by which the troops can go out of the town unseen by the enemy, when they pass to and fro on the relief of the duty in the outworks, or on any other occasion which is to be concealed from the besiegers.

Public Grates, are those passages through the middle of such curtains to which the great roads or public ways lead. The dimensions of these are usually about 13 or 14 feet ligh, and 9 or 10 feet wide, continued through the rampart, with proper recesses for foot passengers to stand in out of the way of whecl carriages.

GAUGE-Line, a line on the common ganging-rod, used for the purpose of gauging liquids. See Gaugrng-Rod.

GAUGL-Point, of a solid measure, is the diameter of a circle, whose area is expressed by the same number as the solid content of that measure. Or it is the diameter of a cylinder, whose altitude is 1 , and its content the same as of that measure. Thus, the solid content of a wine gallon being 231 cubic inches; if a circle be conceived to contain so many square irches, its diameter will be $17 \cdot 15$; which is therefore the gauge-point for wine measure. And an ale-gallon containing 282 cubic inches; by the same rule, the gauge-point for ale measure will be found to be 18.95 . In like manner may the gauge-point for any other measure be determined. Hence it follows, that when the diameter of a cylinder in inches is equal to the gaugepoint in any measure, given likewise in inches, every inch in its length will contain an integer of the same measure. So in a-cylinder whose diameter is $17 \cdot 15$ inclues, every incl in height contains one cntire gallon in wine measure; and in another, whose diameter is 18.95 , every inch in length contains one ale gallon.

G.AUGER, an officer appointed by the commissioners of excise, to gauge, measure, or examine, all casks, tuns, pipes, barrels, hogeheads, of beer, wine, oil, \&c.

GAUGING, the art or act of measuring the capacities or contents of all kinds of vessels, and determining the quantity of fluids, or other matters contained in them. These are principally pipes, tuns, barrels, rundlets, and other casks; also backs, cuolers, vats, \&c.

As to the solisl contents of all prismatical ressels, ats cubes, parallelipipedons, cylinders, \&c, they are found by multiplying the area of the base by their altitude. And the contents of all pyramidal bodis, and cones, are equal to $1-3 \mathrm{~d}$ of the same.

In gauging, it has been usual to divide casks into four variutics or forms, clenominated as follows, from the supposed resemblance they brar to the frustums of solids of the same names: viz.

1. The middle firustum of a spheroid,

2. The middle frustum of a paraloolic spindle,

3. The two equal frustuns of a paraboloid,

4. The two equal frustums of a cone. 
And particular rules, adapted to each of these forms, may be found in most books of gauging, and in my Mensuration, p. 436, \&rc. But as the form is imaginary, and only guessed at, it seldom happens that a true solution is obtained in this waly; besides which, it is very troublesome and inconsenicnt to hare so many rules to put in prac- tice. I shall therefore give here one rule only, from pa. 451 of that book, which is not only general for all casks that are commonly met with, but quite easy, and very accurate, as luaving been often verified and proved by filling ithe casks with a true gallon measure.

General Rule. Add into one sum,

39 times the square of the bung diameter,

25 times the square of the head diameter, and

26 times the product of those diameters;

multiply the sum by the length of the cask, and tive product by the number 00034 ; then this last product divided by 9 will give the wine gallons, and divided by 11 will give the ale gallons.

Or, $\left(39 \mathrm{~B}^{2}+25 \mathrm{H}^{2}+26 \mathrm{BI}\right) \times \frac{\mathrm{x}}{\mathrm{j} 14}$ is the content in iuches;

which being divided by 231 for wine gallons, or by 282 for ale gallons, will be the content.

For Example. If the length of a cask be 40 inches, the bung diameter 32 , and the head diameter 24 .

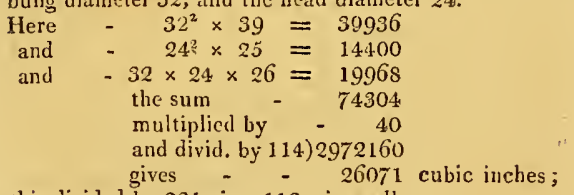

this divided by 231 gives 112 wine gallons,

or divided by 282 gives 92 ale gallons.

But the common practice of gauging is performed mechanically, by means of the gauging or diagonal rod, or the gauging sliding rule, the description and use of which here follow.

Gavging, or Diagonal, Rod, is a rod or rule adapted for determining the contents of casks, by measuring the diagonal only, viz, the diagonal from the bung to the extremity of the opposite stave next the head. It is a square rule, having 4 sides or laces, being usually 4 . feet long, and folding together by means of joints. On one face of the rule is a scale of inclics, for taking the measure of the diagonal; to these are adapted the areas, in ale gallons, of circles to the corresponding diameters, like the lines on the under sides of the three slides in the sliding rule, described below. And on the opposite face are two scales, of alc and wine gallous, expressing the contents of casks having the corresponding diagonals; and these are the lines which chiefly constitute the difference between this instrument and the sliding rule; for all the other lines upon it are the same with those on that instrument, and are to be used in the same manner.

To use the Diargonal Rod. Unfold the rod quite straight and put it in at the bung hole of the cask to be gauged, till its cond arrive at the intersection of the bead and opposite stive, or to the farthest pussible distance from the bung-hole, and note the inclses and parts cut by the midille of the bung; then draw out the rod, and look for the same inclies and parts on the opposite face of it, and annexed to them are the contents of the cask, both in ale and winc gallons.

For Example. Let it be required to find, by this rod, the content of a cask whose diagonal measures 34.4 inches; which answers to the cask in the foregoing example, whose head and bung diameters are 32 and 24 , and length 40 inches; for if to the square of 20 , half the length, be added the square of 28 , half the'sum of the diameters, the square ruot of the sum will be 34.4 ncarly. Now, to this diagonal 34.4 , corresponds, on the rulc, the content 91 ale gallons, or 111 wine gallons; which are but 1 less than the content found by the former general rule above given:

GAUGina Rule, or Sliding Rule, is a sliding rule particularly adapted to the purposes of gauging. It is a square rule, of four faces or sides, three of which are furmished with sliding pieces running in grooves. The lines on these faces are mostly logarithmic ones, or distances which are proportional to the logarithms of the numbers placerl at the ends of then ; which kind of lines was disposcri first upon rulers, by Mr. Edmund Gunter, for expeditiously performing arithmetical operations, using a pair of compasses fur taking ofi and applying the several logarith mic distances. Instead of the compasses, sliding pieces were afterwards added, by Mr. Thomas Ererard, as more certain and convenient in practice, from whom this sliding rule is often called Everard's Rule. For the more particular description and uses of this rule, sec my Mensuration, p. 429 , 4 th ed.

The writers on gauging are, Beyer, 'Kepler, Dechales, Hunt, Everard, Dougherty, Shettleworth, Shirtcliffe, Leaclbetter, Moss, \&c.

GAZONS, in Fortification, turfs, or pieces of fresh earth covered with grass, cut in form of a wedge, about a foot long and half a foot thick, to line or face tlie outside of works made of earth, to keep them up, and prevent their mouldering.

GELLIBRAND ( $\mathrm{H}_{\mathrm{LN} R \mathrm{Y}}$ ), professor of astronomy at Gresham-collegc, was born in London the 27 th of Nov. 1597. He was sent to Trinity-college, Oxford, in 1615 , and took his degree $\mathrm{nn}$ arts 1619 . He then entered into orders, and became curate of Clidddingstone in Kent. Afterwards, taking a great fancy to matlicmatics, by happening to hear one of Sir Henry Saville's lectures in that science, he immediately applied himself to the close study of that noble science; and relinquished his fair prospects in the church. Content with his private patrimony, which was now come to his inheritance by the death of his father, the same year be entered again a student at Oxford, making mathematics his sole empluyment. He made such proficiency in this science befure he procecded $\Lambda$. which was in 1623, that he drew the attention and intimate friendship of $\mathrm{Mr}$. I Ienry Briggs, then lately removed from the geometry professurship in Gresham-college to that of Savilian professor of geometry at Oxford, by the founder Sir Henry Saville, and who, upon the death of Mr. Gunter, procured for our author the professorship of astronomy in Gresham-college, to which he was elected in the begiming of the year 1627 . His friend $\mathrm{M} \mathrm{r}$. Briggs dying in 1630, before he bad finished the introduction to his Trigonometria Britaunica, he recommended the completing and publishing of that work to our author. Gellibrand accordingly added a preface, aud the application of the lugarithms to plane and splierical trigonometry, \&c, and the whole was printed at Goudn, under the care of Aitrian Vlacq, in 1633.

While Mr. Gellibrand was preparing that work, he was brought into trouble in the high-commission court, by Dr. Laud, then bishop of London, on account of an almantc, 

G E N

publislied by William Beale, servant to Mr. Gellibrand, for the yea: 1631, with the approbation of his master. In this ilmanac, the popish saints, then usually put into calcndars, were onitted, and the names of other saints and martyrs, mentioned in the Book of Martyrs, were placed in their stead, as they stand in Fox's calendirs. 'This, it seems, gave much offence to the bishop, and occasioned the prosecution. But when the cause came to be heard, it appeared that other almanacs of the same lind had formerly been printed; upon which, both master and man were acquittcd by Abp. Abbot and the whole court, Laud only excepted: which was afterward made one of the articles against bim on his own trial.

It seems Gellibrand was strongly attached to the old Ptolcmaic system. For when he went over to Holland, about the printing of Briggs's book above mentioned, he had some discuurse with Lansberg, an eminent brother astronomer in Zealiud, who affirming that he was fully persuaded of the truth of the Copeirnican system ; our auther observes, "that this so styled a truth he should receive as an hypothesis; and so be easily led on to the consideration of the imbecility of man's apprebension, as not able rightly to conceive of this admirable opifice of God, or frame of the world, without falling foul of so great an absurdity:" so filmly was he fixed in his adherence to the Ptolemaic system. Gellibrand wrote several things after this, chicfly tending to the improvement of navigation, which would probably have been further advanced by him, had his life been continued longer; but be was untimely carried off by a fever, in 1636 , at 39 years of age.

'The character of Mr. Gellibrand is that of a plain, plodding, industrious, well-intentioned man, with little invention or genius. His writings are chiefly as below.

1. Trigononetria Britannica; or the Doctrine of Triangles, being the $2 \mathrm{~d}$ part of Briggs's work above mentioned. -2 . A small tract concerning the longitude- $-3, A$ discourse on the Variation of the Magnetic Needle; annexed to Wright's Errors in Navigation detected.-4. Institution Trigonomesrical, with its application to astronomy and navigation; 8vo, 1635.-5. Epitome of Navigation, with the necessary tables; Sro.-6. Several manuscripts never published; as, The Doctrine of Eclipses, A Treatise of Lunar Astronomy, A Treatise of Ship-luildting, \&c.

GEMIN], in Astronomy, the Twins; a constellation or sign of the zodiac, the third in order, representing Castor and Pollux; and it is marked thus, $\mathbf{I I}$. The stars in the sign Gemini are. in Ptolemy's catalogue 25, in Tycho's 25 , in Hevelius's 38 , and in the Britannic catalogue 85 .

GLMMA (RENER), commonly called Gemma Frisius, from his native country Fiezland, a Dutch physician and inatliematician, who becanse medical professor at Louvain, where he died in 1558 , at 50 years of age. lle wrote, 1. Methodus Arithmeticæ: 2. De Usu Annuli Astronomici: 3. De Locorum describendorum Ratione, denue bistantiis eorum inveniendis: 4. Demonstrationes Geometrice de usu Radii Astronomici: 5. De Astrolabio Citholices Liber, ace. See further under Frisi.

GLMMa (CORNELUS), son of the lormer, al grood astronomer, was born at louvain in 1535 , and died 1579 . He wrote several tracts, as, De Prodigiosi Specic Naturaque ('ouscte: and one on the star which appeared in 1577 , Altw. 1578, in svo.

GENERA'TE, in Music, is used to signify the operalion of that mechanical power in nature, which every sound has in producing onc or more gencral sounds. 'Thus any given sound, however simple, produces along with itself, its octave and two other sounds extremely sharp, viz, its 12 th above, that is to say, the octave of its fifth; and the other the 17 th above, or, in other words, the double octave of its third major.

Whether we suppose this procreation of sounds to result from an aptitude in the texture and magnitude of certain particles in the air, for conveying to out: cars vibrations that bear those proportions one to another, as being determined at once by the partial and total oscillations of any musical string; or from whatever œeconomy of nature we choose to trace it; the power of one sound thus to produce another, when in action, is said to generate. The same word is applied by Signor Tartini and bis followers, to any two sounds which, simultaneously heard, produce a third.

GENERA'TED, is used by some mathematical writers for whatever is produced by arithmetical operation, or in geonetry by the motion of other magnitudes. Thus 20 is the product generated of 4 and 5 ; $a b$ that of $a$ and $b, 4$, 8,16 , \& c, the powers generated of or from the root 2 , and $a^{2}, a^{3}, a^{4}, \& \mathrm{c}$, those from the root $a$. So also, a circle is generated by the revolution of a linc abuut one of its exiremitiess; a cone by the rotation of a right-angled triangle about its perpendicular; a cylinder by the rotation of a rectangle about one of its sides, or, otherwise, by the motion of a circle in the direction of a right line, and keeping always paralle! to itself.

GiNDiRATING Line or Figure, in Geometry, is that whicls, by any kind of supposed motion, may generate, or produce, some other figure, plane, or solid. Thus a line, according to Euclid, generates a circle; or a right-angle, triangle, a cone, \&r ; - and thus also $A$ rchimedes supposes his spirals to be generated by the motions of generating points and lines. The figure thus generated, is called the Generant.

It is a gencral theorem in geometry, that the measure of any gencrant, or figure produced by any kind of motion of any other figure, or generating quantity, is equal to the product of this ginerating quantity drawn into the length of the path described by its centre of gravity, whatever the kind of motion may be, whether rotatory, or direct, \&rc.In tle modern analysis, or fluxions, all sorts of quantitics are considered as generated by some such motion, and the quantity hereby generated is called a Fluent.

GENERATYON, in Mathematics, is used for the formation or production of any geometrical figure, or other quantities. Such ns of the figures mentioned in the foregoing articles, or the generation of equations, curves, solids, \&c.

Genirator, in Music, significs the principal sound or sounds by which others are produced. Thus, the lowest c fur the treble of the harpsichord, beside's the octave, will strike in attentive car with its $12 \mathrm{~h}$ above, or $\mathrm{G}$ in alt. and with its 17 th above, or $\mathrm{E}$ in alt. The $\mathrm{c}$, therefore is called their generator, the $\mathrm{G}$ and $\mathrm{E}$ its products, or harmonies. But in ihe approximation of chords, for $\mathrm{G}$, its octave below is substituted, which constitutes a tilth from the generator or lowest $\mathrm{c}$; and for $\mathrm{v}$, is likew ise substituted its 1.ith below, which, with the nbove-nemtioned $\mathrm{c}$, forms a third major. 'To the lowest not's, therefore, exchanged for these in alt. by sulbstitusion, the denomimntions of products or harmonies are likewise given, while the c retains the nitme of their gencrator. Bat still, according to the system of 'fartini, two notes in concord, which when sounded, produce $\|$ third, may be termed the concurring generators of that third. Sec Ceneration Ilarmonique, 
par M. Rameau; see also that delineation of Tartini's system called The Power and Principles of Harmony.

GENESIS, in Geometry, means much the sane as generation mentioned above, being the formation of a line, surface, or solid, by the motion or flux of a point, line, or surface; as of a globe by the rotation of a seni-circle about its diameter, \&c. In the genesis of figures, the line or surface which moves, is called the Describent; aud the line round which, or according to which, the rerolution or other motion is made, the Dirigent.

GEOCENTRIC, is said of a planet or its orbit, to denote its having the earth for its centre. 'The moon alone is properly geocentric. And yet the motions of all the planets may be considered in respect of the earth, or as they appear fom the earth, and thence called their geocentric mottons. - Hence also the ter'ms geocentric place, or latitude, or longitude, \&xc, as explained below.

Geocent Ric Place, of a planet, is the place where it appears to us, from the carth; or it is a point in the ecliptic, to whicl a planet, seen from the eartly, is referred.

Geocentric Latitude, of a plánet, is its latitude as scen from the earth; or the inclination of a line, connecting the planet and the earth, to the plane of the earth's (or true) ecliptic. Or it is the angle which the said line, (connecting the planet and the earth) makes with a line drewn to meet a perpendicular let fall from the planet to the plane of the ecliptic.

Geocentric Longitude, of a planet, is the distance measured on the ecliptic, in the order of the signs, between the geocentric place and the first point of $A$ ries.

GEODESI $A$, is properly that part of practical geometry that teaches how to divide or lay out lands and fields, among several owners.

Geodesin is also applied, by some writers, to all measurements in the ficld, and as synonymous with surveying.

Geodesia is defined by Vitalis, as the art of measuring surfaces and solids, not by imaginary lines, as is done in geometry, but by sensible and visible things; or by the sun's rays, \&c.

Geodetical, something relating to the art of measuring the earth or land.

GEOGR $\triangle$ PHER, a person skilled in geography.

GEOGRAPIIICAL, relating to geography, as

Geographical Mile, the sea-mile or minute, being the 6oth part of a degrce of a great circle.

Geograpincal Table. See Map.

GEOGRAPHX, the science that teaches and explains the nature and properties of the earth, as to its figure, place, magnitude, motisns, ceiestial appenrances, \&ce, with the various lines, real or inaginary, on its surface.-Gengraphy is distinguished from Cosmography, as a part from the whole; this latter considering the whole visible world, both heaven and earth. And from Tupograpliy and Chorography, it is distinguished, as the whole from a pait. Golnitz considers gengraphy as either exterior or -interior: but Varenius more justly divides it into general and special; or universal and particular.

General or Universal Grog RAPII, is that which considers the earth in general, without regard to particular countries, or the affections common to the whole globe: as its figure, magnitude, motion, land, sea, \&c.

Special or Particular Geograptiy, is that which contemplates the constitution of the several particular regions, or countries; their bounds, figure, climate, seisons, weather, intabitants, arts, customs, language, \&c.
History of GEoG RAPHY. The study and practice of geography must have commenced at a very carly period of the world. By the accounts we hare remaining, it appears this science was in use among the Babylonians, Arabians, and Egyptians, from whom it passed to the Greeks first of any Luropeans, and from these successivcly to the Romans, and the western nations of Europe. Herodotus relates that the Greeks first learned the pole, the gnomon, and the 12 divisions of the day, flom the Babylonians. But Pliny and Diogenes Laertius assert, that Thales of Miletus, in the 6th century before Christ, first discovered the passage of the sun from tropic to tropic, and it is said was the author of two books, the one on the tropic and the other on the equinox; both probably determined by means of the gnomon; whence be was led ti) the discovery of the fuur seasons of the year, which are dctermined by the equinoxes and solstices. It is however probable that he learned these things of the Egyptians, as well as his division of the year into 365 days. This it is said was invented by the second Mercury, surnamed Trismegistus, who, according to Eusebius, lived about 50 years after the Exodus. Pliny expressly informs us that this discovery was made by observing when the shadow returned to its marks; a sufficient proof that it was by the gnomon. It is also said that Thales constructed a globe, and represented the land and sea upnn a table of brass. Further, that Anaximander, a disciple of 'Thales, first drew the figure of the earth upon a globe; and that Hecatæus, Democritus, Eudoxus, and others, formed geographical maps, and brought them into common use in Greece.-Meton and Euctemon observed the summer solstice at Athens, on the 27 th of June 432 years before Christ, by watching narrowly the sliadow of the gnomon, with the design of fixing the beginning of their cycle of 19 years.

Timocharis and Aristillus, who began their observations about 295 B. C., it secms first attempted to fix the latitudes and longitudes of the fixed stars, by considering their distances from the equator, \&c. One of their observations gave rise to the discovery of the precession of the equinoxes, which was first remarked by Hipparchus abnut 150 years after; who also made use of their method, for delineating the parallels of latitude and the meridians, on the surface of the earth; thus laying the foundation of this science as it now appears. The latitudes and longitudes, thus introduced by Hipparclus, were not bowever much aterided to till Ptolemy's time. Strabo, Vitruvius, and Pliny, have all of them entered into a minute geographical description of the situation of places, according to the length of the shadows of the gnomon, without noticing the longitudes and latitudes.

Niaps at first were little more than rude outlines, and topographical sketches of different countries. The earliest on record were those of Sicsostris, mentioned by Eustathius; who nhserves, that "this Egyptian king, having traversed great part of the earth, recorded his march in maps, and gave coperes of them not only to the ligyptians, but to the Scythians, to their grcal astonishment." Some have imagined witl much probabitity, that the Jews made a map of the Holy Land, when they gave the different portions to the nine tribes at Shiloli: lor Jusliua tells us that they were sent to walk through the land, and that they described it in seven parts in a hook; and Josephus relates that when Joshua sent out people from the different tribes to measure the land, he gave them, as companions, persons well skilled in geometry, who could not be mistaken in the trutl. 
The first Grecian map on record, was that of Anasimander, mentioned by. Strabo, lib. 1, p. 7 , supposed to be the one refisted to by Hipparchus under the designation of the ancient map. Herorlotus minutcly describes a map made by Aristagoras tyrant of Miletus, which will serve to give some idea of the majs of thuse times. He relates, that Aristagoras showed it to Cleomenes king of Sparta, to induce him to attack the king of Persia at Susa, in order to restore the Ionians to their ancient liberty. It was traced upou brass or copper, and seems to have been a mere itinerary, containing the route through the intermediate countries which were to be traversed in that march, with the rivers llalys, the Euphrates, and Tigris, which Herodotus mentions as necessary to be crossed in that expedition. It contained one straight line called the Royal Road or Highway, which took in all the stations or places of encampment, from Sardis to Susa; being 111 in the whole jourmey, and contining 13,500 stadia, or $1687 \frac{1}{2}$ Roman miles of 5000 feet each.

These itincrary maps of the places of encampment were indispensibly necessary in all armies and marches; and indeed war and navigation secm to be the two grand causes of the improvements, both in geography and astronomy. Athenæus cites Bælon as author of a work entitled The encampments of Alexander's march; and likewise Anyntas to the same purpose. Pliny observes that Diognetus and Baton were the surveyors of Alexandcr's marches, and then quotes the exact number of miles according to their mensuration; which he afterwards confirms by the letters of Alexander himself. The same author also remarks, that a copy of this great monarch's surveys was given by Xenocles his treasurcr to Patrocles the geographer, who was admiral of the fleets of Selcucus and Antiochus. His book on geography is often cited both by Strabo and Pliny; and it appears that this author furnished Eratosthenes with the principal materials for constructing his map of the oriental pirt of the world.

The first attempt to reduce geography to a regular system, was made by Eratosthenes, who introduced a regular parallel of latitude, commencing at the straits of Gibraltar, and passing eastwirds through the isle of Rhodes, and so on to the mountains of Inclia, noting all the intermediate places through which it passed. In drawing this line, he was not regulated by the same latitude, but by observjing where the longest day was 14 hours and a half, which Hipparchus afterwards determined was the latitude of 36 degrees. This first parallel tlirough Rliodes was cver after consideres with a degree of preference, in constructing all the ancient maps; and the longitude of the then known world was often attempted to be measured in stadia and miles, according to the extent of that line, by many succecding geographers. Eratosthenes soon after attempted not only to draw other parallels of latitude, but also to trace a meridian at right angles to these, passing through Rhodes and Alexanstria, down to Syene and Meroë; and at length he undertook the arduous task of determining the circumference of the globe, by an actual measurement of a segment of one of its great circles. To find the magnitude of the earth, is indecd a problem which has engiged the attention of astronomers and geographers ever since the spherical figure of it was known. It seems $\Lambda$ naximander was the first among the Creelis who wrote upon this stibject. Archytas of 'f'urentum, a Pythagorean, famous for his skill in mathematics and mechanies, also made soine atiempts in this way; and Dr. Long conjectures that these are the authors of the most ancient opinion that the circumference of the earth is 400,000 stadia: Archimedes makes mention of the antients who estimated the circumference of the earth at only 50,000 stadia.

With respect to the methods of measuring the circumference of the earth, it would appear from what A ristotle says in his treatise $\mathrm{DeC}$ Colo, that they were much the same as those used by the moderns, deficient only in the accuracy of the instrunents. For in that work be says, that difierent stars pass through our zenith, according as our situation is more or less northerly; and that in the southern parts of the carth stars come above our horizon, which are no longer visible if we go northward. Hence it appears that there are two ways of measuring the circumference of the eartls; one by observing stars which pass through the zenith of one place, and do not pass through that of another; the uther, by observing some sturs which comeabove the borizon of me place, and are observed at the same time to be in the horizon of another. The former of these methods, which is the best, was followed by Eratostlenes at Alexandria in Egypt, 250 years before Christ. He knew that at the summer solstice, the sun was vertical to the inhabitants of Syewe, a town on the confines of Lthiopia, uuder the tropic of Cancer, where they had a well made to observe it, at the lotion of which the rays of the sun fell perpendicularly the day of the summer solstice: he observed by the shadow of a wireset perpendicularly in an henispherical bason; how far the sun was on that day at noon distant from the zenith of Alesandria; and found that distance to be equal to the $50 \mathrm{tl}_{1}$ part of a great circle in the heavens. Then supposing Syene and Alexandria under the same meridian, he inferred that the distance between them was the 50th part of a great circle upon the earth; and this distance being by measure 5000 stadia, he concluded that the whole circumterence of the earth was 250,000 stadia. But as this number divided by 360 would give $694 \frac{4}{9}$ stadia to a degree, either Eratusthenes himself, or some of his followers assigned the round number 700 stadia to a degree; which multiplied by 360 , makes the circumference of the earth 252,0n0 stadia; whence both these measures are given by different authors, as that of Eratosthenes.

In the time of Pompey the Great, Posidonius determined the measure of the circumference of the earth by the $2 \mathrm{~d}$ method above hinted by $\Delta$ ristotle, viz, the horizontal observations. Knowing that the star called Canopus wis but just visible in the horizon of Rhorles, and at Alexandria fiuding its meridian height was the 48 th part of a great circle in the heavens, or $7 \frac{\mathrm{T}}{2}$ degrees, answering to the like quantity of a circle on the earth: he by considering these two places under the meridian, and the distance between them 5000 stadia, concluded the circumference of the eartl to be 240,000 stadia ; ' which is the first mcisure of Posidonius. But, according to Strabo, I'osidonius made: the ineasure of the earth to be $180,(100$ stadia, at the rate of 500 stadia to a degree. The reason of this difference is thought to be, that Eratosthencs measured the distance between Rhodes and Alexandria, and found it only 3750 stadia : taking this for a 4.8 th part of the carth's circumference, which is the measure of Posidonins, the whole circumference will be 180,000 stadia. This measure was received by Mlarinus of 'Tyre, and is usually ascribed to Ptolemy. It is to be observed, however, that this methed of cletermining the circumference of the carth is subject to great uncertainty, both on account of the gatat refraction of the stars near the horizon, the dificulty of 
measuring the distance at sea between Rhodes and Alexandria, and by supposirg those places under the same meridian, when they are really very different.

Several geographers afterwards made use of the different. beights of the pole in distant places under the same meridian, to find the dimensions of the earth. Aluout the year 800 , the khalif Almamun had the distance measured between two places that were 2 degrees asunder, and under the same meridian in the plains of Sinjar near the lied Sea. And the result was, that the degree at one time was found equai to 56 miles, and at another $56 \frac{\mathrm{x}}{3}$ or $56 \frac{\mathrm{z}}{3}$ miles.

The next attempt to ascertain the circumference of the earth was in 1525, by Fernelius, a learned philosopher of France. For this purpose, he took the height of the pole at Paris, going from thence directly northwards, till he came to the place where the height of the pole was one degree nore than at that city. The length of the way was measured by the number of revolutions made by one of the wheels of his carriage; and after proper allowances for the declivities and turnings of the road, he concluded that 68 Italian miles werc equal to a degree on the earth. According to these methods many other measurements of the earth's circumference have since that time been made, with inuch greater accuracy: a particular accuunt of wbich is given under the article DEGREE.

'Though the maps of Eratosthenes were the best of his time, they were yet very imperfect and inaccurate. They contained little more than the states of Greece, and the dominions of the successors of Alexander, digested according to the surveys abovenentioned. He had indeed seen, and has quoted, the voyages of Pythias into the great Atlantic ocean, which gave him some faint jea of the western parts of Europe; but so imperfect, that they could not be realised in to the outlines of a chart. Strabo says he was very ignorant of Gaul, Spain, Germany, and Britain; and he was equally ignorant of Italy, the coasts of the Adriatic, Pontus, and all the countries towards the north. Such was the state of geography, and the nature of the maps, before the time of Hipparchus.

War has usually been the occasion of making or improving the maps of countries; and accordingly geography mate great advances from the progress of the Roman arms. In all the provinces occupied by that people, camps were every where constructed at proper intervals, and good roads made for communication between them; and thus were civilisation and surveying carried on according to system, through'the whole extent of that large empire. Lvery new wal produced a new survey and itinerary of the countrics where the scenes of action passed; so that the materials of geography were accumulated by every additional conquest. Polybius observes, that at the beginning of the second Punic war, when Hannibal was preparing his expedition against Rome, the countries through which he was to march were carefully measured by the Romans. And Julius Cæsar caused a general survey of the Roman empire to be made, by a decree of the senate. Three surveyors had this task assigned them, which they completed in 25 years. The Ronan itineraries that are still extant, also show what care and pains they had employed in making surveys in all the different provinces of their empire; and Pliny has filled the $3 \mathrm{~d}$, 4.th, and 5 th books of his Natural History with the Geographical distances that were thus uneasured. Other maps are also still preserved, known by the name of the Pentingerian Tables, published by Welser and Bertius, which give a good speVol. I. cimen of what Vegetius calls the ltincra Picta, for the better direction of their armies in their march.

The Ronan empire had been enlarged to its greatest extent, and all its provinces well known and surveyed, when Ptoleny, about 150 years after Christ, composed his system of geography. The chicf materials he cmployed in composing this work, were the proportions of the gnomon to its shadow, taken by different astrononers at the times of the equinoses and solstices; calculations founded on the length of the longest days ; the measured or computed distances of the principal roads contained in their surreys and itineraries; and the various reports of travellers and navigators. All these were compared together, and digested iuto one nniform body or system; and afterwards were translated by him into a new mathematical language, expressing the different degrees of latitude and longitude, after the invention of Hipparchus, which had continued to be neglected tor 250 years. Ptolemy's system of geography, notwithstanding it was still very imperfect, continued in vogue till the last three or four centurics, within which time the great improvements in astronomy, the many discoveries of new countries by voyagers, and the progress of war and arins, have contributed to bring it to a very considerable degree of perfection; the particulars of which will befound treated under their respective articles in this work.

Among the moderns, the chief authors on the subject of geography are Johanmes de Sacrobosco, or John Halifax, who wrote a trertise on the sphere; Sebastian Munster, in his Cosmographia Universalis, in 1559; Clavius, on the sphere of Sacrobosco; Piccioli's Geographia et $11_{y}$ drographia Reformata; Weigelıus's Speculum Terræ; Dechales's Geography, in his Mundus Mathematicus; Cellarius's Geography ; Cluverius's Introductio in Universan Geographiam; Leibnecht's Elementa Guographice generalis; Stevenius's Compendium Geographicum ; Wolfius's Grographia, in his Elementa Matheseos; Busching's New System of Geograplıy; Gordon's, Salmon's, Guthrie's and Myers's Grammars; Adams's Geography, ancient and modern; and Pinkerton's Geography, lately published in two volumes 4to, with an introduction by Protessor Vince of Cambridge. But the most scientific and systematical work of all is Varenius's Gographia Generalis, with Jurin's additions. And it is much to be wished that some gentleman of proper qualifications would publish a new edition of this admirable performance suited to the present state of the science. Dr. Playfair has recently published a System of Geography, which is in many respects a very valuable performance; and perhajs best of all, in French, Fresnoy's most excellent work.

GEOLOGICAL, relating to the subject of Geology.

GEOLOGY, is that part of natural philosophy which treats of the structure of the earth, in regard to the origin, constitution, and the composition of its solid contents.

GEOMET'ER, or GEOMETRICIAN ; which see.

GEOMETRICAL, something that has a relation to geometry, or done after the manner, or by the means of geometry. As, a geometrical construction, a geometrical curve, a geometrical demonstration, genius, line, method, geometrical strictness, \&c.

G eo metrica c Construction, of an equation, is the draiving of lines and figures, so as to express by them the same general property and relation, as are denoted by the algebraical equation. Sec Construction of Eguations.

'Grometrical Curve or Line, called also an Algebraica 4. $\mathrm{E}$ 


\section{G E O}

one, is that in which the relations between the abscisses and ordinates may be expressed by a finite algebraical equation. See ALgfarical Curves.

Geometrical Lines, as observed by Newton, are distinguished into classes, orders, or genera, accorling to the number of the dimensions of the equation that expresses the relation between the ordindtes and abscisses; or, which comes to the same thing, according to the number of points in which they may be cut by a right line. Thus, a line of the first order, is a right line, since it can be only once cut by another right line, and is expressed by the simple cquation $y+a x+b=0$ : those of the $2 d$, or quadratic order, will be the circle, and the conic sections, since all of these may be cut in two points by a right line, and expressed by the equation $y^{2}+(a x+b) y+c x^{2}+$ $d x+e=0:$ those of the $3 d$ or cubic order, will be such as may be cut in 3 points by a right line, whose most genral equation is $y^{3}+(a x+b) y^{2}+\left(c x^{2}+d x+c\right) y$ $+f x^{3}+g x^{2}+h x+i=0$; as the cubical and Neilian parabola, the cissuid, \&c. And a line of an infinite order, is that which a right line may cut in infinite points: as the spiral, the cyclnid, the quadratrix, and every line that is generated by the infinite revolutions of a radius, or circle, or wheel, \&c. In each of the above equations, $x$ is the absciss, $y$ its corresponding ordinate, making any given angle with it ; and $a, b, c, \delta c$, are given or constant quantitic's, affected with their signs + and -, of which one or more may vanish, be wanting or equal to nothing, provided that by such defect the line or equation does not become one of an inferior order.

It is to be noted that a curve of any kind is denominated by a number next less than the line of the same kind: thus, a curve of the 1st order, (because the right line cannot be reckoned among curves) is the same with a line of the $2 \mathrm{~d}$ order ; and a curve of the $2 \mathrm{~d}$ kind, the same with a line of the $3 \mathrm{~d}$ order, \&c. We ought likewise to obstrve, that it is not so much the equation, as the construction or description, that makes any curve, geometrical or not. Thus, the circle is a geometrical line, not because it may be expressed by an equation, but because its description is a postulate: and it is not the simplicity of the cquation, but the easiness of the description, that is to determine the choice of the lines for the construction of a problem. The equation that expresses a parabola, is more simple than that which expresses a circle; and yet the circle, by reason of its more simple construction, is admitted in preference. Again, the circle and the conic sections, with respect to the dimensions of the equations, are of the same order; and yet the circle is not numbered with them in the construction of problems, but by rason of its simple description is depressed to a lower order, viz, that of a right line; so that it is not improper to express that by a circle, which may be expressed by a right line; but it is a lault to construct that by the conic sections, which may be constructed by a circle. Eisher, therefore, the law must be taken from the dimensions of equations, as observest in a circle, and so the distinction be taken away between plane and solid problems; or the law must be allowed noe to be strictly observed in lines of superiorkinds, but that sone, by reason of their more simple description, may be preferted to others of the same order, and be numbered with lines of inferior orders.

In constructions that are cqually geometrical, the most simple are always to be pueforred: and this law is so universal as to be without exception. But algebraical ex- pressions add nothing to the simplicity of the construction; the bare descriptions of the lines here are only to be considered; and these alone were considered by those geometricians who joined a circle with a right line. And as these are easy or hard, the construction becomes easy or hard: and therefore it is foreign to the nature of the thing, from any other circumstance to establish laws relating to constructions.

lither, therefore, with the ancients, we must exclurte all lines besides the circle, and perliaps the conic sections, out of geometry; or admit all, according to the simplicity of the description. If the trocboid were admitted into geometry, we might by means of it divide an angle $i_{n}$ any given ratio; would it be right therefore to blame those who would make use of this line to divide an angle in the ratio of one number to another; and contend, that you must make use ouly of such lines as are defined by equations, and therefore not of this line, which is not so defined? If, when an angle is proposed to be divided, for instance, into 10001 parts, we should be obliged to bring a curve defined by an equation of more than 100 dimensions to perform the same ; which no body could describe, much less understand; and should prefer this to the troclooid, which is a line well known, and easily described by the motion of a wheel, or circle: who would not see the absurdity?

The troclioid therefore is either not to be admitted at all in geometry, or else, in the construction of problems, $i$ is to be preferred to all lines of a more difficult description; and the reason is the same for other curves. Hence the trisection of an angle by a conchoid, which Archimedes in his Lemmas, and Pappus in his Collections, have preferred to the inventions of all others in this case, must be allowed as good; because we must either exclude all lines, beside the circle and right line, out of geometry, or admit them according to the simplicity of their descriptions; in which case the conchoid yields to none, except the circle. Equatiuns are expressions of arithmetical computation, and properly have no place in geomctry, cxcepting so far as quantitics truly geometricul (that is, lines, surfaces, solids, and proportions) may be said to be some equal to others. Multiplications, divisions, and such like computations, are newly received into geometry, and that unwaily, and contrary to the first design of this science. For whoever considers the construction of problems by a right line and a circle, discovered by the first gcometricians, will easily perceive that geometry was iniroluced for the purpose of avoiding the teclioustess of computation. For which reason the two sciences onght not to be confounded together: the ancients so carefully distinguished between them, that they nerer introduced arithmetical terms into geometry; and the moderns, by blending them, have lost the simplicity in which all the elearance of geometry consists. In short, that is arithmetically more simple, which is determined by lhe more simple equations; but that is geometrically moresimple, which is determined by the more simple drawing of lines; and in geometry that ought to be reckoned hest wiich is geometrically most simple. Newton's Arith. Univers. appendix. See Cunvis.

Giomitu ical. Locrs, or Place, cullad also simply Locus, is the pull or track of some certain geometrical determination, in which it always falls. See Locus.

Geometrical Mledium. Sce Mrdium.

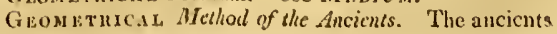


established the higher parts of their geometry on the same principles as the elements of that science, by demonstratinns of the satrie kind: and they were careful not to suppose any thing done, till by a previous problem ilsey had shown that it could be done by actual operation. Much less did they suppose any thing to be done that cannot be conceived; sucls as a line or series to be actually continued to infinity, or a uragnitude diminshed titl it become infinitely less than what it is. The elements into which they resolved inagnitudes were finite, and such as might be conceived to be real. Unbounded liberties have of lite been introduced; by which geometry, which ought to be perfectly clear, is filled with mysteries. Maclaurin's Fluxions, Introd. pa: 39.

Geonetrical Pace, is a measure of 5 feet long.

Geometrical Plan, in Architecture. See Pian.

Geometrical Plane. See Plane.

Geometrical Progression, a progression in which the terms have all successively the same ratio: as $1,2,4,8$, $16, \& c$, where the conmon ratio is 2.-The general and common property of a geometrical progression is, that the prockuct of any two terms, or the square of any one single term, is equal to the product of every other two terins that are taken at an equal distance on both sides from the former. So of these terms, $1,2,4,8,16,32,64$, \& ,

$l \times 64=2 \times 32=4 \times 16=s \times s=64$.

In any geometrical progression, if

$a$ denotes the least term,

$z$ the greatest term,

$r$ the common ratio,

$n$ the number of the terms,

$s$ the sum of the series, or all the terms;

then any of these quantities may be found from the others, by means of these general values, or equations, viz,

$r=\sqrt[n-1]{\frac{z}{a}}$,

$z=a \times r^{\mathrm{n}-1}$,

$a=z \div r^{\mathrm{n}-1}$,

$n=\frac{\log \cdot \frac{r z}{a}}{\log \cdot \frac{r}{r}}=\frac{\log \cdot r+\log \cdot z-\log \cdot a}{\log \cdot r_{.}}$,

$s=\frac{r^{n}-1}{r-1} \times a=\frac{r^{n}-1}{r-1} \times \frac{z}{r^{n-1}}=\frac{r z-a}{r-1}=$

$z^{n-1}-\frac{n}{a^{n-1}} \div z^{\frac{1}{n-1}}-a^{\frac{1}{n-1}}$.

When the series is infinite, then the least term $a$ is nothing, and the sum $s=\frac{r z}{r-1}$. Sce Progression.

Geometrical Proportion, called also simply Proportiotl, is the similitude ur equality of ratios.

1 Thus, if $a: b:: c: d$, or $a: b=c: d$, the terms $a, b, c, d$ are in geometrical proportion; also $6,3,14,7$, are in geometrical proportion, because $6: 3:: 14: 7$, or $6: 3=14: 7$.

In a genmetrical proportion, the product of the extremes, or 1 st and 4th terms, is equal to the product of the means, or the $2 d$ and $3 d$ terms : so $a d=b c$, and

$6 \times 7=3 \times 14=42$. Sce Proportion.

Geom zTrica L Solution, of a problem, is when the problem is directly resolved according to the strict rules and principles of geometry, and by lines that are truly geometrical. This expression is uscd in contradistinction to an arithmetical, or a mechanical, or instrumental solution; the problem being resolved only by a ruler and compasses. - The same term is also employed in opposition to all indirect and inadequate kinds of solutions, as by approximation, infinite scries, \&c. So, we have no geometrical way of finding the quadrature of the circle, the duplicature of the cube, or two mean proportionals; though there are mechanical ways, and others, by infinite series, \&c.

Pappus informs us, that the ancients endeavoured in vain to trisect an angle, and to find out two mean proportionals, by means of the right line and circle. Afterwards they began to consider the properties of scveral other lines; as the conchoid, the cissoid, and the conic sections; and by some of these they endeavoured to resolve some of those problems. At length, having more thoroughly examised the matter, and the conic sections being received into genmetry, they distinguished geometrical problens and solutions into three kinds; viz,

1. Mlane ones, which, deriving their origin from liues on a plane, may be properly resolved by a right line and a circle.-2. Solid ones, which are resolved by lines deriving their original from the consideration of a solid ; that is, of a cone--3. Linear ones, to the solution of which are required lines more compounded. According to this distinction, "we are not to resolve solid problems by other lines than the conic sections; especially if no other lines besides the right line, circle, and the conic sections, must be reccived into geometry. But the moderns, advancing much farther, have received into geometry all lines that can be expressed by equations; and have distinguished, according to the dimensions of the equations, those lines into classes or orders; and have laid it down as a law, not to construct a problem by a line of a higher order, that may be constructed by one of a lower.

GEOMETRICIAN, or GEOMETER, a person skilled in, or professing geometry.

GLOMIETRY, the science or doctrine of local extension, as of lines, surfaccs, and solids, with that of ratios, \&c.-The name geometry literally signifies measuring of the earth, as it was the necessity of measuring the land that first gave occasion to contemplate the principles aud rules of this art; which has since been extended to numberless other speculations; insomuch that, together with arithmetic, geometry forms now the chicf foundation of all the mathematics.

The invention of geometry, like many other things of antiquity, is rariously ascribed.

Herodotus (lib. 2), Diodorus (lib 1), Strabo (lib. 17), and Proclus assign it to the Egyptians, and assert that the annual inundations of the Nile gave occasion to it; for those waters bearing away the bounds and land-marks of estates and farms, covering the face of the ground uniformly with nud, the people, say they, were obliged every year to distinguish and lay out their lands by the consderation of their figure and quantity; atsd thus by $\mathrm{cx}$ perience and habit they formed a methorl or art, which. was the origin of geometry. A farther contemplation of the draught of figures of fields thus laid down, and plotted in proportion, might naturally lead them to the discuvery of some of their excellent and wonderful properties; whicls speculation continually improving, the art successively gained ground, and nuade advances more and more towards perfection.

Josephus however seems to ascribe the invention to the Hebrews: and others of the ancients make Nlercury the inventor. Polyd. Virgil, de Iıvent. Rer. lib. 1, cip. 18.

From Egypt, this science passed into Greece, being carried thither by Thales; where it was much cultivated and improved by himself, as also by Pythagoras, Anaxagoras ol Clazomene, Hlippocratez of Cliios, amil Plato, who 4. E 2 
tistiticel his convictiols of the necessity and importance of geometry the the successful study of philusophy, by this inscription oser the dour of his academy, "Let no one ignosant of grometry enter here." Plato thought the word geunetry too mean a name for this science, and substituted insteat of it the more extensive name of Mensuration; and after him others gave it the title of Pantometry: But eren these are now become tro scanty in their import, tully to comprehend its extent; for it not only inquires into, and demonstrates the quantities of masnitudes, but also their qualities, as the species, figures, ratios, pesicions, transformatiens, descriptions, divisions, the fiuding of their centres, diameters, tangents, asympiotes, curvature, $\mathbb{f}$. Some again define it as the science of inquiring, inventing, and demonstrating all the aflections of magnitude. And Proclus calls it the knowledge of magnitudes and figures, with their limitations; ns also of the-ir ratios, attictions, positions, and motions of every kind.

About 50 vears after Plate, lived Euclid, who collected together all thise theorems which had been invented by his predecessors in Egypt and Greece, and digested then intu $\mathrm{I} 5$ bouks, called the Elements of Geometry; demonstrating and nrranging the whole in a very accurate and perfect mamer. The next to Enclid, of those ancient writers whose works are extant, is Apollonius Pergaus, who flourished in the time of Ptolemy Euergetes, about 230 years before Christ, and about 100 years atter Euclid. He was author of the first and principal work on Conic Sections; on account of which, and his other nccurate and ingenious geometrical writings, he acquired from his patron the imphatical apprllation of "The Great Geometrician." Contemporary with Apollonius, or perhaps a fiw years before him, flourished Archinedes, celebrated for his mechanical inventions at the siege of Syracuse, and not less so fur his very ingenous geometrical compositions.

We can only mention Eudoxus of Cnidus, Archytas of Tarentum, Philolaus, Eratosthenes, Aristarchus of Samos, Dinostratus, the inventor of the quadratrix, Menecimus, his brother and the disciple of Plato, the two Aristeus's, Conon, Thracidius, Nicuteles, Leon, Theudius, Hermoimus, Hero, and Nicomedes the inventor of the conchoil: besides whom, there are many other ancient geometricians, to whom this scirnce has been indebted.

'The Greeks continued their attention to it, even after they were subdued by the Romans. Whereas the Romans thenselves were so little acquainted with it, even in the most flourishing time of their republic, that Tacitus informs us they gave the name of nathematicians to those who pursued the chimeras of divination and judicial astrology. Nor does it appear they were more disposed to cultivate geometry during the decline, and after the fall of the Roman empire. But the case was different with the Greeks; among whom are found many cxcellent geomeIricians since the commencement of the Christian era, and after the translation of the Roman empire. Ptolemy lived under Marcus Aurclius; and we have still extant the works of Pappus of Alexandria, who lived in the time of 'Thcodusius ; the commentary of Eutocius, the Ascalonite, who lised abrut the year of Christ 540 , on Archimedes's mensuration of the circle; and the cummentary on Euclicl, by Proctus, who lived under the empire of Anastasius.

The consequent inundation of ignorance and barbarism was unfavourable to geometry, as well as to the other sciences; and the few who applical themselves to this science, were calumniated as magicians. However, in those times of Furopean dorkness, the Arabians were distinguished as the guardians and pronoters of science; and from the gat w the $1+$ th entury, thes produced many astronomers, geometricians, gergiaphers, $\mathrm{di}^{2}$; fim whom the mathematical sciences were atrain received inte Spain, Italy, and the rest ot kurope, somewhat betore the fear 1 fou. Evmo of the carliest whters atter this period, are Levuardus Pisanus, Lucas Pici-lus or De Burgo, and

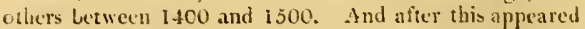
many editions of liuclid, or commentaries upon him: thus, Orubitus finzus, in 1530 , publishod a commentary on the first 6 books; as did Tames Pelctarims, in 1557; and abuut the same lime Nicholas Tartaglia published a commentary on the whole 15 books. There bave been also the sditions, or commentaries, of Commandine, Clavius, Billingsly, Scheubelius, Herlinus, Dasypotius, Ramus, Herigon, Stevinus, Saville, Barruw, Taquet, Ducbales, Furnier, Scarborongh, Keill, Stone, Playfair, and many others; but the completest edition of all the works of Euclid, is that of Dr. Gregory, printed at Oxtord 1703, in Greek and Latiu: the edition of Euclid, by Dr. Rubert Simson of Glasgow, containing the first 6 books, with the 11 th and $12 t h$, is much esteemed for its correctuess. The principal other elementary writers, besides the editors of Luctid, are Borelli, Pardies, Marchett, Wolfius, Simpson, Leslie, Ac. And among those who have gone beyond Euclid in the nature of the Elementary parts of Geometry, may be chietly reckoned, Apollonius, in his Conics, bis Loci Plani, De Sectione Determinata, his Tangencies, Inclinations, Section of a Ratio, Scction of a Space, \&c; Archimedes, in his treatises of the Sphere and Cylinder, the Dimension of the Circle, of Conoids and Spheroids, of Spirals, and the Quadrature of the Parabola; Theodosius, in his Spherics; Screnus, in his Sections of the Cone and $\mathrm{Cy}$ linder: Kepler's Nova Stercometria; Cavallerius's Gcometria Indivisıbilium: Torricelli's Opera Geometrica; Tiviani, in his Divinationes Geometrica, Exercitatio Mathematica, De Locis Solidis, De Maxinis "t Minimis, \&c; Vieta, in his Lfectio Geometrica, Supplemeni. Gcometrix, Sectiones Angulares, Rusponsum aul Prubluma, Apoltonius Gallus, Se; Gregery St. Vincent's Quadratura Circuli; Fermat's Varia Opera Mathematica; Ur. Barrow's Lectiones Geometricæ; Bulliald de Lineis Spiralibus; Cavillerius; Schooten and Gregory's Exercitationes Geometrica, and Gregury's Pars Universalis, \&c; De Billy's treatise De Proportione Harmonica; La Lovern's Geometria reterum promota; Slusins's Mesolabium. Prublemata Solida, dic; Wallis, in his tratises De Cyclovile, Cissoide, Sc; De Proportionibus, De Sectionibus Conicis, Arithmetica Infinitorum, De Centru Gravitatis, De Sectionibus Angularibus, De Angulo Contactas, Cuno-Cuneus, Sc; Hugo De Onerique, in his Analysis Geometrica; Pascal on the Cycloid; Step. Anguli's Prublemata Geometrica; Alcs. Anderson's Suppl. Apollonii liedivivi, Variorum Problematum Practice, Sc; Baronius's Geomet. Prob. \&c; Guido Grandi Geometr. De monstr. Sc; Ghetaldi Apollonius Redivivus, Sc; Ludolph san Colen or a Ceulen, de Circula et Adscriptis, Sic; Snell's Apollonius batavus, Cyclonetricus, \&c; Herberstein's Diotome Circulorum; Palma's Exercit. in Geometriam ; Gulthini Centro-barica; with several others eyually eminent, of more modern date, ns 1)r. Rob. Simson, Mr. Mat. Stewart, The, simpson, John Leslie, Legendre, Sc. Since the introduction of the new geometry, or the geometry of curve lines, as expressed by algebraical equations, in this 
part of geometry, the following names', among many others, are more especially to be respected; viz, Descartes, Schooten, Newton, Maclaurin, Brackenridge, Cramer, Cotes, Waring, \&c, \&.c.-As to the subject of practical geometry, the chilef writers are Beyer, Kepler, Ramus, Clavius, Mallet, Tacquet, Ozunam, Wolfius, Gregory, with insumerable others.

(ieometry is distinguished into theoretical or speculative, and practical.

Theoretical or Speculative Geom ETRY, treats of the various properties and relutions in magnitudes, demonstrating the theorems, \&c. And

Practical Geometry, is that which applies those speculations and theorems to particular uses in the solution of problems, and in the measurements in the ordinary concerms ci life.

Speculative geometry again may be divided into elementary and siblime.

Elcmentary or Common GEom ETRx, is that which is employed in the consideration of right lines and plane surfaces, with the solids generated frum them.

Higher or Subine Geometry, is that which is emplojed in the cunsideration of curve lines, conic sections, and the bodies formed of them. 'This part has been chiefly cultivated by the moderns, by help of the improved state of algebra, and the modern analysis or fluxions.

Descriptive Geometry, a nanc given to a new species of geometry, chiefly treated on by M. Monge. Whon one surface penetrates another, there often result from their intersection curves of double curvature, the description of which is necessary in some of the arts; as, in groined vault-work, and in cutting arch-stones, or woor-cutting, for ornamental work, \&c, the form of which is ciften very fantastical and complicated: it is in the solution of problems relating to these subjects that descriptive geometry is chiefly useful.

Some architects, more versed in geometry than usual with persons of that profession, have long since thrown some light on the first principles of this kind of geometry. There is, for listince, a work by a Jisuit namied Father Courcier, showing low to describe the curves resulting from the mutual penetration of cylindrical, spherical, and conical surfaces: this work was published at Paris in 1663. P. Derand. H. Jiusse, Frazier, \&c, had also contributed a little towards the promotion of this branch of geometry. But Mongr has given it very great extension, not only by proposing and resolving various problems both curious and difficult, but by the invention of several new and interestung theorcms. We can only mention in this place one or two of these. Is, Ist, 'Two right lines being given in space, which are neithe $r$ parallel nor in the same plane, to find in botl of them the points of their least distance, and the pusition of the line joiring these points. $2 \mathrm{~d}$, Three spheres being given in space, to determine the position of the plane which touches them. There are atso curious problems relative to lines of double curvature, and to surfaces resulting from the application of a right line that leans continually upon two or three others given in position iu space. Among the theorems, the following may be inentioned: If a plane surface, given in space, be projected on three plants, the one borizontal, and the two others vertical and perpendicular to each other, the square of that surface will be equal to the sum of the squares of the three surfaces of projection. This theoren is as interesting in the geometry of solids, as that of Pythagoras
(Eucl. i, 47) is in plane geometry. But for more oll this subjec1, reference may be made to Monge's and Lacroix's ingenious works on Geometrie Descriptive.

Geometry of the Compasses, a new species of geometry, more ingenious than profound or useful, lately proposed by the Abbe Mascheroni. Hitherto both the ruler and the compasses have been employed in the solution of problems in plane geometry; and it had not been imagined that such problems could be solved or constructed without the combincd use of these two instruments. But that author has shown a great number of problens, which are rendered very piquant and amusing, by the new condition of employing only the compasses in their solution. Thus: Two points terminating a right line $\cdot$ being given, to find either between or without those two points, any number of points which shall be in the same right line with the former, and which shall make the intervals between them in a given ratio: $2 \mathrm{~d}$, To a given line to draw parallels, or perpendiculars, or lines making given angles with them: $3 \mathrm{~d}$, To inscribe or circumscribe within or about a circle, the various problems whicb are constructible by plane geometry: 4 th, To determine the mean proportional, between two given distances; or to find third or fourth proportionals. All the problems indeed of the Euclidean geometry are thus resolved by the mere intersection of arcs of circles, without drawing a single right line. The author also resolves, by ingenious approximations, many problems which lie beyond the limits of common geometry; such as those relating to duplications, trisections, $\& c$, stitl employing only the compasses. The work above alluded to is entitled, Geometria del Compassa, and of which a French translation was published in 1798, in 1 vol. 8 vo. A chapter on the same practice has also been lately given in Leslie's Geometry.

'T he following problem, proposed by the Emperor $\mathrm{Na}$ poleon to the French mathematicians, with scveral others in this geonetry, are here constructed to show the method.

1. Imperial Problem. To divide the circumference of a circle into four equal parts by the compasses only, let $A D l i B$ be the given circle, whose centre is c. From any point $A$ in its circumference, with a distance equal to the given radius ac, lay off the three successive divisions $A D, D E$, $E B$, and $A B$ will be a diameter. With the centres $A$ and $B$, and radius $A E$ or BD, the chord of two divisions, describe arcs intersecting each other in $\mathbf{P}$; and $\mathrm{PC}$ will be the chord of a quadrant of the given circle; which being applicd

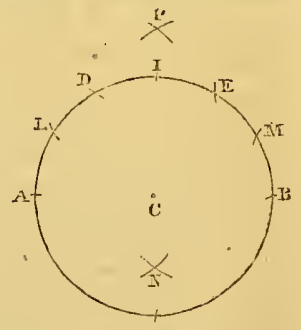
four times to its circumference will divide it as required.

The truth of this is radily shown thus: Because $A B^{2}=4 A C^{2}=A E^{2}+A C^{2}$ (the angle $A E B$ in a semicircle being a right anglc); $\Lambda E^{2}=3 \Lambda C^{2}$. But $A P^{2}=\Lambda E^{2}$ by construction; therefure $A P^{2}=3 A C^{2}$ : and since if $A C$ and $\mathrm{CP}$ were drawn, the angle $\mathrm{ACP}$ would be a right angte, $A P^{2}=A C^{2}+C P^{2}$ : hence $A C^{2}+C P^{2}=3 A C^{2} ;$ and consequently $\mathrm{CP}^{2}=2 \mathrm{~A} \mathrm{c}^{2}$ which is the square of the chord of $90^{\circ}$. Therefore, \&c, Q.E.D.

From the alsove solution the division of the circumference into 8 equal parts is readily derived. For from 
the interscction $\mathrm{P}$ with the radius $\mathrm{A}$ of the given circle, describe an arc intersecting the circumference in $\mathrm{D}$. This intersection will bisect the arc of $90^{\circ}$ above determined.

2. Tu divide the circumference of a circle into five equal parts, or to construct a regular pentagon by means of the compasses only: Let $A B$ be the given circle; then having found the diameter $A B$ and $A I$ the chord of a quadrant or $90^{\circ}$ as before; with centre $\mathrm{I}$ and radius $\mathrm{AC}$, set off the ares IL, LM. From the points $L$ and $M$, with the radius $\mathrm{AI}$, describe ares intersecting at $\mathrm{N}$, on that side of the chord $\mathrm{L} M$ as the centre $\mathrm{c}$ of the circie; and the distance of this intersection from either extremity of the diamerer $\mathrm{A} \mathrm{B}$ will be the side of the pentagon required.

The demonstration is simple.

Nearly to this construction of the pentagon is that given by Ptolemy in his Almagest, for finding the chord of the fifth part of the circumference.

An ingenious friend (Mr. J.B. IVise, of Boyn Hill, near Naidcnhead) has favoured us with a construction, in one simple diagram, for all the regular pulygons inscribed in a circle, of the following number of sides, viz, 3, 4, 5, 6, 7, $8,9,10,12,15,16,18,24,30,40,60, \&$ c.

GEORGIUN SIDUS. See Herschel, and Uranus.

GERBLRT, Pope Silvester the Second, was born of indigent parents at Auvergne, in the early part of the 10 th century. Scarcely had he acquired the first priuciples of the sciences, when he left a country then immersed in ignorance, and travelled into Spain, where he remained scveral years. Having made great progress in the mathematics, he returned into France, where he taught the sciences, which he had learned from the Arabians, particularly arithmetic, which he thus had the bonour of communicating to the western Christians, about the year 960 or 970 . His merit and wisdom afterwards elevated him to the papal chair; which he filled respectably till the time of bis death, in the year 1003 .

GHETALDUS (MARiN Us), a Ragusan nobleman, and very respectable gcometrician, who flourished about the beginning of the 17 th century. He was author of several ingenious works on geometry, after the manner of the ancients; of which the titles and dates of those that are in my possession are the following: 1. Promotus Archimedes, \& c, 4to, Romæ, 1603.-2. Problematum Collectio, 4to, Venct. 1607.-3. Apollonius Redivivus, \&c, 4to, Venet. 1607 and 1613.-4. Supplementum Apollonii Galli, Venet. 1607, 4to,-5. De Resolut. et Composit. Mathemat. in folio, Romæ, 1630. And there might be others.

GIBBOUS, is used for the shape of one state of the enlightened part of the moon, being that in which she appears more than half full or enlightened, which is the time between the first quarter and the full moon, and from the full moon to the last quarter; appearing then gibbous, that is, bunched out, or convex on both sides of the enlightened part; as contradistinguished from the state when slie is less than half full, when she is said to be horned, or a crescent.

GILBERT (Wm.), a physician, who was born at Colchester in 1540, and educated at Cambrilge, but took his degree of M.D. abroad. On his return he was elected a fellow of the college of physicians, and became physician to queen kilizabeth. Dr. Gilbert discovered several of the properties of the loadstone; and in 1660 he published a work in 4to, entitled, De Magnete, magneticisquc Corporibus, et de magno Magnete Tellure, Physiologia nova; in which are many important suggestions for the improvement of navigation. He died in 1603 , conscquiently at 6.3 years of age.

Gl IIBOLS, are the brass rings by which a sea-compass is suspended in its box that usually stands in the binacle.

GIN, in Artillery and Mechanics, is a machine for raising great weights, usually composed of three long legs, \& c.

GIRARD (ALEERT). See an account of bim in the article ALGEBRA.

GIRDLRS, in Architecture, are the largest beams or pieces of tinber supporting the floors. Their ends are usually fastened into the summers, or breast-sunmers; and the joists are framed in at one end to the girders. By the statute lor rebuilding London, no girder is to lie less than 10 inches into the wall, and their ends to be always laid in loam, \&c. The shorter bearings a girder has, and the oftener it is supported by the internal or partition walls, so much the better. The established breadth and depth of a girder, according to its length of bearing, are as in the following tablet:

\begin{tabular}{|c|c|c|c|c|}
\hline \multirow{4}{*}{ 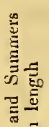 } & From & to & \multicolumn{2}{|c|}{ must be in } \\
\hline & & & Breadth & Depth \\
\hline & 10 feet & 15 feet & 11 inches & 8 inches: \\
\hline & 15 & 18 & 13 & 9 \\
\hline$\infty . \equiv$ & 18 & 21 & 14 & 10 \\
\hline & 21 & 24 & 16 & 12 \\
\hline & 24 & 26 & 17 & 14 \\
\hline
\end{tabular}

GIR'T, in Timber-measuring, is the circumference of a tree; though some use this word for the quarter or 4 th part of the circumference only, on accuint of the great use that is made of it ; for the square of this 4 th part is esteemed and used as equal to the area of the section of the tree; which square therefore multiplied by the length of the tree, is accounted the solid content. This content however is always about one-fourth part less than the true quantity; being nearly equal to what this will he after the tree is hewcd square in the usual way: so that it seems intended to make an allowance for the squaring of the tree.

Grat-Line, is a line on the common or carpenter's sliding rule, employed in computing the contents of trees by means of their girt.

GIVEN, Datum, a term often used in mathematics, and significs something that is supposed to be known.

Thus, if a magnitude be known, or if we can find another equal to it, it is said to be given in magnitude. Or when the position of any thing is known, it is said to be given in position. And when the diameter of a circle is known, the circle is given in magnitude. Or the circle is given in position when its centre is given in position. Whan the kind or species of a figure is known, or remains the same, it is given in specie, \&c.

Huclid wrote a book of Data, or concerning things given, in 95 propositions, usually accompanying his Elements, in the best editions, and which Pappus reckons as one of the best specimens of the analytical works of the ancients.

GLACIS, in Fortification, a sloping bank reaching from the parapet of the counterscarp, or covered-way, to the level side of the ficid, commonly at the distance of about 40 yards.

GLOBL, a round or spherical body, more usually called a sphere, bounded by onc uniform convex surface, every point of which is equally distant from a point within called 
its centre. Euclid defines the globe, or sphere, to be a solid figure described by the revolution of a semi-circle about its diameter, which remains unmoved. Also, its axis is the fixed line or diameter about which the semicircle revolves; and its centre is the same with that of the revolving semi-circle, a diameter of it being any right line that passes through the centre, and terminated both ways by the superficies of the sphere. Eletn.11. def. 14, 15,16, 17 .

Euclid, at the end of the $121 \mathrm{~h}$ book, shows that spheres are to one another in the triplicate ratio of their clianters, that is, their solidities are to one another as the cubes of their diameters. And $A$ rchimedes determines the real magnitudes and measures of the surfaces and solidities of spheres and their segments, in his treatise de Sphara ct Cylindro: viz, 1, That the superficies of any globe is equal to 4 times a great circle of it.-2, That any sphere is equal to $\frac{2}{3}$ of its circumscribing cylinder, or of the cylinder of the same diameter and altitude.-3, That the curve surtace of the segment of a globe, is equal to the circle whose radius is the line drawn from the vertex of the segrient to the circumference of the base.-4, That the content of a solid sector of the globe is fqual to a cone whose altitude is the radius of the globe, and its base equal to the curve superficies or base uf the sector. With many other properties. And from hence are easily deduced these practical rules for the surfaces and solidities of globes and theirscgments ; viz,

1. For the Sunfuce of a Globe, multiply the square of the diameter by $3 \cdot 1416$; or multiply the diameter by the circumference.

2. For the Solidity of a Globe, multiply the cubc of the diameter by 5236 (viz $\frac{x}{6}$ of $3 \cdot 1416$ ) ; or multiply the surface by $\frac{1}{6}$ of the dianneter.

3. For the Surface of a Segment, multiply the diameter of the globe by the altitude of the segment, and the product again by $3 \cdot 1416$.

4. For the Solidity of a Segment, multiply the square of the diameter of the glabe by the difference hetween 3 times that diameter and 2 times the altitude of the scgment, and the product again by $\$ 5236$, or $\frac{r}{6}$ of $3 \cdot 1416$.

Hence, if $d$ denote the diamcter of the globe, $c$ the circumference, $a$ the altitude of any segment, and $p=3.1416$; then

\begin{tabular}{|c|c|c|}
\hline & $p d^{2}=c d$ & \\
\hline & pad & $\frac{1}{6} p a^{2} \times$ \\
\hline
\end{tabular}

Sce the art. SPrere, and my Mensuration, pa. 197, \&c, 2d edit.

The Globe, Terraqueous, is the body or nass of the earth and water together, which is nearly globular.

GLOBn, or irtificial (ilaßE, is more particularly used for a globe of metal, plaster, paper, pasteboard, \&c, on the surfice of which is drawn a map, or representation of cither the heavens or the earth, with the several circles conccived upon them. Anul hence

Glones are of two kinds, terrestrial, and celestial; which are of considerable use in geography and astronomy, by serving to give a lively representation of their principal objects, and for performing and illustrating many of their operations in a manner easy to be perceived by the senses, and so as to be conceived even without any knowledge of the mathematical grounds of those sciences.

Description of the Globes.

The fundamental parts that are common to both globes, are an axis, representing the axis of the world, passing "through the two poles of a spherical shell, represeuting those of the world, which shell makes the borly of the globe, upon the external surface of which is drawu the representation of the whole surface of the earth, sca, rivers, islands, Ec, for the terrestrial glube, and the stars and cunstellations of the hearens, for the celestial one; besides the equinoctial and ecliptic lines, the zodiac, the tropics and polar circles, and a number of meridian lines. These is also a brazen mericlian, being a strong circle of brass, circumscribing the glube, at a small distance from it, quite around, in which the globe is bung by its two poles, upon which it turns round within this circle, which is divided into $\frac{1}{+}$ times 90 degrees, beginning at the equator on both sides, and ending with 90 at the two poles. There are also two small hour circles, of brass, divided into twice 12 hours, and fitted on the meridian round the poles, which carry an index pointing to the hour. The whole is set in a wooden ing, placed parallel to, and representing the horizon, in which the globe slides by the brass meridian, elevating or depressing the pole according to any proposed latitude. There is also a thin slip of brass, calied a Quadrant of Altiturle, made to fit on occasionally upon the brass meridian, at the highest or vertical point, to measure the altitude of any object above the horizon. A magnetic compass is sometimes set underneath. Sce the figure of the globes so mounted, at fig. 1 , plate xiv.

Such is the plain and simple coustruction of the artificial globe, whether celestial or terrestrial, as adapted to the time only for which it is made. But as the angle formed by the equator and ecliptic, as well as their points of intersection, is always changing; to remedy these inconveniences, several contrivances bave been made, so as to adapt the same globes to any other time, either past or to come; as well as other contrivances to answer particular purposes.

Thus, Mr. Sencx, a celebrated maker of ylobes, had a contrivance which, by means of a mut and screw, caused the pole of the equator to revolve about the pole of the ecliptic, by any quantity answering to the precession of the equinoxes, since the time for which the globe was made. Philos. 'Trans. No. 447 , or my $\mathrm{Abr}$. vol. 8 , pa. 176 , also Philos. Trans, vol. 46 , pa. 290.

Mr. Juseph Harris, late assay-master of the Mint, made some contrivances to show the effects of the earth's motions. He fired two horary circles under the brass meridian, to the axis, one at each pole, so as to turu round with the globe, and that meridian served as in index to cut the horary divisions. The globe in this state servè) equally for resolving problems in both noth and south latitudes, as also in places near the' equator ; whereas, in the common construction, the axis and horary circle prevent the brass meridian from being maveable quite round in the horizon. This globe is also adapted for showing how the vicissitudes of day and night, and the alteration of their lengths, are really occasioned by the motion of the earth: for this purpose, he divides the brass meridian, at one of the joles, into months and days, according to the sun's declination, reckoning from the pole. Therefore, by bringing the day of the musth to the horizon, and rectifying the globe according to the time of the day, the horizon will represent the circle separating light and darkness, and the upper half of the globe the illuminated hemisphere, the sun being in the zenith. Mr. Harris also gives an ac- 
count of a cheap machine for showjng luw the annual motion of the carth in its orbit causes ihe change of the sun's declination, without the great expense of an orrery. Philos. I rans. Nio. 456.

The late $\mathrm{Mr}$. George Adams made also some useful inprovements in the construction of the glubes. Besides what is usual, his glubes have a thin brass semi-circle moveable about the poles, with a small thin sliding circle uponit. On the terrestrial globe, the former of these is a moveable meridian, and the latter is the visible horizon of any particular place to which it is set. But on the celestial globe, the semi-circle is a moveable circle of declination, and its small annexed circle an artificial sun or planet. Each globe has a brass wire circle, placed at the limits of the twilight. The terrestrial globe bas many additional circles, as well as the rhumb-lines, for resolving all the necessary geographical and nautical problems: and on the celestial globe are drawn, on each side of the ecliptic, 8 parallel circles, at the distance of une degree from each other, including the zodiac; which are crossed at rightangles by segments of great circles at every 5 th degree of the ecliptic, for the more readily noting the place of the moon or of any planct on the globe. On the strong brass circle of the terrestrial globe, and about $23 \frac{\mathrm{T}}{2}$ degrees on each side of the worth pole, the days of each month are laid down accurding to the sun's declination: and this brass circle is so contrived, that the glube may be placed with the north and south poles in the planc of the horizon, and with the south pole elevated above it. The rquator, on the surface of either globe, serves the purpose of the horary circle, by means of a semi-circular wire placed in the plane of the equator, carrying two indices, one of which is occasionally to be used to point out the time. For a farther account of these globes, with the method of using them, see Mr. Adams's Treatise on their construction and use.

There are also what are called Patent Globes, made by Mr. Neale; by means of which he resolves several astromomical problems, which do not admit of solution by the common globes.

Mr. Ferguson also made scveral improvements of the globes, particularly one for constructing dials, and another called a planetary globe. See Philos. Trans. vol.44, pa. 535, and Ferguson's Astron. pa. 291 and 292.

Lastly, in the Philos. Trans. for 1789 , vol. 79, pa. 1, Mr. Sineaton has proposed some improvements of the celestial globe, especially with respect to the quadrant of altitude, for the resolution of problems relating to the azimuth and altitude. The difficulty, he observes, that has ccurred in fixing a semi-circle, so as to bave a centre in the zenith and nadir points of the globe, at the same time that the meridian is left at liberty to raise the pole to its desired elevation, I suppose, has induced the globe-makers to be contented with the strip of thin flexible brass, called the quadrant of altitude; and it is well known how inperfectly it performs its office. The improvement I have attempted, is in the application of a quadrant of altitude of a more solid construction; which being affixed to a brass socket of some length, and this ground, and made to turn upon an upright stcel spindle, fixed in the zcnith, steadily directs the quadrant, or ratlier arc of altitude, to its true azimuth, without being at liberty to deviate from a vertical circle to the right liand or left : by which means the azimuth and altitude are given with the same exactness as the measure of any other of the great circles. For a more particular description of this mprovement, illus. trated with figures, sce the reference above.

For the practical uses of the globes, see any of the little bouks on this subject.

GI.OBULAR, relating to, or partaking of the property or shape of the globe. As globular chart, globular projection, or globular sailing, \&c.

GLOBULAR Chart, is a representation of the surface, or part of the surface, of the terraqueous globe on a plane: in which the parallels of latitude are circles nearly concentric; and the meridians are curves bending towards the poles; the rhumb-lines heing curves also.

The merits of this chart consist in these particulars, viz, that the distances between places on the same rhumb arc all measured by the same scale of equal parts; and the distance of any two places in the arch of a great circle, is nearly represented in this chart by a straight line.

Land maps also, made accorling to this projection, would have great advantages over those made in any uther nay. But for sea charts for the use of nivigation, Mercator's are preferable, as both the meridians and parallels, as also the rhumbs, are all straight lines.

This projection is not new, though not much noticed till of late. It is mentioned by Ftolemy, in lis Geograpliy; and also by Blundeville, in lis Excrcises.

For globular projection of maps or charts; see MAP.

GLoBULA it Saiting, is the mothou of resolving the cases of sailing on principles deduced from the spherical figure rit the carth. Such as Mercator's sailing, or greatciucle sailing; which see.

GLOSSOCOMON, in Mechanics, is a name given by Heron to a machine, compused of divers dented wheels with pinions, serving to raise huge weights.

GNOMUN, in Astronomy, is an iustrument or apparatus for measuring the altitudes, declinations, $\&$ ce, of the sun and stars. The gnomon is usually a pillar, or column, or pyramid, erected upon level ground, or a pavement. For making the more considerable obsersations, both the ancients and moderns bave made great use of it, especially the former; and many have preferred it to the smaller quadrants, both as more accurate, casier made, and more easily applied.

The most ancient observation of this kind extant, is that made by Pytheas, in the time of Alexander the Great, at Marseilles, where he found the height of the gnomon was in proportion to the meridian sliadow at the summer solstice, as $213 \frac{\mathrm{r}}{8}$ to 600 ; just the samc as Gassendi found it to be, by an observation made at the same place, almost 2000 years after, viz, in the year 1636 . Ricciol. Almag. vol. 1, lib. 3, cap. 14.

Ulugh Beigh, king of Parthia, \&c, used a gnemon in the year 1437, which was 180 Rnman feet bigh. That erected by Ignatius Dante, in the church of St. Petronius, at Bo$\operatorname{logna}$, in the year 1576 , was 67 feet high. M. Cassini erected another of 20 feet high, in the same church, in the year 1655 .

The Egyptian obelisks were also used as gnomons; and it is thought by some modern travellers that this was the very use they were designed and built for; it has also becn found that their four sides stand exactly facing the four cardimal points of the compass. It may be added, that the Spaniards in their conquest of Peru, found pillars of curious and costly workmanship, set up in several places, by the meridian shadows of which their amatas or philo- 
sophers had, by long experience and repeated observations, learned to determine the times of the equinoxes; which seasons of the year were celebrated with great festivity and rich offerings, in honour of the sun. Garcillasso de la Vega, Hist. Pern, lib. 2, cap. 22.

USE of the GNONON, in taking the meridian altitude of the Sun, and thence finding the Latitude of the place.-A meridian line being drawn through the centre of the gnomon, note the point where the shadow of the gnomon terminates when projected along the meridian line, and measure the distance of that extreme point from the centre of the gnomon, which will be the length of its shadow. Then having the height of the gnomon, and the length of the shadow, the sun's altitude is thence easily found.

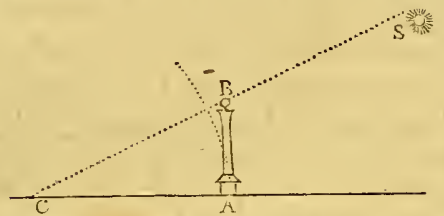

Suppose, ex. gr. A B the gnomon, and Ac the length of the shadow. Here, in the right-angled triangle $A \mathrm{BC}$, are given the base $\mathrm{AC}$, and the perpendicular $\triangle \mathrm{B}$, to find the angle $c$, or the sun's altitude, which will be found by this analogy, as $C_{A}: A D:$ : radius : the tang. of $\angle C$, that is, as the length of the shadow is to the height of the gnomon, so is the radius to the tangent of the sun's altitude above the horizon.-The following example will serve to illustrate this proposition: Pliny says, Nat. Hist. lib.2, cap.72, that at Rome, at the time of tine equinoxes, the shadow is to the gnomon as 8 to 9 ; therefore, as $8: 9:: 1$ or radius : $\frac{9}{4}=1.125$ a tangent, to which answers the angle $48^{\circ} 22^{\circ}$, which is the height of the equator at Rome, and its complement $41^{\circ} 3 \mathrm{~S}^{\prime}$ is therefore the height of the pole, or the latitude of the place.

Riccioli remarks the following defects in the observations of the sun's height, made with the gnomon by the ancients, and some of the moderns; viz, that they neglected the sun's parallax, which makes his apparent altitude less, by the quantity of the parallax, than it would be, if the gnomon were placed at the centre of the earth : $2 \mathrm{~d}$, they neglected also the refraction, by which the apparent height of the sun is a little increased : and 3lly, they made the calculations from the length of the shadow, as if it were terminated by a ray coming from the centre of the sun's disc, whereas the shadow is really terminated by a ray coming from the upper edge of the sun's disc; so that, instead of the height of the sun's centre, their calculations gave the height of the upper edge of his disc. And therefore, to the altitude of the sun found by the gnomon, the sun's parallax must be added, and from the sum must be subtracted the sun's semidiameter, and refraction, which is different at different altitndes; which being done, the correct height of the equator at Rome will be $48^{\circ} 4^{\prime} 13^{\prime \prime}$, the complement of which is the latitude, or $41^{\circ} 53^{\prime} 46^{\prime \prime \prime}$. Ricciol. Geogr. Refor. lib.7, cap. 4.

Thepreceding problem may be resolved more accurately by means of a ray of light let in through a small hole, than by a shadow, thus: Make a circular perforation in a brass plate, to transmit enough of the sun's rays to exhibit his image on the floor, or a stage; fix the platc parallel to the horizon in a high place, proper for observation, the

VoL. I. height of which above the floor let be accurately measured with a plummet. Let the floor, or stage, be perfectly plane and horizontal, and coloured over with some white substance, to show the sun more distinctly. Upon this horizontal plane draw a meridian line passing through the foot or centre of the gnomon, i. e. the point upon which the plummet falls from the centre of the hole; and on this line note the extreme points $I$ and $K$ of the sun's image or diameter, and from each end subtract the image of half the diameter of the aperture, viz $\mathrm{kH}$ and $\mathbf{L I}$ : then will $\mathrm{IL}$ be the image of the sun's diameter, which, when bisected in $\mathrm{B}$, gives the point on which the rays fall from the centre of the sun.

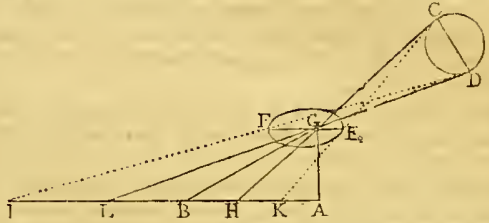

Now having given the line $A \mathrm{~B}$, and the altitude of the gnomon $\Delta G$, besides the right angle $A$, the angle $B$, or the apparent altitude of the sun's centre, is easily found, thus: as $A B: A G$ : : radius : tang. angle $B$.

Gromon, in Dialling, is the style, pin, or cock of a dial, the shadow of which points out the hours. This is always supposed to represent the axis of the world, to which it is therefore parallel, or coincident, the two ends of it pointing straight to the north and south poles of the world.

GNomon, in Geometry, is a figure formed of the two complements, in a parallelogram, together with either of the parallelograms about the diameter. Thus the parall-Jouram AC being divided into four parallelograms by the two lines $D G$, EF parallel to the sides, forming the two complements $\mathrm{AB}$ and $\mathrm{BC}$, with the two DE, FG about the diameter III: then the two gnomons are $\mathrm{AB}+\mathrm{BC}$ †DE , and $A B+B C+F G$.

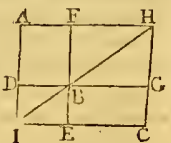

GNomoxic Projection of the Sphere, is the representation of the circles of an hemisphere on a plaue touching it in the vertex, by the eye in the centre, or by lines or rays issuing from the centre of the hemisphere, to all the points in the surface.-In this projection of the sphere, all the great circles are projected into right lines, on the plane, of an indefinite length; and all lesser circles that are parallel to the plane, into circles; but if oblique to the plane, then are they projecterl either into ellipses or hyperbulas, according to their different obliquity. It has its name from gnomonics, or dialling, because the lines on the face of every dial are from a projection of this kind: for if the sphere be projected on any plane, and upon that side of it on which the sun is to shine; also the projected pole be made the centre of the dial, and the axis of the glohe the style or gnomon, and the radius of projection its beight; you will have a dial drawn with all its furniture. See Emerson's Projection of the Spherc.

GNOMONICS, the same as DIALLING; or the art of drawing sun and moon dials, on any given plane; being so called, because it shows how to find the hour of the day or night by the shadow of a gnomon or style. 
GOLDEN Number, is the particular year of the Metonic or Lunar Cycle. See Lunar CYCLE.

To find the Golden Number:

Add 1 to the given year, and divide the sum by 19 , and what renains is the golden number; unless 0 remain, for then 19 is the golden number. Thus, the golden number for the year 1812 is 8 ; as by the operation in the margin.

$$
\begin{aligned}
& 1812 \\
& \text { 19) } \frac{1}{1813}(95 \\
& 171 \\
& \text { Golden } \begin{array}{r}
103 \\
\frac{95}{\mathrm{No.} 8} \\
\hline
\end{array}
\end{aligned}
$$

Golden Pule, a rule so called on account of its excellent use, in arithmetic, and especially in ordinary calculations, by which numbers are found in certain proportions, viz, having three numbers given, to find a 4 th number in proportion. On this account, it is otherwise called The Rule of Three, and The Rule of Proportion. See RuLE of Three.

Having stated, or set down in a line, the three terms, in the order in which they are proportiunal, multiply the $2 \mathrm{~d}$ and $3 \mathrm{~d}$ together, and divide the product by the 1 st, so shall the quotient be the answer, or the 4th term sought.-Thus, if 3 yards of cloth cost a guinea or 21 shillings, what will 20 yards cost. Here the two prices or values must bear the same proportion to each other as the two quantities, or number of yards of cloth, i.e. 3 must bear the same proportion to 20 , as $21 \mathrm{~s}$, the value of the former, must bear to the value $3: 20:: 21: 140 s$. of the latter: and therefore the stating and operation of the numbers will be thus. Then multiplying the $2 \mathrm{~d}$ and $3 \mathrm{~d}$ together, and

3) $\overline{420}$

Quot. 110 s. or $7 l$. dividing the product by the 1 st, it gives $140 s$ or $7 l$. for the answer, being the cost of 20 yards.

GONIOMETRICAL Lines, are lines used for measuring or determining the quantity of angles: such as sines, tangents, secants, versed simes, \&c. Mr. Jones, in the Philos. 'Trans. No. 483, sect. 26 , gave a paper, containing a commodious disposition of cquations for exhibiting the relations of goniometrical lines; from which a multitude of curious theorems may be derived. Sce also Robertson's Elem. of Navigation, vol.1, p. 181 , edit. 4.

GONIOMETRY, a method of measuring angles, so called by M. De Lidgny, who gave several papers, on this methou, in the Memoires of the Royal Acad. an. 1724, $1725,1729$. ML. De Lagny's method of goniometry consists in measuring the angles with a pair of compasses, and that without any scale whatever, except an undivided semicircle. Thus, having any angle drain upon paper, to be measured; produce one of the sides of the angle backwards; tlicn with a pair of fine compisses describe a pretty large semicircle, from the angular point as a centre, cutting the sides of the proposed angle, which will intercept a part of the semicircle. Take then this intercepted part very exactly between the points of the compasses, and turn them successively over upnn the arc of the semicircle, to find how aften it is contained in it, after which there is commonly sonte remainder: then take this remainder in the compasses, and in like manner find how uften it is contained in the last of the integrial parts of the 1st arc, with again some remainder: find in like manner how often this last remainder is contained in the former ; and so on continually, till the remainder become too small to be taken and applied as a measure. By this neans he obtains in series of quotients, or fractional parts, one of another, which being properly reduced into one fraction, give the

\section{G O R}

ratio of the first arc to the semicircle, or of the proposed angle to two right angles, or 180 degrees, and consequently that angle itself in degrees and ninutes.

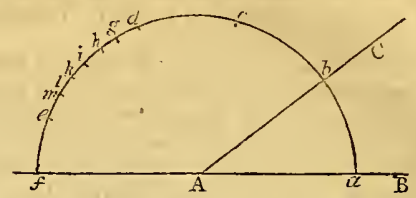

Thus, suppose the angle $\mathrm{B} \boldsymbol{\Delta} \mathrm{C}$ be proposed to be mea. sured. Produce в а out towards $f$; and from the centre $A$ describe the semicircle $a b c f$, in which $a b$ is the measure of the proposed angle. Take $a b$ in the compasses, and apply it 4 times on the semicircle, as at $b, c, d$, and $e$; then take the remainder $f e$, and apply it back upon ed, which is but once, viz at $g$; again take the remainder $g d$, aud apply it 5 times on $g e$, as at $h, i, k, l$, and $m$; lastly, take the remainder me, and it is contained just 2 times in $m l$. Hence the series of quotients is $4,1,5,2$; consequently the 4 th or last arc em is $\frac{1}{2}$ the third $m l$ or $g d$, and therefore the $3 \mathrm{~d}$ arc $g d$ is $\frac{1}{5 \frac{1}{2}}$ or $\frac{2}{15}$ of the $2 \mathrm{~d}$ arc ef; therefore again this $2 \mathrm{~d}$ arc $e f$ is $\frac{1}{1 \frac{2}{1}}$ or $\frac{1}{1} \frac{3}{6}$ of the 1 st arc $a b$; and consequently this 1 st arc $a b$ is $\frac{1}{4 \frac{1}{1} \frac{x}{3}}$ or $\frac{13}{16}$ of the whole semicircle af: But $\frac{x 3}{6}$ of $180^{\circ}$ are $37 \frac{1}{7}$ degrees, or $37^{\circ}$ $\mathrm{s}^{\prime} 34^{\prime \prime}{ }_{7}$, which therefore is the measure of the angle sought. When the operation is nicely performed, this angle may be within 2 or 3 minutes of the truth; though M. De Lagny pretends to measure much nearer than that.

It may be added, that the series of fractions forms what is called a continued fraction. - Thus, in the example above, the continued fraction, and its reduction, will be as follow :

$$
\frac{1}{4}+\frac{1}{1}+\frac{1}{5 \frac{1}{2}}=\frac{1}{4}+\frac{1}{1 \frac{2}{1 T}}=\frac{1}{4_{1}^{1} \frac{1}{3}}=\frac{13}{63}
$$

the quotients being the successive denominators, and 1 always for each mumerntor.

GORGE, or $N e c k$, in Architecture, the narrowest part of the Tuscan or Doric capitals, IJing above the shaft of the pillar, between the astragal and anulets.

It is also a kind of concave moulding, serving for compartments \&c, larger than a scotia, but not so decp.

Gorge, in Fortification, is the entrance into a bastion, or a ravelin, or other out-work.

The Gonge of $a$ Bastion, is what remains of the sicles of the polygon of a place, after cutting of the curtains; in which case it makes an angle in the ccutre of the bastion, viz, the angle made by two adjacent curtains produced to meet within the bastion. - In flat bastions, the gorge is a right line on the curtain, reaching between the two flanks.

GORGE of a Half-moon, or of a Ravelin, is the space between the two ends of their faces next the place.

Gouge of the other out-works, is the interval between their sides. next the great ditch. All the gorges are to be made without parapets: otherwise the besiegers, having taken possession of a work, might make use of them to defend themselves from the shot of the place.- So that they arc only fortified with pallisadoes, to prevent a surprise.

The Demi-Gougi, or Half the Gorge, is that part of 
the polygon between the flank and the centre of the bastion.

GOTHIC, Architecture, is that which deviates from the manner, character, proportions, \& $c$, of the antique; having its ornaments wild and chimerical, and its profiles incorrect. This manner of building came originally from the North, whence it was brought, in the 5 th century, by the Goths into Germany, and has since been introduced into other countries. The first or most ancient style of Gothic building was very solid, heavy, massive and simple, with semicircular arches, \&c: but the more modern style of the Gothic is exceedingly rich, light, and delicate; having an abundance of little whimsical ornaments, with sharp-pointed arches formed by the intersections of different circular segments; also lofty and light spires and steeples, large ramified windows, clustered pillars, \&r. Of this kind are our English catbedrals, and many other old buildings.

GRADUATION, is used for the act of graduating, or dividing any thing into degrees.-For an account of the various methods of graduating mathematical and astronomical instruments, by straight and circular diagonals, and by concentric arcs, \&cc; sce Plain Scale, Nonius, and Vernier. And for an account of Mr. Bird's improved method of dividing astronomical instruments, see MuraL Arch.

Mr. Ramsden, an ingenious mathematical instrumentmaker of London, has lately published, by cncouragement of the commissioners of longitude, an explanation and description of an engine contrived by him for dividing mathematical instruments, accompanied with proper drawings; in consideration of which, the said commissioners granted him the sum of $615 \mathrm{l}$. See his book, 4to, 1777 .

On the subject of dividing a foot into many thousand parts, for mathematical purposes, see Philos. Trans. vol. 2, p. 457,4.59, 541, or my Abr. vol. 1, pa. 161, 195, \&c. And for an account of various otber methods and graduations, see a paper of Mr. Smeaton's in the Philos. Trans. vol. 76 , for the year 1786 , pa. 1 ; being "Observations on the graduation of astronomical instruments; with an explanation of the method invented by the late Mr. Henry Hindley, of York, clock-maker, to divide circles into any given number of parts." Also Mr. Troughton's method and history of such operations, in the vol. for 1809 .

GRAHAM (GEORGE), clock- and watch-maker, the most ingenious and accurate artist in his time, was born at Gratwick, a village in the north of Cumberland, in 1675. He came up to London in 1688, and was put apprentice to a person in that profession; but after being some time with his master, he was received, purely on account of his merit, into the family of the celebrated Mr. l'umpion, who treated him with a kind of parental affection as long as he lived. That Mr. Graham was, without competition, the most eminent of his profession, is but it small part of his character : he was the best general mechanic of his time, and had a complete knowletge of practical astronomy; so that he not only gave to varjous movements for measuring time a degree of perfiction which hasl never before been attained, but invented several astrononical instruments, by which considerable advances have been made in that science: he made great improvements in those which had before been in use; and, by a wonderful manual dexterity, constructed them with greater precisirn and accuracy than perhaps any other person in the world.
A great mural arch in the observatory at Greenwich was made for Dr. Halley, under Mr. Gralıan's immediate inspection, and divided by his own hand: and from this incomparable original, the best foreign instruments of the kind are copies made by Lnglish artists. The sector by which Dr. Bradley first discovered two new motions in the fixed stars, was of our author's invention and fabric. He comprised the whole planetary system within the consass of a small cabinet; from whicl, is a model, all the modern orreries have been constructed. And when the French academicians were sent to the North, to make observations for ascertaining the figure of the earth, Mr. Graham was thought the fittest person in Europe to supply them with instruments; by which means they finished their operations in one year; while those who went to the Soutb, not being so well furnished, were very much embarrassed and retarded in their operations.

Mr. Grabam was many years a member of the Royal Society, to which he communicated several ingenious and important discoveries, viz, from the 31 st to the $42 \mathrm{~d}$ volume of the Philos. Transactions, chiefly on astronomical and philosophical subjects; particularly a kind of horary alteration of the magnetic needle; a quicksilver penduluın, and many curious particulars relating to the true length of the simple pendulum, on which he continued to make experiments till alnost the time of his death, which lappened in 1751 , at 76 years of age.

His temper was not less communicative than his genius was penetrating; and his principal view was the advancement of science, and the benefit of mankind. As he was perfectly sincere, he was above suspicion; and as he was above envy, he was candid.

GRANADO, in Artillery, is a small shell or hollow globe of iron, or other matter, which, being filled with powder, is fired by means of a small fusec, and thrown either by the hand, or a piece of ordnance. As soon as it is kindled, the case flies in pieces, to the great danger of all that stand near it. Granadoes serve to sct fire to close and narrow passages, and are often thrown with the hand among the soldiers, to disorder their ranks; more especially in those posts where they stand thickest, as in trenches, redoubts, lodgments, \&c.

GRANDI (GuIDo), a learned ltalian mathematician, was born at Cremona in 1671 ; appointed professor of mathematics at Pisa in 1714 ; and died in 1742 . In 1690 he published, in 4 to, the demonstration of Viviani's wonderful or quadrable dome, under the title of Gcometrica Divinatio Vivianiorum Problematum ; a work which contains more than its title would lead us to expect, and in which the author remarks many other curiosities in geometry of the same kind, and among others, a portion of the surface of a right cone, which is perfectly quadrable, and to which he gives the name of Vclum Camaldulense, he being a friar of the order of Camaldunes; trom which it scems he did not know the same thing, in a more gcneral form, had before been given by John Bernoulli, in the Lejpsic Acts. In 1701, he puhlished his demonstration if Huygens's theorems on the Logistic Curve, which that atuthor had simply announced without demonstration; being an excellent specimen of the ancient geometrical method; in which piece also, as well as in his letter to the Jesuit Ceva, which follows it, are found several other curious aitd novel particulars.-Another paper of Grandi's is also inserted in the $33 d$ volume of the Philos. Tratus., called it Handful or a Bouquet bf Giometrical Ruscs, being a di... 
sertation on certain curves geometrically described in a circle. This he afterwards enlarged in another treatise, published in 1728, entitled Flores Geometrici ex Rhodonearum, \&c. He was also author of several other miscelIaneous pieces, on the ancient and modern geometry; as, his Quadrature of the Circle and Hyperbola by Infinite Parabolas, in 1703 and 1710; his Dissertation on Infinites of Infinites, \&c, in 1710; an Italian edition of Euclid's Elements; and a posthumous treatıse on Conic Sections, also in the Italian language, in $174 \%$. Grandi it seems was of a turbulent and quarrelsome disposition, being almost always engaged in disputes' on various subjects, geometrical, theological, metaphysical, or philological.

GRAPE-Shot, in Artillery, is a combination of small shot, put into a thick canvass bag, and corded strongly together, so as to form a kind of cylinder, of a diameter suited to the intended piece of ordnance, from the smallest to the largest. The number of shot in a grape varies according to the service, or size of the guns. Of late the grape-shot have been superseded by canister-shot, and by shrapnel shells, \&c, as more convenient and efficacious.

GRAPHOMETER, a mathematical instrument, used in land-surveying \&c, and is otherwise called SEMICI RCLE; which see

GRAVE, in Music, is applied to a sound which is in a low or deep tone. The thicker the chord or string, the more grave the tone or note; and the smaller, the acuter. Notes are supposed to be the more grave, in proportion as the vibrations of the chord are less quick.

GRAVESANDE (IVILIIAM JAMES), a very celebrated Dutch mathematician and philosopher, was born at Boisle-duc, Sept. 27, 1688. He studied the civil law at Leyden, but nathematical learning was his favourite amusement. When he had taken his doctor's degrree, in 1707, he went and setrled at the Hague, wherc he practised at the bar, and cultivated an acquaintance with learned men; with a society of whom he published a periodical review, entitled Le Journal Litteraire, which was continued without interruption from the year 1713 to the year 1722 . The parts of it written or extracted by Gravesande were chiefly those relating to geometry and physics. But he enriched it also with several original pieces entirely of his own composition; viz, Remarks on the Construction of Pneumatical Engines: A Moral Essay on Lying: aud a celebrated Essay on the Collision of Bodies; which, as it opposed the Newtonian philosophy, was attacked by $\mathrm{Dr}$. Clarke, and many other learued men. In 1715 , when the States sent to congratulate George the 1 st, on his accession to the throne, Dr. Gravesande was appointed secretary to the embassy. During his stay in Englant he was admitted a member of the Royal Society, and became intimately acquainted with Sir Isaac Newton. On his return to Holland, he was chosen prolissor of inathematics and astronomy at Leyden; where he had the honour of tirst teaching the Newtonian philosophy, which was then in its infancy. He clied in 1742 , at 54 years of age.

Gravesande was a man amiable in his private character, and respectable in his public one; for fow men of letters have rentered more eminent services to their country. The ministers of the republic consulted him on all occasions when his talents were requisite to assist them, which his skill in calculation often enabled him to do in financial concerns. He was wigreat service as a decipherer, in detecting the secret correspondence of their enemies. $\Lambda$ nd, in his own profession, none ever applicd the powers of na-
G R A

ture with more success, or to more useful purposes.-Of his publications, the principal are,

1. An lntroduction to tbe Newtonian Philosophy; or, a Treatise on the Elements of Physics, confirmed by Experiments. This performance, being only a more perfect copy of his public lectures, was first printed in 1720; and went through many editions, with considerable improvements: the 6th edit. is in English, in 2 large vols. 4to, by Dr. Desaguliers, in $\mathbf{1 7 4 7}$, under the title of Mathematical Elements of Natural Philosophy, confirmed by Experiments.

2. A treatise on the Elements of Algebra, for the use of Young Students; to which is added a Specimen of a Commentary on Newton's Universal Arithmetic; as ulso, A New Rule for determining the Form of an Assumed Infinite Series.-3. An Essay on Perspective: This was written at 19 years of age.-4. A New Theory of the Collision of Bodies.-5. A Course of Logic and Metaphysics. - With several snaller pieces.

His whole mathematical and philosophical works, except the first-mentioned article, were collected and published at Ansterdam, in 2 vols. 4 to, to which is prefixed a critical account of his life and writings, by Professor Allamand.

GRAVIMETER, the name given by M. Guyton, to an instrument for measuring the specific gravities of bodies: he adopts this name rather than either areometer or by drometer, because these latter terms are grounded on the supposition that the liquid is always the thing weighed; whereas, with regard to solids, the liquid is the known term of comparison, to which the unknown weight is referred.

Guyton's gravimeter is executed in glass, and is of a cylindric form, being that which requires the smallest quantity of fluid, and is on that accouut preferable, except so far as it is necessary to deviate for the security of a vertical position. It carries two basins, one of then superior, at the extremity of a thin stem, towards the middle of which, the fixed point of immersion is marked. The other, or lower basin, terminates in a point; it contains the ballast, and is attached to the cylinder by two branches. 'The moveable suspension, by means of a hook, has the inconvenience of sbortening the lever which is to secure the vertical position.

The cylinder of this instrument is 0.71 inches diameter; and 6.55 inches in length. It carries in the upper basin an additional constant wright of 5.115 grains. These dimensions might be increased so as to render it caprable of receiving a much more considerable weight; but this is unnecessary. M. Guyton has added a picce which he calls the plongeur, because in fact it is placed in the lower basin when used, and is consequently entirely immersed in the fluid. It is a bulb of glass loaded with a suthicient quantity of mercury, in order that its total weight maty be equal to the constant additional weight, added to the weight of the volume of water displaced by this piece. It will be readily understond that the weight being determined at the sane temperature at which the instrument was originally adjusted, it will sink to the same mark on the stem, whether it bo loaked with a constant additimal weight in the upper basin, or whether the effect of this weight be produced by the additional piece in the lower dish.

'I'his instyument may be used tor solids, as wetl as fluids, provided the absolute weight of the solid to be cxamined be rather less than the constant additional weight in tlie upper basin, which is 115 grains. 
For liquids of less specific gravity than water, the gravimeter, without the additional weight above mentioned, sveighs about 459 grains, in the dimensions before laid down. It would be easy to limit its weight to the greatest accuracy. We have therefore the range of one-fifth of buoyancy, and consequently the means of ascertaining all the intermediate densities, from water to the most highly rectified spirit, which is known to bear in this respect the ratio of 8 to 10 , with regard to water.

When liquids of greater specific gravity than water are to be tried, the constant weight being applied below, by means of the additional piece, which weighs about 138 grain, the instrument can receive in the upper basin more than 4 times the usual additional weight, without losing the equilibrium of its vertical position. In this state it is capable of showing the specific gravity of the most concentrated acid. It possisses also another property, which is this; namely, that it may be employed as a balance to determine the absolute weight of such borlies as do not exceed its additional load. And the purity of water being known, it will undicate the degrees of rarefaction and condensation, in proportion to its own bulk.

To find the specific gravity of any solid by the gravimeter. Rule: "From the weight in the upper basin, when the instrument is properly immersed in the unknown fluid, take the weight which is placed with the body in the same scale at the like adjustment; the remainder is the absolute weight of the solid. Multiply this by the specific gravity of the fluid, and reserve the product. From the additional weight when the body is placed in the lower basin, take the weight when it was placed in the upper; the remainder will be the loss of weight by inmersion. Divide the reserved by the loss by immersion, and the quotient will be the specific gravity of the solid with regard to distilied water, at the standard temperature and pressure."

To find the specific gravity of a fluid, proceed thus: "To the weight of the gravimeter adrl the weight required in the upper basin, to sink it in the unknown fluid. Again, To the weightof the gravimeter add the weight reqguired in the same manner to sinis it in clistilled water. Divide the first sum by the sccund, and the quotient will be the specific gravity of the fluid in question."

GRAVITATION, the exercise of gravity, or the pressure a body exerts on another body beneath it by its weight. This is sometimes distinguished from gravity. 'Thus, $\mathrm{M}$. Yhupertuis, in his Figure de la Terre, takes gravity for that force by which a body would fall to the earth sujposed at rest; and gravitation for the same, but diminished by the centrifugal force. It is only gravitation, or gravity thus blended witl the centrifugal force, that we can usually measure by our experiments. Methods however have been found to distinguish what remains of the primitive gravity, and what has been destroyed by the centrifugal force.

It is one' of the laws of nature, discovered by Newton, and now received by all philosophers, that every particle of matter in nature gravitates towards every other particle ; which law is the main principle in the Newtonian philosophy. But what is callerl gravitation with respect to the gravitating bosty, is usually called attraction with respect to the body gravititted to. 'The planets, both primary and secontary, as als's the comets, do all gravitate towards the sun, and lowards cach othicr; as well as the sun towards them; and that in propotion to the quantity of matter in each of them.

The Peripatetics \&c hold, that bodies only gravitate or weigh when ont of their natural places, and that gravita- tion ceases when they are restored to the same, the pur pose of nature being then fulfilled; and they maintain that the final cause of this faculty is only to bring elementary bodies to their proper place, where they may rest. But the moderns show that bodies exercise gravity even when at rest, and in their proper places. This is particularly shown of fluids; and it is one of the laws of hydrostatics, demonstrated by Boyle and others, that fluids gravitate in proprio loco, the upper parts pressing on the lower, \&c.

For the laws of gravitation of bodies in fluids specifically lighter or heavier than themselves, see SPEcific GRAVITY. Also for the centre or line or plane of gravitation, see Centre, Line, or Plane.

GPAVITY, in Physics, the natural tendency or inclination of bodies towards the centre. And in this sense gravity agrees with centripetal force.

Gravity however is, by sume, defined more generally as the natural tendency of one body towards another; and again by others still more generally as the mutual tendency of each body, and each particle of a body, towards all others: in which sense the word answers to what is more usually called attraction. Indeed the terms gravity, weight, centripetal force, and attraction, denote in effect all the same thing, only in different views and relations; all which however it is very common to confound, and use promiscuously. But, in propricty, when a body is considered as tending towards the earth, the force with which it so tends is called Gravity, Furce of Gravity, or Gravitating Force; when the body is considered as immediately tending to the centre of the earth, it is called Centripetal Force; but when we consider the earth, or mass to which the body tends, it is called Attraction, or Attractive Force; and when it is considered in respect of an obstacle or another body in the way of its tendency, on which it acts, it is called Weight.

Philosuphers think differently on the subject of gravity. Some consider it as an inactive property or innate power in hodies, by which they endeavour to unite together. Others hold gravity in this sense to be an occult quality, and to be exploded as such out of all sound philosophy. Newton, though be often calls it a vis, power, or property in bodies, yet explains himself, that he means nothing more by the word but the effect or phenomenon: lie does not consider the principle, the cause by which bndies tend downwards, but the tendency itself, which is no occult guality, but a sensible phenomenon, be its causes what they may; whether' a property essential to body, as some make it, or superadded to it, as others; or even an impulse of some body from without, as others.

It is a law of nature long observed, that all bodies near the earth have a gravity or weight, or a tendency towards its centre, nr at least perpendicular to its surface; which law the moderns, and especially Sir I. Newton, from certain observations, have found to be much more extensive, and holding universally with respect to all known bodies and matter in nature. It is therefore at present acknowledged as a principle or law of nature, that all bodies, and all the particles of all boslies, mutually gravitate towards each other: frnm which single principle it is that Newton has lappily deduced all the great phenomena of nature. Hence, Gravity may be distinguished into Particular and General.

Particular Gravite, is that which respects the earth, or by which bodies descend, or tend towards the centre of the earth; the phenomena or properties of which are as follow :- 
1. All circumterrestrial bodies do hereby tend towards a point, which is either accurately or very nearly the centre of magnitude of the terraqueous globe. Not that it is meant that there is really any virtue or charm in the point called the centre, by which it attracts bodies; but because this is the result of the gravitation of bodies towards all the parts of which the earth consists.

2. This point or centre is fixed within the eartl, or at least has been so far as any authentic history reaches. For a consequence of its shifting, though ever so little, would bo the overfowing of the low lands on that side of the globe towards which it should approach. Dr. Halley suggests, that it would well account for the universal deluge, to have the centre of gravitation removed for a time towards the middle of the then inhabited world; for the change of its place but the 2000th part of the radius of the earth, or about two miles, would be sufficient to lay the tops of the highest hills under water.

3. In all places equidistant from the centre of the earth the force of gravity is nearly equal. Indeed all parts of the earth's surface are not at equal distances from the centre, because the equatorial parts are higher than the polar parts by about 17 miles; as has been proved by the necessity of making the pendulum shorter in those places, before it will ribrate seconds. In the new Petersburg Transactions, vol. 6 and $7, \mathrm{M}$. Krafft gives a formula for the proportion of gravity in different latitudes on the earth's surface, which is this :

$$
y=\left(1+0.0052848 \operatorname{sine}^{2} \lambda\right) g \text {; }
$$

where $g$ denotes the gravity at the equator, and $y$ the gravity under any other latitude $\lambda$. On this subject, see also the articles DEgREE, and EARTH.

4. Gravity equally affects all bodies, without regard either to their bulk, figure, or matter : so that, abstracting from the resistance of the medium, the most compact and loose, the greatest and smallest bodies, would all descend through an equal space in the same time; as appears from the quick descent of very light bodies in an exhisusted receiver. The space which bodies do actually fall, in vacuo, is 16 fect in the first second of time, in the latitude of London; and for othertimes, either greater or less than that, the spaces descended from rest are directly proportional to the squares of the times, while the falling body is net far from the carth's surface.

5. This power is the greatest at the carth's surface, from whonce it decreases both upwards and lownwards, but not both ways in the same proportion; for upwards the force of gravity is less, or decreases, as the square of the distance from the contre increases, so that at a double distance from the centre, above the surface, the force would be only 1-4th of what it is at the surface; but below the surface, the power decreases in such sort that its intensity is in the dircet ratio of the distance from the centre; so that at the distance of half a semidianeter from the centre, the force would be but half what it is at the surface; at $\frac{1}{3}$ of a semidianeter the force would be $\frac{T}{3}$, and so on.

6. As all bodies gravitate towards the earth, so does the carth equally gravitate towards all bollics; as well as all bodies towards particular parts of the carth, ins hills, \&c, which has been proved by the attraction a hill has upon a plumb line, insensibly drawing it aside.-Hence the gravitating force of entire bodies consists of those of all their parts: for, by adding or taking away any part of the inatter of a body, its gravity is increased or decreased in the proportion of the quantity of such portion to the whole in ass. Hence also the gravitating powers of bodies, at the same distance from the centre, are proportional to the quaritities of matter in the bodies.

General or Universal Gravitr, is that by which not only the planets, but all the bodies and particles of matter in the universe tend towards one another. The existence of the same principle of gravitation in the superior regions of the heavens, as on the earth, is one of the great discoveries of Newton, who made the proof of it as easy as that on earth. At first it would seem this was only conjecture with him: he observed that all borlies near the earth, and in its atmosphere, had the property of tending directly towards it ; he soon conjectured that it probabiy extended much higher than any distance to which we could reach, or make experiments; and so on, from one distance to another, till he at length saw no reason why it might not extend as far as the moon, by means of which she might be retained in her orbit, as a stone in a sling is retained by the hand; and if so, he next inferred, why might not a similar principle exist in the other'great bodies in the universe, the sun and all the other planets, both primary and secondary, which might all be retained in their orbits, and perform their revolutions, by means of the same universal principle of gravitation.

These conjectures he soon realized and verified by mathematical proofs. Kepler had discovered by contemplating the motions of the planets about the sun, that the area described by a line connecting the sun and planet, as this revolved in its orbit, was always proportional to the time of its description, or that it described equal areas in equal times, in whatever part of its orbit the planet might be, moving always so much the quicker as its distance from the sun was less: And it is also found that the satellites, or secondary planets, observe the same law in revolving about their primaries. But it was soon proved by Newton, that all bodies moving in any curre line described on a plane, and which, by radii drawn to any certain point, describe areas about the point proportional to the times, are impelled or acted on by some power tending towards that point. Consequently the power by which all these planets revolve, and are retained in their orbits, is directed to the centre about which they move, viz, the primary planets to the sun, and the satellites to their several primaries.

Newton also demonstrated, that if several bodies revolve with an cquable motion in several circles about the same centre, and that if the squares of their periodical times be in the same proportion as the cubes of their distances from the common centre, then the centripetal forces of the revolving bodies, by which they tend to their central body, will be in the reciprocal or inverse ratio of the squares of the distances. $\mathrm{Or}$ if bodies revolve in orbits approaching to circles, and the apses of those orbits be at rest, then also the centripetal forces of the revolving bodies will be reciprocally proportional to the squares of the distances. But it lad been agreed on by astionomers, and particularly Kepler, that boul these cases obtain in all the planets. Aud tirerefore he inforred, that the contripetal forces of all the planets, are reciprocally proportional to lhe squares of the distances from the centres of their motions.

On the whole it appears, that the planets arc retained in their orbits by some power which is continually acting upon them: that this power is directed towards the contre of their motions: that the intensity or efficacy of this power increases on an approach towards the centre, and diminishes on receding from the same, and that in the re- 
ciprocal duplicate ratio of the distances: and that, by comparing this centripetal force of the planets with the force of gravity on the earth, they are found to be perfectly alike, as may easily be shown in various instances. For example, in the case of the moon, the nearest of all the planets. The rectilinear spaces described in any given time by a falling body, urged by any powers, reckoning from the beginning of its descent, are proportional to those powers. Consequently the centripetal force of the moon revolving in her orbit, will be to the force of gravity on the surface of the earth, as the space which the moon, would describe in falling during any small time, by her centripetal force towards the earth, if she had no circular motion at all, to the space a body near the earth would describe in falling by its gravity towards the same.

Now by an easy calculation of those two spaces, it appears that the former force is to the latter, as the square of the semi-diameter of the earth is to the square of that of the moon's orbit. The moon's centripetal furce therefore is equal to the force of gravity; and consequently these forces are not different, but they are one and the same : for if they were two distinct forces, bodies acted on by the two powers conjointly would fall-towards the earth with a velocity double to that arising from the sole power of gravity.

It is evident therefore that the moon's centripetal force, by which she is reiained in her orbit, and prevented from flying off in tangents, is the very power of gravity of the earth extended thither. See Newton's Princip. lib. 1, prop. 45 , cor. 2 , and lib. 3 , prop. 3 ; where the numeral calculation may be seen at full length.

The moon therefore gravitates towards the earth, and reciprocally the earth towards the moon. And this is also further confirmed by the phenomena of the tides.

The like reasoning may also be applied to the other planets. For, as the revolutions of the primary planets round the sun, and those of the satellites of Jupiter and Saturn round their primaries, are phenomena of the same kind with the revolution of the moon about the earth ; and as the centripetal powers of the primary are rlirected towards the centre of the sun, and those of the satcllites towards the centres of their primaries; and lastly, as all these powers are reciprocally as the squares of the distances from the centres, it may safely be concluded that the poiver and cause are the same in all.

Therefore, as the monn gravitates towards the earth, and the earth towards the moon; so doall the secondaries to their primaries, and these to their secondaries; and so also do the primaries to the sun, and the sun to the primaries. Newton's Princip. lib. 3, prop. 4, 5, 6; Greg. Astron. lib. 1, sect. 7, prop. 46 and 47 .

The laws of Universal Gravity are the same as those of bodies gravitating to the earth, before laid down.

Cause of Gravitr. Various theories have been advanced by the philosophers of different ages to account for this grand principle. of gravitation. The ancients, who were only acquainted with particular gravity, or the tendency of sublunar bodies towards the earth, aimed no further than a system that might answer the more obvious phenomena of it. Ilowever, some hints are found concerning the gravitation of celestial bodies, in the account given of the doctrine of Thales and his successors; and it would $\mathrm{secm}$ that Pythagoras was still better acquainted with it, to which it is supposed he had a view in what he taucht concerning the Harmony of the Spheres.

Aristotle and the Peripatetics content themselves with referring gravity or weight to a native inclination in heavy bodies to be in their proper place or sphere, the centre of the earth. And Copernicus ascribes it to an innate principle in all parts of matter, by which, when separated from their wholes, they endeavour to return to them again the nearest way. In answer to Aristotle and bis followers, who considered the centre of the earth as the centre of the universe, he observed that it was reasonable to think there was nothing peculiar to the earth in this principle of gravity: that the parts of the sun, noon, and stars, tended likewise to each other, and that their spherical figure wass preserved in their various motions by this power. Copern. Revol. lib. 1, cap. 9. But neither of these systems assigns any physical cause of this great effect : they only amount to this, that bodies descend because they are inclined to descend.

Kepler, in his preface to the commentaries concerning the planet Mars, speaks of gravity as of a power that was mutual between bodies, and says that the earth and moon tend towards each other, and would meet in a point so many times nearer to the earth than to the moon, as the earth is greater than the moon, if their motions did not linder it. He adds, that the tides arise from the gravity of the waters towards the moon. To him we also owe the important discovery of the analogy between the distances of the several planets from the sun, and the periods in which they complete their revolutions, viz, that the squares of their periodic times, are alivays in the same proportion as the cubes of their mean distances from the sun. However, Kepler, Gassendi, Gilbert, and others, ascribe gravity to a certain magnetic attraction of the earth ; conceiving the earth to be one great magnet, continually emitting effluvia, which take hold of all bodies, and draw them towards the earth. But this is inconsistent with the several phenomena.

Descartes and his followers, Rohault, \& c, attribute gravity to an external impulse or trusion of some subtile matter. By the rotation of the earth, say they, all the parts and appendages of it necessarily endenvour to recede from the centre of rotation; but whence they cannot all actually recede, as there is no vacuum or space to receive them. But this hypothesis, founded on the supposition of a pleuum, is overthrown by what has been since proved of the existence of a vacuum.

Dr. Hooke inclines to an opinion much like that of Descartes. Gravity be thinks deducible from the action of a most subtile medium, which easily pervades and penetrates the mrst solid bodies; and which, by some motion it has, detrudes all earthly bodies from it, towards the centre of the earth. Vossius too, and many others, give partly into the Cartesian notion, and suppose gravity to arise from the diurnal rotation of the earth round its axis. See Birch's 11ist. of the Royal Soc. vol. 2, pp. 72 and 91.

Dr. Halley, despairing of any satisfactory theory, chonses to have immediate recourse to the agency of the Deity. So Dr. Clarke, from a view of several properties of gravity, concludes that it is no adventitious effect of any motion, orsubtile matter, but an original and general law impressed by God on all matter, and preserved in it by some effcient power penctrating the very solid and intimate substance of it; being found always proportional, not to the surfaces of bodies or corpuscles, but to their solid quantity and contents. It should therefore be no more incpuired why hodies gravitate, than how they came to be first put in inotion. Annot. in Rohault. l'hys. part 1, cap. 11. 
Grarcsande, in his Introduct. ad Philos. Newton. contends that the cause of gravity is utterly unknown; and that we are to consider it no otherwise than as a law of nature originally and immediately impressed by the Creator, without any dependence on any second law or cause at all. Of this he thinks the three foliowing considerations sufficient proof. 1 . That gravity requires the presence of the gravitating or attracting body : so the satellites of Jupiter, for ex. gravitate towards Jupiter, wherever he may be. 2. That the distance being supposed the same, the velocity with which bodies are inoved by the force of gravity, depends on the quantity of matter in the attracting body; and the velocity is not changed, whatever the mass of the gravitating hody may be. 3 . That if gravity do depend on any known law of motion, it must be some impulse from an extraneous body; sothat as gravity is continual, a continuai stroke must also be required. Now if there be any such matter continually striking on bodies, it must be fluid, and subtile enough to penetrate the substance of all bodies: but how shall a body subtile enough to penetrate the substance of the hardest bodics, and so rare as not sensibly to binder the motion of bodies, be able to impel vast masses towards each other with such force? how does this force increase the ratio of the mass of the body, towards which the other body is moved? whence is it that all bodies move with the same velocity, the distance and body gravitated to being the same? can a fluid which only acts on the surface, either of the bodics themselves, or their internal particles, communicate such a quantity of motion to bodies, which in all bodies shall exactly fullow the proportion of the quantity of matter in them?

Mr. Cotes goes yet furtber. Giving a vicw of Newton's philosophy, he asserts that gravity is to be ranked among the primary qualities of all bodies; and deemed equally essential to matter as extension, mobility, or impenetrability. Prefat. ad Newt. Princip. But Newton himself disclaims this notion; and to show that he does not take gravity to be essential to bodies, he declares his opinion of the cause; choosing to propose it by way of query, not being yet sufficiently satisfied about it for want of experjments. Thus, after having shown that there is a medium in nature vastly more subtile than air, by whose vibrations sound is propagated, by which light communicates heat to bodies, and by the different densities of which the refraction and reflection of light are performed; he proceeds to inquire: "Is not this medium much rarer within the dense budies of the sun, stars, planets, and comets, than in the empty celestial spaces between them ? and in passing from them to greater distances, doth it not grow denser and denser perpetually, and thereby cause the gravity of those great bodies towards one another, and of their parts towards the bodies; crery borly endeavouring to recele from the denser parts of the inedium towards the rarer?

"For if this medium be supposel rarer within the sun's body than at its surface, and rarer there than at the liundredth part of an incls from his body, and rarer there than at the orbit of Saturn; I see no reason why the increase of density-should stop any where, and not rather be continued through all distances from the sun to $\mathrm{Sa}$ turn, and beyond.

"And though this increase of density may at great distances be exceeding slow : yet if the elastic force of this mediuin be exceeding great, it may suffice to impel bodies from the denser parts of the medium towards the rare: with all that power which we call gravity.

" That the elastic force of this medium is exceeding great, may be gathered from theswiftness of its vibrations. Sounds morc about 1140 English feer in a second of time, and in seven or eight minutes of time, they move about one hundred English miles: light mores from the sun to us in about 7 or 8 minutes of time, which distance is about 70000000 English miles; supposing the horizontal parallax of the sun to be about 12 seconds; and the vibrations, or pulses of this medium, that they may cause the alternate fits of easy transmission, and easy reflection, must be swifter than light, and by consequence abore 700000 times swifter than sounds; and therefore the elastic force of this medium, in proportion to its density, must be above $700000 \times 700000$ (that is, above $490000000000)$ times greater than the clastic force of the air is, in proportion to its density : for the velocitics of the pulses of elastic mediums, are in a subduplicate ratio of the elasticities and the rarities of the mediums conjointly.

"As magnetism is stronger in small loadstones than in great ones, in proportion to their bulk; and gravity is stronger on the surface of small planets, than on those of great ones, in proportion to their bulk; and small brodies are agitated much more by electric attraction than great ones: so the smallness of the rays of light may contribute very much to the power of the agent by which they are refracted; and if any one should supposc, that ether (like our air) may contain particles which endeasour to recede from one another (fur I du not know what this ether is), and that its particles are excecdingly smaller than those of air, or even than those of light; the exceeding smallness of such particles may contribute to the greatness of the force, by which they recede from one another, and thercby make that medium exceedingly more rare and elastic than air, and, of consequence, exccedingly less able to resist the motions of projectiles, and exccedingly more able to press upon gross bodies by endeavouring to expand itself." Optics, Query 21, \&c.

M. Le Sage attempts to account for the cause of gravity by the following ingenious hypothesis: "Imagine," says this author, "that through all space numberless corpuscles or atoms, almost iufinitely small, are in perpetual motion: that every corpuscle has its determined direction, and moves for ever in a straight line with a velocity far exceeding that of light. It is cvident that the directions of these corpuscles may be so various, they may be themsclves so small, and their velocity so great, that though they follow each other at vast distances, and leave space, in reality, almost empty; yet they may abound every where in such a manner, that in a portion of time, almost infinitely small, a great number of them shall pass through every point of space whatsoever. On whatever point of space therefore our attention is fixed. we may consider it as a centre, to which the motions of an infinite number of atoms are referred, cither by cliverging therefrom, or converging to it." This constitution of what Le Sage calls the gravific fluid being conccived, suppose a solid body to be plunged into it of any figure whatever, larger than one of the corpuscles, and in some degree, if not entirely, impervious to these particles; this body will remain at rest, or at least without any progressive motion, the impulses of the particles that strike against it boing equal in opposite directions. It may oscillate a little backward 
and forward, but will not be forced any considerable distance from its place.

Now let there be plunged into the gravific fluid, another body, of any figure, and at any distance from the first. These two bodies will immediately begin to move towards each other. For the one serving to protect the other from a certain quantity of the impulsion of the corpuscles, the currents thus left without opposition necessarily produce their effect, and impel the bodies towards each other.

Their motion towards one another will be continually accelerated; and the forces producing that acceleration will increase in proportion as the one body stops more of the currents from falling on the otber; that is, nearly as the squares of the distances dininish.

Again, if the solid particles of which the bodies are made up, be impenetrable to the gravific corpuscles, but the bodies themselves, on account of their porosity, permeable by them in a certain degree, the number of particles that are stopped by each of the bodies will be, cæeteris paribus, proportional to the number of solid particles, that is, to the quantities of matter in the bodies; and hence, in general, the force urging the bodies towards each other, will be directly as their masses, and inversely as the squares of their distances. "Thus, by mechanical action," says Le Sage, " is the Newtonian law of gravitation explained in all its parts."

In objection to this system, we advance the following, urged by Boscorich :- "In this system," says Boscovich, " no particle of the fluid returns to its place, or ever passes a second time through the same point of space. A constant supply of new particles is therefore necessary, as all those that are contuined within the limits of the sensible universe, at any instant, must be replaced before they have entirely escaped from it, and sent forth to traverse for ever the deserts of uninhabited extension. The imagination is terrified at this constant exertion of what cannot be considered as less than creative power employed in producing existencies that, for a limited time, are to be useful; and through all the rest of infinite duration, are to serve no purpose whatsoever."

About the year 1806 several of the clergy, as well as other individuals in this country, being alarmed, it was said, at the efforts which the philosophers on the continent were making to account for the various operations of nature upon inere mechanical principles, with a vicw, as they supposed, to exclude the Deity from any concern in the government of the world, and thereby to lay a foundation for athcism, Mr. Vince, professor of astronomy in the university of Cambridge, was requested to examine the most plausible and generally received hypotheses, which had been framed to account for gravitation, and to give the result of his examination. The inquiry, he tells us, was favourably received, and it was suggested that it might not be improper to offer it to the Royal Society; but on being presented by his friend Dr. Maskelyne, the astronomer-royal, and not mecting that reception which would have been gratifying to the professor's feelings, and have reficted credit on the Society, he in consequence whithdrew it, and submitted the paper to the opinion of the public, in a small pamphlet on this subject, printed at Cambriclge, in 1806.

The systems which he has there considered, are those of Descartes, Newton, Le Sage, and Bernoulli, with a slight mention of some others whose inconsistency ren-

Vol. I. dered any detailed account of them unnecessary, but omitting that of Boscovich.

Speaking of the system of Saussure, (which, by the bye, is that of Le Sage, given above, he observes, that if the corpuscles there mentioned be admitted to move as the author describes them, it will follow that the moving force of each body depends on the surfaces of the bodies, whereas it ought to be as the quantity of matter in each respectively : " this consideration, therefore," continues the professor, " without entering into any further examination of the hypothesis, is sufficient to show that it cannot be admitted."

The hypothesis which Mr. Vince has made the subject of mathematical consideration, is that of our illustrious countryman Newton; who, as has been obscrved, supposed, that if the sun and the planets acted on each other, it must be by some intermediate and invisible substance, such as that of an elastic fluid. The professor then admitting that an elastic fluid is the cause of gravity, lays down, previous to the estimation of its effects on bodies placed in it, the two following facts, as standards, to which his results are to be referred for refutation or support:

1. That the gravitation of a planet varies inversely as the square of jis distance from the sun; that is, whatever be the magnitude or density of the planet, its acceleration towards the sun varies in that ratio.

2. That a force varying inversely as the square of the distance, cannot be compounded of several forces, each of which does not vary in that ratio.

On these grounds, the professor enters on the investigation of the hypothesis; by supposing that the density of the medium is measurable by the number of particles, uniformly diffused on a given square area ; that the variation of density at any distance $d$, from the sun is as $d^{\mathrm{m}}$; and consequently the distance of the particles themselves as $\frac{1}{d^{\frac{1}{2} \mathrm{~m}}}$. Then, admitting that the elastic force of theparticles vary as the $n$th power of their distance, or as $\frac{1}{d^{\frac{x}{2} m n}}$, he at last obtains $\frac{2 m-m n}{3 e} \times a \frac{2 n-m n}{n}-1$, for the accelerative force of the planet towards the sun at the distance $a$ from the same.

"Now it is to be observed," says Mr. Vince, "that this expression is in terms of $e$, the density of the planet; and therefore does not agree with the first given fact or law of gravitation, that law being independent of the density or magnitude. Therefore in this case, the hypothesis is rendered nugatory."

But besides this, he states that the body will, instead of being forced towards the sun, be driven from it, even when the force varies inversely as the square of the distance. Hence, and from what is afterwards demonstrated, he concludes, that it is not possible for any law of variation of density, of the fluid, in terms of the distance from the sun, combined with any law of variation of the repulsive force of the particles of the fluid in terms of their distance, which can satisfy the law of gravitation. And if we were to suppose the law of density to vary in terms of any other quantities besides those expressing the distance from the sun, such quantities must cnter into the law of force, and thereby make a still greater deviation from the law of the inverse square of the distance. Considering the matter therefore, in a mathematical point of view, we 4. $\mathrm{G}$ 
are justified, hesays, in rejecting this hypothesis as the cause of gravitation.

Mr. Playfair however, in revicwing this examination, observes that, this conclusion he (Mr. Vince) next endeavours to extend to all the laws of variation of density and of elasticity tlat can possibly exist, by showing that no one can bc adrhitted that is not capable of being expressed by a single term, and consequently by the nuth or $n$th power of the distance. It is here, if we mistake not, that the error lies. For, though a variation of density or of elasticity, expressed thas, $a x^{m}+b x^{n}$, cannot take place, because the force arising from it would also involve two terms, yet if one of the terms be constant, as if $m=0$, and so the expression $=a+b x^{n}$, then the force would be expressed by one term only, viz, by the fluxion of $b x^{n}$, that is, by a quantity proportional to $x^{n-x}$. Thus, for example, if $x$ be any distance from the sun's centre, $d$ the density of the ether at that distance, and $e$ its elasticity. Let $d$ increase in the direct ratio of $x$, or $d=a x$; and suppose $e=c-\frac{1}{d}=c-\frac{1}{a x}$, where $c$ is a constant quantity, that would be determined, if we knew at what distance from the sun's centre the elasticity of the ether is $=0$. If, for example, it is equal to nothing at the distance $s$ from the sun's centre, or when $x=s, c=\stackrel{1}{a s}$, so that $e=\frac{1}{a s}-\frac{1}{a x}$, and the differential of this being taken, gives $\dot{c}=\frac{\dot{x}}{a x^{2}}$. Now $\dot{c}$ is the difference of elasticity for the change of distance $\dot{x}$; and therefore is the force with which a small spherical body, or a single particle of matter, impervious to the ether, would be impelled toward the sun; and it varies as $\frac{1}{x^{2}}$, or in the inverse ratio of the squares of the distances from the sun. It is therefore POSSIBLE that an elastic fluid may be so constituted as to produce a tendency of one body to another, varying inversely as the squares of the distances of these bodies.-For this purpose, there is only required an elastic fluid, of which the density is as the distance from the sun, and the elasticity as a certain given magnitude diminished by the reciprocal of that distance. There are many other hypotheses concerning the density and elasticity of the fluid, which will give the same result with this; all indeed in which we have these equations, $d=a x^{\mathrm{m}}$, and $e=c-\frac{1}{d^{\frac{1}{\mathrm{~m}}}}$. This is directly contrary to Mr. Vince's conclusion, " that it is not possible for any law of variation of the density of the fluid in terms of the distance from the sun, combined witl any law of variation of the repulsive force of the particles of the fluid in terms of their distance, to satisfy the law of gravitation." See the Edinburgh Review, vol. 13, pa. $101,8 \mathrm{c}$.

Gravity, in Mechanics, denotes the conatus or tendency of bodies towards the centre of the earth. That part of mechanics which considers the equilibrium or motion of bodies arising from gravity or weight, is particularly called Statics.

Gravity is distinguished into absolute and relative.

Al:olute Gravity is that with which a body descends freely and perpendicularly through an unresisting medium. The laws of which see under DEscent or BODifs, Accejeration, Motion, \&c.

Relative GnAvITY is that with which a body descends on an inclined plane, or through a resisting medium, or as opposed by some other resistance. The laws of which, sce under the articles Ixchined Praxe, Descent, Fluid, Resistance, \&c.

Gravity, in Hydrostatics. The law's of bodies gravitating in fluids constitute the doctrinc of Hydrostatics.

Gravity is bere divided into absolute and specific.

Absolute or True Gravity, is the whole. force witl which the body tends downwards.

Specific Gravity, is the relative, comparative, or apparent gravity in any body, in respect of that of an equal bulk or magnitude of another body; denoting that gravity or weight which is peculiar to each species or kind of body, and by which it is distinguished fromall other kinds.

In this sense a body is said to be specifically heavier than another, when under the same bulk it contains a greater weight than that other; and reciprocally, the lutter is said to be specifically lighter than the former. Thus, if there be two equal spheres, cach one foot in diancter; the one of lead, and the other of wood: since the leaden one is found heavier than the wooden one, it is said to be specifically, or in specie, heavier; and the wooden one specifically lighter.

This kind of gravity is by some called relative; in opposition to absolute gravity, which increases in propurtion to the quantity or mass of the body.

Lazus of the SPECIFIC GRAvity of Bodies.

I. If two bodies be equal in bulk, their specific gravitics are to each other as their weights, or as their densities.

II. If two bodies be of the same specific gravity or density, theil absolute weights will be as their magnitudes or bulks.

III. In bodies of the same weight, the specific gravities are reciprncally as their bulks.

IV. 'Ihe specific gravities of all bodies are in a ratio compounded of the direct ratio of their weights, and the reciprocal ratio of their magnitudes. And hence agann the specific gravities are as the densities.

$V$. The absolute gravities or weights of bodies are in the compound ratio of their specific gravitics and nagnitudes or bulks.

VI. The magnitudes of bodies are directly as their weights, and reciprocally as their specific gravities.

VII. A body specifically heavier than a fluid, loses as much of its weight, when jmmersed in it, as is equat to the weiglit of a quantity of the fluid of the same bulk or magnitude.-Hence, since thespecific glavities are as the absolute gravities under the same bulk; the specific gravity of the fluid, will be to that of the body immersed, as the part of the weight lost by the solid, is to the whole weight. And hence the specific gravities of fluids, are as the weights lost by the same solid immersed in them.

VIII. To find the Specific Gravity of a Fluid, or of a Solid.-On one arm of a balance suspend a globe of lead by a fine thread, and to the other fasten an equal weight, which may just balance it in the open air. Immerse tlic globe into the fluid, and observe what weight balances it then, and consequently what weight is lost, which is proportional to the specific gravity as above. And thus the proportion of the specific gravily of one fluid to another, is determined by immersing the globe successively in all the fluids, and observing the weights lost in each, which will be the proportions of the specific gravities of the fluids sought. 
This same operation determines also the specific gravity of the solid imniersed, whether it be a globe or of any other shape or bulh, supposing that of the fluid known. For the specific gravity of the fluid is to that of the solid, as the weight lost is to the whole weight. Hence also may be found the specific gravity of a body that is lighter than the fluid, as follows :

[X. To find the Specific Gravity of a Solid that is ligliter than the fluid, as water, in which it is placed.-Annex to the lighter body another that is much heavier than the fluid, so as the compound mass may sink in the fluid. Weigh the heavier body and the compound mass separately, both in water and out of it; then find how much each loses in water, by subtracting its weight in water from its weight in air; and subtract the less of these remainders from the greater. Then say,

As this last remainder,

Is to the weight of the light body in air,

So is the specific gravity of the fluid,

To the specific gravity of that body.

$\mathrm{X}$. The specific gravities of bodies of equal weight, are reciprocally proportional to the quantities of weight lost in the same fluid. And hence is found the ratio of the specific gravities of solids, by weighing in the same fluids, masses of them that weigh equally in air, and noting the weights lost by each.

The specific gravities of many kinds of bodies, both solid and fluid, have been determined by various authors. Marinus Ghetaldus particularly tried the specific gravities of various bodies, especially metals; which were taken from thence by Oughtred. In the Philos. Trans. are several ample tables of them, by various authors, particularly those of Mr. Davis, vol.45, pa. 416. Some tables of them were also published by P. Mersenne, Muschenbroeck, Ward, Cotes, Emerson, Martin, \&c.

The following table, taken chiefly from Gregory's excellent book of Mechanics, contains the specific gravities of most of the principal bodies, both solid and fluid. The numbers express the avoirdupois ounces in a cubic foot of each body, that of distilled water being just 1000 ounces, which is here made the standard of comparison of all the rest.

\section{Table of the Specific Gravities of Different Bodies.}$$
\text { I. Metals. }
$$

Antimony, crude

A. glass of

Arsenic, glass of, natural

. . molten

native orpiment

Bismuth, molten

native -

Brass, cast, not hammered

$$
\text { - ditto, wire-drawn }
$$$$
\text { cast, common }
$$

Cobalt, molten

- . blue, glass of

Copper, not hammered

. . the sanie wire-drawn

- 4064

. . ore of soft copper, or natural verdigris

Gold, pure, of 24. carats melted, but not hammered

the same hammered

Parisian standard, 22 car. not hammered
Gold, Parisian standard, 22 car, hammered guinea, of Geo. 2. guinea, of Geo. 3. Spanish gold coin Holland ducats trinket standard, 20 car. not hammered the same hammered

Iron, cast

- bar, either hardened or not

Steel, neither tempered nor hardened

. . hardened, but not tempered

- . tempered and hardened

. . ditto, not hardened

Iron, ore prismatic

- ditto spicular

- ditto lenticular

Lead, molten

. . ore of cubic

. . ditto horned

- . ore of black lead

. . ditto, white lead

. . ditto, ditto, vitreous

.. ditto, red lead

.. ditto, saturnite

Manganese, striated

Molybdena

Mercury, solid or congealed

. . . fluent

. . natural calx of

- . . precipitate, per se

- . . precipitate, red

- . brown cinnabar

Nickel, molteir

- ore of, called Kupfernickel of Saxe

Platina, crude, in grains

- . purified, not hammered

. . ditto hammered

. . ditto wire-drawn

- . ditto rolled

Silver, virgin, 12 deniers, fine, not hamnered

- . ditto hammered

- Paris standard

. shilling of Geo. 2.

. shilling of Geo. 3.

. French coin

4.946

6702

3594

5763

5452

9823

9020

4371

8396

8544

7824

7812

24.41

7788

8878

3572

19258

19362

17486
Tin, pnre Cornish, melted, and not hardened

. the same hardened

- of Malacca,'not hardened

- the same hardened

- ore of, red

- ore of, black

- ore of, white

. common

Tungsten

Uranium

Wolfram

Zinc, molten

Beryl, or aqua-marine oriental

\section{Precious Stones.}

Chrysolite, of the jeweller

... of Brazil

Crystal, pure rock of Madagascar

. . . of Brazil

. . Enropean

-

17589

17150

17629

17655

19352

15709

15775

7207

7788

783.3

7840

7818

7816

7355

5218

5012

11352

7587

6072

6745

4059

6558

6027

5925

4756

4738

15632

$1356 \mathrm{~s}$

9230

10871

8390

10218

6902

7807

. 6648

15602

19500

20337

21042

22069

10474

10511

10175

10000

10534

10408

7291

7299

7296

7307

6935

6901

6008

7920

6066

$6+40$

7119

7101 


\section{G R A}

Crystal, ros-ecoloured

. . . yellow

. . . violet, or amethyst

... white amethyst. .

. . Carthaginian

Diam. black

. . rose-coloured oriental

- . . orange ditto

. . . green ditto

. . . blue ditto

. . Brazilian

. . yellow

Emerald, of Peru

Garnet, of Bohemia

- . . of Syria

- . dodecaedral

- volcanic 24 faces

Girasol

Hyacinth, common

Jargon of Ceylon

Quartz, crystallized

- . . in the mass

. . brown crystallized

. . fragile

- . milky

:. fat or greasy

Ruby, oriental

-. Spinell

$\therefore$ Ballas

. Brazillian

Sapphire, oriental

. . ditto white

- : . of Puys

Spar, white sparkling

... red ditto

... green ditto

... blue sparkling

... green and white ditto

... transparent ditto

... adamantine

Topaz, oriental

- pistachio ditto

- Brazilian

- . of Saxe

- white ditto

Vermilion

III. Silicious Stones.

Agate, oriental

. . onyx

-. cloudy

- speckled

- . veined

. . stained

Chalcedony, common

... transparent

- . . veined

. . reddish

. . . blueish

Carnelian, pale onyx

. . . speckled

. . . veined

. . . onyx

- . . stalactite simple
[ $\begin{array}{ll}596 & \text { ] }\end{array}$

G R A

2670 Flint, white

2654 . . black

2654 . . veined

2651

2657

2654

3521

3531

3550

3524

3525

3444

3519

2775

4189

4000 .

4063

2468

4000

3687

4416

2655

2647

2647

2640

2652

2646

4283

3760

3646

3531

3994

3991

4077

3131

2595

2438

2704

2693

3105

2564

3873

4011

4061

3536

3564

3554

4230

2590

2638

2625

2607

2667

2632

2616

2664

2606

2605

2587

... Egyptian

2532

2612

2565

2606.

... spotted

.. onyx

... of Rennes

2587

2054

2609

224.3

. variegated of Limosin

Jade, white

... green

... olive

... from the East Indies

... of Swisserland

Jasper, clear green

- . brownish green

. . red

- . brown

. . yellow

. violet

. . cloudy

. . veined

- . blackish

. blood-coloured

- . lielostrope

. . onyx

- . flowered, red and white

- . red and yellow

- . green and yellow

- . red, green, and grey

- . red, green, and yellow

. . universal

2950

2966

2983

2977

3389

2350

2681

2661

2691

2710

2711

2735

2696

2672

2628

2633

2816

2623

2750

2684

2732

2749

2563

2661

Opal

Pearl, virgin oriental

Pebble ony $x$

-. of Renne

. . English

. . veined

. . stained

Prasium

Sardonyx, pure

- . pale

. . . . speckled

. . . veined

onyx

Schorl, black prismatic, hexaedral

2114

2684

2664

2654

2609

2612

2587

2581

2603

2606

2622

2595

2595

2628

3364

3226

305.4

2923

3156

3256

$\$ 453$

3296

3092

2416

2111

2143

2484

2615

2612

2623

2623

2598

2613

Stone, paving

IV. Various Stones, Earths, \&c.

Alabaster, oriental white 
Alabaster, of Piedmont - . of Malta

- . Spanish saline

. . . of Valencia

- . . of Malaga

- . . of Dalias

Amber, yellow, transparent

- . yellow, opaque

- . red

- - green

Anubergris

Amianthus, long

Asbestos, ripe

Basaltes, from Giant's Causeway

Bitumen, of Judea

Brick

Chalk, Spanish

- coarse Briançon

- British

Gypsum, opaque

. . semi-transparent

. . fine ditto

- . Jhomboidal

. . . ditto 10 faces cuneiform crystallized

Glass, green

. white

- . bottle

. . Leith crystal

. fluid

-. of Bohemia

: of St. Cloud

Granite, red Egyptian

- . grey Egyptian

- beautiful red

- red of Dauphiny

- of Girarduor

- violet of Gy romagny

- green

- radiated

- red of Semur

- . grey of Bretagne

- yellowish

- of Carinthia, blue

Hone, white razor

Lapis, nephriticus

- lazuli,

- hæmatites

- calaminaris

- Judaicus

- Manati

Limestone

- .. white fluor

- green

Marble, green Campanian

- . red

- white Cassara

- . white Parian

Pyrenean

black Biscayan,

Brocatelle

Castilian
2693 Marble, Valencian

2699 . . . white Grenadan
2713 .

2713

2638

2876

2611

1078

1086

1083

1083

926

909

2313

2578

3073

2864

1104

2000

2790

2727

2784

2168

2306

2274

2311

2312

2306

2642

2892

2733

3189

3329

2395

2560

3255

2654

2728

2761

2643

2716

2685

2684

2668

2638

2738

2614

2956

2876

2894

3054

4360

5000

2500

2270

3179

3156

3182

2742

2724

2717

2538

2726

2695

2650

2700

2705

2678

2755

2708

2858

2728

2718

2668

27 ] 4

2649

2516

Obsidian yellow of Florence

Peat, hard

Phosphorus -

Porcelain, Seves

-. . Limoges

Porphyry, red

- . - green

- . . red, from Dauphiny

- . . red, from Cordova

P. - green, from ditto

Pyrites, coppery

. . . . cubical

- . ferruginous cubic

- . ditto round - ditto of St. Domingo -

Serpentine, opake, green Italian

. . . ditto, veined black and olive

. . . ditto, red and black

. . . ditto, fibrous

. . opake penetrated with water

Slate, common

2348

1329

17.14

$2146^{\circ}$

2341

2385

2765

2676

2793

2754

2728

4954

4702

3900

4101

3440

2430

2594

2627

2586

3000

2669

2473

2672

2854

2186

2766

20091

2324.

2478

. . opake

915

2722

2415

2945

2771

2520

2510

2049

2496

2470

1981

2460

2122

2357

2274

2378

2034

2201

2033

199]

2792

2089

2246 
Talc, yellow blacḱ - white

V. Liquors, Oils, \&c.

Acid, sulphuric

\begin{tabular}{|c|c|c|}
\hline \multicolumn{3}{|c|}{ ditto, highly concentrated } \\
\hline muriatic & - & - \\
\hline red acetous & - & - \\
\hline white acetous & - & - \\
\hline distilled ditto & - & - \\
\hline fluoric & - & - \\
\hline acetic & - & - \\
\hline phosphoric & - & - \\
\hline formic & - & \\
\hline citric & - & - \\
\hline senic & - & - \\
\hline $\begin{array}{l}\text { foranges } \\
\text { f gooseberries }\end{array}$ & - & - \\
\hline of grapes & - & - \\
\hline
\end{tabular}

Alcohol, commercial

- highly rectified

Alcohol, mixed with water.

$$
\begin{aligned}
& 15-16 \text { ths alcohol } \\
& 14-16 \text { ths ditto } \\
& 13-16 \text { ths ditto } \\
& 12-16 \text { ths ditto } \\
& 11-16 \text { ths ditto } \\
& 10-16 \text { ths ditto } \\
& 9-16 \text { ths ditto } \\
& 8-16 \text { ths ditto } \\
& 7-16 \text { ths ditto } \\
& 6-16 \text { ths ditto } \\
& 5-16 \text { ths ditto } \\
& 4-16 \text { ths ditto } \\
& 3-16 \text { ths ditto } \\
& 2-16 \text { ths ditto } \\
& 1-16 \text { ths ditto }
\end{aligned}
$$

Ammoniac, liquid

Beer, pale

Cider

Ether, sulphuric

- . nitric

- acetic

Milk, woman's

- cow's

- ass's

- ewe's

- goat's

- mare's

cow's clarified

Oil of filberts

... of walnuts

.. : of hempseed

... of poppies

.. of rape-seed

... of lint-seed

... of poppy-seed

... of whale

... of ben, (a tree in Arabia)

... of beechmast
2655 Oil of cod-fish - _ _ _ $\quad$ - 923

2900 ... of olives

915

2704 . . of almonds, sweet _ _ _ _ 917

... volatile of mint, common

... . of sage,

898

1841

... . of thyme

902

2125

1271

... of rosemary

... . of calamint

1580

1194

1025

1014

1010

1500

1063 .

1558

... of cochlearia

.... . of wormwood

... of tansy

.... of Stragan

... . of Roman camomile

.... of sabine

... . of fennel

... . of fennel-seed

... . of coriander-seed

... . of carraway-seed

... . of dill-seed

... . of anise-seed

... of juniper-seed

... . of cloves

... of cinnamon

902

906

912

943

907

933

99,5

894

929

929

1008

866

905

913

987

858

1036

1044

870

. . . of amber - - - - - 887

... . of the flowers of orange - $\quad$ - 880

... of lavender

894

889

Spirit of wine. "See Alcohol.

Turpentine, liquid

979

985

992

897

1023

1034

1018

739

909

730

866

1020

1032

1036

104.

1035

1034

1019

916

923

926

924

919

940

929

923

912

918
Urine, human

Water, rain -

... distilled

- . sea (average)

- . of Dead-sea

Wine, of Torrins, red -

- white

- Champagne, white

- Paharet

- Xeret

- Malmsey of Madeira

- J.h gundy

- Jurancon

- Bourdeaux

Malaga

- Constance

- Tokay

- Canara

- Port

991

1011

1000

1000

1026

1240

998

785

$99 \mathrm{~s}$

1000

992

1038

992

993

994

1022

1082

$105 \%$

1033

997

VI. Resins, Gums, and Animal Substances, $\& c$.

Aloes, socotrine,

1380

1359

As hatic

$132 \mathrm{~s}$

Bees-wax, yellow

965

969

Bone of an ox

1656

Butter

Calculus humanus

942

1700

$12+0$

14.34

989

Camplior

Copal, opake

1140

1060

1063 
Crassamentum, human blood

Dragon's blood

Elemi

Fat, bee

- . hog's -

- mutzon

. veal

Galbanum

Gamboge

Gum, anmoniac

- Arabic

- Euphorbia

- seraphic

- tragacanth

- bdellium

- scammony of Smyrna

: . ditto of Aleppo

Gunpowder, shıken

... in a loose heap

Honey

Indigo

Irory

Juice of liquorice

. . of acacia

Labdanum

Lard

Mastic

Myrrh

Opium

Scammony. See Gum.

Serum of human blood

Spermaceti

Storax

Tallow

Terra Japonica

Tragacanth. See Gum.

Wax. See Bees-wax. shoemaker's

VII. IVoods.

Alder

Apple-tree

Ash, the trunk of

Bay-iree

Beech

Box, French -

- Dutch -

Campechy wood

Cedar, wilt

- Palestine

- . Indian

- American

Citron

Cocoa-wood -

Cherry-tree

Cork

Cypress, Spanish

Ebony, Anuerican

. Indian

Elder-tree

Elm, trunk of

Filbert-tree

Fir, male

- female

II azel
1126 Jasmin, Spanish

1205 Juniper-tree

1018 Lemon-tree

923 Lignum vitæ

937 Linden-tree

\$24 Logwood. See Campechy.

934

1212

1222

1207

1452

1124

1201

1316

1372

1274

1235

937

836

1745

11.50

769

1826

1723

1515

1186

948

1074

1360

1336

1030

943

1110

942

1398

897

Mastick-tree

Mahogany

Maple

Medlar

Mulberry, Spanish -

Oak, heart of, 60 years old

Olive-tree

Orange-tree -

Pear-tree

Pomegranate-tree

Poplar

Plum-iree - white, Spanish

Quince-tree

Sassafras

Vine

Walnut

Willow

Yew, Dutch

- Spanish

For the Weight and

Gases, see the article GAs.

These numbers being the weight of a cubic foot, or $172 \mathrm{~S}$ cubic inches, of each of the bodies, in avoirdupois ounces, by proportion the quantity in any other weight, or the weight of any other quantity, inay be readily known.

For Example. Required the content of an irregular block of common stone which weighs $1 \mathrm{cwt}$, or $112 \mathrm{lb}$, or 1792 ounces. Here, as $2500: 1792:: 1728$ : $1228 \frac{4}{5}$ cubic inches the content:

Example 2. To find the weight of a bluck of granite, whose length is 63 feet, and breadth and thickness each 12 feet; being the dimensions of one of the stones, of granite, in the wails of Balbeck. Here, $63 \times 12 \times 12=9072$ feet is the content of the stone; therefore as $1: 907 \approx$ : : $3000 \mathrm{oz}_{*}: 27216000 \mathrm{oz}$. or 759 tons $7 \mathrm{cwt} .2$ qris. the weight of the stone.

XI. A body descends in a fuid specifically lighter, $m$ ascends in a fluid specifically heavier, with a force equal to the difference between its weight and that of an equal bulk of the fluid.

XII. $A$ body sinks in a fluid specifically heavier, so far as that the weight of the body is equal to the weight of a quantity of the fluid of the same bulk as the part immersed. Hence, as the specific gravity of the fluid is to that of the body, so is the whole inagnitude of the body, to the magnitude of the part immersed.

XIII. The specific gravities of equal solids, are as their parts immersed in the same fluid.

The several theorems here delivered, are both demonstrable from the principles of mechanics, and are also equally conformable to experiment, which answers exactly. to the calculation; as is abundantly evident from the courses of philosophical experiments, so frequently exhibited; where the laws of specific gravitation are well illustrated.

600

550

498

GREAT BEAR, one of the constellations in the north ern hemisphere. See URsa MAJOR.

GrEat Cincles, of the Globe or Sphere, are those 600

.

.


equal parts or hemispheres, and therefore having the same centre and diameter with the sphere itself. The principal of these are, the equator, the ecliptic, the horizon, the meridians, and the two colures.

Great-Circle Sailing, is the art of conducting a ship along the arc of a great circle. And it is also that part of the theory of navigation which treats of sailing in the arc of a great circle. See NaTrgatron.

GREAVES (Jou N), an eminent astronomer, antiçuary, and linguist, was born in 1602, being the eldest son of John Greaves, rector of Colemore, near Alresford in Hampshire, and master of a gramnar-school, where his son of course was well grounded in the primary rules of literature. He then went to Baliol-college, Oxford, in 1617 ; but afterwards, on account of his skill in philosophy and polite literature, he was the first of five that were elected into Merton-college. Having read over all the ancient Greek and Latin writers, he applied to the study of natural philosophy and mathematics; and having contracted an intimacy with Mr. Briggs, Savilian professor of geometry at Oxford, and Dr. Bainbridge, Savilian professor of astronomy there, he was animated by their examples to prosecute that study with the greatest industry. Not being content, however, with reading the writings of Purbach, Regiomontanus, Copernicus, Tycho Brahé, Kepler, and other celebrated astronomers of that and the preceding age, he inade the ancient Greek, Arabian, and Persian authors familiar to him, having before gained an accurate skill in the oriental languages. These accomplishments procured him the professorship of geometry in Gresham-college London, in 1630 ; and at the same time he held his fellowship of Merton-college.

In a journey to the continent, in 1635 , he visited the celebrated Golius, professor of Arabic at Leyden, and Claud Hardy at Paris, to converse about the Persian language. Hence he passed through Italy, and accurately surveyed the venerable remains of antiquity at Rome, visiting and corresponding everywhere with the most learned men of evcry nation. After visiting Padua, Florence, and Leghorn, be hence embarked for Constantinople, where he arrived in 1638. Thence he passed over to Hhodes, and Alexandria in Egypt, where he staid four or five months, and made a great number of curious observations. He next went to Grand Cairo, and measured the pyramids; and while there he adjusted the measure of the foot, observed by all nations. Hence he rcturned again through Italy, and arrived in England in the year 1640 , having stored his mind with a varicty of curious knowledge, and collected many valuable oriental manuscripts and ancient curiosities. While at Rone he made a particular inquiry into the true state of the ancient weights and measures.

On the death of Dr. John Bainbridge, in 161.3, he was chosen Savilian professor of astronomy at Oxford, and principal reader of Linacre's lecture in iferton-college; an appointment for which he was eminently qualified, from his critical acquaintance witl the works of the ancient and modem astronomers. In 164.5 he proposed a method of reforming the callendar, by omitting the intercalary day for 40 years to come: the paper relating to which, was published by Ir. 'Thomas Smith, in the I'hilos. Trans. for 1699. In 1616, le published his Pyramidographin, or a Description of the Pyramids of Egypt; and, in 1647 , his Discourse on the Roman Foot and Denarius; from which, as from two principles, the measures and weights used by the ancients may be deduced. He also publis he d severa other curious works concerning antiquities, \&c.

Soon after publishing the last-mentioned book, he was ejected, by the parliarient visitors, from the professorship of astronomy and fellowship of Merton-college; $v$ nen the soldiers committed many outrages, breaking open his chests, and destroying many of bis manuscripts; which greatly affected bim. On this occasion be retired to London, where he afterwards married, and prosecuted his studies with great vigour, as appears from several of his philosophical and theological writings. 'This however proved but a transient happiness to him; for he died at London, the 8 th of October 1652 , before he was quite 50 years of age; and left his astronomical instruments to the Savilian library in Oxford, where they are depositcd.

GREEK ORD ERs, in Architecture, are the Doric, Ionic, and Corinthian; in contradistinction to the two Latin orders, viz the Tuscan and Composite.

GREEN, one of the original colours of the rays of light, or of the prismatic colours exhibited by the refraction of the rays of light. This is the pleasantest of all the colours to the sight. And hence it has been inferred as a proof of the wisdom and goodiness of the Deity, that almost all vegetables, clothing the surface of the earth, are green; which they are when growing in the open air; though those in subterraweous places, or places inaccessible to fresh air, are white or yellow. See CHROMATICs, and CoLOURS.

GREGORIAN CALENDAR, so called from Pope Gregory the 13th, is the new or reformed calendar, showing the new and full moons, with the time of Easter, and the other moveable feasts depending on it, by means of epacts disposed through the several months of the Gregorian year.

GREGorian Epoch, is the epoch or time, from which the Gregorian calendar, or computation, took place. This began in the year 1582; so that the year 1800 is the 218 th of this epoch.

Gregorian Telescope, a particular kind of telescope, invented by Mr. James Gregory: See Telescope.

Gregorian Year, the new account, or new style, introduced on the reformation of the calendar, by PopeGregory the 13 th, in the ycar 1582 , and from whom it took its name. This was introduced to reform the old, or Julian year, established by Julius Casar, whicl consisted of 365 days 6 hours, or 365 days and a quarter, that is three years of 365 days each, and the fourth year of 366 days. But as the mean tropical year consists only of $365 \mathrm{ds}$. $5 \mathrm{hrs}$. $4 \mathrm{sm}$. $57 \mathrm{sec}$. the former lost $11 \mathrm{~min}$. $3 \mathrm{sec}$. every year, which in the time of Pope Gregory had amounted to 10 days, and who, by adding these 10 days, brought the account of time to its proper day again, and at the same time appointed that every century alter, a elay more should be added, thereby making the years of the complete centurics, viz, the $17 \mathrm{th}, 18 \mathrm{th}, 19 \mathrm{th}, 21 \mathrm{st}$, which are not exactly divisible by $4, \& \mathrm{c}$, to be common years of 365 days each, instead of leap-y ars of 360 days, which makes the mean Gregorian year equal to $36.5 \mathrm{ds}$. $5 \mathrm{hrs} .45 \mathrm{~m}$. $36 \mathrm{sec}$.

This computation was not introduced into the account of time in lingland, till the year 1752 , when the Julian account had lost 11 days, and therefore the 3d of September was in that year, by act of parliament, accounted the 14 th, thereby restoring the 11 days which bad thus been omitted. See Y liak.

GREGORY (J.I MEs), one of the most cclebrated mil- 
thematicians of the 17 th cenfury, was the son of the Rev. Jolin Gregory, minister of Drumoak in the county of A berdeen, and born at Aberdeen in November 1638 or -9 . His mother was a daughter of Mr. David Anderson of Finzaugh, or Funshaugh, a gentleman who possessed a singular turn for mathematical and mechanical knowledge. This mathematical genius was hereditary in the family of the Andersuns, and from them it appears to have been transmitted to their descendants of the names of Gregory, Reid, \&c. Alexander Anderson, consin-german of the said David, was professor of mathematics at Paris in the beginning of the 17 th century, and published there several valuable and ingenious works; as may be seen in the memoirs of his life and writings, under the article ANDERsow. The mother of James Gregory inherited the genius of her family; and observing in her son, while yet a child, a strong propensity to mathematics, she instructed him herself in the elements of that science. His education in the languages he received at the grammar-school of Aberdeen, and went through the usual course of academical studies in the Marischal college; but he was chiefly delighted with philosophical researches, into which a new door had lately been opened by the key of the mathematics. Galileo, Kepler, Descartes, \&c, were the great masters of this new method: their works therefore became the principal study of young Gregory, who soon began to make improvements on their discoveries in Optics. 'The first of these improvements was the incention of the reflecting telescope; the construction of which instrument he published in his Optica Promota, in 1663, at 24 years of age. This discovery soon attracted the attention of the mathematicians, both of our own and of foreign countries, who immediately perceived its great importance to the sciences of optics and astronomy. But the manner of placing the two specula on the same axis appearing to Newton to be attended with the disadvantage of losing the central rays of the larger speculum, he proposed an improvement on the instrument, by giving an oblique position to the smaller speculum, and placing the cye-glass in the side of the tube. It is observable, however, that the Newtonian construction of that instrument was long abandoned for the original or Gregorian, which is now always used when the instrument is of a moderate size; though Herschel has preferred the Newtonian form for the construction of those immense telescopes, which he has of late so successfully employed in observing the heavens.

About the year 1664 or 1665 , coming to London, he became acquainted with Mr. John Collins, who recommended him to the best optic glass-grinders there, to have his telescope executed. But as this could not be done for want of skill in the artists to grind a plate of metal for the object-speculum into a true parabolic concave, which the design required, he was much discouraged with the disappointment; and after a few imperfect trials made with an ill-polished spherical one, which did not succeed to bis wish, he gave up the pursuit, and resolved to make the tour of Italy, then the mart of mathematical learning, that he might prosecute his favourite study with greater advantage. And the university of Padua being at that time in high reputation for mathematical studies, Mr. Gregory fixed his residence there for some years. Here it was that he published, in 1667, Vera Circuli et Hyperbotæ Quadratura; in which he propounded another discovery of his own, the invention of an infinitely converging scries for the areas of the circle and hyperbolid. Ije sent to VOL. I.
England a copy of this work to his friend Mr. Collins, who communicated it to the Royal Society, where it met with the commendations of Lord Brounker and Dr. Wallis. He reprinted it at Venice the year fullowing, to which he added a new work, entitled Geometrix Pars Universalis, inserviens Quantitatum Curvarum Transmutationi et Mensura; in which he is allowed to have shown, for the first time, a method for the transnutation of curves. These works engaged the notice, and procured the author the correspondence, of the greatest mathematicians of the age, Newton, Huygens, Wallis, and others. An account of this piece was also read by Mr. Collins before the Royal Society, of which Mr. Gregory, being returned from his travels, was chosen a member the same year, and communicated to them an account of a controversy in Italy about the motion of the earth, which was denied by Riccioli and his followers.-Through this channel, in particular, he carried on a dispute with Mr. lluygens on the occasion of his treatise on the quadrature of the circle and hyperbola, to which that great man had started some objections; in the course of which our author produced some improvements of his series. But in this controversy it happened, as it generally does on such occasions, that the antagonists, though setting out with proper coolness, yet grew ton warm in the combat. This was the case here, especially on the side of Gregory, whuse defence was, at his own request, inserted in the Philosophical 'Transactions. It is unnecessary to enter into particulars: suffice it therefore to say, that, in the opinion of I.eilnitz, who allows Mr. Gregory the highest merit for his genius and discoveries, M. Huygens has pointed out, though not errors, some considerable deficiencies in the treatise above mentioned, and shown a much simpler method of attaining the same end.

In 1668, our author published at London another work, entitled, Exercitationes Geometrice, which contributed still much farther to extend his reputation. About this time he was clected professor of mathematics in the university of St. Andrews, an office which he held for six years. During his residence there, he married, in 1669 , Mary, the daughter of George Jameson, the celebrated painter, whom Mr. Walpole has termed the Vandyke of Scotland, and who was fellow-disciple with that great artist in the school of Rubens at Antwerp.

In 1672 , he published The Great and New Art of weighing Vanity: or a Discovery of the Ignorance and Arrogance of the Great and New Artist, in his Pseudaphilosophical Writings. By M. Patrick Mathers, archbedal to the University of St. Andrews. To which are annexed some 'I'entamina de Motu Penduli et Projectorum." Under this fictitious name, our author wrote this little picce to expose the pretended ignorance of $\mathrm{Mr}$. Sinclar, professor at Glasgow, in his hydrostatical writings, and in return for some alleged ill usage of that author to a colleague of Mr. Gregory's. The same year, Newton, on his wonderful discoveries in the nature of light, contrived a new reflecting telescope, and made several objections to Mr. Gregory's. This gave birth to a dispute between those two philosophers, which was carried on during this' and the following year, in the most anicable manner on both sides; Mr. Gregory defending his own construction, so far, as to give his antagonist the whole honour of having made the catoptric telescopes preferatile to the dioptric; and showing, that the imperfections in these instruments were not so much owing to a defoct in 4. $\mathrm{H}$ 
the object-speculum, as to the different refrangibility of the rays of light. In the course of this dispute, our author described a burning concave mirror, which was approved by Newton, and is still in good esteem. Several letters that passed in this dispute, are printed by Dr. Desaguliers, in an Appendix to the English edition of Dr. David Gregory's Elements of Catoptrics and Dioptrics.

Ii $167+, \mathrm{Mr}$. Gregory was called to Edinburøh, to fill the chair of mathematics in that university. This place he had held but little more than a year, whell, in October 1675 , being employed in showing the satellites of Jupiter through a tele'seope to some of his pupils, he was suddenly struck with total blindness, and died a few days after, to the great loss of the mathematical world, at only 36 years of age.

As to his character, Mr. James Gregory was a man of a very acute and penetrating genius. His temper was in some degree an irritable one, as appears from his conduct in the dispute with.Huygens; and, conscious perhaps of his own merits as a discoverer, he seems to have been jealous of losing any portion of his reputation by the improvenients of others upon his inventions. He possessed one of the inost amiable characters of a true philosopher, that of being content with his fortune in his situation. But the most brilliant part of his character is that of his mathenatical genius as an inventor, which was of the first order; as will appear by the following list of his inventions and discoveries. Among many others may be reckoned, his Reflecting Telescope;-Burning Concave Mirror;-Quadrature of the Circle and Hyperbola, by an infinite converging series; - his method for the Transformation of Curves;-a Geometrical Demonstration of lord Brounker's series for Squaring the Hyperbola;-his Demonstration that the Meridian Line is analogous to a scale of Logarithmic Tangents of the Half Complements of the Latitude; - he also invented and demonstrated geometrically, by help of the hyperbola, a very simple converging series for making the logarithms; - he sent to Mr. Collins the solution of the famous Keplerian problem by an infinite series; - he discovered a method of drawing Tangents to Curves geometrically, without any previous calculations; - a rule for the Direct and Inverse method of Tangents, which stands upon the same principle (of exhaustions) with that of fluxions, and differs not much from it in the manner of application; a Series for the length of the Arc of a Circle from the Tangent, and vice versa; as also for the Secant and Logarithricic Tangent and Secant, and vice versa:-these, with others, for measuring the length of the elliptic and hyperbolic eurves, were sent to Mr. Collins, in return for some received from him of Newton's, in which he followed the elegant example of this author, in delivering his series in simple terms, independent of each other. These and other writings of our author are mostly contained in the following works, viz,

1. Optica Promota; 4to, London 1663.

2. Vera Circuli et Hyperbolx Quadratura ; 4to, Padua 1667 and 1668 .

3. Geometrix Pars Universalis; 4to, Parlua 1668.

4. Fixcrcitationes Geomctricx; 4ito, London 1668.

5. The Great and New $\Lambda$ rt of weighing Vanity, \&c. 8 vo, Glasgow 1672.

The rest of his inventions make the subject of several letters and papers, printed cither in the Philos. Trans. vol, 3 ; the Commerc. Epistol. Joh. Collins et $\Lambda$ liorum, $8 v 0,1715$; in the $\Lambda$ ppendix to the English edition of 1)r. David Gregory's lilements of Optics, 8vo, 1735, by Dr.
Desaguliers; and some series in the Exercitatio Gcometrica of the same author, 4to, 1684, Edinburgh; as well as in his little piece on practical geometry.- In different parts of the foregoing works, of James Gregory, are found several ingenious thoughts and mventions, that have sometimes been attributed to later periods and authors. Thus, at the end of the Optica Promota, is a collection of curious astronomical problems, conceived and resolved in a very masterly manner; in a scholium at the end of one of which (pa. 130), is remarked the use to be made of the transits of Venus and Mercury, in determining the sun's parallax; an idea which, I think, has usually been ascribed to Dr. Halley.

GREGORY (DR. DAVID), Savilian professor of astronomy at Oxford, was nephew of the a bove-mentioned $\mathrm{Mr}$. James Gregory, being the eldest son of his brother Mr. David Gregory of Kinardie, a gentleman who had the singular fortune to see three of his suns professors of mathematics, at the same time, in three of the British universities, viz, our author David at Oxford, the second son James at Edinburgh, and the third son Charles at St. Andrews. Our author David, the eldest son, was born at A berdeen in 1661 , where he received the early parts of his education, but completed his studies at Edinburgh; and, being possessed of the mathematical papers of his uncle, soon distinguished limself likewise as the heir of his genius. In the $23 d$ year of his age, be was elected professor of mathematics in the university of Edinburgh; and, in the same year, be published Exercitatio Geometrica de Dimensione Figurarum, sive Specimen Methodi generalis Dimetiendi quasvis Figuras, Edinb. 1684, 4to. He very soon perceived the excellence of the Newtonian philosophy; and bad the inerit of being the first who introduced it into the scluouls, by his public lectures at Edinburgh. "He had (says Mr. Whiston, in the Memoirs of his own Life, i. 32) alrearly caused several of bis scholars to keep acts, as we call then, on several branches of the Newtonian philosophy: while we at Cambridge, poor wretches, were ignominiously studying the fictitious hypothesis of the Cartesian."

On the report of Dr. Bernard's intention of resigning the Savilian professorship of astronomy at Oxford, our author went to London in 1691 ; and being patronised by Newton, and warmly befriended by Mr. Flamsteed the astronomer-royal, he obtained the vacant professorship, for which Dr. Halley was also a competitor. 'This rivalship, however, instead of animosity, laid the foundation of friendship between these eminent men; and Halley soon after became the colleague of Gregory, by obtaining the professorship of geometry in the same university. Our anthor had not long remained in London after his arrival, before he was elected a fellow of the Royal Society; and, previously to his election into the Savilian professorship, had the degree of doctor of physic conferred on him by the university of Oxford.

In 1693, he published in the Philos. Trans. a resolution of the Florentine problem De: Testudine veliformi quadrabili; and he continued to communicate to the public, from time to time, many ingenious mathematical papers through the same chamel.

In $\mathrm{I} 695$, he printed at Oxford, Catoptrica et Dioptrica Spharica Elementa; a wonk which, we are informed in the preface, contains the substance of some of his public lectures. read at Edinburgh, cleven years before. This valuable treatise was" republisled in English, first with addi- 
tions by Dr. William Brown, with the recommendation of Mr. Junes and Dr. Desaguliers; and afterwards by the latter of thrse gentlemen, with an appendix containing an account of the Gregorian and Newtonian telescopes, together with Mr. Hadley's tables for the construction of both those instruments. 1t may not be unwortly of remark, that, in the conclusion of this treatise, there is an observation which shows, that the construction of achromatic telescopes, which Mr. Dollond has carried to such great perfection, had occurred to the mind of David Gregory, from reticcting on the admirable contrivance of nature in combining the different bumours of the eye. The passage is as follows: "Perhaps it would be of service to make the object-lens of a different medium, as we see done in the fabric of the eye; where the crystalline humour (whose power of refracting the ray's of light differs very little from that of glass) is by nature, who never does any thing in vain, joined with the aqueous and vitreous humours (not differing from water as to their power of refraction), in order that the image may be painted as distinct as possible on the botton of the eye."

Iu 1702 our author published at $\mathrm{Oxford}$, in folio, Astronomiæ Physicæ et Geometricæ Elementa; a work which is accounted his master-piece. It is founded on the Newtonian doctrines, and was esteemed by Newton himself as a most excellent explanation and defence of his philosophy. In the following year he gave to the world an edition, in folio, of the works of Euclid, in Greek and Latin; being done in prosecution of a design begun by his predecessor Dr. Bernard, of printing the works of all the ancient mathematicians. In this work, which contains all the treatises that have been attributed to Euclid, Dr. Gregory has been careful to point out such as he found reason, from internal evidence, to believe to be the productions of some inferior geometrician. In prosecution of the same plan, our author engaged soon after, with his colleague Dr. Halley, in the publication of the Conics of Apollonius: but he had proceeded only a little way in this undertaking, when he died at Maidenhead in Berkshire, in 1710 , being the 49 th year of his age only.

Besides those works published in our anthor's life-time, as mentioned above, he had several papers inserted in the Philos. Trans. vol. 18, 19, 21, 24, and 25, particularly a paper on the catenarian curve, first considered by our author. He left also in manuscript, A Short Treatise of the Nature and Arithmetic of Logarithms, which is printed at the end of Keill's translation of Commandine's Euclid; and a Treatise of Practical Geometry, which was afterwards translated, and published in 1745 , by Mr. Maclaurin.

Dr. David Gregory married, in 1695, Elizabeth, the daughter of Mr. Oliphant of Langtown in Scotland. By this lady he had four sons, of whom, the eldest, David, was appointed regius professor of modern history at Oxford by King George the 1st, and died at an advanced age in $176 \%$, after enjoying for many years the dignity of dean of Christchurch in that university.

When David Gregory quitted Edinburgh, he was succeeded in the professorship at that university, by his brother James, likewise an eminent mathematician; who held that office for 33 years, and, retiring in 1725 , was succeeded by the celebrated Maclaurin. A daughter of this professor James Gregory, 'a young lady of great beauty and accomplishments, was the victim of an unfortunate attachment, that furnished the subject of Mallet's wellknown baltid of William and Margaret.
Another brother, Charles, was created professor of ma. thematics at St. Andrews by Queen Anne, in 1707. This office he held with reputation and ability for 32 years; and, resigning in 1739 , was succeeded by his son, who eminently inherited the talents of his family, and died in 1763 . Sone further Particulars of the Family of the Gregorys and Andersons, conmunicated by Dr. Thomas Reid, Professor of Moral Philosophy in the University of Glasgow, a Nephew of the late Di. David Gregory Savillian Professor at Oxford.

Some account of the family of the Gregorys at $A$ berdeen, is given in the Life of the late Dr. John Gregory prefixed to his works, printed at Edinburgh 1788, in four small 8vo volumes.

Who was the author of that Life, or whence he obtainef his information, 1 do not know. 1 have heard it ascribed to Arr. Tytler the younger, whose father was appointe'd one of the guardians of Dr. John Gregory's children. Some additions to what is contained in it, and a few remarks upon it, is all I can furnish on this subject.

Page 3. I know nothing of the education of David Anderson of Finzaugh. He scems to have been a self-taught engineer. Every public work which surpassed the skill of common artists, was committed to the management of D:vid. Such a reputation he acquired by lis success in works of this kind, that with the vulgar he got the byname of Davie do a' thing, that is, in the Scottish dialect, David who could do every thing. By this appellation he is better known than by his proper name. He raised the great bells into the steeple of the principal church : he cut a passage for ships of burden through a ridge of rock under water, which crossed the entrance in to the harbour of Aberdcen. In a long picture gallery at Cullen House, the seat of the earl of Findlater, the wooden ceiling is painted with several of the fables of Ovid's Metamorphoses. 'The colours are still bright, and the representation lively. This present earl's grandfather told ne that this painting was the work of David Anderson my ancestor, whom be acknowledged as a friend and relation of his family.

Such works, while they gave reputation to David, suited ill with his proper business, which was that of a merchant in Aberdeen. In that he succeeded ill; and having give's up mercantile business, from a small remainder of his fortune began a trade of making inalt; and after instructing his wife in the management of the same, loft it to her care, and went into kngland to try his fortune as an engincer; an employment which in his own country he had practised gratuitously. Ilaving in that way realised a fortune which satisfied him, he returned to Aberdeen, where his wife had also marle noney by her malting business.

After making such provision for their children as they thought reasonable, they agreed that the longest liver of the two should enjoy the remainder, and at death should bequeath it to certain purposes in the management of the magistrates of Aberdeen.

The wife happened to be the survivor, and fulfilled what had been concerted with her hisband. Her legacies, well known in Aberdeen, are called after her name Jane Guild's Mortifications, a mortification in Scuts law signifying a bequeathment for some charitable purpose. They consist of sums for different purposes: for orphans, for the ciucation of boys and girls, for unmarried gentlewomen, and for widows; and they still continue to be useful to many in indigent circumstances. She was the daughter of Dr. Guild, a minister of $\Lambda$ berdeen. Besides her moncy, she 1112 
bequeathed a piece of tapestry, wrought by her own hand, and representing the bistory of queen Esther, from a drawing made by her husband. The tapestry continues to ornament the wall of the principal church.

In the same page it is said that Alexander Anderson, professor of mathematics at Paris, was the cousin-german of David above-mentioned. I know not the writer's auItsority for this: I have always understood that they were trothers; but for this I have only family tradition.

Page 4. It is here related that $J$ ames Gregory was instructed in the Elements of Euclid by his mother, the daughter of David Anderson. The account I have heard differs from this. It is, that bis brother David, being ten or eleven years older, had the direction of his education after their father's death, and, when James had finished his course of philosophy, was at a loss to what literary profession he sheruld direct him. After some unsuccessful trials, he put Euclid's Elements into his hand, and finding that he applied to Euclid with great avidity and success, he encouraged and assisted him in his mathematical studies. This tradition agrees with what James Gregory says in the preface to his Optica Promota; where after mentioning his advance to the 26 th proposition, he adds, Ubi diu hæsi ounne spe progrediendi orbatus, sed continuis hortatibus et auxiliis fratris mei Davidis Gregorii, in Mathematicis non parum versati (cui si quid in hisce Scientiis præstitero, me illud debere non inficias ibo) animatus, tandem incidi \&c. Whether David had been instructed in mathematics by his ınother, or had any living instructor, I know not.

Page 5,6. In these two pages I think the merit of Gregory compared with that of Newton in the invention of the catoptric telescope, is put in a light more unfavourable to Newton than is just. Gregory, believing that the imperfection of the dioptric telescrope arose solely from the splerical figure of the glasses, invented his telescope to remedy that imperfection. Being less conversant in the practice of mechanics, he did not attempt to make any model. The specula of his telescope required a degree of polish and a figure which the best opticians of that age were unable to execute. Newton demonstrated that the imperfection of the dioptric telescope arose chiefly from the different refrangibility of the rays of light; he demonstrated also that the catoptric telescope required a degree of polish far beyond what was necessary for the dioptric. He made a nodel of his telescope; and finling that the best polish which the opticians could give, was insufficient, he improved the polish with his own hand, so as to make it answer the intended purpose, and has described most accurately the manner in which he did this. And, had he not given this example of the practicability of making a reflecting tclescope, it is probable that it would have passed as an impracticable idea to this day.

Page 11. To what is said of this Janes Gregory might have been udded, that he was led by analogy to the true law of Refraction, nat knowing that it was discovered by Descartes before (sec Preface to Optica Promota); and that in 1670 having received, in a letter from Collins, a series for the area of the zone of a circle, and as Newton had invented an universal method by which he could square all curves geometrical and mechanical by infinite series of that kind; Gregory after nuch thought discovered this universal method, or an equivalent one. Of this he perfictly satisfied Newton and the other mathernaticians of that time, by a letter to Collins in Feb. 1671. He was strongly solicited by his brother David to publish his Uni- versal Method of Series without delay, but he excused himself on a point of honour; that as Newton was the first inventor, and as he had been led to it by an account of Newton's having such a method, he thought himself bound to wait til! Newton should publish his method. I have seen the letters that passed between the brothers on this subject.

With regard to the controversy betweèn Jantes Gregory and Huygens, 1 take the subject of that controversy to have been, not whether J. Gregory's quadrature of the cir cle by a converging series was just, but whether he had demonstrated, as in one of his propositions he pretended to do, That it is impossible to express perfectly the Area of a Circle in any known algebraical form, besides that of an infinite converging series. Huygens excepted to the demonstration of this proposition, and Gregory defended it; neither of them convinced his antagonist, nor do I know that Leibnitz inproved on what Gregory had done.

Page 12. D. Gregory of hinardie deserved a more particular account than is here given. It is true that he served an apprenticeship to a mercantile house in Holland, but he followed that profession no longer than he was under authority, having a stronger passion for knowledge than for money. He returned to his own country in 1655 , being about 28 years of age, and from that time led the life of a philosopher. Having succeeded to the estate of Kinardie by the death of au elder brother, he lived there to the end of that century. There all his children were born, of whom be harl thirty-two by two wives. Kinardie is above 40 English miles north from Aberdeen, and a few miles from Bamf, upon the river Diveron. He was a jest among the neighbouring gentlemen for his ignorance of what was doing about his own farm, but an oracle in matters of learning and philosophy, and particularly in medicine, which he had studied for his amusement, and begun to practise among his poor neighlours. He acquired such a reputation in that science, that he was afterwards employed by the nobility and gentlemen of that county, but took no fees. His hours of study were singular. Bcing much occupied through the day with those who applied to him as a physician, he went early to bed, rose about two or three in the morning, and, after studying some hours, went to bed again and slept an hour of tivo before breakfast.

He was the first man in that country who had a barometer; and hy some old letters which I have seen, it appeared, that he had corrcsponded with some philosophers on the continent about the changes in the baroneter and in the weather, particularly with Mariotte the Fiench philosopher. He was once in danger of being prosecuted as a conjurer by the Presbytery on account of his barometer. A deputation of that borly having waited upon him to enquire into the ground of certain reports that had come to their ears, he satisfed them so far as to prevent the prosecution of a man known to be so extensively usciul by his knowledge of medicine.-A bout the beginning of the lase century be romoved with his family to Aberdecn, and in the time of Queen Anne's waremployed his thoughts upon an improvement in artillery, in order to matie the shot of great guns more destructive to the enemy, and executed a model of the engine he had conceived. I have conversed with a clock-maker in Aberdeen who was cmployed in making this model; but having made many different pieces by direction, without knowing their intention, or how they were to be put togetlier, be could give no account 
of the whole. After making some experiments with this model, which satisficd him, the old gentleman was so sanguine in the hope of bcing useful to the allies in the war against France, that he set about preparing a field equipage with a view to make a campaign in Flanders, and in the mean time sent his model to his son the Savillian professor, that he might have his and Sir lsaac Newton's opinion of it. His son showed it to Newton, without letting him know that his own father was the inventor. Sir Isaac was much displeased with it, saying, that if it tended as much to the preservation of mankind as to their destruction, the inventor would have deserved a great reward; but as it was contrived solely for destruction, aucl would soon be known by the enemy, he rather deserved to be purished; and urgerl the professor very strongly to destroy it, and if possible to suppress the invention. It is probable the professor followed this advice; for at bis death, which happened soon after, the model was not to be found.

When the rebellion broke out in 1715 , the old gentleman went a second time to Holland, and returned when it was over to Aberdeen, where he died about 1720, aged 93. He left an historical manuscript of the transactions of his own time and country, which my father told me he had read. I was well acquainted with two of this gentleman's sons, and with several of his daughters, besides my own mother. The facts abovementionedare takenfrom what I have occasionally heard from them, and from other persous of his acquaintance.

Page 14. In confirmation of what is said in this page, that the two brothers David and Jannes were the first wlio taught the Newtonian philusnphy in the Scotch Universities; I have by me a Thesis, printed at Edinburgh in 1690 , by James Gregory, who was at that time a professor of philosophy at St. Andrews, and succeeded his brother David in the profession of mathematics at Edinburgh. In this Thesis, alter a dedication to viscount Tarbet, follow the names of twenty-one of his scholars who were candidates for the degree of $A$.. ., then 25 positions or Theses. The first three relate to logic, and the abuse of $i t$ in the Aristotelian and Cartesian philosophy. He defines logic to be the art of making a proper use of things granted, in order to find what is sought, and therefore admits only two Categories in logic, viz, Data and Quresita. The rcmaining 22 positions are a compend of Newton's Principia. 'This Thesis, as was the custom at that time in the Scotch universities, was to be defended in a public disputation, by the candidates, previous to their taking their desrce.

The famous Dr. Pitcairn was a frklow-student and intimate companion of these two Gregorys, and during the vacation of the college was accustomed to go home with them to Kinardie, to their father's house.

David Gregory was appointed a preceptor to the duke of Gloucester, Queen Anne's son; but his chtering on that office was prevented by the death of that prince in the 11 th ycar of his age.

Page 19. D. Gregory's Euclid is said to have been written in prosecution of a design of his predecessor Dr. Bernard, of printing the works of all the ancient mathematicians. This design ought to have been ascribed to Saville, who left in charge to the two professors of his foundation, to print the mathematical works of the ancients, and $I$ think left also a fund for defraying the expense. Wallis did something in consequence of this charge; Gregory and

- Halley performed a great deal; but I think nothing has been done in this desigu by the Savillian professors since their time.
Page 20. Besides what is above mentioned, Dr'. Gregory left in manuscript a Commentary on Newtun's Principia, which Newton valued, and kept by him for many years after the author's death. It is probable that in what relates to astronomy, this commentary may coincide in a great measure with the author's astronomy, which indeed is an excellent commentary upou that part of the Principia.

Page 24. This David Gregory published in Latin, a very good compendium of arithmetic and algebra, with the title Arithmetica et Algebra Compehdium, in Usum Juventutis Academice, Edinb. 1736. He had a design of publishing his uncle's Commentary on the Principia, with extracts from the papers lefi by James Gregory his granduncle; but the expense being too great for his fortune, and lie too gentle a solicitor of the assistance of others, the design was dropped. His son David, yet alive, was master of an East-lndia ship.

Page 40. To the projectors of the society at Aberdeen, ought to have been added John Stewart, professor of mathematics in the Marischal college at Aberdeen. He published an explanation of two treatises of Sir Isaac Newton, viz, his Quadrature of Curves, and his Analysis by Equations of an infinite number of terms. He was an intinate friend of Dr. Reid's.

Another of the first members of that society was $\mathrm{Dr}$. David Skene, who, besides his eminence in the practice of medicine, had applied much to all parts of natural history, particularly to botany, and was a correspondent of the celebrated Linnæus.

Dr. Jobn Gregory and Dr. David Skene were the first who attempted a college of medicine at Aberdeen. The first gave lectures to his pupils in the tbeory and practice of medicine, and in chemistry; the last, in anatomy, materia medica, and midwifery, in order to prepare them for attending the medical college at Edinburgh. T. R. The following additional lines by Mr. James Millar, Professor of Mathematics, Glasgow.

Another instance of the prevalence of mathematical genius in the family of Gregory or Anderson, whether produced by an original and inexplicable determination of the mind, or communicated by the force of example, and the consciousness of an intimate connexion with a reputation already acquired in a particular line, is the celebrated Dr. Reid, professor of moral philosophy in the university of Glasgow ; a nephew, by his mother, of the late Dr. David Gregory, Savillian professor at Oxford.

This gentleman, well known to the public by his moral and metaphysical writings, and remarkable for that liberality, and that ardent spirit of incuiry, which neither overlooks nor undervalues any branch of science, is peculiarly distinguished by his abilities and proficiency in mathematical learning. The objects of literary pursuit are often directed by accidental occurrences. An apprehension of the bad consequences which might result from the philosopby of the late Mr. Hume, induced Dr. Reid to combat the doctrines of that eminent author; and produced a work, which has excited universal attention, and has given a new turn to speculations on that subject. But it is well known to Dr. Reid's literary acquaintance, that these exertions have not diminished the original bent of his genius, nor blunted the edge of his inclination for mathematical researches; whicb, at a very advanced age, he still continues to prosecute with a youthful attachment, and with unremitting assiduity.

It may further be observed, of the extraordinary family above mentioned, that Dr. James Gregory, the picsent 
learned professor of physic and medicine in the university of Edinburgh, is the son of the late Dr. John Gregory, upon the memoirs of whose life the abore remarks have been written by Dr. Reid. The said James has lately published a most ingenious work, entitled, Philos phical and Literary Essajs, in 2 volumes Svu, Lidinb. $179^{2}$; and be appears to be another worthy mheritani of the singular genius of bis family.

Gregory (ST. VINCENT), a very respectable Flemish geometrician, was born at Bruges in 15S4, and became a J'suit at Romeat 20 years of age. He studied mathenatics under the learned Jesuit Chavius. He alterwards became a reputalle prolessor of those sciences himself, and his instructions were solicited by several princes: he was called to Prague by the eniperor Ferdinand the $2 d$; and Philip the 4tb, king of Spain, was desirous of having him to teach mathematics to his son the young prince John of Asturia. He was not less estimable for his virtues, than his skill in the sciences. 1lis well-meant endeavours were very commendable, when bis boly zeal, though for a false religion, led bim to follow the army in Flanders one canpaign, to confess the wounded and dying soldiers, in which he received scveral wounds himself. He died of an apoplexy at Ghent, in 1667 , at 83 years of age.

$\Lambda$ s a writer, Gregory St. Vincent was very diffuse and voluminous, but he was an excellent geometrician. He published, in Latin, three mathematical works, the principal of which was his Opus Geometricum Quadraturæ Circuli, et Sectionum Coni, Antwerp. 1647, 2 vol. folio. Though be has not demonstrated, in this work, the quadrature of the circle, as he pretends to have done, the book nevertheless contains a great number of truths and important discoveries; one of which is this, viz, that if one asymptote of an hyperbola be dividerl into parts in geometrical progression, and from the points of division ordinates bedrainn parallel to the other asymptot', they will divide the space between the asymptote and curve into equal portions; whence it was shown by Mers'nne, that, by taking the continual sums of those parts, there would be obtained areas in arithmetieal progression, adapted to abseisses in geometrical progression, and which therefore were analogous to it system of togarithms.

GRlinade, or Girenado. See Granade.

GRIMaldi (Autir. Franc. Maria), was a learned Itatitin Jesuit, and one of those philosophers of the 17 th century to whom the sciences of optics and astronomy owe great obligations; sciences which be cultivated in compact with his compatriot Riccioli, by diligent experiments and observations. He noticed the spots in the sun, and give to those in the moon names which are still in use, denominating them after the most eminent astronomer's aud philosophers. He made numerous experiments on optics, and some discoveries, which were afterwards confirmed and much farther extended by the brilliast discoveries of Newton. 'Ihose were chicfy contained in lis work, Physico Mathesis de Lmone, Coloribus, et lride, \&c: Bononiæ, 1665 , in 4 to; a work which exhibits a great quantity of eurions experiments and remarks on light and colours; a particular account of which may be seen in my Abridy. of the Phitos. Trans, vol. 1, p. 675. This indeed was Grimaldi's clice merit : for his phitosoplay was but too much arter the manner of his country, which, though it bas given to the world a Galileo, a Torricelli, \&ce, was not the first to shake of the yoke of Aristotle. To his hononr bowever it must be acknowledged, that had he lived in another country, and under other authority than that of his Society, he would have boldly opposed the dogmas of the ancient philosophy. - Grimaldi was cut off at a very early period, in 1663 , being only $4+$ ycars of age.

GRINDING Telescope Glasses, \&c.

GROIN, "ith builders, is the angle made by the intersection of two arches. It is of two kinds, regnlar and irregular; viz, regular when both the archis have the same diameter, but an irregular groin when one arch is a semicircle and the other a semichlipsis. Gruins are ehiefly used in forming arehed roofs, where one hollow arched rautt intersects with another; as in the roofs of most churches, and in some cellars in large bouses.

GRUS, the Crane, one of the new constellations, in the southeru hemisphere; containing, according to Mr. Sharp's catalogue, 13 stars.

Grus is also one of the Arabian constellations, and answers to our Ophiucus, to which they changed this constellation, their religion prohibiting them from drawing any human figures.

GRY, a measure containing $\frac{x}{\mathrm{r}} \mathrm{o}$ of a line. A line is $\frac{\mathrm{r}}{\mathrm{r}} \mathrm{O}$ of a digit, and a digit is $\frac{I}{r o}$ of a foot, and a philosophical foot $\frac{x}{3}$ of a pendulum, whose vibrations, in the latitude of 4.5 degrees, are each performed in one second of time, or $\frac{\mathrm{I}}{6 \sigma}$ of a minute.

GUARDS, a name that has been sometimes applied to the two stars nearest the north pole; being in the hind part of the chariot, at the tail of Ursa Minor, or little bear; one of them being also called the pole-star.

GUERICKE (OTro or OrHo), counsellor to the elector of Brandenbourg and burgomaster of Magdebourg, was born in 1602 , and died in 1686 at Hambourg. He was one of the greatest philosophers of his timc. It was Guericke that invented the air-pump; also the two brass bemispheres, which being applied to cach other, and the air exhausterl, it is said several borses were not able to draw them asunder ; and the marmouset or virunculus of glass which descended in a tube against rain, and ruse agail on the return of serene weather. This last machine fell into disuse on the invention of the barometer, especially after Huygens and Ammtons gave theirs to the world. Gucricke made use of bis marmouset to foretell storms; whence he was looked upon as a sorcerer by the people; so, that the thunder baving one day fallen upon his house, and shivered to pieces several machines which he had employed in his experiments, they failed not to construe the event into a punishment from heaven that was angry with him.-Guericke was author of several works in natural philosophy, the principal of which was his Experimenta Magdeburgica, in folio, which contains his experiments on a vacutum.

GUERI'l"E, in Fortification, a sentry-box; being a small tower of wood, or stone, usually placed on the point of a bastion, or on the angles of the shoulder, to hold a sentincl, who is to take care of the ditch, and watch against a surprise.

GUPULE, in Architecture. Sce GeLa.

GUGLILLMINI (Dominic), an eminent Italian nisthematician and civil engineer, was born at Bologna in 165. His favourite studies were the mathematics and medicine, of which latter faculty he was admitted to the degree of doctor by the university of Bologna, in $167 \mathrm{~s}$. On the appearance of the remarkable comet in the years 1680 and 1681 , he published a treatise 1)e Cometarum Natura et Ortu, \&c, 1681, in which he proposed a rew system on the snbject, which did not, however, meet with the approbation of the scientific world. But his next 
astronomical treatise, containing remarks on the solar eclipse of July 12, 1684 , reflected gruter credit on his knowledge and accuracy of observation. Sool afterivards, the senate of Bologni appointed him principal professor of mathematies in their university, and, in the year 1686 , intendant-general of the rivers of the Bolognese. In consequence of his attention to the dutics of this office, in the year 1690 he published the first part, and in the following year the $2 \mathrm{~d}$ part, of his liydrostatical treatise, entitled Aquarum Fluentium Mensura, Nowo Methodo Inquisita. Some of the observations in this work were attacked by M. Papin, who also entered into a contest with the author on the subject of syphons. Their difference in opinion gave rise totwo letters by Guglitluini, which were printed under the tille of Epistolæ Duæ Hydrostaticæ.

Sig. G. was engaged in settling the differences which arose between the cities of Bologna and Ferrara, respecting the management of the embankment and sluices in their contiguous districts; and received, in reward of his services from his native city, the appointment to a new office in the university, which was that of professur of hydrometry. In the year 1695 he assisted M. Cassini in repairing the meridian line which he had drawn 40 years before in the church. of St. Petronius, at Bologna; on which occasion our author published a memoir, descriptive of the method of laying it down, and establishing its claims to correctness and accuracy. In the year 1697 he published his grand physico-mathematical treatise on the nature of rivers, entitled Della Natura de Fiume, which raised his reputation to the highest pitch for hydraulic science. This reputation procured him the honour of being employed by the dukes of Mantua, of Parma, and Modena, the grand-duke of Tuscany, Pope Clement the 11 th, the republics of Venice and Lucca, \&c, in the invention and construction of the proper hydraulic works in their respective territories.

In the year 1698, our author was induced, by the republic of Venice; to accept of the matlematical chair in the universicy of P'adua; and the senate of Bologna decreed that he should still retain the title of professor in their university, and the emoluments annexed to it. In 1702 he exchanged his mathematical chair at Padua, for the more lucrative one of medicine; after which be pub. lished different tracts on chenical and medical subjects. lle died at Padua in 1710 , in the 551 h year of his age. He had been admitted a member of the Acadeny of Sciences at Paris in 1696 , and was ulso associnte, or correspondent member of the academies of Berlin and Vi(1)na, and of the Royal Society at London.-The whole of his works were printed in a collective form at Geneva, in 1719 , in 2 vols. 4 to ; but the best edition of his treatise on the nature of rivers has been published at Bologna in 17.56 , with the notes of Manfredi.

GUINEA, a gold coin struck in England.-The value or rate of the guinea has varied. It was at first equal to 20 shillings; but by the scarcity of gold it was afterwards advanced to $21 \mathrm{~s}$. 6 $\mathrm{d}$.; and then reduced to $21 \mathrm{~s}$. For some years past $(1812)$ it has disappeared from common circulation, and clandestinely sold at $27 s$. or $28 s$.

The pound weight troy of gold is cut into 44 parts and a half, and rach part mikes is guinca, which is therefore equal to $\frac{2}{8} \mathrm{Ib}$, or $\frac{2}{8} \frac{4}{9} \mathrm{cz}$, or 5 diw ts. $9 \frac{3}{8} \frac{0}{9} \mathrm{gr}$.

'This coin took its name, Gumea, from the circumstance of the gold of which it was first struck being brought from that part of Africa called Guinea, for which reason also it bore the impression of an elephant.
GULA, Guetre, or Gols, in Architecture, a wayy nember whose contour resembles the letter $\mathrm{s}$, commonly called an ogee.

GULBE, in Architecture, the same as Gorge.

GULDIN (FАTILR), was born at St.Gall in 1577, entered into the socicty of the Jesuits in 1597, after abjuring the protestant religion, and died in 1643. Ilaving been sent to Rome to improve his talents, he Lecime protessor of philosophy and mathematics in that city. He also publicly tanght the same scierices at Gratz, and at Vienna, where, in 1635, 1640 , and 1641 , his chief work was printed in a large fulio volume, on the Centre of Gravity, or the Centrobaric Method, being a very elaborate geometrical work.

GULF, or GULPH, in Geography, a part of the occan running up into the land through a narrow passage, or strait, and forning a bay within. As, the Gult of Venice, or Adriatic Sea; the Gulf of Arabia, or of Persia, which is the Red Sea; the Gulf of Constantinople, or the Black Ser ; the Gulf of Mexico, \&c.

GUN, a fire-arm, or weapon of offence, which forcibly discharges a ball or other matter through a cylindrical tube, by means of inflamed gunpowder.

The term gun now includes most of the species of firearms; mortars and pistols being almost the only ones excepted from this denomination. They are divided into great and small guns: the former including all that are usually called camon, ordnance, or artillery; and the latter musquets, firelocks, carbines, musquetoons, blunderbusses, fowling-pieces, \&c.

It is not certainly known at what time these weapons were first invented. And though the introduction of guns into the western part of the world is but' of modern date, comparatively speaking, yet it is certain that in some parts of Asia they had been used for many ages before, though in a very rude and imperfect manner. Philostratus speaks of a city near the river Hyphasis in the Indies, which was said to be impregnable, and that its inhabitants were relations of the gods, because they thew thunder and lightning upon their enemics; and other Greek authors, as also Quintus Curtios, mention the same thing having happened to Alexander the Great. Hunce some have imagined that gums were used by the eastern mations in his time, while others suppose the thunder and lightning alluded to by those authors, were only certain artificial fre-works, or ruckerts, such as we know ate used in the wars by the Intians even in tho prescut day aganst the Europeans, Be this however as it may, it is asserted by many modern travollers, that guns were used in China as far back as the year of Clrist 85 , and have continucd in use cver silice.

'The first hint of the invention of guns in Europe, is in the works of Roger Bacoll, who flourisied in the 13th century. In a trentise writen by him about the year 1280 , he proposes to apply the violent explosive torce of gunpowder to the destruction of a l'mies. And though it is certainly known that the composition of gun-powder is described by Bacon in the said work, yet the mucution has usually, thougi inproperly, been ascribed to Bartholdus Schwaitz, a German monk, who it is said discosered it only in the your 13:0 ; and the invention is related in the foltowing manner. Schwartz having, fur some purpose, pounded nitre, sulphur, and charcoal together, in a mortar, which he atterwards covered imperfectly wilh a stone, a spark of fire accidentally fell into the usurtar, which setting the mixture on fire, the explosion blew the stone 
to a considerable distance. Hence it is probable that Schwartz might be taught the simplest method of applying it in war; for it rather seems that Bacon conceived the manner of using it to be by the violent effort of the flame unconfined, and which is incleed capable of producing astonishing effects. (See Gunpowden.) And the figure and name of mortars, given to a species of old artillery, and their employment, in throwing large stone bullets at an elevation, very much favour this conjecture.

Soon after the time of Schwartz, we find guns commonly employed as instruments of war. Great guns were first used. These were originally made of iron bars soldercd together, and fortified with strong iron hoops or rings; several of which are still to be seen in the Tower of London, and in the Arsend at Woolwich. Others were made of thin sheets of iron rolled together and hooped: and on particular emergencies some have been made of leather, and of lead, with plates of iron or copper. These first pieces werc executed in a rude and imperfect manner, Jike the first essays of most new inventions. Stone balls were thrown out of them, and a small quantity of powder used on account of their weakness. They were of a cylindrical form, without ornaments, and were placed on their carriages by rings.

When, or by whom they were first made, is uncertain. It is known however that the Venetians used cannon at the siege of Claudia Jessa, now called Chioggia, in 1366, which were brought thither by two Germans, with some powder and leaden balls; as also in their wars with the Genoese in 1379. But before that period King Edward the $3 \mathrm{~d}$ made use of camnon at the battle of Cressy in 1346, and at the siege of Calais in 1347 . Cannon were employed by the Turks at the siege of Constantinople, then in possession of the Christians, in 1394, and in that of 1452 , which threw a stone ball of 200lb. weight; but they commonly burst at the first two or three firings. Louis the 12 th had one cast at Tours, of the same size, which threw a ball from the Bastile to Charenton; one of these extraordinary cannon was taken at the siege of Dieu in 1546, by Don John de Castro, and is now in the castle of St. Julian da Barra, 10 miles from Lisbon: the length of it is 20 feet 7 inches, its diameter at the middle 6 feet 3 iuches, and it threw a ball of $100 \mathrm{lb}$. weight: It has neither dolphins, rings, nor button; is of an unusual kind of metal; and it has a barge Indostan inscription on it, which says it was cast in 1400 .

Formerly, it was customary to dignify cannon with uncommon names. Thus, Lewis the $12 \mathrm{kh}$, in 1503 , had 12 brass camnon casi, of an extraordinary size, called atter the numes of the 12 pecrs, of France. The Spanish and Portuguese called them after their saints. The Emperor Charles the 5 th, when he marched against Tunis, founded the 12 Apostles. At Milan there is a 70-pounder, called the Pimontelle; and one at Bois-le-duc, called the Devil. A 60-pounder at Dover-castle, called Queen Elizabeth's pocket-pistol. An 80-pounder in the Tower of London, brought there from Edinburgh-castle, callod Mounts-meg. An 80-pounder in the royal arsenal at Berlin, called the Thunderer. An 80-pounder at Malaga, called the Terrible. Two curious 60-pounders in the arsenal at Bremen, called the Messenger of bad news. And lastly an uncommon 70-pounder in the castle of St. Angelo at Rome, made of the nails that fastened the copper-plates which covered the ancient Pantheon, with this inscription on it, Ex clavis scabalibus porticus Agrippx.
In the beginning of the 15 th century these uncommon names were generally abolished, and the following more universal ones adopted in their stead, viz,

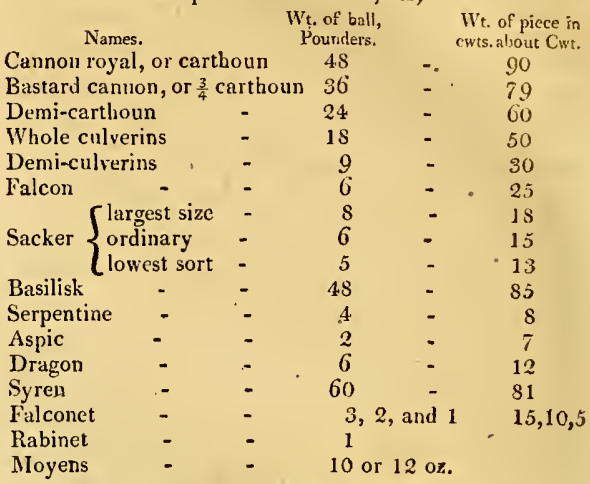

These curious names, of beasts and birds of prey, wcre adopted on account of their swiftness in motion, or of their cruelty; as the falconet, falcon, sacker, and culverin, \&c, for their swiftness in flying; the basilisk, serpentine, aspic, dragon, syren, \&c, for their cruelty. But, at present, cannon take their names from the weight of their proper ball only. Thus, a piece that discharges a cast-iron ball of 24 pounds, is called a 24-pounder; one that carrics a ball of 12 pounds, is called-a 12-pounder: and so of the rest, divided into the following kinds, viz,

Ship-guns, consisting in $42,36,32,24,18,12,9,6$, and 3 -pounders. Garrison-guns, in $42,32,24,18,12$, 9 , and 6 pounders.

Battering-guns, in 24, 18, and 12-pounders.

Field-pieces, in $12,9,6,3,2,1 \frac{1}{2}, 1$, and $\frac{1}{2}$-pounders.

Mortars, it is thought, have be'n at least as ancicnt as cannon. They were employed in the wars of ltaly, to throw balls of red-hot iron, stones, \&c, long before the invention of shells. T'hese last, it is supposed, were of German invention, and the use of them in war shown by the following accident; viz, a citizen of Venlo, at a festival celcbrated in honour of the duke of Cleves, throwing a number of shells, one of them fell on a house and set it on fire, by which misfortune the greatest part of the town was reduced to ashes. The first account of shells used for military purposes, is in 1435 , when Naples was besieged by Charles the 8 th. History informs us, with more certainty, that shells were projected from mortars at the siege of Wachtendonk, in Guelderland, in 1588 , by the earl of Mansfield; aud Cyprian Lucar wrote upon the method of filling and throwing such shells, in his Appendix to the Colloquies of Tartaglia, printed at London in $158 \mathrm{~s}$; where also the compounding and throwing of carcases and various hinds of fure-works is shown.

Mr. Malter, an Englisl engineer, first tanght tho French the art of throwing shells, which they practised at the siege of Motte in 1634. The metliod of firing redhot balls out of mortars was first certaiuly put in practice at the siege of Stralsuud in 1675 by the elector of Brandenburg: though soine say in 1653 at the siege of Bremen.

Another species of ordnance has been long in use, by the nume of howitzer, which is a kind of medium as to its 
length, bctween the cannon and the mortar, and is a very useful piece, for discharging either shells or large balls, which is done either at point-blanc, or at a small elevation. "A new species of ordnance has lately been introduced by the Carron company, and thence called a Carronade, which is only a very short howitzer, and which possesses the advantage of being very light and easy to work. The species of guns before mentioned, are now made chiefly of cast iron; except the howitzer, which is of brass, as well as some cannon and mortars.

Muskets were first used at the siege of Rhege in the year 1521. The Spaniards were the first who armed part of their fuot with these weapons. At first they were very heavy, and could not be used without a rest. They had match-locks, and did execution at a great distance. On their march the soldiers carried only the rests and ammunition, having boys to bear their muskets after them. They were very slow in loading, not only by reason of the nuwieldiness of their pieces, and because they carried the powder and ball separate, but from the time it took to prepare and adjust the match; so that their fire was not near so brisk as ours is now. Afterwards a lighter matchlock musket cane in use: and they carried their ammunition in bandeliers, to which were husg several little cases of wood covered with leather, each containing a charge of powder. The muskets with rests were used as late as the beginning of the civil wars in the time of Charles the 1st. The lighter kind succeeded them, and continued till the beginning of the present century, when they also were disused, and the troups throughunt Europe armed with firelocks. Thèse are usually made of hamnered iron. For the dimensions, cunstruction, and practice of every species of gun, \&c, see the several articles CAN Non, MorTar, \&c. See also Gunvery.

GUNNERY, the art of charging, directing, and exploding fire-arms, as cannon, mortars, muskets, \&c, to the best advantage. Gunnery is sometimes considered as a part of the military art, and sometimes as a part of pyrotechny. To the art of gunnery belongs the knowledge of the force and effect of gunpowder, the dimensions of the pieces, and the proportions of the powder and ball they carry, with the methorls of managing, charging, pointing, spunging. \&c. Also some parts of gunnery are subject to mathematical consideration, which among mathematicians are called absolutely by the name gunnery, viz, the rules and method of computing the range, elevation, quantity of powder, \&c, so as to hit a mark or ubject proposed, and is more particularly called Projectiles; which see.

History of GUNNERY.

Long before the irivention of gunpowder, and of gunnery, properly so called, the art of artillery, or projectiles, was actually in practice. For, not to mention the use of spears, javelins, or stones thrown with the hand, or of bows and arrows, all which are found among the most barbarous and ignorant people, accounts of the larger machines for throwing stones, darts; \&c, are recorded by the most ancient writers. Thus, one of the kings of Judah, 800 years bcfore the Christian æra, erected engines of war on the towers and bulwarks of Jerusalem, for shooting arrows and great stones for the defence of that city. 2 Chron.xxvi. 15. Such machines were afterwarls known among the Greeks and Romats by the names of Ballista, Catapulta, \&c, which produced effects by the action of a spring of a strongly twisted corrlage, furmed of tough and elastic animal substances, no less terrible than the artillery of the moderns.

VOL. I.
Such warlike instruments continued in use down to the 12 th and 13th centuries, and the use of bows still longer; nor is it probable that they were totally laid aside till they were superseded by gunpowder and the modern ordnance.

The first application of gunpowder to military affairs, it scems, was made soon after the year 1300 , for which the proposal of friar Bacon, about the year 1280, for applying its enormous explosion to the destruction of armies, might give the first hint ; and Schwartz, to whom the incention of gunpowder has been errontousiy ascribed, on account of the accident abovementioned under the article $\mathrm{GuN}$, might liase been the first who actually applied it in this way, that is in Europe; for as to Asia, it is probable that the Chinese and Indians had something of the kind many ages before. Thus, only to mention the prohibitiou of firearms in the code of Gentoo laws, printed by the East-India Company.in 1776 , which seems to confirm the suspicion suggested by a passage in Quintus Curtius, that Alexander the Great found some weapons of tbat kind in India: Cannon in the Shanscrit idiom is called sbet-aghnee, or the weapon that kills a hundred men at once.

However, the first pieces of artillery, which were charged with gunpowder and stone bullets of a prodigious size, were of very clumsy and inconvenient structure and weight. Thus, when Mahomet the $2 \mathrm{~d}$ besieged Constantinople in 1453, he battered the walls with stones of this kind, and with pieces of the calibre of 1200 puunds; which could not be fircd more than four times a day. It was howerer soon discovered that "iron bullets, of much less weight than stone ones, would be more efficacious if impelled by greater quantities of stronger powder. 'This oceasioned an alteration in the matter and form of the cannon, which were now cast of brass. Thèse were lighter and more manageable than the former, at the same time that they were stronger in proportion to their bore. This change took place about the close of the 15 th century.

By this means came first into use such powder as is now employed over all Europe, by varying the proportion of the materials. But this change of the proportion was not the only improvement it received. The practice of graining it is doubtless of considerable advantage. At first the powder had been al ways used in the form of fine meal, such as it was reduced to by grinding the materials together. And it is doubtful whether the first graining of powder was intended to increase its strength, or only to render it more convenient for filling into small charges and the loading of small arms, to which alone it was applied for many years, while meal-powder was still used for cannon. But at last the additional strength which the grained powder was found to possess, doubtless from the free passage of the air between the grains, occasioned the meal-powder to be entirely laid aside.

For the last 200 years, the formation of cannon has been very little inuproved; the best pieces of modern artillery differing little in their proportions from those used in the time of Charles the 5 th. Indeed. lighter and shorter pieces have been often proposed and tried; but though they have their advantages in particular cases, it is agreed they are not so efficacious for general service. Yet the size of the pieces has been much diminished; the same purposes being now accomplished by smaller pieces than what were formerly thought necessary. Thus the battering cannon now approves, are those that formerly were called demi cannon, carrying a ball of 24 pounds weight; this weight 4. I 
having been found fully sufficient. The method also of making a breach, by first cutting off the whole wall as low as possible before its upper part is attempted to be beaten down, seems to be a considerable modern improvement in the practical part of gunnery. But the most considerable improvement in the practice of artillery, is the ricochet firing, or the method of firing with small quantities of powder, and elevating the piece but a little, so that the bullet may just go clear of the parapet of the enemy, and drop into their works : for by this means the ball, coming to the ground at a small angle, and with a small velocity, does not bury itself, but bounds or rolls along a great way, destroying all before it. 'This method was first practised by M. Vauban at the siege of Aeth, in the year 1692. A practice of this kind was successfully used by the hing of Prussia at the battle of Rosbach in 1757. He had several six-inch mortars, made with trunnions, and mounted on travelling carriages, which were fired obliquely on the enemy's lines, and a mong their borse. These being charged with only $S$ ounces of powder, and elevated at one degree and a quarter, did great execution : for the shells rolling along the lines with burning fuses made the stoutest of the enemy to fly with precipitation.

The use of fire-arms was however long known before any theory of projectiles was formed. The Italians were the first people who made any attempts at the theory, which they did about the beginning of the 16th century; and among them it appears the first who wrote professedly on the flight of cannon shot, was Nicholas Tartalia, of Brescia, the same author who had so great a share in the invention of the rules for cubic equations. In 1537 he published, at Venice, his Nova Scientia, and in 1546 his Quesiti et Inventioni diversi, in both which he treats professedly on these motions, as well as in another work, translated into English with additions by Cyprian Lucar, under the title of Colloquies concerning the Art of Shooting in great and small Pieces of Irtıllery, and published at London in 1588. Tartalia determined, that the greatest range of a shot was when discharged at an elevation of $45^{\circ}$ : and he asserted, contrary to the opinion of his contemporaries, that no part of the path described by a ball is a right line; though the curvature in the first part of it is so small, that it need not be attended to. He compared it to the surface of the sea; which, though it appears to be a plane, is yet doubtless incurvated runnd the centre of the earth. The same author observes, that he invented the gunuer's quadrant, for laying a piece of ordnance at any point or degree of elevation; and though be had but little opportunity of acquiring any practical knowlerlge by experiments, he yet gave shrewd guesses at the event of some-untried methods.

The philosophers of those times also took part in the questions arisıng on this subject; and many dispules on motion were held, especially in Italy, which continurd till the time of Galileo, and probably gave rise to his celebrated Dialogues on Motion. 'The'se wire not published till the year 1638 ; and in the interval there were published many theories of the motion of military projectiles, as well as inany tables of their comparative ranges; though for the most part very fallacious, and inconsistent with the motions of these bodies.

It is remarkablehowever that, Juring these contests, so few of those who were intrusted with the care of artillery, thought it necessing to bring their theories to the test of experiment. Mr. Robins informs us, in the preface to his New Principles of Gunnery, that he had met with no more than four authors who bad treated experimentally on this subject. The first of these is Collado, in $16+2$, who bas given the ranges of a falconet, carrying a three-pound shot, to every point of the gunner's quadrant, each point being the $12 \mathrm{th}$ part, or $7 \frac{1}{2}^{\circ}$. But from his numbers it is manifest that the piece was not charged with its usual allotment of powder. The result of his trials shows the ranges. at the point-blanc, and the several points of elevation, as below.

\begin{tabular}{|c|c|c|c|c|c|}
\hline \multicolumn{2}{|c|}{$\begin{array}{l}\text { Elevalion } \\
\text { Points. }\end{array}$} & \multicolumn{4}{|r|}{ Range in } \\
\hline 0 & or & 0 & - & - & 268 \\
\hline 1 & - & $7 \frac{1}{2}$ & - & - & 594 \\
\hline 2 & $=$ & 15 & - & - & 794 \\
\hline 3 & - & $22 \frac{\pi}{2}$ & - & - & 954 \\
\hline 4 & - & 30 & - & - & 1010 \\
\hline 5 & - & $37 \frac{\pi}{2}$ & - & - & 1040 \\
\hline 6 & - & 45 & - & - & 1053 \\
\hline 7 & - & $52 \frac{\mathrm{r}}{2}$ & - & betw & the $3 \mathrm{~d}$ and 4 th \\
\hline 8 & - & 60 & - & betw & the $2 \mathrm{~d}$ and $3 \mathrm{~d}$ \\
\hline 9 & - & $67 \frac{\mathrm{I}}{2}$ & " & betw & the 1st and $2 \mathrm{~d}$ \\
\hline 10 & - & 75 & - & betw & the 0 and 1 st \\
\hline 11 & - & $82 \frac{\pi}{2}$ & - & fell & near the picce. \\
\hline
\end{tabular}

The next treatise was by Wm. Burne, in 1643 , in his Art of Shooting in Great Ordnance. His clevations were not regulated by the points of the gunner's quadrant, but by degrees; and he gires the proportions between the ranges at different elevations and the extent of the pointblanc shot, thus: If the extent of the point-blanc shot be represented by 1 , then the proportions of the ranges at several elevations will be as below, viz.

$\begin{array}{cccc}\begin{array}{c}\text { Bourne's } \\ \text { Elevation. }\end{array} & \text { Proportion of Ranges. } \\ 0^{\circ} & - & - & \begin{array}{c}\text { Range. } \\ 5\end{array} \\ 5 & - & - & 2 \frac{2}{9} \\ 10 & - & - & 3 \frac{1}{3} \\ 15 & - & - & 4 \frac{1}{3} \\ 20 & - & - & 4 \frac{5}{6} \\ \text { and the greatest random } & 5 \frac{2}{2} ;\end{array}$

which greatest random, he says, in a calm day is at $42^{\circ}$ elevation; but according to the strength of the wind, and as it favours or opposes the flight of the shot, the eleration may be from $45^{\circ}$ to $36^{\circ}$. He does not inform us with what piece he made his trials; though from his proportions it seems to have been a small one. This however ought to have been mentioned, as the relation between the extent of different ranges varies extremely according to the velocity and density of the bullet.

After him, Eldred and Anderson, both Englishmen also, published treatises on this subject. The former of these was many years guuner of Dover Castle, where most of his experiments were made, the earliest of which are dated in 1611 , though his book was not publinhed till 1646 , and was 'ntitled The Gunner's Glass. His principles were sufficiently simple, and within certitin limits very near the truth, though they were not rigurously so. lle has given the actual range's of difierent perees of artillery at small elevations, all under 10 degrees. Hlis experiments are numerous, and appear to be marle with great care and caution; and he has honestly set down some, which were not reconcilable to his incthod: on the whole he seems to have taken more pains, and to have had a juster knowledge of his business, than is to be found in most of lis practical brethren. 
Galileo's Dialogues on Motion were printed in the year 1646. In these be pointed out the general laws observed by nature in the production and cornposition of motion, and was the first who described the action and effects of gravity on falling bodies: on these principles he determined, that the flight of a cannon-shot, or of any other projectile, would be in the curve of a parabola, unless so far as it should be diverted from that track by the resistance of the air. He also proposed the means of examining the inequalities which arise from thence, and of discovering what sensible effects that resistance would produce in the motion of a bullet at sume given distance from the piece.

Notwithstanding these determinations and hints of Galileo, it seems that those who came after him, never imagined that it was necessary to consider how far the operations of gunnery were affected by this resistance. Instcad of this, they boldly asserted, without making the experiment, that no great variation could arise from the resistance of the air in the flight of shells or cannon shot. In this persuasion they supported themselves chiefly by considering the extreme rarity of the air, compared with those dense and ponderous bodies; and at last it became an almost generally established maxim, that the flighit of these bodies was nearly in the curve of a parabola.

Thus, Rubert Anderson, in his Genuine Use and Effects of the Gunne, published in 1674, and again in his book, To hit a Mark, iu 1690, relates a great many experiments; but pirocecding on the principles of Galileo, he strenuourly asserts that the flight of all bullets is in the curve of a parabola; undertaking to answer all objections that could be brought to the contrary. The same thing was also undertaken by blondel in his Art de jetter les Bombes, published in 1683; where, after long discussion, he concludes, that the variations from the air's resistance are so slight, as not to deserve any notice. The same subject is treated of in the Philos. Trans No. 216 , pa. 6 s, by Dr. Halley; who also, swayed by the very great disproportion betwe'n the density of the air and that of iron or lead, thought it reasonable to believe that the opposition of the air to large metal-shot is scarcely discerrible; though in small and light shot he owns that it must be accounted for.

Though this hypothesis went on smoothly in speculation; yet Añderson, who made a great number of trials, found it impossible to support it without some new modification. For though it does not appear that he ever examined the comparative ranges of either cannon or musket-shot when fired with their isual velocities, yet his experiments on the ranges of shells thrown with velocities that were but small, in comparison of those above mentioned, convinced bim that their whole track was not parabolical. But instead of making the proper inferences from hence, and concluding that the resistance of the air was of considerable efficacy, he framed a rew hypothesis; which was, that the shell or bullet at its first discharge flew to a certain distance in a right line, from the end of which line only it began to describe a parabola: and this right line, which he calls the line of the impulse of the fire, he supposes is the same for all elevations. So that, by assigning a proper length to this line of impulse, it was always in his power to reconcile any two shots made at any Iwo different angles; though the same method could not succeed with three shots; nor indeed does he ever inform us of the event of his experiments when three ranges were tried at one time.
But after the publication of-Newton's Principia, it might have been expected, that the defects of the theory would be ascribed to their true cause, which is the great resistance of the air to such swift motions; as in that work he particularly considered the subject of such motions, and related the result of experiments, made on slow motions at least; by which it appeared, that in such motions the resistance increases as the square of the velocities, and he even hints a suspicion that it will increase above that law in swifter motions, as is now known to be the case. So far however were those who treated this subject scientifically, from making a proper allowance for the resistance of the atmosphere, that they still neglected it, or rather opposed it, and their theories still differed most egregiously from the truth. Huygens alone seems to have attended to this principle: for in the year 1690 he published a treatise on gravity, in which he gave an account of some experiments tending to prove that the track of all projectiles, moving with very swift motions, was widely different from that of a parabola. The rest of the learned generally acquiesced in the justness and sufficiency of Galileo's doctrine, and accordingly very erroneous calculations concerning the ranges of cannon were given. Nor was any farther notice taken of these errors till the year 1716, when.Mr. Ressons, a French officer of artilléry, of great merit and experjence, gave in a memoir to the Royal Academy, importing that, "though it was agreed that theory joined with practice did constitute the perfection of every art; yet experience had taught him that theory was of very little service in the use of mortars: that the works of M. Blondel had justly enough described the several parabolic lines, according to the different degrees of the elevation of the piece; but that practice had conviuced him there was no theory in the cffect of gunpowder; for having endeavoured, with the greatest precision, to point a nortar according to these calculations, he had never been able to establish any solid foundation upon them."-Oneinstance only occurs in which D. Bernoulli applies the doctrine of Newton to the motions of projectiles, in the Com. Acad. Petrop. tom. 2, pa. 338, \&c. Bésides which nothing farther was done in this science till the time of Mr. Benjamin Robins, who published a treatise in 1742, intitled New Principles of Gunnery, in which he treated particularly, not only of the resistanice of the atmosphere, but also of the force of gunpowder, the nature and effects of different guns, and almost every thing else relating to the flight of military projectiles; and indeed he carried the theory of gunnery nearly to its utmost perfection:

The first important consideration which engaged the attention of Mr. Robins, and which is indeed the foundation of all other particulars relating to gunnery, is the cxplosive force of gunpowder. M. Lahire, in the Hist. of the Acad. of Sciences for the year 1702, supposed that this force may be owing to the increased elasticity of the air contained in, and between the grains, in consequence of the beat and fire produced at the time of the explosion: a cause not ádequate to the 200th part of the effect. On the other hand, Mr. Robins determined, by irrefragable experiments, that this force was owing to an elastic fluid, similar to our atmosphere, existing in the powder in all extremely condensed state, which being suddenly disengaged from the powder by the combustion, expanded with an amazing force, and violently impelled the bullet, or whatcver may oppose its expansion. 
In ascertaining the intensity of this force of exploded gunpowder, Mr. Robins bad recourse to several different ways, after the examplc of Mr. Hawksbee, related in the Philos. Trans. No. 295, and his Physico-Mechan. Exper. pa. 81 . One of these is by firing the powder in the air thus: A small quantity of the puwder is placed in the upper part of a glass tube, and the lower part of the tube is immersed in water, the water being made to rise so near the top, that only a small portion of air is lett in that part where the powder is placed: then in this situation the communication between the upper part of the tube and the external air being closed, the powder is fired by means of a burning glass, or otherwise; the water descends upon the explosion, and stands lower in the tube than before, by a space proportioned to the quantity of powder fired.

Another method was by firing the powder in racuo, riz, in an exhausted recciver, by dropping the grains of powder upon a hot iron included in the receiver. By this means a permanent elastic fluid was generated from the fired gunpowder, and the quantity of it was always in proportion to the quantity of powder that was used, as was found by the proportional sinking of the mercurial gage annexed to the air pump. The result of these experiments was, that the weight of the elastic air thus generated, was eyual to $\frac{3}{T O}$ of the compound mass of the gunpowder which yielded it; and that its bulk, when cold and expanded to the rarity of common atmospheric air, was about $\mathbf{2 4 0}$ times the búlk of the powder; and consequently in the same proportion would such fluid at first, if it were cold, exceed the force or elasticity of the atmosphere. But as Mr. Robins found, by another ingenious experiment, that air beated to the extreme degree of the white heat of jron, has its elasticity quadrupled, or is 4 times as strong; he thence inferred that the force of the elastic air generatcd as above, at the monent of the explosion, is at least 4 . times 240 , or 960 , or in round numbers about 1000 times as strong as the elasticity or pressure of the atmosphere, on the same space.

Having thus determined the force of the gunpowder, ot intensity of the agent by which the projectile is to be urged, Mr. Robins next proceeds to determine the effects it will produce, or the velocity with which it will impel a shot of a given weight from a piece of ordnance of given dimensions; which is a problem strictly limited, and perfectly soluble by mathematical rules, and is in general this: Given the first force, and the law of its variation, to determine the velocity with which it will impel a given body in passing through a given space, which is the length of the bore of the gun.

In the solution of this problem, Mr. Robins assumes these two postulates, viz, 1 , 'That the action of the powder on the bullet ceases as soon as the bullet is out of the piece; and 2d, That all the powder of the charge is fired and converted into elastic fluid before the bullet is sensibly moved from its place: assumptions which for good reasons are found to be in many cases very near the truth. It is to be noted also, that the law by which the force of the elastic fluid varic's, is this, viz, that its intensity is directly as its density, or reciprocally proportional to the space it occupies, being so much the stronger as the space is less; a principle well known, and common to all elastic fluids. On these principles then Mr. Robins resolves this problem, hy means of the 39 th prop. of Newton's Principia ill a direct way, and the result is cquivalent to this theo- rem, when the quantities are expressed by algebraic symbols; viz, the velocity of the ball

$v=27130 \sqrt{ }\left(\frac{10 a}{c d} \times \log \cdot \frac{b}{a}\right)=100 \sqrt{ }\left(\frac{223 a d^{2}}{w} \times \log \cdot \frac{b}{a}\right) ;$ where $v$ is the velocity of the ball,

$a$ the length of the charge of powder,

$b$ the whole length of the bore,

$c$ the spec. grav. of the ball, or wt. of a cubic foot of

$d$ the diameter of the bore, the same matter in ounces,

$2 v$ the weight of the ball in ounces.

For Example, Suppose $a=2 \frac{\pi}{8}$ inc., $b=45$ inches, $c=11345 \mathrm{oz}$, for a ball of lead, and $d=\frac{3}{4}$ inches; then $v=27130 \sqrt{ }\left(\frac{7}{2269} \times \log . \frac{120}{7}\right)=1674$ feet per second, the velocity of the ball.

Or, if the wt. of the bullet be $: 0=1 \frac{9}{20} \mathrm{oz} .=\frac{29}{2} \mathrm{oz}$. Then $v=100 \sqrt{ }\left(\frac{1115 \times 189}{29 \times 32} \times \log . \frac{120}{7}\right)=1674$ feet, as before.

"Having in this proposition," says Mr. Robins, "shown how the velocity, which any bullet acquires from the force of powder, may be computed upon the principles of the theory laid down in the preceding propesitions; we shall next show, that the actual velocities, with which bullets of different magnitudes are impelled fiom different pieces, with different quantities of poisder, are really the same with the velocities assigned by these computations; and consequently that this theory of the force of powder, here delivered, does unquestionably ascertain the true action and modification of this enormous power.

"But in order to compare the velocities communicated to bullets by the explosion with the velocitics resulting from the theory by computation; it is necessary that the actual velocities with which bullets move, should be capable of being discovered, which yet is impossible to be done, by any methods hitherto made public. The only ineans hitherto practised by others for that purpose, have been either by observing the time of the flight of the shot through a given space, or by measuring the range of the shot at a given elevation; and thence computing, on the parabolic hypothesis, what velocity would produce this range. The first method labours under this insurmountable,difficulty, that the velocitics of these bodies are olten so swift, and consequently the time observed is so stort, that an imperceptible error in that time may occasion an error in the velocity thus found, of $2,3,4,5$, or 600 feet in a second. The other method is so fallacious, by reason of the resistance of the air (to which inequality the first is also liable), that the velocities thus assigned may not be perhaps the ioth part of the actual velocitics sought.

"' 'o remedy then these inconveniences, I have invented a new method of finding the real velocitics of bullets of all kinds; and this to such a degree of exactness (which may be augmented too at pliasure), that in a bullet moving with a viclocity of 1700 feet in $\mathbf{l}^{\prime \prime}$, the error in the estimation of it need nerer amount 20 its 500 th part; and this without any extraordinary nicety in the construction of the machine."

Mr. Robins then gives an account of the machine by which he measures the vifocities of the balls, which machine is simply. this, viz, a pendulous block of wood suspended fiecly by a horizontal axis, against which block are to be fired the balls whose vclocitics are to be determined. 
"This instrument thus fitted, if the weight of the pendulum be known, and likewise the respective distances of its centre of gravity, and of its centre of oscillation, from its axis of suspension, it will thence be known what motion will be communicated to this pendulum by the percussion of a body of a known weight moving with a known degree of celerity, and striking it in a given point; that is, if the pendulum be supposed at rest before the percussion, it will be known what vibration it ought to nake in consequence of such a determined blow; , and, on the contiary, if the pendulum, bing at rest, is struck by a body of a known weight, and the vibration, which the pendulum makes after the blow, is knowri, the velocity of the striking body may from thence be determined.

"Hence then, if a bullet of a known weight strikes the pendulum, and the vibration, which the pendulum makes in consequence of the stroke, be ascertained; the velocity with which the ball moved, is thence to be known."

Mr. Robins then explains his method of coniputing velocities from experiments with this machine; which methorl is rather troublesome and perplexed, as well as the rulés of Euler and Antoni, who followed him in these investigations; but a much simpler rule is given in my Tracts, vol.2, pa 325, where, such experiments are explained at full length, and this rule is expressed by either of the two following furmulas,

$0=5.6727 \mathrm{cg} \times \frac{p+b}{\text { bir }} \sqrt{ } 0=614.58 \mathrm{cg} \times \frac{p+b}{\text { birn }}$, the velo-

city; where $v$ denotes the velocity of the ball when it strikes the pendulum, $p$ the weight of the pendulum, $b$ the weight of the ball, $c$ the chord of the are described by the vibration to the radius $r, g$ the distance below the axis of motion to the centre of gravity, o the distance to the centrc of oscillation, $i$ the distance to the point of impact, and $n$ the number of oscillations the pendulum will perform inone minute, when made to oscillate in small arcs. "The latter of these two theorems is much the easiest, both because it is free of raticals, and because the value of the radical $\sqrt{ } 0$, in the furmer, is to be first computed from the number $n$, or number of oscillations the pendulum is observed to make.

With such machines' Mr. Robins made a great number of experiments, with musket barecls of different lengths, with balls of various weights, and with difierent charges or quantities of powder. He has set down the results of 61 of these experiments, which nearly agree with the corresponding velocities as computed by his theory of the force of powder, and which therefore establisli that theory on a sure foundation.

From these experiments, as well as from the preceding theory, many important conclusions were deduced by Mr. Robins; and incleed by means of these it is obvious that every thing maty be deternined relative buth to the frue theory of projectilis, and to the practice of artillery. For, by firiug a piece of ordnance, charged in a similar manner, against such a ballistic pendulum from diflerent distances, the velocity lost by passing through such spaces of air will be forund, and consequently the resistance of the air, the ouly circumstance that was wanting to complete the theury of gumnery, or military projectiles; and of this kind 1 have since made a great number of experiments with cannon balls, and have thereby obtained the whole series of resistances to such a ball when moving with every degrec of velocity, from 0 up to 2200 feet per second of tince. In the structure of artillery, they may likcwise be of the greatest use : for heuce may be determined the best lengths of gurs; the proportions of the shot and powder to the several lengths; the thickness of a piece, so as. it may be able to confine, without bursting, any given charge of powder; as also the effect of wads, chambers, placing of the vent, ramming the powder, \&c. For the many other curious circumstances relating to this subject, and the various other improvements in the theory and practice of gunnery, made by $\mathrm{Ml}$ : Robins, consult the first vol. of his Tracts, collected and published by Dr. Wilson, in the year 1761 , and republished with many important notes, and ubservations, by the author of this work, in 1805, where ample infurmation may be obtained.

Soon after the first publication of Robins's New Principles of Guanery, in $17+2$, the learned in several other nations, trading in his-steps, repeated and farther extended the same subject, sometimes varying and enlarging the machinery; particularly Euler in Germany, D'Antoni in Italy, and Messis. D'Arcy and Le Roy in France. But most of these, like Mr. Robins, with small fire-arms, such as muskets and fusils.

But in the year 1775 , in conjunction with several able officers of the royal artillcry, and other ingenious gentlemen, was undertaken a course of experiments with the ballistic pendulum, in 'which we ventured to extend the machinery to cantion shot of 1,2 , and 3 pounds weight. An account of these experiments was published in the Philos. Trans. for 1778, and for which the Royal Society honoured me with the prize of the gold medal. "These were the only experiments that 1 know of which had been made with cannon balls for this purpose, though the conclusions to be deduced from such, are of the greatest importance to those parts of natural philosophy which are dependent on the effects of fired gunpowder; nor do I know of any other practical method of ascertaining the initial velocities within any tolerable degree of the truth. The knowledge of this velocity is of the utmost consequence in gumery: by means of it, together with the law of the resistance of the medium, every thing is determinable relative to that science, for, besides its being an excellent method of trying the strength of diferent kinds of powder, it gives us the law relative to the different quantities of powder, to the different weights of shot, and to the different lengths and sizes of guns. Besides these, there docs not appear to be any thing wanting to answer any inquiry that can be made concerning the Bight and ranges of shot, except the effects arising from the resistance of the medium. In these experiments the weights of the pendulums employed were from 300 to nicar 600 pounds. In that paper is described the method of constructing the machinery, of fiuding the centres of gravity and oscillation of the pendulum, and of making the experiments, which are all set down in the form of a jourual, with all the minute and concomitant circumstances ; as also the investigation of the new and casy rule, given'abuve, for conputing the velocity of the ball from the experiments. The charges of powder were varied from 2 to 8 ounces, and the shot from 1 to near 3 pounds. And from the whole were clearly dedaced thrse principal iuferences, viz,

"1. First, "ithat gunpowder fires almust instantaneonsly. -2 . That the velocities communicated to balls or shot, of the same weight, by different quantitics of powder, are nearly in the subduplicate ratio of those quantities: a small variation, in defect, taking place when the quantities of powder became great. -3 . And when shot of difa 
ferent weights are cmployed, with the same quantity of powder, the velocities communicated to them, are nearly in the reciprocal subduplicate ratio of their weights.4. So that, universally, shot which are of different weights, and impelled by the flame of different quantitics of powder, acquire velocitics which are directly as the square roots of the quantities of powder, and inversely as the square roots of the weights of the shot, nearly. -5 . It would therefore be a great improvement in artillery, to make use of shot of a long form, or of heavier matter ; for thus the momentum of a shot, when fired with the same weight of powder, would be increased in the ratio of the square root of the weight of the shot.-6. It would also be an improvement to diminish the windage; for by so doing, one-third or more of the quantity of powder might be saved.-7. When the improvements mentioned in the last two articles are considered as both taking place, it is evident that about half the quantity of powder might be saved, which is a very considerable object. But important as this saving may be, it seems to be still exceeded by that of the article of the guns; for thus a sinall gun may be made.to have the effect and execution of another of two or three times its size in the present mode, by discharging a shot of two or three times the weight of its natural ball or round shot. And thus a small ship might discharge shot as heavy as those of the greatest now made use of.

"Finally, as the above experiments exhibit the regulations with regard to the weights of powder and balls, when fired from the same piece of ordnance, \&c; so by making similar experiments with a gun, varied in its length, by cutting off from it a certain part before each course of experiments, the effects and general rules for the different lengths of guus may be certainly deturmined by them. In short, the principles on which these experiments were made, are so fruitful in consequences, that, in conjunction with the effects resulting from the resistance of the medium, they seem to be sufficient for answering all the inquiries of the speculative philosopher, as well as those of the practical artillerist.

In the year 1786 was published the first volume of $\mathrm{my}$ Tracts, in which is detailed, at great length, another very extensive course of experiments which were carried on at Woolwich, in the years 1783,1784 , and 1785 , by order of the Duke of Richmond, master-general of the ordnance. The objects of this course were very numerous, but the principal of them were the following:

" 1 . The velocities with which balls are projected by equal charges of powder, from pieces of the same weight and caliber, but of different lengths.

" 2. The velocities with different charges of powder, the weight and length of the gun being the same.

" 3 . The greatest velocity due to guns of different lengths, to be obtained by increasing the charge as far as the resistance of the piece is capable of sustaining.

" 4 . The 'ffect of varying the weight of the piece; every thing clse being the same.

" 5 . The penetration of balls into blocks of wood.

c 6: The ranges and times of flight of balls; to compare them with their initial velocities, for determining the resistance of the medium.

" 7. The effect of wads; of different degrees of ramming; of difierent degrees of windage; of different positions of the vent; of chambers, and trumnions, and every other circumstance necessary to be known for the improvement of artillery."

All these objects were obtajned in a very perfect and accurate manner; excepting only the article of ranges, which were not quite so regular and uniform as might be wished. The balls were most of them of one pound wcight; but the powder was increased from 1 ounce, up till the bore was quite full; and the penduliun was from 600 to $8001 b$. weight. The conclusions from the whole were as follow :

" 1 . That the former law, between the charge and velocity of ball, is again confirmed, viz, that the relocity is directly as the square root of the weight of powder, as far as to about the charge of $S$ ounces : and so it would continue for all charges, were the guns of an indefinite length. But as the length of the charge is increased, and bears i more considerable proportion to the length of the bore, the velocity falls the more short of that proportion.

" 2. That the velocity of the ball increases with the charge to a certain point, which is peculiar to cacl gur, where it is greatest; and that by farther increasing the charge, the velocity gradually diminishes, till the bore is quite full of powder. That this charge for the greatest velocity is greater as the gun is longer, but not greater however in so high a proportion as the length of the gun is ; so that the part of the bore filled with powder bears a less proportion to the whole in the long guns, than it does in the short ones; the part of the whole which is filled being indeed nearly in the reciprocal subduplicate ratio of the length of the empty part. And the other circumstances as in this table.

Charges producing the Greatest Velocity.

\begin{tabular}{c|c|c|c|c}
$\begin{array}{c}\text { Gun } \\
\text { Number }\end{array}$ & $\begin{array}{c}\text { Length of } \\
\text { the bore. }\end{array}$ & $\begin{array}{c}\text { Length } \\
\text { filled. }\end{array}$ & $\begin{array}{c}\text { Part of the } \\
\text { whole. }\end{array}$ & $\begin{array}{c}\text { Wt; of the } \\
\text { powder. }\end{array}$ \\
\cline { 1 - 2 } 1 & $\begin{array}{c}\text { inches. } \\
28 \cdot 5\end{array}$ & $\begin{array}{c}\text { inches. } \\
8 \cdot 2\end{array}$ & $\frac{4}{T^{2}}$ & 12 \\
2 & $38 \cdot 4$ & $9 \cdot 5$ & $\frac{4}{T^{2}}$ & 14 \\
3 & $57 \cdot 7$ & $10^{\circ} 7$ & $\frac{4}{22}$ & 16 \\
4 & 80.2 & $12 \cdot 1$ & $\frac{4}{2 \tau}$ & 18 \\
\hline
\end{tabular}

"3. It appears that the velocity continually increases as the gun is longer, though the increase in velocity is but very small in respect of the increase in length, the velocities being in a ratio somewhat less than that of the square roots of the length of the bore, but somewhat greater than that of the cube roots of the length, and is indeed nearly in the middle of the ratio between the two.

" 4 . The range increases in a much less ratio than the velocity, and indeed is nearly as the square root of the velocity, the gun and elevation being the same. And when this is compared with the property of the vclocity and length of gun in the foregoing paragraph, we perceive that very little is gained in the range by a great increase in the lengtl of the gun, the charge being the same. And indeed the range is nearly as the 5 th root of the length of the bore; which is so small an increase, as to amount only to about $\frac{1}{7}$ th part more range for a double length of gun.

" 5 . It appears that the time of the ball's flight is nearly as the range; the gun and clevation being the same.

" $6.1 \mathrm{t}$ appears also that there is no sensible difference caused in the velocity or range, by varying the weight of the gun, nor by the use of wads, nor by different degrees of ramming, nor by firing the charge of powder in different parts of it. 


\section{G U N}

"7. But a great difference in the velocity arises from a small degree of windage. Incleed with the usual established windage only, namely, about $\frac{1}{2}$ th of the caliber, no less than between $\frac{\pi}{3}$ and $\frac{\pi}{4}$ of the powder escapes and is lost. And as the balls are often smaller than that size, it frequently happens that half the powder is lost by unnecessary windage.

" s. It appears that the resisting force of wood, to balls fired into it, is not constant. And that the depths penetrated by different velocities or charges, are nearly as the logarithins of the charges, instead of being as the charges themselves, or, which is the same thing, as the square of the velocity.

" 9 . These, and most other experiments, show that balls are greatly deflected from the direction they are projected. in; and that so mucb as $300 \mathrm{or} 400$ yards in a range of a mile, or almost $\frac{T}{4}$ of the range, which is nearly a deflection of an angle of 15 degrees.

" 10. Finally, these experiments furnish us with the following concomitant data, to a tolerable degree of accuracy; namely, the dimensious and clevation of the gun, the weight and dimensions of the powder and shot, with the range and time of flight, and the first velocity of the ball. From which it is to be hoped that the measure of the resistance of the air to projectiles, may be determined, and thereby lay the foundation for a true and practical system of guntiery, which may be as well useful in service as in theory."

Since the publication of those Tracts, we have prosecuted the experiments sull farther, from year to year, gradually extending our aim to more objects, and enlarging the guns and machinery, thl] we have arrived at experiments with the 6-pounder guns, and pendulums of 1800 pounds weight. One of the new objects of inquiry, was the resistance the atmosphere makes to military projectiles; to obtain which, the guns have been placed at many difierent distances from the pendulum, against which they are fired, to get the velocity'lost in passing througli those spaces of air ; by which, and the use of the whirling inachine, described in my new 'l'racts, vol. 3, for the slower motions, I have investigated the resistance of the air to given balls moving with all degress of velocity, from 0 up to 2200 feet per second; as well as the resistance for 'many degrees of velocity, to planes and figures of other shapes, and inclined to their path in all varieties of angles; from which 1 have deduced general laws and formulas for all such motions.

Mr. Robins made also similar experiments on the resistance of the air; but being only with musket bullets, on account of their snallness, and of their change of figure by the explusion of the powder, I find they are very inaccurate, and considerably different from those above mentioned, whicl were accurately made with pretty considerable cunnou balts, of iron. For which reason we may omit here the rules and theory deduced from them by Mr. Robins, till others inore correct shall have been established. All these experiments inderd ayree in evincing the very enormus resistance the air makes to the swift motions of military projuctiles, anounting in some cases to 20 or 30 . times the weight of the ball itself; on which account the common rules for projectiles, deduced from the parabolic theory, are of titule or no use in real practice; for, from these experiments it is clearly proved, that the track described by the flight even of the heavirst shot, is neither a parabola, nor yet approaching any thing ncar it, except

\section{GUN}

when they are projected with very small velocities; in so much that some balls, which in the air range only to the distance of one mile, would in vacuo, when projected with the sanc velocity, range above 10 or 20 times as far. For the common rules of the parabolic theory, see Projectiles. See also the articles Force, and Resistance, in this Dictionary.

Mr. Benjamin 'Thompson (now Count Rumford), instituted a very considerable course of experiments of the same kind as those of Mr. Robins, with musket barrels, which was published in the Philos. 'Trans. vol. 71 , for the year 1781. In these experiments, the conclusions of $\mathrm{Mr}$. Robins are grenerally confirmed, and several other curious circumstances in this business are at the same time remarked by Mr. Thompson. This gentleman also pursues a hint thrown out by Mr. Robins relative to the determining the velocity of a ball from the recoil of the pendulous gun itself. Mr. Robins, in prop. 11, remarks that the effect of the explorled powder upon the recoil of the gun, is the same, whether the gun is charged with a ball, or without one; and that the chord, or velocity, of recoil with the powder alone, being subtracted from that of the recoil when charged with both powder and ball, leaves the velocity which is due to the ball alone. Hence Mr. Thomp son observes, that the inference is obvious, viz, that the momentum thus communicated to the gun by the ball alone, being equal to the momentum of the ball, this becoines known; and therefore being divided by the known weight of the ball, the quotient will be its velocity. Mr. Thompron sets a great value on this new rule, the velocities by incans of which, he found lo agree nearly with several of those deduced from the motion of the pendulum; and in the uther cases in which they dificred greatly from thise, he very inconsistently supposes that these latter ones are erroneous. In the experiments however contained in my Tracts, a great multitude of those cases are compared together, and the inaccuracy of that new rule is fully proved

Having in the 9 th prop. compared together a number of computed and experimental velocities of balls, to verify his theory; in the 10th prop. Mr. Robins assigns the changes in the force of poweler, which arise from the different state of the atmosphere, as to heat and moisture, both which he finds have some effect on it, but cspecially the latter. In prop. 11 he invẹstigates the velocity which the flame of gunpowder acquires by expanding itself, supposing it fired in a given picce of artillery, without either a bullet or any other body placed before it. This velocity he finds is upwards of 7000 feet per second. But the celebrated Euler, in his commentary on this part of $\mathrm{Mr}$. Robins's book, thinks it may be still much greater. And in this prop. it is that Mr. Robins declares his opinion, above alluded to, viz, that the effect of the powder upon the recoil of the gun is the same, in all cases, whether fired with a ball, or without one.-In prop. 12 he ascertains the manner in which the flame of powder impels a ball which is laid at a considorable distance from the charge; showing here that the sudden accumalation and density of the fluid against the ball, is the reason that the barrel is so often burst in those cases.-In prop, 13 he enumerates the various kinds of powder, and describes the properest methods of examining its goodness. He here shows that the best proportion of the ingredients, is when the saltpetre is $\frac{3}{4}$ of the whole compound mass of the pown der, and the sulphur and charcoal the other $\frac{x}{4}$ betwece 
them, in equal quantities. In this prop. Mr. Robins takes occasion to remark upon the use of eprouvettes, or nethods of trying powder; condemuing the practice of the English in using what is called the vertical eprouvette; as well as that of the French, in employing a small inortar, with a very large ball, and a small charge of powiler : and instead of these, he strongly recommends the use of his ballistic pendulum, for its great accuracy: but for still greater dispatch, lie tells us he should use another method, which however he reserves to himself, without giving any particular description of it. From what has teen done by Mr. Robins upon this head, several persons have introduced his method of suspending the gun as is pendulum, and noting the quantity of its oscillating recoil when fired with a certain quantity of powder; and of this kind 1 have contrived a machine, which possesses several advantages overall other's, being extremely simple, accurate, and expeditious; so much so indeed, that the weighing out of the powder is the chief part of the trouble. See Gunpowder, and Eprouvette.

The other or ad chapter of Mr. Robins's work, in 8 propositions, treats " of the resistance of the air, and of the track described by the flight of shot and shells." aAnd of these, prop. 1 describes the general principles of the resistance of fluids to solid bodies moving in them. Here Mr. Robins discriminates between continued and compressed fluids, which immediately rush into the space quitted by a hody moving in them, and whose parts yield to the impulse of the body without condensing and accumulating before it; and such fluids as are imperfectly compressed, rushing into a void space with a limited velocity, as in the case of our atmosphere, which cundenses more and more before the ball as this moves quicker, and also presses the less behind it, by following it always with - only a given velocity: hence it happens that the former fluid will resist moving bodies in proportion to the square of the velocity, while the latter resists in a higher proportion.-Proposition 2 is "to determine the resistance of the air to projectiles by experiments." One of the methods for this purpose, is by the ballistic pendulum, placing the gun at different distances from it, by which he finds the velocity lost in passing through certain spaces of air, and consequently the force of resistance to such velocities as the body moves with in the several parts of its path. And another way was by firing balls, with a given velocity, over a large piece of water, in which the fall and plunge of the ball could be seen, and consequently the space it passed over in a given time. By these means $\mathrm{Mr}$. Robins determined the resistances of the air to several different velocities, all which showed that there was a gradual increase of the resistance, over the law of the square of the velocity, as the body moved quicker. In the remaining propositums of this chapter, he proceeds a little further in this subject of the resistance of the air; in which he lays down a rule for the proportion of the resistance between two assigned velocities; and he shows that when a 24-pound ball, fired with its full charge of powder, first issues from the piece, the resistance it meets with from the air is more thun 20 times its weight. He further proves that " the track described by the flight of sloot or shells is neither a parabola, nor nearly a parabola, unless they are projected with small velocities ;" and that " bullets in their flight are not only depressed beneath their original direction by the action of gravity, but are also frequently driven to the right or left of that direction by the action of some other force: and in the 8 th or last proposition, he pretends to show that the deptlis of penetration of balls into firm substances, are as the squares of the velocitics. But this is a mistake; for neither does. it appear that his trials were sufficiently numerous or various, nur were his small leaden balls fit for this purpose; and 1 have found, from a number of trials with iron cannon balls, that the penetrations are in a much lower proportion, and that the resisting force of wood is not uniform. See my Tracts.

In the following small tracts, added to the principles, in this volume, Mr. Robins prosecutes tl:e sulject of the resistance of the air much further, and lays down rules for computing ranges made in the air. But these must be far from accurate, as they are founded on the two following principles, which 1 know, from numerous exjeriments, are erroncous: viz, Ist, "That till the velocity ot the projectile surpasses that of 1100 feet in a second, the resistance may be estecmed to be in the duplicate proportion of the velocity. $2 \mathrm{~d}$, That if the velocity be greater than that of 11 or 1200 feet in a second, then the absolute quantity of that resistance in these greater velocitios will be near 3 times as great, as it should be by a conparison with the smaller velocities." For, instead of passing at once from the law of the square of the velocities, and ever atter being about 3 times as much, my experiments prove that the increase of the resistance above the law of the square of the velocity, takes place at first in the smallest motions, and increases gradually more and more, to a certain point, but never rises so high as to be 3 times that quantity, after which it-decreases again. All which fully appears in the $3 \mathrm{~d}$ vol. of my new Tracts, lately pubîishod.

The additional tracts of Mr. Robins, in the latter part of this volume, which contan many uscful and important mattérs, are numbered and titled as tollows, viz. Number 1 , "Of the resistance of the air. Number 2, Of the resistance of the air; together with the method of computing the motions of bodies projected in that nodium. Number 3 , An account of the experiments, relating to the resistance of the air, exhibited at difierent times before the Royal Society, in the year 1746. Number 4 , Ut the force of fired gunpowder, together with the computation of the velocities thereby communicated to nulitary projectiles. Number 5 , A comparison of the experimental ranges of camnon and mortars with the theory contained in the preceding papers.-Practical Maxims relating to the effects and management of artillery, and the flight of shells and shot, A proposal for increasing the strength of the British navy, by changing all the guns, from the 18 -pounders downwards, into others of equal weight, but of a greater bore." With several letters, and other papirs, "On pointing, or the directing of cannon to strike distant objects; Of the nature and advantage of rifled barrel-picces," \& $c$.

1 have dwelt thus long on Mr. Robins's New Principles of Gunnery, because it is the first work that can be considered as attempting to establisly a practical system of gunnery, and projectiles, on good experiments, on' the uforce of gunpowder, on the resistance of the air, and on the effects of different pieces of artillury. Those ixperiments are however not sufficiently perfict, both on account of the smaltuess of the bullets, and for want of good ranges, to form a proper theory upon. 1 have supplied some of the necessary desiderata for this purpose, viz, the resistance of the air to cannon balls moving with all degrees of velocity, and the velocities communicated by given charges of powder to difierent balls, and from different pieces of 
artillery. But there are still wanting good experiments with different pieces of ordnance, giving the ranges and times of flight, with all varieties of charges, and at all different angles of elevation. A few liowever of those I have obtained, as in the following small table, which are derived from experiments made with a medium une-pounder gun, the iron ball being nearly 2 inches in diameter.

\begin{tabular}{r|c|c|c|c}
\hline Fowder. & $\begin{array}{c}\text { Eleration } \\
\text { of gun. }\end{array}$ & $\begin{array}{c}\text { Velocity of } \\
\text { ball. }\end{array}$ & Range. & $\begin{array}{c}\text { Time } \\
\text { of flight, }\end{array}$ \\
\hline $0 z$ & 0 & feet & feet & \multicolumn{1}{|l}{} \\
2 & 15 & 860 & 4100 & 9 \\
4 & 15 & 1230 & 5100 & 12 \\
8 & 15 & 1640 & 6000 & $14 \frac{\mathrm{I}}{2}$ \\
12 & 15 & 1680 & 6700 & $15 \frac{\mathrm{L}}{2}$ \\
2 & 45 & 860 & 5100 & 21 \\
\hline
\end{tabular}

The celebrated Euler arlded many excellent dissertations on the subject of gunnery, in his translation of Robius's gnnnery into the German language; which were again farther improved in Brown's translation of the same juto English in the year 1777. Sre also Antoni's Examen de la Pusdre; the experiments of MM. d'Arcy and Le Roy, in the Memoirs of the Acadenny in 1751; and D'Arcy's Essai d'une theorie d'artillerie in 1760: my Tracts; and paper on the force of fired gunpowder in the Philos. Trans. for 1778 : and Thompson's paper na the same subject in 1781: but above all, vol. 2 and $S$ of $\mathrm{my}$ new Tracts, containing the concluding deductions drawn from all the experiments made at Woolwich.

Of the common or parabolic theory of gunnery, $\mathrm{Mr}$. Simpson gave a very neat and concise treatise in his Select Exercises. And a very ingenious treatise was also given by Mr. Reuben Burrough, in 1779; containing, not only the problems that are common on this subject, and the method of determining the maximum range on an inclined plane when the piece is situated in that plane; but also when it is placed above or below the given plane; a prohlem of much greater difficulity, and which had not before been publicly given by any other author. Other writers on this part, are Starrat, Gray, Williams, Glenie, \&c.

GUNPOWDER, a composition of nitre, sulphur, and charcoal, mixed together, and usually granulated. This casily takes fire; and when fired, it rarefies or expands with great vehemence, by means of its elastic force.-It is to this powder that we owe all the effect and action of guns, and ordnance of all sorts. So that fortification, with the modern military art, \&c, in a great measure depend upon it. The above definition however is not general, for instead of the nitre, it has lately been discovered that the marine acid answers much better, as to strength, but is dangerous in its use, as too liable to accidental explosion.

The invention of gunpowder is ascribed, by Polydore Virgil, tu a chemist; who having accidentally put some of this comprsition in a mortar, and covered it with a stone, it happened to take fire, and blew up the stone. Theret informs us that the person bere spoken of was a monk of lribourg, named Constantine Anelzen; but Belleforet and nther authors, with more probability, hold it to be Bartholdus Schwartz, or Black, who discovered it, as some sny, about the year 1320; and the first use of it is ascribed to the Venetians, in the ycar 1380 , during the war with the Genoese. But there are earlier accounts of its use, after the accident of Schwartz, as well as before it.

VoL. 1.
For Peter Mexia, in his Various Readings, mentions that the Moors being besieged in 1343, by Alphonsus the 11th, king of Castile, discharged a kind of iron mortars upon them, which made a noise like thunder; and this is seconded by what is related by Don Pedro, bishop of Leon, in his chronicle of King Alphonsus, who reduced Toledo, viz, that in a sea-combat between the king of Tunis and the Moorish king of Seville, about that time, those of Tunis had certain iron tubs or barrels, with which they threw thunderbolts of fire. Ducange adds, that there is mention made of gunpowder in the registers of the chambers of accounts in France as early as the year 1338.

But it appears that Roger Bacon knew of gunpowder near 100 years before Schwartz was born. He tells us, in his Treatise De Secretis Operibus Artis et Naturæ, et de Nullitate Magiæ, cap. 6, (which is supposed by some to have been published at Oxford in 1216, and which was undoubtedly written before his Opus Majus, in 1267), "that from saltpetre, and other ingredients, we are able to make a tire that shall burn at what distance we please." Dr. Plott also in his History of Oxfordshire, pa. 236, assures us that these " other ingredients were explained in is MS. copy of the same treatise, in the hands of Dr. G. Langbain, and seen by Dr. Wallis, to be sulphur and wood coal." In addition to whicls we are told in the life of Friar Bacon in Biographia Britannica, vol. 1, that Bacon himself has divulged the secret of this composition in a cipher, by transposing the letters of the two words in chap. xi. of the said treatise; where it is thus expressed: sed tamen salis petræ LURA MOPE CAN UBRE (i. e. carbonum pulvere) et sulphuris; et sic facies conitrum et corruscationem, si scias artificiuin: and hence the biographer apprehends the words carbonum pulvere were transferred to the 6 th chapter of Langbain's ms. In this same chapter Bacon expressly says that sounds like thunder, and corruscations, may be formed in the air, much nore horrible than those that happen naturally. And farther adds, that there are many ways of doing this, by which a city or an army might be destroyed: and he supposes that by an artifice of this kind Gideon defeated the Nidianites with only 300 men: Judges, chap. $70^{\circ}$ There is also another passage to the same purpose, in the treatise De Scicntia Experimentali. 'See Dr. Jebb's edition of the Opus Majus, pa. 474. See also the life of R. BACON in this Dictionary:

Mr. Robins, in the preface to his gunnery, apprehends that Bacon describes gunpowder not as a new composition first proposed by himself, but as the application of an old one to military purpuses, and that it was known long before lis time.

But M. Dutens carries the antiquity of gunpowder still much higher, and refers to the writings of the ancients themselves for the proof of it. "Virgil," says he, " and his Commentator Servius (Aineid, lib? 6, v. 585), Hyginus (Fabul. 61 and 650), Eustathius (ad Odyss, $\lambda 234$, pa. 1682, lib. 1), La Cerda (in Virgil. loc. cit.), Valerius Flaccus (lib. i. 662), and many other authors (as Raphacl Volatarran. in Commentar. Cornelius Agrippa poster. Oper. de Verbo Dei, c. 100, p'a. 237.-Gruteri Fax Artium Liberal, tom. 2, pa. 1236), speak in such a manner of Salmoneus's attempts to initate thunder, as suggest to us that this prince used for that purpose a composition of ilse nature of gunpowder. Eustathius in particular mentions him on this occasion, as being so vary expert in mechanics, that he formed machines, which imitatcl the noise of thunder ; and the writers of fable, whose surprise in this $4 \mathrm{~K}$ 
respect may be compared to that of the Mexicans when they first beheld the fire-arms of the Spaniards, gire out that Jupiter, incensed at the audacity of this prince, slew him with lightning, as he was employing himself in launching bis thunder. But it is much more natural to suppose that this unfortunate prince, the inventor of gunpowder, gave rise to these fables, by baving accidentally fallen a victim to his own experiments. Dion (Hist. Rom. in Caligula, pa. 662) and Joannes Antiochenus (in Chronico, \&c. a Valesio edita, Paris 1634, pa. 804), report the very same thing of Caligula, assuring us that this emperor imitated thunder and lightning by means of certain machines, which at the same time emitted stones. Themistius also informs us that the Brachmans encountered one another with thunder and lightning, which they had the art of launching from on high at a considerable distance; (Themist. Oratio 27, pa. 337).- $A$ nd in another place he relates, that Hercules and Bacchus, attempting to assail them in a fort where they were entrenched, were so roughly reccived by reiterated strokes of thunder and lightning, that they were obliged to retire, leaving behind then an everlasting monument of the rashness of their enterprise. Agathias the historian reports of Anthemius Traliensis, that having disagreed with his neighbour Zeno the rhetorician, he set fire to his house with thunder and lightning. It appears from all these passages, that the efiects ascribed to these engines of war, especially those of Caligula, Anthenius, and the Indians, could be unly brought about by gunpowder. And what is still more, we find in Julius Africanus a réceipt for an ingenious composition to be thrown upon an enemy, which very nearly resembles that powder. But what places this beyond all cloubt, is a clear and positive passage of an author called Marcus Græcus; whose work in manuscript is in the royal library at Paris, entitled Liber Ignium. Dr. Mead had the same also in manuscript, and a copy of that is now in my hands. (See above.) The author describes several ways of encountering an enemy, by launching fire upon him; and among others gives the following. Mix together one pound of live sulphur, two of charcoal of willow, and 6 of saltpetre ; reducing them to a very fine powder in a marble mortar. He adds, that a certain quantity of this is to be put into a long, narrow, and well compacted cover, and so discharged into the air. Here we have the description of a rocket. The cover with which thunder is imitated, be represents as short, thick, but half-filled, and strongly buund with packthread; which is exactly the form of a cracker. He then treats of difierent methods' of preparing the match, and low one squib may set fire to another in the air, by having it inclosed within it. Iu short, he speaks as clearly of the composition and eflects of gunpowder, as any person in our times could do. I own I have not yet been able precisely to determine when this author lived, but probably. it was before the time of the Arabian physician Mesue, who mentions him, and who flourished in the beginning of the 9th century. Nay, there is reason to believe that he is the same of whom Galen speaks; in which case he will be of antiquity sufficient to support what I advancc." It appear's also from many authors, and many circumstances, that this composition has been known to the Chinese and Indians for thousands of years. Ste what is said on this head under the article GuN.

'To this history of gunpoweler it may be added, that it has lately been cliscovered that saltpetre or nitre is not es- sential to this composition, but that its place may be supplicd by otber substances; for new gunpowder, of double the strength of the old, has lately been made in France, by the chemists in that country, without any nitre at all; and in the year $1790 \mathrm{I}$ tried some of this new powder, that was made at Woolwich, with my eprouvette, when I found it about double the strength of the ordinary sort. This is effected by substituting, instead of the nitre, the like quantity of the marine acid.

But perhaps this new composition may not come into common and general use; both because of the great expense in procuring or making the acid, and of the trouble and danger of preventing it from taking fire by the beat in the operation ; for it is found to catch fire and explode from a very small degree of heat, and without the aid of a 'spark.

As to the Preparation of Gu NPOW DER; there are divers compnsitions of it, with respect to the proportions of the three ingredients, to be met with in pyrotechnical writings ; but the process of making it up is much the same in all.

For some time after the invention of artillery, gunpowder was of a much weaker composítion than that now in use, or that described by Marcus Gracus; which was chiefly owing to the weakness of their first pieces. See Gun and Cannon. Of 23 different compositions, used at different times, and mentioned by Tartaglia in his Ques. and Inv. lib. 3, ques. 5, the first, which was the oldest, contained equal parts of the three ingredients. But when guns of modern structure were introduced, guspowder of the same composition as the present came also into use. In the time of Tartaglia the cannon powder was made of 4 parts of nitre, one of sulphur, and one of charcoal ; and the musket powder of 48 parts of nitre, 7 parts of sulphur, and 8 parts of charcoal; or of 18 parts of nitre, 2 parts of sulphur, and 3 parts of charcoal. But the modern composition is 6 parts of nitre, to one of each of the other two ingredients. Though Mr. Napier says, he finds the strength commonly to be greatest when the proportions are, nitre $3 t b$, charcoal about $90 z$, and sulphur about $30 z$. See his paper on Gunpowder in the Transactions of the Royal Irish Academy, vol. 2. The cannon powder was in meal, and the musket powder grained. And it is certain that the graining of powder, which is a very considerable advantage, is a modern improvement. See the preface to Robins's Math. Tracts, pa.32.

In making gunpowder, regard is to be had to the purity or goodness of the ingredients, as well as the proportions of them; for the strength of the powder depends much on that circumstance, and also on the proper working or mixing them together.

T'o purify the nitre, by taking away the fixt or common salt, and earthy part. Dissolve it in a quantity of hot water over the fire; then filtrate it through a flannel bag, into an open vessel, and set it aside to cool, and to crystallize. These crystals may in like manner be dissolved and crystallized again; and so on, till they become quite pure and white. 'Then put these crystals into a dry kettle over a moderate fire, which gradually increase tilt it begins to smoke, evaporate, lose its humidity, and grow very white: it must be kept continually stirriug with a lackle, lest it should return to its torner figure, by which its greasiness would be taken away: after that, so much water is to be poured into the kettle as will cover the vitre; and when it is dissolved, and reduced to the consistency. of a thick liquor, it must be continually stirred with a ladle till all 
the moisture is again cvaporated, and it be reduced to a dry and white meal.

'The like regard is to be had to the sulphur; choosing that which is in large lumps, clear and perfectly yellow; not very hard, nor compact, but porous; nor yet too much shining; and if, when set on fire, it fieely burns all away, it is a sign of its goodness: so likewise, if it be pressed between two iron plates that are hot enough to make it run, and in the running it appears yellow, and that which remains of a reddish colour, it is then fit for the purpose. But in case it be foul, it may be purified in this manner: melt the sulphur in a large iron ladle, or pot, over a very gentle coal fire, we!l kindlal, but not flaming; then scum off all that rises on the top, and swims upon the sulphur; take it presently after from the fre, and strain it through a double linen cloth, letting it pass leisurely; so will it be pure, the gross matter remaining behind in the cloth.

For the charcoal, the third ingrelient, such should be chosen as is large, clear, and free from knots, well burnt, and cleaving. The charcoal of light woods is mostly preferred, as of willow, and that of the branches or twigs of a moderate thickness, as of an inch or two in diameter. Dogwood is now much esteemed for this purpose. And a method of charring the wood in a large iron cylinder has lately been recommended, and indeed proved, as yielding better charcoal than formerly. The charcoal not only concurs with the sulphur in supplying the inflammable matter, which causes the detonation of the nitre, but also greatly adds to the explosive power of it by the quantity of elastic vapour expelled during its combustion.

These three ingredients, in their purest state, being procured, long experience has shown that they are then to be mixed together in the proportion before mentioned, to have the best effect, viz, three-quarters of the composition to be nitre, and the other quarter made up of equal parts of the other two ingredients; or, which is the same thing, 6 parts nitre, 1 part sulphur, and 1 part charcoal.

But it is not the due proportion of the materials only, which is necessary to the making of good powder; auother circumstance, not less essential, is the mixing them well together: if this be not effectually done, some parts of the composition will have too much nitre in them, and others too little; and in either case there will be a defect of strength in the powder. Robins, pa. 119.

After the materials have been reduced to fine dust, they are mixed together, and moistened with water, or vinegar, or urine, or spirit of wine, \&c, and then beaten together with wooden pestles for 24 hours, either by hand, or by mills, and afterwards pressed into a hard, firm, and solid cake. When dry, it is grained or corned; which is done hy lreaking the cake of powder into small pieces, and so rumuing it through a sieve; by which means the grains may have any size given them, according to the nature of the sicve cmployed, cither finer or coarser; and thus also the dust is separated from the grains, and again mixed with other manufacturing powder, or worked again into rakes.

Powder is smoothed, or glazed, as it is called, for small arms, by the following operation: a hollow cylinder or cask is mounted on an axis, turned by a wheel; this cask is half filled with powder, and turned for. 6 hours; and thus by the mutual friction of the grains of powder it is smoothed, or glazed. The fine mealy part, thus separated or worn off from the rest, is again granulated.
The Nature, Efjects, \&: of Pouder.-When the powiter is prepared as above, it the least spark be struck upon it from a steel and flint, the whole will immediately intlame, and burst forth with extreme violence. The effect is by no means difficult to account for: the charcoal part of the grain upon which the spark falls, catching tire like tinder, the sulphur' and nitre are readily melted, and the former also breaks into flame; the contiguous grains at the sane time undergoing the same decomposition.

Upon this point Sir Isaac Newton reasons thus: The charcoal and sulphur in gunpowder easily take fire, and kindle the nitre; and the spirit of the nitre, being thereby rarefied into vapour, rushes out with an explosion much after the manner that the vapour of water rushes out of an eolipile; the sulphur also, being volatile, is converted into vapour, and augments the explosion: add, that the acid vapour of the sulphur, namely that which distils under it bell into oil of sulphur, entering violently into the fixt body of the nitre, lets loose the spirit of the nitre, and excites a greater fermentation, by which the heat is farther augmented, and the fixt body of the nitre is also rarefied into fume; and the explosion in cunsequence made more vehement and quick.

For if salt of tartar be mixed with gunpowder, and that mixture be warmed till it takes fire, the explosion will be far more violent and rapid than that of gunpowder alone; which cannot proceed from any other cause, than the action of the vapour of the gunipowder upon the salt of tartar, by which that salt is rarefied.

The explosion of gumpowder therefore arises from the violent action, by which all the mixture being quickly and vehemently heated, is rarefied and converted into fume and vapour; which vapour, by the violence of that action becoming so hot as to shine, appears in the form of a flane.

M. Lahire, in the History of the French Acadeny for 1702 , ascribes all the force and effect of gunpowder tu the spring or elasticity of the air inclosed in the several grains of $i t$, and in the intervals or spaces between the grains: the powder being kindled, sets the springs of so many little parcels of air into action, and dilates them alt at once, whence the effect; the powder itself only serving to light a fire which may put the atir in action; after which the whole is performed by the air alone.

But it appears from the experiments and observations of Mr. Robins, that if this air be in its natural state at the time when the powder is fired, the greatest addition its elasticity could acquire from the flame of the explosion, would not amount to five times its usual quantity, and therefore could not suffice for the 200th part of the effort which is exerted by fired powder. To uhderstand the force of gunpowder, it must be consicleres that, whether it be fired in a vacuum or in air, it produces by its explosion a permanent elastic fluid. See Philos. Trans. No. 295; also Hauksuee's Phys. Mechan. Exp. pa. 81. It also appears from experiment, that the elasticity or pressure of the fluid produced by the firing of gunpowder, is, cæteris paribus, directly as its clensity.

In order to determine the elasticity and quantity of this elastic fluid, produced from the explosion of a given quantity of gunpowder, Mr. Robins premises, that the elasticity of this fluid increases by heat, and dininishes by cold, in the same manner as common air; and that the density of this fluid, and consequently its weight, is the same with the weight of an equal bulk of air, having the same elusticity and the same temperature. From these $4 \mathrm{~K} 2$ 
principles, and from the experiments by which they are istablished (for a detail of which we must refer to the bunk itself, so often cited in the preceding articles), he conclides that the fluid produced by the firing of gunpowder is nearly $\mathbf{T}^{3}$ of the weight of the generating powder itself; and that the volume or bulk of this air or fluid, when expanded to the rarity of common atmospheric air, is about $2+4$ times the bulk of the said generating powder; and which 1 have proved to be 1600 times the bulk it occupied in the nitre (Tracts, vol. 3, pa. 303). Count Saluce, in his Mliscel. Phil. Mathem. Soc. Priv. Taurin. pa. 125, makes the proportion as 222 to 1 ; which he says agrees with the computation of Mess. Hauksbee, Amontons, and Belidor.

Hence it appears, that any quantity of powder fired in any confined space, which it adequately filts, exerts at the instant of its explosion against the sides of the vessel cuntaining it, and the bodies it impels before it, a force at least 244 times greater than the elasticity of common air, or, which is the same thing, than the pressure of the atmosphere; and this, without considering the great addition arising from the violent degree of heat with, which it is endued at that time; the quantity of which augmentation is the next head of Mr. Robins's enquiry. He determines that the elasticity of the air is increased in a proportion somewhat greater than that of 4 to 1 , when lieated to the extremest heat of red hot iron; and supposing that the flame of fired gunpowder is not of a less degree of heat, increasing the former number a little more than 4 times, makes nearly 1000 ; which shows that the clasticity of the flame, at the moment of explosion, is about 1000 times stronger than the elasticity of common air, or than the pressure of the atmosphere. But, from the height of the barometer, it is known that the pressure of the atmosphere upon every square inch, is on a medium $14, \frac{3}{4} \mathrm{lb}$; and therefore 1000 times this, or $14750 \mathrm{lb}$, is the force or pressure of the flame of gunpowder, at the in-oment of explosion, upon a square inch, which is very nearly equivalent to 6 tons and a half. And it is proved in my Tracts, vol. 3 , that the furce is more than double of this. This great force however diminishes as the fluid dilates itself, and in that proportion, viz, in proportion to the space it occupies, it being only half the strength when it occupies a double space, one third the strength when triple the space, and so on.

Mr. Robins further supposes the degree of heat above mentioned to be a kind of medium heat; but that in the case of large quautities of powder the beat will be higher, and in very small quantities lower; and that therefore in the former case the force will be somewhat more, and in the latter somewhat less, than 1000 times the force or pressure of the atm sphere.

He further found that the strength of powder is the same in all variations in the density of the atmosphere. But that the moisture of the air has a great effect upon it; for the same quantity which in a dry season would discharge a hullet with a velocity of 1700 feet in one second, will not in damp weather give it a velocity of more than 12 or 1300 feet in a second, or even less, if the powder be bakl, and improperly kept. Robins's Tracts, vol. 1, pa. 101, \&c. Farther, as there is a certain quantity of water which, when mixed with powder, will prevent its firing at alt, it cannot be doubted but every degree of inoisture must abate the violence of the explosion; and hence the cffects of damp powder are not difficult to be accounted for.
It is to be observed, however, that the moisture imbibed by powder does not render it less active when dried again. Indeed, if powder be exposed to very great damps without any caution, or when common salt abounds in it, as often happens through negligence in refining the nitre, in such cases the moisture it imbibes may perhaps be sufficient to dissolve some part of the nitre: which is a permanent damage that no drying can retrieve. But when tolerable care is taken in preserving powder, and the nitre it is composed of has been well purged from common salt, it will retain its force for a long time; and it is said that powder has been known to have been preserved for 50 years without any apparent damage from its age.

'The velocity of expansion of the flame of gunpowder, when fired in a piece of artillery, without either bullet or other body placed before it, is prodigiously great, viz, 7000 feet per second, or upwards, as appears from the experiments of Mr. Robins. But Mr. Bernoulli as well as Euler suspect it is still much greater; though 1 have found it to be nuch the same as Mir. Robins. See my Tracts, vol. 3 , pa. 306 .

It is this prodigious celerity of expansion of the flame of tired gunpowder, which is its peculiar excellence, and the circumstance in which it so eminently surpasses all other inventions, either ancient or modern: for as to the momentum of these projectiles only, many of the warlike machines of the ancients produced this in a degree far surpassing that of our heariest cannon shot or shells; but the great celerity given to these bodies cannot be in the least approached by any other means than the flame of powder.

To prove Gunpowder. 'There are several ways of doing this. 1, By sight: thus, if it be too black, it is a sign that it is moist, or else that it contains too much eharcoal ; so also, if rubbed upon white paper, it blackens it more than guad powder dues: but if it be of a kind of azure colour, somewhat inclining to red, it is a proof of good powder. 2 , By touching : for if in crushing it with the fingers' ends, the grains break easily, and turn into dust, without fecling hard, it has too much coal in it ; or if, in pressing it under the fingers upon a smooth hard board, some grains feel harder than the rest, it denotes that the sulphur is not well mixed with the nitre. Also by thrusting the hand into the parcel of powder, and grasping it, as if to take out a handful, you will feel whether it be dry and equally grained, by its eyading the grasp, and running mustly out of the hand. 3, By burning: and here the method most commonly followerl for this purpose with us, says Mr. Robins, is to fire a small heap of it on a clean board, and to attend nicely to the flame and smoke it produces, as wetl as to the marks it leaves behind on the board: but besides this uncertain method, there are other contrivances made use of, such as powder-triers acting by a spring, commonly sold at the shops, and others again that move a great weight, throwing it upwards, which is a very bad surt of cprouvitte. But these machines, says. Mr. Robins, though more perfect than the common powder-triers, are yet liable to great irregularitics; for as they are all moved by the instantaneous stroke of the flanc, and not by its continued pressure, they do not determine the force of the fired powder with sufficient certainty and uniformity. Another method is to judge from the range given to a large solid ball, thrown from a very short mortar, charged with a small quantity: of powder; which is also an uncertain way, both on account of the great disproportion between the weight of 
the ball and powder, and the unequal resistance of the air; not to mention that it is too tedious to prove large quantities of powder in this way ; for, " if each barrel of powder was to be proved in this manner, the trouble of charging the mortar, and bringing back the ball each time, would he intolerable, and the delay so great, that no business of this kind could ever be. finished; and if a number of burrels are received on the merit of a few, it is great odds, but some bad ones would be among them, which may prove a great disappointınent in time of service." These exceptions do "noways hold," continues Mr. Robins, "against the method by which 1 have tried the comparative strength of different kinds of powder, which has been by the actual velocity given to a hullet, by such a quantity of powder as is usually esteemed a proper clatrge for the piece; and as this velocity, however great, is easily discovered by the motion which the pendulum acquires from the stroke of the bullet, it might seem a good amendment to the method used by the French (viz, that of the small inurtar above-mentioned) to introduce this trial by the pendulum instead of it. But though I am satisfied, that this would be much more accurate, less laborious, and readier than the other, yet, as there is some little attention and caution required in this practice, which might renider it of less dispatch than might be convenient, when a great number of barrels were to be sepa. rately tried, 1 should myself choose to practise another method not less certain, but prodigiously more expeditious; so that I could engage, that the weighing out of a small parcel of powder from each barrel should be the greatest part of the labour; and doubtless three or four hands could, by this means, examine 500 barrels in a morning : besides, the machines for this purpose, as they might be made of cast-iron, would be so very cheap, that they might be multiplied at pleasurè." Robins, page 123 . It is not certainly known what might be the particular construction of the eprouvette here hinted at, but it was probatly a piece of ordnance suspended like a pendulum, as he had made several experiments with a barrel in that manner. Be this however as it may, several persons, from those ideas and experiments of Mr. Robins, have made eprouvettes on this principle, which appears to be the best of any; and on this idea also 1 have lately made a machine for this purpose, which hay several peculiar contrivances, and ailvantages over all others, both in the nature of its motion, and the divisions on its arc, \&c. It is a small cannon, the bore of which is about one inch in diameter, and is usually charged with 2 ounces of powder, and with powder only, as a ball is not necessary, and the strength of the powder is accurately shown by the arc of the gun's recoil. The whole machine is so simple, easy, and expeditious, that, as Mr. Robins observed above, the weighing of the powder is the chief part of the trouble; and so accurate and uniform, that the successive repetitions or firings with the same quantity of the same kind of powder, seldom gave a difference in the recoil of the 100 th part of itself. Sec the construction and use of this eprouvette in my 'Tracts, vol. 3 , tract.35.

To recover damaged Powder. The method of the powder merchants is this: they put part of the powder on a sail-cloth, to which they add an equal weight of what is really good; then with a shovel they mingle it well together, dry it in the sun, and barrel it up, keeping it in a dry and proper place.

Others agrain, if it be very bad, restore it by moistening it with vinegar, water, urine, or brandy; they then beat it fine, sift it, and to every pound of poweler add an ounce, or an ounce and a half, or two ounces (according as it is decayed), of melied nitre ; and afterivards these ingredients are to be moistened and well mixed, so that nothing may be discerried in the composition, which may be known by cutting the mass, and then they granulate it as useful.

In case the powder be quite spoiled, the only way is to extract the saltpetre with water, in the usual way, by boiling, filtrating, evaporating, and crystallizing; and then, with fresh sulphur and clarcoal, to inake it up again.

On the subject of gunpowder, sce also Euler on Robins's Gunnery, Antoni Examen de la Poudre, Baumé's Chemistry, and Thompson's Experiments in the Philos. Trans. for 1781 .

GUNTER (EDmuxD), an excellent English mathematician, was born in Hertfordshire in 1581 . He was educated at Westminster-school under Dr. Busby, and from thence was elected to Christ-church college, Uxford, in 1599, where he took the degree of master of arts in 1606 , and afterwards entered into holy orders; and in 1615 he took the degree of bachelor of divinity. But being particularly distinguished for his mathematical talents, when Mr. Williams resigned the protessorship of astronomy in Gresbam-college, London, Mr. Gunter was chosen to succeed him, the 6ih of March, 1619; whese he greatly distinguished himseli by his lectures and writings, and where he died in 1626 , at only 45 years of age, to the great loss of the mathematical world.

Mr. Gunter was the author of many useful works and inventions. About the year 1606 , he merited the title of an inventor, by the new projection of his Sector, which he then described in a Latin treatise, not printed however till some time afterwards.-ln 16 is he had invented a small portable quadrant, for the more readily finding the hour and azimuth, and other uscful purposes in astronomy.And in 1620 or 1623 , he published his Canon Triangulorum, or Table of Artificial Sines and Tangents, to the radius, $10,000,000$ parts, to every minute of the quadrant, being the first tables of this kind published; together with the first 1000 of Briggs's logarith ms of comınon numbers, which were in later editions extended to 10,000 numbers.-In 1622, he discovered, by experiment made at Deptford, the variation or changeable declination of the magnetic needle; his experiment showing that the declination had varied 5 de'grees in the space of +2 years; and the same was confirmed and established by his successor $\mathrm{Mr}$. Gellibrand.-He applied the logarithms of numbers, and of sines and tangents, to straight lines drawn on a scale or ruler; with which, proportions in comınon numbers and trigonometry were resolved, by the mere application of a pair of compasses; a method founded on this property, that the logarithms of the terms of equal ratios are equidifferent. This was called Gunter's Proportion, and Gunter's Line; and the instrument, in the form of a two-foot scale, is still in common use for navigation and other purposes, and is commonly called the Gunter. He also greatly improved the sector and other instruments, for the same uses; the description of all which he published in 1624.- - lle introduced the common measuring chain, now constantly used in land-surveying, which is thence called Gunter's chain.-Mr. Gunter drew the lines on the dials in Whitchall-garden, and wrote the description and use of them, by the direction of Prince Charles, id a sizalk 
tract; which he afterwards printed at the desire of King James, in 1624 . - He was the first who used the word cosine, for the sine of the complement of an are. He also introduced the use of arithmetical complements into the logarithmical arithmetic, as is witnessed by Briggs, cap. 15, Arith. Log. And it has been said that he first started the idea of the Logaritlmic Curve, which was so called, because the segments of $i$ ts axis are the logarithms of the corresponding ordinates.

His works have been collected, and various editions of them have been published; the 5 th is by Mr. William Leybourne, in 1673 , contuining the Description and Use of the Sector, Cruss-stafi, Bow, Quadrant, and other instruments; with several pieces added by Samuel Foster, Henry Bond, and William Leybourne.

GUNTER's CHarN, the chain in common use for measuring land, according to true or statute measure; so called from Mr. Gunter its reputed inventor. The length of the chain is 66 feet, or 22 yards, or 4 poles of $5 \frac{x}{2}$ yards each; and it is divided into 100 links, of 7.92 inches each. This chain is the most convenient of any instrument for measuring land, because the contents thence computerl are so easily reduced to acres. The reasnn of which is, that an acre of land is just equal to 10 square chains, or 10 chains in lengtl and 1 in breadth, or equal to 100,000 square links. Hence, the dimensions being taken in chains, and multiplied together, it gives the content in square chains; which therefore being divided by 10 , or a figure cut off for decimals, brings the content to acres; after which the decimals are reduced to roods and perches, by multiplying by 4 and 40 . But the better way is to set the dimensions down in links as integers, considering each chain as 100 links; then, having multiplied the dimensions together, producing square links, divide these by 100,000 , that is, cut off five places for decimals, the rest are acres, and the decimals are reduced to roods and perches, as betore.

Example. Suppose in measuring a rectangular picce of ground, its length be 795 links. and its breadth 480 links.

$\frac{33600}{6180}$
Ac. $\frac{3.81600}{3}$
Ro. $\frac{4+264}{40}$
Per. $\frac{40.560}{10.0}$

So the content is 3 ac. 3 roods 10 perches.

GuNTER's LiNe, a Logarithmic line, usually graduated upon scales, scctors, \&c; and so called from its inventor Mr. Gunter. This is otherwise called the line of lines, or line of numbers, and consists of the Jogarithms transferred upon a ruler, \&c, from the tables, by means of a scale of equal parts, which therefore serves to resolve problems instrumentally, in the same manner as logarithms do arithmetically. For, whereas logarithms resolve proportions, or perform multiplication and division, by only addition and subtraction, the same are performed on this line by turning a pair of compasses over this way or that, or by slicling onc slip of wood by the side of another, \& $\mathrm{c}$.

A line of this description has been contrived various ways, for the arlvantage of having it as long as possible. As, first, on the two fect ruler or scale, by Gunter. 'Then, in 1627 the logarithms were drawn by Wingate, on two separate rulers, sliding against each other, to save the use of compasses in resolving proportions. They were also in 1627 applied to concentric circles by Oughtred. Then in a spiral form by $\mathrm{Mr}$. Mlilburne of Yorkshire, about the year 1650 . Also, in 1657 , on the present common sliding rule, by Seth Partridge.

Lastly, Mr. William Nicholson has proposed another disposition of them, on concentric circles, in the Philos. Trans. an. 1787 , pa. 251 . His instrument is eyuivalent to a straight rule of $28 \frac{\mathrm{I}}{2}$ inches iong. It cousists of threc concentric circles, engraved and graduated on a plate of about $1 \frac{1}{2}$ inch in diameter. From the centre proceed two legs, having right-lined edges in the direction of radii; which are moveable either singly, or together. To use this instrument; place the edge of one leg at the antecedent of any proportion, and the other at he consequent, and fix them to that angle: the two legs being then moved together, and the antecedent leg placed at any other number, the other leg gives its consequent in the like position or situation on the lines.

The whole length of the line is divided into two equal intervals, or radii, of 9 larger divisions in each radius, which are numbered from 1 to 10 , the 1 standing at the the begining of the line, because the logarithm of 1 is 0 , and the 10 at the end of each radius; also each of these 9 spaces is subdivided into 10 other parts, unequal according to the logarithms of numbers; the smaller divisions being always 10ths of the larger; thus, if the large divisions be units or ones, the smaller are tenth-parts; if the larger be tens, the smaller are ones; and if the larger be 100 's, the smaller are 10's; \&c.

Use of Gunter's Line. 1. To find the product of two numbers. Extend the compasses from 1 to either of the numbers, and that extent will reach the same way from the other number to the product. Thus, to multiply 7 and 5 together; extend the compasses from $1 \mathrm{t}$ (s) 5 , and that extent will reach from 7 to 35 , the product.

2. To divide one number by another. Extend the compasses from the divisor to 1 , and that extent will reach the same way from the dividend to the quotient. Thus, to divide 35 by 5 ; extend the complasses from 5 to $\mathrm{l}$, and that extent will reach from 35 to 7 , which is the quotient.

3. To find a 4 th proportional to three given numbers; as suppose to 6,9 , and 10 . Extend from 6 to 9 , and that extent will reach from 10 to 15 , which is the 4 th proportional sought. In like manner, a $3 \mathrm{~d}$ proportional is found to two given terms, extending from the 1 st to the $2 \mathrm{~d}$, and then from the $2 \mathrm{~d}$ to the $3 \mathrm{~d}$.

4. To find a mean proportional between two gizen numbers, as suppose between 7 and 28 Extend from 7 to 28 , and bisect that extent; then its half will reach from 7 forward, or from 28 backward, to 14 , the mean proportional between them.-Also, to ex tract the square root, as of 25 , which is only finding a mean proportional between 1 and the given square 25, bisect the distance between 1 and 25 , and the half will reach from 1 to 5 , the root sought.-In like manner the cube or $3 d$ root, or the $4 . t h, 5 t h$, or any ligher root, is found, by taking the extent between 1 and the given power; then take such part of it as is denoted by the index of the root, viz, the 3 \& part for the cube root, the 4 th part for the 4 th root, and so or, and that part will reach from 1 to the root sought.

If the line on the scale or ruler have a slider, this is to bo used instead of the compasses. 
GunTER's QUADRANT, is a quadrant made of wood, brass, or some other substance; being a kind of stereographic projection on the plane of the equinoctial, the eye being supposed in one of the poles: so that the tropic, ecliptic, and horizon, form the arches of circles, but the hour circles other curves, drawn by means of several altitudes of the sun, for some particular latitude every day in the year.

The use of this instrument, is to find the hour of the the day, the sun's azimutb, \&c, and other common problems of the sphere or globe; as also to take the altitude of an object in degrees. See Quadrant.

GunTER's ScaLe, usually called by seamen The Gunter, is a large plain scale, having various lines upon it of great use in worling the cases or questions in Navigation. This scale is usually 2 feet long, and about an inch and a half broad, with various lines upon it, both natural and logarithmic, relating to trigonometry, and navigation, \&cc.

On the one side are the natural lines, arid on the other the artificial or logarithmic ones. The former side is first divided into inches and tenths, and numbered from 1 to 24 inches, running the whole length near one edge. One half the length of this side consists of two plane diagonal scales, for taking off dimensions to three places of figures. On the othcr half or foot of this side, a re contained various lines relating to trigonometry, in the natural numbers, and marked thus, viz,

Rumb, the rumbs or points of the compass,

Chord, the line of chords,

Sine, the line of sines,

Tang. the tangents,

S. $\boldsymbol{T}$. the semitangents,

and at the other end of this half are

Leag. Jeagues, or equal parts,

Rumb. another line of rumbs;
$M$. L. miles of longitude,

Chor. another line of chords.

Also in the middle of this foot are $L$. and $P$. two other lines of equal parts. And all these lines, on this side of the scale, serve for drawing or laying down the figures to the cases in trigonometry and navigation.

On the other side of the scale are the following artificial or logarithmic lines, which serve for working or resolving those càses ; viz,

$S . R$. the sine rumbs,

$T . R$. the tangent rumbs,

Numb. line of numbers,

Sine, Sines,

$V . S$, the versed sines, .

Tang. the tangents,

Meri. Meridional parts,

$E . P$. Equal parts.

The late Mr. John Robertson, clerk and librarian to the Royal Suciety, greatly improved this scale, both as to size and accuracy, for the use of mariners. He extended it to 30 inches long, 2 inches broad, and balf an inch thick; upon which the several lines are very accurately laid down by Mcssrs. Nairne and Blunt, ingenious instrument makers. Mr. Robertson died before his improved scales were published; but the account and description of them were supplied and dravn up by his friend Mr. William Mountaine, and published in 1778 .

GUTTE, or Drops, in Architecture, are ornaments in form of little bells or cones, used in the Doric order, on the architrave, below the tryglyphs. There are usually six of them.

GYMNOSOPHISTS, a set of Indian philosophers, famous in antiquity; so denominated from their going barefoot; as that of Peripatetics was given to those who philosophized walking.

\section{H A I}

H ADLEY (JoH N), a distinguished member of the Royal Society, was elected a fellow in 1717 , and died Feb. 15,1744 . He contributed many useful papers to the Royal Society, which are contained in their Trans. from vol. 32 to vol. 39 inclusive. But he has been chicfly celcbrated for the invention of his reflecting instrument for taking angles, conmonly called the Iladley's Quadrant or Sextant, offered to the Royal Society in 1731, by which his name has been rendered immortal.

HADLEY's Quadrant, Sextunt, \&c, an excellent instrument so called from its inventor John Hadley, esq. See jts descrijtion and use under the article QUADRANT.

HAIL, or HAILSTOXES, an aqueous concretion, usually in form of white or pellucid spberules, descending out of the atmosphere. Hailstones assume various shapes, being sometimes round, at other times pyramidal, crenated, anyular, thin, and flat, and sometimes stellated, with six radii like the small crystals of snow.

It is very difficult to account for the phenomena of hail in a satisfactory manner; and there are various opinions in this head. It is usually conceived that hail is formed of drops of rain, frozen in their passage through the mid-

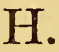

H.

dle region. Others, as the Cartesians, take it for the fragments of a frozen cloud, half melted, and thus precipitated and congealed again. Signor Beccaria supposes, that it is formed in the higher regions of the air, where the cold is intense, and where the electric matter is very copious. In these circumstances, a great number of particles of water are brought near together, where they are frozen, and in their descent they collect other particles; so that the density of the substance of the hailstone becomes less and less, from the centre; this being formed first in the higher regions, and the surface being collected in the lower. Accordingly, in mountains, hailstones as well as drops of rain, are very small; and both agree in this circunstance, that the more intense the electricity is that forms them, the larger they are. It is frequently observed that hail attends thunder and lightning; and hence Beccaria observes, that as motion promotes freezing, so the rapid motion of the electrified clouds may promote that effect in the air. Natural histories furnish us with a great variety of curious instances of extraordinary showers of hail. See the Philos. Trans. No. 203, 229; and Hist. de France, tom. 2, pa. 33 
HALF-MOON, in Fortification, is an outwork having only two faccs, forining together a saliant angle, which is flanked by some part of the place, and of the other bastions. See Demilune and Ravelin.

HALF-TANGENTs, are the tangents of the half arcs. See Scale and Semitangents.

HALLEY (Dr. EDNUND), a most eminent English mathematician, philosopher and astronomer, was born in the parish of St. Leonard, Shoreditch, near London, Oct. 29, 1656. His father, a wealthy citizen and soap-boiler, resolving to improve the promising disposition observed in his son, put him first to St. Paul's sch(o)l, where be soon excelled in all parts of classical learning, and made besides a considerable advance in the mathematics; so that, as Wood observes, he hall perfectly learnt the use of the celestial globe, and could make a complete dial; and we are informed by Halley himseli, that he observed the change of the variation of the magnetic needle at London in 1672, one year before he left schnol. In 1673 he was was sent to Oxford, where he chiefly applied himself to mathematics and astronomy, in which he was greatly assisted by a curious apparatus of instruments, which his father, willing toencourage his son's genius, had purchased for him. At 19 years of age he began to oblige the world with new obscrvations and discoveries (which he continued to do to the end of a very long life), by publishing " A Direct and Geometrical Method of finding the Aphelia and Excentricity of the planets." Besides various particular observations, made from time to time on the celestial phenomena; he had, from his first admission into college, pursued a general scheme for ascertaining the true places af the fixed stars, and by this means to correct the errors of Tycho Brahé. His original view in this was, to carry on the design of that first restorer of astronomy, by completing the catalogue of those stars from his own observations ; but, on further inquiry, finding this province taken up by Hevelius and Flamsteed, he dropped that pursuit, and formed another; which was, to perfect the whole scheme of the heavens, by the addition of the stars which lie so near the south pole, that they could not be observed by those astronomers, as never rising above the horizon either at Dantzick or at Greenwich. With this intention he left the University, before he had taken any degree, and embarked for the island of St. Helena in Nov. 1676, when lie was only 20 years of age, and arrived there after a voyage of three months. He immediately set about his task with such diligence, that he completed his catalogue, aud, returning home, landed in England in Nov. $167 \mathrm{~s}$, after an absence of two years only. The university of $\mathrm{Ox}$ ford inmediately conferred apon hin the degree of $\Lambda . M$. and the Royal Suciety of London elected him one of their inembers.

In 1679 he was selected by the Royal Society to go to Dantzick, to endeavour to adjust a dispute between Hevelius and Mr. Hooke, concerning the preference betiveen plain and glass sights, in astroscopical instruments. He arrived at Dantzick the 26th of May, when he immediatcly, in conjunction with Hevclius, set about their astronomical observations, which they closely continued till the 18 th of July, when Ialley left Dantzick, and returned to England.

In the year 1680 he undertook what is called the grand tour, accompanicd by his friend the celebrated Mr. Nelbon. In the way from Calais to Paris, Mr. Halley had a sight of a remarkable comet, as it then appeared a second time that year, in its return from the sun. He had, the November before, seen it in its descent : and he now hastened to complete his observations upon it, by viewing it from the royal observatory of France. His design in this part of his tour was, to settle a friendly correspondence between the two roy'al astronomers of Greenwich and Paris; and in the nsean time to improve himself under şo great a master as Cassini. From theuce he went to Italy, where he spent a great part of the year $16 \mathrm{~s} 1$; but his affairs calling him home, he theu returned to England.

Soon after his return, he married the daughter of Mr. 'Tooke, auditor of the exchequer, and took up his residence at Islington, where he set up his tube and sextant, and eagerly pursued his favourite study: in the society of this amiable lady he lived happily for five-and-fifty years. In 1683 he published his Theory of the variation of the Magnetical Compass; in which he supposes the whole globe of the earth to be one great magnet, having four magnetical poles, or points of attraction, \&c. The same year also he entered upon a new method of finding the longitude, by an accurate observation of the moon's motion. His pursuits it seeins were now a little interrupted by the death of his father, who having suffered greatly by the fire of London, as well as by a second marriage, into which he bad imprudently entered, was found to have wasted his fortune. Our author however soon resumed his pursuits; for in the beginning of 1684 he turned his thoughts to the subject of Kepler's sesqui-alterate proportion; when, after some meditation, he concluded from it, that the centripetal force must decrease in proportion to the square of the distance reciprocally. He forind himself unable to make it out in any gcometrical way, and therefore, after applying in vain for assistance to $\mathrm{D}$. Hooke and Sir Christopher IVren, he went to Cambridge to Mr. Newton, who fully supplicd him with what be so ardently sought. But Halley having now found an immense treasure in Newton, could not rest, till he had prevailed with the owner to enrich the public with it; and to this interview the world is in some measure indebted for the Principia Mathematica Philosophia Naturatis. That great work was published in 1686 ; and Halley, who had the whole care of the impression, prefixed to it a cliscourse of his own, giving a general account of the astronomical part of the book; and also an elegant copy of verses in Latin.

In 1687 he undertook to explain the cause of a natural phenomenon, which had till then baffled the researches of the ablest geographers. It is observed that the Mediterranean sea never swells in the least, though there is no visible discharge of the prodigious quantity of water that runs into it from nine large rivers, besides several small ones, and the constant spiting in of the current at the mouth of the Straits. His solution of this difficulty gave so much satisfaction to the R. Society, that he was requested to prosecute these ençuiries. He did so; and having shown, by many accurate experiments, how that vast accession of water was acturlly catried off in vapours, raised by the action of the sun and wind upon its surface, he proceeded with the like stccess to point out the mcthed used by nature to returm the said valpours into the sea. This circulation he supposes to be carried on by the winds driving these vapours to the mountains; where being collected, they form springs, which uniting becone rivulets or brooks, and many of thesc again meeting in the valleys, grow into large rivers, cmptying themsclies at 
last into the sea: thus demonstrating, in the most beantiful and satisfactory manner, the way in which the equilibrium of receipt and expense is continually preserved in the universal ocean.

He next ranged in the field of speculative geometry, where, observing some imperfections in the methods before given for constructing solid problems, or equations of the $3 \mathrm{t}$ and 4 th powers, he furnished new rules, which were buth more easy and more elegant than any of the former; together with a new method of finding the number of roots of such equations, and the limits of the same. Mr. Halley next undertook to publish a more correct Ephemeris for the year 168s, there being then great want of proper ephemerides of any tolerable exactness, the conmon ones being justly complained of by Mr. Flamsteed.-In 1691 he published exact tables of the conjunctions of Venus and Hercury; and he afterwards showed one extraordinary use to be made of those tables, viz, for discovering the sun's parallax, and thence the true distance of the earth from the sun.-In 1692, our author produced his tables for showing the value of annuities on lives, calculated from bills of mortality; and his universal theorem for finding the fuci of optic glasses.

But it would be endless to enumerate all his valuable discoveries now communicated to the Royal Socicty, and published in the Philos. Trans. of which, for many years, his pieces were the chief ornament and support. Their various merit is thrown into one view by the writer of his eloge in the Paris Memoirs; who, having mentioned his History of the 'Trade-winds and Monsoons, proceeds in these terms: "This was immediately followed by his estimation of the quantity of vapours which the sun raises from the sea; the circulation of vapours; the origin of fountains; questions on the nature of light and transparent bodies; a determination of the degrees of mortality, in order to adjust the valuation of annuities on lives; and many other works in the sciences, relating to astronomy, geometry, and algebra, optics and dioptrics, ballistics and artillery, speculative and experimental philosophy, natural bistory, antiquities, philology, and criticism; being about 25 or 30 dissertations, which he produced during the 9 or 10 ycars of his residence at London; and all abounding with ideas new, singular, and useful."

In $169 \mathrm{l}$, the Savilliau professorship of astronomy at $\mathrm{Ox}-$ ford being vacant, he applied for that office, but without success. Whiston, in the Memoirs of bis own Life, tells us from Dr. Bentley, that Halley " being thought of for successor to the mathematical chair at Oxford, bishop Stillingfleet was desired to recommend him at court; but hearing that he was a sceptic and a banterer of religion, the bishop scrupled to be concerned, till his chaplain Bentlcy should confer with him on the subject, which he did. But Halley was so sincere in his infidelity, that he would not so much as pretend to believe the Christian religion, though he was likely to lose a professorship: and it was in consequence given to Dr. David Gregory."

Halley had published his Theory of the Variation of the Magnetical Compass, as has bcen already observed, in 1683; which, though it was well received both at home and abroad, he found, upon a review, liable to great and insuperable objections. Yet the phenomena of the variation of the ncedle, upon which it is raised, being so many certain and indisputable facts, he spared no pains to possess himself of all the observations relating to it he could possibly come at. To this end he procured an applica. VoL. I. tion to be made to King William, who appointed bim commander of the Paramour pink, with orders to scarch out by observations the discovery of the rule of variations, and to lay down the longitudes and latitudes of the English settlements in America.-He set out on this attempt on the 24th of November, 1698; but having crossell the litie, his men grew sickly, and his first-lieutenant mutinging, he returned to England in June 1699. Having got the lieutenant tried and cashiered, he set sail a second time in September following, with the same ship, and another of less bulk, of which he had also the command. He now traversed the vast Atlantic ocean from one hemisphere to the other, as far as the ice would permit him to go; and having made his observations at St. Helena, Brazil, Cape Verde, Barbadoes, the Madeiras, the Canaries, the coast of Barbary, and many other latitudes, he arrived in England in Scptember 1700; and the next year published a general chart, showing at one vicw the variation of the compass in all those places.

Captain Halley, as he was now called, had bcen at home little more than half a year, when he was sent by the king, to observe the course of the tides, with the longitude and latitude of the principal head-lands in the British channel; which having exccuted with his usual expedition and accuracy, he published a large map of the channel. Soon after, the Emperor of Germany resolving to make a convenient harbour for shipping in the Adriatic, Captain Halley was sent by Queen Anne to view the two ports on the coast of Dalmatia. He embarked on the $22 \mathrm{~d}$ of November 1702 ; passed over to Holland; and going through Germany to Vienna, be proceeded to Istria; but the Dutch opposing the design, it was laid aside; yet the cmperor made him a present of a rich diamond ring from his finger, and honoured hin with a letter of recommendation, written with his own hand, to Queen Anne. It was not long after his return that lic was sent again on the same business; when passing through Hanover, he supped with the Electoral Prince, who was afterwards King George the 1st, and his sister the queen of Prussia. On his arrival at Vienna, he had likewise the honour the same evening to be presented to the emperor, who sent his chicf engineer to attend him to Istria, where they repaired the fortifications of Trieste, and adderl new ones.

Mr. Halley returned to England in November 1703 ; and the same year he was made professor of geometry in the university of Oxford, instead of Dr. Wallis, then just deceased. On his accession to this office the university was pleased to honour him with the degree of doctor of laws. He was scarcely settled in Oxford, when he began to translate into Latin, from the Arabic, Apollonius de Sectione Rationis; and to restore the two books De Sectione Spatii of the same author, which are lost, from the account given of them by Pappus; and he published the whole work in 1706 . He afterwards had a share in preparing for the press Apollonius's Conics; and ventured to supply the whole 8 th book, the original of which is also lost. To this work be added Screnus on the Section of the Cylinder and Cone, printed from the original Greek, with a Latin translation, and published the whole in folio 1710 . Besides these, the Miscellanea Curiosa, in 3 volumes $8 \mathrm{vo}$, had come out under his direction in 1708 .

In 1713, he succeeded Doctor, afterwards Sir, Hans Sloane, in the office of secretary to the Royal Society. And, on the death of Mr. Flamsteed in 1719, he was appointed to succeed lim at Greenwich as astronomer-royal ; 4. $\mathrm{L}$ 
on whicli occasion, that he might be more at leisure to attend the duties of this important situation, be resigned the office of secretary to the Royal Society in 1721 . Though he was 63 or 64 years of age when he entered on his office at Greenwich, yet for the space of 18 years he watched the heavens with the closest attention, hardly ever missing an observation during all that time, and, without any assistant, performed the whole business of the observatory himself.

On the accession of the late king, the queen consort Caroline made a visit to the Royal Observatory: being pleased with every thing she sav, and understanding the smallness of the astronomer's salary (100l. a year), her majesty very graciously said that she would speak to the king to have it increased; to which Dr. Halley, alarmed, replied, "Pray your majesty do no such thing; for should the salary be increased, it niight become an object of emolument to place there some unqualified needy dependent, to the ruin of the institution." However, understanding that the Dr. had formerly served the crown as a captain in the navy, she soon after obtained a grant of his half-pay for that commission, which he accordingly enjoyed from that time during his life. An offer was also made him of being appointed mathematical preceptor to the Duke of Cumberland; but he declined that honour, on account of his advanced age, and the duties of his office. In 1729 he was chosen a foreign member of the Academy of Sciences at Paris.

About 1737 he was seized with a paralytic disorder in his right hand, which, it is said, was the first attack he ever felt on his constitution: however, he came as usual once a week, till within a very short time of his death, to meet his friends in town on Thursdays, the day of the Royal Society's meeting, at what is still called Dr. Halley's club. Itis paralytical disorder increasing, hisstrength gradually wore away, till be expired Jan. 14, 1742 , in the S6th year of his age; and his corps was interred in the church-yard of Lee near Blackheath.

Besides the works before mentioned, his principal publications are, 1. Catalogus Stellarum Australium. 2. Tabulæ Astronomicæ. 3. The Astronomy of Comets. With a great multitude of papers in the Philos. Trans. from vol. 11 to vol. 60 .

HALIFAX (John). See Holywood.

HALO, or ConoNA, a coloured circle appearing round the body of the sun, moon, or the larger stars.

Naturalists conceive the halo to arise from a refraction of the rays of light, in passing through the fine rare vesiculæo of a thin vapour, towards the top of the atmosphere.

Descartes observes, that the halo never appears when it rains; whence be concludes that this phenomenon is occasioned by the refraction of light in the round particles of ice, which are then floaking in the atmosphere; and to the difierent protuberance of these particles he ascribes the variation in the diameter of the halo. Gassendi supposes, that a halo is occasioned in the same manner as the rainbow ; the rays of light being, in both cases, twice refracted and once reflected within each drop of rain or vapour, and that the difference between then arises entirely from their different situation with respect to the observer. Dechales also 'endeavoured to show that the generation of the halo is similar to that of the rainbow; and that the reason why the colours of the halo are more dilute than those of the rainbow, is owing chicfly to their being formcd, not in large drops of rain, but in very small vapour.
But the most considerable and generally received theory, relating to the generation of balos, is that of Huygens. This celebrated author supposes halos, or circles round the sun, to be formed by small round grains of hail, composed of two different parts, the one of which is transparent, inclosing the other, which is opaque: And this is the geveral structure actually observed in hail. He further supposes that the grains or globules, that form these halos; consisted at first of soft snow, and that they bave been rounded by a continual agitation in the air, and thawed on their outside by the beat of the sun, \&c. And he illustrates lis ideas of their formation by geometrical figures.

Mr. Weidler endeavours to refute Huygens's manner of accounting for halos, by a vast number of small vapours, each with a snowy nucleus, coated round with a transparent covering. He observes, that when the sun paints its image in the atmosphere, and by the force of its rays puts the vapours in motion, and drives them toward the surface, till they are collected in such a quantity, and at such a distance from the sun on each side, that its rays are twice refracted, and twice reflected, they will when they reach the eye exhibit the appearance of a halo, adorned with the colours of the rainbow; which may happen in globular pellucirl vapours without snowy nuclei, as appears by the experiment of hollow glass spheres filled with water: therefure, whenever those spherical vapours are situated as before mentioned, the refractions and refections will happen every where alike, and the figure of a circular crown, with the usual order of colours, will be the consequence. Philos. Trans. No. 458.

Newton's theory of halos may be scen in his Optics, pa. 155. And this curious theory was confirmed by actual observation in June 1692, when the author saw by reflection, in a vessel of stagnated water, three halos, crowns, or rings of colours, about the sun, like three small rainbows concentric to his body. These crowns inclosed one another immediately, so that their colours proceeded in this continual order from the sun outward : bluc, white, red; purple, blue, green, pale yellow, and red; pale blue, pale red. Crowns like these sometimes appear about the moon. The more equal the globules of water or ice are to one another, the more crowns of colours will appear, and the colours will be the more lively. Optics, pa.28s.

There are several ways of exhibiting phenomena similar to these. The flane of a candle, placed in the midst of a steam in cold weather, or set at the distance of some feet from a glass window that has been breathed upon, while the spectator is also at the distance of some feet from another part of the window, or placed behind a glass receiver, when air is admitted into the vacuum within it to a certain density, in eacls of these circumstances will appear to be encompassed by a coloured halo. Also, a quantity of water being thrown up against the sun, will, as it breaks and disperses into drops, form a kind of halo or iris, exhibiting all the colours of the natural rainhow. Musschenbrock obscrved, that when the glass windows of his room were covered with a thin plate of ice on the inside, the moon seen through it was surrounded with a large and varionsly coloured halo; which, upon opening the window, he found arose cntircly from that thin plate of ice, becalise none was observed except through this plate. The same author concludes bis account of coronas with observing, that some density of vapour, or some thickness of the plates of ice, divides the light in its transnission 
either through the small globules or their interstices, into its separate colours; but what that density is, or what the size of the particles which compose the vapour may be, he does not pretend to deternine. Introd. ad Pbil. Nat. pa. 1037 .

It has often been observed that a halo about the sun or moon, does not appear circular and concentric to the luminary, but oval and excentric, with its longest diameter perpendicular to the horizon, and extended from the mon farther downward than upward. Dr. Smith ascribes this phenomenon to the apparent concave vault of the sky being less than a hemisphere. When the angle which the diameter of a balo subtends at the eye is $45^{\circ}$ or $46^{\circ}$, and the bottom of the halo is near the horizon, and consequently its apparent figure is most oval, the apparent vertical diameter is divided by the monn in the proportion of about 2 to 3 or 4 , and is to the horizontal diameter drawn through the moon, as 4 to 3 , nearly.- See farther on the subject of this article, Priestley's Hist. of Discoveries relating to Vision, pa. 596-613; and Smith's Optics, art. $16 \tau, 513,526,527, \& \mathrm{c}$.

HAMEL (JоH $ハ$-BAPTISTE DU), a very learned French philosopher and writer, of the 17 th century, was born in Lower Normandy in 1614 . At 18 years of age he published a treatise, in which he explained, in a very simple manner, and by one or two figures, Theodosius's 3 books on Spherics; to which he added a tract on trigonometry extremely perspicuous, and designed as an introduction to astronomy. He publisbed afterwards various other works on astronomy and philosophy. Natural philosophy, as it was then tanght, was only a collection of vague, knotty, and barren questions; when our author undertook to establish it upon right principles, and published his Astronomia Physica.

In $1666 \mathrm{M}$. Colbert proposed to Louis the 14th a scheme, which was approved of, for establishing a royal academy of sciences; and appointed our author secretary of it. In 1678, his Philosophia Vetus et Nova was printed at Paris in 4 vols, $12 \mathrm{mo}$; and in 1681 it was enlarged and printed there in 6 vols. Hamel wrote several other pieces; and his works in this way were collected and published at Nuremberg 1681 , in 4 volumes 4 to, under the title of Opera Philosophica et Astronomica. These were highly valued at that period, though the improvements in philosophy since that time have rendered them of little or no use in the present.

In 1697, our author resigned his place of secretary of the Royal Academy of Sciences; in which he was succeeded by M. Fontenelle. However, he published, in 1698, Regiæ Scientiarum Academiæ Historia, 4to, in four books; which being much approved, he afterwards augmented with two books more. This work contains an account of the foundation of the Royal Academy of Sciences, and its transactions, from 1666 to 1700 , and is now the most useful of all his works. He was Regius Professor of Philosupby, in which office he was succceded by M. Varignon, at his death, which happened Aug. 6, 1706, in the 93d year of his age.

HAMILTON (Dr. HuGH), a very respectable mathematician, and philosopher, was born at knock, in the county of Dublin, March 26, 1729. He entered Trinitycollege, Dublin, in November 1742, and obtained a fellowship in that college in 1751 , when he was only 22 years of age. In the year 1758 , he published his wellknown and much-admired Treatise on Conic. Sections; and the next year was elected Erasmus Smith's professor of natural philosophy. In the discharge of his duty as professor, he composed and published tour lectures introductory to the study of natural pliilosophy: and in the year 1762 , be wrote three philesophical essays on the ascent of vapours, the formation of clouds, rain, \&c ; on the nature of the aurora borealis, and the tails of comets; and on the principles of mechanics.

Dr. Hamilton resigned his fellowship in 1764 , having accepted the living of Kilmacrean, in the gift of the college: in $176 \tau$, he was collated to the parish of St. Anne's, Dublin. This latter preferment was soon exchanged for the deanery of Armagh. In 1772, he married; and soon after published his essay on the existence and attributes of the Supreme Being. In the year 1792, be made some important improvenents in the consiruction of the barometer; and in 1794, drew up a paper on the power of fixed caustic alkaline salts, to preserve the flesh of animals from putrefaction. All this time he continued to attend sedulously and conscientiously to his duties as a parisb priest. In January 1796, he was consecrated bishop of Clonfert; and about 3 years afterwards be was translated to the see of Ossory, of which he continued bishop nearly scven years. He died Dec. 1,1505 , in the 77 th year of his age.

The principal trait in the disposition of Bistop Hamilton, was unaffected humility : his chief intellectual characteristic, a patient manner of thinking. His theory of the solution of water in air is now explodecl, and his theury of the aurora borealis has yielded to that of M. Libes. (See Aurora Borealis). But his mechanical essays may still be read with pleasure and advantage. Dr. Hamilton's works have been lately collected and published by his son Alexander Hamilton, in 2 vols. 870; of which, the first contains the Latin edition of the Conic Sections.

HANCES, Haxcin Es, or Haxses, in Architecture, are certain intermediate parts of arches between the key or crown and the spring at the bottom, being perhaps about one-third of the arch, and situated nearer the bottom than the top or crown; and are otherwise called the spandrels.

HANDSPIKE, or HAxsPEc, a lever or piece of ash, elm, or other strong wood, for raising by the hand great weights, \&c. It is 5 or 6 feet long, cut thin and crooked at the lower end, that it may be forced more readily between things required to be separated, or under any thing that is to be raised. It is better than a crow of iron, because its length allows a better poise.

HARD Bodies, are such as are absolutely inflexible to any pressure or percussion whatever: differing from soft bodlies, whose parts jield and are easily muved among one another, without restoring themselves again; and from elastic bodies, the parts of which also yield and give way, but presently restore themselves again to their former state and situation. Hence, hard bodies do not bend, or indent, but break. It is probable however there are no bodies in nature that are absolutely or perfectly either hard, soft, or elastic; but all possessing these qualities, more or less, in some degree. M. Bernoulli asserts that bardness, in the common sense, is absolutely impossible, being contrary to the law of continuity. The laws of motion for hard bodies are the same as for soft ones, both being supposed to adhere together on their impact. And these two kinds of bodies might be comprised under the common name of Un-elastic.

HARDENING, the giving a greater degree of hardness to bodies than they had before. There are several ways 
of hardening jron and steel ; as by bammering them, quenching them in cold water, \&c.

Case-HaRDENING, is a superficial conversion of iron into steel, as if it were casing it; or covcring it with a thin coat of harder matter. It is thus periormid: Tuke cowhorn or hoof, well'dried in an oven, and beat it to powder; put equal quantities of this powder and of bay-salt into stale urine, or white-wine vinegar, and mix them well together; cover the iron or steel with this mixture, and wrap it up in loam, or plate iron, so as the mixture may touch every part of the work; then puit it in the fire, and blow the coaks, till the whole lump has acquired a bloodred heat, but no higher; lastiy, take it out, and quench it.-See STEEL, under which article are described otber processes for this purpose.

HARDING, a small new primary planet, called also Juno, discovered by the astronomer $\mathbf{M}$. Harding.

HARDNESS, or RIGIDITY, that quality in bodies by which their parts so cohere as not to yield inward, or give way to an external impulse, without instantly going beyond the distance of their mutual attraction; and therefore are not subject to any motion in respect of cach other, without breaking the body. There were many fanciful opinions among the ancients, concerning the cause of hardness; such as heat, cold, dryness, the hooked figure of the particles of matter. The Cartesians make the hardness of bodies to consist in rest, as that of soft and fuid ones in the motion of their particles.

Newton shows that the primary particles of all bodies, whether solid or fluid, are perfectly hard; and are not capable of being broken or divided by any power in nature. These particles, he maintains, are connected and kept together by an attractive power; and according to the circumstances of this attraction, the body is either hard, or soft, or even fluid. If the particulars be so disposed or fitted for each other, as to touch in large surfaces, the body will be hard; and the more so as those surfaces are the larger. If, on the contrary, they only touch in small surfaces, the body, by the weakness of the attraction, will remain soft. At present, many philosophers are of opinion, that hardness consists in the absence or want of the action of the universal fluid, or elementary fire, among the particles of the body, or a deficiency of what is called latent heat; while on the contrary, fluidity, according to them, consists in the motion of the particles, in consequence of the action of that elementary fire.

Hardness appears to diminish the cohesion of bodies, in some degree, though their frangibility or brittleness does not by any means keep pace with their hardness. Thus, though glass be very liard and very brittle; yet lint is still harder, though less brittle. Among the metals, these two properties seem to be more connected, though even here the connexion is by no means complete: for though steel be both the hardest and most brittle of all the metals; yet lead, which is the softest, is not the most ductile. Neither is hardness connected with the specific gravity of bodies; for a diamond, the hardest substance in nature, is little more than half the weight of the lightest metal. And as little is it connected with the coldness, or electrical properties, or any other quality with which wë are acquainted. Some lodies are rendered hard by cold, and others by different degrces of heat.

Mr. Quist and whers have constructed tables of the lardness of different substances. And the manner of constructing these tables, was by observing the order in which they were able to cut or make any impression upon one another. The following table, extracted from Magellan's edition of Cronstedt's.Mineralogy, was taken from Quist, Bergman, and Kirwan. The first column shows the hatdness, and the second the specific gravity.

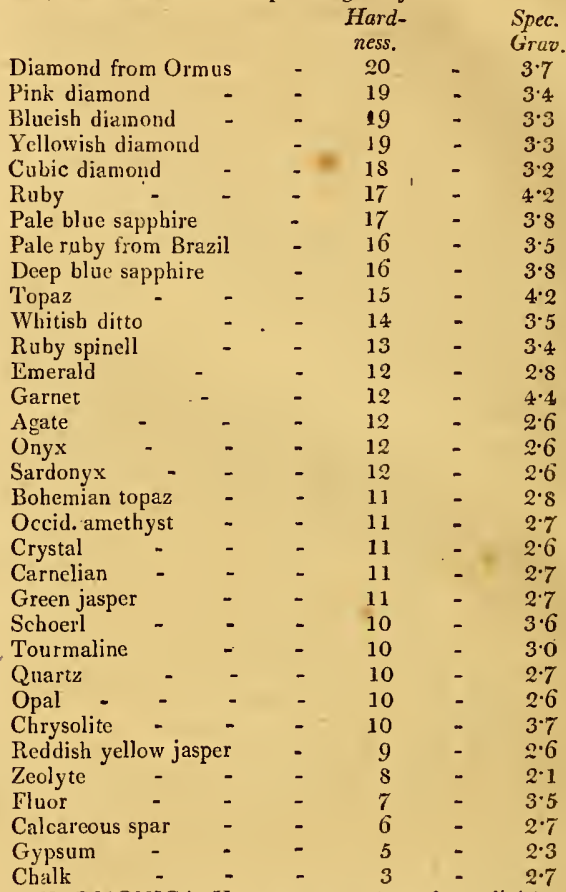

HARMONICA, HARMONICS, a branch or division of the ancient music; being that part which considers the differences and proportions of sounds, with respect to acute and grave; as distinguished from rhythmica, and metrica. Mr. Malcolin has inade a very industrious and learned inquiry into the Harmonica, or barmonic principles of the ancients.

HARMONICA, the name of a musical instrument invented by Dr. Franklin, consisting of the glasses, called musical glasses. - IVe are informed that the first hint of musical glasses is to be found in an old English book, in which a number of various amusements were described. That author directs his pupil to choose half a dozen drinking-glasses; to fill each of them with water in proportion to the gravity or acuteness of the sound which it is intended to give; and having thus adjusted them to each other, he might entertain the company with a church tune. These were perhaps the rude hints which Mr. Puckeridge, an Irish gentleman, afterwards improved, and after him, Mr. E. Delaval, an ingenious menuber of the Royat Society; and finally brought to perfection by the celebrated Franktin. See the history and description in his Letters, particularly in that to lieccaria.

HARMONICAL, or HARMONIC, something relating to Harmony. Thus, 
Harmonical Arithmetic, is so much of the theory and doctrine of numbers, as relates to making the comparisons and reductions of musical intervals, which are expressed by numbers, for finding their mutual relations, compositions, and resolutions.

HAR MONICAL Composition, in its general sense, includes the composition both of harmony and melody; i. e. of music, or song, both in a single part, and in several parts.

HannONICAL Interval, the difference between two sounds, in respect of grave and acute: or that imaginary space terminated with two sounds differing in acuteness or gravity.

HARMONICAL CURve, an imaginary kind of curve, into which it has been supposed that a musical chord would be inflected when put into such a vibratory motion as excited sound. It has been asserted by Dr. Brook Taylor, and others, that if a chord be inflected into any other form than that of the harmonic curve, it will, since those parts which are without this figure are impelled towards it, by an excess of force, and those within it by a deficiency, in a very short time arrive at, or very near, the form of this precise curve. But, as Dr. T. Young very properly remarks, "it would be easy to prove, if this reasoning were allowed, that the form of the curve can be no other than that of the axis, since the tending force is continually impelling the chord towards this line." Philos. Trans. 1800 , pt. 1.- In theory, a musical chord is conceived to be a mathematical line, flexible by the least force, and elastic: and when such chord, at the limit of its vibration, assumes the form of this harmonical curve, it will vibrate to and fro in curves of the same species.According to the investigation of Dr. Smith, the figure contained under the harmonical curve and its base, is of the same species as the figure of sines. From the investigation it follows: 1. That the force by which any small particle of an elastic string is impelled towards the centre of its curvature, is to the tending force, as the length of the particle, is to the radius of curvature. 2. The perpendicular distance of a particle from the string in its quiescent position, is to the string's length, as the same length, is to the radius of curvature into the square of 3.14159 . Hence, if any perpendicular distance $D n$ be put $=d$, the string's length $=s$, and $3 \cdot 14159=c$, then will the radius of curvature $\mathrm{D}=\frac{s s}{\pi c^{2}}$. 3. Each particle of the string will arrive at the axis at the same instant of time. But it is all along supposed that the greatest distance of the string from the axis, bears no sensible proportion to its length. See Smith's Harmon. pa. 248, \&c; also Notes to the Jesuits' Newton, tom. 2, pa. 348.

Hammonical Proportion, or Mrusical Proportion, is that in which the first term is to the third, as the difference of the first and second is to the difference of the $2 \mathrm{~d}$ and $3 \mathrm{~d}$; or when the first, the third, and the said two differences, are in geometrical proportion. Or, four terms are in harmunical proportion, when the 1st is to the 4 th, as the difference of the 1 st and $2 \mathrm{~d}$ is to the difference of the $3 \mathrm{~d}$ and 4 th. Thus, $2,3,6$, are in harmonical proportion, because $2: 6:: 1: 3$. And the four terms $9,12,16,24$ are in harmonical proportion, because $9: 24:: 3: 8$. - If the proportional terms be continued in the former case, they will form an harmonical progression, or series.

1. The reciprocals of an arithmetical progression are in harmonical progression; and, conversely, the reciprocals of harmonicals are arithmeticals. Thus, the reciprocals of the harmonicals $2,3,6$, are $\frac{x}{2}, \frac{x}{3}, \frac{x}{6}$, which are arithmeticals; for $\frac{1}{2}-\frac{1}{3}=\frac{x}{6}$, and $\frac{x}{3}-\frac{1}{6}=\frac{1}{6}$ also: and the reciprocals of the arithmeticals $1,2,3,4$, \&c, are $\frac{x}{1}, \frac{r}{2}, \frac{r}{3}$, $\frac{x}{4}$, \&c, which are harmonicals; for $\frac{1}{1}: \frac{1}{3}:: \frac{x}{1}-\frac{1}{2}: \frac{1}{2}-\frac{1}{3}$; and so on. And, in general, the reciprocals of the arithmeticals $a, a+d, a+2 d, a+3 d, \& \mathrm{c}$, viz,

$\frac{1}{a}, \frac{1}{a+d}, \frac{1}{a+2 d}, \frac{1}{a+3 d}, \& c$, are harmonicals; et $\mathrm{e}$ contra.

2. If three or four numbers in harmonical proportion be either multiplied or divided by some number, the products, or the quotients, will still be in harmonical proportion. Thus, the harmonicals multiplied by 2 give or divided by 2 give

which are also harmonicals.
3. To find a harmoniçal mean proportional between two terms: divide double their product by their sum.

4. To find a $3 \mathrm{~d}$ term in harmonical proportion to two given terms: divide their product by the difference between double the Ist term and the $2 \mathrm{~d}$ term.

5. To find a th term in harmonical proportion to three terms given; divide the product of the 1st and $3 \mathrm{~d}$ by the difference between double the 1 st and the $2 \mathrm{~d}$ term.

Hence, of the two terms 6 and 3 ,

the harmonical mean is 4 , the $3 \mathrm{~d}$ harmonical proportion is 2 , also to $6,4,3$, the 4 th harmonical is 2 .

6. If there be taken an arithmetical meall, and a harmonical mean, between any two terms, the four terms will be in geometrical proportion. Thus, between 6 and 2 , the arithmetical mean is 4 , and

the harmonical mean is 3 ;

and hence $6: 4:: 3: 2$.

7. Also the arithmetical mean, the geometrical mean, and the harmonical mean, between any two terms, are three terms in continued geometrical progression. So, between 6 and 2 , the arithmetical mean is 4 , the geometrical mean is $\sqrt{ } 12$, and the harmonical mean is 3 ; then 4 : $\sqrt{ } 12:: \sqrt{ } 12: 3$.

HARMONY, in Music, the agreeable result of an union of several musical sounds, heard at one and the same time; or the mixture of divers sounds, which together have an agreeable effect on the ear. $-\Lambda \mathrm{s}$ a continued succession of musical sotunds produces melody, so a continued combination of them produces harmony. Among the ancients, however, as sometimes also among the moderns, harmony is used in the strict sense of consonance; and so is equivalent to the symphony.

The words concord and harmony do really signify the same thing; though custom bas made a little difference between them. Concord is the agreeable effect of two sounds in consonance; harmony the effect of any greater number of agreeable sounds in consonance. Again, harmony always implies consonance; but concord is also applied to sounds in succession; though never but where the terms can stand agreeably in consonance. The effect of an agreeable succcssion of several sounds, is called melody; as that of an agreeable consonance is called harmony.

Harmony is well defined, the sum or result of the combination of two or more concords; that is, of three or more simple sounds striking the ear altogether; and different compositions of concords constitute various degrees of barmony. The ancients seem to have been entirely un. 
acquainted with harmony, the soul of the modern music. In all their explications of the melopeia, they say not one word of the concert or harmony of parts. We have instances, indeed, of their joining several voices, or instruments, in consonance; but then these were not so joined, as that each had a distinct and proper melody, so making a succession of various concords; but they were either unisons, or octaves, in every note; and so all performed the same individual melody, and constituted one song. When the parts differ, not in the tension of the whole, but in the difierent relations of the successive notes, it is this that constitutes the modern art of harmony.

H A RMON Y of the Spheres, or Celestial Harmony, a kind of music much spoken of by many of the ancient philosophers and fathers, supposed to be produced by the sweetly tuned motions of the stars and planets. This harmony they attributed to the various proportionate impressions of the heavenly bodies on one another, acting at proper intervals. They think it impossible that such prodigious bodies, moving with such rapidity, should be silent: on the contrary, the atmosphere, continually impelled by them, must yield a set of sonnds proportionate to the inpression it receives; and that consequently, as they run all in different circuits, and with various degrees of velocity, the different tones arising from the diversity of motions, directed by the hand of the Almighty, must form an agreeable symphony or concert. They therefore supposed, that the moon, as being the lowest of the planets, corresponded to $m i$; Mercury, to $f a$; Venus, to sol; the Sun, to la; Mars, to si; Jupiter, to ut; Saturn, to re; and the orb of the fixed stars, as being the bighest of all, to $m i$, or the octave.

It is thought that Pythagoras had a view to the gravitation of celestial bodies, in what he taught concerning the harmony of the spheres. A musical chord gives the same note as one double in length, when the tension or force with which the latter is stretched is quadruple; and the gravity of a planet is quadruple of the gravity of a planet at a double distance. In general, that any musical chord may become unison to a lesser chord of the same kind, its tension must be increased in the same proportion as the square of its length is greater; and that the gravity of a planet may become equal to the gravity of another planet nearer the sun, it must be increased in proportion as the square of $\mathrm{its}$ distance from the sun is greater. If therefore we should suppose musical chords extended from the sun to cach planet, that all these chords might become unison, it would be requisite to increase or diminish their tensions in the same proportions as would be sufficient to render the gravities of the planets equal; and from the similitude of those proportions, the celebrated doctrine of the harmony of the spheres is supposed to have been derivert.

Kepler wrote a large work, in folio, on the harmonies of the world, and particularly of that of the celestial bodics. He first endeavoured to find out some relation between the dimensions of the five regular solids, and the iutervals of the planetary spheres; and imagining that a cube, inscribed in the sphere of Saturn, would touch by its six planes the sphere of Jupiter, and that the other four regular solids in like mamner fitted the intervals that are between the spheres of the other planets, he became persuaded that this was the true reason why the primary planets were precisely six in number, and that the author of the world liad deternined their distances from the sun, the centre of the system, from a regard to this analogy. But afterwards finding that the disposition of the five regular solids among the planetary spheres, was not agreeable to the intervals between their orbits, he endeavoured to discover other schemes of harmony. For this purpose, be compared the motions of the same planet at its greatest and least distances, and of the different planets in their several orbits, as they would appear viewed from the sun; and here he funcied that he found a similitude to the divisions of the octave in music. Lastly, he imagined that if lines were drawn from the earth, to each of the planets, and the planets appended to them, or stretched by weights proportional to the planets, these lines would then sound all the notes in the octave of a musical chord. See his Harmonics; also Plin. lib. 2, cap. 22; Macrob. in Somn. Scip. lib. 2, cap 1; Plutarch. de Animal. Procreatione, è Timieo; and Maclaurin's View of Newton's Discov. book 1, chap. 2.

HAlRQUEBUSS, a bandi-gun, or a fire-arm of a proper length and weight to be borne in the arm. Hanzelet prescribes its proper length to be 40 calibres, or diameters of its bore; and the weight of its ball $1 \mathrm{oz}$. and 7 ; its charge of powder as much.

HARRIOT (Thomas), a very eminent English mathematician and astronomer, was born at Oxford in 1560 , and died at London July 2,1621 , in the 6 lst year of his age. Harriot has hitherto been known to the world only as an algebraist, though a very eminent one; but from his manuscript papers, that have been but lately discovered by Dr. Zach, astronomer to the duke of Saxe-Gotha, it appears that he was not less eminent as an astronnmer and geometrician. Dr. Zach has printed an account of those papers, in the Astronomical Ephemeris of the Royal Academy of Sciences at Berlin, for the year 1788; of which, as it is very curious, and contains a great deal of information, I shall here give a translation, to serve as memoirs concerning the life and writings of this eminent man; afterwards adding only some necessary remarks of my own.

"I here present to the worll," says Dr. Zach, " a short account of some valuable and curious manuscripts, which 1 found in the year 17\$4, at the seat of the earl of Egremont, at Petworth in Sussex, in hopes that this learned and inquisitive age will either think my endeavours about them worthy of its assistance, or else will be thereby induced to attempt some other means of publishing thein. The only undeniable proof 1 can now protuce, of the usefulness of such an undertaking, is by giving a stiecinct report of the contents of these materials, and briefly showing what may be effected by them. And though l come to the performance of such an enterprise with much less abilities than the different parts of it require, yet I trust that my love for truth, my design and zeal to vindicite the honour due to an Englishman, the author of these manuscripts, which are the chief reasons that lave influenced me in this undertaking, will serve as my excuse.

"A predecessor of the family of Lord Egrcmont, viz, that nohle and generous earl of Northumberlaud, named Ilenry Percy, was not only a generous firvourer of all good learning, but also a patron and Mrecenas of the literati of his age. Thomas Ilarriot, the author of the said manuscripts, Robert Hues (well known by his Treatise upon the Globes), and Walter Warner, all three eminent mathematicians, who wcre known to the earl, received from him yearly pensions; so that when the carl was committed pri- 
soner to the Tower of London in the year 1606 , our author, with Hues and Warner, were his constant companions; and were usually called the earl of Northumberland's three Magi.

"Thomas Harriot is a known and celebrated mathematician among the learned of all nations, by his excellent work, Artis Analyticæ Praxis, ad æquationes algebraicas nova expedita et generali methodo, resolvendas, Tractatus posthumus; Lond. 1631 : dedicated to Henry earl of Northumberland; published after his death by Walter Warner. It is remarkable, that the fame and the honour of this truly-great man were constantly attacked by the French mathematicians; who could not endnre that Harriot should in any way diminish the fame of their Vieta and Descartes, especially the latter, who was openly accused of plagiarism from our author. [See Montucla's Histoire des Mlathematiques, part 3 , pa. 485 et seq.-Lettres de M. Descartes, tom. 3, pa. 457 , edit. Paris 1667 , in 4 to.Dictionnaire de Morreri, word Harriot.-Encyclopedie, word Algelra.-Lettres de M. de Voltaire, sur la nation Angloise, lcttre 14.-Memoire de l'Abbé de Gua dans les Mem. de l'Acad. des Sciences de Paris pour 1741.-Jer. Collier's great Historical Dictionary, word Harriot.-Dr. Wallis's preface to his Algebra.-To which may be added the article Algebra, in this dictionary.]

"Descartes published his Geometry 6 years after Harriot's work apjeared, viz, in the year 1637. Sir Charles Cavendish, then ambassador at the French court at Paris, when Descartes's Geometry made its first appearance in public, observed to the famous geometrician Roberval, that these improvements in analysis had been already made these 6 years in England, and showed him afterwards Harriot's Artis Analyticæ Praxis; which, as Roberval was looking over, at every page he cried out, Oui! oui! il l'a vu! Yes! yes! he has seen it! Descartes had also been in England before Harriot's death, and had heard of his new improvements and inventions in analysis. A critical life of this man, which his papers would enable me to publish, will show more clearly what to think upon this matter, which I hope may be discussed to the due honour of our author.

"Now all this relates to Harriot the celebrated analyst; but it lias not hitherto been known that Harriot was an eminent astronomer, both theoretical and practical, which first appears by these manuscripts; among which the most remarkable are 199 observations of the Sun's Spots, with their drawings, calculations, and determinations of the sun's rotation about his axis. There is the greatest probability that Harriot was the first discoverer of these spots, cven before either Galileo or Scheiner. The earliest intelligence we have of the first discovered solar spots, is of one Joh. Fabricius Phrysius, who in the year 1611 published at Wittemberg a small treatise, intitled, De Maculis in Sole observatis et apparente corum cum Sole conversione narratio. Galileo, who is commonly accounterl the first discoverer of the solar spots, published his book, Istoria e Dimonstrazioni intorne alle Machic Solare e loro accidenti, at Rome, in the year 1613. His first observation in this work, is dated June 2, 1612. Angelo de Filiis, the editor of Galileo's work, who wrote the dedication and preface to it, mentions, pa. 3, that Galileo had not only discovered these spots in the month of April in the year 1611, at Rome, in the Quirinal Garden, but had shown them several montlis before (molti mesi innanzi) to his friends in Florence; and that the obser- vations of the disguised $\Lambda$ pelles (the Jesuit Scheiner, a pretencler to this first discovery) were not later than the month of October in the same year; by which the epoch of this discovery was fixed to the beginning of the year 1611. But.a passage in the first letter of Galileo's works, pa. 11, gives a more precise term to this discovery. Galileo there says in plain terms, that he had observed the spots in the sun 18 months before. The date of this letter is May 24, 1612; which brings the true eipoch of this discovery to the month of November, 1610. However, Galileo's first produced observations are only from June 2, 1612, and those of Father Scheiner of the month of October, in the same year. But now it appears from Harriot's manuscrints, that his first observations of these spots are of Dec. 8,1610 . It is not likely that Harriot could have this notice from Galileo, for I do not find this mathematician's name ever quoted in Harriot's papers. But I find him mentioning Josephus a Costa's book 1, chap. 2, of his Natural and Moral History of the West Indies, in which he relates that in Peru there are spots to be seen in the sun which are not to be seen in lurope. It rather seems that Harriot had taken the hint from thence. Besides, it is very likely that Harriot, who lived with so generous a patron to all good learning and improvements, had got the new invention of telescopes in Holland much sooner in England, than they could reach Galileo, who at that time lived at Venice. Harriot's very careful and exact observations of these spots, show also that he was in possession of the best and most improved telescopes of that time; for it appears he had some with magnifying powers of 10, 20, and 30 times. At least there are no earlier observations of the solar spots extant than lis: they run from December \$, 1610, till January 18, 1613 . I compared the corresponding ones with these observed by Galilen, between which I found an exact agreement. Had Harriot had any notion about Galileo's discoveries, he certainly would have also known something about the phases of Venus and Mercury, and especially about the singular shape of Saturn, first discovered by Galileo; but I find not a word in all his papers concerning the particular figure of that planet.

"Of Jupiter's Satellites. 1 found among his papers a large set of observations, with their drawing, position, and calculations of their revolutions and periods. His first observation of those discovered satellites, I find to be of January 16,1610; and they go till February 26, 1612. Galileo pretends to have discovered them January 7,1610 ; so that it is not improbable that Harriot was likewise the first discoverer of these attendants of $\mathrm{Ju}$ piter.

"Among his other observations of the moon, of some eclipses, of the planet Mars, of solsticcs, of refraction, of the dcclination of the needle; there are most remarkable ones of the noted comets of 1607 , and of 1618 , the latter, for there were two this year (see Kcpler de Cometis, pa.49). They were all observed with a cross-staff, by measuring their distances from fixed stars; whence these observations are the more valuable, as comets bad before been but loosely attended to: Kepler bimself observed the comet of 1607 only with the naked eye, pointing out its place by a rough estimation, without the aid of an instrument; and the elements of their orbits could, in defect of better observations, be only calculated by them. The observations of the comet of the year 1607, are of the more importance, cven now for moderu astronomy, as. 


\section{H A R}

this is the same comet that fulfilled Dr. Halley's prediction of its return in the year 1759 . That prediction was only grounded on the elements affurded him by these louse ubservations; for which reason he only assigned the term of its return to the space of a year. The very intricate calculations of the perturbations of this comet, afterwards made by M. Clairaut, reduced the limits to a month's space. But a greater light may now be thrown upon this matter by the more accurate ubservations on this comet by Mr. Harriot. In the month of October 1785, when I conversed on thesubject of Harriot's papers, and especially on this comet, with the very celebrated mathematician M. Lagrange, director of the Royal Academy of Sciences at Berlin, he then suggested to me an idea, which, if brought into execution, will clear up an important point in astronomy. It is well known to astronomers how difficult a matter it is, to determine the mass, or quantity of matter, in the planet Saturn; and how little satisfactory the notions of it are, that have hitherto been formed. The whole theory of the perturbations of comets depending on this uncertain datum, several attempts and trials have been made towards a more exact determination of $\mathrm{it}$ by the most eminent geometricians of this age, and partilarly by Lagrauge himself; but never having been satisficd with the few and uncertain data beretofore obtained for the resolution of this problem, he thought that Harriot's observations on the comet of $160 \bar{t}$, and the modern ones of the same comet in 1759 , would suggest a way of resolving the problem a posteriori ; that of determining by them the elements of its ellipsis. The retardation of the comet comparcd to its period, may clearly be laid to the account of the attraction and perturbation it has suffered in the region of Jupiter and Saturn; and as the part of it belonging to Jupiter is very well knuwn, the remainder must be the share which is due to Saturn; from whence the mass of the latter may be inferred. In consequence of this consideration I have alrcady begun to reduce most of Harriot's observations of this comet, in order to calculate by them the true elements of its orbit on an elliptical hypothesis, to complete M. Lagrange's idea upon this matter.

"I forbear to mention here any more of Harriot's analytical papers, which 1 found in a very great number. They contain several elegant solutions of quadratic, cubic, and bicjuadratic equations; with some other solutions and loca geometrica, that show his eminent qualifications, and will serve to vindicate them against the attacks of several French writers, who refuse him the justice due to his skill and accomplishments, mercly to save Descartes's honour, who yet, by some impartial men of his own nation, was accused of public plagiarism.

"Thomas Harriot was born at Oxford, in the year 1560. After being instructed in the rudinents of languages, be became a coinnioner of St. Mary's-Hlall, where he took the degree of buchelor of arts in 1579 . He had then so distinguished himself by his uncommun skill in mathematics, as to be recommended soon after to Sir Walter Raleigh, as a proper preceptor to him in that science. Accordingly that noble knight became his first patron, took him into his fumily, and allowed him a haridsome pension. In 1584, he went over with Sir Walter's first colony to Virginia; where he was employed in discovering and surveying the country, \&c; maps of which I have found (says Dr. Zach) very neatly executed among his papers. After his return lie published $A$ Bricf and
H A R

True Report of the Newfoundland of Virginia, of the Commodities there found to be raised, \&c ; Lond. 158s. This was reprinted in the $3 \mathrm{~d}$ volume of Hakluyt's Voyages: it was alsu translated into Latin, and printed at Frankfort in the year 1590 . Sir Walter introduced him to the acquaintance of the Earl of Northumberland, who allowed him a yearly pension; Wood says, of 120l. only; but by some of his receipts, which $I$ have found anong his papers, it appear's that he had $300 l$, which indeed was a very large sum at that time. Wood, in his Athen. Oxon. muntions nothing of Harriot's papers, except a manuseript in the library at Sion College, London, entitled Ephemeris Chyrometrica. I got access to this library and manuscripts, and was inced in hopes of finding something more of Harriot's; for most of his observations are dated from Siun Cullege; but I could not find any thing from Harriot himself. I found indeed some other papers of his friends: he mentions, in his observations, one Mr. Standish, at Oxford, and Nathaniel Torperly, who alsu was of the acquaintance of the Earl of Nortbumberland, and had a yearly pension: from the former I found two ubservations of the same comet of $161 \mathrm{~s}$, made in Oxford, which be communicated to Mr. Harriot.

" Thomas Harriot died July 2, 1621. His disease was a cancerous ulcer in the lip, which some pretend he got by a custom he had of holding the mathematical brass instruments, when working, in his mouth. I found several of his letters, and answers to them, from his physician Dr. Alexander Rhead, who in his treatise mentions Harriot's disease. His body was conveyed to St. Christopher's church, in London. Over his grave was soon after erected a monument, with a large and suitable inscription, which was destroyed with the church itself by the dreadful fire of September 1666. He was but 60 years of age."

The peculiar nature and merits of Harrjot's Algebra, we have spoken largely and particularly of, under the article Algebra. As to his manuscripts lately discovered by Dr. Zach, as above mentioned, it is with pleasure I can announce, that they are in a fair train to be published: they have been presented to the university of Oxford, on condition of their printing them; with a view to which, they have been lately put into the bands of an ingenious member of that learned body, to arrange and prepare them for the press. See Wuod's Athenæ Oxoniensis, vol. 1. pa. 390 .

HARIRIS (JOHN), D. D. and F. R. s. was elected into the Royal Society about the year 1696: became one of the secretaries in 1709, and died Sept.7, 1719. Dr. Harris published the first real dictionary of the arts and scicnces, under the title of Lexicon Technicum, in folio, 1699, showing the state of the sciences at that time, a good account of which is given in the Philos. Trans. for 1704, and in my Abridg. vol.5, pa. 149.Some years after, a $2 \mathrm{~d}$ volume was added, in a now alphabet, to include the new improvensents in science. A $3 \mathrm{~d}$ volume was afterwarils added, but supposed to be a compilation by the bookscllers. The whole was a very useful work, and went through several editions. He hail some papers inserted in the Philos. Trans. and publisbed several other useful books: as, a neat tract on Algebra, 8 vo, 1705; and another on Geometry and Plane Trigonometry, being chiclly a translation from the French of Pardics, the 2d edit. 1702.

About this time it seems that Dr. Harris " lived and 
taught mathematics at his house in Amen Corner." Hc wrote also, A New short Treatise of Algebra; with the Geometrical Construction of Equations, as far as the 4th power or Dimeusion: together with a specimen ol the nature and algorith of Fluxions. This tract is announcerl to bave been written primarily for the use of his auditors at the public mathematical lecture set up at the Marine Coffee-house, Birchin-lane, by Charles Cox, Esq. M.P. for Sonthwark. He also published Elements of Plain and Splerical Trigonometry, together with the principles of Spheric Gcometry, and the several projections of the Sphere in Plano. Also The Description and Uses of the Celestial and Terrestrial Globes, and of Collins's Pocket Quadrant, 4 th edition. He was also the author of a Collection of Voyages, 2 vols. fol. 1705 ; of which useful work there have been : some other editions, particularly one in 17.4 , much improved by Dr. John Campbell.

Dr. Harris appears to have been a patron of the mathematical Wm. Jones, Esq. the friend of Sir I. Newton, and father of the late learned Sir Wm. Jones, the celebrated Indian judge, as Mr. Jones dedicated to the Rev. Mr. John Harris his Treatise on Navigation, published in 1702 , in whose house Mr. Jones says he composed his said book.

HARRISON (JoIIN), a most accurate mechanic, the celebrated inventur of the famous time-keeper for ascertaining the longitude at sea, and also of the compound or gridiron pendulum ; was born at Foulby, near Pontefract in Yorkshire, in 1693. His father was a carpenter, in which profession our author assisted; occasionally also, according to the miscellaneous practice of country artists, surveying land, and repairing clocks and watches; and young Harrison always was, from his earliest years, greatly attached to any machinery moving by wheels. In 1700 he removed with his father to Barrow in Lincolnshire; where, though his opportunities of acquiring knowledge were very few, he eagcrly improved every incident fromı which he might collect information; frequently cmploying all or great part of his nights in writing or drawing: and he always acknowledged his obligations to a clergyman who came every Sunday to officiate in the neighbourhood, who lent him a Ms. copy of Professor Sanderson's lectures; which he carefully and neatly transcribed, with all the diagrams. His native genius excrted itself superior to these solitary disadvantages; for in the year 1726 , he had constructed two clocks, cliefly of wood, in which he applied the escapement and compound pendulum of his own invention : these surpassed every thing of the kind then made, scarcely erring a second in a month. In 1728 , he came up to London with the drawings of a machine for determining the longitude at sea, in expectation of being enabled to execute one by the Board of Longitude. On application to Dr. Halley, the astronomer-royal, he referred him to Mr. Gcorge Gralıam, who advised him to make his machine, before applying to that board. Accordingly our author returned home to perform his task; and in 1735 revisited London with his first machine; with which be was sent to Lisbon the next year, to make trial of it. In this short voyage, he corrected the dead reckoning about a degree and a half; a success which procured him both public and private encouragement. About the year 1739 he completed his sccond machine, of a construction much more simple than the former, and which answered much better : this, though not sent to sea, recommended VOL. I.
Mr. Harrison yet stronger to the patronage of his friends and the public. His third macline, which he produced in 1749 , was still less complicitted than the second, and more accurate, as erring only 3 or 4 scconds in a weck. This he conceived to be the ne plus ultra of his attempts; but by endeavouring to improve pocket-watches, he fount? the principles be applied to surpass his expcctations 'so much, as to encourage him to make his fourth timekeeper, which is in the form of a pocket-wateh, about 6 inches diameter. With this time-keeper his son made two voyages, the one to Jamaica, and the other to Barbadoes; in which experiments it corrected the longituile within the nearest limits required by the act of the 12 th of Queen Anne; and the inventor bad therefore, at different times, more than the proposed reward, receiving from $t l_{1}$ Board of Longitude at different times almost 24,000 ? . besides a fcw bundreds from the East India Company, \&c. These four machincs were given up to the Board of Longiturle. The three former were not of any use, as all the advantages gained by nuaking them were comprehended in the last : being worthy hawever of prescrvation, as mechanical curiosities, they are deposited in the Royal Observatory at Greenwich. The fourth machine, emphatically distinguished by the name of the time-keeper, was copied by the ingenious Mr. Kendal; and that duplicate, during a three-years circumnavigation of the globe in the soathern hemisphere by Captain Cook, answered as well as the original.

'The latter part of Mr. Harrison's life was employed in making a fifth improved time-keeper, on the same principles with the preceding one; which, after a ten-weeks trial, in 1772 , at the king's private observatory at Richmond, was found to have erred only $4 \frac{\pi}{2}$ seconds. Within a few years of his death, his constitution visibly declined; and he had frequent fits of the gout, a disorder that never attacked him before his 77 th year. His constitution at last yielding to the infirmities of old age, he died at his house in led-Lion Square, in 1776 , at 83 years of age.

Our author, like many other mere mechanics, found much difficulty in cuclivering his sentiments in writing (at least in the latter periods of his life, when his faculties were much inpaired), in which he adtsered to a peculiar and uncouth phraseology. This was but too evident in his Description concerning such Mechanism as will afiurd a nice or true Mensuration of Time, \&c. 8ro, 1775. In this snall work is included also an account of his new musical scale; being a mechanical division of the octave, according to the proportion which the radius and diameter of the circle have respectively to the circumference. He had in his youth been the leader of a band of church-singers; had a very delicate car for music; and his experiments on sound, with a curious monochord of his own improvement, it has been said were not less accurate than those he was engaged in for the mensuration of time.

HARTSOEISER (NichOLAs), a Dutch philosopher, born at Gouda, in 1656. After receiving a liberal education under his father, who was a minister under the remonstrants, he applied himself assiduously to mathematics and natural philosophy, and became so eminent that Peter the Great invited him to Moscow, but he declined the honour. He became professor of philosophy at Hei= d'elberg, and mathematician to the Elector Palatine. He dicd in 1725. Hartsocker wrote a Course of Natural Philosophy, in 4 to; and some small pieces.

HAUTEFEUILLE (Jors), an ingenious mechanic, 4. M 
born at Orleans in 1674. He made a great progress in mechanics in general, but had a particular taste for clockwork, and made several discoveries in it that were of singular use. It was he, it appears, who determined the method of moderating the vibration of the balance by means of a small steel spring, which has since been used. This discovery he laid before the members of the Academy of Sciences in 1694; and these watches are, by way of eminence, called pendulum watches; not that they have real pendulums, but because they nearly approach to the justness of pendulums. Huygens bas the merit of perfecting this happy invention: but having declared himself the inventor and obtained a patent for making watches with spiral springs, the abbe Feuille opposed the registering of it, and published a piece on the subject against Huygens. He died in 1724, at 50 years of age. Besides the above, he wrote a great many other pieces, most of whicb are small pamphlets, but very curious : as, 1. His Perpetual Pendulum. 2. New Inventions. 3. The Art of Breathing under Water, and the means of preserving a Flame shut up in a Sinall Place. 4, Reflections on machines for raising Water. 5. His Upinion on the different Sentiments of Mallebranche and Regis, relating to the Appearance of the Moon when seen in the Horizon. 6. The Magnetic Balance. 7. A Placet to the king on the Longitude. S. Letter on the Secret of the Longitude. 9. A New System on the Flux and Reflux of the Sea. 10. The means of making Sensible Experiments that prove the Motion of the Earth: and many other pieces.

HAYES (CH A RLEs, Esq.), a very singular person, whose great erudition was so concealed by his modesty, that his name is kuown but to few, though his publications are many. He was born in 1678 , and died in 1760 , at 82 years of age. He became distinguished in 1704 by a Treatise of Fluxions, in folio, being we believe the first treatise on that science ever published in the English language; and the only work to which he ever set his name. In 1710 came out a small 4to pamphlet, in 19 pages, entitled, A New and easy Method of determining the Longitude from observing the Altitudes of the Celestial Bodies. Also, in 1723, he published, The Mloon, a philosophical dialogue; tending to show, that the moon is not an opaque body, but has native light of her own.

To a skill in the Greek and the Latin, as well as, the modern languages, he added the knowledge of the Hebrew : and he published several pieces relating to the translation and chronology of the Scriptures. During a long course of years he had the chief management of thie late African company, being anuually clccted sub-governor. But on the dissolution of that company in 1752 , he retired to Down, in Kent, where he spent most of his hours in study; from whence however he returned, in 1758 , to chambers in Gray's-inn, London, where he died in 1760 , as mentioned above. He left a posthumous work, that was published in Svo, under the title of Chronographia Asiatica et Egyptiaca, \&c.

HEAD, in Architecture. Heads are uscd as an ornament of sculpture or carved work, frequently serving as the key of an arch or platband, and on other occasions.

HEAT, theopposite to cold, being a relative term denoting the property of fire, or of those bodies we denominate hot; being in us a sensation excited by the action of fire.

Heat, as it exists in the hot body, or that which constitutes and denominates a body hot, and enables it to produce such effects on our organs, is variously consider- ed by philosophers : some making it a quality, others a substance, and others only a mechanical affection. The former principle is given and nuantained by Aristotle and the Peripatetics. While the Ejicureans, and other corpuscularians, define heat not as an accident of fire, but as an essential power or property of $j$, the same in reality with it, and only distinguished from fire in the manrer of our conception. Heat, therefore, on their principles, is no other than the volatile substance of fire itself, reduced into atoms, and emitted in a continual stream from ignited bodies; so as not only to warm the objects within its reach, but also, if they be inflammable, to kindle them, turn them into fire, and conspire with them to make flame. In effect, these corpuscles, observe they, flying off from the ignited body, constitute fire while yet comained within the sphere of its flame; but when fled, or got beyond the same, and dispersed every way, so as to escape the apprehension of the eye, and only to be perceived by the feeling, they take the denomination of heat, inasmuch as they excite in us that sensation.

The Cartesians, improving on this doctrine, assert that heat consists in a certain motion of the insensible particles of a body, resembling the motion by which the several parts of our body are agitated by the motion of the heart and blood.

Our latest and best writers of mechanical, experimental, and chemical philosophy, differ very considerably about heat. The chief difference is, whether it be a peculiar property of one certain immutable body, called fire, or phlogiston, or electricity: or whether it inay be produced mechanically in other bodies, by inducing an alteration in their particles. The former tenet, which is as ancient as Democritus, and the system of atoms, had yielded to that of the Cartesians, and other mechanists ; but is now with great address retrieved, and improved on, by some of the latest writers, particularly Homberg, the younger Lemery, Gravesande, Boerhaave in his lectures on fire, Black, Crawford, and other chemical philosophers.

That which is denominated fire, according to Boerhave, is a body sui generis, created such ab origine, unalterable in its nature and propcries, and not either producible de novo from any other body, nor capable of being reduced into any other body, or of ceasing to be fire. This fire, he coutends, is diffused equably every where, and exists alike, or in equal quantity, in all the parts of space, whether void, or possessed by bochies; but that naturally, and in itself, it is perfectly latent and imperceptible; being only discovered by certain effects which it produces, and which are cognizable by our senses. These effects are heat, light, colour, rarefaction, and burning, which arc all indications of firc, as being none of them producible by any other cause; so that wherever we obscrve any of these, we may safely infer the action and presence of fire. But though the effect cannot be without the catloc, yet the fire may remain without any of these effects; any, we mean, gross enough to aficet our senses, or become objects of then : and this, he adds, is the ordinal'y case; there being a concurrence of other circumstances, which are often wanting, necessary to the production of such sensible eflects.

Heat, according to the mechanical philosophers, espccially Bacon, Boyle, and Newton, is considered not as an original inherent property of any particular kind of body; but as mechanically producible in any body. The former, in an express treatise De Forma Calidi, from 
a particular enumeration of the several phenomena and effects of heat, deduces several general properties of it ; and hence he defines heat, an expansive undulatory motion in the minute particles of the bridy; by which they tend, with some rapidity, towards the circumference, and at the same time incline a little upwards.

Mr. Boyle, in a treatise on the mechanical origin of heat and cold, strongly supports the doctrine of the producibility of heat, with new observations and experiments ; as in the instance of a smith briskly hanumering a sinall piece of iron, which, though cold at the commencement, soon becomes exceedingly thot.

This system is also further supported by Newton, who does not conceive fire as any purticular species of body, originally endued with such and such properties. Fire, according to him, is only a body much ignited, that is heated hot, so as to emit light copiously: what else, observes he, is red-hot iron but fire? and what else is a burning charcoal but red-hot wood? or flame itsclf, but redhot smoke? It is certain that flame is only the volatile part of the fuel heated red-hut, i. e. so hot as to shine; and hence only such bodies as are volatile, that is, such as emit a copious fume, will flame; nor will they flame longer than they have fume to burn. In distilling hot spirits, if the head of the still be taken off, the ascending vapours will catch fire from a candle, and turn into a flame. And in the same mannerseveral bodies, much heated by motion, attrition, fermentation, or the like, will emit lucid fumes, which, if they be copious enough, and the heat sufficiently great, will be flame; and the reason why fused metals do not flame, is the smallness of their fume; this is evident, because spilter, which fumes most copiously, does likewise flame. Add, that all flaming bodies, as oil, tallow, wax, wood, pitch, sulphur, \&c, by flaming, waste and vanish into burning smoke. And do not all fixed bodies, when heated beyond a certain degree, emit light, and shine? and is not this emission performed by the vibrating motion of their parts? and do not all bodies, which abound with terrestrial and sulphureous parts, emit light as often as those parts are sufficiently agitated, whether that agitation be made by external fire, or by friction, or percussion, or putrefaction, or by any other cause? Thus, sea-water, in a storm; quicksilver agitated in vacuo; the back of a cat, or the neck of a horse, obliquely rubbed in a dark place; wood, flesh, and fish, while they putrefy; vapours from putrefying waters, usually called ignes fatui ; stacks of moist hay or corn; glow-worms; amber and diamonds by rubbing; fragments of steel struck off with a flint, \&c, all emit light. Are not gross bodies and light convertible into each other, and may not bodics receive much of their activity from the particles of light which enter their composition? I know no body less apt to shine than water; and yet water, by frequent distillations, changes into fixed earth, whicb, by a sufficient heat, may be brought to shine like other bodies.

Let it further be considered, that the sun and stars, according to Newton's conjecture, are no other than great earths vehemently heated; for large bodies, he observes, preserve their heat the longest, their parts heating each other: and why may not great, dense, and fixed bodies, when heated beyond a certain degree, emit light so copiously, as by the emission and reaction of it, and the reflcctions and refractions of the rays within the pores, to grow still hotter, till they arrive at that degree of heat which the sun is of ? Their parts also may be further preserved from funing away, not only by their fixity, but by the vast weight and density of their atmospheres incunshent on them, thus strongly compressing them, and condensing the vapours and exhalations arising from them. Hence we see warm water, in an exhausted receiver, to boil as vehemently as the hottest water exposed to the air; the weight of the incumbent atmosphere, in this latter case, keeping down the vapours, and hindering the ebullition, till it has acquired its utmost degree of heat. So also a mixture of tin and lead, put on a red-hot iron in vacuo, emits a fume and flame; but the same mixture in the open air, by reason of the incumbent atmosphere, does uot emit the least sensible flame.

Thus much for the system of the producibility of heat. On the other hand, M. Homberg, in his Essai du Soufre Principe, holds, that the chemical prisciple or element, sulphur, which is supposed one of the simple, primary, pre-existent ingredients of all natural bodies, is real fire; and consequently that fire is co-eval with body. Mem. de l'Acad. an. 1705.

Dr. Gravesande goes upon much the same principle. According to him, fire enters the composition of all bodies, is contained in all bodics, and may be separated or procured from all bodies, by rubbing them against each other, and thus putting their fire in motion. But fire, he adds, is by no means generated by such motion. Elem. Phys. tom. 2, cap. 1. Heat, in the hot body, he observes, is an agitation of the parts of the body, made by means of the fire contained in it; by such agitation a motion is produced in our bodies, which excites the idea of heat in our minds: so that heat, in respect of us, is nothing but that idea, and in the hot body nothing but motion. If such motion expel the fire in right lincs, it may give us the idea of light; if in a various and irregular motion, only that of heat.

Lemery, the younger, agrees with these two authors, in asserting this absolute and ingenerable nature of fire; but he extends it falther. Not contented with confining it as an element to bodies, he endeavours to show, that it is equably diffused through all space; that it is present in all places, even in the void spaces between the bodies, as well as in the insensible interstices between their parts. And this last sentiment falls in with that of Boerhaave above delivered. Mem. de l'Acad. an. 1713.

Philosophers have lately distinguished heat into absolute and sensible. By absolute beat, or fire, they mean that power or element which, when it is in a certain degree, excites in animals the sensation of heat; and by sensible heat, the same power considered in its relation to the effects it produces: thus, two bodics are said to have equal quantities of sensible heat, when they produce equal eflects upon the mercury in the thermometer; but as bodies of different kinds have different capacities for containing heat, the absolute heat in such bodies will be different, though the sensible heat be the same. Thus, a pound of water and a pound of antimony, of the same temperature, have equal sensible heat; but the former contains a much greater quantity of absolute heat than the lattcr.

M. De Luc bas evinced, by a variety of experiments, that the expansions of mercury between the freezing and boiling points of water, correspond precisely to the quantities of absolute heat applied, and that its contractions are proportionable to the diminution of this element witlin these limits. And hence it may be inferred, that if the mercury were to retain its fluid form, jts contractions would 4. $\mathrm{M} 2$ 
be propurtionable to the decrements of the absolute heat, though the diminution were continued to the point of total privation. But the comparative quantities of absolute heat, which are communicated to different bodies, or separated from them, cannot be determined in a direct manner by the thermometer.

Some philosophers have apprehended that the quantities of absolute beat in bodies, are in proportion to their densities. While other's, as Boerhave, inagined that heat is equally diffused through all bodies, the densest as well as the rarest, and therefore that the quantities of heat in bodies, are in proportion to their bulk or magnitude: and, at his desire, Fahrenheit atternpted to determine the fact by experiment. For this purpose, he took equal quantities of the same fluid, and gave them different degrees of heat; then upon mixing them intimately-together, he found that the temperature of the mixture was a just medium, or arithmetical mean, between the two. But if this experiment be made with water and mercury, in the same circumstances, viz, in equal bulks, the result will be different, as the temperature of the misture will not be a mean between the two, but always nearer to that of the water than to the quicksilver; so that, when the water is the hotter, the temperature of the misture is above the mean, and below it when the water is the colder. And from experiments of this kind it has been inferred, that the comparative quantities of the absolute heats of these fuids, are reciprocally proportional to the changes which are produced in their sensible beats, when they are mixed together at different temperatures: and this fact has been publicly taught, for several ycars, by Dr. Black and Dr. Irvine, in the universities of Edinburgh and Glasgow. This rule however does not apply to those substances whicb, in nixture, excite sensible heat by chemical action.

From the experiments and reasoning employed by $\mathrm{Dr}$. Crawford, it more fully appears, that the quantities of absolute heat in different bodies, are not as their densities; or that equal weights of heterogeneous substances, as air and water, having the same temperature, may contain unequal quantities of absolute heat: he also shows, that if phlogiston be added to a body, a quantity of the absolute heat of that body will be extricated; and if the phlogiston be separated again, an equal quantity of heat will be absorbed. So that heat and phlogiston appear to be two opposite principles in nature. But this ingenious writer bas not presumed absalutely to decide the question that has been long agitated, whether heat be a substance or a quality.-He inclines to the former opinion, lowever, and observes, that if we adopt the opinion, that heat is a distinct substance, or an element sui generis, the phenomena will be found to admit of a simple and obvious interpretation, and to be perfectly agreeable to the analogy of natırc. See Crawford's Experiments and Observations on Animal Heat and the Inflammation of Combustible Bodies. Also Mr. Leslie's ingenious treatise upon heat; Schecle's Experiments on Air and Fire; Thomson's Chemistry, rol. 1; Boerluave's Chemistry; Dary's Essays ; Rumford's Esșays, \&c; Herschel's in Phil. Traus. ; several vols. of the Philos. Magazine, and in Nicholson's Journal.

Animal HЕлт. 'The heat of animals is very various, both according to the diversity of their linds, and the difference of the seasons: accordiugly, zoologists have divided them into hot and cold bluoded, reckoning those to be: hot that are near or above our own temperature, and all others cold whose heat is below ours, and consequently atfect us with the sense of cold; thus making the human species a medium between the hot and cold blooded animals, or at least the lowest order of the lot blooded.

The beat of the human body, in its natural state, according to Dr. Boerhave, is such as to raise the mercury in the thermometer to $92^{\circ}$ or at most to $94^{\circ}$; and Dr. Pitcairn makes the heat of the human skin the same. Indeed it is evident that different parts of the human body, and its different states, as well as the different seasons, will make it show of different-temperatures. Thus, by various experiments at different times, the heat of the luman body is made various by the following autliors:

$$
\begin{aligned}
& \text { Boerhave and Pitcairn - - - } \quad 92^{\circ} \\
& \text { Amontons - } \quad \text { - } \quad \text { - } \quad 91,92 \text {, or } 93 \\
& \text { Sir Isaac Newton - - - _ - - } 95 \frac{x}{3} \\
& \text { Falirenheit and Muschenbroek, the blood - } 96 \\
& \text { Dr. Martine, the skin - } \quad \text { - } \quad \text { - } 97 \text { or } 98 \\
& \text {. - the urine - - - - } 99 \\
& \text { Dr. Hales, the skin - } \quad \text { - } \quad \text { - } \quad \text { - } \quad \text { - } 97 \\
& \text {. . the urine - - } \quad \text { - } \quad \text { - } 103 \\
& \text { Mr. John Hunter, under his congue - } \quad \text { - } 97 \\
& \text { - . . . . in his rectum - - } 98 \frac{\mathrm{r}}{2} \\
& \text { - . . . his urethra at } 1 \text { inch - } 92 \\
& \text { - } 93 \\
& \text { the ball of the thermom. at the bulb of ? } \\
& \text { the urethra } \\
& \} 97
\end{aligned}
$$

For the powers of animals to bear various degrees of heat, see the Philos. Trans. vol. 65, 68, \&c. There is hardly any subject of philosophical investigation that has afforded a greater variety of hypotheses, conjectures, and experiments, than the cause of animal heat. The first opinion which has very generally obtained, is, that the heat of animal bodies is owing to the attrition betwcen the arteries and the blood. All the observations and reasoning brought in favour of this opinion however, only show that the heat and the motion of the arteries are generally proportional to each other; witbout showing which is the cause, and which the effect; or indeed that eitber is the cause or effect of the othcr, since both may be the effects of some other cause. Dr. Douglas, in his Essay on the Generation of Heat in animals, ascribes it solely to the friction of the globules of blood in their circulation through the capillary vessels. Another opinion is, that the Iungs are the fountain of beat in the human body: and this opinion is supported by much the same kind of arguments as the former, and seemingly to little better purpose.

A third. opinion is, that the cause of animal heat is owing to the action of the solid parts upon one another. And as the heart and arteries have the most motion, it has been thought natural to expect that the heat should be owing to this motion. But even this does not seem rery plausible, from the following considerations: 1st, The moving parts, however we term them solid, are neither hard nor dry; which two conditions are absolutcly rcquisite to make them fit to generate hent by attition: $\approx \mathrm{d}$, None of their motions arc swift enough to promise leat in this way. $3 \mathrm{~d}$, They have but little clange of surface in their attritions. And thly, The moveable libes have fat, mucilage, or liquors every way surrounding them, to prevent their being destroyed, or heaterl by attrition.

- Another cause assigned for the heat of our bodics, is that process by which our aliment and fuids are perpetually undergoing some alteration. And this opinion is chicfly supported by Dr. Stesenson, in the Edinburgh Micdical 
Essays, vol. 5, art. 77. The late ingenious Dr. Franklin inclines to this opinion, when he says, that the fluid fire, as well as the fluid air, is attracted by plants in their growth, and becomes consolidated with the other materials of which they are formed, and makes a great part of their substance; that when they come to be digested, and to undergo a kind of fermentation in the vessels, part of the fire, as well as part of the air, recovers its fluid active state again, and diffuses itself on the body digesting and separating it; \&c. Esper. and Obs. on Electricity, pa. 346.

Dr. Mortimer thinks the heat of animais explicable from the phosphorus and air they contain. Phosphorus exists, at least in a dormant state, in animal fuids; and it is also known that they all contain air; it is therefore only necessary to bring the phosphoreal and aerial particles into contact, and heat must of consequence be generated; and were it not for the quantity of aqueous liumours in animals, fatal accensions would frequently happen. Philos. Tlans. No. 476 .

Dr. Black supposes, that animal heat is generated entircly in the lungs, by the action of the air on the principle of inflammability, and is thence diffused over the rest of the body by means of the circulation. But Dr. Leslie urges several arguments against this hypothesis, tending to show that it is repugnant to the known laws of the anjmal machine; and he advances another hypothesis iustead of it, viz, that the subtile principle by chemists termed phlogiston, which enters into the composition of natural bodies, is in consequence of the action of the vascular system gradually evolver through every part of the animal machine, and that during this evolution heat is generated. This opinion, he candidly acknowledges, was first delivered by Dr. Duncan of Edinburgh; and that something similar to it is to be found in Dr. Franklin's works, and in a paper of Dr. Mortimer's in the Philos. Trans.

The last hypothesis we sluall mention, is the very platlsible one of Dr. Crawford, lately published in his Experiments and Observations on Animal Heat. This ingenious gentleman has inferred, from a variety of experiments, that heat and phlogiston, so far from being connected, as most philosophers have jmagined, act in some measure in opposition to each other. By the action of heat on boclies, the force of their attraction of phlogiston is climinished, and by the action of plingiston, a part of thcir absolute heat is expelled. He has also demonstrated, that atmospherical air contains a greater quantity of absolute heat than the air which is expired from the lungs of animals: he makes the proportion of the absolute heat of atmospherical air to that of fixed air, as 67 to 1 ; and the heat of dephlogisticated air to that of atmospherical air, as 4.6 to 1 ; and obscrving that Dr. Priestley has proved, that the power of this dephlogisticated air in supporting animal life, is 5 times as great as that of atmospherical air, he concludes that the quantity of absolute heat contained in any kind of air fit for respiration, is very nearly in proportion to its purity, or to its power of supporting animal life; and since the air exlaterl by respiration, is found to contain only the 67 th part of the heat which was contained in the atmospherical air, previons to inspiration, it is very reasonably inferred, that the latter inust necessarily deposit a very great proportion of its absolute heat in the lungs. Dr. Crawford has also shown, that the blood which passes from the lungs to the heart, by the pulmonary vein, contains more absolute heat than that which passes from the heart to the lungs, by the pulmonary artery; the abso-
Lute heat of florid arterial blood being to that of venous blood, as $11 \frac{\mathrm{r}}{2}$ to 10 : therefore, since the blood which is retarned by the pulmonary vein to the heart has the quantity of its absolute heat increased, it must have acquired this heat in its passage through the lungs; so that in the process of respiration a quantity of absolute heat is separated from the air, and absorbed by the blood. Dr. Priestley has also proved, that in respiration, phlogiston is separated from the blood, and combined with air.

This theory however has been contested and lisputed, and, it has been said, Dr. Crawford's experinents repeated, with contrary results. Though no regular and systematical theory has yet been formed in its stead.

Heat of Combustible and Inflummuble Bodies: Dr. Crawford's theory with respect to the infammation of combustible bodies, is founded on the same principles as his doctrine concerning the beat of animals. According to him, the heat wlich is produced by combustion, is derived from the air, and not from the inflammable body. Inflammable bodies, he says, abound with phlogiston, and contain little absolute heat; the atmosphere, on the contrary, abounds with absolute heat, and contains litule phlogiston. In the process of inflammation, the phlogiston is separated from the infiammable body, and combined with the air; the air is phlogisticated, and affords a great proportion of its absolute heat, which, when extricated suddenly, bursts forth into flane, and produces an intense degree of sensible heat. And since it appears by calculation, that the heat produced by converting atmospherical into fixed air, is such, if it were not dissipated, as would be sufficient to raise the air so changed, to more than 12 times the heat of red-hot iron, it follows, that in the process of inflammation a very great quantity of heat is derived from the air. But, on the contrary, no part of the heat can be derived from the combustible body; because this body, cluring the inflammation, being deprived of its phlogiston, undergoes a change similar to that of the bluod by the process of respiration, in consequence of which its capacity for heat is increased; and thercfore it will not evolve any pait of its absolute lieat, but, like the blood in its passage through the lungs, it will absorb heat. A similar theory of heat lias lately been published by Mr. Elliot. See bis Philosuphical Observations ou the senses of Vision and Hearing; to which is added an Essay on Combustion and Animal Heat, 8vo, 17 so. See CAloric, also Profissor Leslie's late work on heat.

Mr. Ezekiel Walker has given a neat account of the causes and mode of combustion, in the Philos. Magazine, vol. 43, pa. 101, as follows. There are very few bodics in nature that do not contain either thermogen or photogen. All those bodies which are callut combustibles, contain the element of light, which I cali photogen, to avoid the use of ambiguous terms; and all those bodies which are called supporters of combustion, contain the generator of heat, called thrrmogen for the same reason. These elements may be made to unite and produce combustion, or increase the temperature of bodies, by various means; as : 1st, by friction; 2dly, by percussion; $3 \mathrm{dly}$, by pressure; 4 thly, by mixture; 5 thly, by increase of temperature; and. 6 thly, by the functions of living animals and vegetables.

Thermogen and photogen in a condensed state, as in a charged Leyden jar, have so strolig an attraction for each other, that they will pass throngh any of those bodies called conductors, to a very great distance, to be united. 
But when they are united to ponderable matter, they will remain mixed tugether for ages withont producing any effect; but as soon as they are brought within a certain distance of each other, which may be called their striking distance, or their sphere of action, cither combustion takes place, or the temperature of the body, in which they meet, is increased. The following explanations of some wellknown experiments will tend to show the importance of a theory founded on facts.

Experiment 1 . In a memoir read to the National Institute of France, MI. Biot announces the important fact, that a mixture of hydrogen and oxygen gases may be made to explode by mechanical compression only, independently of the electric spark.-Explanation. Oxygen and hydrogen gases would remain mised together, for any length of time, without combining, the thermogen and photogen in them being kept at too great a distance by their bases; but, by mechanical compression only, they are forced within their striking distance, and light and heat are produced on the same principle as combustion is produced by discharging a Leyden jar.

Exper. 2. Take 6 grains of oxygenized muriate of potass, and 3 grains of flour of sulphur, rub them together in a mortar, and a smart detonating noise will be pro-. duced. Continue to rub the mixture hard, and the report will be frequently repeated, accompanied with vivid flashes of light. If the same mixture be wrapped in paper, laid on an anvil, and smartly struck with a hammer, the report will be as loud as that of a pistol.-Explan. These experiments may be explained on the same principle as the last. The two ingredients, though mixed together, produced no effect until the thermogen in one of them and the photogen in the other were forced into union, by friction or percussion. And on the same principle the effects of many other fulminating substances may be explained.

Exper. 3. If an ounce of strong nitrous acid be mixed with about balf its weight of sulphuric acid, and poured into a little oil of turpentine, the whole will immediately burst into flame.-Explan. The thermogen and photogen in these fluids coming immediately into contact, flame is produced, without any mechanical force or increașe of temperature.-Now, from this experiment it appears impossible that water can be a compound of oxygren and hydrogen; for, if it were, these two principles of combustion would burst into flame like the acid and the oil. But no pressure, no increase of temperature, nor any union with combustible matter, can prorluce a single spark of fire from water. In short, nothing combustible is found in its composition.

Exper. 4. Combustion may also be generated by friction. 'Two pieces of hard wood, being smartly rubbed together with great pressure, will soon burst into flame.Explan. The first increment of caloric, by friction, is excited by a portion of the thermogen of the air being forced into union with the photogen in the wood; and by continuing the operation, the temperature of the inflammable principle of the wood is increased till flame is produced.

Exper. 5. When a rod of iron is laid upon an anvil, and hammered with a quick succession of heavy strokes, it soon becomes red-hot. But it cannot be strongly heated a second time by the same means, unless it be first introduced into a fire.-Explan. 'The first stroke of the hammer forces a small portion of the thermogen of the air into contact with the pliotogen of the metal, which generates the first increment of caloric; and by repeated strokes of the hammer the caloric is increased, till the rod becomes red-hot. But the photogen of the metal becomes exhausted by this means, and therefore eannot produce caloric a second time, till it has recovered its combustible property in the fire.-The burning of iron wire in oxygen gas, in a brilliant manner shows how iron loses its photogen, and becomes oxydized.

Exper. 6. Mr. Thomas Wedgwood bas shown, that it has never yet been explained, how friction produces caloric. He took a piece of common window-glass, and beld the edge of it against the edge of a revolviing grit-stone, when that part in contact with the stone became red-hot, and threw oft bot particles which fired gunpowder. The stono and the glass being both incombustible substances, it remains to be explained how caloric was produced. $-E x$ plan. It has before been shown, that the electric machine creates nothing, but by friction only collects the two elements which produce caloric, from the earth and the atmosphere. Though the stone and the gliss, in Mr. W.'s experiment, are both incombustible, yet it is almost a self-evident conclusion, that they collect the two elements of caloric from the air and the earth by friction, in the same manner as the electric machine.

Exper. 7. It is well known, that when flint and steel are smartly struck against each other, a spark always ensues, which is capable of setting fire to tinder or to gunpowder. The spark in this casc, as was long since ascertained by Dr. Hooke, is a small particle of the metal, which is struck off, and takes fre during its passage through the air. This therefore, and all similar cases, belongs to the class of combustion. But light often appears when two bodies are struck against each other, when we are certain that no such thing as combustion can happen, because both the bodies are incombustible. Thus, for instance, sparks are emitted when two quartz stones are struck smartiy against each other, and light is emitted when they are rubbed against each other. Many other hard stones also emit sparks by such collisions.-If they be often made to emit sparks above a sheet of white paper, there are found uinon it a number of sinall black bodies, somewhat like the eggs of flies. These bodies are hard, but friable, and leave a black stain when rubbed on the paper. When viewed with a microscope, they scem to have been melted. Muriatic acid changes their colour to a green, as it does that of lavas. These substances evidently produced the sparks by being heated red-hot.-Explan. It may first be observed, that if the flint be moved over the stone very slowly, no spark is produced, nor will any spark appear if the flint barely touch the steel, though the velocity of the flint be the greatest possible: consequently, caloric cannot be produced by collision or friction, but by velocity and pressure conjointly. $-2 \mathrm{dly}, \mathrm{By}$ the collision of the flint and steel, the thermogen of the atmosphere is forced into union with the plotogen of the metal. The combination thus produced melts the minute particles of steel, struck off by the flint, into small globules. That this metal contains the generator of light is well known, by the burning of iron or stecl wire in oxygen gas.-Sparks are emitted when two quartz stones are struek smartly against each other under water; and Mr. Kirwan aflirms, that sparks are produced by the collision of flint and steel under common spring water.

Kxper.8. From Count Rumford's experiments it appears, that by a moderate degree of friction, the same 
piece of metal afforded so much caloric under water, as to kecp it boiling.-Explan. This experiment may be explained on the same principle as the last. Water, being a better conductor of thermogen than atmospheric air, cannot prevent the production of caloric by the collision or friction of hard bodics.

Heat, in Geograply, is that which relates to the earth. There is a great variety in the heat of different places and seasons. Naturalists have commonly assumed it as a fact, that the nearer any place is to the centre of the earth, the hotter it is found ; but this does not appear to hold strictly true. And if it were, the effect might be otherwise accounted for, and more sutisfactorily, than from their imagined central fire.

Mir. Boyle, who had been at the bottom of some mines himself, with more probability suspects that this degree of heat, at least in some of them, may arise from the peculiar nature of the minerals there produced. And be instances a mineral of the vitriolic kind, dug up in large quantitics, in several parts of England, which, by the bare effusion of common water, will become so hot as almost to take firc. To which may be added, that such places, in the bowels of the earth, usually feel-hot, from the confined and stagnant state of the air in them, in which the heat is retained, for want of a current or change of air to carry the heat off.

On the other hand, on ascending high mountains, the air feels more and more cold and piercing. Thus, the tops of the Pike of Tencriffe, the Alps, and several other mountains, even in the most sultry countries, are found always invested with snow and ice, which the heat is never sufficient to thaw. In some of the mountains of Peru there is no such thing as running water, but all ice : plants vegetate a little about the bottom of the mountains, but near the top no vegetable can live, for the intenseness of the cold. This effect is attributed to the thinness of the air, and the little surface of the earth there is to reflect the rays, as well as the great distance of the general surface of the earth which reflects the rays back into the atmosphere.

As to the diversity in the heat of different climes and seasons, it arises from the different angles under which the sun's rays strike upon the surface of the earth. In the Philos. Trans, $\Lambda \mathrm{br}$, vol. 3, p. 576, Dr. Halley has given a computation of this heat, on the principle, that the simple action of the sun's rays, like uther impulses or strokes, is more or less forcible, according to the sines of the angles of incidence, or to the sines of the sun's altitudes, at different times or places.

Hence it follows, that the time of continuance, or the sun's shining on any place, being taken for a basis, and the sines of the sun's altitueles perpendicularly erected upon it, and a curve line drawn through the extremities of those perpendiculars, the area thus comprehended will be proportional to the collection of all the heat of the sun's beams in that period of time.

Hence it will likewise follow, that at the pole, the collection of all the heat of a tropical day, is proportional to the rectangle or product of the sine of $23 \frac{\pi}{2}$ degrees into 24 hours, or the circumference of a circle, or as $\frac{8}{15}$ into 12 hours, the sine of $23 \frac{x}{2}$ degrees being nearly $\frac{4}{4}$ of radius. Or the polar heat will be equal to that of the sun continuing 12 hours above the horizon at 53 degrees height; and the sun is not 5 hours more elevated than this under the equinoctial.

But as it is the nature of heat to remain in the subject, after the luminary is removed, and particularly in the air, under the equinuctial, the 12 hours absence of the sun abates but little from the effect of his heat in the day; but under the pole, the long absence of the sun for 6 months has so chilled the air, that it is in a manner frozen, and after the sun has risen upon the pole again, it is long befure his beams can make any impression, being obstructed by thick cloudsand fogs.

From the foregoing principle Dr. Halley computes the following table, exhibiting the heat to every loth degree of latitude, for the equinoctial and tropical sun, and from which an estimate may easily be made for the intermediate degrees.

\begin{tabular}{|c|c|c|c|}
\hline \multirow{2}{*}{ Latitude. } & \multicolumn{3}{|c|}{ Sign that the Sun is in. } \\
\hline & $r$ or 2 & ए0 & bo \\
\hline 0 & 20000 & 18341 & 18341 \\
\hline 10 & 19696 & 20296 & $15 S 34$ \\
\hline 20 & 18794 & 21737 & 13166 \\
\hline 30 & 17321 & 22651 & 10124 \\
\hline 40 & 15321 & 23048 & 6944 \\
\hline 50 & 12855 & 22991 & $379 \mathrm{~s}$ \\
\hline 60 & 10000 & 22773 & 1075 \\
\hline 70 & 6840 & 23543 & 0 \\
\hline 80 & 3473 & 24673 & 0 \\
\hline 90 & 0 & 25055 & 0 \\
\hline
\end{tabular}

From the same principles, and table, also are deduced the following corollaries, viz,

1 , 'That the equatorial heat, when the sun becomes vertical, is as twice the square of the radius. -2 , That at the equator the heat is as the sine of the sun's declination.3. That in the frigid zones, when the sun sets not, the heat is as the circumference of a circle into the sine of the altitude at 6 : And consequently, that in the same latitude, these aggregates of heat are as the sines of the sun's declination; and at the same declination of the sun, they are as the sines of the latitudes; and generally they are as the sines of the latitudes into the sines of declination. 4 , 'That the equatorial day's heat is every where as the cosine of the latitude. - 5, In all places where the sun sets, the difference between the sumuner and winter heats, when the declinations are contrary, is equal to a circle into the sine of the altitude at 6 , in the stumer parallel; and consequently those differences are as the rectangles of the sines of the latitude and declinations.- 6 , The tropical sun has the least force of any at the equator; and at the pole it is greatest of all.

Many objections have been urged against this theory of Dr. Halley. Sone have insisted, that the effect of the sun's heat is not in the simple, but in the duplicate ratio of the sines of the angles of incidence; like the law of the impulse of fluids. Ant indeed, the quantity of the sun's direct rays received at any place, being evidently as the sine of the angle of incidence, or of the sun's altitude, if the heat be also proportional to the force with which a ray strikes, like the mechanical action or impulse of any body, then it will follow that the heat nust be in the compound ratio of hoth, that is, as the square of the sinc of the sun's altitude. But this last principle is here only assumed gratis, as we do not know a priori that the heat is proportional to the force of a striking borly ; and it is only experiment that can determine this point.

It is certain that heat communicated by the sun to bo- 
dies on the earth, depends also much upon other circumstances beside the direct force of his rays. These must be modified by our atmosphere, and variously reflected and combined by the action of the earth's surface itself, to produce any remarkable effects of heat. So that, if it were not for these additional circumstances, it is probable the naked heat of the sun would not be very sensible.

Dr. Halley himself was well apprised, that many other circumstances, besides the direct force of the sun's rays, contributed to augment or diminish the effect of this, and the heat resulting from it, in different climates; and therefore no calculation, formed on the preceding theory, can be supposed to correspond exactly with observation and experiment. It has,ailso been objected that, according to the foregoing theory, the greatest heat in the same place, should be at the summer'solstice, and the most extreme cold at the winter solstice; which is contrary to experience. To this objection it may be replied, that heat is not produced in bodies by the sun instantaneously, nor do the effects of his heat cease immediately when his rays are withdrawn; and therefore those parts which are once heated, rctain the heat for some time; which, with the additional heat daily imparted, makes it continue to increase, though the sun declines from us: and this is the reason why July is hotter than June, though the sun bas withdrawn from the summer tropic: as we also find it is generally hotter at one, two, or three in the afternoon, when the sun has declined towards the west, than at noon, when he is on the meridian. As long as the heating particles, which are constantly received, are more numerous than those which fly away or lose their force, the heat of bodies must continually increase. So, after the sun has left the tropic, the number of particles, which heat our atmospliere and carth, constantly increases, because we receive more in the day than we lose at.night, and therefore our heat must also increase. But as the dlays decrease again; and the action of the sun becomes weaker, more particles will be dispersed in the night than are received in the day, by which means the carth and air will gradually cool. Further, those places which are well cooled, require time to be heated again; and therefore January is mostly colder than December, though the sun has withdrawn from the winter tropic, and begun to emit his rays more directly upon us.

But the chicf cause of the difference between the heat of summer and winter is, that in summer the ralys fall more directly, and pass through a less dense part of the atmosphere; and therefore with greater force, or at least in greater number in the same place: and besides, by their long continuance, a much greater degree of heat is imparted by day than can be dispersed by night. For the calculations and opinions of several other philosophers on this head, sce Kicill's Astron. Lect. 8; Ferguson's Astron. chap. 10; Long's Astron. \$777; Memo. Acad. Scienc, 1719 .

A's to the temperature or heat of our atmosplere, it inay be observed that the mercury seldom falls under $16^{\circ}$ in Fahrenheit's thermometer; but we are apt to reckon it very cold at $24^{\circ}$, and it continues chilly to $4.0^{\circ}$ and a little above. However, such colds have been often known to reduce it down to $0^{\circ}$, the beginning of the senle, or nearly the cold produced by a mixture of snow and salt, often near it, and in some places below it. Thus, the degree of the thermometer has been observed at various times and places as follows :

\section{H E A}

\begin{tabular}{|c|c|c|c|}
\hline Placez & Latit. & Yeár & Thermom \\
\hline Pensylvania & $40^{\circ} u^{\prime}$ & 1732 & $5^{\circ}$ \\
\hline Paris & 4850 & $1709 \& 1710$ & $s$ \\
\hline Leyden & 5210 & 1729 & 5 \\
\hline Utrecht & $52 \mathrm{~s}$ & - & + \\
\hline London & 5131 & $1709 \& 1710$ & 0 \\
\hline Copenhagen & 5543 & 1709 & 0 \\
\hline Upsal & 5956 & 1732 & -1 \\
\hline Petersburg & $59 \cdot 56$ & - & -28 \\
\hline Torneo & 6551 & $1736-T$ & -33 \\
\hline Hudson's Bay & 5224 & 1775 & -37 \\
\hline
\end{tabular}

The middle temperature of our atmosphere is about $4 \mathrm{~S}^{\circ}$, being nearly a medium of all the seasons. 'T he French make it somewhat ligher, reckoning it equal to the cave of their royal observatory, or $53^{\circ}$. In cold countries, the air is found agreeable enongh to the inhabitants while it is between 40 and $50^{\circ}$. But in our climate we are best pleased with the beat of the air from 50 to $60^{\circ}$; while in the hot countries the air is generally at a medium about $70^{\circ}$. With us, the air is not reckoned warm till it arrives about $64^{\circ}$, and it is very warm and suliry at $80^{\circ}$. It is to be noted that the foregoing observations are to be understood of the state of the air in the slaade; for as to the heat of bodies acted on by the direct rays of the sun, it is much greater: thus, Dr. Martine found dry earth heated to above $120^{\circ}$; but Dr. Hales found a very hot sun-shine heat in 1727 to be about $140^{\circ}$; and Muschenbroek once observed it so high as $150^{\circ}$; but at Muntpelier the sun was so very hot, on one day in the year 1705 , as to raise M. A montons' thermometer to the mark of boiling water itself, which is our $212^{\circ}$. (See Temperature of the Atmosphere under the article Atmospirere.)

It appears from the register of the thermometer kept at London by Dr. Heberden for 9 jears, viz, from the end of 1763 to the end of 1772 , that the mean beat at $S$ in the morning was $47^{\circ} \cdot 4$; and by another register kept at Hawkhill near Edinburgh, that the nean heat in that place, during the same period of time, was $46^{\circ}$. Also by registers kept in London and at Hawk Lill, for the three year's 1772 , 1773,1774 , it appears, that the mean heat of these thrce years in Lonclon, at 8 in the morning, was $48^{\circ} \cdot 5$, and at 2 in the afternoon $56^{\circ}$, but the mean of both morving and afternoon $52^{\circ} \cdot 2$; while the mean heat at Hawkill for the same.time,

$\begin{array}{lr}\text { at } S \text { in the morning was } & 4.5^{\circ} \cdot 4 \\ \text { and at } 2 \text { in the afternoon } & 50 \cdot 1 \\ \text { and the mean of both } & 4.7 \cdot 7 .\end{array}$

The mean heat of springs near Edinburgh is found to be about $47^{\circ}$, and at London $51^{\circ}$. Philos. 'Trans. vol, 65, art. 4.4.

Lastly, from the meteorological journals of the Royal Socicty, published in the l'hilos. 'Trans, it appears that the mean heights of the thermometer, for the whole years, kept witlout and within the house, are as below:

$$
\begin{aligned}
& \text { Therm. Wishout Therm. Within }
\end{aligned}
$$

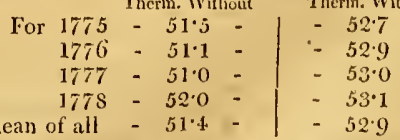

IEAT, Latent, is insensible, concealed, dormant as it were.

HEAVEN, an azure transparent orb investing our earth, where the celestial bodies perform their motions. It is of divers denominations, as the highest or empyrean 


\section{H E I}

heaven, the etherial or starry heaven, the planetary beaven, \& c. Formerly the heavens were considered as solic: substances, or clse as spaces full of solid matter; but Newton bas abundantly shown that the heavens are void of almost all resistance, and consequently of almost all matter: this he proves from the phenomena of the celestial bodies; from the planets persisting in their motions, without any sensible diminution of their velocity; and the comets freely passing in all directions towards all parts of the heavens. Heaven, taken in this general sense, or the whole expanse between our earth and the remotest regions of the fixed stars, may be divided into two very unequal parts, according to the matter occupying them; viz, the atmosphere or acreal heaven, occupied by air; and the ethereal heaven, possessed by a thin and unresisting mcdium, called ether.

HeA $\mathrm{E}$ E is more particularly used, in Astronomy, for an orb, or circular region, of the ethereal heaven.

The ancient astronomers assumed as matly different heavens as they observed different celestial motions. All these they made solid, thinking they could not otherwise sustain the bodies fixed in them; and of a spherical form, as being the most proper for motion. Thus they bad seven heavens for the seven planets; viz, the heavens of the Moon, Mercury, Venus, the Sun, Mars, Jupiter, and Saturn. The 8th was for the fixed stars, which they particularly called the firmament. Ptolemy added a 9 th heaven, which he called the primum mobile. After him two crystalline heavens were added by King Alphonsus, \&c, to account for some irregularities in the motions of the other heavens. $\Lambda$ nd lastly an empyrean heaven was drawn over the whole for the residence of the Deity; which made the number 12. But some admitted many other heavens, according as their different views and hypotbeses required. Eudoxus supposed 23, Calippus 30, Regiomontanus 33, Aristotle 47, and Fracastor no less than 70 . The astrononers however did not much concern themselves whether the heavens they thus allowed, were real or not; provided they served a purpose in accounting for any of the celestial inotions, and agreed with the phenomena.

HEAVINESS, the same as Gravity, which sce.

Heavy bodies rlo not tend precisely to the very centre of the earth, except at the poles and the equator, on account of the spheroidal figure of the carth ; their direction being every where perpendicular to the surface of the splieroid.

HEGIRA, a term in Chronology, signifying the epoch, or account of time, used by the Alahomedans, who begin their computation from the day that Mahomet was forced to make his escape from the city of Mecca, which happened on Friday the 16 th of July 622 .

The years of the hegira are lunar ones, consisting only of 354 days. Hence, to reduce these years to the Julian calendar, that is, to find what Julian ycar a given year of the hegira answers to: reduce the year of the hegira into days, by multiplying by 354 , divide the product by $365 \frac{x}{4}$, and to the quotient add 622 , the year the hegira commenced.

HEIGHT, the third dimension of a body, considered with regard to its clevation above the ground; or any distance above the earth's surface, \& c .

HEIGII, of a figure, the same as its altitude, being the perpendicular from its vertex to the base.

HeIg II t of the pole, \&c. See Altitude of the Pole, \&c. VoL. 1.
H E L

HELIACAL, something relating to the sun. Thus,

HeLIacal Rising, of a star or planet, is when it rises with, or at the same time as, the sum. And heliacal setting, the same as the setting with the sun. Or, a star rises heljacally, when, after it has been in conjunction with the sun, and so invisible, it gets at such a distance from him, as to be seen in the morning before the sun's rising. And it is saill to set heliacally, when it approaches so near the sun as to be hid by lis beams. So that, in strictness, the heliacal rising and setting are only an apparition and occultation.

HELICE MIijor and Minor; the same as Ursa Major and Minor.

HELICOID Parabola, or the Parabolic Spiral, is a curre arising from the supposition that the common or Apollonian parabola is bent or twisted, till the axis come into the periphery of a circle, the ordinate still retaining their places and perpendicular positions with respect to the circle, all these lines still remaining in the same plane. Thus, the axis of a parabola being bent into the circumference $\mathbf{B C D M}$, and the ordinates $C F, D G, \& c$, still perpendicular to it, then the parabola itself, passing through the extremities of the ordinates, is twisted into the curve BFG, \& $x$, called the Helicoid, or Parabolic Spiral. Hence it is evident that all theordinates $\mathrm{Cr}, \mathrm{DG}$, \&cc, tend to the centre of the circle, being perpendicular to the circumference.-Also, the equation of the curve remains the same as when it was a parabola; viz, putting $x=$ any circular absciss $\mathrm{BC}$, and $y=\mathrm{CF}$ the corresponding ordinate, then is $p x=y^{2}$, where $p$ is the parameter of the parabola.

HELIOCENTRIC Place of a Planet, is the place in which a planet would appear to be when viewed from the sun; or the point of the ecliptic, in which a planct viewed from the sun would appear to be. And therefore the heliocentric plâce coincides with the longitude of a planet viewed from the sun.

Helrocentric Latitude of a Planet, is the inclination . of the line drawn between the centre of the sun and the centre of a planet, to the plane of the ecliptic. The greatest heliocentric latitude is equal to the inclination of the planet's orbit to the plane of the ecliptic.

HELIOCOMETE, Comet of the Sun, a phenomenon sometimes observed at the setting of the sun; thus denominated by Sturmius and Pylen, who had seen it, because it seems to make a comet of the sun, being a large tail, or column of light, fixed or attached to that luminary, and dragging after it, at its setting, like the tail of il comet.

HELIOME'TER, or AsTrOMETER; an instrument for measuring, with particular exactness, the diameters of the sun, moon, and stars.- This instrument was invented by $\mathrm{M}$. Bouguer in 1747 , and is a kind of telescope, consisting of two object-glasses of equal focal distance, placed by the side of each other, so that the same eye-glass serves for both. The tube of this instrument is of a conical form, larger at the upper end, which receives the two object-glasses, than at the lower, which is furnished with an eye-glass and micrometer. By the construction $4 \mathrm{~N}$ 
of this instrument, two distinct images of an object are formed in the focus of the eye-glass, whose distance, depending on that of the two object-glasses from each other, may be measured with great accuracy. Mem. Acad. Sci. 1748. Mr. Servington Savery discovered a similar method of improving the micrometer, which was communicated to the Royal Society in 1743 .

HELIOSCOPE, a kind of telescope peculiarly adapted for viewing and observing the sun without hurting the eye. There are various linds of this instrument, usually made by employing coloured glass for the object-or eye-glass, or both; and sometimes only using an cye-glass blackened by bolding it over the smoke or flame of a lamp or candle, which is Huygens's way. Scc Dr. Hooke's treatise on Helioscopes.

HELIOSTATA, an instrument invented by Dr. Gravesande, and so called from its property of fixing the sunbeam in one position, viz, in a horizontal direction across the dark chamber while it is used. See Gravesande's Physices Element. Mathematica, tom. 2, pa. 715, ed.3tia, 1742, for an account of the principles, construction and use of this instrument.

HELISPHERICAL Line, is the rhumb-line in navigation; being so called, because on the globe it winds round the pole heliacally or spirally, approaching always nearer and nearer to it.

HELIX, a spiral line. See SpIral.

HEMICYCLE, in Architecture, a term applied to semicircular vaults.

HEMISPHERE, the half of a sphere or globe, when divided in two by a plane passing through its centre.

Hemisphere, in Astronomy, is particularly used for one half of the mundane sphere. 'The equator divides the sphere into two equal parts, called the northern and southern hemispheres. The horizon also divides the sphere into two equal parts, called the upper and lower bemispheres.

Hemisphere is also used for a map or projection of half the terrestrial globe, or of half the celestial sphere, on a plane; being more frequently called a planisphere. The centre of gravity of a hemisphere, is five-eighths of the radius distant from the vertex. A hemisphere unites the parallel rays at the distance of four-thirds of a diameter from the pole of the glass.

HEMISPH ERES of Magdeburgh, two brass concave bemispheres, one of which is furnished with a cock, by which it may be adjusted to the air-pump. The other has a ring at the middle of its convexity, by means of which it may be suspended. When these two hemispheres are placed together, the air is drawn from within through the medium of the cock by the mechanism of the air-pump; and then, the exhaustion being carried far enough, and the cock turned to prevent the admission of air from without, a considerable force is requisite to separate the hemispheres; the force varying with their great circle. This furnishes an casy and obvious inethod of proving the pressure of the air. Otto Gueriche, the inventor of the air-pump, to whom also is due this invention, made hemispheres of about a foot radius, and found that the atmospheric pressure on them was equivalent to about 5400 pounds.

HEMISPHEROIDAL, in Gcometry, something that approaches to the figure of a hemispliere, but is not justly so.

IIEMITONE, in Music, a half note, or scmi-tone.
HENDECAGON, a figure of eleven sides, or theE decagon; which sce.

HENIOCHAS, or HENIOCHUs, a northern constellation, the same as Auriga, which see.

HEPTAGON, in Genmetry, a figure of seven sides and seven angles. - Irhen those sides and angles are all equal, the heptagon is said to be regular, otherwise it is irregular.-In a regular heptagon, the angle $c$ at the centre is $=51^{\circ} \frac{3}{7}$, the angle $\mathrm{DAB}$ of the polygon is $=128^{\circ} \frac{4}{7}$, and its balf $\mathrm{CAB}=64^{\circ}$. Also the area is = the square of the side $\mathrm{AB}^{2} \times$ 3.6339124 or $=\mathrm{AB}^{2} \times \frac{7}{4} t$, where $t$ is the tangent of the angle $\mathrm{C}_{A} \mathrm{~B}$ of $64^{\mathrm{O}} \frac{2}{7}$ to the radius 1 ; or $t$ is the root of the equation;

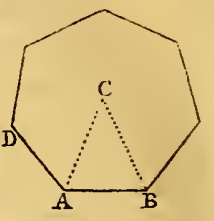
$t^{12}-26 t^{10}+143 t^{3}-245 t^{6}+143 t^{4}-26 t^{2}+1=0$; or $t=\sqrt{ } \frac{1+x}{1-x}=\frac{1+\sqrt{ }\left(1-y^{3}\right)}{y}=\frac{y}{1-\sqrt{\left(1-y^{2}\right)}}$, where the value of $x$ and $y$ are the roots of the equations $x^{6}-\frac{5}{4} x^{4}+\frac{3}{8} x^{2}-\frac{1}{46}=0$,
$y^{6}-\frac{7}{4} y^{4}+\frac{7}{8} y^{2}-\frac{7}{64}=0$.

See my Mensuration, pa. 86 , 4th edition.

HEPTAG ON, in Fortification, a place furtificd or strengthened with seven bastions for its defence.

Heptagona n Numbers, are a kind of polygonal numbers in which the difference of the terns of the corresponding arithmetical progression is 5 . Thus,

Arithmeticals, 1, 6, 11, 16, 21, 26, \&c. Heptagonals, 1, 7, 18, 34, 55, 81, \&c; where the heptagonals are formed by adding continually the terms of the arithmeticals, above them, whose common difference is 5.-One property, among many others, of these heptagonal numbers is, that if any one of them be multiplied by 40 , and to the product be added 9 , the sum will be a square number.

Thus $1 \times 40+9=49=7^{2} ;$

and $7 \times 40+9=289=17^{2}$;

and $18 \times 40+9=729=27^{2}$;

and $34 \times 40+9=1369=37^{2}$; \&c.

Where it is remarkable that the series of squares so formed is $7^{2}, 17^{2}, 27^{2}, 37^{2}$, \&c, the common difference of whose roots is 10 , the double of the common difference of the arithmetical series, from which the heptagonals are formed. -Sce Polygonals.

HEPTANGULAR Figure, in Geometry, is one that has seven angles; and therefore also seven sides.

HERCULLS, in Astronomy, a constellation of the worthern hemisphere, and one of the 48 old constellations mentioned by ancient writers. The stars in this constellation, in Ptolemy's catalogue, are 29; in Tycho's, 28; and in the Britannic catalogue, 113.

HERISSON, in Fortification, a beam armed with iron spikes, having their points turned outward. It is supported in the middle by a stake, having a pivot on which it turns; and serves'as a barrier to block-up a passage.Herissons are frequently placed before gates, especially the posterns of a town or fortress, to secure those passages which must of nccessity be often opened.

HERMANN (J M Es), a learned mathematician of the Academy of Berlin, and member of the Academy of Sciences at Paris, was born at Basil in 1678 . He was a great traveller; and for 6 years was professor of mathematics at Padua. He afterwards went to Russia, being invited thi- 
ther by the Czar in 1724, 'as well as his compatriot Daniel Bernoulli. On his return to his native country, he was appointed professor of morality and natural law at Basil; where lie died in 1733, at 55 years of age. Hermann wrote several mathematical and philosophical pieces, in the Memuirs of difierent Academics, and elscwhere; but bis principal work, is the Phoronomia, or two Books on the Forces and Motions of both Solid and Fluid liodics; 4 to, 1716 : a verylearned work on the new mathematical physics.

HER.METIC, or Hermetical Art, áname given to chemistry, on a supposition that Hermes l'rismegistus was its inventor.

HERMETICAL Philosophy, is that which undertakes to solve and explain all the phenomena of nature, from the three chemical prineiples, salt, sulphur, and mercury. A cousiderable addition wis made to the ancient hermetical philosophy, by the modern doctrine of alkali and acid.

Hermetical Seal, or Hermetical Sealing, a manner of stopping or closing glass vessels, for chenical and other operations, so very clusely, that no substance can possibly exhale or escape. This is usually done by heating the neck of the vessel in the flame of a lamp, with a blow-pipe, till it be ready to melt, and then with a pair of hot pincers twisting it close together.

HERO, or HERoN, the name of two celebrated mathematicians of the Alexandrian school, under Ptolemy Euergetes II ; one called the elder, who wrote a very ingenious treatise on various pneumatics and hydraulic machines, translated into Latin, and published in several editions and forms, since the invention of printing; first a very elegant edition by Commaudine, in 4to, 1583, entitled Heronis Alexandrini Spiritalium Liber. To this was added Quatuor Thoremata Spiritalia, by Juan. Bapt. Aleotti ; translated from Italian into Latin; Amsterdam, 1680, in 4to. The other, called the younger, under the Emperor Heraclius, left a Treatise on Mechanics and Mensuration, published in Latin, at Venice, 1572, in 4to, entitled Heronis Mechanici, liber de Machinis Bellicis, nec non liber de Geodxsia. A Francisco Barocia, patritio Veneto latinitate donati, multis mendis expurgati, et figuris, ac scholiis illustrati.

\section{HERSCHEL. See URANus.}

Herschex's Telescope, a curious one made by that astronomer, being a reflecting one, and the largest ever constructed, 40 feet in length. The figure of it is exhibited in plate $\mathrm{xv}$, and thedescription as follows, abridged from the Philos. Trans, vol. S5, or from my Abridg. vol. 17, pa. 593.

The telescope is placed in a situation due north and south, and the plate delineates the wbole apparatus as seen by a person placed at a convenient distance from it towards thie south-west. From this view the structure is sufficiently understood; and with very little attention, the mode of pointing this immense body to any part of the heavens, will be clearly secn. We shall treat of the chief parts in their order; and first, of the tube itself.

The tube is made of rolled or sheet iron, joined together without rivets, by a seaming similar to that which is used for iron fumcls for stoves; the thickness of the sheets is somewhat less than a 36 th part of an inch, or it may be found more accurately by taking a square foot of it at the weight of 14 pounds. Great care was taken in so joining together the plates, of which the tube is composed, that the cylindrical form should be secured, and then the whole was coated over three i $r$ four times with paint, inside and outside, to secure it against the damp. The tube was formed at a short distance from its present place, and renuved with great ease by 24 men, divided into six sets; so that two men on each side, with a pole of 5 fcet long in their hands, to which was affixed a piece of coarse cloth, 7 feet long, going under the tube, and joined to a pole of 5 feet long, in the hands of two other men, assisted in earrying the tube. The length of the tube is 39 feet 4 inches, the diameter 4 feet 10 inches; and upon a moderate computation it is supposed that a wooden tube for the same purpose would have excceded this in weight by at least 3000 poutds. The length of the iron plate forming the tube, and composed of smaller ones 3 feet 10 inches long, and $23 \frac{1}{2}$ inches broad; is nearly 40 feet, and the breadth 15 feet 4 inches.

The great mirror which, by proper methods, was brought to the lower part of the tube, is made of nietal, $49 \frac{1}{2}$ inches in diameter; but the concave part, or polished surface, is only 48 inches in diameter. Its thickness is $3 \frac{1}{2}$ inches; and when it came from the cast, its weight was 2118 pounds; of which a small quantity must have been lost in polishing. An iron ring, $49 \frac{\mathrm{T}}{2}$ inches in diameter within, 4 inches broad, and $1 \frac{1}{2}$ inch thick, with three strong handles to it, goes round the mirror, and a flat cover of tin is made to correspond with this ring, that the mirror may be preserved from damp; and, by an easy contrivance, it is taken off and fixed on at.pleasure.

At the upper end the tubc is open, and directed to the part of the heavens intended for obscrvation, to which the observer's back is turned, and he, standing on the footboard visible in the plate, looks down the tube, and perccives the object by rays reflccted from the great mirror, through the eye-glass at the opening of the tube. Near the place of the eye-glass is the end of a tin pipe, into which a mouth-piece may be placed; so that, during an observation, a person may direct his voice into this pipe, while his eye is at the glass. This pipe is $1 \frac{\mathrm{I}}{2} \mathrm{inch}$ in diameter; runs down to the bottom of the tube, where it goes into a turning joint, thence into a drawing tube, and out of this into another turuing joint, from which it proceeds by a set of sliding tubes towards the frout of the foundation timber. The use of this tube is to convey the voice of the observer to his assistants; for at the last place it divides itself into two branches, one going into the observatory, the other into the workman's room, ascending into both places through the floor, and being terminated in the usual shape of speaking-trumpets. Though the voice passes in this manner through a tube with many inflections, and not less than $115 \mathrm{feet}$, it requires very little exertion to be well understood.

To direct so immense a body to any part of the heavens at pleasure, much ingenuity, and many mechanical contrivances are evidently necessary. The whole apparatus rests upon rollers, and care was previously taken of the foundation in the ground. This consists of concentrical circular brick walls, the outermost 42 feet, the inmermost 21 feet in diameter; 2 feet 6 inches deep underground; 2 feet 3 inches broad at bottom, and 1 foot 2 inches at the top, capped with paving-stones, about 3 inches thick, and $12 \frac{3}{4}$ inches broad. In the centre is a large post of oak, framed together with braces underground, and walled fast with brick-work, to make it steady. Round this $4 \mathrm{~N} 2$ 
centre, the whole frame is moved horizontally, by means of 20 rollers, 12 upon the outer, and 8 upon the inner wall.

The vertical motion is given to the telescope by means of ropes and pulleys as seen in the plate, passing over the main-beam, supported by the ladders. These ladders are in length 49 feet 2 inches; and there is a moveable gallery with 24 rollers to ease its motion. The small staircase, visible in the plate, is intended for persons. who wish to ascend into the galicry, without being obliged to go up the ladder. The ease with which the horizontal and vertical nutions may be comnunicated to the tube, will be best conceived from a remark of Dr. Herschel's, that, in the year $17 \mathrm{~S} 9$, he several times observed Saturn two or three hours before and after its meridional passage, with one single person to continue, at his directions, the nccessary horizontal and vertical motions.

Upon the platform are visible two rooms, the one called the Observatory, 8 feet 5 inches by 5 feet 5 inches, the other called the Working-room, 6 feet 6 inches by 4 feet 5 inches. To persons in these ruoms, as has been above remarked, the observer can give his directions by means of the speaking-pipes; and in the rooms may be placed things conmonly used in an observatory.

From a view of the plate and the description thus given of it, the reader will form a competent idea of an instrument, which, with proper eye-glasses, magnifies above 6000 times, and is the largest that has ever been made. Astronomers in different parts of the world may be discouraged from continuing their observations, when it should seem that their discoveries must be anticipated by our observer ; but though he has so much the advantage, much is left to their labour and industry. It did not require a telescope of this magnitude to observe the object which was first discovered to be a planet by our astronomer, for it had been seen and taken for a fixed star by many persons in the two last centuries. And the double ring of Saturn, which has indeed been so beautifully observed through Herschel's magnifier, had been already described by Cassini in his Memoirs. Such of our readers as wish for a more particular account of this instrument will find it in the Transactions of the Royal Suciety for 17.95 , second part ; in which there are 18 plates, and 63 pages of letter-press, to give an ample detail of every circumstance relating to joiner's work, carpenter's work, smith's work, \&c : which has attended the formation and erection of this instrument. It was completed on August the 28th, 1789, on which day the sixth satellite of Saturn was discovered.

HERSE, in Fortification, a lattice or portcullice, in the form of a harrow, beset with iron spikes, to block up a gate-way, \&c.

HERSILLON, or little Herse, in Fortification, is a plank armed with iron spikes, for the same use as the herse, and also to impede the marcli of the infantry or cavalry.

HERWART (JoHN GEOR(E), chancellor of Bavaria at the beginning of the 17 th century, was rlescended from a Patrician family of Augsburg. Ile was author of several curious books. 1.Tabulix Arithmetic $æ$ Universales, \&c. Monach. Bavar. in large folio, 1610. This is perhaps a work of the most stapendous magnitude and labour in numeral calculations that ever was exccuted, consisting of a table of numbers closely printed on 999 large folio pages, for readily taking out the products and quotients of all numbers by inspection only; being in fact an immense multiplication-table, of all factors or numbers, both up to 1000 each, or 1 million of products. Many examples of multiplications and divisions are prcfixed, with applications to the calculations of spherical triangles. And, but for the more compendious invention of logarithms, which soon after tock place, Herwart's tables would doubtless still have been in constant use for similar purposes. 2. Herwart also wrote an $A$ pology for the Emperor Louis of Bavaria, against the falsehouds asserted by Bzovius. 3. Chronologia, nova et vera, 1612 and 1626,2 parts, 4to--4. Another very singular book, which was published by his son, John Frederick Herwart, entitled, Admiranda ethicæ Theologiæ Mysteria propolata, de antiquissima veterum Nationum Superstitione, \&c ; 1624, 4to.

HESSE (WILlia M Prince of), rendered his name immortal by his encouragement of learning, by his studies, and by his observations, for many years, of the cclestial bodies. For this purpose, he erected an observatory at Cassel, and furnished it with good instruments, well adaptcd to that design; calling also to his assistance two eminent artists, Christopher Rothmann and Juste Byrge. His observations, which are of a very curious nature, were published at Leyden, in the year $161 \mathrm{~s}$, by Willebrord Snell; and are in part mentioned by Tycho Brabé, as well in his epistles as in the $2 \mathrm{~d}$ volume of his Progymnasmata ; a signal example to all princely' and heroic minds, to undertake the promoting the arts of peace, and advancing this truly noble and celestial science. This prince died in the year 15.97 .

HETERODROMUS Vectis, or Lever, in Mechanics, a lever in which the fulcrum, or point of suspension is between the weight and the power; being the same as what is otherwise called a lever of the first kind.

HETEROGENEOUS, or HeterogeneaL, literally imports things of different natures, or something that consists of parts of different or dissimilar kinds; in opposition to Homogeneons. Thus,

Heterogeneous Bodies, are such as have their parts of unequal density.

Heterogen EOU S Light, that which consists of parts or rays of different refrangibility, reflexibility, and colour. Thus, the common light of the sun, or clouds, is heterogeneous, being a mixture of all sorts of rays.

Heterogenzous Numbers, are mixed numbers, consisting of integers and fractions.

Hete Rog tan zous Particles, are such as are of different kinds, natures, and qualities; of which generally all bodies consist.

Hetx ROG en EOUs 2uantities, in Mathematics, are those which cannot have proportion, or be compared together as to greater and less; being of such different kind and consideration, as that one of them taken any number of times, never equals or exceeds the other. As lines, surfaces, and solids in geometry.

Heterogeneous Surds, are such as have difierent radical signs, as $\sqrt{ } u$ and $\sqrt[3]{ } b^{2}$; or $\sqrt[5]{10}$ and $\sqrt[7]{2} 20$.

HETLROSCII, in Gcography, are such inlaabitants of the earth as have thrir shadows at noon projected always the same way with regard to themselves, or always contrary ways with respect to each other. Thus, all the inhabitants without the torrid zone are lleteroscii, with regard to themselves, since any one such inhabitant has his shadow at noon always the same way, viz, always north of him in north latitude, and always south of him in south 
latitude; or these two situations are Heteroscii to each other, having such shadows projected contrary ways at all times of the year.

HEVELIUS (JoHN), a very celebrated astronomer, and a burgomaster of Dantzick, was born in that city in 1611. He studied mathematics under Peter Cruger, in which he made a wonderful progress. He afterwards spent several years on his travels through Holland, England, Germany, and France', for his improvement in the sciences. On his return, he constructed excellent telescopes himself, and began diligently to observe the heavens, an cmployment he closely followed during the course of a long life, which was terminated only in 1687 , at 76 years of age. Hevelius was author of several remarkable discoveries in the heavens. He was the first who observed the phenomenon called the libration of the moon, and made several other important olservations on the ather planets. He also discovered several fixed stars, which he named the Firmament of Sobieski, in honour of John the 3d, king of Poland. He also framed a large catalogue of the stars, and collected multitudes of the unformed ones into new constellations. His wife was also well skilled in astronomy, and made a part of the observations that were published by her liusband. Our author's principal publications are, his Selenographia, or an exact description of the moon; in which he has engraved all her phases, and remarkable parts, distinguisher by names, and ascertained their respective bounds by the help of telescopes; containing also a delineation of the several visible spots, with the various motions, changes, and appearances, discovered by the telescope, as also in the sun and other planets, 1647 . -In 1654, two epistles; one to the celebrated astronomer Riccioli, concerning the Libration of the Moon; and the other to Bulliald, on the Eclipses of both luminaries. -In 1656, a Dissertation, De Natura Saturni faciei, \&c. -In 1668, his Cometographia, representing the whole nature of comets, their situation, parallaxes, distances, diverse appearances, and surprising motions, with $a$ history of all the comets from the beginning of the world down to the present time; being enriclsed with curious sculpture of his own execution: to which he added a treatise on the planct Mlercury, seen in the sun at Dantzick, May 3, 1661 ; with the history of a new star appearing in the neck of Cetus, and another in the beak of Cygnus; besides an illustration of some astronomical discoveries of the late $\mathrm{Mr}$. Horiox, in his treatise on Venus seen in the sun, November 24,1639 ; with a discourse of some curious Paraselena and Parbelia observed at Dantzick. He sent copies of this work to several members of the Royal Society at London, and among them to Mr. Hooke, in return for which, this gentleman sent to Hevelius a description of the Dioptric Telescope, with an account of the manner of using it; and recommending it to him, as much preferable to telescopes with plain sights. This gave rise to a dispute between them, viz, "whether distances and altitudes could be taken with plain sights any nearer than to a minute." Hooke asserted that they could not ; but that, with an instrument of a span raclius, by the belp of a telescope, they might be determined to the exactness of a second. Hevelius, on the other hand, insisted that, by the advantage of a good ey'e and long practice, he was able with bis instruments to come up even to that exactness; and, appealing to experience and facts, sent by way of challenge 8 distances, each between two different stars, to be cxamined by Hooke. Thus the affair rested for some time with out- ward decency, but not withoutsome inward grudge between the parties.

In 1673 , Hevelius published the first part of his Machina Calestis, as a specimen of the exactness both of his instruments and observations; and sent several copies as presents to his friends in England, but omitting Mr. Hooke. 'This, it is supposed, occasioned that gentleman to print, in 1674, Animadversions on the First Part of the Machina Colestis; in which he treated Hevelius with a very magisterial air, and threw out several unhandsome reflexions, which were greatly resented. This was the cause of a most violent dispute, which arose afterwards to such a beight, and became so notorious, that in $1679 \mathrm{Dr}$. Halley went, at the request of the Royal Society, to exanine both the instruments and the observations made with them. Of both these, Halley gave a favourable account, in a letter to Hevelius; and Hooke managed the controversy so ill, that he was universally condemned, though the preference has since been given to telescopic sights. However, Hevelius could not be prevailed on to make use of them: whether he thought himself too experienced to be informed by a young astronomer, as he considered Hooke; or whether, having made so many observations with plain sights, he was unwilling to alter his method, lest he might bring their exactness into question; or whether, being by long practice accustomed to the use of them, and not thoroughly apprehending the use of the other, nor well understasiding the differençe, is uncertain. Besides Halley's letter, Hevelius received many others in his favour, which he took the opportunity of inserting among the astronomical observations in his Annus Climactericus, printed in 1685. In a lnng preface to this work, he speaks with more confidence and greater indignation than he had done before, and particularly exclaimed against Hooke's dogmatical and magisterial manner of assuming a kind of dictatorship over him. This revived the contest, and excited so much interest as to occasion several other learned men to engage in it. The book itself being sent to the Royal Society, at their request an account of it was given by Dr. Wallis; who, among other things, took notice, that, "Hevelius's observations had been misrepresented, since it appeared from this book, that he could distinguish by plain sights to a small part of a minute.". About the same time Mr. Molyneux also wrote a letter to the Society, in vindication of Hevelius, against Hooke's animadversions. To all which, Hooke drew up a letter in answer, which was read before the socicty, containing many qualifying and accommodating expressions, but still at least exprcssing the superiority of telescopic sights nver plain ones, excellent as the observations were that had been made with these.

Hevelius had published in 1679, the second part of his Machina Cœlestis; but the same year, while he was at a scat in the country, he had the misfortune to have his house at Dantzick burnt down. By this calamity it is said he sustained several thousand pounds damage; having not only his observatory and all his valuable instruments and astronomical apparatus destroyed, but also a great many copies of his Machina Cœlestis, an accident which has made this second part very scarce.

In 1690 , were published a description of the heavens, called, Firmamentum Sobicscianum, in honour of John the $3 \mathrm{~d}$, king of Poland, as above mentioned ; and also Pro'dromus Astronomiæ, et Novæ Tabulæ Solares, una cum Catalogo Fixarum; in which are contained the necessary preliminaries for taking an exact catalogue of the stars. 
Both these works were postbumuus; for Hevelius died the 28 th of January 1687 , exactly 76 years of age, as before observed, and universally admired and respected; abundant evidence of which appears in a collection of letters between bim and many other persons, that was printed at Dantzick in 1683.

HEURAET (VAs), a respectable Dutch mathematician. He contributed some pieces on the nature of equations, in the commentaries published by Schoeten, on the geometry of Descartes; and, by pursuing the analyses of that author, recommended himself to notice by his invention for rectifying the curve of the semicubical parabola. In this, however, he was anticipated by the English mathematician Neil, who had some years before discovered the rectification of that curve. But it is very probable that Van Heuract knew nothing of Neil's discovery; and besides, the two methods are very different from each other.

HEXACHORD, a certain interval or musical concord, usually called a sixth.

HEXAEDRON, or HEXAHEDRON, olle of the five regular or Platonic bodies; being indecd the same as the cube; and is so called from its having 6 faces.-The square of the side or edge of a hexahedron, is one-third of the square of the diameter of the circumscribing sphere: and hence the diameter of a sphere is to the side of its inscribed hexahedron, as $\sqrt{ } 3$ to 1 .

In general, if ${ }_{A}^{A}, \mathrm{~B}$, and $\mathrm{c}$ be put to denote respectively the linear side, the surface, and the solidity of a hexahedron or cube, also $r$ the radius of the inscribed sphere, and $\mathrm{n}$ the radius of the circumscribed one; then we have these general equations or relations:
1. $\mathrm{A}=2 r=\frac{2}{3} \mathrm{R} \sqrt{ } 3=\sqrt{\frac{1}{6} \mathrm{~B}}=\sqrt[3]{\mathrm{c}}$.
2. $\mathrm{B}=24 r^{2}=8 \mathrm{R}^{2}=6 \mathrm{~A}^{2}=6 \sqrt{\mathrm{c}^{2}}$.
3. $\mathrm{c}=8 r^{3}=\frac{8}{9} \mathrm{R}^{3} \sqrt{ } 3=\mathrm{A}^{3} \quad=\frac{\mathrm{T}}{6} \mathrm{~B} \sqrt{ } \frac{1}{6} \mathrm{~B}$.
4: $\mathrm{R}=r \sqrt{ } 3=\frac{1}{2} A \sqrt{ } 3=\frac{1}{2} \sqrt{\frac{1}{2} \mathrm{~B}}=\frac{1}{2} \sqrt{ } 3 \times \sqrt[3]{ } \mathrm{c}$.
5. $r=\frac{\pi}{3} \mathrm{R} \sqrt{ } 3=\frac{1}{2} \mathrm{~A} \quad=\frac{1}{2} \sqrt{\frac{\mathrm{r}}{6} \mathrm{~B}}=\frac{\mathrm{r}}{2} \sqrt{\mathrm{c}} \mathrm{c}$.

HEXAGON, in Geometry, a figure of six sides, and consequently of as many angles. When these are equal, it is a regular hexagon.- The angles of a hexagon are each equal to $120^{\circ}$, and its sides are each equal to the radius of its circumscribing circle. Hence a regular hexagon is inscribed in a circle, by applying the radius 6 times successively to the periphery, and joining with right lines the sevcral divisions. And hence also, to describe a regular hexagon upon a given line, describe an equilateral triangle upon it, the vertex of which will be the centre of the circumscribing circle.

The side of a hexagon being $s$, its area will be $=$ $2.5980762 s^{2}=\frac{3}{2} s^{2} \times$ tang. $60^{\circ}=\frac{3}{2} s^{2} \sqrt{ } 3$.

HEXAson, in Fortification, is a six-lined figuredefended by six hastions.

HEXASTYLE, in the Ancient Architecture, a building with 6 columns in front.

HILRO's Crown, in Hydrostatics. The history of this crown, and of the important hydrostatical proposition which it gave occasion to, is as follows: Hicro, king of Syracuse, having furnished a workman with a quantity of gold for making a crown, suspected that he had been cheated, by the workman using a greater alloy of silver than was necessary in making it; and he aplied to Archimedes to discover the fraud, without defacing the crown.

This cclebrated mathematician was led by chance to a method of detecting the imposture, and of dctermining precisely the quantities of gold and silver composing the crown: for he observed, when bathing in a tub of water, that the water ran over as his body entered it, and he presently concluded that the quantity so running over was equal to the bulk of his body that was immersed. He was so pleased with the discovery, that it is said he ran about naked, crying out, sugriro, sugr, $I$ have found it; and some affirm that he offered a hecatomb to Jupiter for having inspired him with the thought.

On this principle he procured a ball or mass of gold, and another of silver, exactly of the same weight with the crown ; cunsidering, that, if the crown were of pure gold, it would be of equal bulk, and expel an equal quantity of water as the golden ball; and if it were of silver, then it would be of equal bulk and expel an equal quantity of water with the ball of silver; but of intermediate quantity, if it consisted of a mixture of the two, gold and silver. This, upon trial, he found to be the case; and hence, by a comparison of the quantities of water displaced by the three masses, he determined the exact portious of gold and silver in the crown.

Now suppose, for example, that each of the three masses weighed 100 ounces; and that on immersing them severally in water, there were displaced 5 ounces of water by the golden ball, 9 ounces by the silver, and 6 ounces by the compound, or crown; that is, their respective or comparative bulks are as 5,9 , and 6 , the sum of which is 20 .

Then the method of operation is this:

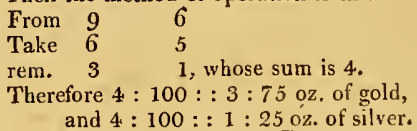

That is, the crowu consisted of 75 ounces of gold, and 25 ounces of silver.

See Cotes Hydros. Lect. p. 81 ; or Martin's Philos. Britan. vol. 1, p. 305, \&c. See also Specific Gravity.

HlGH-Water, that state of the tides when they have flowed to their greatest height, or have ceascd to flow or rise. At high-water the motion commonly ceases for a quarter or half an hour, before it begins to ebb again. The times of high-water of every day of the moon's age, is usually computed from that which is observed on the day of the full or change; viz, by taking 4t-5ths of the moon's age on any day of the month, and adding it to the time of high-water on the day of the full or change; then is the sum ncarly equal to the time of high-water on the day of the month proposed. And as to the times of highlivater, on the day of the full and change of the moon, at many different places; they have been observed as they are sct down in the following table. 
TA В $\mathrm{E}$ of the Times of High-water on the Days of the New and Full Moons, at many different Places.

\begin{tabular}{|c|c|c|c|c|c|c|c|c|c|c|c|c|c|c|}
\hline \multicolumn{2}{|l|}{ ' Nanies of Places. } & \multicolumn{3}{|c|}{ Countries. } & \multicolumn{2}{|c|}{ High-water. } & \multicolumn{3}{|c|}{ Names of Places. } & \multicolumn{3}{|c|}{ Countries. } & \multicolumn{2}{|c|}{ High-water. } \\
\hline Aberdeen . & & Scotland & $\cdot$ & - & & $45^{\mathrm{m}}$ & Dur & & & Scotland & . & - & $2^{\mathrm{h}}$ & $30^{\text {m }}$ \\
\hline Aldborough & & England & - & & 2 & 45 & Dundee & - & & Scotland & . & . & 2 & 15 \\
\hline Alderney I. . & . & England & & . & 12 & 0 & Dungarvan & . & . & Ireland & . & 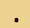 & 4 & 30 \\
\hline Amazons River & & South Ame & erica & . & 6 & 0 & Dungeness & . & & England & . & . & 9 & 45 \\
\hline Amsterdam & & Holland & $\cdot$ & - & 3 & 0 & Dunkirk & - & - & France & - & - & 0 & 0 \\
\hline Anlsterdam I. of & - & South Seas & & - & 8 & 30 & Dunnose & . & - & I. of Wigh & & - & 9 & 45 \\
\hline Andrew's St. . & . & Scotland & . & . & 2 & 15 & Dusky Bay & . & . & N. Zealand & & & 10 & 57 \\
\hline Anholt I. & - & Denmark & - & - & 0 & 0 & Easter Isle & . & - & Chili & & & 2 & 0 \\
\hline Antwerp & - & Flanders & . & . & 6 & 0 & Edystone & - & & English $\mathrm{Cl}$ & hannel & & 5 & 30 \\
\hline Archangel & 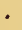 & Russia & - & & 6 & $0^{\circ}$ & Elbe R. & & . & Germany & . & & 0 & 0 \\
\hline Arran I. & & Ireland & - & - & 11 & 0 & Embden & - & & Germany & - & - & 0 & 0 \\
\hline Ashley Riv. • & & Carolina & - & & 0 & 4.5 & Estaples & & - & Fran & - & & II & 0 \\
\hline Augustine St. . & & Florida & . & . & 7 & 30 & Falmouth & & . & England & . & . & 5 & 30 \\
\hline Bajador Ca. . & & Negroland. & & - & 0 & 0 & Flamborough & h H. & • & England & - & - & 4 & 0 \\
\hline Baltimore & & Irela & . & . & 4 & 30 & & . & . & & . & & 7 & 30 \\
\hline Barfleur Ca. & . & France & . & . & 7 & 30 & Flushir & & . & Holland & . & . & 0 & 45 \\
\hline Bayonne & . & France & - & - & 3 & 30 & N. Foreland & & - & land & - & . & 9 & 4.5 \\
\hline$y$-head & - & and. & - & & 0 & 0 & Fouln & & - & & - & . & 6 & 45 \\
\hline N. and S. Bear & - & Labradore & & & 12 & 0 & & & . & England & . & . & 5 & 15 \\
\hline Belfast & - & Ireland . & - & - & 10 & 0 & Fayal Isl. & . & . & Azores & . & . & 2 & 20 \\
\hline Bellisle & - & France & . & & 3 & 30 & ne $R$. & & & & - & . & 3 & 0 \\
\hline Bermudas I. & - & Bahama I. & & c & 7 & 0 & Gibr & & 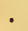 & Spain & . & . & 0 & 0 \\
\hline ick & - & England & . & . & 2 & 30 & C. Good Hop & & • & Caffers & - & - & 3 & 0 \\
\hline kney & - & England & - & & 6 & 0 & Goree (Isle) & & & Atlantic $\mathrm{O}$ & cean & & 1 & 30 \\
\hline Blanco Cap. & - & Negroland & & & 9 & 4.5 & Gran & . & & France & - & - & 7 & 0 \\
\hline et et & . & France & . & . & 3 & 0 & Gravelines & & . & Flanders & . & . & 0 & 0 \\
\hline Bourdeaux & - & Fra & - & - & 3 & 0 & esend & - & - & England & - & . & 1 & 30 \\
\hline gne & - & & . & & 10 & 30 & Groi & . & & Spain & - $\quad$, & & 3 & 3 \\
\hline men & - & any & - & & 6 & 0 & nsey, I. . & . & & English $\mathrm{Ch}$ & hannel & & 1 & 30 \\
\hline Brest & - & Fra & - & & 3 & 45 & $e^{\circ}$. & - & - & Holland & . & - & 8 & 15 \\
\hline Bridlington $\mathrm{B}$. & & Eng & - & - & 3 & 4.5 & Halifax & - & - & $\operatorname{coti}$ & & & 7 & 30 \\
\hline Brill & & Hol & - & & 1 & 30 & aburgh & - & & Ger & . & . & 6 & 0 \\
\hline tol & & Engl & - & & 6 & 4.5 & e Isle & . & . & & - & . & 3 & 30 \\
\hline Buchaness . & & and & - & - & 3 & 0 & $\mathrm{H}$ & - & . & & - & $\cdot$ & 9 & 0 \\
\hline Button's Isles & & Brit. & & & 6 & 50 & pool & - & & & . & . & 3 & 0 \\
\hline Cadiz & . & Spain . & - & & 4 & 30 & Harwich . & . & . & England & . & . & 11 & 15 \\
\hline - & - & & - & & 9 & 0 & e de Grac & & - & & - & $\cdot$ & 9 & 0 \\
\hline ais & - & & . & & 11 & 30 & Head . & · & & & - & . & 1 & 30 \\
\hline ria $\mathbf{I}$. & & & - & & 3 & 0 & orifleur & . & . & Fran & - & . & 9 & 0 \\
\hline C. Cantin & - & & - & & 0 & 0 & & & - & & - & . & 6 & 0 \\
\hline Cape Town & & & . & & 2 & 30 & er R. & & & & & . & 5 & 13 \\
\hline kets & & sey & - & & 8 & 15 & ohn's . & & - & undla & and & . & 6 & 0 \\
\hline hness Po. & - & & - & & 9 & 0 & dian ( $\mathrm{Po}$ & ort) & - & & & . & 4 & 4.5 \\
\hline Town & - & Carolina . & & & 3 & 0 & hnock & & & $\mathrm{h}$ coa & ast & & 0 & 0 \\
\hline Q. Charlotte's S. & . & New Zealan & & & 9 & 0 & & & & Ire & & . & 5 & 15 \\
\hline Cherhourg & • & & & & 7 & 30 & 's End. & & & England & - & - & 7 & 30 \\
\hline I $\mathrm{R}$ & & n's B & Bay & - & 7 & 20 & & & & & . & & 4 & 30 \\
\hline $\operatorname{ar} \quad$. & & Ireland & $\cdot$ & & 4 & 30 & off & & & England & - & - & 9 & 45 \\
\hline rneau . & & & - & & 3 & 0 & & 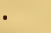 & & ugal & . & . & 2 & 15 \\
\hline et . & & & - & & 2 & 15 & pool & & & & . & & 11 & 15 \\
\hline et Isle. & & & - & & 3 & 0 & Lizard & & & nd & - & . & 7 & 30 \\
\hline - & & & - & & 6 & 30 & Loire (Riv.) & & & Fra & - & - & 3 & 0 \\
\hline C. Corse & & & . & - & 3 & 30 & & & & ind & - & & 3 & 0 \\
\hline mêr & & & - & & 7 & 0 & dy (Isle) & & & England & - & - & 5 & 15 \\
\hline Dartmouth & & land & - & & 6 & 30 & leira & & & Atl. Ocean & & - & 12 & 4 \\
\hline St. David's H. & & & . & & 6 & 0 & & & & & - & & 6 & 0 \\
\hline ope & & & . & & 10 & 30 & Man & & & & - & & 9 & 0 \\
\hline Dort & & & - & & 3 & 0 & Margate & & • & England & . & . & 11 & 15 \\
\hline Duver & & & - & & 11 & 30 & St. Mary's (Is & sle) & & Scilly Isles & & . & 3 & 4.5 \\
\hline Do & & & - & & 1 & 15 & & & & & . & & 5 & 15 \\
\hline Dublin & & Ireland & . & & 9 & 15 & Mount's Bay & & & England & - & - & 4 & 30 \\
\hline
\end{tabular}




\begin{tabular}{|c|c|c|c|c|c|c|c|c|c|c|c|c|c|c|c|}
\hline \multicolumn{3}{|c|}{ Names of Places. } & \multicolumn{3}{|c|}{ Countries. } & \multicolumn{2}{|c|}{ High-water. } & \multicolumn{3}{|c|}{ Names of Places. } & \multicolumn{3}{|c|}{ Countries. } & \multicolumn{2}{|c|}{ High-water. } \\
\hline Nantes & . & . & France & . & & & $0^{\mathrm{m}}$ & Severn (Mout & h.) & & England & . & & $6^{\mathrm{h}}$ & \\
\hline Naze & . & & Norway & . & & 11 & 15 & Sheerness . & & & England & . & . & 0 & 0 \\
\hline Needles & . & & England & . & & 10 & 15 & Sierra Leone & & . & Guinea & . & . & 8 & 15 \\
\hline Newcastle & . & . & England & . & & 3 & 1.5 & Shetland I. & & . & Scotland & . & - & 3 & 0 \\
\hline Nieuport & . & . & Flanders & - & & 12 & 0 & Isle of Sky & & - & Scotland & - & - & 5 & 30 \\
\hline Nore & . & . & England & . & & 0 & 0 & Spurn & & . & England & . & . & 5 & 15 \\
\hline North Cape & . & & Lapland & . & & 3 & 0 & Start Point & & . & England & . & - & 6 & 45 \\
\hline Orfurdness & . & 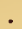 & England & . & & 9 & 45 & Stockton & & . & England & . & . & 5 & 15 \\
\hline Orkneys & . & . & Scotland & . & & 3 & 0 & Sunderland & . & . & England & . & . & 3 & 2) \\
\hline Ostend & . & . & Flanders & . & . & 12 & 0 & Tanua & & . & Pacific Oc & ean & . & 3 & 0 \\
\hline Placentia & . & - & Newfoundl & land & . & 9 & 0 & 'Teneriff & & . & Canaries & . & . & 3 & 0 \\
\hline Plymouth & . & . & England & . & - & 6 & 0 & Texel (Isle) . & & & Holland & . & . & 7 & 30 \\
\hline Portland & . & . & England & & . & 8 & 15 & Thames' Mou & & . & England & . & . & 1 & 30 \\
\hline Porto Praya & . & - & Cape Verde & les & - & 11 & 0 & Tinmouth & & . & England & . & . & 3 & 0 \\
\hline Portsmouth & . & . & England & . & . & 11 & 15 & Torbay & . & . & England & . & & 5 & 15 \\
\hline Quebec & . & & Canada & . & . & 7 & 30 & St. Valery & . & . & France & . & . & 10 & 30 \\
\hline Rhée (Isle) & & . & France & - & . & 3 & 0 & Vanmes & & . & France & . & . & 3 & .45 \\
\hline Resolution ( $\mathrm{P}$ & Bay) & . & Ohitahoo & . & $\therefore$ & 2 & 30 & Ushant & . & . & France & . & . & 4 & 30 \\
\hline Robin Hood': & 's $\mathrm{B}$. & . & Englaud & . & & 3 & 0 & Waterford & . & & Ireland & . & . & 6 & 30 \\
\hline Rochefort & . & . & France & . & . & 4 & 15 & Wells & . & . & England & . & . & 6 & 0 \\
\hline Rochelle & . & . & France & . & $\therefore$ & 3 & 45 & Weymouth & . & . & England & . & . & 7 & 20 \\
\hline Rochester & . & . & England & . & . & 0 & 4.5 & Whitby & & . & England & . & & 3 & 0 \\
\hline Rotterdam & . & . & Holland & . & . & 3 & 0 & Isle of Wight & & & England & . & & 0 & 0 \\
\hline Rouen & . & & France & . & . & 1 & 15 & Winchelsea & & . & England & . & . & 0 & 4.5 \\
\hline Rye & - & & England & - & - & 11 & 15 & Wintertoness & & & England & . & & 9 & 0 \\
\hline Sandwich & & . & England & . & . & 11 & 30 & Yarmouth & & . & England & . & . & 9 & 45 \\
\hline Scarborough & $\mathrm{H}$ & . & England & . & . & 3 & 45 & New York & & . & America & . & & 3 & 0 \\
\hline Scilly Isles & . & . & England & . & & 3 & 45 & Youghalt & . & & Ireland & . & . & 4 & 30 \\
\hline Senegal & . & & Negroland & & & 10 & 30 & Zuric Sea & & & Holland & . & & 3 & 0 \\
\hline
\end{tabular}

HIPS, in Architecture, are those pieces of timber placed at the corners of a roof. These are much longer than the rafters, because of their oblique positien.

Hip means also the angle formed by two parts of the roof, when it rises outwards.

Hrp-Roof, called also Italian Roof, is one in which two parts of the roof meet in an angle, rising outwards : the same angle being called a valley when it sinks inwards.

HIPPARCHUS, a celebrated ancient astronomer, was born at Nice in Bithynia, and fluurished between the 154th and the $163 \mathrm{~d}$ olympiads; that is, between 160 and 135 years before Christ; for in this interval of time it is that his observations are dated. He is accounted the first, who from vague and scattered observations reduced astronomy into a science, and prosecuted the study of it systematically. Pliny often mentions him, and always with great commendation. He was the first, he tells us, who attempted to count the number of the fixed stars; and his catalugue is preserved in Ptolemy's Almagest, where they are all noted according to their longitudes and apparent inagnitudes. The same author places him among those men of a sublime genius, who, by foretelling the eclipses, taught mankind, that they ought not to be alarmed at these phenomena. Thales was the first among the Greeks, who could discover when there was to be an cclipse. Sulpitius Gallus anong the Romans begin to succeed in this kind of prediction; and he gave an essay of his skill very opportunely, the day before a battle was fought. After these, Hipparchus improved the science of eclipses very considerably : making ephemerides, or catalogues of cclipses, for 600 years. Pliny admires him for making a review of all the stars, acquainting us with their situations and magnitudes; for by these means, observes he, posterity will be able to discuver, not only whether

they are born and die, but also whether they change their places, and whether they increase or decrease. He mentions also a new star which was produced in his days; and that by its motion, when it first appeared, he began to doubt whether this did not frequently happen, and whether those stars, which we call fixed, do not likewise move. Hipparchus is also memorable for being the first who discovered the precession of the equinoxes, or a very slow apparent mution of the fixed stars from east to west, by which in a great number of years they will appear to have performed a complete revolution. He endeavoured also to reduce to rule the many discoveries he made, and invented new instruments, hy whicl he marked their magnitudes and places in the heavens: so that by means of them it might be easily observed, not only whether they appear and disappear, but also whether they pass each other, or move, and whether they increase or decrease.

'I he first observations made by' Hipparchus, were in the isle of Rhodes; whence he got the name Rhodius; but afterwards he cultivated this science in Bithyniz and Alexandria only. One of his works is still extant, viz, his Commentary on Aratus's Phenomena. He composed several other works; and on the whole it is agreed, that astronomy is greatly indebted to him, for laying that rational and solid foundation, on which all succceding astronomers have built their superstructure.

HIPPOCRATLS, of Chios, a respectible Grock geometrician, but originally it scens a merchant, who, from his honest and unsuspecting simplicity of nature, being grcatly imposed on, and halt ruined by the knavery of the farmers of the revenue at Byzantium (now Constantinople), suspended his commerce, and repaired to Athens to retrieve his affairs, where he first became ncquainted with geometry. Curiosity, or anuscment, having onc day 
led him to visit one of the schools of philosophy, he so much relished the lectures on geometry, wlich he there heard, that he resolved to devose himself to the study of that science. He soon becane a distinguished geometrician. The quadrature of the lunes, which he discovered, emboldened him to attempt also that of the circle. He adso cumposed Elements of Geometry, which have not however reached our times.

HIRCUS, in Astronomy, a fixed star of the first magnitude, the same with Capella.

Hricus is also used by some writers for a comet, encompassed as it were with a mane, seemingly rough and hairy.

HIRE (PHILIP DE LA), an eminent French mathematician and astronomer, was born at Paris in 1640. His father, who was paiuter to the king, intending him for the same occupation, taught him drawing and such brauches of mathematics as relate to it : but dird when the son was only 17 years of age. Three years after this, he travelled into Italy for improvement in that art, where he spent 4. years. He applied himself also to mathematics, which at last engrossed all his attention. " On his return to Paris, be continued his mathematical studies with great eagerness, and afterwards published some works, which gained him so much reputation, that in $167 \mathrm{~s}$ he was named a nember of the Academy of Sciences.

Colbert the minister having formed a design for a better chart or map of France than any which liad then been conceived, Lahire was appointed, with Picard, to make the necessary observations for that purpose. This occupied him some years in several of the provinces; and was the main object of his peregrinations. But he was not, however, unmindful of other branches of knowledge, for he philosophized on every thing that occurred, and particularly on the phenomena of the variations of the magnetic needle, and refractions, as also on the height of mountains, as determined by the barometer.

In 1683 , Lahire was employed in continuing the meridian line, which. Picard had begun in 1669. He continued it from Paris northward, while Cassini carried it on to the south; but Colbert dying the same year, the work was relinquished before it was finished. Lahire was next employed, with other mombers of the Academy, in taking the necessary levels for the grand agueducts which Louis the 14 th was about to make.

The great number of works published by our author, together with his continual employments, as professor of the Royal College and of the Academy of Architecture, give us some idea of the great labours he underwent. - His lays were always spent in study: his nights very often in astronomical observations; seldom seeking any other relief from his labours, than a change of one for another. In his manner, he had the exterior politeness, circumspection, and prudence of Italy; on which account he ajpeared too reserved in the eyes of his countrymen; though he was always esteemed as a very honest disinterested man. He died in 1718 , at 78 years of age.

Of the numerous works which he published, the principal are, 1. Traité de Mechanique; $1665 .-2$. Nouvelle Methode en Geometrie pour les Sections des Superficies Coniques et Cylindriques; 1673,4 to. -3 . De Cycloide ; $1677,12 \mathrm{mo}-4$. Nouveaux Elemens des Sectiones Coniques : les Lieux Geometriques: la Construction, ou Effection des Equations; 1678, $12 \mathrm{mo}$ - 5 . La Gnomonique, $\& c ; 1682,12$ no -6 . Traité du Nivellement de M.Pi-

VoL. 1. card, avec des additions; $1684 .-7$. Sectiones Coniræ in novem libros distributa; 1685 , folio. This was considered as an original work, and gained the author great reputation all over Europe- 8 . Traité du Mouvement des Eaux, \&c; 1686.-9. T'abulæ Astronomicx; 1687 and 1702, 4to.-10. Ecole des Arpenteurs; $1689 .-11$. Veterum Mathematicorum Opera, Gracè et Iatinè, plcraque sunc primùm edita; 1693 , folio. This edition had been begun by Thevenot; wlio dying, the care of finishing it was committed to Lahire. It shows that our author's strong application to mathematical and astronomical studies had not hindered him from acquiring a very competent knowledge of the Greck tongue. Besides these, and other smaller works, a vast number of his pieces are diffused in Journals, and particularly in the Memoirs of the Academy of Scicnces, viz, from 1666 till the ycar 1718 .

HOBBES (Tнон As), a famous writer and philosopher, was born at Malmsbury in Wiltshire, in 1588 , being the san of a clergyman of that piace. He completed his studies at Oxford, and was afterwards governor to the eldest son of William Cavendish earl of Devonshire, with whom hè travelled through France and Italy, applying himself closely to the study of polite literature. In 1626 his patron the earl of Devonshire died; and in 1628 his son also ; the same year Mr. Hobbes published his translation of Thucydides in English. He soon after went abroad a second time as governor to the son of Sir Gervase Clifton; but shortly after returncd, to resume his concern for the hopes of the Devonshire family, to whom he had so carly attached himself; the countess-dowager having desired to put the young earl under his care, then about 13 years of age. 'This charge was very agreeable to Mr. Hobbes's inclinations, and be discharged the trust with great diligence and fidelity. In 1634 ne accompanied his young pupil to Paris, where he employed his own vacant hours in the study of natural philosophy, frequently conversing with Father Mersenne, a man of great and deserved renown, and who corresponded with almost all the learned in Europe. From Paris be attended his pupil into Italy, where be became acquainted with the celebrated Galileo, who freely communicated his notions to him; and from lience he returned with his ward to England. But afterwards, foreseeing the civil wars, he went to scek a retreat at Paris; where he soon became acquainted with Descartes, with whom he afterwards held a correspondence on several mathematical subjects, as appears from the letters of Mr. Hobbes ptiblished in the works of Descartes.

But when this philosopher printed afterwards his Meditations, whercin he attempted to establish points of the highest conseguence from innate ideas, Mrr. Hobbes took the liberty of dissenting from him, as did also the French king's mathematical professor, the illustrious Peter Gassendi, with whom Mr. Hobbes contracted a very close friendship, which was not interruptcd till the death of the former.

I1 1642, Mr. Hobbes printed his famous book De Cive, which raised him many adversaries, who charged him with instilling principles of a dangerous tendency. Among many illustrious persons who, from the troubles in England, retired to France for safety, was Sir Charles Cavendish, brother to the duke of Ncwcastle: and this gentleman, being well skilled in the mathenatics, proved a constant friend and patron to our atthor; who, by cmbarking in 1645 in a coutroversy about squaring the circle, 40 
was become so famous by it, that in 1647 he was recommended to instruct Charles prince of Wales, afterwards King Clarles the 2d, in mathematical learning. During this he employed his vacant time in composing his Leviathan, which was published in England in 1651. After the publication of this work, he returned to England, and passed the remainder of his long life in a very retired and studious manner, in the house of the earl of Devonshire, mostly at his seat in Derbyshire, but accompanying the earl always to Loudon, fearing to be left out of his immediate protection, lest he should be seized by officers from the parliament or government, on account of the freedom of his opinions in politics and religion. He received great marks of respect from King Charles the $2 d$ at the restoration in 1660 , with a pension of $100 l$. a year. From that time, till his death, he applied limself to his studies, and in opposing the attacks of his adversaries, who were very numerous : in mathematical subjects, disputes rose to a great height betwcen him and Dr. Wallis, on account of his pretended Quadrature of the Circle, Cubature of the Sphere, and Duplication of the Cube, which he obstinately defended without ever acknowledging bis error.

His long life was that of a perfectly honest man; a lover of his country, a good friend, charitable and obliging. He accustomed himself much more to thinking than reading; and was fond of a well-selected, rather than a large library. He had a hatred to the clergy, having been persecuted by them on account of the freedom of his doctrine, and having a very indifferent opinion of their knowledge and their principles. In his last sickness he was very anxious to know whether his disease was curable; and when intimations were given, that he might have ease, but no remedy, he said, "I shall be glad to find a hole to creep out of the world at.' He died the 4th of Dec. 1679, at 91 years of age.

His chiet publications were,

1. An English translation of Thucydides's History of the Grecian War.-2. De Mirabilibus Pecci, and Memoirs of his own Life, both in Latin verse.-3. Elements of Philosophy.-4. Answer to Sir William Davenant's Epistle, or Preface to Gondibert.-5. Human Nature, or the Fundamental Elements of Policy.-6. Elements of Law.7. Leviathan; or the Matter, Form, and Power of a Commonwealth.-8. A Compendium of Aristotle's Rhetoric. -9. A Letter on Liberty and Necessity.-10. The Questions, concerning Necessity and Chance, stated.-11. Six Lessons to the Professors of Matlematics, of the lnstitution of Sir Henry Saville.-12. The Marks of Absurd Geometry, \&c.-13. Dialogues of Natural Philosophy.Besides many other pieces on polity, theology, mathematics, and other miscellaneous subjects, to the number of 41 .

HOBITS, in Gunnery. See Howitz.

HODGSON (JAMES), F. R.s. was a respectable mathematician in the early part of the 18th century, and some time master of the Royal Mathenatical School in Christ's Hospital, London. Besides a multitude of papers in the Philos. 'Trans. from vol. 24 (anno 1704), to vol. 49 inclusive, he was also author of several mathematical works: as, 1. Treatise on Navigation, in 4to, 1706; 2 . System of Mlathematics, in 2 vols. 4 to, 1723 ; 3 . 'The 'l'heory of Jupiter's Satellites, 4to, 1750; 4 . The Doctrine of Fluxions, 4to, $1758: 5$. The Valuation of Anunities upon Lives, 4to; 6 . An Introduction to Chronology. And perbaps some others.-Mr. IJodgson was born 1672; clected F. R. S. 1703; and died June 25, 1755.
HOGSHEAD, a measure, or vessel, of wine or oil ; containing the 4th part of a tun, the half of a pipe, or 63 gallons.

HOLDER (WILLIAM), a learned and philosophical Englishman, was born in Nottinghamshire, educated at Canbridge, and in 1642 became rector of Blechingdon in Oxfordshirc. In 1660 le proceeded D. D., and became afterwards canon of Ely, fellow of the Royal Society, canon of St. Paul's, sub-dean of the royal chapel, and subalmoner to the king. He was a general scholar, a very accomplished person, and a great virtuoso.-Dr. Holder greatly distinguished himself, by giving speech to a young gentleman of the name of Popham, who was born deat. This was effected at his own house at Blechingdon in 1659 ; but the young man losing what he had heen taught by Holder after he was called loome to his friends, he was sent to Dr. Wallis, who brought him to his speech again. Holder published a book, entitled The Elements of Speech; an essay or inquiry into the natural Production of Letters: with an appendix, concerning persons that are dcaf and dumb, 1669, svo. In the appendix be relates how soon, and by what methods, he brought young Popham to speak. In the Philos. Trans.. for July 1670 , was inserted a letter from Dr. Wallis, in which he claims to himself the honour of bringing that gentleman to speak. By way of answer to which, in 1678, Dr. Holder published, in 4to, A Supplement to the Philos. Trans. of July 1670 , with some reflections on Dr. Wallis's letter there inserted. Upon which the latter soon after published A Defence of the Royal Society, and the Philos. Trans. particularly those of July 1670, in answer to the cavils of Dr. William Holder, $1678 ; 4$ to.

Dr. Holder's accomplishments were very general. He was skilled in the theory and practice of music, and wrote A Treatise of the Natural Grounds and Principles of Harmony, 1694, 8vo. He wrote also A Treatise concerning Time, with applications of the Natural Day, Lunar Month, and Solar Year, \&ce, 1694, 8vo. He died at Amen Corner in London, Jan. 24, 1697, and was buried in St. Paul's.

HOLLOW, in Architecture, a concave moulding, about a quarter of a circle, by some called a casement, by others an abacus.

HoL Low-Tower, in Fortification, is a rounding made of the remainder of two brisures, to join the curtin to the crillon, where the small shot are played, that they may not be so much exposed to the vicw of the enemy.

HOLY Thursday, otherwise called Ascension-day, being the 39th day after Faster Sunday, and kept in commemoration of Christ's ascension into heaven.

Hozy Rood, or Holy Cross, a festival kept on the 14th of September, in memory of the exaltation of the holy cross.

HoLx Week, is the last week of Lent, called also Passion week.

HOLYWOOD (Jomn), or Halirax, or Sacrobosco, an eminent mathematician of the 12th century, whose birthplace is variously ascribed. According to Leland, Bale, and Pitts, he was born at Halitax in Yorhshire: accorling to Stanihurst, at IIv] ywood near Dublin; and according to Dempster and Mackenzie, in Nithstale in Scothand: thungh it is possible there might have been more than one of the name. Mackenzie, however, informs us, that having finished his studies, he entered into orders, and became a canon regular of the order of St. Augustin 
in the famous monastery of Holywood in Nithsdale. The English biographers, on the contrary, inform us that he was educated at Oxford. They all agree bowever in asserting, that he spent most of his lite at Paris; where, observes Mackenzie, he was admitted a member of the university, June 5, 1221, under the syndics of the Scotch nation; and soon after was elected professor of mathematics, which he taught with applause for many years. According to the same author, he died in 1256 , as appears from the inscription on his monument in the cloisters of the convent of St. Maturine at Paris.

Holywood was contemporary with Roger Bacon, but probably older by about 20 years. He wrote, 1. De Sphera Mundi; a work often reprinted, and illustrated by varjous commentators.-2. De Anni Ratione, seu de Computo Ecclesiastico.-3. De Algorismo, printed with Comm. Petri Cirvilli Hisp: Paris, 1498. The Algorismus was probably the treatise, in technical verses, which he composed on the Arabian arithmetic.

HOMOCENTRIC, the same as Concentric.

HOMODROMUS Vectis, or Lever, in Mechanics, is a lever in which the weight and power are both on the same side of the fulcrum, as in the lever of the $2 \mathrm{~d}$ and $3 \mathrm{~d}$ kind; being so called because here the weight and power move both in the same direction, whereas in the Heterodromus they move in opposite directions.

HOMOGENEAL, or HOMOGENEOUS, consisting of similar parts, or of the same kind and naturc, in contradistinction from Heterogeneous, where the parts are of difierent kinds. - Natural bodies are usually composed of homogeneous parts, as a diamond, a metal, \&c. But artificial bodies, on the contrary, are assemblages of heterogeneous parts, or parts of different kinds ; as a building, of stone, wood, \&c.

HomogeNeA L Light, is that whose rays are all of one and the same colour, refrangibility, \&c.

Homogeneal Numbers, are those of the sane kind and nature.

Homogen Eal Surds, are such as have one common radical sign; as $\sqrt{ } 27$ and $\sqrt{ } 30$, or $\sqrt{ } a$ and $\sqrt{ } b$, or $2 \sqrt[3]{c}$ and $\sqrt[3]{ } d$.

HOMOGENEOUS Equations, in the doctrine of Fluxions, particularly treated by John Bernoulli, for integrating such forms of two fluxions of the first order, in which the sum of the dimensions of $x$ and $y$, which affect $\dot{x}$ and $\dot{y}$, rise to the same degree in all the terms, then the indeterminates may be separated by a simple substitution, as of $y z$ for $x$ : such for example as the fluxional equations $\left(a x^{2}+x y\right) \dot{x}=\left(b y^{2}+x^{2}\right) \dot{y}$, or $\frac{(a x+y) \dot{x}}{\sqrt{(x x+y y)}}=\frac{(b y+x y)}{\sqrt{ }(x y-y y)}$. Then substituting, in the former of these, $y z$ for $x$, and the fuxion of this for $\dot{x}$, by reduction the equation becomes $\frac{\dot{y}}{y}=\frac{a z^{2}+z}{b-a z^{3}} \dot{z}$, where thc indeterminates are separated, and the fluents may be found.

In like mamner the application is made to such homogeneous equations as the following:

$(a x+b y) \dot{x}+(c x+d y) \dot{y}=0$,

$\left(a x^{2}+b x y+c y^{2}\right) \dot{x}+\left(d x^{2}+e x y+f y^{2}\right) \dot{y}=0$, $\left(a x^{3}+b x^{2} y+c x y^{2}+d y^{3}\right) \dot{x}+\left(e x^{3}+f x^{2} y+g x y^{2}+h y^{3}\right) j=0$
$\&$ \&c.

HOMOGENEUM Adfectionis, a name given by Victa to the second term of a compound or affected equation, being that which makes it adfected.

Homogengum Comparationis, in Algelra, a name given by Vieta to the absolute known number or term in a compound or affected equation. This he places on the right-hand side of the equation, and all the other terns on the left.

HOMOLOGOUS, in Geometry, is applied to the corresponding sides of similar figures, or those that are opposite to equal or corresponding angles, and are so called because they are proportional to each other. For all similar figures have their like sides homologous, or proportional to one another, also their areas or surfaces are homologous, or proportional to the squares of the like sides, and their solid contents homologous, or proportional to the cubes of the same.

HOOF, or Ungula, in Geometry, is a part cut off a cylinder, or cone, \& c, by a plane passing both through the base and part of the curve surface. It has nbtaincd its name from its resemblance to the hoof (ungula) of a horse. For the contents and surfaces of such hoofs, see my Mensuration, pa. $16 \mathrm{I}-183$, 4 th edit.

With respect to the surfaces of conic ungulas formed by planes perpendicular to the base, Father Guido Grandi first remarked, that if a polygon be inscribed in the base of a cone, and if on each side of this polygon a plane be raised perpendicular to the base, the portion of the conical surface cut off towards the axis, is equal to a rectilineal space. 'The portions also of the cone cut off by the above planes, towards the base, are in the same ratio as the segments of the base on which they stand. Indecd, whatever figure be inscribed in the base, if we conceive a right prismatic surface raised from the perimeter of the figure, it will cut off from the conical surface a portion, which will be to it in the same ratio, viz, that of the radius of the base to the slant height of the cone.

HOOKE (Roв $\varepsilon$ RT), a very celebrated mathematician and philosopher, was born, 1635, at Freshwater in the Isle of Wight, where his father was minister. He was jntended for the church ; but being of a weakly constitution, and very subject to the head-ach, all thoughts of that nature were laid aside. Thus left to himself, the boy followed the bent of his genius, which was turned to mechanics; and employed his time in naking little toys, which he cxecuted with wonderful art and dexterity. He had also a good turn for drawing; for which reason, after his father's death, which happened in 1648, he was placed with Sir Peter Lely; but the smell of the oil-colours increasing his head-ach, he quitted painting in a very short time. He was afterwards kindly taken by Dr. Busby into his house, and supported there, while he attended Westminster-school; where he not only acquired a competent share of Greek and Latin, together with an insight ints Hebrew and some other Oriental languages, but also made himself master of a good part of Euclid's Elements; and, as Wood asserts, jnvented 30 different ways of flying.

About the year 1653 he went to Christ-church, Oxford; and in 1655 was introduced to the Philosophical Society there; where, discovering his mechanic genius, he was first cmployed to assist Dr. Willis in his chemical operations, and was afterwards recommended to Mr. Robert Boyle, whom he served several years in the same capacity. He was also instructed in astronomy about this time by Dr. Seth Ward, Savilian professor of that science; and henceforward distinguished himself by many noble inventions and improvements of the mechanical kind. Ile also invented several astronomical instruments, for making observations both at sea and land, and was particularly 
serviceable to Mr. Buyle in completing the invention of the air-pump. In 1662 he was appointed Curator of Experiments to the Royal Society; and when that body was established by royal charter, he was in the list of those who were first named by the council in May 20, 1663; and was admitted accordingly June 3 , with a peculiar exemption from all payments. Sept. 28 of the same year, he was named by Lord Clarendon, chancellor of Oxford, for the degree of M. A.; and Oct. 19 it was ordered that the repositury of the Royal Society should be committed to his care; the white gallery in Gresham-college being appropriated to that use. In May 1664, he began to read the astronomy lecture at Greshain-college, for the professor Dr. Pope, then in Italy; and the same year he was made professor of mechanics to the Royal Society by Sir Joln Cutler, with a salary of $50 l$. per annum, which that gentleman, the founder, settled upon him for life. Jan. 1.1, i665, that society granted a salary also of $30 l$. a year, for his office of curator of experiments for life; and the month of March the same year he was elected professor of geometry in Gresham-college.

In 1665 he published in folio, his Micrographia, or some Philosophical Descriptions of Minute Bodies, made by Magnifying Glasses, with Observations and Inquiries on them. And the same year, during the recess of the Royal Society on account of the plague, he attended Dr. Wilkins and other ingenious gentlemen into Surrey, where they made several experiments. In 1666 he produced to the Royal Society a model for rebuilding the city of London, then destroyed by the great fire, with which the socicty was well pleased; and the lord mayor and aldermen preferred it to that of the city surveyor, though it happened not to be carried into execution. The rebuilding of the city, according to the act of parliament, requiring able persons to set out the ground for the proprietors, Mr. Hooke was appointed one of the surveyors; an employment in which he acquired most part of his estate, as appeared from a large iron chest of money found after his death, locked down with a key in it, and a date of the time, which showed it to have been so shut up above 30 years. "From $166 \mathrm{~s}$. he was engaged for nany years in a warm contest with Hevelius, concerning the difference in accuracy between observing with astronomical instruments with plain and telescopic sights; in which dispute many learned men afterwards engaged, and in wlich Ifooke managed so ill, as to be universally condemned, though it - has since been agreed that he had the better side of the question. In 1671 he attacked Newton's New Theory of Light and Colours; where, though he was obliged to submit in respect to the argument, it is said he came of with more credit. The Royal Society having commenced their meetings at Gresham-college, November 1674, the committee in December allowed him 40 , to erect a turret ower part of his lodgings, for trying his instruments, and making astronomical observations: and the year following he published A Description of Telescopes, and some other instruments made by R. II. with a Postscript, complaining of some injustice done him by their sccretary $\mathrm{Mr}$. Oldenburg, who published the Philosophical Transactions, in regard to his invention of pendulum watches. This charge engaged him in a dispute with that gentleman, which ended in a declaration of the Royal Socicty in their secretary's favour. Mr. (Oldenburg dying in 1677, Mr. Hooke was appointed to supply. his place, and began to take minutes at the meeting in October, but did not publisb the Transactions. Soon after this, he became more reserved than formerly; and though he read his Cutlerian Lecture;, often made experiments, and showed new inventions before the Royal Society, yet he seldom left any account of them to be entered in their register's; designing, as he said, to publish them himself, which bowever he never performed. In 1686, when Newton's work the Principia was published, Hooke laid claim to his discovery concerning the force and action of gravity, wbich was warmly resented by that great philosopher. Hooke, though a great inventor and discoverer himself, was yet so envious and ambitious, that he would fain have been thought the only man who could invent and discover any thing. This made him often lay claim to the inventions and discoveries of other persons; on which occásions, however, as well as in the present case, the thing was generally carricd against him.

In the beginning of the year 1687, his brother's daughter, Mrs. Grace Hooke, who had lived with him several years, died : and he was so affected with grief at her death, that he never recovered it, but was observed from that time to become less active, more melancholy, and more cynical than ever. At the same time, a chancery suit, in which he was concerned with Sir John Cutler, on account of his salary for reading the Cutlerian Lectures, made him uneasy, and increased his disorder. In 1691, he was employed in fiorming the plan of the hospital near Hoxton, founded ty Robert Ask, alderman of London, who appointed Archibishop Tillotson one of his executors; and in December the same year, Hooke was created M. D. by a warrant from that prelate. In July 1696, the chancery suit with Sir Jobn Cutler was detcrmined in his favour, to lis inexpressible satisfaction. His joy on that occasion was found in lis diary thus expressed; DoMsHLGiss a that is, Deo, Optïmo, DIaximo, sit honor, laus, gloria, in sacula saculorum, Amen. "I was born on this day of July 1635 , and God hath given me a new birth : may I never forget his mercies to me! while he gives me breath may I praise him !" In the same year $\mathrm{j} 696$, an order was granted to hin for repeating most of his experiments at the expense of the Royal Society, on a promise of his finishing the accounts, obscrvations, and deductions from them, and of perfecting the description of all the instruments contrived by him: but his increasing jllness, and general decay, rendered him unable to perform it. He continued some years in this wasting condition; and thus languishing till he was quite emaciated, he died March 3,1702 , in his 67 th year, at his lodgings in Gresham-college, and was buricd in St. Helen's church, Bishopsgate-street; his corps being. attended by all the nembers of the Royal Society then in London.

With regard to Mr. Hooke's character, it is not in all respects one of the most amiable. In his person he nuade rather a despicable figure, being but of a short stuture, very crooked, pale, lean, and of a meagre aspect, with dark-brown hair, very long, and hanging over his face, lank and uncut. Suitable to his person, his temper was penurious, melancholy, and mistrustful : and, though possessed of great philosophical knowledge, and strong invention, he had so much ambition, that he would be thought the only man who could invent or discover; and heuce he often laid claim to the inventions and discoveries of others, white he boasted of many of his' own which-he never communicated. In the religious part of his character, he was so exemplary, that he always expressed a great veneration 


\section{H O R}

for the Deity; and seldom received any remarkable benefit in life, or made any considerable discovery in nature, or invented any useful contrivance, or discovered any difficult problem, without noting some professions of acknowledgment to God; as many places in his diary plainly show. His chief publications are,

1. Lectiones Cutlerianæ, or the Cutlerian Lectures.-2. Micrographia, or Descriptions of Minute Bodies made by Magnifying Glasses.-3. A Description of Helioscopes.4. A Description of some Mechanical Improvements of Lamps and Water-poises.-5. Philosophical Collections. -6 . Posthumous Works, collected from his papers by Richard Waller, secretary to the Royal Society. Besides a number of papers in the Philos. Trans. volumes 1, 2, 3, $5,6,9,16,17,22$.

HORARY, something relating to Hours. As,

Horary Circles, hour lines, marking the hours, or drawn at the distance of hours from one another.

Horary MIotion, is the motion or space moved in an hour. Thus, the horary motion of the earth on her asis, is $15^{\circ}$; for, completing her revolution of $360^{\circ}$, in 24 hours, therefore the motion in one hour will be the 24 th part of $360^{\circ}$, which is 15 degrees.

HORIZUN, a great circle of the sphere, dividing the world into two parts, or hemispheres; the one upper, and visible; the other lower, and hid. The borizon is either rational or sensible.

Honizon, Rational, True, or Astronomical, called also simply and absolutely the horizon, is a great circle having its plane passing through the centre of the earth, and its poles are the zenith and nadir. Hence all the points of the horizon, quite around, are at a quadrant distance from the zenith and nadir. Also the meridian and rertical circles cut the horizon at right angles, and into two equal parts.

Hor izon, Apparent, Sensible, or Visible, is a lesser circle of the sphere, parallel to the rational horizon, dividing the visible part of the sphere from the invisible, and whose plane touches the spherical surface of the earth. The sensible horizon is divided into. castern and western; the eastern or ortive being that in which the heavenly borlies rise; and the western, or occidual, being that in which they set.

Horizox, in Gcography, is a circle dividing the visible part of the earth and heavens from that which is invisible. This is peculiurly called sensible or apparent horizon, to distinguish it from the rational or true, which passes through the centre of the earth; as already observed. These two horizons, though distant from one another by the semidiameter of the earth, will appear to coincide when continued to the sphere of the fixed stars; because the eat th compared with this sphere is but a point.

By sensible horizon is also often meant a circle which determines the segment of the surface of the earth, over which the eye can reach; called also the physical horizon. And in this sense we say, a spacious horizon, a narrow or scanty horizon, \&c; depending chiefly on the height the eyc is clevated above the earth.

For, it is evident that the higher the eye is placed, the farther is the visible horizon extended. Thus, if the eye be at $A$, at the height $\triangle D$ above the carth; draw the two tangents $\mathrm{A} h, \mathrm{Ar}$; and let onc of these lines $\mathrm{s} h$, be moved round the point $A$, and in its revolution always touch the surface of the earth; then the other point $h$ will describe the visible horizon $h$ or, \&c. But if the cye be placed
$\mathrm{HOR}$

bigher as at $\mathbf{B}$, the tangents $\mathbf{B} \mathbf{H}$ and $\mathrm{B} \mathbf{R}$ will reach farther; and the visible horizon Hor will be larger.

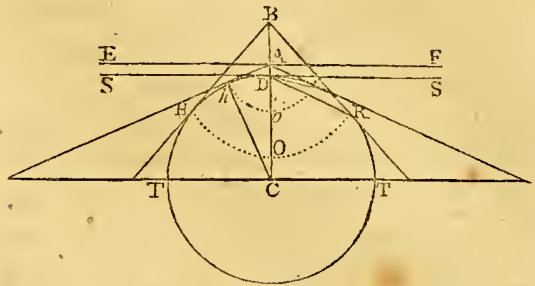

The visible horizon is most accurateiy observed at sea, and is sometimes called the horizon of the sea. In observing this horizon, the risual rays $A / h$ and $A r$ will, on account of the curre surface of the sea, always point a little below the true sensible horizon ss or FF, and consequently below the rational horizon TT, which is parallel to it.

To find the depression of the hurizon of the sea below the true horizon, which varies with the height of the eye, and in a small degree with the variation of the refractive power of the atmosphere, see DePREssion.

As to the right-lined distance, or tangent $A h$, it may be found thus; as radius : sin. $\angle \mathrm{C}:: \mathrm{CA}_{\mathrm{A}}: \mathrm{A} h$, or thus; as radius : $\tan . \angle \mathrm{c}:: \mathrm{ch}: \mathrm{A} h$; either of which will be nearly the same as the arc or curved distance $\mathrm{D} /$. Or, without finding the angle $\epsilon$, thus; the square of $A / h$ is equal to the difference of the squares of $\mathrm{CA}$ and $\mathrm{ch}$, i. e. $\mathrm{A} h h^{2}=\mathrm{CA}^{2}-\mathrm{C} h^{2}=(\mathrm{CA}+\mathrm{C} h) \times(\mathrm{CA}-\mathrm{C} h)=(\mathrm{CA}+\mathrm{C} h)$ $\times A D$, and hence $A h=\sqrt{ }(C A+c h \times A D)$, which is also equal to the curve $\mathrm{D} / \mathrm{n}$ nearly.

The distance on a perfect globe, if the visual rays came to the eye in a straight line, would be as above stated: but by means of the refraction of the atmosphere, distant objects on the horizon appear higher than they really are, or appear less depressed below the true horizon ss, and may be seen at a greater distance, especially on the sea. M. Legendre, in his Nifemoir on Measurements of the Earth, in the Mem. Acad. Sci. for the year 1787, says that, from several experiments, he is induced to allow for refraction a 14 th part of the distance of the place obscrved, expressed in degrees and minutes of a great circle. Thus, if the distance be 14000 toises, the refraction will be 1000 toises, erpual to the 57 th part of a degree, or $1^{\prime} 3^{\prime \prime}$. But the English, and other later measurements, make the allowance a 12th part; which therefore on the same distance will be 1167 toises, or the 49 th part of a degree, equal to $1^{\prime} 13^{\prime \prime}$.

Horizon of the Globe, the broad wooden circle.

IIORIZONTAL, something that relates to the horizon, or that is taken in the horizon, or on a level with or parallel to it. Thus, we say, a horizontal plane, horizontal line, horizontal distance, \&c.

Horizonta L Dial, is one drawn on a plane parallel to the horizon; having its gnomon or style elevated according to the altitude of the pole ol the place it is designed for.

Horizontal Distance, is that estimated in the direction of the horizon.

Horrzontal Line, in Perspective, is a right line drawn through the principal point, parallel to the horizon; or it is the intersection of the horizontal and perspective planes.

Hodrzontal Line, or base of a hill, in Surveying, a 


\section{H O R}

line drawn on the horizontal plane of the bill, or that on which it stands.

Hortzontal Moon. See Apparent Magnitude.

Horizontal Parallax. See Parallax.

Horizuntal Plane, is that which is parallel to the horizon of the place, or not inclined to it.

Horizon tal Plane, in Perspective. See Plane.

Horizontal Projection. See Projectron, and Map.

Horizontal Range, of a piece of ordnance, is the distance it throws its shot on a horizontal plane, whatever be the angle of elevation or direction of the piece. When the piece is pointed parallel to the horizon, the range is then called the point-blank or point-blanc range.-The greatest horizontal range, in the parabolic theory, or in a vacuum, is that made with the piece elevated to 4.5 degrees, and is equal to double the height from which a heavy body must freely fall, to acquire the velocity with which the shot is discharged. Thus, a shot being discharged with the velocity of $v$ feet per second; because gravity generates the velocity $2 \mathrm{~g}$ or $32 \frac{1}{6}$ feet in the first second of time, by falling $16 \frac{\mathrm{I}}{\mathrm{T}}$ or $g$ feet, and because the spaces descended are as the squares of the velocities, therefore as $4 g^{2}: v^{2}:: g: \frac{v^{2}}{4 g}$ the space a body must descend to acquire the velocity $v$ of the shot, or the space due to the velocity $v$; consequently the double of this, or $\frac{v^{2}}{2 g}=\frac{v^{2}}{32 \frac{1}{6}}$ is the greatest horizontal range with the velocity $v$, or at an elevation of 4.5 degrees; which is nearly half the square of a quarter of the velocity.

In other elevations, the horizontal range is as the sine of double the angle of elevation; so that, any other elevation being $e$, it 'will be, as radius $1: \sin .2 e:: \frac{v^{2}}{32 \frac{1}{6}}: \frac{v^{2}}{32 \frac{1}{6}} \times$ sin. $2 e$, the range at the elevation $e$, with the velocity $v$.

But in a resisting medium, like the atmosphere, the actual ranges fall short of those expressed by the above theorems, in so much that with the great velocities, the actual or real ranges may be less than the 10 th or 20 th part of the potential ranges; so that some balls, which actually range but a mile or two, would in vacuo range 20 or 30 miles. And hence also it happens tbat the elevation of the piece, to shoot farthest in the resisting medium, is always below $45^{\circ}$, and gradually the more below it, as the velucity is greater; so that the greater velocities with which balls are discharged from cannon with gun-powder, require an elevation of the gun equal to but about $30^{\circ}$, or little more. And the less the size of the balls is too, the less must this angle of elevation be, to throw them the farthest with a given velocity. See Projectile, and GunNERY.

Homzontal Refraction. See Refraction.

Horizontal Speculum, one to find a hotizon at sea, \& c, when the atmosphere is hazy near the horizon, by which the sight of it is prevented. A speculum of this kind was invented by a Mr. Serson, on the principle of a top spinning, which always keeps its upright position, notwithstanding the motion of the substance it spins upon. This curious instrument, as it bas since been improved by Mr. Smeaton, consists of a well polished inetal specculum, of about 3 inches and a half in diameter, inclosed within a circular rim of brass; so fitted that the centre of gravity of the whole shall fall near the point on which it spins. This is the end of a steel axis runuing through the centre of the speculum, above which it finishes in a square, for

\section{$\mathrm{HOR}$}

the conveniency of fitting a roller on it, which sets it in motion by means of a piece of tape wound about the roller. Various other contrivances to form artificial horizons have been invented by different persons, as glass planes floating on mercury, \&c. See HaDLEY's Qua DI ANT, and several inventions of this sort by Elton, Halley, Leigh, \&c, in the Philos. Trans. vol.37, pa. 273, vol. 38, pa. 167, vol.40, pa. 413,417 , \& c.

HORN-Work, in Fortification, a kind of out-work, advancing towards the field, to cover and defend a curtain, bastion, or other place, suspected to be weaker than the rest ; as also to possess a height ; carrying in the fore-part, or head, two demi-bastions, resembling horns: these horns, epaulments, or shoulderings, being joined by a curtin, shut up on the side by two wings, parallel to each other, are terminated at the gorge of the work, and so present themselves to the enemy.

HOROGRAPHY, the art of making or constructing dials; called also Dialling, Horologiography, Gnomonica, Sciatherica, Photosciatherica, \&c.

HOROLOGIUM, a common name, among ancient writers, for any instrument or machine for measuring the hours. See Clock, Watch, Str-Dial, Chronometer, ClePSYDRA, \&c.

HOROLOGY, that branch of mecbanical science which enables us to measure the portions of time. We judge of the lapse of time by the succession of sensiblc events; and the most convenient and accurate measures of its quantity are derived from motions; either uniform, or else repeated at equal intervals. Of the former kind, the rotation of the earth on its axis is the most exact, and the situation of its-surface with regard to the fixed stars, or less simply; with regard to the sun, constitutes the means for determining the parts of time as they follow each other. Of the latter kind, the rotation of machinery, consisting of wheel-work mored by a weight or spring, and regulated by a pendulum or balance, affords instruments of which the utility is well known. The term horology is at present more particularly confined to the principles on which the art of making clocks and watches is established. A considerable portion of this extended subject of research is given under the article Cцоск. See also Scapement, Watch, \&c.

HOROMETRY, the art of measuring or dividing time by hours, and kecping the account of time.

HOROPTER, in Optics, is a right line drawn through the point where the two optic axes meet, parallel to that which joins the centres of the two eyes, or the two pupils. As the line A B drawn through $c$ the point of concourse of

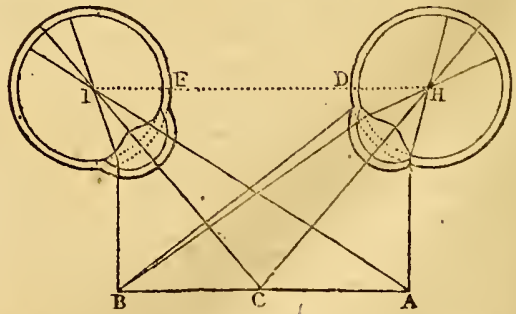

the optic axes of the eyes, and parallel to m joining the centres of the cyes. - This line is called the horopter, because it is found to be the limit of sistinct vision. It has 
several properties in Optics, which are described at large in Aguillonius, Opt. lib. 2, diss. 10.

HOROSCOPE was a mathematical instrument, in inanner of a planisphere; but now disused. It was invented by J. Paduanus, who wrote a special treatise upon it.

HORROR of a Vacuum, an imaginary principle among the nore ancient philosophers, to which they ascribed the ascent of wateriu pumps, and other similar phenomena, which are now known to be occasioned by the weight of the air.

HORROX (JEREMIAH), an eminent English astronomer, was born at Toxteth in Lancashire, about the year 1619. From a grammar school in the country, he was sent to Cambridge, where he spent some time in academical studies. A bout 1633 he began to apply himself to the study of astronomy: but living at that time with his fas ther at Toxteth, in very moderate circumstances, and being destitute of books and other assistances for such studies, he could not make any considerable progress in it. About the year 1636, he formed an acquaintance with Mr. William Crabtree, of Broughton near Manchester, who was engaged in the same studies, with whom a mutual correspondence was carried on till his death; sometimes communicating their imprnvements to $\mathrm{Mr}$. Samuel Foster, professor of geometry at Gresham College in London. Having now obtained a companion in his studies, Mr. Horrox assumed new vigour, procured other instruments and books, and was pursuing his studies and observations with great assiduity, when be was suddenly cut off by death, the $3 \mathrm{~d}$ of Jamuary 1640 , in the $22 \mathrm{~d}$ year of his age.

What we have of his writings is sufficient to show how great a loss the world had by his death. He had just finished his Venus in Sole visa, 1639, a little before, as appears by some of the letters to his friend Mr. Crabtree, by which also it appears that he made his observations on that phenomenon at Hool near Liverpool. This tract, of Venus seen in the Sun, was published at Dantzick in 1668 , by Hevelius, together with his own Mercurius in Sole visus May 3,1661 . His other posthumous works, or rather his imperfect papers, were published by Dr. Wallis, in 1673,4 to, with some account of his life; in which we find he first asserts and promotes the Keplerian astronomy against the hypothesis of Lansberg; which he proves to be inconsistent with itself, and neither agreeing with observations nor theory. He likewise reasons very justly concerning the celestial bodies and their motions, vindicates Tycho Brahé from some objections made to his hypothesis, and gives a new theory of the moon: to which are added the Lunar Numbers of Mr. Flamsteed. There are also extracts from several letters between him and Mr. Crabtree, upon various astronomical subjects; with a catalogue of astronomical observations.

There are two things particularly which will perpetuate the memory of this very extraordinary young man. The one is, that he was the first that ever predicted or saw the planet Venus in the sun; for we do not find that any persons, besides himself and $\mathrm{Mr}$. Crabtree, ever beheld such a phenomenon. Though he was not apprised of the great use that was to be made of this phenomenon, in discovering the parallax and distance of the sun and planets, yet he made from it many useful observations, corrcetions, and improvements in the theory of the motions of Venus. - Secondly, his New Theory of Lunar Motions, which Newton himsclf made the ground-work of all his astronomy, relative to the moon, who always spoke of our author as a genius of the first rank.
HORSE-Sное, in Fortification, is a work somctimes of a round, sometimes of an oval figure, inclosed with a parapet, raised in the ditch of a marshy place, or in low grounds; sometimes also to cover a gate; or to serve as a lodgment for soldiers, to prevent surprises, or to relieve an over-tedious defence.

HORSLEY, (SAMUEL, LL.D. and F. R. S.), a very able mathematician and a learned prelate, was born in October 1732, and died October 4, I s06; consequently about 74 years of age.-He received the elements of his education at Westminster-school, whence lie was removed to the university of Cambridge. There he chiefly applied to the study of mathematics; and not content with carefully consulting the writings of the acutest of the moderns, he recurred to the profoundest of the ancients in that line, and made himself master of their most intricate reasonings. Having taken his degree as master of arts, he accepted an invitation to accompany the late Earl of Aylesford, as private tutor, to Oxford. Here, in 1769 , Dr. Horsley printed his edition of the Geometrical Inclinations of A pollonius, and here he first conceived the design of publishing a complete edition of the works of Sir Isaac Newton, for which he began to prepare the materials. And from that time, it has been more the fashion to cultivate the mathematical sciences at that university. On leaving this place, Dr. Horsley came to London, where he was elected F. R. s. and enriched the Philos. Trans. with many valuable essays, from vol. 57 , to vol. 66 . He was also chosell one of the secretaries in 1773 , on the resignation of Dr. Morton; an office he continued to serve, with the greatest credit to himself and benefit to science, till he thought it proper to resign along with the late president Sir John Pringle, in $177 \mathrm{~s}$. Soon after settling in the metropolis, Dr. Horsley was noticed by Bishop Louth, and was appointed his lordship's domestic chaplain, as well as was presented by that prelate successively to several different livings. In 1776 , Dr. Horstey published proposals for a complete edition of the works of the immortal Newton, with notes, which appeared in 1779 , in 5 vols. 4 to, with an elegant Latin dedication to the king. In $177 \mathrm{~S}$ he preached and publisher a sermon against the principles of materialism, agitated between the doctors Price and Priestley \&c: and on the publication, in 1783, of Dr. Priestley's History of the Corruptions of Christianity, Dr. Horsley again attacked the positions and principles of that writer and his adherents, with great applause: and success, and there ensued an exchange of several pamphlets, \&c. on both sides; which chiefly laid the foundation of Dr. Horsley's elevation to the episcopal dignity in 1788 , assisted probably, by the very celebrated speeches be made in the course of the violent debates, that took place in the Royal Society 2 or 3 years before, on the occasion of the removal of Dr. Hutton frum the office of foreign secretary, when Dr. Horsley, and several other members, forsook, as expressed in his own forcible language, "that temple where Philosophy once reigned, and where Newton presided as her officiating minister." In 1794 , Dr. Ilorsley was translated from St. David's to the see of Rochester, witl the deanery of Westminster; and in 1802, to the lucrative see of St. Asaph. In 1801 he published the first, and in 1803 the last, of 3 octavo volumes of practical mathenatics, for the use of students ; consisting of Euclid's Elements and Data, with notes; the properties and projection of the sphere, and spherical trigonometry; Archimedes on the mensuration of the circle; a tract on the nature and use of logarithms, \&c. Besides 
these, numerous and important were the writings and labours of Dr. Horsley in theology, politics, \&c, too numerous to be here entered into in detail.

Doctor Horsley went down to Brighthelmstone in the latter end of September 1806 , where he had not been two weeks, before he was seized with a complaint in the bowels, then frequent in, the place, which soon turned to a mortification; and terminated his valuable existence on the 4 th day of lis illness. Though Doctor Horsley had reached the 74 th year of his age, the powers both of his body and mind were so vigorous, as to promise still a considerable length of years; indeed he was always of a very remalkably active and cnergetic cast of miud, and firm compact constitution of body. Perhaps no man of the age, possessed more of what is termed recondite learning; or was more profoundly versed in ctassical chronology. As a senator, he ranked in the first class; there were few important discussions in the liouse of lords, in which be did not participate; especially when the topics related to the hicrarchical establishments of the country, or to the French revolution, or to the African slave trade, of which he was a systematic opposer. As an orator, his voice was deep, full-toned, and commanding; his entunciation was distince and clear; and his delivery in other respects, highly advantageous; abating only for a remarkable mixture of certain guttural sounds, such as are almost peculiar to the inhabitants of tbe county of Northumberland; occasionert, he used to say, by the circumstance of a nurse from that part of England being long employed in his father's family.

HOSPITAL, or Hopital (William-Francis $A_{N}$ THONY, marquis of ), a celebrated French mathematiciain, was born of an ancient family in 1661. He was a mathematician almost from his infancy; for being one day at the duke of Rohan's, where, some able mathematicians were speaking of a problem of Pascal's, which appeared to them very difficult, he ventured to say, that he believed be could resolve it. Thry were surprised at such presumption in a boy of 15 : however, in a few days he sent them the solution. M. l'llospital entered early into the army, and was a captain of horse; but being'very shortsighted, and on that accourt exposed to perpetual incolv. veniences and errors, he at length quitted the army, and applied hinself entircly to his favourite amusement.- $\mathrm{He}$ contracted a friendship with Malbranche, and took his opinion upon all occasions. - $\ln 1699$ he was received an honorary member of the Academy of Sciences at Paris. He wis the first person in France who wrote upon Newton's analysis, and on this account was regarded almost as a prodigy. His work was entitled l'Analyse des Infinimens Petits, 1696. Te engaged afterwarels in another mathematical work, in which he included Les Sections Coniques, les Lieux Géometriques, la Construction des Equations, et une Théorie des Courbes Mechaniques: but, a little hefore he bad finished it he was seized with a fever, which carried him off, the 20 l of February 170 4 , at 43 years of age. 'The work was published alter his death, viz, in 1707. Six of his pieces are inserted in different volumes of the Memoirs of the Acadeny.

HOUR, in Clironology, an aliquot part of a natural day, usually the $2+$ th; but sometimes a 12 th paxt. With us, it is the 24 th part of the enth's diurnal rotation, or the lime fiom now to noon, and therefore it answers to 15 degrees of the whole circle of longitude, or of $360^{\circ}$. The hour is clivided by 60 ths, viz, first into 60 minutes, then cach minute into 60 seconds, \&c. 'I'he division of the day into hours is very ancient; as is shown by Kircher, Oedip. Egypt. tom. 2, par. 2, class 7, cap. 8. The most ancient hour is that of the 12 th part of the day. Herodutus, lib. 2, observes, that the Greeks learnt from the Egyptians, among other things, the method of dividing the day into 12 parts. And the astionomers of Cathaya, \&c, still retairi this division.

'The division of the day into 24 hours, was not known to the Romans before the Punic war. Till that time they only regulated their days by the rising and setting of the sun. They divided the 12 hours of their day in four; viz, Prime, which commenced at 6 o'clock, Third at 9, Sixth at 12, and None at 3. They also divided the night into four watches, each containing 3 hours. Various kinds of hours are used by chronologers, astronomers, dialists, \& c. Sometimes too,

Hours are divided into equal and urequial.

Equal Hours, are the 24th parts of a day and night precisely; that is, the time in which the 15 degrees of the equator pass the meridian." These are also called equinoctial hours, because measured on the cquinoctial ; and astronumical, because used by astronomers.

Astronomical Hov Rs, are equal hours, reckoned from noon to noon, in a continued series of $\$ 4$.

Babylonish Hours, are equal hours, reckoned from sunrise in a continued series of 24 .

European Hours, used in civil computation, are cqual hours, reckoned from midnight; 12 from thence till noon, and 12 more from noon till midnight.

Ancient or Jervish Hours, are twelfth parts of the artificial day, or of the night. Hence, as it is only at the time of the equinoxes, that the artificial day is equal to the night, it is then only that the hours of the day are equal to those of the night, or to the 24 th part of the natural day. From the vernal to the autumnal equinos, the hours of the day exceed those of the night; but during the interval between the autumnal and vernal equinoxes, the hours of the night are longer than those of the clay. It is therefore manifest, 'that when it is said the third hour was about nine in the morning, and the ninth about three in the afternoon, this is not to be understood as rigorously exact. The third hour was the middle time between sunrising and noon; which, if the sun rose at five, was half an hour afier eight; if at seven was half an hour after nine, \&c.

The following table exhibits the time of the sun-rising and setting, and the lengtl of the Jewish hour, both of day and night; as calculated for about the middle of every Jewish month, and for the latitude of Jerusalems.

\begin{tabular}{|c|c|c|c|c|c|c|c|c|c|}
\hline \multirow{2}{*}{\multicolumn{4}{|c|}{ Names of the Munths. }} & \multirow{3}{*}{\begin{tabular}{|cc} 
Sun rises. \\
$\begin{array}{c}\text { h. } \\
5\end{array}$ & 46 \\
\end{tabular}} & \multirow{2}{*}{ Sun sets. } & \multicolumn{4}{|c|}{ Length of the Hout } \\
\hline & & & & & & \multicolumn{2}{|c|}{ of dhy. } & \multicolumn{2}{|c|}{ of night. } \\
\hline Nisa & 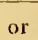 & & - & & $\begin{array}{ll}\overline{\mathrm{n}} & \text { in } \\
6 & 14\end{array}$ & & $\mathrm{~m}_{\mathrm{n}}$ & a. & \\
\hline Ijar, & $\mathrm{Zi}$ & & - & 520 & 640 & ) & 7 & 0 & 53 \\
\hline Sivan & & & - & 50 & 70 & J & 10 & 0 & 50 \\
\hline 'Thка & nez & & - & 456 & 74 & 1 & 11 & 0 & 49 \\
\hline $\mathrm{Ab}$ & - & & - & 510 & 650 & 1 & 8 & 0 & 52 \\
\hline Elul & - & & - & 536 & 624 & 1 & 4 & 0 & 56 \\
\hline T'isli, & $\mathrm{Pr}$ & & - & 66 & $55+$ & 0 & 59 & 1 & -1 \\
\hline Neat & liew & & & (i) 34 & 526 & 0 & 54 & 1 & 6 \\
\hline Chis & & & - & 655 & 55 & 0 & 51 & t & 9 \\
\hline Teb & & . & - & 70 & 50 & 0 & 50 & 1 & 10 \\
\hline Shel & & . & - & 64,2 & 518 & 0 & 53 & 1 & 7 \\
\hline Adar & & & - & 616 & 544 & 0 & 57 & $\mathbf{I}$ & 3 \\
\hline
\end{tabular}


Italian Hou $\mathrm{Rs}$, are equal hours, reckoned from sunset, in a continued series of 2.4 .

Unequal or Temporary Hours, are 12th parts of the artificial day and night. The obliquity of the sphere renders these more or less uneugual at different times; so that they only agree with the equal hours at the times of the equinoxes.

Hour-Circles, or Horarr-Circles, are great circles, meeting in the poles of the globe or world, and crossing the equinoctial or equator at right angles; the same as meridians. They are supposed to be drawn through every 15 th degree of the equinoctial and equator, each answering to an hour, and dividing them into 24 equal parts; and on both globes they are supplied by the meridian hour-circle and index.

How R-Glass, a popular kind of chronometer or clepsydra, serving to measure time by the descent or running of sand, water, \&c, out of one glass vessel into another. The best, it is said, are such as, instcad of sand, have eggshells, wetl dried in the oven, then beaten fine and sifted.

Houn-Lines, on a dial, are lines which arise from the intersections of the plane of the dial, with the several planes of the hour-circles of the sphere; and therefore must be all right lines on a plane dial.

Hoy n-Scale, a divided line on the edge of Collins's quadrant, being only two lines of tangents of 45 degrees each, set together in the middle. Its use, together with the lines of latitude, is to draw the hour-lines of dials that have centres, by means of an equilateral triangle, drawn on the dial-planes.

HOWITZ, or HowITzER, in artillery, a kind of mortar, or something between a cannon and mortar, partaking of the nature of both, being either a very short gun or a long mortar. It is of German invention, and is mounted on a carriage like a travelling gun-carriage, with its trunnions placed nearly in the middle. The howizz is one of the most useful kinds of ordnance, as it can be employed occasionally either as a cannon or mortar, discharging either shells or grape-shot, as well as balls, and so doing great exccution. They are also very easily travelled about from place to place.

HUDDE (JoH N), a burgomaster of Anssterdam, and a respectable mathematician, who died at a great age in 1704. Having been as great in politics as in mathematics, he served his country in distinguished situations, and contributed, by his discoveries, to the advancement of the sciences. He applied himself particularly to the analysis of equations. Onc of his two letters, inserted in Schooten's Commentary on the Geometry of Descartes, contains a very ingenious method for liscovering whether an equation of any dimension has equal roots, and for determining those roots. In the same letters we find Hudde's methods of drawing tangents to curves, and of investigating the maxima and minima in mathematics.

HUES (RoserT), was born at Little Hereford, in Herefordshirc, about 1552, and studied at Oxford. He bccane eminent for his knowledge in geography, navigation, and mathematics; which acquired him particularly the favour of that noble Mrecenas, Henry earl of Northumberland, being one of the carls so called thrce magi; from whom he enjoyed a liberal pension, for the encouragement and promotion of his studies. He died in 1632, at 79 years of age, and was buried at Christ's Church, Oxon.-His publications were chiefly two; viz. 1, Breviarium totius Orbis; printed several times at London, in VoL. I.
Latin and English, in Svo and 12mo.-2. De Globis celest. et terrest. Tract. 2; several times printed in Latin and linglish, in $8 \mathrm{vo}$. The first edition was printed at Londen 1593, in $8 \mathrm{vo}$. It was afterwards inproved by amotations, and illustrated with figures, by Joh. lsaac Pontanus, professor of philosophy at Harderwicke, in Gelderland, ṕrinted at Amsterdam, t617, 1624, \&c, at London, 1639, and at Oxon, 1663, in Svo and $12 \mathrm{mo}$. See Wood, vol. 1 , pa. 489 .

Hues was a man of letters, and had acquired a practical knowledge of maritime affairs, by voyages at sea. A mong other curious particulars, he gives a good account of the attempts that had been made at various times to measure the earth : in the epistle to Sir Walter Raleigh, he enumerates the many'discoveries made by our mariners in different parts of the world. His book was received with great applause, and has been indeed a pattern for such as afterwards treated on the same subject. Among other problems, he solves by the globe, the problem of determining the latitude, from two observations of the sun's altitude, when the time between the two observations is given; and in the last part of his book, he performs the usual questions in navigation, premising a very sensible discourse on the rhumb-lines, opposing the opinion of Gemma Frisius, who had asserted, that they meet in the poles. At the conclusion, he highly praises a treatise of Mr. Thomas Hariot, hoping it would soon be published, in which that author bad treated this subject on geometrical principles, with great sagacity and judgment. But unfortunately that manuscript has not been found among the papers of that author, liately discovered by Dr. Zach.

It was in this book that Hues proposed a curions nautical problen, which has made a considerable sensation in the mathematical world. It was in these words, as translated into English: "The difference of longitude and the distance being given, how to find the rumbe, and the difference of latitude." After which he adds thesę words: "There is not anything in all this art more difficult and hard to be found, than the rumbe out of the distance and difference of longitude given. Neither can it be done upon the glube without long and tedious practice, and many repetítions and mensurations."

The next person who appears to have mentioned this problem, was Willebrord snell, professor of mathematics at Leyden, in his 'Tiphys Batavus, printed in 1624, being the 34 th or last proposition of the first book of that work. Snell solved this problem in an indirect way; and it seeñs probable that his solution was the first that ever was given of it.

The problem was afterwards proposed to the learned world, by the celebrated Dr. Halley, in the year 1696, in the Philos. Trans. vol.19, No. 219; whence it was repeatcd in the $2 \mathrm{~d}$ vol. of the Miscellanea Curiosa in 1708, as be had probably not seen it in either of the fosegoing authors. Dr. Halley proposes it in the following words: "A ship sails from a given latitude, and, having run a certain number of leagues, has altered her longitude by a given angle; it is required to find the course stcered." And he then adds, "The solution hereof would be very acceptable, if not to the public, at least to the author of this tract, being likely to open some further light into the mysterics of geometry."

Since that time, this problem has heen solved in an indirect manner, by several writers on navigation, and $4 \mathrm{P}$ 


\section{H U Y

others:-As by M. Bouguer, in his Nouveau Traité de Navigation; by Mr. Robertson, in the $2 \mathrm{~d}$ vol. of his Elements of Navigation; by Mr. Emerson, in his Theory of Navigation; by Mr. Israel Lyons, in the Nautical Almanack, 1772; and by M. Bezout, in his Traité de Navigation; and lately, Baron Maseres, with the assistance of Mr. Atwood, has given a direct solution of this problem, being the first direct solution that has been given of it. All which solutions may be seen in the 4 th vol. of Baron Maseres's work, entitled Scriptores Logarithmici.

HUMIDITY, or muisture, the power or quality of wetting or moistening other budies, and adhering to them. Fluids are moist to some bodies, and not to others. Thus, quicksilver is not moist in respect to our hands or clothes, and other substances, which it will not adhere to; but it may be called humid in reference to gold, tin; or lead, to the surfaces of which it vill presently adhere, and render them soft and moist. Even water itself, which wets almost every thing, and is the great standard of moisture and humidity, is not capable of wetting all things; for it stands or runs off in globular drops from any thing greased or oiled, or the lcaves of cabbages, and many other plants; and it will not wet the feathers of ducks, geese, swans, and other water-fowl.

HUNDRED, the number of ten times ten, or the square of 10 . The place of hundreds makes the third in order in Arabic or modern numeration, being denoted thus, 100 . In the Roman notation it is denoted by the letter $c$, being the initial of its name, Centum.

HU N DRED Weight, or the great hundred, contains 112 pounds weight. It is subdivided into 4 quarters, and each quarter into $28 \mathrm{lbs}$.

HURTERS, in Fortification, denote pieces of timber, about 6 inches square, placed at the lower end of the platform, next to the parapet, to prevent the wheels of the gun-carriages from damaging the parapet.

HUYGENS (CHRISTIAN), a very eminent astronomer and mathematician, was born at the Hague in Holland, in 1629 , being the son of Constantine Huygens, lord of Zuylichem, who had served three successive princes of Orange in the quality of secretary. He spent his whole life in cultivating the mathematics; and not in the speculative way only, but also in making them subservient to the uses of life. From his infancy he discovered an extraordinary fondness for the mathematics; in a short time made a great progress in them ; and perfected himself in those studies under professor Schooten, at Leyden. In 1649 he went to Holstein and Denmark, in the retinue of Henry. count of Nassan; and was extremely desirous of going to Sweden, to visit Descartes, who was thén in that country with the queen Christina, but the count's short stay in Denmark would not permit him.-In 1651 he gave the world a specimen of his genius for mathematics, in a treatise entitled, Theoremata de Quadratura Hyperbola, Ellipsis, et Circuli, ex dato Portionum Gravitatis Centro; in which he clearly showed what might be expecied from him afterwards.-In 1655 he travelled into France, and took the degrce of LL.D. at Angers. - In 1658 he published his Horologium Oscillatorium, sive de Motn Pendulorum, \&c, at the Hague. He had exhibited in a former work, entitled Brevis lnstitutio de Usn Horologiorum ad inveniendas Longitudines, a model of a new-invented pendulum; bat as some persons, envious of his reputation, were labouring to deprive him of the honour of the invention, he wrote this book to explain the construction of it; and to show that it was very different from the pendulum of astronomers invented by Galileo.-In 1659 he published his Systema Saturninum, \& 2 ; in which he first of any one explained the ring of Saturn, and discovered also one of the satellites belonging to that planet, which had hitherto escaped the eyes of astrunomers: new discoveries, made with glasses of his own forming, which gailued him a high rank among the astronomers of his time.

In 1660 , he took a second journey into France, and the year after passed over into Kngland, where he communicated his art of polishing glasses for telescopes, and was made Fellow of the Royal Society." About this time the air-pump was invented, which received considerable improvements from him. This ycar also he discorered the laws of the collision of elastic bodies; as did also about this time Wallis and Wren, with whom he had a dispute about the honour of this discovery. On his return to France, in 1663, the minister Colbert, being informed of his great merit, settled a considerable pension on him, to engage him to tix at Paris; to which Mr. Huygens consented, and staid there from the year 1666 to 1681 , where he was admitted a momber of the Acaden:y of Sciences. All this time he-spent in mathematical pursuits, wrote several books, which were published from time to time, and invented and perfected several useful instruments anci machines : particularly he had a dispute, about the year 1668, with Mr. James Gregory, concerning the quadrature of the circle and hyperbola of the latter, then just published, in which Huygens it seems had the better side of the question. But continual application gradually impaired his health; and though he had visited his native country twice, viz, in 1670 and 1675 , for the recovery of it, he was now obliged to betake himself to it altogether. Accordiugly he left Paris in 1681 , and retired to his own country, where he spent the remainder of his life in the same pursuits and employments. He died at the Hague, June 8,1695 , in the 67 th year of his age, while his Cosmotheoros, or treatise concerning a plurality of worlds, was printing; so that this work did not appear till 1698 .

Mr. Huygens loved a quict and studious manuer of life, and frequently retired into the country to avoid interroption, but did not contract that moroseness which is so commonly the effect of solitude and retircment. He was one of the purest and most ingenious mathematicians of his age, and indeed of any other; and macle many valuable discoveries. He was the first who discovered Saturn's ring, and a third satellite of that planet, as mentioned above. He invented the means of rendering clocks exact, by applying the pendulum, and of rendering all its vibrations equal, by the cycloid. He brought telescopes to perfection, and mate nany other useful discoveries.

He was the anthor of many excellent works. The principal of these are now contained in two collections, of 2 volumes each, printed in 4 to, under the care of profcssor Gravcsande. The first was at Leyden, in 1682, under the title of Opera Varia; and the second at Amsterdam, in 1728, entitlal Opera Reliqua.

IIYADES, a cluster of 5 stars in the face of the constellation Taurus, or the Bull.

HYALOIDES, the vitreous humour of the eye, container] between the tunica-retina and the uvea.

HYBEIRNAL Occident. Scc Occident.

Hybernal. Oricte. Sec Orient.

HYDATOIDES, the watery humour of the eyc, contained between the cortuea and the uvea. 
HYDRA, a southern constellation, consisting of a number of stars, imagised to represent a water-serpent. The stars in the constellation Hydra, in Ptuleny's catalogue, are 27 ; in Tycho's, 19; in Hevelius's, 31; and in the Britannic catalogue, 60 .

HYDRAULICS, the seience of the motion of water and other fluids, with its application in artifieial waterworks of all kinds.-As to what respeets merely the equilibrium of fluids, or their gravitation or action at rest, belongs to hydrostatics. On removing or destroying that equilibrium, motion ensues; and here hydraulics commence. Hydraulics therelore suppose hydrostatics; and many writers, from the near relation between them, like mechtnics and statics, join the two together, and treat of them eonjointly as one scienee. - The laws of hydraulics are given under the word Flaid. "And the art of raising water, with the several michines employed for that purpose, are described under their several names, Fountain, Hydrncanisterium Pump, Siphon, Syringe, \&e.

The principal writers who have cultivated and improved hydraulics and hydrestatics, are Archimedes, in his Libris de Insidentibus Humido; Hero of Alexandria, in his Liber Spiritualiom; Marinus Ghetaldus, in his Archimedes promotos; Mr. Oughtred; Jo. Ceva, in his Geometria Motus; Ju. Bap. Balianus, De Motu Naturali Gravium, Solidorum et Liquidorum ; Mariotte, in his treatise of the Motion of Water and other Fluids; Boyle, in his Hydrostatical Paradoxes; Fran. Tertius de Lanis, in his Magisterium Naturæ et Artis ; Lamy, in his Traité de l'Equilibre des Liqueurs; Rohault; Dr. Wallis, in bis Mechanics; Dechales; Newton, in his Principia ; Guglielmini, in bis Mensura Aquarum Fluentium ; Herman; Wolfius; Gravesande; Musschenbrock; Leopold; Sehottus, in his Mechanica Hydraulico-Pneumatica; Geo. Andr. Bockler, in his Architectura Curiosa Germanica; August. Rammilleis; Lucas Antonius Portius; Sturmy, in his treatise on the Construction of Mills; Switzer's Hydrostatics; Varignon, in the Mem. Acad. Sci.; Jurin; Belidor; Bernoulli; Desaguliers; Clare; Emerson; Ferguson; Ximenes; Bossu; D'Alembert; Buat ; \&c, \&c.-Sce also Pumps, Stean Engines, \&c.

HYDRAULICO-PNeUMaTiCaL, a term applied by some authors to such engines as raise water by means of the weight or spring of the air.

HY DRAULIC RAM, the uame of a machine by Montgolfier, for raising water.

The ingenious idca of raising water by the moinentum of water itself, was first suggested by Mir. Whitehurst in the Philosophical Transactions for 1775 . The samc principle, in an improved form, has lately been revived in France, and has excited considerable attention both on the continent and in this country. Whatever credit therefore is due to the inventor of the Hydraulic Ram, properly belongs to our countryman Mr. Whiteburst, and Montgolfier can lay claim to nothing more than the inerit of an improver.

Mr. Whitehurst's machine, which is represented in fig. 1. pl. 16, was actually erected at Oulton in Cheshire, and coinpletely answered the expectation of its inventor. AM is the original reservoir, whose surface is on a level with $B$, the bottom of the reservoir $\mathrm{BN}$. The main pipe $\mathbf{A} \mathbf{E}$, is $1 \frac{1}{2}$ inches diameter, and nearly two hundred yards long, and the branch pipe $\mathrm{EF}$ is of such a size that the cork $\mathbf{F}$ is about 16 feet below the surface $M$ of the reservoir. D is a valve box, with its valve $a$, and $\mathrm{c}$ is an air vessel, into which are inserted the extremities $m, n$, of the main pipe, bent downwards to prevent the air from being driven out when the water is forced into jt.

Now, since the difference of level between the cock : and the top of the reservoir Am is 16 feet, on opening the cock $F$ the water will rush out with a velocity of nearly. 30 feet per second. A column of water therefore 200 yards long, is thus put in motion, and though the aperture of the cock $\mathrm{F}$ be small, it must bave a very considerable momentum. Let the cock $F$ be now suddenly stopped, the water must evidently rush through the valve $a$ into the air vessel c, and condense the included air. This condensation must take place every time the cock is opened and shut, and the included air being highly compressed, will press upon the "Iater in the air vessel, and raise it in to the reservoir $\mathrm{BN}$.

From this brief description of Whitehurst's machine, the reader will easily perceive its resemblance to Montgolfier's hydraulic ram, a section of which is represented by fig. 2. $R$ is the reservoir, $\mathrm{Rs}$ the height of the fall, and ST the horizontal tube which conducts the water to the engine $A B n T C$. E and $D$ are two valves, and FG a pipe reaching within a very little of the bottom cs. Now let water descend from the reservoir, it will rush out at the aperture $m n$ till its velocity becomes so great as to force: up the valve E. The water being thus suddenly ehecked, and unable to find a passage at $m n$, will move forwards with great violence and force towards $\mathrm{H}$, and raise the valve $D$. A portion of water being admitted into the vesse] A BC, the impulse of the column of fluid is expended, the valves $\mathrm{D}$ and $\mathrm{E}$ fall, and the water rushes out at $m n$ as befure, when its motion is again stopped, and the same operation repeated which has now bcen described. Every time therefore that the valve $\mathbf{E}$ closes, a portion of water will force its way into the vessel $\mathrm{ABC}$, and condense the air which it contains ; for the jueluded air has no communication witb the atmosphere after the water is higher than the botton of the pipe rg. This condensed air will consequently exest great force on the surface o $p$ of the water, and raise it in the tube $5 \mathrm{G}, \mathrm{t} \omega \mathrm{a}$ height proportioned to the elasticity of the imprisoned air. The external appearance of the cngine, copied from one in the possession of Professor Leslie, is exhibited in fig. 3; where $A B C$ is the air vessel, $F$ the valve box, $G$ the extremity of the valve, and $\mathrm{m} N \mathrm{~N}$ screws for fixing the horizontal tube to the machine. A picce of brass, A, with a small aperture, is screwed on the top, when the engine.is employed, to form a jet of water.

From this description, it will easily be perceived that the only difference between the engines of Montgolfier and Whitehurst is, that the one requires a person to turn the cock, while the other has the advantage of acting spontaneously. Montgolfier assures us (Journal des Mines, vol. 13, No.73) that the honour of this invention does not belong to England, but that he is the sole inventor, and did not receive a hint from any person whatever. We leave it to the reader to determine to what degree of credit these assertions are entitled.

It would appear, from some experiments of Montgolfier, that the effect of the water-ram is equal to between $\frac{x}{2}$ and $\frac{3}{4}$ of the power expended; which renders it superior to most hydraulic engines. -Appendix to Brewster's Ferguson's Lectures, pa. 419.

The principles on which the ram of Montgolfer is constructed, are susceptible of a very extensive application: $4 \mathrm{P} 2$ 
thus, it would be easy to raise other water besids that which runs through the-ram; as for example, with a fall of water we might by a slight modification of this machine raise water from the bottom of wells, or even of mines.

Uther machines analogous to Montgolfier's engine have been invented by Messrs. Sarjeant and Boulton; and the celcbrated hydraulic engine in Chemnitz in Hungary is closely allied to the ram in principle. M. Montgolfici suggests the utility of his invention in the draining of mines; and in that case, the bydraulic ram and the Hungarian machine would become very nearly if not altogether identical as to principle.

HYDRUDYNAMICS, that branch of mechanical science which relates to the motion of non-elastic fluids, and the forces with which they act upron bodies opposed to them.

There is no part of mechanics the true theory of which we are so little acquainted with as that which respects the motion of fluids. Nor is it strange that the knowledge we do possess of this science should be so dubious and confined, when we consider the many powerful obstacles which continualiy present themselves in the cultivation of it. The mass, the figure, and the number of particles of a fluid in notion, are particulars with which we are too superficially acquainted to determine the laws of its motion, with any satisfactory degree of accuracy; and even if we were in possession of the data by which those laws might be determined, it is doubtful whether we should be much farther advanced, as it might be extremely difficult to deduce any convenient and practical results from the intricate and complex cxpressions which might be obtained from the investigation. The greatest mathemati. cians have been forced to yield to the numerous difficulties of these researches, and to confess that the methods so successfully followed in the mechanics of solid bodies, do not affiord any conclusions with respect to fluids, but such as are too gencral and uncertain for the greater part of particular cascs. Whatever we have of this science is almost exclusively due to the labours of modern analysts and geometers; for the only work which has reached us from the ancients is that of Archimedes, in two books, entitled De Insidentibus humido, in which the only enquiries respect the sinking and floating of bodies in flujds, their relative gravities, levities, situations and positions, when in equilibrio. It is indecd true that some hints, as svell as rules, on the motion of fluids, áre contained in a treatise attributed to Sextus Julius Frontinus, inspector of public fountains at Rome, under the emperors Nerva, Cocceius, and T'rajan, entitled, De Aquæ ductibus urbis Roma commentarius; but they are not of sufficient importance $t o$ deserve much attention by a student in this science. Benedict Castelli was the first who opened the way to a true neasure of the flux of waters, in his Treatise Della Mesura dell' Aquá Currenti, which measure he lound to depend on the area of the section and the velocity of the water conjointly. The most valuable and important discoveries and theorems in this department of science, are given in Sir Isaac Newton's Principia, lib.2, pr. 36 , with the comment: Dan. Bernoulli's Hydrodynamique; D'Alembert's 'Iratité des Fluides; D. George Juan's Examen maritino 'Theorico Practico; M. Bossut's Hydrodynamique; Buat's l'rincipes d' Hydraulique, and Mr. Eytelwcin's Ilandbuch der Mechanick und der Hydraulique. 'To which may be added an ingenious pajer on the Motion and Resistance of Fluids, by Mr. Yince, in the Philos. Transact. for 1795 ; and those by the late Dr. Mattlew Young in the Irish Trans. - Sce HsdraULICS.

HYDROGEN, the basis of what has been gencrally called inflammable air, and is one of the components of water.

HYDROGEN Gas, an invisible clastic aeriform fuid uncondensible by any known cold, and is the only form in which pure hyulrogen has ever been exhibited. Tle specific gravity of this gas varies considerably, according to the manner in which it has been procured. In gencral, when pure, it is from 6 to 10 or even to 12 times lighter than common air, so that 8 times may be considered as a general average. $i t$ is one of the most inflammable substances we are acyuainted with; that is to say, it combines with more oxygen than any other body, and occasions more heat by its combustion; it being a well established fact, that hydrogen and oxygen gases, when mixed, produce the most intense beat yet known.

One of the most striking properties of this gas, is that of reducing metallic oxyds to the reguline or nearly reguline state. This is efiected by the union of the hydrogen with the axygen of the metallic oxyd, in consequence of which the hydrogen Joses, its gaseous form, and with the oxygen produces water. The metallic reduction by hydrogen gas appears to have been first noticed by Dr. Pricstley, but the consequent production of water in these cases was suggested by the most important researches of $\mathrm{Mr}$. Cavendish, on the union of hydrogen and oxygen.

From the great levity of hydrogen gas, it has generally been used for filling air-balloons: indeed this appears the only use to which it has at present been applied. The following is the best way of procuring it : Put a quantity of filings of zinc into a vessel which has a glass tube prefised; then pour upon them sulphuric acid, diluted with 6 or 8 times its quantity of water, and an effervescence will immediately take place; the water will be decomposed, and the oxygen of it will become united to the metal, and the hydrogen gas will be disengaged, and may be conveyed into the body of the balloon. This gas can be procured purc only from water, which in all cases must undergo a decomposition. For the usual properties of this gas, the, reacler may consult with much interest Aikins's Chemical Dict.

HY DROGRAPHICAL Charts or MTaps, more usually called sea-charts, are projections of some part of the sca, or coast, for the use of navigation. In these are laid down all the rhumbs or points of the compass, the moridians, parallels, \&c, with the coasts, cupes, islands, rocks, shoals, shallows, \&c, in their proper places, and proportions.

For the construction and use of the seviral kinds of hydrographical maps, see CnART, and SAILING.

HYDROLOGY, is that part of natural history which examines and explaius the naturo and properties of water in general.

IIYDROMETER, an instrument for measuring the propertics and cffects of water, as its density, gratity, force, velocity, \&c. That with which the specitic gravity of water is determined, is olten called an acrometer, or water-poise.

The general principle on which the construction and use of the hydrometer depends, has been illustrated under the article Spccific Gravity; where it is shown that a body specifically lighter than several fluids, will serve to 
find out their specific gravities; because it will sink deepest in the fluids whose specific gravity is the least. In tig. 5 , pl. 14, if $\mathrm{AB}$ represent a small even glass tube, hermetically sealed, having a scale of equal divisions marked upon it, with a bollow ball of about an inch in diameter at bottom, and a smaller ball $\mathrm{c}$ under it, communicating with the first ; and into the little ball be put mercury or small shot, before the tube is sealed, so that it may sink in water below the ball, and Hoat or stand upright, the divisions on the stem showing how far it sinks.- If this instrument be dipped in common water, and sink to $\mathrm{D}$,' it will sink only to some lower point $\mathrm{E}$ in salt water; but in port wine it will sink to sume bigher point $F$, and in brandy perbaps to B. It is evident that an hydrometer of this kind will only show that one liquid is specifically heavier than another; but the true specific weight of any liquid cannot be determined without a celculation for this particular instrument, the tube of which should be truly cylindrical. Besides, these instruments will not serve for fluids whose densities are much different.

Mr. Clarke constructed a new hydrometer, slinwing whether any spirits be proof, or above or below proof, and in what degree. 'I'his instrument was made of a ball of copper (because ivory imbibes spirituous liquors, and glass is apt to break), to which is soldered a brass wire about a quarter of an inch thick ; upon this wire is marked the point to which it exactly sinks in proof spirits; as also two other marks, one above and one below the former, exactly answering to one-tenth above and one-tenth below proof. There are also a number of small weights made to ald to it, so as to answer to the other degrees of strength besides those above, and for determining the specific gravities of different fluids. Philos. Trans. Abr. vol. vii, pa.392.

Dr. Desaguliers contrived an hydrometer for determining the specific gravities of different waters, to such a degree of nicety, that it would show when one kinid of water was but the 40,000 th part heavier than another. It consists of a hollow glass ball of about 3 inches in diameter, charged with shot to a proper degree, and having fixed in it a long and very slcnder wire, of only the 40 th part of an jnch in diameter, and divided into tenths of inches, each tenth auswering to the 40,000th part," as above. See his Exper. Phil as, vol. 2, pa. 234.

Mr. Quin and other persons have also constructed hydrometers, with other and various contrivances, and with different degrees of accuracy; but all nearly on the saine general principles.

But there is one circumstance which deserves particular attention in the construction and graduation of hydrometers, for determining the precise strength of different brandies, and other spirituous liquors. Mr. Reaumur discovered, in making bis spirit-thermometers, that when rectified spirit and water, or phlegm, the other constituent part of brandy, are mixed together, there appears to be a mutual persetration of the two liquors, and not merely juxtaposition of parts ; so that a part of the one fluid scems to be received into the interstices of the other; by which it happens, that if a pint of rectified spirit be added to a pint of water, the mixture will be sensibly less than a quart. The variations hence produced in the bulk of the mixed fluid render the hydrometer, when graduated in the usual way by equal divisions, an erroneous measure of its strength ; because the specific gravity of the compound is found not to correspond to the mean gravity of the two ingredients. M. Montigny constructer a scale for this instrument, in the manner before suggested by Dr. Lewis, on actual obses vation of the sinking or rising of the hydrometer in various mixtures of alcohol and water, made in certain known proportions. Hist. de l'Acad. Roy. des Sci. 1768; also Neumann's Chem. by Lewis, pa.450, note $r$.

M. De Luc has lately published a scheme for the construction of a comparable bydrometer, so that a workman, after having constructed one upon his principles, may make all others similar to each other, and capable of indicating the same degree on the scale, when inmersed in the same liquor of the same temperature. This instrument is proposed to be constructed of a ball of flint glass, communicating with a small hollow cylinder, containing such a quantity ol quicksilver for a ballast, that the instrument may sink nearly to its top, in the most spirituous liquor, when heated as much as possible; to which is also attached a thin silvered tube, for a scale, \&c. The whole description may be seen at large in the Philos. Trans: vol. 68, pa.500. M. Le Roi also published a proposal for constructing comparable bydrometers. See Hist. de l'Acad, des Scien. for 1770, Mèm. 7 .

Mr. Nicholson has made an improvement, by which the hydrometer is adapted to the general purpose of finding the specific gravity, both of solids and fluids (fig. 4, pl. 16). $\quad A$ is a hollow ball of copper, $\mathrm{B}$ a dish affixed to the ball by a short slender stem, D; $\mathrm{C}$ is another dish affixed to the opposite side of the ball by a kind of stirrup. In the instrument actually made, the stem $\mathrm{D}$ is of hardened steel, $\frac{\mathrm{r}}{46}$ of an inch in diameter, and the dish $\mathrm{c}$ is so heavy as in all cases to keep the stem vertical when the instrument is made to float in any liquid. The parts are so adjusted that the addition of 1000 grains in the upper dish B will just sink it in distilled water (at the temperature of $60^{\circ}$ of Fahrenheit's thermometer), so far that the surface shall intersect the middle of the stem $D$.

Let it now be required to find the specific gravity of any fluid. Immerse the instrument in it, and by placing weights in the dish B cause it to float, so that the middle of its stem $D$ shall be cut by the surface of the fluid. Then, is the known weight of the instrument, added to 1000 grains, is to the same known weight added to the weights used in producing the last equilibrium, so is the weight of a quantity of distilled water displaced by the Hoating instrument, to the weight of an equal bulk of the fluid under examination.

Again, let it be required to find the specific gravity of a solid body, whose weight is less than 1000 grains. Place the instrument in distilled water, and put the body in the dish $\mathbf{B}$. Make the adjustment of sinking the instrument to the middle of the stem, by adding weights in the same dish. Subtract those weights from 1000 grains, and the remainder will be the weight of the body. Place now the body in the lower dish $\mathrm{c}$, and add more weight in the upper dish B, till the adjustment is again obtained. The weight last added will be the loss the solid sustains by inmersion, and is the weight of an equal bulk of water. Consequently the specific gravity of the solid is to that of water, as the weight of the borly to the loss occasioned by the immersion. Mr. Nicholson observes, "This instrument was found to be sufficiently accurate to give weights true to less than $\frac{x}{2}{ }^{2}$ th of a grain." Nicholson's Philosophy, vol.2. p. 16.

HYDROMETRIA, HYDROMETRY, the mensuration 
of water and other fluid bodies, their gravity, force, velucity, quantity, \&c ; including both hydrostatics and hydranlics.

HY DROSCOPE, an instrument anciently used for the measure of time. It was a kind of clepsydra or waterclock, consisting of a cylindrical tube, conical at bottom: the cylinder was graduated with divisions, to which the top of the water becoming successirely contiguous, as it trickled out of the vertex of the cone, pointed out the hour.

HYDROSTATICAL Balance, a balance contrived for the exact and easy finding the specific gravities of bodies, both solid and fluid, and thereby of estimating the degree of purity of bodies of all kinds, with the quality and richness of metals, ores, minerals, \&c, and the proportions in any mixture, adulteration, or the like. This is effected by weighing the bolly both in watcr, or other fluid, and out of it; and for this purpose, one of the scales has usually a hook at the bottom, for suspending the body by some very fine thread. And the use of the instrument is founded on this theorem of Archimedes, that any body weighed in water, loses as much of its weight as is equal to the weight of the same bulk of the water. Thus then is known the proportion of the specific gravities of the solid and fluid, or the proportion of their weights under the same bulk, viz, the proportion of the weight of the body weighed out of water, to the difference between the same and its weight in water. Hence also, by doing the same thing for several different solids, with the same fluid, or different fluids with the same sulid, all their specific gravities become known.

This instrument requires but little description. $A \mathrm{~B}$ is a nice balance beam, with its scales $\mathrm{C}$ and $\mathrm{D}$, turning with the small part of a grain, the one of them, $D$, having a hook in the bottom, to receive the loop of a horse hair \& c, e, by which the body $\mathbf{F}$ is suspended. $\mathrm{GH}$ is a

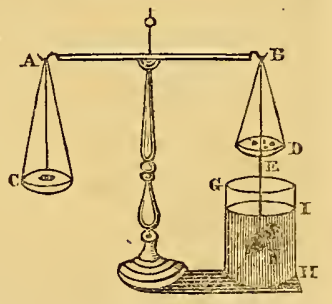
jar of water, in which the body is immersed when weighing. The pieces in the scale $c$ denote the weight of the body out of water; then, upon immerging $i t$, put weights in the scale $D$ to restore the balance again, and their quotient will show the specific gravity of the body. \&

There have been various kinds of the hydrostatical balance, and improvements made on it, by different persons. Thus, Dr. Desaguliers set thrce screws in the foot of the sland, to move any side higher or lower, till the stem be quite upright, which is known by a plummet hanging over a fixed point in the pedestal. Desag. Exp. Philos. vol. 2, pa. 1.96. And for sundry other constructions of this instrument, designed for greateraccuracy than the common sort, see Martin's Phil. Britan. or Gravesande's l'hysices Elem. Math. tom. 1, lib. 3, cap. 3, \& c.

The specific gravities of small weights may be determined by suspending them in loops of horse hair, or fine silken threads, to the hook at the bottom of the scale. Thus, if a guinea suspended in air weigh 129 grains, and on bring immersed in water require $7 \frac{1}{5}$ grains to be put in the scale over it, to restore the equilibrium; we thus find that a quantity of water of equal bulk with the guincat, weighs $7 \frac{1}{5}$ grains, or $7 \cdot 2$; therefore dividing the 129 by the $7 \cdot 2$, the quotient $17 \cdot 88$ shows that the guinea is so many times beavier than its-bulk of water. Whence, if any picce of gold be tried, by weighing it first in air, tuen in water, and if, upon dividing the weight in air by the loss in water, the quotient be $17 \cdot 98$, the gold is good; if the quotinnt be 18 or more, the gold is more fine; but if it be less than $17 \cdot 88$, the gold is too much alloyed with other metal. If silver be tried in the same manner, and found to be 11 times heavier than water, it is very tine; if it be $10 \frac{x}{2}$ times heavier, it is standard; but if less, it is mixed with some lighter metal, such as tin.

When the body, whose specific gravity is sought, is lighter than water, so that it will not quite sink; annex to it a piece of another body heavier than water, so that the mass compounded of the two may sink together. Weigh the denser body, and the compound mass, separately, both in water and out of it, thereby fiading how much each loses in "Iater; and subtract the less of these two losses from the grcater; then say,

As the remainder

is to the iveight of the lighter body in air, so is the specific gravity of weter

to the specific gravity of the lighter body.

HYDROSTATICAL Bellows, a machine for slowing the upward pressure of fluids and the hydro- $e$ is statical paradox. It consists of two thick boards, $\Lambda, D$, each about 16 or 18 inches diameter, more or less, covered or connected firmly with leather round the edges, to open and shut like a common bellows, but without valves; only a pipe, $\mathbf{B}$, about 3 feet high is fixed into the bellows at $e$. If water be poured into the pipe at $\mathrm{c}$, it will run into the hellows, gradually separating the boards, by raising the upper one. And if several weights, as three hundred wcights, be laid upon the upper board, by

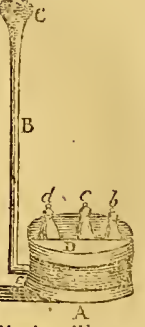
pouring the water in at the pipe till it be full, it will sustain them all, though the water in the pipe should not weigh a quarter of a pound; for the pipe or tube may be as small as we please, provided it be but long enough, the whole eflect depending upon the height, and not at all on the width of the pipe: for the proportion is always this,

As the area of the orifice of the pipe

is to the area of the bellows-board,

so is the weight of water in the pipe

to the weight it will sustain on the board.

Hence if a man stand upon the upper board, and blow into the pipe $B$, he will raise himsell on the buard; and the smaller the pipe, the easier be will be able to raise himsclf; then by putting his finger upon the top of the pipe, lıe can support himself as long as he pleases, provided the bellows be air-tight.

Mr. Ferguson has described' another macline, which may be substituted instead of this common hydrostatical bellows. It is however on the same principle of the hydrostatical paradox; and may be seen in the supplement to his Lectures, pa. 19.

HYDROSTATICAL Paratox, is a principle in hydrostatics, so called because it has a paradoxical appearance at.first view : it is this; that any quantity of water, or other fluid, how small soever, may be made to balance and support any quantity, or any weight, how great soever. 'Jhis is partly illustratcd in the last article, on the 
hydrostatical bellows, where it appears that any weight whatever may be blown up and supported by the breath from a person's mouth. And the principle may be explained as follows: it is well known that water in a pipe or canal, open at both ends, always rises to the same height at both ends, whether those ends be wide or narrow, equal or unequal. Thus, the small pipe $\mathrm{GH}$ being close joined to another open vessel A I, of ally size whatever; by pouring water into one of these, it will rise up in the other, and stand at the same height, or liorizontal line DF in both of them, and that whether they be upright, or inclined in any position. So that all the svater that is in the large vessel from $A$ to $I$, is supported by that which is in the small vessel from $\mathrm{D}$ to I only. And as there is no limit to this latter one, but

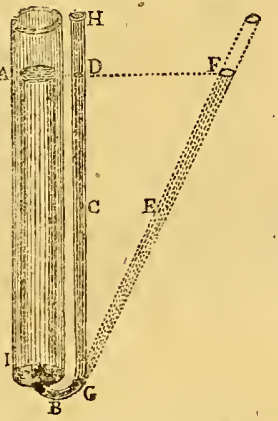
that it may be made as fine even as a hair, it bence evidently appears that any quantity of water inay be thus supported by any other the smallest quantity.

Since then the pressure of fluids is directly as their perpendicular heights, without any regard to their quantities, it appears that whatever the figure or size of the vessels may be, if they are but of equal heights, and the areas of their bottoms equal, the pressures of equal heights of water are equal upon the bottoms of these vessels; even though the one should contain a thousand or ten thousand times as much as the other.

Mr. Ferguson confirms and illustrates this paradox by the following experiment.

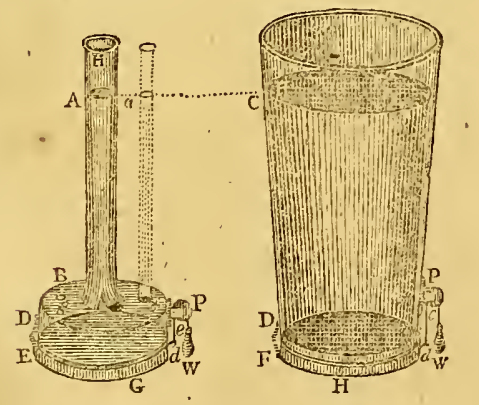

Let two vessels be prepared of equal heights, but very unequal contents, such as $\mathrm{AB}$ and $\mathrm{CD}$; each vessel being open at both ends, and their bottoms $E$ and $F$ of equal widths. Let a brass bottom $\mathrm{G}$ and $\mathrm{H}$ be exactly fitted to each vessel, not to go into it, but for it to stand upon; and let a piece of wet leather be put between each vessel and its brass bottom, to render it as close and tight as possible. Join each bottom to its vessel by a hinge $\mathrm{D}$, so that it may open like the lid of a box; and let each bottom be kept up to its vessel by equal weights w, hung to lines whicb go over the pulleys P, whose blocks are fixed to the sides of the ressel at $f$, and the lines tied to looks at $d$, fixed in the brass bottoms opposite to the hinges $\mathrm{D}$. In this adjustment of parts, hold one vessel upright in the hands over a bason on a table, and causc water to be poured slowly into it, till the pressure of the water bears down its bottom at the side $d$, and raises the weight $\mathrm{E}$; and then part of the water will run out at $d$. Mark the height at which the surface $\mathrm{H}$ of the water stood in the vessel, whien the bottom began to give way at $d$; after which, holding up the other vessel in the same manner, cause water to be poured into it; and it will be seen that when the water rises in this vesse! just as high as it did in the fornser, its bottom will also give way at $d$, and it will also lose part of the water.

The reason of this surprising phenomenon is, that since all parts of a fluid at equal depths below the surface, are equally pressed in all manner of directions, the waterimmediately below the fixed part of $f$ will be pressed as much upward against its lower surface within the vessel, by the action of the column Ag, as it would be by a column of the same height, and of any diameter whatever ; and therefore, since action and reaction are equal and contrary to each other, the water immediately below the surface $\mathrm{g} f$ will be pressed as much downward by it, as if it were immediately touched and pressed by a column of the hieight $\Delta g$, and of the diameter $B f$; and therefore the water in the cavity $\mathrm{B} \mathrm{D} d f$ will be pressed as much downward upon its bottom $G$, as the bottom of the other vessel is pressed by all the water above it. Lectures, pa. 105.

HYDROSTATICS, is that science which treats of the nature, gravity, pressure, and equilibrium of fluids ; and of the weigbing of solids in them. That part of the science of fluids which considers their motions, being included under the article Hydrodynamics. Hydrostatics and hydrodynamics together constitute a branch of philosophy that is justly colisidered as one of the most curious, ingenious, and uscful of any; affording theorems and phenomena not only of the first use and importance, but also surprisingly amusing and pleasant; as appears in the numberless writings on the subject; the principal points of which may be found under the several particular articles of this work, as Pressure, Gravitr, Scc.

The chief writers who have cultivated and improved this sciencé are, Archimedes, in his Libris de Insidentibus Humido; Hern of Alexandria, in his Liber Spiritualium; Marinus Ghetaldus, in lis Archimedes promotus; Oughtred; Jo. Ceva, in his Geometria Motus; Jo. Bap. Balianus De Motu Naturali Gravium, Solidorum et Liquidorum; - Marioke, in his treatise of the Motion of Water and other Fluids; Boyle in his Hydrostatical Paradoxes; Boyle in Tertius de Lanis, in his Magisterium Naturæ et Artis; Lamy, in his 'Traité de l'Equilibre des Liqueurs; Rohault; Dr. Wallis, in his Mechanics; Dechales; Newton, in his Principia ; Gulielmini, in his Mensura Ayuarum Fluentium; Herman; Wolfius; Gravesande; Muschenbroek ; Leopold; Schottus, in his Mechanica Hydruulico-Pneumatica; Geo: Andr. Bockler, in his Architectura Curiosa Germanica; August Rammilleis; Lucas Antonius Portius ; Sivitzer; Várignon, in the Mem. Acad. Sci.; Jurin; Belitlor; Bernoulli; Desaguliers ; Clare; Emerson; Ferguson; Ximenes ; Bossu; D'Alembert; Buat; Vince ; Olinthus Gregory, in his Mechanics, "\&c, \&c.

HYDRUS, or Water Serpent, one of the new southern constellations, including only ten stars. 


\section{H Y G}

\section{[ 664}

HYEMAL Solstice, the same with Winter Solstice. See SOLSTICE.

HYGROMETER, or HYG ROSCOPE, Or NotIONETER, an instrument for measuring the degrees of moisture in the air. There are various kinds of this instrument ; for whatever body either swells by moisture, or shrinks by dryness, is capable of being formed into an hygrometer. Such are wands of most kinds, particularly deal, ash, poplar, \&.c. Such also is catgut, the beard of a wild oat, and a twisted cord, \&c. The best and most usual contrivances for this purpose are as follow.

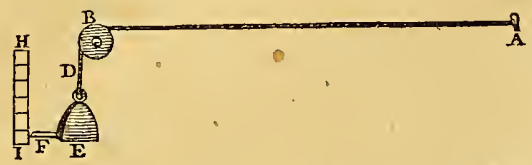

1. Stretch a common cord, or a fiddle-string, $A B D$ along a wall, passing it over a pulley $\mathrm{B}$; fixing it at one end $\mathrm{A}$, and to the other end hanging a weight $\mathrm{E}$, carrying a style or index F. Against the same wall fit a plate of metal $\mathbf{H}$, graduated, or clivided into any number of equal parts; and the hygrometer \& $c$, is complete. For it is matter of constant observation, that moisture sensibly shortens cords and strings; and that, as the moisture evaporates, they return to their former length again.- The like may be said of a fiddle-string: and hence it happens that sucb strings are apt to break in damp weather, if they be not slackencd by the screws of the violin. Hence it follows, that the weight $\mathrm{E}$ will ascend when the air is more moist, and descend again when it becomes drier. By which means the index $\mathrm{F}$ will be carried up and down, and, by pointing to the several divisions on the scale, will show the degrees of moisture or dryness.

2. Or thus, for a more sensible and accuratc hygrome-

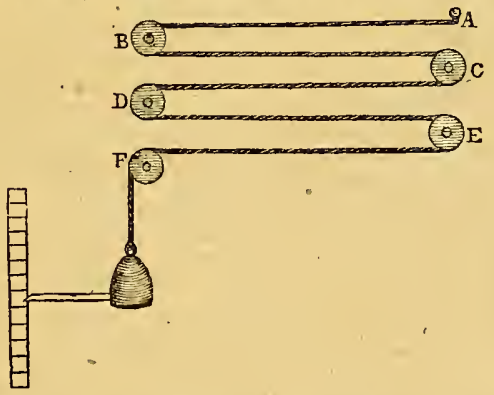

ter: strain a whipcord, or catgut, over several pulleys $b$, $c, D, E, F$; and proceed as before for the rest of the construction. It matters not whether the several parts of the cord be parallel to the horizon, as expressed in the annesed figure, or perpendicular to the same, or in any other position; the advantage of this, over the former method, being merely the having a greater length of cord in the same compass; for the longer the cord, the grcater is the contraction and dilatation, and consequently of the degrees of variation of the index over the scale, for any given change of moisture in the air.

\section{H Y G}

3. Or thus: Fasten a twisted cord, or catgut, $\mathbf{A B}$, by one end at $\mathbf{A}$, sustaining a weight at $B$; carrying an index $\mathrm{c}$ round a circular scale $\mathrm{DE}$ described on a horizontal board or tatle. For a cord or catgut twists itself as it moistens, and untwists again as it dries. Hence, on an increase or decrease of the humidity of the air, the index will show the quantity of twisting or untwisting, and consequently the increase or decrease of moisture or dryness.

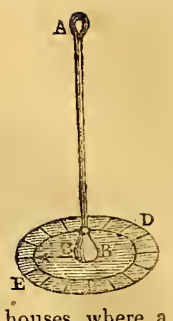

4. Those Dutch toys, called weather houses, where a small image of a man, and another of a woman, are-fixed upon the ends of an index, are constructed on this principle. For the index, being sustained by a cord, or twisted catgut, turus backwards and forwards, sending out the man in wet weather, and the woman in dry.

5. Or thus: Fasten one end of a cord, or catgut, $\mathrm{AB}$, to a hook at $\mathrm{A}$; and to the other end a ball $\mathrm{D}$ of about one pound weight; upon which draw two concentric circles, and divide them into any number of equal parts, for a scale; then fit a style or index $\mathrm{EC}$ into a proper support at $\mathrm{E}$, so as the extremity $\mathrm{c}$ may almost touch the divisions of the ball.-Here the cord twisting or untwisting, as in the former case, will indicate the change of moisture, by the successive application of the divisions of the circular scale, as the ball turns round, to the index $\mathbf{c}$.

6 . Or an hygrometer may be made of the thin boards of ash or fir, by their swelling or contracting. But this, and all the other kinds of this instrument, above described, become in time sensibly less and less accurate; till at last they lose their effect entirely, and suffer no alteration from the weather; in consequence of which, several others of another kind have been invented, much more durable, scrving for niany years with tolerable accuracy. They are as follow :

7. Take the manoscope, described under that article, and instead of the exhausted ball $\mathrm{E}$, substitute a sponge, or other body, that easily imbibes moisture. To prepare the sponge, it may' be proper first to wash it in water very

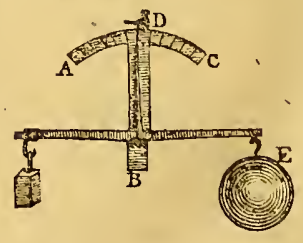
clcan; and, when dry again, in water or vinegar in which there has been dissolved sal ammoniac, or salt of taitar; after which let it dry again-Now, if the air become moist, the sponge will imbibe it and grow heavier, consequently will preponderate, and turn the index towards $c$; on the contrary; when the air becomes drier, the sponge is lighter, and the index turns towards $\mathrm{A}$; and thus showing the state of the air.

8. In the last-mentioned hygrometer, Mr. Gould, in the Philos. Trans. instead of a sponge, reconmends oil of 
vitriol, which grows sensibly lighter or heavier from the degrees of moisture in the air ; so that being saturated in the moistest weather, it afterwards retains or loses its acquired weight, as the air proves mure or less dry. The alteration in this liquor is so great, that in the space of 57 days it has been known to change its weight from 3 drachms to 9; and has shifted a tongue or index of a balance 30 degrecs. So that in this way a pair of scales may afford a very nice hygrometer. The same author suggests, that oil of sulphur or campanam, or oil of tartar per deliquium, or the liquor of fixed nitre, might be used iustead of the oil of vitriol.

9. This halance may be contrived in two ways; by either having the pin in the middle of the beam, with a slender tongue a foot and a half long, pointing to the divisions on an arched plate, as represented in the last figure. Or the scale with the liquor may be hung to the point of the beam near the pin, and the other extremity

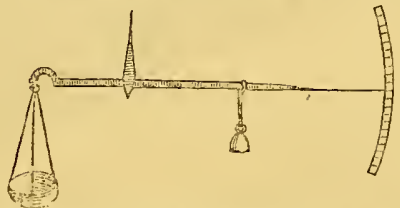

made so long, as to describe a large arch on a board placed . for the purpose; as in the figure here annexed.

10. Mr. Arderon has proposed some inuprovement in the sponge hygrometer. He directs the sponge $\mathrm{A}$ to be so

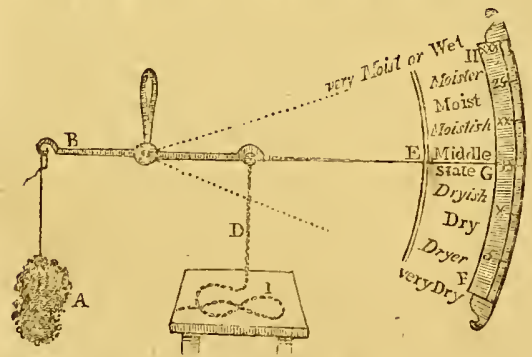

cut, as to contain as large a superficies as possible, and to hang by a fine thread of silk upon the heam of a balance $\mathbf{B}$, and exactly balanced on the other side by another thread of silk at D, strung with the smallest lead shot, at equal distances, so adjusted as to cause an index $\mathrm{E}$ to point at 6 , the middle of a graduated arch.FG $\mathrm{F}$, when the air is in a middle state betwcen the greatest moisture and the greatest dryness. Under this silk so strung with shot, is placed a small table or shelf $\mathrm{s}$, for that part of the silk or sliot to rest upon which is not suspended. When the moisture imbibed by the sponge increases its weight, it will raise the index, with part of the shot, from the table, and vice versa when the air is dry. Phil. Trans. vol.44, pa. 96.

11. From a series of hygroscopical observations, made with an apparatus of deal wood, described in the Philos. Trans. No. 480, Mr. Coniers concludes, 1st, That the wood contracts most in summer, and swells most in winter, but is most liable to change in the spring and fall. 2d, That this motion happens chiefly in the day time, there VOL. I. being scarcely any variation in the night. $3 \mathrm{~d}$, That there is a motion even in dry weather, the wood swelling in the morning, and shrinkiug in the afternoon. 4th, That the woorl, by night as well as by day, usually contracts when the wind is in the north, north-east, and east, both in summer and winter. 5th, That by constant observation of the motion and rest of the wood, with the help of a therinometer, the direction of the wind may be told nearly without a weather-cock. He adds also, that even the time of the year may be known by it; for in spring it moves more and quicker than in winter; in summer it is more contracted than in spring; and has less motion in autumn than in summer.

See an account of a method of constructing these and other hygrometers, in various places of the Philos. Trans. as vol. 11 , pa. 647 and 715 ; vol. 15 , pa. 1032 ; vol. 4,3 , pa. 6 ; vol. 44 , pa. 95,169 , and 184 ; vol. 54 , pa. 259 ; vol. 61, pa. 198; vol. 63, pa. 404, \& c. In my Abridg. also the General Index at the end points out the places of refcrence.

12. Dr. Hooke's hygrometer was made of the beard of a wild oat, set in a small box, with a dial plate and an index. See his Micrographia, pa. 150.

13. The doctors Hales and Desaguliers both contrived another form of sponge bygrometer, on this principle. They made an horizontal axis, having a small part of its

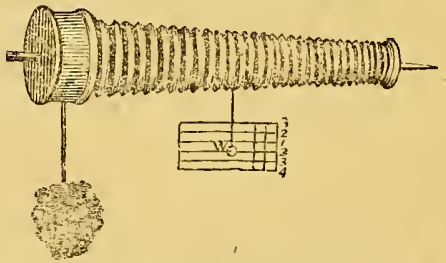

length cylindrical, and the remainder tapering conically with a spiral thread cut in it, after the manner of the fuzee of a watch. 'Thesponge is suspended by a fine silk thread to the cylindrical part of the axis, upon which it winds. This is balanced by a small weight $w$, suspended also by a thread, which winds upon the spiral fuzce. Here, when the sponge grows heavier, in moist weather, it descends and turns the axis, and so draws up the weight, which coming to a thicker part of the axis it becomes a balance to the sponge, and its motion is shown by an attached scalc. And vice versa when the air becomes dricr.-Salt of tartar, or any other salt, or pot-ashes, maly be put into the scale of a balance, and used instead of the sponge. Desag. Exper. Philos. vol. 2, pa. 300.

14. Mr. Ferguson made an hygrometer of a thin deal panncl; and to enlarge the scale, and so render its variations more sensible, he employed a wheel and axle, inaking one cord pass over the axle, which turned a whecl ten times as large, over which passed a line with a weight at the end of it, whose motion was therefore ten times as much as that of the deal pannel. The board should be changed in 3 or 4 years. Sec Philos. Trans. vol. 54, art. 47 .

15. Mr. Smeaton gave also an ingenious and claborate construction of an hygrometer; which may be seen in the Philos. Trans. vol. 61, art. 24.

16. Mr. Deluc's contrivance for an hygrometer is very ingenious, and on this principle. Finding that even ivory. $4 \mathrm{Q}$ 
swells with moisture, and contracts with dryness, he made a small and very thin hollow cylinder of ivory, open only at the upper end, into which is fitted the under or open end of a very fine long glass tube, like that of a thermometer. luro these is introduced some quicksilver, filling the ivory cylinder, and a small part of the length up the glass tule. The consequence is this: when moisture swells the ivory cylinder, its bore or capacity grows larger, and consequently the mercury sinks in the fine glass tube; and vice versa, whicn the air is drier, the ivory contracts, and forces the mercury higher up the glass iube. It is evident that an instrument thus constructed is in fact also a thermometer, and nust necessarily be afficted by the vicissitudes of heat and cold, as well as by thuse of dryness and moisture; or thit it must act as a thermometer as well as an hygrometer. The ingenious contrivances in the structure and mounting of this instrument may be seen in the Philos. Trans. vol. 63, art. 38 ; where it may also be seen how the above imperfection is corrected by some simple and ingenious expedients, emplnyed in the original construction and subsequent use of the instrument; in consequence of which, the variations in the temperature of the air, though they produce their full eflects on the instrument, as a thermometer, do not interfere with or einbarrass its indications as an bygrometer.

17. In the Philos. Trans. for 1791, Mr. Deluc has given a second paper on hygrometry. This has been chiefly occasioned by a memoir of M. de Saussure on the same subject, entitled Essais sur l'Hygrometrie, in 4to, 17 83. In this work $\mathrm{M}$. de $\mathrm{S}$. describes a new hygrometer of his construction, on the following principle.-It is a known fact that a hair will stretch when it is moistened, and contract when dried: and M. de Saussure found, by repeated experiments, that the difference between the greatest extension and contraction, when the hair is properly prepared, and has a weight of about 3 grains suspended by it, is nearly one-40th of its whole length, or one inch in 4.0. This circumstance suggested the idea of a new liygrometer. To render these small variations of the length of the hair perceptible, an apparatus was contrived, in which one of the extremities of the hair is fixed, and the other, bearing the counterpoise abovementioned, surrounds the circumference of a cylinder, which turns upon an axis to which a hand is adapted, marking upon a dial in large divisions the almost insensible motion of this axis. About 12 inches high is recommended as the most convenient and useful: and to render them portable, a contrivance is added, by which the hand and the counterpoise can be occasionally fixed.

But, for all the reasonings of these ingenious philosophers on this interesting suhject, and complete information, see the Philos. Trans. an. 1791, pa.1, and 389; and Deluc's Meteorolngie, vol. 1, an. 1780 ; as also the Monthly Review, vol. 51 , pa. 224 , vol. 71 , pa. 213 , vol. 76 , pa. 316 , vol. 78, pa. 236 , and vol. 6 , of the new series for the year 1791 , pa. 133.

HYGROMETRY, the science of the measurement of the moisture of the atmosplucre. The chicf writings on this science are those of M. Deluc and M. de Saussure, for which sce the last article.

IIYGROSCOPE, is commonly used in the same scnse with Hygrometer. Wolfius, however, regarding the etymology of the word, makes some difference. According to him, the hygroscope only shows that there are alterations of the air in respect of bumidity and dryness, but the hygrometer measures them. A hygroscope therefore is only an indefinite or less accurate hygrometer.

HYPATIA, a very learned and beautiful lady, was born at Alexandria, alout the end of the 4 th century, as she flourished about the year of Christ 430 . She was the daughter of Theon, a celebrated philosopher and mathematician, and president of the famous Alexandrian school. Her father, encouraged by her extraordinary genius, had her not only educated in all the ordinary accomplishments of her sex, but instructed in the most abstruse sciences. She made such great progress in philusophy, geometry, astronomy, and the mathematics in general, that she passed for the most learned person of her time. She published commentaries on Apollonius's Conics, on Diophantus's Arithmetic, and other works. At length she was thought wrorthy to succeed her father in that distinguished and important employment, the government of the school of Alexandria; and to deliver instructinns out of that chair where Ammonius, Hierocles, and many other great men, had taught before; and this at a time when men of great learning abounded both at Alexandria and in many other parts of the Roman empire. Her fame being so extensive, and her worth so universally acknowledged, it was no wonder that she had a crowded auditory. "She explained to her hearers (says Socrates, an ecclesiastical histurian of the 5 th century, born at Constantinople) the several sciences that go under the general name of philusophy; for which reason there was a confluence to her; from all parts, of those who made philosophy their delight and study."

The pupils of this lovely and surprising female, were not less eminent than they were numerous. One of them was the celebrated Synesius, who was afterwards bishop of Ptolemais. This ancient Christian Platonist always expresses the strongest, as well as the most grateful testimony of the virtue of his tutoress; never mentioning her without the most profound respect, and sometimes in terms of affection but, little short of adnration. But it was not Synesius only, and the disciples of the Alexandrian scliool, who admired Hypatia for her virtue and learning: never was woman more caressed by the public, and yet never had woman a inore unspotted character. She was held as an oracle for her wisdom, for which she was consulted by the inagistrates in all important cases; a circumstance which often drew her among the greatest concourse of men, without the least censure of her manners. In short, when Nicephorus intended to pass the highest compliment on the princess Eudocia, he thought he could not do it better than by calling her another Hypatia.

While Hypatia thus reigned the brightest ornament of Alexandria, Orestes was governor of that place for the emperor Theodosius, and Cyril was bishop or patriarch. Orestes, having had a liberal education, could not but admire Hypatia; and as a wise governor often consulted her. This, together with an aversion which Cyril had against Orestes, proved fatal to the lacly. About 500 monks assembling, attacked the governor one day, and wnuld have killed him, had he not been rescued by the townsmen; and the respect which Orestes had for Hypatia causing her to be traduced atnong the Clrristian multitude, they dragged her from her chair, tore her, in pieces, and burnt her limbs. This horrible catastrophe was perpetrated during the lent of the year 416 .

Cyril is strongly suspected of having fomented this tragedy. Cave indecd endeavours to remove the imputation 
of so horrid an action from the patriach; and places it on the Alexandrian mob in general, whom he calls " a very triting inconstant people." But though Cyril should be allowed neither to have been the perpetrator, nor even the contriver of it, yet it is much to be suspected that be did not discountenance it in the manner he ought to have dine: a suspicion which must needs be greatly confirmed by reflecting, that he was so far from censuring the violence committed by the monks upon Orestes, thet lie afterwards received the dead body of Ammonius, one of the most conspicuous in that brutality, who had grievously wounded the governor, and who was justly punished with death. Upon this riotous ruffian, Cyril made a panegyric in the church where he was laid, in wlich he extolled his courage and constancy, as one that had contended for the truth; and changing his name to Thaumasius, or the Admirable, ordered him to be considered as a martyr. "However (continues Socrates), the wisest part of Christians did not approve the zeal which Cyril showed on this man's behalf, being convinced that Ammonius bad justly suffered for his desperate attempt."

HYPERBOLA, one of the conic sections, being that which is made by a plane cutting a cone, so that, entering one side of the cune, and not being parallel tos the opposite side, it may cut the circular base when the opposite side is ever so far produced below the vertex, or shall cut the opposite side of the cone produced above the vertex, or shall make a greater angle with the base than the opposite side of the cone makes; all these three circumstances amounting to the same thing, but in other words.

1. Thus, the figure $\mathrm{DAE}$ is an hyperbola, made by a plane entering the side $\mathrm{V}_{Q}$ of a cone $\mathrm{FVQ}$ at $\mathrm{A}$, and either cutting the base PEQ when the plane is not parallel to VP, and this is ever so far produced; or when the angle $A R Q$ is greater than the angle $V P Q$; or when the plane cuts the oppositeside in B above the vertex.

2. By the hyperbola is sometimes meant the whole plane of the section, and sometimes only the curve line of the section.

3. Hence, the cutting plane meets the opposite cone in $B$, and there forms another hyperbola $d \mathrm{~B} e$, cqual to the former one, and having the

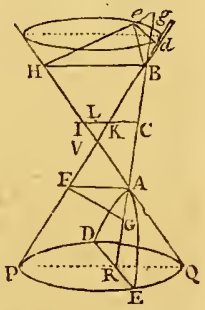
same transverse axis $\mathbf{A} \mathbf{B}$; and the same vertices $\mathbf{A}$ and $\mathbf{B}$. Also the two are called Opposite Hyperbolas.

4. The centre $c$ is the middle of the transverse axis.

5. The semi-conjugate axis is $\mathrm{cr}$, a mean proportional between $\mathrm{cr}$ and $\mathrm{cK}$, the distances to the sides of the opposite cone, when $\mathrm{CI}$ is drawn parallel to the diameter $\mathbf{P Q}$ of the base of the cone. Or the whole conjugate axis is a mean proportional between $\mathbf{A F}$ and $\mathbf{B H}$, which are drawn parallel to the base of the cone.

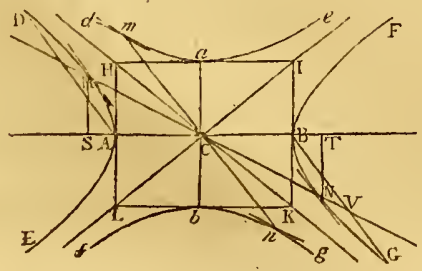

6. If $D A E$ and $P B G$ be two opposite hyperbolas, having the same transverse and conjugate axes $A B$ and $a b$, perpendicularly bisecting each other; and if dae and $f b$ be two other opposite hyperbolas, having the same axes with the two former, but in the contrary order, viz, having $a b$ for their first or transverse axis, and A B for thcir second or conjugate axis: then any two adjacent curves are called Conjugate $\mathrm{H}_{y}$ perbolas, and the wbole figure formed by all the four curves, the figure of the Conjugate Hyperbolas. And if the rectangle fIIKL be inscribed within the four conjugate hyperbolas, touching the vertices $\mathbf{A}, \mathbf{B}, a, b$, and having their sides parallel and equal to the two axes; and if then the two diagonals HCK, ICL, of the parallelogram be drawn, these diagonals are the asymptotes of the curves, being lines that continually approach nearer and ncarer to the curves, without meeting them, except at an infinite distance, where each asymptote and the two adjacent sides of the two conjugate hyperbolas may be sùpposed all to meet; the asymptote being there a common tangent to them both, viz, at that infinite distance.

7. Hence the four hyperbolas, meeting and running into each other at the infinite distance, may be considered as the four parts of one entire curve, having tbe same axes, tangents, and other properties.

8. A diameter in general, is any line, as $M N$, drawn through the centre $\mathrm{c}$, and meeting, or terminated by the opposite legs of the opposite hyperbolas. And if parallel to this diameter there be drawn two tangents, at $m$ and $n$, to the opposite legs of the other two opposite hyperbolas, the line $m c n$ joining the points of contact, is the conjugate diameter to $\mathrm{MN}$, and the two mutually conjugates to each other. Or, if to the points $\mathrm{M}$ or $\mathrm{N}$ there be drawn a tangent, and through the centre $\mathrm{c}$ the line $m n$ parallel to it, that line will be the cunjugate to an. The points where each of these meets the curves, as $M, N, m, n$, are the virtices of the diameters; and the tangents to the curves at the two vertices of any diameter, are parallel to each other, and also to the other or conjugate diameter.

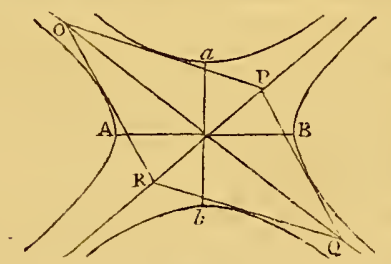

9. Moreover, if those tangents to the four hyperbolas, at the vertices of two conjugate diameters, be produced till they meet, they will form a parallelograin $O P Q R$; and the diagonals $o Q$ and $p r$ of the parallelogram will be the asympiotes of the curves; which therefore pass through the opposite angles of all the parallelograms so inscribed between the curves. Also it is a property of these paraljelograms, that they are all equal to each other, and thereforc equal to the rectangle of the two axes; as will be further noticed below. Further, if these diagonals or asymptotes make a right angle between them, or if the inscribed parallelogram be a square, or if the two axes be equal to each other, then the hyperbola is called a rightangled or an equilateral one.

$$
4 Q 2
$$




\section{H Y P}

10. An ordinate to any diameter, is a line drawn parallel to its conjugate, or to the tangent at its vertex, and terminated by the diameter produced and the curve. So $\mathrm{MS}$ and $\mathrm{TN}$ are ordinates to the axis $\mathrm{AB}$; also $\mathrm{AD}$ and $\mathrm{BG}$ are ordinates to the diameter $\mathrm{MN}$ (last fig. but one). Hence the ordinates to the axis are perpendicular to it; but ordinates to the other diameters are oblique to them.

11. An absciss is a part of any diamcter, contained between its vertex and an ordinate to it; and every ordinate has two abscisses: as $A T$ and $B T$, or MV and $\mathrm{NV}$.

12. The parameter of any diameter, is a third proportional to the diameter and its conjugate. - The parameter of the axis is also equal to the line $\mathrm{AG}$ or $\mathrm{Bg}$ (fig. 1), if $F G$ be drawn to make the angle $A F G=$ the anglc $\mathrm{BAv}$, or the line $\mathrm{Hg}$ to make the angle $\mathrm{Bng}=$ the angle $\mathrm{ABv}$.

13. The focus is the point in the axis where the ordinate is equal to balf the parameter of the axis; as $\mathrm{S}$ and $\mathrm{T}$ (fig. 2) if Ms and TN be half the parameter, or the $3 \mathrm{~d}$ proportional to $\mathrm{cA}$ and $\mathrm{c} a$. Hence there are two foci, one on each side the vertex, or one for each of the opposite hyperbolas. These two points in the axis are called foci, or burning points, because it is found by opticians that rays of light issuing from one of them, and falling upon the curve of the hyperbola, are reflected into lines that verge towards the other point or focus.

To describe an Hyperbola, in various ways.

14. (1st Way by points.)-In the transverse axis AF produced, take the foci $\mathrm{F}$ and $f$, by making $\mathrm{CF}$ and $f=A l$ or $B a$, assume any point $\mathrm{I}$ : Then with the

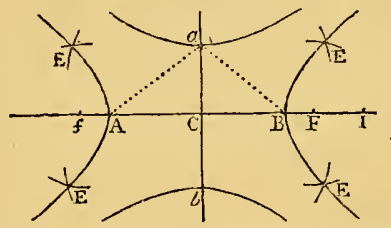

radii $\mathrm{AI}, \mathrm{BI}$, and centres $\mathrm{F}, f$, describe arcs intersecting in $\mathrm{E}$, which will give two points in each of the opposite curves EA E, EDE. In like manner, assuming other points $\mathrm{I}$, as many other points will be found in the curve. Then, with a steady hand, draw the curve line through all the points of intersection E. - In the same manner are to be constructed the other pair of opposite hyperbolas, using the axis $a b$ instead of $\mathrm{A} B$.

15. (2d Way by points, for a Right-angled Hyperbola only.) - On the axis produced if necessary, take any
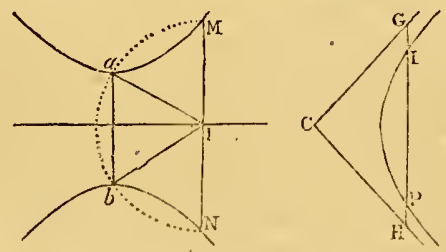

point $\mathbf{I}$, through which draw a perpendicular line, upon which set off $\mathrm{im}$ and $\mathrm{IN}$, equal to the distance $\mathrm{a} a$ or $\mathrm{i} b$, from $I$ to the extremities of the otheraxis; and $M$ and $N$ will be points in the curve.
16. (3d Way by points, to describe the curve through a given point.) - $\mathrm{CG}$ and $\mathrm{CH}$ being the asymptotes, and $\mathrm{P}$ the given point of the curve; through the point $P$ draw any line $\mathrm{GPH}$ between the asymptotes, upon which take $\mathrm{GI}=\mathrm{Pl}$, so shall $\mathrm{I}$ be another point of the curve. And in this manner may any number of points be found, drawing as many lines through the given point $\mathrm{P}$.

17. (4th Way by a continued Motion.) - If one end of a
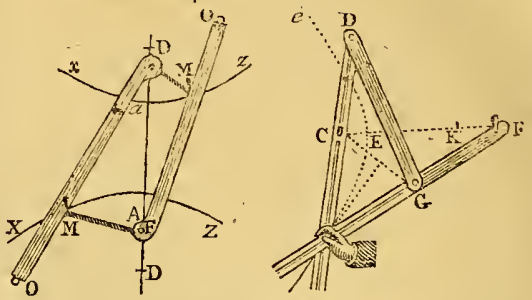

long ruler $f_{\text {Mo }}$ be fastened at the point $f$, by a pin on a plane, so as to turn freely about that point as a centre. Then take a thread Fmo, shorter than the ruler, and fix one end of it in $\mathrm{F}$, and the other to the end 0 of the ruler. Then if the ruler $f$ mo be turned about the fixed point $f$, at the same time kecping the thread omF always tight, and its part mo close to the side of the ruler, by means of the pin $\mathrm{M}$; the curve line $\mathrm{Ax}$ described by the motion of the pin II is one part of an hyperbola. And if the ruler be turned, and move on the other side of the fixed point $F$, the other part $\mathbf{A} \mathbf{Z}$ of the same hyperbola may be described after the same manner.-But if the end of the ruler be fixed in $F$, and that of the thread in $f$, the opposite hyperbola $x a z$ may be described.

18. (5th Way, by a continued Motion.) - Let $\mathrm{c}$ and $\mathrm{F}$ be the two foci, and $\mathrm{E}$ and $\mathrm{K}$ the two vertices of the hyperbola. (See the last fig. above.) Take three rulers CD, $\mathrm{DG}, \mathrm{GF}$, so that $\mathrm{CD}=\mathrm{GF}=\mathrm{EK}$, and $\mathrm{DG}=\mathrm{CF}$; the rulers $\mathrm{CD}$ and $\mathrm{GF}$ being of an indefinite length beyond $\mathrm{C}$ and $\mathrm{G}$, and having slits in them for a pin to move in; and the rulers having boles in them at $\mathrm{c}$ and $\mathrm{F}$, to fasten them to the foci $\mathrm{C}$ and $\mathrm{F}$ by means of pins, and at the points $\mathrm{D}$ and G they are to be joined by the ruler DG. Then, if a pin be put in the slits, viz, the common intersection of the rulers $\mathrm{CD}$ and $\mathrm{GF}$, and moved along, causing the two rulers GF, $C D$, to turn about the foci $\mathbf{C}$ and $F$, that pin will describe the portion $\mathrm{E} e$ of an hyperbola.-The foregoing are a few among various ways given by several authors.

\section{Some of the chief Properties of the Hyperbola.}

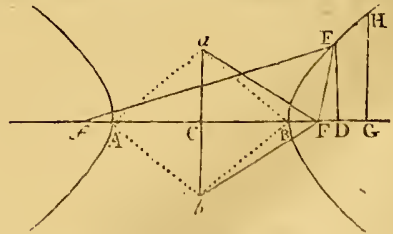

19. (1st) The squares of the ordinates, of any diameter, are to cach other, is the rectangles of their abscisses; i. e. $D E^{2}: G H^{2}:: A D \cdot B D: A G$. BG.

20. As the square of any dianeter, is to the square of its conjugate; so is the rectangle of two abscisses, to the 


\section{H Y P}

$[669$

square of their ordinate. That is, $A B^{2}: a b^{2}:: A D$. BD : $\mathrm{DE}^{2}$.

Or, because the rectangle $A D . B D$ is $=$ the difference of the squares $C D^{2}-C B^{2}$, the same property is,

$$
\begin{aligned}
& \text { As } \mathrm{A} \mathrm{B}^{2}: a b^{2}:: \mathrm{CD}^{2}-\mathrm{CB}^{2}: \mathrm{DE}^{2} \text {, } \\
& \text { Or } \mathrm{AB}: \frac{a b^{2}}{\mathrm{AB}}:: \mathrm{CD}^{2}-\mathrm{CB}^{2}: \mathrm{DE}^{2} \text {, }
\end{aligned}
$$

That is $A B: p:: C^{2}-C^{2}: D^{2}$, where $p$ is the parameter of the diameter $A B$, or the $3 \mathrm{~d}$ proportional to $A B$ and $a b$, or $\frac{a b^{2}}{A B}$.

And hence is deduced the common equation of the byperbola, by which its general nature is expressed. Thus, putting $d=$ the semidiameter $\mathrm{CA}_{\mathrm{A}}$ or $\mathrm{CB}$,

$c=$ its semiconjugate $\mathrm{c} a$ or $\mathrm{c} b$,

$p=$ its parameter or $\frac{2 d^{2}}{c}$,

$x=$ the absciss в $\mathrm{D}$ from the vertex,

$y=$ the ordinate $\mathrm{DE}$, and

$v=$ the absciss CD from the centre:

Then is $d^{2}: c^{2}::(2 d+x) x: y^{2}$,

$$
\begin{aligned}
& \text { or } d^{2}: c^{2}::(d+v) x: y^{2}, \\
& \text { or } d^{2}: c^{2}:: v^{2}-d^{2}: y^{2}, \\
& \text { or } d: p:: v^{2}-d^{2}: y^{2} ; \text { so that } \\
& y^{2}=\frac{2 d x+x^{2}}{d^{2}} \cdot c^{2}=\frac{v^{2}-d^{3}}{d^{3}} \cdot c^{2}=\frac{v^{2}-d^{3}}{d} \cdot p ;
\end{aligned}
$$

of which equations or proportions express the nature of the curve. And hence arises the name byperbola, signifying to exceed, because the ratio of $d^{2}$ to $c^{2}$, or of $d$ to $p$, exceeds that of $2 d x$ to $y^{2}$; that ratio being equal in the parabola, and defective in the ellipse, from which circumstances thcse also take their names.

21. The distance between the centre and the focus, is equal to the distunce between the extremities of the transverse and conjugate axcs. That is, $\mathrm{cF}=\mathrm{A} a$ or $\mathrm{A} b$, where $F$ is the focus.

22. The conjugate semi-axis is a mean proportional between the distances of the focus from both vertices of the transverse. That is, $C a$ is a mean between $A F$ and $B F$, or $\triangle \mathrm{P}: \mathrm{C} a:: \mathrm{C} a: \mathrm{BF}$, or $\mathrm{AF}, \mathrm{BF}=\mathrm{C} a^{2}$.

23. The difference of two lines drawn from the foci, to mcet in any point of the curve, is equal to the transverse axis. That is, $f \mathrm{E}-\mathrm{FE}=\mathrm{AB}$, where $\mathrm{F}$ and $f$ are the two foci.

24. All the parallelograms inscribed between the four conjugate hyperbolas are cqual to one another, and therefore each equal to the rectangle of the two axes. That is, the parallelogram oPQR $=\mathbf{A B} . a b$ (fig. to art. 9).

25 . The difference of the squares of every pair of conjugate diameters, is equal to the same constant quantity, viz, the difference of the squares of the two axes. That is, $\mathrm{MN}^{2}-m n^{2}=\mathrm{AB}^{2}-a b^{2}$, (fig. to art. 6) where $\mathrm{MN}$ and $m n$ are any two conjugate diameters.

26. The rectangles of the parts of two parallel lines, tcrminated by the curve, are to each other, as the rectangles of the parts of any other two parallel lines, any where cutting the forner. Or the rectangles of the parts of two intersecting lines, are as the squares of their parallel diameters, or squares of their parallel tangents.

27. All the rectangles are equal which are made of the segments of any parallel lines, cut by the curve, and limited by the asymptotes, and cach equal to the square of their parallel diameter. That is, $\mathrm{HE} \cdot \mathrm{\textrm {K }}$ or $\mathrm{He} . \mathrm{eK}=\mathrm{CQ}^{2}$ or $C P^{2}$.

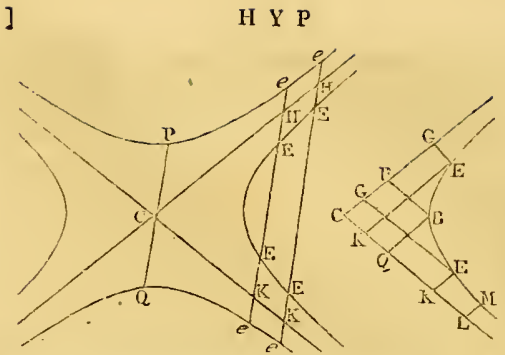

28. All the parallelograms are equal, which are formed between the asymptotes and curve, by lines parallel to the asymptotes. That is, the paral. cCEK $=$ CPRQ.-Hence is obtained anotber method of expressing the nature of the curre by an equation, involving the absciss taken on one asymptote, and ordinate parallel to the other asymptote. Thus, if $x=\mathrm{ck}, y=\mathrm{k \textrm {E }}, a=\mathrm{c} Q$, and $b=\mathrm{BQ}$ the ordinate at the vertex в of the curve; then, by the property in this article, $a b=x y$, or $a: x:: y: b$; that is, the rectangle of the absciss and ordinate is every where of the same magnitude, or any ordinate is reciprocally as its absciss.

29. If the abscisses CQ, cK, CL, \& c, taken on the one asymptote, be in geometrical progression increasing ; ther shall the ordinates $\mathrm{QB}, \mathrm{KF}, \mathrm{L} M$, \& $\mathrm{c}$, parallel to the other asymptote, be a like geometrical progression in the same ratio, but decreasing; and all the rectangles are equal, under every absciss and its ordinate, viz, $\mathrm{CQ} \cdot \mathrm{QB}=\mathrm{CK}$. $\mathrm{\kappa} \mathrm{E}=\mathrm{CL}, \mathrm{LM}, \& \mathrm{C}$.

30. The abscisses $\mathrm{CQ}, \mathrm{CK}, \mathrm{CL}, \& \mathrm{c}$, being taken in geometrical progression; the spaces or asymptotic areas $\mathrm{BQK} \mathrm{r}$, EKLM, \&cc, will be all equal; or, the spaces BQKE, BQLM, $\& \mathrm{c}$, will be in arithmetical progression; and therefore these spaces are the hypcrbolic logarithms of those abscisses.

These, and many other curious properties of the hyperbola, may be seen demonstrated in my Treatise on Conic Sections, and several others. Sce also Conic Secrions.

Acute HyPERBOLA, onc whose asymptotes make an acute angle.

Ambigenal Hyperвol $A$, is that which has one of its infinite legs falling within an angle formed by the asymptotes, and the other falling without that anyle. This is one of Newton's triple hyperbolas of the $2 d$ order. See his Enumeratio Lin. tert. Ord. See also Ambigenal.

Common, or Conic Hyperbola, is that which arises from the section of a cone by a plane; called also the Apollonian hyperbola, being that which was treated on by the first and chief author A pollonius.

Conjugate HYPER BOLAS, are those formed or lying together, and having the same axes, but in a contrary order, viz, the transverse of cach equal the conjugate of the other; as the two Conjugate Hyperbolas ree and EEE in the last figure but one.

Equilateral, or Rectangular Hyper Bola, is that whose two axes are equal to each other, or whose asymptotes make a right angle.-Hence, the property or equation of the equilateral hyperbola, is $y^{2}=a x+x^{2}$, where $a$ is the axis, $x$ the absciss, and $y$ its ordinate; which is similar to the equation of the circle, viz, $y^{2}=a x-x^{2}$, differing only in the sign of the second term, and where $a$ is the diameter of the circle.

Infinite HYPERBOLAs, or HYPERBolas of the higher kinds, are expressed or defined by general equations similar 
H Y P

$[670$

to that of the conic or common hyperbola, but having general exponents, instead of the particular numeral ones, but so as that the sum of those on one side of the equation, is equal to the sum of those on the other side. Such as, $a y^{\mathrm{m}+\mathrm{n}}=b x^{\mathrm{m}}(d+x)^{\mathrm{n}}$, where $x$ and $y$ are the absciss and ordinate to the axis or diameter of the curve; or $x^{\mathrm{m}} y^{\mathrm{n}}=a^{\mathrm{m}+1}$, where the absciss $x$ is taken on one asymptote, and the ordinate $y$ parallel to the other.

As the hyperbola of the first kind, or order, viz the conic hyperbola, has two asymptotes; that of the $2 \mathrm{~d}$ kind or order has three; that of the 3d kind, four; and so on.

Obtuse HYPER BOLA, is that whose asymptotes form an obtuse angle.

Opposite Hyperbolas, are those, pee, Qee, having the same transverse and conjugate axes.

Reciangular HY PERBOLA, the same as equilateral hyperbola.

Hyperbolic Arc, is the arc of an hyperbola.

Put $a=c_{a}$ the semitransverseaxe, $c=\mathrm{c} a$ the semiconjugate, $y=$ an ordinate $\mathbf{P Q}$ to the axe drawn from the end $Q$ of the arc $A Q$, beginning at the vertex $A$ : then putting $q=\frac{a a+c c}{c^{3}}$, $\Lambda=$ the hyp. log. of $\frac{y+\sqrt{ }(c c+y y)}{c}$ $=2.302585093 \times \mathrm{common} \log$. of

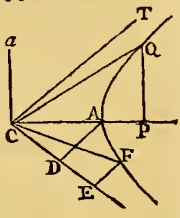
$\frac{y+\sqrt{ }(c c+y y)}{c}, \mathrm{~B}=\frac{y \sqrt{ }(c c+y y)-c c \mathrm{~A}}{2}, \mathrm{c}=$ $\frac{y^{3} \sqrt{ }(c c+y y)-3 c c B}{4}, \& C$; then is the length of the arc $A Q$ expressed by $\mathrm{C} \times\left(\mathrm{A}+\frac{q}{2} \mathrm{~B}-\frac{q^{2}}{2 \cdot 4} \mathrm{C}+\frac{1.3 q^{3}}{2.4 .6} \mathrm{D}-\right.$ $\frac{1.3 .5 q^{4}}{2.4 .6 .8}$ E \&c; or by $\frac{15 c^{2} t+\left(21 c^{2}+19 t^{2}\right) x}{15 c^{2} t+\left(21 c^{2}+9 t^{2}\right) x} \times y$, nearly; where $t$ is the whole transverse axe $2 \mathrm{CA}, c=2 \mathrm{c} a$ the conjugate, $x=A \mathrm{P}$ the absciss, and $y=\mathrm{PQ}$ the ordinate.

These and other rules may be scen demonstrated in my Mensuration, pa. 310 and 313 , 4 th edit.

Hy perbolic Area, that included by the hyperbolic curve and other lines.

Putting $a=\mathrm{c} A$ the semitransverse, $c=\mathrm{c} a$ the semiconjugate, $y=\mathbf{P Q}$ the ordinate, and $v=\mathrm{CP}$ its distance from the centre; then is the

$$
\begin{aligned}
& \text { arca } \triangle \mathrm{PQ}=\frac{1}{2} v y-\frac{x}{2} a c \times \text { hyp. } \log . \text { of } \frac{a y+c v}{a c} ; \\
& \text { sector } \mathrm{CAQ}=\frac{x}{2} a c \times \text { hyp. } \log . \text { of } \frac{a y+c v}{a c} ; \\
& \text { area } \triangle \mathrm{PQ}=2 x y \times\left(\frac{1}{3}-\frac{z^{1}}{1.3 .5}-\frac{z^{z}}{3.5 .7}-\frac{z^{3}}{5.7 .9}-\frac{z^{*}}{7.9 .11} \& \mathrm{c}\right), \\
& \text { where } x=\mathrm{AP} \text {, and } z=\frac{x}{2 a+x} ; \text { or }
\end{aligned}
$$

$\triangle \mathrm{PQ}=\frac{2 c x}{15 a} \times\left[\sqrt{ } 2 a x+4 \sqrt{ }\left(2 a x+\frac{3}{4} x x\right)\right]$ nearly.

Let $\mathrm{CT}$ and $\mathrm{CE}$ be the two asymptotes, and the ordinates DA, EF parallel to the other asymptote CT; then the asymptotic space $A D E F$ or sector $C A F$ is

$$
\begin{aligned}
& =\mathrm{CD} \times \mathrm{DA} \times \text { byp. log. of } \frac{\mathrm{CE}}{\mathrm{CD}} \text {, or } \\
& =\mathrm{CD} \times \mathrm{DA} \times \text { hyp. log. } \frac{\mathrm{DA}}{\mathrm{EF}}, \mathrm{or} \\
& =C D \times D A \times \frac{D E}{C D}-\frac{D E^{2}}{2 C D^{2}}+\frac{D E^{j}}{3 C D^{3}}-\frac{D E^{4}}{4 C D^{4}} \& C \text {; and }
\end{aligned}
$$

this last series was first given by Mercator in his Logarithmotechnia.

Sce my Mcnsuration, pa. 315 , \&c, 4 th edit.
H Y P

Generally, if $x^{\mathrm{m}} y^{\mathrm{n}}=a^{\mathrm{m}+\mathrm{n}}$ be an equation expressing an hyperbola of any order; then its asymptotic area will be $\frac{n}{n-m} x y$; which space therefore is always quadrable, in all the orders of hyperbolas, except the first or common hyperbola only, in which $m$ and $n$ being each 1 , the denominator $n-m$ becomes 0 or nothing.

HYPERBoLic Conoid, a solid formed by the revolution of an hyperbola about its axis, otherwise called an- hyperboloid.

\section{To find the Solid Content of an Hyperboloid.}

Let $\mathrm{AC}$ be the semitransverse of the generating hyperbola, and $\mathrm{Al}$ the height of the solid; then as $2 \mathrm{AC}+\mathrm{AH}$ is to $3 \mathrm{AC}+\mathrm{AH}$, so is the cone of the same base and altitude, to the content of the conoid.
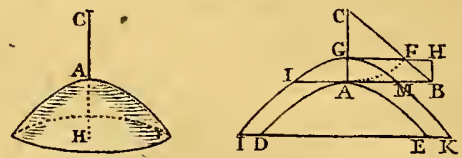

\section{To find the Curve Surface of an Hyperboloid.}

Let $\mathrm{AC}$ be the scmitransverse, and $\mathbf{A} \mathbf{B}$ perpendicular to it, and equal to the semiconjugate of $A \mathrm{DE}$ the generating hyperbola, or section through the axis of the solid. Join $\mathrm{CB}$; make $\mathrm{CF}=\mathrm{CA}$, and on $\mathrm{CA}$ let fall the perpendicular FG; then with the semitransverse $\mathrm{C} G$, and semiconjugate $\mathrm{GII}=\mathrm{AB}$, describe the hyperbola $\mathrm{GIK}$; then as the diameter of a circle is to its circumference, so is the hyperbolic frustum ILAMK to the curve surface of the conoid generated by DAE. See my Mensur. pa. 328, \&c, 4 th edit.

HYPERBoLIC Cylindroid, a solid formed by the revolution of an hyperbola about its conjugate axis, or line through the centre perpendicular to the transverse axis. This solicl is treated of in the Phil.Trans. by SirChristopher Wren, who shows some of its properties, and applies it to the grinding of hyperbolical glasses; affirming that they $m$ ust be formed this way, or not at all. See Philos. 'Trans. vol. 4 , pa. 961 .

Ily PERBolic $\mathrm{Leg}$, of a curve, is that having an asymptute, or tangent at an infinite distance. Newton reduces all curves, both of the first and higher kinds, into hyperbolic and parabolic legs, i.e. such as have asymptotes, and such as have not, or such as have tangents at an infinite distance, and such as have not.

HYPERBOLIC Line, is used by some authors for what is more commonly called the hyperbola itself, being the curve line of that figure; in which sense the surface terminated by it is called the hyperbola.

HY.PERBOLIC Logarithm, a logarithm so called as being similar to the asymptotic spaces of the hyperbola. 'The hyperbolic logarithm of a number, is to the common logarithm, as 2.3025850929940457 to 1 , or as 1 to -4342944819032518 . The first invented logarithıns, by Napier, are of the hyperbolic kind; and so are Kepler's. Sce Logaritum.

HYPERBOLIC Mirror, is one ground into that shape.

HyperborIc Space, the same as Hyperbolic Area.

HYPERBOLICUM Acutum, a solid made by the revolution of the infinite area or space contained between the curve of the hyperbola, and its asymptote. This produces a solid, which though infinitely long and gene- 
rated by an infinite area, is nevertheless equal to a finite solid body; as is denominated by Torricelli, who gave it this name.

HYPERBOLIFORM Figures, are such curves as approach, in their properties, to the nature of the hypcrbula; called also hyperboloides.

HYPERBOLOIDS, are hyperbolas of the higher kind, whose nature is expressed by this equation, $a y^{\mathrm{m}+\mathrm{n}}=$ $b x^{\mathrm{n} 1}(a+x)^{n}$. See IIYYer Bola. It also means the hyperbolic conoid. See that article.

HYPERBOREANS, the most northern nations, or regions, as dwelling beyond or about the wind Boreas : as the Siberians, Samoieds, \& $c$.

HYPERTHYRON, in Architecture, a sort of table, usually placed over gates or doors of the Doric order, above the chambranle, in form of a frize.

HYPETHRE, in Ancient Architecture, two rows of pillars surrounding, and ten at each face of a temple, \&c, with a peristyle within of six columns.

HYPOGEUM, in the Ancient Architecture, a name common to all the parts of a building that are under ground; as the cellars, butteries, \&c.

HYPOMOCHLION, the fulcrum or prop of a lever; or the point which sustains its pressure, when ernployed either in raising or lowering bodies. The hypomocblion is frequently a roller set under the lever; or under stones or pieces of timber, \& c , that they may be the more easily lifted up, or removed.

HYPOTENUSE, or НY РОTHENUSE, in a right-angled triangle, is the side which subtends, or is opposite to the right angle, being al ways the longest of the three sides; as the side $A C$, opposite to the right angle $B$.

It is a celebrated theorem in plane geometry, being the 47 th prop. of the 1 st book of Euclid, that in every rigbt-angled triangle $A B C$, the square formed on the hypotenuse $A C$, is equal to both the two squares formed upon the other two sides $A B$ and $B C$; or that $A C^{2}=A B^{2}+B C^{2}$. This is particularly called

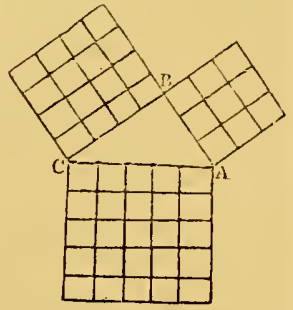
the Pythagorean theorem, from its reputed inventor, Pythagoras, who it is said sacrificed a whole hecatomb to the muses, in gratitude for the discovery. But the same thing is true of circles or any other similar figures, viz, that any figure described on the hypotenuse, is equal to the sum of the two similar figures described on both the other two sides.

HYPOTHENUSE. See Hy poten use.

HYPOTHESIS, in Geometry, or Mathematics, means much the same thing with supposition, being an assumption of something as a condition, upon which to raise a demonstration, or from whicb to draw an inference.

Hypotheses, or postulatums, are considered by Dr. Barrow as propositions assuming or affirming some cvi- dently possible mode, action, or motion of a thing, and that there is the same affinity between hypotheses and problems, as between axioms and theorems: a problem showing the manner, and demnnstrating the possibility of some structure, and an hypotbesis assuming some construction which is manifestly possible.

In disputation, false bypotheses are often employed in order to draw the antagonists into absurdities; and even in geometry, trutls are often to be deduced from sucb false hypotheses.

Every conditional, or hypothetical proposition, may be distinguished into hypothesis and thesis: the first asserts the conditions under which any thing is affirmel or denied; and the latter is the thing itself affirmed or denied. Thus, in the proposition, a triangle is half of a parallelogram, if the Lases and altitudes of the two be equal; the latter part, namely, "if the bases," \&c, is the hypothesis; and the former part, "a triangle is half a parallelogram," the thesis.

In strict logic, we are never to pass from the hypothesis to the thesis: that is, the principle supposed must he proved to be true, before we require the cunsequence to be made.

Нyротнеsis, in Philosophy, denotes a kind of system laid down from our own imagination, by which to account for some plenomenon or appearance of nature. Thus, there are hypotheses to account for the tides, for gravity, for magnetism, for the deluge, \&c. The real and scientific causes of natural things generally lie very deep in obscurity: observation and experiment, the proper means of arriving at them, are in most cases extremely slow; and the human mind in consequence gets very impatient : hence we are often induced to feign or invent something that may seem like the cause, and which is calculated to answer the several phenomena, so that it may possibly be the true catse. Philosuphers are divided as to the use of such fictions or hypotheses, which are much less current now than they were formerly. The latest and best writers are for excluding hypotheses, and standing entirely on observation and experiment. Whatever is not deduced from phenomena, says N(wton, is an hypothesis; and hypotheses, whether metaphysical, or physical, or mechanical, or of occult qualities, have no place in experimental philosophy. Phil. Nat. Prin. Math, in Calce.

Ну ротнеsis is more particularly applied, in astronomy, to the several systems of the heavens; or the divers manners in which different astronomers bave supposed the heavenly bodies to be ranged, or moved. The principal hypotheses are the Ptolemaic, the Tychonic, and the Copernican. This last is now so generally received, and so well established and warranted by observation, that it is thought derogatory to it to call it an bypothesis.

HYYPOTRACHELION, in Architecture, is used for a little frize in the Tuscan and Doric capital, between the astragal and annulets; called also the colerin and gorgerin. The word is applied by some authors in a more general sense, to the neck of any column, or that part of its capital beluw the astragal. 


\section{J.}

J

ACK, in Mechanics, is an instrument in common use for raising heavy timber, or very great weights of any kind; being a certain powerful combination of teeth and pinions; the whole inclosed in a strong wooden stock or frame $\mathrm{BC}$, and moved by a winch or handle IIP; the outside appearing as in the figure annexed.

In fig. $6, \mathrm{pl} .14$, the wheel or rack-work is shown, being the view of the inside when the stock is removed. Though it is not drawn in the just proportions and dimensions, for the rack a m must be supposed at least four times as long in proportion to the wheel $Q$, 'as the figure represents it; and the teeth, which will be then four times more in number to have about 3 in the inch. Now if the handle n $P$ be 7 inches long, the circumference of this radius will be 44 inches, which is the distance or space the power moves through in one revolution of the

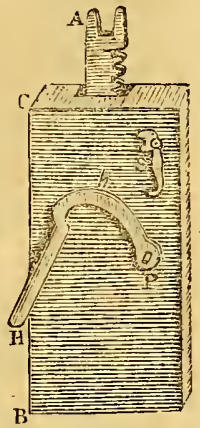
handle: but as the pinion of the bandle has but 4 leaves, and the wheel a suppose 20 teeth, or 5 times the number, therefore to make one revolution of the wheel $Q$, it requires 5 turns of the handle, in which case it passes through 5 times 44 or 220 inches: but the wheel having a pinion $\mathbf{R}$ of 3 leaves, these will raise the rack 3 tecth, or one inch, is the same. space. Hence then, the handle or , power moving 220 times as fast as the weight, will raise or balance a weight of 220 times its own power. And if this be the band of a man, who can sustain 100 pounds weight, lie will, by help of this jack, be able to raise, or sustain a weight or force of $22000 \mathrm{lb}$, or about 10 tons weight. This machine is sometimes oper behind from the bottom almost un to the wheel $\mathrm{Q}$, to let the lower claw, which in that case is turner up as at $\mathrm{B}$, raise any weight. When the weight is drawu or pushed sufficiently high, it is kept from going back by hanging the end of the hook $s$, fixed to a staple, over the curved part of the handle at $h$.

$\mathrm{J}_{\mathbf{A C K}}$ is also the name of a well-known engine in the kitchen, used for turning a spit. Here the weightis the power applier, acting by a set of pulleys; the friction of the parts, and the weight with which the spit is charged, make the force to be overcome; and a steady uniform morion is maintained by means of the fly. See the fig. of this machine, pl. 14, fig. 7 .

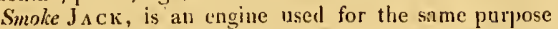
with the common jack, and is so called from jis teing moved by meaus of the smoke, or rarefied air, ascending the chinucy, and striking against the sails of the horizontal whecl $A$ is (plite 14 , fig. 8), which being inclined to the horizon, is mowed about the axis of the wheel, together with the pinion $\mathrm{C}$, which carries the wheels D and $\mathrm{E}$; and 1) carries the chain 1:, which turns the spit. The wheel A is should be placed in the narrow part of the chimucy, where the motion of the smoke is swiftest, and where also the greatest part of it must strike on the sails. The force of this machine depends upon the draught of the chimney, and the strength of the fire.

$\mathbf{J}_{\mathrm{ACK}}-A r c h$, is an arch of one brick thickness.

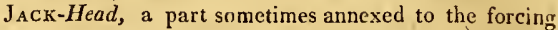
pump.

JACOB's-Staff, a mathematical instrument for taking heights and distances; the same with the Cross-staff; which see.

JAMBS, or JAUMs, in Architecture, are the upright sides of chimneys, from the hearth to the mantle-tree. Also door posts, or the upright posts at the ends of the window frames.

St. JAMES's Day, a festival in the calendar, observed on the 25th of July, in honour of St. James the apostle.

JANUARY, the first month of the year, according to the computation now used in the West, and containing 31 days; so called by the Romans from Janus, one of their divinities, to whom they gave two faces; brcause on the one side, the first day of this month looked towards the new year, and on the other towards the old one. The name may also be derived from Janua, a gate; this month, being the first of the year, may be considered as the gate or entrance of it. January and February were introduced into the year, by Numa Pompilius; Romulus's year beginning with the month of March.

JAUMIS. See JA B B.

ICE, a brittle transparent body, formed of some fluid, frozen or fixed by cold. The specific gravity of ice to water, is various, according to the nature and circumstances of the water, degree of $\operatorname{col}\}, \mathcal{E} c$. Dr. Irving (Plipps's Voyage towards the North Pole) found the densest ice he could meet with about a 14 th part lighter than water. M. de Matran found it, at different trials, 1-14th, 18 th, or 19 th lighter than water ; and when the water was previously purged of air, only a $22 \mathrm{~d}$ part.

The rarefaction of ice has been supposed owing to the air-bubbles produced in ice while frgezing; these, being considerably large in proportion to the water frozen, render the jec so much specifically lighter. It is well known that a considerable quantity of air is lodged in the interstices of water, though it has there little or no elastic property, on account of the disunion of its particles; but on these particles coming closer together, and uniting as the water freezes, light, expansive, and elastic air-bubbies are thus gencrated, and increase in bulk as the cold grows stronger, and by their elastic force bursts to picces any vessel in which the water is closely contained.' But snowwater, or any water long boilcal over the fire, attiords an ice more solid, and witl fener bubles. Pure water loug kept in vacuo atud frozen afterwards there, freczes mucl sooner, on being exposed to the same degree of cold, than water unpurged of its air and set in the open atmosphere. And the ice made of water thus dirested of its air, is much harder, more solid and transparent, and heavier than common ice.

But M. de Mairan, in a dissertation on ice, attributes the increase of the bulk of the water under this form, 
chiefly to a different arrangement of its parts: the icy skin on water being conzosed of filaments which are found to be joined constantly and regularly at an angle of $60^{\circ}$, and which, by this disposition, occupy a greater volume than if they were paraliel. Besides, after ice is formed, he found it continue to expand by cold; a piece of ice, which was at first only a 14th part spucifically lighter than water, on being exposed some days to the frost, became a 12 th part lighter ; and thus he accounts for the bursting of ice in ponds.

It appears from an experiment of Dr. Hooke, in 1663 , that ice refracts the light less than water; wbence he infers, that the lightness of ice, which causes it to swim in water, is not produced merely by the small bubbles which are visible in it, but that it arises from the uniform constitution or general texture of the whole mass : a fact which was afterwards confirmed by M. Lahire. See Hooke's Exper. by Derham, pa. 26, Acad. Per. 1693, Mem. pa. 25.

Sir Robert Barker thus describes the process of making ice in the East Indies, in a country where he never saw any natural ice: On a large plain is dug three or four pits, each about 30 feet square, and 2 feet deep; the bottoms of which are covered, about 8 or 12 inches thick, with sugar-cane, or the stems of the large Indian corn, dried. On this bed are placed in rows a number of small shallow unglazed earthen pans, formed of a very porous earth, a quarter of an inch thick, and about an inch and a quarter deep; which, at the dusk of the evening, they fill with soft water that has been boiled. In the morning before sunrise the ice-makers attend at the pits, and collect what has been frozen in baskets, which they convey to the place of preservation. This is usually prepared in some high and dry situation, by sinking a pit 1 th or 15 feet deep, which they line first with straw, and th $n$ with a coarse kind of blanketing. The ice is deposited in this pit, and beaten down with rammers, till at length its own accumulated cold again freezes it, and it forms one solid mass. The mouth of the pit is well secured from the exterior air with straw and blankets, and a thatched roof is thrown over the whole. Philos. Trans. vol. 65, pa. 252.

For the force of ice and other very interesting particulars relating to it, see the articles FREEZING, CoLD, and CONGELATION.

ICHNOGRAPHY, in Architecture, is a transverse or horizontal section of a building, exhibiting the plot of the whole edifice, and of the several rooms and apartments in any story; together with the thickness of the walls and partitions; the dimensions of the doors, windows, and chimneys; the projectures of the columns and piers, with every thing visible in such a section.

ICH NOGRAPHY, in Fortification, is the plan or representation of the length and breadth of a fortress; the distinct parts of which are marked out, either on the ground itself, or upon paper.

IcunogRAPII, in Perspective, the view of any thing cut off by a plane parallel to the horizon, just by the base or bottom of it; being the same with what is otherwise called tie plan, geonetrical plan, or ground-plot, of any thing, and is opposed to Orthography or Elevation.

ICOSAEDRON, or ICOSAHEDRON, one of the five regular bodies or solids, terminated by twenty equilateral and equal triangles. It may be considered is consisting of 20 equal and similar triangular pyramids, whose vertices meet in the centre of a sphere conceived to circumVol. I. scribe it, and therefore having all their beights and bases cqual : bence the solidity of one of those pyramids multiplied by 20 , the number of them, gives the solid content of the icosaedron.

To form or make the Icosaedron:-Describe upon a card paper, or some other such like substance, 20 equilateral triangles, as in the figure at the article Regular BoDy. Cut it ont by the extreme edges, and cut all the other lines half through, then fold the sides up by these edges, and the solid will be formed.

The linear edge or side of the icosaedron being $A$, then will the surface be $5 A^{2} \sqrt{ } 3=8 \cdot 6602540 A^{2}$, and the solidity $=\frac{5}{6} A^{3} \sqrt{ }^{7+3 \sqrt{ } 5}-2 \cdot 1816950 A^{3}$.

More general equations, and relations, may be seen in my Mensuration, pa. 190, 4th edit.

IDES, in the Roman Calcndar, a name given to a series of 8 days in each month; which, in the full months, March, May, July, and October, commenced on the 15th day; and in the other months, on the 13th day; from thence reckoned backward, so as in those four months to terminate on the 8 th day, and in the rest on the 6 th. These came between the calends and the nones. And this way of counting is still used in the Roman Chancery, and also in the Calendar of the Breviary.

JET D'EAU, a French word, signifying a fountain that throws up water to some height in the air. A jet of water is thrown up by the weight of the column of water above its ajutage, or orifice, up to its source or reservoir; and therefore it would rise to the same height precisely as the head or rescrvoir, if certain causes were removed which prevent it from rising quite so high. For first, the velocity of the lower particles of the jet being greater than that of the upper, the lower water strikes that which is next above it; and as fluids press every way, by its impulse it widens, and consequently shortens the column. Secondly, the water at the top of the jet does not immediately fall off, but forms a kind of ball or head, the weight of whicl depresses the jet; but if the jet be a little inclined, or not quite upright, it will play higher, though it will not in such case be quite so beautiful. Thirdly, the friction against the sides of the pipe and hole of the ajutage, prevents the jet from rising quite so high, and a small one will be more impeded than a large one. And the fourth cause is the resistance of the air, which is proportional to the square of the velocity of the water nearly; and therefore the defect in the height is nearly in the same proportion, which is also the same as the proportion of the heights of the reservoirs above the ajutage. Hence, and from experience, it is found that a jet, properly constructed, rises to different heights according to the height of the reservoir, as in the following table, Of the heights of reservoirs and the heights of their corresponding jets; the former in feet, and the latter in feet, and tenths of a foot.

Heights of Reservoirs and their Jets.

\begin{tabular}{r|c||c|c||c|c||c|c}
\hline Res. & Jet. & Res. & Jet. & Res. & Jet. & Res. & Jet. \\
\hline 5 & $4 \cdot 9$ & 12 & $11 \cdot 6$ & 19 & $17 \cdot 9$ & 26 & $24 \cdot 1$ \\
6 & $5 \cdot 9$ & 13 & $12 \cdot 5$ & 20 & $18 \cdot 8$ & 27 & $24 \cdot 9$ \\
7 & $6 \cdot 8$ & 14 & $13 \cdot 4$ & 21 & $19 \cdot 7$ & 28 & $25 \cdot 8$ \\
8 & $7 \cdot 8$ & 15 & $14 \cdot 3$ & 22 & $20 \cdot 6$ & 29 & $26 \cdot 6$ \\
9 & $8 \cdot 7$ & 16 & $15 \cdot 2$ & 23 & $21 \cdot 5$ & 30 & $27 \cdot 5$ \\
10 & $9 \cdot 7$ & 17 & $16 \cdot 1$ & 24 & $22 \cdot 3$ & 31 & $28 \cdot 3$ \\
11 & $10 \cdot 6$ & 18 & $17 \cdot 0$ & 25 & $23 \cdot 2$ & 32 & $29 \cdot 2$ \\
& \multicolumn{5}{|c|}{} & \multicolumn{4}{|c|}{$\mathbf{R}$} & & \\
\end{tabular}


I G N

\begin{tabular}{c|c||c|c||c|c||c|c} 
Res. & Jet. & Res. & Jet. & Res. & Jet. & Res. & Jet. \\
\hline 33 & $30 \cdot 0$ & 52 & $45 \cdot 2$ & 71 & $59 \cdot 3$ & 90 & $72 \cdot 5$ \\
34 & $30 \cdot 8$ & 53 & $46 \cdot 0$ & 72 & $60 \cdot 0$ & 91 & $73 \cdot 2$ \\
35 & $31 \cdot 6$ & 54 & $46 \cdot 7$ & 73 & $60 \cdot 7$ & 92 & $73 \cdot 8$ \\
36 & $32 \cdot 5$ & 55 & $47 \cdot 5$ & 74 & $61 \cdot 4$ & 93 & $74 \cdot 5$ \\
37 & $33 \cdot 3$ & 56 & $48 \cdot 2$ & 75 & $62 \cdot t$ & 94 & $75 \cdot 2$ \\
38 & $34 \cdot 1$ & 57 & $49 \cdot 0$ & 76 & $62 \cdot 8$ & 95 & $75 \cdot 8$ \\
39 & $34 \cdot 9$ & 58 & $49 \cdot 7$ & 77 & $63 \cdot 5$ & 96 & $76 \cdot 5$ \\
40 & $35 \cdot 7$ & 59 & $50 \cdot 5$ & 78 & $64 \cdot 2$ & 97 & $77 \cdot 2$ \\
41 & $36 \cdot 6$ & 60 & $51 \cdot 2$ & 79 & $64 \cdot 9$ & 98 & $77 \cdot 8$ \\
42 & $37 \cdot 4$ & 61 & $52 \cdot 0$ & 80 & $65 \cdot 6$ & 99 & $78 \cdot 5$ \\
43 & $35 \cdot 1$ & 62 & $52 \cdot 7$ & 81 & $66 \cdot 3$ & 100 & $79 \cdot 1$ \\
44 & $38 \cdot 9$ & 63 & $53 \cdot 5$ & 82 & $67 \cdot 0$ & 110 & $85 \cdot 6$ \\
45 & $39 \cdot 7$ & 64 & $54 \cdot 2$ & 83 & $67 \cdot 7$ & 120 & $91 \cdot 9$ \\
46 & $40 \cdot 5$ & 65 & $54 \cdot 9$ & 84 & $68 \cdot 4$ & 130 & $98 \cdot 0$ \\
47 & $41 \cdot 3$ & 66 & $55 \cdot 7$ & 85 & $69 \cdot 1$ & 140 & 104 \\
48 & $42 \cdot 1$ & 67 & $56 \cdot 4$ & 86 & $69 \cdot 8$ & 150 & 110 \\
49 & $42 \cdot 9$ & 68 & $57 \cdot 1$ & 87 & $70 \cdot 5$ & 160 & 116 \\
50 & $43 \cdot 7$ & 69 & $57 \cdot 8$ & 88 & $71 \cdot 1$ & & \\
51 & $44 \cdot 4$ & 70 & $58 \cdot 6$ & 89 & $71 \cdot 8$ & & \\
\hline
\end{tabular}

By various experiments that have been made by $\mathrm{Ma}$ rintte, Desaguliers, and others, it has been found, that if the reservoir be 5 feet high, a conduct-pipe $1 \frac{3}{4}$ inch diameter will adnit a hole in the ajutage from $\frac{1}{4}$ to $\frac{3}{8}$ of an inch; and so on as in the following table:

$\begin{gathered}\text { Height } \\ \text { Reservoir. }\end{gathered}$
5 feet $\begin{gathered}\text { Diam. of the } \\ \text { Ajutage. }\end{gathered}$

But the size of the pipe is more or less, with the distance.

If it be required to,keep any number of jets of given dimensions playing, by one common conduct-pipe, the diameter of an ajutage must be found that shall be equal to all the small ones that are given, and from this its proper conduct-pipe. Thus, if there be 4 ajutages, each $\frac{3}{4}$ of an inch diameter; then the square of $\frac{3}{4}$ is $\frac{9}{16}$, which multiplied by 4 , the number of them, makes $\frac{36}{2} \frac{6}{6}$, the square root of which is $\frac{6}{4}$ or $1 \frac{1}{2}$, the diameter of an ajutage equal to all the other four; to which in the table answers a pipe of 8 inches diameter. In general, the diameter of the conduct-pipe should be about 6 times that of the ajutage.-See Mariotte's Mouvement des Eaux; Desaguliers's Exper. Philos. vol. 2, pa. 127, \&.c; Clare's Motion of Fluids, pa. 109 ; \&c.

JETTE, the border made round the stilts under a pier, in certain old briclges, being the same with starling, consisting of a strong framing of timber filled with stones, chalk, \&c; to preserve the foundations of the piers from injury.

IGNIS Fatuvs, a common metcor, chiefly seen in dark nights about ineirluw's, marshes, and other moist places, as also in burying-grounds, and near dung-hills. It is known among the people by the appelfations, IVill with a Wisp, and Jack with a lantern. Dr. Shaw describes a remarkable ignis fatuus, which he saw in the Holy Land,

\section{M A}

that was sometimes globular, or in the form of the flame of a candle; and presently afterwards it spread itself so much as to involve the whole company in a pale inofiensive light, and then contract itself again, and suddenly disappear. But in less than a minute it would become visible as before; or, rumning along from one place to another, with a swift progressive motion, would expand itself at certain intervals over more than 2 or 3 acres of the adjacent mountains. The atmosphere had been thick and hazy, and the dew on the liorses' bridles was uncommonly claminy and unctuous. In the same weather he observed those luminous appearances, which skip about the masts and yards of ships at sea, and which the sailors call corpusanse, by a corruption of the Spanish cuerposanto. Shaw's Travels, pa. 363 .

Newton calls it a vapour shining without heat; and supposed it to be of the same nature with the light issuing from putrescent substances. IVillougliby and Ray were of opinion that it is occasioned by shining insects: but all the appearances of it observed by Derham, Beccaria, and others, sufficiently evince that it must be an ignited vapour. Inflammable air has been found to be the most common of all the factitious airs in nature; and that it is the usual product of the putrefaction and decomposition of vegetable substances in water. Signor Volta writes to Dr. Priestley, that he fires inflammable air by the electric spark, even when the electricity is very moderate; and he supposes that this experiment explains the inflammation of the ignes fatui, provided they consist of inflammable air, issuing from marshy ground by help of the electricity of fogs, and by falling stars, which have probably an electrical origin. See Priestley's Obs. on Air, vol. 3, pa. 382 ; the Philos. Trans. Abr. vol. 7, pa. 374, \&c.

IGNITION, properly signifies the setting fire to any substance; but the sense is commonly restrained to that kind of burning which is not accompanied with flame, such as of charcoal, cinders, metals, stones, and other solid substances, The efiects of ignitiun are first to dissipate what is called the phlugiston of the ignited body, after which it is reduced to ashes. Vitrification next follows; and lastly, the substance is totally dissipated in vapour. All these effects, however, depend on the presence of the air; for in vacuo the phlogiston of any substanee cannot be dissipated. Neither can a body which is totally destitute of phlogiston be ignited in such a manner as those which contain it; for as long as the phlogiston remains, the heat is kept up in the body by the action of the external air upon it: but when the phlogiston is entirely evaporated, the air always destroys, instead of augmenting the heat. Philosophers have therefore been greatly embarrassed in cxplaining the phenomena of ignition. See Phlogiston.

ILLUMINATION, the act or effect of a luminous body, or a body that enits light; sometimes also the state of another body that receives it.

Circle of lozuninatron. . Sce Circle.

ILLUMINATIVE Lumar Month, the space of time that the moon is yisible, beneen one conjunction and another.

IMI $\wedge \mathrm{GL}$, in Optics, is the spectre or appearance of an objcct, made either by reflection or refiaction. In all plane mirrors, the image is of the same magnitude as the object; and it appears as far behind the mirror as the object is before it. In convex mirror's, the image appears less that the object; and further distant from the centre of the convesity, than from the point of reflection. Mr. 
Molyneux gives the following rule for finding the diameter of an image, projected in the distinct base of a convex mirror: viz, As the distance of the object from the mirror is to the distance of the image from the glass, so is the dirmeter of the object to the diameter of the image. See Lens, Mirror, Reflection, and Refraction.

IMAGINARY Quantities, or Inpossible Quantities, in Algebra, are the even roots of negative quantities; which expressions are imaginary, or impossible, or opposed to real quantities; as $\sqrt{ }-a a$, or $\sqrt{ }-a^{4}, \& c$. For, as every even power of any quantity whatever, whether positive or negative, is necessarily positive, or laving the sign + , because + by + , or - by - give equally + ; hence it follows that every even power, as the square for instance, which is negative, or having the sign -, has no possible rout; and therefore the even roots of such powers or quantities are said to be impossible or imaginary. The mixt expressions arising from imaginary quantities joined to real ones, are also imaginary; as $a-\sqrt{ }-a u$, or $b+\sqrt{ }-a a$.

The roots of negative quantities were, perhaps, first treated of in Cardan's Algebra. As to the uneven roots of such quantities, he shows that they are negative, and at the same time assigns them : but the even roots of them he rejects, observing that they are nothing as to common use, being neither one thing or other; that is, they are merely inaginary or impossible. And since his time, it has gradually become a part of algebra to treat of the roots of negative quantities. Albert Girard, in his Invention Nouvelle en l'Algebre, pa. 42 , gives names to the three kinds of roots of equations, calling them, greater than nothing, less than nothing, and envelopee, as $\sqrt{ }-3$ : but this was sson after called imaginary or impossible, as appears by Wallis's Algebra, pa. 264 \&c ; where he observes that the square root of a negative quantity, is a mean proportional between a positive and a negative quantity; as $\sqrt{ }-b c$ is the mean proportional between $+b$ and $-c$, or between $-b$ and $+c$; and this he exemplifies by geometrical constructions. See also pa. 313.

The arithmetic of these imaginary' quantities has not yet been generally agreed on; viz, as to the operations of multiplication, division, and involution; some autbors giving the results with + , and others on the contrary with the negative sign -. Thus, Euler, in his Algebra, pa. 106 \&c, makes the square of $\sqrt{ }-3$ to be -3 , of $\sqrt{ }-1$ to be $-1, \& c$; and yet he makes the product of two impossibles, when they are unequal, to be possible and real: as $\sqrt{ }-2 \times \sqrt{ }-3=\sqrt{ } 6$; and $\sqrt{ }-1 \times \sqrt{ }-4=\sqrt{ } 4$ or 2. But how can the cquality or inequality of the factors cause any difference in the signs of the products? If $\sqrt{ }-2 \times \sqrt{ }-3$ be $=\sqrt{ }+6$, how can $\sqrt{ }-3 \times \sqrt{ }-3$, which is the square of $\sqrt{ }-3$, be -3 ? Again, he makes $\sqrt{ }-3 \times \sqrt{ }+5=\sqrt{ }-15$. Also in division, he makes $\sqrt{ }-4 \div \sqrt{ }-1$ to be $=\sqrt{ }+4$ or 2 ; and $\sqrt{ }+3 \div \sqrt{ }-3$ $=\sqrt{ }-1$; also that 1 or $\sqrt{ }+1 \div \sqrt{ }-1=\sqrt{\frac{+1}{-1}}=$ $\sqrt{ }-1$; consequently, multiplying the quotient root $\sqrt{ }-1$ by the divisor $\sqrt{ }-1$, must give the dividend $\sqrt{ }+1$; and yet, by squaring, he makes the square of $\sqrt{ }-1$, or the product $\sqrt{ }-1 \times \sqrt{ }-1$, equal to -1 .

But Emerson makes the product of imaginaries to be imaginary; and for thiş reason, that " otherwise a real product would be raised from impossible factors, which is absurd. Thus, $\sqrt{ }-a \times \sqrt{ }-b=\sqrt{ }-a b$, and $\sqrt{ }-a$ $\times-\sqrt{ }-b=-\sqrt{ }-a b$, \&c. Also $\sqrt{ }-a \times \sqrt{ }-a$ $=-a$, and $\sqrt{ }-a \times-\sqrt{ }-a=+a, \& c . " A$ d thus most of the writers on this part of algebra are pretty equally divided, some making the product of impossibles real, and others imaginary. - in the Phil. Trans. for 1778 , pa. 318 \&c, Mr. Playtair lias given an ingenious dissertation on the Arithmetic of Impossible Quantitics. But this relates chiefly to their applications and uses, and not to the algorithm of them, or rules for their products, quotients, squares, \&c. From some operations however here performed, we learn that he makes the product of $\sqrt{ }-1$ by $\sqrt{ }-1$, or the square of $\sqrt{ }-1$, to be -1 ; and yet in another place he inakes the product of $\sqrt{ }-1$ and $\sqrt{ }\left(1-z^{2}\right)$ to be $\sqrt{ }\left(-1+z^{2}\right)$. This author concludes, "that imaginary expressions are never of use in investigations but when the subject is a property common to the measures both of ratios and of angles; that they never lead to any consequence which might not be drawn from the affinity between those measures; and that they are indeed no more than a particular method of tracing that affinity. The deductions into which they enter are thus reduced to an argument from analogy, but the force of them is not diminished on that account. 'The laws to which this analogy is subject; the cases in which it is perfect, in which it suffers certain alterations, and in which it is wholly interrupted, are capable of being precisely ascertained. Supported on so sure a foundation, the arithmetic of impossible quantities will always remain an useful instrument in the discovery of truth, and may be of service when a more rigid analysis can hardly be applied. For this reason, many researches concerning it, which in themselves might be deemed absurd, are nevertheless not destitute of utility. M. Bernoulli has found, for example, that if $r$ be the radius of a circle, the circumference is $=\frac{4 \log \cdot \sqrt{ }-1}{\sqrt{ }-1} r$. Considered as a quadrature of the circle, this imaginary theorem is wholly insignificant, and would deservedly pass lor an abuse of calculation; at the same time we learn from it, that if in any equation the quantity $\frac{\log \cdot \sqrt{ }-1}{\sqrt{-1}}$ should occur, it may be made to disappear, by the substitution of a circular arch, and a property, common to both the circle and hyperbola, may be obtained. The same is to be observed of the rules which have been invented for the transformation and reduction of impossible quantities * ; they facilitate the operations of this imaginary arithmetic, and thereby lead to the knowledge of the most bcautiful and extensive analogy which the doctrine of quantity has yet exhibited.

* The rules chiefly referred to, are those for reducing the impossible roots of an equation to the form $A+B \sqrt{ }-1$.

Imagina R Roots, of an equation, are those roots or values of the unknown quantity in an equation, which contain some imaginary quantity. So the roots of the equation $x x+a u=0$, are the two imaginary quantities $+\sqrt{ }-a a$ and $-\sqrt{ }-a a$, or $+a \sqrt{ }-1$ and $-a \sqrt{ }-1$; also the two roots of the equation $x x+a x+a a=0$, are the imaginary quantities $\pm \frac{1}{2} a-\frac{1}{2} a \sqrt{ }-3$; and the three roots of the equation $x^{3}-1=0$, or $x^{3}=1$, are 1 , $\frac{-1+\sqrt{ }-3}{2}$, and $\frac{-1-\sqrt{ }-3}{2}$; the first real, and the two latter imaginary. Sometimes the real root of an equation may be expressed by imaginary quantities; as in the irreducible case of cubic equations, when the root is expressed by Cardan's rule; and that happens whenever the equation has no imaginary roots at all; but when it has two imaginary roots, then the only real root is ex$4 \mathrm{R} 2$ 
pressed by that rule in an imaginary form. See my paper on Cubic Equations, in my Tracts, vol. 2, pa. 78 . Philos. Trans. for 1780 , pa. 406 \&c.

Albert Girard first treated expressly on the impossible oi imaginary roots of equations, and stated that every equation has as many roots, either real or imaginary, as the index of the highest power denotes. Thus, the roots of the biquadratic' equation $x^{4}=4 x-3$, are four, which he shows to be two real and two imaginary, viz, $1,1,-1+$ $\sqrt{ }-2$, and $-1-\sqrt{ }-2$; and he renders the relation general, between all the roots and the coefficients of the terms of the equation. See his Invention Nouvelle en l'Algebre, anno 1629 , theor. 2, pa. 40 \&c.

I. Dalembert demonstrated, that every imaginary root of any equation can always be reduced to the form $e+f \sqrt{ }-1$, where $e$ and $f$ are real quantities. And hence it was also shown, that if

one root of an equation be - $\quad-e+f \sqrt{ }-1$, another root of it will always be - $\quad e-f \sqrt{ }-1$ : and hence it appears that the number of the imaginary roots in any equation is always even, if any; $i$. e. either none, or else two', or four, or six, \&cc. Memoirs of the Academy of Berlin, 1746.

To discover how many impossible roots are contained in any proposed equation, Newton gave this rule, in his Algebra, viz, Constitute a series of fractions, whose denominators are the series of natural numbers $1,2,3,4,5$, $\& c$, continued to the number showing the index or exponent of the highest term of the equations, and their numerators the same series of numbers in the contrary order: and divide each of these fractions by that next before it, and place the resulting quotients over the immediate terms of the equation; then under each of the intermediate terms, if its square multiplied by the fraction over it, be greater than the product of the terms on each side of it, place the sign +; but if not, the sign-; and under the first aud last term place the sign + . Then will the equation commonly have as many imaginary roots as there are changes of the underwritten signs, from + to - , and from - to +. So for the equation $x^{3}-4 x^{2}+4 x-6=0$, the series of fractions is $\frac{3}{x}, \frac{2}{2}, \frac{1}{3}$; then the second divided by the first gives $\frac{2}{6}$ or $\frac{\pi}{3}$, and the third divided by the second gives $\frac{1}{3}$ also; hence these quotients placed over the intermediate terms, the whole will stand

$$
\text { thus, } x^{3}-4 x^{\frac{1}{3}}+4 x^{\frac{1}{3}}-6 .
$$

Now because the square of the $2 \mathrm{~d}$ term multiplied by its superscribed fraction, is $\frac{16}{3} x^{4}$, which is greater than $4 x^{4}$ the product of the two adjacent terms, therefore the sign ; is set below the $2 \mathrm{~d}$ term; and because the square of the $3 \mathrm{~d}$ term multiplied by its overwritten fraction, is $\frac{16}{3} x^{2}$, which is less than $24 \cdot x^{2}$ the product of the terms on each side of it, therefore the sign - is placed under that term; also the sign + is set under the first and last terms. Hence the two changes of the underwritten signs ++ -+ , the one from + to - , and the other from - to + , show that the given equation has two impossible roots. When two or more terms are wanting togetler, under the place of the Jst of the deficient terms write the sign under the $2 \mathrm{~d}$ the sign + , under the $3 \mathrm{~d}-$, and so on, always varying the signs, except that under the last of the deficient terms must always be set the sign + , when the adjacent terms on both sides of the deficient terms have contrary signs. $\Lambda$ s in the equation

$$
\begin{gathered}
x^{5}+a^{4} x+\div \div+a^{5}=0 \\
++-+\div+
\end{gathered}
$$

which has four imaginary roots; there being four changes in the signs as written. The autbor remarks, that this rule will sometimes fail of discovering all the impossible roots of an equation, for some equations may have more of such roots than can be found by this rule, though this seldom happens.

Mr. Maclaurin has given some account of this rule of Newton's, together with one of his own, which he says wilt never fail; and the same has also been done by Mr. Campbell; but none of them obtain universally. See Philos. Trans, vol. 34, pa. 104, and vol.35, pa.515. More accurate rules have been since given by Dr. Waring.

The real and imaginary roots of equations may be found from the method of fluxions, applied to the doctrine of maxima and minima, that is, to find such a value of $x$ in an equation, expressing the nature of a curve, made equal to $y$, an abscissa which corresponds to the greatest and least ordinate. But when the equation is above 3 dimensions, the computation is very laborious.- See Stirling's treatise on the lines of the $3 \mathrm{~d}$ order, Schol. pr. 8, pa. 59 \& c.

IMBIBE, is commonly used in the same sense as absorb, viz, where a dry porous body takes up another tbat is moist.

IMIMENSE, that whose amplitude or extension cannot be equalled by any measure whatever, or how often soever repeated.

IMMERSION, the act of plunging into water, or some other fluid.

In mension, in Astronomy, is when a star, or planet comes so near the sun, that it cannot be seen; being as it were enveloped, and bid in the rays of that luminary.

INMERSION also denotes the beginning of an eclipse, or of an occultation, when the body, or any part of it just begins to disappear, either behind the edge of another body, or in its shadow. As, in an eclipse of the moon, when she begins to be darkencd by entering into the shadow of the earth: or the beginning of an eclipse of the sun, when the moon's dise just begins to cover him : or the beginning of the eclipses of any of the satellites, as those of Jupiter, by entering into his shadow : or, lastly, the beginning of an occultation of any star or planet, by passing behind the body of the moon or anotber planet. It all these cases, the darkened body is said to immerge, or to be immerged, or begin to be hid, by entering into the shule. In like manner, when the darkened body begins to appear again, it is said to cmerge, or come out of darkness again.

IMPACT, the simple or single action of one body upon another to put it in motion. Point of impact, is the place or point where a body acts.-Impact is either direct or oblipue: if the borly struck moves in the direction of the stroke, the impact is said to be direct; but if not, it is called an oblique impact.

INIPENETRABILITY, the faculty which a body has to exclude every other body from the place that it occupies: in such manner that two bodies, placed in contact, can never occupy less 'space than that which they filled when they were separate.

Impenetrability, as it respects solid bodies, does not require to be jroved, it is at once obvious; but fluids baving their molccule moveable in all dircetions, and yiclding to every impression exerted on them, their inpenetrability does not manifest itsclf so perceptibly as that 
of solid bodies. Taking the air for an example : it is evident that so long as this fluid is not enclosed, its facility of motion causes it to admit an easy and free passage to all bodies that are urged through it ; and in this case.it cannot be said to be penetrated, but displaced; for, if the air be included within the sides of a vessel, and another body be then presented to take its place, without suffering it to escape, it will exercise its impenetrability in the same manner as solid bodies. This may be sufficiently evinced by the following very easy and simple experiment: Let a vessel be vertically detruded into another vessel filled with water to a'certain height, with its orifice downwards : the surface of the water corresponding with the orifice of the first vessel, is depressed as this vessel itself descends; and this depression may be rendered more sensible by ineans of a little plate or slip of cork, placed so as to float on the surface of the water: nevertheless, this water is not excluded by the air which occupies the immersed vessel; it is always raised within it by a certain quantity which increases as the vessel is immersed to a greater depth, similar to what is observed in the diving-bell; but it is evident that this ascension of the fluid is occasioned by the circumstance that the air is a compressible fluid, and not from any penetration of the water into the bulk of air in the vessel; and therefore its volume is only contracted intor a snaller space by a superior upward pressure of the surrounding water in virtue of its weight.

We must here notice a difficulty which appears to result from this, that when certain bodies are mingled together, the volume of the mixture is less than the sum of the volumes taken separately. This happens, for example, in the mixture of equal parts of alcohol and water; the same also obtains when copper by fusion is mingled with zinc, in order to form the compound metal called brass : it is then observed that the density of the mixture is augmented by about its tenth part. This apparent penetration is owing to the circumstance, that the moleculæ of the two bodies, in consequence of their respective formation, have a stronger attraction than in the two bodies taken separately; there bence results in the figure of the pores such a change as diminishes the space equal to the sum of these pores. On the contrary, in the alloy of silver with copper, a hind of rarefraction is produced, such that the volume of the inixture is somewhat greater than the sum of the capacities of the two bodies previous to fusion.

IMPENETRABLE, that cannot be penetrated.

IMPERFECT Number, is that whose aliquot parts, taken all together, do not make a sum that is equal to the number itself, but either exceed it, or fall short of it ; being an abundant number in the-former case, and a defective number in the latter. Thus, 12 is in abundant imperfect number, because the sum of all its aliquot parts, $1,2,3,4,6$, is 16 , which exceeds the number 12. And 10 is a defective imperfect number, because its aliquot parts, $1,2,5$, taken all together, make only 8 , which is less than the number 10 itself.

IMPERIAL Table, is an instrument made of brass, with a box and needle, and staff, \&c, used for measuring of land.

IMPERVIOUS, not to be pervaded or entered either because of the closeness of the pores, or the particular configuration of its parts. \&c.

IMPETUS, in Mecbanics, force, momentum, motion,
IMPETUs, in Gunnery, is the altitude due to the first force of projection, or the altitude through which a body by falling acquires that velocity. So that, if the velocity be $v$, the impetus or altitude will be $a=v^{2} \div 64 \frac{1}{3}$ fcet.

INPOSSIBLE 2uantity, or Root, the same as

IMAGINARY ones; which see.

Impossible Binomial; see Binomial.

IMPOST, in Architecture, a capital or plinth, to a pillar, or pilaster, or pier, that supports an arch, \&c.

IMPROPER Fraction, is a fraction whose numerator is either equal to, or greater than, its denominator. As $\frac{5}{5}$ or $\frac{5}{3}$ or $\frac{19}{6}$. An improper fraction is reduced to a whole or mixt number, by dividing the numerator by the denominator; the quotient is the integer, and the remainder set over the divisor makes the fractional part, of the value of the original improper fraction. Thus $\frac{5}{5}=1$, and $\frac{5}{3}=1 \frac{2}{3}$, and $\frac{19}{6}=3 \frac{x}{6}$. So that, when the numerator is just equal to the demominator, the improper fraction is exactly equal to unity or 1 ; but when the numerator is the greater, the fraction is greater than 1.

IMPULSE, the single or momentary action or force by which a body is impelled; in contradistinction to continued forces; like the blow of a hammer.

1MPULSIVE, a term applied to actions by impulse.

INACCESSIBLE Height or Distance, is that which cannot be approached, or measured by actual measurement, by reason of some impediment; as water, \&c.-See Heights and Distances.

INCEPTIVE, of Magnitude, a term used by Dr. Wallis, to express such moments, or first principles, as, though of no magnitude themselves, are yet capable of producing such as are. See Infinite, and Indivisible. Thus, a point has no magnitude, but is inceptive of a line, which it produces by its motion. Also a line, though it has not breadth, is yet inceptive of breadth ; that is, it is capable, by its motion, of producing a surface, which has breadth.

INCH, a common English measure, being the 12th part of a foot, or 3 barley-corns in length.

INCIDENCE, or Line of INCIDENCE, in Mechanics, implies the direction or inclination in which one body strikes or acts on another.-In the incursions of two moving bodies, their incidence is said to be direct or oblique, as the directions of their motion make a straight line, or an angle at the point of impact.

Angle of Incridence, by some writers, denotes the angle comprehended between the line of incidence, and a perpendicular to the body acted on at the point of incidence. Thus, suppose $A B$ an incident line, and $\mathrm{BF}$ a perpendicular to

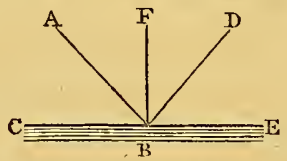
the plane $C B$ at the incident point $B$; then $A R F$ is the angle of incidence, or of inclination.

But, according to Dr. Barrow, and some other writers, the angle of incidence is the complement of the former, or the angle made between the incident line, and the plane acted on, or a tangent at the point of incidence : as the angle $\mathrm{ABC}$.

It is demunstrated by optical writers, 1st, That the angle of incidence of the rays of light, is alsvays equal to the angle of reflection; and that they lie in the same plane. And the same is proved by the writers on mechanics, concerning the reflection of elastic bodies. That is, the $\angle A B F=$ the $\angle F B D$, or the $\angle A B C=$ the $\angle D B E$. - 
2d, That the sines of the angles of incidence and refraction are to each other, either accurately, or very nearly, in a given or constant ratio. - 3dly, That from air into glass, the sine of the angle of incidence is to the sine of the angle of refraction, as 300 to 1.93 , or nearly as 14 to 9 : and, on the other hand, that out of glass into air, the sine ol the angle of incidence, is to the sine of the angle of refraction, as 193 to 300 , or as 9 to 14 nearly.

INCIDENCE of Ectipse. Sce Ecui PSE, and IM Mersion. Aris of IXCIDENCE, is the line FB perpendicular to the reflecting plane at the point of incidence $\mathrm{B}$.

Cathetus of IxCIDENCE. SeeCATHETUS, and ReFLeCTION.

Line of lNCIDENCE, in Catoptrics, denotes a right line, as $\mathbf{A B}$, in which light is propagated from a radiant point $\mathbf{A}$, to a point $\mathrm{B}$, in the surface of a speculum. The same line is also called an incident ray.

Line of INCIDENCE, in Dioptrics, is a right line, as $A B$, in which light is propagated unrefracted, in the same medium, from the radiant point to the surface of the refracting body, CBE.

Point of $1 \mathrm{NCIDLNCE}$, is the point B on the surface of the reflecting or refracting medium, on which the incident ray falls.

Scruples of Incidence. See Scruples.

INCIDENT Ray, is the line or ray $A B$, falling on the surface of any body, at $B$.

INCLINATION, in Geometry, Mechanics, or Physics, denotes the mútual tendency of two lines, planes, or bodies, towards each other; so that their djrections make at the point of concourse some certain angle.

InCLINATION of the Axis of the Earth, is the angle it makes with the plane of the ecliptic ; or the angle between the planes of the equator and ecliptic.

INCLINATION of a Line to a Plane, is the acute angle, as CDE, which the line $C D$ makes with another line $\mathrm{DE}$ drawn in the plane through the incident point $D$ and the foot of a perpendicular-E, from any point of the line, upon the plane.

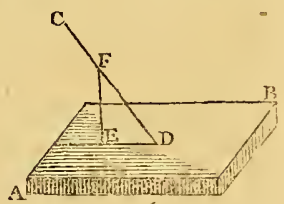

INCLI N A Tion of un Incident Ray, is the angle of inclination, or angle of incidence.

Inclination of the Magnetical Needle. Sce Dipping Needle.

IńCLINATION of Meridians, in Dialling, is the angle that the hour-line on the globe, which is perpendicular to the dial-plane, makes with the meridian.

InCLINatiun of the Orbit of a Planet, is the angle formed by the planes of the ecliptic and of the orbit of the planet. 'The quantity of this inclination for the several planets, is as follows, viz.

$\begin{array}{lllrrr}\text { Mercury } & - & - & 6^{\circ} & 54^{\prime} & 0^{\prime \prime} \\ \text { Venus } & - & - & 3 & 23 & 35 \\ \text { Earth } & - & - & 0 & 0 & 0 \\ \text { Moon } & - & - & 5 & 18 & 0 \\ \text { Mars } & - & - & 1 & 51 & 0 \\ \text { Jupiter } & - & - & 1 & 18 & 56 \\ \text { Saturn } & - & - & 2 & 29 & 50 \\ \text { Herschel } & - & - & 0 & 46 & 20\end{array}$

Incunarion of a Plane, in Dialling, is the arch of a vertical circle, perpendicular both to the plane and the horizon, and intercepted between them.

InCLINATION of a Planet, is the arch or angle com- prehended between the ecliptic and the place of the planet in its orbit. The greatest inclination, or declination, is the same as the inclination of the orbit; which see above.

Inclixation of a Reflected Ray, is the angle which a ray after reflection makes with the axis of inclination; as the angle $F B D$, in the last fig. lut one.

Inclination of Two Planes, is the angle made by two lines drawn in those planes perpendicnlar to their coinmon intersection, and meeting in any point of that intersection.

Angtc of lnclination, is the same as what is otherwise called the angle of incidence.

Argument of InCLIMATION. See Allgument.

INCLINATIONS (Problem of ), is one of the last works of Apollonius, described by Pappus, as consisting of two books. Of these, restorations have been attempted by several of the moderns, with various success. 1st, By Ghetaldus, in two books entitled Apollonius Redivivus, 4to, Venice, 1607 \& 1613.-2d, Alex. Anderson, Supplem. Apol. Rediv. Inclin. Paris, 1612, \& 1615, 4to.-3d, Horsley, Apol. Inclin. lib. 2, Oxon, 1770, 4to.-4th, Reuben Burrow published an English edition in 1779,4 to.

INCLINED Plune, in Mlechanics, is a plane inclined to the horizon, or making an angle with it. It is one of the simple mechanic powers, and the double inclined plane makes the wedge.

1. The power gained by the inclined plane, is in proportion as the length of the plane is to its beight, or as radius to the sine of its inclination; that is, a given weight hanging frecly, wiil balance upon tbe plane another weight, that shall be greater in that proportion. So, when the greater weiglit $w$ on the plane, is balanced by the less weight $w$, hanging perpendicularly, then is $w: \mathrm{w}:: \mathrm{BC}$ : $A C:: \sin . \angle \Lambda$ : radius. $O_{l}$, in other words, the relative gravity of a boty upon the plane, or its force in descending down the plane, is to its ab-

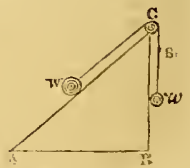
solute gravity or weight, in the same proportion of the height of the plane to its length, or of the sine of inclination to radius.

2. Hence therefore the relative gravities of the same body, on different inclined planes, or their forces, to descend down the planes, are to each other, as the sines of the anglés of inclination, to radius $I$, or directly as the lieights of the planes, and inversely as their lengths.

3. Hence, if the planes have the same height, and the absolute weiglits of the bodies be directly proportional to the lengths of the planes, then the forces to desccnd will be equal. Consequently, if the bodies be then con-

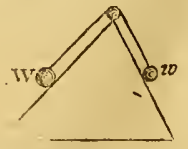
nected by a string acting parallel to the planes, they will exactly balance each other; as in the annexed figurc.

4. The relative force of gravity on the plane being in a constant ratio to the absolute weight of the body, viz, as sine of inclination to radius; therefore all the laws relating to the perpendicular free descents of bodics by gravily, hold equally true for the descents on inclined planes; sucli as, that the motion is a uniformly accelerated one; that the velocities are dircetly as the times, and the spaces as the square of either of them; using only the relative force upon the plane for the absolute weight of the body, or instead of $32 \frac{1}{6}$ fect, the velocity generated by gravity in the first second of time, using $32 \frac{\mathrm{T}}{6} \mathrm{~s}$, where $s$ is the sine of the inclination to the radius 1 .

5. The velocity acquired by a body in descending 
I $\mathrm{N} \mathrm{C}$

down an inclined plane $\mathrm{Ac}$, when the body arrives at $A$, is the same as the velocity acquired by descending freely down the perpendicular altitude $\mathbf{\text { в }}$, when it arrives at $\mathbf{B}$. But the times are very different; for the time of descending down the inclined plane, is greater than down the perpendicular, in the sane proportion

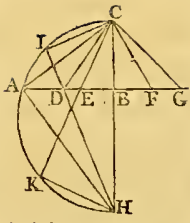
as the length of the plane $\mathrm{AC}$, is to the height $\mathrm{cв}$ : and so the time of descending from any point $\mathrm{c}$ to a horizontal line or plane $A B G$ \& $c$, down any oblique line, or inclined plane, is directly proportional to the length of that plane, $\mathrm{CA}$, or $\mathrm{CD}$, or $\mathrm{CE}$, or $\mathrm{CB}$, or $\mathrm{CP}$, \& $\mathrm{C}$.

6. Hence, if there be drawn $A \mathrm{H}$ perpendicular to $\mathrm{AC}$, meeting $\mathrm{CB}$ prorluced in $\mathrm{H}$; then the time of descending down any plane $\mathrm{C} A$, is equal to the time of descending down the perpendicular cir. So that, if upon cri, as a diameter, a circle be described, the times of descent will be exactly equal, down every chord in the circle, beginning at $\mathrm{c}$, and terminating any where in the circumference, as $\mathrm{CI}, \mathrm{CA}, \mathrm{CK}, \mathrm{CH}, \& \mathrm{c}$, or beginning any where in the circumference, and ending at the lowest point of the circle, as $\mathrm{CH}, \mathrm{IH}, \mathrm{All}, \mathrm{KH}$, Src. ·

7. When bodies ascend inclined planes, their motion is uniformly retarded; and all the former laws for descents, or the generation of motion, hold equally true for ascents, or the destruction of the same.

INCLINED Towers, are towers inclined, or leaning from the perpendicular, as those of Pisa or Bologna in Italy. See Towers.

INCLINERS, are inclined dials. See Diat.

INCOMMENSURABLE, Lines, or Numbers, or Quantities in general, are sucb as have no common measure, or no line, number, or quantity of the same kind, that will measure or divide them both without a remainder. Thus, the numbers 15 and 16 are incommensurable, because, though 15 can be measured by 3 and 5 , and 16 by 2,4 , and 8 , there is yet no single number that will divide or measure them both. - Euclid demonstrates (prop. 117, lib. 10) that the side of a square and its diagonal are incommensurable to each other. And Pappus, prop. 17, lib. 4, speaks of incommensurable angles.

Incommensurable in Power, is said of quantities whose $2 \mathrm{~d}$ powers, or squares, are incommensurable. $A \mathrm{~s}$ $\sqrt{ } 2$ and $\sqrt{ } 3$, whose squares are 2 and 3 , which are incominensurable. It is commonly supposed that the diameter and circumference of a circle are incommensurable to each other; at least their commensurability has never been proved. And Dr. Barrow surmises even that they are infinitely incommensurable, or that all possible puwers of them are incommensurable.

INCOMPOSITE Numbers, are the same with those called by Euclid prime numbers, being such as are not composed by the multiplication together of other numbers. As $3,5,7,11$, \&c.

INCOMI'R LSSIBLE, incapable of being squeezed into less room.

INCOMPRESSIBILITY, incapacity to be compressed into less room.-During a long period, water was considered as a fluid perfectly unelastic, that is, incompressible or unyickding: and this opinion was corroborated by an experiment of the Academy del Cimento in Italy. By that experiment, the members of the academy endeavoured to ascertain whether water was capable of being com-
I $\mathrm{N} \mathrm{C}$

pressed in any degrce. For this purpose, they filled a hollow metallic globe with that fluid, and closed the orifice very accurately. This ball was then forcibly pressed in a proper machine; but no contraction could be observed, neither indeed was the apparatus capable of showing small degrees of compression: hence however they concluded that water was not capable of compression. This opinion prevailed till the year 1761, when Mr. Canton discovered the compressibility of water, and of other liquids. He took a glass tube, having a hollow ball at one end, but open at top; he filled the ball and part of the tube with water, which he had deprived of air as much as possible; he then placed the glass thus filled under the receiver of an air-pump; and on exhausting the receiver, which removed the pressure of the atmospliere from over the water and the glass vessel, the consequence was, that the water rose a little way in the tube, that is, it expanded itself. He then placed the apparatus under the receiver of a condensing engine, and on forcing the air into it, which inereased the pressure on the water, a diminution of bulk visibly took place, the water descending a little in the tube. "In this manner," says Mr. Canton, "I have found by repeated trials, when the heat of the air has been about $50^{\circ}$, and the mercury at a mean height in the barometer, that the water will expand and rise in the tube, by removing the weight of the atmosphere, one part in 21740 , and will be as much compressed under the weight of an additional atmosphere. Therefore the compression of water by twice the weight of the atmosphere, is one part in 10870. Water has also the remarkable property of being more compressible in winter than in sum. 'mer, which is contrary to what I have observed both in spirits of wine and oil of olives." By the same means, and in the same circumstances, Mr. Canton ascertained compressibility in some other fluids, the results of which are as below : viz,

\begin{tabular}{lccc} 
Compression of & & \multicolumn{3}{c}{ Millionth parts. } \\
Spirit of wine & - & - & 66 \\
Oil of olives & - & - & 48 \\
Rain-water & - & - & 46 \\
Sea-water & - & - & 40 \\
Mercury & - & - & 3
\end{tabular}

INCRLMENT, is the small increase of a variable quantity. Newton, in his Treatise on Fluxions, calls these- by the name Moments, and observes that they are proportional to the velocity or rate of increase of the flowing or variable quantities, in an indefinitely small time; he denotes them by subjoining a cipher 0 , to the varying quantity whose moment or increment it is ; thus $x 0$ is the moment of $x$. In the doctrine of increments, by Dr. Broolic: Taylor and Mr. Emerson, they are denoted by points bekw the variable quantities; 'as $x$ Some have also represented them by accents underneath the letter, as $x$; but it is now more usual to express them by accents over the same letter; as $x^{\prime}$.

INCREMENTS, Method of, a branch of Analytics, in which a calculus is founded on the properties of the successive values of variable quantities, and their differences, on increments. The inventor of this method was the learned Dr. Taylor, who, in the year 1715 , published a treatise upnn it; and aftcrwards gave some further ac. count and explication of it in the P'hilos. Trans. as applied to the finding the sums of serics. Another ingenious and easy treatise on the same, was published by Mr. Lmerson, in the year 1763 . The method is nearly allied to New- 


\section{N C}

ton's doctrine of fluxions, and arises out of it. Also the differential method of Mr. Stirling, which he applies to the summation and interpolation of series, is of the same nature as the method of increments, but not so general and extensive.

From the method of increments, Mr. Emerson observes, " the principal foundation of the method of fluxions may be easily derived. For, as in the method of increments, the increment may be of any magnitude, so in the method of fluxions, it mast be supposed infinitely small; whence all preceding and successive valucs of the variable quantity will be equal, from which equality the rules for performing the principal operations of fluxions are immediately deduced. That I may give the reader," continues he, "a more perfect idea of the nature of this method: suppose the abscissa of a curve to be divicled into any number of equal parts, each part of which is called the increment of the abscissa; and imagine so many parallclograms to be erected thereon; either circumscribing the curvilineal figure, or inscribed in it; then the finding the sum of all these parallelograms belongs to the method of increments. But if the parts of the abscissa be taken infinitely small, then these parallelograms degenerate into the curve; and then it becomes the method of fluxions, to find the sum of all, or the area of the curve. So that the method of increments finds the sum of any number of finite quantities; and the method of fluxions the sum of any infinite number of infinitely small ones: and this is the essential difference betweers these two methods." Again, "There is such a near rclation between the method of fluxions, and that of increments, that many of the rules for the one, with little variation, serve alșo for the other. And here, as in the method of fluxions, some questions may he solved, and the integrals found, in finite terms; while in others we areforced to have recourse to infinite series for a solution. And the like difficulties will occur in the method of increments, as usually happen in fuxions. For while some fuxionary quantities have no fluents, but what are expressed by series; so also some increments have no integrals, but what infinite series afford; which will often, as in fluxions, diverge and become useless."

By means of the method of increments, many curious and useful problems are casily resolved, which scarcely admit of a solution in any other way. As, suppose several series of quantities be given, whose terms are all formed according to some certain law, which is also given; the method of increments will determine a general series, which comprehends all particular cases, and from, which all of that hind may be found. The method of increments is also of great use in finding any term of a series proposed; having the law given by which the terms are formed; which will cither be expressed in finite quantitics, or by an infinite series. Another application of the method of increments, is to find the summation of series, which it will often effect in finite terms. And when the sum of a serics cannot be had in finite terms, we must have recourse to infinite serics; for the integral being expressed by such a series, the sum of a competent number of its terms will give the sum of the serjes required. This is equivalent to transforming one serics into another, converging quicker : and sometimes a very few terms of this series will give the sum of the series sought.

Definitions in the Mcthod of Increments.

1. When a quantity is considered as increasing, or decreasing, by certain steps or degrees, it is called an Inte- gral.-2. The increase of any quantity from its present value, to the next succeeding valuc, is called an increment: or, if it decrcases, a decrement.-3. The increase of any increment, is the second increment; and the increase of the $2 \mathrm{~d}$ increment, is the $3 \mathrm{~d}$ increment; and so on. -4. Succeeding values, are the several values of the integral, succeeding each other in regular order, from the present value; and preceding vąlues, are such as arise before the present value. All these arc called by the general term Factors.-5. A perfect quantity is such as contains any number of successive values without intermission; and a defective quantity, is that which wants some of the successive values. Thus $x x x$ is a perfect quantity; and $x x x$, an imperfect or defective one.

$$
245
$$

Notation. This, according to Mr. Emersori's method, is as follows; 1 . Simple integral quantities are denoted by any letters whatever, as $z, y, x, u, \& c .-2$. The several values of a simple integral, are denoted by the same letter with small figures under them: so if $z$ be an jutegral, then $z, z, z, z, \& c$, are the present values, and the $1 \mathrm{st}$, $2 \mathrm{~d}, 3 \mathrm{~d},{ }^{-1} \& \mathrm{c},{ }^{2}, \mathbf{s u c c s s i v e}^{3}$ valucs of it; and the preceding values are denoted by figures with negative signs, thus $z, z, z, z$, are the 1 st, $2 \mathrm{~d}, 3 \mathrm{~d}$, 4 th preceding values; and $-1-2-3--4$

the figure denoting any value, is the characteristic.

3. The increments are denoted with the same letters, and points under them: thus, $x$ is the increment of $x$, and $z$ is the increment of $z$. Also $x$ is the increment of $x$; and $x$ of $x, \& c$.

4. "The $2 \mathrm{~d}, 3 \mathrm{~d}$, and other increments, arc denoted with two, three, or more points : so $z$ is the $2 d$ increment of $z$, and $z$ is the $3 \mathrm{~d}$ increment of $z$, and so onl. And these are denominated increments of such an order, according to the number of points.

5. If $x$ be any increment, then [x] is the integral of it; also ${ }^{2}[x]$ denotes the integral of $[x]$, or the $2 \mathrm{~d}$ integral of $x$; and ${ }^{3}[x]$ is the $3 \mathrm{~d}$ integral of $x$, or an integral of the $3 \mathrm{~d}$ order, \&c.

6. Quantities written thus, $x \ldots x$ mean the same as $x \times x \times x$, or signify that the quantitics are continued from the first to the last, without break or interruption.

To find the Increment of any integral, or variable quantity.

Rule 1 . If the proposed quantity be not fractional, and be a perfect integral, consisting of the successive values of the variable quantity, which increases uniformly: Multiply the proposed integral by the number of factors, and cliange the lowcst factor for an increment. So the increment of $a-3 x+6 z$ is $-3 x+6 z$; for the increment of the constant quantity $a$ is 0 or nothing. So likewise,

The incrcment of $c x x \times x$, is $4 c_{x} \times x \times$.

The increment of $a x x_{x}^{1} x^{2}$, is $3 a x x^{\prime} x^{2}$.

The increment of $a x-2-2$, is $3 a x-1$

The increment of $x^{-3-2-1}$, is $(m+n+1) \cdot x x \ldots x$. $-m=1-m+1$ n

Rule 2. In fractional quantities, where the denominator is perfect, and the variable quantity increases uniformly. Multiply the proposed integral by the number of factors, and by the constant increment with a negative sign, and take the next succeeding value into the denominator. Thus,

The increment of $\frac{a}{x \ldots x}$, is $\frac{-5 a x}{x \ldots x}$. 


\section{I $\mathrm{N} \mathrm{C}$}

The increment of $\frac{c}{x \ldots x x}$, is $\frac{-9 c x}{x \ldots x}$.

Rule 3. The increment of any power, as $x^{\mathrm{n}}$ is $(x+x)^{n}-x^{n}$; that is, the difference between the present value $x^{\mathrm{n}}$ and the next succeeding value $(x+x)^{\mathrm{n}}$. And generally, the increment of any quantity whitever, is found by subtracting the present value, or the given quantity, froin its next succeeding value.' Also by expanding the compound quantity in a series, and subtracting $x^{n}$ from it, the increment will be either

$=n \dot{x}^{n-1} x+\frac{n . n-1}{2} x^{n-2} x^{2}+\frac{n 2 . n-1 . n-2}{2.3} x^{\mathrm{n}-3} x^{3} \& \mathrm{c}$, or

$=x^{n} \times: \frac{n x}{x}+\frac{n \cdot n-1}{2} \cdot \frac{x^{2}}{x^{3}}+\frac{n \cdot n-1 \cdot n-2}{2 \cdot 3} \cdot \frac{x^{3}}{x^{3}} \& \mathrm{c}$.

So the increment of $x^{4}$, is $(x+x)^{1}-x^{4}=$ $4 x^{3} x+6 x^{2} x^{2}+4 x x^{3}+x^{4}$.

The increment of $\frac{1}{x^{3}}$ or $x^{-3}$,

is $\frac{1}{(x+x)^{3}}-\frac{1}{x^{3}}=(x+x)^{-3}-x^{-3}$

$=-3 x^{-4} x+6 x^{-5} x^{2}-10 x^{-6} x^{3} \& \mathrm{c}$

$=-\frac{3 x}{x^{3}}+\frac{6 x^{2}}{x^{5}}-\frac{10 x^{3}}{x^{5}} \& \mathrm{c}$.

The increment of $a^{\mathrm{x}}, a$ being constant, is $a^{x}-a^{x}=a^{x+x}-a^{x}=a^{x} \cdot\left(a^{x}-1\right)$.

The increment of $\frac{2}{a^{x}}$ is $\frac{1}{a_{1}^{x}}-\frac{1}{a^{x}}$

$=\frac{a^{x}-a^{x}}{a^{x} a^{\mathrm{x}}}=\frac{a^{\mathrm{x}}-a^{x}+x}{a^{\mathrm{x}}} \frac{x}{a^{x}+\not x}=\frac{1-a^{x}}{a^{x}+\underline{x}}$.

The increment of $x z$ is $x z-x z=$

$(x+x) \cdot(z+z)-x z \stackrel{11}{=} z x+x z+x z$.

And so on for any form of integral whatever, subtracting the given quantity from its next succeeding value. So,

The iucrement of the $\log$. of $x$ is $\log . x-\log . x$

$=\log \cdot x+x-\log \cdot x=\log \cdot \frac{x+x}{x}$, which, by the nature of logarithms, is $\frac{x}{x}-\frac{x^{3}}{2 x^{3}}+\frac{x^{3}}{3 \cdot x^{3}}-\frac{x^{4}}{4 \cdot x^{4}} \& \mathrm{c}$.

Schol. Hence may be deduced the principles and rules of fluxions; fur the method of fluxions is only a particular case of the methed of increments, fluxions being infinitely small increments; therefore, if in any form of increw ments, the increment be taken infinitely small, the form or expression will be clanges inta a fluxional one. Thus, in $z x+x z+x z$, which is the increment of the rectangle $x \approx$, if $x$ and $z$ be changed for $\dot{x}$ and $\dot{z}$, the expression will become $z \dot{x}+x \dot{z}+\dot{x} \dot{z}$ for the fluxion of $x z$, or only $z \dot{x}+x \dot{z}$, because $\dot{x} \dot{z}$ is infinitely less than the rest.

So likewise, if $x$ be changed for $\dot{x}$ in this

$n x^{n-1} x+\frac{n \cdot n-1}{2} x^{n-2} x^{2}+\frac{n \cdot n-1 \cdot n \cdot-2}{2 \cdot 3} x^{n-3} x^{3}$

$\& \mathrm{c}$, which is the increment of $x^{\mathrm{n}}$, it becomes

$n x^{n-1} \dot{x}+\frac{n \cdot n-1}{2} x^{n-2} \dot{x}^{2}+\frac{n \cdot n-1 \cdot n-2}{2 \cdot 3} x^{n-3} \dot{x}^{3}$

$\& c$, or $n x^{n-1} \dot{x}$, for the fluxion of the power $x^{n}$, as all the ternis after the first will be nothing, because $\dot{x}^{2}$ and $\dot{x}^{3} \& c$ are infinitely less than $\dot{x}$. And thus may all the other forms of fluxions be derived from the corresponding increments. And in like manner, the finding of the integrals, is only a more general way of finding fluents, as appears in what fullows.

Vol. I.

\section{N C}

To find the Integral of any given Increment.

Rule 1 . When the variable quantity increases uniform$\mathrm{J}_{y}$, and the proposed integral cousists of the successive values of it multiplied together, or is a perfect increment not fractional: Miltiply the given increment by the next preceding value of the variable quantity, then divide by the new number of factors, and by the constant increment.

Example. Thus, the integral of $4 c x x x x$ is cxxxx.

The integral of $3 a x x \quad x$ is $a x x \quad x$.

Rule 2. In a fractional expression, where the variable quantity increases uniformly, and the denominator is perfect, containing the successive values of the variable quantity: Expunge the greatest value of the variable letter, then divide by the new number of factors, and by the constant increment with a negative sign. So,

The integral of $\frac{a x}{x \ldots x}$ is $\frac{-a}{5 x \cdots x}$.

The integral of $\frac{-c x^{5}}{x \cdots x^{2}}$ is $\frac{c x^{4}}{x \ldots \cdot x^{5}}$.

Rule 3. Varions other particular rules are given, but these and the two foregoing are all best included in the following general table of the most useful forms of increments and integrals, to be used in the same way as the similar table of fuxions and fluents, to which these correspond.

\begin{tabular}{|c|c|c|}
\hline Forms. & Increments. & Integrals. \\
\hline 1 & $\begin{array}{l}x \text { when constant } \\
\text { not constánt }\end{array}$ & $\begin{array}{l}x, \text { or } x \text {, or } x, \text { or } x \& c . \\
x \text { only. }\end{array}$ \\
\hline 2 & 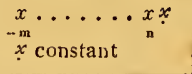 & $\frac{\underset{m-1}{x \ldots \ldots}{ }_{n}^{x}}{m+n}$ \\
\hline 3 & $\frac{a x}{x \ldots \ldots x}$ & $-a$ \\
\hline 3 & $x$ constant & $(m+n-2) \underset{-m}{x} \ldots \ldots x$ \\
\hline 4 & $z x+x z$ & $x z$ \\
\hline 5 & $\frac{z x-x z}{\substack{z z \\
1}}$ & $\frac{x}{z}$ \\
\hline 6 & $\begin{array}{l}a^{\mathrm{x}} \\
x \text { given }\end{array}$ & $\frac{a^{x}}{a^{x}-1}$ \\
\hline
\end{tabular}

Integrals, when found from given increments, are corrected in the very same way as tluents, when found from given fluxions, viz, instead of each several variable quantity in the integral, substituting such a determinate value of them as they are known to have in sume particular case; and then subtracting each side of the resulting equation from the corresponding side of the integral, the remaining equation will be the correct state of the integrals.

For an example of the use of the method of increments, suppose it were required to find the sum of any number of terms of the scries $1.2+2.3+3.4 \& \mathrm{c}$,

Let $x$ be the number of the terms, and $z$ the sum of them. 
Then, by the progression of the series, the last, or the $x$ term, is $x x$, and the next term after that will be $x x$, that is $z=x \dot{x}$, where $x=1$. Hence the integral is $z=\frac{1}{3} x^{2} x=\frac{x}{3} x \cdot x+1 \cdot x+2$, which is the sum of $x$ terms of the given series. So if the number of terms $x$ be 10 , this becomes $\frac{1}{3} \cdot 10 \cdot 11 \cdot 12=440$, which is the sum of 10 terms of the given series $1.2+2.3+3.4 \mathrm{~d} c$. Or, when $x=100$, the sum of 100 terms of the same series is $\frac{1}{3}, 100 \cdot 101.102=100 \cdot 101 \cdot 34=343400$.

Again, to find the sum $z$ of $n$ terms of the series

$\frac{1}{1.3 .5}+\frac{1}{3.5 .7}+\frac{1}{5.7 .9}$ \&c.

Here the $n$th term is $\frac{1}{2 n-1.2 n+1.2 n+3}$.

Put $x=2 n-1 ;$ then is $x=2 n=2$, and the $n$th term is $\frac{1}{x, x+2 \cdot x+4}=\frac{1}{x x x}$; and the $n+1$ th term or $\approx$ is $\frac{1}{x x x}$; the general integral of which is $z=\frac{-1}{a x x x}=\frac{-1}{4 x x}$. But this wants a correction; for when $n=0$, then $x=-1$, and the sum $z=0$, and the integral becomes $z$ or $0=\frac{-1}{4.1 .3}=\frac{-1}{12}$; that is $-\frac{1}{12}$ is the correction, which being subtracted, the corrcet state of the integrals becomes $z=\frac{1}{12}-\frac{1}{4 x .5}=\frac{1}{12}-\frac{1}{4 \cdot 2 n+1 \cdot 2 n+3}$, which is the sum of $n$ terms of the proposed series. And when $n$ is infinite, the latter fraction is nothing, and the sum of the infinite series, or the infinite number of the terms, is accurately $\frac{1}{12}$.

When $n=100$, the sum of 100 terms of the serics becomes $z=\frac{1}{12}-\frac{1}{4.201 .203}=\frac{1}{12}-\frac{1}{163.212}$.

For more ample information and application on this science, see Emerson's Incremeuts, Taylor's Methodus Incrementoruin, and Stirling's Summatio et Interpolatio Scrierum.

INCURVATION of the Rays of Light. See LIGnt, and REFRACTION.

INDEFINITE, Indeterminate, that which has no certain bounds, or to which the human mind cantrot affix any. Descartes uses the word, in his Philosophy, instead of infinite, both in numbers and quantities, to signify an inconceivable number, or number so great, that an unit cannot be added to it; and a quantity so great, as not to be capable of any addition. 'Thus, he says, the sturs, visib) and invisible, are in number indefinite, and not, as the ancients held, infinite; and that quantity may be divided into an indefinite number of parts, not an infinite number.-Indefinite is now commonly used for indetcrminate, number or quantity, that is, a number or quantity in general, in contradistinction from some particular known and given one.

IN DETERMINED, or IN DETERMINA Te, in Grometry, is under:tond of a quantity, which has no ccrtain or definite bounds.

INDETER⿻上丨т Analysis, that particular branch of analysis which treats of the solution of indeterminate problems.

I DETUMMINATE Prollem, is that which admits of innumerable different solutions, and sometimes perhaps only of a grcat many different answers; otherwise called an unlimited problem. In problens of this kind the number of unknown quantitics concerned, is greater than the number of the conditions or equations by which they are to be found; from which it happens, that gencrally some orher conditions or quantities are assumed, to supply the defect, which being taken at plcasure, give the same number of answers as varieties in those assumptions. As, if it were required to find two square numbers whose ditference shall be a given quautity $d$. Here, if $x^{2}$ and $y^{2}$ denotc the two squares, then will $x^{2}-y^{2}=d$, by the question, which is only one equation, for finding two quantities. Now by assuming a third quantity $z$, so that $z=x+y$ the sum of the two roots; then is $x=\frac{x^{2}+d}{2 x}$, and $y=$ $\frac{z^{2}-d}{2 z}$, wlich are the two roots, having the difference of their squares equal to the given quantity $d$, and are expressed by means of an assumed quantity $z$; so that there will be as many answers to the question, as there can be taken values of the indeterminate quantity $z$, that is, inuumerable.

Again, If it were required to divide 25 into two such parts, that one of thein may be divisible by 2 , and the otber by 3 . Then, putting one of the parts sought $=2 x$, and the other $=3 y$, we shall have $2 x+3 y=25$; one cquation as before with two unknown quantities. By riduction, $x=\frac{25-3 y}{2}$; from which we may conclude in the first place, that $3 y$ must be less than 25 ; and consequently $y$ is less than 8 . By division, $x=12-y+\frac{1-y}{3}$; from which it is evident that $1-y$, or rather $y-1$, must be divisible by 2 . Let us therefore assume a third quantiry $z$, such that $y-1=2 z$; and we get $y=2 z+1$; so that $x=12-2 z-1-z=11-3 z$. Now since $y$ cannot be greater than 8 , we must not substitutc any numbers for $z$ which would make $2 z+1$ greater than 8 . Consequently $z$ must be less than 4; that is to say, $z$ cannot the taken greater than 3 ; for which reasons, are obtaincd the following answers :

1 If $z=0$; then $y=1$, and $x=11$.

2 If $z=1$; then $y=3$, and $x=8$.

3 If $z=2$; then $y=5$, and $x=5$.

4. If $z=3$; then $y=7$, and $x=2$.

Hence, and from the equation it appears, that the two parts souglit are I. $22+3$, Ir. $16+9$, 111. $10+15$, Iv. $4+21$, the several sums of which are evidently the number given 25 .

Diophantus was the first writer on inclerminate prom blems, viz, in his Arithmetic or Algebra, which was first publisted in 1575 by Xilander, and afterwards in 1621 by Bachet, with a large commontary, and many additions to it. His book is wholly upon this subject; whence it hat happened, that such kinel of questions hise been called by the name of Diophantine problems. Firunt, Descartes, Frenicle in France, and Wallis snd others in England, particularly cultivated this branch of alyctbra, ou which they held a correspondence, proposing difficult questions to each other; an jnstance of which are those two curious ones, pruposed by M. Furmat, as a challenge to all the mathemuticians of Europe, viz, 1st, To find a cube number which added to all its aliquot parts shall make a square number; and $2 \mathrm{~d}$, 'To find a square number which added to al I its aliquot parts shall mahe 
cubic number; which problems were answered after several ways by Dr. Wallis, as well as some others of a different nature. See the letters that passed letween Dr. Wallis, the lord Brounker, Sir Kenelm Digby, \& $x$, in the doctor's Works; and the WV orks of Fermat, which were collected and published by his son. Most authors m algebra have also treated more or less on this part of it, but more especially kersey, Prestet, Ozanam, Kirkby, \&c. But afterwards, mathematicians seemed to have forgotten such questions, if they did not even despise them as useless, when Euler drew their attention by some. excellent compositions, demonstrating some general theorems, which had only been known by induction. M. Lagrange has also taken up the subject, having resolved very difficult problems in a general way, and discovered more direct metlieds than heretofore. The $2 \mathrm{~d}$ volume of the French translation of Fuler's Algebra contains an elementary treatise on this brancl of analysis, and, with La. grange's additions, an excellent theory of it ; treating very generally of indeterminate problems, of the first and second degree, of solutions in whole numbers, of the method of indeterminate coefficients, \&.c. Also, much in the same way were such indeterminate problems treated by the Indian algebraists; as may be scen by the account of their algebra given in my 'Tracts, vol. 2, pa. 151, \&c.

Finally, Mr. John Leslie has given, in the $2 \mathrm{~d}$ volume of the Edinburgh Philos. Transactions, an ingenious paper on the resolution of indeterminate problems, resolving them by a new and general principle. "The doctrine of indeterminate equations," says Mr. Leslie, " has been scldom treated in a form equally systematic with the other parts of algebra. The solutions commonly given are devoid of uniformity, and often require a variety of assumptions. The object of this paper is to resolve the complicated expressions which we obtain in the solution of indeterminate problems, into simple cquations, and to do so, without traming a number of assumptions, by help of a single principle, which, though extremely simple, admits of a very extensive application."

"Let $\Lambda \times$ B be any compound quantity equal to another, $\mathrm{C} \times \mathrm{D}$, and let $m$ be ary rational number assumed at pleasure; it is manifest that, taking equimultiples, $\mathrm{A} \times m_{\mathrm{B}}=\mathrm{C} \times m \mathrm{D}$. If therefore we suppose that $\mathbf{A}=m \mathrm{D}$, it must follow that $m_{\mathrm{B}}=\mathrm{c}$, or $\mathrm{B}=\frac{\mathbf{c}}{m}$. Thus two equations of a lower dimension are obtained. If these be capable of farther decomposition, we may assume the multiples $n$ and $p$, and form four equations still more simple. By the repeated application of this principle, a highler equation, admitting of divisors, will be resolved into those of the first order, the number of which will be one greater than that of the multiples assunied.

For example, in the problem, to find two rational numbers, the difference of the squares of which shall be a giren number. Let the given number be the product of $a$ and $b$; then by the hypothesis, $x^{2}-y^{2}=a b$; but these compound quantities admit of an easy resolution, for $(x+y)$ $\times(x-y)=a \times b$. If therefore we suppose $x \times y=m a$, we shall obtain $x-y=\frac{b}{m}$; where $m$ is arbitrary, and if rational, $x$ and $y$ must also be rational. Hence the resolution of these two equations gives the valuer of $x$ and $y$, the numbers sought, in terms of $m$; viz,

$x=\frac{m^{2} a+b}{2 m}$, and $y=\frac{m^{2} a-b}{2 m}$.
INDEX, in Arithmetic, is the same with what is otherwise called the characteristic or the exponent of a logarithm; being that which shows of how many places the absolute or natural number belonging to the logarithm consists, and of what nature it is, whether an integer or a fraction; the index being less by 1 than the number of integer figures in the natural number, and is positive for integer or whole numbers, but negative in fractions, or in the denoninator of a fraction; and in decimals, the negative index is 1 more than the number of ciphers in the decimal, after the point, and befnre the- first significant figure; or, still more generally, the index shows now far the first figure of the natural number is distant from the place of units, either towards the left hand, as in whole numbers, or towards the right, as in decimals; these opposite cases being marked by the correspondent signs + and - of opposite affections, the sign - being set over the index, and not before it, because it is this index only which is understood as negative, and not the decimal part of the logarithm. Thus, in this logarithm $2 \cdot 4.23409 \%$, the figures of whose natural number are 2651 , the 2 is the index, and being positive, it shows that the first figure of the number must be two places removed from the units place, or that there will be thrce places of integers, the number of these places being always 1 more than the index; so that the natural number will be $265 \cdot 1$. But if the same index be negative, thus $\overline{2} \cdot 4234097$, it shows that the natural number is a rlccimal, and that the first significant figure of it is in the $2 \mathrm{~d}$ place from units, or that there is one cipher at the beginning of the decimal, being 1 less than the negative index; and consequently that the natural number of the logarithm in this case is 02651 . Hence, by varying the natural num-

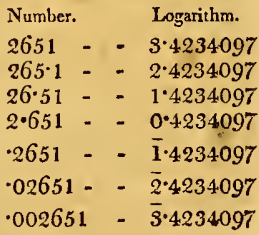
ber, with respect to the decimal places in it, as in the former of the two columns here annexed, the index of their logaritlm will vary as in the $2 \mathrm{~d}$ column.

Mr. 'Tuwnley introduced a peculiar way of noting these indices, when they become negative, or express decimal figures, which is now much in use, especially in the log. sines and tangents, \&ce, viz, by taking, instead of the true index, its arithmetical complement to 10 ; so that, in this way, the logarithn $2 * 4234097$ is written $8 * 4234097$. For the addition and subtraction of indices, see LOGARITHM.

I $\mathrm{DEx}$ of a Globe, is a little stile fitted on to the northpole, and turning round with it, pointing out the divisions of the lour-circle.

IN DEx of a 2uantity, in Arithmetic and Algebra, otherwise called the exponent, is the number that shows to what power it is understood to be raised: as in $10^{3}$, or $a^{3}$, the figure 3 is the index or exponent of the power, signifying that the root or quantity, 10 or $a$, is raised to the $3 \mathrm{~d}$ power. See this fully trented under kx posent.

INDICTION, or Roman INDICTION, a kind of epoch, or manner of counting time, among the Romans; containing a cycle or revolution of 15 years. The popes have dated their acts by the year of the indiction, which was fixed to the 1st of January anno domini 813 , ever since Charlemagne made them sovereign; before that time, they dated them by the years of the emperors.-At the time of $4 \mathrm{~S}:$ 
reforming the calendar, the year 1582 was reckuned the 10 th year of the indiction; so that beginning to reckon from hence, and dividing the number of years elapsed between that time and this, by 15 , the remainder, with the addition of 10 , rejecting 15 if the sum be more, will be the year of the indiction.

But the indiction will be easier found thus: Add 3 to the given year of Christ; divide the sum by 15 , and the remainder after the division, will be the year of the indiction: if there be no remainder, the indiction is 15 . In either of these ways, the indiction for the year 1795 is 13 .

INDIVISIBLES, are those indefinitely small elements, or principles, into which any body or figure may ultimately be divided. - A line is said. to consist of points, a surface of parallel lines, and a solid of parallel surfaces: and because each of these elements is supposed indivisible, if in any figure a line be drawn perpendicularly through all the elements, the number of points in that line, will be the same as the number of the elements.

Whence it appears, that a parallelogram, or a prism, or a cylinder, is resolvable into elements, as indivisibles, all equal to each other, parallel, and like or similar to the hase; for which reasun, one of these elements multiplied by the number of them, that is the base of the figure multiplied by its height, gives the area or content. And a triangle is resolvable into lines parallel to the base, but decreasing in arithmetical progression; so also do the circles, which constitute the parabolic conoid, as well as those which constitute the plane of a circle, or the surface of a cone. In all which cases, as the last or least term of the arithmetic progression is 0 , and the length of the figure the same thing as the number of the terms, therefore the greatest term, or base, being multiplied by the length of the figute, half the product is the sum of the whole, or the content of the figure.

And in any other figure or solid, if the law of the decrease of the elements be known, and thence the relation of the sum to the greatest term, which is the base, the whole number of them being the altitude of the figure, then the said sum of the elements is always the content. A cylinder may also be resolved in to cylindrical curve surface's, having all the same height, and continually decreasing inwards, as the circles of the base decrease, on which they insist.

This way of considering magnitudes, is called the method of indivisibles, which is unly the ancient method of exhaustions, a little disguiserl and contracted. And it is found of good use, both in computing the contents of figures in a very short and easy way, as above instanced, and in shortening other demonstrations in matthematics; an instance of which may here be given in that celebrated proposition of Archimedes, that a sphere is two-thirds of its circumscribed cylinder. 'Thus, suppose a cylinder, a hemisphere, and an inverted cone, having all the same base and altitude, and cut by an infinite number of planes all parallel to the base, of which EFe 11 is one; it is evident that the square of Es, the radius of the cylinder, is every where equal to the square of si, the radius of the sphere; and also that the square of ti $\mathrm{I}$, or of s:", is equal to the sum of the squarers of $1 F$ and 15 , or of $1 \mathrm{l}$ and $1 \mathrm{k}$, because $x k=1$ is ; that is, $3 x^{2}=1 \mathrm{~F}^{2}+$ $1 \mathrm{~K}$, in every pesition; but $\mathrm{te}$ is the rudius of the cylinder, if the corre- sponding radius of the sphere, and ik that of the cone; and the circular sections of these bodies, are as the spuares of their radii; therefore the section of tbe cylinder is every where equal to the sum of the sections of the hemisphere and cone; and, as the number of all thuse sections, which is the common height of the figures, is the sane, therefore all the sections, or elements, of the cylinder, will be equal to the sum of all those of the hemisphere and cone tahen together; that is, the cylinder is equal to both the hemisphere and cone: but as the cone itself is cqual to one-third part of the cylinder; therefore the hemisphere is equal to the other twu-thirds of it.

The method of indivisibles was introduced by Cavalerius, in 1635 , in his Geometria indivisibiliun. The same was also pursued by Torricelii in lis Works, printed $16+4:$ and again by Cavalerius himself in another treatise, publislied in 1647 .

INEQUALITY OPTIC. See OPTIC Inequality.

INERTIA of IJatter, in Philosophy, is defined by Newton to be a power implanted in all matter, by which it resists any change endearoured to be made in its actual state; that is, by which it becomes difficult to alter its state, either of rest or motion. This power then, agrees with the vis resistendi, or power of resisting, by which every body endeavours, as much as it can, to persevere in its own state, whether of rest or uniform rectilinear motion; which power is still proportional to the body, or to the quantity of matter in it, the same as the weight, or gravity of the body; and yet it is quite different from, and even independent of the force of gravity, and would be and act just the same if the bocly were clevoid of gravity. Thus, a body by this furce resists the same in all directions, upwards, or downwards, or obliquely; whereas gravity acts only downwards.

Bodies only exert this puwer in changes brought in their state by some vis impressa, or force impressed on them. And the exercise of this power is, in different respects, both resistance and impetus; resistance, so far as the body opposes a force impressed on it to change its state; and impctus, as the same body endeavuurs to change the state of the resisting obstacle. Phil. Nat. Princ, Math lib. 1.

Inertia, the same great author clsewhere obstrves, is a passive principle, by which bodies persist in their motion or rest, and receive motion, in proportion to the furce impressing it, and resist as nuch is they are resisted. See RESISTANCE.

INLR'IA Vis. Sec Vis Inertia.

INFINITE, is applied ti quantities which are either greater or less than any assignable ones. In which sense it diffirs but little fiem the terms Indefinite and Inteterminate. Thus, an

INF1N1TE, or Infinitely great line, denotes only an indefinite or indeterminate line; or a line to which ne cortain bounds or limits are prescribed.

INFIN ITE 2umtities. Though the idea of magnitude inlinitely great, or such als excerds any. assignable quantity, does include a negation of limits, yet such magnitudes are not all equal among themselses; lout besides infinite length, and intinite arra, these are no lens than three several sorts of infinte s)liclity: all of which are quantities sui generis; and those of ench specis's are in given proportions. - Intinte length, ur a line infinitely long, may be considered, either as beginning at at point, and so infinitely catcueded one wily : or else both ways from the same peint. -As to infinite surfice or area, any right line infinitely 
exteuded both ways on a plane infinitely extended every way, divides that plane into two equal parts, one on each side of the line. But if from any point in such a plane, two right lines be infinitely exterded, making an angle between them; the infinite area, intercepted between these infinite right lines, is to the whole infinite plane, as that angle is to 4 right angles. And if two infinite and parallel lines be drawn at a given clistance on such an infinite plane, the area intercepted between them will be likewise infinite; but yet it will be infinitely less than the whole plane; and even infinitely less than the angular or sectoral space, intercepted between two infinite lines, that are inclined, thougin at never so small an angle; because in the one case, the given finite distance of the parallel lines diminishes the intinity in one of the dimensions; whereas in a sector, there is infinity in both dimensions. And thus there are two species of infinity in surfaces, the one infiniely greater than the other.

In like manner there are species of infinites in solids, according as only one, or two, or as all their three dimensions, are infinite; which, though they be all infinitely greater than a firite solid, yet are they in succession infinitely greater than each other.-Some farther properties of infinite quaitities are as follow : the ratio between a finite and infinite quantixy, is an infinite ratio.-If a finite quantity be multiplied by an infinitely small one, the product will be an infinitely small one; but if the former be divided by the latter, the quoticnt will be infinitely great. - On the contrary, a finite quantity being multiplied by an infinitely great one, the product is infinitely great ; but the formcr divided by the latter, the quotient will be infinitely littli.-The product or quotient of an infinitely great or au infinitely small quantity, by a finite one, is respectively infinitely great, or infinitcly little.-An infinitely great multiplied by an infinitely little, is a finite quantity; but the former divided by the latter, the quotientis infinitely infinite.-The mean proportional between infinitely great, and infinitel hittle, is finite.

Arilhmetic of Infinitrs. See Arithmetic. Also Wallis's treatise on this subject; and another by limerson, at the beginning of his Conic Sections; also Bul!iald's treatise Arithmetica Infinitorum.

IN EINTE Decimals, such as do not terminate, but go on without end; as $333 \& \mathrm{c}=\frac{1}{3}$, or $\cdot 142857 \& \mathrm{c}=\frac{1}{7}$. See Repetend.

INFINITEIY Infinile Fractions, or all the powers of the fractions whose numerator is 1 ; which are all together equal to unity, as is demonstrated by Dr. Wood, in Hook's Philus. Coll. No 3, pa. 45 ; where some curious properties are deduced from the same.

INFINITr Series, a series considered as infinitcly coutinued as to the number of its terms. See Series.

INFINITESIMALS, are certain infinitely or indefinitely small parts; as also the method of computing by them.- In the inethod of infinitesimals, the elcinent by which any quantity increases or decreases, is supposed to bc infinitely small, and is generally expressed by two or more terins, some of which are infinitely less than the rest, which being neglected as of no importance, the remaining terms form what is called the Difference of the proposcil quantity. The torms that are neglected in this manner, as intinitely less than the other terms of the element, are the very same which arise in consequence of the acceleration, or retardation, of the generating motion, during the intinitely small time in which the element is generated; so that the remaining terms express the element that would have been produced in that time, if the generating motion bad continued uniform. 'Therefore, those differences are accurately in the same ratio to each other, as the generating motions or fluxions. And hence, though in this inethod, infinitesimal parts of the elements are neglected, the conclusions are accurately true without even an infinitely small error, and agree preciscly with those that are deduced by the method of fluxions.

But however safe and convenient this methoủ may be, some will alway's scruple to admit infinitely little quantities, and infinite orders and infinitesimals, into a scicuce that boasts of the most evident and accurate principles, is well as of the most rigid demonstrations. In order to avoid such suppositions, Newton considers the simultaneous increments of the flowing quantitics as finite, and then investigates the ratio which io the limit of the various proportions which those increnents bear to each other, while lie supposes them to decrease together till they vanish; which ratio is the same with the ratio of the fluxions. See Maclaurin's Fluxions, in the Introduc. pa. 39 \&c, also art. 495 in 502 .

INFLAMMABILITY, that property of bodies by which they kindle, or catch fire.

INFLECIION, in Optics, called also Diffiaction, and Deflection of the rays of light, is a property of them, by reasun of which, wher they come within a certain distance of any body, they are either bent from the body, or towards it; being a kind of inperfect reflection or refraction. Sonie writers ascribe the first discovery of this property to Grimaldi, wbo published an account of it, in his treatise De Lumine, Coloribus, et Iride, printed in 1666 . But Dr. Hooke also claims the discovery of it, and communicated his observations on this subject to the Róyal Society, in 1672. He shows that this property differs both from reflection and refraction; and that it seems to depend on the unequal density of the constituent parts of the ray, by which the light is dispersed from the place of condensation, and rarefied or gradually diverged into a quadrant; and this deflection, he observes, is male towards the superficies of the opaque borly perpendicularly. Newton discovered, by experiments, this inflection of the ray's of light; which may be seen in his Optics.

MI. Labire observed, that when we look at a candle, or any luminous body, with our eycs nearly shut, rays of light are extended from it, in several directions, to a. considerable distance, like the tails of comets. The true cause of this phenomenon, which has exercised the sagacity of Descartes, Rohault, and others, seems to be, that the light passing among the eyelashes, in this situation of the eye, is inflected by its near approach to then, and therefore enters the eye in a great variety of directions. He also observes, that he found that the beams of the stars being observed, in a deep valley, to pass near the brow of a hill, are always more refracted than if there were no such hill, or the observation was made on.the top of it ; as if the rays of light were bent duwn intos a curve, by palssing near the surface of the mountain.

Point of In FLection, or of contrary flexure, in a curve, is the point or place in the curve where it begins to bend or turn a contrary way; or which separates the concave part from the convex part, and lying between the two ; or where the curve changes from-concave to convex, or from convex to concave, on the same sille of the curve; such as the point $\mathrm{E}$ in the annexed figures; where the former of 
the two is concave towards the axis a $D$ from $A$ to $E$, and convex from $\mathrm{B}$ to $\mathrm{F}$; but, on the contrary, the latter figure is convex froin $\Lambda$ to $\mathrm{E}$, and concave from $\mathrm{E}$ to $\mathrm{F}$.
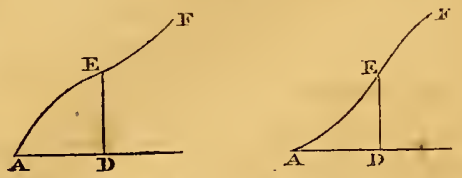

There are various ways of finding the point of inflexion; but the following, which is new, seems to be the simplest and easiest of all. From the nature of curvature it is evident that, while a curve is concave towards an axis, the fluxion of the ordinate decreases, or is in a decreasing ratio, with regard to the fluxion of the absciss; but, on the contrary, that the said fluxion increases, or is in an increasing ratio to the fluxion of the absciss, where the curve is convex towards the axis; hence it follows that those two fiuxions are in a constant ratio at the point of inflection, where the curve is neither concare nor conrcx, That is, if $x=A \mathrm{D}$ the absciss, and $y=\mathrm{DE}$ the ordinate, then $\dot{x}$ is to $\dot{y}$ in a constant ratio, or $\frac{\dot{x}}{y}$ or $\frac{\dot{y}}{\dot{x}}$ is a constant quantity. But constant quantities have no fluxion, or their fluxion is equal to nothing; so that in this case the fuxion of $\frac{\dot{x}}{\dot{y}}$ or of $\frac{\dot{y}}{\dot{x}}$ is equal to nothing. And hence ive have this general rule: viz,

Put the given equation of the curve into fluxions; from which cquation of the fluxions find $\frac{\dot{x}}{\dot{y}}$ or $\frac{\dot{y}}{\dot{x}}$; then take the fuxion of this ratio or fraction, and put it equal to 0 or nothing; and from this last equation find also the value of the same $\frac{\dot{x}}{\dot{y}}$ or $\frac{\dot{y}}{\dot{x}}$ : then put this latter value equal to the former, which will be an equation from whence, and the first given equation of the curve, $x$ and $y$ will be determined, being the absciss or ordinate answering to the point of inflection in the curve.

Or, putting the fluxion of $\frac{\dot{x}}{\dot{y}}$ equal to 0 , that is, $\frac{\dot{y} \dot{y}-\dot{x} \ddot{y}}{y^{2}}=0$, or $\ddot{x} \dot{y}-\dot{x} \bar{y}=0$, or $\ddot{x} \dot{y}=\dot{x} \ddot{y}$, or $\dot{x}: \dot{y}:: \bar{x}: \ddot{y}$, that is, the $2 d$ fluxions have the same ratio as the 1 st fluxjons, which is a constant ratio; and therefore if $x$ be constant, or $\ddot{x}=0$, then shall $\dot{y}$ be $=0$ also; which gives another rule, viz; Take both the 1st and $2 \mathrm{~d}$ fluxions of the given equation of the curve, in which make both $\bar{x}$ and $\bar{y}=0$, and the resulting equations will determine the values of $x$ and $y$, or absciss and ordinate to the point of inflection.

For cxample, if it be required to find the point of inflcction in the curve whose equation is $a x^{2}=a^{2} y+x^{2} y$. Now the fluxion of this is $2 a x \dot{x}=a^{2} \dot{y}+2 x y \dot{x}+x^{2} \dot{y}$, which gives $\frac{\dot{x}}{y}=\frac{a^{2}+x^{2}}{2 a x-2 x y}$. Then the fluxion of this again raade $=0$, gives $2 x \dot{x}(a x-x y)=\left(a^{2}+x^{2}\right) \cdot(a \dot{x}-y \dot{x}-x \dot{y})$; and this gives again $\frac{\dot{x}}{\dot{y}}=\frac{a^{2}+a^{2}}{a^{3}-x^{3}} \times \frac{x}{a-y}$. Lastly, this value of $\frac{\dot{x}}{y}$ put $=$ the former, gives

$\frac{x^{5}+x^{2}}{a^{2}-x^{3}} \cdot \frac{x}{a-y}=\frac{a^{2}+x^{2}}{2 x} \cdot \frac{1}{a-y}$; and hence $2 x^{2}=$ $a^{2}-5^{2}$, or $3 x^{2}=a^{2}$, and $x=a \sqrt{\frac{2}{3}}$, the absciss.
Hence also, from the original equation, $y=\frac{a x^{2}}{n^{2}+x^{2}}=\frac{\frac{1}{3} a^{3}}{\frac{4}{3} a^{2}}=\frac{1}{4} a$, the ordinate to the point of inflection sought.

When the curve has but one point of inflection, it will be determined by a simple equation, as above; but when there are several points of inflection, by the curve bending several times from the one side to the otber, the resulting cquation will be of a degree corresponding to them, and its roots will determine the abscisses or ordinates to the sume. Other methods of determining the points of inflection in curves, may be scen in most books on the doctrine of thuxjons.

T'o know whether a curre be concave or convex towarils any point assigned in the axis; find the value of $\ddot{y}$ at that point; then if this value be positive, the curve will be convex towards the axis, but if $\mathrm{jt}$ be negative, it will be concave.

INFORMED Stars, or IN For M Es Stella, are such stars as have not been reduced into any constellation; otherwise called Sporades.-A great many of this kind were left by the ancient astronomers; but Hevelius and some others of the moderns have provided for the greater part of then, by making new constellations. And I think that the later celestial globes made in England, have the constellations contrived to include the old unformed stars.

INGINEER. Sec ENGINEER.

INGRESS, in Astronomy, the sun's entrance into one of the signs, cspecially Aries.

INNOCENTS $D a y$, a feast celebrated on the 28 th day of December, in commemoration of the infants said to be murdered by Herod.

INORDINATE Proportion, is where the order of the terms compared, is disturbed or irregular. As, for cxample, in two ranks of numbers, three in cach rank, viz, in one rank, - - - - $2,3,9$, and in the other rank - - $8,24,36$, which are proportional, the former to the latter, hut in a different order, viz, - $\quad$ - $2: 3:: 24: 36$, and - $\quad-\quad 3: 9:: 8: 24$, then, casting out the mean terms in each rank, it is concluded that - - - $2: 9:: \mathrm{s}: 36$, that is, the first is to the $3 \mathrm{~d}$ in the first rank, as the first is to the $3 d$ in the $2 d$ rank.

INSCRIBED Figure, is one that has all its angular points touching the sides of another figure in which the former is said to be inscribed.

Inscri B E Hyperbola, is one that lies wholly within the angle of its asymptotes; as the common or conical hyperbola doth.

INSTANT, otlicrwise called a moment, an infinitely small part of duration, or in which we perccive no succession, or which takes up the time of only one idca in our mind. It is a maxim in mechanics, that no natural eflect can be produced in an instant, or without some definite time; also that the greater the time, the greater the eflect. And hence may appear the reason, why a burthen scems lighter to a person, the faster he carrics it; and why, the faster a person slides or skates on the ice, the less liable it is to break, or bend.

INS'IITUTE is a name which lias lately been substituted for academy or school. When royalty was lately abolished in France, the several royal academies were abolinhed, and were all condensed into one general acadeiny, under the name of the 
Autional Instritute, or New Academy of Aris and Sciences. This academy was founded on a decree of the $n$ new government constitution in the year 1795. It consists of 268 members, half of whom reside in Paris, the obtuer half in the departments; and to them are added 24 foreigners, as honorary members. The acalemy is divided into 3 classes, each class into scctions, each section coutaining 12 members.

1st Class. Mathematics and natural philosophy. 'J'his class is divided into 10 sections. 1. Mathematics. 2. Mcchanical arts. 3. Astronomy. 4. Experimental plilosophy. 5. Chemistry. 6. Natural kistory. 7. Botany. 8. Anatomy and animal history. 9. Medicine and surgery. 10. Animal economy, and the veterinary science.

$2 \mathrm{~d}$ Class. Morality and politics. This class consists of 6 sections. 1. Analysis of sensations and ideas. 2. Morals. 3. Legislature. 4. Political economy. 5. History. 6. Geography.

3d Class. Literature and the fine arts, consisting of $\mathrm{S}$ sections. 1. Universal grammar. 2. Ancient languages. 3. Poctry. 4. Antiquities. 5. Painting. 6. Sculpture. 7. Architecture. 8. Music.

For each class a particular room in the Louvre is appropriated. No person can be a member of two classes at the same time: but a member of ne class may be present at the meetings of any other. Each class prints, yearly, an accuunt of its transactions.

Four times a-year public meetings are held, when the three classes meet together. At the end of each year, they give a circumstantial account to the legislative body, of the prongress made in that y'ar in the arts and sciences. The prizes given annually by each class, are published at certain times. The sums requisite for the support of the institution, are decreed yearly by the legislative body, on a requisition made by the executive directory.

The first 48 members were chosen by the exceutive directory, to whom the choice of the remaining members was contidecl. T'o the members resident in Paris is reserved the choice, both of the department and foreign members. On a vacancy in any class, three candidates are named by the class, for the choice of the body at large. Jiach class is to have, at its place of meeting, a collection of the products, both of nature and art, and a library, according to its particular wants. 'The regulations of the institution, with respect to the times of meeting, and its employmens, are to be dratwn up by the body at large, and laitl belore the legislative assembly.

INSULATS, or INSULATED, a term appliced to a column or vilier exlifice, which stauls ature, or free and detached from any adjacent wall, \&c, like an island in the se'al.

Insulare D, in Flectricity, is a term applied to bodies that inc supported by electrics, or nom-conductors; so that their communication with the carth, by conducting sulistances, is interrupte:].

INSURANCl, the same as Assurance; which see.

INTACIA, are right lines to which curves do continually approach, and yet can never meet them, more usually called Asvniptotes.

INTE(iLRS, are whole numbers, as contra-distinguished from lractions. Integer's may be considered as numbers which refer to unity, as a whole to a part.

JNTEGRAL Number, an integer; not a fraction.

INTEgra1. Calculus, in the New Analysis, is the reverse of the differential calculus, and is the finding the integral from a given differential ; being similar to the inverse method of fluxions, or the finding the fluent to a given fluxion.

The rules for the integration of differentials, like those for the extraction of roots, are established by inspection of the direct process by which, from given functions, differentials are derived: thus, the differential of $x^{\mathrm{m}}$ or $d\left(x^{\mathrm{m}}\right)$, is $m x^{\mathrm{m}-1} d x$; therefore, from $m x^{\mathrm{m}-1} d x$, the integral may be found from this process: increase the index $m-1$, or power of the valiable quantity, by unity or 1 , and divide by the index so increased, and by the differential of the variable quantity, for the integral required. Let $d^{-1}$, be the sign of the operation by which the integral is obtained, or the reverse of that operation which $d$ indicates; then will $d^{-1}\left(m x^{m-1} d x\right)=x^{m}$; or, $d^{-1} d\left(x^{m}\right)=x^{m}$. Also, $d^{-1}\left(x^{\frac{m}{n}} d x\right)=\frac{x_{n}^{-}+1}{\frac{m}{n}+1}$; or $\frac{n x^{\frac{m+n}{n}}}{m+n}$. Again the integrals of $a^{x} d x, e^{x} d x$; are $a^{x}, e^{x}$; and are obtained on the very same grounds of inspection as the above.

The differentials of $a+b x, a+b x, a^{\prime \prime}+b x$, \&c, or $d(a+b x), d(\dot{a}+b x), d(a \vec{a}+b x), \&$ c. are $d b x, d b x, d b x$, \&c. Hence inversely, the integrals are $d^{-1}(d b x), d^{-1}(d b x)$ $d^{-1}(d b x)$ \&c: where it is to be noted, that the constant quantities $a, a, a^{\prime \prime}, \& c$, are the corrections of the integrals; and are determined in the same manner as those in the inverse method of fluxions, from the particular nature of the problem under discussion.

It may be here observed, that as in the analysis of finites, any quantity may be raised to any degree or power; but vice versa, the root cannot be extracted out of any number required: so in the analysis of infinites, any variable or flowing quantity may be difierenced, but vice versa, any differential cannot be integrated. And as, in the analy'sis of finites, we are not yet arrived at a method of extracting the ronts of all equations, so neither has the integral calculus arrived at its perfection: and as in the former we are obliged to have recourse to approximation, so in the latter we have recourse to infinite series, where we cannot attain to a perfect integration. For the several authors on this calculus, see Fruxions.

INTEGRANT" Parts, in Philosophy, are the similar parts of a bocly, or parts of the same nature with the whole; as filings of iron are the integrant parts of iron, having the same nature and propertics with the bar or mass they were filed of from.

IN'TENSITY, or INTrNsion, in Physics, is the degree or rate of the power or cnergy of any quality; as heat, cold, \&c. The: intensity of qualities, as gravity, light, heat, \&c, vary in the reciprocal ratio of the squares of the distances from the centre of the radiating quality.

IN'I'ERCALARY Day, denotes the odd day inserted in the leap-year. Sec Bissextile.

JN'liRCEP'TED Axis, in curves, the same with what is otherwise called the absciss or abscissa.

INTIRCOLUMNATION, Or INTERCOLUMNIATION, is the space betiveen column and column, which is alivays to be priportional to the height and bulk of the columns. From a medium, some authors bave laisl down, the following proportions: in the Tuscan order, the intercolumanativn to be 4 diameters of the body of the column below; in the Doric 3 , in the lonic $2 \frac{x}{2}$, in the Corinthian $2 \frac{x}{4}$, and in the Composite $1 \frac{x}{2}$.

INTERLST, is a sum reckoned for the loan or for- 
bearance of another sum, or principal, lent for, or due at, a certain time, according to some certain rate or proportion; being estimated usually at so much per cent. or by the 100 . This forms a particular rule in arithmetic. The highest legal interest now allowed inEngland, is after the rate of 5 per cent. per annum, or the $20 t h$ part of the princi$\mathrm{pal}$ for the space of a year, and so in proportion for other times, either greater or less : except in the case of pawnbrokers, to whom it has lately been made legal to take a much higher intcrest, for one of the worst and most destructive purposes that can be suffered in any state.

Interest is either Simplc or Compound.

Simple INTEREST, is that which is counted and allowed upon the principal only, for the whole time of forbearance.-The sum of the principal and interest is called the amount.

As the interest of any sum, for any time, is directly proportional to the principal sum and time; therefore the intcrest of 1 pound for one ycar being multiplied by any proposed principal sum, and by the time of its forbearance, in years and parts, will be its interest for that time. That is, if

$r=$ the rate of intercst of $1 l$. per annum,

$p=$ any principal sum lent,

$t=$ the time it is lent for, and

$a=$ the amount, or sum of principal and interest;

then is prt $=$ the interest of the sum $p$, for the time $t$, at the ratc $r$; and conscquently $p+p r t=p \times(1+r t)=a$, is the amount of the same for that timc. And from this general formula, other theorems can easily be deduced for finding any of the quantities above-nsentioned; which collected all together, will be as follow:

$$
\begin{array}{ll}
\text { 1st, } a=p+p r t & \text { the amount, } \\
\text { 2d, } p=\frac{a}{1+r t} & \text { the principal, } \\
3 \mathrm{~d}, r=\frac{a-p}{p t} & \text { the rate, } \\
4 \mathrm{~h}, t=\frac{a-p}{\mu r} & \text { the time. }
\end{array}
$$

For Example, lit it be required to find, in what time any principal sum will double itself, at any rate of simple interest. In this case we may use the 1 st theorcm $a=p$ $+p r t$, in which the amount $a$ must be $=2 p$, or double the principal, i. e. $p+p r t=2 p$; and hence $t=\frac{1}{r}$;

where $r$ bcing the interest of $1 \mathbf{l}$. for one year, it follows that the time of doubling at simple interest, is equal to the quutient of any sum divided by its interest for one year. So that, if the rate of interest be 5 per cent. then $100 \div 5$ $=20$, is the time of doubling.-Or the th theorem immediately gives $t=\frac{a-p}{p r}=\frac{-2 p-p}{p r}=\frac{2-1}{r}=\frac{1}{r}$.

For some compendious tables and rules for computing the interest on moncy, sce my small book of Arithmetic.

Compound INT TRES'r, called also Interest-upon-Interest, is that which is counted, not only on the principal sum lent, but also for its interest, as it becomes duc, at the end of each statid lime of payment.

Though it be not lawful to lend money at compound interest, yot in purchatsing ammoitics, pensions, \&c, and leases in reversion, it is usual to allow compound interest to the purchaser fir his ready money; and therefore it is necessary to understind this sulject.

Besides the quantities concerned in simple interest, viz, the principal $p$, the rate or interest of $1 l$. Lor 1 year $r$, the amount $a$, and the time $t$, there is another quantity employed in compound interest, viz, the ratio of the rate of interest, which is the amount of $1 l$. for 1 time of payment, and which here let be denoted by $\mathrm{R}, \mathrm{viz}, \mathrm{R}=1+r$. Then, the particular amounts for the several times may be thus computed, viz, As 1 pound, is to its amount for any time, so is any proposed principal sum, to its amount for the same time; i. e.

1 l.: $\mathrm{R}:: p \quad: p \mathrm{R}$ the 1 st year's amount,

1l.: $\mathrm{R}:: p \mathrm{R}: p \mathrm{R}^{2}$ the $2 \mathrm{~d}$ year's amount,

ll.: $\mathrm{R}:: p \mathrm{R}^{2}: p \mathrm{R}^{3}$ the $3 \mathrm{~d}$ year's amount, and so on.

Therefore in general, $p_{\mathrm{R}^{\mathrm{t}}}=a$, is the amount for the $t$ year, or $t$ time of payment. Whence the following general theorems are deduced:

$$
\begin{aligned}
& \text { 1st, } a=p \mathrm{R}^{\mathrm{t}} \text { the amount, } \\
& \text { 2d, } p=\frac{a}{\mathrm{R}^{\mathrm{t}}} \quad \text { the principal, } \\
& \text { 3d, } \mathrm{R}=\sqrt[t]{\frac{a}{p}} \quad \text { the ratio, } \\
& \text { 4th, } t=\frac{\log \text {. of } a-\log \text {. of } p}{\log \text {. of } \mathrm{R}} \text { the time. }
\end{aligned}
$$

From which any one of the quantities may befound, when the rest are given.

For example, suppose it were required to find, in low many years any principal sum will double itself, at any proposed rate of interest. In this case nust be employed the 4th theorem, where $a$ will be $=2 p$, and then it is $t=\frac{1 . a-1 \cdot p}{\log . \mathbf{R}}=\frac{1.2 p-1 \cdot p}{\log \cdot \mathbf{R}}=\frac{\log \cdot 2}{\log \cdot \mathrm{R}}$.

So, if the rate of interest be 5 per cent. per annum; then $\mathrm{r}=1+\cdot 05=1 \cdot 05$, and hence

\begin{tabular}{|c|c|c|c|}
\hline & & $\left\{\begin{array}{c}\text { At Simple Interest } \\
\text { leats. }\end{array}\right.$ & $\begin{array}{c}\text { At Comp. Interest. } \\
\text { Years. }\end{array}$ \\
\hline & & 100 & 69.6003 \\
\hline & & 50 & 35.0028 \\
\hline & & 40 & $28 \cdot 0701$ \\
\hline & per cent. per all. & $33 \frac{1}{3}$ & $23 \cdot 4.408$ \\
\hline $3 \frac{\pi}{2}$ & interest, 11 . or & $28 \frac{4}{7}$ & $20 \cdot 1488$ \\
\hline & any other sum & 25 & $17 \cdot 6730$ \\
\hline & will doublc in & $22 \frac{2}{9}$ & $15 \cdot 7+473$ \\
\hline & & 20 & $14: 2067$ \\
\hline & & $16 \dot{0}_{3}^{2}$ & 11.8957 \\
\hline & & $14 \frac{2}{7}$ & $10 \cdot 2448$ \\
\hline & & $12 \frac{1}{2}$ & $9 \cdot 0065$ \\
\hline & & $11 \frac{\pi}{9}$ & $80 \$ 32$ \\
\hline & & 10 & $7 \cdot 2725$ \\
\hline
\end{tabular}

$t=\frac{\log \cdot 2}{\log .1 \cdot 05}=\frac{.3010300}{.0211893}=14 \cdot 2067$ nearly; that is, any sum doubles in $14 \frac{x}{5}$ years nearly, at the rate of 5 per cent. per annum compound interest.

Hence, and from the like question in simple interest, above given, are deduced the times in which any sun doubles itself, at several rate's of interest, both simple and compound : viz,

The following 'Table will very much facilitate the calculation of the compound interest for any sum, for any number of jears, al various ritu's of inter'st.

'The use of this table, which contains all the powers $\mathrm{r}^{\mathrm{t}}$, to the $201 \mathrm{~h}$ power, or the amounts of 12 . is chictly to calculate the interest, wre the amount, of any principal sum, for any time, not more than 20 years. for cxample, required to find to how much $523 l$. will amount in 15 years, at the rate of $5 l$. per cent. per amum compound interest. 
In the table, on the line 15 , and in the column 5 per cent, is the amount of $1 l$, viz. this multiplied by the principal gives the amount or and therefore the interest is The Amount of $1 \mathrm{l}$. in any Number of Years.

\begin{tabular}{c|c|c|c|c|c|c}
\hline Yrs. & 3 & $3 \frac{x}{2}$ & 4 & $4 \frac{x}{2}$ & 5 & 6 \\
\hline 1 & $1 \cdot 0300$ & $1 \cdot 0350$ & $1 \cdot 0400$ & $1 \cdot 0450$ & $1 \cdot 0500$ & $1 \cdot 0600$ \\
2 & $1 \cdot 0609$ & 10712 & $1 \cdot 0816$ & $1 \cdot 0920$ & $1 \cdot 1025$ & $1 \cdot 1236$ \\
3 & $1 \cdot 0927$ & $1 \cdot 1037$ & $1 \cdot 1249$ & $1 \cdot 1412$ & $1 \cdot 1576$ & $1 \cdot 1910$ \\
4 & $1 \cdot 1255$ & $1 \cdot 1475$ & $1 \cdot 1699$ & $1 \cdot 1925$ & $1 \cdot 2155$ & $1 \cdot 2625$ \\
5 & $1 \cdot 1593$ & $1 \cdot 1877$ & $1 \cdot 2167$ & $1 \cdot 2462$ & $1 \cdot 2763$ & $1 \cdot 3382$ \\
6 & $1 \cdot 1941$ & $1 \cdot 2293$ & $1 \cdot 2653$ & $1 \cdot 3023$ & $1 \cdot 3401$ & $1 \cdot 4185$ \\
7 & $1 \cdot 2299$ & $1 \cdot 2723$ & $1 \cdot 3159$ & $1 \cdot 3609$ & $1 \cdot 4071$ & $1 \cdot 5036$ \\
8 & $1 \cdot 2668$ & $1 \cdot 3168$ & $1 \cdot 3686$ & $1 \cdot 4221$ & $1 \cdot 4775$ & $1 \cdot 5939$ \\
9 & $1 \cdot 3045$ & $1 \cdot 3629$ & $1 \cdot 4233$ & $1 \cdot 461$ & $1 \cdot 5513$ & $1 \cdot 6995$ \\
10 & $1 \cdot 3439$ & $1 \cdot 4106$ & $1 \cdot 4802$ & $1 \cdot 5530$ & $1 \cdot 6289$ & $1 \cdot 7909$ \\
11 & $1 \cdot 3842$ & $1 \cdot 4600$ & $1 \cdot 5395$ & $1 \cdot 6229$ & $1 \cdot 7103$ & $1 \cdot 8383$ \\
12 & $1 \cdot 4258$ & $1 \cdot 5111$ & $1 \cdot 6010$ & $1 \cdot 6959$ & $1 \cdot 7959$ & $2 \cdot 0122$ \\
13 & $1 \cdot 4685$ & $1 \cdot 5640$ & $1 \cdot 6651$ & $1 \cdot 7722$ & $1 \cdot 8356$ & $2 \cdot 1329$ \\
$1+$ & $1 \cdot 5126$ & $1 \cdot 6187$ & $1 \cdot 7317$ & $1 \cdot 9519$ & $1 \cdot 9799$ & $2 \cdot 2609$ \\
15 & $1 \cdot 5580$ & $1 \cdot 6753$ & $1 \cdot 8009$ & $1 \cdot 9353$ & $2 \cdot 0789$ & $2 \cdot 3466$ \\
16 & $1 \cdot 6047$ & $1 \cdot 7340$ & $1 \cdot 8730$ & $2 \cdot 0224$ & $2 \cdot 1829$ & $2 \cdot 5404$ \\
17 & $1 \cdot 6528$ & $1 \cdot 7947$ & $1 \cdot 9479$ & $2 \cdot 1134$ & $2 \cdot 2920$ & $2 \cdot 6928$ \\
18 & $1 \cdot 7024$ & $1 \cdot 8575$ & $2 \cdot 0258$ & $2 \cdot 2085$ & $2 \cdot 4066$ & $2 \cdot 8543$ \\
19 & $1 \cdot 7535$ & $1 \cdot 9225$ & $2 \cdot 1068$ & $2 \cdot 3079$ & $2 \cdot 5270$ & $2 \cdot 0256$ \\
20 & $1 \cdot 8061$ & $1 \cdot 9898$ & $2 \cdot 1911$ & $2 \cdot 4117$ & $2 \cdot 6533$ & $2 \cdot 2071$ \\
\hline
\end{tabular}

See Annuities; Discount; Reversion; Smart's Tables of Interest; the Philos. 'Trans. vol. 6, pa. 50s; and most books on Arithmetic.

INTERIOR Figure, Angle of. See Axgle.

Interior Polygon. See Polygon.

Interior Talus. See Talus.

Internal Angles, are the angles made within any figure, by the sides of it. In a triangle $A \mathrm{Bc}$, the two angles
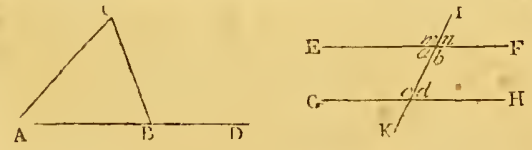

$\mathbf{A}$ and $\mathrm{C}$ are peculiarly called internal and opposite, in respect of the external angle $\mathrm{CBD}$, which is equal to them both together.

IN'ER N A A Angle is also applied to the two angles formed between two parallels, by a line intersecting those parallels, on each sidc of the intersecting line. Such are the angles $a, b, c, d$, formed between the parallels $\mathbf{E F}$ and $(i n$, on each sicle of the intersecting line.-The two adjacent internal angles $a$ and $b$, or $c$ and $d$, are together equal to two right angles.

InTERNa $\mathrm{L}$ and Opposite Augles, is also applied to the two angles $a$ and $b$, which are respectively equal to the two $n$ and $m$, called the external and opposite angles. Also the alternate internal angles are equal to each other; viz, $a=d$, and $b=c$.

INTERPOLATION, in the modern Algebra, is nsed for finding an intermediate term of a series, its place in the series being given. The method of interpolation was first inveuted by MIr: Briggs, and by him applied to the calculation of logarithms, \&c, in his Aritlnmetica Logarithmica, VoL. 1. and, his Trigonometria Britamnica; where he explains, and fully applies the method of interpolation by difference's. His principles were followed by Reginal and Mouton in France, and by Cotes and others in England. Wallis employed the method of interpolation in several parts of his works; as his Arithmetic ol infinites, and his Algebra, for quadratures, \&c. The same was also happily applied by Newton in various ways : by it he investigated his binoinial theorem, and quadratures of the circle, ellipse, and hyperbola: see Wallis's Algebra, chap. 85 , \&c. Newton also, in lemma 5, lib. 3 Princip. gave a most clegant solution of the problem for drawing a curve line through the extremities of any number of given ordinates; and in the subsequent proposition, he applied the solution of this problem to that of finding, from certain observed places of a comet, its place at any given intermediate time. And Dr. Waring, who adds, that a solution still more elegart, on sume accounts, has been since discovered by Mess. Nichol and Stirling, has also resolved the same problem, and rendered it more general, without having recourse to finding the successive differences. Philos. Trans, vol. 69, part 1, art. 7 . Mr. Stirling indeed pursued this branch as a distinct science, in a separate treatise, viz, Tractatus de Summatione et Interpolatione Serierum Infinitarum, in the year 1730 .

When the 1st, 2d, or other successive differences of the terms of a series become at last equal, the interpolation of any term of such a series, may be found by Newton's Differential Method. When the algebraic equation of a series is given, the term required, whether it be a primary or intermediate one, may be found by the resolution of affected equations; but when that equation is not given, as it often happens, the value of the term sought must be exhibited by a converging seriss, or by the quadrature of curves. See Stirling, ut supra, pa. 86. Nleyer, in Act. Petr. tom. 2, pa. 180.

A general theorem for interpolating any term is as follows: Let $A$ denote any term of an equidistant series of terms, and $a, b, c, \& c$, the first of the Ist, $2 d, 3 \mathrm{~d}$, \& e, orders of differences; then the term $z$, whose distance from $\Lambda$ is expressed by $x$, will be this, viz, Theorem 1 , $z=\Lambda+x a+x \cdot \frac{x-1}{2} b+x \cdot \frac{x-1}{2} \cdot \frac{x-2}{3} c \& c$.

Hence, if any of the orders of differences become equal to one another, or $=0$, this series for the interpolaterl term will break off, and terminate, otberwise it will run out in an infinite series.

Example. To find the 20th term of the series of cubes $1,8,2 \pi, 64,125, \& c$, or $1^{3}, 2^{3}, 3^{3}, 4^{3}, 5^{3}, \& c$.

Set down the series in a column, and take their continual differences as here annexed, where the 4 th difterences, and all after it become $=0$, also $\mathrm{A}=1, a=7$, $b=12, c=6$, and $x=19 ;$ therefore the 20 th term sought is barely

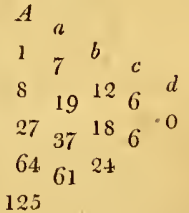

$z=1+19 \times 7+19 \times \frac{19}{2} \times 12+19 \cdot \frac{18}{2} \cdot \frac{17}{3} \cdot 6$

$=1+133+2052+5814=8000$.

Theor. 2. In any series of equidistant terms, $a, b, c, d$, \&cc, whose first differences are small; to find any lerm wanting in that series, having any number of terms given. Take the cquation which stands against the number of a $\mathrm{T}$ 


\section{N T}

[ 690

given terms, in the following table; and by reducing the equation, that term will be found.

\begin{tabular}{c|l} 
No. & Equations. \\
1 & $a-b=0$ \\
2 & $a-2 b+c=0$ \\
3 & $a-3 b+3 c-d=0$ \\
4 & $a-4 b+6 c-4 d+e=0$ \\
5 & $a-5 b+10 c-10 d+5 e-f=0$ \\
6 & $a-6 b+15 c-20 d+15 e-6 f+g=0$ \\
\&c. & \&c. \\
$n$ & $a-n b+n \cdot \frac{n-1}{2} c-n \cdot \frac{n-1}{2} \cdot \frac{n-2}{3} d, \& c=0:$
\end{tabular}

where it is evident that the coefficients in any equation, are the unciæ of a binomial $1+1$ raised to the power denoted by the number of the equation.

Example. Given the logarithms of 101, 102, 104, and 105 ; to find the log. of 103.

Here are 4 quantities given; therefore we must take the 4th equation $a-4 b+6 c-4 d+e=0$, in which it is the middle quantity or term $c$ that is to be found, because 103 is in the middle among the numbers 101, 102, 104, 105; then that equation gives the value of, $c$ as follows, viz $c=\frac{4 \cdot(b+a)-(a+e)}{6}$.

Now the logs. of the given numbers will be thus:

$$
\begin{aligned}
& 2 \cdot 0043214=a \\
& 2 \cdot 0086002=b \\
& 2 \cdot 0170333=d \\
& 2 \cdot 0211893=e \\
& \overline{4.0256335=b}+d \\
& 4 \\
& 16 \cdot 1025340=4 \cdot(b+d) \\
& \text { subtr. } \frac{4.0255107}{6)}= \\
& 2.0128372 \text { the log. of } 103 \text {. }
\end{aligned}
$$

Theor. 3. When the terms $a, b, c, d$; \&c, are at unequal distances from each other; to find any intermediate one of these terms, the rest being given.

Let $p, q, r, s, \& c$, be the several distances of those terms from each other; then let

$$
\begin{aligned}
& \mathrm{B}=\frac{b-a}{p} ; \mathrm{C}=\frac{\mathrm{B} 1-\mathrm{B}}{p+q} ; \mathrm{D}=\frac{\mathrm{C} 1-\mathrm{C}}{p+q+r} ; \mathrm{E}=\frac{\mathrm{D} 1-\mathrm{D}}{p+q+r+s} ; \\
& \mathrm{B} 1=\frac{c-b}{q} ; \mathrm{C} 1=\frac{\mathrm{B} 2-\mathrm{B} 1}{q+r} ; \mathrm{D} 1=\frac{\mathrm{C} 2-\mathrm{C} 1}{q+r+s} ;
\end{aligned}
$$$$
\text { B } 2=\frac{d-c}{r} ; C 2=\frac{B 3-B 2}{r+s} ;
$$$$
\text { В3 }=\frac{e-d}{s} \text {; }
$$

\&c \&c $\quad$ \&c. Then the term $z$, whose distance from the beginning is $x$, will be

$z=a+\mathbf{B} x+\mathbf{C} x \cdot(x-p)+\mathrm{D} x \cdot(x-p) \cdot(x-j-g)$ $+\mathrm{E} x \cdot(x-p) \cdot(x-p-q) \cdot(x-p-q-r)+\& \mathbf{c}$ to be continued to as many terms as there arc terms in the given series.

By this series may be found the place of a comet, or the sun, or any other object at a given time; by knowing the places of the same for several other given times. Other methods of interpolation may be found in the Philos. Trans. number 362; or Stirling's Summation and Interpolation of Series.

INTERSCENDENT, in Algebra, is applied to quantities, when the exponents of their powers are radical quantitics. Thus $x^{\sqrt{ } 2}, x^{\sqrt{ } a}, \& c$, are interscendent quantitics. See Function.
$1 \mathrm{~N} \mathrm{~T}$

INTERSECTION, the cutting of one line, or plane, by another; or the point or line in which two lines or two planes cut each otlier.-The mutual intersection of two planes is a right line. The centre of a circle, or conic section, \&c, is in the intersection of two diameters; and the central point of a quadrangle, is the intersection of two diagonals.

INTERSTELLAR, intervening between the stars; a word used by some authors, to express those parts of the uniyerse, that are without and beyond the limits of our solar system.-In the interstellar regions, it is supposed there are several other systems of planets, moving round the fixed stars, as the centres of their respective notions. And if it be true, as it is not improbable, that each fixed star is thus a sun to some habitable orbs, or earths, that move round it, the interstellar world will be infinitely the greatest part of the universe.

INTERTIES, ur lN ter Duces, in Architecture, those small pieces of timber which lie horizontally between the summers, or between them and the cell or rising plate.

INTERVAL, in Music, the difference between two sounds, in respect of acute and grave. Authors distinguish several divisions of an interval, as first into Simple and Compound. The

- Simple Interval is that without parts, or division; such are the octave, and all that are within it; as the $2 \mathrm{~d}$, $3 \mathrm{~d}, 4 \mathrm{th}, 5 \mathrm{th}, 6 \mathrm{th}$, and $7 \mathrm{th}$, with their varieties.

Compound I T TER RAL consists of several lesser intervals; as the 9 th, 10 th, 11 th, \&c, with their varieties.

The simple interval was by the ancients called a diastem, and the compound they called a system.

An interval is also divided into just or true, and into false. The

Just or True INTERVALS, are such as all those above mentioned, with their varieties, whether major or minor.

False INTERVALS, are the diminutive or superfluous ones.

An interval is also divided into the consonance and dissonance; which sce.

INTESTINE MIotion of the parts of fuids, that which is among its corpuscles or component parts. When the attracting corpuscles of any fluid are elastic, they must necessarily produce an intestine motion; and that, greater or Jess, according to the degrees of their elasticity and attractive force. For, two elastic particles, after meeting, will fly from each other, with the same degree of velocity with which they met; abstracting from the resistance of the medium. But when, in leaping back from each other, they approach other particles, thcir velocity will be increased.

INTONATION, in Music, the action of sounding the notes in the scule with the voice, or any other given order of "musical tones.-Intonation may be either true or false ; either too bigh or too low, either too sharp or too flat; and then this word intonation attended with an epithet must be understood concerning the manner of performing the notes.

INTRADOS, the interior and lower side, or curve, of an arch. In contradistinction from the extrados, or exterior curve, of line on the upper side of the arch.. Sec my Tracts, vol. 1.

INTRENCHMENT, in Fortification, any work that shelters a post against the attacks of an cnemy. It is genelally taken for a ditch, or trench with a parapet. Intrenclments are sometimes made of fascincs with carth 
thrown over them, of gabions, luogsheads, or bags filled with earth, to cover the men from the enemy's fire.

INVERSE, is applied to a manner of working the rule of three, or proportion, which seems to go backward, i.e. reverse or contrary to the order of the common and direct rule: so that, whereas, in the direct rule, more requires more, or less requires less; in the inverse rule, on the contrary, more requires less, or less requires more.-For instance, in the direct rule it is said, If 3 yards of cloth cost 20 shillings, how much will 6 yards cost ? the answer is 40 shillings; where more yards require more money, and less yards require less money. But in the inverse rule it is said, If 20 men perform a piece of work in 6 days, in how many days will 40 men perform as much ? where the answer is 3 days; and here the more men require the less time, and the fewer men the more time.

IN verse Method of Fluxions, is the method of finding fluents, from the fluxions being given; and is similar to what the foreign mathematicians call the calculus integralis. See Fuuents.

Invers e Method of Tangents, is the method of finding the curve belonging to a given tangent; as opposed to the direct method, or the finding the tangent to a given curve. - As, to find a curve whose subtangent is a third proportional to $r-y$ and $y$, or whose subtangent is equal to the semiordinate, or whose subnormal is a constant quantity. The solution of this problem depends chiefly on the inverse method of fluxions. See Tangent. See also MLontucla, v. 3, pa. 164.

Inverse Proportion, or Inverse Ratio, is that in which more requires less, or less requires more. As for instance, in the case of light, or heat from a luminous object, the light received is less at a greater distince, and greater at a less distance; so that here more, as to distance, gives less, as to light, and less distance gives more light. This is usually expressed by the term inversely, or reciprocally; as in the case above, where the light is inversely, or reciprocally as the square of the distance; or in the inverse or reciprocal duplicate ratio of the distance.

INVERSION, Invertendo, or by Inversion, by the 14.th def. of Euclid, lib. 5 , is inverting the terms of a proportion, by changing the antecedents into consequents, and the consequents into antecedents. As in these, $a: b:: c: d$, then by inversion $b: a:: d: c$.

INVESTIGATION, the searching or finding any thing out, by means of certain steps, traces, or ways.

INVOLU'TE Figure or Curce, is that which is traced out by the outer extremity of a string as it is folded or wrapped upon another figure, or as it is unwound from off it. The involute of a cycloid, is also a cycloid equal to the former, which was first discovered by Huygens, and by means of which he contrived to make a pendulum vibrate in the curve of a cycloid, and so, theoretically at least, vibratc always in equal times, whether the arch of vibration were great or small; which is a property of that curve. Sec Evolute.

INVOLUTION, in Arithmetic and Algebra, is the raising of powers from a given root; as opposed to Evolution, which is the extracting, or developing of roots from given powers. So the involution of the number 3 , or its powcrs, are thus raised:

3 - or $3^{x}$ or 3 is the 1 st power, or root,

$3 \times 3$ or $3^{2}$ or 9 is the $2 \mathrm{~d}$ power, or square,

$3 \times 3 \times 3$ or $3^{3}$ or 27 is the $3 \mathrm{~d}$ power, or cube,

and so on. - And hence, to find any power of a given root, or quantity, let the root be multiplied by itself a number of times which is one less than the nunber of the incles; i. c. once multiplied for the $2 \mathrm{~d}$ root, twice for the $3 \mathrm{~d}$ root, thrice for the 4 th root, \&c.

So also, in algebra, to involve the binomial $a+b$, or raise its powers.

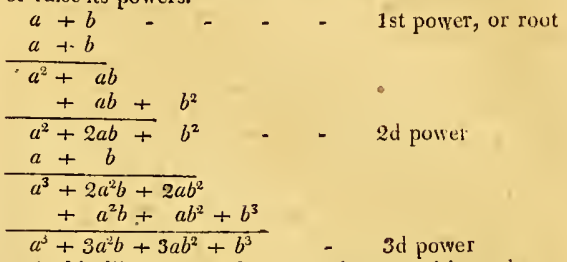

And in like manner for any other quantities, whatever the number of their terms may be. But compound algebraic quantities are best involved by the Brмom IA L Theorein; which see.

Simple quantities are involved, by raising the numeral coefficients to the given power, and the literal quantities are raised by multiplying their indices by that of the root; that is, the raising of powers is performed by the multiplication of indices, the same as the multiplication of logarithms. Thus,

The $2 \mathrm{~d}$ power of $a$ is $a^{2}$.

The $2 \mathrm{~d}$ power of $2 a^{2}$ is $2^{2} a^{2 \times 2}$ or $4 a^{4}$.

The $3 \mathrm{~d}$ power of $3 a^{2} b^{3}$ is $27 a^{6} b^{9}$.

The $3 \mathrm{~d}$ power of $a^{\frac{1}{2}} b^{\frac{2}{3}}$ is $a^{\frac{3}{2}} b^{2}$.

The $n$th power of $a^{\mathrm{mt}} c^{\mathrm{P}}$ is $a^{\mathrm{mn}} c^{\mathrm{pn}}$ or $\left(a^{\mathrm{ms}} c^{\mathrm{P}}\right)^{\mathrm{n}}$.

INWARD Flanking Angle, in Fortification, is that male by the curtin and the razant flanking line of defence.

JOINTS, in Architecture, are the separations between the stones or bricks; which may be filled with mortar, plaster, or cement.

Joint, in Carpentry, \&rc, is applied to several manners of assembling, setting, or fixing pieces of wood together. As by a mortise joint, a dove-tail joint, \& $c$.

Universal JoINT, in Mechanics, an excellent invention of Dr. Hooke, adapted to all kinds of motions and fiexures; of which he has given a large account in his Cutlerian Lectures, printed in 1678. This seems to have given occasion to the gimbols used in suspending the sca compass; the mechanism of which is the same with that of Desiguliers's rolling lamp.

Join-Lives, are such as continue during the same time, or that exist together. See LifE-Annuities.

JOIS'TS, or JoYsts, those pieces of timber framed into the girders and summers, and on which the boarding of floors is laid. They are of varions kinds: as, 1. Conmon Joists, being those which are framed level or flush with the upper surface of the girders, but are seldom of equal depth. 2. Trimming Joists, those which are framed into two other joists, that other joists may be framed into them, where the opening is made for a stair-case, or a chimney bearth. 3. Binding Joists, those which are laid across from girder to girder, and about 3 or 4 inches lower than their upper surface, in order that other joists, called 4. Bridging Joists, may be laid over them parallel to the girders. 5. Ceiling Joists, which are turned into the biuding joists, and are generally made slender, having but little weight to support.-'The chief or flooring joists, should be abuut 8 inches deep, the common breadth of a $4 \mathrm{~T} 2$ 
deal board; their breadth more or less, according to their length, viz, from 2 to 4 inches broad, or different lengths from 6 to 12 feet.

"JONES (WILIIAM), F. K. s. a very eminent mathematician, was born at the foot of Bodavon mountain [Mynydd Bodafon] in the parish of Lianfitangel tre'r Bard, in the lsle of Anglesy, North Wales, in the year 1675. His father's name was John George, his surname being the proper name of his father. For it is a custom in several parts of Wales for the proper name of the father to become the suruame of his children. Juhn George the father was commonly called Sion Siors of Llanbabo, to which place he moved, and where his children were brought up. Accorlingly our author, whose proper name was William, took the surname of Jones from the proper name of lis father, who was a farmer, and of a good fimily, being descended from Hwfa ap Cynddelw, one of the 15 tribes of North Wales. He gave his two sons the common school education of the country, reading, writing, and accounts, in Einglish, and the Latin Grammar. Harry his second son took to the farming business; but Willian the eldest, having an extraordinary turn for mathenatical studies, determined to try his fortune abroad from a place where the same was but of little service to him. He accordingly came to London, accompanied by a young man, Rowland Williams, afterwards an eminent perfumer in Wych-street. The report in the country is, that Mr. Jones soon got into a merchant's counting bouse, and so gained the esteem of his master, that he gave him the command of a ship for a West India voyage; and that upon his return he set up a mathematical school, and published his book of Navigation; and that upon the death of the merchant he married bis widow : that, lord Macclesfield's son being his pupil, he was made secretary to the chancellor, and one of the deputy tellers of the exchequer:- and they have a story of an Italian wedding, which caused great disturbance in lord Macclesfield's family, but was compromised by Mr. Jones; which gave rise to a saying, "that Macclesfield was the making of Joncs, and Jones the making of Macclesfield." "The foregoing account of Mr. Jones, 1 found among the papers of the late Mr. John Robertson, Jibrarian and clerk to the Royal Society, who had been well known to Mr. Jones, and possessed many of his papers.

Mr. Jones having by his industry acquired a competent fortune, lived upon it as a private gentleman for many years, in the latter part of his life, in babits of intimacy with Sir Isaac Newton and others the most eminent matbematicians and philosophers of his time; and died July 3, 1749 , at 74 years of age, being one of the vice-presidents of the Royal Society; leaving at his death one daughter, and a son very young, who was the late Sir William Jones, one of the judges in India, and highly rstecmed for his great abilities, extensive learning, \&r.-Mr. Jones's publications were,

1. A new Compendium of the whole Art of Navigation, \&c; in small Svo, London, 1702 . This is a neat little piece, and derlicated to the Rev. Mr. Jobn Harris, the author of the Lexicon Technicum, or Universal Dictionary of Arts and Scicnces, in whose house Mr. Jones says he composed lis book.

2. Synopsis Palinariorum Mathescos: or a New lntroduction to the Mathematics, \&c; 8vo, London, 1706; being a very neat ant uscful compendium of all the inathematical sciences, in about 300 pages.
His papers in the Philos. Trans. are the following:

3. A Compendious Disposition of Equations for exhibiting the relations of Gonionetrical Lines; vol. 44: pa. 560 .

4. A Tract on Logarithms; vol. 61, pa. 455 .

5. Properties of the Conic Sections, deduced by a compendious metbod; vol. 63 , pa. 340 .

In all these works of Mr. Jones, a remarkable neatness, brevity, and accuracy, ercry where prevail. He scemed to delight in a very short and comprehensive mode of expression and arrangement; in so much that, sometimes what be bas contriced to express in two or three pages, woulet occupy a little volume in the ordinary stjle of writing.

Mr. lones, it is said, possessed the best mathematical library in lingland; scarcely any book of that kind but what was there to be found. IIe had collected also a great quantity of manuscript papers and letters of former mathematicians, which have often proved useful to writers of their lives, \&c. After bis death, these were dispersed, and fell into different persons' hands; many of them, as iwell as of Mr. Jones's own papers, were possessed by the late Mr. John Robertson, before mentioned, at whose cleath I purcliased a considerảble quantity of then. From such collections as these it was that Mr. Jones was enabled to gise that first and elegant cdition, in $4 \mathrm{to}, 1711$, of severat of Newton's papers, that might otherwise bave been lost, entitled, Analysis per quantitatum Series, Fluxiones, ac Differentias: cum Enumeratione Lincarum Tertii Ordinis.

IONIC Column, or Order, the $3 \mathrm{~d}$ of the five orders, or columns, of architecture. The first idea of this order was given by the people of Ionia; who, according to Vitruvius, formed it on the model of a young wonjan, dressed in her hair, and of an easy elegant shape, as the Doric had been formed on the modei of a strong iobust man. This column is a medium between the massive, and the more delicate orders, the simple and the rich. It is distinguished from the composite, by having none of the leaves of acanthus in its capital; and from the Tuscan, Doric, and Corinthian, by the volutes, or rams horns, which adorn its capital; and from the Tuscan and Doric, by the channels, or fluting, in is slatt. The height of this column is 18 modules, or 9 -diameters of the column taken at the bottom: indeed at first its height was but 16 modules; but, to rculer it more beautiful than the Doric, its height was augmented by adding a base to it, which was unknown in the Doric. M. Lcclerc makes its entablature to be 4 modules and 10 minutes, and its pedestal 6 modules; so that the whole order makes 28 modules 10 minutes

JOURNAL, in Merchants' Accounts, is a book into which every particular article is posted out of the wastebook, according to the order of time, specifying the debtor and creditor in each account aud transaction.

JoU RNAL, in Mlaritime Affairs, is a register kept by the pilot, and others, noticing cvery thing that happens to the ship, from day to day, and from bour to hour, with regard to the winds, the rhumbs or courses, the knots or rate of running, the rake, soundings, astronomical observations, for the latitudes and longitudes, \&c; to enable then to aljust the reckoning, and determine the place where the ship is. In all sem journals, the day, or what is calted the 2.4 bours, is divided into twice 12 hours; thuse before noon marked A.m. for ante meridiem, and those from noon to milnight markod P.M. post meridicm, or afternoon. 
There are various ways of keeping a sea journal, according to the different notions of mariners concerning the articles to be entered. Some writers direct the keeping such a kind of journal as is only an abstract of each day's transactions, specifying the weather, what ships or lands were seen, accidents on board, the latitude, longitude, meridional distance, conrse, and run: these particulars are to be drawn from the ship's log-book, or from that kept by the person himself. Other authors recommend the keeping only of one account, including the log-book, and all the work of each day, with the deductions drawn from it.

JoURNAL is also used for the title of several books published at stated times; giving accounts and abstracts of the new books that are published, with the new improvements daily made in arts and sciences. The first journal of this kind was, the Journal des Sçavans, printed at Paris: the design was set on foot for the ease of such as are too busy, or too indolent, to read the entire books themselves. It seems a ready way of satisfying a man's curiosity, and becoming learned on casy terms: and so useful has it becu found, that it has been executed in most other countries, shough under a great variety of titles.

Of this kind are the Acta Eruditorum of Leipsic; the Nouvelles de la Republique des Lettres of M. Bayle, \&c; the Bibliotheque Universelle, Choisie, et Ancienne et Moderne, of M.Leclerc; the Memnirs de Trevoux, \&c. In 1692, Juncker printed in Latin, An Historical Treatise of the Journals of the Learned, published in the several parts of Europe; and Wolfius, Struvius, Morhoff, Fabricius, \&c, have done something of the same kind.

The Philosophical Transactions of London, and those of Edinburgh; the Memoirs of the Royal Academy of Sciences; those of the Academy of Belles Lettres; the Miscellanea Nature Curiosorum: the Experiments of the Acarlemy del Cimento, the Acta Philo-exoticorum Nature et Artis, which appeared from March 1686 to April 1687 , and which are a history of the Academy of Bresse; the Misccllanea Berolinensia, or Memoirs of the Academy of Berlin; the Commentaries of the Academy of Petersburg; the Memoirs of the Institute at Bologna; the Acta Literaria Suecix; the Memoirs of the Royal Academy of Stockholm, begun in 1740; the Commentarii Societatis Regix Gottingensis, begun in $1750, \& c, \& c$, are not so properly juarmals, though they are frequently ranked in the number.

Juncker and Wolfius give the honour of the first invention of journals to Photius. His Bibliotheca, however, is not altogether of the same nature with the modern journals; nor was his design the same. It consists of abridgments and extracts of books which he had read during his embassy in Persia. M. Salo first began the Journal des Sçavans at Paris, in 1665 , under the name of the Sieur de Hedonville; but his death soon after interrupted the work. The abbé Gallois then took it up, and he, in the year 1674, gave way to the abbé de la Roque, who continued it nine years, and was succeeded by M. Cousin, who carried it on till the year 1702, when the abbé Bignon instituted a new society, and committed the care of continuing the journal to them, who improved and publisbed it under a new form. This society is still continued, and M. de Loyer has had the inspection of the journal; which is no longer the work of any single author, but of a great number. The other French journals are the Memoirs and Conferences of Arts and Sciences, by M. Dennis, during the years 1672,1673 , and 1674 ; New Discoveries in all the parts of Physic, by M. de Blegny; the journal of Physic, begun in 1684 , and some whers, discontinued almost as suon as begun. Rozier's Journal de Physique, begun in July 1771 , and continued till, iu the year 1780 , there were 19 vols. quarto.

The Nouvelles de la Republique des Lettres, News from the Republic of Lettres, were begun by M. Bayle in 1684 , and carried on by him till the year 1687 , when $M$. Bayle being disabled by sickness, his friends, M. Bernard and M. de Laroque, took them up, and continued them till 1699. After an interruption of nine ycars, M. Bernard resumed the work, and continued it till the year 1710. The History of the Works of the Learned, by M. Basnage, was begun in the year 1686, and ended in 1710 . The Universal Historical Library, by M. Leclerc, was continued to the year 1693, and contained twenty-five volumes. The Bibliotheque Choisie of the same author, began in 1703. The Mercury of France is one of the most ancient journals of that country, and is contimued by different persons : the Memoirs of a History of Sciences, and Arts, usually called Memoires des Trevoux, from the place where they are printed, began in 1701. The Essays of Literature reached but to a 12 th volume in 1702,1703 , and 1704; these only take notice of ancient authors. The Journal Literaire, by Father Hugo, began and ended in 1705. At Hansurgh they liave made two attempts for a French journal, but the desiga failed : an Ephemerides Sçavantes has also been undertaken, but that soon disappearerl. A Journal des Sçavans, by ML. Dartis, appeared in 1694, but was discontinued the year following. That of M. Chauvin, begun at Berlin in 1696, held out three years; and an essay of the same kind was made at Geneva. To these may be added, the Journal Literaire begun at the Hague 1715, and that of Verdun, and the Memoires Literaires de la Grande Bretagne by M. de Laroche; the Bibliotheque Angloise, and Journal Britannique, which are confined to English books alone. The Italian journals are, that of Abbot Nazari, which continued from 1668 to 1681 , and was printed at Rome. That of Venice began in 1671 , and ended at the same time with the other: the authors were Peter Moretti and Fraricis Miletti. The Journal of Parma, by Roberti and Father Bacchini, was declined in 1690 , and resumed again in 1692. The Journal of Ferrara, hy the abbe de Latorre, began and ended in 1691. La Galerio di Mlinerva, begun in 1696 , is the work of a society of men of letters. Seignior Apostolo Zeno, secretary to that society, began another Journal in 1710 , under the protection of the grand cluke: it is printed at Venice, and several persons of distinction are concerned in it.

The Fasti Euriditi della Bibliotheca Volante, were published at Parma. There has appeared since, in Italy; the Giornale dei Letterati.

The principal among the Latin journals, is that of Leipsic, under the title of Acta Eruditorum, begun in 1682: P.P. Manzani began another at Parma. 'The Nova Literaria Maris Balthici lasted from 1698 to 1708 . 'The Nova Literaria Germaniæ, collectcd at Hamburgh, began in 1703 . The Acta Literaria ex Manuscriptis, and the Bibliotheca Curiosa, begun in 1705, and ended in 1707, are the works of Struvius. Messrs. Kuster and Sike, in 1697 , began a Biblitotheca Novorum Librorum, and continued it for two 
years. Since that time, there have been many Latin journals; such, besides others, is the Commentarii de Rebus in Scientia Naturali et Medicina gestis, by M. Lndwig. The Swiss journal, called Nova Literaria Helvetix, was begun in 1702 , by M. Scheuchzer; and the Acta Medica Hafnensia, published by T. Bartholin, make five volumes from the year 1671 to 1679 . There are two Low-Dutch journals; the one under the title of Boockzal van Europe, which was begun at Rotterdam in 1692 , by Peter Rabbus; and continued from 1702 to 1708 , by Sewel and Gavern: the other was conducted by a physician, called Ruiter, who began it in 1710 . The German jourials of best note are, the Monathlichen Unterredungen, which continued from 1689 to 1698 . The Bibliotheca Curiosa, began in 1\%04, and ended in 1707 , both by M. Tenzel. The Magazin d'Hambourg, commenced in 1748: the Physicalische Belustigunzen, or Plilosophical Amusements, begun at Berlin in 1751. The Journal of lianover began in 1700 , and continued for two years by. AI. Eccard, under the direction of M. Leibnitz, and afterwards carried on by others. The Theological Journal, published by M. Loescher, uniler the title of Altes und Neues, that is, OHd and New. A third at Leipsic and Francfort, the authors Mess. Walterck, Krause, and Groschuffius; and a fourth at Hall, by M. Turk.

The English journals are, 'The History of the Works of the Learned, begun at London in 1699. Censura Temporum, in 1708. About the same time there appeared two new ones, the one under the title of Mlemoirs of Literature, containing little more than an English translation of some articles in the foreign journals, by M. De Laroche; the other a collection of loose tracts, entitled, Bibliotheca Curiosa, or a Miscellany. These, however, with some others, are now no more, but are succeeded by the Annual Register, which began in 1758; the New Annual Register, begun in 1780; the Monthly Review, which began in the year $174 \dot{9}$, and gives a character of all English literary publications, with the most considerable of the foreign ones: the Critical Review, which began in 1756, and is nearly on the same plan: as also the London Review, by Dr. Kenrick, from 1775 to 1780 ; Maty's Review, from Feb. 1782, to Aug. 1786 ; the English Review begun in Jan. 1783; and the Analytical Review begun in Mlay 1788 , both now extinct. The Ldinburgh Review begun in 1802, the Quarterly Review in 1508, and the New Quarterly in 1813, all these published four times a year; also Tilloch's Philosophical Magazine, begun 1798; Nicholson's Philosophical Journal in 1797 , but ended with 18 I3; and 'Thomson's Annals of Literature; all published monthly. Besides these, we have several inonthly pamphlets, called Magazines, which, together with a cbronological series of occurrences, contain letters from correspondents, communicating extraordinary discoveries in nature and art, with controversial pieces on all subjects. Of these, the principal are those called, the Gentleman's Magazine, which began with the year 1731 ; the London Magazine, which began a few months after, and bas lately been discontinued; the Universal Magazine, which is nearly of as olld a date; and best of all the Monthly Magazine began in $\mathbf{1 7 9 6 .}$

IRIS, anotlser mane for the RAINBow; which see.

Iris also, denotes the striped variegated circle round the pupil of the eyc, formed of a duplicature of the uvea. In different subjects, the iris is of several very different colours; whence the eye is called grey, or black, \&c. In its middle is a perforation, through which appears a small black speck, called the sight, pupil, or apple of the eye, round which the iris forms a ring.

IRIs is also applied to those changeable colours, which sometimes appear in the glasses of telescopes, microscopes, Sc ; so called from their similitude to a rainbow.-The sanc appellation is also given to that coloured spectrum, which a triangular prismatic glass will project on a wall, when placed at a proper angle in the sun-beans.

Inis Marina, the Sea-Rainborv. This tlegant appearance is generally scen after a violent storm, in which the sea water has been in vast emotions. The celestial rainbow however has great advantage over the marine one, in the brightness and variety of the colours, and in their distinctness one from the other; for in the sea-rainbow, there are scarce any other colours than a dusky yellow on the part next the sun, and a pale green on the op. posite side. The other colours are not so bright, or so distinct, as to be well determined; hut the sea-rainbows are more frequent and more numerous than the others: it is not unconmon to sce 20 or 30 of them at a time at noonday.

IRRADIATION, the emission of subtile effluvia, in all directions, like the rays of the sun.

IRRATIONAL Numbers, or 2uantitics, are the same as surds, or such roots as cannot be accurately extracted, being incommensurable to unity. See Sunds.

IRIREUCIBLE Case, in Algebra, is used for that case of cubic equations where the root, accordiug to Cardan's rule, appears under an impossible or imaginary form, and yet is real; viz, in the form $x^{3}-a x= \pm c$, having $\frac{1}{27} a^{3}$ greater than $\frac{1}{4} c^{2}$, or $4 a^{3}$ greater than $27 c^{2}$. Thus, in the equation $x^{3}-15 x=4$, the root, according to Cardan's rule, is $x=\sqrt[3]{ }(2+\sqrt{ }-121)+\sqrt[V]{ }(2-\sqrt{ }-121)$, which is in the form of an impossible expression, and yet it is equal to the quantity $4:$

$$
\begin{aligned}
& \text { for } \sqrt[3]{ }(2+\sqrt{ }-121)=2+\sqrt{ }-1, \\
& \text { and } \sqrt{ }(2-\sqrt{ }-121)=2-\sqrt{ }-1, \\
& \text { therefore their sum is } x=4 \text {. }
\end{aligned}
$$

The other two roots of the equation are also real.

Algebraists, for almost three centuries, have: in vain endeavoured to resolve this case, and to bring it under a real form; aud the problem is not less celebrated inong them, than the squaring of the circle is among geometricians. It is to be observed, that, as in some oiher cases of cubic equations, the valuc of the root, though rational, is found under an irrational or surd form; because the root in this case is compounded of two equal surds witl contrary signs, which destroy each other; as if $x=2+\sqrt{ } 3+2-\sqrt{ } 3$, then $x=4$. In like manner, in the irreducible case, where the root is rational, there are two equal imaginary quantities, with contrary signs, joined to real ifuantities; so that the inaginary quantities destroy each other; as in the case above of the root of the equation $x^{3}-15 x=4$, which was found to be $2+\sqrt{ }-1+2-\sqrt{ }-1=4$. It is remarkable that this case always happens, viz one root, by Cardan's rule, in an impossible form, wheneser the equation has three real roots, and no inpossible ones, but at no time else.

If we were possessed of a gencral rule for accurately extracting the cube root of a binomial radical quantity, it is evident we might resolve the irreducible case generally, which consists of two of such cubic binomial roots. But 
the labours of the algebraists, from Cardan's down to the present time, have not been able to remove this difficulty. Dr. Wallis thought that he had discovered such a rule; but, like most others, it is merely tentative, and can only succeed in certain particular circumstances.

It has been sometimes asserted that, in the irreducible case of cubic equation, if the coefficients of all the terms be rational, the equation will have at least one rational root: which may be demonstrated in this manner.

The given equation being $x^{3}-a x=c$, where the coefficients $a$ and $c$ are rational.

Let $\pm 2 m$ denote one of the roots; then $\mp m+n\}$ may denote the other two roots.

Hence $a$ or $m^{2}-n^{2}-4 m^{2}$ is rational: but $4 m^{2}$ is rational, therefore $m^{2}-n^{2}$ is rational.

Again, $c=2 m$. $\left(m^{2}-n^{2}\right)$ is rational:

but $m^{2}-n^{2}$ is rational, therefore $2 m$ is rational, which is one of the roots.

One of the most convenient methods of resolving such cubic equations, is by means of tables of sines, \&c. See the article Cuxic Equations.

Mr. Maseres, cursitor baron of the exchequer, has lately deduced, by a long train of algebraical reasoning, from Newton's celebrated binomial theorem, an infinite series, which will resolve this case, without any mention of either impossible or negative quantities. And I have also discovered several other series which will do the same thing, in all cases whatever; both inserted in the Phil. Trans. See Cardan's Algebra; the articles Algebra, Cubic Equations; Wallis's Algebra, chap. 48; De Moivre in the Appendix to Sanderson's Algebra, pa. 744; Philos. Trans. vol. 68 , part 1, art. 42 , and vol. 70, pa. 387 ; and my New Tracts, vol. 2, pa. 98 , \&c.

IRREGULAR, something that deviates from the common forms or rules. Thus, we say an irregular fortification, an irregular building, \&c.

Irregular Figure, in Geometry, whether plane or solid, is that whose sides, as well as angles, are not all equal and similar among themselves.

IRREGULARITIES in the Moon's motion. See Moon.

ISAGONE, in Geometry, is sumetimes used for a figure consisting of equal angles.

ISLAND, or ISLE, a tract of dry land encompassed by water; whether by the sea, a river, or lake, \&c. In which sense 1sland stands contradistinguished from Continent, or terra firma; like Great Britain, lreland, Jersey, Sicily, Minorca, \&c.

Some naturalists imagine that islands were formed at the deluge: others think they have been rent and separated from the continent by violent storms, inundations, and earthquakes; while others are thrown up by volcanoes, or otherwise grow or emerge from the sea.

Varenius thinks most of these opinions true in some instances, and believes that there have been islands produced each of these ways. St. Helena, Ascension, and other steep rocky islands, he supposes have become so, by the sea's overflowing their neighbouring champaigns. By the heaping up huge quantities of sand, \&r, he thinks the islands of Zealand, Japan, \& c, were formed: Sumatra and Ceylon, and most of the East-India islands, he rather thinks were rent off from the main land, as England probably was from France. It is also certain that some have cmerged from the bottom of the sea; as Santorini formerly, and thrce other isles near it lately; the last in 1707, which rose from the bottom of the sea, after an earthquake: the ancients bad a tradition that Delos rose from the bottom of the sea; and Seneca observes that the island Therasia rose out of the Egcan sea in his time, of which the mariners were eye witnesses; as they have heen within these 10 years, in the sea between Norway and Iceland, as also in the Atlantic ucean, where an island has just emerged, near St. Michael's. The late circumnavigators too have made it probable, that many of the South sea islands have bad their foundations, of coral rock, gradually increasing, and growing out of the sea.

ISLES, or rather Ailes, in Architecture, the wings or sides of a building.

ISOCHRONAL, or Isocn RoNous, is applied to such vibrations of a penduluin as are performed in equal times. Of which kind are all the vibrations of the same pendulum in a cycloidal curve, and in a circle nearly, whether the arcsit describes be longer or shorter; for when it describes a shorter arc, it moves so much the slower; and when a long one, proportionably faster.

IsOCHRONAL Line, is that in which a heavy body is supposed to descend with a uniform velocity, or without any acceleration. Leibnitz, in the $\Lambda$ ct. Erud. Lips. for April 1689 , has a discourse on the Ininea. Isochrona, in which he shows, that a beavy body, with the velocity acquired by its descent from any height, may descend from the same point by an infinite number of isochronal curves, which are all of the same species, differing from one another only in the magnitude of their parameters (such as are all the quadratocubical paraboloids), and corsequently similar to one another. He shows also, how to find a line, in which a heavy body descending, shall recede uniformly from a given point, or approach uniformly to it.

ISOMERIA, in Algebra, a terin of Vieta, denoting the freeing an equation from fractions; which is done by reducing all the fractions to one common denominator, and then multiplying each member of the equation by that common denominator, that is rejecting it out of them all.

ISOPERIMETRICAI. Figures, are such as have equal perimeters, or circumferences. It is demonstrated in geometry, that among isoperimetrical figures, that is-always the greatest, which contains the most sides or angles. Whence it follows, that the circle is the most capacious of all figures which have the same perimeter with it.--That of two isoperimetrical triangles, which have the same base, and one of them two sides equal, and the other unequal; that is the greater whose sides are equal. That of isoperimetrical figures, whose sides are equal in number, that is the greatest which is equilateral, and equiangular. Hence arises the solution of that popular problem, To make the hedging or walling, which will fence in a certain given quantity of land, also to fence in any other greater quantity of the same. For let $x$ be one side of the rectangle that will contain the quantity $a a$ of acres; then will $\frac{a a}{x^{\circ}}$ be its other side, and double their sum, viz, $2 x+\frac{2 a a}{x}$, will be the perimeter of the rcctangle: let also $b b$ bc any greater number of acres, in the forin of a square, then is $b$ one side of it, and $4 b$ its perimeter, which nust be equal to that of the rectangle; and hence the equation $2 x+\frac{2 a a}{3}$ $=4 b$, or $x^{2}+a^{2}=2 b x$, in which quadratic equation the two roots are $x=b \pm \sqrt{ }\left(b^{2}-a^{2}\right)$, which are the lengths 
of the two dimensions of the rectangle, viz, whose area $b^{2}$, is in any proportion less than the square $a^{2}$, of the same perimeter. As, for example, if one side of a square be 10 , and one side of a rectangle be 19 , but the otber only 1 ; such square and parallelogram witl be isoperimetrical, viz, each perimeter 40 ; jet the area of the square is 100 , and of the parallelogram only 19 .

Isoperimerrical lines alid figures have greatly engaged the attention of mathematicians at all times. The 5 th book of Pappus's Collections is chiefly on this subject; where a great variety of curious and important properties are demonstrated, both of planes and solids, some of which were then old in his time, and many new ones of his own. lndeed it seems he has here brought together, into this book, all the properties relating to isoperimetrical figures then known, and their different degrees of capacity.

The analysis of the general problem concerning figures that, among all those of the same perimeter, produce maxima and minima, was given by Mr. James Bernoulli, from computations that involve $2 \mathrm{~d}$ and $3 \mathrm{~d}$ fluxions. And several enquiries of this nature have been since prosecuted in like manner, but not always with equal success. Mr. Maclaurin, to vindicate the doctrine of Auxions from the imputation of uncertainty or obscurity, has illustrated this subject, which is considered as one of the most abstruse parts of this doctrine, by giving the resolution and composition of these problems by first fluxions only; and in a manner that suggests a synthetic demonstration, serving to verify the solution. See Maclaurin's Fluxions, pa. 486 ; Analysis Magni Problematis Isoperimetrici Act. Erud. Lips. 1701, pa. 213; Mem. Acad. Scienc. 1705, 1706, 1718 ; and the works of John Bernoulli, tom. 1, pa. 202, 208, 424, and tom.2, pa. 235; where is contained what he and his brother James published on this problem. Mr. Joln Bernoulli, in his first paper, considered only two small successive sides of the curve; whereas the true method of resolving this problem in general, requires the considering three such small sides, as may be perceived by examining the two solutions.

II. Luler has also published, on this subject, many profound researches, in the Petersburg commentaries; and there was printed at Lausanne, in 1744 , a pretty large work upon it, entitled, Methodus inveniendi lincas curvas, maxımi minimive proprietate gaudentes: sive Sulutio problematis isoperimetrici in tatissimo sensu accepti.-M. Cramer too, in the Berlin Memoirs for 1752 , has given a paper, in which he proposes to demonstrate in general, what can be desnonstrated only of regular figures in the elements of gcomctry, viz, that the circle is the greatest of all isopetimetrical figures, regular or irregular-M. Lhuilher has some elementary problems on isoperimeters, in his Polygonometry; and a very considerable tract on this subject is given in my Course of Mathematics, vol. 3 , pa. $3 \uparrow-53$.

On this head, see also Simpson's Tracts, pa. 98 ; and the Philus. 'Trans. vol, 49 and 50.

lSOSCLLES Triangle, is a triangle that has two sides equal. In the 5 th prop. of Euclid's ist book, which prop. is usually called the pons asinorum, or asses bridge, it is denonstrated, that the angles, $a$ and $b$, at the base of the isosceles triangle, are equal to each other; and thit if the equal sides be produced, the two angles, $c$ and $d$, below the base, will also be equal. It is also inferred, that every equilateral triangle is also equiangular.

Other properties of this figure are, that the perpendicular AP, from the vertex to the base, bisects the base, the vertical angle, and also the whole triangle. And that if a line be drawn from the vertex to any point in the base, the square of this line, together with the rectangle of the segments of the base, will be equal to the square of one of the equal sides of the triangle. Also, if the rertical angles of two isosceles triangles be equal, the two triangles will be equiangular.

ISTHMUS, in Geography, a narrow neck or slip of land, that joins two other large tracts together, and separating two seas, or two parts of the same sea. The most remarkable isthmuses are, that of Panama or Straits of Darien, connecting north and south America; that of Suez, whicb joins Asia and Africa; that of Corinth, or Peloponnesus, in the Horea ; that of Crim Tartary, otherwise called Taurica Chersonesus; that of the Peninsula Romania and Erisso, or the isthmus of the Thracian Chersonesus, 12 furlongs brond, and which Xerxes undertonk to cut through. The ancients hat several designs of cutting the istlmus of Corinth, which is a rocky hillock, about 10 miles over; but without effect, the invention of sluices being not then known. There have alsu been attempts for cutting the isthmus of Suez, to make a communication between the Mediterranean and the Red-sea.

JULIAN Calendar, is that depending on, and connected with the Julian Year and account of time; so called from Julius Crsar, by whom it was established. See CALENDAR.

J LIAN Epoch, is that of the institution of the Julian reformation of the calendar, which began the 46 th year before Christ.

Julian Period, is a cycle of 7980 consecutive years, invented by Julius Scaliger, from whom it was named; though some say his name was Joscph Scaliger, and that it was called the Julian period, because he made use of Julian years. This period is formed by multiplying continually together the three following cycles, viz, that of the stin of 28 years, that of the moon of 19 years, and that of the indiction of 15 years; so that this epoch, though but artificial or lcigned, is yet of good use; in that every year within the period is distinguishable by a cerfain peculiar character; for the year of the sun, moon, and indiction, will not be the same again till the whole 7980 years have revolved. Scaliger fixed the beginning of this period 764 years before the creation, or rather the period naturally reduces to that ycar, taking the numbers of the three given cycles as he then found them; and accounting 3950 years from the creation to the birth of Christ, this makes the 1st year of the Christian cra answer to the $4.7 \mathrm{t}+\mathrm{th}$ year of the Julian period ; therefore, to find the yen of this period, answering to any proposed year of Christ, to the constant number +713 , add the given year of Christ, and the sum will be the year of the Julian period: thus, to 4.713 adding 1791 , the sum 6504 is the year of this period for the year of Christ 1791. Ilence the first revolution of the Julian period will not be completed till the year of Christ $326 \%$, after which a new revolution of this period will commence.

But the year of the Julian period may be found for any time, from the numbers of the three cycles that compose it, without making use of the given year of Christ; thus, inultiply the 

numbers $\left\{\begin{array}{l}4845 \\ 4200 \\ 6916\end{array}\right\}$
$\left.\begin{array}{l}\text { respeetively by } \\ \text { the year of the }\end{array}\right\}$
sun,
moon,
indiction:

then add the three produets together, and divide the sum by 7980 ; so shall the remainder, after division, be the year of the Julian periud corresponding to the given years of the ocher three eycles. 'lhus, for the year 1791, the years of the solar, lunar, and indiction eycles, are 8,6 , and 9 ; therctore multiplying by these, \&c, according to the rule, thus

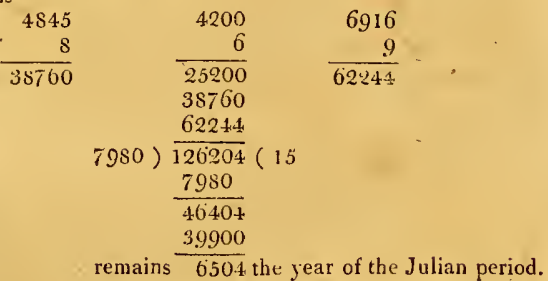

JULian Yerr, is the old account of the ycur, established by Julius Cæsar, and consisted of $365 \frac{1}{4}$ days. 'This year continued in use in all Europe, till it was superseded in most parts by the new or Gregorian account, in the year 1582. In England however it continued to be used till the year 1752, when it was abolished by aet of parliament, and eleven days added to the account, to bring it up to the new style. In Russia, the old or Julian year and style are still in use.

JULY, the 7 th month of the year, consisting of 31 days; about the 21 st of which the sun usually enters the sign $\Omega$ leo. It was so named by Mark Antony, from Julius Cæsar, who was born in this month.

JUNGIUS (Јонснг), a learned Gernan, who was born at Lubeck. He studied at Giessen, where he applied to mathematies and metaphysies, and wrote Latin tragedies. He was some time professor of mathematies, but quitting that station, he studied physic at Augsburg, and took his doetor's degree at Paclua. He was esteemed a vẹry learned man, being autlior of several valuable works, and dierl at Hamburg, in 1657, where his Geometria Empyrica was published, in 1669,4 to.

Dr. Bireh (Hist. Royal Soe. vol. 2, pa. 342) says, Jungius was reetor of the Gymnasium at Hamburg. In the original draught of a letter of Mr. (afterwards Dr.) John Pell, to Sir Charles Cavendyshe, dated at Amsterdam, 1644 , that eminent mathematician recommends Dr. Jungius and John Adolphus Tassins, correetor of the same Gymnasium, to the acquaintanee of Sir Charles, who was then at Hamburg, as both mathematicians, of whose abilities and worth he had a great opinion. He remarked that Jungius had many things in print. As Apodictica, and Apodidactica. That Tassius had, two years before, reprinted Jungius's Geometria Empyrica in 5 sheets of paper, but without diagrams: that Tassius was a very courteous, affable, open man; Jungius a little more reserved. He added, "The most barbarous nations had something to worship; and there are few men that have not some idol, some man or woman, whom they esteem and admire above all the rest of mankind; and Jungius is mine. For I esteem of meu more or less, as I find them more or less rational; and therefore having seen something of Jungius's writing, wherein be seemed to me to make a true and better use of his reason, and to manage that diVol. I. vine instrument of instruments, with more dexterity and skill, than any other son of Adam, alt other writers must pardon me, if I profess to expect more solidity in Jungits's writings, than in any other man now liviug. If you lind but the one half of what I imagine to be in Jungius, you will never be able to relish any other modern philosopher." Sir Charles Cavendyshe accordingly, in a lutter to Mr. Pell, without a date, but reecived by the latter it Amsterdam, Sept. $\frac{19}{2}, 1644$, mentions his having seen Jungius, whom he found very free, and who, he observer, preferred the analyties of the antients before Vieta's by letters, as being more subject to errors and mistakes, though more facile and quick of dispatel.

Mr. Oldenburg, secretary of the Royal Society, real io the Society a Latin letter to him, fiom Dr.Martin Fogelius of Hamburg, dated there Jan. $166 \frac{8}{9}$, containing an account of some manuseripts lift by Jungius, enncerning motion, and Apollonius de Loeis Planis, in 2 books; of the former of which subjects were trausmitted the chief beads, and the method observed in the latter: the writer of which letter expressed his inclination to publish those writings, if encouragement were given to him. It does not appear that any thing was done in consequence of it, but the latter may be-seen in the letter-book of the Royal Soeiety, vol. 3 , pa. 5 .

JUNO, a name given to one of the small newly-discovered planets, viz, that found by M. Harding in the year 1804. See Astronomy, Hardin r, Excentricity, \&c.

JUPITER, 4 , one of the superior planets, remarkable for its splendour, being the brightest of all, except sometimes the planet Venus, and is much the largest of all the planets. Jupiter is situated between Mars and Saturn, being the 9 th in order of the primary planets from the sun. His diameter is more than 10 times the diameter of the earth, and therefore his magnitude more than 1000 times. His real diameter is 89170 miles. His annual revolution about the sun is almost 12 years, viz, 4350 days 14 hours 39 minutes 2 scconds, going at the rate of more than 25 thousand miles per hour, at the mean distance of 490 millions of miles; and he revolves about his own axis in the short space of 9 hours $56^{\circ}$ minutes, by which his cquatorial parts are earried round at the amazing rate of $26^{\circ}$ thousand miles per hour, which is about 25 times faster than the like partş of our earth revolve.

Jupiter is surrourded by faint substanecs, called zones or belts, in which so many ehanges appear, that they are generally ascribed to elouds: for some of them have been first interrupted and broken, and then have vanished entirely. They have sometimes been observed of different breadths, and afterwards have all become nearly of the same breadth. Large spots have been seen in these belts ; and when a belt vanishes, the contiguous spots disappear with it. The broken ends of some belts have oftea been observed to revolve in the same time with the spots; only those nearer the equator in somewhat less time than those nearer the poles; perhaps on account of the sun's greater heat near the equator, which is parallel to the belts and course of the spots. Several large spots, which appear round at one time, grow oblong by degrees, and then divide into two or three round spots. The periodical time of the spots near the equator is 9 hours 50 minutes, but of those near the poles 9 hours 56 minutes. See Dr. Smitl's Opties, $\$ 1105$ and 1109.

The axis of Jupiter is so nearly perpendieular to his orbit, that he hats no sensible change of seasons; which is 4. U 
a great advantage, and considered as wisely ordered by the Author of Nature. For, if the axis of this planet were inclined any considerable number of degrees, just so many degrees round each pole would in their turn be almost 6 years together in darkness. And, as each degree of a great circle on Jupiter contains about 706 miles, it is easy to judge what vast tracts of land would be rendered uninhabitable by any considerable inclination of his axis.

The difference between the equatorial and polar diameters of Jupiter, is upwards of 60010 miles; the former buing to the latter as 13 to 12 : so that his poles are more than 3000 miles nearer his centre than the equator is. This happens from his quick motion round his axis; for the fluids, together with the light particles, which they can carry or wash away with them, recede from the poles which are at rest, towards the equator where the motion is quickest, until there be a sufficient number accumulated to make up the deficiency of gravity lost by the centrifugal force, which always arises from a quick motion round an axis : and when the deficiency of weight or gravity of the particles is made up by a sufficient accumulation, there is then an equilibrium, and the equatorial parts rise no higher.

Jupiter's orbit is $1^{\circ} 18^{\prime} 56^{\prime \prime}$ inclined to the ecliptic. The place of his aphelion $11^{\circ} \mathrm{s}^{\prime}$ of $\bumpeq$, the place of his ascending node $7^{\circ} 29^{\prime}$ of $\sigma_{\sigma}$, and that of his south or descending node $7^{\circ} 29^{\prime}$ of $h^{\circ}$. The excentricity of his orbit is $\frac{\pi}{2}$ of his mean distance from the sun.

The sun appears to Jupiter but the 48 th part so large as to us; and his light and heat are in the same small proportion, but compensated by the quick returns of them, and by 4 moons, some of them larger than our earth, which revolve about him; so that there is scarce any part of this huge planet but what is, during the whole night, enlightened by one or more of these moons, except his poles, whence only the farthest moons can be seen, and where their light is not wanted, because the sun constantly circulates in or near the horizon, and is very probably kept in view of both poles by the refraction of Jupiter's atmosphere, which, if it be like ours, has certainly refractive power enough for that purpose. This planet, seen from its nearest moon, appears 1000 times as large as our moun does to us; increasing and waning in all her monthly shapes, every $42 \frac{\pi}{2}$ hours. The periods, distances, in semidiameters of Jupiter, and angles of the orbits of these moons, seen from the earth, are as follow :

\begin{tabular}{|c|c|c|c|}
\hline No. & $\begin{array}{l}\text { Periods round } \\
\text { Jupiter. } \\
1^{\mathrm{d}} 18^{\mathrm{h}} 36^{\mathrm{m}}\end{array}$ & $\begin{array}{c}\text { Distances. } \\
5 \frac{2}{3}\end{array}$ & $\begin{array}{l}\text { Angles of } \\
\text { orbits. } \\
3^{\prime} 55^{\prime \prime}\end{array}$ \\
\hline 2 & $\begin{array}{lll}3 & 13 & 15\end{array}$ & $9^{3}$ & $6 \quad 14$ \\
\hline 3 & $\begin{array}{lll}7 & 3 & 59\end{array}$ & $14 \frac{x}{3}$ & 958 \\
\hline 4 & $1618 \quad 30$ & $25 \frac{1}{3}$ & 1730 \\
\hline
\end{tabular}

The three nearest moons of Jupiter fall into his shadow, and are eclipsed in every revolution: but the orbit of the th satellite is so much inclined, that it passeth by its opposition to Jupiter, without falling into his shadow, two years in every six. By these eclipses, astronomers have not only discovered that the sun's light takes up 8 minutes of time in coming to us; but liave also by them determined the longitudes of places on this earth, with greater certainty and facility, than by any other method yet known. The outermost of these satellites will appear nenrly as large as the moon does to us. Sce M. Laplace's Theory of Jupiter's Satellit's, in the Memoires de l'Acad. and is the Connoissance des 'Te'mps for 1792, pa. 273.
The English astronomer Mr. Pound long since observed, that the irregularities of the three interior satellites of Jupiter were repeated in a period of 437 days; and this observation is found io be just to the present time. For the following curious circumstance has now been established, viz, that

$\begin{array}{rlllllll}247 & \text { revolutions of the } 1 \text { st occupy } & 437^{\mathrm{d}} & 3^{\mathrm{h}} & 44^{\mathrm{m}} \\ 123 & - & - & 2 \mathrm{l} & - & 437 & 3 & 4.2 \\ 61 & - & - & 3 \mathrm{l} & - & 437 & 3 & 36 \\ 26 & - & - & 4 \mathrm{th} & - & 435 & 14 & 16\end{array}$

This naturally led mathematicians to examine their motions, and see in what manner their relative positions, or configurations, as they are called, corresponded to this period; and it is found, that the mean longitude of the 1st satellite,

minus' 3 times that of the $2 \mathrm{~d}$,

plus 2 times that of the $3 \mathrm{~d}$, always amounted to 180 degrees.

'This requires that the mean motion of the first, added to twice that of the $3 \mathrm{~d}$, shall be equal to 3 times the mean motion of the 2d.-This correspondence of the mean motions is of itscif a singular thing, and the odds or chance against its probability seems infinitely great; and when we add to this the particular positions of the satellites in any one moment, which is necessary for the above constant relation of their longitudes, the inprobability of the coincidence, as a thing quite fortuitons, becomes infinitely greater. Doubts were at first entertained of the coincidence, because it was not indeed accurate to a second. The result of the investigation is curious. When the consequences of mutual gravitation are followed out, we find, that though neither the primitive motions of projection, nor the points of the orbit from which the satellites were projected, were precisely such as suited these observed relations of their revolutions, and their contemporaneous longitudes; yet, if they differed from them only by very minute quantities, the mutual gravitations of the satellites would in time bring them into those positions, and those states of mean motion, that would induce the observed relations; and when they are once reduced, they will be continued for ever.

Though there be $\mathrm{s}$ primary planets below Jupiter, yet an eje placed on his surface would never perceive any of them; unless perhaps as spots passing over the sun's disc, when they happen to come between the eye and the sun. The parallax of the sun, vicwed from Jupiter, will scarce be sensible, being not much above 20 seconds; and the sun's apparent sliameter in Jupiter, but about 6 minutes. Dr. Gregory adds, that an astronomer in Jupiter would easily distinguish two kinds of planets, four nearer him, viz his satellites, and two more remote, viz the sun and Saturn; the former however will fall vastly short of the sun in brightness, notwithstanding the great disproportion in the distances and apparent magnitude.

JUIRIN (Dr. JAMES), a very distinguished person in several walks of titerature, particularly medicine, mathematics, and philosophy, which be cultivnted with cqual success. He was secretary of the Royal Society in London, as well as president of the College of Physicians there, at the time of his death, which happened March 22, 1750. Doctor Jurin was nuthor of several ingeniouy compositions ; particularly An lissay upon Distinct and lndistinct Vision ; printed at the end of the $2 d$ volume of Dr. Smith's System of Optics: also several controversial papers; agninst Michellotti, on the momentum of running 
waters ; against Robins, on distinct vision ; against Bishop Berkeley, on the doctrine of fluxions; and against the partisans of Leibnitz, on the forces of moving bodies; \&c. His papers inserted in the Philos. Traus, are the following:

1. On the Suspension of Water in Capillary Tubes: vol. 30 , pa. $739 .-2$. Observations on the Mlotion of Running Water: pa. 748.-3. On an old Roman Inscription: pa. 813.4. A Discourse on the Power of the Heart: pa. 863 and $929 .-5$. On the Specific Gravity of Human Blood, pa. 1000.-6. Defence of his Doctrine of the Power of the Heart against the Objections of Dr. Keill : pa. 1039.-7. On the Action of Glass Tubes upon Water and Quicksilver : pa.1083.-8. On the Specific
Gravity of Solids when weighed in Water : vol.31, pa.223. -9. On the Motion of Rumning Water, against Michellotti : vol. 32, pa. 179.-10. Remarkable Instance of the Small-pox : vol. 32, pa. 191.-11. Inoculated and Natural Small-pox compared: vol. 32, pa. 213.-12. On Meteorological Diaries: vol. 32, pa. 422.-13. On the Measure and Motion of Running Water: vol. 41, pa. 5 and 65.-14. Meteorological Observations in CharlesTown : vol. 42, pa. 491.-15. On the Action of Springs : vol. 43 , pa. $46 .-16$. On the Force of Bodies in Motion : pa. 423.-17. Dynamic Principles, or Meteorological Principles of Mechanies: vol.66, pa. 103.

\section{$\mathrm{K} \wedge \mathrm{N}$}

K ISTNER ( thematician; the soth year of his age, having filled the chair of professor of mathematics and natural philosophy in the university of Göttingen from the year 1756 , being as it were the Patriarch of European mathematicians. From his pen we have several elementary works on different branches of the mathematics, which have all met witb great success; his Elements of Arithmetic, of Plane and Spherical Trigonometry, and of Perspective, having passed through five editions between the years 1758 and 1794. He was one of those who have the most materially contributed to introduce a sound method into the study of mathematics. The catalogue of his different works fills above nine pages in the last edition of Meusel's German Literature. Among the number are the translations of several important works from the French, the English, and the LowDutch. His History of the Mathematies, in 4 volumes, may be considered as a descriptive catalogue of bis own library: for he possessed a precious collection of all the most rare and valuable works in the mathematical departments.

Kæstner's mode of life was somewhat singular: during the latter years of his life he never went abroad except on Sundays, when he regularly attended the sermons at his parish-church, and on the days when the Royal Literary Society at Göttingen held their sittings, A few months before his death, he was afflicted with a paralytic stroke in his right hand; but so assiduous and indefatigable was he in the prosecution of his studies, that he begall to write with his left; and so continued till the time of his decease.

KANG Hl, one of the greatest princes of China, who reigned in the 17 th century. He, wishing to have a chart of his empire and its environs, employed for that purpose several European missionaries, to whose labour we are indebted for the grand chart of China, first published in 1735, in France, by M. Duhalde; and since with some corrections by Danville. He also caused to be translated into the Chinese and Tartar languages, several treatises on astronomy, and some astronomical tables; as also the Elements of Euclid, which he very much admired. Verbiost calculated for him, in 1683, a tahle of all the eclipses of the sun and moon that would happen in the course of

\section{K E I}

2000 years, See Astron. Europaa by Verbiost. The invention of logarithms, and the tables of sines, delighted him beyond all expression: he soon caused a small edition of them to be made, that he always carried about with him, and which after his death were sent as a curiosity to the library of the jesuits at Lyons. He also very nuch admired the European art of writing; but his ears being accustomed to the grave inusic and monotony of his country, he could never relish the lively and animating music of the Europeans; what we call harmony seemed to him only a confused noise. He had several sons, who were instructed in most of the European sciences, at their own request, thus imitating the example given them by their father.

KEILL (Dr. JOHN), an eminent mathematician and philosopher, was born at Edinburgh in 1671 , and studied in the university of that city. His genius leading him to the mathematics, he made a great progress under David Gregory the professor there, who was one of the first that had embraced and publicly taught the Newtonian philosoplyy. In 1694 he followed his tutor to Oxford, where, being admitted of Baliol-college, he ohtained one of the Scotch exhilitions in that college. It is said he was the first who taught Newton's principles by the experiments on which they are founded: and this it seems he did by an apparatus of instruments of his own providing; by which means he acquired a great reputation in the university. The first public specimen he gave of his skill in mathematical and philosophical knowledge, was his Examination of Dr. Burnet's Theory of the Earth : with Remarks on Mr. Whiston's New Theory; which appeared in 1698. These theories were defended by their respective authors; which drew from him, in 1699, An Examination of the Reflections on the Theory of the Earth, together with A Defence of the. Remarks on Mr. Whiston's New Theory. Dr. Burnet was a man of great humanity, moderation, and candour ; and it was therefore supposed that Keill had treated him too roughly, consillering the great disparity of years between them. Keill however left the doctor in possession of that which has since been thought the great characteristic and excellence of his work; and though he disclaimed him as a philosopher, yet allowed him to be a man of a fine imagiluation. 


\section{K E I}

The year following, Dr. Millington, Sedleian professor of natural philosophy in Oxford, who had been appointed physician to King William, substituted Keill as his deputy, to read the lectures in the public school. This office he discharged with great reputation; and, the term of enjoying the Scotch exhibition at Baliol-college now expiring, he accepted an invitation from Dr. Aldrich, dean of Christchurch, to reside there.

In 1701 he published his celebrated treatise, entitled Introductio ad Veram Physicam, which is supposed to be the best and most useful of all his performances. The first edition of this book contained only fourteen lectures; but to the second, in 1705, he added two more. This work was deservedly esteemed, both at home and abroad, as the best introduction to the Principia, or the new mechanical philosophy, and was reprinted in different places; also a new edition in English was printed at London in 1736 , at the instance of M. Maupertuis, who was then in England.

Being made Fellow of the Royal Society, he publisbed, in the Philos. Trans. 1708, a paper on the Laws of Attraction, and its physical principles: and being offended at a passage in the Acta Eruditorum of Leipsic, where Newton's claim to the first invention of the metbod of fluxions was called in question, he warmly vindicated that claim against Leibnitz. In 1709 lie went to New-England as treasurer of the Palatines; and soon after his return in 1710 , he was chosen Savillian professor of astronomy at Oxford. In 1711 , being attacked by Leibnitz, he entered the lists with that mathematician, in the dispute concerning the invention of fluxions. Leibuitz wrote a letter to Dr. Hans Sloane, then secretary to the Royal Society, requiring Keill, in effect, to make him satisfaction for the injury he had done him in his paper relating to the passage in the Acta Eruditorum; he protested, that he was far from assuming to himself Newton's method of fuxions; and therefore desired that Keill might be obliged to retract his false assertion. On the other hand, Keill desired that he might be permitted to justify what he had asserted; and he made his defence to the approbation of Newton, and other members of the Society. A copy of this was sent to Leibnitz; who, in a second letter, remonstrated still more against Keill's want of candour and sincerity; adding, that it was not fit for one of his age and experience to engage in a dispute with an upstart, who acted without any autbority from Newton, and desiring that the Royal Society would enjoin him to silence. On this, a special commitice was appointed; who, after examining the facts, concluded their report with " declaring Newton to be the inventor of fuxions: and that Mr. Keill, in asserting the same, had been in no respect injurious to M. Leibnitz." The whole proceedings on this matter may be seen in Collins's Commercium Epistolicum, with many valuable papers of Newton, Leibnitz, Gregory, and other mathematicians. In the mean time Keill behaved himself with great firmness and spirit; which be also sbowed afterwards in a Latin epistle, written in 1720 , to John Bernoulli, mathematical professor at Basil, on account of the same usage shown to Newton: in the title-page of which he put the arms of Scotland, viz, a thistle, witb this motto, Nemo me impune lacessit.

$\Delta$ bout the year 1711 , several objections being urged against Newton's philosopliy, in'support of Descartes's nutions of a plenum, Keill published a paper in the Philos. Trans, on the Rarity of Matter, and the 'lenuity of its
Composition. While he was engaged in this dispute, Queen Anne was pleased to appoint him her decipherer; and he continued in that place under King George the 1 st till the year 1716 . The university of Oxford conferred on him the degree of M.D.in 1713; and two years after, he published an edition of Commandine's Enclid, with additions of his own. In 17 is he published his Introductio ad Verain Astronomiam ; which was afterwards, at the request of the duchess of Chandos, translated by himself into English ; and, with several emendations, published in 1721, under the title of An Introduction to True Astronomy, \&c. This was his last gift to the public ; for being that summer seized with a violent fever, it terminated his life Sept. 1, in the 50th year of his age.His papers in the Philos. Trans, above alluded to, are contained in volumes 26 and 29 .

KEıL (Dr. James), an eminent physician and philosopher, and younger brother of Dr. John Keill above mentioned, was also born in Scotland, in 1673 . Having travelled abroad, on his return be read lectures on anatomy with great applause in the universities of Oxford and Cambridge, by the latter of which he had the degree of M. D. conferred upon him. In 1703 hesettled at Northampton as a plissician, where be died of a cancer in the mouth in 1719 . His publications are

I. An English translation of Lemery's Chemistry.

2. On Animal Secretion, the quantity of Blood in the Human Body, and on Muscular Motion.

3. A treatise on Anatomy.

4. Some pieces in the Philos. Trans. vols. 25 and 30.

KEPLER (JoHN), a very eminent astronomer and mathematician, was born at Wiel, in the duchy of Wirtenberg, in 1571. He was the disciple of Mæstlinus, a learned mathematician and astronomer, of whom be learned those sciences, and became afterwards professor of them to three successive emperors, viz, Matthias, Rudolphus, and Ferdinand the $2 \mathrm{~d}$.

To this sagacious philosopher we owe the first discovery of the great laws of the planetary motions, viz, that the planets describe areas that are always proportional to the times; that they move in elliptical orbits, having the sun in one focus; and that the squares of their periodic times, are proportional to the cubes of their mean distances; which are now generally known by the name of Kepler's Laws. As this great man stands as it were at the head of the modern astronomers, we shall cnter at some length intu his history, and clicely from the words of that celcbrated mathematician Mr. Maclaurin.

Kepler had a particular passion for finding analogies and harmonies in nature, after the manner of the Pythagorrans and Platonists; and to this disposition we owe such valuable discoveries, as are more than sufficient to excuse his conccits. Three things, he tells us, he anxiously sought to find out the reason of, from Jis carly youth; viz, Why the planets were 6 in number? Why the dimensions of their orbits were such as Copernicus had described from observations? And what was the analogy or law of their revolutions? He sought for the reasons of the two first of these, in the properties of numbers and plane finures, without success: but at length roflecting, that while the plane regular figures may be infinite in number, the regular solids are only five, as Euclid had long ago demonstrated; he imagined, that certain mysteries in nature might correspond with this remarkable limitation inherent in the essences of things; and the rather, as he found 
that the Pythagoreans had made great use of those five regular solids in their philosophy. He therefore endeavoured to find some relation between the dimensions of these solids and the intervals of the planetary spheres; thus, imagining that a cube, inscribed in the sphere of Saturn, would touch by its six planes the sphere of Jupiter; and that the other four regular solids in like manner fitted the intervals that are between the spheres of the other planets : he at length became persuaded that this was the true reason why the primary planets were preciscly six in number, and that the Author of the World had deternined their distances from the sun, the centre of the system, from a regard to this analogy. Being thus possessed, as be thought, of the grand secret of the Pythagoreans, and greatly pleased with his discovery, he publisherl it in 1596, under the title of Mysterium Cosmographicum: and was for some time so charmed with it, that lie said he would not give up the honour of having invented what was contained in that book, for the electorate of Saxony.

Kepler sent a copy of this book to Tycho Brahé, who did not approve of those abstract speculations concerning the system of the world, but wrote to Kepler, first to lay a solid foundation in observations, and then, by ascending from them, w endeavour to come at the causes of things. Tycho, however, pleased with his genius, was very desirous of having Kepler with hin to assist him in his labours: and having settled, under the protection of the emperor, in Bohemia, where he passed the last years of his life, after having left his native country on account' of some ill usage, le prevailed upon Kepler to leave the university of Gratz, and remove into Bobemia, with his family and library, in the year 1600 . But Tycho dying the next year, the task of arranging the observations devolved upon Kepler, and from that time he had the title of Mathematician to the Emperor all his life, and gained continually more and more reputation by his works. The emperor Rudolph ordered him to finish the tables of Tycho Brahé; which were to be called the Rudolphine Tables, \&c. Kepler applied diligently to the work: but unhappy are those learned men who depend on the good-humour of the intendants of the finances; the treasurcrs were so ill-affected towards our author, that he could not publish these tables till 1627. He died at Ratisbon, in 1630, where he was soliciting the payment of the arrears of his pension.

Kepler made many important discoveries from Tycho's observations, as well as from his own. He found, that astronomers had erred, from the first rise of the science, in ascribing always circular orbits and uniform motions to the planets; that, on the contrary, each of them moves in an ellipsis which has one of its foci in the sun: that the motion of each is really unequable, and varies so, that a ray supposed to be always drawn from the planet to the sun describes equal areas in equal times.

It was some years later before he discovered the analogy existing between the distances of the several planets Irom the sun, and the periods in which they complete their revolutinns. He easily saw, that the higher planets not only moved in greater circuits, but also slower than those near the sun; so that, on a double account, their periodic times were greater. Saturn, for example, revolves at the distance from the sun $9 \frac{\pi}{2}$ times greater than the earth's distance from it ; and the orbit of Saturn is therefore in the same proportion: but as the earth revolves in one year, so, if their velocities were equal, Saturn ought to re- volve in 9 years and a half; whereas the periodic time of Saturn is about 29 years. The periodic times of the planets increase, therefore, in a greater proportion than their distances from the sun: but yet not in so great a proportion as the squares of those distances; for if that were the law of the motions, (the square of $9 \frac{1}{2}$ being $90 \frac{x}{4}$,) the periodic time of Saturn ought to be above 90 years. A mean proportion between that of the distances of the planets, and that of the squares of those distances, is the true proportion of the periodic times; as the mean betiveen $9 \frac{1}{2}$ and its square $90 \frac{\pi}{4}$, gives the perindic time of Saturn in years. Kepler, after having committed several mistakes in determining this analogy, hit upon it at last, Nay 15, 1618; for he is so particular as to mention the precise day when he found that "The squares of the periodic times were always in the same proportion as the cubes of their mean distances from the sun."

When Kepler saw, according to better observations, that his disposition of the five regular solids among the planetary spheres, was not agreeable to the intervals between their orbits, he endeavoured to discover other schemes of harmony; for which purpose, he compared the motions of the same planet at its greatest and least distances, and of the different planets in their several orbits, as they would appear viewed from the sun; and here he fancied that he found a similitude to the divisions of the octave in music. These were the dreams of this ingenious man, which he was so fond of, that, hearing of the discovery of four new planets (the satellites of Jupiter) by Galileo, he owns that his first reflections were from a concern how he could save his favourite scheme, which was threatened by this addition to the number of the planets. The same attachment led him into a wrong judgment concerning the sphere of the fixed stars : for being obliged, by lis doctrine, to allow a vast superiority to the sun in the universe, he restrains the fixed stars within very narrow linits : nor disl he consider them as suns, placed in the centres of their several systems, having planets revolving around them; as the other followers of Copernicus bad allowed them to be, from their having light in themselves, from their immense distances, and from the analogy of uature. Not contented with these harmonies, which he had learned from the observations of Tycho, he gave himself the liberty to imagine several other analogies, that have no foundation in nature, and are overthrown by the best observations. 'Thus from the opinions of Kepler, though most justly admired, we are taught the danger of espousing principles, or hypotheses, borrowed from abstract sciences, and of applying them, with such freedom, to natural enquirics.

A more recent instance of this fondness for discovering analogies between matters of abstract speculation, and the constitution of nature, we find in Huygens, one of the greatest geometricians and astronomers any age has produced: when he had discovered that satellite of $\mathrm{Sa}$ turn, which from him is still called the Huygenian satellite, this, with our moon, and the four satellites of Jupiter, completed the number of six secondary planets then discovered in the system; and because the number of primary planets was also six, and this number is called by mathematicians a perfect number (being equal to the sum of its aliquot parts, 1, 2, 3, ) Huygens was hence induced to believe that the number of the planets was complete, and that it was in vain to look for any more. This is not mentioned to lessen the credit of this great man, who: 
never perhaps reasoned in such a manner on any other occasion; but only to show, by another instance, how ill grounded reasonings of this kind have always proved. For, not long after, the celebrated Cassini discovered four more satellites about Saturn, not to mention the three others that have been discovered belonging to that planet by $\mathrm{Dr}$. Herschel, with another new primary planet and its 6 satellites, besides the other new planets lately discovered. The same Cassini having found that the analogy, discovered by Kepler, between the periodic times and the distances from the centre, takes place in the lesser systems of Jupiter and Saturn, as well as in the great solar system; his observations overturned that groundless analogy which had been imagined between the number of the planets, both primary and secondary, and the number six : but established, at the same time, that harmony in their motions, which will afterwards appear to flow from one real principle extended throughout the universe.

But to return to Kepler; his great sagacity, and continual meditations on the planetary motions, suggested to him some views of the true principles from which these motions flow. In his preface to the commentaries concerning the planet Mars, he speaks of gravity as of a power that was mutual between bodies, and tells us, that the earth and moon tend tuwards each other, and would meet in a point, so many times nearer to the carth than to the moon, as the earth is greater than the moon, if their motions did not prevent it. He adds also that the tides arise from the gravity of the waters towards the moon. But not having notions sufficiently just of the laws of motion, it seems he was not able to make the best use of these thoughts; nor does it appear that he adhered to them steadily, since in his Epitome of Astronomy, published many years after, he proposes a physical account of the planetary motions, derived from different principles.

He supposes, in that treatise, that the motion of the sun on his axis, is preserved by some inherent vital principle; that a certain virtue, or immaterial image of the sun, is diffused with his rays into the ambient spaces, and, revolving with the body of the sun on his axis, takes hold of the planets, and carries them along with it in the same direction; like as a loadstone turned round near a magnetic needle, makes it turn round at the same time. The planet, according to him, by its inertia, endeavours to continue in its place, and the action of the sun's image and this inertia are in a perpetual struggle. He adds, that this action of the sun, like his light, decreases as the distance increases; and therefore moves the same planet with greater celerity when acarer the sun, than at a greater distance. To account for the planet's approaching towards the sun as it descends from the aphelion to the perihelion, and receding from the sun while it ascends to the aphelion again, he supposes that the sun attracts one part of each planet, and repels the opposite part; and that the part attracted is turned towards the sun in the descent, and the other towards the sun in the ascent. By suppositions of this kind, he endeavoured to account for all tbe other varieties of the celestial motions.

But, now that the laws of motiou are better known than they were in Kepler's time, it is easy to show the fallacy of every part of this account of the planetary motions. 'I'he planet does not endeavour to stop in consequence of its inertia, but to persevere in its motion in a right line. An attructive force makes it descend from the apliclion to the peribelion in a curve concave tuwards the sun: but the repelling force, which he supposed to begin at the perihelion, would cause it to ascend in a figure convex towards the sun. There will be occasion to show afterwards from Sir Isaac Newton, how an attraction or gravitation towards the sun, alone produces the effects, which, according to Kepler, required both an attractive and repelling force; and that the virtue which he ascribed to the sun's image, propagated in to the planetary regions, is unnecessary, as it could be of no use for this effect, though it were actmitted. For now his own prophecy, with which he concludes his book, is verified; where he tells us, that, "the discovery of such things was reserved for the succeeding ages, when the Author of Nature would be pleased to reveal these mysteries."-The works of this celebrated author are many and valuable; as,

1. His Cosmographical Mystery, 1596.

2. Optical Astronomy, in 1604 .

3. Account of a New Star in Sagittarius, 1605.

4. New Astronomy; or, Celestial Physics, in Commentaries on the planet Mars.

5. Dissertations; with the Nuncius Siderius of Galileo, 1610.

6. New Gauging of Wine Casks, 1615. Said to be written on occasion of an erroneous measurement of the wine at his marriage by the revenue officer.

7. New Ephemerides, from 1617 to 1620 .

8. Copernican System, three first books of the, 1618 .

9. Harmony of the World; and 3 books of Comets, 1619.

10. Cosmographical Mýstery, 2d edit. with Notes, 1621 .

11. Cojernican Astronomy; the last 3 books, 1622.

12. Logarithms, 1624; and the Supplement, in 1625.

13. His Astronomical Tables, called the Rudolphine Tables, in honour of the emperor Rudolphus, his great and learned patron, in 1627.

14. Epitome of the Copernican Astronomy, 1635.

Besides these, he wrote several pieces on various other branches, as Chronology, Geometry of Solids, Trigonometry, and an excellent treatise of Dioptrics, for that time. His numerous manuscripts were purchased from his representatives, by Hevelius, and were probably consumed by a fire at the observatory of the latter, in the year 1679 , which destroyed many of his valuable books and instruments.

KEPLER's LAw', are those laws of the planctary motions discovered by Kepler. These discoverjes in the mundane system, are commonly accounted two, viz. 1st, That the planets describe about the sun, areas that are proportional to the times in which they are described, namely, by a line connecring the sun and planet; and $2 d$, That the squares of the times of revolution, are as the cubes of the mean distances of the planets from the sur. Kepler discovered also that the orbits of the planets are elliptical.

These discoveries of Kepler, however, were only found out by many trials, in searching among a great number of astronomical obscrvations and revolutions, what rules and laws were found to obtain. On the other hand, Newton has demonstrated, a priori, all these laws, showing that they must obtain in the mundane system, from the laws of gravitation and cenrripetal force; viz, the first of these laws resulting from a centripetal force urging the planets towards the sun, and the $2 \mathrm{~d}$, from the centripetal force being in an iuverse ratio of the square of the distance. And the elliptic form of the orbits, from a projectile force regulated by a centripetal onc. 
KEPLER's Problem, is the determining the true from the mean anomaly of a planet, or the determining its place, in its elliptic orbit, answering to any given time; and so named from the celebrated astronomer Kcpler, who first proposell it. See ANomaLx.

The general state of the problem is this: To find the position of a right linc, which, passing through one of the foci of an ellipsis, shall cut off an area which shall be in any given proportion to the whole area of the cllipsis; which results from this property, that such a line sweeps areas that are proportional to the times. Nany solutions have beén given of this problem, some direct and geometrical, others not: viz, by Kepler, Bulliald, Ward, Newton, Keill, Machin, \&c. See Newton's Princip. lib. 1. prop. 31, Keili's Astroṇ. Lect, 23, Philos. Trans. Abr. vol. 8 , pa. 177 , \& $\mathrm{c}$.

In the last of these places, Mr. Machin observes, that many attempts have been made at different times, but with no great success, towards the solution of the problem proposed by Kepler: To divide the area of a semicircle into given parts, by a line drawn from a given point in the diameter, in order to find a universal rule for the motion of a body in an elliptic orlit. For, anong the several methods offered, some are only true in speculation, but are really of no service; others are not different from his own, which he judgcd improper. And as to the rest, thicy are all so limited and confined to particular conditions and circumstances, as still to leave the problem in general untouched. To be more particular; it is evident, that all constructions by mechanical curves are seeming solutions only, being in reality unapplicablc; that the roots of infinite series are, on account of thcir known limitations in all respects, so far from being sufficient rules, that they scrve for little more than exercises in a method of calculation. And then, as to the universal method, which proceeds by a continued correction of the errors of a false position, it is no method of solution at all in itsclf; bccause, unless there be some antecedent rulc or hypothesis to bcgin the operation (as suppose that of an uniform motion about the upper focus, for the orbit of a planct; or that of a motion in a parabola for the perihclion part of the orbit of a comet, or something of a similar nature, it would be impossible to proceed one step in it. But as no gencral rule has ever yet been laid down, to assist this method, so as to make it always operate, it is the same in effect as if therc were no method at all. And accordingly in experience it is found, that there is no rule now subsisting but what is absolutely useless in the elliptic orbits of comets; for in such cases there is no other way to procced but that which was used by Kepler: to compute a table for some part of the orbit, and in it examine if the time to which the place is required, will fall out any where in that part. So that, upon the whole, it appears cvident, that this problem, contrary to the rcceived opinion, has ncver yet been advanced one step towarls its true solution.

Mr. Michin then proceels to give his own solution of this problem, which is particularly neccssary in orbits of a great excentricity; and he illustrates his method by examples for the orbits of Venus, of Mercury, of the comet of the year 1682 , and of the great comet of the year 1680 , sufficiently showing the universality of the method.

$\mathrm{KFY}$, in Music, is a certain fundamental notc, or tone, to which the wholc piece, be it concerto, sonata, cantata, \& $c$ c, is accommodated; and with which it usually begins, and always ends, - See $C_{L E F}$.
Kers dcnote also, in an organ, or harpsichord, \&c, the pieccs of wood or ivory which are struck by the fingers, in playing upon the instrument.

KEY STON E, is the middle voussoir, or the arch stone in the top, or immediately over the centre of an arch.-The length of the keystone, or thickness of the archivolt at top, is allowed by the best architects, to be about the 15 th or 16 th part of the span.

KIIDERKLN, a kind of liquid measure, containing two firkins, or 18 gallons, beer-measure.

K1NG-piece, or KING-post, is a piece of timber set upright in the iniddle, between two principal rafters, and having struts or braces going from it to the middle of each rafter.

KIRBY (JosuUA), was the eldest of the five sons of Mr. John Kirby; who was originally a schoolmaster at Orford; but afterwards took an actual survey of the county of Suffolk in the year 1732 , \&c, and published a small map of it; as also the Suffolk Traveller, 1735, $12 \mathrm{mo}$; a new and enlarged edition of which was published by subscription in 1764 , in which the name of "Joshua Kirby, Est. désigner in perspective to his majesty," occurs for 50 copies. Mr. John Kiroy died at Ipswich, Dec. 13, 1753 , aged 63 .

Our author Joshua was born in 1716 , at Parham, near Wickham-Market; and settled as a house-painter at Ipsivich about the year 1738. He always had a genius for drawing, and published 12 prints of castles, ancient churches and monuments, in Suffolk, with a small pamphlet, containing further illustrations of them. But the study which led him to eminence, was that of the art of perspective, in his improvement of which it may almost be said that he invented a ncw art; so simple was his method, in comparison with the systems at that time in use. Ile had made a considerable progress in his treatise, when he met with Dr. Brook Taylur's book, which contributed to the perfecting of those rules, by which he rendered this art so easy, that on the publication of his work he was requested by the Society of Artists to read lectures; for which he received the thanks of its incmbers, in the public papers.

Having obtained the friendship and intimacy of Sir Joshua Reynolds, Mr. Hogarth, and other eminent artists, he quitted Ipswich, and removed to London; where he was patronized by the earl of Butc, who introduced him to his present majesty, when prince of Wales, and was appointed clerk of the works at Kew. By his majesty's patronage he published, in 1761, his splendisl work, The Perspective of Architecture, in 2 vols. folio; bcing a masterly performance, and the elegant designs with which it is illustrated reflect honour on the artists of our country.In 1766 , in conjunction with his brother William, then of Witnisham, in the county of Suffolk, attorncy at law (who died Sept. 25, 1791, aged 72), he published an improved edition of their father's map of Suffolk, on a larger scale. Mr. K. was a member both of the Royal and Autiquarian Societies; and, at the first forming of the Royal Academy, he was president of the Society for Artists, from which that institution cmanated. In the year 1768 he published a 3 d clition of his Treatise on Perspective. MIr. K. died June 20,1774 , aged 58, and was buried in Kew churchyard, leaving his widow, who died the year after, and a daughter, the celebrated Mrs. Trimmer, of Old Brentford, csteemed for numerous works for the instruction of youth. KIRCH (Christian FrEDERIC), of Berlin, a cele- 
brated astronomer, was born at Guben in 1694 . He acquired great reputation in the observatories of Dantzic and Berlin. Godfrey Kirch his father, and Mary his mother, also acquired considerable reputation by their astronomical observations. This family corresponded with all the learned societies of Europe, and their astronomical works are in great repute.

KIRCHER (ATHaNasius), a noted plilosopher and mathematician, was born at Fulde in 1601. He entered into the society of the Jesuits in 1618, and taught philosophy, mathematics, the Hebrew and Syriac languages, in the university of Wirtsburg, with great applause, till the year 1631; when be retired to France, on account of the ravages committed by the Swedes in Franconia, and lived some time at Avignon. He was afterwards called to Rome, where he taught mathematics in the Roman college, collected a rich cabinet of machines and antiquities, and died in 1680 , in the soth year of his age.

The quantity of his works is immense, amounting to 22 volumes in folio, 11 in quarto, and three in octavo; enough to employ a man for a great part of his life even to transcribe them. Most of them are rather curious than useful; many of them visionary and fanciful; and it is not to be wondered at if they are not always accompanied with the greatest exactness and precision. The principal of them are,

1. Prælusiones Magneticæ.

2. Primitiæ Gnomonicæ Catoptricæ.

3. Ars magna Lucis et Umbræ.

4. Musurgia Universalis.

5. Obeliscus Pamphilius.

6. Oedipus Ægyptiacus; 4 volumes folio.

7. Itinerarium Extaticum.

8. Obeliscus Ægyptiacus; 4 volumes folio.

9. Mundus Subterraneus.

10. China lllustrata.

KNOT, a tye, or complication of a rope, cord, or string, or of the ends of two together. There are divers sorts of kuots used for different purposes, which may be explained by showing the figures of them open, or undrawn, thus. 1. Fig. 1, plate 17 , is a Thumb knot. This is the simplest of all. It is used to tye at the end of a rope, to prevent its opening out: it is also used by taylors, \&c, at the end of their thread.

Fig. 2, a Loop knot. Used to join pieces of rope, \&c, together.-Fig. 3, a Draw knot, which is the same as the last; only one end or both return the same way back, as $a b c d$. By drawing at $a$, the part $b c d$ comes tbrough, and the knot is loosed.-Fig. 4, a Ring keot. This serves also to join pieces of cord, \&c, together.-Fig. 5 is another knot for tying cords together. This is used when any cord is often to be loosed.-Fig.6, a Running knot, to draw any thing close. By pulling at the end $a$, the cord is drawn througb the loop $b$, and the part $c d$ is drawn close about a beam, \&c.-Fig. 7 is a nother knot, to tye any thing 10 it post. And here the end may be put through as often as you please.-Fig. 8, a Very small knot. A thumb knot is first made at the end of each piece, and then the end of the other is passed through it. Thus, the cord $a c$ runs through the loop $d$, and $b d$ through $c$; and then drawn close by pulling at $a$ and $b$. If the ends $e$ and $f$ be drawn, the knot will be loosed again.-Fig. 9, a Fisher's knot, or Water knot. This is the same as the $4 \mathrm{t} \mathrm{l}$, only the ends are to be put twice through the ring; which in the former was but once; and then drawn close.-Fig. 10, a Meshing knot, for nets; and is to be drawn close.-Fig. 11, a Barber's knot, or a knot for cawls of wigs; and is to be drawn close. -Fig. 12, a Bowline knot. When this is drawn close, it makes a loop that will not slip, as fig. 7 ; and serves to hitch over any thing.-Fig. 13, a Wale knot, which is made with the three strands of a rope, so that it cannot slip. When the rope is put through a hole, this knot keeps it from slipping through. When the three strands are wrought round once or twice more, after the same manner, it is called crowning. By this means the knot is made larger and stronger. A thumb knot, No. 1, may be applied to the same use as this.

KNOTS mean also the divisions of the $\log$ line, used at sea. These are usually $T$ fathoms, or 42 feet asunder; but should be $8 \frac{1}{3}$ fathoms, or 50 feet. And then, as many kuots as the $\log$ line runs out in half a minute, so many miles does the ship sail in an hour; supposing her to keep going at an equal rate, and allowing for yaws, leeway, \&c.

KOENIG (SAMUEL), a learned philosopher and mathematician, was a Swiss by birth, and came early into eminence by his mathematical abilities. He was professor of philosophy and natural law at Franeker, and atterwards at the Hague, where he became also librarian to the stadtholder, and to the princess of Orange; and where he died in 1757 .

The Academy of Berlin enrolled him among ber members; but afterwards expelled him on the following occasion. Maupertuis, the president, had inserted in the volume of the Memoirs for 1746 , a discourse on the Laws of Motion; which Koenig not only attacked, but also attributed the memoir to Leibnitz: Maupertuis, stung with the imputation of plagiarism, engaged the Acarlemy of Berlin to call upon him for his proof; which Koenig failing to produce, he was struck out of the academy. All Europe was interested in the quarrel which this occasioned betwcen Koenig and Maupertuis. The former appealed to the public; and his appeal, written with the animation of resentment, procured him many friends. He was author of some other works, and liad the character of being one of the best mathematicians of the age. 
$\mathbf{L}^{\mathrm{A}}$ ABEL, a long thin brass ruler, with a small sight at one end, and a central hole at the other; commonly used with a tangent-line on the edge of a circumferentor, to take altitudes, and other angles.

LACAILLE (Nicholas Lewis), an eminent French mathematician and astronomer, was born at Rumigny in the diocese of Rheims, in 1713. His father having quitted the army, in which he had served, amused himself in his retirement witl studying mathematics and mechanics, in which he proved the happy author of several inventions of considerable use to the public. From this example of his father, our author almost in his infancy took a fancy to mechanics, and at school discovered very early tokens of genius. In 1729 he came to Paris, where he studied the classics, philosophy, and mathematics. He afterwards studied divinity in the college de Navarre, with the view of embracing the ecclesiastical life: however he never entered into priest's orders, apprehending that his astronomical studies, to which he had become much devoted, might too much interfere with his religious duties. His attachment to astronomy soon connected him with the celebrated Cassini, who procured him an apartment in the observatory; where, assisted by the counsels of this celebrated man, he soon acquired a name among the astronomers. In 1739 he was engaged with M. Cassini de Thury, son to M. Cassini, in verifying the meridian through the whole extent of France: and in the same year he was named professor of mathematics in the college of Mazarine. In 1741 our author was admitted into the Academy of Sciences as an adjoint member for astronomy; and had many excellent papers inserted in their Memoirs; beside which be published several useful treatises, viz, Elements of Geometry, Astronomy, Mechanics, and Optics. He also carefully computed all the eclipses of the sun and moon that bad happened since the Christian æra, which were printed in the work entitled l'Art de verifier les Dates, \&c, Paris 1750 , in 4to. He alsocompiled a volume of astronomical ephemerides for the years 1745 to 1755 ; another for the years 1755 to 1765 ; and a third for the years 1765 to 1775 : as also the most correct solar tables of any; andan excellent work entitled Astronomix fundamenta novissimis solis et stellarum observationibus stabilita.

'Having gone through a series of seven years' astronomical observations in his own observatory in the Mazarine college, he formed the project of going to observe the southern stars at the Cape of Good Hope: being countenanced by the French court, he set out upon this expedition in 1750, and in two years he observed the places of about 10 thousand stars in the southern hemisphere that are not visible in our latitudes, as well as many other important elements, viz, the parallaxes of the sun, moon, and some of the planets, the obliquity of the ecliptic, the refractions, \&c. Having thus executed the purpose of his voyage, and no present opportunity offering for his return, he thought of employing the vacant time in another arduous attempt, namely that of taking the measure of the carth, as he had already done that of the heavens. This indeed had becn done before by many learned men both in Furope and America; some determining the quantity of VOL. I. a degree at the equator, and others at the arctic circle: but it had not yet been decided whether in the southern parallels of latitude the same dimensions obtained as in the northern. His labours were rewarded with the satisfaction be wished for; having determined a distance of 410814 feet from a place called Klip-Fontyn to the Cape, by means of a base of 38802 feet, three times actually measured: he discovered a new secret of nature, namely, that the radii of the parallels in south latitude are not of the same length as those of the corresponding parallels in north latitude. About the 23d degree of south latitude he found a degree on the meridian to contain 342222 Paris feet. Agreeably to the orders from the: court of Versailles, he fixed the situation of the Isles of France and of Bourbon. While at the Cape, he observed a wonderful effect of the atmosphere in some states of it: though the sky at the Cape be generally pure and serene, yet when the south-east wind blows, which is pretty often, it is attended with some strange and even terrible effects: the stars look larger, and appear to dance; the moon has an undulating tremor; and the planets have a.sort of beard like consets.

M. Lacaille returned to France in the autumn of 1754 , after an absence of 4 years; loaded, not with the spoils of the east, but with those of the southern heavens, before then almost unknown to astronomers. On his return, he first drew up a reply to some strictures which the celebrated Euler had published relative to the meridian: after which he settled the results of the comparison of his observations for the parallaxes, with those of other astronomers: that of the sun he fixed at $9 \frac{1^{\prime \prime}}{2}$; of the moon at $56^{\prime}$ $56^{\prime \prime}$; of Mars in his opposition, $36^{\prime \prime}$; of Venus $38^{\prime \prime}$. He also settled the laws by which astronomical refractions are varied by the different density or rarity of the air, by heat or cold, and by dryness or moisture. And lastly he showed an easy and practicable method of finding the longitude at sea by means of the moon. His fame being now generally established, he was elected a member of most of the academies and societies of Europe, as London, Bologna, Petersburg, Berlin, Stockholm, and Göttingen.

In 1760 our author was attacked with a severe fit of the gout; which however did not interrupt the course of his studies; for he then planned a new and large work, intended as a history of astronomy through all ages, with a comparison of the ancient and modern observations, and the construction and use of the instruments employed in making them. Towards the latter part of 1761 , his constitution became greatly reduced; though his mind continued unaffected, and he resolutely persisted in his studies to the last; death only putting an end to his labours the 21 st of March 1762 , at 49 years of age; after having committed bis manuscripts to the care and discretion of his esteemed friend M. Maraldi.

Besides the publications before mentioned, and perhaps some others also, he had a vast number of papers inserted in the volumes of the Meinoirs of the French Acadeny of Sciences, much too numerous indeed, though very important, to be here all mentioned particularly; suffice it thereforc just to distinguish the years of those volumes in $4 \mathrm{X}$ 
which his pieces are to be found, by the following list of them, viz, 1741, 1742, 1743, 1744, 1745, 1746, 1747, $1748,1749,1750,1751,1752,1753,1754,1755,1756$, $1757,1758,1759,1760,1761,1763$; in all or most of which years there are two or three or more of his papers.

LACERTA, Lizard, one of the new constellations of the northern hemisphere, added by Hevelius to the 48 old ones, near Cepheus and Cassiopeia. This constellation contains, in Hevelius's - catalogue 10 stars, and in Flamsteed's 16 .

LACUNAR, an arched roof or cieling; more especially the planking or flooring above the porticos.

LADY-Day, the 25th of March, being the Annunciation of the Holy Virgin.

LAGNY (ThoMas FANTET DE), an eminent French mathematician, was born at Lyons. Fournier's Euclid, and Pelletier's Algebra, by chance falling in his way, developed his genius for the mathematics. It was in vain that his father designed him for the law; he went to Paris to deliver himself wholly up to the study of his favourite science; and in 1697, the Abbé Bignon, protector-genera] of letters, got him appointed professor-royal of hydrography at Rochefort. Soon after, the duke of Orleans, then regent of France, fixed him at Paris, and made him subdirector of the general bank, in which he lost the greatest part of his fortune in the failure of that establishment. He had been received into the ancient academy in 1696 ; on the renewal of which he was named associate-geometrician in 1699, and pensioner in 1723. After a life spent in close application, he died, April 12, 1734.

De Lagny particularly excelled in arithmetic, algebra, and geometry, in which he made many improvements and discoveries. $\mathrm{He}$, as well as Leibnitz, invented a binary arithmetic, in which only two figures are concerned. He rendered much easier the resolution of algebraic equations, especially the irreducible case in cubic equations; and the numeral resolution of the higher powers, by means of short approximating theorems.-He delivered the measures of angles in a new science, called Goniometry; in which he neasured angles by a pair of compasses, without scales, or tables, to great exactness; and thus gave a new appearance to trigonometry.-Cyclometry, or the measure of the circle, was also an object of his attention; and he calculated, by means of infinite series, the ratio of the eircumference of a circle to its diameter, to 120 places of figures.-He gave a general theorem for the tangents of multiple arcs: with many other curious or useful improvements, which are found in the great multitude of his papers, that are printed in the different volumes of the Memoirs of the Academy of Sciences, viz, in almost every volume, from the year 1699 to 1729 .

LAGRANGE (JosepH Lours), a most illustrious mathematician and philosopher, was born at Turin, Nov.25, 1736, and dicel at Paris, Aprit 1813. His father was treasurer of war; but falling into poverty through unfortunate specalations, induced our author to endeavour to render himself distinguished in literature. His taste however for mathematics did not appear early. He was passionately devoted to Cicero and Vurgil, before he could relish Archimedes and Newton. Ilc soon however became an enthu-iastic achuirr of the geonetry of the ancients, which he at first preferrod to the modern analysis. But a memoir which the celebrated Halley had composed to demonstrate the superiority of the analytic method, had the effect of teaching him his true path to glory. He devoted bimself to this new study with the same success that he had had to synthesis; and his application was so de. cided, that at the age of 16 , he became professor of mathematics in the royal military school at 'Turin.

The youth of the professor however had no effect in hindering the pupils, all older than himself, from profiting, by his lessons. Sume of them he distinguished as his' friends; and this early association laid the foundation of an important institution, the Academy of Turin, which published, in 1759, a first volume, under the title of Actes de la Societé Priveé. In those acts it appears that the young Lagrange directed the philosophical researches of Cigna the anatomist, and the labours of the Chevalier de Saluces. He furnished to Foncenex the aualytical part of his memoirs, leaving to him the task of develuping the reasoning upon which the formulas depended. But Lagrange, while he abandoned insulated theorems, published at the same time, under his own name, theories which lie promised to develope further. Thus, alter giving new formulas of maxima and minima, in all cases, after having shown the insufficiency of the known methods, he announced that he would treat this subject, which he considered as insportant, in a work which he was preparing, in which would be deduced, from the same principles, all the mechanical properties of bodies, whether solid or fluid, thereby laying, at the age of 23 , the foundation of those great works which constitute the admiration of philosophers.

Newton had undertaken to submit the motions of fluids to calculation, and had made researches on the propagation of suund; but his principles were insufficient, and his suppositions sometimes erroneous. Lagrange demonstrated this, and founded his researches on the known laws of dynamics, by considering only in the air the particles which are in a straight line, thereby reducing the problem to that of vibrating chords. He demonstrated, that, whatever figure is given to the chord, the duration of the oscillations is always the same: a truth derived liom experiment, but which Dalembert considered as very difficult, if not impossible, to demonstrate. He then passed to the propagation of sound, treated of simple and compound echoes, of the mixture of sounds, of the possibility of their spreading in the same space without interfering with each other, and demonstrated rigorously the generation of harmonious sounds.

Euler saw the merit of the new method, and adopted it as an object of his profoundest meditations; but Dalembert did not yield the point in dispute. In his private letters, as well as in his printed memoirs, he proposed numerous objections, to which Lagrange afterwards answered. The first notice of Euter was to make Lagrange an associate of the Berlin academy. When he announced to him this nomination on the 20th of October, 1759, he said, "Your sulution of the problem of isoperimetres leaves nothing to desire; and I am happy that this subject, with which I have been almost solily vecupiod since my first attempts, has been carried by you to the highest degree of perfection. The importance of the point has induced mo to draw up, with your unsistance, "n analytical solution of it. But I shall not publish it till you have published the sequel of your rearenches, that I may not deprive you of any part of the glory which is your due."

Dalembert considered it impossible to subject to calctlation the motions of a fluid inclosed in a vessel, unless 
this vessel had a ertain figure. Lagrange demonstrated the contrary; except in the case when the fluid divides itself into different masses. But even then we may determine the places where the fluid divides itself into different portions, and determine the motion of each as if it were alone. Dalembert thought, that in a fluid mass, such as the earth may have been at its origin, it was not necessary for the different beds to be on a level; but Lagrange shows, that the equations of Dalembert are themselves equations of beds on a level. In combating Dalembert with all the respect due to a mathematician of his rank, he often employed very beautiful theorems, for which he had been indebted to his adversary. Dalembert, on his side, added to the delicacy of Lagrange. "Your problem appeared to me so beautiful," says he in a letter to Lagrange, "that I have sought for another solution of it. I have found a simpler method of arriving at your elegant formula."

The Academy of Sciences of Paris proposed, at this time (1764), as the subject of a prize, the theory of the libration of the moon, i. e. they demanded the cause why the moon, in revolving round the earth, always turns the same face to it, some variations excepted, observed by astronomers, and of which Cassini had first explained the phenomenon. The point was to calculate all the phenomena, and to deduce them from the principle of universal gravitation. Such a subject was an appeal to the genius of Lagrange, an opportunity furnished to apply his analytical principles and discoveries. The hope of Dalembert was not disappointed, and the memoir of Lagrange is one of his finest pieces. We see in it the germ of his Mecanique Analytique. Dalembert wrote to bim: "I have read with as much pleasure as advantage, your excellent paper on the moon's librations, so worthy of the prize which it has obtained."

This success encouraged the Academy to propose, as a prize, the theory of the satellites of Jupiter. Euler, Clairaut, and Dalembert, employed themselves about the problem of these bodies on the principle of the movements of the moon. Bailly applied the theory of Clairaut to the problem of the satcllites, and it led him to some interesting results. But his theory was insufficient; the earth has only one moon, while Jupiter has four, which continue to act upon each other, and vary their positions in their revolutions. The problem was one of six bodies : but Lagrange attacked the difficulty and overcame it, demonstrating the cause of the inequalities observed by astronomers, and pointing out some others too trivial to be ascertained by observations. The shortness of the time allowed, and the multiplicity of the calculations, analytical and numerical, did not permit him to exhaust the subject entirely in a first memoir. He was sensible of this himself, and promised further results, which his other labours always prevented him from completing; but twenty-four years after, M. Laplace took up that difficult theory, and completed it.

About the same time a problem of a different kind drew the attention of MI. Lagrange. Fermat, a great mathematician, had left some remarkable theorems respecting the properties of numbers, which it seems he discovered by induction. He promised the demonstrations of them, but at his death no trace of them could be found. Many mathematicians employed themselves on the theorems of Fermat; but none were successful. Euler alone had penetrated into that difficult path ; but Lagrange, in demonstrating or rectifying some opinions of Euler, resolved a problem which gave him a key to all the others; and from which be deduced the complete solution of equations of the second degree, with two indeterminates which must be whole numbers.

His residence at Turin was not agreeable to him. He saw no peison who cultivated the mathematics with success. He was impatient to visit Paris. M. de Caraccioli, with whom he lived in the greatest intimacy, was appointed ambassador to London, and was to pass through Paris on his way, where he intended to spend some time. He proposed this journey to M. Lagrange, who accepted it with joy, and was received at Paris, as he had a right to expect, by Dalembert, Cląiraut, Condorcet, Fontaine, Nollet, Marie, and the other mathematicians. Falling dangerously ill after a dinner, in the ltalian style, given by Nollet, he was not able to accompany his friend to London, who was obliged to leave him in a furnished lodging under the care of an agent.

This incident changed his projects, and he thought of returning to Turin, when he understood that the academy of Berlin was threatened with the loss of Euler, who intended to return to Petersburg. Dalembert suggested the idea of putting Lagrange in the place of Euler, and Euler himself pointed out Lagrange as the only man capable of filling his place. Lagrange was therefore appointed, and he received a pension of 1500 Prussian crowns, about $250 l$. with the title of director of the academy in regard to the physico-mathematical sciences. Euler and Ligrange, in the place of Maupertuis, receiver only half his salary, but Frederick had no respect for the sciences, though he considered himself obliged to protect them as a king.

Lagrange took possession of his new situation in $\mathbf{1 7 6 6}$. He was well received by the king; but he soon perceived that the Germans do not like foreigners to occupy situations in their country. He applied to the study of their language, and devoted himself to mathematics. "The king," sail he, " treated me well ; I thought that he preferred me to Eulcr, who was something of a devotee, while I took no part in the disputes about worship; and did not contradict the opinion of any one."

It will not be expected that I should follow Lagrange in the important researches with which he filled the Berlin Memoirs; and even some volumes of the Memoirs of the Turin Academy, which was indebted to him for its existence. All the space that could be devoted to this biography, would be insufficient to give even an imperfect idea of his labours. Some of these nemoirs are of such extent and importance, that they might pass for great separate works, yet they constituted a part only of what these 20 years enabled him to produce. He now composed his Mecanique Analytique, and he was anxious to have it printed at Paris; but he experienced great diffculty in finding a bookscller who would undertake to publish a work which, the more sublime the theory, the fewer readers would be found capable of appreciating. At length onc was found, and the work was ably edited by $M$. Legendre, who was repaid by the esteem and the gratitude of the author. The death of Frederick occasioned great changes in Prussia, and still greater were to be apprehended. Philosophers were no longer respected as heretofore, and these causes, with the approaching publication of the Mecanique Analytique, were sufficient to bring lim back to Paris.

The successor of Frederick, though he did not much $4 \mathrm{X}_{2}$ 
interest himself in the sciences, made some difficulty in allowing a philosopber to depart, whom his predecessor hadinvited, and whom he had honoured with his particular esteem. After some delay, M. Lagrange obtained liberty to depart, but it was stipulated that he should still give some memoirs to the Berlin academy, and the volumes of 1792,1793 , and 1803 , show that he kept his promise.

In 1787 then, Lagrange returned to Paris, to take his seat in the Academy of Sciences, of which he had been a foreign member for 15 years. To give him the right of voting in all their deliberations, this title was changed into that of Veteran Pensionary. The queen treated him with regard, and considered him as a German. He had been recommended to her from Vienna; and he obtained, in consequence, a lodging in the Louvre, where he lived happy till the revolution.

The satisfaction he enjoyed in his new residence, did not however appear outwardly. He was affable and kind when interrogated, but he spoke little, and appeared absent and melancholy. In companies which must have been suited to his taste, among the most distinguished men of all countries who met, at the house of the illustrious Lavoisier, he was seen musing, with his head reclined against a window, where nothing seemed to attract his attention. He acknowledged, himself, that his enthusiasm was gone, that he had lost even his taste for mathematics. When informed that any mathematician was employed on a particular object, "so much the better," he would say, "I had begun it, it will now be unnecessary for me to finish it." But he had merely changed the object of his studies. Metaphysics, the history of human nature, of different religions, the general theory of languages, medicine, and botany, divided his leisure hours. Surrounded by chemists who were reforming the theory and language of the science, he made himself acquainted with their discoveries, which gave to facts formerly isolated, that connexion which distinguishes the different parts of mathematics. In this philosophical repose he passed his time till the revolution, without adding any thing to his matbematical discoveries.

The revolution gave philosophers the power of making a great and difficult innovation in the establishment of a system of weights and measures, founded on nature, and perfectly analogous to the decimal scale of numbers. Lagrange was one of the commissioners whom the Academy charged with the execution of that task, and be was one of its most ardent promoters, for he wished to see the decimal system adopted in all its simplicity.

When the academy was suppressed, the commission charged with the establishment of the new system was interrupted for a time. In order to purify it, the names of Lavoisicr, Borda, Laplace, Coulomb, Brisson, and Delambre, were struck out. Lagrange was retaincd, as president; though he could not help expressing his regret at the dismission of so many of his associates; but unless the suppression had been tntal, it could scarcely have extended to him. Besides, he was known to be whslly devoted to the sciences; he had 110 place either in the civil department or the administration; and the moderation of his character hisd prevented him from expressing what he could not but think in secret. The horrors, however, attending the revolution, made a strong impression on his sensible mind. In a conversation with M. Delambre, the day after the atrocious and absurd sentence which, con- trary to every thing like justice, bad thrown all lovers of the sciences into mourning, by cutting off the most illustrious philosopher in Europe, Lavoisier: "It bas cost them but a moment," said he, " to cut off that head; but a hundred years perhaps will not be sufficient to produce another like it." Some months before they had had a sinilar conversation in the cabinet of Lavoisier, on account of the death of the unfortunate Bailly. Helamented the dreadful consequences of the dangerous experiment which the irench had attempted. The projection of their plans of amelioration appeared to him no certain proofs of greatness in the human mind: "If you wish tosee it truly" great," added he, " enter into the cabinet of Newton, employed in decomposing light, or in explaining the system of the universe."

For some time he regretted that he had not listened to the advice of his friends, who at the commencement of the troubles had recommended hin to seek an asylum, which it would have been so easy to find. As long as the revolution seemed only to threaten the pension which he enjoyed in France, he had neglected that consideration, from a feeling of curiosity to be upon the spot of one of those great convulsions which it is always more prudent to observe at a distance. "It was your own choice," said he several times to himself. It was to no purpose that a special decree of the Constituent Assembly had ensured the payment of his pension, because the depreciation of the paper currency rendered it illusory. He bad been named member of the board of consultation, appointed to examine and reward useful inventions, and he had been appointed one of the administrators of the Mint. This commission offered him few objects to fix his attention, and in no degree removed his apprehensions. It was aguin proposed to draw him to Berlin, and to restore him to his former situation, and he agreed to the proposal. Herault de Sechelles, to whom he applied for a passport, offered him, for greater security, a mission to Prussia; but Madame Lagrange, his wife, would not consent to quit her country; a repugnance which at that dime be considered as a misfortune, though it proved a source of fortune and new glory.

The Norman school, of which he was named professor, but which had only an eṕhemeral existence, scarcely gave him time to explain his ideas respecting the foundation of arithmetic and algebra, and their application to geometry. The Polytechnic school, a happier idea, had a more durable success: and, among the best effects which it produced, we may place that of having restored Lagrange to analysis. There he had an opportunity of developing those ideas, the germ of which was to be found in two memoirs published in 1772 , and the object of which was to explain the true metaphysics of the differential and integral calculus. He composed his Analytical Functions, and his Lectures on that Calculus, of which he published screral editions. He likewise published his Treatise on the Numerical Solution of Equations, with notes on several points of the theory of algebraic equations.

The desire of multiplying useful applications induced him to undertake a new edition of the Mecanique Analytique. His project was to develope the most useful parts of it. He laboured on it with all the ardour and intellectual power which lie coull have applied at any period of his life. Bat this application occasioned a degree of fatigue which threw hin into a fainting fit, and he was found in that state by Madame Lagrange. Mis head in 


\section{A G}

falling had struck against the corner of a table, and the shock had not restored him to his senses. This was a warning to take more care of himself, and he thought so at first; but he was too anxious to finish his work, the printing of which was not completed at the period of his decease. The first volume had appeared some time before his death. It had been followed by a new edition of his Fonctious Analy tiques. So much labour exhausted him. Towards the end of March (1813) a fever came on, he lost his appetite, his sleep was uneasy, and his waking was accompanied by alarming 'swoonings. He perceived his danger; but, preserving his serenity, he studied what passed within him, and, as if he were assisting at a great and uncommon experiment, he bestowed all his attention on it. Friendship conducted to his house, on the Sth of April, MM. Lacepede, Monge, and Chaptal, who wrote down the principal points of a conversation which was his last; and the following passages, within inverted commas, are copied from the manuscript of $\mathrm{M}$. Chaptal.

"He received them with tenderness and cordiality. I was very ill, my friends (said he), the day before yesterday; I perceived myself dying, my body becane weaker, my mental and physical powers were graduatly declining; I'observed with pleasure the gradual diminution of my strength; and I arrived at the point without pain, without regret, and by a very gentle declivity. Death is not to be feared, and when it comes without violence, it is a last function which is neither painful nor disagreeable." He then explained to them his ideas respecting life, the seat of which be considered as spread over the whole body, in every organ, and all parts of the machine, which in his case became cqually feebler in every part by the same degree. "A little longer, and there would have been no functions, death would have overspread the whole body, for death is mercly the absolute repose of the body; I wished to die," added he with greater force, "I found a pleasure in it; but my wife did not wish it. I should have preferred at that time a wife less kind, less eager to restore iny strength, and who would have allowed me gently to have finished my career. I have performed my task, I have acquired some celebrity in the mathematics, I have hated nobody, I have done no ill; it is now proper to finish."

As he exerted himself too much, especially in these last words, his friends, notwithstanding the interest with which they had listened to him, proposed to retire; but he retained them, and brgan to relate to them the history of his life, his labours, his success, his residence at Berlin, (where he had often told us what he had scen near a king, ) of his arrival at Paris, the tranquillity he had enjoyed at first, the anxiety which he suffered during the revolution, and how he had been tinally rewarded by a powerful monarch, capable of appreciating his worth, who had loaded him with honours and dignities, and who had even lately sent him the granil ribbon of the Imperial Or.ler of Re-Union. And, it may be added, who, after having given him during his life the most unequivocal proufs of the highest esteem, has since, says MI. Delambre, done more for his widow and his brother, than Frederick had done for him all the time he was director of his academy.

He had beenambitious noither of riches nor honour; but he harl received both with respectiul gratitude, and he rejoiced at the acq'inition for the glory of the sciences. He had not lost all hope of cure; he belicved only that his convalescence would be long. He offered, when he reco- vered his strength, to go and dine at $M$. Lacepede's country-house, with MM. Monge and Chaptal, and proposed to give them details respecting his life, which could no where else be found. During this conversation, which lasted more than two hours, his memory often failed bim; be made vain eflorts to recover names and dates, but his discourse was always connected, full of strong thoughts and bold expressions. This exercise of his faculties wasted the whole remains of his strength. Scarcely had his friends left him, when be fell into a fainting fit, and he died two days after, on the 10th of April, 1813.

Lagrange was of a delicate but good constitution. His tranquillity, his moderation, and an austere and frugal regimen, from which he rarely deviated, prolonged his life to the age of 76 years, 4 months, and 15 days. He was twice married : first at Berlin, for the purpose of being on a footing with the rest of the academicians, none of whom were bachelors. He afterwards married, in France, Mademoiselle Lemonnier, daughter of the celebrated astronomer of that name. The countess Lagrange, daughter, grand-daughter, and niece, of members of the Academy of Sciences, was deserving of the name which he gave her. This advantage, in her eyes, made up for the difference of their ages, and she soon felt for him the tenderest regard. He was so grateful that he could scarcely bear to be separated from her, and it was on her account alone that he felt any regret at relinquishing life,

Though he had a venerable figure, indicating his excellent characteristics, he would never allow his portrait to be drawn. More than once, by a very excusable piece of address, persons have been introduced during the meeting of the Institute to take a sketch of him without bis knowledge.-Geritle and even timid in conversation, he took a pleasure in asking questions, either to draw out others, or to add their reflections to his own vast knowledge. When he spoke, it was always in a tone of doubt, and his first words usually were, "I do not know." He respected the opinions of others, and was very far from laying down bis own as a rule. Yet it was not easy to make him change them. Sometimes he even defended thein with a degree of heat which continued to increase till he was sensible of some alteration in himself; be then inmediately resumed his usual tranquillity.

Among the master-pieces which the world owes to his genius, his Mecanique is the most remarkable and the most important. His Fonctions Analytiques loold only the second place, notwithstanding the capability of the principal idea, and the beauty of the developernents. A notation less cominodious, and calculations nore embarrassing, though more luminous, will prevent mathematicians from employing, except in difficult and doubtful cases, his symbols and names. It is sufficient that he has proved the legitimacy of the more expeditious processes of the differential and integral calculus, and he has himself followed the ordinary notation in the second edition of his Mecanique. This great work is entirely founded on the culculus of variations, of which he was the inventor. The whole flows from a single formula, and from a principle known before his time; but the utility of it was far from being susprcted. This sublime composition includes all his preceding labours which could be connected with it. It is distinguished likewise by the philosuphical spirit which reigns from one end of it to the other. The gcometrical law of the celestial motions are deduced from 
simple mechanical and analytical considerations. From those problems, which serve to analy'se the true system of the world, the author passes to questions more difficult and complicated, which show the extent of his resources. We sce there his new theory of the variations of arbitrary constant quantities in the motion of the planets, which so much distinguished the Memoirs of the Institute, where it proved that the author, at the age of 75 , had not sunk from the rank which he had filled during so long a period in the opinion of all mathematicians.

Finally, it is remarked, that Legrange was successively the founder of the Academy of Turin, director during 20 years of the Berlin Academy, foreign associate of the Academy of Sciences of Paris, member of the Imperial Institute, and of the Board of Longitude; and he was besides, senator and count of the empire, grand officer of the Legion of Honour, and Grand Cross of the Imperial Urder of Re-Union.

Abridged from Delambre's Eloge of Lagrange.

LAKE, a collection of water, inclosed in the cavity of some inland place, of a considerable extent and depth; as the lake of Geneva, \&c.

LALANDE (Joseph Jerome le Français De), an eminent professor of astronomy in the college of. France, was born at Bourg, in the department of l'Aix, July 11 , 1732, and he died at Paris, April 4, 1807, in the 75th year of his age. He was intended for the bar, and went to Paris for the study of Jurisprudence, when the view of the observatory inspired him with notions which deranged his former plan, and became the ruling passion of his life. He was kindly reccived by the cclebrated astronomer le Monnier, by whose lessons he made such a rapid advance, that at the age of 19 he was nominated commissioner from the Academy of Sciences, to go to Berlin, to determine the parallax of the moon, while M. Lacaille was to perform the same operation at the Cape of Good-Hope. Soon after his return, he was appointed a member of the Academy of Sciences; from which time, every volume of their Memoirs contained some of his compositions, which were not limited to astronomical obscrvations. He published the French edition of Halley's Tables, and the history of the comet of 1759 , besides furnishing Clairaut with many calculations for the theory of the same. Being charged in 1760 with the compilation of the Connoissance des Temps, he quite changed the form of the work, giving it that which it bears at present; of which collection, he edited the 32 volumes from 1775 to 1807 .

In 1764 appearea the first edition of his great work, the Traité Astronomique, a classical work, which he has since perfected, in 4 vols 4 to. He composed all the astronomical articles for the Yverdun Encyclopedia, and new cast the whole for the Encyclopedie Methodique.

To his written instructions he joined, during 46 years, oral lessons. In 1761 he succeeded Delille in the astronomical chair at the college of France. In the midst of his labours, he drew up his Voyage d'Italic, his Traite des Canaux, and his Bibliographic Astronomique, a vast catalogue of all the works which have appeared on this science. In 1802, M. Lalande published the latter part of the new edition of Montucla's History of Mathematics, in 4 vols 4 to ; most of the last two volumes being prepared from Montucla's papers, with the assistance of Laplace, Lacroix, and other mathenaticians.

In the year 1793, he published Abrégé de Navigation, containing many valuable rules and tables. The Connois- sance des Temps, from the year 7 to 12 , or 1799 to 1804 , contains his new catalogue of more than 12,000 stars. A few years before his death, viz, in 1802, he published a small collection for the pocket, of Tables of Logarithms, Sines, and Tangents, on the plan of Lacaille, tut much inferior. And during the latter years of his life. he published annually a concise history of astronomy for the current year, which exhibited a summary of the most remarkable facts, discoveries, and inventions connected with the subjcct.

Lalande was certainly ań excellent astronomer, and an active promoter of that scicnce; but he had very little 'taste, and a very confined knowledge of inathematics in general. He considered himself, however, as the father of the mathematical sciences generally, aud at his death, he founded the prize of a medal, which the Institute annually awards to the author of the best astronomical memoir, or the maker of the most curious observations. To the extraordinary arour and activity of his character, he united a love for truth that approached the borders of fanticism. To him every degree of concealment appeared unworthy of an honest man; and he therefore, without any reserve, uttered whatever inght be his sentiments on all occasions. The high consideration which he obtained, would probably, with prudence and circunspection, have secured him an enviable respect to the end of his days: but the habit of speaking his mind, which he did not refrain from in the most stormy times, and on topics when he might, and indeed ought to have been slent, together with his. bluntness in sometımes opposing thr established systems of others, animated aganst him a crowd of detractors and enemies. His long and durable services in matters of science, were thus in a measure forgotien on neglected, in the contemplation of his dangerous ipeculations, and of the imprudent zeal with which he promulgated his opinions.

By his will, M. Lalande ordered his body to be dissected, and the skeleton to be placed in the Mluseun of Natural History. His relations however, regardless of the injunction, caused the body to be interred a few days after his death; on which occasion, the funeral was attended by the members of the National Institute.

LAMBERT (Jo 1 N HENRY), aneminent mathematician, was a native of Mulhausen, in Alsace, where he was born, April 28, 1728. His education was rather confuned, but he gave early indications of genius. At a very early period, he invented an arithmetical machine, a mercurial pendulum that vibrated for 27 minutes, arithmetical scales, and a machine for drawing in perspective. From $174 \mathrm{~s}$ to 1756 , he was employed as tutor to the grandsons of M. le comte de Salis, at Coire. In 1757, the tutor and his pupils had passed the year in Holland, and he there published his trcatise on the passage of Light. They afterwards travelled to Paris, where he was introduced to Dalembert. In 1759 , he published his Perspective at Zurich. From 1753 to 1760 , he had several picces published in the Acta Helvetica. In 1759, his Photometry was printed at Augsburg. In 1760 , he collected the different pieces, still in a fugitive state, of his Novum Organum; but which was not published till 1764 . In 1761 , his treatise on the Properties of the Orbits of Comets was printed at Augsburg. He was admitted a pensioner of the Berlin Academy in 1765 ; from which time, to his death, in 1777 , he furnished many memoirs for the several volumes of that academy.-The scientific knowledge of this gentleman 
was very considerable; and he had great independence of mind, with a very warm and vivid imagination.

LAMMAS-DAY, the 1st of August; so called, according to some, because lambs then grow out of season, as bejng too large. Others derive it from a Saxon word, signifying loaf-mass, because on that day our forefathers made an offering of bread prepared with new wheat. It is celebrated by the Romish church in memory of St.Peter's imprisonment.

LAMPEDIAS, a kind of bearded comet, resembling a burning lamp, being of several shapes; for sometimes its flame or blaze runs tapering upwards like a sword, and sometimes it is double or treble pointed.

LANI)LN (Jons), an eminent mathematician, was born at Peakirk, near Peterborough in Northamptonshire, in January 1719 . He became very early a proficient in the mathematics, for we find him a very rcspectable contributar to the Ladies' Diary in 1744; and he was soon among the foremost of those who then contributed to the support of that small but valuable publication, in which almost every English mathematician, who has arrived at any degree of $\mathrm{eminence}$ for the best part of that century, has contended tor fame at one time or other of his life. Mr. Landen continued his contributions to it at times, under various signatures, till within a few years of his death.

It has been frequently observed, that the histories of literary men consist chietly of the history of their writings; and the observation was never more fully verified, than in the present article concerning Mr. Landen.

In the 48 th volume of the Philosophical Transactions, for the year 1754, Mr. Landen gave An investigation of some theorems which suggest several very remarkable properties of the Circle, and are at the same time of considerable use in resolving fractions, the denominators of which are ccrtain multinomials, into more simple ones, and by that means facilitate the computation of flucnts.

In the year 1755 , he published a volume of about 160 pages, entitled Mathematical Lacubrations. The title to this publication was made choice of, as a means of informing the world, that the study of the mathematics was at that time rather the pursuit of bis leisure buors, than his principal enployment : and indeed it continued to be so, during the greatest part of his life; for about the year 1762 he was appointed agent to Ear\} Fitzwilliam, an employment which be resigned only two years before his death. These lucubrations contain a variety of tracts relative to the rectification of curve lines, the summation of series, the finding of fluents, and many other points in the higher parts of the mathematics.

A bout the latter end of the year 1757 , or the beginning of 1758 , he putlished proposals for printing by subscription The Resicual Analysis, a new branch of the algebraic art: and in 1758 he published a small tract, entitled $\mathrm{A}$ Discourse on the Residual Analysis; in which he resolved a variety of problems, to which the nuthod of fluxions had usually been applied, ty a mode of reasoning entircly new : he also compared these solutions with others deriver from the fluxionary method; and showed that the solutions by his new mishod were commonly more iratural and elegant than the fluxiunary ones.

In the 5 lst volume of the Philosophical Transactions, for the year 1760 , he gave a new methorl of computing the sums of a great number of infinite series.

In 1764, he published the first book of The Residual Analysis. In this trentise, besides explaining the prin- ciples which his new analysis was founded on, he applied it, in a variety of problems, to drawing tangents, and finding the properties of curve lines; to describing their involutes and evolutes, finding the radius of curvature, their greatest and least ordinates, and points of contrary flexurc; to the determination of their cusps, and the drawing of asymptotes: and he proposed, in a second book, to extend the application of this new analysis to a great variety of mechanical and physical subjects. The papers which were to have formed this book lay long by him; but he never found leisure to put them in order for the press.

In the year 1766 , Mr. Landen was elected a Fellow of the Royal Society. And in the 58th volume of the Philosophical Transactions, for the year 1768 , he gave a specimen of a new method of comparing curvilinear areas; by means of which many areas are compared, that did not appear to be comparable by any other method: a circumstance of no small importance in that part of natural philosophy which relates to the doctrine of motion.

In the 60th volume of the same work, for the year 1770 , he gave some new theorems for computing the whole areas of curve lines, where the ordinates are expressed by fractious of a certain form, in a more concise and elegant manner than bad been done by Cotes, De Mojure, and others who had considered the subject before him.

In the 6 Ist volume, for 1771 , he has investigated several new and useful theorems for computing certain fluents, which are assignable by arcs of the conic sections; a subject which had been considered before, both by Maclaurin and Dalembert; but some of the theorems that were given by these celebrated mathematicians, being in part expressed by the difference between an hyperbolic arc and its tangent, and that difference being not directly attainable when the arc and its tangent both become infinite, as they will do when the whole fluent is wanted, though such fluent be finite; these theorems therefore fail in such cases, and the computation becomes impracticable without further belp. This defect Mr. Landen removed, by assigning the limit of the difference between the hyperbolic arc and its tangent, while the point of contact is supposed to be removed to an infinite distance from the vertex of the curve. And he concludes the paper by stating a curious and remarkable property relating to pendulous bodies, which is deducible from those theorems. In the same year he published Animadversions on Dr. Stewart's Computation of the Sun's Distance from the Earth.

In the 65th volume of the Philosophical Trausactions, for 1775 , he gave the investigation of a general theorem, which he had promised in 1771 , for finding the length of any curve of a conic hyperbola by means of two elliptic arcs: and he observes, that by the theorems there investigated, both the elastic curve and the curve of equable recess from a given point, may be constructed in those cases where Maclaurin's elegant method fails.

In the 67 th volume, for 1777 , he gave a new theory of the motion of bodies revolving about an axis in free space, when that motion is disturbed by some extraneous force, either percussive or accelerative. At this time he did not know that the subject bad been treated by any person before him, and he considered only the motion of a sphere, spheroid, and cylinder. After the publication of this paper however he was informed, that the doctrine of rotatory motion had been considered by Dalembert; and on procuring that author's Opuscules Mathematiques, he there learned that Dalembert was not the only one who 
had considered the matter before him; for Dalembert there speaks of a mathematician, though be does not mention his name, who, after reading what had been written on the subject, doubted whether there could be any solid whatever, besides the sphere, in which any line, passing through the centre of gravity, will be a permanent axis of rotation. In consequence of this, Mr. Landen took up the subject again; and though be did not then give a solution to the general problem, viz, " to determine the motions of a body of any form whatever, revolving without restraint about any axis passing through its centre of gravity," he fully removed every doubt of the kind which had been started by the person alluded to by Dalembert, and pointed out several bodies which, under certain dimensions, have that remarkable property. This paper is given, among many others equally curious, in a volume of Memoirs, which he published in the year 1780 . That volume is also enriched with a very extensive appendix, containing theorems for the calculation of fluents; which are more complete and extensive than those that are found in any author before him.

In 1781,1782 , and 1783 , he published three small tracts on the summation of converging series; in which he explained and slowed the extent of some theorems which had been given for that purpose by De Moivre, Stirling, and his old friend Thomas Simpson, in answer to some things which he thought had been written injurious to the fame of those excellent mathematicians. It was the opinion of some, that Mr. Landen did not show less mathematical skill in explaining and illustrating these theorems, than he has done in bis writings on original subjects; and that the authors of them were as little aware of the extent of their own theorems, as the rest of the world were, before Mr. Landen's ingenuity made it obvious to all.

About the beginning of the year 1782, Mr. Landen had made such inprovements in his thcory of rotatory motion, as enabled him, he thought, to give a solution of the general probiem mentioned above; but finding the result of it to differ very materially from the result of the solution which bad been given of it by Dalembert, and not being able to,see clearly where that gentleman in his opinion had erred, he did not venture to make his own solution public. In the course of that year, however, having procured the Memoirs of the Berlin Academy for 1757 , which contain M. Euler's solution of the problem, he found that this gentleman's solution gave the same result as had been deduced by Dalembert; but the perspicuity of Euler's manner of writing enabled him to discover where he had differed from 'his own, which the obscurity of the other did not do. The agreement, however, of two writers of such established reputation as Euler and Dalembert made him long dubious of the truth of his own solution, and induced him to revise the process again and again with the utmost circumspection; and being every time more convinced that his own solution was right, and theirs wrong, he at length gave it to the public, in the 75 th volume of the Philosophical Transactions, for 1785 .

The extreme difficulty of the subject, joined to the concise manner in which Mr. Landen had been obliged to give his solution, to confine it within proper limits for the Transactions, rendered it too difficult, or at least too laborious a task for most mathematicians to read it; and this circumstance, joined to the established reputation of Euler and Dalembert, induced many to think that their solution was right, and Mr. Landen's wrong; and there did not want attempts to prove it; particularly a long and ingenious paper by the learned Mr. Wildbore, a gentleman of very distinguished talents and experitnce in such calculations : this paper is given in tibe soth volume of the Philosophical Transactions, for the year 1790 , in which he agrces with the solutions of Euler and Dalembert, and against that of $\mathrm{Mr}$. Landen. This determined the latter to revise and extend his solution, and give it at greater length, to render it more generally understood. About this time also he met by chance with the late Frisi's Cosmographiæ Physicæ et Mathematicæ; in the second part of which there is a solution of this problem, agreeing in the result with those of Euler and Dalembert. Here Mr. Landen learned that Euler had revised the solution which he bad given formerly in the Berlin Memoirs, and given it another form, and at greater length, in a volume published at Rostoch and Gryphiswald in 1765, entitled, Theoria Motûs Corporum Solidorum seu Rigidorum. Having therefore procured this book, Mr. Landen found the same principles employed in it, and of course the same conclusion resulting from them, as in M. Euler's former solution of the problem. But notwithstanding that there were thus a coincidence of at least four most respectable mathematicians against him, Mr. Landen was still persuaded of the truth of his own solution, and prepared to defend it. And as he was convinced of the necessity of explaining his ideas on the subject more fully, so he now found it necessary to lose no time in setting about it. He had for several years been severely afflicted with the stone in the bladder, and towards the latter part of bis life to such a degree as to be confined to his hed for more than a month at a time: yet even this dreadful disorder did not extinguish his ardour for mathematical studies; for the second volume of his Memoirs, lately published, was written and revised during the intervals of his disorder. This volume, besides a solution of the general problem concerning rotatory motion, contains the resolution of the problem relating to the motion of a top; with an investigation of the motion of the equinoxes, in which Mr. Landen has first of any one pointed out the cause of Sir lsaac Newton's mistake in his solution of this celebrated problem; and some other papers of considerable importance. He just liverl to see this work finished, and received a copy of it the day before his death, which happened on the 15 th of January 1790 , at Milton, near Peterborough, in the 71 st year of his age.

LANTERN (MaGic). See Magic Lantern.

LARBOARD, the left-hand side of a ship, when a person stands with his face towards the head.

LARMILR, in Architecture, a flat square nember of the cornice below the cimasium, and jets out farthest ; being so called from its use, which is to disperse the water, and cause it to fall at a distance from the wall, drop by drop, or, as it were, by tears; larme in Froncb signifying a tear.

LATENT / Heat, is that which is insensible, inactive, dormant, \&c.

LATElid Equation, in Algebra, is the same with simple cquation. It has but one root, and may be constructed by right lines only.

LATITUDE, in Geography, or Navigation, the distance of a place from the cquator; or an arch of the meridian, intercepted between its zenith and the equator. Hence the latitude is either worth or south, according as the place is 
on the north or south side of the equator: thus London is said to be in $51^{\circ} 31^{\prime}$ of north latitude. Circles parallel to the equator are called parallels of latitude, because they show the latitudes of places by their intersections with the meridian. The latitude of a place is equal to the elevation of the pole above the horizon of the place: and hence these two terms are used indiffereñtly for eách other. This will be evident from-the figure, where the circle $\mathrm{zHQP}$ is the meridian, $z$ the zenith of the place, Ho the horizon, EQ the equator, and $P$ the pole, then is $\mathbf{z E}$ the latitude, and $P o$ the elevation of the pole above the horizon. And bccause $P E=z o$, being each a quadrantal

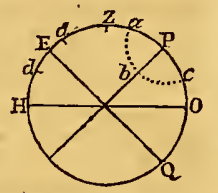
arc, if the common part $\mathrm{PZ}$ be taken from both, there will remain the latitude $Z E=$ Po the elevation of the pole.Hence we have a method of measuring the circumference of the earth, or of determining the quantity of a degree on its surface; for by measuring directly northward or southward, till the pole be one degree higher or lower, we have the number of miles in a degree of a great circle on the surface of the earth; and consequently multiplying that by 360 , will give the number of miles round the whole circumference of the earth. The knowledge of the latitude of the place, is of the utmost consequence, in geography, navigation, and astronomy; it may be proper therefore to lay down some of the best modes of determining it, both by sea and land.

1st. One method is, to find the height of the pole, to which the latitude is equal, by means of the pole star, or any other circumpolar star, thus: Either draw a true meridian line, or find the times when the star is on the meridian, both above and below the pole; then at these times, with a quadrant, or other fit instrument, take the altitudes of the star; or take the same when the star comes upon your meridian line; which will be the greatest and least altitude of the star: then shall half the sum of the two be the elevation of the pole, or the latitude sought.-For, if $a b c$ be the path of the star about the pole $\mathbf{P}, z$ the zenith, and 110 the horizon: then is ao the altitude of the star upon the meridian when above the pole, and co the same when below the pole; hence, because $a \mathbf{P}=c \mathbf{P}$, therefore $u 0+c 0=20 \mathrm{P}$, hence the height of the pole $\mathrm{OP}$, or latitude of $z$, is equal to half the sum of $a o$ and $c o$.

$2 \mathrm{~d}$. A second method is by means of the declination' of the sun, or a star, and one meridian altitude of the same, thus: Having with a quadrant, or other instrument, observed the zenith distance $z d$ of the luminary; or else its altitude $n d$, and taken its complement $z d$; then to this zenith distance, add the declination $d \mathrm{E}$ when the luminary and place are on the same side of the equator, or subtract it when on different sides, and the sum or difference will be the latitude $\mathrm{E} z$ sought. But note, that all altitudes observed, must be corrected for refraction and the dip of the horizon, and for the semidiameter of the sun, when that is the luminary observed.

Many other methods of observing and computing the latitude may be seen in Robertson's Navigation; see book 5 and book 9. See also the Nautical Almanac for 1771; and Mr. Brinkley's tables and rules for the same purpose, in the Nautical Almanac for the year 1797. Mr. Richard Graham contrived an ingenious instrument for taking the latitude of a place at any time of the day. See Philos. l'rans. No.435, or my Abr. vol. 7. pa. 673.

VoL. I.
LAtitu De, in Astronomy, as of a star or planet, is its distance from the ecliptic, being an arch of a circle of latitude, reckoned from the ecliptic towards its poles, either north or south. Hence, the astronomical latitude is quite different from the geographical, the former measuring from the ecliptic, and the latter from the equator, so that this latter answers to the declination in astronomy, which measures from the equinoctial. The sun has no latitude, being always in the ecliptic; but all the stars have their several latitudes, and the planets are continually changing their latitudes, sometimes north and sometimes south, crossing the ecliptic from the one side to the other; the points in which they cross the ecliptic being called thenodes of the the planet, and in these points it is that they may appear to pass over the face of the sun, or behind his body, viz, when they come both to this point of the ecliptic at the same time,

Circle of LA TITEDE, is a great circle passing through the poles of the ecliptic, and consequently perpendicular to it, as the meridians are perpendicular to the equator, and pass through its poles.

LATITUDE, of the Moon, North ascending, is when she proceeds from the ascending node towards her northern limit, or greatest elongation.

LATITU DE, North descending, is when the moon returns from her northern limit towards the descending node.

LATIT U DE, South descending, is when she proceeds from the descending node towards her southern limit.

LatirudE, South ascending, is when she returns from her southern limit towards her ascending node. And the same is to be understood of the planets.

Heliocentric LATITUDE of a planet, is lts latitude, or distance from the ecliptic, sucl as it would appear from the sun.-This, when the planet comes to the same point of its orbit, is always the same, or unchangeable.

Geocentric LATITUDE of a planet, is its latitude as seen from the earth.-This, though the planet be in the same point of its orbit, is not always the same, but alters according to the position of the earth, in respect to the plantet. The latitude of a star is altered only by the aberration of light, and the secular variation of latitude.

Difference of LATITUDE, is an arc of the meridian, or the nearest distance between the parallels of latitude of two places. When the two latitudes are of the same name, either both nurth or both south, subtract the less latitude from the greater, to give the difference of latitude; but when they are of different names, add them together for the difference of latifude.

Middle La TITUDE, is the middle point between two latitudes or places; and is found by taking half the sum of the two.

Parallax of Latitude. See Parallax.

Refraction of Latitude. Sce Refraction.

LATUS Rectum, in Conic Sections, the same with parameter; which sec.

Latus Transversim, of the hyperbola, is the right line between the vertices of the two opposite sections; or that part of their common axis lying between the two opposite cones; as the line DE. It is the same as the transverse axis of the hyperbola, or opposite hyperbolas.

La tus Primarium, a right line, $\mathrm{DD}$, or $E E$, drawn through the vertex of the section of a cone, within the same, and parallel to the base.

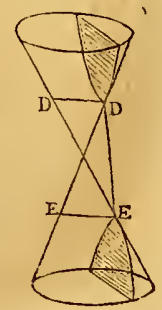


LEAGUE, an extent of three miles in length. A nautical teague, or three nautical miles, is the 20 th part of a degree of a great circle.

LEAP-Yrar, the same as BISSExtre; which see. It is so called from its leaping a day more that year than in a common year; consisting of 366 days, and a common year only of 365 . This happens every 4 th year, except only such complete centuries as are not exactly divisible by 4 ; such as the 17 th, 18 th, 19 th, 21 st $\&$ c, centuries, because $17,18,19,21, \& c$, cannot be divided by 4 without a remainder.

To find Leap-Year, \&c. Divide the number of the year by 4 ; then if 0 remain, it is leap-year; but if 1,2 , or 3 remain, it is so many after leap-year. Or the rule is sometimes thus expressed, in these two memorial verses:

Divide by 4 ; what's left shall be,

For leap-year 0 ; for past 1,2 , or 3 .

Thus if it be required to know what year 1814 is:

$$
\text { then 4) } \begin{aligned}
1314 & (4.53 \\
& 2 \text { remains: }
\end{aligned}
$$

so that 2 remaining, shows that 1814 is the $2 d$ year after leap-year. And to find what year 1816 is:

$$
\text { then 4) } 1816 \text { ( } 454
$$

here 0 remaining, shows that 1816 is a leap-year.

LEE, a term in Navigation, signifying that side, or quarter, which is from the wiud.

$L_{E E}-W_{A Y}$, of a ship, is the angle made by the point of the compass steered on, and the real line of the ship's way, occasioned by contrary winds and a rough sea. All ships are apt to make some lee-way; so that something must be allowed for it, in casting up the log-board. But the letway made by different ships, under similar circumstances of wind and sails, is different; and even the same ship, with different lading, and having more or less sail set, will have mure or less lee-way. The usual allowances for it are these, as they were given by Mr. John Buckler, to the late ingenious Mr. William Jones, who first published them in 1702 in his Compendium of Practical Navigation. 1st, When a ship is close-hauled, has all her sails set, the sea smooth, and a moderate gate of wind, it is then súpposed she makes little or nolee-way. 2d, Allow 1 point, when it blows so fresh that the small sails are taken in. 3d, Allow 2 points, when the top-sail must be close reefed. 4th, Allow $2 \frac{1}{2}$ points, when one topsail must be handed. 5 th, Allow $3 \frac{1}{2}$ points, when both topsails must be taken in. 6 th, Allow 4 points, when the fore-course is handed. 7 th, Allow 5 points, when trying under the mainsail only. 8 th, Allow 6 points, when both main and fore-courses are taken in. 9th, Allow 7 points, when the ship tries a hull, or with all sails handed.

When the wind has blown hard in either quarter, and shifts across the meridian into the next quarter, the leeway will be lessened. But in all these cases, respect must be had to the roughness of the sea, and the trim of the ship. And bence the mariner will be able to correct his course.

LEGS, of a Triangle. When one side of a triangle is taken as the base, the other two are sometimes called the legs. The term is more frequently used for the base and perpendicular of a right-angled triangle, or the two sicles about the riglat angle.

Hyperbolic LEGs, are the parts of a curve line that partake of the nature of the hyperbola, or having asymptotes.

LEIBNIT\% (GoDVILY-WILLIAM), an eminent mathematician and philosopher, was born at Leipsic in Saxony in 1646. At the age of 15 , he applied himself to the

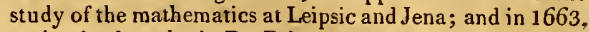
maintained a thesis De Principis Individuationis. The year following he was admitted master of arts. He read with great attention the Greek philosophers; and endeavoured to reconcile Plato with Aristotle, as be afterwards did Aristotle with Descartes. But the study of the law was his principal view; in which faculty he was admitted bachelor in 1665 . The year following he would have taken the degree of doctor; but was refused it on pretence that he was too young, though in reality because he had raised himself several enemies by rejecting the principles of Aristotle and the schoolmen. On this he repaired to Altorf, where he maintained a thesis De Casibus Perplexis, with such applause, that he had the degree of doctor conferred on him.

In 1672 he went to Paris, to manage some affairs at the French court for the baron Boinebourg. Here he became acquainted with all the literati, and made farther and considerable progress in the study of mathematics and philosophy, chiefly, as he says, by the works of Pascal, Gregory St. Vincent, and Huygens. In this course, having observed the imperfection of Pascal's a rithmetical machine, he invented a new one, as he called it, which was approved of by the minister Colbert, and the Academy of Sciences, in which he was offered a seat as a member, but refused the offers made to hin, as it would have been necessary to embrace the Catholic religion.

In 1673 he came over to England; where he became acquainted with $\mathrm{Mr}$. Oldenburg, secretary of the Royal Society, and Mr. John Collins, a distinguished member of the Society; from whom it seems he received some hints of the inethod of fluxions, which had been invented, in 1664 or 1665 , by the then Mr. Isaac Newton, which probably laid the foundation of his Differential Calculus. The same year he returned to France, where he resided till 1676, when be again passed through England and Holland, in his journey to Hanover, where he proposed to settle.

In 1700 he was admitted a member of the Royal Academy of Sciences at Paris: and in the same year the elector of Brandenburg, afterwards king of Prussia, founded an academy at Berlin by his advice; of which he was appointed perpetual president, though bis affairs would not permit him to reside constantly at that place. Besides the office of privy counsellor of justice, which the elector of Hanover had given him, the emperor appointed him, in 1711 , aulic counsellor; and the czar made bim privy counsellor of justice, with a pension of 1000 ducats. Leibnitz undertook at the same time to establish an academy of sciences at Vienna; but the plague prevented the execution of it. However, the emperor, as a mark of his favour, settled a pension on him of 2000 forins, and promised him one of 4000 if he would come and reside at Vienna; an offer he was inclined to comply with, but was prevented by his death.

The electur of Hanover being raised to the throne of Great Britain, Leibnitz visited that place the latter cnd of 1714, when he received particular marks of friendship from the king, and was frequently at court. He now was engaged in a dispute with Dr. Samuel Clurke, on the subjects of free will, the reality of space, and other philosophical subjects; which was conducted with great candour and learning; and the papers that were published by Clarke, will ever be estecmed by men of genius and learn- 
ing. The controversy ended only with the death of Leibnitz, Nov. 14, 1716 , which was occasioned by the gout and stone, in the 70 th year of his age.

In person be was of a middle stature, and a thin habit of body. He had a studious air, and a sweet aspect, though near-sighted. He was indefatigably industrious to the end of his life : he ate and drank little: hunger alone marked the time of his meals, and his diet was plain and strong. He had a very good memory, and it was said could repeat the Æneid from beginning to end. What he wanted to remember, he wrote down, and never read it afterwards. He was never married, nor ever seems to lave entertained the idea but once, when he was about 50 years old ; and the lady desiring time to consider of it, gave him an opportunity of doing the same: he used to say, "that marriage was a good thing, but a wise man ought to consider of it all his life."

Leibnitz was author of a great multitude of writings; several of which were published separately, and many others in the memoirs of different academies. He invented a binary arithmetic, and many other ingenious matters. His claim to the invention of fluxions, has been spoken of under that article. Hanschius collected, with great care, every thing that Leibnitz had said, in different passages of his works, on the principles of philosophy; and formed of them a complete system, under the title of G. G. Leibnitzii Principia Philosophiæ more geometrico demonstrata \&c, 1728, in 4to. A collection of our author's letters appeared in 1734 and 1735 , entitled, Epistolæ ad diversos theologici, juridici, medici, philosophici, mathematici, historici, et philologici argumenti e Mss. auctoris: cún annotationibus suis primum divulgavit Christian Cortholtus. But all his works were collected, distributed into classes by $M$. Dutens, and published at Geneva in 6 large volumes 4 to, in 1768 , entitled, Gothotredi Guillelmi Leibnitii Opera Omnia \& c.'

Leibnitzian Philosopily, or the philosoply of Leibnitz, is a system formed and published by its author in the last century, partly in emendation of the Cartesian, and partly in opposition to the Newtonian doctrine. In this philosophy, the author setained the Cartesian subtile matter, with the vortices and universal plenum; and he represented the universe as a machine that should proceed for ever, by the laws of mechanism, in the most perfect state, by an absolute inviolable necessity. After Newton's philosophy was published, in 1687, Leibnitz printed an essay on the celestial motions in the Act. Erud. 1689 , where he admits the circulation of the ether with Descartes, and of gravity with Newton; though he has not reconciled these principles, nor shown how gravity arose from the impulse of this ether, nor bow to account for the planetary revolutions in their respective orbits. His system is also defective, as it does not reconcile the circulation of the ether with the free motions of the comets in all directions, or with the obliquity of the planes of the planetary orbits; nor resolve other objections to which the hypothesis of the vortices and a plenum is liable.

Soon after the period just mentioned, the dispute commenced concerning the invention of the method of fluxions, which led M. Leibnitz to take a very decided part in opposition to the plilosophy of Newton. From the goodness and wisdom of the Deity, and his principle of is sufficient reason, he concluded, that the universe was a perfect work, or the best that could possibly have been made; and that other things, which are evil or incommodious, were permitted as necessary consequences of what was best : that the material system, considered as a perfect machine, can never fall into disorder, or require to be set right; and to suppose that God interposes in it, is to lessen the skill of the author, and the perfection of his work. He expressly charges an impious tendency on the philosophy of Newton, because he asserts, that the fabric of the universe and course of nature could not continue for ever in its present state, but in process of time would require to be re-established or renewed by the hand of its first framer. The perfection of the universe, in consequence of which it is capable of continuing for ever by mechanical laws in its present state, led M. Leibnitz to distinguish between the quantity of motion and the force of bodies; and, while he owns, in opposition to Descartes, that the former varies, to maintain that the quantity of force is for ever the same in the universe ; and to measure the forces of bodies by the squares of their velocities.

M. Leibuitz proposes two principles as the foundation of all our knowledge; the first, that it is impossible for a thing ta be, and not to be at the same time, which he says is the foundation of speculative truth; and secondly, that nothing is without a sufficient reason why it should be so, rather than otherwise; and by this principle he says we make a transition from abstracted truths to natural philosophy. Hence he concludes that the mind is naturally determined, in its volitions and elections, by the greatest apparent good, and that it is impossible to make a choice between things perfectly like, which he calls Indiscernibles; whence he infers, that two things perfectly like could not have been produced even by the Deity himself: and one reason why he rejects a vacuum, is because the parts of it must be supposed perfectly like to each other. For the same reason too, he rejects atoms, and all similar parts of matter, to each of which, though divisible ad infinitum, he ascribes a Monad (Act. Lipsiæ 1698, pa. 435) or active kind of principle, endued with perception and appetite. The essence of substance he places in action or activity, or, as he expresses it, in something that is between acting and the faculty of acting. He affirms that absolute rest is impossible, and holds that motion, or a sort of nisus, is essential to all material substances. Fach monad he describes as representative of the whole universe from its point of sight; and yet he tells us, in one of his letters, that matter is not a substance, but a substantiatum, ur phenomené bien fondé. See also Maclaurin's View of Newton's Philosophical Discoveries, book 1, chap. 4.

LEMMA, is a term chiefly used by mathematicians, and signifies a proposition, previously laid down to prepare the way for the more easy apprehension of the demonstration of some theorem, or the construction of some problem.

LEMNISCATE, the name of a curve in the form of the figure of 8 . If we call $A \mathbf{P}, x ; \mathbf{P Q}, y$, and the constant line $\mathrm{AB}$ or $A \mathbf{c}, a$; theequation $a y=x \sqrt{ }\left(a^{2}-x^{2}\right)$, or $a^{2} y^{2}=a^{2} x^{2}-x^{4}$, expressing a line of the 4th degree, will denote a lemniscate, having a double point in the point $A$. There may be other lemmiscates, as the ellipse of Cassini, \& $c$; but that above defined is the simplest of them.

It casily appears that this curve is quadrable. For 4. $\mathrm{Y} 2$ 


\section{E N}

[ 710

L E N

since $a y=x \sqrt{ }\left(a^{2}-x^{2}\right)$, therefore the fluxion of the curve or $y \dot{x}$ is $=\frac{x}{a} \dot{x} \sqrt{ }\left(a^{2}-x^{2}\right)$; the fluent of which is $\frac{\pi}{3} a^{2}-\frac{1}{3 a} \cdot\left(a^{2}-x^{2}\right)^{\frac{3}{2}}$ for the general area of the curve; which, when $x$ is $=a$, becomes barely $\frac{\mathrm{x}}{3} a^{2}=\mathrm{AQB}$.

LENS, a piece of glass or other transparent substance, having its two surfaces so formed that the rays of light, in passing through it, have their direction changed, and made to converge and tend to a point beyond the lens, or to become parallel after converging or diverging, or lastly to diverge as if they had proceeded from a point before the lens. Some lenses are convex, or thicker in the middle; others concave, or thinner in the middle; while others are plano-convex, or plano-concave; and some again are convex on one side and concave on the other, which are called meniscuses, the properties of which see under that word. When the particular figure is not considered, a lens that is thickest in the middle is called, a convex lens; and that which is thinnest in the middle is called a concave lens, without further distinction. These several forms of lenses are represented in the annexed figure:

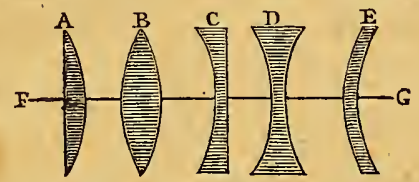

where $\mathbf{A}, \mathbf{B}$ are convex lenses, and $\mathbf{C}, \mathbf{D}, \mathbf{E}$ are concave ones; also $\mathbf{A}$ is 'a plano-convex, $\mathbf{B}$ is' convexo-convex, $\mathrm{C}$ is plano-concave, $\mathrm{D}$ is concavo-concave, and $\mathrm{E}$ is a medisscus.

In every lens, the right line perpendicular to the two surfaces, is called the Axis of the lens, as F G; the points where the axis cuts the surface, are called the Vertices of the lens; also the middle point between them is called the Centre; and the distance between them, the Diameter. Some confine lenses within the diameter of half an inch; and such as exceed that thickness, they call Lenticular Glasses.-Lenses are either blown or ground.

Blown LENSEs, are small globules of glass, melted in the flame of a lamp or taper. See Microspope.

Ground LENSES, are such as are ground or rubbed into the desired shape, and then polished. For a method of grinding them, and description of a machine for that purpose, see Philos. Trans. vol. xli. pa. 555, or my Abr. viii, 451 .

Maurolyc first delivered something relative to the nature of lenses; but we are chiefly indebted to Kepler for exexplaining the doctrine of refraction through mediums of different forms, the chief substance of which may be comprehended in the cases following: .

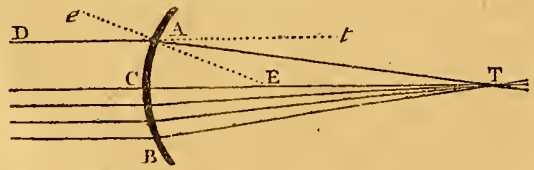

Let $\mathrm{D} \Lambda$ be a ray of light falling on a convex dense medium, having its centre at $\mathrm{E}$. When the ray isrives at $A$, it will not proceed in the same direction $\Lambda t$; but it will be there bent, and thrown into a direction $\mathrm{AT}$, nearer the perpendicular AE. In the same manner, another ray falling on $B$, at. an equal distance on the other side of the vertex $c$, and parallel to the former ray $D A$, will be refracted into the same point T. And it will also be found, that all the intermecliate parallel rays will converge to the same point, very nearly.

On the other hand, if the rays fall parallel on the inside of this denser medium, as in the figure below, they will tend from the perpendicular EAf; and converge to a point $T$ in the air, or any rarer medium. Also the ray incident on $\mathrm{s}$, at the same distance from the vertex $\mathrm{c}$, will converge to the same place $T$, together with all the intermediati parallel rays.

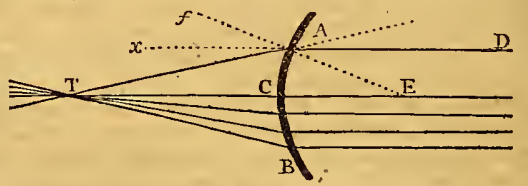

Since therefore rays are made to converge when they pass either from a rarer or a denser medium terminated by a convex surface, and converge again when they pass from the same medium convex towards the rarer, a lens which is conves on both sides must, on both accounts, make parallel rays converge to a point beyond it. Thus, the parallel rays between $\mathbf{A}$ and $\mathbf{B}$, falling on

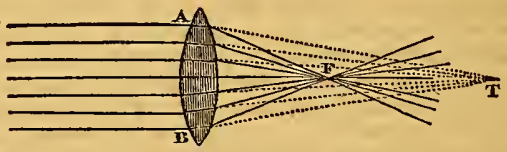

the convex surface of the glass $\mathbf{A B}$, would in that dense medium have converged to $\mathrm{T}$; but that medium being terminated by another convex surface, they will be made more converging, and be collected at some place $F$, nearer to the lens.

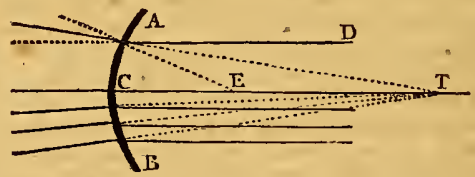

Again, to explain the effects of a concave glass, let $A B$ be the concave side of a dense medium, the centre of concavity being at $\mathrm{E}$. In this case, DA will be refracted towards the perpendicular EA; and so likewise will the ray incident at $\mathrm{B}$; in consequence of which they will diverge from one another within the dense medium. The intermediate rays will also diverge more or less, as they recede from the axis Tc; which, being in the perpendicular, will go straight on.

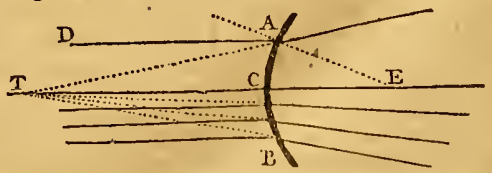

If the rays be parallel within the dense medium, they will diverge when they pass from thence into a rarer me- 


\section{E N}

dium, through a concave surface. For the ray DA will be refracted from the perpendicular $\mathrm{AE}$, as will also the ray that is incident at $\mathbf{B}$, together with all the intermediate rays, in proportion to their distance from the axis or central ray $\mathrm{Tc}$.

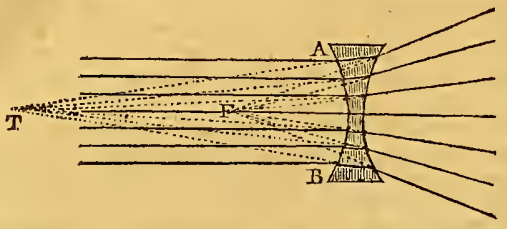

Therefore, if a dense medium, as the glass $\mathrm{AB}$, be terminated by two concave surfaces, parallel rays passing through it will be made to diverge by both the sides of it. Thus the first surface $\mathbf{A} \mathbf{B}$ will inake them diverge as if they had come from the point $\mathrm{r}$; and with the effect of the second surface added to this, they will diverge as from a nearer point, $\mathrm{F}$.

It was Kepler, who by these investigations first gave a clear explanation of the effects of lenses, in making the rays of a pencil of light converge or diverge. He showed that a plano-convex lens makes rays, that were parallel to $i$ ts axis, mect at the distance of the diameter of the sphere of convexity ; but that if both sides of the lens be equally convex, the rays will have their focus at the distance of the radius of the circle corresponeling to that degree of convexity. But he did not investigate any rule for the foci of lenses unequally convex. He only says, in general, that they will fall somewhere in the medium, between the foci belonging to the two different degrees of convexity. It is to Cavalerius that we owe this investigation; who Jaid down this rule: As the sum of both the diameters is to one of them, so is the other to the distance of the focus. And it is to be noted that all these rules, conccrning convex lenses, are applicable to those that are concave, with this difference, that the focus is on the contrary side of the glass. Sce Muntucla, vol. 2, pa. 176; or Priestley's Hist. of Visioll, pa. 65, 4to.

On this pronciple it was not difficult to find the foci of pencils of rays issuing from any point in the axis of the lens; sisce those that are parallel will meet in the focus; and if they issue from the focus, they will be parallel on the other side. If they issue from a point between the focus and the glass, they will continue to diverge after passing the lens, but less than before; while those that come from beyond the focus, will converge after passing the glass, and will meet in a place beyond the opposite focus. This plilosopher particularly observed, that rays which issue from twice the distance of the focus, will meet at the same distance on the other side. The most important of these observations have been already illustrated by proper figures, and from them the rest inay be casily conceived. Later optical writers bave assigned the distances at which rays will ineet, that issue from any other place in the axis of a lens; but Kepler was too much intent upon his astronomical and other pursuits, to pay much attention to geometry. Moutucla gives the following rule concerning this subject: As the excess of the distance of the object from the glass, above the distance of the focus, is to the distance of the focus; so is this distance, to the place of convergency beyond the glass. And

\section{E N}

the satne rule will find the point of divergency, when the rays issue from any place between the lens and the focus; for then the excess of the distance of the object from the glass, above that of the focus, is negative, which is the same distance taken the contrary way. Montucla, vol. 2, pa. 177 .

And from the principle above-mentioned, it will not be difficult to understand the application of lenses, in the rationale of telescopes and microscopes. And on these principles also is founded the structure of refracting burning glisses, by which the sun's light and heat are exceedingly augmented in the focus of the lens, whether convex or plano-convex; since the rays, falling parallel to the axis of the lens, are reduced into a much narrower compass; so that it is no wonder they burn some bodies, melt others, and produce other extraordinary phenomena.

In the Philos. T'rans. vol. x vii. 960 , or my Abr. iii, 593 , Dr. Halley gives an ingenious investigation of the foci of rays refracted through any lenses, nearly as follows:

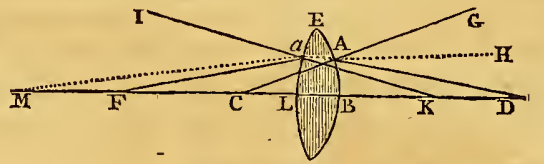

Let BEL, be a double convex lens, $\mathrm{c}$ the centre of the segment $\mathbf{E B}$, and $\mathrm{K}$ the centre of the segment EL; BL the thickness or diameter of the lens, and $D$ a point in the axis ; it is required to find the point $\mathrm{r}$, or focus, where the rays proceeding from $D$ shall be collected, after being refracted through the lens at $A$ and $a$, points very near to the axis $\mathrm{BL}$. Put the distance $\mathrm{DA}$ or $\mathrm{DB}=d$, the radius $\mathrm{CA}_{\text {or }} \mathbf{\mathrm { B }}=r$, and the radius $\mathrm{K} a$ or $\mathrm{KL}=\mathrm{R}$; also the thickness of the lens BL $=t$, and $m$ to $n$ the ratio of the sine of the angle of incidence DAG to the sine of the refracted angle $\mathrm{HAG}$ or CAM; or $m$ to $n$ will be the ratio of those angles themselves nearly, since very small angles are to each other in the same ratio as their sines. Hence $m$ is as the angle DAG or DAC, $n$ is as the angle HA.G or MAC,

and because in this case the sides are as their opposite angles, therefore DC: $\mathrm{DA}:: \angle \mathrm{DAC}: \angle \mathrm{c}$, or $d+r: d:: m$ : $\frac{d m}{d+r}$ which is as the $\angle \mathrm{c}$; from this take $n$ or the $\angle M A C$, and there remains $\frac{d m-\frac{d n-r n}{d}+r}{r}$ as the $\angle M$; hence again $\angle \mathrm{M}: \angle \mathrm{C}:: \mathrm{CA}: \mathrm{MA}$ or MB, that is $\frac{d m-d n-r n}{d+r}: \frac{d m}{d+r}::$ $r: \frac{m d r}{(m-n) \cdot d-n r}=M \Lambda$ or $M \mathrm{~B}$; which shows in what point the rays would be collected after one refraction, viz, when $n r$ is less than $(m-n) . d$. But when $n r$ is $=(n-n) \cdot d$, the point would he at an infinite distance, or the rays will be parallel to the axis; and when $n r$ is grcater than $(m-n) \cdot d$, then $\mathbf{M}$ B is negative, or $\mathrm{M}$ falls on the other sicle of the lens beyoud $D$, and the rays still continac to diverge after the first refraction.

The point $\mathrm{m}$ bcing now found, to or from which the rays proceed after the first refraction, and BM - BL being thus given, which call $\mathrm{D}$, by a process like the former it follows that $\mathbf{F} \mathbf{L}$, or the focal distance sought, is equal to $\frac{n \mathbf{D R}}{(m-n), \mathrm{D}+m \mathbf{R}}=f$. And here, instead of $\mathbf{D}$ substituting: 
$\mathrm{MB}-\mathrm{LB}$ or $\frac{m d r}{(m-n) \cdot d-n r}-t$, and putting $p$ for $\frac{n}{m-n}$, the same theorem will become

$(m p d r-n d t+n p r t) \times \mathbf{R}$ $\overline{m d r+m d \mathbf{R}-m p r \mathbf{R}-(m-n) \cdot d t+n r t}=f$, the focal distance sought, in its most general form, including thethickness of the lens; being the uriversal rule for the foci of double convex glasses exposed to diverging rays.

But if $t$ the thickness of the lens be rejected, as not sensible, the rule will be much shorter, viz,

\section{$\frac{p d r \mathbf{R}}{d r+d \mathbf{R}-p r \mathbf{R}}=f$.}

If therefore the lens consist of glass, whose refraction is as 3 to 2, it will be $\frac{2 d r \mathbf{R}}{d r+d \mathbf{R}-2 r \mathrm{R}}=f$. And if it be of water, whose refraction is as 4 to 3 , it will be $\frac{3 d r R}{d r+d \mathbf{R}-3 r \mathbf{R}}$ $=f$. But, if the lens could be made of diamond, whose refraction is as 5 to 2 , it would be $\frac{2 d r R}{3 d r+3 d R-2 r R}=f$.

If the incident rays, instead of diverging, be converging, the distance $\mathrm{DE}$ or $d$ will be negative, and then the theorem for a double convex glass leus will be $\frac{-2 d \mathbf{R}}{-d r-d \mathbf{R}-2 r \mathbf{R}}$ or $\frac{2 d r \mathbf{R}}{d r+d \mathbf{R}+2 r \mathbf{R}}=f$, in which case therefore the focus is always on the other side of the glass.

And if the rays be parallel, as coming from an infinite distance, or nearly so, then will $d$ be negative, as well as the terms in the theorem in which it is tound; and therefore the othei term $p r$ will be nothing, in respect to those infinite terms; and by omitting it, the theorem will be $\frac{p d r R}{d r+d \mathbf{R}}=\frac{p r \mathbf{R}}{r+\mathbf{R}}=f$, or $\frac{2 r \mathbf{R}}{r+\mathbf{R}}=f$ for glass.

And here if $r=\mathrm{R}$, or the two sides of the glass be of equal convexity, this last will become barely $\frac{2 \gamma^{3}}{2 r}$ or only $r=f$ the focus, which therefore is in the centre of the convexity of the lens.

If the lens be a meniscus of glass; then, making $r$ negative, the theorem is

$\frac{-2 d r \mathbf{R}}{-d r+d \mathbf{R}+2 r \mathbf{R}}$ or $\frac{2 d r \mathbf{R}}{d r-d} \frac{2 r \mathbf{R}-2}{\mathbf{R}}=f$ for diverging rays,

$\frac{-2 d r \mathrm{R}}{-d r+d \mathbf{R}-2 r \mathrm{R}}$ or $\frac{2 d r \mathrm{R}}{d r-d \mathbf{R}+2 r \mathrm{R}}=f$ for converging rays, and $\frac{-2 r R}{-r+\mathbf{R}}$ or $\frac{2 r R}{r-R}=f$ for parallel rays.

If the lens be a double concave glass, $r$ and $\mathbf{R}$ will be both negative, and then the theorem becomes

$\frac{-2 d r \mathbf{R}}{d r+d \mathbf{R}+2 r \mathbf{R}}=f$ for diverging rays, always negative;

$\frac{-2 d r \mathbf{R}}{d r+d \mathbf{R}-2 r \mathbf{R}}=f$ for converging rays;

and $\frac{-2 r \mathbf{R}}{r+\mathbf{R}}=f$ for parallel rays.

And here, if the radii of curvature $r$ and $k$ be cqual, this last will be barely $-r=f$ for parallel rays falling on a double concave glass of equal curvature.

Lastly, when the lens is a plano-convex glass; then, $r$ being infinite, the theorem becomes

$$
\begin{aligned}
\frac{-2 d \mathrm{R}}{d-2 \mathrm{R}} & =f \text { for diverging rays, } \\
\frac{2 t \mathrm{R}}{d+2 \mathrm{n}} & =f \text { for converging rays, } \\
\text { and } 2 \mathrm{n} & =f \text { for parallel rays. }
\end{aligned}
$$

The theorems for parallel rays, as coming from an in fuite distance, take place in the common refracting telescopes. And those for converging rays are chiefly of use to determine the focus resulting from any sort of lens placed in a telescope, between the focus of theobject-glass and the glass itself; the distance between the said focus of the object-glass and the interposed lens being made $=-d$; while those for diverging rays are chicfly of use in microscopes, reading-glasses, and other cases in which near objects are viewed.

lt is evident that the foregoing general theorem will serve to find any of the other circumstances, as well as the focus, by considering this as given. Thus, for instance, suppose it be required to find the distance at which an object being placed, it shall by a given lens be represented as large as the object itself; which is of singular use in viewing and drawing them, by transmitting the image througl a glass in a dark room, as in the camera obscura, which gives not only the true figure and shades, but the colours themselves as vivid as the life. Now in this case $d$ is $=f$, which makes the theorem become $p d r \mathbf{R}=d^{2} r+d^{2} \mathrm{R}-p d r \mathrm{R}$, and this gives $d=\frac{2 p r R}{r+R}$. But if the two convexities belong to equal spheres, so as that $r=\mathrm{R}$, then it is $d=p r$, or $=2 r$ when the lens is glass. So that if the object be placed at the diameter of the sphere distant from the lens, then the focus will be as far distant on the other side, and the image as large as the object. But if the glass were a plano-convex, the same distance would be just twice as much.

Again, recurring to the first general theorem, including $t$, the thickness of the lens; let the lens be a whole sphere; then $t=2 r$, and $r=\mathbf{R}$; and hence the theorem reduces to $\frac{m p d r-2 n d r-2 n p r^{2}}{2 n d+2 n r-m p r}=f$.

And here if $d$ be infinite, the theorem contracts to $\frac{m p-2 n}{2 n} r$ or $\frac{2 n-m}{2 m-2 n} r=f$; or for glass $\frac{1}{2} r=f$ : showing that a sphere of glass collects the sun's rays at half the radius of the sphere without it. And for a sphere of water, the focus is at the distance of a whole radius.

For another example; when a hemisphere is exposed to parallel rays; then $d$ and $\mathbf{R}$ being infinite, and $t=r$, the theorem becomes $\frac{m p-n}{m} r,=\frac{n n}{m^{2}-m n} r=f$. That is, in glass it is $\frac{3}{7} r$, and in water $\frac{9}{4} r$.

Several other corollaries may be deduced from the foregoing principles. As follows:

1st. That the thickness of the lens, being very small, the focus will remain the same, when either side is exposed to the rays.

2d. If a luminous body be placed in a focus belind a lens, whether plano-convex, or convex on both sides; or whether cqually or unequally so; the rays become parallel after refraction, as the refracted rays become what were before the incident rays. And hence, by means of a convex lens, or a little glass bubble full of water, a very intense light may be projected to a great distance. Which furnishes us with the structure of a lamp or lantern, to throw an intense light to an immense distance: for a lens, convex on both sides, being placed opposite to a concare mirror, and a lighted candle or wick being placed in the common focus of both, the rays reflected back from the mirror to the lens will be parallel to each other; and after refraction will converge, till they concur at the dis- 
lance of the radius, after which they will again diverge. But the candle being likewise in the focus of the lens, the rays it throws on the lens will be parallel; and theretore a very intense light meeting with another equally intense, at the distance of the diameter from the lens, the light will be surprising: and though it afterwards decrease, yet the parallel and diverging rays going a long way together, it will be very great at a great distanee. Lanterns of this kind are of considerable service in the night time, to discover remote objects.

If it be required to bave the light, at the same time, transmitted to several places, as through several streets, \&e, the number of lenses and mirrors must be increased.

3. The images of objects are shown inverted in the focus of a convex lens: nor is the focus of the sun's rays any thing else, in effeet, but the image of the sun inverted. Hence, in solar eclipses, the sun's image, eclipsed as it is, may be burnt by a large lens on a board, \& c, and exhibit a very entertaining phenomenon.

4 th. If a eoneave mirror be so placed, as that an inverted image, formed by refraction through a lens, be found between the centre and the focus, or even beyond the centre, it will again be inverted by reflection, and so appear erect; in the first case beyond the centre, and in the latter between the centre and the focus. And on these principles the camera obscura is eunstructed.

5 th. The image of an objeet, delineated beyond a convex lens, is of the same magnitude, as it would be of, were the object to shine into a dark room through a small hole, upon a wall, at the same distance from the hole, as the focus is from the lens. - Whon an objeet is less distant from a lens than the focus of parallet ray's, the distance of the image is greater than that of the object; otherwise, the distance of the image is less than that of the object: in the former case therefore, the image is larger than the object; in the latter, it is less.

When the images are less than the objects, they appear more distinet and vivid; beeause then nore rays arre accumulated into a given space. But if the images be made greater than the objects, they will not alpeal distinctly; because in that case there are fewer riays which met after refraction in the same point; whence it happens, that rays proceeding from different points of all oljict, terminate in the same point of an image, which is the cause of confusion. Hence it appears that the same aperture of a lens may be admitted in every case, if we would keep off the rays which produce confusion. However, thrugh the image be then more distinct, when no rays are admitted but those near the axis, y't for want of rays the image is apt to be lim.

6 . If the eye be placed in the focus of a convex lens, an object viewed through it, appears erect, and enlarged in the ratio of the distance of the objeet from the eye, to that of the eye from the leus, if it be near; but infinitely if remote.

7 th. An object viewed through a eoneave lens, appears crect, and diminished in a ratio compounded of the ratios of the space in the axis between the point of inciclenee, and the point to which an oblique ray would pass without refraction, to the space in the axis between the eye and the middle of the object; and the spaee in the same axis tertween the eye and the point of incidenee, to the space hetween the midsle of the object and the point to which the oblique ray would pass without refraction.

Finally, it may be observed, that the very small magnifying glasses used in microscopes, most properly come un- der the denomination of lens, as thicy most approach to the figure of the lentil, a seed of the vetch or pea kind, from whence the name is derived; but the reading glasses, and burning glasses, and all that magnify, cone under the same denomination; for their surtaces are conve'x, though less so. A drop of water is a lens, and it will serve as one ; and many have used it by way of lens in their microscopes. A drop of any transparent fluid, euclosed between two eoneave glasses, acquires the shape of a lens, and has all its properties. The erystalline humour of the eye is a lens exactly of this kind; it is a small quantity of a translucent fluid, contained between two concave and transparent membranes, ealled the coats of the eye; and it acts as the lens made of water would do, in an equal degree of convexity.

LEO, the Lion, a considerable constellation of the northern hemisphere, being one of the 48 old constellations, and the 5th sign of the zodiac. It is marked thus $\Omega$, as a rude sketch of the animal. The Greeks fabled that this was the Nenæan lion, which had dropped from the moon, but being slain by Hercules, was raised to the heavens by Jupitcr, in commemoration of the dreadful conflict, and in honour of that hero. But the hieroglyphieal meaning of this sign, so depicted by the Egyptians long before the invention of the fables of IIercules, was probably no inore than to signify, hy the fury of the lion, the violent heats occasioned by the sun when he eritered that part of the ecliptic.-The stars in the constellation Leo, in Ptolemy's catalogue are 27 , besid's 8 unformed unes, now counted in the constellation ('oma Berenices; in Tycho's 30, in that of Hevelius 49, and in Flimsteed's 95 ; one of then, ot the first magnitude, in the breast of the Lion, is ealled Regulus, and Cor Leonis, or Lion's Heart.

Leo Minor, the Litlle Lion, a constellation of the northeru hemisphere, and one of the new ones that were formed out of what were left by the ancients, under the name of stellæ informes, or unformed stars, and added to the 48 old ones. It contains 53 stars in Flamsteed's catalogue.

Cor LEON Is, Lion's heart, a fixed star, of the first magnitude, in the sign leo; called also regulus, basilicus, \&e.

LEPUS, the Hare, a constellation of the southern beinisphere, and one of the 48 old constellations. The Grecks fabled, that this animal was placed in the heavens, near Orion, as being one of the animals which he hunted. But it is probable their masters, the Egyptians, had some other meaning in this hieroglyphic. The stars in the constellation lepus, in Ptolemy's catalogue are 12, in Tycho's 13, and in Flamsteed's 19.

LEUCtPPUS, a celebrated Greek philosopher and mathematician, who flourished about the $42 \mathrm{Sth}$ year before Christ. He was the first author of the famous system of atoms and vacuums, and of the hypothesis of storms; since attributerl to the moderns.

LEVEL, an instrument used to make a line parallel to the horizon, and to continue it out at pleasure; and by this means to find the true level, or the difterenee of ascent or deseent between two or more places, for conveying water, draining feins, \&c. There are several instruments, of different constructions and materials invented for the perfeetion of levelling, as may be seen in Lahire's and Picard's treatises of levelling, in Biron's treatise on mathematical instruments, as also in the Philos. Trans. and the Memoires de l'Acad. \&c. But they maty be reduced to the following kinds. 
Water-Level, that which shows the horizontal line by means of a surface of water or other fluid; founded on this principle, that water always places itself level or Iorizontal. The most simple kind is made of a long wooden trough or canal; which being equally filled with water, its surface shows the line of level. And this is the chorobates of the ancients, described by Vitruvius, Jib. viii, cap. 6 . The water-level is also made with two cups fitted to the two ends of a straight tube, about an inch diameter, and 3 or 4 feet long, by means of which the water communicates from the one cup to the other; and this pipe being moveable on its stand by means of a ball and socket, when the two cups show equally full of water, their two surfaces mark the line of Jevel.

This instrument, instead of cups, may also be made with two short cylinders of glass 3 or 4 inches long, fastened to each extremity of the pipe with wax or mastic. The pipe is filled with common or coloured water, which shows itself through the cylinders, by means of which the line of level is determined; the height of the water, with respect to the centre of the earth, bcing always the same in both cylinders. This level, though very simple, is however very commodious for levelling small distances. See the method of preparing and using a water-level, and a mercurial level, annexed to Davis's quadrant, for the same purpose, by Mr. Leigh, in Philos. Trans. vol. xl. 417, or my Abr. viii, 260.

Air-LeveL, is that which shows the line of level by meails of a bubble of air, enclosed with some fluid in a glass tube, of an indeterminate-length and thickness, and having its two ends hermetically sealed : an invention, it is said, of $\mathbf{M}$. Thevenot. When the bubble fixes itself at a certain mark, made exactly in the middle of the tube, the case or ruler in which it is fixed, is then level; and when it is not level, the bubble will rise to onc end. This glasstube may be set in another of brass, having an aperture in the middle, where the bubble of air nay be observed. The liqunr with which the tube is filled, is usually either oil of tartar, or aqua secunda; those not being liable to freeze as common water, nor to rarefaction and condensation as spirit of wine is.

There is one of these instruments with sights, being an improvement on that last described, which, by the addition of other apparatus, becomes more exact and commodious. It consists of an air-level, No. $1,(f i g .1, p l .18)$ about $\mathrm{s}$ inches loug, and about two thirds of an inch in diameter, set in a brass tube, 2 , having an aperture in the middle $c$. The tubes are carried in a strong straight ruler, of a foot long; at the ends of which are fixed two sights, 3, 3, exactly perpendicular to the tubes, and of an equal height, having a square hole, formed by two fillets of brass crossing each other at right angles; in the middle of which is drilled a very small hole, through which a point on a level with the instrument is seen. The brass tube is fastened to the ruler by means of two screws; the one of which, marked 4 , serves to raise or depress the tube at pleasure, for bringing it towards a level; the top of the ball and socket is rivetted to a small ruler that springs, one end of which is fastened with springs to the great ruler, and at the other end is a screw, 5 , scrving to raise and depress the instrument when nearly level. But this instrument is less commodious than the following one: for though the holes be cver so small, yet they will still take in too great a space to determine the point of level precisely.

Fig. 2, is a Level with Telescopic Sights, first invented by
Mr. Huygens. It is like the last; with only this difference, that instead of plain sights, it carries a telescope, to determine exactly a point of level at a considerable distance. The screw 3, is for raising or lowering a little fork, which carries the hair, and making it agree with the bubble of air when the instrument is level; and the screw 4 , is for making the bubble of air, $\mathrm{D}$ or $\mathrm{E}$, agrec with the telescope. The whole is fitted to a ball and socket, or otherwise moved by joints and screws.-It may be observed that a telescope may be added to any kind of level, by applying it upon, or parallel to, the base or ruler, when there is occasion to take the level of remote objects: and it possesses this advantage, that it may be inverted by turning the ruler and telescope half round; and if then the hair cut the same point that it did before, the operation is just. Many varieties and improvements of this instrument have been made by the more moderu opticians.

Dr. Desaguliers proposed a nachine for taking the difference of level, which contained the principles both of a barometer and thermometer ; but it is not accurate in practice: Philus. Trans. vol. xxxii, pa. 165, or my Abr. vol.vii, 4.9. Fig. 3, 4, 5, 6 .

Mr. Hadley too has contrived a Spirit Level to be fixed to a quadrant, for taking a meridian altitude at sea, when the horizon is not visible. See the description and figure of it in the Philos. Trans. vol. xxxviii, 167, or Abr. vii, 620. Various other spirit levels, and mercurial levels, are also invented and used on different occasions.

Reflecting LEVEL, that made by means of a pretty long surface of water, representing the same object inverted, which we see erect by the eye; so that the point where these two objects appear to meet, is on a level with the place where the surface of the water is found. This is the invention of M. Mariotte.

There is another reflecting level, consisting of a polishod metal mirror, placed a little before the object glass of a telescope, suspended perpendicularly. This mirror must be set at an angle of 45 degrees; in which case the perpendicular line of the telescope becomes a horizontal line, or a line of level. Which is the invention of $\mathbf{M}$. Cassini.

Artillery Foot-LeveL, is in form of a square (fig. 7 ), having its two legs or branches of an equal length; at the junction of which is a small hole, by which hangs a plummet playing on a perpendicular line in the iniddle of a quadrant, which is divided both ways from that point into 45 degrees. This instrument may be used on other occasions, by placing the ends of its two branches on a plane; for when the plummet plays perpendicularly over the middle division of the quadrant, the plane is then level. To use it in gunnery, place the two ends on the piece of artillery, which may be raised to any proposed height, and the plummet will cut the degree above the level. But this supposes the outside of the camnon is parallel to its axis, which is not always the case; and therefore another instrument is employed either to set the piece level, or clevate it at any angle: namely a small quadrant, with one of its radii continued out pretty long, which being put into the inside of the cylindrical bore, the plum met shonvs the angle of elevation, or the line of level. Sce Gunner's Quadiant.

Carpenter's, Bricklayer's, or Pavior's Levex, consists of a long ruler, in the middle of which is fitted at right angles another broasler piece, at the top of which is fastened a plunmet, which when it hangs over the middle line of the 
ad or upright piece, shows that the base or long ruler is horizontal or level. Fig. S.

Mason's LEVEL, is composed of 3 rules, so jointed as to form an isosceles triangle, somewhat like a Roman A; from the vertex of which is suspended a plummet, which hangs directly over a mark in the middle of the base, when this is horizontal or level. Fig. S.

Plumb or Pendulum Leve L, said to be invented by $\mathrm{M}$. Picard; fig. 10. This shows the horizontal line by means of another line perpendicular to that described by a plummet or pendulum. It consists of two legs or branches, joined at right angles, the one of which, of about 18 inches long, carries a thread and plummet; the thread being hung near the top of the branch, at the point 2 . The middle of the branch where the thread passes is hollow, so that it may hang free every-iwhere: but towards the bottom, where there is a small blade of silver, on which a line is drawn perpendicular to the telescope, the said cavity is covered by two pieces of brass, with a piece of glass $G$, to see the plummet through, forming a kind of case, to prevent the wind from agitating the thread. The telescope, of a proper length, is fixed to the other leg of the instrument, at right angles to the perpendicular, and having a hair stretched horizontally across the focus of the objectglass, which determines the point of level, when the string of the plummet liangs against the line on the silver blade : the whole being fixed by a ball and socket to its stand.

Fig. 12, is a Balance Lever; which being suspended by the ring, the two sig'nts, when in equilibrio, will be horizontal, or in a level.

Some other leve's are also represented in pl. 18.

LEVELLING, the art or act of finding a line parallel to the horizon 3.t one or more stations, to determine the height or depth of one place with respect to another; for laying out grounds even, regulating descents, draining morasses, cov.ducting water, \&c.

'Two or m.ore places are on a true level when they are equally distint from the centre of the earth. Also one place is high 1er than another, or out of level with it, when it is farther from the ccntre of the earth: and a line equally distant fro'm that centre in all its points, is called the line of True I sevel. Hence, because the earth is round, that line mus'c be a curve, and make a

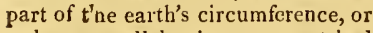
at least parallel to it, or concentrical with it; as the line BCEG, which has all its points equally distant from $\mathrm{A}$ the ce ntre of the earth; considering it as : a perfect globe.

B' at the line of sight BDF \& $c$, given by 1 he operations of levels, is a tangen $t$, or a right line perpendicular to

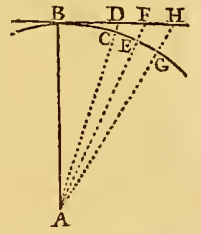
the : semidiameter of the earth at the point of contact B, ris ing always higher above the true line of level, the farther to ic distance is, this being called the Apparent Line of

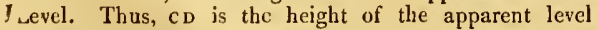
above the true level, at the distance $\mathrm{BC}$ or $\mathrm{BD}$; also $\mathrm{EF}$ is the excess of height at $\mathrm{E}$; and $\mathrm{GH}$ at $\mathrm{G}$; \&c. The difference, it is evident, is always equal to the excess of the secant of the arch of distance above the radius of the earth.

The common methods of levelling are sufficient for laying pavements of walks, or for conveying water to small distances, \&cc: but in more extensive opcrations, as in VoL. I. levelling the bottoms of canals, which are to convey water to the distance of many miles, and such like, the difference between the true and the apparent level must be taken into the account.

Now the difference $\mathrm{cD}$ between the true and apparent level, at any distance $\mathrm{BC}$ or $\mathrm{BD}$, may be found thus : By a well-known property of the circle $2 \mathrm{AC}+\mathrm{CD}: \mathrm{BD}:: \mathrm{BD}$ : $C D$; or because the diameter of the earth is so great with respect to the line $\mathrm{CD}$ at all distances to which an operation of levelling commonly extends, that $2 \mathrm{AC}$ may be safely: taken for $2 \mathrm{AC}+\mathrm{CD}$ in that proportion without any sensible error, it will be $2 A C: B D:: B D: C D$, which therefore is $=\frac{\mathbf{B D}^{2}}{2 \mathrm{AC}}$ or $\frac{\mathbf{B C}^{3}}{2 \mathrm{AC}}$ nearly; that is, the difference between the true and apparent level, is equal to the square of the distance between the places, divided by the diameter of the earth; and consequently it is always proportional to the square of the distance.

Now the diameter of the earth being nearly 7914 miles; if we first take $\mathrm{BC}=1$ mile, then the excess $\frac{\mathrm{BC}^{2}}{2 \mathrm{AC}}$ becomes $\frac{1}{7912}$ of a mile, which is 8 inches, the height of the apparent above the true level at the distance of one mile. Hence, proportioning the excesses in altitude according to the squares of the distances, the following table is obtained, which shows the height of the apparent above the true level for every 100 yards of distance on the one hand, and for every mile on the other.

\begin{tabular}{|c|c|}
\hline Dist. or $\mathbf{B C}$ & $\begin{array}{l}\text { Dif. of level, } \\
\text { or } \mathbf{c s} \text {. }\end{array}$ \\
\hline Yards. & Inches. \\
\hline 100 & $\begin{array}{l}0.026 \\
0.103\end{array}$ \\
\hline $\begin{array}{l}200 \\
300\end{array}$ & $\begin{array}{l}0.103 \\
0.231\end{array}$ \\
\hline 400 & 0.4111 \\
\hline 500 & 0.643 \\
\hline 600 & 0.925 \\
\hline 700 & $1 \cdot 260$ \\
\hline 800 & $1 \cdot 654$ \\
\hline 900 & $2 \cdot 081$ \\
\hline 1000 & $2 \cdot 570$ \\
\hline 1100 & $3 \cdot 110$ \\
\hline 1200 & 3.701 \\
\hline 1300 & $4 \cdot 344$ \\
\hline 1400 & 5.038 \\
\hline 1500 & 5.784 \\
\hline 1600 & 6.580 \\
\hline 1700 & $7 \cdot 4 \cdot 25$ \\
\hline
\end{tabular}

\begin{tabular}{|c|cc|}
\hline Dist. or вc. & \multicolumn{2}{|c|}{$\begin{array}{c}\text { Dif. of levcl, } \\
\text { or } \mathbf{C D} .\end{array}$} \\
\cline { 1 - 3 } Miles. & Feet. & Irches. \\
$\frac{t}{4}$ & 0 & $0 \frac{\pi}{2}$ \\
$\frac{1}{2}$ & 0 & 2 \\
$\frac{3}{4}$ & 0 & $4 \cdot \frac{1}{2}$ \\
1 & 0 & 8 \\
2 & 2 & 8 \\
3 & 6 & 0 \\
4 & 10 & 7 \\
5 & 16 & 7 \\
6 & 23 & 11 \\
7 & 32 & 6 \\
8 & 42 & 6 \\
9 & 53 & 9 \\
10 & 66 & 4 \\
11 & 80 & 3 \\
12 & 95 & 7 \\
13 & 112 & 2 \\
14 & 130 & 1 \\
\hline
\end{tabular}

By means of these tables of reductions, we can now Jevel to almost any distance at one operation, which the ancients could not do but by a great multitude; for, being unacquainted with the correction answering to particular distances, they only levelled from one 20 yards to another, when they had occasion to continue the work to any considerable extent.

This table will answer several useful purposes. Thus, first, to find the height of the apparent above the true level, at any distance. If the given distance be contained in the table, the correction of level is found on the same line with it: thus at the distance of 1000 yards, the correction is 2.57 , or two inches and a half nearly; and at the distance of 10 miles, it is 66 feet 4 inches. But if 4. $\mathrm{Z}$ 
the exact distance be not found in the table, then multiply the square of the distance in yards by 2.57 , and divide by $3,000,000$, or cut off 6 places on the right for decimals, and the rest will be inches: or multiply the square of the distance in miles by 66 feet 4 inches, and divide by 100 . 2ndly, To find the extent of the visible horizon, or how far can be seen from any given height, on a horizontal plane, as at sea, \&c: Suppose the eye of an observer, on the top of a ship's mast at sea, be at the height of 130 fcet above the water, he will then see about 1 miles all around. Or from the top of a cliff by the sea-side, the height of which is 66 feet, a person may see to the distance of near 10 miles on the surface of the sea. Also, when the top of a hill, or the light in a lighthouse, or such like, whose height is 130 feet, first comes into the view of an eye on board a ship; the table shows that the distance of the ship from it is 14 miles, if the eye be at the surface of the water; but if the height of the eye. in the ship be 80 feet, then the distance will be increased by near 11 miles, making in all about 25 miles, distance.

$3 \mathrm{dly}$, Suppose a spring to be on one side of a hill, and a house on an opposite hill, with a valley between them; and that the spring seen from the house appears, by a le-velling instrument, to be on a level with the foumdation of the house, which suppose is at a mile distance from it; then is the spring $S$ inches above the true level of the house; and this difference would be about sufficient for the water to be brought in pipes from the spring to the house.

4, h, If the height or clistance exceed the limits of the table: Then, first, if the distance be given, divide it by 2 , or by 3 , or by $4, \& c$, till the quotient come within the distances in the table; then take out the height answering to the quotient, and multiply it by the square of the divisor, that is by 4 , or 9 , or 16 , \&c, for the height required: So, if the top of a hill be just seen at the distance of 40 miles; then 40 divided by 4 gives 10 , to which in the table answers $66 \frac{\mathrm{r}}{3}$ feet, which being multiplied by 16 , the square of 4 , gives $1061 \frac{1}{3}$ feet for the height of the hill. But when the beight is given, divide it by one of these square numbers $4,9,16,25, \& c$, till the quotient come within the limits of the table, and multiply the quotient by the square root of the divisor, that is by 2 , or 3 , or 4 , or 5 , \&c, for the distance sought: So when the top of the peak of Teneriff, said to be almost 3 miles or 15,840 feet high, just comes into view at sea; divide 15,840 by 225 , or the square of 15 , and the quotient is 70 nearly ; which answers in the table, by proportion, to about $10 \frac{2}{7}$ miles; then multiplying $10 \frac{2}{T}$ by 15 , gives $154 \frac{2}{7}$ miles, for the distance of the hill.

\section{On the Praclice of Levelling.}

The operation of levelling is as follows. Suppose the height of the point $A$ on the top of a mountain, above that of $\mathrm{B}$, at the foot of it, be required. Place the level about the middle distance at $\mathrm{D}$, and set up pickets, poles, or staffs, at $A$ and $B$, where persons must attend with signals for raising and lowering, on the said poles, little marks of pasteboard or other matter. 'The level having been placed horizontally by the bubble, sce, look towaris the staff $\mathbf{A E}$, and cause the person there toraise or lower the mark, till it appear through the telescope, or sighis, \&c, at $\mathbf{E}$ : then measure exactly the perpendicular height of the point $\mathrm{E}$ above the point $\boldsymbol{A}$, which suppose 5 feet 8 inches, set it down in your book. Then direct your sight the other way, towards the pole $B$, and cause the person there to raise or lower his mark, till it appear in the visual line as before at $\mathrm{c}$; and measuring the height of $\mathrm{C}$ above $\mathrm{B}$, which suppose 15 feet 6 inches; set this down in your, book also, immediately above the number of the first observation. Then subtract the one from the other, and the remainder 9 feet 10 inches, will be the difference of level between $A$ and $x$, or the height of the point $A$ above the point $B$.

If the point $\mathrm{D}$, where the instrumient is fixed, be exactly in the middle between the points $\mathrm{A}$ and $\mathrm{B}$, there will be no necessity for relucing the apparent level to the true one, the visual ray on both sides being raised equally above the true level. But if not, each lieight must be corrected or reduced according to its distance, before the one corrected height is subtracted from the other; as in the case following.

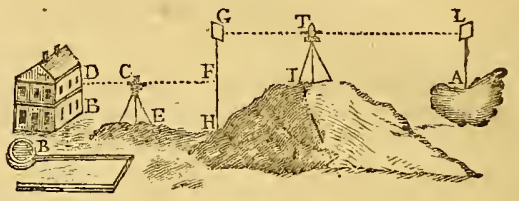

When the distance is very considerable, or irregular, so that the operation cannot be effected at once placing of the level; or when it is required to know if there be a sufficient descent for conveying water from the spring $A$ to the point $B$; it will be necessary to perform this at several operations. Having chosen a proper place for the first station, as at $1, \mathrm{fix}$ a pole at the point $\mathrm{A}$ near the spring, with a proper mark to slide up and down it, as $\mathbf{L}$; and measure the distance from $A$ to $I$. Now the level being adjusted in the point $\mathrm{I}$, let the mark $\mathrm{I}$ be raised or lowered till it is scen through the telescope or sights of the level, and measure the height $\mathrm{AL}$. Then having fixed another pole at 11 , direct the level to it, and cause the mark $G$ to be moved up or down till it appear through the instrument : then measure the height $\mathrm{GH}$, and the distauce from I to $\mathrm{I}$; noting them down in the book. This done, remove the level forwards to some other eminence as E, from which the pole it may be viewed, as also another pole at $\mathrm{D}$; and having adjusted the level in the point $\mathrm{E}$, look back to the pole 11 ; mantaging the mark as before, till the visual ray will give the point $\mathbf{F}$; then measuring the distance $\mathrm{H}$ and the height $\mathrm{nF}$, note them down in the book. And here turn the level to look at the next pole $\mathrm{D}$, and the visual ray will give the point $\mathrm{D}$; there measure the height of $\mathrm{D}$, and the ilistance $\mathrm{EB}$, cntering them in the book as before. And thus procecd from one station to another, till the whole is completed.

But all these heights must be corrected or reduced by the foregoing table, according to their respective distances; and the whole, both distances and heights, with their corrections, cntered in the book in the following manner. 
L E V

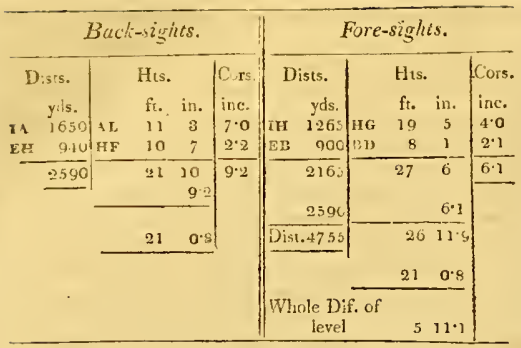

IIaving summed up all the columns, add those of the distances logether, and the whole distance from $A$ to $B$ is 4755 yards, or 2 miles, and 3 quarters nearly. Then, the sums of the corrections taken from the sums of the apparent heights, leave the two corrected beights; the one of which being taken from the other, leaves 5 feet 11.1 inc. for the true difference of level sought between the two places $A$ and $B$, which is at the rate of an inch and half nearly to every 100 yards, a quantity more than suffcient to cause the water to run from the spring to the bouse.

Or, the operation may be otherwise performed, thus : Instead of placing the level between cvery two poles, and taking both back-sights and fore-sighis; plant it first at the spring $\mathrm{A}$, and thence observe the level to the first pole; then remove it to this pole, and observe the $2 \mathrm{~d}$ pole; next move it to the $2 d$ pole, and obscrve the $3 \mathrm{~d}$ pole; and so on, from one pole to another, always taking forward sights or observations only. Then at the last, add all the corrected heights together, and the sum will be the whole difference of level sought.

Dr. Halley suggested a new method of levelling, performed wholly by means of the barometer, the mercury being suspended at so much the less beight, as the place is farther remote from the centre of the earth; and hence the different heights of the mercury in two places, give the difference of level. This method is, in fact, no other than the method of measuring altitudes by the barometer, which has lately been so successfully practised and perfected by M. Deluc and others; but though it serves very well for the heights of hills, and other considerable altitudes, it is not accurate enough for determining small altitudes, to inches and parts. Sec the Barometrical Measurement of Altitudes.

Leveruing Poles, or Stroes, are instruments used in levelling, for carrying the marks to be observed, and at the same time to measure the heights of those marks from the ground. They usually consist cach of two long wooden rulcr's, made to slide over each other, and divided into feet and inches, \&c.

LEVER, a straight bar of iron or wood, \&c, supposed to be inflexible, supported on a fulcrum or prop by a single point, about which all the parts are moveable. The lever is the first of those simple machines called mechanical powcrs, being the simplest of them all; and is chiefly used for raising great weights to small heights.

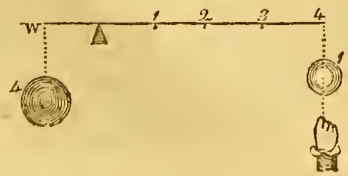

L. $E V$

The lever is of three kinds. First the common sont. where the weight intended to be raised is at one end of it, our strength or another weight called the power is at the other end, and the prop or fulcrum is between thrm both. In stirring up the tire with a poker, we make use of this lever; the poker is the lever, it rests on one of the bars of the grate as a prop, the incumbent fuel is the weight to be overcome, and the pressure of the hand on the other cnd is the force or power. In this, as in all the othir machincs, we bave only to increase the distance Letween the force and the prop, or to decrease the distance between the weight and the prop, to give the operator the greater power or effect. Tho this kind of lever may also be referred all scissars, pincers, snufficrs, \& $c$. The steel-yard and the common balance are also leyers of this kind.

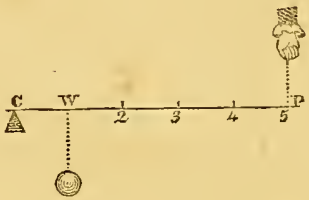

In the lever of the $2 \mathrm{~d}$ kind the prop is at one end, the force or power at the other, and the weight to be raised is between them. Thus, in raising a water-plug in the streets, the workman puts his iron bar or lever through the ring or hole of the plug, till the cnd of it reaches the ground on the other sicle; then making that the prop, he lifts the plug by his force or strength applied at the other end of the lever. In this lever too, the nearer the weight is to the prop, or the farther the power from the prop, the greater is the effect. To this $2 \mathrm{~d}$ kind of lever may also be referted the oars and rudder of a boat, the masts of a ship, cutting knives fixed at onc end, and doors, whose hinges serve as a fulcrum.

In the lever of the third kind, the power acts between the weight and the prop; such as a ladder raised by a man somewhere betwcen the two ends, to rear it against a wall, or a pair of tongs, \&x.

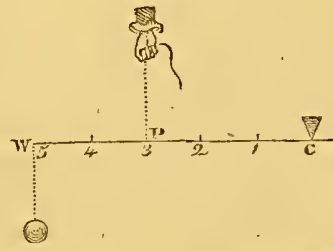

It is by this kind of lever also that the muscular motions of animals are performed, the muscles being inserted much nearer to the centrc of motion, than the point where is placed the centre of gravity of the weight to be raised; so that the power of the muscle is many times greater than the weight it is able to sustain. And in this thirl kind of lever, to produce a balance between the jower and weight, the power or force must exceed the weight, in the same proportion as it is nearer the prop than the weight is; whereas, in the other two kinds, the power is less than the weight, in the same proportion as its distance is greater; that is, universally, the power and weight are 4. Z 2 


\section{E V}

\section{[. 72}

each of them reciprocally as their distance from the prop; as is demonstrated below.

Some anthors make a 4 th kind, of what is called a bended lever; such as a hammer in drawing a nail, \&c.

In all levers, the universal property is, that the effect of either the weight or the power, to turn the lever about the fulcrum, is directly as its force and its distance from the prop, that is as $d f$, where $d$ denotes the distance, and $f$ the force, strength, or weight, \&c, of the agent. For since it is proved that at a double distance it will have a double effect, at a triple distance a triple effect, and so on; also since a donble force produces a double effect, a triple force a triple effect, and so on; therefore universally the effect is as of the product of the two. In like manner, if $D$ be the distance of another power or agent, whose intensity is $F$, then is DF the effect of this also to move the lever. And if these two agents act against each other on the lever, and their effects be supposed equal, or the lever kept in equilibrio by the equal and contrary effects of these two agents; then is $\mathrm{DF}=d f$, which equation resolves into this analogy, viz, $\mathrm{D}: d:: f: \mathbf{F}$; that is, the distances of the agents from the prop, are reciprocally or inversely as their forces, or the power is to the weight, as the distance of the latter from the prop is to the distance of the former.

Writers on mechanics commonly demonstrate this proportion in a very absurd manner, viz, by supposing the lever put into motion about the prop, and then inferring that, because the momenta of two bodies are equal, when placed upon the lever at such distances, that these distances are reciprocally proportional to the weights of the bodies, and that therefore this is also the proportion in case of an equilibrium; which is an attempt absurdly to demonstrate a thing, supposing the contrary, that a body is at rest, by supposing it to be in motion. I shall therefore give here a universal demonstration of the property, on the pure principles of rest and pressure, or force only. Thus, let Pw be a lever, $c$ the prop, and $\mathbf{P}$ and $w$ any two forces acting on the lever at the points $P$ and $w$, in the directions Po, wo; then if $C E$ and $C D$ be the perpendicular distances of the directions of these forces from the prop $c$, it is to be demonstrated that $\mathbf{P}: \mathrm{w}:$ : CD : CE. In order to which join $\mathrm{co}$, and draw св parallel to wo,

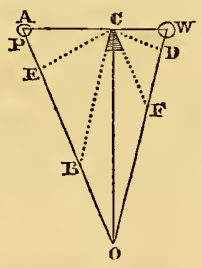
and $\mathrm{Cr}$ parallel to po. Then will co, the perpendicular to $\mathbf{P w}$, be the direction of the pressure on the prop, otherwise there conld be no equilibrium, for the directions of three forces that keep each other in equilibrium, must necessarily meet in the same point. And because any three forces that keep each other in equilibrium, are proportional to the three sides of a triangle formed by drawing lines parallel to the directions of these forces; therefore the forces on $\mathrm{P}, \mathrm{C}$, and $\mathrm{w}$, are as the three lines $\mathrm{BO}, \mathrm{CO}$, $\mathrm{CB}_{\mathrm{B}}$, which are in the sume direction, or parallel to them; that is, the force $P$ is to the force $w$, as Bo or its equal $c F$ is to $\mathrm{CB}$. But the two triangles $\mathbf{C D F}$, and have their like sides proportional,

$$
\text { viz, } \mathrm{CF}: \mathrm{C} \text { B : : CD : CE; }
$$

and because it was $\mathrm{CF}: \mathrm{CB}:: \mathrm{P}: \mathrm{w}$;

therefore by equality $P: W:: C D: C E$;

that is, each force is reciprocally proportional to the distance of its direction from the fulcrum. And it will be

\section{E V}

found that this demonstration serves also for the other kinds of levers, by drawing the lines as directed. H(nce, if any given force $P$ be applied to a lever at $A$; its effect upon the lever, to turn it about the centre of motion $\mathrm{c}$, is as the length of the arm $C_{A}$, and the sine of the angle of direction $\mathrm{CAE}_{\mathrm{E}}$. For the perp. $\mathrm{CE}$ is as $\mathrm{CA}_{\mathrm{A}} \times \sin . \angle \mathrm{A}$.

It having been objected to the principle of this demonstration, that it inferred the stability of the lever, or the line $\mathrm{Pw}$, from the theorem that three forces meeting in a point, and keeping that point, or a body there, stable or fixed, are proportional to the three sides of a triangle which are parallel to their directions; which is a misconception. For, it is only meant that the point $o$ is stable by that theorem. As to the stability of the lever PW, that is inferred from its being perp. to the given line oc, and limited by the two lines op, ow, given in position.-Since the time when the above demonstration was printed in the first edition of this Dictionary, 1 have seen another similar one that had been given in Dr. Hamilton's Essays.

In any analogy, because the product of the extremes is equal to that of the means; therefure the product of the power by the distance of its direction is equal to the product of the weight by the distance of its direction. That is, $P \times C E=w \times C D$.

If the lever, with the two weights fixed to it, be made to move about the centre $\mathrm{c}$; the momentum of the power will be equal to that of the weight; and the weights will be reciprocally proportional to their velocities.

When the two forces act perpendicularly on the lever, as two weights \&c; then, in case of an equilibrium,

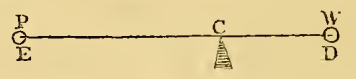

coincides with $\mathrm{P}$, and $\mathrm{D}$ with $\mathrm{W}$; and the distances $\mathrm{CP}$, Cw, taken on the lever, or the distances of the power and weight, from the fulcrum, are reciprocally proportional to the power and weight.

In a straight lever, when kept in equilibrio by a weight and power acting perpendicularly upon it; then, of these three, the power, weight, and pressure on the prop, any one is as the distance of the other two.

And hence too P + W: P:': ED:CD, and $F+W: W::$ : $: C P$;

that is, the sum of the weights is to either of them, as the sum of their distances is to the distance of the other.

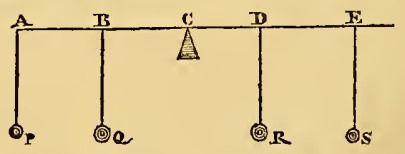

Also, if several weights $\mathbf{P}, \mathrm{Q}, \mathrm{R}, \mathrm{s}$, \&c, act on a straight lever, and keep it in eqnilibrio; then the sum of the products on nue side of the prop, will be cqual to the sum on the other side, made by multiplying each weight by its distance from the prop; viz, P. $A C+Q \cdot B C=R \cdot D C+s$. $\mathbf{E c}+$ \& $\mathrm{c}$.

Hitherto the lever has been considered as a mathematical line void of weight or gravity. But when its weight is considered, it is to be done thus: Find the weight and the centre of gravity of the lever alone, and then consider it as a mathematical line, but having an equal weight suspended by that contre of gravity; and so combine its effect with those of the other weights, as above. 
On the foregoing principles depends the nature of scales and beams for weighing all bodies. For, if the distances be equal, then will the weights be equal also; which gives the eunstruction of the common scales. And the Roman statera, or steel-yard, is also a lever, but of unequal arms or distances, so contrived that one weight only may scrve to weigh a great many, by sliding it backwards and forwards to different distances upon the longer arm of the lever. See BaLANCE, \&c.

Also, on the principle of the lever depend almost all other mechanical powers and effects. See WHEEL-ANDAXLe, Pulleet, Wedge, Screw, \&c.

LEVITY, the privation or want of weight in any body, when compared with another that is heavier; in which sense it stands opposed to gravity. Thus cork, and most sorts of wood that float in water, have levity with respect to water, that is, are less heavy. The schools maintained that there was such a thing as positive and absolute levity; and to this they imputed the rise and buoyancy of bodies lighter in specie than the bodies in which they rise and float. But it is now well known that this happens only in consequence of the heavier and denser fluid, which, by its superior gravity, gains the lowest place, and raises up the lighter body by a force which is equal to the difference of their gravities. It was denonstrated by Archimedes, that a solid body will float any-where in a fluid of the same specific gravity as itself; and that a lighter budy will always be raised up in it.

LEUWENHOEK (AN'rony), a celebrated Dutch philosopher, was born at Delft in 1632; and acquired a great reputation throughout all Europe, by his experiments and discoveries in natural history, by means of the miscroscope. He particularly excelled in making glasses for microscopes and spectacles; and he was a member of most of the literary societies of Europe; to whom he sent many memoirs. Those in the Philosophical Transactions, and in the Paris Memoirs, extend through many volumes; the former were extracted, and published at Leyden, in 1722 . He died in 1723 , at 91 years of age.

LEYBOURN (WIXIAM), a respectable and very useful mathematician, of the 17 th century, died about the year 1690. He was, it seems, originally a printer in London; but by industry became a considerable proficient in mathematics, and published a number of very useful and popular books, on the practical parts of those sciences. Those of them that are in my possession, are the following: 1. Compleat Surveyor, in folio, 1653, afterwards re-published and improverl by Cunn. -2 . Nine Geometrical Exercises, in 4to, 1669.-3. Dialling, in 4to, 1687; the same in folio, $1700 .-4$. Cursus Mathematicus, 2 vols folio, 1690, then beld in much esteem.-5. Recreations, in folio, 1694.-6. Arithmetic, 8 vo, 1700.-7. On the Gunter's Quadrant, $12 \mathrm{mo}, 1731$. There may be other editions of these works, and probably earlier ones of several of them, as well as of other works which 1 have not seen, such as one entitled the Trader's Guide.

J.EYDEN Puna, in Electricity, is a glass phial or jar, coated both within and witlout with tin-foil, or some other conducting substance, that it may be charged, and thus employed in a variety of useful and entertaining experiments. Or even flat glass, or any other shape, so coated and used, has also received the same denomination. $\Lambda$ lso a vacuum produced in such a jar, \&c, has been named the Leyden Vacuum.
The Leyden phial has been so called, because it is said that M. Cunæus, a native of Leyden, first contrived, about the close of the year 1745 , to accumulate the electrical power in glass, and to use it in the same manner as at present. But Dr. Priestley asserts that this discovery was first made by Von Isleist, dean of the cathedral in Camin; who, on the 4 th of November 1745 , sent an account of it to Dr. Lieberkubn at Berlin : however, those to whom Kleist's account was communicated, could not succeed in performing his experiments. The chief circumstances of this discovery are stated by Dr. Priestley in his Hist. of Electricity, vol. 1, pa. 191, \&c.

\section{LIBRA. See Balance.}

LIBRA is also one of the $4 \mathrm{~S}$ old constellations, and the 7 th sign of the zodiac, being opposite to Aries, and marked like a part of a pair of scales, thus $\bumpeq$. The figure of the balance was probably given to this part of the ecliptic, because when the sun arrives at this part, which is at the time of the autumnal equinox, the days and nights are equal, as if weighed in a balance. The stars in this constellation are, according to Ptolemy 17, Tycho 10, Hevelius 20 , and Flamsteed 51 .

Libra also denotes the ancient Roman pound, which was divided into 12 unciæ, or ounces, and the ounce into 24 scruples. It seems the mean weight of the scruple was nearly equal to $17 \frac{1}{2}$ grains Troy, and consequently the libra, or pound, equal to 5040 grains. It was also the naine of a gold coin, equal in value to 20 denarii. See Philos. Trans. vol. 61, pa. 462.

The French livre is derived from the Roman libra, as this was from the Sicilians, who called it litra. This was used in France for the proportions of their coin till about the year 1100 , their sols being so proportioned as that 20 of them were equal to the libra. By degrees it became a term of account, and every thing of the value of 20 sols was called a livre.

LIBRATION, of the Moon, is an apparent irregularity in her motion, by which she seems to librate, or waver, about her own axis, at one time towards the east, and at another towards the west. See Moon, and Evection. Hence it is that some parts, near the moon's western edge, at one time recede from the centre of the disc, while those on the other or eastern side approach nearer to it; and, on the contrary, at another time the western parts are seen to be nearer the centre, and the eastern parts farther from it: by which means it happens that some of those parts, which were before visible, set and hide themselves in the hinder or invisible side of the moon, and afterwards return and appear again, on the nearer or visible side.

This libration of the moon was first discovered by $\mathrm{He}$ velius, in the year 1654; and it is owing to her equable rotation round her own axis, once in a mouth, in conjunction with her unequal motion in the perimeter of her orbit round the earth. For if the moon moved in a circle, having its centre coinciding with the centre of the earth, while it turned on its axis in the precise time of its period round the earth, then the plane of the same lunar meridian would always pass through the earth, and the same face of the moon would be constantly and exactly turned towards us. But since the real motion of the moon is about a point at a considerable distance from the centre of the earth, that motion is very unequal, as seen from bence, the plane of no one meridian constantly passing through the carth.

The libration of the moon is of three kinds. 
1st, Her libration in longiturle, or a seeming vibrating motion according to the order of the signs of the zodiac. This libratson is nothing twice in eacis periotical month, viz, when the moon is in her apogeum, and when in her perigeum; for in buth these case's the plane of her meridian, which is turned towards us, is directed alike towards the earth.

$2 \mathrm{~d}$, Her libration in latitude; which arises from hence, that her axis not teing perpendicular to the plane of her orbit, but inclined to it, sometimes one of her pols's, and sometimes the other will decline, as it were, or dip a little towards the earth, and consequenly she will appear to librate a little, and to show sometimes more of her spots, and sometimes less of them, towards each pole; which libration, depending on the position of the moon, in respect to the nodes of les orbit, and her axis being nearly perpendicular to the plane of the icliptic, is very properly said to be in latitude: and this also is completed in the space of the moon's periodical month, or rather while the moon is returning again to the same position, in respect of her nodes.

3d, There is also a third kind of libration; by which it happens that though another part of the moon be not really turned to the earth, as in the fornite libration, yet another is illuminated by the sun. For since the moms axis is nearly perpendicular to the plane of the ecliptic, when she is most southerly, in respect of the north pole of the ecliptic, some parts near to it will be illummated by the sun; while, on the contrary, the somb pole will be in darkness. In this case therefore, it the sun be in the same line with the moon's southern limit, then, as she proceeds from conjunction with the sun towards her ascending node, she will appear to dip her nurthern polar, parts a little into the dark bemispliere, and to raise her southern polar parts as much into the light one: and the contrary to this will happen two weeks after, while the new moon is descending from her northern limit; for then her northern polar parts will appear to emerge out of darkness, and the southern polar parts to dip into it: which seening libration, or rather these effects of the former libration in jatitude, depending on the light of the sun, will be completed in the moon's synodical month. Greg. Astron. lib. 4 , sect. 10 .

LiBRATION of the Earth, is a term applied by some astronomers to that motion, by which the earth is so retained in its orbit, that its axis continues constantly parallel to the axis of the world. This Copernicus calls the motion of libration, which may be thus illustrated : Suppose a globe, with its axis parallel to that of the earth, painted on a flag at the hearl of a mast, moveable on its axis, and constantly driven by an east wind, while it sails round an island, it is evilent that the painted globe will be so librated, as that its axis will be paratlel to that of the world, in every siruation of the ship.

LIFE-ANNUITIES, are such periodical payments as depend on the continuance of some particular life or lives; and they may be distinguished into annuities that commence inmodiately, and such as conmence at some future period, called reversionary life-annuities.
The value, or present worth, of an annuity for any proposed life or lives, it is evident, depends on two circumstances, the interest of money, and the chance or expectatson of the continuance of Infe. On the furmer only, it has been shown, under the article ANNurtes, iepends the value or present worth of an annuity certain, or that is not subject to the continuance of a life, or other contingency; but the expectation of life being a thing not certain, but only posscssing a certain chance, it is evident that the value of the certain annuity: as stated abose, must be diminished in proportion as the expuctancy is befow certainty: thus, if the present value of an annuity certain be any surn, as suppose $100 t$. ant the value or expectancy of the life be $\frac{1}{2}$, then the value of the lite-annuity will be only half of the former, or $50 l$; and if the value of the life be only $\frac{x}{3}$, the value of the life-annuity will be but $\frac{1}{3}$ of 100 , that is $33 \%$. 6. $8 d$; and so on.

The measure of the value or expectancy of hife, depends on the proportion of the number of persons that die, out of a given number, in the tine proposed; thus, if 50 persons die, out of 100 , in any proposed time, then, half the number only remaining alive, any one person has an equal chance to live or die in that time, or the value of his life for that time is $\frac{1}{2}$; but it $\frac{2}{3}$ of the number die in the time proposed, or only $\frac{1}{3}$ remain alive, then the value of any ones life is $\frac{x}{3}$; and if $\frac{3}{4}$ or the number die, or only $\frac{x}{4} \mathrm{re-}$ matn alive, tiles the value of any life is but $\frac{1}{4}$; and so on. lis these properticiss then must the value of the annuity certin be uminshed, to give the value of the like lite annuity.

It is plain therefore that, in this business, it is neccssary to know the value of life at all the different ages, from some table of observations on the mortality of mankind, which may show the proportion of the persons living, out of a given number, at the end of any limited time; or from some certain hypothesis, or assumed principle. Now various tables and hypotheses of this kind have been given by the writers on this subject, as Dr. Halley, Mr. Demoirre, Mr. Thomas Simpson, Mr. Dodson, Mr. Kerseboom, Mr. Parcieus, Dr. Price, Mr. Morgan, Mr. Baron Maseres, Mr. Baily, and many others. But the same table of probabilities of life will not suit all places; for long experience has shown that all places are not equaliy liealthy, or that the proportion of the number of persons that die annually, is different for different places. Dr. Halley conputed a table of the annual deaths as drawn from the bills of mortality of the city of Breslaw in Germany : Mr. Smart and Mlr. Simpson from those of London; Dr. Price fiom those of Northaupton; Mr, Kersseboom from those of the provinces of 1 lulland and West-Friesland; and M. Parcieux from the lists of the French tontines, or long annuities; and all these are found to differ from one another. It may not therefore be improper to insert bere a comparative view of the principal tables that have been given of this kind, as below; where the first column shows the age, and the other columns the number of persons living at that age, out of 1000 born, of of the age (), in the first line of each column. 
ТАвLE I.-Showing the Number of Persons liting at all Ages, out of 1000 that had been born at seteral Places, viz.

\begin{tabular}{|c|c|c|c|c|c|c|c|c|c|c|c|}
\hline Ages. & Viemna. & Berlin. & London. & Norwich. & $\begin{array}{c}\text { North- } \\
\text { ampton. }\end{array}$ & Breslaw. & $\begin{array}{c}\text { Branden- } \\
\text { burg. }\end{array}$ & $\begin{array}{l}\text { Holy- } \\
\text { Cruss. }\end{array}$ & Holland. & France. & $\begin{array}{c}\text { Vaud, } \\
\text { Switzerland. }\end{array}$ \\
\hline 0 & 1000 & 1000 & $10(10$ & 1000 & 1000 & 1000 & 1000 & 1000 & 1000 & 1000 & 1000 \\
\hline 1 & $5+2$ & 633 & 680 & 798 & 738 & 769 & 775 & 882 & 80.4 & 805 & 811 \\
\hline 2 & 471 & 528 & 548 & 651 & 628 & 658 & 718 & 762 & 764 & 777 & 765 \\
\hline 3 & 4.30 & $4 \times 5$ & 492 & 595 & 585 & 614 & 687 & 717 & 736 & 750 & 735 \\
\hline 4 & 400 & $43+$ & 452 & 566 & 562 & 585 & 654 & 682 & 709 & 727 & 715 \\
\hline 5 & 377 & 403 & 4.26 & 54.4 & 544 & 563 & 64.2 & 659 & 689 & 711 & 701 \\
\hline 6 & 357 & 387 & 410 & 526 & 530 & 540 & 622 & 636 & 676 & 697 & 688 \\
\hline 7 & $3+4$ & 376 & 397 & 511 & 518 & 532 & 607 & 618 & 664 & 686 & 677 \\
\hline 8 & 337 & 367 & 388 & 500 & 510 & 523 & 595 & 601 & 652 & 676 & 667 \\
\hline 9 & 331 & 361 & 380 & 490 & 504 & 315 & 585 & 595 & $6+6$ & 667 & 659 \\
\hline 10 & 326 & 356 & 373 & 481 & 4.98 & 508 & 577 & 589 & 639 & 660 & 653 \\
\hline 11 & 322 & 353 & 367 & 474 & 493 & 502 & 570 & 585 & 633 & 654 & 648 \\
\hline 12 & 318 & 350 & 361 & 469 & 488 & 497 & 56. & 581 & 627 & 649 & $6+3$ \\
\hline 13 & 314 & 347 & 356 & $46+$ & 484 & 492 & 559 & 577 & 621 & 644 & 639 \\
\hline 14 & 310 & $3+4$ & 351 & 460 & 4.80 & 485 & 554 & 573 & 616 & 639 & 635 \\
\hline 1.5 & 306 & $3: 1$ & 347 & 455 & 475 & 48.3 & 549 & $56^{\prime}$ & 611 & 635 & 631 \\
\hline 16 & 302 & 338 & 343 & 451 & 470 & 479 & $5+4$ & 565 & 606 & 631 & 626 \\
\hline 17 & 299 & 335 & 338 & 446 & 4.65 & 474 & 539 & 560 & 601 & 626 & 622 \\
\hline 18 & 295 & 332 & 334 & $4+2$ & 459 & 470 & 535 & 555 & 596 & 621 & $61 \mathrm{~s}$ \\
\hline 19) & 231 & 328 & 329 & 4.37 & 453 & 465 & 531 & 550 & 590 & 616 & 614 \\
\hline 20 & 287 & 324 & 325 & 432 & 447 & 461 & 527 & 545 & 584 & 610 & 610 \\
\hline 21 & $28+$ & 320 & 321 & 426 & 440 & 456 & 522 & 539 & 577 & 604 & $600^{\circ}$ \\
\hline 22 & 280 & 315 & 316 & 421 & 433 & 451 & 517 & 532 & 571 & 593 & 602 \\
\hline 23 & 276 & 310 & 310 & 415 & 426 & $4+6$ & 512 & 525 & 566 & 592 & 597 \\
\hline 24 & 273 & 305 & 305 & 409 & 419 & 441 & 507 & 518 & 559 & 586 & $592^{\circ}$ \\
\hline 25 & 269 & 297 & 299 & $40 t$ & +12 & 436 & 502 & 512 & 551 & 580 & 587 \\
\hline 26 & 265 & 293 & 29.4 & 398 & 405 & 431 & 498 & 506 & 543 & $57+$ & 532 \\
\hline 27 & 261 & 287 & 288 & 392 & 398 & 426 & 495 & 501 & 535 & 568 & 577 \\
\hline 28 & 250 & 281 & 283 & 385 & 391 & 421 & 492 & 496 & 526 & 562 & 572 \\
\hline 29 & 251 & 275 & 278 & 378 & 354 & 415 & 489 & 491 & 517 & 556 & 567 \\
\hline 30 & 247 & 269 & 272 & 372 & 378 & 409 & 486 & 456 & 508 & 550 & 563 \\
\hline 31 & 243 & 264 & 266 & 366 & 372 & 403 & 482 & 481 & 499 & 544 & 558 \\
\hline 32 & 239 & 250 & 260 & 361 & 366 & 397 & 477 & 476 & 490 & 538 & 553 \\
\hline 33 & 235 & 254 & $25 \cdot 4$ & 355 & 360 & 391 & 472 & 471 & 482 & 532 & 54.8 \\
\hline 34 & 2.31 & 2.19 & 248 & 350 & 354 & 384 & 4.67 & 466 & 474 & 526 & 544 \\
\hline 35 & 226 & $2+3$ & 242 & $3+4$ & 348 & 377 & 462 & 460 & 467 & 520 & 539 \\
\hline 36 & 221 & 237 & 230 & 338 & 342 & 370 & 4.56 & 454 & 460 & 514 & 533 \\
\hline 37 & 216 & 230 & 230 & 333 & 336 & 363 & 4.50 & 4.47 & 453 & 508 & 527 \\
\hline 38 & 211 & 223 & 224 & 327 & 330 & 356 & $44 h$ & 440 & 446 & 503 & 520 \\
\hline 39 & 205 & 216 & 218 & 322 & 324 & 349 & 438 & 433 & 439 & 497 & 513 \\
\hline 40 & 199 & 209 & 214 & 317 & 317 & $3+2$ & 432 & 126 & 4.32 & 492 & 506 \\
\hline 41 & 194 & 20.3 & 207 & 311 & 310 & 335 & 4.27 & 418 & 425 & 457 & 500 \\
\hline 43 & 189 & 197 & 201 & 306 & 303 & 328 & 422 & 410 & 419 & 482 & 494 \\
\hline 43 & 185 & 192 & 194 & 300 & 296 & 321 & 417 & 401 & 413 & 476 & 488 \\
\hline 45 & 181 & 187 & 187 & 294 & 289 & 314 & 412 & 393 & 4.07 & 471 & 482 \\
\hline+5 & 176 & 182 & 180 & 287 & 282 & 307 & 407 & 356 & 4.00 & 466 & 476 \\
\hline 46 & 171 & 177 & 174 & 281 & 275 & 299 & 400 & 379 & 393 & 460 & 4.69 \\
\hline 4.7 & 165 & 172 & 107 & 274 & 268 & 291 & 394 & 372 & 386 & 455 & $4.61=$ \\
\hline 48 & 159 & 167 & 159 & 268 & 261 & 283 & 388 & 365 & 378 & $4+9$ & 4.51 \\
\hline 49 & 153 & 162 & 153 & 261 & 254 & 275 & 381 & 359 & 370 & $4+3$ & $4+1$ \\
\hline 50 & 147 & 157 & 147 & 255 & 247 & 267 & 374 & 353 & 362 & 436 & 431 \\
\hline 51 & 112 & 152 & $1+1$ & $2+8$ & 239 & 259 & 307 & $3+7$ & 3.4 & 429 & 422 \\
\hline 52 & 137 & 147 & 135 & 242 & 232 & 250 & 359 & 340 & 345 & 422 & 414 \\
\hline 53 & 13.3 & $1+2$ & 130 & 2.35 & 225 & 241 & 351 & 333 & 3.30 & 414 & 4.06 \\
\hline 54 & 128 & 1.37 & 12.5 & 228 & 218 & 232 & $3+3$ & 320 & 327 & 400 & 397 \\
\hline 55 & 123 & 132 & 120 & 221 & 211 & 224 & 334 & 318 & 318 & 397 & 388 \\
\hline 56 & 117 & 127 & 116 & 213 & 20.4 & 216 & 324 & $310^{\circ}$ & 309 & 388 & 377 \\
\hline 57 & 111 & 121 & 111 & 200 & 197 & 209 & $31+$ & 301 & 300 & 379 & $30 \mathrm{t}$ \\
\hline 58 & 106 & 115 & 106 & 199 & 190 & 201 & 304 & 292 & 291 & 369 & 348 \\
\hline 59 & 101 & 109 & 101 & 191 & 183 & 193 & 293 & 283 & $28 \%$ & 359 & 3.31 \\
\hline
\end{tabular}




\begin{tabular}{|c|c|c|c|c|c|c|c|c|c|c|c|}
\hline Ages. & Viensa. & Berlin. & London. & Norwich. & $\begin{array}{l}\text { Norili- } \\
\text { ampion. }\end{array}$ & Breslaw. & $\begin{array}{c}\text { Branden- } \\
\text { burg. }\end{array}$ & $\begin{array}{l}\text { Holy- } \\
\text { Cross. }\end{array}$ & Holland. & France. & $\begin{array}{c}\text { Vaud, } \\
\text { Switzerland. }\end{array}$ \\
\hline 60 & 96 & 103 & 96 & 184 & 176 & 196 & 232 & 273 & 273 & 349 & 314 \\
\hline 61 & 91 & 97 & 92 & 177 & 169 & 178 & 271 & 263 & 264 & 339 & 299 \\
\hline 62 & 87 & 92 & 87 & 169 & 162 & 170 & 260 & 253 & 255 & 329 & 286 \\
\hline 63 & 82 & 88 & 83 & $16 \mathrm{t}$ & 155 & 163 & 24.8 & 243 & 245 & 318 & 274 \\
\hline 64 & 77 & 84 & 78 & 153 & 14.8 & 155 & 236 & 233 & 235 & 307 & 262 \\
\hline 65 & 72 & 80 & 74. & 144 & 141 & 147 & 224 & 223 & 225 & 296 & 250 \\
\hline 66 & 67 & 75 & 70 & 136 & 134. & 140 & 213 & 213 & 215 & 285 & 236 \\
\hline 67 & 62 & 70 & 65 & 128 & 127 & 132 & 202 & 203 & 205 & 273 & 220 \\
\hline 68 & .57 & 65 & 61 & 119 & 120 & 124 & 190 & 193 & 195 & 260 & 202 \\
\hline 69 & 52 & 60 & 56 & 111 & 113 & 117 & 17 & 182 & 185 & 246 & 184 \\
\hline 70 & . 48 & 55 & 52 & 103 & 106 & 109 & 16 & 171 & 175 & 232 & 168 \\
\hline 71 & 44 & 51 & 47 & 94 & 99 & 101 & 153 & 161 & 165 & 218 & 153 \\
\hline 72 & 40 & 47 & 43 & 86 & 92 & 93 & 138 & 151 & 155 & 195 & 140 \\
\hline 73 & 36 & 43 & 39 & 79 & 85 & 85 & 12 & 142 & 145 & 185 & 129 \\
\hline 74 & $3 \dot{3}$ & 39 & 3.5 & 71 & 78 & 77 & 107 & 134 & 135 & 173 & 119 \\
\hline 75 & 30 & 35 & 32 & 64 & 71 & 69 & 93 & 126 & 125 & 158 & 109 \\
\hline 76 & 27 & 32 & $2 S$ & 57 & 64 & 61 & 80 & 119 & 114 & 144 & 98 \\
\hline 77 & 24 & 29 & 25 & 50 & 58 & 53 & $6 \mathrm{~s}$ & 112 & 103 & 129 & 85 \\
\hline 78 & 21 & 26 & 22 & 43 & 52 & 4.5 & 59 & 105 & 92 & 115 & $7 \mathrm{I}$ \\
\hline 79 & 18 & 23 & 19 & 37 & 46 & 38 & 51 & 98 & 82 & 102 & 58 \\
\hline 80 & 16 & 20 & 17 & 32 & 40 & 32 & 44 & 90 & 72 & 88 & 46 \\
\hline 81 & 14 & 18 & 14 & 27 & 34 & 26 & 38 & 81 & 62 & 75 & 36 \\
\hline 82 & 12 & 10 & 12 & 23 & 28 & 22 & 32 & 71 & 5.3 & 63 & 29 \\
\hline 83 & 10 & 14. & 10 & 19 & 23 & 18 & 25 & 61 & 45 & 53 & 24 \\
\hline 84 & 8 & 12 & 8 & 16 & 19 & 15 & 21 & 51 & 38 & 44 & 20 \\
\hline 85 & 7 & 10 & 7 & 13 & 16 & 12 & 15 & 41 & 31 & 36 & 17 \\
\hline 86 & 6 & 8 & 6 & 10 & 13 & 9 & 11 & 32 & 25 & 28 & 14 \\
\hline 87 & 5 & 7 & 5 & 8 & 11 & 6 & 3 & 24 & 19 & 21 & 11 \\
\hline $8 S$ & 4 & 6 & 4 & 6 & 8 & 4 & 6 & 17 & 14 & 16 & 9 \\
\hline 89 & 3 & 5 & 3 & 5 & 6 & 2 & 4 & 11 & 10 & 12 & 7 \\
\hline 90 & 2 & 4 & 2 & 4 & 4 & l & 3 & 7 & 7 & 8 & 5 \\
\hline
\end{tabular}

These tables show that the mortality and chance of life are various in different places; and that therefore, to obtain a sufficient accuracy in this business, it is necessary to adapt a table of probabilities or chances of life, to every place for which annuities are to be calculated; or at least one set of tables for large towns, and another for country places, as well as for the supposition of different rates of interest.

Several of the foregoing tables, as they commeneed with different numbers, are here reduced to the same number at the begimning, viz, 1000 persons, by which means we are enabled by inspection, at any age, to compare the numbers together, and immediately perceive the relative degrees of vitality at the several places. The tables are also arranged according to the degree of vitality among them; the least, or that at Vienna, first; and the rest in their order, to the highest, which is the province of Vaud in Switzerland. The authorities on which these tables depend, are as they here follow. The first, taken from Dr. Price's Observations on Reversionary payments, is formed from the bilis at Vienna, for $\delta$ years, as given by Mr. Susmilch, in his Gottliclie Ordnung; the 2d, for Berlin, from the same, as formed from the bills there for 4 years, viz, from 1752 to 1755 ; the $3 \mathrm{~d}$, from Dr. Price, showing the true probabilities of life in London, formed from the bills for ten years, viz, from 1759 to 1768 ; the 4th, for Norwich, formcd by Dr. Price from the bills for
30 years, viz, from 1740 to 1769 ; the 5 th, by the same, from the bills for Northampton ; the 6 th, as deduced by Dr. Halley, from the bills of mortality at Breslaw; the 7 th shows the probabilities of life in a country parish in Brandenburg, formed from the bills for 50 years, viz, from 1710 to 1759 , as given by Mr. Susmilch; the Sth shows the probabilities of life in the parish of Holy-Cross, near Slirewsbury, formed from a register kept by the Rev. Mr. Garsuch, for 20 years, from 1750 to 1770 ; the 9 th, for Holland, was formed by M. Kersseboom, from the registers of certain annuities for lives granted by the government of Holland, which had been kept there for 125 years, in which the ages of the several annuitants dying during that period had been truly entered; the 10 th, for France, were formed by M. Parcieux, from the lists of the French tontines, or long annuities, and verified by a comparison with the mortuary registers of several religinus houses for both sexes; and the 11 th, or last, for the district of Vaud in Switzerland, was formed by Dr. Price from the registers of 43 parishes given by M. Muret, in the Bern Memoirs for the year 1766 .

Now from such lists as the foregoing, various tables have been formed for the valuation of annuities on single and joint lives, at several rates of interest, in which the value is shown by inspection.-The following are thuse that are given by Mr. Simpson, in his Select Exercises, as deduced from the London bills of mortality. 


\section{I F}

[ 729$]$

L I F

TABLE 11.-Showing the Value of an Annuity on One Life, or Number of Years Annuity in the Value, supposing

Money to bear Interest at the several Rates of 3, 4, and 5 per cent:

\begin{tabular}{|c|c|c|c|c|c|c|c|}
\hline Age. & $\begin{array}{c}\text { Years value at } \\
3 \text { per cent. }\end{array}$ & $\begin{array}{l}\text { Years vahue at } \\
4 \text { per cent. }\end{array}$ & $\begin{array}{l}\text { Years value at } \\
5 \text { per cent. }\end{array}$ & Age. & $\begin{array}{l}\text { Years value at } \\
3 \text { per cent. }\end{array}$ & $\begin{array}{l}\text { Years value at } \\
4 \text { per cent. }\end{array}$ & $\begin{array}{l}\text { Years ralue at } \\
5 \text { per cent. }\end{array}$ \\
\hline 6 & 18.8 & 16.2 & $14 \cdot 1$ & 41 & $13 \cdot 0$ & $11 \cdot 4$ & $10 \cdot 2$ \\
\hline 7 & 18.9 & 16.3 & $14 \cdot 2$ & 42 & 12.8 & $11 \cdot 2$ & $10 \cdot 1$ \\
\hline 8 & 19.0 & $16 \cdot 4$ & $1 \div 3$ & 43 & $12 \cdot 6$ & $11 \cdot 1$ & $10 \cdot 0$ \\
\hline 9 & $19 \cdot 0$ & $16 \cdot 4$ & $14 \cdot 3$ & 44 & 12.5 & $11 \cdot 0$ & $9 \cdot 9$ \\
\hline 10 & $19 \cdot 0$ & 164 & $14: 3$ & 45 & $12 \cdot 3$ & $10 \cdot 8$ & $9 \cdot 8$ \\
\hline 11. & $19 \cdot 0$ & 164 & $14 \cdot 3$ & 46 & $12 \cdot 1$ & $10 \cdot 7$ & $9 \cdot 7$ \\
\hline 12 & $18 \cdot 9$ & $16 \cdot 3$ & $14 \cdot 2$ & 47 & $11 \cdot 9$ & 10.5 & $9 \cdot 5$ \\
\hline 13 & $18 \cdot 7$ & 16.2 & $14 \cdot 1$ & 48 & $11 \cdot 8$ & $10 \cdot 4$ & $9 \cdot 4$ \\
\hline 4 & 18.5 & $16^{\circ} 0$ & 14.0 & 49 & $11 \cdot 6$ & $10 \cdot 2$ & $9 \cdot 3$ \\
\hline 15 & $18 \cdot 3$ & 15.8 & $13 \cdot 9$ & 50 & $11 \cdot 4$ & $10 \cdot 1$ & $9 \cdot 2$ \\
\hline 16 & 18.1 & $15 \cdot 6$ & $13 \cdot 7$ & 51 & $11 \cdot 2$ & 0.9 & $9 \cdot 0$ \\
\hline 17 & $17 \cdot 9$ & 15.4 & 13.5 & 52 & 11.0 & $9 \cdot 8$ & $8 \cdot 9$ \\
\hline 18 & $17 \cdot 6$ & 15.2 & 13.4 & 53 & $10 \cdot 7$ & $9 \cdot 6$ & $8 \cdot 8$ \\
\hline 19 & $17 \cdot 4$ & $15^{\circ} 0$ & 13.2 & 54 & $10 \cdot 5$ & $9 \cdot 4$ & $8 \cdot 6$ \\
\hline 20 & $17 \cdot 2$ & $14 \cdot 3$ & $13 \cdot 0$ & 55 & $10 \cdot 3$ & $9 \cdot 3$ & $8 \cdot 5$ \\
\hline 21 & $17 \cdot 0$ & $14 \cdot 7$ & $12 \cdot 9$ & 56 & $10 \cdot 1$ & $9 \cdot 1$ & $8 \cdot 4$ \\
\hline 22 & 16.8 & $14 \cdot 5$ & 127 & 57 & $9 \cdot 9$ & 8.9 & $8 \cdot 2$ \\
\hline 23 & 16.5 & $14 \cdot 3$ & $12 \cdot 6$ & 58 & $9^{\circ} 6$ & $8 \cdot 7$ & $S \cdot 1$ \\
\hline 24 & 16.3 & $14 \cdot 1$ & $1 \cdot 2 \cdot 4$ & 59 & $9 \cdot 4$ & $8 \cdot 6$ & $8 \cdot 0$ \\
\hline 25 & $16 \cdot 1$ & 14.0 & $12 \cdot 3$ & 60 & $9 \cdot 2$ & 8.4 & $7 \cdot 9$ \\
\hline 26 & $15 \cdot 9$ & 13.8 & $12 \cdot 1$ & 61 & $8 \cdot 9$ & $8 \cdot 2$ & $7 \cdot 7$ \\
\hline 27 & $15 \cdot 6$ & $13 \cdot 6$ & $12 \cdot 0$ & 62 & 8.7 & $8 \cdot 1$ & $7 \cdot 6$ \\
\hline 28 & $15 \cdot 4$ & $13 \cdot 4$ & 11.8 & 63 & $8 \cdot 5$ & $7 \cdot 9$ & $7 \cdot 4$ \\
\hline 29 & $15 \cdot 2$ & 13.2 & I] 7 & 64 & $8 \cdot 3$ & $7 \cdot 7$ & $7 \cdot 3$ \\
\hline 30 & $15 \cdot 0$ & $13 \cdot 1$ & $11 \cdot 6$ & 65 & $8 \cdot 0$ & $7 \cdot 5$ & $7 \cdot 1$ \\
\hline 31 & $14 \cdot 8$ & $12 \cdot 9$ & $11 \cdot 4$ & 66 & $7 \cdot 8$ & $7 \cdot 3$ & $6 \cdot 9$ \\
\hline 32 & $14 \cdot 6$ & 12.7 & $11 \cdot 3$ & 67 & $7 \cdot 6$ & $7 \cdot 1$ & 6.7 \\
\hline 33 & $14 \cdot 4$ & $12 \cdot 6$ & $11 \cdot 2$ & 68 & $7 \cdot 4$ & $6 \cdot 9$ & $6 \cdot 6$ \\
\hline 34 & 142 & $12 \cdot 4$ & 11.0 & 69 & $7 \cdot 1$ & $6 \cdot 7$ & $6 \cdot 4$ \\
\hline 35 & $14 \cdot 1$ & 12.3 & 10.9 & 70 & $6 \cdot 9$ & $6 \cdot 5$ & $6 \cdot 2$ \\
\hline 36 & $13 \cdot 9$ & $12 \cdot 1$ & 10.8 & 71 & $6 \cdot 7$ & $6 \cdot 3$ & $6 \cdot 0$ \\
\hline 37 & 13.7 & 11.9 & $10^{\prime} 6$ & 72 & 6.5 & $6 \cdot 1$ & $5 \cdot 8$ \\
\hline 38 & 13.5 & $11 \cdot 8$ & 10.5 & 73 & $6 \cdot 2$ & $5 \cdot 9$ & $5 \cdot 6$ \\
\hline 39 & $13 \cdot 3$ & $11 \cdot 6$ & $10^{\circ} 4$ & 74 & $5 \cdot 9$ & $5 \cdot 6$ & $5 \cdot 4$ \\
\hline 40 & 13.2 & 11.5 & 10.3 & 75 & $5 \cdot 6$ & $5 \cdot 4$ & $5 \cdot 2$ \\
\hline
\end{tabular}

TABLE III.-Showing the Value of an Anmuity for Two Joint Lives, that is, for as long as they exist together.

\begin{tabular}{|c|c|c|c|c|c|c|c|c|c|}
\hline $\begin{array}{l}\text { Age of } \\
\text { Younger. }\end{array}$ & $\begin{array}{l}\text { Age of } \\
\text { Elder. }\end{array}$ & $\begin{array}{l}\text { Value at } \\
3 \text { per cent. }\end{array}$ & $\begin{array}{l}\text { Value at } \\
4 \text { per cent. }\end{array}$ & $\begin{array}{l}\text { Value at } \\
5 \text { per cent. }\end{array}$ & $\begin{array}{c}\text { Age of } \\
\text { Younger. }\end{array}$ & $\begin{array}{l}\text { Age of } \\
\text { Elder. }\end{array}$ & $\begin{array}{l}\text { Value at } \\
3 \text { per cent. }\end{array}$ & $\begin{array}{l}\text { Value at } \\
4 \text { per cent. }\end{array}$ & $\begin{array}{l}\text { Value at } \\
5 \text { per cert. }\end{array}$ \\
\hline \multirow{14}{*}{10} & 10 & $14 \cdot 7$ & $13 \cdot 0$ & $11 \cdot 6$ & \multirow{7}{*}{15} & 4.5 & $9 \cdot 6$ & 8.8 & $8 \cdot 0$ \\
\hline & 15 & $14 \cdot 3$ & $12 \cdot 7$ & $11 \cdot 3$ & & 50 & $8 \cdot 9$ & $8 \cdot 2$ & $7 \cdot 5$ \\
\hline & 20 & 13.8 & $12 \cdot 2$ & $10 \cdot 8$ & & 55 & $8 \cdot 2$ & $7 \cdot 6$ & $7 \cdot 0$ \\
\hline & 25 & 13.1 & $11 \cdot 6$ & $10 \cdot 2$ & & 60 & $7 \cdot 5$ & $7 \cdot 0$ & $6 \cdot 5$ \\
\hline & 30 & $12 \cdot 3$ & $10 \cdot 9$ & $9 \cdot 7$ & & 65 & $6 \cdot 5$ & $6 \cdot 4$ & $6 \cdot 0$ \\
\hline & .35 & $11 \cdot 5$ & $10 \cdot 2$ & $9 \cdot 1$ & & 70 & $6 \cdot 0$ & $5 \cdot 7$ & $5 \cdot 4$ \\
\hline & 40 & $10 \cdot 7$ & $9 \cdot 6$ & $8 \cdot 6$ & & 75 & $5 \cdot 2$ & $5 \cdot 0$ & 4.8 \\
\hline & 45 & $10 \cdot 0$ & $9 \cdot 0$ & $8 \cdot 1$ & \multirow{13}{*}{20} & 20 & $12 \cdot 8$ & $11 \cdot 3$ & $10 \cdot 1$ \\
\hline & 50 & $9 \cdot 3$ & 8.4 & $7 \cdot 6$ & & 25 & $12 \cdot 2$ & 10.8 & $9 \cdot 7$ \\
\hline & 55 & $B \cdot 6$ & $7 \cdot 8$ & $7 \cdot 1$ & & 30 & 11.6 & $10 \cdot 3$ & $9 \cdot 2$ \\
\hline & 60 & $7 \cdot 8$ & $7 \cdot 2$ & $6 \cdot 6$ & & 35 & $10 \cdot 9$ & $9 \cdot 8$ & $8 \cdot 8$ \\
\hline & 65 & $6 \cdot 9$ & 6.5 & $6 \cdot 1$ & & 40 & $10 \cdot 2$ & $9 \cdot 2$ & $8 \cdot 4$ \\
\hline & 70 & $6 \cdot 1$ & $5 \cdot 8$ & $5 \cdot 5$ & & 45 & $9 \cdot 5$ & $8 \cdot 6$ & $7 \cdot 9$ \\
\hline & 75 & $5 \cdot 3$ & $5 \cdot 1$ & $4 \cdot 9$ & & 50 & $8 \cdot 8$ & $8^{\circ} 0$ & $7 \cdot 4$ \\
\hline \multirow{6}{*}{15} & 15 & $13 \cdot 9$ & $12 \cdot 3$ & $11^{\circ} 0$ & & 55 & $8 \cdot 1$ & $7 \cdot 5$ & $6 \cdot 9$ \\
\hline & 20 & $13 \cdot 3$ & $11 \cdot 8$ & 10.5 & & 60 & $7 \cdot 4$ & $6 \cdot 9$ & $6 \cdot 4$ \\
\hline & 25 & $12 \cdot 6$ & $11 \cdot 2$ & $10 \cdot 1$ & & $65^{\circ}$ & $6 \cdot 7$ & $6 \cdot 3$ & $5 \cdot 9$ \\
\hline & 30 & $11 \cdot 9$ & $10 \cdot 6$ & $9 \cdot 5$ & & 70 & $6 \cdot 0$ & $5 \cdot 7$ & $5 \cdot 4$ \\
\hline & 35 & $11 \cdot 2$ & $10^{\circ} 0$ & $9 \cdot 0$ & & 75 & $5 \cdot 2$ & $5 \cdot 0$ & $4 \cdot 8$ \\
\hline & 40 & $10 \cdot 4$ & $0 \cdot 4$ & 8.5 & & & \multicolumn{3}{|c|}{$5 \mathrm{~A}$} \\
\hline
\end{tabular}


L I F

[ 730 ]

L I F

\begin{tabular}{|c|c|c|c|c|c|c|c|c|c|}
\hline $\begin{array}{c}\text { Age of } \\
\text { Younger. }\end{array}$ & $\begin{array}{l}\text { Age of } \\
\text { Eider. }\end{array}$ & $\begin{array}{l}\text { Value at } \\
3 \text { per cent. }\end{array}$ & $\begin{array}{l}\text { Value at } \\
4 \text { per ceut. }\end{array}$ & $\begin{array}{l}\text { Value at } \\
5 \text { per cent. }\end{array}$ & $\begin{array}{c}\text { Age of } \\
\text { Younger. }\end{array}$ & $\begin{array}{l}\text { Age of } \\
\text { Eider. }\end{array}$ & $\begin{array}{l}\text { Value at } \\
3 \text { per cent. }\end{array}$ & $\begin{array}{l}\text { Value at } \\
4 \text { per cent. }\end{array}$ & $\begin{array}{l}\text { Value at } \\
5 \text { per cent. }\end{array}$ \\
\hline \multirow[b]{2}{*}{25} & $\begin{array}{l}25 \\
30 \\
35 \\
40\end{array}$ & $\begin{array}{l}11 \cdot 5 \\
11 \cdot 3 \\
10 \cdot 7 \\
10 \cdot 0\end{array}$ & $\begin{array}{r}10 \cdot 5 \\
10 \cdot 1 \\
9 \cdot 6 \\
9 \cdot 1\end{array}$ & $\begin{array}{r}9 \cdot 4 \\
9 \cdot 0 \\
8 \cdot 6 \\
8 \cdot 2\end{array}$ & 40 & $\begin{array}{l}60 \\
65 \\
70 \\
75 \\
\end{array}$ & $\begin{array}{l}7 \cdot 0 \\
6 \cdot 4 \\
5 \cdot 7 \\
5 \cdot 0 \\
\end{array}$ & $\begin{array}{l}6 \cdot 4 \\
5 \cdot 9 \\
5 \cdot 1 \\
4 \cdot 8 \\
\end{array}$ & $\begin{array}{l}6 \cdot 0 \\
5 \cdot 5 \\
5 \cdot 1 \\
4 \cdot 6 \\
\end{array}$ \\
\hline & $\begin{array}{l}45 \\
50 \\
55 \\
60 \\
65 \\
70 \\
75 \\
\end{array}$ & $\begin{array}{l}9 \cdot 4 \\
8 \cdot 7 \\
8 \cdot 0 \\
7 \cdot 3 \\
6 \cdot 6 \\
5 \cdot 9 \\
5 \cdot 1 \\
\end{array}$ & $\begin{array}{l}8 \cdot 5 \\
7 \cdot 9 \\
7 \cdot 4 \\
6 \cdot 8 \\
6 \cdot 2 \\
5 \cdot 6 \\
4 \cdot 9 \\
\end{array}$ & $\begin{array}{r}7 \cdot 8 \\
7 \cdot 3 \\
6 \cdot 8 \\
6 \cdot 3 \\
5 \cdot 8 \\
5 \cdot 3 \\
4 \cdot 7 \\
\end{array}$ & 4.5 & $\begin{array}{l}45 \\
50 \\
55 \\
60 \\
65 \\
70 \\
75 \\
\end{array}$ & $\begin{array}{l}\cdot 3 \\
7 \cdot 9 \\
7 \cdot 4 \\
6 \cdot 8 \\
6 \cdot 3 \\
5 \cdot 6 \\
-\quad 4.9 \\
\end{array}$ & $\begin{array}{l}7 \cdot 4 \\
7 \cdot 1 \\
6 \cdot 7 \\
6 \cdot 3 \\
5 \cdot 8 \\
5 \cdot 3 \\
4 \cdot 7 \\
\end{array}$ & $\begin{array}{l}6 \cdot 7 \\
6 \cdot 5 \\
6 \cdot 2 \\
5 \cdot 8 \\
5 \cdot 4 \\
5 \cdot 0 \\
4 \cdot 5 \\
\end{array}$ \\
\hline \multirow[t]{2}{*}{30} & $\begin{array}{l}30 \\
35 \\
40 \\
45 \\
50 \\
55\end{array}$ & $\begin{array}{r}10 \cdot 8 \\
10 \cdot 3 \\
9 \cdot 7 \\
9 \cdot 1 \\
8 \cdot 5 \\
7 \cdot 9\end{array}$ & $\begin{array}{l}9 \cdot 6 \\
9 \cdot 2 \\
8 \cdot 8 \\
8 \cdot 3 \\
7 \cdot 8 \\
7 \cdot 3\end{array}$ & $\begin{array}{l}8 \cdot 6 \\
8 \cdot 3 \\
8 \cdot 0 \\
7 \cdot 6 \\
7 \cdot 2 \\
6 \cdot 7 \\
6 \cdot 2\end{array}$ & 50 & $\begin{array}{r}50 \\
55 \\
60 \\
65 \\
70 \\
75 \\
\end{array}$ & $\begin{array}{l}7 \cdot 6 \\
7 \cdot 2 \\
6 \cdot 7 \\
6 \cdot 2 \\
5 \cdot 5 \\
4 \cdot 8 \\
\end{array}$ & $\begin{array}{l}6 \cdot 8 \\
6 \cdot 5 \\
6 \cdot 1 \\
5 \cdot 7 \\
5 \cdot 2 \\
4 \cdot 6 \\
\end{array}$ & $\begin{array}{l}6 \cdot 2 \\
6 \cdot 0 \\
5 \cdot 7 \\
5 \cdot 3 \\
4 \cdot 9 \\
4 \cdot 4 \\
\end{array}$ \\
\hline & $\begin{array}{l}60 \\
65 \\
70 \\
75 \\
\end{array}$ & $\begin{array}{l}7 \cdot 2 \\
6 \cdot 5 \\
5 \cdot 8 \\
5 \cdot 1 \\
\end{array}$ & $\begin{array}{l}6 \cdot 7 \\
6 \cdot 1 \\
5 \cdot 5 \\
4 \cdot 9 \\
\end{array}$ & $\begin{array}{r}6 \cdot 2 \\
5 \cdot 7 \\
-\quad 5 \cdot 2 \\
4 \cdot 7 \\
\end{array}$ & 55 . & $\begin{array}{l}55 \\
60 \\
65 \\
70\end{array}$ & $\begin{array}{l}6 \cdot 9 \\
6 \cdot 5 \\
6 \cdot 0 \\
5 \cdot 4\end{array}$ & $\begin{array}{l}6 \cdot 2 \\
5 \cdot 9 \\
5 \cdot 6 \\
5 \cdot 1\end{array}$ & $\begin{array}{l}5 \cdot 7 \\
5 \cdot 5 \\
5 \cdot 2 \\
4 \cdot 8\end{array}$ \\
\hline \multirow[t]{2}{*}{35} & $\begin{array}{l}35 \\
40 \\
45 \\
50 \\
55 \\
60\end{array}$ & $\begin{array}{l}9 \cdot 9 \\
9 \cdot 4 \\
8 \cdot 9 \\
8 \cdot 3 \\
7 \cdot 7 \\
7 \cdot 1\end{array}$ & $\begin{array}{l}8 \cdot 9 \\
8 \cdot 5 \\
8 \cdot 1 \\
7 \cdot 6 \\
7 \cdot 1 \\
6 \cdot 5\end{array}$ & $\begin{array}{l}8 \cdot 0 \\
7 \cdot 7 \\
7 \cdot 4 \\
7 \cdot 0 \\
6 \cdot 6 \\
6 \cdot 1\end{array}$ & 60 & $\begin{array}{l}75 \\
60 \\
65 \\
70 \\
75 \\
\end{array}$ & $\begin{array}{r}4 \cdot 7 \\
6 \cdot 1 \\
5 \cdot 7 \\
5 \cdot 2 \\
4 \cdot 6 \\
\end{array}$ & $\begin{array}{l}4 \cdot 5 \\
5 \cdot 6 \\
5 \cdot 3 \\
4 \cdot 9 \\
4 \cdot 4 \\
\end{array}$ & $\begin{array}{l}4 \cdot 3 \\
5 \cdot 2 \\
4 \cdot 9 \\
4 \cdot 6 \\
4 \cdot 2 \\
\end{array}$ \\
\hline & $\begin{array}{l}65 \\
70 \\
75 \\
\end{array}$ & $\begin{array}{l}6 \cdot 4 \\
5 \cdot 7 \\
5 \cdot 0\end{array}$ & $\begin{array}{l}6 \cdot 0 \\
5 \cdot 4 \\
4 \cdot 8 \\
\end{array}$ & $\begin{array}{l}5 \cdot 6 \\
5 \cdot 1 \\
4 \cdot 6\end{array}$ & 65 & $\begin{array}{l}65 \\
70 \\
75 \\
\end{array}$ & $\begin{array}{l}5 \cdot 4 \\
4 \cdot 9 \\
4 \cdot 4 \\
\end{array}$ & $\begin{array}{l}5 \cdot 0 \\
4 \cdot 6 \\
4 \cdot 2 \\
\end{array}$ & $\begin{array}{l}4 \cdot 7 \\
4 \cdot 4 \\
4 \cdot 0 \\
\end{array}$ \\
\hline \multirow{2}{*}{40} & $\begin{array}{l}40 \\
4.5\end{array}$ & $\begin{array}{l}9 \cdot 1 \\
8 \cdot 7\end{array}$ & $\begin{array}{l}8 \cdot 1 \\
7 \cdot 8\end{array}$ & $\begin{array}{l}7 \cdot 3 \\
7 \cdot 1\end{array}$ & 70 & $\begin{array}{l}70 \\
75 \\
\end{array}$ & $\begin{array}{l}4 \cdot 6 \\
4 \cdot 2 \\
\end{array}$ & $\begin{array}{l}4 \cdot 4 \\
4 \cdot 0\end{array}$ & $\begin{array}{l}4 \cdot 2 \\
3 \cdot 9 \\
\end{array}$ \\
\hline & $\begin{array}{l}50 \\
5.5\end{array}$ & $\begin{array}{l}8 \cdot 2 \\
7 \cdot 6\end{array}$ & $\begin{array}{l}7 \cdot 4 \\
6 \cdot 9\end{array}$ & $\begin{array}{l}6 \cdot 8 \\
6 \cdot 4\end{array}$ & 75 & 75 & 3.8 & 3.7 & 3.6 \\
\hline
\end{tabular}

Ta BLE IV.-For the Value of an Annuity on the Longer of Two Given Lives.

\begin{tabular}{|c|c|c|c|c|c|c|c|c|c|}
\hline $\begin{array}{c}\text { Age of } \\
\text { Younger. }\end{array}$ & $\begin{array}{l}\text { Age of } \\
\text { Elder. }\end{array}$ & $\begin{array}{l}\text { Value at } \\
3 \text { per cent. }\end{array}$ & $\begin{array}{l}\text { Value at } \\
4 \text { per cent. }\end{array}$ & $\begin{array}{l}\text { Value at } \\
5 \text { per cent. }\end{array}$ & $\begin{array}{c}\text { Age of } \\
\text { Younger. }\end{array}$ & $\begin{array}{l}\text { Age of } \\
\text { Eller. }\end{array}$ & $\begin{array}{l}\text { Value at } \\
3 \text { per cent. }\end{array}$ & $\begin{array}{l}\text { Value at } \\
4 \text { per cent. }\end{array}$ & $\begin{array}{l}\text { Value at } \\
3 \text { per cent. }\end{array}$ \\
\hline \multirow{14}{*}{10} & 10 & $23 \cdot 4$ & $19 \cdot 9$ & $17 \cdot 1$ & \multirow{6}{*}{15} & 50 & $20 \cdot 7$ & $17 \cdot 6$ & $15 \cdot 4$ \\
\hline & 15 & $22 \cdot 9$ & $19 \cdot 5$ & : $16 \cdot 8$ & & 55 & $20 \cdot 4$ & $17 \cdot 4$ & $15 \cdot 3$ \\
\hline & 20 & $22 \cdot 5$ & $19 \cdot 1$ & $16 \cdot 6$ & & 60 & $20 \cdot 1$ & $17 \cdot 2$ & $15 \cdot 2$ \\
\hline & 25 & $22 \cdot 2$ & $18 \cdot 8$ & $16 \cdot 4$ & & 65 & $19 \cdot 8$ & $16 \cdot 9$ & $15 \cdot 0$ \\
\hline & 30 & $21 \cdot 9$ & $18 \cdot 6$ & $16 \cdot 2$ & & 70 & $19 \cdot 4$ & $16 \cdot 6$ & $14 \cdot 7$ \\
\hline & 35 & $21 \cdot 6$ & $18 \cdot 4$ & $16 \cdot 1$ & & 75 & 18.9 & $16 \cdot 3$ & $14 \cdot 4$ \\
\hline & -40 & $21 \cdot 4$ & $18 \cdot 3$ & $16^{\circ} 0^{\prime}$ & \multirow{12}{*}{20} & 20 & $21 \cdot 6$ & $18 \cdot 3$ & 15.9 \\
\hline & 45 & $21 \cdot 2$ & $18 \cdot 2$ & $15 \cdot 9$ & & 25 & $21 \cdot 1$ & $17 \cdot 9$ & $15 \cdot 5$ \\
\hline & 50 & $20 \cdot 9$ & $18 \cdot 0$ & $15 \cdot 8$ & & 30 & $20 \cdot 7$ & $17 \cdot 6$ & $15 \cdot 3$ \\
\hline & 55 & $20 \cdot 7$ & $17 \cdot 8$ & $15 \cdot 7$ & & 35 & $20 \cdot 4$ & $17 \cdot 4$ & $15 \cdot 1$ \\
\hline & 60 & $20 \cdot 4$ & $17 \cdot 6$ & $15 \cdot 5$ & & 40 & $20 \cdot 1$ & $17 \cdot 2$ & $15 \cdot 0$ \\
\hline & 65 & $20 \cdot 1$ & $17 \cdot 4$ & $15 \cdot 3$ & & 4.5 & $19 \cdot 9$ & $17 \cdot 0$ & $14 \cdot 9$ \\
\hline & 70 & $19 \cdot 8$ & $17 \cdot 2$ & $15 \cdot 1$ & & 50 & $19 \cdot 6$ & $16 \cdot 8$ & $14 \cdot 7$ \\
\hline & 75 & $19 \cdot 5$ & $16 \cdot 9$ & $14 \cdot 8$ & & 55 & $19 \cdot 4$ & $16 \cdot 6$ & 14.5 \\
\hline \multirow{7}{*}{15} & 15 & $22 \cdot 8$ & $19 \cdot 3$ & $16 \cdot 7$ & & 60 & $19 \cdot 1$ & $16 \cdot 3$ & $14 \cdot 3$ \\
\hline & 20 & $22 \cdot 3$ & $18 \cdot 9$ & $16 \cdot 4$ & & 65 & 18.7 & $16 \cdot 0$ & $I+\cdot 1$ \\
\hline & 25 & $21 \cdot 9$ & $18 \cdot 6$ & $16 \cdot 2$ & & 70 & 18.2 & $15 \cdot 7$ & $13 \cdot 8$ \\
\hline & 30 & $21 \cdot 6$ & $18 \cdot 3$ & $16 \cdot 0$ & & 75 & $17 \cdot 7$ & $15 \cdot 3$ & $13 \cdot 5$ \\
\hline & 35 & $21 \cdot 3$ & $18 \cdot 1$ & $15 \cdot 9$ & \multirow{3}{*}{25} & 25 & $2(0) 3$ & $17 \cdot 4$ & $15 \cdot 1$ \\
\hline & 40 & $21 \cdot 1$ & $17 \cdot 9$ & $15 \cdot 7$ & & 30 & $19 \cdot 8$ & $17 \cdot 0$ & $14 \cdot 9$ \\
\hline & 4.5 & $20 \cdot 9$ & $17 \cdot 8$ & $15 \cdot 6$ & & 35. & $19 \cdot 4$ & $16 \cdot 7$ & $14 \cdot 7$ \\
\hline
\end{tabular}


L I F

\begin{tabular}{|c|c|c|c|c|c|c|c|c|c|}
\hline $\begin{array}{c}\text { Age of } \\
\text { Younger. }\end{array}$ & $\begin{array}{l}\text { Age of } \\
\text { Eider. }\end{array}$ & $\begin{array}{l}\text { Value at } \\
3 \text { percent. }\end{array}$ & $\begin{array}{l}\text { Vidue at } \\
4 \text { per cent. }\end{array}$ & $\begin{array}{l}\text { Value at } \\
5 \text { per cent. }\end{array}$ & $\mid \begin{array}{c}\text { Age of } \\
\text { Younger. }\end{array}$ & $\begin{array}{l}\text { Age of } \\
\text { Eilder. }\end{array}$ & $\begin{array}{l}\text { Value at } \\
{ }^{3} \text { per cent. }\end{array}$ & $\begin{array}{l}\text { Value at } \\
{ }_{4}^{4} \text { per cent. }\end{array}$ & $\begin{array}{l}\text { Value at } \\
5 \text { per cent. }\end{array}$ \\
\hline \multirow{8}{*}{25} & 40 & $19 \cdot 2$ & $16 \cdot 5$ & $14 \cdot 5$ & \multirow{3}{*}{40} & 65 & $14 \cdot 9$ & $13 \cdot 1$ & $11 \cdot 8$ \\
\hline & 45 & $18 \cdot 9$ & $16 * 3$ & $14 \cdot 3$ & & $70^{\circ}$ & $14 \cdot 5$ & 12.7 & $11 \cdot 4$ \\
\hline & 50 & $18 \cdot 7$ & $16 \cdot 1$ & $14 \cdot 2$ & & 75 & $14 \cdot 0$ & $12 \cdot 3$ & $11 \cdot 0$ \\
\hline & 55 & $18 \cdot 4$ & $15 \cdot 9$ & 140 & \multirow{7}{*}{45} & 4.5 & $16 \cdot 2$ & $14 \cdot 2$ & $12 \cdot 8$ \\
\hline & 60 & $18 \cdot 0$ & $15 \cdot 6$ & $13 \cdot 9$ & & 50 & $15 \cdot 7$ & $13 \cdot 3$ & 12.5. \\
\hline & 65 & $17 \cdot 6$ & $15 \cdot 3$ & $13 \cdot 6$ & & 55 & $15 \cdot 2$ & $13 \cdot 1$. & $12 \cdot 1$ \\
\hline & 70 & $17 \cdot 2$ & $15 \cdot 0$ & $13 \cdot 3$ & & 60 & $14 \cdot 7$ & $12 \cdot 9$ & $11 \cdot 7$ \\
\hline & 75 & $10 \cdot 7$ & $14 \cdot 6$ & $12 \cdot 9$ & & 65 & $14 \cdot 1$ & $12 \cdot 5$ & $11 \cdot 4$ \\
\hline \multirow{9}{*}{301} & 30 & $19 \cdot 3$ & $16 \cdot 6$ & 14.5 & & 70 & $13 \cdot 6$ & $12 \cdot 0$ & $11 \cdot 0$ \\
\hline & 35 & $18 \cdot 8$ & $16 \cdot 2$ & $14 \cdot 2$ & & 75 & $13 \cdot 1$ & $11 \cdot 6$ & $10 \cdot 6$ \\
\hline & 40 & $18 \cdot 4$ & $1.5 \cdot 9$ & $14 \cdot 0$ & \multirow{6}{*}{50} & 50 & $15 \cdot 6$ & $13 \cdot 3$ & $12 \cdot 1$ \\
\hline & 4.5 & $18.1^{1}$ & $15 \cdot 6$ & $13 \cdot 8$ & & 55 & $14 \cdot 5$ & $12 \cdot 9$ & $11 \cdot 7$ \\
\hline & 50 & $17 \cdot 8$ & $15 \cdot 4$ & $13 \cdot 6$ & & 60 & $13 \cdot 9$ & $12 \cdot 4$ & $11 \cdot 3$ \\
\hline & 5.3 & $17 \cdot 4$ & $15 \cdot 1$ & $13 \cdot 4$ & & 65 & $13 \cdot 3$ & $12 \cdot 0$ & $10 \cdot 9$ \\
\hline & 60 & $17 \cdot 0$ & $14 \cdot 8$ & $13 \cdot 2$ & & 70 & $12 \cdot 8$ & $11 \cdot 5$ & $10 \cdot 5$ \\
\hline & 65 & $16 \cdot 6$ & 14.5 & $\begin{array}{l}12 \cdot 9 \\
12 \cdot 6\end{array}$ & & 75 & $12 \cdot 3$ & $11 \cdot 0$ & $10 \cdot 1$ \\
\hline & $\begin{array}{l}70 \\
75\end{array}$ & $\begin{array}{l}16 \cdot 1 \\
15 \cdot 6\end{array}$ & $\begin{array}{l}14 \cdot 1 \\
13 \cdot 7\end{array}$ & $\begin{array}{l}12 \cdot 6 \\
12 \cdot 2\end{array}$ & \multirow{5}{*}{55} & 35 & $13 \cdot 6$ & $12 \cdot 4$ & $11 \cdot 3$ \\
\hline \multirow{9}{*}{35} & 35 & 18.3 & $15 \cdot 4$ & $3 \cdot 8$ & & 60 & $13 \cdot 0$ & $\cdot 11 \cdot 9$ & $10 \cdot 9$ \\
\hline & 40) & $17 \cdot 8$ & $15 \cdot 4$ & 13.5 & & 65 & $12 \cdot 4$ & $11 \cdot 3$ & $10 \cdot 5$ \\
\hline & 4.5 & $17 \cdot 4$ & $15 \cdot 1$ & $13 \cdot 3$ & & 70 & $11 \cdot 8$ & $10 \cdot 8$ & $10 \cdot 0$ \\
\hline & 50 & $17 \cdot 1$ & 14.8 & $13 \cdot 1$ & & 75 & $11 \cdot 3$ & $10 \cdot 3$ & $9 \cdot 5$ \\
\hline & 55 & $16 \cdot 7$ & $14 \cdot 5$ & $12 \cdot 9$ & \multirow{4}{*}{60} & 60 & $12 \cdot 2$ & $11 \cdot 2$ & $10 \cdot 5$ \\
\hline & 60 & $16 \cdot 3$ & $14 \cdot 2$ & $12 \cdot 7$ & & 65 & $11 \cdot 5$ & $10 \cdot 6$ & $10^{\circ} 0$ \\
\hline & 65 & $15 \cdot 8$ & 13.8 & $12 \cdot 4$ & & 70 & $10 \cdot 9$ & $10 \cdot 1$ & $9 \cdot 5$ \\
\hline & 70 & $15 \cdot 3$ & $13 \cdot 4$ & $12 \cdot 0$ & & 75 & $10 \cdot 3$ & $9 \cdot 5$ & $9 \cdot 0$ \\
\hline & 75 & $14 \cdot 8$ & $13 \cdot 0$ & $11 \cdot 6$ & \multirow{3}{*}{65} & 65 & $10 \cdot 7$ & $10 \cdot 0$ & $9 \cdot 4$ \\
\hline \multirow{5}{*}{40} & 40 & $17 \cdot 3$ & $15 \cdot 0$ & $13 \cdot 3$ & & 70 & $10 \cdot 0$ & $9 \cdot 4$ & $8 \cdot 9$ \\
\hline & 4.5 & $16 \bullet 8$ & $14 \cdot 6$ & $13 \cdot 0$ & & 7.5 & $9 \cdot 3$ & $8 \cdot 7$ & $8 \cdot 3$ \\
\hline & 50 & $16 \cdot 3$ & $14 \cdot 2$ & $12 \cdot 7$ & \multirow{2}{*}{70} & 70 & $y=2$ & $8 \cdot 6$ & 8.2 \\
\hline & 5.5 & $15 \cdot 9$ & $13 \cdot 9$ & $12 \cdot 4$ & & 75 & $8 \cdot 4$ & $7 \cdot 9$ & $7 \cdot 6$ \\
\hline & 60 & $15 \cdot 4$ & $13 \cdot 5$ & $12 \cdot 1$ & 75 & 75 & $7 \cdot 6$ & $7 \cdot 2$ & $6 \cdot 9$ \\
\hline
\end{tabular}

The uses of these tables may be exemplified in the following problems.

Pro B. 1. To find the Probability or Proportion of Chance, that a Person of a Given Age continues in being a proposed number of years.-Thus, suppose the age be 40 , and the number of years proposed 15; then, to calculate by the table of the probabilities for London, in tab. 1. against 40 years stands 214, and against 55 years, the age to which the person must arrive, stands 120 , which shows that, of 214 persons who attain to the age of 40 , only 120 of them reach the age of 55 , and consequently 94 die between the ages of 40 and 55: It is evident therefore that the odds for 2ttaining the proposed age of 55 , are as 120 to 94 , or as 9 to 7 nearly.

Proв. 2. To find the Value of an Annuity for a proposed Life.-This problem is resolved from tab. 2, by looking against the given age, and under the proposed rate of interest; then the corresponding quantity shows the number of years purchase required. For example, if the given age be 36 , the rate of interest 4 per cent, and the proposed annuity 250 : then in the table it appears that the value is 12.1 years purchase, or 12.1 times $250 l$, that is $3025 l$.

After the same manner the answer will be found in any other case falling within the limits of the table. But as thcre may sometimes be occasion to know the values of lives computed at higher rates of interest than those in the table, the two following practical rules are subjoined; by which the problem is resolved independently of tables.
Rule 1. When the given age is not less than 4.5 years nor greater than 85 , subtract it from 92 ; then multiply the remainder by the perpetuity, and divide the product by the said remaincer added to $2 \frac{x}{2}$ times the perpetuity; so shall the quotient be the number of years purchase required. Where it is to be noted, that by the perpetuity is meant the number of years purchase of the fee-simple; found by dividing 100 by the rate per cent at which interest is reckoned.

$E x$. Let the given age be 50 years, and the rate of interest 10 per cent. Then subtracting 50 from 92 , there remains 42 ; which multiplied by 10 the perpetuity, gives 420 ; and this divided by 67 , the remainder increased by $2 \frac{1}{2}$ times 10 the perpetuity, gives $6 \cdot 3$ nearly, for the number of years purchase. Therefore, supposing the annuity to be $100 l$, its value in present money will be $630 l$.

Rule 2. When the age is between 10 and 45 years; take 8 tenths of what it wants of 4.5 , which divide by the rate per cent increased by 1.2 ; then if the quotient be added to the value of a life of 45 years, found by the preceding rule, there will be obtained the number of years purchase in this case. For example, let the proposed age be 20 years, and the rate of interest 5 per cent. Here then, taking 20 from 45 , there remains $25 ; \frac{8}{\text { Io }}$ of which is 20 ; which divided by 6.2 , gives 3.2 ; and this added to $9 \cdot 8$, the value of a life of 45 , found by the former rule, gives 13 for the number of years purchase that a life of 20 ought to be valued at.

And the conclusions derived by these rules, Mr. Simp5 A 2 


\section{I F}

son adds, are so near the true values, computed from real observations, as seldom to differ from then by more than $\frac{1}{T^{\circ}}$ ol ${ }^{2} \overline{0}$ of one year's purchase.

The observations here alluded to, are those which are fuunded on the London bills of mortality: and a similar method of solution, accommodated to the Breslaw obscrvations, will be as follows, viz. "Multiply the difference between the given age and 85 years by the perpetuity, and divide the product by $\mathcal{S}$, tentlis of the said difference increased by duuble the perpetuity, for the answer." Which, from $\&$ to 80 years of age, will commonly come within less than $\frac{x}{8}$ of a year's purchase of the truth.

Proв.3. To find the Value of an Annuity for Tivo Joint Lives, that is, for us long as they both continue in being together.-In table 3 , find the younger age, or that ncarest to it, in column 1, and the ligher age in column 2; then against this last is the number of years purchase in the proper column for the interest. E.r. Suppose the two ages be 20 and 35 years; then the value

is $10^{\circ} .9$ years purcliase at 3 per cent.

or $9 \cdot \mathrm{S} \quad$ - $\quad$ at 4 per cent.

or 8.8 - - at 5 per cent.

Proв.4. To find the Value of the Annuity for the Longest of Two Lives, that is, for as long as ejther of them continues in being.-In table 4, col. 1 , find the age of the youngest life, or the nearest to it, in col. 1 , and the age of the elder in col. 2: then against this last is the answer in the proper column of interest.-Ex. So, if the two ages be 15 and 40 ; then the value of the annuity upon the longest of two such lives

is $21 \cdot 1$ years purchase at 3 per cent.

$\begin{array}{llll}\text { or } 17.9 & - & - & 4 \text { per cent. } \\ \text { or } 15.7 & - & - & 5 \text { per cent. }\end{array}$

In the last two problems, if the younger age, or the rate of interest, be not exactly found in the tables, the nearest to them may be taken, and then by proportion the value for the true numbers will be nearly found.

Rules and tables for the values of three lives, \&c, may also be secn in Simpson, in Baron Maseres's, and in Bailey's Annuities, \&c. All these calculations have been made from tables of the real mortuary registers, differing unequally at the several ages: but rules have also been given on other principles, as by Demoivre, on the supposition that the decrements of life are equal at all ages; an assumption not much differing from the truth, from 7 to 70 years of age.

LiF E-ANNUities, payable half-yearly, sc.-These are worth more than those that are payable yearly, as computed by the foregoing rules and tables, on the two following accounts: First, that parts of the payments are reccived sooner; and $2 \mathrm{dly}$, there is a chance of rcceiving some part or parts of a whole year's payment more than when the payments are obly made annually. Mr. Simpson, in his Select Exercises, pa. 283, observes, that the value of these two advantages put together, will always amount to $\frac{1}{4}$ of a year's purchase for half-yearly payments, and to $\frac{3}{8}$ of a year's purchase for quarterly payments; and Mr. Mitseres, at page $233 \& \mathrm{c}$ of his Annuities, by a very elaborate calculation, finds the former difference to be nearly $\frac{1}{4}$ also. But Dr. Price, in an essay in the Philos. Trans. vol. 66, pa. 109, stiltes the same differences only

at ${ }_{70}^{2}$ for half-yearly payments, and $\frac{3}{30}$ for quarterly payments:

And the doctor then adds some algebraical theorems for such calculations.

\section{I G}

Life-ANnuries, secured by Land.-These differ from other life-anuuities only in this, that the annuity is to be paid up to the very day of the death of the age in question, or of the person on whose life the annuity is granted. To obtain the more exact value therefore of such an annuity, a small quantity must be added to the same as compnted by the foregoing rules and oliservations, which is different according as the payments are yearly, halfyearly, or quarterly, \&c ; and are thus stated by Dr. Price in his essay quoted above; viz, the addition

$$
\begin{aligned}
& \text { is } \frac{y}{2 n} \text { for anmual payments, } \\
& \text { or } \frac{h}{4 n} \text { for half-ycarly payments, } \\
& \text { or } \frac{q}{8 n} \text { for quarterly payments : }
\end{aligned}
$$

where $n$ is the complement of the given age, or what it wants of 86 years; and $y, h, q$ are the respective values of an annuity certain for $n$ years, payable yearly, halfyearly, or quarterly. And, by numeral examples, it is found that the first of these additional quantities is about $\frac{2}{10}$, the second $\frac{1}{T}$, and the Sd half a tenth of one year's purchase.

Complement of Lire. See Conplement.

Exprciation of LIFE. See Expsectation.

Insurance or 'Assurance on Lives. Sce Assurance.

LIGHT, that principle by which objects are made perceptible to our sense of seeing; or the sensition occasioned in the mind by the view of luminous objects. The nature of light has been a subject of speculation from the first dawnings of philosophy. Some of the earliest philosophers doubted whether objects became visible by means of any thing proceeding from them, or from the eye of the spectator. But this opinion was qualified by Empedocles and Plato, who maintained, that vision was occasioned by particles continually flying off from the surfaces of bodies, which meet with others proceeding from the eye; while the effect was ascribed by Pythagoras solely to the particles proceeding from the external objects, and entering the pupil of the eye. But Aristotle defines liglit to be the act of a trinsparent body, considered as such : and he observes that light is not fire, nor any matter radiating from the luminous budy, and transmitted through the transparent one.

'The Cartesians have refined considerably on this notion: they maintain that light, as it exists in the luminous body, is only a power or faculty of exciting in us a very clear and vivid sensation; or that it is an invisible fluid present at all times and in all places, but requiring to be set in motion, by a body innited or otherwise properly qualified to make objects visible to us.

Father Malbranche explains the nature of light from a supposed analogy between it and sound.-Thus, he supposes, all the parts of a luminous body are in a rapid motion, which, by rery quick pulses, are constantly compressing the subtle matter between the luminous bouly and the eye, and cxcites vibrations of pression: and as these vibrations are greater, the body appears more luminous; but as they are quicker or slower, the body is of this or that colour.

The Newtonians maintain, that light is not a fluid per se, but consists of a great number of very small particles, thrown off from the luminous body by a. repulsive power with an immense velocity, and in all dircctions: and these particles, they also assert, are emitted in right lines: 


\section{I G}

which rectilinear motion they preserve till they are turned out of their path by some of the following causes, viz, by the attraction of some other body near which they pass, which is called Inflection; or by passing obliquely through a medium of different density, which is called Refraction; or by being turned aside by the opposition of some intervening body, which is called Reflection; or, lastly, by being totally stopped by some substance into which they pentrate, and which is called their Extinction. A succession of these particles following one another, in an exact straight line, is called a Ray of Light ; and this ray, in whatever manner its direction may be changed, wliether by refraction, reflection, or inflection, always preserves a rectilinear course till it be again changed; neither, say they, is it possible to make it move in the arch of a circle, ellipsis, or other curve. For the above properties of the rays of light, see the several words, Refraction, ReFLEC'TON, \&c.

The velocity of the particles and rays of light is truly astonishiug, amounting to near 2 hundred thousand miles in a second of time, which is near a million times greater than the velocity of a cannon-ball: and this amazing motion of light has been manifested in various ways ${ }_{2}$ but chietly from the eclipses of Jupiter's satellites. It was first observed by Roemer, that the eclipses of those satellites happen sometimes sooner, and sometimes later, than the times given by the tables of them; and that the observed time was before or after the computed time, according as the earth was nearer to, or farther from Jupiter. Hence Roemer and Cassini both concluded that this circumstance depended on the distance of Jupiter from the earth ; and that, to account for it, they must suppose that the light was about 14 minutes in crussing the earth's orbit. This conclusion however was afterward abandoned and attacked by Cassini himself. But Roemer's opinion found an able advocate in Dr. Halley; who removed Cassini's difficulty, and left Roemer's conclusion in its full force. Yet, in a memoir presented to the academy in 1707, M. Maraldi endeavoured to strengthen Cassini's arguments; when Roemer's doctrine found a new defender in Mr. Pound. See Philos. Trans. number 136, also my Abridg. vol. 6, pa. 349 and 386 , and Groves, Phys. Elem. number 2636: and it has since been found, by repeated experiments, that when the eartls is exactly between $\mathrm{J}_{L \text { - }}$ piter and the sun, lis satellites are seen eclipsed about $S \frac{r}{4}$ minutes sooner than they could be according to the tables; but when the earth is nearly in the opposite point of its orlit, these eclipses happen about $8 \frac{1}{4}$ minutes later than the tables predict them. Hence then it is certain that the motion of light is not instantaneous, but that it takes up about $16 \frac{x}{2}$ minutes of time to pass over a space equal to the diameter of the earth's orbit, which is at least 190 millions of miles in length, or at the rate of near 200,000 miles per second, as above stated. Hence it is easy to know the time in which light travels to the earth, from the moon, or any of the other planets, or even from the fixed stars if ever their distances be known; but these distances are so immensely great, that from, the nearest of them, supposed to be Sirius, the dog-star, it requires many years for light to travel to the earth: and it has even been conjectured that there are many stars whose light have not yet arrived at us since their creation.

It was Galilco who first conceived the notion of measuring the velocity of light; and a description of his contrivance for this purpose, is given in bis Treatise on Me- chanics, pa. 39. He had two men with lights covered; the one was to observe when the other uncovered his light, and to exhibit his own the moinent he perccived it. This rude experiment was tried at the distance of a mile, but without success, as may naturally be imagined: and the members of the acalemy Del Cinento repeated the experiment, and placed their observers, to as little purpose, at the distance of 2 miles.

But our excellent astronomer, Dr. Bradley, afterwards found nearly the same velocity of light as Ruemer, from his accurate obscrvations, and most ingenious thcory, to account for some apparent motions in the fixed stars; for an account of which, sce Aberration of light. By a long series of these observations, he found the difference between the true and apparent place of several fixed stars, for different times of the year; which difference could no otherwise be accounted for, than from the progressive motion of the rays of light. From the mean quantity of this difference he found, that the ratio of the velocity of light to the velocity of the earth in its orbit, was as 103 I 3 to 1 , or that light moves 10313 times faster than the earth moves in its orbit about the sun; and as this latter motion is at the rate of $18 \frac{x}{2} \frac{x}{2}$ miles per second nearly, it follows that the former, or the relocity of light, is at the rate of about 195000 miles in a second; a motion according to which it will require just $8^{\prime} \bar{t}^{\prime \prime}$ to move from the sun to the earth', or about 95 millions of miles.

It was also inferred, from the foregoing principles, that light proceeds with the same velocity from all the stars. And hence it follows, that the motion of light, in its path through the immense space above our atmosphere, is equable or uniform. And since the different methods of determining the velocity of light thus agree in the result, it is reasonable to conclude that, in the same medium, light is propagated, after it has been reflected, with the same velocity as before. For an account of Mr. Melville's hypothesis of the different velocities of differently coloured rays, see Colour.

To the doctrine concerning the materiality of light, and its amazing velocity, several objections have been made; of which the most considerable is, That as rays of light are continually passing in different directions from every visible point, they must necessarily interfere with each other in such a manner, as entirely to confound all distinct perception of objects, if not quite to destroy the whole sense of seeing : not to meution the continual waste of substance which a constant emission of particles must occasion in the luminous bolly, and thereby since the creation must have greatly diminished the matter in the sun and stars, as well as increased the bulk of the earth and planets by the vast quantity of particles of light absorbed by them in so long a period of time.

But it has been replied, that if light were not a body, but consisted in mere pression or pulsion, it could never be propagated in right lines, but would be continually inflected ad umbram. Thus Sir I. Newton: "A pressure on a fluid medium, i. e. a motion propagated by sucb a medium, beyond an obstacle, "which impedes any part of its motion, cannot be propagated in right lines, hut will be always inflecting and diffusing itself every way, to the quiescent medium beyond that obstacle. The power of gravity tends downwards ; but the pressure of water arising from it tends every way with an equable force, and is propagated with equal ease and equal strength, in curves, as in straight lines. Waves, on the surface of the water, gliding 
by the extrenses of any very large obstacle, inflect and dilate themselves, still diffusing gradually into the quiescent water beyond that obstacle. 'The waves, pulses, or vibrations of the air, wherein sound consiststs, are manifestly inflected, though not so considerably as the waves of water; and sounds are propagated with equal ease, through crooked tubes, and through straight lines; but light was never known to move in any curve, nor to inflect itself ad umbram."

It must be acknowledged however, that many philosophers, both Linglish and foreigners, have recurred to the ypinion, that light consists of vibrations propagated from the luminous body, through a suhtle etherial medium. The ingenious Dr. Franklin, in a letter dated April 23, 1752, expresses his dissatisfaction with the doctrine of light consisting of particles of matter continually driven off from the sun's surface, with such an astonishing velocity. "Must not," says he, "the sinallest portion conccivable, have, with such a motion, a force exceeding that of a 24 pounder discharged from a cannon? Must not the sun diminish exceedingly by such a waste of matter; and the planets, instead of drawing nearer to him, as some have feared, recede to grcater distances through the diminished attraction? Yet these particles, with this amazing motion, will not drive before them, or remove, the least and slightest dust they meet with; and the sun appears to continue of his ancient dimensions, and his attendants move in their ancient orbits." He therefore conjectures that all the plienomena of light may be more properly solved, by supposing all space filled with a subtle elastic fluid, which is not visible when at rest, but which, by its vibrations, affects that fine sense in the eye, as those of the air affect the grosser organs of the ear; and even that different degrees of the vibration of this medium may cause the appearances of different colours. Franklin's Exper. and Observ. 1769 , pa. 264 .

The celebrated Euler has also maintained the same hypothesis, in his Theoria Lucis et Colorum. In the summary of his arguments against the common opinion, recited in Acad. Berl. 1752 , pa. 271 , besides the objections above mentioned, he doubts the possibility, of particles of matter, moring with the amazing velocity of light, and penetrating transparent substances with so much ease. In whatever manner they are transmitted, those bodies must have pores, disposed in right lines, and in all possible directions, to serve as canals for the passage of the rays: but such a structure must take away all solid matter from those bidies, and all coherence among their parts, if they do contain any solid matter.

Doctor Horsley has taken considerable pains to obviate the difficulties startcd by Dr. Franklin. Supposing that the diameter of each particle of light does not exceed one millionth of one millionth of an inch, and that the density of each particle is even 3 times that of iron, and also that the light of the sun reaches the earth in $7^{\prime}$, at the distance of 22919 of the earth's semidiameters, he calculates that the momentum or force of motion in each particle of light coming from the sun, is less than that in an iron ball of a quaster of an inch diameter, moving at the rate of less than an inch in 12 thousand millions of millions of years. And hence he concludes, that a particle of matter, which probably is larger than any particle of light, moving with the velocity of light, has a force of Inotion, which, instead of exceeding the force of a 24pounder discliarged from. a cannon, is ahmost infmitcly less than that of the smallest shot discharged from a pocket-pistol, or less than any that art can create. He also thinks it possible, that light may be produced by a continual emission of matter from the sun, without any such waste of his substance as sbould sensibly contract his dimensions, or alter the motions of the planets, withis any moderate length of time. In proof of this, he observes that, for the production of any of the phenomens of light, it is not necessary that the emanation from the sun should be continual, in a strict mathematical sense, or without any interval; and likewise that part of the light whicb issnes from the sun, is continually returned to him by reflection from the planets, as well as other light from the suns of other systems. He proceeds, by calculation, to show, that in $385,130,000$ years, the sun would lose but the $13232 \mathrm{~d}$ part of his matter, and consequently of the gravitation towards him, at any given distance; which is an alteration much too small to discover itself in the motion of the earth, or of any of the pianets. He further computes that the greatest stroke which the retina of a common eje sustains, when turned directly to the sun in a bright day, does not exceed that which would be given by an iron shot, a quarter of an inch diameter, and moving only at the rate of $16 \frac{3}{6}$ inches in a year; whereas the ordinary struke is less than the 208tth part of this. See Philos. Trans. vol. 60 and 61.

In answer to the dificulty respecting the interference of the particles of light with each other, Mr. Melville observes (Edinb. Ess, vol. 2), there is probably no physical point in the risible horizon, that does not send rays to every other point, unless where opaque bodies interposc. Light, in its passage from one system to another, often passes through toirents of light issuing from other suns and systems, without ever interfering, or being diverted from its course, either by it, or by the particles of that elastic medium, which has been supposed by some diffused through all the mundane space. To account for this fact, he supposes that the particles of light are incomparably rare, eren when they are the most donse, or that their dianeters are incomparubly less than thcir distauce from one another : which obviates the objection urged by Euler and others against the matcriality of light, from its influence in disturbing the freed om and perpetuity of the celestial motions. Buscorich and some others solve the difficulty concerning the non-interference of the particles of light, by supposing that each particle is cndued with an insuperable impulsive force; but in this ease, their spheres of impulsion would be more likcly to interfere, and on that account they would be more liable to disturb one another.

Mr. Canton shows (Philos. Trans. vol. 5s, pa. 3.4), that the difficulty of the interference will vamish, if a very small portion of time be allowed between the cmission of every particle and the next that follows in the same direction. Suppose, for instance, that a lucid point in the sun's surface emits 150 particles in a second of time, which, he observes, will be more than sufficient to gise continual light to the eje, without the least appearance of intermission; yet still the particles of sucl a ray, on account of their great velocity, will be more than 1000 miles bchind each other, a space sufficient to allow others to pass in all directions without any perceptible interruption. And if we adopt the conclusions drawn from the experiments on the duration of the sensations excited by light, by the chevalier D'Arcy, in the Acad. Scienc. 1765, who 


\section{I G}

states it at the 7 th part of a second, an interval of more than 20,000 miles may be admitted betwecn every two successive particles.

The doctrine of the materiality of light is further confirmed by those experiments, which show, that the colour and invard texture of some bodies are changed by being exposed to the light.

Of the Momentum, or Force, of the Particles of Light. Some writers have attempted to prove the materiality of light, by determining the momentum of their component particles, or by showing that they have a force, so as, by: their impulse, to give motion to light bodies. M. Homberg, Ac. Par. 170 s, Hist. pa. 25, imagined, that he could not only disperse pieces of amianthus, and other light substances, by the impulse of the solar ray's, but also that by throwing them upon the end of a kind of lever, connected with the spring of a watch, he could make it move sensibly quicker; from wiich, and from other experiments, lie inferred the weight of the particles of light. And Hartsoecker made pretensions of the same nature. But M. Du Fay and MI. Mairan made other experiments of a more accurate kind, without the effects which the former had imagined, and which even proved that the effects mentioned by them were owing to currents of heated air produced by the burning glasses used in their experiments, or some other causes which they had overlooked.

Dr. Priestley, howerer, informs us, that Mr. Michell endeavoured to ascertain the momentum of light with still greater accuracy, and that his endeavours were not altogether without success. Having found that the instrument he used, accquired, from the impulse of the rays of light, a relocity of an inch in a second of time, he inferred that the quantity of matter containcd in the rays falling upon the instrunent in that time, anounted to no more than the 12 hundred millionth part of a grain. In the experiment, the light was collected from a surface of about 3 square feet; and as this surface reflected only about the half of what fell upon it, the quantity of matter contained in the solar rays, incident upon a square foot and a half of surface, in a second of tinc, ought to be no more than the 12 hundred millionth part of a grain, or upon one square foot only, the 18 hundred millionth part of a grain. But as the density of the rays of light at the surface of the sun, is 45000 times grcater than at the earth, there ought to issue from a square foot of the sun's surface, in one sccond of time, the 40 thousandth part of a grain of matter; that is, a little more than 2 grains a day, or about $4,752,000$ grains, which is about 670 pounds avoirdupois, in 6000 years, the time since the creation; a quantity which would have shortened the sun's semidiameter by no more than aljout 10 feet, if it be supposed of no greater density than water only.

The Expansion or Extension of any portion of light, is inconceivable. Dr. Hooke shows that it is as unlimited as the universe; this he proves from the immense distance of many of the fixed stars, which only become visible to the eye by the best telescopes. Nor, adds he, are they only the grcat bodies of the sun or stars that are thus liable to disperse their light through the vast expanse of the universe, but the smallest spark of a lucid body must do the same, even the smallest globule struck from a steel by a fint.

The Intensity of different lights, or of the same light in different circumstances, affords a curious subject of speculation. M.Bouguer, in his Traité de Optique, found that when one light is from 60 to 80 times less than another, its presence or absence will not be perceived by all ordinary eye; that the moon's light, when she is $19^{\circ} 16^{\prime}$ high above the horizonl, is but about $\frac{1}{3}$ of her light at $66^{\circ} 11^{\prime} \mathrm{high}$; and when one limb just touched the horizon, her light is but the 2000th part of her light at $66^{\circ} 1 \mathrm{l}^{\prime}$ ligh ; and that hence light is diminished in the proportion of 3 to 1 by traversing 7\$69 toises of dense air. He found also, that the centre of the sur's disc is considerably more luminous than the edges of it; whereas both the primary and secondary planets are more luminous at their edges than near their centres: That, further, the light of the sun is about 300,000 times greater than that of the moon; and therefore it is no wonder that philosophers have had so little success in their attempts to collect the light of the moon with burning-glasses; for, should one of the largest of them even increase the light 1000 times, it will still leave the light of the moon in the focus of the glass, 300 times less than the iutensity of the common light of the sun.

Dr. Smith, in his Optics, vol. 1, pa. 29, thought he had proved that the light of the full moon would be only the 90,900 th part of the full day light, if no rays were lost at the moon. But Mr. Robins, in his Tracts, vol. 2, pa. 225 , shows that this is too great by onc half. And Mr. Michell, by a more easy and accurate mode of computation, found that the density of the sun's light on the surface of the moon is but the 45,000 th part of the density at the sun; and that therefore, as the moon is nearly of the same apparent magnitude as the sun, if she reflected to us all the light received on her surface, it would be only the 45,000 th part of our day light, or that which we receive from the sun. Admitting therefore, with M. Bouguer, that the moon's light is only the 300,000 th part of the day or sun's light, Mr. Michell concludes that the moon reflects no more than between the 6 th and 7 th part of what she receives.

Dr. Gravesande says, a Iucid body is that which emits or gives fire a motion in right lines, and makes the difference between light and heat to consist in this, that to produce the former, the fiery particles must enter the eye in a rectilinear motion, which is not required in the latter: on the contrary, an irregular motion seems more proper for it, as appears from the rays coming directly from the sun to the tops of mountains, which bave not near that effect with those in the valley, agitated with an irregular inotion, by several reflections.

Sir I. Newton observes, that bodies and light act mutually on each other; bodies on light, in emitting, reflecting, refracting, and inflecting it; and light on bodies, by heating them, and putting their parts into a vibrating motion, in which heat principally consists. For all fixed bodies, he observes, when heated beyond a certain degree, do emit light, and shine; which shining \&c appears to be owing to the vibrating motion of their parts; and all bodies, abounding in earthy and sulphureous particles, if sufficiently agitated, emit light, which way socver that agitation be effected. Thus, sea water shines in a storm ; quicksi]ver, when shaken in vacuo; cats or horses, when rubberl in the dark; and wood, fish, and fiesh, when putrefied.

Light proceeding from putrescent animal and vegetablc substances, as well as from glow-worms, is mentioned by Aristotle. And Bartholin mentions four kinds of luminous insects, two of which have wings : but in hot climatcs it is said they are found in much greater numbers, and of 


\section{I G}

different species. Columna observes, that their light is not extinguished immediately on the death of the animal. The first distinct account that occurs of light procceding from putrescent animal flesh, is that which is given by Fabricius ab Aquapendente in 1592, de Visione \&c, pa. 45. And Bartholin relates also a similar appearance in his treatise De Luce Animalium, which liappened at Montpelier in $16+1$.

Mr. Boyle speaks of a piece of shining rotten wood which was extinguished in vacuo; but on re-admitting the air, it reviled again, and shone as before; though he could not perceive that it was increased in condensed air. But in Birch's History of the Royal Society, vol. 2, pa. 254 , there is an account of the light of a shining fish, which was rendered more vivid by putting the fish into a condensing engine. Whitings were the fish commonly used by Mr. Boyle in his experiments : though in a discourse read before the Royal Society in 1681 , it was asserted that, of all fishy substances, the eggs of lobsters, after they liad been boiled, slone the brigbtest. Birch's Hist. vol. 2, pa. 70. In $1672 \mathrm{Mr}$. Boyle accidentally observed light issuing from flesh meat; and, among other remarks on this subject, he observes that extreme cold extinguishes the light of shining wood; probably because extreme cold checks the putrefaction, which is the cause of the light. The shell fish called pholas, is remarkable for its luminous quality. The luminousness of the sea lias been also a subject of frequent observation. See IG is Fatuus, Philosplorus, and Petrefaction, \&c.

Mr. Hawksbee, and many writers on the subject of electricity since his time, have produced a great variety of instances of the artificial production of light, by the attrition of bodies naturally not luminous; as of amber rubbed on woollen cloth in vacuo; of glass on woollen, of glass on glass, of oyster shells on woollen, and of woollen on woollen, all in vacuo. On the several experiments of this kind, he makes the following reflections: that different sorts of bodies afford light of various kinds, with regard both to colour and force; that the effects of an attrition are various, according to the different preparations and treatment of the bodies which are to endure it; and that bodies which bave yielded a particular light, may be brought by friction to yield no more of that light.

M. Bernoulli found by experiment, that mercury amalgamated with tin, and rubbed on glass, produced a consislerable light in the air; that gold subbed on glass, exhibited the same in a greater degrce; but that the most exquisite light of all was produced by the attrition of a diamond, this being equally vivid with that of a burning coal briskly agitated with the bellows. See ElectraCITY, \&c.

Of the Attraction of Light. That the particles of light are attracted by those of other bodles, is evident from numerous experiments. This phenomenon was observed by Sir I. Newton, who found, by repeated trials, that the rays of light, in their passage near the edges of bodies, are diverted out of the right lines, and always inflected or bent towards those bodies, whether they be opaque or transparent, as pieces of metals, the edges of knives, broken glasses, \&c. See Intrection and Raysi The curious observations that had been male on this subject by I). Hooke and Grinaldi, led Sir I. Newton to repeat and diversily their experiments, and to pursuc them much farther than they had done. For a particular account of his experiment and olservations, sce bis treatise on Optics, pit. $2 y: 3$, Sc.
This action of bodies on light is found to exert itself at a sensible distance, though it always increases as the distance is diminished; as appears very sensibly in the passage of a ray between the edges of two thin planes at different apertures; which is attended with this peculiar circunistance, that the attraction of one edge is increased as the other is brought nearer to it. The rays of light, in their passage out of glass into a vacuum, are not only inflected towards the glass, but if they fall too obliquely, they will revert back again to the glass, and be tutally reflected. Now the cause of this reflection cannut be attributed to-any lesistance of the vacuum, but must be entirely owing to some force or power in the glass, which attracts or draws back the rays as they were passing into the vacuum. And this appears to be further confirmed from hence, that if you wet the back surface of the glass with water, oil, honey, or a solution of quicksilver, then the rays which would otherwise have been reflecterl, will pervade and pass through that liquor; which shows that the rays are not reflected till they come to that back surface of the glass, nor even till they begin to go out of it; for if, at their going out, they fall into any of the aforesaid mediums, they will not then be reflected, but will persist in their former course, the attraction of the glass being in this case counterbalanced by that of the liquor.

M. Maraldi prosecuted experiments similar to those of Sir 1. Newton on inflected light. And his observations. chiefly respect the inflection of light towards other bodies, by which their sliadows are partially illuminated. Acad. Paris 1723, Mem. pa. 159. See also Priestley's Hist. pa. 521 , \&c.

M. Mairan, without attempting the discovery of new facts, endeavoured to explain the old ones, by the hypothesis of an atmosphere surrounding all bodies; and consequently two reflections and refractions of light that impinges on them, one at the surface of the atmosphere, and the other at the surface of the body itsclf. This atmosphere he supposed to be of a variable densiry and refractive power, like the air.

M. Dutour succeeded Mairan, and imagined that he could account for all the phenomena by the lielp of an atmosphere of a uniform density, but of a less refractive power than the air surrounding all bodies. Dutour also varied the Newtonian experinents, and discovered more than three fringes in the colours produced by the inflection of light. He further concludes that the refracting atmospheres, surrounding all kinds of bodies, are of the same size; for when he used a great variety of substances, and of different dimensions, he always found coloured streaks of the same dimensions. - He also observes, that this hypothesis contradicts an observation of Sir I. Newton, viz, that thuse rays are the most inflected which pass the nearest to any body. Mem. de Math. et de Phys. vol. 5, pa. 650, or Priestley's Hist. pá. 531.

M. Lecat found that objects sometimes appear magnified by means of the inflection of light: Looking at a distant steeple, when a wire, of a less dianeter than the pupil of his eye, was held pretty near to it, and drawing it several times between that object and his eye, he was surpriscd to find that every time the wire passed bofore his eye, the stecple seemed to cliange its place, and some hills beyond the stceple secmed to have the same motion, just as if a lens had been drawn between them and his eye. This discovery led him to several others depending on the inflection of the rays of light. 'Thus, he inaguificd small 
objects, as the head of a pin, by viewing them through a small hole in a card; so that the rays which formed the image must necessarily pass so near the circumference of the hole, as to be attracted by it. He exhibited also other appearances of a similar nature. Traité des Sens, pa. 299. Priestley, ubi supra, pa. 537.

Reflection and Refroction of Light. From the mutual attraction between the particles of light and other bodies, arise two other grand phenomena, besides the inflection of light, which are called the reflection and refraction of light. It is well known that the determination of borlies in motion, especially clastic ones, is changed by the interposition of other bodies in their way: thus also light, impinging on the surfaces of bodies, should also be turned out of its course, and beaten back or reflected, so as, like other striking bodies, to make the angle of. its reflection equal to the angle of incidence. This, it is found by cxperience, ligbt does; and yet the cause of this effect is different from that just now assigned: for the rays of light are not reflected by striking on the very parts of the reflecting bodies, but by some power equally diffused over the whole surface of the body, by which it acts on the limbt, either attracting or repelling it, without contact: by which same power, in other circumstances, the rays are refracted; and by which also the rays are first emittcl from the luninous body; as Newton fully proves by a great variety of arguments. Sce Petilection and Refraction.

That great author proves, that all those ray's which are reflcctcil, do not really touch the body, though they approach infinitely near to it; and that those which strike on the parts of solid bodics, adhere to them, and are as it were extinguished and lost. Since the reflection of the rays is ascribed to the action of the whole surface of the budy without contact, if it be asked, bow it happens that all the ray's are not reflected from every surface; but that, while some are reflected, others pass through, and are refracted? the answer given by Newton is as follows:Every ray of light, in its passage through any refracting surface, is put into a certain transicnt coustitution or state, which in the progress of the ray returus at equal intervals, and disposes the ray at cwery return to be casily transmitted through the next refracting surface, and between the seturns to be easily reflected by it: which alteration of reflection and transmission it appears is propagated from every surface, and to all distances. IV hat kind of action or disposition this is, and whether it consists in a circulating or vibrating motion of the ray, or the meditim, or something clse, be does not enquire; but allows those who are foud of hypotheses to suppose, that the rays of light, by impingeing on any reflecting or refracting surface, excite vibrations in the refecting or refracting medium, and by that means agitate the solid parts of the body. These vibrations, thus produced in the medium, move faster than the rays, so as to overtake them; and when any ray is in that part of the vibration which conspires with jts motion, its velocity is increasfo, and so it easily breaks through a refracting surface; but when it is in a contrary part of the vibration, which impeles its motion, it is casily reflected ; and thus cvery ray is successively disposed to be easily reflected or transmitted by every vibration which meets it. These returns in the disposition of any ray to be reflected, he calls Fits of Lasy Reflection; and the returns in the disposition to be transmitted, be calls Fits of Easy Transmission; also the space between the returns, the lnterval of the Fits. Hence then the reason why the surlaces VOL. I. of all thick transparcut bodies reflect part of the light incident on them, and refract the rest, is, that some rays at their incidence are in lits of easy reflection, and others of easy transmission. For the properties of reflected Light, see RevLection, Mirton, \&c.

Again, a ray of light, passing out of one medium into another of different density, and in its passage making an oblique angle with the surface that separates the mediuns, will be refracted, or turned out of its direction; because the rays are more strongly attracted by a denser than by a rarer nedium. That these rays are not refracted by striking on the solid parts of bodics, but that this is effected without a real contact, and by the same force by which they are emitted and reflected, only exerting itself differently in different circunstances, is proved in a great measure by the same arguments by which it is demonstrated that refection is performed withoul contact. See REF k ACtion, LeNs, Coloun, Vision, \&c.

Chemical Properties of Light.-The chemical properties of light are yet but very imperfectly known, though there is reason to believe that it is an agent of great importance in many of the principal changes that are going on in the visible world, and even in the experiments of the laboratory its effects are often clearly demonstrable.

The process of rapitl combustion is always attended with the evolution of light along with heat, the source of botl of which is ascribed by Lavoisier to the oxygen, which is knowis to be essential to combustion; so that according to this eminent philosopher, while any substance is burning, the oxygen alone unites with the combustible substance, forming an oxyd, acid, \&c, according to circumstances; and both the light and heat which previously existed in the oxygen are now set at liberty, and appear in the form of visible flame.

But there are many weighty objections to the latter part of this hypothesis (sce Oxygen and Combustion), so that many chemists have returned to the old opinion, that the light is derived from the burning body, and not from the oxygen that supports combustions; an opinion which oll the whole better agress with the observed phenomena.

The sun's rays, the great source of light and heat to our world, have been fuund by Dr. Herschel to contain, bisides all the common coluured rays, separable by the prism, a nunber of rays of mere huat, giving no light, still less refrangible than the red rays of the spectrum, and therefore cxtending beyond them, and producing a greater heat than any of the visible rays. Since that time it has teen discovered, as appears both by Dr. Wollaston, Philosophical Trans. for 1802, and Mr. Ritter, Philosophical Juirnal, 8 vis, vols' 4 and 8 , that beyond, the opposite, or violet side of the prism, are certain other rays, also invisible, which do not effect the thermometer, but which produce some of the chemical effects of the visible rays of light, particularly of the violet rays to which they are contiguous. These therefore appear to be, if the expression may be allowed, invisible rays of light, or rays which, thongh they do not illuminate, agrce in chemical properties with those that do, and appear to be more than any other, separate from solar heat.

The must rapid and most unexceptionable chemical effects of pure light hitherto discovered, has been the change of colours of white luna cornea, to a purplish brown, or slate colour. This change takes place without requiring the least increase of temperature, or in any way being affected by heat or cold, and it occurs cqually well $5 \mathrm{~B}$ 
in close as in open vessels, and only on the surface immcdiately exposed to the light. Akin's Chem. Dict.

LIGHTNING, a large bright flame, shooting swiftly through the atmospherc, of momentary or very short duration, and rommonly.attended with thunder.

Some philosophers accounted for this awful natural phenomenon in this manner, viz, that an inflammable substance is formed of the particles of sulphur, nitre, and other combustible matter, which are exhaled from the earth, and carried into the higher regions of the atmosphere, and that by the collision of two clouds, or otberwise, this substance takes firc, and darts out into a train of light, larger or smaller according to the strength and quantity of the materials. And others bave explained the plicnomenon of lightning by the fermentation of sulphureous substances with nitrous acids. See Tuunder.

But it is now universally allowed, that lightning is really an electrical explosion or phenomenon. Philosophers bad not proceeded far in their experiments and enquiries on this subject, before they perceived the obvious analogy between lightning and clectricity, and they produced many arguments to evince their similarity. But the method of proving this hypothesis beyond a duubt, was first proposed by Dr. Franklin, who, about the close of the year 1749 , conccived the practicability of drawing lightning down from the clouds. Various circumstances of resemblance between lightning and electricity were remarked by this ingenious philosopher, ant have been abundantly con* firmed by later discoveries, such as the following: Flashes of lightning are usually seen crooked and waving in the air; so the electric spark drawn from an irregular body at some distance, and when it is drawn by an irregular body, or through a space in which the best conductors are disposed in an irregular manner, always exbibits the same appearance: lightning strikes the bighest and most pointed objects in its course, in preference to others, as hills, trees, spires, masts of stips, \&c; so all pointed conductors receive and throw off the electric fluid more readily than those that are terminated by flat surfaces: ligbtning is observed to take and follow the readiest and best conductor; and the same is the case with electricity in the discharge of the Leyden phial; whence the doctor infers, that in a thunder-storm, it would be safer to have nue's clothes wet than dry; liglitning burns, dissolves metals, rends some bodies, sumetimès strikes persons blind, destroys animal life, depriv's magnets of their virtue, or reverses their poles; and all these are well-known properties of elęctricity.

But lightning also gives polarity to the magnetic needle, as well as to all bodics that have any thing of iron in them, as bricks \&c; and by observing afterwards which way the magnetic poles of these bodies lie, it may thence be known in what direction the stroke passed. Persons are somotimes killed by lightning, without exhibiting any visible marks of injury; and in this case Sig. Beccaria supposes that the lightning does not really touch them, but only produces a sudden vacuum near tlem, and the air rusbing violently out of their lungs to supply it, they cannot recover their breath again: and in proof of this opinion he alleges, that the lungs of such persons are found flaccid ; whereas these are found inflated when the persons are really killed by the: electric shock. 'This hypothesis however is controverted by 1)r. Priestley.

To demonstrate by actual experinent, the identity of the electric fluid with the matter of lightuing, Dr. Frank- lin contrived to bring lightning from the lsearens, by means of a paper kite, properly fitted up for the purpose, with a long fine wire string, and calleil ai electrical kite, which he raised when a thunder-sturm was perceived to be coming on: and with the clectricity thus obtained, he charged phials, kindled spirits, and performed all other such electrical expcriments as are usually exhibited by an excited glass globe or cylinder. This happened in June 1752, a month after the electricians in France, in pursuauce of the method which lie liad-before proposed, had verified the same theory. The most active of these were Messr's. Dalibard and Delor, followed by Ml. Mazeas and M. Mlonnier.

In $A p r i l$ and June 1753 , Dr. Franklin discovered that the air is sometimes electrified negatively, as well as sometimes positively; and he even found that the clouds would change from positive to ncgative electricity several times in the course of one thunder-gust. This curious and important discovery he soon perceived was capable of being applied to practical use in life, and in consequence proposed a method, which he soon accomplished, of sccuring buildings from being damaged by lightning, by means of CoNDUctors: which see.

Nor lad, the English philosophers been inattentive to this subject: but, for want of proper opportunities of trying the necessary cxperiments, and from some other unfavourable circumstances, they had failed of success. Mr. Canton, however, succeeded in July 1752 ; and in the following month Dr. Bevis and Mr. Wilson observed nearly the same appearances as Mr. Canton had done before. By a number of experiments MIr. Canton also soon after obscrved that some clouds were in a positive, while some were in a negative state of electricity; and that the electricity of his conductor would sometimes change, from one state to the other, five or six times in less than half an hour.

But Sig. Beccaria discovered this variable state of thunder clouls, before he knew that it had been obscried by Dr. Franklin or any other person; and he has given a very exact and particular account of the external ajpcarances of these clouds. From the observations of lis apparatus within doors, and of the lightuing abroad, he inferred, that the quantity of electric matter in a common thunder-storm, is inconceivably great, considering how many pointed bodies, as spires, trees, \& c, are continually drawing it off, and what a prodigious quantity is repeatedly discharged to or from the earth. 'This natter is in such abundance, that he thinks it impussibie for any cloud or number of clouds to contain it all, so as cizher to receive or discharge it. lle observes also, that during the progress and increase of the storm, tunugh the lightning frequently struck to the carth, the same clouds were the nex moment ready to make a still greater dischatrge, and his apparatus contmoed to be as much affected as ever; so that the clouds must have received at one part, in the same moment when a discharge was made from them in another. And trom the whole he conclurles, that the clouds serve as conductors to cunvey the electric fluid from those parts of the carth that are orerloaded with it, to those that arc cxhausted of it. The same cause by which a cloud is first raised, from vapours dispersed in the atmospliere, draws to it those that arc aiready formed, and still continues to form new ones, till the whole collected mass extends so fit as to ranch a part of the eartl where there is a deficiency of the electric fluid, and where the clectric matter will discharge itscli on the earth. A chan- 
nel of communication being thus formed, a fresh supply of electric matter is raised from the overloaded part, which continues to be conveyed by the medium of the clouds, till the equilibrium of the fluid is restored between the two places of the earth. Sig. Beccaria observes, that a wind always blows from the place from which the thunder-cloud proceeds; and it is plain that the sudden accumulation of such a prodigious quantity of vapours must displace the air, and repel it on all sides. Indeed many observations of the descent of lightning confirm his theory of the manner of its ascent; for it often throws before it the parts of conducting bodies, and distributes them along the resisting medium, through which it must force its passage; and on this principle, the longest flashes of lightning seem to be made, by forcing into its way part of the vapours in the air. One of the chief reasons why these flashes make so long a rumbling, is that they are occasioned by the vast length of a vacuum made by the passage of the electric matter: for though the air collapses the moment after it has passed, and that the vibration, on which the sound depends, commences at the same noment; yet when the flash is directed towards the person who hears the report, the vibrations excited at the nearer end of the track, will reach his ear much sooner than those from the more remote end; and the sound will, without any echo or repercussion, continue till all the vibrations have successively reached him.

How it happens that particular parts of the earth, or the clouds, come into the opposite states of positive and negative electricity, is a question not absolutely determined: though it is easy to conceive that when particular clouls, or different parts of the earth, possess opposite electricities, a discharge will take place within a certain distance; or the one will strike into the other, and in the discharge a flash of lightning will be seen. Mr. Canton querics whether the clonds do not become possessed of electricity by the gradual heating and cooling of the air'; and whether air suddenly rarefied, may not give electric fire to clouds and vapours passing through it, and air suddenly condensed receive electric fire from them.-Mr. Wilcke supposes, that the air contracts its electricity in the same manner that sulphur and other substances do, when they are heated and cooled in contact with various bodies. Thus, the air being heated or cooled near the earth, gives electricity to the earth, or receives it from it; and the electrified air, being conveyed upwards by various means, communicates its electricity to the clouds.-Others have queried, whether, since thunder commonly happens in a sultry state of the air, when it seems charged with sulphureous vapours, the eletric mattér then in the clouds may not be generated hy the fermentation of sulphurcous vapours with mincral or acid vapours in the air.

With regard to places of safety in times of thunder and lightning, Dr. Franklin's advice is, to sit in a chair in the middle of a room, provided it be not under a metal lustre suspended by a chain, and laying the feet on another chair. "It is still better," he says, "to bring two or three mattresses or berls into the middle of the room, and folding them double, to place the chairs upon them; for as they are not so good conductors as the walls, the lightning will not' be sulikely to pass through them: but the safest place of any, is in a hammock hung by silken cords, at an equal distance from all the sides of a room. Dr. Priestley however obscrves, that the place of most perfect safety must be a cellar, and cspecially the middle of it; for when a person is lower than the surface of the earth, the lightning must strike it before it can possibly reach him. In the fields, the most secure place is within a few yards of a tree, but not quite near it. Beccaria cautions persons not always to trust too much to the ncighbourhood of a higher or better conductor than their own body; since he has repeatedly found that the lightning by no means descends in one undivided track, but that bodies of various kinds conduct their share of it at the same time, in proportion to their quantity and conducting power. See Franklin's Letters, Beccaria's Lettre dell' Ellettriccssimo; Priestley's Hiśt. of Electric., and Lord Mahon's Principles of Electricity.

Lord Mahon observes that damage may be done by lightning, not only by the main stroke and lateral explosion, but also by what he calls the returning stroke; by which is meant the sudden violent return of that part of the natural share of electricity which had been gradually expelled from some body or bodies, by the superinduced elastic electrical pressure of the electrical atmosphere of a thunder-cloud.

Artificial Licrining, an imitation of real or natural lightning by gunpowder, aurum fulminans, phosphorus, \&ce, but especially the last, between which and lightning there is much more resemblance than the others. Phosphorus, when newly made, gives a sort of artificial lightning visible in the dark, wbich would surprise those not used to such a phenomenon. It is usual to keep this preparation under water; and if it is desired to see the corruscations to the greatest advartage, it should be kept in a deep cylindrical glass, not more than three quarters filled wirls water. At times the phosphorus will send up corruscations, which will pierce through the incumbent water, and expand themselves with great brightness in the upper or empty part of the glass, and much resembling lightning. The phosphorus, while burning, acts the part of a corrosive, and when it goes out resolves into a menstruum, which dissolves gold, iron, ind other metals; and lightning, in like manner, melts the same substances.

LlkE Quantities, or.Similar 2uantities, in Algebra, are such as are expressed by the same powers of the same letters, or equally repeated in each quantity; though the numeral coefficients may be different.

Thus $4 a$ and $5 a$ are like quantities,

as are also $3 a^{2}$ and $12 a^{2}$,

and also $6 b x y^{2}$ and $10 b x y^{2}$.

But $4 a$ and $5 b$, or $3 a^{2} b$ and $10 a^{2} b^{2}$, \&c, are unlike quantities; because they have not the same dimensions throughont, nor are the letters equally repeated. - Like quantities can be united into one quantity, by addition or subtraction; but unlike quantities can only be added or subtracted by placing the signs of these operations between them.

Like Signs, in Algebra, are the same signs, either both positive or both negative. But when one is positive and the other negative, they are unlike signs.

So, $+3 a b$ and $+5 c d$ bave like signs,

as have also $-2 a^{2} c$ and $-2 a x^{2}$;

but $+3 a b$ and $-5 c d$ bave unlike signs, as also - $2 a x$ and $3 a x$.

Li к F Figures, or Arcs, \&c, are the same as similar figures, arcs, \&c. See Similar. All like figures have their homologous lines in the same ratio. Also like plane figures are in the duplicate ratio, or as the squares of their lomologous lines or sides; and like solid figures are in the 
triplicate ratio, or as the cubes of their homologous lines or sides.

LILLY (W1LLAM), a noted English astrologer, born in Leicestershire in 1602. His father was not able to gire him any farther education than common reading and writing; but young Lilly being of a forward temper, and endued with shrewd wit, he resolved to push his fortune in London, where he arrived in 1620, and, for a present support, articled himself as a servant to a mantuic-maker in the parish of St. Clement Dancs. But in 1624 be moved a step higher, by entering into the service of $\mathrm{M}_{\mathrm{r}}$. Wright in the Strand, master of the Salters company, who not being able to write, Lilly among other offices kept his books. On the death of his master, in 1627 , Lilly paid his addresses to the widow, whom he married with a fortune of 1000 l. Being now his own master, he pursued. the bent of his inclinations, which led him to follow the puritanical preachers: but afterwards, turning his mind to judicial astrology, in 1632 he becane pupil, in that art, to one Evans, a profligate Welsh parson; and the next year gave the public a specimen of his skill, by an intimation that the ling had chesen an mulucky horoscope for the coronation in Scotland. In i634, getting a manuscript copy of the Ars Nuticia of Comelius Agrippa, with alterations, he followed the doctrine of the magic circle, and the invocation of spirits, with great eagerness, and practised it for some time; after which he treated the mystery of recovering stolen goods, \& $\mathrm{c}$ with great contempt, claiming a supernatural sight, and the gift of prophetical predictions; all which he well knew how to turn to good advantage.

Mean while, he had buried his first wife, purchased a. moiety of 13 houses in the Strand, and married a second wife, who, joining to an extravagant temper a termagant spirit, which, notwithstanding his skill in magic, he could not lay, made him unhappy, and greatly reduced his circumstances. With this uncomfortable yokemate he removed, in 1636 , to Hersham in Surrey, where he staid till $164 \mathrm{t}$; when, seeing a prospect of tishing in troubled waters, he returned to Londow. Here having purchased several curious books in this art, which were found on pulling down the house of another nstrologer, he studied them incessantly, finding out secrets contained in them, which were written in an imperfect Greek character; and in 1644, publisticd his Mcrlinus Anglicus, an almanac, which he continued, annually till his death, and several other astrological works; devoting his pen, and other labours, sometimes to the king's party, and sometimes to that of the parliament, but mostly to the lattes, raising his fortune by favourable predictious to both parties, sometimes by presents, and sometimes by pensions: thus, in 1048 , the council of state gave him in moncy $50 \%$. and a pension of $100 \%$. per anmum, which he received for two yeare, and then resigned it on some disgust. liyghis advice and contrivance, the king atiompted several tincs to make his cacape from his continemut : he procured and sent the aquafortis and files to cut the iron bars of his prison wiudows at Carisbrook'castle; but still advising and writing for the other party at the same time. Mean while he read public lectures on astrology, in 1648 and $16 \% 9$, for the improvement of young sturlents in that art; amol in short, plied his business so well, that in 1651 and 1652 he laid out near $2000 l$. for lands and a house at Hersham.

During the siege of Colchester, he and Booker were sent for thither, to encourage the soldiers; which they did by. assuring them that the town would soon be taken; which proved true in the event.- Having, in 1650 , written publicly that the parliament should not continue, but a new government arise; agreably to which, in his almanac for 1653 , he asserted that the parliament stood upon a ticklish foundation, and that the commonalty and soldiery would join together against them. Upon which he was summoned before the committee of plundered ministers; but, receiving notice of it before the arrival of the messenger, he applied to his friend Lenthal the speaker, who pointed ont the uffensive passages. He immediately altered them; attended the committee next morning, with 6 copies printed, which six alone he acknowledged to be his; and by that means came off with only 13 days custody by the serjeant at arms. This year lie wals engaged in a dispute with Mr. Thomas Gataker.- In 1665 he was indicted at Hicks's-hall, for giving judgment on stolen goods; but was acquitted. And in 1659, he received from the king of Sweden, a present of a gold chain and medal, worth about $50 l$. on account of his having mentioned that n.onarcl with great respect in bis almanacs of 165 , and 1655 . - After the Restoration, in 1660 , being taken into custody, and examined by a committce of the house of commons, touching the execution of Charles the 1st, he declared, that Roberrt Spavin, then secretary to Cromwell, dining with him soon after the fact, assured him it was done by cornet Joyce. The same year hesued out his pardon under the broad seal of England; and afterwards continued in Lundon till 1665 ; when, on the raging of the plague there, lie retired to lis estate at Hersham. Here he applied himsell to the study of physic, laving, by means of his frienil Elias Ashmole, procured from archbishop Sheldon a licence to practise it, which he did, as well as astrology, from thence till the time of his death.-In October 1666 he was examined before a committee of the house of cummons concerning the fire of London, which happened in September that year. A little before lis death, he adnpted for his son, by the name of Merlin Junior, one Henry Coley, a taylor by trade; and at the same time gave him the impression of his almanac, which had been printed for 36 years successively. This Coley became afterwards a celebrated astrologer, publishiog in his own name, almanacs, and books of astrology, particularly one entitled A Kicy to Astrology. 1.illy died of a palsy in 1681 , at 79 years of age; and lis friend Mr. Aslmole placed a monument orer his grave in the church of Walton-upon-Thanes.

Lilly was author of many works. His O bservations on the Life and Death of Charles late King of England, if we overlook the astrological nousense, may be read with as much satisfaction as more eclebrated histories; lilly being not only very well intormerl, but stritty impartial. This work, with the Lives of Lilly and Ashmole, written by themselves, were published in one volume, $8 \% 0$, in 1774 , by Mr. Burman. His other works were principally as follows: 1. Norlinus Anglicus junior-2. Supernatural Sight. - 3. The White King's Prophecy.-4. lingland's Prophetical Mlerlin: all printed in $164+,-5$. The Starry in essenger, 1645.-6. Cullection of Proplucies, $16+6 .-$ 7. A Comment on the White King's Prophicey, 16.46.-s. The Nativities of Archbishop Latud and Thomas liart of Straford, 1646. -9. Christian Astrology, 164. : on this piece he read his lectures in 164.8 , mentioned above -10 . The 'Third book of Nativities, $1647-11$. 'The World's Cat tastrophe, 1647.- 12. The Prophecies of Ambrose Mer- 
lin, with a Key, 1647.-13. Trithemius, or the Government of the IVorld ky Presiding Angels, 1647.-14. A treatise of the Three Suns seen in the winter of 1647 , printed in 1648.-15. Monarchy or no Monarchy, 1651.-16. Observations on the Life and Death of Charles, late king of Englatud, 1651; and again in 1651, with the title of Mr. William Lilly's True History of King James and King Charles the 1st, \& c. -17. Amnus Tenebrosus; or, the Black Year. 'This drew him into the dispute with Gataker, which Lilly carried on in his Almanac in 165 .

LIMB, the ontermost border, or graduated edge, of a quadrant, astrolabe, or such like mathematical instrument. The word is'also used for the arch of the primitive circle, in any projection of the sphere in plano.

Li M e also signifies the outermost border or edge of the sun or the moon; as the upper limb, or edge; the lower limb; the preceding limb, or side; the following limb.Astronomers observe the upper or lower limb of the sun or moon, to find their true height, or that of the centre, which differs from the others by the semidiameter of the disc.

LIMBERS, in Artillery, a sort of advanced train, joined to the carriage of a cunnon on a march. It is composed of two shafts, wide enough to receive a horse between them, called the fillet horse: these shafts are joined by two bars of wookl, and a bolt of iron at one end, and mounted on a pair of rather small whecls. On the axlctree rises a strong iron spike, which is put into a bole in the hinder part of the train of the gun-carriage, to draw it by. But when a gun is in action, the limbers are taken off, and run out behind it. - See the dimensions and figure of it in Müller's 'Treatise of Artillery, pa. 187.

LIMIT, is a term used by mathematicians, for some determinate quantity, to which a variable one continually approaches, and may come nearer to it than by any given difference, but can never go beyond it; in wbich sense a circle may be said to be the limit of all its inscribed and circumscribed polygons : because these, by increasing the number of their sides, may be made to approach so near to the true area of the circle, that the difference shall be less than any assignable quantity.

Limir's of the roots of EqUATIONs, in Algebra, form a part of that science, by means of which the solution of cquations is sometimes much facilitated, particularly in those cases where we can only proceed by approximations; for, knowing that a root must lie between certain limits, that is, that it is greater than one known quantity, and less than another, we are led to a near approximate value in the first instance, which, without this consideration, we should hate perhaps discovered only after a tedious operation.

To find a limit grenter than the greatest root of an equation.

Let $a, b, c$, acc, be roots of an equation; transform it into anoiher whose roots are $a-e, b-e, c-e, \& \mathrm{c}$; and if by trial, such a value of $e$ be found, that all the terms of the transformed equation be positive, all its roots are negative; and consequently $e$ is greater than the greatest root of the proposed equation.

Suppose, for example, it were requircd to find a number greater than the greatest root of the equation $x^{3}-5 x^{2}+$ $7 x-1=0$.

Assume $x=y+e$, and we have

$$
\left.\begin{array}{r}
x^{3}=y^{3}+3 e y^{2}+3 e^{2} y+e^{3} \\
-5 x^{2}=-5 y^{2}-10 e y-5 e^{2} \\
+7 x=0 \quad+7 y+7 e \\
-1=0
\end{array}\right\}=0
$$

in which equation, if 3 be substituted for $e$, each of the quantities $e^{3}-5 e^{2}+7 e-1,3 e^{2}-10 e+7,3 e-5$, is positive, or all the values of 7 are negative, therefore 3 is greater than the greatest value of $x$.

Again, to find a limit less than the last root of the equation $x^{3}-3 x+72=0$.

When the signs of these roots are changed, this equation becomes $x^{3}-3 x-72=0$.

$$
\begin{aligned}
& \text { Assume } x=y+\epsilon \text {, then } \\
& \left.\begin{array}{rr}
x^{3}=y^{3}+3 e y^{2}+3 e^{2} y+e^{3} \\
-3 x= & -3 y-3 e \\
-72= & -72
\end{array}\right\}=0 .
\end{aligned}
$$

and here, if 5 be substituted for $e$, every term becomes positive, consequently 5 is greater than the greatest ruot of the equation $\dot{x}^{3}-3 x-72=0$, and -5 less than the least root of the equation $x^{3}-3 x+72=0$.

And in this manner may be found the limits of the roots of equations of any dimensions. For more on this subject, see Maclaurin's Algebra, and Wood's Algebra.

Lia I of Distinct Vision, in Optics. See Distinct Vision.

Limit of a Planet, has been sometimes used for its greatest heliocentric latitude.

Li MITED Problem, denotes a problem that has but one solution, or some determinate number of solutions: as, to describe a circle through three given points that do not lie in a right line, which is limited to one solution only; to divide a parallelogram into two equal parts by a line parallel to one side, which admits of two solutions, according as the line is parallel to the length or breadth of the parallelogram; or to divide a triangle in any ratio by a line parallel to one side, which is limited to three solutions, as the line may be parallel to any of the three sides.

LINE, in Geometry, a quantity ex tended in length only, without either breadth or thickness. A line is sometimes considered as generated by the flux or motion of a point; and sometimes as the limit or termination of a superficies, but not as any part of that surface, however small.

Lines are either Right or Curved. A Right, or straight line, is the nearest distance between two points, which are its extremes or ends; or it is a, line which has in every part of it the same direction or position. But a Curve Line has in every part of it a different direction, and is not the shortest distance between its extremes or ends.

Right Lines are all of the same species; but curves are of an infinite number of different sorts. As many may be conceived as there are different compound motions, or as many as there may be different relations between their ofdinates, and abscisses. See Curves.

Again, Curve Lines are usually divided into geometrical and mechanical.

Geometrical Lines are those which may be found exactly in all their parts. See Geometrical Line.

Mechanicallines are such as are not determined exactly in all their parts, but only nearly, or tentatively.

Descartes, and his followers, define geometrical lines to be those which may be expressed by an algebraical equation of a determinate or finite degree, called its locus. And mechanical lines, such as cannot be expressed by such an equation. But others distinguish the same lines by the name Algebraical and Transcendental.

Lines are also divided into orders, by Newton, according to the number of intersections which may be made by them and a right line, viz, the $1 \mathrm{st}, 2 \mathrm{~d}, 3 \mathrm{~d}, 4 \mathrm{th}$, \&ce, order, according as they may be cut by a right line, in 1, or 2, or 3 , or 4 , \& 8 , points. In this way of considering thens, the right line only is of the lst order, being but one in 
number; the $2 \mathrm{~d}$ order contains 4 curves only, being such as may be cut from a cune by a plane, viz, the circle, the ellipse, the hyperbola, and the parabola; the lines of the $3 d$ order have been enumerated by Newton, in a particular treatise, who makes their number amount to 72 ; but Mr. Stirling found 4 others, and Mr. Stone 2 more; though it is disputed by some whether the last two ought to be accounted diffirent from some of Newton's, or not. See Newton's Enumer. Lin. Tertii Ordin. also Stirling's Lineæ Tert. Ordin. Newtonianæ Oxon. 1717, 8vo. and Philos. Trans. No.456, \&c. Again,

Algebraical Lines are divided into different orders according to the power or degree of their equations. So, the simple equation $a+b y+c x=0$, or equation of the Ist degrce, deñntes the Ist order or right line; the equation $u+b y+c x+d y^{2}+e r y+f x^{2}=0$, of the $2 \mathrm{~d}$ degree, denotes the lines of the $2 d$ order; and the equation $a+b y+c x+d y^{2}+e x y+f x^{2}+g y^{3}+h x y^{2}+i x^{2} y+$ $h x^{3}=0$, of the $3 \mathrm{~d}$ degree, expresses the lines of the $3 \mathrm{~d}$ order; and so on. See Cramer's Introd. à. l'Analyse des Lignes Courbes.

Lines, considered with regard to their positions, are either Parallel, Perpendicular, or Oblique. The construction of which, see under the respective terms.

LINE also denotes a French measure of length, being the 12 th part of an inch, or the 144 th part of a foot.

In Astronomy,

Lin $\mathrm{E}$ of the Apses, or Apsides, the line joining the two apses, or the longer axis of the orbit of a planet.

Fiducial Line, the index line or edge of the ruler, which passes through the middle of an astrolabe, or other instrument, on which the sights are fitted, and marking the divisions.

Iorizontal Line, a line parallel to the horizon.

Live of the Nodes, that which joins the nodes of the orbit of a planet, being the common section of the plane of the orbit with the plane of the ecliptic.

\section{In Dialling,}

Horizontal Line, is the common section of the horizon and the dial-plate.

Horary, or Huur Lines, are the common intersections of the hour-circles of the spliere with the plane of the dial.

Equinoctial Line, is the common intersection of the equinoctial and the plane of the dial.

In Fortification, Line is sometimes used for a ditch, bordered with its parapet: ard sometimes for a row of gabions, or sacks of earth, extended lengthwise on the ground, to serve as a shelter against the enemy's fire. When the trenches have been carried on within 30 paces of the glacis, they draw two lines, one on the right, and the other on the left, for a place of arms.

Lines are commonly made to shut up an avenue or entrance to some place; the sides of the entrance being covered by rivers, woods, mountains, morasses, or other obstructions, not easy to be passed over by an army. When they are constructed in an open country, they are carried round the place to be defended, and resemble the lines surrounding a camp, called lines of circumvallation. Lines are also thrown up to stop the progress of an army; but the term is mostly used for the line covering a pass which can only be attucked in front.

When lines are made to cover a camp, nr a large tract of land, where a consislerable body of troops is posted, the work is not made in one straight, or uniformly bending line; but, at certain distances, the lines project in saliant angles, called redents, redans, or flankers, towards the enemy. The distance between these angles is commonly between the limits of 200 and 260 yards; the ordinary flight of a musket ball, point blank, being commonly within those limits; though muskets a little elevated will do effectual service at the distance of 360 yards.

Fundamental Line, is the first line drawn for the plan of a place, and which shows its area.

Central Line, is the line drawn from the angle of the centre to the angle of the bastion.

Line of Defence, \&c. See Defence, \&c.

Line of Approach, or Attack, signities the work which the besiegers carry on under cover, to gain the moat, and the body of the place.

Line of Circumvallation, is a line or trench cut by the besiegers, within canñon-shot of the place, ranging round the camp, and securing its quarters against any relief to be brought to the besicged.

Line of Contravallation, is a ditch borclered with a parapet, serving to cover the besiegers on the side next the place, and to stop the sallies of the garrison.

Lines of Communication are those which run from one work to another.

Line of the Base, is that which joins the points of the two nearest bastions.

To Line a work, signifies to face it, as with brick or stone; for example, to strengthen a rampart with a firm wall, or to encompass a parapet or moat with good turf, \&c.

LrNe, in Geography and Navigation, is emplatically used for the equator or equinoctial line.

In Perspective,

'The Geometrical Line, is a right line drawn in any manner on the geometrical plane.

Terrestrial or Fundamental Line, is the common intersection of the geometrical plane and plane of the picture.

Line of the Front, is any line parallel to the terrestrial line.

Vertical Line, is the section of the vertical and draft planes.

Visual Line, is the line or ray conceived to pass from the object to the eye.

Objective Line, is any line drawn on the geometrical plane, whose representation is sought for in the draught or picture.

Line of Measures, is used by Oughtred, and others, to denote the diameter of the primitive circle, in the projection of the sphere in plano, or that line in which falls the diameter of any circle to be projected.

Linear NuMBERs, are such as have relation to length only; such, for example, as express one side of a plane figure; and when the plane figure is a square, the linear number is called a root.

Linear Problem, is one that can be solved geometrically by the intersection of two right lines. I'his is called a simple problem, and is capable of only one solution.

LIQUID, a fluid which wets or smears such bodies as are immersed in it, arising from some configuration of its particles, which disposes them to adhere to the surfaces of bodies contiguous to them. Thus, water, oil, milk, \&c, are liquids, as well as tluids; but quicksilver is not a liquid, but simply a fluid.

LISLE (W1LL1AM DE), a learned French geographer, was born at Paris in 1675 . His father being much occu- 


\section{O C}

pied in the same pursuit, young Lisle began at 9 years of age to draw maps, and soon made a considerable progress in this art. In 1699 be first distinguished himself to the public, by giving a map of the world, and other pieces, which procured him a place in the Academy of Sciences, 1702. He was afterwards appointed geographer to the king, with a pension, and had the honour of instructing the king himself in geography, for whose particular use he drew up several works. Delisle's reputation was so great, that scarcely any history or travels came out without the embellishment of his maps. Nor was his name less celebrated abroad than in his own country. Many sovereigns in vain attempted to draw him out of France. The Czar Peter, when at Paris on his travels, paid him a visit, to communicate to bim some remarks upon Muscovy; but more especially, says Fontenelle, to learn from him, better than he could any-where else, the extent and situation of his own dominions. Delisle died of an apoplexy in 1726, at 51 years of age. Besides the excellent maps he published, he wrote many pieces in the Memoirs of the Academy of Sciences.

LISLE (JOSEPH-Nicrol AS DE), an eminent astronomer and geographer, was born at Paris in 1688 . He was brother of the above-mentioned William, and had the same education with him under their father, in geography and astronomy. At the age of 18 be made an accurate observation of the great total eclipse of the stin in 1706 ; which may be considered as the beginning of his public career. He was admitted in the astronomical department of the Academy in 1714. In 1724 he visited England, and was much esteemed by Nervton and Halley; and he was also chosen a Fellow of the Royal Society. In 1726 he was invited to Petersburg, where he had the charge of the observatory, with a considerable pension. He returned to Paris in 1747 , where he filled the place of professor to the Royal College, and where he formed the astronomers Laland and Messier, illustrious pupils of so great a master. M. Delisle died in $\mathbf{1 7 6 8 .}$ His principal works are, his History of Astronomy, 173S, in 2 vols 4to; and $4+$ memoirs in the volumes of the Academy, from the year 1714 to 1766 .

LIST, or LISTEL, a small square moulding, serving to crown or accompany larger mouldings; or on occasion to separate the flutings of columns.

Literal Algebila. Sce Aqgebra.

LIZARD, in Astronomy. See Lacerta.

LOADSTONE, or MIANET; which see.

LOCAL Problem, is one that is capable of an infinite number of different solutions; because the point, which is to solve the problem, may be indifferently taken within a a certain extent; as suppose any-where in such a line, within such a plane figure, \&cc, which is called a Geometrical Locus.

A local problem is Simple, when the point sought is in a right line; Plane, when the point sought is in the circumference of a circle; Solid, when it is in the circumference of a conic section; or Sursolid, when the point is in the perimeter of a line of a higher kind.

Local Motron, or Loco-Motion, the change of place: Sec Motion.

LOCI, the plural of Locus, which see.

LOCI PlaNi, or Loca Plana, one of the last works of Apollonius, that have been restored by Schooten, in 1656; by Fermat, in 1679; and by Simson, in 1749 .
LOCK, for Canals. See Canaz.

LOCUS, is some line by which a local or indeterminate problem is solved; or a line of which any point may equally solve an indeterminate problem. Loci are expressed by algebraic equations of different orders, according to the riature of the locus. If the equation, is constructed by a right line, it is called Locus ad Rectum; if by a circle, Locus ad Circulum; if by a parabola, Locus ad Parabolam; if by an ellipsis, Locis ad Ellipsim; and so on,

The loci of such equations as are right lines or circles, the ancients called Plane Loci; and of those that are conic sections, Solid Loci; but such as are curves of a higher order, Sursolid Loci. The moderns distinguist the loci into orders according to the dimensions of the equations by which they are expressed, or the number of the powers of indeterminate or unknown quantities in any one term: thus, the equation

$a y=b x+c$ denotes a locus of the 1st order, but $y^{2}=a x$, or $=a x-x^{2}, 8 c$, a locus of the $2 \mathrm{~d}$ order, and $y^{3}=a^{2} x$, or $=a x^{2}-x^{3}, \& c$, a locus of the $3 \mathrm{~d}$ order, and so on; where $x$ and $y$ are unknown or indeterminate quantities, and the others known or determinate ones; also $x$ denotes the absciss, and $y$ the ordinate of the curve or line which is the locus of the equation.

For instance, suppose two variable or indeterminate right lines A $\mathrm{P}$, $A Q$, making any given angle $P A Q$ between them, where they are supposed to commence, and to extend indefinitely both

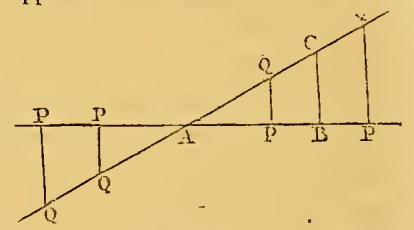
ways from the point $\mathrm{A}$ : then calling any $\mathrm{AP}, x$, and its corresponding ordinate $\mathrm{PQ}, y$, continually changing its position by moving parallel to itself along the indefinite line $\mathrm{AP}$; also in the line $\mathrm{AP}$ assume $\mathrm{AB}=a$, and from $\mathrm{B}$ draw $\mathrm{BC}$ parallel to $\mathrm{PQ}$ and $=b$ : then the indefinite line $A Q$ is called in general a Geometrical Locus, and in particular the Jocus of the equation $y=\frac{b x}{a}$; for whatever point $Q$ is, the triangles $A B C, A P Q$ are always similar, and therefore $\mathrm{AB}: \mathrm{BC}:: \mathrm{AP}: \mathrm{PQ}$, that is $a: b:: x: y$, thcrefore $a y=b x$ is the equation to the right line $A Q$, or $A Q$ is the locus ot the equation $a y=b x$.

Again, if $A Q$ be a parabola, the nature of which is such, that $\mathrm{AB}: \mathrm{AP}:: \mathrm{BC}^{2}: \mathrm{PQ}^{2}$, ol $a: x:: b^{2}: y^{2}$, and therefore $a y^{2}=b^{2} x$ is the equation which has the parabola fur its locus, or the parabola is the locus to every equation of this form $a y^{2}=b^{2} x$.

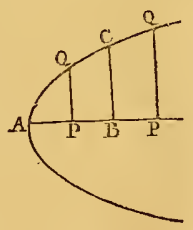

Or-if $A Q$ be a circle, having its radius $\mathrm{AB}=a$, the nature of which is this, that $\mathrm{PQ}^{2}=A \mathrm{P} \cdot \mathrm{PD}$, or $y^{2}=x \cdot(2 a-x)$ or $=2 a x-x^{2}$; the refore the locus of the equation of this form $y^{2}=2 a x-x^{2}$, is a circle.

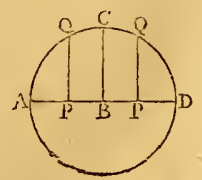


In like manner it will appear, that the ellipse is the locus to the equation $t^{2} y^{2}=c^{2} \times\left(t x-x^{2}\right)$, and the hyperbola the locus to the equation $t^{2} y^{2}=c^{2} \times\left(t x+x^{2}\right)$; where $t$ is the transversc, and $c$ the conjugate asis of the ellipse or hyperbola.

All equations whose loci are of the first order, may be reduced to one of the 4 following forms: Ist, $y=$ $\frac{l x}{a} ; 2 \mathrm{~d}, y=\frac{b x}{a}+c ; 3 \mathrm{~d}, y=\frac{l x}{a}-c ; 4 t h, y=c-\frac{b x}{a}$ where the letter $c$ denntes the distance that the ordinates commence from the line $A \mathrm{P}$, either on the one side or the other of it, according as the sign of that quantity is + or -

All loci of the 2 degree are conic sections, viz, either the parabola, the circle, ellipsis, or hyperbola. Therefore when an equation is given, whose locus is of the $2 \mathrm{~d}$ degree, and it is required to draw that locus, or, which is the same thing, to construct the equation generally; bring over all the terms of the equation to one side, so that the other side be 0 ; Wen to know which of the conic sections it denotes, there will be two general cases, viz, either when the rectangle $x y$ is in the equation, or when it is not in it.

Case 1. When the term $x y$ is not in the proposed equation. Then, 1 st, if only one of the squares $x^{2}, y^{2}$ be found in it, the locus will be a parabola. 2d, If both the squares be in it, and if they have the same sign, the locus witl be a circle or an ellipse. $3 \mathrm{~d}$, But if the signs of the squares $x^{2}, y^{2}$ be different, the locus will be an hyperbola, or the opposite hyperbolas.

Case 2. When the rectangle $x y$ is in the proposed equation; then $1 \mathrm{st}$, If neither of the squares $x^{2}, y^{2}$, or nnly one of them be in the cquation, the locus will be an byperbola letween the asymptotes. $2 \mathrm{~d}$, If both $x^{2}$ and $y^{2}$ be in it, having different signs, the locus will be an hyperbola, having the abscisses on its diameter. $3 d$, If both the squares be in it, and with the same sign, then if the coefficient of $x^{2}$ be greater than the square of half the coeffcient of $x y$, the locus will be an cllipse ; if equal, a parabola; and if less, an hyperbola.

This method of determining geometric loci, by reducing them to the most compound or gencral cquations, was first published by Mr. Craig, in his Treatise on the Quadrature of Curves, in 1693 . It is explaincd at large in the $7 \mathrm{th}$ and 8 th books of l'Hospital's Conic Sections. This subject is also particularly illustrated in Maclaurinn's Algebra. The method of Descartes for finding the loci of equations of the $2 d$ urder, is by extracting the root of the equation. Sec his Gcometry; as also Stirling's ll lustratio Linearum Tertii Ordinis. The doctrine of these loci is likewise well treated by De Witt in lis Llementa Curvarum. And Bartholomeus Intieri, in his Aditus ad Nova Arcana Gcometrica delegenda, lias shown how to find the loci of equations of the ligher orders. Mr. Stirling too, in his treatise above-mentioned, has given an example or two of finding the loci of equations of 3 dimensions. Euclid, Apollonius, Aristaus, Format, Viviani, have also written on the subject of loci.

LOG, in Navigation, is a piece of thin board, of a sectoral or quadrantal form, loaded in the circular side with Iead sufficient to make it swim upright in the water; to which is fastened a line of a bout 150 fathoms, or 300 yards long, called the log-line, divided into certain spaces, called knots, and wound on a recl turning very freely, for the line ti) wind casily off.
The use of the log, or log-line, is to measure the velocity of the ship, or rate at which she runs, which is done from time to time, as the foundation on which the ship's reckoning, or finding her place, iskept; and the practice is, to heave the $\log$ into the sea, with the line tied to it, and by observing how much of the line is run off the rcel, while the ship sails, during the space of lialf a minute, which time is measured by a sand-glass made to run that time viry exactly. About 10 fathoms of stray or waste line is left next the log before the knoting or counting commence, that space being usually allowed to carry the $\log$ out of the eddy of the ship's wake.

The using of the log for finding the velocity of the ship, is called heaving the $\log$; and is thus performed: One man holds the reel, and another the half-minute glass; an officer of the watch throws the log over the ship's stern, on the lee-side, and when he observes the stray line, and the first mark is going ofi, he cries Turn ! when the glassholder instantly turns the glass crying out Done! then watching the glass, the moment it is run out he says Slop! upon which the reel being quichly stopt, the list mark run off shows the number of knots, and the distance of that mark from the reel is estimated in fathoms : then the knots and fathoms together show the distance run in laalf a minute, or the distance per hour nearly, by considering the knots as miles, and the fathoms as decimals of a mile: Thus, if 7 knots and 4 fathoms be observed, then the ship runs at the rate of $7 \cdot 4$ miles an bour.

It follows, thercfore, that the leugth of each knot, or division of the line, ought to be the same part of a sea mile, as balf a minute is of an loour, that is $\frac{x}{2 \delta}$ th part. Now it is found that a degree of the meridian conlains nearly 366,000 feet, therefore $\frac{1}{60}$ of this, or a nautical mile, will be 6100 fect ; the ${ }_{T^{2}}^{\frac{x}{2}}$ th of which, or 51 feet nearly, should be the length of each knot, or division of the log-line. But, because it is safer to bave the rechoning rather before the ship, than after $\mathrm{it}$, it is thercfore usual now to make each knot equal to $S$ fathoms or 48 feet. But the knots are made sometimes to contain only 42 feet; which method of dividing the log-line is founded on the supposition, that 60 milcs, of 5000 feet eacb, make a dcgrce; for $\frac{1}{1} \frac{1}{2}$ th of 5000 is $41 \frac{2}{3}$, or in round numbers 42 feet. And though many mariners find by experience that this length of the knot is too short, yet rather than quit the old way, they use sand-glasses for half-minute ones that run only 24 or 25 seconds. The sand, or half-minute glass, may be tried by a pendulum vibrating seconds, in the following manner: On a hook or peg, hang a thread or fine string that bas a musket-ball fixed to one end, carefully measuring between the centre of the ball and the string's loop over the nail $39 \frac{\mathrm{r}}{8}$ inches, being the length of a second pendulum; then make it swing or vibrate very small arches, and count one for erery time it passes under the nail, beginning at the second time it passes; and the number of swings made during the time the glass is running out, shows the seconds in the glass.

It is not known who was the jurentor of this method of measuring the ship's way, or her rate of sailing; but no mention of jt occurs till the year 1607 , in an last-India voyage, published by Purchas; and from that time ity name occurs in other royages in his collections; after which it became more known, being noticed both by our own authors, and by foreigners; is by Gunter in 1623 ; Snellius, in 1624; Metius, in 1631; Uughtred, in 1633; Ilerigone, in 1634; Saltonstall, in 1636; Norwool, iu 


\section{O G}

1637 ; Fournier, in 1643 ; and almost all the succeeding writers on navigation of every country. Various improvements have lately been made on this instrument by different persons. See Robertson's Navigation, the Prelace, pa. v. Also Hist. Math. tom. iv, 525- 537.

LoG-Board, in Navigation, a board or table usually divided into 5 columns: in the first of which is entered the hour of the day; in the $2 \mathrm{~d}$, the course steered; in the $3 \mathrm{~d}$, the number of knots run off the reel each time of heaving the $\log$; in the 4 th, the direction of the wind; and in the 5 th, observations on the weather, variation of the compass, \&c.

LoG-Book, a book ruled in columns like the log-hoard, into which the account on this is transcribed every day at noon; from whence, after it is corrected, $S c_{s}$, it is entered in to the journal.

LOGARITHM, from the Greek $i . s \% s 5$ ratio, and asfuss number; q. d. ratio of numbers, or perliaps rather number of ratios; the indices of the ratios of numbers to one another; or a series of numbers in arithmetical proportion, corresponding to as many others in geometrical proportion, in such sort that 0 -corresponds to, or is the index of 1 , in the geometricals. They have been devised for the ease of large arithmetical calculations.

Thus,

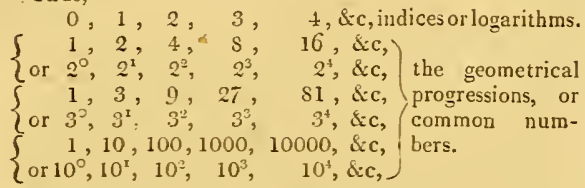

Where the same indices, or logarithms, serve equally for any geometric series; and from which it is evident, that there may be an endless variety of sets of logaritbms to the same common numbers, by varying the 2d term 2, or 3 , or 10 ; \&c of the geometric series; as this will change the original series of terms whose indices are the numbers $1,2,3, \& \mathrm{c}$; and, by interpolation, the wbole systen of numbers may be made to enter the geometrical series, and receive their proportional logarithms, whether integers or deciunals.

Or the logarithm of any given number, is the indes of such a power of some other number, as is equal to the given one. So if $\mathrm{v}$ be $=r^{n}$, then the logaritbm of $\mathrm{s}$ is $n$, which may be either positive or negative, and $r$ any number whatever, according to the different sjstems of logarithms. When $\mathrm{s}$ is 1 , then $n$ is $=0$, whatever the value of $r$ is; and consequently the logarithm of $I$ is always 0 in every system of logarithms. When $n=1$, then $\mathrm{v}=r$; consequently the root $r$ is alwass the number whose logarithm is 1 , in every system. When $r=2.718281828+59$ $\& c$, the indices are the byperbolic logarithms; so that $n$ is always the hyperbolic logarithm of $(2 \cdot T 1 \mathrm{~S} \mathrm{sc})^{\mathrm{n}}$. But in the common logarithms $r=10$; so that the common logarithm of any number, is the index of that power of 10 which is equal to the said number; so the common logarithm of $\mathrm{x}=10^{n}$, is $n$ the index of the power of 10 ; for example, 1000 , being the $3 \mathrm{~d}$ power of $1(1)$, has 3 for its logarithm; and if 50 be $=10^{\mathrm{s} t \mathrm{~s}}$, then is 1.69597 the common logarithm of 50 . And hence it follows that this decimal scries of terms

VกL.I.

\section{O G}

$1000,100,10,1, \cdot 1,0 \mathrm{~J}, .001$, or $10^{3}, 10^{2}, 10^{1}, 10^{\circ}, 10^{-1}, 10^{-2}, 10^{-3}$, have $3,2,1,0,-1,-2,-3$, respectively for the logarithms of those terms.

The logarithm of a number contained between any two terms of the first series, is included between the two corresponding terms of the latter; and therefore that $\log 2-$ rithm will consist of the same indes, whether positive or negative, as the smaller of those two terms, together with a decimal fraction, which will always be positive. So the number 50 falling between 10 and 100 , its logarithm will fall between 1 and 2 , being indeed equal to 1.69897 near$1 y$ : also the number ' 05 falling between the terms $\cdot 1$ and 01 , its logarithm will fall between -1 and -2 , and is indeed $=-2+\cdot 69897$, the index of the less term together with the decimal 69897 . The index is also called the characteristic of the logarithms, and is alway's an integer, either positive or negative, or else $=0$; and it shows what place is occupied by the first significant figure of the given number, either above or. below the place of units, being in the former case + or pasitive; in the latter - or negative.

When the iudes of a logarithm is negative, the sign is commonly set over it, to distinguish it from the decimal part, which, being the logarithm found in the tables, is always positive: so $-2+69897$, or the logaritbm of $\cdot 05$, is written thus $\overline{2} \cdot 69897$. But on some occasions it is convenient to reduce the whole expression to a negative form; which is done by making the cbaracteristic less by 1 , and taking the arithmetical complement of the decimal, that is, beginning at the left band, subtract each figure from 9, except the last significant figure, which is subtracted from 10; so shall the remainders form the logarithm wholly negative: thus the logaritim of $\cdot 05$, which is $\overline{2} \cdot 6989 \mathrm{~T}$ or $-2+6989 \mathrm{~T}$, is also expressed by -1.30103 , which is all negative. It is also sometimes thought more culvenient to express such logarithms entirely as positive, namely by only joining to the tabular decimal the complement of the index to 10 ; and in this way the above logarithm is expressed by 8.69897 ; which is only increasing the indices in the scale by 10 .

The Properties of Logarithms, - From the definition of logarithms, either as being the indices of a series of geometricals, or as the indices of the power of the same root, it follows, tbat the multiplication of the numbers will answer to the addition of their logaritbms; the division of numbers, to the subtraction of their logarithms; the raising of powers, to the multiplying the logarithm of the ruot by the index of the power; and the extracting of roots, to the dividing the logarithm of the given number by the index of the root required to be extracted.

So, 1st,

Log, $a b$ or of $a \times b$ is $=\log . a+\log . b$,

Log. Is or of $3 \times 6$ is $=\log .3+\log .6$,

Log. $5 \times 9 \times 73$ is $=\log .5+\log .9+\log .73$.

Secondly,

Log. $a \div b \quad$ is $=$ lng. $a-\log . b$,

Log. $18 \div 6$. is $=\log .18-\log .6$,

$\log .79 \times 5 \div 9$ is $=\log .79+\log .5-\log .9$

Log. $\frac{1}{2}$ or $1 \div 2$ is $=1.1-1.2=0-1.2=-1.2$,

Log $\frac{1}{f}$ or $1 \div n$ is $=1.1-1 . n=-1 . n$.

$5 \mathrm{C}$ 


\section{O G}

Thirdly,

Log. $r^{\mathrm{n}}$ is $=n 1 . r ; \log . r^{\frac{1}{n}}$ or of $\mathrm{Z} r$ is $=\frac{1}{n} 1 . r$;

Log. $r^{\frac{m}{n}}$ is $=\frac{m}{n} 1 . r ; \log \cdot 2^{6}$ is $=61.2 ; \log \cdot 2^{\frac{\pi}{3}}$ or

of $\sqrt[3]{2}$ is $=\frac{i}{3} 1.2 ;$ and $\log .2^{\frac{3}{5}}$ is $=\frac{3}{5} 1.2$.

So that any number and its reciprocal have the same logarithm, but with contrary signs; and the sum of the logarithms of any number and its reciprocal, or complement, is equal to 0 . For other particulars, see the Introduction to my Logarithms.

History and Construction of Logarithms.-The properties of logarithms hitherto mentioned, or of aritbmetical indices to powers or geometricals, with their various uses and properties, as above-mentioned, are taken notice of by Stifelius, in his Arithmetic; and indeed they were not unknown to the ancients; but they come all far short of the use of Ingarithms in trigonometry, as first discovercd by John Napier, baron of Merchiston in Scotland, and published at Edinburgh in 1614, in his Mirifici Logarithmorum Canonis Descriptio; which containcd a large canon of logarithms, with the description and uses of them; but their construction was reserved till the scusc of the learned concerning his invention should be known. This work was translated into English by the celebrated Mr. Edward Wright, and published by his son in 1616 . In the year 1619 , Robert Napicr, son of the inventor of logarithms, published a new edition of his late father's work, together with the promised construction of the logarithms, with other miscellaneous pieces written by his father and Mr. Briggs. And in the same year, 1619, Mr. John Speidell published his New Logarithns, being an improved furm of Napier's.

All these tables were of the kind that have since been called hyperbolical, because the numbers express the areas between the asymptote and curve of the hyperbola. And logarithms of this kind were also soon after published by several other persons; as by Ursinus in 1619, Kepler in $162+$, and some others.

On the first publication of Napier's logarithms, Henry Brigas, then professor of geometry in Gresham-college in Lnndon, immediately applied himself to the study and improvement of them, and soon published the logarithms of the first 1000 numbers, but on a new scale, which he had invented, viz, in wlich the logarithm of the ratio of 10 to 1 is 1 , the logarithm of the same ratio in Napier's system being 2.3025 \& \& ; and in 1624, Briggs published his Arithmetica Logarithmica, containing the logarithms of 30,000 natural numbers, to 14 places of figures besides the index, in a form that Napier and lye bad agreed upon together, being the present form of logarithms; also in 1633 was published, to the same extent of figures, his 'l'rigonometria Britannica, containing the natural and logarithmic sincs, tangents, \& $c$.

With varions and gradual improvements, logarithms were alsn published successively, by Gunter in 1620 , Wingate in 1624, Henrion in 1626, Miller and Norwood in 1631, Cavalerius in 1632 and 1643 , Vlacq and Rowe in 1 (i33. Frobenius in 1634, Newton in 165s, Caramuel in 1670, Slicrwin in 1706, Gardiner in 1742, and Dodson's Autilogarithmir. Canon in the same yeur, Taylor in 1793, besides many others of lesser note; not to mention the accurate and comprehensive tables in the Tables Portative in 1793 , \&ce, those of Borda and Delambre in 1801 , and

\section{O G}

in my own Logaritbms lately published, where a complete history of this science may be seen, with the rarious ways of constructing them, that have been invented by different authors.

In Napier's construction of logarithms, the natural numbers, and their logarithms, as he sometimes called them, or at other times the artificial numbers, are supposed to arise, or to be generated, by the mutions of points, describing two lines, of which the one is the natural number, and the other its logaritlum, or artificial. Thus, he conceived the line or length of the raclius to be described, or run over, by a point moving along it in such a manner, that in equal portions of time it generaterd, or cut off, parts in a decreasing geometrical progression, leaving the sereral remainders, or sines, in geometrical progression also; while another point described equal parts of an indefinite line, in the same equal portions of time; so that the respective sums of these, or the whole line generated, were always the arithmeticals or logarithms of the aforesaid natural sines. In this idea of the generation of the logarithms and numbers, Napier assumed 0 as the logarithm of the greatest sine or radius; and next be limited his system, not by assuming a particular value to some assigned number, or part of the radius, bit by supposing that the two generating points, which, by their motions along the two lines, described the natural numbers and logarithms, should bave their velocities equal at the beginning of those lines. And this is the reason that, in bis table, the natural sines and their logarithms, at the complcte qualrant, have equal differences or increments; and this is also the reason why bis scale of logarithms happens accidentally to agree with what have since been called the hyperbolical logarithms, which have likewise numeral differences equal to those of their natural numbers at the beginning; except only that these latter increase with the watural numbers, while his on the contrary decrease; the lugarithm of the ratio of 10 to 1 being the same in both, namely $2.3025 \$ 509$ \&c.

Having thus limited his system, Napier procecds, in the posthumous work of 1619 , to explain his construction of the logarithmic canon. This he effects in various ways, but chiefly by generating, in a very-casy manner, a scries of proportional numbers, and their aritlumeticals or logarithms; and then finding, by proportion, the logarithns to the natural sincs from those of the natural numbers, among the original proportionals; a particular account of which may be seen in my book of logarithms ahove mentioned.

The nicthods above alluded to, relate to Napjer's or the hyperbolical system of logarithms, and indeed are in a manner peculiar to that kind of them. But in an appendix to the posthumous work, mention is made of other methods, by which the common logarithms, agreed on by him and Briggs, maly be constructed, and which it apjears were written after that agreement. One of these methods is as follows: Having assumed 0 for the longarithm of 1 , and 10000 \&c, for the logarithm of 10 ; this logarithm of 10 , and the successire quotients, are to be divided ten times by 5 , by which divisions there will be obtained these other ten logarithms, namely 2000000000,400000000 , $80000000,16000000,3200000,640000,125000,25600$, 5120,1024 ; then this last logarithm, and its "quoticuts, being divided ten times by 2 , will give these other ten lo. garithms, 
viz, 5 t $2,256,128,64,32,16,8,4,2,1$. Also the numbers answcring to these twenty logarithms are to be found in this manner, viz, Extract the 5 th root of 10 (with ciphers), then the 5th root of that root, and so on for ten continual extractions of the 5th root: so shall these ten roots be the natural numbers belonging to the first ten logarithms above found, in flividing continually by 5 . Next, out of the last 5 th rout is to be extracted the square root, then the square root of this last root, and so on for ten successive extractions of the square root: so shall these last ten roots be the natural numbers corresponding to the logarithms or quotients arising from the last ten divisions by the number 2 . And from these twenty logarithms, $1,2,4,8, \& c$, and their natural numbers, the author observes that other logarithms and their numbers may be formed, namely by adding the logarithms, and multiplying their corresponding numbers. But, besides the inmense labour of this method, it is evident that this process would generate rather an antilogarithmic canon, such as Dodson's, than the table of Briggs.

Napier next mentions another method of deriving a few of the primitive numbers and their logarithins, namcly, by taking continually geometrical means, first betwcen 10 and 1 , then between 10 and this meall, and again betwcen 10 and the last mean, and so on; and then taking the arithmetical means between their corresponcling logarithms.

He then lays down various relations between numbers and their logarithms, such as, that the products and quoifents of nunbers, answer to the sums and differences of their logarithins; and that the powers and roots of numbers, answer to the products and quotients of the logarithms when nultiplied or divided by the index of the power or root, \&c; as also that, of any two numbers, whose logarithms are given, if each number be raised to the nower denoted by the logarithm of the other, the two results will be equal; thus, if $x$ be the logarithm of any number $\mathrm{x}$, and $y$ the logarithm of $\mathrm{x}$, then is $\mathrm{x}^{\mathrm{y}}=\mathrm{x}^{\mathrm{x}}$. Napier then adverts to another method of making the lugarithms to a few of the prime integer numbers, which is well adapted for the construction of the common table of logarithms: this method easily follows from what has been said above, depending on this property, that the logarithm of any number in this scale, is one less than the number of places or figures contained in that power of the given number whose exponent is 10000000000 , or the logarith $\mathrm{m}$ of 10 , at least as to integer numbers, for they really differ by a fraction, as is shown by Mr. Briggs in his illustrations of these properties; printed at the end of the Appendix to the Construction of Logarithms.

Kepler gave. a construction of logarithrns somewhat varied from Napier's. His work is divided into two parts : In the tirst, he raises a regular and purely mathematical system of proportions, and the measures of them, demonstrating both the nature and principles of the construction of logarithms, which he calls the Nleasures of Ratios: and in the second part, he applies those principles in the actual construction of his table, which contains only 1000 numbers and their logarithms. The fundamental principles are briefly these: That at the beginning of the logarithms, their increments or differences are equal to those of the natural numbers: that the natural numbers may be considered as the decreasing cosines of iticreasing arcs: and that the secants of those arcs at the beginuing have the same difierences as the cosines, and thercfore the same differences as the logarithms. Then, since the secants are the reciprucals of the cusines if the same arcs, from the foregoing principles, he establishes the firllowing method of raising the first 100 logarithms, to the numbers $1000,999,99 \mathrm{~s}, \mathrm{sc}$, to 900 ; viz, in this manner: Divide the radius 1000 , increased with seven ciphers, by each of these numbers separately, and the quotients will be the secants of those arcs which have the divisors for their cosines; continuing the division to the Sth figure, as it is in that place only that the arithmetical and gcometrical means differ. Then by adding continually the arithmetical means between every two successive secauts, the sums will be the series of logarithms. Or by adding continually every "two secants, the successive. sums will be the series of the doublc logarithms. He then derives all the other logarithms from these first 100 , by common principles.

Briggs first adverts to the methods mentioned above, in the Appendix to Napier's Construction, which methods were common to both these authurs, and had doubtless been jointly agreed on by them. He then gives an example of computing a logarithm by the property, that the logarithm is one less than the number of places or figures contained in that power of the given number whose exponent is the logarithm of 10 with ciphers. Briggs next treats of the other general method of finding the logarithms of prime numbers, which he thinks is an easier way tban the former, at least when many figures are required. 'This method consists in taking a great number of continued geometrical meanś between $j$ and the given number whose logarithın is required; that is, first extracting the square root of the given number, then the root of the 1st root, the root of the $2 \mathrm{~d}$ root, the root of the $3 \mathrm{~d}$ root, and so on, till the last root shall exceed $I$ by a very small decimal, greater or less according to the intended number of places to be in the logarithm sought: then finding the lugarithm of this small number, by easy methods described alterwards, he doubles it as often as he made extractions of the square ruot, or, which is the same thing, he inultiplies it by such power of 2 as is denoted by the said number of extractions, and the result is the required lugarithm of the given number; as is evident from the nature of logarithms.

But, as the extraction of so many roots is a very trouLlesome operation, our author devises some ingenious methods of abridging that labour, chiefly by a proper application of the several orders of the differences of numbers, forming the first instance of what may be called The Differential Method; for a particular description of which, sce my Logarithms, above quoted, pa. 65 , sc.

Mr. James Gregory, in his Vera Circuli Hyperbolse Quadratura, printed at Padua in 1667 , having approximated to the hyperbolic asymptotic spaces by means of a series of inscribed and circumscribed polygons, from thence shows how to compute the logarithms, which are analogous to the areas of those spaces: and thus the quadrature of the hyperbolic spaces became the same thing as the computation of the logarithms. He here alsu lays down various methods to abridge the computation, with the assistance of some properties of numbers themselves, by which the logarithms of all prime numbers under 1000 may be computed, each by one multiplication, two divisions, and the extraction of the square rout. And the same subject is furtber pursued in his Exercitationes Geometrica." In this latter place, hc first tinds an algcbraic expression, in an infinite $5 \mathrm{C} \stackrel{2}{2}$ 
series, for the logarithm of $\frac{1+a}{1}$, and then likewise for the logarithm of $\frac{1}{1-a}$; and as the one series bas all its terms positive, while those of the other are alternately positive and negative, by adding the two together, every $2 \mathrm{~d}$ term is cancelled, and the double of the other terms gives the logarithm of the product of

$\frac{1+a}{1}$ and $\frac{1}{1-a}$, or the logarithm of $\frac{1+a}{1-a}$, that is, of the ratio of $1-a$ to $1+a$ :

Thus, he finds,

$$
\begin{aligned}
& \text { first } a-\frac{1}{2} a^{2}+\frac{1}{3} a^{3}-\frac{1}{4} a^{4} \& \mathrm{c}=\log \text {. of } \frac{1+a}{1}, \\
& \text { and } a+\frac{1}{2} a^{2}+\frac{1}{3} a^{3}+\frac{1}{4} a^{4} \& \mathrm{c}=\log \text {. of } \frac{1}{1-a}, \\
& \text { theref. } 2 a+\frac{2}{3} a^{3}+\frac{2}{5} a^{5}+\frac{2}{7} a^{7} \& \mathrm{c}=\log \text {. of } \frac{1+a}{1-a} .
\end{aligned}
$$

Which may be accounted Mr. James Gregory's method of making logarithms.

In 1668, Nicholas Mereator published his Logarithmotechnia, sive Methodus Construendi Logarithmos, nova, accurata. et facilis; in which he delivers a new and ingenious method for computing the logarithms on principles purely arithmetical; and here, in his modes of thinking and expression, he closely follows the celebrated Kepler, in his writings on the same subject; accounting logarithm, as the measures of ratios, or as the number of ratiunculæ contained in the ratio which any number bears to unity. Purely from these priniciples then, the number of the equal ratiunculæ contained in some one ratio, as of 10 to 1 , being supposed given, our author shows how the logarithrn, or measure, of any other ratio may be found. But this, however, only by-the-bye, as not being the principal method he intends to teach, as his last and best. Having shown then, that these logarithms, or numbers of small ratios, or measures of ratios, maly be all properly represented by numbers, and that of 1 , or the ratio of equality, the logarithm or measure being always 0 , the logarithm of 10 , or the measure of the ratio of 10 to 1 , is most conveniently represented by 1 with any number of ciphers; he then proceeds to show how the measures of all other ratios may be found from this last supposition: and he explains these principles by some examples in numbers.

In the latter part of the work, Mercator treats of bis other method, given by an infinite series of algebraic terms, which are collected in numbers by common addition only. He here squares the hyperbola, and finally finds that the hyperbolic logarithm of $1+a$, is equal to the infinite series $a-\frac{1}{2} a^{2}+\frac{1}{3} a^{3}-\frac{1}{4} a^{4} \& \mathrm{c}$; which may be considered as Mlercator's quadrature of the hyperbola, or his general expression of an hyperbolic logarithm, in an infinite series.

And this metlitd was further improved by Dr. Wallis, in the Philos. Trans. for the year 1668 ; by showing that the hyp. $\log$. of the ratio of 1 to $1-a$ is equal to the infinite seriss $a+\frac{1}{2} a^{2}+\frac{1}{3} a^{3}+\frac{1}{4} a^{4}+\& c$. The celebrated Newton invented also the same series for the quadrature of the hyperbola, and the construction of logarithms, and that before they were given by Gregory and Mercator, though unknown to one anotluer, as appears by his letter to Mr. Oldenburg, dated October 24, 1676. 'The explanation and construction of the lengarithms are also further pursued in his Flusions, publislsed in $\mathbf{1 7 5 6}$ by $\mathrm{Mr}$. Colson.

Dr. Ilalley, in the Philos. Trans. for the year 1695, gave a very ingenious essay on the construction of logarithms, entitled, "A most compendious and facile methou for constructing the logarithms, and exemplified and demonstrated from the nature of numbers, without any regard to the hyperbola, with a speedy method for finding the number from the given logarithm."

Instead of the more ordinary definition of logarithms, viz, 'numeroruin proportionalium æquidifferentes comites," the learned author adopts this other, "numeri rationum exponentes,' as better adapted to the principle on which logarith uns are bere constructed, considering them as the number of ratiunculæ contained in the given ratios whose logarithms are in question. In this way he first arrives at the logarithmic series before given by Newton and others, and afterwards, by various combinations and sections of the ratios, be derives others, converging still faster than the former. Thus he found the logarithms of several ratios, as beluw, viz, when multiplied by the modulus peculiar to the scale of logarithms,

$$
\begin{aligned}
& q-\frac{1}{2} q^{2}+\frac{1}{3} q^{3}-\frac{1}{4} q^{4} \& c \text {, the log. of } 1 \text { to } 1+q \text {, } \\
& q+\frac{1}{2} q^{2}+\frac{1}{3} q^{3}+\frac{1}{4} q^{4} \delta c c \text {, the log. of } 1 \text { to } 1-q \text {, } \\
& \frac{x}{a}-\frac{x^{3}}{2 a^{2}}+\frac{x^{3}}{3 a^{3}}-\frac{x^{2}}{4 a^{3}} \& c \text {, the log. of } a \text { to } b \text {, or } \\
& \frac{x}{b}+\frac{x^{2}}{2 b^{3}}+\frac{x^{3}}{3 b^{3}}+\frac{x^{4}}{4 b^{4}} \& \mathrm{c} \text {, the saine log. of } a \text { to } b \text {, or } \\
& \frac{2 x}{z}+\frac{2 x^{3}}{3 z^{3}}+\frac{2 x^{5}}{5 x^{3}}+\frac{2 x^{7}}{7 z^{7}} \& \mathrm{c} \text {, the same log. of } a \text { to } b \text {, } \\
& \frac{x^{3}}{2 z^{3}}+\frac{x^{4}}{4 z^{4}}+\frac{x^{6}}{6 z^{6}}+\frac{x^{8}}{8 z^{8}} \& c \text {, the log. of } \sqrt{ } a b \text { to } \frac{\mathrm{r}}{2} z \text {, or } \\
& \frac{1}{y^{3}}+\frac{1}{3 y^{6}}+\frac{1}{5 y^{10}}+\frac{1}{7 y^{14}} \& c \text {, the same } \log \text { of } \sqrt{ } a b \text { to } \frac{x}{z} z \text {; }
\end{aligned}
$$

where $a, b, q$, are any quantities, and the values of $x, y, z$, are thus, viz, $x=b-a, z=b+a, y=a b+\frac{\mathrm{r}}{4} z^{2}$.

Dr. Halley also, the first who performed the reverse of the problem, by assigning the number to a given logarithm; viz,

$$
\begin{aligned}
& \frac{b}{a}=1+l+\frac{x}{2} l^{2}+\frac{1}{2 \cdot 3} l^{3}+\frac{1}{2 \cdot 3 \cdot 4} l^{4} \& \mathrm{c} \text {, or } \\
& a=1-l+\frac{x}{2} l^{2}-\frac{1}{2 \cdot 3} l^{3}+\frac{1}{2 \cdot 3 \cdot 4} l^{4} \& c \text {. where } l \text { is the }
\end{aligned}
$$

logarithm of the ratio of $a$ the less, to $b$ the greater of any two terms.

Mr. Abraham Sharp of Yorkshire made many calculations and improvements in logarithms, \&c. The most remarkable of these were, his quadrature of the circle to 72 places of figures, and his computation of logarithms to 61 figures, viz, for all numbers to 100 , and for all prime numbers to 1100 .

The celcbrated Mr. Roger Cotes gave to the world a learned tract on the nature and construction of logarithıns: this was first printed in the Philos. Trans. No. $3 \hat{\partial} \mathrm{S}$, and afterwards with his Harmonia Mensurarum in 1722 , under the title Lngometria. This tract however has justly been complained of, as very obscure and intriente, and the principle is something between that of kiepler and the method of fluxions. Hle invented the terms modulus and modular ratio, this being the ratio

of $1+\frac{1}{1}+\frac{1}{2}+\frac{1}{2 \cdot 3}+\frac{1}{2 \cdot 3 \cdot 4}+\frac{1}{2 \cdot 3 \cdot 4 \cdot 5}$ \&c to 1 or of 1 to $1-\frac{1}{1}+\frac{1}{2}-\frac{1}{2.3}+\frac{1}{2.3 .4}-\frac{1}{4.3 .4 .3} \& \mathrm{c}$; that is the ratio of 2.718281828 .459 \&c to 1 , or the ratio of 1 to $0.367979441171 \& \mathrm{c}$; 
the modulus of any system being the measure or logarithm of that ratio, whicls in the hyp. logarithms is 1 , which in Briggs's or the common logarithms is 0.434294481903 \&c.

The learned Dr. Brook Taylor gave another method of computing logarithms in the Philos. Trans. No. 352, which is founded on these three principles, viz, Ist, That the sum of the logarithms of any two numbers is the logarithm of the product of those numbers; $2 d$, That the logarithm of 1 is 0 , and consequently that the nearer any number is to 1 , the nearer will its logaritbm be to 0 ; $3 \mathrm{~d}$, That the product of two numbers or factors, of which the one is greater, and the other less than 1 , is nearer to 1 , than that factor is which is on the same side of 1 with itself; so of the two numbers $\frac{2}{3}$ and $\frac{4}{3}$, the product $\frac{8}{9}$ is less than 1 , but yet nearer to it than $\frac{2}{3}$ is, which is also less than 1.-And on these principles he founds an ingenious, though not very obvious, approximation to the logarithms of given numbers.

In the Philos. Trans. a Mr. Jobn Long gave a method of constructing logarithms, by means of a small table, something in the manner of one of Briggs's methods for the sanie purpose.

Also in the Philos. Trans. vol. 61, a tract on the construction of logarithms is given by the ingenious $\mathrm{Mr}$. William Jones. In this method, all numbers are considered as some certain powers of a constant determined root: thus, any number $x$ is considered as the $z$ power of any root $r$, or $x=r^{z}$ is takeu as a general expression for all numbers in terms of the constant root $r$ and a variable exponent $z$. Now the index $z$ being the logarithm of the number $x$, therefore to find this logarithm, is the same thing as to find what power of the radix $r$ is equal to the number $x$.

An elegant tract on logarithms, as a comment on Dr. Halley's method, was also given by Mr. Jones in his Synopsis Palmariorum Matheseos, publishedin the year 1706 .

In the year 1742, Mr. James Dodson published his Antilngarithmic Canon, containing all logarithms under 100,000 , and their corresponding natural numbers to cleven places of figures, with all their differences and the proportional parts; the whole arranged in the order contrary to that used in the common tables of numbers and logarithms, the exact logarithms being here placed first, and their corresponding nearest numbers in the columns opposite to them.

And in $1767, \mathrm{Mr}$. Andrew Reid published an "Essay on logarithms," in which he shows the computation of logarithms from principles depending on the binomial theorem, and on the nature of the exponents of powers, the logarithms of numbers being here considered as the exponents of the powers of 10 . In this way he brings out the usual series for logarithns, and exemplifies Dr. Halley's construction of them. But for the particulars of this, and the methods given by the other authors, we must refer to the historical preface to my treatise on logarithms.

Besides the authors above-mentioned, many others have treated on the subject of logarithms; ameng the principal of whom are, Leibnitz, Euler, Maclaurin, Wolfius, Keill, Lagrange, aud professor Simson in an ingenious geometrical tract on logarithms, contained in his posthumous works, elegantly printed at Glasgow in the year 1776 , at the expense of the learned Earl Stanhope, and by his lerdship disposed of in presents anoug gentlemen most eminent for mathematical learning.

For the description and uses of logarithms in numeral calculations, with the various methods of constructing them, see the historical intreduction to my Logarithms pa. 124 et seq.

Briggs's or Common Logariturs, are those that have 1 for the logarithm of 10 , or which have 0.4342944819 \&c for the modulus; as has been explained above.

Hyperbolic Loganitir Ms, are those that were computed by the inventor Napier, and called also sometimes natural logarithins, having 1 for their modulus, or $2 \cdot 302585092994$ $\& \mathrm{c}$ for the logarithm of 10 . These have since been called hyperbolical logarithms, because they are analogous to the areas of a right-angled hyperbola between the asymptotes and the curve. See Logarithims, also HyPERBola and Asymptotic Space.

Logistic Logaritums, are certain logarithms of sexagesimal numbers or fractions, useful in astronomical calculations. The logistic logarithm of any number of seconds, is the difference between the common logarithm of that number and the logarithm of 3600 , the seconds in I degree.

The chief use of the table of logistic logarithms, is for' the ready computing a proportional part in minutes and seconds, when two terms of the proportion are minutes and seconds, or hours and minutes, or other such sexagesimal numbers. See the introd. to my Logarithms, pa. 14.4.

Imaginary LogarithM, a term used in the Log. of imaginary and negative quantities; such as $-a$, or $\sqrt{ }-a^{2}$ or $a \sqrt{ }-1$. The fluents of certain imaginary expres. sions are also imaginary logarithms; as of $\frac{\dot{x}}{x \sqrt{-1}}$, or of $\frac{a \dot{x}}{c x \sqrt{ }-2}$, \&c. See Euler Analys. Infin. vol. i. pa. 72, 74 . It is well known that the expression $\frac{\dot{x}}{x}$ represents the fluxion of the logarithm of $x$, and therefore the fluent of $\frac{\dot{x}}{x}$ is the logarithm of $x$; and hence the fluent of $\frac{\dot{x}}{x \sqrt{-1}}$ is the imaginary logarithm of $x$.

However, when these imaginary logarithms occur in the solutions of problems, they may be transformed into circular arcs or sectors; that is, the imaginary logarithm, or imaginary hyperbolic sector, becomes a real circular sector. See Bernoulli Oper. tom. i, pa. 400, and pa. 512. Maclaurin's Fluxions, art. 762 . Cotes's Harmon. Mens. pa. 45. Walmesley, Anal. des Mes. pa.63.

LOGARITHMIC, or Logistic CURve, a curve so called from its properties and uses, in explaining and constructing the logarithms, because its ordinates are in geometrical progression, while the abscisses are in arithmetical progression; so that the abscisses are as the logarithms of the corresponding ordinates. And hence the curve will be constructed in this manner: Upon any right line, as an axis, take the equal parts $A B, B C, C D, \& C$, or the arithmetical progression $A B, A C, A D, \& C$; and at the points $A, B, C, D, \& c$, erect the perpendicular ordinates $A P, B Q$, CR, DS, \&C, in a geometrical progression; so is the curve line drawn tlurough all the points $P, Q, R, s, \& c$, the logarithmic, or logistic curve; so called, because any absciss $\triangle B$, is as the logarithm of its ordinate $B Q$. So that the axis $\mathrm{ABC} \& \mathrm{C}$ is an asymptote to the curve.

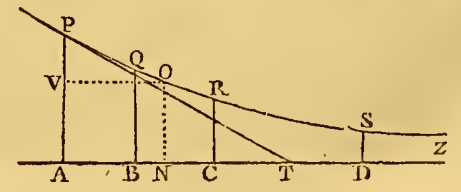


Hence, if any absciss $A \mathbb{N}=x$, its ordinate $\mathrm{No}=y$, $A \mathrm{P}=1$, and $a=$ a certain constant quantity, or the modulus of the logarithms; then the equation of the curve is $x=a \times \log$. of $y=\log . y^{\text {a }}$.

And if the fuxion of this equation be taken, it will be $\dot{x}=\frac{a \dot{y}}{a} ;$ which gives this proportion,

$$
\dot{y}: \dot{x}:: y: a
$$

but in any curve $\dot{y}: \dot{x}:: y$ : the subtangent $\mathbf{A ~ T}$; therefore the subtangent of this curve is cvery-where equal to the constant yuantity $a$, or the modulus of the logarithms.

To find the Area contnined between two ordinates. Here the fluxion of the area $\dot{A}$ or $y \dot{x}$ is $y \times \frac{a \dot{y}}{y}=a \dot{y}$; and the correct fluent is $\mathrm{A}=a \times(\mathrm{AP}-y)=a \times(\mathrm{AP}-\mathrm{NO})$ $=a \times \mathrm{PV}=\mathrm{AT} \times \mathrm{PV}$. That is, the area $\mathrm{APON}$ between any two ordinates, is cyual to the rectangle of the constant subtangent and the difference of the ordinates. And hence, when the absciss is infinitely long, or the farther ordinate equal to nothing, then the infinitely long area $\mathrm{APZ}$ is equal $\mathrm{AT} \times \mathrm{AP}$, or double the triangle $\mathrm{APT}$.

To find the Solid formed by the curve revolved about its xis Az'. The fluxion of the solid is $\dot{s}=p y^{2} \dot{x}=$ $\eta y^{2} \times \frac{a j}{y}=p a y j$, where $p$ is $=3.1416$; and the correct fluent is $s=\frac{1}{2} p a \times\left(\mathrm{AP}^{2}-y^{2}\right)=\frac{\mathrm{T}}{2} p \times \mathrm{AT} \times\left(\mathrm{AP}^{2}-\mathrm{NO} \mathrm{O}^{2}\right)$, which is half the difference betwcen two cylinders of the common altitude $a$ or $\mathrm{AT}$, and the radii of their bases $\mathrm{AP}$, No. And hence, supposing the solid infinitely long towards $z$, where $y$ or the ordinate is nothing, the infiuitely long solid will be equal to $\frac{1}{2} p a \times A^{2}=\frac{1}{2} p \times A T \times A P^{2}$, or half the cylinder on the same base and its altitude AT.

It has bcen said that Gunter gave the first idea of a curve whose abscisses are in arithmetical progression, while the corresponding ordinates are in geometrical progression, or whose abscisses are the logarithms of their ordinates; but $I$ so not find it noticel in any part of his writings. This curve was afterwards considered by others, and named the logarithmic or logistic curve, by Huygens, in his Dissertatio de Causa Gravitatis, where he enumerates all the principal properties of $i t$, showing its analogy to logarithms. Nany other learned men have also treated of its properties; particularly Ja. Gregory, in the preface to his Geometriæ Pars Universalis \&c; Le Seur and Jacquicr, in their Comment on Newton's Principia; Dr. John Keill, in the elegant little Tract on Logarithms subjoined to his edition of Euclid's Elements ; and Francis Maseres Esq. Cursitor Baron of the Exchequer, in his ingenious Treatise on Trigonometry: see also Bernoulli's Discourse in the Acta Eruditorum for the year 1696, pa.216; Guido Grando's Denonstratio Theorematum Huygeneanorum circa Logisticam seu Loga rithmicam Lineam; and Emerson on Curve Lines, pa. 19--1t is indced ratler extraordinary that this curve was not sooner announced to the public, since it results immediately from Napier's manner of conceiving the generation of logarithms, by only supposing the lines which represent the natural numbers as placed at right angles to that upon which the logarithms are taken.

This curve greatly facilitates the conception of logarithms, and affords an almost intuitive proof of the very important property of their fluxions, or very small increments, namely', that the fluxion of the number is to the fluxion of the logarithm, as the number is to the subtangent; as alsn this property, that if three numbers be taken iery nearly equal, so that their ratios may differ but it little from a ratio of equality, as the three numbers $10000000,10000001,10000002$, their differences will be very nearly proportional to the logarithms of the ratios of those numbers to each other: all which follows fron! the logarithmic arcs differing very little from their chords, when they are taken very small. And the coustant subtangent of this.curve is what was afterwards by Cotes called the modulus of the system of logarithms.

LogARITHMIC, or Logistic Spiral, is a curve constructed as follows. Divide the arch of a circle into any number: of equal parts $\mathrm{AB}, \mathrm{BD}, \mathrm{DE}$, \& $\mathrm{C}$; and upon the radii drawn to the points of division take $\mathrm{c} b, \mathrm{c} d, \mathrm{ce}, \& \mathrm{c}$, in a geometrical progression; sa is the curve $\mathrm{s} b d e$ \& $\mathrm{c}$ the logarithmic spiral; being thus called, because it is evident that $\mathrm{AB}, \mathrm{AD}, \mathrm{AE}$, \&c , being arithmeticals, are as the logarithms of $\mathrm{cA}, \mathrm{c} b, \varepsilon d, \mathrm{ce}, \& \mathrm{c}$ which are geo-

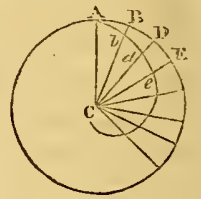
metricals; and a spiral, because it winds continually about the centre c, coming continually nearer, but without ever really falling into it. In the Philos. Trans. Dr. Halley bas happily applied this curve to the division of the meridian line in Mercator's chart. See alo Cotes's Harmonia Mens., Guido Grando's Demonst. Theor. Huygen, the Acta Erudit. 1691, and Emerson's Curves, \&c.

LOGISTICS, or LOGISTICAL $\Lambda_{\text {R ITI METIC, a name }}$ sometimes employed for the arithmetic of sexagesimal fractions, used in astronomical computations. This nume was perhaps taken from a Grcets trcatise of Barlæmus, a monk, who wrote a book on Sexagesimal Mlultiplication, which he called logistic. Vossius places this author about the year 1350 , but he mistakes the work for a treatise on algebra. The same term however bas been used for the rules of computations in algebra, and in other species of arithmetic: witness the logistics of Vieta and other writers.

Shakerly, in his Tabula Britannicx, has a table of logarithms adapted to sexagesimal fractions, which he calls logistical logarithms; and the expeditions arithmetic, obtained by means of them, -he calls logistical arithmetic.

Logistic al Curre, Line, or Spiral, the same as the Logarithmic ; which see.

LONG (RogER), D. D. master of Pembroke-hall in Cambridge, Lowndes's professor of astronomy in that university, \&c. He was author of a well-known and machapproved treatise of astronomy, and the inventor of a remarkably curious astronomical machine. This was a hollow splere, of 18 feet diameter, in which more than 30 persons might sit converiently. Within sice the surface, which represented the heavens, was painted the stars and constellations, with the zodiac, meridians, and axis paralke to the axis of the world, upon which it was easily turned round by a winch. He died, December $16,17 \% 0$, at 91 ycars of age.

A few years before his death, Mr. Jones gave some anecdotes of Dr. Long, as follows: "He is now in the 88 th year of his age, and for his ycars regete and active. He was lately put in nomination for the office of vice-chancellor: he executed that trust once before, I think in the year 1737 . He is a very ingenious person, and sometimes very facetious. At the public commencement, in the year 1713 , Dr. Creene (mister of Bennet-college, and afterwards bishop of IHy) being then vice-chancellor, Mr. Long was pitched upon for the tripos performance; it was. 


\section{I, O N}

$[751]$

L O N

witty and bumourous, and has passed through divers editions. Some that remembered the delivery of it, told me, that in addressing the vice-chancellor (whom the university wags usually styled Miss Greene), the tripos-orator, being a native of Norfolk, and assuming the Norfolk dialect, instead of saying Domine Vice-Cancellarie, archly pronounced the words thus, Domina Vice-Cancellaria; which occasioned a general smile in that great auditory. His friend the late Mr. Bonfoy of Ripton related to me the following little incident: "That he and Dr. Lung walking together in Cambridge in a dusky evening, and coming to a short post fixed in the pavement, which Mr. Bonfoy in the midst of chat and inattention took to be a boy standing in his way, he said in a hurry, 'Get out of my way, boy!' ' That boy, Sir,' said the doctor very calruly and slily, "is a post-boy, who turns out of his way for nobody.' I could recollect several other ingenious repartees if there svere occasion. One thing is remarkable, he never was a hale and hearty man, always of a tender and delicate constitution, yet took great care of it: his common drink water; he always dines with the fellows in the hall. Of late years he left off eating flesh; in the room thereof, puddings, vegetables, \&cc; sometimes a glass or two of wine."

LONGIMETRY, the art of measuring lengths or distances, both accessible and inaccessible, forming a part of what is called heights and distances, being an application of geometry and trigonometry to such measurements. As to accessible lengths, they are easily measured by the actual application of a rod, a chain, or wheel, or some other measure of length.

But inaccessible lengths require the practice and properties of geometry and trigonometry, either in the measurement and construction, or in the computation. For example, Suppose it were required to know the length or distance between the two places $\mathrm{A}$ and $\mathrm{B}$, to which places there is free access, but not to the intermediate parts, on account of water or some other impediment; measure therefore, from $\mathrm{A}$ and $\mathrm{B}$, the distances to any convenient place $\mathrm{c}$, which suppose to be thus, viz. $\mathrm{AC}=735$, and $\mathrm{EC}=810$ links; and let the angle at $\mathrm{C}$, taken with a theodolite or other instrument, be $55^{\circ} 40^{\prime}$. From these measures the length or distance $A \mathrm{~B}$ may be determined, either by geometrical construction, or by trigonometrical computation. Thus, first lay down an angle $\mathrm{c}=55^{\circ} 40^{\prime}$, and upon its legs set off, from any convenient scale of equal parts, $\mathrm{C} \Lambda=735$, and $\mathrm{CB}=8+0$; then measure the distance between the points $A$ and $\mathbf{B}$ by the same scale of eqqual parts, which will be found to be 740 nearly. Or
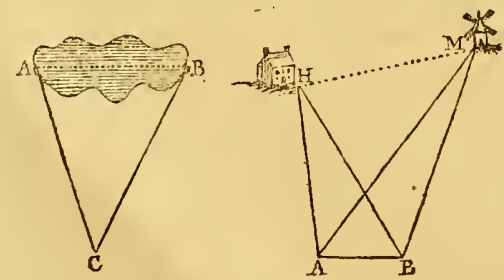

thus by calculation,

$840180^{\circ}-55^{\circ} 40^{\prime}=124^{\circ} 20^{\prime}$, its half $62^{\circ} 10^{\prime}$, 735

Sum $\quad 1575$

Dif. 105
Tang. $62^{\circ} 10^{\prime}$

Tang. $7111_{1}^{1 \pi}$

s. Sum or $\angle \Lambda=99^{\circ} 21 \frac{1}{5} \frac{\pi}{4}$

to s. $\angle \mathrm{c}=5540$.

So $\mathrm{BC}=840$

To $\mathrm{AB}=741 \cdot 2$

For a 2d Example-Suppose it were required to find the distance between two inaccessible objects, as between the house and mill, is and $M$; first measure any convenient line on the ground, as A B, 300 yards; then at the station $\mathrm{A}$ take the angles $\mathrm{BAM}=5 \mathrm{~S}^{\circ} 20^{\prime}$, and $\mathrm{MA} \mathbf{H}=$ $37^{\circ}$; also at the station $B$ take the angles $A B H=$ $53^{\circ} 30^{\prime}$, and $11 \mathrm{BM}=45^{\circ} 15^{\prime}$; hence the distance or length M may be found, either by geometrical construction, or by trignnometrical calculation, thus :

First draw a line A B of the given length of 300 , by a convenient scale of equal parts; then at the point $A$ lay down the angles BAM and MA $A$ of the magnitudes above given; and also at the point $\mathrm{B}$ the given angles $\mathrm{ABn}$ and H BM; then by applying the length $\mathrm{HM}$ to the same scale of equal parts, it is found to be nearly 480 yards.

Otherwise, by calculation. First, by adding and subtracting the angles, there is found as below :

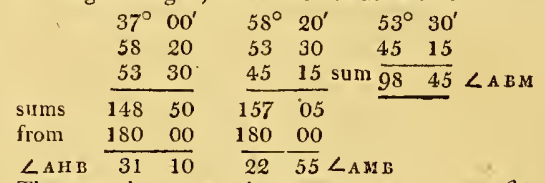

Then, as sin. $\mathbf{A H B}: \sin$. $\mathbf{A B H}:: \mathbf{A B}: \mathbf{A H}=465.9776$, and, as sin. $\mathrm{AMB}: \sin$ А $\mathrm{BM}:: \mathrm{AB}: \mathrm{AM}=761 \cdot 4655$, their sum is $\overline{1227 \cdot 4431}$ and their diff. 295.4879

Then as $\operatorname{sum} A M+A H$ : to dif. $A M-A H:$ : tang. $\frac{T}{2} A H M+\frac{T}{2} A M 11=71^{\circ} 30^{\prime}$, to tang. $\mathrm{I}$ AHM $-\frac{\mathrm{T}}{2} \mathrm{AMH}=3544$

the dif. of which is $A M H=\overline{3546 \text {. }}$

Lastly, as s. Ами : s. мА II : : Ан : нм $=479.7933$, the distance sought.

LONGITUDE of the Earth, is sometimes used to denote its extent from wcst to east, according to the direction of the equator : by which it stands contra-distinguished from the latitude of the earth, which denotes its extent from one pole to the other.

Longitude of a Place, in Geography, is its longitudinal distance from some first meridian, which is assumed arbitrarily by different nations: the English call the first meridian, that which passes over the Royal Observatory at Greenwich; the French take the meridian of Paris for the first meridian; the Spaniards, Madrid; and so most other nations reckon that the first meridian which passes over their metropolis.

The longitude of any place is therefore an arch of the equator intercepted between the meridian of that place, and the first meridian.

LONGITUDE in the Heavens, as of a star, \&c, is an arch of the ecliptic, counted from the beginning of Aries, to the place where it is cut by a circle perpendicular to it, and passing through the place of the star.

LONGITUDE of the Sun or Star from the next equinoctial point, is the degrees they are distant from the beginning of Aries or Libra, either before or after then ; which can never excecd 180 degrecs. 
Lon G I Tu DE, Geocentric, Heliocentric, \&c, the longitude of a planet as seen from the earth, or from the sun. See the respective terms.

LoNGITUDE, in Navigation, is the distance of a ship, or place, east or west, from some other place or meridian, counted in degrees of the equator. When this distance is counted in leagues, or miles, or in degrees of the meridian, and not in those proper to the parallel of latitude, it is usually called departure.

An easy practicable method of finding the longitude at sea, is the only thing wanted to render the art of navigation perfect, and is a problem that has greatly perplexed mathematicians for the last two centuries: accordingly most of the commercial nations of Europe have offered great rewards for the discovery of it; and in consequence very considerable advances have been made towards a perfect solution of the problem, especially by the English.

In the year 1598, the government of Spain offered a reward of 1000 crowns for the solution of this problem; and soon after the States of Holland offered 10 thousand florins for the same. Encouraged by such offers, in 1635, M. John Morin, professor of mathematics at Paris, proposed to cardinal Richlieu, a method of resolving it ; and though the commissioners, who wcre appointed to examine this method, on account of the imperfect state of the lunar tables, judged it insufficient, cardinal Mazarin, in 1645, procured for the author a pension of 2000 livres.

In 1714 an act was passed in the British parliament, allowing $2000 l$. towards making experiments; and also offering a reward to the person who should discover the longitude at sea, proportioned to the degree of accuracy that might be attained by such discovery ; viz, a reward of $10,000 l$. if it determines the longitude to one degree of a great circle, or 60 geographical miles; $15,000 l$. if it determines the same to two-thirds of that distance; and $20,000 l$. ' if it determines it to half that distance; with other regulations and encouragements. 12 Anu. cap. 15. See also stat. 14 Geo: 2 , cap. 39 , and 26 Geo. 2, cap. 25. But, by stat. Geo. 3, all former acts concerning the longitude at sea are repealed, except so much of them as relates to the appointment and authority of the commissioners, and such clauses as relate to the publishing of nautical almanacs, and other useful tables; and it enacts, that any person who shall discover a method for finding the longitude by means of a time-keeper, the principles of which not having hitherto been made public, shall be entitlerl to the reward of $5000 l$. if it shall enable a ship to keep her longitude, during a voyage of 6 months, within 60 geographical miles, or one degree of a great circle; to $7500 l$. if within 40 geographical miles, or two-thirds of a degree of a great circle; or to a reward of $10,000 l$. if within 30 geographical miles, or balf a degree of a great circle. But if the method shall be by means of improved solar and lunar tables, the author of them shall be entitled to a reward of $5000 l$. if they show the distance of the moon from the sun and stars within $15^{\prime \prime}$ of a degree, answering to about $7^{\prime}$ of longitude, after making an allowance of half a degree for the errors of observation, and after comparison with astronomical observations for a period of $18 \frac{\pi}{2}$ years, or during the period of the irregularities of the lunar motions. Or that in case any other method shall be proposed for finding the longitude at sea, besides those before-mentioned, the author shall be entitled to 5000 l. If it shall determine the longitude within one degree of a great circle, or 60 grographical miles; to 7500$)$. if within two-thirds of that distance; and to $10,000 l$. if within balf the said distance.

Accordingly, many attempts have been made for such discovery, and several ways proposed, with various degrees of success. These however have been chiefly directed to methods of determining the difference of time between any two points on the earth; for the longitude of any place being an arc of the equator intercepted between two meridians, and this arc being proportional to the time required by the sun to more from the one meridian to the other, at the rate of 4 minutes of time to one degree of the arc, it follows that the difference of time being known, and turned into degrees according to that proportion, it will give the longitude.

This measurement of time has been attempted by some persons by means of clocks, watches, and other automata: for if a clock or watch were contrived to go uniformly at all seasons, and in all places and situations; such a machine being regulated, for instance, to London or Greenwich time, would always show the time of the day at London or Greenwich, wherever it should be carried to; then the time of the day at this place being found by observations, the difference between these two times woyld give the difference of longitude, according to the proportion of one degree to 4 minutes of time.

Gemma Frisius, in his tract De Principiis Astronomiæ et Geographiæ, printed at Antwerp in 1530, it secms, first suggested.the method of finding the longitude at sea by means of watches, or time-keepers; which machines, be says, were then but lately invented. And soon after, the same was attempted by Metius, and some others; but the state of watch-making was then too imperfect for that purpose. Dr. Hooke and Mr. Huygens also, about the year 1664, applied the invention of the pendulum-spring to watches; and employed it for the purpose of discovering the longitude at sea. Some disputes however between Dr. Hooke and the English ministry prevented any experiments from being made with watches constructed by him; but many experiments were made with some constructed by Huygens; particularly Major Holmes, in a voyage from the coast of Guinea in 1665 , by one of these watches predicted the longitude of the island of Fuego to a great degree of accuracy. This success enconraged Huygens to improve the structure of his watches, (sec Philos. Trans. for May 1669); but experience soon convinced him, that unless methods could be discovered for preserving the regular motion of such machines, and preventing the effects of heat and cold, and other disturbing causes, they could never answer the intention of discovering the longitude, and on this account his attempts failed.

'The first person who turned his thoughts this way, after the public encouragement held out by the act of 171 , was Henry Sully, an Englishinan; who, in the same year, printed at Vienna, a small tract on the subject of watchmaking; and afterwards removing to Paris, he employed himself there in improving time-kecpers for the discovery of the longitude. It is said he greatly diminished the friction in the machine, and rendered uniform that which remained: and to him is principally to be attributed what is yet known of watch-making in France: for the celebrated Julier le Roy was his pupil, and to him owed most of his inventions, which he afterwards perfected and executed: and this gentleman, with his son, and M. Berthoud, are the principal persons in France who have 
turned their thoughts this way since the time of Sully. Several watches made by these last two artists, lave been tried at sea, it is said with good success, and many accounts have been published of these trials, at considerable length.

In the year 1726 our countryman, Mr. John Harrison, produced a time-keeper of his own construction, which did not err above one second in a month, for 10 years together : and in the year $\mathbf{1 7 3 6}$ he had a machine tried in a voyage to and from Lisbon; which was the means of corrccting an error of almost a degree and a half in the computation of the ship's reckoning. In consequence of this success, Mr. Harrison received public encouragement to proceed, and he made thrce other time-keepers, each more accurate than the former, which were finished successively in the years 1739,1758 , and 1761 ; the last of which proved so much to his own satisfaction, that be applied to the commissioners of longitude to have this instrument tried in a voyage to some port in the WestIndies, according to the directions of the statute of the 12th of Anne above cited. Accordingly, Mr. William Harrison, son of the inventor, embarked in November $\mathbf{1 7 6 1}$, on a voyage for Jamaica, with this 4 th time-keeper or watch; and on his arrival there, the longitude, as shown by the time-keeper, differed but one geographical mile and a quarter from the true longitude, deduced from astronomical observations. The same gentleman returned to England, with the time-keeper, in March 1762; when he found that it had erred, in the 4 months, no more than $1^{\prime} 54^{\prime \prime} \frac{r}{2}$ in time, or $28 \frac{5}{8}$ minutes of longitude ; whereas the act requires no greater exactness than 30 geographical miles, or minutes of a great circle, in such a voyage. $\mathrm{Mr}$. Harrison now claimed the whole reward of $20,000 l$, offered by the said act: but some doubts arising in the minds of the commissioners, conccrning the true situation of the island of Jamaica, and the manner in which the time at that place had been found, as well as at Portsmouth; and it being farther suggested by soine, that though the time-keeper happened to be right at Jamaica, and after its return to England, it was by no means a proof that it had been always so in the intermediate times; another trial was therefure proposed, in a voyage to the island of Barbadoes, in which precautions were taken to obviate as many of these objections as possible. Accordingly, the commissioners previously sent out proper persons to make astronomical observations at that island, which, when compared with other corresponding ones made in England, would determine, beyond a doubt, its true situation: and Mr. William Harrison again set out with his father's timekeeper, in March 1764, the watch having been compared with equal altitudes at Portsmouth, before lig set out, and he arrived at Barbadoes about the middle of May; where, on comparing it again by equal altitudes of the sun, it was found to show the difference of longitude, between Portsmouth and Barbadoes, to be $3^{\mathrm{h}} 55^{\mathrm{m}} 3^{\mathrm{s}}$; the true difference of longitude between these places, by astronomical observations, being $3^{\text {h }} 54^{\mathrm{m}} 20^{3}$; so that the error of the watch was $43^{\prime}$, or $10^{\prime} 4.5^{\prime \prime}$ of longitude. In consequence of this, and the former trials, Mr. Harrison received one moiety of the reward offered by the 12th of Queen Anne, after explaining the principles on which his watch was constructed, and delivering this as well as the three former to the commissioners of the longitude, for the use of the public: and he was promised the other moiety of the reward, when other time-kecpers should be made, on the Voz. I. same principles, either by himself or others, performing equally well with that which he had last made. In the mean time, this last time-keeper was sent down to the Royal Observatory at Greenwich, to be tried there under the direction of the Rev. Dr. Maskelyne, the astronomerroyal. But it did not appear, during this trial, that the watch went with the regularity that was expected; from which it was apprehended, that the performance even of the same watch, was not at all times equal; and consequently that little certainty could be expected in the performance of different ones. Moreover, the watch was now found to go faster than during the voyage to and from Barbarloes, by 18 or 19 seconds in 24 hours : but this circumstance was accounted for by Mr. Harrison; who informs us that he had altered the rate of its going by trying some experiments, which he had not time to finish before he was ordered to deliver up the watch to the Board. Soon after this trial, the commissioners of longitude agreed with Mr. Kendal, one of the watch-makers appointed by them to receive Mr. Harrison's discuveries, to make another watch on the same construction witli this, to determine whether such watches could be made from the account which Mr. Harrison nad given, by other persons, as well as himself. The event proved the affirmative; for the watch produced by Mr. Kendal, in consequence of this agreement, went considerably better than Mr Harrison's did. Mr. Kendal's watch was sent out with Capt. Cook, in his $2 \mathrm{~d}$ voyage towards the south pole and round the globe, in the years $1772,1773,1774$, and 1775; when the only fault found in the watch was, that its rate of going was continually accelerated; though in this trial, of 3 years and a half, it never amounted to $14^{\prime \prime \frac{1}{2}}$ a day. The consequence was, that the house of commons in 1774 , to whom an appeal had been made, were pleased to order the $2 \dot{d}$ moiety of the reward to be given to Mr. Harrison, and to pass the act above-mentioned. Mr. Harrison had also at different times received some other sums of money, as encouragements to him to continue his endeavours, from the Board of Longitude, and from the India Company, as well as from many individuals. Mr. Arnold, Mr. Mudge, Mr. Erushaw, Mr. Hardy, and some other persons have since also made several very good watches for the same purpose.

Others have proposed various astronomical methods for finding the longitude, which chicfly depend on having an ephemeris or almanac suited to the meridian of some place, as Greenwich for instance, to which the nautical almanac is adapted, that shall contain for every day computations of the times of all remarkable celestial motions and appearances, as adapted to that meridian. So that, if the hour and minute be known when any of the same phenomena are observed in any other place, whose longitude is desired, the difference between this time and that to which the time of the said phenomenon was calculated and set down in the almanac, will be known, and consequently the difference of longitude also becomes known, between that place and Greenwich, allowing at the rate of 15 degrees to an hour.

Now it is easy to find the time at any place, by means of the altitude or azimuth of the sun or stars; which time it is necessary to find by the same means, both in these astronomical modes of determining the longitude, and in the former by a time-keeper; and it is the difference between that time, so determined, and the time at Grcenwich, known cither by the time-kceper or by the astrono-

$$
5 \mathrm{D}
$$




\section{O N}

[ 754$]$

\section{O N}

mical observations of celestial phenomena, which gives the difference of longitude, at the rate above-mentioned. But here the great difficulty is, that there are only a few phenomena proper, or capable of being thus observed; for all slow motions, such as belong to the planet Saturn for instance, are quite excluded, as affording too small a difference, in a considerable space of time, to be properly observed; indeed there are no phenomena in the heavens proper for this purpose, except the eclipses or motions of Jupiter's satellites, and the eclipses or motions of the moon, viz, such as her distance from the sun or certain fixed stars lying near her path, or her longitude or place in the zodiac, \&c.

1st, That by the eclipses of the moon is very easy, and sufficiently accurate, if they did but happen often, as every night. . For at the moment when the beginning, or middle, or end of an eclipse is observed by a telescope, there is no more to be done but to determine the time by observing the altitude or azimuth of some known star; which time being compared with that in the tables, set down for the happening of the same phenomenon at Greenwich, gives the difference in time, and consequently of longitude sought. But as the beginning or end of an eclipse of the moon cannot generally be observed nearer than one minute, and sometimes 2 or 3 minutes of time, the longitude cannot certainly be determined by this method, from a single observation, nearer than one degree of longitude. However, by two or more observations, as of the beginning and end \&c, a much greater degree of exactness may be attained.

$2 \mathrm{~d}$, The moon's place in the zodiac is a phenomenon more frequent than that of her eclipses; but then the observation of it is difficult, and the calculus perplexed and intricate, by reason of two parallaxes; so that it is hardly practicable, to any tolerable degree of accuracy.

3d, But the moon's distances from the sun, or certain fixed stars, are phenomena to be observed many times in almost every night, and afford a good practical method of determining the longitude of a ship at almost any time; either by computing, from thence, the moon's true place, to compare with the same in the almanac; or by comparing her observed distance itself with the same as there set down.

It is said that the first person who recommended the finding the longitude from this observed distance between the moon and some star, was John Werner, of Nuremberg, who printed his annotations on the first book of Ptolemy's Geography in 1514. And the same thing was recommended in 1524, by Peter Apian, professor of mathematics at Ingolstadt; also about 1530, by Oronce Finé, of Briançon; and the same year by the celebrated Kepler, and by Gemma Frisins, at Antwerp; and in 1560, by Nonius or Pedro Nunez.

Nor were the English mathematicians behind hand on this head. In $1665 \mathrm{Sir}$ Jonas Moore prevailed on king Charles the $2 \mathrm{~d}$ to erect the Royal Observatory at Greenwich, and to appoint Mr. Flamsteed his astronomical observer, with this express command, that he should apply himsclf with the utmost care and diligence to the rectifying the table of the motions of the heavens, and the places of the fixed stars, in order to find out the longitude at sea, tor perfecting the art of navigation. And to the fidelity and industry with which Mr. Flamstecd executed lis commission, it is that we are chicfly indebted for that cusious theory of the moon, which was afterwards formicd by the immortal Newton. This incomparable philosopher made the best possible use of the observations with which he was furnished; but as these were interrupted and imperfect, his theory would sometimes differ from the heavens by 5 minutes or morè.

Dr. Halley bestowed much time on the same object; and a starry zodiac was published under his direction, containing all the stars to which the moon's appulse can be observed; but for want of correct tables, and proper jnstruments, he could not proceed in making the necessary observations. In a paper on this subject, in the Philos. Trans. No.421, he expresses his hope, that the instrument just invented by Mr. Hadley might be applied to taking angles at sea with the desired accuracy. This great astronomer, and after him Lacaille, and others, have reckoned the best astronomical method for finding the longitude at sea, to be that in which the distance of the moon from the sun or from a star is used; for the moon's daily motion being about 13 degrees, her hourly mean motion is above half a degree, or one minute of a degree in two minutes of time; so that an error of one minute of a degree in position will produce an error of 2 minutes in time, or half a degree in longitude. Now from the great improvements made by Newton in the theory of the moon, and more lately by Euler and others on his principies, Professor Mayer of Gottingen, was enabled to calculate lunar tables more correct than any former ones; having so far succeeded as to give the moon's place within one minute of the truth, which has been proved by a comparison of the tables with the observations made at the Greenwich observatory by the late Dr. Bradley, and by Dr. Maskelyne, the astronomer royal; and the same have been still further improved under his direction, by the late Mr. Charles Mason, by several new equations, and the whole computed to tenths of a second. 'These new tables, when compared with the above-mentioned series of observations, a proper allowance being made for the unavoidable error of observation, seem to give always the moon's longitude in the heavens correctly within 30 seconds of a degree; which greatest error, added to a possible error of one minute in taking the moon's distance from the sun or a star at sea, will at a medium only produce an error of 42 minutes of longitude. Further successive improvements in them have since been made, by the labours of Messieurs Lagrange, Laplace, Lalande, Delambre, Burg, and Vince; by which means it is thought the astronomical tables have been brought to the most correct form that the present state of astronomical knowledge will permit.

To facilitate the use of the tables, Dr. Maskelyne proposed a nautical ephemeris, the scheme of which was adopted by the commissioners of longitude, and first executed in the year 1707 ; since which time it has been regularly continued, and published as far as for the 'year 1816. But as the rules that were given in the appendix to one of those publications, for correcting the eflects of refraction and parallax, were thought too difficult for gcneral use, they have bcen reduced to tables. So that, by the help of the ephemeris, these tables, and other's that are also provided by the Board of Longitude, the calculations relating to the longitude, which could not be performod by the most expert mathematician in less than 4 hours, may now be completed with great case and accuracy in half an hour.

As this method of determining the longitude depends on thic use of the tables amually published for this purpose, 
those who wish for further information, are referred to the instructions that accompany them, and particularly to those that are annexed to the Tables requisite to be used with the Astronomical and Nautical Ephemeris, 3d edit. 1802. See Lunar Method, below.

4th, The phenomena of Jupiter's satellites have commonly been preferred to those of the moon, for finding the longitude; because they are less liable to parallaxes than these are, and besides they afford a very commodious observation whenever the planet is above the horizon. Their motion is very sivift, and must be calculated for every hour. 'These satellites of Jupiter were no sooner announced by Galileo, in his Syderius Nuncius, first printed at Venice in 1610 , than the frequency of their eclipses recornmended them for this purpose; and among those who treated on this subject, none was more successful than Cassini. This great astronomer published, at Bologna, in 1688 , tables for calculating the appearances of their eclipses, with directions for finding the longitudes of places by them ; and being invited to France by Louis the 14th, he there, in the year 1693, published more correct tables of the same. But the mutual attractions of the satellites rendering their motions very irregular, those tables soon became useless for this purpose; insomuch that they require to be renewed from time to time; a service which has been performed by several ingenious astronomers, as Dr. Pound, Dr. Bradley, M. Cassini the son, and more especially by $\mathrm{Mr}$. Wargentin, whose tables are much esteemed, and have been published in several places, as well as in the Nautical Almanacs for 1771 and 1779.

Now, to find the longitude by these satellites; with a good telescope observe some of their phenomena, as the conjunction of two of them, or of one of them with Jupiter, \&cc; and at the same time find the hour and minute, from the altitudes of the stars, or by means of a clock or watch, previously regulated for the place of observation; then, consulting tables of the satellites, observe the time when the same appearance happens in the meridian of the place for which the tables are calculated; and the difference of time, as before, will give the longitude.

The eclipses of the first and second of Jupiter's satellites are the most proper for this purpose; and as they happen almost daily, they afford a ready means of determining the longitude of places at land, having indeed contributed much to the modern improvements in geography; and if it were possible to observe them with proper telescopcs, in a ship under sail, they would be of great service in ascertaining its longitude from time to time. To obviate the inconvenience to which these observations are liable from the motions of the ship, a Mr. Irwin invented what he called a marine chair; this was tried by Dr. Maskelyne, in his voyage to Barbadoes, when it was not found that any bemefit could be derived from the use of it. And indeed, considering the great power requisite in a telescope proper for these observations, and the violence, as well as irreguIarities in the motion of a ship, it is to be feared that the complete management of a telescope on ship-board, will always remain among the desiderata in this part of nautical science. $\Lambda$ nd further, since all methods that depend on the phenomena of the heavens, have also this other defect, that they cannot be observed at all times, this renders the improvement of time-keepers an object of the more importance.

Many other schemes and proposals have been made by different persons, but most of them of very little or no use; sucl as by the space between the flash and report of a great gun, proposed by Messrs. Whiston and Ditton; and another proposed by Mr. Whiston, by means of the inclinatory or dipping needle; besides a method by the variation of the magnetic-needle, \&c, \& c.

For approximate constructions for clearing the lunar distances from the effects of parallax and refraction, the reader may consult Dr. Kelly's Spherics and Nautical Astronomy, \&c. And for more detajled information respecting the above, and other methods of ascertaining the longitucle, see Dr. Mackay's and Mr. Mendoza's valuable works; also Vince's Astronomy, vol.2, pa. 515, \&c, and O. Gregory's Astronomy, pa. 446 to 470.

LONGITUDE of Motion, is a term used by Dr. Wallis for the measure of motion, estimated according to its line of direction; or it is the distance or length gone through by the centre of any moving body, as it moves on in a right line.-The same anthor calls the measure of any motion, estimated according to the line of direction of the vis motrix, the altitude of it.

LONGOMIONTANUS (CHRISTIAN), a learned astronomer, born in Denmark in 1562, in the village of Longomontum, whence he took his name. Vossius, by mistake, calls him Christopher. He was the son of a poor ploughman, and he was obliged to suffer, during his studies, all the hardships to which he could be exposed, dividing his time, like the philosopher Cleanthes, between the cultivation of the earth and the lessons be received from the minister of the place. At length, at 15 years of age he stole away from his family, and went to Wiburg, where there was a college, in which he spent 11 years; and though he was obliged to earn his livelihood as he could, his close application to study, enabled him to make a great progress in learning, particularly in the mathematical sciences.

Hence he went to Copenhagen; where the professors of that university soon conceived a very high opinion of him, and recommended him to the celebrated Tycho Brahé, with whom Longomontanus lived 8 years, and was of great service to him in his observations and calculations. At length, being very desirous of obtaining a professor's chair in Denmark, Tycho Brahé consented, with some difficulty, to his leaving him, giving him a discharge filled with the highest testimonies of his esteem, and furnishing him with money for the expense of his long journey from Germany, whither Tycho had retired. He accordingly obtained a professorship of mathematics in the university of Copenbagen in 1605, the duty of which he discharged very worthily till his death, which happened in 1647 , at 85 ycars of age.

Longomontanus was author of several works, which show great talents. in mathematics and astronomy. The most distinguished of which, is his Astronomica Danica, first printeo in 4to, 1621, and afterwards in folio in 1640, with augmentations. He amused himself with endeavouring to square the circle, and pretended that he had made the discovery of it; but our countryman Dr. John Pell attacked him warmly on that subject, and proved that he was mistaken.-It is remarkable that, obscure as his villago and father were, he contrived to dignify and eternize tliem both; for he took his name from his village,' and in the title page to some of his works he wrote himself Christianus Longomontanus Severini filius, his father's name being Severin or Severinus.

LOXODROMIC CuRve, or SPIRAL, is the same as the rhumb line, or path of a ship sailing always on the same $5 \mathrm{D} 2$ 
course in an oblique direction, or making always the same angle with every meridian. It is a species of Jogarithmic spiral, described on the surface of the sphere, having the meridians for its radii.

LOXODROMICS, the art or method of oblique sailing, by the loxodromic or rhumb line.

LOZENGE, an oblique-angled parallelogram; being otherwise called a rhombus, or a rhomboides.

LUBIENIETSKI (STANISLAUS), a Polish gentleman, born at Cracow, in 1623, and educated with great care by his father. He was learned in astronomy, and became a celebrated Socinian minister. He took great pains to obtain a toleration from the German princes for his Socinian brethren. His endeavours however were all in vain; being himself persecuted by the Lutheran ministers, and banished from place to place; till at length he was banished out of the world, with bis two daughters, by poison, in 1675 , his wife narrowly escaping.

We have, of his writing, A History of the Reformation in Poland; and a Treatise on Comets, entitled Theatrum Cometicum, printed at Amsterdam in 2 volumes folio; which is a most elaborate work, containing a minute historical account of every single comet that had been seen or recorded.

LUCIDA CoRON in the northern crown. See Conon a Borealis.

Lucida Hydra. See Cor Hydra.

LuCIDA LYR the constellation Lyra.

LUCIFER, a name given to the planet Venus, when she appears in the morning before sunrise.

LUDOLPH van Ceulew. See Ceulen.

LUMINARIES, a term used for the sun and moon, by way of eminence, for their extraordinary lustre, and the great quantity of light they give us.

LUNA, the Moon; which see.

LUNAR, something relating to the moon.

Lunar Cycle, or Cycle of the Moon. See Cycle.

Lun A Distance, in nautical astronomy, a term denoting the distance of the moon from the sun, or from a fixed star lying nearly in the line of its path. The measurement of the apparent lunar distances, and the determination of the true lunar distances from thence, are of great use in determining the longitude at sea, or on land.

Luna M Method for the Longitude, a method of keeping or finding the longitude by means of the moon's motions, particularly by her observed distances from the sun and stars; for which; see the article LONGITUDE.

This method consists in taking and reducing the distance between the moon and the sun or a fixed star, in order to find the longitude at sea; which has been already explained generally under the article LONGITUDE; but the great utility and importance of the subject require here a further and more minute explanation of its principles and operations, with an account of the different methods that have been devised for obtaining the solution; and I shall conclude the article with a very simple projection, invented by Dr. Kelly, which is found sufficiently correct for the common purposes of nautical practice, and even where the greatest accuracy is required, it is found useful as a guide or check to calculation.

The lunar method of finding the longitude at sea, is the greatest modern improvement in navigation. In order to form a clear and comprehensive idea of the subject, it should be first considered, that in the most practical inethods of finding the longitude at sea by celestial observatious, the moon is the chief instrument; for the quickness of her motion renders her peculiarly well adapted for measuring small portions of correspondent time.-Now, as she is seen in the same part of the heavens nearly, at the same instant of absolute time, from all parts of the earth where she is visible, and as she is continually and seusibly changing her place, it is evident that if two correspondent observers vere to note the precise moment of their respective times, when she is seen in any particular part of the heavens the difference between those times would show the difference of longitude.

In every method of finding the longitude by the moon, the first object is to be able to ascertain the part of the heavens where she is :-this is easily seen at the time of her eclipses, or at the occultation of a fixed star, and these were naturally the first methods resorted to ; but they occur too seldom for general use: the moon's place however may be marked with equal precision, by taking her distance from some fixed object, whose latitude and longitude are known; the stars in or near the zodiac are preferred, as the nearer such objects are to the moon's patll, the quicker will be her motion with respect to them : and though her motion is not unifurm, yet, during the short space of time that she is near any star, it may be considered as such.

It may be observed, that if two persons under different meridians were to mark the moon's place, and also their relative times of observation, they might thence tell their difference of longitude; but they could not communicate their observations sufficiently soon for practical purposes; but even admitting the possibility of this, it were necessary that the longitude of one place should be known, in order to determine that of the other. Now the Nautical Almanac is calculated to supply all these wants. This work may be considered a perpetual observer, that communicates universally and instantaneously certain celestial appearances, as they take place at Greenwich observatory. Here the distances are given between the moon and the sun, and certain remarkable stars in or near the zodiac, for every three hours; and any intermediate distance, or time, may be thence found by the rule of proportion, with sufficient accuracy. If therefore, under any meridian, a lunar distance be observed, the difference between the time of observation and the time in the almanac, when the same distance was to take place at Greenwich, will show the longitude. For example, if the observed distance between the sun and monn be $50^{\circ}$ at 8 o'clock, but by the almanac the same distance of $50^{\circ}$ will take place at Greenwich at 6 , it is evident that the difference between the observed and computed time is 2 hours, and therefore the longitude is $30^{\circ}$; and it is also clear that the longitude is east, the time being most advanced at the place of observation.

A method so apparently simple must have been long since adopted, but for two difficulties which occurred: one the want of proper instruments; which now bas been happily supplied by the iuvention and subsequent improvement of Hadley's quadrant; and the other, correct lunar tables; for the inoon, though so near and so conspicuous to the earth, has always perplexed astronomers more than any other celestial body. The various inequalities of her motions were never properly understood, until Sir Isaac Newton discovered the physical laws which govern them; and from his thcory professor Mayer formod 
lunar tables, from which those tables, in the Nautical Almanac, of the lunar distances were calculated, under the direction of Dr. Maskelyne for many years. In 1806 the French Board of Longitude published new Lunar Tables, calculated by Du Burg, from the theory of Laplace and the observations of Dr. Maskelyne; and from these tables the lunar distances in the Nautical Almanac of 1813 , and the following years, are computed.

The above two difficulties having been obviated, a third seems still to remain; and though this is in some measure removed by the application of the Nautical Almanac and requisite tables, yet still the calculation is more tedious than might be wished; nor is it possible to render it much shorter, as the problem necessarily comprehends the solutions of two spheric triangles: this arises from the circumstance of the observed distances between the heavenly bodies not being the true distances; for the altitucies of those bodies are more or less affected both by refraction and parallax; and though these effects only operate in a vertical direction, yet that which changes the altitude of two bodies, must also cbange the distance between them. This is evident from the consideration, that the al titude of a celestial object is an arc of an aziruuth circle intercepted between the object and the horizon, and as all azimuth circles incline gradually to each other, from the horizon to the zenith, where they meet, it is plain that the more two bodies are apparently raised, the less will be their apparent distance asunder, and the contrary.

It has been already stated that the heavenly bodies are raised by refraction, and depressed by parallax; and that these effects are greatest in the horizon, and gradually diminish to the zenith, where they become nothing. Refraction depends on altitude alone, but parallax depends on both altitude and distance from the earth. All celestial objects, except the moon, are more affected by refraction than by parallax, and therefore appear above their true places; but the moon is always seen, excepting in the zenith, below her true place, being more affected by parallax than by refraction, on account of her proximity to the earth.

These effects of parallax and refraction, though counteracting each other, seldom do it so equally as to render all correction unnecessary. Sometimes the apparent distance is nearly a whole degree more or less than the true distance; and the principal cause of so great a difference is the moon's parallax; for this body, which is the chief guide to the longitude, is also the great cause of error in the distances, and is therefore the principal object of correction.

In making a lunar observation, four persons are usually employed, one of whom takes the distance, two the altitudes, and the fourth notes the time. These things should be performed at the same instant; and if the observation be repeated several times, and a mean taken, the work is likely to be the more correct; and great care is here necessary, for an error in this part of the operation, particularly in taking the distance, will pervade the subsequent parts of the work, and will of course produce a wrong solution. The manner of adjusting the instruments, and of making the observations, is best taught by practice. 'Those who wish for written instructions on the subject, are referred to the British Mariner's Guide by Dr. Maskelyne, to Dr. Mackay's book on the Longitude, or to professor Vince's Practical Astronomy.
Of correcting the Altitudes of the observed Objects. - When a lunar observation is made, the first object is to clear the altitudes from semidiameter, dip, refraction, and parallax, according to usual practice. 'The moon's parallax in altitude must next be calculated : by saying, "As radius is to the sine of her zenith distance, so is the sine of her horizontal' parallax (as given in the Nautical Almanac) to the sine of her parallax in alitude.

In correcting the moon's altitude, an allowance should be made for the augmentation of her semidianeter, which gradually takes place from the horizon to the zenith. This increase is given, in the 4 th of the Requisite Tables, for every 5 degrees of altitude, which correction is to be added to her horizontal semidiameter given in the Nautical Almanac.

The augmentation of the moon's semidiameter is caused by her being nearer to the spectator in the zenith than in the horizon, by a semidiameter of the earth-for the apparent magnitude of a body is in the inverse ratio of its distance from the observer; and as the earth's semidiameter bears a very sensible proportion to the moon's distance, she is seen under the greatest angle in the zenith, which angle gradually diminishes to the horizon.

There are other corrections of the altitudes, which may be necessary in cases of peculiar nicety, but which are seldom noticed at sea. These are, an allowance for the contraction of the vertical semidiameters of the sun and moori by refraction; a correction of the moon's parallax, supposing the earth an oblate spheroid; a correction for the refraction according to the actual state of the atmosphere, as shown by a thermometer and barometer, and not according to the mean astronomical refraction which is commonly used. These corrections, though perbaps necessary towards the perfection of this problem, being very small, and frequently counteracting each other, are generally considered of little consequence in nautical practice, where greater errors are unavoidable.

From the corrected Altitudes to find the true Distance.-It is easy to conceive that by a lunar observation, the three sides of a spheric triangle are measured in the beavens, which sides are the apparent co-altitudes of the observed bodies, and their apparent distance asunder.

The co-altitudes or zenith distances being corrected, the question is, to find the true distance between the observed bodies; but here only two things are given, and therefore it cannot be performed until the angle at the zenith is known, which is determined from the three given sides of the triangle, by the rules of spheric trigonometry. See KELIY's Spherics, pa. 182.

As the effects of parallax, refraction, \&.c, operate only in a vertical direction, it is evident tlat the corrections of the zenith distances, or containing sides, will not change the included anglc at the zenith; and therefore three things are now known, namely, the corrected zenith distances and the included angle, whence the other side is determined by spherics, and this side is the true distance sought.

$A$ general View of the different Methods of working the lunar Observätions.-Few problems have ever been mole investigated and studicd, than that of clearing the lunar distances, and many ingenious methods have been devised for contracting the operation. These methods are founded on some of the following general principles. The first is spheric trigonometry, as before explained; the second is 


\section{U U}

the doctrine of proportional errors, by which the effects that the errors in the altitudes produce on the distance, are solved by fluxions, or by the differential calculus; and a third principle has been lately discovered, which is founded on the properties of a quadrangle inscribed in a circle, as explained and exemplified by the inventor, Dr. Andrew, in his Astronomical and Nautical Tables.

Various methods of working the lunar observations have been devised, chiefly by Halley, Euler, Mayer, Maskelyne, Dunthorn, Lyons, Witchell, Burrow, Borda, Wales, Mackay, Kelly, Gerrard, Andrew, and Mendoza. The methods of the two lasi authors appear to be the most concise, but all are sufficiently correct, and seamen generally prefer such as they have first learnt. It may indeed be observed, that operations which appear the most concise, are not always the most expeditiously performed; as much depends on the number and variety of tables required, and the manner of applying them. No method lias hitherto obtained an exclusiye preference over the rest, nor does it appear possible to reduce the calculation to a conciseness to answer the general wants or wishes of seamen; and hence, other modes have been devised of obtaining approximate solutions by projection or graphic operation.

Graphic Operations.-The first graphic method for clearing the distances, was that published by Lacaille, called the Chassis de Reduction, which has since been copied by Lalande, Mackay, and others. It is an orthographic projection, consisting of a great number of lines accurately drawn, and various scales for obtaining the different corrections.

On similar principles, and for the same purpose, the late Mr. James Fergusson, teacher of navigation and examiner in that science to the East-India Company, constructed a Rotula, or longitude instrument, which did great credit to him as a man of science.

Another graphic operation, of a different description, was executed by Mr. George Margetts, and published in 1790. It consists of 70 large plates, containing numerous lines drawn from the solution of lunar distances in Dr. Shepherd's Tables. By Margett's Longitude Tables, the solution of a lunar observation may be obtained in about one-fourth of the time required by calculation; and the answer, though not given as perfectly accurate, is sufficiently so for the general purposes of navigation.

Dr. Kelly has devised an orthographic projection founded on the fluxional analogies of spheric triangles, which is published in his Introduction to Spherics and Nautical Astronomy, where an investigation of its principles is given (pa. 195. edit. 2, 3, and 4), with a demonstration, showing that, in proper altitudes, it cannot essentially err, but must give the true distance within a few seconds. The simplicity of this projection is extremely curious, as giving an approximate solution of a complicated problcm, by drawing four right lines only, from the scale of chords, and it must therefore be very useful, cspecially where expedition is required.

Since the first appearance of this projection, which was in 1795 , several mathematicians have turnce their. attention to the subject, and various ingenious plans have been proposed for shortening and simplifying the operation, among which may be mentioned a Rotula by Mr. B. Donue of Bristol, which solves the problem with great accuracy.

\section{U N}

The following is an abstract of Kelly's Projection, in. sertedhere by permission of the author.

$E x a m .1$. Given the apparent distance between the moon, and thesun, or astar - $\quad-30^{\circ}$ The moon's altitude $\quad-\quad$ - $\quad$ - $\quad-20$ The star's altitude - $\quad-\quad-\quad-\quad-\quad 40$ The moon's horizontal parallax - - $\quad 1$ To find the true distance.

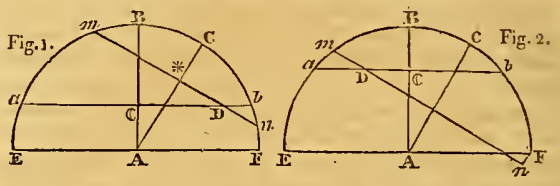

With the sweep of $60^{\circ}$, from a scale of chords, describe a semicircle, and bisect it by the perpendicular AB. On the right hand side of this line, from $\mathrm{B}$ to $\mathrm{c}$, lay off $30^{\circ}$, the apparent distance, from the same scale. Also the moon's altitude, $20^{\circ}$, from $\mathrm{E}$ to $a$, and from $\mathrm{F}$ to $b$; and draw $a b$. Then lay off the sun's co-altitude, $50^{\circ}$, both ways from $c$ to $m$ and $n$, and draw $m n$, intersecting $a b$ in D.-Then is $D$ \& the mean correction, to be measured on the scale of chords, calling every degree on the scale a minute; which must be reduced to the true correction by the following general rule: first observing that if this line $D$ f falls on the right-hand side, as in fig. 1 , the correction is negative or subtractive; but if on the left, as in fig. 2 , the correction is positive or additive.

RuLE. Multiply the mean correction, $\mathrm{D} \&$, by the given horizontal parallax, and divide the product by 62 when the correction is subtractive, but by 53 when it is additive. Then the quotient will be the true correction. Thus, in the first case D \& measures $41 \frac{\pi}{4}$, or $41^{\prime} 15^{\prime \prime}$, and being subtractive, it is multiplied by $60^{\prime}$, and divided by 62 , which gives the true correction $39^{\prime} 55^{\prime \prime}$, being only one second more than by calculation; hence the true distance is $29^{\circ} 20^{\prime} 5^{\prime \prime}$.

Examp. 2. Given the apparent distance $30^{\circ}$

The moon's altitude $\quad 40$

'The sun or star's altitude 20

The moon's horizontal parallax 1

To find the true distance.

Describe the semicircle as before (or a greater arc to $n$ if wanted, as in fig. 2), and lay off the apparent distance $\mathrm{BC}=30^{\circ}$. Make $\mathrm{E} a$ and $\mathrm{F} b=40^{\circ}$ the moon's altitude, and draw $a b$. Lay off the star's co-altitude $70^{\circ}$, from $\mathrm{c}$ to $m$ and $n$, and drawn $m n$. Then is $\mathrm{D} \mathbb{Q}$ the mean correction, which here measures $24^{\prime} 10^{\prime \prime}$; and being multiplied by 60 , and divided by 53 , it gives $27^{\prime} 21^{\prime \prime}$ for the true correction, being only $3^{\prime \prime}$ more than the result by calculation; and the correction being additive, it gives $30^{\circ} 27^{\prime} 21^{\prime \prime}$ for the true distance.

This projection will appear still more simple by drawing the triangle only, and laying off the altitudes from the line of sines. Thus, in fig. 1 , project the angle $\mathbf{B} A \mathrm{C}=30^{\circ}$, and make $A Q=$ sine of $20^{\circ}$, also $A *=40^{\circ}$. Thers perpendicular lines from $\mathbb{a}$ and $*$ will meet in $\mathrm{D}$ as before.

From the foregoing projection, the following general rules are obvious, for estimating the correction of a lunar observation without any operation. -1 . When the moon is lower than the star, the correction is always subtractive; but when it is the higher body, beyond a certain extent, 
and within a certain limit as to distance, the correction is additive.-2. When the sine of the moon's altitude is to that of the star's, as radius is to the cosine of the distance, then the true and apparent distances are nearly equal: but when the moon's altitude is greater than the star's, beyond that proportion, the correction is additive; except when the distance is about $90^{\circ}$, or more, and then it is subtractive. -3 . Though the correction additive does not occur so frequently as the subtractive, nor is it in general so great, yet it admits of a widervariation, being sometimes above $1^{\circ}$; but the subtractive correction is never quite so much.

The author of this construction further observes that, "In solving the lunar problem by this projection, a slate will perhaps be found more convenient than paper; and a large scale of chords is, of course, preferable to a small one.-That, in the foregoing examples, round numbers have heen chosen for the sake of simplicity; but fractional quantities might have been adopted with equal accuracy. It is not however presumed that the results of all Iunar problems will be alike correct by this method, but they will be found seldom to vary above half a minute from the true distance; which cannot be deemed of much importance, when it is considered that greater inaccuracies are perhaps unavoidable in the observations. It is extremely difficult, for instance, to ascertain within a minute the true point of contact between the moon and the sun or star. Their altitudes too are often doubtful, on account of the changes of refraction, and the haziness of the horizon: to which may be added the imperfection of instruments, the incompetency of observers, and even the inaccuracy of the Iunar tables themselves, which have been hitherto only in a progressive state of improvement.

It is well known that, from various unavoidable causes, all astronomical computations are but approximations to perfect accuracy; and that in this science difficulties and obstructions are much greater at sea than at land. lt is also allowed, that of all the operations in nautical astronomy, the lunar problem is the most embarrassing, and the most liable to crror; and it is likewise agreed that the best mode of diminishing such uncertainties, is by multiplying the observations, and taking a meth of many trials. Hence an approximate method, like the present, which. cannot essentially err, and which is performed with so much expedition, must be highly useful, as affording the most time for repeating the observations. Even where no such repetitions are required, this projection may be advantageously used as a substitute for troublesome computations, particularly in the common course of natical practice. It may indecd be observed, that the more urgent duties of seamen seldom allow thrm sufficient leisure for tedious numcrical operations. Besides, it seems rather a vain pursuit to sacrifice so much time in search of a few seconds, where there are probably errors of whole minutes in the data. It may even be observed, that long and laborious calculations contain within themselves many sources of error, to which projection is not exposed."

Lunar $M T o m$, is either periodical, synodical, or illuminative: which see; also Montrr.

LUNaR Year, consists of 354 days, or 12 synodical months, of $29 \frac{\pi}{2}$ days each. See YEAr. In the carly ages, the lunar year was used by all nations; the variety of course being more frequent and conspicuous in this planct, and consequently better known to men, than those of any other. The Romans regulated thcir year, in part, by the moon, cven till the time of Julius Cæsar. The

Jews too had their lunar month and year.

Lun a R Dial, Eelipse, Horoscope, and Rainbow. See the severál substantives.

LUNATION, the period or time between one new moon and another; it is also called the synodical month, consisting of 29 days $12 \mathrm{hrs} .44 \mathrm{~m}$. 3 sec. 11 thirds; exceeding the periodical month by 2 ds. 5 hrs. $0 \mathrm{~m}$. $55 \mathrm{sec}$.

LUNE, or LUNULA, or little moon, is a geometrical figure, in form of a crescent, terminated by the arcs of two circles that intersect each other within.

Though the quadrature of the whole circle has never been effected, yet many of its parts have been squared. The first of these partial quadratures was that of the lunula, given by Hippocrates of Scion, or Chios; who, from being a shipwrecked merchant, commenced geomctrician. But though the quadrature of the lune be generally ascribed to Hippocrates, yet Proclus expressly says it was found out by. Oenopidas of the same place. See Heinius in Mem. de YAcad. de Berlin, tom. ii. pa, 410, where he gives a dissertation concerning this Oenopidas. See also CIRCLE, and Quadrature.

'The lune of Hippocrates is this: Let $A B C$ be' a semicircle, having its centre $\mathrm{E}$, and $\mathrm{A} D \mathrm{DC}_{\mathrm{a}}$ quadrant, having its centre $\mathbf{F}$; then the figure $A B C D A$, contained between the arcs of the semicircle and quadrant, is his lune; and it is equal to the right-angled triangle $A C F$, as is thus easily proved. Since $A F^{2}=2 A E^{2}$, that is, the square of the radius of the quadrant equal to double the square of the radius of the semicircle; therefore the quadrantal area $\triangle D C F A$ is = the semicircle $A B C E A$; from each of these take away the common space $\triangle D_{C E A}$, and there remains the. triangle $A C F=$ the lunc $A B C D A$.
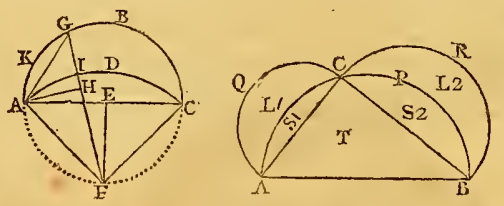

Another property of this lune is, that if FG be any line. drawn from the point $F$, and $A F$ perpendicular to it; then is the intercepted part of the lune $A G I A=$ the triangle $\Lambda G H$, cut off by the chord line $A G$; or in gencral, that the small segment $\Lambda \mathrm{KG}_{A}$ is equal to the trilineal AIHA. For, the angle $A F G$ being at the centre of the one circle, and at the circumference of the other, the arcs cut off $\mathrm{AG}, \mathrm{AI}$. are similar to the wholes $A B C, A D C$; therefure the small. seg. $A K G A$ is to the semisegment $A I 1$, as the whole semicircle $A B C A$, to the scnisegment or quadrant $\triangle D C F$, that is in a ratio of equality.

Again, if $A$ BC (fig. 2 ) be a triangle, right-angled at $\mathrm{C}_{9}$ and if semicircles be described on the three sides as diameters; then the triangle $T$ ( $A \mathrm{BC}$ ) is equal to the sum of the two lunes $\mathrm{L} 1, \mathrm{~L} 2$. For, the greatest semicircle is equal to the sum of both the other two; from the greatest semicircle take away the segments $\mathrm{s} I$ and $\mathrm{s} 2$, and there remains. the triangle $\mathrm{r}$; also from the two less semicircles take arvay the same two segments $\mathrm{s} 1$ and $\mathrm{s} 2$, and there remains the two lunes $\mathrm{L} 1$ and $\mathrm{L} 2$; therefore the triangle $\mathrm{T}=\mathrm{L} 1+\mathrm{L} 2$, the two lunes.

LUNETTE, in Fortification, an inveloped counterguard, or mound of earth, made beyond the second ditch, oppo-n 
site to the place of arms; differing from the ravelins only in their situation. Lunettes are usually made in wet ditches, and serve the same purpose as fausse-brays, to defend the passage of the ditcli.

LUPUS, the Wolf, a southern constellation, joined to the Centaur, containing together 19 stars in Ptolemy's catalogue, but $2+$ in the Britannic catalogue.

LYNX, a constellation of the northern hemisphere, composed by Hevelius out of the unformed stars. In his catalogue it consists of 19 stars, but in the Britannic of 44 .

LYONS (ISRAEL), a respectable mathematician and botanist, was the.son of a Polish Jew silversmith, and teacher of Hebrew at Cambridge in England, where be had come to settle, and where young Lyons was born, 1739 . He was a very extraordinary young man for parts and ingenuity; and showed very early in life a great inclination to learning, particularly mathematics, on which account he was much patronized by Dr. Sinith, master of Trinity-college. About $\mathbf{1 7 5 5}$ he began to study botany, which he continued occasionally till his death; in which he made a considerable progress, and could remember not only the Linniean names of almost all the English plants, but even the synonyma of the old botanists; and he had prepared large materials for a Flora Cantabrigiensis, describing fully every part of each plant from the specimen, without being obliged to consult, or being liable to be misled by, former authors.

In 1758 , he obtained much celebrity by publishing A Treatise on Fluxions, dedicated to his.patron, Dr. Smith ; and in 1763 , Fasciculus Plantarum circa Cantabrigiam, \&c. In the same year, or the year before, he read lectures on botany at Oxford with great applause, to at least $60 \mathrm{pu}-$ pils; but be could not be prevailed on to make a long ab. sence from Cambridge.

Mr. Lynns was some time employed as one of the computers of the Nautical Almanac; besides which, he received frequent presents from the Board of Longitude for his own inventions.- He had studied the English history; and could quote whole passages from the monkish writers verbatim. He could read Latin and French with ease, but wrote the former indifferently. He was appointed by the Board of Longitude to sail with Capt. Pbipps, in his voyage towards the north pole, in 1773 , as astronomical observator ; which office he discharged much to the satisfaction of his employers. After bis return from this voyage, he married, and settled in London, where he died of the meazles about two years after.

At the time of his death he was engaged in preparing for the press, a complete edition of all the works of the learned Dr. Halley; a work very much wated.-His Calculations in Spherical Trigonometry abridged, were printed in the Philos. Trans. rol. 65, for the year 1775 , pa.470.-After his death, his name appeared in the titlepage of A Geographical Dictionary, the astronomical parts of which were said to be "taken from the papers of the late Mr. Israel Lyons of Cambridge, author of several valuable mathematical productions, and astronomer in Lord Mulgrave's voyage to the northern hemisphere."The astronomical and other mathematical calculations, printed in the account of Captain Phipps's voyage towards the north pole, mentioned above, were made by Mr. Lyons. This appeared afterwards, by the acknowledgment of Captain Phipps, when Dr. Horsley detected a material error in some part of them, in his Remarks on the Observations made in the late Voyage, \&c, 1774.

The Scholar's Instructor, or Hebrew Grammar, by Israel Lyons, teacher of the Hebrew tongue in the University of Cambridge, the $2 \mathrm{~d}$ edit. \&c, $1757,8 \mathrm{vo}$, was the production of his father; as was also another treatise printed at the Cambridge press, under the title of Observations and Enquiries relating to various parts of Scripture History, 1761 .

LYRA, the Harp, a constellation in the northern bemisphere; containing 10 stars in Ptolemy's catalogue, 11 in Tycho's, 17 in Hevelius's, and 21 in the Britannic catslogue.

\section{ADDENDUM.}

After line 17 , col..2, page 341, on the nature of comets, add:-Dr. Herschel, in several volumes of the Philos. Trans. states his opinions of the nature and constitution of cumets. His hypothesis is, that they are formed of the condensed nebulous matter diffused through the universal space, and tying within the sphere of the sun's activity; which thus gives them a revolutionary motion in an elliptic orbit. Of the same opinion also is Count Laplace, as appears by a learned memoir on the subject, in the Connoissance des Temps for the year 1816 .

\section{ERRATA.}

P. 135, col.2, end of the 6 from the bottom, for $\mathrm{N}^{2}$, read $2 \mathrm{~N}$.

- 269, - 1, lin. 24, 25, for Chichester, read Chesier.

- 480, - 2, end of line 28 , for $i t$, read $j l$.

- $484,-1$, line 20 , for plate viii, read plate $x$.

- 484, - 2, line 5, for $k$, read $\mathbf{~}$.

- $698,-1$, lise 28 , for $\frac{1}{2}$, read $\frac{1}{2 r}$.

END OF THE FIRST VOLUMX, 


\section{A I R-BALLOON S.}

Montgolticr's.

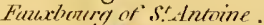

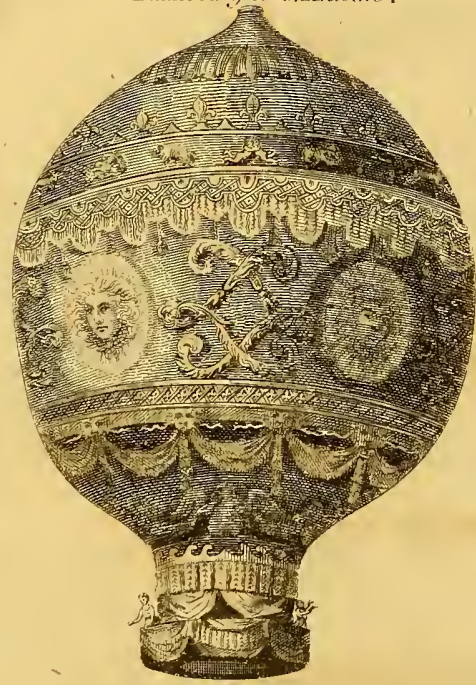

Montgolfier's

Fauxbourg if 5 ! Grmain

Cluarles \& Robert's.

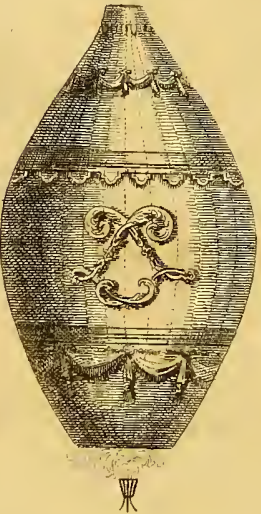

Champ de Afars.

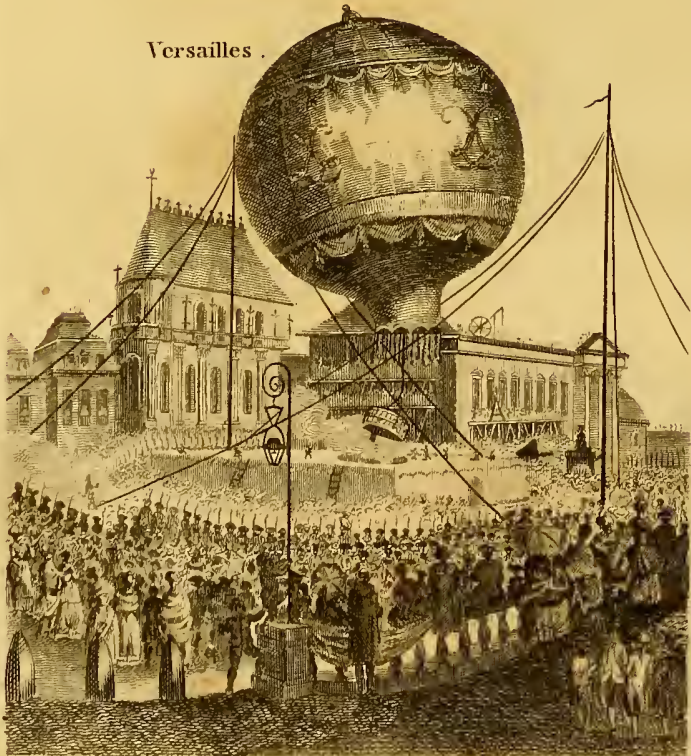

Blanchard's

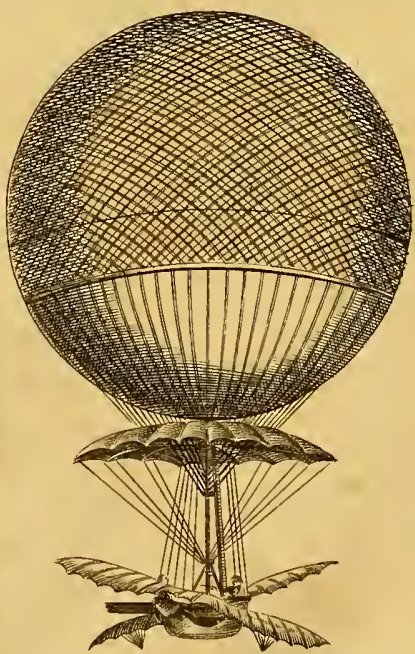



Fig. 1. AIR GUN.

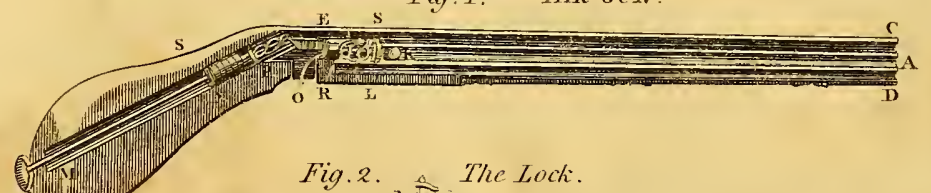

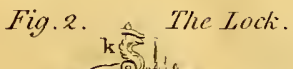
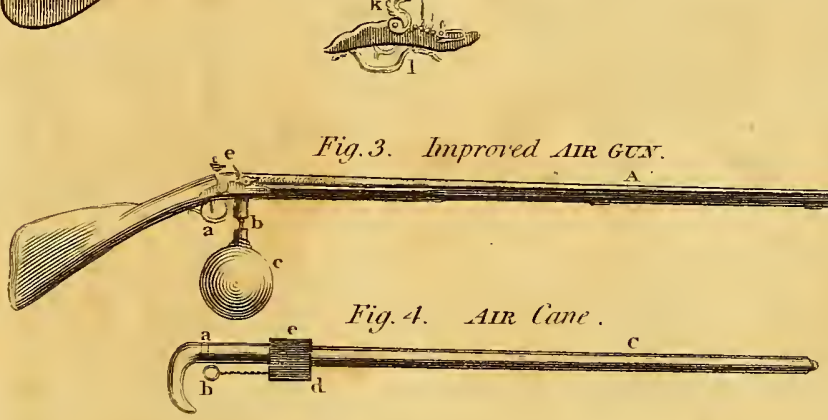

Fig.5. Magazine -HR Grv.
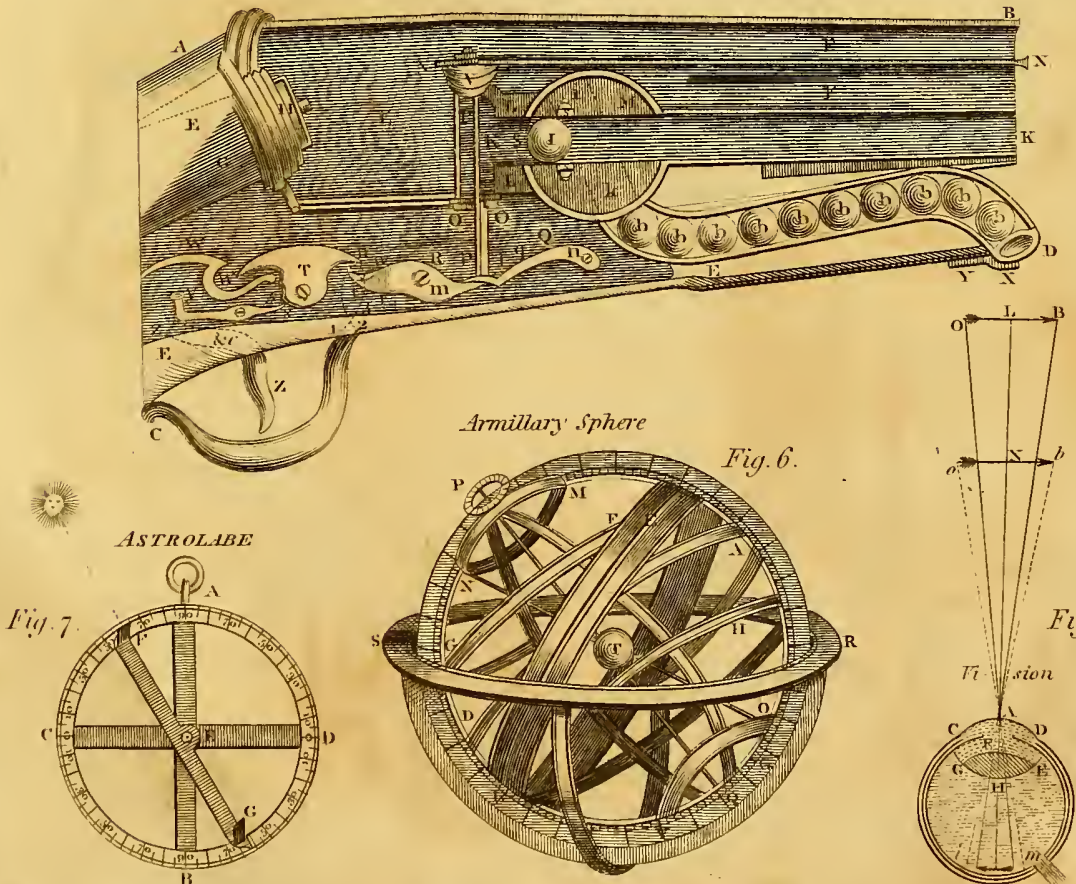

Armillary sphere
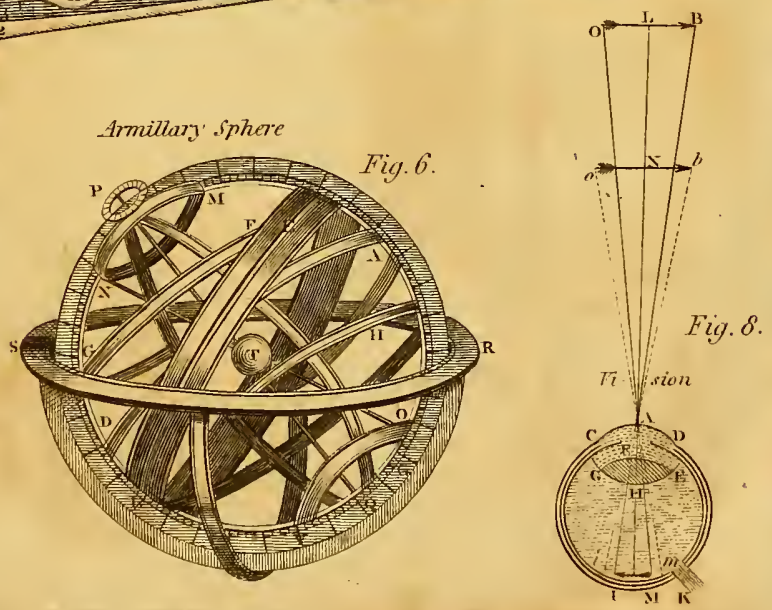



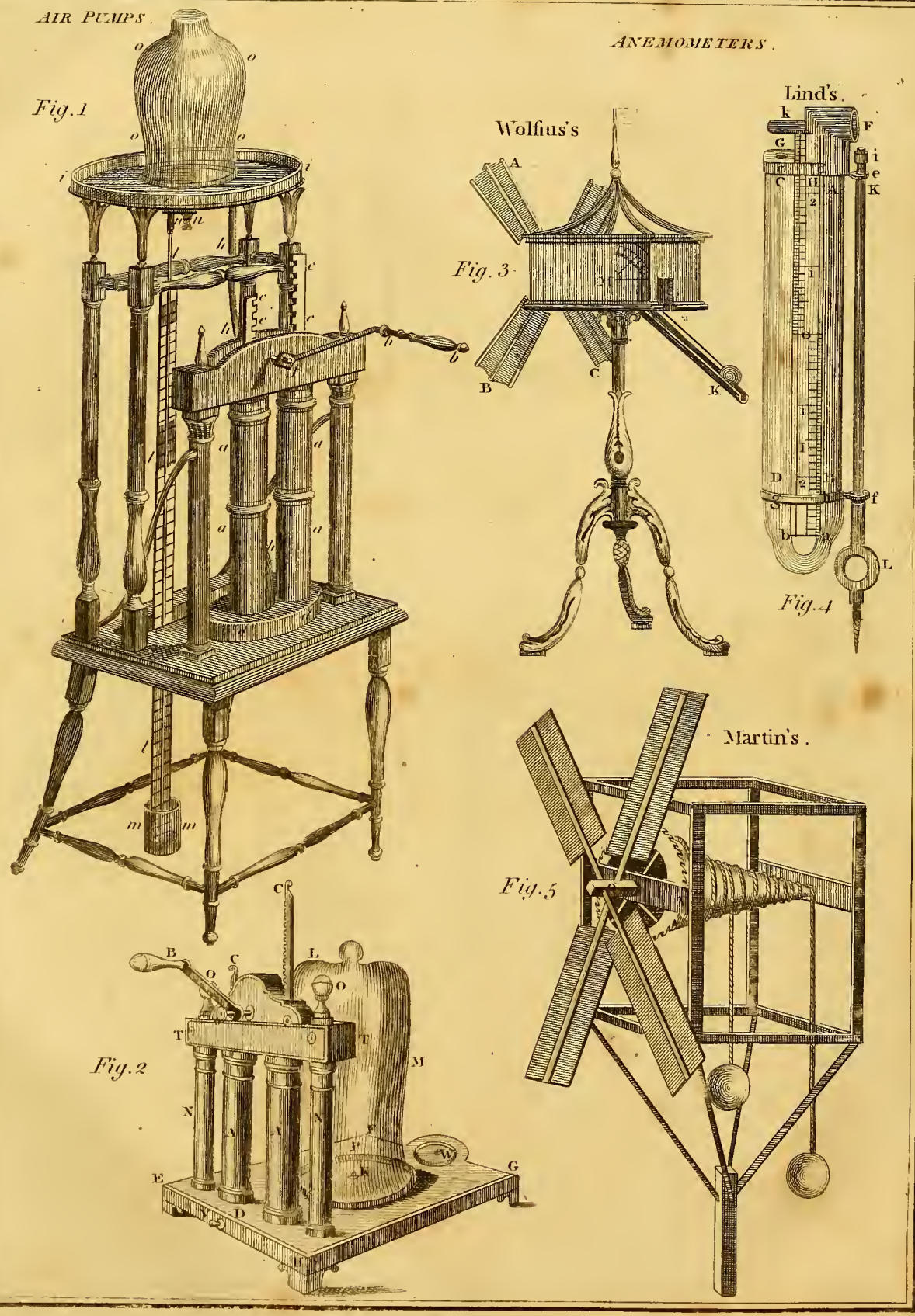





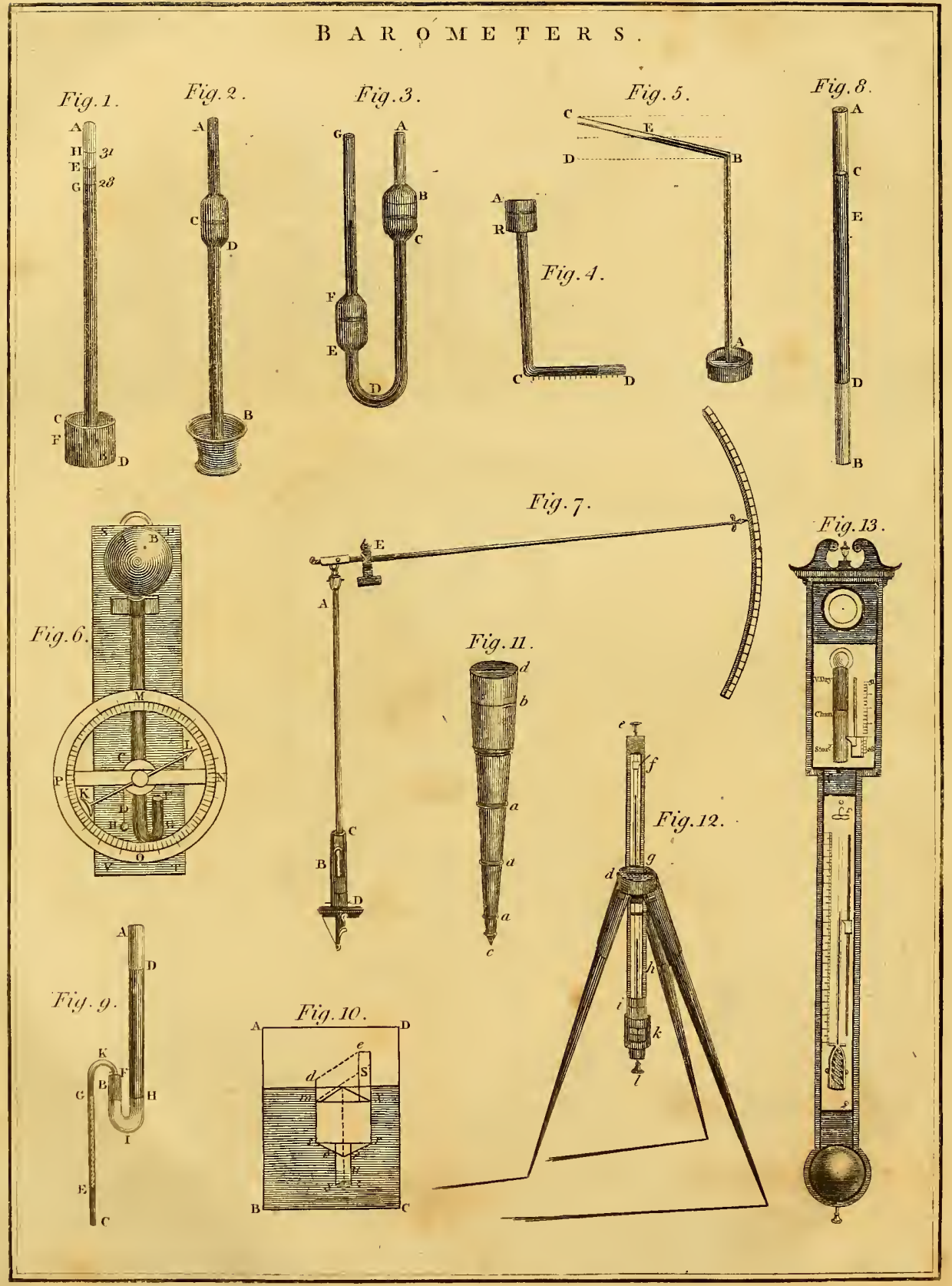



BdLIISTA.
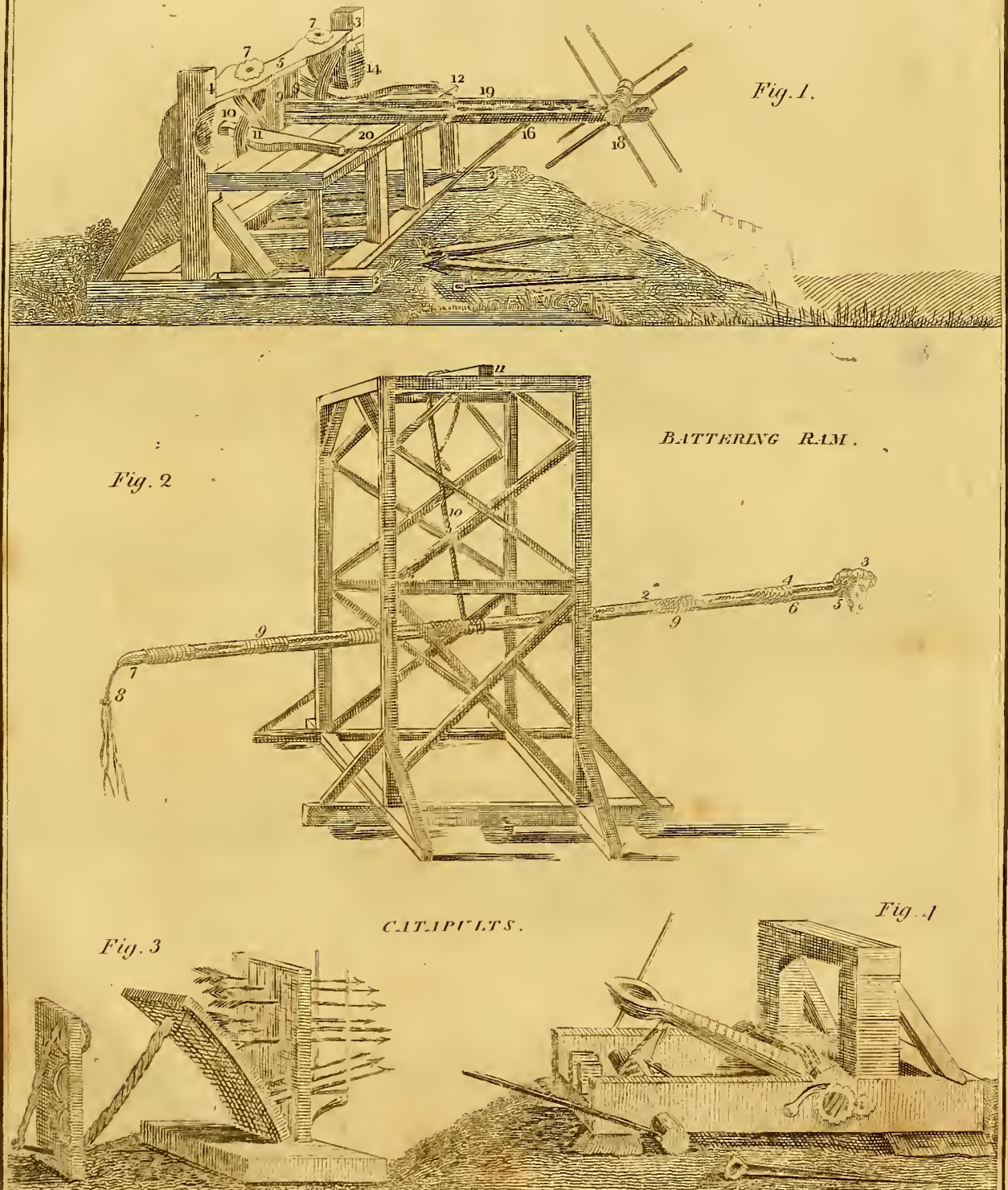

Cathetits.

Fig. 1 



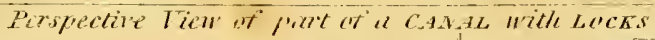

Fily. 1 .

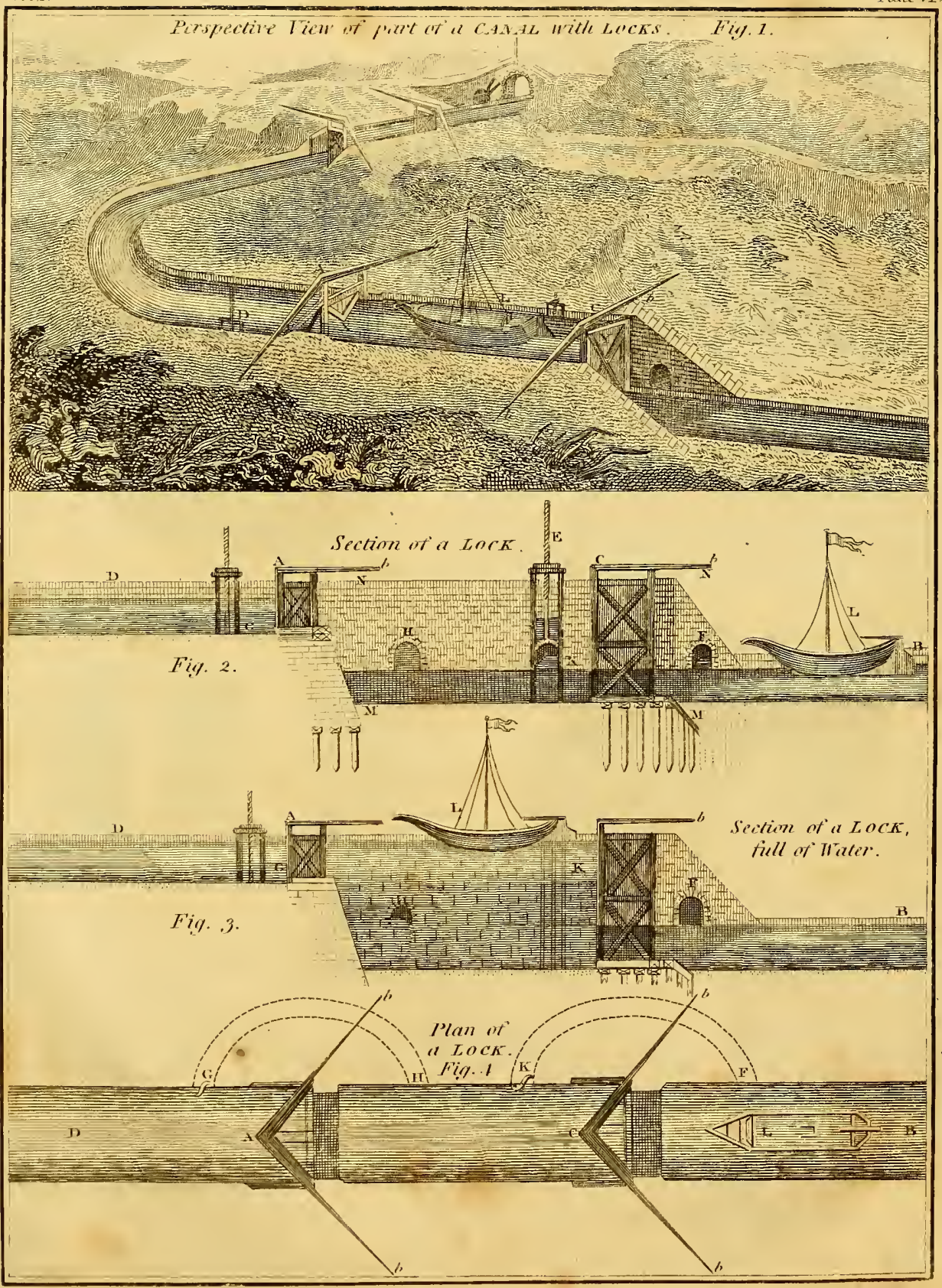


Plan \& Eletatien of a Donte, constnucted withet cintring
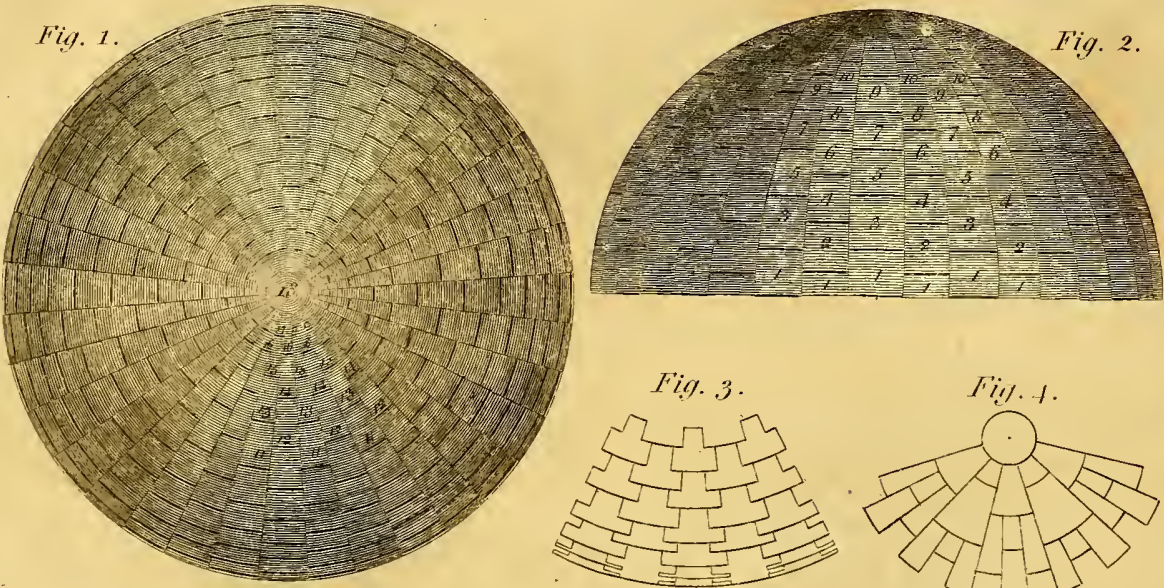

Für.

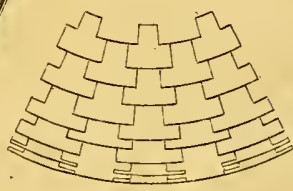

liil. 1 .

Fig. 3 .

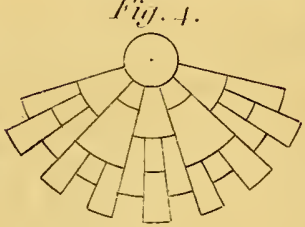

GALVANISM

Fil. .5.
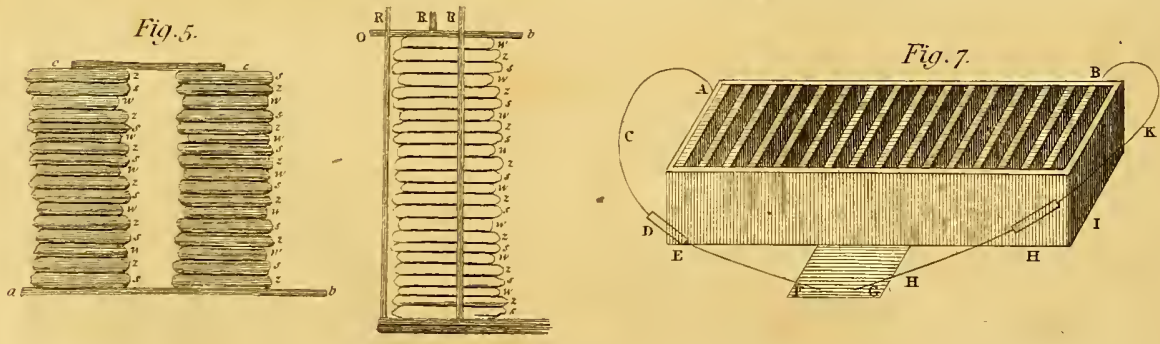

Electrical Bells

Circular Instruments

Fia.8.

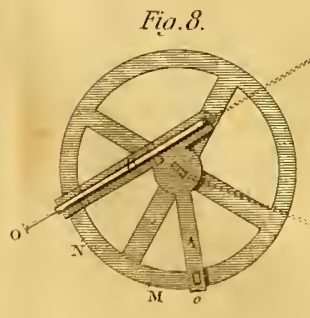

QI

$=0 \mathrm{~s}$

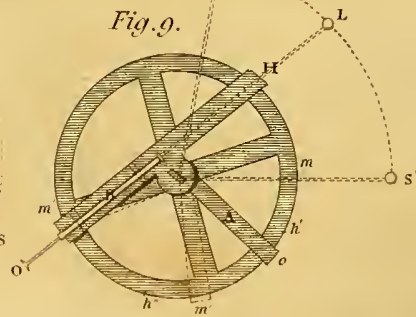

Fia.1o.

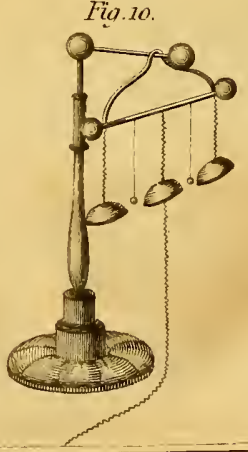



Fig. 1

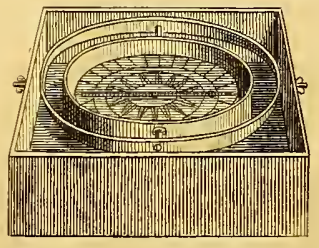

Azimith COALASS.

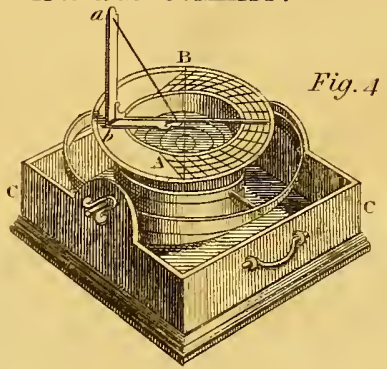

Proportional Compalses

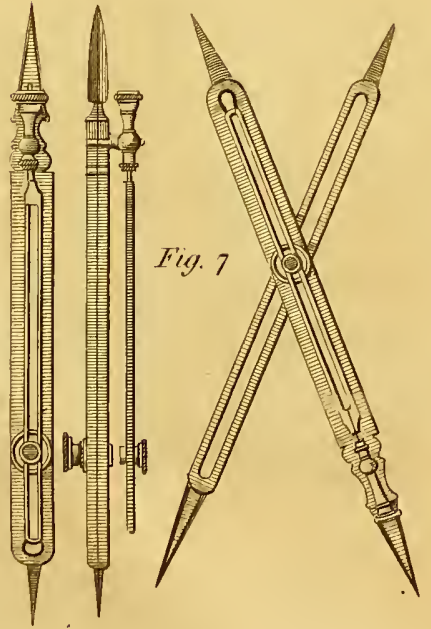

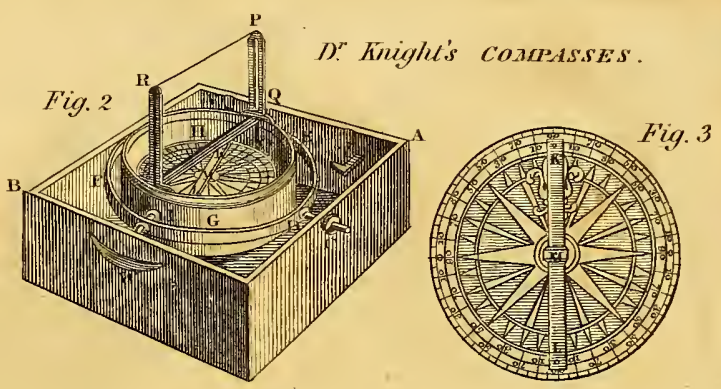

Coareass Card.

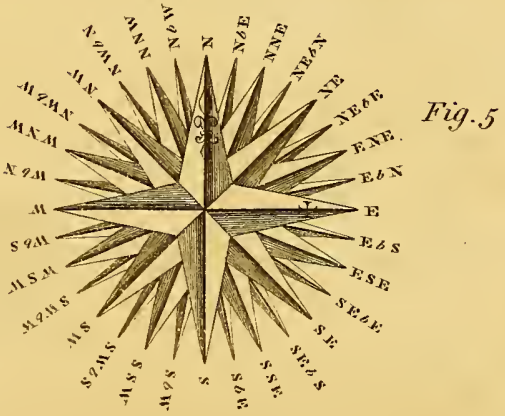

Elliptic compajes.

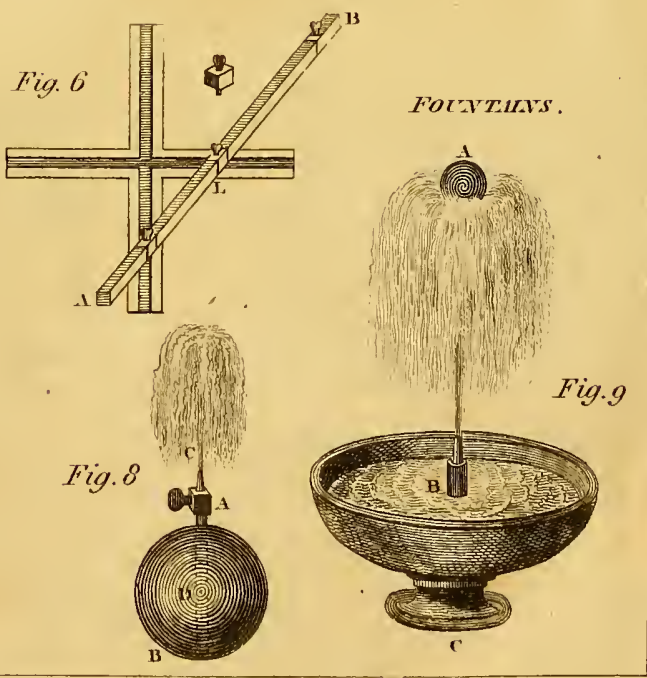




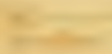

, .

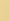




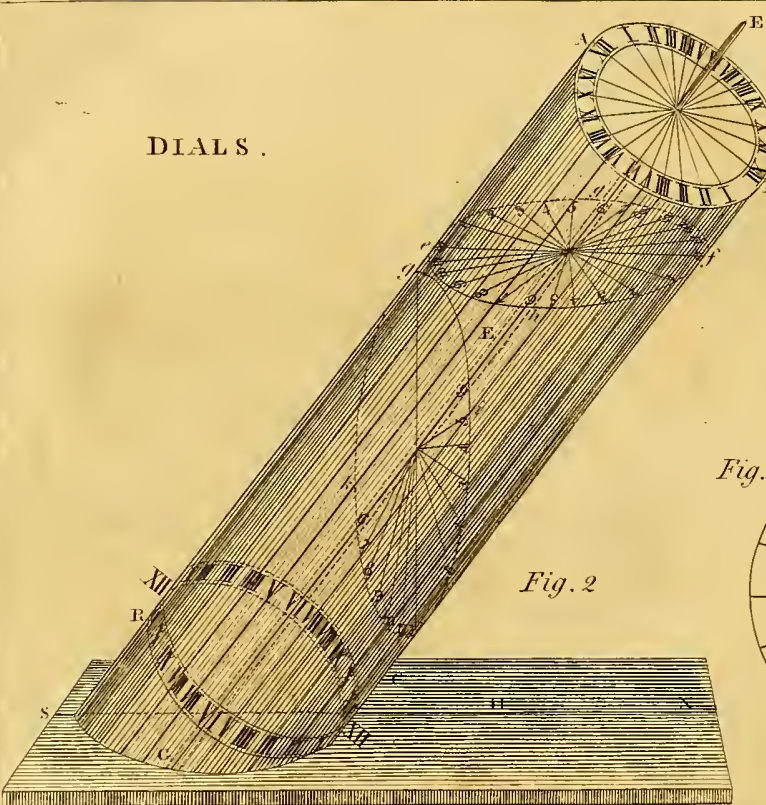

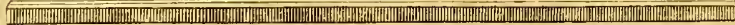
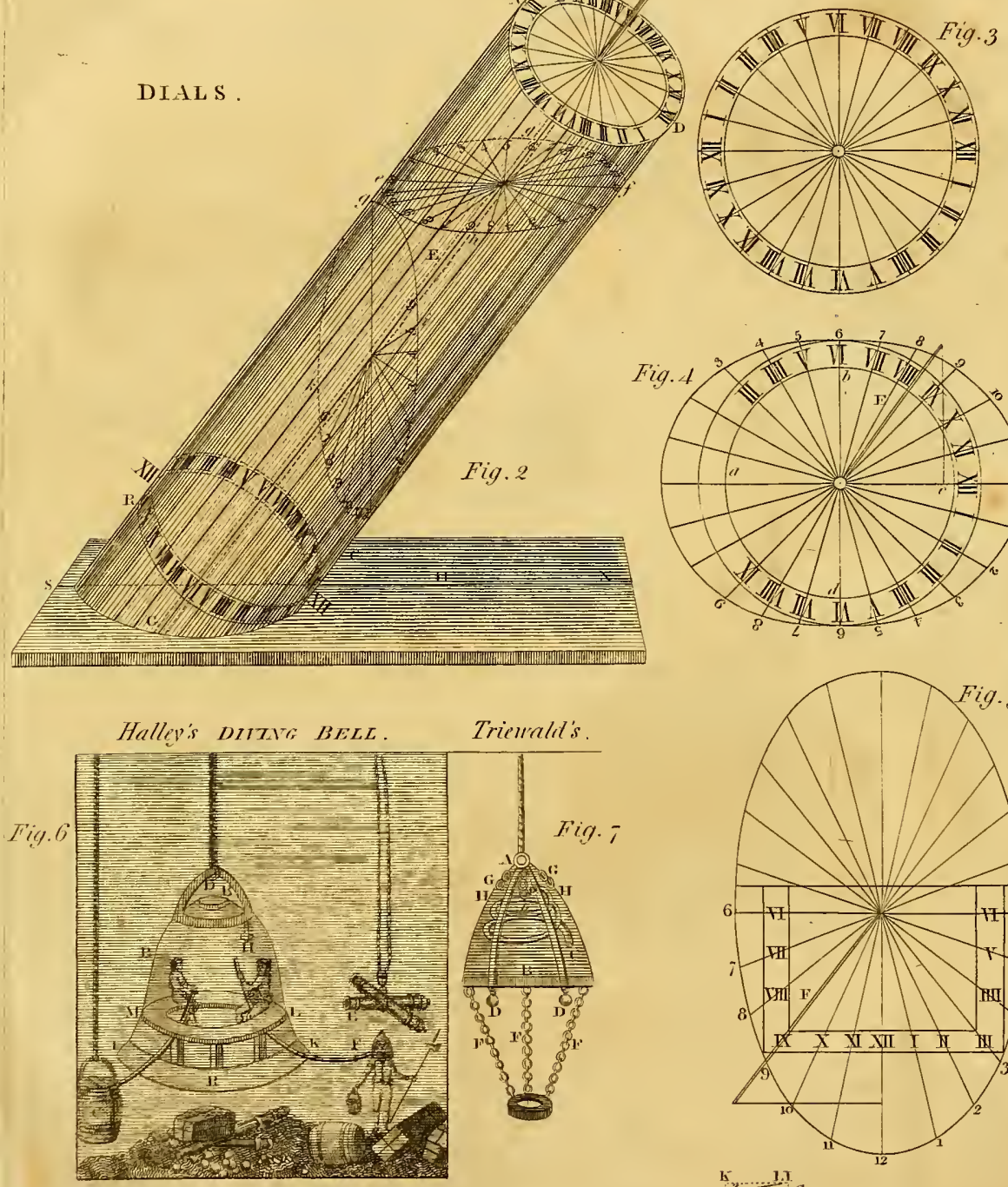

Triemald's
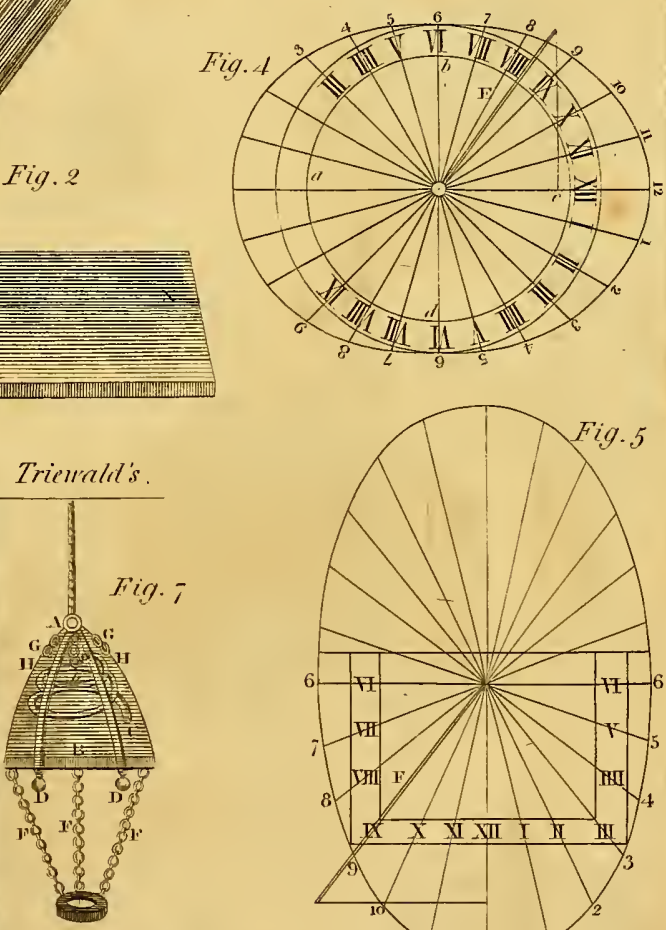

Profile of a Fortincention

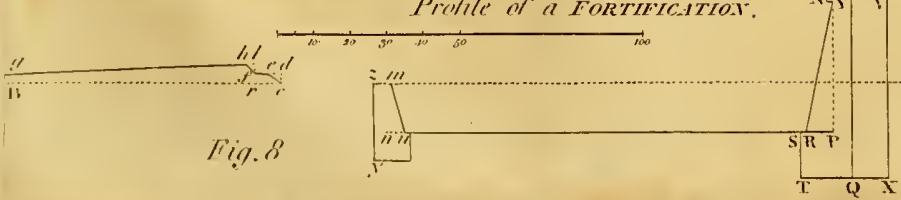





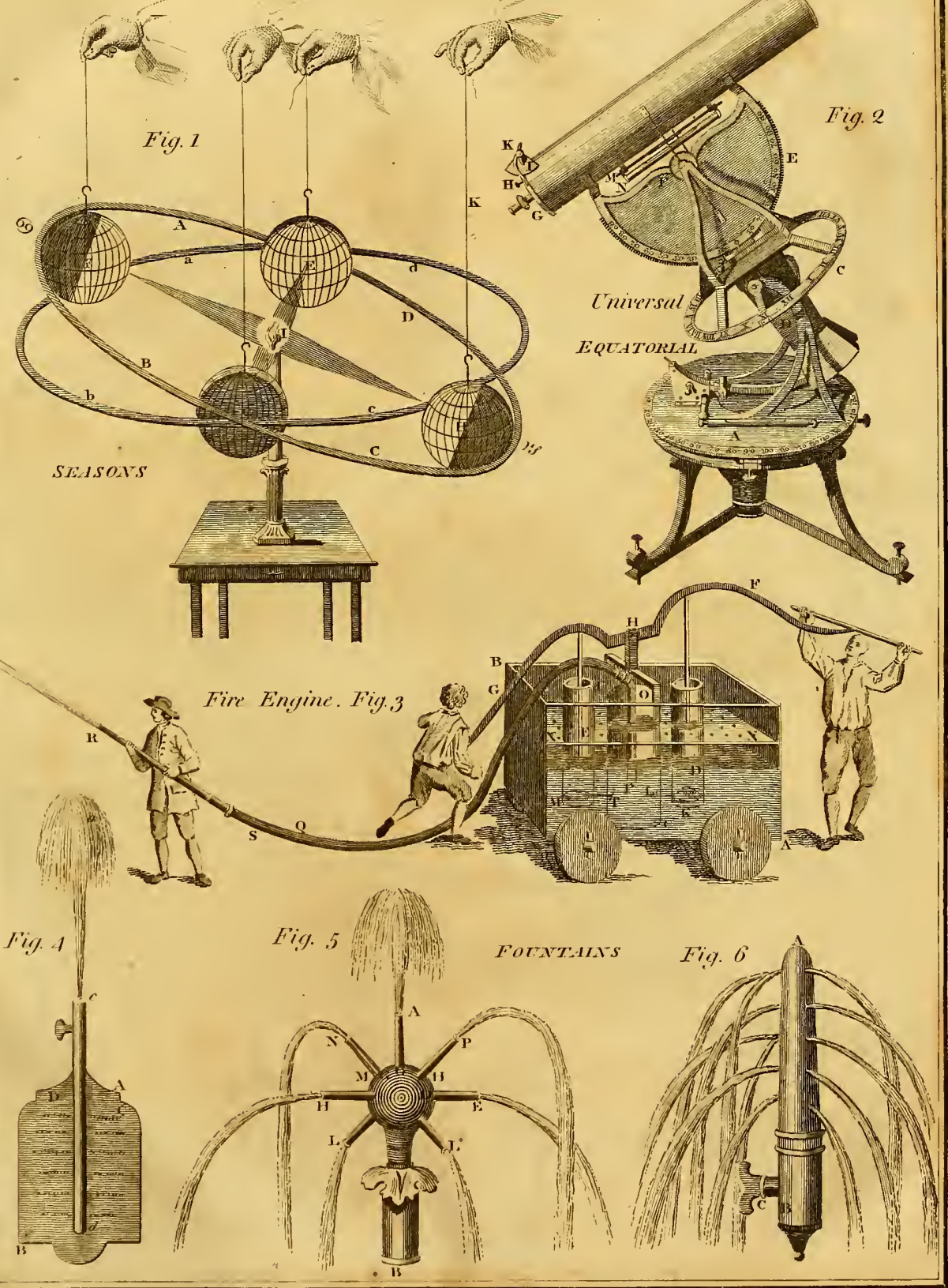





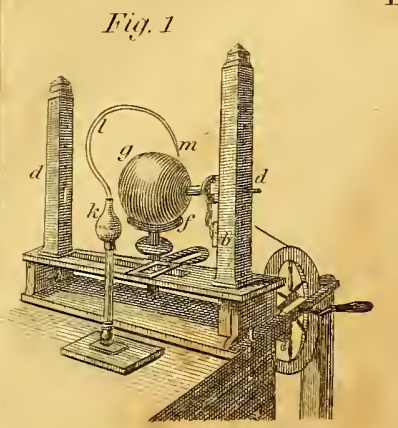

EIECTRICAJ MACHINES .
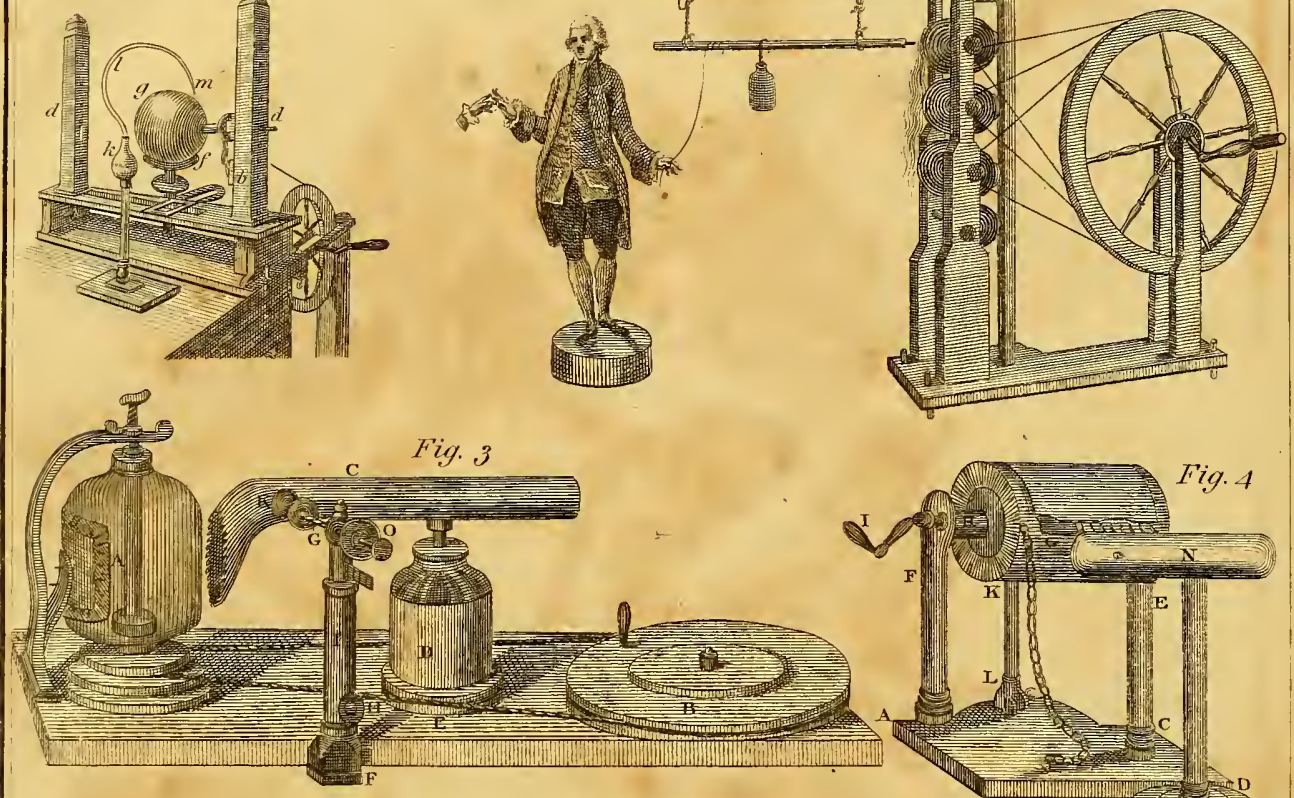

Cinton's

ELECTRONETER
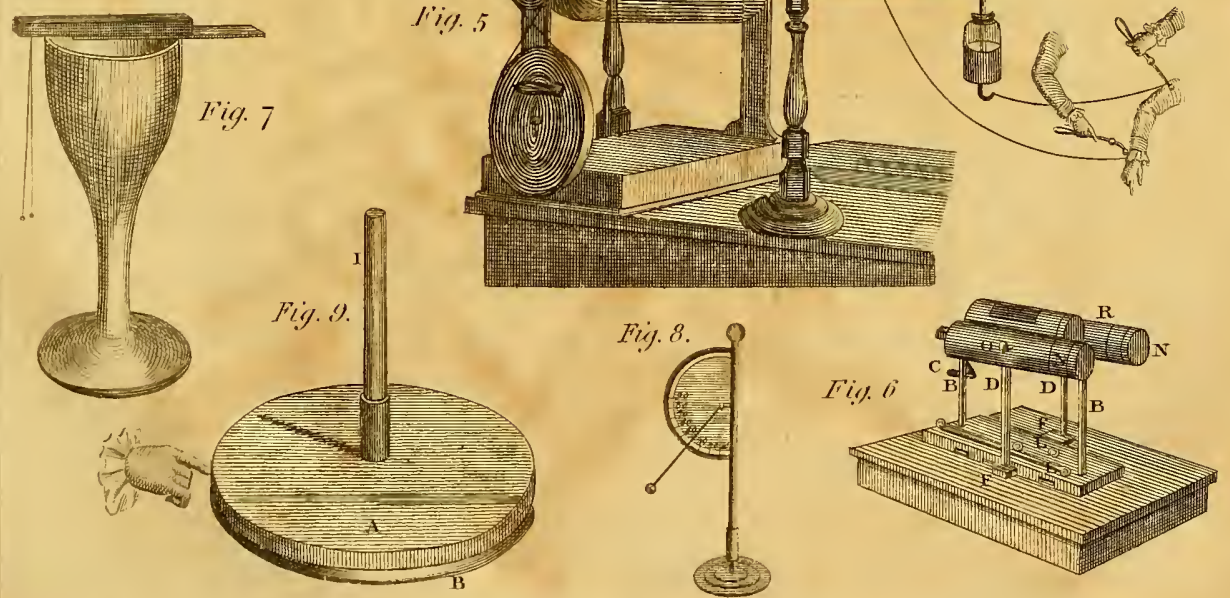


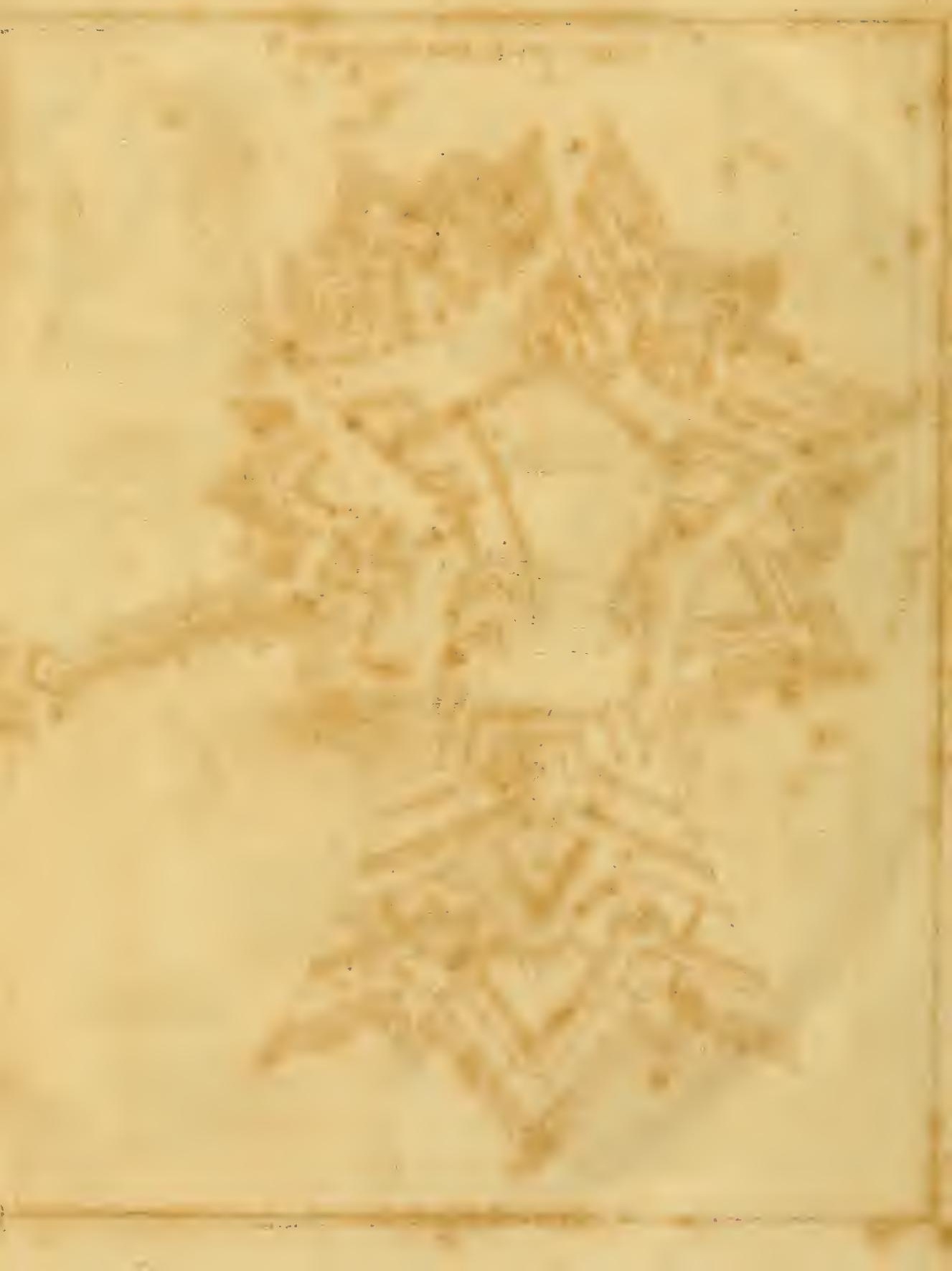




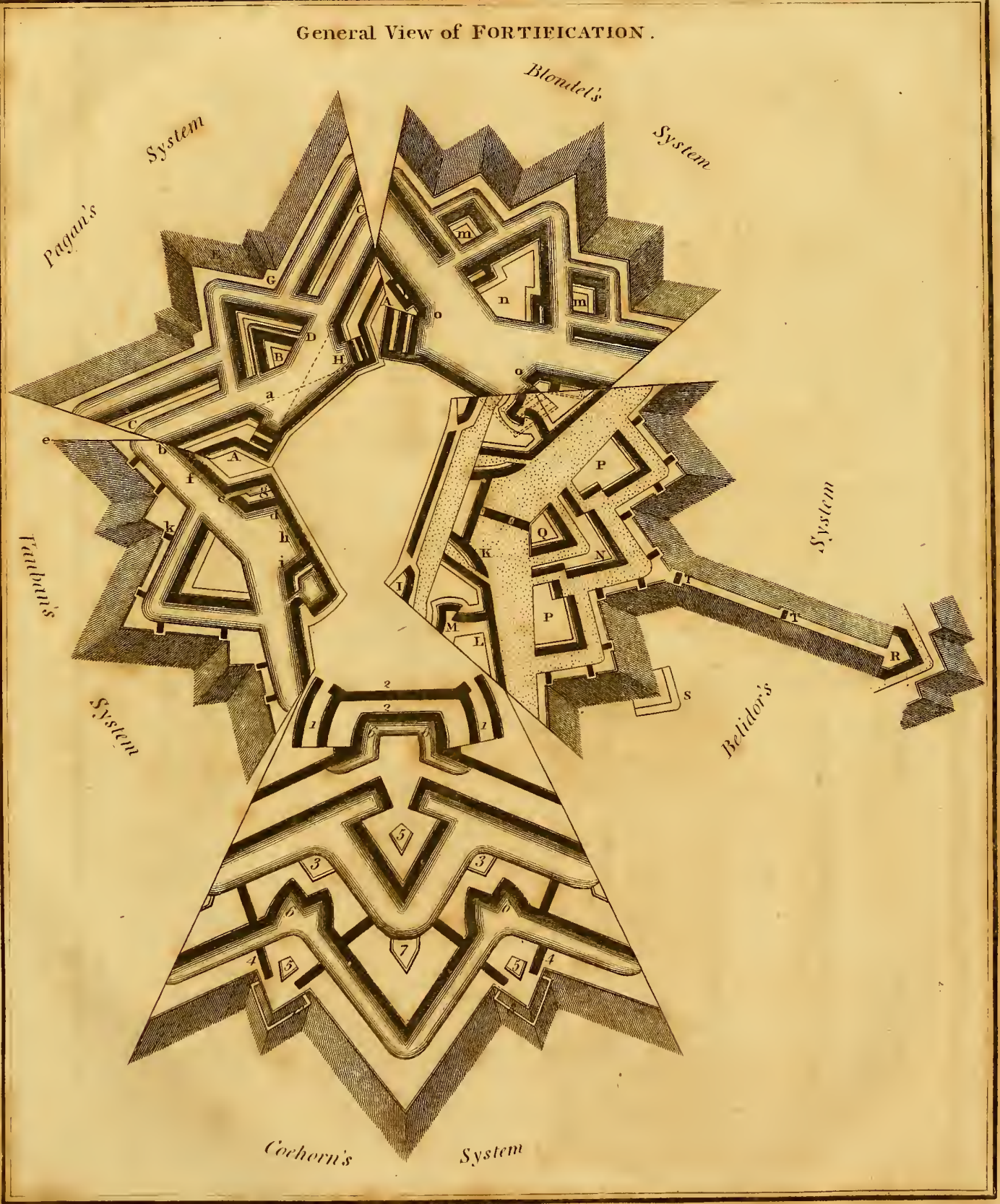




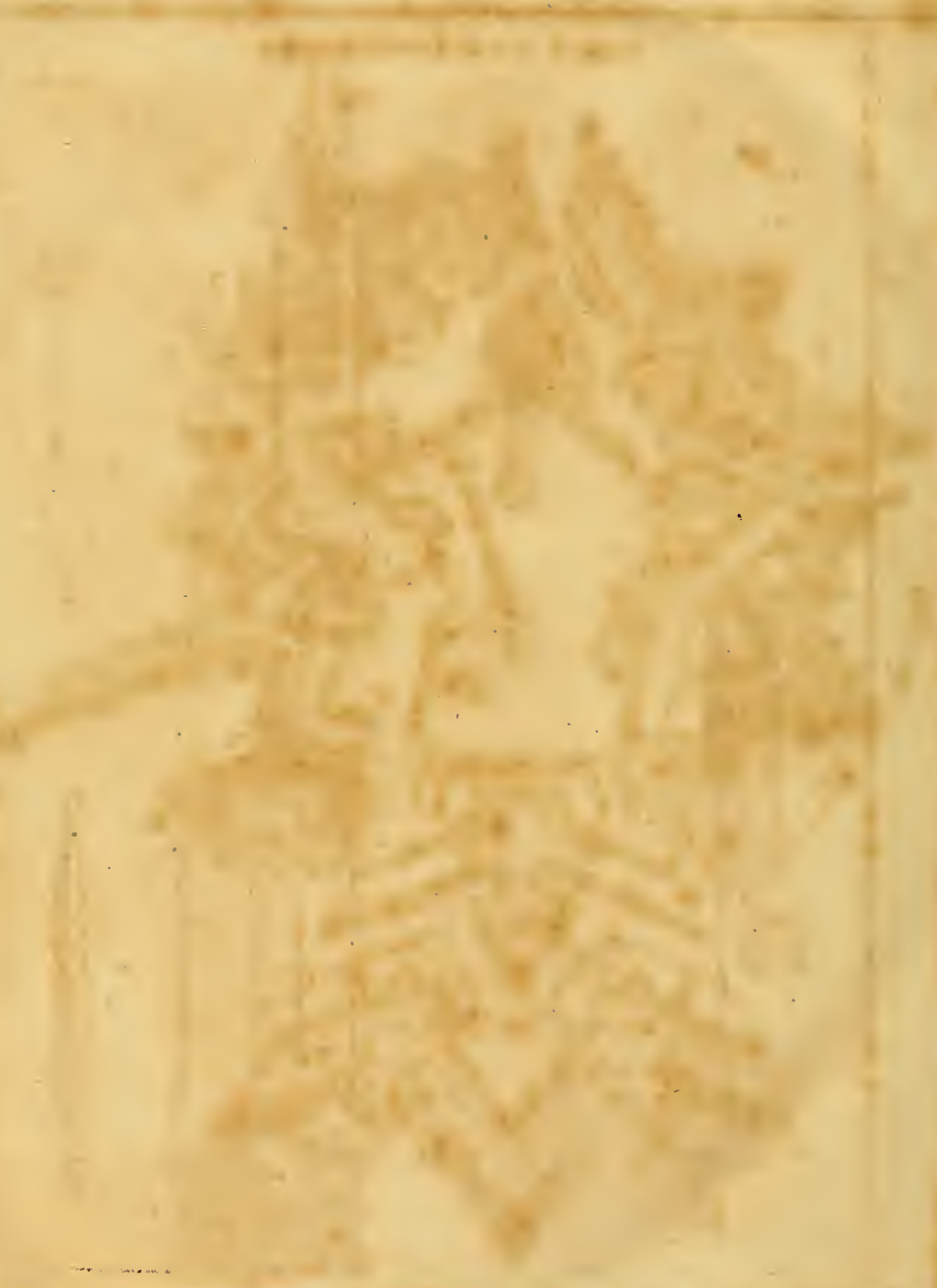




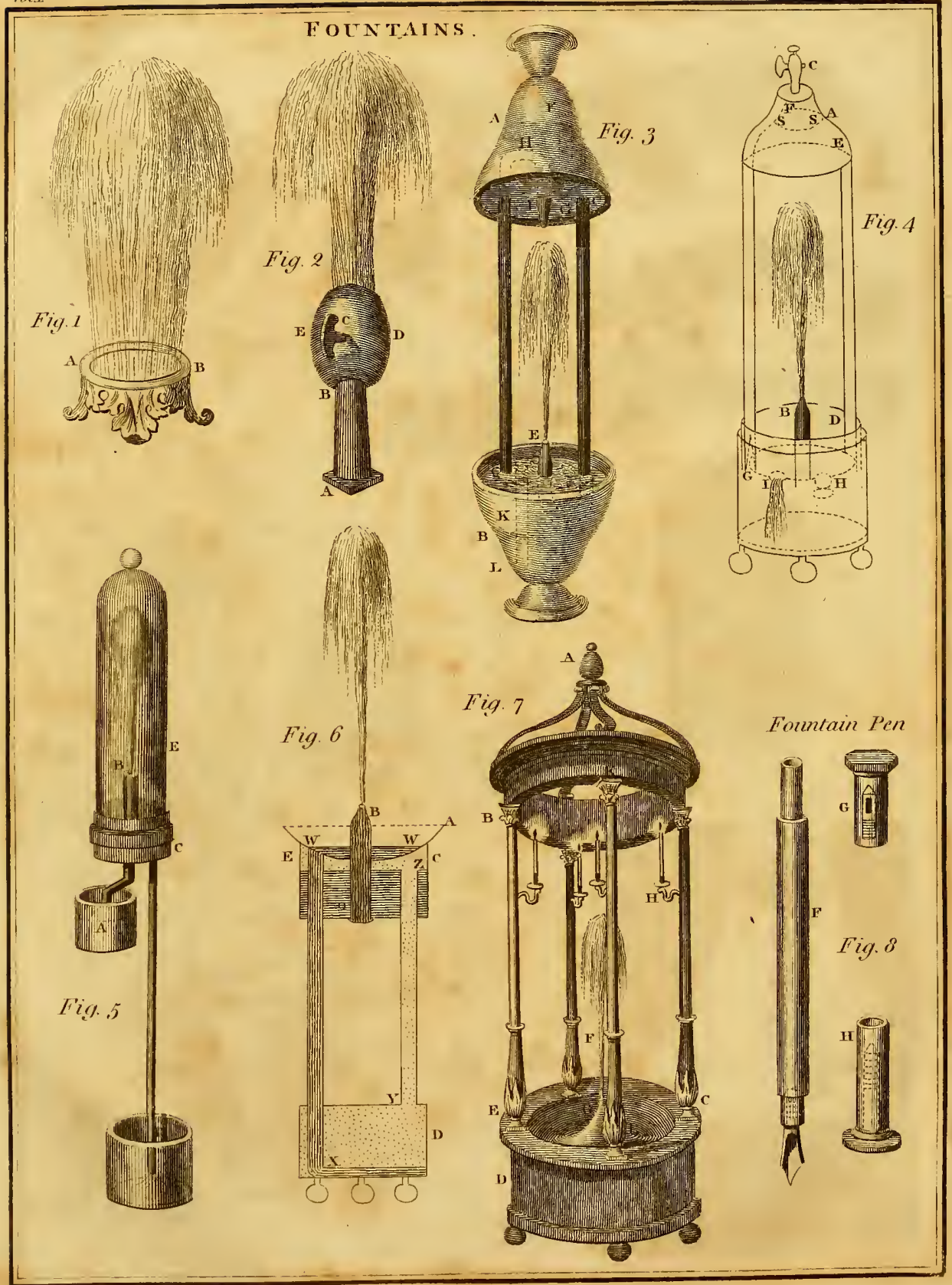


Lo 7

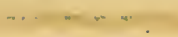
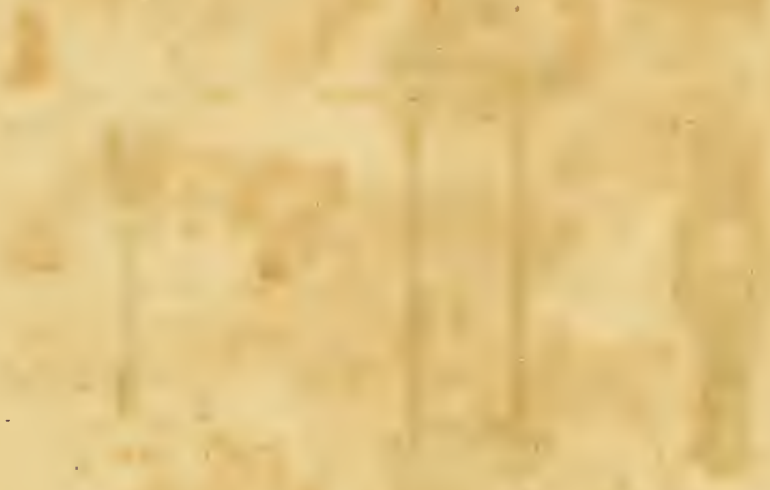

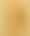
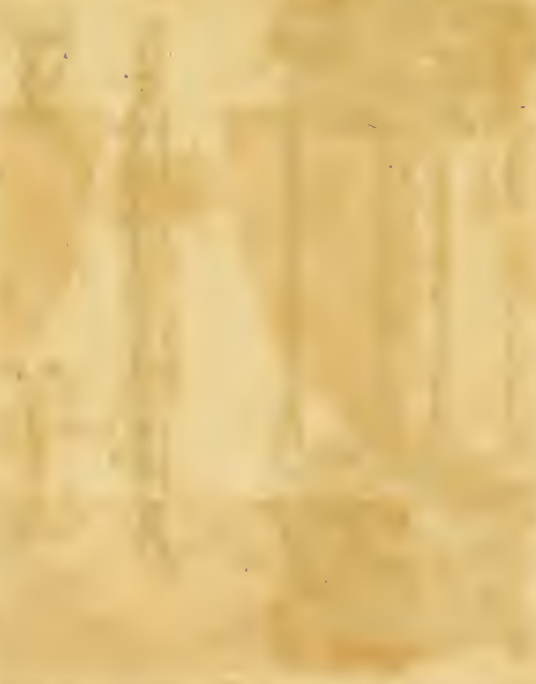


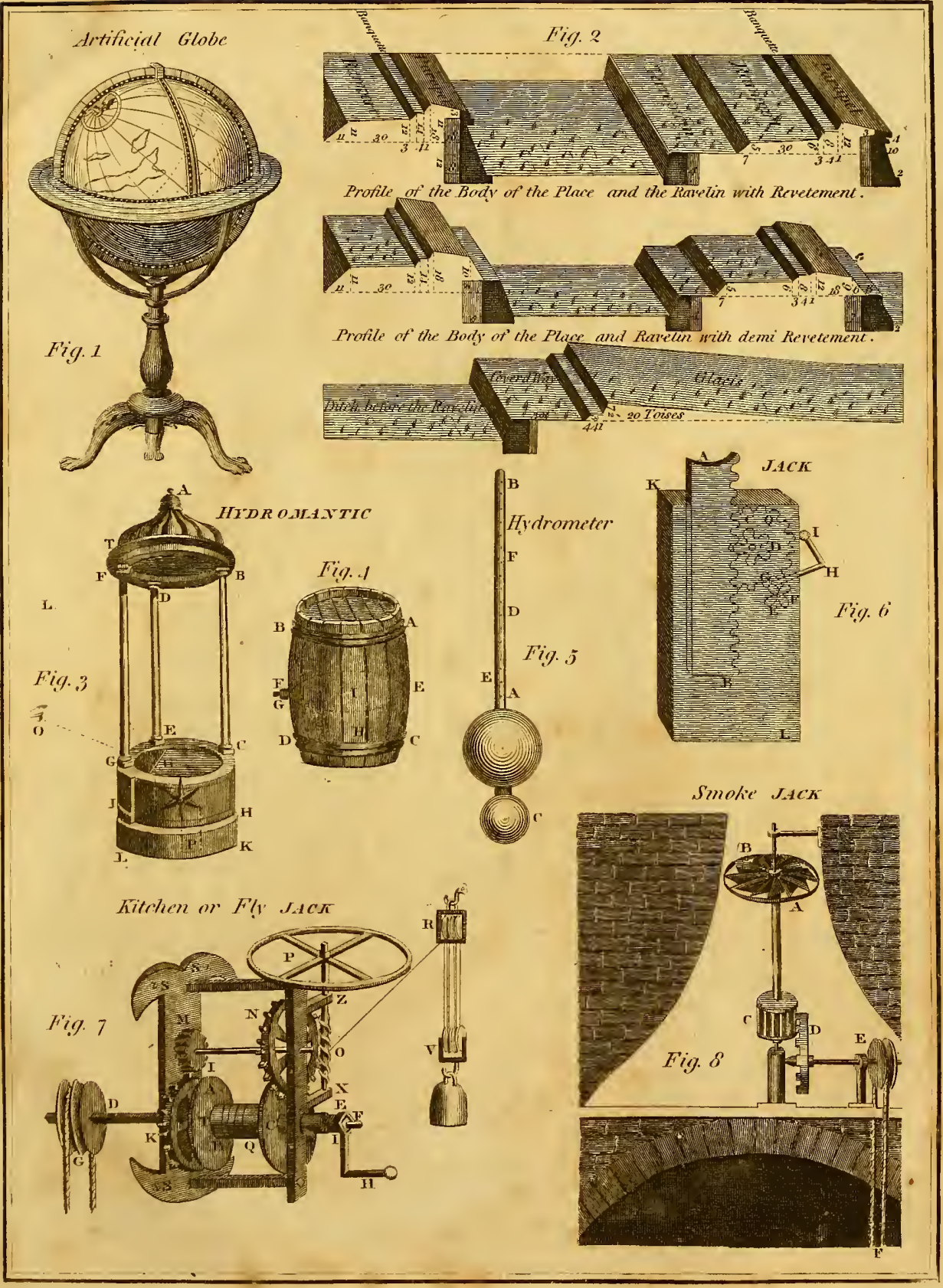



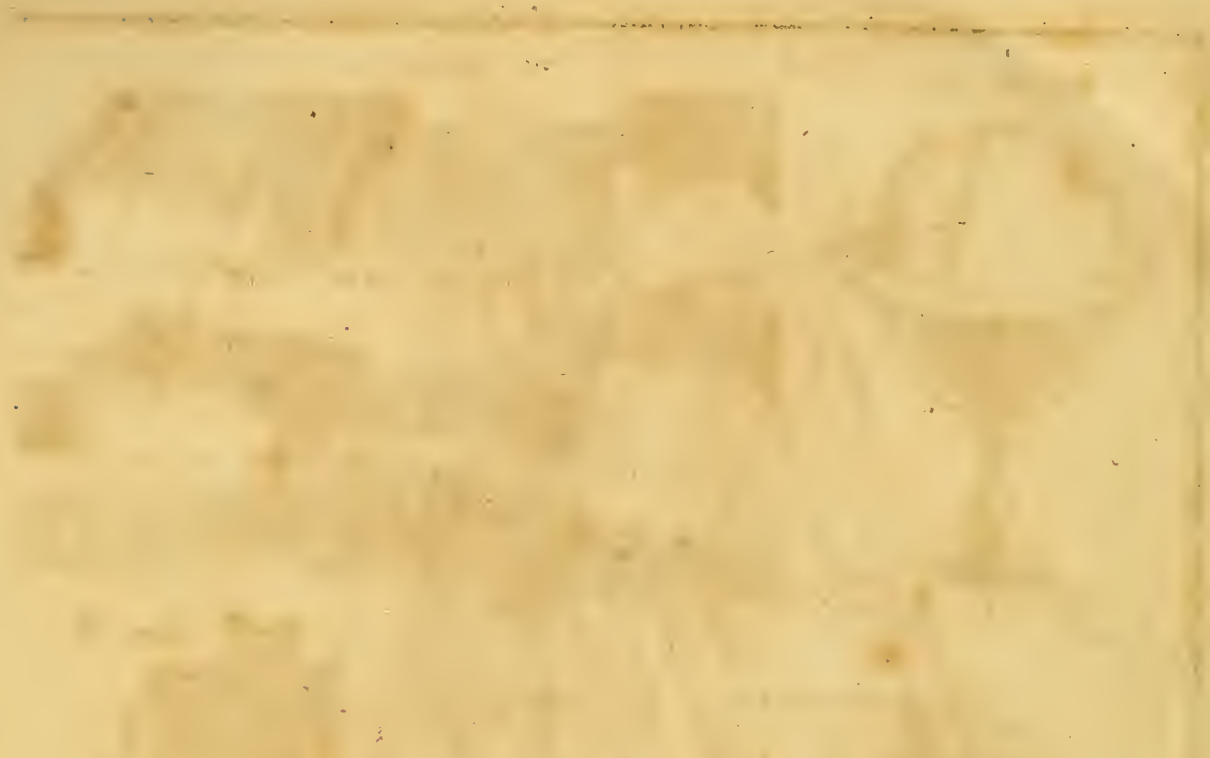

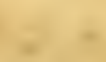

$\sqrt{3}$

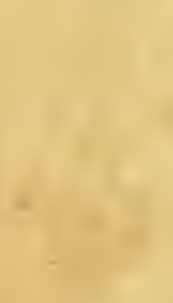

1
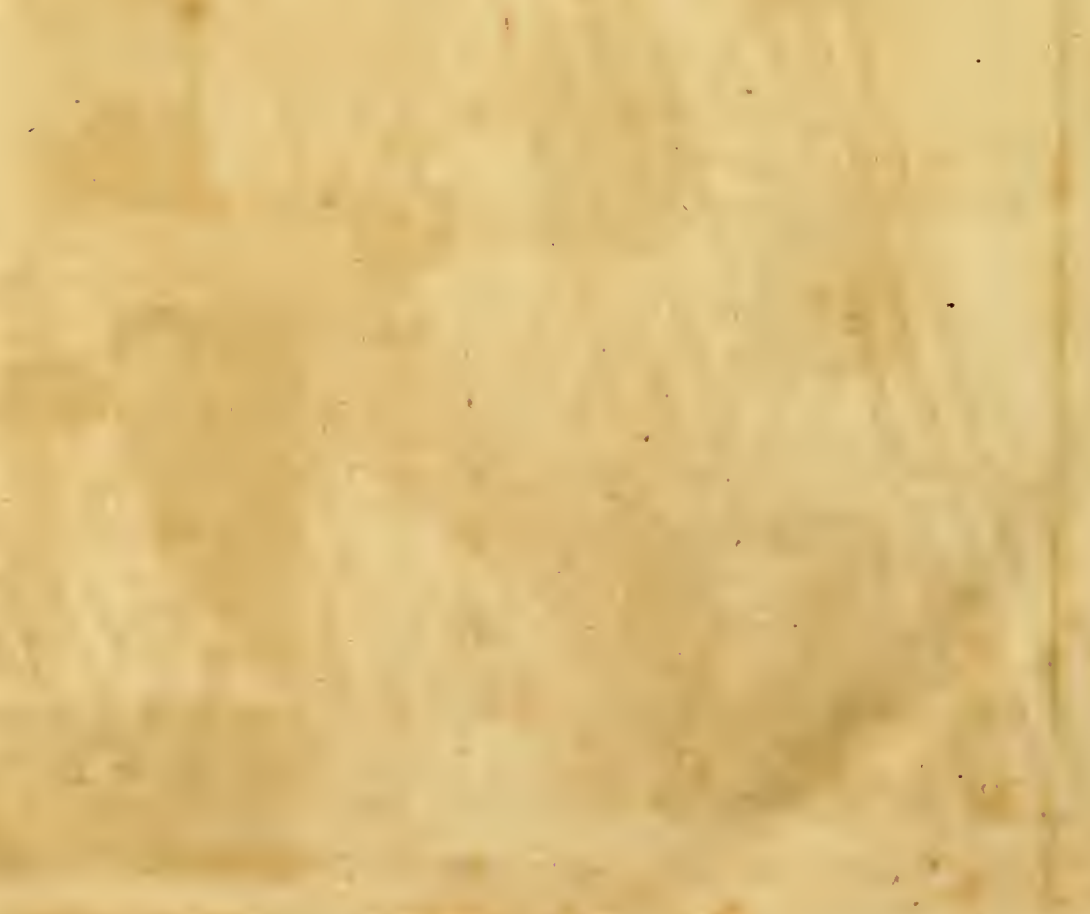
Herschel's Telescope.

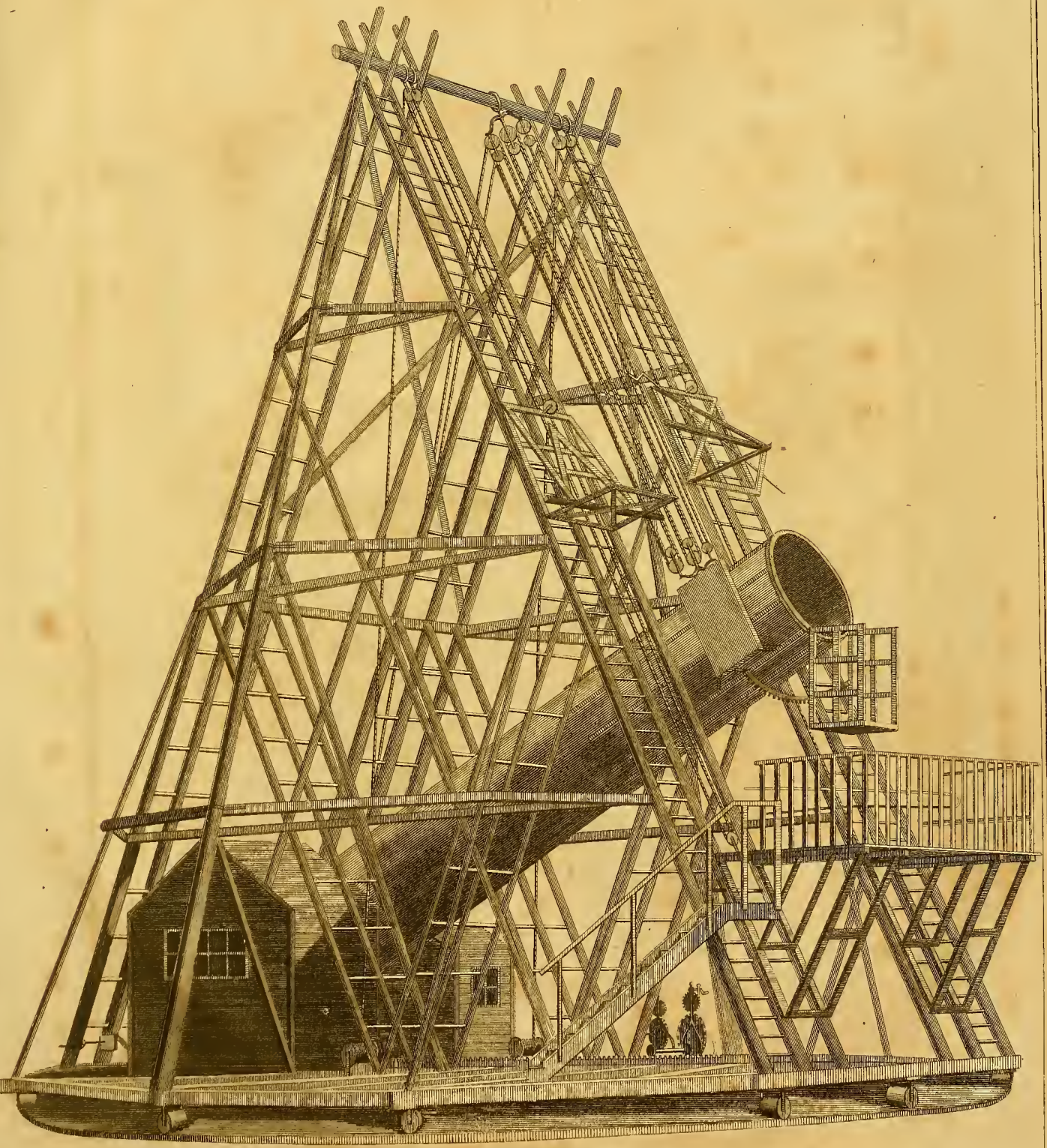




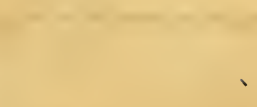

$\checkmark$

.

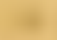

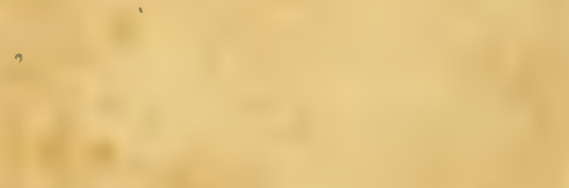

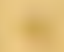

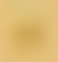

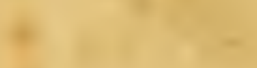

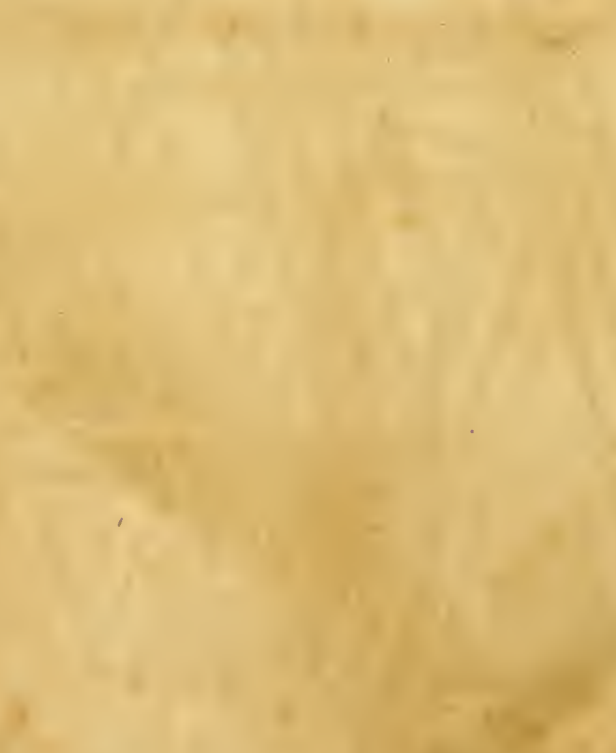




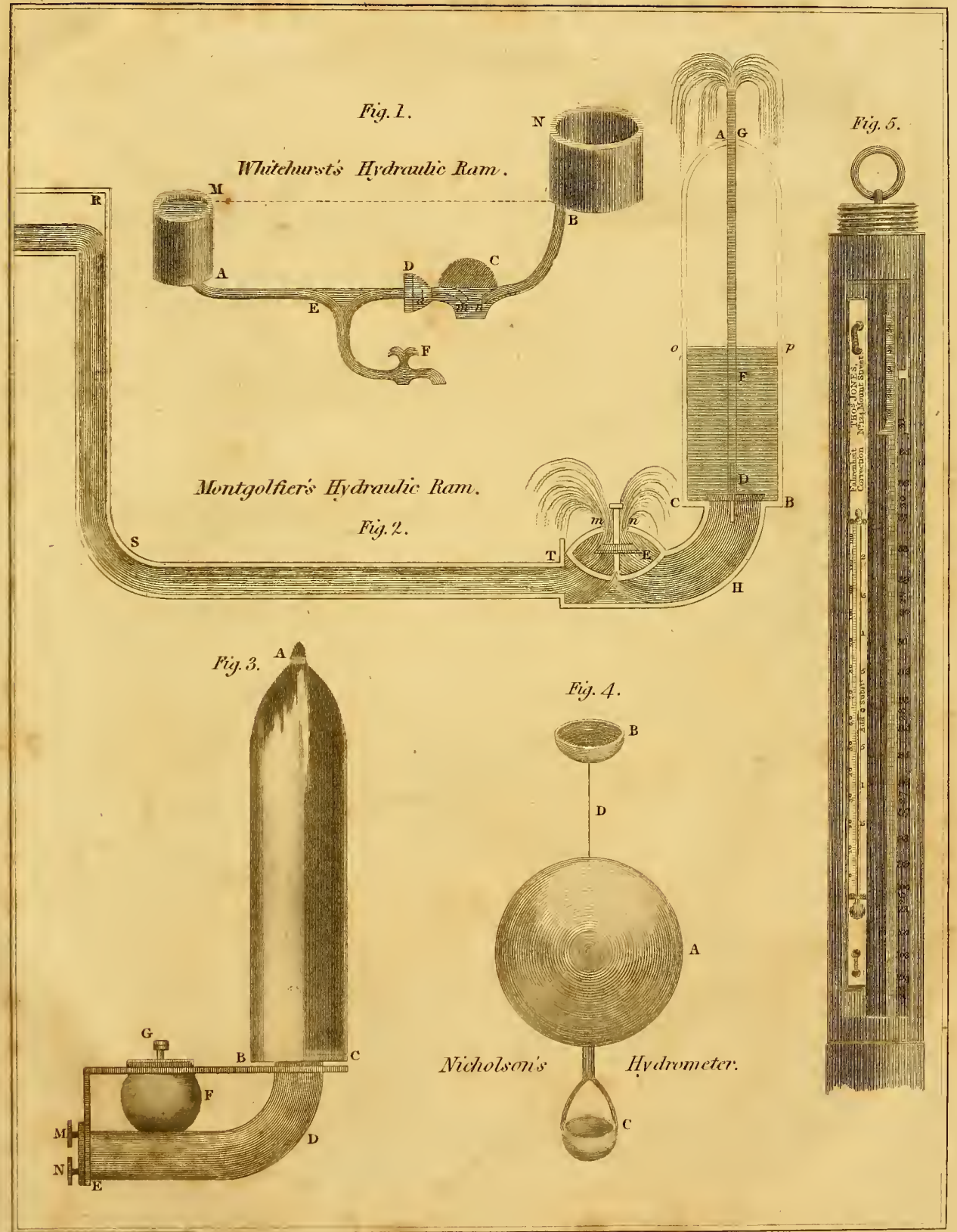


(1)

-

$-$

$+2$

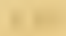
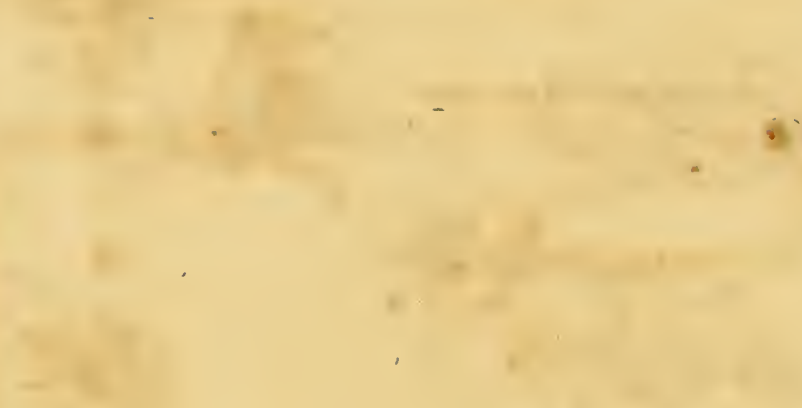

a

$+$
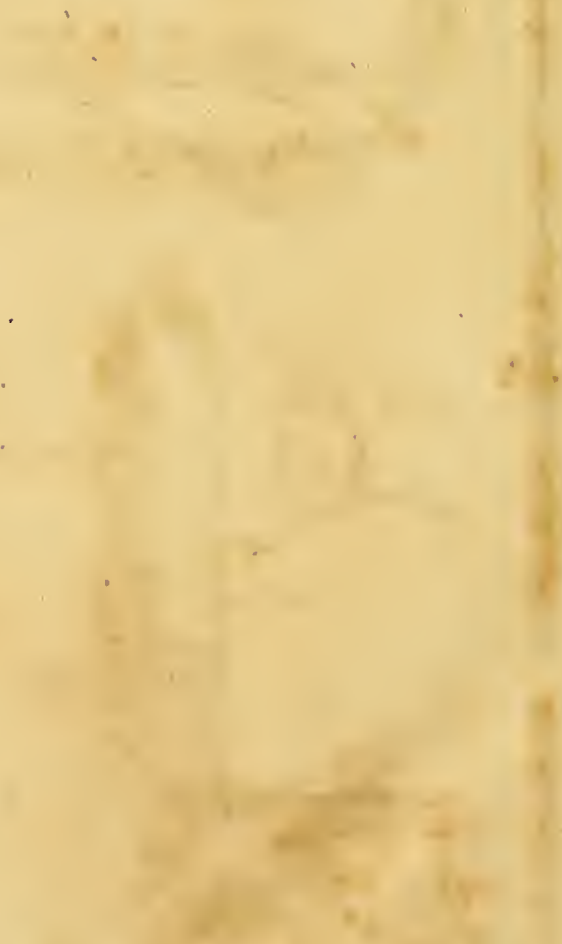

$\sqrt{2}+x^{2}$ 
KNOTS of different kinds.
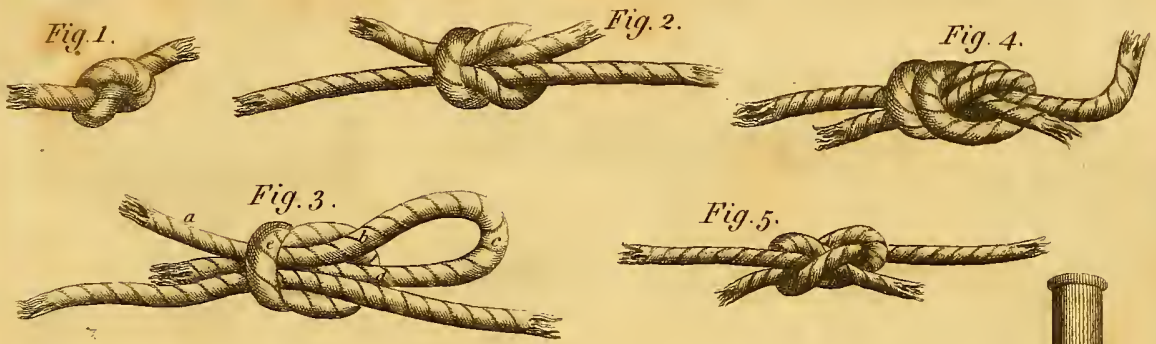

Fig. 5 .
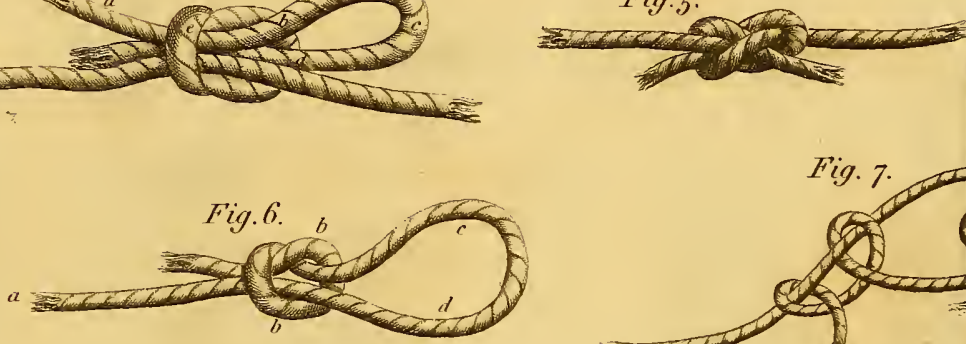

lig. 9.

Fig.8.
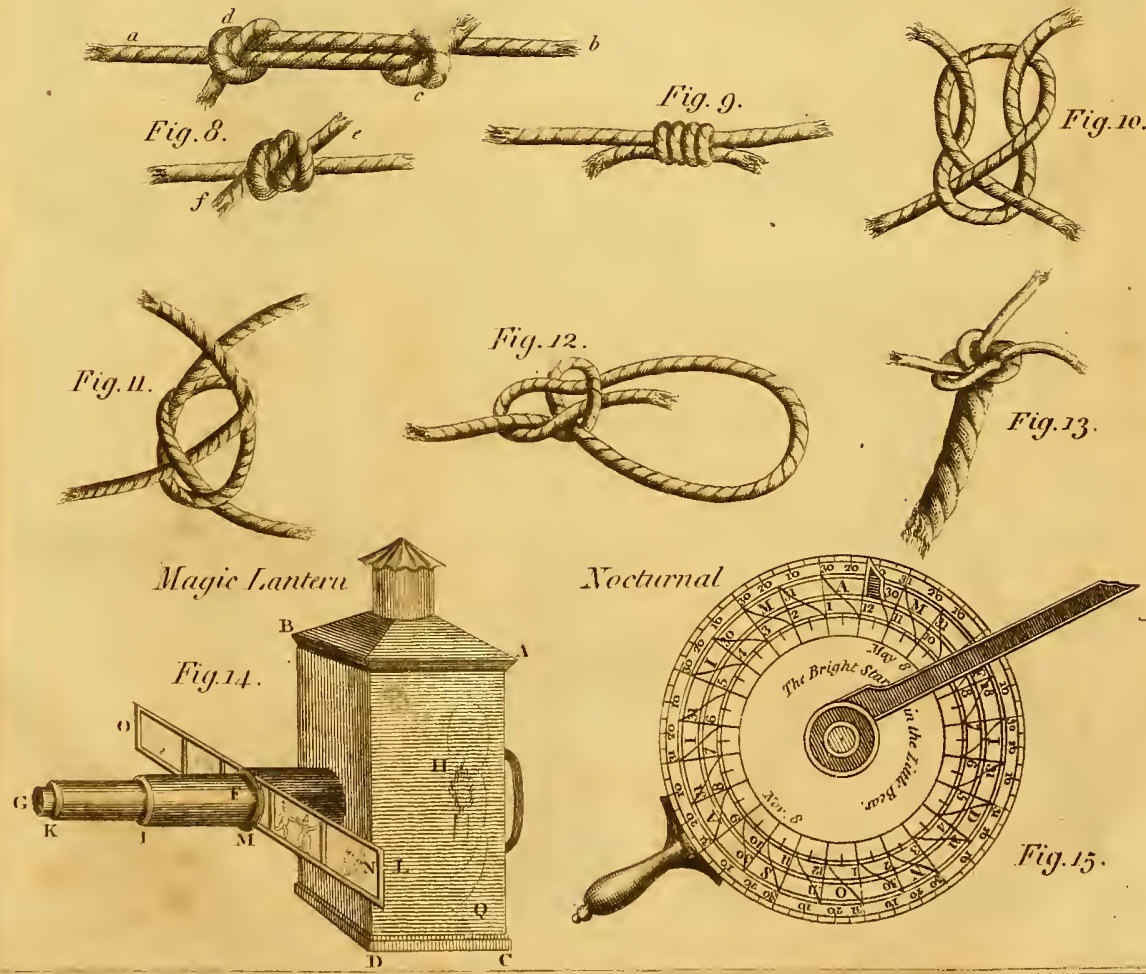

I. E V F I S .

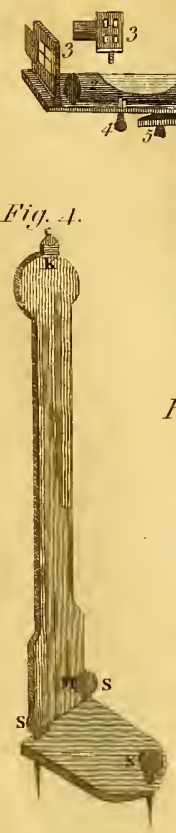

Fig. 1.

Lii 7iriels

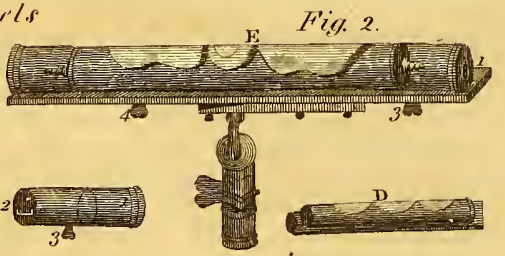

Fig. 3.

$\cos _{2},-x^{2}$

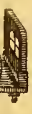

Fii). $b$.

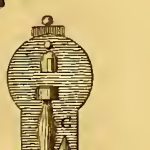

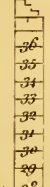

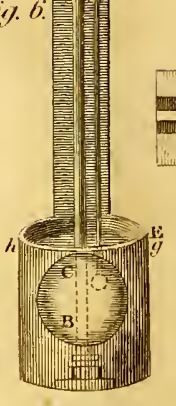

1.1. Fig. 5

Penelulum or 2 Plumb Lerer.

lig. 10

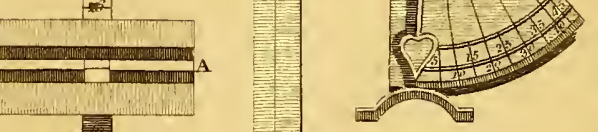

A

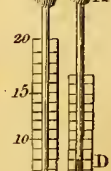

时
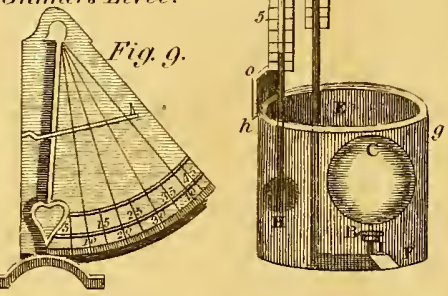

Carpenter's \& Provints Tevels.
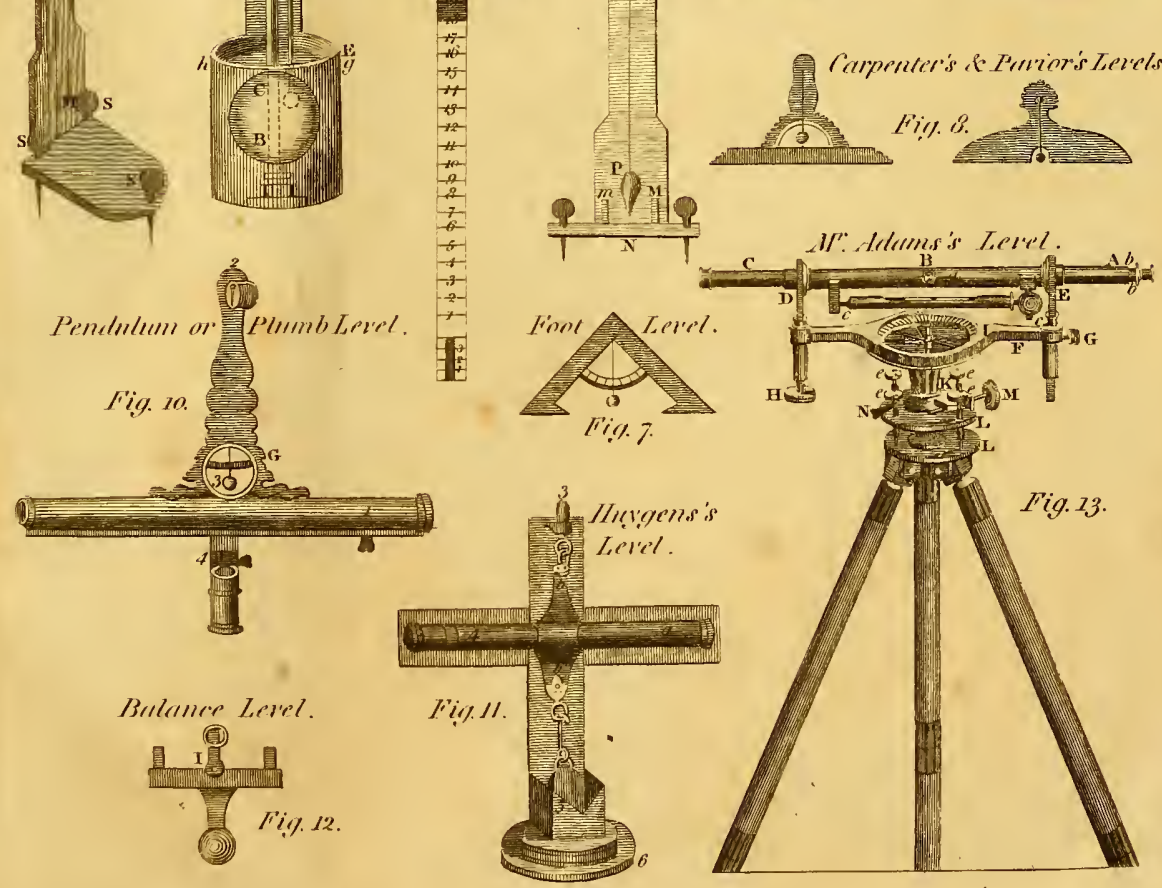

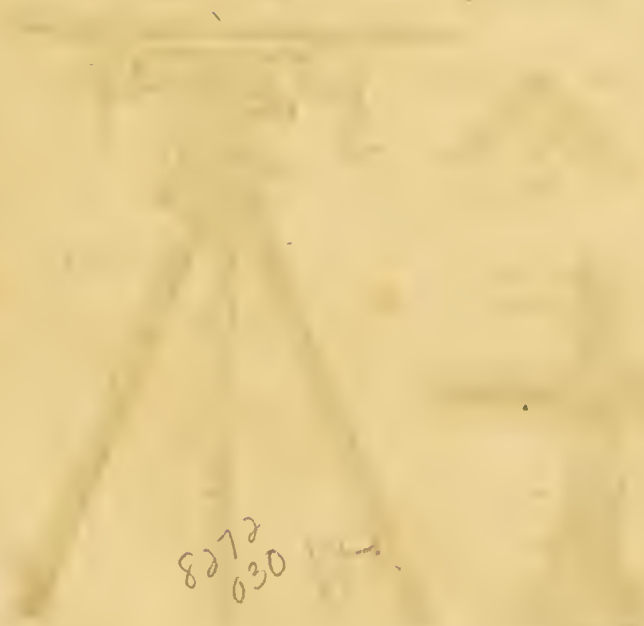

AC:

AMNR! ?

DEC C. $25 \%$ 

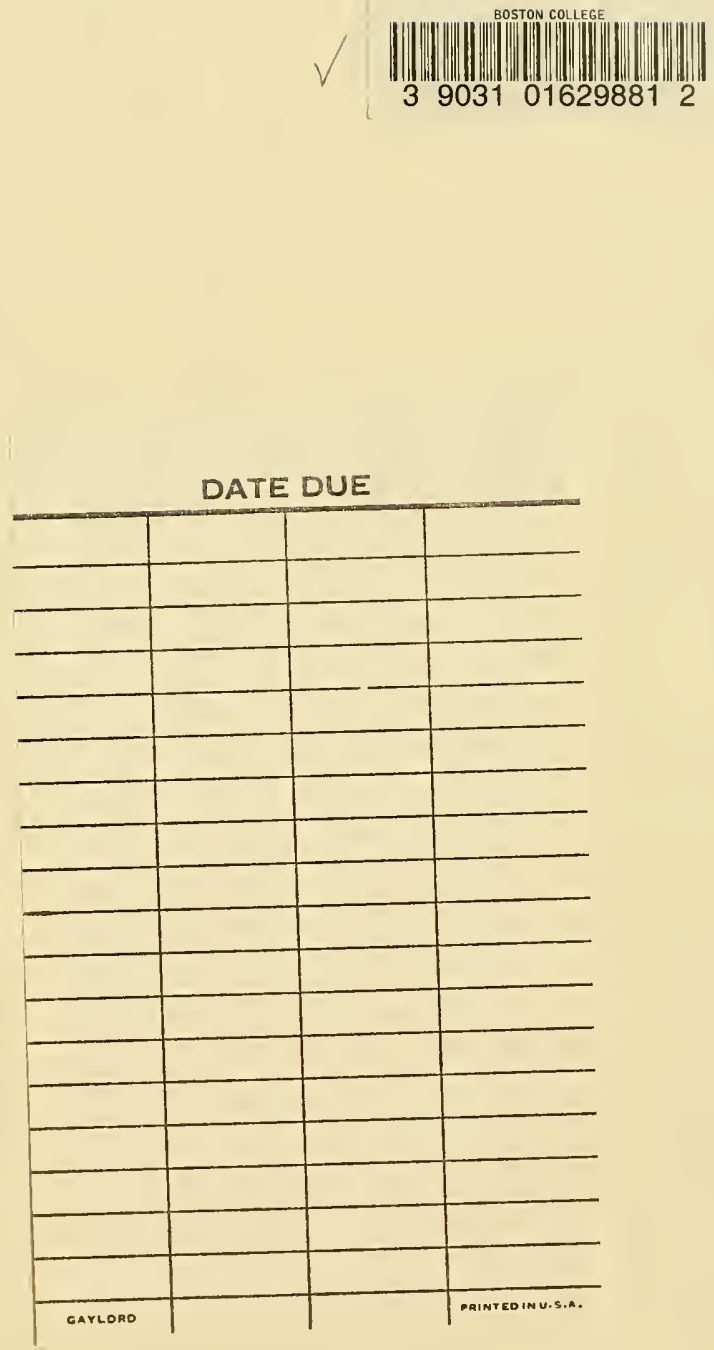
